\title{
The methylation landscape and its role in domestication and gene regulation in the chicken
}

Andrey Höglund, Rie Henriksen, Jesper Fogelholm, Allison M. Churcher, Carlos M. Guerrero-Bosagna, Alvaro Martinez-Barrio, Martin Johnsson, Per Jensen and Dominic Wright

The self-archived postprint version of this journal article is available at Linköping University Institutional Repository (DiVA):

http://urn.kb.se/resolve?urn=urn:nbn:se:liu:diva-170139

N.B.: When citing this work, cite the original publication.

The original publication is available at www.springerlink.com:

Höglund, A., Henriksen, R., Fogelholm, J., Churcher, A. M., Guerrero-Bosagna, C. M., Martinez-Barrio, A., Johnsson, M., Jensen, P., Wright, D., (2020), The methylation landscape and its role in domestication and gene regulation in the chicken, Nature Ecology \& Evolution. https://doi.org/10.1038/s41559-020-01310-1

Original publication available at:

https://doi.org/10.1038/s41559-020-01310-1

Copyright: Springer Nature

http://www.springer.com/ 
The Methylation Landscape and its role in Domestication and Gene Regulation in the Chicken

Andrey Höglund ${ }^{1}$, Rie Henriksen ${ }^{1}$, Jesper Fogelholm ${ }^{1}$, Allison M. Churcher ${ }^{2}$, Carlos M. Guerrero-Bosagna ${ }^{1,3}$, Alvaro Martinez-Barrio ${ }^{4}$, Martin Johnsson, ${ }^{5,6}$, Per Jensen $^{1}$, Dominic Wright ${ }^{*}$

${ }^{1}$ AVIAN Behavioural Genomics and Physiology Group, Linköping University, Linköping 58183, Sweden

${ }^{2}$ NBIS, Umeå University, Department of Molecular Biology, 901 87, Umeå Sweden

${ }^{3}$ Dept of Organismal Biology, Evolutionary Biology Centrum, Uppsala University, S-75236 Uppsala, Sweden

${ }^{4}$ NBIS, Uppsala University, SciLifeLab, Box 596, S-75124 Uppsala, Sweden

5 The Roslin Institute and Royal (Dick) School of Veterinary Studies, The University of Edinburgh, Midlothian, EH25 9RG, Scotland, United Kingdom.

${ }^{6}$ Department of Animal Breeding and Genetics, Swedish University of Agricultural Sciences, Box 7023, 75007 Uppsala, Sweden.

*Corresponding author: email dominic.wright@liu.se, ORCID 0000-0003-23292635 


\begin{abstract}
Domestication is one of the strongest examples of artificial selection, and has produced some of the most extreme within-species phenotypic variation known. In the case of the chicken, it has been hypothesised that DNA methylation may play a mechanistic role in the domestication response. By inter-crossing wild-derived Red Junglefowl with domestic chickens we mapped Quantitative Trait Loci for hypothalamic methylation (methQTL), gene expression (eQTL) and behaviour. We find large, stable methylation differences, with 6179 cis and 2973 trans methQTL identified. Over $46 \%$ of the trans effects were genotypically controlled by five loci, mainly associated with increased methylation in the Junglefowl genotype. In a third of eQTL we find that there is a correlation between gene expression and methylation, whilst statistical causality analysis reveals multiple instances where methylation is driving gene expression, as well as the reverse. We also show that methylation is correlated to some aspects of behavioural variation in the intercross. In conclusion, our data suggest a role of methylation in the regulation of gene expression underlying the domesticated phenotype of the chicken.
\end{abstract}


Introduction

Domestication has occurred in a range of different plants and animals, and generally leads to extreme phenotypic variation from the original wild progenitor phenotype ${ }^{1,2}$. In the case of the chicken, domestication first occurred around $8 \mathrm{k}$ years ago ${ }^{3,4}$. The greatest changes in phenotypic variation, however, occurred only in the past few hundred years, a common occurrence among domesticated species ${ }^{5-7}$, with the chicken separately selected in the last $\sim 50$ years as broiler (for increased meat production) and layer (for increased egg production) breeds. In the case of the layer chicken, this recent selection has led to genetic changes in reproduction ${ }^{8}$, brain composition ${ }^{9}$, bone allocation ${ }^{10}$, growth ${ }^{11}$, sexual ornament size ${ }^{12}$, and anxiety-related and other behaviours, amongst others 13-15. More recently, methylation differences between Red Junglefowl (the wild progenitor to the domestic chicken) selected for high and low tameness ${ }^{16}$ and an excess of mutations in $\mathrm{CpG}$ sites ${ }^{17}$ have also been reported in chickens. In the case of the chicken, evidence exists for transgenerational effects on behaviour, with a potential epigenetic basis ${ }^{18}$. However, no direct evidence is available to our knowledge on how epigenetic and genetic components interact to modulate gene expression in the chicken. Disentangling DNA methylation-related epigenetic modifications and their effects on transcription will provide valuable information to understand the domestication process in the chicken and the potential role of DNA methylation.

Epigenome alterations and DNA methylation in particular are key regulators of eukaryotic genomes, and can lead to heritable phenotypic and transcriptomic changes. The relationship between DNA methylation and gene expression is complex. The canonical view is that $\mathrm{CpG}$ methylation in promoter regions directly represses transcription factor binding, thereby inhibiting transcription 19,20. Recent work, however, has reported that DNA methylation can also increase transcription factor binding ${ }^{21}$. Additionally, DNA methylation in other genomic regions such as enhancers, exons and splicing sites has also been found to yield complex patterns of transcriptomic expression. In vertebrates, enhancers regulated by DNA methylation are linked to the coordinated transcriptional and epigenomic regulation of developmental genes 
22. Additionally, there are genomic regions that concentrate mediator and cohesion components called 'super-enhancers' ${ }^{23}$. These super enhancers have altered methylation status in tumours, with methylation being inversely correlated to gene expression ${ }^{24}$. In mammals and honey bees exonic regions which are hypermethylated in relation to adjacent regions are correlated with increased gene expression ${ }^{25}$. In humans, CpG methylation is shown to be higher in alternative splicing sites ${ }^{26}$.

Local DNA variation can also modify DNA methylation ${ }^{27-30}$, with sequence polymorphisms modifying the extent of $\mathrm{CpG}$ methylation. In this manner, epigenetic modifications can act as the mechanism by which DNA variants influence gene expression, and ultimately phenotypic variation 31. By using genome-wide inter-population variation it is possible to identify DNA polymorphisms that underpin the extent of local DNA methylation. Quantitative Trait Loci (QTL) studies with DNA methylation can be performed in this manner, by either inter-crossing populations with different levels of DNA methylation (a linkage-based approach) or by using within-population variation in DNA methylation in a single population (a linkage disequilibrium based approach). Both methods work by assessing local DNA methylation in discrete windows throughout the genome, then correlating these with molecular SNP markers also spaced throughout the genome to identify genomic regions that underpin quantitative variation in DNA methylation. Although powerful, very few studies have used this approach, which has only been performed in human cell lines ${ }^{32}$, blood ${ }^{33,34}$ and brain tissue ${ }^{35}$, and Arabidopsis ${ }^{36}$. By combining methylation QTL studies with simultaneous expression QTL studies using the same individuals, it is possible to detect regions that control both methylation and transcriptomic variation, and even statistically assess the likelihood that methylation status is potentially causal to gene expression and vice-versa for each individual region and gene.

To address the extent to which DNA methylation and related gene expression is affected by domestication, we conducted a DNA methylation 
quantitative trait locus (methQTL) analysis using an advanced intercross between domestic (White Leghorn) chickens and Red Junglefowl. The hypothalamic transcriptome and methylome of 124 individuals were assayed and DNA methylation and expression QTL were jointly mapped and compared, along with a variety of domestication-related anxiety behaviours. In this manner, not only was methylation over the entire autosomal genome covered, but this was performed on an individual basis, allowing inter-individual variation in DNA methylation for any given region to be assessed.

\section{RESULTS}

\section{The Regulation of Methylation, Gene Expression and Behaviour} Abundant Genetic Regulation of Methylation in the Chicken

Both local (cis) and trans methQTL were detected in the intercross, with 6179 cis and 2973 trans methQTL identified (see Supplementary Table 1 and Figure 1). In comparison, 1123 expression QTL were detected in the same tissue from the same intercross individuals, indicating that genetic variation in the regulation of DNA methylation between wild and domestic genotypes appears to be highly abundant in the chicken in both cis and trans forms. Of the cis methQTL, there was a modest but significant enrichment of alleles with a greater effect on methylation being derived from the Red Junglefowl genotype (3235 methQTL with the greater effect derived from RJF alleles, 2944 methQTL with the greater effect from WL alleles, chi-squared test, $p$-value $=0.0002$ ). In the case of cis regulation, this was not only limited to small discrete regions, but in some cases stretched over several kilobases to form consistently methylated blocks (i.e. multiple adjacent $1 \mathrm{~kb}$ regions were controlled by the same genetic region -see Supplementary Table 2). In total 879 cis methQTL mapped to 77 different SNP markers, with these regions stretching over $\sim 13 \mathrm{~kb}$ on average. The largest of these consistently methylated blocks consisted of 186 cis methQTL mapping to a SNP marker on chromosome 1 in three discrete blocks, covering a 204kb region at $137 \mathrm{Mb}(137.574 \mathrm{Mb}-137.778 \mathrm{Mb})$. All these blocks were associated with increased methylation from the RJF allele. In general, where these blocks occur they are significantly more likely to show an increased methylation in the wild 
(RJF) genotype compared to the domestic White Leghorn (WL) genotype (58 regions to 24; chi- square $p$-value $<2 \mathrm{e}-5$ ). Similarly, the methQTL within these blocks were significantly more likely to have a greater effect from the RJF allele (675 methQTL with a greater effect from RJF alleles, 183 methQTL with a greater effect from WL alleles; chi-square $p$-value $<3 \mathrm{e}-63$ ).

\section{Trans MethQTL Hotspots}

Trans acting methQTL were less abundant than cis methQTL, however the trans methQTL were found to cluster together into a small number of regions (referred to as hotspots hereafter). Thirteen hotspots were identified on nine chromosomes (see Supplementary Table 3 and Figure 1), with these accounting for $\sim 60 \%$ of all trans methQTL (1792 of the 2973 trans methQTL were present in these 13 hotspots). The largest of these hotspots contained 561 trans methQTL, while the top five trans hotspots accounted for 1375 trans methQTL in total (see Supplementary Table 3). There was a significant bias towards trans methQTL possessing a larger allelic effect derived from Red Junglefowl in these hotspots (986 RJF methQTL, 806 WL methQTL, p-value=2.2e-5). To more finely analyse the trans methQTL hotspot regions, we firstly checked if there were any specific epistatic interactions between the trans QTL in the hotspot and the methylation phenotypes they control. Very little epistasis was apparent in the hotspot regions, with less than 7\% of trans methQTL in these hotspots possessing a nominally significant $(\mathrm{P}<0.05)$ epistatic interaction, and less than $1 \%$ of trans methQTL with a significant epistatic interaction (see Supplementary Table 3). One hotspot on chromosome 5 did have more significant epistasis (with 12 of the 98 trans methQTL exhibiting a significant epistatic interaction). We also calculated the minimum common overlap region between the confidence intervals of every trans methQTL in the hotspot to identify any candidate genes 
that could be present (see Supplementary Table 3). We performed a GO

enrichment analysis using all the genes present in each hotspot, and also

identified which of these genes possessed an eQTL in the hypothalamus, with 11

eQTL found spread over these 13 hotspots. These genes were PATZ1, LIMK2,

CAPN3, ACOT1L, GANC, ERH, ADAM20, DAP, ROPN1L, CMBL, EPGN. Taken as a

whole, the hotspots were significantly enriched for five GO terms (alpha-linolenic acid metabolism, linoleic acid metabolism, arachidonic acid metabolism, ether lipid metabolism, glycerophospholipid metabolism), related to acid and lipid metabolism, though this was principally driven by just six genes.

\section{Methylation Regulation of Gene Expression}

By overlapping autosomal methQTL $(\mathrm{n}=8689)$ with autosomal expression QTL $(n=1123)$ and then using methylation to predict gene expression for the resulting overlaps, we identified candidate regions where methylation putatively is controlling gene expression and vice versa. A total of 3694 significant overlaps were identified using this approach, with these representing 435 unique expression microarray probesets and 1988 unique methylation windows (with multiple methylation windows often overlapping a given probeset, see Supplementary table 4). Where multiple methQTL overlapped with an eQTL, we then used a multiple regression to combine all methylation windows associated with the expression of a given probeset (see Supplementary Table 5). All nonsignificant methylation windows were then dropped sequentially (starting with the least significant) until only significant correlations remained. This resulted in 1093 overlaps being retained, representing 795 methylation windows that significantly correlated with one or more of the 435 expression probesets.

To compare the relative impact of genomic variation on DNA methylation and gene expression, all of the 795 methylation windows that were significant in the multiple regression model were then modelled with genotype at the eQTL included as a covariate. In this case 136 of the methylation windows no longer 
explained any additional variation in gene expression (no significant difference in a likelihood ratio test between the linear models of genotype with and without methylation), leaving 890 overlaps with 659 methylation windows that significantly increased the explained variance of the combined gene expression model. These represented 306 unique probesets (i.e. methylation significantly increased the total variance explained in a probeset model that also included eQTL genotype, see Supplementary table 5), with these comprising of 201 annotated genes. We can see that methylation explains much less variation in gene expression than genomic variation does (average proportion of gene expression attributable to methylation is $7.7 \%$, average proportion of gene expression attributable to genotype plus sex is $15 \%$ ), but there is nevertheless an appreciable effect (see Extended Data 1).

\section{$\underline{\text { Statistical Directionality/ Correlation Based Tests of Causal Models }}$}

When two traits share a common genetic marker (SNP), it is possible to use these markers as a causal 'anchor' to assess how these traits relate both to one another and to the anchor marker ${ }^{37}$. Analyses can then be performed which weigh the correlations between the traits themselves and between the traits and marker to assess the probability of how these traits are linked. In this way, it is possible to weigh the probability that one of the traits is causal to the other, or whether they are both independently linked to the anchor SNP. Using the Network Edge Orientation (NEO) method devised by Aten et al ${ }^{37}$ that uses a network-based approach to look at these different correlations and assess their relationship, it is possible to try and define the relationships between the 306 probesets and 659 methylation windows identified above. Using the NEO software we inferred the likelihood of whether the probeset expression in question was statistically causally associated to methylation, the reverse, or whether methylation and probeset expression were independent of one another. Note that when we refer to statistical causation, this is not the same as true functional validation, but rather whether the correlation networks between the traits and the SNP genotypes support the statistical models of SNP genotypes controlling the methylation level of the window in question, and this methylation, in turn, 
controlling the probeset expression. Alternatively, the SNP genotype may control probeset expression, which in turn controls methylation at the window (see methods). To distinguish between these two types, we refer to the statistical causality analysis technique devised by Aten et al as 'statistical directionality' to reduce the confusion with actual functional validation using wet-lab techniques. Of the 890 overlaps that were assessed, we could infer statistical directionality in 110 of the overlaps, representing 98 probesets (69 annotated genes and 23 ESTs) and 95 methylation windows (see Supplementary Table 6). Of these, 36 probesets supported gene expression causing changes in methylation and 74 probesets supported methylation causing gene expression changes. Of these 110 suggestive or significant-models, 88 were local (cis) methylation and eQTL interactions and 22 involved either trans eQTL or methQTL.

\section{Identification of Putative Causal SNPs}

For the 88 methylation-probeset regions that were significant using statistical directionality and that were also cis acting it was possible to scan the methylation sequence reads for each region for putatively causal SNPs. Using an allelic imbalance test these SNPs should display an excess of the 'high' methylation allele in heterozygotes, whilst also being in linkage with the QTL locus. A total of 55 SNPs called from the sequence reads had a cis-methQTL effect (i.e. were significantly correlated in a linear model with the respective DNA methylation phenotype). Of these, 36 SNPs (covering 14 of the methylation windows) also displayed a significant allelic imbalance (see Supplementary Table 7). These SNPs can be further refined by assessing the genotypic effect of SNPs and comparing to the effect of the imputed QTL genotype. If these SNPs are causal the SNP should be a similar or more significant predictor of methylation. These are then either the causal quantitative trait nucleotides for the methQTL and the corresponding eQTL, or are in linkage with the actual causal SNP and also closely mirror the allele ratio of the causal SNP. Twelve genes contained one or more SNPs where heterozygote individuals (as determined by either the local methylation reads directly or the genotype at the methQTL locus) displayed allelic imbalance (see Supplementary Table 7). As an example, in Figure 2 we show the location of the SNPs for the $1 \mathrm{~kb}$ region on chromosome 7 at 27835000 - 
$27836000 \mathrm{bp}$, their proximity to CpG islands, and their proximity to selected predicted transcription factor binding sites. The four strongest candidates are NEK3 (SNP 182303631bp on chromosome 1), ENSGAL0000023564 (SNP 99945095bp on chromosome 2), MYLK (SNP 27344378bp on chromosome 7), and DPP10 (27835914bp on chromosome 7). In each instance, the genotypic effect taken directly from the SNP allele genotype is more significant than the imputed QTL genotype from the methQTL mapping procedure for those reads (see Supplementary Table 7).

\section{Methylation and Behaviour}

Previously in this intercross we have identified 25 genes as being significant candidate genes to behavioural phenotypes, using the same method of statistical directionality analysis demonstrated here 9,13-15, with these behaviours representing anxiety-related behaviours, as well as predictability/ habituation behaviour. Predictability behaviour refers to the degree of intra-individual variation present (i.e how repeatable/ predictable a given individual is in their behavioural response to a particular test). We have previously shown that domestic WL genotypes display more intra-individual variability than wild RJF genotypes ${ }^{38}$. Of these 25 genes, 14 were present in the subset of 435 probesets that possessed both eQTL and methQTL that overlapped and were correlated, with this being a close to significant enrichment (hypergeometric test, $\mathrm{p}$-value= 0.058). Therefore, we estimated the association of the behaviours linked with these genes with gene expression and methylation in a multiple linear regression model. Of the 14 genes, 5 genes had significant associations between methylation and behaviour (see Supplementary Table 8). Two genes (ITGBL, LOC10013671/Loc770352) that correlated with predictability behavioural phenotypes were correlated with methylation, whilst one of the genes that was a candidate for freezing behaviour/ tonic immobility (CA8), one gene that was a candidate for social reinstatement behaviour (TTRAP) and two genes that correlated with open field activity (ADAM10 and LOC10013671/Loc770352 once again) also showed a correlation between behaviour and methylation. We then used the statistical directionality analysis to test the association between behaviour and methylation in the 12 behavioural traits that correlated with 
these five genes. Although none were significant, they did pass the 0.3 suggestive threshold suggested by 39,40 in 8 instances (see Supplementary Table 9, encompassing four social reinstatement traits, one open field trait, one tonic immobility trait and one predictability trait).

\section{Comparison of Cis methQTL with Red Junglefowl Selection Lines}

To assess whether the methylation differences observed between genotypes in our intercross were replicated in a separate population of selected Red Junglefowl individuals, we used data previously available from Belteky and colleagues ${ }^{16}$. These RJF ( $\left.n=12\right)$ had been selected for either high fear or low fear of humans for five generations, as a means to try and mimic the effects of early domestication. If the differences we observe in our wild $\mathrm{x}$ domestic intercross are also present in these selected individuals it could imply that very early tameness selection could be responsible for the differences we observe. Genomewide methylation data for 6 high and 6 low selected individuals was generated previously and the data available online ${ }^{16}$. The locations of suggestive and significant cis methQTL from the most variable methylated regions we identified in the wild $\mathrm{x}$ domestic intercross were then lifted from the selected Red Junglefowl data. For each $1 \mathrm{~kb}$ window, a t-test was used to identify significant differences between high fear and low fear selected Red Junglefowl individuals. Of the 1073 regions analysed, $571 \mathrm{~kb}$ windows showed nominally $(\mathrm{p}<0.05)$ significant differences between high fear and low fear groups, however none of the p-values passed an experiment-wide significance threshold (see Supplementary Table 10).

\section{Methylation Landscape in the Chicken}

Typically, when examining patterns of methylation with gene expression on a genome-wide level gene expression is considered with regard to the rest of the genome. I.e. methylation patterns of genes with greater or lesser expression relative to the rest of the transcriptome present in the tissue under consideration are investigated. This has shown that methylation at the Transcription Start Site (TSS) is decreased in highly expressed genes (for example see the Great Tit genome ${ }^{41}$ ). To verify that the chicken has a similar pattern we divided the 
transcriptome into high and low expressed genes, relative to the overall gene expression present. Methylation both immediately up-and down-stream from these genes was then averaged across all genes in these categories. A similar pattern to the Great Tit is seen in our data, with decreased methylation found at the TSS of highly expressed genes (see Figure 3A). The large number of individual methylomes in our dataset also allows us to examine the interpopulation variation in methylation and its relationship with gene expression on a gene-by-gene basis. To test this, a $+/-2 \mathrm{Mb}$ region was considered around each gene in the transcriptome, with all methylation windows present individually correlated with expression of the gene under consideration. The most significant window was then retained, with a permutation test used to determine experiment-wide significance. Using this method, we identified 6689 genes with a significant correlation with a local $1 \mathrm{~kb}$ methylation window. Of these, 3460 genes were positively correlated and 3229 were negatively correlated with methylation (see Figure 3E). By overlapping these results with the analysis of methylation patterns in high and low expressed genes we see that both categories (high and low expressed genes) have negative and positive local correlations (see Figure 3D). We also find that genes with a negative correlation with local methylation have lower methylation at the TSS than those with a positive local correlation (Figure 3B, C). No effect was found if the local correlation was located upstream or downstream of the gene body (figure 3C).

\section{DISCUSSION}

Using a wild $\mathrm{x}$ domestic intercross to map the effects of chicken domestication, we find that selection appears to have induced extreme changes not only in the phenotype and transcriptome, but also in its regulation by DNA methylation, with continuous stretches of the genome being hypo-methylated via local cisacting elements. The presence of large trans-acting methylation hotspots is also particularly striking. This is evidence that certain key regions control a huge amount of the variation in DNA methylation in the domestic chicken, at least in the case of the intercross presented here. Although to our knowledge this is the 
first evidence that chicken domestication may be regulated via such trans hotspots, this pattern of massive pleiotropy affecting trans loci has also been found in plants. Studies in Arabidopsis ${ }^{27}$ demonstrate that the gene CMT2 (a DNA (cytosine-5)-methyltransferase) accounts for approximately 21\% of significant associations ${ }^{36}$. Overall, these results show that domestication appears to have had several large-scale effects on the methylome of the chicken, with the caveat that QTL results pertain directly to the actual individuals used in the inter-cross used, and may not be entirely representative of the overall populations they are taken from, depending on the degree of genetic variation present in the founders. These methylation changes also appear to affect gene expression, with around one third of all the eQTL detected in the intercross showing a significant correlation with a methylated region. Using a more stringent statistical directionality analysis we identify 74 probesets that support a role for local methylation altering gene expression, and 36 probesets that support a role for gene expression altering local methylation. Although such tests are still not the same as true functional assessments of causality, they do help us to start assessing which genes are either partially regulated by or are regulating DNA methylation in the chicken. In this way, we demonstrate an important role for DNA methylation in chicken domestication, and identify genes that appear to be affected as well as the potentially causal SNPs that underpin such variation in DNA methylation. Finally, we also find evidence that DNA methylation is associated with the regulation of behavioural traits in the chicken.

By looking at inter-individual variation in methylation and how it correlates with gene expression, we find that around half of the correlations between DNA methylation and gene expression were positive, with the locations of these peak correlated windows being highly dispersed around the genes and not just limited to the promoter regions. This supports recent findings regarding the role of DNA methylation in gene expression, with DNA methylation being involved in increasing transcription factor binding activity ${ }^{21}$ and also being present in enhancer regions ${ }^{7}$, therefore outside promoter regions ${ }^{42}$. Here we use inter-individual variation to show that such complex regulation is present through-out the genome, and can lead to both positive and negative correlations 
between DNA methylation and gene expression. Such a large amount of positive correlations between DNA methylation and gene expression are also seen in the few other methylation QTL studies that have been performed. Using human blood as a tissue, Bonder and colleagues ${ }^{34}$ found that one third of all methylation/ transcription correlations were positive. A similar split between positive and negative correlations was also seen in a methylation QTL study using Arabidopsis ${ }^{43}$.

The results shown here based on inter-individual variation do have some caveats however. Unlike the statistical directionality analysis performed on the overlapping methylation and expression QTL, these only represent correlations between gene expression and local methylation. Therefore, in many cases these are not the actual causal region regulating gene expression, but are in linkage or linkage disequilibrium with the causal region(s). Furthermore, the methylation windows used represent the total methylation observed in a $1 \mathrm{~kb}$ region. In the case of windows where multiple CpGs are methylated it is possible that the actual causal methylated SNP does not have a similar methylation pattern compared to surrounding CpGs (see ${ }^{44}$ for a classical example of such an effect), either causing the true association to be missed, or to reverse the correlation from positive to negative or vice-versa. However, in general, methylated DNA marks tend to strongly correlate with one another in local regions of around 400$1000 \mathrm{bp} 45-47$, whilst a more recent study found differentially methylated regions to be around $1700 \mathrm{bp}$ on average ${ }^{48}$. This suggests that such isolated CpGs may be less of a problem in our data.

The large trans methQTL hotspots contained 13 differentially expressed genes that could be considered as potential candidates for these hotspots. Five of these genes were related to cancer susceptibility $\left(A C O T 1 L^{49}, E R H^{50}, D A P\right.$ 51, $C M B L^{52}, E P G N^{53,54}$ ), whilst five have previously been identified as having their expression modified by methylation (CAPN3 55 , DAP 51, ROPN1L ${ }^{56-58}, E G F^{59}$, $E P G N^{53}$ ). However, two of the candidates (CAPN3 and ADAM20 at two neighbouring chromosome 5 hotspots) were also identified as being significant using the statistical directionality analysis, with gene expression driving 
methylation changes in the significant models in both cases. This would be consistent with a gene driving methylation changes throughout the genome, making them strong candidates for further analysis. CAPN3 has been associated with multiple production traits in domestic animals, including in geese 60 . ADAM20 encodes a membrane-anchored protein involved in cell-cell and cellmatrix interactions that has also been associated with fertilization in domestic animals and embryonic development 61,62 .

Gene expression has the potential to modify DNA methylation and viceversa. Genetic variants may recruit DNA methylation or affect histone modifications ${ }^{27-29}$, with these being potential mechanisms by which phenotypic change is effected. We see that around one third of our strongest candidate genes statistically support this pattern, with gene expression driving methylation in 36 instances, and methylation driving gene expression in 74 instances. Examples of DNA methylation changes as a consequence of gene expression are not solely restricted to the chicken, with Meng and colleagues ${ }^{43}$ finding a similar pattern of causality in Arabidopsis methQTL candidates, with $20 \%$ of their causal candidates appearing to show gene expression driving methylation. Similarly, the transcription factor PU.1 forms a complex with Dnmt3a/b to induce changes in DNA methylation around the binding sites ${ }^{63}$. Examples of gene expression changes as a cause of methylation are found in transcription factor binding sites that preferentially bind methylated DNA ${ }^{21}$, whilst DNA methylation can also act to stabilize gene regulation 64 .

The combination of statistical directionality analysis with allelic imbalance identified candidate SNPs for 14 of the methylation/gene expression complexes, with 12 of these having an annotated gene. Of these, six genes (DPP10, PAPSS2, KLF12, MYLK, NSUN4, DDX18) have previous links with DNA methylation as a regulator or a role in methylation regulation ${ }^{65-70 .}$. Of note, NSUN4 is a rRNA methyltransferase of the mitochondrial ribosome, whilst PAPSS2 has been shown to contain a methylation QTL in a human GWAS assay ${ }^{45}$. Further, four genes have roles in neurological diseases or cognitive phenotypes (DPP10 - autism, ADHD and brain development, NEK3 - neurodegeneration ${ }^{71}$, 
DDX18 - opioid susceptibility ${ }^{72}$, FAM196B - epilepsy susceptibility ${ }^{73}$ ). In particular, DPP10 has an association between DNA methylation and ADHD 74 , and adverse cognitive effects due to prenatal alcohol exposure ${ }^{75}$. Structural variation at this gene is associated with autism ${ }^{76}$. It has also been found that histone H3 lysin 4 trimethylated in humans, chimpanzees and macaques, potentially playing a role in cognition ${ }^{65}$. Two of the genes were also previously implicated in the regulation of production traits in domestic animals, with MYLK in particular being differentially methylated between fast and slow-growing broiler chickens ${ }^{42}$. NEK3 was one of the strongest candidates in the candidate SNP analysis and plays a role in microtubule acetylation in neurons ${ }^{77}$, modulates signaling in the prolactin receptor 78 and is also associated with neuronal degeneration ${ }^{71}$. The links with cognition also further indicates a role for DNA methylation in the regulation of behaviour, bolstering the correlations observed between DNA methylation and behaviour in this study. Such a role for methylation is also seen in other studies on birds. Temperature-related changes related to onset of seasonal breeding also alters blood DNA methylation in Great Tits ${ }^{79}$, whilst the gene $D R D 4$ that is correlated with exploratory behaviour also shows differential methylation in selected lines ${ }^{80}$.

To date no studies have shown direct evidence of genomic variation causing epigenetic inheritance in wild birds ${ }^{81}$. Although the study presented here is not performed on birds in a natural environment, it does show that domestication has led to a strong degree of heritable epigenetic variation via genomic regulation in the chicken. These strong inter-population differences could be indicative of a role for DNA methylation in speciation. However, an important caveat that applies to all QTL mapping, especially linkage-based QTL mapping, is that the results are most strictly applicable to the actual populations (and even just the individuals) used in the actual analysis. As such, the differences observed may be specific to the particular Red Junglefowl and White Leghorn sub-populations used, rather than all domestic birds (in the case of the White Leghorn) or wild Red Junglefowl in general (in the case of the Red Junglefowl subpopulation used). For example, in the twelve albeit highly inbred and selected Red Junglefowl birds that were selected for high and low fear of 
humans, no evidence of common cis methQTL effects at the loci identified in the intercross were seen between the high and low fear birds. This could indicate that the methQTL identified in the intercross presented here are the product of long-term not short-term selection, or that the populations used are sufficiently different that they have different patterns of methylation due to either recent environmental and selection pressures or long-standing genetic differences.

Other evidence for a role of DNA methylation in speciation comes from analyses with house sparrow populations, with increased global methylation seen in older populations ${ }^{82}$. House sparrows also exhibit a correlation between geographic distances between populations, bill length and global DNA methylation ${ }^{83}$. Other indirect evidence for genetic variation underlying methylation variation comes from early exploratory behaviour in selected lines of Great Tits ${ }^{84}$. Although these birds exhibited heritable variation in exploration behaviour, no QTL were identified in two separate GWAS studies 85,86. However, these lines do differ in DNA methylation at the DRD4 promoter ${ }^{80}$.

\section{Conclusion}

In conclusion, we detect large and wide-spread genetic influences on DNA methylation in our wild $\mathrm{x}$ domestic inter-cross, indicating an important role for methylation in chicken domestication. Our population-level analysis of DNA methylation reveals a rich landscape of regulatory effects in the chicken, where DNA methylation is not just suppressing expression at the transcription start site, but also positively associated with expression through other regulatory elements. In this fashion, DNA methylation is highly influential in regulating the variation in gene expression present in this intercross. The regulation of DNA methylation variation under domestication in the chicken is genetically complex, but key signatures of domestication are observed and are functionally important in the methylome of the chicken. There are large trans-acting hotspots that affect DNA methylation of many regions in concert, whilst relatively long continuous methylated regions are also de-methylated during domestication in the chicken. Furthermore, DNA methylation appears to be the driver of expression at some 
loci, but also responsive to gene expression at other loci. Brain DNA methylation in the chicken is thus both complex and rife for further exploration. To our knowledge, this is the first evidence of the extent polymorphisms affect DNA methylation in domestication, and of the important role DNA methylation plays as a mechanism of transcriptional modulation in domestication.

\section{Acknowledgements}

The research was carried out within the framework of the Swedish Centre of Excellence in Animal Welfare Science, and the Linköping University Neuronetwork. SNP genotyping was performed by the Uppsala Sequencing Center. Bioinformatic support was provided by NBIS (National Bioinformatics Infrastructure Sweden). The project was supported by grants from the European Research Council (Advanced grant GENEWELL and Consolidator grant FERALGEN 772874), the Swedish Research Council (VR), Carl Tryggers Stiftelse, and the Linköping University Neuro-network.

\section{METHODS}

Materials \& Methods

Population sample

A total of 124 adult chickens (55 females and 69 males) from an 8th generation advanced intercross were used. The parental of the cross were 3 White Leghorn females that have been maintained since the 1960s and 1 Red Junglefowl male originating from Thailand. A detailed description of rearing and housing conditions can be found elsewhere (see Johnsson et al ${ }^{12}$ ). A seven-piece brain dissection was performed on day 212 and the tissues flash-frozen in liquid nitrogen and stored at $-80^{\circ} \mathrm{C}$ until RNA and DNA isolation. The study was approved by the Local Ethical Committee of the Swedish National Board of Laboratory Animals.

\section{RNA \& DNA isolation}

To assess gene expression levels mRNA isolation was performed on hypothalamus tissue by homogenizing the samples with Ambion TRI Reagent (Life Technologies) and following the manufacturer's protocol. A detailed description of cDNA 
synthesis and microarray preparations can be found elsewhere (see Johnsson et $\mathrm{al}^{15}$ ). To assess DNA methylation levels DNA was isolated from the TRI Reagenthomogenate from the RNA extraction, using DNeasy Blood \& Tissue Kit (Qiagen) with these additional steps prior to following the manufacturer's protocol: $125 \mu \mathrm{l}$ ice cold 99\% ethanol was added to $250 \mu \mathrm{l}$ TRI Reagent-homogenate; the samples were vortexed, incubated on ice for $5 \mathrm{~min}$ and centrifuged at 12000 RPM for 10 min at room temperature. Past this step the manufacturer's protocol was followed.

\section{Methylated DNA Immunoprecipitation (MeDIP)}

$4 \mu \mathrm{g}$ DNA was fragmented on a Bioruptor (Diagenode) with the following sonication settings; high for 30s and off 90s, for 4-6 cycles. Fragmentation verification was done on a 2\%-agarose gel with GeneRuler Low Range ladder (Thermofisher), with a desired fragmentation size of 500 - 1000bp. To exclude any un-fragmented genomic DNA a spin-column (100 - 10000bp) from PCR Purification Kit (Qiagen) was used following the manufacturer's protocol. The MeDIP protocol developed by Guerrero-Bosagana and colleagues ${ }^{87}$ was used with the following modifications; $10 \mu \mathrm{g}$ monoclonal mouse anti-5-methylcytidine (Diagenode) antibody was incubated for $2 \mathrm{~h}$ at $4^{\circ} \mathrm{C}$ on a rotating platform. Post MeDIP the methylated DNA was whole genome amplified using GenomPlex WGA2 Kit (Sigma-Aldrich) following the manufacturer's protocol and lastly purified with PCR Purification Kit (Qiagen). The WGA protocol used has been developed to reduce bias by using only a low number of cycles (14). In addition, a standardized starting amount of DNA was taken from all samples before proceeding with the WGA. Regions that are resistant to WGA may be missed, however this will be the same for all samples (i.e. some methQTL may be missed, but the ones that are detected will be unaffected). This protocol therefore means that only $\mathrm{CpG}$ methylation is measured, and not other more rare methylation motifs. It does cover both dense, less dense, and repeat regions throughout the genome. However, due to the nature of the antibody enrichment it does not give single base resolution, but is around $150 \mathrm{bp}$ at best and more usually around $500-800 \mathrm{bp}$, leading to assessment of methylation in windows instead of by single base 88 . Sequencing was performed by the National Genomics Infrastructure (NGI) in Uppsala Sweden on an IonProton machine (Thermo Fisher Scientific) using a 
fragment read sequencing approach and aligned using the Torrent Suite software (version 4.4.1). The sequence data had an average of $3.4 \mathrm{X} \pm 0.97$ coverage (based on the total genome size), $136 \mathrm{bp} \pm 15$ average read length, 23.8 million $\pm 5.2 \mathrm{raw}$ reads and 22 quality score \pm 1 .

Data cleanup

Because the lack of post-alignment tools working with IonTorrent Suite BAM alignment files, the initial data cleanup step was done using a custom script. We remove reads that map to multiple places in the chicken genome and mark PCR duplicates.

We considered multi-mapped reads as those with 0 MAPQ score and they are accounted for but not written to the new BAM output.

The algorithm to filter and mark PCR duplicates in Ion Torrent data handles forward and reverse strands separately. In the forward strand, first, collect all strands that start at the given position; second, after sorting by 3 ' position, the longest strand will not count as a duplicate; finally, we marked all shorter reads without a B adapter as duplicated in the BAM file within this strand except the one that was kept with the same 3' length. A schematic depiction of this forward strand process is as follows:

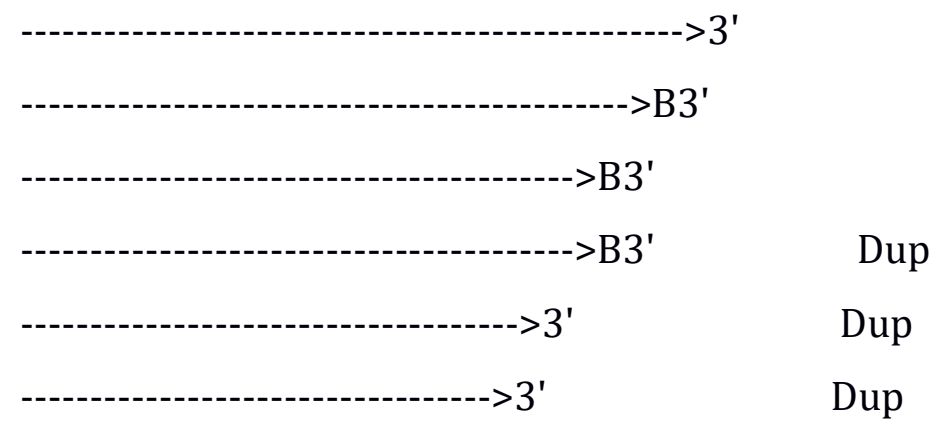

In the reverse strand, we build a table of reads given that in a certain position, the longer read is not the duplicate one. This table facilitates the process because only the 0 -base position, marking the 3 ' end of the alignment, and the CIGAR line are known from the BAM file. A schematic depiction of this reverse strand process for reads starting in the same 5' position is as follows: $3 '<$

B3'< 
After processing all the BAM files, on average, 58\% percent of reads remained following the removal of multi-mapped reads and mark PCR duplicates.

\section{Data QC}

Reads with low quality map scores (q-values less than 10) were filtered out using SAMtools (version 1.3) ${ }^{89}$ prior to data QC. The extend, shift and window size parameters were set to 800,0 and 100 respectively. All reads mapping to the same genomic loci were retained by setting the 'uniq' parameter to 0 because PCR duplicates had already been removed in the data cleanup step. Default values for the 'nit' and 'nrit' parameters were used, and the 'empty_bins' and 'rank' parameters were set to 'TRUE'.

\section{DNA methylation phenotyping}

In order to QTL map DNA methylation differences in the study population, the chicken reference genome was divided into $1000 \mathrm{bp}$ windows, starting at the first nucleotide on each chromosome adding $1000 \mathrm{bp}$ until the end of the chromosome. Sequence read aligning with each methylation window were counted. To normalize the phenotype values between individuals each window's count was divided by the total number of sequence read for each individual, respectively, then multiplied by $1 \times 10^{7}$ for readability purposes. The GRCg6a reference genome was used, generating $1050176 \quad$ windows (https://www.ncbi.nlm.nih.gov/assembly/GCF 000002315.6). Outlier removal within each window accounting all individuals was performed with the R-package 'outliers' version 0.14 using the Chi-square test. Any test with a p-value less than 1e-9 was used to reject values, no more than two values per window. 
methQTL mapping

615 autosomal SNP markers were used to generate a map of length $\sim 9668 \mathrm{cM}$, average distance of $\sim 16 \mathrm{cM}$ between markers. Location and genotypes of the markers can be found elsewhere (see Johnsson et al ${ }^{10}$. DNA methylation QTL mapping, methQTL mapping, was performed with Haley-Knott regression using the R-package R/qtl ${ }^{90}$. Cis-acting methQTL were mapped in an interval $100 \mathrm{cM}$ around each methylation window, expanding to a flanking marker at least 50cM both upstream and downstream. Trans-acting methQTL were mapped to all markers genome-wide. Rearing batch was included as an additive covariate and sex was included as an additive and (where significant) interactive covariate. Through permutation tests LOD-thresholds were generated using the npermfunction in R/qtl set to 1000 for 1000 randomly chosen methylation windows in 10 different seed-iterations, for sex and non-sex-interactions respectively. The 95th percentile LOD-score was used as the significant threshold and the 80th percentile was used as the suggestive threshold. methQTL peak position was determined using refineqtl-function and the confidence intervals using lodintfunction (R/qtl package), with a 1.8 LOD-score drop from the peak position expanding to the closest markers. The threshold for suggestive cis-methQTL was 4.1 LOD and 5.53 LOD for standard and sex interaction, respectively, whilst the threshold for significance for cis methQTL was 4.48 LOD and 6.03 LOD (non-sex and sex-interaction). The suggestive threshold for trans methQTL was 5.6 LOD and 7.27 LOD (nonsex and sex interaction respectively), whilst the threshold for significant trans methQTL was 6.8 LOD and 9.01 LOD (nonsex and sex interaction).

\section{methQTL continuous blocks and trans-hotspots}

Suggestive cis methQTL originating from the same marker were named cis methQTL blocks. A minimum of four cis methQTL methylation windows had to be adjacent to each other within a distance of up to 5000bp to count as a block. Suggestive and significant trans methQTL originating from the same marker, all with an overlapping confidence interval and with a minimum of 30 methylation windows were considered to be trans methQTL hotspots. Only methQTL on the autosomes were considered. Additionally, for the trans methQTL methylation windows an epistatic QTL interaction was performed to assess if the underlying 
genomic region had any influence on the methylation levels. This was tested by using the closest genetic markers genotype to the methylation window and the QTL marker using the makeqtl and fitqtl functions (R/qtl package). Interactions with $\mathrm{p}$-value $<0.05$ were considered as suggestive, whilst $\mathrm{p}$-values $<0.001$ were significant.

\section{Overlap between eQTL and methQTL}

From a previous study (see Johnsson et al., 2016), 1123 eQTL (1064 cis, 59 trans) were identified from the same individuals and tissue used in this study. Overlapping regions between eQTL and methQTL were investigated to elucidate the relationship between DNA methylation and gene expression levels. Regions chosen from the eQTL were based on the confidence intervals of each eQTL with a 1.8 LOD-score drop from the peak position expanded to the closest markers. The intervals from the methQTL were the intervals for the $1 \mathrm{~kb}$ methylation windows. The overlapping regions were tested for correlation (see model 1).

(1) methylation gene expression + batch + sex + error

To control for multiple testing correction for these overlaps a Principal Component analysis was performed on all the gene expression probesets overlapping with any methylation window, 1091 in total. 16 PCs explained 1\% or more of the variation, therefore the significance threshold for these initial overlaps between methQTL and eQTL phenotypes was set at 0.05/16 $=0.003$. Additionally, each significant association was tested with and without DNA methylation to elucidate how much the DNA methylation affected the model (models $2 a$ and $b$ below):

(2a) gene_expression methylation + eqtl_genotype + sex + batch + error

(2b) gene_expression eqtl_genotype + sex + batch + error 


\section{Combined multiple methylation phenotypes model}

As multiple methylation phenotypes were frequently correlated with an expression probeset phenotype, all such methylation predictors were combined in a single model for each of these expression phenotypes. The model tested the methylation phenotypes, with the weakest non-significant methylation phenotypes discarded one by one until only those that were significant predictors in the combined model remained. Furthermore, the significant multiple methylation models were tested with and without all methylation predictors to elucidate the total effect of DNA methylation on gene expression for that particular probeset. A likelihood ratio test was used on the two models to test if there was a significant increase in the R-squared of the full model (3a and $3 b$ ).

(3a) gene_expression methylation $1+$ methylation $2+\ldots+$ eqtl_genotype + sex + batch + error

(3b) gene_expression eqtl_genotype + sex + batch + error

\section{Statistical Directionality/ Causality Assessment using NEO}

All of the methylation phenotypes that were significant in the combined model were then taken forward to test for statistical causality with gene expression. This was performed using a network edge orienting (NEO) method based on the NEO R-package (R version 3.3.5) (Aten et al., 2008). This method not only assesses causality between gene expression and methylation, but also enables determination of whether methylation is causing changes in gene expression or whether gene expression is causing changes in methylation. NEO uses the genetic SNP markers from each QTL as anchors for orientating the edges for the network and integrating the traits of interest, gene expression and DNA methylation, with Structural Equation Model comparisons (SEM). The edge orientation is summarized with a Local SEM-based Edge Orienting score (LEO), comparing it to the next best model as well (NB). In the cases where both the eQTL and methQTL shared the genetic marker a LEO.NB.CPA threshold of $>0.8$ was used as significant. When the QTL had separate genetic markers LEO.NB.OCA score of $>0.3$ was used as significant. Direction of causality was tested as follows: 
(4) eQTL marker -> gene expression -> DNA methylation <- methQTL marker

(5) eQTL marker -> gene expression <- DNA methylation <- methQTL marker

(6) eQTL marker -> gene expression -> DNA methylation

(7) gene expression <- DNA methylation <- methQTL marker

(8) gene expression <- shared QTL marker -> DNA methylation

(9) gene expression -> DNA methylation <- methQTL marker

(10) eQTL marker -> gene expression <- DNA methylation

\section{Allelic imbalance/ Epi-allelic Imbalance analysis}

Allelic imbalance (AI) occurs when regulatory elements affect gene expression where alleles are expressed in different ratios in the same individual. In RNA sequencing it is possible to detect $\mathrm{AI}$ in heterozygote regions. As the MeDIP-seq protocol bind to methylated cytosine in a $\mathrm{CpG}$ it should be possible to detect epiallelic imbalance when comparing methylated regions in heterozygote state, i.e. a heterozygote locus should show a 50:50 ratio if the DNA methylation levels are the same, any deviation from that ratio would indicate an epiallelic imbalance.

From the NEO-analysis any cis methQTL phenotype (1kb methylation window) was used to test for epiallelic imbalance. The genotype for the regions was assessed in two ways; (1) from the meQTL peak QTL position - imputed with the sim.geno function (R/qtl R-package) with step $=1$ and error.prob $=10 e-9$, and (2) using the bcftools call -c function. Polymorphic positions (SNPs) and the allele counts within the methylation windows were located using the function scanForHeterozygotes (AllelicImbalance R-package). A multiple linear regression was performed on the observed genotypes from both the genotype calculated from both the vcf calling and the QTL peak, sex and batch as predictors and the observed allele count as response variable (i.e. two regressions were performed for each SNP, one using the QTL-peak genotype, the other using the genotypes called from the SNP itself). Regression with p-values $<0.05$ were kept as candidates. From these candidates the heterozygote genotypes were used to calculate an epiallelic imbalance with a chi-square test (chi-square p-value $<0.05$ ), with heterozygotes again called from both the QTL-peak and the SNP itself. 
Ascertainment bias can be an issue with sequence data whereby SNPs are more likely to be called when they are the same as the genomic reference allele rather than the alternate genomic allele, with this being a particular problem with low coverage SNPs. To test for this, the SNP with the strongest allelic imbalance from each of the 14 discrete methylation windows containing one or more SNPs in allelic imbalance were tested. Of these 14 SNPs, five had the higher frequency allele derived from the alternate allele (chr1.119143447, chr2.99945095, chr6.22860234, chr7.27344378, chr7.28598812), so ascertainment bias was not an issue for these five SNPs. Of the remaining nine SNPs, these were tested for allelic imbalance both with all individuals and also with individuals that only had at least seven reads, to remove the issue of low coverage individuals biasing the estimate. In all cases, the SNPs without low coverage individuals still displayed significant allelic imbalance, implying that ascertainment bias is not an issue with the SNP data (see Supplementary Table 11).

\section{DNA methylation levels $\pm 2 \mathrm{Mb}$ up- and downstream of each gene}

Initially, an assessment of gene expression with methylation was performed for all genes that were $4 \mathrm{~kb}$ or larger and more than $10 \mathrm{~kb}$ away from the start or end of a chromosome (Figure 3A). All applicable genes were subdivided into high expression genes (1st quartile) and low expression genes (4th quartile), with the average methylation for each of these two classes plotted from $10 \mathrm{~kb}$ upstream of the TSS and from the TTS to $10 \mathrm{~kb}$ downstream ( $\mathrm{n}=5201$ genes). To further assess the relationship between gene expression and methylation, we tested for correlations between DNA methylation levels and gene expression levels genomewide on a gene-by-gene basis using a general linear model, with the DNA methylation levels as predictor and the probeset expression levels as the response variable. Regions tested were divided into $2 \mathrm{Mb}$ upstream, $2 \mathrm{Mb}$ downstream and within gene. These regions were further divided into $1 \mathrm{~kb}$ methylation windows. The analysis was performed for all available autosomes. The most significant correlation was retained for each expression probeset, with a total of 29740 probesets tested. As some genes are represented by multiple probesets, one gene could be composed of both positive and negative correlations with methylation in certain instances. A permutation tests was performed to generate a significant $p$ - 
value threshold by selecting 1000 probesets randomly and shuffling their expression values before running the regression test retaining the top p-value. This was done 500 times. The 95th percentile was used as the significant threshold, generating a p-value $<6.9 \mathrm{e}-4$.

Behavioural Correlations with Methylation

Several behavioural assays relating to anxiety were performed on the individuals used in this study, with these being open field behaviour (degree of movement when in a novel arena), social reinstatement behaviour (time spent associating with conspecifics in a novel arena), and tonic immobility behaviour (a form of freezing behaviour). Further details of the assays can be found in 13-15. A fourth behavioural assay was based on the previous three, and measured the degree of within-individual variation for each of the measurements (termed predictability, though more strictly within-individual variation), as each test was performed twice for each individual ${ }^{38}$. These behaviours have previously been mapped for QTL, with these QTL then overlapped with eQTL, followed by correlating gene expression and behaviour and to identify a total of 25 candidate genes that putatively different aspects of these behaviours. These genes and the behavioural traits that they are correlated with are shown in Supplementary Table 8. Any of these genes that were also identified as being correlated with methylation were therefore tested for potential effects between the methylation phenotype that correlated with gene expression and the behavioural measure that correlated with gene expression. The following three linear models were used to assess the relationship between behaviour and methylation:

(11) behaviour $\sim$ methylation + gene expression + sex + batch + error

(12) behaviour $\sim$ methylation + sex + batch + error

(13) behaviour $\sim$ gene expression + sex + batch + error

In addition, a NEO causal analysis was also performed using the five genes that possessed one or more significant correlations. In this case the same procedure as 
outlined above was performed, but with the behavioural phenotype in place of the gene expression phenotype.

\section{Comparison of Cis methQTL with Red Junglefowl Selection Lines}

MeDip-seq data was available from Belteky and colleagues ${ }^{16}$, consisting of RJF $(n=12)$ selected for either high fear or low fear of humans for five generations. Genome-wide methylation data for 6 high and 6 low selected individuals was taken from an online repository ${ }^{16}$. The locations of suggestive and significant cis methQTL from the 200k most variable methylated regions identified in the wild $\mathrm{x}$ domestic intercross $(n=1073)$ were then lifted from the selected Red Junglefowl data. For each $1 \mathrm{~kb}$ window, a t-test was used to identify significant differences between high fear and low fear selected Red Junglefowl individuals. Suggestive significance was assumed with a nominal $p<0.05$, whilst an experiment-wide $p$ value (incorporating a Bonferroni multiple testing correction) occurred with $\mathrm{p}<$ $4.7 \times 10^{-5}$.

\section{Competing Interests Statement}

None of the authors have any competing interests.

Ethical Statement

The study was approved by the local Ethical Committee of the Swedish National Board for Laboratory Animals, ethical permit Dnr 50-13.

Author Contributions

DW and PJ conceived the study. DW, AH, MJ, RH, JF collected data. AH, RH, CMGB, AMC, AM-B, MJ, JF performed the analyses. DW, AH, RH, PJ wrote the initial draft of the manuscript, all authors revised and contributed to the initial and final drafts.

\section{Data Availability}

Microarray data for the chicken hypothalamus tissue are available at E-MTAB-3154 in ArrayExpress. DNA methylation and behavioural phenotypes (https://doi.org/10.6084/m9.figshare.12803873), genotypes (https://doi.org/10.6084/m9.figshare.12803876) and a readymade QTL crossfile (https://doi.org/10.6084/m9.figshare.12803870) are available via Figshare 


\section{References}

1 Jensen, P. \& Wright, D. in Genetics and behavior of domestic animals (eds T. Grandin \& M.J. Deesing) 41-80 (Academic Press, 2014).

2 Wright, D. The Genetic Architecture of Domestication in Animals. Bioinformatics and Biology Insights 9, 11-20, doi:10.4137/BBI.S28902 (2015).

3 Fumihito, A., Miyake, T., Sumi, S. I., Ohno, S. \& Kondo, N. One species of the red jungelfowl (Gallus-gallus gallus) suffices as the matriarchic ancestor of all domestic breeds. Proceedings of the National Academy of Sciences of the USA 91, 12505-12509 (1994).

4 Fumihito, A. et al. Monophyletic origin and unique dispersal patterns of domestic fowls. Proceedings of the National Academy of Sciences 93, 67926795 (1996).

5 Frantz, L. A. \& Larson, G. in Hybrid Communities: Biosocial Approaches to Domestication and Other Trans-species Relationships (Taylor \& Francis, 2018).

6 Larson, G. et al. Current perspectives and the future of domestication studies. Proceedings of the National Academy of Sciences 111, 6139-6146, doi:10.1073/pnas.1323964111 (2014).

7 Larson, G. \& Fuller, D. Q. The Evolution of Animal Domestication. Annual Review of Ecology, Evolution, and Systematics 45, 115-136, doi:10.1146/annurev-ecolsys-110512-135813 (2014).

8 Johnsson, M., Jonsson, K. B., Andersson, L., Jensen, P. \& Wright, D. Quantitative Trait Locus and Genetical Genomics Analysis Identifies Putatively Causal Genes for Fecundity and Brooding in the Chicken. G3: Genes/Genomes/Genetics, doi:10.1534/g3.115.024299 (2015).

9 Henriksen, R., Johnsson, M., Andersson, L., Jensen, P. \& Wright, D. The domesticated brain: genetics of brain mass and brain structure in an avian species. Scientific Reports 6, doi:10.1038/srep34031 (2016).

10 Johnsson, M., Jonsson, K. B., Andersson, L., Jensen, P. \& Wright, D. Genetic Regulation of Bone Metabolism in the Chicken: Similarities and Differences to Mammalian Systems. PLoS Genetics 11, e1005250, doi:10.1371/journal.pgen.1005250 (2015).

11 Johnsson, M. et al. Genetical genomics of growth in a chicken model. BMC Genomics 19, 72, doi:10.1186/s12864-018-4441-3 (2018).

12 Johnsson, M. et al. A Sexual Ornament in Chickens Is Affected by Pleiotropic Alleles at HAO1 and BMP2, Selected during Domestication. PLoS Genetics 8, e1002914, doi:10.1371/journal.pgen.1002914 (2012).

13 Fogelholm, J. et al. Genetical Genomics of Tonic Immobility in the Chicken. Genes 10, 341 (2019).

14 Johnsson, M. et al. Genetics and Genomics of Social Behavior in a Chicken Model. Genetics 209, 209-221, doi:10.1534/genetics.118.300810 (2018).

15 Johnsson, M., Williams, M. J., Jensen, P. \& Wright, D. Genetical Genomics of Behavior: A novel chicken genomic model for anxiety behavior. Genetics 202, 327-340 (2016).

16 Bélteky, J. et al. Epigenetics and early domestication: differences in hypothalamic DNA methylation between red junglefowl divergently 
selected for high or low fear of humans. Genetics Selection Evolution 50, 13 (2018).

17 Pértille, F. et al. Mutation dynamics of CpG dinucleotides during a recent event of vertebrate diversification. Epigenetics 14, 685-707 (2019).

18 Nätt, D. et al. Heritable genome-wide variation of gene expression and promoter methylation between wild and domesticated chickens. BMC genomics 13, 59 (2012).

19 Gaston, K. \& Fried, M. CpG methylation has differential effects on the binding of YY1 and ETS proteins to the bi-directional promoter of the Surf-1 and Surf-2 genes. Nucleic acids research 23, 901-909 (1995).

20 Mann, I. K. et al. CG methylated microarrays identify a novel methylated sequence bound by the CEBPB| ATF4 heterodimer that is active in vivo. Genome research 23, 988-997 (2013).

21 Yin, Y. et al. Impact of cytosine methylation on DNA binding specificities of human transcription factors. Science 356, eaaj2239 (2017).

22 Bogdanović, O. et al. Active DNA demethylation at enhancers during the vertebrate phylotypic period. Nature genetics 48, 417-426 (2016).

23 Murtha, M. \& Esteller, M. Extraordinary cancer epigenomics: thinking outside the classical coding and promoter box. Trends in cancer 2, 572584 (2016).

24 Heyn, H. et al. Epigenomic analysis detects aberrant super-enhancer DNA methylation in human cancer. Genome biology 17, 11 (2016).

25 Ruden, D. M. et al. Epigenetics as an answer to Darwin's "special difficulty," Part 2: natural selection of metastable epialleles in honeybee castes. Frontiers in genetics 6, 60 (2015).

26 Anastasiadou, C., Malousi, A., Maglaveras, N. \& Kouidou, S. Human epigenome data reveal increased $\mathrm{CpG}$ methylation in alternatively spliced sites and putative exonic splicing enhancers. DNA and cell biology 30, 267275 (2011).

27 Kasowski, M. et al. Extensive variation in chromatin states across humans. Science 342, 750-752 (2013).

28 Kilpinen, H. et al. Coordinated effects of sequence variation on DNA binding, chromatin structure, and transcription. Science 342, 744-747 (2013).

29 McVicker, G. et al. Identification of genetic variants that affect histone modifications in human cells. Science 342, 747-749 (2013).

30 Guerrero-Bosagna, C. in Seminars in cell \& developmental biology. (Elsevier).

31 Furey, T. S. \& Sethupathy, P. Genetics Driving Epigenetics. Science 342, 705-706, doi:10.1126/science.1246755 (2013).

32 Bell, J. T. et al. DNA methylation patterns associate with genetic and gene expression variation in HapMap cell lines. Genome biology 12, R10 (2011).

33 Imgenberg-Kreuz, J. et al. DNA methylation mapping identifies gene regulatory effects in patients with systemic lupus erythematosus. Annals of the Rheumatic Diseases 77, 736-743, doi:10.1136/annrheumdis-2017212379 (2018).

34 Bonder, M. J. et al. Disease variants alter transcription factor levels and methylation of their binding sites. Nature genetics 49, 131 (2017). 
35 Gibbs, J. R. et al. Abundant quantitative trait loci exist for DNA methylation and gene expression in human brain. PLoS genetics 6, e1000952 (2010).

36 Dubin, M. J. et al. DNA methylation in Arabidopsis has a genetic basis and shows evidence of local adaptation. elife 4, e05255 (2015).

37 Aten, J. E., Fuller, T. F., Lusis, A. J. \& Horvath, S. Using genetic markers to orient the edges in quantitative trait networks: the NEO software. $B M C$ Systems Biology 2, 34 (2008).

38 Henriksen, R. et al. Intra-individual behavioural variability: a trait under genetic control. bioRxiv, doi: https://doi.org/10.1101/795864, doi:doi: https://doi.org/10.1101/795864 (2019).

39 Farber, C. R. et al. Genetic dissection of a major mouse obesity QTL (Carfhg2): integration of gene expression and causality modeling. Physiological genomics 37, 294-302 (2009).

40 Plaisier, C. L. et al. A systems genetics approach implicates USF1, FADS3, and other causal candidate genes for familial combined hyperlipidemia. PLoS genetics 5, e1000642 (2009).

41 Laine, V. N. et al. Evolutionary signals of selection on cognition from the great tit genome and methylome. Nature Communications 7, 10474, doi: $10.1038 /$ ncomms 10474

https://http://www.nature.com/articles/ncomms10474 - supplementaryinformation (2016).

$42 \mathrm{Hu}, \mathrm{Y}$. et al. Comparison of the genome-wide DNA methylation profiles between fast-growing and slow-growing broilers. PloS one 8 (2013).

43 Meng, D. et al. Limited contribution of DNA methylation variation to expression regulation in Arabidopsis thaliana. PLoS genetics 12, e1006141 (2016).

44 Watt, F. \& Molloy, P. L. Cytosine methylation prevents binding to DNA of a HeLa cell transcription factor required for optimal expression of the adenovirus major late promoter. Genes \& Development 2, 1136-1143, doi:10.1101/gad.2.9.1136 (1988).

45 Zhang, W., Spector, T. D., Deloukas, P., Bell, J. T. \& Engelhardt, B. E. Predicting genome-wide DNA methylation using methylation marks, genomic position, and DNA regulatory elements. Genome biology 16, 1414, doi:10.1186/s13059-015-0581-9 (2015).

46 Bell, J. T. et al. DNA methylation patterns associate with genetic and gene expression variation in HapMap cell lines. Genome Biol 12, R10 (2011).

47 Eckhardt, F. et al. DNA methylation profiling of human chromosomes 6, 20 and 22. Nature genetics 38, 1378-1385, doi:10.1038/ng1909 (2006).

48 Perzel Mandell, K. A. et al. Characterizing the dynamic and functional DNA methylation landscape in the developing human cortex. bioRxiv, 823781, doi:10.1101/823781 (2019).

49 Wang, F. et al. ACOT1 expression is associated with poor prognosis in gastric adenocarcinoma. Human pathology 77, 35-44 (2018).

50 Pang, K. et al. The ERH gene regulates migration and invasion in 5637 and T24 bladder cancer cells. BMC cancer 19, 225 (2019).

51 Yamaguchi, S., Asao, T., Nakamura, J.-i., Ide, M. \& Kuwano, H. High frequency of DAP-kinase gene promoter methylation in colorectal cancer specimens and its identification in serum. Cancer letters 194, 99-105 (2003). 
52 Kasai, N. et al. Anti-tumor efficacy evaluation of a novel monoclonal antibody targeting neutral amino acid transporter ASCT2 using patientderived xenograft mouse models of gastric cancer. American journal of translational research 9, 3399 (2017).

53 Arrieta, 0. et al. (American Society of Clinical Oncology, 2015).

54 Sanchez-Carbayo, M. et al. 772: Identification of Genetic Signatures Associated with TP53 Status in Patients with Bladder Cancer. The Journal of Urology 173, 209-210 (2005).

55 Hair, B. Y. et al. Body mass index associated with genome-wide methylation in breast tissue. Breast cancer research and treatment 151, 453-463 (2015).

56 Case, C. (Google Patents, 2019).

57 Wang, K.-S. et al. A novel locus for body mass index on 5p15. 2: a metaanalysis of two genome-wide association studies. Gene 500, 80-84 (2012).

58 Morahan, J. M., Yu, B., Trent, R. J. \& Pamphlett, R. A genome-wide analysis of brain DNA methylation identifies new candidate genes for sporadic amyotrophic lateral sclerosis. Amyotrophic Lateral Sclerosis 10, 418-429 (2009).

59 Singh, B., Carpenter, G. \& Coffey, R. J. EGF receptor ligands: recent advances. F1000Research 5 (2016).

$60 \mathrm{Xu}, \mathrm{H}$. et al. Characterization of the Goose CAPN3 Gene and its Expression Pattern in Muscle Tissues of Sichuan White Geese at Different Growth Stages. The Journal of Poultry Science, 0170150 (2018).

61 Sha, Y.-W. et al. Sperm-egg fusion disorder in a Chinese male patient was associated with a rare ADAM20 variant. Oncotarget 9, 2086 (2018).

62 Sigdel, A., Liu, L., Abdollahi-Arpanahi, R., Aguilar, I. \& Peñagaricano, F. Genetic dissection of reproductive performance of dairy cows under heat stress. Animal Genetics n/a, doi:10.1111/age.12943.

63 Suzuki, M. et al. Site-specific DNA methylation by a complex of PU. 1 and Dnmt3a/b. Oncogene 25, 2477 (2006).

64 Maurano, M. T. et al. Role of DNA methylation in modulating transcription factor occupancy. Cell reports 12, 1184-1195 (2015).

65 Shulha, H. P. et al. Human-specific histone methylation signatures at transcription start sites in prefrontal neurons. PLoS biology $\mathbf{1 0}$, e1001427-e1001427, doi:10.1371/journal.pbio.1001427 (2012).

66 Zhang, D. et al. Genetic Control of Individual Differences in Gene-Specific Methylation in Human Brain. The American Journal of Human Genetics 86, 411-419, doi:https://doi.org/10.1016/j.ajhg.2010.02.005 (2010).

67 Du, Y., Chen, Y., Wang, F. \& Gu, L. miR-137 plays tumor suppressor roles in gastric cancer cell lines by targeting KLF12 and MY01C. Tumor Biology 37, 13557-13569 (2016).

68 Liu, J., Morgan, M., Hutchison, K. \& Calhoun, V. D. A study of the influence of sex on genome wide methylation. PloS one 5, e10028-e10028, doi:10.1371/journal.pone.0010028 (2010).

69 Cámara, Y. et al. MTERF4 regulates translation by targeting the methyltransferase NSUN4 to the mammalian mitochondrial ribosome. Cell metabolism 13, 527-539, doi:10.1016/j.cmet.2011.04.002 (2011). 
70 Wang, L. et al. CARM1 Methylates Chromatin Remodeling Factor BAF155 to Enhance Tumor Progression and Metastasis. Cancer Cell 25, 21-36, doi:https://doi.org/10.1016/j.ccr.2013.12.007 (2014).

71 Forcella, M. et al. Neuronal specific and non-specific responses to cadmium possibly involved in neurodegeneration: A toxicogenomics study in a human neuronal cell model. Neurotoxicology 76, 162-173, doi:10.1016/j.neuro.2019.11.002 (2020).

72 Cheng, Z. et al. Genome-wide scan identifies opioid overdose risk locus close to MCOLN1. Addiction biology 25, e12811, doi:10.1111/adb.12811 (2020).

73 Harrison, V.et al. Compound heterozygous deletion of NRXN1 causing severe developmental delay with early onset epilepsy in two sisters. American Journal of Medical Genetics Part A 155, 2826-2831, doi:10.1002/ajmg.a.34255 (2011).

74 Heinrich, H. et al. Attention, cognitive control and motivation in ADHD: Linking event-related brain potentials and DNA methylation patterns in boys at early school age. Scientific Reports 7, 3823, doi:10.1038/s41598017-03326-3 (2017).

75 Frey, S. et al. Prenatal Alcohol Exposure Is Associated With Adverse Cognitive Effects and Distinct Whole-Genome DNA Methylation Patterns in Primary School Children. Frontiers in Behavioral Neuroscience 12, doi:10.3389/fnbeh.2018.00125 (2018).

76 Marshall, C. R. et al. Structural variation of chromosomes in autism spectrum disorder. American journal of human genetics 82, 477-488, doi:10.1016/j.ajhg.2007.12.009 (2008).

77 Chang, J., Baloh, R. H. \& Milbrandt, J. The NIMA-family kinase Nek3 regulates microtubule acetylation in neurons. J Cell Sci 122, 2274-2282, doi:10.1242/jcs.048975 (2009).

78 Miller, D., Forrester, K., Leonard, C., Salo, P. \& Bray, R. C. ACL deficiency impairs the vasoconstrictive efficacy of neuropeptide $Y$ and phenylephrine in articular tissues: a laser speckle perfusion imaging study. Journal of Applied Physiology 98, 329-333, doi:10.1152/japplphysiol.00514.2004 (2005).

79 Viitaniemi, H. M. et al. Seasonal variation in genome-wide DNA methylation patterns and the onset of seasonal timing of reproduction in great tits. Genome biology and evolution 11, 970-983 (2019).

80 Verhulst, E. C. et al. Evidence from pyrosequencing indicates that natural variation in animal personality is associated with DRD 4 DNA methylation. Molecular ecology 25, 1801-1811 (2016).

81 Sepers, B. et al. Avian ecological epigenetics: pitfalls and promises. Journal of Ornithology, 1-21 (2019).

82 Schrey, A. W. et al. Epigenetic variation may compensate for decreased genetic variation with introductions: a case study using house sparrows (Passer domesticus) on two continents. Genetics Research International 2012 (2012).

83 Riyahi, S. et al. Natural epigenetic variation within and among six subspecies of the house sparrow, Passer domesticus. Journal of Experimental Biology 220, 4016-4023 (2017). 
84 van Oers, K. \& Naguib, M. Avian personality. Animal personalities: behavior, physiology, and evolution, 66-95 (2013).

85 Santure, A. W. et al. Replicated analysis of the genetic architecture of quantitative traits in two wild great tit populations. Molecular Ecology 24, 6148-6162 (2015).

86 Kim, J. M. et al. A high - density SNP chip for genotyping great tit (Parus major) populations and its application to studying the genetic architecture of exploration behaviour. Molecular ecology resources 18, 877-891 (2018).

87 Guerrero-Bosagna, C., Settles, M., Lucker, B. \& Skinner, M. K. Epigenetic transgenerational actions of vinclozolin on promoter regions of the sperm epigenome. PloS one 5 (2010).

88 Weber, M. et al. Chromosome-wide and promoter-specific analyses identify sites of differential DNA methylation in normal and transformed human cells. Nature genetics 37, 853-862 (2005).

$89 \mathrm{Li}, \mathrm{H}$. et al. The sequence alignment/map format and SAMtools. Bioinformatics 25, 2078-2079 (2009).

90 Broman, K. W., Wu, H., Sen, S. \& Churchill, G. A. R/qtl: QTL maping in experimental crosses. Bioinformatics 19, 889-890 (2003).

Figure Legends

Figure 1. Circle plot showing the position of (A) continuous cis methQTL blocks, and (B-D) three of the trans methQTL hotspots. In (A) blocks in the grey shaded area indicate a locus with a larger effect from the domestic allele, whilst blocks in the red shaded area indicates a larger effect from the RJF allele. (B-D) show the trans effects arising from three trans methQTL hotspots on chromosomes 15, 2 and 3 (one hotspot per frame), with the lines connecting the different trans genomic regions regulated via the hotspot.

Figure 2. Significant NEO Causal methQTL/eQTL complex on chromosome 7 at $27 \mathrm{Mb}$. A) Mean +/- s.e. for eQTL and methQTL genotype effects at this locus $(n=124)$. B) Comparison of methQTL effects with the genotype taken directly from the SNP present within the methQTL $1 \mathrm{~kb}$ region (vcf) and the genotype taken from the predicted QTL peak (peak), with the phenotype restricted to the sequencing reads overlapping the SNP region, median +/- quartiles shown $(\mathrm{n}=124)$. C) MethQTL $1 \mathrm{~kb}$ region, showing the normalised read coverage for the three different genotypes (corrected for number of individuals in each genotype class). Locations of the significant SNPs are marked, as are several classes of predicted Transcription Factor Binding Sites and CpG locations. D) Allelic imbalance for heterozygous individuals for each of the putatively causal SNPs, mean +/- s.e. shown ( $n=52$ for SNP 27835914).

Figure 3: Median methylation for regions upstream of the TSS, in the gene body, and downstream of the TTS. (A) Genes subdivided into the highest expressed (1 $1^{\text {st }}$ quartile) and lowest expressed genes ( $4^{\text {th }}$ quartile), $n=5201$. (B) The degree of methylation subdivided into those that possess a positive correlation and those 
that possess a negative correlation with methylation when considering interindividual variation (genes with a significant correlation with methylation used, $\mathrm{n}=5425$ ). (C) Positively and negatively correlated genes further subdivided by location of methylation-gene expression correlation (genes with a significant correlation with methylation used, $n=5425$ ). (D) Positively and negatively correlated genes subdivided into highly expressed ( $1^{\text {st }}$ quartile) and low expressed genes ( $4^{\text {th }}$ quartile) (genes with a significant correlation with methylation used, with high and low expressed genes then subsetted out, $n=2650$ ). (E) Location of correlation and sign (positively or negatively correlated) between methylation and gene expression for all experiment-wide significantly correlated genes, $\mathrm{n}=6689$. 
A

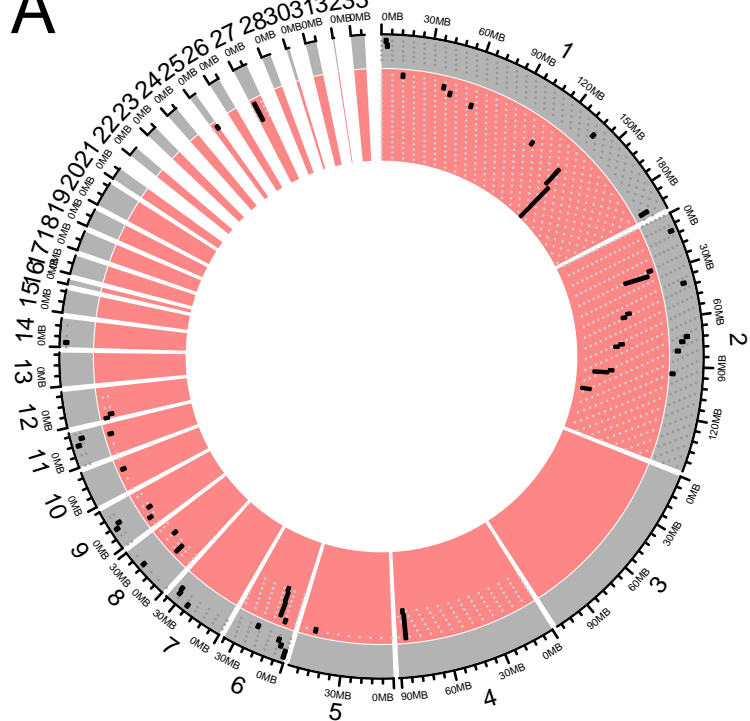

C

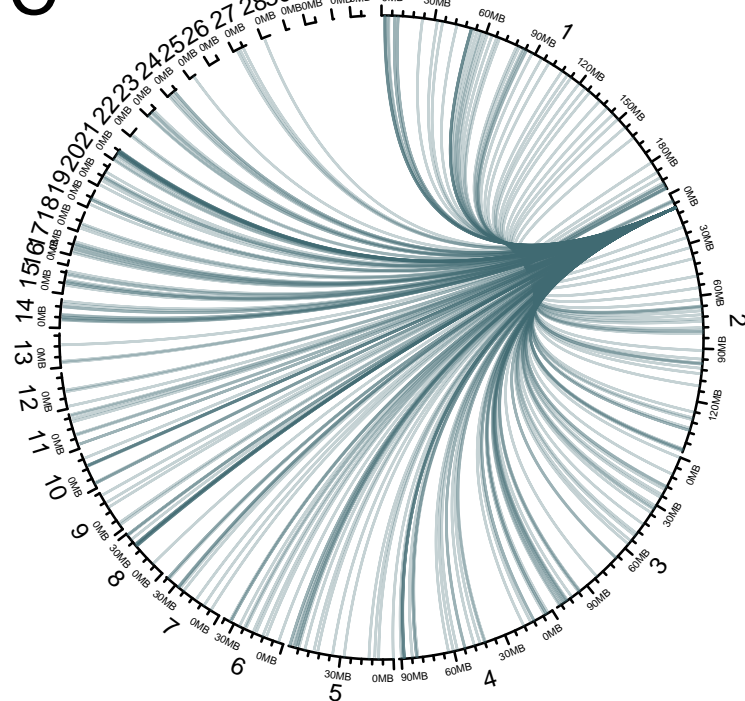

B

D

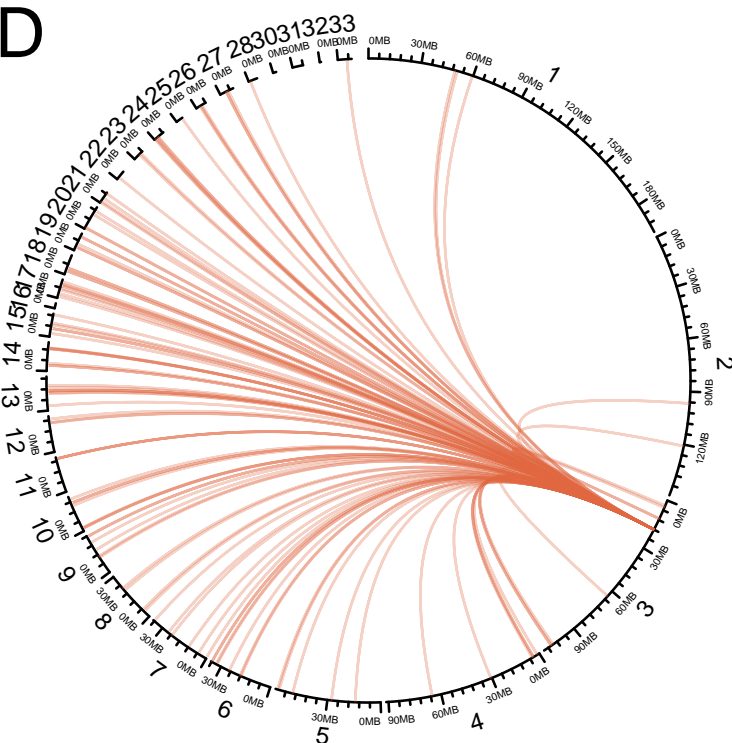


A
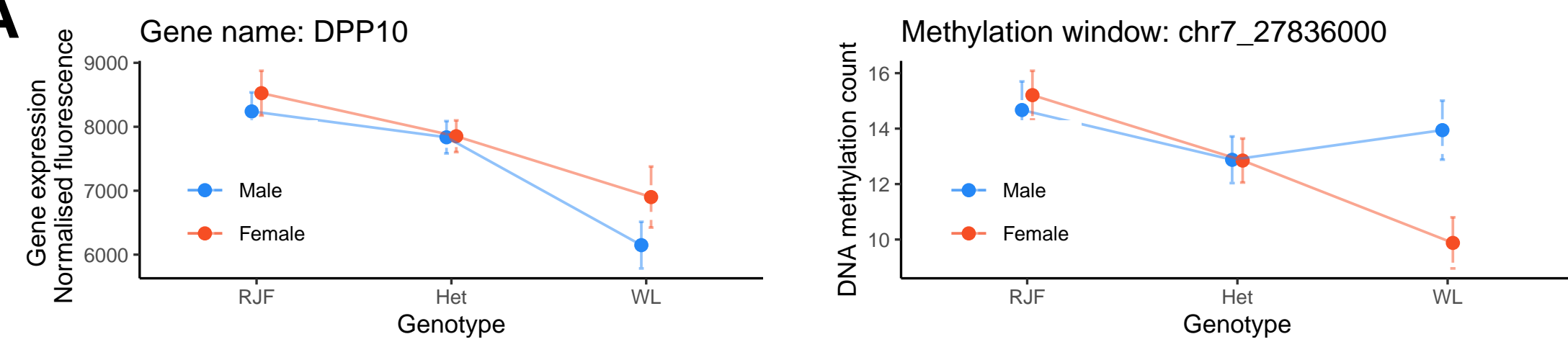

B

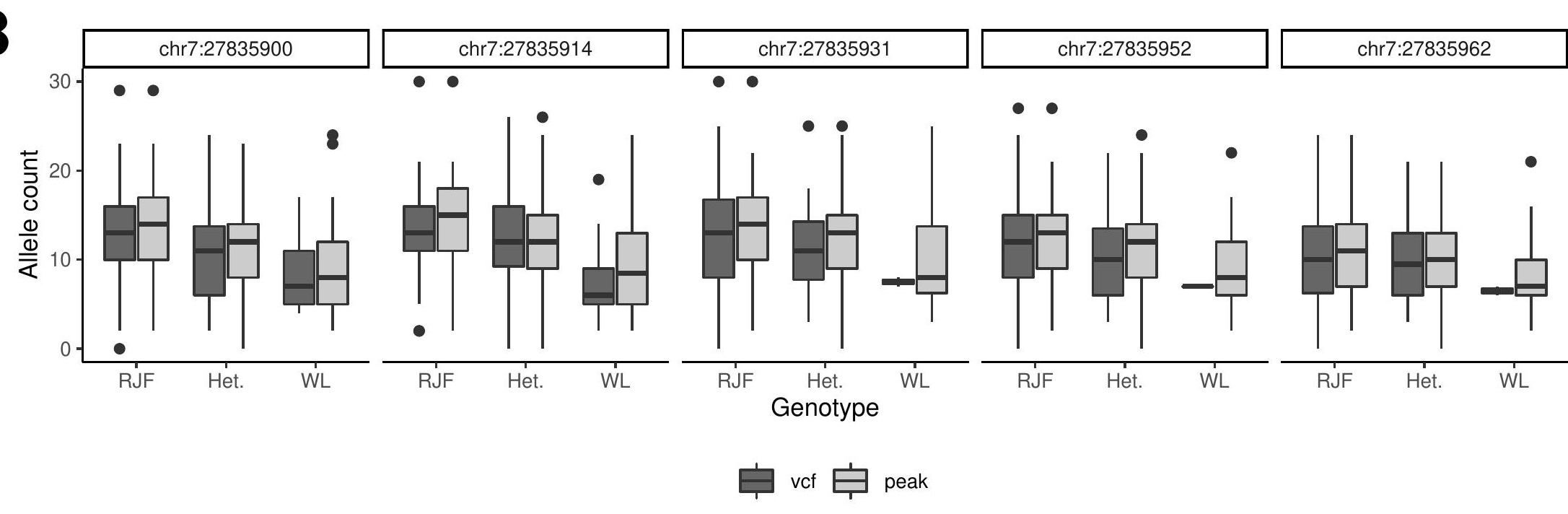

C

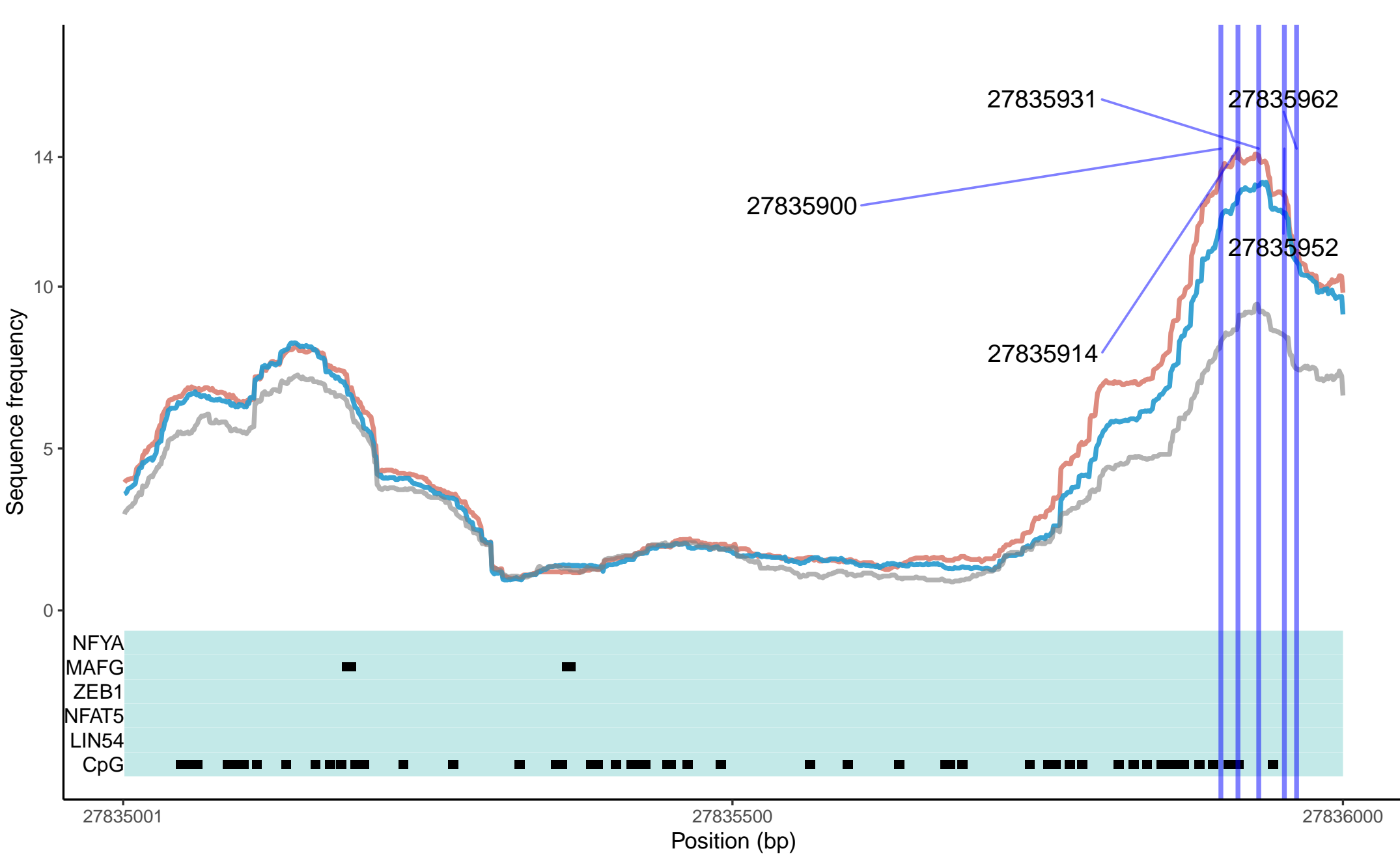

- RJF - Het. - WL

D

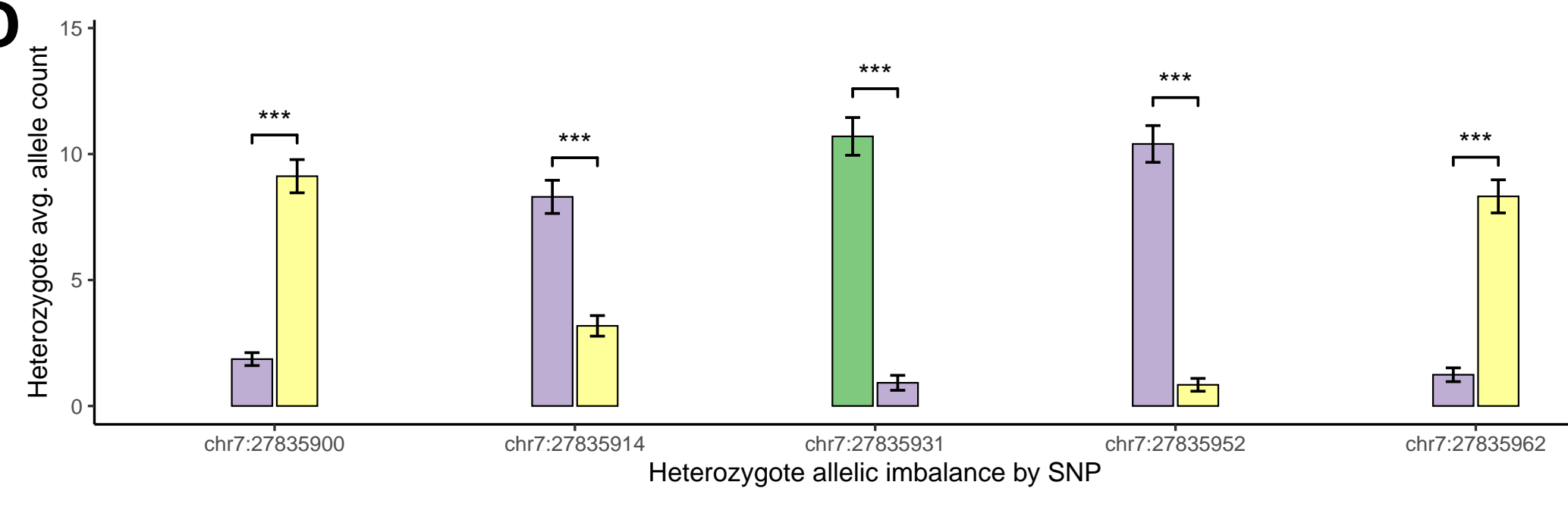

SNPs $\square$ A $\square$ C $\square$ G $\square$ T 
Supplementary Table 1. cis and trans methQTL. Position, type (cis/trans), LOD scol

\begin{tabular}{|c|c|c|}
\hline chr marker pos & phenotype lod & sexint \\
\hline $1 \mathrm{rbl} / 449$ & 50 chr1_180000 & 5.58 nonsex \\
\hline $1 \mathrm{rbl} 449$ & 50 chr1_181000 & 9.78 nonsex \\
\hline $1 \mathrm{rbl} 449$ & 50 chr1_192000 & 11.1 nonsex \\
\hline $1 \mathrm{rbl} 449$ & 50 chr1_193000 & 5.09 nonsex \\
\hline $1 \mathrm{rbl} 449$ & 50 chr1_194000 & 7.02 nonsex \\
\hline $1 \mathrm{rb} / 449$ & 50 chr1_221000 & 9.06 nonsex \\
\hline $1 \mathrm{rbl} 449$ & 50 chr1_222000 & 5.45 nonsex \\
\hline $1 \mathrm{rbl} 449$ & 50 chr1_244000 & 8.66 nonsex \\
\hline $1 \mathrm{rbl} 449$ & 50 chr1_255000 & 10.75 nonsex \\
\hline $1 \mathrm{rb} / 449$ & 50 chr1_256000 & 6.51 nonsex \\
\hline $1 \mathrm{rb} / 449$ & 50 chr1_257000 & 5.39 nonsex \\
\hline $1 \mathrm{rbl} 449$ & 50 chr1_258000 & 10.73 nonsex \\
\hline 1 rbl449 & 50 chr1_303000 & 5.1 nonsex \\
\hline $1 \mathrm{rb} / 449$ & 50 chr1_195000 & 4.8 nonsex \\
\hline $1 \mathrm{rb} / 449$ & 50 chr1_220000 & 5.08 nonsex \\
\hline $1 \mathrm{rb} / 449$ & 50 chr1_245000 & 4.53 nonsex \\
\hline $1 \mathrm{rbl} 449$ & 50 chr21_43310 & 5.63 nonsex \\
\hline $1 \mathrm{rbl} / 449$ & 50 chr25_25790। & 4.98 nonsex \\
\hline 1 X1_366532 & 50 chr1_445600 & 4.73 nonsex \\
\hline 1 X1_366532 & 50 chr1_490100 & 3.69 nonsex \\
\hline 1 rbl449 & 56 chr6_238470 & 5.25 nonsex \\
\hline $1 \mathrm{rb} / 449$ & 78 chr1_860520। & 5.51 nonsex \\
\hline 1 X1_366532 & 85 chr3_508230। & 5.95 nonsex \\
\hline 1 rbl449 & 95 chr1_175647। & 8.64 sex \\
\hline 1 Gg_rs138261 & 157 chr1_714100। & 3.84 nonsex \\
\hline 1 X1_406616 & 165 chr1_163000। & 4.97 nonsex \\
\hline 1 X1_406616 & 166 chr1_105900 & 6.86 nonsex \\
\hline 1 X1_406616 & 167 chr1_106000। & 7.16 nonsex \\
\hline 1 X1_406616 & 167 chr1_106100 & 7.03 nonsex \\
\hline 1 X1_406616 & 170 chr12_99830 & 7.7 nonsex \\
\hline 1 X1_406616 & 179 chr1_107700 & 4.3 nonsex \\
\hline 1 X1_406616 & 183 chr1_202400। & 5.9 nonsex \\
\hline 1 X1_406616 & 183 chr1_194164! & 5.77 nonsex \\
\hline 1 X1_406616 & 217 chr1_132300 & 7.78 nonsex \\
\hline 1 X1_406616 & 217 chr1_290500 & 6.11 nonsex \\
\hline 1 X1_406616 & 217 chr1_106200 & 4.87 nonsex \\
\hline 1 X1_406616 & 217 chr1_133100 & 3.42 nonsex \\
\hline 1 X1_406616 & 217 chr1_193300 & 2.82 nonsex \\
\hline 1 X1_406616 & 218 chr1_108500 & 5.75 nonsex \\
\hline 1 X1_406616 & 220 chr1_329900। & 3.93 nonsex \\
\hline 1 Gg_rs140803 & 226 chr1_208100। & 4.71 nonsex \\
\hline
\end{tabular}




\begin{tabular}{|c|c|c|c|}
\hline 1 Gg_rs140803। & 229 chr3_664490। & 6.31 nonsex & trans \\
\hline 1 Gg_rs140803 & 230 chr1_118780 & 7.46 sex & cis \\
\hline 1 Gg_rs140803 & 232 chrZ_224520। & 9.13 sex & trans \\
\hline 1 Gg_rs140803 & 234 chr1_194400 & 5.36 sex & cis \\
\hline 1 Gg_rs140803 & 234 chr1_198300 & 4.71 nonsex & cis \\
\hline 1 Gg_rs140803। & 234 chr1_571500 & 7.03 nonsex & cis \\
\hline 1 Gg_rs140803। & 234 chr1_154100 & 4.37 nonsex & cis \\
\hline 1 Gg_rs140803। & 234 chr1_289800। & 3.73 nonsex & cis \\
\hline 1 Gg_rs140803। & 234 chr1_327300। & 3.45 nonsex & cis \\
\hline 1 Gg_rs140803 & 234 chr1_435000। & 4.22 nonsex & cis \\
\hline 1 Gg_rs151869 & 234 chr1_311000। & 2.38 nonsex & cis \\
\hline 1 Gg_rs140803। & 236 chr1_639500। & 5.17 nonsex & cis \\
\hline 1 Gg_rs140803। & 236 chr1_162300 & 3.99 nonsex & cis \\
\hline 1 Gg_rs151869 & 237 chr1_284200। & 7.65 nonsex & cis \\
\hline 1 Gg_rs151869 & 238 chr1_214900। & 30.29 nonsex & cis \\
\hline 1 Gg_rs151869 & 238 chr1_284600। & 11.09 nonsex & cis \\
\hline 1 Gg_rs151869 & 238 chr1_461200। & 7.75 sex & cis \\
\hline 1 Gg_rs151869 & 238 chr1_132620 & 6.42 nonsex & cis \\
\hline 1 Gg_rs151869 & 239 chr1_333300। & 7.03 nonsex & cis \\
\hline 1 Gg_rs151869 & 239 chr1_285900। & 4.04 nonsex & cis \\
\hline 1 Gg_rs151869 & 239 chr1_258000। & 4.29 nonsex & cis \\
\hline 1 Gg_rs151869 & 239 chr1_711100 & 4.02 nonsex & cis \\
\hline 1 Gg_rs151869 & 240 chr1_193800 & 5.66 nonsex & cis \\
\hline 1 Gg_rs151869 & 240 chr1_264500 & 8.64 nonsex & cis \\
\hline 1 Gg_rs151869 & 240 chr1_333400। & 8.3 nonsex & cis \\
\hline 1 Gg_rs151869 & 240 chr1_345500 & 12.76 nonsex & cis \\
\hline 1 Gg_rs151869 & 240 chr1_453400। & 4.9 nonsex & cis \\
\hline 1 Gg_rs151869 & 240 chr1_552100। & 5.62 nonsex & cis \\
\hline 1 Gg_rs151869 & 240 chr1_785400। & 6.17 sex & cis \\
\hline 1 Gg_rs151869 & 240 chr1_343200। & 5.63 nonsex & cis \\
\hline 1 Gg_rs151869 & 240 chr1_230400 & 4.08 nonsex & cis \\
\hline 1 Gg_rs151869 & 240 chr1_284100 & 4.22 nonsex & cis \\
\hline 1 Gg_rs151869 & 240 chr1_510200। & 3.93 nonsex & cis \\
\hline 1 Gg_rs151869 & 240 chr1_799600 & 3.89 nonsex & cis \\
\hline 1 Gg_rs151869 & 240 chr1_118680 & 4.42 nonsex & cis \\
\hline 1 RBL4976 & 240 chr1_343700 & 8.34 nonsex & cis \\
\hline 1 RBL4976 & 240 chr1_344000 & 10.81 nonsex & cis \\
\hline 1 RBL4976 & 240 chr1_344300 & 15.97 nonsex & cis \\
\hline 1 RBL4976 & 240 chr1_344600 & 7.79 nonsex & cis \\
\hline 1 RBL4976 & 240 chr1_345200 & 15.93 nonsex & cis \\
\hline 1 RBL4976 & 240 chr1_345300 & 6.92 nonsex & cis \\
\hline 1 RBL4976 & 240 chr1_231700 & 3.9 nonsex & cis \\
\hline 1 RBL4976 & 241 chr1_215000 & 15.21 nonsex & cis \\
\hline
\end{tabular}




\begin{tabular}{|c|c|c|c|}
\hline 1 RBL4976 & 241 chr1_231500। & 7.7 nonsex & cis \\
\hline 1 RBL4976 & 241 chr1_342100। & 8.28 nonsex & cis \\
\hline 1 RBL4976 & 241 chr1_342900। & 7.61 nonsex & cis \\
\hline 1 RBL4976 & 241 chr1_344400 & 7.29 nonsex & cis \\
\hline 1 RBL4976 & 242 chr1_344900। & 4.06 nonsex & cis \\
\hline 1 RBL4976 & 242 chr1_230200। & 7.82 nonsex & cis \\
\hline 1 RBL4976 & 242 chr1_232800 & 8.54 nonsex & cis \\
\hline 1 RBL4976 & 242 chr1_263800 & 8.88 nonsex & cis \\
\hline 1 RBL4976 & 242 chr1_342400। & 6.69 sex & cis \\
\hline 1 RBL4976 & 242 chr1_342600 & 16.27 nonsex & cis \\
\hline 1 RBL4976 & 242 chr1_342800 & 9.18 nonsex & cis \\
\hline 1 RBL4976 & 242 chr1_343400। & 16.47 nonsex & cis \\
\hline 1 RBL4976 & 242 chr1_343500। & 6.61 nonsex & cis \\
\hline 1 RBL4976 & 242 chr1_343600। & 8.97 nonsex & cis \\
\hline 1 RBL4976 & 242 chr1_343900। & 9.73 sex & cis \\
\hline 1 RBL4976 & 242 chr1_344500। & 8.64 nonsex & cis \\
\hline 1 RBL4976 & 242 chr1_345400। & 8.77 nonsex & cis \\
\hline 1 RBL4976 & 242 chr1_629300। & 6.43 nonsex & cis \\
\hline 1 RBL4976 & 242 chr1_634800। & $6.25 \mathrm{sex}$ & cis \\
\hline 1 RBL4976 & 242 chr1_343800 & 3.76 nonsex & cis \\
\hline 1 RBL4976 & 242 chr1_264000। & 4.99 nonsex & cis \\
\hline 1 RBL4976 & 242 chr1_264800 & 4.28 nonsex & cis \\
\hline 1 RBL4976 & 242 chrW_41930 & 9.17 sex & trans \\
\hline 1 Gg_rs147885 & 242 chr1_344200। & 13.57 nonsex & cis \\
\hline 1 Gg_rs147885 & 242 chr1_345100। & 12.02 nonsex & cis \\
\hline 1 Gg_rs147885 & 243 chr1_278600 & 5.94 sex & cis \\
\hline 1 Gg_rs147885. & 243 chr1_278800 & 5.31 nonsex & cis \\
\hline 1 Gg_rs147885 & 243 chr1_284000। & 6.31 nonsex & cis \\
\hline 1 Gg_rs147885 & 243 chr1_369200। & 3.14 nonsex & cis \\
\hline 1 Gg_rs147885 & 243 chr1_455700। & 6.35 nonsex & cis \\
\hline 1 Gg_rs147885 & 243 chr1_515500 & 7.41 nonsex & cis \\
\hline 1 Gg_rs147885 & 243 chr1_515600 & 10.74 nonsex & cis \\
\hline 1 Gg_rs147885 & 243 chr1_140600 & 3.12 nonsex & cis \\
\hline 1 Gg_rs147885 & 243 chr1_410400 & 4.37 nonsex & cis \\
\hline 1 Gg_rs147885 & 243 chr1_633300। & 4.89 nonsex & cis \\
\hline 1 Gg_rs147885 & 243 chr31_43090। & 7.56 sex & trans \\
\hline 1 Gg_rs147885 & 243 chr33_44410 & 5.54 nonsex & trans \\
\hline 1 Gg_rs147885 & 243 chr1_140457। & 2.8 nonsex & trans \\
\hline 1 Gg_rs147885 & 244 chr1_265300 & 9.52 nonsex & cis \\
\hline 1 Gg_rs147885 & 244 chr1_214800 & 4.24 nonsex & cis \\
\hline 1 Gg_rs147885 & 244 chr1_503600 & 3.1 nonsex & cis \\
\hline 1 Gg_rs147885 & 244 chr1_706300। & 4.71 nonsex & cis \\
\hline 1 Gg_rs151869 & 245 chr1_342500। & 13.79 nonsex & cis \\
\hline
\end{tabular}




\begin{tabular}{|c|c|c|}
\hline 1 Gg_rs151869 & 245 chr1_343300। & 14.28 nonsex \\
\hline 1 RBL4976 & 245 chr1_264600 & 8.17 nonsex \\
\hline 1 RBL4976 & 245 chr1_631200। & 10.81 nonsex \\
\hline 1 Gg_rs147885 & 245 chr1_453500 & 4.89 nonsex \\
\hline 1 Gg_rs147885 & 245 chr1_488900। & 4.1 nonsex \\
\hline 1 Gg_rs147885 & 245 chr1_132130। & 4.19 nonsex \\
\hline 1 Gg_rs151869 & 246 chr1_293600। & 5.06 nonsex \\
\hline 1 Gg_rs147885 & 246 chr1_639900। & 3.89 nonsex \\
\hline 1 Gg_rs138261 & 246 chr1_458200 & 5.45 nonsex \\
\hline 1 Gg_rs151869 & 247 chr1_249300 & 4.31 nonsex \\
\hline 1 RBL4976 & 247 chr1_661800। & 6.97 nonsex \\
\hline 1 RBL4976 & 247 chr1_997900। & 3.97 nonsex \\
\hline 1 RBL4976 & 247 chr1_126710 & 3.71 nonsex \\
\hline 1 Gg_rs138261 & 247 chr1_367100। & 8.66 nonsex \\
\hline 1 Gg_rs138261 & 247 chr1_100540। & 8.85 nonsex \\
\hline 1 Gg_rs138261 & 247 chr1_102280 & 5.72 nonsex \\
\hline 1 Gg_rs138261 & 247 chr1_665900। & 3.63 nonsex \\
\hline 1 Gg_rs138261 & 247 chr1_991100। & 3.85 nonsex \\
\hline 1 RBL4976 & 248 chr1_729500। & 4.04 nonsex \\
\hline 1 Gg_rs138261 & 248 chr1_748100। & 5.88 nonsex \\
\hline 1 Gg_rs138261 & 248 chr1_784600। & 7.53 nonsex \\
\hline 1 RBL4976 & 249 chr1_711300 & 3.19 nonsex \\
\hline 1 Gg_rs138261 & 249 chr1_711200। & 6.15 nonsex \\
\hline 1 Gg_rs138261 & 249 chr1_785300। & 5.42 nonsex \\
\hline 1 Gg_rs138261 & 249 chr1_759500। & 4.12 nonsex \\
\hline 1 Gg_rs138261 & 250 chr1_257900 & 6.12 nonsex \\
\hline 1 Gg_rs138261 & 250 chr1_461300 & 5.56 nonsex \\
\hline 1 Gg_rs138261 & 250 chr1_694200। & 4.59 nonsex \\
\hline 1 Gg_rs138261 & 250 chr1_718700 & 4.36 nonsex \\
\hline 1 Gg_rs138261 & 250 chr1_724400 & 4.34 nonsex \\
\hline 1 Gg_rs138261 & 250 chr1_776300। & 4.21 nonsex \\
\hline 1 Gg_rs138261 & 251 chr1_631100। & 9.39 nonsex \\
\hline 1 Gg_rs138261 & 251 chr1_130380 & 3.73 nonsex \\
\hline 1 chr1.929557C & 252 chr1_506800 & 5.23 nonsex \\
\hline 1 chr1.929557C & 253 chr1_844300। & 12.8 nonsex \\
\hline 1 chr1.929557C & 253 chr1_727600। & 3.78 nonsex \\
\hline 1 chr1.929557C & 253 chr1_801200। & 4.97 nonsex \\
\hline 1 chr1.929557C & 254 chr1_132250 & 8.46 nonsex \\
\hline 1 chr1.929557C & 255 chr1_599900। & 4.96 sex \\
\hline 1 Gg_rs147969. & 255 chr1_700600 & 5 nonsex \\
\hline 1 Gg_rs147969. & 255 chr1_852800 & 10.22 nonsex \\
\hline 1 Gg_rs147969. & 255 chr1_852900। & 5.34 nonsex \\
\hline 1 Gg_rs147969. & 255 chr1_482000। & 3.73 nonsex \\
\hline
\end{tabular}




\begin{tabular}{|c|c|c|}
\hline 1 Gg_rs147969. & 255 chr1_515700। & 5.25 nonsex \\
\hline 1 Gg_rs147969. & 255 chr1_878200 & 3.91 nonsex \\
\hline 1 Gg_rs147969. & 255 chr31_60720। & 7.39 nonsex \\
\hline 1 Gg_rs147969. & 255 chr31_29970। & 5.41 nonsex \\
\hline 1 Gg_rs147969. & 255 chr31_30550। & 6.15 nonsex \\
\hline 1 Gg_rs147969. & 255 chr31_30580 & 5.47 nonsex \\
\hline 1 Gg_rs147969. & 255 chr31_32290। & 5.69 nonsex \\
\hline 1 Gg_rs147969. & 255 chr31_32900। & 6.24 nonsex \\
\hline 1 Gg_rs147969. & 255 chr31_34050। & 6.21 nonsex \\
\hline 1 Gg_rs147969. & 255 chr31_36490। & 6.01 nonsex \\
\hline 1 Gg_rs147969. & 255 chr31_37150। & 6.15 nonsex \\
\hline 1 Gg_rs147969. & 255 chr31_38530। & 5.78 nonsex \\
\hline 1 Gg_rs147969. & 255 chr31_43190। & 5.96 nonsex \\
\hline 1 Gg_rs147969. & 255 chr31_59490। & 6.36 nonsex \\
\hline 1 Gg_rs147969. & 255 chr31_60430। & 5.73 nonsex \\
\hline 1 Gg_rs147969. & 255 chr31_60440। & 6.07 nonsex \\
\hline 1 Gg_rs147969. & 255 chr31_61200। & 6.7 nonsex \\
\hline 1 Gg_rs147969. & 255 chr33_36340। & 5.77 nonsex \\
\hline 1 Gg_rs147969. & 255 chr33_36370। & 6.68 nonsex \\
\hline 1 Gg_rs147969. & 255 chr33_44400। & 5.71 nonsex \\
\hline 1 Gg_rs147969. & 255 chr33_44520। & 5.43 nonsex \\
\hline 1 X1_1130108€ & 255 chr1_575700। & 6.43 nonsex \\
\hline 1 X1_1130108E & 255 chr1_227800 & 7.36 nonsex \\
\hline 1 X1_1130108E & 255 chr31_57660। & 6.66 nonsex \\
\hline 1 X1_1130108€ & 255 chr31_38880 & 6.04 nonsex \\
\hline 1 X1_1130108E & 255 chr31_42310 & 6.27 nonsex \\
\hline 1 X1_1130108E & 256 chr1_227900। & 9.71 nonsex \\
\hline 1 X1_1130108E & 256 chr1_230300। & 10.14 nonsex \\
\hline 1 X1_1130108E & 256 chr1_231600। & 9.53 nonsex \\
\hline 1 X1_1130108E & 256 chr1_232900। & 9.97 nonsex \\
\hline 1 X1_1130108€ & 256 chr1_695100। & 6.24 nonsex \\
\hline 1 X1_1130108€ & 256 chr13_55900। & 6.66 nonsex \\
\hline 1 X1_1130108€ & 256 chr31_31860। & 6.49 nonsex \\
\hline 1 X1_1130108E & 256 chr31_36750। & 6.66 nonsex \\
\hline 1 X1_1130108E & 257 chr1_732200। & 6.7 nonsex \\
\hline 1 X1_1130108€ & 257 chr1_128110 & 5.19 sex \\
\hline 1 X1_1130108€ & 257 chr1_135940। & 3.46 nonsex \\
\hline 1 RBL4976 & 258 chr1_342700। & 7.04 nonsex \\
\hline 1 X1_1130108E & 258 chr1_130810 & 5.88 nonsex \\
\hline 1 X1_1130108€ & 258 chr1_122670 & 5.32 nonsex \\
\hline 1 X1_1130108€ & 258 chr31_41180 & 7 nonsex \\
\hline 1 X1_1130108€ & 258 chr31_35900। & 6.03 nonsex \\
\hline 1 Gg_rs151869 & 259 chr1_265200। & 7.26 nonsex \\
\hline
\end{tabular}




\begin{tabular}{|c|c|c|c|}
\hline 1 X1_1130108€ & 259 chr33_44390। & 6.81 nonsex & trans \\
\hline 1 X1_1130108€ & 259 chr1_208650 & 5.62 nonsex & trans \\
\hline 1 Gg_rs138324! & 259 chr1_142310 & $6.08 \operatorname{sex}$ & cis \\
\hline 1 Gg_rs147937 & 259 chr1_117720 & 5.98 nonsex & cis \\
\hline 1 Gg_rs151869 & 260 chr1_228000। & 4.86 nonsex & cis \\
\hline 1 RBL4976 & 260 chr1_345600 & 8.57 nonsex & cis \\
\hline 1 X1_1130108€ & 260 chr1_314300 & 6.8 nonsex & cis \\
\hline 1 X1_1130108€ & 260 chr1_132520। & 5.25 nonsex & cis \\
\hline 1 Gg_rs138261 & 261 chr1_698500। & 5.4 nonsex & cis \\
\hline 1 X1_1130108€ & 261 chr1_115780 & 4.61 nonsex & cis \\
\hline 1 X1_1130108E & 261 chr1_130240। & 7.86 nonsex & cis \\
\hline 1 X1_1130108€ & 261 chr1_118650 & 5.28 nonsex & cis \\
\hline 1 X1_1130108€ & 262 chr1_129820। & 5.49 nonsex & cis \\
\hline 1 X1_1130108€ & 262 chr31_58300। & 5.85 nonsex & trans \\
\hline 1 Gg_rs138324! & 262 chr1_113820 & 19.54 nonsex & cis \\
\hline 1 Gg_rs138324! & 262 chr1_119290। & 6.78 sex & cis \\
\hline 1 X1_1130108E & 263 chr31_31630। & 6.1 nonsex & trans \\
\hline 1 Gg_rs138324 & 263 chr1_113830 & 11.23 nonsex & cis \\
\hline 1 X1_1130108€ & 264 chr1_711600 & 3.84 nonsex & cis \\
\hline 1 Gg_rs138324! & 264 chr1_131560। & 4.93 nonsex & cis \\
\hline 1 Gg_rs138324! & 265 chr1_113810 & 7.73 nonsex & cis \\
\hline 1 Gg_rs138324! & 265 chr1_121300 & 9.31 nonsex & cis \\
\hline 1 Gg_rs138324! & 265 chr1_128320 & 5.47 sex & cis \\
\hline 1 Gg_rs138324। & 266 chr1_119090 & 5.74 sex & cis \\
\hline 1 Gg_rs138324! & 267 chr1_119530 & 5.69 nonsex & cis \\
\hline 1 Gg_rs138324! & 267 chr1_124170 & 8.92 sex & cis \\
\hline 1 Gg_rs138324! & 267 chr1_115840 & 4.78 nonsex & cis \\
\hline 1 Gg_rs138324! & 267 chr5_180040 & 5.59 sex & trans \\
\hline 1 Gg_rs138324 & 268 chr1_132220। & 8.65 nonsex & cis \\
\hline 1 Gg_rs138324! & 268 chrZ_125640। & 8.25 sex & trans \\
\hline 1 Gg_rs138324! & 270 chr1_754300 & 5.81 sex & cis \\
\hline 1 Gg_rs138324 & 270 chr1_794800 & 5.63 sex & cis \\
\hline 1 Gg_rs138324 & 270 chr1_115810 & 8.44 nonsex & cis \\
\hline 1 Gg_rs138324! & 270 chr1_117050। & 8.23 nonsex & cis \\
\hline 1 Gg_rs138324! & 270 chr1_116270 & 4.44 nonsex & cis \\
\hline 1 Gg_rs138324! & 270 chr1_131370 & 4.26 nonsex & cis \\
\hline 1 Gg_rs138324 & 270 chr1_133270 & 3.98 nonsex & cis \\
\hline 1 Gg_rs138324 & 270 chr1_134450 & 5.24 nonsex & cis \\
\hline 1 Gg_rs138324 & 270 chr31_30990। & 7.27 sex & trans \\
\hline 1 Gg_rs138324! & 270 chrW_10000 & 7.83 sex & trans \\
\hline 1 Gg_rs138324! & 271 chr1_132380 & 7.92 nonsex & cis \\
\hline 1 Gg_rs138324! & 271 chr1_137040 & 3.95 nonsex & cis \\
\hline 1 Gg_rs138324। & 271 chr1_138830 & 3.5 nonsex & cis \\
\hline
\end{tabular}




\begin{tabular}{|c|c|c|c|}
\hline 1 Gg_rs138324 & 271 chrZ_547500। & 7.49 sex & trans \\
\hline 1 Gg_rs138324! & 272 chr1_134640। & 8.05 sex & cis \\
\hline 1 Gg_rs138324। & 274 chr1_126700 & 6.5 nonsex & cis \\
\hline 1 Gg_rs138261 & 275 chr1_785500 & 4.47 nonsex & cis \\
\hline 1 Gg_rs138324। & 275 chr1_119520 & 6.61 nonsex & cis \\
\hline 1 Gg_rs138324! & 275 chr1_135460 & 11.12 nonsex & cis \\
\hline 1 Gg_rs138324! & 275 chr1_130550 & 5.19 nonsex & cis \\
\hline 1 Gg_rs138324। & 276 chr1_132410 & 7.82 nonsex & cis \\
\hline 1 Gg_rs138324 & 277 chr1_136280 & $4.5 \operatorname{sex}$ & cis \\
\hline 1 Gg_rs147937 & 277 chr1_132390। & 4.78 nonsex & cis \\
\hline 1 Gg_rs147937 & 278 chr1_124680 & 4.56 nonsex & cis \\
\hline 1 Gg_rs147937 & 278 chr1_128950। & 4.31 nonsex & cis \\
\hline 1 Gg_rs147937 & 278 chr1_130600 & 4.52 nonsex & cis \\
\hline 1 Gg_rs147937 & 279 chr1_138640 & 7.13 nonsex & cis \\
\hline 1 Gg_rs147937 & 279 chr1_136700। & 5.05 sex & cis \\
\hline 1 Gg_rs147937 & 279 chr1_138630 & 5.83 nonsex & cis \\
\hline 1 Gg_rs147937 & 279 chr1_173200 & 4.5 nonsex & cis \\
\hline 1 Gg_rs147937 & 280 chr1_192480 & 6.5 sex & cis \\
\hline 1 Gg_rs147937 & 280 chr1_846300। & 4.37 nonsex & cis \\
\hline 1 Gg_rs147937 & 280 chr1_113240 & 4.49 nonsex & cis \\
\hline 1 Gg_rs147937 & 280 chr2_951450। & 6.07 nonsex & trans \\
\hline 1 Gg_rs147937 & 281 chr8_157460। & 5.42 nonsex & trans \\
\hline 1 Gg_rs147937 & 282 chr1_365800। & 5.18 nonsex & cis \\
\hline 1 Gg_rs147937 & 282 chr1_374700। & 5.51 nonsex & cis \\
\hline 1 Gg_rs147937 & 282 chr1_554800 & 5.22 sex & cis \\
\hline 1 Gg_rs147937 & 282 chr1_846100। & 6.38 sex & cis \\
\hline 1 Gg_rs147937 & 282 chr1_888000। & 6.81 nonsex & cis \\
\hline 1 Gg_rs147937 & 282 chr1_888100। & 5.45 nonsex & cis \\
\hline 1 Gg_rs147937 & 282 chr1_106840 & 5.26 nonsex & cis \\
\hline 1 Gg_rs147937 & 282 chr1_108510। & 4.86 nonsex & cis \\
\hline 1 Gg_rs147937 & 282 chr1_120920 & $5.54 \operatorname{sex}$ & cis \\
\hline 1 Gg_rs147937 & 282 chr1_124620 & 5.95 sex & cis \\
\hline 1 Gg_rs147937 & 282 chr1_133840 & 5.26 nonsex & cis \\
\hline 1 Gg_rs147937 & 282 chr1_138590। & 6.63 sex & cis \\
\hline 1 Gg_rs147937 & 282 chr1_141260। & 5.91 nonsex & cis \\
\hline 1 Gg_rs147937 & 282 chr1_191070। & 5.72 sex & cis \\
\hline 1 Gg_rs147937 & 282 chr1_193580 & 6.27 nonsex & cis \\
\hline 1 Gg_rs147937 & 282 chr1_199530। & 5.09 nonsex & cis \\
\hline 1 Gg_rs147937 & 282 chr1_262900। & 4.24 nonsex & cis \\
\hline 1 Gg_rs147937 & 282 chr1_435100। & 4.19 nonsex & cis \\
\hline 1 Gg_rs147937 & 282 chr1_475600 & 5.06 nonsex & cis \\
\hline 1 Gg_rs147937 & 282 chr1_622700। & 4.23 nonsex & cis \\
\hline 1 Gg_rs147937 & 282 chr1_623400। & 4.51 nonsex & cis \\
\hline
\end{tabular}




\begin{tabular}{|c|c|c|c|}
\hline 1 Gg_rs147937 & 282 chr1_699300। & 4.53 nonsex & cis \\
\hline 1 Gg_rs147937 & 282 chr1_745500 & 4.47 nonsex & cis \\
\hline 1 Gg_rs147937 & 282 chr1_850600 & 4.24 nonsex & cis \\
\hline 1 Gg_rs147937 & 282 chr1_925600। & 4.12 nonsex & cis \\
\hline 1 Gg_rs147937 & 282 chr1_934700। & 4.71 nonsex & cis \\
\hline 1 Gg_rs147937 & 282 chr1_949300। & 4.2 nonsex & cis \\
\hline 1 Gg_rs147937 & 282 chr1_964500 & 4.14 nonsex & cis \\
\hline 1 Gg_rs147937 & 282 chr1_104260 & 4.11 nonsex & cis \\
\hline 1 Gg_rs147937 & 282 chr1_108560 & 4.15 nonsex & cis \\
\hline 1 Gg_rs147937 & 282 chr1_114310 & 4.31 nonsex & cis \\
\hline 1 Gg_rs147937 & 282 chr1_120930। & 4.31 nonsex & cis \\
\hline 1 Gg_rs147937 & 282 chr1_129570 & 4.11 nonsex & cis \\
\hline 1 Gg_rs147937 & 282 chr1_132630। & 4.26 nonsex & cis \\
\hline 1 Gg_rs147937 & 282 chr1_134350 & 4.84 nonsex & cis \\
\hline 1 Gg_rs147937 & 282 chr1_134830 & 4.43 nonsex & cis \\
\hline 1 Gg_rs147937 & 282 chr1_137540 & 4.95 nonsex & cis \\
\hline 1 Gg_rs147937 & 282 chr1_138610 & 4.47 nonsex & cis \\
\hline 1 Gg_rs147937 & 282 chr1_138650। & 5.22 nonsex & cis \\
\hline 1 Gg_rs147937 & 282 chr1_146280 & 4.57 nonsex & cis \\
\hline 1 Gg_rs147937 & 282 chr1_147790। & 4.58 nonsex & cis \\
\hline 1 Gg_rs147937 & 282 chr1_152170 & 4.73 nonsex & cis \\
\hline 1 Gg_rs147937 & 282 chr1_158740 & 4.46 nonsex & cis \\
\hline 1 Gg_rs147937 & 282 chr1_172870 & 4.29 nonsex & cis \\
\hline 1 Gg_rs147937 & 282 chr1_178900 & 4.58 nonsex & cis \\
\hline 1 Gg_rs147937 & 282 chr1_179570। & 4.11 nonsex & cis \\
\hline 1 Gg_rs147937 & 282 chr1_186770 & 4.42 nonsex & cis \\
\hline 1 Gg_rs147937 & 282 chr1_193170 & 4.25 nonsex & cis \\
\hline 1 Gg_rs147937 & 282 chr1_197960। & 4.21 nonsex & cis \\
\hline 1 Gg_rs147937 & 282 chr1_201880 & 4.03 nonsex & cis \\
\hline 1 Gg_rs147937 & 282 chr4_546820। & 6.82 nonsex & trans \\
\hline 1 Gg_rs147937 & 282 chr7_206350। & 7.61 sex & trans \\
\hline 1 Gg_rs147937 & 282 chr7_253550। & 6.29 sex & trans \\
\hline 1 Gg_rs147937 & 282 chr9_980800। & 7.7 sex & trans \\
\hline 1 Gg_rs147937 & 282 chr11_15929| & 7.36 nonsex & trans \\
\hline 1 Gg_rs147937 & 282 chrZ_4246301 & $7.51 \mathrm{sex}$ & trans \\
\hline 1 Gg_rs147937 & 282 chrZ_4891801 & $7.51 \mathrm{sex}$ & trans \\
\hline 1 Gg_rs147937 & 282 chr2_456420 & 6.42 sex & trans \\
\hline 1 Gg_rs147937 & 282 chr2_844030। & 6.52 sex & trans \\
\hline 1 Gg_rs147937 & 282 chr2_133103। & 7.61 sex & trans \\
\hline 1 Gg_rs147937 & 282 chr1_369920। & 5.99 nonsex & trans \\
\hline 1 Gg_rs147937 & 282 chr1_443280 & 5.84 nonsex & trans \\
\hline 1 Gg_rs147937 & 282 chr4_382150। & 5.47 nonsex & trans \\
\hline 1 Gg_rs147937 & 282 chr4_757890। & 5.78 nonsex & trans \\
\hline
\end{tabular}




\begin{tabular}{|c|c|c|}
\hline 1 Gg_rs147937 & 282 chr5_744100। & 5.64 nonsex \\
\hline 1 Gg_rs147937 & 282 chr5_335940। & 6.03 nonsex \\
\hline 1 Gg_rs147937 & 282 chr5_374960। & 5.67 nonsex \\
\hline 1 Gg_rs147937 & 282 chr7_339350। & 5.79 nonsex \\
\hline 1 Gg_rs147937 & 282 chr11_69170। & 5.68 nonsex \\
\hline 1 Gg_rs147937 & 282 chr11_79140। & 5.55 nonsex \\
\hline 1 Gg_rs147937 & 282 chr13_81340 & 5.78 nonsex \\
\hline 1 Gg_rs147937 & 282 chrZ_1887201 & 5.73 nonsex \\
\hline 1 Gg_rs147937 & 282 chrZ_247700। & 5.75 nonsex \\
\hline 1 Gg_rs147937 & 282 chr1_590930। & 6.07 nonsex \\
\hline 1 Gg_rs147937 & 282 chr1_676800। & 5.94 nonsex \\
\hline 1 Gg_rs147937 & 282 chr1_921880 & 6.65 nonsex \\
\hline 1 Gg_rs147937 & 282 chr1_150723। & 5.65 nonsex \\
\hline 1 Gg_rs147937 & 282 chr1_156908। & 5.6 nonsex \\
\hline 1 Gg_rs147937 & 282 chr1_158082 & 6.96 nonsex \\
\hline 1 Gg_rs147937 & 282 chr1_174383। & 6.29 nonsex \\
\hline 1 Gg_rs147937 & 282 chr2_131980 & 5.79 nonsex \\
\hline 1 Gg_rs147937 & 282 chr2_508810 & 5.86 nonsex \\
\hline 1 Gg_rs147937 & 282 chr2_992810। & 5.48 nonsex \\
\hline 1 Gg_rs147937 & 282 chr2_105751। & 5.64 nonsex \\
\hline 1 Gg_rs147937 & 282 chr2_116387। & 5.81 nonsex \\
\hline 1 Gg_rs147937 & 282 chr3_731090। & 6.22 nonsex \\
\hline 1 Gg_rs147937 & 282 chr3_736490। & 5.52 nonsex \\
\hline 1 Gg_rs147937 & 282 chr3_793000। & 5.85 nonsex \\
\hline 1 Gg_rs147937 & 282 chr4_133690। & 6.47 nonsex \\
\hline 1 Gg_rs147937 & 283 chr1_104610 & 4.68 nonsex \\
\hline 1 Gg_rs147937 & 283 chr1_151150 & 5.7 sex \\
\hline 1 Gg_rs147937 & 283 chr1_539900। & 4.47 nonsex \\
\hline 1 Gg_rs147937 & 283 chr1_111460। & 4.31 nonsex \\
\hline 1 Gg_rs147937 & 283 chr1_170180 & 5.12 nonsex \\
\hline 1 Gg_rs147937 & 283 chr8_158440 & 5.55 nonsex \\
\hline 1 Gg_rs147937 & 283 chrZ_5864701 & 5.57 nonsex \\
\hline 1 Gg_rs147937 & 284 chr1_993100। & 5.39 sex \\
\hline 1 Gg_rs147937 & 284 chr1_135070। & 4.72 nonsex \\
\hline 1 Gg_rs147937 & 284 chr1_157030 & 4.21 nonsex \\
\hline 1 Gg_rs147937 & 284 chr1_177530। & 4.89 nonsex \\
\hline 1 Gg_rs147937 & 284 chr1_183470 & 4.37 nonsex \\
\hline 1 Gg_rs147937 & 284 chr11_13907। & 5.71 nonsex \\
\hline 1 Gg_rs147937 & 284 chr14_77980। & 6.02 nonsex \\
\hline 1 Gg_rs147937 & 284 chr21_54420। & 6.17 nonsex \\
\hline 1 Gg_rs147937 & 284 chr1_861490। & 6 nonsex \\
\hline 1 Gg_rs147937 & 285 chr1_141250 & 5.97 nonsex \\
\hline 1 Gg_rs147937 & 285 chr1_678300। & 4.12 nonsex \\
\hline
\end{tabular}




\begin{tabular}{|c|c|c|c|}
\hline 1 Gg_rs147937 & 285 chr1_137750। & 4.54 nonsex & cis \\
\hline 1 Gg_rs147937 & 285 chr1_145790 & 5.34 nonsex & cis \\
\hline 1 Gg_rs147937 & 285 chr1_173640 & 4.28 nonsex & cis \\
\hline 1 Gg_rs147937 & 285 chr1_191550 & 3.95 nonsex & cis \\
\hline 1 Gg_rs147937 & 285 chr4_722400। & 6.99 nonsex & trans \\
\hline 1 Gg_rs147937 & 285 chr1_431220। & 6.1 nonsex & trans \\
\hline 1 Gg_rs147937 & 286 chr1_142320 & 7.06 sex & cis \\
\hline 1 Gg_rs147937 & 286 chr1_146220 & 7.77 nonsex & cis \\
\hline 1 Gg_rs147937 & 286 chr1_108210 & 4.54 nonsex & cis \\
\hline 1 Gg_rs147937 & 286 chr3_836150। & 5.83 nonsex & trans \\
\hline 1 Gg_rs147937 & 287 chr1_142240 & 6.51 sex & cis \\
\hline 1 Gg_rs138324! & 288 chr1_124290। & 4.41 nonsex & cis \\
\hline 1 Gg_rs147937 & 288 chr1_148870 & 6.11 nonsex & cis \\
\hline 1 Gg_rs151948. & 288 chr1_146500 & 4.18 nonsex & cis \\
\hline 1 Gg_rs147937 & 289 chr1_142810 & 8.19 nonsex & cis \\
\hline 1 Gg_rs151948. & 290 chr1_136980 & 4.98 nonsex & cis \\
\hline 1 Gg_rs151948. & 290 chr1_148880 & 5.48 sex & cis \\
\hline 1 Gg_rs151948. & 290 chr1_121052। & 6.82 sex & trans \\
\hline 1 Gg_rs151948. & 293 chr1_158750 & 4.91 nonsex & cis \\
\hline 1 Gg_rs151948. & 294 chr1_148120। & 5.78 sex & cis \\
\hline 1 Gg_rs151948. & 297 chr1_147990। & 11.08 nonsex & cis \\
\hline 1 Gg_rs151948. & 297 chr1_149060। & 4.45 nonsex & cis \\
\hline 1 Gg_rs151948. & 300 chr1_147980 & 7.98 nonsex & cis \\
\hline 1 Gg_rs151948. & 300 chr1_154960 & 4.41 nonsex & cis \\
\hline 1 Gg_rs151948. & 301 chr1_147860 & 4.25 nonsex & cis \\
\hline 1 Gg_rs151948. & 302 chr1_146360। & 5.09 nonsex & cis \\
\hline 1 Gg_rs151948. & 303 chr1_150090 & 4.77 nonsex & cis \\
\hline 1 Gg_rs151948. & 305 chr1_148000 & 8.09 nonsex & cis \\
\hline 1 Gg_rs151948. & 305 chr1_151340 & 4.97 nonsex & cis \\
\hline 1 Gg_rs151948. & 306 chr1_147970। & 3.71 nonsex & cis \\
\hline 1 Gg_rs151948. & 310 chr1_141550 & 4.33 nonsex & cis \\
\hline 1 Gg_rs147937 & 315 chr1_163370 & 4.84 nonsex & cis \\
\hline 1 Gg_rs138283 & 322 chr1_182457। & 6.14 nonsex & trans \\
\hline 1 Gg_rs138283 & 328 chr11_18013। & 5.48 nonsex & trans \\
\hline 1 Gg_rs138283 & 330 chr1_163340 & 4.55 nonsex & cis \\
\hline 1 Gg_rs138283| & 331 chr1_164490 & 4.69 nonsex & cis \\
\hline 1 Gg_rs137990. & 339 chr1_163320 & 5.06 nonsex & cis \\
\hline 1 Gg_rs137990 & 339 chr1_176330। & 4.71 nonsex & cis \\
\hline 1 Gg_rs137990. & 341 chr1_179450। & 6.34 nonsex & cis \\
\hline 1 Gg_rs137990. & 342 chr1_165470 & 5.49 nonsex & cis \\
\hline 1 Gg_rs137990. & 343 chr1_198730 & 4.72 nonsex & cis \\
\hline 1 Gg_rs147981: & 347 chr1_204820। & 6.69 nonsex & cis \\
\hline 1 Gg_rs147981 & 349 chr1_205580। & 5.03 nonsex & cis \\
\hline
\end{tabular}




\begin{tabular}{|c|c|c|c|}
\hline 1 Gg_rs147981: & 352 chr1_205360। & 8.06 nonsex & cis \\
\hline 1 Gg_rs147981: & 353 chr1_206340 & 6.16 nonsex & cis \\
\hline 1 Gg_rs147981: & 353 chr1_644790। & 5.7 sex & trans \\
\hline 1 Gg_rs147981: & 358 chr1_201820 & 3.09 nonsex & cis \\
\hline 1 Gg_rs147981: & 359 chr1_220340। & 8.05 nonsex & cis \\
\hline 1 X1_2401256€ & 370 chr1_173520। & 4.18 nonsex & cis \\
\hline 1 X1_2401256€ & 371 chr6_402300 & 5.36 sex & trans \\
\hline 1 X1_2401256€ & 372 chr1_242660। & 4.53 sex & cis \\
\hline 1 X1_2401256€ & 372 chr1_165810 & 3.76 nonsex & cis \\
\hline 1 X1_2401256€ & 372 chr1_169260 & 4.16 nonsex & cis \\
\hline 1 X1_2401256€ & 372 chr1_218490। & 4.37 nonsex & cis \\
\hline 1 X1_2401256€ & 372 chrZ_352900। & 7.55 sex & trans \\
\hline 1 X1_2401256€ & 372 chr1_431910। & 5.88 nonsex & trans \\
\hline 1 X1_2401256€ & 373 chr1_189110 & 4.32 nonsex & cis \\
\hline 1 X1_2401256€ & 375 chr1_202020। & 5.48 nonsex & cis \\
\hline 1 X1_2401256€ & 375 chr1_154616। & 7.55 nonsex & trans \\
\hline 1 X1_2401256€ & 375 chr3_895600। & 8.59 sex & trans \\
\hline 1 X1_2401256€ & 375 chr1_405040 & 6.36 nonsex & trans \\
\hline 1 X1_2401256€ & 376 chr1_176540। & 5.21 nonsex & cis \\
\hline 1 X1_2401256€ & 376 chr1_167500 & 5.81 nonsex & cis \\
\hline 1 X1_2401256€ & 376 chr1_170990। & 4.99 nonsex & cis \\
\hline 1 X1_2401256€ & 376 chr1_536800 & 6.45 nonsex & trans \\
\hline 1 X1_2401256€ & 377 chr1_187690 & 4.46 nonsex & cis \\
\hline 1 X1_2401256€ & 377 chr1_232930। & 3.95 nonsex & cis \\
\hline 1 X1_2401256€ & 378 chr1_193770 & 5.72 nonsex & cis \\
\hline 1 X1_2401256€ & 379 chr1_228810 & 5.26 nonsex & cis \\
\hline 1 Gg_rs147998. & 385 chr1_224410 & 4.34 nonsex & cis \\
\hline 1 Gg_rs147998. & 388 chr1_244130। & 4.8 nonsex & cis \\
\hline 1 Gg_rs147998. & 391 chr1_239420। & 3.78 nonsex & cis \\
\hline 1 Gg_rs147998. & 391 chr1_518400। & 7.98 sex & trans \\
\hline 1 X1_27115232 & 416 chr1_241200 & 4.41 nonsex & cis \\
\hline 1 X1_27115232 & 416 chr1_253440 & 3.91 nonsex & cis \\
\hline 1 X1_27115232 & 416 chr1_255600। & 4.31 nonsex & cis \\
\hline 1 X1_27115232 & 425 chr1_240480 & 4.98 nonsex & cis \\
\hline 1 hrm_chr1.29l & 432 chr1_295750। & 4.58 nonsex & cis \\
\hline 1 hrm_chr1.29l & 433 chr1_294590। & 5.22 nonsex & cis \\
\hline 1 hrm_chr1.29l & 439 chr1_295740। & 3.88 nonsex & cis \\
\hline 1 hrm_chr1.29l & 442 chr1_278520। & 5.3 nonsex & cis \\
\hline 1 hrm_chr1.29l & 442 chr1_285470। & 5.61 nonsex & cis \\
\hline 1 hrm_chr1.29l & 442 chr1_276640। & 4.18 nonsex & cis \\
\hline 1 X1_30454963 & 444 chr1_260730 & 4.81 nonsex & cis \\
\hline 1 X1_30454963 & 444 chr1_294790। & 20.43 nonsex & cis \\
\hline 1 X1_30454963 & 444 chr1_294800। & 6.05 nonsex & cis \\
\hline
\end{tabular}




\begin{tabular}{|c|c|c|c|}
\hline $1 \times 130454963$ & 444 chr1 295480 & 4.97 nonsex & cis \\
\hline 1 Gg_rs148039 & 468 chr1_293510 & 5.1 nonsex & cis \\
\hline 1 X32_1 & 490 chr1_246140 & 4.37 nonsex & cis \\
\hline $1 \times 32 \_1$ & 490 chr1_338970। & 3.55 nonsex & cis \\
\hline $1 \times 32 \_1$ & 490 chr2_417000 & 5.09 nonsex & trans \\
\hline $1 \times 32 \_1$ & 492 chr1_276190। & 3.85 nonsex & cis \\
\hline $1 \times 32 \_1$ & 494 chr1_257510 & 5.79 sex & cis \\
\hline $1 \times 32 \_1$ & 503 chr1_299090। & 4.1 nonsex & cis \\
\hline $1 \times 32 \_1$ & 503 chr4_300550। & 6.24 nonsex & trans \\
\hline $1 \times 32 \_1$ & 503 chr2_742360 & 5.9 nonsex & trans \\
\hline $1 \times 32 \_1$ & 511 chr1_293970। & 3.66 nonsex & cis \\
\hline $1 \times 32 \_1$ & 512 chr1_326780 & 4.35 nonsex & cis \\
\hline $1 \times 33 \_2$ & 576 chr1_307560। & 5.5 nonsex & cis \\
\hline 1 X33_2 & 588 chr1_338460। & 3.59 nonsex & cis \\
\hline $1 \times 35.5 \mathrm{Mb}$ & 598 chr1_334320। & 5.94 nonsex & cis \\
\hline $1 \times 35.5 \mathrm{Mb}$ & 615 chr31_26260। & 7.48 sex & trans \\
\hline $1 \times 35.5 \mathrm{Mb}$ & 638 chr1_330620। & 6.49 nonsex & cis \\
\hline $1 \times 35.5 \mathrm{Mb}$ & 641 chr1_335550 & 5.78 nonsex & cis \\
\hline $1 \times 35.5 \mathrm{Mb}$ & 643 chr1_323160। & 6.28 nonsex & cis \\
\hline $1 \times 35.5 \mathrm{Mb}$ & 647 chr1_359350। & 5.71 nonsex & cis \\
\hline $1 \times 36.15 \mathrm{Mb}$ & 703 chr22_13900। & $11.6 \mathrm{sex}$ & trans \\
\hline $1 \times 36.15 \mathrm{Mb}$ & 703 chr25_49900। & 10.48 sex & trans \\
\hline $1 \times 36.15 \mathrm{Mb}$ & 703 chr25_57700। & $11.03 \mathrm{sex}$ & trans \\
\hline $1 \times 36.15 \mathrm{Mb}$ & 703 chr30_26200। & 8.62 sex & trans \\
\hline $1 \times 36.15 \mathrm{Mb}$ & 703 chr30_26300। & 7.42 sex & trans \\
\hline $1 \times 36.15 \mathrm{Mb}$ & 705 chr1_317250 & 6.48 nonsex & cis \\
\hline $1 \times 36.15 \mathrm{Mb}$ & 705 chr1_339960। & 4.67 nonsex & cis \\
\hline $1 \times 36 \_45$ & 708 chr1_348090। & 5.38 nonsex & cis \\
\hline 1 X36_9 & 718 chr11_15402 & 8.12 nonsex & trans \\
\hline 1 X36_9 & 721 chr3_392280 & 5.6 nonsex & trans \\
\hline 1 X36_9 & 722 chr3_663500। & 6.62 sex & trans \\
\hline 1 X1_37164711 & 751 chr1_355370। & 11.8 nonsex & cis \\
\hline 1 X1_37164711 & 752 chr1_355180। & 6.56 nonsex & cis \\
\hline 1 X1_37164711 & 752 chr1_353080 & 5.16 nonsex & cis \\
\hline 1 Gg_rs137516 & 752 chr1_363610 & 4.09 nonsex & cis \\
\hline $1 \times 36 \_45$ & 754 chr1_348050। & 4.68 nonsex & cis \\
\hline 1 X1_37164711 & 754 chr1_352690। & 5.26 nonsex & cis \\
\hline 1 Gg_rs137516 & 755 chr1_369130। & 2.62 nonsex & cis \\
\hline 1 Gg_rs148123 & 756 chr1_384330। & 8.97 sex & cis \\
\hline 1 Gg_rs148123 & 757 chr1_358770 & 6.17 nonsex & cis \\
\hline 1 Gg_rs148123 & 757 chr1_359010 & 8.35 nonsex & cis \\
\hline 1 Gg_rs148123 & 757 chr1_364960। & 3.06 nonsex & cis \\
\hline 1 Gg_rs137516 & 758 chr1_364150। & 7.39 nonsex & cis \\
\hline
\end{tabular}




\begin{tabular}{|c|c|c|}
\hline 1 Gg_rs137516 & 758 chr1_377800। & 7.29 nonsex \\
\hline 1 Gg_rs148123 & 759 chr1_359020। & 9.7 nonsex \\
\hline 1 Gg_rs152374 & 760 chr1_377310। & 7.06 nonsex \\
\hline 1 Gg_rs152374 & 760 chr1_377940। & 16.86 nonsex \\
\hline 1 Gg_rs152374 & 760 chr1_359870। & 5.17 nonsex \\
\hline 1 Gg_rs152374 & 760 chr1_377370। & 5.05 nonsex \\
\hline 1 Gg_rs152374 & 763 chr1_380590। & 5.6 nonsex \\
\hline 1 Gg_rs148123 & 763 chr1_358970। & 6.53 nonsex \\
\hline 1 Gg_rs148123 & 763 chr1_360990। & 9.89 nonsex \\
\hline 1 Gg_rs148123 & 763 chr1_367500। & 9.22 nonsex \\
\hline 1 Gg_rs148123 & 763 chr1_368360। & 7.15 sex \\
\hline 1 Gg_rs148123. & 763 chr1_369090। & 6.74 nonsex \\
\hline 1 Gg_rs148123 & 763 chr1_375720। & 6.75 nonsex \\
\hline 1 Gg_rs148123. & 763 chr1_367940। & 5.31 nonsex \\
\hline 1 Gg_rs148123 & 763 chr1_379170। & 5.28 nonsex \\
\hline 1 Gg_rs152374 & 764 chr1_377100। & 5.32 nonsex \\
\hline 1 Gg_rs148123 & 764 chr1_367210। & 7.96 nonsex \\
\hline 1 Gg_rs148123 & 764 chr1_376680 & 7.88 nonsex \\
\hline 1 Gg_rs148123 & 764 chr1_379690। & 8.51 sex \\
\hline 1 Gg_rs148123 & 764 chr1_375700। & 5.24 nonsex \\
\hline 1 Gg_rs152393 & 764 chr1_368530। & 14.51 nonsex \\
\hline 1 Gg_rs152374 & 765 chr1_369100। & 7.45 nonsex \\
\hline 1 Gg_rs148123. & 765 chr1_376830। & 3.44 nonsex \\
\hline 1 Gg_rs148123 & 766 chr1_379680। & 7.1 nonsex \\
\hline 1 Gg_rs148123 & 766 chr1_369370। & 4.52 nonsex \\
\hline 1 Gg_rs148123 & 767 chr1_379090। & 6.18 nonsex \\
\hline 1 Gg_rs152374 & 768 chr1_378430। & 2.92 nonsex \\
\hline 1 Gg_rs148123 & 769 chr1_361700। & 5.47 nonsex \\
\hline 1 Gg_rs148123 & 769 chr1_398710। & 5.48 nonsex \\
\hline 1 Gg_rs148123 & 770 chr1_360220। & 5.63 nonsex \\
\hline 1 Gg_rs152393 & 770 chr1_374560। & 8.17 nonsex \\
\hline 1 Gg_rs152393 & 770 chr1_369050। & 5.72 nonsex \\
\hline 1 Gg_rs152393 & 771 chr1_395780। & 6.13 nonsex \\
\hline 1 Gg_rs152393। & 772 chr1_386100। & 8.94 nonsex \\
\hline 1 Gg_rs148147. & 772 chr1_386040। & 6.15 nonsex \\
\hline 1 Gg_rs152393' & 773 chr1_392440। & 13.39 nonsex \\
\hline 1 Gg_rs148147. & 773 chr1_401900। & 7.04 nonsex \\
\hline 1 Gg_rs148147. & 774 chr1_382120। & 5.59 nonsex \\
\hline 1 Gg_rs148147. & 774 chr1_394620। & 5.51 nonsex \\
\hline 1 Gg_rs148147. & 775 chr1_403500। & 5.75 nonsex \\
\hline 1 Gg_rs148159 & 775 chr1_397570। & 5.15 nonsex \\
\hline 1 Gg_rs148147. & 776 chr1_393880 & 6.19 nonsex \\
\hline 1 Gg_rs148147. & 776 chr4_401490। & 6.88 nonsex \\
\hline
\end{tabular}




\begin{tabular}{|c|c|c|c|}
\hline 1 Gg_rs148147. & 777 chr1_395810। & 6.25 nonsex & cis \\
\hline 1 Gg_rs148147. & 777 chr1_386730। & 5.61 nonsex & cis \\
\hline 1 Gg_rs148147. & 777 chr1_391660। & 5.42 nonsex & cis \\
\hline 1 Gg_rs148147. & 778 chr1_396230। & 10.1 nonsex & cis \\
\hline 1 Gg_rs148147. & 778 chr1_399200। & 7.08 nonsex & cis \\
\hline 1 Gg_rs148147. & 778 chr1_404370 & 5.59 nonsex & cis \\
\hline 1 Gg_rs148147. & 778 chr1_395790 & 5.15 nonsex & cis \\
\hline 1 Gg_rs148147. & 778 chr1_405250 & 4.66 nonsex & cis \\
\hline 1 Gg_rs148147. & 779 chr1_396210 & 7.54 nonsex & cis \\
\hline 1 Gg_rs148147. & 780 chr1_403460 & 4.79 nonsex & cis \\
\hline 1 Gg_rs148147. & 782 chr1_388370। & 4.32 nonsex & cis \\
\hline 1 Gg_rs148147. & 782 chr1_404040 & 4.55 nonsex & cis \\
\hline 1 Gg_rs148147. & 783 chr1_406650 & 11.41 nonsex & cis \\
\hline 1 Gg_rs148147. & 783 chr1_406600। & 3.45 nonsex & cis \\
\hline 1 Gg_rs148147. & 784 chr1_396220। & 6.18 nonsex & cis \\
\hline 1 Gg_rs148147. & 784 chr1_404970। & 7.65 nonsex & cis \\
\hline 1 Gg_rs148147. & 784 chr1_409150 & 4.02 nonsex & cis \\
\hline 1 Gg_rs148147. & 784 chr1_396170। & 3.09 nonsex & cis \\
\hline 1 Gg_rs148147. & 784 chr1_401130। & 4.13 nonsex & cis \\
\hline 1 Gg_rs148147. & 784 chr1_405060। & 4.27 nonsex & cis \\
\hline 1 Gg_rs148147. & 784 chr1_410350 & 2.93 nonsex & cis \\
\hline 1 Gg_rs148147. & 784 chrW_16720 & 7.34 sex & trans \\
\hline 1 Gg_rs148147. & 785 chr1_402400 & 7.49 nonsex & cis \\
\hline 1 Gg_rs148147. & 785 chr1_409500। & 5.09 nonsex & cis \\
\hline 1 Gg_rs148147. & 785 chr1_403440। & 5.81 nonsex & cis \\
\hline 1 Gg_rs148147. & 786 chr1_404610 & 7.23 nonsex & cis \\
\hline 1 Gg_rs148147. & 786 chr1_406090। & 9.21 nonsex & cis \\
\hline 1 Gg_rs148147. & 786 chr1_406100। & 11.07 nonsex & cis \\
\hline 1 Gg_rs148159 & 786 chr1_360250। & 3.97 nonsex & cis \\
\hline 1 Gg_rs148159 & 786 chr1_390840। & 5.56 nonsex & cis \\
\hline 1 Gg_rs148159 & 786 chr1_390310 & 5.5 nonsex & cis \\
\hline 1 Gg_rs148147. & 787 chr1_403490। & 5.89 nonsex & cis \\
\hline 1 Gg_rs148159 & 787 chr1_407120। & 8.52 nonsex & cis \\
\hline 1 Gg_rs148159 & 788 chr1_399860। & 3.73 nonsex & cis \\
\hline 1 Gg_rs148159 & 788 chr1_399940। & 5.15 sex & cis \\
\hline 1 Gg_rs148159 & 788 chr1_408070। & 7.25 nonsex & cis \\
\hline 1 Gg_rs148159 & 788 chr1_411810 & 10.05 nonsex & cis \\
\hline 1 Gg_rs148159 & 788 chr1_430220। & 7.11 nonsex & cis \\
\hline 1 Gg_rs148159 & 789 chr1_411770 & 6.95 nonsex & cis \\
\hline 1 Gg_rs148159 & 789 chr1_411780 & 20.23 nonsex & cis \\
\hline 1 Gg_rs148159 & 789 chr1_411790 & 16.2 nonsex & cis \\
\hline 1 Gg_rs148159 & 789 chr1_411800 & 11.5 nonsex & cis \\
\hline 1 Gg_rs148159 & 789 chr1_412350 & 9.9 nonsex & cis \\
\hline
\end{tabular}




\begin{tabular}{|c|c|c|c|}
\hline 1 Gg_rs148159 & 789 chr1_428700। & 10.3 nonsex & cis \\
\hline 1 Gg_rs148159 & 789 chr1_428850। & 11.85 nonsex & cis \\
\hline 1 Gg_rs148159 & 789 chr1_364350 & 2.15 nonsex & cis \\
\hline 1 Gg_rs148159 & 789 chr1_395400। & 2.11 nonsex & cis \\
\hline 1 Gg_rs148159 & 789 chr1_401780 & 2.42 nonsex & cis \\
\hline 1 Gg_rs148159 & 789 chr1_411160। & 5.17 nonsex & cis \\
\hline 1 Gg_rs148159 & 789 chr1_416720। & 4.77 nonsex & cis \\
\hline 1 Gg_rs148159 & 789 chr1_424290। & 3.97 nonsex & cis \\
\hline 1 Gg_rs148159 & 789 chr1_427160। & 5.55 nonsex & cis \\
\hline 1 Gg_rs148159 & 789 chr1_433440। & 5.1 nonsex & cis \\
\hline 1 Gg_rs148159 & 789 chr1_434870 & 2.68 nonsex & cis \\
\hline 1 Gg_rs148159 & 789 chr1_439700। & 4.65 nonsex & cis \\
\hline 1 Gg_rs148159 & 789 chr2_144825। & 6.83 sex & trans \\
\hline 1 Gg_rs148159 & 789 chr4_792830। & 6.01 nonsex & trans \\
\hline 1 Gg_rs148159 & 789 chr2_846000। & 5.16 nonsex & trans \\
\hline 1 Gg_rs148159 & 790 chr1_411150 & 6.82 nonsex & cis \\
\hline 1 Gg_rs148159 & 791 chr1_404850। & 6.21 nonsex & cis \\
\hline 1 Gg_rs148159 & 791 chr1_851000। & 4.65 nonsex & trans \\
\hline 1 Gg_rs148159 & 792 chr1_384610 & $5.28 \mathrm{sex}$ & cis \\
\hline 1 Gg_rs148159 & 792 chr1_406110 & 13.78 nonsex & cis \\
\hline 1 Gg_rs148159 & 792 chr1_443300। & 5.42 nonsex & cis \\
\hline 1 Gg_rs148159 & 792 chrZ_5313701 & 3.93 nonsex & trans \\
\hline 1 Gg_rs148159 & 795 chr1_385050। & 6.23 nonsex & cis \\
\hline 1 Gg_rs138586 & 796 chr1_444920। & 6.34 nonsex & cis \\
\hline 1 Gg_rs138586. & 797 chr1_410270। & 6.32 sex & cis \\
\hline 1 Gg_rs138586. & 797 chr1_428330। & 5.7 nonsex & cis \\
\hline 1 Gg_rs138586 & 799 chr2_304530। & 6.39 sex & trans \\
\hline 1 Gg_rs138586 & 799 chrZ_2129301 & 5.67 nonsex & trans \\
\hline 1 Gg_rs138586 & 800 chr1_428690 & 6.38 sex & cis \\
\hline 1 Gg_rs138586. & 800 chr4_185360। & 6.05 nonsex & trans \\
\hline 1 Gg_rs138586. & 801 chr1_443460। & 3.89 nonsex & cis \\
\hline 1 Gg_rs138586 & 801 chr4_401210 & 5.07 sex & trans \\
\hline 1 Gg_rs138586. & 802 chr1_442390। & 4.04 nonsex & cis \\
\hline 1 Gg_rs138586 & 802 chr1_445200 & 5.33 nonsex & cis \\
\hline 1 Gg_rs138586 & 802 chr7_381000 & 5.83 sex & trans \\
\hline 1 Gg_rs138586. & 802 chr8_216410 & 4.29 sex & trans \\
\hline 1 Gg_rs138586. & 802 chr12_49540। & 6.25 sex & trans \\
\hline 1 Gg_rs138586 & 802 chr3_178940। & 6.02 sex & trans \\
\hline 1 Gg_rs138586 & 802 chr3_662360। & 6.31 sex & trans \\
\hline 1 Gg_rs138586 & 805 chr2_879430। & 6.8 sex & trans \\
\hline 1 Gg_rs138586 & 806 chr1_442380 & 5.01 sex & cis \\
\hline 1 Gg_rs138586. & 806 chr1_464100 & 4.52 sex & cis \\
\hline 1 Gg_rs138586. & 807 chr9_130630। & 6.17 nonsex & trans \\
\hline
\end{tabular}




\begin{tabular}{|c|c|c|c|}
\hline 1 Gg_rs138586. & 810 chrW_50580 & $8 \operatorname{sex}$ & trans \\
\hline 1 Gg_rs138586. & 811 chr11_44900। & 6.37 nonsex & trans \\
\hline 1 Gg_rs138586 & 817 chr1_406450 & 4.71 nonsex & cis \\
\hline 1 Gg_rs148186. & 826 chr1_459470। & 5.23 nonsex & cis \\
\hline 1 Gg_rs148186. & 834 chr1_364990। & 4.63 nonsex & cis \\
\hline 1 Gg_rs148186. & 844 chr1_461750 & 10.75 nonsex & cis \\
\hline 1 Gg_rs148186. & 844 chr1_461760। & 10.09 nonsex & cis \\
\hline 1 Gg_rs148186. & 844 chr1_461770 & 6.43 nonsex & cis \\
\hline 1 Gg_rs148186. & 844 chr1_461790। & 7.98 nonsex & cis \\
\hline 1 Gg_rs148186. & 844 chr1_468890 & 6.21 nonsex & cis \\
\hline 1 Gg_rs148186. & 844 chr1_458280 & 4.02 nonsex & cis \\
\hline 1 Gg_rs148186. & 844 chr1_459560। & 3.9 nonsex & cis \\
\hline 1 Gg_rs148186. & 844 chr1_461260। & 4.44 nonsex & cis \\
\hline 1 Gg_rs148186. & 844 chr1_461780 & 4.3 nonsex & cis \\
\hline 1 Gg_rs148186. & 844 chr1_461820 & 4.01 nonsex & cis \\
\hline 1 Gg_rs148186. & 845 chr1_461640 & 9.15 nonsex & cis \\
\hline 1 Gg_rs148186 & 845 chr1_461720। & 7.05 nonsex & cis \\
\hline 1 Gg_rs148186. & 845 chr1_461550। & 4.37 nonsex & cis \\
\hline 1 Gg_rs148186 & 847 chr1_461560। & 4.09 nonsex & cis \\
\hline 1 Gg_rs148186. & 848 chr1_461680 & 5.3 nonsex & cis \\
\hline 1 Gg_rs148186. & 848 chr1_461740 & 9.89 nonsex & cis \\
\hline 1 Gg_rs148186. & 848 chr4_103960। & 6.11 nonsex & trans \\
\hline 1 Gg_rs148186. & 850 chr1_461630 & 8.21 sex & cis \\
\hline 1 Gg_rs148186. & 850 chr1_461660 & 7.8 nonsex & cis \\
\hline 1 Gg_rs148186. & 850 chr1_407750 & 4.19 nonsex & cis \\
\hline 1 Gg_rs148186. & 851 chr1_461250। & 4.73 nonsex & cis \\
\hline 1 Gg_rs148186. & 851 chr1_461540 & 4.06 nonsex & cis \\
\hline 1 Gg_rs148186. & 851 chr1_461710 & 4.92 nonsex & cis \\
\hline 1 Gg_rs148186. & 852 chr1_461650 & 4.47 nonsex & cis \\
\hline 1 Gg_rs148186. & 853 chr1_461690। & 5.76 nonsex & cis \\
\hline 1 Gg_rs148186. & 853 chr1_461570 & 4.36 nonsex & cis \\
\hline 1 Gg_rs148186. & 854 chr1_460490। & 4.83 nonsex & cis \\
\hline 1 Gg_rs148186. & 854 chr4_402780 & 5.82 nonsex & trans \\
\hline 1 Gg_rs148186. & 855 chr1_451200 & 4.4 nonsex & cis \\
\hline 1 Gg_rs148186. & 857 chr1_102480 & 7.13 nonsex & trans \\
\hline 1 Gg_rs148186. & 858 chr1_470410 & 4.77 nonsex & cis \\
\hline 1 Gg_rs148186. & 860 chr1_447450 & 5.29 nonsex & cis \\
\hline 1 Calle_cGLA1 & 861 chrW_58830c & 10.14 sex & trans \\
\hline 1 Calle_cGLA1 & 863 chr1_479620 & 7.75 nonsex & cis \\
\hline 1 Calle_cGLA1 & 863 chr2_382000। & 6.99 nonsex & trans \\
\hline 1 Gg_rs138635 & 866 chr1_505770 & 4.28 nonsex & cis \\
\hline 1 Calle_cGLA1 & 868 chr1_480240 & 5.34 nonsex & cis \\
\hline 1 Calle_cGLA1 & 869 chr1_935020। & 7.25 nonsex & \\
\hline
\end{tabular}




\begin{tabular}{|c|c|c|c|}
\hline 1 Calle_cGLA1 & 874 chr1_500200। & 6.05 sex & cis \\
\hline 1 Calle_cGLA1 & 874 chr1_474750 & 4.34 nonsex & cis \\
\hline 1 Calle_cGLA1 & 878 chr1_479610 & 7.45 nonsex & cis \\
\hline 1 Calle_cGLA1 & 883 chr1_505210 & 5.72 sex & cis \\
\hline 1 Calle_cGLA1 & 884 chr1_458890। & 6.59 sex & cis \\
\hline 1 Calle_cGLA1 & 887 chr1_478740 & 6.12 nonsex & cis \\
\hline 1 Gg_rs148208. & 889 chr2_368810 & 6.61 nonsex & trans \\
\hline 1 Gg_rs148208. & 890 chr1_478920। & 8.58 nonsex & cis \\
\hline 1 Gg_rs148208. & 891 chr1_478910। & 3.86 nonsex & cis \\
\hline 1 Gg_rs148208. & 891 chr1_524360। & 4.33 nonsex & trans \\
\hline 1 Gg_rs138635 & 895 chr10_16301। & 5.71 nonsex & trans \\
\hline 1 Gg_rs138635 & 898 chr1_492350। & 4.05 nonsex & cis \\
\hline 1 Gg_rs138635 & 906 chr1_490950 & 15.41 nonsex & cis \\
\hline 1 Gg_rs138635 & 909 chr1_490940। & 11.92 nonsex & cis \\
\hline 1 rbl1791 & 925 chr1_501450। & 4.53 nonsex & cis \\
\hline 1 rbl1791 & 927 chr1_505420 & 5.82 nonsex & cis \\
\hline 1 rbl1791 & 929 chr1_502510 & 4.77 sex & cis \\
\hline 1 rbl1791 & 930 chr1_505440। & 5.8 nonsex & cis \\
\hline 1 rbl1791 & 931 chr1_514790। & 4.43 nonsex & cis \\
\hline 1 rbl1791 & 933 chr1_500980 & 4.13 nonsex & cis \\
\hline 1 rbl1791 & 934 chr1_505050। & 4.5 nonsex & cis \\
\hline 1 rbl1791 & 934 chr1_507500 & 6.33 nonsex & cis \\
\hline 1 rbl1791 & 934 chr1_499530। & 4.18 nonsex & cis \\
\hline 1 rbl1791 & 938 chr1_515260। & 5.99 nonsex & cis \\
\hline 1 Gg_rs136528 & 964 chr1_537690। & 4.6 nonsex & cis \\
\hline 1 Gg_rs136528 & 964 chr1_525000। & 3.76 nonsex & cis \\
\hline 1 Gg_rs136528 & 964 chr1_545960। & 4.29 nonsex & cis \\
\hline 1 Gg_rs136528 & 964 chr1_530420। & 4.03 nonsex & cis \\
\hline 1 X1_5881184ع & 964 chr1_536810 & 4.06 nonsex & cis \\
\hline 1 Gg_rs136528 & 965 chr1_530390। & 7.2 nonsex & cis \\
\hline 1 Gg_rs136528 & 966 chr1_532830 & 5.64 nonsex & cis \\
\hline 1 Gg_rs148254. & 966 chr1_528900 & 5 nonsex & cis \\
\hline 1 Gg_rs148254. & 966 chr1_527980 & 2.96 nonsex & cis \\
\hline 1 X1_56996295 & 966 chr1_538200। & 4.93 nonsex & cis \\
\hline 1 chr1.573778C & 966 chr1_529370। & 5.21 nonsex & cis \\
\hline 1 chr1.573778c & 966 chr1_548490। & 4.97 nonsex & cis \\
\hline 1 Gg_rs148254. & 968 chr1_184396। & 4.91 nonsex & trans \\
\hline 1 Gg_rs148254. & 970 chr1_529890। & 6.12 nonsex & cis \\
\hline 1 Gg_rs148254 & 972 chr1_525390। & 5.3 nonsex & cis \\
\hline 1 Gg_rs148254. & 973 chr1_532820 & 7.83 nonsex & cis \\
\hline 1 chr1.573778C & 978 chr1_532720 & 4.64 nonsex & cis \\
\hline 1 chr1.573778C & 978 chr1_551810 & 6.16 sex & cis \\
\hline 1 X1_56996295 & 979 chr1_536990। & 9.88 nonsex & cis \\
\hline
\end{tabular}




\begin{tabular}{|c|c|c|c|}
\hline 1 X1_56996295 & 981 chr1_544980 & 3.55 nonsex & cis \\
\hline 1 X1_5881184E & 983 chr1_497900। & 2.64 nonsex & cis \\
\hline 1 X1_5881184ع & 983 chr1_555590। & 3.08 nonsex & cis \\
\hline 1 X1_5881184ع & 986 chr3_462670 & 5.81 nonsex & trans \\
\hline 1 X1_58811848 & 987 chr1_537230। & 4.2 nonsex & cis \\
\hline 1 X1_5881184ع & 988 chr1_545970। & 5.01 nonsex & cis \\
\hline 1 X1_5881184E & 989 chr1_537000 & 3.77 nonsex & cis \\
\hline 1 X1_5881184ع & 994 chr1_543640 & 5.12 nonsex & cis \\
\hline 1 X1_5881184ع & 995 chr1_566040। & 6.5 nonsex & cis \\
\hline 1 X1_58811848 & 998 chr1_536040 & 6.47 nonsex & cis \\
\hline 1 Gg_rs148323. & 1023 chr1_517010 & 4.54 nonsex & cis \\
\hline 1 Gg_rs148323. & 1023 chr25_35040। & 5.9 nonsex & trans \\
\hline 1 Gg_rs148323. & 1027 chr1_589810 & 6.57 nonsex & cis \\
\hline 1 Gg_rs148323. & 1027 chr1_597380 & 5.87 sex & cis \\
\hline 1 Gg_rs148323. & 1027 chr1_592880 & 4.31 nonsex & cis \\
\hline 1 Gg_rs148323. & 1027 chr1_595990। & 4.07 nonsex & cis \\
\hline 1 Gg_rs148323. & 1029 chrZ_416270। & 7.11 sex & trans \\
\hline 1 Gg_rs148323. & 1031 chr1_596130 & 4.05 nonsex & cis \\
\hline 1 Gg_rs138763 & 1073 chrW_30260 & 9.23 sex & trans \\
\hline 1 Gg_rs138763 & 1075 chr1_607530 & 7.15 nonsex & cis \\
\hline 1 Gg_rs138763 & 1082 chr1_611530 & 4.99 nonsex & cis \\
\hline 1 Gg_rs138763 & 1083 chr1_612330 & 6.23 sex & cis \\
\hline 1 Gg_rs138763 & 1085 chr1_612420 & 7.51 nonsex & cis \\
\hline 1 Gg_rs138763 & 1085 chr1_594420 & 3.92 nonsex & cis \\
\hline 1 Gg_rs138763 & 1085 chr2_835020 & 8.58 sex & trans \\
\hline 1 Gg_rs138763 & 1088 chr1_623550। & 5.51 nonsex & cis \\
\hline 1 Gg_rs138763 & 1088 chr2_716230 & 5.69 nonsex & trans \\
\hline 1 Gg_rs138763 & 1089 chr1_622360 & 6.35 nonsex & cis \\
\hline 1 Gg_rs148372 & 1093 chr1_621510 & 4.64 nonsex & cis \\
\hline 1 Gg_rs148351 & 1097 chr1_652450। & 4.85 nonsex & cis \\
\hline 1 Gg_rs148351 & 1100 chr1_621450 & 4.73 nonsex & cis \\
\hline 1 Gg_rs148351 & 1100 chr1_621480 & 8.88 nonsex & cis \\
\hline 1 Gg_rs148351 & 1100 chr1_625730 & 3.67 nonsex & cis \\
\hline 1 Gg_rs148351 & 1101 chr1_621320। & 4.84 nonsex & cis \\
\hline 1 Gg_rs148351 & 1102 chr1_621420 & 7.74 nonsex & cis \\
\hline 1 Gg_rs148351 & 1104 chr1_621530। & 6.84 nonsex & cis \\
\hline 1 Gg_rs148351 & 1104 chr1_621440। & 6.95 nonsex & cis \\
\hline 1 Gg_rs148351 & 1105 chr1_621410 & 5.72 nonsex & cis \\
\hline 1 Gg_rs148351 & 1105 chr1_635080 & 6.22 nonsex & cis \\
\hline 1 Gg_rs148351 & 1106 chr1_621520। & 5 nonsex & cis \\
\hline 1 Gg_rs148363। & 1107 chr1_634870 & 4.96 nonsex & cis \\
\hline 1 Gg_rs148363। & 1107 chr1_639180 & 6.45 nonsex & cis \\
\hline 1 Gg_rs148363 & 1107 chr1_621500। & 4.86 nonsex & cis \\
\hline
\end{tabular}




\begin{tabular}{|c|c|c|c|}
\hline 1 Gg_rs148363। & 1108 chr1_639090 & 6.2 nonsex & cis \\
\hline 1 Gg_rs148363। & 1112 chr1_631890 & 6.59 sex & cis \\
\hline 1 Gg_rs148363 & 1112 chr1_634310 & 5.83 sex & cis \\
\hline 1 Gg_rs148363। & 1112 chr1_634850 & 6.33 nonsex & cis \\
\hline 1 Gg_rs148363 & 1115 chr1_640170 & 11.02 nonsex & cis \\
\hline 1 Gg_rs148363। & 1115 chr1_642230 & 8.32 nonsex & cis \\
\hline 1 Gg_rs148363। & 1116 chr1_639070 & 6.53 nonsex & cis \\
\hline 1 Gg_rs148363। & 1116 chr1_639080 & 13.93 nonsex & cis \\
\hline 1 Gg_rs148363। & 1118 chr1_640680 & 6.37 nonsex & cis \\
\hline 1 X1_6802129C & 1119 chr1_640250। & 6.71 nonsex & cis \\
\hline 1 Gg_rs148372 & 1120 chr1_621400 & 4.39 nonsex & cis \\
\hline 1 Gg_rs148372 & 1120 chrZ_209000। & 8.57 sex & trans \\
\hline 1 Gg_rs148372 & 1122 chr1_646410 & 4.48 nonsex & cis \\
\hline 1 Gg_rs148372 & 1123 chr1_654140 & 9.02 nonsex & cis \\
\hline 1 Gg_rs148372 & 1126 chr1_645740 & 6.4 nonsex & cis \\
\hline 1 Gg_rs148372 & 1126 chr1_651220 & 6.21 sex & cis \\
\hline 1 Gg_rs148372 & 1130 chr1_655130 & 7.11 nonsex & cis \\
\hline 1 Gg_rs148372 & 1130 chr1_647060 & 4.73 nonsex & cis \\
\hline 1 X1_6802129C & 1131 chr1_655100 & 5.9 nonsex & cis \\
\hline 1 X1_6802129C & 1138 chr1_648570 & 6.51 nonsex & cis \\
\hline 1 X1_6802129C & 1139 chr1_649570। & 4.77 nonsex & cis \\
\hline 1 X1_6802129C & 1141 chr1_645110 & $6.25 \operatorname{sex}$ & cis \\
\hline 1 X1_6802129C & 1150 chr1_659970 & 7.29 nonsex & cis \\
\hline 1 X1_6802129C & 1154 chr1_659980 & 3.07 nonsex & cis \\
\hline 1 X1_6802129C & 1158 chr1_655120 & 4.72 nonsex & cis \\
\hline 1 X1_6802129C & 1159 chr1_327310 & 9.99 nonsex & trans \\
\hline 1 X1_6802129C & 1160 chr1_214000 & 10.28 nonsex & trans \\
\hline 1 X1_6802129C & 1164 chr1_215000 & 10.68 nonsex & trans \\
\hline 1 X1_6802129C & 1165 chr1_327320 & 11.18 nonsex & trans \\
\hline 1 X1_6802129C & 1166 chr1_327270 & 10.18 nonsex & trans \\
\hline 1 X1_6802129C & 1166 chr1_327300 & 9.05 nonsex & trans \\
\hline 1 X1_6802129C & 1168 chr1_327260 & 16.22 nonsex & trans \\
\hline 1 X1_6802129C & 1171 chr1_212000 & 7.12 nonsex & trans \\
\hline 1 X1_6802129C & 1173 chr1_327290। & 7.1 nonsex & trans \\
\hline 1 X1_71708614 & 1193 chr1_685820 & 5.61 nonsex & cis \\
\hline 1 X1_71708614 & 1194 chr1_676400 & 5.28 sex & cis \\
\hline 1 X1_71708614 & 1194 chr1_775070 & 6.46 sex & cis \\
\hline 1 X1_71708614 & 1205 chr1_714470 & 5.66 sex & cis \\
\hline 1 X1_71708614 & 1205 chr1_659470 & 3.07 nonsex & cis \\
\hline 1 X1_71708614 & 1205 chr1_713610 & 3.96 nonsex & cis \\
\hline 1 Gg_rs148495 & 1327 chr1_847440 & 4.12 nonsex & cis \\
\hline 1 Gg_rs148495 & 1327 chr30_54900 & 8.98 sex & trans \\
\hline 1 Gg_rs148495 & 1327 chr6_149980। & 5.57 nonsex & trans \\
\hline
\end{tabular}




\begin{tabular}{|c|c|c|c|}
\hline 1 Gg_rs148495 & 1327 chr8_264830 & 6.07 nonsex & trans \\
\hline 1 Gg_rs148495 & 1327 chr21_32770। & 6.1 nonsex & trans \\
\hline 1 Gg_rs148495 & 1345 chr1_890220 & 4.96 nonsex & cis \\
\hline 1 Gg_rs139018 & 1349 chr1_918910 & 4.69 nonsex & cis \\
\hline 1 Gg_rs136480 & 1359 chr4_921800 & 5.46 nonsex & trans \\
\hline 1 Gg_rs139018 & 1362 chr1_906510 & 4.85 nonsex & cis \\
\hline 1 X1_88934871 & 1363 chr1_879620 & 4.34 nonsex & cis \\
\hline 1 X1_88934871 & 1367 chr1_866790 & 7.57 nonsex & cis \\
\hline 1 X1_88934871 & 1370 chr1_883390 & 3.02 nonsex & cis \\
\hline 1 X1_88934871 & 1371 chr1_929360 & 3.3 nonsex & cis \\
\hline 1 X1_88934871 & 1372 chr4_259570। & 6.02 nonsex & trans \\
\hline 1 Gg_rs138974. & 1376 chr1_878190। & 4.23 nonsex & cis \\
\hline 1 Gg_rs136480 & 1381 chr1_877740 & 4.83 nonsex & cis \\
\hline 1 X1_88934871 & 1390 chr1_937060। & 4.25 nonsex & cis \\
\hline 1 Gg_rs138974. & 1391 chr1_871350 & 5.57 nonsex & cis \\
\hline 1 Gg_rs138974 & 1391 chr1_878250 & 8.86 nonsex & cis \\
\hline 1 Gg_rs138974. & 1391 chr8_291770। & 4.88 nonsex & trans \\
\hline 1 Gg_rs136480 & 1393 chr1_877730 & 4.13 nonsex & cis \\
\hline 1 Gg_rs136480 & 1393 chr1_881270। & 3.78 nonsex & cis \\
\hline 1 Gg_rs136480 & 1393 chr5_267600 & 4.65 nonsex & \\
\hline 1 Gg_rs138974 & 1394 chr1_867110 & 7.56 nonsex & cis \\
\hline 1 Gg_rs136480 & 1394 chr1_868860 & 4.38 nonsex & cis \\
\hline 1 X1_88934871 & 1396 chr1_859950 & 3.31 nonsex & cis \\
\hline 1 Gg_rs148589. & 1397 chr1_999660। & 4.61 nonsex & cis \\
\hline 1 X1_88934871 & 1398 chr1_870970 & 5.68 nonsex & cis \\
\hline 1 X1_88934871 & 1398 chr14_16086। & 5 nonsex & trans \\
\hline 1 Gg_rs138974 & 1399 chr1_889230 & 5.21 nonsex & cis \\
\hline 1 Gg_rs148584 & 1399 chr1_895130 & 7.35 nonsex & cis \\
\hline 1 Gg_rs148584 & 1403 chr1_903470 & 4.44 nonsex & cis \\
\hline 1 Gg_rs139018 & 1404 chr1_919520 & 7.93 nonsex & cis \\
\hline 1 Gg_rs139018 & 1404 chr4_563830 & 5.93 nonsex & trans \\
\hline 1 Gg_rs139018 & 1405 chr1_905580 & 4.62 nonsex & cis \\
\hline 1 Gg_rs139018 & 1405 chr1_919460 & 4.49 nonsex & cis \\
\hline 1 Gg_rs139018 & 1406 chr1_891190 & 4.44 nonsex & cis \\
\hline 1 Gg_rs139018 & 1406 chr1_894200 & 4.2 nonsex & cis \\
\hline 1 Gg_rs139018 & 1408 chr1_915170 & 7.02 nonsex & cis \\
\hline 1 Gg_rs139018 & 1408 chr1_924300 & 9.87 nonsex & cis \\
\hline 1 Gg_rs139018 & 1408 chr1_912390। & 4.28 nonsex & cis \\
\hline 1 Gg_rs139018 & 1408 chr1_927920 & 4.29 nonsex & cis \\
\hline 1 Gg_rs139018 & 1408 chr1_153750 & 7.28 nonsex & trans \\
\hline 1 Gg_rs139018 & 1409 chr1_903420 & 4.26 nonsex & cis \\
\hline 1 Gg_rs148584 & 1409 chr1_902100 & 5.02 nonsex & cis \\
\hline 1 Gg_rs148584. & 1410 chr1_926110 & 6.2 nonsex & cis \\
\hline
\end{tabular}




\begin{tabular}{|c|c|c|c|}
\hline 1 Gg_rs148584 & 1411 chr1_898040 & 5.1 nonsex & cis \\
\hline 1 Gg_rs148584 & 1411 chr1_854520 & 6.9 sex & cis \\
\hline 1 Gg_rs148584 & 1411 chr1_860340 & $7.3 \mathrm{sex}$ & cis \\
\hline 1 Gg_rs148584 & 1411 chr1_870950 & 6.62 sex & cis \\
\hline 1 Gg_rs148584. & 1411 chr1_873100 & 6.97 nonsex & cis \\
\hline 1 Gg_rs148584. & 1411 chr1_883660 & 4.94 nonsex & cis \\
\hline 1 Gg_rs148584. & 1411 chr1_884140 & 5.5 nonsex & cis \\
\hline 1 Gg_rs148584 & 1411 chr1_900090 & 5.95 sex & cis \\
\hline 1 Gg_rs148584 & 1411 chr1_912690 & 5.15 nonsex & cis \\
\hline 1 Gg_rs148584 & 1411 chr1_853140 & 4.51 nonsex & cis \\
\hline 1 Gg_rs148584. & 1411 chr1_889560 & 4.73 nonsex & cis \\
\hline 1 Gg_rs148584. & 1411 chr1_899660 & 5.02 nonsex & cis \\
\hline 1 Gg_rs148584 & 1411 chr1_919560 & 4.6 nonsex & cis \\
\hline 1 Gg_rs148584 & 1411 chr1_920970 & 4.51 nonsex & cis \\
\hline 1 Gg_rs148584 & 1411 chr1_925680 & 4.99 nonsex & cis \\
\hline 1 Gg_rs148584. & 1411 chr1_925710 & 4.54 nonsex & cis \\
\hline 1 Gg_rs148584 & 1411 chr1_928300 & 5.05 nonsex & cis \\
\hline 1 Gg_rs148584 & 1411 chr1_938830 & 4.8 nonsex & cis \\
\hline 1 Gg_rs148584 & 1411 chr1_747000 & 7.52 sex & trans \\
\hline 1 Gg_rs148584. & 1411 chr4_549220 & 5.85 nonsex & trans \\
\hline 1 Gg_rs148584. & 1411 chrZ_189460। & 7.71 sex & trans \\
\hline 1 Gg_rs148584 & 1411 chrZ_315800। & 7.99 sex & trans \\
\hline 1 Gg_rs148584 & 1411 chr2_834530 & 6.21 nonsex & trans \\
\hline 1 Gg_rs148584. & 1411 chr3_326250 & 5.74 nonsex & trans \\
\hline 1 Gg_rs148584. & 1411 chr3_874840 & 6.29 nonsex & trans \\
\hline 1 X1_88934871 & 1412 chr1_869340 & 5.47 nonsex & cis \\
\hline 1 Gg_rs148584. & 1412 chr1_919530 & 7.57 nonsex & cis \\
\hline 1 Gg_rs148584 & 1412 chr1_888890 & 6.57 nonsex & cis \\
\hline 1 Gg_rs148584 & 1412 chr1_882960 & 4.39 nonsex & cis \\
\hline 1 Gg_rs148584. & 1412 chr1_921130 & 4.61 nonsex & cis \\
\hline 1 Gg_rs139018 & 1413 chr1_877870 & 5.79 nonsex & cis \\
\hline 1 Gg_rs148584. & 1413 chr1_924080 & 4.9 nonsex & cis \\
\hline 1 Gg_rs148584 & 1413 chr1_926130। & 5.39 nonsex & cis \\
\hline 1 Gg_rs148589. & 1414 chr1_893150 & 5.73 nonsex & cis \\
\hline 1 Gg_rs148589. & 1414 chr1_896140 & 4.94 nonsex & cis \\
\hline 1 Gg_rs148589. & 1414 chr1_898200 & 4.59 nonsex & cis \\
\hline 1 Gg_rs148589. & 1414 chr1_967760 & 4.96 nonsex & cis \\
\hline 1 Gg_rs148589. & 1415 chr1_103864। & 6.07 sex & cis \\
\hline 1 Gg_rs148589. & 1415 chr1_931020 & 5.18 nonsex & cis \\
\hline 1 Gg_rs148589. & 1416 chr1_927060। & 3.61 nonsex & cis \\
\hline 1 Gg_rs148589. & 1418 chr1_931410 & 4.32 nonsex & cis \\
\hline 1 Gg_rs148589. & 1418 chr4_887200 & 5.95 nonsex & trans \\
\hline 1 Gg_rs148589. & 1418 chr5_571810 & 6.1 nonsex & trans \\
\hline
\end{tabular}




\begin{tabular}{|c|c|c|c|}
\hline 1 Gg_rs148589. & 1427 chr1_933280 & 7.19 nonsex & cis \\
\hline 1 Gg_rs148589. & 1431 chr1_944590 & 6.72 sex & cis \\
\hline 1 Gg_rs148589. & 1431 chr10_82920 & 6.04 nonsex & trans \\
\hline 1 Gg_rs148589. & 1431 chr1_193543। & 5.86 nonsex & trans \\
\hline 1 Gg_rs148589. & 1433 chr1_895250 & 4.4 nonsex & cis \\
\hline 1 Gg_rs148589. & 1433 chr1_921910 & 4.5 nonsex & cis \\
\hline 1 Gg_rs148589. & 1436 chr7_230410 & 6.31 nonsex & trans \\
\hline 1 Gg_rs136480 & 1437 chr1_871250 & 4.43 nonsex & cis \\
\hline 1 Gg_rs148589. & 1437 chr1_912410 & 4.75 nonsex & cis \\
\hline 1 Gg_rs148589. & 1438 chr1_847840 & 6.3 sex & cis \\
\hline 1 Gg_rs148589. & 1438 chr6_253420 & 6.36 nonsex & trans \\
\hline 1 Gg_rs148589. & 1439 chr1_100730 & 4.68 nonsex & cis \\
\hline 1 Gg_rs148589. & 1440 chr1_902160 & 5.12 nonsex & cis \\
\hline 1 Gg_rs139018 & 1441 chr1_919050 & 5.08 nonsex & cis \\
\hline 1 Gg_rs148589. & 1441 chr1_933020 & 6.14 nonsex & cis \\
\hline 1 Gg_rs138974. & 1442 chr1_869180 & 4.64 nonsex & cis \\
\hline 1 Gg_rs139018 & 1442 chr1_938990। & 4.67 nonsex & cis \\
\hline 1 Gg_rs148589. & 1443 chr1_920020 & 4.83 nonsex & cis \\
\hline 1 Gg_rs139018 & 1444 chr1_924060 & 5.43 nonsex & cis \\
\hline 1 Gg_rs148589. & 1444 chr1_893740 & 5.22 nonsex & cis \\
\hline 1 Gg_rs148589. & 1444 chr1_933150 & 5.6 nonsex & cis \\
\hline 1 Gg_rs148589. & 1444 chr4_867250 & 7.19 nonsex & trans \\
\hline 1 Gg_rs139018 & 1445 chr1_905670 & 4.68 nonsex & cis \\
\hline 1 Gg_rs148589. & 1446 chr1_100646। & 5.13 nonsex & cis \\
\hline 1 Gg_rs148589. & 1449 chr1_927340 & 6.43 nonsex & cis \\
\hline 1 Gg_rs139018 & 1450 chr1_873150 & 4.72 nonsex & cis \\
\hline 1 Gg_rs148589. & 1451 chr1_100458। & 5.88 nonsex & cis \\
\hline 1 Gg_rs148589. & 1452 chr1_100457। & 8.65 nonsex & cis \\
\hline 1 Gg_rs148584 & 1453 chr1_895410 & 5.85 nonsex & cis \\
\hline 1 Gg_rs148589. & 1455 chr1_943850 & 6.74 nonsex & cis \\
\hline 1 Gg_rs139018 & 1456 chr1_924760 & 6.56 nonsex & cis \\
\hline 1 Gg_rs139109. & 1459 chr1_983840 & 8.46 nonsex & cis \\
\hline 1 Gg_rs139109. & 1468 chr1_100027। & 6.02 nonsex & cis \\
\hline 1 Gg_rs139109. & 1468 chr1_101932 & 4.78 nonsex & cis \\
\hline 1 Gg_rs139109. & 1470 chr1_103059 & 7.25 nonsex & cis \\
\hline 1 Gg_rs139109. & 1471 chr1_976770 & 5.42 nonsex & cis \\
\hline 1 snp.26.313.2، & 1471 chr1_105993। & 5.38 nonsex & cis \\
\hline 1 Gg_rs139109. & 1474 chr1_994530। & 5.69 nonsex & cis \\
\hline 1 Gg_rs139109. & 1474 chr1_100462 & 5.64 nonsex & cis \\
\hline 1 Gg_rs139109. & 1475 chr1_100793। & 6.69 nonsex & cis \\
\hline 1 Gg_rs139109. & 1477 chr1_100370 & 5.73 nonsex & cis \\
\hline 1 Gg_rs139109. & 1479 chr1_941800 & 4.14 nonsex & cis \\
\hline 1 Gg_rs139109. & 1479 chr1_102224। & 5.12 nonsex & cis \\
\hline
\end{tabular}




\begin{tabular}{|c|c|c|c|}
\hline 1 Gg_rs139109. & 1480 chr1_100028। & 5.71 nonsex & cis \\
\hline 1 Gg_rs139109. & 1480 chr1_105559। & 6.13 nonsex & cis \\
\hline 1 Gg_rs139109. & 1480 chr1_103926। & 5.48 nonsex & cis \\
\hline 1 Gg_rs139109. & 1481 chr1_101332 & 4.59 nonsex & cis \\
\hline 1 X1_1115558C & 1481 chr1_116627। & 4.68 nonsex & cis \\
\hline 1 Gg_rs139109. & 1483 chr1_102571। & 6.37 nonsex & cis \\
\hline 1 Gg_rs139109. & 1483 chr1_942290। & 4.44 nonsex & cis \\
\hline 1 Gg_rs139109. & 1486 chr1_955680। & 4.24 nonsex & cis \\
\hline 1 Gg_rs139109. & 1500 chr1_100171। & 7.35 nonsex & cis \\
\hline 1 Gg_rs139109. & 1501 chr1_102817। & 5.77 nonsex & cis \\
\hline 1 Gg_rs139109. & 1502 chr1_947550। & $5.41 \mathrm{sex}$ & cis \\
\hline 1 Gg_rs139109. & 1503 chr1_100666। & 6.23 sex & cis \\
\hline 1 Gg_rs139109. & 1503 chr1_101132 & 5.52 nonsex & cis \\
\hline 1 Gg_rs139109. & 1503 chr1_102267। & 5.31 nonsex & cis \\
\hline 1 Gg_rs139109. & 1504 chr1_100832 & 7.06 nonsex & cis \\
\hline 1 Gg_rs139109. & 1504 chr1_103083। & 5.41 nonsex & cis \\
\hline 1 snp.26.313.2، & 1505 chr1_103457। & 8 sex & cis \\
\hline 1 snp.26.313.2، & 1506 chr1_102764। & 7.23 nonsex & cis \\
\hline 1 snp.26.313.2 & 1506 chr1_102765। & 6.87 nonsex & cis \\
\hline 1 snp.26.313.2 & 1506 chr1_109618 & 6.72 sex & cis \\
\hline 1 snp.26.313.2 & 1506 chr1_104196। & 4.63 nonsex & cis \\
\hline 1 snp.26.313.2 & 1507 chr1_102404! & 5.91 nonsex & cis \\
\hline 1 snp.26.313.2، & 1507 chr1_995220। & 4.88 nonsex & cis \\
\hline 1 snp.26.313.2 & 1507 chr1_102918 & 4.99 nonsex & cis \\
\hline 1 snp.26.313.2 & 1507 chr1_103765। & 5.07 nonsex & cis \\
\hline 1 snp.26.313.2 & 1508 chr1_103872 & 6 nonsex & cis \\
\hline 1 snp.26.313.2 & 1508 chr1_104377। & 6.04 sex & cis \\
\hline 1 snp.26.313.2، & 1508 chr1_105302 & 6.65 nonsex & cis \\
\hline 1 snp.26.313.2، & 1508 chr1_100353। & 4.78 nonsex & cis \\
\hline 1 snp.26.313.2 & 1508 chr1_103153। & 4.94 nonsex & cis \\
\hline 1 snp.26.313.2 & 1508 chr1_104572 & 5.39 nonsex & cis \\
\hline 1 snp.26.313.2 & 1508 chr2_479200 & 6.94 nonsex & trans \\
\hline 1 snp.26.313.2 & 1509 chr1_105735। & 6.51 sex & cis \\
\hline 1 snp.26.313.2 & 1510 chr1_102403। & 7.98 sex & cis \\
\hline 1 snp.26.313.2، & 1510 chr1_102406। & 5.51 nonsex & cis \\
\hline 1 snp.26.313.2، & 1510 chr1_102758 & 5.32 nonsex & cis \\
\hline 1 snp.26.313.2، & 1510 chr1_105403। & 5.94 nonsex & cis \\
\hline 1 snp.26.313.2 & 1510 chr1_972490 & 4.68 nonsex & cis \\
\hline 1 snp.26.313.2 & 1510 chr1_972500 & 4.89 nonsex & cis \\
\hline 1 snp.26.313.2 & 1510 chr1_102401। & 4.75 nonsex & cis \\
\hline 1 snp.26.313.2 & 1510 chr1_104884 & 4.51 nonsex & cis \\
\hline 1 snp.26.313.2 & 1510 chr1_105400 & 4.95 nonsex & cis \\
\hline 1 snp.26.313.2 & 1510 chr1_106038। & 4.86 nonsex & cis \\
\hline
\end{tabular}




\begin{tabular}{|c|c|c|c|}
\hline 1 snp.26.313.2 & 1510 chr5_456670 & 6.61 nonsex & trans \\
\hline 1 snp.26.313.2 & 1510 chr1_965740 & 6.42 nonsex & trans \\
\hline 1 snp.26.313.2 & 1510 chr1_970450। & 7.4 nonsex & trans \\
\hline 1 snp.26.313.2 & 1510 chr1_142764। & 6.31 nonsex & rans \\
\hline 1 snp.26.313.2 & 1511 chr1_999760। & 4.87 nonsex & cis \\
\hline 1 snp.26.313.2 & 1512 chr1_103697। & 7.06 sex & cis \\
\hline 1 snp.26.313.2 & 1513 chr1_106868 & 6.43 nonsex & cis \\
\hline 1 snp.26.313.2 & 1513 chr3_481550 & 7.64 nonsex & trans \\
\hline 1 snp.26.313.2 & 1515 chr1_102484! & $6.11 \mathrm{sex}$ & cis \\
\hline 1 snp.26.313.2 & 1515 chr1_102630 & 7.87 nonsex & cis \\
\hline 1 X1_1115558C & 1515 chr1_105767। & 6.09 nonsex & cis \\
\hline 1 X1_1115558C & 1516 chr1_105231। & 6.09 nonsex & cis \\
\hline 1 X1_1115558C & 1516 chr1_109103। & 5.36 nonsex & cis \\
\hline 1 X1_1115558C & 1516 chr1_104593। & 5.26 nonsex & cis \\
\hline 1 X1_1115558C & 1516 chr1_107992 & 4.6 nonsex & cis \\
\hline 1 X1_1115558C & 1517 chr1_108516। & $6.91 \mathrm{sex}$ & cis \\
\hline 1 X1_1115558C & 1517 chr1_108257। & 4.56 nonsex & cis \\
\hline 1 X1_1115558C & 1518 chr1_107986। & 4.73 nonsex & cis \\
\hline 1 X1_1115558C & 1518 chr11_18458 & 8.36 sex & trans \\
\hline 1 X1_1115558C & 1518 chr5_683300 & 6.47 nonsex & trans \\
\hline 1 X1_1115558C & 1519 chr1_109648 & 6.24 nonsex & cis \\
\hline 1 X1_1115558C & 1519 chr1_112354! & 4.46 nonsex & cis \\
\hline 1 X1_1115558C & 1519 chr1_117533। & 5.03 nonsex & cis \\
\hline 1 X1_1115558C & 1520 chr1_107987। & 8.21 nonsex & cis \\
\hline 1 X1_1115558C & 1521 chr1_107319। & 15.08 sex & cis \\
\hline 1 X1_1115558C & 1521 chr1_108800 & 11.29 nonsex & cis \\
\hline 1 X1_1115558C & 1521 chr1_110176। & 6.27 nonsex & cis \\
\hline 1 X1_1115558C & 1521 chr1_111231। & 5.43 nonsex & cis \\
\hline 1 X1_1115558C & 1521 chr1_111235। & 15.1 nonsex & cis \\
\hline 1 X1_1115558C & 1521 chr1_111236। & 17.65 nonsex & cis \\
\hline 1 X1_1115558C & 1521 chr1_111238 & 7.39 nonsex & cis \\
\hline 1 X1_1115558C & 1521 chr1_111239| & 9.01 nonsex & cis \\
\hline 1 X1_1115558C & 1521 chr1_102679। & 4.85 nonsex & cis \\
\hline 1 X1_1115558C & 1521 chr1_111129| & 4.34 nonsex & cis \\
\hline 1 X1_1115558C & 1521 chr10_56430। & $6.97 \mathrm{sex}$ & trans \\
\hline 1 X1_1115558C & 1525 chr1_109781। & 6.29 nonsex & cis \\
\hline 1 X1_1115558C & 1525 chr1_111234! & 13.51 nonsex & cis \\
\hline 1 X1_1115558C & 1525 chr1_111237। & 8.95 nonsex & cis \\
\hline 1 X1_1115558C & 1527 chr1_111232 & 14.46 nonsex & cis \\
\hline 1 X1_1115558C & 1528 chr1_111233। & 13.5 sex & cis \\
\hline 1 X1_1115558C & 1528 chr1_110954! & 4.68 nonsex & cis \\
\hline 1 X1_1115558C & 1531 chr1_111572 & 4.49 nonsex & cis \\
\hline 1 X1_1115558C & 1535 chr1_108412 & 5.46 sex & cis \\
\hline
\end{tabular}




\begin{tabular}{|c|c|c|c|}
\hline 1 X1_1115558C & 1535 chrZ_920500। & 7.66 sex & trans \\
\hline 1 X1_1115558C & 1537 chr1_109478। & 4.62 nonsex & cis \\
\hline 1 X1_1115558C & 1552 chr1_112723। & 8.67 nonsex & cis \\
\hline 1 X1_1167836؟ & 1561 chr1_106280 & 5.33 nonsex & cis \\
\hline 1 X1_1167836؟ & 1591 chr1_114576। & 6.44 nonsex & cis \\
\hline 1 X1_1167836؟ & 1595 chr1_115042 & 10.26 nonsex & cis \\
\hline 1 X1_1167836؟ & 1595 chr1_110558। & 4.61 nonsex & cis \\
\hline 1 X1_11700171 & 1598 chr1_115080 & 5.92 sex & cis \\
\hline 1 X1_1167836؟ & 1600 chr1_100482 & 5.66 sex & cis \\
\hline 1 X1_11700171 & 1603 chr1_115403। & 5.49 nonsex & cis \\
\hline 1 X1_1182262C & 1608 chr1_116953। & 4.77 nonsex & cis \\
\hline 1 X1_1182262C & 1611 chr1_140509| & 6.52 nonsex & trans \\
\hline 1 X1_1182262C & 1612 chr27_71280 & 6.23 nonsex & trans \\
\hline 1 X1_1182262C & 1613 chr1_121032 & 4.18 nonsex & cis \\
\hline 1 X1_1182262C & 1615 chr1_116758। & 6.3 sex & cis \\
\hline 1 X1_1182262C & 1615 chr1_116961। & 4.61 nonsex & cis \\
\hline 1 X1_1182262C & 1616 chr1_116311 & 7.77 nonsex & cis \\
\hline 1 X1_1182262C & 1616 chr1_117287। & 4.7 nonsex & cis \\
\hline 1 X1_1167836؟ & 1617 chr1_108144! & 7.18 sex & cis \\
\hline 1 X1_1182262C & 1617 chr1_113876 & 4.14 nonsex & cis \\
\hline 1 X1_1182262C & 1618 chr1_117797। & 4.47 nonsex & cis \\
\hline 1 X1_1182262C & 1618 chr10_13117। & 5.12 nonsex & trans \\
\hline 1 X1_1182262C & 1619 chr1_116790 & 7.27 nonsex & cis \\
\hline 1 X1_1182262C & 1620 chr1_117237। & 6.92 nonsex & cis \\
\hline 1 X1_1182262C & 1621 chr11_16330 & 6.57 sex & trans \\
\hline 1 X1_1182262C & 1622 chr1_119143। & 4.69 nonsex & cis \\
\hline 1 X1_1182262C & 1623 chr1_116981 & 5.18 nonsex & cis \\
\hline 1 X1_1182262C & 1623 chr1_117064! & 10.15 nonsex & cis \\
\hline 1 X1_1182262C & 1623 chr1_117180 & 5.82 sex & cis \\
\hline 1 X1_1182262C & 1623 chr1_115725। & 3.72 nonsex & cis \\
\hline 1 X1_1182262C & 1623 chr1_117115। & 3.75 nonsex & cis \\
\hline 1 Gg_rs148779. & 1625 chr1_122389| & 3.75 nonsex & cis \\
\hline 1 Gg_rs148779. & 1626 chr1_115985 & 5.72 sex & cis \\
\hline 1 Gg_rs148779. & 1626 chr1_118211 & 4.86 sex & cis \\
\hline 1 Gg_rs148779. & 1626 chr1_118644। & 5.07 nonsex & cis \\
\hline 1 Gg_rs148779. & 1626 chr1_119144! & 10.07 nonsex & cis \\
\hline 1 Gg_rs148779. & 1626 chr1_116149| & 3.58 nonsex & cis \\
\hline 1 Gg_rs148779. & 1627 chr1_118205। & $5.6 \mathrm{sex}$ & cis \\
\hline 1 Gg_rs148779. & 1632 chrZ_266390। & 6.97 sex & trans \\
\hline 1 X1_1182262C & 1635 chr1_121340 & 3.74 nonsex & cis \\
\hline 1 X1_12585307 & 1645 chr1_126449! & 4.67 nonsex & cis \\
\hline 1 Gg_rs139298. & 1646 chr1_121317। & 6.41 nonsex & cis \\
\hline 1 Gg_rs139298 & 1646 chr1_112442 & 4.74 nonsex & cis \\
\hline
\end{tabular}




\begin{tabular}{|c|c|c|c|}
\hline 1 snp.146.19.5! & 1667 chr1_781000 & 7.85 sex & trans \\
\hline 1 X1_1182262C & 1672 chr1_123821 & 4.56 nonsex & cis \\
\hline 1 snp.146.19.5! & 1676 chr1_124619 & 5.07 nonsex & cis \\
\hline 1 snp.146.19.5! & 1678 chr1_121769 & 6.24 nonsex & cis \\
\hline 1 snp.146.19.5! & 1678 chr1_123882 & 5.88 nonsex & cis \\
\hline 1 snp.146.19.5! & 1678 chr1_123833। & 4.59 nonsex & cis \\
\hline 1 snp.146.19.5! & 1679 chr1_123394। & 3.63 nonsex & cis \\
\hline 1 snp.146.19.5! & 1680 chr1_124173। & 5.95 nonsex & cis \\
\hline 1 snp.146.19.5! & 1682 chr1_124618 & 4.32 nonsex & cis \\
\hline 1 snp.146.19.5! & 1684 chr1_135541। & 6.18 sex & cis \\
\hline 1 snp.146.19.5! & 1685 chr1_132844। & 4.47 nonsex & cis \\
\hline 1 snp.146.19.5! & 1686 chr1_124016। & 5.08 nonsex & cis \\
\hline 1 snp.146.19.5! & 1688 chr1_123223। & 5.47 sex & cis \\
\hline 1 snp.146.19.5! & 1688 chr1_127010 & 5.57 sex & cis \\
\hline 1 snp.146.19.5! & 1688 chr1_131442 & 6.27 sex & cis \\
\hline 1 snp.146.19.5! & 1688 chr1_117627। & 3.74 nonsex & cis \\
\hline 1 snp.146.19.5! & 1688 chr1_120075। & 4.72 nonsex & cis \\
\hline 1 snp.146.19.5! & 1688 chr1_120327। & 4.92 nonsex & cis \\
\hline 1 snp.146.19.5! & 1688 chr1_123283। & 4.61 nonsex & cis \\
\hline 1 snp.146.19.5! & 1688 chr1_124172 & 4.45 nonsex & cis \\
\hline 1 snp.146.19.5! & 1688 chr1_124856। & 4.48 nonsex & cis \\
\hline 1 snp.146.19.5! & 1688 chr4_549180 & 7.81 sex & trans \\
\hline 1 snp.146.19.5! & 1688 chr22_24810 & 7.68 sex & trans \\
\hline 1 snp.146.19.5! & 1688 chr4_271860 & 5.92 nonsex & trans \\
\hline 1 snp.146.19.5! & 1688 chr4_280070 & 6.07 nonsex & rans \\
\hline 1 snp.146.19.5! & 1688 chr4_912920। & 5.84 nonsex & trans \\
\hline 1 snp.146.19.5! & 1690 chr1_124004। & 5.79 nonsex & cis \\
\hline 1 snp.146.19.5! & 1690 chr1_124020 & 3.14 nonsex & cis \\
\hline 1 snp.146.19.5! & 1690 chr28_92800 & 6.12 nonsex & trans \\
\hline 1 snp.146.19.5! & 1691 chr23_55680 & 7.64 sex & trans \\
\hline 1 snp.146.19.5! & 1697 chr1_123170 & 6.69 nonsex & cis \\
\hline 1 snp.146.19.5! & 1698 chr33_67400 & 7.17 nonsex & trans \\
\hline 1 X1_12837468 & 1712 chr1_130171 & 5.59 nonsex & cis \\
\hline 1 X1_12837468 & 1713 chr1_129312 & 4.8 nonsex & cis \\
\hline 1 Gg_rs148852 & 1718 chr1_127201। & 4.57 nonsex & cis \\
\hline 1 X1_12837468 & 1720 chr1_126629 & 4.42 nonsex & cis \\
\hline 1 X1_12837468 & 1720 chr2_100758 & 6.26 nonsex & trans \\
\hline 1 X1_12837468 & 1725 chr1_130174! & 4.32 nonsex & cis \\
\hline 1 X1_12837468 & 1727 chr1_126937। & 4.14 nonsex & cis \\
\hline 1 X1_12837468 & 1727 chr1_1276361 & 4.73 nonsex & cis \\
\hline 1 Gg_rs148850 & 1728 chr1_126659 & 6 nonsex & cis \\
\hline 1 Gg_rs148850 & 1729 chr1_128935। & 12.14 nonsex & cis \\
\hline 1 Gg_rs148852 & 1729 chr1_129694। & 6.59 nonsex & cis \\
\hline
\end{tabular}




\begin{tabular}{|c|c|c|c|}
\hline 1 Gg_rs148852 & 1729 chr1_129822 & 5.24 nonsex & cis \\
\hline 1 Gg_rs148852 & 1729 chr1_127570 & 4.47 nonsex & cis \\
\hline 1 Gg_rs148852 & 1729 chr1_129673। & 5.14 nonsex & cis \\
\hline 1 Gg_rs148852 & 1729 chr1_129770 & 4.6 nonsex & cis \\
\hline 1 Gg_rs148852 & 1729 chr1_130336। & 5.19 nonsex & cis \\
\hline 1 X1_12837468 & 1732 chr1_130442 & 4.15 nonsex & cis \\
\hline 1 X1_12837468 & 1733 chr1_130587। & 4.75 nonsex & cis \\
\hline 1 Gg_rs148853. & 1733 chr1_126709। & 8.29 nonsex & cis \\
\hline 1 Gg_rs148853. & 1733 chrW_36060 & 8.53 sex & trans \\
\hline 1 X1_12837468 & 1734 chr1_130565। & 4.23 nonsex & cis \\
\hline 1 X1_1341656c & 1738 chr1_130063। & 6.17 sex & cis \\
\hline 1 X1_1341656C & 1739 chr1_131192 & 6.71 nonsex & cis \\
\hline 1 X1_1341656C & 1739 chr1_129633। & 3.76 nonsex & cis \\
\hline 1 X1_1341656C & 1741 chr1_131263। & 6.95 nonsex & cis \\
\hline 1 Gg_rs139396 & 1758 chr1_140880 & 5.36 sex & cis \\
\hline 1 Gg_rs139396 & 1758 chr1_133193। & 5.02 nonsex & cis \\
\hline 1 Gg_rs154270 & 1759 chr1_133244। & 7.74 nonsex & cis \\
\hline 1 Gg_rs154270 & 1759 chr1_133788। & 4.12 nonsex & cis \\
\hline 1 Gg_rs154270 & 1760 chr1_133542। & 4.15 nonsex & cis \\
\hline 1 Gg_rs154270 & 1761 chr1_128969। & 7.02 sex & cis \\
\hline 1 Gg_rs139396 & 1762 chr1_131968। & 4.04 nonsex & cis \\
\hline 1 Gg_rs154270 & 1763 chr1_133943। & 4.4 nonsex & cis \\
\hline 1 Gg_rs139396 & 1764 chr1_139143। & 5.63 sex & cis \\
\hline 1 Gg_rs154270 & 1765 chr1_133191। & 7 nonsex & cis \\
\hline 1 Gg_rs139396 & 1766 chr1_133450 & 4.98 nonsex & cis \\
\hline 1 Gg_rs154270 & 1767 chr1_134539। & 5.34 nonsex & cis \\
\hline 1 Gg_rs148910. & 1771 chr5_375770 & 7.21 sex & trans \\
\hline 1 Gg_rs148910. & 1773 chrZ_672210। & $6.04 \operatorname{sex}$ & trans \\
\hline 1 Gg_rs148159 & 1775 chr1_363860 & 2.89 nonsex & cis \\
\hline 1 Gg_rs148910. & 1775 chr1_136185 & 6.21 nonsex & cis \\
\hline 1 Gg_rs148910. & 1775 chr1_134801। & $6.81 \mathrm{sex}$ & cis \\
\hline 1 Gg_rs148910. & 1775 chr1_135072 & 6.11 nonsex & cis \\
\hline 1 Gg_rs148910. & 1775 chr1_135684। & 5.53 nonsex & cis \\
\hline 1 Gg_rs148910. & 1776 chr1_134200 & 4.51 nonsex & cis \\
\hline 1 Gg_rs148910. & 1776 chr1_135213। & 4.8 nonsex & cis \\
\hline 1 Gg_rs148910. & 1776 chr1_135843। & 5.16 nonsex & cis \\
\hline 1 Gg_rs148910. & 1777 chr1_133995। & 6.99 nonsex & cis \\
\hline 1 Gg_rs148910. & 1779 chr1_133676। & 4.76 nonsex & cis \\
\hline 1 Gg_rs148910. & 1780 chr1_133896। & 4.99 nonsex & cis \\
\hline 1 Gg_rs148910. & 1781 chr1_138209। & 8.03 sex & cis \\
\hline 1 Gg_rs148910. & 1782 chr1_136389 & 4.91 nonsex & cis \\
\hline 1 Gg_rs148910. & 1783 chr1_133697। & 6.39 sex & cis \\
\hline 1 Gg_rs148934 & 1786 chr1_136991। & 4.73 nonsex & cis \\
\hline
\end{tabular}




\begin{tabular}{|c|c|c|}
\hline 1 Gg_rs148934 & 1787 chr1_135735। & 5.82 nonsex \\
\hline 1 Gg_rs148934! & 1787 chr1_137382। & 6.07 nonsex \\
\hline 1 Gg_rs148954 & 1787 chr1_139254! & 4.03 nonsex \\
\hline 1 Gg_rs148934! & 1788 chr1_138541। & 5.94 nonsex \\
\hline 1 Gg_rs148934! & 1788 chr1_138281। & 4.71 nonsex \\
\hline 1 Gg_rs148934! & 1789 chr1_138835। & 7.82 nonsex \\
\hline 1 Gg_rs148934 & 1789 chr1_137871। & 3.9 nonsex \\
\hline 1 Gg_rs148934 & 1791 chr1_136403। & 6.88 sex \\
\hline 1 Gg_rs148934 & 1791 chr1_138286। & 7.07 nonsex \\
\hline 1 Gg_rs148934! & 1791 chr1_138387। & 5.3 nonsex \\
\hline 1 Gg_rs148934! & 1792 chr1_138285। & 5.3 nonsex \\
\hline 1 Gg_rs148934 & 1792 chr1_138290। & 6.55 nonsex \\
\hline 1 Gg_rs148934! & 1792 chr1_137788। & 4.83 nonsex \\
\hline 1 Gg_rs148934 & 1792 chr1_1378381 & 4.14 nonsex \\
\hline 1 Gg_rs148934 & 1793 chr1_136500 & 7.17 nonsex \\
\hline 1 Gg_rs148934 & 1793 chr1_138288 & 5.88 nonsex \\
\hline 1 Gg_rs148934 & 1794 chr1_136739| & 4.54 nonsex \\
\hline 1 Gg_rs148934 & 1794 chr1_137768 & 4.39 nonsex \\
\hline 1 Gg_rs148934 & 1795 chr1_136511। & 5.22 nonsex \\
\hline 1 Gg_rs148934 & 1795 chr1_137610। & 8.68 nonsex \\
\hline 1 Gg_rs148934 & 1795 chr1_137629 & 19.85 nonsex \\
\hline 1 Gg_rs148934 & 1795 chr1_137656। & 11.5 nonsex \\
\hline 1 Gg_rs148934 & 1795 chr1_137682 & 11.7 nonsex \\
\hline 1 Gg_rs148934 & 1795 chr1_137686। & 11.2 nonsex \\
\hline 1 Gg_rs148934! & 1795 chr1_137716। & 14.66 nonsex \\
\hline 1 Gg_rs148934 & 1796 chr1_137581। & 7.77 nonsex \\
\hline 1 Gg_rs148934 & 1796 chr1_137594। & 10.53 nonsex \\
\hline 1 Gg_rs148934 & 1796 chr1_137596। & 11.39 nonsex \\
\hline 1 Gg_rs148934 & 1796 chr1_137597। & 10.26 nonsex \\
\hline 1 Gg_rs148934! & 1796 chr1_137601। & 21.57 nonsex \\
\hline 1 Gg_rs148934! & 1796 chr1_137624। & 11.53 nonsex \\
\hline 1 Gg_rs148934! & 1796 chr1_137625। & 11.15 nonsex \\
\hline 1 Gg_rs148934 & 1796 chr1_137641। & 11.45 nonsex \\
\hline 1 Gg_rs148934 & 1796 chr1_137648। & 11.67 nonsex \\
\hline 1 Gg_rs148934 & 1796 chr1_137649| & 11.23 nonsex \\
\hline 1 Gg_rs148934 & 1796 chr1_137650। & 10.88 nonsex \\
\hline 1 Gg_rs148934! & 1796 chr1_137652 & 11.68 nonsex \\
\hline 1 Gg_rs148934 & 1796 chr1_137653। & 10.35 nonsex \\
\hline 1 Gg_rs148934 & 1796 chr1_137678। & 12.09 nonsex \\
\hline 1 Gg_rs148934 & 1796 chr1_137680 & 11.65 nonsex \\
\hline 1 Gg_rs148934 & 1796 chr1_137696| & 10.03 nonsex \\
\hline 1 Gg_rs148934 & 1796 chr1_137708। & 10.55 nonsex \\
\hline 1 Gg_rs148934 & 1796 chr1_137709! & 11.02 nonsex \\
\hline
\end{tabular}




\begin{tabular}{|c|c|c|}
\hline 1 Gg_rs148934! & 1796 chr1_137710 & 12.66 nonsex \\
\hline 1 Gg_rs148934! & 1796 chr1_137711। & 11.03 nonsex \\
\hline 1 Gg_rs148934! & 1796 chr1_137712। & 11.4 nonsex \\
\hline 1 Gg_rs148934! & 1796 chr1_137713। & 11.54 nonsex \\
\hline 1 Gg_rs148934! & 1796 chr1_137726। & 9.81 nonsex \\
\hline 1 Gg_rs148934 & 1796 chr1_137739| & 11.17 nonsex \\
\hline 1 Gg_rs148934 & 1796 chr1_137743। & 11.15 nonsex \\
\hline 1 Gg_rs148934 & 1796 chr1_137747। & 18.69 nonsex \\
\hline 1 Gg_rs148934! & 1796 chr1_138867। & 4.23 nonsex \\
\hline 1 Gg_rs148934 & 1797 chr1_136430। & 4.97 nonsex \\
\hline 1 Gg_rs148934! & 1797 chr1_137582 & 12.1 nonsex \\
\hline 1 Gg_rs148934 & 1797 chr1_137583। & 7.53 nonsex \\
\hline 1 Gg_rs148934! & 1797 chr1_137595। & 12.69 nonsex \\
\hline 1 Gg_rs148934 & 1797 chr1_137598| & 11.89 nonsex \\
\hline 1 Gg_rs148934 & 1797 chr1_137599| & 14.05 nonsex \\
\hline 1 Gg_rs148934 & 1797 chr1_137600 & 14.13 nonsex \\
\hline 1 Gg_rs148934 & 1797 chr1_137602 & 18.72 nonsex \\
\hline 1 Gg_rs148934! & 1797 chr1_137611। & 11.58 nonsex \\
\hline 1 Gg_rs148934 & 1797 chr1_137612। & 6.53 nonsex \\
\hline 1 Gg_rs148934 & 1797 chr1_137622। & 10.07 nonsex \\
\hline 1 Gg_rs148934! & 1797 chr1_137623। & 13.09 nonsex \\
\hline 1 Gg_rs148934! & 1797 chr1_1376261 & 11.6 nonsex \\
\hline 1 Gg_rs148934! & 1797 chr1_137627। & 12.04 nonsex \\
\hline 1 Gg_rs148934 & 1797 chr1_137628। & 14.6 nonsex \\
\hline 1 Gg_rs148934 & 1797 chr1_137630। & 20.13 nonsex \\
\hline 1 Gg_rs148934 & 1797 chr1_137634। & 5.22 nonsex \\
\hline 1 Gg_rs148934 & 1797 chr1_137644। & 17.4 nonsex \\
\hline 1 Gg_rs148934 & 1797 chr1_137651। & 11.52 nonsex \\
\hline 1 Gg_rs148934 & 1797 chr1_137654। & 12 nonsex \\
\hline 1 Gg_rs148934 & 1797 chr1_137655। & 14.8 nonsex \\
\hline 1 Gg_rs148934 & 1797 chr1_137657। & 20.05 nonsex \\
\hline 1 Gg_rs148934! & 1797 chr1_137661। & 14.44 nonsex \\
\hline 1 Gg_rs148934 & 1797 chr1_137666। & 8.69 nonsex \\
\hline 1 Gg_rs148934 & 1797 chr1_137667। & 12.12 nonsex \\
\hline 1 Gg_rs148934 & 1797 chr1_137669। & 7.63 nonsex \\
\hline 1 Gg_rs148934 & 1797 chr1_137674! & 17.46 nonsex \\
\hline 1 Gg_rs148934! & 1797 chr1_137679| & 11.19 nonsex \\
\hline 1 Gg_rs148934 & 1797 chr1_137681। & 11.75 nonsex \\
\hline 1 Gg_rs148934 & 1797 chr1_137683! & 10.91 nonsex \\
\hline 1 Gg_rs148934 & 1797 chr1_137684! & 13.1 nonsex \\
\hline 1 Gg_rs148934! & 1797 chr1_137685। & 15.45 nonsex \\
\hline 1 Gg_rs148934! & 1797 chr1_137687। & 20.63 nonsex \\
\hline 1 Gg_rs148934! & 1797 chr1_137695। & 5.8 nonsex \\
\hline
\end{tabular}




\begin{tabular}{|c|c|c|}
\hline 1 Gg_rs148934! & 1797 chr1_137698| & 7.13 nonsex \\
\hline 1 Gg_rs148934 & 1797 chr1_137714। & 12.06 nonsex \\
\hline 1 Gg_rs148934! & 1797 chr1_137715। & 14.88 nonsex \\
\hline 1 Gg_rs148934 & 1797 chr1_137717। & 19.34 nonsex \\
\hline 1 Gg_rs148934 & 1797 chr1_137725। & 6.06 nonsex \\
\hline 1 Gg_rs148934! & 1797 chr1_137728। & 7.61 nonsex \\
\hline 1 Gg_rs148934 & 1797 chr1_137729| & 9.84 nonsex \\
\hline 1 Gg_rs148934 & 1797 chr1_137738। & 10.68 nonsex \\
\hline 1 Gg_rs148934 & 1797 chr1_137740। & 11.54 nonsex \\
\hline 1 Gg_rs148934 & 1797 chr1_137741। & 11.01 nonsex \\
\hline 1 Gg_rs148934 & 1797 chr1_137742 & 11.49 nonsex \\
\hline 1 Gg_rs148934 & 1797 chr1_137744! & 11.31 nonsex \\
\hline 1 Gg_rs148934! & 1797 chr1_137745। & 13.04 nonsex \\
\hline 1 Gg_rs148934 & 1797 chr1_137746। & 15.17 nonsex \\
\hline 1 Gg_rs148934 & 1797 chr1_137748। & 18.61 nonsex \\
\hline 1 Gg_rs148934 & 1797 chr1_137769। & 10.67 nonsex \\
\hline 1 Gg_rs148934 & 1797 chr1_137791। & 14.07 nonsex \\
\hline 1 Gg_rs148934 & 1797 chr1_139238। & 11.53 nonsex \\
\hline 1 Gg_rs148934 & 1797 chr1_132737। & 4.33 nonsex \\
\hline 1 Gg_rs148934 & 1797 chr1_137767। & 5.11 nonsex \\
\hline 1 Gg_rs148934 & 1797 chr3_782750 & 5.76 nonsex \\
\hline 1 Gg_rs148934 & 1798 chr1_137584। & 7.86 nonsex \\
\hline 1 Gg_rs148934 & 1798 chr1_137589| & 13.65 nonsex \\
\hline 1 Gg_rs148934 & 1798 chr1_137605। & 12.38 nonsex \\
\hline 1 Gg_rs148934 & 1798 chr1_137609। & 7.31 nonsex \\
\hline 1 Gg_rs148934 & 1798 chr1_137613। & 8.76 nonsex \\
\hline 1 Gg_rs148934 & 1798 chr1_137615। & 15.89 nonsex \\
\hline 1 Gg_rs148934 & 1798 chr1_137617। & 14.17 nonsex \\
\hline 1 Gg_rs148934 & 1798 chr1_137618 & 16.44 nonsex \\
\hline 1 Gg_rs148934 & 1798 chr1_137631। & 14.54 nonsex \\
\hline 1 Gg_rs148934! & 1798 chr1_137658। & 17.96 nonsex \\
\hline 1 Gg_rs148934! & 1798 chr1_137665। & 10 nonsex \\
\hline 1 Gg_rs148934 & 1798 chr1_137668। & 7.29 nonsex \\
\hline 1 Gg_rs148934 & 1798 chr1_137688। & 17.84 nonsex \\
\hline 1 Gg_rs148934 & 1798 chr1_137697। & 10.75 nonsex \\
\hline 1 Gg_rs148934 & 1798 chr1_137699| & 9.84 nonsex \\
\hline 1 Gg_rs148934! & 1798 chr1_137701। & 14.71 nonsex \\
\hline 1 Gg_rs148934 & 1798 chr1_137703। & 14.78 nonsex \\
\hline 1 Gg_rs148934 & 1798 chr1_137704! & 14.85 nonsex \\
\hline 1 Gg_rs148934 & 1798 chr1_137718। & 14.8 nonsex \\
\hline 1 Gg_rs148934 & 1798 chr1_137720 & 12.94 nonsex \\
\hline 1 Gg_rs148934 & 1798 chr1_137721। & 17.19 nonsex \\
\hline 1 Gg_rs148934! & 1798 chr1_137727। & 10.77 nonsex \\
\hline
\end{tabular}




\begin{tabular}{|c|c|c|}
\hline 1 Gg_rs148934| & 1798 chr1_137731। & 15.52 nonsex \\
\hline 1 Gg_rs148934 & 1798 chr1_1377331 & 14.23 nonsex \\
\hline 1 Gg_rs148934 & 1798 chr1_137734! & 15.49 nonsex \\
\hline 1 Gg_rs148934! & 1798 chr1_137770 & 6.25 nonsex \\
\hline 1 Gg_rs148934 & 1798 chr1_137771। & 6.84 nonsex \\
\hline 1 Gg_rs148934 & 1798 chr1_137777। & 13.14 nonsex \\
\hline 1 Gg_rs148934 & 1798 chr1_137790। & 9.73 nonsex \\
\hline 1 Gg_rs148934 & 1798 chr1_137375। & 4.42 nonsex \\
\hline 1 Gg_rs148934 & 1798 chr1_142187| & 5.12 nonsex \\
\hline 1 Gg_rs148934 & 1798 chr10_10400 & 5.88 nonsex \\
\hline 1 Gg_rs148934 & 1799 chr1_137574! & 13.87 nonsex \\
\hline 1 Gg_rs148934! & 1799 chr1_137576। & 11.94 nonsex \\
\hline 1 Gg_rs148934 & 1799 chr1_137579| & 12.49 nonsex \\
\hline 1 Gg_rs148934 & 1799 chr1_137585। & 11 nonsex \\
\hline 1 Gg_rs148934 & 1799 chr1_137586। & 13.87 nonsex \\
\hline 1 Gg_rs148934 & 1799 chr1_137587। & 11.77 nonsex \\
\hline 1 Gg_rs148934 & 1799 chr1_137590। & 13.24 nonsex \\
\hline 1 Gg_rs148934 & 1799 chr1_137593। & 13.66 nonsex \\
\hline 1 Gg_rs148934 & 1799 chr1_137603। & 13.07 nonsex \\
\hline 1 Gg_rs148934 & 1799 chr1_137608। & 12.22 nonsex \\
\hline 1 Gg_rs148934 & 1799 chr1_137614। & 10.31 nonsex \\
\hline 1 Gg_rs148934 & 1799 chr1_137619| & 12.43 nonsex \\
\hline 1 Gg_rs148934! & 1799 chr1_137621। & 13.68 nonsex \\
\hline 1 Gg_rs148934! & 1799 chr1_137633। & 11.92 nonsex \\
\hline 1 Gg_rs148934! & 1799 chr1_137643। & 13.72 nonsex \\
\hline 1 Gg_rs148934 & 1799 chr1_137647। & 12.23 nonsex \\
\hline 1 Gg_rs148934 & 1799 chr1_137659 & 13.41 nonsex \\
\hline 1 Gg_rs148934 & 1799 chr1_137670। & 9.41 nonsex \\
\hline 1 Gg_rs148934! & 1799 chr1_137671। & 15.22 nonsex \\
\hline 1 Gg_rs148934! & 1799 chr1_137672 & 12.01 nonsex \\
\hline 1 Gg_rs148934! & 1799 chr1_137673। & 11.1 nonsex \\
\hline 1 Gg_rs148934 & 1799 chr1_137675। & 11.47 nonsex \\
\hline 1 Gg_rs148934! & 1799 chr1_137689| & 13.29 nonsex \\
\hline 1 Gg_rs148934 & 1799 chr1_137691। & 13.54 nonsex \\
\hline 1 Gg_rs148934! & 1799 chr1_137693। & 15.25 nonsex \\
\hline 1 Gg_rs148934 & 1799 chr1_137694। & 10.96 nonsex \\
\hline 1 Gg_rs148934! & 1799 chr1_137700 & 12.24 nonsex \\
\hline 1 Gg_rs148934! & 1799 chr1_137707| & 14.34 nonsex \\
\hline 1 Gg_rs148934! & 1799 chr1_137749| & 14.29 nonsex \\
\hline 1 Gg_rs148934! & 1799 chr1_137751। & 12.18 nonsex \\
\hline 1 Gg_rs148934! & 1799 chr1_137756। & 11.17 nonsex \\
\hline 1 Gg_rs148934 & 1799 chr1_137772 & 9.71 nonsex \\
\hline 1 Gg_rs148934 & 1799 chr1_137773। & 14.08 nonsex \\
\hline
\end{tabular}




\begin{tabular}{|c|c|c|c|}
\hline 1 Gg_rs148934! & 1799 chr1_137774! & 11.46 nonsex & cis \\
\hline 1 Gg_rs148934 & 1799 chr1_137786। & 6.34 nonsex & cis \\
\hline 1 Gg_rs148934 & 1799 chr1_139021। & 5.78 nonsex & cis \\
\hline 1 Gg_rs148934 & 1799 chr1_141590 & 6.14 nonsex & cis \\
\hline 1 Gg_rs148934 & 1799 chr1_124122 & 16.21 nonsex & trans \\
\hline 1 Gg_rs148934 & 1800 chr1_137575। & 14.57 nonsex & cis \\
\hline 1 Gg_rs148934! & 1800 chr1_137578। & 14.89 nonsex & cis \\
\hline 1 Gg_rs148934! & 1800 chr1_137591। & 11.3 nonsex & cis \\
\hline 1 Gg_rs148934! & 1800 chr1_137592 & 11.45 nonsex & cis \\
\hline 1 Gg_rs148934 & 1800 chr1_137604। & 14.51 nonsex & cis \\
\hline 1 Gg_rs148934 & 1800 chr1_137606। & 13.67 nonsex & cis \\
\hline 1 Gg_rs148934! & 1800 chr1_137607। & 14.26 nonsex & cis \\
\hline 1 Gg_rs148934! & 1800 chr1_137616। & 12.35 nonsex & cis \\
\hline 1 Gg_rs148934! & 1800 chr1_137620 & 12.52 nonsex & cis \\
\hline 1 Gg_rs148934! & 1800 chr1_137632 & 14.85 nonsex & cis \\
\hline 1 Gg_rs148934 & 1800 chr1_137642 & 11.5 nonsex & cis \\
\hline 1 Gg_rs148934 & 1800 chr1_137645। & 11.49 nonsex & cis \\
\hline 1 Gg_rs148934 & 1800 chr1_137646। & 11.33 nonsex & cis \\
\hline 1 Gg_rs148934 & 1800 chr1_137660 & 13.64 nonsex & cis \\
\hline 1 Gg_rs148934 & 1800 chr1_137662 & 13.75 nonsex & cis \\
\hline 1 Gg_rs148934 & 1800 chr1_137663। & 13.79 nonsex & cis \\
\hline 1 Gg_rs148934 & 1800 chr1_137664। & 12.5 nonsex & cis \\
\hline 1 Gg_rs148934 & 1800 chr1_137677। & 11.72 nonsex & cis \\
\hline 1 Gg_rs148934 & 1800 chr1_137690 & 13.19 nonsex & cis \\
\hline 1 Gg_rs148934 & 1800 chr1_137692 & 13.15 nonsex & cis \\
\hline 1 Gg_rs148934! & 1800 chr1_137705। & 11.7 nonsex & cis \\
\hline 1 Gg_rs148934 & 1800 chr1_137706 & 11.74 nonsex & cis \\
\hline 1 Gg_rs148934 & 1800 chr1_137719| & 13.47 nonsex & cis \\
\hline 1 Gg_rs148934 & 1800 chr1_137722 & 13.95 nonsex & cis \\
\hline 1 Gg_rs148934 & 1800 chr1_137723। & 15.21 nonsex & cis \\
\hline 1 Gg_rs148934 & 1800 chr1_137724! & 12.58 nonsex & cis \\
\hline 1 Gg_rs148934 & 1800 chr1_137730 & 10.98 nonsex & cis \\
\hline 1 Gg_rs148934 & 1800 chr1_137735 & 12.23 nonsex & cis \\
\hline 1 Gg_rs148934 & 1800 chr1_137736। & 11.26 nonsex & cis \\
\hline 1 Gg_rs148934 & 1800 chr1_137737। & 12.9 nonsex & cis \\
\hline 1 Gg_rs148934 & 1800 chr1_137750 & 14.69 nonsex & cis \\
\hline 1 Gg_rs148934! & 1800 chr1_137752 & 13.9 nonsex & cis \\
\hline 1 Gg_rs148934 & 1800 chr1_137753। & 14.86 nonsex & cis \\
\hline 1 Gg_rs148934 & 1800 chr1_137754! & 11.23 nonsex & cis \\
\hline 1 Gg_rs148934 & 1800 chr1_137757। & 10.92 nonsex & cis \\
\hline 1 Gg_rs148934 & 1800 chr1_137778। & 13.73 nonsex & cis \\
\hline 1 Gg_rs148934 & 1800 chr1_137785। & 4.97 nonsex & cis \\
\hline 1 Gg_rs148934! & 1800 chr1_138820 & 7.95 sex & cis \\
\hline
\end{tabular}




\begin{tabular}{|c|c|c|c|}
\hline 1 Gg_rs148934 & 1800 chr1_137442। & 4.43 nonsex & cis \\
\hline 1 Gg_rs148934 & 1800 chr1_138611। & 4.28 nonsex & cis \\
\hline 1 Gg_rs148934| & 1800 chr1_139451। & 5.25 nonsex & cis \\
\hline 1 Gg_rs148934। & 1800 chr10_10610 & 6.71 nonsex & trans \\
\hline 1 Gg_rs148934! & 1801 chr1_137676। & 10.73 nonsex & cis \\
\hline 1 Gg_rs148934 & 1801 chr1_137702 & 10.02 nonsex & cis \\
\hline 1 Gg_rs148934| & 1801 chr1_137732 & 10.16 nonsex & cis \\
\hline 1 Gg_rs148934। & 1801 chr1_137755। & 10.63 nonsex & cis \\
\hline 1 Gg_rs148934| & 1801 chr1_1377581 & 8.15 nonsex & cis \\
\hline 1 Gg_rs148934 & 1801 chr1_141420 & 5.22 nonsex & cis \\
\hline 1 Gg_rs148934 & 1801 chr1_141456। & 5.08 nonsex & cis \\
\hline 1 Gg_rs148934 & 1801 chr1_141538। & 7.88 nonsex & cis \\
\hline 1 Gg_rs148934! & 1801 chr1_141540 & 8.16 nonsex & cis \\
\hline 1 Gg_rs148934 & 1801 chr1_141582 & 6.58 nonsex & cis \\
\hline 1 Gg_rs148934 & 1801 chr1_139815। & 3.73 nonsex & cis \\
\hline 1 Gg_rs148934। & 1802 chr1_137779 & 8.81 nonsex & cis \\
\hline 1 Gg_rs148934! & 1802 chr1_140973। & 6.19 sex & cis \\
\hline 1 Gg_rs148934| & 1802 chr1_141417। & 7.96 nonsex & cis \\
\hline 1 Gg_rs148934 & 1802 chr1_141459। & 7.95 nonsex & cis \\
\hline 1 Gg_rs148934 & 1802 chr1_141584। & 5.13 nonsex & cis \\
\hline 1 Gg_rs148934| & 1802 chr1_141585। & 6.5 nonsex & cis \\
\hline 1 Gg_rs148934 & 1802 chr1_137760 & 5.32 nonsex & cis \\
\hline 1 Gg_rs148934। & 1802 chr1_141421। & 4.86 nonsex & cis \\
\hline 1 Gg_rs148934! & 1802 chr1_141455 & 4.91 nonsex & cis \\
\hline 1 Gg_rs148934! & 1803 chr1_137588। & 6.55 nonsex & cis \\
\hline 1 Gg_rs148934| & 1803 chr1_141419 & 4.92 nonsex & cis \\
\hline 1 Gg_rs148934 & 1803 chr1_138614। & 4.41 nonsex & cis \\
\hline 1 Gg_rs148934! & 1803 chr1_136908। & 4.62 nonsex & cis \\
\hline 1 Gg_rs148954. & 1804 chr1_139940 & 4.44 nonsex & cis \\
\hline 1 Gg_rs148954. & 1804 chr1_145294। & 4.54 nonsex & cis \\
\hline 1 Gg_rs148934 & 1806 chr1_137201। & 6.7 sex & cis \\
\hline 1 Gg_rs148954. & 1806 chr5_106940 & 8.54 sex & trans \\
\hline 1 Gg_rs148954 & 1808 chr1_138182 & 4.91 nonsex & cis \\
\hline 1 Gg_rs148954 & 1810 chr1_138436। & 6.16 sex & cis \\
\hline 1 Gg_rs148954. & 1810 chr1_139915। & 4.71 nonsex & cis \\
\hline 1 Gg_rs148934 & 1820 chr1_141428। & 4.65 nonsex & cis \\
\hline 1 Gg_rs148954. & 1820 chr1_139716। & 6.67 nonsex & cis \\
\hline 1 Gg_rs148954 & 1820 chr1_140928। & 7.86 sex & cis \\
\hline 1 Gg_rs148954 & 1820 chr1_142966। & 6.12 sex & cis \\
\hline 1 Gg_rs148934 & 1821 chr1_141445। & 5.64 nonsex & cis \\
\hline 1 Gg_rs148934 & 1821 chr1_141448। & 4.84 nonsex & cis \\
\hline 1 Gg_rs148934 & 1821 chr1_141556। & 4.85 nonsex & cis \\
\hline 1 Gg_rs148934! & 1822 chr1_141574! & 4.86 nonsex & cis \\
\hline
\end{tabular}




\begin{tabular}{|c|c|c|}
\hline 1 Gg_rs148954. & 1824 chr1_140166। & 6.2 nonsex \\
\hline 1 Gg_rs148984 & 1828 chr1_141370 & 7.87 nonsex \\
\hline 1 Gg_rs148973. & 1829 chr1_141190। & 8.96 nonsex \\
\hline 1 Gg_rs148984 & 1830 chr1_140937। & 5.35 nonsex \\
\hline 1 Gg_rs148973. & 1832 chr1_145411। & 5.08 nonsex \\
\hline 1 Gg_rs148984 & 1833 chr1_145040 & 9.04 nonsex \\
\hline 1 Gg_rs148973. & 1834 chr1_141562। & 10.79 nonsex \\
\hline 1 Gg_rs148973. & 1834 chr1_141605। & 8.71 nonsex \\
\hline 1 Gg_rs148973. & 1835 chr1_1414361 & 8.39 nonsex \\
\hline 1 Gg_rs148984 & 1835 chr1_141604! & 5.67 nonsex \\
\hline 1 Gg_rs148973. & 1836 chr1_140934! & 7.2 nonsex \\
\hline 1 Gg_rs148973. & 1836 chr1_141353। & 9.14 nonsex \\
\hline 1 Gg_rs148973. & 1836 chr1_141384! & 5.42 nonsex \\
\hline 1 Gg_rs148973. & 1836 chr1_141561। & 4.47 nonsex \\
\hline 1 Gg_rs148973. & 1837 chr1_144189| & 6.41 nonsex \\
\hline 1 Gg_rs148973. & 1837 chr1_140994। & 3.5 nonsex \\
\hline 1 Gg_rs148973. & 1838 chr1_141352 & 8.7 nonsex \\
\hline 1 Gg_rs148973. & 1839 chr1_141437। & 8.73 nonsex \\
\hline 1 Gg_rs148973. & 1839 chr1_141503। & 7.43 nonsex \\
\hline 1 Gg_rs148973. & 1839 chr1_141563। & 8.23 nonsex \\
\hline 1 Gg_rs148973. & 1839 chr1_144171। & 10.37 nonsex \\
\hline 1 Gg_rs148973. & 1839 chr1_145480 & 5.94 sex \\
\hline 1 Gg_rs148973. & 1839 chr1_1442461 & 5.56 nonsex \\
\hline 1 Gg_rs148973. & 1840 chr1_141301। & 6.44 nonsex \\
\hline 1 Gg_rs148973. & 1841 chr1_141219| & 7.24 nonsex \\
\hline 1 Gg_rs148973. & 1841 chr1_141291। & 5.44 nonsex \\
\hline 1 Gg_rs148973. & 1841 chr1_141360। & 5.57 nonsex \\
\hline 1 Gg_rs148973. & 1841 chr1_141390। & 5.55 nonsex \\
\hline 1 Gg_rs148973. & 1841 chr1_141496। & 8.49 nonsex \\
\hline 1 Gg_rs148973. & 1841 chr1_141509। & 6.03 nonsex \\
\hline 1 Gg_rs148973. & 1841 chr1_141621। & 5.84 nonsex \\
\hline 1 Gg_rs148973. & 1841 chr1_144190। & 3.75 nonsex \\
\hline 1 Gg_rs148973. & 1842 chr1_141302। & 4.97 nonsex \\
\hline 1 Gg_rs148973. & 1843 chr1_141272 & 5.39 nonsex \\
\hline 1 Gg_rs148973. & 1843 chr1_141287। & 8.59 nonsex \\
\hline 1 Gg_rs148973. & 1843 chr1_141288 & 7.2 nonsex \\
\hline 1 Gg_rs148973. & 1843 chr1_141295। & 8.52 nonsex \\
\hline 1 Gg_rs148973. & 1843 chr1_141296। & 6.47 nonsex \\
\hline 1 Gg_rs148973. & 1843 chr1_141297। & 6.99 nonsex \\
\hline 1 Gg_rs148973. & 1843 chr1_141300 & 5.67 nonsex \\
\hline 1 Gg_rs148973. & 1843 chr1_141332। & 10.16 nonsex \\
\hline 1 Gg_rs148973. & 1843 chr1_141333! & 9.1 nonsex \\
\hline 1 Gg_rs148973. & 1843 chr1_141337। & 6.47 nonsex \\
\hline
\end{tabular}




\begin{tabular}{|c|c|c|}
\hline 1 Gg_rs148973. & 1843 chr1_141345। & 7.95 nonsex \\
\hline 1 Gg_rs148973. & 1843 chr1_141355। & 5.64 nonsex \\
\hline 1 Gg_rs148973. & 1843 chr1_141359! & 8.05 nonsex \\
\hline 1 Gg_rs148973. & 1843 chr1_141361। & 7.24 nonsex \\
\hline 1 Gg_rs148973. & 1843 chr1_141362। & 6.98 nonsex \\
\hline 1 Gg_rs148973. & 1843 chr1_141363। & 7.06 nonsex \\
\hline 1 Gg_rs148973. & 1843 chr1_141364! & 7.34 nonsex \\
\hline 1 Gg_rs148973. & 1843 chr1_141373। & 6.13 nonsex \\
\hline 1 Gg_rs148973. & 1843 chr1_141377। & 10.31 nonsex \\
\hline 1 Gg_rs148973. & 1843 chr1_141388| & 6.77 nonsex \\
\hline 1 Gg_rs148973. & 1843 chr1_141389| & 7.77 nonsex \\
\hline 1 Gg_rs148973. & 1843 chr1_141391। & 7.95 nonsex \\
\hline 1 Gg_rs148973. & 1843 chr1_141392 & 6.17 nonsex \\
\hline 1 Gg_rs148973. & 1843 chr1_141404! & 6.07 nonsex \\
\hline 1 Gg_rs148973. & 1843 chr1_141472। & 6.27 nonsex \\
\hline 1 Gg_rs148973. & 1843 chr1_141473। & 6.43 nonsex \\
\hline 1 Gg_rs148973. & 1843 chr1_141483। & 8.85 nonsex \\
\hline 1 Gg_rs148973. & 1843 chr1_141484! & 11.08 nonsex \\
\hline 1 Gg_rs148973. & 1843 chr1_141488| & 5.86 nonsex \\
\hline 1 Gg_rs148973. & 1843 chr1_141507। & 6.97 nonsex \\
\hline 1 Gg_rs148973. & 1843 chr1_141508। & 7.83 nonsex \\
\hline 1 Gg_rs148973. & 1843 chr1_141510 & 7.34 nonsex \\
\hline 1 Gg_rs148973. & 1843 chr1_141511। & 6.41 nonsex \\
\hline 1 Gg_rs148973. & 1843 chr1_141512 & 6.17 nonsex \\
\hline 1 Gg_rs148973. & 1843 chr1_141523। & 6.57 nonsex \\
\hline 1 Gg_rs148973. & 1843 chr1_141524! & 5.85 nonsex \\
\hline 1 Gg_rs148973. & 1843 chr1_141597। & 5.88 nonsex \\
\hline 1 Gg_rs148973. & 1843 chr1_141606। & 5.79 nonsex \\
\hline 1 Gg_rs148973. & 1843 chr1_141629| & 8.07 nonsex \\
\hline 1 Gg_rs148973. & 1843 chr1_141631। & 4.89 nonsex \\
\hline 1 Gg_rs148973. & 1843 chr1_141633। & 10.61 nonsex \\
\hline 1 Gg_rs148973. & 1843 chr1_141634। & 11.6 nonsex \\
\hline 1 Gg_rs148973. & 1843 chr1_141049| & 2.79 nonsex \\
\hline 1 Gg_rs148973. & 1843 chr1_141286। & 5.41 nonsex \\
\hline 1 Gg_rs148973. & 1843 chr1_141292 & 5.22 nonsex \\
\hline 1 Gg_rs148973. & 1843 chr1_141365! & 5.8 nonsex \\
\hline 1 Gg_rs148973. & 1843 chr1_141372 & 4.64 nonsex \\
\hline 1 Gg_rs148973. & 1843 chr1_141385! & 4.59 nonsex \\
\hline 1 Gg_rs148973. & 1843 chr1_141402 & 4.79 nonsex \\
\hline 1 Gg_rs148973. & 1843 chr1_141403! & 5.17 nonsex \\
\hline 1 Gg_rs148973. & 1843 chr1_141434! & 4.85 nonsex \\
\hline 1 Gg_rs148973. & 1843 chr1_141486 & 4.44 nonsex \\
\hline 1 Gg_rs148973. & 1843 chr1_141504! & 4.55 nonsex \\
\hline
\end{tabular}




\begin{tabular}{|c|c|c|}
\hline 1 Gg_rs148973. & 1843 chr1_141560। & 4.47 nonsex \\
\hline 1 Gg_rs148973. & 1843 chr1_141569। & 5.35 nonsex \\
\hline 1 Gg_rs148973. & 1843 chr1_146887। & 4.56 nonsex \\
\hline 1 Gg_rs148973. & 1844 chr1_141334! & 6.84 nonsex \\
\hline 1 Gg_rs148973. & 1844 chr1_141338। & 7.42 nonsex \\
\hline 1 Gg_rs148973. & 1844 chr1_141358। & 7.06 nonsex \\
\hline 1 Gg_rs148973. & 1844 chr1_141376। & 6.34 nonsex \\
\hline 1 Gg_rs148973. & 1844 chr1_141485। & 7.13 nonsex \\
\hline 1 Gg_rs148973. & 1844 chr1_141489| & 8.32 nonsex \\
\hline 1 Gg_rs148973. & 1844 chr1_142072 & 5.33 nonsex \\
\hline 1 Gg_rs148973. & 1844 chr1_141265। & 8.87 nonsex \\
\hline 1 Gg_rs148973. & 1844 chr1_141323। & 6.76 nonsex \\
\hline 1 Gg_rs148984 & 1845 chr1_141317। & 6.14 sex \\
\hline 1 Gg_rs148984 & 1846 chr1_145049| & 7.41 nonsex \\
\hline 1 Gg_rs148984 & 1846 chr1_145298। & 7.13 nonsex \\
\hline 1 Gg_rs148984 & 1847 chr1_144697। & 7.58 nonsex \\
\hline 1 Gg_rs148984 & 1847 chr1_144841। & 3.88 nonsex \\
\hline 1 Gg_rs148984 & 1847 chr1_144945। & 9.42 nonsex \\
\hline 1 Gg_rs148984 & 1847 chr1_145151। & 4.51 nonsex \\
\hline 1 Gg_rs148984 & 1847 chr1_145344। & 4.84 nonsex \\
\hline 1 Gg_rs148984 & 1847 chr1_145554! & 4.28 nonsex \\
\hline 1 Gg_rs148984 & 1848 chr1_145206। & 5.21 nonsex \\
\hline 1 Gg_rs148973. & 1849 chr1_141264! & 10.72 nonsex \\
\hline 1 Gg_rs107286. & 1849 chr1_144968। & 3.66 nonsex \\
\hline 1 Gg_rs148973. & 1850 chr1_141318। & 9.41 nonsex \\
\hline 1 Gg_rs148973. & 1850 chr1_141319| & 9.36 nonsex \\
\hline 1 Gg_rs148973. & 1850 chr1_141268। & 6.73 nonsex \\
\hline 1 Gg_rs148973. & 1850 chr1_141320। & 7.13 nonsex \\
\hline 1 Gg_rs148973. & 1850 chr1_141274! & 5.58 nonsex \\
\hline 1 Gg_rs107286. & 1850 chr1_141324। & 11.38 nonsex \\
\hline 1 Gg_rs107286. & 1850 chr1_141259| & 8.23 nonsex \\
\hline 1 Gg_rs107286. & 1850 chr1_141271। & 7.59 nonsex \\
\hline 1 Gg_rs107286. & 1850 chr1_141329| & 9.17 nonsex \\
\hline 1 Gg_rs107286. & 1850 chr1_141480 & 8.28 nonsex \\
\hline 1 Gg_rs107286. & 1850 chr1_145487। & 6.92 nonsex \\
\hline 1 Gg_rs107286. & 1850 chr1_141258! & 5.72 nonsex \\
\hline 1 Gg_rs107286. & 1850 chr1_141330 & 6.06 nonsex \\
\hline 1 Gg_rs107286. & 1850 chr1_141481। & 5.79 nonsex \\
\hline 1 Gg_rs107286. & 1850 chr1_145301। & 4.74 nonsex \\
\hline 1 Gg_rs107286. & 1852 chr1_145493। & 5.01 nonsex \\
\hline 1 Gg_rs107286. & 1853 chr1_145361। & 6.35 nonsex \\
\hline 1 Gg_rs107286. & 1856 chr1_144154! & 5.24 nonsex \\
\hline 1 Gg_rs107286. & 1857 chr1_145297। & 4.66 nonsex \\
\hline
\end{tabular}




\begin{tabular}{|c|c|c|c|}
\hline 1 Gg_rs107286. & 1864 chr1_145488। & 7.03 nonsex & cis \\
\hline 1 Gg_rs139535 & 1874 chr1_165727। & 6.96 sex & cis \\
\hline 1 Gg_rs139535 & 1875 chr1_146987। & 6.96 sex & cis \\
\hline 1 snp.23.342.18 & 1875 chr1_148777। & 3.83 nonsex & cis \\
\hline 1 Gg_rs139535 & 1876 chr1_146091। & 7.37 sex & cis \\
\hline 1 Gg_rs137793. & 1876 chr1_148807। & 4.92 nonsex & cis \\
\hline 1 Gg_rs139535 & 1879 chr1_149134। & 4 nonsex & cis \\
\hline 1 Gg_rs139535 & 1881 chr1_148662 & $5.2 \mathrm{sex}$ & cis \\
\hline 1 Gg_rs139535 & 1881 chr1_151962 & 5.99 nonsex & cis \\
\hline 1 Gg_rs139535 & 1882 chr1_147866। & 5.28 nonsex & cis \\
\hline 1 Gg_rs139535 & 1885 chr1_147310 & 6.63 nonsex & cis \\
\hline 1 Gg_rs139535 & 1888 chr1_147190 & 4.93 nonsex & cis \\
\hline 1 Gg_rs139535 & 1888 chr1_147191। & 5.92 nonsex & cis \\
\hline 1 Gg_rs139535 & 1888 chr1_147311। & 5.67 sex & cis \\
\hline 1 Gg_rs139535 & 1888 chr1_147189 & 4.15 nonsex & cis \\
\hline 1 Gg_rs139535 & 1890 chr1_157850 & 4.14 nonsex & cis \\
\hline 1 Gg_rs139535 & 1891 chr2_726380 & 6.96 sex & trans \\
\hline 1 Gg_rs139535 & 1892 chr1_147365। & 5.68 nonsex & cis \\
\hline 1 Gg_rs139535 & 1892 chr1_155393। & 6.42 sex & cis \\
\hline 1 Gg_rs139535 & 1893 chr1_146401। & 4.13 nonsex & cis \\
\hline 1 Gg_rs139535 & 1894 chr1_148841। & 5.12 nonsex & cis \\
\hline 1 Gg_rs139535 & 1895 chr2_112835 & 7.75 sex & trans \\
\hline 1 snp.23.342.1§ & 1902 chr2_192160 & 5.92 nonsex & trans \\
\hline 1 snp.23.342.1§ & 1903 chr17_45960। & 5.82 nonsex & trans \\
\hline 1 snp.23.342.18 & 1903 chr3_448790 & 5.8 nonsex & trans \\
\hline 1 snp.23.342.18 & 1904 chr1_156529। & 4.02 nonsex & cis \\
\hline 1 snp.23.342.18 & 1907 chrW_12050 & 8.74 sex & trans \\
\hline 1 snp.23.342.18 & 1907 chrW_12860 & 8.65 sex & trans \\
\hline 1 snp.23.342.18 & 1907 chrW_47850 & 7.73 sex & trans \\
\hline 1 Gg_rs139567 & 1908 chr1_148050 & 6.77 nonsex & cis \\
\hline 1 Gg_rs139567 & 1908 chr1_148180 & 6.7 nonsex & cis \\
\hline 1 Gg_rs139567 & 1908 chr1_155326। & 5.51 nonsex & cis \\
\hline 1 Gg_rs139567 & 1908 chr1_155760 & 5.19 nonsex & cis \\
\hline 1 Gg_rs139567 & 1908 chr13_24700 & 6.25 nonsex & trans \\
\hline 1 Gg_rs139567 & 1909 chr1_156376। & 4.44 nonsex & cis \\
\hline 1 Gg_rs139567 & 1910 chrW_21890 & 7.66 sex & trans \\
\hline 1 Gg_rs137793. & 1914 chr1_155448। & 4.56 nonsex & cis \\
\hline 1 Gg_rs137793. & 1915 chr1_155492 & 4.4 nonsex & cis \\
\hline 1 Gg_rs137793. & 1916 chr1_155302 & 11.05 nonsex & cis \\
\hline 1 Gg_rs137793. & 1916 chr1_155549। & 4.9 nonsex & cis \\
\hline 1 Gg_rs137793. & 1918 chr1_155523। & 5.5 nonsex & cis \\
\hline 1 Gg_rs137793. & 1919 chr1_155546। & 5.4 nonsex & cis \\
\hline 1 Gg_rs137793. & 1920 chr1_156268। & 5.77 nonsex & cis \\
\hline
\end{tabular}




\begin{tabular}{|c|c|c|c|}
\hline 1 Gg_rs137793. & 1920 chr1_153102 & 5.14 nonsex & cis \\
\hline 1 Gg_rs137793. & 1920 chr1_156390 & 4.29 nonsex & cis \\
\hline 1 Gg_rs137793. & 1921 chr1_154834। & 5.2 nonsex & cis \\
\hline 1 Gg_rs137793. & 1922 chr1_155715। & 4.76 sex & cis \\
\hline 1 Gg_rs137793. & 1923 chr1_155895। & 4.85 nonsex & cis \\
\hline 1 Gg_rs137793. & 1923 chr3_214310 & 10.44 sex & trans \\
\hline 1 Gg_rs137793. & 1924 chr1_168902 & 5.9 nonsex & cis \\
\hline 1 Gg_rs139652. & 1935 chr1_157382 & 5.52 nonsex & cis \\
\hline 1 Gg_rs137969| & 1940 chr3_304290। & 7.72 sex & trans \\
\hline 1 Gg_rs139652. & 1941 chr5_562290 & 9.22 sex & trans \\
\hline 1 Gg_rs139652 & 1942 chr1_159670 & 4.85 nonsex & cis \\
\hline 1 Gg_rs139652 & 1943 chr1_170816। & 5.71 sex & cis \\
\hline 1 Gg_rs139652. & 1947 chr1_156588 & 7.77 nonsex & cis \\
\hline 1 Gg_rs139652 & 1949 chr1_170046। & 4.84 nonsex & cis \\
\hline 1 Gg_rs139652. & 1951 chr1_168726। & 7.48 sex & cis \\
\hline 1 Gg_rs154918. & 1955 chr1_167035। & 4.97 nonsex & cis \\
\hline 1 Gg_rs154918. & 1957 chr1_167686। & 5.95 nonsex & cis \\
\hline 1 Gg_rs154918. & 1958 chr1_164163। & 4.99 nonsex & cis \\
\hline 1 Gg_rs154918. & 1970 chr1_163487। & 4.17 nonsex & cis \\
\hline 1 Gg_rs154918. & 1970 chr1_163615। & 4.57 nonsex & cis \\
\hline 1 Gg_rs154918. & 1970 chr1_164162 & 4.19 nonsex & cis \\
\hline 1 Gg_rs154918. & 1970 chr1_167801। & 4.81 nonsex & cis \\
\hline 1 X1_17441654 & 1994 chrZ_102840। & 7.52 nonsex & trans \\
\hline 1 X1_1785128c & 2017 chr1_175597। & 3.81 nonsex & cis \\
\hline 1 X1_1785128؟ & 2020 chr1_173487। & 8.29 nonsex & cis \\
\hline 1 X1_1785128؟ & 2020 chr1_174117| & 4.59 nonsex & cis \\
\hline 1 Gg_rs139760 & 2023 chr1_173377| & 5.09 nonsex & cis \\
\hline 1 Gg_rs139760 & 2027 chrZ_2325401 & 5.82 nonsex & trans \\
\hline 1 Gg_rs139760 & 2028 chr1_173226। & 4.35 nonsex & cis \\
\hline 1 Gg_rs139760 & 2029 chr1_173589 & 4.61 nonsex & cis \\
\hline 1 X1_1785128c & 2029 chr1_173486! & 14.19 nonsex & cis \\
\hline 1 Gg_rs139760 & 2030 chr1_176333। & 5.11 nonsex & cis \\
\hline 1 Gg_rs139760 & 2032 chr1_173477। & 5.62 nonsex & cis \\
\hline 1 Gg_rs139760 & 2033 chr2_131215। & 7.17 nonsex & trans \\
\hline 1 Gg_rs139760 & 2040 chr1_175527। & 4.44 nonsex & cis \\
\hline 1 Gg_rs139760 & 2040 chr1_174241। & 3.97 nonsex & cis \\
\hline 1 Gg_rs139760 & 2040 chr1_174711। & 4.27 nonsex & cis \\
\hline 1 Gg_rs139760 & 2040 chr1_174862 & 4.55 nonsex & cis \\
\hline 1 Gg_rs139760 & 2040 chrZ_658470। & 7.37 sex & trans \\
\hline 1 Gg_rs139760 & 2040 chr27_19840। & 6.29 nonsex & trans \\
\hline 1 X1_1785128؟ & 2040 chr1_171822 & 5.93 sex & cis \\
\hline 1 X1_1785128؟ & 2044 chr1_174703। & 4.17 nonsex & cis \\
\hline 1 X1_1785128c & 2046 chr1_1733961 & 7.32 nonsex & cis \\
\hline
\end{tabular}




\begin{tabular}{|c|c|c|c|}
\hline 1 X1_1785128G & 2048 chr1_172387। & 7.57 nonsex & cis \\
\hline 1 X1_1785128c & 2048 chr1_174482 & 4.28 nonsex & cis \\
\hline 1 Gg_rs135559: & 2056 chr1_177009। & 6.73 nonsex & cis \\
\hline 1 Gg_rs135559: & 2056 chr1_174595। & 4.57 nonsex & cis \\
\hline 1 Gg_rs135559: & 2057 chr1_175578। & 4.74 nonsex & cis \\
\hline 1 Gg_rs135559: & 2059 chr1_172667। & 6.28 nonsex & cis \\
\hline 1 Gg_rs135559: & 2060 chr1_176580 & 4.63 nonsex & cis \\
\hline 1 Gg_rs135559: & 2061 chr1_177074! & 3.78 nonsex & cis \\
\hline 1 Gg_rs135559: & 2063 chr1_176976। & 3.97 nonsex & cis \\
\hline 1 Gg_rs135559: & 2063 chr1_234710 & 8.15 sex & trans \\
\hline 1 Gg_rs135559: & 2063 chrZ_557920। & 8.11 sex & trans \\
\hline 1 Gg_rs135559: & 2064 chr1_177071। & 5.97 sex & cis \\
\hline 1 Gg_rs135559: & 2064 chr1_181752 & 5.47 sex & cis \\
\hline 1 Gg_rs135559: & 2066 chr1_177296। & 4.34 nonsex & cis \\
\hline 1 Gg_rs135559: & 2066 chrW_48950c & 9.28 sex & trans \\
\hline 1 Gg_rs135559: & 2067 chr14_14935। & 7.98 sex & trans \\
\hline 1 Gg_rs135559: & 2069 chr1_175447। & 4.98 nonsex & cis \\
\hline 1 Gg_rs135559: & 2071 chr1_172217। & 5.72 nonsex & cis \\
\hline 1 snp.4.489.26 & 2072 chr1_179820 & $6.33 \operatorname{sex}$ & cis \\
\hline 1 snp.4.489.26 & 2072 chr2_566900। & 8.88 sex & trans \\
\hline 1 snp.4.489.26 & 2078 chr1_179056। & 8.66 nonsex & cis \\
\hline 1 snp.4.489.26 & 2078 chr1_180242 & 4.37 nonsex & cis \\
\hline 1 snp.4.489.26 & 2078 chr33_68050 & 14.13 sex & trans \\
\hline 1 snp.4.489.26 & 2078 chrZ_515200। & 7.57 sex & trans \\
\hline 1 snp.4.489.26 & 2079 chr1_178825। & 5.93 nonsex & cis \\
\hline 1 snp.4.489.26 & 2079 chr1_179091। & 3.93 nonsex & cis \\
\hline 1 snp.4.489.26 & 2080 chr1_178988। & 7.31 nonsex & cis \\
\hline 1 snp.4.489.26 & 2081 chr1_181301। & 5.97 nonsex & cis \\
\hline 1 snp. 4.489 .26 < & 2081 chrZ_463840। & 6.09 nonsex & trans \\
\hline 1 snp.4.489.26 & 2082 chr1_180160। & 6.92 nonsex & cis \\
\hline 1 snp.4.489.26 & 2082 chr1_180789| & 5.55 nonsex & cis \\
\hline 1 X1_18598868 & 2083 chr1_180065। & 5.05 nonsex & cis \\
\hline 1 X1_18448663 & 2084 chr1_183309। & 5.11 nonsex & cis \\
\hline 1 X1_18448663 & 2084 chr1_173693। & 4.3 nonsex & cis \\
\hline 1 X1_18389794 & 2088 chr1_179753। & 7.14 nonsex & cis \\
\hline 1 X1_18389794 & 2088 chr1_180251। & $6.95 \operatorname{sex}$ & cis \\
\hline 1 X1_18389794 & 2088 chr1_181012 & 6.85 nonsex & cis \\
\hline 1 X1_18389794 & 2088 chr1_184845। & 4.18 nonsex & cis \\
\hline 1 X1_18389794 & 2088 chr1_186038। & 3.86 nonsex & cis \\
\hline 1 X1_18389794 & 2088 chr31_27580 & 8.76 sex & trans \\
\hline 1 X1_18389794 & 2088 chrZ_363420। & 7.96 sex & trans \\
\hline 1 X1_18389794 & 2088 chr1_757400 & 6.2 nonsex & trans \\
\hline 1 X1_18389794 & 2088 chr4_581460। & 6.38 nonsex & trans \\
\hline
\end{tabular}




\begin{tabular}{|c|c|c|c|}
\hline 1 X1_18448663 & 2089 chr1_181876। & 6.04 nonsex & cis \\
\hline 1 X1_18448663 & 2090 chr1_181883। & 5.63 nonsex & cis \\
\hline 1 X1_18598868 & 2091 chr1_181882 & 4.33 nonsex & cis \\
\hline 1 X1_18448663 & 2092 chr1_181887। & 8.5 nonsex & cis \\
\hline 1 X1_18448663 & 2093 chr1_189105। & 4.18 nonsex & cis \\
\hline 1 X1_18448663 & 2093 chrZ_589160। & 6.63 nonsex & trans \\
\hline 1 X1_18448663 & 2094 chr1_183285। & 5.99 nonsex & cis \\
\hline 1 X1_18448663 & 2094 chr1_183504। & 5.36 nonsex & cis \\
\hline 1 X1_18448663 & 2094 chr1_182212 & 5.36 nonsex & cis \\
\hline 1 X1_18448663 & 2094 chr1_182213। & 4.67 nonsex & cis \\
\hline 1 X1_18448663 & 2094 chr1_183280 & 5.7 nonsex & cis \\
\hline 1 X1_18448663 & 2094 chr1_185115। & 4.07 nonsex & cis \\
\hline 1 X1_18448663 & 2094 chr1_182860 & 4.05 nonsex & cis \\
\hline 1 X1_18448663 & 2094 chr1_183279| & 4.79 nonsex & cis \\
\hline 1 X1_18448663 & 2094 chr5_135310 & 5.79 nonsex & trans \\
\hline 1 X1_18448663 & 2095 chr1_174031। & 6.31 nonsex & cis \\
\hline 1 X1_18598868 & 2095 chr1_182166। & 5.9 nonsex & cis \\
\hline 1 X1_18598868 & 2095 chr1_177381। & 4.8 nonsex & cis \\
\hline 1 X1_18598868 & 2095 chr1_179002 & 4.7 nonsex & cis \\
\hline 1 X1_18598868 & 2095 chr1_181772 & 5.48 nonsex & cis \\
\hline 1 X1_18598868 & 2095 chr1_182067। & 4.75 nonsex & cis \\
\hline 1 X1_18598868 & 2095 chr1_183293। & 5.44 nonsex & cis \\
\hline 1 X1_18598868 & 2095 chr2_144318। & 8.59 sex & trans \\
\hline 1 X1_18598868 & 2095 chr1_115478। & 5.98 nonsex & trans \\
\hline 1 X1_18598868 & 2095 chr3_169720 & 5.79 nonsex & trans \\
\hline 1 X1_18598868 & 2096 chr1_183257। & 6.41 nonsex & cis \\
\hline 1 X1_18598868 & 2097 chr1_183258। & 6.82 sex & cis \\
\hline 1 X1_18598868 & 2098 chr1_182304। & 16.7 nonsex & cis \\
\hline 1 X1_18598868 & 2099 chr1_180016। & 4.41 nonsex & cis \\
\hline 1 X1_18598868 & 2101 chr1_181677। & 5.66 sex & cis \\
\hline 1 X1_18598868 & 2102 chr1_186346। & 4.17 nonsex & cis \\
\hline 1 X1_18598868 & 2103 chr1_185450 & 6.69 sex & cis \\
\hline 1 X1_18598868 & 2103 chr1_181296। & 4.75 nonsex & cis \\
\hline 1 X1_18448663 & 2108 chr1_180056। & 7.46 nonsex & cis \\
\hline 1 X1_18598868 & 2108 chr1_181304। & 4.26 nonsex & cis \\
\hline 1 X1_18598868 & 2108 chr1_184469। & 5.15 nonsex & cis \\
\hline 1 X1_18389794 & 2111 chrZ_609430। & 7.47 sex & trans \\
\hline 1 X1_18448663 & 2112 chr1_185328। & 4.66 nonsex & cis \\
\hline 1 X1_18448663 & 2118 chr1_179847। & 7.68 nonsex & cis \\
\hline 1 X1_18598868 & 2120 chr1_182473। & 5.95 nonsex & cis \\
\hline 1 snp.4.489.26 & 2122 chr1_178813। & 5.06 nonsex & cis \\
\hline 1 X1_18598868 & 2122 chr1_182703। & 6.25 nonsex & cis \\
\hline 1 X1_19033467 & 2129 chr1_178917। & 5.71 nonsex & cis \\
\hline
\end{tabular}




\begin{tabular}{|c|c|c|}
\hline 1 X1_19202981 & 2129 chr3_109981। & 8.14 sex \\
\hline 1 X1_19202981 & 2130 chrW_48390 & 8.26 sex \\
\hline 1 X1_19202981 & 2131 chr28_13550। & 9.3 sex \\
\hline 1 X1_19033467 & 2132 chr1_189679| & 4.64 nonsex \\
\hline 1 X1_19202981 & 2132 chr30_15650। & 10.31 sex \\
\hline 1 X1_19033467 & 2132 chr2_349440। & 6.67 nonsex \\
\hline 1 X1_19033467 & 2133 chr1_189823। & 4.53 nonsex \\
\hline 1 X1_19202981 & 2155 chrZ_899000 & 10.16 nonsex \\
\hline 1 X1_19202981 & 2158 chr7_908000। & 7.81 nonsex \\
\hline 1 X1_19033467 & 2160 chr1_187623। & 4.7 nonsex \\
\hline 1 X1_19202981 & 2160 chr1_190455। & 6.59 nonsex \\
\hline 1 X1_19202981 & 2161 chr1_187104! & 5.81 nonsex \\
\hline 1 snp.317.20.1: & 2164 chr1_191248। & 6.17 nonsex \\
\hline 1 X1_19527164 & 2167 chr1_188738| & 5.93 nonsex \\
\hline 1 X1_19527164 & 2174 chr1_191773। & 5.21 nonsex \\
\hline 1 X1_19527164 & 2176 chr2_148122। & 8.27 nonsex \\
\hline 1 X1_19527164 & 2177 chr1_194235। & 4.27 nonsex \\
\hline 1 X1_19527164 & 2178 chr1_187976। & 5.17 nonsex \\
\hline 1 X1_19527164 & 2178 chr8_299680 & 6.39 nonsex \\
\hline 1 snp.317.20.1: & 2180 chr1_193738। & 5.85 nonsex \\
\hline 1 snp.317.20.1: & 2182 chr3_971430। & 6.41 nonsex \\
\hline 1 X1_19527164 & 2183 chr1_190661। & 6.53 sex \\
\hline 1 X1_19527164 & 2183 chr1_188212 & 5.27 nonsex \\
\hline 1 snp.317.20.1: & 2183 chr1_195082 & 5.27 nonsex \\
\hline 1 X1_19527164 & 2184 chr7_269370। & 6.99 nonsex \\
\hline 1 X1_19527164 & 2185 chr7_282730 & 6.62 nonsex \\
\hline 1 snp.317.20.1: & 2187 chr1_193682 & 4.75 nonsex \\
\hline 1 X1_19527164 & 2188 chr1_190683। & 8.3 nonsex \\
\hline 1 X1_19527164 & 2188 chr1_194072 & 4.7 nonsex \\
\hline 1 X1_19527164 & 2190 chr1_190997। & 4.64 nonsex \\
\hline 1 snp.317.20.1: & 2190 chr1_193736। & 6.05 nonsex \\
\hline 1 X1_19527164 & 2193 chr1_188617| & 4.47 nonsex \\
\hline 1 snp.317.20.1: & 2194 chr1_191153। & 6.84 nonsex \\
\hline 1 snp.317.20.1: & 2195 chr1_190989| & 4.35 nonsex \\
\hline 1 X1_19527164 & 2197 chr1_194189| & 4.66 nonsex \\
\hline 1 snp.317.20.1: & 2197 chr1_193923। & 4.66 nonsex \\
\hline 1 snp.317.20.1: & 2198 chr1_193884! & 5.16 nonsex \\
\hline 1 snp.317.20.1: & 2200 chr1_193896। & 5.87 nonsex \\
\hline 1 X1_19527164 & 2203 chr1_192122। & 5.17 nonsex \\
\hline 1 X1_19527164 & 2205 chr1_193615। & 4.44 nonsex \\
\hline 1 X1_19527164 & 2205 chr1_194060। & 4.3 nonsex \\
\hline 1 X1_19527164 & 2211 chr1_190909| & 4.33 nonsex \\
\hline 1 X1_19527164 & 2211 chr1_191032। & 4.52 nonsex \\
\hline
\end{tabular}




\begin{tabular}{|c|c|c|c|}
\hline 1 X1_19527164 & 2211 chr1_193801। & 3.76 nonsex & cis \\
\hline 1 X1_19527164 & 2211 chr1_194066। & 4.4 nonsex & cis \\
\hline 1 X1_19527164 & 2211 chr1_194068। & 4.15 nonsex & cis \\
\hline 1 X1_19527164 & 2211 chr1_194070। & 3.94 nonsex & cis \\
\hline 1 X1_19527164 & 2211 chr1_194071। & 3.98 nonsex & cis \\
\hline 1 X1_19527164 & 2211 chr1_194177। & 4.1 nonsex & cis \\
\hline 1 X1_19527164 & 2211 chr1_194187। & 4.12 nonsex & cis \\
\hline 1 X1_19527164 & 2211 chr1_194221। & 4.33 nonsex & cis \\
\hline 1 X1_19527164 & 2211 chr1_194225। & 4.43 nonsex & cis \\
\hline 1 X1_19527164 & 2211 chr1_194236। & 4.49 nonsex & cis \\
\hline 1 X1_19527164 & 2211 chr1_194238। & 4.58 nonsex & cis \\
\hline 1 X1_19527164 & 2211 chr1_194239। & 4.41 nonsex & cis \\
\hline 1 X1_19527164 & 2211 chr1_194241। & 4.25 nonsex & cis \\
\hline 1 X1_19527164 & 2211 chr7_194370। & 7.33 nonsex & trans \\
\hline 1 snp.317.20.1: & 2211 chr1_193761। & 9.82 nonsex & cis \\
\hline 1 snp.317.20.1: & 2211 chr1_193790। & 6.79 nonsex & cis \\
\hline 1 snp.317.20.1: & 2211 chr1_194425। & 3.74 nonsex & cis \\
\hline 1 snp.317.20.1: & 2211 chr1_193591। & 4.84 nonsex & cis \\
\hline 1 snp.317.20.1: & 2211 chr1_193912 & 4.19 nonsex & cis \\
\hline 1 snp.317.20.1: & 2211 chr1_194392 & 4.56 nonsex & cis \\
\hline 1 snp.317.20.1: & 2211 chr1_195151। & 5.41 nonsex & cis \\
\hline 1 snp.317.20.1: & 2211 chrZ_182820। & 7.71 sex & trans \\
\hline 1 snp.317.20.1: & 2211 chr2_214540। & 5.97 nonsex & trans \\
\hline 1 snp.317.20.1: & 2211 chr2_588060। & 6.49 nonsex & trans \\
\hline 1 X1_19527164 & 2228 chr1_192123। & 4.68 nonsex & cis \\
\hline 1 X1_19527164 & 2230 chr1_193102 & 3.6 nonsex & cis \\
\hline 1 snp.317.20.1: & 2235 chr1_191463। & 4.91 sex & cis \\
\hline 1 snp.317.20.1: & 2241 chr1_194055। & 5.44 nonsex & cis \\
\hline 1 snp.317.20.1: & 2243 chr1_194057। & 5.6 nonsex & cis \\
\hline 1 X1_19527164 & 2245 chr1_191950 & 4.8 nonsex & cis \\
\hline 1 snp.317.20.1: & 2246 chr1_194056। & 5.23 nonsex & cis \\
\hline 1 X1_2004005€ & 2287 chr1_193678। & 5.98 nonsex & cis \\
\hline 1 X1_2004005€ & 2317 chr1_196394। & 4.37 nonsex & cis \\
\hline 1 X1_2004005€ & 2317 chr1_191634! & 2.41 nonsex & cis \\
\hline 1 X1_2004005€ & 2317 chr1_195795। & 5.57 nonsex & cis \\
\hline 1 X1_2004005€ & 2317 chr9_174960 & 3.54 nonsex & trans \\
\hline 1 X1_2004005€ & 2317 chr5_297130 & 2.98 nonsex & trans \\
\hline 1 X1_2004005€ & 2317 chr7_299660। & 2.66 nonsex & trans \\
\hline 1 X1_2004005€ & 2317 chr33_61840 & 4.09 nonsex & trans \\
\hline 1 X1_2004005€ & 2317 chrZ_171080। & 3.03 nonsex & trans \\
\hline 1 X1_2004005€ & 2346 chr3_659610 & 3.55 nonsex & trans \\
\hline 1 X1_2004005€ & 2347 chr20_10891। & 2.86 nonsex & trans \\
\hline 1 Gg_rs149396 & 2348 chr1_195780। & 3.67 nonsex & cis \\
\hline
\end{tabular}




\begin{tabular}{|c|c|c|c|}
\hline 1 Gg_rs149396 & 2350 chr1_197002 & 3.89 nonsex & cis \\
\hline 1 Gg_rs149396 & 2350 chr6_172470। & 4.99 nonsex & trans \\
\hline 1 Gg_rs149396 & 2350 chr20_12610 & 4.54 nonsex & trans \\
\hline 1 Gg_rs149396 & 2352 chr1_197013। & 4.7 nonsex & cis \\
\hline 1 Gg_rs149396 & 2352 chr4_781200। & 5.13 sex & trans \\
\hline 1 Gg_rs149396 & 2352 chr5_360010 & 7.08 sex & trans \\
\hline 1 Gg_rs149396 & 2352 chr3_274350 & 6.29 sex & trans \\
\hline 1 Gg_rs149396 & 2352 chr3_671910 & 7.49 sex & trans \\
\hline 1 Gg_rs149396 & 2352 chr2_104826। & 4.92 nonsex & trans \\
\hline 2 X2_3527379 & 0 chr2_502500 & 5.11 nonsex & cis \\
\hline 2 Gg_rs141315 & 1 chr18_30860। & 4.29 nonsex & trans \\
\hline 2 Gg_rs141315 & 2 chr2_502400। & 5.14 nonsex & cis \\
\hline 2 X2_3527379 & 3 chr6_270630। & 6.71 sex & trans \\
\hline 2 Gg_rs141315 & 4 chr31_50740। & 5.33 nonsex & trans \\
\hline 2 Gg_rs141315 & 4 chr31_50750। & 4.81 nonsex & trans \\
\hline 2 Gg_rs141315 & 6 chr16_24200। & 5.17 nonsex & trans \\
\hline 2 Gg_rs141315 & 6 chr31_49910। & 5.16 nonsex & trans \\
\hline 2 Gg_rs141315 & 11 chr1_173320 & 6.11 nonsex & trans \\
\hline 2 Gg_rs141315 & 12 chr8_300430 & 6.74 sex & trans \\
\hline 2 Gg_rs141315 & 17 chr12_16851 & 6.75 sex & trans \\
\hline 2 X2_3527379 & 18 chr2_508300 & 5.64 nonsex & cis \\
\hline 2 Gg_rs141323 & 33 chr2_562100 & 5.51 nonsex & cis \\
\hline 2 Gg_rs141323 & 36 chr1_197421 & 8.22 nonsex & trans \\
\hline 2 Gg_rs141323 & 41 chr2_539500 & 6.26 nonsex & cis \\
\hline 2 Gg_rs141323 & 41 chr2_582700 & 6.33 nonsex & cis \\
\hline 2 Gg_rs141323 & 41 chr2_599900 & 5.75 sex & cis \\
\hline 2 Gg_rs141323 & 41 chr2_585000 & 4.29 nonsex & cis \\
\hline 2 Gg_rs141323 & 41 chr2_591300 & 4.36 nonsex & cis \\
\hline 2 Gg_rs141323 & 41 chr2_745600 & 5.39 nonsex & cis \\
\hline 2 Gg_rs141323 & 57 chr2_610400 & 5.36 nonsex & cis \\
\hline 2 Gg_rs141315 & 69 chr20_13316 & 4.58 nonsex & trans \\
\hline 2 Gg_rs141339: & 72 chr2_710100 & 6.13 nonsex & cis \\
\hline 2 Gg_rs150605. & 72 chr2_701100 & 7.86 sex & cis \\
\hline 2 Gg_rs141339: & 73 chr2_721400 & 6.96 nonsex & cis \\
\hline 2 Gg_rs141339: & 74 chr2_732300 & 6.76 nonsex & cis \\
\hline 2 Gg_rs141339: & 74 chr2_701800 & 3.95 nonsex & cis \\
\hline 2 Gg_rs150605. & 74 chr4_482100 & 5.94 nonsex & trans \\
\hline 2 Gg_rs150605 & 74 chr4_604440 & 4.54 nonsex & trans \\
\hline 2 Gg_rs141339: & 75 chr2_705600 & 5.9 nonsex & cis \\
\hline 2 Gg_rs141339: & 76 chr2_701900 & 8.72 nonsex & cis \\
\hline 2 Gg_rs141339: & 76 chr2_732400 & 7 nonsex & cis \\
\hline 2 Gg_rs150605 & 78 chr2_722200 & 5.48 nonsex & cis \\
\hline 2 Gg_rs141339: & 79 chr2_422800। & 4.58 nonsex & cis \\
\hline
\end{tabular}




\begin{tabular}{|c|c|c|}
\hline 2 Gg_rs141339: & 79 chr2_609200 & 4.33 nonsex \\
\hline 2 Gg_rs141339: & 79 chr2_132980 & 4.64 nonsex \\
\hline 2 Gg_rs141339: & 80 chr2_617900 & 4.7 nonsex \\
\hline 2 Gg_rs141339: & 80 chr2_692600 & 5.18 nonsex \\
\hline 2 Gg_rs141339: & 80 chr1_409690 & 5.86 nonsex \\
\hline 2 Gg_rs141339: & 81 chr2_705500। & 7.9 nonsex \\
\hline 2 Gg_rs141339: & 81 chr2_713500 & 4.96 nonsex \\
\hline 2 Gg_rs141339: & 81 chr8_231360। & 6.02 nonsex \\
\hline 2 Gg_rs141339: & 81 chr3_943600 & 6.35 nonsex \\
\hline 2 Gg_rs141339: & 82 chr2_822300 & 5.62 nonsex \\
\hline 2 Gg_rs141339: & 82 chr2_721000। & 5.21 nonsex \\
\hline 2 Gg_rs141339: & 82 chr6_945300 & 6.36 nonsex \\
\hline 2 Gg_rs141339: & 82 chr2_217500 & 5.71 nonsex \\
\hline 2 Gg_rs141339: & 82 chr2_984320 & 5.82 nonsex \\
\hline 2 Gg_rs141339: & 83 chr10_31090। & 5.66 nonsex \\
\hline 2 Gg_rs141339: & 84 chr2_833200। & 5.15 nonsex \\
\hline 2 Gg_rs141339: & 84 chr2_513000। & 4.34 nonsex \\
\hline 2 Gg_rs141339: & 84 chr12_63700। & 5.96 nonsex \\
\hline 2 Gg_rs141339: & 85 chr2_844800 & 4.51 nonsex \\
\hline 2 Gg_rs141339: & 85 chr2_113580 & 4.23 nonsex \\
\hline 2 Gg_rs141339: & 86 chr2_765100 & 9.28 nonsex \\
\hline 2 Gg_rs141339: & 86 chr2_937400 & 4.76 nonsex \\
\hline 2 Gg_rs141339: & 86 chr2_114140 & 4.4 nonsex \\
\hline 2 Gg_rs141339: & 86 chrZ_4824401 & 5.94 nonsex \\
\hline 2 Gg_rs141339: & 86 chr2_661920। & 6.32 nonsex \\
\hline 2 Gg_rs141339: & 87 chr4_876120 & 6 nonsex \\
\hline 2 Gg_rs141339: & 87 chr8_285850 & 5.94 nonsex \\
\hline 2 Gg_rs141339: & 88 chr2_893200 & 4.33 nonsex \\
\hline 2 Gg_rs141339: & 88 chr1_628240 & 5.87 nonsex \\
\hline 2 Gg_rs141339: & 89 chr1_771560। & 6.3 nonsex \\
\hline 2 Gg_rs141339: & 89 chr1_120453। & 7.8 sex \\
\hline 2 Gg_rs141339: & 90 chr10_13616। & 5.86 nonsex \\
\hline 2 Gg_rs141339: & 91 chr2_495700 & 6.56 nonsex \\
\hline 2 Gg_rs141339: & 91 chr2_695300। & 6.78 nonsex \\
\hline 2 Gg_rs141339: & 91 chr2_726700। & 5.24 nonsex \\
\hline 2 Gg_rs141339: & 91 chr2_741600 & 5.57 nonsex \\
\hline 2 Gg_rs141339: & 91 chr2_822200। & 7.47 sex \\
\hline 2 Gg_rs141339: & 91 chr2_131120 & 6.78 sex \\
\hline 2 Gg_rs141339: & 91 chr2_163000 & 4.18 nonsex \\
\hline 2 Gg_rs141339 & 91 chr2_200000 & 4.36 nonsex \\
\hline 2 Gg_rs141339: & 91 chr2_170300 & 4.81 nonsex \\
\hline 2 Gg_rs141339: & 91 chr2_559800 & 4.17 nonsex \\
\hline 2 Gg_rs141339: & 91 chr2_615100। & 4.18 nonsex \\
\hline
\end{tabular}




\begin{tabular}{|c|c|c|}
\hline 2 Gg_rs141339 & 91 chr2_652500। & 4.56 nonsex \\
\hline 2 Gg_rs141339: & 91 chr2_724700 & 3.85 nonsex \\
\hline 2 Gg_rs141339: & 91 chr2_741700 & 4.14 nonsex \\
\hline 2 Gg_rs141339: & 91 chr2_771400 & 4.28 nonsex \\
\hline 2 Gg_rs141339: & 91 chr2_106040 & 4.54 nonsex \\
\hline 2 Gg_rs141339: & 91 chr2_115970 & 4.21 nonsex \\
\hline 2 Gg_rs141339 & 91 chr6_263300 & 6.4 nonsex \\
\hline 2 Gg_rs141339: & 91 chr6_338690 & 7.03 nonsex \\
\hline 2 Gg_rs141339: & 91 chrZ_161270। & 7.75 sex \\
\hline 2 Gg_rs141339: & 91 chrZ_191510। & 8.56 sex \\
\hline 2 Gg_rs141339: & 91 chr1_765520 & 6.22 nonsex \\
\hline 2 Gg_rs141339: & 91 chrZ_556610। & 6.85 sex \\
\hline 2 Gg_rs141339: & 91 chr4_883190। & 5.93 nonsex \\
\hline 2 Gg_rs141339: & 91 chr5_140200 & 7.14 nonsex \\
\hline 2 Gg_rs141339: & 91 chr5_737100 & 5.6 nonsex \\
\hline 2 Gg_rs141339: & 91 chr5_256470 & 6.18 nonsex \\
\hline 2 Gg_rs141339: & 91 chr6_235130 & 5.76 nonsex \\
\hline 2 Gg_rs141339: & 91 chr8_246800 & 5.83 nonsex \\
\hline 2 Gg_rs141339: & 91 chr8_250680 & 5.59 nonsex \\
\hline 2 Gg_rs141339: & 91 chr10_12720 & 5.73 nonsex \\
\hline 2 Gg_rs141339: & 91 chr10_19902 & 6.74 nonsex \\
\hline 2 Gg_rs141339: & 91 chr20_10621 & 6.87 nonsex \\
\hline 2 Gg_rs141339: & 91 chr27_43480 & 5.77 nonsex \\
\hline 2 Gg_rs141339: & 91 chr1_620270 & 5.53 nonsex \\
\hline 2 Gg_rs141339: & 91 chr1_628230 & 6.34 nonsex \\
\hline 2 Gg_rs141339: & 91 chr1_957570 & 5.93 nonsex \\
\hline 2 Gg_rs141339: & 91 chr1_126394। & 6.88 nonsex \\
\hline 2 Gg_rs141339: & 91 chr1_134366 & 5.96 nonsex \\
\hline 2 Gg_rs141339: & 91 chr3_280800 & 5.34 nonsex \\
\hline 2 Gg_rs141339: & 92 chr2_781500 & 4.62 nonsex \\
\hline 2 Gg_rs141339: & 92 chr2_794600 & 4.28 nonsex \\
\hline 2 Gg_rs141339: & 92 chr2_838400 & 4.27 nonsex \\
\hline 2 Gg_rs141339: & 92 chr4_809620 & 7.57 nonsex \\
\hline 2 Gg_rs141339: & 92 chr6_343850 & 6.9 sex \\
\hline 2 Gg_rs141339: & 92 chr7_259760 & 8.81 sex \\
\hline 2 Gg_rs141339. & 92 chrZ_308150। & 7.72 sex \\
\hline 2 Gg_rs141339: & 92 chr1_293840 & 6.01 nonsex \\
\hline 2 Gg_rs141339: & 92 chr5_543410 & 6.15 nonsex \\
\hline 2 Gg_rs141339: & 92 chr5_590920 & 6.31 nonsex \\
\hline 2 Gg_rs141339: & 92 chr6_256270 & 6.05 nonsex \\
\hline 2 Gg_rs141339: & 92 chr10_18255। & 5.77 nonsex \\
\hline 2 Gg_rs141339 & 92 chr12_11591। & 5.41 nonsex \\
\hline 2 Gg_rs141339; & 92 chr14_26240। & 6.32 nonsex \\
\hline
\end{tabular}




\begin{tabular}{|c|c|c|}
\hline 2 Gg_rs141339: & 92 chr23_37620। & 5.81 nonsex \\
\hline 2 Gg_rs141339: & 92 chr1_718070 & 6.48 nonsex \\
\hline 2 Gg_rs141339: & 92 chrZ_567380। & 5.74 nonsex \\
\hline 2 Gg_rs141339: & 92 chr1_121268 & 6.1 nonsex \\
\hline 2 Gg_rs141339: & 92 chr2_414030 & 5.42 nonsex \\
\hline 2 Gg_rs141339: & 93 chr2_146800 & 5.36 nonsex \\
\hline 2 Gg_rs141339: & 93 chr2_340400 & 5.91 nonsex \\
\hline 2 Gg_rs141339: & 93 chr2_457200 & 6.13 nonsex \\
\hline 2 Gg_rs141339: & 93 chr2_629800 & 6.29 nonsex \\
\hline 2 Gg_rs141339: & 93 chr2_238700 & 4.54 nonsex \\
\hline 2 Gg_rs141339: & 93 chr2_373000। & 4.91 nonsex \\
\hline 2 Gg_rs141339: & 93 chr2_661300 & 4.48 nonsex \\
\hline 2 Gg_rs141339: & 93 chr2_958800 & 4.33 nonsex \\
\hline 2 Gg_rs141339: & 93 chr1_522820 & 7.62 nonsex \\
\hline 2 Gg_rs141339: & 93 chr6_134040 & 6.26 nonsex \\
\hline 2 Gg_rs141339: & 93 chr9_564800। & 5.88 nonsex \\
\hline 2 Gg_rs141339: & 93 chr10_89080 & 6.26 nonsex \\
\hline 2 Gg_rs141339: & 93 chr11_22560। & 6.59 nonsex \\
\hline 2 Gg_rs141339: & 93 chr17_51530 & 6.36 nonsex \\
\hline 2 Gg_rs141339: & 93 chrZ_397000 & 6.23 nonsex \\
\hline 2 Gg_rs141339: & 93 chr2_446720 & 6.27 nonsex \\
\hline 2 Gg_rs141339: & 93 chr2_729820 & 5.7 nonsex \\
\hline 2 Gg_rs141339: & 93 chr2_138322 & 6.02 nonsex \\
\hline 2 Gg_rs150605 & 93 chr2_122830 & 3.41 nonsex \\
\hline 2 Gg_rs150605 & 93 chr14_15271। & 5.11 nonsex \\
\hline 2 Gg_rs150605 & 93 chr2_658880 & 6.08 nonsex \\
\hline 2 Gg_rs150605 & 93 chr2_795190 & 5.72 nonsex \\
\hline 2 Gg_rs150605 & 93 chr3_85000 & 5.33 nonsex \\
\hline 2 Gg_rs150605 & 93 chr3_948640। & 4.71 nonsex \\
\hline 2 Gg_rs141339: & 94 chr2_373500। & 5.23 nonsex \\
\hline 2 Gg_rs141339: & 94 chr2_740300। & 8.89 nonsex \\
\hline 2 Gg_rs141339: & 94 chr2_361300 & 4.97 nonsex \\
\hline 2 Gg_rs141339: & 94 chr2_696400 & 5.19 nonsex \\
\hline 2 Gg_rs141339: & 94 chr2_769300। & 4.59 nonsex \\
\hline 2 Gg_rs141339: & 94 chr2_824900। & 4.66 nonsex \\
\hline 2 Gg_rs141339: & 94 chr6_346480 & 7.83 nonsex \\
\hline 2 Gg_rs141339: & 94 chr1_336770 & 6.25 nonsex \\
\hline 2 Gg_rs141339: & 94 chrZ_415830। & 6.23 nonsex \\
\hline 2 Gg_rs141339: & 94 chr4_285200। & 6.54 nonsex \\
\hline 2 Gg_rs150605 & 94 chr2_765200 & 10.31 nonsex \\
\hline 2 Gg_rs150605 & 94 chr2_650200 & 4.46 nonsex \\
\hline 2 Gg_rs150605 & 94 chr2_732000 & 4.6 nonsex \\
\hline 2 Gg_rs150605 & 94 chr2_742800 & 4.84 nonsex \\
\hline
\end{tabular}




\begin{tabular}{|c|c|c|c|}
\hline 2 Gg_rs150605 & 94 chr2_777800। & 3.79 nonsex & cis \\
\hline 2 Gg_rs150605 & 94 chr6_166120 & 7.78 nonsex & trans \\
\hline 2 Gg_rs150605 & 94 chrZ_327890। & 8.2 sex & trans \\
\hline 2 Gg_rs150605 & 94 chr4_814570 & 5.56 nonsex & trans \\
\hline 2 Gg_rs150605 & 94 chr8_748000 & 5.23 nonsex & trans \\
\hline 2 Gg_rs150605 & 94 chr10_13735। & 5.95 nonsex & trans \\
\hline 2 Gg_rs150605 & 94 chr10_13746! & 4.8 nonsex & trans \\
\hline 2 Gg_rs150605 & 94 chr10_18254। & 5.14 nonsex & trans \\
\hline 2 Gg_rs150605 & 94 chr20_55500 & 6.11 nonsex & trans \\
\hline 2 Gg_rs150605. & 94 chr20_65410 & 6.26 nonsex & trans \\
\hline 2 Gg_rs150605 & 94 chr27_22660 & 5.48 nonsex & trans \\
\hline 2 Gg_rs150605 & 94 chrZ_894700। & 5.82 nonsex & trans \\
\hline 2 Gg_rs150605 & 94 chr1_635510 & 5.59 nonsex & trans \\
\hline 2 Gg_rs150605 & 94 chr1_123956। & 5.89 nonsex & trans \\
\hline 2 Gg_rs150605 & 94 chr2_987040 & 5.32 nonsex & trans \\
\hline 2 Gg_rs150605 & 94 chr3_327940 & 5.78 nonsex & trans \\
\hline 2 Gg_rs141339: & 95 chr2_451100 & 6.82 sex & cis \\
\hline 2 Gg_rs141339: & 95 chr2_486000 & 5.7 nonsex & cis \\
\hline 2 Gg_rs150605 & 95 chr2_585100 & 6.04 nonsex & cis \\
\hline 2 Gg_rs150605 & 95 chr2_638600 & 5.52 nonsex & cis \\
\hline 2 Gg_rs150605 & 95 chr2_676300 & 6.32 nonsex & cis \\
\hline 2 Gg_rs150605 & 95 chr2_797700 & 6.33 nonsex & cis \\
\hline 2 Gg_rs150605 & 95 chr2_833500 & 14.61 nonsex & cis \\
\hline 2 Gg_rs150605 & 95 chr2_780300 & 3.55 nonsex & cis \\
\hline 2 Gg_rs150605 & 95 chr2_808400 & 4.99 nonsex & cis \\
\hline 2 Gg_rs150605. & 95 chr2_863100 & 4.3 nonsex & cis \\
\hline 2 Gg_rs150605 & 95 chr2_115980 & 3.88 nonsex & cis \\
\hline 2 Gg_rs150605 & 95 chr2_160170 & 4.54 nonsex & cis \\
\hline 2 Gg_rs150605 & 95 chr2_171410 & 4.53 nonsex & cis \\
\hline 2 Gg_rs150605 & 95 chr5_106090 & 9.18 nonsex & trans \\
\hline 2 Gg_rs150605 & 95 chr12_13370 & 7.12 nonsex & trans \\
\hline 2 Gg_rs150605 & 95 chr1_194553। & 5.95 sex & trans \\
\hline 2 Gg_rs150605 & 95 chr1_371200 & 6.71 nonsex & trans \\
\hline 2 Gg_rs150605 & 95 chr1_421000 & 6.65 nonsex & trans \\
\hline 2 Gg_rs150605 & 95 chr1_752300 & 5.22 nonsex & trans \\
\hline 2 Gg_rs150605 & 95 chr4_536480 & 5.93 nonsex & trans \\
\hline 2 Gg_rs150605 & 95 chr4_883380 & 5.6 nonsex & trans \\
\hline 2 Gg_rs150605 & 95 chr5_430970 & 6.75 nonsex & trans \\
\hline 2 Gg_rs150605 & 95 chr5_504100 & 5.67 nonsex & trans \\
\hline 2 Gg_rs150605 & 95 chr6_285800 & 5.15 nonsex & trans \\
\hline 2 Gg_rs150605 & 95 chr7_243620 & 4.73 nonsex & trans \\
\hline 2 Gg_rs150605 & 95 chr8_218990 & 5.54 nonsex & trans \\
\hline 2 Gg_rs150605 & 95 chr8_223600 & 5.21 nonsex & trans \\
\hline
\end{tabular}




\begin{tabular}{|c|c|c|}
\hline 2 Gg_rs150605 & 95 chr9_406400। & 6.22 nonsex \\
\hline 2 Gg_rs150605 & 95 chr12_97370 & 5.28 nonsex \\
\hline 2 Gg_rs150605 & 95 chr17_25960। & 5.77 nonsex \\
\hline 2 Gg_rs150605 & 95 chr20_57760 & 7.15 nonsex \\
\hline 2 Gg_rs150605 & 95 chr21_51210 & 6.51 nonsex \\
\hline 2 Gg_rs150605 & 95 chr21_58570 & 5.38 nonsex \\
\hline 2 Gg_rs150605 & 95 chr22_46500 & 6.03 nonsex \\
\hline 2 Gg_rs150605 & 95 chr24_19220 & 5.84 nonsex \\
\hline 2 Gg_rs150605 & 95 chrZ_247750। & 6.33 nonsex \\
\hline 2 Gg_rs150605. & 95 chr1_576410 & 6.13 nonsex \\
\hline 2 Gg_rs150605 & 95 chrZ_670780। & 6.22 nonsex \\
\hline 2 Gg_rs150605 & 95 chr1_145499| & 6.44 nonsex \\
\hline 2 Gg_rs150605 & 95 chr1_186681। & 6.33 nonsex \\
\hline 2 Gg_rs150605 & 95 chr1_196837। & 6.28 nonsex \\
\hline 2 Gg_rs150605 & 95 chr2_661660 & 5.68 nonsex \\
\hline 2 Gg_rs150605 & 95 chr2_708030 & 6.19 nonsex \\
\hline 2 Gg_rs150605 & 95 chr2_130460 & 5.54 nonsex \\
\hline 2 Gg_rs150605 & 95 chr2_148492 & 5.84 nonsex \\
\hline 2 Gg_rs150605 & 95 chr3_183760 & 6.4 nonsex \\
\hline 2 Gg_rs150605 & 95 chr3_249920 & 5.64 nonsex \\
\hline 2 Gg_rs150605 & 95 chr3_824760 & 5.99 nonsex \\
\hline 2 Gg_rs141339: & 96 chr2_445400 & 5.23 nonsex \\
\hline 2 Gg_rs141339: & 96 chr2_543300 & 4.93 nonsex \\
\hline 2 Gg_rs150605 & 96 chr2_913200 & 6.44 sex \\
\hline 2 Gg_rs150605 & 96 chr2_104760 & 5.4 sex \\
\hline 2 Gg_rs150605 & 96 chr2_104920 & 5.12 nonsex \\
\hline 2 Gg_rs150605 & 96 chr2_147700 & 5.5 nonsex \\
\hline 2 Gg_rs150605 & 96 chr2_585500 & 4.86 nonsex \\
\hline 2 Gg_rs150605 & 96 chr2_586800 & 4.31 nonsex \\
\hline 2 Gg_rs150605 & 96 chr2_624400 & 4.65 nonsex \\
\hline 2 Gg_rs150605 & 96 chr4_581220 & 7.66 nonsex \\
\hline 2 Gg_rs150605 & 96 chr5_560620 & 5.26 sex \\
\hline 2 Gg_rs150605 & 96 chr6_321320 & 6.08 nonsex \\
\hline 2 Gg_rs150605 & 96 chr7_348900 & 9.38 nonsex \\
\hline 2 Gg_rs150605 & 96 chr8_213000 & 6.9 nonsex \\
\hline 2 Gg_rs150605 & 96 chr1_824790 & 8.54 nonsex \\
\hline 2 Gg_rs150605 & 96 chr1_194656 & 6.93 sex \\
\hline 2 Gg_rs150605 & 96 chr1_195399। & 7.41 nonsex \\
\hline 2 Gg_rs150605 & 96 chr4_270300 & 6.6 nonsex \\
\hline 2 Gg_rs150605 & 96 chr1_763600 & 5.56 nonsex \\
\hline 2 Gg_rs150605 & 96 chr1_946500 & 6.82 nonsex \\
\hline 2 Gg_rs150605 & 96 chr1_317060 & 5.74 nonsex \\
\hline 2 Gg_rs150605 & 96 chr1_347170 & 6.77 nonsex \\
\hline
\end{tabular}




\begin{tabular}{|c|c|c|}
\hline 2 Gg_rs150605 & 96 chr1_514810। & 4.95 nonsex \\
\hline 2 Gg_rs150605 & 96 chr4_807620 & 5.65 nonsex \\
\hline 2 Gg_rs150605 & 96 chr4_814550 & 6.01 nonsex \\
\hline 2 Gg_rs150605 & 96 chr4_884330 & 5.95 nonsex \\
\hline 2 Gg_rs150605 & 96 chr5_345290 & 5.38 nonsex \\
\hline 2 Gg_rs150605 & 96 chr5_549180 & 5.61 nonsex \\
\hline 2 Gg_rs150605 & 96 chr6_182520 & 5.43 nonsex \\
\hline 2 Gg_rs150605 & 96 chr6_228520 & 6.82 nonsex \\
\hline 2 Gg_rs150605 & 96 chr7_268010 & 6.03 nonsex \\
\hline 2 Gg_rs150605 & 96 chr7_277760 & 5.17 nonsex \\
\hline 2 Gg_rs150605 & 96 chr7_279410 & 6.51 nonsex \\
\hline 2 Gg_rs150605 & 96 chr8_212170 & 5.96 nonsex \\
\hline 2 Gg_rs150605 & 96 chr8_213560 & 5.87 nonsex \\
\hline 2 Gg_rs150605 & 96 chr8_257890 & 5.3 nonsex \\
\hline 2 Gg_rs150605 & 96 chr9_146790 & 5.12 nonsex \\
\hline 2 Gg_rs150605 & 96 chr11_19756। & 5.59 nonsex \\
\hline 2 Gg_rs150605 & 96 chr14_48360 & 5.62 nonsex \\
\hline 2 Gg_rs150605 & 96 chr14_53270 & 6.38 nonsex \\
\hline 2 Gg_rs150605 & 96 chr14_13806। & 6.87 nonsex \\
\hline 2 Gg_rs150605 & 96 chr15_11529 & 4.88 nonsex \\
\hline 2 Gg_rs150605 & 96 chr17_38880 & 5.81 nonsex \\
\hline 2 Gg_rs150605 & 96 chr17_43640 & 6.17 nonsex \\
\hline 2 Gg_rs150605 & 96 chr17_62990 & 5.48 nonsex \\
\hline 2 Gg_rs150605 & 96 chr21_11900 & 6.84 nonsex \\
\hline 2 Gg_rs150605 & 96 chr21_45880 & 5.8 nonsex \\
\hline 2 Gg_rs150605 & 96 chr21_49950 & 5.59 nonsex \\
\hline 2 Gg_rs150605 & 96 chr21_57020 & 5.84 nonsex \\
\hline 2 Gg_rs150605 & 96 chr23_42800 & 6.81 nonsex \\
\hline 2 Gg_rs150605 & 96 chr24_34510 & 4.95 nonsex \\
\hline 2 Gg_rs150605 & 96 chr25_26870 & 6.44 nonsex \\
\hline 2 Gg_rs150605 & 96 chr1_593360 & 6.07 nonsex \\
\hline 2 Gg_rs150605 & 96 chr1_680770 & 6.39 nonsex \\
\hline 2 Gg_rs150605 & 96 chr1_844980 & 5.56 nonsex \\
\hline 2 Gg_rs150605 & 96 chr1_882860 & 5.62 nonsex \\
\hline 2 Gg_rs150605 & 96 chr1_197291। & 6.59 nonsex \\
\hline 2 Gg_rs150605 & 96 chr2_487750 & 5.93 nonsex \\
\hline 2 Gg_rs150605 & 96 chr2_685280 & 5.32 nonsex \\
\hline 2 Gg_rs150605 & 96 chr2_801060 & 5.98 nonsex \\
\hline 2 Gg_rs150605 & 96 chr2_958920 & 5.6 nonsex \\
\hline 2 Gg_rs150605 & 96 chr2_984450 & 4.94 nonsex \\
\hline 2 Gg_rs150605 & 96 chr3_569860 & 6.05 nonsex \\
\hline 2 Gg_rs150605 & 96 chr3_106368। & 6.02 nonsex \\
\hline 2 Gg_rs150605 & 96 chr3_109011। & 6.18 nonsex \\
\hline
\end{tabular}




\begin{tabular}{|c|c|c|}
\hline 2 Gg_rs150605 & 96 chr4_182560। & 6.28 nonsex \\
\hline 2 Gg_rs150605 & $97 \mathrm{chr} 2 \_646200$ & 4.69 nonsex \\
\hline 2 Gg_rs150605 & 97 chr2_956300 & 4.4 nonsex \\
\hline 2 Gg_rs150605 & 97 chr2_624300 & 3.84 nonsex \\
\hline 2 Gg_rs150605 & 97 chr2_693400 & 4.48 nonsex \\
\hline 2 Gg_rs150605 & 97 chr2_735900 & 4.55 nonsex \\
\hline 2 Gg_rs150605 & 97 chr2_946600 & 5.21 nonsex \\
\hline 2 Gg_rs150605 & 97 chr5_279210 & 8.12 nonsex \\
\hline 2 Gg_rs150605. & 97 chr6_266270 & 6.8 nonsex \\
\hline 2 Gg_rs150605 & 97 chr7_356580 & 6.69 nonsex \\
\hline 2 Gg_rs150605 & 97 chr7_357040 & $6.13 \operatorname{sex}$ \\
\hline 2 Gg_rs150605 & 97 chr10_27740 & 5.33 nonsex \\
\hline 2 Gg_rs150605 & 97 chr10_13538 & 6.92 nonsex \\
\hline 2 Gg_rs150605 & 97 chr14_61970 & 7.37 nonsex \\
\hline 2 Gg_rs150605 & 97 chr13_28020 & 5.42 nonsex \\
\hline 2 Gg_rs150605 & 97 chr21_63390। & 5.4 nonsex \\
\hline 2 Gg_rs150605 & 97 chrZ_563670। & 8.83 sex \\
\hline 2 Gg_rs150605 & 97 chr2_136700 & 6.25 nonsex \\
\hline 2 Gg_rs150605 & 97 chr3_672450 & 7.6 nonsex \\
\hline 2 Gg_rs150605 & 97 chr3_104805। & 7.46 nonsex \\
\hline 2 Gg_rs150605 & 97 chr1_199200 & 5.41 nonsex \\
\hline 2 Gg_rs150605 & 97 chr1_512660 & 6.48 nonsex \\
\hline 2 Gg_rs150605 & 97 chr1_512900 & 5.34 nonsex \\
\hline 2 Gg_rs150605 & 97 chr1_513330 & 6.06 nonsex \\
\hline 2 Gg_rs150605 & 97 chr5_120000 & 5.31 nonsex \\
\hline 2 Gg_rs150605 & 97 chr5_138660 & 5.73 nonsex \\
\hline 2 Gg_rs150605 & 97 chr6_103140 & 6.24 nonsex \\
\hline 2 Gg_rs150605. & 97 chr7_552100 & 5.09 nonsex \\
\hline 2 Gg_rs150605 & 97 chr7_157980 & 5.13 nonsex \\
\hline 2 Gg_rs150605 & 97 chr8_222460 & 5.78 nonsex \\
\hline 2 Gg_rs150605 & 97 chr8_287910 & 5.96 nonsex \\
\hline 2 Gg_rs150605 & 97 chr8_292090 & 5.49 nonsex \\
\hline 2 Gg_rs150605 & 97 chr10_32070 & 5.64 nonsex \\
\hline 2 Gg_rs150605 & 97 chr10_13327। & 6.32 nonsex \\
\hline 2 Gg_rs150605 & 97 chr11_83300 & 5.72 nonsex \\
\hline 2 Gg_rs150605 & 97 chr11_19129 & 6.15 nonsex \\
\hline 2 Gg_rs150605 & 97 chr13_34920 & 6.4 nonsex \\
\hline 2 Gg_rs150605 & 97 chr15_79710 & 6.55 nonsex \\
\hline 2 Gg_rs150605 & 97 chr15_10927 & 5.18 nonsex \\
\hline 2 Gg_rs150605 & 97 chr17_56920 & 6.96 nonsex \\
\hline 2 Gg_rs150605 & 97 chr17_67480 & 5.43 nonsex \\
\hline 2 Gg_rs150605 & 97 chr19_48530 & 4.53 nonsex \\
\hline 2 Gg_rs150605 & 97 chr20_11080। & 6.24 nonsex \\
\hline
\end{tabular}




\begin{tabular}{|c|c|c|}
\hline 2 Gg_rs150605 & 97 chr20_37980। & 5.71 nonsex \\
\hline 2 Gg_rs150605 & 97 chr20_91640 & 5.28 nonsex \\
\hline 2 Gg_rs150605 & 97 chr21_76200 & 5.04 nonsex \\
\hline 2 Gg_rs150605 & 97 chr21_26710 & 5.44 nonsex \\
\hline 2 Gg_rs150605 & 97 chr21_44980 & 5.21 nonsex \\
\hline 2 Gg_rs150605 & 97 chr21_50480 & 6.19 nonsex \\
\hline 2 Gg_rs150605 & 97 chr23_33970 & 6.3 nonsex \\
\hline 2 Gg_rs150605 & 97 chr27_38410 & 4.97 nonsex \\
\hline 2 Gg_rs150605 & 97 chr27_59450 & 5 nonsex \\
\hline 2 Gg_rs150605. & 97 chrZ_207300। & 6.72 nonsex \\
\hline 2 Gg_rs150605 & 97 chr1_608470 & 5.28 nonsex \\
\hline 2 Gg_rs150605 & 97 chr1_992210 & 5.25 nonsex \\
\hline 2 Gg_rs150605 & 97 chr1_140650 & 6.13 nonsex \\
\hline 2 Gg_rs150605 & 97 chr1_190156। & 5.6 nonsex \\
\hline 2 Gg_rs150605 & 97 chr1_196839| & 5.91 nonsex \\
\hline 2 Gg_rs150605 & 97 chr2_910090 & 5.03 nonsex \\
\hline 2 Gg_rs150605 & 97 chr2_101065। & 6.1 nonsex \\
\hline 2 Gg_rs150605 & 97 chr2_129449 & 5.53 nonsex \\
\hline 2 Gg_rs150605 & 97 chr3_494110 & 5.13 nonsex \\
\hline 2 Gg_rs150605 & 97 chr3_510010 & 6.89 nonsex \\
\hline 2 Gg_rs150605 & 97 chr4_500800 & 5.07 nonsex \\
\hline 2 Gg_rs150605 & 97 chr4_116830 & 5.68 nonsex \\
\hline 2 Gg_rs150605 & 98 chr2_609700 & 5.15 nonsex \\
\hline 2 Gg_rs150605 & 98 chr2_553400 & 4.24 nonsex \\
\hline 2 Gg_rs150605 & 98 chr2_589900 & 4.87 nonsex \\
\hline 2 Gg_rs150605. & 98 chr2_592300 & 5.22 nonsex \\
\hline 2 Gg_rs150605 & 98 chr2_750300 & 4.33 nonsex \\
\hline 2 Gg_rs150605 & 98 chr2_796500 & 4.97 nonsex \\
\hline 2 Gg_rs150605 & 98 chr2_829700 & 5.4 nonsex \\
\hline 2 Gg_rs150605 & 98 chr2_847000 & 4.2 nonsex \\
\hline 2 Gg_rs150605 & 98 chr2_928300 & 3.98 nonsex \\
\hline 2 Gg_rs150605 & 98 chr2_962800 & 4.86 nonsex \\
\hline 2 Gg_rs150605 & 98 chr2_998300 & 4.43 nonsex \\
\hline 2 Gg_rs150605 & 98 chr2_129200 & 4.22 nonsex \\
\hline 2 Gg_rs150605 & 98 chr5_577380 & 7.33 nonsex \\
\hline 2 Gg_rs150605 & 98 chr15_63430 & 7.38 nonsex \\
\hline 2 Gg_rs150605 & 98 chr21_50470 & 7.05 nonsex \\
\hline 2 Gg_rs150605 & 98 chr1_787760 & 7.5 nonsex \\
\hline 2 Gg_rs150605 & 98 chrZ_641490। & 7.36 sex \\
\hline 2 Gg_rs150605 & 98 chr2_323260 & 6.51 sex \\
\hline 2 Gg_rs150605 & 98 chr2_147751 & 6.09 nonsex \\
\hline 2 Gg_rs150605 & 98 chr3_321340 & 6.97 nonsex \\
\hline 2 Gg_rs150605 & 98 chr3_351750 & 7.65 sex \\
\hline
\end{tabular}




\begin{tabular}{|c|c|c|}
\hline 2 Gg_rs150605 & 98 chr4_138140। & 6.19 nonsex \\
\hline 2 Gg_rs150605 & 98 chr1_209300 & 5.98 nonsex \\
\hline 2 Gg_rs150605 & 98 chr4_632060 & 5.6 nonsex \\
\hline 2 Gg_rs150605 & 98 chr4_838740 & 5.44 nonsex \\
\hline 2 Gg_rs150605 & 98 chr5_839900 & 5.87 nonsex \\
\hline 2 Gg_rs150605 & 98 chr5_566070 & 6.18 nonsex \\
\hline 2 Gg_rs150605 & 98 chr5_592010 & 6.3 nonsex \\
\hline 2 Gg_rs150605 & 98 chr6_123870 & 5.68 nonsex \\
\hline 2 Gg_rs150605 & 98 chr6_320930 & 5.62 nonsex \\
\hline 2 Gg_rs150605 & 98 chr7_102430 & 5.85 nonsex \\
\hline 2 Gg_rs150605 & 98 chr7_281900 & 5.06 nonsex \\
\hline 2 Gg_rs150605 & 98 chr8_210820 & 5.19 nonsex \\
\hline 2 Gg_rs150605 & 98 chr8_218790 & 4.88 nonsex \\
\hline 2 Gg_rs150605 & 98 chr8_222610 & 5.48 nonsex \\
\hline 2 Gg_rs150605 & 98 chr10_13863। & 5.22 nonsex \\
\hline 2 Gg_rs150605 & 98 chr11_23920 & 5.72 nonsex \\
\hline 2 Gg_rs150605 & 98 chr11_24390। & 6.03 nonsex \\
\hline 2 Gg_rs150605 & 98 chr12_10625। & 6.04 nonsex \\
\hline 2 Gg_rs150605 & 98 chr13_12812 & 6.03 nonsex \\
\hline 2 Gg_rs150605 & 98 chr14_73400 & 5.99 nonsex \\
\hline 2 Gg_rs150605 & 98 chr14_76810 & 4.64 nonsex \\
\hline 2 Gg_rs150605 & 98 chr14_11991। & 5.34 nonsex \\
\hline 2 Gg_rs150605 & 98 chr15_71760 & 7.21 nonsex \\
\hline 2 Gg_rs150605 & 98 chr17_79410 & 5.81 nonsex \\
\hline 2 Gg_rs150605 & 98 chr18_22650 & 6.69 nonsex \\
\hline 2 Gg_rs150605 & 98 chr18_48740 & 5.78 nonsex \\
\hline 2 Gg_rs150605 & 98 chr19_22150 & 5.36 nonsex \\
\hline 2 Gg_rs150605. & 98 chr19_49240 & 6.28 nonsex \\
\hline 2 Gg_rs150605 & 98 chr21_37090 & 5.98 nonsex \\
\hline 2 Gg_rs150605 & 98 chr21_58550 & 4.85 nonsex \\
\hline 2 Gg_rs150605 & 98 chr21_62590 & 6.29 nonsex \\
\hline 2 Gg_rs150605 & 98 chr23_29130 & 6.25 nonsex \\
\hline 2 Gg_rs150605 & 98 chr24_47870 & 5.5 nonsex \\
\hline 2 Gg_rs150605 & 98 chr24_49860 & 6.1 nonsex \\
\hline 2 Gg_rs150605 & 98 chrZ_204300। & 5.65 nonsex \\
\hline 2 Gg_rs150605 & 98 chr1_567270 & 5.08 nonsex \\
\hline 2 Gg_rs150605 & 98 chr1_664810 & 5.59 nonsex \\
\hline 2 Gg_rs150605 & 98 chrZ_488920। & 6.15 nonsex \\
\hline 2 Gg_rs150605 & 98 chr1_111577| & 5.38 nonsex \\
\hline 2 Gg_rs150605 & 98 chr1_119788 & 4.81 nonsex \\
\hline 2 Gg_rs150605 & 98 chr1_127533। & 5.4 nonsex \\
\hline 2 Gg_rs150605. & 98 chr1_180309| & 4.53 nonsex \\
\hline 2 Gg_rs150605 & 98 chr1_193515। & 5.38 nonsex \\
\hline
\end{tabular}




\begin{tabular}{|c|c|c|}
\hline 2 Gg_rs150605 & 98 chr1_196987। & 6.14 nonsex \\
\hline 2 Gg_rs150605 & 98 chr2_201470 & 6.12 nonsex \\
\hline 2 Gg_rs150605 & 98 chr2_729670 & 5.57 nonsex \\
\hline 2 Gg_rs150605 & 98 chr2_812150 & 6.66 nonsex \\
\hline 2 Gg_rs150605 & 98 chr2_104150 & 5.59 nonsex \\
\hline 2 Gg_rs150605 & 98 chr2_111085 & 5.5 nonsex \\
\hline 2 Gg_rs150605 & 98 chr2_136699। & 6.6 nonsex \\
\hline 2 Gg_rs150605 & 98 chr2_149104! & 5.69 nonsex \\
\hline 2 Gg_rs150605 & 98 chr3_104370 & 5.84 nonsex \\
\hline 2 Gg_rs150605 & 98 chr3_306930। & 5.58 nonsex \\
\hline 2 Gg_rs150605 & 98 chr3_675190। & 5.51 nonsex \\
\hline 2 Gg_rs150605 & 98 chr3_958590। & 5.95 nonsex \\
\hline 2 Gg_rs150605 & 98 chr3_100631। & 5.41 nonsex \\
\hline 2 Gg_rs150605 & 98 chr3_104192 & 5.67 nonsex \\
\hline 2 Gg_rs150605 & 98 chr3_105382 & 5.56 nonsex \\
\hline 2 Gg_rs150605 & 98 chr3_108317। & 6 nonsex \\
\hline 2 Gg_rs150605 & 98 chr4_382900। & 5.38 nonsex \\
\hline 2 Gg_rs150605 & 99 chr2_440500 & 7.29 nonsex \\
\hline 2 Gg_rs150605 & 99 chr4_448170 & 5.83 nonsex \\
\hline 2 Gg_rs150605 & 100 chr2_150500 & 5.45 nonsex \\
\hline 2 Gg_rs150605 & 100 chr14_62340 & 5.9 nonsex \\
\hline 2 Gg_rs150605 & 100 chr15_50260 & 5.59 nonsex \\
\hline 2 Gg_rs150605 & 100 chr23_48050 & 5.14 nonsex \\
\hline 2 Gg_rs150605 & 100 chr2_263870 & 5.61 nonsex \\
\hline 2 Gg_rs150605. & 100 chr2_554680 & 5.31 nonsex \\
\hline 2 Gg_rs150605 & 100 chr2_135503। & 5.26 nonsex \\
\hline 2 Gg_rs150605. & 101 chr2_140580 & 4.83 nonsex \\
\hline 2 Gg_rs150605 & 101 chr4_890960। & 6.71 nonsex \\
\hline 2 Gg_rs150605 & 101 chr5_452460 & 5.57 nonsex \\
\hline 2 Gg_rs150605 & 101 chr17_15530। & 5.97 nonsex \\
\hline 2 Gg_rs150605 & 101 chr1_801990। & 5.74 nonsex \\
\hline 2 Gg_rs150605 & 101 chr1_149422 & 5.1 nonsex \\
\hline 2 Gg_rs150605. & 101 chr2_980690। & 5.11 nonsex \\
\hline 2 Gg_rs150605 & 101 chr3_180970 & 5.32 nonsex \\
\hline 2 Gg_rs150605 & 102 chr2_708100 & 4.3 nonsex \\
\hline 2 Gg_rs150605. & 102 chr2_172890 & 3.75 nonsex \\
\hline 2 Gg_rs150605. & 102 chr5_548720 & 6.46 nonsex \\
\hline 2 Gg_rs150605 & 102 chr1_196000 & 5.44 nonsex \\
\hline 2 Gg_rs150605 & 102 chr5_539140। & 4.9 nonsex \\
\hline 2 Gg_rs150605 & 102 chr9_283400। & 5.79 nonsex \\
\hline 2 Gg_rs150605 & 102 chr14_45300 & 6.41 nonsex \\
\hline 2 Gg_rs150605 & 102 chr15_63530 & 4.63 nonsex \\
\hline 2 Gg_rs150605. & 102 chrZ_2065701 & 6.18 nonsex \\
\hline
\end{tabular}




\begin{tabular}{|c|c|c|}
\hline 2 Gg_rs150605 & 102 chrZ_802960। & 5.75 nonsex \\
\hline 2 Gg_rs150605 & 102 chr3_107027। & 5.93 nonsex \\
\hline 2 Gg_rs150605 & 103 chr2_828600 & 5.4 nonsex \\
\hline 2 Gg_rs150605 & 103 chr2_962900। & 5.57 nonsex \\
\hline 2 Gg_rs150605 & 103 chr2_142630 & 4.14 nonsex \\
\hline 2 Gg_rs150605 & 103 chr11_78610 & 5.64 nonsex \\
\hline 2 Gg_rs150605 & 103 chr11_15657। & 5.21 nonsex \\
\hline 2 Gg_rs150605 & 103 chr21_58580। & 6.89 nonsex \\
\hline 2 Gg_rs150605 & 103 chr1_134963। & 5.49 nonsex \\
\hline 2 Gg_rs150605. & 103 chr1_155540 & 5.97 nonsex \\
\hline 2 Gg_rs150605 & 103 chr2_126164। & 4.83 nonsex \\
\hline 2 Gg_rs150605 & 103 chr3_430180 & 5.31 nonsex \\
\hline 2 Gg_rs150605 & 104 chr2_142470 & 6.92 sex \\
\hline 2 Gg_rs150605 & 104 chr9_224140। & 6.52 nonsex \\
\hline 2 Gg_rs150605 & 104 chr6_327170 & 5.82 nonsex \\
\hline 2 Gg_rs150605 & 104 chr24_82700। & 5.37 nonsex \\
\hline 2 Gg_rs150605 & 104 chrZ_397690। & 6.93 nonsex \\
\hline 2 Gg_rs150605 & 104 chr1_114139| & 6.75 nonsex \\
\hline 2 Gg_rs150605 & 104 chr3_199690 & 5.88 nonsex \\
\hline 2 Gg_rs150605 & 104 chr3_233580 & 5.72 nonsex \\
\hline 2 Gg_rs150605 & 104 chr4_118800 & 5.3 nonsex \\
\hline 2 Gg_rs150605 & 105 chr1_934400। & 5.3 nonsex \\
\hline 2 Gg_rs150605 & 105 chr4_422120। & 5.21 nonsex \\
\hline 2 Gg_rs150605 & 105 chr4_538420। & 5.89 nonsex \\
\hline 2 Gg_rs150605 & 105 chr4_883370। & 7.22 nonsex \\
\hline 2 Gg_rs150605. & 105 chr8_209850। & 5.78 nonsex \\
\hline 2 Gg_rs150605 & 105 chr11_17245। & 6.01 nonsex \\
\hline 2 Gg_rs150605 & 105 chr15_12937। & 5.57 nonsex \\
\hline 2 Gg_rs150605 & 105 chr1_832500। & 5.91 nonsex \\
\hline 2 Gg_rs150605 & 105 chr1_943450। & 6.53 nonsex \\
\hline 2 Gg_rs150605 & 105 chr2_485400 & 6.21 nonsex \\
\hline 2 Gg_rs150605 & 105 chr2_666250 & 6.89 nonsex \\
\hline 2 Gg_rs150605 & 105 chr2_745040 & 6.15 nonsex \\
\hline 2 Gg_rs150605 & 105 chr3_547400 & 5.88 nonsex \\
\hline 2 Gg_rs150605 & 105 chr3_103739! & 5.83 nonsex \\
\hline 2 Gg_rs150605 & 106 chr2_795300 & 8.41 sex \\
\hline 2 Gg_rs150605 & 106 chr2_126540 & 3.96 nonsex \\
\hline 2 Gg_rs150605 & 106 chr2_142560 & 4.26 nonsex \\
\hline 2 Gg_rs150605 & 106 chr1_428860 & 5.1 nonsex \\
\hline 2 Gg_rs150605 & 106 chr4_900360। & 6.15 nonsex \\
\hline 2 Gg_rs150605 & 106 chr9_176020 & 6.11 nonsex \\
\hline 2 Gg_rs150605 & 106 chr1_548620 & 5.21 nonsex \\
\hline 2 Gg_rs150605 & 106 chr1_163075। & 5.48 nonsex \\
\hline
\end{tabular}




\begin{tabular}{|c|c|c|c|}
\hline 2 Gg_rs150605. & 106 chr2_397800 & 6.51 nonsex & trans \\
\hline 2 Gg_rs150605. & 106 chr2_148271। & 5.05 nonsex & trans \\
\hline 2 Gg_rs150605 & 107 chr2_800600 & 6.14 nonsex & cis \\
\hline 2 Gg_rs150605 & 107 chr2_553200 & 4.44 nonsex & cis \\
\hline 2 Gg_rs150605. & 107 chr2_998400। & 4.41 nonsex & cis \\
\hline 2 Gg_rs150605. & 107 chr2_172810 & 4.51 nonsex & cis \\
\hline 2 Gg_rs150605. & 107 chr4_417270 & $6.88 \mathrm{sex}$ & trans \\
\hline 2 Gg_rs150605 & 107 chr21_60760। & 8.29 sex & trans \\
\hline 2 Gg_rs150605. & 107 chr5_592870 & 7.08 nonsex & rans \\
\hline 2 Gg_rs150605. & 107 chr10_27050। & 6.3 nonsex & ans \\
\hline 2 Gg_rs150605 & 107 chr18_39530। & 5.99 nonsex & trans \\
\hline 2 Gg_rs150605 & 107 chr19_58260। & 6.06 nonsex & trans \\
\hline 2 Gg_rs150605. & 107 chr1_107890 & 5.64 nonsex & trans \\
\hline 2 Gg_rs150605 & 107 chr2_387940। & 6.13 nonsex & trans \\
\hline 2 Gg_rs150605. & 108 chr2_780700। & 5.95 nonsex & cis \\
\hline 2 Gg_rs150605 & 108 chr2_808600 & 6.1 nonsex & cis \\
\hline 2 Gg_rs150605. & 108 chr2_130500। & 4.79 nonsex & cis \\
\hline 2 Gg_rs150605 & 108 chr1_529430 & 7.54 sex & trans \\
\hline 2 Gg_rs150605. & 108 chr4_884280 & 6.11 nonsex & rans \\
\hline 2 Gg_rs150605 & 108 chr6_237380 & 5.92 nonsex & trans \\
\hline 2 Gg_rs150605 & 108 chr8_292130। & 6.11 nonsex & trans \\
\hline 2 Gg_rs150605. & 108 chr23_88700। & 6.43 nonsex & trans \\
\hline 2 Gg_rs150605 & 108 chr1_847950 & 6.16 nonsex & trans \\
\hline 2 Gg_rs150605 & 108 chr4_398200। & 5.01 nonsex & trans \\
\hline 2 Gg_rs150605. & 109 chr2_788800 & 8.21 sex & cis \\
\hline 2 Gg_rs150605. & 109 chr2_649200 & 4.89 nonsex & cis \\
\hline 2 Gg_rs150605. & 109 chr2_773100 & 4.45 nonsex & cis \\
\hline 2 Gg_rs150605. & 109 chr1_176500 & 6.14 nonsex & trans \\
\hline 2 Gg_rs150605 & 109 chr1_231780 & 6.24 nonsex & trans \\
\hline 2 Gg_rs150605 & 109 chr2_781190। & 6.86 nonsex & trans \\
\hline 2 Gg_rs150605 & 110 chr3_952540 & 7.77 nonsex & trans \\
\hline 2 Gg_rs150605. & 111 chr2_160620 & 6.29 nonsex & cis \\
\hline 2 Gg_rs150605. & 111 chr22_13810 & $8.3 \operatorname{sex}$ & trans \\
\hline 2 Gg_rs150605. & 111 chr28_14610 & 7.9 nonsex & trans \\
\hline 2 Gg_rs150605. & 111 chr14_12224! & 6.13 nonsex & trans \\
\hline 2 Gg_rs150605. & 112 chr2_788700 & 4.96 nonsex & cis \\
\hline 2 Gg_rs150605 & 112 chr2_847400 & 5.6 nonsex & cis \\
\hline 2 Gg_rs150605 & 113 chr2_618960। & 6.22 nonsex & trans \\
\hline 2 Gg_rs141391. & 125 chr2_148360 & 5.37 nonsex & cis \\
\hline 2 Gg_rs150638. & 126 chr2_936500। & 4.25 nonsex & cis \\
\hline 2 Gg_rs141391. & 128 chr2_127070 & 5.35 nonsex & cis \\
\hline 2 Gg_rs141391. & 129 chr2_104100 & 4.38 nonsex & cis \\
\hline 2 Gg_rs150638. & 132 chr2_104800 & 4.93 nonsex & cis \\
\hline
\end{tabular}




\begin{tabular}{|c|c|c|c|}
\hline 2 Gg_rs141391. & 133 chr2_121980! & 9.28 nonsex & cis \\
\hline 2 Gg_rs141391. & 134 chr2_103450 & 7.52 sex & cis \\
\hline 2 Gg_rs141391. & 137 chr2_105180 & 5.08 nonsex & cis \\
\hline 2 Gg_rs141392. & 137 chr2_131080 & 5.98 nonsex & cis \\
\hline 2 Gg_rs141391. & 138 chr2_990200। & 10.15 nonsex & cis \\
\hline 2 Gg_rs141392. & 138 chr2_953000। & 5.46 nonsex & cis \\
\hline 2 Gg_rs141391. & 139 chr2_129430। & 6.59 sex & cis \\
\hline 2 Gg_rs150638. & 139 chr2_238890 & 7.99 sex & trans \\
\hline 2 Gg_rs141391. & 141 chr2_105060 & 5.95 nonsex & cis \\
\hline 2 Gg_rs141392. & 142 chr4_469900। & 5.13 nonsex & trans \\
\hline 2 Gg_rs141392. & 144 chr2_103130 & $5.2 \mathrm{sex}$ & cis \\
\hline 2 Gg_rs141391. & 145 chr2_100440। & 6.91 nonsex & cis \\
\hline 2 Gg_rs141391. & 146 chr2_964300। & 18.73 nonsex & cis \\
\hline 2 Gg_rs141391. & 146 chr2_964500। & 10.72 nonsex & cis \\
\hline 2 Gg_rs141391. & 146 chr2_994200। & 4.06 nonsex & cis \\
\hline 2 Gg_rs141391. & 146 chr2_103700 & 8.38 sex & cis \\
\hline 2 Gg_rs141391. & 146 chr2_103910 & 6.41 nonsex & cis \\
\hline 2 Gg_rs141391. & 146 chr2_103920 & 5.63 nonsex & cis \\
\hline 2 Gg_rs141391. & 146 chr2_105120 & 5.12 nonsex & cis \\
\hline 2 Gg_rs141391. & 146 chr2_105380 & 5.35 sex & cis \\
\hline 2 Gg_rs141391. & 146 chr2_116250 & 7.56 sex & cis \\
\hline 2 Gg_rs141391. & 146 chr2_116260 & 5.49 sex & cis \\
\hline 2 Gg_rs141391. & 146 chr2_118230 & 6.77 nonsex & cis \\
\hline 2 Gg_rs141391. & 146 chr2_127570 & 6.85 nonsex & cis \\
\hline 2 Gg_rs141391. & 146 chr2_979500। & 4.6 nonsex & cis \\
\hline 2 Gg_rs141391. & 146 chr2_992800 & 4.64 nonsex & cis \\
\hline 2 Gg_rs141391. & 146 chr2_999400। & 4.5 nonsex & cis \\
\hline 2 Gg_rs141391. & 146 chr2_106590। & 3.94 nonsex & cis \\
\hline 2 Gg_rs141391. & 146 chr2_110390 & 4.07 nonsex & cis \\
\hline 2 Gg_rs141391. & 146 chrZ_1804401 & 7.16 sex & trans \\
\hline 2 Gg_rs141392. & 146 chr2_940000। & 7.42 nonsex & cis \\
\hline 2 Gg_rs141392. & 146 chr2_107370। & 5.78 nonsex & cis \\
\hline 2 Gg_rs141392. & 146 chr2_113450 & 3.87 nonsex & cis \\
\hline 2 Gg_rs141392. & 146 chr2_964100। & 12.83 nonsex & cis \\
\hline 2 Gg_rs141392. & 146 chr2_964200। & 19.56 sex & cis \\
\hline 2 Gg_rs141392. & 146 chr2_969100। & 4.96 nonsex & cis \\
\hline 2 Gg_rs141392. & 146 chr2_969400। & 4.78 nonsex & cis \\
\hline 2 Gg_rs141392. & 146 chr2_130550 & 5.05 nonsex & cis \\
\hline 2 Gg_rs141392. & 147 chr2_963900। & 9.12 sex & cis \\
\hline 2 Gg_rs141392. & 147 chr2_964000 & 6.96 nonsex & cis \\
\hline 2 Gg_rs150638. & 147 chr2_916800 & 8.75 nonsex & cis \\
\hline 2 Gg_rs150638. & 148 chr2_947500 & 5.46 sex & cis \\
\hline 2 Gg_rs150638. & 148 chr2_960700 & 9.36 nonsex & cis \\
\hline
\end{tabular}




\begin{tabular}{|c|c|c|c|}
\hline 2 Gg rs150638. & $148 \mathrm{chr} 2128490$ & 10.42 nonsex & cis \\
\hline 2 Gg_rs150638. & 148 chr2_128570 & 4.52 nonsex & cis \\
\hline 2 Gg_rs150638. & 148 chr2_131510 & 4.62 nonsex & cis \\
\hline 2 Gg_rs150638. & 149 chr2_961800 & 6.57 nonsex & cis \\
\hline 2 Gg_rs150638. & 149 chr2_113780 & 4.27 nonsex & cis \\
\hline 2 Gg_rs150638. & 150 chr2_130070 & 13.9 nonsex & cis \\
\hline 2 Gg_rs150638. & 150 chr2_150620 & $6.02 \mathrm{sex}$ & cis \\
\hline 2 Gg_rs150638. & 150 chr2_131710 & 4.78 nonsex & cis \\
\hline 2 Gg_rs150638. & 151 chr2_130990 & 3.54 nonsex & cis \\
\hline 2 Gg_rs150638. & 152 chr2_114220 & 11.32 nonsex & cis \\
\hline 2 Gg_rs150638. & 152 chr2_130400 & 7.04 nonsex & cis \\
\hline 2 Gg_rs150638. & 152 chr2_130540 & 10.89 nonsex & cis \\
\hline 2 Gg_rs150638. & 152 chr2_130290 & 4.92 nonsex & cis \\
\hline 2 Gg_rs150638. & 152 chr2_153710 & 4.63 nonsex & cis \\
\hline 2 Gg_rs150638. & 153 chr2_943200 & 6.83 nonsex & cis \\
\hline 2 Gg_rs141391. & 154 chrW_40370 & $9.28 \mathrm{sex}$ & trans \\
\hline 2 Gg_rs141392. & 154 chr2_940800 & 5.39 nonsex & cis \\
\hline 2 Gg_rs141392. & 154 chr2_118600 & 4.82 nonsex & cis \\
\hline 2 Gg_rs150638. & 154 chr2_148410 & 7.32 nonsex & cis \\
\hline 2 Gg_rs150638. & 154 chr2_818000 & $6.62 \operatorname{sex}$ & cis \\
\hline 2 Gg_rs150638. & 154 chr2_121890 & 5.04 nonsex & cis \\
\hline 2 Gg_rs150676. & 154 chr2_135090 & 4.66 nonsex & cis \\
\hline 2 Gg_rs141391. & 155 chr2_149670 & 6.07 sex & cis \\
\hline 2 Gg_rs141391. & 155 chr2_112090 & 5.8 nonsex & cis \\
\hline 2 Gg_rs150638. & 155 chr2_961900 & 6.32 nonsex & cis \\
\hline 2 Gg_rs150638. & 155 chr2_147800 & 4.28 nonsex & cis \\
\hline 2 Gg_rs150638. & 155 chr2_583400 & 8.39 sex & trans \\
\hline 2 Gg_rs150652 & 155 chr2_964900 & 5.37 sex & cis \\
\hline 2 Gg_rs150652 & 156 chr2_111190। & 6.13 nonsex & cis \\
\hline 2 Gg_rs150652 & 156 chr2_132550 & 5.62 nonsex & cis \\
\hline 2 Gg_rs150652 & 157 chr2_135080 & 4.81 nonsex & cis \\
\hline 2 Gg_rs150652 & 157 chr3_101351। & 10.43 sex & trans \\
\hline 2 Gg_rs150652 & 158 chr2_133240 & 8.45 nonsex & cis \\
\hline 2 Gg_rs150652 & 158 chr2_134450 & 5.05 nonsex & cis \\
\hline 2 Gg_rs150652 & 158 chr2_134720 & 6.41 nonsex & cis \\
\hline 2 Gg_rs150652 & 158 chr2_142070 & 11.69 nonsex & cis \\
\hline 2 Gg_rs150652 & 158 chr2_143390। & 11.36 nonsex & cis \\
\hline 2 Gg_rs150652 & 158 chr2_146320 & 5.53 nonsex & cis \\
\hline 2 Gg_rs150652 & 158 chr1_397730 & 8.02 sex & trans \\
\hline 2 Gg_rs150652 & 159 chr2_142060 & 8.3 nonsex & cis \\
\hline 2 Gg_rs150652 & 160 chr2_135640 & 5.68 sex & cis \\
\hline 2 Gg_rs150652 & 162 chr2_147180 & 4.07 nonsex & cis \\
\hline 2 Gg_rs150652 & 162 chr2_142210 & 4.4 nonsex & cis \\
\hline
\end{tabular}




\begin{tabular}{|c|c|c|c|}
\hline 2 Gg_rs150652 & 162 chr2_143980 & 4.3 nonsex & cis \\
\hline 2 Gg_rs150652 & 162 chrZ_5003901 & 6.67 sex & trans \\
\hline 2 Gg_rs150652 & 162 chr2_101667। & 7.34 sex & trans \\
\hline 2 Gg_rs150652 & 162 chr8_281700। & 5.45 nonsex & trans \\
\hline 2 Gg_rs150652 & 162 chr9_168740। & 6.4 nonsex & trans \\
\hline 2 Gg_rs150652 & 163 chr2_158420 & 5.32 sex & cis \\
\hline 2 Gg_rs150652 & 163 chr2_130780 & 4.65 nonsex & cis \\
\hline 2 Gg_rs150652 & 163 chr2_142120 & 4.85 nonsex & cis \\
\hline 2 Gg_rs150652 & 164 chr2_144670 & 5.29 nonsex & cis \\
\hline 2 Gg_rs150652 & 164 chr2_970800 & 4.55 nonsex & cis \\
\hline 2 Gg_rs150652 & 164 chr2_143150 & 4.26 nonsex & cis \\
\hline 2 Gg_rs150652 & 164 chr2_143370 & 2.88 nonsex & cis \\
\hline 2 Gg_rs150652 & 164 chr3_786000। & 7.72 sex & trans \\
\hline 2 Gg_rs150652 & 165 chr2_151200 & 4.12 nonsex & cis \\
\hline 2 Gg_rs150652 & 166 chr2_146070 & 6.11 nonsex & cis \\
\hline 2 Gg_rs150652 & 166 chr2_163980 & 6.3 nonsex & cis \\
\hline 2 Gg_rs150652 & 166 chr2_147400 & 5.23 nonsex & cis \\
\hline 2 Gg_rs150652 & 166 chr1_134520 & 8.28 sex & trans \\
\hline 2 Gg_rs150652 & 167 chr2_164130। & 5.38 nonsex & cis \\
\hline 2 Gg_rs150652 & 168 chr3_372200। & 6.27 nonsex & trans \\
\hline 2 Gg_rs150652 & 169 chr2_146060 & 6.59 nonsex & cis \\
\hline 2 Gg_rs150652 & 169 chr2_170670 & 6.11 nonsex & cis \\
\hline 2 Gg_rs150652 & 170 chr2_159590 & 7.61 sex & cis \\
\hline 2 Gg_rs150652 & 170 chr2_171640 & 6.49 nonsex & cis \\
\hline 2 Gg_rs150652 & 170 chr2_151370 & 5.27 nonsex & cis \\
\hline 2 Gg_rs150652 & 170 chr2_151900 & 5.17 nonsex & cis \\
\hline 2 Gg_rs150676. & 171 chr3_865030। & 6.7 nonsex & trans \\
\hline 2 Gg_rs150676. & 172 chr2_167200 & 7.95 sex & cis \\
\hline 2 Gg_rs150676. & 173 chr2_196310 & 6.02 sex & cis \\
\hline 2 Gg_rs150676. & 173 chr2_155400 & 4.57 nonsex & cis \\
\hline 2 Gg_rs150676. & 173 chr2_163370 & 6.65 nonsex & cis \\
\hline 2 Gg_rs150676. & 174 chr2_163590 & 6.85 sex & cis \\
\hline 2 Gg_rs150676. & 174 chr2_155610 & 4.73 nonsex & cis \\
\hline 2 Gg_rs150676. & 174 chr1_181192 & 6.95 nonsex & trans \\
\hline 2 Gg_rs150676. & 175 chr2_103170 & 5.1 nonsex & cis \\
\hline 2 Gg_rs150676. & 175 chr2_145700 & 4.86 nonsex & cis \\
\hline 2 Gg_rs150676. & 175 chrZ_6411201 & 8.62 sex & trans \\
\hline 2 Gg_rs150676. & 176 chr2_156270 & 6.42 nonsex & cis \\
\hline 2 Gg_rs150676. & 176 chr2_170680 & 6.71 sex & cis \\
\hline 2 Gg_rs150676. & 176 chr2_148460 & 5.59 nonsex & cis \\
\hline 2 Gg_rs150676. & 176 chr1_120024! & 6.58 nonsex & trans \\
\hline 2 Gg_rs150676. & 177 chr2_159090। & 4.44 nonsex & cis \\
\hline 2 Gg_rs150676. & 177 chr2_164340 & 4.81 nonsex & cis \\
\hline
\end{tabular}




\begin{tabular}{|c|c|c|c|}
\hline 2 Gg_rs150676. & 178 chr2_156280 & 7.04 nonsex & cis \\
\hline 2 Gg_rs150676. & 178 chr2_123320 & 5.21 nonsex & cis \\
\hline 2 Gg_rs150676. & 178 chr2_170720 & 3.08 nonsex & cis \\
\hline 2 Gg_rs150676. & 179 chr2_167560 & 9.51 nonsex & cis \\
\hline 2 Gg_rs150676. & 179 chr2_157440 & 3.63 nonsex & cis \\
\hline 2 Gg_rs150676. & 179 chr2_190020 & 4.76 nonsex & cis \\
\hline 2 Gg_rs150676. & 180 chr2_180790 & $2.98 \mathrm{sex}$ & cis \\
\hline 2 Gg_rs150676. & 180 chr2_168540 & 3.52 nonsex & cis \\
\hline 2 Gg_rs150676. & 180 chrZ_3934101 & 7.77 sex & trans \\
\hline 2 Gg_rs150676. & 180 chrZ_1847701 & 6.59 nonsex & trans \\
\hline 2 Gg_rs150676. & 182 chr2_165040 & 4.58 nonsex & cis \\
\hline 2 Gg_rs150676. & 183 chr2_158960। & 4.64 nonsex & cis \\
\hline 2 Gg_rs150676. & 183 chr3_467140। & 9.22 sex & trans \\
\hline 2 Gg_rs150676. & 184 chr2_162340 & 5.33 nonsex & cis \\
\hline 2 Gg_rs150676. & 185 chr2_177150 & 4.34 nonsex & cis \\
\hline 2 Gg_rs150676. & 189 chr2_177210 & 6.04 nonsex & cis \\
\hline 2 Gg_rs150692 & 196 chr2_181110 & 5.42 sex & cis \\
\hline 2 Gg_rs150692 & 196 chr2_186530 & 5.08 nonsex & cis \\
\hline 2 Gg_rs150692 & 203 chr2_182580 & 7.83 nonsex & cis \\
\hline 2 Gg_rs150692 & 203 chr2_182590। & 5.9 nonsex & cis \\
\hline 2 Gg_rs150692 & 203 chr2_190750 & 3.99 sex & cis \\
\hline 2 Gg_rs150692 & 203 chr2_193810 & 4.69 nonsex & cis \\
\hline 2 Gg_rs150692 & 203 chr11_19452। & 8.73 sex & trans \\
\hline 2 Gg_rs150692 & 203 chr13_18567। & 8.34 sex & trans \\
\hline 2 Gg_rs150692 & 203 chr22_15530। & 9.96 sex & trans \\
\hline 2 Gg_rs150692 & 203 chr5_224030। & 5.82 nonsex & trans \\
\hline 2 Gg_rs150692 & 203 chr32_60500। & 7 nonsex & trans \\
\hline 2 Gg_rs150700. & 203 chr28_33180। & $10.16 \mathrm{sex}$ & trans \\
\hline 2 Gg_rs150700. & 204 chr2_181350 & 3.91 nonsex & cis \\
\hline 2 Gg_rs150692 & 208 chr2_160530 & 5.53 sex & cis \\
\hline 2 Gg_rs150692 & 208 chr2_182600 & 8.21 nonsex & cis \\
\hline 2 Gg_rs150700. & 209 chr2_158770 & 6.1 nonsex & cis \\
\hline 2 Gg_rs150700. & 209 chr2_174370 & 4.46 sex & cis \\
\hline 2 Gg_rs150700. & 210 chr2_171540 & 5.37 nonsex & cis \\
\hline 2 Gg_rs150676. & 212 chr2_172020 & 7.51 nonsex & cis \\
\hline 2 snp.2.314.16! & 217 chr2_220690 & 6.32 nonsex & cis \\
\hline 2 snp.2.314.16! & 218 chr2_211380 & 4.66 nonsex & cis \\
\hline 2 snp.2.314.16! & 218 chr2_211870 & 4.49 nonsex & cis \\
\hline 2 snp.2.314.16! & 219 chr2_199980 & 4.36 nonsex & cis \\
\hline 2 snp.2.314.16! & 220 chrW_43570 & 10.36 sex & trans \\
\hline 2 snp.2.314.16! & 222 chr2_216030 & 8.75 nonsex & cis \\
\hline 2 snp.2.314.16! & 222 chr2_219010 & 5.33 nonsex & cis \\
\hline 2 snp.2.314.16! & 225 chr2_218630। & 4.75 sex & cis \\
\hline
\end{tabular}




\begin{tabular}{|c|c|c|c|}
\hline 2 snp.2.314.16! & 225 chrZ_6491501 & 8.07 sex & trans \\
\hline 2 snp.2.314.16! & 229 chr3_675310 & $8.3 \mathrm{sex}$ & trans \\
\hline 2 snp.2.314.16! & 230 chr2_219740 & $4.5 \mathrm{sex}$ & cis \\
\hline 2 snp.2.314.16! & 232 chr2_210640 & 13.76 nonsex & cis \\
\hline 2 snp.2.314.16! & 232 chr2_212710 & 4.91 nonsex & cis \\
\hline 2 snp.2.314.16! & 232 chrW_16610 & 9.17 sex & trans \\
\hline 2 snp.2.314.16! & 238 chr2_215230। & 6.19 nonsex & cis \\
\hline 2 snp.2.314.16! & 238 chr2_240120। & 5.93 nonsex & cis \\
\hline 2 snp.2.314.16! & 241 chr2_214470 & 4.73 nonsex & cis \\
\hline 2 snp.2.314.16! & 247 chr2_239330। & 5.82 nonsex & cis \\
\hline 2 X2_23979784 & 252 chr2_225640। & 4.38 nonsex & cis \\
\hline 2 X2_23979784 & 258 chr2_260590। & 5.88 nonsex & cis \\
\hline 2 Gg_rs150782. & 260 chr2_242450। & 4.98 nonsex & cis \\
\hline 2 Gg_rs150782. & 261 chr2_267760। & 6.39 nonsex & cis \\
\hline 2 X2_23979784 & 262 chr2_310960। & 4.59 nonsex & cis \\
\hline 2 X2_23979784 & 267 chr2_310120 & 5.49 nonsex & cis \\
\hline 2 X2_23979784 & 267 chr2_235280। & 4.39 nonsex & cis \\
\hline 2 X2_23979784 & 267 chr2_239010। & 4.56 nonsex & cis \\
\hline 2 X2_23979784 & 267 chr2_310400। & 3.32 nonsex & cis \\
\hline 2 X2_23979784 & 268 chr2_223920। & 5.14 nonsex & cis \\
\hline 2 X2_23979784 & 268 chr2_291490। & 4.6 nonsex & cis \\
\hline 2 X2_23979784 & 268 chr3_370280 & 5.84 nonsex & trans \\
\hline 2 X2_23979784 & 269 chr2_310700 & 6.2 nonsex & cis \\
\hline 2 X2_23979784 & 269 chr2_310630। & 3.66 nonsex & cis \\
\hline 2 X2_23979784 & 272 chr2_310380 & 6.24 nonsex & cis \\
\hline 2 X2_23979784 & 273 chr2_310350। & 5.1 nonsex & cis \\
\hline 2 X2_23979784 & 273 chr2_310390। & 3.54 nonsex & cis \\
\hline 2 X2_23979784 & 273 chr2_310720 & 4.55 nonsex & cis \\
\hline 2 X2_23979784 & 273 chr33_66700। & 5.23 nonsex & trans \\
\hline 2 X2_23979784 & 274 chr2_279640। & 4.64 nonsex & cis \\
\hline 2 X2_23979784 & 275 chr19_10183। & 5.81 nonsex & trans \\
\hline 2 X2_23979784 & 276 chr2_231930 & 5.67 sex & cis \\
\hline 2 X2_23979784 & 276 chr2_310370। & 4.34 nonsex & cis \\
\hline 2 X2_23979784 & 276 chr2_310680 & 3.67 nonsex & cis \\
\hline 2 X2_23979784 & 276 chr2_310320 & 3.58 nonsex & cis \\
\hline 2 X2_23979784 & 277 chr2_311240। & 7.45 nonsex & cis \\
\hline 2 X2_23979784 & 277 chr2_219660। & 4.04 nonsex & cis \\
\hline 2 X2_23979784 & 277 chr2_292430। & 3.64 nonsex & cis \\
\hline 2 X2_23979784 & 278 chr2_310690। & 3.48 nonsex & cis \\
\hline 2 X2_23979784 & 278 chr2_20000 & 4.96 nonsex & trans \\
\hline 2 X2_23979784 & 279 chr2_310130 & 3.41 nonsex & cis \\
\hline 2 X2_23979784 & 280 chr2_304390। & 6.68 nonsex & cis \\
\hline 2 X2_23979784 & 280 chr2_310150। & 6.48 nonsex & cis \\
\hline
\end{tabular}




\begin{tabular}{|c|c|c|c|}
\hline 2 X2_23979784 & 280 chr2_310670 & 6.3 nonsex & cis \\
\hline 2 X2_23979784 & 280 chr2_310900 & 3.91 nonsex & cis \\
\hline 2 X2_23979784 & 280 chr2_310920। & 5.09 nonsex & cis \\
\hline 2 X2_23979784 & 280 chr2_310930। & 6.2 nonsex & cis \\
\hline 2 X2_23979784 & 280 chr2_310950 & 4.45 nonsex & cis \\
\hline 2 X2_23979784 & 280 chr2_311230। & 7.28 nonsex & cis \\
\hline 2 X2_23979784 & 280 chr2_301290। & 3.48 nonsex & cis \\
\hline 2 X2_23979784 & 280 chr2_310140 & 3.52 nonsex & cis \\
\hline 2 X2_23979784 & 280 chr2_310160। & 3.67 nonsex & cis \\
\hline 2 X2_23979784 & 280 chr2_310180 & 3.56 nonsex & cis \\
\hline 2 X2_23979784 & 280 chr2_310360। & 3.53 nonsex & cis \\
\hline 2 X2_23979784 & 280 chr2_310640 & 3.52 nonsex & cis \\
\hline 2 X2_23979784 & 280 chr2_310660 & 3.77 nonsex & cis \\
\hline 2 X2_23979784 & 280 chr2_310880 & 3.63 nonsex & cis \\
\hline 2 X2_23979784 & 280 chr2_310940। & 3.64 nonsex & cis \\
\hline 2 X2_23979784 & 280 chr2_311200 & 3.7 nonsex & cis \\
\hline 2 X2_23979784 & 280 chr2_311220। & 3.85 nonsex & cis \\
\hline 2 X2_23979784 & 280 chr33_66670। & 6.33 nonsex & trans \\
\hline 2 X2_23979784 & 280 chr32_36400। & 5.39 nonsex & trans \\
\hline 2 X2_23979784 & 280 chr2_19000 & 5.32 nonsex & trans \\
\hline 2 X2_23979784 & 281 chr2_309920। & 3.83 nonsex & cis \\
\hline 2 X2_23979784 & 281 chr2_310870 & 3.49 nonsex & cis \\
\hline 2 X2_23979784 & 282 chr2_311210 & 3.55 nonsex & cis \\
\hline 2 X2_2601738C & 282 chr2_310170 & 3.51 nonsex & cis \\
\hline 2 X2_2601738C & 282 chr2_310650 & 3.24 nonsex & cis \\
\hline 2 X2_2601738C & 284 chr2_257210 & 4.89 nonsex & cis \\
\hline 2 X2_2601738C & 284 chr2_272180 & 4.36 nonsex & cis \\
\hline 2 X2_2601738C & 284 chr2_304960। & 4.32 nonsex & cis \\
\hline 2 X2_2601738C & 285 chr2_260200। & 6.7 nonsex & cis \\
\hline 2 X2_2601738C & 285 chr2_264940। & 5.32 nonsex & cis \\
\hline 2 Gg_rs150782. & 286 chr2_238420 & 5.08 nonsex & cis \\
\hline 2 Gg_rs150782. & 286 chr2_258110 & 6.03 nonsex & cis \\
\hline 2 Gg_rs150782. & $288 c h r 2 \_250760$ & 3.77 sex & cis \\
\hline 2 Gg_rs150782. & $288 c h r 2 \_269600$ & 5.49 nonsex & cis \\
\hline 2 Gg_rs150782. & 288 chr2_236890। & 3.74 nonsex & cis \\
\hline 2 Gg_rs150782. & 288 chr2_239740। & 4.63 nonsex & cis \\
\hline 2 Gg_rs150782. & 288 chr2_244940। & 4.13 nonsex & cis \\
\hline 2 Gg_rs150782. & 288 chr2_261270 & 4.37 nonsex & cis \\
\hline 2 Gg_rs150782. & 288 chr2_279590। & 4.22 nonsex & cis \\
\hline 2 Gg_rs150782. & 288 chr2_285010। & 4.23 nonsex & cis \\
\hline 2 Gg_rs150782. & 288 chr2_309640। & 4.61 nonsex & cis \\
\hline 2 Gg_rs150782. & 288 chr2_313710 & 5.08 nonsex & cis \\
\hline 2 Gg_rs150782. & 288 chrZ_2177701 & 7.6 sex & trans \\
\hline
\end{tabular}




\begin{tabular}{|c|c|c|}
\hline 2 Gg_rs150782. & 289 chr2_241470। & 3.79 nonsex \\
\hline 2 Gg_rs150782. & 290 chr2_279080। & 3.62 nonsex \\
\hline 2 Gg_rs150782. & 290 chr2_106601। & 5.98 nonsex \\
\hline 2 Gg_rs150782. & 291 chr2_313790। & 7.33 nonsex \\
\hline 2 Gg_rs150782. & 292 chr2_312550। & 4.48 nonsex \\
\hline 2 Gg_rs150782. & 293 chr2_285410 & 7.78 nonsex \\
\hline 2 Gg_rs150782. & 294 chr2_289360। & 5.26 nonsex \\
\hline 2 Gg_rs150782. & 294 chr2_307250। & 8.61 nonsex \\
\hline 2 Gg_rs150782. & 294 chr2_288120। & 3.99 nonsex \\
\hline 2 Gg_rs150782. & 294 chr2_307260। & 4.17 nonsex \\
\hline 2 Gg_rs150782. & 296 chr2_279650। & 5.42 nonsex \\
\hline 2 Gg_rs150782. & 296 chr2_240450। & 4.05 nonsex \\
\hline 2 X2_2601738C & 298 chr2_254730। & 13.47 nonsex \\
\hline 2 Gg_rs150782. & 298 chr2_241480 & 5.15 nonsex \\
\hline 2 Gg_rs150782. & 298 chr2_291960। & 4.43 nonsex \\
\hline 2 Gg_rs150782. & 299 chr2_279680। & 3.65 nonsex \\
\hline 2 X2_2601738C & 301 chr2_284500। & 9.02 nonsex \\
\hline 2 Gg_rs150782. & 301 chr2_280500। & 5.87 nonsex \\
\hline 2 Gg_rs150782. & 301 chr2_297360। & 7.52 nonsex \\
\hline 2 Gg_rs150782. & 301 chr2_279660। & 4.52 nonsex \\
\hline 2 Gg_rs150782. & 303 chr2_285590। & 7.73 nonsex \\
\hline 2 Gg_rs150782. & 304 chr2_280490। & 9.33 nonsex \\
\hline 2 Gg_rs150782. & 305 chr2_269880 & 5.66 nonsex \\
\hline 2 Gg_rs150782. & 309 chr2_316290। & 6.7 sex \\
\hline 2 Gg_rs150782. & 310 chr2_280510 & 5.85 nonsex \\
\hline 2 X2_23979784 & 311 chr2_288320। & 3.52 nonsex \\
\hline 2 Gg_rs150782. & 311 chr2_239940। & 5.93 nonsex \\
\hline 2 Gg_rs150782. & 313 chr2_272090। & 6.41 nonsex \\
\hline 2 Gg_rs150782. & 313 chr2_293190। & 8.11 nonsex \\
\hline 2 snp.5.86.557? & 321 chr2_318010। & 6.52 nonsex \\
\hline 2 snp.5.86.557? & 330 chr3_104485। & 9.74 sex \\
\hline 2 snp.5.86.557? & 331 chr2_330640। & 7.85 nonsex \\
\hline 2 snp.5.86.557? & 331 chr2_293200। & 5.91 nonsex \\
\hline 2 snp.5.86.557? & 334 chr2_345940। & $6.4 \operatorname{sex}$ \\
\hline 2 snp.5.86.557? & 334 chr10_74630। & 6.46 nonsex \\
\hline 2 snp.5.86.557? & 338 chr1_824000 & 8.09 sex \\
\hline 2 snp.5.86.557? & 338 chr1_825000 & 11.03 sex \\
\hline 2 snp.5.86.557 & 338 chr3_952790। & 9.09 sex \\
\hline 2 snp.5.86.557? & 341 chr2_330510। & 4.61 nonsex \\
\hline 2 snp.5.86.557؛ & 341 chr28_14970। & 7.99 sex \\
\hline 2 snp.5.86.557 & 342 chr27_35800 & 7.8 sex \\
\hline 2 snp.5.86.557? & 347 chr2_335320। & 4.13 nonsex \\
\hline 2 snp.5.86.557亿 & 347 chr1_148742। & 6.75 nonsex \\
\hline
\end{tabular}




\begin{tabular}{|c|c|c|c|}
\hline 2 snp.5.86.557? & 348 chr2_338380। & $7.91 \mathrm{sex}$ & cis \\
\hline 2 snp.5.86.557؛ & 348 chr2_338830। & 4.91 nonsex & cis \\
\hline 2 snp.5.86.557? & 348 chr2_340000 & 5.09 nonsex & cis \\
\hline 2 snp.5.86.557? & 348 chr2_343340 & 4.96 nonsex & cis \\
\hline 2 snp.5.86.557? & 348 chr2_331120। & 4.48 nonsex & cis \\
\hline 2 snp.5.86.557? & 348 chr2_331910 & 4.21 nonsex & cis \\
\hline 2 snp.5.86.557؛ & 348 chr2_336820। & 3.96 nonsex & cis \\
\hline 2 snp.5.86.557? & 348 chr2_365000। & 5.02 nonsex & cis \\
\hline 2 snp.5.86.557؛ & 348 chr2_365880 & 4.95 nonsex & cis \\
\hline 2 snp.5.86.557 & 348 chr2_419360। & 4.09 nonsex & cis \\
\hline 2 snp.5.86.557؛ & 348 chr28_97100। & 9.1 sex & trans \\
\hline 2 snp.5.86.557? & 348 chr1_386440। & 5.87 nonsex & trans \\
\hline 2 snp.5.86.557? & 348 chr1_103952। & 5.32 nonsex & trans \\
\hline 2 snp.5.86.557؛ & 353 chr2_332860। & $7.21 \mathrm{sex}$ & cis \\
\hline 2 snp.5.86.557 & 353 chr2_335620। & 4.27 nonsex & cis \\
\hline 2 snp.5.86.557? & 353 chr2_356200। & 3.66 nonsex & cis \\
\hline 2 snp.5.86.557? & 358 chr2_340780 & 4.82 nonsex & cis \\
\hline 2 snp.5.86.557? & 359 chr16_67100। & 7.94 sex & trans \\
\hline 2 snp.5.86.557? & 360 chr2_342100। & 4.48 nonsex & cis \\
\hline 2 snp.5.86.557? & 362 chr2_330560। & 5.23 nonsex & cis \\
\hline 2 snp.5.86.557? & 365 chr2_364720 & 4.52 nonsex & cis \\
\hline 2 snp.5.86.557? & 365 chr30_15570 & 9.84 sex & trans \\
\hline 2 snp.5.86.557? & 367 chr25_13460 & 7.55 sex & trans \\
\hline 2 snp.5.86.557? & 368 chr2_400730 & 4.38 sex & cis \\
\hline 2 snp.5.86.557? & 368 chr14_22000। & 7.86 sex & trans \\
\hline 2 snp.5.242.10t & 408 chr2_387280 & 4.55 nonsex & cis \\
\hline 2 X2_40263015 & 423 chr2_420040। & 3.85 nonsex & cis \\
\hline 2 X2_40263015 & 427 chr2_411610 & 4.5 nonsex & cis \\
\hline 2 X2_40263015 & 432 chr2_413560। & 4.92 nonsex & cis \\
\hline 2 X2_40263015 & 433 chr2_403820। & 5.43 sex & cis \\
\hline 2 X2_40263015 & 433 chr2_409690। & 5.89 nonsex & cis \\
\hline 2 X2_40263015 & 434 chr2_405900। & 4.6 nonsex & cis \\
\hline $2 \times 2 \_40263015$ & 434 chr2_406050। & 4.47 nonsex & cis \\
\hline 2 X2_40263015 & 435 chr2_405930। & 7.89 nonsex & cis \\
\hline 2 X2_40263015 & 435 chr2_406120। & 7.98 nonsex & cis \\
\hline 2 X2_40263015 & 435 chr2_405980 & 5.76 nonsex & cis \\
\hline $2 \times 2 \_41000405$ & 436 chr2_405950। & 10.88 nonsex & cis \\
\hline $2 \times 2 \_41000405$ & 437 chr2_405920। & 9.63 nonsex & cis \\
\hline $2 \times 2 \_41000405$ & 437 chr2_405940। & 10.54 nonsex & cis \\
\hline $2 \times 2 \_41000405$ & 437 chr2_406040। & 19.77 nonsex & cis \\
\hline $2 \times 2 \_41000405$ & 438 chr2_405990। & 13.5 nonsex & cis \\
\hline $2 \times 2 \_41000405$ & 438 chr2_406020। & 26.04 nonsex & cis \\
\hline $2 \times 2 \_41000405$ & 438 chr3_313370। & 5.74 sex & trans \\
\hline
\end{tabular}




\begin{tabular}{|c|c|c|c|}
\hline $2 \times 2 \_41000405$ & 439 chr2_406030। & 25.16 nonsex & cis \\
\hline $2 \times 2 \_41000405$ & 439 chr2_416440। & $5.1 \mathrm{sex}$ & cis \\
\hline $2 \times 2 \_41000405$ & 440 chr2_406010 & 24.19 nonsex & cis \\
\hline 2 X2_41000405 & 440 chr2_406090। & 14.14 nonsex & cis \\
\hline $2 \times 2 \_41000405$ & 440 chr2_416390। & 6.76 sex & cis \\
\hline 2 X2_41000405 & 442 chr2_406000। & 15.1 nonsex & cis \\
\hline $2 \times 2 \_41000405$ & 443 chr2_406080 & 14.51 nonsex & cis \\
\hline 2 X2_41000405 & 443 chr2_409180 & 6.04 sex & cis \\
\hline $2 \times 2 \_41000405$ & 443 chr2_409700। & 19.16 nonsex & cis \\
\hline 2 X2_41000405 & 443 chr2_416400 & 8 sex & cis \\
\hline 2 X2_41000405 & 443 chr2_430920। & 6.37 sex & cis \\
\hline $2 \times 2 \_41000405$ & 443 chr2_440430। & 4.91 sex & cis \\
\hline $2 \times 2 \_41000405$ & 444 chr2_405910 & 12.88 nonsex & cis \\
\hline $2 \times 2 \_41000405$ & 444 chr2_408480 & 6.82 nonsex & cis \\
\hline 2 X2_41000405 & 444 chr2_414690 & 5.08 nonsex & cis \\
\hline 2 X2_41000405 & 445 chr2_405820। & 7.78 nonsex & cis \\
\hline $2 \times 2 \_41000405$ & 445 chr2_405870। & 7.6 nonsex & cis \\
\hline $2 \times 2 \_41000405$ & 445 chr2_406100। & 7.23 nonsex & cis \\
\hline $2 \times 2 \_41000405$ & 445 chr2_416410 & 7.41 nonsex & cis \\
\hline 2 X2_41000405 & 446 chr2_405860। & 11.59 nonsex & cis \\
\hline 2 X2_41000405 & 446 chr2_436900। & 5.43 nonsex & cis \\
\hline 2 X2_41000405 & 446 chr2_414700 & 5.17 nonsex & cis \\
\hline 2 X2_41000405 & 447 chr2_428030। & 5.34 nonsex & cis \\
\hline 2 Gg_rs150910. & 450 chr2_424310 & 6.07 nonsex & cis \\
\hline 2 Gg_rs150910. & 452 chr2_430900। & 6.39 nonsex & cis \\
\hline 2 Gg_rs150910. & 452 chr1_172959। & 6.37 nonsex & trans \\
\hline 2 Gg_rs150910. & 453 chr2_427770 & 6.11 nonsex & cis \\
\hline 2 Gg_rs150910. & 455 chr2_423910 & 6.56 nonsex & cis \\
\hline 2 Gg_rs150910. & 455 chr2_426430 & 4.53 nonsex & cis \\
\hline 2 Gg_rs150910. & 455 chr21_51730 & 8.54 nonsex & trans \\
\hline 2 Gg_rs150910. & 471 chr2_421250 & 7.08 nonsex & cis \\
\hline 2 Gg_rs150910. & 471 chr2_488060। & 4.55 nonsex & cis \\
\hline 2 Gg_rs150910. & 474 chr2_424290। & 4.92 nonsex & cis \\
\hline 2 Gg_rs150910. & 476 chr2_435470 & 7.71 nonsex & cis \\
\hline 2 Gg_rs150910. & 478 chr2_440900 & 6.74 nonsex & cis \\
\hline 2 Gg_rs150910. & 479 chr2_480110 & 5.4 nonsex & cis \\
\hline 2 Gg_rs150910. & 481 chr2_413540। & 5.32 nonsex & cis \\
\hline 2 Gg_rs150910. & 481 chr2_434190। & 4.55 nonsex & cis \\
\hline 2 Gg_rs150910. & 482 chr2_440170 & 5.11 nonsex & cis \\
\hline 2 Gg_rs150910. & 482 chr2_146057। & 6.99 nonsex & trans \\
\hline 2 X2_41000405 & 483 chr2_422790 & 6.18 nonsex & cis \\
\hline 2 Gg_rs150910. & 483 chr2_421720 & 5.55 nonsex & cis \\
\hline 2 Gg_rs150910. & 483 chr2_426830 & 5.89 nonsex & cis \\
\hline
\end{tabular}




\begin{tabular}{|c|c|c|c|}
\hline 2 Gg_rs150910. & 484 chr2_437790। & 6.49 nonsex & cis \\
\hline $2 \times 2 \_41000405$ & 485 chr2_416990। & 7.04 sex & cis \\
\hline 2 Gg_rs150910. & 485 chr2_421140 & 7.38 nonsex & cis \\
\hline 2 Gg_rs150910. & 487 chr2_435860। & 6.68 sex & cis \\
\hline 2 Gg_rs150910. & 487 chr2_435330। & 4.69 nonsex & cis \\
\hline 2 Gg_rs150910. & 488 chr2_421290। & 7.57 nonsex & cis \\
\hline 2 Gg_rs150910. & 489 chr2_423530। & 6.35 nonsex & cis \\
\hline 2 Gg_rs150944. & 489 chr2_465300। & 6.92 nonsex & cis \\
\hline 2 Gg_rs150944. & 490 chr2_603680 & 5.93 nonsex & cis \\
\hline 2 Gg_rs150944. & 494 chr2_442760। & 8.9 nonsex & cis \\
\hline 2 Gg_rs150944. & 494 chr2_423450। & 5.77 nonsex & cis \\
\hline 2 Gg_rs150944. & 495 chr2_438220। & 6.15 nonsex & cis \\
\hline 2 Gg_rs150944. & 497 chr2_432650। & 7.11 nonsex & cis \\
\hline 2 Gg_rs150944. & 498 chr2_458440। & 6.31 nonsex & cis \\
\hline 2 Gg_rs150944. & 499 chr3_963190। & 7.89 nonsex & trans \\
\hline 2 Gg_rs150944. & 500 chr2_451530। & 5.45 nonsex & cis \\
\hline 2 Gg_rs150944. & 502 chr2_467500। & 7.03 nonsex & cis \\
\hline 2 Gg_rs150944. & 502 chr2_443120। & 4.89 nonsex & cis \\
\hline 2 Gg_rs150944. & 503 chr2_433320। & 6.34 nonsex & cis \\
\hline 2 Gg_rs150944. & 506 chr2_421160। & 5.27 nonsex & cis \\
\hline 2 Gg_rs150944. & 507 chr2_459550। & $9.43 \operatorname{sex}$ & cis \\
\hline 2 Gg_rs150944. & 507 chr2_418900 & 5.07 nonsex & cis \\
\hline 2 Gg_rs150944. & 507 chr2_443920। & 4.93 nonsex & cis \\
\hline 2 Gg_rs150944. & 507 chr2_459710 & 5.25 nonsex & cis \\
\hline 2 Gg_rs150944. & 508 chr2_470560। & 6.14 nonsex & cis \\
\hline 2 Gg_rs150944. & 508 chr2_467490। & 5.63 nonsex & cis \\
\hline 2 Gg_rs150944. & 512 chr2_451040 & 6.33 nonsex & cis \\
\hline 2 Gg_rs150944. & 513 chr2_444210 & 5.94 nonsex & cis \\
\hline 2 Gg_rs150944. & 519 chr2_450630 & 6.93 nonsex & cis \\
\hline 2 Gg_rs150944. & 519 chr2_456690। & 13.78 nonsex & cis \\
\hline 2 Gg_rs150944. & 519 chr2_456720 & 6.44 nonsex & cis \\
\hline 2 Gg_rs150944. & 519 chr2_456920। & 10.8 nonsex & cis \\
\hline 2 Gg_rs150944. & 519 chr2_549380। & 6.2 nonsex & cis \\
\hline 2 Gg_rs150944. & 519 chr2_607380 & 7.91 sex & cis \\
\hline 2 Gg_rs150944. & 519 chrZ_2535901 & 7.79 sex & trans \\
\hline 2 Gg_rs150944. & 519 chr1_118241। & 8.44 sex & trans \\
\hline 2 Gg_rs150944. & 519 chr2_303270 & 8.83 sex & trans \\
\hline 2 Gg_rs150944. & 521 chr2_471100 & 6.53 sex & cis \\
\hline 2 Gg_rs150944. & 525 chr2_445100 & 8.68 nonsex & cis \\
\hline 2 Gg_rs150944. & 525 chr2_470580 & 5.23 nonsex & cis \\
\hline 2 Gg_rs150944. & 526 chr2_445090 & 9.04 nonsex & cis \\
\hline 2 Gg_rs150944. & 527 chr2_515700 & 4.8 nonsex & cis \\
\hline 2 Gg_rs150944. & 527 chr23_18170। & 8.56 sex & trans \\
\hline
\end{tabular}




\begin{tabular}{|c|c|c|c|}
\hline 2 Gg_rs150944. & 528 chr2_443690 & 8.6 nonsex & cis \\
\hline 2 Gg_rs150944. & 529 chr2_455370। & 3.92 nonsex & cis \\
\hline 2 Gg_rs150944. & 532 chrZ_1936701 & 8.41 sex & trans \\
\hline 2 Gg_rs150944. & 533 chr2_470300 & 5.84 nonsex & cis \\
\hline 2 Gg_rs150944. & 534 chrZ_193660। & 8.11 sex & trans \\
\hline 2 snp.20.360.5: & 554 chr2_572380 & 4.71 nonsex & cis \\
\hline 2 GG_rs150996 & 555 chr2_567590। & 5.02 nonsex & cis \\
\hline 2 snp.20.360.5: & 555 chr2_480310 & 8.77 nonsex & cis \\
\hline 2 Gg_rs141832 & 556 chrZ_6865401 & 6.89 nonsex & trans \\
\hline 2 snp.20.360.5: & 556 chr2_555210 & 4.39 nonsex & cis \\
\hline 2 GG_rs150996 & 557 chrZ_5576501 & 6.54 nonsex & trans \\
\hline 2 Gg_rs141832 & 557 chr2_569320। & 4.38 nonsex & cis \\
\hline 2 snp.213.19.4l & 560 chr2_502540 & 5.46 nonsex & cis \\
\hline 2 snp.213.19.41 & 560 chr2_527160 & 5.36 nonsex & cis \\
\hline 2 snp.213.19.4l & 560 chr2_530290। & 6.49 nonsex & cis \\
\hline 2 snp.213.19.4l & 560 chr2_531770 & 7.13 sex & cis \\
\hline 2 snp.213.19.4l & 560 chr2_519500। & 4.57 nonsex & cis \\
\hline 2 Gg_rs141832 & 560 chr2_510740 & 7.25 sex & cis \\
\hline 2 Gg_rs141832 & 560 chr2_527010 & 23.35 nonsex & cis \\
\hline 2 Gg_rs141832 & 560 chr2_527020 & 16.48 nonsex & cis \\
\hline 2 Gg_rs141832 & 560 chr2_527030 & 12.36 nonsex & cis \\
\hline 2 Gg_rs141832 & 560 chr2_527040 & 27.63 nonsex & cis \\
\hline 2 Gg_rs141832 & 560 chr2_527100 & 6.45 nonsex & cis \\
\hline 2 Gg_rs141832 & 560 chr2_527110 & 5.87 nonsex & cis \\
\hline 2 Gg_rs141832 & 560 chr2_527150 & 28.95 nonsex & cis \\
\hline 2 Gg_rs141832 & 560 chr2_534090। & 6.48 nonsex & cis \\
\hline 2 Gg_rs141832 & 560 chr5_666000 & 7.63 sex & trans \\
\hline 2 Gg_rs141832 & 560 chrZ_6137901 & 7.71 sex & trans \\
\hline 2 Gg_rs141832 & 561 chr2_488040। & 6.78 nonsex & cis \\
\hline 2 Gg_rs141832 & 561 chr2_527000। & 12.53 nonsex & cis \\
\hline 2 Gg_rs141832 & 561 chr2_527140 & 14.34 nonsex & cis \\
\hline 2 snp.20.360.5: & 561 chr2_527050। & 11.15 nonsex & cis \\
\hline 2 snp.20.360.5: & 561 chr2_534080 & 8.53 nonsex & cis \\
\hline 2 snp.20.360.5: & 561 chr2_549920। & 6.84 nonsex & cis \\
\hline 2 snp.20.360.5: & 561 chr2_549930। & 7.06 nonsex & cis \\
\hline 2 snp.20.360.5: & 561 chr2_563810 & 5.62 nonsex & cis \\
\hline 2 snp.20.360.5: & 561 chr2_542610 & 4.67 nonsex & cis \\
\hline 2 snp.20.360.5: & 561 chr2_541320 & 5.07 nonsex & cis \\
\hline 2 snp.20.360.5: & 561 chr2_543080। & 3.93 nonsex & cis \\
\hline 2 snp.20.360.5: & 561 chr2_549400। & 5.07 nonsex & cis \\
\hline 2 snp.20.360.5: & 561 chr26_39300। & 5.8 nonsex & trans \\
\hline 2 snp.20.360.5: & 561 chrW_36050c & 8.16 sex & trans \\
\hline 2 snp.20.360.5: & 562 chr2_480300। & 4.9 nonsex & cis \\
\hline
\end{tabular}




\begin{tabular}{|c|c|c|c|}
\hline 2 snp.20.360.5: & 562 chr2_488050। & 7.24 nonsex & cis \\
\hline 2 snp.20.360.5: & 562 chr2_496120 & 5.7 nonsex & cis \\
\hline 2 snp.20.360.5: & 562 chr2_496630। & 6.07 sex & cis \\
\hline 2 snp.20.360.5: & 562 chr2_498810 & 5.67 nonsex & cis \\
\hline 2 snp.20.360.5: & 562 chr2_510510 & 6.09 sex & cis \\
\hline 2 snp.20.360.5: & 562 chr2_514840 & 5.78 sex & cis \\
\hline 2 snp.20.360.5: & 562 chr2_526720। & 6 sex & cis \\
\hline 2 snp.20.360.5: & 562 chr2_549300। & 5.76 sex & cis \\
\hline 2 snp.20.360.5: & 562 chr2_495760। & 3.71 nonsex & cis \\
\hline 2 snp.20.360.5: & 562 chr2_541310 & 4.03 nonsex & cis \\
\hline 2 snp.20.360.5: & 562 chr15_95760। & 5.89 nonsex & trans \\
\hline 2 snp.20.360.5: & $563 \mathrm{chr} 2$ 526990 & 11.99 nonsex & cis \\
\hline 2 snp.20.360.5: & 563 chr2_549820 & 6.23 nonsex & cis \\
\hline 2 snp.20.360.5: & 563 chr2_549950। & 9.13 nonsex & cis \\
\hline 2 snp.20.360.5: & 563 chr2_558760। & 7.75 nonsex & cis \\
\hline 2 snp.20.360.5: & 564 chr2_532200। & 4.62 nonsex & cis \\
\hline 2 snp.20.360.5: & 565 chr2_477630 & 4.44 nonsex & cis \\
\hline 2 snp.20.360.5: & 565 chr2_519510 & 3.93 nonsex & cis \\
\hline 2 snp.20.360.5: & 566 chr2_543590। & 6.29 nonsex & cis \\
\hline 2 snp.20.360.5: & 566 chr2_558570 & 4.92 nonsex & cis \\
\hline 2 snp.20.360.5: & 566 chr2_549980 & 4.48 nonsex & cis \\
\hline 2 snp.20.360.5: & 567 chr2_475400 & 5.35 sex & cis \\
\hline 2 snp.20.360.5: & 567 chr2_515020 & 6.94 sex & cis \\
\hline 2 snp.20.360.5: & 567 chr2_549960। & 6.6 nonsex & cis \\
\hline 2 snp.20.360.5: & 567 chr2_606930। & 4.59 sex & cis \\
\hline 2 snp.20.360.5: & 567 chr5_193600 & 5.76 sex & trans \\
\hline 2 snp.20.360.5: & 567 chr3_223510 & 7.67 sex & trans \\
\hline 2 Gg_rs141832 & 568 chr2_549970 & 5.49 nonsex & cis \\
\hline 2 snp.20.360.5: & 570 chr2_588560 & 6.39 sex & cis \\
\hline 2 snp.20.141.7l & 579 chr2_583180। & 11.9 nonsex & cis \\
\hline 2 snp.20.141.7( & 580 chr2_592920 & 8.01 nonsex & cis \\
\hline 2 snp.20.141.7( & 580 chr2_574460 & 4.46 nonsex & cis \\
\hline 2 snp.20.141.7l & 580 chr2_584550। & 4.63 nonsex & cis \\
\hline 2 snp.20.141.7l & 581 chr2_582890। & 5.67 sex & cis \\
\hline 2 snp.20.141.7l & 582 chr2_543700। & 3.79 nonsex & cis \\
\hline 2 snp.20.141.7l & 584 chr2_503590। & 4.24 sex & cis \\
\hline 2 snp.20.141.7l & 584 chr2_526550। & 5.22 sex & cis \\
\hline 2 snp.20.141.7l & 584 chr2_587970। & $4.9 \operatorname{sex}$ & cis \\
\hline 2 snp.20.141.7l & 584 chr7_223990। & 9.64 sex & trans \\
\hline 2 snp.20.141.7( & 584 chr11_20100। & $5.59 \mathrm{sex}$ & trans \\
\hline 2 snp.20.141.7( & 584 chr12_95310 & $13.54 \mathrm{sex}$ & trans \\
\hline 2 snp.20.141.7( & 584 chr27_38500। & 5.51 sex & trans \\
\hline 2 snp.20.141.7l & 584 chr31_20890। & 7.51 sex & trans \\
\hline
\end{tabular}


2 snp.20.141.7(

2 snp.20.141.7(

2 snp.20.141.7(

2 snp.20.141.71

2 snp.20.141.7(

2 snp.20.141.7(

2 snp.20.141.7(

2 snp.20.141.7(

2 snp.20.141.7(

2 snp.20.141.7(

2 snp.20.141.7(

2 snp.20.141.7(

2 snp.20.141.7(

2 snp.20.141.7(

2 snp.20.141.7(

2 snp.20.141.7(

2 snp.20.141.7(

2 snp.20.141.7(

2 snp.20.141.7(

2 Gg_rs151120

2 Gg_rs151120

2 Gg_rs151120

2 Gg_rs151120

2 Gg_rs142004

2 Gg_rs142004

2 Gg_rs142004

2 Gg_rs142004

2 Gg_rs142004

2 Gg_rs142004

2 Gg_rs142004

2 Gg_rs142004

2 Gg_rs142004

2 Gg_rs142004

2 Gg_rs142004

2 Gg_rs142004

2 Gg_rs151162

2 Gg_rs151154

2 Gg_rs151162

2 Gg_rs151154

2 Gg_rs151154

2 Gg_rs151154

2 Gg_rs151154

2 Gg_rs151154
584 chr33_36140।

584 chr2_965770।

584 chr3_634930।

584 chr3_962610

585 chr2_510790।

585 chr2_603880

585 chr2_590380।

585 chr2_597510।

585 chr28_12300।

586 chr2_490700।

586 chr2_491080।

586 chr2_593380।

586 chr2_597460।

586 chr2_574470।

586 chr28_40440।

587 chr2_618120

587 chr2_604920।

588 chr2_598640।

588 chr32_23900।

671 chr2_666130।

671 chr2_674220।

672 chr2_851290।

676 chr2_836850।

680 chr2_677170।

683 chr2_722570।

686 chr2_673090।

687 chr2_686510

688 chr6_130600।

689 chr2_681440।

689 chr2_744790।

689 chr2_747720

689 chr2_691210।

689 chr2_789720।

690 chr2_681430।

690 chr2_744890।

690 chr2_751550।

691 chr2_744780।

691 chr2_823120।

692 chr2_683530।

692 chr2_710450।

692 chr2_709690।

692 chr2_680800।

692 chr15_99850।
7.41 sex

5.99 sex

6.32 sex

6.01 sex

5.73 sex

5.25 sex

3.17 nonsex cis

3.93 nonsex cis

3.89 sex trans

3.99 sex cis

3.94 sex cis

7.79 nonsex cis

5.46 sex cis

3.95 nonsex cis

7.83 sex

5.6 sex cis

4.48 nonsex cis

6.98 nonsex cis

9.12 sex trans

4.08 nonsex cis

4.55 nonsex cis

4.41 nonsex cis

5.42 nonsex cis

3.73 nonsex cis

4.54 nonsex cis

5.96 sex cis

4.01 nonsex cis

5.94 nonsex trans

4.58 nonsex cis

6.38 sex cis

7.52 nonsex cis

4.05 nonsex cis

4.83 nonsex cis

8.03 nonsex cis

5.96 sex cis

5.41 nonsex cis

4.28 nonsex cis

4.75 nonsex cis

5.66 nonsex cis

10.64 nonsex cis

6.56 nonsex cis

4.41 nonsex cis

5.68 nonsex trans 


\begin{tabular}{|c|c|c|c|}
\hline 2 Gg rs151154 & $693 \mathrm{chr} 2688540$ & $6.31 \mathrm{sex}$ & cis \\
\hline 2 Gg_rs151154 & 693 chr2_738870 & $5.61 \mathrm{sex}$ & cis \\
\hline 2 Gg_rs151154 & 693 chr2_738880 & 6.39 sex & cis \\
\hline 2 Gg_rs151154 & 693 chr2_744700 & 8.14 nonsex & cis \\
\hline 2 Gg_rs151154 & 693 chr2_744710 & 8.01 nonsex & cis \\
\hline 2 Gg_rs151154 & 693 chr2_744740 & 8.54 nonsex & cis \\
\hline 2 Gg_rs151154 & 693 chr2_744810 & 9.72 nonsex & cis \\
\hline 2 Gg_rs151154 & 693 chr2_744910 & 9.46 nonsex & cis \\
\hline 2 Gg_rs151154 & 693 chr2_750730 & 5.66 nonsex & cis \\
\hline 2 Gg_rs151154 & 693 chr2_688610 & 4.75 nonsex & cis \\
\hline 2 Gg_rs151154 & 693 chr2_718780 & 4.99 nonsex & cis \\
\hline 2 Gg_rs151162. & 693 chr2_873130 & 4.43 nonsex & cis \\
\hline 2 Gg_rs151162. & 693 chr2_667050 & 4.28 nonsex & cis \\
\hline 2 Gg_rs151162. & 694 chr2_699280 & 7.38 nonsex & cis \\
\hline 2 Gg_rs151162. & 694 chr2_744840 & 6.1 nonsex & cis \\
\hline 2 Gg_rs151162. & 694 chr2_744940 & 6.2 nonsex & cis \\
\hline 2 Gg_rs151162. & 694 chr2_744850 & 3.27 nonsex & cis \\
\hline 2 Gg_rs151162. & 695 chr2_718770 & 6.05 nonsex & cis \\
\hline 2 Gg_rs151162. & 695 chr2_747700 & 18.45 nonsex & cis \\
\hline 2 Gg_rs151162. & 695 chr4_909440 & 8.63 sex & trans \\
\hline 2 Gg_rs151162. & 696 chr2_718750 & 6.61 nonsex & cis \\
\hline 2 Gg_rs151162. & 696 chr2_718760 & 6.48 nonsex & cis \\
\hline 2 Gg_rs151162. & 696 chr2_742540 & 6.11 nonsex & cis \\
\hline 2 Gg_rs151162. & 697 chr2_715810 & 6.6 nonsex & cis \\
\hline 2 Gg_rs151162. & 697 chr2_718630 & 7.31 nonsex & cis \\
\hline 2 Gg_rs151162. & 697 chr2_721770 & 6.66 sex & cis \\
\hline 2 Gg_rs151162. & 697 chr3_627500 & 7.23 sex & trans \\
\hline 2 Gg_rs151162. & 698 chr2_718740 & 7.66 nonsex & cis \\
\hline 2 Gg_rs151162. & 698 chr2_718790 & 7.2 nonsex & cis \\
\hline 2 Gg_rs151154 & 699 chr2_745910 & 5.96 nonsex & cis \\
\hline 2 Gg_rs151162. & 699 chr2_709330 & 5.65 nonsex & cis \\
\hline 2 Gg_rs151162. & 699 chr2_718600 & 11.84 nonsex & cis \\
\hline 2 Gg_rs151162. & 699 chr2_690710 & 4.6 nonsex & cis \\
\hline 2 Gg_rs151162. & 699 chr2_693230 & 4.74 nonsex & cis \\
\hline 2 Gg_rs151162. & 699 chr2_718690 & 4.75 nonsex & cis \\
\hline 2 Gg_rs142004 & 700 chr2_747650 & 11.24 nonsex & cis \\
\hline 2 Gg_rs151162. & 700 chr2_718610 & 8.79 nonsex & cis \\
\hline 2 Gg_rs151162. & 700 chr2_741810 & 6.19 sex & cis \\
\hline 2 Gg_rs151162. & 700 chr2_744520 & 6.11 nonsex & cis \\
\hline 2 Gg_rs151162. & 700 chr2_744580 & 12.65 nonsex & cis \\
\hline 2 Gg_rs151162. & 700 chr2_752370 & 11.75 nonsex & cis \\
\hline 2 Gg_rs142004 & 701 chr2_665110 & 5.46 nonsex & cis \\
\hline 2 Gg_rs151162. & 701 chr2_723050 & 6.47 nonsex & cis \\
\hline
\end{tabular}




\begin{tabular}{|c|c|c|c|}
\hline 2 Gg_rs151162. & 701 chr2_755290। & 5.25 nonsex & cis \\
\hline 2 Gg_rs151162. & 701 chr2_676900। & 4.83 nonsex & cis \\
\hline 2 Gg_rs151162. & 701 chr2_669120। & 5.72 nonsex & cis \\
\hline 2 Gg_rs142004 & 702 chr2_718680 & 4.75 nonsex & cis \\
\hline 2 Gg_rs151162. & 702 chr2_718570 & 7.26 nonsex & cis \\
\hline 2 Gg_rs151162. & 702 chr2_722070 & 7.32 nonsex & cis \\
\hline 2 Gg_rs151162. & 702 chr2_747640 & 11.56 nonsex & cis \\
\hline 2 Gg_rs151162. & 702 chr2_754840। & 7.69 nonsex & cis \\
\hline 2 Gg_rs151162. & 702 chr2_687810 & 5.75 nonsex & cis \\
\hline 2 Gg_rs151162 & 702 chr2_718640 & 4.75 nonsex & cis \\
\hline 2 Gg_rs151162. & 703 chr2_718620। & 8.43 nonsex & cis \\
\hline 2 Gg_rs151162. & 703 chr2_747630 & 13.56 nonsex & cis \\
\hline 2 Gg_rs151162. & 703 chr2_683210 & 7.88 nonsex & cis \\
\hline 2 Gg_rs151162 & 703 chr2_687530 & 5.03 nonsex & cis \\
\hline 2 Gg_rs151183 & 703 chr2_675220। & 4.77 nonsex & cis \\
\hline 2 Gg_rs142061. & 704 chr2_751020। & 5.65 nonsex & cis \\
\hline 2 Gg_rs142061. & 705 chr2_666850 & 5.5 nonsex & cis \\
\hline 2 Gg_rs142061. & 705 chr2_744590। & 6.22 nonsex & cis \\
\hline 2 Gg_rs142061. & 705 chr2_747520। & 5.2 nonsex & cis \\
\hline 2 Gg_rs151183 & 706 chr13_49320। & 7 nonsex & trans \\
\hline 2 Gg_rs142061. & 707 chr2_755590। & 4.81 nonsex & cis \\
\hline 2 Gg_rs151183 & 707 chr2_718580 & 5.05 nonsex & cis \\
\hline 2 Gg_rs142061. & 709 chr2_761600 & 7.82 sex & cis \\
\hline 2 Gg_rs142061. & 709 chr2_695390। & 4.46 nonsex & cis \\
\hline 2 Gg_rs142061. & 711 chr2_761540 & 11.13 nonsex & cis \\
\hline 2 Gg_rs142061. & 711 chr2_746400 & 4.53 nonsex & cis \\
\hline 2 Gg_rs142061. & 712 chr2_761590। & 12.47 nonsex & cis \\
\hline 2 Gg_rs142061. & 712 chr2_842050। & 4.85 nonsex & cis \\
\hline 2 Gg_rs142061. & 713 chr2_722310 & 6.04 nonsex & cis \\
\hline 2 Gg_rs142061. & 713 chr2_757130। & 4.87 nonsex & cis \\
\hline 2 Gg_rs142061. & 713 chr2_765830 & 5.34 nonsex & cis \\
\hline 2 Gg_rs142061. & 713 chr2_775000 & 5.24 nonsex & cis \\
\hline 2 Gg_rs142061. & 713 chr3_299070। & 6.87 sex & trans \\
\hline 2 Gg_rs142061. & 713 chr3_512030। & 8.28 sex & trans \\
\hline 2 Gg_rs142061. & 713 chr1_394160। & 6.16 nonsex & trans \\
\hline 2 Gg_rs142061. & 713 chrZ_614170। & 6.25 nonsex & trans \\
\hline 2 Gg_rs151183 & 713 chr2_767140 & 4.63 nonsex & cis \\
\hline 2 Gg_rs151183 & 713 chr2_746390 & 5.18 nonsex & cis \\
\hline 2 Gg_rs151183 & 713 chr2_748730। & 4.39 nonsex & cis \\
\hline 2 Gg_rs151183 & 713 chr2_775090। & 4.77 nonsex & cis \\
\hline 2 Gg_rs151183 & 713 chr2_868300 & 5.42 nonsex & cis \\
\hline 2 Gg_rs151183 & 713 chr6_153010 & 6.25 nonsex & trans \\
\hline 2 Gg_rs151183 & 713 chrZ_4641201 & 6.38 nonsex & trans \\
\hline
\end{tabular}




\begin{tabular}{|c|c|c|c|}
\hline 2 Gg_rs151183 & 713 chr1_167503। & 6.27 nonsex & trans \\
\hline 2 Gg_rs151183 & 714 chr2_761580 & 8.41 nonsex & cis \\
\hline 2 Gg_rs151183 & 714 chr2_724560 & 4.36 nonsex & cis \\
\hline 2 Gg_rs151183 & 714 chr2_847780 & 4.87 nonsex & cis \\
\hline 2 Gg_rs142061. & 715 chr2_792000 & 4.79 nonsex & cis \\
\hline 2 Gg_rs151183 & 715 chr2_663040। & 7.15 nonsex & cis \\
\hline 2 Gg_rs151183 & 715 chr2_775020 & 9.97 nonsex & cis \\
\hline 2 Gg_rs151183 & 715 chr2_760040 & 5.04 nonsex & cis \\
\hline 2 Gg_rs151183 & 715 chr2_881510 & 5.08 nonsex & cis \\
\hline 2 Gg_rs151183 & 715 chr1_625880 & 6.49 nonsex & trans \\
\hline 2 Gg_rs151183. & 715 chrZ_615010। & 7.1 nonsex & trans \\
\hline 2 Gg_rs151183 & 715 chr3_616850 & 6.64 nonsex & trans \\
\hline 2 Gg_rs142061. & 716 chr2_805640 & 4.9 nonsex & cis \\
\hline 2 Gg_rs142061. & 716 chr2_826320। & 4.48 nonsex & cis \\
\hline 2 Gg_rs142061. & 716 chr2_895510। & 4.52 nonsex & cis \\
\hline 2 Gg_rs151183 & 716 chr2_676940। & 4.32 nonsex & cis \\
\hline 2 Gg_rs151183 & 716 chr2_762610 & 5.33 nonsex & cis \\
\hline 2 Gg_rs151183 & 716 chr2_196570 & 6.96 nonsex & trans \\
\hline 2 Gg_rs151183: & 716 chr3_970290। & 6.51 nonsex & trans \\
\hline 2 Gg_rs151231. & 716 chr2_859880 & 4.93 nonsex & cis \\
\hline 2 Gg_rs142061. & 717 chr2_761520। & 7.16 sex & cis \\
\hline 2 Gg_rs142061. & 717 chr2_724870 & 5.32 nonsex & cis \\
\hline 2 Gg_rs142061. & 717 chr2_835180 & 5.42 nonsex & cis \\
\hline 2 Gg_rs142061. & 717 chr1_975940। & 6.14 nonsex & trans \\
\hline 2 Gg_rs142061. & 717 chr3_532650। & 5.38 nonsex & trans \\
\hline 2 Gg_rs151183 & 717 chr2_719360। & 5.21 nonsex & cis \\
\hline 2 Gg_rs151183 & 717 chr2_725750 & 4.71 nonsex & cis \\
\hline 2 Gg_rs151183 & 717 chr2_745270 & 4.81 nonsex & cis \\
\hline 2 Gg_rs151183 & 717 chr7_834100। & 7.09 nonsex & trans \\
\hline 2 Gg_rs142061. & 718 chr2_761530। & 4.74 nonsex & cis \\
\hline 2 Gg_rs142061. & 718 chr2_800110 & 5.65 nonsex & cis \\
\hline 2 Gg_rs142061. & 718 chr2_655620 & 5.45 nonsex & cis \\
\hline 2 Gg_rs142061. & 718 chr2_722640। & 4.97 nonsex & cis \\
\hline 2 Gg_rs142061. & 718 chr2_759900। & 5.38 nonsex & cis \\
\hline 2 Gg_rs142061. & 718 chr2_782040। & 4.58 nonsex & cis \\
\hline 2 Gg_rs151183 & 718 chr2_719960। & 5.75 nonsex & cis \\
\hline 2 Gg_rs142082 & 718 chr2_790800 & 6.69 nonsex & cis \\
\hline 2 Gg_rs142082 & 718 chr2_890060। & 5.33 nonsex & cis \\
\hline $2 \mathrm{chr} 2.846684 \mathrm{c}$ & 718 chr2_810080 & 5.35 nonsex & cis \\
\hline 2 Gg_rs151231. & 718 chr2_795050। & 5.27 nonsex & cis \\
\hline 2 Gg_rs142061. & 719 chr1_323810 & 7.1 nonsex & trans \\
\hline 2 Gg_rs142082 & 719 chr2_853970 & 9.57 nonsex & cis \\
\hline 2 Gg_rs142082 & 719 chr2_805860 & 5.76 nonsex & cis \\
\hline
\end{tabular}




\begin{tabular}{|c|c|c|c|}
\hline 2 Gg_rs142082 & 719 chr2_790220। & 7.21 nonsex & cis \\
\hline 2 Gg_rs142082 & 719 chr2_791590। & 8.9 nonsex & cis \\
\hline 2 Gg_rs142082 & 719 chr2_826390। & 5.81 nonsex & cis \\
\hline 2 Gg_rs142082 & 719 chr2_662130 & 5.3 nonsex & cis \\
\hline 2 Gg_rs142082 & 719 chr2_760050 & 5.03 nonsex & cis \\
\hline 2 Gg_rs142082 & 719 chr2_761930। & 4.79 nonsex & cis \\
\hline 2 Gg_rs142082 & 719 chr2_847570। & 5.09 nonsex & cis \\
\hline 2 Gg_rs142082 & 719 chr2_890240। & 4.43 nonsex & cis \\
\hline 2 Gg_rs151225. & 719 chr2_842800। & 11.63 nonsex & cis \\
\hline 2 Gg_rs151231 & 719 chr2_804420। & 8.94 nonsex & cis \\
\hline 2 Gg_rs151231. & 719 chr2_833210 & 7.52 nonsex & cis \\
\hline 2 Gg_rs151231 & 719 chr3_318610 & 6.23 nonsex & trans \\
\hline 2 Gg_rs142082 & 720 chr2_805900। & 7.1 nonsex & cis \\
\hline 2 Gg_rs142082 & 720 chr2_845440 & 10.05 nonsex & cis \\
\hline 2 Gg_rs142082 & 720 chr2_894950। & 6.93 nonsex & cis \\
\hline 2 Gg_rs142082 & 720 chr2_903200। & 6.12 nonsex & cis \\
\hline 2 Gg_rs142082 & 720 chr2_791810 & 5.06 nonsex & cis \\
\hline 2 Gg_rs142082 & 720 chr2_820260। & 4.66 nonsex & cis \\
\hline 2 Gg_rs142082 & 720 chr2_855820 & 4.55 nonsex & cis \\
\hline 2 Gg_rs142082 & 720 chr2_899550। & 4.92 nonsex & cis \\
\hline 2 Gg_rs142082 & 720 chrZ_2363001 & 7.88 sex & trans \\
\hline 2 Gg_rs142082 & 720 chr4_318700। & 7.12 nonsex & trans \\
\hline 2 Gg_rs142082 & 720 chr5_259130 & 7.16 nonsex & trans \\
\hline 2 Gg_rs142082 & 720 chr6_488200। & 6.99 nonsex & trans \\
\hline $2 \mathrm{chr} 2.846684 \mathrm{c}$ & 720 chr2_826070। & 4.41 nonsex & cis \\
\hline 2 Gg_rs151231. & 720 chr2_745430 & 4.75 nonsex & cis \\
\hline 2 Gg_rs142082 & 721 chr2_796160। & 5.61 nonsex & cis \\
\hline 2 Gg_rs142082 & 721 chr2_813930। & 6.14 nonsex & cis \\
\hline 2 Gg_rs142082 & 721 chr2_835830। & 4.95 nonsex & cis \\
\hline 2 Gg_rs142082 & 721 chr2_897000। & 6.84 nonsex & cis \\
\hline 2 Gg_rs142082 & 721 chr2_912140। & $6.61 \mathrm{sex}$ & cis \\
\hline 2 Gg_rs142082 & 721 chr2_818090। & 7.06 nonsex & cis \\
\hline 2 Gg_rs142082 & 721 chr2_694810 & 4.62 nonsex & cis \\
\hline 2 Gg_rs142082 & 721 chr2_730580। & 4.38 nonsex & cis \\
\hline 2 Gg_rs142082 & 721 chr2_802500। & 5.33 nonsex & cis \\
\hline 2 Gg_rs142082 & 721 chr2_820150। & 4.85 nonsex & cis \\
\hline 2 Gg_rs142082 & 721 chr2_856320 & 4.41 nonsex & cis \\
\hline 2 Gg_rs142082 & 721 chr2_889620 & 4.03 nonsex & cis \\
\hline 2 Gg_rs142082 & 721 chr2_897340। & 4.44 nonsex & cis \\
\hline 2 Gg_rs142082 & 721 chr1_471360। & 6.38 nonsex & trans \\
\hline 2 Gg_rs142082 & 721 chr4_554300। & 6.16 nonsex & trans \\
\hline 2 Gg_rs142082 & 721 chr5_336260। & 6.12 nonsex & trans \\
\hline 2 Gg_rs142082 & 721 chr5_470740 & 6.2 nonsex & trans \\
\hline
\end{tabular}




\begin{tabular}{|c|c|c|}
\hline 2 Gg_rs142082 & 721 chr6_870200। & 6.26 nonsex \\
\hline 2 Gg_rs151231. & 721 chr2_805870। & 4.55 nonsex \\
\hline 2 Gg_rs142082 & 722 chr2_787900। & 6.26 nonsex \\
\hline 2 Gg_rs142082 & 722 chr2_798150 & 6.32 sex \\
\hline 2 Gg_rs142082 & 722 chr2_805810। & 7.37 nonsex \\
\hline 2 Gg_rs142082 & 722 chr2_884770। & 5.17 nonsex \\
\hline 2 Gg_rs142082 & 722 chr2_915570। & 5.84 nonsex \\
\hline 2 Gg_rs142082 & 722 chr2_917670। & 5.09 sex \\
\hline 2 Gg_rs142082 & 722 chr2_790770। & 4.57 nonsex \\
\hline 2 Gg_rs142082 & 722 chr2_796860। & 4.58 nonsex \\
\hline 2 Gg_rs142082 & 722 chr2_879880। & 4.53 nonsex \\
\hline 2 Gg_rs142082 & 722 chr2_900570। & 4.54 nonsex \\
\hline 2 Gg_rs142082 & 722 chr1_281130। & 6.39 nonsex \\
\hline 2 Gg_rs142082 & 722 chr4_292270। & 6.06 nonsex \\
\hline 2 Gg_rs142082 & 722 chr7_760000 & 5.78 nonsex \\
\hline 2 Gg_rs142082 & 722 chr7_954000 & 6.57 nonsex \\
\hline 2 Gg_rs142082 & 723 chr2_804070। & 5.46 nonsex \\
\hline 2 Gg_rs142082 & 723 chr2_874800। & 8.63 sex \\
\hline 2 Gg_rs142082 & 723 chr2_918380। & 5.88 sex \\
\hline 2 Gg_rs142082 & 723 chr2_682660। & 4.87 nonsex \\
\hline 2 Gg_rs142082 & 723 chr2_759670 & 4.56 nonsex \\
\hline 2 Gg_rs142082 & 723 chr2_877600। & 4.65 nonsex \\
\hline 2 Gg_rs142082 & 723 chr2_906180 & 4.1 nonsex \\
\hline 2 Gg_rs142082 & 723 chr2_913590। & 3.99 nonsex \\
\hline 2 Gg_rs142082 & 723 chr1_470710 & 6.51 nonsex \\
\hline 2 Gg_rs142082 & 723 chr3_861660। & 7.65 sex \\
\hline 2 Gg_rs142082 & 723 chr4_417820 & 5.81 nonsex \\
\hline 2 Gg_rs142082 & 723 chr5_794900। & 5.06 nonsex \\
\hline 2 Gg_rs142082 & 723 chr6_173350 & 6.89 nonsex \\
\hline 2 Gg_rs142082 & 723 chr6_205980 & 5.96 nonsex \\
\hline 2 Gg_rs151231. & 723 chr2_920030। & 5.51 nonsex \\
\hline 2 Gg_rs142082 & 724 chr2_659390। & 4.72 nonsex \\
\hline 2 Gg_rs142082 & 724 chr2_791190। & 5.56 nonsex \\
\hline 2 Gg_rs142082 & 724 chr2_797390। & 7.78 nonsex \\
\hline 2 Gg_rs142082 & 724 chr2_803480 & 7.23 nonsex \\
\hline 2 Gg_rs142082 & 724 chr2_803500। & 7.2 nonsex \\
\hline 2 Gg_rs142082 & 724 chr2_803510 & 10.87 nonsex \\
\hline 2 Gg_rs142082 & 724 chr2_803520। & 7.75 nonsex \\
\hline 2 Gg_rs142082 & 724 chr2_803530। & 10.05 nonsex \\
\hline 2 Gg_rs142082 & 724 chr2_803540। & 8.45 nonsex \\
\hline 2 Gg_rs142082 & 724 chr2_803550। & 6.34 nonsex \\
\hline 2 Gg_rs142082 & 724 chr2_804210 & 5.23 nonsex \\
\hline 2 Gg_rs142082 & 724 chr2_818170। & 5.89 nonsex \\
\hline
\end{tabular}




\begin{tabular}{|c|c|c|c|}
\hline 2 Gg_rs142082 & 724 chr2_856940। & 3.94 nonsex & cis \\
\hline 2 Gg_rs142082 & 724 chr2_878390। & 6.25 sex & cis \\
\hline 2 Gg_rs142082 & 724 chr2_683330 & 4.32 nonsex & cis \\
\hline 2 Gg_rs142082 & 724 chr2_788710 & 4.64 nonsex & cis \\
\hline 2 Gg_rs142082 & 724 chr2_799950। & 4.23 nonsex & cis \\
\hline 2 Gg_rs142082 & 724 chr2_856300। & 3.97 nonsex & cis \\
\hline 2 Gg_rs142082 & 724 chr2_858400। & 4.32 nonsex & cis \\
\hline 2 Gg_rs142082 & 724 chr2_873180 & 4.43 nonsex & cis \\
\hline 2 Gg_rs142082 & 724 chr2_874150। & 4.43 nonsex & cis \\
\hline 2 Gg_rs142082 & 724 chr2_882410 & 4.52 nonsex & cis \\
\hline 2 Gg_rs142082 & 724 chr2_894060। & 4.34 nonsex & cis \\
\hline 2 Gg_rs142082 & 724 chr2_903300। & 4.7 nonsex & cis \\
\hline 2 Gg_rs142082 & 724 chr2_906820। & 4.49 nonsex & cis \\
\hline 2 Gg_rs142082 & 724 chr2_907230। & 4.35 nonsex & cis \\
\hline 2 Gg_rs142082 & 724 chr1_366020। & 6.88 sex & trans \\
\hline 2 Gg_rs142082 & 724 chr5_430910 & 6 nonsex & trans \\
\hline 2 Gg_rs142082 & 724 chr1_626430। & 7.9 nonsex & trans \\
\hline 2 Gg_rs142082 & 724 chr2_490000। & 6.37 nonsex & trans \\
\hline 2 Gg_rs142082 & 724 chr2_514650 & 5.96 sex & trans \\
\hline 2 Gg_rs142082 & 724 chr2_554650। & 6.95 nonsex & trans \\
\hline 2 Gg_rs142082 & 724 chr3_253680 & 6.3 sex & trans \\
\hline 2 Gg_rs142082 & 724 chr3_935450। & 8.62 sex & trans \\
\hline 2 Gg_rs142082 & 724 chr4_573400। & 6.83 nonsex & trans \\
\hline 2 Gg_rs142082 & 724 chr4_480330 & 5.1 nonsex & trans \\
\hline 2 Gg_rs142082 & 724 chr4_520010 & 5.07 nonsex & trans \\
\hline 2 Gg_rs142082 & 724 chr5_294350। & 5.71 nonsex & trans \\
\hline 2 Gg_rs142082 & 724 chr6_743300 & 5.53 nonsex & trans \\
\hline 2 Gg_rs151231. & 724 chr2_945360। & 4.95 nonsex & cis \\
\hline 2 Gg_rs142082 & 725 chr2_668770 & 5.9 sex & cis \\
\hline 2 Gg_rs142082 & 725 chr2_788810 & 5.99 nonsex & cis \\
\hline 2 Gg_rs142082 & 725 chr2_789610 & 8.34 nonsex & cis \\
\hline 2 Gg_rs142082 & 725 chr2_791200। & 5.21 sex & cis \\
\hline 2 Gg_rs142082 & 725 chr2_796560। & 7.58 nonsex & cis \\
\hline 2 Gg_rs142082 & 725 chr2_796810 & 5.58 sex & cis \\
\hline 2 Gg_rs142082 & 725 chr2_828920। & 5.47 nonsex & cis \\
\hline 2 Gg_rs142082 & 725 chr2_799870। & 4.44 nonsex & cis \\
\hline 2 Gg_rs142082 & 725 chr2_876910। & 4.76 nonsex & cis \\
\hline 2 Gg_rs142082 & 725 chr1_105970 & 6.79 nonsex & trans \\
\hline 2 Gg_rs142082 & 725 chr4_202030। & 7.88 sex & trans \\
\hline 2 Gg_rs142082 & 725 chr6_742400 & 7.37 sex & trans \\
\hline 2 Gg_rs142082 & 725 chr3_959180। & 7.09 nonsex & trans \\
\hline 2 Gg_rs142082 & 725 chr1_364690 & 5.83 nonsex & trans \\
\hline 2 Gg_rs142082 & 725 chr4_358470। & 5.27 nonsex & trans \\
\hline
\end{tabular}




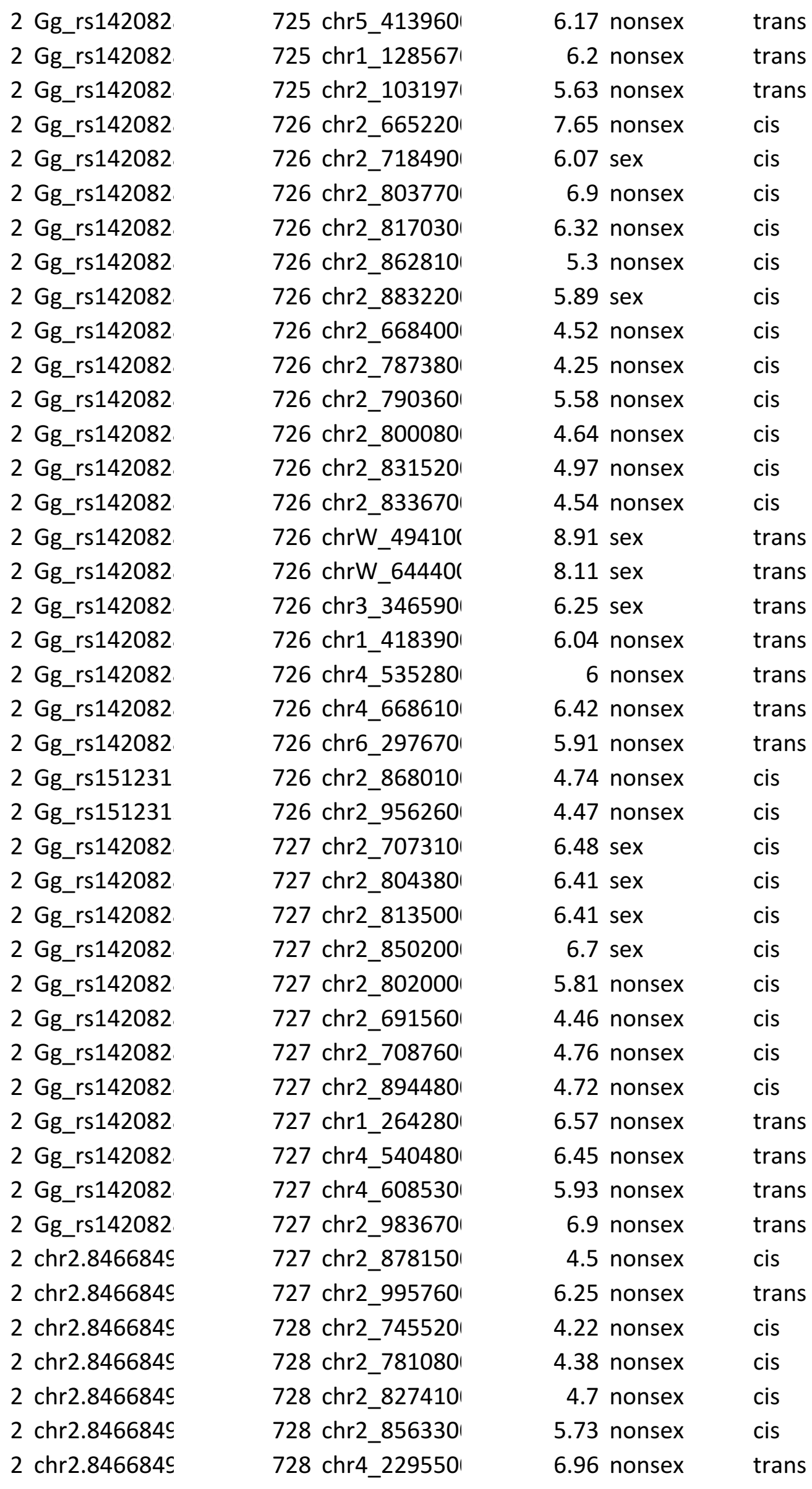




\begin{tabular}{|c|c|c|}
\hline 2 chr2.846684c & 728 chr5_312020। & $8.2 \mathrm{sex}$ \\
\hline $2 \mathrm{chr} 2.846684 \mathrm{c}$ & 728 chr5_428930। & 7.93 sex \\
\hline 2 chr2.846684c & 728 chr5_934400। & 6.76 nonsex \\
\hline 2 chr2.846684c & 728 chr7_210870 & 6.01 nonsex \\
\hline $2 \mathrm{chr} 2.846684 \mathrm{c}$ & 728 chr11_79520। & 5.84 nonsex \\
\hline 2 chr2.846684c & 728 chrZ_6438101 & 6.02 nonsex \\
\hline 2 chr2.846684c & 728 chr3_130110 & 6.64 nonsex \\
\hline 2 Gg_rs151231. & 728 chr1_706300। & 6.18 nonsex \\
\hline 2 chr2.846684c & 729 chr2_834730। & 4.98 nonsex \\
\hline $2 \mathrm{chr} 2.846684 \mathrm{c}$ & 729 chr2_848870 & 5.8 nonsex \\
\hline $2 \mathrm{chr} 2.846684 \mathrm{c}$ & 729 chr2_852310 & 5.7 nonsex \\
\hline $2 \mathrm{chr} 2.846684 \mathrm{c}$ & 729 chr2_885120। & 5.25 nonsex \\
\hline $2 \mathrm{chr} 2.846684 \mathrm{c}$ & 729 chr2_712150 & 4.24 nonsex \\
\hline $2 \mathrm{chr} 2.846684 \mathrm{c}$ & 729 chr2_745210 & 4.31 nonsex \\
\hline $2 \mathrm{chr} 2.846684 \mathrm{c}$ & 729 chr2_746750 & 4.21 nonsex \\
\hline 2 chr2.846684c & 729 chr2_813050। & 4.99 nonsex \\
\hline $2 \mathrm{chr} 2.846684 \mathrm{c}$ & 729 chr2_817580 & 4.74 nonsex \\
\hline $2 \mathrm{chr} 2.846684 \mathrm{c}$ & 729 chr2_827390। & 5.12 nonsex \\
\hline $2 \mathrm{chr} 2.846684 \mathrm{c}$ & 729 chr2_831180 & 4.98 nonsex \\
\hline $2 \mathrm{chr} 2.846684 \mathrm{c}$ & 729 chr2_833520। & 5.18 nonsex \\
\hline $2 \mathrm{chr} 2.846684 \mathrm{c}$ & 729 chr2_859460। & 5.12 nonsex \\
\hline $2 \mathrm{chr} 2.846684 \mathrm{c}$ & 729 chr4_284320। & 6.79 nonsex \\
\hline 2 chr2.846684c & 729 chrZ_7328001 & 5.85 nonsex \\
\hline $2 \mathrm{chr} 2.846684 \mathrm{c}$ & 729 chr1_112721। & 6.47 nonsex \\
\hline 2 chr2.846684c & 729 chr2_634700। & 5.91 nonsex \\
\hline 2 snp.12.170.1: & 729 chr2_782790। & 4.37 nonsex \\
\hline 2 snp.12.170.1: & 729 chr1_965130। & 5.95 nonsex \\
\hline 2 chr2.846684c & 730 chr2_840370। & 4.33 nonsex \\
\hline $2 \mathrm{chr} 2.846684 \mathrm{c}$ & 730 chr2_846100। & 6.55 nonsex \\
\hline $2 \mathrm{chr} 2.846684 \mathrm{c}$ & 730 chr2_854090। & $9.19 \operatorname{sex}$ \\
\hline $2 \mathrm{chr} 2.846684 \mathrm{c}$ & 730 chr2_860830 & 6.88 nonsex \\
\hline $2 \mathrm{chr} 2.846684 \mathrm{c}$ & 730 chr2_835380। & 8.22 nonsex \\
\hline $2 \mathrm{chr} 2.846684 \mathrm{c}$ & 730 chr2_677560। & 4.47 nonsex \\
\hline $2 \mathrm{chr} 2.846684 \mathrm{c}$ & 730 chr2_813900। & 5.77 nonsex \\
\hline 2 chr2.846684c & 730 chr4_263130। & 5.95 nonsex \\
\hline 2 chr2.846684c & 730 chr5_299490। & 6.01 nonsex \\
\hline $2 \mathrm{chr} 2.846684 \mathrm{c}$ & 730 chr7_902300। & 5.82 nonsex \\
\hline $2 \mathrm{chr} 2.846684 \mathrm{c}$ & 730 chr20_60270। & 6.51 nonsex \\
\hline $2 \mathrm{chr} 2.846684 \mathrm{c}$ & 730 chr1_647230। & 5.84 nonsex \\
\hline $2 \mathrm{chr} 2.846684 \mathrm{c}$ & 730 chr3_646900। & 6.44 nonsex \\
\hline 2 snp.12.170.1: & 730 chr2_848880 & 5.67 nonsex \\
\hline 2 snp.12.170.1: & 730 chr2_820760। & 6.61 nonsex \\
\hline 2 snp.12.170.1: & 730 chr2_821560। & 6 nonsex \\
\hline
\end{tabular}




\begin{tabular}{|c|c|c|c|}
\hline 2 snp.12.170.1: & 730 chr2_833450। & 7.94 nonsex & cis \\
\hline 2 snp.12.170.1: & 730 chr2_846370 & 6.95 nonsex & cis \\
\hline 2 snp.12.170.1: & 730 chr2_760480 & 5.39 nonsex & cis \\
\hline 2 snp.12.170.1: & 730 chr2_787990। & 5.36 nonsex & cis \\
\hline 2 snp.12.170.1: & 730 chr2_789840। & 4.34 nonsex & cis \\
\hline 2 snp.12.170.1: & 730 chr2_791770 & 4.52 nonsex & cis \\
\hline 2 snp.12.170.1: & 730 chr2_799320। & 4.23 nonsex & cis \\
\hline 2 snp.12.170.1: & 730 chr2_820860। & 4.76 nonsex & cis \\
\hline 2 snp.12.170.1: & 730 chr2_826080 & 4.69 nonsex & cis \\
\hline 2 snp.12.170.1: & 730 chr2_855750 & 4.44 nonsex & cis \\
\hline 2 snp.12.170.1: & 730 chr3_986430। & 6.94 nonsex & trans \\
\hline 2 snp.12.170.1: & 730 chr4_411190। & 5.96 nonsex & trans \\
\hline 2 snp.12.170.1: & 730 chr4_436360 & 6.26 nonsex & trans \\
\hline 2 snp.12.170.1: & 730 chr5_851000 & 6.19 nonsex & trans \\
\hline 2 snp.12.170.1: & 730 chr8_108480 & 6.46 nonsex & trans \\
\hline 2 snp.12.170.1: & 730 chr1_574960। & 5.84 nonsex & trans \\
\hline 2 snp.12.170.1: & 730 chr1_853290 & 6.05 nonsex & trans \\
\hline 2 snp.12.170.1: & 730 chr2_567670 & 6.21 nonsex & trans \\
\hline 2 Gg_rs151231. & 730 chr2_931660। & 5.91 nonsex & cis \\
\hline 2 Gg_rs151231. & 730 chr2_962430। & 5.05 nonsex & cis \\
\hline $2 \mathrm{chr} 2.846684 \mathrm{c}$ & 731 chr2_861320। & 6.5 nonsex & cis \\
\hline $2 \mathrm{chr} 2.846684 \mathrm{c}$ & 731 chr2_820750। & 8.56 nonsex & cis \\
\hline 2 chr2.846684c & 731 chr2_883640। & 4.93 nonsex & cis \\
\hline 2 chr2.846684c & 731 chr10_54810 & 7.26 nonsex & trans \\
\hline $2 \mathrm{chr} 2.846684 \mathrm{c}$ & 731 chr1_128960। & 7.38 nonsex & trans \\
\hline $2 \mathrm{chr} 2.846684 \mathrm{c}$ & 731 chr2_491370। & 6.05 nonsex & trans \\
\hline 2 snp.12.170.1: & 731 chr2_840850। & 5.9 nonsex & cis \\
\hline 2 snp.12.170.1: & 731 chr2_846060। & 4.59 nonsex & cis \\
\hline 2 snp.12.170.1: & 731 chr2_918110। & 4.6 nonsex & cis \\
\hline 2 snp.12.170.1: & 731 chr1_120097। & 6.15 nonsex & trans \\
\hline 2 Gg_rs151225. & 731 chr2_798890। & 6.09 nonsex & cis \\
\hline 2 Gg_rs151231 & 731 chr2_809960। & 5.06 nonsex & cis \\
\hline 2 Gg_rs151231. & 731 chr2_838130। & 7.52 nonsex & cis \\
\hline 2 Gg_rs151231. & 731 chr2_741220। & 4.67 nonsex & cis \\
\hline 2 Gg_rs151231. & 731 chr2_793440। & 4.73 nonsex & cis \\
\hline 2 Gg_rs151231. & 731 chr2_822080 & 5.53 nonsex & cis \\
\hline 2 Gg_rs151231. & 731 chr2_832400। & 5.47 nonsex & cis \\
\hline 2 Gg_rs151231. & 731 chr2_833530। & 4.52 nonsex & cis \\
\hline 2 Gg_rs151231. & 731 chr2_853530। & 4.77 nonsex & cis \\
\hline 2 Gg_rs151231. & 731 chr2_926450। & 4.54 nonsex & cis \\
\hline 2 Gg_rs151231. & 731 chr1_260300 & 5.92 nonsex & trans \\
\hline 2 Gg_rs151225. & 732 chr2_857510 & 5.04 nonsex & cis \\
\hline 2 Gg_rs151225. & 732 chr2_874230। & 4.39 nonsex & cis \\
\hline
\end{tabular}




\begin{tabular}{|c|c|c|c|}
\hline 2 Gg_rs151231. & 733 chr2_840070। & 5.92 nonsex & cis \\
\hline 2 Gg_rs151231 & 733 chr2_827340। & 6.72 nonsex & cis \\
\hline 2 Gg_rs151231. & 733 chr2_852630। & 6.14 nonsex & cis \\
\hline 2 Gg_rs151231. & 733 chr2_770370। & 4.32 nonsex & cis \\
\hline 2 Gg_rs151231. & 733 chr2_806010 & 4.7 nonsex & cis \\
\hline 2 Gg_rs151231. & 733 chr2_814000। & 5.19 nonsex & cis \\
\hline 2 Gg_rs151231. & 733 chr2_841130। & 5.26 nonsex & cis \\
\hline 2 Gg_rs151231. & 733 chr2_846230। & 4.92 nonsex & cis \\
\hline 2 Gg_rs151231 & 733 chr2_855690। & 4.33 nonsex & cis \\
\hline 2 Gg_rs151231. & 733 chr2_878610 & 4.5 nonsex & cis \\
\hline 2 Gg_rs151231. & 733 chr7_149800 & 6.18 nonsex & trans \\
\hline 2 Gg_rs151231. & 733 chr2_471100। & 6.13 nonsex & trans \\
\hline 2 Gg_rs151231. & 733 chr3_866980 & 6.93 nonsex & trans \\
\hline 2 Gg_rs151231. & 734 chr2_810670। & 5.99 nonsex & cis \\
\hline 2 Gg_rs151231. & 734 chr2_835220। & 7.55 sex & cis \\
\hline 2 Gg_rs151231. & 734 chr2_848080। & 11.8 nonsex & cis \\
\hline 2 Gg_rs151231. & 734 chr2_850080। & 17.23 nonsex & cis \\
\hline 2 Gg_rs151231. & 734 chr2_853540। & 7.22 nonsex & cis \\
\hline 2 Gg_rs151231. & 734 chr2_853770। & 7.79 nonsex & cis \\
\hline 2 Gg_rs151231 & 734 chr2_854460। & 5.37 nonsex & cis \\
\hline 2 Gg_rs151231. & 734 chr2_709180 & 4.19 nonsex & cis \\
\hline 2 Gg_rs151231. & 734 chr2_787110 & 4.68 nonsex & cis \\
\hline 2 Gg_rs151231. & 734 chr2_861330। & 5.29 nonsex & cis \\
\hline 2 Gg_rs151231. & 734 chr2_880290। & 4.77 nonsex & cis \\
\hline 2 Gg_rs151231. & 734 chr2_883520। & 4.71 nonsex & cis \\
\hline 2 Gg_rs151231. & 734 chr13_68290। & 6.91 sex & trans \\
\hline 2 Gg_rs151231. & 734 chr1_163680 & 7.57 nonsex & trans \\
\hline 2 Gg_rs151231 & 735 chr2_842860। & 8.89 nonsex & cis \\
\hline 2 Gg_rs151231. & 735 chr2_841020। & 5.99 nonsex & cis \\
\hline 2 Gg_rs151231. & 735 chr2_859650। & 6.13 nonsex & cis \\
\hline 2 Gg_rs151231. & 735 chr2_881560 & 6.3 sex & cis \\
\hline 2 Gg_rs151231. & 735 chr2_900610 & 8.43 nonsex & cis \\
\hline 2 Gg_rs151231. & 735 chr2_749210 & 4.97 nonsex & cis \\
\hline 2 Gg_rs151231. & 735 chr2_936190। & 4.48 nonsex & cis \\
\hline $2 \mathrm{chr} 2.846684 \mathrm{c}$ & 736 chr2_910410। & 4.58 nonsex & cis \\
\hline 2 Gg_rs151231. & 736 chr2_877740। & 6.38 nonsex & cis \\
\hline 2 Gg_rs151231. & 736 chr2_837140। & 5.13 nonsex & cis \\
\hline 2 Gg_rs151231. & 736 chr2_896470। & 4.92 nonsex & cis \\
\hline 2 Gg_rs151265 & 736 chr2_880590। & 5.91 nonsex & cis \\
\hline 2 Gg_rs151265 & 736 chr2_886960। & 8.42 nonsex & cis \\
\hline 2 Gg_rs151265 & 736 chr2_779200। & 4.56 nonsex & cis \\
\hline 2 snp.12.170.1: & 737 chr10_88070। & 6.01 nonsex & trans \\
\hline 2 Gg_rs151225. & 737 chr2_853760। & 4.92 nonsex & cis \\
\hline
\end{tabular}




\begin{tabular}{|c|c|c|c|}
\hline 2 Gg_rs151231. & 737 chr2_857800। & 6.43 nonsex & cis \\
\hline 2 Gg_rs151231. & 737 chr2_859870। & 6.01 nonsex & cis \\
\hline 2 Gg_rs151231. & 737 chr2_862670 & 5 nonsex & cis \\
\hline 2 Gg_rs151251 & 737 chr2_863240 & 6.15 nonsex & cis \\
\hline 2 Gg_rs151251 & 737 chr2_906870। & 5.29 nonsex & cis \\
\hline 2 Gg_rs151251 & 737 chr2_887900। & 6.96 nonsex & cis \\
\hline 2 Gg_rs151251 & 737 chr2_846750। & 4.79 nonsex & cis \\
\hline 2 Gg_rs151265 & 737 chr2_839520। & 4.32 nonsex & cis \\
\hline 2 snp.12.170.1: & 738 chr2_842520 & 5.14 nonsex & cis \\
\hline 2 Gg_rs151251 & 738 chr2_857220। & 7.7 nonsex & cis \\
\hline 2 Gg_rs151251 & 738 chr2_881590। & 5.17 nonsex & cis \\
\hline 2 Gg_rs151251 & 738 chr2_859680। & 13.39 nonsex & cis \\
\hline 2 Gg_rs151251 & 738 chr2_880530। & 4.8 nonsex & cis \\
\hline 2 Gg_rs151251 & 739 chr2_850550। & 4.2 nonsex & cis \\
\hline 2 Gg_rs151251 & 739 chr2_874870। & 5.06 nonsex & cis \\
\hline 2 Gg_rs151251 & 740 chr2_874410 & 5.38 nonsex & cis \\
\hline 2 Gg_rs151251 & 741 chr2_854420। & 4.17 nonsex & cis \\
\hline 2 Gg_rs151251 & 741 chr2_922320। & 5.26 nonsex & cis \\
\hline 2 Gg_rs151265 & 741 chr2_842670। & 5.2 nonsex & cis \\
\hline 2 Gg_rs142223 & 741 chr2_967770। & 5.45 nonsex & cis \\
\hline 2 Gg_rs151251 & 742 chr2_859640। & 10.6 nonsex & cis \\
\hline 2 Gg_rs151251 & 742 chr2_876520। & 4.51 nonsex & cis \\
\hline 2 Gg_rs151251 & 742 chr2_893320। & 5.23 nonsex & cis \\
\hline 2 Gg_rs151251 & 742 chrZ_1252201 & 6.48 nonsex & trans \\
\hline 2 Gg_rs151265 & 742 chr2_845710 & 6.46 nonsex & cis \\
\hline 2 Gg_rs151265 & 742 chr2_783080। & 4.69 nonsex & cis \\
\hline 2 Gg_rs151265 & 743 chr2_859150। & 6.11 nonsex & cis \\
\hline 2 Gg_rs151265 & 743 chr2_882430 & 6.45 nonsex & cis \\
\hline 2 Gg_rs151265 & 743 chr2_855360। & 6.19 nonsex & cis \\
\hline 2 Gg_rs151265 & 743 chr2_886970। & 5.31 nonsex & cis \\
\hline 2 Gg_rs151265 & 743 chr4_139380 & 6.04 nonsex & trans \\
\hline 2 Gg_rs151265 & 744 chr2_887530। & 8.93 nonsex & cis \\
\hline 2 Gg_rs151265 & 744 chr2_888060। & 9.18 nonsex & cis \\
\hline 2 Gg_rs151265 & 744 chr2_892130। & 12.72 nonsex & cis \\
\hline 2 Gg_rs151265 & 745 chr2_781360 & 5.19 nonsex & cis \\
\hline 2 Gg_rs151265 & 745 chr2_850070 & 7.3 sex & cis \\
\hline 2 Gg_rs151265 & 745 chr2_855970 & 8.12 nonsex & cis \\
\hline 2 Gg_rs151265 & 745 chr2_862390। & 5.62 sex & cis \\
\hline 2 Gg_rs151265 & 745 chr2_884800 & 5.55 nonsex & cis \\
\hline 2 Gg_rs151265 & 745 chr2_887520। & 7.18 nonsex & cis \\
\hline 2 Gg_rs151265 & 745 chr2_888410 & 7.12 nonsex & cis \\
\hline 2 Gg_rs151265 & 745 chr2_889630। & 6.91 nonsex & cis \\
\hline 2 Gg_rs151265 & 745 chr2_889710 & 5.75 nonsex & cis \\
\hline
\end{tabular}




\begin{tabular}{|c|c|c|c|}
\hline 2 Gg rs151265 & 745 chr2 889870 & 5.54 nonsex & cis \\
\hline 2 Gg_rs151265 & 745 chr2_891840। & 6.71 sex & cis \\
\hline 2 Gg_rs151265 & 745 chr2_891850 & 5.56 sex & cis \\
\hline 2 Gg_rs151265 & 745 chr2_892030। & 6.56 nonsex & cis \\
\hline 2 Gg_rs151265 & 745 chr2_892920। & 9.91 nonsex & cis \\
\hline 2 Gg_rs151265 & 745 chr2_842550। & 4.58 nonsex & cis \\
\hline 2 Gg_rs151265 & 745 chr2_850850 & 4.29 nonsex & cis \\
\hline 2 Gg_rs151265 & 745 chr2_858690 & 3.76 nonsex & cis \\
\hline 2 Gg_rs151265 & 745 chr2_871880 & 4.67 nonsex & cis \\
\hline 2 Gg_rs151265 & 745 chr2_878910। & 3.98 nonsex & cis \\
\hline 2 Gg_rs151265 & 745 chr2_881240। & 4.57 nonsex & cis \\
\hline 2 Gg_rs151265 & 745 chr2_883370। & 4.39 nonsex & cis \\
\hline 2 Gg_rs151265 & 745 chr4_329440। & 5.88 nonsex & trans \\
\hline 2 Gg_rs151265 & 745 chr7_255480 & 5.71 nonsex & trans \\
\hline 2 Gg_rs151265 & 745 chr2_955200 & 6.28 nonsex & trans \\
\hline 2 Gg_rs151265 & 746 chr2_878300। & 7.99 sex & cis \\
\hline 2 Gg_rs151265 & 746 chr2_892340। & 8.14 nonsex & cis \\
\hline 2 Gg_rs151265 & 747 chr2_889350। & 5.77 nonsex & cis \\
\hline 2 Gg_rs151265 & 748 chr2_891440। & 6.96 nonsex & cis \\
\hline 2 Gg_rs151265 & 748 chr2_894710 & $5.56 \operatorname{sex}$ & cis \\
\hline 2 Gg_rs151265 & 748 chr2_922560। & 5.5 sex & cis \\
\hline 2 Gg_rs151265 & 750 chr2_889670। & 6.4 nonsex & cis \\
\hline 2 Gg_rs151265 & 752 chr2_890230। & 6.26 nonsex & cis \\
\hline 2 Gg_rs151265 & 752 chr2_894000। & 6.87 nonsex & cis \\
\hline 2 Gg_rs151265 & 753 chr2_889480। & 7.65 nonsex & cis \\
\hline 2 Gg_rs151265 & 754 chr2_877570। & 4.44 nonsex & cis \\
\hline 2 Gg_rs142223 & 754 chr2_953190। & 5.25 sex & cis \\
\hline 2 Gg_rs151251 & 755 chr2_883540। & 5.38 nonsex & cis \\
\hline 2 Gg_rs151265 & 755 chr2_900140। & 11.88 nonsex & cis \\
\hline 2 Gg_rs151265 & 755 chr2_915850। & 5.11 nonsex & cis \\
\hline 2 Gg_rs151265 & 757 chr2_934080। & 7.62 sex & cis \\
\hline 2 Gg_rs142223 & 758 chr2_965880 & 4.08 nonsex & cis \\
\hline 2 Gg_rs151265 & 759 chr2_931240। & 7.8 sex & cis \\
\hline 2 Gg_rs142209: & 759 chr2_916670। & 4.6 nonsex & cis \\
\hline 2 Gg_rs142223 & 759 chr2_920540 & 7.6 nonsex & cis \\
\hline 2 Gg_rs142189. & 762 chr2_906040। & 5.92 nonsex & cis \\
\hline 2 Gg_rs142189. & 762 chr2_906360। & 12.12 nonsex & cis \\
\hline 2 Gg_rs142189. & 762 chr2_919300। & 5.75 nonsex & cis \\
\hline 2 Gg_rs142223 & 762 chr2_916660। & 8.72 nonsex & cis \\
\hline 2 Gg_rs142223 & 762 chr2_962840। & 5.2 nonsex & cis \\
\hline 2 Gg_rs151265 & 763 chr2_887460। & 3.93 nonsex & cis \\
\hline 2 Gg_rs142189. & 763 chr2_818040। & 5.88 nonsex & cis \\
\hline 2 Gg_rs142189. & 763 chr2_818110 & 11.22 nonsex & cis \\
\hline
\end{tabular}




\begin{tabular}{|c|c|c|c|}
\hline 2 Gg_rs142189. & 763 chr2_903650। & 4.69 nonsex & cis \\
\hline 2 Gg_rs142223 & 763 chr2_919710। & 5.85 nonsex & cis \\
\hline 2 Gg_rs142223 & 763 chr2_919360। & 4.38 nonsex & cis \\
\hline 2 Gg_rs142189. & 764 chr2_818190। & 6.42 nonsex & cis \\
\hline 2 Gg_rs142189. & 764 chr2_898000। & 6.53 nonsex & cis \\
\hline 2 Gg_rs142189. & 764 chr2_905370। & 4.69 nonsex & cis \\
\hline 2 Gg_rs142223 & 764 chr2_920550। & 4.32 nonsex & cis \\
\hline 2 Gg_rs142223 & 764 chr2_966960। & 5.73 sex & cis \\
\hline 2 Gg_rs142189. & 765 chr2_907360। & 5.21 nonsex & cis \\
\hline 2 Gg_rs142189. & 765 chr2_818180 & 4.58 nonsex & cis \\
\hline 2 Gg_rs142189. & 765 chrZ_7883101 & 9.47 sex & trans \\
\hline 2 Gg_rs142189. & 766 chr2_900970। & 5.43 nonsex & cis \\
\hline 2 Gg_rs142189. & 766 chr2_906240। & 7.69 sex & cis \\
\hline 2 Gg_rs142189. & 766 chr2_923050। & 6.44 nonsex & cis \\
\hline 2 Gg_rs142189. & 768 chr2_909650। & 5.55 sex & cis \\
\hline 2 Gg_rs142189. & 769 chr2_838480 & $6.69 \mathrm{sex}$ & cis \\
\hline 2 Gg_rs142189. & 769 chr2_900480। & 5.24 nonsex & cis \\
\hline 2 Gg_rs142189. & 769 chr2_903270। & 7.15 nonsex & cis \\
\hline 2 Gg_rs142189. & 770 chr2_901260। & 5.62 sex & cis \\
\hline 2 Gg_rs142189. & 770 chr2_903540। & 6.64 sex & cis \\
\hline 2 Gg_rs142189. & 770 chr2_915890। & 6.05 sex & cis \\
\hline 2 Gg_rs142189. & 771 chr2_798800। & 5.45 sex & cis \\
\hline 2 Gg_rs142189. & 771 chr2_835300 & 5.37 sex & cis \\
\hline 2 Gg_rs142189. & 771 chr2_906160। & 5.87 sex & cis \\
\hline 2 Gg_rs142189. & 771 chr2_906440। & 4.89 nonsex & cis \\
\hline 2 Gg_rs142189. & 771 chrZ_6358101 & 7.92 sex & trans \\
\hline 2 Gg_rs142189. & 773 chr2_906530। & 5.22 sex & cis \\
\hline 2 Gg_rs142189. & 774 chr2_898500। & 8.15 nonsex & cis \\
\hline 2 Gg_rs142189. & 774 chr2_904710 & 9.31 nonsex & cis \\
\hline 2 Gg_rs107232. & 774 chr2_970890। & 4.37 sex & cis \\
\hline 2 Gg_rs142189. & 775 chr2_915880 & 6.15 sex & cis \\
\hline 2 Gg_rs142189. & 775 chr2_907010। & 4.09 nonsex & cis \\
\hline 2 Gg_rs142189. & 775 chr2_914110 & 5.7 nonsex & cis \\
\hline 2 Gg_rs142189. & 775 chr2_920790। & 5.25 nonsex & cis \\
\hline 2 Gg_rs142189. & 776 chr2_922240 & 6.17 nonsex & cis \\
\hline 2 Gg_rs107232. & 776 chr2_967750। & 5.03 sex & cis \\
\hline 2 Gg_rs142189. & 777 chr2_908010 & 3.76 nonsex & cis \\
\hline 2 Gg_rs142209: & 777 chr2_914120। & 6.44 nonsex & cis \\
\hline 2 Gg_rs142209: & 777 chr2_941850। & 4.53 nonsex & cis \\
\hline 2 Gg_rs142189. & 778 chr2_915420। & 5.84 nonsex & cis \\
\hline 2 Gg_rs142209: & 778 chr2_942220 & 4.73 sex & cis \\
\hline 2 Gg_rs142209: & 778 chr2_920530। & 8.55 nonsex & cis \\
\hline 2 Gg_rs142209: & 778 chr2_919420। & 3.41 nonsex & cis \\
\hline
\end{tabular}




\begin{tabular}{|c|c|c|}
\hline 2 Gg_rs142223 & 778 chr2_912990। & 4.14 nonsex \\
\hline 2 Gg_rs107232. & 778 chr2_100252 & 4.48 nonsex \\
\hline 2 Gg_rs142209: & 779 chr2_912980 & 5.42 nonsex \\
\hline 2 Gg_rs142223 & 780 chr2_910070। & 6.3 nonsex \\
\hline 2 Gg_rs142209: & 781 chr2_928260। & 4.44 nonsex \\
\hline 2 Gg_rs142209: & 782 chr2_918690। & 9.4 nonsex \\
\hline 2 Gg_rs142209: & 782 chr2_921420। & 7.12 nonsex \\
\hline 2 Gg_rs142209: & 782 chr2_969660। & 3.81 nonsex \\
\hline 2 Gg_rs142209: & 783 chr2_926190। & 5.35 nonsex \\
\hline 2 Gg_rs142209: & 783 chr2_921450। & 5.7 nonsex \\
\hline 2 Gg_rs142209: & 783 chr2_929030। & 5.44 nonsex \\
\hline 2 Gg_rs142209: & 783 chr2_942020। & 5.67 nonsex \\
\hline 2 Gg_rs142209: & 785 chr2_921430। & 9.21 nonsex \\
\hline 2 Gg_rs142209: & 785 chr2_941520। & 10.38 nonsex \\
\hline 2 Gg_rs142209: & 785 chr2_942750। & 10.26 nonsex \\
\hline 2 Gg_rs142209: & 785 chr2_943660। & 6.15 sex \\
\hline 2 Gg_rs142209: & 785 chr2_963190। & 6.65 nonsex \\
\hline 2 Gg_rs142209: & 785 chr2_963210। & 5.87 nonsex \\
\hline 2 Gg_rs142209: & 785 chr2_963490। & 6.48 nonsex \\
\hline 2 Gg_rs142209: & 785 chr2_963820। & 6.55 nonsex \\
\hline 2 Gg_rs142209: & 785 chr2_964030। & 5.61 nonsex \\
\hline 2 Gg_rs142209: & 785 chr2_964080। & 6.25 nonsex \\
\hline 2 Gg_rs142209: & 785 chr2_964100। & 5.34 nonsex \\
\hline 2 Gg_rs142209' & 785 chr2_968260। & 9.21 nonsex \\
\hline 2 Gg_rs142209: & 785 chr2_926520। & 3.99 nonsex \\
\hline 2 Gg_rs142209: & 785 chr2_963010। & 4.51 nonsex \\
\hline 2 Gg_rs142209: & 785 chr2_963150। & 4.78 nonsex \\
\hline 2 Gg_rs142209: & 785 chr2_963480 & 4.78 nonsex \\
\hline 2 Gg_rs142209: & 785 chr2_963510। & 4.78 nonsex \\
\hline 2 Gg_rs142209: & 785 chr2_963560। & 4.28 nonsex \\
\hline 2 Gg_rs142209: & 785 chr2_963790। & 4.47 nonsex \\
\hline 2 Gg_rs142209: & 785 chr2_963840। & 4.72 nonsex \\
\hline 2 Gg_rs142209: & 785 chr2_964010। & 4.61 nonsex \\
\hline 2 Gg_rs142209: & 786 chr2_963100। & 5.58 nonsex \\
\hline 2 Gg_rs142209: & 786 chr2_963110 & 5.77 nonsex \\
\hline 2 Gg_rs142209: & 786 chr2_963170। & 5.11 nonsex \\
\hline 2 Gg_rs142209: & 786 chr2_963530। & 5.79 nonsex \\
\hline 2 Gg_rs142209: & 786 chr2_963800। & 7.4 nonsex \\
\hline 2 Gg_rs142209: & 786 chr2_963870। & 5.12 nonsex \\
\hline 2 Gg_rs142209: & 786 chr2_963880 & 5.63 nonsex \\
\hline 2 Gg_rs142209: & 786 chr2_963910। & 5.66 nonsex \\
\hline 2 Gg_rs142209: & 786 chr2_963940। & 6.69 nonsex \\
\hline 2 Gg_rs142209: & 786 chr2_944740। & 4.36 nonsex \\
\hline
\end{tabular}




\begin{tabular}{|c|c|c|c|}
\hline 2 Gg_rs142209: & 786 chr2_963120। & 4.33 nonsex & cis \\
\hline 2 Gg_rs142209: & 786 chr2_963730। & 4.75 nonsex & cis \\
\hline 2 Gg_rs142209: & 786 chr2_963830। & 4.24 nonsex & cis \\
\hline 2 Gg_rs142223 & 786 chr2_963570। & 4.5 nonsex & cis \\
\hline 2 Gg_rs142223 & 786 chr2_963770। & 4.89 nonsex & cis \\
\hline 2 Gg_rs142223 & 786 chr2_963930। & 4.9 nonsex & cis \\
\hline 2 Gg_rs142223 & 787 chr2_936500। & 24.21 nonsex & cis \\
\hline 2 Gg_rs142223 & 787 chr2_936510। & 26.95 nonsex & cis \\
\hline 2 Gg_rs142223 & 787 chr2_941590। & 18.17 nonsex & cis \\
\hline 2 Gg_rs142223 & 787 chr2_944150। & 10.43 nonsex & cis \\
\hline 2 Gg_rs142223 & 787 chr2_944160। & 10.23 nonsex & cis \\
\hline 2 Gg_rs142223 & 788 chr2_881040। & 6.55 sex & cis \\
\hline 2 Gg_rs142223 & 788 chr2_936060। & 5.37 nonsex & cis \\
\hline 2 Gg_rs142223 & 788 chr2_936070। & 9.9 nonsex & cis \\
\hline 2 Gg_rs142223 & 788 chr2_941530। & 9.8 nonsex & cis \\
\hline 2 Gg_rs142223 & 788 chr2_941840। & 8.56 nonsex & cis \\
\hline 2 Gg_rs142223 & 788 chr2_942210। & 8.35 nonsex & cis \\
\hline 2 Gg_rs142223 & 788 chr2_943600। & 13.55 nonsex & cis \\
\hline 2 Gg_rs142223 & 788 chr2_944170। & 8.14 nonsex & cis \\
\hline 2 Gg_rs142223 & 788 chr2_944200। & 11.82 nonsex & cis \\
\hline 2 Gg_rs142223 & 788 chr2_944210 & 7.38 nonsex & cis \\
\hline 2 Gg_rs142223 & 788 chr2_957070। & 5.87 nonsex & cis \\
\hline 2 Gg_rs142223 & 788 chr2_959780। & 5.07 nonsex & cis \\
\hline 2 Gg_rs142223 & 788 chr2_960550। & 29.97 nonsex & cis \\
\hline 2 Gg_rs142223 & 788 chr2_962780। & 7.84 nonsex & cis \\
\hline 2 Gg_rs142223 & 788 chr2_941630। & 4.55 nonsex & cis \\
\hline 2 Gg_rs142223 & 788 chr2_942620। & 4.71 nonsex & cis \\
\hline 2 Gg_rs142223 & 788 chr2_943500। & 4.75 nonsex & cis \\
\hline 2 Gg_rs142223 & 788 chr2_944860। & 4.17 nonsex & cis \\
\hline 2 Gg_rs142223 & 788 chr2_975850। & 4.33 nonsex & cis \\
\hline 2 Gg_rs142223 & 788 chrW_48510 & 7.59 sex & trans \\
\hline 2 Gg_rs142223 & 789 chr2_943590। & 16.46 nonsex & cis \\
\hline 2 Gg_rs142223 & 789 chr2_962060। & 6.25 nonsex & cis \\
\hline 2 Gg_rs142223 & 789 chr2_975500। & 5.92 nonsex & cis \\
\hline 2 Gg_rs142223 & 789 chr2_927720। & 4.22 nonsex & cis \\
\hline 2 Gg_rs142223 & 789 chr2_1005281 & 3.41 nonsex & cis \\
\hline 2 Gg_rs142223 & 790 chr2_904430। & 7.2 nonsex & cis \\
\hline 2 Gg_rs142223 & 790 chr2_940410। & 5.2 nonsex & cis \\
\hline 2 Gg_rs142223 & 790 chr2_948800। & 8.11 nonsex & cis \\
\hline 2 Gg_rs142223 & 790 chr2_944960। & 3.98 nonsex & cis \\
\hline 2 Gg_rs142223 & 790 chr2_947980। & 4.76 nonsex & cis \\
\hline 2 Gg_rs142223 & 791 chr2_944190। & 8.76 nonsex & cis \\
\hline 2 Gg_rs142223 & 791 chr2_956250 & 9.38 nonsex & cis \\
\hline
\end{tabular}




\begin{tabular}{|c|c|c|}
\hline 2 Gg_rs142223 & 791 chr2_960540। & 9.67 nonsex \\
\hline 2 Gg_rs142223 & 791 chr2_960570। & 5.93 nonsex \\
\hline 2 Gg_rs142223 & 791 chr2_949610। & 5.12 nonsex \\
\hline 2 Gg_rs142223 & 792 chr2_961120। & 6.35 nonsex \\
\hline 2 Gg_rs142223 & 792 chr2_941600। & 7.89 nonsex \\
\hline 2 Gg_rs142223 & 792 chr2_968270। & 6.66 nonsex \\
\hline 2 Gg_rs142223 & 792 chr2_941310। & 4.26 nonsex \\
\hline 2 Gg_rs142223 & 792 chr2_943110। & 4.61 nonsex \\
\hline 2 Gg_rs142223 & 792 chr2_965350। & 4.58 nonsex \\
\hline 2 Gg_rs142223 & 793 chr2_944750। & 6.13 nonsex \\
\hline 2 Gg_rs142223 & 793 chr2_967190। & 5.1 nonsex \\
\hline 2 Gg_rs107232. & 793 chr2_944880 & 8.92 nonsex \\
\hline 2 Gg_rs107232. & 793 chr2_944870। & 4.4 nonsex \\
\hline 2 Gg_rs107232 & 794 chr2_953060। & 6.67 nonsex \\
\hline 2 Gg_rs107232. & 794 chr2_928690। & 8.31 sex \\
\hline 2 Gg_rs107232. & 794 chr2_953960। & 7.07 nonsex \\
\hline 2 Gg_rs107232. & 794 chr2_947240। & 4.92 nonsex \\
\hline 2 Gg_rs107232. & 794 chr2_948920। & 4.9 nonsex \\
\hline 2 Gg_rs107232. & 795 chr2_944070। & 7.48 nonsex \\
\hline 2 Gg_rs107232. & 795 chr2_954740। & 4.24 nonsex \\
\hline 2 Gg_rs107232. & 795 chr2_976660। & 3.6 nonsex \\
\hline 2 Gg_rs107232 & 796 chr2_926470। & 6.34 nonsex \\
\hline 2 Gg_rs107232 & 796 chr2_941690। & 7.4 nonsex \\
\hline 2 Gg_rs107232 & 797 chr2_925900। & 5.11 nonsex \\
\hline 2 Gg_rs107232. & 797 chr2_944670। & 6.67 sex \\
\hline 2 Gg_rs107232. & 797 chr2_946570। & 5.88 nonsex \\
\hline 2 Gg_rs107232. & 797 chr2_959190। & 6.45 nonsex \\
\hline 2 Gg_rs107232. & 797 chr2_971110 & 5.33 nonsex \\
\hline 2 Gg_rs107232. & 797 chr2_971330। & 4.05 nonsex \\
\hline 2 Gg_rs107232. & 797 chr2_999280। & 5.17 nonsex \\
\hline 2 Gg_rs107232. & 797 chr2_906710। & 4.27 nonsex \\
\hline 2 Gg_rs107232. & 797 chr2_924230। & 4.85 nonsex \\
\hline 2 Gg_rs107232. & 797 chr2_960640। & 4.49 nonsex \\
\hline 2 Gg_rs107232. & 797 chr2_976240। & 4.14 nonsex \\
\hline 2 Gg_rs107232. & 798 chr2_945330। & 4.31 nonsex \\
\hline 2 Gg_rs107232. & 798 chr2_958750। & 5.41 nonsex \\
\hline 2 Gg_rs107232. & 799 chr2_101574। & 6.36 nonsex \\
\hline 2 Gg_rs107232. & 799 chr2_943330। & 5.06 nonsex \\
\hline 2 Gg_rs107232. & 799 chr2_976530। & 4.61 nonsex \\
\hline 2 Gg_rs107232. & 800 chr2_925160। & 6.2 nonsex \\
\hline 2 Gg_rs107232 & 800 chr2_944910। & 7.1 nonsex \\
\hline 2 Gg_rs107232. & 800 chr2_970700। & 8.53 nonsex \\
\hline 2 Gg_rs107232. & 800 chr2_970900। & $5.98 \mathrm{sex}$ \\
\hline
\end{tabular}




\begin{tabular}{|c|c|c|c|}
\hline 2 Gg rs 107232 & $800 \mathrm{chr} 2961740$ & 5.45 nonsex & cis \\
\hline 2 Gg_rs107232 & 800 chr2_975580। & 4.77 nonsex & cis \\
\hline 2 Gg_rs107232. & 801 chr2_934730। & 4.41 nonsex & cis \\
\hline 2 Gg_rs142223 & 802 chr2_965530। & 8.89 nonsex & cis \\
\hline 2 Gg_rs107232 & 802 chr2_997700। & 5.63 nonsex & cis \\
\hline 2 Gg_rs107232. & 803 chr2_969570। & 8.96 nonsex & cis \\
\hline 2 Gg_rs107232 & 803 chr2_972280। & 4.35 nonsex & cis \\
\hline $2 \times 2 \_10635231$ & 803 chr2_982010 & 4.65 nonsex & cis \\
\hline 2 snp.397.7.57! & 805 chr2_101116। & 3.67 nonsex & cis \\
\hline 2 snp.397.7.57! & 806 chr2_990860। & 4.7 nonsex & cis \\
\hline 2 snp.397.7.57! & 806 chr2_974220। & 4.66 nonsex & cis \\
\hline 2 snp.397.7.57! & 806 chr2_983020। & 4.73 nonsex & cis \\
\hline 2 Gg_rs142223 & 807 chr2_996760। & 4.13 nonsex & cis \\
\hline 2 snp.397.7.57! & 807 chr2_983510 & 3.46 nonsex & cis \\
\hline 2 snp.397.7.57! & 808 chr2_984390। & 4.69 nonsex & cis \\
\hline 2 snp.397.7.57! & 809 chr2_984050। & 8.52 nonsex & cis \\
\hline 2 snp.397.7.57! & 809 chr2_980860। & 4.12 nonsex & cis \\
\hline 2 snp.397.7.57! & 809 chr2_988610 & 4.77 nonsex & cis \\
\hline 2 snp.397.7.57! & 810 chr2_978580 & 4.05 nonsex & cis \\
\hline 2 snp.397.7.57! & 811 chr2_980590। & 6.44 nonsex & cis \\
\hline 2 snp.397.7.57! & 811 chr2_100833। & 5.14 nonsex & cis \\
\hline 2 snp.397.7.57! & 811 chr2_977830। & 4.34 nonsex & cis \\
\hline 2 snp.397.7.57! & 811 chr2_105594! & 4.31 nonsex & cis \\
\hline 2 snp.397.7.57! & 812 chr2_996620। & 4.92 nonsex & cis \\
\hline 2 snp.397.7.57! & 812 chr2_101677। & 3.84 nonsex & cis \\
\hline 2 snp.397.7.57! & 813 chr2_983000। & 5.5 nonsex & cis \\
\hline 2 snp.397.7.57! & 813 chr2_981750। & 4.44 nonsex & cis \\
\hline 2 Gg_rs142253 & 813 chr27_25660। & 6.65 nonsex & trans \\
\hline 2 snp.397.7.57! & 814 chr2_986000। & 3.84 nonsex & cis \\
\hline 2 snp.397.7.57! & 814 chr2_991300। & 11.29 nonsex & cis \\
\hline 2 snp.397.7.57! & 814 chr2_101908। & 5.82 nonsex & cis \\
\hline 2 snp.397.7.57! & 814 chr2_991870 & 5.32 nonsex & cis \\
\hline 2 snp.397.7.57! & 814 chr2_105232 & 4.33 nonsex & cis \\
\hline 2 snp.397.7.57! & 815 chr2_993510 & 5.84 nonsex & cis \\
\hline 2 snp.397.7.57! & 815 chr2_981890। & 4.22 nonsex & cis \\
\hline 2 Gg_rs142253 & 815 chr2_106175। & 4.87 nonsex & cis \\
\hline 2 Gg_rs142253 & 815 chr2_100144! & 6.07 nonsex & cis \\
\hline 2 snp.397.7.57! & 816 chr2_999430। & 3.9 nonsex & cis \\
\hline 2 snp.397.7.57! & 816 chr2_999520। & 5.32 nonsex & cis \\
\hline 2 Gg_rs142253 & 816 chr2_978280 & 7.86 nonsex & cis \\
\hline 2 Gg_rs142253 & 816 chr2_101099| & 5.3 nonsex & cis \\
\hline 2 Gg_rs142253 & 816 chr2_984870 & 4.26 nonsex & cis \\
\hline 2 Gg_rs142253 & 816 chr2_990370। & 4.97 nonsex & cis \\
\hline
\end{tabular}




\begin{tabular}{|c|c|c|}
\hline 2 Gg_rs142253 & 816 chr2_999460। & 4.1 nonsex \\
\hline 2 Gg_rs142253 & 817 chr2_998100। & 9.19 nonsex \\
\hline 2 Gg_rs142253 & 817 chr2_101213। & 7.06 nonsex \\
\hline 2 Gg_rs142253 & 818 chr2_100049| & 7.34 nonsex \\
\hline 2 Gg_rs142253 & 818 chr2_100062। & 6.06 nonsex \\
\hline 2 Gg_rs142253 & 818 chr2_102958। & 5.67 sex \\
\hline 2 Gg_rs142253 & 818 chr2_997610। & 4.5 nonsex \\
\hline 2 Gg_rs142253 & 819 chr2_990090। & 4.12 nonsex \\
\hline 2 Gg_rs142253 & 819 chr2_997970। & 5.72 nonsex \\
\hline 2 Gg_rs142253 & 819 chr2_998120। & 5.33 nonsex \\
\hline 2 Gg_rs142253 & 819 chr2_999810। & 5.12 nonsex \\
\hline 2 Gg_rs142253 & 819 chr2_100050 & 8.16 nonsex \\
\hline 2 Gg_rs142253 & 819 chr2_100055। & 6.93 sex \\
\hline 2 Gg_rs142253 & 819 chr2_100905। & 5.55 nonsex \\
\hline 2 Gg_rs142253 & 819 chr2_100939| & 7.05 nonsex \\
\hline 2 Gg_rs142253 & 819 chr2_101605। & 5.03 nonsex \\
\hline 2 Gg_rs142253 & 819 chr2_902440। & 4.24 nonsex \\
\hline 2 Gg_rs142253 & 819 chr2_967150। & 4.31 nonsex \\
\hline 2 Gg_rs142253 & 819 chr2_977450। & 4.32 nonsex \\
\hline 2 Gg_rs142253 & 819 chr2_989070। & 4.65 nonsex \\
\hline 2 Gg_rs142253 & 819 chr2_100497। & 3.88 nonsex \\
\hline 2 Gg_rs142253 & 819 chr2_100622 & 4.16 nonsex \\
\hline 2 Gg_rs142253 & 819 chr2_100690। & 4.97 nonsex \\
\hline 2 Gg_rs142253 & 819 chr2_101650 & 4.03 nonsex \\
\hline 2 X2_10700517 & 819 chr2_115824! & 7.5 sex \\
\hline 2 Gg_rs142253 & 821 chr2_960420। & 5.15 nonsex \\
\hline 2 Gg_rs142253 & 821 chr2_995090। & 5 nonsex \\
\hline 2 Gg_rs142253 & 824 chr2_993190। & 5.07 nonsex \\
\hline 2 X2_10635231 & 824 chr2_999270। & 7.75 nonsex \\
\hline 2 Gg_rs142253 & 825 chr2_991280। & 6.12 nonsex \\
\hline 2 X2_10635231 & 826 chr2_101945। & 5.04 nonsex \\
\hline 2 X2_10635231 & 826 chr2_100507। & 5.78 nonsex \\
\hline 2 X2_10635231 & 827 chr2_996030। & 5.26 nonsex \\
\hline 2 X2_10635231 & 827 chr2_999400। & 12 nonsex \\
\hline 2 X2_10635231 & 827 chr2_102081। & 4.79 nonsex \\
\hline 2 X2_10635231 & 827 chr2_102593। & $5.24 \mathrm{sex}$ \\
\hline 2 X2_10635231 & 827 chr2_987060। & 5.63 nonsex \\
\hline 2 X2_10635231 & 827 chr2_100297। & 3.88 nonsex \\
\hline 2 X2_10635231 & 828 chr2_998400। & 6.24 nonsex \\
\hline 2 X2_10635231 & 828 chr2_920420। & 5.1 nonsex \\
\hline 2 X2_10635231 & 828 chr2_981320। & 4.12 nonsex \\
\hline 2 X2_10635231 & 829 chr2_1010261 & 4.84 nonsex \\
\hline $2 \times 2 \_10635231$ & 829 chr2_998110। & 4.68 nonsex \\
\hline
\end{tabular}




\begin{tabular}{|c|c|c|c|}
\hline $2 \times 2 \_10635231$ & 830 chr2_986770। & 5.53 nonsex & cis \\
\hline $2 \times 2 \_10635231$ & 830 chr2_101935। & 5.09 nonsex & cis \\
\hline $2 \times 2 \_10635231$ & 831 chr2_991560। & 5.34 nonsex & cis \\
\hline 2 X2_10635231 & 832 chr2_994700। & 6.29 nonsex & cis \\
\hline 2 X2_10635231 & 832 chr2_999650। & 7.54 nonsex & cis \\
\hline $2 \times 2 \_10635231$ & 832 chr2_990000। & 4.54 nonsex & cis \\
\hline $2 \times 2 \_10635231$ & 833 chr2_981570। & 6.24 nonsex & cis \\
\hline $2 \times 2 \_10635231$ & 833 chr2_989060। & 5.11 nonsex & cis \\
\hline $2 \times 2 \_10700517$ & 834 chr2_986680 & 5.68 nonsex & cis \\
\hline 2 X2_10700517 & 834 chr2_100557। & 5.42 nonsex & cis \\
\hline 2 X2_10635231 & 835 chr2_917920। & 4.22 nonsex & cis \\
\hline 2 X2_10700517 & 836 chr2_100061। & 6.45 nonsex & cis \\
\hline $2 \times 2 \_10700517$ & 836 chr2_100401। & 4.21 nonsex & cis \\
\hline $2 \times 2 \_10700517$ & 836 chr2_100912 & 4.74 nonsex & cis \\
\hline $2 \times 2 \_10700517$ & 836 chr2_101571। & 4.49 nonsex & cis \\
\hline $2 \times 2 \_10700517$ & 837 chr2_995810 & 5.42 nonsex & cis \\
\hline 2 X2_10700517 & 837 chr2_101243। & 8.41 nonsex & cis \\
\hline $2 \times 2 \_10700517$ & 837 chr7_338810 & 7.6 sex & trans \\
\hline 2 X2_10700517 & 838 chr2_983980। & 5.65 nonsex & cis \\
\hline 2 X2_10700517 & 838 chr2_992010। & 6.85 nonsex & cis \\
\hline $2 \times 2 \_10700517$ & 838 chr2_996420 & 5.61 nonsex & cis \\
\hline $2 \times 2 \_10700517$ & 838 chr2_996430। & 4.92 nonsex & cis \\
\hline $2 \times 2 \_10700517$ & 838 chr2_980830 & 4.58 nonsex & cis \\
\hline 2 X2_10700517 & 838 chr2_988930। & 4.8 nonsex & cis \\
\hline 2 X2_10700517 & 838 chr2_996340। & 4.26 nonsex & cis \\
\hline $2 \times 2 \_10700517$ & 838 chr2_997340। & 3.93 nonsex & cis \\
\hline $2 \times 2 \_10700517$ & 838 chr2_101016! & 3.78 nonsex & cis \\
\hline $2 \times 2 \_10700517$ & 839 chr2_101682 & 4.98 nonsex & cis \\
\hline 2 X2_10700517 & 840 chr2_987550। & 4.72 nonsex & cis \\
\hline $2 \times 2 \_10700517$ & 840 chr2_100020 & 5.76 nonsex & cis \\
\hline 2 X2_10700517 & 840 chr2_990790। & 3.84 nonsex & cis \\
\hline 2 X2_10700517 & 842 chr2_978210 & 3.94 nonsex & cis \\
\hline 2 X2_10700517 & 843 chr2_100172। & 4.26 nonsex & cis \\
\hline $2 \times 2 \_10700517$ & 845 chr2_102449। & 9.16 nonsex & cis \\
\hline 2 X2_10700517 & 845 chr2_101034। & 3.59 nonsex & cis \\
\hline $2 \times 2 \_10700517$ & 846 chr2_100506। & 4.54 nonsex & cis \\
\hline $2 \times 2 \_10700517$ & 846 chr2_101261। & 4.32 nonsex & cis \\
\hline 2 X2_10700517 & 848 chr2_103016। & 4.16 nonsex & cis \\
\hline 2 snp.397.7.57! & 849 chr2_982030। & 4.29 nonsex & cis \\
\hline 2 X2_10700517 & 850 chr2_102358 & 4.66 nonsex & cis \\
\hline $2 \times 2 \_10635231$ & 851 chr2_908490। & 4.5 nonsex & cis \\
\hline $2 \times 2 \_10700517$ & 851 chr2_101832 & 4.64 nonsex & cis \\
\hline $2 \times 2 \_10700517$ & 861 chr2_104164! & 4.83 nonsex & cis \\
\hline
\end{tabular}




\begin{tabular}{|c|c|c|c|}
\hline 2 Gg_rs151424 & 874 chr2_110535। & 7.09 nonsex & cis \\
\hline 2 Gg_rs142189. & 879 chr2_907190। & 4.59 nonsex & cis \\
\hline 2 Gg_rs151424 & 882 chr2_110468। & 6.81 sex & cis \\
\hline 2 Gg_rs142329 & 884 chr2_104393। & 5.89 nonsex & cis \\
\hline 2 Gg_rs142329 & 885 chr2_104352 & 6.6 nonsex & cis \\
\hline 2 Gg_rs151424 & 888 chr2_111119! & 5.9 nonsex & cis \\
\hline 2 Gg_rs142329 & 894 chr2_107994। & 6.3 nonsex & cis \\
\hline 2 Gg_rs142329 & 920 chr2_108300 & 4.45 nonsex & cis \\
\hline 2 Gg_rs142329 & 920 chr2_108373। & 10.72 nonsex & cis \\
\hline 2 Gg_rs142329 & 920 chr2_109483। & 7.21 nonsex & cis \\
\hline 2 Gg_rs142329 & 920 chr2_110214! & 7.19 nonsex & cis \\
\hline 2 Gg_rs142329 & 921 chr2_108376 & 6.54 sex & cis \\
\hline 2 Gg_rs142329 & 922 chr2_110552। & 3.93 nonsex & cis \\
\hline 2 Gg_rs151424 & 923 chr2_110189| & 5.67 nonsex & cis \\
\hline 2 Gg_rs151424 & 924 chr2_111024! & 5.19 nonsex & cis \\
\hline 2 Gg_rs151424 & 924 chr2_117306। & 5.77 sex & cis \\
\hline 2 Gg_rs151424 & 925 chr2_110332 & 5.25 nonsex & cis \\
\hline 2 Gg_rs151424 & 925 chr2_110714! & 24.52 nonsex & cis \\
\hline 2 Gg_rs151424 & 925 chr2_110799। & 14.22 nonsex & cis \\
\hline 2 Gg_rs151424 & 925 chr8_313400। & 7.07 sex & trans \\
\hline 2 Gg_rs151424 & 926 chr2_110715। & 21.37 nonsex & cis \\
\hline 2 Gg_rs151424 & 926 chr2_112422 & 4.21 nonsex & cis \\
\hline 2 Gg_rs151424 & 927 chr2_1105361 & 10.69 nonsex & cis \\
\hline 2 Gg_rs151424 & 927 chr2_111731। & 8.55 nonsex & cis \\
\hline 2 Gg_rs151424 & 927 chr2_110602 & 5.31 nonsex & cis \\
\hline 2 Gg_rs151429: & 927 chr2_111455। & 6.91 nonsex & cis \\
\hline 2 Gg_rs151424 & 928 chr2_111674! & 6.22 nonsex & cis \\
\hline 2 Gg_rs151424 & 928 chr2_110025। & 3.99 nonsex & cis \\
\hline 2 Gg_rs151429: & 928 chr2_111732 & 7.36 nonsex & cis \\
\hline 2 Gg_rs151429: & 928 chr2_111764। & 9.03 nonsex & cis \\
\hline 2 Gg_rs151429: & 928 chr2_111735। & 5.45 nonsex & cis \\
\hline 2 Gg_rs151424 & 929 chr2_1104981 & 5.97 nonsex & cis \\
\hline 2 Gg_rs151429: & 929 chr2_111617। & 5.91 nonsex & cis \\
\hline 2 Gg_rs151429: & 929 chr2_111730 & 8.32 nonsex & cis \\
\hline 2 Gg_rs151429: & 929 chr2_111738 & 5.59 nonsex & cis \\
\hline 2 Gg_rs151429: & 929 chr2_111743। & 8.42 nonsex & cis \\
\hline 2 Gg_rs151429: & 929 chr2_111744! & 7.32 nonsex & cis \\
\hline 2 Gg_rs151429: & 929 chr2_111752 & 9.5 nonsex & cis \\
\hline 2 Gg_rs151429: & 929 chr2_111739| & 4.58 nonsex & cis \\
\hline 2 Gg_rs151429: & 929 chr2_111746 & 4.86 nonsex & cis \\
\hline 2 Gg_rs151429: & 929 chr2_111759! & 5.22 nonsex & cis \\
\hline 2 Gg_rs151429: & 929 chr2_111765। & 4.47 nonsex & cis \\
\hline 2 Gg_rs151429: & 930 chr2_111734! & 9.77 nonsex & cis \\
\hline
\end{tabular}




\begin{tabular}{|c|c|c|c|}
\hline 2 Gg_rs151429: & 930 chr2_111745। & 11.27 nonsex & cis \\
\hline 2 Gg_rs151429: & 930 chr2_111755। & 8.89 nonsex & cis \\
\hline 2 Gg_rs151429: & 930 chr2_111763। & 7.8 nonsex & cis \\
\hline 2 Gg_rs151429: & 930 chr2_109506। & 5.01 nonsex & cis \\
\hline 2 Gg_rs151429: & 930 chr2_110549! & 4.78 nonsex & cis \\
\hline 2 Gg_rs151429: & 930 chr2_111194! & 4.15 nonsex & cis \\
\hline 2 Gg_rs151429: & 930 chr2_111663। & 4 nonsex & cis \\
\hline 2 Gg_rs151429: & 930 chr1_790910 & 5.89 nonsex & trans \\
\hline 2 Gg_rs151429: & 930 chr5_519660 & 5.83 nonsex & trans \\
\hline 2 Gg_rs151429: & 930 chr6_331690। & 5.69 nonsex & trans \\
\hline 2 Gg_rs151429: & 930 chr12_98130। & 5.77 nonsex & trans \\
\hline 2 Gg_rs151429: & 930 chr14_72820 & 5.23 nonsex & trans \\
\hline 2 Gg_rs151429: & 931 chr33_54970। & 6.16 sex & trans \\
\hline 2 Gg_rs151429: & 931 chr3_818650। & 6.29 sex & trans \\
\hline 2 Gg_rs151429: & 932 chr2_111753। & 8.98 nonsex & cis \\
\hline 2 Gg_rs151429: & 933 chr2_111742 & 11.47 nonsex & cis \\
\hline 2 Gg_rs151429: & 933 chr2_111198। & 4.97 nonsex & cis \\
\hline 2 Gg_rs151429: & 934 chr2_111729। & 9.75 nonsex & cis \\
\hline 2 Gg_rs151429: & 935 chr2_111741। & 5.04 nonsex & cis \\
\hline 2 Gg_rs151429: & 936 chr2_111615। & 7.1 nonsex & cis \\
\hline 2 Gg_rs151429: & 936 chr2_111648। & 6.61 sex & cis \\
\hline 2 Gg_rs151429: & 936 chr2_111123। & 4.27 nonsex & cis \\
\hline 2 RBL1120 & 936 chr2_111618 & 6.39 nonsex & cis \\
\hline 2 RBL1120 & 938 chr2_112271। & 5.32 nonsex & cis \\
\hline 2 RBL1120 & 942 chr2_116072 & 5.65 sex & cis \\
\hline 2 RBL1120 & 943 chr8_263030। & 5.49 nonsex & trans \\
\hline 2 Gg_rs151445 & 949 chr2_114475। & 4.83 nonsex & cis \\
\hline 2 Gg_rs151445 & 951 chr2_111740 & 4.7 nonsex & cis \\
\hline 2 Gg_rs151445 & 951 chr3_104455। & 6.38 sex & trans \\
\hline 2 Gg_rs151445 & 952 chr31_21390। & 7.73 sex & trans \\
\hline 2 Gg_rs151445 & 953 chr31_21420 & 8.06 sex & trans \\
\hline 2 Gg_rs151445 & 955 chr2_111450 & 5.57 nonsex & cis \\
\hline 2 Gg_rs151445 & 955 chr2_112301। & 5.7 sex & cis \\
\hline 2 Gg_rs151445 & 955 chr2_114660। & 6.42 nonsex & cis \\
\hline 2 Gg_rs151445 & 955 chrW_35040 & 7.63 sex & trans \\
\hline 2 Gg_rs151445 & 955 chr3_700600 & 6.02 nonsex & trans \\
\hline 2 Gg_rs151445 & 955 chr3_236220। & 5.75 nonsex & trans \\
\hline 2 Gg_rs151429: & 956 chr2_108283। & 3.95 nonsex & cis \\
\hline 2 Gg_rs151445 & 956 chr2_113886 & 4.54 nonsex & cis \\
\hline 2 Gg_rs151445 & 956 chr2_114191। & 6.05 sex & cis \\
\hline 2 Gg_rs151445 & 956 chr2_115659 & 5.42 sex & cis \\
\hline 2 Gg_rs151445 & 956 chr2_1084981 & 4.53 nonsex & cis \\
\hline 2 Gg_rs151445 & 956 chr2_115732। & 4.13 nonsex & cis \\
\hline
\end{tabular}




\begin{tabular}{|c|c|c|c|}
\hline 2 Gg_rs151445 & 957 chr2_117556। & 5.89 sex & cis \\
\hline 2 Gg_rs151445 & 959 chr2_114192। & 5.68 nonsex & cis \\
\hline 2 rbl2524 & 960 chr2_115886 & 6.79 nonsex & cis \\
\hline 2 rbl2524 & 960 chr2_107882 & 5.97 sex & cis \\
\hline 2 rbl2524 & 960 chr2_110803। & 4.82 nonsex & cis \\
\hline 2 rbl2524 & 961 chr2_110130। & 5.97 sex & cis \\
\hline 2 rbl2524 & 961 chr2_114799। & 4.47 nonsex & cis \\
\hline 2 rbl2524 & 969 chr2_115657। & 4.67 nonsex & cis \\
\hline 2 Gg_rs151465. & 973 chr1_721170 & 8.24 sex & trans \\
\hline 2 Gg_rs151465. & 974 chr2_117246। & 6.18 nonsex & cis \\
\hline 2 Gg_rs151465. & 980 chr2_111544। & 4.9 nonsex & cis \\
\hline 2 Gg_rs151465. & 980 chr2_117250 & 4.49 nonsex & cis \\
\hline 2 Gg_rs151465. & 981 chr2_117451। & 6.68 nonsex & cis \\
\hline 2 Gg_rs151465. & 985 chr2_117663। & 4.77 nonsex & cis \\
\hline 2 Gg_rs142424 & 988 chr2_117666। & 5.84 nonsex & cis \\
\hline 2 Gg_rs142424. & 989 chr2_121718। & 4.4 nonsex & cis \\
\hline 2 snp.21.121.2: & 989 chr2_121817। & 4.53 nonsex & cis \\
\hline 2 Gg_rs142424. & 991 chr2_116563। & 5.31 sex & cis \\
\hline 2 snp.21.121.2: & 992 chr2_118093। & 5.6 nonsex & cis \\
\hline 2 Gg_rs142424. & 993 chr2_117822 & 3.89 nonsex & cis \\
\hline 2 Gg_rs142424. & 993 chr2_120475। & 4.75 nonsex & cis \\
\hline 2 Gg_rs142424. & 994 chr2_118090 & 6.64 nonsex & cis \\
\hline 2 snp.21.121.2: & 996 chr2_121143। & 6.86 nonsex & cis \\
\hline 2 snp.21.121.2: & 998 chr2_120413। & 4.91 nonsex & cis \\
\hline 2 snp.21.121.2: & 998 chr4_429890। & 7.14 sex & trans \\
\hline 2 snp.21.121.2: & 999 chr2_120205। & 8.05 nonsex & cis \\
\hline 2 snp.21.121.2: & 999 chr2_120178। & 3.57 nonsex & cis \\
\hline 2 snp.21.121.2: & 1001 chr2_121273। & 7.75 nonsex & cis \\
\hline 2 snp.21.121.2: & 1002 chr2_119621। & 6.22 sex & cis \\
\hline 2 snp.21.121.2: & 1002 chr2_120230 & 5.16 nonsex & cis \\
\hline 2 snp.21.121.2: & 1003 chr2_124034! & 5.8 nonsex & cis \\
\hline 2 snp.21.121.2: & 1003 chr2_117471 & 4.12 nonsex & cis \\
\hline 2 snp.21.121.2: & 1004 chr2_120430 & 6.05 nonsex & cis \\
\hline 2 snp.21.121.2: & 1004 chr2_120242 & 4.67 nonsex & cis \\
\hline 2 snp.21.121.2: & 1006 chr2_123136। & 4.68 nonsex & cis \\
\hline 2 snp.21.121.2: & 1007 chr2_121857। & 7.68 nonsex & cis \\
\hline 2 snp.21.121.2: & 1007 chr2_120218 & 5.85 nonsex & cis \\
\hline 2 Gg_rs151445 & 1010 chr2_117224! & 4.74 nonsex & cis \\
\hline 2 Gg_rs142487। & 1011 chr2_123188 & 8.99 nonsex & cis \\
\hline 2 Gg_rs151526 & 1013 chr2_122359! & 6.28 nonsex & cis \\
\hline 2 Gg_rs142487 & 1013 chr2_123014। & 9.43 nonsex & cis \\
\hline 2 Gg_rs142487 & 1014 chr2_122665। & 4.84 nonsex & cis \\
\hline 2 Gg_rs151526 & 1016 chr2_121422 & 7.45 nonsex & cis \\
\hline
\end{tabular}




\begin{tabular}{|c|c|c|c|}
\hline 2 Gg_rs142487 & 1017 chr2_121202 & $6.8 \mathrm{sex}$ & cis \\
\hline 2 Gg_rs142487 & 1019 chr2_122966। & 4.15 nonsex & cis \\
\hline 2 Gg_rs151526 & 1020 chr2_121717। & 5.41 nonsex & cis \\
\hline 2 Gg_rs151526 & 1020 chr2_122797। & 3.91 nonsex & cis \\
\hline 2 Gg_rs151526 & 1025 chr2_123000 & 3.78 nonsex & cis \\
\hline 2 Gg_rs151526 & 1032 chr2_125870 & 5.9 nonsex & cis \\
\hline 2 Gg_rs151526 & 1032 chr2_124292 & 7.16 nonsex & cis \\
\hline 2 Gg_rs142487 & 1032 chr2_122381। & 5.74 nonsex & cis \\
\hline 2 Gg_rs142487 & 1032 chr2_122557। & 7.6 nonsex & cis \\
\hline 2 Gg_rs142487 & 1032 chr2_123189! & 10.12 nonsex & cis \\
\hline 2 Gg_rs142487 & 1032 chr2_123362 & 5.56 nonsex & cis \\
\hline 2 Gg_rs142487 & 1032 chr2_1242931 & 5.27 sex & cis \\
\hline 2 Gg_rs142487 & 1032 chr2_125142 & 6.74 sex & cis \\
\hline 2 Gg_rs142487| & 1032 chr2_125154! & 6.4 nonsex & cis \\
\hline 2 Gg_rs142487| & 1032 chr2_125669। & 6.04 sex & cis \\
\hline 2 Gg_rs142487| & 1032 chr2_125976। & 5.37 nonsex & cis \\
\hline 2 Gg_rs142487 & 1032 chr2_126131। & 4.36 nonsex & cis \\
\hline 2 Gg_rs142487 & 1032 chr2_126239। & 5.56 nonsex & cis \\
\hline 2 Gg_rs142487 & 1032 chr2_121401। & 4.18 nonsex & cis \\
\hline 2 Gg_rs142487 & 1032 chr2_122660 & 4.13 nonsex & cis \\
\hline 2 Gg_rs142487| & 1032 chr2_123074! & 4.16 nonsex & cis \\
\hline 2 Gg_rs142487| & 1032 chr2_124356! & 3.58 nonsex & cis \\
\hline 2 Gg_rs142487| & 1032 chr2_124709। & 4.18 nonsex & cis \\
\hline 2 Gg_rs142487 & 1032 chr2_125137। & 4.08 nonsex & cis \\
\hline 2 Gg_rs142487 & 1032 chr2_125406 & 3.44 nonsex & cis \\
\hline 2 Gg_rs142487| & 1032 chr2_125974! & 4.82 nonsex & cis \\
\hline 2 Gg_rs142487| & 1032 chr2_126130 & 4.8 nonsex & cis \\
\hline 2 Gg_rs142487| & 1032 chr2_127144! & 4.23 nonsex & cis \\
\hline 2 Gg_rs142487 & 1032 chrZ_813610। & 7.26 sex & trans \\
\hline 2 Gg_rs142487 & 1032 chr2_301760 & 4.26 nonsex & trans \\
\hline 2 Gg_rs142487 & 1033 chr2_126042 & 5.68 nonsex & cis \\
\hline 2 Gg_rs142487 & 1033 chr2_121858। & 4.41 nonsex & cis \\
\hline 2 Gg_rs142487 & 1033 chr2_1226861 & 3.64 nonsex & cis \\
\hline 2 Gg_rs142487| & 1033 chr2_125164! & 4.63 nonsex & cis \\
\hline 2 Gg_rs142487| & 1034 chr2_1255361 & 11.09 nonsex & cis \\
\hline 2 Gg_rs142487 & 1034 chr2_123327। & 4.5 nonsex & cis \\
\hline 2 Gg_rs142487 & 1035 chr2_123197। & 5.57 nonsex & cis \\
\hline 2 Gg_rs142487 & 1035 chr2_122494! & 4.61 nonsex & cis \\
\hline 2 Gg_rs142487 & 1035 chr2_122661। & 3.82 nonsex & cis \\
\hline 2 Gg_rs142487 & 1035 chr2_125970 & 4.86 nonsex & cis \\
\hline 2 Gg_rs142487| & 1036 chr2_122117। & 3.9 nonsex & cis \\
\hline 2 Gg_rs142487| & 1036 chr2_123073। & 3.84 nonsex & cis \\
\hline 2 Gg_rs142487| & 1036 chr2_125133। & 4.86 nonsex & cis \\
\hline
\end{tabular}




\begin{tabular}{|c|c|c|c|}
\hline 2 Gg_rs142487। & 1036 chr2 125513 & 4.11 nonsex & cis \\
\hline 2 Gg_rs142487| & 1037 chr2_125609। & $7.21 \mathrm{sex}$ & cis \\
\hline 2 Gg_rs142487| & 1037 chr2_124230 & 4.61 nonsex & cis \\
\hline 2 Gg_rs142487| & 1037 chr2_125606। & 5.43 nonsex & cis \\
\hline 2 Gg_rs151526 & 1038 chr2_124309। & 4.02 nonsex & cis \\
\hline 2 Gg_rs142487 & 1038 chr2_122799। & 3.7 nonsex & cis \\
\hline 2 Gg_rs142487| & 1039 chr2_121300 & 4.33 nonsex & cis \\
\hline 2 Gg_rs151546: & 1042 chr2_130605। & 3.88 nonsex & cis \\
\hline 2 Gg_rs151546: & 1043 chr2_130607। & 4.68 nonsex & cis \\
\hline 2 Gg_rs151546 & 1047 chr2_127652 & 3.7 nonsex & cis \\
\hline 2 Gg_rs151546 & 1049 chr2_127291। & 4.6 nonsex & cis \\
\hline 2 Gg_rs151546: & 1049 chr33_47330 & 8.86 nonsex & trans \\
\hline 2 Gg_rs151546: & 1049 chr4_254400। & 7.65 sex & trans \\
\hline 2 Gg_rs151546: & 1050 chr1_130200 & 4.75 nonsex & trans \\
\hline 2 Gg_rs151546: & 1050 chr28_11610 & 6 nonsex & trans \\
\hline 2 Gg_rs151546! & 1051 chr2_126720 & 4.81 nonsex & cis \\
\hline 2 Gg_rs151546: & 1051 chr2_126950 & 21.9 nonsex & cis \\
\hline 2 Gg_rs151546 & 1051 chr2_126951। & 5.12 nonsex & cis \\
\hline 2 Gg_rs151546! & 1051 chr2_126989। & 6.98 nonsex & cis \\
\hline 2 Gg_rs151546 & 1051 chr2_127397। & $6.4 \operatorname{sex}$ & cis \\
\hline 2 Gg_rs151546: & 1051 chr2_130654। & $5.91 \mathrm{sex}$ & cis \\
\hline 2 Gg_rs151546 & 1051 chr2_127655। & 4.19 nonsex & cis \\
\hline 2 Gg_rs151546 & 1051 chr1_107300 & 5.91 nonsex & trans \\
\hline 2 Gg_rs151546 & 1051 chr1_107400 & 6.3 nonsex & trans \\
\hline 2 Gg_rs151546: & 1051 chr5_581130 & 6 nonsex & trans \\
\hline 2 Gg_rs151546 & 1051 chr10_20299| & 5.98 nonsex & trans \\
\hline 2 Gg_rs151546 & 1051 chr12_95000 & 5.82 nonsex & trans \\
\hline 2 Gg_rs151546: & 1051 chr18_92790। & 4.88 nonsex & trans \\
\hline 2 Gg_rs151546: & 1051 chrZ_25000 & 5.48 nonsex & trans \\
\hline 2 Gg_rs151546 & 1054 chr2_127671। & 5.48 nonsex & cis \\
\hline 2 Gg_rs151546 & 1055 chr2_126948। & 4.72 nonsex & cis \\
\hline 2 Gg_rs151546: & 1059 chr2_1267361 & 5.03 nonsex & cis \\
\hline 2 Gg_rs151546: & 1060 chr2_127194! & 6.78 sex & cis \\
\hline 2 Gg_rs151546! & 1064 chr2_126663। & 4.64 nonsex & cis \\
\hline 2 Gg_rs142487| & 1073 chr2_127467। & 6.14 nonsex & cis \\
\hline 2 Gg_rs137949: & 1087 chr2_134053। & 4.38 nonsex & cis \\
\hline 2 rbl2639 & 1090 chr2_132475। & 5.41 nonsex & cis \\
\hline 2 Gg_rs142536 & 1091 chr2_136144! & 4.51 nonsex & cis \\
\hline 2 Gg_rs137949: & 1095 chr2_134786! & 4.47 nonsex & cis \\
\hline 2 Gg_rs151559. & 1100 chr2_132339। & 13.6 nonsex & cis \\
\hline 2 rbl2639 & 1101 chr2_129624! & 4.31 nonsex & cis \\
\hline 2 rbl2639 & 1102 chr2_131370 & 4.74 nonsex & cis \\
\hline 2 Gg_rs142536 & 1107 chr2_148320। & 2.99 nonsex & cis \\
\hline
\end{tabular}


2 rbl2639

2 rbl2639

2 rbl2639

2 rbl2639

2 rbl2639

2 rbl2639

2 rbl2639

2 rbl2639

2 rbl2639

2 Gg_rs151559

2 Gg_rs151559

2 Gg_rs151559

2 Gg_rs151559

2 Gg_rs142536

2 Gg_rs151559

2 Gg_rs151559

2 Gg_rs151559

2 Gg_rs151559

2 Gg_rs151559

2 Gg_rs151559

2 Gg_rs151559

2 Gg_rs137949

2 Gg_rs137949

2 Gg_rs137949

2 Gg_rs137949,

2 Gg_rs137949

2 Gg_rs137949

2 Gg_rs137949

2 Gg_rs137949

2 Gg_rs142536

2 Gg_rs 142536

2 Gg_rs142536

2 Gg_rs142536

2 Gg_rs 142536

2 Gg_rs 142536

2 Gg_rs142536

2 Gg_rs 142536

2 Gg_rs 142536

2 Gg_rs142536

2 Gg_rs 142536

2 Gg_rs 142536

2 Gg_rs 142536

2 Gg_rs142536
1110 chr2_128543।

1119 chr2_131590।

1119 chr2_132028

1119 chr2_125935

1119 chr2_135675।

1119 chrz_163250।

1120 chr2_1303231

1121 chr2_132099!

1121 chr2_129607।

1122 chr2 129609

1122 chr2_129761।

1122 chr2_132535।

1123 chr2_131519!

1123 chr2_138146।

1124 chr2_131420।

1125 chr2_130285

1125 chr2_129760।

1126 chr2_1323181

1127 chr2_1323381

1127 chr2_131366।

1128 chr2_134392

1128 chr2_132170।

1129 chr2_1325231

1131 chr2_128795।

1131 chr2_1324331

1131 chr2_132554!

1132 chr2_132674!

1133 chr2_134660।

1133 chrW_59740

1136 chr2_136235

1136 chr2_136361।

1136 chr2_132962

1136 chr2_136716।

1137 chr2_1352531

1137 chr2_135685।

1137 chr2_136739!

1137 chrW_41390(

1138 chr2_136373!

1138 chrW_34760

1139 chr2_1366581

1139 chr2_141101।

1139 chr2_136129!

1139 chr2_136499!
4.08 nonsex cis

5.61 sex cis

5.33 nonsex cis

4.21 nonsex cis

3.59 nonsex cis

5.19 nonsex trans

6.3 nonsex cis

5.4 nonsex cis

4.44 nonsex cis

6.34 nonsex cis

10.12 nonsex cis

7.39 nonsex cis

8.4 nonsex cis

3.73 nonsex cis

5.95 nonsex cis

6.54 nonsex cis

4.26 nonsex cis

6.2 nonsex cis

9.12 sex cis

4.92 nonsex cis

5.55 nonsex cis

4.23 nonsex cis

7.19 nonsex cis

4.83 nonsex cis

8.62 nonsex cis

6.99 sex cis

4.25 nonsex cis

5.27 nonsex cis

$9.87 \mathrm{sex} \quad$ trans

8.04 nonsex cis

7.38 sex cis

4.24 nonsex cis

4.27 nonsex cis

5.62 nonsex cis

7.84 nonsex cis

4.57 nonsex cis

7.6 sex trans

4.32 nonsex cis

8.65 sex trans

5.87 sex cis

4.21 nonsex cis

4.66 nonsex cis

3.98 nonsex cis 


\begin{tabular}{|c|c|c|c|}
\hline 2 Gg_rs142536 & 1139 chrW_35150 & 9.13 sex & trans \\
\hline 2 Gg_rs142536 & 1141 chr2_136233। & 22.14 nonsex & cis \\
\hline 2 Gg_rs142536 & 1141 chr2_137380 & 9.6 nonsex & cis \\
\hline 2 Gg_rs142536 & 1142 chr2_136073। & 5.22 nonsex & cis \\
\hline 2 Gg_rs142536 & 1142 chr2_136441। & 3.2 nonsex & cis \\
\hline 2 Gg_rs142536 & 1143 chr2_137789 & 5.28 nonsex & cis \\
\hline 2 Gg_rs137615. & 1145 chr2_136732 & 4.67 nonsex & cis \\
\hline 2 Gg_rs142536 & 1146 chr2_136240 & 3.7 nonsex & cis \\
\hline 2 Gg_rs142536 & 1147 chr2_138237। & 5.28 nonsex & cis \\
\hline 2 Gg_rs142536 & 1150 chr2_136751। & 3.63 nonsex & cis \\
\hline 2 Gg_rs161553 & 1150 chr2_136367। & 6.29 sex & cis \\
\hline 2 Gg_rs142536 & 1151 chr2_142853। & 5.64 sex & cis \\
\hline 2 Gg_rs161553 & 1152 chr2_137890 & 4.98 nonsex & cis \\
\hline 2 Gg_rs161553 & $1153 \mathrm{chr} 2 \_132320$ & 5.7 sex & cis \\
\hline 2 Gg_rs161553 & 1154 chr2_141055 & 7.31 nonsex & cis \\
\hline 2 Gg_rs161553 & 1156 chr2_143987। & 5.8 nonsex & cis \\
\hline 2 Gg_rs161553 & 1157 chr2_134867। & 4.07 nonsex & cis \\
\hline 2 Gg_rs161553 & 1157 chr2_136684। & 4.83 nonsex & cis \\
\hline 2 Gg_rs161553 & 1157 chr2_146757। & 4.54 nonsex & cis \\
\hline 2 Gg_rs161553 & 1158 chr2_141083। & 5.17 nonsex & cis \\
\hline 2 Gg_rs161553 & 1160 chr2_145785 & 6.26 nonsex & cis \\
\hline 2 Gg_rs161553 & 1160 chr2_140959 & 4.83 nonsex & cis \\
\hline 2 Gg_rs161553 & 1160 chr2_141307। & 3.99 nonsex & cis \\
\hline 2 Gg_rs161553 & 1162 chr2_143541। & 4.14 nonsex & cis \\
\hline 2 Gg_rs161553 & 1162 chr2_146026। & 4.86 nonsex & cis \\
\hline 2 Gg_rs161553 & 1162 chr2_146629 & 4.62 nonsex & cis \\
\hline 2 Gg_rs161553 & 1162 chr2_146668। & 4.36 nonsex & cis \\
\hline 2 Gg_rs161553 & 1165 chr2_137788 & 3.54 nonsex & cis \\
\hline 2 Gg_rs161553 & 1165 chr2_138238। & 4.5 nonsex & cis \\
\hline 2 Gg_rs161553 & 1166 chr2_140105। & 7.01 sex & cis \\
\hline 2 Gg_rs161553 & 1167 chr2_138247। & 4.74 nonsex & cis \\
\hline 2 Gg_rs161553 & 1168 chr2_138503। & 4.45 nonsex & cis \\
\hline 2 Gg_rs142536 & 1169 chr2_137794। & 3.92 nonsex & cis \\
\hline 2 Gg_rs161553 & 1169 chr2_137786 & 7.11 nonsex & cis \\
\hline 2 Gg_rs161553 & 1170 chr2_131068। & 4.65 nonsex & cis \\
\hline 2 Gg_rs151670 & 1173 chr2_138785 & 6.95 nonsex & cis \\
\hline 2 Gg_rs137615. & 1174 chr2_142887। & 4.4 nonsex & cis \\
\hline 2 Gg_rs142580. & 1175 chr2_144673। & 9.34 sex & cis \\
\hline 2 Gg_rs151670 & 1176 chr2_138820 & 6.51 nonsex & cis \\
\hline 2 Gg_rs151670 & 1183 chr2_148521। & 4.13 nonsex & cis \\
\hline 2 Gg_rs151670 & 1184 chr2_142461। & 6.18 sex & cis \\
\hline 2 Gg_rs151670 & 1185 chr2_131132 & 5.94 sex & cis \\
\hline 2 Gg_rs151670 & 1185 chr2_131134! & 5.74 sex & cis \\
\hline
\end{tabular}




\begin{tabular}{|c|c|c|c|}
\hline 2 Gg_rs151670 & 1185 chr2_131136। & 5.81 sex & cis \\
\hline 2 Gg_rs151670 & 1185 chr2_140050। & 5.79 sex & cis \\
\hline 2 Gg_rs151670 & 1185 chr2_120237। & 7.41 sex & trans \\
\hline 2 Gg_rs151670 & 1186 chr2_145711। & 4.53 nonsex & cis \\
\hline 2 Gg_rs151670 & 1187 chr2_142398। & 5.3 nonsex & cis \\
\hline 2 Gg_rs137615. & 1188 chr2_138671। & 7.7 nonsex & cis \\
\hline 2 Gg_rs137615. & 1188 chr2_139696। & 7.29 sex & cis \\
\hline 2 Gg_rs137615. & 1188 chr2_142894! & 5.9 nonsex & cis \\
\hline 2 Gg_rs137615. & 1188 chr2_145473। & 6.64 nonsex & cis \\
\hline 2 Gg_rs137615. & 1188 chr2_148325। & 4.33 nonsex & cis \\
\hline 2 Gg_rs137615. & 1188 chr30_68800। & 6.75 sex & trans \\
\hline 2 Gg_rs137615. & 1189 chr2_138673। & 6.9 sex & cis \\
\hline 2 Gg_rs137615. & 1189 chr2_139393। & 6.61 nonsex & cis \\
\hline 2 Gg_rs137615. & 1189 chr2_145038। & 5.79 nonsex & cis \\
\hline 2 Gg_rs137615. & 1189 chr2_148179। & 5.7 sex & cis \\
\hline 2 Gg_rs137615. & 1189 chr2_148188। & $7.01 \mathrm{sex}$ & cis \\
\hline 2 Gg_rs137615. & 1189 chr2_138708। & 4.96 nonsex & cis \\
\hline 2 Gg_rs137615. & 1189 chr2_145858। & 5.3 nonsex & cis \\
\hline 2 Gg_rs137615. & 1190 chr2_148160। & 4.5 nonsex & cis \\
\hline 2 Gg_rs137615. & 1190 chr2_139237। & 8.05 nonsex & cis \\
\hline 2 Gg_rs137615. & 1190 chr2_139258। & 7.3 nonsex & cis \\
\hline 2 Gg_rs137615. & 1190 chr2_139272 & 12.69 nonsex & cis \\
\hline 2 Gg_rs137615. & 1190 chr2_140107। & 6.39 nonsex & cis \\
\hline 2 Gg_rs137615. & 1190 chr2_140358। & 6.35 nonsex & cis \\
\hline 2 Gg_rs137615. & 1190 chr2_140435। & 8.95 nonsex & cis \\
\hline 2 Gg_rs137615. & 1190 chr2_140436। & 13.67 nonsex & cis \\
\hline 2 Gg_rs137615. & 1190 chr2_140506। & 8.2 sex & cis \\
\hline 2 Gg_rs137615. & 1190 chr2_141578। & 5.51 nonsex & cis \\
\hline 2 Gg_rs137615. & 1190 chr2_141585। & 5.31 nonsex & cis \\
\hline 2 Gg_rs137615. & 1190 chr2_142060 & 5.46 sex & cis \\
\hline 2 Gg_rs137615. & 1190 chr2_143179। & 6.1 nonsex & cis \\
\hline 2 Gg_rs137615. & 1190 chr2_144661। & 6.42 nonsex & cis \\
\hline 2 Gg_rs137615. & 1190 chr2_145859। & 14.64 nonsex & cis \\
\hline 2 Gg_rs137615. & 1190 chr2_145875। & 7.35 sex & cis \\
\hline 2 Gg_rs137615. & 1190 chr2_146218। & 7.53 nonsex & cis \\
\hline 2 Gg_rs137615. & 1190 chr2_148147। & 5.52 nonsex & cis \\
\hline 2 Gg_rs137615. & 1190 chr2_148408। & 6.32 nonsex & cis \\
\hline 2 Gg_rs137615. & 1190 chr2_138893। & 4.28 nonsex & cis \\
\hline 2 Gg_rs137615. & 1190 chr2_140458। & 4.27 nonsex & cis \\
\hline 2 Gg_rs137615. & 1190 chr2_140856 & 4.24 nonsex & cis \\
\hline 2 Gg_rs137615. & 1190 chr2_142467। & 4.49 nonsex & cis \\
\hline 2 Gg_rs137615. & 1190 chr2_142649। & 5.08 nonsex & cis \\
\hline 2 Gg_rs137615. & 1190 chr2_144903। & 3.85 nonsex & cis \\
\hline
\end{tabular}




\begin{tabular}{|c|c|c|}
\hline 2 Gg_rs137615 & 1190 chrW_53050( & 8.4 sex \\
\hline 2 Gg_rs137615 & 1190 chrZ_103570। & 8.26 sex \\
\hline 2 Gg_rs137615 & 1190 chr6_201680। & 4.34 nonsex \\
\hline 2 Gg_rs151590 & 1190 chr2_148920 & 8.16 sex \\
\hline 2 Gg_rs137615 & 1191 chr2_139695। & 6 nonsex \\
\hline 2 Gg_rs137615. & 1191 chr2_139115। & 5.06 nonsex \\
\hline 2 Gg_rs137615 & 1191 chrW_53060r & 9.06 sex \\
\hline 2 Gg_rs142580. & 1191 chr2_139248। & 9.42 nonsex \\
\hline 2 Gg_rs142580. & 1192 chr2_141700 & 5.87 nonsex \\
\hline 2 Gg_rs142580. & 1192 chr2_142376। & 9.28 nonsex \\
\hline 2 Gg_rs142580. & 1192 chr2_144856। & 4.69 nonsex \\
\hline 2 Gg_rs151590 & 1192 chr2_148971। & 5.39 nonsex \\
\hline 2 Gg_rs142580. & 1193 chr2_140624। & 17.26 nonsex \\
\hline 2 Gg_rs142580. & 1193 chr2_141217। & 6.18 nonsex \\
\hline 2 Gg_rs142580. & 1193 chr2_142193। & 6.12 sex \\
\hline 2 Gg_rs142580. & 1193 chr2_144694! & 6.56 nonsex \\
\hline 2 Gg_rs142580. & 1193 chr2_147681। & 5.48 nonsex \\
\hline 2 Gg_rs142580. & 1193 chr2_148793। & 5.65 nonsex \\
\hline 2 Gg_rs142580. & 1193 chr2_142394! & 4.44 nonsex \\
\hline 2 Gg_rs142580. & 1193 chr2_138997। & 4.17 nonsex \\
\hline 2 Gg_rs142580. & 1193 chr2_139712। & 4.22 nonsex \\
\hline 2 Gg_rs142580. & 1193 chr2_142705। & 3.89 nonsex \\
\hline 2 Gg_rs142580. & 1193 chr2_146881। & 4.4 nonsex \\
\hline 2 Gg_rs142580. & 1193 chr5_437000 & 8.5 sex \\
\hline 2 Gg_rs142580. & 1193 chr11_41850 & 8 sex \\
\hline 2 Gg_rs142580. & 1193 chr13_25520। & 6.53 nonsex \\
\hline 2 Gg_rs142580. & 1193 chr13_24100 & 7.56 sex \\
\hline 2 Gg_rs142580. & 1193 chrZ_715050। & 6.21 nonsex \\
\hline 2 Gg_rs151590 & 1193 chr2_147470 & 4.75 nonsex \\
\hline 2 Gg_rs151590 & 1193 chr2_148681। & 3.68 nonsex \\
\hline 2 Gg_rs151590 & 1193 chr2_148994। & 4.34 nonsex \\
\hline 2 Gg_rs151590 & 1194 chr2_146612 & 6.79 nonsex \\
\hline 2 Gg_rs151590 & 1194 chr2_148407। & 9.84 nonsex \\
\hline 2 Gg_rs151590 & 1194 chr2_148733। & 5.72 nonsex \\
\hline 2 Gg_rs151590 & 1194 chr2_148792। & 8.47 nonsex \\
\hline 2 Gg_rs151590 & 1194 chr2_148923। & 5.46 nonsex \\
\hline 2 Gg_rs151590 & 1194 chr2_148995। & 7.97 nonsex \\
\hline 2 Gg_rs151590 & 1194 chr2_149007। & 7.6 nonsex \\
\hline 2 Gg_rs151590 & 1194 chr2_141185। & 4.42 nonsex \\
\hline 2 Gg_rs151590 & 1194 chr2_144680 & 5.06 nonsex \\
\hline 2 Gg_rs151590 & 1194 chr2_148457। & 4.75 nonsex \\
\hline 2 Gg_rs151590 & 1194 chr2_148956। & 4.17 nonsex \\
\hline 2 Gg_rs151590 & 1194 chr2_148973। & 4.72 nonsex \\
\hline
\end{tabular}




\begin{tabular}{|c|c|c|c|}
\hline 2 Gg_rs151590 & 1194 chr2_149008। & 4.49 nonsex & cis \\
\hline 2 Gg_rs151590 & 1194 chr4_695770 & 6.58 nonsex & trans \\
\hline 2 Gg_rs142580. & 1197 chr2_148145। & 4.3 nonsex & cis \\
\hline 2 Gg_rs151590 & 1200 chr2_141756। & 5.46 nonsex & cis \\
\hline 2 Gg_rs151590 & 1202 chr2_143184! & 11.49 nonsex & cis \\
\hline 2 Gg_rs151590 & 1202 chr2_140433। & 4.73 nonsex & cis \\
\hline 2 Gg_rs151590 & 1202 chr1_178151। & 7.84 sex & trans \\
\hline 2 Gg_rs142580. & 1204 chr2_138802 & 4.09 nonsex & cis \\
\hline 2 Gg_rs151590 & 1205 chr4_894870 & 5.91 nonsex & trans \\
\hline 2 Gg_rs142580. & 1206 chr3_693990 & 7.85 sex & trans \\
\hline 2 Gg_rs142580. & 1208 chr2_143699। & 6.47 sex & cis \\
\hline 2 Gg_rs151590 & 1209 chr2_139985 & 7.13 nonsex & cis \\
\hline 2 Gg_rs142580. & 1210 chr2_140290 & 4.3 nonsex & cis \\
\hline 2 Gg_rs142580. & 1211 chr1_179193। & $7.1 \mathrm{sex}$ & trans \\
\hline 2 Gg_rs151590 & 1212 chr2_104640 & 7.31 sex & trans \\
\hline 2 Gg_rs137615. & 1214 chr2_143978। & 5.89 nonsex & cis \\
\hline 2 Gg_rs142580. & 1214 chr2_142987। & $5.93 \mathrm{sex}$ & cis \\
\hline 2 Gg_rs142580. & 1214 chr2_142913। & 4.32 nonsex & cis \\
\hline 2 Gg_rs151590 & 1215 chr2_141174! & $5.68 \operatorname{sex}$ & cis \\
\hline 2 Gg_rs142580. & 1216 chr2_146352 & 9.24 nonsex & cis \\
\hline 2 Gg_rs137615. & 1217 chr2_141314। & 5.36 nonsex & cis \\
\hline 2 Gg_rs151590 & 1217 chr2_141183! & 6.79 nonsex & cis \\
\hline 2 Gg_rs137615. & 1218 chr2_141781। & 5.5 nonsex & cis \\
\hline 2 Gg_rs137615. & 1218 chr2_140214। & 4.64 nonsex & cis \\
\hline 2 Gg_rs142580. & 1218 chr2_142462 & 4.78 nonsex & cis \\
\hline 2 Gg_rs137615. & 1219 chr2_142615 & 3.98 nonsex & cis \\
\hline 2 Gg_rs142580. & 1219 chr2_142686 & 4.65 nonsex & cis \\
\hline 2 Gg_rs142580. & 1219 chr3_354970 & 9.62 sex & trans \\
\hline 2 Gg_rs151590 & 1219 chr2_141775 & 5.46 nonsex & cis \\
\hline 2 Gg_rs137615 & 1220 chr2_143575। & 5.55 nonsex & cis \\
\hline 2 Gg_rs142580. & 1221 chr2_143178। & 6.91 nonsex & cis \\
\hline 2 Gg_rs151590 & 1221 chr2_146206। & 6.59 sex & cis \\
\hline 2 Gg_rs137615. & 1222 chr2_136769। & 7.39 nonsex & cis \\
\hline 2 Gg_rs151590 & 1223 chr30_15690 & 12.93 sex & trans \\
\hline 2 Gg_rs137615. & 1226 chr2_138750 & 5.7 nonsex & cis \\
\hline 2 Gg_rs137615. & 1226 chr2_145786। & 4.48 nonsex & cis \\
\hline 2 Gg_rs151670 & 1232 chr2_139553। & 6.92 sex & cis \\
\hline 2 Gg_rs137615. & 1232 chr2_139927। & 5.13 nonsex & cis \\
\hline 3 X3_4521634 & 0 chr3_379200 & 6.37 sex & cis \\
\hline 3 Gg_rs152823 & 215 chr3_428800 & 6.08 sex & trans \\
\hline 3 Gg_rs152823 & 215 chr10_16270 & 6.58 sex & trans \\
\hline 3 Gg_rs152823 & 216 chr15_46840 & 5.56 sex & trans \\
\hline 3 Gg_rs152823 & 216 chr1_493040 & 7.3 sex & trans \\
\hline
\end{tabular}




\begin{tabular}{|c|c|c|}
\hline 3 Gg_rs152823 & 216 chr24_88200। & $6.12 \operatorname{sex}$ \\
\hline 3 Gg_rs152823 & 216 chrZ_818900। & 6.04 sex \\
\hline 3 Gg_rs152823 & 217 chr24_43500। & $6.16 \mathrm{sex}$ \\
\hline 3 Gg_rs152823 & 217 chr15_48170 & $6.37 \mathrm{sex}$ \\
\hline 3 Gg_rs152823 & 217 chr12_18673। & 5.43 sex \\
\hline 3 Gg_rs152823 & 218 chr6_248390। & 6.91 sex \\
\hline 3 Gg_rs152823 & 218 chrZ_818270। & 4.23 nonsex \\
\hline 3 Gg_rs152823 & 218 chrZ_585550। & 6 sex \\
\hline 3 Gg_rs152823 & 218 chr21_33500। & 6.06 sex \\
\hline 3 Gg_rs152823 & 219 chr19_22350। & $5.2 \mathrm{sex}$ \\
\hline 3 Gg_rs152823 & 219 chr8_201000। & 6.34 sex \\
\hline 3 Gg_rs152823 & 219 chr4_388200। & 3.51 nonsex \\
\hline 3 Gg_rs152823 & 219 chr21_32530। & $6.6 \operatorname{sex}$ \\
\hline 3 Gg_rs152823 & 219 chr24_13800। & 6.25 sex \\
\hline 3 Gg_rs152823 & 219 chr20_59700। & 4.06 nonsex \\
\hline 3 Gg_rs152823 & 219 chr3_112400। & $6.2 \operatorname{sex}$ \\
\hline 3 Gg_rs152823 & 219 chr23_37560। & 5.92 sex \\
\hline 3 Gg_rs152823 & 219 chr10_20070 & 5.73 sex \\
\hline 3 Gg_rs152823 & 219 chrZ_827400। & 6.26 sex \\
\hline 3 Gg_rs152823 & 219 chr6_330940। & 6.16 sex \\
\hline 3 Gg_rs152823 & 219 chr17_11010। & 6.01 sex \\
\hline 3 Gg_rs152823 & 219 chr21_64000। & 3.73 nonsex \\
\hline 3 Gg_rs152823 & 219 chr17_82380 & 6.08 sex \\
\hline 3 Gg_rs152823 & 219 chr4_455500 & 6.15 sex \\
\hline 3 Gg_rs152823 & 219 chr20_85200। & 5.88 sex \\
\hline 3 Gg_rs152823 & 219 chrZ_1344001 & 7.68 sex \\
\hline 3 Gg_rs152823 & 219 chr10_15559| & 3.86 nonsex \\
\hline 3 Gg_rs152823 & 219 chr3_314100 & 5.58 sex \\
\hline 3 Gg_rs152823 & 219 chr4_377300। & 5.7 sex \\
\hline 3 Gg_rs152823 & 219 chr7_118090। & 6.19 sex \\
\hline 3 Gg_rs152823 & 219 chr11_19349| & 6.11 sex \\
\hline 3 Gg_rs152823 & 219 chr26_24700। & 5.67 sex \\
\hline 3 Gg_rs152823 & 219 chr14_12862 & 6.12 sex \\
\hline 3 Gg_rs152823 & 219 chr11_19326। & 6.6 sex \\
\hline 3 Gg_rs152823 & 219 chr10_15620 & 6.43 sex \\
\hline 3 Gg_rs152823 & 219 chr18_40270 & 4.11 nonsex \\
\hline 3 Gg_rs152823 & 219 chrZ_134700। & 8.65 sex \\
\hline 3 Gg_rs152823 & 219 chr6_358730 & 6.54 sex \\
\hline 3 Gg_rs152823 & 219 chr13_11011। & 7.11 sex \\
\hline 3 Gg_rs152823 & 219 chr15_77800 & 8.6 sex \\
\hline 3 Gg_rs152823 & 219 chrZ_818970। & 5.96 sex \\
\hline 3 Gg_rs152823 & 219 chr5_586490। & 5.92 sex \\
\hline 3 Gg_rs152823 & 219 chrZ_305000 & 8.2 sex \\
\hline
\end{tabular}




\begin{tabular}{|c|c|c|}
\hline 3 Gg_rs152823 & 219 chr3_104754! & $5.99 \mathrm{sex}$ \\
\hline 3 Gg_rs152823 & 219 chr7_112000 & 4.73 nonsex \\
\hline 3 Gg_rs152823 & 219 chr6_176100 & 5.17 sex \\
\hline 3 Gg_rs152823 & 219 chr27_36670। & $6.38 \mathrm{sex}$ \\
\hline 3 Gg_rs152823 & 219 chr26_36770 & 5.89 sex \\
\hline 3 Gg_rs152823 & 219 chr14_13058। & 6.84 sex \\
\hline 3 Gg_rs152823 & 219 chr14_32120। & 6.06 sex \\
\hline 3 Gg_rs152823 & 219 chr10_19493। & 4.82 nonsex \\
\hline 3 Gg_rs152823 & 219 chrZ_821000। & 6.25 sex \\
\hline 3 Gg_rs152823 & 219 chr8_394100। & 3.56 nonsex \\
\hline 3 Gg_rs152823 & 219 chr14_12591। & 6.12 sex \\
\hline 3 Gg_rs152823 & 219 chr8_216800। & 5.51 sex \\
\hline 3 Gg_rs152823 & 219 chr19_85680 & 6.46 sex \\
\hline 3 Gg_rs152823 & 219 chr27_49850। & 5.92 sex \\
\hline 3 Gg_rs152823 & 219 chr15_68440। & 6.28 sex \\
\hline 3 Gg_rs152823 & 219 chr18_21530। & 7.09 sex \\
\hline 3 Gg_rs152823 & 219 chr12_16710 & 5.97 sex \\
\hline 3 Gg_rs152823 & 219 chr18_23020। & 6.26 sex \\
\hline 3 Gg_rs152823 & 219 chr28_93800। & 6.62 sex \\
\hline 3 Gg_rs152823 & 219 chr17_27300। & 6.1 sex \\
\hline 3 Gg_rs152823 & 219 chr23_48200। & 3.89 nonsex \\
\hline 3 Gg_rs152823 & 219 chr11_19337। & 5.89 sex \\
\hline 3 Gg_rs152823 & 219 chr19_84740 & 6.37 sex \\
\hline 3 Gg_rs152823 & 219 chr19_41110 & 4.07 nonsex \\
\hline 3 Gg_rs152823 & 219 chr5_498720 & 6.17 sex \\
\hline 3 Gg_rs152823 & 219 chr17_73130 & 6.5 sex \\
\hline 3 Gg_rs152823 & 219 chr10_17259 & 6.57 sex \\
\hline 3 Gg_rs152823 & 219 chr13_12763। & 6.29 sex \\
\hline 3 Gg_rs152823 & 219 chr17_49460। & 6 sex \\
\hline 3 Gg_rs152823 & 219 chr21_11570। & 5.66 sex \\
\hline 3 Gg_rs152823 & 219 chr13_14096। & 6.28 sex \\
\hline 3 Gg_rs152823 & 219 chr15_12619| & 6.03 sex \\
\hline 3 Gg_rs152823 & 219 chr27_38600। & 3.82 nonsex \\
\hline 3 Gg_rs152823 & 219 chr14_31640। & 5.78 sex \\
\hline 3 Gg_rs152823 & 219 chrZ_125000 & 8.26 sex \\
\hline 3 Gg_rs152823 & 219 chr3_105894। & 5.71 sex \\
\hline 3 Gg_rs152823 & 219 chrZ_124000 & 5.98 sex \\
\hline 3 Gg_rs152823 & 219 chrZ_408000 & 6.9 sex \\
\hline 3 Gg_rs152823 & 219 chrZ_7287801 & 6.75 sex \\
\hline 3 Gg_rs152823 & 219 chr20_95180। & 6.13 sex \\
\hline 3 Gg_rs152823 & 219 chr14_12618। & 5.98 sex \\
\hline 3 Gg_rs152823 & 219 chr20_11787। & 5.81 sex \\
\hline 3 Gg_rs152823 & 219 chr13_95190। & $6.41 \mathrm{sex}$ \\
\hline
\end{tabular}




\begin{tabular}{|c|c|c|}
\hline 3 Gg_rs152823 & 219 chrZ_223380। & 6.36 sex \\
\hline 3 Gg_rs152823 & 219 chrZ_819110। & 5.93 sex \\
\hline 3 Gg_rs152823 & 219 chrZ_122000 & 7.88 sex \\
\hline 3 Gg_rs152823 & 219 chrZ_134600। & 7.7 sex \\
\hline 3 Gg_rs152823 & 219 chr6_358370। & $6.52 \mathrm{sex}$ \\
\hline 3 Gg_rs152823 & 219 chr9_196940 & 4.19 nonsex \\
\hline 3 Gg_rs152823 & 219 chrZ_8087001 & 6.23 sex \\
\hline 3 Gg_rs152823 & 219 chr1_506350। & 6.43 sex \\
\hline 3 Gg_rs152823 & 219 chr14_42960। & 3.66 nonsex \\
\hline 3 Gg_rs152823 & 219 chr13_26600। & 6.2 sex \\
\hline 3 Gg_rs152823 & 219 chr9_108220 & 4.59 nonsex \\
\hline 3 Gg_rs152823 & 219 chr5_578320 & 6.63 sex \\
\hline 3 Gg_rs152823 & 219 chr17_58800। & 6.58 sex \\
\hline 3 Gg_rs152823 & 219 chrZ_557760। & 5.89 sex \\
\hline 3 Gg_rs152823 & 219 chr5_142560 & 7.26 sex \\
\hline 3 Gg_rs152823 & 219 chr4_134900 & 4.14 nonsex \\
\hline 3 Gg_rs152823 & 219 chr2_966320 & $6.12 \mathrm{sex}$ \\
\hline 3 Gg_rs152823 & 219 chr13_11008। & 5.42 sex \\
\hline 3 Gg_rs152823 & 219 chr10_17256। & 6.63 sex \\
\hline 3 Gg_rs152823 & 219 chr25_36740। & 6.03 sex \\
\hline 3 Gg_rs152823 & 219 chr9_236360। & 3.66 nonsex \\
\hline 3 Gg_rs152823 & 219 chr13_99450। & 7.01 sex \\
\hline 3 Gg_rs152823 & 219 chr22_13870 & 5.6 sex \\
\hline 3 Gg_rs152823 & 219 chrZ_8177101 & 6.22 sex \\
\hline 3 Gg_rs152823 & 219 chr7_523500 & 3.89 nonsex \\
\hline 3 Gg_rs152823 & 219 chrZ_8189301 & 6.02 sex \\
\hline 3 Gg_rs152823 & 219 chr17_77730। & 6.57 sex \\
\hline 3 Gg_rs152823 & 219 chr9_236000। & 5.98 sex \\
\hline 3 Gg_rs152823 & 219 chr26_29290। & 7.15 sex \\
\hline 3 Gg_rs152823 & 219 chrZ_818950। & 6.51 sex \\
\hline 3 Gg_rs152823 & 219 chrZ_8161301 & 7.27 sex \\
\hline 3 Gg_rs152823 & 219 chr19_21120 & 5.6 sex \\
\hline 3 Gg_rs152823 & 219 chr15_19360। & 6.19 sex \\
\hline 3 Gg_rs152823 & 219 chrZ_143600। & $6.6 \mathrm{sex}$ \\
\hline 3 Gg_rs152823 & 219 chr9_100580 & 6.22 sex \\
\hline 3 Gg_rs152823 & 219 chr2_120850। & 5.02 nonsex \\
\hline 3 Gg_rs152823 & 219 chr13_11196। & 6.24 sex \\
\hline 3 Gg_rs152823 & 219 chr17_95690। & $6.1 \mathrm{sex}$ \\
\hline 3 Gg_rs152823 & 219 chr12_16462 & 5.48 sex \\
\hline 3 Gg_rs152823 & 219 chr1_595530। & 6.27 sex \\
\hline 3 Gg_rs152823 & 220 chr7_360930। & 4.27 nonsex \\
\hline 3 Gg_rs152823 & 220 chrZ_8194901 & 5.97 sex \\
\hline 3 Gg_rs152823 & 221 chr9_147540। & 4.23 nonsex \\
\hline
\end{tabular}




\begin{tabular}{|c|c|c|}
\hline 3 Gg_rs152823 & 221 chr24_24170। & 3.61 nonsex \\
\hline 3 Gg_rs152823 & 223 chr4_651880 & 4.02 nonsex \\
\hline 3 Gg_rs152823 & 224 chr6_326290। & 4.26 nonsex \\
\hline 3 Gg_rs152823 & 225 chrZ_204600। & 5.49 nonsex \\
\hline 3 Gg_rs152823 & 226 chr3_105997। & 4.37 nonsex \\
\hline 3 Gg_rs152823 & 226 chr18_39130। & 4.26 nonsex \\
\hline 3 Gg_rs152823. & 226 chrZ_156800। & 5.81 nonsex \\
\hline 3 Gg_rs152823 & 226 chr7_232890। & 4.75 nonsex \\
\hline 3 Gg_rs152823. & 227 chr8_525900। & 4.13 nonsex \\
\hline 3 Gg_rs152823 & 228 chr3_111590 & 5.81 sex \\
\hline 3 Gg_rs152823. & 228 chr33_52040। & 4.87 nonsex \\
\hline 3 Gg_rs152823. & 228 chr24_26330। & 4.84 nonsex \\
\hline 3 Gg_rs152823 & 228 chr5_276710 & 4.08 nonsex \\
\hline 3 Gg_rs152823. & 231 chr7_258410 & 5.9 nonsex \\
\hline 3 Gg_rs152823 & 235 chr6_181250 & 5.25 nonsex \\
\hline 3 Gg_rs152823. & 236 chr3_637010 & 5.02 nonsex \\
\hline 3 Gg_rs152823 & 238 chr4_306420। & 5.88 nonsex \\
\hline 4 Gg_rs144576. & 312 chr2_133161। & 8.07 sex \\
\hline 4 Gg_rs144576. & 313 chr2_133872 & 8.92 sex \\
\hline 4 Gg_rs144576. & 314 chrW_58840 & 8.52 sex \\
\hline 4 Gg_rs144576. & 314 chr1_117285। & 8.71 sex \\
\hline 4 Gg_rs144576. & 314 chr1_158847। & 8.04 sex \\
\hline 4 Gg_rs144576. & 314 chr2_544360 & 7.74 sex \\
\hline 4 Gg_rs144576. & 314 chr2_124884! & 7.86 sex \\
\hline 4 Gg_rs144576. & 315 chr1_104987। & 8.02 sex \\
\hline 4 Gg_rs144576. & 315 chr1_191077। & 7.94 sex \\
\hline 4 Gg_rs144576. & 315 chr3_755340 & 7.78 sex \\
\hline 4 Gg_rs144576. & 315 chr4_111860 & 7.81 sex \\
\hline 4 Gg_rs144576. & 316 chr1_107847। & 8.75 sex \\
\hline 4 Gg_rs144576. & 316 chr1_186230 & 8.04 sex \\
\hline 4 Gg_rs144576. & 317 chr2_147593। & 8.54 sex \\
\hline 4 Gg_rs144576. & 317 chr5_265500 & 6.08 nonsex \\
\hline 4 Gg_rs144576. & 317 chr5_205510 & 6.12 nonsex \\
\hline 4 Gg_rs144576. & 317 chrZ_5440001 & 6.12 nonsex \\
\hline 4 Gg_rs144576. & 318 chr3_584630। & 6.51 nonsex \\
\hline 4 Gg_rs144576. & 320 chr2_122100 & 5.99 nonsex \\
\hline 4 Gg_rs144576. & 320 chr2_731300 & 5.79 nonsex \\
\hline 4 Gg_rs144576. & 321 chrW_51570 & 7.63 sex \\
\hline 4 Gg_rs144576. & 321 chr1_786680 & 7.23 sex \\
\hline 4 Gg_rs144576. & 321 chr1_128876 & 5.61 nonsex \\
\hline 4 Gg_rs144576. & 321 chr3_592510। & 6.2 nonsex \\
\hline 4 Gg_rs144576. & 321 chr3_923110। & 5.54 nonsex \\
\hline 4 Gg_rs144576. & 322 chr1_103877। & 6.13 nonsex \\
\hline
\end{tabular}




\begin{tabular}{|c|c|c|}
\hline 4 Gg_rs144576. & 322 chr2_726670 & 5.58 nonsex \\
\hline 4 Gg_rs144576. & 322 chr2_762300। & 6.17 nonsex \\
\hline 4 Gg_rs144576. & 323 chrZ_715990। & 6.51 nonsex \\
\hline 4 Gg_rs144576. & 324 chr1_993080 & 6.45 nonsex \\
\hline 4 Gg_rs144576. & 324 chr3_630710 & 8.17 sex \\
\hline 4 Gg_rs144576. & 324 chr2_698600 & 6.32 nonsex \\
\hline 4 Gg_rs144576. & 325 chr3_743890 & 7.42 sex \\
\hline 4 Gg_rs144576. & 327 chr3_888930। & 9.08 sex \\
\hline 4 Gg_rs144576. & 327 chr5_417450 & 6.05 nonsex \\
\hline 4 Gg_rs144576. & 329 chr2_110439। & 5.73 nonsex \\
\hline 4 Gg_rs144576. & 330 chr2_139532 & 8.22 sex \\
\hline 4 Gg_rs144576. & 331 chr1_183876। & 6.91 nonsex \\
\hline 4 Gg_rs144576. & 332 chr2_499260। & 5.92 nonsex \\
\hline 4 Gg_rs144576. & 333 chr3_694000 & 6.37 nonsex \\
\hline 4 Gg_rs144576. & 334 chr2_952260 & 5.76 nonsex \\
\hline 4 Gg_rs144576. & 334 chr3_324790। & 6.47 nonsex \\
\hline 4 Gg_rs144576. & 335 chrW_48960 & 7.97 sex \\
\hline 4 Gg_rs144576. & 339 chr3_434710 & 6.47 nonsex \\
\hline 4 Gg_rs156084. & 429 chrW_29210 & 7.51 sex \\
\hline 4 Gg_rs156084 & 433 chr4_659950 & 3.72 nonsex \\
\hline 4 Gg_rs156084 & 433 chrZ_414700। & 7.33 sex \\
\hline 4 Gg_rs156084 & 433 chr18_98370 & 7.64 nonsex \\
\hline 4 Gg_rs156084 & 436 chr4_665650 & 6.34 sex \\
\hline 4 Gg_rs156084 & 436 chr4_665660 & 4.78 nonsex \\
\hline 4 Gg_rs156084 & 437 chr4_687080 & 5.7 nonsex \\
\hline 4 Gg_rs156084 & 439 chr4_665820 & 7.16 nonsex \\
\hline 4 Gg_rs144874 & 442 chr4_685840 & 6.69 nonsex \\
\hline 4 Gg_rs144874 & 442 chr4_679600 & 6.27 nonsex \\
\hline 4 Gg_rs144874 & 443 chr4_669740। & 6.47 nonsex \\
\hline 4 Gg_rs144874 & 443 chr4_674310 & 9.66 nonsex \\
\hline 4 Gg_rs144874 & 443 chr4_681060 & 8.46 sex \\
\hline 4 Gg_rs144874 & 443 chr4_676630 & 6.35 nonsex \\
\hline 4 Gg_rs144874 & 443 chr4_687470 & 3.47 nonsex \\
\hline 4 Gg_rs144874 & 444 chr4_656840 & 5.77 sex \\
\hline 4 Gg_rs144874 & 444 chr4_686410 & 5.11 nonsex \\
\hline 4 Gg_rs144874 & 446 chr4_606590। & 4.2 sex \\
\hline 4 Gg_rs144874 & 446 chr4_672710 & 6.26 nonsex \\
\hline 4 Gg_rs144874 & 446 chr4_691760 & 6.98 sex \\
\hline 4 Gg_rs144874 & 448 chr4_666250 & 4.65 nonsex \\
\hline 4 Gg_rs144874 & 448 chr4_686060। & 5.22 nonsex \\
\hline 4 Gg_rs144874 & 448 chr4_670280 & 3.85 nonsex \\
\hline 4 Gg_rs144874 & 448 chr4_687200 & 5.29 nonsex \\
\hline 4 Gg_rs144874 & 448 chr4_689170। & 4.83 nonsex \\
\hline
\end{tabular}




\begin{tabular}{|c|c|c|}
\hline 4 Gg_rs144874! & 448 chrZ_865400। & 8.83 sex \\
\hline 4 Gg_rs144874 & 449 chr4_671570 & 4.21 nonsex \\
\hline 4 Gg_rs144874 & 450 chr4_686050। & 3.51 nonsex \\
\hline 4 Gg_rs144874 & 451 chr4_683160। & 6.42 nonsex \\
\hline 4 Gg_rs144874 & 452 chr4_680600 & 3.61 nonsex \\
\hline 4 Gg_rs144874 & 452 chr4_682300 & 2.41 nonsex \\
\hline 4 Gg_rs144874 & 458 chr4_683300 & 2.85 nonsex \\
\hline 4 Gg_rs144874 & 460 chr4_692520। & 3.25 nonsex \\
\hline 4 chr4.7183334 & 460 chr4_697090। & 5.97 sex \\
\hline 4 chr4.7183334 & 460 chr4_702480 & 5.13 nonsex \\
\hline 4 chr4.7183334 & 463 chr4_697050। & 4.86 nonsex \\
\hline 4 chr4.7183334 & 464 chr4_697080। & 4.17 nonsex \\
\hline 4 chr4.7183334 & 464 chr4_711750 & 4.4 nonsex \\
\hline 4 chr4.7183334 & 464 chr4_736380 & 4.68 nonsex \\
\hline 4 chr4.7183334 & 464 chrW_50350c & 7.38 sex \\
\hline 4 chr4.7183334 & 464 chr4_168710 & 4.94 nonsex \\
\hline 4 chr4.7183334 & 465 chr4_695810 & 4.26 nonsex \\
\hline 4 chr4.7183334 & 465 chr4_695820 & 4.18 nonsex \\
\hline 4 chr4.7183334 & 465 chr4_703160। & 4.06 nonsex \\
\hline 4 chr4.7183334 & 465 chr4_712620 & 4.52 nonsex \\
\hline 4 chr4.7183334 & 466 chr4_695780 & 5.74 nonsex \\
\hline 4 chr4.7183334 & 466 chr4_706470 & 4.96 nonsex \\
\hline 4 chr4.7183334 & 466 chr4_737180 & 3.72 nonsex \\
\hline 4 chr4.7183334 & 468 chr4_712740 & 5.14 nonsex \\
\hline 4 chr4.7183334 & 469 chr4_761490। & 5.28 nonsex \\
\hline 4 Gg_rs144887. & 469 chr4_737450। & 4.78 nonsex \\
\hline 4 Gg_rs144887. & 469 chr4_697040। & 4.4 nonsex \\
\hline 4 Gg_rs144887. & 469 chr4_735420। & 4.79 nonsex \\
\hline 4 chr4.7183334 & 470 chr4_732370 & 8.75 nonsex \\
\hline 4 chr4.7183334 & 471 chr4_734110 & 4.16 nonsex \\
\hline 4 chr4.7183334 & 471 chr1_146573। & 5.64 nonsex \\
\hline 4 Gg_rs144887. & 471 chr4_695700। & 3.79 nonsex \\
\hline 4 chr4.7183334 & 472 chr4_704690। & 5.61 nonsex \\
\hline 4 chr4.7183334 & 472 chr2_129271। & 5.8 nonsex \\
\hline 4 Gg_rs144887. & 473 chr4_720640 & 4.83 nonsex \\
\hline 4 Gg_rs144887. & 473 chr4_695790। & 5.07 nonsex \\
\hline 4 Gg_rs144887. & 475 chr4_731210 & 6.04 nonsex \\
\hline 4 Gg_rs144887. & 475 chr4_734870 & 6.99 nonsex \\
\hline 4 Gg_rs144887. & 475 chr4_711780 & 4.36 nonsex \\
\hline 4 Gg_rs144887. & 475 chr4_718830 & 4.74 nonsex \\
\hline 4 Gg_rs144887. & 476 chr4_728220। & 7.41 nonsex \\
\hline 4 Gg_rs144887. & 476 chr4_731120। & 4.99 nonsex \\
\hline 4 Gg_rs144887. & 476 chr4_736930 & 15.73 nonsex \\
\hline
\end{tabular}




\begin{tabular}{|c|c|c|c|}
\hline 4 Gg_rs144887. & 477 chr4_710720। & 6.2 nonsex & cis \\
\hline 4 Gg_rs144887. & 477 chr4_736920। & 16.4 nonsex & cis \\
\hline 4 chr4.7183334 & 478 chr4_731620। & 5.8 nonsex & cis \\
\hline 4 chr4.7183334 & 478 chr4_732990। & 6.84 sex & cis \\
\hline 4 chr4.7183334 & 478 chr4_712650 & 5.83 nonsex & cis \\
\hline 4 chr4.7183334 & 478 chr4_699450 & 5.07 nonsex & cis \\
\hline 4 Gg_rs144887. & 478 chr4_714120 & 6.42 nonsex & cis \\
\hline 4 snp.131.95.11 & 478 chr4_695680 & 3.95 nonsex & cis \\
\hline 4 Gg_rs144887. & 480 chr4_730900 & 4.68 nonsex & cis \\
\hline 4 chr4.7183334 & 482 chr4_737660 & 5.21 nonsex & cis \\
\hline 4 snp.131.95.11 & 482 chr4_738400 & 2.31 nonsex & cis \\
\hline 4 snp.454.6.31؛ & 482 chr4_730870 & 5.92 nonsex & cis \\
\hline 4 chr4.7183334 & 483 chr4_740970 & 5.23 nonsex & cis \\
\hline 4 snp.454.6.31؛ & 484 chr33_72510 & 7.7 sex & trans \\
\hline 4 snp.131.95.11 & 485 chr23_20360। & 9.35 sex & trans \\
\hline 4 snp.131.95.11 & 486 chr4_783190 & 3.19 nonsex & cis \\
\hline 4 snp.131.95.11 & 486 chr3_524370 & 7.4 sex & trans \\
\hline 4 Gg_rs144887. & 487 chr4_729790। & 5.79 nonsex & cis \\
\hline 4 snp.131.95.11 & 487 chr4_734980 & 7.98 sex & cis \\
\hline 4 snp.131.95.11 & 487 chr4_739270 & 5.86 sex & cis \\
\hline 4 snp.131.95.11 & 487 chr4_742040 & 6.68 sex & cis \\
\hline 4 snp.131.95.11 & 487 chr4_745990 & 5.94 nonsex & cis \\
\hline 4 snp.131.95.11 & 487 chr4_760750 & 6.41 sex & cis \\
\hline 4 snp.131.95.11 & 487 chr4_761130 & 6.07 sex & cis \\
\hline 4 snp.131.95.11 & 487 chr4_778030 & 5.76 sex & cis \\
\hline 4 snp.131.95.11 & 487 chr4_789840 & 5.25 sex & cis \\
\hline 4 snp.131.95.11 & 487 chr4_804500 & 5.9 sex & cis \\
\hline 4 snp.131.95.11 & 487 chr4_818440 & 6.12 sex & cis \\
\hline 4 snp.131.95.11 & 487 chr4_682790 & 4.01 nonsex & cis \\
\hline 4 snp.131.95.11 & 487 chr4_739190। & 3.6 nonsex & cis \\
\hline 4 snp.131.95.11 & 487 chr4_744730 & 5.67 nonsex & cis \\
\hline 4 snp.131.95.11 & 487 chr4_746270 & 3.63 nonsex & cis \\
\hline 4 snp.131.95.11 & 487 chr4_751500 & 5.33 nonsex & cis \\
\hline 4 snp.131.95.11 & 487 chr4_769590 & 3.7 nonsex & cis \\
\hline 4 snp.131.95.11 & 487 chr4_786420 & 4.93 nonsex & cis \\
\hline 4 snp.131.95.11 & 487 chr4_380270 & 8.5 sex & trans \\
\hline 4 snp.131.95.11 & 487 chr8_192150 & 7.65 sex & trans \\
\hline 4 snp.131.95.11 & 487 chr25_28820 & $9.91 \mathrm{sex}$ & trans \\
\hline 4 snp.131.95.11 & 487 chr30_15530 & 10.93 sex & trans \\
\hline 4 snp.131.95.11 & 487 chr1_128686। & 8.13 sex & trans \\
\hline 4 snp.131.95.11 & 487 chr3_963890 & 9.44 sex & trans \\
\hline 4 snp.131.95.11 & 487 chr3_970670 & 7.77 nonsex & trans \\
\hline 4 snp.131.95.11 & 487 chr1_299530। & 6.4 nonsex & \\
\hline
\end{tabular}




\begin{tabular}{|c|c|c|c|}
\hline 4 snp.131.95.11 & 487 chr1_307380 & 4.14 nonsex & trans \\
\hline 4 snp.131.95.11 & 487 chr8_124000। & 6.36 nonsex & trans \\
\hline 4 snp.131.95.11 & 487 chr2_808200। & 5.59 nonsex & trans \\
\hline 4 snp.131.95.11 & 487 chr2_242760। & 5.47 nonsex & trans \\
\hline 4 snp.131.95.11 & 487 chr3_589960। & 5.72 nonsex & \\
\hline 4 snp.131.95.11 & 487 chr3_714840 & 5.76 nonsex & \\
\hline 4 snp.131.95.11 & 488 chr4_705370। & 6.14 sex & cis \\
\hline 4 snp.131.95.11 & 489 chr4_695720। & 4.87 nonsex & cis \\
\hline 4 snp.131.95.11 & 489 chr4_762760। & 3.28 nonsex & cis \\
\hline 4 snp.454.6.31؛ & 490 chr4_738640 & 5.95 nonsex & cis \\
\hline 4 snp.454.6.31؛ & 491 chr4_748300। & 7.67 nonsex & cis \\
\hline 4 snp.454.6.31؛ & 491 chr1_378000। & 6.47 nonsex & trans \\
\hline 4 Gg_rs138210. & 491 chr4_784090। & 4.75 nonsex & cis \\
\hline 4 Gg_rs144887. & 492 chr4_734720। & 5.34 nonsex & cis \\
\hline 4 snp.454.6.31؛ & 492 chr4_747170। & 6.13 nonsex & cis \\
\hline 4 snp.454.6.31؛ & 492 chr2_837190। & 6.66 nonsex & \\
\hline 4 Gg_rs138210. & 492 chr4_758980 & 6.55 nonsex & cis \\
\hline 4 Gg_rs138210. & 492 chr4_752410 & $6.12 \operatorname{sex}$ & cis \\
\hline 4 Gg_rs138210. & 492 chr4_755760। & 17.03 nonsex & cis \\
\hline 4 Gg_rs138210. & 493 chr4_748410 & 11.82 nonsex & cis \\
\hline 4 Gg_rs138210. & 493 chr4_748420 & 9.45 nonsex & cis \\
\hline 4 Gg_rs138210. & 493 chr4_758990। & 7.52 nonsex & cis \\
\hline 4 Gg_rs138210. & 493 chr4_776920। & 5.76 sex & cis \\
\hline 4 Gg_rs138210. & 493 chr4_747860। & 5.02 nonsex & cis \\
\hline 4 Gg_rs138210. & 493 chr4_758470। & 4.81 nonsex & cis \\
\hline 4 Gg_rs138210. & 493 chr4_758930। & 5.1 nonsex & cis \\
\hline 4 Gg_rs138210. & 493 chr3_104466। & 8.1 sex & trans \\
\hline 4 snp.454.6.31؛ & 495 chr4_763490। & 5.87 sex & cis \\
\hline 4 Gg_rs138210. & 495 chr4_759700। & 5 nonsex & cis \\
\hline 4 Gg_rs138210. & 495 chr2_887400। & 6.71 nonsex & trans \\
\hline 4 Gg_rs138210. & 496 chr4_736430। & 7.15 nonsex & cis \\
\hline 4 Gg_rs138210. & 497 chr4_765560। & 7.6 nonsex & cis \\
\hline 4 Gg_rs138210. & 497 chr4_767840। & 12.86 nonsex & cis \\
\hline 4 Gg_rs138210. & 501 chr4_744480 & 6 nonsex & cis \\
\hline 4 Gg_rs138210. & 501 chr4_788630। & 7.35 nonsex & cis \\
\hline 4 Gg_rs138210. & 501 chr4_788360। & 8.71 nonsex & cis \\
\hline 4 Gg_rs156277 & 502 chr4_790820 & 5.12 nonsex & cis \\
\hline 4 Gg_rs156277 & 506 chr4_793910 & 18.58 nonsex & cis \\
\hline 4 Gg_rs156277 & 506 chr4_796860। & 5.83 nonsex & cis \\
\hline 4 Gg_rs156277 & 509 chr4_789690। & 5.24 nonsex & cis \\
\hline 4 Gg_rs156277 & 509 chr4_796970। & 4.39 nonsex & cis \\
\hline 4 Gg_rs156277 & 509 chr4_798230। & 4.58 nonsex & cis \\
\hline 4 Gg_rs156277 & 511 chr4_800050। & 4.42 nonsex & cis \\
\hline
\end{tabular}




\begin{tabular}{|c|c|c|c|}
\hline 4 Gg_rs156277 & 513 chr4_805490। & 3.61 nonsex & cis \\
\hline 4 Gg_rs156277 & 520 chr4_807600 & 5.96 nonsex & cis \\
\hline 4 Gg_rs144966 & 540 chr4_809600 & 6.98 nonsex & cis \\
\hline 4 Gg_rs144966 & 541 chr4_809390 & 4.87 nonsex & cis \\
\hline 4 Gg_rs144966 & 545 chr4_723030 & 7.11 sex & cis \\
\hline 4 Gg_rs156310. & 545 chr31_21680 & 11.22 sex & trans \\
\hline 4 Gg_rs156310. & 546 chr4_909470 & 6.73 sex & cis \\
\hline 4 Gg_rs156310. & 548 chr4_860430 & 4.03 nonsex & cis \\
\hline 4 Gg_rs145002 & 559 chr2_130107। & 8.39 sex & trans \\
\hline 4 Gg_rs145002 & 562 chr4_832260 & 6.36 nonsex & cis \\
\hline 4 Gg_rs145002 & 562 chrZ_459140। & 7.57 sex & trans \\
\hline 4 Gg_rs145002 & 562 chrZ_7059301 & 8.62 sex & trans \\
\hline 4 Gg_rs145002 & 563 chrZ_141610। & 7.84 sex & trans \\
\hline 4 Gg_rs145002 & 563 chr2_270010 & 8.97 sex & trans \\
\hline 4 Gg_rs145002 & 564 chr4_813760 & 5.76 sex & cis \\
\hline 4 Gg_rs145002 & 564 chr4_848470 & 4.36 nonsex & cis \\
\hline 4 Gg_rs145002 & 565 chr4_836220 & 4.26 nonsex & cis \\
\hline 4 Gg_rs145002 & 565 chrZ_6040301 & 7.63 sex & trans \\
\hline 4 Gg_rs145002 & 566 chr4_841670 & 3.61 nonsex & cis \\
\hline 4 Gg_rs145002 & 566 chrZ_798670। & 8.15 sex & trans \\
\hline 4 Gg_rs145002 & 566 chr2_608530 & 4.62 nonsex & trans \\
\hline 4 Gg_rs145002 & 567 chr4_830560 & 3.87 nonsex & cis \\
\hline 4 Gg_rs145002 & 567 chrZ_137050। & 6.95 sex & trans \\
\hline 4 Gg_rs145002 & 569 chr4_756280 & 5.96 sex & cis \\
\hline 4 Gg_rs145002 & 569 chr4_762920 & 5.85 sex & cis \\
\hline 4 Gg_rs145002 & 569 chr4_840020 & 7.42 nonsex & cis \\
\hline 4 Gg_rs145002 & 569 chr4_844680 & 6.85 sex & cis \\
\hline 4 Gg_rs145002 & 569 chr4_856710 & 5.37 nonsex & cis \\
\hline 4 Gg_rs145002 & 569 chr4_856720 & 6.94 nonsex & cis \\
\hline 4 Gg_rs145002 & 569 chr4_856820 & 4.95 nonsex & cis \\
\hline 4 Gg_rs145002 & 569 chr4_856850 & 4.98 nonsex & cis \\
\hline 4 Gg_rs145002 & 569 chr4_856860 & 8.75 nonsex & cis \\
\hline 4 Gg_rs145002 & 569 chr4_856870 & 8.76 nonsex & cis \\
\hline 4 Gg_rs145002 & 569 chr4_856880 & 9.79 nonsex & cis \\
\hline 4 Gg_rs145002 & 569 chr4_856890 & 15.13 nonsex & cis \\
\hline 4 Gg_rs145002 & 569 chr4_856900 & 7.03 nonsex & cis \\
\hline 4 Gg_rs145002 & 569 chr4_856910 & 6.46 nonsex & cis \\
\hline 4 Gg_rs145002 & 569 chr4_856920 & 6.07 nonsex & cis \\
\hline 4 Gg_rs145002 & 569 chr4_856950 & 5.81 nonsex & cis \\
\hline 4 Gg_rs145002 & 569 chr4_856960 & 7.3 nonsex & cis \\
\hline 4 Gg_rs145002 & 569 chr4_856970 & 9.84 nonsex & cis \\
\hline 4 Gg_rs145002 & 569 chr4_856980 & 15.16 nonsex & cis \\
\hline 4 Gg_rs145002 & 569 chr4_856990। & 5.91 nonsex & cis \\
\hline
\end{tabular}




\begin{tabular}{|c|c|c|}
\hline 4 Gg_rs145002 & 569 chr4_857050। & 7.4 nonsex \\
\hline 4 Gg_rs145002 & 569 chr4_857060। & 5.6 nonsex \\
\hline 4 Gg_rs145002 & 569 chr4_857160। & 7.07 nonsex \\
\hline 4 Gg_rs145002 & 569 chr4_857240। & 4.46 nonsex \\
\hline 4 Gg_rs145002 & 569 chr4_857290। & 5.16 nonsex \\
\hline 4 Gg_rs145002 & 569 chr4_857350। & 6.65 nonsex \\
\hline 4 Gg_rs145002 & 569 chr4_857360। & 5.06 nonsex \\
\hline 4 Gg_rs145002 & 569 chr4_857370। & 5.72 nonsex \\
\hline 4 Gg_rs145002 & 569 chr4_857380 & 11.02 nonsex \\
\hline 4 Gg_rs145002 & 569 chr4_857390। & 8.91 nonsex \\
\hline 4 Gg_rs145002 & 569 chr4_857400। & 9.28 nonsex \\
\hline 4 Gg_rs145002 & 569 chr4_857570। & 8.49 nonsex \\
\hline 4 Gg_rs145002 & 569 chr4_857580 & 6.96 nonsex \\
\hline 4 Gg_rs145002 & 569 chr4_857700। & 4.23 sex \\
\hline 4 Gg_rs145002 & 569 chr4_857830। & 6.45 nonsex \\
\hline 4 Gg_rs145002 & 569 chr4_857860। & 11.19 nonsex \\
\hline 4 Gg_rs145002 & 569 chr4_857870। & 9.2 nonsex \\
\hline 4 Gg_rs145002 & 569 chr4_857880 & 9.61 nonsex \\
\hline 4 Gg_rs145002 & 569 chr4_857890। & 5.86 nonsex \\
\hline 4 Gg_rs145002 & 569 chr4_857910। & 5.09 nonsex \\
\hline 4 Gg_rs145002 & 569 chr4_857980। & 6.35 nonsex \\
\hline 4 Gg_rs145002 & 569 chr4_857990। & 6.84 nonsex \\
\hline 4 Gg_rs145002 & 569 chr4_858210 & 7.53 nonsex \\
\hline 4 Gg_rs145002 & 569 chr4_858220। & 5.5 nonsex \\
\hline 4 Gg_rs145002 & 569 chr4_858360। & 5.76 nonsex \\
\hline 4 Gg_rs145002 & 569 chr4_858490। & 5.59 nonsex \\
\hline 4 Gg_rs145002 & 569 chr4_858580 & 5.46 nonsex \\
\hline 4 Gg_rs145002 & 569 chr4_864240। & 10.77 sex \\
\hline 4 Gg_rs145002 & 569 chr4_864250। & 9.38 nonsex \\
\hline 4 Gg_rs145002 & 569 chr4_864260। & 6.16 nonsex \\
\hline 4 Gg_rs145002 & 569 chr4_864310। & 11.99 nonsex \\
\hline 4 Gg_rs145002 & 569 chr4_864320। & 8.42 nonsex \\
\hline 4 Gg_rs145002 & 569 chr4_864330। & 8.01 sex \\
\hline 4 Gg_rs145002 & 569 chr4_864340। & 9.07 nonsex \\
\hline 4 Gg_rs145002 & 569 chr4_864350। & 5.48 nonsex \\
\hline 4 Gg_rs145002 & 569 chr4_864360। & 7.32 nonsex \\
\hline 4 Gg_rs145002 & 569 chr4_864370। & 9.85 nonsex \\
\hline 4 Gg_rs145002 & 569 chr4_864380। & $8.3 \operatorname{sex}$ \\
\hline 4 Gg_rs145002 & 569 chr4_864390। & 7.18 nonsex \\
\hline 4 Gg_rs145002 & 569 chr4_864400। & 7.23 nonsex \\
\hline 4 Gg_rs145002 & 569 chr4_864440। & 8.21 nonsex \\
\hline 4 Gg_rs145002 & 569 chr4_864450। & 5.32 nonsex \\
\hline 4 Gg_rs145002 & 569 chr4_864460। & 5.84 sex \\
\hline
\end{tabular}




\begin{tabular}{|c|c|c|}
\hline 4 Gg_rs145002 & 569 chr4_864470। & 9.58 nonsex \\
\hline 4 Gg_rs145002 & 569 chr4_864480। & 9.76 nonsex \\
\hline 4 Gg_rs145002 & 569 chr4_864490। & 7.37 sex \\
\hline 4 Gg_rs145002 & 569 chr4_864500। & 7.15 sex \\
\hline 4 Gg_rs145002 & 569 chr4_864520। & 10.5 sex \\
\hline 4 Gg_rs145002 & 569 chr4_864530। & 7.01 sex \\
\hline 4 Gg_rs145002 & 569 chr4_864540। & 6.74 sex \\
\hline 4 Gg_rs145002 & 569 chr4_864550। & 6.42 sex \\
\hline 4 Gg_rs145002 & 569 chr4_864570। & 10.49 sex \\
\hline 4 Gg_rs145002 & 569 chr4_864580 & 10.47 sex \\
\hline 4 Gg_rs145002 & 569 chr4_864590। & 8.86 sex \\
\hline 4 Gg_rs145002 & 569 chr4_864610। & 7.87 sex \\
\hline 4 Gg_rs145002 & 569 chr4_864620। & 6.3 sex \\
\hline 4 Gg_rs145002 & 569 chr4_864700। & 5.95 nonsex \\
\hline 4 Gg_rs145002 & 569 chr4_881810। & 7.49 sex \\
\hline 4 Gg_rs145002 & 569 chr4_836110। & 3.47 nonsex \\
\hline 4 Gg_rs145002 & 569 chr4_848690। & 3.47 nonsex \\
\hline 4 Gg_rs145002 & 569 chr4_856620। & 4.3 nonsex \\
\hline 4 Gg_rs145002 & 569 chr4_856630। & 4.07 nonsex \\
\hline 4 Gg_rs145002 & 569 chr4_856680 & 4.23 nonsex \\
\hline 4 Gg_rs145002 & 569 chr4_856760। & 3.86 nonsex \\
\hline 4 Gg_rs145002 & 569 chr4_856830। & 4.51 nonsex \\
\hline 4 Gg_rs145002 & 569 chr4_856930। & 4.59 nonsex \\
\hline 4 Gg_rs145002 & 569 chr4_856940। & 4.37 nonsex \\
\hline 4 Gg_rs145002 & 569 chr4_857000। & 3.83 nonsex \\
\hline 4 Gg_rs145002 & 569 chr4_857030। & 4.13 nonsex \\
\hline 4 Gg_rs145002 & 569 chr4_857280 & 3.75 nonsex \\
\hline 4 Gg_rs145002 & 569 chr4_857530। & 4.02 nonsex \\
\hline 4 Gg_rs145002 & 569 chr4_857550। & 4.26 nonsex \\
\hline 4 Gg_rs145002 & 569 chr4_857560। & 4.6 nonsex \\
\hline 4 Gg_rs145002 & 569 chr4_857590। & 5.14 nonsex \\
\hline 4 Gg_rs145002 & 569 chr4_857760। & 3.73 nonsex \\
\hline 4 Gg_rs145002 & 569 chr4_857770। & 4.57 nonsex \\
\hline 4 Gg_rs145002 & 569 chr4_857900। & 3.99 nonsex \\
\hline 4 Gg_rs145002 & 569 chr4_857950। & 4.1 nonsex \\
\hline 4 Gg_rs145002 & 569 chr4_858380। & 4.36 nonsex \\
\hline 4 Gg_rs145002 & 569 chr4_858510। & 3.97 nonsex \\
\hline 4 Gg_rs145002 & 569 chr4_858680। & 4.73 nonsex \\
\hline 4 Gg_rs145002 & 569 chr4_858700। & 3.81 nonsex \\
\hline 4 Gg_rs145002 & 569 chr4_858710। & 3.96 nonsex \\
\hline 4 Gg_rs145002 & 569 chr4_858770। & 4.88 nonsex \\
\hline 4 Gg_rs145002 & 569 chr4_858870। & 4.19 nonsex \\
\hline 4 Gg_rs145002 & 569 chr4_858890। & 3.73 nonsex \\
\hline
\end{tabular}




\begin{tabular}{|c|c|c|}
\hline 4 Gg_rs145002 & 569 chr4_858990। & 3.98 nonsex \\
\hline 4 Gg_rs145002 & 569 chr4_859120। & 3.84 nonsex \\
\hline 4 Gg_rs145002 & 569 chr4_864040। & 4.01 nonsex \\
\hline 4 Gg_rs145002 & 569 chr4_864230। & 4.22 nonsex \\
\hline 4 Gg_rs145002 & 569 chrZ_2322001 & 7.57 sex \\
\hline 5 Gg_rs156490. & 0 chr22_13800 & 5.93 nonsex \\
\hline 5 Gg_rs156490. & 4 chr5_802000 & 4.62 nonsex \\
\hline 5 Gg_rs145097. & 16 chr5_894000 & 7.88 nonsex \\
\hline 5 Gg_rs145097. & 19 chr5_116300 & 6.53 sex \\
\hline 5 Gg_rs145155. & 69 chr5_137780 & 3.82 nonsex \\
\hline 5 Gg_rs156661 & 71 chr2_120081। & 6.89 nonsex \\
\hline 5 Gg_rs145155 & 72 chr5_117990 & 7.19 sex \\
\hline 5 Gg_rs145155. & 76 chr5_121810 & 5.15 nonsex \\
\hline 5 Gg_rs145155. & 77 chr5_102220 & 4.89 sex \\
\hline 5 Gg_rs145155. & 77 chr5_104870 & 5.09 nonsex \\
\hline 5 Gg_rs145155. & 78 chr5_125320 & 5.6 nonsex \\
\hline 5 Gg_rs145155. & 79 chr5_752000 & 5.04 nonsex \\
\hline 5 Gg_rs145155. & 80 chr5_121200 & 3.55 nonsex \\
\hline 5 Gg_rs145155. & 83 chr10_14593। & 5.72 nonsex \\
\hline 5 Gg_rs145155. & 92 chr5_118420 & 9.95 nonsex \\
\hline 5 Gg_rs145155. & 92 chr5_119080 & 5.67 sex \\
\hline 5 Gg_rs145155. & 92 chr5_120800 & 4.35 nonsex \\
\hline 5 Gg_rs145155 & 92 chr5_122150 & 5.65 nonsex \\
\hline 5 Gg_rs145155 & 92 chr5_125790 & 5.5 nonsex \\
\hline 5 Gg_rs145155. & 92 chr5_126900 & 8.05 nonsex \\
\hline 5 Gg_rs145155. & 92 chr5_890100। & 3.92 nonsex \\
\hline 5 Gg_rs145155. & 92 chr5_111940 & 3.04 nonsex \\
\hline 5 Gg_rs145155. & 92 chr5_118810 & 3.68 nonsex \\
\hline 5 Gg_rs156661 & 92 chr5_121210 & 4.37 sex \\
\hline 5 Gg_rs145155 & 93 chr5_986400। & 6.05 nonsex \\
\hline 5 Gg_rs145155. & 93 chr5_145490 & 5.92 nonsex \\
\hline 5 Gg_rs145155. & 93 chr5_118700 & 4.36 nonsex \\
\hline 5 Gg_rs145155. & 94 chr5_169170 & 4.91 nonsex \\
\hline 5 Gg_rs145155. & 94 chr4_770470 & 6.25 nonsex \\
\hline 5 Gg_rs156661 & 94 chr5_105350 & 4.78 nonsex \\
\hline 5 Gg_rs156661 & 94 chr5_129070 & 4.32 nonsex \\
\hline 5 Gg_rs156661 & 94 chr5_105030 & 4.38 nonsex \\
\hline 5 Gg_rs156661 & 95 chr5_128860 & 7.86 nonsex \\
\hline 5 Gg_rs156661 & 95 chr5_129760 & 8.26 nonsex \\
\hline 5 Gg_rs156661 & 95 chr5_134810 & 10.07 nonsex \\
\hline 5 Gg_rs156661 & 95 chr5_137580 & 5.71 sex \\
\hline 5 Gg_rs156661 & 95 chr5_138110 & 5.93 nonsex \\
\hline 5 Gg_rs156661 & 95 chr5_117970 & 5.38 nonsex \\
\hline
\end{tabular}




\begin{tabular}{|c|c|c|c|}
\hline 5 Gg_rs156661 & 96 chr5_128970 & 3.83 nonsex & cis \\
\hline 5 Gg_rs156661 & 96 chr5_129470 & 4.69 nonsex & cis \\
\hline 5 Gg_rs156661 & 96 chr5_135600 & 4.87 nonsex & cis \\
\hline 5 Gg_rs156661 & 96 chr5_136500 & 5.98 nonsex & cis \\
\hline 5 Gg_rs156661 & 96 chr5_137970 & 5.81 nonsex & cis \\
\hline 5 Gg_rs156661 & 96 chr5_137990 & 5.14 nonsex & cis \\
\hline 5 Gg_rs156661 & 97 chr5_129060 & 4.17 nonsex & cis \\
\hline 5 Gg_rs156661 & 97 chr1_720760 & 6.03 nonsex & trans \\
\hline 5 Gg_rs156661 & 99 chr5_128940 & 4.96 nonsex & cis \\
\hline 5 Gg_rs156661 & 99 chr5_131030 & 3.84 sex & cis \\
\hline 5 Gg_rs156661 & 100 chr2_129228। & 3.91 nonsex & trans \\
\hline 5 Gg_rs156661 & 103 chr5_136730 & 5.22 nonsex & cis \\
\hline 5 Gg_rs156661 & 103 chr5_138920 & 8.2 nonsex & cis \\
\hline 5 Gg_rs156661 & 103 chr5_141940 & 5.89 nonsex & cis \\
\hline 5 Gg_rs156661 & 103 chr5_139120 & 4.58 nonsex & cis \\
\hline 5 Gg_rs156661 & 104 chr5_178100 & 4.22 nonsex & cis \\
\hline 5 Gg_rs145155. & 105 chr5_41000 & 4.97 nonsex & cis \\
\hline 5 Gg_rs156661 & 105 chr5_155400 & 6.19 nonsex & cis \\
\hline 5 Gg_rs145155. & 106 chr5_109010 & 6.54 nonsex & cis \\
\hline 5 Gg_rs145155. & 106 chr5_144260 & 8.2 nonsex & cis \\
\hline 5 Gg_rs156661 & 106 chr5_131080 & 5.29 nonsex & cis \\
\hline 5 Gg_rs156661 & 106 chr5_120750 & 4.62 nonsex & cis \\
\hline 5 Gg_rs156661 & 107 chr5_109270 & 4.76 nonsex & cis \\
\hline 5 Gg_rs156661 & 108 chr5_101750 & 5.14 nonsex & cis \\
\hline 5 Gg_rs156661 & 108 chr5_136480 & 5.08 nonsex & cis \\
\hline 5 Gg_rs156661 & 108 chr2_129203। & 4.56 nonsex & trans \\
\hline 5 Gg_rs145155. & 109 chr5_106400 & 6.27 nonsex & cis \\
\hline 5 Gg_rs145155. & 109 chr5_120770 & 4.51 sex & cis \\
\hline 5 Gg_rs156661 & 109 chr5_164530 & 4.14 nonsex & cis \\
\hline 5 Gg_rs145189. & 110 chr5_161770 & 5.8 nonsex & cis \\
\hline 5 Gg_rs145155. & 111 chr5_121140 & 6.64 nonsex & cis \\
\hline 5 Gg_rs156661 & 111 chr5_122460 & 4.24 nonsex & cis \\
\hline 5 Gg_rs145155. & 112 chr5_112770 & $6.32 \operatorname{sex}$ & cis \\
\hline 5 snp.98.79.911 & 112 chr5_161510 & 6.19 sex & cis \\
\hline 5 Gg_rs145189. & 113 chr5_203270 & 5.69 nonsex & cis \\
\hline 5 Gg_rs145155. & 114 chr5_118610 & 4.23 nonsex & cis \\
\hline 5 Gg_rs156661 & 114 chr5_139460 & 3.24 nonsex & cis \\
\hline 5 Gg_rs145189. & 115 chr5_148730 & 4.97 nonsex & cis \\
\hline 5 Gg_rs145189. & 115 chr5_190520 & 4.87 nonsex & cis \\
\hline 5 Gg_rs145189. & 115 chr17_98940 & 6.19 nonsex & trans \\
\hline 5 Gg_rs145189. & 118 chr5_119730 & 5.36 sex & cis \\
\hline 5 Gg_rs145189. & 118 chr31_25960। & 8.91 sex & trans \\
\hline 5 Gg_rs145189. & 118 chrW_43560 & 7.63 sex & trans \\
\hline
\end{tabular}




\begin{tabular}{|c|c|c|c|}
\hline 5 Gg_rs145189. & 118 chr2_252960। & 8.06 sex & trans \\
\hline 5 Gg_rs145189. & 119 chr5_135960। & 3.72 nonsex & cis \\
\hline 5 Gg_rs145189. & 120 chr5_148900 & 7.27 sex & cis \\
\hline 5 Gg_rs145189. & 120 chr4_597910 & 7.66 nonsex & trans \\
\hline 5 Gg_rs145189. & 121 chr5_145720 & $6.06 \mathrm{sex}$ & cis \\
\hline 5 Gg_rs145189. & 121 chr5_146990 & 7.76 sex & cis \\
\hline 5 Gg_rs145189. & 121 chr5_168560 & 5.43 nonsex & cis \\
\hline 5 Gg_rs145189. & 121 chr5_180940 & 5.54 sex & cis \\
\hline 5 Gg_rs145189. & 121 chr5_186050 & 6.68 sex & cis \\
\hline 5 Gg_rs145189. & 121 chr5_186090 & 4.26 sex & cis \\
\hline 5 Gg_rs145189. & 121 chr5_186100 & 5.79 sex & cis \\
\hline 5 Gg_rs145189. & 121 chr5_147010 & 4.16 nonsex & cis \\
\hline 5 Gg_rs145189. & 121 chr5_157590 & 3.18 nonsex & cis \\
\hline 5 Gg_rs145189. & 121 chr5_158160 & 4.34 nonsex & cis \\
\hline 5 Gg_rs145189. & 121 chr5_161310 & 3.97 nonsex & cis \\
\hline 5 Gg_rs145189. & 121 chr5_167090 & 4.53 nonsex & cis \\
\hline 5 Gg_rs145189. & 122 chr5_180950 & 4.03 nonsex & cis \\
\hline 5 Gg_rs145189. & 123 chr5_181470 & 4.45 nonsex & cis \\
\hline 5 Gg_rs145189. & 123 chr2_499150 & 6.62 sex & trans \\
\hline 5 Gg_rs145189. & 124 chr5_176700 & 3.96 nonsex & cis \\
\hline 5 Gg_rs145189. & 124 chr5_173720 & 4.87 nonsex & cis \\
\hline 5 Gg_rs145189. & 124 chr5_174210 & 3.96 nonsex & cis \\
\hline 5 Gg_rs145189. & 126 chr5_159490 & 3.22 nonsex & cis \\
\hline 5 snp.98.79.911 & 126 chr5_109650 & 5.94 sex & cis \\
\hline 5 snp.98.79.911 & 127 chrZ_627110। & 7.85 sex & trans \\
\hline 5 Gg_rs145189. & 128 chr5_178840 & 3.4 nonsex & cis \\
\hline 5 snp.98.79.911 & 129 chr4_721020 & 8.18 sex & trans \\
\hline 5 Gg_rs145189. & 131 chr5_176170 & 3.66 nonsex & cis \\
\hline 5 snp.98.79.911 & 131 chr5_959800 & 4.24 sex & cis \\
\hline 5 snp.98.79.91( & 131 chr5_157090 & 3.82 sex & cis \\
\hline 5 snp.98.79.911 & 131 chr5_230700 & 4 sex & cis \\
\hline 5 snp.98.79.911 & 131 chr30_12270 & 8.14 sex & trans \\
\hline 5 snp.98.79.911 & 131 chr1_233600 & 5.86 nonsex & trans \\
\hline 5 snp.98.79.911 & 133 chr5_167250 & 6.4 nonsex & cis \\
\hline 5 snp.98.79.911 & 137 chr5_183670 & 4.14 nonsex & cis \\
\hline 5 snp.98.79.911 & 141 chr30_29600। & $5.67 \mathrm{sex}$ & trans \\
\hline 5 Gg_rs156795 & 176 chr5_338340 & 6.17 nonsex & cis \\
\hline 5 Gg_rs156795 & 178 chr5_226030 & 5.24 sex & cis \\
\hline 5 Gg_rs156795 & 178 chr5_244500 & 6.86 nonsex & cis \\
\hline 5 Gg_rs156795 & 179 chr5_184360 & 5.41 nonsex & cis \\
\hline 5 Gg_rs156795 & 179 chr5_333720 & 3.89 nonsex & cis \\
\hline 5 Gg_rs156795 & 180 chr5_167810 & 3.87 nonsex & cis \\
\hline 5 Gg_rs156795 & 180 chr5_329890 & 4.06 nonsex & cis \\
\hline
\end{tabular}




\begin{tabular}{|c|c|c|c|}
\hline 5 Gg_rs156795 & 180 chr5_336130। & 4.26 nonsex & cis \\
\hline 5 Gg_rs156795 & 181 chr5_244300 & 6.59 nonsex & cis \\
\hline 5 Gg_rs156795 & 181 chr5_334180 & 4.16 nonsex & cis \\
\hline 5 Gg_rs156795 & 181 chr5_351960 & 4.21 nonsex & cis \\
\hline 5 Gg_rs156795 & 181 chr6_122410 & 8.92 sex & trans \\
\hline 5 Gg_rs156795 & 182 chr5_216010 & 5.57 nonsex & cis \\
\hline 5 Gg_rs156795 & 182 chr5_217550 & 5.16 nonsex & cis \\
\hline 5 Gg_rs156795 & 182 chr5_323900 & 6.19 nonsex & cis \\
\hline 5 Gg_rs156795 & 182 chr5_217110 & 4.11 nonsex & cis \\
\hline 5 Gg_rs156795 & 182 chr5_219680 & 4.27 nonsex & cis \\
\hline 5 Gg_rs156795 & 182 chr5_352760 & 3.69 nonsex & cis \\
\hline 5 Gg_rs156795 & 182 chr4_906900। & 12.92 sex & trans \\
\hline 5 Gg_rs156795 & 184 chr3_105358। & 7.31 sex & trans \\
\hline 5 Gg_rs156795 & 185 chr5_583500 & 10.05 sex & trans \\
\hline 5 Gg_rs156795 & 186 chr3_960350। & 7.81 sex & trans \\
\hline 5 Gg_rs156795 & 188 chr5_207890 & 4.27 nonsex & cis \\
\hline 5 Gg_rs156795 & 188 chr4_494860 & 8.89 sex & trans \\
\hline 5 Gg_rs156795 & 188 chr3_489690। & $11.32 \mathrm{sex}$ & trans \\
\hline 5 Gg_rs156795 & 189 chr5_244490। & 11.51 nonsex & cis \\
\hline 5 Gg_rs156795 & 189 chr6_245200। & 9.17 sex & trans \\
\hline 5 Gg_rs156795 & 190 chr6_269360। & 10.61 sex & trans \\
\hline 5 Gg_rs156795 & 190 chr2_130870 & 9.19 sex & trans \\
\hline 5 Gg_rs156795 & 191 chr5_241560 & $6.8 \mathrm{sex}$ & cis \\
\hline 5 Gg_rs156795 & 191 chr5_248560। & 7.42 nonsex & cis \\
\hline 5 Gg_rs156795 & 191 chr5_248480 & 4.88 nonsex & cis \\
\hline 5 Gg_rs156795 & 191 chr6_121820 & 9.66 sex & trans \\
\hline 5 Gg_rs156795 & 191 chr3_303750। & 16.33 sex & trans \\
\hline 5 Gg_rs156859. & 192 chr22_41600। & 8.47 sex & trans \\
\hline 5 Gg_rs156859. & 192 chr26_13510 & 6.58 nonsex & trans \\
\hline 5 Gg_rs156859. & 194 chr6_239990। & 8.53 sex & trans \\
\hline 5 Gg_rs156859. & 194 chr14_55580 & 7.94 sex & trans \\
\hline 5 Gg_rs156859. & 195 chr28_25030। & 7.06 sex & trans \\
\hline 5 Gg_rs156859. & 195 chr28_39660। & 7.1 sex & trans \\
\hline 5 Gg_rs156859. & 195 chr1_126747। & 8.63 sex & trans \\
\hline 5 Gg_rs156859. & 195 chr4_136300। & 9.54 sex & trans \\
\hline 5 Gg_rs156859. & 195 chr18_22100। & 6.06 nonsex & trans \\
\hline 5 Gg_rs156859. & 196 chr5_436870 & 8.37 sex & trans \\
\hline 5 Gg_rs156859. & 197 chr7_221290। & 6.39 nonsex & trans \\
\hline 5 Gg_rs156859. & 197 chr22_51920। & 6.77 sex & trans \\
\hline 5 Gg_rs156859. & 197 chr23_54410। & 7.36 sex & trans \\
\hline 5 Gg_rs156859. & 197 chr1_121158। & 7.49 sex & trans \\
\hline 5 Gg_rs156859. & 198 chr2_686650। & 8.12 sex & trans \\
\hline 5 Gg_rs156859. & 198 chr22_52250। & 6.6 nonsex & trans \\
\hline
\end{tabular}




\begin{tabular}{|c|c|c|}
\hline 5 Gg_rs156859. & 198 chr25_15060। & 5.35 nonsex \\
\hline 5 Gg_rs156859. & 199 chr18_99060। & 5.27 nonsex \\
\hline 5 Gg_rs156859. & 200 chr1_671000 & 8.98 sex \\
\hline 5 Gg_rs156859. & 200 chr5_500850 & 7.48 sex \\
\hline 5 Gg_rs156859. & 200 chr13_25970। & $10.51 \mathrm{sex}$ \\
\hline 5 Gg_rs156859. & 200 chr22_51770 & 8.07 sex \\
\hline 5 Gg_rs156859. & 200 chr22_53180 & 7.82 sex \\
\hline 5 Gg_rs156859. & 200 chr27_53580। & 7.46 sex \\
\hline 5 Gg_rs156859. & 200 chr28_24550। & 4.81 sex \\
\hline 5 Gg_rs156859. & 200 chr28_31740। & $5.51 \mathrm{sex}$ \\
\hline 5 Gg_rs156859. & 200 chr1_794910 & 7.47 sex \\
\hline 5 Gg_rs156859. & 200 chrZ_5373101 & 8.53 sex \\
\hline 5 Gg_rs156859. & 200 chr4_175800 & 7.79 sex \\
\hline 5 Gg_rs156859. & 200 chr20_10094! & 7.26 nonsex \\
\hline 5 Gg_rs156859. & 200 chr21_44260। & 6.35 nonsex \\
\hline 5 Gg_rs156859. & 200 chr22_38030। & 6.26 nonsex \\
\hline 5 Gg_rs156859. & 201 chr5_532000 & 8.66 sex \\
\hline 5 Gg_rs156859. & 201 chr5_578600 & 11.89 sex \\
\hline 5 Gg_rs156859. & 201 chr5_580920। & 8.06 sex \\
\hline 5 Gg_rs156859. & 201 chr7_285260। & 7.99 sex \\
\hline 5 Gg_rs156859. & 201 chr13_23970। & 6.65 nonsex \\
\hline 5 Gg_rs156859. & 201 chr13_27180 & 9.05 sex \\
\hline 5 Gg_rs156859. & 201 chr13_27300। & 8.54 sex \\
\hline 5 Gg_rs156859. & 201 chr24_40900। & 9.98 sex \\
\hline 5 Gg_rs156859. & 201 chr26_43590। & 6.44 sex \\
\hline 5 Gg_rs156859. & 201 chr27_37100। & 7.05 sex \\
\hline 5 Gg_rs156859. & 201 chr27_56780 & 7.84 sex \\
\hline 5 Gg_rs156859. & 201 chr1_935110। & 8.47 sex \\
\hline 5 Gg_rs156859. & 201 chr3_104574! & 7.24 sex \\
\hline 5 Gg_rs156859. & 201 chr4_122300। & 7.29 sex \\
\hline 5 Gg_rs156859. & 201 chr19_87300। & 6.03 nonsex \\
\hline 5 Gg_rs156859. & 201 chr28_33030 & 4.94 nonsex \\
\hline 5 Gg_rs156859. & 201 chr31_19070। & 5.62 nonsex \\
\hline 5 Gg_rs156859. & 202 chr1_311000 & 7.47 sex \\
\hline 5 Gg_rs156859. & 202 chr1_515000 & 8.07 sex \\
\hline 5 Gg_rs156859. & 202 chr1_560000 & 8.47 sex \\
\hline 5 Gg_rs156859. & 202 chr1_564000 & 8.69 sex \\
\hline 5 Gg_rs156859. & 202 chr1_696000 & 7.85 sex \\
\hline 5 Gg_rs156859. & 202 chr5_590220। & 9.39 sex \\
\hline 5 Gg_rs156859. & 202 chr6_240450 & 6.39 sex \\
\hline 5 Gg_rs156859. & 202 chr7_221310 & 6.08 sex \\
\hline 5 Gg_rs156859. & 202 chr7_285180 & 8.57 sex \\
\hline 5 Gg_rs156859. & 202 chr9_502400। & 6.67 sex \\
\hline
\end{tabular}




\begin{tabular}{|c|c|c|}
\hline 5 Gg_rs156859. & 202 chr9_150000। & 8.31 sex \\
\hline 5 Gg_rs156859. & 202 chr14_66560 & 9.06 sex \\
\hline 5 Gg_rs156859. & 202 chr24_34200। & 12.14 sex \\
\hline 5 Gg_rs156859. & 202 chr26_24300। & 8.76 sex \\
\hline 5 Gg_rs156859. & 202 chr28_24480। & 9.24 sex \\
\hline 5 Gg_rs156859. & 202 chr28_24990। & 5.29 sex \\
\hline 5 Gg_rs156859. & 202 chr28_32550 & 8.17 sex \\
\hline 5 Gg_rs156859. & 202 chr33_49900। & 8.22 sex \\
\hline 5 Gg_rs156859. & 202 chr33_49960। & 7.45 sex \\
\hline 5 Gg_rs156859. & 202 chr33_76720। & 11.88 sex \\
\hline 5 Gg_rs156859. & 202 chr18_10502 & 5.65 nonsex \\
\hline 5 Gg_rs156859. & 203 chr1_72000 & 7.22 sex \\
\hline 5 Gg_rs156859. & 203 chr1_438000 & 8.68 sex \\
\hline 5 Gg_rs156859. & 203 chr1_664000 & 8.37 sex \\
\hline 5 Gg_rs156859. & 203 chr5_487000 & 9.29 sex \\
\hline 5 Gg_rs156859. & 203 chr5_578630 & 8.61 sex \\
\hline 5 Gg_rs156859. & 203 chr6_234980 & 7.79 sex \\
\hline 5 Gg_rs156859. & 203 chr13_27270 & 8.5 sex \\
\hline 5 Gg_rs156859. & 203 chr26_43650। & 9.31 sex \\
\hline 5 Gg_rs156859. & 203 chr28_25010। & 7.26 sex \\
\hline 5 Gg_rs156859. & 203 chrW_81700 & 10.41 sex \\
\hline 5 Gg_rs156859. & 203 chr1_194561। & 7.94 sex \\
\hline 5 Gg_rs156859. & 203 chr2_660680 & 8.71 sex \\
\hline 5 Gg_rs156859. & 203 chr3_105533। & 8.61 sex \\
\hline 5 Gg_rs156859. & 203 chr4_116900। & 9.54 sex \\
\hline 5 Gg_rs156859. & 204 chr4_792470 & 13.27 sex \\
\hline 5 Gg_rs156859. & 204 chr5_393530। & 9.16 sex \\
\hline 5 Gg_rs156859. & 204 chr13_18370 & 8.5 sex \\
\hline 5 Gg_rs156859. & 204 chr13_21810 & 8.07 sex \\
\hline 5 Gg_rs156859. & 204 chr13_26200। & 10.63 sex \\
\hline 5 Gg_rs156859. & 204 chr22_41110 & 8.24 sex \\
\hline 5 Gg_rs156859. & 204 chr1_794950 & 9.09 sex \\
\hline 5 Gg_rs156859. & 204 chr1_897430। & 8.81 sex \\
\hline 5 Gg_rs156859. & 204 chr20_98630। & 6.15 nonsex \\
\hline 5 Gg_rs156859. & 204 chr24_56240। & 6.27 nonsex \\
\hline 5 Gg_rs156859. & 205 chr10_14946। & 9.39 sex \\
\hline 5 Gg_rs156859. & 205 chr14_55640। & 8.84 sex \\
\hline 5 Gg_rs156859. & 205 chr1_814530। & 9.72 sex \\
\hline 5 Gg_rs156859. & 205 chr4_164800 & 8.11 sex \\
\hline 5 RBL3041 & 206 chr5_261490। & 12.26 nonsex \\
\hline 5 RBL3041 & 206 chr5_255610 & 5.6 nonsex \\
\hline 5 RBL3041 & 206 chr1_42000 & 9.53 sex \\
\hline 5 RBL3041 & 206 chr4_895770। & 9.99 sex \\
\hline
\end{tabular}




\begin{tabular}{|c|c|c|}
\hline 5 RBL3041 & 206 chr13_17389| & $9.41 \mathrm{sex}$ \\
\hline 5 RBL3041 & 206 chr15_55500। & 11.56 sex \\
\hline 5 RBL3041 & 206 chr15_63980 & 9.09 sex \\
\hline 5 RBL3041 & 206 chr15_67460। & 10.86 sex \\
\hline 5 RBL3041 & 206 chr15_12580 & 10.07 sex \\
\hline 5 RBL3041 & 206 chr25_36830 & 9.26 sex \\
\hline 5 RBL3041 & 206 chr26_46160। & 10 sex \\
\hline 5 RBL3041 & 206 chr26_48800 & 9.52 sex \\
\hline 5 RBL3041 & 206 chr27_37020। & 9.52 sex \\
\hline 5 RBL3041 & 206 chr28_28650। & 9.37 sex \\
\hline 5 RBL3041 & 206 chr28_30300। & 9.29 sex \\
\hline 5 RBL3041 & 206 chr1_196989| & 12.24 sex \\
\hline 5 RBL3041 & 206 chr2_995000 & 10.46 sex \\
\hline 5 RBL3041 & 207 chr5_365650। & 7 sex \\
\hline 5 RBL3041 & 207 chr5_268670 & 4.99 nonsex \\
\hline 5 RBL3041 & 207 chr5_516870 & 10.56 sex \\
\hline 5 RBL3041 & 207 chr6_324520। & 9.52 sex \\
\hline 5 RBL3041 & 207 chr6_357780 & 9.09 sex \\
\hline 5 RBL3041 & 207 chr13_16130। & 9.53 sex \\
\hline 5 RBL3041 & 207 chr13_16569| & 9.29 sex \\
\hline 5 RBL3041 & 207 chr14_47680। & 9.33 sex \\
\hline 5 RBL3041 & 207 chr14_13150। & 9.02 sex \\
\hline 5 RBL3041 & 207 chr15_47200 & 10.24 sex \\
\hline 5 RBL3041 & 207 chr15_68280 & 9.64 sex \\
\hline 5 RBL3041 & 207 chr30_49300। & 7.28 nonsex \\
\hline 5 RBL3041 & 207 chr23_41860। & 9.19 sex \\
\hline 5 RBL3041 & 207 chr23_52630। & 9.39 sex \\
\hline 5 RBL3041 & 207 chr1_139997। & 9.82 sex \\
\hline 5 RBL3041 & 208 chr1_510610 & 9.35 sex \\
\hline 5 RBL3041 & 208 chr7_662300। & 9.44 sex \\
\hline 5 RBL3041 & 208 chr12_18804। & 8.47 sex \\
\hline 5 RBL3041 & 208 chr14_13496। & 8.6 sex \\
\hline 5 RBL3041 & 208 chr15_68270 & 11.73 sex \\
\hline 5 RBL3041 & 208 chr15_81470। & 8.89 sex \\
\hline 5 RBL3041 & 208 chr23_41620 & 8.89 sex \\
\hline 5 RBL3041 & 209 chr5_589450। & 10.59 sex \\
\hline 5 RBL3041 & 209 chr15_70890 & 8.39 sex \\
\hline 5 RBL3041 & 209 chr15_80690 & 8.03 sex \\
\hline 5 RBL3041 & 209 chr15_10162। & 8.71 sex \\
\hline 5 RBL3041 & 209 chr23_40200। & 8.02 sex \\
\hline 5 RBL3041 & 209 chr27_46490। & 10.22 sex \\
\hline 5 RBL3041 & 209 chr2_735800 & 8.46 sex \\
\hline 5 RBL3041 & 209 chr2_141240 & 8.4 sex \\
\hline
\end{tabular}




\begin{tabular}{|c|c|c|}
\hline 5 RBL3041 & 209 chr3_409170। & $8.58 \mathrm{sex}$ \\
\hline 5 RBL3041 & 209 chr3_992100। & $8.88 \mathrm{sex}$ \\
\hline 5 RBL3041 & 210 chr6_696000 & 7.78 sex \\
\hline 5 RBL3041 & 210 chr7_665200। & $9.51 \mathrm{sex}$ \\
\hline 5 RBL3041 & 211 chr13_12839। & 8.81 sex \\
\hline 5 RBL3041 & 212 chr5_271600 & 4.47 nonsex \\
\hline 5 RBL3041 & 212 chr5_106200 & 7.57 sex \\
\hline 5 RBL3041 & 212 chr7_120280 & 8.28 sex \\
\hline 5 RBL3041 & 212 chr4_129200। & 7.86 sex \\
\hline 5 RBL3041 & 212 chr3_854400। & 5.37 nonsex \\
\hline 5 RBL3041 & 213 chr6_246500 & 5.54 nonsex \\
\hline 5 RBL3041 & 214 chr9_102750 & 7.41 sex \\
\hline 5 RBL3041 & 214 chr13_96010 & 9.01 sex \\
\hline 5 RBL3041 & 214 chr2_433170 & 8.89 sex \\
\hline 5 RBL3041 & 215 chr13_84600। & 7.95 sex \\
\hline 5 RBL3041 & 216 chr4_898480 & 8.61 sex \\
\hline 5 RBL3041 & 216 chr14_47710 & 8.51 sex \\
\hline 5 RBL3041 & 216 chr3_662810 & 8.57 nonsex \\
\hline 5 RBL3041 & 217 chr5_231290। & $6.81 \mathrm{sex}$ \\
\hline 5 RBL3041 & 217 chr5_368390। & 6.95 sex \\
\hline 5 RBL3041 & 217 chr5_264320। & 5.32 nonsex \\
\hline 5 RBL3041 & 217 chr5_283520। & 5.85 nonsex \\
\hline 5 RBL3041 & 217 chr9_361000 & 8.72 sex \\
\hline 5 RBL3041 & 218 chr5_139660 & 9.01 sex \\
\hline 5 RBL3041 & 218 chr2_326460। & 9.6 sex \\
\hline 5 RBL3041 & 218 chr4_124100 & 8.91 sex \\
\hline 5 RBL3041 & 219 chr13_12841। & 9.69 sex \\
\hline 5 Gg_rs135852 & 227 chr5_306540। & 5.53 nonsex \\
\hline 5 Gg_rs135852 & 227 chr20_50960। & 6.47 nonsex \\
\hline 5 Gg_rs135852 & 228 chr3_525640। & 8.16 sex \\
\hline 5 Gg_rs135852 & 229 chr5_308300। & 4.31 nonsex \\
\hline 5 Gg_rs135852 & 232 chr5_302650। & 7.7 sex \\
\hline 5 Gg_rs135852 & 232 chr5_308310 & 5.72 nonsex \\
\hline 5 Gg_rs135852 & 232 chr5_295640। & 4.95 nonsex \\
\hline 5 Gg_rs135852 & 232 chr5_304620। & 4.76 nonsex \\
\hline 5 Gg_rs135852 & 232 chr5_307270 & 5.07 nonsex \\
\hline 5 Gg_rs135852 & 232 chr5_308680 & 4.34 nonsex \\
\hline 5 Gg_rs135852 & 233 chr5_287140। & 4.84 nonsex \\
\hline 5 Gg_rs135852 & 233 chr5_302820। & 4.97 nonsex \\
\hline 5 X5_36025717 & 233 chr5_348210 & 4.71 nonsex \\
\hline 5 Gg_rs135852 & 234 chr5_295260। & 4.53 nonsex \\
\hline 5 GG_rs156965 & 234 chr5_328940। & 6.44 nonsex \\
\hline 5 GG_rs156965 & 234 chr5_343050। & 5.49 nonsex \\
\hline
\end{tabular}




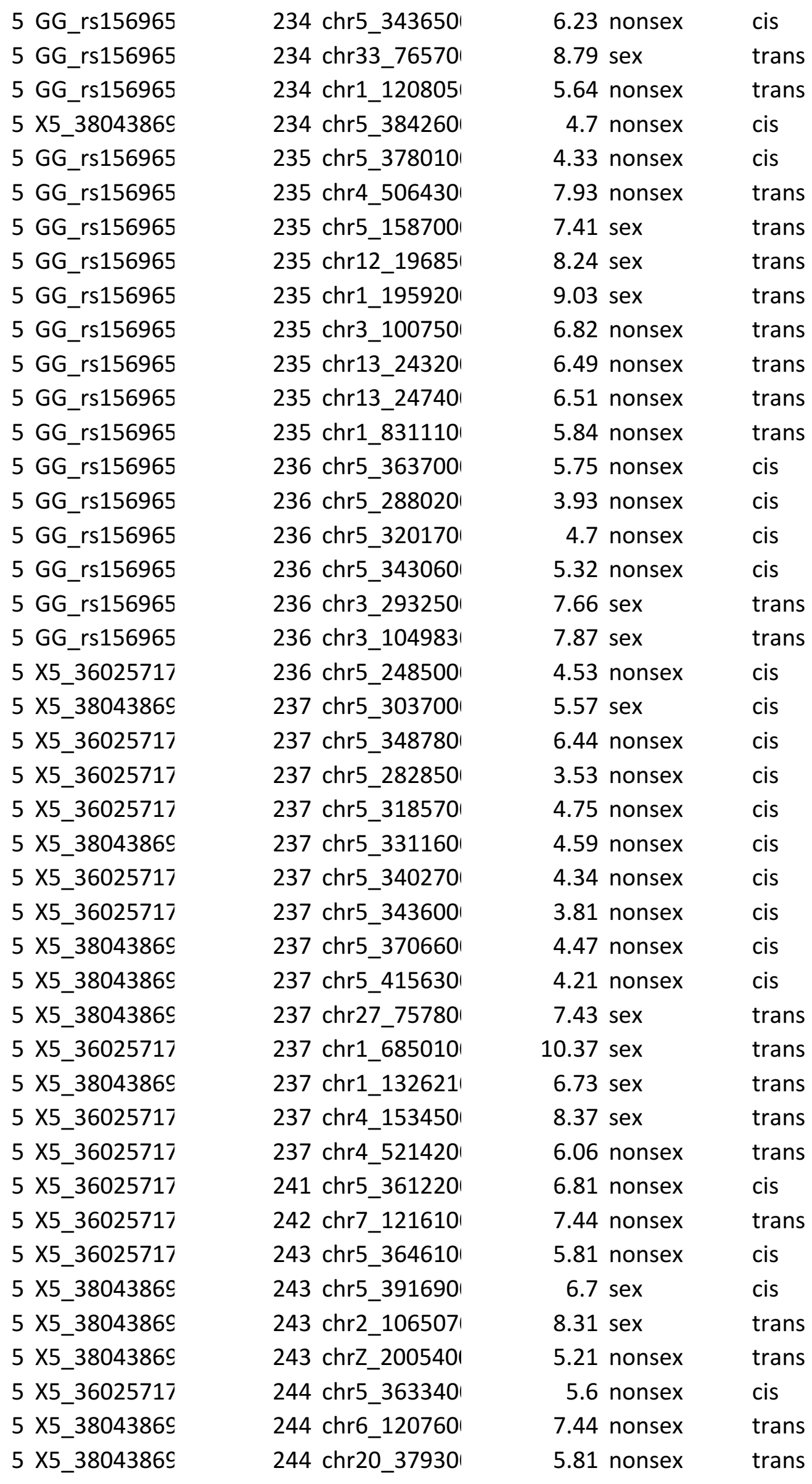




\begin{tabular}{|c|c|c|c|}
\hline 5 X5_3804386؟ & 245 chr5_358260। & 5.94 sex & cis \\
\hline $5 \times 5$ X56025717 & 246 chr5_257660। & 7.47 sex & cis \\
\hline 5 GG_rs157021 & 249 chr5_393080 & 4.8 nonsex & cis \\
\hline 5 GG_rs157021 & 251 chr5_373140 & 5.71 sex & cis \\
\hline 5 GG_rs157021 & 251 chr5_358470 & 5.25 nonsex & cis \\
\hline 5 GG_rs157021 & 253 chr5_369750 & 8.03 nonsex & cis \\
\hline 5 GG_rs157021 & 257 chr5_364510 & 3.79 nonsex & cis \\
\hline 5 GG_rs157021 & 257 chr5_377660। & 4.13 nonsex & cis \\
\hline 5 GG_rs157021 & 258 chr5_374150 & 3.73 nonsex & cis \\
\hline 5 GG_rs157021 & 259 chr5_387680 & 6.08 sex & cis \\
\hline 5 GG_rs157021 & 259 chr5_446450 & 5.54 nonsex & cis \\
\hline 5 GG_rs157021 & 260 chr1_155901। & 6.08 nonsex & trans \\
\hline 5 GG_rs157021 & 280 chr5_383940। & 5.54 nonsex & cis \\
\hline 5 Gg_rs157184 & 281 chr5_397550। & 6.32 sex & cis \\
\hline 5 Gg_rs157060. & 289 chr5_413510 & 4.54 nonsex & cis \\
\hline 5 Gg_rs157060. & 290 chr5_441270 & 6.34 nonsex & cis \\
\hline 5 chr5.4325034 & 290 chr5_444910 & 4.11 nonsex & cis \\
\hline 5 chr5.4325034 & 296 chr5_413520। & 7.42 nonsex & cis \\
\hline 5 Gg_rs157060. & 297 chr5_413530। & 6.59 nonsex & cis \\
\hline 5 Gg_rs157060. & 297 chr5_405590। & 4.6 nonsex & cis \\
\hline 5 Gg_rs157060. & 305 chr5_441810 & 4.73 nonsex & cis \\
\hline 5 Gg_rs157060. & 306 chr5_449000 & 5.87 nonsex & cis \\
\hline 5 chr5.4325034 & 306 chr5_440120 & 3.4 nonsex & cis \\
\hline 5 chr5.4325034 & 307 chr5_397540 & 10.56 nonsex & cis \\
\hline 5 chr5.4325034 & 307 chr5_162040। & 5.73 nonsex & trans \\
\hline 5 chr5.4325034 & 308 chr5_397530। & 13.45 nonsex & cis \\
\hline 5 chr5.4325034 & 308 chr5_400420 & 4.14 nonsex & cis \\
\hline 5 chr5.4325034 & 308 chr5_434470 & 4.07 nonsex & cis \\
\hline 5 chr5.4325034 & 308 chr5_460790 & 4.54 nonsex & cis \\
\hline 5 chr5.4325034 & 308 chr5_478490। & 4.55 nonsex & cis \\
\hline 5 chr5.4325034 & 308 chr12_19891। & 5.7 nonsex & trans \\
\hline 5 chr5.4325034 & 308 chr13_10545। & 6.56 nonsex & trans \\
\hline 5 chr5.4325034 & 309 chr5_434050। & 4.57 nonsex & cis \\
\hline 5 chr5.4325034 & 309 chr5_461500 & 4.97 nonsex & cis \\
\hline 5 Gg_rs157135. & 309 chr9_788400। & 7.14 sex & trans \\
\hline 5 Gg_rs157135. & 310 chr5_404090। & 5.89 nonsex & cis \\
\hline 5 Gg_rs157135. & 310 chr5_428430 & 4.81 nonsex & cis \\
\hline 5 Gg_rs157135. & 310 chr5_435310 & 7.66 nonsex & cis \\
\hline 5 Gg_rs157135. & 310 chr5_461490। & 6.49 sex & cis \\
\hline 5 Gg_rs157135. & 310 chr5_470670 & 5.06 sex & cis \\
\hline 5 Gg_rs157135. & 310 chr5_395970 & 4.77 nonsex & cis \\
\hline 5 Gg_rs157135. & 310 chr5_399130। & 4.55 nonsex & cis \\
\hline 5 Gg_rs157135. & 310 chr5_420030 & 5.28 nonsex & cis \\
\hline
\end{tabular}




\begin{tabular}{|c|c|c|c|}
\hline 5 Gg_rs157135. & 310 chr5_420040 & 4.79 nonsex & cis \\
\hline 5 Gg_rs157135. & 310 chr5_434140। & 4.82 nonsex & cis \\
\hline 5 Gg_rs157135. & 310 chr5_179510 & 5.57 nonsex & trans \\
\hline 5 Gg_rs157135. & 311 chr5_429190। & 4.86 nonsex & cis \\
\hline 5 Gg_rs157135. & 311 chr5_431750 & 6.13 sex & cis \\
\hline 5 Gg_rs157135. & 312 chr5_435430 & 7.18 nonsex & cis \\
\hline 5 Gg_rs157135. & 312 chr5_435420 & 4.76 nonsex & cis \\
\hline 5 Gg_rs157135. & 312 chr5_443170 & 4.81 nonsex & cis \\
\hline 5 Gg_rs157135. & 312 chr5_448430 & 4.13 nonsex & cis \\
\hline 5 chr5.4325034 & 313 chr5_447750 & 4.71 nonsex & cis \\
\hline 5 chr5.4325034 & 313 chr5_405610 & 4.2 nonsex & cis \\
\hline 5 Gg_rs157135. & 313 chr5_424010 & 6.72 sex & cis \\
\hline 5 Gg_rs157135. & 313 chr5_440490 & 6.17 nonsex & cis \\
\hline 5 Gg_rs157135. & 313 chr5_465120 & 6.46 nonsex & cis \\
\hline 5 Gg_rs157135. & 313 chr5_405470 & 4.51 nonsex & cis \\
\hline 5 chr5.4325034 & 314 chr5_441450 & 6.05 nonsex & cis \\
\hline 5 Gg_rs157135. & 314 chr5_461550 & 7.95 nonsex & cis \\
\hline 5 Gg_rs157135. & 314 chr5_462900 & 5.72 nonsex & cis \\
\hline 5 Gg_rs157184 & 314 chr5_459060। & 4.46 nonsex & cis \\
\hline 5 Gg_rs157184 & 314 chr5_463020 & 5.7 nonsex & cis \\
\hline 5 chr5.4325034 & 315 chr5_442290 & 3.74 nonsex & cis \\
\hline 5 Gg_rs157184 & 315 chr5_441440 & 6.31 sex & cis \\
\hline 5 Gg_rs157184 & 315 chr5_446120 & 5.55 sex & cis \\
\hline 5 Gg_rs157184 & 315 chr5_449670 & 5.65 nonsex & cis \\
\hline 5 Gg_rs157184 & 315 chr5_457650 & 5 nonsex & cis \\
\hline 5 Gg_rs157184 & 316 chr5_441800 & 5.31 sex & cis \\
\hline 5 Gg_rs157184 & 316 chr5_447870 & 3.89 nonsex & cis \\
\hline 5 Gg_rs157184 & 317 chr5_440830 & 5.18 nonsex & cis \\
\hline 5 Gg_rs157184 & 317 chr5_449690 & 5.37 nonsex & cis \\
\hline 5 Gg_rs157184 & 318 chr5_388560 & $5.84 \operatorname{sex}$ & cis \\
\hline 5 Gg_rs157184 & 318 chr5_395620 & 5.16 sex & cis \\
\hline 5 Gg_rs157184 & 318 chr5_449910 & 5.44 sex & cis \\
\hline 5 Gg_rs157184 & 318 chr5_452630 & 6.72 sex & cis \\
\hline 5 Gg_rs157184 & 318 chr5_457250 & 5.52 sex & cis \\
\hline 5 Gg_rs157184 & 318 chr5_461290। & 5.73 sex & cis \\
\hline 5 Gg_rs157184 & 318 chr5_530500 & 5.17 sex & cis \\
\hline 5 Gg_rs157184 & 318 chr5_447350 & 4.19 nonsex & cis \\
\hline 5 Gg_rs157184 & 318 chr5_453370 & 3.68 nonsex & cis \\
\hline 5 Gg_rs157184 & 318 chr5_469890। & 4.24 nonsex & cis \\
\hline 5 Gg_rs157184 & 318 chr13_18223। & 7.95 sex & trans \\
\hline 5 Gg_rs157184 & 318 chr2_293000। & 6.67 sex & trans \\
\hline 5 Gg_rs157184 & 318 chrZ_453810। & 5.86 nonsex & trans \\
\hline 5 Gg_rs157184 & 322 chr5_451190। & 8.51 nonsex & cis \\
\hline
\end{tabular}




\begin{tabular}{|c|c|c|c|}
\hline 5 Gg_rs157184 & 323 chr5_407630। & 6.01 sex & cis \\
\hline 5 Gg_rs157184 & 324 chr5_398420। & 6.03 nonsex & cis \\
\hline 5 Gg_rs157184 & 325 chr5_409870 & 6.2 sex & cis \\
\hline 5 Gg_rs157184 & 325 chr5_450800 & 5.92 sex & cis \\
\hline 5 Gg_rs157184 & 325 chr5_451280 & 5.82 sex & cis \\
\hline 5 Gg_rs157184 & 325 chr5_452480 & 5.4 sex & cis \\
\hline 5 Gg_rs157184 & 326 chr5_460420 & 5.93 sex & cis \\
\hline 5 Gg_rs157184 & 327 chrW_31730 & 8.17 sex & trans \\
\hline 5 Gg_rs157184 & 330 chr5_467450 & 4.5 nonsex & cis \\
\hline 5 Gg_rs157184 & 331 chr5_445650 & 4.87 nonsex & cis \\
\hline 5 Gg_rs157184 & 332 chr5_462950। & 4.58 nonsex & cis \\
\hline 5 Gg_rs157184 & 334 chr5_440510 & 5.22 nonsex & cis \\
\hline 5 Gg_rs157184 & 335 chr5_464630 & 4.69 nonsex & cis \\
\hline 5 chr5.4325034 & 336 chr5_445160 & 4.72 nonsex & cis \\
\hline 5 Gg_rs157184 & 336 chr5_458900 & 5.68 nonsex & cis \\
\hline 5 Gg_rs157269 & 344 chr5_474460 & 6.86 nonsex & cis \\
\hline 5 Gg_rs157269 & 345 chr5_485370 & 7.15 nonsex & cis \\
\hline 5 Gg_rs157269 & 347 chr5_485990। & 5.96 nonsex & cis \\
\hline 5 Gg_rs157269 & 347 chr5_486560। & 5.87 nonsex & cis \\
\hline 5 Gg_rs157269 & 348 chr5_481390। & 5.29 nonsex & cis \\
\hline 5 Gg_rs157269 & 348 chr5_484980 & 5.69 nonsex & cis \\
\hline 5 Gg_rs157269 & 349 chr31_19550। & 4.91 nonsex & trans \\
\hline 5 Gg_rs157269 & 352 chr5_483100 & 4.64 nonsex & cis \\
\hline 5 Gg_rs157269 & 352 chr5_494680 & 4.77 nonsex & cis \\
\hline 5 Gg_rs157269 & 356 chr5_488410 & 6.66 nonsex & cis \\
\hline 5 Gg_rs157269 & 356 chr31_34670। & 5.63 nonsex & trans \\
\hline 5 Gg_rs157269 & 358 chr5_494580 & 8.96 nonsex & cis \\
\hline 5 Gg_rs157269 & 358 chr5_494590। & 9.01 nonsex & cis \\
\hline 5 Gg_rs157269 & 358 chr5_486640 & 3.09 nonsex & cis \\
\hline 5 Gg_rs157269 & 358 chr5_494630। & 4.56 nonsex & cis \\
\hline 5 Gg_rs157269 & 358 chr5_494710 & 5.72 nonsex & cis \\
\hline 5 Gg_rs157269 & 358 chr5_502470 & 4.32 nonsex & cis \\
\hline 5 Gg_rs157269 & 359 chr5_499250 & 4.36 nonsex & cis \\
\hline 5 Gg_rs157269 & 360 chr5_473910 & 5.12 nonsex & cis \\
\hline 5 Gg_rs157269 & 361 chr5_506230। & 5.22 nonsex & cis \\
\hline 5 GG_rs145495 & 361 chr5_525360। & 4.62 nonsex & cis \\
\hline 5 Gg_rs135913. & 364 chr5_507900। & 7.39 nonsex & cis \\
\hline 5 Gg_rs135913. & 365 chr5_512690। & 7.23 nonsex & cis \\
\hline 5 Gg_rs135913. & 365 chr5_507200 & 5.91 nonsex & cis \\
\hline 5 Gg_rs135913. & 367 chr5_510210 & 7.69 nonsex & cis \\
\hline 5 Gg_rs135913. & 367 chr5_510340 & 7.36 nonsex & cis \\
\hline 5 Gg_rs135913. & 369 chr5_408870 & $5.62 \mathrm{sex}$ & cis \\
\hline 5 Gg_rs135913. & 369 chr5_512780 & 7.82 nonsex & cis \\
\hline
\end{tabular}




\begin{tabular}{|c|c|c|c|}
\hline 5 Gg_rs135913. & 369 chr5_510320 & 4.14 nonsex & cis \\
\hline 5 Gg_rs135913. & 369 chr5_511790। & 4.03 nonsex & cis \\
\hline 5 Gg_rs135913. & 369 chr5_512480 & 4.49 nonsex & cis \\
\hline 5 Gg_rs135913. & 369 chr5_513520 & 3.58 nonsex & cis \\
\hline 5 Gg_rs135913. & 369 chr5_514360 & 3.62 nonsex & cis \\
\hline 5 Gg_rs135913. & 370 chr5_511380 & 7.93 nonsex & cis \\
\hline 5 Gg_rs135913. & 370 chr5_510190 & 4.9 nonsex & cis \\
\hline 5 Gg_rs135913. & 370 chr5_512040 & 3.62 nonsex & cis \\
\hline 5 Gg_rs135913. & 371 chr5_513830 & 9.77 nonsex & cis \\
\hline 5 Gg_rs135913. & 373 chr5_519410 & 6.88 nonsex & cis \\
\hline 5 Gg_rs135913. & 373 chr5_514390। & 6.22 nonsex & cis \\
\hline 5 Gg_rs135913. & 374 chr5_514400 & 7.98 nonsex & cis \\
\hline 5 Gg_rs135913. & 374 chr5_514840 & 5.76 nonsex & cis \\
\hline 5 Gg_rs135913. & 374 chr5_524540 & 5.1 nonsex & cis \\
\hline 5 GG_rs145495 & 374 chr5_526600 & 3.5 nonsex & cis \\
\hline 5 GG_rs145495 & 375 chr5_515420 & 8.24 nonsex & cis \\
\hline 5 GG_rs145495 & 376 chr5_519180 & 3.15 nonsex & cis \\
\hline 5 GG_rs145495 & 376 chr5_530020 & 4.13 nonsex & cis \\
\hline 5 GG_rs145495 & 377 chr5_521860 & 7.35 nonsex & cis \\
\hline 5 GG_rs145495 & 378 chr5_503100 & 5.48 nonsex & cis \\
\hline 5 GG_rs145495 & 378 chrZ_6484501 & $8.08 \mathrm{sex}$ & trans \\
\hline 5 GG_rs145495 & 379 chr5_519230 & 4.52 nonsex & cis \\
\hline 5 GG_rs145495 & 380 chr5_523540। & 3.54 nonsex & cis \\
\hline 5 GG_rs145495 & 380 chr5_524960 & $6.52 \operatorname{sex}$ & cis \\
\hline 5 GG_rs145495 & 380 chr5_536780 & 4.93 nonsex & cis \\
\hline 5 GG_rs145495 & 380 chr3_310930। & 4.98 nonsex & trans \\
\hline 5 GG_rs145495 & 383 chr5_522040। & 10.29 nonsex & cis \\
\hline 5 GG_rs145495 & 387 chr5_514980 & 5.66 nonsex & cis \\
\hline 5 GG_rs145495 & 388 chrZ_2915401 & 7.82 sex & trans \\
\hline 5 GG_rs145495 & 391 chrZ_3813501 & 9.41 sex & trans \\
\hline 5 GG_rs145495 & 394 chr5_527000 & 7.6 nonsex & cis \\
\hline 5 GG_rs145495 & 395 chr5_529080 & 5.58 nonsex & cis \\
\hline 5 GG_rs145495 & 400 chr5_537530। & 6.25 nonsex & cis \\
\hline 5 X5_56411157 & 409 chr5_584390। & 6.89 sex & cis \\
\hline 5 X5_56411157 & 410 chr22_52020। & 7.78 sex & trans \\
\hline 5 X5_56411157 & 410 chr30_74400। & 8.13 sex & trans \\
\hline 5 X5_56411157 & 410 chr2_651220 & 8.53 sex & trans \\
\hline 5 X5_56411157 & 411 chr5_581170। & 6.24 sex & cis \\
\hline 5 X5_56411157 & 416 chr5_551240 & 4.68 nonsex & cis \\
\hline 5 X5_56411157 & 419 chr5_558960। & 5.92 nonsex & cis \\
\hline 5 X5_56411157 & 419 chr5_551130 & 4.69 nonsex & cis \\
\hline 5 X5_56411157 & 419 chr5_551180 & 4.59 nonsex & cis \\
\hline $5 \times 5 \_56411157$ & 419 chr5_555290 & 3.96 nonsex & cis \\
\hline
\end{tabular}




\begin{tabular}{|c|c|c|c|}
\hline $5 \times 5 \_56411157$ & 421 chr5_551230 & 6.52 nonsex & cis \\
\hline 5 X5_56411157 & 432 chr32_92000 & 8.1 sex & trans \\
\hline $5 \times 5 \_56411157$ & 434 chr30_11920। & 11.36 sex & trans \\
\hline $5 \times 5 \_56411157$ & 437 chr5_175000 & 9.05 sex & trans \\
\hline 5 X5_56411157 & 437 chr26_50200। & 8.75 sex & trans \\
\hline 5 X5_56411157 & 439 chr15_25300। & 14.18 sex & trans \\
\hline 5 Gg_rs157434 & 442 chr27_37000। & 10.69 sex & trans \\
\hline 5 Gg_rs157434 & 442 chr33_35850। & 10.76 sex & trans \\
\hline 5 Gg_rs157434 & 443 chr25_12940। & 9.22 sex & trans \\
\hline 5 Gg_rs157434 & 443 chr25_19550। & 8.8 sex & trans \\
\hline 5 Gg_rs157434 & 444 chr31_45140। & 10.88 sex & trans \\
\hline 5 Gg_rs157434 & 444 chr33_32600। & 14.42 sex & trans \\
\hline 5 Gg_rs157434 & 445 chr25_35180 & 9.5 sex & trans \\
\hline 5 Gg_rs157434 & 451 chr9_184510 & 6.46 sex & trans \\
\hline 5 Gg_rs157434 & 452 chr5_571960 & 6.03 sex & cis \\
\hline 5 Gg_rs157434 & 452 chr5_545940 & 4.42 nonsex & cis \\
\hline 5 Gg_rs157434 & 452 chr5_580700 & 4.47 nonsex & cis \\
\hline 5 Gg_rs157434 & 452 chr27_78180। & 7.52 sex & trans \\
\hline 6 Gg_rs157502 & 0 chr6_101900 & 7.25 nonsex & cis \\
\hline 6 Gg_rs157502 & 0 chr6_105200 & 8.3 nonsex & cis \\
\hline 6 Gg_rs157502 & 0 chr6_164000 & 5.5 nonsex & cis \\
\hline 6 Gg_rs157502 & 0 chr6_650000 & 4.16 nonsex & cis \\
\hline 6 Gg_rs157502 & 0 chr6_682000 & 3.87 nonsex & cis \\
\hline 6 Gg_rs157502 & 0 chr6_743000 & 3.84 nonsex & cis \\
\hline 6 Gg_rs157502 & 0 chr6_108000 & 3.98 nonsex & cis \\
\hline 6 Gg_rs157502 & 0 chr6_186300 & 4.64 nonsex & cis \\
\hline 6 Gg_rs157502 & 0 chr6_198300 & 4.48 nonsex & cis \\
\hline 6 Gg_rs157502 & 0 chr6_215800 & 4.7 nonsex & cis \\
\hline 6 Gg_rs157520 & 2 chr6_186100। & 7.86 nonsex & cis \\
\hline 6 Gg_rs157520 & 2 chr6_186200। & 9.15 nonsex & cis \\
\hline 6 Gg_rs157520 & 2 chr6_338100 & 5.24 sex & cis \\
\hline 6 Gg_rs157520 & 2 chr6_443400 & 7.15 nonsex & cis \\
\hline 6 Gg_rs157520 & 2 chr6_443600 & 6.53 nonsex & cis \\
\hline 6 Gg_rs157520 & 11 chr6_221700 & 6.77 nonsex & cis \\
\hline 6 Gg_rs157502 & 12 chr6_105900 & 10.19 nonsex & cis \\
\hline 6 Gg_rs157502 & 13 chr6_102500 & 12.91 nonsex & cis \\
\hline 6 Gg_rs157520 & 13 chr6_445000 & 9.8 nonsex & cis \\
\hline 6 Gg_rs157502 & 14 chr6_101800 & 10.06 nonsex & cis \\
\hline 6 Gg_rs157502 & 14 chr6_106000 & 9.7 nonsex & cis \\
\hline 6 Gg_rs157502 & 15 chr6_104600 & 9.8 nonsex & cis \\
\hline 6 Gg_rs157502 & 15 chr6_105300 & 11.01 nonsex & cis \\
\hline 6 Gg_rs157520 & 15 chr6_444900 & 7.44 nonsex & cis \\
\hline 6 Gg_rs157520 & 16 chr6_445400 & 3.58 nonsex & cis \\
\hline
\end{tabular}




\begin{tabular}{|c|c|c|c|}
\hline 6 Gg_rs157502 & 17 chr6_102400। & 7.19 nonsex & cis \\
\hline 6 Gg_rs157502 & 18 chr6_742000 & 3.98 nonsex & cis \\
\hline 6 Gg_rs157520 & 18 chr6_185200 & 4.52 nonsex & cis \\
\hline 6 Gg_rs157502 & 19 chr6_104700 & 5.87 nonsex & cis \\
\hline 6 Gg_rs157502 & 19 chr6_179700 & 4.01 nonsex & cis \\
\hline 6 Gg_rs157520 & 19 chr6_444100 & 5.36 nonsex & cis \\
\hline 6 Gg_rs157502 & 20 chr6_105500 & 3.45 nonsex & cis \\
\hline 6 Gg_rs157502 & 21 chr6_105400 & 6.4 nonsex & cis \\
\hline 6 Gg_rs157520 & 21 chr6_442200 & 6.42 nonsex & cis \\
\hline 6 Gg_rs157502 & 22 chr6_106100 & 5.72 nonsex & cis \\
\hline 6 Gg_rs157502 & 22 chr6_886000 & 4.23 nonsex & cis \\
\hline 6 Gg_rs157502 & 23 chr6_104800 & 4.32 nonsex & cis \\
\hline 6 Gg_rs157520 & 23 chr6_447200 & 4.22 nonsex & cis \\
\hline 6 Gg_rs157502 & 24 chr6_101600 & 4.02 nonsex & cis \\
\hline 6 Gg_rs157520 & 24 chr6_444800 & 4.8 nonsex & cis \\
\hline 6 Gg_rs157520 & 27 chr6_443300 & 4.34 nonsex & cis \\
\hline 6 X6_2930562 & 33 chr30_89900 & 9.35 sex & trans \\
\hline 6 X6_2930562 & 35 chr6_442300 & 3.89 nonsex & cis \\
\hline 6 X6_2930562 & 39 chr6_185100 & 4.42 nonsex & cis \\
\hline 6 X6_2930562 & 41 chr6_558600 & 5.05 nonsex & cis \\
\hline 6 X6_2930562 & 41 chr6_606400 & 4.47 nonsex & cis \\
\hline 6 X6_2930562 & 42 chr25_28180 & 6.32 nonsex & trans \\
\hline 6 Gg_rs157568. & 56 chr6_414000 & 6.37 nonsex & cis \\
\hline 6 Gg_rs157568. & 64 chr6_432300 & 4.76 nonsex & cis \\
\hline 6 Gg_rs157568. & 64 chr6_437600 & 6.29 sex & cis \\
\hline 6 Gg_rs157568. & 64 chr6_452600 & 7.29 nonsex & cis \\
\hline 6 Gg_rs157568. & 64 chr6_367900 & 4.94 nonsex & cis \\
\hline 6 Gg_rs157591. & 64 chr6_523200 & 5.07 sex & cis \\
\hline 6 Gg_rs157568. & 65 chr6_438100 & 10.44 nonsex & cis \\
\hline 6 Gg_rs157568. & 66 chr6_523300 & 4.66 nonsex & cis \\
\hline 6 Gg_rs157568. & 66 chr6_438200 & 7.79 nonsex & cis \\
\hline 6 Gg_rs157591. & 67 chr6_482600 & 3.6 nonsex & cis \\
\hline 6 Gg_rs157596. & 67 chr6_523000 & 5.73 nonsex & cis \\
\hline 6 Gg_rs157591. & 69 chr6_517600 & 6.33 nonsex & cis \\
\hline 6 Gg_rs157596. & 69 chr6_478000 & 5.75 sex & cis \\
\hline 6 Gg_rs157596. & 71 chr6_577100 & 7.37 nonsex & cis \\
\hline 6 Gg_rs157591. & 74 chr6_519700 & 4.25 nonsex & cis \\
\hline 6 Gg_rs157654 & 77 chr6_576400 & 4.37 nonsex & cis \\
\hline 6 Gg_rs157654 & 81 chr6_672800 & 8.22 nonsex & cis \\
\hline 6 Gg_rs157654 & 81 chr15_96250 & 10.09 sex & trans \\
\hline 6 Gg_rs157654 & 82 chr6_582800 & 4.19 nonsex & cis \\
\hline 6 Gg_rs157654 & 84 chr6_394200 & 5.79 sex & cis \\
\hline 6 Gg_rs157654 & 84 chr6_671800 & 5.27 nonsex & cis \\
\hline
\end{tabular}




\begin{tabular}{|c|c|c|c|}
\hline 6 Gg_rs157654 & 84 chr6_603700। & 4.76 nonsex & cis \\
\hline 6 Gg_rs157654 & 85 chr6_672400 & 7.28 nonsex & cis \\
\hline 6 Gg_rs157654 & 85 chr6_777200 & 4.8 nonsex & cis \\
\hline 6 Gg_rs157654 & 86 chr6_444300 & 7.44 nonsex & cis \\
\hline 6 Gg_rs157654 & 86 chr6_609900 & 8.16 nonsex & cis \\
\hline 6 Gg_rs157654 & 86 chr6_665400 & 27.96 nonsex & cis \\
\hline 6 Gg_rs157654 & 86 chr6_665900 & 9.67 nonsex & cis \\
\hline 6 Gg_rs157654 & 86 chr6_672100 & 9.64 nonsex & cis \\
\hline 6 Gg_rs157654 & 86 chr6_672200 & 9.79 nonsex & cis \\
\hline 6 Gg_rs157654 & 86 chr6_672300 & 14.97 nonsex & cis \\
\hline 6 Gg_rs157654 & 86 chr6_672700 & 7.19 nonsex & cis \\
\hline 6 Gg_rs157654 & 86 chr6_681800 & 7.96 nonsex & cis \\
\hline 6 Gg_rs157654 & 86 chr6_685700। & 5.78 nonsex & cis \\
\hline 6 Gg_rs157654 & 86 chr6_447300 & 4.67 nonsex & cis \\
\hline 6 Gg_rs157654 & 86 chr6_582400 & 4.09 nonsex & cis \\
\hline 6 Gg_rs157654 & 86 chr6_610100 & 4.89 nonsex & cis \\
\hline 6 Gg_rs157654 & 86 chr6_621000 & 5.47 nonsex & cis \\
\hline 6 Gg_rs157654 & 86 chr6_637100 & 4.39 nonsex & cis \\
\hline 6 Gg_rs157654 & 86 chr6_658100 & 4.21 nonsex & cis \\
\hline 6 Gg_rs157654 & 86 chr6_678200 & 3.79 nonsex & cis \\
\hline 6 Gg_rs157654 & 86 chr6_980400 & 4.27 nonsex & cis \\
\hline 6 Gg_rs157654 & 88 chr6_671700 & 6.51 nonsex & cis \\
\hline 6 Gg_rs157654 & 88 chr1_672870 & 7.98 sex & trans \\
\hline 6 Gg_rs157654 & 89 chr6_633500 & 7.39 nonsex & cis \\
\hline 6 Gg_rs157654 & 89 chr6_684300 & 4.51 nonsex & cis \\
\hline 6 Gg_rs157654 & 90 chr6_732400 & 5.18 nonsex & cis \\
\hline 6 Gg_rs157654 & 92 chr6_635000 & 6.08 sex & cis \\
\hline 6 Gg_rs157654 & 92 chrZ_3072601 & 7.08 nonsex & trans \\
\hline 6 Gg_rs157730. & 92 chr6_929500। & 5.7 nonsex & cis \\
\hline 6 Gg_rs157654 & 94 chr6_735800 & 11.29 nonsex & cis \\
\hline 6 Gg_rs157654 & 94 chr6_958500 & 7.94 sex & cis \\
\hline 6 Gg_rs145688 & 95 chr6_631600। & 6.42 sex & cis \\
\hline 6 Gg_rs145688. & 95 chr6_946200 & 8.3 nonsex & cis \\
\hline 6 Gg_rs145688. & 95 chr6_946100 & 5.36 nonsex & cis \\
\hline 6 Gg_rs145688. & 96 chr6_730900 & 4.02 nonsex & cis \\
\hline 6 Gg_rs145688 & 97 chr6_755600 & 6.77 nonsex & cis \\
\hline 6 Gg_rs145688 & 97 chr6_564400 & 5.29 nonsex & cis \\
\hline 6 Gg_rs145688 & 97 chrZ_2373001 & 9.71 sex & trans \\
\hline 6 Gg_rs145688 & 98 chr6_752300 & 22.43 nonsex & cis \\
\hline 6 Gg_rs145688 & 98 chr6_708000 & 4.92 nonsex & cis \\
\hline 6 X6_2930562 & 99 chr6_558500। & 4.09 nonsex & cis \\
\hline 6 Gg_rs145688 & 99 chr6_944300। & 4.74 nonsex & cis \\
\hline 6 Gg_rs145688. & 100 chr6_755800 & 9.32 nonsex & cis \\
\hline
\end{tabular}




\begin{tabular}{|c|c|c|c|}
\hline 6 Gg_rs145688 & 100 chr6_576000 & 4.51 nonsex & cis \\
\hline 6 Gg_rs145688 & 100 chr1_141431। & 5.36 nonsex & trans \\
\hline 6 Gg_rs145688. & 102 chr2_813740 & 6.46 nonsex & trans \\
\hline 6 Gg_rs145688. & 104 chr6_848300। & 5.16 nonsex & cis \\
\hline 6 Gg_rs145688 & 105 chr6_658700 & $4.28 \mathrm{sex}$ & cis \\
\hline 6 Gg_rs145688 & 105 chr6_740200 & 8.05 sex & cis \\
\hline 6 Gg_rs145688 & 105 chr6_895400 & 4.37 sex & cis \\
\hline 6 Gg_rs145688 & 105 chr6_938800 & 5.17 sex & cis \\
\hline 6 Gg_rs145688 & 105 chr6_954500 & 5.54 sex & cis \\
\hline 6 Gg_rs145688 & 105 chr6_960300 & 5.76 sex & cis \\
\hline 6 Gg_rs145688. & 105 chr4_472700 & 7.09 sex & trans \\
\hline 6 Gg_rs145688 & 105 chr4_699290। & 8.7 sex & trans \\
\hline 6 Gg_rs145688. & 105 chr7_142450 & 8.11 sex & trans \\
\hline 6 Gg_rs145688 & 105 chrZ_2575801 & 7.6 sex & trans \\
\hline 6 Gg_rs145688 & 105 chrZ_3327301 & 7.63 sex & trans \\
\hline 6 Gg_rs145688. & 105 chrZ_4248401 & 8.88 sex & trans \\
\hline 6 Gg_rs145688 & 105 chrZ_6429301 & 7.89 sex & trans \\
\hline 6 Gg_rs145688 & 105 chrZ_6495401 & 8.9 sex & trans \\
\hline 6 Gg_rs145688 & 105 chrZ_665800। & 7.54 sex & trans \\
\hline 6 Gg_rs145688 & 105 chr3_492870 & 8.01 sex & trans \\
\hline 6 Gg_rs145688 & 105 chr1_629840। & 5.78 nonsex & trans \\
\hline 6 Gg_rs145688 & 105 chr2_911590। & 5.28 nonsex & trans \\
\hline 6 Gg_rs145688 & 105 chr3_806400। & 6.31 nonsex & trans \\
\hline 6 Gg_rs145688. & 106 chr6_847600 & 5.62 nonsex & cis \\
\hline 6 Gg_rs145688 & 106 chr6_783000 & 5.6 nonsex & cis \\
\hline 6 Gg_rs145688 & 107 chrW_40550 & 8.05 sex & trans \\
\hline 6 Gg_rs145688 & 107 chrZ_2380501 & 7.82 sex & trans \\
\hline 6 Gg_rs145688 & 107 chrZ_4893501 & 7.59 sex & trans \\
\hline 6 Gg_rs145688 & 107 chr1_161906। & 7.72 sex & trans \\
\hline 6 Gg_rs145688. & 107 chr3_510170। & 5.59 nonsex & trans \\
\hline 6 Gg_rs145688 & 108 chr6_693200 & 7.27 nonsex & cis \\
\hline 6 Gg_rs145688 & 108 chr6_693300 & 7.65 nonsex & cis \\
\hline 6 Gg_rs145688 & 108 chr6_693500 & 5.27 nonsex & cis \\
\hline 6 Gg_rs145688 & 108 chr6_693700 & 5.43 nonsex & cis \\
\hline 6 Gg_rs145688. & 108 chr6_831100 & 3.96 nonsex & cis \\
\hline 6 Gg_rs145688 & 108 chrZ_514660। & 6.37 nonsex & trans \\
\hline 6 Gg_rs157730. & 108 chr6_691500 & 4.53 nonsex & cis \\
\hline 6 Gg_rs157730. & 108 chrZ_2127301 & 7.96 sex & trans \\
\hline 6 Gg_rs145688 & 109 chr6_756800 & 6.19 sex & cis \\
\hline 6 Gg_rs145688 & 109 chr6_846500 & 4.38 sex & cis \\
\hline 6 Gg_rs145688. & 109 chr6_820900 & 4.62 nonsex & cis \\
\hline 6 Gg_rs157730. & 109 chr6_917400 & 5.09 nonsex & cis \\
\hline 6 Gg_rs157730. & 109 chr6_990700। & 9.79 nonsex & cis \\
\hline
\end{tabular}




\begin{tabular}{|c|c|c|c|}
\hline 6 Gg_rs145688 & 110 chr6_693400 & 6.65 nonsex & cis \\
\hline 6 Gg_rs157730. & 110 chr6_987800। & 5.42 nonsex & cis \\
\hline 6 Gg_rs157730. & 110 chr6_958600 & 4.58 nonsex & cis \\
\hline 6 Gg_rs157730. & 111 chr6_944500 & $5.39 \operatorname{sex}$ & cis \\
\hline 6 Gg_rs157730. & 111 chrZ_709830। & 7.53 sex & trans \\
\hline 6 Gg_rs157730. & 111 chr2_143312 & 7.83 sex & trans \\
\hline 6 Gg_rs157730. & 112 chr6_837100 & 6.21 nonsex & cis \\
\hline 6 Gg_rs157730. & 112 chr6_652600 & 3.91 nonsex & cis \\
\hline 6 Gg_rs157730. & 112 chr6_822300। & 3.67 nonsex & cis \\
\hline 6 Gg_rs157730. & 112 chr1_139884। & 7.82 sex & trans \\
\hline 6 Gg_rs157730. & 113 chr6_935100 & 18.79 nonsex & cis \\
\hline 6 Gg_rs157730. & 113 chr1_139883। & 7.32 sex & trans \\
\hline 6 Gg_rs157730. & 113 chr2_107632 & 6.17 nonsex & trans \\
\hline 6 Gg_rs157730. & 114 chr6_921500 & 7.99 nonsex & cis \\
\hline 6 Gg_rs157730. & 114 chr6_921600। & 5.75 nonsex & cis \\
\hline 6 Gg_rs157730. & 115 chr6_921400 & 7.85 nonsex & cis \\
\hline 6 Gg_rs157730. & 115 chr6_433800 & 4.72 nonsex & cis \\
\hline 6 Gg_rs157730. & 115 chr1_135568। & $11.18 \mathrm{sex}$ & trans \\
\hline 6 Gg_rs157730. & 116 chr6_693000 & 5.05 nonsex & cis \\
\hline 6 Gg_rs157730. & 117 chr6_608800 & 5.17 nonsex & cis \\
\hline 6 Gg_rs157730. & 117 chr6_839800 & 3.66 nonsex & cis \\
\hline 6 Gg_rs157730. & 118 chr6_577200। & 4.78 nonsex & cis \\
\hline 6 Gg_rs145688 & 119 chr6_779600 & 6.26 nonsex & cis \\
\hline 6 Gg_rs145688 & 119 chr6_780000। & 8.49 nonsex & cis \\
\hline 6 Gg_rs157730. & 119 chr6_951700 & 4.05 nonsex & cis \\
\hline 6 Gg_rs157770 & 120 chr6_780100 & 5.08 nonsex & cis \\
\hline 6 Gg_rs157770 & 120 chr6_101680 & 4.69 nonsex & cis \\
\hline 6 Gg_rs157770 & 121 chr6_991800 & 5.51 nonsex & cis \\
\hline 6 Gg_rs157770 & 121 chr6_102070 & 4.71 nonsex & cis \\
\hline 6 Gg_rs157770 & 122 chr6_779700 & 7.34 nonsex & cis \\
\hline 6 Gg_rs157770 & 122 chr6_890200। & 4.97 nonsex & cis \\
\hline 6 Gg_rs157770 & 122 chr6_106370 & 5.31 nonsex & cis \\
\hline 6 Gg_rs157770 & 122 chr4_337110 & 10.04 sex & trans \\
\hline 6 Gg_rs157770 & 123 chr6_239740 & 4.53 nonsex & cis \\
\hline 6 Gg_rs157770 & 123 chr8_101500। & 5.87 nonsex & trans \\
\hline 6 Gg_rs157770 & 124 chr6_937200। & 4.2 nonsex & cis \\
\hline 6 Gg_rs157770 & 124 chr6_104070 & 4.9 nonsex & cis \\
\hline 6 Gg_rs157770 & 125 chr6_108000 & 5.53 nonsex & cis \\
\hline 6 Gg_rs157770 & 126 chr6_103830 & 5.28 nonsex & cis \\
\hline 6 Gg_rs157770 & 126 chr10_20500। & 8.13 sex & trans \\
\hline 6 Gg_rs157770 & 127 chr6_107960। & 5.48 sex & cis \\
\hline 6 Gg_rs157770 & 127 chr6_108920 & 5.46 nonsex & cis \\
\hline 6 Gg_rs157770 & 127 chr6_110470 & 7.02 sex & cis \\
\hline
\end{tabular}




\begin{tabular}{|c|c|c|c|}
\hline 6 Gg_rs157770 & 127 chr6_111220 & 6.34 sex & cis \\
\hline 6 Gg_rs157770 & 127 chr6_140460 & 5.68 sex & cis \\
\hline 6 Gg_rs157770 & 127 chr6_535300। & 4 nonsex & cis \\
\hline 6 Gg_rs157770 & 127 chr6_104030 & 4.31 nonsex & cis \\
\hline 6 Gg_rs157770 & 127 chr6_106090 & 3.56 nonsex & cis \\
\hline 6 Gg_rs157770 & 127 chr6_107820 & 4.07 nonsex & cis \\
\hline 6 Gg_rs157770 & 127 chr12_97800। & 5.13 nonsex & trans \\
\hline 6 Gg_rs157770 & 127 chr12_10169। & 5.04 nonsex & trans \\
\hline 6 Gg_rs157770 & 128 chr5_216710 & 5.51 nonsex & trans \\
\hline 6 Gg_rs157770 & 132 chrW_43320 & $12.36 \mathrm{sex}$ & trans \\
\hline 6 Gg_rs157770 & 134 chr6_104620 & 7.53 nonsex & cis \\
\hline 6 Gg_rs157770 & 137 chr6_110410 & 7.82 sex & cis \\
\hline 6 Gg_rs157770 & 137 chr6_110700 & 7.92 sex & cis \\
\hline 6 Gg_rs157770 & 139 chr6_117880 & 9.08 sex & cis \\
\hline 6 Gg_rs157770 & 139 chr6_110980 & 4.38 nonsex & cis \\
\hline 6 Gg_rs157770 & 139 chr1_197432। & 10.12 sex & trans \\
\hline 6 Gg_rs157770 & 140 chr6_110990 & 5.61 nonsex & cis \\
\hline 6 Gg_rs157770 & 141 chr6_117950 & 4.35 nonsex & cis \\
\hline 6 Gg_rs157770 & 142 chr6_111210 & 7.13 nonsex & cis \\
\hline 6 Gg_rs157770 & 142 chr6_111110 & 5.56 nonsex & cis \\
\hline 6 Gg_rs157770 & 143 chr6_110450 & 9.3 nonsex & cis \\
\hline 6 Gg_rs157770 & 143 chr6_110710 & 6.83 nonsex & cis \\
\hline 6 Gg_rs157770 & 143 chr6_110780 & 6.35 nonsex & cis \\
\hline 6 Gg_rs157770 & 143 chr6_110950 & 7.64 nonsex & cis \\
\hline 6 Gg_rs157770 & 143 chr6_111200 & 9.72 nonsex & cis \\
\hline 6 Gg_rs157770 & 143 chr6_110420 & 4.67 nonsex & cis \\
\hline 6 Gg_rs157770 & 144 chr6_110180 & 4.91 nonsex & cis \\
\hline 6 Gg_rs157770 & 144 chr6_110460 & 8.88 nonsex & cis \\
\hline 6 Gg_rs157770 & 144 chr6_110480 & 10.72 nonsex & cis \\
\hline 6 Gg_rs157770 & 146 chr6_110400 & 9.03 nonsex & cis \\
\hline 6 Gg_rs157770 & 146 chr6_110790 & 9.14 nonsex & cis \\
\hline 6 Gg_rs157770 & 146 chr6_110940 & 6.79 nonsex & cis \\
\hline 6 Gg_rs157770 & 146 chr6_111190 & 8.43 nonsex & cis \\
\hline 6 Gg_rs157770 & 146 chr6_117650 & 5.22 nonsex & cis \\
\hline 6 Gg_rs157770 & 146 chr6_117870 & 6.39 nonsex & cis \\
\hline 6 Gg_rs157770 & 146 chr6_117960 & 6.52 nonsex & cis \\
\hline 6 Gg_rs157770 & 147 chr6_111120 & 8.1 nonsex & cis \\
\hline 6 Gg_rs157770 & 147 chr6_128100 & 4.46 nonsex & cis \\
\hline 6 Gg_rs157770 & 148 chr6_118140 & 9.35 nonsex & cis \\
\hline 6 Gg_rs157770 & 148 chr6_127910 & 7.74 nonsex & cis \\
\hline 6 Gg_rs157770 & 150 chr6_110690 & 9.3 nonsex & cis \\
\hline 6 Gg_rs157770 & 152 chr6_110190। & 10.28 nonsex & cis \\
\hline 6 Gg_rs157770 & 152 chr6_111000 & 7.96 nonsex & cis \\
\hline
\end{tabular}




\begin{tabular}{|c|c|c|}
\hline 6 Gg_rs157770 & 152 chr6_117660 & 9.09 nonsex \\
\hline 6 Gg_rs157816. & 153 chr6_110390 & 9.22 nonsex \\
\hline 6 Gg_rs157816. & 154 chr6_117860 & 9.97 nonsex \\
\hline 6 Gg_rs157816. & 154 chr6_128110 & 6.27 nonsex \\
\hline 6 Gg_rs157816. & 155 chr6_110490 & 7.22 nonsex \\
\hline 6 Gg_rs157816. & 156 chr6_117740 & 6.08 nonsex \\
\hline 6 Gg_rs157816. & 157 chr6_110570 & 7.27 nonsex \\
\hline 6 Gg_rs157816. & 157 chr6_117750 & 6.55 nonsex \\
\hline 6 Gg_rs157816. & 157 chr6_117780 & 5.67 nonsex \\
\hline 6 Gg_rs157830. & 157 chr6_120070 & 5.73 nonsex \\
\hline 6 Gg_rs157816. & 158 chr6_110290 & 7.21 nonsex \\
\hline 6 Gg_rs157816. & 158 chr6_110680 & 6.49 nonsex \\
\hline 6 Gg_rs157816. & 158 chr6_117760 & 7.15 nonsex \\
\hline 6 Gg_rs157816. & 158 chr6_208330 & 7.53 nonsex \\
\hline 6 Gg_rs157816. & 158 chr6_110270 & 6.2 nonsex \\
\hline 6 Gg_rs157816. & 158 chr6_110580 & 6.01 nonsex \\
\hline 6 Gg_rs157816. & 158 chr6_110640 & 6.06 nonsex \\
\hline 6 Gg_rs157816. & 158 chr6_118210 & 6 nonsex \\
\hline 6 Gg_rs157816. & 158 chr6_118270 & 5.21 nonsex \\
\hline 6 Gg_rs157816. & 158 chr6_127840 & 5.73 nonsex \\
\hline 6 Gg_rs157816. & 159 chr6_117680 & 7.39 nonsex \\
\hline 6 Gg_rs157816. & 159 chr6_208350 & 6.52 nonsex \\
\hline 6 Gg_rs157816. & 159 chr6_117670 & 5.15 nonsex \\
\hline 6 Gg_rs157816. & 159 chr6_118280 & 5.41 nonsex \\
\hline 6 Gg_rs157816. & 159 chr6_118340 & 5.83 nonsex \\
\hline 6 Gg_rs157816. & 159 chr6_127160 & 6.31 nonsex \\
\hline 6 Gg_rs157816. & 160 chr6_110310 & 6.48 nonsex \\
\hline 6 Gg_rs157816. & 160 chr6_110610 & 7.12 nonsex \\
\hline 6 Gg_rs157816. & 160 chr6_110530 & 6.34 nonsex \\
\hline 6 Gg_rs157816. & 161 chr6_110340 & 7.46 nonsex \\
\hline 6 Gg_rs157816. & 161 chr6_110500 & 6.4 nonsex \\
\hline 6 Gg_rs157816. & 161 chr6_110510 & 7 nonsex \\
\hline 6 Gg_rs157816. & 161 chr6_110590 & 6.28 nonsex \\
\hline 6 Gg_rs157816. & 161 chr6_110660 & 6.94 nonsex \\
\hline 6 Gg_rs157816. & 162 chr6_110360 & 5.7 nonsex \\
\hline 6 Gg_rs157816. & 162 chr6_117830 & 5.21 nonsex \\
\hline 6 Gg_rs157816. & 163 chr6_117810 & 6.87 nonsex \\
\hline 6 Gg_rs157816. & 163 chr6_117840 & 5.99 nonsex \\
\hline 6 Gg_rs157816. & 163 chr6_117770 & 4.92 nonsex \\
\hline 6 Gg_rs157816. & 163 chr6_117850 & 4.77 nonsex \\
\hline 6 Gg_rs157816. & 163 chr6_125460 & 4.74 nonsex \\
\hline 6 Gg_rs157816. & 163 chr6_127820 & 5.47 nonsex \\
\hline 6 Gg_rs157816. & 164 chr6_110320 & 5.29 nonsex \\
\hline
\end{tabular}




\begin{tabular}{|c|c|c|}
\hline 6 Gg_rs157816. & 164 chr6_111130 & 7.97 nonsex \\
\hline 6 Gg_rs157816. & 164 chr6_110300 & 5.3 nonsex \\
\hline 6 Gg_rs157816. & 165 chr6_111030 & 6.29 nonsex \\
\hline 6 Gg_rs157816. & 165 chr6_118290 & 4.42 nonsex \\
\hline 6 Gg_rs157816. & 166 chr6_110370 & $7.63 \mathrm{sex}$ \\
\hline 6 Gg_rs157816. & 166 chr6_110800 & 7.37 nonsex \\
\hline 6 Gg_rs157816. & 166 chr6_111010 & 8.15 nonsex \\
\hline 6 Gg_rs157816. & 166 chr6_111150 & 8.37 nonsex \\
\hline 6 Gg_rs157816. & 166 chr6_117700 & 6.5 nonsex \\
\hline 6 Gg_rs157816. & 166 chr6_117790 & 6.03 sex \\
\hline 6 Gg_rs157816. & 166 chr6_110540 & 4.53 nonsex \\
\hline 6 Gg_rs157816. & 166 chr6_110600 & 4.65 nonsex \\
\hline 6 Gg_rs157816. & 166 chr6_127350 & 4.32 nonsex \\
\hline 6 Gg_rs157816. & 167 chr6_110670 & 6.96 sex \\
\hline 6 Gg_rs157816. & 167 chr6_110820 & 7.29 nonsex \\
\hline 6 Gg_rs157816. & 167 chr6_110850 & 8.18 nonsex \\
\hline 6 Gg_rs157816. & 167 chr6_115990 & 3.73 nonsex \\
\hline 6 Gg_rs157816. & 168 chr6_110560 & 5.95 nonsex \\
\hline 6 Gg_rs157816. & 168 chr6_110830 & 7.83 nonsex \\
\hline 6 Gg_rs157816. & 168 chr6_110840 & 7.17 nonsex \\
\hline 6 Gg_rs157816. & 168 chr6_110860 & 6.69 nonsex \\
\hline 6 Gg_rs157816. & 168 chr6_110870 & 7.4 nonsex \\
\hline 6 Gg_rs157816. & 168 chr6_111140 & 8 nonsex \\
\hline 6 Gg_rs157816. & 169 chr6_110880 & 7.21 nonsex \\
\hline 6 Gg_rs157816. & 169 chr6_110890 & 7.1 nonsex \\
\hline 6 Gg_rs157816. & 169 chr6_110900 & 7.39 nonsex \\
\hline 6 Gg_rs157816. & 169 chr6_111020 & 7.93 nonsex \\
\hline 6 Gg_rs157816. & 169 chr6_110630 & 4.84 nonsex \\
\hline 6 Gg_rs157816. & 170 chr6_110810 & 7.81 nonsex \\
\hline 6 Gg_rs157816. & 170 chr6_127520 & 6.19 nonsex \\
\hline 6 Gg_rs157816. & 170 chr6_129770 & 6.07 nonsex \\
\hline 6 Gg_rs157816. & 170 chr6_110920 & 4.56 nonsex \\
\hline 6 Gg_rs157816. & 171 chr6_111170 & 4.57 nonsex \\
\hline 6 Gg_rs157816. & 171 chr6_124070 & 4.1 nonsex \\
\hline 6 Gg_rs157816. & 172 chr6_117730 & 6.32 sex \\
\hline 6 Gg_rs157816. & 173 chr6_113410 & 5.89 sex \\
\hline 6 Gg_rs157816. & 173 chr6_118300 & 4.29 nonsex \\
\hline 6 Gg_rs157816. & 173 chr6_127370 & 4.57 nonsex \\
\hline 6 Gg_rs157816. & 173 chr6_131260 & 4.65 nonsex \\
\hline 6 Gg_rs157816. & 174 chr6_111040 & 5.86 nonsex \\
\hline 6 Gg_rs157816. & 175 chr6_208320 & 4.26 nonsex \\
\hline 6 Gg_rs157816. & 176 chr6_136780 & 5.66 nonsex \\
\hline 6 Gg_rs157816. & 180 chr6_111160 & 5.37 nonsex \\
\hline
\end{tabular}




\begin{tabular}{|c|c|c|c|}
\hline 6 Gg_rs157816. & 180 chr6_120270 & 6.25 nonsex & cis \\
\hline 6 Gg_rs157816. & 180 chr6_120790 & 5.14 nonsex & cis \\
\hline 6 Gg_rs157816. & 180 chr6_129860 & 7.22 sex & cis \\
\hline 6 Gg_rs157816. & 180 chr6_135100 & 5.51 nonsex & cis \\
\hline 6 Gg_rs157816. & 180 chr6_135350 & $5.53 \mathrm{sex}$ & cis \\
\hline 6 Gg_rs157816. & 180 chr6_136770 & 7.29 sex & cis \\
\hline 6 Gg_rs157816. & 180 chr6_151590 & 5.78 sex & cis \\
\hline 6 Gg_rs157816. & 180 chr6_156990 & 7.02 nonsex & cis \\
\hline 6 Gg_rs157816. & 180 chr6_162690 & 6.9 sex & cis \\
\hline 6 Gg_rs157816. & 180 chr6_210210 & 6.19 sex & cis \\
\hline 6 Gg_rs157816. & 180 chr6_222130 & 6.62 sex & cis \\
\hline 6 Gg_rs157816. & 180 chr6_140010 & 5.25 nonsex & cis \\
\hline 6 Gg_rs157816. & 180 chr6_117630 & 3.86 nonsex & cis \\
\hline 6 Gg_rs157816. & 180 chr6_135190 & 4.03 nonsex & cis \\
\hline 6 Gg_rs157816. & 180 chr6_139020 & 4.35 nonsex & cis \\
\hline 6 Gg_rs157816. & 180 chr6_140210 & 4.06 nonsex & cis \\
\hline 6 Gg_rs157816. & 180 chr6_161710 & 4.22 nonsex & cis \\
\hline 6 Gg_rs157816. & 180 chr6_169790 & 4.18 nonsex & cis \\
\hline 6 Gg_rs157816. & 180 chr6_214500 & 4.45 nonsex & cis \\
\hline 6 Gg_rs157816. & 180 chr1_173410 & 7.42 sex & trans \\
\hline 6 Gg_rs157816. & 180 chr7_284390। & 5.94 nonsex & trans \\
\hline 6 Gg_rs157816. & 180 chr3_287000 & 6.92 sex & trans \\
\hline 6 Gg_rs157816. & 181 chr6_117610 & 5.85 nonsex & cis \\
\hline 6 Gg_rs157816. & 181 chr6_151580 & 11.02 nonsex & cis \\
\hline 6 Gg_rs157816. & 181 chr6_155530 & 4.81 sex & cis \\
\hline 6 Gg_rs157816. & 181 chr6_216800 & 4.63 sex & cis \\
\hline 6 Gg_rs157816. & 181 chr6_127150 & 5.87 nonsex & cis \\
\hline 6 Gg_rs157816. & 181 chr6_802500 & 4.37 nonsex & cis \\
\hline 6 Gg_rs157816. & 181 chr6_114470 & 4.3 nonsex & cis \\
\hline 6 Gg_rs157816. & 181 chr6_135730 & 5.24 nonsex & cis \\
\hline 6 Gg_rs157816. & 181 chr6_139010 & 4.3 nonsex & cis \\
\hline 6 Gg_rs157816. & 181 chr6_148660 & 4.86 nonsex & cis \\
\hline 6 Gg_rs157816. & 181 chr12_45250। & 7.46 sex & trans \\
\hline 6 Gg_rs157830. & 181 chr6_147020 & 6.26 nonsex & cis \\
\hline 6 Gg_rs157830. & 181 chr6_151250 & 6.47 sex & cis \\
\hline 6 Gg_rs157830. & 181 chr6_152690 & 7.27 sex & cis \\
\hline 6 Gg_rs157830. & 181 chr6_158440 & 6.72 nonsex & cis \\
\hline 6 Gg_rs157830. & 181 chr6_145840 & 4.25 nonsex & cis \\
\hline 6 Gg_rs157816. & 182 chr6_118090 & 4.42 nonsex & cis \\
\hline 6 Gg_rs157830. & 182 chr6_128060 & 4.8 nonsex & cis \\
\hline 6 Gg_rs157830. & 182 chr6_152680 & 7.2 nonsex & cis \\
\hline 6 Gg_rs157830. & 182 chr6_153610 & 4.89 nonsex & cis \\
\hline 6 Gg_rs157830. & 182 chr6_127980 & 4.18 nonsex & cis \\
\hline
\end{tabular}




\begin{tabular}{|c|c|c|c|}
\hline 6 Gg_rs157830. & 182 chr6_128020 & 4.04 nonsex & cis \\
\hline 6 Gg_rs157830. & 182 chr6_128960। & 4.2 nonsex & cis \\
\hline 6 Gg_rs157830. & 182 chr4_224850 & 5.78 nonsex & trans \\
\hline 6 Gg_rs145756 & 182 chr6_153690 & 5.7 sex & cis \\
\hline 6 Gg_rs145756 & 182 chr6_149550 & 5.14 nonsex & cis \\
\hline 6 Gg_rs145756 & 182 chr6_149560 & 4.51 nonsex & cis \\
\hline 6 Gg_rs145756 & 182 chr3_938100। & 5.26 nonsex & trans \\
\hline 6 Gg_rs157830. & 183 chr6_154410 & 18.34 nonsex & cis \\
\hline 6 Gg_rs145756 & 183 chr6_139220 & 5.71 sex & cis \\
\hline 6 Gg_rs145756 & 183 chr6_161480 & 5.81 nonsex & cis \\
\hline 6 Gg_rs145756 & 183 chr6_139940। & 4.4 nonsex & cis \\
\hline 6 Gg_rs145756 & 183 chr6_160250 & 4.44 nonsex & cis \\
\hline 6 Gg_rs145756 & 183 chr6_169760 & 4.99 nonsex & cis \\
\hline 6 Gg_rs157830. & 184 chr6_140000 & 12.78 nonsex & cis \\
\hline 6 Gg_rs145756 & 184 chr6_165100। & 4.75 nonsex & cis \\
\hline 6 Gg_rs145756 & 184 chr6_151260 & 6.27 nonsex & cis \\
\hline 6 Gg_rs145756 & 184 chr6_151550 & 7.6 nonsex & cis \\
\hline 6 Gg_rs145756 & 184 chr6_158680 & 5.27 nonsex & cis \\
\hline 6 Gg_rs145756 & 184 chr6_157370 & 4.32 nonsex & cis \\
\hline 6 Gg_rs157816. & 185 chr6_871100। & 5.45 nonsex & cis \\
\hline 6 Gg_rs157816. & 185 chr6_168630 & 4.83 nonsex & cis \\
\hline 6 Gg_rs157816. & 185 chr6_170780 & 6.95 nonsex & cis \\
\hline 6 Gg_rs157830. & 185 chr6_172720 & 4.87 sex & cis \\
\hline 6 Gg_rs145756 & 185 chr6_155800। & 9.36 nonsex & cis \\
\hline 6 Gg_rs145756 & 185 chr6_131170 & 5.19 nonsex & cis \\
\hline 6 Gg_rs145756 & 186 chr6_207050। & 4.97 nonsex & cis \\
\hline 6 Gg_rs145756 & 186 chr6_150650। & 9.04 nonsex & cis \\
\hline 6 Gg_rs145756 & 186 chr6_227430 & 4.42 nonsex & cis \\
\hline 6 Gg_rs157816. & 187 chr6_850500। & $4.51 \mathrm{sex}$ & cis \\
\hline 6 Gg_rs157874 & 187 chr6_167720 & 4.9 nonsex & cis \\
\hline 6 Gg_rs157874 & 187 chr6_167750 & 4.38 nonsex & cis \\
\hline 6 Gg_rs157874 & 187 chr6_216380 & 4.51 nonsex & cis \\
\hline 6 Gg_rs157874 & 187 chr6_217290। & 4.54 nonsex & cis \\
\hline 6 Gg_rs157874 & 187 chr6_223480 & 4.84 nonsex & cis \\
\hline 6 Gg_rs157874 & 188 chr6_157580 & 5.8 nonsex & cis \\
\hline 6 Gg_rs157874 & 188 chr6_162290। & 5.5 nonsex & cis \\
\hline 6 Gg_rs157874 & 188 chr6_189080 & $5.9 \operatorname{sex}$ & cis \\
\hline 6 Gg_rs157874 & 188 chr6_210190। & 5.94 nonsex & cis \\
\hline 6 Gg_rs157874 & 188 chr6_165310 & 4.65 nonsex & cis \\
\hline 6 Gg_rs157874 & 188 chr6_166210 & 4.61 nonsex & cis \\
\hline 6 Gg_rs157874 & 188 chr6_180630। & 4.38 nonsex & cis \\
\hline 6 Gg_rs157874 & 188 chr6_188360 & 5.16 nonsex & cis \\
\hline 6 Gg_rs157874 & 188 chr1_839100। & 7.53 sex & trans \\
\hline
\end{tabular}




\begin{tabular}{|c|c|c|c|}
\hline 6 Gg_rs157874 & 189 chr3_948590 & 7.49 sex & trans \\
\hline 6 Gg_rs157874 & 190 chr6_167340 & 8.52 sex & cis \\
\hline 6 Gg_rs157874 & 190 chr6_172670 & 5.57 nonsex & cis \\
\hline 6 Gg_rs157874 & 190 chr6_183860 & 5.1 nonsex & cis \\
\hline 6 Gg_rs157874 & 190 chr6_186820 & 7.43 nonsex & cis \\
\hline 6 Gg_rs157874 & 190 chr6_189090 & $7.03 \mathrm{sex}$ & cis \\
\hline 6 Gg_rs157874 & 190 chr6_189880 & 5.54 nonsex & cis \\
\hline 6 Gg_rs157874 & 190 chr6_194340 & 5.65 sex & cis \\
\hline 6 Gg_rs157874 & 190 chr6_194480 & 8.37 nonsex & cis \\
\hline 6 Gg_rs157874 & 190 chr6_196700 & 6.37 nonsex & cis \\
\hline 6 Gg_rs157874 & 190 chr6_198100 & 6.28 sex & cis \\
\hline 6 Gg_rs157874 & 190 chr6_203180 & 5.21 sex & cis \\
\hline 6 Gg_rs157874 & 190 chr6_226150 & 5.4 nonsex & cis \\
\hline 6 Gg_rs157874 & 190 chr6_274270 & 5.49 sex & cis \\
\hline 6 Gg_rs157874 & 190 chr6_138250 & 4.17 nonsex & cis \\
\hline 6 Gg_rs157874 & 190 chr6_183410 & 4.46 nonsex & cis \\
\hline 6 Gg_rs157874 & 190 chr6_184190 & 4.55 nonsex & cis \\
\hline 6 Gg_rs157874 & 190 chr6_186060 & 4.63 nonsex & cis \\
\hline 6 Gg_rs157874 & 190 chr6_186910 & 4.55 nonsex & cis \\
\hline 6 Gg_rs157874 & 190 chr6_198850 & 4.57 nonsex & cis \\
\hline 6 Gg_rs157874 & 190 chr6_200040 & 4.34 nonsex & cis \\
\hline 6 Gg_rs157874 & 190 chr6_205100 & 4.91 nonsex & cis \\
\hline 6 Gg_rs157874 & 190 chr6_236940 & 4.46 nonsex & cis \\
\hline 6 Gg_rs157874 & 190 chr4_813470 & 7.37 sex & trans \\
\hline 6 Gg_rs157874 & 191 chr6_193260 & 6.76 nonsex & cis \\
\hline 6 Gg_rs157874 & 191 chr6_194380 & 5.06 sex & cis \\
\hline 6 Gg_rs157874 & 191 chr6_215040 & 6.16 nonsex & cis \\
\hline 6 Gg_rs157874 & 191 chr6_169050 & 4.39 nonsex & cis \\
\hline 6 Gg_rs157874 & 191 chr6_176860 & 4.25 nonsex & cis \\
\hline 6 Gg_rs157874 & 191 chr6_182250 & 4.33 nonsex & cis \\
\hline 6 Gg_rs157874 & 191 chr6_198940 & 4.52 nonsex & cis \\
\hline 6 Gg_rs157874 & 191 chr2_139640 & 5.52 nonsex & trans \\
\hline 6 Gg_rs157874 & 192 chr6_199510 & 6.6 nonsex & cis \\
\hline 6 Gg_rs157874 & 192 chr6_203170 & 5.72 nonsex & cis \\
\hline 6 Gg_rs157874 & 192 chr6_204500 & 5.34 nonsex & cis \\
\hline 6 Gg_rs157874 & 193 chr6_171670 & 5.35 nonsex & cis \\
\hline 6 Gg_rs157874 & 193 chr6_172950 & 7.13 nonsex & cis \\
\hline 6 Gg_rs157874 & 193 chr6_184540 & 8.72 nonsex & cis \\
\hline 6 Gg_rs157874 & 193 chr6_188980 & 16.05 nonsex & cis \\
\hline 6 Gg_rs157874 & 193 chr6_190380 & 6.05 nonsex & cis \\
\hline 6 Gg_rs157874 & 193 chr6_197700 & 5.89 sex & cis \\
\hline 6 Gg_rs157874 & 193 chr6_200610 & 5.6 sex & cis \\
\hline 6 Gg_rs157874 & 193 chr6_201100 & 6.45 nonsex & cis \\
\hline
\end{tabular}




\begin{tabular}{|c|c|c|}
\hline 6 Gg_rs157874 & 193 chr6_203190। & 5.01 nonsex \\
\hline 6 Gg_rs157874 & 193 chr6_203560 & 5.69 nonsex \\
\hline 6 Gg_rs157874 & 193 chr6_210950। & 5.63 sex \\
\hline 6 Gg_rs157874 & 193 chr6_247870 & 5.66 sex \\
\hline 6 Gg_rs157874 & 193 chr6_209870। & 4.62 nonsex \\
\hline 6 Gg_rs135785 & 193 chr6_197570। & 5.08 nonsex \\
\hline 6 Gg_rs157874 & 194 chr6_179170। & 5.52 nonsex \\
\hline 6 Gg_rs157874 & 194 chr6_180620 & 8.61 nonsex \\
\hline 6 Gg_rs157874 & 194 chr6_193160 & 9.19 nonsex \\
\hline 6 Gg_rs157874 & 194 chr6_194400 & 9.65 sex \\
\hline 6 Gg_rs157874 & 194 chr6_207420। & 6.11 nonsex \\
\hline 6 Gg_rs157874 & 194 chr6_207720। & 5.58 sex \\
\hline 6 Gg_rs157874 & 194 chr6_214570 & 7.42 nonsex \\
\hline 6 Gg_rs157874 & 194 chr6_179520। & 4.71 nonsex \\
\hline 6 Gg_rs157874 & 194 chr6_183500 & 4.63 nonsex \\
\hline 6 Gg_rs157874 & 194 chr6_201760। & 4.64 nonsex \\
\hline 6 Gg_rs157874 & 194 chr6_202560। & 4.48 nonsex \\
\hline 6 Gg_rs157874 & 194 chr6_214750 & 4.43 nonsex \\
\hline 6 Gg_rs157874 & 194 chr6_221070। & 4.57 nonsex \\
\hline 6 Gg_rs157874 & 195 chr6_185880 & 7.58 sex \\
\hline 6 Gg_rs157874 & 195 chr6_186340 & 6.58 nonsex \\
\hline 6 Gg_rs157874 & 195 chr6_189870 & 6.58 sex \\
\hline 6 Gg_rs157874 & 195 chr6_195940। & 6.98 nonsex \\
\hline 6 Gg_rs157874 & 195 chr6_196280 & 6.37 nonsex \\
\hline 6 Gg_rs157874 & 195 chr6_203010 & 6.24 sex \\
\hline 6 Gg_rs157874 & 195 chr6_211050 & 6.05 nonsex \\
\hline 6 Gg_rs157874 & 195 chr6_214710 & 6.83 nonsex \\
\hline 6 Gg_rs157874 & 195 chr6_217960। & 6.56 nonsex \\
\hline 6 Gg_rs157874 & 195 chr6_120260 & 4.44 nonsex \\
\hline 6 Gg_rs157874 & 195 chr6_202390। & 4.79 nonsex \\
\hline 6 Gg_rs135785 & 195 chr6_185890 & 5.47 nonsex \\
\hline 6 Gg_rs135785 & 195 chr6_190490। & 4.65 nonsex \\
\hline 6 Gg_rs157874 & 196 chr6_187840 & 14.44 nonsex \\
\hline 6 Gg_rs157874 & 196 chr6_190390। & 6.9 sex \\
\hline 6 Gg_rs157874 & 196 chr6_193250 & 9.19 nonsex \\
\hline 6 Gg_rs157874 & 196 chr6_202570। & 6.23 sex \\
\hline 6 Gg_rs157874 & 196 chr6_210530 & 8.06 nonsex \\
\hline 6 Gg_rs157874 & 196 chr6_215230 & 9.03 sex \\
\hline 6 Gg_rs135785 & 196 chr6_203220 & 4.78 nonsex \\
\hline 6 Gg_rs157874 & 197 chr6_224940। & 5.32 nonsex \\
\hline 6 Gg_rs157874 & 197 chr6_195950 & 5.49 nonsex \\
\hline 6 Gg_rs157874 & 197 chr6_229190। & 7.96 nonsex \\
\hline 6 Gg_rs157874 & 197 chr6_229310। & 6.86 nonsex \\
\hline
\end{tabular}




\begin{tabular}{|c|c|c|}
\hline 6 Gg_rs157874 & 197 chr6_216810 & 4.4 nonsex \\
\hline 6 X6_18893102 & 197 chr6_199010 & 5.82 nonsex \\
\hline 6 Gg_rs135785 & 197 chr6_201250 & 5.64 nonsex \\
\hline 6 Gg_rs135785 & 197 chr6_214690। & 5.19 nonsex \\
\hline 6 Gg_rs135785 & 197 chr6_228220 & 6.13 nonsex \\
\hline 6 Gg_rs157874 & 198 chr6_199830 & 5.65 nonsex \\
\hline 6 X6_18893102 & 198 chr6_196290। & 5.98 nonsex \\
\hline 6 X6_18893102 & 198 chr6_227420 & 8.07 nonsex \\
\hline 6 X6_18893102 & 199 chr6_187550 & 11.36 nonsex \\
\hline 6 X6_18893102 & 199 chr6_190800 & 5.78 nonsex \\
\hline 6 X6_18893102 & 199 chr6_204790। & 4.93 nonsex \\
\hline 6 X6_18893102 & 199 chr6_210990। & 5.09 nonsex \\
\hline 6 X6_18893102 & 199 chr6_223870 & 5.13 nonsex \\
\hline 6 Gg_rs135785 & 199 chr6_213320 & 6.8 nonsex \\
\hline 6 Gg_rs135785 & 199 chr6_228730 & 5.43 nonsex \\
\hline 6 X6_18893102 & 200 chr6_212390। & 5.2 nonsex \\
\hline 6 X6_18893102 & 200 chr6_197560 & 13.74 nonsex \\
\hline 6 X6_18893102 & 201 chr6_201260। & 4.92 nonsex \\
\hline 6 X6_18893102 & 201 chr6_214620 & 10.74 nonsex \\
\hline 6 X6_18893102 & 201 chr6_214760 & 4.47 nonsex \\
\hline 6 X6_18893102 & 202 chr6_222740। & 7.8 sex \\
\hline 6 X6_18893102 & 202 chr6_227500 & 5.91 nonsex \\
\hline 6 X6_18893102 & 202 chr6_229450। & 5.89 nonsex \\
\hline 6 X6_18893102 & 203 chr6_206190। & 5.23 nonsex \\
\hline 6 X6_18893102 & 203 chr6_218600 & 5.07 sex \\
\hline 6 X6_18893102 & 203 chr6_234710 & 7.65 sex \\
\hline 6 Gg_rs145824 & 203 chr6_283260 & 5.59 sex \\
\hline 6 X6_18893102 & 204 chr6_190790। & 5.79 sex \\
\hline 6 X6_18893102 & 205 chr6_186410 & 4.84 nonsex \\
\hline 6 X6_18893102 & 205 chr6_212360। & 5.99 sex \\
\hline 6 X6_18893102 & 205 chr6_235550। & 6.98 sex \\
\hline 6 X6_18893102 & 206 chr6_222440। & 4.05 nonsex \\
\hline 6 Gg_rs145824 & 206 chr6_218290। & 5.16 sex \\
\hline 6 Gg_rs145824 & 206 chr6_222280 & 16.98 nonsex \\
\hline 6 X6_18893102 & 208 chr6_221960। & 4.74 nonsex \\
\hline 6 X6_18893102 & 209 chr6_222730। & 8.71 sex \\
\hline 6 X6_18893102 & 209 chr6_222790। & 6.5 nonsex \\
\hline 6 Gg_rs145824 & 209 chr6_223160। & 5.91 nonsex \\
\hline 6 Gg_rs135785 & 211 chr6_190520 & 3.51 nonsex \\
\hline 6 Gg_rs135785 & 212 chr6_197870 & 6.53 nonsex \\
\hline 6 Gg_rs135785 & 212 chr6_214780 & 5.11 nonsex \\
\hline 6 Gg_rs135785 & 212 chr6_226920। & 4.48 nonsex \\
\hline 6 Gg_rs135785 & 212 chr6_228040। & 4.88 nonsex \\
\hline
\end{tabular}




\begin{tabular}{|c|c|c|c|}
\hline 6 Gg_rs135785 & 213 chr6_220930 & 4.43 nonsex & cis \\
\hline 6 Gg_rs135785 & 214 chr6_225590। & 6.04 nonsex & cis \\
\hline 6 Gg_rs135785 & 214 chr6_201950 & 4.23 nonsex & cis \\
\hline 6 Gg_rs135785 & 214 chr6_205320। & 2.89 nonsex & cis \\
\hline 6 Gg_rs135785 & 215 chr6_201750 & 4.75 nonsex & cis \\
\hline 6 Gg_rs135785 & 215 chr6_201560 & 9.19 nonsex & cis \\
\hline 6 Gg_rs135785 & 215 chr6_210520 & 6.35 nonsex & cis \\
\hline 6 Gg_rs135785 & 215 chr6_236440 & 6.19 nonsex & cis \\
\hline 6 Gg_rs135785 & 215 chr6_238090। & 5.74 nonsex & cis \\
\hline 6 Gg_rs135785 & 215 chr6_228610 & 4.32 nonsex & cis \\
\hline 6 Gg_rs135785 & 215 chr6_235980 & 4.98 nonsex & cis \\
\hline 6 Gg_rs135785 & 218 chr6_235620। & 4.41 nonsex & cis \\
\hline 6 Gg_rs135785 & 219 chr6_235630 & 4.74 nonsex & cis \\
\hline 6 Gg_rs135785 & 220 chr6_228050। & 5.29 nonsex & cis \\
\hline 6 Gg_rs135785 & 221 chr6_200740। & 8.42 nonsex & cis \\
\hline 6 Gg_rs135785 & 222 chr6_202310 & 6.52 nonsex & cis \\
\hline 6 Gg_rs135785 & 222 chr6_205370 & 3.78 nonsex & cis \\
\hline 6 Gg_rs135785 & 226 chr6_190020 & 5.05 nonsex & cis \\
\hline 6 X6_25762392 & 232 chr6_258450। & 4.72 nonsex & cis \\
\hline 6 X6_25762392 & 235 chr6_245600 & 5.6 nonsex & cis \\
\hline 6 X6_25762392 & 235 chr6_254340 & 8.5 nonsex & cis \\
\hline 6 X6_25762392 & 235 chr6_256430 & 6.7 nonsex & cis \\
\hline 6 X6_25762392 & 235 chr6_260500 & 7.36 nonsex & cis \\
\hline 6 X6_25762392 & 235 chr6_251590। & 5.18 nonsex & cis \\
\hline 6 X6_25762392 & 235 chr6_255380 & 4.19 nonsex & cis \\
\hline 6 X6_25762392 & 235 chr6_263260। & 4.62 nonsex & cis \\
\hline 6 X6_25762392 & 235 chr6_273260। & 4.47 nonsex & cis \\
\hline 6 Gg_rs158051 & 235 chr6_267570 & 4.32 nonsex & cis \\
\hline 6 X6_25762392 & 236 chr6_245150। & 4.23 nonsex & cis \\
\hline 6 X6_25762392 & 236 chr6_245850। & 5.12 nonsex & cis \\
\hline 6 X6_25762392 & 236 chr6_247810 & 4.6 nonsex & cis \\
\hline 6 X6_25762392 & 236 chr6_257030 & 3.55 nonsex & cis \\
\hline 6 X6_25762392 & 236 chr27_66980। & 6.46 nonsex & trans \\
\hline 6 X6_25762392 & 237 chr6_251250। & 5.34 nonsex & cis \\
\hline 6 X6_25762392 & 237 chr6_264370 & 4.66 nonsex & cis \\
\hline 6 X6_25762392 & 237 chr6_255180 & 4.04 nonsex & cis \\
\hline 6 X6_25762392 & 238 chr6_258220 & 6.28 nonsex & cis \\
\hline 6 X6_25762392 & 238 chr6_259980 & 7.2 nonsex & cis \\
\hline 6 X6_25762392 & 238 chr6_256320 & 4.26 nonsex & cis \\
\hline 6 X6_25762392 & 238 chr6_258180 & 4.11 nonsex & cis \\
\hline 6 X6_25762392 & 238 chr6_258230। & 4.47 nonsex & cis \\
\hline 6 X6_25762392 & 239 chr6_254140 & 4.48 nonsex & cis \\
\hline 6 X6_25762392 & 239 chr6_255900। & 4.72 nonsex & cis \\
\hline
\end{tabular}




\begin{tabular}{|c|c|c|c|}
\hline 6 X6_25762392 & 239 chr6_259490। & 3.71 nonsex & cis \\
\hline $6 \times 6 \_25762392$ & 239 chr6_262060। & 4.18 nonsex & cis \\
\hline $6 \times 6$ X625762392 & 240 chr6_254530। & 4.85 nonsex & cis \\
\hline $6 \times 6 \_25762392$ & 240 chr6_261170 & 6.56 nonsex & cis \\
\hline $6 \times 6 \_25762392$ & 240 chr6_261310 & 11.65 nonsex & cis \\
\hline 6 X6_25762392 & 240 chr6_257250 & 4.26 nonsex & cis \\
\hline 6 X6_25762392 & 241 chr6_240760 & 5.61 nonsex & cis \\
\hline 6 X6_25762392 & 241 chr6_252520। & 4.97 nonsex & cis \\
\hline $6 \times 6$ X6_25762392 & 241 chr6_259250। & 5.28 nonsex & cis \\
\hline $6 \times 6$ X625762392 & 242 chr6_240770 & 6.05 nonsex & cis \\
\hline 6 X6_25762392 & 242 chr6_252500 & 5.38 nonsex & cis \\
\hline 6 X6_25762392 & 242 chr6_257330। & 7.63 nonsex & cis \\
\hline 6 X6_25762392 & 242 chr6_258340 & 5.64 nonsex & cis \\
\hline 6 X6_25762392 & 242 chr6_261020। & 3.72 nonsex & cis \\
\hline 6 X6_25762392 & 242 chr6_261300। & 5.16 nonsex & cis \\
\hline 6 X6_25762392 & 242 chr6_172770 & 4.47 nonsex & cis \\
\hline 6 X6_25762392 & 242 chr6_262840 & 4.01 nonsex & cis \\
\hline 6 X6_25762392 & 242 chr6_262920। & 3.63 nonsex & cis \\
\hline 6 X6_25762392 & 242 chr6_263360। & 4.42 nonsex & cis \\
\hline 6 X6_25762392 & 242 chr6_264030। & 4.15 nonsex & cis \\
\hline 6 X6_25762392 & 242 chrZ_1059301 & 5.8 nonsex & trans \\
\hline 6 X6_25762392 & 243 chr6_260300 & 7.27 nonsex & cis \\
\hline 6 X6_25762392 & 244 chr6_244170 & 5.07 nonsex & cis \\
\hline $6 \times 6 \_25762392$ & 244 chr6_245420। & 5.36 nonsex & cis \\
\hline 6 X6_25762392 & 244 chr6_259430 & 5.65 nonsex & cis \\
\hline 6 X6_25762392 & 244 chr6_240740 & 4.86 nonsex & cis \\
\hline 6 X6_25762392 & 245 chr6_254000 & 7.99 nonsex & cis \\
\hline 6 X6_25762392 & 245 chr6_257350 & 5.13 nonsex & cis \\
\hline $6 \times 6$ X6_25762392 & 247 chr6_264390। & 4.7 nonsex & cis \\
\hline 6 X6_25762392 & 247 chr6_290640 & 3.47 nonsex & cis \\
\hline 6 Gg_rs158077 & 247 chr6_282090। & 5.49 nonsex & cis \\
\hline 6 Gg_rs158077 & 247 chr6_289570 & 3.11 nonsex & cis \\
\hline 6 Gg_rs158077 & 248 chr6_290040। & 3.85 nonsex & cis \\
\hline 6 Gg_rs158077 & 248 chr6_291280 & 3.85 nonsex & cis \\
\hline 6 X6_25762392 & 249 chr6_262510 & 6.34 sex & cis \\
\hline 6 Gg_rs158051 & 249 chr6_265040। & 5.42 nonsex & cis \\
\hline 6 Gg_rs158051 & 249 chr6_252920। & 5.02 nonsex & cis \\
\hline 6 Gg_rs158051 & 249 chr6_269650। & 3.73 nonsex & cis \\
\hline 6 Gg_rs158051 & 250 chr6_265020 & 7.58 nonsex & cis \\
\hline 6 Gg_rs158051 & 250 chr6_267990। & 6.28 nonsex & cis \\
\hline 6 Gg_rs158051 & 250 chr6_245790। & 4.9 nonsex & cis \\
\hline 6 Gg_rs158051 & 250 chr6_258790। & 5.33 nonsex & cis \\
\hline 6 Gg_rs158051 & 250 chr6_265510 & 4.99 nonsex & cis \\
\hline
\end{tabular}




\begin{tabular}{|c|c|c|}
\hline 6 Gg_rs158077 & 250 chr6_260920। & 5.63 nonsex \\
\hline 6 Gg_rs158051 & 251 chr6_257340। & 6.37 nonsex \\
\hline 6 Gg_rs158051 & 251 chr6_255860 & 3.47 nonsex \\
\hline 6 Gg_rs158051 & 252 chr6_267360। & 7.61 nonsex \\
\hline 6 Gg_rs158051 & 253 chr6_248490। & 5.64 nonsex \\
\hline 6 Gg_rs158051 & 253 chr6_258320 & 7.79 nonsex \\
\hline 6 Gg_rs158051 & 254 chr6_272060 & 6.49 nonsex \\
\hline 6 Gg_rs158051 & 254 chr6_289830। & 4.59 nonsex \\
\hline 6 Gg_rs158051 & 255 chr6_264230 & 6.01 sex \\
\hline 6 Gg_rs158051 & 255 chr6_265270 & 6.5 nonsex \\
\hline 6 Gg_rs158051 & 255 chr6_265530। & 5.47 nonsex \\
\hline 6 Gg_rs158051 & 255 chr6_265900 & 6.3 nonsex \\
\hline 6 Gg_rs158051 & 255 chr6_295650 & 6.14 sex \\
\hline 6 Gg_rs158051 & 255 chr6_265260। & 5.14 nonsex \\
\hline 6 Gg_rs158051 & 255 chr6_264850 & 4.65 nonsex \\
\hline 6 Gg_rs158051 & 257 chr6_264720। & 5.94 nonsex \\
\hline 6 Gg_rs158051 & 257 chr6_266160। & 6.12 nonsex \\
\hline 6 Gg_rs158077 & 257 chr6_265690 & 6.66 sex \\
\hline 6 Gg_rs158077 & 258 chr6_286910 & 4.16 nonsex \\
\hline 6 Gg_rs158077 & 259 chr6_286730 & 5.44 nonsex \\
\hline 6 Gg_rs158077 & 261 chr6_282080। & 7.26 nonsex \\
\hline 6 Gg_rs158077 & 262 chr6_275290। & 6.28 nonsex \\
\hline 6 Gg_rs158077 & 262 chr6_265140 & 4.66 nonsex \\
\hline 6 Gg_rs158077 & 265 chr6_289500। & 10.88 nonsex \\
\hline 6 Gg_rs158077 & 266 chr6_268280 & 5.18 nonsex \\
\hline 6 Gg_rs158077 & 266 chr6_252790। & 5.22 nonsex \\
\hline 6 Gg_rs158077 & 266 chr6_264930। & 5.02 nonsex \\
\hline 6 Gg_rs158051 & 275 chr6_276750 & 6.56 sex \\
\hline 6 Gg_rs158077 & 275 chr6_252670 & 6.25 nonsex \\
\hline 6 Gg_rs158051 & 280 chr6_264760। & 4.42 nonsex \\
\hline 6 Gg_rs145922 & 286 chr6_263240। & 5.32 nonsex \\
\hline 6 Gg_rs145922 & 288 chr6_304280 & 3.63 nonsex \\
\hline 6 Gg_rs145922 & 292 chr6_312840। & 4.99 nonsex \\
\hline 6 Gg_rs145922. & 293 chr6_285810 & 4.97 sex \\
\hline 6 Gg_rs145922. & 297 chr6_308720। & 5.15 nonsex \\
\hline 6 Gg_rs145922. & 298 chr6_307560। & 4.45 nonsex \\
\hline 6 Gg_rs145922. & 300 chr6_307450 & 8.84 nonsex \\
\hline 6 Gg_rs145922. & 300 chr6_313340 & 5.39 nonsex \\
\hline 6 Gg_rs145922. & 300 chr6_314040 & 5.02 nonsex \\
\hline 6 Gg_rs145922. & 300 chr6_317680 & 5.84 sex \\
\hline 6 Gg_rs145922. & 300 chr6_318160 & 7.07 nonsex \\
\hline 6 Gg_rs145922. & 300 chr6_345470 & 3.8 nonsex \\
\hline 6 Gg_rs145922. & 300 chr6_302620 & 3.62 nonsex \\
\hline
\end{tabular}




\begin{tabular}{|c|c|c|c|}
\hline 6 Gg_rs145922. & 300 chr6_308320 & 3.28 nonsex & cis \\
\hline 6 Gg_rs145922. & 300 chr6_313550। & 4.8 nonsex & cis \\
\hline 6 Gg_rs145922. & 300 chr6_315080 & 4.62 nonsex & cis \\
\hline 6 Gg_rs145922 & 300 chr1_843230। & 4.69 nonsex & trans \\
\hline 6 Gg_rs145922. & 305 chr2_275160 & 4.86 nonsex & trans \\
\hline 6 Gg_rs145922. & 306 chr6_313230 & 2.96 nonsex & cis \\
\hline 6 Gg_rs158177. & 317 chr6_323740 & 5.34 nonsex & cis \\
\hline 6 Gg_rs158177. & 323 chr6_327890। & 4.23 nonsex & cis \\
\hline 6 Gg_rs158177. & 324 chr6_323390। & 4.7 nonsex & cis \\
\hline 6 Gg_rs158177. & 324 chr31_21310। & 8.66 sex & trans \\
\hline 6 Gg_rs158177. & 325 chr6_324640 & 4.76 nonsex & cis \\
\hline 7 Gg_rs158261 & 0 chr7_643000 & 4.81 nonsex & cis \\
\hline 7 Gg_rs158261 & 0 chr7_208300। & 5.11 nonsex & cis \\
\hline 7 Gg_rs158261 & 0 chr4_394000 & 6.71 sex & trans \\
\hline 7 Gg_rs158261 & 0 chr6_230820। & 7.85 sex & trans \\
\hline 7 Gg_rs158261 & 0 chr13_15540 & 18.25 sex & trans \\
\hline 7 Gg_rs158261 & 0 chr5_387670। & 4.43 nonsex & trans \\
\hline 7 Gg_rs158261 & 1 chr28_29840 & 7.67 sex & trans \\
\hline 7 Gg_rs158261 & 1 chr3_104453! & 9.27 sex & trans \\
\hline 7 Gg_rs158261 & 3 chr7_202700 & 8.03 nonsex & cis \\
\hline 7 Gg_rs158261 & 3 chr11_18439! & 8.39 sex & trans \\
\hline 7 Gg_rs158261 & 3 chr2_782680 & 8.48 sex & trans \\
\hline 7 Gg_rs158284 & 5 chr7_267100। & 5.14 nonsex & cis \\
\hline 7 Gg_rs158261 & 6 chr7_761000 & 4.79 nonsex & cis \\
\hline 7 Gg_rs158284 & 7 chr7_232000। & 7.71 nonsex & cis \\
\hline 7 Gg_rs158284 & 7 chr7_340020। & 6.17 nonsex & trans \\
\hline 7 Gg_rs158261 & 8 chr7_903000 & 3.78 nonsex & cis \\
\hline 7 Gg_rs158284 & 8 chr7_147400 & 5.28 nonsex & cis \\
\hline 7 Gg_rs158284 & 8 chr7_572000 & 5.24 nonsex & cis \\
\hline 7 Gg_rs158284 & 8 chr30_83500। & 9.81 sex & trans \\
\hline 7 Gg_rs158284 & 8 chr2_123192। & 5.7 nonsex & trans \\
\hline 7 Gg_rs158284 & 9 chr7_145100 & 6.69 nonsex & cis \\
\hline 7 Gg_rs158284 & 9 chr7_173400। & 6.84 nonsex & cis \\
\hline 7 Gg_rs158284 & 9 chr7_641000 & 4.72 nonsex & cis \\
\hline 7 Gg_rs158284 & 9 chr7_181800 & 5.62 nonsex & cis \\
\hline 7 Gg_rs158284 & 9 chr7_356290। & 8.02 sex & trans \\
\hline 7 Gg_rs158284 & 9 chr10_18044। & 7.32 sex & trans \\
\hline 7 Gg_rs158284: & 9 chr1_148800 & 6.56 nonsex & trans \\
\hline 7 Gg_rs158284 & 10 chr7_164100 & 6.07 nonsex & cis \\
\hline 7 Gg_rs158284 & 10 chr7_169100 & 6.07 sex & cis \\
\hline 7 Gg_rs158284 & 10 chr7_169700 & 7.01 sex & cis \\
\hline 7 Gg_rs158284 & 10 chr7_186000 & 6.94 nonsex & cis \\
\hline 7 Gg_rs158284: & 10 chr7_187600 & 6.05 nonsex & cis \\
\hline
\end{tabular}




\begin{tabular}{|c|c|c|c|}
\hline 7 Gg_rs158284 & 10 chr7_198800। & 5.65 nonsex & cis \\
\hline 7 Gg_rs158284 & 10 chr7_212200 & 7.4 sex & cis \\
\hline 7 Gg_rs158284 & 10 chr7_217400 & 6.55 nonsex & cis \\
\hline 7 Gg_rs158284 & 10 chr7_220600 & 5.88 nonsex & cis \\
\hline 7 Gg_rs158284 & 10 chr7_465000 & 5.28 nonsex & cis \\
\hline 7 Gg_rs158284 & 10 chr7_975000 & 4.93 nonsex & cis \\
\hline 7 Gg_rs158284 & 10 chr7_998000 & 4.42 nonsex & cis \\
\hline 7 Gg_rs158284 & 10 chr7_101900 & 4.81 nonsex & cis \\
\hline 7 Gg_rs158284 & 10 chr7_106800 & 5.47 nonsex & cis \\
\hline 7 Gg_rs158284 & 10 chr7_114400 & 5.35 nonsex & cis \\
\hline 7 Gg_rs158284 & 10 chr7_142900 & 3.66 nonsex & cis \\
\hline 7 Gg_rs158284 & 10 chr7_151400 & 4.32 nonsex & cis \\
\hline 7 Gg_rs158284 & 10 chr7_154900 & 4.41 nonsex & cis \\
\hline 7 Gg_rs158284 & 10 chr7_164000 & 4.62 nonsex & cis \\
\hline 7 Gg_rs158284 & 10 chr7_171000 & 4.1 nonsex & cis \\
\hline 7 Gg_rs158284 & 10 chr7_177800 & 4.51 nonsex & cis \\
\hline 7 Gg_rs158284 & 10 chr7_186700 & 5.66 nonsex & cis \\
\hline 7 Gg_rs158284 & 10 chr7_188200 & 4.76 nonsex & cis \\
\hline 7 Gg_rs158284 & 10 chr7_226000 & 4.6 nonsex & cis \\
\hline 7 Gg_rs158284 & 10 chr7_232300 & 3.6 nonsex & cis \\
\hline 7 Gg_rs158284 & 10 chr7_637000 & 4.41 nonsex & cis \\
\hline 7 Gg_rs158284 & 10 chr7_823800 & 4.18 nonsex & cis \\
\hline 7 Gg_rs158284 & 10 chr7_858600 & 4.44 nonsex & cis \\
\hline 7 Gg_rs158284 & 10 chr7_118330 & 4.82 nonsex & cis \\
\hline 7 Gg_rs158284 & 10 chr1_677000 & 9.14 sex & trans \\
\hline 7 Gg_rs158284 & 10 chr4_270660 & 8.41 sex & trans \\
\hline 7 Gg_rs158284 & 10 chr4_911160 & 9.28 sex & trans \\
\hline 7 Gg_rs158284 & 10 chr5_206510 & 8.01 sex & trans \\
\hline 7 Gg_rs158284 & 10 chr5_522630 & 7.36 nonsex & trans \\
\hline 7 Gg_rs158284 & 10 chr5_564740 & 7.74 sex & trans \\
\hline 7 Gg_rs158284 & 10 chr15_55730 & 10.63 sex & trans \\
\hline 7 Gg_rs158284 & 10 chr25_18420 & 8.61 sex & trans \\
\hline 7 Gg_rs158284 & 10 chr27_65120 & 10.18 sex & trans \\
\hline 7 Gg_rs158284 & 10 chr30_30100 & 10.09 sex & trans \\
\hline 7 Gg_rs158284 & 10 chr4_224380 & 5.87 nonsex & trans \\
\hline 7 Gg_rs158284 & 10 chr4_652340 & 6.09 nonsex & trans \\
\hline 7 Gg_rs158284 & 10 chr4_700500 & 5.99 nonsex & trans \\
\hline 7 Gg_rs158284 & 10 chr5_401460 & 6.14 nonsex & trans \\
\hline 7 Gg_rs158284 & 10 chr6_189380 & 6.17 nonsex & trans \\
\hline 7 Gg_rs158284 & 10 chr8_120590 & 5.98 nonsex & trans \\
\hline 7 Gg_rs158284 & 10 chr8_126130 & 6.02 nonsex & trans \\
\hline 7 Gg_rs158284 & 10 chr10_64250 & 6.21 nonsex & trans \\
\hline 7 Gg_rs158284 & 10 chr22_50050 & 5.91 nonsex & trans \\
\hline
\end{tabular}




\begin{tabular}{|c|c|c|}
\hline 7 Gg_rs158284 & 10 chr31_48830। & 5.62 nonsex \\
\hline 7 Gg_rs158284 & 10 chr1_104248। & 6.23 nonsex \\
\hline 7 Gg_rs158284 & 10 chr1_115734। & 5.93 nonsex \\
\hline 7 Gg_rs158284 & 10 chr1_164334। & 5.32 nonsex \\
\hline 7 Gg_rs158284 & 10 chr1_172386 & 6.49 nonsex \\
\hline 7 Gg_rs158284 & 10 chr2_117550 & 5.83 nonsex \\
\hline 7 Gg_rs158284 & 10 chr2_434710 & 6 nonsex \\
\hline 7 Gg_rs158284 & 10 chr2_763020 & 6.07 nonsex \\
\hline 7 Gg_rs158284 & 10 chr2_933730 & 5.57 nonsex \\
\hline 7 Gg_rs158284 & 10 chr2_106134! & 5.9 nonsex \\
\hline 7 Gg_rs158284 & 10 chr2_112577। & 6.89 nonsex \\
\hline 7 Gg_rs158284 & 10 chr2_119995। & 5.85 nonsex \\
\hline 7 Gg_rs158284 & 10 chr2_122072 & 6.13 nonsex \\
\hline 7 Gg_rs158284 & 10 chr3_291060 & 6.27 nonsex \\
\hline 7 Gg_rs158284 & 10 chr3_708970 & 5.85 nonsex \\
\hline 7 Gg_rs158284 & 10 chr3_906270 & 5.82 nonsex \\
\hline 7 Gg_rs158284 & 10 chr4_159510 & 5.78 nonsex \\
\hline 7 Gg_rs158284 & 11 chr7_350000 & 4.73 nonsex \\
\hline 7 Gg_rs158284 & 11 chr7_169900 & 4.52 nonsex \\
\hline 7 Gg_rs158284 & 11 chr7_178700 & 4.52 nonsex \\
\hline 7 Gg_rs158284 & 12 chr7_176500 & 5.97 nonsex \\
\hline 7 Gg_rs158284 & 12 chr5_430800 & 6.43 nonsex \\
\hline 7 Gg_rs158284 & 13 chr1_878600 & 6.11 nonsex \\
\hline 7 Gg_rs158284 & 14 chr7_187500 & 5.29 nonsex \\
\hline 7 Gg_rs158284 & 14 chr2_832000 & 5.96 nonsex \\
\hline 7 Gg_rs158284 & 15 chr7_925000 & 3.59 nonsex \\
\hline 7 Gg_rs158284 & 15 chr7_949300 & 4.68 nonsex \\
\hline 7 Gg_rs158284 & 16 chr7_108600 & 5.13 nonsex \\
\hline 7 Gg_rs158284 & 19 chr7_335520 & 5.94 nonsex \\
\hline 7 Gg_rs158284 & 19 chr3_618730 & 6.35 nonsex \\
\hline 7 Gg_rs158284 & 20 chr7_517200 & 4.65 nonsex \\
\hline 7 Gg_rs158261 & 31 chr18_11194। & 4.95 nonsex \\
\hline 7 Gg_rs165755. & 44 chr7_390700 & 6.33 nonsex \\
\hline 7 Gg_rs158322 & 44 chr7_383900 & 6.13 nonsex \\
\hline 7 Gg_rs146014. & 54 chr7_362900 & 5.29 nonsex \\
\hline 7 Gg_rs146014 & 56 chr7_352700 & 3.16 nonsex \\
\hline 7 Gg_rs165755. & 58 chr7_397600 & 4.4 sex \\
\hline 7 Gg_rs165755. & 58 chr7_400100 & 11.98 nonsex \\
\hline 7 Gg_rs165755. & 58 chr7_400200 & 35.27 nonsex \\
\hline 7 Gg_rs165755. & 58 chr7_395100 & 3.28 nonsex \\
\hline 7 Gg_rs165755. & 58 chr7_400400 & 3.6 nonsex \\
\hline 7 Gg_rs146014 & 58 chr3_268760 & 5.33 nonsex \\
\hline 7 Gg_rs146014 & 59 chr7_379800। & 3.77 nonsex \\
\hline
\end{tabular}


7 Gg_rs158322

7 Gg_rs158322

7 Gg_rs165755

7 Gg_rs165755

7 Gg_rs158322

7 Gg_rs158322

7 Gg_rs158345

7 Gg_rs158345

7 Gg_rs158353.

7 Gg_rs158353.

7 Gg_rs158353.

7 Gg_rs158353

7 Gg_rs158353.

7 Gg_rs158353.

7 Gg_rs158353.

7 Gg_rs146071

7 Gg_rs146071

7 Gg_rs146071

7 Gg_rs146071

7 Gg_rs146071

7 Gg_rs146071

7 Gg_rs146071

7 Gg_rs146071

7 Gg_rs146071

7 Gg_rs146071

7 Gg_rs146071

7 Gg_rs146071

7 Gg_rs146071

7 Gg_rs146071

7 Gg_rs146071

7 Gg_rs146071

7 Gg_rs146071

7 Gg_rs146071

7 Gg_rs146071

7 Gg_rs146071

7 Gg_rs158548

7 Gg_rs158548

7 Gg_rs158548

7 Gg_rs137816

7 Gg_rs137816

7 Gg_rs137816

7 Gg_rs137816

7 Gg_rs137816
60 chr7_381200

61 chr1_142190।

62 chr7_400300।

63 chr7_386100।

68 chrW_48490(

71 chr7_358800

82 chr5_193930।

84 chr1_216610

87 chr2_116099।

88 chr7_911800।

91 chr7_450000।

91 chr7_559300।

91 chr7_553300।

93 chr10_49390।

97 chr7_107040।

105 chr7_107080

105 chr7_925300

105 chr7_979300।

105 chr7_107060।

105 chr7_111770

106 chr7_925000।

106 chr7_107070

106 chr7_925400।

106 chr7_112820

106 chr13_94800

106 chrW_48300(

106 chr2_126288

107 chr7_118350

108 chr7_113690।

108 chr5_470600।

109 chr7_110710

109 chr5_103530।

110 chr7_110890।

116 chr11_10656

117 chr3_228100।

167 chr1_158740।

169 chr7_202170।

170 chr7_174460।

176 chr8_260000

176 chr8_295000

176 chr8_301000

176 chr8_315000

176 chr24_64260।
4.36 nonsex cis

5.84 nonsex trans

4.57 nonsex cis

5.7 nonsex cis

8.54 sex trans

5.48 nonsex cis

3.39 nonsex trans

7.4 sex trans

8.07 sex trans

4.76 nonsex cis

4.81 sex cis

5.72 nonsex cis

4.31 nonsex cis

6.32 sex trans

4.51 nonsex cis

5.54 nonsex cis

4.89 nonsex cis

4.8 nonsex cis

4.83 nonsex cis

5.71 nonsex cis

5.92 nonsex cis

6.99 nonsex cis

4.91 nonsex cis

4.91 nonsex cis

7.77 sex trans

8.16 sex trans

7.74 sex trans

5.74 nonsex cis

8 nonsex cis

5.29 nonsex trans

5.13 nonsex cis

12.43 sex trans

4.55 nonsex cis

7.32 sex trans

9.05 sex trans

8.28 sex trans

7.71 nonsex cis

9.88 sex cis

8.43 sex trans

7.29 nonsex trans

7.34 nonsex trans

7.64 nonsex trans

8.34 nonsex trans 


\begin{tabular}{|c|c|c|}
\hline 7 Gg_rs137816 & 176 chr24_64270। & 10.45 nonsex \\
\hline 7 Gg_rs137816 & 176 chr24_64490। & 8.25 nonsex \\
\hline 7 Gg_rs137816 & 176 chr25_12770 & 8.14 nonsex \\
\hline 7 Gg_rs137816 & 176 chr30_16030 & 6.7 nonsex \\
\hline 7 Gg_rs137816 & 176 chr31_97000 & 9.72 nonsex \\
\hline 7 Gg_rs137816 & 176 chr31_98000 & 10.31 nonsex \\
\hline 7 Gg_rs137816 & 176 chr31_10600। & 8.09 nonsex \\
\hline 7 Gg_rs137816 & 176 chr31_12400 & 7.82 nonsex \\
\hline 7 Gg_rs137816 & 176 chr31_19910। & 7.79 nonsex \\
\hline 7 Gg_rs137816 & 176 chr31_19990। & 7.27 nonsex \\
\hline 7 Gg_rs137816 & 176 chr31_28490। & 7.59 nonsex \\
\hline 7 Gg_rs137816 & 176 chr31_28500। & 9.09 nonsex \\
\hline 7 Gg_rs137816 & 176 chr31_28520। & 8.98 nonsex \\
\hline 7 Gg_rs137816 & 176 chr31_31110 & 8.08 nonsex \\
\hline 7 Gg_rs137816 & 176 chr31_32030। & 8.34 nonsex \\
\hline 7 Gg_rs137816 & 176 chr31_32040। & 9.1 nonsex \\
\hline 7 Gg_rs137816 & 176 chr31_32050। & 9.02 nonsex \\
\hline 7 Gg_rs137816 & 176 chr31_32060। & 9.86 nonsex \\
\hline 7 Gg_rs137816 & 176 chr31_32100। & 7.83 nonsex \\
\hline 7 Gg_rs137816 & 176 chr31_32240 & 8.95 nonsex \\
\hline 7 Gg_rs137816 & 176 chr31_32450। & 10.11 nonsex \\
\hline 7 Gg_rs137816 & 176 chr31_32460। & 9.98 nonsex \\
\hline 7 Gg_rs137816 & 176 chr31_32500। & 7.33 nonsex \\
\hline 7 Gg_rs137816 & 176 chr31_32540। & 6.79 nonsex \\
\hline 7 Gg_rs137816 & 176 chr31_32550। & 8.49 nonsex \\
\hline 7 Gg_rs137816 & 176 chr31_33410। & 8.05 sex \\
\hline 7 Gg_rs137816 & 176 chr31_33440। & 8.07 nonsex \\
\hline 7 Gg_rs137816 & 176 chr31_34420। & 7.86 nonsex \\
\hline 7 Gg_rs137816 & 176 chr31_34720। & 8.77 nonsex \\
\hline 7 Gg_rs137816 & 176 chr31_34730। & 8.33 nonsex \\
\hline 7 Gg_rs137816 & 176 chr31_35000। & 8.56 nonsex \\
\hline 7 Gg_rs137816 & 176 chr31_35010। & 9.58 nonsex \\
\hline 7 Gg_rs137816 & 176 chr31_35330। & 7.69 nonsex \\
\hline 7 Gg_rs137816 & 176 chr31_35360। & 8.26 sex \\
\hline 7 Gg_rs137816 & 176 chr31_35770। & 8.3 nonsex \\
\hline 7 Gg_rs137816 & 176 chr31_35810 & 9.71 nonsex \\
\hline 7 Gg_rs137816 & 176 chr31_35820। & 8.27 nonsex \\
\hline 7 Gg_rs137816 & 176 chr31_36000। & 7.65 nonsex \\
\hline 7 Gg_rs137816 & 176 chr31_36010। & 7.25 nonsex \\
\hline 7 Gg_rs137816 & 176 chr31_37280 & 8.33 sex \\
\hline 7 Gg_rs137816 & 176 chr31_37480 & 7.46 nonsex \\
\hline 7 Gg_rs137816 & 176 chr31_38590। & 7.12 nonsex \\
\hline 7 Gg_rs137816 & 176 chr31_39090। & 7.6 nonsex \\
\hline
\end{tabular}




\begin{tabular}{|c|c|c|}
\hline 7 Gg_rs137816 & 176 chr31_39580। & 8.39 nonsex \\
\hline 7 Gg_rs137816 & 176 chr31_39590 & 8.76 nonsex \\
\hline 7 Gg_rs137816 & 176 chr31_39600। & 8.1 nonsex \\
\hline 7 Gg_rs137816 & 176 chr31_40310 & 8.35 nonsex \\
\hline 7 Gg_rs137816 & 176 chr31_41780 & 8.27 nonsex \\
\hline 7 Gg_rs137816 & 176 chr31_41790। & 8.44 nonsex \\
\hline 7 Gg_rs137816 & 176 chr31_42050। & 8.66 nonsex \\
\hline 7 Gg_rs137816 & 176 chr31_42060। & 10.07 nonsex \\
\hline 7 Gg_rs137816 & 176 chr31_42150 & 8.22 nonsex \\
\hline 7 Gg_rs137816 & 176 chr31_42200। & 7.81 nonsex \\
\hline 7 Gg_rs137816 & 176 chr31_42210 & 9.24 nonsex \\
\hline 7 Gg_rs137816 & 176 chr31_42230। & 7.87 nonsex \\
\hline 7 Gg_rs137816 & 176 chr31_43040। & 7.34 nonsex \\
\hline 7 Gg_rs137816 & 176 chr31_44100। & 7.65 nonsex \\
\hline 7 Gg_rs137816 & 176 chr31_44430। & 7.17 nonsex \\
\hline 7 Gg_rs137816 & 176 chr31_44510 & 7.51 nonsex \\
\hline 7 Gg_rs137816 & 176 chr31_44560। & 8.11 nonsex \\
\hline 7 Gg_rs137816 & 176 chr31_44570। & 9.55 nonsex \\
\hline 7 Gg_rs137816 & 176 chr31_44600। & 8.04 nonsex \\
\hline 7 Gg_rs137816 & 176 chr31_54590। & 10.6 nonsex \\
\hline 7 Gg_rs137816 & 176 chr31_54880 & 8.95 nonsex \\
\hline 7 Gg_rs137816 & 176 chr31_54900 & 9.9 nonsex \\
\hline 7 Gg_rs137816 & 176 chr31_54910 & 12.48 nonsex \\
\hline 7 Gg_rs137816 & 176 chr31_54930। & 9.69 nonsex \\
\hline 7 Gg_rs137816 & 176 chr31_54940। & 7.5 nonsex \\
\hline 7 Gg_rs137816 & 176 chr31_55030। & 9.68 nonsex \\
\hline 7 Gg_rs137816 & 176 chr31_55040। & 9.46 nonsex \\
\hline 7 Gg_rs137816 & 176 chr31_55050। & 12.4 nonsex \\
\hline 7 Gg_rs137816 & 176 chr31_55070। & 9.71 nonsex \\
\hline 7 Gg_rs137816 & 176 chr31_55170। & 9.89 nonsex \\
\hline 7 Gg_rs137816 & 176 chr31_55180 & 11.04 nonsex \\
\hline 7 Gg_rs137816 & 176 chr31_55190। & 11.92 nonsex \\
\hline 7 Gg_rs137816 & 176 chr31_55200। & 9.35 nonsex \\
\hline 7 Gg_rs137816 & 176 chr31_55210। & 8.35 nonsex \\
\hline 7 Gg_rs137816 & 176 chr31_55310 & 10.27 nonsex \\
\hline 7 Gg_rs137816 & 176 chr31_55330। & 8.87 nonsex \\
\hline 7 Gg_rs137816 & 176 chr31_55440। & 9.23 nonsex \\
\hline 7 Gg_rs137816 & 176 chr31_55450। & 10 nonsex \\
\hline 7 Gg_rs137816 & 176 chr31_55480 & 10.14 nonsex \\
\hline 7 Gg_rs137816 & 176 chr31_55580 & 9.91 nonsex \\
\hline 7 Gg_rs137816 & 176 chr31_55600 & 10.92 nonsex \\
\hline 7 Gg_rs137816 & 176 chr31_55610 & 9.8 nonsex \\
\hline 7 Gg_rs137816 & 176 chr31_55680 & 7.74 nonsex \\
\hline
\end{tabular}




\begin{tabular}{|c|c|c|}
\hline 7 Gg_rs137816 & 176 chr31_55720। & 10.22 nonsex \\
\hline 7 Gg_rs137816 & 176 chr31_55740। & 12.94 nonsex \\
\hline 7 Gg_rs137816 & 176 chr31_55750। & 10.44 nonsex \\
\hline 7 Gg_rs137816 & 176 chr31_55840। & 9.29 nonsex \\
\hline 7 Gg_rs137816 & 176 chr31_57370। & 8.5 nonsex \\
\hline 7 Gg_rs137816 & 176 chr31_58140। & 8.45 nonsex \\
\hline 7 Gg_rs137816 & 176 chr31_58680। & 7.64 nonsex \\
\hline 7 Gg_rs137816 & 176 chr31_59180। & 8.09 nonsex \\
\hline 7 Gg_rs137816 & 176 chr31_60250। & 8.5 nonsex \\
\hline 7 Gg_rs137816 & 176 chr31_60650। & 8.76 nonsex \\
\hline 7 Gg_rs137816 & 176 chr32_30200। & 8.57 sex \\
\hline 7 Gg_rs137816 & 176 chr33_29400। & 7.71 nonsex \\
\hline 7 Gg_rs137816 & 176 chr33_29770 & 7.65 nonsex \\
\hline 7 Gg_rs137816 & 176 chr33_36640। & 7.62 nonsex \\
\hline 7 Gg_rs137816 & 176 chr33_44690। & 7.8 nonsex \\
\hline 7 Gg_rs137816 & 176 chr8_244000 & 6.66 nonsex \\
\hline 7 Gg_rs137816 & 176 chr8_257000 & 6.46 nonsex \\
\hline 7 Gg_rs137816 & 176 chr8_272000 & 6.14 nonsex \\
\hline 7 Gg_rs137816 & 176 chr8_282000 & 7.01 nonsex \\
\hline 7 Gg_rs137816 & 176 chr8_306000 & 6.84 nonsex \\
\hline 7 Gg_rs137816 & 176 chr24_64320। & 6.99 nonsex \\
\hline 7 Gg_rs137816 & 176 chr24_64480 & 7.04 nonsex \\
\hline 7 Gg_rs137816 & 176 chr25_12780 & 7.2 nonsex \\
\hline 7 Gg_rs137816 & 176 chr31_99000 & 7.04 nonsex \\
\hline 7 Gg_rs137816 & 176 chr31_12500। & 7.2 nonsex \\
\hline 7 Gg_rs137816 & 176 chr31_13200। & 6.72 nonsex \\
\hline 7 Gg_rs137816 & 176 chr31_19630। & 7.14 nonsex \\
\hline 7 Gg_rs137816 & 176 chr31_19980 & 6.95 nonsex \\
\hline 7 Gg_rs137816 & 176 chr31_28070। & 7.15 nonsex \\
\hline 7 Gg_rs137816 & 176 chr31_28080। & 6.23 nonsex \\
\hline 7 Gg_rs137816 & 176 chr31_28510। & 6.88 nonsex \\
\hline 7 Gg_rs137816 & 176 chr31_30400। & 6.7 nonsex \\
\hline 7 Gg_rs137816 & 176 chr31_30740। & 6.86 nonsex \\
\hline 7 Gg_rs137816 & 176 chr31_31000। & 7.4 nonsex \\
\hline 7 Gg_rs137816 & 176 chr31_31100। & 7.4 nonsex \\
\hline 7 Gg_rs137816 & 176 chr31_31560। & 6.98 nonsex \\
\hline 7 Gg_rs137816 & 176 chr31_31870। & 6.72 nonsex \\
\hline 7 Gg_rs137816 & 176 chr31_31880 & 7.12 nonsex \\
\hline 7 Gg_rs137816 & 176 chr31_32020। & 6.42 nonsex \\
\hline 7 Gg_rs137816 & 176 chr31_32250। & 6.52 nonsex \\
\hline 7 Gg_rs137816 & 176 chr31_32260। & 7.05 nonsex \\
\hline 7 Gg_rs137816 & 176 chr31_32280 & 6.64 nonsex \\
\hline 7 Gg_rs137816 & 176 chr31_32420। & 7.06 nonsex \\
\hline
\end{tabular}




\begin{tabular}{|c|c|c|}
\hline 7 Gg_rs137816 & 176 chr31_32430। & 6.32 nonsex \\
\hline 7 Gg_rs137816 & 176 chr31_32440। & 7.4 nonsex \\
\hline 7 Gg_rs137816 & 176 chr31_32910। & 6.93 nonsex \\
\hline 7 Gg_rs137816 & 176 chr31_33330। & 6.18 nonsex \\
\hline 7 Gg_rs137816 & 176 chr31_33490। & 6.55 nonsex \\
\hline 7 Gg_rs137816 & 176 chr31_33670। & 6.67 nonsex \\
\hline 7 Gg_rs137816 & 176 chr31_34310 & 6.32 nonsex \\
\hline 7 Gg_rs137816 & 176 chr31_34410 & 7.41 nonsex \\
\hline 7 Gg_rs137816 & 176 chr31_34430। & 6.68 nonsex \\
\hline 7 Gg_rs137816 & 176 chr31_34510 & 6.66 nonsex \\
\hline 7 Gg_rs137816 & 176 chr31_34660। & 6.26 nonsex \\
\hline 7 Gg_rs137816 & 176 chr31_34700। & 5.12 nonsex \\
\hline 7 Gg_rs137816 & 176 chr31_34840 & 6.75 nonsex \\
\hline 7 Gg_rs137816 & 176 chr31_34950। & 7.26 nonsex \\
\hline 7 Gg_rs137816 & 176 chr31_34960। & 7.09 nonsex \\
\hline 7 Gg_rs137816 & 176 chr31_34980 & 6.56 nonsex \\
\hline 7 Gg_rs137816 & 176 chr31_35020। & 7.15 nonsex \\
\hline 7 Gg_rs137816 & 176 chr31_35030। & 6.52 nonsex \\
\hline 7 Gg_rs137816 & 176 chr31_35320। & 6.94 nonsex \\
\hline 7 Gg_rs137816 & 176 chr31_35680 & 6.32 nonsex \\
\hline 7 Gg_rs137816 & 176 chr31_35800। & 7.97 nonsex \\
\hline 7 Gg_rs137816 & 176 chr31_35830। & 6.97 nonsex \\
\hline 7 Gg_rs137816 & 176 chr31_35920। & 7.38 nonsex \\
\hline 7 Gg_rs137816 & 176 chr31_36190। & 6.92 nonsex \\
\hline 7 Gg_rs137816 & 176 chr31_37310। & 6.3 nonsex \\
\hline 7 Gg_rs137816 & 176 chr31_37450। & 6.54 nonsex \\
\hline 7 Gg_rs137816 & 176 chr31_37550। & 8.57 nonsex \\
\hline 7 Gg_rs137816 & 176 chr31_38220। & 7.32 nonsex \\
\hline 7 Gg_rs137816 & 176 chr31_38450। & 7.25 nonsex \\
\hline 7 Gg_rs137816 & 176 chr31_38680। & 6.29 nonsex \\
\hline 7 Gg_rs137816 & 176 chr31_38890। & 6.73 nonsex \\
\hline 7 Gg_rs137816 & 176 chr31_39100। & 7.47 nonsex \\
\hline 7 Gg_rs137816 & 176 chr31_39550। & 6.04 nonsex \\
\hline 7 Gg_rs137816 & 176 chr31_40560। & 6.88 nonsex \\
\hline 7 Gg_rs137816 & 176 chr31_41210 & 6.36 nonsex \\
\hline 7 Gg_rs137816 & 176 chr31_41260। & 6.74 nonsex \\
\hline 7 Gg_rs137816 & 176 chr31_41390। & 6.64 nonsex \\
\hline 7 Gg_rs137816 & 176 chr31_42040। & 7.51 nonsex \\
\hline 7 Gg_rs137816 & 176 chr31_42070। & 7.15 nonsex \\
\hline 7 Gg_rs137816 & 176 chr31_42160। & 6.33 nonsex \\
\hline 7 Gg_rs137816 & 176 chr31_42240। & 7.23 nonsex \\
\hline 7 Gg_rs137816 & 176 chr31_42480 & 6.63 nonsex \\
\hline 7 Gg_rs137816 & 176 chr31_42490। & 6.75 nonsex \\
\hline
\end{tabular}




\begin{tabular}{|c|c|c|}
\hline 7 Gg_rs137816 & 176 chr31_43010। & 6.72 nonsex \\
\hline 7 Gg_rs137816 & 176 chr31_43120 & 6.56 nonsex \\
\hline 7 Gg_rs137816 & 176 chr31_43510 & 5.95 nonsex \\
\hline 7 Gg_rs137816 & 176 chr31_44090। & 6.1 nonsex \\
\hline 7 Gg_rs137816 & 176 chr31_44400। & 6.88 nonsex \\
\hline 7 Gg_rs137816 & 176 chr31_44500। & 7.02 nonsex \\
\hline 7 Gg_rs137816 & 176 chr31_44540। & 6.21 nonsex \\
\hline 7 Gg_rs137816 & 176 chr31_54800 & 6.92 nonsex \\
\hline 7 Gg_rs137816 & 176 chr31_54950। & 6.54 nonsex \\
\hline 7 Gg_rs137816 & 176 chr31_55060। & 6.89 nonsex \\
\hline 7 Gg_rs137816 & 176 chr31_55350। & 7.37 nonsex \\
\hline 7 Gg_rs137816 & 176 chr31_55500। & 6.74 nonsex \\
\hline 7 Gg_rs137816 & 176 chr31_55760। & 7.17 nonsex \\
\hline 7 Gg_rs137816 & 176 chr31_57170। & 6.45 nonsex \\
\hline 7 Gg_rs137816 & 176 chr31_57280 & 7.35 nonsex \\
\hline 7 Gg_rs137816 & 176 chr31_57480 & 7.08 nonsex \\
\hline 7 Gg_rs137816 & 176 chr31_57630 & 6.23 nonsex \\
\hline 7 Gg_rs137816 & 176 chr31_57670। & 5.95 nonsex \\
\hline 7 Gg_rs137816 & 176 chr31_58130। & 6.52 nonsex \\
\hline 7 Gg_rs137816 & 176 chr31_58360। & 6.84 nonsex \\
\hline 7 Gg_rs137816 & 176 chr31_59270। & 6.96 nonsex \\
\hline 7 Gg_rs137816 & 176 chr31_59480 & 7.16 nonsex \\
\hline 7 Gg_rs137816 & 176 chr31_59670। & 7.42 nonsex \\
\hline 7 Gg_rs137816 & 176 chr31_60360। & 7.24 nonsex \\
\hline 7 Gg_rs137816 & 176 chr31_60690। & 6.35 nonsex \\
\hline 7 Gg_rs137816 & 176 chr33_29320। & 6.4 nonsex \\
\hline 7 Gg_rs137816 & 176 chr33_29390। & 7.49 nonsex \\
\hline 7 Gg_rs137816 & 176 chr33_29430। & 6.9 nonsex \\
\hline 7 Gg_rs137816 & 176 chr33_36360। & 6.54 nonsex \\
\hline 7 Gg_rs137816 & 177 chr31_54600। & 8.96 nonsex \\
\hline 7 Gg_rs137816 & 177 chr31_54790। & 9.71 nonsex \\
\hline 7 Gg_rs137816 & 177 chr31_55020। & 9.32 nonsex \\
\hline 7 Gg_rs137816 & 177 chr31_55300। & 9.47 nonsex \\
\hline 7 Gg_rs137816 & 177 chr31_55430। & 9.27 nonsex \\
\hline 7 Gg_rs137816 & 177 chr31_55470 & 8 nonsex \\
\hline 7 Gg_rs137816 & 177 chr31_55590। & 9.98 nonsex \\
\hline 7 Gg_rs137816 & 177 chr31_55620। & 8.68 nonsex \\
\hline 7 Gg_rs137816 & 177 chr31_55710। & 9.71 nonsex \\
\hline 7 Gg_rs137816 & 177 chr31_54640। & 6.85 nonsex \\
\hline 7 Gg_rs137816 & 177 chr31_54760। & 6.58 nonsex \\
\hline 7 Gg_rs137816 & 178 chr31_54890 & 9.3 nonsex \\
\hline 7 Gg_rs137816 & 178 chr31_55160। & 9.3 nonsex \\
\hline 7 Gg_rs137816 & 178 chr31_55290। & 8.06 nonsex \\
\hline
\end{tabular}




\begin{tabular}{|c|c|c|}
\hline 7 Gg_rs137816 & 178 chr31_55320 & 9.09 nonsex \\
\hline 7 Gg_rs137816 & 178 chr31_55340। & 9.97 nonsex \\
\hline 7 Gg_rs137816 & 178 chr31_55460। & 10.73 nonsex \\
\hline 7 Gg_rs137816 & 178 chr31_55700। & 8.87 nonsex \\
\hline 7 Gg_rs137816 & 178 chr31_55730। & 10.89 nonsex \\
\hline 7 Gg_rs137816 & 178 chr31_55820। & 6.78 nonsex \\
\hline 7 Gg_rs137816 & 179 chr31_54920। & 10.08 nonsex \\
\hline 7 Gg_rs137816 & 179 chr31_55010। & 8.3 nonsex \\
\hline 7 Gg_rs137816 & 179 chr31_55560। & 8.18 nonsex \\
\hline 7 Gg_rs137816 & 179 chr31_55570। & 8.98 nonsex \\
\hline 7 Gg_rs137816 & 179 chr31_55830। & 7.93 nonsex \\
\hline 7 Gg_rs137816 & 180 chr7_193900। & 5.06 nonsex \\
\hline 7 Gg_rs137816 & 180 chr31_55150। & 8.2 nonsex \\
\hline 7 Gg_rs137816 & 180 chr31_54870 & 7.64 nonsex \\
\hline 7 Gg_rs137816 & 180 chr31_55280 & 7 nonsex \\
\hline 7 Gg_rs137816 & 180 chr31_55420। & 7.7 nonsex \\
\hline 7 Gg_rs137816 & 180 chr31_55690। & 7.16 nonsex \\
\hline 7 Gg_rs137816 & 180 chr31_55800। & 6.88 nonsex \\
\hline 7 Gg_rs137816 & 180 chr31_55850। & 7.08 nonsex \\
\hline 7 Gg_rs137816 & 181 chr31_55920। & 7.65 nonsex \\
\hline 7 Gg_rs137816 & 181 chr31_55390। & 6.82 nonsex \\
\hline 7 Gg_rs137816 & 181 chr31_55530। & 6.62 nonsex \\
\hline 7 Gg_rs137816 & 183 chr7_234330। & 5.54 nonsex \\
\hline 7 Gg_rs137816 & 183 chr31_55120। & 6.68 nonsex \\
\hline 7 Gg_rs137816 & 189 chr7_234000। & 5.16 nonsex \\
\hline 7 Gg_rs158674 & 190 chr7_248740। & 5.67 nonsex \\
\hline 7 Gg_rs137816 & 191 chr7_236590। & 3.43 nonsex \\
\hline 7 X7_24150587 & 192 chr7_182110 & 6.37 nonsex \\
\hline 7 X7_24150587 & 192 chr7_228870 & 8.12 sex \\
\hline 7 X7_24150587 & 193 chr7_220180 & 6.02 nonsex \\
\hline 7 Gg_rs158674 & 194 chr7_233700। & 8.48 nonsex \\
\hline 7 X7_24150587 & 195 chr7_251950। & 8.51 sex \\
\hline 7 X7_24150587 & 196 chr7_243140। & 5.71 nonsex \\
\hline 7 X7_24150587 & 196 chr7_245690 & 5.44 nonsex \\
\hline 7 Gg_rs158674 & 196 chr7_241800। & 6.92 nonsex \\
\hline 7 X7_24150587 & 197 chr7_235120। & 8.73 sex \\
\hline 7 X7_24150587 & 197 chr7_228590। & 5.06 nonsex \\
\hline 7 X7_24150587 & 197 chr2_789160। & 9.25 sex \\
\hline 7 X7_24150587 & 198 chr7_238400। & 6.55 sex \\
\hline 7 X7_24150587 & 199 chr7_240940। & 6.75 sex \\
\hline 7 X7_24150587 & 199 chr7_242980 & 6.34 nonsex \\
\hline 7 Gg_rs158650 & 199 chr7_236050। & 7.75 nonsex \\
\hline 7 X7_24150587 & 200 chr7_247310 & 6.17 sex \\
\hline
\end{tabular}




\begin{tabular}{|c|c|c|c|}
\hline 7 X7 24150587 & 200 chr7 251790 & 5.5 nonsex & cis \\
\hline 7 X7_24150587 & 201 chr7_233350। & 6.14 nonsex & cis \\
\hline 7 X7_24150587 & 201 chr7_235300। & 11.61 nonsex & cis \\
\hline 7 X7_24150587 & 201 chr7_245810 & 5.25 nonsex & cis \\
\hline 7 X7_24150587 & 201 chr7_233880 & 4.9 nonsex & cis \\
\hline 7 X7_24150587 & 202 chr7_228380। & 5.88 nonsex & cis \\
\hline 7 X7_24150587 & 202 chr7_228730। & 5.75 nonsex & cis \\
\hline 7 X7_24150587 & 202 chr7_234870 & 7.17 nonsex & cis \\
\hline 7 X7_24150587 & 203 chr7_232900। & 11.23 nonsex & cis \\
\hline 7 X7_24150587 & 203 chr7_235200। & 10.37 nonsex & cis \\
\hline 7 Gg_rs158674 & 205 chr7_242740 & 7.68 sex & cis \\
\hline 7 X7_24150587 & 207 chr7_253070। & 5.21 nonsex & cis \\
\hline 7 Gg_rs158674 & 207 chr7_253130। & 5.01 nonsex & cis \\
\hline 7 X7_24150587 & 208 chr7_238860। & 6.11 nonsex & cis \\
\hline 7 X7_24150587 & 209 chr7_249520। & 7.71 sex & cis \\
\hline 7 X7_24150587 & 209 chr7_255440। & 8.72 sex & cis \\
\hline 7 Gg_rs158650 & 209 chr7_250640। & 4.67 nonsex & cis \\
\hline 7 X7_24150587 & 210 chr7_242780 & 6.84 sex & cis \\
\hline 7 Gg_rs158650 & 210 chr7_243860। & 7.76 sex & cis \\
\hline 7 Gg_rs158650 & 210 chr7_244240। & 5.2 nonsex & cis \\
\hline 7 Gg_rs158650 & 210 chr1_944630। & $7.4 \mathrm{sex}$ & trans \\
\hline 7 Gg_rs158650 & 211 chr7_238750 & 4.97 nonsex & cis \\
\hline 7 Gg_rs158650 & 211 chr7_239370। & 10.83 sex & cis \\
\hline 7 Gg_rs158650 & 211 chr7_249960। & 8.04 nonsex & cis \\
\hline 7 Gg_rs158650 & 211 chr7_248590। & 4.3 nonsex & cis \\
\hline 7 Gg_rs158650 & 211 chr7_251130 & 4.73 nonsex & cis \\
\hline 7 Gg_rs158674 & 211 chr7_259090। & 5.34 nonsex & cis \\
\hline 7 Gg_rs158650 & 212 chr7_235580 & 4.92 nonsex & cis \\
\hline 7 Gg_rs158650 & 212 chr7_241310 & 7.47 nonsex & cis \\
\hline 7 Gg_rs158650 & 212 chr7_247980 & 8.18 sex & cis \\
\hline 7 Gg_rs158650 & 212 chr7_248570 & 11.97 sex & cis \\
\hline 7 Gg_rs158650 & 212 chr7_249110 & 5.69 nonsex & cis \\
\hline 7 Gg_rs158650 & 212 chr7_250740। & 5.52 nonsex & cis \\
\hline 7 Gg_rs158650 & 212 chr7_251510 & 11.49 nonsex & cis \\
\hline 7 Gg_rs158650 & 212 chr7_249230। & 5.05 nonsex & cis \\
\hline 7 Gg_rs158650 & 213 chr7_235750। & 8.21 nonsex & cis \\
\hline 7 Gg_rs158650 & 213 chr7_240170 & 11.96 nonsex & cis \\
\hline 7 Gg_rs158650 & 213 chr7_241470। & 4.76 nonsex & cis \\
\hline 7 Gg_rs158674 & 214 chr7_235310 & 6.03 nonsex & cis \\
\hline 7 Gg_rs158674 & 214 chr7_240450। & 5.1 nonsex & cis \\
\hline 7 Gg_rs158674 & 214 chr7_241020 & 5.08 nonsex & cis \\
\hline 7 Gg_rs158674 & 214 chr7_241320 & 8.99 nonsex & cis \\
\hline 7 Gg_rs158674 & 214 chr7_241590। & 5.28 nonsex & cis \\
\hline
\end{tabular}




\begin{tabular}{|c|c|c|}
\hline 7 Gg_rs158674 & 214 chr7_243500। & 4.59 nonsex \\
\hline 7 Gg_rs158674 & 214 chr7_245620 & 8.92 nonsex \\
\hline 7 Gg_rs158674 & 214 chr7_245880 & $6.23 \mathrm{sex}$ \\
\hline 7 Gg_rs158674 & 214 chr7_248560। & 10.31 nonsex \\
\hline 7 Gg_rs158674 & 214 chr7_250580 & 5.49 sex \\
\hline 7 Gg_rs158674 & 214 chr7_253410 & 7.86 nonsex \\
\hline 7 Gg_rs158674 & 214 chr7_254910। & 7.92 nonsex \\
\hline 7 Gg_rs158674 & 214 chr7_241370। & 4.39 nonsex \\
\hline 7 Gg_rs158674 & 214 chr7_245630 & 3.37 nonsex \\
\hline 7 Gg_rs158674 & 214 chr7_245640। & 4.53 nonsex \\
\hline 7 Gg_rs158674 & 214 chr7_246020। & 4.19 nonsex \\
\hline 7 Gg_rs158674 & 214 chr7_246820 & 3.66 nonsex \\
\hline 7 Gg_rs158674 & 215 chr7_236330। & 6.04 nonsex \\
\hline 7 Gg_rs158674 & 215 chr7_243540 & 9.31 nonsex \\
\hline 7 Gg_rs158674 & 215 chr7_250470। & 5.57 nonsex \\
\hline 7 Gg_rs158674 & 215 chr7_253490। & 3.95 nonsex \\
\hline 7 Gg_rs158674 & 216 chr7_241430। & 8.17 nonsex \\
\hline 7 Gg_rs158674 & 216 chr7_236040। & 8.1 nonsex \\
\hline 7 Gg_rs158674 & 216 chr7_238580। & 6.48 nonsex \\
\hline 7 Gg_rs158674 & 216 chr7_238760। & 6.62 sex \\
\hline 7 Gg_rs158674 & 216 chr7_242020। & 10.19 nonsex \\
\hline 7 Gg_rs158674 & 216 chr7_242040। & 33.31 nonsex \\
\hline 7 Gg_rs158674 & 216 chr7_246010 & 4.9 nonsex \\
\hline 7 Gg_rs158674 & 216 chr7_253400। & 7.14 nonsex \\
\hline 7 Gg_rs158674 & 216 chr7_253540। & 5.05 nonsex \\
\hline 7 Gg_rs158650 & 217 chr7_245830। & 5.83 nonsex \\
\hline 7 Gg_rs158650 & 217 chr7_251960। & 14.27 nonsex \\
\hline 7 Gg_rs158674 & 217 chr7_236790। & 6.79 sex \\
\hline 7 Gg_rs158674 & 217 chr7_238720। & 8.77 sex \\
\hline 7 Gg_rs158674 & 217 chr7_241180 & 11.17 nonsex \\
\hline 7 Gg_rs158674 & 217 chr7_250550 & 5.49 nonsex \\
\hline 7 Gg_rs158674 & 217 chr7_253060। & 6.77 nonsex \\
\hline 7 Gg_rs158674 & 217 chr7_242820 & 4.55 nonsex \\
\hline 7 Gg_rs158674 & 218 chr7_249010। & 6.38 nonsex \\
\hline 7 Gg_rs158674 & 218 chr7_237140। & 6.13 nonsex \\
\hline 7 Gg_rs158674 & 218 chr7_239030। & 5.52 nonsex \\
\hline 7 Gg_rs158674 & 218 chr7_242050। & 8.87 nonsex \\
\hline 7 Gg_rs158674 & 218 chr7_242490। & 22.3 nonsex \\
\hline 7 Gg_rs158674 & 218 chr7_244710 & 9.61 nonsex \\
\hline 7 Gg_rs158674 & 218 chr7_246640। & 5.97 nonsex \\
\hline 7 Gg_rs158674 & 218 chr7_250950। & 18.23 nonsex \\
\hline 7 Gg_rs158674 & 218 chr7_253530। & 5.65 nonsex \\
\hline 7 Gg_rs158674 & 218 chr7_254140 & 9.91 nonsex \\
\hline
\end{tabular}




\begin{tabular}{|c|c|c|}
\hline 7 Gg_rs158674 & 218 chr7_254900। & 8.86 nonsex \\
\hline 7 Gg_rs158674 & 218 chr7_246390। & 4.27 nonsex \\
\hline 7 X7_24150587 & 219 chr7_236800 & 7.5 sex \\
\hline 7 X7_24150587 & 219 chr7_241390। & 5.56 nonsex \\
\hline 7 Gg_rs158650 & 219 chr7_258630। & 7.01 nonsex \\
\hline 7 Gg_rs158650 & 219 chr7_237920। & 4.89 nonsex \\
\hline 7 Gg_rs158674 & 219 chr7_254890। & 6.3 nonsex \\
\hline 7 Gg_rs158674 & 219 chr7_240860। & 7.95 nonsex \\
\hline 7 Gg_rs158674 & 219 chr7_240950। & 9.13 nonsex \\
\hline 7 Gg_rs158674 & 219 chr7_246690। & 5.95 nonsex \\
\hline 7 Gg_rs158674 & 219 chr7_240160। & 3.91 nonsex \\
\hline 7 X7_24150587 & 220 chr7_237270। & 6.93 nonsex \\
\hline 7 X7_24150587 & 220 chr7_245910। & 6.56 nonsex \\
\hline 7 X7_24150587 & 220 chr7_243970। & 4.96 nonsex \\
\hline 7 X7_24150587 & 220 chr7_246440। & 5.52 nonsex \\
\hline 7 X7_24150587 & 220 chr7_254350। & 4.59 nonsex \\
\hline 7 Gg_rs158650 & 220 chr7_239380। & 9.53 nonsex \\
\hline 7 Gg_rs158650 & 220 chr7_244250। & 7.51 nonsex \\
\hline 7 Gg_rs158674 & 220 chr7_246430। & 5.76 nonsex \\
\hline 7 Gg_rs158674 & 220 chr7_247540। & 6.34 nonsex \\
\hline 7 Gg_rs158674 & 220 chr7_250650। & 7.53 nonsex \\
\hline 7 Gg_rs158674 & 220 chr7_255810 & 5.52 nonsex \\
\hline 7 Gg_rs158674 & 220 chr7_245070। & 4.92 nonsex \\
\hline 7 Gg_rs158674 & 220 chr7_246630। & 5.38 nonsex \\
\hline 7 Gg_rs158674 & 220 chr7_256040। & 4.86 nonsex \\
\hline 7 Gg_rs146200 & 220 chr7_266430। & 4.27 nonsex \\
\hline 7 X7_24150587 & 221 chr7_235030। & 5.76 nonsex \\
\hline 7 X7_24150587 & 221 chr7_236500। & 7.43 nonsex \\
\hline 7 X7_24150587 & 221 chr7_251490। & 6.82 nonsex \\
\hline 7 X7_24150587 & 221 chr7_255230। & 6.51 nonsex \\
\hline 7 X7_24150587 & 221 chr7_235960। & 5.49 nonsex \\
\hline 7 X7_24150587 & 221 chr7_237230। & 4.61 nonsex \\
\hline 7 X7_24150587 & 221 chr7_237240। & 4.82 nonsex \\
\hline 7 Gg_rs158650 & 221 chr7_254360। & 7.93 nonsex \\
\hline 7 Gg_rs158674 & 221 chr7_237970। & 7.92 nonsex \\
\hline 7 Gg_rs158674 & 221 chr7_239010। & 9.88 nonsex \\
\hline 7 Gg_rs158674 & 221 chr7_240290। & 7.75 nonsex \\
\hline 7 Gg_rs158674 & 221 chr7_236190। & 5.48 nonsex \\
\hline 7 Gg_rs158674 & 221 chr7_237290। & 6.78 nonsex \\
\hline 7 Gg_rs158674 & 221 chr7_239020। & 5.06 nonsex \\
\hline 7 Gg_rs146200 & 221 chr7_244610 & 6.75 nonsex \\
\hline 7 Gg_rs146200 & 221 chr7_249630। & 5.67 nonsex \\
\hline 7 X7_24150587 & 222 chr7_137270। & 4.25 nonsex \\
\hline
\end{tabular}




\begin{tabular}{|c|c|c|c|}
\hline 7 X7_24150587 & 222 chr7_261630। & 5.83 nonsex & cis \\
\hline 7 Gg_rs146200 & 222 chr7_237980। & 6.62 sex & cis \\
\hline 7 Gg_rs146200 & 222 chr7_246090। & 6.98 nonsex & cis \\
\hline 7 Gg_rs146200 & 222 chr7_256610 & 6.69 nonsex & cis \\
\hline 7 Gg_rs146200 & 222 chr7_261500। & 5.51 nonsex & cis \\
\hline 7 Gg_rs146200 & 222 chr7_247960। & 5.28 nonsex & cis \\
\hline 7 Gg_rs146200 & 223 chr7_226740। & 7.64 nonsex & cis \\
\hline 7 Gg_rs146200 & 223 chr7_228340। & 6.56 nonsex & cis \\
\hline 7 Gg_rs146200 & 223 chr7_233970। & 6.97 nonsex & cis \\
\hline 7 Gg_rs146200 & 223 chr7_239940। & 7.74 nonsex & cis \\
\hline 7 Gg_rs146200 & 223 chr7_234060। & 5.26 nonsex & cis \\
\hline 7 Gg_rs146200 & 223 chr7_257680। & 6.01 nonsex & cis \\
\hline 7 Gg_rs146200 & 223 chr7_259660। & 5.75 nonsex & cis \\
\hline 7 Gg_rs146200 & 224 chr7_236460। & 6.52 nonsex & cis \\
\hline 7 Gg_rs146200 & 224 chr7_234420। & 5.27 nonsex & cis \\
\hline 7 Gg_rs146200 & 224 chr7_236300। & 5.6 nonsex & cis \\
\hline 7 Gg_rs146200 & 225 chr7_268380। & 5.63 nonsex & cis \\
\hline 7 Gg_rs146200 & 225 chr2_897140। & 8.42 sex & trans \\
\hline 7 Gg_rs146200 & 225 chr9_171800 & 6.19 nonsex & trans \\
\hline 7 Gg_rs146200 & 226 chr7_263340। & 5.25 nonsex & cis \\
\hline 7 Gg_rs146200 & 227 chr7_231900। & 4.84 nonsex & cis \\
\hline 7 Gg_rs146200 & 228 chr7_283470। & 5.54 sex & cis \\
\hline 7 Gg_rs146200 & 228 chr7_289400। & 4.88 nonsex & cis \\
\hline 7 Gg_rs146200 & 229 chr7_269160। & 6.14 nonsex & cis \\
\hline 7 Gg_rs146200 & 229 chr7_292570। & 4.88 nonsex & cis \\
\hline 7 Gg_rs158722 & 230 chr7_236240। & 5.19 nonsex & cis \\
\hline 7 Gg_rs158722 & 230 chr7_269670। & 6.04 nonsex & cis \\
\hline 7 Gg_rs158722 & 230 chr7_282070। & 5.85 nonsex & cis \\
\hline 7 Gg_rs146225 & 230 chr7_280680 & 8.92 nonsex & cis \\
\hline 7 Gg_rs158722 & 231 chr7_268680 & 5.82 sex & cis \\
\hline 7 Gg_rs158722 & 231 chr7_275200। & 6.91 nonsex & cis \\
\hline 7 Gg_rs158722 & 231 chr7_231950। & 5.35 nonsex & cis \\
\hline 7 Gg_rs158722 & 231 chr7_257840। & 5.67 nonsex & cis \\
\hline 7 Gg_rs158722 & 231 chr7_268230। & 5.6 nonsex & cis \\
\hline 7 Gg_rs158722 & 231 chr7_270850 & 5.95 nonsex & cis \\
\hline 7 Gg_rs158722 & 231 chr7_295620। & 5.35 nonsex & cis \\
\hline 7 Gg_rs158722 & 231 chr2_238860। & 8.35 nonsex & trans \\
\hline 7 Gg_rs158722 & 232 chr7_273690। & 8.48 nonsex & cis \\
\hline 7 Gg_rs158722 & 232 chr7_275080 & 4.83 nonsex & cis \\
\hline 7 Gg_rs158722 & 232 chr7_278600 & 5.46 nonsex & cis \\
\hline 7 Gg_rs158722 & 233 chr7_269600। & 6.6 nonsex & cis \\
\hline 7 Gg_rs158722 & 233 chr7_270860। & 7.78 nonsex & cis \\
\hline 7 Gg_rs158722 & 233 chr7_272940। & 5.04 nonsex & cis \\
\hline
\end{tabular}




\begin{tabular}{|c|c|c|c|}
\hline 7 Gg_rs158722 & 233 chr7_290190। & 5.62 nonsex & cis \\
\hline 7 Gg_rs158722 & 233 chr7_303160 & 4.52 nonsex & cis \\
\hline 7 Gg_rs158722 & 233 chr18_45120। & 6.45 nonsex & trans \\
\hline 7 Gg_rs158722 & 233 chr33_28000। & 6.6 nonsex & trans \\
\hline 7 Gg_rs158722 & 233 chr33_28330। & 6.14 nonsex & trans \\
\hline 7 Gg_rs158722 & 234 chr7_267790। & 5.09 nonsex & cis \\
\hline 7 Gg_rs158722 & 234 chr7_277410 & 5.12 nonsex & cis \\
\hline 7 Gg_rs158722 & 234 chrW_63280c & 7.16 sex & trans \\
\hline 7 Gg_rs158722 & 235 chr7_273300। & 7.87 nonsex & cis \\
\hline 7 Gg_rs158722 & 235 chr7_273450। & 6.69 nonsex & cis \\
\hline 7 Gg_rs158722 & 235 chr7_281540 & 6.72 nonsex & cis \\
\hline 7 Gg_rs158722 & 235 chr7_302010 & 4.56 nonsex & cis \\
\hline 7 Gg_rs158722 & 235 chrW_51010 & 8.78 sex & trans \\
\hline 7 Gg_rs158722 & 236 chr7_267500। & 6.57 nonsex & cis \\
\hline 7 Gg_rs158722 & 236 chr7_272230। & 3.2 nonsex & cis \\
\hline 7 Gg_rs158722 & 236 chr7_276260। & 6.03 nonsex & cis \\
\hline 7 Gg_rs158722 & 236 chr7_277170। & 5.81 nonsex & cis \\
\hline 7 Gg_rs158722 & 236 chr7_271340। & 5.32 nonsex & cis \\
\hline 7 Gg_rs158722 & 236 chr7_281930। & 5.03 nonsex & cis \\
\hline 7 Gg_rs158722 & 237 chr7_283180 & 7.67 nonsex & cis \\
\hline 7 Gg_rs158722 & 237 chr7_271930। & 3.36 nonsex & cis \\
\hline 7 Gg_rs158722 & 237 chr7_285560। & 4.62 nonsex & cis \\
\hline 7 Gg_rs158722 & 238 chr7_289110 & 5.26 nonsex & cis \\
\hline 7 Gg_rs158722 & 238 chr7_271200। & 4.89 nonsex & cis \\
\hline 7 Gg_rs158722 & 239 chr7_274930। & 4.76 sex & cis \\
\hline 7 Gg_rs158722 & 239 chr7_292130। & 5.09 nonsex & cis \\
\hline 7 Gg_rs158783. & 239 chr7_296480 & 4.2 nonsex & cis \\
\hline 7 Gg_rs158722 & 240 chr7_273510। & 8.48 sex & cis \\
\hline 7 Gg_rs158722 & 241 chr7_282410। & 6.22 nonsex & cis \\
\hline 7 Gg_rs158722 & 241 chr7_276430। & 4.61 nonsex & cis \\
\hline 7 Gg_rs158722 & 241 chr7_281940। & 4.91 nonsex & cis \\
\hline 7 Gg_rs146225 & 242 chr7_281090। & 7.53 nonsex & cis \\
\hline 7 Gg_rs158722 & 243 chr7_278360। & 3.24 nonsex & cis \\
\hline 7 Gg_rs146225 & 244 chr7_282210। & 5.67 nonsex & cis \\
\hline 7 Gg_rs146225 & 244 chr7_285810 & 4.77 nonsex & cis \\
\hline 7 Gg_rs146225 & 245 chr7_280610 & 6.04 nonsex & cis \\
\hline 7 Gg_rs146225 & 245 chr7_292890। & 5.39 nonsex & cis \\
\hline 7 Gg_rs146225 & 245 chr7_280620। & 4.77 nonsex & cis \\
\hline 7 Gg_rs146225 & 246 chr7_286000। & 4.99 nonsex & cis \\
\hline 7 Gg_rs146225 & 246 chr7_290950। & 5.29 sex & cis \\
\hline 7 Gg_rs146225 & 246 chr7_332830। & 4.73 sex & cis \\
\hline 7 Gg_rs146225 & 246 chrZ_215470। & 5.95 nonsex & trans \\
\hline 7 Gg_rs146225 & 247 chr7_280780 & 5.29 nonsex & cis \\
\hline
\end{tabular}




\begin{tabular}{|c|c|c|c|}
\hline 7 Gg_rs146225 & 247 chr7_239320। & 4.61 nonsex & cis \\
\hline 7 Gg_rs146225 & 247 chr7_249190। & 4.16 nonsex & cis \\
\hline 7 Gg_rs146225 & 247 chr7_278450। & 4.33 nonsex & cis \\
\hline 7 Gg_rs146225 & 247 chr7_282580। & 4.88 nonsex & cis \\
\hline 7 Gg_rs146225 & 247 chr1_331600। & 7.15 sex & trans \\
\hline 7 Gg_rs146225 & 247 chr1_529730 & 5.81 nonsex & trans \\
\hline 7 Gg_rs146225 & 247 chr12_13350। & 5.9 nonsex & trans \\
\hline 7 Gg_rs146225 & 247 chr2_639480 & 6.57 nonsex & trans \\
\hline 7 Gg_rs146225 & 247 chr2_643510 & 5.82 nonsex & trans \\
\hline 7 Gg_rs146225 & 249 chr7_283230। & 6.19 nonsex & cis \\
\hline 7 Gg_rs146225 & 250 chr7_280020। & 3.05 nonsex & cis \\
\hline 7 Gg_rs146225 & 252 chr7_291520। & 5.63 nonsex & cis \\
\hline 7 Gg_rs146225 & 255 chr7_290530। & 7.58 sex & cis \\
\hline 7 Gg_rs158783. & 255 chr7_291760। & 5.45 nonsex & cis \\
\hline 7 Gg_rs158783. & 255 chr7_294520। & 6.51 nonsex & cis \\
\hline 7 Gg_rs158722 & 256 chr7_283970। & 8.06 nonsex & cis \\
\hline 7 Gg_rs158783. & 256 chr7_295390। & 4.93 nonsex & cis \\
\hline 7 Gg_rs158783. & 258 chr7_286200। & 7.73 sex & cis \\
\hline 7 Gg_rs158783. & 258 chr7_289510। & 7.92 nonsex & cis \\
\hline 7 Gg_rs158783. & 259 chr7_298520। & 5.83 nonsex & cis \\
\hline 7 Gg_rs158783. & 260 chr7_296570। & 5.26 nonsex & cis \\
\hline 7 Gg_rs158783. & 261 chr7_286210 & 4.31 nonsex & cis \\
\hline 7 Gg_rs158783. & 261 chr7_288660। & 5.24 nonsex & cis \\
\hline 7 Gg_rs158783. & 261 chr7_293010। & 7.11 nonsex & cis \\
\hline 7 Gg_rs158783. & 262 chr7_290640। & 5.95 nonsex & cis \\
\hline 7 Gg_rs158783. & 262 chr7_295100। & 9.63 nonsex & cis \\
\hline 7 Gg_rs158783. & 262 chr7_295350। & 5.3 nonsex & cis \\
\hline 7 Gg_rs158783. & 262 chr7_286790। & 3.93 nonsex & cis \\
\hline 7 Gg_rs158783. & 262 chr7_291190। & 4.74 nonsex & cis \\
\hline 7 Gg_rs158783. & 263 chr7_292560। & 6.5 sex & cis \\
\hline 7 Gg_rs158783. & 265 chr7_288540। & 5.77 nonsex & cis \\
\hline 7 Gg_rs158783. & 265 chr7_291000। & 8.42 nonsex & cis \\
\hline 7 Gg_rs158783. & 265 chr7_293370। & 6.8 sex & cis \\
\hline 7 Gg_rs158783. & 266 chr7_288000। & 4.27 nonsex & cis \\
\hline 7 Gg_rs158783. & 266 chr7_291580 & 4.22 nonsex & cis \\
\hline 7 Gg_rs158783. & 267 chr7_285990। & 10.24 nonsex & cis \\
\hline 7 Gg_rs158783. & 267 chr7_291700। & 5.34 nonsex & cis \\
\hline 7 Gg_rs158783. & 267 chr7_292260। & 8.46 nonsex & cis \\
\hline 7 Gg_rs158783. & 267 chr7_293000। & 7.59 nonsex & cis \\
\hline 7 Gg_rs158783. & 267 chr7_287010। & 4.9 nonsex & cis \\
\hline 7 Gg_rs158783. & 267 chr7_287270। & 4.37 nonsex & cis \\
\hline 7 Gg_rs158783. & 268 chr7_289720। & 6.65 nonsex & cis \\
\hline 7 Gg_rs158783. & 268 chr7_294700। & 4.77 nonsex & cis \\
\hline
\end{tabular}




\begin{tabular}{|c|c|c|c|}
\hline 7 Gg_rs158783. & 268 chr7_298840। & 3.99 nonsex & cis \\
\hline 7 Gg_rs158783. & 268 chr7_300360। & 4.63 nonsex & cis \\
\hline 7 Gg_rs158783. & 269 chr7_291710 & 10.61 nonsex & cis \\
\hline 7 Gg_rs158808. & 271 chr7_317120। & 3.84 nonsex & cis \\
\hline 7 Gg_rs158808. & 272 chr7_291460। & 7.48 nonsex & cis \\
\hline 7 Gg_rs158808. & 272 chr7_291850 & 8.3 nonsex & cis \\
\hline 7 Gg_rs158808. & 272 chr7_292230। & 6.79 sex & cis \\
\hline 7 Gg_rs158808. & 272 chr7_292740। & 6.59 nonsex & cis \\
\hline 7 Gg_rs158808. & 273 chr7_302170। & 8.85 nonsex & cis \\
\hline 7 Gg_rs158808. & 273 chr7_294550। & 4.65 nonsex & cis \\
\hline 7 Gg_rs158808. & 273 chr7_301580 & 4.72 nonsex & cis \\
\hline 7 Gg_rs158808. & 273 chr7_306600। & 4.2 nonsex & cis \\
\hline 7 Gg_rs158808. & 274 chr7_289740 & 5.92 nonsex & cis \\
\hline 7 Gg_rs158808. & 274 chr7_295490। & 6.73 sex & cis \\
\hline 7 Gg_rs158808. & 274 chr7_320750। & 7.65 nonsex & cis \\
\hline 7 Gg_rs158808. & 275 chr7_320770 & 9.7 nonsex & cis \\
\hline 7 Gg_rs158808. & 275 chr7_302610 & 4.18 nonsex & cis \\
\hline 7 Gg_rs158808. & 276 chr7_298740। & 7.65 sex & cis \\
\hline 7 Gg_rs158808. & 276 chr7_310940। & 5.8 nonsex & cis \\
\hline 7 Gg_rs158808. & 276 chr7_283340। & 4.51 nonsex & cis \\
\hline 7 Gg_rs158808. & 276 chr6_929300। & 8.07 nonsex & trans \\
\hline 7 Gg_rs158808. & 277 chr7_301590। & 6.37 nonsex & cis \\
\hline 7 Gg_rs158808. & 277 chr7_301940। & 6.67 nonsex & cis \\
\hline 7 Gg_rs158808. & 277 chr7_302090। & 4.29 nonsex & cis \\
\hline 7 Gg_rs158808. & 277 chr7_306080 & 4.71 nonsex & cis \\
\hline 7 Gg_rs158808. & 278 chr7_302590। & 5.77 nonsex & cis \\
\hline 7 Gg_rs158808. & 278 chr7_307260। & 7.49 sex & cis \\
\hline 7 Gg_rs158808. & 278 chr7_307920। & 12.33 sex & cis \\
\hline 7 Gg_rs158808. & 278 chr7_293890। & 4.37 nonsex & cis \\
\hline 7 Gg_rs158808. & 278 chr7_297130। & 4.3 nonsex & cis \\
\hline 7 Gg_rs158808. & 278 chr7_304330। & 3.61 nonsex & cis \\
\hline 7 Gg_rs158808. & 278 chr7_307540 & 4.34 nonsex & cis \\
\hline 7 Gg_rs158808. & 278 chr7_307910। & 4.08 nonsex & cis \\
\hline 7 Gg_rs158808. & 278 chr7_308280 & 4.82 nonsex & cis \\
\hline 7 Gg_rs158808. & 278 chr7_312780 & 4.14 nonsex & cis \\
\hline 7 Gg_rs158808. & 278 chr7_313470 & 4.07 nonsex & cis \\
\hline 7 Gg_rs158808. & 278 chr7_320240। & 4.31 nonsex & cis \\
\hline 7 Gg_rs158808. & 278 chr5_446230 & 5.88 nonsex & trans \\
\hline 7 Gg_rs158808. & 279 chr7_249990। & 4.34 nonsex & cis \\
\hline 7 Gg_rs158808. & 284 chr7_313680 & 4.66 nonsex & cis \\
\hline 7 Gg_rs158808. & 284 chr7_320710 & 15.38 nonsex & cis \\
\hline 7 Gg_rs158808. & 285 chr7_320760। & 10.03 nonsex & cis \\
\hline 7 Gg_rs158808. & 286 chr7_312660। & 8.08 nonsex & cis \\
\hline
\end{tabular}




\begin{tabular}{|c|c|c|c|}
\hline 7 Gg_rs158808. & 286 chr7_320690। & 7.43 sex & cis \\
\hline 7 Gg_rs158808. & 286 chr7_320700। & 11.8 nonsex & cis \\
\hline 7 Gg_rs158808. & 286 chr7_320780 & 9.12 nonsex & cis \\
\hline 7 Gg_rs158808. & 288 chr7_320730। & 14.43 nonsex & cis \\
\hline 7 Gg_rs158808. & 288 chr7_320740 & 13.34 nonsex & cis \\
\hline 7 Gg_rs158808. & 291 chr7_320720। & 12.08 nonsex & cis \\
\hline 7 Gg_rs158808. & 293 chr7_344080। & 4.49 nonsex & cis \\
\hline 7 Gg_rs136003. & 297 chr7_328580। & 8.53 nonsex & cis \\
\hline 7 Gg_rs158883 & 297 chr7_328570। & 7.78 nonsex & cis \\
\hline 7 Gg_rs158883 & 297 chr7_328600। & 6.15 nonsex & cis \\
\hline 7 Gg_rs136003. & 298 chr7_328610 & 8.03 sex & cis \\
\hline 7 Gg_rs136003. & 298 chr7_328620। & 9.1 nonsex & cis \\
\hline 7 Gg_rs158883 & 298 chr7_336630। & 4.74 nonsex & cis \\
\hline 7 Gg_rs136003. & 299 chr7_328630। & 6.78 nonsex & cis \\
\hline 7 Gg_rs158883 & 302 chr7_328640। & 10.18 nonsex & cis \\
\hline 7 Gg_rs136003. & 303 chr7_328560। & 10.17 nonsex & cis \\
\hline 7 Gg_rs136003. & 303 chr7_328590। & 9.1 nonsex & cis \\
\hline 7 Gg_rs136003. & 303 chr7_328660। & 11.58 nonsex & cis \\
\hline 7 Gg_rs136003. & 304 chr7_328650। & 10.6 nonsex & cis \\
\hline 7 Gg_rs136003. & 314 chr7_340410 & 5.83 nonsex & cis \\
\hline 7 Gg_rs158883 & 320 chr7_347280 & 6.47 nonsex & cis \\
\hline 7 Gg_rs158883 & 320 chr11_84800 & 8.37 sex & trans \\
\hline 7 Gg_rs158883 & 320 chr15_53840 & 8.26 sex & trans \\
\hline 7 Gg_rs158883| & 321 chr7_347270। & 7.51 nonsex & cis \\
\hline 7 Gg_rs158883 & 324 chr7_346100। & 4.55 nonsex & cis \\
\hline 7 Gg_rs158883 & 325 chr1_100686 & 6.63 nonsex & trans \\
\hline 7 Gg_rs158905. & 329 chr7_348470। & 7.41 nonsex & cis \\
\hline 7 Gg_rs136003. & 330 chr7_353290। & 5.33 nonsex & cis \\
\hline 7 Gg_rs158905. & 334 chr7_355300। & 6.91 nonsex & cis \\
\hline 8 RBL4827 & 0 chr8_243200 & 7.54 sex & cis \\
\hline 8 RBL4827 & 0 chr8_358300 & 6.77 nonsex & cis \\
\hline 8 RBL4827 & 0 chr8_280700। & 4.85 nonsex & cis \\
\hline 8 RBL4827 & 0 chr8_324100। & 4.54 nonsex & cis \\
\hline 8 RBL4827 & 0 chr8_339300। & 5.1 nonsex & cis \\
\hline 8 RBL4827 & 0 chrZ_3635201 & 8.32 sex & trans \\
\hline 8 RBL4827 & 0 chr2_256490। & 7.86 sex & trans \\
\hline 8 RBL4827 & 0 chr2_688800। & 7.41 sex & trans \\
\hline 8 RBL4827 & 0 chr1_159382। & 5.85 nonsex & trans \\
\hline 8 RBL4827 & 0 chr1_162132 & 5.17 nonsex & trans \\
\hline 8 RBL4827 & 0 chr3_707890 & 5.86 nonsex & trans \\
\hline 8 RBL4827 & 0 chr3_744580 & 6.27 nonsex & trans \\
\hline 8 RBL4827 & 1 chr8_222700 & 4.92 nonsex & cis \\
\hline 8 RBL4827 & 3 chr8_664000 & 6.03 sex & cis \\
\hline
\end{tabular}




\begin{tabular}{|c|c|c|c|}
\hline 8 RBL4827 & 3 chr8_373300। & 9.71 nonsex & cis \\
\hline 8 RBL4827 & 3 chr3_889570 & 7.05 nonsex & trans \\
\hline 8 RBL4827 & 3 chr4_633200 & 7.31 nonsex & trans \\
\hline 8 RBL4827 & 4 chr8_324600 & 6.44 nonsex & cis \\
\hline 8 RBL4827 & 4 chr8_357900 & 4.87 nonsex & cis \\
\hline 8 RBL4827 & 5 chrZ_5981701 & 5.85 nonsex & trans \\
\hline 8 RBL4827 & 6 chr1_269310 & 6.35 nonsex & trans \\
\hline 8 RBL4827 & 6 chr1_165153। & 6.11 nonsex & trans \\
\hline 8 RBL4827 & 7 chr8_345700 & 5.92 nonsex & cis \\
\hline 8 RBL4827 & 7 chr2_134658। & 6.65 nonsex & trans \\
\hline 8 RBL4827 & 9 chr1_143192 & 6.99 nonsex & trans \\
\hline 8 RBL4827 & 12 chr8_270800 & 3.84 nonsex & cis \\
\hline 8 Gg_rs146387 & 51 chr8_131170 & 5.45 nonsex & cis \\
\hline 8 Gg_rs146387 & 53 chr8_152980 & 5.45 nonsex & cis \\
\hline 8 Gg_rs146387 & 54 chr8_120460 & 6.8 sex & cis \\
\hline 8 Gg_rs146387 & 55 chr8_189070। & 5.16 nonsex & cis \\
\hline 8 Gg_rs146387 & 55 chrZ_3643301 & 9.24 sex & trans \\
\hline 8 Gg_rs146387 & 55 chr1_881680 & 8.64 sex & trans \\
\hline 8 Gg_rs146387 & 55 chrZ_6019901 & 9.44 sex & trans \\
\hline 8 Gg_rs146387 & 56 chr1_175952। & 8.07 sex & trans \\
\hline 8 Gg_rs146387 & 58 chr8_890500 & 7.11 sex & cis \\
\hline 8 Gg_rs146387 & 58 chrZ_5095701 & 8.09 sex & trans \\
\hline 8 Gg_rs146387 & 59 chrZ_6967401 & 8.56 sex & trans \\
\hline 8 chr8_20.5 & 59 chr8_766700। & 3.65 nonsex & cis \\
\hline 8 Gg_rs146387 & 60 chr8_808400। & 5.5 sex & cis \\
\hline 8 Gg_rs146387 & 61 chrZ_2786401 & 9.99 sex & trans \\
\hline 8 Gg_rs146387 & 61 chr1_126894! & 5.25 nonsex & trans \\
\hline 8 Gg_rs146387 & 65 chrZ_724500। & 7.47 sex & trans \\
\hline 8 Gg_rs146387 & 68 chr8_143830 & 5.22 nonsex & cis \\
\hline 8 Gg_rs146387 & 69 chr8_196760। & 5.48 sex & cis \\
\hline 8 Gg_rs146387 & 69 chr8_218150 & 6.1 sex & cis \\
\hline 8 Gg_rs146387 & 69 chr8_828700। & 4.53 nonsex & cis \\
\hline 8 Gg_rs146387 & 69 chrZ_1829301 & 9.29 sex & trans \\
\hline 8 Gg_rs146387 & 69 chrZ_799550। & 8.42 sex & trans \\
\hline 8 Gg_rs146387 & 69 chr1_101959| & 5.56 nonsex & trans \\
\hline 8 Gg_rs146387 & 69 chr3_533910। & 5.4 nonsex & trans \\
\hline 8 Gg_rs146387 & 71 chr8_751700 & 3.55 nonsex & cis \\
\hline 8 Gg_rs146387 & 71 chr8_851400 & 4.55 nonsex & cis \\
\hline 8 Gg_rs146387 & 73 chr8_140270 & 4.1 nonsex & cis \\
\hline 8 Gg_rs146387 & 78 chr8_715700। & 4.55 nonsex & cis \\
\hline 8 Gg_rs146387 & 79 chr8_113250 & 4.85 nonsex & cis \\
\hline 8 Gg_rs166258. & 80 chr8_113200 & 5.71 nonsex & cis \\
\hline 8 Gg_rs166258. & 80 chr8_113100 & 5.31 nonsex & cis \\
\hline
\end{tabular}




\begin{tabular}{|c|c|c|c|}
\hline 8 Gg rs166258. & 80 chr8 113110 & 5.26 nonsex & cis \\
\hline 8 Gg_rs166258 & 80 chr8_113130 & 5.1 nonsex & cis \\
\hline 8 Gg_rs166258 & 81 chr8_113320 & 5.83 nonsex & cis \\
\hline 8 Gg_rs166258. & 81 chr8_113410 & 5.82 nonsex & cis \\
\hline 8 Gg_rs166258. & 81 chr8_113910 & 5.63 nonsex & cis \\
\hline 8 Gg_rs166258. & 81 chr8_113790 & 5.71 nonsex & cis \\
\hline 8 Gg_rs166258. & 81 chr8_113170 & 4.88 nonsex & cis \\
\hline 8 Gg_rs166258. & 82 chr8_113120 & 5.36 nonsex & cis \\
\hline 8 Gg_rs166258 & 82 chr8_113260 & 5.57 nonsex & cis \\
\hline 8 Gg_rs166258. & 82 chr8_113310 & 5.66 nonsex & cis \\
\hline 8 Gg_rs166258. & 82 chr8_158910 & 6.66 sex & cis \\
\hline 8 Gg_rs166258. & 82 chr8_113300 & 4.71 nonsex & cis \\
\hline 8 Gg_rs166258 & 82 chr8_113920 & 4.9 nonsex & cis \\
\hline 8 Gg_rs159102 & 82 chr8_846700। & 5.34 sex & cis \\
\hline 8 Gg_rs166258. & 83 chr8_113220 & 5.47 nonsex & cis \\
\hline 8 Gg_rs166258. & 83 chr8_113400 & 5.49 nonsex & cis \\
\hline 8 Gg_rs166258. & 83 chr8_113620 & 5.67 nonsex & cis \\
\hline 8 GG_rs166276 & 83 chr8_168690 & 4.96 sex & cis \\
\hline 8 Gg_rs166258 & 84 chr8_113290 & 5.49 nonsex & cis \\
\hline 8 Gg_rs166258 & 84 chr8_114150 & 4.47 nonsex & cis \\
\hline 8 Gg_rs166258. & 85 chr8_113420 & 6.2 nonsex & cis \\
\hline 8 Gg_rs166258. & 85 chr8_113560 & 7.07 nonsex & cis \\
\hline 8 Gg_rs166258. & 85 chr8_113650। & 6.67 nonsex & cis \\
\hline 8 Gg_rs166258. & 85 chr8_113590। & 4.32 nonsex & cis \\
\hline 8 Gg_rs166258 & 85 chr8_113640 & 4.76 nonsex & cis \\
\hline 8 Gg_rs166258 & 86 chr8_113600 & 5.57 nonsex & cis \\
\hline 8 Gg_rs166258. & 86 chr8_114080 & 4.75 nonsex & cis \\
\hline 8 Gg_rs166258 & 86 chr4_309450। & 9.88 sex & trans \\
\hline 8 Gg_rs166258. & 87 chr8_811300 & 4.31 nonsex & cis \\
\hline 8 Gg_rs166258. & 88 chr4_524620। & 7.26 sex & trans \\
\hline 8 Gg_rs166258 & 88 chrZ_148000 & 7.27 sex & trans \\
\hline 8 Gg_rs166258 & 89 chr8_846500 & 9.81 sex & cis \\
\hline 8 Gg_rs166258. & 89 chr8_846600 & 10.44 sex & cis \\
\hline 8 Gg_rs166258. & 89 chr8_899400। & 10.67 nonsex & cis \\
\hline 8 Gg_rs166258. & 89 chr8_120320 & 5.92 sex & cis \\
\hline 8 Gg_rs166258 & 89 chr8_140580 & 5.63 nonsex & cis \\
\hline 8 Gg_rs166258 & 89 chr8_199340। & 4.11 nonsex & cis \\
\hline 8 Gg_rs166258 & 89 chr8_816400। & 3.84 nonsex & cis \\
\hline 8 Gg_rs166258 & 89 chr8_836200। & 5.16 nonsex & cis \\
\hline 8 Gg_rs166258 & 89 chr8_859400। & 4.43 nonsex & cis \\
\hline 8 Gg_rs166258 & 89 chr8_195140 & 4.06 nonsex & cis \\
\hline 8 GG_rs166276 & 89 chr8_899500। & 19.28 nonsex & cis \\
\hline 8 Gg_rs159102 & 89 chr8_102330। & 13.69 nonsex & cis \\
\hline
\end{tabular}




\begin{tabular}{|c|c|c|c|}
\hline 8 Gg_rs159102 & 89 chr8_899700। & 3.58 nonsex & cis \\
\hline 8 Gg_rs159102 & 89 chr8_110970 & 3.53 nonsex & cis \\
\hline 8 Gg_rs159126 & 89 chr8_125800 & 4.17 nonsex & cis \\
\hline 8 Gg_rs159126 & 89 chr8_195650 & 3.91 nonsex & cis \\
\hline 8 Gg_rs146415 & 90 chr8_815100 & 6.64 nonsex & cis \\
\hline 8 Gg_rs146415 & 90 chr8_846400 & 4.3 nonsex & cis \\
\hline 8 Gg_rs146415 & 90 chr8_879200 & 6.61 nonsex & cis \\
\hline 8 Gg_rs146415 & 90 chr8_161970 & 4.41 nonsex & cis \\
\hline 8 Gg_rs146415 & 90 chr8_127420 & 4.29 nonsex & cis \\
\hline 8 Gg_rs146415 & 90 chr10_12733। & 6.36 sex & trans \\
\hline 8 Gg_rs146415 & 90 chr11_14752। & $6.36 \mathrm{sex}$ & trans \\
\hline 8 Gg_rs159126 & 91 chr8_128850 & 8.24 nonsex & cis \\
\hline 8 Gg_rs159126 & 91 chr8_163660। & 5.41 sex & cis \\
\hline 8 Gg_rs159126 & 91 chr8_878500। & 4.07 nonsex & cis \\
\hline 8 Gg_rs159126 & 91 chr8_139440 & 3.22 nonsex & cis \\
\hline 8 Gg_rs159126 & 91 chr8_149650 & 3.43 nonsex & cis \\
\hline 8 Gg_rs159126 & 91 chr8_183760। & 4.44 nonsex & cis \\
\hline 8 Gg_rs159126 & 92 chr8_131930 & 10.34 nonsex & cis \\
\hline 8 Gg_rs159126 & 92 chr8_136680 & 4.87 nonsex & cis \\
\hline 8 Gg_rs159126 & 92 chr8_144480 & 13.02 nonsex & cis \\
\hline 8 Gg_rs159126 & 92 chr8_144760 & 8.05 nonsex & cis \\
\hline 8 Gg_rs159126 & 92 chr8_762800 & 4.65 nonsex & cis \\
\hline 8 Gg_rs159126 & 92 chr8_158590 & 3.61 nonsex & cis \\
\hline 8 Gg_rs159126 & 92 chr8_158970। & 3.26 nonsex & cis \\
\hline 8 Gg_rs159126 & 92 chr8_159960। & 3.61 nonsex & cis \\
\hline 8 Gg_rs159126 & 92 chr8_164260 & 3.63 nonsex & cis \\
\hline 8 Gg_rs159126 & 92 chr3_935500। & 7.59 sex & trans \\
\hline 8 Gg_rs159126 & 93 chr8_156350 & 11.84 nonsex & cis \\
\hline 8 Gg_rs159126 & 93 chr8_859500। & 4.96 nonsex & cis \\
\hline 8 Gg_rs159126 & 94 chr8_146520। & 7.29 nonsex & cis \\
\hline 8 GG_rs166276 & 95 chr8_154950। & 5.12 nonsex & cis \\
\hline 8 Gg_rs146415 & 95 chr8_136480 & 5.77 nonsex & cis \\
\hline 8 Gg_rs159199. & 97 chr8_912800। & 5.45 nonsex & cis \\
\hline 8 Gg_rs159199، & 97 chr8_146050 & 5.78 nonsex & cis \\
\hline 8 Gg_rs146475 & 97 chr8_139860। & 4.44 nonsex & cis \\
\hline 8 Gg_rs159199. & 98 chr8_102160 & 4.8 nonsex & cis \\
\hline 8 Gg_rs159199. & 99 chr8_982700। & 9.64 nonsex & cis \\
\hline 8 Gg_rs159199. & 99 chr8_141180 & 5.8 nonsex & cis \\
\hline 8 Gg_rs159199. & 100 chr8_166410 & 6.33 sex & cis \\
\hline 8 Gg_rs159199. & 100 chrZ_1475801 & 8.43 sex & trans \\
\hline 8 Gg_rs159199، & 101 chr8_156650 & 6.58 nonsex & cis \\
\hline 8 Gg_rs159199. & 101 chr8_168430। & 5.9 nonsex & cis \\
\hline 8 Gg_rs159199. & 101 chr8_172640। & 5.96 nonsex & cis \\
\hline
\end{tabular}




\begin{tabular}{|c|c|c|c|}
\hline 8 Gg_rs159199. & 101 chr8_179410। & 4.51 nonsex & cis \\
\hline 8 Gg_rs159199. & 101 chr8_181610 & 6.52 nonsex & cis \\
\hline 8 Gg_rs159199. & 101 chr8_181620। & 9.85 nonsex & cis \\
\hline 8 Gg_rs159199. & 101 chr8_141080 & 4.41 nonsex & cis \\
\hline 8 Gg_rs159199. & 101 chr8_180300 & 4.38 nonsex & cis \\
\hline 8 Gg_rs159199. & 101 chr8_184750 & 4.46 nonsex & cis \\
\hline 8 Gg_rs159199. & 101 chr8_185860। & 5.25 nonsex & cis \\
\hline 8 Gg_rs159199. & 101 chr8_189210 & 5.19 nonsex & cis \\
\hline 8 Gg_rs159199. & 102 chr8_159330 & 7.96 nonsex & cis \\
\hline 8 Gg_rs159199. & 102 chr8_167980 & 6.98 nonsex & cis \\
\hline 8 Gg_rs159199. & 102 chr8_188690। & 4.94 nonsex & cis \\
\hline 8 Gg_rs159199. & 102 chr8_190610 & 4.12 nonsex & cis \\
\hline 8 Gg_rs159199. & 102 chr8_199840 & 5.16 nonsex & cis \\
\hline 8 Gg_rs159199. & 103 chr8_930100। & 7.01 nonsex & cis \\
\hline 8 Gg_rs159199. & 103 chr8_982800। & 7.56 nonsex & cis \\
\hline 8 Gg_rs159199. & 103 chr8_180080 & 4.51 nonsex & cis \\
\hline 8 Gg_rs146475 & 103 chr8_177650 & 5.36 nonsex & cis \\
\hline 8 Gg_rs146475 & 103 chr8_183500 & 4.92 nonsex & cis \\
\hline 8 Gg_rs146475 & 103 chr8_185870 & 6.44 nonsex & cis \\
\hline 8 Gg_rs146475 & 103 chr8_188380 & 13.84 nonsex & cis \\
\hline 8 Gg_rs146475 & 103 chr8_188390। & 21.62 nonsex & cis \\
\hline 8 Gg_rs146475 & 103 chr8_188400 & 35.85 nonsex & cis \\
\hline 8 Gg_rs146475 & 103 chr8_191150 & 5.16 nonsex & cis \\
\hline 8 Gg_rs146475 & 103 chr8_159560। & 4.47 nonsex & cis \\
\hline 8 Gg_rs146475 & 103 chr8_162450 & 5.07 nonsex & cis \\
\hline 8 chr8_20.5 & 103 chr8_144500 & 4.27 nonsex & cis \\
\hline 8 chr8_20.5 & 103 chr8_146620 & 4.67 nonsex & cis \\
\hline 8 Gg_rs146475 & 104 chr8_857500। & 5.32 nonsex & cis \\
\hline 8 Gg_rs146475 & 104 chr8_930000। & 5.64 nonsex & cis \\
\hline 8 Gg_rs146475 & 104 chr8_188350। & 35.54 nonsex & cis \\
\hline 8 Gg_rs146475 & 104 chr8_188370 & 34.59 nonsex & cis \\
\hline 8 Gg_rs146475 & 104 chr8_189480 & 5.02 nonsex & cis \\
\hline 8 Gg_rs146475 & 104 chr8_195320। & 7.11 nonsex & cis \\
\hline 8 Gg_rs146475 & 104 chr8_865600। & 4.36 nonsex & cis \\
\hline 8 Gg_rs146475 & 104 chr8_998600। & 4.99 nonsex & cis \\
\hline 8 Gg_rs146475 & 104 chr8_194220 & 4.94 nonsex & cis \\
\hline 8 Gg_rs146475 & 104 chr8_195430 & 5.03 nonsex & cis \\
\hline 8 Gg_rs146475 & 104 chrZ_6483901 & 7.5 sex & trans \\
\hline 8 Gg_rs146475 & 105 chr8_191140 & 4.72 nonsex & cis \\
\hline 8 Gg_rs146475 & 105 chr8_195380 & 6.84 nonsex & cis \\
\hline 8 Gg_rs146475 & 105 chr8_194200 & 5.33 nonsex & cis \\
\hline 8 Gg_rs146475 & 105 chr8_199240 & 5.03 nonsex & cis \\
\hline 8 Gg_rs146475 & 105 chrZ_314700। & 7.59 sex & trans \\
\hline
\end{tabular}




\begin{tabular}{|c|c|c|c|}
\hline 8 Gg_rs146475 & 105 chrZ_7250701 & 7.77 sex & trans \\
\hline 8 Gg_rs146475 & 106 chr8_188360 & 23.19 nonsex & cis \\
\hline 8 Gg_rs146475 & 106 chr8_326000 & 5.05 nonsex & trans \\
\hline 8 Gg_rs146475 & 108 chr8_205120। & 4.8 sex & cis \\
\hline 8 Gg_rs146475 & 109 chr8_146510 & 4.81 nonsex & cis \\
\hline 8 Gg_rs146475 & 110 chr8_177490 & 4.6 nonsex & cis \\
\hline 8 Gg_rs146475 & 110 chr8_196410 & 4.42 nonsex & cis \\
\hline 8 Gg_rs146475 & 111 chr8_194800 & 7.21 sex & cis \\
\hline 8 chr8_20.5 & 114 chr8_194920 & 5.22 nonsex & cis \\
\hline 8 chr8_20.5 & 114 chr8_124440 & 4.24 nonsex & cis \\
\hline 8 chr8_20.5 & 115 chrZ_626810। & 7.19 sex & trans \\
\hline 8 chr8_20.5 & 116 chr8_190940। & 5.8 nonsex & cis \\
\hline 8 chr8_20.5 & 116 chr8_193430 & 4.16 nonsex & cis \\
\hline 8 chr8_20.5 & 123 chr8_188340 & 5.7 nonsex & cis \\
\hline 8 chr8_20.5 & 123 chr8_196280 & 4.55 nonsex & cis \\
\hline 8 chr8_20.5 & 123 chr8_232460। & 4.15 nonsex & cis \\
\hline 8 chr8_20.5 & 125 chr8_198550। & 5.12 nonsex & cis \\
\hline 8 chr8_20.5 & 126 chr8_157930 & 5.14 nonsex & cis \\
\hline 8 X8_2162738E & 131 chr8_193240 & 5.07 nonsex & cis \\
\hline 8 X8_2162738€ & 131 chr8_192500 & 5.21 nonsex & cis \\
\hline 8 X8_2162738E & 132 chr8_194070। & 5.68 sex & cis \\
\hline 8 X8_21627386 & 132 chr8_211150 & 6.45 nonsex & cis \\
\hline 8 X8_2162738E & 132 chr8_211180 & 6.37 nonsex & cis \\
\hline 8 X8_2162738E & 132 chr8_157920 & 4.6 nonsex & cis \\
\hline 8 X8_2162738€ & 132 chr8_211190। & 5.48 nonsex & cis \\
\hline 8 X8_21627386 & 132 chrZ_188780। & 7.42 sex & trans \\
\hline 8 X8_21627386 & 133 chr8_211170 & 6.25 nonsex & cis \\
\hline 8 X8_2162738E & 133 chr8_211160। & 5.63 nonsex & cis \\
\hline 8 X8_21627386 & 133 chr8_211200। & 5.37 nonsex & cis \\
\hline 8 X8_2162738€ & 134 chr8_177690 & 5.65 sex & cis \\
\hline 8 X8_2162738€ & 134 chr8_124590। & 4.49 nonsex & cis \\
\hline 8 X8_21627386 & 134 chr8_142090 & 4.93 nonsex & cis \\
\hline 8 X8_2162738€ & 135 chr8_215840 & 3.91 nonsex & cis \\
\hline 8 X8_21627386 & 135 chr8_242340। & 3.58 nonsex & cis \\
\hline 8 X8_2162738E & 135 chr4_429700। & 5.66 nonsex & trans \\
\hline 8 X8_2162738E & 135 chr6_215690 & 6.59 nonsex & trans \\
\hline 8 X8_2162738€ & 137 chr8_197530 & 5.53 sex & cis \\
\hline 8 X8_2162738€ & 137 chr8_215640 & 5.76 nonsex & cis \\
\hline 8 X8_2162738€ & 137 chrZ_3209601 & 5.56 nonsex & trans \\
\hline 8 X8_2162738E & 138 chr1_203010 & 5.13 nonsex & trans \\
\hline 8 X8_2162738E & 139 chr13_24620 & 5.17 nonsex & trans \\
\hline 8 X8_2162738E & 139 chr2_553480 & 5.92 nonsex & trans \\
\hline 8 X8_21627386 & 140 chr8_169120 & 3.78 nonsex & cis \\
\hline
\end{tabular}




\begin{tabular}{|c|c|c|c|}
\hline 8 X8_2162738€ & 143 chr4_403390। & 5.56 sex & trans \\
\hline 8 X8_2162738€ & 147 chr8_238490। & 5.3 nonsex & cis \\
\hline 8 X8_2162738E & 148 chr8_219380 & 4.7 nonsex & cis \\
\hline 8 X8_2162738E & 151 chr2_122102। & 6.07 nonsex & trans \\
\hline 8 X8_2162738E & 152 chr5_112200 & 6.87 nonsex & trans \\
\hline 8 X8_2162738€ & 156 chr8_188320। & 4.58 sex & cis \\
\hline 8 X8_2162738E & 160 chr8_180060। & 5.86 sex & cis \\
\hline 8 Gg_rs146522 & 165 chr8_124050। & $6.91 \mathrm{sex}$ & cis \\
\hline 8 Gg_rs146522 & 166 chr8_238520। & 8.19 sex & cis \\
\hline 8 Gg_rs146522 & 167 chr8_115070। & 6.45 sex & cis \\
\hline 8 Gg_rs146522 & 167 chr7_144040 & 5.84 nonsex & trans \\
\hline 8 Gg_rs146522 & 168 chr8_991000। & 7.25 sex & cis \\
\hline 8 Gg_rs146522 & 168 chr8_248140। & $6.07 \mathrm{sex}$ & cis \\
\hline 8 Gg_rs146522 & 169 chr8_101310 & 5.57 nonsex & cis \\
\hline 8 Gg_rs146522 & 169 chr8_124710 & 6.01 sex & cis \\
\hline 8 Gg_rs146522 & 170 chr8_877300। & 5.79 sex & cis \\
\hline 8 Gg_rs146522 & 172 chr8_886000। & 6.4 sex & cis \\
\hline 8 Gg_rs146522 & 172 chr8_996100। & 5.73 sex & cis \\
\hline 8 Gg_rs146522 & 172 chr8_117070 & 6.08 sex & cis \\
\hline 8 Gg_rs146522 & 172 chr8_119230। & 6.83 sex & cis \\
\hline 8 Gg_rs146522 & 172 chr8_240660। & 7.12 sex & cis \\
\hline 8 Gg_rs146522 & 172 chr8_244690। & 6.72 nonsex & cis \\
\hline 8 Gg_rs146522 & 172 chr8_244710 & 6 sex & cis \\
\hline 8 Gg_rs146522 & 172 chr8_246160। & 6.03 sex & cis \\
\hline 8 Gg_rs146522 & 172 chr8_301080 & 6.41 sex & cis \\
\hline 8 Gg_rs146522 & 172 chr8_867800। & 4.37 nonsex & cis \\
\hline 8 Gg_rs146522 & 172 chr8_191160। & 4.46 nonsex & cis \\
\hline 8 Gg_rs146522 & 172 chr1_236440 & $8 \operatorname{sex}$ & trans \\
\hline 8 Gg_rs146522 & 172 chr1_273210 & 7.25 sex & trans \\
\hline 8 Gg_rs146522 & 172 chr1_277590। & 9.28 sex & trans \\
\hline 8 Gg_rs146522 & 172 chr1_280770 & 7.76 sex & trans \\
\hline 8 Gg_rs146522 & 172 chr1_411900 & 7.62 sex & trans \\
\hline 8 Gg_rs146522 & 172 chr1_417240 & 8.46 sex & trans \\
\hline 8 Gg_rs146522 & 172 chr7_170810 & 6.28 nonsex & trans \\
\hline 8 Gg_rs146522 & 172 chr20_15980 & 5.96 nonsex & trans \\
\hline 8 Gg_rs146522 & 172 chr2_995370। & 5.8 nonsex & trans \\
\hline 8 Gg_rs146522 & 174 chr8_238190। & 11.22 nonsex & cis \\
\hline 8 Gg_rs146522 & 174 chr31_28890। & 6.59 nonsex & trans \\
\hline 8 Gg_rs146522 & 176 chrZ_2865301 & 6.04 nonsex & trans \\
\hline 8 Gg_rs146522 & 179 chrZ_1797701 & 6.24 nonsex & trans \\
\hline 8 Gg_rs146522 & 180 chr8_238450 & 6.58 nonsex & cis \\
\hline 8 X8_29856293 & 236 chr8_294880 & 5.17 sex & cis \\
\hline 8 X8_29856293 & 238 chr8_262550। & 5.28 nonsex & cis \\
\hline
\end{tabular}




\begin{tabular}{|c|c|c|c|}
\hline 8 X8_29856293 & 239 chr8_283980। & 7.37 nonsex & cis \\
\hline 8 X8_29856293 & 242 chr8_298360। & 5.92 nonsex & cis \\
\hline 8 X8_29856293 & 243 chr8_298890। & 7.24 nonsex & cis \\
\hline 8 X8_29856293 & 244 chr8_291560। & 5.85 sex & cis \\
\hline 9 Gg_rs159444 & 0 chr9_158100 & 3.89 nonsex & cis \\
\hline 9 Gg_rs159444 & 0 chr9_225000। & 5.79 nonsex & cis \\
\hline 9 Gg_rs159444 & 0 chr5_532440। & 8.33 sex & trans \\
\hline 9 Gg_rs137413' & 2 chr9_197700 & 4.86 nonsex & cis \\
\hline 9 Gg_rs137413 & 4 chr9_229800 & 5.23 nonsex & cis \\
\hline 9 Gg_rs137413: & 4 chr9_147200 & 3.9 nonsex & cis \\
\hline 9 Gg_rs137413 & 4 chr9_207300। & 4.95 nonsex & cis \\
\hline 9 Gg_rs159444: & 7 chr9_237000। & 5.48 nonsex & cis \\
\hline 9 Gg_rs159444 & 7 chr9_195800 & 7.57 nonsex & cis \\
\hline 9 Gg_rs146603. & 7 chr9_170500 & 5.23 sex & cis \\
\hline 9 Gg_rs146603. & 7 chr9_312300। & 5.01 sex & cis \\
\hline 9 Gg_rs146603. & 8 chr9_252800 & 6.69 nonsex & cis \\
\hline 9 Gg_rs146603. & 8 chr9_265700। & 6.24 nonsex & cis \\
\hline 9 Gg_rs146603. & 8 chr9_270700। & 5.48 nonsex & cis \\
\hline 9 Gg_rs146603. & 8 chr9_170600 & 3.83 nonsex & cis \\
\hline 9 Gg_rs146603. & 8 chr9_260200। & 3.37 nonsex & cis \\
\hline 9 Gg_rs146603. & 8 chr9_265400। & 4.43 nonsex & cis \\
\hline 9 Gg_rs146603. & 8 chr9_280000 & 4.55 nonsex & cis \\
\hline 9 Gg_rs146603. & 21 chr9_285200 & 8.95 nonsex & cis \\
\hline 9 Gg_rs146603. & 21 chr9_285300 & 14.49 nonsex & cis \\
\hline 9 Gg_rs137670 & 22 chr9_311200 & 3.93 nonsex & cis \\
\hline 9 Gg_rs159669 & 26 chr9_307100 & 3.63 nonsex & cis \\
\hline 9 Gg_rs159669 & 33 chr9_347700 & 12.6 nonsex & cis \\
\hline 9 Gg_rs137670 & 39 chr9_378500 & 5.16 nonsex & cis \\
\hline 9 Gg_rs146695. & 46 chr9_119850 & 5.98 sex & cis \\
\hline 9 Gg_rs146695. & 51 chr9_431100 & 6.98 nonsex & cis \\
\hline 9 Gg_rs146695. & 56 chr9_367700 & 6.43 sex & cis \\
\hline 9 Gg_rs146695. & 57 chr9_431900 & 5.43 nonsex & cis \\
\hline 9 Gg_rs146695. & 62 chr9_431800 & 4.41 nonsex & cis \\
\hline 9 Gg_rs137644 & 63 chr9_862100। & 6.41 sex & cis \\
\hline 9 Gg_rs137644 & 65 chr9_868000 & 6.04 nonsex & cis \\
\hline 9 Gg_rs137644 & 70 chr9_689900। & 5.33 nonsex & cis \\
\hline 9 Gg_rs159599 & 71 chr9_453300 & 6.14 nonsex & cis \\
\hline 9 GG_rs159573 & 71 chr9_108150 & 5.27 nonsex & cis \\
\hline 9 GG_rs159573 & 72 chr9_594200। & 5.08 nonsex & cis \\
\hline 9 Gg_rs159599 & 73 chr9_633300। & 4.84 nonsex & cis \\
\hline 9 Gg_rs159599 & 73 chr9_715300 & 4.74 nonsex & cis \\
\hline 9 Gg_rs159599 & 73 chr9_833500। & 4.87 nonsex & cis \\
\hline 9 GG_rs159573 & 73 chr9_594300। & 5.19 nonsex & cis \\
\hline
\end{tabular}




\begin{tabular}{|c|c|c|}
\hline 9 Gg_rs146717 & 73 chr9_867700। & 7.69 nonsex \\
\hline 9 Gg_rs159599 & 75 chr9_603300 & 5.21 nonsex \\
\hline 9 Gg_rs159599 & 76 chr9_630500 & 8.31 nonsex \\
\hline 9 Gg_rs159599 & 78 chr9_804200 & 5.4 nonsex \\
\hline 9 Gg_rs159599 & 78 chr9_566000 & 4.63 nonsex \\
\hline 9 Gg_rs146661 & 79 chr9_677900 & 12.86 nonsex \\
\hline 9 Gg_rs159599 & 80 chr9_629000 & 5.41 nonsex \\
\hline 9 Gg_rs159599 & 81 chr9_571000 & 4.94 nonsex \\
\hline 9 Gg_rs159599 & 81 chr9_612700 & 3.88 nonsex \\
\hline 9 Gg_rs159599 & 82 chr9_558500 & 4.84 nonsex \\
\hline 9 Gg_rs159599 & 82 chr9_113040 & 4.65 nonsex \\
\hline 9 Gg_rs159599 & 83 chr9_594700। & 6.48 nonsex \\
\hline 9 Gg_rs159599 & 83 chr9_644400। & 13.61 nonsex \\
\hline 9 Gg_rs159599 & 84 chr9_376500। & 4.55 nonsex \\
\hline 9 Gg_rs159599 & 86 chr9_433300। & 5.31 nonsex \\
\hline 9 Gg_rs159599 & 86 chr9_545700 & 11.77 nonsex \\
\hline 9 Gg_rs159599 & 86 chr9_545800 & 12.03 nonsex \\
\hline 9 Gg_rs159599 & 86 chr9_545900 & 11.61 nonsex \\
\hline 9 Gg_rs159599 & 86 chr9_546000 & 11.53 nonsex \\
\hline 9 Gg_rs159599 & 86 chr9_546100 & 10.81 nonsex \\
\hline 9 Gg_rs159599 & 86 chr9_546200 & 11.09 nonsex \\
\hline 9 Gg_rs159599 & 86 chr9_546300। & 11.98 nonsex \\
\hline 9 Gg_rs159599 & 86 chr9_546400 & 11.65 nonsex \\
\hline 9 Gg_rs159599 & 86 chr9_546500 & 11.16 nonsex \\
\hline 9 Gg_rs159599 & 86 chr9_546600 & 11.8 nonsex \\
\hline 9 Gg_rs159599 & 86 chr9_575700 & 8.83 nonsex \\
\hline 9 Gg_rs159599 & 86 chr9_575800 & 7.57 nonsex \\
\hline 9 Gg_rs159599 & 86 chr9_579900। & 5.66 nonsex \\
\hline 9 Gg_rs159599 & 86 chr9_589300 & 7.69 nonsex \\
\hline 9 Gg_rs159599 & 86 chr9_605000। & 4.81 nonsex \\
\hline 9 Gg_rs159599 & 86 chr9_612600 & 5.73 nonsex \\
\hline 9 Gg_rs159599 & 86 chr9_630400। & 9 nonsex \\
\hline 9 Gg_rs159599 & 86 chr9_634900। & 8.98 nonsex \\
\hline 9 Gg_rs159599 & 86 chr9_651500। & 5.52 nonsex \\
\hline 9 Gg_rs159599 & 86 chr9_657900 & 11.59 nonsex \\
\hline 9 Gg_rs159599 & 86 chr9_777300 & 5.34 nonsex \\
\hline 9 Gg_rs159599 & 86 chr9_128480 & 4.46 nonsex \\
\hline 9 Gg_rs159599 & 86 chr9_643200 & 3.82 nonsex \\
\hline 9 Gg_rs159599 & 86 chr9_646900 & 3.8 nonsex \\
\hline 9 Gg_rs159599 & 86 chr9_650900 & 3.85 nonsex \\
\hline 9 Gg_rs159599 & 86 chr9_654000 & 3.8 nonsex \\
\hline 9 Gg_rs159599 & 86 chr9_727600 & 3.92 nonsex \\
\hline 9 Gg_rs159599 & 86 chr9_760100 & 4.67 nonsex \\
\hline
\end{tabular}




\begin{tabular}{|c|c|c|c|}
\hline 9 Gg_rs159599 & 86 chr9_120410। & 4.25 nonsex & cis \\
\hline 9 Gg_rs146661 & 87 chr9_641400 & 7.39 nonsex & cis \\
\hline 9 Gg_rs146661 & 87 chr9_646800 & 14.24 nonsex & cis \\
\hline 9 Gg_rs146661 & 87 chr9_678700 & 23.8 nonsex & cis \\
\hline 9 Gg_rs146661 & 87 chr9_618000 & 4.62 nonsex & cis \\
\hline 9 Gg_rs146661 & 87 chr9_768700 & 4.61 nonsex & cis \\
\hline 9 GG_rs159573 & 87 chr9_435500 & 5.43 nonsex & cis \\
\hline 9 GG_rs159573 & 87 chr9_610500 & 5.99 sex & cis \\
\hline 9 GG_rs159573 & 87 chr9_720500 & 7.99 nonsex & cis \\
\hline 9 GG_rs159573 & 87 chr9_835100 & 5.72 nonsex & cis \\
\hline 9 GG_rs159573 & 87 chr9_630800 & 4 nonsex & cis \\
\hline 9 GG_rs159573 & 87 chr9_753500 & 4.45 nonsex & cis \\
\hline 9 GG_rs159573 & 87 chr4_204670 & 7.73 sex & trans \\
\hline 9 Gg_rs159599 & 88 chr9_621300 & 18.59 nonsex & cis \\
\hline 9 Gg_rs146661 & 88 chr9_633900 & 7.04 nonsex & cis \\
\hline 9 Gg_rs146661 & 88 chr9_678500 & 8.03 nonsex & cis \\
\hline 9 Gg_rs146661 & 88 chr9_678600 & 35.03 nonsex & cis \\
\hline 9 Gg_rs146661 & 88 chr9_761600 & 4.47 nonsex & cis \\
\hline 9 GG_rs159573 & 88 chr9_850300 & 7.91 sex & cis \\
\hline 9 GG_rs159573 & 88 chr9_709300 & 4.15 nonsex & cis \\
\hline 9 GG_rs159573 & 88 chr9_794900 & 4.97 nonsex & cis \\
\hline 9 GG_rs159573 & 88 chr9_804100 & 4.51 nonsex & cis \\
\hline 9 Gg_rs159599 & 89 chr9_550300 & 5.35 nonsex & cis \\
\hline 9 Gg_rs146661 & 89 chr9_837300 & 8.6 nonsex & cis \\
\hline 9 Gg_rs146661 & 89 chr9_741700 & 4.03 nonsex & cis \\
\hline 9 GG_rs159573 & 89 chr9_715200 & 8.6 nonsex & cis \\
\hline 9 GG_rs159573 & 89 chr9_672800 & 4.37 nonsex & cis \\
\hline 9 Gg_rs159599 & 90 chr9_570800 & 5.94 nonsex & cis \\
\hline 9 Gg_rs159599 & 90 chr9_580100 & 5.05 sex & cis \\
\hline 9 Gg_rs159599 & 90 chr9_757300 & 5.37 nonsex & cis \\
\hline 9 Gg_rs159599 & 90 chr9_608600 & 4.6 nonsex & cis \\
\hline 9 Gg_rs146661 & 90 chr9_637900 & 5.45 nonsex & cis \\
\hline 9 Gg_rs146661 & 90 chr9_375100 & 3.63 nonsex & cis \\
\hline 9 GG_rs159573 & 90 chr9_592200 & 6.46 nonsex & cis \\
\hline 9 GG_rs159573 & 90 chr9_735600 & 7.96 nonsex & cis \\
\hline 9 GG_rs159573 & 90 chr9_774800 & 5.27 nonsex & cis \\
\hline 9 GG_rs159573 & 90 chr9_619300 & 5.37 nonsex & cis \\
\hline 9 Gg_rs146717 & 90 chr9_851600 & 5.67 nonsex & cis \\
\hline 9 Gg_rs159599 & 91 chr9_575900 & 4.43 nonsex & cis \\
\hline 9 Gg_rs159599 & 91 chr9_845200 & 4.36 nonsex & cis \\
\hline 9 Gg_rs159599 & 91 chr1_260420 & 6.41 nonsex & trans \\
\hline 9 Gg_rs146661 & 91 chr9_605100 & 5.06 nonsex & cis \\
\hline 9 Gg_rs146661 & 91 chr9_690200। & 4.81 nonsex & cis \\
\hline
\end{tabular}




\begin{tabular}{|c|c|c|c|}
\hline 9 GG_rs159573 & 91 chr9_992600। & 5.02 nonsex & cis \\
\hline 9 GG_rs159573 & 91 chr9_729800 & 10.04 nonsex & cis \\
\hline 9 GG_rs159573 & 91 chr9_622000। & 4.26 nonsex & cis \\
\hline 9 GG_rs159573 & 91 chr9_700500 & 4.57 nonsex & cis \\
\hline 9 GG_rs159573 & 91 chr9_790100 & 4.65 nonsex & cis \\
\hline 9 Gg_rs159599 & 92 chr9_645500। & 4.28 nonsex & cis \\
\hline 9 Gg_rs159599 & 92 chr9_647100 & 4.27 nonsex & cis \\
\hline 9 Gg_rs146661 & 92 chr9_816000। & 6.33 nonsex & cis \\
\hline 9 GG_rs159573 & 92 chr9_720200 & 8.08 nonsex & cis \\
\hline 9 GG_rs159573 & 92 chr9_654300 & 6.27 nonsex & cis \\
\hline 9 GG_rs159573 & 92 chr9_734400 & 9.01 nonsex & cis \\
\hline 9 GG_rs159573 & 92 chr9_654200 & 5.36 nonsex & cis \\
\hline 9 GG_rs159573 & 92 chr9_756900 & 4.83 nonsex & cis \\
\hline 9 GG_rs159573 & 92 chr9_796400 & 4.47 nonsex & cis \\
\hline 9 GG_rs159573 & 92 chr9_845100 & 4.24 nonsex & cis \\
\hline 9 Gg_rs137644 & 92 chr9_874500। & 4.33 nonsex & cis \\
\hline 9 Gg_rs146717 & 92 chr9_838500। & 4.49 nonsex & cis \\
\hline 9 Gg_rs159599 & 93 chr9_644700। & 5.42 nonsex & cis \\
\hline 9 GG_rs159573 & 93 chr9_835900। & 8.06 nonsex & cis \\
\hline 9 GG_rs159573 & 93 chr9_728300 & 4.57 nonsex & cis \\
\hline 9 GG_rs159573 & 93 chr9_754600 & 5 nonsex & cis \\
\hline 9 Gg_rs137644 & 93 chr9_110420 & 4.27 nonsex & cis \\
\hline 9 Gg_rs146717 & 93 chr9_644300 & 6.05 nonsex & cis \\
\hline 9 Gg_rs146717 & 93 chr9_108100 & 6.36 nonsex & cis \\
\hline 9 Gg_rs137644 & 94 chr9_106360 & 5.65 nonsex & cis \\
\hline 9 Gg_rs137644 & 94 chr9_885200। & 5.43 nonsex & cis \\
\hline 9 Gg_rs137644 & 94 chr9_751000 & 5.06 nonsex & cis \\
\hline 9 Gg_rs137644 & 94 chr9_104830 & 5.22 nonsex & cis \\
\hline 9 Gg_rs137644 & 94 chr1_913950। & 7.08 nonsex & trans \\
\hline 9 Gg_rs137644 & 95 chr9_509600 & 7.03 nonsex & cis \\
\hline 9 Gg_rs137644 & 95 chr9_644500। & 7.08 nonsex & cis \\
\hline 9 Gg_rs137644 & 95 chr9_882100 & 7.14 sex & cis \\
\hline 9 Gg_rs137644 & 95 chr9_103140। & 8.28 sex & cis \\
\hline 9 Gg_rs137644 & 95 chr9_106500। & 6.44 nonsex & cis \\
\hline 9 Gg_rs137644 & 95 chr9_744600 & 4.42 nonsex & cis \\
\hline 9 Gg_rs137644 & 95 chr9_763800 & 4.27 nonsex & cis \\
\hline 9 Gg_rs137644 & 95 chr9_101720 & 5.17 nonsex & cis \\
\hline 9 Gg_rs137644 & 95 chr9_123910 & 5.71 nonsex & cis \\
\hline 9 Gg_rs137644 & 95 chr9_125730 & 5.54 nonsex & cis \\
\hline 9 Gg_rs137644 & 95 chr9_126070 & 3.99 nonsex & cis \\
\hline 9 Gg_rs137644 & 95 chr2_668120 & 6.91 nonsex & trans \\
\hline 9 Gg_rs137644 & 96 chr9_742100। & 6.16 sex & cis \\
\hline 9 Gg_rs137644 & 96 chr9_869200। & 5.86 nonsex & cis \\
\hline
\end{tabular}




\begin{tabular}{|c|c|c|c|}
\hline 9 Gg rs137644 & 96 chr9 919900। & 7.16 nonsex & cis \\
\hline 9 Gg_rs137644 & 96 chr9_108990 & 11.04 nonsex & cis \\
\hline 9 Gg_rs137644 & 96 chr9_662800 & 5.07 nonsex & cis \\
\hline 9 Gg_rs137644 & 96 chr9_666100 & 4.52 nonsex & cis \\
\hline 9 Gg_rs137644 & 96 chr9_780000 & 4.37 nonsex & cis \\
\hline 9 Gg_rs137644 & 96 chr9_164050 & 5.61 nonsex & cis \\
\hline 9 Gg_rs137644 & 97 chr9_656800 & 5.21 nonsex & cis \\
\hline 9 Gg_rs137644 & 97 chr9_872000 & 6 nonsex & cis \\
\hline 9 Gg_rs137644 & 97 chr9_885900। & 8.08 sex & cis \\
\hline 9 Gg_rs137644 & 97 chr9_903100। & 9.96 nonsex & cis \\
\hline 9 Gg_rs137644 & 97 chr9_905000। & 5.68 nonsex & cis \\
\hline 9 Gg_rs137644 & 97 chr9_913700। & 7.37 nonsex & cis \\
\hline 9 Gg_rs137644 & 97 chr9_927500। & 10.91 nonsex & cis \\
\hline 9 Gg_rs137644 & 97 chr9_105840 & 9.32 nonsex & cis \\
\hline 9 Gg_rs137644 & 97 chr9_105940 & 5.59 sex & cis \\
\hline 9 Gg_rs137644 & 97 chr9_105970 & 5.95 nonsex & cis \\
\hline 9 Gg_rs137644 & 97 chr9_106460 & 6.06 nonsex & cis \\
\hline 9 Gg_rs137644 & 97 chr9_114230 & 7.17 sex & cis \\
\hline 9 Gg_rs137644 & 97 chr9_116100 & 7.59 sex & cis \\
\hline 9 Gg_rs137644 & 97 chr9_122790 & 8.06 nonsex & cis \\
\hline 9 Gg_rs137644 & 97 chr9_871900। & 4.86 nonsex & cis \\
\hline 9 Gg_rs137644 & 97 chr9_874300 & 5.22 nonsex & cis \\
\hline 9 Gg_rs137644 & 97 chr9_908800 & 5.06 nonsex & cis \\
\hline 9 Gg_rs137644 & 97 chr9_969200। & 4.1 nonsex & cis \\
\hline 9 Gg_rs137644 & 97 chr9_991900। & 4.78 nonsex & cis \\
\hline 9 Gg_rs137644 & 97 chr9_100100 & 5.43 nonsex & cis \\
\hline 9 Gg_rs137644 & 97 chr9_101130 & 4.5 nonsex & cis \\
\hline 9 Gg_rs137644 & 97 chr9_104110 & 5.13 nonsex & cis \\
\hline 9 Gg_rs137644 & 97 chr9_109120 & 5.22 nonsex & cis \\
\hline 9 Gg_rs137644 & 97 chr9_113080 & 4.44 nonsex & cis \\
\hline 9 Gg_rs137644 & 97 chr9_114150 & 4.62 nonsex & cis \\
\hline 9 Gg_rs137644 & 97 chr9_120140 & 4.88 nonsex & cis \\
\hline 9 Gg_rs137644 & 97 chr9_122360 & 4.86 nonsex & cis \\
\hline 9 Gg_rs137644 & 97 chr1_318530। & 7.77 sex & trans \\
\hline 9 Gg_rs137644 & 98 chr9_105900 & 7.5 nonsex & cis \\
\hline 9 Gg_rs137644 & 98 chr9_114180 & 10.39 nonsex & cis \\
\hline 9 Gg_rs137644 & 98 chr9_115430 & 8.15 nonsex & cis \\
\hline 9 Gg_rs137644 & 98 chr9_118480 & 8.46 nonsex & cis \\
\hline 9 Gg_rs137644 & 98 chr9_159920। & 6.49 nonsex & cis \\
\hline 9 Gg_rs137644 & 98 chr9_898100। & 5.46 nonsex & cis \\
\hline 9 Gg_rs137644 & 98 chr9_116870 & 4.17 nonsex & cis \\
\hline 9 Gg_rs137644 & 99 chr9_114590 & 5.14 nonsex & cis \\
\hline 9 Gg_rs137644 & 99 chr9_102200। & 5.08 nonsex & cis \\
\hline
\end{tabular}




\begin{tabular}{|c|c|c|c|}
\hline 9 Gg_rs137644 & 99 chr9_115080 & 5.01 nonsex & cis \\
\hline 9 Gg_rs146717 & 100 chr9_834100। & 4.54 nonsex & cis \\
\hline 9 Gg_rs146717 & 100 chr9_108130 & 6.3 nonsex & cis \\
\hline 9 Gg_rs146717 & 100 chr9_110490 & 5.55 nonsex & cis \\
\hline 9 Gg_rs146717 & 100 chr9_100120 & 7.51 sex & cis \\
\hline 9 Gg_rs146717 & 100 chr9_107110 & 5.8 nonsex & cis \\
\hline 9 Gg_rs146717 & 100 chr9_114240 & 5.25 nonsex & cis \\
\hline 9 Gg_rs146717 & 100 chr9_780100। & 5.08 nonsex & cis \\
\hline 9 Gg_rs146717 & 100 chr9_872100 & 4.54 nonsex & cis \\
\hline 9 Gg_rs146717 & 100 chr9_103900 & 5.2 nonsex & cis \\
\hline 9 Gg_rs146717 & 100 chr9_105980 & 4.45 nonsex & cis \\
\hline 9 Gg_rs146717 & 100 chr9_135390 & 4.88 nonsex & cis \\
\hline 9 Gg_rs159733 & 100 chr9_132220। & 5.43 nonsex & cis \\
\hline 9 Gg_rs146717 & 101 chr9_481900 & 5.69 nonsex & cis \\
\hline 9 Gg_rs146717 & 101 chr9_483800। & 6.42 sex & cis \\
\hline 9 Gg_rs146717 & 101 chr9_558400। & 7.72 nonsex & cis \\
\hline 9 Gg_rs146717 & 101 chr9_100090। & 9.47 nonsex & cis \\
\hline 9 Gg_rs146717 & 101 chr9_109610 & 7.9 nonsex & cis \\
\hline 9 Gg_rs146717 & 101 chr9_114870 & 6.83 sex & cis \\
\hline 9 Gg_rs146717 & 101 chr9_126600 & 6.6 nonsex & cis \\
\hline 9 Gg_rs146717 & 101 chr9_132170 & 6.02 sex & cis \\
\hline 9 Gg_rs146717 & 101 chr9_484000। & 5.1 nonsex & cis \\
\hline 9 Gg_rs146717 & 101 chr9_601100 & 4.77 nonsex & cis \\
\hline 9 Gg_rs146717 & 101 chr9_652400। & 4.77 nonsex & cis \\
\hline 9 Gg_rs146717 & 101 chr9_833400। & 4.91 nonsex & cis \\
\hline 9 Gg_rs146717 & 101 chr9_871300। & 5.32 nonsex & cis \\
\hline 9 Gg_rs146717 & 101 chr9_106420 & 4.85 nonsex & cis \\
\hline 9 Gg_rs146717 & 101 chr1_250300। & 6.17 nonsex & trans \\
\hline 9 Gg_rs146717 & 101 chr4_597940। & 6.3 nonsex & trans \\
\hline 9 Gg_rs136083 & 101 chr9_131900 & 4.27 nonsex & cis \\
\hline 9 Gg_rs146717 & 102 chr9_700700 & 5.15 nonsex & cis \\
\hline 9 Gg_rs146717 & 102 chr9_933000। & 5.38 nonsex & cis \\
\hline 9 Gg_rs146717 & 102 chr9_993000। & 6.32 nonsex & cis \\
\hline 9 Gg_rs146717 & 102 chr9_998700। & 4.54 nonsex & cis \\
\hline 9 Gg_rs146717 & 102 chr9_104220 & 5.74 nonsex & cis \\
\hline 9 Gg_rs146717 & 102 chr9_108140 & 12.2 nonsex & cis \\
\hline 9 Gg_rs146717 & 102 chr9_117450। & 4.8 nonsex & cis \\
\hline 9 Gg_rs146717 & 102 chr9_126740 & 10.94 nonsex & cis \\
\hline 9 Gg_rs146717 & 102 chr9_126750 & 6.4 nonsex & cis \\
\hline 9 Gg_rs146717 & 102 chr9_128190। & 8.26 sex & cis \\
\hline 9 Gg_rs146717 & 102 chr9_128210 & 5.83 nonsex & cis \\
\hline 9 Gg_rs146717 & 102 chr9_128580 & 4.94 nonsex & cis \\
\hline 9 Gg_rs146717 & 102 chr9_128640। & 4.51 nonsex & cis \\
\hline
\end{tabular}




\begin{tabular}{|c|c|c|c|}
\hline 9 Gg_rs146717 & 102 chr9_130040। & 6.8 nonsex & cis \\
\hline 9 Gg_rs146717 & 102 chr9_129370 & 6.15 nonsex & cis \\
\hline 9 Gg_rs146717 & 102 chr9_490500। & 4.5 nonsex & cis \\
\hline 9 Gg_rs146717 & 102 chr9_630600 & 4.7 nonsex & cis \\
\hline 9 Gg_rs146717 & 102 chr9_804600 & 4.45 nonsex & cis \\
\hline 9 Gg_rs146717 & 102 chr9_872200। & 4.57 nonsex & cis \\
\hline 9 Gg_rs146717 & 102 chr9_100110 & 4.96 nonsex & cis \\
\hline 9 Gg_rs146717 & 102 chr9_102250 & 4.66 nonsex & cis \\
\hline 9 Gg_rs146717 & 102 chr9_107770 & 4.82 nonsex & cis \\
\hline 9 Gg_rs146717 & 102 chr9_123820 & 4.05 nonsex & cis \\
\hline 9 Gg_rs146717 & 102 chr9_128440 & 4.08 nonsex & cis \\
\hline 9 Gg_rs146717 & 102 chr9_135420 & 4.63 nonsex & cis \\
\hline 9 Gg_rs146717 & 102 chr9_140170 & 4.15 nonsex & cis \\
\hline 9 Gg_rs146717 & 102 chr9_151990। & 4.37 nonsex & cis \\
\hline 9 Gg_rs146717 & 102 chr9_152510 & 4.58 nonsex & cis \\
\hline 9 Gg_rs146717 & 102 chrZ_177100। & 5.43 sex & trans \\
\hline 9 Gg_rs146717 & 102 chrZ_4781201 & 7.49 sex & trans \\
\hline 9 Gg_rs136083: & 102 chr9_127560 & 4.48 nonsex & cis \\
\hline 9 Gg_rs136083 & 102 chr9_147960। & 4.43 nonsex & cis \\
\hline 9 Gg_rs166674 & 105 chr9_924000। & 4.38 nonsex & cis \\
\hline 9 Gg_rs166674 & 105 chr2_243120 & 5.62 nonsex & trans \\
\hline 9 Gg_rs166674 & 106 chr9_114170 & 10.01 nonsex & cis \\
\hline 9 Gg_rs166674 & 110 chr9_110510 & 4.66 nonsex & cis \\
\hline 9 Gg_rs166674 & 111 chr9_917000। & 9.24 nonsex & cis \\
\hline 9 Gg_rs166674 & 112 chr9_923000। & 4.95 nonsex & cis \\
\hline 9 Gg_rs159733 & 112 chr9_137700 & 4.49 nonsex & cis \\
\hline 9 Gg_rs137644 & 113 chr9_888100 & 4.65 nonsex & cis \\
\hline 9 Gg_rs159733 & 113 chr9_122970 & 6.64 nonsex & cis \\
\hline 9 Gg_rs159733 & 114 chr9_132880 & 5.34 nonsex & cis \\
\hline 9 Gg_rs159733 & 115 chr9_134290। & 12.09 nonsex & cis \\
\hline 9 Gg_rs159733 & 115 chr9_134300 & 20.2 nonsex & cis \\
\hline 9 Gg_rs159733 & 115 chr9_134310 & 17.39 nonsex & cis \\
\hline 9 Gg_rs159733 & 115 chr9_134250 & 14.65 nonsex & cis \\
\hline 9 Gg_rs159733 & 116 chr9_134260 & 15.8 nonsex & cis \\
\hline 9 Gg_rs159733 & 117 chr9_144330 & 4.22 nonsex & cis \\
\hline 9 Gg_rs159733 & 118 chr9_134320 & 13.75 nonsex & cis \\
\hline 9 Gg_rs159733 & 118 chr9_137340 & 4.15 nonsex & cis \\
\hline 9 Gg_rs159733 & 118 chr9_146120 & 4.3 nonsex & cis \\
\hline 9 Gg_rs136083 & 120 chr9_556100 & 7.63 sex & cis \\
\hline 9 Gg_rs136083 & 120 chr9_145270 & 5.79 nonsex & cis \\
\hline 9 Gg_rs136083: & 120 chr9_147610 & 7.15 nonsex & cis \\
\hline 9 Gg_rs136083: & 120 chr9_134680 & 3.82 nonsex & cis \\
\hline 9 Gg_rs136083: & 120 chr9_142750। & 3.45 nonsex & cis \\
\hline
\end{tabular}




\begin{tabular}{|c|c|c|c|}
\hline 9 Gg_rs136083: & 121 chrZ_896900। & 6.94 sex & trans \\
\hline 9 Gg_rs136083 & 122 chr9_147590। & 6.3 sex & cis \\
\hline 9 Gg_rs136083: & 125 chr1_816530। & 8.38 sex & trans \\
\hline 9 Gg_rs136083 & 126 chr1_119600 & 7.62 sex & trans \\
\hline 9 Gg_rs136083 & 128 chr9_108190। & 5.47 sex & cis \\
\hline 9 Gg_rs159781. & 134 chr9_131490 & 6.7 sex & cis \\
\hline 9 Gg_rs159781. & 134 chr9_167960। & 4.66 nonsex & cis \\
\hline 9 Gg_rs159781. & 137 chr9_169850 & 7.99 sex & cis \\
\hline 9 Gg_rs159781. & 137 chr9_171930। & 3.87 nonsex & cis \\
\hline 9 Gg_rs159781. & 139 chr9_637600 & 3.59 nonsex & cis \\
\hline 9 Gg_rs159781. & 145 chr9_169150। & 6.86 nonsex & cis \\
\hline 9 Gg_rs159781. & 145 chr9_173830 & 4.22 nonsex & cis \\
\hline 9 Gg_rs159781. & 154 chr9_173820 & 4.96 nonsex & cis \\
\hline 9 Gg_rs159781. & 157 chr9_169190। & 5.11 nonsex & cis \\
\hline 9 X9_20745674 & 171 chr9_173060 & 5.73 nonsex & cis \\
\hline 9 X9_20745674 & 192 chr9_185600 & 4.7 nonsex & cis \\
\hline 9 Gg_rs146817. & 195 chr9_233910 & 4.02 nonsex & cis \\
\hline 9 X9_20745674 & 196 chr9_217170 & 5.75 sex & cis \\
\hline 9 X9_20745674 & 198 chr9_214150। & 6.24 nonsex & cis \\
\hline 9 X9_20745674 & 198 chr9_217650। & 4.39 nonsex & cis \\
\hline 9 X9_20745674 & 200 chr9_222960। & 4.87 nonsex & cis \\
\hline 9 Gg_rs146817. & 204 chr9_183990। & 3.34 nonsex & cis \\
\hline 9 Gg_rs146817. & 206 chr9_222530। & 3.65 nonsex & cis \\
\hline 9 Gg_rs146817. & 207 chr9_222490। & 6.85 nonsex & cis \\
\hline 9 Gg_rs146817. & 207 chr9_222970। & 6.16 nonsex & cis \\
\hline 9 Gg_rs146817. & 207 chr9_216620। & 3.97 nonsex & cis \\
\hline 9 Gg_rs146817. & 207 chr9_220710 & 4.51 nonsex & cis \\
\hline 9 Gg_rs146817. & 207 chr9_222520। & 4.39 nonsex & cis \\
\hline 10 Gg_rs149412 & 0 chr10_11960। & 5.48 nonsex & cis \\
\hline 10 Gg_rs149412 & 0 chr10_12480 & 5.39 nonsex & cis \\
\hline 10 Gg_rs149412 & 0 chr10_12690। & 5.72 nonsex & cis \\
\hline 10 Gg_rs149412 & 0 chr10_11710 & 4.66 nonsex & cis \\
\hline 10 Gg_rs149412 & 0 chr10_12740 & 3.95 nonsex & cis \\
\hline 10 Gg_rs149412 & 6 chr10_12540 & 5.16 nonsex & cis \\
\hline 10 Gg_rs149412 & 9 chr10_12750 & 4.28 nonsex & cis \\
\hline 10 Gg_rs149412 & 11 chr10_11970 & 4.29 nonsex & cis \\
\hline 10 Gg_rs149412 & 11 chr10_12510 & 4.55 nonsex & cis \\
\hline 10 Gg_rs149412 & 13 chr10_12720 & 4.7 nonsex & cis \\
\hline 10 Gg_rs149412 & 14 chr10_12490 & 4.27 nonsex & cis \\
\hline 10 Gg_rs140016 & 85 chr1_111652 & 7.09 nonsex & trans \\
\hline 10 Gg_rs140016 & 87 chr10_40200 & 4.58 nonsex & cis \\
\hline 10 Gg_rs140016 & 94 chr10_44220 & 5.23 nonsex & cis \\
\hline 10 Gg_rs140016 & 96 chr10_35480 & 5.63 nonsex & cis \\
\hline
\end{tabular}




\begin{tabular}{|c|c|c|c|}
\hline 10 Gg_rs140016 & 97 chr10_42740। & 5.4 nonsex & cis \\
\hline 10 Gg_rs140018 & 98 chr10_40300 & 9.71 nonsex & cis \\
\hline 10 Gg_rs140018 & 98 chr10_41950 & 9.75 nonsex & cis \\
\hline 10 Gg_rs140018 & 98 chr10_41960 & 7.78 nonsex & cis \\
\hline 10 Gg_rs140018 & 98 chr10_66560 & 6.6 nonsex & cis \\
\hline 10 Gg_rs140018 & 98 chr10_40510 & 4.56 nonsex & cis \\
\hline 10 Gg_rs140018 & 98 chr10_58180 & 4.87 nonsex & cis \\
\hline 10 Gg_rs140018 & 98 chr10_66810 & 4.55 nonsex & cis \\
\hline 10 Gg_rs140018 & 98 chr2_527600 & 6.82 nonsex & trans \\
\hline 10 Gg_rs140020 & 99 chr10_37500 & 5.82 nonsex & cis \\
\hline 10 Gg_rs140020 & 99 chr10_38590 & 7.39 nonsex & cis \\
\hline 10 Gg_rs140020 & 99 chr10_38600 & 6.98 nonsex & cis \\
\hline 10 Gg_rs140020 & 99 chr10_44750 & 7.16 sex & cis \\
\hline 10 Gg_rs140020 & 99 chr10_50120 & 8.15 sex & cis \\
\hline 10 Gg_rs140020 & 99 chr10_40490। & 4.64 nonsex & cis \\
\hline 10 Gg_rs140020 & 99 chr10_41250 & 4.51 nonsex & cis \\
\hline 10 Gg_rs140020 & 99 chr10_41260 & 4.58 nonsex & cis \\
\hline 10 Gg_rs140020 & 99 chr10_44050 & 4.78 nonsex & cis \\
\hline 10 Gg_rs140020 & 99 chr10_50720 & 5.44 nonsex & cis \\
\hline 10 Gg_rs140020 & 99 chr10_10820 & 4.51 nonsex & cis \\
\hline 10 Gg_rs140020 & 99 chr7_266090। & 6.22 nonsex & trans \\
\hline 10 Gg_rs140020 & 101 chr10_48080 & 24.49 nonsex & cis \\
\hline 10 Gg_rs140018 & 102 chr10_51770 & 5.28 nonsex & cis \\
\hline 10 Gg_rs140018 & 102 chr9_222510 & 6.98 nonsex & trans \\
\hline 10 Gg_rs140018 & 103 chr10_42000 & 3.87 nonsex & cis \\
\hline 10 Gg_rs140018 & 103 chr10_69990। & 5.64 nonsex & cis \\
\hline 10 Gg_rs140020 & 103 chr10_43850 & 6.48 sex & cis \\
\hline 10 Gg_rs140018 & 104 chr10_79540। & 6.76 nonsex & cis \\
\hline 10 Gg_rs140020 & 104 chr10_48090। & 30.99 nonsex & cis \\
\hline 10 Gg_rs140018 & 105 chr10_70600 & 6.44 nonsex & cis \\
\hline 10 Gg_rs140018 & 105 chr10_68490। & 5.54 nonsex & cis \\
\hline 10 Gg_rs140018 & 106 chr10_57630 & 8.02 nonsex & cis \\
\hline 10 Gg_rs140020 & 106 chr10_50040 & 6.05 nonsex & cis \\
\hline 10 Gg_rs140020 & 107 chr10_29830। & 6.53 nonsex & cis \\
\hline 10 Gg_rs140018 & 108 chr10_58590। & 3.53 nonsex & cis \\
\hline 10 Gg_rs140031 & 109 chr10_56160। & 6.57 nonsex & cis \\
\hline 10 Gg_rs140016 & 110 chr10_39400 & 5.44 nonsex & cis \\
\hline 10 Gg_rs140031 & 110 chr10_76990 & 7.4 nonsex & cis \\
\hline 10 Gg_rs140031 & 111 chr10_71600 & 8.94 sex & cis \\
\hline 10 Gg_rs140031 & 112 chrZ_8705001 & 8.15 sex & trans \\
\hline 10 Gg_rs140031 & 113 chr10_72700 & 4.97 nonsex & cis \\
\hline 10 Gg_rs140031 & 114 chr1_105251। & 7.91 sex & trans \\
\hline 10 Gg_rs140031 & 115 chr10_12016। & 4.84 nonsex & cis \\
\hline
\end{tabular}




\begin{tabular}{|c|c|c|}
\hline 10 Gg_rs140031. & 115 chr9_326100। & 5.95 nonsex \\
\hline 10 Gg_rs140031 & 116 chr4_909790। & 7.77 sex \\
\hline 10 Gg_rs140031 & 118 chr10_58980 & 3.88 nonsex \\
\hline 10 Gg_rs140031 & 118 chr10_49850। & 3.91 nonsex \\
\hline 10 Gg_rs140031 & 118 chr12_59010 & 6.95 nonsex \\
\hline 10 Gg_rs140031 & 118 chr1_147957। & 7.4 sex \\
\hline 10 Gg_rs140031 & 119 chr10_86790। & 3.24 nonsex \\
\hline 10 Gg_rs140031 & 119 chr2_142057। & 7.21 sex \\
\hline 10 X10_798626€ & 121 chr10_68600 & 5.65 nonsex \\
\hline 10 X10_798626€ & 121 chr3_871640 & 6 nonsex \\
\hline 10 X10_952577c & 123 chr10_70380 & 5.14 nonsex \\
\hline 10 X10_798626€ & 127 chr10_52330। & 5.65 nonsex \\
\hline 10 Gg_rs140060. & 134 chr27_43200। & 6.84 nonsex \\
\hline 10 Gg_rs140082 & 134 chr10_12504! & 4.75 nonsex \\
\hline 10 Gg_rs140060. & 140 chr10_92680 & 6.21 sex \\
\hline 10 Gg_rs149477 & 146 chr2_127375। & 9.43 sex \\
\hline 10 Gg_rs149477 & 147 chr2_983060। & 9.29 sex \\
\hline 10 Gg_rs149477 & 148 chr1_195819| & 6.96 sex \\
\hline 10 Gg_rs149477 & 148 chr1_196037। & 7.29 sex \\
\hline 10 Gg_rs149477 & 151 chr2_104343। & 7.28 sex \\
\hline 10 Gg_rs149477 & 152 chr2_119043। & 7.79 sex \\
\hline 10 Gg_rs149477 & 156 chr10_12390। & 4.56 nonsex \\
\hline 10 Gg_rs149477 & 157 chr10_56920। & 5.98 nonsex \\
\hline 10 Gg_rs140082 & 160 chr5_464650 & 8.08 nonsex \\
\hline 10 Gg_rs140082 & 164 chr10_14489! & 5.97 nonsex \\
\hline 10 Gg_rs140082. & 164 chrZ_3458201 & 9.71 sex \\
\hline 10 Gg_rs149477 & 165 chr10_93970। & 5.02 nonsex \\
\hline 10 Gg_rs140082. & 166 chr10_13980 & 4.83 nonsex \\
\hline 10 Gg_rs140082 & 167 chr10_13131। & 4.69 nonsex \\
\hline 10 Gg_rs140082 & 168 chr5_286490। & 8.51 sex \\
\hline 10 Gg_rs140082. & 168 chr11_11594। & 8.69 sex \\
\hline 10 Gg_rs140082. & 172 chr10_12817। & 11.79 nonsex \\
\hline 10 Gg_rs140082. & 176 chr11_84700। & 8.79 sex \\
\hline 10 Gg_rs149498. & 177 chr10_14254! & 5.43 nonsex \\
\hline 10 Gg_rs149498 & 178 chr10_13776। & 25.49 nonsex \\
\hline 10 Gg_rs149498 & 181 chr10_14175। & 5.76 nonsex \\
\hline 10 Gg_rs149498 & 181 chr10_14251। & 14.37 nonsex \\
\hline 10 Gg_rs149498 & 181 chr10_13937। & 3.98 nonsex \\
\hline 10 Gg_rs149498 & 182 chr10_13771। & 33.97 nonsex \\
\hline 10 Gg_rs149498 & 182 chr10_13772 & 41.62 nonsex \\
\hline 10 Gg_rs149498 & 182 chr10_13775। & 22.26 nonsex \\
\hline 10 Gg_rs149498 & 182 chr10_13988। & 5.08 nonsex \\
\hline 10 Gg_rs149498 & 182 chr10_14172। & 13.35 nonsex \\
\hline
\end{tabular}




\begin{tabular}{|c|c|c|c|}
\hline 10 Gg_rs149498. & 182 chr10_14174! & 10.44 nonsex & cis \\
\hline 10 Gg_rs149498 & 183 chr10_13770 & 35.24 nonsex & cis \\
\hline 10 Gg_rs149498 & 183 chr10_13773। & 34.13 nonsex & cis \\
\hline 10 Gg_rs149498 & 183 chr10_13777। & 26.79 nonsex & cis \\
\hline 10 Gg_rs149498 & 183 chr10_14173। & 7.61 nonsex & cis \\
\hline 10 Gg_rs149498 & 183 chr10_14252 & 13.88 nonsex & cis \\
\hline 10 Gg_rs149498 & 185 chr10_14250। & 10.11 nonsex & cis \\
\hline 10 Gg_rs149498 & 189 chr10_13774! & 25.54 nonsex & cis \\
\hline 10 Gg_rs149498 & 190 chr10_13769| & 22.7 nonsex & cis \\
\hline 10 Gg_rs149498 & 191 chr10_13935। & 3.82 nonsex & cis \\
\hline 10 Gg_rs149498. & 191 chr10_14205। & 3.97 nonsex & cis \\
\hline 10 Gg_rs149498. & 192 chr8_263810। & 8.84 sex & trans \\
\hline 10 Gg_rs149498. & 196 chrZ_6890201 & 9.74 sex & trans \\
\hline 10 GG_rs149515 & 205 chr10_13317। & 8.64 sex & cis \\
\hline 10 GG_rs149515 & 206 chr1_293400। & 10.31 sex & trans \\
\hline 10 GG_rs149515 & 209 chr10_14633। & 7.51 sex & cis \\
\hline 10 GG_rs149515 & 209 chr10_15211। & 8.77 sex & cis \\
\hline 10 GG_rs149515 & 210 chr10_13716। & 8.01 sex & cis \\
\hline 10 GG_rs149515 & 213 chr10_15866। & 7 sex & cis \\
\hline 10 GG_rs149515 & 218 chr10_19262। & 3.95 nonsex & cis \\
\hline 10 GG_rs149515 & 224 chr10_14809। & 6.83 sex & cis \\
\hline 10 GG_rs149515 & 226 chr10_15439। & 7.49 sex & cis \\
\hline 10 GG_rs149515 & 227 chr10_20554! & 4.89 nonsex & cis \\
\hline 10 GG_rs149515 & 229 chr10_15495। & 10.03 sex & cis \\
\hline 11 X11_4341055 & 7 chr2_211730 & 6 nonsex & trans \\
\hline 11 X11_4341055 & 11 chr1_490620 & 6.05 nonsex & trans \\
\hline 11 Gg_rs140198. & 14 chr11_55250। & 5.01 nonsex & cis \\
\hline 11 Gg_rs140198. & 14 chr11_21050। & 4.14 nonsex & cis \\
\hline 11 Gg_rs140198 & 15 chrZ_445840 & 7.39 sex & trans \\
\hline 11 Gg_rs140198. & 17 chr11_19360। & 6.13 sex & cis \\
\hline 11 Gg_rs140198. & 17 chr11_18300 & 3.54 nonsex & cis \\
\hline 11 Gg_rs140198. & 17 chr11_19900। & 4.11 nonsex & cis \\
\hline 11 Gg_rs140198. & 17 chr11_21060 & 4.14 nonsex & cis \\
\hline 11 Gg_rs140198. & 17 chr11_27210 & 3.56 nonsex & cis \\
\hline 11 Gg_rs140198 & 17 chr11_27230 & 4.67 nonsex & cis \\
\hline 11 Gg_rs140198 & 17 chr11_27250 & 4.89 nonsex & cis \\
\hline 11 Gg_rs140198 & 17 chr11_34610 & 4.17 nonsex & cis \\
\hline 11 Gg_rs140198 & 17 chr11_38220 & 4.02 nonsex & cis \\
\hline 11 Gg_rs140198 & 17 chr11_48730 & 3.84 nonsex & cis \\
\hline 11 Gg_rs140198. & 17 chr11_66280 & 4.18 nonsex & cis \\
\hline 11 Gg_rs140198 & 17 chr8_318700 & 6.92 sex & trans \\
\hline 11 Gg_rs140198 & 17 chr2_787420 & 7.17 sex & trans \\
\hline 11 Gg_rs140198 & 17 chr11_17716। & 4.03 nonsex & trans \\
\hline
\end{tabular}




\begin{tabular}{|c|c|c|}
\hline 11 Gg_rs140198. & 17 chrZ_606680। & 6.77 nonsex \\
\hline 11 Gg_rs140198 & 17 chr3_154710 & 6.61 nonsex \\
\hline 11 Gg_rs140198 & 18 chr11_52700 & 4.08 nonsex \\
\hline 11 Gg_rs140198 & 18 chr11_57980 & 3.9 nonsex \\
\hline 11 Gg_rs140198 & 18 chr2_999840 & 5.43 nonsex \\
\hline 11 Gg_rs140198 & 19 chr11_53000 & 3.85 nonsex \\
\hline 11 Gg_rs140198 & 20 chr11_58070 & 4.64 nonsex \\
\hline 11 Gg_rs140198 & 20 chr11_59610 & 4.51 nonsex \\
\hline 11 Gg_rs140198 & 20 chr17_74510 & 5.63 nonsex \\
\hline 11 Gg_rs140198 & 20 chr3_863500 & 6.03 nonsex \\
\hline 11 Gg_rs140198. & 21 chr11_17850 & 3.96 nonsex \\
\hline 11 Gg_rs140198. & 21 chr13_10842। & 5.91 nonsex \\
\hline 11 Gg_rs140198. & 22 chr11_18540 & 4.33 nonsex \\
\hline 11 Gg_rs140198. & 22 chr11_59380 & 5.12 nonsex \\
\hline 11 Gg_rs140198. & 23 chr11_63770 & 5.19 nonsex \\
\hline 11 Gg_rs140198 & 23 chr11_77680 & 4.46 nonsex \\
\hline 11 Gg_rs140198. & 25 chr11_76600 & 4.5 nonsex \\
\hline 11 Gg_rs140198. & 25 chr4_214240 & 7.67 nonsex \\
\hline 11 Gg_rs140198 & 27 chr11_45410 & 4.6 nonsex \\
\hline 11 X11_6450003 & 27 chr11_71260 & 7.52 sex \\
\hline 11 X11_6450003 & 31 chr3_104960 & 6.38 nonsex \\
\hline 11 Gg_rs149618 & 31 chr11_12183! & 6.03 sex \\
\hline 11 X11_6450003 & 33 chr11_64120 & 4.23 nonsex \\
\hline 11 X11_6450003 & 35 chr11_72030 & 4.87 nonsex \\
\hline 11 Gg_rs149618 & 36 chr11_63690 & 3.8 nonsex \\
\hline 11 Gg_rs149618. & 36 chr11_79530 & 4.09 nonsex \\
\hline 11 Gg_rs149618. & 36 chr11_79620 & 4.15 nonsex \\
\hline 11 Gg_rs149618. & 36 chr27_42650 & 5.69 nonsex \\
\hline 11 Gg_rs149630 & 72 chr11_91350 & 4.8 nonsex \\
\hline 11 Gg_rs149630 & 75 chr11_87270 & 5.1 nonsex \\
\hline 11 Gg_rs140250' & 84 chr11_11578। & 5.52 sex \\
\hline 11 Gg_rs140250' & 88 chr11_11603। & 3.07 nonsex \\
\hline 11 Gg_rs140250 & 88 chr7_182250। & 8.09 sex \\
\hline 11 Gg_rs140250 & 88 chr15_30770 & 7.04 sex \\
\hline 11 Gg_rs140250 & 92 chr11_11607! & 3.68 nonsex \\
\hline 11 Gg_rs140250 & 92 chr3_390700 & 6.25 nonsex \\
\hline 11 Gg_rs140262 & 96 chr11_12652 & 4.25 nonsex \\
\hline 11 Gg_rs140250 & 97 chr11_11982 & 7.69 nonsex \\
\hline 11 Gg_rs149653. & 97 chr11_13594! & 6.76 nonsex \\
\hline 11 Gg_rs149653 & 98 chr11_13592 & 8.18 nonsex \\
\hline 11 Gg_rs149653 & 98 chr11_13482 & 5.41 nonsex \\
\hline 11 Gg_rs149653 & 98 chr11_12699| & 4.48 nonsex \\
\hline 11 Gg_rs149653 & 99 chr11_13363। & 4.78 nonsex \\
\hline
\end{tabular}




\begin{tabular}{|c|c|c|}
\hline 11 Gg_rs149653. & 99 chr6_718700। & 6.65 nonsex \\
\hline 11 Gg_rs149653 & 100 chr11_12243। & 25.14 nonsex \\
\hline 11 Gg_rs149653 & 101 chr11_14340। & 4.07 nonsex \\
\hline 11 Gg_rs140262 & 101 chr11_12476। & 28.78 nonsex \\
\hline 11 Gg_rs149653 & 102 chr11_14008। & 4.45 nonsex \\
\hline 11 Gg_rs149653 & 102 chr11_13046। & 5.26 nonsex \\
\hline 11 Gg_rs149653 & 103 chr11_12700 & 4.47 nonsex \\
\hline 11 Gg_rs140262 & 104 chr11_13194! & 4.71 nonsex \\
\hline 11 Gg_rs149653 & 105 chr11_11611। & 4.46 nonsex \\
\hline 11 Gg_rs140262 & 105 chr11_13946। & 6.17 nonsex \\
\hline 11 Gg_rs140262 & 105 chr11_12850 & 4.82 nonsex \\
\hline 11 Gg_rs149653. & 107 chr11_13598। & 10.78 nonsex \\
\hline 11 Gg_rs149653. & 107 chr11_14731। & 4.38 nonsex \\
\hline 11 Gg_rs149653. & 108 chr11_13605। & 9.45 nonsex \\
\hline 11 Gg_rs149653. & 108 chr11_14833। & 6.42 nonsex \\
\hline 11 Gg_rs140262 & 108 chr13_61160 & 6.76 nonsex \\
\hline 11 Gg_rs149653 & 109 chr11_13601। & 7.12 nonsex \\
\hline 11 Gg_rs140262 & 109 chr11_13768। & 4.14 nonsex \\
\hline 11 Gg_rs149653. & 111 chr11_13593। & 6.31 nonsex \\
\hline 11 Gg_rs140262 & 112 chr11_14395। & 4.66 nonsex \\
\hline 11 Gg_rs149653 & 114 chr11_13602 & 7.12 nonsex \\
\hline 11 Gg_rs140262 & 114 chr11_14373। & 8.87 nonsex \\
\hline 11 Gg_rs149653 & 116 chr11_14597। & 3.72 nonsex \\
\hline 11 RBL3647 & 119 chr11_15433। & 5.49 nonsex \\
\hline 11 RBL3647 & 123 chrZ_521460। & 7.96 sex \\
\hline 11 RBL3647 & 125 chr11_15651। & 7.71 nonsex \\
\hline 11 RBL3647 & 125 chr11_15656। & 6.76 nonsex \\
\hline 11 RBL3647 & 125 chr11_15662 & 6.12 nonsex \\
\hline 11 RBL3647 & 125 chr11_15278। & 4.33 nonsex \\
\hline 11 RBL3647 & 125 chr11_15650। & 4.1 nonsex \\
\hline 11 RBL3647 & 125 chr5_125910 & 5.67 nonsex \\
\hline 11 RBL3647 & 126 chr11_15770 & 6.23 nonsex \\
\hline 11 RBL3647 & 126 chr2_683680 & 7.35 sex \\
\hline 11 RBL3647 & 127 chr11_11864! & 10.5 nonsex \\
\hline 11 RBL3647 & 127 chr11_11868। & 16.59 nonsex \\
\hline 11 RBL3647 & 127 chr11_14809। & 7.29 nonsex \\
\hline 11 RBL3647 & 127 chr11_15663। & 7.64 nonsex \\
\hline 11 RBL3647 & 127 chr11_12710 & 5.08 nonsex \\
\hline 11 RBL3647 & 127 chr4_373880 & 7.58 sex \\
\hline 11 RBL3647 & 128 chr11_11865 & 9.49 nonsex \\
\hline 11 RBL3647 & 128 chr11_11869| & 16.07 nonsex \\
\hline 11 RBL3647 & 128 chr11_15655। & 6.42 nonsex \\
\hline 11 RBL3647 & 128 chr11_12442। & 4.32 nonsex \\
\hline
\end{tabular}




\begin{tabular}{|c|c|c|c|}
\hline 11 RBL3647 & 128 chr11_16339| & 5.03 nonsex & cis \\
\hline 11 RBL3647 & 128 chr11_17350। & 4.23 nonsex & cis \\
\hline 11 Gg_rs149653. & 129 chr11_14808| & 3.84 nonsex & cis \\
\hline 11 RBL3647 & 129 chr11_11866। & 10.19 sex & cis \\
\hline 11 RBL3647 & 129 chr11_11867। & 7.77 sex & cis \\
\hline 11 RBL3647 & 129 chr11_13600 & 5.4 nonsex & cis \\
\hline 11 RBL3647 & 129 chr11_13664। & 4.44 nonsex & cis \\
\hline 11 RBL3647 & 129 chr11_15661। & 4.02 nonsex & cis \\
\hline 11 RBL3647 & 129 chr11_15665। & 5.2 nonsex & cis \\
\hline 11 RBL3647 & 129 chr9_194600 & 5.91 sex & trans \\
\hline 11 Gg_rs140262 & 130 chr11_11740 & 4.66 nonsex & cis \\
\hline 11 RBL3647 & 130 chr11_13603। & 6.95 nonsex & cis \\
\hline 11 RBL3647 & 130 chr11_16347। & 5.69 nonsex & cis \\
\hline 11 RBL3647 & 130 chr11_14308। & 4.9 nonsex & cis \\
\hline 11 RBL3647 & 131 chr11_15279| & 6.59 sex & cis \\
\hline 11 RBL3647 & 131 chr11_15741। & 6.9 nonsex & cis \\
\hline 11 RBL3647 & 131 chr11_14853। & 4.5 nonsex & cis \\
\hline 11 RBL3647 & 131 chrZ_972500। & 7.87 sex & trans \\
\hline 11 RBL3647 & 131 chr5_443540 & 6.63 nonsex & trans \\
\hline 11 Gg_rs149669 & 131 chr11_13940। & 6.25 nonsex & cis \\
\hline 11 Gg_rs140262. & 132 chr11_14876। & 5.25 nonsex & cis \\
\hline 11 Gg_rs149669 & 132 chr11_15384। & 5.95 nonsex & cis \\
\hline 11 Gg_rs149669 & 132 chr11_13604! & 7.13 nonsex & cis \\
\hline 11 Gg_rs149669 & 132 chr11_17339! & 7.79 nonsex & cis \\
\hline 11 Gg_rs149669 & 133 chr11_15780 & 4.71 nonsex & cis \\
\hline 11 Gg_rs149669 & 133 chr6_428100 & 6.85 nonsex & trans \\
\hline 11 Gg_rs140262 & 134 chr11_12509। & 4.57 nonsex & cis \\
\hline 11 Gg_rs149669 & 134 chr11_14390। & 5.76 nonsex & cis \\
\hline 11 Gg_rs149669 & 134 chr11_13550। & 6.01 nonsex & cis \\
\hline 11 Gg_rs149669 & 134 chr11_15792 & 7.64 sex & cis \\
\hline 11 Gg_rs149669 & 134 chr11_12613। & 5.67 nonsex & cis \\
\hline 11 Gg_rs149669 & 134 chr11_13235। & 5.1 nonsex & cis \\
\hline 11 Gg_rs149669 & 134 chr11_13811। & 4.83 nonsex & cis \\
\hline 11 Gg_rs149669 & 134 chrZ_586960। & 8.06 sex & trans \\
\hline 11 Gg_rs149669 & 134 chr3_887700 & 8.01 sex & trans \\
\hline 11 Gg_rs149669 & 135 chr11_13082 & 4.31 nonsex & cis \\
\hline 11 Gg_rs149669 & 135 chr11_14748। & 4.85 nonsex & cis \\
\hline 11 Gg_rs149669 & 135 chr11_15790। & 5.43 nonsex & cis \\
\hline 11 Gg_rs149669 & 135 chr11_16769। & 5.21 nonsex & cis \\
\hline 11 Gg_rs149669 & 135 chr11_18048। & 4.21 nonsex & cis \\
\hline 11 Gg_rs149669 & 135 chr3_867890 & 6.06 nonsex & trans \\
\hline 11 Gg_rs149669 & 136 chr11_17091। & 6.44 nonsex & cis \\
\hline 11 Gg_rs149669 & 136 chr11_17530। & 6.96 nonsex & cis \\
\hline
\end{tabular}




\begin{tabular}{|c|c|c|}
\hline 11 Gg_rs149669 & 136 chr11_13762। & 5.01 nonsex \\
\hline 11 Gg_rs149669 & 136 chr11_15788 & 5.2 nonsex \\
\hline 11 Gg_rs149669 & 136 chr11_16375। & 4.37 nonsex \\
\hline 11 Gg_rs149669 & 136 chr11_16694! & 5.39 nonsex \\
\hline 11 Gg_rs149669 & 136 chr11_16897। & 4.57 nonsex \\
\hline 11 Gg_rs149669 & 136 chr11_16986। & 4.62 nonsex \\
\hline 11 Gg_rs149669 & 136 chr13_10067। & 5.32 nonsex \\
\hline 11 Gg_rs149669 & 136 chr18_10337। & 6.39 nonsex \\
\hline 11 Gg_rs149669 & 136 chr3_752560। & 5.82 nonsex \\
\hline 11 Gg_rs149669 & 137 chr11_15246। & 5.72 nonsex \\
\hline 11 Gg_rs149669 & 137 chr11_16373। & 5.79 nonsex \\
\hline 11 Gg_rs149669 & 137 chr11_16431। & 5.69 nonsex \\
\hline 11 Gg_rs149669 & 137 chr11_16826। & 5.58 nonsex \\
\hline 11 Gg_rs149669 & 137 chr11_16837। & 5.5 sex \\
\hline 11 Gg_rs149669 & 137 chr11_16849| & 6.38 nonsex \\
\hline 11 Gg_rs149669 & 137 chr11_16938। & 5.9 nonsex \\
\hline 11 Gg_rs149669 & 137 chr11_16975। & 5.5 nonsex \\
\hline 11 Gg_rs149669 & 137 chr11_17066। & 4.76 nonsex \\
\hline 11 Gg_rs149669 & 137 chr11_17197। & 7.05 nonsex \\
\hline 11 Gg_rs149669 & 137 chr11_11338। & 5.25 nonsex \\
\hline 11 Gg_rs149669 & 137 chr11_13378। & 4.67 nonsex \\
\hline 11 Gg_rs149669 & 137 chr11_13425। & 4.81 nonsex \\
\hline 11 Gg_rs149669 & 137 chr11_15469। & 4.63 nonsex \\
\hline 11 Gg_rs149669 & 137 chr11_15565। & 4.17 nonsex \\
\hline 11 Gg_rs149669 & 137 chr11_16618। & 4.59 nonsex \\
\hline 11 Gg_rs149669 & 137 chr11_16625। & 5.18 nonsex \\
\hline 11 Gg_rs149669 & 137 chr11_16765। & 4.18 nonsex \\
\hline 11 Gg_rs149669 & 137 chr11_17055। & 4.51 nonsex \\
\hline 11 Gg_rs149669 & 137 chrW_36040c & 8.09 sex \\
\hline 11 Gg_rs149669 & 137 chrZ_181700। & 6.37 nonsex \\
\hline 11 Gg_rs149669 & 137 chr1_149434। & 6.66 nonsex \\
\hline 11 Gg_rs149669 & 137 chr1_365350 & 5.98 nonsex \\
\hline 11 Gg_rs149669 & 137 chr4_500510 & 6.65 nonsex \\
\hline 11 Gg_rs149669 & 137 chr5_248580 & 5.91 nonsex \\
\hline 11 Gg_rs149669 & 137 chr8_926000 & 5.66 nonsex \\
\hline 11 Gg_rs149669 & 137 chr12_19991। & 6.1 nonsex \\
\hline 11 Gg_rs149669 & 137 chrZ_164650। & 5.45 nonsex \\
\hline 11 Gg_rs149669 & 137 chrZ_3709201 & 5.79 nonsex \\
\hline 11 Gg_rs149669 & 137 chr1_635790। & 5.88 nonsex \\
\hline 11 Gg_rs149669 & 137 chr1_707920 & 5.84 nonsex \\
\hline 11 Gg_rs149669 & 137 chr2_104884! & 5.99 nonsex \\
\hline 11 Gg_rs149669 & 141 chr3_277280 & 5.72 nonsex \\
\hline 11 Gg_rs149669 & 144 chr11_16752। & 4.4 nonsex \\
\hline
\end{tabular}




\begin{tabular}{|c|c|c|c|}
\hline 11 Gg_rs149669 & 147 chr11_16796। & 5.09 nonsex & cis \\
\hline 11 Gg_rs149669 & 147 chr27_77860। & 8.64 sex & trans \\
\hline 11 RBL2392 & 189 chr11_10838| & 5.77 sex & cis \\
\hline 12 Gg_rs140312 & 0 chr12_24880 & 3.51 nonsex & cis \\
\hline 12 Gg_rs140312 & 0 chr12_17410 & 3.91 nonsex & cis \\
\hline 12 Gg_rs140312 & 0 chr12_21890। & 2.7 nonsex & cis \\
\hline 12 Gg_rs149706 & 2 chr12_48100। & 5.13 nonsex & cis \\
\hline 12 Gg_rs149706 & 2 chr12_48800 & 5.12 nonsex & cis \\
\hline 12 Gg_rs149706 & 3 chr12_47800 & 4.73 nonsex & cis \\
\hline 12 Gg_rs149706 & 3 chr12_48400 & 4.71 nonsex & cis \\
\hline 12 Gg_rs149706 & 3 chr12_48500 & 4.67 nonsex & cis \\
\hline 12 Gg_rs149711 & 24 chr2_344790। & 5.83 nonsex & trans \\
\hline 12 Gg_rs149711 & 26 chr12_27230 & 9.28 nonsex & cis \\
\hline 12 Gg_rs149711 & 26 chr12_27310 & 11.69 nonsex & cis \\
\hline 12 Gg_rs149711 & 27 chr12_27170 & 11.62 nonsex & cis \\
\hline 12 Gg_rs149711 & 27 chr12_27240 & 10.12 nonsex & cis \\
\hline 12 Gg_rs149711 & 27 chr12_27320 & 14.79 nonsex & cis \\
\hline 12 Gg_rs149711 & 27 chr12_26690 & 4.29 nonsex & cis \\
\hline 12 Gg_rs149711 & 29 chr12_36210 & 2.09 sex & cis \\
\hline 12 Gg_rs149711 & 29 chr4_909360 & 4.75 nonsex & trans \\
\hline 12 Gg_rs149711 & 30 chr12_27210 & 9.53 nonsex & cis \\
\hline 12 Gg_rs149711 & 30 chr12_27290 & 6.94 nonsex & cis \\
\hline 12 Gg_rs136214 & 31 chr12_27280 & 9.42 nonsex & cis \\
\hline 12 Gg_rs136214 & 31 chr12_26950 & 10.36 nonsex & cis \\
\hline 12 Gg_rs136214 & 31 chr12_27590 & 7.41 nonsex & cis \\
\hline 12 Gg_rs136214 & 31 chr12_27580 & 4.76 nonsex & cis \\
\hline 12 Gg_rs136214 & 31 chr12_27600 & 5.83 nonsex & cis \\
\hline 12 Gg_rs136214 & 32 chr12_27200। & 7.92 nonsex & cis \\
\hline 12 Gg_rs136214 & 35 chr3_101570 & 5.58 nonsex & trans \\
\hline 12 Gg_rs136214 & 39 chr12_33540। & 4.01 nonsex & cis \\
\hline 12 Gg_rs136214 & 42 chr12_49980 & 5.76 sex & cis \\
\hline 12 Gg_rs136214 & 42 chr3_598180 & 6.35 nonsex & trans \\
\hline 12 Gg_rs136214 & 44 chr12_27530। & 7.15 nonsex & cis \\
\hline 12 Gg_rs136214 & 46 chr12_27390। & 5.75 nonsex & cis \\
\hline 12 Gg_rs136094 & 48 chr12_43740 & 5.15 nonsex & cis \\
\hline 12 Gg_rs136094 & 51 chr12_35730 & 4.58 nonsex & cis \\
\hline 12 Gg_rs136094 & 54 chr12_32910 & 5.54 nonsex & cis \\
\hline 12 Gg_rs136094 & 54 chr9_861100 & 5.37 nonsex & trans \\
\hline 12 Gg_rs136094 & 55 chr12_45840 & 17.62 nonsex & cis \\
\hline 12 Gg_rs136094 & 56 chr12_45850 & 9.85 nonsex & cis \\
\hline 12 Gg_rs136094 & 58 chr12_33970 & 5.63 nonsex & cis \\
\hline 12 Gg_rs136094 & 58 chr12_35250 & 6.32 nonsex & cis \\
\hline 12 Gg_rs136094 & 58 chr12_52780 & 6.56 nonsex & cis \\
\hline
\end{tabular}




\begin{tabular}{|c|c|c|}
\hline 12 Gg_rs136094 & 58 chr5_285550 & 8.21 sex \\
\hline 12 Gg_rs136094 & 60 chr12_35330 & 4.58 nonsex \\
\hline 12 Gg_rs136094 & 60 chr7_223170 & 8.09 nonsex \\
\hline 12 Gg_rs136094 & 60 chr7_226090 & 5.89 nonsex \\
\hline 12 Gg_rs136094 & 61 chr12_36630 & 5.35 nonsex \\
\hline 12 Gg_rs136094 & 61 chr12_46240 & $6.66 \operatorname{sex}$ \\
\hline 12 Gg_rs136094 & 62 chr12_46880 & 7.2 sex \\
\hline 12 Gg_rs136094 & 62 chr1_449020 & 8.73 sex \\
\hline 12 Gg_rs149791 & 144 chr2_893960। & 6.02 nonsex \\
\hline 12 Gg_rs149791 & 150 chr17_17380 & 6.08 nonsex \\
\hline 12 Gg_rs140408 & 150 chr12_11001। & 4.63 nonsex \\
\hline 12 Gg_rs149791 & 151 chr1_113539| & 6.39 nonsex \\
\hline 12 Gg_rs140408 & 152 chr12_76430 & 4.27 nonsex \\
\hline 12 Gg_rs140408 & 152 chrZ_191280। & 7.07 sex \\
\hline 12 Gg_rs140408 & 152 chr33_59410। & 5.73 nonsex \\
\hline 12 Gg_rs140408 & 157 chr12_88250। & 4.56 nonsex \\
\hline 12 Gg_rs140408 & 158 chrW_16700 & 7.92 sex \\
\hline 12 Gg_rs149803 & 159 chrW_50000 & 7.73 sex \\
\hline 12 Gg_rs149803 & 161 chrZ_668660। & 7.39 sex \\
\hline 12 Gg_rs149803 & 163 chr1_44000 & 8.19 nonsex \\
\hline 12 Gg_rs149803 & 165 chr12_12173। & 4.38 nonsex \\
\hline 12 Gg_rs149803 & 165 chr27_26320। & 8.17 sex \\
\hline 12 Gg_rs149803 & 165 chr1_595470 & $7.28 \mathrm{sex}$ \\
\hline 12 Gg_rs149803 & 168 chr23_24130। & 6.54 nonsex \\
\hline 12 Gg_rs149803 & 169 chrW_18150 & 9.35 sex \\
\hline 12 Gg_rs149803 & 173 chrZ_5244101 & 8.33 sex \\
\hline 12 Gg_rs149803 & 174 chr8_143400 & 6.81 nonsex \\
\hline 12 X12_140511€ & 192 chr12_13761। & 7.25 nonsex \\
\hline 12 X12_140511€ & 202 chr12_14333। & 4.68 nonsex \\
\hline 12 GG_rs156686 & 221 chr12_18503। & 5.22 nonsex \\
\hline 12 Gg_rs156655 & 231 chr19_50560। & 6.89 nonsex \\
\hline 12 Gg_rs156655 & 233 chr12_13767। & 3.98 nonsex \\
\hline 12 Gg_rs156655 & 234 chr12_15649। & 4.04 nonsex \\
\hline 12 GG_rs156686 & 235 chr5_373850 & 5.82 nonsex \\
\hline 12 GG_rs156686 & 237 chr12_18228। & 7.15 nonsex \\
\hline 12 GG_rs156686 & 237 chr12_18258। & 4.67 nonsex \\
\hline 12 GG_rs156686 & 237 chr7_166640 & 5.78 nonsex \\
\hline 12 GG_rs156686 & 237 chr24_27150। & 5.64 nonsex \\
\hline 12 GG_rs156686 & 238 chr12_18215। & 11.2 nonsex \\
\hline 12 GG_rs156686 & 238 chr12_18219। & 7.26 nonsex \\
\hline 12 GG_rs156686 & 238 chr12_18841। & 4.28 nonsex \\
\hline 12 GG_rs156686 & 239 chr12_18213। & 14.33 nonsex \\
\hline 12 GG_rs156686 & 239 chr12_17940। & 5.12 nonsex \\
\hline
\end{tabular}




\begin{tabular}{|c|c|c|c|}
\hline 12 GG_rs156686 & 239 chr12_18216। & 4.51 nonsex & cis \\
\hline 12 GG_rs156686 & 239 chr12_184381 & 4.84 nonsex & cis \\
\hline 12 GG_rs156686 & 240 chr12_19203। & 4.81 nonsex & cis \\
\hline 12 Gg_rs149849 & 241 chr12_16909। & $6.03 \mathrm{sex}$ & cis \\
\hline 12 Gg_rs149849 & 241 chr12_18194। & 5.61 sex & cis \\
\hline 12 Gg_rs149849 & 242 chr12_17912 & 6.14 nonsex & cis \\
\hline 12 Gg_rs149849 & 242 chr12_17932 & 5.76 nonsex & cis \\
\hline 12 Gg_rs149849 & 242 chr12_18402 & 4.36 nonsex & cis \\
\hline 12 Gg_rs149849 & 242 chr12_19129| & 6.64 sex & cis \\
\hline 13 Gg_rs149872 & 0 chr13_10610 & 7.41 sex & cis \\
\hline 13 Gg_rs149872 & 0 chr13_11550। & 6.97 sex & cis \\
\hline 13 Gg_rs149872 & 0 chr13_11690। & 6.38 sex & cis \\
\hline 13 Gg_rs149872 & 0 chr13_12210 & 6.93 sex & cis \\
\hline 13 Gg_rs149872 & 0 chr13_15250 & 11.9 sex & cis \\
\hline 13 Gg_rs149872 & 0 chr13_10500 & 5.19 nonsex & cis \\
\hline 13 Gg_rs149872 & 0 chr13_11100। & 6.2 nonsex & cis \\
\hline 13 Gg_rs149872 & 0 chr13_12800 & 6.48 nonsex & cis \\
\hline 13 Gg_rs149872 & 0 chr13_14220 & 6.8 nonsex & cis \\
\hline 13 GG_rs156764 & 0 chr13_14230 & 11.56 nonsex & cis \\
\hline 13 GG_rs156764 & 0 chr13_14210 & 13.54 nonsex & trans \\
\hline 13 Gg_rs149872 & 1 chr13_11140। & 6.18 nonsex & cis \\
\hline 13 GG_rs156764 & 1 chr13_14190। & 12.02 sex & trans \\
\hline 13 Gg_rs149872 & 2 chr13_10620। & 7.11 nonsex & cis \\
\hline 13 GG_rs156764 & 2 chr13_14140 & 5.02 nonsex & cis \\
\hline 13 GG_rs156764 & 3 chr13_10420 & 6.04 nonsex & cis \\
\hline 13 GG_rs156764 & 4 chr13_10640 & 6.67 sex & cis \\
\hline 13 Gg_rs149872 & 6 chr13_15300 & 7.39 sex & cis \\
\hline 13 GG_rs156764 & 6 chr13_11120। & 6.82 sex & cis \\
\hline 13 GG_rs156764 & 6 chr13_11680 & 6.28 sex & cis \\
\hline 13 GG_rs156764 & 6 chr13_12320। & 5.59 nonsex & cis \\
\hline 13 Gg_rs149872 & 7 chr13_10510 & 8.05 nonsex & cis \\
\hline 13 Gg_rs149872 & 7 chr13_11560। & 6.23 nonsex & cis \\
\hline 13 GG_rs156764 & 7 chr13_10530। & 4.87 nonsex & cis \\
\hline 13 GG_rs156764 & 8 chr13_15090 & 19.22 nonsex & cis \\
\hline 13 Gg_rs149872 & 9 chr13_15080 & 10.51 nonsex & cis \\
\hline 13 Gg_rs149872 & 9 chr13_15000। & 8.81 sex & trans \\
\hline 13 GG_rs156764 & 9 chr13_14310 & 3.23 nonsex & cis \\
\hline 13 Gg_rs149872 & 10 chr13_15010 & 10.43 sex & trans \\
\hline 13 GG_rs156764 & 10 chr13_14650 & 4.73 nonsex & cis \\
\hline 13 Gg_rs149910 & 55 chr13_10292 & 5.99 nonsex & cis \\
\hline 13 Gg_rs149910 & 78 chr13_12409! & 6.8 sex & cis \\
\hline 13 Gg_rs149910 & 78 chr13_61070 & 3.98 nonsex & cis \\
\hline 13 Gg_rs149910 & 78 chr13_61150 & 4.1 nonsex & cis \\
\hline
\end{tabular}




\begin{tabular}{|c|c|c|c|}
\hline 13 Gg_rs149910 & 81 chr13_52600। & 7.09 nonsex & cis \\
\hline 13 Gg_rs149910 & 82 chr13_44290 & 4.89 sex & cis \\
\hline 13 X13_555545؟ & 84 chr13_67880 & 4.1 nonsex & cis \\
\hline 13 X13_555545؟ & 85 chr13_62090 & 3.22 nonsex & cis \\
\hline 13 X13_555545@ & 85 chr3_462110 & 8.41 sex & trans \\
\hline 13 X13_555545؟ & 88 chr13_63370 & 5.71 nonsex & cis \\
\hline 13 X13_555545؛ & 88 chr13_87070 & 5.2 nonsex & cis \\
\hline 13 X13_555545؟ & 88 chr13_63380 & 4.4 nonsex & cis \\
\hline 13 X13_555545؟ & 88 chr13_65210 & 5.02 nonsex & cis \\
\hline 13 X13_555545@ & 88 chr13_82810 & 5.25 nonsex & cis \\
\hline 13 X13_555545؛ & 88 chr13_102281 & 4.6 nonsex & cis \\
\hline 13 X13_555545@ & 90 chr13_60230 & 5.4 nonsex & cis \\
\hline 13 X13_555545@ & 90 chr13_89020 & 6.44 nonsex & cis \\
\hline 13 X13_555545؟ & 90 chr1_279730 & 6.51 nonsex & trans \\
\hline 13 X13_555545c & 91 chr13_59450। & 7.64 nonsex & cis \\
\hline 13 X13_555545c & 91 chr13_64850 & 7.03 nonsex & cis \\
\hline 13 X13_555545؟ & 92 chr13_65650 & 4.65 nonsex & cis \\
\hline 13 X13_555545؟ & 93 chr13_51540 & 6.71 nonsex & cis \\
\hline 13 Gg_rs149910 & 94 chr13_10489! & 4.47 nonsex & cis \\
\hline 13 X13_555545؟ & 94 chr13_79470 & 4.7 nonsex & cis \\
\hline 13 X13_555545؛ & 94 chr2_37000 & 6.87 nonsex & trans \\
\hline 13 X13_555545؛ & 95 chr2_475030। & 6.73 nonsex & trans \\
\hline 13 X13_5555459 & 96 chr13_80510 & 5.69 nonsex & cis \\
\hline 13 X13_555545@ & 96 chr1_126527। & 9.82 sex & trans \\
\hline 13 X13_8702725 & 96 chr13_10205। & 11.76 sex & cis \\
\hline 13 X13_8702725 & 97 chr13_82350। & 5.44 nonsex & cis \\
\hline 13 Gg_rs140586 & 107 chr13_10375। & 4.64 nonsex & cis \\
\hline 13 X13_8702725 & 108 chr13_90630। & 6.46 nonsex & cis \\
\hline 13 Gg_rs140586 & 110 chr13_10201। & 13.12 nonsex & cis \\
\hline 13 Gg_rs140586 & 111 chr13_10687। & 5.67 nonsex & cis \\
\hline 13 Gg_rs140586 & 111 chr13_10706। & 5.74 nonsex & cis \\
\hline 13 Gg_rs140586 & 111 chr13_10073। & 5.41 nonsex & cis \\
\hline 13 Gg_rs140586 & 111 chrZ_254670। & 7.34 sex & trans \\
\hline 13 Gg_rs140586 & 111 chr2_126973। & 7.48 sex & trans \\
\hline 13 Gg_rs140586 & 113 chr13_10480 & 5.51 nonsex & cis \\
\hline 13 X13_5555459 & 121 chr13_62660। & 4.93 nonsex & cis \\
\hline 13 Gg_rs149956 & 121 chr13_83310 & 5.42 nonsex & cis \\
\hline 13 Gg_rs149956 & 123 chr13_12317। & 5.8 nonsex & cis \\
\hline 13 Gg_rs149956 & 127 chr13_11544! & 6.22 nonsex & cis \\
\hline 13 Gg_rs149956. & 127 chr13_77670 & 4.83 nonsex & cis \\
\hline 13 Gg_rs149956 & 128 chr13_80900 & 5.63 sex & cis \\
\hline 13 Gg_rs149956 & 129 chr13_75250। & 5.53 nonsex & cis \\
\hline 13 Gg_rs149956. & 130 chr13_49220 & 5.72 nonsex & cis \\
\hline
\end{tabular}




\begin{tabular}{|c|c|c|c|}
\hline 13 Gg_rs149956. & 130 chr13_11573। & 8.36 nonsex & cis \\
\hline 13 Gg_rs149956 & 130 chr13_85940। & 4.75 nonsex & cis \\
\hline 13 Gg_rs149956. & 131 chr13_78180 & 6.84 sex & cis \\
\hline 13 Gg_rs149956. & 133 chr13_65710 & 5.89 nonsex & cis \\
\hline 13 Gg_rs149956. & 133 chr13_66020 & 5.19 nonsex & cis \\
\hline 13 Gg_rs149956 & 133 chr13_59000 & 4.23 nonsex & cis \\
\hline 13 Gg_rs149956 & 133 chr13_60580 & 4.48 nonsex & cis \\
\hline 13 Gg_rs149956. & 133 chr13_61130। & 4.62 nonsex & cis \\
\hline 13 Gg_rs149956. & 133 chr13_63440 & 3.97 nonsex & cis \\
\hline 13 Gg_rs149956. & 133 chr13_67220 & 4.55 nonsex & cis \\
\hline 13 Gg_rs149956 & 133 chr13_79020। & 4.94 nonsex & cis \\
\hline 13 Gg_rs149956 & 133 chr13_10647। & 4.15 nonsex & cis \\
\hline 13 Gg_rs149956 & 133 chr13_12737। & 4.19 nonsex & cis \\
\hline 13 Gg_rs149956. & 133 chr14_12059! & 6.95 nonsex & trans \\
\hline 13 Gg_rs149956. & 133 chr12_87330। & 5.8 nonsex & trans \\
\hline 13 Gg_rs149956 & 133 chrZ_715000 & 6.23 nonsex & \\
\hline 13 Gg_rs149956 & 133 chr2_918500 & 6.08 nonsex & trans \\
\hline 13 Gg_rs149956 & 133 chr2_976500। & 5.6 nonsex & trans \\
\hline 13 Gg_rs149956 & 133 chr3_357780 & 5.86 nonsex & trans \\
\hline 13 Gg_rs149956 & 133 chr3_974660। & 5.9 nonsex & trans \\
\hline 13 Gg_rs149956 & 135 chr13_59170। & 4.2 nonsex & cis \\
\hline 13 Gg_rs149956 & 136 chr4_156870 & 5.58 nonsex & trans \\
\hline 13 Gg_rs149956 & 137 chr4_326460। & 5.94 nonsex & trans \\
\hline 13 Gg_rs149956 & 138 chr13_81220 & 4.27 nonsex & cis \\
\hline 13 Gg_rs149956 & 139 chr13_12419| & 4.35 nonsex & cis \\
\hline 13 Gg_rs149956 & 139 chr5_149400 & 6.52 nonsex & trans \\
\hline 13 Gg_rs149956 & 140 chr13_16428। & 5.06 nonsex & cis \\
\hline 13 Gg_rs149956 & 144 chr13_11617। & 4.86 nonsex & cis \\
\hline 13 Gg_rs149956. & 148 chr13_59160। & 4.9 nonsex & cis \\
\hline 13 X13_129794€ & 154 chr13_12667। & 5.36 nonsex & cis \\
\hline 13 X13_129794€ & 162 chr13_12639! & 5.97 nonsex & cis \\
\hline 13 X13_129794€ & 166 chr13_14642 & 4.49 nonsex & cis \\
\hline 13 X13_129794€ & 168 chr16_66500 & 8.7 sex & trans \\
\hline 13 X13_129794€ & 169 chr13_15811। & 6.04 sex & cis \\
\hline 13 X13_129794€ & 169 chr4_911570। & 12.98 sex & trans \\
\hline 13 X13_129794E & 169 chr1_849140। & 6.31 nonsex & trans \\
\hline 13 X13_129794E & 170 chr13_66310 & $6.84 \operatorname{sex}$ & cis \\
\hline 13 X13_129794E & 174 chr7_324720 & 7.94 sex & trans \\
\hline 13 X13_129794€ & 175 chr13_15474! & 8.01 nonsex & cis \\
\hline 13 X13_129794€ & 175 chrW_49400 & 8.87 sex & trans \\
\hline 13 X13_129794€ & 175 chr2_132802 & 7.9 sex & trans \\
\hline 13 X13_129794€ & 178 chrW_60560 & 7.95 sex & trans \\
\hline 13 X13_129794E & 180 chr13_13675। & 4.92 nonsex & cis \\
\hline
\end{tabular}




\begin{tabular}{|c|c|c|c|}
\hline 13 Gg_rs140628. & 181 chr13_15498। & 6.95 nonsex & cis \\
\hline 13 Gg_rs140628 & 192 chr13_14692 & 5.55 sex & cis \\
\hline 13 Gg_rs140628 & 201 chr3_745300 & 7.87 sex & trans \\
\hline 13 Gg_rs140640 & 202 chr5_464000 & 8.25 sex & trans \\
\hline 13 Gg_rs140640 & 203 chr13_15600 & 4.23 nonsex & cis \\
\hline 13 Gg_rs140640 & 203 chr13_16976। & 4.93 nonsex & cis \\
\hline 13 Gg_rs140640 & 203 chr3_403720 & 7.63 sex & trans \\
\hline 13 Gg_rs140640 & 204 chr3_110175। & 7.33 sex & trans \\
\hline 13 Gg_rs140640 & 205 chr13_16561। & 6.17 nonsex & cis \\
\hline 13 Gg_rs140640 & 205 chr4_376830। & 7.3 sex & trans \\
\hline 13 Gg_rs140640. & 206 chr13_13383। & 7.49 sex & cis \\
\hline 13 Gg_rs140640. & 207 chr3_605500। & 7.46 sex & trans \\
\hline 14 Gg_rs150035 & 4 chr14_11830 & 4.83 nonsex & cis \\
\hline 14 Gg_rs135304. & 5 chrW_17100 & 5.7 nonsex & trans \\
\hline 14 Gg_rs150035 & 9 chr14_22000। & 9.38 nonsex & cis \\
\hline 14 Gg_rs150035 & 15 chr14_18610 & 4.79 nonsex & cis \\
\hline 14 Gg_rs135309. & 17 chr14_26510 & 10.04 nonsex & cis \\
\hline 14 Gg_rs135309. & 18 chr14_26480 & 16.35 nonsex & cis \\
\hline 14 Gg_rs135309. & 18 chr14_26490 & 19.89 nonsex & cis \\
\hline 14 Gg_rs135309. & 19 chr14_26470 & 19.66 nonsex & cis \\
\hline 14 Gg_rs135309. & 21 chr14_26500 & 22.44 nonsex & cis \\
\hline 14 Gg_rs135309. & 22 chr14_26920 & 3.84 nonsex & cis \\
\hline 14 Gg_rs135311 & 24 chr14_27590 & 3.22 nonsex & cis \\
\hline 14 Gg_rs135311 & 24 chr14_30310 & 3.02 nonsex & cis \\
\hline 14 Gg_rs135311 & 24 chr14_34460 & 4.4 nonsex & cis \\
\hline 14 Gg_rs135311 & 24 chr21_54790। & 6.68 nonsex & trans \\
\hline 14 Gg_rs150035 & 25 chr14_20250। & 4.29 nonsex & cis \\
\hline 14 Gg_rs135311 & 25 chr14_34160। & 4.43 nonsex & cis \\
\hline 14 Gg_rs135311 & 25 chr14_37770 & 4.09 nonsex & cis \\
\hline 14 Gg_rs135311 & 25 chr14_26160। & 4.07 nonsex & cis \\
\hline 14 Gg_rs135311 & 25 chr14_34150। & 3.37 nonsex & cis \\
\hline 14 Gg_rs135311 & 25 chr14_34290। & 3.75 nonsex & cis \\
\hline 14 Gg_rs135311 & 25 chr14_34320 & 4.6 nonsex & cis \\
\hline 14 Gg_rs135311 & 25 chr14_36620। & 3.8 nonsex & cis \\
\hline 14 Gg_rs135311 & 25 chr14_40290 & 2.74 nonsex & cis \\
\hline 14 Gg_rs135311 & 25 chr1_158500 & 6.33 nonsex & trans \\
\hline 14 Gg_rs135311 & 25 chr5_590090 & 6.68 nonsex & trans \\
\hline 14 Gg_rs135311 & 25 chr14_54930 & 6.29 nonsex & trans \\
\hline 14 Gg_rs135311 & 28 chr14_29680 & 3.83 nonsex & cis \\
\hline 14 Gg_rs135311 & 30 chr14_18600 & 3.98 nonsex & cis \\
\hline 14 Gg_rs135311 & 30 chr14_37460 & 4.39 nonsex & cis \\
\hline 14 Gg_rs150035 & 31 chr14_14790 & 4.01 nonsex & cis \\
\hline 14 Gg_rs140717 & 32 chr14_43380 & 4.94 nonsex & cis \\
\hline
\end{tabular}




\begin{tabular}{|c|c|c|}
\hline 14 Gg_rs140717 & 32 chr5_436360। & 7.25 nonsex \\
\hline 14 Gg_rs140717 & 33 chr14_17270 & 3.9 nonsex \\
\hline 14 Gg_rs140717 & 33 chr14_40310 & 2.95 nonsex \\
\hline 14 Gg_rs140717 & 34 chr14_39580 & 19.97 nonsex \\
\hline 14 Gg_rs140717 & 35 chr14_34960 & 4.24 nonsex \\
\hline 14 Gg_rs140717 & 36 chr14_40160 & 6.38 nonsex \\
\hline 14 Gg_rs140717 & 36 chr14_46000 & 3.36 nonsex \\
\hline 14 Gg_rs140717 & 36 chr13_50900 & 14.76 sex \\
\hline 14 Gg_rs140717 & 36 chr13_51000 & 14.02 sex \\
\hline 14 Gg_rs140717 & 40 chr14_41240 & 5.56 nonsex \\
\hline 14 Gg_rs140717 & 48 chr2_704140 & 4.3 nonsex \\
\hline 14 Gg_rs135319. & 75 chr14_47560 & 4.24 nonsex \\
\hline 14 Gg_rs135319. & 79 chr14_47570 & 5.14 nonsex \\
\hline 14 Gg_rs135319. & 81 chr14_58520 & 5.49 nonsex \\
\hline 14 Gg_rs135319. & 94 chr5_586400। & 6.43 nonsex \\
\hline 14 Gg_rs135319. & 96 chr14_56710 & 5.2 nonsex \\
\hline 14 Gg_rs135319. & 96 chr14_58640। & 5.05 nonsex \\
\hline 14 Gg_rs140765 & 123 chr14_82030। & 3.96 nonsex \\
\hline 14 Gg_rs140765 & 127 chr14_82070। & 5.39 nonsex \\
\hline 14 Gg_rs140765 & 128 chr14_92740। & 5.47 nonsex \\
\hline 14 Gg_rs140765 & 130 chr14_82020। & 4.68 nonsex \\
\hline 14 Gg_rs140765. & 130 chr14_94450 & 7.11 nonsex \\
\hline 14 Gg_rs140765 & 131 chr14_79400। & 8.04 nonsex \\
\hline 14 Gg_rs140765 & 133 chr14_87840। & 6 sex \\
\hline 14 Gg_rs140765 & 134 chr14_90010। & 8.12 nonsex \\
\hline 14 Gg_rs140765 & 134 chr14_91450। & 5.02 sex \\
\hline 14 Gg_rs140765 & 134 chr14_94250 & 5.44 nonsex \\
\hline 14 Gg_rs140765 & 134 chr14_96970। & 8.26 nonsex \\
\hline 14 Gg_rs140765 & 134 chr14_89900। & 4.8 nonsex \\
\hline 14 Gg_rs140765 & 134 chr14_97410। & 5.22 nonsex \\
\hline 14 Gg_rs140765 & 136 chr14_86860 & 4.4 nonsex \\
\hline 14 Gg_rs140765. & 143 chr14_96310 & 8.35 nonsex \\
\hline 14 Gg_rs140765. & 144 chr14_86970 & 4.58 nonsex \\
\hline 14 Gg_rs140765. & 149 chr14_83340 & 5.7 nonsex \\
\hline 14 Gg_rs140765 & 150 chr14_11524। & 7.26 sex \\
\hline 14 Gg_rs140765 & 156 chr14_95810 & 5.96 sex \\
\hline 14 Gg_rs150026 & 218 chr14_11915। & 9.81 sex \\
\hline 14 Gg_rs150026 & 223 chr14_10822 & 5.69 nonsex \\
\hline 14 Gg_rs150026 & 223 chr14_13772 & 4.48 nonsex \\
\hline 14 Gg_rs150026 & 224 chr14_12185। & 6.53 nonsex \\
\hline 14 Gg_rs150026 & 228 chr1_270100। & 6.4 nonsex \\
\hline 14 Gg_rs150026 & 233 chr14_13373। & 5.93 nonsex \\
\hline 14 Gg_rs150026 & 236 chr14_12726। & 4.48 nonsex \\
\hline
\end{tabular}




\begin{tabular}{|c|c|c|}
\hline 14 Gg_rs150026. & 247 chr14_15067। & 4.35 nonsex \\
\hline 14 Gg_rs150026 & 249 chr2_840390। & 8.35 nonsex \\
\hline 14 Gg_rs150026 & 252 chr14_14621। & 7.48 nonsex \\
\hline 14 rbl1871 & 258 chr7_278020। & 7.12 sex \\
\hline 14 rbl1871 & 258 chr13_24680 & 7.45 nonsex \\
\hline 14 rbl1871 & 258 chrZ_358540। & 7.93 sex \\
\hline 14 rbl1871 & 259 chrZ_2868501 & 8.68 sex \\
\hline 14 rbl1871 & 259 chrZ_6836701 & 7.56 sex \\
\hline 14 rbl1871 & 260 chr14_14618। & 5 nonsex \\
\hline 14 rbl1871 & 260 chr5_295150 & 8.02 sex \\
\hline 14 rbl1871 & 260 chr13_81140। & 8.04 sex \\
\hline 14 rbl1871 & 260 chrZ_1908101 & 8.24 sex \\
\hline 14 rbl1871 & 260 chr1_881670 & 8.47 sex \\
\hline 14 rbl1871 & 260 chrZ_4208301 & 7.59 sex \\
\hline 14 rbl1871 & 260 chrZ_4433501 & 7.8 sex \\
\hline 14 rbl1871 & 260 chr2_956060। & 8.56 sex \\
\hline 14 rbl1871 & 261 chr8_159300 & 8.33 sex \\
\hline 14 rbl1871 & 261 chrZ_100980। & 7.54 sex \\
\hline 14 rbl1871 & 261 chr1_490610 & 5.87 nonsex \\
\hline 14 rbl1871 & 261 chr6_876100। & 5.88 nonsex \\
\hline 14 rbl1871 & 262 chr14_14928। & 4.97 nonsex \\
\hline 14 rbl1871 & 262 chr6_185960। & 7.68 sex \\
\hline 14 rbl1871 & 262 chrZ_3027701 & 7.82 sex \\
\hline 14 rbl1871 & 262 chr1_981910। & 8.98 sex \\
\hline 14 rbl1871 & 262 chrZ_4295301 & 8.43 sex \\
\hline 14 rbl1871 & 263 chr1_316240। & 7.47 sex \\
\hline 14 rbl1871 & 263 chrZ_2097901 & 8.62 sex \\
\hline 14 rbl1871 & 263 chrZ_2954301 & 7.92 sex \\
\hline 14 rbl1871 & 263 chr1_711220 & 7.36 sex \\
\hline 14 rbl1871 & 263 chr1_753550। & 7.85 sex \\
\hline 14 rbl1871 & 263 chr4_105420 & 7.45 sex \\
\hline 14 rbl1871 & 264 chr1_527290 & 7.31 sex \\
\hline 14 rbl1871 & 264 chrZ_9997001 & 7.25 sex \\
\hline 14 rbl1871 & 264 chrZ_3015701 & 7.48 sex \\
\hline 14 rbl1871 & 264 chr1_577500 & 8.41 sex \\
\hline 14 rbl1871 & 264 chr4_149860। & 7.61 sex \\
\hline 14 rbl1871 & 264 chr13_24170 & 6.12 nonsex \\
\hline 14 rbl1871 & 265 chr14_14638। & 3.98 nonsex \\
\hline 14 rbl1871 & 265 chr4_645410। & 8.3 sex \\
\hline 14 rbl1871 & 265 chr5_296100। & 7.57 sex \\
\hline 14 rbl1871 & 265 chrZ_194050। & 8.63 sex \\
\hline 14 rbl1871 & 265 chrZ_2874101 & 7.2 sex \\
\hline 14 rbl1871 & 266 chr14_14990। & 5.8 nonsex \\
\hline
\end{tabular}




\begin{tabular}{|c|c|c|}
\hline 14 rbl1871 & 266 chr14_15273। & 5.8 nonsex \\
\hline 14 rbl1871 & 266 chr1_100190। & 7.58 sex \\
\hline 14 rbl1871 & 266 chr1_295510 & 7.87 sex \\
\hline 14 rbl1871 & 266 chr1_453070 & 7.29 sex \\
\hline 14 rbl1871 & 266 chr4_487720 & 7.09 sex \\
\hline 14 rbl1871 & 266 chr13_60390। & 7.42 sex \\
\hline 14 rbl1871 & 266 chrZ_1775101 & 7.76 sex \\
\hline 14 rbl1871 & 266 chrZ_1846101 & 7.72 sex \\
\hline 14 rbl1871 & 266 chrZ_2067101 & 7.41 sex \\
\hline 14 rbl1871 & 266 chrZ_2113201 & 6.78 sex \\
\hline 14 rbl1871 & 266 chrZ_3008301 & 8.06 sex \\
\hline 14 rbl1871 & 266 chrZ_329600। & 7.6 sex \\
\hline 14 rbl1871 & 266 chrZ_3385301 & 7.22 sex \\
\hline 14 rbl1871 & 266 chrZ_3614701 & 12.06 sex \\
\hline 14 rbl1871 & 266 chr1_867210। & 8.76 sex \\
\hline 15 Gg_rs157609 & 0 chr15_16840 & 6.67 nonsex \\
\hline 15 Gg_rs157609 & 0 chr15_28300 & 6.68 sex \\
\hline 15 Gg_rs157609 & 0 chr15_28310 & 5.22 sex \\
\hline 15 Gg_rs157609 & 0 chr15_28280 & 4.34 nonsex \\
\hline 15 Gg_rs157609 & 0 chr15_28290। & 3.76 nonsex \\
\hline 15 Gg_rs157609 & 0 chr1_897100। & 7.15 sex \\
\hline 15 Gg_rs140867. & 0 chr15_15350 & 5.33 nonsex \\
\hline 15 Gg_rs140871 & 0 chr15_28410 & 4.59 nonsex \\
\hline 15 Gg_rs140871 & 1 chr3_872990। & 7.56 sex \\
\hline 15 Gg_rs157609 & 3 chr15_38000। & 4.19 nonsex \\
\hline 15 Gg_rs140871. & 3 chr15_17270। & 4.54 nonsex \\
\hline 15 Gg_rs140871. & 4 chr15_15630 & 4.8 nonsex \\
\hline 15 Gg_rs140871. & 4 chr15_18300। & 4.39 nonsex \\
\hline 15 Gg_rs150194 & 5 chr15_24300 & 5.37 nonsex \\
\hline 15 Gg_rs157637 & 10 chr15_23710 & 3.45 nonsex \\
\hline 15 Gg_rs150194 & 17 chr11_12486। & 6.26 nonsex \\
\hline 15 Gg_rs150194 & 21 chr15_35970 & 4.94 nonsex \\
\hline 15 Gg_rs150194 & 21 chr15_23520। & 3.99 nonsex \\
\hline 15 Gg_rs150194 & 21 chr15_42690 & 4.44 nonsex \\
\hline 15 Gg_rs150194 & 29 chr15_27590 & 3.85 nonsex \\
\hline 15 Gg_rs150238 & 78 chr7_269200 & 9.98 sex \\
\hline 15 Gg_rs150238 & 78 chr1_164280 & 8.51 sex \\
\hline 15 Gg_rs150238 & 78 chr1_740290 & 9.26 sex \\
\hline 15 Gg_rs150238 & 78 chr1_804350 & 9.34 sex \\
\hline 15 Gg_rs150238 & 78 chr4_270730 & 8.62 sex \\
\hline 15 Gg_rs150238 & 78 chr1_329220। & 8.46 sex \\
\hline 15 Gg_rs150238 & 78 chrW_63450c & 9.45 sex \\
\hline 15 Gg_rs150238. & 78 chr4_194840 & 8.75 sex \\
\hline
\end{tabular}




\begin{tabular}{|c|c|c|}
\hline 15 Gg_rs150238. & 78 chr1_160219! & 9.24 sex \\
\hline 15 Gg_rs150238 & 78 chrZ_419170। & 8.37 sex \\
\hline 15 Gg_rs150238 & 78 chr2_458320 & 8.33 sex \\
\hline 15 Gg_rs150238 & 79 chr1_944360। & 8.36 sex \\
\hline 15 Gg_rs150238 & 79 chr2_398320 & 8.5 sex \\
\hline 15 Gg_rs150238 & 79 chrZ_4909001 & 8.58 sex \\
\hline 15 Gg_rs150238. & 79 chr4_382480 & 7.73 sex \\
\hline 15 Gg_rs150238 & 79 chr1_610960 & 8.23 sex \\
\hline 15 Gg_rs150238 & 79 chrW_25900( & 8.36 sex \\
\hline 15 Gg_rs150238 & 79 chrW_19010r & 9.67 sex \\
\hline 15 Gg_rs150238. & 79 chr3_753800 & 10.4 sex \\
\hline 15 Gg_rs150238 & 79 chr19_17740 & 8.39 sex \\
\hline 15 Gg_rs150238. & 79 chr1_153661। & 8.23 sex \\
\hline 15 Gg_rs150238. & 79 chr1_257860 & 8.7 sex \\
\hline 15 Gg_rs150238. & 79 chrW_17130r & 8.69 sex \\
\hline 15 Gg_rs150238 & 79 chr1_102872। & 8.46 sex \\
\hline 15 Gg_rs150238 & 79 chr2_123130 & 7.88 sex \\
\hline 15 Gg_rs150238 & 79 chrZ_4901001 & 8.35 sex \\
\hline 15 Gg_rs150238 & 79 chr2_405970 & 8.08 sex \\
\hline 15 Gg_rs150238 & 79 chrW_15670c & 9.14 sex \\
\hline 15 Gg_rs150238 & 79 chr1_155662 & 8.72 sex \\
\hline 15 Gg_rs150238 & 79 chrW_19730c & 8.47 sex \\
\hline 15 Gg_rs150238 & 79 chr2_233710 & 8.33 sex \\
\hline 15 Gg_rs150238 & 80 chr15_89990। & 6.56 sex \\
\hline 15 Gg_rs150238 & 80 chr5_340100 & 8.59 sex \\
\hline 15 Gg_rs150238. & 80 chr2_743860 & 7.84 sex \\
\hline 15 Gg_rs150238 & 80 chr2_510160 & 8.51 sex \\
\hline 15 Gg_rs150238 & 80 chr1_187951। & 7.69 sex \\
\hline 15 Gg_rs150238 & 80 chr1_167468। & 9.34 sex \\
\hline 15 Gg_rs150238. & 80 chrW_47410c & 8.52 sex \\
\hline 15 Gg_rs150238. & 80 chrW_10470c & 12 sex \\
\hline 15 Gg_rs150238 & 80 chr1_181868। & 8.22 sex \\
\hline 15 Gg_rs150238. & 80 chr3_535940 & 8.3 sex \\
\hline 15 Gg_rs150238. & 80 chr1_142791। & 8.65 sex \\
\hline 15 Gg_rs150238 & 80 chr4_224330 & 8.15 sex \\
\hline 15 Gg_rs150238 & 80 chrW_98700c & 9.68 sex \\
\hline 15 Gg_rs150238 & 80 chr2_148731। & 8.12 sex \\
\hline 15 Gg_rs150238 & 80 chr3_812890 & 10.27 sex \\
\hline 15 Gg_rs150238 & 80 chr1_151228। & 8.69 sex \\
\hline 15 Gg_rs150238 & 80 chr1_148437। & 8.52 sex \\
\hline 15 Gg_rs150238. & 80 chr3_798690 & 8.01 sex \\
\hline 15 Gg_rs150238 & 80 chrZ_4395401 & 8.12 sex \\
\hline 15 Gg_rs150238. & 80 chr1_194097। & 8.96 sex \\
\hline
\end{tabular}




\begin{tabular}{|c|c|c|}
\hline 15 Gg_rs150238. & 80 chr6_253990 & 8.05 sex \\
\hline 15 Gg_rs150238 & 80 chr3_698000 & 8.51 sex \\
\hline 15 Gg_rs150238 & 80 chr3_316750 & 8.05 sex \\
\hline 15 Gg_rs150238 & 80 chr2_712050 & 8.54 sex \\
\hline 15 Gg_rs150238 & 80 chr4_281410 & 7.96 sex \\
\hline 15 Gg_rs150238 & 80 chrW_10240c & 9.24 sex \\
\hline 15 Gg_rs150238. & 80 chr4_251100। & 7.71 sex \\
\hline 15 Gg_rs150238 & 80 chr1_841200 & 9.11 sex \\
\hline 15 Gg_rs150238 & 80 chr9_818700 & 7.75 sex \\
\hline 15 Gg_rs150238 & 80 chr2_954360। & 7.97 sex \\
\hline 15 Gg_rs150238. & 80 chr2_455870 & 7.7 sex \\
\hline 15 Gg_rs150238 & 80 chr5_326120 & 8.56 sex \\
\hline 15 Gg_rs150238. & 80 chr12_76480 & 10 sex \\
\hline 15 Gg_rs150238. & 80 chrW_52820( & 8.42 sex \\
\hline 15 Gg_rs150238. & 80 chr3_106007! & 8.36 sex \\
\hline 15 Gg_rs150238 & 80 chr3_733330 & 8.33 sex \\
\hline 15 Gg_rs150238 & 81 chrW_18530c & 8.94 sex \\
\hline 15 Gg_rs150238 & 81 chrW_50030c & 9.23 sex \\
\hline 15 Gg_rs150238 & 81 chr1_850810 & 8.06 sex \\
\hline 15 Gg_rs150238 & 81 chr2_952400। & 10.06 sex \\
\hline 15 Gg_rs150238 & 81 chr4_568130। & 8.19 sex \\
\hline 15 Gg_rs150238 & 81 chr1_151005! & 7.82 sex \\
\hline 15 Gg_rs150238 & 81 chr24_61400! & 8.32 sex \\
\hline 15 Gg_rs150238 & 81 chr1_153310 & 8.34 sex \\
\hline 15 Gg_rs150238 & 81 chr2_168420 & 8.03 sex \\
\hline 15 Gg_rs150238. & 81 chr3_654960। & 8.02 sex \\
\hline 15 Gg_rs150238. & 81 chr1_178220 & 7.81 sex \\
\hline 15 Gg_rs150238 & 81 chr2_595050 & 7.94 sex \\
\hline 15 Gg_rs150238 & 81 chr11_64080। & 8.8 sex \\
\hline 15 Gg_rs150238. & 81 chr1_741590 & 9.06 sex \\
\hline 15 Gg_rs150238. & 81 chr2_738310 & 7.66 sex \\
\hline 15 Gg_rs150238 & 81 chr3_616140 & 8.05 sex \\
\hline 15 Gg_rs150238. & 81 chr1_175210 & 7.68 sex \\
\hline 15 Gg_rs150238. & 81 chr6_753000 & 9.33 sex \\
\hline 15 Gg_rs150238 & 81 chr1_706100। & 8.06 sex \\
\hline 15 Gg_rs150238 & 81 chr4_182870 & 9.18 sex \\
\hline 15 Gg_rs150238 & 81 chr4_195000। & 7.71 sex \\
\hline 15 Gg_rs150238 & 81 chr1_565660 & 7.66 sex \\
\hline 15 Gg_rs150238 & 81 chr3_881800 & 7.57 sex \\
\hline 15 Gg_rs150238 & 81 chrW_95800c & 12.66 sex \\
\hline 15 Gg_rs150238 & 81 chr2_107938। & 8.46 sex \\
\hline 15 Gg_rs150238 & 81 chr3_532400! & 7.92 sex \\
\hline 15 Gg_rs150238. & 81 chr1_128405। & 7.62 sex \\
\hline
\end{tabular}




\begin{tabular}{|c|c|c|}
\hline 15 Gg_rs150238. & 82 chr15_97830। & 5.56 nonsex \\
\hline 15 Gg_rs150238 & 82 chr5_448220 & 7.77 sex \\
\hline 15 Gg_rs150238 & 82 chr5_498080 & 7.69 sex \\
\hline 15 Gg_rs150238 & 82 chr28_59700 & 9.84 sex \\
\hline 15 Gg_rs150238 & 82 chrW_50570( & $14.26 \mathrm{sex}$ \\
\hline 15 Gg_rs150238 & 82 chr2_282990 & 7.85 sex \\
\hline 15 Gg_rs150238 & 82 chr2_716170 & 8.52 sex \\
\hline 15 Gg_rs150238 & 82 chr17_10607। & 7.98 sex \\
\hline 15 Gg_rs150238 & 82 chr2_142065। & 7.6 sex \\
\hline 15 Gg_rs150238 & 82 chr9_186030 & 8.15 sex \\
\hline 15 Gg_rs150238. & 82 chr2_689340 & $8.58 \mathrm{sex}$ \\
\hline 15 Gg_rs150238. & 82 chrW_44080c & 12.03 sex \\
\hline 15 Gg_rs150238. & 82 chr4_562220 & 8.25 sex \\
\hline 15 Gg_rs150238. & 82 chr3_753450 & 7.57 sex \\
\hline 15 Gg_rs150238. & 82 chr1_656710। & 7.46 sex \\
\hline 15 Gg_rs150238 & 82 chr2_105439! & 8.13 sex \\
\hline 15 Gg_rs150238 & 82 chr10_10890 & 8.65 sex \\
\hline 15 Gg_rs150238 & 82 chr1_150681। & 7.51 sex \\
\hline 15 Gg_rs150238 & 82 chr1_164414! & 8.57 sex \\
\hline 15 Gg_rs150238 & 82 chr5_248800 & 7.5 sex \\
\hline 15 Gg_rs150238 & 82 chrZ_4134201 & 8.61 sex \\
\hline 15 Gg_rs150238. & 82 chr1_475320 & 8.59 sex \\
\hline 15 Gg_rs150238 & 82 chr2_133390 & 7.82 sex \\
\hline 15 Gg_rs150238 & 82 chrW_54960c & $12.41 \mathrm{sex}$ \\
\hline 15 Gg_rs150238 & 82 chr2_1084381 & 8.64 sex \\
\hline 15 Gg_rs150238. & 82 chr13_17336 & 7.66 sex \\
\hline 15 Gg_rs150238. & 82 chr1_734500 & 7.57 sex \\
\hline 15 Gg_rs150238. & 82 chr4_412630। & 8.21 sex \\
\hline 15 Gg_rs150238 & 82 chr1_103404! & 7.53 sex \\
\hline 15 Gg_rs150238. & 82 chr2_148748। & 7.68 sex \\
\hline 15 Gg_rs150238. & 82 chr2_136910 & 7.92 sex \\
\hline 15 Gg_rs150238. & 83 chr1_525900। & 8.38 sex \\
\hline 15 Gg_rs150238. & 83 chr1_602900। & 8.1 sex \\
\hline 15 Gg_rs150238. & 83 chr1_930970। & 8.87 sex \\
\hline 15 Gg_rs150238 & 83 chr3_107908। & 8.2 sex \\
\hline 15 Gg_rs150238 & 83 chr3_109104! & 10.03 sex \\
\hline 15 Gg_rs150238 & 83 chr1_818480 & 7.78 sex \\
\hline 15 Gg_rs150238 & 83 chr4_201620 & 8.6 sex \\
\hline 15 Gg_rs150238 & 83 chr6_768100 & 7.48 sex \\
\hline 15 Gg_rs150238 & 83 chr4_230360। & 7.8 sex \\
\hline 15 Gg_rs150238 & 83 chr4_264310 & 8.6 sex \\
\hline 15 Gg_rs150238 & 83 chr1_101386 & 7.87 sex \\
\hline 15 Gg_rs150238 & 83 chr4_279350 & 8.07 sex \\
\hline
\end{tabular}




\begin{tabular}{|c|c|c|}
\hline 15 Gg_rs150238. & 83 chr1_143010 & 8.82 sex \\
\hline 15 Gg_rs150238 & 83 chr3_938000। & 7.84 sex \\
\hline 15 Gg_rs150238 & 83 chr7_803100। & 7.31 sex \\
\hline 15 Gg_rs150238 & 83 chrZ_5929901 & 7.92 sex \\
\hline 15 Gg_rs150238 & 83 chr4_723650 & 7.97 sex \\
\hline 15 Gg_rs150238 & 83 chr3_918370 & 7.84 sex \\
\hline 15 Gg_rs150238. & 83 chr1_150541। & 8.58 sex \\
\hline 15 Gg_rs150238 & 83 chrZ_4984601 & 7.63 sex \\
\hline 15 Gg_rs150238 & 83 chr3_110138ı & 7.38 sex \\
\hline 15 Gg_rs150238 & 83 chr1_213670 & 9.59 sex \\
\hline 15 Gg_rs150238. & 83 chrW_19850c & 9.74 sex \\
\hline 15 Gg_rs150238 & 83 chrZ_4369401 & 7.99 sex \\
\hline 15 Gg_rs150238. & 83 chr1_777970 & 7.5 sex \\
\hline 15 Gg_rs150238. & 84 chr1_916200 & 8.34 sex \\
\hline 15 Gg_rs150238. & 84 chr6_351140 & 7.39 sex \\
\hline 15 Gg_rs150238 & 84 chr27_49230। & 10.57 sex \\
\hline 15 Gg_rs150238 & 84 chr2_687460 & 7.48 sex \\
\hline 15 Gg_rs150238 & 84 chr2_953090। & 8.76 sex \\
\hline 15 Gg_rs150238 & 84 chrZ_618100। & 7.85 sex \\
\hline 15 Gg_rs150238 & 84 chr1_105898। & 8.62 sex \\
\hline 15 Gg_rs150238 & 84 chr2_516860 & 7.59 sex \\
\hline 15 Gg_rs150238 & 84 chr1_153057। & 8.02 sex \\
\hline 15 Gg_rs150238 & 84 chr1_151794! & 7.61 sex \\
\hline 15 Gg_rs150238 & 84 chr1_127922। & 7.67 sex \\
\hline 15 Gg_rs150238 & 84 chrZ_616150। & 7.24 sex \\
\hline 15 Gg_rs150238. & 84 chr6_281300 & 9.52 sex \\
\hline 15 Gg_rs150238 & 84 chr2_543630 & 7.31 sex \\
\hline 15 Gg_rs150238 & 84 chr1_159279| & 7.27 sex \\
\hline 15 Gg_rs150238 & 84 chrZ_453510। & 7.56 sex \\
\hline 15 Gg_rs150238. & 84 chr3_817430 & 7.58 sex \\
\hline 15 Gg_rs150238. & 84 chr1_125785 & 7.4 sex \\
\hline 15 Gg_rs150238. & 84 chr4_411350 & 7.39 sex \\
\hline 15 Gg_rs150238. & 84 chrW_52000 & 9.62 sex \\
\hline 15 Gg_rs150238. & 84 chr3_813040 & 9.26 sex \\
\hline 15 Gg_rs150238 & 84 chrZ_6494601 & 8.66 sex \\
\hline 15 Gg_rs150238 & 84 chr2_724030 & 8.31 sex \\
\hline 15 Gg_rs150238 & 84 chr4_277720 & 7.6 sex \\
\hline 15 Gg_rs150238 & 84 chr1_179379! & 7.59 sex \\
\hline 15 Gg_rs150238 & 84 chr6_186600 & 8.31 sex \\
\hline 15 Gg_rs150238 & 85 chrZ_156410। & 8.02 sex \\
\hline 15 Gg_rs150238 & 85 chr1_109380 & 7.02 sex \\
\hline 15 Gg_rs150238 & 85 chr2_722790 & 7.56 sex \\
\hline 15 Gg_rs150238. & 85 chr1_157079ı & 7.31 sex \\
\hline
\end{tabular}




\begin{tabular}{|c|c|c|}
\hline 15 Gg_rs150238. & 85 chr2_952330 & 7.78 sex \\
\hline 15 Gg_rs150238 & 85 chr2_121680 & 7.78 sex \\
\hline 15 Gg_rs150238 & 85 chr1_163703। & 8.82 sex \\
\hline 15 Gg_rs150238 & 85 chrZ_6453001 & 7.67 sex \\
\hline 15 Gg_rs150238 & 85 chrW_64670 & 8.32 sex \\
\hline 15 Gg_rs150238 & 85 chrZ_5900601 & 7.22 sex \\
\hline 15 Gg_rs150238. & 85 chr2_330410 & 7.78 sex \\
\hline 15 Gg_rs150238 & 85 chr3_845770 & 7.33 sex \\
\hline 15 Gg_rs150238 & 85 chr1_138865। & 7.6 sex \\
\hline 15 Gg_rs150238 & 85 chrW_23940r & 7.76 sex \\
\hline 15 Gg_rs150238. & 85 chr1_165612 & 7.96 sex \\
\hline 15 Gg_rs150238 & 85 chr1_917600। & 7.41 sex \\
\hline 15 Gg_rs150238. & 85 chr5_481200 & 8.46 sex \\
\hline 15 Gg_rs150238. & 86 chr1_106270 & 7.44 sex \\
\hline 15 Gg_rs150238. & 86 chr3_339720 & 7.25 sex \\
\hline 15 Gg_rs150238 & 86 chr2_134763। & 8.7 sex \\
\hline 15 Gg_rs150238 & 86 chr1_1231381 & 7.8 sex \\
\hline 15 Gg_rs150238 & 86 chr1_146032। & 7.97 sex \\
\hline 15 Gg_rs150238 & 86 chr3_901590। & 8.34 sex \\
\hline 15 Gg_rs150238 & 86 chr1_954610 & 7.62 sex \\
\hline 15 Gg_rs150238 & 86 chr1_161386! & 7.72 sex \\
\hline 15 Gg_rs150238 & 87 chrW_22500c & 9.75 sex \\
\hline 15 Gg_rs150238 & 87 chrZ_5007001 & 7.55 sex \\
\hline 15 Gg_rs150238 & 87 chrZ_2977301 & 7.64 sex \\
\hline 15 Gg_rs150238 & 87 chr2_780770 & 7.65 sex \\
\hline 15 Gg_rs150238. & 87 chr1_103645। & 7.84 sex \\
\hline 15 Gg_rs150238 & 87 chr1_156059 & 8.86 sex \\
\hline 15 Gg_rs150238 & 87 chr2_375560। & 7.48 sex \\
\hline 15 Gg_rs150238 & 87 chr1_965650। & 7.23 sex \\
\hline 15 Gg_rs150238. & 87 chr3_598120 & 7.61 sex \\
\hline 15 Gg_rs150238. & 87 chr4_722700 & 7.15 sex \\
\hline 15 Gg_rs150238. & 88 chr5_424520 & 8.19 sex \\
\hline 15 Gg_rs150238. & 88 chrW_47980( & 9.36 sex \\
\hline 15 Gg_rs150238. & 88 chr3_108822। & 7.91 sex \\
\hline 15 Gg_rs150238 & 88 chr1_416670 & 7.05 sex \\
\hline 15 Gg_rs150238 & 88 chr4_253030। & 7.97 sex \\
\hline 15 Gg_rs150238 & 88 chr3_1035981 & 7.33 sex \\
\hline 15 Gg_rs150238 & 88 chr3_861810 & 7.57 sex \\
\hline 15 Gg_rs150238 & 88 chr1_157087। & 9.85 sex \\
\hline 15 Gg_rs150238 & 88 chr5_593490। & 7.29 sex \\
\hline 15 Gg_rs150238 & 89 chr7_165400 & 6.37 sex \\
\hline 15 Gg_rs150238 & 89 chr2_791420 & 8.41 sex \\
\hline 15 Gg_rs150238. & 89 chr1_583020। & 7.49 sex \\
\hline
\end{tabular}




\begin{tabular}{|c|c|c|}
\hline 15 Gg_rs150238. & 89 chr1_182873। & 7.55 sex \\
\hline 15 Gg_rs150238 & 89 chr1_763290 & 7.14 sex \\
\hline 15 Gg_rs150238 & 89 chr1_916100 & 7.24 sex \\
\hline 15 Gg_rs150238 & 89 chr5_587710 & 7.9 sex \\
\hline 15 Gg_rs150238 & 90 chr15_78160 & 5.67 sex \\
\hline 15 Gg_rs150238 & 90 chr7_710400 & 10.56 sex \\
\hline 15 Gg_rs150238 & 90 chr12_89200 & 7.43 sex \\
\hline 15 Gg_rs150238 & 90 chrW_50240( & 8.08 sex \\
\hline 15 Gg_rs150238 & 90 chrZ_102010। & 7.54 sex \\
\hline 15 Gg_rs150238 & 90 chr1_939760 & 7.41 sex \\
\hline 15 Gg_rs150238. & 90 chr3_697800 & 5.6 nonsex \\
\hline 15 Gg_rs150238. & 90 chr14_12117। & 7.47 sex \\
\hline 15 Gg_rs150238. & 90 chr1_114744। & 7.26 sex \\
\hline 15 Gg_rs150238. & 90 chr1_188435। & 7.48 sex \\
\hline 15 Gg_rs150238. & 90 chr1_146186। & 7.17 sex \\
\hline 15 Gg_rs150238 & 90 chr1_115044। & 7.4 sex \\
\hline 15 Gg_rs150238 & 90 chrZ_374050। & 7.19 sex \\
\hline 15 Gg_rs150238 & 90 chr2_122586 & 7.22 sex \\
\hline 15 Gg_rs150238 & 90 chr1_120430 & 7.91 sex \\
\hline 15 Gg_rs150238 & 90 chr1_528840 & 7.23 sex \\
\hline 15 Gg_rs150238 & 90 chr1_499970 & 7.29 sex \\
\hline 15 Gg_rs150238 & 90 chr3_186570 & 7.47 sex \\
\hline 15 Gg_rs150238 & 91 chr2_145324! & 7.14 sex \\
\hline 15 Gg_rs150238 & 91 chr1_831980 & 8.91 sex \\
\hline 15 Gg_rs150238 & 92 chr5_254600 & 7.45 sex \\
\hline 15 Gg_rs150238. & 92 chr1_160156। & 8.39 sex \\
\hline 15 Gg_rs150238. & 92 chr1_113854। & 7.3 sex \\
\hline 15 Gg_rs150238. & 93 chr12_67860। & 7.58 sex \\
\hline 15 Gg_rs150238 & 93 chr5_404250 & 8.12 sex \\
\hline 15 Gg_rs150238. & 93 chr1_115362 & 7.27 sex \\
\hline 15 Gg_rs150238. & 93 chrZ_5956201 & 7.36 sex \\
\hline 15 Gg_rs150238. & 93 chr1_110163। & 7.36 sex \\
\hline 15 Gg_rs150238. & 93 chr2_413250। & 7.8 sex \\
\hline 15 Gg_rs150238. & 93 chr2_977240। & 7.21 sex \\
\hline 15 Gg_rs150238 & 93 chr28_60000 & 7.14 sex \\
\hline 15 Gg_rs150238 & 93 chr2_115539! & 7.94 sex \\
\hline 15 Gg_rs150238 & 94 chr7_153940 & 7.89 sex \\
\hline 15 Gg_rs150238 & 94 chr1_110928। & 7.33 sex \\
\hline 15 Gg_rs150238 & 94 chr1_158270 & 7.46 sex \\
\hline 15 Gg_rs150238 & 94 chr2_102281। & 7.7 sex \\
\hline 15 Gg_rs150238 & 94 chr3_378590 & 7.15 sex \\
\hline 15 Gg_rs150238 & 94 chr3_217340 & 7.56 sex \\
\hline 15 Gg_rs150238. & $94 \mathrm{chr} 2 \_433200$ & 8.15 sex \\
\hline
\end{tabular}




\begin{tabular}{|c|c|c|c|}
\hline 15 Gg_rs150238. & 95 chr15_10805। & 6.35 sex & cis \\
\hline 15 Gg_rs150238 & 95 chr1_110929। & 7.24 sex & trans \\
\hline 15 Gg_rs150238 & 95 chr2_126867। & 8.91 sex & trans \\
\hline 15 Gg_rs150238 & 96 chr6_356600 & 7.77 sex & ans \\
\hline 15 Gg_rs150238 & 96 chr3_1092331 & $7.18 \mathrm{sex}$ & ans \\
\hline 15 Gg_rs150238 & 96 chr1_137096। & 7.4 sex & ans \\
\hline 15 Gg_rs150238 & 96 chrW_45080( & 7.93 sex & trans \\
\hline 15 Gg_rs150238 & 96 chr1_348590 & 7.95 sex & trans \\
\hline 15 Gg_rs150238 & 96 chr4_713200 & 8.35 sex & \\
\hline 15 Gg_rs150238 & 96 chr1_160066। & 7.25 sex & ans \\
\hline 15 Gg_rs150238. & 96 chr3_104684! & 7.28 sex & \\
\hline 15 Gg_rs150238. & 96 chr2_718910 & 7.34 sex & trans \\
\hline 15 Gg_rs150238. & 97 chr5_500070 & 10.17 sex & trans \\
\hline 15 Gg_rs150238. & 97 chr14_14746। & 7.53 sex & trans \\
\hline 15 Gg_rs150238. & 97 chr1_108537। & 8.19 sex & trans \\
\hline 15 Gg_rs150238 & 97 chr3_1074361 & 8.84 sex & \\
\hline 15 Gg_rs150238 & 97 chr5_404260 & 7.29 sex & trans \\
\hline 15 Gg_rs150238 & 97 chr2_143953। & 7.53 sex & trans \\
\hline 15 Gg_rs150238 & 97 chrZ_602170। & 8.27 sex & \\
\hline 15 Gg_rs150238 & 97 chr1_121958। & 8.01 sex & \\
\hline 15 Gg_rs150238 & 97 chr19_86000 & 8.84 sex & \\
\hline 15 Gg_rs150238. & 97 chr4_869560। & 8.47 sex & trans \\
\hline 15 Gg_rs150238 & 98 chr1_683600 & 9.24 sex & trans \\
\hline 15 Gg_rs150238 & 98 chr7_332990। & 7.56 sex & \\
\hline 15 Gg_rs150238 & 98 chrZ_301170। & 7.61 sex & \\
\hline 15 Gg_rs150238. & 98 chr1_129372। & 8.9 sex & \\
\hline 15 Gg_rs150238. & 98 chr3_221390। & 7.29 sex & trans \\
\hline 15 Gg_rs150238. & 98 chr2_118914। & 8.37 sex & tra \\
\hline 15 Gg_rs150238 & 98 chrW_34540c & 7.31 sex & \\
\hline 15 Gg_rs150238. & 98 chr3_449650 & 7.75 sex & \\
\hline 15 Gg_rs150238. & 98 chr4_362830। & 7.18 sex & \\
\hline 15 Gg_rs150238. & 98 chr8_104260। & 7.77 sex & trans \\
\hline 15 Gg_rs150238. & 98 chr1_202760। & 7.01 sex & trans \\
\hline 15 Gg_rs150238. & 98 chr1_812300। & 7.97 sex & trans \\
\hline 15 Gg_rs150238 & 98 chr3_700370 & 7.73 sex & \\
\hline 15 Gg_rs150238 & 98 chr1_168983 & 7.17 sex & trans \\
\hline 15 Gg_rs150238 & 98 chrW_20980( & 8.47 sex & trans \\
\hline 15 Gg_rs150238 & 98 chrW_57190( & 7.54 sex & trans \\
\hline 15 Gg_rs150238 & 98 chr1_837290 & 7.26 sex & trans \\
\hline 15 Gg_rs150238 & 99 chr7_657700 & 7.7 sex & \\
\hline 15 Gg_rs150238 & 99 chr28_17500 & 7.42 sex & trans \\
\hline 15 Gg_rs150238 & 99 chr2_137815। & 9.06 sex & trans \\
\hline 15 Gg_rs150238 & 99 chr1_124357। & 7.98 sex & \\
\hline
\end{tabular}




\begin{tabular}{|c|c|c|}
\hline 15 Gg_rs150238. & 99 chr2_123701। & 7.51 sex \\
\hline 15 Gg_rs150238 & 99 chr31_77800। & 7.17 sex \\
\hline 15 Gg_rs150238 & 99 chr1_103071। & 8.33 sex \\
\hline 15 Gg_rs150238 & 100 chr28_18500 & 7.34 sex \\
\hline 15 Gg_rs150238 & 100 chr1_915260 & 7.51 sex \\
\hline 15 Gg_rs150238 & 100 chr1_121910 & 7.72 sex \\
\hline 15 Gg_rs150238. & 100 chr2_132252 & 7.24 sex \\
\hline 15 Gg_rs150238 & 100 chr1_831970 & 8.57 sex \\
\hline 15 Gg_rs150238 & 100 chr1_128447| & 9.6 sex \\
\hline 15 Gg_rs150238 & 100 chr7_875000 & 7.34 sex \\
\hline 15 Gg_rs150238. & 100 chr2_131675। & 8.75 sex \\
\hline 15 Gg_rs150238 & 100 chr1_134449! & 7.62 sex \\
\hline 15 Gg_rs150238. & 100 chr2_111968। & 7.44 sex \\
\hline 15 Gg_rs150238. & 100 chrZ_5262201 & 7.55 sex \\
\hline 15 Gg_rs150238. & 100 chr2_516230 & 7.25 sex \\
\hline 15 Gg_rs150238 & 100 chr1_118912 & 8.08 sex \\
\hline 15 Gg_rs150238 & 100 chr7_101300 & 7.3 sex \\
\hline 15 Gg_rs150238 & 100 chr3_667020 & 8.49 sex \\
\hline 15 Gg_rs150238 & 100 chr2_153270 & 7.27 sex \\
\hline 15 Gg_rs150238 & 100 chr2_440320 & 7.66 sex \\
\hline 15 Gg_rs150238 & 100 chr1_137350 & 7.21 sex \\
\hline 15 Gg_rs150238 & 101 chr15_80200 & 5.92 nonsex \\
\hline 15 Gg_rs150238 & 101 chr6_355390 & 8.88 sex \\
\hline 15 Gg_rs150238 & 101 chrZ_2280301 & $7.4 \mathrm{sex}$ \\
\hline 15 Gg_rs150238 & 101 chr2_791320 & 7.83 sex \\
\hline 15 Gg_rs150238. & 101 chr4_231600 & 7.59 sex \\
\hline 15 Gg_rs150238 & 101 chr2_893370 & 7.84 sex \\
\hline 15 Gg_rs150238 & 101 chrW_92400( & 16.3 sex \\
\hline 15 Gg_rs150238 & 101 chr4_553560 & 7.78 sex \\
\hline 15 Gg_rs150238. & 101 chrW_35580 & 7.2 sex \\
\hline 15 Gg_rs150238. & 101 chr1_121725। & 7.79 sex \\
\hline 15 Gg_rs150238. & 101 chr2_903040। & 7.54 sex \\
\hline 15 Gg_rs150238. & 101 chr1_699580 & 7.86 sex \\
\hline 15 Gg_rs150238. & 101 chr27_24580 & 7.41 sex \\
\hline 15 Gg_rs150238 & 101 chr4_274120 & $7.28 \mathrm{sex}$ \\
\hline 15 Gg_rs150238 & 101 chr3_840480 & 7.61 sex \\
\hline 15 Gg_rs150238 & 101 chr6_182340 & 10.77 sex \\
\hline 15 Gg_rs150238 & 101 chr1_110106। & 8.02 sex \\
\hline 15 Gg_rs150238 & 101 chrW_22100( & 7.32 sex \\
\hline 15 Gg_rs150238 & 101 chr2_838750 & 7.5 sex \\
\hline 15 Gg_rs150238 & 102 chr15_77670 & 6.34 sex \\
\hline 15 Gg_rs150238 & 102 chr28_13700। & 7.18 sex \\
\hline 15 Gg_rs150238. & 102 chr28_14600। & 7.37 sex \\
\hline
\end{tabular}




\begin{tabular}{|c|c|c|}
\hline 15 Gg_rs150238. & 102 chr2_133187। & 7.84 sex \\
\hline 15 Gg_rs150238 & 102 chr11_18120। & 7.49 sex \\
\hline 15 Gg_rs150238 & 102 chr3_100774! & 7.46 sex \\
\hline 15 Gg_rs150238 & 102 chr1_150711। & $9.11 \mathrm{sex}$ \\
\hline 15 Gg_rs150238 & 102 chr2_884690 & 7.98 sex \\
\hline 15 Gg_rs150238 & 102 chr1_106085। & 7.36 sex \\
\hline 15 Gg_rs150238 & 102 chr1_115045। & 8.65 sex \\
\hline 15 Gg_rs150238 & 102 chr16_18980 & 9.33 sex \\
\hline 15 Gg_rs150238 & 102 chr1_181200। & 7.54 sex \\
\hline 15 Gg_rs150238 & 102 chrW_66120( & 9.35 sex \\
\hline 15 Gg_rs150238. & 102 chr1_115606। & 7.8 sex \\
\hline 15 Gg_rs150238. & 102 chr1_115801। & 7.58 sex \\
\hline 15 Gg_rs150238. & 102 chr4_828230। & 7.43 sex \\
\hline 15 Gg_rs150238. & 102 chr1_103644! & 7.28 sex \\
\hline 15 Gg_rs150238. & 102 chr1_115361। & 7.36 sex \\
\hline 15 Gg_rs150238 & 103 chr4_651800 & 7.74 sex \\
\hline 15 Gg_rs150238 & 103 chr24_62290। & 8.22 sex \\
\hline 15 Gg_rs150238 & 103 chrZ_566700। & 7.91 sex \\
\hline 15 Gg_rs150238 & 103 chr2_528870 & 8.27 sex \\
\hline 15 Gg_rs150238 & 103 chr1_162429 & 8.71 sex \\
\hline 15 Gg_rs150238 & 103 chr2_137955 & 7.63 sex \\
\hline 15 Gg_rs150238 & 103 chr1_728260 & 8.32 sex \\
\hline 15 Gg_rs150238 & 103 chr1_162758 & 7.53 sex \\
\hline 15 Gg_rs150238 & 103 chr1_152131। & 7.32 sex \\
\hline 15 Gg_rs150238 & 103 chr9_228100 & 7.39 sex \\
\hline 15 Gg_rs150238. & 103 chrZ_3355701 & 7.5 sex \\
\hline 15 Gg_rs150238. & 103 chr1_186800 & 7.38 sex \\
\hline 15 Gg_rs150238. & 103 chrZ_6825701 & 7.84 sex \\
\hline 15 Gg_rs150238 & 103 chr33_28310 & 7.64 sex \\
\hline 15 Gg_rs150238. & 103 chrW_35170 & 9.18 sex \\
\hline 15 Gg_rs150238. & 103 chr1_152927। & 7.76 sex \\
\hline 15 Gg_rs150238. & 103 chr2_123415। & 7.35 sex \\
\hline 15 Gg_rs150238. & 103 chr1_134780 & 7.67 sex \\
\hline 15 Gg_rs150238. & 103 chr1_115823। & 7.65 sex \\
\hline 15 Gg_rs150238 & 103 chr1_130641। & 7.71 sex \\
\hline 15 Gg_rs150238 & 103 chrW_11390 & 9.02 sex \\
\hline 15 Gg_rs150238 & 104 chrW_18820 & 14.2 sex \\
\hline 15 Gg_rs150238 & 104 chrW_53770 & 8.43 sex \\
\hline 15 Gg_rs150238 & 104 chr2_133376 & 7.43 sex \\
\hline 15 Gg_rs150238 & 104 chr1_118892 & 8.17 sex \\
\hline 15 Gg_rs150238 & 104 chr1_151275। & 7.8 sex \\
\hline 15 Gg_rs150238 & 104 chr1_153850 & 8.35 sex \\
\hline 15 Gg_rs150238 & 104 chr1_143430। & 7.79 sex \\
\hline
\end{tabular}




\begin{tabular}{|c|c|c|}
\hline 15 Gg_rs150238. & 104 chrZ_6938501 & 8.31 sex \\
\hline 15 Gg_rs150238 & 104 chrW_91500 & 7.67 sex \\
\hline 15 Gg_rs150238 & 104 chr1_162438। & 8.19 sex \\
\hline 15 Gg_rs150238 & 104 chr1_108320 & 7.36 sex \\
\hline 15 Gg_rs150238 & 104 chrW_23550 & 13.61 sex \\
\hline 15 Gg_rs150238 & 104 chr4_678120 & 7.67 sex \\
\hline 15 Gg_rs150238 & 104 chrZ_319780। & 7.72 sex \\
\hline 15 Gg_rs150238 & 104 chr3_102501। & 7.68 sex \\
\hline 15 Gg_rs150238 & 105 chr15_63610 & 5.77 sex \\
\hline 15 Gg_rs150238 & 105 chr6_353320 & 8.03 sex \\
\hline 15 Gg_rs150238. & 105 chr28_53100। & 8.28 sex \\
\hline 15 Gg_rs150238. & 105 chr2_119495। & 8.03 sex \\
\hline 15 Gg_rs150238. & 105 chr3_412300 & 7.39 sex \\
\hline 15 Gg_rs150238. & 105 chr1_728540। & 7.54 sex \\
\hline 15 Gg_rs150238. & 105 chr2_129241। & 8.35 sex \\
\hline 15 Gg_rs150238 & 105 chr2_133230 & 8.4 sex \\
\hline 15 Gg_rs150238 & 105 chr1_115624। & 8.06 sex \\
\hline 15 Gg_rs150238 & 105 chr2_121965। & 8.77 sex \\
\hline 15 Gg_rs150238 & 105 chr5_214640 & 7.9 sex \\
\hline 15 Gg_rs150238 & 105 chr1_136705। & 7.53 sex \\
\hline 15 Gg_rs150238 & 105 chr1_143358। & 7.98 sex \\
\hline 15 Gg_rs150238. & 105 chr10_18420 & 7.36 sex \\
\hline 15 Gg_rs150238 & 105 chrZ_2974401 & 8.02 sex \\
\hline 15 Gg_rs150238 & 105 chr1_421440। & 7.95 sex \\
\hline 15 Gg_rs150238 & 105 chr3_353400 & 7.74 sex \\
\hline 15 Gg_rs150238. & 106 chr31_39250। & 7.54 sex \\
\hline 15 Gg_rs150238. & 106 chrW_10400 & 8.35 sex \\
\hline 15 Gg_rs150238. & 106 chrZ_861400। & 9.26 sex \\
\hline 15 Gg_rs150238 & 106 chr1_115645। & 7.61 sex \\
\hline 15 Gg_rs150238. & 106 chr1_124895। & 7.68 sex \\
\hline 15 Gg_rs150238. & 106 chr1_162377। & 7.56 sex \\
\hline 15 Gg_rs150238. & 106 chr3_402470 & 7.52 sex \\
\hline 15 Gg_rs150238. & 106 chr2_962760। & 8.16 sex \\
\hline 15 Gg_rs150238. & 106 chr2_128780 & 8.2 sex \\
\hline 15 Gg_rs150238 & 106 chr13_57620 & 7.85 sex \\
\hline 15 Gg_rs150238 & 106 chr6_128290 & 7.81 sex \\
\hline 15 Gg_rs150238 & 106 chrW_66430 & 8.93 sex \\
\hline 15 Gg_rs150238 & 106 chrZ_7276301 & 7.4 sex \\
\hline 15 Gg_rs150238 & 106 chr3_642240 & 8.27 sex \\
\hline 15 Gg_rs150238 & 106 chr1_415480 & 8.24 sex \\
\hline 15 Gg_rs150238 & 106 chr2_722370 & 7.94 sex \\
\hline 15 Gg_rs150238 & 106 chr27_24470। & 7.73 sex \\
\hline 15 Gg_rs150238 & 106 chr1_142173। & 8.72 sex \\
\hline
\end{tabular}




\begin{tabular}{|c|c|c|}
\hline 15 Gg_rs150238. & 107 chr12_14003। & 9.12 sex \\
\hline 15 Gg_rs150238 & 107 chrW_17640 & 8.05 sex \\
\hline 15 Gg_rs150238. & 107 chrW_16340 & $9.2 \mathrm{sex}$ \\
\hline 15 Gg_rs150238 & 107 chr3_409000 & 7.79 sex \\
\hline 15 Gg_rs150238 & 107 chr1_115354। & 7.69 sex \\
\hline 15 Gg_rs150238. & 107 chrW_58530c & 7.56 sex \\
\hline 15 Gg_rs150238. & 107 chrZ_6518401 & 7.52 sex \\
\hline 15 Gg_rs150238. & 107 chr2_149026 & 7.8 sex \\
\hline 15 Gg_rs150238. & 107 chrW_12350 & 9.55 sex \\
\hline 15 Gg_rs150238 & 107 chrW_10340 & 8.34 sex \\
\hline 15 Gg_rs150238. & 107 chr1_164415। & 7.64 sex \\
\hline 15 Gg_rs150238 & 107 chr2_791400। & 7.79 sex \\
\hline 15 Gg_rs150238. & 107 chr1_606470 & 7.85 sex \\
\hline 15 Gg_rs150238. & 107 chr3_223020। & 7.4 sex \\
\hline 15 Gg_rs150238. & 107 chr4_279410। & 8.65 sex \\
\hline 15 Gg_rs150238 & 108 chr7_579300 & 8.71 sex \\
\hline 15 Gg_rs150238 & 108 chr7_843400 & 7.97 sex \\
\hline 15 Gg_rs150238 & 108 chrZ_125010। & 8.35 sex \\
\hline 15 Gg_rs150238 & 108 chrZ_2384701 & 8.21 sex \\
\hline 15 Gg_rs150238 & 108 chr4_378470 & 7.94 sex \\
\hline 15 Gg_rs150238 & 108 chrW_58220 & 7.84 sex \\
\hline 15 Gg_rs150238 & 108 chr1_323010 & 7.58 sex \\
\hline 15 Gg_rs150238 & 108 chrW_97100 & 10.61 sex \\
\hline 15 Gg_rs150238 & 108 chr1_107877। & 7.85 sex \\
\hline 15 Gg_rs150238 & 108 chr2_325190 & 8.05 sex \\
\hline 15 Gg_rs150238. & 108 chrW_65030 & 7.52 sex \\
\hline 15 Gg_rs150238. & 108 chrW_66830 & 7.65 sex \\
\hline 15 Gg_rs150238. & 108 chrW_13370 & 7.74 sex \\
\hline 15 Gg_rs150238 & 108 chr4_193990। & 7.81 sex \\
\hline 15 Gg_rs150238. & 108 chr1_443120 & 7.73 sex \\
\hline 15 Gg_rs150238. & 108 chrW_19230 & 9.49 sex \\
\hline 15 Gg_rs150238. & 109 chr15_79610 & 8.14 sex \\
\hline 15 Gg_rs150238. & 109 chr23_53620। & 8.17 sex \\
\hline 15 Gg_rs150238. & 109 chr27_18000 & 10.12 sex \\
\hline 15 Gg_rs150238 & 109 chrZ_542400। & 9.14 sex \\
\hline 15 Gg_rs150238 & 109 chr1_923450। & 8.21 sex \\
\hline 15 Gg_rs150238 & 109 chrW_22060 & 8.08 sex \\
\hline 15 Gg_rs150238 & 109 chr4_713090 & 7.97 sex \\
\hline 15 Gg_rs150238 & 109 chrW_25490( & 7.93 sex \\
\hline 15 Gg_rs150238 & 109 chrW_13160 & 9.17 sex \\
\hline 15 Gg_rs150238 & 109 chrW_17400 & 8.34 sex \\
\hline 15 Gg_rs150238 & 109 chr3_912780 & 7.73 sex \\
\hline 15 Gg_rs150238. & 109 chr1_735320। & 8.28 sex \\
\hline
\end{tabular}




\begin{tabular}{|c|c|c|}
\hline 15 Gg_rs150238. & 109 chrW_57760 & 9.02 sex \\
\hline 15 Gg_rs150238 & 109 chr1_129267। & 7.63 sex \\
\hline 15 Gg_rs150238 & 109 chr33_38510 & 7.94 sex \\
\hline 15 Gg_rs150238 & 109 chrW_59290 & 8.25 sex \\
\hline 15 Gg_rs150238 & 110 chr15_91910 & 9.27 nonsex \\
\hline 15 Gg_rs150238. & 110 chr7_335000 & 9.41 sex \\
\hline 15 Gg_rs150238. & 110 chrZ_167200। & 8.84 sex \\
\hline 15 Gg_rs150238. & 110 chr2_959860। & 8.01 sex \\
\hline 15 Gg_rs150238 & 110 chrW_28490( & 9.73 sex \\
\hline 15 Gg_rs150238 & 110 chrW_28350 & 7.9 sex \\
\hline 15 Gg_rs150238. & 110 chrW_57500 & 7.86 sex \\
\hline 15 Gg_rs150238. & 110 chrW_63770 & 9.23 sex \\
\hline 15 Gg_rs150238. & 110 chr4_741020 & 9.66 sex \\
\hline 15 Gg_rs150238. & 110 chr22_50160। & 8.07 sex \\
\hline 15 Gg_rs150238. & 110 chr4_307210 & 8.35 sex \\
\hline 15 Gg_rs150238 & 110 chrW_66290( & 8.37 sex \\
\hline 15 Gg_rs150238 & 110 chrW_39210 & 8.54 sex \\
\hline 15 Gg_rs150238 & 110 chrW_19120 & 13.81 sex \\
\hline 15 Gg_rs150238 & 110 chrW_33970 & 10.36 sex \\
\hline 15 Gg_rs150238 & 110 chr2_405830 & 8.8 sex \\
\hline 15 Gg_rs150238 & 110 chrW_55290( & 11.05 sex \\
\hline 15 Gg_rs150238. & 110 chr1_156708। & 7.71 sex \\
\hline 15 Gg_rs150238 & 111 chr27_59160। & 8.54 sex \\
\hline 15 Gg_rs150238 & 111 chrW_10350 & 11.64 sex \\
\hline 15 Gg_rs150238 & 111 chrW_55760 & 8.41 sex \\
\hline 15 Gg_rs150238. & 111 chr10_98100। & 7.86 sex \\
\hline 15 Gg_rs150238. & 111 chrW_52860c & 8.25 sex \\
\hline 15 Gg_rs150238. & 111 chrW_23960 & 10.19 sex \\
\hline 15 Gg_rs150238 & 111 chrW_64170 & 9.02 sex \\
\hline 15 Gg_rs150238. & 111 chrW_11810 & 9.86 sex \\
\hline 15 Gg_rs150238. & 112 chr31_39400। & 8.14 sex \\
\hline 15 Gg_rs150238. & 112 chrW_44800 & 8.45 sex \\
\hline 15 Gg_rs150238. & 112 chrW_43930 & 8.65 sex \\
\hline 15 Gg_rs150238. & 112 chr2_657050। & 8.5 sex \\
\hline 15 Gg_rs150238 & 112 chrW_12630 & 8.26 sex \\
\hline 15 Gg_rs150238 & 112 chrW_27630 & 7.86 sex \\
\hline 15 Gg_rs150238 & 112 chrZ_449660। & 9.3 sex \\
\hline 15 Gg_rs150238 & 112 chrW_28430 & 8.61 sex \\
\hline 15 Gg_rs150238 & 112 chr1_103129! & 8.76 sex \\
\hline 15 Gg_rs150238 & 113 chrW_50930 & 8.46 sex \\
\hline 15 Gg_rs150238 & 113 chrW_40260 & 8.25 sex \\
\hline 15 Gg_rs150238 & 113 chrW_66630 & 9.04 sex \\
\hline 15 Gg_rs150238. & 113 chr1_162106 & 8 sex \\
\hline
\end{tabular}




\begin{tabular}{|c|c|c|}
\hline 15 Gg_rs150238. & 113 chrW_29990( & 9.45 sex \\
\hline 15 Gg_rs150238 & 113 chrW_31350 & 8.45 sex \\
\hline 15 Gg_rs150238 & 114 chr7_925100। & 9.3 sex \\
\hline 15 Gg_rs150238 & 114 chrW_48160 & 10.28 sex \\
\hline 15 Gg_rs150238 & 114 chr3_107568। & 10.05 sex \\
\hline 15 Gg_rs150238. & 114 chr4_187270 & 8.88 sex \\
\hline 15 Gg_rs150238. & 114 chr2_406060 & 10.95 sex \\
\hline 15 Gg_rs150238. & 114 chrW_57620 & 8.23 sex \\
\hline 15 Gg_rs150238. & 114 chrW_13440 & 9.65 sex \\
\hline 15 Gg_rs150238 & 114 chr2_746060 & 9.52 sex \\
\hline 15 Gg_rs150238. & 114 chrW_16480 & 8.82 sex \\
\hline 15 Gg_rs150238 & 114 chrW_57490( & 8.34 sex \\
\hline 15 Gg_rs150238. & 114 chrW_35240 & 8.5 sex \\
\hline 15 Gg_rs150238. & 114 chr4_194000 & 8.43 sex \\
\hline 15 Gg_rs150238. & 115 chrW_47770 & 9.76 sex \\
\hline 15 Gg_rs150238 & 115 chrW_47810 & 8.65 sex \\
\hline 15 Gg_rs150238 & 115 chrW_12310 & 12.97 sex \\
\hline 15 Gg_rs150238 & 115 chrW_34140 & 8.78 sex \\
\hline 15 Gg_rs150238 & 115 chrW_38940 & 10.57 sex \\
\hline 15 Gg_rs150238 & 115 chr18_97000 & 8.68 sex \\
\hline 15 Gg_rs150238 & 115 chrW_24100 & 9.38 sex \\
\hline 15 Gg_rs150238 & 115 chrW_12360 & 8.93 sex \\
\hline 15 Gg_rs150238 & 115 chrW_22260 & 10.65 sex \\
\hline 15 Gg_rs150238 & 116 chr15_79690 & 6.18 nonsex \\
\hline 15 Gg_rs150238 & 116 chrW_10250 & 8.67 sex \\
\hline 15 Gg_rs150238. & 116 chrW_53300( & 9.01 sex \\
\hline 15 Gg_rs150238 & 116 chrW_62160c & 9.04 sex \\
\hline 15 Gg_rs150238. & 116 chrW_52810 & 9.4 sex \\
\hline 15 Gg_rs150238 & 116 chrW_54400 & 9.82 sex \\
\hline 15 Gg_rs150238. & 116 chrW_12730 & 12.74 sex \\
\hline 15 Gg_rs150238. & 117 chrW_10410 & 14.53 sex \\
\hline 15 Gg_rs150238. & 117 chrW_17810 & 8.87 sex \\
\hline 15 Gg_rs150238. & 117 chrW_87100 & 10.34 sex \\
\hline 15 Gg_rs150238. & 117 chr1_154452 & 8.86 sex \\
\hline 15 Gg_rs150238 & 117 chrW_11440 & 10.22 sex \\
\hline 15 Gg_rs150238 & 118 chrW_66130c & 9.36 sex \\
\hline 15 Gg_rs150238 & 118 chrW_13110 & 10.95 sex \\
\hline 15 Gg_rs150238 & 119 chrW_13490 & 11.63 sex \\
\hline 15 Gg_rs150238 & 119 chrW_46600 & 10.03 sex \\
\hline 15 Gg_rs150238 & 120 chrW_65180 & 9.54 sex \\
\hline 15 Gg_rs150238 & 120 chrW_35180 & $9.61 \mathrm{sex}$ \\
\hline 15 Gg_rs150238 & 121 chrW_17200 & 9.67 sex \\
\hline 15 Gg_rs150238. & 122 chrW_56890( & 11.41 sex \\
\hline
\end{tabular}




\begin{tabular}{|c|c|c|}
\hline 15 Gg_rs150238. & 122 chrW_43420 & 12.19 sex \\
\hline 15 Gg_rs150238 & 123 chr2_553600 & 10.92 sex \\
\hline 15 Gg_rs150238 & 124 chrW_46410 & 11.18 sex \\
\hline 15 Gg_rs150238 & 125 chrW_66050 & 11.75 sex \\
\hline 15 Gg_rs140951 & 126 chrW_23660 & $12.85 \mathrm{sex}$ \\
\hline 15 Gg_rs140951 & 129 chr15_10583। & 6.81 nonsex \\
\hline 15 Gg_rs140951 & 130 chr4_187250 & 11.66 sex \\
\hline 15 Gg_rs140951 & 132 chr15_10524! & 6.53 nonsex \\
\hline 15 Gg_rs140951 & 136 chrW_24730c & 11.18 sex \\
\hline 15 Gg_rs140951 & 137 chr15_10853। & 7.98 sex \\
\hline 15 Gg_rs140951 & 140 chrW_19630 & 8.56 sex \\
\hline 15 Gg_rs140951 & 140 chr2_145402 & 7.38 nonsex \\
\hline 15 Gg_rs140951 & 142 chrW_65220 & 9.18 sex \\
\hline 15 Gg_rs140951 & 144 chr12_97850। & 5.42 nonsex \\
\hline 15 Gg_rs140951 & 145 chr15_12171। & 4.34 nonsex \\
\hline 15 Gg_rs140951 & 165 chr2_168040 & 5.64 nonsex \\
\hline 15 Gg_rs140951 & 167 chrW_69000 & 8.06 sex \\
\hline 15 Gg_rs140959 & 184 chr15_11697। & 5.63 nonsex \\
\hline 15 Gg_rs150257. & 188 chr2_352800 & 6.28 nonsex \\
\hline 15 Gg_rs140959 & 189 chr5_566670 & 3.89 nonsex \\
\hline 15 Gg_rs150262 & 202 chr15_12345। & 8.01 sex \\
\hline 16 Gg_rs150267 & 0 chr16_17970 & 4.35 sex \\
\hline 16 Gg_rs150267 & 0 chr16_24510 & 5.63 sex \\
\hline 16 Gg_rs150267 & 0 chr16_27260। & 5.26 sex \\
\hline 16 Gg_rs150267 & 0 chr16_22990। & 4.53 nonsex \\
\hline 16 Gg_rs150267 & 0 chr16_23240। & 3.14 nonsex \\
\hline 16 Gg_rs150267 & 0 chr16_24090 & 2.97 nonsex \\
\hline 16 Gg_rs150267 & 0 chr16_25400। & 4.95 nonsex \\
\hline 16 Gg_rs150267 & 0 chrW_59850r & 7.51 sex \\
\hline 16 Gg_rs150267 & 0 chr1_143208! & 7.42 sex \\
\hline 16 Gg_rs150267 & 0 chr1_179560। & 5.55 nonsex \\
\hline 16 Gg_rs150267 & 0 chrZ_153560। & 5.29 nonsex \\
\hline 16 Gg_rs150267 & 0 chrZ_6934301 & 5.81 nonsex \\
\hline 16 Gg_rs150267 & 0 chr2_118529! & 5.31 nonsex \\
\hline 16 Gg_rs150267 & 0 chr3_197200 & 6.42 nonsex \\
\hline 16 Gg_rs150267 & 0 chr3_997000। & 5.79 nonsex \\
\hline 16 Gg_rs140966 & 1 chr16_22940। & 4.19 nonsex \\
\hline 16 Gg_rs140966 & 1 chr16_23050। & 4.8 nonsex \\
\hline 16 Gg_rs140966 & 1 chr16_23060। & 4.39 nonsex \\
\hline 16 Gg_rs140966 & 1 chr16_23070। & 4.07 nonsex \\
\hline 16 Gg_rs140966 & 1 chr4_676380 & 7.05 sex \\
\hline 16 Gg_rs140966 & 1 chr4_720340। & 6.23 nonsex \\
\hline 16 Gg_rs140966 & 1 chr1_153825। & 6.02 nonsex \\
\hline
\end{tabular}




\begin{tabular}{|c|c|c|}
\hline 16 Gg_rs140966 & 1 chr2_388040 & 5.71 nonsex \\
\hline 17 Gg_rs150351 & 0 chr5_206000 & 11.44 sex \\
\hline 17 Gg_rs150351 & 0 chr25_30310। & 5.57 sex \\
\hline 17 Gg_rs150351 & 0 chr27_65550 & 7.2 sex \\
\hline 17 Gg_rs150351 & 0 chr27_71560 & 8.93 sex \\
\hline 17 Gg_rs150351 & 0 chr4_909280 & 4.43 nonsex \\
\hline 17 Gg_rs150351 & 0 chr3_176710 & 5.71 nonsex \\
\hline 17 Gg_rs141048 & 0 chr17_92900। & 4.34 nonsex \\
\hline 17 Gg_rs141048 & 0 chr8_30000 & 7.34 sex \\
\hline 17 Gg_rs150351 & 1 chr17_16420 & 6.81 nonsex \\
\hline 17 Gg_rs150351 & 1 chr14_15412 & 9.06 sex \\
\hline 17 Gg_rs150351 & 1 chr23_61340। & 7.93 sex \\
\hline 17 Gg_rs150351 & 1 chr24_64710। & 8.78 sex \\
\hline 17 Gg_rs150351 & 1 chr25_25700 & 8.44 sex \\
\hline 17 Gg_rs150351 & 1 chr25_33570। & 7.14 sex \\
\hline 17 Gg_rs150351 & 1 chr3_138040 & 5.82 nonsex \\
\hline 17 Gg_rs141048 & 1 chr7_211080 & 7.44 sex \\
\hline 17 Gg_rs141048 & 1 chr8_10000 & 7.76 sex \\
\hline 17 Gg_rs141048 & 1 chr11_20020। & 8.03 sex \\
\hline 17 Gg_rs141048 & 1 chr11_20032! & 9.43 sex \\
\hline 17 Gg_rs141048 & 1 chr13_59500 & 8.59 sex \\
\hline 17 Gg_rs137449 & 42 chr17_47170 & 6.98 nonsex \\
\hline 17 Gg_rs137449 & 43 chr1_831500 & 9.34 nonsex \\
\hline 17 Gg_rs137449 & 47 chr25_20880 & 10.94 sex \\
\hline 17 Gg_rs137449 & 49 chr27_55160 & 12.94 sex \\
\hline 17 Gg_rs137449 & 49 chr3_936730। & 7.57 nonsex \\
\hline 17 Gg_rs137449 & 50 chr10_14469। & 7.43 nonsex \\
\hline 17 Gg_rs137449 & 51 chr4_761850। & 9.61 sex \\
\hline 17 Gg_rs137449 & 52 chr6_120120 & 9.96 sex \\
\hline 17 Gg_rs137449 & 52 chrZ_6931401 & 9.25 sex \\
\hline 17 Gg_rs137449 & 53 chr7_429900। & 9.03 sex \\
\hline 17 Gg_rs137449 & 54 chrZ_254100। & 8.57 sex \\
\hline 17 Gg_rs137449 & 54 chr1_128850 & 7.72 nonsex \\
\hline 17 Gg_rs137449 & 54 chr2_434580 & 7.32 nonsex \\
\hline 17 Gg_rs137449 & 55 chr6_107180 & 5.44 nonsex \\
\hline 17 Gg_rs137449 & 56 chr17_71400 & 7.04 sex \\
\hline 17 Gg_rs137449 & 58 chr17_39220 & 7.47 sex \\
\hline 17 Gg_rs137449 & 58 chrZ_276800। & 9.13 sex \\
\hline 17 Gg_rs137449 & 58 chrZ_3456201 & 8.38 sex \\
\hline 17 Gg_rs137449 & 58 chrZ_3467101 & 8.99 sex \\
\hline 17 Gg_rs137449 & 58 chr2_604560 & 6.9 nonsex \\
\hline 17 Gg_rs137449 & 61 chr17_45820 & 5.74 nonsex \\
\hline 17 Gg_rs137449 & 64 chr17_41600 & 5.91 nonsex \\
\hline
\end{tabular}




\begin{tabular}{|c|c|c|}
\hline 17 Gg_rs137449 & 66 chr1_612600 & 8.11 nonsex \\
\hline 17 Gg_rs137449 & 67 chr1_121326। & 8.39 nonsex \\
\hline 17 Gg_rs137449 & 68 chr17_46790। & 10.43 nonsex \\
\hline 17 Gg_rs137449 & 68 chr17_48180 & 6.6 nonsex \\
\hline 17 Gg_rs137449 & 68 chr17_54900 & 6.82 nonsex \\
\hline 17 Gg_rs137449 & 69 chr17_46780 & 13.07 nonsex \\
\hline 17 rbl4379 & 86 chr17_47220 & 7.39 sex \\
\hline 17 rbl4379 & 89 chr1_514630 & 8.38 sex \\
\hline 17 rbl4379 & 89 chrZ_3063201 & 8.6 sex \\
\hline 17 rbl4379 & 90 chr1_164080 & 7.34 nonsex \\
\hline 17 rbl4379 & 90 chr1_869730 & 5.86 nonsex \\
\hline 17 rbl4379 & 91 chr1_211700 & 5.74 nonsex \\
\hline 17 rbl4379 & 91 chr4_562810 & 5.65 nonsex \\
\hline 17 rbl4379 & 92 chrZ_7190201 & 6.35 sex \\
\hline 17 rbl4379 & 92 chrZ_4777301 & 6.15 nonsex \\
\hline 17 rbl4379 & 92 chr2_106347। & 5.62 nonsex \\
\hline 17 rbl4379 & 94 chr8_242980। & 7.9 sex \\
\hline 17 Gg_rs140992 & 132 chr17_71360। & 4.56 nonsex \\
\hline 17 Gg_rs150304 & 133 chr17_86380 & 4.11 nonsex \\
\hline 17 Gg_rs140992 & 137 chr17_71540। & 6.07 nonsex \\
\hline 17 Gg_rs140992 & 138 chr1_190107। & 5.46 nonsex \\
\hline 17 Gg_rs140981 & 154 chr5_280160 & 7.05 sex \\
\hline 17 Gg_rs140972 & 177 chr17_94770 & 4.43 sex \\
\hline 17 Gg_rs140972 & 178 chr17_99190। & 4.09 nonsex \\
\hline $18 \times 18 \_4226902$ & 13 chr4_241900 & 11.4 sex \\
\hline $18 \times 18 \_4226902$ & 26 chr10_71500। & 7.13 sex \\
\hline $18 \times 18 \_4226902$ & 32 chrZ_3400201 & 6.93 sex \\
\hline $18 \times 18 \_4226902$ & 37 chr2_335850। & 6.23 sex \\
\hline $18 \times 18 \_4226902$ & 49 chr18_39520 & 5.75 sex \\
\hline $18 \times 18 \_4226902$ & 49 chr18_40090। & 4.24 nonsex \\
\hline $18 \times 18 \_4226902$ & 49 chr18_40080 & 4.17 nonsex \\
\hline $18 \times 18 \_4226902$ & 49 chr18_41500 & 3.7 nonsex \\
\hline $18 \times 18 \_4226902$ & 49 chr2_127261। & 7.25 sex \\
\hline $18 \times 18 \_4226902$ & 50 chr5_186620 & 7.37 sex \\
\hline $18 \times 18 \_4226902$ & 53 chr18_40070 & 3.7 nonsex \\
\hline $18 \times 18 \_4226902$ & 53 chr4_378000 & 6.83 sex \\
\hline $18 \times 18 \_4226902$ & 53 chr15_82520 & 5.98 nonsex \\
\hline $18 \times 18 \_4226902$ & 54 chr18_52290 & 5.63 sex \\
\hline $18 \times 18 \_4226902$ & 55 chr13_17810 & 7.6 sex \\
\hline $18 \times 18 \_4226902$ & 56 chr18_37520 & 5.23 nonsex \\
\hline $18 \times 18 \_4226902$ & 57 chrW_46540( & 10.62 sex \\
\hline 18 Gg_rs150414 & 109 chr4_849390। & 7.89 sex \\
\hline 18 Gg_rs150414 & 115 chrW_46930 & 9.19 sex \\
\hline
\end{tabular}




\begin{tabular}{|c|c|c|c|}
\hline 18 Gg_rs150414 & 118 chr1_124073। & 8.27 sex & trans \\
\hline 19 Gg_rs141150 & 0 chr19_14830 & 4.26 nonsex & cis \\
\hline 19 Gg_rs150444 & 14 chr19_12980 & 5.46 nonsex & cis \\
\hline 19 Gg_rs150444 & 14 chr19_28550 & 4.7 nonsex & cis \\
\hline 19 Gg_rs150444 & 17 chr19_14600 & 5.41 nonsex & cis \\
\hline 19 Gg_rs150444 & 19 chr19_14860 & 6.06 sex & cis \\
\hline 19 Gg_rs141168 & 27 chr19_10185! & 6.54 sex & cis \\
\hline 19 Gg_rs141191 & 62 chrZ_3232301 & 9.02 sex & trans \\
\hline 19 Gg_rs141195 & 63 chr4_852980 & 7.99 sex & trans \\
\hline 19 Gg_rs141195 & 67 chr19_39450 & 5.09 nonsex & cis \\
\hline 19 Gg_rs141191 & 68 chr2_182860 & 9.99 sex & trans \\
\hline 19 Gg_rs141191 & 70 chr12_11248। & 10.25 sex & trans \\
\hline 19 Gg_rs141195 & 71 chr19_37440 & 4.66 nonsex & cis \\
\hline 19 Gg_rs150470 & 74 chr19_39440। & 7.2 sex & cis \\
\hline 19 Gg_rs150470 & 74 chr19_10195। & 7.56 sex & cis \\
\hline 19 Gg_rs150470 & 74 chr19_10196। & 7.56 sex & cis \\
\hline 19 Gg_rs150470 & 74 chr1_333850 & 9.08 sex & trans \\
\hline 19 Gg_rs150470 & 74 chrW_36630c & 9.48 sex & trans \\
\hline 19 Gg_rs150470 & 75 chr4_403030 & 9.48 sex & trans \\
\hline 19 Gg_rs150470 & 75 chr1_853100 & 9.4 sex & trans \\
\hline 19 Gg_rs150470 & 76 chr19_30820 & 6.62 sex & cis \\
\hline 19 Gg_rs150470 & 76 chr9_194810 & 9.77 sex & trans \\
\hline 19 Gg_rs150470 & 76 chr12_13890 & 10.9 sex & trans \\
\hline 19 Gg_rs150470 & 76 chr1_634570 & 9.09 sex & trans \\
\hline 19 Gg_rs150470 & 77 chr19_14760 & 6.52 sex & cis \\
\hline 19 Gg_rs150470 & 77 chr19_16470। & 7.33 sex & cis \\
\hline 19 Gg_rs150470 & 77 chr19_18540 & 7.88 sex & cis \\
\hline 19 Gg_rs150470 & 77 chr19_26110। & 6.41 sex & cis \\
\hline 19 Gg_rs150470 & 77 chr19_26400 & 8.37 sex & cis \\
\hline 19 Gg_rs150470 & 77 chr19_31030। & 7.74 sex & cis \\
\hline 19 Gg_rs150470 & 77 chr19_31310 & 6.86 sex & cis \\
\hline 19 Gg_rs150470 & 77 chr19_31610 & 7.18 sex & cis \\
\hline 19 Gg_rs150470 & 77 chr19_37790। & 7.02 sex & cis \\
\hline 19 Gg_rs150470 & 77 chr19_39790। & 6.72 sex & cis \\
\hline 19 Gg_rs150470 & 77 chr19_44780 & 7.16 sex & cis \\
\hline 19 Gg_rs150470 & 77 chr19_44790 & 5.61 nonsex & cis \\
\hline 19 Gg_rs150470 & 77 chr19_51130 & 8.61 sex & cis \\
\hline 19 Gg_rs150470 & 77 chr19_52730 & 6.41 sex & cis \\
\hline 19 Gg_rs150470 & 77 chr19_64290 & 6.97 sex & cis \\
\hline 19 Gg_rs150470 & 77 chr19_66110 & 6.6 sex & cis \\
\hline 19 Gg_rs150470 & 77 chr19_66260 & 6.99 sex & cis \\
\hline 19 Gg_rs150470 & 77 chr19_67820 & 6.41 sex & cis \\
\hline 19 Gg_rs150470 & 77 chr19_67850 & 6.9 sex & cis \\
\hline
\end{tabular}




\begin{tabular}{|c|c|c|c|}
\hline 19 Gg_rs150470 & 77 chr19_68800। & 7.26 sex & cis \\
\hline 19 Gg_rs150470 & 77 chr19_77340 & 7.5 sex & cis \\
\hline 19 Gg_rs150470 & 77 chr19_79210 & 8.33 sex & cis \\
\hline 19 Gg_rs150470 & 77 chr19_85770 & 7.13 sex & cis \\
\hline 19 Gg_rs150470 & 77 chr19_98160 & 7.03 sex & cis \\
\hline 19 Gg_rs150470 & 77 chr19_10044! & 6.76 sex & cis \\
\hline 19 Gg_rs150470 & 77 chr19_10094! & 6.22 sex & cis \\
\hline 19 Gg_rs150470 & 77 chr19_42310 & 4.02 nonsex & cis \\
\hline 19 Gg_rs150470 & 77 chr19_64910 & 4.24 nonsex & cis \\
\hline 19 Gg_rs150470 & 77 chr1_253960 & 8.03 sex & trans \\
\hline 19 Gg_rs150470 & 77 chr1_298450। & 8.9 sex & trans \\
\hline 19 Gg_rs150470 & 77 chr4_432970 & 9.22 sex & trans \\
\hline 19 Gg_rs150470 & 77 chr5_179100 & 7.76 sex & trans \\
\hline 19 Gg_rs150470 & 77 chr5_296210 & 8.56 sex & trans \\
\hline 19 Gg_rs150470 & 77 chr5_456980 & 8.92 sex & trans \\
\hline 19 Gg_rs150470 & 77 chr5_514320 & 8.01 sex & \\
\hline 19 Gg_rs150470 & 77 chr5_547660 & 8.44 sex & trans \\
\hline 19 Gg_rs150470 & 77 chr5_594610 & 8.33 sex & trans \\
\hline 19 Gg_rs150470 & 77 chr6_132770 & 8.55 sex & trans \\
\hline 19 Gg_rs150470 & 77 chr6_187960 & 8.25 sex & trans \\
\hline 19 Gg_rs150470 & 77 chr7_240540 & 9.63 sex & \\
\hline 19 Gg_rs150470 & 77 chr10_65700। & 8.19 sex & trans \\
\hline 19 Gg_rs150470 & 77 chr10_82380 & 8.44 sex & trans \\
\hline 19 Gg_rs150470 & 77 chr12_12257! & 9.31 sex & trans \\
\hline 19 Gg_rs150470 & 77 chr15_88730 & 8.34 sex & trans \\
\hline 19 Gg_rs150470 & 77 chr22_28760। & 11.9 sex & rans \\
\hline 19 Gg_rs150470 & 77 chr1_816810 & 8.49 sex & trans \\
\hline 19 Gg_rs150470 & 77 chr1_852150। & 9.6 sex & trans \\
\hline 19 Gg_rs150470 & 77 chr1_107530 & 9.44 sex & trans \\
\hline 19 Gg_rs150470 & 77 chr1_123107। & 10.65 sex & trans \\
\hline 19 Gg_rs150470 & 77 chr1_135906। & 8.01 sex & trans \\
\hline 19 Gg_rs150470 & 77 chr1_179126। & 6.68 sex & trans \\
\hline 19 Gg_rs150470 & 77 chr1_188466। & 8.51 sex & trans \\
\hline 19 Gg_rs150470 & 77 chr1_188468। & 8.56 sex & trans \\
\hline 19 Gg_rs150470 & 77 chr1_188469। & 8.74 sex & trans \\
\hline 19 Gg_rs150470 & 77 chr1_188470 & 8.44 sex & trans \\
\hline 19 Gg_rs150470 & 77 chr1_188471। & 8.34 sex & trans \\
\hline 19 Gg_rs150470 & 77 chr1_188472 & 8.59 sex & trans \\
\hline 19 Gg_rs150470 & 77 chr1_188473। & 8.56 sex & trans \\
\hline 19 Gg_rs150470 & 77 chr4_175650 & 9.43 sex & trans \\
\hline 19 Gg_rs150470 & 77 chr1_393110 & 5.92 nonsex & trans \\
\hline 19 Gg_rs150470 & 77 chr7_115290 & 5.82 nonsex & trans \\
\hline 20 Gg_rs151697. & 8 chrZ_151540। & 8.52 sex & trans \\
\hline
\end{tabular}




\begin{tabular}{|c|c|c|c|}
\hline 20 Gg_rs151697. & 11 chr20_77200। & 5.13 nonsex & cis \\
\hline 20 Gg_rs151697. & 11 chr20_12680 & 5.44 nonsex & cis \\
\hline 20 Gg_rs151697. & 13 chr20_15800 & 10.37 nonsex & cis \\
\hline 20 Gg_rs151697. & 13 chr1_152476! & 5.79 nonsex & trans \\
\hline 20 Gg_rs151697. & 14 chr20_13430 & 5.45 nonsex & cis \\
\hline 20 Gg_rs151697. & 15 chr20_14550 & 7.03 nonsex & cis \\
\hline 20 Gg_rs151697. & 15 chr20_14570 & 5.72 sex & cis \\
\hline 20 Gg_rs151697. & 15 chr20_45910 & 5.65 nonsex & cis \\
\hline 20 Gg_rs151697. & 15 chr20_13370 & 4.81 nonsex & cis \\
\hline 20 Gg_rs151697. & 15 chr20_14000 & 4.52 nonsex & cis \\
\hline 20 Gg_rs151697. & 15 chr20_14480 & 4.85 nonsex & cis \\
\hline 20 Gg_rs151697. & 15 chr20_14560 & 4.6 nonsex & cis \\
\hline 20 Gg_rs151697. & 15 chr20_29250 & 4.19 nonsex & cis \\
\hline 20 Gg_rs151697. & 20 chr20_29130 & 4.93 nonsex & cis \\
\hline 20 Gg_rs151697. & 21 chr20_13890। & 4.51 nonsex & cis \\
\hline 20 Gg_rs151697. & 21 chr20_14360 & 3.48 nonsex & cis \\
\hline 20 Gg_rs151697. & 21 chr8_269520 & 5.72 nonsex & trans \\
\hline 20 Gg_rs151697. & 22 chr20_42150 & 5.47 nonsex & cis \\
\hline 20 Gg_rs151697. & 22 chr2_170800 & 5.42 nonsex & trans \\
\hline 20 Gg_rs151697. & 23 chr20_29830 & 4.37 nonsex & cis \\
\hline 20 Gg_rs151697. & 26 chr20_17020 & 3.97 nonsex & cis \\
\hline 20 Gg_rs151697. & 27 chr20_25830। & 6.67 nonsex & cis \\
\hline 20 Gg_rs151697. & 27 chr20_28260 & 5.02 nonsex & cis \\
\hline 20 Gg_rs151697. & 28 chr20_42290 & 7.03 nonsex & cis \\
\hline 20 Gg_rs151697. & 28 chr20_29460 & 5.79 nonsex & cis \\
\hline 20 Gg_rs151697. & 28 chr20_42440 & 6.03 nonsex & cis \\
\hline 20 Gg_rs151697. & 29 chr20_20030। & 8.59 nonsex & cis \\
\hline 20 Gg_rs142708 & 31 chr20_20070। & 7.76 nonsex & cis \\
\hline 20 Gg_rs142708 & 32 chr20_20020 & 5.42 nonsex & cis \\
\hline 20 Gg_rs142708 & 33 chr20_32610 & 5.7 nonsex & cis \\
\hline 20 Gg_rs142708 & 34 chr20_20200। & 5.68 nonsex & cis \\
\hline 20 Gg_rs142708 & 35 chr20_20060। & 5.75 nonsex & cis \\
\hline 20 Gg_rs142708 & 36 chr20_37150। & 5.14 nonsex & cis \\
\hline 20 Gg_rs142708 & 37 chr20_36320। & 7.63 nonsex & cis \\
\hline 20 Gg_rs142708 & 38 chr20_20140 & 4.89 nonsex & cis \\
\hline 20 Gg_rs142708 & 38 chr20_20190 & 4.93 nonsex & cis \\
\hline 20 Gg_rs142708 & 38 chr20_45510 & 4.2 nonsex & cis \\
\hline 20 Gg_rs142708 & 40 chr20_14150 & 5.45 sex & cis \\
\hline 20 Gg_rs142708 & 40 chr20_36880 & 6.95 nonsex & cis \\
\hline 20 Gg_rs142708 & 40 chr20_42340 & 4.71 nonsex & cis \\
\hline 20 Gg_rs142708 & 41 chr8_198190 & 6.4 nonsex & trans \\
\hline 20 Gg_rs142708 & 42 chr20_28460। & 3.84 nonsex & cis \\
\hline 20 Gg_rs142708 & 44 chr20_60150 & 4.18 nonsex & cis \\
\hline
\end{tabular}




\begin{tabular}{|c|c|c|c|}
\hline 20 Gg_rs142708 & 45 chr20_59980। & 4.28 nonsex & cis \\
\hline 20 Gg_rs142708 & 46 chr20_29840 & 3.88 nonsex & cis \\
\hline 20 Gg_rs142708 & 48 chr20_34410 & 5.21 nonsex & cis \\
\hline 20 Gg_rs142708 & 48 chr20_61130 & 6.65 nonsex & cis \\
\hline 20 Gg_rs142708 & 48 chr20_19400 & 2.58 nonsex & cis \\
\hline 20 Gg_rs142708 & 49 chr20_59240 & 4.07 nonsex & cis \\
\hline 20 Gg_rs142708 & 50 chr20_35660 & 4.42 nonsex & cis \\
\hline 20 Gg_rs142708 & 51 chr20_18210 & 3.33 nonsex & cis \\
\hline 20 Gg_rs142708 & 52 chr20_61360 & 4.65 nonsex & cis \\
\hline 20 Gg_rs151715 & 56 chr6_214550 & 6.46 nonsex & trans \\
\hline 20 Gg_rs151715 & 57 chr5_703000। & 7.69 sex & rans \\
\hline 20 Gg_rs151715 & 57 chr4_484980 & 5.61 nonsex & trans \\
\hline 20 GG_rs151718 & 57 chr20_83100। & 7.5 nonsex & cis \\
\hline 20 GG_rs151718 & 57 chr20_83090। & 5.71 nonsex & cis \\
\hline 20 Gg_rs151715 & 58 chr8_145760। & 7.52 sex & trans \\
\hline 20 Gg_rs151715 & 59 chr20_40280 & 4.47 nonsex & cis \\
\hline 20 Gg_rs151715 & 59 chr20_41400 & 4.85 nonsex & cis \\
\hline 20 Gg_rs151715 & 59 chr7_151080 & 7.5 sex & trans \\
\hline 20 GG_rs151718 & 60 chr20_38440 & 6.01 nonsex & cis \\
\hline 20 GG_rs151718 & 60 chr20_40370 & 5.43 nonsex & cis \\
\hline 20 GG_rs151718 & 60 chr20_39980 & 5.14 nonsex & cis \\
\hline 20 GG_rs151718 & 60 chr20_40380 & 4.98 nonsex & cis \\
\hline 20 GG_rs151718 & 60 chr20_44690 & 5.02 nonsex & cis \\
\hline 20 GG_rs151718 & 60 chr4_702750 & 7.19 sex & trans \\
\hline 20 GG_rs151718 & 60 chr7_132300 & 7.02 sex & trans \\
\hline 20 GG_rs151718 & 60 chr7_319530 & 7.55 sex & trans \\
\hline 20 GG_rs151718 & 60 chr2_653260। & 7.52 sex & trans \\
\hline 20 GG_rs151718 & 61 chr20_44740। & 6.54 nonsex & cis \\
\hline 20 GG_rs151718 & 69 chr20_68020 & 4.91 nonsex & cis \\
\hline 20 Gg_rs142708 & 77 chr20_58630। & 4.95 nonsex & cis \\
\hline 20 GG_rs151718 & 77 chr20_68330। & 5.31 nonsex & cis \\
\hline 20 Gg_rs142708 & 81 chr20_59680 & 5.08 nonsex & cis \\
\hline 20 Gg_rs151737. & 83 chr20_60070। & 5.25 nonsex & cis \\
\hline 20 Gg_rs151744 & 83 chr20_70130। & 4.54 nonsex & cis \\
\hline 20 GG_rs151718 & 84 chr20_45180 & 4.52 nonsex & cis \\
\hline 20 Gg_rs151737. & 84 chr20_61140 & 6.57 nonsex & cis \\
\hline 20 Gg_rs151744 & 84 chr20_70140 & 4.85 nonsex & cis \\
\hline 20 Gg_rs151737. & 86 chr20_69590 & 6.85 nonsex & cis \\
\hline 20 Gg_rs151737. & 86 chr20_66900 & 4.47 nonsex & cis \\
\hline 20 Gg_rs151737. & 87 chr20_60210 & 4.38 nonsex & cis \\
\hline 20 Gg_rs142708 & 89 chr20_35670 & 3.71 nonsex & cis \\
\hline 20 Gg_rs151744 & 89 chr20_71230 & 4.21 nonsex & cis \\
\hline 20 Gg_rs151737 & 91 chr20_58310 & 5.26 nonsex & cis \\
\hline
\end{tabular}




\begin{tabular}{|c|c|c|c|}
\hline 20 Gg_rs151737. & 91 chr20_60200। & 7.71 nonsex & cis \\
\hline 20 Gg_rs151737 & 92 chr20_61000 & 6.31 sex & cis \\
\hline 20 Gg_rs151744 & 94 chr20_76910 & 5.87 nonsex & cis \\
\hline 20 Gg_rs151737 & 96 chr20_62820 & 4.05 nonsex & cis \\
\hline 20 Gg_rs151737 & 108 chr20_56300 & 7.93 nonsex & cis \\
\hline 20 Gg_rs151737. & 108 chr20_60950। & 6.85 nonsex & cis \\
\hline 20 Gg_rs151737. & 108 chr20_61560। & 6.76 nonsex & cis \\
\hline 20 Gg_rs151737. & 108 chrW_26170 & 8.29 sex & trans \\
\hline 20 Gg_rs151737 & 109 chr20_62380 & 4.62 nonsex & cis \\
\hline 20 Gg_rs151737 & 110 chr20_34910। & 4.32 nonsex & cis \\
\hline 20 Gg_rs151744 & 112 chr20_66570। & 8.2 nonsex & cis \\
\hline 20 Gg_rs151744 & 115 chr20_66500। & 5.99 sex & cis \\
\hline 20 Gg_rs151744 & 115 chr20_67390 & 6.27 sex & cis \\
\hline 20 Gg_rs151744 & 115 chr20_69880 & 8.62 nonsex & cis \\
\hline 20 Gg_rs151744 & 115 chr20_70650 & 7.79 nonsex & cis \\
\hline 20 Gg_rs151744 & 115 chr20_73820 & 7.26 nonsex & cis \\
\hline 20 Gg_rs151744 & 115 chr20_66910 & 4.03 nonsex & cis \\
\hline 20 Gg_rs151744 & 120 chr20_73830 & 11.33 nonsex & cis \\
\hline 20 Gg_rs151744 & 122 chr20_72650। & 4.4 nonsex & cis \\
\hline 20 Gg_rs151744 & 124 chr20_58330। & 6.56 nonsex & cis \\
\hline 20 Gg_rs151744 & 127 chr20_70220। & 6.36 nonsex & cis \\
\hline 20 Gg_rs151744 & 129 chr20_58320 & 7 nonsex & cis \\
\hline 20 Gg_rs151737. & 130 chr20_61920। & 4.05 nonsex & cis \\
\hline 20 Gg_rs151744 & 131 chr20_70060। & $6.01 \mathrm{sex}$ & cis \\
\hline 20 Gg_rs151744 & 131 chr20_70440। & 7.51 nonsex & cis \\
\hline 20 Gg_rs151751. & 135 chr20_58350। & 7.98 sex & cis \\
\hline 20 Gg_rs151751. & 138 chr20_61120 & 5.72 nonsex & cis \\
\hline 20 Gg_rs151751. & 140 chr20_70450। & 5.5 nonsex & cis \\
\hline 20 Gg_rs151751 & 142 chr20_70430। & $5.7 \operatorname{sex}$ & cis \\
\hline 20 Gg_rs151751. & 142 chr20_70880 & 5.95 sex & cis \\
\hline 20 Gg_rs151751. & 144 chr20_71630 & 5.87 sex & cis \\
\hline 20 Gg_rs151751 & 145 chr20_54040 & 5.78 sex & cis \\
\hline 20 Gg_rs151751. & 146 chr20_58160। & 6.53 sex & cis \\
\hline 20 Gg_rs151751. & 146 chr20_70970। & 6.05 sex & cis \\
\hline 20 Gg_rs151751 & 149 chr20_69090। & $5.71 \mathrm{sex}$ & cis \\
\hline 20 Gg_rs151779. & 229 chr20_11920। & 5.08 nonsex & cis \\
\hline 20 Gg_rs151779. & 237 chr20_12060। & 4.7 nonsex & cis \\
\hline 20 Gg_rs151779. & 241 chr20_11581। & 4.22 nonsex & cis \\
\hline 20 Gg_rs161757. & 241 chr20_12608। & 4.99 nonsex & cis \\
\hline 20 GG_rs142804 & 243 chr20_10783। & 5.41 nonsex & cis \\
\hline 20 Gg_rs161757. & 243 chr20_12766। & 5.57 nonsex & cis \\
\hline 20 Gg_rs161757. & 244 chr7_271800। & 5.74 nonsex & trans \\
\hline 20 GG_rs142804 & 245 chr1_180414! & 6.07 nonsex & \\
\hline
\end{tabular}




\begin{tabular}{|c|c|c|}
\hline 20 GG_rs142804 & 248 chr20_13430। & 7.39 nonsex \\
\hline 20 GG_rs142804 & 248 chr20_13713! & 5.38 sex \\
\hline 20 GG_rs142804 & 248 chr20_12216। & 4.09 nonsex \\
\hline 20 GG_rs142804 & 248 chr2_882950। & 8.28 sex \\
\hline 20 GG_rs142804 & 248 chr12_18856। & 5.79 nonsex \\
\hline 20 GG_rs142804 & 249 chr1_123445। & 7.46 nonsex \\
\hline 20 Gg_rs161757. & 249 chr20_12057। & 6.17 nonsex \\
\hline 20 Gg_rs161757. & 249 chr20_12709। & 4.78 nonsex \\
\hline 20 Gg_rs161757. & 249 chr5_531240 & 6.34 nonsex \\
\hline 20 Gg_rs161757 & 250 chr20_12609। & 7.38 nonsex \\
\hline 20 Gg_rs161757. & 250 chr6_101500 & 6.13 nonsex \\
\hline 20 Gg_rs161757 & 250 chr14_10547। & 6.15 nonsex \\
\hline 20 Gg_rs161757 & 250 chr1_696460। & 5.65 nonsex \\
\hline 20 Gg_rs161757. & 251 chr20_13141। & 6.08 nonsex \\
\hline 20 Gg_rs142808 & 252 chr1_841310। & 7.52 sex \\
\hline 20 Gg_rs142808 & 252 chr1_841400। & 7.44 sex \\
\hline 21 GG_rs151812 & 5 chrZ_520210। & 7.93 sex \\
\hline 21 GG_rs151812 & 6 chr13_10841। & 5.92 nonsex \\
\hline 21 GG_rs151812 & 7 chr21_41820। & 4.29 nonsex \\
\hline 21 GG_rs151812 & 7 chr1_168988। & 6.16 nonsex \\
\hline 21 GG_rs151812 & 8 chrZ_5202001 & 9.95 sex \\
\hline 21 GG_rs151812 & 9 chr21_19200। & 5.17 nonsex \\
\hline 21 GG_rs151812 & 9 chr21_19780 & 5.53 nonsex \\
\hline 21 GG_rs151812 & 9 chr21_28750। & 4.72 nonsex \\
\hline 21 GG_rs151812 & 9 chr21_32150। & 5.22 nonsex \\
\hline 21 GG_rs151812 & 9 chr6_230740 & 7.9 sex \\
\hline 21 GG_rs151812 & 9 chrZ_119400। & 8.65 sex \\
\hline 21 GG_rs151812 & 9 chrZ_5399101 & 7.14 sex \\
\hline 21 GG_rs151812 & 9 chr3_393850 & 7.25 sex \\
\hline 21 GG_rs151812 & 9 chr19_29680। & 6.34 nonsex \\
\hline 21 GG_rs151812 & 9 chr1_111283। & 6.23 nonsex \\
\hline 21 GG_rs151812 & 9 chr2_139002। & 6.04 nonsex \\
\hline 21 GG_rs151812 & 10 chr21_20390। & 4.4 nonsex \\
\hline 21 GG_rs151812 & 10 chr2_116555। & 6.21 nonsex \\
\hline 21 GG_rs151812 & 10 chr3_616380 & 6.27 nonsex \\
\hline 21 GG_rs151812 & 11 chr19_96740 & 6.29 nonsex \\
\hline 21 GG_rs151812 & 11 chr21_60000 & 6.27 nonsex \\
\hline 21 GG_rs151812 & 12 chr21_22060 & 4.57 nonsex \\
\hline 21 Gg_rs151816 & 13 chr21_57300 & 4.31 nonsex \\
\hline 21 Gg_rs151816 & 13 chr21_95500 & 4.36 nonsex \\
\hline 21 Gg_rs151816 & 14 chr14_13490 & 6.43 nonsex \\
\hline 21 Gg_rs151816 & 16 chr21_37140 & 5.78 nonsex \\
\hline 21 Gg_rs151816 & 16 chr14_44420 & 6.62 nonsex \\
\hline
\end{tabular}




\begin{tabular}{|c|c|c|}
\hline 21 Gg_rs151816 & 16 chr23_42500 & 6.06 nonsex \\
\hline 21 Gg_rs151816 & 16 chr3_105400 & 5.82 nonsex \\
\hline 21 Gg_rs151821 & 27 chrZ_6252901 & 7.57 sex \\
\hline 21 Gg_rs151821 & 28 chr21_14950 & 4.73 nonsex \\
\hline 21 Gg_rs151821 & 28 chr2_126150 & 7.74 nonsex \\
\hline 21 Gg_rs151821 & 29 chr21_37720 & 3.48 nonsex \\
\hline 21 Gg_rs151821 & 29 chrZ_193160। & 6.76 nonsex \\
\hline 21 Gg_rs151821 & 30 chr7_144980 & 6.75 nonsex \\
\hline 21 Gg_rs151821 & 34 chr21_41490 & 5.36 nonsex \\
\hline 21 Gg_rs151821 & 36 chr21_23640 & 5.75 nonsex \\
\hline 21 GG_rs151812 & 37 chr2_250550 & 6.41 nonsex \\
\hline 21 Gg_rs151821 & 37 chr21_14690। & 7.28 nonsex \\
\hline 21 Gg_rs151816. & 38 chr6_183070 & 6.22 nonsex \\
\hline 21 Gg_rs151816. & 39 chr21_24570 & 4.95 nonsex \\
\hline 21 Gg_rs151816. & 40 chr19_53170 & 7.26 nonsex \\
\hline 21 GG_rs151812 & 41 chr21_46130 & 5.71 nonsex \\
\hline 21 Gg_rs151842 & 44 chr21_59990। & 6.33 nonsex \\
\hline 21 Gg_rs151839 & 46 chr21_46330 & 6.48 nonsex \\
\hline 21 Gg_rs151839 & 46 chr13_26570। & 7.76 nonsex \\
\hline 21 Gg_rs151839 & 47 chr21_48420 & 7.02 nonsex \\
\hline 21 Gg_rs151839 & 48 chr21_47430 & 8.41 nonsex \\
\hline 21 Gg_rs151839 & 49 chr21_41510 & 5.68 nonsex \\
\hline 21 Gg_rs151839 & 50 chr24_11050 & 6.97 nonsex \\
\hline 21 Gg_rs151839 & 53 chr5_248570 & 8.13 nonsex \\
\hline 21 Gg_rs151839 & 54 chr5_368460 & 6.66 nonsex \\
\hline 21 Gg_rs151839 & 55 chr2_145350 & 6.81 nonsex \\
\hline 21 Gg_rs151839 & 59 chr4_896660। & 5.95 nonsex \\
\hline 21 Gg_rs151839 & 60 chr21_48390 & 3.55 nonsex \\
\hline 21 Gg_rs151839 & 60 chr21_21370 & 4.96 nonsex \\
\hline 21 Gg_rs151839 & 61 chr4_484950 & 6.15 nonsex \\
\hline 21 Gg_rs151839 & 63 chr4_685420 & 7.54 sex \\
\hline 21 Gg_rs151839 & 63 chr6_108650 & 6.84 nonsex \\
\hline 21 Gg_rs151839 & 65 chr21_49200 & 9.15 sex \\
\hline 21 Gg_rs151839 & 65 chr21_17800 & 4.77 nonsex \\
\hline 21 Gg_rs151839 & 66 chr30_14500। & 6.29 nonsex \\
\hline 21 Gg_rs151839 & 67 chr21_48100। & 8.28 sex \\
\hline 21 Gg_rs151839 & 67 chr17_10700 & 7.22 nonsex \\
\hline 21 Gg_rs151842 & 71 chr26_17890 & 9.01 sex \\
\hline 21 Gg_rs151842 & 72 chr12_20073। & 8.69 sex \\
\hline 21 Gg_rs151842 & 72 chr1_602240 & 7.74 sex \\
\hline 21 Gg_rs151842 & 74 chrZ_170860। & 7.8 sex \\
\hline 21 Gg_rs151842 & 74 chrZ_418210। & 7.63 sex \\
\hline 21 Gg_rs151842 & 78 chrZ_436000। & 8.23 sex \\
\hline
\end{tabular}




\begin{tabular}{|c|c|c|}
\hline 21 Gg_rs151842 & 85 chr31_22750 & 9.89 sex \\
\hline 21 Gg_rs151842 & 86 chr33_77730। & 25.29 sex \\
\hline 21 Gg_rs151842 & 87 chr14_16043। & 9.69 sex \\
\hline 21 rbl2878 & 109 chr2_226900 & 6.57 nonsex \\
\hline 23 Gg_rs142875 & 0 chr6_320540 & 5.84 nonsex \\
\hline 23 Gg_rs142875 & 0 chr6_352820। & 5.16 nonsex \\
\hline 23 Gg_rs142875 & 0 chr1_181413। & 4.74 nonsex \\
\hline 23 Gg_rs151926 & 6 chr23_61300। & 4.22 nonsex \\
\hline 23 Gg_rs151926 & 9 chr23_60200। & 5.03 nonsex \\
\hline 23 Gg_rs151926 & 9 chr5_543560। & $6.73 \operatorname{sex}$ \\
\hline 23 Gg_rs151926. & 9 chrW_28390r & 7.85 sex \\
\hline 23 Gg_rs151926. & 9 chrW_63410 & 8.94 sex \\
\hline 23 Gg_rs151926. & 10 chrW_52240( & 8.26 sex \\
\hline 23 Gg_rs151926. & 10 chrW_61040( & 8.61 sex \\
\hline 23 Gg_rs151926. & 11 chrW_25430c & 10.25 sex \\
\hline 23 Gg_rs151926. & 11 chrW_27470c & 9.77 sex \\
\hline 23 Gg_rs151926. & 11 chrW_28400( & 8.66 sex \\
\hline 23 Gg_rs151926. & 11 chrW_33120( & 8.33 sex \\
\hline 23 Gg_rs151926. & 12 chr23_78400 & 7.81 sex \\
\hline 23 Gg_rs151926 & 12 chrW_61020c & 9 sex \\
\hline 23 Gg_rs151940 & 14 chrW_12740c & 7.63 sex \\
\hline 23 Gg_rs151940 & 15 chr4_837870 & 5.92 nonsex \\
\hline 23 Gg_rs151940 & 15 chr6_214320 & 5.93 nonsex \\
\hline 23 Gg_rs151940 & 16 chr26_12220 & 9.28 sex \\
\hline 23 Gg_rs151940 & 16 chr2_116235! & 7.03 sex \\
\hline 23 Gg_rs151940 & 16 chr1_363700 & 5.79 nonsex \\
\hline 23 RBL4501 & 56 chr23_30760 & 4.99 nonsex \\
\hline 24 Gg_rs142928 & 0 chr24_12690 & 6.21 nonsex \\
\hline 24 Gg_rs142928 & 0 chr24_11550। & 5.41 nonsex \\
\hline 24 Gg_rs142928 & 0 chr1_447000 & 7.96 sex \\
\hline 24 Gg_rs142928 & 0 chr10_87600। & 7.61 sex \\
\hline 24 Gg_rs142928 & 0 chr11_17760। & 7.39 sex \\
\hline 24 Gg_rs142928 & 0 chr11_16384! & 5.1 sex \\
\hline 24 Gg_rs142928 & 0 chr13_81540। & 4.13 sex \\
\hline 24 Gg_rs142928 & 0 chr24_50920। & 7.82 sex \\
\hline 24 Gg_rs142928 & 0 chr27_64500। & 7.9 sex \\
\hline 24 Gg_rs142928 & 0 chr28_63900। & 6.43 nonsex \\
\hline 24 Gg_rs142928 & 0 chr3_465300। & 7.77 sex \\
\hline 24 Gg_rs142928 & 1 chr24_11150। & 4.89 nonsex \\
\hline 24 Gg_rs142928 & 5 chr24_27060। & 4.2 nonsex \\
\hline 24 Gg_rs142928 & 5 chr2_681740 & 8.27 sex \\
\hline 24 Gg_rs142931 & 15 chr1_195728। & 7.58 sex \\
\hline 24 Gg_rs142931 & 16 chr2_239020। & 6.91 nonsex \\
\hline
\end{tabular}




\begin{tabular}{|c|c|c|c|}
\hline 24 Gg_rs142928 & 19 chr24_11090। & 3.01 nonsex & cis \\
\hline 24 Gg_rs142928 & 19 chr24_11110 & 2.79 nonsex & cis \\
\hline 24 GG_rs161944 & 19 chr24_18030 & 8.61 sex & cis \\
\hline 24 GG_rs161944 & 22 chr24_41050 & 4.08 nonsex & cis \\
\hline 24 GG_rs161944 & 22 chr12_13542। & $7.57 \mathrm{sex}$ & trans \\
\hline 24 GG_rs161944 & 22 chrW_63710c & 8.84 sex & trans \\
\hline 24 GG_rs161944 & 31 chr24_43000 & 8.3 sex & cis \\
\hline 24 GG_rs161944 & 37 chr1_330270 & 7.9 nonsex & trans \\
\hline 25 GG_rs167237 & 0 chr33_36900 & 10.42 sex & trans \\
\hline 25 GG_rs167237 & 0 chr1_167259 & 4.64 nonsex & trans \\
\hline 25 GG_rs167237 & 16 chr26_63300 & 8.63 nonsex & \\
\hline 25 snp.2347.3.31 & 73 chr25_31960 & 10.37 nonsex & cis \\
\hline 25 Gg_rs166878 & 92 chr33_66060 & 5.22 nonsex & trans \\
\hline 25 snp.2347.3.31 & 92 chr25_31830। & 11.98 nonsex & cis \\
\hline 25 Gg_rs166878 & 100 chr25_44600 & 4.35 sex & cis \\
\hline 25 Gg_rs166878 & 100 chr25_25860 & 5.68 sex & cis \\
\hline 25 Gg_rs166878 & 100 chr25_25940। & 3.35 nonsex & cis \\
\hline 25 Gg_rs166878 & 100 chr25_25820 & 2.3 nonsex & cis \\
\hline 25 Gg_rs166878 & 100 chr25_25880 & 2.96 nonsex & cis \\
\hline 25 Gg_rs166878 & 100 chr6_240300 & 3.65 nonsex & trans \\
\hline 25 Gg_rs166878 & 100 chr9_151250 & 4.39 nonsex & ans \\
\hline 25 Gg_rs166878 & 100 chr18_90250। & 4.95 nonsex & trans \\
\hline 25 Gg_rs166878 & 100 chr23_21990। & 3.63 nonsex & trans \\
\hline 25 Gg_rs166878 & 100 chr27_71510 & 2.9 nonsex & trans \\
\hline 25 Gg_rs166878 & 100 chr33_63040। & 3.21 nonsex & trans \\
\hline 25 X25_133616ع & 100 chr25_29970। & 2.3 nonsex & cis \\
\hline 25 snp.2347.3.31 & 100 chr25_14730 & 5.84 sex & cis \\
\hline 25 snp.2347.3.31 & 100 chr25_31840। & 22.8 nonsex & cis \\
\hline 25 snp. 2347.3 .31 & 100 chr25_31850 & 25.3 nonsex & cis \\
\hline 25 snp.2347.3.31 & 100 chr25_31860। & 19 nonsex & cis \\
\hline 25 snp.2347.3.31 & 100 chr25_31870 & 12.7 nonsex & cis \\
\hline 25 snp.2347.3.31 & 100 chr25_31920 & 15.96 nonsex & cis \\
\hline 25 snp.2347.3.31 & 100 chr25_31930 & 20.89 nonsex & cis \\
\hline 25 snp.2347.3.31 & 100 chr25_31940। & 25.17 nonsex & cis \\
\hline 25 snp.2347.3.31 & 100 chr25_31950। & 22.64 nonsex & cis \\
\hline 25 snp.2347.3.31 & 100 chr25_34050। & $5.75 \operatorname{sex}$ & cis \\
\hline 25 snp.2347.3.31 & 100 chr25_33620 & 3 nonsex & cis \\
\hline 25 Gg_rs166878 & 121 chr25_29430। & 3.49 nonsex & cis \\
\hline 25 snp. 2347.3 .31 & 124 chr25_31900। & 3.81 nonsex & cis \\
\hline 25 snp.2347.3.31 & 129 chr25_31890। & 4.61 nonsex & cis \\
\hline 25 snp.2347.3.31 & 130 chr25_31910 & 7.89 nonsex & cis \\
\hline 25 snp.2347.3.31 & 130 chr25_31980 & 4.7 nonsex & cis \\
\hline 25 snp.2347.3.31 & 135 chr25_31970। & 5.54 nonsex & cis \\
\hline
\end{tabular}




\begin{tabular}{|c|c|c|c|}
\hline 25 Gg_rs166823 & 186 chr25_13440। & 6.35 sex & cis \\
\hline 25 Gg_rs166823 & 190 chr5_395320 & 7.34 sex & trans \\
\hline 25 Gg_rs166823 & 191 chr25_24990। & 6.74 sex & cis \\
\hline 25 Gg_rs166823 & 191 chr15_10681। & 6.58 nonsex & trans \\
\hline 25 Gg_rs166823 & 191 chrZ_412440। & 5.09 nonsex & trans \\
\hline 26 Gg_rs137248 & 0 chr26_34600। & 3.8 nonsex & cis \\
\hline 26 Gg_rs137248 & 0 chr26_36600। & 4.52 nonsex & cis \\
\hline 26 Gg_rs137248 & 0 chr26_60800। & 3.73 nonsex & cis \\
\hline 26 Gg_rs137248 & 0 chr26_14290। & 3.19 nonsex & cis \\
\hline 26 Gg_rs137248 & 0 chr31_25390। & 5.5 nonsex & trans \\
\hline 26 Gg_rs137248 & 2 chr26_66700। & 5.97 nonsex & cis \\
\hline 26 Gg_rs137246. & 4 chr4_252980 & 7.22 sex & trans \\
\hline 26 Gg_rs154660 & 8 chr26_15000 & 3.7 nonsex & cis \\
\hline 26 Gg_rs154660 & 10 chr31_67800 & 7.68 sex & trans \\
\hline 26 Gg_rs154660 & 10 chrW_18810 & 10.88 sex & trans \\
\hline 26 Gg_rs154660 & 11 chr26_18200 & 3.78 nonsex & cis \\
\hline 26 Gg_rs154660 & 11 chr4_521810 & 6.66 sex & trans \\
\hline 26 Gg_rs154660 & 11 chr5_551120 & 6.62 sex & trans \\
\hline 26 Gg_rs154660 & 11 chr23_11880 & 7.58 sex & trans \\
\hline 26 Gg_rs154660 & 11 chrW_89500( & 7.36 sex & trans \\
\hline 26 Gg_rs154660 & 11 chrW_92900( & 8.9 sex & rans \\
\hline 26 Gg_rs154660 & 11 chrW_18390( & 9.74 sex & trans \\
\hline 26 Gg_rs154660 & 11 chrW_18980( & 9.01 sex & trans \\
\hline 26 Gg_rs154660 & 13 chrW_24910c & 7.3 sex & trans \\
\hline 26 Gg_rs154660 & 19 chr11_13197। & 6.67 sex & trans \\
\hline 26 X26_1308001 & 19 chr3_533980 & 7.33 sex & rans \\
\hline 26 GG_rs162045 & 138 chrZ_5242701 & 5.9 nonsex & trans \\
\hline 27 RBL280 & 0 chr27_22450। & 11.11 nonsex & cis \\
\hline 27 RBL280 & 0 chr27_22460। & 8.67 nonsex & cis \\
\hline 27 RBL280 & 0 chr27_22500। & 11.14 nonsex & cis \\
\hline 27 RBL280 & 0 chr27_22510 & 12.98 nonsex & cis \\
\hline 27 RBL280 & 0 chr27_22520 & 9.49 nonsex & cis \\
\hline 27 RBL280 & 0 chr27_22540। & 12.66 nonsex & cis \\
\hline 27 RBL280 & 0 chr27_23280। & 10.87 nonsex & cis \\
\hline 27 RBL280 & 0 chr27_23330। & 16.34 nonsex & cis \\
\hline 27 RBL280 & 0 chr27_23340। & 17.46 nonsex & cis \\
\hline 27 RBL280 & 0 chr27_23390। & 6.15 nonsex & cis \\
\hline 27 RBL280 & 0 chr27_23430। & 9.31 nonsex & cis \\
\hline 27 RBL280 & 0 chr27_23880 & 15.75 nonsex & cis \\
\hline 27 RBL280 & 0 chr27_24290। & 15.22 nonsex & cis \\
\hline 27 RBL280 & 0 chr27_24300। & 13.24 nonsex & cis \\
\hline 27 RBL280 & 0 chr27_24810 & 6.39 nonsex & cis \\
\hline 27 RBL280 & 0 chr27_24880 & 7.52 nonsex & cis \\
\hline
\end{tabular}


27 RBL280

27 RBL280

27 RBL280

27 RBL280

27 RBL280

27 RBL280

27 RBL280

27 RBL280

27 RBL280

27 RBL280

27 RBL280

27 RBL280

27 RBL280

27 RBL280

27 RBL280

27 RBL280

27 RBL280

27 RBL280

27 RBL280

27 RBL280

27 RBL280

27 Gg_rs152418

27 Gg_rs152418

27 Gg_rs152418

27 Gg_rs152418

27 Gg_rs152418

27 Gg_rs152418

27 Gg_rs152418

27 Gg_rs152418

27 Gg_rs152418

27 Gg_rs152418

27 Gg_rs152418

27 Gg_rs152418

27 Gg_rs152418

27 Gg_rs152418

27 Gg_rs152418

27 Gg_rs152418

27 Gg_rs152418

27 Gg_rs152418

27 Gg_rs152418

27 Gg_rs152418

27 Gg_rs152418

27 Gg_rs152418
0 chr27_25090।

0 chr27_25100।

0 chr27_26010।

0 chr27_27990।

0 chr27_28250।

0 chr27_28680

0 chr27_28760।

0 chr27_21960।

0 chr27_21980

0 chr27_22260।

0 chr27_22370।

0 chr27_22530।

0 chr27_22550।

0 chr27_23440।

0 chr27_23740।

0 chr27_24010।

0 chr27_24230।

0 chr27_24430

0 chr27_24630।

0 chr27_24840।

0 chr27_28960।

1 chr27_23240

1 chr27_21970।

1 chr27_22220।

1 chr27_22310

1 chr27_22320।

1 chr27_22350

1 chr27_22570।

1 chr27_23250।

1 chr27_23270।

1 chr27_23350।

1 chr27_23400।

1 chr27_23890।

1 chr27_24240।

1 chr27_24250।

1 chr27_24340।

1 chr27_27970।

1 chr27_28570

1 chr27_29370।

1 chr27_24740।

1 chr27_24920।

1 chr27_24990।

1 chr27_25190।
7.99 nonsex cis

8.24 nonsex cis

5.6 nonsex cis

6.13 nonsex cis

8.34 nonsex cis

9.06 sex cis

5.11 nonsex cis

4.87 nonsex cis

5.6 nonsex cis

4.71 nonsex cis

4.68 nonsex cis

4.44 nonsex cis

4.52 nonsex cis

4.36 nonsex cis

4.57 nonsex cis

4.79 nonsex cis

4.61 nonsex cis

3.67 nonsex cis

5.14 nonsex cis

4.54 nonsex cis

4.93 nonsex cis

6.38 nonsex cis

6.43 nonsex cis

11.67 nonsex cis

7.42 nonsex cis

8.86 nonsex cis

12.72 nonsex cis

9.76 nonsex cis

7.4 nonsex cis

5.55 sex cis

11.32 nonsex cis

7.58 nonsex cis

11.2 nonsex cis

12.24 nonsex cis

13.76 nonsex cis

5.53 nonsex cis

5.48 nonsex cis

7.85 nonsex cis

6.65 nonsex cis

5.19 nonsex cis

4.87 nonsex cis

4.41 nonsex cis

4.81 nonsex cis 
27 Gg_rs152418

27 Gg_rs152418

27 Gg_rs152418

27 Gg_rs152418

27 Gg_rs152418

27 Gg_rs152418

27 Gg_rs152418

27 Gg_rs152418

27 Gg_rs152418

27 Gg_rs152418

27 RBL280

27 Gg_rs152418

27 Gg_rs152418

27 RBL280

27 Gg_rs152418

27 RBL280

27 RBL280

27 Gg_rs152418

27 Gg_rs152418

27 Gg_rs152418

27 Gg_rs152418

28 Gg_rs152462

28 X28_1608048

$28 \times 28 \_160804 \varepsilon$

$28 \times 28 \_1608048$

28 Gg_rs152476

28 Gg_rs143061

28 Gg_rs143061

28 Gg_rs143061

28 Gg_rs152476

28 Gg_rs152476

28 Gg_rs143072

28 Gg_rs143072

28 Gg_rs143072

28 Gg_rs143072

28 Gg_rs143072

28 Gg_rs143072

28 X28_3803332
1 chr27_27370

1 chr27_27980

1 chr27_28230।

1 chr27_29130।

1 chr27_29290।

1 chr4_612940।

1 chr8_224000

1 chr8_225000

1 chrW_21810

2 chrW_49280

4 chr1_138350

4 chr4_911730।

4 chr5_388910

6 chrZ_587750

7 chr27_28690।

8 chr27_28720।

8 chr4_414200।

8 chr27_27100।

8 chr1_108700

9 chr12_39110

10 chr1_148392

1 chr1_136760

27 chrW_62410

28 chr4_560320।

32 chr1_173130।

52 chrZ_144070।

59 chr28_25430।

63 chr3_271440।

64 chrZ_365600।

68 chr4_506170।

71 chrZ_300840।

98 chrZ_649360।

102 chr1_213910।

106 chr3_101405।

107 chrW_55380(

109 chr2_104785।

113 chrW_46450(

130 chr1_701960।
5.53 nonsex cis

4.56 nonsex cis

5.18 nonsex cis

5.76 nonsex cis

4.83 nonsex cis

7.36 nonsex trans

10.18 sex trans

10.08 sex trans

6.31 nonsex trans

7.82 nonsex trans

7.55 sex trans

9.33 sex trans

7.92 sex trans

10.88 sex trans

5.83 nonsex cis

5.11 nonsex cis

6.53 nonsex trans

4.04 nonsex cis

8.85 sex trans

10.22 sex trans

7.16 nonsex trans

5.76 nonsex trans

7.38 sex trans

7.35 sex trans

5.67 nonsex trans

8.06 sex trans

6.73 sex trans

6.41 nonsex trans

9.21 sex trans

8.73 sex trans

8.5 sex trans

8.06 sex trans

6.79 nonsex trans

7.85 sex trans

14.42 sex trans

7.6 sex trans

7.78 sex trans

6.45 nonsex trans 
re, effect size, $r$-squared, confidence intervals in $\mathrm{cM}$ and the nearest markers to the confiden

\begin{tabular}{|c|c|c|c|c|c|c|}
\hline Cl & QTL_thr & QTL_dir & variance_per & ve_est & e_SE & dominance_e \\
\hline chr1:50-115 & significant & WL & 17.03 & 6.22 & 1.19 & -0.08 \\
\hline chr1:50-110 & significant & WL & 29.45 & 7.7 & 1.08 & -1.71 \\
\hline chr1:50-102 & significant & WL & 30.26 & 7.71 & 0.99 & -0.67 \\
\hline chr1:50-114 & significant & WL & 14.89 & 6.94 & 1.4 & 0.36 \\
\hline chr1:50-113 & significant & WL & 20.67 & 3.58 & 0.6 & 0.13 \\
\hline chr1:50-107 & significant & WL & 26.34 & 4.8 & 0.7 & 0.67 \\
\hline chr1:50-113 & significant & WL & 15.97 & 7.14 & 1.38 & 0.2 \\
\hline chr1:50-109 & significant & WL & 25.71 & 7.23 & 1.08 & -0.46 \\
\hline chr1:50-107 & significant & WL & 29.35 & 7.25 & 0.95 & -0.7 \\
\hline chr1:50-108 & significant & WL & 18.61 & 4.56 & 0.8 & -0.3 \\
\hline chr1:50-114 & significant & WL & 15.87 & 6.45 & 1.26 & 0.8 \\
\hline chr1:50-105 & significant & WL & 30.08 & 6.22 & 0.82 & 0.86 \\
\hline chr1:50-122 & significant & WL & 15.77 & 6.89 & 1.39 & 0.56 \\
\hline chr1:50-121 & significant & WL & 14.81 & 7.77 & 1.62 & 0.51 \\
\hline chr1:50-123 & significant & WL & 15.59 & 7.84 & 1.58 & 0.19 \\
\hline chr1:50-121 & suggestive & WL & 14.76 & 8.19 & 1.75 & -0.52 \\
\hline chr1:50-119 & suggestive & RJF & 18.46 & -0.59 & 0.12 & -0.3 \\
\hline chr1:50-124 & suggestive & RJF & 17 & -1.49 & 0.31 & -0.52 \\
\hline chr1:50-127 & significant & WL & 16.09 & 1.37 & 0.33 & -1.03 \\
\hline chr1:50-116 & suggestive & WL & 12.26 & 2.85 & 0.69 & 0.36 \\
\hline chr1:50-125 & suggestive & WL & 16.85 & 1.84 & 0.38 & 0.58 \\
\hline chr1:50-129 & suggestive & WL & 18.27 & 2.02 & 0.39 & -0.11 \\
\hline chr1:50-129 & suggestive & WL & 18.59 & 2.27 & 0.42 & 0.3 \\
\hline chr1:50-126 & suggestive & WL & 26.1 & 2.85 & 0.51 & 0.03 \\
\hline chr1:116-268 & 3 suggestive & WL & 12.58 & 2.19 & 0.52 & 0.07 \\
\hline chr1:127-245 & 5 significant & WL & 15.21 & 2.38 & 0.64 & -1.59 \\
\hline chr1:133-228 & 3 significant & WL & 20.83 & 6.64 & 1.35 & -2.07 \\
\hline chr1:135-228 & 3 significant & WL & 21.72 & 11.3 & 2.25 & -3.94 \\
\hline chr1:134-228 & 3 significant & WL & 21.43 & 11.64 & 2.34 & -4.05 \\
\hline chr1:134-228 & 3 significant & WL & 23.92 & 2.36 & 0.41 & 3.24 \\
\hline chr1:130-242 & 2 suggestive & WL & 14.46 & 3.32 & 0.81 & -0.57 \\
\hline chr1:142-246 & 5 significant & WL & 18.54 & 1.83 & 0.43 & -1.27 \\
\hline chr1:144-233 & 3 suggestive & WL & 18.38 & 17.09 & 4.09 & -12.62 \\
\hline chr1:169-228 & 3 significant & WL & 24.94 & 2.39 & 0.46 & -1.43 \\
\hline chr1:157-287 & 7 significant & WL & 19.96 & 1.68 & 0.4 & -1.53 \\
\hline chr1:149-232 & 2 significant & WL & 16.51 & 7.35 & 1.93 & -5.7 \\
\hline chr1:141-245 & 5 significant & RJF & 11.25 & -5.06 & 1.34 & -6.6 \\
\hline chr1:139-246 & 5 suggestive & RJF & 9.66 & -1.78 & 0.5 & -1.85 \\
\hline chr1:136-253 & 3 significant & WL & 18.64 & 2.48 & 0.47 & 1.02 \\
\hline chr1:147-246 & 5 significant & RJF & 11.94 & -1.83 & 0.44 & -0.43 \\
\hline chr1:136-251 & 1 significant & RJF & 15.48 & -1.49 & 0.33 & 0.29 \\
\hline
\end{tabular}


chr1:171-244 suggestive RJF

chr1:187-242 significant RJF

chr1:220-237 significant RJF

chr1:139-246 suggestive RJF

chr1:222-248 significant RJF

chr1:175-253 significant RJF

chr1:150-248 significant RJF

chr1:162-244 suggestive RJF

chr1:155-251 suggestive RJF

chr1:147-249 suggestive RJF

chr1:50-424 suggestive RJF

chr1:163-253 significant

chr1:220-265 suggestive

chr1:223-249 significant

chr1:236-242 significant

chr1:224-248 significant

chr1:223-248 significant

chr1:182-277 significant

chr1:226-251 significant

chr1:171-287 suggestive

chr1:167-274 suggestive

chr1:182-266 suggestive

chr1:221-253 significant

chr1:236-265 significant

chr1:229-251 significant

chr1:237-248 significant

chr1:220-261 significant

chr1:228-257 significant

chr1:236-246 significant

chr1:235-268 significant

chr1:226-302 significant

chr1:179-254 significant

chr1:218-268 suggestive

chr1:162-266 suggestive

chr1:224-282 suggestive

chr1:236-248 significant

chr1:237-246 significant

chr1:237-247 significant

chr1:236-248 significant

chr1:237-247 significant

chr1:236-268 significant

chr1:223-342 suggestive

chr1:235-242 significant
19.2

24

21.63

17.14

15.89

22.8

13.64

12.63

12.02

14.1

6.85

17.23

13.55

24.03

66.23

32.27

24.58

20.98

22.91

12.93

14.7

13.75

18.35

26.45

26.38

37.65

16.65

18.69

19.96

17.37

12.96

14.5

13.54

13.4

14.94

26.26

31.91

44.61

24.87

44.44

22.61

12.36

42.16
$-1.1$

$-2.02$

$-0.07$

$-3.36$

$-1.8$

$-0.97$

$-1.09$

$-1.03$

$-1.54$

$-1$

$-1.22$

$-2.07$

$-1.07$

$-1.52$

3.8

$-1.7$

$-2$

$-2.24$

$-1.78$

$-1.54$

$-0.67$

$-1.47$

$-1.51$

10.37

$-1.98$

10.12

$-2.12$

$-2.13$

$-1.91$

6.39

4.53

$-2.7$

$-1.8$

$-1.44$

$-1.35$

5.82

4.05

13.43

6

14.24

4.89

4.22

1.47
0.27

0.45

0.23

0.7

0.39

0.17

0.25

0.25

0.38

0.22

0.45

0.44

0.26

0.25

0.24

0.22

0.34

0.4

0.3

0.4

0.17

0.35

0.3

1.63

0.3

1.22

0.44

0.41

0.63

1.28

1.15

0.64

0.42

0.36

0.3

0.9

0.54

1.4

0.96

1.49

0.83

1.11

0.16
1.38

1.75

$-0.63$

$-0.56$

0.32

0.26

0.23

$-0.5$

$-0.45$

$-0.13$

$-1.15$

0.66

0.18

$-0.72$

0.09

0.17

0.13

$-0.73$

0.2

1.14

0.53

0.56

0.55

$-4.11$

0.08

$-2.2$

0.44

$-0.91$

3.13

$-2.61$

$-3.15$

$-2.12$

$-0.35$

0.73

0.21

$-1.01$

$-1.05$

$-3.31$

$-0.78$

$-3.55$

$-0.34$

$-3.27$

$-0.69$ 


\begin{tabular}{|c|c|c|c|c|c|}
\hline chr1:236-265 significant & WL & 24.7 & 6.43 & 1.06 & -2.07 \\
\hline chr1:236-248 significant & WL & 26.14 & 5.61 & 0.87 & -1.09 \\
\hline chr1:236-249 significant & WL & 24.33 & 5.93 & 0.96 & -0.89 \\
\hline chr1:236-273 significant & WL & 23.66 & 5.85 & 0.97 & -0.43 \\
\hline chr1:230-341 suggestive & WL & 12.62 & 4.97 & 1.21 & -2.51 \\
\hline chr1:236-265 significant & WL & 25.04 & 6.51 & 1.07 & -2.19 \\
\hline chr1:236-264 significant & WL & 27 & 7.27 & 1.14 & -2.73 \\
\hline chr1:236-266 significant & WL & 27.68 & 4.79 & 0.75 & -2.17 \\
\hline chr1:235-248 significant & WL & 19.98 & 2.43 & 2.03 & -0.51 \\
\hline chr1:238-247 significant & WL & 45.32 & 13.17 & 1.36 & -3.62 \\
\hline chr1:237-249 significant & WL & 28.68 & 5.33 & 0.78 & -0.91 \\
\hline chr1:238-246 significant & WL & 45.72 & 13 & 1.34 & -3.61 \\
\hline chr1:236-276 significant & WL & 21.74 & 7.34 & 1.31 & -1.73 \\
\hline chr1:237-248 significant & WL & 28.15 & 5.64 & 0.83 & -0.85 \\
\hline chr1:236-245 significant & WL & 28.17 & 2.43 & 1.84 & -0.61 \\
\hline chr1:237-264 significant & WL & 27.23 & 6.87 & 1.05 & -1.52 \\
\hline chr1:237-265 significant & WL & 27.62 & 7.43 & 1.12 & -1.42 \\
\hline chr1:235-255 significant & RJF & 21.07 & -3.95 & 0.7 & -1.15 \\
\hline chr1:156-268 significant & WL & 20.62 & 0.95 & 0.37 & 1.08 \\
\hline chr1:227-291 suggestive & WL & 12.24 & 3.33 & 0.84 & -1.7 \\
\hline chr1:235-289 significant & WL & 16.64 & 4.04 & 0.87 & -1.83 \\
\hline chr1:235-265 suggestive & WL & 14.38 & 2.41 & 0.57 & -1.15 \\
\hline chr1:237-247 suggestive & RJF & 11.61 & -0.4 & 0.19 & 1.98 \\
\hline chr1:238-247 significant & WL & 38.85 & 8.19 & 0.95 & -0.93 \\
\hline chr1:238-247 significant & WL & 35.46 & 6.36 & 0.79 & -0.63 \\
\hline chr1:219-248 suggestive & RJF & 19.58 & -0.24 & 0.36 & 0.64 \\
\hline chr1:228-250 significant & RJF & 16.5 & -1.51 & 0.31 & 0.62 \\
\hline chr1:232-249 significant & RJF & 20.76 & -1.67 & 0.31 & -1.1 \\
\hline chr1:156-253 significant & RJF & 10.12 & -2.52 & 0.68 & -1.49 \\
\hline chr1:236-247 significant & WL & 19.94 & 1.04 & 0.19 & 0.27 \\
\hline chr1:219-249 significant & WL & 23.45 & 2.91 & 0.48 & 0.02 \\
\hline chr1:242-248 significant & WL & 31.68 & 2.83 & 0.37 & 0.12 \\
\hline chr1:225-295 suggestive & RJF & 10.66 & -1.91 & 0.5 & 0.07 \\
\hline chr1:174-252 significant & RJF & 14.63 & -1.54 & 0.34 & 0.14 \\
\hline chr1:235-252 significant & RJF & 16.48 & -1.34 & 0.28 & 0.03 \\
\hline chr1:237-246 suggestive & WL & 23.51 & 0.28 & 1.19 & -1.22 \\
\hline chr1:235-266 suggestive & WL & 18.31 & 3.82 & 0.82 & -2.52 \\
\hline chr1:152-263 suggestive & WL & 9.77 & 0.44 & 0.48 & -2.7 \\
\hline chr1:236-264 significant & WL & 28.8 & 10.25 & 1.54 & -3.9 \\
\hline chr1:176-277 suggestive & WL & 14.39 & 1.64 & 0.37 & 0.12 \\
\hline chr1:194-294 suggestive & RJF & 10.55 & -2.01 & 0.53 & -0.16 \\
\hline chr1:233-255 significant & RJF & 15.95 & -1.09 & 0.23 & -0.46 \\
\hline chr1:237-262 significant & WL & 39.16 & 15.03 & 1.74 & -3.87 \\
\hline
\end{tabular}




\begin{tabular}{|c|c|c|c|c|c|}
\hline chr1:237-262 significant & WL & 40.32 & 15.35 & 1.74 & -4.29 \\
\hline chr1:236-265 significant & WL & 25.93 & 7.75 & 1.25 & -2.86 \\
\hline chr1:237-266 significant & RJF & 32.11 & -3.48 & 0.46 & 0.29 \\
\hline chr1:223-268 significant & RJF & 15.79 & -2.93 & 0.62 & 0.62 \\
\hline chr1:197-264 significant & RJF & 12.96 & -1.51 & 0.35 & -0.14 \\
\hline chr1:171-289 suggestive & RJF & 13.53 & -1.35 & 0.32 & 0.31 \\
\hline chr1:222-279 significant & RJF & 16.04 & -1.56 & 0.32 & -0.07 \\
\hline chr1:180-264 significant & RJF & 12.52 & -1.41 & 0.34 & -0.07 \\
\hline chr1:222-261 significant & RJF & 17.26 & -2.4 & 0.5 & 0.79 \\
\hline chr1:218-264 significant & RJF & 14.04 & -1.17 & 0.26 & -0.13 \\
\hline chr1:236-266 significant & RJF & 21.21 & -2.05 & 0.38 & -1.47 \\
\hline chr1:222-264 suggestive & RJF & 13.08 & -2.14 & 0.51 & 0.06 \\
\hline chr1:206-316 significant & RJF & 11.22 & -1.96 & 0.5 & 0.06 \\
\hline chr1:238-260 significant & RJF & 26.14 & -2.58 & 0.4 & -0.83 \\
\hline chr1:221-262 significant & RJF & 26.85 & -1.83 & 0.28 & 0.4 \\
\hline chr1:226-287 significant & RJF & 18.02 & -1.9 & 0.37 & -0.69 \\
\hline chr1:139-267 suggestive & WL & 12.06 & 2.14 & 0.53 & -0.15 \\
\hline chr1:136-271 suggestive & WL & 12.63 & 1.06 & 0.32 & -1.22 \\
\hline chr1:220-279 significant & RJF & 12.73 & -2.1 & 0.5 & -0.16 \\
\hline chr1:234-265 significant & RJF & 19.28 & -2.2 & 0.42 & 0.13 \\
\hline chr1:242-263 significant & RJF & 23.5 & -1.63 & 0.26 & -0.38 \\
\hline chr1:152-289 suggestive & RJF & 9.69 & -2.52 & 0.71 & -1.01 \\
\hline chr1:228-267 significant & RJF & 20 & -2.09 & 0.4 & 0.99 \\
\hline chr1:227-285 significant & RJF & 18.14 & -1.33 & 0.26 & -0.22 \\
\hline chr1:205-254 suggestive & RJF & 14.05 & -1.41 & 0.34 & -1.02 \\
\hline chr1:236-264 significant & RJF & 20.22 & -2.23 & 0.41 & -0.58 \\
\hline chr1:224-259 significant & RJF & 18.33 & -1.93 & 0.37 & 0.04 \\
\hline chr1:169-258 significant & RJF & 15.64 & -1.96 & 0.42 & -0.55 \\
\hline chr1:237-262 suggestive & WL & 14.94 & 1.64 & 0.37 & -0.35 \\
\hline chr1:238-289 significant & RJF & 14.24 & -1.15 & 0.34 & 1.61 \\
\hline chr1:246-265 suggestive & RJF & 14.32 & -0.81 & 0.18 & -0.26 \\
\hline chr1:245-259 significant & RJF & 28.63 & -2.61 & 0.37 & -0.05 \\
\hline chr1:169-289 suggestive & RJF & 12.83 & -1.25 & 0.32 & -1.03 \\
\hline chr1:140-262 significant & WL & 17.15 & 1.81 & 0.37 & -0.46 \\
\hline chr1:237-262 significant & RJF & 37.22 & -2.45 & 0.29 & -0.09 \\
\hline chr1:236-268 suggestive & WL & 12.68 & 2.5 & 0.6 & -0.07 \\
\hline chr1:226-263 significant & RJF & 16.73 & -1.45 & 0.3 & -0.46 \\
\hline chr1:241-261 significant & RJF & 26.92 & -1.95 & 0.3 & 0.57 \\
\hline chr1:191-269 suggestive & RJF & 15.55 & -3.66 & 0.9 & -1.55 \\
\hline chr1:246-270 significant & WL & 15.89 & 1.41 & 0.29 & 0.16 \\
\hline chr1:247-264 significant & WL & 29.71 & 2.28 & 0.31 & 0.04 \\
\hline chr1:237-268 significant & WL & 17.37 & 2.56 & 0.51 & -0.68 \\
\hline chr1:218-314 suggestive & RJF & 12.23 & -1.58 & 0.38 & -0.68 \\
\hline
\end{tabular}




\begin{tabular}{|c|c|c|c|c|c|}
\hline chr1:240-273 significant & WL & 16.96 & 1.09 & 0.22 & 0.16 \\
\hline chr1:237-268 suggestive & WL & 13.39 & 1.32 & 0.33 & 1.02 \\
\hline chr1:245-267 significant & WL & 23.71 & 4.13 & 0.68 & -0.27 \\
\hline chr1:243-267 suggestive & WL & 18.08 & 4.05 & 0.79 & 0.18 \\
\hline chr1:243-266 suggestive & WL & 20.2 & 11.71 & 2.12 & 1.4 \\
\hline chr1:244-265 suggestive & WL & 18.21 & 6.78 & 1.31 & -0.18 \\
\hline chr1:245-270 suggestive & WL & 18.92 & 7.81 & 1.48 & 0.86 \\
\hline chr1:237-265 suggestive & WL & 19.39 & 5.56 & 1 & -0.18 \\
\hline chr1:243-267 suggestive & WL & 20.46 & 11.6 & 2.09 & 0.42 \\
\hline chr1:245-269 suggestive & WL & 19.98 & 5.11 & 0.94 & 0.54 \\
\hline chr1:244-267 suggestive & WL & 20.17 & 11.56 & 2.09 & 0.61 \\
\hline chr1:244-265 suggestive & WL & 19.27 & 5.1 & 0.96 & -0.02 \\
\hline chr1:244-269 suggestive & WL & 19.28 & 2.48 & 0.46 & 0.45 \\
\hline chr1:236-271 suggestive & WL & 19.95 & 5.16 & 0.92 & -0.2 \\
\hline chr1:244-268 suggestive & WL & 19.04 & 4.9 & 0.92 & 0.08 \\
\hline chr1:238-272 suggestive & WL & 20.1 & 4.85 & 0.89 & 0.42 \\
\hline chr1:245-270 suggestive & WL & 21.71 & 4.59 & 0.79 & -0.02 \\
\hline chr1:242-268 suggestive & WL & 19.22 & 5.02 & 0.94 & -0.07 \\
\hline chr1:244-267 significant & WL & 21.89 & 10.36 & 1.79 & 0.38 \\
\hline chr1:168-270 suggestive & WL & 19.02 & 7.44 & 1.42 & -1.3 \\
\hline chr1:239-267 suggestive & WL & 17.63 & 2.11 & 0.41 & 0 \\
\hline chr1:245-267 significant & WL & 20.37 & 2.02 & 0.36 & -0.48 \\
\hline chr1:236-266 significant & WL & 23.74 & 6.3 & 1.04 & -0.85 \\
\hline chr1:239-271 significant & WL & 21.73 & 6.12 & 1.06 & 0.02 \\
\hline chr1:236-272 suggestive & WL & 19.43 & 3.12 & 0.57 & -0.09 \\
\hline chr1:243-273 suggestive & WL & 20.66 & 4.35 & 0.78 & -0.09 \\
\hline chr1:237-265 significant & WL & 29.33 & 11.45 & 1.62 & -2.42 \\
\hline chr1:237-265 significant & WL & 30.38 & 11.93 & 1.64 & -2.1 \\
\hline chr1:237-266 significant & WL & 28.76 & 11.72 & 1.68 & -2.01 \\
\hline chr1:237-266 significant & WL & 29.79 & 11.77 & 1.64 & -2.25 \\
\hline chr1:244-269 significant & WL & 20.32 & 2.41 & 0.45 & -0.7 \\
\hline chr1:239-273 significant & WL & 21.72 & 5.24 & 0.91 & -0.26 \\
\hline chr1:244-275 suggestive & WL & 21.16 & 5.61 & 0.99 & 0.11 \\
\hline chr1:245-274 significant & WL & 21.66 & 3.92 & 0.68 & 0.27 \\
\hline chr1:246-275 significant & WL & 21.66 & 1.81 & 0.32 & 0.67 \\
\hline chr1:247-286 suggestive & RJF & 17.21 & -2.12 & 0.73 & -1.93 \\
\hline chr1:219-313 significant & RJF & 11.9 & -2.04 & 0.51 & -0.56 \\
\hline chr1:236-275 significant & WL & 22.97 & 6.17 & 1.05 & -0.94 \\
\hline chr1:244-276 significant & WL & 19.23 & 1.75 & 0.33 & 0.63 \\
\hline chr1:246-279 significant & WL & 17.64 & 1.38 & 0.28 & 0.71 \\
\hline chr1:245-274 significant & WL & 22.57 & 5.67 & 0.96 & -0.13 \\
\hline chr1:238-275 suggestive & WL & 19.8 & 4.22 & 0.78 & -0.17 \\
\hline chr1:236-273 significant & WL & 22.39 & 7.76 & 1.34 & -2.97 \\
\hline
\end{tabular}




\begin{tabular}{|c|c|c|c|c|c|}
\hline chr1:244-274 significant & WL & 21.79 & 5.58 & 0.97 & -0.17 \\
\hline chr1:235-278 suggestive & RJF & 17.15 & -2.31 & 0.49 & -1.79 \\
\hline chr1:257-289 significant & RJF & 18.82 & -3.13 & 0.62 & -1.99 \\
\hline chr1:257-292 significant & RJF & 18.56 & -1.03 & 0.2 & -0.79 \\
\hline chr1:235-288 significant & WL & 15.31 & 5.51 & 1.26 & -3.45 \\
\hline chr1:236-274 significant & WL & 26.56 & 4.49 & 0.68 & -0.67 \\
\hline chr1:239-275 significant & WL & 21.03 & 1.62 & 0.29 & -0.23 \\
\hline chr1:247-279 significant & WL & 16.58 & 0.94 & 0.19 & 0.3 \\
\hline chr1:237-295 significant & WL & 16.83 & 1.09 & 0.25 & -0.71 \\
\hline chr1:238-291 significant & RJF & 14.32 & -1.52 & 0.34 & 0.3 \\
\hline chr1:246-276 significant & WL & 23.27 & 4.06 & 0.67 & -0.03 \\
\hline chr1:245-285 significant & WL & 16.42 & 2 & 0.41 & 0.26 \\
\hline chr1:233-290 significant & RJF & 16.55 & -2.37 & 0.49 & -0.34 \\
\hline chr1:239-277 suggestive & WL & 19.05 & 3.65 & 0.69 & 0.31 \\
\hline chr1:257-276 significant & WL & 45.93 & 1.98 & 0.19 & 0.04 \\
\hline chr1:257-279 significant & RJF & 19.49 & -1.64 & 0.44 & -0.44 \\
\hline chr1:236-277 suggestive & WL & 18.85 & 5.07 & 0.97 & -0.98 \\
\hline chr1:257-279 significant & WL & 31.14 & 1.34 & 0.18 & 0.1 \\
\hline chr1:200-296 suggestive & RJF & 12.08 & -2.13 & 0.53 & -0.38 \\
\hline chr1:257-288 significant & WL & 16.03 & 1.51 & 0.34 & -0.62 \\
\hline chr1:257-279 significant & WL & 23.28 & 1.21 & 0.2 & -0.16 \\
\hline chr1:257-279 significant & RJF & 27.62 & -2.95 & 0.45 & 0.71 \\
\hline chr1:257-280 suggestive & WL & 17.95 & 0.37 & 0.83 & 1.67 \\
\hline chr1:257-278 suggestive & WL & 18.46 & 0.99 & 0.64 & 0.63 \\
\hline chr1:257-280 significant & RJF & 18.15 & -1.65 & 0.32 & 0.28 \\
\hline chr1:257-279 significant & RJF & 27.62 & -0.98 & 0.18 & -0.49 \\
\hline chr1:257-293 significant & RJF & 15.98 & -1.24 & 0.28 & 0.46 \\
\hline chr1:258-273 suggestive & RJF & 18.24 & -1.6 & 0.6 & -3.44 \\
\hline chr1:257-277 significant & RJF & 24.63 & -3.28 & 0.5 & 0.14 \\
\hline chr1:262-272 suggestive & RJF & 19.35 & -0.75 & 0.28 & -0.14 \\
\hline chr1:263-276 suggestive & WL & 19.06 & 0.34 & 0.45 & 1.23 \\
\hline chr1:262-275 suggestive & RJF & 18.7 & -0.8 & 0.6 & 3.27 \\
\hline chr1:260-279 significant & WL & 26.92 & 1.69 & 0.27 & -0.65 \\
\hline chr1:261-278 significant & WL & 26.32 & 0.99 & 0.15 & 0.31 \\
\hline chr1:257-286 suggestive & WL & 15.08 & 0.89 & 0.21 & -0.45 \\
\hline chr1:257-349 significant & WL & 14.03 & 1.4 & 0.32 & -0.23 \\
\hline chr1:257-280 suggestive & RJF & 13.64 & -1.41 & 0.38 & 1.07 \\
\hline chr1:259-280 significant & RJF & 17.67 & -0.9 & 0.18 & 0.13 \\
\hline chr1:257-275 suggestive & WL & 23.42 & 2.32 & 1.02 & 0.37 \\
\hline chr1:264-274 suggestive & WL & 12.21 & 0.26 & 0.11 & 1.06 \\
\hline chr1:261-282 significant & RJF & 25.22 & -2.59 & 0.41 & -0.33 \\
\hline chr1:257-338 suggestive & WL & 13.5 & 1.24 & 0.29 & 0.73 \\
\hline chr1:257-295 suggestive & WL & 11.57 & 1.94 & 0.5 & -0.62 \\
\hline
\end{tabular}




\begin{tabular}{|c|c|c|c|c|c|}
\hline chr1:264-276 suggestive & WL & 17.3 & 0.06 & 0.22 & 0.41 \\
\hline chr1:258-281 significant & RJF & 25.18 & -1.85 & 0.54 & 1.28 \\
\hline chr1:257-310 significant & RJF & 20.14 & -2.02 & 0.38 & -1.22 \\
\hline chr1:239-308 significant & RJF & 13.29 & -2.01 & 0.47 & -1.24 \\
\hline chr1:258-292 significant & RJF & 19.87 & -1.77 & 0.32 & -0.57 \\
\hline chr1:258-288 significant & RJF & 31.94 & -3.11 & 0.43 & 0.55 \\
\hline chr1:257-341 significant & WL & 16.46 & 1.25 & 0.26 & 0.34 \\
\hline chr1:257-293 significant & RJF & 22.66 & -2.23 & 0.38 & -0.63 \\
\hline chr1:257-293 suggestive & RJF & 13.55 & -3.75 & 0.93 & -0.1 \\
\hline chr1:257-295 suggestive & RJF & 15.27 & -2.6 & 0.56 & -0.84 \\
\hline chr1:260-295 suggestive & RJF & 14.9 & -1.76 & 0.4 & -1.88 \\
\hline chr1:258-313 suggestive & RJF & 14.26 & -1.66 & 0.38 & -0.52 \\
\hline chr1:257-294 significant & WL & 14.43 & 0.57 & 0.25 & -1.19 \\
\hline chr1:262-306 significant & RJF & 22.14 & -2.5 & 0.43 & -0.64 \\
\hline chr1:262-294 suggestive & RJF & 15.65 & -1.34 & 0.56 & 2.32 \\
\hline chr1:271-295 significant & RJF & 18.98 & -2.03 & 0.39 & -1.83 \\
\hline chr1:270-293 suggestive & RJF & 15.01 & -1.24 & 0.32 & -1.92 \\
\hline chr1:270-291 significant & RJF & 21.22 & -4.54 & 0.9 & -3.15 \\
\hline chr1:257-289 suggestive & RJF & 14.22 & -1.42 & 0.37 & -2.14 \\
\hline chr1:257-293 suggestive & RJF & 14.97 & -1.47 & 0.36 & -1.99 \\
\hline chr1:274-292 suggestive & RJF & 19.39 & -0.46 & 0.48 & -4.24 \\
\hline chr1:275-291 suggestive & RJF & 18.14 & -1.85 & 0.65 & -5.36 \\
\hline chr1:277-290 significant & RJF & 17.12 & -1.69 & 0.52 & -3.99 \\
\hline chr1:278-290 significant & RJF & 17.92 & -0.66 & 0.37 & -3.22 \\
\hline chr1:275-293 suggestive & RJF & 17.09 & -2.98 & 0.85 & -3.69 \\
\hline chr1:277-292 significant & RJF & 20.65 & -1.23 & 0.66 & -3.29 \\
\hline chr1:278-289 significant & RJF & 21.41 & -1.41 & 0.38 & -3.44 \\
\hline chr1:278-288 significant & RJF & 18.04 & -0.61 & 0.33 & -2.79 \\
\hline chr1:278-290 significant & RJF & 16.9 & -0.58 & 0.63 & -5.21 \\
\hline chr1:277-290 significant & RJF & 15.67 & -0.36 & 0.36 & -2.88 \\
\hline chr1:278-286 suggestive & RJF & 18 & -1.55 & 0.96 & -3 \\
\hline chr1:277-291 suggestive & WL & 19.79 & 0.63 & 0.48 & -0.7 \\
\hline chr1:275-290 significant & RJF & 17.15 & -0.16 & 0.3 & -2.49 \\
\hline chr1:273-291 significant & RJF & 20.81 & -0.93 & 0.48 & 3.42 \\
\hline chr1:274-289 significant & RJF & 19.36 & -1.07 & 0.25 & -1.85 \\
\hline chr1:277-292 suggestive & RJF & 19.09 & -1.17 & 0.42 & -1.91 \\
\hline chr1:279-290 significant & RJF & 20.16 & -0.93 & 0.33 & -2.97 \\
\hline chr1:278-292 significant & RJF & 16.76 & -0.76 & 0.48 & -3.95 \\
\hline chr1:276-290 suggestive & WL & 14.19 & 1.23 & 0.99 & -6.04 \\
\hline chr1:277-293 suggestive & RJF & 14.4 & -1.29 & 0.38 & -2.48 \\
\hline chr1:275-291 significant & RJF & 16.77 & -1.49 & 0.38 & -2.61 \\
\hline chr1:278-290 suggestive & RJF & 14.24 & -0.08 & 0.37 & -2.67 \\
\hline chr1:278-290 significant & RJF & 14.89 & -1.06 & 0.62 & -4.76 \\
\hline
\end{tabular}




\begin{tabular}{|c|c|c|c|c|c|}
\hline chr1:277-289 significant & RJF & 14.26 & -0.87 & 0.43 & -3.35 \\
\hline chr1:277-289 suggestive & RJF & 15.28 & -0.59 & 0.55 & -4.21 \\
\hline chr1:271-310 suggestive & RJF & 14.21 & -0.91 & 0.21 & -0.95 \\
\hline chr1:273-294 suggestive & RJF & 13.84 & -1.09 & 0.51 & -3.68 \\
\hline chr1:274-288 significant & RJF & 15.15 & -1.45 & 0.4 & -2.76 \\
\hline chr1:277-290 suggestive & RJF & 13.08 & -0.46 & 0.3 & -2.22 \\
\hline chr1:277-292 suggestive & RJF & 13.5 & -0.98 & 0.36 & -2.48 \\
\hline chr1:277-290 suggestive & RJF & 13.39 & -0.69 & 0.3 & -2.15 \\
\hline chr1:277-292 suggestive & RJF & 14.11 & -0.43 & 0.35 & -2.61 \\
\hline chr1:277-289 suggestive & RJF & 13.87 & -0.97 & 0.43 & -3.22 \\
\hline chr1:276-288 suggestive & RJF & 13.63 & -0.69 & 0.39 & -2.9 \\
\hline chr1:277-288 suggestive & RJF & 13.74 & -1.46 & 1.65 & -12.04 \\
\hline chr1:273-301 suggestive & RJF & 14.63 & -1.22 & 0.3 & -1.61 \\
\hline chr1:267-292 significant & WL & 16.24 & 0.57 & 0.34 & -2.08 \\
\hline chr1:257-291 significant & WL & 14.64 & 0.68 & 0.29 & -1.42 \\
\hline chr1:276-292 significant & WL & 16.57 & 1.31 & 0.45 & -2.12 \\
\hline chr1:270-311 significant & RJF & 15.25 & -2.01 & 0.44 & -1.43 \\
\hline chr1:271-305 significant & RJF & 17.08 & -1.85 & 0.4 & 0.35 \\
\hline chr1:277-293 significant & RJF & 15.49 & -0.76 & 0.32 & -2.4 \\
\hline chr1:273-319 significant & WL & 14.53 & 0.97 & 0.37 & -1.78 \\
\hline chr1:273-306 significant & RJF & 15.96 & -1.7 & 0.37 & -0.26 \\
\hline chr1:257-303 suggestive & RJF & 15.06 & -1.42 & 0.33 & 0.2 \\
\hline chr1:276-290 suggestive & RJF & 13.66 & -0.18 & 0.2 & -1.51 \\
\hline chr1:277-289 significant & RJF & 15.04 & -0.54 & 0.26 & -1.97 \\
\hline chr1:277-290 suggestive & RJF & 13.93 & -1.39 & 0.49 & -3.36 \\
\hline chr1:277-292 suggestive & RJF & 14.94 & -0.7 & 0.54 & -4.09 \\
\hline chr1:277-291 suggestive & RJF & 13.82 & -0.41 & 0.34 & -2.51 \\
\hline chr1:278-292 suggestive & RJF & 13.89 & -0.55 & 0.33 & -2.46 \\
\hline chr1:278-292 suggestive & RJF & 13.7 & -0.46 & 0.3 & -2.17 \\
\hline chr1:277-286 significant & RJF & 21.47 & -2.04 & 0.57 & -5.19 \\
\hline chr1:278-287 suggestive & RJF & 22.83 & -1.2 & 0.54 & -1.73 \\
\hline chr1:266-294 suggestive & RJF & 20.59 & -3.05 & 0.57 & -0.66 \\
\hline chr1:277-290 suggestive & RJF & 24.55 & -2.15 & 0.58 & -2.96 \\
\hline chr1:278-286 significant & RJF & 23.47 & -0.91 & 0.36 & -3.67 \\
\hline chr1:278-286 suggestive & RJF & 18.26 & -0.37 & 0.48 & -0.98 \\
\hline chr1:276-287 suggestive & RJF & 17.05 & -1.05 & 0.66 & -1.78 \\
\hline chr1:277-289 suggestive & RJF & 21.15 & -1.12 & 0.41 & -1.75 \\
\hline chr1:275-294 suggestive & RJF & 21.26 & -2.98 & 0.73 & -2.68 \\
\hline chr1:278-288 suggestive & RJF & 23.95 & -1.59 & 0.56 & -2.12 \\
\hline chr1:277-291 suggestive & RJF & 19.91 & -0.69 & 0.27 & -2.44 \\
\hline chr1:276-291 suggestive & RJF & 19.47 & -0.92 & 0.34 & -2.95 \\
\hline chr1:276-289 suggestive & RJF & 18.17 & -1.55 & 0.49 & -4.04 \\
\hline chr1:278-290 suggestive & RJF & 18.67 & -0.73 & 0.32 & -2.83 \\
\hline
\end{tabular}




\begin{tabular}{|c|c|c|c|c|c|}
\hline chr1:277-289 suggestive & RJF & 18.86 & -0.57 & 0.37 & -3.27 \\
\hline chr1:277-288 suggestive & RJF & 17.8 & -0.75 & 0.33 & -2.98 \\
\hline chr1:278-288 suggestive & RJF & 18.55 & -0.94 & 0.35 & -3.05 \\
\hline chr1:274-289 suggestive & WL & 18.41 & 1.03 & 0.58 & -4 \\
\hline chr1:279-289 suggestive & RJF & 17.96 & -0.39 & 0.33 & -2.87 \\
\hline chr1:278-291 suggestive & RJF & 18.36 & -0.8 & 0.35 & -2.98 \\
\hline chr1:277-289 suggestive & RJF & 19.05 & -0.97 & 0.3 & -2.53 \\
\hline chr1:278-291 suggestive & RJF & 14.81 & -0.29 & 0.23 & -2.02 \\
\hline chr1:278-288 suggestive & RJF & 18.77 & -0.18 & 0.42 & -3.59 \\
\hline chr1:277-286 suggestive & RJF & 19.91 & -1.55 & 0.44 & -3.78 \\
\hline chr1:278-286 suggestive & RJF & 19.51 & -0.92 & 0.56 & -4.99 \\
\hline chr1:278-288 suggestive & RJF & 21.01 & -1.35 & 0.36 & -3.19 \\
\hline chr1:278-290 suggestive & RJF & 18.44 & -0.51 & 0.27 & -2.33 \\
\hline chr1:278-290 suggestive & WL & 17.82 & 0.11 & 0.34 & -2.76 \\
\hline chr1:278-288 significant & RJF & 21.74 & -0.94 & 0.45 & -4.45 \\
\hline chr1:278-289 suggestive & RJF & 20.59 & -0.32 & 0.27 & -2.52 \\
\hline chr1:277-286 suggestive & RJF & 17.96 & -0.63 & 0.31 & -2.71 \\
\hline chr1:277-291 suggestive & RJF & 19.31 & -2.14 & 0.59 & -4.82 \\
\hline chr1:275-291 suggestive & RJF & 17.35 & -1.04 & 0.31 & -2.44 \\
\hline chr1:277-289 suggestive & RJF & 18.69 & -0.93 & 0.44 & -3.87 \\
\hline chr1:276-289 suggestive & RJF & 18.56 & -0.7 & 0.29 & -2.58 \\
\hline chr1:279-289 suggestive & RJF & 19.76 & -0.64 & 0.63 & -5.71 \\
\hline chr1:276-289 suggestive & RJF & 18.04 & -0.49 & 0.4 & -3.45 \\
\hline chr1:277-291 suggestive & RJF & 19.24 & -1.67 & 0.55 & -4.74 \\
\hline chr1:278-290 suggestive & RJF & 21.07 & -0.97 & 0.28 & -2.55 \\
\hline chr1:277-294 significant & RJF & 14.94 & -0.53 & 0.35 & -2.72 \\
\hline chr1:278-295 suggestive & RJF & 17.83 & -0.28 & 0.64 & -1.19 \\
\hline chr1:274-295 significant & RJF & 14.66 & -1.75 & 0.55 & -3.78 \\
\hline chr1:276-295 suggestive & RJF & 14.26 & -1.14 & 0.4 & -2.81 \\
\hline chr1:277-292 significant & RJF & 16.98 & -0.56 & 0.43 & -3.51 \\
\hline chr1:278-292 suggestive & RJF & 18.09 & -0.87 & 0.47 & -4.06 \\
\hline chr1:277-294 suggestive & RJF & 11.81 & -1.87 & 0.54 & -4.27 \\
\hline chr1:278-297 suggestive & RJF & 17.55 & -2.11 & 0.8 & -3.14 \\
\hline chr1:263-307 significant & RJF & 15.57 & -2.16 & 0.46 & -1.58 \\
\hline chr1:274-308 suggestive & RJF & 14.1 & -1.38 & 0.47 & -3.21 \\
\hline chr1:276-295 significant & RJF & 15.35 & -0.3 & 0.23 & -1.83 \\
\hline chr1:277-303 suggestive & RJF & 13.9 & -0.51 & 0.49 & -3.67 \\
\hline chr1:277-293 suggestive & RJF & 18.48 & -0.32 & 0.32 & -2.77 \\
\hline chr1:276-293 suggestive & RJF & 19.52 & -0.78 & 0.4 & -3.56 \\
\hline chr1:277-294 suggestive & RJF & 19.23 & -1.58 & 0.49 & -4.19 \\
\hline chr1:277-292 suggestive & RJF & 19.16 & -0.88 & 0.42 & -3.8 \\
\hline chr1:276-307 significant & WL & 18.58 & 1.12 & 0.43 & -2.59 \\
\hline chr1:274-306 suggestive & RJF & 12.99 & -1.11 & 0.42 & -2.89 \\
\hline
\end{tabular}




\begin{tabular}{|c|c|c|c|c|c|}
\hline chr1:270-311 suggestive & RJF & 14.85 & -2.14 & 0.47 & -0.34 \\
\hline chr1:272-306 significant & RJF & 17.49 & -2.31 & 0.46 & -1.64 \\
\hline chr1:276-304 suggestive & RJF & 13.67 & -1.11 & 0.5 & -3.62 \\
\hline chr1:276-299 suggestive & RJF & 13.03 & -0.77 & 0.54 & -3.81 \\
\hline chr1:275-294 significant & RJF & 20.67 & -1.17 & 0.28 & -2.47 \\
\hline chr1:277-294 suggestive & RJF & 19.27 & -1.28 & 0.48 & -4.25 \\
\hline chr1:264-309 significant & RJF & 21.86 & -4.03 & 0.79 & -0.37 \\
\hline chr1:276-305 significant & RJF & 24.41 & -1.69 & 0.27 & -0.68 \\
\hline chr1:276-305 suggestive & RJF & 13.65 & -0.56 & 0.35 & -2.62 \\
\hline chr1:277-300 suggestive & RJF & 17.87 & -0.75 & 0.3 & -2.55 \\
\hline chr1:261-311 significant & RJF & 20.55 & -3.74 & 0.72 & -0.59 \\
\hline chr1:256-344 suggestive & WL & 14.46 & 0.87 & 0.19 & 0.06 \\
\hline chr1:263-312 significant & RJF & 19.34 & -3.38 & 0.65 & -0.49 \\
\hline chr1:257-322 significant & WL & 12.86 & 1.79 & 0.44 & 0.11 \\
\hline chr1:274-311 significant & RJF & 23.76 & -2.39 & 0.39 & -0.7 \\
\hline chr1:272-320 significant & WL & 15.6 & 1.45 & 0.31 & 0.61 \\
\hline chr1:272-320 suggestive & RJF & 16.88 & -2.27 & 0.63 & 0.79 \\
\hline chr1:275-310 suggestive & RJF & 20.68 & -2.28 & 0.59 & -2.16 \\
\hline chr1:272-313 significant & RJF & 16.06 & -2.49 & 0.53 & -0.12 \\
\hline chr1:281-313 suggestive & WL & 19.02 & 0.14 & 0.35 & -0.36 \\
\hline chr1:291-311 significant & WL & 33.29 & 3.14 & 0.42 & -0.06 \\
\hline chr1:276-317 suggestive & RJF & 15 & -3.18 & 0.7 & -0.5 \\
\hline chr1:287-316 significant & WL & 25.49 & 2.72 & 0.47 & -0.66 \\
\hline chr1:273-325 significant & WL & 14.62 & 0.8 & 0.25 & -0.84 \\
\hline chr1:284-325 significant & WL & 13.98 & 0.8 & 0.22 & -0.46 \\
\hline chr1:283-324 significant & WL & 16.31 & 0.81 & 0.19 & -0.35 \\
\hline chr1:277-333 significant & WL & 15.56 & 2.34 & 0.56 & -0.71 \\
\hline chr1:275-323 significant & WL & 24.04 & 2.16 & 0.37 & -0.26 \\
\hline chr1:272-328 significant & WL & 15.22 & 1.73 & 0.37 & 0.26 \\
\hline chr1:270-342 significant & WL & 12.21 & 2.2 & 0.54 & 0.33 \\
\hline chr1:273-356 significant & WL & 12.69 & 1.24 & 0.3 & 0.94 \\
\hline chr1:271-346 significant & WL & 14.86 & 0.26 & 0.23 & -1.52 \\
\hline chr1:308-343 suggestive & WL & 19.35 & 0.44 & 0.12 & -0.59 \\
\hline chr1:314-339 suggestive & RJF & 17.61 & -0.05 & 0.06 & -0.5 \\
\hline chr1:310-355 suggestive & WL & 15.4 & 2.05 & 0.44 & 0.11 \\
\hline chr1:316-357 suggestive & WL & 15.63 & 1.84 & 0.39 & 1.01 \\
\hline chr1:317-350 significant & WL & 16.62 & 1.52 & 0.32 & -0.09 \\
\hline chr1:302-345 significant & WL & 16.01 & 2.37 & 0.51 & -0.27 \\
\hline chr1:302-351 significant & WL & 19.06 & 1.29 & 0.24 & -0.22 \\
\hline chr1:321-355 significant & WL & 17.66 & 1.5 & 0.3 & 0.12 \\
\hline chr1:320-359 suggestive & WL & 15.02 & 2.32 & 0.52 & 1.51 \\
\hline chr1:321-372 significant & RJF & 19.51 & -2.06 & 0.38 & 0.11 \\
\hline chr1:334-370 suggestive & WL & 16.38 & 1.74 & 0.36 & -0.02 \\
\hline
\end{tabular}




\begin{tabular}{|c|c|c|c|c|c|}
\hline chr1:344-363 significant & WL & 25.37 & 1.95 & 0.3 & -0.1 \\
\hline chr1:345-364 significant & WL & 20.39 & 2.65 & 0.5 & 0.71 \\
\hline chr1:346-370 suggestive & RJF & 19.08 & -1.8 & 0.56 & -2.63 \\
\hline chr1:319-380 significant & RJF & 10.29 & -1.61 & 0.47 & 1.15 \\
\hline chr1:346-373 significant & WL & 25.33 & 2.64 & 0.43 & 0.53 \\
\hline chr1:349-383 suggestive & RJF & 14.11 & -1.48 & 0.35 & -1.47 \\
\hline chr1:350-381 suggestive & RJF & 17.59 & -2.24 & 0.5 & -2.07 \\
\hline chr1:358-384 suggestive & RJF & 15.27 & -2.59 & 0.7 & -2.73 \\
\hline chr1:327-386 significant & RJF & 12.92 & -2.2 & 0.61 & -3.02 \\
\hline chr1:359-385 suggestive & RJF & 14.21 & -2.19 & 0.52 & -2.38 \\
\hline chr1:359-387 significant & RJF & 14.78 & -1.76 & 0.41 & -1.82 \\
\hline chr1:363-396 suggestive & RJF & 17.25 & -0.03 & 0.72 & 0.9 \\
\hline chr1:360-380 suggestive & RJF & 19.19 & -1.81 & 0.49 & -3.62 \\
\hline chr1:355-386 suggestive & RJF & 13.9 & -0.38 & 0.22 & -1.46 \\
\hline chr1:363-388 significant & RJF & 17.93 & -1.24 & 0.25 & -1.11 \\
\hline chr1:366-385 significant & RJF & 21 & -2.35 & 0.47 & -3.58 \\
\hline chr1:367-382 suggestive & RJF & 26.46 & -4.44 & 0.9 & -6.17 \\
\hline chr1:361-386 suggestive & RJF & 19.7 & -1.3 & 0.31 & -2.29 \\
\hline chr1:358-389 significant & RJF & 16.91 & -2.98 & 0.62 & -2.7 \\
\hline chr1:366-389 significant & RJF & 18.58 & -1.56 & 0.31 & -1.48 \\
\hline chr1:363-390 suggestive & RJF & 15.49 & -1.74 & 0.4 & -2.17 \\
\hline chr1:359-387 suggestive & RJF & 20.29 & -1.11 & 0.2 & -0.99 \\
\hline chr1:364-396 suggestive & RJF & 13.98 & -1.29 & 0.46 & -2.86 \\
\hline chr1:362-400 suggestive & RJF & 12.51 & -2.05 & 0.49 & -1.14 \\
\hline chr1:366-397 significant & RJF & 16.88 & -1.32 & 0.28 & -1.41 \\
\hline chr1:363-397 suggestive & RJF & 15.69 & -1.5 & 0.32 & -1.33 \\
\hline chr1:368-428 suggestive & RJF & 13.57 & -1.56 & 0.36 & -0.68 \\
\hline chr1:373-405 significant & RJF & 15.94 & -3.52 & 0.87 & -0.19 \\
\hline chr1:371-407 suggestive & RJF & 12.29 & -1.26 & 0.34 & -0.18 \\
\hline chr1:383-403 suggestive & RJF & 25.38 & -1.32 & 0.36 & -0.82 \\
\hline chr1:404-425 suggestive & WL & 15.01 & 1.1 & 0.44 & -1.18 \\
\hline chr1:384-436 suggestive & WL & 13.49 & 2.53 & 0.7 & 0.2 \\
\hline chr1:400-427 suggestive & WL & 14.76 & 1.67 & 0.54 & -0.74 \\
\hline chr1:383-461 significant & WL & 14.64 & 1.18 & 0.39 & -0.64 \\
\hline chr1:397-495 suggestive & RJF & 14.13 & -1.34 & 0.36 & 0.45 \\
\hline chr1:399-486 significant & RJF & 16.81 & -2.04 & 0.47 & 0.37 \\
\hline chr1:408-536 suggestive & RJF & 12.65 & -2.05 & 0.48 & -0.82 \\
\hline chr1:424-475 significant & RJF & 17.41 & -2.42 & 0.48 & -0.99 \\
\hline chr1:424-444 significant & RJF & 18.14 & -1.78 & 0.34 & -0.44 \\
\hline chr1:421-529 suggestive & RJF & 14.26 & -1.84 & 0.42 & -0.38 \\
\hline chr1:413-465 significant & WL & 15.84 & 2.51 & 0.53 & 0.74 \\
\hline chr1:434-456 significant & WL & 52.19 & 4.3 & 0.39 & 0.33 \\
\hline chr1:422-463 significant & WL & 19.84 & 1.89 & 0.35 & 0.52 \\
\hline
\end{tabular}




\begin{tabular}{|c|c|c|c|c|c|}
\hline chr1:418-477 significant & RJF & 16.5 & -1.71 & 0.36 & -0.25 \\
\hline chr1:397-501 suggestive & RJF & 14.23 & -2.25 & 0.53 & -0.13 \\
\hline chr1:468-533 significant & RJF & 13.94 & -2.11 & 0.56 & -0.87 \\
\hline chr1:468-536 suggestive & RJF & 11.6 & -1.81 & 0.65 & -1.71 \\
\hline chr1:470-531 suggestive & RJF & 16.42 & -1.63 & 0.4 & -0.67 \\
\hline chr1:472-537 suggestive & RJF & 12.09 & -0.87 & 0.36 & -1.3 \\
\hline chr1:476-528 suggestive & RJF & 18.95 & -1.65 & 0.52 & -2.09 \\
\hline chr1:484-529 suggestive & RJF & 13.89 & -0.66 & 0.36 & -1.66 \\
\hline chr1:480-529 significant & RJF & 20.13 & -1.27 & 0.29 & -0.85 \\
\hline chr1:483-528 suggestive & RJF & 19.14 & -1.73 & 0.38 & -0.75 \\
\hline chr1:471-541 suggestive & RJF & 12.28 & -2.37 & 0.65 & -0.79 \\
\hline chr1:477-538 significant & RJF & 14.53 & -1.69 & 0.48 & -1.11 \\
\hline chr1:546-593 significant & WL & 17.81 & 1.46 & 0.28 & 0.15 \\
\hline chr1:515-677 significant & WL & 11.26 & 1.88 & 0.51 & 0.87 \\
\hline chr1:549-666 significant & WL & 16.05 & 1.25 & 0.27 & -0.16 \\
\hline chr1:606-639 suggestive & WL & 23.03 & 0.31 & 0.05 & -0.26 \\
\hline chr1:584-662 significant & WL & 20 & 2.22 & 0.42 & -0.63 \\
\hline chr1:588-666 significant & WL & 16.77 & 2.55 & 0.62 & 0.66 \\
\hline chr1:592-663 significant & WL & 17.67 & 1.55 & 0.3 & -0.68 \\
\hline chr1:592-667 significant & RJF & 12.9 & -0.29 & 0.76 & 4.5 \\
\hline chr1:688-710 significant & WL & 34.26 & 0.01 & 0.07 & 0.01 \\
\hline chr1:686-709 significant & WL & 31.01 & 0.01 & 0.05 & 0 \\
\hline chr1:688-712 significant & RJF & 32.3 & 0 & 0.04 & -0.03 \\
\hline chr1:684-709 suggestive & RJF & 26.33 & -0.02 & 0.06 & 0.05 \\
\hline chr1:680-713 suggestive & WL & 22.8 & 0.03 & 0.07 & 0.02 \\
\hline chr1:687-708 significant & WL & 20.85 & 2.3 & 0.43 & 0.06 \\
\hline chr1:661-719 significant & WL & 15.66 & 1.91 & 0.44 & 0.19 \\
\hline chr1:653-745 significant & RJF & 17.82 & -1.32 & 0.32 & -0.44 \\
\hline chr1:713-729 significant & WL & 22.31 & 0.06 & 0.01 & -0.06 \\
\hline chr1:719-730 suggestive & WL & 18.14 & 0.24 & 0.05 & -0.33 \\
\hline chr1:719-731 suggestive & WL & 21.63 & 2.1 & 0.63 & -3.39 \\
\hline chr1:734-756 significant & RJF & 34.53 & -2.34 & 0.31 & 0.75 \\
\hline chr1:732-773 significant & RJF & 20.22 & -2.6 & 0.5 & 0.84 \\
\hline chr1:734-772 significant & RJF & 16.26 & -1.97 & 0.41 & -0.28 \\
\hline chr1:719-885 significant & WL & 11.56 & 2.43 & 0.6 & 0.83 \\
\hline chr1:648-771 significant & RJF & 14.69 & -2 & 0.54 & -0.65 \\
\hline chr1:730-796 significant & RJF & 14.98 & -2.14 & 0.47 & 0.46 \\
\hline chr1:549-885 suggestive & WL & 8.31 & 1.35 & 0.41 & 0.06 \\
\hline chr1:750-775 significant & RJF & 26.43 & -2.51 & 0.41 & -0.82 \\
\hline chr1:750-776 significant & RJF & 19.42 & -1.51 & 0.28 & -0.07 \\
\hline chr1:750-771 significant & RJF & 25.09 & -4.69 & 0.82 & 2.17 \\
\hline chr1:728-786 suggestive & WL & 9.95 & 1.17 & 0.31 & 0.16 \\
\hline chr1:751-771 significant & RJF & 23.62 & -2.05 & 0.34 & -0.89 \\
\hline
\end{tabular}




\begin{tabular}{|c|c|c|c|c|c|}
\hline chr1:751-775 significant & RJF & 23.35 & -1.95 & 0.32 & -0.17 \\
\hline chr1:753-769 significant & RJF & 30.14 & -5.81 & 0.83 & 0.33 \\
\hline chr1:752-772 significant & WL & 23.06 & 2 & 0.34 & 0.58 \\
\hline chr1:757-770 significant & RJF & 46.34 & -3.39 & 0.34 & -0.03 \\
\hline chr1:755-778 suggestive & RJF & 17.39 & -2.41 & 0.52 & 0.77 \\
\hline chr1:751-774 significant & RJF & 16.79 & -1.31 & 0.27 & -0.16 \\
\hline chr1:751-777 significant & RJF & 18.69 & -1.29 & 0.25 & -0.34 \\
\hline chr1:753-772 significant & RJF & 21.49 & -4.2 & 0.76 & 0.31 \\
\hline chr1:755-769 significant & WL & 30.67 & 2.67 & 0.39 & -0.76 \\
\hline chr1:755-772 significant & RJF & 28.87 & -2.2 & 0.34 & 0.54 \\
\hline chr1:753-776 significant & RJF & 22.71 & -3.54 & 0.99 & 2.99 \\
\hline chr1:753-774 significant & RJF & 21.98 & -3.75 & 0.67 & 0.39 \\
\hline chr1:754-774 significant & RJF & 21.57 & -2.44 & 0.42 & -0.39 \\
\hline chr1:752-777 significant & RJF & 17.12 & -1.68 & 0.35 & 0.28 \\
\hline chr1:751-780 suggestive & RJF & 17.44 & -3.41 & 0.69 & 0.36 \\
\hline chr1:755-787 significant & RJF & 17.48 & -1.08 & 0.23 & 0.31 \\
\hline chr1:752-776 significant & RJF & 25.49 & -2.66 & 0.42 & -0.39 \\
\hline chr1:752-776 significant & RJF & 25.34 & -3.38 & 0.53 & -0.34 \\
\hline chr1:755-775 significant & RJF & 26.36 & -1.34 & 0.45 & 2.39 \\
\hline chr1:755-780 significant & RJF & 17.66 & -2.23 & 0.45 & 0.09 \\
\hline chr1:754-772 significant & RJF & 40.94 & -2.87 & 0.31 & -0.7 \\
\hline chr1:754-777 significant & RJF & 23.46 & -3.57 & 0.59 & -0.18 \\
\hline chr1:718-787 suggestive & WL & 11.76 & 1.6 & 0.4 & 0.16 \\
\hline chr1:754-780 significant & RJF & 22.65 & -2.76 & 0.49 & 0.6 \\
\hline chr1:752-793 significant & RJF & 15.1 & -1.31 & 0.28 & -0.15 \\
\hline chr1:754-781 significant & RJF & 19.95 & -2.44 & 0.45 & -0.4 \\
\hline chr1:645-786 suggestive & WL & 9.9 & 2.53 & 0.72 & -0.23 \\
\hline chr1:755-799 suggestive & RJF & 17.62 & -2.29 & 0.49 & 0.76 \\
\hline chr1:754-792 significant & RJF & 17.18 & -1.74 & 0.45 & 1.61 \\
\hline chr1:754-787 significant & RJF & 17.32 & -1.73 & 0.36 & 0.41 \\
\hline chr1:753-782 significant & RJF & 23.05 & -1.48 & 0.25 & -0.9 \\
\hline chr1:751-788 suggestive & RJF & 17.51 & -1.58 & 0.31 & -0.4 \\
\hline chr1:750-788 significant & RJF & 18.26 & -1.94 & 0.37 & -0.13 \\
\hline chr1:755-791 significant & RJF & 24.19 & -1.74 & 0.28 & -0.06 \\
\hline chr1:752-796 significant & RJF & 16.59 & -1.89 & 0.38 & -0.16 \\
\hline chr1:756-794 significant & RJF & 32.61 & -2.36 & 0.3 & -0.18 \\
\hline chr1:754-796 significant & RJF & 20.86 & -2.56 & 0.46 & 0.17 \\
\hline chr1:752-794 suggestive & RJF & 15.5 & -0.93 & 0.21 & -0.57 \\
\hline chr1:754-788 suggestive & RJF & 16.14 & -2.41 & 0.5 & 0.4 \\
\hline chr1:756-796 significant & RJF & 17.65 & -2.18 & 0.43 & -0.21 \\
\hline chr1:755-800 significant & RJF & 16.75 & -2.22 & 0.49 & -0.99 \\
\hline chr1:757-796 significant & RJF & 19.09 & -3.07 & 0.58 & -0.22 \\
\hline chr1:765-798 suggestive & RJF & 19.22 & -1.48 & 0.39 & -2.16 \\
\hline
\end{tabular}




\begin{tabular}{|c|c|c|c|c|c|}
\hline chr1:758-800 significant & RJF & 19.17 & -1.73 & 0.33 & -0.35 \\
\hline chr1:754-797 significant & RJF & 17.99 & -3.06 & 0.6 & 0.04 \\
\hline chr1:757-796 significant & RJF & 16.84 & -2.71 & 0.55 & 0.54 \\
\hline chr1:766-787 significant & WL & 30.22 & 2.3 & 0.32 & -0.53 \\
\hline chr1:764-798 significant & RJF & 22.19 & -1.78 & 0.31 & -0.26 \\
\hline chr1:751-788 significant & WL & 17.32 & 1.5 & 0.29 & -0.15 \\
\hline chr1:756-884 significant & RJF & 16.6 & -1.2 & 0.25 & 0.25 \\
\hline chr1:755-795 suggestive & RJF & 15.37 & -1.13 & 0.25 & 0.4 \\
\hline chr1:766-786 significant & WL & 23.83 & 2.28 & 0.37 & 0.04 \\
\hline chr1:764-796 suggestive & RJF & 15.43 & -1.35 & 0.29 & -0.33 \\
\hline chr1:759-796 suggestive & RJF & 14.23 & -1.18 & 0.26 & -0.25 \\
\hline chr1:764-797 suggestive & RJF & 14.68 & -1.21 & 0.26 & -0.05 \\
\hline chr1:772-787 significant & RJF & 32.81 & -2.25 & 0.29 & 0.46 \\
\hline chr1:755-831 suggestive & WL & 11.89 & 1.92 & 0.48 & 0.02 \\
\hline chr1:774-793 significant & WL & 20.26 & 1.85 & 0.34 & -0.57 \\
\hline chr1:774-785 significant & WL & 24.31 & 2.24 & 0.36 & 0.55 \\
\hline chr1:767-793 significant & WL & 13.78 & 2.29 & 0.53 & 0.53 \\
\hline chr1:768-832 significant & WL & 10.79 & 1.24 & 0.34 & -0.39 \\
\hline chr1:767-790 significant & WL & 14.11 & 2.85 & 0.66 & -0.76 \\
\hline chr1:765-819 significant & WL & 14.21 & 1.03 & 0.25 & -0.55 \\
\hline chr1:748-921 significant & WL & 10.01 & 1.83 & 0.5 & -0.04 \\
\hline chr1:774-787 suggestive & WL & 16.71 & 0.38 & 0.2 & -1.78 \\
\hline chr1:765-795 significant & RJF & 23.87 & -1.87 & 0.33 & 0.89 \\
\hline chr1:766-798 significant & WL & 16.57 & 2.41 & 0.49 & -0.24 \\
\hline chr1:770-795 significant & RJF & 18.94 & -2.45 & 0.46 & 0.14 \\
\hline chr1:774-800 significant & RJF & 21.67 & -1.38 & 0.24 & -0.41 \\
\hline chr1:770-795 significant & RJF & 27.25 & -2.98 & 0.44 & 0.07 \\
\hline chr1:775-797 significant & RJF & 31.05 & -2.63 & 0.35 & -0.2 \\
\hline chr1:729-799 significant & WL & 11.52 & 1.94 & 0.5 & -1.07 \\
\hline chr1:751-798 significant & WL & 17.19 & 1.78 & 0.36 & -0.01 \\
\hline chr1:760-798 significant & RJF & 17.53 & -1.29 & 0.26 & -0.29 \\
\hline chr1:767-800 significant & RJF & 17.82 & -2.53 & 0.49 & -0.17 \\
\hline chr1:769-796 significant & RJF & 26.01 & -2.1 & 0.34 & -0.64 \\
\hline chr1:771-800 significant & WL & 12.47 & 1.13 & 0.28 & -0.6 \\
\hline chr1:775-794 significant & WL & 16.86 & 1.16 & 0.49 & -2.44 \\
\hline chr1:776-796 significant & RJF & 22.83 & -1.98 & 0.34 & -0.52 \\
\hline chr1:784-792 significant & RJF & 27.42 & -1.82 & 0.25 & -0.13 \\
\hline chr1:768-797 significant & RJF & 21.65 & -2.52 & 0.43 & -0.28 \\
\hline chr1:785-795 significant & RJF & 21.33 & -0.95 & 0.16 & -0.14 \\
\hline chr1:787-789 significant & RJF & 51.22 & -3.77 & 0.33 & 0.47 \\
\hline chr1:787-791 significant & RJF & 44.42 & -3.08 & 0.31 & 0.24 \\
\hline chr1:787-792 significant & RJF & 31.83 & -2.44 & 0.31 & 0.27 \\
\hline chr1:786-794 significant & RJF & 30.72 & -1.78 & 0.28 & -1.12 \\
\hline
\end{tabular}




\begin{tabular}{|c|c|c|c|c|c|}
\hline chr1:786-796 significant & RJF & 31.3 & -2.03 & 0.28 & -0.07 \\
\hline chr1:787-795 significant & RJF & 35.09 & -3.92 & 0.48 & 0.27 \\
\hline chr1:722-827 suggestive & WL & 6.79 & 1.85 & 0.72 & -2.07 \\
\hline chr1:721-869 suggestive & WL & 7.31 & 1.7 & 0.58 & -1.11 \\
\hline chr1:767-831 significant & WL & 8.06 & 1.09 & 0.38 & -1.02 \\
\hline chr1:780-806 significant & RJF & 15.95 & -1.4 & 0.28 & -0.06 \\
\hline chr1:785-825 significant & RJF & 15.94 & -1.27 & 0.27 & -0.25 \\
\hline chr1:786-825 suggestive & RJF & 13.31 & -1.05 & 0.25 & -0.31 \\
\hline chr1:774-797 significant & RJF & 17.49 & -2.67 & 0.52 & -0.13 \\
\hline chr1:756-799 significant & RJF & 16.94 & -1.77 & 0.38 & -0.7 \\
\hline chr1:730-828 suggestive & WL & 9.44 & 2.17 & 0.62 & -0.84 \\
\hline chr1:784-797 suggestive & RJF & 15.8 & -1.49 & 0.33 & -0.33 \\
\hline chr1:786-794 suggestive & WL & 22.02 & 1.38 & 0.49 & -3.81 \\
\hline chr1:786-795 suggestive & RJF & 19.43 & -0.65 & 0.15 & -0.57 \\
\hline chr1:778-797 suggestive & RJF & 16.61 & -0.79 & 0.3 & -1.67 \\
\hline chr1:785-799 significant & RJF & 20.63 & -2.52 & 0.44 & -0.16 \\
\hline chr1:767-809 significant & RJF & 20.36 & -2.53 & 0.49 & -1.09 \\
\hline chr1:785-799 suggestive & RJF & 15.39 & -0.41 & 0.36 & -2.21 \\
\hline chr1:779-824 significant & RJF & 17.17 & -0.3 & 0.34 & -1.07 \\
\hline chr1:784-799 significant & RJF & 38.54 & -2.51 & 0.29 & -0.26 \\
\hline chr1:781-827 suggestive & RJF & 16.9 & -2.14 & 0.43 & 0.9 \\
\hline chr1:785-799 suggestive & WL & 9.73 & 0.4 & 0.3 & -1.72 \\
\hline chr1:768-835 significant & RJF & 17.99 & -1.31 & 0.33 & -1.24 \\
\hline chr1:774-826 significant & RJF & 19.02 & -2.44 & 0.7 & -1.67 \\
\hline chr1:781-823 significant & WL & 18.14 & 4.92 & 0.98 & -2.69 \\
\hline chr1:784-833 significant & RJF & 17.71 & -1.6 & 0.34 & 0.25 \\
\hline chr1:794-817 suggestive & WL & 19.73 & 2.48 & 0.59 & -3.9 \\
\hline chr1:792-822 suggestive & WL & 13.19 & 1.85 & 0.48 & -2.93 \\
\hline chr1:794-820 significant & WL & 20.34 & 3.57 & 0.65 & -2.59 \\
\hline chr1:791-824 suggestive & RJF & 19.64 & -0.15 & 0.13 & -0.67 \\
\hline chr1:782-831 significant & WL & 13.1 & 2.59 & 0.63 & -0.69 \\
\hline chr1:794-824 suggestive & WL & 16.78 & 6.71 & 1.45 & -4.9 \\
\hline chr1:793-824 suggestive & WL & 13.77 & 1.3 & 0.32 & -0.24 \\
\hline chr1:792-830 significant & RJF & 17.75 & -1.54 & 0.43 & -0.81 \\
\hline chr1:795-827 significant & WL & 19.2 & 5.34 & 1.04 & -2.43 \\
\hline chr1:797-826 suggestive & WL & 14.49 & 5.69 & 1.28 & -3.95 \\
\hline chr1:797-817 suggestive & WL & 20.25 & 6.91 & 1.3 & -6.32 \\
\hline chr1:796-817 suggestive & WL & 19.14 & 6.27 & 1.18 & -4.64 \\
\hline chr1:799-821 suggestive & WL & 20.45 & 6.46 & 1.33 & -8.14 \\
\hline chr1:796-823 suggestive & WL & 21.57 & 4.99 & 0.9 & -4.88 \\
\hline chr1:794-839 suggestive & WL & 16.7 & 2.27 & 0.56 & -0.86 \\
\hline chr1:799-829 suggestive & WL & 15.33 & 4.04 & 0.91 & -3.9 \\
\hline chr1:793-832 suggestive & RJF & 20.13 & -0.36 & 0.16 & -0.67 \\
\hline
\end{tabular}




\begin{tabular}{|c|c|c|c|c|c|}
\hline chr1:798-824 suggestive & WL & 9.81 & 0.57 & 0.26 & -2.15 \\
\hline chr1:796-828 suggestive & WL & 20.42 & 0.08 & 0.02 & -0.1 \\
\hline chr1:791-839 suggestive & WL & 14.64 & 0.71 & 0.35 & -2.06 \\
\hline chr1:792-868 suggestive & RJF & 14.31 & -2.15 & 0.48 & -0.87 \\
\hline chr1:795-880 suggestive & RJF & 14.99 & -1.42 & 0.33 & -1.03 \\
\hline chr1:832-857 significant & RJF & 31.78 & -3.9 & 0.52 & 0.94 \\
\hline chr1:826-856 significant & RJF & 30.98 & -6.47 & 0.88 & -0.2 \\
\hline chr1:826-860 significant & RJF & 20.54 & -5.93 & 1.06 & 1.73 \\
\hline chr1:831-859 significant & RJF & 25.66 & -7.56 & 1.24 & 4.47 \\
\hline chr1:832-870 significant & RJF & 20.56 & -3.28 & 0.59 & 0.23 \\
\hline chr1:817-872 suggestive & RJF & 13.79 & -3.49 & 0.8 & 0.24 \\
\hline chr1:799-870 significant & RJF & 13.38 & -2.88 & 0.67 & -0.72 \\
\hline chr1:822-869 significant & RJF & 15.11 & -7.74 & 1.75 & 4.3 \\
\hline chr1:823-871 significant & RJF & 14.48 & -3.37 & 0.74 & 0.33 \\
\hline chr1:819-876 suggestive & RJF & 13.79 & -5.63 & 1.29 & -0.48 \\
\hline chr1:835-863 significant & RJF & 28.17 & -3.77 & 0.54 & -0.32 \\
\hline chr1:828-866 significant & RJF & 21.59 & -4.06 & 0.68 & -0.55 \\
\hline chr1:823-872 significant & RJF & 14.85 & -7.94 & 1.83 & 4.83 \\
\hline chr1:822-888 suggestive & RJF & 13.86 & -2.96 & 0.69 & 1.2 \\
\hline chr1:832-872 significant & RJF & 17.52 & -1.64 & 0.35 & -1.14 \\
\hline chr1:834-866 significant & RJF & 29.98 & -5.39 & 0.75 & 1.26 \\
\hline chr1:824-865 suggestive & RJF & 19.9 & -1.07 & 0.26 & -1.75 \\
\hline chr1:830-867 significant & RJF & 23.35 & -2.07 & 0.69 & 3.17 \\
\hline chr1:832-871 significant & RJF & 23.95 & -3.54 & 0.58 & -0.97 \\
\hline chr1:819-878 significant & RJF & 13.84 & -1.93 & 0.55 & -2.57 \\
\hline chr1:827-884 significant & RJF & 15.68 & -3.16 & 0.67 & -0.15 \\
\hline chr1:822-885 suggestive & RJF & 13.6 & -2.54 & 0.59 & -0.1 \\
\hline chr1:824-888 significant & RJF & 15.56 & -2.73 & 0.59 & -1.32 \\
\hline chr1:829-885 suggestive & RJF & 14.17 & -2.23 & 0.5 & -0.86 \\
\hline chr1:823-886 significant & RJF & 17.94 & -3.52 & 0.69 & -0.95 \\
\hline chr1:819-886 suggestive & RJF & 13.94 & -2.77 & 0.63 & -0.41 \\
\hline chr1:817-889 significant & RJF & 15.18 & -2.76 & 0.6 & -0.85 \\
\hline chr1:787-885 suggestive & RJF & 18.27 & -3.05 & 0.59 & -0.51 \\
\hline chr1:793-889 suggestive & RJF & 13.96 & -1.55 & 0.35 & -0.19 \\
\hline chr1:816-886 suggestive & RJF & 20.22 & -1.38 & 0.28 & -1.18 \\
\hline chr1:810-924 significant & RJF & 13.68 & -1.92 & 0.49 & -1.69 \\
\hline chr1:813-887 significant & RJF & 14.98 & -2.28 & 0.5 & -0.71 \\
\hline chr1:781-888 suggestive & RJF & 15.33 & -0.99 & 0.23 & -0.47 \\
\hline chr1:833-886 significant & RJF & 22.09 & -1.4 & 0.31 & -0.49 \\
\hline chr1:822-888 suggestive & RJF & 18.63 & -0.6 & 0.27 & -1.15 \\
\hline chr1:793-921 significant & RJF & 11.77 & -1.77 & 0.49 & -0.08 \\
\hline chr1:846-889 significant & RJF & 16.11 & -1.36 & 0.48 & -1.37 \\
\hline chr1:846-890 significant & RJF & 21.3 & -0.95 & 0.2 & -0.18 \\
\hline
\end{tabular}




\begin{tabular}{|c|c|c|c|c|c|}
\hline chr1:859-890 significant & WL & 18.87 & 4.08 & 1 & -5.79 \\
\hline chr1:842-888 significant & WL & 14.31 & 1.3 & 0.39 & 0.53 \\
\hline chr1:854-886 significant & RJF & 23.94 & -1.84 & 0.38 & -0.57 \\
\hline chr1:854-923 suggestive & RJF & 18.73 & -2.47 & 0.75 & -0.94 \\
\hline chr1:868-909 significant & WL & 21.37 & 0.75 & 0.68 & 0.2 \\
\hline chr1:859-906 significant & RJF & 17.79 & -1.81 & 0.43 & -0.47 \\
\hline chr1:871-909 suggestive & WL & 20.83 & 1.87 & 0.47 & -3.69 \\
\hline chr1:885-895 significant & RJF & 26.77 & -1.79 & 0.4 & -1.19 \\
\hline chr1:879-898 suggestive & RJF & 13.3 & -1 & 0.42 & -1.11 \\
\hline chr1:886-917 suggestive & RJF & 14.42 & -1.15 & 0.41 & 2.66 \\
\hline chr1:848-911 suggestive & RJF & 16.72 & -0.63 & 0.19 & -0.41 \\
\hline chr1:848-911 significant & RJF & 13.73 & -1.21 & 0.4 & -0.69 \\
\hline chr1:897-916 significant & WL & 41.83 & 4.62 & 0.56 & -0.35 \\
\hline chr1:896-922 significant & WL & 32.13 & 2.21 & 0.32 & -0.37 \\
\hline chr1:902-962 significant & WL & 14.5 & 2.59 & 0.58 & 0.59 \\
\hline chr1:910-944 significant & RJF & 18.56 & -1.04 & 0.2 & 0.05 \\
\hline chr1:913-946 suggestive & WL & 15.47 & 0.06 & 0.51 & 1.52 \\
\hline chr1:913-954 significant & RJF & 18.63 & -1.12 & 0.21 & 0.14 \\
\hline chr1:910-959 suggestive & RJF & 14.95 & -1 & 0.22 & -0.04 \\
\hline chr1:911-949 suggestive & RJF & 14.08 & -3.78 & 0.86 & 0.91 \\
\hline chr1:923-952 significant & WL & 14.55 & 2.43 & 0.53 & -0.53 \\
\hline chr1:921-947 significant & WL & 20.15 & 2.27 & 0.41 & 0.01 \\
\hline chr1:920-958 suggestive & WL & 14.28 & 1.9 & 0.43 & 0.13 \\
\hline chr1:917-958 significant & RJF & 19.61 & -1.9 & 0.36 & 0.54 \\
\hline chr1:940-100 significant & WL & 14.96 & 1.42 & 0.3 & 0.07 \\
\hline chr1:947-999 suggestive & WL & 12.32 & 1.81 & 0.43 & -0.14 \\
\hline chr1:943-100 significant & WL & 14.66 & 1.68 & 0.38 & -0.32 \\
\hline chr1:949-985 significant & WL & 13.47 & 1.56 & 0.39 & -0.76 \\
\hline chr1:946-102 significant & WL & 11.8 & 2.67 & 0.67 & -0.05 \\
\hline chr1:948-993 significant & WL & 22 & 2.72 & 0.47 & -0.73 \\
\hline chr1:947-997 significant & RJF & 17.63 & -1.6 & 0.31 & -0.28 \\
\hline chr1:939-100 significant & WL & 15.66 & 2.43 & 0.59 & -1.4 \\
\hline chr1:905-101 significant & WL & 9.75 & 2.29 & 0.71 & -1.16 \\
\hline chr1:936-100 significant & WL & 15.19 & 4.33 & 1.01 & -2.31 \\
\hline chr1:950-100 significant & WL & 16.41 & 3.4 & 0.76 & -1.39 \\
\hline chr1:944-100 significant & WL & 15.72 & 2.79 & 0.63 & -0.95 \\
\hline chr1:953-995 suggestive & WL & 15.54 & 0.1 & 0.39 & -2.61 \\
\hline chr1:966-995 significant & RJF & 20.29 & -2.29 & 0.42 & -0.5 \\
\hline chr1:923-996 significant & RJF & 17.54 & -1.93 & 0.38 & -0.64 \\
\hline chr1:966-996 significant & RJF & 24.65 & -1.81 & 0.29 & -0.48 \\
\hline chr1:949-998 significant & WL & 15.63 & 4.17 & 0.93 & -1.15 \\
\hline chr1:952-999 significant & WL & 20.32 & 2.4 & 0.62 & -2.47 \\
\hline chr1:964-996 significant & WL & 30.13 & 2.61 & 0.37 & -0.35 \\
\hline
\end{tabular}




\begin{tabular}{|c|c|c|c|c|c|}
\hline chr1:948-100 suggestive & WL & 12.15 & 1.28 & 0.37 & -0.92 \\
\hline chr1:909-101 significant & WL & 9.12 & 2 & 0.78 & -2.59 \\
\hline chr1:961-101 suggestive & WL & 10.29 & 1.57 & 0.87 & -4.46 \\
\hline chr1:968-100 suggestive & RJF & 18.43 & -0.8 & 0.36 & -3.1 \\
\hline chr1:951-101 significant & WL & 14.14 & 2.68 & 0.69 & -1.84 \\
\hline chr1:960-101 significant & WL & 16.63 & 1.49 & 0.45 & -2.3 \\
\hline chr1:946-101 significant & WL & 12.13 & 1.37 & 0.42 & -1.48 \\
\hline chr1:953-102 significant & WL & 15.52 & 2.38 & 0.57 & -1.36 \\
\hline chr1:966-101 significant & RJF & 18.05 & -1.95 & 0.37 & -1.2 \\
\hline chr1:959-102 significant & WL & 16.98 & 2.1 & 0.53 & -2.26 \\
\hline chr1:996-103 suggestive & RJF & 14.67 & -1.74 & 0.42 & -1.53 \\
\hline chr1:1000-10 suggestive & WL & 19.22 & 0.14 & 0.04 & -0.18 \\
\hline chr1:1010-10 significant & WL & 20.99 & 1.43 & 0.27 & -0.4 \\
\hline chr1:1004-10 suggestive & WL & 19.58 & 2.32 & 0.5 & -1.34 \\
\hline chr1:1003-10 suggestive & WL & 14.29 & 1.71 & 0.42 & -0.59 \\
\hline chr1:1003-10 significant & WL & 13.17 & 2.58 & 0.62 & -0.4 \\
\hline chr1:1011-10 suggestive & WL & 16.47 & 0.12 & 0.66 & 0.33 \\
\hline chr1:1002-10 suggestive & WL & 13.31 & 1.6 & 0.39 & -0.2 \\
\hline chr1:1060-10 suggestive & WL & 10.64 & 0.67 & 0.23 & -1.34 \\
\hline chr1:1058-10 significant & WL & 21.17 & 1.78 & 0.4 & -1.22 \\
\hline chr1:1061-10 significant & WL & 15.68 & 1.99 & 0.41 & 0.73 \\
\hline chr1:1064-10 significant & WL & 20.03 & 2.2 & 0.48 & 1.06 \\
\hline chr1:1070-10 significant & WL & 24.3 & 2.19 & 0.45 & -1.42 \\
\hline chr1:1057-10 suggestive & WL & 13.48 & 1.32 & 0.35 & 1.44 \\
\hline chr1:1069-10 suggestive & WL & 27.1 & 2.33 & 0.48 & -1.57 \\
\hline chr1:1066-10 significant & WL & 17.81 & 1.53 & 0.34 & -0.57 \\
\hline chr1:1071-10 suggestive & WL & 18.2 & 1.15 & 0.28 & 1.56 \\
\hline chr1:1065-11 significant & WL & 19.74 & 1.83 & 0.45 & -1.47 \\
\hline chr1:1066-11 suggestive & RJF & 14.68 & -2.08 & 0.56 & -0.69 \\
\hline chr1:1086-11 significant & WL & 15.53 & 0.98 & 0.26 & 1.37 \\
\hline chr1:1094-11 significant & RJF & 16.1 & -2.1 & 0.49 & 1.12 \\
\hline chr1:1095-11 significant & RJF & 27.57 & -2.27 & 0.35 & 0.6 \\
\hline chr1:1092-11 significant & RJF & 12.71 & -1.16 & 0.28 & -0.29 \\
\hline chr1:1093-11 significant & RJF & 16.38 & -2.94 & 0.72 & 2.17 \\
\hline chr1:1094-11 significant & RJF & 24.29 & -3.79 & 0.63 & 0.9 \\
\hline chr1:1094-11 significant & RJF & 21.73 & -4.87 & 0.93 & 2.43 \\
\hline chr1:1093-11 significant & RJF & 22.13 & -3.1 & 0.58 & 1.31 \\
\hline chr1:1094-11 significant & RJF & 18.07 & -2.98 & 0.62 & 1.13 \\
\hline chr1:1094-11 significant & WL & 18.4 & 1.61 & 0.31 & 0.15 \\
\hline chr1:1091-11 significant & RJF & 15.64 & -2.15 & 0.54 & 1.55 \\
\hline chr1:1096-11 significant & RJF & 15.57 & -2.22 & 0.47 & -1.13 \\
\hline chr1:1089-11 significant & WL & 19.87 & 1.93 & 0.36 & 0.07 \\
\hline chr1:1091-11 significant & RJF & 15.05 & -2.69 & 0.62 & 0.67 \\
\hline
\end{tabular}




\begin{tabular}{|c|c|c|c|c|c|}
\hline chr1:1096-11 significant & WL & 19.13 & 1.61 & 0.3 & 0.24 \\
\hline chr1:1105-11 significant & WL & 21.84 & 1.36 & 0.7 & 0.63 \\
\hline chr1:1105-11 suggestive & WL & 19.37 & 1.44 & 0.38 & -0.98 \\
\hline chr1:1104-11 significant & WL & 20.93 & 2.58 & 0.49 & -0.47 \\
\hline chr1:1106-11 significant & WL & 32.31 & 3.47 & 0.46 & 0.28 \\
\hline chr1:1102-11 significant & RJF & 24.66 & -1.79 & 0.29 & -0.91 \\
\hline chr1:1094-11 significant & WL & 20.02 & 2.28 & 0.44 & -0.39 \\
\hline chr1:1105-11 significant & WL & 36.81 & 3.37 & 0.41 & -0.28 \\
\hline chr1:1105-11 significant & WL & 17.14 & 1.66 & 0.33 & -0.15 \\
\hline chr1:1072-11 significant & WL & 19.19 & 3.28 & 0.61 & -1.6 \\
\hline chr1:1060-11 suggestive & RJF & 13.68 & -2.53 & 0.65 & -0.25 \\
\hline chr1:1112-11 suggestive & RJF & 19.94 & -0.03 & 0.22 & -0.04 \\
\hline chr1:1108-11 significant & WL & 14.74 & 2.22 & 0.6 & 0.85 \\
\hline chr1:1116-11 significant & RJF & 27.33 & -3.13 & 0.49 & 0.04 \\
\hline chr1:1119-11 significant & RJF & 21.11 & -2.98 & 0.62 & -0.77 \\
\hline chr1:1117-11 significant & RJF & 20.53 & -2.96 & 0.73 & -1.21 \\
\hline chr1:1118-11 significant & WL & 22.17 & 2.9 & 0.52 & -0.2 \\
\hline chr1:1114-11 significant & WL & 15.66 & 1.34 & 0.3 & -0.08 \\
\hline chr1:1114-11 significant & WL & 19.53 & 2.48 & 0.5 & 0.3 \\
\hline chr1:1117-11 significant & WL & 20.9 & 3.09 & 0.55 & -0.56 \\
\hline chr1:1117-11 significant & WL & 15.62 & 1.46 & 0.32 & 0.03 \\
\hline chr1:1121-12 significant & WL & 20.16 & 4.36 & 0.84 & -2.54 \\
\hline chr1:1121-11 significant & RJF & 23.29 & -3.75 & 0.68 & -0.51 \\
\hline chr1:1116-12 suggestive & RJF & 10.37 & -2.7 & 0.77 & -0.21 \\
\hline chr1:1115-12 suggestive & WL & 14.39 & 2.39 & 0.54 & -0.03 \\
\hline chr1:1120-11 significant & WL & 28.94 & 11.55 & 1.63 & -3.85 \\
\hline chr1:1120-11 significant & WL & 29.71 & 12.18 & 1.69 & -3.94 \\
\hline chr1:1123-11 significant & WL & 29.84 & 10.12 & 1.41 & -1.99 \\
\hline chr1:1124-11 significant & WL & 30.51 & 11.54 & 1.58 & -2.05 \\
\hline chr1:1119-11 significant & WL & 28.47 & 4.79 & 0.69 & -1.04 \\
\hline chr1:1117-11 significant & WL & 26 & 13.49 & 2.12 & -1.62 \\
\hline chr1:1123-11 significant & WL & 40.12 & 6.26 & 0.69 & -1.87 \\
\hline chr1:1114-12 suggestive & WL & 20.23 & 5.84 & 1.07 & -0.93 \\
\hline chr1:1114-12 suggestive & WL & 19.83 & 5.86 & 1.09 & -0.92 \\
\hline chr1:1147-12 significant & WL & 17.54 & 8.09 & 1.68 & -4.18 \\
\hline chr1:1168-12 suggestive & WL & 15.12 & 4.79 & 1.45 & -3.89 \\
\hline chr1:1172-12 significant & RJF & 20.14 & -0.7 & 1.58 & 6.13 \\
\hline chr1:1192-12 suggestive & RJF & 18.94 & -1.01 & 1.33 & 0.33 \\
\hline chr1:1155-12 suggestive & RJF & 10.71 & -4.76 & 1.31 & 2.91 \\
\hline chr1:1181-12 suggestive & WL & 13.08 & 1.88 & 0.95 & -7.85 \\
\hline chr1:1304-13 suggestive & WL & 14.01 & 1.54 & 0.35 & -0.61 \\
\hline chr1:1312-13 suggestive & WL & 27.26 & 0.35 & 0.07 & -0.29 \\
\hline chr1:1308-13 suggestive & WL & 18.36 & 1.39 & 0.27 & -0.33 \\
\hline
\end{tabular}




\begin{tabular}{|c|c|c|c|c|c|}
\hline chr1:1303-13 suggestive & RJF & 19.94 & -2.05 & 0.53 & -2.54 \\
\hline chr1:1307-13 suggestive & RJF & 19.11 & -1.57 & 0.36 & -1.37 \\
\hline chr1:1297-13 significant & WL & 14.86 & 1.23 & 0.27 & -0.05 \\
\hline chr1:1306-14 significant & RJF & 14.28 & -1.97 & 0.45 & -0.29 \\
\hline chr1:1329-14 suggestive & RJF & 14.02 & -1.13 & 0.28 & -0.91 \\
\hline chr1:1302-14 suggestive & WL & 12.56 & 1.45 & 0.34 & 0.08 \\
\hline chr1:1314-14 suggestive & RJF & 11.35 & -1.49 & 0.38 & -0.19 \\
\hline chr1:1342-14 significant & RJF & 21.21 & -2.26 & 0.4 & -1.1 \\
\hline chr1:1319-14 suggestive & RJF & 9.6 & -1.97 & 0.55 & -0.5 \\
\hline chr1:1338-14 suggestive & RJF & 10.5 & -1.27 & 0.39 & -1.32 \\
\hline chr1:1345-14 suggestive & RJF & 18.7 & -1.12 & 0.21 & -0.23 \\
\hline chr1:1347-14 suggestive & WL & 13.92 & 1.65 & 0.39 & -0.41 \\
\hline chr1:1349-14 significant & WL & 15.79 & 2.52 & 0.53 & -0.19 \\
\hline chr1:1363-14 significant & RJF & 14.43 & -1.58 & 0.36 & 0.25 \\
\hline chr1:1366-14 significant & RJF & 18.67 & -2.08 & 0.42 & 0.63 \\
\hline chr1:1371-14 significant & RJF & 26.66 & -1.23 & 0.18 & -0.02 \\
\hline chr1:1368-14 suggestive & RJF & 16.4 & -1.07 & 0.22 & 0.05 \\
\hline chr1:1360-14 suggestive & WL & 14.08 & 3.53 & 0.83 & -0.84 \\
\hline chr1:1364-14 suggestive & RJF & 12.38 & -1.63 & 0.41 & 0.45 \\
\hline chr1:1363-14 suggestive & RJF & 15.54 & -1.19 & 0.27 & -0.78 \\
\hline chr1:1372-14 significant & RJF & 23.9 & -1.26 & 0.21 & 0.09 \\
\hline chr1:1366-14 suggestive & RJF & 14.75 & -1.97 & 0.43 & -0.23 \\
\hline chr1:1349-14 suggestive & RJF & 11.33 & -1.27 & 0.37 & -1.24 \\
\hline chr1:1366-14 suggestive & RJF & 14.71 & -1.25 & 0.28 & -0.4 \\
\hline chr1:1364-14 significant & RJF & 17.54 & -1.64 & 0.35 & 0.73 \\
\hline chr1:1357-14 suggestive & WL & 15.79 & 5.23 & 1.18 & -1.83 \\
\hline chr1:1369-14 significant & RJF & 15.6 & -1.73 & 0.38 & 0.19 \\
\hline chr1:1356-14 significant & WL & 18.4 & 2.48 & 0.49 & 0.64 \\
\hline chr1:1358-14 suggestive & RJF & 14.7 & -1.55 & 0.36 & -0.67 \\
\hline chr1:1394-14 significant & RJF & 24.19 & -1.85 & 0.3 & -0.19 \\
\hline chr1:1395-14 suggestive & RJF & 17.84 & -0.92 & 0.2 & 0.62 \\
\hline chr1:1395-14 suggestive & RJF & 15.21 & -0.81 & 0.18 & 0.39 \\
\hline chr1:1368-14 suggestive & RJF & 14.71 & -1.7 & 0.37 & -0.07 \\
\hline chr1:1396-14 suggestive & RJF & 14.18 & -1.42 & 0.33 & 0.69 \\
\hline chr1:1375-14 suggestive & RJF & 14.03 & -1.85 & 0.43 & 0.61 \\
\hline chr1:1400-14 significant & RJF & 22.63 & -2.34 & 0.4 & 0.44 \\
\hline chr1:1403-14 significant & RJF & 30.66 & -3.88 & 0.56 & 1.62 \\
\hline chr1:1398-14 suggestive & RJF & 14.57 & -2.34 & 0.53 & 0.73 \\
\hline chr1:1398-14 suggestive & RJF & 14.72 & -1.8 & 0.41 & 0.68 \\
\hline chr1:1402-14 significant & RJF & 23.78 & -1.56 & 0.26 & 0.25 \\
\hline chr1:1386-14 suggestive & RJF & 14.18 & -1.18 & 0.26 & 0 \\
\hline chr1:1396-14 significant & RJF & 16.64 & -3.27 & 0.69 & -1.07 \\
\hline chr1:1396-14 significant & RJF & 20.09 & -2.44 & 0.44 & 0.26 \\
\hline
\end{tabular}




\begin{tabular}{|c|c|c|c|c|c|}
\hline chr1:1338-14 significant & RJF & 17.19 & -2.01 & 0.42 & -0.8 \\
\hline chr1:1407-14 significant & RJF & 22.54 & -0.64 & 0.33 & -0.12 \\
\hline chr1:1408-14 significant & RJF & 22.26 & -0.38 & 0.44 & -0.33 \\
\hline chr1:1408-14 significant & RJF & 21.28 & -1.15 & 0.43 & 0.7 \\
\hline chr1:1397-14 significant & RJF & 22.59 & -1.84 & 0.32 & -0.54 \\
\hline chr1:1401-14 significant & RJF & 16.57 & -1.69 & 0.4 & -1.25 \\
\hline chr1:1402-14 significant & RJF & 18.4 & -2.08 & 0.45 & -1.35 \\
\hline chr1:1403-14 suggestive & RJF & 19.73 & -2.64 & 0.54 & -0.58 \\
\hline chr1:1396-14 significant & RJF & 17.37 & -1.78 & 0.36 & -0.32 \\
\hline chr1:1399-14 significant & RJF & 15.26 & -1.2 & 0.27 & -0.5 \\
\hline chr1:1400-14 significant & RJF & 16.03 & -1.7 & 0.38 & -0.74 \\
\hline chr1:1402-14 significant & RJF & 16.79 & -1.75 & 0.36 & 0.5 \\
\hline chr1:1399-14 significant & RJF & 15.39 & -1.54 & 0.38 & -1.2 \\
\hline chr1:1399-14 suggestive & RJF & 15.1 & -1.33 & 0.3 & 0.5 \\
\hline chr1:1396-14 significant & RJF & 16.38 & -1.88 & 0.5 & -2.07 \\
\hline chr1:1402-14 significant & RJF & 15.18 & -0.99 & 0.22 & 0.39 \\
\hline chr1:1359-14 significant & RJF & 16.41 & -1.96 & 0.41 & 0.68 \\
\hline chr1:1351-14 significant & RJF & 16.02 & -1.4 & 0.3 & -0.35 \\
\hline chr1:1404-14 suggestive & RJF & 23.61 & -0.26 & 0.42 & -0.13 \\
\hline chr1:1408-14 suggestive & RJF & 19.2 & -1.59 & 0.3 & -0.1 \\
\hline chr1:1409-14 suggestive & WL & 20.31 & 0.01 & 0.43 & 0.7 \\
\hline chr1:1409-14 suggestive & RJF & 18.27 & -0.23 & 0.6 & 0.32 \\
\hline chr1:1402-14 suggestive & RJF & 20.29 & -1.16 & 0.21 & -0.27 \\
\hline chr1:1403-14 suggestive & RJF & 18.97 & -1.5 & 0.32 & -1.05 \\
\hline chr1:1405-14 suggestive & RJF & 20.56 & -1.06 & 0.19 & -0.09 \\
\hline chr1:1359-14 significant & RJF & 13.56 & -1.39 & 0.32 & -0.22 \\
\hline chr1:1396-14 significant & RJF & 22.6 & -2.8 & 0.46 & -0.59 \\
\hline chr1:1401-14 significant & RJF & 20.68 & -2.47 & 0.45 & -0.87 \\
\hline chr1:1404-14 suggestive & RJF & 14.8 & -1.32 & 0.29 & -0.21 \\
\hline chr1:1403-14 significant & RJF & 14.55 & -1.25 & 0.27 & -0.37 \\
\hline chr1:1383-14 significant & RJF & 17.78 & -1.74 & 0.34 & -0.14 \\
\hline chr1:1401-14 significant & RJF & 15.86 & -2.38 & 0.5 & 0.06 \\
\hline chr1:1398-14 significant & RJF & 17.41 & -2.88 & 0.57 & -0.31 \\
\hline chr1:1394-14 significant & RJF & 18.24 & -1.65 & 0.32 & -0.58 \\
\hline chr1:1402-14 significant & RJF & 15.86 & -1.89 & 0.4 & -0.62 \\
\hline chr1:1386-14 suggestive & RJF & 15.02 & -1.03 & 0.25 & -0.83 \\
\hline chr1:1402-14 significant & RJF & 15.92 & -1.76 & 0.38 & -0.78 \\
\hline chr1:1409-14 suggestive & RJF & 18.99 & -1.2 & 0.39 & 0.91 \\
\hline chr1:1398-14 significant & RJF & 16.63 & -1.25 & 0.26 & 0.2 \\
\hline chr1:1369-14 significant & WL & 12.48 & 3.34 & 0.88 & -1.62 \\
\hline chr1:1349-14 suggestive & RJF & 14.57 & -0.8 & 0.18 & -0.19 \\
\hline chr1:1412-14 suggestive & RJF & 19.58 & -1.78 & 0.36 & 0.98 \\
\hline chr1:1412-14 suggestive & RJF & 20.11 & -0.99 & 0.24 & 1.07 \\
\hline
\end{tabular}




\begin{tabular}{|c|c|c|c|c|c|}
\hline chr1:1411-14 significant & RJF & 23.03 & -1.69 & 0.29 & 0.39 \\
\hline chr1:1413-14 significant & RJF & 20.93 & -2.21 & 0.42 & 0.21 \\
\hline chr1:1409-14 suggestive & RJF & 19.42 & -1.54 & 0.29 & -0.29 \\
\hline chr1:1410-14 suggestive & RJF & 18.99 & -1.33 & 0.29 & -1.19 \\
\hline chr1:1405-14 suggestive & RJF & 13.97 & -1.54 & 0.38 & -1.14 \\
\hline chr1:1409-14 suggestive & RJF & 14.75 & -1.06 & 0.26 & -0.8 \\
\hline chr1:1409-14 suggestive & RJF & 19.72 & -1.53 & 0.28 & -0.07 \\
\hline chr1:1360-14 suggestive & RJF & 12.67 & -1.72 & 0.41 & -0.18 \\
\hline chr1:1402-14 suggestive & RJF & 14.95 & -1.36 & 0.3 & -0.52 \\
\hline chr1:1410-14 significant & RJF & 19.21 & -0.78 & 0.38 & -0.13 \\
\hline chr1:1410-14 suggestive & RJF & 19.52 & -0.87 & 0.16 & -0.14 \\
\hline chr1:1409-14 suggestive & RJF & 14.86 & -1.23 & 0.27 & -0.01 \\
\hline chr1:1401-14 significant & RJF & 15.59 & -1.8 & 0.41 & -1.02 \\
\hline chr1:1394-14 significant & RJF & 14.99 & -1.42 & 0.31 & -0.27 \\
\hline chr1:1409-14 significant & RJF & 18.45 & -1.18 & 0.22 & -0.1 \\
\hline chr1:1343-15 significant & RJF & 12.76 & -1.62 & 0.39 & 0.18 \\
\hline chr1:1379-14 suggestive & RJF & 14.01 & -1.61 & 0.37 & 0.36 \\
\hline chr1:1363-14 suggestive & RJF & 14.81 & -1.74 & 0.38 & 0.03 \\
\hline chr1:1403-14 significant & RJF & 17.02 & -1.67 & 0.34 & 0.54 \\
\hline chr1:1409-14 suggestive & RJF & 15.23 & -1.17 & 0.25 & -0.22 \\
\hline chr1:1404-14 significant & RJF & 15.77 & -1.54 & 0.32 & 0.33 \\
\hline chr1:1404-14 suggestive & RJF & 19.2 & -1.41 & 0.26 & -0.42 \\
\hline chr1:1397-14 suggestive & RJF & 13.37 & -1.79 & 0.41 & -0.06 \\
\hline chr1:1402-14 suggestive & RJF & 14.86 & -2.1 & 0.46 & 0.12 \\
\hline chr1:1399-14 significant & RJF & 16.31 & -1.62 & 0.33 & -0.19 \\
\hline chr1:1395-15 suggestive & RJF & 13.44 & -1.22 & 0.29 & -0.21 \\
\hline chr1:1396-15 suggestive & RJF & 14.75 & -0.88 & 0.19 & 0.11 \\
\hline chr1:1398-15 significant & RJF & 19.98 & -1.95 & 0.36 & -0.02 \\
\hline chr1:1399-15 suggestive & RJF & 14.47 & -1.65 & 0.37 & -0.38 \\
\hline chr1:1413-15 significant & RJF & 16.48 & -2.82 & 0.62 & 1.2 \\
\hline chr1:1401-14 significant & RJF & 20.8 & -3.03 & 0.58 & 1.8 \\
\hline chr1:1420-15 significant & RJF & 18.38 & -1.73 & 0.34 & -0.26 \\
\hline chr1:1440-15 suggestive & RJF & 17.22 & -1.5 & 0.32 & -0.01 \\
\hline chr1:1411-15 suggestive & RJF & 13.33 & -0.99 & 0.24 & -0.25 \\
\hline chr1:1427-15 significant & RJF & 18.84 & -1.16 & 0.29 & 0.82 \\
\hline chr1:1443-15 suggestive & RJF & 15.76 & -1.94 & 0.41 & -0.35 \\
\hline chr1:1435-15 significant & RJF & 16.49 & -1.77 & 0.36 & -0.29 \\
\hline chr1:1430-15 suggestive & RJF & 16.41 & -1.37 & 0.41 & 1.26 \\
\hline chr1:1443-15 suggestive & RJF & 17 & -1.11 & 0.24 & 0.11 \\
\hline chr1:1441-15 significant & RJF & 18.71 & -1.24 & 0.32 & 1 \\
\hline chr1:1444-15 significant & RJF & 17.08 & -1.47 & 0.34 & 0.47 \\
\hline chr1:1400-15 suggestive & RJF & 12.94 & -0.09 & 0.23 & 1.2 \\
\hline chr1:1446-15 significant & RJF & 15.66 & -1.21 & 0.28 & 0.24 \\
\hline
\end{tabular}




\begin{tabular}{|c|c|c|c|c|c|}
\hline chr1:1450-15 significant & RJF & 17.86 & -1.53 & 0.32 & 0.14 \\
\hline chr1:1449-15 significant & RJF & 18.93 & -1.71 & 0.46 & 1.43 \\
\hline chr1:1445-15 suggestive & RJF & 17.43 & -1.97 & 0.4 & -0.34 \\
\hline chr1:1441-15 suggestive & RJF & 14.74 & -0.46 & 0.26 & 1.22 \\
\hline chr1:1449-15 suggestive & RJF & 14.68 & -1.49 & 0.41 & 0.57 \\
\hline chr1:1454-15 significant & RJF & 18.44 & -1.82 & 0.37 & 0.35 \\
\hline chr1:1439-15 suggestive & RJF & 13.88 & -0.98 & 0.4 & 1.47 \\
\hline chr1:1451-15 suggestive & RJF & 13.57 & -0.18 & 0.21 & 1.1 \\
\hline chr1:1472-15 significant & WL & 23.08 & 2.23 & 0.37 & 0.71 \\
\hline chr1:1471-15 significant & RJF & 18.58 & -1.08 & 0.3 & 1.04 \\
\hline chr1:1466-15 suggestive & RJF & 16.98 & -0.05 & 0.45 & 3.21 \\
\hline chr1:1450-15 significant & RJF & 19.62 & -1.7 & 0.35 & -0.72 \\
\hline chr1:1453-15 significant & RJF & 17.67 & -1.71 & 0.38 & 0.34 \\
\hline chr1:1459-15 suggestive & RJF & 17.48 & -1.73 & 0.4 & 0.5 \\
\hline chr1:1467-15 significant & RJF & 22.23 & -2.46 & 0.47 & 0.44 \\
\hline chr1:1463-15 significant & RJF & 17.08 & -1.53 & 0.39 & 0.8 \\
\hline chr1:1465-15 significant & RJF & 23.51 & -0.87 & 0.33 & 1.88 \\
\hline chr1:1465-15 significant & RJF & 22.31 & -2.48 & 0.46 & 0.93 \\
\hline chr1:1468-15 significant & RJF & 21.48 & -1.78 & 0.37 & 1.06 \\
\hline chr1:1459-15 significant & RJF & 20.65 & -1.21 & 0.36 & 2.1 \\
\hline chr1:1458-15 suggestive & RJF & 15.4 & -2.07 & 0.45 & -0.17 \\
\hline chr1:1468-15 significant & RJF & 19.11 & -2.8 & 0.53 & -0.02 \\
\hline chr1:1459-15 suggestive & RJF & 15.95 & -2.33 & 0.5 & 0.35 \\
\hline chr1:1474-15 suggestive & RJF & 16.64 & -1.48 & 0.44 & 1.71 \\
\hline chr1:1476-15 suggestive & RJF & 16.61 & -1.28 & 0.34 & 1.09 \\
\hline chr1:1471-15 significant & RJF & 19.56 & -2.14 & 0.43 & 0.71 \\
\hline chr1:1476-15 suggestive & RJF & 19.72 & -1.65 & 0.51 & 1.74 \\
\hline chr1:1486-15 significant & RJF & 21.67 & -2.22 & 0.44 & 1.1 \\
\hline chr1:1463-15 significant & RJF & 16.2 & -1.02 & 0.22 & -0.59 \\
\hline chr1:1468-15 suggestive & RJF & 16.32 & -2.41 & 0.51 & 0.21 \\
\hline chr1:1480-15 significant & RJF & 17.63 & -1.69 & 0.47 & 1.84 \\
\hline chr1:1473-15 suggestive & RJF & 22.57 & -1.79 & 0.42 & 1.81 \\
\hline chr1:1480-15 significant & RJF & 20.34 & -2.32 & 1 & 2.63 \\
\hline chr1:1504-15 significant & RJF & 24.94 & -3.92 & 0.79 & 3.04 \\
\hline chr1:1504-15 significant & RJF & 18.31 & -2.42 & 0.61 & 2.1 \\
\hline chr1:1485-15 significant & WL & 17.72 & 2.17 & 0.44 & 1.49 \\
\hline chr1:1481-15 significant & RJF & 19.12 & -2.96 & 0.65 & 1.8 \\
\hline chr1:1502-15 suggestive & RJF & 15.18 & -1.75 & 0.39 & 0.43 \\
\hline chr1:1503-15 significant & RJF & 16.17 & -1.38 & 0.28 & -0.22 \\
\hline chr1:1505-15 suggestive & RJF & 15.36 & -2.15 & 0.56 & 1.62 \\
\hline chr1:1502-15 suggestive & RJF & 15.13 & -1.08 & 0.33 & 1.22 \\
\hline chr1:1465-15 suggestive & RJF & 16.06 & -2.57 & 0.56 & 0.55 \\
\hline chr1:1490-15 suggestive & RJF & 16.43 & -1.9 & 0.45 & 0.93 \\
\hline
\end{tabular}




\begin{tabular}{|c|c|c|c|c|c|}
\hline chr1:1505-15 suggestive & RJF & 21.44 & -0.77 & 0.38 & 2.64 \\
\hline chr1:1503-15 suggestive & RJF & 20.86 & -2.7 & 0.57 & 1.66 \\
\hline chr1:1503-15 suggestive & RJF & 23.8 & -2.38 & 0.48 & 1.67 \\
\hline chr1:1504-15 suggestive & RJF & 19.6 & -0.88 & 0.28 & 1.52 \\
\hline chr1:1481-15 suggestive & RJF & 16.35 & -2.68 & 0.69 & 1.98 \\
\hline chr1:1482-15 significant & RJF & 22.54 & -1.59 & 0.51 & 2.37 \\
\hline chr1:1483-15 significant & RJF & 20.27 & -2.1 & 0.41 & 0.53 \\
\hline chr1:1503-15 suggestive & RJF & 22.31 & -1.19 & 0.36 & 2.22 \\
\hline chr1:1502-15 suggestive & RJF & 18.02 & -1.12 & 0.5 & 3.2 \\
\hline chr1:1481-15 significant & RJF & 22.86 & -2.1 & 0.35 & 0.05 \\
\hline chr1:1504-15 significant & RJF & 18.12 & -0.97 & 0.38 & 1.61 \\
\hline chr1:1470-15 significant & RJF & 18.44 & -2.09 & 0.43 & -0.28 \\
\hline chr1:1458-15 significant & RJF & 16.44 & -1.91 & 0.47 & 0.39 \\
\hline chr1:1504-15 significant & RJF & 16 & -1.63 & 0.41 & 0.41 \\
\hline chr1:1454-15 suggestive & RJF & 14.76 & -2.04 & 0.59 & 0.9 \\
\hline chr1:1505-15 significant & RJF & 21.63 & -1.07 & 0.72 & 3.76 \\
\hline chr1:1456-15 suggestive & RJF & 14.31 & -1.53 & 0.39 & 0.18 \\
\hline chr1:1493-15 suggestive & RJF & 15.49 & -1.49 & 0.48 & 1.22 \\
\hline chr1:1477-15 suggestive & WL & 25.92 & 0.23 & 0.08 & -0.19 \\
\hline chr1:1505-15 suggestive & RJF & 20.34 & -0.48 & 0.51 & 3.34 \\
\hline chr1:1504-15 significant & RJF & 20.53 & -2.34 & 0.48 & 0.01 \\
\hline chr1:1453-15 suggestive & RJF & 15.11 & -1.38 & 0.35 & 0.17 \\
\hline chr1:1510-15 significant & RJF & 16.81 & -0.1 & 0.22 & 1.37 \\
\hline chr1:1511-15 significant & RJF & 25.95 & -2.49 & 0.46 & 0.52 \\
\hline chr1:1516-15 significant & RJF & 42.83 & -2.51 & 0.54 & 1.16 \\
\hline chr1:1514-15 significant & RJF & 33.77 & -2.57 & 0.38 & 0.21 \\
\hline chr1:1514-15 significant & RJF & 20.56 & -1.85 & 0.48 & 1.34 \\
\hline chr1:1513-15 significant & RJF & 17.27 & -2.98 & 0.67 & 0.32 \\
\hline chr1:1517-15 significant & RJF & 41.48 & -5.13 & 0.56 & -1.68 \\
\hline chr1:1517-15 significant & RJF & 45.31 & -5.03 & 0.49 & -1.78 \\
\hline chr1:1516-15 significant & RJF & 23.66 & -1.73 & 0.3 & -0.25 \\
\hline chr1:1516-15 significant & RJF & 28.34 & -1.71 & 0.27 & -0.28 \\
\hline chr1:1504-15 significant & RJF & 16.44 & -1.03 & 0.31 & 0.74 \\
\hline chr1:1511-15 suggestive & RJF & 14.48 & -1.64 & 0.39 & -0.14 \\
\hline chr1:1512-15 suggestive & RJF & 21.9 & -4.16 & 0.79 & -1.14 \\
\hline chr1:1512-15 significant & RJF & 20.66 & -3.11 & 0.89 & 3.03 \\
\hline chr1:1517-15 significant & RJF & 38.94 & -4.65 & 0.56 & -1.09 \\
\hline chr1:1515-15 significant & RJF & 27.57 & -2.68 & 0.4 & -1.36 \\
\hline chr1:1518-15 significant & RJF & 40.34 & -7.45 & 0.95 & 0.94 \\
\hline chr1:1518-15 significant & RJF & 38.63 & -2.36 & 0.81 & 2.36 \\
\hline chr1:1505-15 significant & RJF & 15.45 & -2.81 & 0.67 & 0.04 \\
\hline chr1:1514-15 suggestive & RJF & 15.02 & -2.09 & 0.52 & 0.1 \\
\hline chr1:1505-15 suggestive & WL & 17.77 & 1.33 & 0.59 & 2.38 \\
\hline
\end{tabular}




\begin{tabular}{|c|c|c|c|c|c|}
\hline chr1:1513-15 suggestive & WL & 20.11 & 0.11 & 0.21 & -0.52 \\
\hline chr1:1510-15 suggestive & WL & 14.61 & 1.42 & 0.4 & -0.56 \\
\hline chr1:1513-16 significant & RJF & 20.75 & -2.38 & 0.49 & 0.2 \\
\hline chr1:1518-16 suggestive & WL & 13.84 & 1.34 & 0.3 & 0.35 \\
\hline chr1:1557-16 significant & RJF & 20.71 & -2.27 & 0.4 & -0.33 \\
\hline chr1:1575-16 significant & WL & 31.01 & 2.54 & 0.35 & -0.3 \\
\hline chr1:1563-16 significant & WL & 15.39 & 1.09 & 0.24 & 0.52 \\
\hline chr1:1569-16 suggestive & WL & 19.12 & 1.94 & 0.51 & -0.78 \\
\hline chr1:1560-16 suggestive & RJF & 16.73 & -1.02 & 0.45 & -1.68 \\
\hline chr1:1565-16 significant & WL & 16.69 & 1.86 & 0.4 & -0.62 \\
\hline chr1:1559-16 significant & WL & 13.75 & 1.84 & 0.43 & -0.83 \\
\hline chr1:1545-16 suggestive & WL & 17.58 & 1.29 & 0.28 & -1.19 \\
\hline chr1:1545-16 suggestive & WL & 18.55 & 1.39 & 0.26 & -0.26 \\
\hline chr1:1572-16 suggestive & WL & 12.96 & 1.63 & 0.41 & -1.08 \\
\hline chr1:1573-16 significant & WL & 18.93 & 1.7 & 0.52 & -3.04 \\
\hline chr1:1535-16 suggestive & RJF & 14.62 & -1.48 & 0.32 & 0.25 \\
\hline chr1:1598-16 significant & WL & 24.2 & 2.8 & 0.45 & -0.61 \\
\hline chr1:1548-16 significant & RJF & 14.79 & -1.46 & 0.32 & 0.48 \\
\hline chr1:1546-16 suggestive & WL & 18.22 & 1.5 & 0.36 & -0.95 \\
\hline chr1:1565-16 suggestive & WL & 13.5 & 0.76 & 0.19 & -0.52 \\
\hline chr1:1582-16 significant & WL & 14.89 & 2.57 & 0.57 & 0.46 \\
\hline chr1:1596-16 suggestive & WL & 16.86 & 1.19 & 0.25 & -0.67 \\
\hline chr1:1604-16 significant & WL & 23.15 & 1.52 & 0.26 & 0.56 \\
\hline chr1:1603-16 significant & RJF & 22.27 & -2.3 & 0.39 & 0.17 \\
\hline chr1:1601-16 suggestive & WL & 18.56 & 2.53 & 0.52 & -2.22 \\
\hline chr1:1602-16 significant & RJF & 15.21 & -1.47 & 0.31 & -0.16 \\
\hline chr1:1604-16 significant & RJF & 17.41 & -2.64 & 0.53 & -0.19 \\
\hline chr1:1608-16 significant & RJF & 29.9 & -1.63 & 0.22 & 0.4 \\
\hline chr1:1604-16 suggestive & RJF & 18.58 & -2.29 & 0.69 & -2.13 \\
\hline chr1:1595-16 suggestive & WL & 12.64 & 1.96 & 0.48 & -0.81 \\
\hline chr1:1601-16 suggestive & RJF & 12.89 & -2.38 & 0.64 & -1.71 \\
\hline chr1:1595-16 suggestive & WL & 12.42 & 1.38 & 0.36 & -1.01 \\
\hline chr1:1613-16 suggestive & RJF & 18.57 & -0.88 & 0.46 & -1.91 \\
\hline chr1:1602-16 suggestive & WL & 16.49 & 1.22 & 0.46 & -1.02 \\
\hline chr1:1603-16 significant & WL & 16.82 & 2.81 & 0.57 & -0.93 \\
\hline chr1:1615-16 significant & RJF & 31.16 & -4.64 & 0.65 & -1.07 \\
\hline chr1:1607-16 suggestive & RJF & 12.39 & -1.61 & 0.39 & 0.04 \\
\hline chr1:1614-16 suggestive & RJF & 18.73 & -1.69 & 0.69 & -2 \\
\hline chr1:1606-16 suggestive & RJF & 14.57 & -0.06 & 0.43 & 0.17 \\
\hline chr1:1599-16 suggestive & WL & 11.93 & 1.44 & 0.35 & 0.34 \\
\hline chr1:1614-16 significant & RJF & 15.21 & -3.11 & 0.67 & 0.54 \\
\hline chr1:1607-16 significant & WL & 20.68 & 3.89 & 0.7 & 0.26 \\
\hline chr1:1612-17 significant & WL & 15.8 & 1.08 & 0.23 & -0.35 \\
\hline
\end{tabular}




\begin{tabular}{|c|c|c|c|c|c|}
\hline chr1:1638-17 suggestive & RJF & 22.26 & -0.23 & 0.32 & 0.64 \\
\hline chr1:1564-17 suggestive & WL & 13.34 & 1.09 & 0.25 & 0.01 \\
\hline chr1:1650-17 significant & WL & 15.91 & 0.63 & 0.18 & 0.28 \\
\hline chr1:1650-17 significant & RJF & 20.15 & -2.86 & 0.55 & 0.68 \\
\hline chr1:1652-17 significant & WL & 18.05 & 1.61 & 0.37 & 0.25 \\
\hline chr1:1653-17 suggestive & WL & 14.65 & 1.1 & 0.32 & 0.44 \\
\hline chr1:1647-17 suggestive & RJF & 12.38 & -2.03 & 0.5 & 1.01 \\
\hline chr1:1659-17 significant & WL & 19.12 & 1.14 & 0.41 & 1.61 \\
\hline chr1:1647-17 suggestive & WL & 14.67 & 1.11 & 0.28 & 0.01 \\
\hline chr1:1669-16 suggestive & WL & 19.71 & 0.19 & 0.47 & 0.28 \\
\hline chr1:1667-17 suggestive & WL & 15.2 & 0.29 & 0.37 & 1.94 \\
\hline chr1:1660-17 significant & RJF & 16.96 & -2.52 & 0.56 & 0.2 \\
\hline chr1:1669-17 suggestive & WL & 18.24 & 1.54 & 0.51 & 0.72 \\
\hline chr1:1673-16 suggestive & WL & 18.49 & 1.4 & 0.46 & -0.19 \\
\hline chr1:1671-17 significant & RJF & 20.65 & -0.86 & 0.54 & 4.11 \\
\hline chr1:1666-17 suggestive & WL & 12.52 & 0.63 & 0.24 & 0.61 \\
\hline chr1:1669-17 significant & WL & 15.99 & 0.77 & 0.24 & 0.52 \\
\hline chr1:1668-17 significant & WL & 16.43 & 0.88 & 0.27 & 0.62 \\
\hline chr1:1673-16 suggestive & WL & 15.73 & 1.14 & 0.48 & 1.68 \\
\hline chr1:1668-17 suggestive & WL & 15.19 & 0.75 & 0.47 & 2.09 \\
\hline chr1:1663-17 significant & WL & 15.29 & 1.69 & 0.46 & 0.48 \\
\hline chr1:1676-16 suggestive & RJF & 24.87 & -0.09 & 0.13 & 0.19 \\
\hline chr1:1674-17 suggestive & WL & 24.25 & 0.04 & 0.01 & -0.04 \\
\hline chr1:1676-16 suggestive & WL & 19.36 & 0.3 & 0.31 & 1.9 \\
\hline chr1:1673-16 suggestive & WL & 20.07 & 0.08 & 0.22 & 1.45 \\
\hline chr1:1664-17 suggestive & WL & 19.36 & 0.23 & 0.04 & -0.09 \\
\hline chr1:1670-17 significant & WL & 19.26 & 1.35 & 0.3 & 0.15 \\
\hline chr1:1662-17 suggestive & WL & 10.82 & 0.74 & 0.2 & -0.2 \\
\hline chr1:1669-17 suggestive & WL & 20.19 & 0.28 & 0.07 & 0.19 \\
\hline chr1:1673-17 suggestive & WL & 24.48 & 0.4 & 0.08 & -0.55 \\
\hline chr1:1656-17 significant & WL & 19.53 & 1.64 & 0.39 & 0.61 \\
\hline chr1:1651-17 suggestive & WL & 21.35 & 0.15 & 0.03 & -0.11 \\
\hline chr1:1689-17 significant & WL & 16.71 & 1.64 & 0.33 & -0.14 \\
\hline chr1:1669-17 suggestive & WL & 13.42 & 1.19 & 0.28 & -0.69 \\
\hline chr1:1696-17 suggestive & WL & 14.85 & 1.15 & 0.26 & -0.54 \\
\hline chr1:1697-17 suggestive & WL & 14.77 & 1.64 & 0.36 & -0.18 \\
\hline chr1:1705-17 suggestive & WL & 19.5 & 1.24 & 0.32 & -2.09 \\
\hline chr1:1705-17 suggestive & WL & 14.77 & 1.64 & 0.4 & -1.54 \\
\hline chr1:1712-17 suggestive & RJF & 13.56 & -0.28 & 0.21 & -1.36 \\
\hline chr1:1708-17 significant & RJF & 15.74 & -1.7 & 0.37 & -0.46 \\
\hline chr1:1716-17 significant & RJF & 19.83 & -1.53 & 0.29 & -0.55 \\
\hline chr1:1717-17 significant & RJF & 34.62 & -2.26 & 0.28 & 0.08 \\
\hline chr1:1708-17 significant & WL & 21.49 & 2.25 & 0.39 & -0.35 \\
\hline
\end{tabular}




\begin{tabular}{|c|c|c|c|c|c|}
\hline chr1:1709-17 significant & WL & 17.46 & 1.54 & 0.34 & -1.31 \\
\hline chr1:1702-17 suggestive & WL & 14.91 & 1.15 & 0.26 & -0.65 \\
\hline chr1:1704-17 significant & WL & 16.71 & 1.13 & 0.24 & -0.72 \\
\hline chr1:1710-17 significant & WL & 15.66 & 2.43 & 0.55 & -1.7 \\
\hline chr1:1708-17 significant & WL & 17.36 & 1.79 & 0.38 & -1.25 \\
\hline chr1:1697-17 suggestive & WL & 14 & 1.32 & 0.3 & -0.42 \\
\hline chr1:1709-17 suggestive & WL & 14.87 & 1.23 & 0.36 & -1.94 \\
\hline chr1:1713-17 significant & WL & 24.66 & 2.28 & 0.37 & -1.13 \\
\hline chr1:1718-17 suggestive & WL & 13.94 & 1.15 & 0.21 & -1.14 \\
\hline chr1:1703-17 suggestive & WL & 14.43 & 1.23 & 0.27 & -0.22 \\
\hline chr1:1713-17 significant & RJF & 17.71 & -1.7 & 0.51 & -1.27 \\
\hline chr1:1720-17 significant & WL & 19.94 & 3.27 & 0.62 & -1.59 \\
\hline chr1:1712-17 suggestive & RJF & 11.74 & -1.76 & 0.44 & -0.46 \\
\hline chr1:1730-17 significant & RJF & 21.45 & -1.87 & 0.33 & -0.35 \\
\hline chr1:1753-17 suggestive & WL & 17.92 & 1.75 & 0.4 & -1.24 \\
\hline chr1:1749-17 suggestive & WL & 16.45 & 1.32 & 0.41 & -1.97 \\
\hline chr1:1750-17 significant & RJF & 24.04 & -1.7 & 0.27 & -0.05 \\
\hline chr1:1735-17 significant & RJF & 14.03 & -1.84 & 0.42 & -0.45 \\
\hline chr1:1743-17 significant & RJF & 14.22 & -1.76 & 0.4 & -0.15 \\
\hline chr1:1752-17 significant & WL & 21.43 & 1.6 & 0.45 & -3.04 \\
\hline chr1:1737-17 suggestive & RJF & 13.46 & -1.36 & 0.32 & -0.54 \\
\hline chr1:1746-17 significant & WL & 14.62 & 1.04 & 0.23 & 0.16 \\
\hline chr1:1750-17 suggestive & WL & 18.04 & 1.07 & 0.68 & -3.24 \\
\hline chr1:1748-17 significant & WL & 21.59 & 5.24 & 0.97 & -2.34 \\
\hline chr1:1739-17 suggestive & RJF & 15.35 & -1.27 & 0.27 & -0.45 \\
\hline chr1:1732-17 suggestive & WL & 15.64 & 2.58 & 0.57 & -1.22 \\
\hline chr1:1763-17 suggestive & RJF & 21.44 & -0.15 & 0.03 & -0.15 \\
\hline chr1:1759-17 suggestive & RJF & 11.08 & -0.64 & 0.75 & -0.92 \\
\hline chr1:257-235 suggestive & WL & 6.3 & 1.52 & 0.55 & -0.83 \\
\hline chr1:1755-17 significant & WL & 20.32 & 2.04 & 0.42 & -0.62 \\
\hline chr1:1766-17 significant & WL & 21.63 & 0.82 & 0.23 & -1.01 \\
\hline chr1:1768-17 significant & WL & 20.06 & 2.3 & 0.43 & 0.21 \\
\hline chr1:1768-17 significant & WL & 17.86 & 1.78 & 0.45 & -1.15 \\
\hline chr1:1751-17 suggestive & WL & 14.85 & 3.61 & 0.97 & -1.92 \\
\hline chr1:1758-17 suggestive & WL & 16.16 & 2.96 & 0.75 & -1.34 \\
\hline chr1:1763-17 significant & WL & 17.22 & 1.77 & 0.45 & -1 \\
\hline chr1:1762-17 significant & WL & 21.47 & 2.39 & 0.53 & -1.51 \\
\hline chr1:1733-17 suggestive & WL & 15.63 & 1.24 & 0.33 & -0.67 \\
\hline chr1:1763-17 suggestive & WL & 14.76 & 1.71 & 0.48 & -1.17 \\
\hline chr1:1763-17 significant & WL & 23.36 & 2.42 & 0.45 & -1.03 \\
\hline chr1:1749-18 suggestive & WL & 15 & 2.15 & 0.47 & 0.84 \\
\hline chr1:1749-17 significant & WL & 19.12 & 2.22 & 1.09 & -0.01 \\
\hline chr1:1765-18 suggestive & WL & 14.14 & 1.67 & 0.43 & -0.71 \\
\hline
\end{tabular}




\begin{tabular}{|c|c|c|c|c|c|}
\hline chr1:1771-18 significant & RJF & 17.1 & -0.89 & 0.21 & 0.45 \\
\hline chr1:1772-18 significant & WL & 17.71 & 2.04 & 0.4 & 0.61 \\
\hline chr1:1737-18 significant & WL & 11.61 & 1.65 & 0.49 & -0.35 \\
\hline chr1:1774-18 significant & WL & 17.2 & 1.91 & 0.45 & -1.1 \\
\hline chr1:1733-18 suggestive & WL & 14.97 & 1.51 & 0.35 & -0.29 \\
\hline chr1:1780-18 significant & WL & 23.42 & 3.24 & 0.67 & -2.16 \\
\hline chr1:1772-18 suggestive & WL & 12.28 & 2.79 & 0.68 & 0.29 \\
\hline chr1:1782-18 significant & WL & 21.61 & 5.33 & 1.06 & -0.16 \\
\hline chr1:1781-18 significant & WL & 22.64 & 3.37 & 0.71 & -2.1 \\
\hline chr1:1779-18 significant & WL & 17.49 & 2.46 & 0.55 & -0.82 \\
\hline chr1:1781-18 significant & WL & 17.28 & 3.05 & 0.62 & -0.08 \\
\hline chr1:1782-18 significant & WL & 21.43 & 3.99 & 0.74 & -0.48 \\
\hline chr1:1780-18 significant & WL & 16.03 & 2.38 & 0.71 & -2.41 \\
\hline chr1:1756-18 suggestive & WL & 12.97 & 2.29 & 0.52 & 0.24 \\
\hline chr1:1783-17 significant & WL & 22.21 & 1.71 & 0.36 & -1.19 \\
\hline chr1:1781-18 significant & WL & 19.22 & 3.51 & 0.69 & -0.4 \\
\hline chr1:1783-18 significant & WL & 15.13 & 3.31 & 0.78 & -0.8 \\
\hline chr1:1780-18 significant & RJF & 14.98 & -2.9 & 0.63 & -1.19 \\
\hline chr1:1781-18 significant & RJF & 17.36 & -2.26 & 0.45 & -0.33 \\
\hline chr1:1785-18 significant & RJF & 27.33 & -14.52 & 2.2 & -1.53 \\
\hline chr1:1788-18 significant & RJF & 51.79 & -24.17 & 2.26 & 3.07 \\
\hline chr1:1786-18 significant & RJF & 34.54 & -21.97 & 2.89 & 1.17 \\
\hline chr1:1787-18 significant & RJF & 34.63 & -73.49 & 9.54 & 2.91 \\
\hline chr1:1785-18 significant & RJF & 33.82 & -21.29 & 2.84 & 0.79 \\
\hline chr1:1786-18 significant & RJF & 41.78 & -22.02 & 2.53 & 2.96 \\
\hline chr1:1784-18 significant & RJF & 24.84 & -9.39 & 1.52 & -1.08 \\
\hline chr1:1787-18 significant & RJF & 31.6 & -76.78 & 10.52 & -3.23 \\
\hline chr1:1787-18 significant & RJF & 33.81 & -79.84 & 10.51 & 1.19 \\
\hline chr1:1786-18 significant & RJF & 30.93 & -70.31 & 9.86 & -0.12 \\
\hline chr1:1790-18 significant & RJF & 54.53 & -28.8 & 2.52 & 2.57 \\
\hline chr1:1787-18 significant & RJF & 34.14 & -84.79 & 11.09 & 0.96 \\
\hline chr1:1787-18 significant & RJF & 33.23 & -64.92 & 8.61 & -1.31 \\
\hline chr1:1788-18 significant & RJF & 34.31 & -19.02 & 2.43 & -3.5 \\
\hline chr1:1787-18 significant & RJF & 34.5 & -69.56 & 9.04 & 1.14 \\
\hline chr1:1787-18 significant & RJF & 33.34 & -70.55 & 9.37 & 0.25 \\
\hline chr1:1787-18 significant & RJF & 32.63 & -67.47 & 9.14 & 0.54 \\
\hline chr1:1787-18 significant & RJF & 34.48 & -74.7 & 9.72 & 2.65 \\
\hline chr1:1786-18 significant & RJF & 31.26 & -66.52 & 9.25 & -0.98 \\
\hline chr1:1787-18 significant & RJF & 35.42 & -61.48 & 7.76 & -1.86 \\
\hline chr1:1787-18 significant & RJF & 34.54 & -73.34 & 9.51 & 0.19 \\
\hline chr1:1786-18 significant & RJF & 30.87 & -22.13 & 3.07 & -3.02 \\
\hline chr1:1787-18 significant & RJF & 31.77 & -79.66 & 11 & 1.16 \\
\hline chr1:1787-18 significant & RJF & 32.93 & -76.43 & 10.23 & -0.24 \\
\hline
\end{tabular}




\begin{tabular}{|c|c|c|c|c|c|}
\hline chr1:1788-18 significant & RJF & 36.69 & -59.39 & 7.29 & -1.29 \\
\hline chr1:1787-18 significant & RJF & 32.97 & -82.24 & 10.98 & -1.85 \\
\hline chr1:1787-18 significant & RJF & 33.74 & -67.99 & 8.86 & -2.54 \\
\hline chr1:1787-18 significant & RJF & 34.22 & -60.21 & 7.92 & 2.38 \\
\hline chr1:1786-18 significant & RJF & 30.19 & -19.53 & 2.75 & -2.36 \\
\hline chr1:1787-18 significant & RJF & 33.12 & -67.25 & 8.86 & -4.34 \\
\hline chr1:1786-18 significant & RJF & 33.24 & -78.19 & 10.41 & -0.28 \\
\hline chr1:1789-18 significant & RJF & 49.4 & -24.34 & 2.37 & 1.99 \\
\hline chr1:1781-18 suggestive & RJF & 13.36 & -0.35 & 0.25 & 1.27 \\
\hline chr1:1782-18 significant & RJF & 16.43 & -1.29 & 0.42 & 1.62 \\
\hline chr1:1787-18 significant & RJF & 35.53 & -31.77 & 3.95 & -4.83 \\
\hline chr1:1785-18 significant & RJF & 23.75 & -30.4 & 4.97 & -8.7 \\
\hline chr1:1788-18 significant & RJF & 36.32 & -49.45 & 6 & -7.12 \\
\hline chr1:1787-18 significant & RJF & 34.7 & -41.56 & 5.28 & -4.39 \\
\hline chr1:1789-18 significant & RJF & 39.74 & -45.44 & 5.23 & -3.39 \\
\hline chr1:1787-18 significant & RJF & 40.07 & -27.4 & 3.19 & 1.18 \\
\hline chr1:1789-18 significant & RJF & 49 & -35.76 & 3.44 & -0.73 \\
\hline chr1:1787-18 significant & RJF & 34.18 & -29.52 & 3.77 & -4.73 \\
\hline chr1:1784-18 significant & RJF & 20.96 & -32.76 & 5.81 & -10.14 \\
\hline chr1:1786-18 significant & RJF & 30.43 & -62.97 & 8.85 & -3.97 \\
\hline chr1:1788-18 significant & RJF & 37.51 & -42.91 & 5.08 & -7.01 \\
\hline chr1:1787-18 significant & RJF & 34.24 & -55.9 & 7.33 & 1.67 \\
\hline chr1:1788-18 significant & RJF & 35.33 & -41.12 & 5.16 & -4.96 \\
\hline chr1:1788-18 significant & RJF & 41.18 & -34.32 & 3.89 & 0.5 \\
\hline chr1:1790-18 significant & RJF & 51.65 & -36.04 & 3.29 & -0.02 \\
\hline chr1:1783-18 significant & RJF & 17.27 & -6.06 & 1.24 & -0.38 \\
\hline chr1:1788-18 significant & RJF & 46.31 & -41.69 & 4.24 & 1.2 \\
\hline chr1:1787-18 significant & RJF & 33.95 & -64.95 & 8.37 & -6.1 \\
\hline chr1:1788-18 significant & RJF & 35.16 & -36.03 & 4.52 & -4.43 \\
\hline chr1:1789-18 significant & RJF & 41.21 & -41.1 & 4.6 & -1.74 \\
\hline chr1:1790-18 significant & RJF & 51.24 & -32.73 & 3.05 & 1.88 \\
\hline chr1:1789-18 significant & RJF & 40.44 & -29.58 & 3.32 & -2.98 \\
\hline chr1:1784-18 significant & RJF & 27 & -8.04 & 1.24 & -0.47 \\
\hline chr1:1787-18 significant & RJF & 35.6 & -31.5 & 3.92 & -4.15 \\
\hline chr1:1785-18 significant & RJF & 24.09 & -34.37 & 5.59 & -8.71 \\
\hline chr1:1788-18 significant & RJF & 46.42 & -44.27 & 4.46 & 0.19 \\
\hline chr1:1787-18 significant & RJF & 33.2 & -70.23 & 9.34 & -0.41 \\
\hline chr1:1787-18 significant & RJF & 34.18 & -59.07 & 7.54 & -6.27 \\
\hline chr1:1787-18 significant & RJF & 32.51 & -59.2 & 7.95 & -3.05 \\
\hline chr1:1788-18 significant & RJF & 37.7 & -36.35 & 4.33 & -3.83 \\
\hline chr1:1789-18 significant & RJF & 42.68 & -42.9 & 4.66 & -2.11 \\
\hline chr1:1790-18 significant & RJF & 52.6 & -31.08 & 2.82 & 2.07 \\
\hline chr1:1784-18 significant & RJF & 18.77 & -6.14 & 1.16 & -1.71 \\
\hline
\end{tabular}




\begin{tabular}{|c|c|c|c|c|c|}
\hline chr1:1785-18 significant & RJF & 22.7 & -32.69 & 5.52 & -9.36 \\
\hline chr1:1788-18 significant & RJF & 35.32 & -38.44 & 4.81 & -5.12 \\
\hline chr1:1788-18 significant & RJF & 41.59 & -37.53 & 4.19 & -1.15 \\
\hline chr1:1790-18 significant & RJF & 50.05 & -35.5 & 3.36 & 0.5 \\
\hline chr1:1784-18 significant & RJF & 19.45 & -7.14 & 1.32 & -2.16 \\
\hline chr1:1785-18 significant & RJF & 23.98 & -34.56 & 5.63 & -9.33 \\
\hline chr1:1787-18 significant & RJF & 29.73 & -34.01 & 4.79 & -7.19 \\
\hline chr1:1787-18 significant & RJF & 31.91 & -73.73 & 10.13 & 0.79 \\
\hline chr1:1787-18 significant & RJF & 33.87 & -54.69 & 7.13 & -1.36 \\
\hline chr1:1786-18 significant & RJF & 32.84 & -72.49 & 9.68 & -3.56 \\
\hline chr1:1787-18 significant & RJF & 33.78 & -52.87 & 6.92 & -1.03 \\
\hline chr1:1787-18 significant & RJF & 33.39 & -44.82 & 5.94 & -0.57 \\
\hline chr1:1788-18 significant & RJF & 37.77 & -42.74 & 5.1 & -4.46 \\
\hline chr1:1788-18 significant & RJF & 42.34 & -31.51 & 3.49 & 0.71 \\
\hline chr1:1790-18 significant & RJF & 48.72 & -37.01 & 3.58 & -0.69 \\
\hline chr1:1787-18 significant & RJF & 31.89 & -24.71 & 3.32 & -4.37 \\
\hline chr1:1788-18 significant & RJF & 39.7 & -23.58 & 2.76 & 0.78 \\
\hline chr1:1787-18 significant & WL & 34.21 & 2.96 & 0.39 & -0.14 \\
\hline chr1:1787-18 suggestive & RJF & 14.03 & -0.04 & 0.3 & 1.75 \\
\hline chr1:1784-18 significant & RJF & 16.66 & -6.58 & 1.34 & -2.14 \\
\hline chr1:1786-18 suggestive & WL & 18.82 & 1.58 & 0.43 & 2.7 \\
\hline chr1:1785-18 significant & RJF & 24.58 & -33.85 & 5.44 & -7.54 \\
\hline chr1:1787-18 significant & RJF & 38.05 & -48.32 & 5.72 & -2.57 \\
\hline chr1:1788-18 significant & RJF & 35.22 & -33.28 & 4.16 & -4.92 \\
\hline chr1:1786-18 significant & RJF & 22.79 & -7.95 & 1.33 & -1.89 \\
\hline chr1:1786-18 significant & RJF & 26.88 & -34.48 & 5.22 & -7.14 \\
\hline chr1:1789-18 significant & RJF & 42.47 & -54.37 & 5.81 & -7.77 \\
\hline chr1:1787-18 significant & RJF & 39.1 & -50.29 & 5.83 & -2.66 \\
\hline chr1:1788-18 significant & RJF & 43.77 & -38.49 & 4.08 & -0.59 \\
\hline chr1:1789-18 significant & RJF & 40.41 & -30.75 & 3.46 & -4.5 \\
\hline chr1:1789-18 significant & RJF & 47.18 & -31.22 & 3.12 & -0.37 \\
\hline chr1:1787-18 significant & RJF & 29.55 & -10.65 & 1.5 & -2.26 \\
\hline chr1:1786-18 significant & RJF & 22.69 & -29.19 & 4.9 & -9.69 \\
\hline chr1:1789-18 significant & RJF & 46.93 & -29.93 & 2.99 & -0.95 \\
\hline chr1:1787-18 significant & RJF & 31.74 & -26.88 & 3.61 & -5.74 \\
\hline chr1:1787-18 significant & RJF & 29.41 & -34.82 & 4.93 & -7.35 \\
\hline chr1:1789-18 significant & RJF & 40.19 & -50.58 & 5.68 & -7.12 \\
\hline chr1:1787-18 significant & RJF & 40.67 & -53.51 & 6.04 & -1.06 \\
\hline chr1:1788-18 significant & RJF & 40.47 & -36.85 & 4.2 & 0.09 \\
\hline chr1:1788-18 significant & RJF & 40.84 & -31.72 & 3.56 & -3.32 \\
\hline chr1:1788-18 significant & RJF & 36.84 & -35.03 & 4.26 & -3.69 \\
\hline chr1:1790-18 significant & RJF & 45.51 & -29.56 & 3 & -2.32 \\
\hline chr1:1787-18 significant & RJF & 31.81 & -27.72 & 3.72 & -5.38 \\
\hline
\end{tabular}




\begin{tabular}{|c|c|c|c|c|c|}
\hline chr1:1789-18 significant & RJF & 41.83 & -53.87 & 5.86 & -6.39 \\
\hline chr1:1787-18 significant & RJF & 39.41 & -53.32 & 6.15 & -2.4 \\
\hline chr1:1789-18 significant & RJF & 41.89 & -36.37 & 4.01 & -0.54 \\
\hline chr1:1784-18 significant & RJF & 19.85 & -21.42 & 3.93 & -6.83 \\
\hline chr1:1785-18 significant & RJF & 21.79 & -24.14 & 4.18 & -7.4 \\
\hline chr1:1787-18 significant & RJF & 37.27 & -45.7 & 5.52 & -2 \\
\hline chr1:1786-18 significant & RJF & 29.31 & -35.27 & 5.03 & -6.8 \\
\hline chr1:1781-18 suggestive & RJF & 14.14 & -0.98 & 0.26 & 0.5 \\
\hline chr1:1788-18 suggestive & WL & 15.49 & 0.22 & 0.38 & 2.47 \\
\hline chr1:1787-18 suggestive & RJF & 18.71 & -8.38 & 1.6 & -4.17 \\
\hline chr1:1789-18 significant & RJF & 38.39 & -31.72 & 3.7 & -5.75 \\
\hline chr1:1788-18 significant & RJF & 34.08 & -33.27 & 4.28 & -4.47 \\
\hline chr1:1788-18 significant & RJF & 35.1 & -22.03 & 2.77 & -2.73 \\
\hline chr1:1788-18 significant & RJF & 31.47 & -40.54 & 5.43 & -11.97 \\
\hline chr1:1789-18 significant & RJF & 37.45 & -37.84 & 4.45 & -8.08 \\
\hline chr1:1789-18 significant & RJF & 33.25 & -45.41 & 5.89 & -9.77 \\
\hline chr1:1788-18 significant & RJF & 36.75 & -35.13 & 4.3 & -0.7 \\
\hline chr1:1789-18 significant & RJF & 37.11 & -33.15 & 4 & -1.84 \\
\hline chr1:1789-18 significant & RJF & 36.2 & -31 & 3.78 & -6.4 \\
\hline chr1:1789-18 significant & RJF & 34.3 & -20.37 & 2.6 & -3.03 \\
\hline chr1:1788-18 significant & RJF & 29.43 & -39.84 & 5.58 & -12.71 \\
\hline chr1:1788-18 significant & RJF & 34.51 & -44.01 & 5.64 & -1.71 \\
\hline chr1:1789-18 significant & RJF & 37.54 & -28.19 & 3.35 & -2.73 \\
\hline chr1:1788-18 significant & RJF & 34.06 & -33.87 & 4.36 & -4.68 \\
\hline chr1:1788-18 significant & RJF & 37.69 & -48.99 & 5.84 & -3.2 \\
\hline chr1:1789-18 significant & RJF & 33.8 & -31.56 & 4.05 & -3.35 \\
\hline chr1:1790-18 significant & RJF & 36.81 & -37.21 & 4.47 & -8.2 \\
\hline chr1:1788-18 significant & RJF & 27.46 & -39.47 & 5.82 & -13.13 \\
\hline chr1:1789-18 significant & RJF & 40.67 & -52.38 & 5.78 & -8.9 \\
\hline chr1:1789-18 significant & RJF & 33.86 & -46.17 & 5.91 & -8.86 \\
\hline chr1:1787-18 significant & RJF & 31.35 & -43.67 & 5.94 & -5.2 \\
\hline chr1:1787-18 significant & RJF & 32.45 & -40.58 & 5.47 & -1.58 \\
\hline chr1:1790-18 significant & RJF & 36.44 & -37.08 & 4.48 & -8.49 \\
\hline chr1:1789-18 significant & RJF & 37.37 & -35.1 & 4.17 & -6.35 \\
\hline chr1:1789-18 significant & RJF & 40.65 & -25.98 & 2.9 & -3.13 \\
\hline chr1:1788-18 significant & RJF & 31.39 & -15.51 & 2.12 & -1.77 \\
\hline chr1:1789-18 significant & RJF & 33.52 & -44.23 & 5.62 & -12.21 \\
\hline chr1:1789-18 significant & RJF & 38.52 & -32.9 & 3.86 & -1.19 \\
\hline chr1:1789-18 significant & RJF & 38.92 & -31.8 & 3.67 & -5.56 \\
\hline chr1:1789-18 significant & RJF & 34.67 & -33.92 & 4.3 & -4.7 \\
\hline chr1:1787-18 significant & RJF & 32.12 & -13.18 & 1.78 & -1.36 \\
\hline chr1:1788-18 significant & RJF & 27.95 & -39.26 & 5.7 & -13.79 \\
\hline chr1:1789-18 significant & RJF & 38.3 & -41.73 & 4.84 & -7.67 \\
\hline
\end{tabular}




\begin{tabular}{|c|c|c|c|c|c|}
\hline chr1:1789-18 significant & RJF & 32.33 & -43.13 & 5.71 & -8.89 \\
\hline chr1:1786-18 significant & RJF & 19.32 & -6.89 & 1.28 & -3.24 \\
\hline chr1:1783-18 significant & WL & 18.43 & 2.37 & 0.45 & 0.86 \\
\hline chr1:1786-18 significant & RJF & 18.73 & -25.98 & 4.89 & -7.87 \\
\hline chr1:1789-18 significant & RJF & 42.29 & -29.35 & 3.13 & -3.71 \\
\hline chr1:1789-18 significant & RJF & 38.39 & -34.48 & 4 & -5.85 \\
\hline chr1:1789-18 significant & RJF & 39.28 & -24.45 & 2.83 & -1.66 \\
\hline chr1:1787-18 significant & RJF & 31.16 & -37.34 & 5.16 & -1.36 \\
\hline chr1:1789-18 significant & RJF & 31.77 & -33.58 & 4.46 & -6.89 \\
\hline chr1:1790-18 significant & RJF & 38.5 & -35.37 & 4.12 & -5.28 \\
\hline chr1:1789-18 significant & RJF & 36.52 & -33.98 & 4.11 & -5.54 \\
\hline chr1:1789-18 significant & RJF & 38.01 & -24.73 & 2.92 & -2.45 \\
\hline chr1:1789-18 significant & RJF & 33.7 & -36.87 & 4.74 & -7.46 \\
\hline chr1:1789-18 significant & RJF & 34.08 & -30.45 & 3.85 & -4.9 \\
\hline chr1:1790-18 significant & RJF & 38.64 & -33.84 & 3.89 & -6.37 \\
\hline chr1:1789-18 significant & RJF & 32.05 & -40.29 & 5.36 & -7.58 \\
\hline chr1:1787-18 significant & RJF & 32.23 & -44.08 & 5.95 & -1.7 \\
\hline chr1:1788-18 significant & RJF & 30.7 & -31.84 & 4.35 & -4.27 \\
\hline chr1:1789-18 significant & RJF & 36.72 & -33.56 & 4.06 & -4.93 \\
\hline chr1:1789-18 significant & RJF & 36.32 & -34.81 & 4.23 & -5.45 \\
\hline chr1:1789-18 significant & RJF & 36.47 & -24.05 & 2.93 & -2.68 \\
\hline chr1:1789-18 significant & RJF & 34.53 & -21.41 & 2.71 & -3.56 \\
\hline chr1:1789-18 significant & RJF & 32.29 & -33.45 & 4.44 & -4.08 \\
\hline chr1:1789-18 significant & RJF & 35.83 & -33.34 & 4.12 & -4.43 \\
\hline chr1:1789-18 significant & RJF & 34.92 & -33.36 & 4.17 & -5.48 \\
\hline chr1:1787-18 significant & RJF & 32.37 & -38.93 & 5.24 & -1.42 \\
\hline chr1:1789-18 significant & RJF & 32.31 & -32.82 & 4.31 & -6.1 \\
\hline chr1:1789-18 significant & RJF & 36.18 & -36.24 & 4.39 & -6.84 \\
\hline chr1:1790-18 significant & RJF & 36.86 & -34.89 & 4.2 & -4.98 \\
\hline chr1:1789-18 significant & RJF & 39.57 & -25.62 & 2.93 & -2.43 \\
\hline chr1:1789-18 significant & RJF & 34.74 & -18.79 & 2.38 & -2.42 \\
\hline chr1:1789-18 significant & RJF & 30.45 & -41.23 & 5.61 & -13.29 \\
\hline chr1:1788-18 significant & RJF & 33.93 & -42.51 & 5.51 & -2.45 \\
\hline chr1:1789-18 significant & RJF & 31.02 & -29.43 & 3.98 & -5.25 \\
\hline chr1:1789-18 significant & RJF & 35.1 & -27.36 & 3.43 & -2.35 \\
\hline chr1:1790-18 significant & RJF & 38.46 & -33.96 & 3.92 & -6.32 \\
\hline chr1:1790-18 significant & RJF & 36.82 & -36.13 & 4.34 & -5.81 \\
\hline chr1:1789-18 significant & RJF & 39.22 & -23.99 & 2.78 & -1.39 \\
\hline chr1:1788-18 significant & RJF & 31.54 & -17.43 & 2.35 & -3.28 \\
\hline chr1:1787-18 significant & RJF & 30.75 & -40.18 & 5.61 & -2.29 \\
\hline chr1:1788-18 significant & RJF & 37.31 & -24.7 & 3 & -0.55 \\
\hline chr1:1786-18 significant & RJF & 15.21 & -9.78 & 2.1 & -4.01 \\
\hline chr1:1789-18 significant & RJF & 22.93 & -0.82 & 0.48 & 0.72 \\
\hline
\end{tabular}




\begin{tabular}{|c|c|c|c|c|c|}
\hline chr1:1780-18 significant & RJF & 13.95 & -1.06 & 0.28 & 0.45 \\
\hline chr1:1782-18 suggestive & RJF & 13.28 & -1.26 & 0.37 & 0.86 \\
\hline chr1:1779-18 significant & WL & 15.93 & 2.32 & 0.48 & 0.61 \\
\hline chr1:1788-18 significant & RJF & 19.82 & -3.98 & 0.73 & -1.84 \\
\hline chr1:1788-18 significant & RJF & 29.23 & -32.61 & 4.62 & -4.09 \\
\hline chr1:1788-18 significant & RJF & 27.24 & -39.72 & 5.91 & -8.94 \\
\hline chr1:1788-18 significant & RJF & 28.06 & -38.75 & 5.67 & -8.6 \\
\hline chr1:1787-18 significant & RJF & 29.11 & -9.31 & 1.32 & -2.72 \\
\hline chr1:1787-18 significant & RJF & 22.83 & -11.72 & 1.96 & -2.64 \\
\hline chr1:1786-18 significant & RJF & 15.03 & -18.15 & 3.9 & -7.1 \\
\hline chr1:1786-18 significant & RJF & 14.78 & -17.76 & 3.86 & -6.99 \\
\hline chr1:1786-18 significant & RJF & 22.02 & -25.32 & 4.33 & -5.53 \\
\hline chr1:1788-18 significant & RJF & 22.73 & -31.83 & 5.32 & -10.72 \\
\hline chr1:1787-18 significant & RJF & 18.85 & -23.76 & 4.47 & -8.15 \\
\hline chr1:1771-18 suggestive & RJF & 11.32 & -0.78 & 0.38 & 1.42 \\
\hline chr1:1786-18 significant & RJF & 23.47 & -21.45 & 3.56 & -2.7 \\
\hline chr1:1785-18 suggestive & WL & 16.92 & 0.49 & 0.55 & 1.3 \\
\hline chr1:1786-18 significant & RJF & 21.63 & -20.3 & 3.48 & -4.9 \\
\hline chr1:1786-18 significant & RJF & 21.63 & -20.98 & 3.6 & -4.9 \\
\hline chr1:1783-18 significant & RJF & 14.67 & -23.52 & 5.14 & -7.48 \\
\hline chr1:1785-18 significant & RJF & 17.99 & -16.62 & 3.21 & -3.35 \\
\hline chr1:1786-18 significant & RJF & 14.78 & -2.37 & 0.53 & -1.52 \\
\hline chr1:1786-18 significant & RJF & 13.24 & -16.73 & 3.95 & -11.67 \\
\hline chr1:1787-18 significant & RJF & 13.51 & -17.1 & 3.99 & -12.02 \\
\hline chr1:1786-18 significant & RJF & 16.97 & -20.06 & 4 & -6.7 \\
\hline chr1:1785-18 significant & RJF & 13.16 & -21.2 & 4.97 & -11.68 \\
\hline chr1:1786-18 suggestive & RJF & 12.86 & -0.96 & 0.35 & 1.21 \\
\hline chr1:1784-18 suggestive & RJF & 13.08 & -0.93 & 0.27 & 0.56 \\
\hline chr1:1770-18 significant & WL & 13.85 & 1.76 & 0.44 & 0.04 \\
\hline chr1:1787-18 suggestive & RJF & 14.08 & -1 & 0.44 & 1.48 \\
\hline chr1:1785-18 significant & WL & 18.78 & 0.28 & 0.55 & 1.21 \\
\hline chr1:1800-18 suggestive & RJF & 25.67 & 0 & 0.02 & 0 \\
\hline chr1:1795-18 significant & RJF & 16.5 & -1.05 & 0.32 & 0.79 \\
\hline chr1:1799-18 significant & RJF & 20.1 & -0.26 & 0.55 & 1.68 \\
\hline chr1:1800-18 suggestive & RJF & 15.93 & -1.39 & 0.32 & 0.05 \\
\hline chr1:1787-18 significant & RJF & 13.07 & -9.51 & 2.23 & -4.23 \\
\hline chr1:1795-18 significant & RJF & 19.5 & -1.94 & 0.44 & 0.52 \\
\hline chr1:1806-18 significant & RJF & 22.55 & -2.52 & 0.8 & -1.86 \\
\hline chr1:1790-18 suggestive & RJF & 19.22 & -0.1 & 0.49 & 0.34 \\
\hline chr1:1783-18 significant & RJF & 13.06 & -9.08 & 2.16 & -1.27 \\
\hline chr1:1787-18 significant & RJF & 13.64 & -9.73 & 2.22 & -4.24 \\
\hline chr1:1786-18 suggestive & RJF & 11.5 & -10.29 & 2.58 & -3.74 \\
\hline chr1:1786-18 suggestive & RJF & 12.16 & -7.88 & 1.92 & -3.36 \\
\hline
\end{tabular}




\begin{tabular}{|c|c|c|c|c|c|}
\hline chr1:1802-18 significant & RJF & 15.06 & -2.43 & 0.58 & 0.33 \\
\hline chr1:1814-18 significant & RJF & 20.81 & -0.54 & 0.1 & -0.4 \\
\hline chr1:1814-18 significant & RJF & 24.43 & -2.99 & 0.48 & -0.72 \\
\hline chr1:1813-18 significant & WL & 16.91 & 1.47 & 0.31 & 0.91 \\
\hline chr1:1811-18 suggestive & RJF & 15.42 & -1.64 & 0.35 & -0.06 \\
\hline chr1:1820-18 significant & RJF & 26.36 & -2.02 & 0.31 & -0.15 \\
\hline chr1:1823-18 significant & RJF & 28.76 & -5.62 & 0.76 & -2 \\
\hline chr1:1821-18 significant & RJF & 22.85 & -6.91 & 1.07 & -2.5 \\
\hline chr1:1823-18 significant & RJF & 24.58 & -5.22 & 0.81 & -1.93 \\
\hline chr1:1821-18 significant & RJF & 17.93 & -5.22 & 1 & -1.06 \\
\hline chr1:1820-18 significant & WL & 21.92 & 1.86 & 0.32 & -0.05 \\
\hline chr1:1825-18 significant & RJF & 26.75 & -5.21 & 0.77 & -2.08 \\
\hline chr1:1820-18 significant & RJF & 14.87 & -5.76 & 1.13 & -1.81 \\
\hline chr1:1820-19 suggestive & RJF & 14.37 & -3.81 & 0.86 & -2.06 \\
\hline chr1:1821-18 significant & WL & 20.26 & 2.15 & 0.39 & 0.03 \\
\hline chr1:1814-19 suggestive & WL & 11.83 & 2.55 & 0.64 & 0.18 \\
\hline chr1:1824-18 significant & RJF & 23.03 & -8.78 & 1.31 & -2.28 \\
\hline chr1:1825-18 significant & RJF & 22.97 & -8.85 & 1.32 & -2.12 \\
\hline chr1:1824-18 significant & RJF & 19.78 & -8.53 & 1.39 & -1.88 \\
\hline chr1:1825-18 significant & RJF & 21.84 & -9.4 & 1.45 & -2.21 \\
\hline chr1:1827-18 significant & WL & 31.13 & 2.61 & 0.36 & -0.43 \\
\hline chr1:1822-18 significant & WL & 19.58 & 2.45 & 0.52 & -0.31 \\
\hline chr1:1820-18 significant & RJF & 17.7 & -2.09 & 0.4 & -0.44 \\
\hline chr1:1823-18 significant & RJF & 17.47 & -8.49 & 1.5 & -2.29 \\
\hline chr1:1825-18 significant & RJF & 23.5 & -2.15 & 0.36 & 0.13 \\
\hline chr1:1823-18 significant & RJF & 17.42 & -10.86 & 2.12 & -0.51 \\
\hline chr1:1824-18 significant & RJF & 17.48 & -15.76 & 3.02 & -2.04 \\
\hline chr1:1823-18 significant & RJF & 17.53 & -14.69 & 2.82 & -2.22 \\
\hline chr1:1827-18 significant & RJF & 26.89 & -4.19 & 0.63 & -1.02 \\
\hline chr1:1824-18 significant & RJF & 19.05 & -14.72 & 2.7 & -1.57 \\
\hline chr1:1823-18 significant & RJF & 19.11 & -4.87 & 0.91 & -0.44 \\
\hline chr1:1817-18 significant & WL & 12.99 & 1.98 & 0.48 & -0.2 \\
\hline chr1:1821-18 significant & RJF & 13.48 & -6.48 & 1.33 & -0.1 \\
\hline chr1:1829-18 significant & RJF & 16.95 & -8.8 & 1.72 & -1.95 \\
\hline chr1:1834-18 significant & RJF & 26.8 & -7 & 1.05 & -1.65 \\
\hline chr1:1831-18 significant & RJF & 23.04 & -11.03 & 1.83 & -3.44 \\
\hline chr1:1830-18 significant & RJF & 26.67 & -11.89 & 1.79 & -2.88 \\
\hline chr1:1828-18 significant & RJF & 21.21 & -20.41 & 3.63 & -7.62 \\
\hline chr1:1828-18 significant & RJF & 21.67 & -16.43 & 2.77 & -3.16 \\
\hline chr1:1829-18 significant & RJF & 15.5 & -7.81 & 1.49 & -0.68 \\
\hline chr1:1832-18 significant & RJF & 29.75 & -7.07 & 0.96 & -1.4 \\
\hline chr1:1832-18 significant & RJF & 28.65 & -5.43 & 0.79 & -0.69 \\
\hline chr1:1831-18 significant & RJF & 21.34 & -4.72 & 0.83 & -0.62 \\
\hline
\end{tabular}




\begin{tabular}{|c|c|c|c|c|c|}
\hline chr1:1829-18 significant & RJF & 25.47 & -4.84 & 0.76 & -0.29 \\
\hline chr1:1827-18 significant & RJF & 15.49 & -8.17 & 1.56 & -0.71 \\
\hline chr1:1831-18 significant & RJF & 25.78 & -18.42 & 2.86 & -5.4 \\
\hline chr1:1829-18 significant & RJF & 22.8 & -16.74 & 2.77 & -2.7 \\
\hline chr1:1830-18 significant & RJF & 22.71 & -21.55 & 3.66 & -7.82 \\
\hline chr1:1827-18 significant & RJF & 22.34 & -14.89 & 2.51 & -0.77 \\
\hline chr1:1828-18 significant & RJF & 22.43 & -18.46 & 3.02 & -3.36 \\
\hline chr1:1830-18 significant & RJF & 17.7 & -5.34 & 0.97 & -0.65 \\
\hline chr1:1833-18 significant & RJF & 31.71 & -11.04 & 1.48 & -2.68 \\
\hline chr1:1828-18 significant & RJF & 21.86 & -12.62 & 2.18 & -3.56 \\
\hline chr1:1831-18 significant & RJF & 25.01 & -20.42 & 3.25 & -7.23 \\
\hline chr1:1829-18 significant & RJF & 24.14 & -14.14 & 2.21 & -2.43 \\
\hline chr1:1827-18 significant & RJF & 20 & -6.57 & 1.19 & -0.65 \\
\hline chr1:1830-18 significant & RJF & 19.61 & -5.74 & 1.05 & -2.12 \\
\hline chr1:1831-18 significant & RJF & 20.31 & -5.9 & 1.06 & -2.09 \\
\hline chr1:1830-18 significant & RJF & 18.52 & -4.48 & 0.8 & -0.26 \\
\hline chr1:1832-18 significant & RJF & 26.16 & -6.16 & 0.91 & -0.97 \\
\hline chr1:1833-18 significant & RJF & 33.64 & -6.61 & 0.85 & -0.88 \\
\hline chr1:1831-18 significant & RJF & 19.53 & -4.1 & 0.76 & -0.84 \\
\hline chr1:1826-18 significant & RJF & 22.33 & -10.61 & 1.79 & -3.12 \\
\hline chr1:1831-18 significant & RJF & 25.19 & -20.78 & 3.29 & -7.21 \\
\hline chr1:1828-18 significant & RJF & 22.58 & -14.92 & 2.44 & -3.18 \\
\hline chr1:1826-18 significant & RJF & 20.74 & -6.51 & 1.16 & -0.51 \\
\hline chr1:1828-18 significant & RJF & 17.39 & -8.52 & 1.54 & -1.61 \\
\hline chr1:1831-18 significant & RJF & 20.6 & -6.33 & 1.11 & -1.88 \\
\hline chr1:1828-18 significant & RJF & 19.52 & -5.43 & 1.01 & -1.86 \\
\hline chr1:1828-18 significant & RJF & 18.81 & -6.67 & 1.24 & -1.56 \\
\hline chr1:1823-18 significant & RJF & 15.88 & -7.42 & 1.4 & -0.62 \\
\hline chr1:1832-18 significant & RJF & 25.88 & -6.6 & 1.02 & -1.38 \\
\hline chr1:1822-18 significant & RJF & 16.5 & -2.78 & 0.57 & -0.33 \\
\hline chr1:1831-18 significant & RJF & 32.13 & -7.72 & 1.03 & -2.45 \\
\hline chr1:1834-18 significant & RJF & 34.82 & -5.79 & 0.72 & -1.07 \\
\hline chr1:1815-18 suggestive & WL & 9.38 & 1.81 & 0.51 & 0.89 \\
\hline chr1:1828-18 significant & RJF & 18.09 & -5.26 & 1.03 & -2.1 \\
\hline chr1:1830-18 significant & RJF & 16.45 & -4.48 & 0.9 & -0.17 \\
\hline chr1:1826-18 significant & RJF & 18.74 & -8.9 & 1.67 & -1.35 \\
\hline chr1:1824-18 suggestive & RJF & 14 & -1.07 & 0.23 & -0.07 \\
\hline chr1:1825-18 suggestive & RJF & 12.86 & -7.2 & 1.54 & -0.78 \\
\hline chr1:1829-18 suggestive & RJF & 15.41 & -4.79 & 1.01 & 0.02 \\
\hline chr1:1828-18 significant & RJF & 14.98 & -3.44 & 0.71 & 0.48 \\
\hline chr1:1824-18 significant & RJF & 13.45 & -7.7 & 1.59 & -0.85 \\
\hline chr1:1829-18 significant & RJF & 15.14 & -2.91 & 0.64 & -1.32 \\
\hline chr1:1824-18 suggestive & RJF & 12.74 & -8.17 & 1.75 & -1.1 \\
\hline
\end{tabular}




\begin{tabular}{|c|c|c|c|c|c|}
\hline chr1:1825-18 suggestive & RJF & 12.55 & -7.35 & 1.59 & -1 \\
\hline chr1:1826-18 significant & RJF & 17.73 & -5.38 & 1.05 & -1.13 \\
\hline chr1:1829-18 significant & WL & 15.06 & 0.44 & 0.23 & -1.25 \\
\hline chr1:1829-18 significant & RJF & 21.57 & -5.58 & 0.99 & -3 \\
\hline chr1:1829-18 significant & RJF & 23.55 & -5.4 & 0.89 & -0.91 \\
\hline chr1:1828-18 significant & RJF & 22.65 & -14.67 & 2.48 & -4.61 \\
\hline chr1:1828-18 significant & RJF & 20.6 & -15.26 & 2.76 & -5.97 \\
\hline chr1:1829-18 significant & RJF & 22.35 & -6.05 & 1.04 & -2.69 \\
\hline chr1:1831-18 significant & RJF & 26.28 & -6.07 & 0.93 & -0.58 \\
\hline chr1:1826-18 significant & WL & 16.72 & 1.5 & 0.35 & -0.84 \\
\hline chr1:1830-18 significant & RJF & 25.77 & -4.93 & 0.73 & -1.12 \\
\hline chr1:1826-18 significant & RJF & 20.82 & -4.32 & 0.75 & -0.79 \\
\hline chr1:1829-18 significant & RJF & 18.75 & -4.56 & 2.29 & -3.89 \\
\hline chr1:1832-18 significant & WL & 23.4 & 1.86 & 0.31 & 0.21 \\
\hline chr1:1825-18 significant & RJF & 22.99 & -1.72 & 0.29 & -0.91 \\
\hline chr1:1825-18 significant & RJF & 24.35 & -2.21 & 0.36 & -0.05 \\
\hline chr1:1832-18 significant & WL & 13.41 & 2.09 & 0.5 & -0.06 \\
\hline chr1:1840-18 significant & RJF & 29.1 & -3.11 & 0.46 & 0.25 \\
\hline chr1:1825-18 significant & WL & 15.41 & 2.66 & 0.57 & 0.45 \\
\hline chr1:1830-18 significant & RJF & 16.35 & -2.39 & 0.5 & -0.13 \\
\hline chr1:1822-18 suggestive & RJF & 14.68 & -1.79 & 0.41 & -1.09 \\
\hline chr1:1818-18 significant & RJF & 16.9 & -0.73 & 0.16 & 0.27 \\
\hline chr1:1831-18 significant & RJF & 30.1 & -9.81 & 1.31 & -1.38 \\
\hline chr1:1822-18 significant & WL & 12.76 & 1.45 & 0.35 & 0.16 \\
\hline chr1:1831-18 significant & RJF & 25.7 & -11.55 & 1.71 & 0.19 \\
\hline chr1:1831-18 significant & RJF & 26.38 & -7.45 & 1.11 & -0.38 \\
\hline chr1:1826-18 significant & RJF & 20.75 & -8.07 & 1.42 & -1.46 \\
\hline chr1:1827-18 significant & RJF & 21.49 & -8.34 & 1.44 & -1.18 \\
\hline chr1:1829-18 significant & RJF & 15.84 & -3.24 & 0.65 & 0.2 \\
\hline chr1:1835-18 significant & RJF & 32.42 & -9.48 & 1.2 & -0.26 \\
\hline chr1:1847-18 significant & RJF & 22.96 & -7.21 & 1.13 & 1.43 \\
\hline chr1:1831-18 significant & RJF & 23.55 & -8.55 & 1.4 & 1.63 \\
\hline chr1:1847-18 significant & RJF & 25.29 & -7.51 & 1.11 & 1.4 \\
\hline chr1:1848-18 significant & RJF & 23.76 & -6.38 & 1 & 1.14 \\
\hline chr1:1847-18 significant & RJF & 22 & -2.15 & 0.37 & 0.1 \\
\hline chr1:1849-18 significant & RJF & 16.97 & -6.44 & 1.23 & 0.9 \\
\hline chr1:1848-18 significant & RJF & 17.88 & -6.45 & 1.2 & 1.27 \\
\hline chr1:1849-18 significant & RJF & 16.56 & -6.36 & 1.23 & 1.69 \\
\hline chr1:1823-18 suggestive & RJF & 15.97 & -1.31 & 0.29 & 0.54 \\
\hline chr1:1824-18 suggestive & RJF & 16.86 & -3.41 & 0.73 & 1.16 \\
\hline chr1:1848-18 significant & RJF & 20.77 & -1.86 & 0.34 & 0.37 \\
\hline chr1:1848-18 suggestive & RJF & 16.99 & -2.27 & 0.48 & 0.87 \\
\hline chr1:1829-18 significant & RJF & 15.47 & -1.21 & 0.26 & 0.21 \\
\hline
\end{tabular}




\begin{tabular}{|c|c|c|c|c|c|}
\hline chr1:1843-18 significant & RJF & 18.72 & -2.03 & 0.37 & -0.2 \\
\hline chr1:1853-19 significant & WL & 19.57 & 0.42 & 0.59 & -0.63 \\
\hline chr1:1854-18 significant & RJF & 19.19 & -2.81 & 0.68 & 1.67 \\
\hline chr1:1854-19 suggestive & RJF & 12.7 & -1.58 & 0.4 & 0.31 \\
\hline chr1:1852-19 significant & WL & 21.02 & 1.26 & 0.54 & 1.15 \\
\hline chr1:1850-19 significant & RJF & 14.81 & -1.78 & 0.44 & 0.56 \\
\hline chr1:1850-19 suggestive & RJF & 12.69 & -1.31 & 0.43 & 0.94 \\
\hline chr1:1861-19 significant & RJF & 16.61 & -2.48 & 0.91 & 3.23 \\
\hline chr1:1860-19 significant & WL & 18.94 & 1.35 & 0.29 & -0.2 \\
\hline chr1:1865-19 significant & RJF & 17.43 & -2.4 & 0.72 & 2.31 \\
\hline chr1:1866-18 significant & WL & 21.17 & 2.53 & 0.46 & 0.35 \\
\hline chr1:1870-19 significant & RJF & 16.72 & -2.35 & 0.54 & 0.32 \\
\hline chr1:1875-19 significant & RJF & 19.56 & -2.51 & 0.53 & 0.44 \\
\hline chr1:1873-18 suggestive & WL & 17.83 & 1.92 & 0.65 & 2.65 \\
\hline chr1:1868-19 significant & RJF & 14.21 & -2.98 & 0.73 & 0.09 \\
\hline chr1:1870-19 suggestive & RJF & 13.85 & -2.35 & 0.91 & -5.69 \\
\hline chr1:1866-19 suggestive & RJF & 21.47 & -0.19 & 0.59 & -0.5 \\
\hline chr1:1871-19 significant & RJF & 18.5 & -2.33 & 0.45 & -1.24 \\
\hline chr1:1864-19 significant & RJF & 19.28 & -0.28 & 0.41 & -1.17 \\
\hline chr1:1861-19 suggestive & RJF & 13.14 & -2.53 & 0.61 & -2.35 \\
\hline chr1:1869-19 significant & RJF & 15.96 & -2.05 & 0.49 & -2.68 \\
\hline chr1:1866-19 suggestive & WL & 20.34 & 0.29 & 0.44 & 0.97 \\
\hline chr1:1890-19 suggestive & RJF & 18.41 & -1.12 & 0.3 & -1.91 \\
\hline chr1:1882-19 suggestive & RJF & 18.39 & -1.13 & 0.23 & -0.92 \\
\hline chr1:1881-19 suggestive & RJF & 19.18 & -1.17 & 0.22 & -0.47 \\
\hline chr1:1864-19 suggestive & RJF & 13.22 & -1.32 & 0.31 & -0.92 \\
\hline chr1:1898-19 suggestive & RJF & 11.46 & -2.31 & 0.62 & -5.42 \\
\hline chr1:1897-19 suggestive & RJF & 10.34 & -1.95 & 0.57 & -5.07 \\
\hline chr1:1897-19 suggestive & RJF & 15.17 & -1.97 & 0.7 & -5.9 \\
\hline chr1:1895-19 significant & RJF & 22.05 & -1.72 & 0.31 & 0.17 \\
\hline chr1:1905-19 significant & WL & 21.6 & 2.64 & 0.46 & 0.46 \\
\hline chr1:1889-19 significant & WL & 16.16 & 1.66 & 0.33 & -0.2 \\
\hline chr1:1873-19 significant & WL & 16.3 & 2.48 & 0.52 & -0.36 \\
\hline chr1:1898-19 significant & RJF & 20.61 & -0.57 & 0.24 & 1.55 \\
\hline chr1:1892-19 suggestive & WL & 14.26 & 1.64 & 0.39 & -0.46 \\
\hline chr1:1905-19 suggestive & WL & 6.59 & 1.06 & 0.22 & -0.79 \\
\hline chr1:1891-19 significant & RJF & 13.97 & -1.25 & 0.27 & -0.35 \\
\hline chr1:1903-19 suggestive & WL & 14.9 & 4.3 & 1.05 & -1.16 \\
\hline chr1:1908-19 significant & WL & 32.96 & 4.51 & 0.63 & -0.51 \\
\hline chr1:1901-19 significant & WL & 15.75 & 3.21 & 0.73 & -0.63 \\
\hline chr1:1898-19 significant & RJF & 17.98 & -2.06 & 0.43 & 0.11 \\
\hline chr1:1906-19 significant & WL & 16.99 & 3.07 & 0.65 & -0.1 \\
\hline chr1:1910-19 significant & RJF & 17.54 & -2.01 & 0.41 & -0.09 \\
\hline
\end{tabular}




\begin{tabular}{|c|c|c|c|c|c|}
\hline chr1:1911-19 significant & RJF & 15.45 & -1.88 & 0.54 & 1.52 \\
\hline chr1:1891-19 significant & RJF & 13.61 & -1.89 & 0.44 & -0.39 \\
\hline chr1:1900-19 significant & RJF & 16 & -1.1 & 0.26 & 0.33 \\
\hline chr1:1896-19 suggestive & RJF & 15.04 & -1.15 & 1.23 & 0.85 \\
\hline chr1:1893-19 suggestive & RJF & 13.91 & -1.09 & 0.26 & 0.11 \\
\hline chr1:1912-19 suggestive & RJF & 25.02 & -1.77 & 0.31 & -1 \\
\hline chr1:1910-19 significant & RJF & 16.88 & -1.13 & 0.23 & -0.71 \\
\hline chr1:1924-19 significant & WL & 17.83 & 5.13 & 1.1 & 1.62 \\
\hline chr1:1924-19 suggestive & RJF & 24.55 & -2.06 & 0.34 & -1.61 \\
\hline chr1:1924-19 significant & RJF & 28.15 & -3.67 & 0.54 & -2.82 \\
\hline chr1:1929-19 significant & RJF & 14.49 & -5.5 & 1.21 & -6.25 \\
\hline chr1:1940-19 suggestive & RJF & 18.39 & -4.48 & 1.02 & -4.22 \\
\hline chr1:1920-19 significant & RJF & 23.51 & -1.72 & 0.3 & -0.65 \\
\hline chr1:1941-19 suggestive & RJF & 15.02 & -2.33 & 0.56 & -2.9 \\
\hline chr1:1942-19 significant & RJF & 21.78 & -4.73 & 0.94 & -5.75 \\
\hline chr1:1911-19 suggestive & RJF & 12.02 & -1.55 & 0.4 & -1.57 \\
\hline chr1:1923-19 significant & RJF & 15.01 & -1.68 & 0.37 & -0.58 \\
\hline chr1:1913-19 suggestive & RJF & 13.65 & -2.35 & 0.53 & -1.33 \\
\hline chr1:1953-19 suggestive & RJF & 13.94 & -2.12 & 0.47 & -1.36 \\
\hline chr1:1956-19 significant & RJF & 15.28 & -2.19 & 0.47 & -1.42 \\
\hline chr1:1952-19 suggestive & RJF & 14.09 & -2.8 & 0.63 & -2.22 \\
\hline chr1:1951-19 significant & RJF & 15.6 & -1.89 & 0.44 & -0.26 \\
\hline chr1:1988-20 significant & RJF & 16.97 & -11.74 & 1.97 & -13.44 \\
\hline chr1:1987-20 suggestive & WL & 11.61 & 1.74 & 0.44 & -0.1 \\
\hline chr1:2000-20 significant & RJF & 24.09 & -1.71 & 0.28 & 0.19 \\
\hline chr1:1991-20 significant & WL & 13.72 & 1.97 & 0.44 & 0.15 \\
\hline chr1:1985-20 significant & WL & 16.1 & 1.9 & 0.4 & 0.65 \\
\hline chr1:2007-20 suggestive & WL & 12.8 & 0.97 & 0.2 & 0.77 \\
\hline chr1:2000-20 suggestive & WL & 14.34 & 1.54 & 0.34 & 0.36 \\
\hline chr1:2000-20 significant & WL & 15.22 & 1.38 & 0.3 & 0.14 \\
\hline chr1:2014-20 significant & RJF & 40.01 & -2.4 & 0.27 & 0.1 \\
\hline chr1:2005-20 significant & RJF & 16.88 & -1.43 & 0.29 & 0.22 \\
\hline chr1:2006-20 significant & WL & 18.67 & 1.57 & 0.32 & 0.94 \\
\hline chr1:2014-20 significant & WL & 22.94 & 1.39 & 0.25 & -0.74 \\
\hline chr1:2014-21 significant & RJF & 13.96 & -2.31 & 0.54 & 1.38 \\
\hline chr1:2013-20 suggestive & WL & 13.64 & 1.8 & 0.43 & -0.59 \\
\hline chr1:2010-20 significant & WL & 14.52 & 1.51 & 0.34 & 0.36 \\
\hline chr1:2013-20 significant & WL & 14.75 & 2.28 & 0.49 & 0.41 \\
\hline chr1:2022-20 suggestive & WL & 17.39 & 0.47 & 0.48 & -0.34 \\
\hline chr1:2021-20 suggestive & WL & 20.81 & 0.53 & 0.1 & -0.29 \\
\hline chr1:2016-20 significant & WL & 19.55 & 0.7 & 0.71 & -1.15 \\
\hline chr1:2007-20 suggestive & WL & 13.73 & 0.92 & 0.21 & -0.18 \\
\hline chr1:2006-20 significant & WL & 23.58 & 2.12 & 0.35 & 0.04 \\
\hline
\end{tabular}




\begin{tabular}{|c|c|c|c|c|c|}
\hline chr1:2012-20 significant & RJF & 23.41 & -1.75 & 0.3 & -0.86 \\
\hline chr1:2002-20 suggestive & RJF & 14.27 & -3.09 & 0.7 & -0.86 \\
\hline chr1:1994-20 significant & RJF & 19.15 & -1.6 & 0.38 & -1.12 \\
\hline chr1:2012-21 suggestive & WL & 14.01 & 1.76 & 0.41 & -1.08 \\
\hline chr1:2014-20 significant & WL & 14.91 & 1.64 & 0.36 & -0.83 \\
\hline chr1:2008-20 significant & RJF & 19.57 & -3.03 & 0.57 & 0.21 \\
\hline chr1:2033-20 suggestive & RJF & 15.11 & -2.26 & 0.54 & -0.65 \\
\hline chr1:2043-20 significant & WL & 12.72 & 1.53 & 0.45 & 0.94 \\
\hline chr1:1996-20 significant & WL & 13.37 & 1.9 & 0.45 & -1.16 \\
\hline chr1:2054-20 suggestive & RJF & 25.47 & -2.19 & 0.97 & -5.33 \\
\hline chr1:2056-20 suggestive & WL & 25.7 & 0.12 & 0.02 & -0.15 \\
\hline chr1:2050-20 suggestive & RJF & 19.83 & -1.49 & 0.51 & -1.17 \\
\hline chr1:2049-20 suggestive & RJF & 17.56 & -2.33 & 0.78 & -2.21 \\
\hline chr1:2052-20 suggestive & RJF & 14.89 & -2.78 & 0.64 & -0.26 \\
\hline chr1:2057-20 significant & WL & 10.98 & 1.39 & 0.22 & -1.42 \\
\hline chr1:2055-20 suggestive & RJF & 25.11 & -1.91 & 0.65 & -3.01 \\
\hline chr1:2044-20 significant & WL & 15.67 & 1.04 & 0.22 & -0.49 \\
\hline chr1:1995-21 significant & WL & 16.15 & 1.46 & 0.32 & -1.11 \\
\hline chr1:2048-21 significant & WL & 18.27 & 0.12 & 0.85 & 0.56 \\
\hline chr1:2053-20 suggestive & WL & 24.89 & 0.22 & 0.04 & -0.21 \\
\hline chr1:2073-20 significant & RJF & 26.37 & -1.46 & 0.26 & -0.28 \\
\hline chr1:2068-21 significant & WL & 14.96 & 1.83 & 0.4 & -1.31 \\
\hline chr1:2072-20 significant & WL & 38.6 & 0.58 & 0.07 & -0.71 \\
\hline chr1:2073-20 suggestive & RJF & 19.76 & -0.11 & 0.45 & 0.12 \\
\hline chr1:2068-20 significant & WL & 18.58 & 2.41 & 0.45 & -0.96 \\
\hline chr1:2071-21 suggestive & WL & 13.31 & 3.05 & 0.73 & -0.78 \\
\hline chr1:2070-21 significant & WL & 23.12 & 1.66 & 0.28 & -0.55 \\
\hline chr1:2070-21 significant & WL & 19.23 & 2.1 & 0.4 & -1.32 \\
\hline chr1:2069-21 suggestive & WL & 16 & 1.11 & 0.25 & -1.46 \\
\hline chr1:2070-21 significant & WL & 21.44 & 2.47 & 0.47 & -0.1 \\
\hline chr1:2069-21 significant & WL & 17.26 & 1.49 & 0.3 & -0.4 \\
\hline chr1:2068-21 significant & RJF & 16.31 & -1.62 & 0.37 & -0.28 \\
\hline chr1:2071-21 significant & WL & 17.11 & 2.01 & 0.41 & -1.21 \\
\hline chr1:2068-21 suggestive & WL & 13.97 & 1.21 & 0.27 & -0.51 \\
\hline chr1:2070-21 significant & RJF & 23.15 & -0.98 & 0.27 & -1.16 \\
\hline chr1:2082-21 significant & RJF & 21.99 & -1.36 & 1.02 & -3.75 \\
\hline chr1:2077-21 significant & RJF & 21.4 & -1.8 & 0.48 & -1.9 \\
\hline chr1:2073-21 suggestive & WL & 13.97 & 1.59 & 0.4 & -1.77 \\
\hline chr1:2083-21 suggestive & WL & 12.8 & 0.88 & 0.26 & -1.29 \\
\hline chr1:2086-21 suggestive & WL & 27.68 & 0.19 & 0.03 & -0.19 \\
\hline chr1:2084-21 suggestive & WL & 23.56 & 0.02 & 0.21 & -0.05 \\
\hline chr1:2083-21 suggestive & WL & 20.12 & 1.18 & 0.24 & -1.26 \\
\hline chr1:2083-21 suggestive & WL & 20.97 & 0.63 & 0.13 & -0.75 \\
\hline
\end{tabular}




\begin{tabular}{|c|c|c|c|c|c|}
\hline chr1:2077-21 significant & WL & 19.65 & 1.44 & 0.27 & -0.8 \\
\hline chr1:2072-21 significant & WL & 18.34 & 2.33 & 0.48 & 0.07 \\
\hline chr1:2075-21 significant & WL & 14.33 & 0.82 & 0.19 & -0.03 \\
\hline chr1:2081-21 significant & WL & 25.38 & 2.09 & 0.32 & -0.52 \\
\hline chr1:2078-21 suggestive & WL & 13.54 & 1.07 & 0.34 & 0.91 \\
\hline chr1:2083-21 suggestive & WL & 17.99 & 1.84 & 0.34 & -1.72 \\
\hline chr1:2081-21 significant & RJF & 19.73 & -1.99 & 0.44 & -0.83 \\
\hline chr1:2080-21 significant & WL & 17.96 & 2.43 & 0.48 & -1.21 \\
\hline chr1:2079-21 significant & WL & 17.59 & 1.7 & 0.33 & -0.83 \\
\hline chr1:2081-21 significant & WL & 15.73 & 1.27 & 0.27 & -0.84 \\
\hline chr1:2080-21 significant & WL & 18.66 & 2.01 & 0.39 & -0.28 \\
\hline chr1:2084-21 suggestive & WL & 13.95 & 2.47 & 0.57 & -1.64 \\
\hline chr1:2074-21 suggestive & WL & 13.64 & 1.54 & 0.35 & -0.83 \\
\hline chr1:2080-21 significant & WL & 15.76 & 1.33 & 0.29 & -0.1 \\
\hline chr1:2085-21 suggestive & WL & 19.3 & 1.43 & 0.27 & -1 \\
\hline chr1:2083-21 significant & WL & 20.75 & 1.55 & 0.29 & -1.23 \\
\hline chr1:2080-21 significant & RJF & 19.37 & -1 & 0.3 & -1.23 \\
\hline chr1:2072-21 suggestive & WL & 16.07 & 0.17 & 0.32 & -2.13 \\
\hline chr1:2070-21 significant & WL & 15.7 & 1.7 & 0.36 & -1.1 \\
\hline chr1:2071-21 significant & RJF & 17.33 & -2.21 & 0.66 & -2.37 \\
\hline chr1:2082-21 significant & WL & 15.85 & 1.64 & 0.34 & -0.81 \\
\hline chr1:2079-21 significant & RJF & 18.11 & -1.43 & 0.29 & 0.25 \\
\hline chr1:2092-21 suggestive & WL & 27.06 & 2.48 & 0.52 & -4.1 \\
\hline chr1:2072-21 suggestive & WL & 19.76 & 0.77 & 0.18 & -1.2 \\
\hline chr1:2080-21 suggestive & WL & 19.34 & 1.24 & 0.32 & -2.12 \\
\hline chr1:2082-21 significant & WL & 20.98 & 0.79 & 0.14 & -0.34 \\
\hline chr1:2073-21 significant & WL & 22.24 & 0.36 & 0.25 & 0.29 \\
\hline chr1:2090-21 significant & RJF & 45.52 & -3.44 & 0.36 & -0.04 \\
\hline chr1:2075-21 significant & WL & 14.61 & 2.3 & 0.5 & -1.23 \\
\hline chr1:2073-21 suggestive & WL & 18.39 & 0.89 & 0.54 & -0.49 \\
\hline chr1:2069-21 suggestive & WL & 13.26 & 1.24 & 0.29 & -0.94 \\
\hline chr1:2081-21 significant & RJF & 20.91 & -0.18 & 0.54 & 1.43 \\
\hline chr1:2067-21 suggestive & RJF & 16.01 & -1.39 & 0.42 & -1.24 \\
\hline chr1:2076-21 significant & WL & 22.36 & 3.16 & 0.53 & -1.05 \\
\hline chr1:2066-21 suggestive & WL & 13.63 & 1.51 & 0.35 & -0.61 \\
\hline chr1:2068-21 significant & WL & 15.21 & 1.33 & 0.29 & -1.2 \\
\hline chr1:2080-21 suggestive & WL & 19.71 & 0.25 & 0.51 & 0.37 \\
\hline chr1:2074-21 suggestive & WL & 14.92 & 1.62 & 0.36 & -0.48 \\
\hline chr1:2066-21 significant & RJF & 20.6 & -1.48 & 0.29 & -0.13 \\
\hline chr1:2071-21 significant & WL & 14.45 & 1.16 & 0.28 & 0.15 \\
\hline chr1:2067-21 suggestive & RJF & 15.35 & -2.36 & 0.68 & -1.31 \\
\hline chr1:2005-21 significant & RJF & 16.56 & -1.46 & 0.33 & -0.22 \\
\hline chr1:2100-21 significant & RJF & 16.77 & -3.78 & 1.06 & -3.67 \\
\hline
\end{tabular}




\begin{tabular}{|c|c|c|c|c|c|}
\hline chr1:2113-21 suggestive & RJF & 23.67 & -0.1 & 0.17 & -0.06 \\
\hline chr1:2097-21 suggestive & WL & 8.43 & 2.1 & 0.42 & -4.29 \\
\hline chr1:2116-21 suggestive & WL & 27.77 & 0.21 & 0.04 & -0.32 \\
\hline chr1:2102-21 significant & WL & 15.38 & 2.64 & 0.61 & -3.48 \\
\hline chr1:2119-21 significant & WL & 30.23 & 0.65 & 0.1 & -1.04 \\
\hline chr1:2111-21 suggestive & WL & 20.95 & 1.72 & 0.49 & -4.81 \\
\hline chr1:2069-21 suggestive & RJF & 14.42 & -1.7 & 0.41 & -0.3 \\
\hline chr1:2131-21 significant & RJF & 25.95 & -0.42 & 0.22 & -2.15 \\
\hline chr1:2121-22 suggestive & RJF & 20.98 & -1.6 & 0.79 & -5.98 \\
\hline chr1:2123-22 suggestive & WL & 12.75 & 2.52 & 0.6 & -0.55 \\
\hline chr1:2130-22 significant & RJF & 17.65 & -1.91 & 0.49 & -1.41 \\
\hline chr1:2132-22 significant & RJF & 15.86 & -2.27 & 0.65 & -2.02 \\
\hline chr1:2131-22 significant & RJF & 16.8 & -1.79 & 0.37 & -0.14 \\
\hline chr1:2121-22 suggestive & RJF & 14.33 & -1.52 & 0.34 & -0.3 \\
\hline chr1:2132-22 suggestive & RJF & 15.29 & -2.98 & 0.64 & -1.89 \\
\hline chr1:2151-22 suggestive & RJF & 22.31 & -1.95 & 0.39 & -2.62 \\
\hline chr1:2142-22 suggestive & WL & 13.55 & 8.46 & 2.09 & -1.21 \\
\hline chr1:2144-22 suggestive & RJF & 15.6 & -1.74 & 0.38 & -1.32 \\
\hline chr1:2151-22 suggestive & RJF & 18.75 & -1.84 & 0.37 & -1.72 \\
\hline chr1:2143-22 significant & RJF & 17.99 & -1.72 & 0.35 & -0.02 \\
\hline chr1:2150-22 suggestive & RJF & 20.05 & -2.24 & 0.41 & -1.23 \\
\hline chr1:2153-22 suggestive & RJF & 20.34 & -1.68 & 0.59 & -0.13 \\
\hline chr1:2150-22 significant & RJF & 16.4 & -1.41 & 0.3 & -1.23 \\
\hline chr1:2147-22 significant & RJF & 16.61 & -1.4 & 0.29 & -0.84 \\
\hline chr1:2156-22 suggestive & RJF & 21.18 & -2.9 & 0.51 & -1.9 \\
\hline chr1:2160-22 suggestive & RJF & 20.37 & -1.65 & 0.36 & -2.4 \\
\hline chr1:2147-22 suggestive & RJF & 15.42 & -1.23 & 0.26 & -0.46 \\
\hline chr1:2158-22 significant & RJF & 24.34 & -1.31 & 0.22 & 0.13 \\
\hline chr1:2150-22 significant & WL & 15.53 & 11.3 & 2.52 & -0.42 \\
\hline chr1:2148-22 suggestive & RJF & 15.21 & -2 & 0.45 & 0.11 \\
\hline chr1:2155-22 significant & RJF & 19.45 & -2.04 & 0.38 & -0.6 \\
\hline chr1:2155-22 suggestive & RJF & 14.87 & -2.53 & 0.55 & -1.3 \\
\hline chr1:2158-22 significant & RJF & 21.24 & -2.33 & 0.4 & -0.73 \\
\hline chr1:2151-22 suggestive & RJF & 14.23 & -1.45 & 0.32 & -0.91 \\
\hline chr1:2155-22 significant & WL & 15.69 & 9.62 & 2.16 & -0.81 \\
\hline chr1:2152-22 suggestive & RJF & 14.77 & -1.36 & 0.31 & 0.22 \\
\hline chr1:2159-22 significant & RJF & 17.25 & -1.56 & 0.33 & 0.04 \\
\hline chr1:2160-22 significant & RJF & 19.44 & -3.13 & 0.58 & -1.32 \\
\hline chr1:2157-22 significant & RJF & 16.97 & -1.79 & 0.36 & -0.33 \\
\hline chr1:2158-22 significant & WL & 14.5 & 1.6 & 0.37 & -0.18 \\
\hline chr1:2155-22 significant & WL & 14.6 & 10.52 & 2.44 & -0.51 \\
\hline chr1:2148-22 suggestive & RJF & 14.63 & -0.74 & 0.2 & 0.47 \\
\hline chr1:2161-22 suggestive & RJF & 15.07 & -1.48 & 0.32 & -0.23 \\
\hline
\end{tabular}




\begin{tabular}{|c|c|c|c|c|c|}
\hline chr1:2158-22 suggestive & WL & 12.95 & 1.66 & 0.43 & -0.45 \\
\hline chr1:2163-22 significant & WL & 14.6 & 11.8 & 2.62 & 1.71 \\
\hline chr1:2159-22 significant & WL & 13.97 & 11.71 & 2.72 & 0.39 \\
\hline chr1:2163-22 significant & WL & 13.02 & 13.9 & 3.26 & 2.38 \\
\hline chr1:2157-22 suggestive & WL & 13.41 & 10.49 & 2.48 & 0.87 \\
\hline chr1:2158-22 suggestive & WL & 13.83 & 10.08 & 2.37 & 0.11 \\
\hline chr1:2163-22 significant & WL & 13.65 & 14.21 & 3.29 & 1.21 \\
\hline chr1:2157-22 significant & WL & 14.69 & 11.75 & 2.7 & -0.32 \\
\hline chr1:2157-22 significant & WL & 15 & 11.64 & 2.63 & -0.02 \\
\hline chr1:2159-22 significant & WL & 15.11 & 12.61 & 2.82 & 0.31 \\
\hline chr1:2164-22 significant & WL & 15.19 & 13.82 & 3 & 1.9 \\
\hline chr1:2162-22 significant & WL & 14.82 & 11.83 & 2.68 & 0.09 \\
\hline chr1:2161-22 significant & WL & 14.14 & 13.43 & 3.03 & 2.09 \\
\hline chr1:2167-22 significant & RJF & 23.75 & -2.77 & 0.46 & -2.07 \\
\hline chr1:2175-22 significant & RJF & 30.3 & -4.11 & 0.58 & -0.75 \\
\hline chr1:2175-22 significant & WL & 22.27 & 2.09 & 0.37 & 0.2 \\
\hline chr1:2147-22 significant & WL & 12.64 & 2.97 & 0.72 & 0.52 \\
\hline chr1:2158-22 significant & RJF & 16.36 & -1.62 & 0.34 & -0.43 \\
\hline chr1:2158-22 suggestive & RJF & 13.78 & -1.69 & 0.39 & -0.21 \\
\hline chr1:2158-22 suggestive & RJF & 15.48 & -2.46 & 0.55 & -2.31 \\
\hline chr1:2171-22 significant & RJF & 17.99 & -2.95 & 0.58 & -0.7 \\
\hline chr1:2170-22 suggestive & RJF & 14.96 & -0.74 & 0.39 & -0.42 \\
\hline chr1:2172-22 suggestive & RJF & 19.59 & -1.9 & 0.35 & -0.91 \\
\hline chr1:2166-22 suggestive & RJF & 20.19 & -2.49 & 0.47 & -2.54 \\
\hline chr1:2163-22 significant & RJF & 15.63 & -1.06 & 0.25 & 0.26 \\
\hline chr1:2158-22 suggestive & RJF & 12.28 & -1.26 & 0.31 & -0.32 \\
\hline chr1:2142-22 suggestive & RJF & 16.38 & -0.23 & 0.28 & -0.42 \\
\hline chr1:2163-22 significant & RJF & 14.85 & -7.9 & 1.71 & 1.86 \\
\hline chr1:2162-22 significant & RJF & 15.15 & -7.77 & 1.69 & 2.35 \\
\hline chr1:2170-22 suggestive & RJF & 15.08 & -1.25 & 0.33 & 0.75 \\
\hline chr1:2158-23 significant & RJF & 14.07 & -7.24 & 1.67 & 2.46 \\
\hline chr1:2255-23 significant & RJF & 17.83 & -0.61 & 0.29 & 1.94 \\
\hline chr1:2279-23 significant & RJF & 14.31 & -2.19 & 0.48 & -0.58 \\
\hline chr1:2274-23 significant & WL & 8.26 & 3.41 & 1.2 & -2.35 \\
\hline chr1:2283-23 significant & RJF & 18.09 & -1.3 & 0.26 & 0.25 \\
\hline chr1:2273-23 suggestive & WL & 11.79 & 3.28 & 1.02 & -3.1 \\
\hline chr1:2277-23 suggestive & WL & 10.39 & 3.21 & 1.11 & -3.17 \\
\hline chr1:2257-23 suggestive & WL & 9.08 & 2.31 & 0.8 & -1.9 \\
\hline chr1:2277-23 suggestive & RJF & 13.4 & -0.94 & 0.22 & -0.59 \\
\hline chr1:2267-23 suggestive & WL & 5.58 & 2.29 & 0.94 & -3.59 \\
\hline chr1:2268-23 suggestive & WL & 10.54 & 2.04 & 0.71 & -2.21 \\
\hline chr1:2187-23 suggestive & WL & 8.82 & 3.33 & 1.26 & -3.48 \\
\hline chr1:2298-23 suggestive & WL & 12.24 & 2.26 & 0.57 & 0.34 \\
\hline
\end{tabular}




\begin{tabular}{|c|c|c|c|c|c|c|}
\hline \multicolumn{2}{|c|}{ chr1:2282-23 suggestive } & WL & 12.82 & 2.51 & 0.65 & 1.01 \\
\hline \multicolumn{2}{|c|}{ chr1:2337-23 significant } & WL & 16.48 & 4 & 0.83 & -2.13 \\
\hline \multicolumn{2}{|c|}{ chr1:2335-23 suggestive } & WL & 15.22 & 1.71 & 0.38 & -1.12 \\
\hline \multirow{2}{*}{\multicolumn{2}{|c|}{$\begin{array}{l}\text { chr1:2336-23 significant } \\
\text { chr1:2339-23 suggestive }\end{array}$}} & WL & 15.31 & 3.8 & 0.84 & 0.74 \\
\hline & & WL & 17.17 & 3.13 & 0.68 & -2.56 \\
\hline \multicolumn{2}{|c|}{ chr1:2342-23 suggestive } & WL & 22.58 & 2.18 & 0.44 & -2.05 \\
\hline \multicolumn{2}{|c|}{ chr1:2344-23 suggestive } & WL & 20.66 & 3.3 & 0.63 & -2.2 \\
\hline \multicolumn{2}{|c|}{ chr1:2345-23 suggestive } & WL & 24.11 & 5.45 & 1.02 & -3.87 \\
\hline \multicolumn{2}{|c|}{ chr1:2341-23 suggestive } & WL & 16.45 & 1.99 & 0.44 & -1.64 \\
\hline chr2:0-25 & significant & RJF & 17.24 & -2.14 & 0.44 & -0.64 \\
\hline chr2:0-26 & suggestive & RJF & 14.66 & -0.12 & 0.68 & -3.99 \\
\hline chr2:0-32 & significant & RJF & 16.88 & -2.34 & 0.47 & -0.46 \\
\hline chr2:0-27 & suggestive & WL & 21.94 & 4.71 & 1.03 & -6.07 \\
\hline chr2:0-28 & suggestive & WL & 17.58 & 37.73 & 8.91 & -31.72 \\
\hline chr2:0-30 & suggestive & WL & 16.02 & 27.99 & 6.59 & -19.2 \\
\hline chr2:0-34 & significant & WL & 17.15 & 4.59 & 1.58 & -8.19 \\
\hline chr2:0-30 & suggestive & WL & 16.91 & 22.29 & 5.29 & -17.82 \\
\hline chr2:0-32 & suggestive & WL & 19.17 & 9.61 & 2.6 & -12.87 \\
\hline chr2:0-35 & suggestive & WL & 20.71 & 3.93 & 0.8 & -3.18 \\
\hline chr2:0-74 & suggestive & WL & 18.79 & 2.97 & 0.98 & -2.74 \\
\hline chr2:0-97 & significant & RJF & 16.44 & -1.43 & 0.31 & -0.59 \\
\hline chr2:3-67 & significant & RJF & 17.86 & -2.75 & 0.58 & -0.18 \\
\hline chr2:17-58 & significant & RJF & 25.66 & -0.07 & 0.01 & -0.08 \\
\hline chr2:17-64 & significant & RJF & 20.16 & -1.67 & 0.39 & 0.75 \\
\hline chr2:20-59 & significant & RJF & 20.38 & -2.39 & 0.54 & 0.9 \\
\hline chr2:14-60 & significant & RJF & 17.8 & -1.35 & 0.83 & 1.26 \\
\hline chr2:23-60 & suggestive & RJF & 14.62 & -1.3 & 0.33 & 0.18 \\
\hline chr2:10-65 & suggestive & RJF & 14.87 & -1.51 & 0.44 & 0.71 \\
\hline chr2:19-63 & significant & RJF & 17.89 & -1.32 & 0.27 & -0.23 \\
\hline chr2:14-85 & significant & RJF & 16 & -1.03 & 0.22 & -0.2 \\
\hline chr2:0-95 & suggestive & WL & 14.63 & 1.16 & 0.65 & -3.5 \\
\hline chr2:11-96 & significant & RJF & 17.67 & -1.73 & 0.38 & -1.82 \\
\hline chr2:50-110 & significant & RJF & 21.91 & -2.02 & 0.39 & -1.55 \\
\hline chr2:31-103 & significant & RJF & 19.32 & -1.58 & 0.31 & -0.03 \\
\hline chr2:55-108 & significant & RJF & 18.49 & -1.58 & 0.36 & -2.09 \\
\hline chr2:3-115 & suggestive & RJF & 11.92 & -2.28 & 0.56 & -1.07 \\
\hline chr2:51-117 & suggestive & RJF & 16.26 & -0.48 & 0.39 & -2.52 \\
\hline chr2:51-122 & suggestive & RJF & 13.57 & 0 & 0.45 & -2.46 \\
\hline chr2:47-111 & significant & RJF & 17.31 & -1.53 & 0.31 & -0.19 \\
\hline chr2:57-97 & significant & RJF & 25.6 & -2.65 & 0.42 & -1.73 \\
\hline chr2:56-107 & significant & RJF & 21.24 & -1.8 & 0.34 & -1.71 \\
\hline chr2:50-117 & significant & RJF & 15.66 & -2.01 & 0.42 & -0.69 \\
\hline chr2:57-97 & suggestive & RJF & 14.55 & -0.31 & 0.26 & -1.59 \\
\hline
\end{tabular}




\begin{tabular}{|c|c|c|c|c|c|c|}
\hline chr2:57-99 & suggestive & RJF & 13.84 & -0.69 & 0.35 & -2.04 \\
\hline chr2:57-112 & significant & RJF & 15 & -1.57 & 0.47 & -2.58 \\
\hline chr2:57-104 & significant & RJF & 15.41 & -1.44 & 0.46 & -2.63 \\
\hline chr2:54-110 & significant & RJF & 16.95 & -1.49 & 0.32 & -1.29 \\
\hline chr2:62-96 & suggestive & RJF & 17.78 & -0.76 & 0.41 & -2.87 \\
\hline chr2:62-100 & significant & RJF & 24.09 & -1.74 & 0.3 & 0.17 \\
\hline chr2:57-98 & significant & WL & 15.99 & 0.11 & 0.38 & -2.26 \\
\hline chr2:63-95 & suggestive & RJF & 18.9 & -0.47 & 0.35 & -2.43 \\
\hline chr2:63-97 & suggestive & RJF & 20.18 & -0.74 & 0.34 & -2.47 \\
\hline chr2:62-106 & significant & RJF & 17.66 & -2.55 & 0.51 & -1.96 \\
\hline chr2:60-116 & significant & RJF & 16.87 & -1.61 & 0.33 & -0.84 \\
\hline chr2:58-97 & suggestive & RJF & 20.65 & -0.18 & 0.34 & -2.49 \\
\hline chr2:60-102 & suggestive & RJF & 18.58 & -0.48 & 0.24 & -1.69 \\
\hline chr2:63-111 & suggestive & RJF & 18.46 & -0.15 & 0.27 & -1.84 \\
\hline chr2:60-98 & suggestive & RJF & 17.88 & -0.53 & 0.33 & -2.27 \\
\hline chr2:58-107 & significant & RJF & 16.93 & -1.93 & 0.4 & -1.42 \\
\hline chr2:56-97 & suggestive & RJF & 13.88 & -1.02 & 0.33 & -1.79 \\
\hline chr2:66-97 & suggestive & RJF & 19.17 & -1.03 & 0.27 & -1.76 \\
\hline chr2:63-96 & significant & RJF & 15.06 & -1.93 & 0.48 & -2.28 \\
\hline chr2:62-118 & suggestive & RJF & 14.29 & -0.62 & 0.4 & -2.39 \\
\hline chr2:68-94 & significant & RJF & 26.45 & -2.2 & 0.32 & -0.5 \\
\hline chr2:62-97 & significant & WL & 16 & 0.81 & 0.76 & -4.08 \\
\hline chr2:65-103 & suggestive & RJF & 14.9 & -1.16 & 0.45 & -2.68 \\
\hline chr2:64-98 & suggestive & WL & 10.82 & 0.73 & 0.44 & -2.54 \\
\hline chr2:66-96 & suggestive & RJF & 20.72 & -1.38 & 0.45 & -3.29 \\
\hline chr2:68-94 & suggestive & RJF & 19.6 & -0.54 & 0.28 & -2.03 \\
\hline chr2:68-97 & suggestive & WL & 19.17 & 0.49 & 0.37 & -2.21 \\
\hline chr2:68-98 & suggestive & RJF & 14.58 & -0.39 & 0.51 & -3.07 \\
\hline chr2:69-94 & suggestive & RJF & 19.51 & -0.78 & 0.4 & -2.84 \\
\hline chr2:69-96 & suggestive & RJF & 20.77 & -0.22 & 0.5 & -3.59 \\
\hline chr2:64-98 & suggestive & RJF & 24.45 & -1.69 & 0.44 & -2.29 \\
\hline chr2:69-97 & suggestive & WL & 19.47 & 0.26 & 0.21 & -1.3 \\
\hline chr2:70-96 & significant & RJF & 20.99 & -0.83 & 0.32 & -2.43 \\
\hline chr2:72-98 & significant & WL & 22.16 & 1.02 & 0.34 & -1.66 \\
\hline chr2:75-103 & significant & RJF & 17.09 & -4.03 & 0.81 & -0.67 \\
\hline chr2:73-96 & significant & WL & 18.19 & 0.14 & 0.38 & -2.41 \\
\hline chr2:70-96 & significant & RJF & 24.04 & -2.04 & 0.55 & 0.01 \\
\hline chr2:66-96 & significant & WL & 21.92 & 0.51 & 0.48 & -0.64 \\
\hline chr2:72-97 & suggestive & RJF & 13.96 & -0.56 & 0.58 & -3.41 \\
\hline chr2:71-106 & suggestive & RJF & 14.68 & -0.21 & 0.59 & -3.5 \\
\hline chr2:68-114 & significant & RJF & 16.05 & -0.38 & 0.34 & -2.17 \\
\hline chr2:71-99 & suggestive & RJF & 13.79 & -0.79 & 0.41 & -2.44 \\
\hline chr2:66-97 & suggestive & RJF & 14.27 & -0.42 & 0.5 & -3.01 \\
\hline
\end{tabular}




\begin{tabular}{|c|c|c|c|c|c|c|}
\hline chr2:69-96 & significant & RJF & 15.52 & -0.17 & 0.35 & -2.13 \\
\hline chr2:66-110 & suggestive & RJF & 12.38 & -1.96 & 0.47 & -1.53 \\
\hline chr2:62-96 & suggestive & RJF & 14.14 & -0.37 & 0.36 & -2.1 \\
\hline chr2:72-114 & significant & WL & 14.65 & 0.7 & 0.55 & -2.67 \\
\hline chr2:74-96 & significant & RJF & 15.39 & -0.31 & 0.26 & -1.64 \\
\hline chr2:69-121 & suggestive & RJF & 14.47 & -0.72 & 0.44 & -2.6 \\
\hline chr2:71-96 & suggestive & WL & 21.13 & 0.11 & 0.28 & -1.95 \\
\hline chr2:71-97 & significant & RJF & 21.96 & -0.15 & 0.28 & -2.15 \\
\hline chr2:69-93 & suggestive & RJF & 15.23 & -0.5 & 0.39 & -0.3 \\
\hline chr2:79-96 & suggestive & RJF & 17.07 & -0.3 & 0.37 & -1.19 \\
\hline chr2:70-96 & suggestive & RJF & 20.59 & -0.19 & 0.49 & -3.53 \\
\hline chr2:73-95 & suggestive & WL & 10.81 & 0.49 & 0.6 & 0.24 \\
\hline chr2:75-97 & suggestive & WL & 19.77 & 0.06 & 0.4 & -2.68 \\
\hline chr2:70-95 & significant & RJF & 22.86 & -1.23 & 0.43 & -3.38 \\
\hline chr2:59-96 & suggestive & RJF & 18.37 & -1.03 & 0.34 & -2.25 \\
\hline chr2:73-97 & suggestive & WL & 20.3 & 0.18 & 0.52 & -3.54 \\
\hline chr2:75-97 & suggestive & RJF & 18.45 & -1.01 & 0.55 & -3.9 \\
\hline chr2:66-97 & suggestive & RJF & 19.31 & -0.92 & 0.44 & -3.14 \\
\hline chr2:75-106 & suggestive & RJF & 18.61 & -0.18 & 0.36 & -2.47 \\
\hline chr2:69-96 & suggestive & RJF & 18.85 & -0.13 & 0.29 & -2.01 \\
\hline chr2:75-97 & suggestive & WL & 21.91 & 0.27 & 0.36 & -2.46 \\
\hline chr2:70-95 & significant & RJF & 22.44 & -0.26 & 0.11 & -0.82 \\
\hline chr2:67-95 & suggestive & RJF & 18.25 & -0.04 & 0.55 & -3.72 \\
\hline chr2:80-96 & suggestive & WL & 18.53 & 0.13 & 0.27 & -1.68 \\
\hline chr2:72-95 & suggestive & RJF & 20.95 & -1.5 & 0.68 & -5.06 \\
\hline chr2:72-97 & suggestive & RJF & 19.34 & -0.18 & 0.29 & -2.03 \\
\hline chr2:74-96 & suggestive & WL & 22.53 & 0.95 & 0.43 & -2.54 \\
\hline chr2:73-96 & suggestive & RJF & 19.7 & -0.02 & 0.52 & -3.56 \\
\hline chr2:77-95 & suggestive & WL & 16.52 & 0.17 & 0.34 & -2.08 \\
\hline chr2:68-110 & significant & WL & 15.4 & 1.04 & 0.44 & -1.8 \\
\hline chr2:73-126 & suggestive & WL & 14.22 & 1.33 & 0.41 & -1.05 \\
\hline chr2:67-110 & suggestive & RJF & 14.49 & -0.31 & 0.45 & -2.66 \\
\hline chr2:68-98 & significant & RJF & 23.36 & -0.46 & 0.41 & -3.31 \\
\hline chr2:67-97 & suggestive & WL & 22.5 & 0.88 & 0.45 & -1.14 \\
\hline chr2:76-97 & suggestive & RJF & 27.01 & -1.22 & 0.69 & -2.79 \\
\hline chr2:76-96 & suggestive & RJF & 17.63 & -0.29 & 0.32 & -0.13 \\
\hline chr2:70-106 & suggestive & RJF & 18.73 & -0.2 & 0.31 & -2.19 \\
\hline chr2:62-101 & suggestive & WL & 19.87 & 0.3 & 0.42 & -2.72 \\
\hline chr2:70-98 & suggestive & WL & 19.84 & 0.05 & 0.17 & -1.17 \\
\hline chr2:72-108 & suggestive & RJF & 19.96 & -0.79 & 0.39 & -2.83 \\
\hline chr2:63-103 & suggestive & WL & 19.1 & 0.61 & 0.43 & -2.5 \\
\hline chr2:72-103 & suggestive & RJF & 17.91 & -0.08 & 0.39 & -2.55 \\
\hline chr2:77-98 & suggestive & WL & 20.4 & 0.57 & 0.59 & -3.87 \\
\hline
\end{tabular}




\begin{tabular}{|c|c|c|c|c|c|c|}
\hline chr2:79-122 & suggestive & WL & 18.51 & 0.97 & 0.6 & -3.39 \\
\hline chr2:70-98 & suggestive & WL & 21.15 & 0.35 & 0.22 & -1.32 \\
\hline chr2:76-108 & suggestive & WL & 15.05 & 0.41 & 0.35 & -2.12 \\
\hline chr2:77-105 & suggestive & WL & 19.72 & 0.41 & 0.45 & -2.88 \\
\hline chr2:64-108 & suggestive & WL & 17.86 & 0.94 & 0.58 & -3.17 \\
\hline chr2:63-114 & significant & WL & 17.35 & 0.36 & 0.37 & -2.14 \\
\hline chr2:73-104 & significant & RJF & 19.08 & -0.4 & 0.29 & -2.09 \\
\hline chr2:73-120 & significant & WL & 19.73 & 1.16 & 0.3 & -0.94 \\
\hline chr2:66-120 & significant & WL & 19.79 & 1.14 & 0.36 & -1.52 \\
\hline chr2:70-116 & suggestive & RJF & 15.01 & -0.03 & 0.18 & -1.03 \\
\hline chr2:61-134 & significant & WL & 15.73 & 0.61 & 0.33 & -1.57 \\
\hline chr2:63-117 & suggestive & WL & 14.97 & 0.36 & 0.52 & -2.81 \\
\hline chr2:65-114 & suggestive & RJF & 14.31 & -0.58 & 0.45 & -2.71 \\
\hline chr2:70-105 & significant & WL & 23.12 & 0.18 & 0.33 & -2.42 \\
\hline chr2:77-106 & suggestive & WL & 20.27 & 0.09 & 0.51 & -3.51 \\
\hline chr2:66-108 & suggestive & WL & 18.99 & 0.09 & 0.3 & -1.98 \\
\hline chr2:72-112 & suggestive & RJF & 20.02 & -0.94 & 0.36 & -2.59 \\
\hline chr2:72-104 & suggestive & WL & 20.73 & 0.42 & 0.36 & -2.36 \\
\hline chr2:64-105 & suggestive & WL & 20.45 & 0.24 & 0.56 & -3.8 \\
\hline chr2:73-114 & suggestive & WL & 14.26 & 0.68 & 0.44 & -2.58 \\
\hline chr2:70-111 & suggestive & RJF & 19.17 & -0.32 & 0.44 & -3.18 \\
\hline chr2:68-105 & suggestive & RJF & 18.7 & -0.11 & 0.27 & -1.84 \\
\hline chr2:74-114 & suggestive & WL & 19.14 & 0.68 & 0.42 & -2.41 \\
\hline chr2:58-129 & suggestive & RJF & 9.6 & -0.24 & 0.36 & -1.67 \\
\hline chr2:54-122 & suggestive & RJF & 14.22 & -0.22 & 0.44 & -2.54 \\
\hline chr2:65-115 & suggestive & WL & 16.72 & 0.3 & 0.37 & -2.17 \\
\hline chr2:58-121 & suggestive & RJF & 15.74 & -0.18 & 0.37 & -2.25 \\
\hline chr2:69-117 & suggestive & WL & 14.85 & 0.85 & 0.46 & -2.13 \\
\hline chr2:60-123 & suggestive & RJF & 13.44 & -0.42 & 0.48 & -2.71 \\
\hline chr2:74-117 & significant & WL & 16.3 & 0.81 & 0.4 & -1.88 \\
\hline chr2:69-114 & significant & WL & 25.76 & 2 & 0.41 & -1.36 \\
\hline chr2:69-119 & significant & RJF & 15.67 & -0.39 & 0.4 & -2.48 \\
\hline chr2:71-118 & significant & WL & 16.29 & 0.83 & 0.33 & -1.42 \\
\hline chr2:73-123 & suggestive & WL & 14.56 & 1.55 & 0.4 & -0.58 \\
\hline chr2:68-117 & suggestive & RJF & 14.74 & -0.97 & 0.4 & -2.37 \\
\hline chr2:78-117 & significant & WL & 23.13 & 0.95 & 0.49 & -3.12 \\
\hline chr2:76-116 & suggestive & WL & 18.85 & 0.55 & 0.49 & -2.97 \\
\hline chr2:76-111 & suggestive & WL & 14.93 & 0.94 & 0.38 & -1.91 \\
\hline chr2:78-111 & suggestive & WL & 20.59 & 0.4 & 0.27 & -1.62 \\
\hline chr2:71-114 & significant & RJF & 28.33 & -2.03 & 0.28 & -0.74 \\
\hline chr2:64-123 & suggestive & WL & 13.36 & 1.55 & 0.63 & -2.28 \\
\hline chr2:66-123 & suggestive & WL & 14.29 & 1.58 & 0.55 & -1.81 \\
\hline chr2:44-117 & significant & RJF & 14.76 & -1.8 & 0.44 & -1.91 \\
\hline
\end{tabular}




\begin{tabular}{|c|c|c|c|c|c|c|}
\hline chr2:61-118 & significant & WL & 11.1 & 0.03 & 0.29 & -1.42 \\
\hline chr2:71-116 & significant & WL & 21.44 & 0.01 & 0.4 & -2.87 \\
\hline chr2:44-115 & suggestive & RJF & 14.99 & -0.78 & 0.55 & -1.16 \\
\hline chr2:63-120 & suggestive & RJF & 16.03 & -0.03 & 0.47 & -2.89 \\
\hline chr2:62-108 & suggestive & RJF & 15.83 & -0.73 & 0.38 & -2.34 \\
\hline chr2:69-123 & suggestive & WL & 17.6 & 0.29 & 0.44 & -2.68 \\
\hline chr2:62-119 & suggestive & WL & 13.76 & 0.76 & 0.44 & -1.97 \\
\hline chr2:58-112 & suggestive & WL & 14.11 & 0.3 & 0.46 & -2.48 \\
\hline chr2:60-118 & suggestive & WL & 17.22 & 0.69 & 0.45 & -2.46 \\
\hline chr2:63-116 & suggestive & WL & 17.8 & 1.43 & 0.63 & -3.12 \\
\hline chr2:67-116 & suggestive & WL & 15.73 & 0.25 & 0.36 & -2.04 \\
\hline chr2:63-126 & suggestive & WL & 13.68 & 0.87 & 0.51 & -2.67 \\
\hline chr2:59-125 & suggestive & RJF & 15.89 & -0.59 & 0.42 & -2.65 \\
\hline chr2:56-122 & suggestive & WL & 16.9 & 0.38 & 0.55 & -3.25 \\
\hline chr2:60-120 & suggestive & WL & 15.59 & 0.94 & 0.53 & -2.6 \\
\hline chr2:63-122 & suggestive & WL & 17.22 & 0.59 & 0.38 & -2.03 \\
\hline chr2:62-124 & suggestive & WL & 19.06 & 1.53 & 0.6 & -0.71 \\
\hline chr2:75-120 & significant & WL & 16.61 & 0.56 & 0.42 & -2.21 \\
\hline chr2:64-114 & significant & WL & 18.04 & 1.8 & 0.64 & -2.85 \\
\hline chr2:55-111 & significant & RJF & 17.62 & -1.44 & 0.43 & -2.62 \\
\hline chr2:58-119 & significant & WL & 19.26 & 1.87 & 0.41 & -0.8 \\
\hline chr2:71-119 & significant & WL & 20.08 & 2.13 & 0.43 & -0.51 \\
\hline chr2:76-112 & significant & RJF & 38.34 & -1.59 & 0.18 & -0.12 \\
\hline chr2:27-114 & significant & RJF & 11.21 & -1.3 & 0.34 & -0.97 \\
\hline chr2:64-121 & significant & WL & 15.16 & 0.5 & 0.53 & -2.85 \\
\hline chr2:71-118 & suggestive & WL & 13.65 & 0.26 & 0.23 & -1.11 \\
\hline chr2:58-121 & suggestive & RJF & 12.65 & -0.69 & 0.32 & -1.72 \\
\hline chr2:66-114 & significant & WL & 13.77 & 0.34 & 0.35 & -1.77 \\
\hline chr2:67-122 & suggestive & WL & 14.42 & 0.95 & 0.58 & -2.74 \\
\hline chr2:71-116 & significant & RJF & 26.66 & -0.19 & 0.43 & -3.64 \\
\hline chr2:71-113 & significant & WL & 21.19 & 0.52 & 0.49 & -3.27 \\
\hline chr2:58-122 & suggestive & WL & 17.33 & 0.02 & 0.63 & -2.21 \\
\hline chr2:67-118 & suggestive & WL & 19.8 & 1.04 & 0.59 & -3.44 \\
\hline chr2:75-122 & suggestive & WL & 20.11 & 0.37 & 0.27 & -1.7 \\
\hline chr2:62-119 & suggestive & RJF & 16.25 & -0.1 & 0.63 & -3.88 \\
\hline chr2:67-115 & suggestive & WL & 17.96 & 0.3 & 0.36 & -2.18 \\
\hline chr2:67-117 & suggestive & WL & 17.63 & 0.52 & 0.44 & -2.53 \\
\hline chr2:67-115 & suggestive & WL & 19.49 & 0.08 & 0.51 & -3.48 \\
\hline chr2:72-115 & suggestive & WL & 17.61 & 0.58 & 0.43 & -2.4 \\
\hline chr2:47-114 & suggestive & WL & 16.17 & 0.05 & 0.52 & -3.14 \\
\hline chr2:50-119 & suggestive & RJF & 14.5 & -1.15 & 0.39 & -2.19 \\
\hline chr2:71-118 & suggestive & WL & 17.01 & 0.84 & 0.4 & -1.93 \\
\hline chr2:67-113 & suggestive & WL & 16.24 & 0.18 & 0.31 & -1.82 \\
\hline
\end{tabular}




\begin{tabular}{|c|c|c|c|c|c|c|}
\hline chr2:70-122 & suggestive & WL & 19.43 & 1.86 & 0.69 & -3.33 \\
\hline chr2:51-114 & suggestive & WL & 16.29 & 0.66 & 0.48 & -2.58 \\
\hline chr2:63-117 & suggestive & WL & 17.12 & 0.94 & 0.51 & -2.7 \\
\hline chr2:67-118 & suggestive & WL & 20.95 & 0.84 & 0.51 & -3.17 \\
\hline chr2:72-114 & suggestive & WL & 19.58 & 1.19 & 0.87 & -5.43 \\
\hline chr2:65-119 & suggestive & RJF & 16.31 & -0.14 & 0.54 & -3.4 \\
\hline chr2:76-120 & suggestive & WL & 18.08 & 0.68 & 0.43 & -2.37 \\
\hline chr2:73-119 & suggestive & WL & 18.43 & 1.06 & 0.37 & -1.61 \\
\hline chr2:70-115 & suggestive & WL & 12.4 & 0.84 & 0.54 & -3.19 \\
\hline chr2:76-119 & suggestive & WL & 18.67 & 0.58 & 0.63 & -3.91 \\
\hline chr2:77-126 & suggestive & WL & 16.47 & 0.42 & 0.21 & -1.17 \\
\hline chr2:74-121 & suggestive & WL & 19.23 & 0.27 & 0.4 & -2.57 \\
\hline chr2:78-117 & suggestive & WL & 19.62 & 0.67 & 0.42 & -2.49 \\
\hline chr2:75-118 & suggestive & WL & 19.15 & 0.31 & 0.5 & -3.25 \\
\hline chr2:66-116 & suggestive & WL & 17.24 & 0.42 & 0.42 & -2.44 \\
\hline chr2:70-121 & suggestive & WL & 18.79 & 0.19 & 0.39 & -2.53 \\
\hline chr2:62-110 & suggestive & RJF & 16.58 & -0.29 & 0.34 & -2.22 \\
\hline chr2:73-124 & suggestive & WL & 17.92 & 1.12 & 0.56 & -2.91 \\
\hline chr2:69-115 & suggestive & WL & 19.56 & 1.17 & 0.6 & -3.37 \\
\hline chr2:70-119 & suggestive & WL & 17.42 & 0.3 & 0.42 & -2.5 \\
\hline chr2:76-115 & suggestive & WL & 18.2 & 0.62 & 0.28 & -1.41 \\
\hline chr2:71-132 & significant & WL & 15.1 & 0.16 & 0.31 & -1.72 \\
\hline chr2:70-125 & suggestive & WL & 14.31 & 1.05 & 1.25 & -6.46 \\
\hline chr2:81-132 & significant & RJF & 19.5 & -0.67 & 0.53 & -1.24 \\
\hline chr2:80-120 & suggestive & WL & 16.68 & 0.92 & 0.7 & -0.89 \\
\hline chr2:80-121 & significant & WL & 16.37 & 0.6 & 0.44 & -2.36 \\
\hline chr2:74-119 & significant & WL & 17.56 & 1.74 & 0.72 & -3.37 \\
\hline chr2:67-113 & significant & WL & 15.53 & 0.27 & 0.29 & -1.57 \\
\hline chr2:74-120 & suggestive & WL & 14.37 & 0.99 & 0.28 & -0.64 \\
\hline chr2:72-120 & suggestive & WL & 15.09 & 1.11 & 0.64 & -3.07 \\
\hline chr2:85-120 & significant & WL & 23.86 & 0.64 & 0.23 & -1.32 \\
\hline chr2:47-114 & suggestive & WL & 16.86 & 0.79 & 0.55 & -0.94 \\
\hline chr2:79-122 & significant & WL & 19.05 & 0.49 & 0.33 & -1.92 \\
\hline chr2:87-109 & significant & WL & 28.39 & 0.41 & 0.38 & -3.13 \\
\hline chr2:84-116 & significant & WL & 21.82 & 1.03 & 0.43 & -2.49 \\
\hline chr2:85-117 & significant & WL & 25.27 & 0.81 & 0.27 & -1.62 \\
\hline chr2:82-121 & suggestive & WL & 22 & 1.61 & 1.02 & -2.24 \\
\hline chr2:76-113 & significant & WL & 22.52 & 0.58 & 0.36 & -2.41 \\
\hline chr2:75-111 & significant & WL & 20.39 & 0.02 & 0.32 & -2.23 \\
\hline chr2:79-124 & suggestive & WL & 17.8 & 0.77 & 0.36 & -1.85 \\
\hline chr2:83-116 & suggestive & WL & 20.55 & 1.19 & 0.57 & -3.35 \\
\hline chr2:71-117 & suggestive & WL & 18.31 & 0.45 & 0.49 & -3 \\
\hline chr2:81-116 & suggestive & WL & 21.57 & 0.38 & 0.36 & -2.4 \\
\hline
\end{tabular}




\begin{tabular}{|c|c|c|c|c|c|c|}
\hline chr2:68-118 & suggestive & WL & 15.75 & 0.71 & 0.56 & -2.96 \\
\hline chr2:75-117 & suggestive & WL & 18.25 & 0.71 & 0.45 & -2.51 \\
\hline chr2:77-117 & suggestive & WL & 19.1 & 1.6 & 0.49 & -2.01 \\
\hline chr2:83-114 & suggestive & WL & 18.61 & 0.66 & 0.42 & -2.41 \\
\hline chr2:59-114 & suggestive & RJF & 16.97 & -0.11 & 0.37 & -2.36 \\
\hline chr2:73-116 & suggestive & WL & 17.93 & 0.37 & 0.37 & -2.21 \\
\hline chr2:60-113 & suggestive & WL & 17.74 & 1.19 & 0.54 & -2.65 \\
\hline chr2:62-116 & suggestive & RJF & 21.26 & -0.85 & 0.39 & -2.97 \\
\hline chr2:63-113 & significant & RJF & 19.28 & -0.13 & 0.22 & -1.51 \\
\hline chr2:69-112 & suggestive & WL & 16.08 & 0.31 & 0.36 & -2.08 \\
\hline chr2:85-116 & suggestive & WL & 20.35 & 1.33 & 0.53 & -2.79 \\
\hline chr2:80-118 & suggestive & WL & 18.7 & 0.5 & 0.29 & -1.64 \\
\hline chr2:81-118 & suggestive & WL & 18.64 & 0.51 & 0.39 & -2.34 \\
\hline chr2:76-122 & suggestive & WL & 17.08 & 0.72 & 0.33 & -1.6 \\
\hline chr2:72-127 & suggestive & WL & 16.3 & 0.04 & 0.49 & -3 \\
\hline chr2:66-115 & suggestive & WL & 16.57 & 0.38 & 0.46 & -2.76 \\
\hline chr2:73-116 & suggestive & WL & 17.89 & 1.22 & 0.5 & -2.42 \\
\hline chr2:81-116 & significant & WL & 20.44 & 0.58 & 0.39 & -2.39 \\
\hline chr2:81-115 & suggestive & WL & 22.03 & 1.13 & 0.31 & -1.37 \\
\hline chr2:72-121 & suggestive & WL & 15.84 & 1 & 0.52 & -2.47 \\
\hline chr2:80-118 & suggestive & WL & 18.44 & 1.77 & 0.54 & -2.14 \\
\hline chr2:65-117 & suggestive & WL & 19.44 & 0.67 & 0.49 & -2.94 \\
\hline chr2:76-120 & suggestive & WL & 17.74 & 1 & 0.42 & -2.02 \\
\hline chr2:71-112 & significant & WL & 21.34 & 0.67 & 0.43 & -2.7 \\
\hline chr2:78-124 & suggestive & WL & 18.68 & 1.15 & 0.73 & -4.2 \\
\hline chr2:82-112 & suggestive & WL & 18.08 & 0.87 & 0.29 & -1.2 \\
\hline chr2:69-113 & suggestive & WL & 18.18 & 0.63 & 0.44 & -2.52 \\
\hline chr2:78-116 & suggestive & WL & 21.17 & 1.52 & 0.51 & -2.6 \\
\hline chr2:71-115 & suggestive & WL & 15.63 & 0.62 & 0.55 & -2.96 \\
\hline chr2:79-119 & suggestive & WL & 20.39 & 0.71 & 0.33 & -1.88 \\
\hline chr2:73-116 & suggestive & WL & 19.1 & 0.68 & 0.43 & -2.55 \\
\hline chr2:77-114 & suggestive & WL & 19.67 & 0.34 & 0.39 & -2.52 \\
\hline chr2:76-115 & suggestive & RJF & 17.82 & -0.03 & 0.43 & -2.79 \\
\hline chr2:63-124 & suggestive & WL & 17.84 & 0.72 & 0.44 & -2.4 \\
\hline chr2:83-116 & suggestive & WL & 21.03 & 1.55 & 0.47 & -2.12 \\
\hline chr2:82-127 & suggestive & WL & 18.95 & 0.82 & 0.37 & -1.97 \\
\hline chr2:72-119 & suggestive & $W L$ & 17.37 & 1.36 & 0.68 & -3.4 \\
\hline chr2:68-118 & suggestive & RJF & 18.94 & -0.04 & 0.57 & -3.84 \\
\hline chr2:74-116 & suggestive & WL & 17.89 & 0.61 & 0.34 & -1.84 \\
\hline chr2:67-119 & suggestive & WL & 15.93 & 0.35 & 0.43 & -2.4 \\
\hline chr2:73-116 & suggestive & WL & 19.22 & 2.07 & 0.57 & -2.07 \\
\hline chr2:65-115 & suggestive & WL & 18.65 & 0.36 & 0.17 & -0.91 \\
\hline chr2:73-124 & suggestive & WL & 19.14 & 0.65 & 0.37 & -2.13 \\
\hline
\end{tabular}




\begin{tabular}{|c|c|c|c|c|c|c|}
\hline chr2:76-116 & suggestive & WL & 19.79 & 0.12 & 0.33 & -2.24 \\
\hline chr2:77-117 & significant & RJF & 14.93 & -0.1 & 0.51 & -3.04 \\
\hline chr2:65-122 & significant & WL & 14.5 & 0.12 & 0.46 & -2.6 \\
\hline chr2:75-125 & suggestive & WL & 12.84 & 1.62 & 0.49 & -1.01 \\
\hline chr2:81-120 & suggestive & WL & 14.53 & 0.62 & 0.32 & -1.46 \\
\hline chr2:68-116 & significant & WL & 15.27 & 1.08 & 0.35 & -1.16 \\
\hline chr2:70-117 & significant & WL & 16.51 & 0.79 & 0.41 & -2.1 \\
\hline chr2:90-111 & significant & WL & 24.95 & 1.93 & 0.49 & -2.45 \\
\hline chr2:92-116 & significant & WL & 22.15 & 0.51 & 0.3 & -1.95 \\
\hline chr2:89-116 & suggestive & WL & 21.7 & 1.31 & 0.39 & -1.8 \\
\hline chr2:85-117 & suggestive & WL & 20.14 & 1.44 & 0.56 & -1.79 \\
\hline chr2:85-118 & suggestive & WL & 17.68 & 0.65 & 0.4 & -2.19 \\
\hline chr2:86-113 & significant & WL & 22.25 & 0.97 & 0.54 & -3.45 \\
\hline chr2:92-115 & significant & WL & 22.62 & 1.07 & 0.45 & -2.8 \\
\hline chr2:84-115 & suggestive & WL & 18.04 & 1.4 & 0.58 & -2.81 \\
\hline chr2:83-117 & suggestive & WL & 17.65 & 0.7 & 0.37 & -1.98 \\
\hline chr2:92-110 & suggestive & RJF & 18.74 & -0.08 & 0.31 & -0.39 \\
\hline chr2:69-117 & suggestive & WL & 20.16 & 0.85 & 0.3 & -1.46 \\
\hline chr2:89-118 & significant & WL & 24.23 & 1.83 & 0.53 & -2.76 \\
\hline chr2:92-114 & significant & WL & 23.68 & 0.45 & 0.29 & -2.04 \\
\hline chr2:81-119 & suggestive & WL & 17.94 & 0.67 & 0.51 & -2.95 \\
\hline chr2:68-116 & suggestive & WL & 21.01 & 0.45 & 0.42 & -2.76 \\
\hline chr2:76-113 & suggestive & WL & 17.88 & 0.81 & 0.4 & -2.03 \\
\hline chr2:90-117 & suggestive & WL & 19.36 & 0.08 & 0.31 & -2.1 \\
\hline chr2:73-124 & suggestive & WL & 17.16 & 0.6 & 0.44 & -2.48 \\
\hline chr2:81-120 & suggestive & WL & 18.84 & 1.02 & 0.62 & -3.54 \\
\hline chr2:76-115 & significant & WL & 19.73 & 1.35 & 0.51 & -2.59 \\
\hline chr2:79-120 & suggestive & WL & 16.8 & 1.05 & 0.58 & -2.98 \\
\hline chr2:82-122 & suggestive & WL & 16.11 & 0.73 & 0.37 & -1.87 \\
\hline chr2:76-117 & suggestive & WL & 18.83 & 1.24 & 0.43 & -1.94 \\
\hline chr2:79-113 & suggestive & WL & 19.19 & 1.04 & 0.56 & -3.21 \\
\hline chr2:84-115 & suggestive & WL & 18.17 & 0.05 & 0.48 & -3.1 \\
\hline chr2:80-116 & suggestive & WL & 18.64 & 1.59 & 0.5 & -2.04 \\
\hline chr2:74-118 & suggestive & WL & 20.46 & 1.7 & 0.85 & -4.94 \\
\hline chr2:76-119 & suggestive & WL & 17.83 & 0.69 & 0.43 & -2.47 \\
\hline chr2:65-116 & significant & WL & 20.18 & 1.21 & 0.54 & -3 \\
\hline chr2:76-121 & suggestive & WL & 20.66 & 0.44 & 0.46 & -3.04 \\
\hline chr2:85-117 & suggestive & WL & 20.33 & 0.26 & 0.43 & -2.94 \\
\hline chr2:71-118 & suggestive & WL & 17.1 & 0.09 & 0.43 & -2.7 \\
\hline chr2:88-119 & suggestive & WL & 21.8 & 0.5 & 0.23 & -1.42 \\
\hline chr2:77-116 & suggestive & WL & 17.94 & 1.51 & 0.61 & -2.86 \\
\hline chr2:12-120 & suggestive & WL & 15.2 & 0.44 & 0.81 & -4.52 \\
\hline chr2:92-112 & suggestive & WL & 20.46 & 0.25 & 0.45 & -3.08 \\
\hline
\end{tabular}




\begin{tabular}{|c|c|c|c|c|c|c|}
\hline chr2:77-118 & suggestive & WL & 18.39 & 0.44 & 0.34 & -1.98 \\
\hline chr2:78-122 & suggestive & WL & 17.03 & 0.69 & 0.61 & -3.5 \\
\hline chr2:70-116 & suggestive & WL & 16.89 & 0.65 & 0.75 & -4.37 \\
\hline chr2:87-116 & suggestive & WL & 18.02 & 0.16 & 0.32 & -1.98 \\
\hline chr2:87-118 & suggestive & WL & 17.36 & 0.85 & 0.42 & -2.14 \\
\hline chr2:80-111 & suggestive & WL & 20.35 & 0.91 & 0.67 & -4.21 \\
\hline chr2:91-115 & significant & WL & 20.46 & 0.02 & 0.46 & -3.27 \\
\hline chr2:68-121 & suggestive & WL & 15.72 & 0.77 & 0.4 & -1.95 \\
\hline chr2:76-113 & suggestive & WL & 16.5 & 0.47 & 0.42 & -2.36 \\
\hline chr2:86-116 & suggestive & WL & 19.95 & 0.88 & 0.25 & -1.15 \\
\hline chr2:72-121 & suggestive & WL & 17.56 & 0.49 & 0.58 & -3.48 \\
\hline chr2:73-117 & suggestive & WL & 17.11 & 0.34 & 0.37 & -2.22 \\
\hline chr2:88-116 & suggestive & WL & 20.07 & 0.31 & 0.51 & -3.41 \\
\hline chr2:75-118 & suggestive & WL & 18.3 & 0.84 & 0.54 & -3.08 \\
\hline chr2:80-119 & suggestive & WL & 19.13 & 0.33 & 0.57 & -3.72 \\
\hline chr2:72-119 & suggestive & WL & 16.38 & 0.28 & 0.35 & -2.03 \\
\hline chr2:78-117 & suggestive & WL & 19.9 & 0.8 & 0.6 & -3.69 \\
\hline chr2:65-116 & suggestive & RJF & 17.81 & -0.43 & 0.47 & -3.18 \\
\hline chr2:72-119 & suggestive & WL & 17.11 & 0.4 & 0.63 & -3.79 \\
\hline chr2:87-115 & significant & WL & 21.89 & 0.92 & 0.68 & -4.49 \\
\hline chr2:64-122 & suggestive & WL & 16.79 & 0.73 & 0.54 & -2.95 \\
\hline chr2:86-119 & suggestive & WL & 18.68 & 0.7 & 0.5 & -2.95 \\
\hline chr2:93-118 & significant & WL & 17.18 & 0.9 & 0.38 & -1.77 \\
\hline chr2:80-119 & suggestive & WL & 13.59 & 0.84 & 0.47 & -2.13 \\
\hline chr2:92-117 & significant & WL & 15.79 & 1 & 0.43 & -1.91 \\
\hline chr2:92-117 & significant & WL & 17.31 & 0.57 & 0.5 & -2.9 \\
\hline chr2:68-119 & suggestive & WL & 14.81 & 1.77 & 0.54 & -1.49 \\
\hline chr2:94-115 & significant & WL & 15.84 & 1.14 & 0.43 & -1.83 \\
\hline chr2:93-120 & significant & WL & 16.48 & 1.83 & 0.49 & -1.48 \\
\hline chr2:73-123 & suggestive & WL & 14.2 & 1.85 & 0.54 & -1.25 \\
\hline chr2:70-119 & significant & WL & 13.57 & 0.42 & 0.46 & -2.34 \\
\hline chr2:92-123 & significant & WL & 15.48 & 0.45 & 0.25 & -1.26 \\
\hline chr2:80-152 & significant & WL & 14.37 & 0.74 & 0.39 & -1.81 \\
\hline chr2:60-117 & suggestive & WL & 14.04 & 0.74 & 0.86 & -4.52 \\
\hline chr2:95-109 & significant & WL & 23.46 & 0.16 & 0.27 & -2 \\
\hline chr2:92-118 & significant & WL & 23.88 & 2.11 & 0.7 & -3.93 \\
\hline chr2:93-115 & significant & WL & 23.01 & 1.6 & 0.74 & -4.57 \\
\hline chr2:94-114 & significant & WL & 23.54 & 0.88 & 0.41 & -2.71 \\
\hline chr2:95-115 & suggestive & RJF & 15.62 & -0.3 & 0.32 & -0.48 \\
\hline chr2:92-118 & suggestive & WL & 21.23 & 1.22 & 0.44 & -1.05 \\
\hline chr2:92-115 & suggestive & WL & 20.01 & 1.02 & 0.36 & -1.74 \\
\hline chr2:92-113 & significant & WL & 22.57 & 1.05 & 0.68 & -4.52 \\
\hline chr2:67-110 & suggestive & RJF & 24.06 & -1.03 & 0.56 & -4.56 \\
\hline
\end{tabular}




\begin{tabular}{|c|c|c|c|c|c|c|}
\hline chr2:71-110 & significant & RJF & 19.62 & -0.19 & 0.13 & -0.93 \\
\hline chr2:91-120 & suggestive & WL & 19.69 & 1.56 & 0.43 & -1.59 \\
\hline chr2:93-118 & suggestive & WL & 18.74 & 0.82 & 0.42 & -2.25 \\
\hline chr2:90-115 & suggestive & WL & 17.42 & 0.62 & 0.58 & -3.44 \\
\hline chr2:92-119 & suggestive & WL & 19.46 & 1.13 & 0.42 & -2.08 \\
\hline chr2:93-114 & suggestive & WL & 20.15 & 1.07 & 0.37 & -1.82 \\
\hline chr2:95-116 & suggestive & WL & 20.52 & 1.06 & 0.38 & -1.93 \\
\hline chr2:93-117 & suggestive & WL & 18.93 & 0.19 & 0.25 & -1.57 \\
\hline chr2:89-117 & suggestive & WL & 18.2 & 0.72 & 0.48 & -2.8 \\
\hline chr2:71-117 & suggestive & RJF & 19.14 & -0.08 & 0.62 & -4.19 \\
\hline chr2:92-116 & suggestive & WL & 16.58 & 0.21 & 0.31 & -1.84 \\
\hline chr2:89-116 & suggestive & WL & 17.49 & 1.88 & 0.68 & -2.89 \\
\hline chr2:64-115 & suggestive & WL & 16.57 & 0.9 & 0.63 & -3.37 \\
\hline chr2:73-116 & suggestive & WL & 18.34 & 0.97 & 0.34 & -1.45 \\
\hline chr2:67-118 & suggestive & WL & 17.38 & 0.5 & 0.7 & -4.21 \\
\hline chr2:92-117 & suggestive & WL & 19.08 & 0.3 & 0.08 & -0.29 \\
\hline chr2:93-119 & suggestive & WL & 19.78 & 1.14 & 0.4 & -1.95 \\
\hline chr2:93-114 & significant & WL & 19.5 & 0.69 & 0.48 & -2.92 \\
\hline chr2:92-115 & suggestive & WL & 19.82 & 0.14 & 0.39 & -2.65 \\
\hline chr2:93-117 & suggestive & WL & 19.57 & 1.34 & 0.44 & -2.02 \\
\hline chr2:50-118 & suggestive & RJF & 15.74 & -0.16 & 0.43 & -2.61 \\
\hline chr2:84-122 & suggestive & WL & 17.67 & 1.25 & 0.45 & -1.96 \\
\hline chr2:94-113 & significant & WL & 22.56 & 1.36 & 0.47 & -2.63 \\
\hline chr2:91-112 & suggestive & RJF & 19.27 & -0.24 & 0.28 & -1.93 \\
\hline chr2:93-113 & significant & WL & 21.99 & 0.02 & 0.54 & -3.92 \\
\hline chr2:92-116 & suggestive & WL & 19.09 & 1.41 & 0.84 & -4.82 \\
\hline chr2:71-117 & suggestive & WL & 18 & 0.52 & 0.86 & -5.31 \\
\hline chr2:93-115 & suggestive & WL & 20.51 & 0.66 & 0.3 & -1.68 \\
\hline chr2:89-117 & significant & WL & 19.8 & 0.98 & 0.42 & -2.25 \\
\hline chr2:78-133 & suggestive & WL & 16.3 & 0.48 & 0.57 & -3.25 \\
\hline chr2:93-117 & suggestive & WL & 20.27 & 2.35 & 0.71 & -3.17 \\
\hline chr2:92-111 & suggestive & WL & 20.45 & 0.17 & 0.05 & -0.23 \\
\hline chr2:85-118 & suggestive & WL & 18.38 & 0.97 & 0.53 & -2.88 \\
\hline chr2:94-113 & suggestive & WL & 19.81 & 0.65 & 0.77 & -5.07 \\
\hline chr2:93-114 & suggestive & WL & 13.9 & 0.89 & 0.38 & -1.96 \\
\hline chr2:93-115 & suggestive & WL & 16.41 & 0.38 & 0.41 & -2.39 \\
\hline chr2:91-122 & suggestive & WL & 18.37 & 0.19 & 0.25 & -1.53 \\
\hline chr2:94-113 & suggestive & WL & 15.28 & 1.06 & 0.48 & -2.67 \\
\hline chr2:86-112 & suggestive & WL & 16.41 & 0.63 & 0.32 & -1.7 \\
\hline chr2:93-121 & suggestive & WL & 15.95 & 0.72 & 0.4 & -1.98 \\
\hline chr2:93-120 & suggestive & WL & 16.61 & 0.71 & 0.4 & -2.14 \\
\hline chr2:93-120 & suggestive & WL & 13.9 & 0.33 & 0.37 & -2.02 \\
\hline chr2:89-120 & suggestive & WL & 17.56 & 0.72 & 0.28 & -1.31 \\
\hline
\end{tabular}




\begin{tabular}{|c|c|c|c|c|c|c|}
\hline chr2:93-115 & suggestive & WL & 20.37 & 0.68 & 0.31 & -1.71 \\
\hline chr2:94-115 & suggestive & WL & 20.13 & 0.31 & 0.24 & -1.49 \\
\hline chr2:93-114 & suggestive & WL & 18.24 & 0.49 & 0.38 & -2.22 \\
\hline chr2:93-112 & suggestive & RJF & 20.77 & 0 & 0.22 & -1.61 \\
\hline chr2:93-120 & suggestive & WL & 18.74 & 0.95 & 0.5 & -2.74 \\
\hline chr2:92-118 & suggestive & WL & 17.94 & 0.86 & 0.26 & -1.01 \\
\hline chr2:93-115 & significant & WL & 21.51 & 0.53 & 0.21 & -1.18 \\
\hline chr2:93-114 & suggestive & WL & 18.96 & 0.93 & 0.94 & -5.81 \\
\hline chr2:91-116 & suggestive & WL & 19.15 & 0.94 & 0.57 & -3.3 \\
\hline chr2:94-116 & suggestive & WL & 18.45 & 1.13 & 0.43 & -2.04 \\
\hline chr2:92-117 & suggestive & WL & 18.4 & 0.88 & 0.34 & -1.6 \\
\hline chr2:94-120 & suggestive & WL & 19.51 & 0.67 & 0.28 & -1.5 \\
\hline chr2:93-119 & suggestive & WL & 16.76 & 0.52 & 0.48 & -2.86 \\
\hline chr2:94-115 & suggestive & WL & 18.05 & 0.65 & 0.48 & -2.84 \\
\hline chr2:87-112 & suggestive & WL & 18.31 & 0.59 & 0.54 & -3.24 \\
\hline chr2:94-114 & suggestive & WL & 19.47 & 1.3 & 0.52 & -2.66 \\
\hline chr2:92-117 & suggestive & WL & 17.53 & 1.23 & 0.6 & -3.11 \\
\hline chr2:94-113 & significant & WL & 23.39 & 0.36 & 0.4 & -2.87 \\
\hline chr2:92-116 & suggestive & WL & 18.74 & 0.06 & 0.54 & -3.63 \\
\hline chr2:92-117 & significant & WL & 18.29 & 0.83 & 0.56 & -3.19 \\
\hline chr2:92-119 & suggestive & WL & 19.41 & 1.69 & 0.53 & -2.26 \\
\hline chr2:86-119 & suggestive & WL & 18.46 & 1.15 & 0.42 & -1.91 \\
\hline chr2:83-123 & suggestive & WL & 17.28 & 1.23 & 0.37 & -1.32 \\
\hline chr2:93-117 & suggestive & WL & 18.12 & 0.19 & 0.35 & -2.24 \\
\hline chr2:93-120 & suggestive & WL & 17.07 & 0.77 & 0.69 & -4.05 \\
\hline chr2:92-118 & suggestive & WL & 17.69 & 0.24 & 0.38 & -2.29 \\
\hline chr2:89-121 & significant & WL & 16.12 & 0.18 & 0.21 & -1.17 \\
\hline chr2:92-118 & suggestive & WL & 20.94 & 1.02 & 0.26 & -1.06 \\
\hline chr2:92-118 & suggestive & WL & 18.09 & 0.52 & 0.47 & -2.83 \\
\hline chr2:92-119 & suggestive & WL & 19.92 & 1.35 & 0.41 & -1.77 \\
\hline chr2:94-118 & suggestive & WL & 19.07 & 0.44 & 0.25 & -1.39 \\
\hline chr2:92-120 & suggestive & WL & 15.95 & 0.27 & 0.34 & -2 \\
\hline chr2:92-126 & suggestive & WL & 17.01 & 0.37 & 0.3 & -1.71 \\
\hline chr2:92-119 & suggestive & WL & 17.59 & 0.81 & 0.34 & -1.61 \\
\hline chr2:72-124 & suggestive & WL & 14.6 & 1.85 & 0.73 & -2.75 \\
\hline chr2:64-124 & suggestive & WL & 12.91 & 0.32 & 0.3 & -1.43 \\
\hline chr2:92-118 & significant & WL & 20.42 & 1.3 & 0.66 & -3.94 \\
\hline chr2:64-117 & suggestive & RJF & 17.94 & -0.38 & 0.45 & -2.99 \\
\hline chr2:81-118 & suggestive & WL & 16.13 & 0.44 & 0.4 & -2.22 \\
\hline chr2:91-120 & suggestive & WL & 18.62 & 0.82 & 0.47 & -2.66 \\
\hline chr2:92-120 & suggestive & WL & 20.91 & 0.69 & 0.3 & -1.66 \\
\hline chr2:93-120 & suggestive & WL & 15.52 & 0.21 & 0.47 & -2.72 \\
\hline chr2:94-119 & suggestive & WL & 9.81 & 0.34 & 0.37 & -2.39 \\
\hline
\end{tabular}




\begin{tabular}{|c|c|c|c|c|c|c|}
\hline chr2:92-119 & suggestive & WL & 12.24 & 0.97 & 0.31 & -1.28 \\
\hline chr2:93-119 & suggestive & WL & 19.54 & 0.79 & 0.66 & -4.12 \\
\hline chr2:93-120 & significant & WL & 16.51 & 0.82 & 0.42 & -2.21 \\
\hline chr2:91-140 & significant & WL & 18.04 & 0.82 & 0.38 & -1.96 \\
\hline chr2:76-124 & suggestive & WL & 14.04 & 1.17 & 0.57 & -2.38 \\
\hline chr2:93-119 & suggestive & WL & 18.16 & 1.17 & 0.64 & -3.52 \\
\hline chr2:60-122 & suggestive & WL & 17.38 & 1.94 & 1.11 & -5.83 \\
\hline chr2:93-119 & significant & WL & 21.58 & 0.35 & 0.59 & -4.21 \\
\hline chr2:86-119 & suggestive & WL & 17.77 & 0.46 & 0.56 & -3.4 \\
\hline chr2:93-120 & suggestive & WL & 19.33 & 0.07 & 0.48 & -3.22 \\
\hline chr2:79-122 & suggestive & WL & 16.25 & 1.17 & 0.56 & -2.61 \\
\hline chr2:94-118 & suggestive & RJF & 17.01 & -0.34 & 0.44 & -2.87 \\
\hline chr2:69-119 & significant & RJF & 21.98 & -1.45 & 0.61 & -3.92 \\
\hline chr2:88-123 & suggestive & WL & 20.94 & 0.85 & 0.33 & -1.82 \\
\hline chr2:73-118 & suggestive & WL & 18.9 & 1.05 & 0.57 & -3.14 \\
\hline chr2:61-121 & suggestive & WL & 17.71 & 0.26 & 0.5 & -3.11 \\
\hline chr2:94-123 & significant & WL & 17.54 & 0.51 & 0.24 & -1.47 \\
\hline chr2:92-119 & suggestive & WL & 21.81 & 1.39 & 0.55 & -3.11 \\
\hline chr2:92-119 & suggestive & WL & 18.56 & 0.2 & 0.5 & -3.31 \\
\hline chr2:86-120 & suggestive & WL & 18.62 & 0.46 & 0.45 & -2.75 \\
\hline chr2:80-124 & suggestive & WL & 17.57 & 0.56 & 0.28 & -1.41 \\
\hline chr2:94-126 & suggestive & WL & 16.35 & 1.22 & 0.41 & -1.64 \\
\hline chr2:87-127 & suggestive & WL & 17.1 & 0.88 & 0.43 & -2.15 \\
\hline chr2:92-120 & suggestive & WL & 18.99 & 0.56 & 0.7 & -4.46 \\
\hline chr2:82-119 & significant & RJF & 22.55 & -0.21 & 0.44 & -3.4 \\
\hline chr2:92-123 & suggestive & WL & 18.84 & 1.48 & 0.64 & -3.26 \\
\hline chr2:92-122 & suggestive & WL & 19.65 & 0.81 & 0.44 & -2.5 \\
\hline chr2:93-125 & suggestive & WL & 17.46 & 1.71 & 0.73 & -3.59 \\
\hline chr2:93-123 & suggestive & WL & 18.68 & 1.49 & 0.35 & -0.86 \\
\hline chr2:93-120 & suggestive & WL & 19.95 & 0.68 & 0.52 & -3.32 \\
\hline chr2:93-120 & suggestive & WL & 19.95 & 0.94 & 0.34 & -1.69 \\
\hline chr2:91-120 & suggestive & WL & 21.25 & 0.79 & 0.51 & -3.29 \\
\hline chr2:86-120 & suggestive & RJF & 19.52 & -0.4 & 0.38 & -2.69 \\
\hline chr2:92-125 & suggestive & WL & 18.95 & 1.17 & 0.35 & -1.36 \\
\hline chr2:92-120 & suggestive & WL & 18.39 & 0.81 & 0.7 & -4.22 \\
\hline chr2:85-121 & significant & WL & 25.95 & 2.13 & 0.66 & -3.07 \\
\hline chr2:68-130 & suggestive & WL & 13.24 & 0.68 & 0.46 & -2.1 \\
\hline chr2:76-126 & suggestive & WL & 14.11 & 2.78 & 1.13 & -4.19 \\
\hline chr2:93-127 & suggestive & WL & 16.71 & 0.59 & 0.45 & -2.44 \\
\hline chr2:62-122 & suggestive & WL & 19.05 & 1.25 & 0.53 & -2.82 \\
\hline chr2:77-121 & suggestive & WL & 19.44 & 0.21 & 0.27 & -1.75 \\
\hline chr2:93-123 & suggestive & WL & 16.49 & 1 & 0.52 & -2.61 \\
\hline chr2:93-121 & suggestive & WL & 16.26 & 0.15 & 0.32 & -1.99 \\
\hline
\end{tabular}




\begin{tabular}{|c|c|c|c|c|c|c|}
\hline chr2:93-122 & suggestive & WL & 20.72 & 0.79 & 0.39 & -2.3 \\
\hline chr2:70-125 & suggestive & WL & 16.45 & 1.19 & 0.76 & -3.98 \\
\hline chr2:80-124 & significant & WL & 19.46 & 1.18 & 0.38 & -1.69 \\
\hline chr2:94-125 & suggestive & RJF & 14.04 & -0.02 & 0.41 & -2.31 \\
\hline chr2:86-157 & suggestive & WL & 14.19 & 0.64 & 0.26 & -1.03 \\
\hline chr2:91-123 & suggestive & WL & 14.34 & 1.58 & 1.07 & -5.19 \\
\hline chr2:72-121 & suggestive & WL & 21.1 & 0.77 & 0.71 & -4.84 \\
\hline chr2:87-126 & suggestive & RJF & 25.4 & -0.43 & 0.21 & -1.5 \\
\hline chr2:93-121 & suggestive & RJF & 21.92 & -0.05 & 0.34 & -2.49 \\
\hline chr2:73-124 & suggestive & WL & 20.01 & 0.3 & 0.12 & -0.65 \\
\hline chr2:92-124 & suggestive & WL & 18.82 & 0.63 & 0.47 & -2.77 \\
\hline chr2:79-125 & suggestive & WL & 19.21 & 0.43 & 0.34 & -2.04 \\
\hline chr2:70-122 & suggestive & RJF & 17.28 & 0 & 0.43 & -2.77 \\
\hline chr2:72-123 & suggestive & RJF & 19.13 & -0.15 & 0.42 & -2.9 \\
\hline chr2:87-129 & significant & WL & 18.94 & 1.06 & 0.25 & -0.63 \\
\hline chr2:92-124 & significant & WL & 18.15 & 2.26 & 0.76 & -3.43 \\
\hline chr2:54-129 & significant & WL & 14.85 & 0.17 & 0.41 & -2.37 \\
\hline chr2:74-125 & suggestive & WL & 23.25 & 0.2 & 0.65 & -4.79 \\
\hline chr2:75-124 & suggestive & WL & 18.92 & 0.87 & 0.47 & -2.64 \\
\hline chr2:91-123 & suggestive & RJF & 18.37 & -0.49 & 0.5 & -3.42 \\
\hline chr2:78-125 & suggestive & WL & 19.08 & 1.17 & 0.45 & -2.23 \\
\hline chr2:92-122 & suggestive & WL & 19.6 & 0.5 & 0.6 & -3.83 \\
\hline chr2:82-122 & suggestive & RJF & 18.57 & -0.11 & 0.24 & -1.61 \\
\hline chr2:61-124 & suggestive & WL & 15.55 & 0.04 & 0.46 & -2.71 \\
\hline chr2:93-124 & significant & WL & 25.1 & 2.66 & 0.79 & -2.43 \\
\hline chr2:74-129 & significant & WL & 15.55 & 1.43 & 0.41 & -1.13 \\
\hline chr2:72-127 & suggestive & WL & 13.89 & 0.51 & 0.37 & -1.77 \\
\hline chr2:92-124 & suggestive & WL & 18.85 & 1.41 & 0.72 & -3.94 \\
\hline chr2:72-125 & suggestive & WL & 18.45 & 0.15 & 0.29 & -1.9 \\
\hline chr2:78-123 & suggestive & RJF & 19.84 & -0.16 & 0.38 & -2.69 \\
\hline chr2:92-123 & suggestive & WL & 21.58 & 0.06 & 0.52 & -3.73 \\
\hline chr2:68-130 & significant & WL & 18.08 & 0.39 & 0.34 & -2.08 \\
\hline chr2:84-124 & suggestive & WL & 23.68 & 1.57 & 0.5 & -2.33 \\
\hline chr2:75-128 & significant & WL & 21.67 & 1.5 & 0.45 & -2.22 \\
\hline chr2:87-134 & suggestive & WL & 18.37 & 0.96 & 0.36 & -1.67 \\
\hline chr2:91-133 & suggestive & WL & 14.14 & 0.48 & 0.35 & -1.71 \\
\hline chr2:92-134 & significant & WL & 16.42 & 2.12 & 0.59 & -1.76 \\
\hline chr2:77-139 & suggestive & WL & 17.96 & 1.35 & 0.44 & -1.84 \\
\hline chr2:92-156 & suggestive & WL & 13.36 & 1.08 & 0.27 & 0.39 \\
\hline chr2:110-153 & 3 suggestive & RJF & 13.63 & -0.98 & 0.33 & -1.36 \\
\hline chr2:101-190 & suggestive & WL & 14.84 & 0.85 & 0.19 & -0.41 \\
\hline chr2:107-157 & suggestive & WL & 13.29 & 1.77 & 0.42 & 0.13 \\
\hline chr2:110-158 & 3 significant & WL & 16.22 & 2.92 & 0.61 & -0.63 \\
\hline
\end{tabular}




\begin{tabular}{|c|c|c|c|c|c|}
\hline chr2:117-156 significant & WL & 26.64 & 2.88 & 0.43 & -1.15 \\
\hline chr2:113-153 significant & WL & 22.99 & 2.77 & 1.41 & -1.31 \\
\hline chr2:116-155 significant & WL & 16.62 & 3.4 & 0.81 & 1.99 \\
\hline chr2:118-164 significant & WL & 19.25 & 2.12 & 0.41 & 0.12 \\
\hline chr2:123-159 significant & WL & 30.28 & 3 & 0.42 & -0.4 \\
\hline chr2:118-161 significant & WL & 17.86 & 1.53 & 0.3 & -0.26 \\
\hline chr2:120-158 significant & WL & 20.87 & 2.55 & 0.79 & -2.06 \\
\hline chr2:128-161 suggestive & WL & 24.83 & 1.97 & 0.43 & -2.28 \\
\hline chr2:121-154 significant & WL & 19.4 & 2.8 & 0.53 & 0.11 \\
\hline chr2:122-158 suggestive & WL & 17.01 & 0.96 & 0.25 & 0.92 \\
\hline chr2:113-154 significant & RJF & 16.86 & -3.4 & 0.89 & -1.59 \\
\hline chr2:124-152 significant & WL & 22.59 & 4.49 & 0.78 & 0.46 \\
\hline chr2:136-147 significant & WL & 50.04 & 7.03 & 0.68 & 1.41 \\
\hline chr2:129-149 significant & WL & 32.72 & 4.58 & 0.64 & 1.16 \\
\hline chr2:118-160 significant & WL & 13.97 & 2.55 & 0.6 & 0.31 \\
\hline chr2:133-147 significant & WL & 26.23 & 1.04 & 0.55 & -0.04 \\
\hline chr2:127-152 significant & WL & 20.82 & 2.13 & 0.38 & 0.01 \\
\hline chr2:122-151 significant & WL & 18.78 & 2.26 & 0.45 & 0.33 \\
\hline chr2:122-154 significant & WL & 17.26 & 2.18 & 0.44 & -0.35 \\
\hline chr2:118-154 suggestive & WL & 17.81 & 0.69 & 0.95 & 1.05 \\
\hline chr2:129-166 significant & WL & 24.38 & 0.69 & 0.46 & -0.09 \\
\hline chr2:118-201 suggestive & WL & 18.25 & 0.81 & 0.47 & 0.17 \\
\hline chr2:129-154 significant & RJF & 21.27 & -2.37 & 0.42 & -0.38 \\
\hline chr2:125-153 significant & WL & 22.43 & 2.89 & 0.49 & -0.68 \\
\hline chr2:121-197 significant & WL & 15.49 & 2.03 & 0.43 & -0.45 \\
\hline chr2:123-167 significant & WL & 15.79 & 2.55 & 0.54 & -0.69 \\
\hline chr2:126-157 significant & WL & 15.32 & 1.95 & 0.42 & -0.56 \\
\hline chr2:125-173 significant & RJF & 13.55 & -2.6 & 0.61 & 0.16 \\
\hline chr2:128-153 suggestive & RJF & 13.54 & -1.79 & 0.41 & 0.45 \\
\hline chr2:127-160 suggestive & WL & 12.95 & 0.51 & 0.36 & -0.7 \\
\hline chr2:125-157 significant & WL & 24.04 & 2.06 & 0.35 & 0.2 \\
\hline chr2:128-155 significant & RJF & 19.25 & -1.87 & 0.35 & 0.28 \\
\hline chr2:121-176 suggestive & RJF & 13.1 & -2.76 & 0.65 & 0.07 \\
\hline chr2:136-151 significant & WL & 37.63 & 3.23 & 0.38 & -0.16 \\
\hline chr2:136-146 significant & WL & 51.4 & 2.55 & 0.53 & 1.8 \\
\hline chr2:127-160 significant & WL & 16.55 & 2.19 & 0.45 & -0.08 \\
\hline chr2:120-161 significant & WL & 16.19 & 2.43 & 0.52 & 0.04 \\
\hline chr2:129-160 significant & WL & 16.71 & 1.37 & 0.28 & -0.73 \\
\hline chr2:121-155 significant & WL & 28.17 & 1.7 & 1.04 & -0.34 \\
\hline chr2:126-156 significant & WL & 21.98 & 3.49 & 0.65 & 1.12 \\
\hline chr2:129-157 significant & WL & 27.19 & 2.95 & 0.44 & -0.1 \\
\hline chr2:126-154 suggestive & RJF & 18.13 & -0.5 & 0.42 & -0.22 \\
\hline chr2:123-153 significant & WL & 28.43 & 2.43 & 0.37 & 1 \\
\hline
\end{tabular}




\begin{tabular}{|c|c|c|c|c|c|}
\hline chr2:127-155 significant & WL & 31.55 & 2.97 & 0.4 & 0.12 \\
\hline chr2:101-156 suggestive & WL & 15.28 & 1.41 & 0.35 & 0.98 \\
\hline chr2:93-168 significant & WL & 15.57 & 2.59 & 0.57 & -1.59 \\
\hline chr2:128-158 significant & WL & 21.47 & 2.41 & 0.42 & -0.53 \\
\hline chr2:129-159 suggestive & WL & 13.66 & 1.09 & 0.3 & 0.94 \\
\hline chr2:147-156 significant & WL & 39.85 & 2.47 & 0.28 & -0.96 \\
\hline chr2:134-160 suggestive & WL & 19.65 & 1.97 & 0.58 & -1.86 \\
\hline chr2:120-162 significant & WL & 16.07 & 1.87 & 0.39 & -0.44 \\
\hline chr2:101-172 significant & RJF & 11.96 & -2.11 & 0.54 & -0.47 \\
\hline chr2:133-159 significant & WL & 31.91 & 3.24 & 0.42 & -0.28 \\
\hline chr2:131-172 significant & WL & 22.34 & 2.73 & 0.47 & -0.86 \\
\hline chr2:129-159 significant & WL & 31.67 & 4.49 & 0.59 & -1.05 \\
\hline chr2:123-171 significant & WL & 15.28 & 2.73 & 0.58 & -0.95 \\
\hline chr2:107-172 suggestive & WL & 14.94 & 2.42 & 0.53 & -0.48 \\
\hline chr2:131-193 significant & WL & 21.33 & 1.85 & 0.33 & -0.7 \\
\hline chr2:132-166 significant & WL & 10.29 & 1.73 & 0.26 & -0.01 \\
\hline chr2:126-168 significant & WL & 17.47 & 1.87 & 0.38 & 0.13 \\
\hline chr2:116-198 significant & WL & 14.54 & 1.33 & 0.29 & -0.54 \\
\hline chr2:128-172 significant & WL & 21.24 & 2.38 & 0.41 & -0.5 \\
\hline chr2:133-168 suggestive & WL & 19.61 & 2.41 & 0.69 & -3.26 \\
\hline chr2:74-194 significant & WL & 15.41 & 1.43 & 0.34 & 0.68 \\
\hline chr2:127-201 suggestive & WL & 14.65 & 1.43 & 0.32 & 0.07 \\
\hline chr2:126-172 suggestive & WL & 18.98 & 2.4 & 0.55 & -2.46 \\
\hline chr2:121-173 significant & WL & 17.98 & 1.51 & 0.29 & -0.21 \\
\hline chr2:118-192 significant & WL & 18.58 & 0.98 & 0.19 & -0.27 \\
\hline chr2:125-197 suggestive & WL & 12.82 & 1.72 & 0.4 & -0.29 \\
\hline chr2:124-168 suggestive & WL & 24.14 & 2.73 & 0.58 & -2.72 \\
\hline chr2:95-196 suggestive & WL & 15.47 & 0.59 & 0.92 & 2.35 \\
\hline chr2:112-193 significant & WL & 16.86 & 1.51 & 0.31 & 0.45 \\
\hline chr2:114-206 significant & WL & 16.21 & 1.69 & 0.36 & 0.5 \\
\hline chr2:126-187 suggestive & WL & 14.97 & 1.55 & 0.33 & -0.02 \\
\hline chr2:148-177 suggestive & WL & 28.71 & 3.03 & 0.61 & -1.73 \\
\hline chr2:150-171 significant & WL & 25.49 & 3.09 & 0.48 & -0.21 \\
\hline chr2:125-187 significant & WL & 16.06 & 3.16 & 0.67 & -1.02 \\
\hline chr2:148-177 significant & WL & 19.69 & 2.25 & 0.44 & 0.88 \\
\hline chr2:151-177 significant & WL & 32.95 & 2.57 & 0.34 & 0.43 \\
\hline chr2:150-172 significant & RJF & 32.82 & -3.59 & 0.47 & 0.69 \\
\hline chr2:147-176 significant & WL & 17.58 & 1.6 & 0.32 & -0.42 \\
\hline chr2:133-170 suggestive & WL & 22.75 & 1.97 & 0.45 & -2.04 \\
\hline chr2:135-176 significant & WL & 25.46 & 2.76 & 0.45 & 0.84 \\
\hline chr2:125-175 significant & WL & 18.09 & 1.11 & 0.43 & 0.46 \\
\hline chr2:152-187 significant & RJF & 13.96 & -1.72 & 0.4 & -0.47 \\
\hline chr2:154-179 suggestive & WL & 14.56 & 1.11 & 0.28 & 0.82 \\
\hline
\end{tabular}




\begin{tabular}{|c|c|c|c|c|c|}
\hline chr2:151-175 suggestive & WL & 14.71 & 0.82 & 0.18 & -0.17 \\
\hline chr2:157-171 suggestive & WL & 11.23 & 0.14 & 0.4 & 0.38 \\
\hline chr2:153-171 suggestive & WL & 23.78 & 1.19 & 0.24 & -0.81 \\
\hline chr2:154-179 suggestive & WL & 17.96 & 1.27 & 0.25 & -0.32 \\
\hline chr2:153-174 suggestive & WL & 21.1 & 1.68 & 0.3 & -0.43 \\
\hline chr2:156-217 suggestive & WL & 17.81 & 1.45 & 0.48 & 2.28 \\
\hline chr2:152-200 suggestive & WL & 15.68 & 1.13 & 0.25 & 0.34 \\
\hline chr2:153-183 significant & WL & 16.26 & 1.03 & 0.21 & -0.34 \\
\hline chr2:153-184 significant & RJF & 17.51 & -1.38 & 0.28 & -0.33 \\
\hline chr2:151-194 significant & WL & 14.95 & 1.07 & 0.23 & -0.33 \\
\hline chr2:150-190 significant & RJF & 13.95 & -2.09 & 0.46 & 0.08 \\
\hline chr2:153-215 suggestive & RJF & 10.09 & -3.06 & 0.93 & -1.86 \\
\hline chr2:153-172 suggestive & WL & 24.21 & 1.47 & 0.37 & -2.39 \\
\hline chr2:151-198 suggestive & WL & 13.66 & 0.93 & 0.23 & -0.61 \\
\hline chr2:157-186 significant & WL & 19.43 & 2.05 & 0.38 & 0.25 \\
\hline chr2:157-184 significant & RJF & 19.94 & -3.28 & 0.6 & -0.54 \\
\hline chr2:154-191 significant & WL & 17.24 & 1.8 & 0.36 & -0.14 \\
\hline chr2:148-175 suggestive & WL & 25.36 & 1.55 & 0.44 & -2.93 \\
\hline chr2:156-191 significant & WL & 17.29 & 1.89 & 0.4 & 0.99 \\
\hline chr2:157-192 suggestive & WL & 18.94 & 1.48 & 0.28 & -0.65 \\
\hline chr2:156-196 significant & WL & 20.45 & 2.33 & 0.46 & 1.2 \\
\hline chr2:156-193 significant & WL & 17.72 & 1.46 & 0.29 & -0.87 \\
\hline chr2:156-189 significant & WL & 22.04 & 4.52 & 0.93 & -1.79 \\
\hline chr2:157-190 significant & WL & 19.78 & 1.45 & 0.31 & 1.16 \\
\hline chr2:155-199 significant & WL & 16.62 & 1.75 & 0.38 & 0.62 \\
\hline chr2:151-194 significant & WL & 16.33 & 1.78 & 0.39 & 0.74 \\
\hline chr2:153-200 suggestive & WL & 19.63 & 2.21 & 0.43 & -0.56 \\
\hline chr2:123-189 significant & WL & 22.62 & 1.69 & 0.42 & -0.49 \\
\hline chr2:158-193 suggestive & WL & 18.32 & 2.21 & 0.57 & -1.27 \\
\hline chr2:155-193 suggestive & WL & 14.27 & 1.88 & 0.42 & 0.06 \\
\hline chr2:156-194 significant & WL & 20.37 & 1.31 & 0.26 & 1.1 \\
\hline chr2:160-186 suggestive & WL & 19.99 & 0.93 & 0.41 & -0.85 \\
\hline chr2:152-199 significant & WL & 15.27 & 2.32 & 0.55 & -1.17 \\
\hline chr2:156-196 suggestive & WL & 21.81 & 1.47 & 0.26 & -0.01 \\
\hline chr2:162-190 significant & WL & 16.28 & 0.54 & 0.16 & 1 \\
\hline chr2:155-198 significant & WL & 14.51 & 1.68 & 0.35 & 0.36 \\
\hline chr2:165-192 suggestive & WL & 19.05 & 1.4 & 0.53 & 0.05 \\
\hline chr2:160-194 significant & WL & 20.2 & 2.35 & 0.42 & 0.89 \\
\hline chr2:156-192 significant & WL & 21.47 & 1.01 & 0.81 & -2.98 \\
\hline chr2:156-198 suggestive & WL & 17.78 & 2.1 & 0.47 & 2.31 \\
\hline chr2:159-191 suggestive & WL & 21.42 & 1.58 & 0.28 & 0.46 \\
\hline chr2:164-190 suggestive & RJF & 14.05 & -1.34 & 0.55 & 2.88 \\
\hline chr2:157-202 significant & WL & 16.21 & 1.39 & 0.29 & 0.53 \\
\hline
\end{tabular}




\begin{tabular}{|c|c|c|c|c|c|}
\hline chr2:164-194 significant & WL & 22.76 & 2.4 & 0.41 & 0.23 \\
\hline chr2:154-193 significant & WL & 17.45 & 1.28 & 0.26 & 0.74 \\
\hline chr2:154-199 significant & RJF & 10.48 & -2.75 & 0.73 & -0.65 \\
\hline chr2:168-192 significant & WL & 29.43 & 2.16 & 0.32 & 1.35 \\
\hline chr2:155-200 suggestive & RJF & 12.44 & -2.56 & 0.62 & -0.83 \\
\hline chr2:160-199 significant & WL & 15.4 & 0.87 & 0.18 & 0.28 \\
\hline chr2:158-234 significant & RJF & 10.22 & -4.02 & 1.15 & -3.1 \\
\hline chr2:167-208 significant & RJF & 11.92 & -2.2 & 0.55 & 0.04 \\
\hline chr2:168-190 suggestive & WL & 13.73 & 0.3 & 0.43 & 0.39 \\
\hline chr2:169-193 suggestive & WL & 11.2 & 1.35 & 0.23 & 0.27 \\
\hline chr2:159-202 suggestive & WL & 15.32 & 1.81 & 0.39 & 0.29 \\
\hline chr2:170-195 suggestive & RJF & 14.62 & -1.3 & 0.49 & 2.51 \\
\hline chr2:171-193 suggestive & WL & 28.24 & 1.16 & 0.4 & 2.53 \\
\hline chr2:167-201 significant & WL & 17.53 & 1.69 & 0.33 & 0.19 \\
\hline chr2:157-247 suggestive & WL & 14.37 & 2.04 & 0.46 & -0.11 \\
\hline chr2:164-208 significant & WL & 17.73 & 1.75 & 0.34 & 0.23 \\
\hline chr2:169-221 significant & WL & 16.69 & 0.98 & 0.71 & -0.16 \\
\hline chr2:155-233 significant & WL & 16.44 & 2.33 & 0.58 & -0.01 \\
\hline chr2:199-217 significant & RJF & 24.99 & -2.85 & 0.5 & -0.53 \\
\hline chr2:198-216 significant & RJF & 19.39 & -2.69 & 0.65 & 0.47 \\
\hline chr2:192-213 suggestive & RJF & 13.71 & -0.96 & 0.98 & 1.9 \\
\hline chr2:185-238 suggestive & WL & 15.92 & 2.56 & 0.55 & 1.18 \\
\hline chr2:194-224 suggestive & RJF & 27.25 & -0.34 & 0.05 & -0.3 \\
\hline chr2:193-221 significant & RJF & 25.91 & -0.23 & 0.04 & -0.3 \\
\hline chr2:194-217 suggestive & RJF & 30.91 & -1.64 & 0.23 & -1.36 \\
\hline chr2:190-213 suggestive & WL & 19.31 & 1.11 & 0.35 & -0.87 \\
\hline chr2:192-222 suggestive & RJF & 22.51 & -1.36 & 0.23 & -0.96 \\
\hline chr2:194-217 suggestive & RJF & 31.23 & -2.3 & 0.33 & -2.31 \\
\hline chr2:191-215 suggestive & RJF & 13.29 & -0.78 & 1.1 & 4.51 \\
\hline chr2:191-222 suggestive & RJF & 18.03 & -0.77 & 0.52 & -0.65 \\
\hline chr2:198-241 significant & RJF & 25.51 & -3.14 & 0.55 & -0.45 \\
\hline chr2:195-222 significant & RJF & 19.46 & -2.94 & 0.69 & -4.58 \\
\hline chr2:176-221 suggestive & RJF & 13.72 & -2.04 & 1.99 & 0.98 \\
\hline chr2:186-250 significant & WL & 16.91 & 3.64 & 0.74 & 2.03 \\
\hline chr2:166-246 significant & RJF & 18.1 & -3.33 & 0.64 & -0.68 \\
\hline chr2:183-243 significant & WL & 18.96 & 1.61 & 0.32 & 0.32 \\
\hline chr2:157-267 significant & WL & 14.67 & 2.03 & 0.48 & 0.39 \\
\hline chr2:157-253 suggestive & WL & 14.01 & 1.48 & 0.39 & -0.18 \\
\hline chr2:170-261 suggestive & WL & 13.74 & 2.03 & 0.49 & 0.42 \\
\hline chr2:211-249 suggestive & WL & 14.36 & 0.51 & 0.21 & 2.13 \\
\hline chr2:210-250 significant & WL & 27.33 & 4 & 0.62 & 1.36 \\
\hline chr2:204-249 significant & WL & 17.68 & 2.24 & 0.46 & 0.74 \\
\hline chr2:210-259 significant & WL & 15.62 & 1.29 & 0.67 & -0.85 \\
\hline
\end{tabular}




\begin{tabular}{|c|c|c|c|c|c|}
\hline chr2:219-246 suggestive & RJF & 16.83 & -1.54 & 0.43 & -1.21 \\
\hline chr2:219-245 suggestive & WL & 26.1 & 0.72 & 0.43 & 3.8 \\
\hline chr2:215-274 significant & WL & 15.29 & 1 & 0.9 & -2.78 \\
\hline chr2:218-247 significant & RJF & 39.18 & -2.92 & 0.46 & 1.65 \\
\hline chr2:213-259 significant & RJF & 16.35 & -1.65 & 0.49 & 1.08 \\
\hline chr2:220-248 suggestive & WL & 14.66 & 2.24 & 0.78 & 7.45 \\
\hline chr2:215-266 significant & WL & 19.17 & 2.78 & 0.56 & 0.33 \\
\hline chr2:206-256 significant & WL & 16.73 & 1.29 & 0.29 & 1.81 \\
\hline chr2:210-277 significant & RJF & 14.79 & -1.66 & 0.43 & 0.25 \\
\hline chr2:211-301 significant & WL & 15.66 & 2.11 & 0.5 & -0.1 \\
\hline chr2:218-327 suggestive & RJF & 12.44 & -1.72 & 0.42 & -0.08 \\
\hline chr2:233-328 significant & RJF & 15.37 & -1.94 & 0.46 & 1.66 \\
\hline chr2:227-307 significant & WL & 15.37 & 2.85 & 0.6 & 0.26 \\
\hline chr2:239-322 significant & RJF & 19.4 & -2.71 & 0.51 & 0.81 \\
\hline chr2:221-332 significant & RJF & 14.38 & -3.99 & 0.87 & -0.02 \\
\hline chr2:234-326 significant & RJF & 16.8 & -4.74 & 0.93 & 0.43 \\
\hline chr2:227-306 suggestive & RJF & 14.41 & -1.25 & 0.31 & -0.75 \\
\hline chr2:235-325 significant & RJF & 14.96 & -1.94 & 0.43 & 0.71 \\
\hline chr2:214-325 suggestive & RJF & 11 & -4.76 & 1.22 & 0.68 \\
\hline chr2:239-323 significant & RJF & 16.4 & -1.47 & 0.3 & -0.08 \\
\hline chr2:228-315 suggestive & WL & 15.02 & 1.35 & 0.37 & 1.29 \\
\hline chr2:245-286 suggestive & RJF & 18.04 & -0.1 & 0.58 & 4.49 \\
\hline chr2:239-320 significant & RJF & 18.89 & -4.63 & 0.85 & 0.19 \\
\hline chr2:232-320 significant & RJF & 11.82 & -9.1 & 2.27 & 4.86 \\
\hline chr2:241-324 significant & RJF & 19.12 & -8.59 & 1.55 & 1.22 \\
\hline chr2:236-324 significant & RJF & 16.05 & -4.76 & 0.97 & -0.27 \\
\hline chr2:216-329 suggestive & RJF & 11.63 & -5.02 & 1.25 & -0.28 \\
\hline chr2:234-321 significant & RJF & 14.84 & -3.23 & 0.71 & -0.36 \\
\hline chr2:236-326 suggestive & RJF & 16.34 & -4.57 & 0.91 & 0.18 \\
\hline chr2:246-328 significant & RJF & 15.64 & -2.65 & 0.58 & 1.4 \\
\hline chr2:254-287 suggestive & WL & 18.03 & 0.38 & 0.11 & -0.76 \\
\hline chr2:246-302 suggestive & WL & 18.89 & 0.79 & 0.5 & 1.71 \\
\hline chr2:234-326 significant & RJF & 13.59 & -9.09 & 2 & 1.94 \\
\hline chr2:229-329 significant & RJF & 11.61 & -7.02 & 1.69 & 0.93 \\
\hline chr2:235-324 significant & RJF & 12.04 & -4.6 & 1.13 & 1.57 \\
\hline chr2:249-320 significant & RJF & 22.25 & -8.37 & 1.36 & 1.24 \\
\hline chr2:244-323 suggestive & RJF & 13.85 & -1.3 & 0.3 & 0.36 \\
\hline chr2:247-327 suggestive & RJF & 12.47 & -2.87 & 0.88 & 3.76 \\
\hline chr2:217-321 suggestive & RJF & 11.22 & -5.21 & 1.33 & -1.21 \\
\hline chr2:241-324 suggestive & RJF & 15.46 & -9.59 & 1.96 & 2.55 \\
\hline chr2:221-322 suggestive & RJF & 11.08 & -5.55 & 1.43 & -1.25 \\
\hline chr2:261-327 significant & RJF & 21.29 & -1.71 & 0.3 & 0.9 \\
\hline chr2:252-318 significant & RJF & 19.83 & -10.7 & 1.88 & 1.48 \\
\hline
\end{tabular}




\begin{tabular}{|c|c|c|c|c|c|}
\hline chr2:248-317 significant & RJF & 19.54 & -9.34 & 1.67 & 1.4 \\
\hline chr2:215-323 significant & RJF & 12.74 & -5.74 & 1.34 & -0.19 \\
\hline chr2:250-321 significant & RJF & 16.18 & -7.17 & 1.45 & 0.28 \\
\hline chr2:248-318 significant & RJF & 18.84 & -10.75 & 1.94 & 1.95 \\
\hline chr2:240-320 significant & RJF & 14.42 & -5.38 & 1.18 & -0.38 \\
\hline chr2:257-315 significant & RJF & 21.97 & -7.41 & 1.22 & 0.36 \\
\hline chr2:229-334 suggestive & RJF & 11.57 & -1.33 & 0.37 & -0.85 \\
\hline chr2:231-330 suggestive & RJF & 11.24 & -6.95 & 1.7 & 1.05 \\
\hline chr2:241-323 suggestive & RJF & 12.17 & -5.04 & 1.21 & 0.29 \\
\hline chr2:213-327 significant & RJF & 11.5 & -4.43 & 1.1 & -0.62 \\
\hline chr2:220-321 significant & RJF & 11.14 & -5.72 & 1.45 & -1.41 \\
\hline chr2:213-333 significant & RJF & 11.4 & -3.34 & 0.82 & 0.64 \\
\hline chr2:244-323 suggestive & RJF & 12.53 & -4.43 & 1.05 & 0.15 \\
\hline chr2:237-326 suggestive & RJF & 12.09 & -4.21 & 1.02 & 0.73 \\
\hline chr2:215-329 significant & RJF & 11.51 & -5.96 & 1.44 & 1.18 \\
\hline chr2:216-327 significant & RJF & 12.03 & -4.33 & 1.06 & -0.57 \\
\hline chr2:244-321 significant & RJF & 12.74 & -5.18 & 1.21 & 0.33 \\
\hline chr2:247-317 significant & RJF & 19.39 & -10.32 & 1.84 & 1.82 \\
\hline chr2:257-301 suggestive & WL & 17.77 & 0.26 & 0.07 & -0.46 \\
\hline chr2:249-317 suggestive & RJF & 16.82 & -7 & 1.38 & 0.26 \\
\hline chr2:214-327 significant & RJF & 12.54 & -5.98 & 1.41 & 0.51 \\
\hline chr2:214-330 suggestive & RJF & 11.59 & -5.93 & 1.47 & 0.43 \\
\hline chr2:214-330 significant & RJF & 11.72 & -5.45 & 1.34 & 1 \\
\hline chr2:214-329 suggestive & RJF & 11.7 & -5.49 & 1.35 & 0.75 \\
\hline chr2:214-334 suggestive & RJF & 10.85 & -5.43 & 1.4 & 1.35 \\
\hline chr2:253-318 significant & RJF & 16.17 & -1.13 & 0.24 & -0.34 \\
\hline chr2:263-328 suggestive & RJF & 14.67 & -0.97 & 0.26 & -0.87 \\
\hline chr2:255-329 significant & RJF & 14.4 & -1.44 & 0.32 & -0.18 \\
\hline chr2:257-319 significant & RJF & 21.57 & -2.04 & 0.35 & 0.31 \\
\hline chr2:241-318 significant & RJF & 16.61 & -0.91 & 0.22 & -0.8 \\
\hline chr2:246-319 significant & WL & 17.08 & 2.95 & 0.6 & -0.07 \\
\hline chr2:259-308 significant & WL & 19.04 & 2.74 & 0.51 & -0.53 \\
\hline chr2:213-364 suggestive & RJF & 12.98 & -0.28 & 0.61 & 0.48 \\
\hline chr2:240-311 significant & RJF & 18.49 & -1.05 & 0.21 & -0.25 \\
\hline chr2:238-320 suggestive & WL & 12.86 & 3.85 & 0.92 & -0.39 \\
\hline chr2:261-318 significant & WL & 15.2 & 3.51 & 0.75 & -0.31 \\
\hline chr2:228-316 suggestive & WL & 13.7 & 2.49 & 0.57 & -0.11 \\
\hline chr2:265-316 significant & WL & 14.58 & 3.49 & 0.76 & -0.84 \\
\hline chr2:281-316 suggestive & RJF & 14.43 & -1.42 & 0.43 & -1.48 \\
\hline chr2:237-313 suggestive & WL & 14.49 & 1.92 & 0.43 & -0.14 \\
\hline chr2:251-321 significant & WL & 15.75 & 3.52 & 0.75 & -1.12 \\
\hline chr2:257-308 significant & WL & 16.64 & 1.93 & 0.39 & -0.41 \\
\hline chr2:262-309 suggestive & RJF & 11.62 & -0.15 & 0.65 & -0.5 \\
\hline
\end{tabular}




\begin{tabular}{|c|c|c|c|c|c|}
\hline chr2:250-326 suggestive & WL & 12.9 & 3.6 & 0.86 & -1.68 \\
\hline chr2:250-327 suggestive & RJF & 12.4 & -2.78 & 0.7 & -0.36 \\
\hline chr2:270-319 suggestive & WL & 19.66 & 1.6 & 0.34 & -1.66 \\
\hline chr2:261-318 significant & WL & 23.66 & 2.86 & 0.47 & -1.18 \\
\hline chr2:281-324 significant & WL & 15.29 & 1.96 & 0.42 & -0.7 \\
\hline chr2:261-315 significant & RJF & 24.91 & -2.11 & 0.35 & -0.45 \\
\hline chr2:254-319 significant & RJF & 17.44 & -1.7 & 0.37 & -0.58 \\
\hline chr2:283-316 significant & WL & 26.48 & 4.25 & 0.64 & -0.53 \\
\hline chr2:243-326 suggestive & WL & 13.47 & 2.37 & 0.55 & -1.19 \\
\hline chr2:251-327 suggestive & WL & 14.07 & 2.5 & 0.56 & -0.72 \\
\hline chr2:251-324 significant & RJF & 17.94 & -2.67 & 0.53 & 0.13 \\
\hline chr2:247-327 suggestive & WL & 13.84 & 1.79 & 0.41 & -0.35 \\
\hline chr2:257-314 significant & RJF & 37.81 & -3.22 & 0.37 & 0.97 \\
\hline chr2:261-324 significant & WL & 16.64 & 3.61 & 0.74 & -0.05 \\
\hline chr2:243-327 significant & WL & 14.83 & 4.18 & 0.94 & 0.39 \\
\hline chr2:247-325 suggestive & RJF & 11.29 & -1.74 & 0.56 & -1.69 \\
\hline chr2:262-318 significant & RJF & 26.5 & -3.5 & 0.56 & -1.27 \\
\hline chr2:266-325 significant & RJF & 18.91 & -2.17 & 0.41 & 0.36 \\
\hline chr2:251-322 significant & RJF & 22.57 & -1.81 & 0.3 & 0.26 \\
\hline chr2:260-329 significant & RJF & 14.8 & -2.22 & 0.51 & -0.42 \\
\hline chr2:260-324 significant & RJF & 23.37 & -2.22 & 0.37 & 0.34 \\
\hline chr2:281-323 significant & RJF & 27.1 & -3.4 & 0.52 & -0.4 \\
\hline chr2:252-330 significant & RJF & 17.59 & -2.31 & 0.46 & 0.05 \\
\hline chr2:264-331 suggestive & WL & 18.91 & 0.59 & 0.78 & -2.09 \\
\hline chr2:259-337 significant & RJF & 16.57 & -1.29 & 0.27 & 0.06 \\
\hline chr2:251-360 suggestive & RJF & 11.73 & -1.98 & 0.52 & 1.34 \\
\hline chr2:247-338 significant & RJF & 16.23 & -1.45 & 0.33 & -0.48 \\
\hline chr2:254-342 significant & RJF & 16.32 & -1.63 & 0.34 & -0.15 \\
\hline chr2:261-339 significant & RJF & 20.73 & -1.47 & 0.26 & 0.15 \\
\hline chr2:280-379 significant & RJF & 15.3 & -2.61 & 0.63 & 0.45 \\
\hline chr2:315-374 suggestive & WL & 21.15 & 0.6 & 0.11 & -0.49 \\
\hline chr2:305-375 significant & WL & 22.99 & 1.99 & 0.41 & 0.12 \\
\hline chr2:297-372 significant & RJF & 17.71 & -1.65 & 0.53 & -1.06 \\
\hline chr2:315-371 suggestive & RJF & 19.53 & -1.08 & 0.69 & -0.87 \\
\hline chr2:314-366 suggestive & RJF & 19.47 & -1.22 & 0.39 & -0.95 \\
\hline chr2:328-377 suggestive & WL & 23.52 & 1.31 & 0.23 & -1.44 \\
\hline chr2:329-373 significant & WL & 30.51 & 1.34 & 0.19 & -1.41 \\
\hline chr2:328-374 significant & WL & 26.77 & 1.38 & 0.22 & -1.24 \\
\hline chr2:315-368 significant & RJF & 15.48 & -2.04 & 0.52 & 0.09 \\
\hline chr2:330-380 suggestive & WL & 24.54 & 1.69 & 0.28 & -1.22 \\
\hline chr2:329-375 suggestive & WL & 24.18 & 2.15 & 0.37 & -1.47 \\
\hline chr2:309-378 suggestive & RJF & 13.66 & -2.67 & 0.74 & -0.11 \\
\hline chr2:324-370 significant & RJF & 21.94 & -2.28 & 0.48 & -0.08 \\
\hline
\end{tabular}




\begin{tabular}{|c|c|c|c|c|c|}
\hline chr2:327-367 significant & RJF & 24.92 & -2.64 & 0.83 & -0.75 \\
\hline chr2:329-376 significant & RJF & 16.2 & -1.77 & 0.5 & -0.47 \\
\hline chr2:323-375 significant & RJF & 17.02 & -2.48 & 0.63 & -0.21 \\
\hline chr2:321-383 significant & RJF & 16.65 & -2.41 & 0.6 & 0.01 \\
\hline chr2:320-378 suggestive & RJF & 15.19 & -1.45 & 0.45 & -0.58 \\
\hline chr2:326-381 significant & RJF & 14.2 & -2.81 & 0.69 & 0.71 \\
\hline chr2:326-386 significant & RJF & 13.43 & -2.01 & 0.55 & 0.11 \\
\hline chr2:325-373 significant & RJF & 16.61 & -1.2 & 0.24 & 0.71 \\
\hline chr2:322-380 significant & RJF & 15.89 & -1.64 & 0.38 & 0.28 \\
\hline chr2:328-379 suggestive & RJF & 13.87 & -0.67 & 0.46 & -1.57 \\
\hline chr2:336-371 significant & WL & 28.31 & 1.03 & 0.15 & -0.89 \\
\hline chr2:331-363 suggestive & RJF & 19.56 & -1.51 & 0.41 & -0.59 \\
\hline chr2:325-377 suggestive & RJF & 17.8 & -1.86 & 0.39 & 0.59 \\
\hline chr2:324-383 significant & WL & 22.43 & 0.42 & 1.01 & -0.27 \\
\hline chr2:322-389 significant & RJF & 14.11 & -2.2 & 0.55 & 0.38 \\
\hline chr2:325-392 suggestive & RJF & 12.6 & -2.43 & 0.78 & -0.48 \\
\hline chr2:325-390 significant & RJF & 16.04 & -2.15 & 0.53 & 0.15 \\
\hline chr2:330-381 suggestive & WL & 24.71 & 0.91 & 0.17 & -0.63 \\
\hline chr2:327-396 significant & RJF & 15.03 & -2.42 & 0.53 & 1.32 \\
\hline chr2:304-392 significant & WL & 15.75 & 1.91 & 0.53 & 0.51 \\
\hline chr2:321-401 suggestive & RJF & 14.01 & -3.2 & 0.78 & 0.89 \\
\hline chr2:331-385 significant & WL & 27.22 & 0.94 & 0.15 & -0.71 \\
\hline chr2:332-391 suggestive & WL & 21.82 & 0.92 & 0.16 & -0.81 \\
\hline chr2:321-422 suggestive & RJF & 13.84 & -1.93 & 1.28 & 0.12 \\
\hline chr2:328-394 suggestive & WL & 21.95 & 1.56 & 0.29 & -1.14 \\
\hline chr2:374-427 significant & RJF & 15.43 & -1.79 & 0.39 & 0.03 \\
\hline chr2:376-510 significant & RJF & 12.28 & -3.84 & 0.94 & 0.94 \\
\hline chr2:399-454 suggestive & WL & 15.01 & 1.77 & 0.4 & 0.89 \\
\hline chr2:421-500 significant & WL & 15.65 & 2.75 & 0.58 & 1.08 \\
\hline chr2:416-513 significant & WL & 17.89 & 0.28 & 0.61 & -0.27 \\
\hline chr2:419-505 significant & WL & 18.21 & 2 & 0.38 & 0.11 \\
\hline chr2:403-478 suggestive & WL & 15.3 & 9.91 & 2.18 & 2.92 \\
\hline chr2:409-476 suggestive & WL & 14.83 & 9 & 2.01 & 2.72 \\
\hline chr2:417-448 significant & WL & 24.05 & 6.78 & 1.09 & -0.28 \\
\hline chr2:418-454 significant & WL & 24.92 & 6.13 & 0.97 & -0.15 \\
\hline chr2:413-472 significant & WL & 18.54 & 13.36 & 2.59 & 2.59 \\
\hline chr2:422-454 significant & WL & 31.66 & 7.98 & 1.09 & -0.1 \\
\hline chr2:421-450 significant & WL & 29.15 & 7.65 & 1.1 & -1.12 \\
\hline chr2:422-453 significant & WL & 31.21 & 10.89 & 1.51 & 0.34 \\
\hline chr2:428-447 significant & WL & 50.16 & 11.51 & 1.05 & -0.66 \\
\hline chr2:428-448 significant & WL & 38.59 & 7.81 & 0.92 & 0 \\
\hline chr2:432-445 significant & WL & 60.73 & 13.59 & 1.01 & -0.78 \\
\hline chr2:427-447 suggestive & WL & 18.21 & 0.22 & 0.59 & -2.26 \\
\hline
\end{tabular}




\begin{tabular}{|c|c|c|c|c|c|}
\hline chr2:432-445 significant & WL & 59.7 & 19.56 & 1.47 & -2.75 \\
\hline chr2:415-467 significant & RJF & 16.72 & -2.35 & 0.8 & -2.88 \\
\hline chr2:434-446 significant & WL & 57.7 & 11.63 & 0.9 & -0.97 \\
\hline chr2:430-449 significant & WL & 40.61 & 17.74 & 1.97 & -3.2 \\
\hline chr2:427-463 significant & WL & 21.91 & 3.09 & 0.77 & -1.67 \\
\hline chr2:432-449 significant & WL & 42.76 & 18.45 & 1.96 & -3.12 \\
\hline chr2:433-450 significant & WL & 40.63 & 8.92 & 0.98 & -1.24 \\
\hline chr2:432-465 suggestive & WL & 20.05 & 2.78 & 1.03 & -2.48 \\
\hline chr2:436-446 significant & WL & 50.12 & 4.99 & 0.46 & -0.43 \\
\hline chr2:432-452 significant & WL & 25.39 & 4.66 & 0.89 & -2.86 \\
\hline chr2:433-454 significant & WL & 21 & 3.45 & 0.68 & -1.76 \\
\hline chr2:433-490 suggestive & WL & 16.33 & 0.72 & 0.91 & -2.41 \\
\hline chr2:430-451 significant & WL & 37.79 & 13.73 & 1.62 & -2.53 \\
\hline chr2:431-493 significant & WL & 21.88 & 2.71 & 0.48 & 0.16 \\
\hline chr2:423-470 significant & WL & 16.42 & 2 & 0.41 & -1.1 \\
\hline chr2:426-462 significant & WL & 24.78 & 5.75 & 0.93 & -0.31 \\
\hline chr2:425-460 significant & WL & 24.13 & 10.26 & 1.67 & -1.41 \\
\hline chr2:425-469 significant & WL & 22.75 & 7.08 & 1.21 & -0.15 \\
\hline chr2:431-481 significant & WL & 23.72 & 3.57 & 0.6 & -0.2 \\
\hline chr2:429-453 significant & WL & 34.06 & 11.79 & 1.5 & -2.78 \\
\hline chr2:420-501 significant & WL & 16.95 & 2.61 & 0.52 & -0.66 \\
\hline chr2:414-488 significant & WL & 16.29 & 1.85 & 0.38 & -0.89 \\
\hline chr2:427-491 significant & WL & 16.14 & 2.8 & 0.57 & -0.91 \\
\hline chr2:426-488 significant & RJF & 19.3 & -1.34 & 0.25 & 0.01 \\
\hline chr2:433-493 significant & WL & 20.8 & 1.22 & 0.22 & 0.28 \\
\hline chr2:425-499 suggestive & WL & 20.25 & 0.98 & 0.18 & -0.28 \\
\hline chr2:437-495 significant & WL & 18.45 & 1.53 & 0.28 & -0.55 \\
\hline chr2:435-489 significant & WL & 21.17 & 1.23 & 0.21 & 0.03 \\
\hline chr2:444-494 suggestive & WL & 15.4 & 1.68 & 0.37 & -0.51 \\
\hline chr2:449-479 significant & WL & 25.72 & 1.15 & 0.25 & -1.98 \\
\hline chr2:434-503 significant & WL & 21.76 & 1.96 & 0.34 & -0.38 \\
\hline chr2:447-513 suggestive & WL & 14.77 & 1.3 & 0.38 & -1.87 \\
\hline chr2:433-515 significant & RJF & 15.43 & -1.39 & 0.29 & -0.22 \\
\hline chr2:435-510 significant & RJF & 23.1 & -1.12 & 0.19 & -0.44 \\
\hline chr2:432-518 significant & WL & 20.24 & 1.42 & 0.26 & 0.32 \\
\hline chr2:445-528 significant & WL & 16.3 & 1.48 & 0.31 & -0.4 \\
\hline chr2:420-538 significant & WL & 15.87 & 0.89 & 0.19 & -0.39 \\
\hline chr2:437-546 significant & RJF & 14.37 & -1.57 & 0.35 & -0.2 \\
\hline chr2:437-528 suggestive & WL & 15.35 & 1.62 & 0.35 & -0.22 \\
\hline chr2:447-537 suggestive & WL & 19.16 & 1.21 & 0.29 & -1.65 \\
\hline chr2:425-518 significant & WL & 18.81 & 2.17 & 0.41 & -0.84 \\
\hline chr2:436-552 suggestive & WL & 15.91 & 1.72 & 0.36 & 0.06 \\
\hline chr2:418-547 significant & WL & 17.36 & 1.74 & 0.36 & -0.79 \\
\hline
\end{tabular}




\begin{tabular}{|c|c|c|c|c|c|}
\hline chr2:447-516 significant & WL & 19.06 & 1.48 & 0.28 & 0.18 \\
\hline chr2:433-510 significant & WL & 20.9 & 1.49 & 0.5 & -0.76 \\
\hline chr2:436-527 significant & WL & 20.69 & 2.38 & 0.43 & -0.7 \\
\hline chr2:445-539 suggestive & WL & 18.34 & 1.08 & 0.65 & 0.32 \\
\hline chr2:365-554 significant & RJF & 13.08 & -2.13 & 0.5 & -0.31 \\
\hline chr2:445-534 significant & WL & 19.63 & 1.25 & 0.23 & -0.2 \\
\hline chr2:435-541 significant & WL & 17.19 & 2.39 & 0.48 & 0.26 \\
\hline chr2:449-542 significant & WL & 20.15 & 2.05 & 0.45 & 0.08 \\
\hline chr2:456-540 significant & WL & 15.94 & 2.99 & 0.63 & -2.47 \\
\hline chr2:449-532 significant & WL & 23.58 & 2.81 & 0.54 & -0.15 \\
\hline chr2:447-554 significant & WL & 15.54 & 2.33 & 0.5 & -1.06 \\
\hline chr2:443-534 significant & WL & 17.95 & 4.18 & 0.87 & -1.35 \\
\hline chr2:446-543 significant & WL & 20.11 & 2.72 & 0.52 & -1.11 \\
\hline chr2:451-536 significant & WL & 18.53 & 2.61 & 0.51 & -1.24 \\
\hline chr2:480-545 significant & WL & 22.95 & 1.3 & 0.23 & -1.48 \\
\hline chr2:467-539 significant & WL & 16.55 & 2.24 & 0.45 & -1.49 \\
\hline chr2:462-538 significant & WL & 20.2 & 2.36 & 0.46 & -0.42 \\
\hline chr2:463-545 significant & WL & 15.54 & 2.5 & 0.56 & -0.89 \\
\hline chr2:465-538 significant & WL & 19.83 & 3.13 & 0.61 & -1.11 \\
\hline chr2:468-546 significant & WL & 17.16 & 3.98 & 0.85 & -1.35 \\
\hline chr2:486-529 significant & WL & 28.62 & 1.17 & 0.44 & 0.7 \\
\hline chr2:473-548 significant & WL & 16.73 & 2.22 & 0.45 & -1.68 \\
\hline chr2:466-545 suggestive & WL & 16.06 & 2.04 & 0.47 & -0.44 \\
\hline chr2:464-547 significant & WL & 16.76 & 1.77 & 0.44 & 0.15 \\
\hline chr2:475-543 significant & WL & 19.75 & 2.03 & 0.41 & -0.51 \\
\hline chr2:463-541 significant & WL & 17.86 & 4.36 & 0.94 & -0.77 \\
\hline chr2:479-538 significant & WL & 19.72 & 1.93 & 0.4 & -0.19 \\
\hline chr2:481-537 significant & WL & 19.42 & 2.18 & 0.48 & -0.04 \\
\hline chr2:490-532 significant & WL & 22.32 & 2.05 & 0.47 & 0.59 \\
\hline chr2:502-531 significant & WL & 39.62 & 2.9 & 0.36 & -0.56 \\
\hline chr2:488-529 significant & WL & 20.68 & 2.55 & 0.55 & 0.16 \\
\hline chr2:499-529 significant & WL & 32.65 & 3.64 & 0.56 & -0.02 \\
\hline chr2:498-534 significant & WL & 20.01 & 1.75 & 0.32 & -1.07 \\
\hline chr2:497-536 significant & WL & 25.35 & 0.25 & 0.53 & 0.01 \\
\hline chr2:498-529 suggestive & WL & 17.2 & 0.29 & 0.88 & 0.36 \\
\hline chr2:494-530 suggestive & RJF & 26.56 & -0.2 & 0.96 & 0.87 \\
\hline chr2:498-531 suggestive & WL & 27.34 & 0.29 & 0.53 & 1.02 \\
\hline chr2:485-535 significant & WL & 21.44 & 0.58 & 0.45 & -0.27 \\
\hline chr2:487-539 significant & WL & 26.38 & 4.04 & 0.69 & -0.59 \\
\hline chr2:486-551 significant & WL & 17.32 & 2.32 & 0.49 & -0.94 \\
\hline chr2:494-540 significant & WL & 27.14 & 3.66 & 0.61 & -0.65 \\
\hline chr2:492-552 suggestive & WL & 15.84 & 1.75 & 0.38 & -0.8 \\
\hline chr2:496-547 suggestive & WL & 26.23 & 0.09 & 0.52 & 1.51 \\
\hline
\end{tabular}




\begin{tabular}{|c|c|c|c|c|c|}
\hline chr2:477-542 significant & WL & 25.54 & 2.37 & 0.4 & -0.5 \\
\hline chr2:454-554 suggestive & WL & 12.32 & 1.24 & 0.36 & 0.05 \\
\hline chr2:495-552 suggestive & WL & 17.38 & 0.36 & 0.44 & -0.12 \\
\hline chr2:484-555 significant & WL & 16.96 & 1.25 & 0.25 & -1.13 \\
\hline chr2:494-556 suggestive & WL & 16.45 & 1.3 & 0.63 & -1.14 \\
\hline chr2:535-570 suggestive & RJF & 13.01 & -0.52 & 0.4 & -1.76 \\
\hline chr2:478-570 suggestive & WL & 14.6 & 1.97 & 0.45 & -2.06 \\
\hline chr2:538-570 significant & WL & 26.35 & 2.65 & 0.4 & -1.6 \\
\hline chr2:550-565 suggestive & WL & 11.36 & 2.02 & 0.38 & -2.32 \\
\hline chr2:474-579 suggestive & WL & 14.11 & 1.98 & 0.44 & -0.67 \\
\hline chr2:552-561 suggestive & WL & 15.58 & 0.74 & 0.18 & -1.27 \\
\hline chr2:541-605 significant & RJF & 14.09 & -2.08 & 0.55 & 0 \\
\hline chr2:553-561 significant & WL & 18.29 & 2 & 0.41 & -0.73 \\
\hline chr2:552-582 significant & RJF & 17.85 & -2.2 & 0.5 & 0.11 \\
\hline chr2:554-565 significant & WL & 20.86 & 2.51 & 0.46 & -1.01 \\
\hline chr2:555-572 significant & WL & 23.26 & 1.69 & 1.1 & 1.78 \\
\hline chr2:553-563 significant & RJF & 15.19 & -1.17 & 0.47 & -1.24 \\
\hline chr2:550-566 significant & WL & 22.53 & 2.75 & 0.52 & -1.37 \\
\hline chr2:556-562 significant & RJF & 57.18 & -6.77 & 0.63 & 0.13 \\
\hline chr2:556-562 significant & RJF & 45.28 & -4.5 & 0.57 & -0.61 \\
\hline chr2:555-569 significant & RJF & 36.16 & -3.91 & 0.56 & 0.05 \\
\hline chr2:556-561 significant & RJF & 62.63 & -9.21 & 0.74 & 0.59 \\
\hline chr2:555-571 significant & RJF & 21.04 & -0.72 & 0.16 & -0.1 \\
\hline chr2:553-573 significant & RJF & 19.09 & -0.87 & 0.2 & -0.03 \\
\hline chr2:556-561 significant & RJF & 64.63 & -9.81 & 0.75 & 0.71 \\
\hline chr2:555-581 significant & RJF & 20.71 & -2.04 & 0.41 & 0.22 \\
\hline chr2:558-560 suggestive & WL & 24.22 & 0.13 & 0.02 & -0.15 \\
\hline chr2:554-566 suggestive & RJF & 15.57 & -0.08 & 0.78 & 0.09 \\
\hline chr2:550-567 significant & WL & 20.82 & 1.35 & 0.33 & 0.48 \\
\hline chr2:555-569 significant & RJF & 35.83 & -2.17 & 0.32 & -0.1 \\
\hline chr2:553-569 significant & RJF & 39.94 & -4.58 & 0.62 & -0.22 \\
\hline chr2:552-570 significant & RJF & 32.72 & -2.8 & 0.43 & -0.56 \\
\hline chr2:554-571 significant & RJF & 26.36 & -2.63 & 0.45 & -0.21 \\
\hline chr2:552-567 significant & RJF & 22.36 & -2.64 & 0.53 & -0.54 \\
\hline chr2:553-570 significant & RJF & 22.33 & -3.61 & 0.67 & -0.1 \\
\hline chr2:537-574 significant & WL & 18.09 & 2.55 & 0.5 & -1.13 \\
\hline chr2:546-589 significant & RJF & 15.48 & -1.59 & 0.48 & -1.12 \\
\hline chr2:553-571 significant & RJF & 16.68 & -0.72 & 0.16 & -0.05 \\
\hline chr2:551-572 significant & RJF & 13.11 & -2.1 & 0.57 & -0.37 \\
\hline chr2:552-569 significant & WL & 16.42 & 2.81 & 0.58 & -1.47 \\
\hline chr2:544-566 suggestive & WL & 18.95 & 0.05 & 0.01 & -0.05 \\
\hline chr2:560-568 suggestive & WL & 9.75 & 1.75 & 0.32 & -2.31 \\
\hline chr2:526-572 significant & WL & 16.41 & 2.3 & 0.51 & -0.11 \\
\hline
\end{tabular}




\begin{tabular}{|c|c|c|c|c|c|}
\hline chr2:553-569 significant & WL & 23.13 & 1.7 & 0.37 & 0.93 \\
\hline chr2:551-569 significant & WL & 18.65 & 3.56 & 0.72 & -0.3 \\
\hline chr2:554-571 significant & WL & 20.08 & 4.66 & 1.27 & -3.34 \\
\hline chr2:554-571 significant & WL & 18.93 & 2.05 & 0.39 & -0.84 \\
\hline chr2:553-570 significant & RJF & 19.82 & -2.2 & 0.57 & -0.73 \\
\hline chr2:556-571 suggestive & RJF & 19.23 & -0.53 & 0.49 & 0.76 \\
\hline chr2:560-571 significant & WL & 19.89 & 2.56 & 0.61 & -2.49 \\
\hline chr2:554-571 suggestive & WL & 19.13 & 4.43 & 0.94 & -1.68 \\
\hline chr2:554-604 suggestive & RJF & 12.78 & -1.43 & 0.41 & -0.35 \\
\hline chr2:553-572 suggestive & RJF & 13.73 & -0.67 & 0.16 & 0.07 \\
\hline chr2:560-571 suggestive & WL & 19.53 & 1.45 & 0.27 & -1 \\
\hline chr2:559-570 significant & RJF & 34.57 & -3.19 & 0.46 & -0.64 \\
\hline chr2:554-572 significant & RJF & 20.09 & -1.96 & 0.42 & -0.48 \\
\hline chr2:550-572 significant & RJF & 28.57 & -3.56 & 0.6 & -0.57 \\
\hline chr2:561-572 significant & RJF & 24.58 & -2.61 & 0.48 & -0.47 \\
\hline chr2:554-601 significant & RJF & 15.59 & -2.52 & 0.59 & 0.02 \\
\hline chr2:542-588 suggestive & RJF & 14.46 & -1.02 & 0.38 & -1.19 \\
\hline chr2:554-580 suggestive & RJF & 12.69 & -0.08 & 0.25 & -1.3 \\
\hline chr2:553-611 significant & RJF & 19.69 & -1.15 & 0.26 & -0.35 \\
\hline chr2:553-597 significant & RJF & 16.06 & -1.94 & 0.5 & -0.69 \\
\hline chr2:552-600 suggestive & RJF & 14.37 & -1.79 & 0.47 & -0.46 \\
\hline chr2:560-574 suggestive & WL & 16.81 & 1.86 & 0.69 & -2.75 \\
\hline chr2:556-583 significant & RJF & 20.27 & -1.66 & 0.7 & 2.11 \\
\hline chr2:557-598 significant & RJF & 19.94 & -2.62 & 0.54 & -0.34 \\
\hline chr2:553-574 suggestive & WL & 14.19 & 1.81 & 0.83 & -3.46 \\
\hline chr2:560-572 suggestive & WL & 16.94 & 2.24 & 0.86 & -4.9 \\
\hline chr2:561-573 suggestive & WL & 21.2 & 0.92 & 0.44 & -2.46 \\
\hline chr2:550-599 significant & RJF & 15.52 & -2.17 & 0.58 & -0.48 \\
\hline chr2:555-588 suggestive & WL & 18.35 & 2.57 & 0.82 & -2.11 \\
\hline chr2:561-597 significant & RJF & 31 & -1.14 & 0.19 & -0.39 \\
\hline chr2:569-601 significant & RJF & 23.64 & -1.96 & 0.35 & 0.19 \\
\hline chr2:556-595 significant & RJF & 14.21 & -1.19 & 0.33 & -0.38 \\
\hline chr2:561-607 suggestive & RJF & 13.54 & -1.35 & 0.37 & -0.4 \\
\hline chr2:551-598 significant & RJF & 17.26 & -3.42 & 1.03 & 1.87 \\
\hline chr2:550-603 suggestive & RJF & 12.91 & -1.69 & 0.55 & -0.91 \\
\hline chr2:577-605 suggestive & RJF & 14.43 & -2.78 & 0.7 & 1.1 \\
\hline chr2:582-589 significant & WL & 17.54 & 1.61 & 0.37 & -2.09 \\
\hline chr2:578-590 significant & RJF & 16.55 & -2.65 & 0.75 & 1.62 \\
\hline chr2:582-587 significant & WL & 30.27 & 1.97 & 0.29 & -1.93 \\
\hline chr2:582-588 suggestive & WL & 18.38 & 1.51 & 0.3 & -1.46 \\
\hline chr2:582-585 significant & WL & 39.08 & 0.26 & 0.03 & -0.26 \\
\hline chr2:582-588 suggestive & WL & 18.47 & 2.36 & 0.48 & -2.22 \\
\hline chr2:581-588 suggestive & WL & 24.27 & 1.61 & 0.27 & -1.5 \\
\hline
\end{tabular}




\begin{tabular}{|c|c|c|c|c|c|}
\hline chr2:583-587 suggestive & WL & 24.03 & 0.79 & 0.14 & -0.79 \\
\hline chr2:580-588 suggestive & RJF & 19.41 & -2.48 & 0.89 & 2.28 \\
\hline chr2:580-588 suggestive & RJF & 20.29 & -2.81 & 0.68 & 1.26 \\
\hline chr2:578-589 suggestive & RJF & 17.41 & -2.34 & 0.76 & 0.66 \\
\hline chr2:580-590 significant & RJF & 18.69 & -1.57 & 0.5 & 2.28 \\
\hline chr2:576-590 suggestive & RJF & 17.05 & -3.07 & 1.09 & 3.61 \\
\hline chr2:537-606 suggestive & RJF & 10.88 & -2.88 & 0.81 & 0.27 \\
\hline chr2:576-598 suggestive & RJF & 13.25 & -2.28 & 0.55 & 0.63 \\
\hline chr2:581-594 suggestive & WL & 13.4 & 2.53 & 0.67 & -2.44 \\
\hline chr2:573-606 suggestive & RJF & 13.13 & -13.74 & 3.4 & 7.17 \\
\hline chr2:576-605 suggestive & RJF & 12.84 & -15.18 & 3.87 & 10.32 \\
\hline chr2:578-592 significant & RJF & 24.68 & -3.55 & 0.64 & -0.28 \\
\hline chr2:578-590 suggestive & RJF & 17.86 & -2.09 & 0.85 & 3.22 \\
\hline chr2:564-608 suggestive & RJF & 13.2 & -0.74 & 0.25 & -0.46 \\
\hline chr2:581-590 suggestive & WL & 24.05 & 1.23 & 0.21 & -1.11 \\
\hline chr2:577-599 suggestive & RJF & 17.06 & -1.06 & 0.72 & 2.79 \\
\hline chr2:561-602 significant & RJF & 14.38 & -1.46 & 0.43 & -0.69 \\
\hline chr2:577-600 significant & RJF & 20.29 & -2.5 & 0.45 & 1.02 \\
\hline chr2:581-598 suggestive & WL & 24.2 & 0.34 & 0.06 & -0.37 \\
\hline chr2:655-689 significant & RJF & 13.95 & -2.52 & 0.6 & -0.31 \\
\hline chr2:657-686 significant & WL & 15.42 & 2.13 & 0.46 & -1.21 \\
\hline chr2:658-689 suggestive & WL & 14.3 & 1.8 & 0.4 & -0.97 \\
\hline chr2:651-717 significant & WL & 17.16 & 2.01 & 0.41 & -0.32 \\
\hline chr2:622-703 suggestive & RJF & 11.89 & -1.69 & 0.42 & 0.21 \\
\hline chr2:651-722 suggestive & WL & 13.84 & 1.49 & 0.36 & -0.96 \\
\hline chr2:676-691 suggestive & WL & 19.61 & 1.02 & 0.84 & -5.66 \\
\hline chr2:674-693 significant & RJF & 13.63 & -1.45 & 0.38 & -1.05 \\
\hline chr2:676-717 suggestive & WL & 19.48 & 1.26 & 0.25 & -0.78 \\
\hline chr2:673-707 significant & RJF & 15.11 & -2.08 & 0.45 & 0.39 \\
\hline chr2:677-699 significant & RJF & 20.53 & -1.27 & 0.91 & 4.85 \\
\hline chr2:675-704 significant & WL & 23.77 & 1 & 0.17 & -0.35 \\
\hline chr2:663-701 suggestive & RJF & 13.56 & -1.25 & 0.29 & -0.35 \\
\hline chr2:676-722 significant & WL & 15.86 & 0.83 & 0.21 & -0.75 \\
\hline chr2:674-701 significant & RJF & 24.83 & -3.25 & 0.52 & 0.62 \\
\hline chr2:678-700 suggestive & RJF & 18.6 & -1.51 & 0.9 & 4.8 \\
\hline chr2:674-705 significant & RJF & 16.61 & -2.39 & 0.48 & 0 \\
\hline chr2:677-706 suggestive & RJF & 13.95 & -1.9 & 0.49 & 1.43 \\
\hline chr2:676-712 significant & WL & 14.85 & 0.41 & 0.15 & -0.79 \\
\hline chr2:669-703 significant & RJF & 18.38 & -1.99 & 0.39 & -0.53 \\
\hline chr2:685-697 significant & RJF & 31.23 & -0.87 & 0.12 & -0.09 \\
\hline chr2:678-704 significant & RJF & 20.45 & -1.8 & 0.32 & -0.36 \\
\hline chr2:675-708 suggestive & WL & 14.9 & 0.83 & 0.22 & -0.73 \\
\hline chr2:679-704 suggestive & WL & 17.95 & 0.19 & 0.37 & -2.6 \\
\hline
\end{tabular}




\begin{tabular}{|c|c|c|c|c|c|}
\hline chr2:689-703 significant & RJF & 19.92 & -1.73 & 0.56 & -3.02 \\
\hline chr2:643-706 suggestive & RJF & 18.33 & -0.06 & 0.56 & -0.08 \\
\hline chr2:655-707 significant & RJF & 20.71 & -0.3 & 0.39 & -0.2 \\
\hline chr2:688-698 significant & RJF & 24.82 & -1.69 & 0.27 & 0.48 \\
\hline chr2:690-701 significant & RJF & 24.75 & -0.81 & 0.13 & -0.32 \\
\hline chr2:686-699 significant & RJF & 27.05 & -2.18 & 0.33 & 0.37 \\
\hline chr2:690-698 significant & RJF & 28.22 & -1.35 & 0.19 & 0.03 \\
\hline chr2:691-698 significant & RJF & 26.22 & -1.09 & 0.15 & 0.11 \\
\hline chr2:683-701 significant & RJF & 17.01 & -1.89 & 0.36 & 0.18 \\
\hline chr2:686-705 significant & WL & 16.04 & 1.24 & 0.33 & -1.3 \\
\hline chr2:681-705 significant & WL & 16.82 & 1.55 & 0.32 & -0.29 \\
\hline chr2:685-707 significant & WL & 15.03 & 0.17 & 0.3 & -1.85 \\
\hline chr2:678-718 suggestive & WL & 14.48 & 0.42 & 0.27 & -1.57 \\
\hline chr2:678-701 significant & WL & 23.86 & 0.88 & 0.16 & -0.64 \\
\hline chr2:684-704 significant & RJF & 19.61 & -1.44 & 0.26 & -0.07 \\
\hline chr2:686-703 significant & RJF & 19.71 & -1.13 & 0.21 & -0.38 \\
\hline chr2:679-709 suggestive & RJF & 11.35 & -1.55 & 0.45 & 1.17 \\
\hline chr2:673-708 significant & WL & 19.76 & 1.84 & 0.34 & -0.53 \\
\hline chr2:689-701 significant & WL & 49.33 & 1.9 & 0.18 & -0.63 \\
\hline chr2:690-706 suggestive & RJF & 27.02 & -0.12 & 0.03 & -0.13 \\
\hline chr2:680-707 significant & WL & 21.6 & 2.88 & 0.51 & -0.62 \\
\hline chr2:683-708 significant & WL & 21.22 & 2.52 & 0.45 & -0.64 \\
\hline chr2:680-711 significant & WL & 19.86 & 0.61 & 0.14 & -0.6 \\
\hline chr2:677-708 significant & WL & 21.23 & 1.35 & 0.34 & -1.84 \\
\hline chr2:689-708 significant & WL & 23.3 & 1.67 & 0.28 & 0.03 \\
\hline chr2:688-709 significant & WL & 21.5 & 1.7 & 0.6 & -2.05 \\
\hline chr2:688-709 suggestive & RJF & 22.84 & -1.08 & 0.44 & -1.96 \\
\hline chr2:678-709 significant & WL & 23.46 & 2.09 & 0.35 & -0.42 \\
\hline chr2:677-709 significant & WL & 22.49 & 2.13 & 0.37 & -0.69 \\
\hline chr2:682-712 significant & WL & 18.91 & 2.3 & 0.43 & -0.16 \\
\hline chr2:672-717 significant & WL & 18.32 & 1.71 & 0.36 & -1.08 \\
\hline chr2:688-707 significant & WL & 34.02 & 3.03 & 0.38 & -0.11 \\
\hline chr2:663-714 suggestive & WL & 15.06 & 1.54 & 0.36 & -0.86 \\
\hline chr2:671-721 suggestive & WL & 15.46 & 1.53 & 0.35 & -0.76 \\
\hline chr2:676-716 significant & WL & 14.81 & 2.56 & 0.55 & 0.05 \\
\hline chr2:677-708 significant & WL & 32.79 & 1.46 & 0.2 & -0.54 \\
\hline chr2:688-713 significant & WL & 25.28 & 3.26 & 0.53 & 1.32 \\
\hline chr2:677-711 suggestive & WL & 19.32 & 0.84 & 0.48 & -1.62 \\
\hline chr2:677-712 significant & WL & 19.19 & 1.14 & 0.24 & -0.83 \\
\hline chr2:675-709 significant & WL & 34.46 & 4.64 & 0.58 & -0.41 \\
\hline chr2:685-709 significant & WL & 33.26 & 3.33 & 0.47 & -1.94 \\
\hline chr2:660-715 significant & WL & 15.16 & 3.13 & 0.64 & 0.26 \\
\hline chr2:679-727 significant & WL & 19.85 & 1.48 & 0.33 & -1.39 \\
\hline
\end{tabular}




\begin{tabular}{|c|c|c|c|c|c|}
\hline chr2:682-717 significant & RJF & 15.83 & -2.34 & 0.49 & 0.12 \\
\hline chr2:678-736 significant & WL & 15.54 & 1.78 & 0.46 & -1.67 \\
\hline chr2:656-727 significant & WL & 17.15 & 2.6 & 0.55 & -1.29 \\
\hline chr2:675-723 suggestive & WL & 14.56 & 1.91 & 0.42 & -0.02 \\
\hline chr2:683-719 significant & WL & 19.99 & 2.66 & 0.5 & 1.09 \\
\hline chr2:679-720 significant & WL & 21.1 & 1.35 & 0.27 & -1.02 \\
\hline chr2:677-721 significant & WL & 30.13 & 1.51 & 0.21 & -0.42 \\
\hline chr2:674-716 significant & WL & 21.75 & 1.46 & 0.27 & -0.87 \\
\hline chr2:678-719 significant & WL & 16.75 & 1.76 & 0.36 & -0.19 \\
\hline chr2:674-737 suggestive & WL & 13.85 & 1.47 & 0.35 & -0.68 \\
\hline chr2:689-719 significant & WL & 23.05 & 3.33 & 0.57 & 0.95 \\
\hline chr2:688-714 significant & WL & 33.78 & 2.49 & 0.32 & -0.1 \\
\hline chr2:661-726 significant & WL & 20.74 & 2.49 & 0.44 & -0.08 \\
\hline chr2:659-731 suggestive & WL & 14.66 & 1.29 & 0.36 & -1.37 \\
\hline chr2:638-736 suggestive & WL & 13.18 & 1.58 & 0.37 & -0.36 \\
\hline chr2:675-729 significant & WL & 16.2 & 1.64 & 0.34 & 0.55 \\
\hline chr2:658-723 significant & WL & 15.96 & 1.72 & 0.38 & -0.52 \\
\hline chr2:682-728 significant & WL & 18.07 & 2.21 & 0.44 & -0.33 \\
\hline chr2:683-722 suggestive & WL & 15.58 & 1.34 & 0.3 & -0.37 \\
\hline chr2:681-729 suggestive & WL & 20.57 & 1.79 & 0.42 & -1.83 \\
\hline chr2:691-737 suggestive & WL & 15.63 & 2.47 & 0.55 & 1.44 \\
\hline chr2:662-731 suggestive & WL & 15.29 & 1.58 & 0.38 & -0.9 \\
\hline chr2:699-719 significant & RJF & 24.39 & -4.79 & 0.92 & -0.77 \\
\hline chr2:655-720 significant & WL & 14.1 & 0.95 & 0.29 & -1.09 \\
\hline chr2:703-719 significant & RJF & 33.28 & -4.42 & 0.59 & 0.87 \\
\hline chr2:688-725 significant & WL & 14.81 & 1.17 & 0.29 & -0.74 \\
\hline chr2:705-718 significant & RJF & 36.85 & -5.45 & 0.72 & 2.55 \\
\hline chr2:699-749 significant & WL & 15.52 & 1.15 & 0.28 & -0.73 \\
\hline chr2:701-741 significant & WL & 20.02 & 1.64 & 0.3 & 0.55 \\
\hline chr2:700-721 suggestive & WL & 16.34 & 2.34 & 0.49 & -0.21 \\
\hline chr2:701-720 significant & WL & 17.13 & 1.33 & 0.26 & 0.29 \\
\hline chr2:706-735 significant & WL & 17.59 & 1.8 & 0.36 & -0.21 \\
\hline chr2:700-722 suggestive & RJF & 22.37 & -0.66 & 0.47 & -1.64 \\
\hline chr2:706-717 suggestive & WL & 26.34 & 1.18 & 0.35 & 0.77 \\
\hline chr2:705-721 suggestive & WL & 20.38 & 1.03 & 0.19 & -0.14 \\
\hline chr2:704-722 suggestive & WL & 16.05 & 1.21 & 0.23 & -0.31 \\
\hline chr2:701-729 significant & WL & 14.98 & 1.2 & 0.27 & -0.31 \\
\hline chr2:703-722 significant & WL & 16.75 & 1.63 & 0.33 & -0.22 \\
\hline chr2:697-723 suggestive & WL & 14.44 & 1.27 & 0.28 & 0.26 \\
\hline chr2:702-736 suggestive & WL & 15.54 & 1.22 & 0.26 & 0.03 \\
\hline chr2:698-722 suggestive & WL & 17.99 & 1.54 & 0.33 & 1.21 \\
\hline chr2:703-721 suggestive & WL & 20.42 & 1.62 & 0.34 & -1.05 \\
\hline chr2:704-721 suggestive & WL & 13.67 & 2.03 & 0.36 & 0.22 \\
\hline
\end{tabular}




\begin{tabular}{|c|c|c|c|c|c|}
\hline chr2:706-720 suggestive & WL & 20.46 & 0.43 & 0.22 & 1.64 \\
\hline chr2:707-721 significant & RJF & 26.75 & -1.48 & 0.24 & 0.58 \\
\hline chr2:704-727 significant & WL & 14.72 & 0.74 & 0.25 & -1.06 \\
\hline chr2:699-737 suggestive & WL & 16.11 & 1.94 & 0.41 & 0.93 \\
\hline chr2:706-724 significant & WL & 16.31 & 1.03 & 0.37 & -1.8 \\
\hline chr2:708-723 significant & WL & 22.94 & 2.18 & 0.39 & -0.89 \\
\hline chr2:709-722 significant & WL & 30.29 & 2.84 & 0.4 & -0.49 \\
\hline chr2:696-733 significant & WL & 16.1 & 1.73 & 0.38 & -0.74 \\
\hline chr2:701-737 significant & WL & 16.76 & 1.14 & 0.26 & -0.68 \\
\hline chr2:701-728 suggestive & WL & 20.8 & 2.41 & 0.43 & -0.06 \\
\hline chr2:706-738 suggestive & WL & 16.12 & 1.66 & 0.29 & -0.47 \\
\hline chr2:705-724 suggestive & WL & 20.28 & 1.47 & 0.28 & -0.65 \\
\hline chr2:704-749 significant & WL & 16.44 & 1.44 & 0.31 & -0.4 \\
\hline chr2:701-737 significant & WL & 14.64 & 1.51 & 0.39 & -1 \\
\hline chr2:704-729 suggestive & WL & 14.45 & 1.11 & 0.35 & -1.35 \\
\hline chr2:698-729 suggestive & WL & 14.14 & 1.03 & 0.43 & -2.01 \\
\hline chr2:703-738 significant & WL & 16.94 & 2.03 & 0.43 & -0.7 \\
\hline chr2:705-728 suggestive & WL & 21.59 & 1.88 & 0.39 & -1.43 \\
\hline chr2:705-726 suggestive & WL & 20.51 & 1.55 & 0.33 & -1.11 \\
\hline chr2:700-745 suggestive & WL & 15.68 & 1.07 & 0.22 & -0.09 \\
\hline chr2:699-729 significant & RJF & 22.42 & -4.92 & 1.39 & -0.86 \\
\hline chr2:699-736 significant & WL & 16.48 & 1.44 & 0.33 & -0.82 \\
\hline chr2:704-734 significant & WL & 16.7 & 0.7 & 0.21 & -0.94 \\
\hline chr2:704-731 suggestive & WL & 18.22 & 1.06 & 0.23 & -0.65 \\
\hline chr2:700-737 suggestive & WL & 16.12 & 1.07 & 0.24 & -0.58 \\
\hline chr2:697-739 significant & WL & 15.64 & 1.13 & 0.23 & 0.35 \\
\hline chr2:701-735 suggestive & WL & 15.1 & 1.35 & 0.34 & -0.88 \\
\hline chr2:700-745 suggestive & WL & 15.56 & 1.01 & 0.24 & -0.51 \\
\hline chr2:702-736 suggestive & WL & 21.75 & 2.28 & 0.42 & 1.55 \\
\hline chr2:694-733 significant & RJF & 15.4 & -2.64 & 0.58 & -1.35 \\
\hline chr2:702-736 significant & WL & 17.78 & 1.12 & 0.24 & -0.45 \\
\hline chr2:696-739 significant & WL & 16.71 & 1.7 & 0.36 & -0.52 \\
\hline chr2:697-737 suggestive & WL & 15.17 & 1.33 & 0.3 & -0.34 \\
\hline chr2:698-738 significant & WL & 17 & 1.69 & 0.38 & -0.84 \\
\hline chr2:697-738 suggestive & WL & 15.43 & 1.39 & 0.31 & -0.21 \\
\hline chr2:683-733 significant & WL & 16.37 & 0.58 & 0.2 & -1.02 \\
\hline chr2:703-738 significant & WL & 19.96 & 1.61 & 0.29 & -0.04 \\
\hline chr2:705-754 significant & WL & 16.17 & 1.54 & 0.32 & -0.06 \\
\hline chr2:707-739 significant & WL & 17.02 & 1.76 & 0.37 & -0.88 \\
\hline chr2:701-739 significant & WL & 16.89 & 1.27 & 0.26 & -0.36 \\
\hline chr2:705-736 suggestive & WL & 19.61 & 1.38 & 0.43 & -2.36 \\
\hline chr2:707-737 significant & WL & 26.15 & 1.87 & 0.34 & -1.89 \\
\hline chr2:705-738 significant & WL & 17.79 & 1.44 & 0.29 & -0.69 \\
\hline
\end{tabular}




\begin{tabular}{|c|c|c|c|c|c|}
\hline chr2:709-761 significant & WL & 21.91 & 2.63 & 0.47 & 0.65 \\
\hline chr2:708-737 significant & WL & 24.5 & 2.11 & 0.37 & -1.65 \\
\hline chr2:702-734 significant & WL & 17.75 & 1.47 & 0.31 & -1.05 \\
\hline chr2:705-740 significant & WL & 16.29 & 1.43 & 0.3 & 0.24 \\
\hline chr2:700-739 suggestive & WL & 15.37 & 1.52 & 0.33 & 0 \\
\hline chr2:692-748 suggestive & WL & 14.72 & 1.02 & 0.24 & -0.71 \\
\hline chr2:686-738 suggestive & WL & 14.83 & 0.84 & 0.2 & -0.6 \\
\hline chr2:703-759 suggestive & WL & 13.25 & 0.8 & 0.21 & -0.76 \\
\hline chr2:708-736 significant & WL & 35.01 & 2.53 & 0.32 & 0.25 \\
\hline chr2:710-737 significant & WL & 24.6 & 2.07 & 0.35 & -1.38 \\
\hline chr2:705-738 significant & WL & 23.07 & 1.64 & 0.29 & -1.06 \\
\hline chr2:709-739 suggestive & WL & 20.2 & 2.61 & 0.51 & -1.67 \\
\hline chr2:707-736 significant & WL & 20.32 & 2.21 & 0.44 & -2.08 \\
\hline chr2:712-739 significant & WL & 28.58 & 3.01 & 0.43 & -0.78 \\
\hline chr2:707-729 significant & WL & 20.64 & 1.51 & 0.33 & -1.65 \\
\hline chr2:708-737 significant & WL & 18.73 & 1.91 & 0.42 & -1.79 \\
\hline chr2:707-739 significant & WL & 15.71 & 1.43 & 0.31 & -0.71 \\
\hline chr2:702-737 suggestive & WL & 13.42 & 0.35 & 0.11 & -0.56 \\
\hline chr2:701-739 suggestive & WL & 14.78 & 1.03 & 0.23 & 0.32 \\
\hline chr2:683-737 significant & WL & 15.14 & 1.06 & 0.28 & -1.27 \\
\hline chr2:710-734 suggestive & WL & 19.21 & 0.84 & 0.52 & -1.84 \\
\hline chr2:706-735 suggestive & WL & 21.55 & 1.14 & 0.23 & -1.13 \\
\hline chr2:697-734 suggestive & WL & 21.8 & 1.54 & 0.32 & -1.6 \\
\hline chr2:707-735 suggestive & WL & 20.63 & 1.31 & 0.28 & -1.48 \\
\hline chr2:683-741 suggestive & RJF & 14.2 & -1.55 & 0.34 & 0.04 \\
\hline chr2:697-738 suggestive & WL & 14.29 & 0.59 & 0.22 & -1.15 \\
\hline chr2:708-737 significant & WL & 17.99 & 1.61 & 0.38 & -1.77 \\
\hline chr2:701-742 significant & WL & 19.46 & 2.2 & 0.42 & -1 \\
\hline chr2:705-738 significant & WL & 16.03 & 0.82 & 0.22 & -1 \\
\hline chr2:712-729 significant & WL & 21.61 & 1.79 & 0.34 & -1.38 \\
\hline chr2:715-730 significant & WL & 20.86 & 2.09 & 0.54 & -2.58 \\
\hline chr2:711-742 significant & WL & 22.27 & 3.47 & 0.59 & -0.7 \\
\hline chr2:709-737 suggestive & WL & 15.02 & 0.87 & 0.43 & -2.67 \\
\hline chr2:689-739 suggestive & WL & 13.52 & 0.91 & 0.28 & -1.37 \\
\hline chr2:710-737 significant & WL & 16.96 & 1.45 & 0.32 & -1.19 \\
\hline chr2:705-737 significant & WL & 15.43 & 1.32 & 0.32 & -1.3 \\
\hline chr2:704-755 suggestive & WL & 14.43 & 2.42 & 0.57 & -1.47 \\
\hline chr2:704-753 suggestive & WL & 13.47 & 1.55 & 0.41 & -1.41 \\
\hline chr2:707-738 suggestive & WL & 14.3 & 1.2 & 0.35 & -1.59 \\
\hline chr2:713-734 suggestive & WL & 19.03 & 0.82 & 0.24 & -1.63 \\
\hline chr2:711-731 suggestive & WL & 18.65 & 1.15 & 0.28 & -1.54 \\
\hline chr2:713-737 suggestive & WL & 19.1 & 1.31 & 0.36 & -2.2 \\
\hline chr2:709-735 suggestive & WL & 18.63 & 0.96 & 0.23 & -1.19 \\
\hline
\end{tabular}




\begin{tabular}{|c|c|c|c|c|c|}
\hline chr2:696-735 suggestive & WL & 20 & 1.23 & 0.3 & -1.7 \\
\hline chr2:704-755 suggestive & WL & 14.65 & 1.15 & 0.26 & -0.68 \\
\hline chr2:710-740 significant & WL & 20.08 & 1.54 & 0.29 & -0.7 \\
\hline chr2:715-730 suggestive & WL & 20.2 & 1.45 & 0.38 & -1.58 \\
\hline chr2:714-736 significant & WL & 23.3 & 1.96 & 0.36 & -1.53 \\
\hline chr2:706-738 significant & WL & 16.81 & 0.98 & 0.3 & -1.72 \\
\hline chr2:714-735 significant & WL & 18.69 & 1.13 & 0.25 & -1.11 \\
\hline chr2:696-729 suggestive & WL & 16.33 & 1.04 & 0.72 & -3.78 \\
\hline chr2:705-746 suggestive & WL & 14.77 & 1.17 & 0.31 & -1.39 \\
\hline chr2:706-737 suggestive & WL & 15.28 & 1.41 & 0.34 & -1.26 \\
\hline chr2:703-754 suggestive & WL & 14.84 & 1.11 & 0.26 & -0.81 \\
\hline chr2:692-738 suggestive & WL & 15.06 & 1 & 0.26 & -1.1 \\
\hline chr2:714-737 suggestive & WL & 20.35 & 0.69 & 0.19 & -1.18 \\
\hline chr2:714-738 suggestive & WL & 19.81 & 0.94 & 0.2 & -0.98 \\
\hline chr2:713-740 suggestive & WL & 18.78 & 1.28 & 0.31 & -1.67 \\
\hline chr2:715-729 suggestive & WL & 20.32 & 0.88 & 0.19 & -1.06 \\
\hline chr2:716-729 significant & WL & 18.23 & 0.89 & 0.24 & -1.34 \\
\hline chr2:716-729 significant & WL & 27.16 & 0.92 & 0.35 & -1.68 \\
\hline chr2:705-729 suggestive & WL & 18.76 & 0.81 & 0.35 & -1.91 \\
\hline chr2:714-738 significant & WL & 16.49 & 1.58 & 0.4 & -1.89 \\
\hline chr2:703-755 significant & WL & 14.95 & 1.83 & 0.45 & -1.79 \\
\hline chr2:715-734 significant & WL & 15.73 & 1.03 & 0.26 & -1.11 \\
\hline chr2:714-735 suggestive & WL & 13.59 & 1.28 & 0.54 & -3.07 \\
\hline chr2:711-731 suggestive & WL & 13.55 & 0.51 & 0.28 & -1.65 \\
\hline chr2:716-728 suggestive & WL & 21.22 & 0.86 & 0.23 & -1.52 \\
\hline chr2:717-724 suggestive & WL & 24.13 & 0.7 & 0.46 & -3.31 \\
\hline chr2:716-735 suggestive & WL & 18.76 & 1.08 & 0.27 & -1.47 \\
\hline chr2:715-738 suggestive & WL & 16.78 & 0.94 & 0.29 & -1.7 \\
\hline chr2:716-734 significant & WL & 21.92 & 1.43 & 0.32 & -1.89 \\
\hline chr2:715-735 suggestive & WL & 19.71 & 1.16 & 0.27 & -1.38 \\
\hline chr2:704-748 suggestive & WL & 14.11 & 1.26 & 0.37 & -1.56 \\
\hline chr2:716-733 significant & WL & 16.02 & 1.44 & 0.45 & -2.47 \\
\hline chr2:716-739 significant & WL & 18.56 & 3.39 & 0.71 & -2.54 \\
\hline chr2:717-728 significant & WL & 24.75 & 2.45 & 0.44 & -2.22 \\
\hline chr2:716-743 significant & WL & 23.13 & 14.36 & 2.38 & -0.01 \\
\hline chr2:716-744 significant & WL & 23.04 & 15.97 & 2.65 & -0.03 \\
\hline chr2:718-729 significant & WL & 32.81 & 14.42 & 1.88 & -0.73 \\
\hline chr2:716-740 significant & WL & 24.56 & 17.36 & 2.76 & -0.27 \\
\hline chr2:718-739 significant & WL & 30.79 & 15.26 & 2.09 & -0.25 \\
\hline chr2:717-739 significant & WL & 26.46 & 16.8 & 2.54 & -0.89 \\
\hline chr2:715-767 significant & WL & 20.78 & 6.86 & 1.23 & 0.14 \\
\hline chr2:713-733 significant & RJF & 17.61 & -3.1 & 0.62 & -0.2 \\
\hline chr2:715-743 significant & WL & 19.01 & 3.78 & 0.7 & -0.88 \\
\hline
\end{tabular}




\begin{tabular}{|c|c|c|c|c|c|}
\hline chr2:713-753 significant & RJF & 13.54 & -1.01 & 0.28 & -0.87 \\
\hline chr2:718-729 significant & WL & 19.95 & 0.39 & 0.37 & -2.23 \\
\hline chr2:714-735 suggestive & WL & 14.24 & 0.94 & 0.27 & -1.31 \\
\hline chr2:714-740 significant & WL & 15.73 & 1.78 & 0.39 & -0.95 \\
\hline chr2:716-740 suggestive & WL & 14.25 & 2.55 & 0.68 & -2.76 \\
\hline chr2:713-731 suggestive & WL & 13.17 & 1.31 & 0.37 & -1.51 \\
\hline chr2:713-740 suggestive & WL & 14.81 & 1.32 & 0.32 & -1.12 \\
\hline chr2:717-738 suggestive & WL & 14.95 & 1.31 & 0.42 & -2.24 \\
\hline chr2:717-751 suggestive & WL & 15 & 0.95 & 0.25 & -1.07 \\
\hline chr2:716-739 suggestive & WL & 15.38 & 1.75 & 0.43 & -1.61 \\
\hline chr2:716-753 suggestive & WL & 14.62 & 1.74 & 0.45 & -1.82 \\
\hline chr2:716-737 significant & WL & 16.01 & 0.94 & 0.26 & -1.32 \\
\hline chr2:717-740 significant & WL & 15.15 & 1.29 & 0.43 & -2.4 \\
\hline chr2:698-738 suggestive & WL & 14.78 & 0.78 & 0.57 & 3.5 \\
\hline chr2:717-729 suggestive & WL & 22.5 & 0.63 & 0.63 & -4 \\
\hline chr2:717-731 suggestive & WL & 19.86 & 1.52 & 0.38 & -2.23 \\
\hline chr2:718-728 significant & WL & 25.14 & 0.99 & 0.24 & -1.78 \\
\hline chr2:719-729 suggestive & WL & 20.81 & 1.55 & 0.35 & -1.91 \\
\hline chr2:717-729 suggestive & RJF & 19.5 & -0.59 & 0.6 & -4.13 \\
\hline chr2:717-729 significant & WL & 22.44 & 1.77 & 0.41 & -2.56 \\
\hline chr2:718-729 suggestive & RJF & 20.45 & -0.19 & 0.47 & -2.7 \\
\hline chr2:718-728 suggestive & WL & 26.13 & 1.38 & 0.38 & -2.64 \\
\hline chr2:717-736 significant & WL & 22.05 & 1.24 & 0.26 & -1.42 \\
\hline chr2:717-729 suggestive & WL & 17.38 & 0.48 & 0.35 & -2.45 \\
\hline chr2:716-740 suggestive & WL & 17.08 & 1.08 & 0.39 & -2.49 \\
\hline chr2:720-728 suggestive & WL & 18.72 & 0.59 & 0.25 & -1.76 \\
\hline chr2:718-729 suggestive & WL & 18.03 & 1.09 & 0.31 & -1.88 \\
\hline chr2:716-752 suggestive & WL & 14.59 & 1.5 & 0.39 & -1.38 \\
\hline chr2:718-729 suggestive & WL & 19.28 & 2.74 & 0.7 & -3.65 \\
\hline chr2:716-736 significant & WL & 19.18 & 0.9 & 0.19 & -0.92 \\
\hline chr2:718-739 significant & WL & 26.38 & 2.01 & 0.32 & -1.01 \\
\hline chr2:717-733 suggestive & WL & 17.37 & 0.87 & 0.72 & -3.29 \\
\hline chr2:717-737 significant & WL & 24.45 & 1.74 & 0.28 & -0.49 \\
\hline chr2:719-736 suggestive & WL & 18.09 & 2.05 & 0.64 & -3.39 \\
\hline chr2:716-736 significant & WL & 17.9 & 1.15 & 0.28 & -1.47 \\
\hline chr2:714-736 significant & WL & 15.07 & 2.09 & 0.62 & -3.04 \\
\hline chr2:714-738 significant & WL & 15.65 & 1.68 & 0.45 & -2.1 \\
\hline chr2:718-736 suggestive & WL & 20.47 & 0.86 & 0.21 & -1.4 \\
\hline chr2:719-729 suggestive & WL & 24.82 & 1.95 & 0.59 & -3.09 \\
\hline chr2:717-733 suggestive & WL & 23.15 & 0.96 & 0.46 & -2.83 \\
\hline chr2:719-737 significant & WL & 22.73 & 1.88 & 0.39 & -2.25 \\
\hline chr2:717-736 suggestive & WL & 17.96 & 1.11 & 0.29 & -1.72 \\
\hline chr2:716-740 suggestive & WL & 17.69 & 1.84 & 0.42 & -1.81 \\
\hline
\end{tabular}




\begin{tabular}{|c|c|c|c|c|c|}
\hline chr2:718-738 suggestive & WL & 20.06 & 1.25 & 0.38 & -2.56 \\
\hline chr2:716-738 suggestive & WL & 20.31 & 1.1 & 0.28 & -1.69 \\
\hline chr2:717-731 suggestive & WL & 18.82 & 1.3 & 0.35 & -2.1 \\
\hline chr2:717-734 significant & WL & 23.84 & 1.87 & 0.37 & -2.04 \\
\hline chr2:719-731 suggestive & WL & 18.94 & 1.39 & 0.5 & -2.91 \\
\hline chr2:718-736 significant & WL & 21.5 & 1.31 & 0.28 & -1.55 \\
\hline chr2:717-740 significant & WL & 20.29 & 1.71 & 0.33 & -1.08 \\
\hline chr2:715-745 significant & WL & 17.08 & 1.88 & 0.42 & -1.65 \\
\hline chr2:718-745 suggestive & RJF & 18.5 & -0.09 & 0.34 & -1.97 \\
\hline chr2:704-737 suggestive & WL & 14.82 & 1.26 & 0.32 & -1.19 \\
\hline chr2:709-739 suggestive & WL & 14.18 & 1.49 & 0.41 & -1.74 \\
\hline chr2:715-752 significant & WL & 18.39 & 1.9 & 0.37 & 0.01 \\
\hline chr2:714-739 significant & WL & 14.78 & 1.54 & 0.37 & -1.35 \\
\hline chr2:713-751 significant & WL & 15.99 & 2.32 & 0.5 & -1.36 \\
\hline chr2:717-739 suggestive & WL & 14.82 & 2.34 & 0.56 & -1.94 \\
\hline chr2:720-731 suggestive & WL & 17.14 & 1.33 & 0.23 & -1.15 \\
\hline chr2:720-730 suggestive & WL & 12.39 & 0.52 & 0.16 & -1.24 \\
\hline chr2:716-736 suggestive & RJF & 19.62 & -0.13 & 0.67 & -3.83 \\
\hline chr2:715-736 suggestive & WL & 19.12 & 1.25 & 0.26 & -1.07 \\
\hline chr2:717-735 suggestive & WL & 19.23 & 2.12 & 0.46 & -2.02 \\
\hline chr2:716-736 suggestive & WL & 19.83 & 1.57 & 0.38 & -2.31 \\
\hline chr2:716-734 suggestive & WL & 19.16 & 0.66 & 0.17 & -1.01 \\
\hline chr2:715-750 significant & WL & 15.25 & 1.92 & 0.44 & -1.32 \\
\hline chr2:705-739 suggestive & WL & 13.58 & 1.11 & 0.26 & -0.55 \\
\hline chr2:691-737 suggestive & WL & 19.06 & 0.64 & 0.45 & -2.5 \\
\hline chr2:717-738 suggestive & WL & 19.09 & 1.04 & 0.43 & -2.35 \\
\hline chr2:715-734 suggestive & WL & 19.25 & 2.4 & 0.72 & -3.45 \\
\hline chr2:715-739 significant & WL & 20.17 & 2.4 & 0.7 & -3.08 \\
\hline chr2:716-737 significant & WL & 18.4 & 1.03 & 0.21 & -0.54 \\
\hline chr2:701-738 suggestive & WL & 14.52 & 1.09 & 0.26 & -0.82 \\
\hline chr2:706-736 suggestive & WL & 15.28 & 1.26 & 0.3 & -1.06 \\
\hline chr2:698-750 suggestive & WL & 14.73 & 1.64 & 0.36 & -0.74 \\
\hline chr2:716-746 suggestive & WL & 18.64 & 1.02 & 0.27 & -1.66 \\
\hline chr2:714-735 suggestive & WL & 20.03 & 1.66 & 0.41 & -2.44 \\
\hline chr2:708-738 suggestive & WL & 18.96 & 1.53 & 0.35 & -1.63 \\
\hline chr2:719-737 suggestive & WL & 20.73 & 1.42 & 0.29 & -1.39 \\
\hline chr2:715-747 suggestive & WL & 14.52 & 0.81 & 0.23 & -0.98 \\
\hline chr2:718-736 suggestive & WL & 19.61 & 1.51 & 0.32 & -1.36 \\
\hline chr2:713-738 suggestive & WL & 13.94 & 1.04 & 0.25 & -0.58 \\
\hline chr2:715-736 suggestive & WL & 14.32 & 0.95 & 0.29 & -1.4 \\
\hline chr2:714-735 suggestive & WL & 15.18 & 1.19 & 0.33 & -1.51 \\
\hline chr2:710-757 significant & WL & 18.53 & 1.41 & 0.29 & -0.82 \\
\hline chr2:700-736 suggestive & WL & 22.01 & 1.49 & 0.29 & -1.11 \\
\hline
\end{tabular}




\begin{tabular}{|c|c|c|c|c|c|}
\hline chr2:716-734 suggestive & WL & 25.81 & 0.55 & 0.37 & -0.98 \\
\hline chr2:720-735 suggestive & WL & 23.42 & 1.69 & 0.65 & -4.29 \\
\hline chr2:713-738 suggestive & WL & 21.64 & 1.96 & 0.39 & -1.69 \\
\hline chr2:716-736 suggestive & WL & 18.75 & 0.94 & 0.21 & -0.91 \\
\hline chr2:717-736 suggestive & WL & 18.78 & 1.48 & 0.38 & -2.06 \\
\hline chr2:717-738 suggestive & WL & 12.13 & 1.36 & 0.28 & -0.96 \\
\hline chr2:718-736 suggestive & WL & 21.24 & 1.07 & 0.22 & -0.92 \\
\hline chr2:717-739 suggestive & WL & 19.37 & 1.18 & 0.32 & -1.78 \\
\hline chr2:714-739 significant & WL & 16.65 & 1.71 & 0.39 & -1.31 \\
\hline chr2:714-735 significant & WL & 19.06 & 1.28 & 0.26 & -0.85 \\
\hline chr2:715-736 significant & WL & 18.14 & 1.17 & 0.27 & -1.13 \\
\hline chr2:717-737 significant & WL & 17.25 & 0.64 & 0.23 & -1.45 \\
\hline chr2:714-737 suggestive & WL & 13.82 & 0.88 & 0.31 & -1.56 \\
\hline chr2:714-738 suggestive & WL & 14.24 & 0.97 & 0.24 & -0.72 \\
\hline chr2:699-738 suggestive & WL & 13.97 & 0.93 & 0.27 & -1.17 \\
\hline chr2:714-737 significant & WL & 16.41 & 1.26 & 0.28 & -0.9 \\
\hline chr2:718-737 significant & WL & 15.27 & 0.97 & 0.26 & -1.19 \\
\hline chr2:702-736 significant & WL & 16.93 & 1.08 & 0.29 & -1.38 \\
\hline chr2:715-734 significant & WL & 16.46 & 1.14 & 0.33 & -1.73 \\
\hline chr2:717-744 significant & WL & 17.34 & 1.63 & 0.33 & 0.06 \\
\hline chr2:716-741 significant & WL & 17.18 & 1.34 & 0.27 & -0.02 \\
\hline chr2:716-739 suggestive & WL & 21.54 & 1.34 & 0.24 & -0.71 \\
\hline chr2:711-739 suggestive & WL & 16.22 & 0.58 & 0.13 & -0.63 \\
\hline chr2:709-730 suggestive & WL & 20.69 & 0.85 & 0.21 & -1.27 \\
\hline chr2:716-736 suggestive & WL & 18.9 & 1.78 & 0.37 & -1.39 \\
\hline chr2:716-739 suggestive & WL & 14.85 & 1.44 & 0.34 & -0.83 \\
\hline chr2:717-750 suggestive & WL & 19.46 & 1.76 & 0.35 & -1.07 \\
\hline chr2:704-737 significant & RJF & 14.67 & -2.33 & 0.51 & -0.25 \\
\hline chr2:718-735 significant & WL & 21.05 & 1.76 & 0.32 & -0.65 \\
\hline chr2:715-739 significant & WL & 28.73 & 1.02 & 0.56 & -1.14 \\
\hline chr2:717-741 significant & WL & 22.02 & 1.15 & 0.2 & -0.32 \\
\hline chr2:726-735 significant & WL & 26.12 & 2.49 & 0.4 & -1.03 \\
\hline chr2:715-738 suggestive & WL & 14.75 & 1.33 & 0.3 & -0.61 \\
\hline chr2:715-738 significant & WL & 19.13 & 1.67 & 0.32 & -0.56 \\
\hline chr2:721-737 suggestive & WL & 18.99 & 1.38 & 0.33 & -1.7 \\
\hline chr2:719-735 suggestive & WL & 19.69 & 1.82 & 0.45 & -2.52 \\
\hline chr2:717-732 suggestive & WL & 19.42 & 1.18 & 0.39 & -2.57 \\
\hline chr2:723-731 suggestive & WL & 21.35 & 0.85 & 0.26 & -1.81 \\
\hline chr2:716-734 suggestive & WL & 17.8 & 1.14 & 0.26 & -1.26 \\
\hline chr2:721-738 suggestive & WL & 21.22 & 1.42 & 0.28 & -1.13 \\
\hline chr2:716-735 significant & WL & 18.9 & 1.32 & 0.25 & -0.38 \\
\hline chr2:719-738 significant & WL & 21.35 & 1.98 & 0.35 & -0.45 \\
\hline chr2:720-738 significant & WL & 19.66 & 2.3 & 0.42 & -0.41 \\
\hline
\end{tabular}




\begin{tabular}{|c|c|c|c|c|c|}
\hline chr2:725-735 significant & WL & 24.75 & 2.09 & 0.33 & -0.18 \\
\hline chr2:725-734 significant & WL & 21.86 & 2.09 & 0.37 & -0.89 \\
\hline chr2:708-739 significant & WL & 17.99 & 1.71 & 0.35 & -0.76 \\
\hline chr2:724-737 significant & WL & 17.84 & 0.78 & 0.2 & -0.94 \\
\hline chr2:708-738 suggestive & WL & 14.74 & 0.8 & 0.3 & -1.65 \\
\hline chr2:710-761 suggestive & WL & 15.23 & 2.04 & 0.45 & -0.89 \\
\hline chr2:719-737 suggestive & WL & 13.17 & 0.84 & 0.25 & -1.12 \\
\hline chr2:716-744 significant & WL & 16.14 & 1.43 & 0.3 & 0.19 \\
\hline chr2:715-739 significant & RJF & 14.96 & -2.63 & 0.55 & 0.4 \\
\hline chr2:726-736 suggestive & WL & 14.97 & 0.6 & 0.18 & -0.91 \\
\hline chr2:710-740 suggestive & WL & 22.34 & 1.72 & 0.31 & -0.93 \\
\hline chr2:713-736 suggestive & WL & 19.23 & 1.56 & 0.3 & -0.65 \\
\hline chr2:725-730 suggestive & WL & 20.58 & 0.83 & 0.2 & -1.13 \\
\hline chr2:714-736 suggestive & WL & 20.4 & 1.62 & 0.29 & -0.11 \\
\hline chr2:725-734 suggestive & WL & 20.95 & 1.67 & 0.38 & -2.13 \\
\hline chr2:714-735 suggestive & WL & 19.44 & 1.07 & 0.24 & -1.07 \\
\hline chr2:718-741 suggestive & WL & 19.71 & 1.58 & 0.29 & 0.05 \\
\hline chr2:721-739 suggestive & WL & 20.16 & 1.03 & 0.19 & -0.48 \\
\hline chr2:715-737 significant & WL & 15.36 & 1.05 & 0.25 & -0.89 \\
\hline chr2:707-754 suggestive & WL & 16.12 & 1.21 & 0.27 & 0.61 \\
\hline chr2:718-741 significant & WL & 19.7 & 1.89 & 0.36 & -1.21 \\
\hline chr2:720-735 significant & WL & 26.46 & 2.08 & 0.32 & -0.76 \\
\hline chr2:716-739 significant & WL & 15.96 & 0.9 & 0.21 & -0.73 \\
\hline chr2:718-737 significant & WL & 22.94 & 1.37 & 0.29 & -1.56 \\
\hline chr2:713-739 significant & WL & 22.74 & 1.06 & 0.2 & -0.84 \\
\hline chr2:716-737 suggestive & WL & 18.79 & 0.69 & 0.15 & -0.67 \\
\hline chr2:715-740 significant & WL & 19.02 & 1.67 & 0.31 & 0.02 \\
\hline chr2:721-757 suggestive & WL & 15.37 & 2.43 & 0.56 & -1.55 \\
\hline chr2:715-738 suggestive & WL & 15.09 & 0.92 & 0.2 & 0.04 \\
\hline chr2:723-736 suggestive & WL & 19.84 & 1.39 & 0.27 & -0.94 \\
\hline chr2:701-736 significant & WL & 19.61 & 1.36 & 0.32 & -1.59 \\
\hline chr2:711-738 significant & WL & 16.92 & 1.84 & 0.38 & 0.44 \\
\hline chr2:711-743 significant & WL & 23.83 & 2.89 & 0.47 & -0.45 \\
\hline chr2:717-739 significant & WL & 15.35 & 1.09 & 0.26 & -0.86 \\
\hline chr2:713-738 significant & WL & 15.6 & 2.81 & 0.64 & -1.75 \\
\hline chr2:711-737 significant & WL & 16.69 & 1.82 & 0.37 & -0.82 \\
\hline chr2:706-766 significant & WL & 18.12 & 1.29 & 0.25 & -0.07 \\
\hline chr2:719-738 suggestive & WL & 15.01 & 1.99 & 0.51 & -1.89 \\
\hline chr2:710-740 suggestive & WL & 15.66 & 1.41 & 0.32 & -0.81 \\
\hline chr2:716-739 suggestive & WL & 14.04 & 1.17 & 0.3 & -1.03 \\
\hline chr2:712-739 suggestive & WL & 18.71 & 1.58 & 0.34 & -1.34 \\
\hline chr2:713-738 significant & WL & 17.02 & 1.58 & 0.34 & -0.99 \\
\hline chr2:707-740 suggestive & WL & 14.97 & 1.49 & 0.37 & -1.29 \\
\hline
\end{tabular}




\begin{tabular}{|c|c|c|c|c|c|}
\hline chr2:704-737 significant & WL & 19.7 & 2.96 & 0.58 & -1.8 \\
\hline chr2:710-740 significant & WL & 21.95 & 2.77 & 0.48 & 0.06 \\
\hline chr2:716-739 significant & WL & 20.38 & 1.98 & 0.41 & -1.75 \\
\hline chr2:719-739 suggestive & WL & 14.62 & 0.87 & 0.26 & -1.21 \\
\hline chr2:706-740 significant & WL & 15.9 & 1.02 & 0.25 & -0.97 \\
\hline chr2:707-739 significant & WL & 17.5 & 1.37 & 0.28 & -0.68 \\
\hline chr2:716-743 significant & WL & 17.49 & 1.23 & 0.25 & -0.62 \\
\hline chr2:715-739 significant & WL & 16.44 & 1.16 & 0.26 & -0.81 \\
\hline chr2:709-743 suggestive & WL & 14.53 & 1.64 & 0.36 & 0.2 \\
\hline chr2:708-750 suggestive & WL & 15.36 & 1.78 & 0.38 & -0.24 \\
\hline chr2:708-738 suggestive & WL & 20.16 & 2.43 & 0.44 & -0.54 \\
\hline chr2:710-746 suggestive & WL & 19.78 & 1.52 & 0.28 & -0.29 \\
\hline chr2:718-738 suggestive & WL & 22.25 & 1.47 & 0.27 & -1.04 \\
\hline chr2:715-744 significant & WL & 19.67 & 2.62 & 0.48 & -0.52 \\
\hline chr2:720-740 significant & WL & 23.94 & 0.73 & 0.61 & -3.27 \\
\hline chr2:726-739 significant & WL & 35.34 & 2.24 & 0.28 & -0.04 \\
\hline chr2:731-738 significant & WL & 46.61 & 3.57 & 0.35 & 0.35 \\
\hline chr2:718-748 significant & WL & 23.06 & 3.28 & 0.54 & -0.47 \\
\hline chr2:717-741 significant & WL & 23.71 & 2.34 & 0.38 & -0.94 \\
\hline chr2:714-746 significant & WL & 17.73 & 1.82 & 0.38 & -1.02 \\
\hline chr2:715-743 suggestive & WL & 14.1 & 0.89 & 0.25 & -0.99 \\
\hline chr2:718-758 suggestive & WL & 15.74 & 1.48 & 0.33 & -0.73 \\
\hline chr2:716-744 significant & WL & 16.92 & 1.67 & 0.33 & -0.3 \\
\hline chr2:720-747 significant & WL & 16 & 1.38 & 0.3 & -0.63 \\
\hline chr2:713-751 suggestive & WL & 15.5 & 1.9 & 0.4 & -0.46 \\
\hline chr2:727-739 suggestive & RJF & 22.31 & -0.73 & 0.45 & -2.48 \\
\hline chr2:720-740 significant & WL & 24.01 & 1.44 & 0.23 & -0.16 \\
\hline chr2:715-743 significant & WL & 27.73 & 4.13 & 0.61 & 0.28 \\
\hline chr2:718-743 significant & WL & 18.9 & 2.28 & 0.43 & -0.8 \\
\hline chr2:709-749 significant & WL & 20.01 & 2.23 & 0.41 & -0.32 \\
\hline chr2:717-752 significant & WL & 20.41 & 1.21 & 0.53 & -2.44 \\
\hline chr2:731-739 significant & WL & 26.3 & 2.26 & 0.39 & -1.87 \\
\hline chr2:700-751 significant & WL & 16.19 & 0.71 & 0.18 & -0.8 \\
\hline chr2:717-750 suggestive & WL & 14.69 & 1.32 & 0.3 & -0.55 \\
\hline chr2:706-770 suggestive & WL & 14.68 & 1.11 & 0.26 & -0.78 \\
\hline chr2:719-751 significant & WL & 19.78 & 1.88 & 0.34 & 0.17 \\
\hline chr2:706-752 suggestive & WL & 16.52 & 2.2 & 0.45 & 0.16 \\
\hline chr2:714-755 significant & WL & 15.46 & 1.1 & 0.27 & -1.04 \\
\hline chr2:713-757 significant & WL & 18.79 & 1.66 & 0.33 & -0.47 \\
\hline chr2:726-755 significant & WL & 25.2 & 1.9 & 0.35 & -1.35 \\
\hline chr2:714-761 suggestive & WL & 14.63 & 1.97 & 0.51 & -1.32 \\
\hline chr2:718-747 suggestive & WL & 17.86 & 1.5 & 0.33 & -1.27 \\
\hline chr2:713-752 suggestive & WL & 15.36 & 1.65 & 0.35 & -0.07 \\
\hline
\end{tabular}




\begin{tabular}{|c|c|c|c|c|c|}
\hline chr2:709-754 significant & WL & 19.76 & 1.93 & 0.36 & 0.43 \\
\hline chr2:711-754 significant & WL & 18.08 & 1.79 & 0.34 & -0.05 \\
\hline chr2:721-754 suggestive & WL & 15.13 & 2.33 & 0.51 & -0.91 \\
\hline chr2:716-751 significant & WL & 18.87 & 1.32 & 0.26 & -0.38 \\
\hline chr2:711-774 significant & WL & 16.15 & 1 & 0.22 & -0.4 \\
\hline chr2:724-754 significant & WL & 21.03 & 1.56 & 0.3 & -0.7 \\
\hline chr2:720-756 suggestive & WL & 14.73 & 1.58 & 0.37 & -0.72 \\
\hline chr2:703-758 suggestive & WL & 14.29 & 1.18 & 0.33 & -0.98 \\
\hline chr2:716-755 suggestive & WL & 15.72 & 2.56 & 0.53 & -0.1 \\
\hline chr2:716-744 significant & WL & 22.67 & 1.35 & 0.22 & 0.3 \\
\hline chr2:721-752 significant & WL & 16.88 & 1.26 & 0.28 & -0.72 \\
\hline chr2:732-748 significant & WL & 37.74 & 3.54 & 0.42 & -0.12 \\
\hline chr2:703-753 suggestive & WL & 15.47 & 2.1 & 0.46 & -0.41 \\
\hline chr2:721-758 suggestive & RJF & 13.21 & -1.13 & 0.27 & -0.83 \\
\hline chr2:706-755 significant & WL & 16.61 & 1.75 & 0.37 & -0.44 \\
\hline chr2:726-749 significant & WL & 17.99 & 2.84 & 0.56 & -0.03 \\
\hline chr2:720-775 significant & RJF & 14.23 & -1.11 & 0.28 & -1.11 \\
\hline chr2:732-768 significant & WL & 17.63 & 2.59 & 0.51 & 0.29 \\
\hline chr2:711-758 significant & WL & 14.88 & 2.72 & 0.61 & -0.58 \\
\hline chr2:693-806 significant & WL & 10.54 & 1.41 & 0.37 & 0.6 \\
\hline chr2:737-750 significant & WL & 31.7 & 3.58 & 0.48 & -0.54 \\
\hline chr2:732-764 suggestive & RJF & 15.06 & -1.28 & 0.29 & 0.47 \\
\hline chr2:733-756 significant & WL & 17.48 & 1.98 & 0.4 & 0.07 \\
\hline chr2:736-759 suggestive & WL & 12.79 & 1.69 & 0.3 & 0.41 \\
\hline chr2:731-761 significant & WL & 19.97 & 2.52 & 0.49 & -0.8 \\
\hline chr2:735-757 suggestive & WL & 15.01 & 1.57 & 0.36 & -0.54 \\
\hline chr2:726-762 significant & WL & 19.31 & 2.32 & 0.43 & -0.1 \\
\hline chr2:732-753 significant & WL & 20.41 & 1.72 & 0.33 & -0.65 \\
\hline chr2:729-761 significant & WL & 18.22 & 2.69 & 0.5 & 0.93 \\
\hline chr2:734-758 significant & WL & 16.72 & 1.83 & 0.37 & -0.2 \\
\hline chr2:715-755 suggestive & WL & 18.8 & 1.55 & 0.3 & -0.26 \\
\hline chr2:734-754 significant & WL & 26.82 & 1.94 & 0.29 & -0.22 \\
\hline chr2:735-751 significant & WL & 28.72 & 1.91 & 0.29 & -0.58 \\
\hline chr2:735-754 significant & WL & 37.27 & 3.22 & 0.4 & -0.72 \\
\hline chr2:723-755 significant & WL & 16.68 & 1.11 & 0.31 & -1.33 \\
\hline chr2:731-756 significant & WL & 23.09 & 1.45 & 0.37 & -0.68 \\
\hline chr2:736-755 significant & WL & 24.44 & 2.62 & 0.44 & -1.09 \\
\hline chr2:736-758 significant & WL & 18.49 & 0.06 & 0.59 & -2.03 \\
\hline chr2:736-760 significant & WL & 18.51 & 2.41 & 0.48 & -0.46 \\
\hline chr2:735-758 significant & WL & 22.42 & 2.83 & 0.47 & 1 \\
\hline chr2:737-755 significant & WL & 22.31 & 3.08 & 0.52 & -0.18 \\
\hline chr2:732-754 significant & WL & 22.54 & 2.18 & 0.4 & -0.77 \\
\hline chr2:737-758 significant & WL & 18.4 & 3.45 & 0.67 & -0.53 \\
\hline
\end{tabular}




\begin{tabular}{|c|c|c|c|c|c|}
\hline chr2:732-754 significant & WL & 18.4 & 1.91 & 0.42 & -1.19 \\
\hline chr2:737-760 significant & WL & 21.45 & 0.3 & 0.49 & -0.52 \\
\hline chr2:712-774 significant & WL & 18.27 & 1.05 & 0.8 & -1.89 \\
\hline chr2:734-754 significant & WL & 21.41 & 3 & 0.55 & -0.68 \\
\hline chr2:741-754 significant & WL & 30.52 & 3.59 & 0.49 & 0.82 \\
\hline chr2:715-766 suggestive & WL & 14.41 & 1.67 & 0.36 & -0.02 \\
\hline chr2:723-757 suggestive & WL & 14.48 & 1.66 & 0.42 & -0.97 \\
\hline chr2:732-756 suggestive & WL & 12.93 & 0.9 & 0.33 & -1.4 \\
\hline chr2:724-754 significant & WL & 15.75 & 1.1 & 0.31 & -1.16 \\
\hline chr2:718-755 suggestive & WL & 13.7 & 1.34 & 0.48 & -2.04 \\
\hline chr2:738-756 significant & WL & 15.35 & 1.98 & 0.46 & -0.84 \\
\hline chr2:732-759 suggestive & WL & 14.9 & 0.93 & 0.27 & -0.99 \\
\hline chr2:732-753 suggestive & WL & 19.17 & 3.71 & 0.74 & -1.25 \\
\hline chr2:735-757 suggestive & WL & 18.79 & 1.6 & 0.33 & -0.71 \\
\hline chr2:735-758 suggestive & WL & 20.66 & 1.82 & 0.33 & -0.14 \\
\hline chr2:725-759 significant & WL & 25.03 & 0.37 & 0.51 & -0.49 \\
\hline chr2:738-759 significant & WL & 25.62 & 2.69 & 0.42 & 0.28 \\
\hline chr2:740-773 significant & RJF & 19.17 & -2.39 & 0.45 & -0.2 \\
\hline chr2:731-760 significant & WL & 22.35 & 2.28 & 0.42 & -0.8 \\
\hline chr2:739-761 suggestive & WL & 18.15 & 0.14 & 0.47 & -1.86 \\
\hline chr2:736-776 significant & WL & 18.09 & 0.87 & 0.76 & -2 \\
\hline chr2:736-764 significant & WL & 20.4 & 2.41 & 0.44 & 0.04 \\
\hline chr2:736-769 significant & RJF & 19.02 & -1.37 & 0.27 & 0.48 \\
\hline chr2:736-769 significant & WL & 21.25 & 2.36 & 0.45 & -0.89 \\
\hline chr2:740-769 significant & RJF & 21.98 & -2.61 & 0.46 & 0.47 \\
\hline chr2:738-786 suggestive & RJF & 13.16 & -1.11 & 0.26 & 0.1 \\
\hline chr2:732-798 significant & WL & 14.22 & 0.8 & 0.5 & 1.47 \\
\hline chr2:712-779 significant & WL & 15.75 & 0.72 & 0.18 & -0.61 \\
\hline chr2:737-770 significant & WL & 31.14 & 3.02 & 0.44 & -0.87 \\
\hline chr2:737-782 suggestive & WL & 15.17 & 2.57 & 0.55 & 0.45 \\
\hline chr2:740-787 significant & WL & 22.06 & 1.08 & 0.73 & -1.53 \\
\hline chr2:737-809 significant & WL & 12.52 & 2.36 & 0.57 & 0.76 \\
\hline chr2:732-793 significant & WL & 20.28 & 0.69 & 0.45 & -1.18 \\
\hline chr2:718-804 suggestive & WL & 12.19 & 2.91 & 0.72 & 0.91 \\
\hline chr2:741-797 significant & WL & 22.27 & 2.09 & 0.37 & -0.39 \\
\hline chr2:738-781 significant & WL & 17.11 & 2.11 & 0.48 & 1.86 \\
\hline chr2:747-779 significant & WL & 32.54 & 2.84 & 0.38 & 1.25 \\
\hline chr2:732-791 significant & WL & 17.73 & 1.24 & 0.25 & 0.25 \\
\hline chr2:745-796 significant & WL & 24.14 & 4.23 & 0.7 & -0.42 \\
\hline chr2:732-804 significant & WL & 9.39 & 4.54 & 1 & 0.04 \\
\hline chr2:736-778 significant & RJF & 13.14 & -1.09 & 0.26 & 0.07 \\
\hline chr2:714-779 significant & WL & 17.99 & 4.95 & 1.02 & -2.4 \\
\hline chr2:753-776 significant & WL & 31.58 & 4.31 & 0.6 & -1.64 \\
\hline
\end{tabular}




\begin{tabular}{|c|c|c|c|c|c|}
\hline chr2:732-781 suggestive & WL & 14.41 & 1.36 & 0.33 & 1.12 \\
\hline chr2:739-802 significant & WL & 14.38 & 2.27 & 0.51 & -0.17 \\
\hline chr2:738-809 significant & WL & 11.76 & 1.72 & 0.54 & -1.57 \\
\hline chr2:749-778 significant & WL & 19.88 & 4.43 & 0.86 & -2.31 \\
\hline chr2:749-780 significant & WL & 19.97 & 2.66 & 0.48 & -0.32 \\
\hline chr2:741-781 suggestive & WL & 14.29 & 1.81 & 0.47 & 1.96 \\
\hline chr2:737-807 suggestive & WL & 13.73 & 1.65 & 0.41 & -0.55 \\
\hline chr2:746-804 significant & WL & 18.44 & 1.9 & 1.1 & -0.35 \\
\hline chr2:747-784 significant & WL & 16.21 & 2.53 & 0.52 & 0.9 \\
\hline chr2:713-784 suggestive & WL & 14.95 & 2.35 & 0.52 & -0.71 \\
\hline chr2:751-782 significant & WL & 15.98 & 0.1 & 0.42 & 0.18 \\
\hline chr2:749-783 significant & WL & 17.33 & 1.62 & 0.32 & -0.32 \\
\hline chr2:750-779 significant & WL & 23.29 & 0.68 & 0.63 & 0.17 \\
\hline chr2:754-796 significant & WL & 20.42 & 1.8 & 0.34 & 1.12 \\
\hline chr2:748-781 significant & RJF & 17.73 & -0.07 & 0.77 & -0.34 \\
\hline chr2:759-781 significant & RJF & 21.62 & -2.29 & 0.55 & 0.31 \\
\hline chr2:754-778 significant & WL & 17.06 & 1.33 & 0.28 & 0.78 \\
\hline chr2:748-778 significant & WL & 23.1 & 1.89 & 0.32 & 0.44 \\
\hline chr2:760-777 suggestive & WL & 18.73 & 1.79 & 0.45 & 2 \\
\hline chr2:754-781 significant & WL & 21.53 & 2.01 & 0.52 & -0.65 \\
\hline chr2:753-780 significant & RJF & 19.71 & -0.04 & 0.86 & 1.1 \\
\hline chr2:750-784 significant & RJF & 18.37 & -0.65 & 0.64 & 1.2 \\
\hline chr2:759-778 suggestive & RJF & 18.04 & -2.09 & 0.65 & 0.38 \\
\hline chr2:761-781 significant & RJF & 18.66 & -3.36 & 0.74 & -0.04 \\
\hline chr2:757-783 significant & RJF & 16.28 & -1.1 & 0.25 & 0.83 \\
\hline chr2:759-779 suggestive & RJF & 16.23 & -0.54 & 0.36 & -0.34 \\
\hline chr2:759-800 suggestive & RJF & 17.34 & -2.36 & 0.56 & 0.69 \\
\hline chr2:762-785 significant & RJF & 25.62 & -2.12 & 0.33 & 0.02 \\
\hline chr2:762-783 significant & RJF & 28.41 & -2.31 & 0.35 & 1.03 \\
\hline chr2:744-807 significant & WL & 13.89 & 1.7 & 0.98 & 2.08 \\
\hline chr2:765-786 significant & RJF & 19.45 & -4.28 & 0.92 & -1.69 \\
\hline chr2:748-801 suggestive & RJF & 12.73 & -0.76 & 0.19 & 0.55 \\
\hline chr2:751-787 significant & WL & 17.97 & 1.54 & 0.31 & 0.88 \\
\hline chr2:751-795 significant & WL & 16.55 & 1.71 & 0.35 & 0.6 \\
\hline chr2:755-795 significant & WL & 18.59 & 2.3 & 0.48 & 1.9 \\
\hline chr2:741-809 significant & WL & 14.2 & 1.36 & 0.8 & 1.19 \\
\hline chr2:754-804 suggestive & RJF & 11.96 & -1.41 & 0.39 & 1.12 \\
\hline chr2:747-803 significant & WL & 17.49 & 1.81 & 0.35 & 0.09 \\
\hline chr2:736-803 suggestive & WL & 12.91 & 0.96 & 0.23 & 0.2 \\
\hline chr2:762-802 significant & RJF & 17.35 & -2.06 & 0.42 & 0.54 \\
\hline chr2:738-801 suggestive & WL & 12.89 & 0.72 & 0.63 & 2.35 \\
\hline chr2:754-795 significant & WL & 23.86 & 1.62 & 0.29 & -0.76 \\
\hline chr2:709-815 suggestive & WL & 10.57 & 1.69 & 0.45 & 0.48 \\
\hline
\end{tabular}




\begin{tabular}{|c|c|c|c|c|c|}
\hline chr2:738-839 suggestive & RJF & 13.65 & -0.89 & 0.28 & 1.03 \\
\hline chr2:766-810 suggestive & RJF & 13.76 & -0.64 & 0.3 & 1.59 \\
\hline chr2:742-803 significant & RJF & 16.13 & -1.06 & 0.27 & 1 \\
\hline chr2:765-802 significant & RJF & 20.08 & -1.01 & 0.26 & 1.27 \\
\hline chr2:763-808 significant & WL & 14.86 & 1.74 & 0.38 & 0.81 \\
\hline chr2:773-803 significant & RJF & 28.89 & -2.32 & 0.33 & -0.24 \\
\hline chr2:772-793 significant & RJF & 22.63 & -1.95 & 0.37 & 1.19 \\
\hline chr2:763-803 suggestive & RJF & 12.97 & -1.83 & 0.46 & 0.59 \\
\hline chr2:769-804 significant & RJF & 17.54 & -2.11 & 0.42 & -0.58 \\
\hline chr2:767-795 significant & RJF & 18.94 & -2.51 & 0.49 & 0.54 \\
\hline chr2:770-804 significant & WL & 18.1 & 1.81 & 0.36 & 0.91 \\
\hline chr2:773-793 significant & WL & 18.25 & 2.23 & 0.43 & 0.99 \\
\hline chr2:777-790 significant & RJF & 28.46 & -2.2 & 0.32 & 0.33 \\
\hline chr2:779-790 significant & RJF & 30.79 & -4.41 & 0.59 & -1.45 \\
\hline chr2:779-802 significant & RJF & 31.12 & -2.33 & 0.33 & 0.79 \\
\hline chr2:779-804 suggestive & RJF & 19.96 & -1.89 & 0.43 & -0.62 \\
\hline chr2:778-805 significant & RJF & 21.33 & -16.8 & 3.04 & -11.13 \\
\hline chr2:778-807 significant & RJF & 19.05 & -23.73 & 4.59 & -15.2 \\
\hline chr2:778-807 significant & RJF & 20.57 & -23.88 & 4.34 & -13.82 \\
\hline chr2:778-807 significant & RJF & 20.8 & -16.09 & 2.92 & -9.9 \\
\hline chr2:777-808 significant & RJF & 18.19 & -20.77 & 4.16 & -14.44 \\
\hline chr2:778-807 significant & RJF & 20.06 & -26.36 & 4.93 & -17.05 \\
\hline chr2:777-808 significant & RJF & 17.31 & -21.09 & 4.39 & -15.59 \\
\hline chr2:780-795 significant & RJF & 26.73 & -3.25 & 0.47 & 0.09 \\
\hline chr2:774-803 suggestive & RJF & 13.56 & -2.32 & 0.54 & -1.08 \\
\hline chr2:776-809 significant & RJF & 14.95 & -24.27 & 5.54 & -18.41 \\
\hline chr2:777-809 significant & RJF & 15.68 & -17.45 & 3.97 & -15.21 \\
\hline chr2:777-808 significant & RJF & 15.53 & -18.41 & 4.06 & -13.55 \\
\hline chr2:777-810 significant & RJF & 15.62 & -22.89 & 5 & -15.62 \\
\hline chr2:776-810 suggestive & RJF & 14.26 & -12.16 & 2.99 & -11.56 \\
\hline chr2:776-796 significant & RJF & 15.06 & -8.51 & 2.08 & -8.6 \\
\hline chr2:776-809 significant & RJF & 15.56 & -23.52 & 5.2 & -16.97 \\
\hline chr2:777-810 significant & RJF & 15.12 & -18.87 & 4.4 & -16.76 \\
\hline chr2:777-809 significant & RJF & 18.02 & -23.35 & 4.71 & -16.24 \\
\hline chr2:777-808 significant & RJF & 18.57 & -27.6 & 5.39 & -17.29 \\
\hline chr2:777-809 significant & RJF & 16.61 & -17.59 & 3.73 & -12.53 \\
\hline chr2:777-809 significant & RJF & 18.43 & -16.53 & 3.25 & -10.44 \\
\hline chr2:779-806 significant & RJF & 23.34 & -7.86 & 1.32 & -4.5 \\
\hline chr2:776-810 significant & RJF & 16.5 & -20.09 & 4.33 & -15.39 \\
\hline chr2:777-809 significant & RJF & 17.89 & -25.75 & 5.14 & -16.51 \\
\hline chr2:777-808 significant & RJF & 18.1 & -26.07 & 5.16 & -15.91 \\
\hline chr2:778-806 significant & RJF & 21.18 & -18.71 & 3.43 & -13.13 \\
\hline chr2:773-810 suggestive & RJF & 14.64 & -1.21 & 0.3 & 0.79 \\
\hline
\end{tabular}




\begin{tabular}{|c|c|c|c|c|c|}
\hline chr2:775-870 suggestive & RJF & 14.18 & -17.75 & 4.12 & -12.11 \\
\hline chr2:777-870 significant & RJF & 15.37 & -22.65 & 5.01 & -15.61 \\
\hline chr2:776-873 suggestive & RJF & 13.87 & -19.16 & 4.55 & -14.32 \\
\hline chr2:776-874 suggestive & RJF & 14.48 & -20.8 & 4.66 & -13.62 \\
\hline chr2:776-810 significant & RJF & 15.48 & -21.92 & 4.72 & -14.01 \\
\hline chr2:777-810 significant & RJF & 15.6 & -15.22 & 3.23 & -9.21 \\
\hline chr2:785-793 significant & RJF & 57.89 & -4.33 & 0.33 & -0.48 \\
\hline chr2:783-794 significant & RJF & 60.28 & -7.12 & 0.51 & -0.58 \\
\hline chr2:780-793 significant & RJF & 47.44 & -2.67 & 0.26 & 0.13 \\
\hline chr2:773-796 significant & WL & 31.5 & 1.35 & 0.18 & 0.57 \\
\hline chr2:776-800 significant & WL & 30.52 & 1.57 & 0.21 & 0.66 \\
\hline chr2:775-795 significant & WL & 21.28 & 0.09 & 0.19 & 0.38 \\
\hline chr2:774-804 significant & WL & 17.7 & 3.51 & 0.7 & 0.02 \\
\hline chr2:780-796 significant & WL & 30.17 & 3.43 & 0.48 & 0.43 \\
\hline chr2:781-801 significant & RJF & 30.17 & -3.26 & 0.48 & 0.95 \\
\hline chr2:774-796 significant & WL & 26.66 & 3.73 & 0.56 & 0.96 \\
\hline chr2:778-795 significant & WL & 26.63 & 2 & 0.31 & 0.82 \\
\hline chr2:777-793 significant & WL & 38.61 & 1.41 & 0.16 & 0.51 \\
\hline chr2:777-799 significant & WL & 25.96 & 1.91 & 0.3 & 0.95 \\
\hline chr2:781-796 significant & WL & 35.4 & 2.2 & 0.27 & 0.46 \\
\hline chr2:777-794 significant & WL & 23.27 & 0.66 & 0.11 & 0.21 \\
\hline chr2:774-804 significant & WL & 19.33 & 1.28 & 0.29 & 1.67 \\
\hline chr2:764-809 significant & WL & 17.07 & 1.4 & 0.28 & 0.61 \\
\hline chr2:786-792 significant & RJF & 67.09 & -3.05 & 0.21 & 0.94 \\
\hline chr2:755-798 significant & WL & 24.96 & 4.97 & 0.85 & -1.55 \\
\hline chr2:775-805 significant & RJF & 15.34 & -1.22 & 0.33 & 1.04 \\
\hline chr2:776-809 significant & RJF & 14.68 & -0.88 & 0.21 & 0.47 \\
\hline chr2:767-807 significant & WL & 14.73 & 1.19 & 0.25 & 0.5 \\
\hline chr2:778-805 suggestive & RJF & 14.1 & -0.7 & 0.23 & 0.95 \\
\hline chr2:766-810 significant & WL & 14.54 & 1.22 & 0.36 & 1.94 \\
\hline chr2:786-802 suggestive & RJF & 15.55 & -1.13 & 0.21 & -1.21 \\
\hline chr2:786-797 significant & WL & 43.81 & 3.27 & 0.33 & 0.78 \\
\hline chr2:776-809 significant & RJF & 19.98 & -0.99 & 0.24 & 1.11 \\
\hline chr2:775-809 significant & WL & 19.53 & 3.09 & 0.58 & 1.64 \\
\hline chr2:758-807 suggestive & RJF & 13.74 & -1.18 & 0.3 & 0.65 \\
\hline chr2:752-866 suggestive & WL & 11.78 & 1.23 & 0.31 & 0.76 \\
\hline chr2:762-802 significant & RJF & 22.23 & -1.1 & 0.2 & 0.45 \\
\hline chr2:772-806 significant & RJF & 16.52 & -1 & 0.23 & 0.57 \\
\hline chr2:777-801 significant & RJF & 24.95 & -1.08 & 0.21 & 0.82 \\
\hline chr2:745-810 suggestive & RJF & 13.24 & -1.42 & 0.37 & 0.66 \\
\hline chr2:774-806 significant & RJF & 15.85 & -0.74 & 0.2 & 0.71 \\
\hline chr2:776-803 significant & WL & 26.31 & 1.66 & 0.25 & 0.28 \\
\hline chr2:781-809 significant & RJF & 28.27 & -3.17 & 0.47 & 0.38 \\
\hline
\end{tabular}


chr2:782-807 significant RJF

chr2:779-805 significant RJF

chr2:775-808 significant RJF

chr2:777-807 significant RJF

chr2:780-808 significant RJF

chr2:779-807 significant RJF

chr2:758-808 suggestive RJF

chr2:780-863 significant RJF

chr2:777-806 significant RJF

chr2:778-810 significant RJF

chr2:773-808 significant RJF

chr2:776-805 significant RJF

chr2:757-808 suggestive RJF

chr2:779-808 significant

chr2:783-806 significant

chr2:778-809 significant

chr2:772-844 significant

chr2:781-859 significant

chr2:778-806 significant

chr2:772-809 suggestive

chr2:737-805 suggestive

chr2:779-806 significant

chr2:782-806 significant

chr2:768-804 significant

chr2:790-801 significant

chr2:787-807 significant

chr2:778-806 significant

chr2:790-806 significant

chr2:773-807 significant

chr2:791-807 significant

chr2:763-806 suggestive

chr2:775-815 significant

chr2:783-808 suggestive

chr2:781-807 suggestive

chr2:776-810 suggestive

chr2:786-808 significant

chr2:789-808 significant

chr2:782-810 significant

chr2:776-816 significant

chr2:771-810 significant

chr2:783-810 significant

chr2:792-808 significant

chr2:786-809 significant

WL
28.48

18.4

16.89

19.8

23.58

19.78

13.17

14.62

14.12

19.45

15.89

27.03

14.16

21.29

26.13

22.6

16.11

15.7

23.02

13.95

12.3

20.93

23.41

17.1

21.88

19.11

21.28

17.51

13.86

16.75

14.48

16.34

14.85

14.16

14.79

18.11

19.54

16.91

15.1

19.74

22.14

26.4

19.03
$-0.71$

$-1.18$

$-1.31$

$-0.98$

$-1.22$

$-1.8$

$-0.72$

$-0.83$

$-1.37$

$-2.03$

$-1.1$

$-1.99$

$-1.27$

$-1.44$

$-2.52$

$-2.53$

$-1.4$

$-1.24$

$-2.73$

$-1$

1.09

$-2.04$

$-1.69$

$-1.23$

$-1.7$

$-1.56$

$-2.02$

$-1.56$

1.96

$-0.78$

$-1.44$

$-1.09$

$-1.1$

$-1.14$

$-0.95$

$-2.13$

$-1.29$

$-1.24$

$-2.05$

$-1.76$

$-1.38$

$-2.64$

2.14
0.11

0.29

0.28

0.25

0.2

0.32

0.21

0.25

0.32

0.38

0.31

0.3

0.31

0.26

0.44

0.44

0.29

0.26

0.45

0.24

0.33

0.41

0.28

0.25

0.35

0.31

0.37

0.32

0.47

0.27

0.35

0.23

0.25

0.34

0.24

0.42

0.29

0.26

0.46

0.32

0.25

0.4

0.92
0.22

1.1

0.19

1.08

$-0.19$

$-0.23$

0.61

0.98

0.46

$-0.11$

1.19

0.31

0.54

0.28

$-0.26$

0.44

0.02

0.02

0.01

0.42

1.31

1.25

0.14

$-0.12$

$-1.01$

0.59

0.52

0.42

1.31

1.47

0.67

0.32

0.38

1.27

0.59

$-0.02$

1.23

0.32

0.73

$-0.06$

0.52
0.19

2.27 


\begin{tabular}{|c|c|c|c|c|c|}
\hline chr2:779-826 significant & RJF & 17.78 & -1.5 & 0.3 & 0.21 \\
\hline chr2:790-813 significant & RJF & 15.51 & -0.6 & 0.3 & 1.82 \\
\hline chr2:774-859 suggestive & RJF & 13.97 & -1.4 & 0.39 & 1.37 \\
\hline chr2:783-810 significant & RJF & 27.53 & -1.95 & 0.3 & 0.09 \\
\hline chr2:787-851 significant & RJF & 17.31 & -2.15 & 0.45 & 0.8 \\
\hline chr2:789-836 significant & RJF & 26.07 & -2.38 & 0.37 & 0.32 \\
\hline chr2:745-849 significant & WL & 13.45 & 2.83 & 0.66 & 0.35 \\
\hline chr2:785-864 suggestive & RJF & 13.67 & -2.52 & 0.59 & 0.03 \\
\hline chr2:756-848 significant & WL & 10.19 & 0.71 & 0.31 & 1.1 \\
\hline chr2:752-875 significant & WL & 14.68 & 1.88 & 0.42 & -0.22 \\
\hline chr2:790-862 suggestive & RJF & 14.26 & -1.54 & 0.41 & 1.64 \\
\hline chr2:788-854 suggestive & RJF & 14.81 & -0.78 & 0.18 & -0.17 \\
\hline chr2:776-865 suggestive & RJF & 12.28 & -0.72 & 0.27 & 1.15 \\
\hline chr2:747-867 suggestive & WL & 11.45 & 1.48 & 0.39 & 0.34 \\
\hline chr2:791-855 significant & RJF & 15.45 & -2.07 & 0.45 & 0.08 \\
\hline chr2:802-814 significant & WL & 26.58 & 2.01 & 0.31 & 0.26 \\
\hline chr2:785-821 suggestive & RJF & 13.63 & -2.13 & 0.49 & 0.74 \\
\hline chr2:800-835 suggestive & RJF & 15.94 & -1.81 & 0.51 & -1.86 \\
\hline chr2:786-847 suggestive & WL & 13.81 & 2.17 & 0.51 & 0.34 \\
\hline chr2:806-826 significant & RJF & 21.17 & -2.85 & 0.51 & 0.1 \\
\hline chr2:801-818 significant & RJF & 16.79 & -2.43 & 0.5 & -0.44 \\
\hline chr2:800-858 suggestive & RJF & 14.37 & -1.86 & 0.41 & 0.63 \\
\hline chr2:801-848 suggestive & RJF & 14.71 & -1.53 & 0.34 & 0.52 \\
\hline chr2:801-836 significant & WL & 16.38 & 1.43 & 0.3 & 0.27 \\
\hline chr2:787-863 significant & WL & 12.92 & 2.34 & 0.56 & -1.16 \\
\hline chr2:800-861 significant & RJF & 18.34 & -1.9 & 0.37 & 0.61 \\
\hline chr2:801-831 suggestive & RJF & 14.54 & -1.54 & 0.34 & -0.06 \\
\hline chr2:802-851 suggestive & RJF & 17.64 & -4.02 & 0.96 & -2.2 \\
\hline chr2:791-866 significant & WL & 12.23 & 3.01 & 0.73 & -1.29 \\
\hline chr2:802-847 significant & RJF & 32.61 & -4.63 & 0.61 & 1.04 \\
\hline chr2:801-861 significant & RJF & 18.28 & -1.71 & 0.32 & 0.14 \\
\hline chr2:800-861 significant & RJF & 16.96 & -2.53 & 0.51 & 0.96 \\
\hline chr2:800-862 significant & RJF & 14.24 & -0.76 & 0.24 & 1.21 \\
\hline chr2:803-859 significant & WL & 18.22 & 2.09 & 0.4 & -0.34 \\
\hline chr2:800-863 suggestive & RJF & 13.61 & -1.72 & 0.4 & 0.73 \\
\hline chr2:779-862 significant & RJF & 15.01 & -1.84 & 0.42 & -0.02 \\
\hline chr2:801-851 significant & RJF & 17.53 & -1.52 & 0.29 & 0.3 \\
\hline chr2:787-871 significant & WL & 11.82 & 2.01 & 0.49 & -0.1 \\
\hline chr2:801-870 significant & WL & 15.81 & 1.95 & 0.4 & -0.22 \\
\hline chr2:801-844 significant & RJF & 23.28 & -2 & 0.33 & 0.25 \\
\hline chr2:795-870 significant & RJF & 17.03 & -2.08 & 0.43 & 0.2 \\
\hline chr2:800-859 suggestive & RJF & 14.16 & -1.7 & 0.39 & 0.35 \\
\hline chr2:787-856 significant & RJF & 16.03 & -1.85 & 0.41 & -0.13 \\
\hline
\end{tabular}




\begin{tabular}{|c|c|c|c|c|c|}
\hline chr2:802-870 significant & WL & 12.88 & 3.35 & 0.79 & -1.91 \\
\hline chr2:805-851 significant & RJF & 28.14 & -2.51 & 0.36 & 0.77 \\
\hline chr2:806-848 significant & RJF & 22.57 & -2.58 & 0.45 & 0.08 \\
\hline chr2:811-855 significant & WL & 23.05 & 3.74 & 0.62 & -0.89 \\
\hline chr2:792-847 significant & RJF & 19.31 & -2.75 & 0.51 & 1.71 \\
\hline chr2:808-855 suggestive & RJF & 18.72 & -1.7 & 0.38 & -0.25 \\
\hline chr2:803-860 significant & RJF & 14.66 & -2.06 & 0.44 & 0.63 \\
\hline chr2:792-850 significant & RJF & 13.95 & -2.09 & 0.47 & 0.54 \\
\hline chr2:814-853 significant & RJF & 18.4 & -1.73 & 0.33 & 0.34 \\
\hline chr2:807-853 significant & RJF & 16.84 & -1.69 & 0.37 & -0.47 \\
\hline chr2:813-865 significant & RJF & 17.1 & -1.58 & 0.32 & 0.31 \\
\hline chr2:812-852 significant & WL & 25.73 & 3.11 & 0.49 & -0.37 \\
\hline chr2:813-855 significant & RJF & 22.68 & -1.9 & 0.37 & 1.17 \\
\hline chr2:814-858 significant & RJF & 18.28 & -1.01 & 0.22 & -0.39 \\
\hline chr2:812-841 significant & RJF & 22.61 & -1.78 & 0.3 & 0.42 \\
\hline chr2:813-851 significant & RJF & 16.99 & -2.31 & 0.47 & 0.66 \\
\hline chr2:804-835 suggestive & RJF & 14.22 & -0.98 & 0.31 & -0.93 \\
\hline chr2:805-849 suggestive & RJF & 14.67 & -1.5 & 0.41 & -0.83 \\
\hline chr2:787-859 suggestive & RJF & 14.34 & -1.62 & 0.39 & -0.32 \\
\hline chr2:809-856 significant & RJF & 15.5 & -0.91 & 0.23 & -0.39 \\
\hline chr2:813-857 suggestive & RJF & 12.46 & -0.94 & 0.26 & -0.45 \\
\hline chr2:791-868 suggestive & RJF & 13.38 & -1.33 & 0.3 & 0.64 \\
\hline chr2:794-862 significant & RJF & 15.89 & -1.28 & 0.27 & 0.2 \\
\hline chr2:789-862 suggestive & RJF & 12.66 & -1.03 & 0.27 & -0.37 \\
\hline chr2:813-862 suggestive & RJF & 19.87 & -3.51 & 0.72 & -0.26 \\
\hline chr2:781-862 significant & RJF & 16.81 & -1.16 & 0.26 & -0.27 \\
\hline chr2:793-858 significant & RJF & 16.6 & -1.59 & 0.33 & 0.14 \\
\hline chr2:813-855 significant & RJF & 16.58 & -1.55 & 0.36 & -0.48 \\
\hline chr2:814-854 significant & RJF & 24.57 & -3.14 & 0.5 & 1.33 \\
\hline chr2:805-862 significant & RJF & 19.89 & -1.1 & 0.21 & 0.01 \\
\hline chr2:795-859 significant & RJF & 16.59 & -2.49 & 0.51 & 0.9 \\
\hline chr2:792-861 significant & RJF & 18.89 & -1.61 & 0.33 & 1.64 \\
\hline chr2:815-864 significant & RJF & 17.09 & -2.63 & 0.53 & 0.25 \\
\hline chr2:816-847 significant & RJF & 35.39 & -3.6 & 0.45 & 0.05 \\
\hline chr2:802-866 significant & RJF & 16.17 & -1.77 & 0.39 & -0.43 \\
\hline chr2:810-861 suggestive & RJF & 17.53 & -1.96 & 0.46 & -1.07 \\
\hline chr2:801-872 significant & RJF & 18.69 & -2.42 & 0.48 & -0.44 \\
\hline chr2:787-869 suggestive & RJF & 13.3 & -1.97 & 0.47 & 0.1 \\
\hline chr2:807-860 significant & RJF & 20.2 & -1.98 & 0.36 & 0.32 \\
\hline chr2:814-875 significant & RJF & 17.13 & -2.25 & 0.48 & -0.57 \\
\hline chr2:804-863 suggestive & RJF & 14.05 & -2 & 0.48 & -0.59 \\
\hline chr2:814-857 significant & RJF & 16.26 & -4.19 & 0.87 & 1.76 \\
\hline chr2:816-867 significant & RJF & 15.67 & -2.23 & 0.52 & -1.06 \\
\hline
\end{tabular}




\begin{tabular}{|c|c|c|c|c|c|}
\hline chr2:813-856 significant & RJF & 18.43 & -1.76 & 0.36 & -0.44 \\
\hline chr2:807-864 significant & RJF & 16.81 & -2.02 & 0.44 & -0.76 \\
\hline chr2:806-863 significant & RJF & 17.93 & -1.67 & 0.33 & 0.05 \\
\hline chr2:814-865 significant & RJF & 20.37 & -2.35 & 0.43 & 1.29 \\
\hline chr2:816-860 significant & RJF & 23.95 & -2.19 & 0.37 & -0.3 \\
\hline chr2:802-862 significant & RJF & 15.33 & -1.8 & 0.4 & -0.14 \\
\hline chr2:796-862 significant & RJF & 20.41 & -2.27 & 0.43 & -0.54 \\
\hline chr2:815-871 significant & RJF & 16.43 & -1.37 & 0.3 & -0.54 \\
\hline chr2:804-865 significant & RJF & 18.74 & -1.63 & 0.35 & -0.91 \\
\hline chr2:801-860 significant & RJF & 17.26 & -3.74 & 0.73 & 0.37 \\
\hline chr2:778-875 suggestive & RJF & 13.54 & -1.68 & 0.42 & -0.77 \\
\hline chr2:813-865 significant & RJF & 20.97 & -2.72 & 0.48 & 0.87 \\
\hline chr2:796-871 suggestive & RJF & 13.97 & -1.25 & 0.29 & -0.11 \\
\hline chr2:815-865 significant & RJF & 15.93 & -1.65 & 0.35 & 0.4 \\
\hline chr2:815-870 significant & RJF & 15.05 & -1.84 & 0.4 & -0.03 \\
\hline chr2:800-858 significant & RJF & 17.58 & -4.09 & 0.8 & 1.29 \\
\hline chr2:816-857 significant & RJF & 26.52 & -5.11 & 0.78 & 0.45 \\
\hline chr2:814-859 suggestive & RJF & 24.38 & -2.28 & 0.79 & -2.37 \\
\hline chr2:814-866 significant & RJF & 18.46 & -3.44 & 0.67 & -0.54 \\
\hline chr2:805-865 significant & RJF & 21.98 & -3.06 & 0.54 & -0.62 \\
\hline chr2:814-864 significant & RJF & 18.68 & -4.68 & 0.89 & 0.77 \\
\hline chr2:815-860 significant & RJF & 16.34 & -3.3 & 0.68 & 0.41 \\
\hline chr2:800-871 significant & RJF & 14.94 & -1.91 & 0.41 & 1.13 \\
\hline chr2:802-864 significant & RJF & 16.18 & -3.5 & 0.73 & 0.76 \\
\hline chr2:787-865 suggestive & RJF & 13.84 & -2.33 & 0.62 & 3.27 \\
\hline chr2:795-875 suggestive & RJF & 13.55 & -2.91 & 0.76 & -1.79 \\
\hline chr2:790-873 suggestive & RJF & 13.09 & -3.13 & 0.75 & -0.16 \\
\hline chr2:815-869 significant & WL & 16.82 & 5.62 & 1.14 & -1.72 \\
\hline chr2:814-872 significant & RJF & 15.81 & -2.5 & 0.55 & -0.81 \\
\hline chr2:813-868 significant & WL & 18.55 & 4.17 & 0.8 & -2.73 \\
\hline chr2:792-877 suggestive & RJF & 13.12 & -2.21 & 0.52 & 0.7 \\
\hline chr2:795-874 suggestive & RJF & 13.46 & -2.32 & 0.54 & 0.66 \\
\hline chr2:814-876 significant & RJF & 14.07 & -2.44 & 0.56 & -0.48 \\
\hline chr2:825-866 significant & RJF & 27.84 & -3.86 & 0.57 & -0.28 \\
\hline chr2:801-877 suggestive & WL & 12.08 & 2.89 & 0.74 & -2.48 \\
\hline chr2:788-874 significant & RJF & 15.23 & -1.79 & 0.39 & 0.76 \\
\hline chr2:813-873 significant & RJF & 14.43 & -2.64 & 0.6 & 1.98 \\
\hline chr2:815-878 suggestive & RJF & 13.73 & -2.15 & 0.49 & 0.04 \\
\hline chr2:797-879 significant & RJF & 14.31 & -1.96 & 0.44 & 0.76 \\
\hline chr2:811-877 significant & RJF & 15.32 & -4.23 & 0.91 & 0.72 \\
\hline chr2:804-876 suggestive & RJF & 14.23 & -0.11 & 0.54 & -3.93 \\
\hline chr2:807-882 significant & RJF & 14.59 & -4.49 & 0.99 & -0.25 \\
\hline chr2:790-894 suggestive & WL & 13.51 & 4.68 & 1.12 & -3.39 \\
\hline
\end{tabular}


chr2:834-947 suggestive WL

chr2:761-929 suggestive RJF

chr2:858-928 suggestive RJF

chr2:844-945 significant RJF

chr2:855-929 significant RJF

chr2:861-936 significant RJF

chr2:867-941 significant RJF

chr2:890-933 significant

chr2:893-930 significant

chr2:882-927 significant

chr2:883-928 significant

chr2:884-928 significant

chr2:881-929 suggestive

chr2:884-938 significant

chr2:888-930 significant

chr2:888-929 suggestive

chr2:875-956 significant

chr2:921-926 significant

chr2:911-926 significant

chr2:878-927 suggestive

chr2:923-926 significant

chr2:877-937 suggestive

chr2:884-937 significant

chr2:887-939 significant

chr2:886-950 significant

chr2:895-948 significant

chr2:881-937 significant

chr2:857-952 suggestive

chr2:884-956 significant

chr2:897-939 significant

chr2:894-962 significant

chr2:872-964 significant

chr2:921-941 significant

chr2:905-938 significant

chr2:903-967 significant

chr2:895-941 significant

chr2:899-940 significant

chr2:922-950 significant

chr2:897-970 significant

chr2:894-958 significant

chr2:885-940 significant

chr2:875-968 significant

chr2:927-938 significant
14.56

11.5

19.56

17.32

19.39

18.37

19.54

14.97

32.12

23.44

22.98

21.07

13.13

18.51

17.23

17.39

17.64

56.24

38.75

22.74

53.14

13.7

32.59

24.13

16.87

19.99

19.11

13.3

21.25

26.56

16.56

17.3

19.33

25.25

17.56

25.35

22.4

28.54

14.78

16.2

17.11

14.45

29.51
2.15

$-1.33$

$-1.07$

$-1.44$

$-1.38$

$-1.29$

$-1.9$

2.2

3.42

$-3.81$

$-2.67$

2.27

1.41

2.41

1.36

$-0.16$

2.49

4.37

3.94

$-1.17$

2.37

0.82

2.66

$-2.2$

1.3

$-2.35$

$-1.53$

1.98

$-2.67$

$-3.53$

$-1.54$

3.16

1.54

$-3.24$

$-3.89$

$-2.57$

$-2.75$

$-2.12$

$-2.87$

$-0.97$

$-1.49$

$-2.48$

$-2.35$
0.48

0.34

0.48

0.41

0.29

0.27

0.37

0.52

0.45

0.64

0.45

0.69

0.37

0.47

0.29

0.61

0.58

0.33

0.44

0.73

0.2

0.26

0.35

0.35

0.29

0.42

0.28

0.47

0.45

0.52

0.33

0.64

0.29

0.5

0.75

0.4

0.45

0.3

0.61

0.21

0.31

0.55

0.33
0.17

0.42

$-2.09$

$-2.28$

$-1.19$

$-0.81$

$-1.03$

$-0.99$

0.55

0.21

0.36

$-2.95$

$-0.85$

$-0.25$

$-0.49$

$-4.04$

$-1.88$

$-0.25$

$-0.61$

$-5.1$

$-0.49$

$-1.03$

0.06

$-0.89$

$-0.85$

0.42

$-0.19$

$-0.17$

0.06

0.24

$-0.58$

1.23

$-0.27$

1.24

0.74

0.74

0.43

0.3

0.35

$-0.33$

$-0.25$

$-0.35$

$-0.27$ 


\begin{tabular}{|c|c|c|c|c|c|}
\hline chr2:927-938 significant & RJF & 33.85 & -3.41 & 0.43 & 0.11 \\
\hline chr2:927-937 significant & RJF & 27.18 & -2.26 & 0.34 & -0.38 \\
\hline chr2:925-941 significant & RJF & 24.69 & -4.4 & 0.7 & -0.25 \\
\hline chr2:928-939 significant & WL & 16.37 & 1.07 & 0.57 & 3.61 \\
\hline chr2:889-989 significant & WL & 16.22 & 1.01 & 0.22 & -0.6 \\
\hline chr2:893-970 suggestive & WL & 14.16 & 1.3 & 0.36 & 1.16 \\
\hline chr2:886-953 significant & RJF & 12.03 & -3.09 & 0.71 & 0.47 \\
\hline chr2:928-938 suggestive & WL & 19.57 & 0.04 & 0.34 & 2.63 \\
\hline chr2:929-940 suggestive & RJF & 19.44 & -0.1 & 0.35 & 2.74 \\
\hline chr2:928-937 suggestive & WL & 19.01 & 0.29 & 0.5 & 3.71 \\
\hline chr2:927-938 suggestive & WL & 18.58 & 0.81 & 0.45 & 3.14 \\
\hline chr2:928-937 suggestive & RJF & 17.4 & -0.45 & 0.37 & 2.71 \\
\hline chr2:929-970 suggestive & RJF & 20.52 & -0.74 & 0.25 & -0.98 \\
\hline chr2:903-942 suggestive & WL & 20.79 & 0.96 & 0.49 & -1.74 \\
\hline chr2:923-961 significant & RJF & 28.18 & -2.21 & 0.32 & 0.08 \\
\hline chr2:927-945 significant & RJF & 30.82 & -3.33 & 0.43 & -0.15 \\
\hline chr2:889-960 significant & WL & 14.47 & 1.31 & 0.27 & -0.3 \\
\hline chr2:927-954 significant & RJF & 29.24 & -2.56 & 0.36 & 0.14 \\
\hline chr2:890-963 significant & RJF & 15.96 & -2.62 & 0.54 & 0.8 \\
\hline chr2:928-949 significant & RJF & 22.04 & -1.33 & 0.25 & -0.56 \\
\hline chr2:927-955 significant & RJF & 19.07 & -1.2 & 0.81 & 1.44 \\
\hline chr2:853-952 significant & RJF & 12.82 & -1.12 & 0.27 & -0.35 \\
\hline chr2:888-965 significant & WL & 19.64 & 1.99 & 0.37 & -0.16 \\
\hline chr2:877-954 significant & RJF & 17.3 & -1.71 & 0.37 & 0.93 \\
\hline chr2:932-953 significant & WL & 18.53 & 0.11 & 0.47 & 0.12 \\
\hline chr2:933-956 suggestive & RJF & 18.25 & -0.95 & 0.24 & -1.18 \\
\hline chr2:881-967 significant & RJF & 15.33 & -1.24 & 0.49 & -2.11 \\
\hline chr2:884-965 significant & RJF & 15.12 & -1.97 & 0.42 & 0.85 \\
\hline chr2:879-959 suggestive & RJF & 19.42 & -1.24 & 0.51 & -0.59 \\
\hline chr2:895-959 suggestive & RJF & 23.97 & -0.18 & 0.34 & 0.09 \\
\hline chr2:923-959 suggestive & RJF & 25.34 & -0.22 & 0.24 & 0.03 \\
\hline chr2:928-959 significant & WL & 17.68 & 1.62 & 0.32 & -1.44 \\
\hline chr2:880-969 suggestive & WL & 19 & 4.06 & 0.95 & 0.21 \\
\hline chr2:947-959 significant & WL & 19.99 & 2.69 & 0.48 & -1.92 \\
\hline chr2:947-957 suggestive & WL & 9.69 & 0.74 & 0.24 & -1.98 \\
\hline chr2:948-963 suggestive & WL & 17.62 & 1.3 & 0.24 & -0.86 \\
\hline chr2:932-960 suggestive & WL & 19.07 & 0.58 & 0.37 & -2.91 \\
\hline chr2:893-972 suggestive & WL & 13.34 & 1.58 & 0.4 & 0.73 \\
\hline chr2:899-975 significant & RJF & 14.69 & -3.03 & 0.67 & 0.47 \\
\hline chr2:939-974 suggestive & WL & 19.39 & 2.42 & 0.59 & -1.92 \\
\hline chr2:946-974 suggestive & WL & 17.52 & 1.56 & 0.54 & -2.62 \\
\hline chr2:944-968 significant & WL & 14.46 & 1.31 & 0.31 & -1.54 \\
\hline chr2:944-968 suggestive & WL & 13.57 & 2.24 & 0.51 & -1.55 \\
\hline
\end{tabular}




\begin{tabular}{|c|c|c|c|c|c|}
\hline chr2:947-978 suggestive & WL & 19.23 & 0.99 & 0.31 & -0.1 \\
\hline chr2:929-972 significant & WL & 18.2 & 1.84 & 0.45 & 1.17 \\
\hline chr2:947-970 significant & WL & 22.14 & 1.66 & 0.47 & 1.41 \\
\hline chr2:947-971 significant & WL & 19.83 & 3.14 & 0.73 & 1.56 \\
\hline chr2:950-971 significant & WL & 16.13 & 1.85 & 0.39 & -0.92 \\
\hline chr2:955-976 suggestive & WL & 19.32 & 0.07 & 0.24 & 0.12 \\
\hline chr2:947-975 suggestive & WL & 14.55 & 1.65 & 0.49 & 0.74 \\
\hline chr2:950-999 suggestive & WL & 13.76 & 2.33 & 0.54 & -0.83 \\
\hline chr2:959-991 suggestive & WL & 23.61 & 0.07 & 0.34 & 1.12 \\
\hline chr2:961-991 significant & WL & 17.9 & 1.28 & 0.46 & 1.33 \\
\hline chr2:970-100 significant & WL & 16.19 & 0.8 & 0.17 & -0.72 \\
\hline chr2:970-990 significant & RJF & 15.18 & -1.5 & 0.33 & 0.58 \\
\hline chr2:968-991 significant & WL & 21.63 & 2.07 & 0.46 & 0.35 \\
\hline chr2:967-100 significant & RJF & 15.2 & -1.84 & 0.39 & 1.05 \\
\hline chr2:962-102 significant & WL & 16.58 & 1.35 & 0.32 & 0.43 \\
\hline chr2:966-103 suggestive & RJF & 13.79 & -0.77 & 0.2 & -0.19 \\
\hline chr2:963-104 significant & WL & 13.74 & 1.73 & 0.57 & 1.42 \\
\hline chr2:982-100 suggestive & RJF & 17.44 & -0.33 & 0.45 & 2.62 \\
\hline chr2:978-101 significant & WL & 18.29 & 1.47 & 0.29 & -0.27 \\
\hline chr2:969-101 suggestive & RJF & 12.74 & -4.6 & 1.15 & 0 \\
\hline chr2:965-101 significant & WL & 15.23 & 1.57 & 0.38 & 0.28 \\
\hline chr2:985-100 significant & RJF & 21.24 & -2.75 & 0.52 & -0.13 \\
\hline chr2:986-101 significant & WL & 21.53 & 1.38 & 0.32 & 0.87 \\
\hline chr2:987-101 significant & WL & 16.12 & 1.74 & 0.4 & 0.21 \\
\hline chr2:985-101 suggestive & WL & 22.77 & 0.11 & 0.4 & 3.03 \\
\hline chr2:984-101 significant & RJF & 25.19 & -2.61 & 0.41 & 1.04 \\
\hline chr2:985-102 suggestive & WL & 12.25 & 2.6 & 0.76 & 0.75 \\
\hline chr2:988-101 significant & WL & 24.2 & 2.5 & 0.5 & 1.06 \\
\hline chr2:984-102 suggestive & WL & 18.94 & 1.8 & 0.58 & 1.05 \\
\hline chr2:982-102 significant & WL & 16.44 & 2.47 & 0.57 & 0.41 \\
\hline chr2:988-101 significant & WL & 17.95 & 0.38 & 0.44 & 2.42 \\
\hline chr2:958-104 suggestive & WL & 13.14 & 2.13 & 0.5 & -0.78 \\
\hline chr2:989-102 significant & WL & 17.91 & 1.37 & 0.28 & -0.09 \\
\hline chr2:985-102 significant & WL & 14.81 & 1.79 & 0.53 & 1.07 \\
\hline chr2:984-104 suggestive & WL & 14.48 & 0.48 & 0.3 & 1.27 \\
\hline chr2:987-102 significant & WL & 19.93 & 1.98 & 0.43 & 0.85 \\
\hline chr2:966-104 significant & WL & 16.34 & 1.63 & 0.4 & 0.59 \\
\hline chr2:945-103 suggestive & WL & 14.35 & 1.76 & 0.4 & -0.53 \\
\hline chr2:984-104 significant & WL & 23.54 & 1.98 & 0.34 & -0.66 \\
\hline chr2:987-104 significant & WL & 14.49 & 1.28 & 0.28 & 0.47 \\
\hline chr2:990-103 significant & WL & 24.96 & 2.74 & 0.42 & -0.59 \\
\hline chr2:990-104 significant & WL & 14.68 & 1.66 & 0.37 & 0.12 \\
\hline chr2:992-104 significant & RJF & 20.13 & -2.44 & 0.44 & -0.44 \\
\hline
\end{tabular}




\begin{tabular}{|c|c|c|c|c|c|}
\hline chr2:1002-10 significant & WL & 20.96 & 0.48 & 0.36 & -1.14 \\
\hline chr2:996-104 suggestive & WL & 13.31 & 0.89 & 0.2 & -0.09 \\
\hline chr2:997-104 significant & RJF & 16.22 & -1.73 & 0.35 & -0.35 \\
\hline chr2:985-105 suggestive & WL & 12.35 & 2.76 & 0.68 & 0.75 \\
\hline chr2:1002-10 significant & WL & 12.77 & 2.84 & 0.71 & 1.46 \\
\hline chr2:1011-10 significant & WL & 18.79 & 2.21 & 0.42 & 0.52 \\
\hline chr2:1014-10 significant & WL & 21.44 & 3.68 & 0.64 & -0.48 \\
\hline chr2:1005-10 significant & WL & 18.56 & 1.64 & 0.31 & 0.37 \\
\hline chr2:1009-10 significant & WL & 24.49 & 1.09 & 0.18 & -0.17 \\
\hline chr2:1015-10 significant & WL & 30.16 & 2.86 & 0.41 & -1.27 \\
\hline chr2:1015-10 significant & WL & 18.62 & 2.25 & 0.43 & 0.25 \\
\hline chr2:1021-10 suggestive & WL & 17.17 & 2.9 & 0.64 & 0.2 \\
\hline chr2:1025-10 significant & WL & 22.08 & 0.87 & 0.38 & -0.7 \\
\hline chr2:1022-10 significant & WL & 20.9 & 2.15 & 0.39 & -0.44 \\
\hline chr2:1014-10 suggestive & WL & 19.58 & 1.56 & 0.52 & -1.11 \\
\hline chr2:1012-10 significant & WL & 17.94 & 2.05 & 0.4 & -0.14 \\
\hline chr2:1018-10 significant & WL & 14.7 & 2.07 & 0.46 & -0.4 \\
\hline chr2:1016-10 significant & WL & 18.25 & 2.17 & 0.42 & -0.07 \\
\hline chr2:1004-10 suggestive & RJF & 14.24 & -2 & 0.45 & -0.22 \\
\hline chr2:1002-10 significant & WL & 13.44 & 2.09 & 0.47 & 0.44 \\
\hline chr2:1013-10 suggestive & WL & 14.28 & 1.14 & 0.27 & -0.4 \\
\hline chr2:1015-10 suggestive & WL & 12.08 & 2.07 & 0.51 & 0.44 \\
\hline chr2:987-104 suggestive & WL & 13.49 & 1.58 & 0.37 & -0.71 \\
\hline chr2:1012-10 significant & WL & 13.97 & 2.35 & 0.53 & 0.36 \\
\hline chr2:1003-10 significant & WL & 11.47 & 1.47 & 0.36 & 0.07 \\
\hline chr2:1004-10 significant & WL & 16.24 & 1.56 & 0.33 & 0.43 \\
\hline chr2:1008-10 significant & RJF & 15.89 & -1.99 & 0.42 & -0.39 \\
\hline chr2:1015-10 significant & WL & 13.98 & 2.12 & 0.47 & 0.41 \\
\hline chr2:1017-10 suggestive & WL & 12.51 & 1.17 & 0.75 & -1.99 \\
\hline chr2:1007-10 suggestive & WL & 14.6 & 1.23 & 0.3 & 0.92 \\
\hline chr2:1017-10 significant & WL & 18.94 & 2.07 & 0.39 & 0.02 \\
\hline chr2:1004-10 suggestive & WL & 14.65 & 1.18 & 0.26 & -0.23 \\
\hline chr2:959-104 suggestive & WL & 12.34 & 1.14 & 0.29 & -0.45 \\
\hline chr2:1013-10 significant & WL & 15.1 & 2.28 & 0.48 & 0.1 \\
\hline chr2:1014-10 significant & WL & 33.31 & 1.84 & 0.25 & -0.56 \\
\hline chr2:1006-10 significant & WL & 14.54 & 0.88 & 0.19 & -0.16 \\
\hline chr2:1017-10 significant & WL & 18.01 & 0.89 & 0.19 & -0.57 \\
\hline chr2:994-104 significant & WL & 14.84 & 1.52 & 0.32 & 0.3 \\
\hline chr2:1002-10 suggestive & WL & 12.91 & 1.33 & 0.31 & 0.23 \\
\hline chr2:1007-10 significant & WL & 16.32 & 1.1 & 0.23 & 0.39 \\
\hline chr2:970-104 suggestive & WL & 12.81 & 2.08 & 0.5 & -0.67 \\
\hline chr2:1000-10 suggestive & WL & 12.99 & 1.59 & 0.39 & -0.39 \\
\hline chr2:994-104 significant & WL & 15.25 & 1.42 & 0.33 & -0.89 \\
\hline
\end{tabular}




\begin{tabular}{|c|c|c|c|c|c|}
\hline chr2:1008-10 suggestive & WL & 13.81 & 1 & 0.25 & -0.61 \\
\hline chr2:1014-10 significant & WL & 22.53 & 3.19 & 0.71 & -1.65 \\
\hline chr2:1006-10 significant & WL & 14.76 & 1.1 & 0.25 & -0.31 \\
\hline chr2:1014-10 significant & WL & 17.45 & 2.08 & 0.41 & 0.12 \\
\hline chr2:991-111 suggestive & WL & 12.75 & 1.03 & 0.25 & 0 \\
\hline chr2:1005-10 significant & WL & 11.58 & 2.63 & 0.66 & -0.61 \\
\hline chr2:990-107 suggestive & WL & 13.53 & 0.98 & 0.28 & -0.94 \\
\hline chr2:998-108 suggestive & WL & 11.34 & 7.4 & 1.96 & 4.68 \\
\hline chr2:1002-10 significant & WL & 14.11 & 8.53 & 1.92 & 3.52 \\
\hline chr2:1032-10 suggestive & RJF & 12.11 & -3.2 & 0.79 & -1.61 \\
\hline chr2:1033-10 significant & RJF & 15.13 & -2.54 & 0.55 & -0.17 \\
\hline chr2:1042-10 significant & WL & 27.34 & 0.41 & 0.09 & -0.55 \\
\hline chr2:1039-10 suggestive & WL & 24.01 & 2.11 & 0.49 & -1.28 \\
\hline chr2:1040-10 suggestive & WL & 16 & 0.44 & 0.12 & -0.37 \\
\hline chr2:1041-10 suggestive & WL & 19.53 & 1.06 & 0.23 & -0.71 \\
\hline chr2:1041-10 significant & WL & 16.1 & 3.88 & 0.89 & -1.56 \\
\hline chr2:1048-10 significant & WL & 55.08 & 5.25 & 0.43 & 0.22 \\
\hline chr2:1044-10 significant & WL & 17.3 & 2.31 & 0.52 & -1.1 \\
\hline chr2:1044-10 significant & WL & 22.4 & 3.4 & 0.62 & -1.16 \\
\hline chr2:1045-10 significant & WL & 20.75 & 0.68 & 0.29 & 0.34 \\
\hline chr2:1043-10 suggestive & WL & 19.16 & 0.75 & 0.37 & 0.24 \\
\hline chr2:1040-10 suggestive & WL & 14.28 & 2.91 & 0.76 & -1.71 \\
\hline chr2:1045-10 suggestive & WL & 19.55 & 1.54 & 0.37 & -1.39 \\
\hline chr2:1045-10 suggestive & WL & 20.76 & 2.03 & 0.43 & -1.26 \\
\hline chr2:1041-10 suggestive & WL & 19.32 & 1.42 & 0.3 & -0.84 \\
\hline chr2:1042-10 suggestive & WL & 19.66 & 0.15 & 0.03 & -0.11 \\
\hline chr2:1045-10 suggestive & WL & 19.4 & 1.15 & 0.24 & -0.5 \\
\hline chr2:1039-10 suggestive & WL & 16.31 & 0.76 & 0.16 & -0.14 \\
\hline chr2:1044-10 suggestive & WL & 16.35 & 0.46 & 0.11 & -0.31 \\
\hline chr2:1039-10 significant & WL & 18.46 & 2.21 & 0.44 & -0.35 \\
\hline chr2:1045-10 significant & RJF & 15.9 & -1.67 & 0.35 & -0.57 \\
\hline chr2:1040-10 significant & RJF & 16.64 & -2.28 & 0.46 & -0.7 \\
\hline chr2:1040-10 significant & WL & 21.48 & 2.48 & 0.61 & -0.98 \\
\hline chr2:1032-10 significant & WL & 14.41 & 2.32 & 0.51 & 0.01 \\
\hline chr2:999-110 significant & WL & 14.55 & 2.01 & 0.45 & 1 \\
\hline chr2:1063-11 suggestive & WL & 14.76 & 0.54 & 0.12 & 0.01 \\
\hline chr2:1057-11 significant & WL & 14.68 & 1.34 & 0.29 & 0.03 \\
\hline chr2:1027-11 significant & WL & 12.71 & 1.58 & 0.42 & 0.5 \\
\hline chr2:1064-11 suggestive & WL & 13.55 & 1.47 & 0.34 & 0.21 \\
\hline chr2:1084-11 significant & WL & 37.4 & 3.02 & 0.36 & -0.34 \\
\hline chr2:1069-11 significant & WL & 13.41 & 1.54 & 0.36 & 0.3 \\
\hline chr2:1075-11 significant & WL & 15.28 & 1.25 & 0.34 & -1.44 \\
\hline chr2:1051-12 suggestive & WL & 7.75 & 1.32 & 0.56 & 1.27 \\
\hline
\end{tabular}




\begin{tabular}{|c|c|c|c|c|c|}
\hline chr2:1079-11 suggestive & RJF & 13.54 & -2.51 & 0.59 & 1.07 \\
\hline chr2:1091-11 significant & WL & 18.63 & 0.42 & 0.58 & 1.42 \\
\hline chr2:1094-11 significant & RJF & 16.96 & -1.59 & 0.37 & -1.17 \\
\hline chr2:1090-11 significant & WL & 14.16 & 0.56 & 0.46 & 2.65 \\
\hline chr2:1084-11 significant & WL & 11.93 & 2.36 & 0.62 & 1.08 \\
\hline chr2:1089-11 suggestive & WL & 10.88 & 0.91 & 0.51 & 3.22 \\
\hline chr2:1099-11 significant & WL & 20.65 & 1.69 & 0.34 & 0.99 \\
\hline chr2:1090-11 significant & WL & 16.81 & 2.93 & 0.7 & 2.46 \\
\hline chr2:1096-11 suggestive & WL & 14.42 & 1.62 & 0.38 & 0.58 \\
\hline chr2:1088-11 significant & RJF & 20.35 & -3.35 & 0.6 & 0.38 \\
\hline chr2:1104-11 significant & RJF & 30.37 & -1.05 & 0.15 & 0.11 \\
\hline chr2:1084-11 significant & WL & 23.36 & 3.58 & 0.61 & 0.74 \\
\hline chr2:1096-11 significant & RJF & 26.6 & -1.37 & 0.22 & -0.68 \\
\hline chr2:1084-11 significant & WL & 11.4 & 2.51 & 0.63 & -0.61 \\
\hline chr2:1092-11 significant & WL & 19.12 & 2.87 & 0.58 & 1.66 \\
\hline chr2:1092-11 significant & RJF & 21.1 & -3.15 & 0.56 & 1.19 \\
\hline chr2:1087-11 suggestive & RJF & 14.6 & -0.91 & 0.2 & 0 \\
\hline chr2:1096-11 significant & WL & 19.59 & 3.49 & 0.64 & 0.27 \\
\hline chr2:1097-11 significant & WL & 28.22 & 2.02 & 0.66 & 1.67 \\
\hline chr2:1093-11 significant & RJF & 16.41 & -2.44 & 0.51 & -0.37 \\
\hline chr2:1085-11 significant & RJF & 17.67 & -1.94 & 0.38 & 0.1 \\
\hline chr2:1083-11 suggestive & RJF & 14.01 & -1.43 & 0.36 & -0.76 \\
\hline chr2:1091-11 significant & WL & 23.17 & 1.57 & 0.27 & 0.25 \\
\hline chr2:1094-11 significant & RJF & 16.23 & -2.14 & 0.49 & -1.17 \\
\hline chr2:1122-11 significant & WL & 26.97 & 3.4 & 0.51 & -1.24 \\
\hline chr2:1126-11 significant & RJF & 22.03 & -3.69 & 0.74 & -1.18 \\
\hline chr2:1078-11 significant & WL & 14.18 & 2.21 & 0.49 & -0.56 \\
\hline chr2:1120-11 significant & RJF & 16.91 & -2.39 & 0.48 & 0.56 \\
\hline chr2:1127-11 suggestive & RJF & 13.07 & -0.71 & 0.18 & -1.19 \\
\hline chr2:1127-11 significant & WL & 24.34 & 3.15 & 0.53 & 0.29 \\
\hline chr2:1123-11 significant & WL & 22.84 & 0.21 & 0.46 & 0.72 \\
\hline chr2:1091-11 significant & WL & 13.76 & 1.35 & 0.35 & 0.67 \\
\hline chr2:1127-11 significant & WL & 13.75 & 1.48 & 0.39 & -1.61 \\
\hline chr2:1126-11 significant & WL & 18.14 & 1.44 & 0.28 & -0.32 \\
\hline chr2:1083-11 significant & WL & 23.55 & 2.52 & 0.41 & -0.74 \\
\hline chr2:1126-11 suggestive & RJF & 15.21 & -2.95 & 0.68 & -0.53 \\
\hline chr2:1134-11 suggestive & WL & 15.01 & 0.51 & 0.4 & -3.12 \\
\hline chr2:1128-11 significant & WL & 14.81 & 1.12 & 0.26 & 0.22 \\
\hline chr2:1134-11 suggestive & WL & 7.5 & 3.45 & 0.93 & -7.38 \\
\hline chr2:1129-11 significant & WL & 17.76 & 1.05 & 0.68 & -1.38 \\
\hline chr2:1134-11 significant & WL & 14.4 & 2.54 & 0.58 & -1.68 \\
\hline chr2:1133-11 suggestive & RJF & 15.31 & -2.78 & 0.61 & -0.25 \\
\hline chr2:1121-11 suggestive & WL & 13.35 & 1.65 & 0.38 & -0.15 \\
\hline
\end{tabular}




\begin{tabular}{|c|c|c|c|c|c|}
\hline chr2:1136-11 significant & WL & 8.75 & 2.95 & 0.8 & -6.62 \\
\hline chr2:1136-11 significant & RJF & 54.4 & -5.15 & 0.43 & 0.12 \\
\hline chr2:1134-11 significant & RJF & 29.91 & -2.18 & 0.31 & -0.04 \\
\hline chr2:1133-11 significant & RJF & 16.5 & -2.68 & 0.53 & 0.68 \\
\hline chr2:1080-11 suggestive & WL & 11.12 & 1.6 & 0.42 & -0.33 \\
\hline chr2:1131-11 significant & RJF & 17.22 & -1.3 & 0.29 & -0.43 \\
\hline chr2:1131-12 significant & RJF & 14.64 & -1.74 & 0.43 & 0.21 \\
\hline chr2:1098-11 suggestive & WL & 11.94 & 1.1 & 0.37 & 1.06 \\
\hline chr2:1133-11 suggestive & RJF & 16.51 & -1.21 & 0.36 & -1.35 \\
\hline chr2:1087-11 significant & WL & 11.42 & 1.47 & 0.38 & 0.01 \\
\hline chr2:1132-11 significant & RJF & 17.59 & -1.91 & 1.13 & -0.31 \\
\hline chr2:1094-11 significant & WL & 16.56 & 1.43 & 0.94 & 0.83 \\
\hline chr2:1128-11 suggestive & WL & 14.56 & 1.36 & 0.3 & -0.34 \\
\hline chr2:1130-11 significant & RJF & 17.43 & -1.2 & 0.95 & 0.53 \\
\hline chr2:1135-11 significant & WL & 22.05 & 2.31 & 0.42 & 0.89 \\
\hline chr2:1138-11 significant & WL & 17.95 & 1.66 & 0.32 & 0.1 \\
\hline chr2:1134-11 significant & RJF & 13.61 & -2.19 & 0.5 & 0.33 \\
\hline chr2:1135-12 significant & RJF & 15.93 & -1.31 & 0.28 & -0.03 \\
\hline chr2:1136-11 significant & WL & 14.27 & 1.13 & 0.27 & 0.55 \\
\hline chr2:1137-11 significant & WL & 17.06 & 1.69 & 0.38 & 0.99 \\
\hline chr2:1142-11 significant & RJF & 20.21 & -4.49 & 0.87 & -2.03 \\
\hline chr2:1139-11 significant & RJF & 16.32 & -2.1 & 0.44 & 0.05 \\
\hline chr2:1136-11 suggestive & RJF & 13.38 & -2.4 & 0.58 & -0.78 \\
\hline chr2:1144-11 suggestive & RJF & 14.23 & -1.74 & 0.42 & -0.85 \\
\hline chr2:1146-11 significant & RJF & 16.37 & -1.44 & 0.3 & 0.44 \\
\hline chr2:1140-11 significant & RJF & 15.59 & -2.51 & 0.54 & 0.92 \\
\hline chr2:1140-11 suggestive & RJF & 14.7 & -1.65 & 0.37 & -0.32 \\
\hline chr2:1139-11 suggestive & RJF & 12.14 & -1.92 & 0.56 & -1.58 \\
\hline chr2:1145-11 significant & RJF & 14.93 & -1.58 & 0.34 & -0.17 \\
\hline chr2:1148-11 significant & RJF & 22.29 & -1.34 & 0.43 & 1.75 \\
\hline chr2:1141-12 significant & RJF & 15.73 & -1.79 & 0.38 & 0.09 \\
\hline chr2:1139-12 significant & RJF & 14.43 & -2.54 & 0.56 & 0.03 \\
\hline chr2:1111-12 significant & WL & 13.25 & 1.84 & 0.43 & -0.73 \\
\hline chr2:1139-12 significant & RJF & 21.87 & -2.54 & 0.44 & -0.48 \\
\hline chr2:1144-11 suggestive & WL & 13.93 & 0.07 & 0.17 & -1.07 \\
\hline chr2:1144-12 significant & RJF & 20.04 & -3.99 & 0.74 & -0.52 \\
\hline chr2:1138-12 suggestive & RJF & 14.32 & -1.02 & 0.28 & 0.41 \\
\hline chr2:1156-12 significant & WL & 29.24 & 1.43 & 0.47 & 1.5 \\
\hline chr2:1150-11 significant & RJF & 19.3 & -1.18 & 0.27 & 0.86 \\
\hline chr2:1167-11 suggestive & RJF & 13.26 & -0.18 & 0.25 & 1.62 \\
\hline chr2:1171-11 suggestive & RJF & 20.43 & -2.32 & 0.67 & 3.14 \\
\hline chr2:1168-11 suggestive & RJF & 18.45 & -0.2 & 0.05 & -0.36 \\
\hline chr2:1154-12 suggestive & RJF & 18.6 & -0.22 & 0.05 & -0.25 \\
\hline
\end{tabular}




\begin{tabular}{|c|c|c|c|c|c|}
\hline chr2:1149-11 suggestive & RJF & 18.79 & -0.16 & 0.04 & -0.23 \\
\hline chr2:1173-11 suggestive & RJF & 19.17 & -1.18 & 0.39 & 1.83 \\
\hline chr2:1173-12 suggestive & RJF & 23.06 & -0.97 & 0.46 & 3.49 \\
\hline chr2:1174-12 suggestive & RJF & 15.04 & -0.73 & 0.27 & 1.27 \\
\hline chr2:1166-12 significant & RJF & 17.01 & -0.97 & 0.23 & 0.53 \\
\hline chr2:1172-12 significant & RJF & 24.12 & -2.3 & 0.44 & 0.64 \\
\hline chr2:1176-12 significant & RJF & 23.32 & -1.74 & 0.56 & 2.45 \\
\hline chr2:1167-12 significant & RJF & 19.41 & -1.3 & 0.29 & 0.37 \\
\hline chr2:1172-12 significant & RJF & 19.81 & -2.13 & 0.41 & 0.13 \\
\hline chr2:1169-12 suggestive & RJF & 14.48 & -1 & 0.34 & 1.02 \\
\hline chr2:1143-12 suggestive & RJF & 21.49 & -1.93 & 0.35 & -0.52 \\
\hline chr2:1177-12 significant & RJF & 22.38 & -0.79 & 0.34 & 0.68 \\
\hline chr2:1170-12 significant & RJF & 21.2 & -1.3 & 0.24 & -0.15 \\
\hline chr2:1173-12 significant & WL & 19.12 & 1.84 & 0.35 & 0.6 \\
\hline chr2:1169-12 suggestive & RJF & 18.84 & -1.71 & 0.49 & 0.16 \\
\hline chr2:1174-12 significant & RJF & 22.49 & -1.46 & 0.33 & 0.26 \\
\hline chr2:1151-12 significant & RJF & 16.74 & -1.35 & 0.28 & -0.64 \\
\hline chr2:1152-12 significant & RJF & 17.75 & -1.14 & 0.29 & 0.67 \\
\hline chr2:1167-12 significant & RJF & 14.55 & -0.91 & 0.25 & 0.49 \\
\hline chr2:1187-12 significant & RJF & 25.25 & -1.1 & 0.18 & -0.21 \\
\hline chr2:1186-12 significant & RJF & 23.57 & -2.81 & 0.52 & 0.43 \\
\hline chr2:1187-12 significant & RJF & 37.38 & -2.61 & 0.32 & -0.57 \\
\hline chr2:1187-12 significant & RJF & 20.95 & -1.89 & 0.38 & 0.36 \\
\hline chr2:1186-12 significant & RJF & 20.94 & -1.82 & 0.35 & 0.05 \\
\hline chr2:1187-12 significant & RJF & 28.25 & -2.26 & 0.43 & 1.21 \\
\hline chr2:1188-12 significant & RJF & 39.38 & -1.15 & 0.13 & -0.3 \\
\hline chr2:1188-12 significant & RJF & 25.85 & -0.87 & 0.32 & 0.36 \\
\hline chr2:1185-12 significant & RJF & 17.06 & -1.22 & 0.24 & -0.5 \\
\hline chr2:1175-12 significant & RJF & 16.77 & -1.63 & 0.33 & -0.4 \\
\hline chr2:1135-12 suggestive & RJF & 17.68 & -0.84 & 0.28 & 0.38 \\
\hline chr2:1187-12 significant & RJF & 19.92 & -2.95 & 0.58 & -0.13 \\
\hline chr2:1173-12 significant & RJF & 21.2 & -2.08 & 0.39 & -0.14 \\
\hline chr2:1187-12 significant & RJF & 41.83 & -2.83 & 0.35 & 0.44 \\
\hline chr2:1182-12 significant & RJF & 23.36 & -2.83 & 0.56 & -0.07 \\
\hline chr2:1188-12 significant & RJF & 23.98 & -1.41 & 0.39 & 1.85 \\
\hline chr2:1177-12 significant & RJF & 18.26 & -1.57 & 0.3 & -0.78 \\
\hline chr2:1167-12 significant & WL & 20.41 & 1.48 & 0.31 & -0.43 \\
\hline chr2:1134-12 suggestive & RJF & 14.23 & -0.97 & 0.26 & 0.3 \\
\hline chr2:1177-12 suggestive & RJF & 14.52 & -1.48 & 0.39 & 0.43 \\
\hline chr2:1169-12 significant & RJF & 14.2 & -2.07 & 0.46 & -1.17 \\
\hline chr2:1152-12 suggestive & RJF & 14.41 & -1.18 & 0.28 & 0.05 \\
\hline chr2:1186-12 significant & RJF & 17.13 & -1.59 & 0.36 & 0.17 \\
\hline chr2:1176-12 suggestive & RJF & 13.27 & -2.34 & 0.55 & -1.31 \\
\hline
\end{tabular}




\begin{tabular}{|c|c|c|c|c|c|}
\hline chr2:1188-12 suggestive & RJF & 10.63 & -0.44 & 0.19 & 1.43 \\
\hline chr2:1188-12 suggestive & RJF & 21.66 & -0.33 & 0.24 & 0.06 \\
\hline chr2:1187-12 suggestive & RJF & 14.65 & -1.47 & 0.33 & -1.37 \\
\hline chr2:1177-12 significant & RJF & 24.06 & -5.55 & 0.94 & -0.63 \\
\hline chr2:1186-12 significant & RJF & 19.75 & -1.96 & 0.39 & -0.01 \\
\hline chr2:1151-12 significant & RJF & 16.91 & -1.26 & 0.26 & -0.35 \\
\hline chr2:1185-12 suggestive & RJF & 8.79 & -1.52 & 0.25 & 0.33 \\
\hline chr2:1188-12 significant & RJF & 28.9 & -2.36 & 0.35 & -0.37 \\
\hline chr2:1188-12 significant & RJF & 18.64 & -1.53 & 0.35 & 0.61 \\
\hline chr2:1186-12 significant & RJF & 28.63 & -1.57 & 0.24 & -0.11 \\
\hline chr2:1189-12 significant & RJF & 15.7 & -1.18 & 0.25 & -0.9 \\
\hline chr2:1170-12 significant & RJF & 17.56 & -4.39 & 0.89 & -0.94 \\
\hline chr2:1190-12 significant & RJF & 47.18 & -2.71 & 0.28 & -0.02 \\
\hline chr2:1188-12 significant & RJF & 20.4 & -1.59 & 0.32 & 0.15 \\
\hline chr2:1153-12 significant & RJF & 19.87 & -1.43 & 0.3 & -0.07 \\
\hline chr2:1178-12 significant & WL & 21.48 & 3.39 & 0.68 & -0.64 \\
\hline chr2:1187-12 significant & RJF & 18.28 & -2.4 & 0.47 & -1.04 \\
\hline chr2:1185-12 significant & RJF & 18.61 & -1.63 & 0.32 & -0.46 \\
\hline chr2:1190-12 suggestive & RJF & 14.9 & -0.86 & 0.19 & -0.72 \\
\hline chr2:1131-12 suggestive & RJF & 13.81 & -1.12 & 0.26 & -0.21 \\
\hline chr2:1186-12 suggestive & RJF & 14.45 & -2.12 & 0.52 & 0.06 \\
\hline chr2:1120-12 significant & WL & 13.33 & 4.35 & 1.38 & -3.01 \\
\hline chr2:1188-12 suggestive & RJF & 14.68 & -1.26 & 0.31 & -1.61 \\
\hline chr2:1187-12 suggestive & RJF & 26.66 & -1.32 & 0.22 & -0.48 \\
\hline chr2:1188-12 suggestive & RJF & 25.23 & 0 & 0.01 & -0.01 \\
\hline chr2:1187-12 significant & RJF & 21.25 & -4.71 & 0.84 & -3.96 \\
\hline chr2:1188-12 suggestive & RJF & 24.35 & -1.04 & 0.37 & -0.03 \\
\hline chr2:1190-12 suggestive & RJF & 18.45 & -0.61 & 0.15 & -1.12 \\
\hline chr2:1166-12 significant & RJF & 16.01 & -0.99 & 0.22 & -0.05 \\
\hline chr2:1165-12 significant & WL & 12.53 & 2.44 & 0.64 & 0.05 \\
\hline chr2:1168-12 suggestive & RJF & 14.17 & -5.85 & 1.34 & -0.93 \\
\hline chr2:1186-12 significant & RJF & 21.58 & -2.28 & 0.45 & 0.5 \\
\hline chr2:1187-12 significant & WL & 28.94 & 1.11 & 0.18 & -0.28 \\
\hline chr2:1186-12 significant & RJF & 19.06 & -1.65 & 0.37 & 0.53 \\
\hline chr2:1189-12 significant & RJF & 26.55 & -1.81 & 0.28 & -0.45 \\
\hline chr2:1186-12 significant & RJF & 17.87 & -4.71 & 0.93 & -1.31 \\
\hline chr2:1190-12 significant & RJF & 24.26 & -3.88 & 0.63 & -0.68 \\
\hline chr2:1184-12 significant & RJF & 23.84 & -4.88 & 0.79 & -1.82 \\
\hline chr2:1186-12 suggestive & RJF & 15.07 & -1.53 & 0.35 & -0.28 \\
\hline chr2:1187-12 significant & RJF & 17.04 & -1.56 & 0.34 & -0.12 \\
\hline chr2:1188-12 significant & RJF & 16.12 & -1.79 & 0.39 & -1.73 \\
\hline chr2:1186-12 suggestive & RJF & 14.19 & -3.35 & 0.76 & -1.28 \\
\hline chr2:1182-12 significant & RJF & 15.77 & -5.71 & 1.44 & 1.91 \\
\hline
\end{tabular}


chr2:1185-12 suggestive RJF

chr2:1189-12 suggestive RJF

chr2:1187-12 suggestive RJF

chr2:1186-12 significant RJF

chr2:1188-12 significant RJF

chr2:1187-12 significant RJF

chr2:1187-12 suggestive RJF

chr2:1167-12 suggestive RJF

chr2:1188-12 suggestive RJF

chr2:1188-12 suggestive RJF

chr2:1187-12 significant RJF

chr2:1187-12 significant RJF

chr2:1169-12 suggestive RJF

chr2:1188-12 suggestive RJF

chr2:1188-12 suggestive RJF

chr2:1165-12 significant RJF

chr2:1170-12 suggestive RJF

chr2:1186-12 significant RJF

chr2:1182-12 suggestive RJF

chr2:1188-12 significant RJF

chr2:1187-12 significant RJF

chr2:1186-12 significant RJF

chr2:1169-12 significant RJF

chr2:1167-12 suggestive RJF

chr2:1186-12 significant RJF

chr2:1150-12 suggestive RJF

chr2:1187-12 significant RJF

chr2:1188-12 suggestive RJF

chr2:1181-12 significant RJF

chr2:1187-12 significant RJF

chr2:1187-12 significant RJF

chr2:1178-12 significant RJF

chr2:1186-12 significant

chr2:1189-12 significant

chr2:1171-12 significant

chr2:1186-12 suggestive

chr2:1174-12 significant

chr2:1166-12 suggestive

chr3:0-13 significant

chr3:197-231 suggestive

chr3:201-232 suggestive

chr3:197-239 suggestive

chr3:202-229 significant
15.08

21.53

14.05

18.14

34.05

15.64

25.14

13.87

19.36

24.19

19.97

22.4

14.34

22.75

22.22

18.88

18.68

14.71

17.78

28.73

17.54

19.63

17.22

14.82

15.28

13.29

15.08

25.33

16.79

16.49

21.28

18.8

20.21

29.31

16.7

14.7

19.86

14.15

20.71

18.95

20.99

17.33

22.32
$-4.21$

$-1.09$

$-1.59$

$-3.21$

$-2.34$

$-1.67$

$-2.32$

$-1.44$

$-1.34$

$-1.87$

$-1.97$

$-2.04$

$-1.48$

$-0.69$

$-0.17$

$-1.15$

$-1.58$

$-1.61$

$-1.45$

$-3.1$

$-1.99$

$-1.37$

$-3.16$

$-1.94$

$-1.81$

$-1.92$

$-1.44$

$-0.76$

$-3.23$

$-1.59$

$-1.82$

$-1.19$

$-1.67$

0

$-1.78$

$-2.48$

$-2.1$

$-1.78$

$-7.64$

1.42

$-1.53$

0.44

$-1.38$
1.03

0.19

0.36

0.64

0.32

0.35

0.48

0.33

0.26

0.46

0.65

0.35

0.33

0.32

0.23

0.22

0.45

0.45

0.46

0.6

0.39

0.36

0.65

0.43

0.39

0.47

0.32

0.3

0.74

0.32

0.33

0.49

0.34

0.04

0.38

0.75

0.79

0.51

2.46

1.69

1.53

1.36

1.38
0.58

$-0.68$

$-1.53$

$-0.75$

$-0.13$

$-0.77$

$-0.65$

$-0.47$

$-1.41$

$-0.13$

1.86

$-0.8$

$-0.81$

0.77

$-0.13$

$-0.52$

1.47

0.77

$-0.02$

1.98

$-1.35$

1.13

$-0.98$

$-1.34$

$-0.82$

$-0.28$

$-0.38$

0.59

0.43

$-0.94$

$-0.65$

2.38

0.23

$-0.01$

$-0.15$

1.78

4.16

0.9

$-17.37$

1.02

1.34

1.54

0.41 
chr3:198-234 suggestive RJF

chr3:197-233 suggestive RJF

chr3:200-231 suggestive RJF

chr3:201-235 suggestive RJF

chr3:184-231 suggestive RJF

chr3:203-236 suggestive RJF

chr3:194-244 suggestive WL

chr3:201-237 suggestive RJF

chr3:202-230 suggestive WL

chr3:203-237 suggestive RJF

chr3:206-236 suggestive RJF

chr3:203-252 suggestive

chr3:203-234 suggestive

chr3:207-241 suggestive

chr3:202-245 suggestive

chr3:202-233 suggestive

chr3:206-233 suggestive

chr3:206-235 suggestive

chr3:207-236 suggestive

chr3:205-231 suggestive

chr3:209-233 suggestive

chr3:203-241 suggestive

chr3:205-230 suggestive

chr3:205-234 suggestive

chr3:203-237 suggestive

chr3:206-229 significant

chr3:203-246 suggestive

chr3:202-235 suggestive

chr3:204-236 suggestive

chr3:203-238 suggestive

chr3:205-238 suggestive

chr3:202-234 suggestive

chr3:204-238 suggestive

chr3:204-235 significant

chr3:207-232 suggestive

chr3:202-245 suggestive

chr3:211-232 significant

chr3:207-234 suggestive

chr3:205-231 significant

chr3:208-231 significant

chr3:209-239 suggestive

chr3:209-234 suggestive

chr3:212-234 significant
20.12

13.17

18.7

19.73

17.17

21.45

10.03

12.94

17.81

16.65

19.7

12.13

20.15

20.4

13.55

20.36

18.72

18.11

15.89

19.34

19.86

11.52

19.32

19.9

19.47

17.59

13.15

18.31

18.76

18.63

20.03

17.66

19.99

21.42

20.2

14.08

20.76

20.59

21.61

26.32

14.62

19.26

19.4
$-1.57$

$-0.01$

$-0.63$

$-0.1$

$-1.43$

$-0.75$

2.86

$-0.51$

0.11

$-0.98$

$-0.52$

4.41

$-0.21$

$-2.51$

3.48

$-0.49$

$-0.35$

1.12

$-0.54$

0.84

0.8

4.9

1.01

0.16

$-0.97$

0.68

3.31

$-0.17$

0.03

$-1.08$

$-1.76$

1.77

$-1.58$

$-0.61$

$-0.11$

5.13

0.01

$-0.08$

0.14

$-0.95$

$-0.85$

1.27

$-0.81$
1.17

1.06

1.7

1.1

1.7

1.12

0.64

0.95

1.25

1.41

1.23

1.17

1.21

2.28

0.79

1.34

1.75

1.43

1.33

1.33

1.19

1.19

1.13

1.5

1.82

0.92

0.79

1.44

1.15

1.14

1.89

1.83

1.15

1.65

1.11

1.16

1.16

1.25

1.09

1.19

2.3

1.34

1.22
1.04

0.15

0.76

1.28

$-0.97$

2.17

$-1.44$

$-0.4$

$-1.4$

0.88

1.79

$-4.13$

1.19

$-0.64$

$-1.46$

2.48

1.48

$-0.16$

1.07

0.7

$-0.21$

$-3.4$

0.74

2.23

0.35

$-0.57$

$-2.39$

0.45

$-0.53$

1.75

0.7

$-0.16$

1.35

$-0.29$

1.28

$-2.58$

0.41

0.22

1.04

$-0.46$

$-0.03$

$-0.98$

0.82 


\begin{tabular}{|c|c|c|c|c|c|}
\hline chr3:207-235 suggestive & WL & 19.12 & 0.59 & 1.49 & -1.09 \\
\hline chr3:205-236 significant & WL & 15.7 & 3.66 & 0.78 & -2.61 \\
\hline chr3:203-240 suggestive & RJF & 17.12 & -0.17 & 1.68 & -1.93 \\
\hline chr3:204-232 suggestive & RJF & 19.8 & -0.09 & 1.27 & 0.73 \\
\hline chr3:209-233 suggestive & RJF & 18.45 & -0.8 & 1.28 & 1.5 \\
\hline chr3:205-228 suggestive & WL & 21.97 & 0.84 & 1.27 & 0.81 \\
\hline chr3:206-237 suggestive & RJF & 19.22 & -0.8 & 1.39 & 1.23 \\
\hline chr3:204-241 suggestive & WL & 16.04 & 3.54 & 0.79 & -3.21 \\
\hline chr3:205-231 suggestive & RJF & 14.13 & -0.38 & 1.22 & 0.62 \\
\hline chr3:204-246 suggestive & WL & 11.68 & 4.25 & 1.09 & -3.55 \\
\hline chr3:202-238 suggestive & RJF & 20.12 & -1.37 & 1.39 & 1.09 \\
\hline chr3:201-237 suggestive & WL & 17.87 & 1.54 & 1.11 & -3.2 \\
\hline chr3:206-233 significant & WL & 21.24 & 0.4 & 1.54 & -0.64 \\
\hline chr3:206-236 suggestive & RJF & 18.6 & -0.02 & 1.7 & 1.12 \\
\hline chr3:201-232 suggestive & WL & 19.81 & 0.41 & 1.09 & 0.67 \\
\hline chr3:208-232 significant & RJF & 22.27 & -0.05 & 2.4 & -0.68 \\
\hline chr3:205-233 suggestive & WL & 19.83 & 0.56 & 1.11 & -0.17 \\
\hline chr3:205-231 suggestive & RJF & 20.06 & -0.9 & 1.86 & 1.95 \\
\hline chr3:207-234 suggestive & RJF & 21.25 & -1.03 & 1.43 & 2.26 \\
\hline chr3:206-236 suggestive & WL & 20.13 & 2.1 & 1.74 & -1.51 \\
\hline chr3:199-242 suggestive & WL & 13.44 & 3.11 & 0.77 & -2.77 \\
\hline chr3:203-239 suggestive & RJF & 19.03 & -0.82 & 1.56 & 0.4 \\
\hline chr3:208-237 suggestive & RJF & 20.11 & -0.89 & 1.49 & 0.95 \\
\hline chr3:203-241 suggestive & WL & 13.31 & 3.29 & 0.77 & -2.46 \\
\hline chr3:205-235 suggestive & WL & 19.99 & 0.26 & 1.36 & 2.15 \\
\hline chr3:207-233 suggestive & WL & 21.2 & 1 & 1.5 & 0.87 \\
\hline chr3:209-235 suggestive & RJF & 20.69 & -0.2 & 1.32 & 1.04 \\
\hline chr3:206-240 suggestive & WL & 19.32 & 0.06 & 1.23 & 1.32 \\
\hline chr3:205-238 suggestive & WL & 19.43 & 0.31 & 1.52 & -0.18 \\
\hline chr3:205-232 suggestive & RJF & 18.24 & -0.3 & 1.34 & 0.96 \\
\hline chr3:204-231 suggestive & WL & 19.82 & 0.42 & 1.43 & 1.25 \\
\hline chr3:207-235 suggestive & RJF & 19.19 & -0.68 & 1.22 & 0.28 \\
\hline chr3:208-240 significant & WL & 12.6 & 4.72 & 1.21 & -4.62 \\
\hline chr3:206-238 suggestive & RJF & 19.29 & -0.17 & 1.51 & -0.45 \\
\hline chr3:208-231 significant & RJF & 19.81 & -0.4 & 0.95 & 0.67 \\
\hline chr3:203-232 suggestive & RJF & 18.55 & -0.48 & 1.8 & -1.33 \\
\hline chr3:207-233 suggestive & RJF & 14.72 & -1.14 & 1.83 & 0.88 \\
\hline chr3:205-237 significant & WL & 13.51 & 2.09 & 1.35 & -3.28 \\
\hline chr3:205-235 significant & RJF & 16.17 & -0.37 & 1.01 & 0.58 \\
\hline chr3:207-235 suggestive & WL & 19.13 & 0.82 & 1.35 & 0.3 \\
\hline chr3:204-235 suggestive & WL & 18.88 & 0.63 & 1.45 & 1.33 \\
\hline chr3:208-241 suggestive & WL & 17.7 & 0.57 & 1.32 & -0.07 \\
\hline chr3:205-232 significant & WL & 20.09 & 0.19 & 1.17 & -0.23 \\
\hline
\end{tabular}




\begin{tabular}{|c|c|c|c|c|c|}
\hline chr3:203-236 suggestive & RJF & 14.85 & -0.6 & 1.99 & 1.06 \\
\hline chr3:207-234 suggestive & WL & 15.24 & 0.37 & 1.1 & 0.04 \\
\hline chr3:210-232 significant & WL & 19.77 & 0.12 & 1.04 & 0.17 \\
\hline chr3:208-231 significant & RJF & 17.65 & -0.01 & 1.32 & 0.66 \\
\hline chr3:205-230 suggestive & WL & 21 & 0.8 & 1.22 & 1.49 \\
\hline chr3:204-246 suggestive & WL & 13.55 & 3.47 & 0.82 & -3.04 \\
\hline chr3:206-240 suggestive & WL & 15.99 & 0.34 & 1.14 & 0.48 \\
\hline chr3:206-233 suggestive & WL & 20.61 & 0.58 & 1.41 & 0.45 \\
\hline chr3:204-239 suggestive & WL & 11.75 & 3.53 & 0.89 & -2.9 \\
\hline chr3:205-236 suggestive & RJF & 19.43 & -0.8 & 1.83 & 0.3 \\
\hline chr3:208-240 significant & WL & 15.54 & 5.9 & 1.35 & -5.46 \\
\hline chr3:204-238 suggestive & RJF & 20.93 & -2 & 1.38 & 0.76 \\
\hline chr3:203-233 suggestive & RJF & 21.39 & -0.42 & 1.5 & 2 \\
\hline chr3:199-240 suggestive & WL & 12.54 & 1.08 & 1.02 & -0.73 \\
\hline chr3:206-235 significant & RJF & 23.02 & -0.66 & 1.35 & 1.1 \\
\hline chr3:201-240 suggestive & WL & 13.99 & 3.99 & 0.91 & -2.71 \\
\hline chr3:203-232 suggestive & RJF & 19.71 & -0.49 & 1.41 & 1.55 \\
\hline chr3:201-237 suggestive & WL & 17.53 & 0.5 & 1.46 & -1.38 \\
\hline chr3:208-235 suggestive & RJF & 21.25 & -1.06 & 1.21 & 0.47 \\
\hline chr3:195-236 suggestive & RJF & 19.97 & -0.01 & 1.12 & 0.56 \\
\hline chr3:206-240 suggestive & WL & 12.06 & 3.87 & 0.97 & -3.15 \\
\hline chr3:208-230 significant & RJF & 22.34 & -0.57 & 1.12 & 1.64 \\
\hline chr3:206-233 suggestive & WL & 18.29 & 0.41 & 1.86 & -0.51 \\
\hline chr3:205-230 suggestive & RJF & 15.24 & -0.14 & 1.54 & 1.53 \\
\hline chr3:206-244 suggestive & WL & 13.19 & 3.35 & 0.78 & -1.89 \\
\hline chr3:204-237 suggestive & RJF & 12.78 & -0.54 & 1.61 & 0.49 \\
\hline chr3:208-234 suggestive & RJF & 21.26 & -0.94 & 1.28 & 1.64 \\
\hline chr3:205-235 suggestive & WL & 19.23 & 0.08 & 1.48 & 0.02 \\
\hline chr3:208-232 significant & WL & 22.54 & 1 & 1.35 & -1.44 \\
\hline chr3:204-231 suggestive & WL & 14.45 & 0.41 & 0.97 & 0.6 \\
\hline chr3:207-233 significant & RJF & 17.69 & -1.02 & 1.15 & 0.34 \\
\hline chr3:204-236 suggestive & RJF & 17.16 & -0.25 & 1.61 & 0.44 \\
\hline chr3:203-235 suggestive & RJF & 19.35 & -0.71 & 1.91 & 1.66 \\
\hline chr3:207-232 suggestive & RJF & 16.52 & -0.44 & 1.1 & 1.18 \\
\hline chr3:200-236 significant & WL & 19.93 & 0.51 & 1.12 & 0.16 \\
\hline chr3:207-240 significant & WL & 16.51 & 3.35 & 0.78 & -3.77 \\
\hline chr3:209-232 suggestive & WL & 19.86 & 1.56 & 1.23 & -1.3 \\
\hline chr3:207-239 suggestive & WL & 19.58 & 0.68 & 1.48 & -0.78 \\
\hline chr3:206-237 suggestive & RJF & 17.84 & -1.86 & 1.98 & -0.34 \\
\hline chr3:203-234 suggestive & RJF & 20.13 & -1.44 & 1.68 & 1.22 \\
\hline chr3:208-243 significant & WL & 14.47 & 4 & 0.95 & -3.67 \\
\hline chr3:203-241 suggestive & WL & 12.32 & 0.97 & 1.1 & 0.28 \\
\hline chr3:202-248 significant & WL & 13.6 & 3.98 & 0.93 & -3.3 \\
\hline
\end{tabular}




\begin{tabular}{|c|c|c|c|c|c|}
\hline chr3:203-249 suggestive & WL & 11.97 & 3.81 & 0.95 & -2.75 \\
\hline chr3:202-248 suggestive & WL & 13.57 & 3.68 & 0.85 & -2.17 \\
\hline chr3:205-247 suggestive & WL & 14.4 & 3.3 & 0.77 & -2.66 \\
\hline chr3:206-245 significant & WL & 11.04 & 3.7 & 0.73 & -2.25 \\
\hline chr3:203-249 suggestive & WL & 13.82 & 4.93 & 1.09 & -2.59 \\
\hline chr3:207-249 suggestive & WL & 14.27 & 3.21 & 0.8 & -3.29 \\
\hline chr3:206-244 significant & WL & 14.43 & 4.19 & 0.79 & -2.1 \\
\hline chr3:197-247 suggestive & WL & 15.63 & 4.06 & 0.86 & -2.1 \\
\hline chr3:204-247 suggestive & WL & 13.73 & 4.04 & 0.98 & -3.53 \\
\hline chr3:210-247 suggestive & WL & 18.02 & 0.07 & 1.55 & -0.34 \\
\hline chr3:205-251 significant & WL & 15.42 & 7.13 & 1.56 & -5.79 \\
\hline chr3:200-250 significant & WL & 15.36 & 3.77 & 0.81 & -2.73 \\
\hline chr3:203-251 suggestive & WL & 13.04 & 4.11 & 0.97 & -2.85 \\
\hline chr3:206-249 significant & WL & 17.64 & 5.2 & 1.01 & -3.11 \\
\hline chr3:206-258 significant & WL & 15.82 & 3.84 & 0.82 & -2.35 \\
\hline chr3:200-259 suggestive & WL & 14.29 & 3.44 & 0.77 & -1.89 \\
\hline chr3:206-264 suggestive & WL & 16.38 & 6.91 & 1.52 & -6.15 \\
\hline chr4:299-342 suggestive & WL & 21.47 & 1.79 & 0.35 & -1.69 \\
\hline chr4:300-339 suggestive & WL & 26.17 & 1.24 & 0.22 & -0.87 \\
\hline chr4:301-349 suggestive & WL & 15.08 & 0.91 & 0.15 & -0.45 \\
\hline chr4:300-347 suggestive & WL & 26.45 & 3.14 & 0.5 & -1.76 \\
\hline chr4:300-339 suggestive & WL & 24.1 & 1.57 & 0.28 & -1.12 \\
\hline chr4:298-351 suggestive & WL & 24.02 & 1.42 & 0.27 & -1.08 \\
\hline chr4:300-346 suggestive & WL & 23.03 & 2.63 & 0.45 & -1.29 \\
\hline chr4:304-342 suggestive & WL & 24.02 & 0.92 & 0.35 & -2.58 \\
\hline chr4:302-350 suggestive & WL & 24.38 & 2.17 & 0.4 & -1.66 \\
\hline chr4:302-343 suggestive & WL & 23.89 & 2.04 & 0.4 & -1.15 \\
\hline chr4:302-345 suggestive & WL & 24.07 & 2.74 & 0.52 & -2.13 \\
\hline chr4:305-343 suggestive & WL & 26.66 & 3.84 & 0.67 & -3.42 \\
\hline chr4:302-339 suggestive & WL & 24.32 & 2.1 & 0.37 & -1.04 \\
\hline chr4:305-344 suggestive & WL & 26.44 & 2.13 & 0.37 & -1.88 \\
\hline chr4:303-348 suggestive & WL & 19.81 & 1.71 & 0.33 & -1.08 \\
\hline chr4:302-344 suggestive & WL & 19.61 & 1.48 & 0.27 & -0.66 \\
\hline chr4:303-343 suggestive & WL & 16.37 & 1.44 & 0.29 & -1.2 \\
\hline chr4:304-348 suggestive & WL & 21.11 & 1.87 & 0.34 & -1.16 \\
\hline chr4:306-340 suggestive & WL & 19.86 & 1.43 & 0.28 & -1.12 \\
\hline chr4:306-343 suggestive & WL & 18.51 & 1.3 & 0.26 & -0.92 \\
\hline chr4:310-338 suggestive & WL & 19.14 & 0.6 & 0.14 & -0.85 \\
\hline chr4:309-338 suggestive & WL & 23.48 & 2.18 & 0.42 & -1.63 \\
\hline chr4:307-344 suggestive & WL & 17.73 & 2.04 & 0.47 & 1.28 \\
\hline chr4:310-344 suggestive & WL & 20.53 & 1.33 & 0.24 & -0.46 \\
\hline chr4:310-345 suggestive & WL & 18.03 & 0.86 & 0.16 & -0.26 \\
\hline chr4:306-348 suggestive & WL & 20.24 & 1.45 & 0.27 & -0.9 \\
\hline
\end{tabular}




\begin{tabular}{|c|c|c|c|c|c|}
\hline chr4:309-347 suggestive & WL & 18.53 & 2.11 & 0.4 & -0.52 \\
\hline chr4:305-346 suggestive & WL & 20.09 & 1.83 & 0.35 & 0.29 \\
\hline chr4:307-346 significant & WL & 16.84 & 1.08 & 0.19 & -0.23 \\
\hline chr4:305-347 suggestive & WL & 20.66 & 1.37 & 0.24 & -0.63 \\
\hline chr4:309-345 suggestive & WL & 25.22 & 2.2 & 0.45 & -2.85 \\
\hline chr4:305-346 suggestive & WL & 20.82 & 1.08 & 0.23 & 0.57 \\
\hline chr4:305-346 suggestive & WL & 23.41 & 2.57 & 0.49 & -2.42 \\
\hline chr4:308-346 suggestive & WL & 27.84 & 1.84 & 0.32 & -1.45 \\
\hline chr4:308-349 suggestive & WL & 18.37 & 1.42 & 0.27 & -0.79 \\
\hline chr4:305-352 suggestive & WL & 18.46 & 1.6 & 0.33 & 0.33 \\
\hline chr4:300-350 suggestive & WL & 25.61 & 2.01 & 0.36 & -0.99 \\
\hline chr4:307-351 suggestive & WL & 21.89 & 1.29 & 0.23 & -0.78 \\
\hline chr4:301-354 suggestive & WL & 18.28 & 1.03 & 0.2 & -0.49 \\
\hline chr4:309-355 suggestive & WL & 19.9 & 3.13 & 0.57 & -0.91 \\
\hline chr4:305-356 suggestive & WL & 17.31 & 1.49 & 0.33 & 0.58 \\
\hline chr4:310-356 suggestive & WL & 19.91 & 1.62 & 0.3 & -0.76 \\
\hline chr4:313-359 suggestive & WL & 10.95 & 1.48 & 0.25 & -0.72 \\
\hline chr4:302-366 suggestive & WL & 17.97 & 0.92 & 0.18 & -0.49 \\
\hline chr4:422-441 suggestive & RJF & 17.14 & -0.22 & 0.11 & 0.54 \\
\hline chr4:398-446 suggestive & WL & 12.9 & 1.76 & 0.58 & -0.55 \\
\hline chr4:426-433 suggestive & RJF & 19.1 & -0.32 & 0.42 & 0.14 \\
\hline chr4:431-433 significant & RJF & 24.38 & -0.15 & 0.02 & -0.15 \\
\hline chr4:424-448 suggestive & RJF & 20.31 & -1.53 & 0.77 & 0.86 \\
\hline chr4:421-451 significant & WL & 14.93 & 3.43 & 0.84 & 0.24 \\
\hline chr4:425-454 significant & RJF & 17.69 & -1.44 & 0.28 & -0.87 \\
\hline chr4:406-454 significant & RJF & 21.49 & -3.16 & 0.77 & 0.99 \\
\hline chr4:425-461 significant & RJF & 20.02 & -1.52 & 0.28 & -0.25 \\
\hline chr4:423-462 significant & RJF & 18.77 & -1.8 & 0.38 & 0.4 \\
\hline chr4:424-460 significant & RJF & 19.88 & -2.87 & 0.59 & 0.71 \\
\hline chr4:435-456 significant & RJF & 28.8 & -2.12 & 0.33 & 0.16 \\
\hline chr4:435-461 significant & RJF & 24.48 & -1.2 & 0.74 & 1.7 \\
\hline chr4:422-458 significant & RJF & 19.69 & -2.13 & 0.43 & 0.37 \\
\hline chr4:419-461 suggestive & WL & 11.57 & 1.36 & 0.39 & -0.4 \\
\hline chr4:435-464 suggestive & RJF & 18.46 & -0.42 & 0.54 & 1.42 \\
\hline chr4:430-461 suggestive & RJF & 16.26 & -2.84 & 0.59 & -1.39 \\
\hline chr4:438-462 suggestive & WL & 14.12 & 0.92 & 0.93 & -0.02 \\
\hline chr4:436-462 significant & WL & 20.13 & 3.05 & 0.58 & 0.01 \\
\hline chr4:438-462 significant & WL & 22.42 & 1.29 & 0.96 & 1.16 \\
\hline chr4:436-465 significant & WL & 15.6 & 2.35 & 0.56 & -0.56 \\
\hline chr4:438-464 significant & WL & 16.86 & 1.87 & 0.45 & -0.82 \\
\hline chr4:437-465 significant & WL & 12.41 & 1.47 & 0.4 & -0.47 \\
\hline chr4:438-461 significant & RJF & 16.89 & -2.4 & 0.48 & -1.35 \\
\hline chr4:440-462 significant & WL & 16.31 & 1.39 & 0.31 & -0.12 \\
\hline
\end{tabular}




\begin{tabular}{|c|c|c|c|c|c|}
\hline chr4:444-460 significant & WL & 22.6 & 0.13 & 0.61 & 0 \\
\hline chr4:435-465 significant & WL & 14.21 & 1.68 & 0.39 & 0.16 \\
\hline chr4:434-472 suggestive & WL & 11.43 & 0.68 & 0.2 & -0.28 \\
\hline chr4:440-463 significant & RJF & 20.9 & -2.26 & 0.41 & -0.66 \\
\hline chr4:427-471 suggestive & WL & 11.98 & 1.83 & 0.48 & -0.14 \\
\hline chr4:407-526 suggestive & WL & 8.33 & 2.57 & 0.88 & -0.63 \\
\hline chr4:422-540 significant & WL & 8.26 & 1.63 & 0.88 & -2.45 \\
\hline chr4:407-565 significant & WL & 8.67 & 1.65 & 0.51 & 0.01 \\
\hline chr4:439-482 suggestive & RJF & 16.25 & -1.92 & 0.84 & 1.39 \\
\hline chr4:439-480 significant & RJF & 13.96 & -1.44 & 0.32 & 0.08 \\
\hline chr4:443-481 suggestive & RJF & 14.5 & -1.72 & 0.38 & -0.33 \\
\hline chr4:439-480 significant & RJF & 13.21 & -2.07 & 0.49 & -0.66 \\
\hline chr4:445-480 suggestive & WL & 13.87 & 1.93 & 0.44 & -0.1 \\
\hline chr4:436-480 suggestive & WL & 14.55 & 1.2 & 0.27 & 0.35 \\
\hline chr4:451-483 suggestive & WL & 10.73 & 1.89 & 0.34 & -0.37 \\
\hline chr4:445-480 suggestive & RJF & 15.72 & -2.3 & 0.49 & -0.09 \\
\hline chr4:435-482 suggestive & RJF & 13.89 & -1.77 & 0.46 & 1.27 \\
\hline chr4:440-480 significant & RJF & 13.48 & -2.05 & 0.48 & 0.46 \\
\hline chr4:439-482 suggestive & RJF & 13 & -2.72 & 0.64 & -0.36 \\
\hline chr4:441-484 significant & WL & 14.12 & 1.74 & 0.39 & 0.08 \\
\hline chr4:450-480 significant & RJF & 18.42 & -1.72 & 0.38 & 1.23 \\
\hline chr4:452-509 significant & RJF & 16.1 & -1.91 & 0.4 & -0.41 \\
\hline chr4:443-483 suggestive & RJF & 12.2 & -2.68 & 0.69 & -1.57 \\
\hline chr4:456-483 significant & WL & 17.02 & 3.03 & 0.64 & 1.82 \\
\hline chr4:455-485 significant & RJF & 17.43 & -1.83 & 0.36 & 0.21 \\
\hline chr4:453-480 significant & RJF & 14.95 & -2.41 & 0.53 & -0.95 \\
\hline chr4:452-483 significant & RJF & 14.32 & -3.08 & 0.69 & 0.84 \\
\hline chr4:454-483 significant & RJF & 15.8 & -1.39 & 0.3 & 0.47 \\
\hline chr4:460-482 significant & WL & 27.53 & 3.63 & 0.55 & -0.99 \\
\hline chr4:456-484 suggestive & WL & 14 & 3.92 & 0.88 & 0.34 \\
\hline chr4:458-486 suggestive & WL & 17.82 & 1.64 & 0.32 & 0.77 \\
\hline chr4:453-528 suggestive & RJF & 12.55 & -2.85 & 0.75 & 1.86 \\
\hline chr4:459-504 significant & WL & 18.73 & 2 & 0.39 & 0.8 \\
\hline chr4:457-480 suggestive & WL & 18.99 & 4.44 & 0.91 & -2.55 \\
\hline chr4:459-484 significant & WL & 16.33 & 1.12 & 0.26 & 0.79 \\
\hline chr4:460-483 significant & RJF & 17.04 & -3.23 & 0.71 & 2.12 \\
\hline chr4:461-481 significant & RJF & 19.76 & -2.66 & 0.51 & -1.02 \\
\hline chr4:462-482 significant & RJF & 22.77 & -3.09 & 0.53 & 0.98 \\
\hline chr4:459-509 suggestive & WL & 14.64 & 1.07 & 0.24 & 0.18 \\
\hline chr4:462-483 significant & WL & 15.81 & 1.65 & 0.35 & 0.43 \\
\hline chr4:465-484 significant & WL & 23.37 & 1.73 & 0.29 & 0.27 \\
\hline chr4:460-485 significant & RJF & 16.7 & -3.8 & 0.77 & -0.12 \\
\hline chr4:466-480 significant & WL & 43.52 & 2.97 & 0.31 & 0.63 \\
\hline
\end{tabular}




\begin{tabular}{|c|c|c|c|c|c|}
\hline chr4:464-502 significant & WL & 19.38 & 1.95 & 0.39 & 1.1 \\
\hline chr4:468-482 significant & WL & 44.75 & 2.68 & 0.27 & 0.1 \\
\hline chr4:458-510 significant & WL & 17.68 & 2.65 & 0.52 & -0.34 \\
\hline chr4:462-496 significant & WL & 21.91 & 2.88 & 0.78 & 3.53 \\
\hline chr4:456-508 significant & WL & 18.46 & 1.41 & 0.27 & 0.08 \\
\hline chr4:458-506 significant & WL & 16.55 & 2.2 & 0.45 & 0.54 \\
\hline chr4:466-485 significant & WL & 19.58 & 0.92 & 0.21 & 0.97 \\
\hline chr4:450-526 suggestive & RJF & 10.55 & -0.97 & 0.36 & 0.89 \\
\hline chr4:463-505 suggestive & WL & 13.75 & 2.33 & 0.52 & 0.22 \\
\hline chr4:459-513 suggestive & WL & 13 & 3.4 & 0.79 & -0.04 \\
\hline chr4:454-569 suggestive & RJF & 7.37 & -1.89 & 0.63 & 0.07 \\
\hline chr4:464-508 significant & WL & 18.55 & 2.76 & 0.52 & 1.12 \\
\hline chr4:458-529 suggestive & WL & 12.26 & 2.28 & 0.55 & 0.34 \\
\hline chr4:477-502 suggestive & RJF & 23.47 & -0.5 & 0.08 & -0.41 \\
\hline chr4:480-500 suggestive & RJF & 29.02 & -1.93 & 0.32 & -2.34 \\
\hline chr4:467-528 significant & RJF & 11.03 & -1.73 & 0.55 & 0.93 \\
\hline chr4:480-490 suggestive & WL & 23.86 & 0.22 & 0.45 & 3.37 \\
\hline chr4:465-503 significant & WL & 16.65 & 1.94 & 0.39 & 0.16 \\
\hline chr4:483-501 significant & WL & 24.9 & 4 & 0.8 & 5.11 \\
\hline chr4:479-491 significant & RJF & 19.26 & -0.36 & 0.55 & 3.4 \\
\hline chr4:482-496 suggestive & WL & 21.57 & 1.36 & 0.49 & 2.59 \\
\hline chr4:477-491 significant & RJF & 19.45 & -2.46 & 0.53 & 1.04 \\
\hline chr4:483-491 suggestive & WL & 20.97 & 1.33 & 0.61 & 3.76 \\
\hline chr4:482-490 suggestive & WL & 19.3 & 0.93 & 0.62 & 4.66 \\
\hline chr4:482-491 suggestive & RJF & 18.88 & -0.07 & 0.53 & 3.38 \\
\hline chr4:483-491 suggestive & WL & 17.43 & 0.1 & 0.62 & 3.91 \\
\hline chr4:481-491 suggestive & WL & 19.24 & 0.94 & 0.55 & 3.65 \\
\hline chr4:481-491 suggestive & WL & 20.2 & 1.42 & 0.5 & 3.55 \\
\hline chr4:478-500 suggestive & WL & 13.35 & 0.94 & 0.44 & 2.66 \\
\hline chr4:479-504 significant & RJF & 12.47 & -0.44 & 0.36 & 1.74 \\
\hline chr4:481-497 significant & WL & 18.41 & 2.21 & 0.42 & 0.73 \\
\hline chr4:479-491 significant & RJF & 12.37 & -1.69 & 0.49 & 0.82 \\
\hline chr4:482-505 significant & WL & 16.36 & 2.59 & 0.53 & 2.2 \\
\hline chr4:479-491 suggestive & RJF & 12.74 & -1.25 & 0.48 & 1.61 \\
\hline chr4:482-511 suggestive & WL & 16.33 & 1.6 & 0.38 & 1.98 \\
\hline chr4:483-490 suggestive & WL & 26.65 & 1.53 & 0.58 & 5.2 \\
\hline chr4:483-489 suggestive & WL & 24.47 & 0.58 & 0.61 & 5.03 \\
\hline chr4:482-500 suggestive & RJF & 29.96 & -0.69 & 0.1 & -0.73 \\
\hline chr4:483-499 significant & RJF & 33.18 & -1.37 & 0.21 & -1.73 \\
\hline chr4:483-490 suggestive & WL & 25.86 & 0.67 & 0.43 & 3.54 \\
\hline chr4:481-491 significant & WL & 28.96 & 2.28 & 0.71 & 6.47 \\
\hline chr4:481-490 significant & WL & 24.64 & 1.55 & 0.34 & 2.66 \\
\hline chr4:481-489 suggestive & WL & 20.46 & 0.83 & 0.28 & 2.14 \\
\hline
\end{tabular}




\begin{tabular}{|c|c|c|c|c|c|}
\hline chr4:479-491 suggestive & RJF & 14 & -0.32 & 0.39 & 2.17 \\
\hline chr4:484-494 suggestive & WL & 19.73 & 1.48 & 0.39 & 2.73 \\
\hline chr4:482-490 suggestive & WL & 18.7 & 0.22 & 0.46 & 3.3 \\
\hline chr4:482-491 suggestive & WL & 18.3 & 0.7 & 0.4 & 2.88 \\
\hline chr4:480-489 suggestive & WL & 18.92 & 0.88 & 0.37 & 2.68 \\
\hline chr4:481-490 suggestive & WL & 19.21 & 0.59 & 0.23 & 1.66 \\
\hline chr4:482-492 suggestive & RJF & 19.73 & -0.02 & 0.6 & 4.07 \\
\hline chr4:461-510 significant & RJF & 15.11 & -0.79 & 0.58 & 3.07 \\
\hline chr4:464-552 suggestive & RJF & 10.38 & -0.92 & 0.29 & 0.37 \\
\hline chr4:476-506 significant & WL & 18.52 & 2.3 & 0.44 & 0.31 \\
\hline chr4:480-506 significant & RJF & 23.18 & -2.42 & 0.46 & 1.33 \\
\hline chr4:471-503 suggestive & WL & 20.22 & 1.89 & 0.34 & 0.86 \\
\hline chr4:477-533 suggestive & WL & 15.72 & 1.58 & 0.34 & 0.06 \\
\hline chr4:465-508 significant & WL & 15.55 & 2.08 & 0.46 & 1.09 \\
\hline chr4:481-503 significant & WL & 19.42 & 2.05 & 0.37 & 0.46 \\
\hline chr4:476-500 suggestive & WL & 20.97 & 1.57 & 0.3 & 1.37 \\
\hline chr4:476-505 significant & WL & 21.3 & 3.57 & 0.64 & -0.09 \\
\hline chr4:489-502 significant & WL & 20.27 & 2.47 & 0.63 & -1.01 \\
\hline chr4:490-498 significant & WL & 44.83 & 8.37 & 0.84 & -0.45 \\
\hline chr4:492-502 significant & WL & 35.29 & 2.78 & 0.35 & -0.2 \\
\hline chr4:489-502 significant & WL & 28.4 & 2.55 & 0.36 & 0.45 \\
\hline chr4:489-506 significant & WL & 24.28 & 3.46 & 0.56 & 0.32 \\
\hline chr4:489-505 suggestive & WL & 19.12 & 2.43 & 0.56 & -0.3 \\
\hline chr4:472-505 significant & WL & 16.86 & 1.42 & 0.29 & 0.41 \\
\hline chr4:478-506 suggestive & WL & 16.13 & 2.2 & 0.46 & 0.96 \\
\hline chr4:470-509 significant & WL & 16.52 & 1.82 & 0.38 & 1.19 \\
\hline chr4:479-502 suggestive & RJF & 25.78 & -0.67 & 0.11 & -0.43 \\
\hline chr4:488-507 suggestive & WL & 19.26 & 0.08 & 0.76 & 3.79 \\
\hline chr4:469-515 significant & WL & 16.78 & 1.86 & 0.38 & 0.5 \\
\hline chr4:480-510 suggestive & WL & 21.79 & 2.3 & 0.41 & 1.46 \\
\hline chr4:489-510 significant & WL & 22.95 & 1.89 & 0.32 & 0.21 \\
\hline chr4:490-510 significant & RJF & 23.58 & -2.8 & 0.49 & 0.82 \\
\hline chr4:489-507 significant & RJF & 35.41 & -1.92 & 0.23 & -0.18 \\
\hline chr4:460-528 significant & WL & 18.06 & 1.44 & 0.28 & 0.33 \\
\hline chr4:489-523 significant & WL & 20.84 & 1.56 & 0.27 & 0.02 \\
\hline chr4:489-524 significant & WL & 25.01 & 1.58 & 0.26 & -0.09 \\
\hline chr4:467-526 significant & WL & 15.17 & 2.12 & 0.46 & 0.04 \\
\hline chr4:496-518 significant & WL & 47.05 & 3.58 & 0.35 & 0.1 \\
\hline chr4:488-528 significant & WL & 18.47 & 2.06 & 0.4 & 0.32 \\
\hline chr4:497-527 significant & RJF & 17.05 & -1.18 & 0.29 & 1.14 \\
\hline chr4:482-533 significant & WL & 14.24 & 2.77 & 0.61 & 0.24 \\
\hline chr4:486-531 significant & WL & 14.96 & 1.88 & 0.4 & 0.07 \\
\hline chr4:485-527 significant & WL & 14.92 & 2.64 & 0.58 & 0.48 \\
\hline
\end{tabular}


chr4:491-537 suggestive RJF

chr4:499-534 significant RJF

chr4:528-557 significant RJF

chr4:529-555 significant RJF

chr4:530-552 significant RJF

chr4:529-550 significant RJF

chr4:532-555 significant RJF

chr4:526-569 suggestive RJF

chr4:549-569 suggestive RJF

chr4:547-569 significant

chr4:553-569 suggestive

chr4:553-569 suggestive

chr4:554-569 suggestive

chr4:554-569 suggestive

chr4:553-569 suggestive

chr4:551-569 suggestive

chr4:549-569 suggestive

chr4:555-569 suggestive

chr4:549-569 significant

chr4:556-569 suggestive

chr4:553-569 suggestive

chr4:547-569 suggestive

chr4:556-569 suggestive

chr4:559-569 significant

chr4:554-569 suggestive

chr4:557-569 significant

chr4:558-569 significant

chr4:556-569 significant

chr4:556-569 significant

chr4:554-569 significant

chr4:556-569 significant

chr4:561-569 significant

chr4:559-569 significant

chr4:560-569 significant

chr4:563-569 significant

chr4:555-569 significant

chr4:557-569 significant

chr4:558-569 significant

chr4:557-569 significant

chr4:556-569 significant

chr4:561-569 significant

chr4:563-569 significant

chr4:553-569 significant
12.28

18.3

22.36

16.2

20.45

30.83

17.95

13.09

23.25

19.87

15.51

20.86

18.76

27.51

18.54

14.55

14.53

13.85

12.41

20.24

15.42

13.07

17.09

19.54

19.49

24.09

21.59

17.1

21.54

15.67

16.27

27.38

27.32

29.99

42.48

21.86

20.51

19.36

18.81

23.2

29.74

42.71

18.7
$-2.27$

$-2.56$

$-2.88$

$-3.74$

$-2.58$

$-0.54$

$-1.59$

$-1.22$

$-1.88$

2.75

$-0.2$

$-0.39$

0.09

$-1.96$

$-0.45$

1.46

0.94

$-0.25$

1.84

0.21

$-0.33$

$-1.55$

$-0.48$

1.13

$-2.16$

1.5

0.99

$-6.39$

$-8.75$

$-8.45$

$-8.4$

$-8.8$

$-12.31$

$-8.04$

$-14.21$

$-6.7$

$-9.04$

$-6.83$

$-10.67$

$-3.6$

$-7.76$

$-14.11$

$-5.34$
0.55

0.52

0.56

0.82

0.48

0.08

0.38

0.81

0.79

0.51

0.43

0.31

0.43

0.44

0.42

0.4

0.24

0.58

0.54

0.33

0.36

0.36

0.49

0.28

0.62

0.29

0.43

1.25

1.58

1.83

1.74

1.35

1.92

1.16

1.53

1.19

1.7

1.28

2.12

0.64

1.1

1.52

1.09
0.04

1.26

0.43

$-0.26$

$-2.7$

$-0.46$

$-2.07$

2.98

$-1.53$

$-0.37$

$-1.07$

0.2

$-0.93$

$-1.15$

$-2$

0.77

0.33

$-0.56$

0.82

$-1.13$

2.22

0.63

$-0.77$

$-1.24$

$-1.3$

0.65

$-2.03$

1.91

$-0.59$

$-0.31$

1.35

0.56

$-0.35$

0.34

2.11

$-0.08$

$-0.65$

0.75

$-0.5$

$-0.43$

0.97

2.01

$-0.95$ 


\begin{tabular}{|c|c|c|c|c|c|}
\hline chr4:559-569 significant & RJF & 22.77 & -7.18 & 1.18 & 1.74 \\
\hline chr4:555-569 significant & RJF & 17.83 & -5.42 & 1.09 & 0 \\
\hline chr4:556-569 significant & RJF & 22.29 & -7.52 & 1.32 & -0.06 \\
\hline chr4:553-569 significant & RJF & 14.27 & -7.22 & 1.61 & 0.55 \\
\hline chr4:555-569 significant & RJF & 17.06 & -9.61 & 2.06 & -0.87 \\
\hline chr4:556-569 significant & RJF & 20.87 & -4.82 & 0.87 & 0.29 \\
\hline chr4:555-569 significant & RJF & 16.22 & -7.53 & 1.61 & -0.33 \\
\hline chr4:557-569 significant & RJF & 18.01 & -8.67 & 1.67 & 1.32 \\
\hline chr4:562-569 significant & RJF & 33.12 & -9.95 & 1.34 & 0.24 \\
\hline chr4:559-569 significant & RJF & 27.69 & -12.75 & 1.99 & -0.71 \\
\hline chr4:559-569 significant & RJF & 28.54 & -12.99 & 1.91 & 1.32 \\
\hline chr4:561-569 significant & RJF & 26.84 & -9.47 & 1.47 & 0.74 \\
\hline chr4:557-569 significant & RJF & 22.35 & -10.62 & 1.92 & -0.91 \\
\hline chr4:549-569 suggestive & WL & 12.71 & 3.9 & 1.04 & 0.5 \\
\hline chr4:557-569 significant & RJF & 20.14 & -7.04 & 1.26 & 1.32 \\
\hline chr4:562-569 significant & RJF & 33.56 & -10.46 & 1.4 & 0.27 \\
\hline chr4:560-569 significant & RJF & 28.54 & -10.57 & 1.64 & -1.21 \\
\hline chr4:559-569 significant & RJF & 29.5 & -14.92 & 2.17 & 0.82 \\
\hline chr4:554-569 significant & RJF & 18.46 & -8.09 & 1.59 & -0.24 \\
\hline chr4:558-569 significant & RJF & 16.56 & -10.37 & 2.13 & 1.49 \\
\hline chr4:557-569 significant & RJF & 19.91 & -7.01 & 1.26 & 1.51 \\
\hline chr4:557-569 significant & RJF & 21.63 & -4.94 & 0.88 & 0.15 \\
\hline chr4:558-569 significant & RJF & 22.9 & -6.24 & 1.02 & 1.54 \\
\hline chr4:556-569 significant & RJF & 17.64 & -7.09 & 1.47 & -0.62 \\
\hline chr4:556-569 significant & RJF & 18.07 & -5.94 & 1.12 & 1.56 \\
\hline chr4:556-569 significant & RJF & 17.59 & -5.63 & 1.08 & 1.46 \\
\hline chr4:556-569 significant & RJF & 17.23 & -7.11 & 1.43 & 0.33 \\
\hline chr4:560-569 significant & RJF & 30.72 & -3.42 & 0.65 & -1.21 \\
\hline chr4:564-569 significant & RJF & 26.77 & -2 & 0.29 & 0.19 \\
\hline chr4:563-569 significant & RJF & 18.74 & -2.16 & 0.39 & 0.83 \\
\hline chr4:565-569 significant & RJF & 33.28 & -2.98 & 0.39 & -0.16 \\
\hline chr4:561-569 significant & RJF & 25.07 & -2.17 & 0.35 & -0.08 \\
\hline chr4:560-569 significant & RJF & 23.28 & -3.89 & 0.73 & -0.61 \\
\hline chr4:564-569 significant & RJF & 27.14 & -4.29 & 0.66 & -0.13 \\
\hline chr4:562-569 significant & RJF & 17.45 & -2.94 & 0.58 & 0.54 \\
\hline chr4:562-569 significant & RJF & 22.53 & -3.74 & 0.69 & -0.85 \\
\hline chr4:564-569 significant & RJF & 28.07 & -3.15 & 0.48 & -0.53 \\
\hline chr4:561-569 significant & RJF & 23.53 & -2.53 & 0.49 & -0.64 \\
\hline chr4:562-569 significant & RJF & 22.17 & -2.77 & 0.48 & 0.17 \\
\hline chr4:563-569 significant & RJF & 21.1 & -2.43 & 0.43 & -0.08 \\
\hline chr4:562-569 significant & RJF & 24.45 & -2.11 & 0.35 & -0.13 \\
\hline chr4:557-569 significant & RJF & 16.98 & -1.57 & 0.32 & 0.19 \\
\hline chr4:559-569 suggestive & RJF & 18.63 & -3.01 & 0.65 & 0.33 \\
\hline
\end{tabular}




\begin{tabular}{|c|c|c|c|c|c|}
\hline chr4:563-569 significant & RJF & 27.36 & -2.58 & 0.38 & -0.1 \\
\hline chr4:561-569 significant & RJF & 28.59 & -3 & 0.44 & -0.01 \\
\hline chr4:558-569 significant & RJF & 22.78 & -2.1 & 0.44 & -0.46 \\
\hline chr4:561-569 significant & RJF & 22.31 & -2.34 & 0.46 & -0.16 \\
\hline chr4:564-569 significant & RJF & 30.61 & -3.25 & 0.57 & -0.91 \\
\hline chr4:558-569 significant & RJF & 21.01 & -2.82 & 0.59 & -0.45 \\
\hline chr4:556-569 significant & RJF & 20.28 & -1.71 & 0.41 & -0.7 \\
\hline chr4:557-569 significant & RJF & 19.53 & -1.35 & 0.32 & -0.53 \\
\hline chr4:562-569 significant & RJF & 29.01 & -3.57 & 0.63 & -1.84 \\
\hline chr4:563-569 significant & RJF & 29.18 & -5.18 & 0.92 & -1.48 \\
\hline chr4:561-569 significant & RJF & 25.74 & -3.28 & 0.61 & -0.48 \\
\hline chr4:561-569 significant & RJF & 24.41 & -1.7 & 0.38 & -0.76 \\
\hline chr4:557-569 significant & RJF & 19.06 & -1.75 & 0.43 & -0.87 \\
\hline chr4:561-569 significant & RJF & 18.07 & -1.67 & 0.31 & 0.64 \\
\hline chr4:555-569 significant & RJF & 23.92 & -1.52 & 0.34 & -0.52 \\
\hline chr4:550-569 significant & RJF & 11.93 & -1.91 & 0.47 & 0.78 \\
\hline chr4:551-569 suggestive & WL & 11.84 & 3.49 & 0.87 & -0.69 \\
\hline chr4:553-569 significant & RJF & 14.11 & -6.89 & 1.57 & 0.41 \\
\hline chr4:556-569 significant & RJF & 13.29 & -6.96 & 1.59 & 1.76 \\
\hline chr4:552-569 suggestive & RJF & 13.76 & -4.93 & 1.14 & 0.13 \\
\hline chr4:553-569 suggestive & RJF & 12.72 & -9.18 & 2.28 & -0.43 \\
\hline chr4:555-569 significant & RJF & 14.39 & -4.01 & 0.87 & 0.77 \\
\hline chr4:554-569 significant & RJF & 15.24 & -8.48 & 1.84 & 1.23 \\
\hline chr4:554-569 significant & RJF & 13.95 & -5.87 & 1.32 & 0.55 \\
\hline chr4:550-569 suggestive & RJF & 12.77 & -7.63 & 1.94 & -0.9 \\
\hline chr4:553-569 suggestive & RJF & 13.56 & -7.03 & 1.7 & -0.64 \\
\hline chr4:548-569 suggestive & RJF & 12.11 & -7.18 & 1.76 & 0.56 \\
\hline chr4:551-569 significant & RJF & 12.81 & -5.82 & 1.43 & -0.59 \\
\hline chr4:553-569 suggestive & RJF & 13.78 & -6.03 & 1.41 & -0.17 \\
\hline chr4:555-569 significant & RJF & 14.52 & -7.19 & 1.56 & 1.06 \\
\hline chr4:554-569 significant & RJF & 16.88 & -9.98 & 2.07 & 0.55 \\
\hline chr4:548-569 suggestive & RJF & 12.02 & -7 & 1.7 & 1.14 \\
\hline chr4:555-569 significant & RJF & 15.27 & -9.59 & 2.18 & -0.58 \\
\hline chr4:554-569 suggestive & RJF & 12.68 & -5.58 & 1.32 & 0.53 \\
\hline chr4:551-569 suggestive & RJF & 13.38 & -3.34 & 0.81 & -0.23 \\
\hline chr4:555-569 significant & RJF & 13.95 & -7.16 & 1.68 & -0.67 \\
\hline chr4:553-569 suggestive & RJF & 12.88 & -6.63 & 1.6 & 0.03 \\
\hline chr4:555-569 significant & RJF & 15.31 & -4.53 & 0.95 & 1.31 \\
\hline chr4:551-569 suggestive & RJF & 12.42 & -5.38 & 1.34 & -0.16 \\
\hline chr4:554-569 suggestive & RJF & 12.92 & -3.29 & 0.76 & 1.13 \\
\hline chr4:555-569 significant & RJF & 15.44 & -6 & 1.32 & -0.37 \\
\hline chr4:554-569 significant & RJF & 13.67 & -4.45 & 1 & 1.32 \\
\hline chr4:554-569 suggestive & RJF & 12.13 & -6.88 & 1.75 & -0.48 \\
\hline
\end{tabular}




\begin{tabular}{|c|c|c|c|c|c|c|}
\hline \multicolumn{2}{|c|}{ chr4:554-569 suggestive } & RJF & 12.88 & -5.89 & 1.43 & -0.16 \\
\hline \multicolumn{2}{|c|}{ chr4:552-569 suggestive } & RJF & 12.53 & -7 & 1.72 & -0.04 \\
\hline \multicolumn{2}{|c|}{ chr4:555-569 suggestive } & RJF & 13.65 & -1.58 & 0.39 & -0.19 \\
\hline \multicolumn{2}{|c|}{ chr4:558-569 suggestive } & RJF & 14.41 & -1.65 & 0.38 & 0.18 \\
\hline \multicolumn{2}{|c|}{ chr4:559-569 suggestive } & WL & 16.32 & 0.68 & 0.39 & -1.2 \\
\hline chr5:0-7 & suggestive & RJF & 19.56 & -0.15 & 0.03 & -0.13 \\
\hline $\operatorname{chr} 5: 0-17$ & significant & RJF & 14.76 & -3.06 & 0.85 & 1.11 \\
\hline $\operatorname{chr} 5: 6-27$ & significant & WL & 24.46 & 2.69 & 0.5 & 0.38 \\
\hline chr5:0-30 & significant & RJF & 19.4 & -0.74 & 0.76 & 1.42 \\
\hline $\operatorname{chr} 5: 46-118$ & suggestive & WL & 10.65 & 1.01 & 0.28 & 0.54 \\
\hline chr5:61-103 & suggestive & WL & 18.58 & 0.35 & 0.21 & 1.65 \\
\hline chr5:58-95 & suggestive & WL & 19.83 & 0.03 & 0.48 & 2.44 \\
\hline chr5:61-110 & significant & WL & 16.62 & 1.04 & 0.27 & 1.35 \\
\hline chr5:61-120 & suggestive & WL & 15.61 & 2.55 & 0.64 & 1.76 \\
\hline chr5:60-118 & significant & WL & 15.45 & 1.97 & 0.42 & 0.8 \\
\hline chr5:61-99 & significant & RJF & 17.46 & -3.51 & 0.69 & 0.05 \\
\hline chr5:64-108 & significant & WL & 16.24 & 0.62 & 0.25 & 1.6 \\
\hline chr5:58-126 & significant & WL & 11.82 & 3.03 & 0.76 & 1.09 \\
\hline chr5:65-100 & suggestive & WL & 18.91 & 0.42 & 0.18 & 1.33 \\
\hline chr5:72-96 & significant & RJF & 30.34 & -4.23 & 0.58 & 0.41 \\
\hline chr5:68-98 & suggestive & RJF & 18.61 & -0.83 & 0.96 & 0.78 \\
\hline chr5:61-136 & significant & WL & 14.62 & 3.13 & 0.72 & 1.68 \\
\hline $\operatorname{chr} 5: 65-118$ & significant & WL & 18.83 & 1.71 & 0.34 & 0.95 \\
\hline chr5:65-103 & significant & RJF & 18.22 & -4.68 & 0.92 & -1.53 \\
\hline chr5:69-95 & significant & WL & 25.64 & 2.25 & 0.35 & 0.19 \\
\hline chr5:64-109 & suggestive & WL & 13.54 & 0.42 & 0.26 & 1.61 \\
\hline chr5:57-156 & suggestive & WL & 10.48 & 1.64 & 0.44 & 0.06 \\
\hline chr5:64-119 & significant & WL & 12.37 & 1 & 0.25 & 0.52 \\
\hline chr5:64-131 & significant & WL & 12.69 & 3.34 & 0.85 & 1.17 \\
\hline chr5:67-109 & significant & RJF & 19.24 & -3.58 & 0.66 & 0.7 \\
\hline chr5:69-117 & significant & RJF & 19.57 & -1.9 & 0.35 & -0.02 \\
\hline chr5:65-115 & suggestive & RJF & 14.6 & -1.36 & 0.32 & 0.64 \\
\hline chr5:62-115 & significant & WL & 15.9 & 1.42 & 0.31 & 0.84 \\
\hline chr5:68-109 & suggestive & WL & 19.26 & 1.13 & 0.29 & 1.72 \\
\hline chr5:64-115 & significant & WL & 15.67 & 1.39 & 0.32 & 0.92 \\
\hline chr5:59-117 & significant & WL & 13.94 & 1.52 & 0.36 & 0.58 \\
\hline chr5:65-113 & suggestive & WL & 13.35 & 1.33 & 0.31 & 0.71 \\
\hline chr5:70-111 & significant & WL & 23.96 & 2.68 & 0.43 & 0.83 \\
\hline chr5:78-110 & significant & RJF & 25.54 & -1.53 & 0.24 & 0.47 \\
\hline chr5:80-102 & significant & RJF & 29.87 & -3.58 & 0.49 & -0.19 \\
\hline chr5:67-109 & significant & WL & 18 & 1.63 & 0.37 & 0.23 \\
\hline chr5:72-107 & significant & RJF & 19.01 & -2.66 & 0.49 & -0.3 \\
\hline chr5:68-115 & significant & RJF & 16.33 & -1.85 & 0.36 & 0.2 \\
\hline
\end{tabular}




\begin{tabular}{|c|c|c|c|c|c|c|}
\hline chr5:63-116 & significant & WL & 12.99 & 1.17 & 0.28 & 0.41 \\
\hline chr5:71-110 & suggestive & RJF & 15.56 & -2.89 & 0.62 & -0.74 \\
\hline chr5:73-109 & suggestive & RJF & 16.21 & -2.83 & 0.59 & -0.58 \\
\hline chr5:69-114 & significant & RJF & 19.05 & -1.94 & 0.36 & 0.14 \\
\hline chr5:73-107 & significant & RJF & 19.12 & -2.86 & 0.53 & -0.16 \\
\hline chr5:78-113 & suggestive & RJF & 16.57 & -1.79 & 0.36 & 0.21 \\
\hline chr5:64-119 & significant & WL & 13.77 & 1.71 & 0.4 & -0.71 \\
\hline chr5:94-109 & suggestive & WL & 19.93 & 0.19 & 0.12 & 0.92 \\
\hline chr5:61-114 & significant & WL & 16.51 & 1.63 & 0.44 & 2.05 \\
\hline chr5:74-121 & suggestive & WL & 12.85 & 0.63 & 0.43 & 0.83 \\
\hline chr5:75-127 & suggestive & WL & 12.45 & 3.73 & 1.05 & -3.93 \\
\hline chr5:72-121 & significant & WL & 16.26 & 1.23 & 0.27 & 0.62 \\
\hline chr5:90-116 & significant & WL & 24.6 & 2.34 & 0.37 & 0.54 \\
\hline chr5:75-121 & significant & WL & 19.06 & 2.2 & 0.42 & -0.6 \\
\hline chr5:65-133 & significant & WL & 14.97 & 1.08 & 0.24 & 0.38 \\
\hline chr5:70-131 & significant & WL & 13.48 & 1.44 & 0.33 & -0.47 \\
\hline chr5:62-127 & suggestive & RJF & 14.8 & -0.14 & 0.03 & -0.04 \\
\hline chr5:75-119 & significant & RJF & 17.61 & -1.42 & 0.28 & 0.7 \\
\hline chr5:69-120 & significant & WL & 20.31 & 1.38 & 0.25 & 0.36 \\
\hline chr5:69-118 & significant & WL & 24.46 & 1.39 & 0.23 & 0.56 \\
\hline chr5:64-126 & significant & WL & 15.68 & 3.25 & 0.69 & 0.74 \\
\hline chr5:56-143 & suggestive & WL & 13.14 & 0.93 & 0.32 & 1.48 \\
\hline chr5:62-136 & significant & WL & 13.54 & 2.48 & 0.59 & 0.88 \\
\hline chr5:65-136 & suggestive & WL & 14.14 & 1.76 & 0.54 & 2.37 \\
\hline chr5:73-124 & suggestive & RJF & 16.22 & -1.36 & 0.28 & 0.32 \\
\hline chr5:70-138 & suggestive & WL & 13.41 & 2.19 & 0.55 & -1.67 \\
\hline chr5:66-154 & significant & WL & 17.22 & 2.22 & 0.47 & 1.19 \\
\hline chr5:59-129 & suggestive & WL & 12.58 & 1.7 & 0.58 & 2.28 \\
\hline chr5:64-148 & significant & WL & 11.39 & 1.8 & 0.45 & 0.15 \\
\hline chr5:75-144 & significant & RJF & 15.98 & -2.32 & 0.49 & 0.26 \\
\hline chr5:68-127 & significant & WL & 20.22 & 3.79 & 0.68 & 1.29 \\
\hline chr5:58-145 & significant & WL & 10.63 & 2.14 & 0.55 & -0.04 \\
\hline chr5:64-148 & suggestive & WL & 15.67 & 3.15 & 0.82 & 3.07 \\
\hline chr5:78-149 & suggestive & RJF & 16.95 & -0.88 & 0.65 & 1.21 \\
\hline chr5:64-140 & suggestive & WL & 16.5 & 0.89 & 0.27 & 1.62 \\
\hline chr5:60-150 & significant & WL & 13.23 & 3.68 & 0.87 & 1.06 \\
\hline chr5:50-159 & suggestive & WL & 7.92 & 1.59 & 0.48 & -0.12 \\
\hline chr5:100-128 & significant & WL & 15.74 & 1.76 & 0.39 & 1.1 \\
\hline chr5:100-143 & suggestive & WL & 15.21 & 0.23 & 0.37 & 2.63 \\
\hline chr5:95-131 & suggestive & RJF & 19.09 & -2.73 & 0.51 & 0.13 \\
\hline chr5:100-144 & suggestive & WL & 16.6 & 4.44 & 1.09 & 5.22 \\
\hline chr5:108-147 & suggestive & RJF & 27.56 & -1.14 & 0.21 & -1.37 \\
\hline chr5:104-138 & suggestive & RJF & 11.76 & -1.8 & 0.29 & -0.8 \\
\hline
\end{tabular}




\begin{tabular}{|c|c|c|c|c|c|}
\hline chr5:108-141 suggestive & RJF & 24.71 & -1.65 & 0.31 & -1.95 \\
\hline chr5:97-146 suggestive & WL & 12.6 & 3.74 & 0.9 & 1.42 \\
\hline chr5:108-142 significant & WL & 23.58 & 3.03 & 0.88 & 3.36 \\
\hline chr5:111-126 significant & WL & 23.79 & 1.19 & 0.29 & 2.31 \\
\hline chr5:109-137 significant & WL & 20.08 & 4.28 & 1.55 & 7.04 \\
\hline chr5:110-128 significant & RJF & 24.6 & -1.49 & 0.83 & 4.44 \\
\hline chr5:110-168 significant & RJF & 18.22 & -2.32 & 0.46 & -0.84 \\
\hline chr5:111-144 suggestive & WL & 18.04 & 4.69 & 1.36 & 7.32 \\
\hline chr5:110-143 significant & WL & 21.02 & 1.3 & 1.42 & -4.85 \\
\hline chr5:103-150 suggestive & WL & 13.23 & 10 & 4.51 & -1.06 \\
\hline chr5:108-145 significant & WL & 17.43 & 5.43 & 2.43 & -2.73 \\
\hline chr5:110-136 significant & WL & 14.22 & 1.99 & 0.45 & 0.75 \\
\hline chr5:111-157 suggestive & WL & 10.9 & 2.96 & 0.77 & 0.85 \\
\hline chr5:110-150 significant & WL & 14.68 & 2.63 & 0.67 & 2.78 \\
\hline chr5:102-160 suggestive & RJF & 13.32 & -1.18 & 0.27 & -0.29 \\
\hline chr5:110-145 suggestive & WL & 15.36 & 3.25 & 0.85 & 3.94 \\
\hline chr5:110-151 significant & WL & 13.86 & 4.07 & 0.98 & 2.57 \\
\hline chr5:108-149 suggestive & WL & 14.74 & 2.43 & 0.65 & 2.97 \\
\hline chr5:113-146 suggestive & WL & 21.08 & 2.35 & 1.15 & 5.65 \\
\hline chr5:94-152 significant & WL & 12.9 & 2.94 & 0.71 & -0.76 \\
\hline chr5:108-161 significant & WL & 15.97 & 3.42 & 0.81 & 3.29 \\
\hline chr5:111-158 suggestive & WL & 12.8 & 2.7 & 0.67 & 1.98 \\
\hline chr5:96-158 suggestive & WL & 10.37 & 1.91 & 0.51 & -0.32 \\
\hline chr5:110-147 suggestive & WL & 18.41 & 1.91 & 0.57 & 2.54 \\
\hline chr5:109-145 suggestive & WL & 17.1 & 0.24 & 0.52 & -0.22 \\
\hline chr5:51-166 suggestive & WL & 10.66 & 1.14 & 0.3 & 0.24 \\
\hline chr5:109-146 suggestive & RJF & 24.12 & -1.7 & 0.45 & -1.66 \\
\hline chr5:82-160 suggestive & WL & 11.29 & 1.8 & 0.46 & 0.61 \\
\hline chr5:108-155 suggestive & WL & 13.51 & 3.97 & 1.32 & 4.08 \\
\hline chr5:83-152 suggestive & WL & 12.37 & 2.35 & 1.15 & 3.26 \\
\hline chr5:113-152 suggestive & WL & 13.7 & 4.81 & 1.65 & 5.5 \\
\hline chr5:117-143 suggestive & RJF & 25.91 & -0.24 & 0.05 & -0.18 \\
\hline chr5:113-148 suggestive & WL & 18.81 & 0.23 & 0.36 & 2.57 \\
\hline chr5:115-155 significant & WL & 21.12 & 5.73 & 1.03 & 0.96 \\
\hline chr5:106-166 suggestive & RJF & 14.06 & -1.94 & 0.44 & 0.42 \\
\hline chr5:118-157 suggestive & RJF & 17.79 & -1.06 & 0.29 & -1.38 \\
\hline chr5:161-195 significant & RJF & 16.64 & -2.1 & 0.43 & -1.38 \\
\hline chr5:163-198 suggestive & RJF & 16.52 & -1 & 0.6 & -0.54 \\
\hline chr5:160-203 significant & RJF & 20.65 & -2.42 & 0.43 & -1.08 \\
\hline chr5:158-190 significant & RJF & 16.83 & -1.12 & 0.23 & -1.1 \\
\hline chr5:169-193 suggestive & RJF & 12.75 & -1.36 & 0.41 & -2.06 \\
\hline chr5:146-198 suggestive & RJF & 13.05 & -1.24 & 0.29 & -0.86 \\
\hline chr5:170-192 suggestive & RJF & 13.34 & -1.44 & 0.4 & -1.99 \\
\hline
\end{tabular}




\begin{tabular}{|c|c|c|c|c|c|}
\hline chr5:167-195 suggestive & RJF & 13.61 & -1.8 & 0.41 & -1.38 \\
\hline chr5:174-197 significant & RJF & 21.53 & -2.12 & 0.38 & -0.88 \\
\hline chr5:169-197 suggestive & RJF & 13.95 & -0.94 & 0.21 & -0.66 \\
\hline chr5:166-197 suggestive & RJF & 14.19 & -1.75 & 0.44 & -2.13 \\
\hline chr5:176-192 suggestive & RJF & 27.66 & -2.29 & 0.41 & -2.04 \\
\hline chr5:164-190 significant & RJF & 17.76 & -2.88 & 0.61 & -0.35 \\
\hline chr5:175-191 significant & RJF & 17.22 & -0.79 & 0.16 & -0.58 \\
\hline chr5:176-193 significant & RJF & 19.99 & -1.53 & 0.28 & -1.06 \\
\hline chr5:167-199 suggestive & RJF & 13.99 & -2.4 & 0.54 & -1.33 \\
\hline chr5:175-199 significant & RJF & 14.59 & -2.81 & 0.62 & -1.59 \\
\hline chr5:177-230 suggestive & RJF & 12.78 & -1.27 & 0.31 & -1.04 \\
\hline chr5:180-187 significant & RJF & 37.39 & -0.74 & 0.09 & -0.8 \\
\hline chr5:179-193 suggestive & RJF & 23.39 & -1.98 & 0.38 & -1.7 \\
\hline chr5:178-193 significant & RJF & 29.2 & -1.74 & 0.25 & -1.63 \\
\hline chr5:177-196 suggestive & RJF & 23.78 & -1.54 & 0.31 & -1.98 \\
\hline chr5:169-223 suggestive & RJF & 13.38 & -1.38 & 0.33 & -1.37 \\
\hline chr5:178-210 suggestive & RJF & 26.31 & -2.89 & 0.48 & -1.92 \\
\hline chr5:177-198 significant & RJF & 30.48 & -0.08 & 0.01 & -0.09 \\
\hline chr5:172-199 significant & RJF & 32.92 & -2.72 & 0.37 & -0.81 \\
\hline chr5:178-203 suggestive & RJF & 25.96 & -0.88 & 0.15 & -0.71 \\
\hline chr5:177-208 significant & RJF & 28.14 & -2 & 0.29 & -1.51 \\
\hline chr5:179-207 suggestive & RJF & 24.79 & -2.43 & 0.42 & -2.35 \\
\hline chr5:176-225 suggestive & RJF & 18.13 & -0.92 & 0.18 & -0.65 \\
\hline chr5:174-229 significant & RJF & 20.78 & -2.7 & 0.5 & -1 \\
\hline chr5:171-252 significant & RJF & 14.66 & -3.15 & 0.75 & -0.65 \\
\hline chr5:169-226 suggestive & RJF & 26.27 & -1.28 & 0.21 & -0.74 \\
\hline chr5:177-203 significant & RJF & 38.06 & -0.6 & 0.07 & -0.44 \\
\hline chr5:173-206 suggestive & RJF & 23.66 & -1.41 & 0.24 & -1.11 \\
\hline chr5:177-207 suggestive & RJF & 18.73 & -1.95 & 0.37 & -1.26 \\
\hline chr5:183-206 suggestive & RJF & 25.25 & -0.64 & 0.1 & -0.52 \\
\hline chr5:184-207 suggestive & RJF & 23.85 & -2.47 & 0.45 & -2.31 \\
\hline chr5:183-207 suggestive & RJF & 21.82 & -3.13 & 0.56 & -2.32 \\
\hline chr5:184-208 suggestive & RJF & 21.77 & -1.89 & 0.35 & -1.85 \\
\hline chr5:185-208 suggestive & RJF & 25.39 & -3.9 & 0.65 & -3.31 \\
\hline chr5:186-209 suggestive & RJF & 27.42 & -1.19 & 0.25 & -1.72 \\
\hline chr5:180-206 suggestive & RJF & 18.96 & -0.71 & 0.14 & -0.34 \\
\hline chr5:186-208 suggestive & RJF & 24.93 & -3.26 & 0.56 & -1.3 \\
\hline chr5:184-207 suggestive & RJF & 20.48 & -1.31 & 0.24 & -0.99 \\
\hline chr5:186-207 suggestive & RJF & 21.69 & -3.33 & 0.61 & -2.91 \\
\hline chr5:187-207 suggestive & RJF & 23.53 & -4.72 & 0.86 & -4.35 \\
\hline chr5:187-206 suggestive & RJF & 23.8 & -1.26 & 0.24 & -1.5 \\
\hline chr5:190-205 suggestive & RJF & 25.03 & -1.93 & 0.38 & -2.53 \\
\hline chr5:186-206 suggestive & RJF & 21.32 & -0.82 & 0.15 & -0.73 \\
\hline
\end{tabular}


chr5:184-209 suggestive RJF chr5:185-208 suggestive RJF chr5:196-205 suggestive RJF chr5:192-207 suggestive RJF chr5:192-204 significant RJF chr5:192-206 suggestive RJF chr5:194-206 suggestive RJF chr5:192-206 suggestive RJF chr5:192-207 suggestive RJF chr5:190-206 significant RJF chr5:190-205 suggestive RJF chr5:197-205 suggestive RJF chr5:193-207 suggestive RJF chr5:190-205 significant RJF chr5:194-207 suggestive RJF chr5:188-206 suggestive RJF chr5:192-206 suggestive RJF chr5:192-205 significant RJF chr5:193-206 suggestive RJF chr5:196-208 suggestive RJF chr5:193-209 significant RJF chr5:192-206 significant RJF chr5:193-206 suggestive RJF chr5:191-206 significant RJF chr5:189-208 suggestive RJF chr5:191-207 suggestive RJF chr5:193-207 suggestive RJF chr5:193-206 suggestive RJF chr5:192-206 suggestive RJF chr5:192-208 suggestive RJF chr5:190-208 suggestive RJF chr5:191-210 suggestive RJF chr5:191-207 suggestive RJF chr5:190-209 suggestive RJF chr5:193-208 suggestive RJF chr5:194-208 suggestive RJF chr5:195-207 suggestive RJF chr5:193-208 suggestive RJF chr5:193-207 significant RJF chr5:190-208 suggestive RJF chr5:188-209 suggestive RJF chr5:192-208 suggestive RJF chr5:192-208 suggestive RJF

$\begin{array}{rrrr}17.57 & -0.48 & 0.1 & -0.45 \\ 17.25 & -1.65 & 0.33 & -1.3 \\ 26.78 & -2.31 & 0.38 & -2.62 \\ 23.71 & -1.99 & 0.34 & -1.83 \\ 31.55 & -2.12 & 0.29 & -1.5 \\ 24.98 & -2.7 & 0.43 & -1.71 \\ 24.91 & -3.32 & 0.57 & -3.06 \\ 24.2 & -1.61 & 0.27 & -1.37 \\ 15.95 & -2.07 & 0.44 & -1.51 \\ 18.36 & -2.03 & 0.47 & -2.08 \\ 24 & -0.64 & 0.11 & -0.57 \\ 27.09 & -0.16 & 0.03 & -0.16 \\ 24.79 & -3.32 & 0.57 & -2.71 \\ 22.63 & -1.2 & 0.2 & -0.97 \\ 20.94 & -4.52 & 0.81 & -3.62 \\ 20.32 & -5.62 & 1.01 & -3.25 \\ 26.71 & -3.04 & 0.48 & -2.9 \\ 35.01 & -5.06 & 0.66 & -3.94 \\ 25.54 & -3 & 0.51 & -2.63 \\ 25.2 & -2.64 & 0.42 & -1.96 \\ 21.86 & -1.47 & 0.28 & -1.61 \\ 28.04 & -1.27 & 0.22 & -1.55 \\ 26.96 & -2.52 & 0.4 & -2.11 \\ 29.75 & -4.08 & 0.58 & -3.55 \\ 21.12 & -5.02 & 0.98 & -4.13 \\ 22.75 & -3.36 & 0.63 & -3.7 \\ 23.81 & -4.47 & 0.73 & -3.36 \\ 26.82 & -2.42 & 0.39 & -2.31 \\ 23.16 & -5.71 & 1.04 & -5.95 \\ 23.37 & -3.99 & 0.71 & -2.97 \\ 19.57 & -2.15 & 0.39 & -1.42 \\ 16.6 & -1.17 & 0.25 & -1.03 \\ 18.72 & -13.59 & 2.93 & -16.53 \\ 23.84 & -1.78 & 0.3 & -1.53 \\ 25.18 & -4.85 & 0.82 & -4.63 \\ 26.03 & -3.85 & 0.64 & -3.9 \\ 26.86 & -3.87 & 0.61 & -3.12 \\ 24.81 & -5.26 & 0.89 & -4.09 \\ 28.52 & -3.83 & 0.57 & -3.51 \\ 20.51 & -3.87 & 0.79 & -3.92 \\ 19.77 & -4.28 & 0.86 & -4.25 \\ 26.03 & -2.27 & 0.39 & -1.05 \\ 21.57 & -1.58 & 0.31 & -1.38\end{array}$




\begin{tabular}{|c|c|c|c|c|c|}
\hline chr5:194-207 suggestive & RJF & 26.25 & -2.82 & 0.5 & -2.29 \\
\hline chr5:194-207 suggestive & RJF & 27.63 & -3.71 & 0.62 & -4.1 \\
\hline chr5:196-206 significant & RJF & 34.65 & -2.37 & 0.31 & -2.17 \\
\hline chr5:189-209 suggestive & RJF & 26.96 & -1.33 & 0.21 & -1.07 \\
\hline chr5:192-208 significant & RJF & 27.79 & -6.97 & 1.09 & -6.04 \\
\hline chr5:185-210 suggestive & RJF & 16.87 & -1.17 & 0.26 & -1.07 \\
\hline chr5:188-208 suggestive & RJF & 25.61 & -5.34 & 0.85 & -3.42 \\
\hline chr5:189-207 suggestive & RJF & 25.93 & -1.87 & 0.31 & -1.81 \\
\hline chr5:187-207 suggestive & RJF & 22.55 & -2.95 & 0.54 & -2.89 \\
\hline chr5:194-206 significant & RJF & 34.09 & -3.18 & 0.42 & -2.74 \\
\hline chr5:188-209 suggestive & RJF & 18.78 & -1.89 & 0.36 & -1.43 \\
\hline chr5:190-210 suggestive & RJF & 21.97 & -0.67 & 0.12 & -0.59 \\
\hline chr5:187-210 suggestive & RJF & 25.46 & -1.79 & 0.3 & -1.79 \\
\hline chr5:194-208 suggestive & RJF & 25.17 & -2.2 & 0.35 & -1.51 \\
\hline chr5:191-208 suggestive & RJF & 27.64 & -4.25 & 0.65 & -3.38 \\
\hline chr5:189-209 suggestive & RJF & 25.22 & -2.16 & 0.36 & -1.99 \\
\hline chr5:190-209 suggestive & RJF & 23.71 & -3.65 & 0.63 & -3.05 \\
\hline chr5:189-210 suggestive & RJF & 25.68 & -1.92 & 0.34 & -1.03 \\
\hline chr5:190-209 significant & RJF & 28.14 & -2.88 & 0.45 & -2.53 \\
\hline chr5:189-210 suggestive & RJF & 21.86 & -1.17 & 0.22 & -0.94 \\
\hline chr5:187-208 significant & RJF & 27.71 & -0.86 & 0.12 & -0.6 \\
\hline chr5:191-209 suggestive & RJF & 23.79 & -1.95 & 0.37 & -2.35 \\
\hline chr5:191-209 suggestive & RJF & 26.13 & -4.61 & 0.73 & -2.74 \\
\hline chr5:190-209 suggestive & RJF & 26 & -3.64 & 0.65 & -4.01 \\
\hline chr5:195-208 suggestive & RJF & 28.79 & -4.35 & 0.66 & -4.07 \\
\hline chr5:193-210 significant & RJF & 34.94 & -1.77 & 0.22 & -1.22 \\
\hline chr5:190-211 suggestive & RJF & 26.81 & -2.61 & 0.4 & -1.93 \\
\hline chr5:193-219 suggestive & RJF & 24.08 & -3.61 & 0.64 & -3.44 \\
\hline chr5:192-211 suggestive & RJF & 23.41 & -1.8 & 0.31 & -1.07 \\
\hline chr5:191-221 significant & RJF & 29.46 & -2.94 & 0.46 & -2.08 \\
\hline chr5:192-221 suggestive & RJF & 23.3 & -0.57 & 0.1 & -0.48 \\
\hline chr5:189-219 suggestive & RJF & 25.47 & -1.65 & 0.28 & -1.22 \\
\hline chr5:192-223 suggestive & RJF & 24.88 & -2.26 & 0.4 & -1.95 \\
\hline chr5:192-236 suggestive & RJF & 19.25 & -1.94 & 0.37 & -0.95 \\
\hline chr5:191-218 suggestive & RJF & 19.09 & -1.76 & 0.38 & -2.25 \\
\hline chr5:192-224 suggestive & RJF & 25.7 & -2.82 & 0.46 & -1.12 \\
\hline chr5:192-220 suggestive & RJF & 24.85 & -4.51 & 0.75 & -3.74 \\
\hline chr5:190-219 suggestive & RJF & 26.36 & -1.26 & 0.2 & -0.85 \\
\hline chr5:181-220 suggestive & RJF & 22.71 & -1.58 & 0.28 & -1.22 \\
\hline chr5:192-226 significant & RJF & 32.08 & -2.21 & 0.31 & 0.37 \\
\hline chr5:186-225 suggestive & RJF & 16.78 & -2.26 & 0.47 & 0.14 \\
\hline chr5:185-224 suggestive & RJF & 24.79 & -4.05 & 0.75 & -3.58 \\
\hline chr5:192-222 suggestive & RJF & 26.09 & -4.24 & 0.76 & -3.36 \\
\hline
\end{tabular}


chr5:193-225 suggestive RJF

chr5:193-223 suggestive RJF

chr5:190-222 suggestive RJF

chr5:192-221 suggestive RJF

chr5:188-223 suggestive RJF

chr5:191-221 suggestive RJF

chr5:197-222 suggestive RJF

chr5:184-223 significant RJF

chr5:186-225 suggestive RJF

chr5:180-223 suggestive RJF

chr5:193-222 suggestive RJF

chr5:189-223 significant RJF

chr5:188-224 suggestive RJF

chr5:195-219 significant RJF

chr5:180-224 suggestive RJF

chr5:196-222 suggestive RJF

chr5:191-224 suggestive RJF

chr5:198-223 suggestive RJF

chr5:187-225 suggestive RJF

chr5:185-224 suggestive RJF

chr5:194-220 suggestive RJF

chr5:186-223 suggestive RJF

chr5:197-224 suggestive RJF

chr5:191-223 suggestive RJF

chr5:198-225 suggestive RJF

chr5:191-219 suggestive RJF

chr5:201-223 suggestive RJF

chr5:187-224 suggestive RJF

chr5:202-219 suggestive RJF

chr5:202-220 suggestive RJF

chr5:200-221 suggestive RJF

chr5:201-222 suggestive RJF

chr5:202-221 significant RJF

chr5:201-219 suggestive RJF

chr5:202-221 suggestive RJF

chr5:205-219 significant RJF

chr5:203-223 suggestive RJF

chr5:203-221 suggestive RJF

chr5:203-223 suggestive RJF

chr5:202-221 suggestive RJF

chr5:204-220 significant RJF

chr5:203-220 suggestive RJF

chr5:203-220 suggestive RJF
25.9

28.85

24.89

28.16

26.91

24.98

26.08

25.44

25.24

24.97

23.62

30.98

27.36

20.83

14.84

27.65

25.19

25.9

26.3

26.18

25.98

25.07

27.01

26.23

20.71

25.36

25.77

26.59

27.15

28.06

25.14

25.16

32.09

26.17

25.96

31.25

25.68

25.27

25.33

24

30.68

25.64

24.89
$-2.91$

$-2.08$

$-3.48$

$-4.16$

$-2.74$

$-1.11$

$-2.09$

$-1.53$

$-1.47$

$-1.86$

$-1.74$

$-3.01$

$-2.81$

$-1.91$

$-1.68$

$-3.19$

$-1.24$

$-1.81$

$-1.12$

$-2.48$

$-1.24$

$-1.83$

$-2.96$

$-4.44$

$-1.66$

$-1.8$

$-1.68$

$-0.79$

$-1.82$

$-1.5$

$-1.51$

$-1.58$

$-6.03$

$-1.37$

$-2.32$

$-0.31$

$-1.35$

$-6.42$

$-2.56$

$-1.75$

$-3.02$

$-2.5$

$-1.21$
0.54

0.38

0.62

0.79

0.48

0.2

0.39

0.25

0.27

0.33

0.31

0.45

0.48

0.45

0.37

0.57

0.21

0.29

0.2

0.44

0.23

0.34

0.49

0.86

0.3

0.32

0.29

0.13

0.34

0.28

0.34

0.28

0.95

0.27

0.41

0.05

0.29

1.29

0.51

0.34

0.49

0.47

0.22
$-2.14$

$-1.81$

$-2.22$

$-3.34$

$-1.01$

$-0.55$

$-2.13$

$-0.9$

$-1.31$

$-1.22$

$-1.07$

$-1.99$

$-1.94$

$-2.27$

$-1.03$

$-3.04$

$-0.86$

$-0.87$

$-0.96$

$-1.36$

$-0.9$

$-1.36$

$-2.1$

$-3.57$

$-0.91$

$-0.66$

$-1.27$

$-0.52$

$-1.19$

$-0.45$

$-1.35$

$-0.74$

$-4.66$

$-0.93$

$-1.54$

$-0.26$

$-1.37$

$-4.9$

$-2.72$

$-1.34$

$-2.42$

$-0.46$

$-1.09$ 


\begin{tabular}{|c|c|c|c|c|c|}
\hline chr5:203-220 suggestive & RJF & 26.45 & -2.4 & 0.47 & -0.69 \\
\hline chr5:203-221 suggestive & RJF & 26.05 & -1.59 & 0.36 & -0.51 \\
\hline chr5:204-220 suggestive & RJF & 24.76 & -4.19 & 0.72 & -2.49 \\
\hline chr5:205-220 significant & RJF & 29.11 & -0.73 & 0.13 & -0.72 \\
\hline chr5:205-218 suggestive & RJF & 27.8 & -3.01 & 0.54 & -1.59 \\
\hline chr5:203-221 suggestive & RJF & 14.57 & -1.63 & 0.35 & -0.38 \\
\hline chr5:210-217 suggestive & RJF & 24.44 & -0.17 & 0.03 & -0.14 \\
\hline chr5:205-219 suggestive & RJF & 26.12 & -2.65 & 0.55 & -2.5 \\
\hline chr5:206-219 suggestive & RJF & 24.66 & -0.84 & 0.16 & -0.72 \\
\hline chr5:204-221 suggestive & RJF & 17.73 & -1.36 & 0.34 & -1.77 \\
\hline chr5:204-221 suggestive & RJF & 18.29 & -0.98 & 0.27 & -1.63 \\
\hline chr5:204-222 suggestive & RJF & 23.6 & -1.55 & 0.32 & -0.87 \\
\hline chr5:205-222 suggestive & RJF & 28.11 & -5.71 & 0.95 & -3.11 \\
\hline chr5:206-222 suggestive & RJF & 27.56 & -1.47 & 0.36 & -1.68 \\
\hline chr5:204-223 suggestive & RJF & 24.64 & -2.23 & 0.41 & -1.59 \\
\hline chr5:203-223 suggestive & RJF & 26.19 & -3.67 & 0.85 & -4.09 \\
\hline chr5:202-225 suggestive & RJF & 25.55 & -3.05 & 0.53 & -1.49 \\
\hline chr5:204-225 significant & RJF & 26.11 & -0.67 & 0.1 & -0.32 \\
\hline chr5:201-228 suggestive & RJF & 19.9 & -1.95 & 0.39 & -1.25 \\
\hline chr5:202-227 suggestive & RJF & 20.52 & -3.25 & 0.63 & -1.38 \\
\hline chr5:185-230 suggestive & RJF & 17.06 & -1.93 & 0.43 & 0.69 \\
\hline chr5:184-229 significant & RJF & 17.77 & -1.41 & 0.28 & -0.65 \\
\hline chr5:203-225 suggestive & RJF & 26.07 & -2.88 & 0.51 & -1.98 \\
\hline chr5:203-227 suggestive & RJF & 25.2 & -1.62 & 0.33 & -1.05 \\
\hline chr5:202-226 suggestive & RJF & 25.98 & -0.6 & 0.1 & -0.41 \\
\hline chr5:204-225 suggestive & RJF & 26 & -3.86 & 0.74 & -2.51 \\
\hline chr5:202-229 suggestive & RJF & 25.85 & -2.18 & 0.47 & -2.01 \\
\hline chr5:214-243 significant & RJF & 17.79 & -1.89 & 0.39 & -0.56 \\
\hline chr5:215-245 suggestive & RJF & 19.82 & -1.24 & 0.24 & -0.47 \\
\hline chr5:220-232 suggestive & WL & 24.66 & 0.21 & 0.5 & -2.76 \\
\hline chr5:215-245 significant & RJF & 14.4 & -4.79 & 1.07 & -0.1 \\
\hline chr5:223-232 significant & RJF & 24.48 & -0.8 & 0.67 & -0.97 \\
\hline chr5:223-236 significant & RJF & 18.96 & -4.46 & 0.84 & 0.31 \\
\hline chr5:223-247 significant & RJF & 15.9 & -1.95 & 0.42 & 1.22 \\
\hline chr5:225-235 significant & WL & 15.87 & 1.42 & 0.3 & 0.1 \\
\hline chr5:221-245 significant & RJF & 17.15 & -2.53 & 0.51 & 0.19 \\
\hline chr5:221-235 suggestive & RJF & 14.83 & -2.22 & 0.52 & -0.79 \\
\hline chr5:222-248 significant & RJF & 16.11 & -2.35 & 0.51 & -0.59 \\
\hline chr5:220-244 significant & WL & 16.15 & 2.55 & 0.55 & -1.61 \\
\hline chr5:216-248 significant & RJF & 15.6 & -2.86 & 0.64 & -1.07 \\
\hline chr5:223-255 significant & WL & 13.93 & 2.16 & 0.52 & -1.59 \\
\hline chr5:219-238 significant & RJF & 20.68 & -2 & 0.37 & -0.4 \\
\hline chr5:213-244 significant & RJF & 17.92 & -1.14 & 0.22 & 0.07 \\
\hline
\end{tabular}




\begin{tabular}{|c|c|c|c|c|c|}
\hline chr5:217-251 significant & RJF & 19.78 & -2.22 & 0.41 & -0.06 \\
\hline chr5:220-242 suggestive & RJF & 24.72 & -1.35 & 0.25 & -0.79 \\
\hline chr5:218-247 suggestive & RJF & 18.59 & -0.79 & 0.15 & 0.1 \\
\hline chr5:214-248 significant & RJF & 15.63 & -0.51 & 0.12 & -0.22 \\
\hline chr5:233-255 suggestive & RJF & 14.58 & -1.07 & 0.28 & -0.75 \\
\hline chr5:233-242 significant & WL & 23.24 & 5.09 & 0.81 & 0.68 \\
\hline chr5:234-244 suggestive & RJF & 23.44 & -2.41 & 0.46 & -1.5 \\
\hline chr5:234-246 suggestive & RJF & 26.28 & -0.53 & 0.11 & -0.47 \\
\hline chr5:234-238 suggestive & RJF & 28.46 & -3.05 & 0.58 & -2.19 \\
\hline chr5:224-245 suggestive & RJF & 22.08 & -1.55 & 0.31 & -1.06 \\
\hline chr5:233-237 suggestive & RJF & 20.87 & -1.54 & 0.33 & -1.3 \\
\hline chr5:233-238 suggestive & RJF & 20.98 & -1.57 & 0.33 & -1.26 \\
\hline chr5:234-249 suggestive & RJF & 18.91 & -0.55 & 0.14 & -0.59 \\
\hline chr5:225-253 significant & RJF & 17.82 & -2.94 & 0.6 & -0.94 \\
\hline chr5:146-256 suggestive & RJF & 12.27 & -0.99 & 0.24 & -0.04 \\
\hline chr5:221-253 significant & RJF & 15.23 & -1.28 & 0.28 & -0.21 \\
\hline chr5:215-243 significant & RJF & 17.45 & -1.46 & 0.29 & 0.22 \\
\hline chr5:234-244 suggestive & WL & 24.53 & 3.18 & 0.65 & -3.29 \\
\hline chr5:234-243 suggestive & RJF & 24 & -1.26 & 0.36 & -1.86 \\
\hline chr5:215-253 suggestive & RJF & 14.99 & -1.94 & 0.52 & -1.75 \\
\hline chr5:221-258 suggestive & RJF & 18.63 & -0.66 & 0.6 & 0.19 \\
\hline chr5:223-250 significant & RJF & 20.78 & -2.21 & 0.41 & -0.54 \\
\hline chr5:216-258 suggestive & RJF & 11.97 & -2.23 & 0.57 & 1.25 \\
\hline chr5:227-257 significant & RJF & 16.13 & -1.46 & 0.31 & 0.47 \\
\hline chr5:220-252 significant & RJF & 15.47 & -2.15 & 0.51 & -1.14 \\
\hline chr5:220-253 suggestive & WL & 14.46 & 1.43 & 0.32 & -0.48 \\
\hline chr5:216-252 suggestive & RJF & 12.99 & -1.86 & 0.44 & -0.17 \\
\hline chr5:217-247 suggestive & RJF & 14.82 & -1.72 & 0.42 & -1.1 \\
\hline chr5:214-269 suggestive & RJF & 14.32 & -1.29 & 0.31 & -0.46 \\
\hline chr5:237-246 suggestive & RJF & 23.53 & -0.08 & 0.02 & -0.08 \\
\hline chr5:236-242 significant & RJF & 31.91 & -1.25 & 0.29 & -1.21 \\
\hline chr5:236-250 suggestive & RJF & 22.01 & -0.97 & 0.53 & -1.43 \\
\hline chr5:233-246 suggestive & WL & 26.41 & 0.82 & 0.52 & -2.74 \\
\hline chr5:233-247 suggestive & RJF & 19.79 & -2.36 & 0.43 & 0.28 \\
\hline chr5:234-254 significant & RJF & 21.51 & -1.7 & 0.32 & -0.77 \\
\hline chr5:226-253 significant & RJF & 22.57 & -1.37 & 0.27 & -1.04 \\
\hline chr5:226-258 significant & RJF & 18.47 & -2.14 & 0.42 & 0.9 \\
\hline chr5:236-254 suggestive & RJF & 20.04 & -0.86 & 0.51 & -2.77 \\
\hline chr5:236-253 suggestive & RJF & 24.65 & -0.72 & 0.19 & -0.9 \\
\hline chr5:231-289 suggestive & RJF & 10.6 & -1.47 & 0.32 & 1.01 \\
\hline chr5:214-277 significant & RJF & 17.18 & -1.39 & 0.31 & -0.74 \\
\hline chr5:235-257 suggestive & RJF & 21.35 & -1.39 & 0.27 & -0.87 \\
\hline chr5:218-289 suggestive & RJF & 17.52 & -1.54 & 0.3 & 0.21 \\
\hline
\end{tabular}




\begin{tabular}{|c|c|c|c|c|c|}
\hline chr5:231-287 suggestive & RJF & 18.68 & -1.36 & 0.52 & -0.97 \\
\hline chr5:233-290 suggestive & WL & 20.47 & 1.19 & 0.48 & -2.81 \\
\hline chr5:203-287 suggestive & RJF & 13.38 & -0.9 & 0.21 & -0.15 \\
\hline chr5:232-282 suggestive & RJF & 17.56 & -0.64 & 0.46 & -0.49 \\
\hline chr5:233-283 significant & RJF & 15.6 & -1.39 & 0.29 & -0.51 \\
\hline chr5:240-278 significant & RJF & 24.17 & -2.89 & 0.49 & 0.25 \\
\hline chr5:239-291 suggestive & WL & 12.8 & 1.79 & 0.43 & 0.34 \\
\hline chr5:241-285 suggestive & RJF & 12.9 & -1.96 & 0.44 & -0.78 \\
\hline chr5:235-279 suggestive & WL & 12.9 & 3.34 & 0.97 & -1.68 \\
\hline chr5:248-275 significant & RJF & 20.19 & -2.43 & 0.63 & -2.11 \\
\hline chr5:245-279 significant & RJF & 18.37 & -1.88 & 0.36 & -0.54 \\
\hline chr5:248-281 suggestive & RJF & 20.09 & -0.29 & 0.05 & -0.17 \\
\hline chr5:216-317 suggestive & RJF & 13.89 & -1.41 & 0.32 & -0.2 \\
\hline chr5:236-343 suggestive & RJF & 19.4 & -1.29 & 0.47 & -1.73 \\
\hline chr5:259-323 suggestive & WL & 13.51 & 1.59 & 0.37 & 0.43 \\
\hline chr5:265-316 significant & RJF & 18.15 & -1.18 & 0.25 & -0.86 \\
\hline chr5:262-346 suggestive & RJF & 13.79 & -2.7 & 0.62 & -0.51 \\
\hline chr5:277-314 significant & WL & 23.45 & 2.75 & 0.5 & 1.6 \\
\hline chr5:279-315 significant & WL & 21.23 & 2.64 & 0.47 & -0.01 \\
\hline chr5:275-317 significant & RJF & 15.14 & -2.59 & 0.57 & 0.51 \\
\hline chr5:281-344 significant & RJF & 14.86 & -2.78 & 0.66 & -2.05 \\
\hline chr5:273-335 significant & RJF & 19.27 & -2.14 & 0.45 & -1.63 \\
\hline chr5:277-339 suggestive & WL & 11.63 & 1.49 & 0.45 & 1.36 \\
\hline chr5:287-313 significant & RJF & 32.43 & -2.83 & 0.38 & -0.78 \\
\hline chr5:297-332 suggestive & WL & 18.8 & 1.59 & 0.42 & -2.21 \\
\hline chr5:300-315 significant & RJF & 39.22 & -2.45 & 0.28 & -0.63 \\
\hline chr5:289-338 suggestive & RJF & 13.88 & -2.29 & 0.55 & 1.3 \\
\hline chr5:283-317 suggestive & RJF & 13.25 & -2.48 & 0.56 & -0.14 \\
\hline chr5:287-340 significant & RJF & 15.5 & -3.08 & 0.66 & 0.08 \\
\hline chr5:294-315 significant & RJF & 15.44 & -1.57 & 0.35 & 0.6 \\
\hline chr5:283-316 suggestive & WL & 18.82 & 1.97 & 0.43 & -1.65 \\
\hline chr5:290-315 suggestive & WL & 21.51 & 0.99 & 0.36 & -2.6 \\
\hline chr5:278-314 significant & RJF & 15.43 & -1.98 & 0.43 & 0.4 \\
\hline chr5:283-338 significant & RJF & 16.5 & -2.96 & 0.61 & -0.61 \\
\hline chr5:289-313 suggestive & WL & 22.35 & 3.25 & 0.68 & -3.58 \\
\hline chr5:284-315 significant & RJF & 19.59 & -2.12 & 0.4 & 0.79 \\
\hline chr5:276-319 significant & WL & 16.29 & 2.6 & 0.55 & 0.42 \\
\hline chr5:306-315 significant & RJF & 24.76 & -1.97 & 0.33 & -0.79 \\
\hline chr5:306-317 significant & RJF & 21.41 & -2.54 & 0.74 & -2.21 \\
\hline chr5:305-314 suggestive & WL & 16.25 & 1.63 & 0.53 & -2.84 \\
\hline chr5:301-317 significant & RJF & 16.19 & -2.43 & 0.52 & -0.51 \\
\hline chr5:295-320 suggestive & RJF & 15 & -1.09 & 0.25 & -0.54 \\
\hline chr5:293-317 significant & RJF & 17.77 & -1.1 & 0.22 & 0.23 \\
\hline
\end{tabular}




\begin{tabular}{|c|c|c|c|c|c|}
\hline chr5:289-328 significant & RJF & 15.96 & -1.74 & 0.38 & -0.64 \\
\hline chr5:304-318 significant & RJF & 16.22 & -2.48 & 0.52 & 0.54 \\
\hline chr5:296-316 suggestive & WL & 18.43 & 2.38 & 0.46 & 0.18 \\
\hline chr5:296-327 significant & WL & 16.39 & 1.73 & 0.38 & 0.65 \\
\hline chr5:306-317 suggestive & RJF & 20.23 & -3.08 & 0.99 & -3.5 \\
\hline chr5:296-347 significant & RJF & 22.98 & -3.09 & 0.52 & -0.23 \\
\hline chr5:286-344 significant & RJF & 15.39 & -1.92 & 0.41 & -0.48 \\
\hline chr5:299-335 significant & RJF & 15.68 & -3.04 & 0.63 & 0.44 \\
\hline chr5:279-343 suggestive & RJF & 14.04 & -1.72 & 0.39 & 0.3 \\
\hline chr5:276-340 suggestive & RJF & 14.85 & -1.09 & 0.24 & 0.35 \\
\hline chr5:287-354 suggestive & RJF & 13.98 & -2.5 & 0.57 & 0.78 \\
\hline chr5:295-328 significant & RJF & 20.93 & -2.5 & 0.54 & 0.3 \\
\hline chr5:287-329 significant & WL & 19.31 & 2.23 & 0.42 & -0.2 \\
\hline chr5:286-344 significant & RJF & 20.29 & -1.92 & 0.35 & 0.19 \\
\hline chr5:288-349 suggestive & RJF & 14.29 & -1.52 & 0.34 & 0.43 \\
\hline chr5:292-341 significant & RJF & 19 & -2.28 & 0.43 & 0.45 \\
\hline chr5:306-328 significant & WL & 23.85 & 2.89 & 0.52 & 1.69 \\
\hline chr5:295-338 significant & RJF & 17.45 & -1.71 & 0.34 & 0.55 \\
\hline chr5:275-350 significant & RJF & 12.72 & -2.38 & 0.58 & 0.26 \\
\hline chr5:285-341 significant & RJF & 15.74 & -1.91 & 0.42 & 0.54 \\
\hline chr5:263-346 suggestive & WL & 11.87 & 2.44 & 0.63 & 0.89 \\
\hline chr5:288-341 suggestive & RJF & 18.86 & -2.25 & 0.56 & -1.58 \\
\hline chr5:286-356 suggestive & RJF & 17.17 & -2.23 & 0.77 & -1.51 \\
\hline chr5:285-337 significant & RJF & 17.79 & -1.45 & 0.28 & -0.24 \\
\hline chr5:290-347 significant & WL & 14.5 & 3.87 & 1.03 & -3.05 \\
\hline chr5:268-340 significant & RJF & 16.99 & -1.6 & 0.97 & -1.31 \\
\hline chr5:293-351 suggestive & RJF & 12.93 & -2.12 & 0.51 & 0.03 \\
\hline chr5:279-335 significant & WL & 16.75 & 2.01 & 0.44 & -0.79 \\
\hline chr5:288-340 significant & RJF & 16.96 & -1.1 & 0.22 & -0.33 \\
\hline chr5:313-333 significant & RJF & 19.29 & -2.22 & 0.8 & -3.3 \\
\hline chr5:313-336 suggestive & RJF & 17.12 & -2.4 & 0.73 & -2.75 \\
\hline chr5:312-339 suggestive & RJF & 17.32 & -1.96 & 0.61 & -1.19 \\
\hline chr5:314-338 significant & RJF & 21.13 & -2.48 & 0.64 & -2.45 \\
\hline chr5:313-337 significant & RJF & 16.26 & -3.11 & 1.05 & -3.46 \\
\hline chr5:313-335 significant & RJF & 18.95 & -2.7 & 1.01 & -3.26 \\
\hline chr5:315-333 suggestive & RJF & 17.24 & -0.7 & 0.68 & -2.66 \\
\hline chr5:313-338 suggestive & WL & 12.99 & 1.75 & 0.49 & -1.52 \\
\hline chr5:309-335 suggestive & WL & 12.54 & 1.89 & 0.46 & 0 \\
\hline chr5:307-340 suggestive & RJF & 14.53 & -1.99 & 0.56 & -2.75 \\
\hline chr5:315-335 suggestive & RJF & 23.86 & -2.03 & 0.51 & -2.77 \\
\hline chr5:312-332 suggestive & RJF & 21.38 & -2.85 & 0.66 & -2.26 \\
\hline chr5:314-331 suggestive & WL & 12.28 & 1.38 & 0.34 & -1.48 \\
\hline chr5:314-337 significant & RJF & 25.01 & -2.26 & 0.36 & -1.54 \\
\hline
\end{tabular}




\begin{tabular}{|c|c|c|c|c|c|}
\hline chr5:306-340 significant & RJF & 19.52 & -2.06 & 0.69 & -0.89 \\
\hline chr5:313-340 significant & WL & 19.66 & 1.46 & 0.28 & -0.11 \\
\hline chr5:306-342 significant & RJF & 19.94 & -3.36 & 1 & -2.39 \\
\hline chr5:313-339 suggestive & WL & 18.81 & 2.68 & 0.63 & -0.66 \\
\hline chr5:312-343 significant & RJF & 18.39 & -1.26 & 0.51 & -1.43 \\
\hline chr5:274-344 suggestive & RJF & 17.8 & -0.79 & 0.53 & -1.1 \\
\hline chr5:313-340 suggestive & WL & 17.95 & 2.29 & 0.6 & -0.76 \\
\hline chr5:316-338 suggestive & WL & 7.77 & 0.76 & 0.16 & -0.49 \\
\hline chr5:271-362 suggestive & RJF & 14.01 & -1.51 & 0.39 & -1.55 \\
\hline chr5:294-349 suggestive & WL & 13.82 & 1.7 & 0.41 & -0.7 \\
\hline chr5:282-357 suggestive & WL & 13.71 & 1.24 & 0.29 & 0.43 \\
\hline chr5:294-358 suggestive & WL & 13.83 & 0.71 & 0.16 & -0.03 \\
\hline chr5:272-361 suggestive & RJF & 12.22 & -1.43 & 0.35 & 0.1 \\
\hline chr5:271-359 suggestive & RJF & 13.94 & -2.22 & 0.5 & -0.27 \\
\hline chr5:274-364 suggestive & RJF & 13.8 & -1.8 & 0.43 & 0.44 \\
\hline chr5:326-368 significant & WL & 18.44 & 3.02 & 0.59 & -0.99 \\
\hline chr5:325-365 significant & RJF & 18.43 & -1.96 & 0.37 & 0.44 \\
\hline chr5:329-364 significant & RJF & 17.79 & -2.93 & 0.58 & -0.14 \\
\hline chr5:329-364 significant & RJF & 17.34 & -2.21 & 0.44 & 0.09 \\
\hline chr5:330-366 significant & RJF & 16.38 & -3.3 & 0.69 & -0.24 \\
\hline chr5:331-366 significant & RJF & 17.85 & -2.88 & 0.59 & -0.53 \\
\hline chr5:334-364 suggestive & WL & 14.71 & 5.82 & 1.58 & -7.09 \\
\hline chr5:333-365 suggestive & RJF & 15.12 & -2.2 & 0.48 & -0.06 \\
\hline chr5:336-403 suggestive & RJF & 16.04 & -1.6 & 0.34 & 0.24 \\
\hline chr5:340-364 significant & RJF & 21.88 & -3.2 & 0.59 & -0.91 \\
\hline chr5:341-362 suggestive & WL & 17.95 & 3.92 & 1.08 & -6.09 \\
\hline chr5:347-365 significant & RJF & 27.4 & -4.35 & 0.64 & -0.03 \\
\hline chr5:345-363 significant & RJF & 28.35 & -4.06 & 0.59 & 1.23 \\
\hline chr5:338-368 suggestive & WL & 10.63 & 2.05 & 0.54 & -0.67 \\
\hline chr5:338-366 significant & RJF & 14.94 & -2.31 & 0.49 & 0.3 \\
\hline chr5:344-367 significant & RJF & 18.24 & -2.35 & 0.44 & 0.17 \\
\hline chr5:344-366 suggestive & RJF & 14.67 & -1.2 & 0.28 & -0.37 \\
\hline chr5:335-371 suggestive & WL & 14.02 & 1.03 & 0.23 & 0.02 \\
\hline chr5:337-378 significant & RJF & 16.31 & -1.54 & 0.32 & -0.17 \\
\hline chr5:339-378 significant & RJF & 16.69 & -1.17 & 0.26 & -0.44 \\
\hline chr5:335-406 suggestive & RJF & 14.66 & -1.48 & 0.33 & -0.77 \\
\hline chr5:339-376 significant & RJF & 21.83 & -3.96 & 0.69 & -1.43 \\
\hline chr5:346-376 significant & RJF & 22.87 & -1.88 & 0.33 & -0.92 \\
\hline chr5:343-378 significant & RJF & 19.24 & -2.11 & 0.46 & -2.04 \\
\hline chr5:361-374 significant & WL & 24.21 & 3.21 & 0.54 & -0.77 \\
\hline chr5:358-375 significant & RJF & 23.45 & -3.22 & 0.54 & -1.11 \\
\hline chr5:362-376 suggestive & WL & 18.65 & 0.02 & 0.4 & 0.46 \\
\hline chr5:361-376 significant & RJF & 24.74 & -2.46 & 0.4 & -1.1 \\
\hline
\end{tabular}




\begin{tabular}{|c|c|c|c|c|c|}
\hline chr5:361-377 significant & WL & 13.72 & 2.51 & 0.6 & -0.97 \\
\hline chr5:362-377 significant & WL & 13.8 & 1.6 & 0.43 & -1.21 \\
\hline chr5:361-377 significant & WL & 15.28 & 2.06 & 0.47 & -0.77 \\
\hline chr5:360-376 significant & WL & 12.41 & 1.58 & 0.44 & -0.95 \\
\hline chr5:359-377 significant & WL & 12.25 & 2.66 & 0.72 & -1.51 \\
\hline chr5:362-376 significant & WL & 25.27 & 1.78 & 0.31 & -0.99 \\
\hline chr5:359-382 suggestive & RJF & 16.49 & -2.71 & 0.57 & -1.1 \\
\hline chr5:361-392 suggestive & WL & 12.21 & 1.79 & 0.45 & -0.54 \\
\hline chr5:365-378 significant & RJF & 27.5 & -2.67 & 0.38 & -0.75 \\
\hline chr5:360-397 significant & WL & 20.01 & 2.46 & 0.47 & -0.82 \\
\hline chr5:361-393 significant & RJF & 18.92 & -1.58 & 0.3 & -0.6 \\
\hline chr5:363-390 significant & RJF & 22.77 & -3.03 & 0.5 & -0.42 \\
\hline chr5:360-395 significant & RJF & 16.38 & -2.86 & 0.58 & -1.23 \\
\hline chr5:357-395 suggestive & RJF & 16.19 & -2.5 & 0.57 & -1.92 \\
\hline chr5:355-410 suggestive & WL & 10.81 & 2.07 & 0.54 & 0 \\
\hline chr5:364-395 significant & RJF & 23.19 & -1.5 & 0.27 & 0.38 \\
\hline chr5:360-416 significant & WL & 10.43 & 1.27 & 0.39 & -0.53 \\
\hline chr5:360-411 significant & WL & 12.82 & 2.15 & 0.52 & -0.22 \\
\hline chr5:363-392 significant & RJF & 23.02 & -1.7 & 0.28 & -0.39 \\
\hline chr5:346-398 significant & RJF & 18.03 & -1.22 & 0.24 & -0.65 \\
\hline chr5:359-393 suggestive & RJF & 14.4 & -0.08 & 0.33 & 0.87 \\
\hline chr5:353-397 suggestive & RJF & 15.26 & -1.6 & 0.36 & -1.09 \\
\hline chr5:361-408 significant & WL & 12.28 & 2.93 & 0.75 & -0.36 \\
\hline chr5:365-396 significant & RJF & 21.17 & -1.05 & 0.49 & 0.47 \\
\hline chr5:371-401 significant & RJF & 16.05 & -1.44 & 0.29 & -0.4 \\
\hline chr5:371-393 suggestive & RJF & 16.74 & -1.1 & 0.29 & -1.53 \\
\hline chr5:375-397 significant & RJF & 31.42 & -2.39 & 0.34 & 0.38 \\
\hline chr5:373-406 significant & RJF & 18.13 & -1.25 & 0.26 & 0.26 \\
\hline chr5:377-402 suggestive & WL & 17.05 & 0.17 & 0.34 & 0.24 \\
\hline chr5:372-405 suggestive & RJF & 17.75 & -0.56 & 0.42 & 0 \\
\hline chr5:374-414 significant & RJF & 20.63 & -2.32 & 0.44 & 0.38 \\
\hline chr5:374-417 suggestive & RJF & 16.11 & -1.61 & 0.34 & 0.02 \\
\hline chr5:371-436 suggestive & RJF & 16.09 & -2.42 & 0.52 & 0.02 \\
\hline chr5:394-439 suggestive & RJF & 19.6 & -3.23 & 0.68 & -3.62 \\
\hline chr5:397-444 suggestive & RJF & 22 & -1.91 & 0.47 & -3.38 \\
\hline chr5:398-440 suggestive & RJF & 23.61 & -1.14 & 0.22 & -1.32 \\
\hline chr5:400-441 suggestive & RJF & 19.13 & -1.09 & 0.37 & -3.33 \\
\hline chr5:397-439 suggestive & RJF & 19.03 & -1.72 & 0.36 & -1.74 \\
\hline chr5:396-436 suggestive & RJF & 14.23 & -11.63 & 2.48 & -3.59 \\
\hline chr5:408-449 significant & WL & 19.41 & 1.84 & 0.37 & -0.95 \\
\hline chr5:399-436 suggestive & RJF & 15.05 & -17.39 & 3.68 & -5.72 \\
\hline chr5:400-436 suggestive & RJF & 14.61 & -16.89 & 3.62 & -5.78 \\
\hline chr5:400-438 significant & WL & 13.6 & 1.65 & 0.38 & 0.43 \\
\hline
\end{tabular}




\begin{tabular}{|c|c|c|c|c|c|}
\hline chr5:406-437 significant & RJF & 19.51 & -30.71 & 5.42 & -0.14 \\
\hline chr5:403-441 suggestive & RJF & 24.22 & -0.8 & 0.19 & -1.52 \\
\hline chr5:407-443 significant & RJF & 30.92 & -0.3 & 0.05 & -0.37 \\
\hline chr5:407-451 suggestive & RJF & 22.77 & -2.37 & 0.51 & -3.3 \\
\hline chr5:401-452 suggestive & RJF & 22.15 & -1.9 & 0.37 & -1.86 \\
\hline chr5:404-452 significant & RJF & 29.68 & -1.67 & 0.28 & -2.17 \\
\hline chr5:431-452 suggestive & RJF & 27.2 & -1.68 & 0.28 & -1.89 \\
\hline chr5:430-452 suggestive & RJF & 26.77 & -2.19 & 0.37 & -2.67 \\
\hline chr5:432-452 suggestive & RJF & 24.87 & -0.81 & 0.14 & -0.96 \\
\hline chr5:428-452 suggestive & RJF & 23.6 & -0.76 & 0.15 & -0.89 \\
\hline chr5:433-452 suggestive & RJF & 28.09 & -2.47 & 0.4 & -3 \\
\hline chr5:435-452 significant & RJF & 35.65 & -0.97 & 0.13 & -1.11 \\
\hline chr5:435-452 suggestive & RJF & 26.51 & -0.99 & 0.16 & -1.06 \\
\hline chr5:435-452 suggestive & WL & 21.01 & 4.3 & 0.84 & 0.61 \\
\hline chr5:445-452 suggestive & WL & 19.96 & 1.63 & 0.43 & 2.53 \\
\hline chr5:401-452 suggestive & RJF & 14.88 & -0.08 & 0.1 & 0.54 \\
\hline chr5:440-452 suggestive & WL & 14.26 & 0.36 & 0.36 & 2.21 \\
\hline chr5:446-452 suggestive & RJF & 24 & 0 & 0.02 & 0.02 \\
\hline chr6:0-28 significant & WL & 23.49 & 14.02 & 2.32 & 1.77 \\
\hline significant & WL & 26.31 & 12.09 & 1.85 & 1.36 \\
\hline significant & RJF & 18.4 & -1.75 & 0.35 & -0.71 \\
\hline significant & WL & 13.85 & 5.24 & 1.31 & -3.19 \\
\hline suggestive & WL & 13.33 & 1.11 & 0.26 & -0.06 \\
\hline suggestive & WL & 12.82 & 3.52 & 0.89 & -1.77 \\
\hline suggestive & WL & 13.22 & 4.67 & 1.21 & -3.04 \\
\hline suggestive & RJF & 15.63 & -1.01 & 0.25 & -0.88 \\
\hline significant & WL & 14.7 & 1.12 & 0.25 & -0.32 \\
\hline suggestive & RJF & 15.5 & -1.58 & 0.35 & -0.69 \\
\hline significant & RJF & 24.24 & -3.75 & 0.59 & -0.48 \\
\hline significant & RJF & 28.41 & -3.86 & 0.56 & -0.7 \\
\hline suggestive & WL & 17.65 & 1.43 & 0.61 & -3.8 \\
\hline significant & WL & 23.12 & 9.33 & 1.55 & 0.93 \\
\hline significant & WL & 21.35 & 11.3 & 1.98 & 1.13 \\
\hline significant & WL & 21.21 & 1.98 & 0.35 & -0.02 \\
\hline significant & WL & 31.02 & 12.62 & 1.72 & 0.67 \\
\hline significant & WL & 36.9 & 13.8 & 1.64 & 0.47 \\
\hline significant & WL & 29.23 & 12.18 & 1.74 & 1.49 \\
\hline significant & WL & 29.85 & 11.58 & 1.61 & 0.63 \\
\hline significant & WL & 29.07 & 17.37 & 2.45 & 0.75 \\
\hline significant & WL & 28.96 & 12.17 & 1.73 & 0.29 \\
\hline significant & WL & 31.91 & 14.16 & 1.88 & 0.71 \\
\hline significant & WL & 22.28 & 14.19 & 2.4 & 1.2 \\
\hline suggestive & WL & 10.79 & 6.4 & 1.65 & 2.28 \\
\hline
\end{tabular}




\begin{tabular}{|c|c|c|c|c|c|c|}
\hline chr6:0-40 & significant & WL & 21.4 & 19.52 & 3.35 & -0.65 \\
\hline chr6:0-42 & significant & WL & 13.32 & 4.64 & 1.16 & -2.37 \\
\hline chr6:0-59 & significant & WL & 13.84 & 1.69 & 0.39 & -0.16 \\
\hline chr6:0-45 & significant & WL & 17.18 & 19.9 & 3.88 & 1.33 \\
\hline chr6:0-50 & significant & WL & 12.49 & 1.91 & 0.47 & -0.24 \\
\hline chr6:0-45 & significant & WL & 15.46 & 14.98 & 3.12 & 1.09 \\
\hline chr6:0-50 & suggestive & WL & 10.39 & 6.42 & 1.66 & 1.31 \\
\hline chr6:0-45 & significant & WL & 18.02 & 20.27 & 3.81 & 0.6 \\
\hline chr6:0-52 & significant & WL & 18.23 & 4.43 & 0.85 & 0.37 \\
\hline chr6:0-46 & significant & WL & 16.02 & 17.49 & 3.51 & 1.08 \\
\hline chr6:0-44 & suggestive & WL & 13.15 & 1.27 & 0.3 & -0.12 \\
\hline chr6:0-52 & significant & WL & 11.89 & 8.2 & 1.98 & 2.04 \\
\hline chr6:0-51 & significant & WL & 11.61 & 15.1 & 3.67 & 1.65 \\
\hline chr6:0-52 & significant & WL & 11.26 & 7.27 & 1.79 & 1.13 \\
\hline chr6:0-51 & significant & WL & 12.73 & 13.68 & 3.16 & 1.67 \\
\hline chr6:0-56 & significant & WL & 11.2 & 14.12 & 3.54 & 3.11 \\
\hline chr6:17-46 & significant & WL & 28.93 & 0.1 & 0.05 & -0.06 \\
\hline chr6:0-54 & suggestive & WL & 12.98 & 12.55 & 2.93 & -7.5 \\
\hline chr6:2-59 & significant & WL & 14.65 & 1.83 & 0.41 & -0.66 \\
\hline chr6:26-62 & significant & WL & 16.34 & 4.91 & 1.09 & -0.8 \\
\hline chr6:7-65 & significant & WL & 14.29 & 2.52 & 0.62 & -0.03 \\
\hline chr6:21-56 & suggestive & WL & 19.34 & 0.39 & 0.08 & -0.5 \\
\hline chr6:41-82 & significant & WL & 19.85 & 2.7 & 0.59 & 1.24 \\
\hline chr6:45-74 & significant & RJF & 15.65 & -4.17 & 0.92 & -0.34 \\
\hline chr6:50-85 & significant & WL & 20.59 & 2.47 & 0.83 & 2.08 \\
\hline chr6:52-69 & significant & RJF & 23.31 & -2.99 & 0.5 & 0.42 \\
\hline chr6:47-81 & significant & WL & 16.68 & 0.98 & 0.21 & 0.03 \\
\hline chr6:42-79 & suggestive & RJF & 15.66 & -2.56 & 1.67 & -1.76 \\
\hline chr6:52-69 & significant & WL & 31.44 & 2.92 & 0.39 & -1.02 \\
\hline chr6:47-76 & significant & RJF & 15.46 & -3.48 & 0.81 & -0.57 \\
\hline chr6:51-74 & significant & WL & 24.33 & 2.33 & 0.39 & -0.1 \\
\hline chr6:47-85 & suggestive & RJF & 11.97 & -0.93 & 0.23 & -0.01 \\
\hline chr6:49-79 & significant & RJF & 18.33 & -2.44 & 0.54 & -1 \\
\hline chr6:52-77 & significant & WL & 20.94 & 2.6 & 0.48 & 0.14 \\
\hline chr6:39-80 & suggestive & WL & 19 & 1.7 & 0.39 & -1.11 \\
\hline chr6:65-81 & significant & RJF & 23.59 & -2.68 & 0.46 & -0.17 \\
\hline chr6:66-103 & suggestive & RJF & 13.27 & -1.07 & 0.47 & -1.89 \\
\hline chr6:31-109 & suggestive & WL & 12.55 & 1.45 & 0.43 & 0.54 \\
\hline chr6:72-111 & significant & WL & 24.47 & 4.58 & 0.86 & 1.16 \\
\hline chr6:71-95 & significant & WL & 28.97 & 0.04 & 0.02 & -0.04 \\
\hline chr6:56-106 & suggestive & WL & 13.88 & 2.9 & 0.69 & -0.4 \\
\hline chr6:73-94 & significant & WL & 18.8 & 1.96 & 0.57 & -2.7 \\
\hline chr6:73-102 & significant & WL & 16.7 & 11.66 & 3.44 & 9.45 \\
\hline
\end{tabular}




\begin{tabular}{|c|c|c|c|c|c|c|}
\hline chr6:72-100 & significant & WL & 16.03 & 1.9 & 0.4 & -1.23 \\
\hline chr6:76-99 & significant & WL & 22.73 & 14.66 & 3.5 & 10.97 \\
\hline chr6:72-102 & significant & WL & 15.57 & 1.64 & 0.41 & 0.52 \\
\hline chr6:78-97 & significant & WL & 24.12 & 11.04 & 2.02 & 1.09 \\
\hline chr6:79-100 & significant & WL & 24.92 & 5.42 & 1.06 & 2.24 \\
\hline chr6:82-90 & significant & WL & 63.19 & 5.22 & 0.4 & 0.47 \\
\hline chr6:79-95 & significant & WL & 29.86 & 4 & 0.56 & -1.35 \\
\hline chr6:78-95 & significant & WL & 29.88 & 14.55 & 2.21 & -0.03 \\
\hline chr6:80-93 & significant & WL & 30.23 & 11.8 & 1.82 & 0.78 \\
\hline chr6:79-94 & significant & WL & 41.06 & 10.9 & 1.38 & 2.79 \\
\hline chr6:77-96 & significant & WL & 23.17 & 12.34 & 2.29 & 1.12 \\
\hline chr6:80-93 & significant & WL & 24.19 & 3.88 & 0.61 & -1.58 \\
\hline chr6:74-100 & significant & WL & 17.41 & 3.17 & 0.64 & -0.16 \\
\hline chr6:79-100 & significant & WL & 15.85 & 7.53 & 1.86 & 1.69 \\
\hline chr6:75-96 & significant & RJF & 13.5 & -1.87 & 0.49 & -0.37 \\
\hline chr6:73-100 & significant & WL & 15.58 & 1.85 & 0.42 & 0.14 \\
\hline chr6:76-95 & significant & WL & 18.3 & 3.2 & 0.62 & -2.07 \\
\hline chr6:76-101 & suggestive & WL & 14.72 & 1.63 & 0.43 & 0.48 \\
\hline chr6:75-102 & suggestive & WL & 14.35 & 2.02 & 0.46 & -0.54 \\
\hline chr6:72-103 & significant & RJF & 12.96 & -1.96 & 0.52 & -0.13 \\
\hline chr6:74-99 & suggestive & WL & 14.4 & 1.48 & 0.34 & -0.25 \\
\hline chr6:75-102 & significant & WL & 20.88 & 8.64 & 2.11 & 5.58 \\
\hline chr6:75-98 & suggestive & WL & 24.44 & 1.39 & 0.24 & -0.98 \\
\hline chr6:76-101 & significant & WL & 22.8 & 3.33 & 0.57 & -0.54 \\
\hline chr6:72-107 & suggestive & WL & 15.17 & 2.6 & 0.56 & -1.38 \\
\hline chr6:76-139 & significant & RJF & 16.96 & -1.29 & 0.28 & 0.06 \\
\hline chr6:73-109 & suggestive & WL & 19.28 & 1.82 & 0.56 & -1.61 \\
\hline chr6:77-110 & suggestive & WL & 17.66 & 1.51 & 0.27 & -0.98 \\
\hline chr6:74-119 & significant & WL & 18.36 & 1.65 & 0.32 & -0.09 \\
\hline chr6:70-108 & significant & WL & 30.01 & 3.14 & 0.44 & -1.13 \\
\hline chr6:74-116 & significant & WL & 20.8 & 0.64 & 0.92 & -0.64 \\
\hline chr6:75-110 & suggestive & WL & 17.87 & 0.62 & 0.29 & -1.24 \\
\hline chr6:75-114 & significant & WL & 24.6 & 3.36 & 0.54 & 0.09 \\
\hline chr6:69-121 & significant & WL & 15.64 & 1.69 & 0.37 & -0.67 \\
\hline chr6:69-111 & suggestive & WL & 11.89 & 2.35 & 0.57 & 0.03 \\
\hline chr6:68-109 & significant & WL & 18.85 & 1.66 & 0.37 & -1.48 \\
\hline chr6:70-116 & significant & WL & 16.26 & 1.79 & 0.37 & -0.03 \\
\hline chr6:75-114 & suggestive & RJF & 21.22 & -0.28 & 0.64 & -1.61 \\
\hline chr6:89-108 & significant & WL & 52.29 & 3.72 & 0.33 & 0.64 \\
\hline chr6:77-111 & suggestive & WL & 15.47 & 1.42 & 0.33 & -0.84 \\
\hline chr6:19-111 & suggestive & WL & 13.35 & 2.42 & 0.62 & -0.14 \\
\hline chr6:78-112 & suggestive & WL & 14.44 & 1.21 & 0.42 & -2.01 \\
\hline chr6:89-111 & significant & WL & 28.26 & 1.92 & 0.29 & -0.63 \\
\hline
\end{tabular}




\begin{tabular}{|c|c|c|c|c|c|c|}
\hline chr6:72-115 & suggestive & WL & 14.3 & 1.59 & 0.35 & -0.14 \\
\hline chr6:89-113 & suggestive & WL & 16.53 & 1.43 & 1.76 & 11.93 \\
\hline chr6:92-110 & suggestive & WL & 20.25 & 0.89 & 0.35 & -2.42 \\
\hline chr6:90-110 & significant & WL & 17.14 & 1.18 & 0.26 & -0.8 \\
\hline chr6:78-124 & suggestive & RJF & 14.67 & -2.08 & 0.83 & -4.2 \\
\hline chr6:99-111 & significant & WL & 25.46 & 0.04 & 0.25 & 0.23 \\
\hline chr6:95-121 & significant & WL & 13.96 & 1.44 & 0.96 & -1.67 \\
\hline chr6:90-117 & suggestive & WL & 17.24 & 0.43 & 0.91 & -1.82 \\
\hline chr6:98-119 & suggestive & RJF & 18.57 & -0.13 & 0.69 & -2.47 \\
\hline chr6:93-109 & significant & RJF & 19.24 & -0.6 & 0.56 & -2.9 \\
\hline chr6:96-111 & suggestive & RJF & 22.03 & -0.79 & 0.5 & -1.64 \\
\hline chr6:96-111 & suggestive & WL & 27.42 & 0.54 & 0.48 & -0.43 \\
\hline chr6:97-108 & suggestive & WL & 25.58 & 1.07 & 0.46 & 3.7 \\
\hline chr6:98-112 & suggestive & RJF & 19.64 & -0.34 & 0.43 & -0.84 \\
\hline chr6:100-111 & suggestive & WL & 20.6 & 0.18 & 0.37 & -0.89 \\
\hline chr6:97-111 & suggestive & WL & 20.18 & 0.09 & 0.41 & -0.28 \\
\hline chr6:100-111 & suggestive & WL & 20.46 & 0.07 & 0.45 & -0.85 \\
\hline chr6:100-110 & suggestive & RJF & 26.04 & -0.56 & 0.41 & -1.21 \\
\hline chr6:100-109 & suggestive & RJF & 22.28 & -0.37 & 0.34 & -0.56 \\
\hline chr6:98-111 & suggestive & WL & 24.34 & 0.03 & 0.46 & -1.01 \\
\hline chr6:94-110 & suggestive & WL & 19.03 & 1.11 & 0.25 & -0.94 \\
\hline chr6:75-117 & suggestive & WL & 16.85 & 1.21 & 0.25 & 0.58 \\
\hline chr6:99-109 & suggestive & WL & 20.78 & 0.55 & 0.23 & -1.62 \\
\hline chr6:90-116 & significant & WL & 18.4 & 1.72 & 0.33 & -0.25 \\
\hline chr6:91-113 & significant & WL & 17.45 & 1.38 & 0.31 & -1.16 \\
\hline chr6:98-111 & suggestive & WL & 14.72 & 0.73 & 0.41 & 3.32 \\
\hline chr6:93-121 & suggestive & WL & 20.6 & 0.18 & 0.25 & 0.55 \\
\hline chr6:97-119 & suggestive & WL & 22.27 & 0.08 & 0.35 & 0.04 \\
\hline chr6:100-117 & suggestive & RJF & 23.44 & -0.84 & 0.44 & -1.59 \\
\hline chr6:92-118 & suggestive & WL & 17.74 & 0.66 & 0.24 & -1.42 \\
\hline chr6:88-117 & significant & RJF & 21.8 & -2.58 & 0.45 & 0.57 \\
\hline chr6:72-120 & significant & RJF & 23.53 & -4.56 & 0.76 & 1.06 \\
\hline chr6:92-132 & significant & RJF & 16.59 & -3.46 & 0.77 & 1.94 \\
\hline chr6:91-124 & significant & RJF & 17.01 & -2.06 & 0.45 & 1.16 \\
\hline chr6:77-127 & suggestive & RJF & 12.97 & -1.73 & 0.42 & -0.69 \\
\hline chr6:95-119 & suggestive & WL & 15.31 & 1.61 & 0.33 & -1.09 \\
\hline chr6:91-142 & suggestive & $W L$ & 14.07 & 1.04 & 0.23 & 0.2 \\
\hline chr6:95-141 & suggestive & RJF & 14.12 & -0.32 & 0.41 & 0.24 \\
\hline chr6:92-122 & significant & RJF & 17.96 & -1.9 & 0.44 & -1.76 \\
\hline chr6:95-130 & suggestive & RJF & 12.95 & -1.8 & 0.65 & -2.09 \\
\hline chr6:72-122 & suggestive & WL & 14.92 & 2.06 & 0.47 & 0.88 \\
\hline chr6:89-124 & significant & WL & 16.08 & 2.06 & 0.45 & 1 \\
\hline chr6:101-122 & significant & WL & 28.16 & 2.22 & 0.33 & -0.6 \\
\hline
\end{tabular}




\begin{tabular}{|c|c|c|c|c|c|}
\hline chr6:46-123 significant & RJF & 19.17 & -3.25 & 0.61 & 0.55 \\
\hline chr6:92-122 significant & WL & 17.82 & 1.94 & 0.4 & -0.67 \\
\hline chr6:76-118 suggestive & WL & 14.33 & 1.39 & 0.31 & -0.17 \\
\hline chr6:90-121 suggestive & WL & 17.93 & 0.88 & 0.89 & -1.43 \\
\hline chr6:105-119 suggestive & RJF & 21.29 & -0.22 & 0.2 & -0.18 \\
\hline chr6:102-119 suggestive & RJF & 24.41 & 0 & 0.32 & 0.34 \\
\hline chr6:102-120 significant & WL & 20.16 & 1.59 & 0.29 & 0.18 \\
\hline chr6:46-124 suggestive & RJF & 13.39 & -1.98 & 0.48 & 0.65 \\
\hline chr6:106-126 suggestive & RJF & 12.65 & -2.15 & 0.54 & 0.76 \\
\hline chr6:106-120 suggestive & RJF & 23.36 & -1.35 & 0.66 & -0.35 \\
\hline chr6:110-117 significant & WL & 50.23 & 3.16 & 0.29 & -0.11 \\
\hline chr6:107-120 suggestive & RJF & 23.62 & -0.72 & 0.4 & -1.73 \\
\hline chr6:109-121 suggestive & WL & 20.44 & 1.5 & 0.35 & -1.61 \\
\hline chr6:108-121 significant & RJF & 25.34 & -3.83 & 0.6 & 0.17 \\
\hline chr6:100-123 significant & RJF & 18.95 & -2.3 & 0.44 & -0.76 \\
\hline chr6:108-124 significant & RJF & 24.03 & -3.9 & 0.63 & -0.73 \\
\hline chr6:95-132 suggestive & WL & 15.16 & 0.94 & 0.24 & 0.94 \\
\hline chr6:108-122 significant & WL & 32.7 & 0.3 & 0.3 & -0.15 \\
\hline chr6:96-135 significant & RJF & 16.52 & -3.21 & 0.69 & 0.98 \\
\hline chr6:107-139 significant & RJF & 16.22 & -2.44 & 0.55 & 1.33 \\
\hline chr6:97-143 suggestive & RJF & 11.61 & -1.31 & 0.4 & 1.16 \\
\hline chr6:89-140 significant & RJF & 14.82 & -2.42 & 0.55 & 0.73 \\
\hline chr6:88-145 significant & RJF & 14.15 & -4.02 & 0.85 & -0.8 \\
\hline chr6:90-141 significant & RJF & 19.73 & -1.21 & 0.22 & -0.32 \\
\hline chr6:105-151 significant & RJF & 12.51 & -4 & 1.12 & 3.05 \\
\hline chr6:92-146 suggestive & RJF & 13.58 & -2.46 & 0.55 & -0.29 \\
\hline chr6:108-156 suggestive & RJF & 13.67 & -1.17 & 0.29 & 0.24 \\
\hline chr6:109-146 significant & RJF & 16.73 & -2.33 & 0.54 & 0.84 \\
\hline chr6:106-152 significant & RJF & 13.93 & -1.92 & 0.48 & 0.59 \\
\hline chr6:107-141 significant & RJF & 20.9 & -0.5 & 0.09 & -0.12 \\
\hline chr6:105-147 significant & RJF & 15.45 & -2.13 & 0.45 & -0.55 \\
\hline chr6:110-144 significant & WL & 16.06 & 0.63 & 0.21 & -0.82 \\
\hline chr6:116-136 suggestive & RJF & 27.09 & -0.77 & 0.12 & -0.67 \\
\hline chr6:115-144 suggestive & RJF & 13.76 & -0.69 & 0.17 & -0.65 \\
\hline chr6:112-147 suggestive & RJF & 18.74 & -1.48 & 0.31 & 0.46 \\
\hline chr6:107-149 significant & RJF & 14.21 & -0.82 & 0.61 & 3.13 \\
\hline chr6:114-150 significant & RJF & 15.67 & -2.2 & 0.47 & -1.38 \\
\hline chr6:108-152 significant & RJF & 18.39 & -2.64 & 0.57 & 0.91 \\
\hline chr6:116-147 significant & RJF & 17.36 & -2.24 & 0.45 & -0.11 \\
\hline chr6:119-142 suggestive & RJF & 25.61 & -0.03 & 0.04 & 0.01 \\
\hline chr6:119-149 significant & RJF & 18.17 & -3.13 & 0.75 & -1.78 \\
\hline chr6:118-147 significant & RJF & 18.34 & -2.07 & 0.41 & -0.02 \\
\hline chr6:121-149 significant & RJF & 22.67 & -4.78 & 0.89 & -0.42 \\
\hline
\end{tabular}




\begin{tabular}{|c|c|c|c|c|c|}
\hline chr6:120-148 significant & RJF & 20.97 & -4.61 & 0.89 & -1.05 \\
\hline chr6:120-145 suggestive & RJF & 18.36 & -1.41 & 0.34 & -1.63 \\
\hline chr6:119-151 suggestive & RJF & 13.37 & -0.68 & 0.4 & 1.87 \\
\hline chr6:117-147 significant & RJF & 14.23 & -1.55 & 0.45 & 1.26 \\
\hline chr6:113-158 suggestive & RJF & 11.65 & -3.43 & 0.95 & 1.22 \\
\hline chr6:119-151 significant & RJF & 13.82 & -1.4 & 0.36 & 0.43 \\
\hline chr6:117-149 suggestive & RJF & 16.53 & -2.99 & 0.61 & -0.34 \\
\hline chr6:118-148 suggestive & RJF & 16.68 & -1.85 & 0.57 & 2.15 \\
\hline chr6:119-152 suggestive & RJF & 18.3 & -1.21 & 0.44 & 2.16 \\
\hline chr6:125-146 significant & RJF & 32.96 & -0.34 & 0.04 & -0.32 \\
\hline chr6:123-154 significant & RJF & 23.9 & -2.34 & 0.38 & -0.46 \\
\hline chr6:123-160 significant & RJF & 24.21 & -7.69 & 1.37 & -3.35 \\
\hline chr6:123-162 significant & RJF & 24.45 & -8.48 & 1.48 & -2.89 \\
\hline chr6:123-159 significant & RJF & 27.13 & -8.75 & 1.49 & -2.38 \\
\hline chr6:118-165 suggestive & RJF & 14.14 & -5.3 & 1.35 & 1.79 \\
\hline chr6:125-153 significant & RJF & 28.29 & -0.07 & 0.01 & -0.07 \\
\hline chr6:120-163 significant & RJF & 17.65 & -4.14 & 0.85 & -0.08 \\
\hline chr6:119-169 suggestive & RJF & 13.74 & -8.56 & 2.11 & 1.44 \\
\hline chr6:123-161 significant & RJF & 20.84 & -4.64 & 0.91 & 1.29 \\
\hline chr6:120-166 significant & RJF & 16.89 & -10.74 & 2.39 & 2.62 \\
\hline chr6:123-161 significant & RJF & 25.58 & -7.18 & 1.26 & 2.52 \\
\hline chr6:122-164 significant & RJF & 19.49 & -5.27 & 1.04 & 0.88 \\
\hline chr6:121-165 significant & RJF & 18.94 & -9.4 & 1.89 & 1.2 \\
\hline chr6:122-163 significant & RJF & 21.79 & -8.62 & 1.59 & 1.35 \\
\hline chr6:124-161 significant & RJF & 26.36 & -10.69 & 1.84 & 3.89 \\
\hline chr6:119-170 suggestive & RJF & 14.02 & -4.06 & 0.98 & 0.62 \\
\hline chr6:121-172 significant & RJF & 14.71 & -7.07 & 1.58 & -0.98 \\
\hline chr6:123-162 significant & RJF & 23.95 & -6.1 & 1.13 & 2.48 \\
\hline chr6:124-162 significant & RJF & 28.86 & -4.47 & 0.68 & 0.48 \\
\hline chr6:123-167 significant & RJF & 24.24 & -4.68 & 0.76 & -0.76 \\
\hline chr6:123-166 significant & RJF & 24.22 & -4.92 & 0.85 & 0.66 \\
\hline chr6:122-171 significant & RJF & 18.84 & -8.55 & 1.71 & 0.68 \\
\hline chr6:123-167 significant & RJF & 22.91 & -10.37 & 1.84 & 1.1 \\
\hline chr6:121-174 significant & RJF & 15.03 & -7.23 & 1.63 & -0.1 \\
\hline chr6:122-169 significant & RJF & 18.29 & -3.89 & 0.75 & -0.7 \\
\hline chr6:121-171 significant & RJF & 18.3 & -4.82 & 0.95 & -0.23 \\
\hline chr6:122-171 significant & RJF & 21.26 & -5.41 & 0.99 & 0.18 \\
\hline chr6:120-180 suggestive & RJF & 12.75 & -6.51 & 1.62 & 0.27 \\
\hline chr6:123-172 significant & RJF & 23.62 & -9.59 & 1.72 & 2.13 \\
\hline chr6:122-174 significant & RJF & 19.92 & -7.92 & 1.53 & 0.87 \\
\hline chr6:122-178 significant & RJF & 22.18 & -6.63 & 1.14 & -1.41 \\
\hline chr6:123-180 significant & RJF & 21.87 & -7.97 & 1.51 & 1.89 \\
\hline chr6:122-184 significant & RJF & 17.67 & -3.29 & 0.69 & 0.38 \\
\hline
\end{tabular}




\begin{tabular}{|c|c|c|c|c|c|}
\hline chr6:123-181 significant & RJF & 19.88 & -6.79 & 1.38 & 1.84 \\
\hline chr6:121-183 significant & RJF & 18.53 & -5.27 & 1.43 & -1.81 \\
\hline chr6:122-182 significant & RJF & 20.07 & -5.41 & 1.39 & -1.84 \\
\hline chr6:121-183 significant & RJF & 13.59 & -4.66 & 1.38 & -0.64 \\
\hline chr6:123-184 significant & RJF & 17.19 & -7.56 & 2.31 & -3.84 \\
\hline chr6:122-182 significant & RJF & 14.73 & -6 & 1.91 & -2.4 \\
\hline chr6:124-180 significant & RJF & 17.51 & -8.12 & 2.38 & -3.6 \\
\hline chr6:124-182 significant & RJF & 15.86 & -8.6 & 2.63 & -3.47 \\
\hline chr6:123-183 significant & RJF & 13.66 & -3.67 & 1.27 & -1.8 \\
\hline chr6:125-187 significant & RJF & 14.48 & -2.95 & 0.72 & 0.33 \\
\hline chr6:125-182 significant & RJF & 17.52 & -12.25 & 3.78 & -6.81 \\
\hline chr6:125-184 significant & RJF & 16.59 & -3.39 & 0.99 & -1.25 \\
\hline chr6:125-182 significant & RJF & 17.41 & -12.44 & 3.65 & -5.42 \\
\hline chr6:126-182 significant & RJF & 18 & -3.27 & 0.85 & -0.68 \\
\hline chr6:124-180 significant & RJF & 15.76 & -6.07 & 1.87 & -2.45 \\
\hline chr6:124-183 significant & RJF & 14.8 & -8.09 & 2.86 & -5.14 \\
\hline chr6:124-183 significant & RJF & 14.79 & -7.64 & 2.16 & -1.28 \\
\hline chr6:125-183 significant & RJF & 15.12 & -4.72 & 1.38 & -1.19 \\
\hline chr6:123-183 suggestive & RJF & 13.36 & -6.47 & 2.16 & -2.42 \\
\hline chr6:125-183 suggestive & RJF & 14.41 & -4.94 & 1.41 & -0.67 \\
\hline chr6:127-182 significant & RJF & 18.35 & -7.41 & 1.99 & -2.31 \\
\hline chr6:125-182 significant & RJF & 16.37 & -11.25 & 3.56 & -5.83 \\
\hline chr6:124-185 suggestive & RJF & 14.03 & -6.67 & 2.4 & -4.11 \\
\hline chr6:123-183 significant & RJF & 13.7 & -6.28 & 2.53 & -5.23 \\
\hline chr6:126-184 significant & RJF & 15.14 & -7.15 & 2.04 & -1.54 \\
\hline chr6:126-187 significant & RJF & 16.81 & -4.1 & 1.37 & -2.8 \\
\hline chr6:128-182 significant & RJF & 16.99 & -4.85 & 1.4 & -1.75 \\
\hline chr6:129-180 significant & RJF & 18.3 & -5 & 1.5 & -2.77 \\
\hline chr6:127-182 significant & RJF & 16.48 & -7.49 & 1.9 & -0.67 \\
\hline chr6:135-180 significant & RJF & 19.64 & -7.78 & 2.12 & -3.58 \\
\hline chr6:131-184 significant & RJF & 17.29 & -9.09 & 2.73 & -4.45 \\
\hline chr6:135-180 significant & RJF & 18.91 & -6.17 & 1.55 & -1.23 \\
\hline chr6:132-182 significant & RJF & 16.8 & -11.36 & 3.57 & -6.18 \\
\hline chr6:135-183 significant & RJF & 18.8 & -11.12 & 3 & -3.94 \\
\hline chr6:132-184 significant & RJF & 15.8 & -7.03 & 2.07 & -2.25 \\
\hline chr6:132-185 significant & RJF & 14.88 & -6.83 & 2.02 & -1.7 \\
\hline chr6:138-180 significant & RJF & 18.91 & -8.43 & 2.28 & -3.29 \\
\hline chr6:138-184 significant & RJF & 17.19 & -10.79 & 3.24 & -5.23 \\
\hline chr6:132-184 suggestive & RJF & 14.19 & -5.83 & 2.03 & -3.21 \\
\hline chr6:132-186 suggestive & RJF & 14.01 & -3.35 & 1.07 & -1.11 \\
\hline chr6:134-188 significant & RJF & 13.78 & -1.18 & 0.4 & -0.56 \\
\hline chr6:131-183 significant & RJF & 15.82 & -4.86 & 1.25 & -0.32 \\
\hline chr6:137-185 significant & RJF & 15.72 & -5.72 & 1.81 & -2.65 \\
\hline
\end{tabular}




\begin{tabular}{|c|c|c|c|c|c|}
\hline chr6:142-186 significant & RJF & 22.69 & -12.18 & 3.33 & -8.01 \\
\hline chr6:138-184 significant & RJF & 15.8 & -8.72 & 2.82 & -4.59 \\
\hline chr6:141-187 significant & RJF & 18.88 & -5.37 & 1.57 & -2.91 \\
\hline chr6:137-186 suggestive & RJF & 13.56 & -8.16 & 2.94 & -4.64 \\
\hline chr6:147-182 significant & RJF & 22.4 & -15.82 & 4.01 & -10.47 \\
\hline chr6:143-187 significant & RJF & 21.67 & -14.95 & 4.24 & -10.07 \\
\hline chr6:143-186 significant & RJF & 23.74 & -14.47 & 3.87 & -9.8 \\
\hline chr6:146-185 significant & RJF & 24.83 & -16.6 & 4.33 & -11.16 \\
\hline chr6:144-182 significant & RJF & 19.26 & -7.38 & 1.83 & -1.65 \\
\hline chr6:145-182 suggestive & RJF & 18.23 & -7.18 & 2.22 & -6.25 \\
\hline chr6:137-184 suggestive & RJF & 13.88 & -5.06 & 1.83 & -3.23 \\
\hline chr6:139-185 suggestive & RJF & 14.41 & -5.7 & 1.83 & -2.23 \\
\hline chr6:136-185 suggestive & RJF & 13.2 & -4.04 & 1.45 & -2.23 \\
\hline chr6:148-183 significant & RJF & 21.23 & -12.35 & 3.09 & -7.32 \\
\hline chr6:145-187 significant & RJF & 21.76 & -17.93 & 5.07 & -12.09 \\
\hline chr6:144-185 significant & RJF & 24.24 & -15.67 & 4.06 & -9.96 \\
\hline chr6:126-194 suggestive & RJF & 10.58 & -1.16 & 0.51 & -0.96 \\
\hline chr6:145-182 significant & RJF & 18.61 & -4.22 & 1.19 & -1.88 \\
\hline chr6:146-186 significant & RJF & 23.7 & -17.22 & 4.52 & -10.87 \\
\hline chr6:146-187 significant & RJF & 21.71 & -18.15 & 5.27 & -13.26 \\
\hline chr6:146-187 significant & RJF & 20.56 & -16.25 & 4.9 & -12.05 \\
\hline chr6:146-186 significant & RJF & 22.62 & -16.9 & 4.65 & -11.25 \\
\hline chr6:145-186 significant & RJF & 24 & -16.02 & 4.19 & -10.25 \\
\hline chr6:148-187 significant & RJF & 22.29 & -17.38 & 5.06 & -13.47 \\
\hline chr6:148-187 significant & RJF & 21.93 & -16.36 & 4.68 & -11.66 \\
\hline chr6:147-187 significant & RJF & 22.78 & -13.31 & 3.75 & -9.63 \\
\hline chr6:148-186 significant & RJF & 24.14 & -12.82 & 3.48 & -9.44 \\
\hline chr6:145-184 significant & RJF & 15.49 & -4.83 & 1.77 & -3.82 \\
\hline chr6:148-186 significant & RJF & 23.98 & -17.73 & 4.78 & -12.55 \\
\hline chr6:147-188 significant & RJF & 19.6 & -8.36 & 2.47 & -5.15 \\
\hline chr6:150-189 significant & WL & 19.25 & 1.17 & 0.37 & 0.87 \\
\hline chr6:143-184 significant & RJF & 14.95 & -5.71 & 1.86 & -2.71 \\
\hline chr6:145-184 significant & RJF & 15.15 & -6.29 & 2.05 & -3.1 \\
\hline chr6:141-186 suggestive & RJF & 13.44 & -2.66 & 0.82 & -0.64 \\
\hline chr6:153-182 significant & RJF & 20.34 & -7.71 & 2.34 & -8.02 \\
\hline chr6:153-186 suggestive & WL & 18.91 & 2.24 & 0.94 & 4.14 \\
\hline chr6:148-186 suggestive & RJF & 14.36 & -4.48 & 1.41 & -1.52 \\
\hline chr6:145-185 significant & RJF & 15.28 & -2.12 & 0.67 & -0.95 \\
\hline chr6:152-189 significant & WL & 15.54 & 0.95 & 0.6 & 2.19 \\
\hline chr6:151-182 significant & RJF & 19.34 & -3.83 & 1.07 & -1.77 \\
\hline chr6:148-188 suggestive & RJF & 14.32 & -2.73 & 0.94 & -1.48 \\
\hline chr6:155-191 significant & WL & 18.72 & 1.76 & 0.52 & 0.97 \\
\hline chr6:153-186 significant & RJF & 17.76 & -6.46 & 1.92 & -3.29 \\
\hline
\end{tabular}




\begin{tabular}{|c|c|c|c|c|c|}
\hline chr6:162-197 significant & WL & 20.38 & 3.43 & 0.68 & -0.84 \\
\hline chr6:163-199 significant & WL & 16.7 & 1.42 & 0.44 & 0.81 \\
\hline chr6:167-189 significant & WL & 23.15 & 3.18 & 0.84 & 3.2 \\
\hline chr6:159-188 significant & WL & 17.63 & 1 & 0.45 & 1.61 \\
\hline chr6:162-195 suggestive & WL & 18.21 & 2.33 & 0.83 & 3.08 \\
\hline chr6:164-195 significant & WL & 23.58 & 2.33 & 0.46 & 0.35 \\
\hline chr6:165-189 significant & WL & 19.01 & 2.9 & 1.03 & 1.71 \\
\hline chr6:165-192 significant & WL & 22.76 & 2.15 & 0.54 & 0.97 \\
\hline chr6:165-199 significant & WL & 22.39 & 3.83 & 0.94 & 1.94 \\
\hline chr6:164-195 significant & WL & 20.51 & 2.01 & 0.95 & 4.71 \\
\hline chr6:164-202 significant & WL & 21.48 & 1.44 & 0.61 & 3.01 \\
\hline chr6:163-197 significant & WL & 17.65 & 1.83 & 0.54 & 0.87 \\
\hline chr6:151-186 suggestive & RJF & 13.33 & -3.14 & 1.17 & -1.94 \\
\hline chr6:156-203 suggestive & WL & 13.76 & 2.83 & 1.13 & 2.35 \\
\hline chr6:153-198 suggestive & WL & 14.9 & 1.56 & 0.53 & 0.85 \\
\hline chr6:154-193 suggestive & WL & 13.95 & 1.57 & 0.69 & 1.7 \\
\hline chr6:158-191 suggestive & WL & 13.78 & 0.91 & 0.52 & 1.7 \\
\hline chr6:153-197 suggestive & WL & 13.7 & 1.21 & 0.55 & 1.45 \\
\hline chr6:156-199 suggestive & WL & 15 & 0.86 & 0.54 & 1.92 \\
\hline chr6:163-182 suggestive & WL & 23.7 & 2.26 & 0.56 & 1.72 \\
\hline chr6:163-186 suggestive & WL & 18.84 & 0.04 & 0.01 & -0.04 \\
\hline chr6:163-194 suggestive & WL & 22.04 & 2.75 & 0.69 & 1.8 \\
\hline chr6:150-188 significant & RJF & 18.24 & -11.37 & 3.87 & -9.64 \\
\hline chr6:167-188 significant & WL & 32.23 & 3.65 & 0.59 & 0.02 \\
\hline chr6:159-199 suggestive & WL & 15.43 & 5.61 & 1.58 & 1.15 \\
\hline chr6:157-201 suggestive & WL & 14.73 & 2.11 & 0.67 & 0.35 \\
\hline chr6:148-189 significant & RJF & 18.53 & -6.51 & 2.01 & -4.17 \\
\hline chr6:151-189 suggestive & WL & 14.06 & 1.15 & 0.47 & 1.02 \\
\hline chr6:156-196 suggestive & WL & 14.47 & 1.69 & 0.62 & 1.15 \\
\hline chr6:151-193 significant & WL & 16.54 & 2.44 & 0.72 & 1 \\
\hline chr6:152-197 suggestive & WL & 14.63 & 2.91 & 0.86 & 0.64 \\
\hline chr6:152-194 significant & WL & 15.99 & 1.53 & 0.56 & 1.25 \\
\hline chr6:166-195 suggestive & WL & 22.59 & 2.85 & 0.79 & 2.5 \\
\hline chr6:162-191 significant & WL & 20.4 & 2.37 & 0.6 & 1.07 \\
\hline chr6:167-202 significant & WL & 20.64 & 3.29 & 0.92 & 2.31 \\
\hline chr6:166-187 significant & WL & 22.3 & 2.37 & 0.8 & 2.58 \\
\hline chr6:165-190 significant & WL & 21.43 & 2.21 & 0.49 & 0.56 \\
\hline chr6:154-195 suggestive & WL & 14.25 & 2.19 & 0.69 & 1.02 \\
\hline chr6:153-194 suggestive & RJF & 13 & -12.18 & 5.12 & -10.64 \\
\hline chr6:155-188 significant & RJF & 16.09 & -9.45 & 3.05 & -6.7 \\
\hline chr6:165-186 significant & WL & 23.03 & 2.46 & 0.54 & 0.78 \\
\hline chr6:157-189 significant & WL & 16.61 & 2.76 & 0.67 & 0.19 \\
\hline chr6:153-190 suggestive & RJF & 14.08 & -6.89 & 2.4 & -4.88 \\
\hline
\end{tabular}




\begin{tabular}{|c|c|c|c|c|c|}
\hline chr6:154-191 suggestive & RJF & 13.75 & -9.81 & 3.3 & -5.71 \\
\hline chr6:154-189 significant & RJF & 14.22 & -7.83 & 2.74 & -5.72 \\
\hline chr6:161-188 suggestive & WL & 19.25 & 1.91 & 0.54 & 1.26 \\
\hline chr6:163-203 suggestive & WL & 19 & 1.68 & 0.5 & 1.21 \\
\hline chr6:159-190 significant & WL & 16.97 & 1.7 & 0.4 & 0.04 \\
\hline chr6:157-191 significant & WL & 14.6 & 2.35 & 0.58 & -0.09 \\
\hline chr6:176-190 suggestive & WL & 17.46 & 3.47 & 0.78 & -0.16 \\
\hline chr6:181-186 significant & WL & 48.96 & 4.34 & 0.46 & -0.31 \\
\hline chr6:177-192 suggestive & WL & 18.74 & 1.11 & 0.53 & 2.52 \\
\hline chr6:167-191 significant & WL & 19.3 & 3.61 & 0.77 & -0.15 \\
\hline chr6:173-205 suggestive & WL & 14.81 & 1.78 & 0.51 & 0.59 \\
\hline chr6:164-196 suggestive & WL & 14.89 & 1.51 & 0.42 & 0.34 \\
\hline chr6:171-192 significant & WL & 16.62 & 2.23 & 0.52 & -0.09 \\
\hline chr6:175-188 significant & WL & 37.08 & 4.29 & 0.59 & -0.05 \\
\hline chr6:168-202 significant & WL & 15.39 & 2.1 & 0.66 & 1.23 \\
\hline chr6:170-194 significant & WL & 19.93 & 1.91 & 0.45 & 0.55 \\
\hline chr6:176-191 significant & WL & 24.04 & 3.24 & 0.62 & 0.14 \\
\hline chr6:180-197 significant & WL & 17.08 & 1.55 & 0.46 & 0.95 \\
\hline chr6:163-200 suggestive & WL & 14.27 & 2.02 & 0.6 & 0.72 \\
\hline chr6:166-195 significant & WL & 17.6 & 2.31 & 0.72 & 1.33 \\
\hline chr6:159-222 significant & WL & 15.75 & 1.29 & 0.46 & 1 \\
\hline chr6:160-196 significant & WL & 21.38 & 3.05 & 0.7 & 0.61 \\
\hline chr6:168-214 significant & WL & 15.78 & 2.23 & 0.68 & 1.45 \\
\hline chr6:177-196 significant & WL & 27.73 & 2.61 & 0.54 & 1.2 \\
\hline chr6:166-199 significant & WL & 16.53 & 1.58 & 0.38 & 0.08 \\
\hline chr6:169-217 significant & WL & 14.89 & 1.57 & 0.43 & 0.33 \\
\hline chr6:166-201 significant & WL & 25.77 & 3.21 & 0.59 & 0.25 \\
\hline chr6:160-205 suggestive & WL & 13.48 & 2.95 & 0.79 & 0 \\
\hline chr6:164-228 suggestive & WL & 14.73 & 2.55 & 0.69 & 0.12 \\
\hline chr6:165-200 significant & WL & 15.56 & 3.36 & 0.77 & 0.35 \\
\hline chr6:175-204 suggestive & WL & 13.77 & 2.64 & 0.73 & 1.27 \\
\hline chr6:159-204 suggestive & WL & 14.61 & 2.14 & 0.53 & 0.54 \\
\hline chr6:172-235 suggestive & WL & 13.98 & 2.09 & 0.47 & -0.35 \\
\hline chr6:158-215 suggestive & WL & 15.51 & 2.09 & 0.48 & 0.2 \\
\hline chr6:171-202 significant & WL & 18.91 & 1.8 & 0.47 & 1.42 \\
\hline chr6:170-204 significant & WL & 17.83 & 1.83 & 0.46 & 1.18 \\
\hline chr6:171-202 suggestive & WL & 19.47 & 3.01 & 0.69 & 0.89 \\
\hline chr6:180-204 significant & WL & 19.35 & 2.64 & 0.57 & 0.93 \\
\hline chr6:178-205 significant & WL & 15.7 & 1.39 & 0.38 & 0.89 \\
\hline chr6:181-197 significant & WL & 15.53 & 2.24 & 0.48 & -0.78 \\
\hline chr6:169-200 significant & WL & 14.69 & 2.71 & 0.6 & -0.56 \\
\hline chr6:169-204 significant & WL & 17 & 1.31 & 0.31 & 0.56 \\
\hline chr6:182-197 suggestive & WL & 23.7 & 2.14 & 0.43 & 1.31 \\
\hline
\end{tabular}




\begin{tabular}{|c|c|c|c|c|c|}
\hline chr6:183-198 suggestive & WL & 23.64 & 1.19 & 0.46 & 2.33 \\
\hline chr6:184-197 significant & WL & 26.59 & 3.21 & 0.6 & 0.71 \\
\hline chr6:184-199 significant & WL & 18.6 & 2.11 & 0.46 & 0.57 \\
\hline chr6:176-201 significant & WL & 17.21 & 3.63 & 0.79 & 0.47 \\
\hline chr6:184-199 significant & WL & 24.05 & 2.63 & 0.48 & 0.59 \\
\hline chr6:185-198 significant & WL & 22.87 & 4.18 & 0.79 & -0.2 \\
\hline chr6:183-202 significant & WL & 18.57 & 1.74 & 0.39 & 0.64 \\
\hline chr6:185-201 significant & WL & 18.51 & 3.08 & 0.67 & -1.3 \\
\hline chr6:185-198 significant & WL & 26.64 & 3.87 & 0.62 & -0.03 \\
\hline chr6:185-199 significant & WL & 20.67 & 2.2 & 0.46 & 0.81 \\
\hline chr6:186-203 significant & WL & 19.36 & 3.53 & 0.75 & -1.46 \\
\hline chr6:177-201 suggestive & WL & 17.23 & 2.2 & 0.53 & -0.33 \\
\hline chr6:168-200 significant & WL & 18.03 & 2.26 & 0.47 & 0.21 \\
\hline chr6:185-199 significant & WL & 17.67 & 3 & 0.78 & 0.44 \\
\hline chr6:173-202 suggestive & WL & 14.17 & 2.29 & 0.51 & -0.81 \\
\hline chr6:182-207 suggestive & WL & 15.26 & 1.54 & 0.39 & 0.57 \\
\hline chr6:184-199 significant & WL & 15.17 & 1.81 & 0.39 & -0.42 \\
\hline chr6:162-202 significant & WL & 15.63 & 2.26 & 0.48 & -0.83 \\
\hline chr6:180-204 suggestive & WL & 14.45 & 1.86 & 0.48 & 0.83 \\
\hline chr6:174-201 suggestive & WL & 15.45 & 1.23 & 0.32 & 0.62 \\
\hline chr6:183-202 suggestive & WL & 14.81 & 1.27 & 0.35 & 0.69 \\
\hline chr6:181-200 significant & WL & 16.61 & 2.23 & 0.5 & 0.4 \\
\hline chr6:174-198 significant & WL & 15.05 & 2.66 & 0.58 & -0.62 \\
\hline chr6:184-200 suggestive & WL & 23.59 & 1.81 & 0.4 & 1.16 \\
\hline chr6:184-201 significant & WL & 22.05 & 2.13 & 0.45 & 1.01 \\
\hline chr6:172-205 suggestive & WL & 16.25 & 3.23 & 0.83 & -0.75 \\
\hline chr6:183-202 significant & WL & 20.39 & 2.33 & 0.49 & 0.83 \\
\hline chr6:184-204 significant & WL & 14.98 & 2.19 & 0.49 & -0.27 \\
\hline chr6:184-236 suggestive & WL & 14.22 & 2.52 & 0.63 & 0.6 \\
\hline chr6:183-227 suggestive & WL & 14.7 & 2.37 & 0.59 & 0.66 \\
\hline chr6:184-205 suggestive & WL & 14.76 & 1.48 & 0.43 & 1.09 \\
\hline chr6:185-201 suggestive & WL & 16.18 & 0.38 & 0.31 & 1.86 \\
\hline chr6:186-204 significant & WL & 20.9 & 3.71 & 0.71 & 0.5 \\
\hline chr6:184-204 significant & WL & 17.97 & 2.35 & 0.54 & 1.11 \\
\hline chr6:184-205 significant & WL & 17.75 & 1.6 & 0.38 & 0.77 \\
\hline chr6:183-211 significant & WL & 17.06 & 2.34 & 0.49 & 0.14 \\
\hline chr6:184-203 significant & RJF & 22.75 & -2.39 & 0.4 & 0.73 \\
\hline chr6:186-200 significant & RJF & 26.39 & -4.7 & 0.7 & 2.11 \\
\hline chr6:187-199 significant & WL & 43.53 & 3.31 & 0.37 & 0.36 \\
\hline chr6:185-204 significant & WL & 19.65 & 1.75 & 0.34 & 0.01 \\
\hline chr6:187-202 significant & WL & 19 & 2.15 & 0.61 & 0.32 \\
\hline chr6:185-203 suggestive & WL & 17.99 & 2.42 & 0.55 & -0.97 \\
\hline chr6:185-205 significant & WL & 20.63 & 1.57 & 0.33 & 0.54 \\
\hline
\end{tabular}




\begin{tabular}{|c|c|c|c|c|c|}
\hline chr6:186-205 significant & WL & 16.16 & 1.76 & 0.49 & 1.35 \\
\hline chr6:185-221 significant & WL & 18.57 & 2.42 & 0.49 & -0.02 \\
\hline chr6:181-225 suggestive & WL & 18.37 & 3.62 & 0.83 & 1.31 \\
\hline chr6:181-204 suggestive & WL & 17.6 & 2.02 & 0.61 & 0.39 \\
\hline chr6:169-223 suggestive & WL & 15.42 & 1.61 & 0.43 & 0.91 \\
\hline chr6:184-231 significant & WL & 16.96 & 0.83 & 0.17 & -0.09 \\
\hline chr6:185-211 significant & WL & 17.9 & 2.39 & 0.51 & 0.3 \\
\hline chr6:183-204 significant & WL & 26.08 & 3.01 & 0.51 & 0.64 \\
\hline chr6:187-204 significant & WL & 27.64 & 4.48 & 0.73 & 0.91 \\
\hline chr6:187-203 significant & WL & 28.92 & 3.26 & 0.58 & 0.27 \\
\hline chr6:185-227 significant & WL & 19.5 & 2.64 & 0.6 & 1.38 \\
\hline chr6:185-205 significant & WL & 17.16 & 7.72 & 1.63 & -1.97 \\
\hline chr6:181-204 significant & WL & 23.05 & 3.49 & 0.62 & 0.22 \\
\hline chr6:184-220 suggestive & WL & 15.41 & 1.01 & 0.29 & 0.73 \\
\hline chr6:174-234 suggestive & WL & 15.09 & 1.32 & 0.32 & 0.4 \\
\hline chr6:162-239 suggestive & WL & 15.31 & 2.58 & 0.6 & 0.31 \\
\hline chr6:185-243 suggestive & WL & 14.34 & 1.45 & 0.34 & 0.06 \\
\hline chr6:167-224 suggestive & WL & 14.7 & 1.75 & 0.41 & 0.1 \\
\hline chr6:166-220 suggestive & WL & 14.92 & 2.05 & 0.54 & 1 \\
\hline chr6:186-210 significant & WL & 23.24 & 2.85 & 0.66 & 1.87 \\
\hline chr6:185-226 significant & WL & 20.39 & 2.21 & 0.46 & 0.77 \\
\hline chr6:176-214 suggestive & WL & 20.03 & 2.24 & 0.54 & 1.01 \\
\hline chr6:184-205 significant & WL & 20.82 & 3.13 & 0.57 & -0.09 \\
\hline chr6:182-205 significant & RJF & 19.15 & -2.22 & 0.41 & 0.77 \\
\hline chr6:185-209 significant & WL & 18.57 & 3.71 & 0.97 & 0.31 \\
\hline chr6:185-214 significant & WL & 18.3 & 4.18 & 0.8 & -0.6 \\
\hline chr6:186-228 significant & WL & 20.72 & 2.84 & 0.53 & 0.22 \\
\hline chr6:184-233 significant & WL & 20.11 & 2.78 & 0.67 & 1.95 \\
\hline chr6:171-212 suggestive & WL & 14.19 & 2.87 & 0.65 & -0.79 \\
\hline chr6:173-235 suggestive & WL & 15.26 & 1.56 & 0.43 & 0.96 \\
\hline chr6:187-232 significant & WL & 16.82 & 2.31 & 0.46 & -0.73 \\
\hline chr6:184-232 suggestive & WL & 15.14 & 1.85 & 0.41 & -0.7 \\
\hline chr6:188-205 significant & WL & 38.06 & 4.4 & 0.56 & 0.71 \\
\hline chr6:182-212 significant & WL & 20.38 & 3.35 & 0.7 & -0.07 \\
\hline chr6:188-227 significant & WL & 25.46 & 3.11 & 0.56 & 1.15 \\
\hline chr6:180-214 suggestive & WL & 19.14 & 2.62 & 0.58 & 0.6 \\
\hline chr6:185-228 significant & WL & 24.28 & 3.72 & 0.68 & 0.96 \\
\hline chr6:185-206 significant & WL & 26.33 & 3 & 1 & 3.88 \\
\hline chr6:185-229 suggestive & WL & 14.89 & 1.88 & 0.41 & -0.53 \\
\hline chr6:166-230 significant & WL & 16.07 & 2.14 & 0.5 & 0.46 \\
\hline chr6:183-232 significant & WL & 16.17 & 6.2 & 1.31 & -1.08 \\
\hline chr6:186-229 significant & WL & 21.49 & 5.01 & 0.92 & 0.59 \\
\hline chr6:182-214 significant & WL & 19.4 & 1.68 & 0.42 & 1.24 \\
\hline
\end{tabular}




\begin{tabular}{|c|c|c|c|c|c|}
\hline chr6:184-229 suggestive & WL & 13.14 & 2.54 & 0.6 & -0.26 \\
\hline chr6:185-235 significant & WL & 16.04 & 2.82 & 0.62 & 0.28 \\
\hline chr6:185-231 significant & WL & 17.38 & 1.65 & 0.33 & -0.57 \\
\hline chr6:184-236 significant & WL & 15.17 & 2.79 & 0.65 & -1.95 \\
\hline chr6:183-234 significant & WL & 18.62 & 3.11 & 0.6 & -1.27 \\
\hline chr6:182-234 significant & WL & 16.3 & 1.26 & 0.3 & 0.44 \\
\hline chr6:184-233 significant & WL & 16.29 & 3.07 & 0.65 & -0.33 \\
\hline chr6:185-224 significant & WL & 22.31 & 3.39 & 0.64 & 0.94 \\
\hline chr6:188-225 significant & WL & 29.15 & 2.19 & 0.31 & -0.54 \\
\hline chr6:184-236 significant & WL & 16.02 & 1.92 & 0.4 & -0.84 \\
\hline chr6:186-232 suggestive & WL & 13.75 & 3.12 & 0.71 & -0.77 \\
\hline chr6:182-235 significant & WL & 14.9 & 2.16 & 0.49 & 0.08 \\
\hline chr6:186-214 significant & WL & 15.63 & 0.84 & 0.23 & 0.51 \\
\hline chr6:183-227 significant & WL & 19.59 & 1.76 & 0.34 & -0.96 \\
\hline chr6:184-231 significant & WL & 16.82 & 1.79 & 0.38 & -0.98 \\
\hline chr6:183-214 significant & WL & 15.96 & 1.72 & 0.37 & -0.25 \\
\hline chr6:190-223 significant & WL & 35.55 & 1.63 & 0.21 & 0.21 \\
\hline chr6:181-230 significant & WL & 15.81 & 1.75 & 0.42 & 0.58 \\
\hline chr6:192-214 significant & WL & 30.17 & 3.78 & 0.53 & -0.64 \\
\hline chr6:166-241 suggestive & WL & 14.22 & 1.78 & 0.4 & -0.42 \\
\hline chr6:190-219 significant & WL & 24.24 & 3.2 & 0.66 & -0.38 \\
\hline chr6:188-232 significant & WL & 18.23 & 3.14 & 0.61 & -0.63 \\
\hline chr6:184-231 significant & WL & 18.7 & 1.52 & 0.32 & 0.34 \\
\hline chr6:192-225 significant & RJF & 16.91 & -2.05 & 0.41 & 0.83 \\
\hline chr6:176-214 suggestive & WL & 16.41 & 5.38 & 1.17 & -1.32 \\
\hline chr6:197-210 significant & WL & 22.67 & 0.1 & 0.02 & -0.09 \\
\hline chr6:191-217 significant & WL & 17.35 & 2.61 & 0.6 & -1.1 \\
\hline chr6:193-232 suggestive & WL & 19.08 & 0.5 & 1.19 & 1.31 \\
\hline chr6:194-240 significant & RJF & 16.24 & -2.68 & 0.56 & 0.68 \\
\hline chr6:194-214 suggestive & WL & 18.94 & 3.59 & 1.24 & 2.52 \\
\hline chr6:194-213 significant & RJF & 21.89 & -0.76 & 0.94 & 1.82 \\
\hline chr6:195-214 significant & RJF & 13.77 & -1.32 & 0.31 & 0.85 \\
\hline chr6:197-211 suggestive & RJF & 17.39 & -2.52 & 1.46 & 3.23 \\
\hline chr6:203-209 significant & RJF & 46.65 & -4.86 & 0.48 & 0.76 \\
\hline chr6:189-226 significant & WL & 15.46 & 1.71 & 0.37 & -0.29 \\
\hline chr6:197-222 significant & WL & 25.9 & 3.21 & 0.62 & -0.71 \\
\hline chr6:192-229 significant & WL & 20.74 & 2.55 & 0.47 & -0.14 \\
\hline chr6:196-225 significant & RJF & 17.99 & -2.8 & 0.55 & 0.04 \\
\hline chr6:195-242 suggestive & RJF & 11.56 & -1.84 & 0.47 & 0.64 \\
\hline chr6:188-232 significant & WL & 20.04 & 2.35 & 0.43 & 0.43 \\
\hline chr6:190-235 significant & WL & 16.17 & 2.01 & 0.41 & -0.11 \\
\hline chr6:195-232 significant & RJF & 14.6 & -1.98 & 0.44 & -0.09 \\
\hline chr6:185-235 significant & WL & 15.77 & 4.16 & 0.9 & -1.99 \\
\hline
\end{tabular}




\begin{tabular}{|c|c|c|c|c|c|}
\hline chr6:181-224 suggestive & WL & 14.81 & 1.59 & 0.36 & 0.49 \\
\hline chr6:193-227 significant & WL & 19.98 & 1.67 & 0.31 & -0.15 \\
\hline chr6:186-233 suggestive & WL & 13.81 & 2.29 & 0.51 & -0.56 \\
\hline chr6:146-233 suggestive & RJF & 9.92 & -1.56 & 0.46 & 1.09 \\
\hline chr6:183-234 significant & WL & 16.16 & 1.7 & 0.4 & -1.31 \\
\hline chr6:209-226 significant & WL & 28.87 & 1.53 & 0.22 & -0.46 \\
\hline chr6:208-228 significant & WL & 20.98 & 2.3 & 0.43 & -1.27 \\
\hline chr6:211-230 significant & WL & 20.47 & 2.66 & 0.49 & 0.41 \\
\hline chr6:209-228 significant & WL & 18.74 & 3.09 & 0.59 & 0.51 \\
\hline chr6:191-237 suggestive & WL & 14.69 & 2 & 0.46 & -1.12 \\
\hline chr6:208-227 significant & WL & 15.63 & 1.87 & 0.43 & 1.25 \\
\hline chr6:209-235 suggestive & WL & 14.66 & 2.95 & 0.65 & 0.17 \\
\hline chr6:208-236 significant & WL & 15.5 & 3.03 & 0.64 & -0.27 \\
\hline chr6:211-236 significant & WL & 17.42 & 2.28 & 0.5 & -1.64 \\
\hline chr6:189-233 significant & WL & 25.91 & 4.06 & 0.63 & -0.72 \\
\hline chr6:188-236 significant & WL & 20.26 & 3.29 & 0.61 & -1.32 \\
\hline chr6:193-250 significant & RJF & 12.59 & -1.82 & 0.48 & 1.34 \\
\hline chr6:184-246 suggestive & WL & 15.34 & 2.38 & 0.54 & -1.26 \\
\hline chr6:182-277 suggestive & WL & 13.2 & 2.61 & 0.73 & -0.43 \\
\hline chr6:215-248 significant & WL & 16.4 & 3.05 & 0.96 & -2.44 \\
\hline chr6:222-247 significant & WL & 24.92 & 2.74 & 0.57 & -1.19 \\
\hline chr6:221-256 significant & WL & 19.96 & 2.53 & 0.56 & -0.56 \\
\hline chr6:220-248 significant & WL & 22.05 & 3.47 & 0.77 & -1.35 \\
\hline chr6:215-281 significant & WL & 15.65 & 2.83 & 0.73 & -0.7 \\
\hline chr6:210-272 significant & WL & 13.03 & 2.12 & 0.55 & 0.08 \\
\hline chr6:214-252 significant & WL & 13.94 & 2.08 & 0.6 & -0.72 \\
\hline chr6:214-254 suggestive & WL & 13.57 & 1.9 & 0.58 & -0.84 \\
\hline chr6:210-298 significant & WL & 13.68 & 2.31 & 0.54 & -0.12 \\
\hline chr6:217-251 suggestive & WL & 12.62 & 1.49 & 0.4 & -0.18 \\
\hline chr6:218-251 significant & WL & 15.05 & 1.75 & 0.83 & -3.19 \\
\hline chr6:220-250 significant & WL & 14.08 & 1.6 & 0.7 & -2.25 \\
\hline chr6:212-261 suggestive & WL & 10.88 & 1.66 & 0.55 & -0.65 \\
\hline chr6:223-249 suggestive & WL & 19.09 & 0.64 & 0.37 & 2.73 \\
\hline chr6:221-266 significant & WL & 16.5 & 2.1 & 0.54 & -0.68 \\
\hline chr6:217-251 significant & WL & 14.76 & 1.76 & 0.47 & -0.46 \\
\hline chr6:218-251 significant & WL & 13.12 & 2.93 & 0.95 & -1.59 \\
\hline chr6:225-250 significant & WL & 19.93 & 3.08 & 0.6 & 0.43 \\
\hline chr6:226-247 significant & WL & 22.63 & 3.04 & 0.76 & -2.18 \\
\hline chr6:221-257 significant & WL & 14.04 & 2.16 & 0.59 & -0.5 \\
\hline chr6:220-259 significant & WL & 13.42 & 1.67 & 0.45 & -0.22 \\
\hline chr6:221-250 suggestive & WL & 14.83 & 1.46 & 0.46 & -0.94 \\
\hline chr6:221-250 significant & WL & 14.97 & 3.54 & 1.22 & -3.12 \\
\hline chr6:222-251 significant & WL & 15.34 & 1.85 & 0.48 & -0.49 \\
\hline
\end{tabular}




\begin{tabular}{|c|c|c|c|c|c|}
\hline chr6:221-253 suggestive & WL & 12.54 & 1.59 & 0.39 & 0.53 \\
\hline chr6:223-250 significant & WL & 13.92 & 1.6 & 0.42 & -0.2 \\
\hline chr6:224-256 significant & WL & 16.26 & 2.62 & 0.71 & -1.06 \\
\hline chr6:228-250 significant & WL & 21.42 & 2.34 & 0.52 & -0.73 \\
\hline chr6:231-250 significant & WL & 34.06 & 4.47 & 0.66 & -0.7 \\
\hline chr6:222-283 suggestive & WL & 14.27 & 1.35 & 0.4 & -0.58 \\
\hline chr6:228-253 significant & RJF & 18.24 & -1.78 & 0.41 & 0.34 \\
\hline chr6:227-252 significant & WL & 16.72 & 2.07 & 0.43 & 0.71 \\
\hline chr6:227-255 significant & WL & 17.75 & 2.41 & 0.52 & 0.24 \\
\hline chr6:230-252 significant & RJF & 19.29 & -1.94 & 0.38 & -0.32 \\
\hline chr6:230-249 significant & WL & 17.98 & 2.1 & 0.53 & -0.86 \\
\hline chr6:236-251 significant & RJF & 24.48 & -4.62 & 0.77 & -1.35 \\
\hline chr6:230-251 significant & WL & 18.71 & 2.02 & 0.41 & 0.35 \\
\hline chr6:228-256 significant & WL & 12.7 & 2.4 & 0.59 & 0.69 \\
\hline chr6:229-251 significant & WL & 17.32 & 3.32 & 0.79 & -0.6 \\
\hline chr6:230-251 suggestive & RJF & 15.25 & -2.17 & 0.67 & -4.07 \\
\hline chr6:225-250 significant & WL & 13.37 & 1.67 & 0.68 & -1.94 \\
\hline chr6:224-253 suggestive & WL & 12.59 & 2.14 & 0.84 & -2.05 \\
\hline chr6:224-251 significant & WL & 14.87 & 1.27 & 0.55 & -1.88 \\
\hline chr6:227-254 significant & WL & 14.22 & 2.52 & 0.85 & -1.9 \\
\hline chr6:234-254 suggestive & RJF & 13.34 & -2.01 & 0.38 & -1.47 \\
\hline chr6:235-252 significant & RJF & 23.48 & -2.33 & 0.45 & 0.27 \\
\hline chr6:227-266 significant & RJF & 16.84 & -2.3 & 0.59 & 0.74 \\
\hline chr6:236-272 significant & RJF & 17.86 & -3.42 & 0.69 & -1.09 \\
\hline chr6:227-256 significant & WL & 18.44 & 2.7 & 0.67 & -1.08 \\
\hline chr6:233-280 significant & RJF & 15.95 & -2.86 & 0.6 & -1.04 \\
\hline chr6:237-269 significant & RJF & 24.95 & -2.39 & 0.38 & -1.74 \\
\hline chr6:233-283 significant & RJF & 16.94 & -3.12 & 0.63 & -1.99 \\
\hline chr6:235-270 significant & RJF & 14.7 & -1.62 & 0.55 & 1.29 \\
\hline chr6:226-297 significant & WL & 10.73 & 2.57 & 0.7 & 0.34 \\
\hline chr6:221-281 significant & WL & 16.7 & 2.45 & 0.49 & 0.11 \\
\hline chr6:220-309 suggestive & WL & 10.16 & 1.91 & 0.52 & -0.12 \\
\hline chr6:223-283 significant & WL & 12.72 & 2.87 & 0.69 & 0.79 \\
\hline chr6:209-281 significant & WL & 12.93 & 2.26 & 0.53 & -0.09 \\
\hline chr6:234-288 suggestive & RJF & 19.55 & -4.26 & 0.94 & -3.7 \\
\hline chr6:229-286 significant & WL & 16.91 & 2.32 & 0.48 & -0.18 \\
\hline chr6:236-283 significant & RJF & 15.98 & -2.99 & 0.64 & 0.24 \\
\hline chr6:224-293 significant & WL & 11.82 & 4.58 & 1.14 & -0.1 \\
\hline chr6:233-278 significant & WL & 23.26 & 2.81 & 0.48 & -0.25 \\
\hline chr6:228-294 significant & WL & 19.11 & 1.67 & 0.35 & -0.83 \\
\hline chr6:235-286 significant & RJF & 15.5 & -2.52 & 0.57 & 0.87 \\
\hline chr6:235-291 significant & RJF & 16.99 & -2.26 & 0.47 & 0.39 \\
\hline chr6:237-283 significant & RJF & 14.45 & -2.18 & 0.45 & -0.54 \\
\hline
\end{tabular}




\begin{tabular}{|c|c|c|c|c|c|}
\hline chr6:236-283 significant & RJF & 18.5 & -3.48 & 0.67 & -0.01 \\
\hline chr6:238-276 significant & RJF & 20.31 & -2.6 & 0.47 & -0.32 \\
\hline chr6:220-317 suggestive & WL & 11.51 & 1.73 & 0.44 & 0.32 \\
\hline chr6:245-281 significant & WL & 24.15 & 2.8 & 0.59 & -2.66 \\
\hline chr6:240-285 significant & RJF & 18.59 & -1.81 & 0.35 & -0.12 \\
\hline chr6:243-275 significant & RJF & 24.46 & -1.85 & 0.3 & 0.08 \\
\hline chr6:244-273 significant & RJF & 21.41 & -1.44 & 0.26 & -0.53 \\
\hline chr6:237-278 suggestive & RJF & 15.48 & -2.04 & 0.44 & -0.13 \\
\hline chr6:245-289 significant & RJF & 19.69 & -2.48 & 0.71 & -3.04 \\
\hline chr6:247-277 significant & RJF & 20.78 & -1.53 & 0.28 & 0.33 \\
\hline chr6:246-283 significant & RJF & 17.61 & -2.77 & 0.54 & -0.02 \\
\hline chr6:247-285 significant & RJF & 20.63 & -2.38 & 0.43 & -0.23 \\
\hline chr6:247-284 significant & RJF & 19.41 & -0.33 & 0.37 & 0.24 \\
\hline chr6:242-281 significant & RJF & 16.97 & -2.29 & 0.46 & -0.66 \\
\hline chr6:250-293 significant & RJF & 15.83 & -2.35 & 0.5 & -0.1 \\
\hline chr6:246-286 significant & RJF & 19.43 & -3.62 & 0.68 & -0.07 \\
\hline chr6:243-282 significant & RJF & 19.95 & -3.06 & 0.57 & -1.27 \\
\hline chr6:247-281 significant & RJF & 21.77 & -3.48 & 0.7 & -2.31 \\
\hline chr6:245-289 suggestive & WL & 13.93 & 0.94 & 0.22 & -0.27 \\
\hline chr6:253-278 significant & RJF & 18.13 & -2.75 & 0.54 & 0.21 \\
\hline chr6:245-280 significant & WL & 22.53 & 2.12 & 0.35 & 0.18 \\
\hline chr6:239-282 significant & RJF & 20.5 & -2.37 & 0.43 & 0.1 \\
\hline chr6:242-290 significant & RJF & 15.82 & -3.72 & 0.79 & -0.52 \\
\hline chr6:252-280 significant & RJF & 32.07 & -3.15 & 0.41 & -0.41 \\
\hline chr6:243-292 significant & RJF & 17.07 & -3.54 & 0.72 & -1.29 \\
\hline chr6:235-292 significant & RJF & 17.08 & -2.6 & 0.52 & -0.24 \\
\hline chr6:244-291 significant & RJF & 16.5 & -2.63 & 0.54 & -0.07 \\
\hline chr6:248-307 suggestive & RJF & 18.33 & -1.62 & 0.68 & 2.26 \\
\hline chr6:245-307 significant & RJF & 17.26 & -3.35 & 0.72 & 1.23 \\
\hline chr6:244-311 suggestive & RJF & 14.08 & -1.27 & 0.29 & 0.1 \\
\hline chr6:248-309 significant & RJF & 16.62 & -1.77 & 0.42 & -1.03 \\
\hline chr6:249-313 suggestive & WL & 11.85 & 2.17 & 0.55 & 0.16 \\
\hline chr6:267-316 significant & RJF & 16.46 & -2.03 & 0.42 & 0.07 \\
\hline chr6:254-311 suggestive & RJF & 16.31 & -0.74 & 0.54 & -1.48 \\
\hline chr6:270-308 significant & RJF & 17.34 & -2.14 & 0.48 & -0.96 \\
\hline chr6:271-314 significant & RJF & 14.9 & -1.86 & 0.44 & -0.67 \\
\hline chr6:280-308 significant & WL & 27.53 & 2.26 & 0.33 & -0.67 \\
\hline chr6:277-312 significant & WL & 17.75 & 2.9 & 0.57 & -0.87 \\
\hline chr6:276-309 significant & RJF & 16.37 & -2.1 & 0.49 & -1.13 \\
\hline chr6:277-306 suggestive & RJF & 19.49 & -2.15 & 0.69 & -3.16 \\
\hline chr6:280-308 significant & RJF & 23.01 & -3.38 & 0.58 & -0.26 \\
\hline chr6:277-324 significant & WL & 12.15 & 1.66 & 0.5 & -2.24 \\
\hline chr6:223-315 suggestive & WL & 12.54 & 1.6 & 0.41 & -1.15 \\
\hline
\end{tabular}




\begin{tabular}{|c|c|c|c|c|c|}
\hline chr6:283-310 suggestive & WL & 11.09 & 0.02 & 0.41 & -2.23 \\
\hline chr6:276-311 significant & RJF & 16.06 & -1.99 & 0.48 & -1.12 \\
\hline chr6:277-314 suggestive & RJF & 15.74 & -2.02 & 0.43 & -0.04 \\
\hline chr6:280-317 suggestive & WL & 15.76 & 1.85 & 0.41 & -1.26 \\
\hline chr6:287-323 suggestive & WL & 15.94 & 2.23 & 0.5 & -1.76 \\
\hline chr6:219-325 suggestive & WL & 9.9 & 1.41 & 0.39 & -0.69 \\
\hline chr6:280-325 significant & RJF & 16.67 & -1.93 & 0.46 & 1.63 \\
\hline chr6:307-325 suggestive & WL & 14.21 & 1.43 & 0.33 & 0.39 \\
\hline chr6:309-325 suggestive & RJF & 15.58 & -3.57 & 1.05 & 4.85 \\
\hline chr6:314-325 suggestive & WL & 27.18 & 0.51 & 0.09 & -0.55 \\
\hline chr6:314-325 significant & WL & 16.1 & 4.48 & 0.94 & 0.27 \\
\hline significant & WL & 15.52 & 1.08 & 0.22 & -0.49 \\
\hline suggestive & RJF & 17.14 & -2.56 & 0.67 & -1.68 \\
\hline suggestive & WL & 21.82 & 0.92 & 0.17 & -0.54 \\
\hline suggestive & WL & 25.17 & 0.41 & 0.07 & -0.42 \\
\hline significant & WL & 48.72 & 0.49 & 0.05 & -0.43 \\
\hline suggestive & WL & 14.92 & 1.17 & 0.26 & -0.05 \\
\hline suggestive & WL & 24.58 & 2.72 & 0.47 & -2.27 \\
\hline suggestive & WL & 29.01 & 1 & 0.15 & -0.81 \\
\hline significant & RJF & 24.18 & -2.35 & 0.56 & -2.29 \\
\hline suggestive & WL & 25.15 & 3.41 & 0.56 & -2.59 \\
\hline suggestive & WL & 24.07 & 1.63 & 0.53 & -0.15 \\
\hline suggestive & RJF & 14.19 & -3.33 & 0.77 & 2.26 \\
\hline significant & WL & 13.42 & 2.01 & 0.48 & -0.1 \\
\hline significant & WL & 21.43 & 2.46 & 0.41 & 0.42 \\
\hline suggestive & RJF & 19.44 & -0.97 & 0.35 & 2.26 \\
\hline significant & WL & 11.48 & 1.74 & 0.48 & 0.19 \\
\hline significant & RJF & 17.02 & -2.11 & 0.42 & 0.53 \\
\hline significant & RJF & 16.25 & -1.72 & 0.35 & 0.66 \\
\hline significant & WL & 29.24 & 2.26 & 0.36 & -2.23 \\
\hline suggestive & RJF & 17.2 & -0.51 & 0.26 & 1.73 \\
\hline significant & RJF & 21.55 & -4.35 & 0.79 & 2.3 \\
\hline significant & RJF & 22.21 & -2.13 & 0.36 & 0.52 \\
\hline suggestive & RJF & 15.66 & -1.36 & 0.5 & 2.77 \\
\hline significant & RJF & 18.68 & -2.7 & 0.59 & 2.45 \\
\hline suggestive & WL & 25.73 & 1.3 & 0.3 & -1.42 \\
\hline suggestive & WL & 23.57 & 0.65 & 0.13 & -0.37 \\
\hline suggestive & RJF & 21.43 & -0.64 & 0.38 & 2.85 \\
\hline significant & RJF & 18.76 & -2.98 & 0.54 & 0.58 \\
\hline significant & RJF & 20.06 & -2.64 & 0.81 & -0.93 \\
\hline significant & RJF & 22.72 & -2.28 & 0.54 & 1.33 \\
\hline significant & RJF & 20.73 & -4.41 & 0.75 & 0.5 \\
\hline significant & RJF & 19.9 & -3.26 & 0.61 & 1.32 \\
\hline
\end{tabular}




\begin{tabular}{|c|c|c|c|c|c|c|}
\hline chr7:5-27 & significant & RJF & 18.86 & -1.32 & 0.33 & 1.68 \\
\hline chr7:7-25 & significant & RJF & 23.99 & -1.32 & 0.4 & 1.13 \\
\hline chr7:4-25 & significant & RJF & 20.54 & -3.02 & 0.54 & 1.17 \\
\hline chr7:3-26 & significant & RJF & 19.07 & -2.33 & 0.44 & 0.83 \\
\hline chr7:3-29 & significant & RJF & 17.18 & -3.09 & 0.61 & 0.89 \\
\hline chr7:3-29 & significant & RJF & 15.81 & -1.06 & 0.23 & 0.56 \\
\hline chr7:4-28 & suggestive & RJF & 14.95 & -1.46 & 0.35 & 1.08 \\
\hline chr7:5-23 & significant & RJF & 16.08 & -1.61 & 0.35 & 0.94 \\
\hline chr7:2-27 & significant & RJF & 18.39 & -3.52 & 0.68 & 0.96 \\
\hline chr7:6-28 & significant & RJF & 17.41 & -0.4 & 0.2 & 1.29 \\
\hline chr7:2-35 & suggestive & WL & 11.95 & 1.15 & 0.28 & -0.03 \\
\hline chr7:0-26 & suggestive & RJF & 14.8 & -1.6 & 0.37 & 0.91 \\
\hline chr7:0-26 & suggestive & RJF & 14.42 & -1.86 & 0.43 & 1.17 \\
\hline chr7:2-27 & suggestive & RJF & 14.62 & -1.81 & 0.38 & 0.48 \\
\hline chr7:2-26 & suggestive & RJF & 13.74 & -1.27 & 0.49 & 2.56 \\
\hline chr7:3-31 & suggestive & RJF & 15.33 & -2.44 & 0.54 & 1.12 \\
\hline chr7:5-24 & significant & RJF & 18.38 & -1.81 & 0.35 & 0.78 \\
\hline chr7:3-31 & suggestive & RJF & 16.05 & -2.34 & 0.56 & 2.12 \\
\hline chr7:3-25 & suggestive & RJF & 14.69 & -1.03 & 0.32 & 1.6 \\
\hline chr7:0-35 & suggestive & WL & 11.81 & 1.43 & 0.36 & 0.24 \\
\hline chr7:3-24 & suggestive & RJF & 14.43 & -0.36 & 0.27 & 1.67 \\
\hline chr7:3-32 & suggestive & WL & 14.17 & 0.21 & 0.3 & 1.71 \\
\hline chr7:6-30 & suggestive & RJF & 15.04 & -0.32 & 0.38 & 2.35 \\
\hline chr7:5-29 & significant & WL & 15.99 & 0.01 & 0.23 & 1.46 \\
\hline chr7:7-20 & suggestive & WL & 28.3 & 1.96 & 0.29 & -1.2 \\
\hline chr7:6-23 & suggestive & RJF & 25.86 & -1.5 & 0.56 & 2.46 \\
\hline chr7:7-18 & suggestive & WL & 28.91 & 1.69 & 0.26 & -1.36 \\
\hline chr7:6-23 & suggestive & RJF & 25.18 & -2.73 & 0.59 & 1.6 \\
\hline chr7:6-27 & significant & RJF & 23.7 & -0.32 & 0.25 & 2.05 \\
\hline chr7:4-25 & suggestive & WL & 25.01 & 1.08 & 0.18 & -0.7 \\
\hline chr7:7-18 & significant & WL & 31.72 & 3.2 & 0.48 & -2.47 \\
\hline chr7:7-20 & suggestive & WL & 27.53 & 0.86 & 0.14 & -0.82 \\
\hline chr7:7-18 & significant & WL & 30.85 & 0.7 & 0.11 & -0.8 \\
\hline chr7:8-21 & suggestive & WL & 31.39 & 1.16 & 0.17 & -1.07 \\
\hline chr7:5-24 & suggestive & RJF & 19.48 & -0.8 & 0.3 & 2.02 \\
\hline chr7:4-27 & suggestive & RJF & 20.22 & -0.07 & 0.36 & 2.68 \\
\hline chr7:6-21 & suggestive & RJF & 19.61 & -0.77 & 0.33 & 2.31 \\
\hline chr7:6-26 & suggestive & RJF & 20.28 & -0.44 & 0.36 & 2.66 \\
\hline chr7:6-23 & suggestive & RJF & 20.37 & -0.51 & 0.19 & 1.34 \\
\hline chr7:6-25 & suggestive & RJF & 19.87 & -0.67 & 0.26 & 1.78 \\
\hline chr7:7-27 & suggestive & RJF & 19.9 & -0.87 & 0.31 & 2.05 \\
\hline chr7:5-25 & suggestive & RJF & 19.12 & -0.97 & 0.41 & 2.9 \\
\hline chr7:6-22 & suggestive & RJF & 19.67 & -0.41 & 0.39 & 2.81 \\
\hline
\end{tabular}




\begin{tabular}{|c|c|c|c|c|c|c|}
\hline chr7:6-23 & suggestive & WL & 18.82 & 0.17 & 0.33 & 2.27 \\
\hline chr7:6-22 & suggestive & RJF & 20.45 & -0.94 & 0.33 & 2.27 \\
\hline chr7:7-22 & suggestive & RJF & 19.41 & -0.45 & 0.28 & 2.04 \\
\hline chr7:4-28 & suggestive & RJF & 16.81 & -0.53 & 0.26 & 1.69 \\
\hline chr7:5-27 & suggestive & RJF & 20.07 & -1.44 & 0.32 & 1.68 \\
\hline chr7:7-27 & suggestive & RJF & 19.23 & -0.65 & 0.36 & 2.56 \\
\hline chr7:6-24 & suggestive & RJF & 19.88 & -1.83 & 0.36 & 1.16 \\
\hline chr7:7-25 & suggestive & RJF & 19.91 & -0.36 & 0.31 & 2.29 \\
\hline chr7:4-28 & suggestive & RJF & 18.28 & -0.92 & 0.28 & 1.63 \\
\hline chr7:5-28 & suggestive & RJF & 18.45 & -1.09 & 0.3 & 1.73 \\
\hline chr7:6-25 & suggestive & RJF & 22.4 & -1.34 & 0.38 & 2.58 \\
\hline chr7:6-27 & suggestive & RJF & 18.69 & -0.61 & 0.26 & 1.81 \\
\hline chr7:3-24 & suggestive & RJF & 19.18 & -0.65 & 0.2 & 1.26 \\
\hline chr7:6-20 & suggestive & RJF & 20.73 & -1.33 & 0.38 & 2.43 \\
\hline chr7:5-25 & suggestive & RJF & 19.43 & -1.84 & 0.47 & 2.57 \\
\hline chr7:5-23 & suggestive & RJF & 18.64 & -1.07 & 0.4 & 2.68 \\
\hline chr7:5-23 & suggestive & RJF & 19.05 & -0.62 & 0.36 & 2.57 \\
\hline chr7:6-30 & significant & RJF & 16.07 & -0.65 & 0.39 & 2.46 \\
\hline chr7:2-31 & significant & WL & 15.02 & 0.8 & 0.39 & 2.11 \\
\hline chr7:3-36 & suggestive & RJF & 14.94 & -1.33 & 0.29 & -0.01 \\
\hline chr7:3-31 & significant & RJF & 19.43 & -2.13 & 0.43 & 1.59 \\
\hline chr7:7-32 & suggestive & RJF & 20.92 & -0.86 & 0.27 & 1.85 \\
\hline chr7:6-31 & suggestive & RJF & 20.22 & -0.91 & 0.43 & 3.04 \\
\hline chr7:0-36 & significant & RJF & 17.34 & -3.65 & 0.73 & 1.47 \\
\hline chr7:5-28 & suggestive & RJF & 19.72 & -0.41 & 0.34 & 2.46 \\
\hline chr7:2-39 & suggestive & WL & 12.24 & 2.08 & 0.62 & 1.67 \\
\hline chr7:0-32 & significant & RJF & 15.41 & -0.02 & 0.29 & 1.82 \\
\hline chr7:4-35 & significant & WL & 16.63 & 1.7 & 0.48 & 1.96 \\
\hline chr7:6-32 & suggestive & RJF & 17.55 & -0.12 & 0.34 & 2.4 \\
\hline chr7:5-33 & suggestive & RJF & 20.11 & -0.63 & 0.33 & 2.36 \\
\hline chr7:5-36 & suggestive & RJF & 14.58 & -0.67 & 0.36 & 2.09 \\
\hline chr7:0-55 & suggestive & WL & 15.47 & 1.54 & 0.33 & -0.31 \\
\hline chr7:25-76 & significant & RJF & 18.75 & -2.47 & 0.47 & 0.26 \\
\hline chr7:25-76 & significant & RJF & 18.04 & -1.74 & 0.34 & 0.4 \\
\hline chr7:32-70 & significant & WL & 15.71 & 1.77 & 0.35 & -0.54 \\
\hline chr7:17-76 & suggestive & WL & 10.55 & 2.01 & 0.57 & 0.94 \\
\hline chr7:39-73 & suggestive & WL & 14.77 & 2.67 & 0.75 & 0.57 \\
\hline chr7:47-67 & significant & WL & 35.8 & 3.97 & 0.49 & -0.09 \\
\hline chr7:54-63 & significant & WL & 72.89 & 5.68 & 0.32 & -0.22 \\
\hline chr7:33-76 & suggestive & WL & 11.38 & 2.7 & 0.82 & 1.96 \\
\hline chr7:42-75 & suggestive & WL & 12.38 & 1.82 & 0.44 & -0.62 \\
\hline chr7:39-72 & suggestive & WL & 17.87 & 1.37 & 0.27 & -0.65 \\
\hline chr7:33-78 & significant & WL & 12.86 & 2.56 & 0.67 & 1.24 \\
\hline
\end{tabular}




\begin{tabular}{|c|c|c|c|c|c|c|}
\hline chr7:41-77 & significant & WL & 14.44 & 2.41 & 0.54 & 0.42 \\
\hline chr7:44-70 & suggestive & WL & 19.41 & 0.79 & 0.22 & -1.48 \\
\hline chr7:36-77 & significant & WL & 15.53 & 2.06 & 0.44 & -0.18 \\
\hline chr7:43-79 & significant & WL & 18.67 & 0.96 & 0.19 & 0.31 \\
\hline chr7:41-80 & suggestive & WL & 11.71 & 1.14 & 0.21 & -1.16 \\
\hline chr7:21-90 & suggestive & RJF & 14.69 & -1.77 & 0.39 & -0.06 \\
\hline chr7:73-89 & suggestive & RJF & 11.48 & -0.87 & 0.44 & 2.64 \\
\hline chr7:69-91 & suggestive & WL & 22.45 & 1.66 & 0.3 & -1.03 \\
\hline chr7:69-109 & suggestive & WL & 22.98 & 2.27 & 0.53 & -2.14 \\
\hline chr7:72-106 & significant & RJF & 15.01 & -1.32 & 0.75 & -2.54 \\
\hline chr7:85-105 & significant & WL & 16.22 & 1.63 & 0.49 & -2.18 \\
\hline chr7:84-102 & significant & RJF & 19.08 & -1.76 & 0.43 & -0.29 \\
\hline chr7:87-100 & suggestive & RJF & 14.66 & -1.17 & 0.41 & -0.73 \\
\hline chr7:84-105 & suggestive & WL & 19.97 & 1.7 & 0.64 & -2.51 \\
\hline chr7:73-106 & significant & WL & 13.82 & 1.35 & 0.59 & 1.44 \\
\hline chr7:86-116 & significant & RJF & 17.08 & -4.94 & 1.24 & -0.35 \\
\hline chr7:85-117 & significant & RJF & 15.22 & -4.03 & 1.09 & -0.41 \\
\hline chr7:95-118 & significant & WL & 14.55 & 2.23 & 0.48 & -1.21 \\
\hline chr7:83-116 & significant & RJF & 15.44 & -3.88 & 1.03 & -0.18 \\
\hline chr7:95-112 & significant & WL & 17.99 & 2.36 & 0.54 & -0.16 \\
\hline chr7:88-114 & significant & RJF & 18.41 & -7.13 & 1.73 & -1.03 \\
\hline chr7:94-114 & significant & RJF & 21.62 & -9.96 & 2.28 & -2.26 \\
\hline chr7:85-115 & significant & RJF & 15.66 & -6.67 & 1.77 & -0.79 \\
\hline chr7:98-119 & significant & WL & 15.4 & 2.39 & 0.56 & -0.46 \\
\hline chr7:103-118 & suggestive & WL & 24.52 & 0.02 & 0.02 & -0.06 \\
\hline chr7:88-114 & suggestive & WL & 17.38 & 1.22 & 0.21 & -1.09 \\
\hline chr7:102-114 & suggestive & RJF & 24.32 & -0.14 & 0.19 & -0.51 \\
\hline chr7:104-117 & significant & WL & 18.9 & 1.78 & 0.53 & 1.01 \\
\hline chr7:104-117 & significant & RJF & 24.95 & -4.42 & 0.85 & -0.17 \\
\hline chr7:95-117 & suggestive & WL & 17.58 & 2.13 & 0.43 & -2.09 \\
\hline chr7:104-119 & significant & RJF & 16.06 & -3.66 & 1.02 & -1.14 \\
\hline chr7:104-118 & significant & WL & 36.59 & 0.02 & 0.02 & -0.02 \\
\hline chr7:104-122 & significant & RJF & 14.93 & -2.98 & 0.69 & 0.95 \\
\hline chr7:106-122 & suggestive & WL & 22.12 & 3.63 & 0.67 & -2.89 \\
\hline chr7:105-125 & suggestive & RJF & 24.5 & -0.22 & 0.14 & 0.05 \\
\hline chr7:163-174 & suggestive & WL & 23.13 & 0.14 & 0.31 & 0.14 \\
\hline chr7:164-178 & significant & RJF & 23.58 & -3.07 & 0.79 & -1.42 \\
\hline chr7:166-189 & significant & WL & 29.39 & 4.62 & 0.97 & -0.3 \\
\hline chr7:155-186 & suggestive & WL & 24.89 & 6.06 & 1.82 & 0.46 \\
\hline chr7:174-192 & suggestive & WL & 22.96 & 4.88 & 1.31 & 3.62 \\
\hline chr7:174-188 & suggestive & WL & 22.25 & 6.17 & 1.49 & 3.18 \\
\hline chr7:174-188 & significant & WL & 22.82 & 7.59 & 1.72 & 3.3 \\
\hline chr7:173-190 & significant & WL & 25.47 & 5.06 & 1.01 & 1.35 \\
\hline
\end{tabular}




\begin{tabular}{|c|c|c|c|c|c|}
\hline chr7:171-185 significant & WL & 31.48 & 8.36 & 1.58 & 3.66 \\
\hline chr7:173-188 significant & WL & 23.6 & 8.5 & 1.93 & 4.51 \\
\hline chr7:174-189 significant & WL & 23.93 & 5.79 & 1.44 & 4.25 \\
\hline chr7:170-190 suggestive & WL & 20.77 & 3.57 & 0.78 & 0.65 \\
\hline chr7:171-186 significant & WL & 28.09 & 2.9 & 0.49 & 0.21 \\
\hline chr7:173-187 significant & WL & 28.74 & 3.73 & 0.73 & 1.85 \\
\hline chr7:174-188 significant & WL & 23.75 & 5.47 & 1.24 & 2.74 \\
\hline chr7:173-190 significant & WL & 21.83 & 3.27 & 0.72 & 1.29 \\
\hline chr7:174-189 significant & WL & 23.24 & 6.91 & 1.67 & 4.15 \\
\hline chr7:173-190 suggestive & WL & 21.59 & 5.08 & 1.06 & 0.84 \\
\hline chr7:174-190 significant & WL & 23.26 & 4.06 & 0.96 & 2.09 \\
\hline chr7:174-187 significant & WL & 27.03 & 2.98 & 0.6 & 1.22 \\
\hline chr7:169-186 significant & WL & 27.85 & 8.11 & 1.77 & 4.51 \\
\hline chr7:173-190 significant & WL & 23.45 & 5.83 & 1.25 & 2.2 \\
\hline chr7:174-188 significant & WL & 25.22 & 5.3 & 1.08 & 1.58 \\
\hline chr7:169-187 significant & WL & 27.33 & 6.82 & 1.32 & 2.1 \\
\hline chr7:171-186 significant & WL & 28.05 & 8.7 & 1.77 & 3.53 \\
\hline chr7:166-187 significant & WL & 30.51 & 4.71 & 0.93 & 2.15 \\
\hline chr7:169-189 significant & WL & 23.58 & 7.34 & 1.53 & 1.95 \\
\hline chr7:174-186 significant & WL & 28.08 & 3.33 & 0.79 & 2.53 \\
\hline chr7:170-187 significant & WL & 30.58 & 6.57 & 1.14 & 1.32 \\
\hline chr7:173-187 significant & WL & 30.87 & 5.68 & 1.08 & 2.24 \\
\hline chr7:169-188 suggestive & WL & 21.32 & 2.62 & 0.57 & 0.72 \\
\hline chr7:156-190 significant & WL & 22.15 & 2.36 & 0.58 & 1.11 \\
\hline chr7:169-186 significant & WL & 26.71 & 8.82 & 1.87 & 3.68 \\
\hline chr7:155-187 suggestive & WL & 24.17 & 6.3 & 1.85 & -0.56 \\
\hline chr7:174-188 significant & WL & 24.16 & 6.97 & 1.57 & 3.42 \\
\hline chr7:174-189 significant & WL & 23.19 & 9 & 1.89 & 2.65 \\
\hline chr7:168-188 significant & WL & 26.63 & 7.65 & 1.55 & 2.68 \\
\hline chr7:174-188 significant & WL & 25.5 & 5.28 & 1.06 & 1.39 \\
\hline chr7:168-187 significant & WL & 27.01 & 5.34 & 1.31 & 4.13 \\
\hline chr7:170-186 significant & WL & 29.26 & 9.74 & 1.88 & 3.56 \\
\hline chr7:173-188 significant & WL & 22.88 & 6.59 & 1.49 & 2.9 \\
\hline chr7:174-186 suggestive & WL & 24.97 & 6.55 & 2 & 0.08 \\
\hline chr7:173-188 significant & WL & 24.15 & 5.91 & 1.18 & 1.47 \\
\hline chr7:170-185 significant & WL & 29.86 & 5.3 & 1.11 & 2.99 \\
\hline chr7:167-189 significant & WL & 24.96 & 3.17 & 0.8 & 2.45 \\
\hline chr7:173-188 significant & WL & 22.45 & 7.27 & 1.48 & 1.31 \\
\hline chr7:174-189 suggestive & WL & 21.82 & 7.7 & 1.92 & 4.47 \\
\hline chr7:154-186 suggestive & WL & 24.65 & 5.91 & 1.8 & 0.5 \\
\hline chr7:173-188 significant & WL & 22.2 & 7.54 & 1.48 & 0.46 \\
\hline chr7:174-190 suggestive & WL & 21.74 & 7.29 & 1.94 & 5.15 \\
\hline chr7:173-188 significant & WL & 22.77 & 6.67 & 1.83 & 5.79 \\
\hline
\end{tabular}




\begin{tabular}{|c|c|c|c|c|c|}
\hline chr7:169-187 significant & WL & 26.34 & 7.08 & 1.57 & 3.62 \\
\hline chr7:168-186 significant & WL & 26.79 & 7.46 & 1.55 & 3.12 \\
\hline chr7:173-188 significant & WL & 24.81 & 4.13 & 0.78 & 0.39 \\
\hline chr7:173-189 significant & WL & 23.54 & 10.26 & 1.96 & 1.52 \\
\hline chr7:169-187 significant & WL & 26.02 & 7.26 & 1.61 & 3.56 \\
\hline chr7:167-188 significant & WL & 25.79 & 7.56 & 1.58 & 2.84 \\
\hline chr7:168-187 significant & WL & 27.26 & 8 & 1.76 & 4.19 \\
\hline chr7:170-186 significant & WL & 30.29 & 9.07 & 1.7 & 3.33 \\
\hline chr7:173-188 significant & WL & 22.92 & 3.93 & 0.99 & 3.02 \\
\hline chr7:164-188 significant & WL & 24.97 & 3.61 & 0.84 & 1.84 \\
\hline chr7:170-186 significant & WL & 28.56 & 7.63 & 1.61 & 3.95 \\
\hline chr7:173-187 significant & WL & 23.9 & 3.93 & 0.8 & 0.89 \\
\hline chr7:173-189 significant & WL & 20.68 & 6.24 & 1.27 & 0.84 \\
\hline chr7:173-189 significant & WL & 21.17 & 7 & 1.38 & 0.76 \\
\hline chr7:174-189 suggestive & WL & 21.86 & 6.89 & 1.77 & 4.41 \\
\hline chr7:173-189 significant & WL & 21.41 & 3 & 0.74 & 1.77 \\
\hline chr7:167-187 significant & WL & 25.78 & 4.57 & 1.08 & 2.82 \\
\hline chr7:169-185 significant & WL & 29.31 & 8.57 & 1.74 & 4.13 \\
\hline chr7:166-190 significant & WL & 23.59 & 6.98 & 1.8 & 5.41 \\
\hline chr7:174-189 significant & WL & 30.69 & 12.22 & 2.49 & 7.69 \\
\hline chr7:175-190 significant & WL & 27.19 & 10.34 & 2.75 & 10.6 \\
\hline chr7:175-190 significant & WL & 30.38 & 10.7 & 2.23 & 6.3 \\
\hline chr7:175-188 significant & WL & 36.6 & 11.57 & 2.58 & 12.53 \\
\hline chr7:174-190 significant & WL & 28.76 & 11.45 & 2.49 & 7.5 \\
\hline chr7:174-192 suggestive & WL & 23.33 & 12.16 & 3.14 & 8.6 \\
\hline chr7:175-190 significant & WL & 29.44 & 12.69 & 2.71 & 7.77 \\
\hline chr7:174-190 significant & WL & 29.23 & 9.92 & 2.19 & 6.48 \\
\hline chr7:175-188 significant & WL & 36.1 & 10.97 & 2.47 & 12.08 \\
\hline chr7:173-189 significant & WL & 28.91 & 13.09 & 2.81 & 8.21 \\
\hline chr7:175-190 significant & WL & 30.12 & 12.04 & 2.54 & 7.35 \\
\hline chr7:175-189 significant & WL & 33.04 & 9.14 & 1.79 & 5.39 \\
\hline chr7:175-187 significant & WL & 35.11 & 8.48 & 2.2 & 12.02 \\
\hline chr7:174-190 significant & WL & 27.79 & 7.78 & 1.8 & 5.85 \\
\hline chr7:174-192 significant & WL & 25.54 & 12.05 & 2.74 & 6.68 \\
\hline chr7:176-189 significant & WL & 31.1 & 13.28 & 2.75 & 8.29 \\
\hline chr7:174-189 significant & WL & 27.52 & 8.21 & 2.55 & 11.63 \\
\hline chr7:175-191 significant & WL & 28.47 & 13.72 & 2.99 & 8.16 \\
\hline chr7:175-190 significant & WL & 30.52 & 10.91 & 2.22 & 5.94 \\
\hline chr7:174-189 significant & WL & 29.91 & 12.2 & 2.53 & 7.45 \\
\hline chr7:175-190 significant & WL & 30.24 & 14.15 & 3.05 & 9.45 \\
\hline chr7:175-188 significant & WL & 32.72 & 8.66 & 2.69 & 15.32 \\
\hline chr7:173-190 significant & WL & 28.75 & 8.06 & 2.03 & 8.08 \\
\hline chr7:173-191 significant & WL & 24.24 & 9.95 & 2.99 & 11.11 \\
\hline
\end{tabular}




\begin{tabular}{|c|c|c|c|c|c|}
\hline chr7:176-189 significant & WL & 31.02 & 14.48 & 2.93 & 8.12 \\
\hline chr7:176-187 significant & WL & 37.21 & 12.51 & 2.66 & 12.82 \\
\hline chr7:174-189 significant & WL & 29.91 & 10.81 & 2.16 & 6.03 \\
\hline chr7:175-190 significant & WL & 28 & 11.72 & 3.09 & 12.39 \\
\hline chr7:174-187 significant & WL & 25.71 & 8.22 & 2.1 & 6.98 \\
\hline chr7:174-188 significant & WL & 24.54 & 7.15 & 1.51 & 2.89 \\
\hline chr7:173-187 significant & WL & 22.86 & 6.82 & 1.74 & 4.81 \\
\hline chr7:174-190 significant & WL & 24.47 & 5.23 & 1.1 & 1.75 \\
\hline chr7:173-187 significant & WL & 24.12 & 4.76 & 0.87 & 0.31 \\
\hline chr7:175-187 significant & WL & 25.62 & 9.78 & 2.1 & 4.75 \\
\hline chr7:176-190 suggestive & RJF & 27.23 & -0.85 & 0.46 & -0.74 \\
\hline chr7:173-189 significant & WL & 21.78 & 3.74 & 0.92 & 2.33 \\
\hline chr7:170-188 significant & WL & 22.1 & 6.52 & 1.41 & 2.11 \\
\hline chr7:173-190 significant & WL & 22.6 & 4.92 & 1.06 & 1.38 \\
\hline chr7:174-190 significant & WL & 22.98 & 6.72 & 1.39 & 1.68 \\
\hline chr7:167-191 suggestive & WL & 20.01 & 7.8 & 1.98 & 3.99 \\
\hline chr7:167-190 suggestive & WL & 19.65 & 4.45 & 1.02 & 1.05 \\
\hline chr7:172-191 suggestive & WL & 18.91 & 5.91 & 1.41 & 1.51 \\
\hline chr7:167-188 suggestive & WL & 21.56 & 6.49 & 1.45 & 1.83 \\
\hline chr7:165-188 suggestive & WL & 22.19 & 8.97 & 2.68 & 8.31 \\
\hline chr7:173-190 suggestive & WL & 20.79 & 4.57 & 1.02 & 1.3 \\
\hline chr7:165-190 suggestive & WL & 20.85 & 5.09 & 1.43 & 4.01 \\
\hline chr7:168-189 suggestive & WL & 21.46 & 4.66 & 1 & 1.01 \\
\hline chr7:174-189 suggestive & WL & 21.27 & 3.24 & 0.93 & 2.79 \\
\hline chr7:173-190 suggestive & WL & 21.61 & 4.66 & 1.37 & 4.47 \\
\hline chr7:173-190 suggestive & WL & 20.38 & 6.32 & 1.79 & 4.86 \\
\hline chr7:167-191 suggestive & WL & 21.63 & 6.54 & 1.8 & 5.12 \\
\hline chr7:173-191 suggestive & WL & 21.31 & 6.04 & 1.65 & 4.43 \\
\hline chr7:167-190 suggestive & WL & 21.3 & 7.99 & 1.91 & 3.74 \\
\hline chr7:166-194 suggestive & WL & 18.89 & 5.83 & 1.46 & 2.34 \\
\hline chr7:166-191 suggestive & WL & 20.81 & 3.25 & 0.71 & 0.69 \\
\hline chr7:168-188 suggestive & WL & 20.8 & 4.6 & 1.01 & 0.83 \\
\hline chr7:167-191 suggestive & WL & 20.91 & 6.55 & 1.44 & 1.48 \\
\hline chr7:173-189 significant & WL & 21.76 & 7.72 & 1.58 & 1.22 \\
\hline chr7:166-188 significant & WL & 23.17 & 4.7 & 1.13 & 2.49 \\
\hline chr7:166-190 suggestive & WL & 21.39 & 5.11 & 1.14 & 1.24 \\
\hline chr7:150-191 suggestive & WL & 21.95 & 9.6 & 3.23 & 11.51 \\
\hline chr7:174-188 suggestive & WL & 23.14 & 10.9 & 3.09 & 9.35 \\
\hline chr7:167-191 suggestive & WL & 20.15 & 7.39 & 2.17 & 5.9 \\
\hline chr7:165-190 suggestive & WL & 20.28 & 1.24 & 0.31 & 0.58 \\
\hline chr7:174-191 suggestive & WL & 21.7 & 1.74 & 0.36 & 0.15 \\
\hline chr7:174-192 suggestive & WL & 21.78 & 10.86 & 3.53 & 11.91 \\
\hline chr7:165-191 suggestive & WL & 21.22 & 5.36 & 1.29 & 2.37 \\
\hline
\end{tabular}




\begin{tabular}{|c|c|c|c|c|c|}
\hline chr7:168-191 suggestive & WL & 19.94 & 6.86 & 1.99 & 5.19 \\
\hline chr7:174-190 significant & WL & 22.94 & 4.92 & 1.07 & 1.4 \\
\hline chr7:166-187 suggestive & WL & 21.99 & 5.21 & 1.37 & 3.24 \\
\hline chr7:169-190 suggestive & WL & 19.22 & 4.95 & 1.21 & 1.58 \\
\hline chr7:167-190 suggestive & WL & 20.42 & 5.9 & 1.72 & 4.76 \\
\hline chr7:166-193 suggestive & WL & 19.89 & 4.8 & 1.39 & 3.82 \\
\hline chr7:165-191 suggestive & WL & 19.97 & 6.67 & 2.21 & 7.13 \\
\hline chr7:166-189 significant & WL & 22.8 & 6.3 & 1.42 & 2.33 \\
\hline chr7:174-191 suggestive & WL & 21.03 & 9.06 & 2.66 & 7.74 \\
\hline chr7:173-190 suggestive & WL & 19.2 & 5.16 & 1.08 & 0.36 \\
\hline chr7:166-190 suggestive & WL & 19.65 & 9.38 & 2.57 & 5.7 \\
\hline chr7:155-193 suggestive & WL & 17.22 & 4.28 & 1.15 & 1.26 \\
\hline chr7:173-192 suggestive & WL & 19.49 & 6.46 & 1.45 & 1.54 \\
\hline chr7:173-190 suggestive & WL & 20.87 & 5.43 & 1.23 & 1.97 \\
\hline chr7:166-186 suggestive & WL & 22.21 & 4.41 & 0.99 & 1.32 \\
\hline chr7:165-190 suggestive & WL & 21.49 & 3.36 & 0.85 & 1.63 \\
\hline chr7:167-191 suggestive & WL & 22.16 & 5.04 & 1.08 & 1.06 \\
\hline chr7:168-190 suggestive & WL & 20.44 & 4.88 & 1.31 & 2.98 \\
\hline chr7:173-189 suggestive & WL & 21.34 & 7.17 & 2.23 & 7.51 \\
\hline chr7:176-190 suggestive & WL & 20.34 & 0.27 & 0.05 & -0.18 \\
\hline chr7:161-188 significant & WL & 25.53 & 2.76 & 0.62 & 1.27 \\
\hline chr7:171-190 suggestive & WL & 21.88 & 3.53 & 0.71 & 0.1 \\
\hline chr7:174-188 suggestive & WL & 23.83 & 10.68 & 3.09 & 10.21 \\
\hline chr7:173-188 suggestive & WL & 20.67 & 6.52 & 1.59 & 3.03 \\
\hline chr7:167-189 suggestive & WL & 19.35 & 4.28 & 0.99 & 0.93 \\
\hline chr7:169-196 suggestive & WL & 19.83 & 6.3 & 1.56 & 2.5 \\
\hline chr7:173-188 significant & WL & 24.3 & 5.13 & 1.02 & 1.46 \\
\hline chr7:174-191 suggestive & WL & 21.71 & 6.05 & 1.36 & 2.17 \\
\hline chr7:173-189 significant & WL & 20.58 & 6.05 & 1.22 & 0.54 \\
\hline chr7:165-191 suggestive & WL & 19.29 & 6.94 & 1.92 & 4.28 \\
\hline chr7:170-190 suggestive & WL & 19.26 & 8.12 & 1.74 & 1.04 \\
\hline chr7:168-188 significant & WL & 22.8 & 4.01 & 1.08 & 3.19 \\
\hline chr7:156-189 suggestive & WL & 18.71 & 1.5 & 0.44 & 1.06 \\
\hline chr7:173-189 suggestive & WL & 21.58 & 7.23 & 1.64 & 2.13 \\
\hline chr7:165-191 suggestive & WL & 20 & 6.36 & 1.72 & 3.8 \\
\hline chr7:167-189 suggestive & WL & 20.94 & 7.19 & 1.71 & 2.73 \\
\hline chr7:167-192 suggestive & WL & 20.36 & 8.03 & 2.03 & 4.03 \\
\hline chr7:173-189 significant & WL & 24.15 & 2.9 & 0.69 & 1.46 \\
\hline chr7:167-190 suggestive & WL & 21.69 & 5.47 & 1.23 & 1.77 \\
\hline chr7:167-188 suggestive & WL & 19.52 & 3.07 & 0.76 & 1.13 \\
\hline chr7:173-190 suggestive & WL & 21.88 & 6.43 & 1.63 & 4 \\
\hline chr7:166-190 suggestive & WL & 20.79 & 2.16 & 0.6 & 1.55 \\
\hline chr7:174-190 suggestive & WL & 21.79 & 3.11 & 0.92 & 2.73 \\
\hline
\end{tabular}




\begin{tabular}{|c|c|c|c|c|c|}
\hline chr7:171-190 suggestive & WL & 21.03 & 3.18 & 0.71 & 0.68 \\
\hline chr7:168-191 suggestive & WL & 19.17 & 7.66 & 1.73 & 1.66 \\
\hline chr7:173-192 suggestive & WL & 16.92 & 6.52 & 1.39 & -0.38 \\
\hline chr7:173-192 suggestive & WL & 19.45 & 3.45 & 0.78 & 0.42 \\
\hline chr7:166-191 suggestive & WL & 20.77 & 5.09 & 1.43 & 4.02 \\
\hline chr7:174-189 suggestive & WL & 21.22 & 4.09 & 1.31 & 4.64 \\
\hline chr7:164-189 suggestive & WL & 20.3 & 2.83 & 0.7 & 0.96 \\
\hline chr7:173-193 suggestive & WL & 21.6 & 10.42 & 2.57 & 5.15 \\
\hline chr7:174-192 suggestive & WL & 21.03 & 11.04 & 3.69 & 12.62 \\
\hline chr7:174-192 suggestive & WL & 21.83 & 4.66 & 1.79 & 7.46 \\
\hline chr7:174-193 suggestive & WL & 22.99 & 10.98 & 2.83 & 7.44 \\
\hline chr7:174-192 suggestive & WL & 21.6 & 11.82 & 3.64 & 11.6 \\
\hline chr7:173-193 suggestive & WL & 22.53 & 11.53 & 2.83 & 6.15 \\
\hline chr7:174-190 suggestive & WL & 20.55 & 1.99 & 0.67 & 2.3 \\
\hline chr7:172-189 suggestive & WL & 20.76 & 5.14 & 1.14 & 1.73 \\
\hline chr7:168-191 suggestive & WL & 21.55 & 6.08 & 1.42 & 2.44 \\
\hline chr7:165-192 suggestive & WL & 19.6 & 6.47 & 1.71 & 3.39 \\
\hline chr7:164-189 suggestive & WL & 19.53 & 4.53 & 1.53 & 4.62 \\
\hline chr7:174-192 suggestive & WL & 20.4 & 8.18 & 2.45 & 7.13 \\
\hline chr7:174-190 suggestive & WL & 21.19 & 4.62 & 1.41 & 4.53 \\
\hline chr7:173-190 suggestive & WL & 21.56 & 6.31 & 1.39 & 1.54 \\
\hline chr7:169-187 suggestive & WL & 22.63 & 6.68 & 1.47 & 1.81 \\
\hline chr7:174-188 suggestive & WL & 22.26 & 6.48 & 1.55 & 3.34 \\
\hline chr7:171-188 suggestive & WL & 21.85 & 5.15 & 1.18 & 1.94 \\
\hline chr7:165-191 suggestive & WL & 20.19 & 5.62 & 1.55 & 3.59 \\
\hline chr7:173-191 suggestive & WL & 19.38 & 5.59 & 1.46 & 2.96 \\
\hline chr7:167-189 significant & WL & 22.52 & 3.17 & 1.04 & 4.09 \\
\hline chr7:166-189 significant & WL & 22.12 & 2.73 & 0.69 & 1.46 \\
\hline chr7:174-191 suggestive & WL & 21.47 & 10.58 & 3.49 & 11.77 \\
\hline chr7:174-191 significant & WL & 27.55 & 6.69 & 2.1 & 9.75 \\
\hline chr7:173-191 significant & WL & 28.47 & 10.16 & 2.25 & 7.1 \\
\hline chr7:175-192 significant & WL & 28.27 & 9.49 & 2.02 & 5.2 \\
\hline chr7:175-191 significant & WL & 29.15 & 12.58 & 2.9 & 9.53 \\
\hline chr7:175-191 significant & WL & 28.01 & 10.49 & 2.69 & 10.31 \\
\hline chr7:174-191 significant & WL & 24.81 & 6.48 & 2.2 & 9.69 \\
\hline chr7:175-190 significant & WL & 30.47 & 9.44 & 2.09 & 6.97 \\
\hline chr7:173-192 significant & WL & 26.31 & 13.01 & 2.98 & 8.14 \\
\hline chr7:175-190 significant & WL & 29.84 & 12.42 & 2.73 & 8.5 \\
\hline chr7:174-193 suggestive & WL & 21.35 & 6.22 & 1.84 & 5.64 \\
\hline chr7:174-194 suggestive & WL & 21.41 & 5.39 & 1.39 & 2.85 \\
\hline chr7:175-191 significant & WL & 28.62 & 13.1 & 2.81 & 7.36 \\
\hline chr7:175-192 significant & WL & 28.66 & 9.22 & 2.03 & 5.79 \\
\hline chr7:175-192 significant & WL & 24.8 & 11.44 & 3.6 & 14.87 \\
\hline
\end{tabular}




\begin{tabular}{|c|c|c|c|c|c|}
\hline chr7:174-192 significant & WL & 27.8 & 8.19 & 2.13 & 8.13 \\
\hline chr7:174-191 significant & WL & 29.31 & 10.6 & 2.24 & 6.67 \\
\hline chr7:175-190 significant & WL & 32.06 & 10.09 & 2.58 & 12.1 \\
\hline chr7:176-191 significant & WL & 26.92 & 11.77 & 3.41 & 14.48 \\
\hline chr7:175-190 significant & WL & 32.38 & 9.18 & 2 & 7.61 \\
\hline chr7:175-194 suggestive & WL & 21.39 & 10.64 & 3.55 & 12.68 \\
\hline chr7:174-191 significant & WL & 30.01 & 7.17 & 2.09 & 10.34 \\
\hline chr7:175-193 significant & WL & 25.5 & 13.36 & 3.91 & 15.29 \\
\hline chr7:175-193 significant & WL & 25.18 & 13.57 & 3.97 & 15.29 \\
\hline chr7:175-193 significant & WL & 27.54 & 10.22 & 2.24 & 5.8 \\
\hline chr7:175-194 significant & WL & 24.63 & 14.46 & 4.58 & 18.56 \\
\hline chr7:167-202 suggestive & WL & 16.96 & 3.08 & 0.84 & 0.99 \\
\hline chr7:175-194 significant & WL & 25.23 & 10.12 & 2.89 & 10.76 \\
\hline chr7:175-195 suggestive & WL & 23.78 & 13.35 & 4.21 & 16.11 \\
\hline chr7:175-196 suggestive & WL & 21.83 & 12.47 & 3.98 & 13.82 \\
\hline chr7:175-194 suggestive & WL & 24.13 & 14.48 & 4.7 & 18.82 \\
\hline chr7:174-195 suggestive & WL & 22.34 & 13.09 & 4.21 & 15.29 \\
\hline chr7:174-196 suggestive & WL & 21.59 & 11.91 & 3.37 & 9.37 \\
\hline chr7:174-196 suggestive & WL & 22.81 & 5.99 & 1.46 & 2.89 \\
\hline chr7:174-197 suggestive & WL & 23.81 & 4.2 & 1.16 & 3.62 \\
\hline chr7:174-197 suggestive & WL & 21.36 & 11.36 & 3.44 & 10.64 \\
\hline chr7:175-197 suggestive & WL & 20.88 & 9.94 & 2.62 & 5.74 \\
\hline chr7:173-202 suggestive & WL & 17.8 & 0.95 & 0.48 & 1.9 \\
\hline chr7:174-199 suggestive & WL & 20.73 & 8.79 & 2.83 & 9.13 \\
\hline chr7:169-211 suggestive & WL & 14.67 & 1.35 & 0.42 & 0.63 \\
\hline chr7:174-222 significant & WL & 16.48 & 1.56 & 0.32 & -0.22 \\
\hline chr7:156-231 suggestive & RJF & 10.25 & -2.2 & 0.65 & 0.51 \\
\hline chr7:168-231 significant & WL & 17.04 & 3.22 & 0.66 & -0.4 \\
\hline chr7:176-225 suggestive & WL & 21.56 & 3.08 & 0.64 & -1.67 \\
\hline chr7:169-232 significant & WL & 16.31 & 2.01 & 0.42 & 0.7 \\
\hline chr7:176-227 significant & WL & 23.81 & 5 & 0.85 & -1.63 \\
\hline chr7:176-230 significant & WL & 23.7 & 3.92 & 0.69 & -0.98 \\
\hline chr7:176-230 significant & WL & 16.92 & 2.03 & 0.41 & -0.11 \\
\hline chr7:175-224 significant & WL & 14.78 & 1.84 & 0.39 & -0.07 \\
\hline chr7:176-222 significant & WL & 20.06 & 2.91 & 0.53 & 0.44 \\
\hline chr7:176-225 significant & WL & 25.24 & 3.74 & 0.68 & -1.66 \\
\hline chr7:174-230 significant & WL & 15.15 & 2.88 & 0.62 & -0.36 \\
\hline chr7:178-212 suggestive & WL & 24.85 & 1.86 & 0.41 & -1.8 \\
\hline chr7:176-223 suggestive & WL & 20.09 & 4.3 & 0.85 & -1.29 \\
\hline chr7:176-220 suggestive & WL & 21.25 & 3.14 & 0.63 & -1.4 \\
\hline chr7:179-221 significant & WL & 19.62 & 2.95 & 0.55 & 0.86 \\
\hline chr7:179-226 significant & WL & 22.94 & 1.97 & 0.36 & -0.96 \\
\hline chr7:176-222 suggestive & WL & 19.24 & 2.01 & 0.4 & -0.38 \\
\hline
\end{tabular}




\begin{tabular}{|c|c|c|c|c|c|}
\hline chr7:178-223 significant & WL & 17.71 & 2.01 & 0.4 & -0.2 \\
\hline chr7:179-234 significant & WL & 18.67 & 3.04 & 0.57 & -0.37 \\
\hline chr7:187-225 significant & WL & 33.57 & 2.9 & 0.39 & -0.94 \\
\hline chr7:179-237 significant & WL & 16.75 & 2.54 & 0.51 & 0.13 \\
\hline chr7:176-240 significant & WL & 15.96 & 2.83 & 0.6 & 0.1 \\
\hline chr7:179-213 significant & WL & 18.4 & 1.83 & 0.36 & -0.57 \\
\hline chr7:180-233 significant & WL & 18.67 & 3.03 & 0.6 & -0.91 \\
\hline chr7:185-227 significant & WL & 22.12 & 2.03 & 0.35 & -0.32 \\
\hline chr7:188-212 significant & WL & 32.79 & 3.92 & 0.54 & -1.54 \\
\hline chr7:188-222 significant & WL & 31.08 & 3.92 & 0.55 & -1.1 \\
\hline chr7:185-222 significant & WL & 23.63 & 2.87 & 0.53 & -0.16 \\
\hline chr7:183-228 significant & WL & 16.61 & 3.93 & 0.78 & 1 \\
\hline chr7:182-229 significant & WL & 15.56 & 3.33 & 0.69 & 0.34 \\
\hline chr7:192-226 significant & WL & 20.26 & 2.59 & 0.48 & -0.45 \\
\hline chr7:194-219 significant & WL & 24.73 & 3.33 & 0.61 & -1.1 \\
\hline chr7:195-221 significant & WL & 27.33 & 3.02 & 0.48 & -0.71 \\
\hline chr7:185-224 significant & WL & 15.34 & 2.24 & 0.48 & 0.24 \\
\hline chr7:178-220 suggestive & WL & 21.49 & 2.2 & 0.58 & -2.44 \\
\hline chr7:195-219 significant & WL & 24.07 & 2.71 & 0.55 & -1.58 \\
\hline chr7:190-220 significant & WL & 16.53 & 2.03 & 0.41 & -0.16 \\
\hline chr7:193-231 suggestive & WL & 22.97 & 2.54 & 0.49 & -1.42 \\
\hline chr7:197-220 significant & RJF & 16.55 & -1.36 & 0.34 & 1.17 \\
\hline chr7:192-218 significant & WL & 31.45 & 3.94 & 0.61 & -1.71 \\
\hline chr7:200-221 significant & WL & 25.28 & 2.06 & 0.32 & 0.42 \\
\hline chr7:177-227 suggestive & WL & 14.53 & 1.7 & 0.38 & 0.45 \\
\hline chr7:190-223 significant & WL & 15.41 & 1.34 & 0.28 & 0.07 \\
\hline chr7:189-223 significant & WL & 17.93 & 2.18 & 0.43 & 0.27 \\
\hline chr7:189-226 significant & RJF & 16.66 & -2.61 & 0.55 & 0.55 \\
\hline chr7:201-219 significant & RJF & 23.68 & -2.68 & 0.44 & -1.03 \\
\hline chr7:201-222 significant & WL & 25.89 & 3.08 & 0.61 & -1.57 \\
\hline chr7:209-219 significant & WL & 35.57 & 5.73 & 0.9 & -1.67 \\
\hline chr7:189-223 significant & RJF & 19.04 & -2.5 & 0.49 & 0.81 \\
\hline chr7:198-222 significant & WL & 18.39 & 1.49 & 0.29 & 0.57 \\
\hline chr7:203-220 significant & RJF & 34.57 & -4.61 & 0.59 & 0.53 \\
\hline chr7:191-222 significant & WL & 16.82 & 1.71 & 0.35 & 0.09 \\
\hline chr7:206-222 significant & RJF & 25.8 & -3.17 & 0.52 & 1.35 \\
\hline chr7:206-217 significant & RJF & 35.26 & -2.46 & 0.31 & 0.55 \\
\hline chr7:200-226 significant & RJF & 16.06 & -2.21 & 0.48 & 0.66 \\
\hline chr7:190-223 significant & WL & 19.99 & 3.18 & 0.65 & -1.89 \\
\hline chr7:203-220 significant & RJF & 17.17 & -1.44 & 0.3 & 0.28 \\
\hline chr7:194-222 significant & RJF & 17.06 & -2.26 & 0.47 & 0.64 \\
\hline chr7:201-219 significant & RJF & 27.69 & -1.47 & 0.21 & -0.2 \\
\hline chr7:195-224 significant & WL & 17.73 & 2.01 & 0.4 & -0.39 \\
\hline
\end{tabular}




\begin{tabular}{|c|c|c|c|c|c|}
\hline chr7:204-220 significant & RJF & 14.91 & -1.98 & 0.44 & 0.64 \\
\hline chr7:206-219 significant & RJF & 27.9 & -3.01 & 0.45 & 0.62 \\
\hline chr7:197-228 significant & RJF & 20.3 & -0.62 & 0.57 & -0.85 \\
\hline chr7:193-220 significant & WL & 31.35 & 2.87 & 0.39 & 0.24 \\
\hline chr7:199-222 suggestive & RJF & 17.87 & -0.21 & 0.47 & 1 \\
\hline chr7:200-222 significant & WL & 25.26 & 4.58 & 0.72 & 0.22 \\
\hline chr7:197-221 significant & WL & 24.83 & 4.53 & 0.72 & -0.44 \\
\hline chr7:188-223 suggestive & WL & 14.63 & 1.6 & 0.35 & 0.1 \\
\hline chr7:197-222 suggestive & RJF & 11.76 & -2.03 & 0.51 & 0.02 \\
\hline chr7:199-221 significant & RJF & 14.73 & -1.12 & 0.24 & -0.07 \\
\hline chr7:190-228 suggestive & RJF & 13.66 & -1.09 & 0.26 & 0.47 \\
\hline chr7:190-224 suggestive & RJF & 12.3 & -0.97 & 0.25 & 0.32 \\
\hline chr7:183-224 significant & WL & 20.02 & 3.66 & 0.74 & -1.89 \\
\hline chr7:197-221 significant & WL & 28.83 & 3.79 & 0.56 & -0.83 \\
\hline chr7:189-223 significant & WL & 18.65 & 2.92 & 0.56 & 0.66 \\
\hline chr7:176-229 suggestive & WL & 13.55 & 3.55 & 0.88 & -1.48 \\
\hline chr7:189-222 significant & WL & 25.93 & 2.43 & 0.38 & 0.7 \\
\hline chr7:188-223 significant & WL & 25.52 & 3.35 & 0.57 & -1.66 \\
\hline chr7:194-224 significant & WL & 20.51 & 3.52 & 0.64 & -0.81 \\
\hline chr7:199-223 significant & RJF & 20.73 & -0.25 & 0.45 & 1.13 \\
\hline chr7:203-222 significant & WL & 31.11 & 5.16 & 0.72 & -0.99 \\
\hline chr7:213-218 significant & WL & 69.1 & 8.4 & 0.5 & -0.07 \\
\hline chr7:201-235 significant & RJF & 15.66 & -1.92 & 0.43 & 0.99 \\
\hline chr7:193-224 significant & WL & 22.59 & 5.12 & 0.86 & -0.17 \\
\hline chr7:195-233 significant & WL & 16.78 & 3.01 & 0.61 & 0.11 \\
\hline chr7:186-225 significant & WL & 18.6 & 2.56 & 0.51 & 1.1 \\
\hline chr7:195-222 significant & WL & 39.58 & 4.18 & 0.47 & -0.51 \\
\hline chr7:192-224 significant & WL & 21.55 & 5.66 & 1.58 & -6.47 \\
\hline chr7:203-224 significant & WL & 26.67 & 3.15 & 0.72 & -3.19 \\
\hline chr7:197-222 significant & WL & 31.93 & 4.42 & 0.58 & -0.61 \\
\hline chr7:195-233 significant & RJF & 17.2 & -1.74 & 0.35 & 0.35 \\
\hline chr7:187-227 significant & WL & 20.7 & 3.4 & 0.61 & -0.84 \\
\hline chr7:200-234 significant & RJF & 15.1 & -1.63 & 0.35 & -0.12 \\
\hline chr7:187-228 significant & WL & 20.19 & 3.23 & 0.59 & -0.11 \\
\hline chr7:188-226 significant & WL & 19.29 & 4.06 & 0.76 & 0.8 \\
\hline chr7:192-229 significant & WL & 16.77 & 3.06 & 0.63 & -0.57 \\
\hline chr7:195-231 significant & WL & 25.82 & 3.79 & 0.58 & -0.1 \\
\hline chr7:198-222 significant & WL & 53.53 & 3.79 & 0.32 & -0.08 \\
\hline chr7:190-224 significant & WL & 27.89 & 4.05 & 0.59 & 0.34 \\
\hline chr7:193-230 significant & WL & 18.39 & 2.53 & 0.49 & 0.69 \\
\hline chr7:209-222 significant & WL & 46.76 & 5.08 & 0.5 & 0.16 \\
\hline chr7:199-229 significant & WL & 17.85 & 4.05 & 0.79 & -0.12 \\
\hline chr7:194-225 significant & WL & 27.99 & 4.38 & 0.62 & 0.28 \\
\hline
\end{tabular}




\begin{tabular}{|c|c|c|c|c|c|}
\hline chr7:200-231 significant & WL & 25.71 & 5.34 & 0.82 & -0.28 \\
\hline chr7:176-234 suggestive & WL & 12.96 & 3.19 & 0.74 & -0.38 \\
\hline chr7:177-228 significant & WL & 21.83 & 4.49 & 0.91 & -2.69 \\
\hline chr7:192-231 significant & WL & 17.6 & 2.61 & 0.52 & 0.14 \\
\hline chr7:191-234 significant & WL & 20.2 & 4.03 & 0.74 & -0.92 \\
\hline chr7:182-239 suggestive & WL & 14.91 & 2.4 & 0.52 & 0.61 \\
\hline chr7:185-237 significant & WL & 17.67 & 2.98 & 0.57 & 0.06 \\
\hline chr7:192-232 significant & WL & 22.48 & 4.8 & 0.81 & -0.82 \\
\hline chr7:197-229 significant & WL & 25.95 & 3.06 & 0.47 & 0.16 \\
\hline chr7:176-230 significant & WL & 18.12 & 3.16 & 0.63 & -0.69 \\
\hline chr7:196-238 suggestive & RJF & 12.98 & -1.18 & 0.36 & 1.18 \\
\hline chr7:183-231 significant & WL & 20.28 & 2.32 & 0.43 & 0.89 \\
\hline chr7:181-248 significant & WL & 19.12 & 2.14 & 0.42 & -0.57 \\
\hline chr7:186-231 suggestive & WL & 15.14 & 1.67 & 0.37 & -0.2 \\
\hline chr7:188-234 significant & WL & 17.7 & 2.66 & 0.54 & -0.76 \\
\hline chr7:183-240 suggestive & WL & 13.76 & 2.56 & 0.56 & -0.25 \\
\hline chr7:194-230 significant & WL & 27.95 & 3.07 & 0.46 & -0.79 \\
\hline chr7:194-248 significant & WL & 21.79 & 1.73 & 0.31 & -0.29 \\
\hline chr7:189-234 significant & WL & 16.48 & 2.86 & 0.59 & -0.3 \\
\hline chr7:194-230 significant & WL & 17.9 & 4.32 & 0.85 & -0.83 \\
\hline chr7:187-234 significant & WL & 20.24 & 2.07 & 0.37 & 0.05 \\
\hline chr7:193-234 significant & WL & 15.54 & 3.72 & 0.78 & -0.81 \\
\hline chr7:184-246 suggestive & WL & 14.11 & 2.48 & 0.56 & 0.11 \\
\hline chr7:183-239 suggestive & WL & 15.47 & 2.91 & 0.62 & 0.08 \\
\hline chr7:181-235 suggestive & WL & 13.12 & 2.95 & 0.67 & -0.38 \\
\hline chr7:192-242 significant & RJF & 12.73 & -2.23 & 0.54 & 0.23 \\
\hline chr7:186-245 significant & WL & 15.98 & 3.81 & 0.81 & -1.11 \\
\hline chr7:182-233 significant & WL & 21.35 & 2.29 & 0.4 & -0.01 \\
\hline chr7:176-230 significant & WL & 19.31 & 2.09 & 0.39 & 0.86 \\
\hline chr7:190-240 significant & WL & 20.3 & 2.42 & 0.45 & -0.35 \\
\hline chr7:182-234 significant & WL & 16.3 & 2.83 & 0.58 & 0.18 \\
\hline chr7:185-254 suggestive & WL & 14.5 & 3.5 & 0.77 & 0.1 \\
\hline chr7:184-247 suggestive & WL & 14.37 & 2.18 & 0.48 & 0.09 \\
\hline chr7:190-243 significant & WL & 20.41 & 3.83 & 0.68 & -0.17 \\
\hline chr7:193-233 significant & WL & 20.16 & 4.96 & 0.89 & 0.38 \\
\hline chr7:192-232 significant & WL & 24.91 & 2.63 & 0.41 & -0.41 \\
\hline chr7:186-232 significant & WL & 20.77 & 2.19 & 0.4 & -0.53 \\
\hline chr7:186-242 significant & WL & 13.92 & 4.69 & 1.09 & -2.03 \\
\hline chr7:183-243 significant & WL & 17.97 & 3.16 & 0.61 & -0.1 \\
\hline chr7:180-239 significant & WL & 13.88 & 3.62 & 0.81 & -0.01 \\
\hline chr7:186-234 significant & WL & 19.99 & 2.11 & 0.39 & -0.3 \\
\hline chr7:199-245 significant & RJF & 17.2 & -3.44 & 0.68 & -0.16 \\
\hline chr7:184-255 suggestive & WL & 11.03 & 1.86 & 0.7 & -2.56 \\
\hline
\end{tabular}




\begin{tabular}{|c|c|c|c|c|c|}
\hline chr7:187-249 significant & WL & 16.52 & 3.77 & 0.79 & -0.93 \\
\hline chr7:207-235 suggestive & WL & 20.03 & 3.17 & 0.84 & -2.22 \\
\hline chr7:201-231 significant & WL & 22.16 & 2.39 & 0.41 & 0.02 \\
\hline chr7:190-232 significant & WL & 20.32 & 2.09 & 0.39 & -0.67 \\
\hline chr7:197-244 significant & RJF & 17.48 & -3.25 & 0.65 & -0.03 \\
\hline chr7:185-233 suggestive & WL & 16.93 & 1.89 & 0.38 & 0.05 \\
\hline chr7:216-235 significant & WL & 23.45 & 3.34 & 0.57 & -1.03 \\
\hline chr7:216-231 significant & WL & 21.23 & 2.08 & 0.37 & 0.21 \\
\hline chr7:216-231 significant & WL & 22.39 & 2.16 & 0.38 & -0.55 \\
\hline chr7:217-233 significant & WL & 24.05 & 2.11 & 0.36 & -0.67 \\
\hline chr7:176-231 suggestive & WL & 17.15 & 1.88 & 0.38 & 0.47 \\
\hline chr7:199-233 significant & WL & 18.47 & 2.57 & 0.48 & -0.09 \\
\hline chr7:207-232 significant & WL & 18.79 & 1.45 & 0.29 & -0.37 \\
\hline chr7:217-232 significant & WL & 20.83 & 2.16 & 0.39 & -0.18 \\
\hline chr7:217-235 suggestive & WL & 16.95 & 2.42 & 0.48 & 0.26 \\
\hline chr7:217-235 significant & WL & 18.27 & 1.81 & 0.36 & -0.38 \\
\hline chr7:220-232 significant & WL & 18.43 & 2.02 & 0.38 & 0.35 \\
\hline chr7:220-231 suggestive & WL & 26.75 & 2.45 & 0.42 & 1.79 \\
\hline chr7:219-241 suggestive & WL & 19.18 & 1.29 & 0.25 & -0.6 \\
\hline chr7:217-241 significant & WL & 16.95 & 3.48 & 0.72 & -1 \\
\hline chr7:216-247 suggestive & WL & 15.86 & 1.27 & 0.28 & -0.5 \\
\hline chr7:206-257 suggestive & RJF & 17.16 & -0.86 & 0.5 & 1.74 \\
\hline chr7:221-251 suggestive & WL & 16 & 1.37 & 0.35 & -1.05 \\
\hline chr7:218-247 significant & WL & 17.95 & 2.69 & 0.52 & -0.5 \\
\hline chr7:216-245 suggestive & WL & 14.96 & 2.37 & 0.57 & -1.29 \\
\hline chr7:217-252 suggestive & WL & 14.83 & 1.94 & 0.42 & -0.65 \\
\hline chr7:192-249 significant & WL & 17.77 & 1.93 & 0.38 & 0.15 \\
\hline chr7:216-244 significant & WL & 16.53 & 2.75 & 0.58 & -2.25 \\
\hline chr7:218-253 significant & WL & 25.18 & 2.22 & 0.36 & 0.27 \\
\hline chr7:220-245 suggestive & WL & 18.29 & 2.76 & 0.89 & -3.65 \\
\hline chr7:221-247 significant & WL & 20.56 & 1.91 & 0.34 & -0.45 \\
\hline chr7:177-254 significant & WL & 15.57 & 2.36 & 0.49 & -0.36 \\
\hline chr7:195-245 significant & WL & 17.86 & 2.51 & 0.53 & -2.07 \\
\hline chr7:221-259 significant & WL & 16.19 & 2.42 & 0.5 & 0.54 \\
\hline chr7:221-247 significant & WL & 18.24 & 2.04 & 0.39 & 0.01 \\
\hline chr7:220-269 significant & WL & 16.73 & 1.87 & 0.43 & -1.89 \\
\hline chr7:221-245 significant & WL & 23.91 & 1.62 & 0.26 & -0.7 \\
\hline chr7:224-247 significant & WL & 25.62 & 1.89 & 0.29 & -0.28 \\
\hline chr7:220-245 suggestive & WL & 14.95 & 2.84 & 0.61 & -0.69 \\
\hline chr7:222-246 significant & WL & 16.72 & 1.2 & 0.24 & -0.36 \\
\hline chr7:223-243 significant & WL & 19.99 & 4 & 0.71 & -1.05 \\
\hline chr7:226-244 significant & WL & 22.63 & 2.66 & 0.43 & 0.04 \\
\hline chr7:223-247 suggestive & WL & 16.22 & 1.45 & 0.31 & 0.34 \\
\hline
\end{tabular}




\begin{tabular}{|c|c|c|c|c|c|}
\hline chr7:220-246 significant & WL & 17.88 & 1.84 & 0.36 & -0.73 \\
\hline chr7:220-242 suggestive & WL & 14.19 & 2.12 & 0.55 & -2.56 \\
\hline chr7:222-246 suggestive & WL & 19.93 & 1.81 & 0.33 & -0.66 \\
\hline chr7:223-244 suggestive & WL & 19.75 & 2.52 & 0.45 & -1.04 \\
\hline chr7:223-244 suggestive & WL & 18.66 & 3.24 & 0.61 & -1.45 \\
\hline chr7:224-253 significant & WL & 16.55 & 2.92 & 0.59 & -0.41 \\
\hline chr7:224-245 significant & WL & 16.38 & 1.4 & 0.28 & -0.01 \\
\hline chr7:227-243 suggestive & WL & 9.59 & 0.05 & 0.27 & 2.44 \\
\hline chr7:227-242 significant & WL & 25.21 & 2.48 & 0.39 & -0.64 \\
\hline chr7:224-242 significant & WL & 21.38 & 3 & 0.54 & -1.79 \\
\hline chr7:226-242 significant & WL & 20.77 & 4 & 0.7 & -1.5 \\
\hline chr7:224-243 suggestive & WL & 13.98 & 2.65 & 0.63 & -2.34 \\
\hline chr7:230-239 suggestive & WL & 13.83 & 0.14 & 0.11 & 1.14 \\
\hline chr7:227-241 significant & WL & 20.33 & 2.03 & 0.36 & -0.74 \\
\hline chr7:229-262 significant & RJF & 10.81 & -1.81 & 0.51 & -1.07 \\
\hline chr7:230-247 significant & WL & 19.85 & 1.86 & 0.37 & 0.97 \\
\hline chr7:226-244 significant & WL & 18.28 & 2.28 & 0.44 & -1.1 \\
\hline chr7:226-254 significant & WL & 17.12 & 2.88 & 0.57 & -0.78 \\
\hline chr7:228-251 significant & WL & 15.74 & 1.23 & 0.26 & 0.41 \\
\hline chr7:229-246 significant & WL & 24.61 & 2.41 & 0.39 & -0.74 \\
\hline chr7:230-259 significant & RJF & 11.68 & -2.18 & 0.59 & -1.03 \\
\hline chr7:224-261 significant & RJF & 15.61 & -1.88 & 0.41 & 0.76 \\
\hline chr7:227-275 significant & WL & 17.5 & 2.95 & 0.62 & -2.03 \\
\hline chr7:222-251 significant & WL & 15.61 & 2.42 & 0.51 & -0.8 \\
\hline chr7:218-265 significant & RJF & 15.99 & -1.95 & 1.16 & -2.79 \\
\hline chr7:228-276 significant & WL & 16.19 & 2.86 & 0.59 & -0.76 \\
\hline chr7:225-273 significant & WL & 13.35 & 1.83 & 0.48 & -1.24 \\
\hline chr7:224-254 significant & WL & 25.3 & 3.49 & 0.85 & -2.89 \\
\hline chr7:195-255 significant & WL & 18.76 & 2.78 & 0.54 & -1.24 \\
\hline chr7:230-259 significant & RJF & 14.99 & -1.99 & 0.44 & -0.29 \\
\hline chr7:223-253 suggestive & WL & 15.03 & 1.84 & 0.39 & -0.03 \\
\hline chr7:227-254 significant & WL & 21.99 & 3.36 & 0.57 & -0.53 \\
\hline chr7:227-268 suggestive & RJF & 9.99 & -1.81 & 0.49 & -0.08 \\
\hline chr7:232-258 significant & WL & 18.28 & 1.43 & 0.28 & -0.62 \\
\hline chr7:220-269 significant & WL & 14.96 & 2.1 & 0.45 & -0.89 \\
\hline chr7:231-256 significant & WL & 19.7 & 1.51 & 0.28 & 0.14 \\
\hline chr7:232-261 significant & WL & 17.19 & 1.7 & 0.36 & -1.32 \\
\hline chr7:229-255 significant & WL & 15.91 & 1.44 & 0.31 & -0.03 \\
\hline chr7:225-258 significant & WL & 16.14 & 2.73 & 0.56 & -0.93 \\
\hline chr7:238-268 suggestive & WL & 17.71 & 2.08 & 0.63 & -3.23 \\
\hline chr7:237-258 suggestive & WL & 15.92 & 1.55 & 0.55 & -2.76 \\
\hline chr7:238-255 suggestive & WL & 14.72 & 0.76 & 0.17 & -0.98 \\
\hline chr7:237-258 significant & WL & 17.77 & 2.21 & 0.44 & -0.23 \\
\hline
\end{tabular}




\begin{tabular}{|c|c|c|c|c|c|}
\hline chr7:240-253 significant & WL & 15.46 & 0.99 & 0.24 & -1.04 \\
\hline chr7:240-255 suggestive & WL & 14.3 & 0.84 & 0.23 & -1.1 \\
\hline chr7:239-257 suggestive & WL & 14.42 & 0.99 & 0.23 & -0.65 \\
\hline chr7:227-255 significant & WL & 16.51 & 1.53 & 0.32 & 0.14 \\
\hline chr7:243-253 suggestive & WL & 23.01 & 0.38 & 0.38 & 0.77 \\
\hline chr7:240-256 suggestive & WL & 18.99 & 1.25 & 0.24 & -0.55 \\
\hline chr7:239-258 suggestive & WL & 19.43 & 1.79 & 0.34 & -0.83 \\
\hline chr7:244-252 suggestive & WL & 21.19 & 1.94 & 0.37 & -1.68 \\
\hline chr7:240-254 suggestive & WL & 18.81 & 2.08 & 0.4 & -1.26 \\
\hline chr7:238-258 significant & RJF & 20.07 & -2.3 & 0.42 & 0.72 \\
\hline chr7:230-296 suggestive & RJF & 10.31 & -2.17 & 0.59 & -0.25 \\
\hline chr7:231-268 significant & WL & 18.02 & 1.3 & 0.25 & -0.31 \\
\hline chr7:239-270 significant & WL & 21.53 & 1.98 & 0.48 & -2.4 \\
\hline chr7:231-289 significant & WL & 15.25 & 1.28 & 0.27 & -0.13 \\
\hline chr7:237-274 significant & WL & 18.69 & 1.81 & 0.35 & 0.44 \\
\hline chr7:232-271 significant & RJF & 22.71 & -2.06 & 0.35 & -0.55 \\
\hline chr7:226-277 significant & WL & 14.02 & 1.6 & 0.38 & 1.17 \\
\hline chr7:238-270 significant & WL & 23.75 & 4.02 & 1.05 & -2.94 \\
\hline chr7:239-273 significant & WL & 23.67 & 2.29 & 0.37 & 0.12 \\
\hline chr7:242-272 significant & WL & 17.85 & 2.69 & 0.53 & -0.7 \\
\hline chr7:250-277 significant & WL & 17.38 & 3.25 & 0.65 & -0.23 \\
\hline chr7:229-277 significant & WL & 14.75 & 2.39 & 0.53 & 0.4 \\
\hline chr7:251-271 significant & WL & 17.04 & 2.53 & 0.5 & -0.18 \\
\hline chr7:252-269 significant & RJF & 22.85 & -2.22 & 0.37 & -0.31 \\
\hline chr7:256-274 significant & RJF & 19.02 & -2.03 & 0.37 & -0.02 \\
\hline chr7:256-271 significant & WL & 29.19 & 3.64 & 0.51 & 0.05 \\
\hline chr7:252-270 significant & WL & 16.85 & 3.95 & 0.78 & 0.62 \\
\hline chr7:252-273 suggestive & RJF & 12.96 & -1.13 & 0.28 & -0.64 \\
\hline chr7:253-275 significant & RJF & 15.72 & -1.06 & 0.22 & 0.06 \\
\hline chr7:252-279 significant & WL & 20.93 & 4.22 & 0.95 & -2.85 \\
\hline chr7:251-277 significant & WL & 18.65 & 2.74 & 0.52 & 0.5 \\
\hline chr7:252-275 significant & RJF & 25.27 & -2.5 & 0.39 & -0.47 \\
\hline chr7:255-277 significant & WL & 21.99 & 1.91 & 0.57 & -1.06 \\
\hline chr7:254-293 significant & WL & 14.32 & 2.21 & 0.5 & -0.29 \\
\hline chr7:255-292 significant & RJF & 14.13 & -1.72 & 0.39 & 0.23 \\
\hline chr7:253-276 significant & WL & 29.53 & 5.58 & 0.78 & 0.93 \\
\hline chr7:253-286 significant & WL & 16.71 & 3.87 & 0.78 & 0.34 \\
\hline chr7:253-280 significant & WL & 25.44 & 1.96 & 0.31 & 0.45 \\
\hline chr7:252-277 significant & RJF & 23.33 & -2.54 & 0.42 & -0.3 \\
\hline chr7:255-292 significant & RJF & 15.74 & -2.17 & 0.46 & 0.11 \\
\hline chr7:255-288 significant & WL & 13.78 & 1.87 & 0.42 & 0.15 \\
\hline chr7:254-286 significant & RJF & 20.25 & -2.8 & 0.52 & -1.38 \\
\hline chr7:253-291 significant & RJF & 14.73 & -4.15 & 0.93 & 0.88 \\
\hline
\end{tabular}




\begin{tabular}{|c|c|c|c|c|c|}
\hline chr7:254-285 significant & WL & 12.91 & 1.99 & 0.48 & -0.43 \\
\hline chr7:231-300 suggestive & RJF & 14.54 & -1.43 & 0.32 & 0.41 \\
\hline chr7:254-284 significant & WL & 29.22 & 2.41 & 0.34 & 0.19 \\
\hline chr7:242-303 suggestive & WL & 12 & 1.13 & 0.32 & -0.46 \\
\hline chr7:252-290 significant & WL & 21.87 & 2.74 & 0.48 & 1.81 \\
\hline chr7:255-291 significant & WL & 22.92 & 2.42 & 0.4 & 1.13 \\
\hline chr7:260-291 suggestive & WL & 21.07 & 0.58 & 0.94 & 2.06 \\
\hline chr7:247-293 significant & WL & 18.46 & 2.02 & 0.39 & 1.28 \\
\hline chr7:264-290 significant & WL & 26.27 & 3.65 & 0.56 & 0.71 \\
\hline chr7:264-293 suggestive & WL & 14.1 & 1.91 & 0.51 & 2.47 \\
\hline chr7:246-302 suggestive & WL & 15 & 1.67 & 0.37 & -0.12 \\
\hline chr7:254-294 suggestive & RJF & 13.45 & -2.5 & 0.58 & -0.78 \\
\hline chr7:260-290 significant & WL & 18.8 & 1.51 & 0.29 & -0.07 \\
\hline chr7:254-292 significant & WL & 20.51 & 2.19 & 0.43 & 0.63 \\
\hline chr7:265-299 significant & WL & 22.68 & 2.22 & 0.38 & 1.55 \\
\hline chr7:268-295 significant & WL & 29.38 & 2.09 & 0.3 & 0.98 \\
\hline chr7:259-297 significant & RJF & 13.44 & -2.62 & 0.59 & -0.83 \\
\hline chr7:264-289 significant & WL & 24.49 & 0.58 & 1.07 & 0.46 \\
\hline chr7:265-293 significant & WL & 19.18 & 3.14 & 0.59 & 0.69 \\
\hline chr7:267-291 suggestive & WL & 15.09 & 0.12 & 0.61 & 4.03 \\
\hline chr7:268-292 significant & RJF & 25.3 & -0.17 & 0.22 & 1.89 \\
\hline chr7:265-291 significant & WL & 20.72 & 2.67 & 0.49 & -0.05 \\
\hline chr7:267-290 significant & RJF & 21.67 & -2.54 & 0.48 & 0.74 \\
\hline chr7:262-292 significant & WL & 14.06 & 2.74 & 0.61 & 0.58 \\
\hline chr7:264-296 significant & WL & 15.63 & 2.73 & 0.58 & 0.42 \\
\hline chr7:270-291 significant & RJF & 19.18 & -2.47 & 0.51 & 0.73 \\
\hline chr7:269-291 significant & WL & 23.09 & 0.89 & 0.66 & 2.33 \\
\hline chr7:274-287 significant & RJF & 36.61 & -0.45 & 0.83 & 3.45 \\
\hline chr7:270-293 suggestive & WL & 14.74 & 1.13 & 0.33 & 1.78 \\
\hline chr7:264-295 significant & RJF & 14.45 & -2.58 & 0.57 & -0.7 \\
\hline chr7:263-296 suggestive & RJF & 12.5 & -2.26 & 0.56 & -1.45 \\
\hline chr7:265-297 significant & WL & 14.27 & 2.5 & 0.55 & 0.65 \\
\hline chr7:269-292 suggestive & RJF & 13.97 & -2.34 & 0.67 & 1.74 \\
\hline chr7:264-296 significant & RJF & 16 & -1.51 & 0.36 & 0.68 \\
\hline chr7:268-299 suggestive & WL & 13.68 & 1.45 & 0.34 & 0.98 \\
\hline chr7:268-298 suggestive & WL & 13.96 & 2.38 & 0.63 & 2.76 \\
\hline chr7:268-296 suggestive & WL & 14.7 & 1.59 & 0.37 & 1.34 \\
\hline chr7:269-292 suggestive & WL & 18.92 & 0.37 & 0.38 & 2.99 \\
\hline chr7:270-293 suggestive & WL & 14.86 & 0.2 & 0.28 & 1.86 \\
\hline chr7:265-301 significant & RJF & 15.17 & -1.5 & 0.34 & 0.2 \\
\hline chr7:272-293 significant & WL & 41.45 & 3.22 & 0.35 & 0.42 \\
\hline chr7:270-297 significant & WL & 29.22 & 2.52 & 0.36 & 1.61 \\
\hline chr7:269-298 significant & WL & 24.31 & 2.72 & 0.44 & 0.88 \\
\hline
\end{tabular}




\begin{tabular}{|c|c|c|c|c|c|c|}
\hline \multicolumn{2}{|c|}{ chr7:264-301 significant } & WL & 21.17 & 1.88 & 0.55 & -0.49 \\
\hline \multicolumn{2}{|c|}{ chr7:270-296 significant } & WL & 33.16 & 4 & 0.51 & 0.76 \\
\hline \multicolumn{2}{|c|}{ chr7:267-298 significant } & WL & 27.2 & 1.5 & 0.22 & 0.7 \\
\hline \multicolumn{2}{|c|}{ chr7:269-299 significant } & WL & 37.64 & 6.01 & 0.71 & 2.3 \\
\hline \multicolumn{2}{|c|}{ chr7:268-300 significant } & WL & 34.26 & 4.23 & 0.52 & 1.26 \\
\hline \multicolumn{2}{|c|}{ chr7:267-304 significant } & WL & 29.63 & 3.06 & 0.42 & 1.09 \\
\hline \multicolumn{2}{|c|}{ chr7:257-330 suggestive } & RJF & 11.97 & -2.91 & 0.72 & -1.04 \\
\hline \multicolumn{2}{|c|}{ chr7:270-332 significant } & WL & 19.67 & 2.93 & 0.58 & -0.11 \\
\hline \multicolumn{2}{|c|}{ chr7:272-336 significant } & WL & 18.05 & 2.78 & 0.55 & -0.11 \\
\hline \multicolumn{2}{|c|}{ chr7:268-336 significant } & WL & 16.08 & 1.79 & 0.38 & 0.92 \\
\hline \multicolumn{2}{|c|}{ chr7:282-323 significant } & WL & 21.24 & 0.51 & 0.84 & -1.54 \\
\hline \multicolumn{2}{|c|}{ chr7:281-325 significant } & WL & 22.68 & 2 & 0.37 & -0.24 \\
\hline \multicolumn{2}{|c|}{ chr7:269-334 significant } & RJF & 14.09 & -2.18 & 0.62 & 1.87 \\
\hline \multicolumn{2}{|c|}{ chr7:275-332 significant } & WL & 17.76 & 2.22 & 0.44 & 0.72 \\
\hline \multicolumn{2}{|c|}{ chr7:289-328 significant } & WL & 28.2 & 2.41 & 0.36 & -0.32 \\
\hline \multicolumn{2}{|c|}{ chr7:291-332 significant } & WL & 28.64 & 2.32 & 0.39 & -0.68 \\
\hline \multicolumn{2}{|c|}{ chr7:291-329 significant } & WL & 26.82 & 3.36 & 0.6 & -1.05 \\
\hline \multicolumn{2}{|c|}{ chr7:292-322 significant } & WL & 31.79 & 5.51 & 0.79 & -0.4 \\
\hline \multicolumn{2}{|c|}{ chr7:293-322 significant } & WL & 30.43 & 5.83 & 0.91 & -1.14 \\
\hline \multicolumn{2}{|c|}{ chr7:294-330 significant } & RJF & 18.39 & -3.14 & 0.61 & -0.62 \\
\hline \multicolumn{2}{|c|}{ chr7:316-333 significant } & RJF & 20.32 & -2.54 & 0.47 & -1.56 \\
\hline \multicolumn{2}{|c|}{ chr7:317-327 suggestive } & RJF & 26.25 & -0.26 & 0.05 & -0.27 \\
\hline \multicolumn{2}{|c|}{ chr7:317-326 suggestive } & RJF & 26.24 & -0.6 & 0.11 & -0.45 \\
\hline \multicolumn{2}{|c|}{ chr7:314-332 significant } & RJF & 23.52 & -2.05 & 0.33 & -0.16 \\
\hline chr7:302-3 & suggestive & RJF & 14.45 & -1.61 & 0.36 & 0.32 \\
\hline chr7:316-3 & suggestive & RJF & 20.39 & -0.37 & 0.1 & -0.61 \\
\hline chr7:292-3 & significant & WL & 22.63 & 2.47 & 0.48 & 1.11 \\
\hline chr7:297-3 & significant & RJF & 16.57 & -1.32 & 0.31 & 0.27 \\
\hline chr7:322-3 & significant & RJF & 22.28 & -1.99 & 0.35 & -0.01 \\
\hline chr8:0-9 & significant & RJF & 24.25 & -2.11 & 0.72 & -1.84 \\
\hline chr8:0-9 & significant & RJF & 21.79 & -2.28 & 0.4 & -0.07 \\
\hline chr8:0-7 & suggestive & RJF & 16.49 & -1.85 & 0.38 & 0.43 \\
\hline chr8:0-8 & suggestive & RJF & 15.34 & -2.01 & 0.43 & 1.09 \\
\hline chr8:0-12 & significant & RJF & 16.92 & -1.85 & 0.39 & -0.15 \\
\hline chr8:0-9 & suggestive & RJF & 19.66 & -0.2 & 0.22 & 0.02 \\
\hline chr8:0-8 & suggestive & RJF & 25.05 & -1.08 & 0.45 & -1.42 \\
\hline chr8:0-6 & suggestive & WL & 23.88 & 1.69 & 0.55 & -3.85 \\
\hline chr8:0-12 & suggestive & RJF & 17.93 & -1.58 & 0.31 & -0.27 \\
\hline chr8:0-14 & suggestive & RJF & 16.14 & -1.56 & 0.31 & 0.35 \\
\hline chr8:0-9 & suggestive & RJF & 18.75 & -1.57 & 0.3 & 0.88 \\
\hline chr8:0-12 & suggestive & RJF & 20.74 & -1.52 & 0.28 & 0.1 \\
\hline chr8:0-14 & suggestive & RJF & 16.13 & -1.6 & 0.35 & -0.35 \\
\hline chr8:0-16 & suggestive & RJF & 19.62 & -0.07 & 0.45 & -1.3 \\
\hline
\end{tabular}




\begin{tabular}{|c|c|c|c|c|c|c|}
\hline chr8:0-12 & significant & RJF & 29.58 & -2.79 & 0.39 & 0.7 \\
\hline chr8:0-13 & suggestive & RJF & 21.87 & -0.97 & 0.2 & -0.61 \\
\hline chr8:0-13 & suggestive & RJF & 22.99 & -1.54 & 0.3 & -0.71 \\
\hline chr8:0-15 & significant & RJF & 20.43 & -1.66 & 0.31 & -0.03 \\
\hline chr8:0-17 & suggestive & RJF & 15.97 & -1.76 & 0.38 & -0.04 \\
\hline chr8:0-18 & suggestive & RJF & 15.29 & -0.86 & 0.18 & -0.12 \\
\hline chr8:0-19 & suggestive & RJF & 19.33 & -1.4 & 0.26 & 0.18 \\
\hline chr8:0-19 & suggestive & RJF & 18.74 & -2.06 & 0.41 & -0.13 \\
\hline chr8:0-36 & significant & RJF & 17.7 & -1.64 & 0.36 & -0.54 \\
\hline chr8:0-19 & suggestive & RJF & 19.78 & -2.06 & 0.38 & 0.31 \\
\hline chr8:0-46 & suggestive & RJF & 18.91 & -1.09 & 0.25 & -0.65 \\
\hline chr8:0-51 & significant & WL & 9.79 & 1.6 & 0.45 & -0.13 \\
\hline chr8:34-78 & suggestive & WL & 13.1 & 0.95 & 0.25 & -0.81 \\
\hline chr8:38-80 & suggestive & WL & 14.43 & 1.24 & 0.37 & -1.77 \\
\hline chr8:42-80 & suggestive & WL & 18.87 & 0.78 & 0.5 & -0.13 \\
\hline chr8:41-122 & suggestive & WL & 14.81 & 1.15 & 0.33 & -1.57 \\
\hline chr8:45-82 & suggestive & RJF & 21 & -0.08 & 0.25 & -0.05 \\
\hline chr8:45-78 & suggestive & WL & 23.74 & 1.14 & 0.67 & -1.16 \\
\hline chr8:45-74 & suggestive & WL & 22.29 & 0.28 & 0.43 & -0.66 \\
\hline chr8:45-76 & suggestive & WL & 23.5 & 0.54 & 0.53 & -0.08 \\
\hline chr8:46-75 & significant & WL & 20.21 & 0.43 & 0.52 & -0.48 \\
\hline chr8:46-80 & suggestive & WL & 20.54 & 0.05 & 0.5 & -0.34 \\
\hline chr8:48-76 & suggestive & WL & 22.89 & 0.27 & 0.38 & 0.18 \\
\hline chr8:39-132 & suggestive & WL & 12.31 & 1.72 & 0.44 & 0.02 \\
\hline chr8:43-80 & suggestive & WL & 17.9 & 2.75 & 0.7 & 1.02 \\
\hline chr8:49-76 & significant & WL & 24.93 & 0.54 & 0.35 & 0.38 \\
\hline chr8:48-79 & suggestive & WL & 16.85 & 1.28 & 0.37 & -2.07 \\
\hline chr8:50-78 & suggestive & WL & 20.93 & 0.01 & 0.44 & 0.49 \\
\hline chr8:48-93 & significant & WL & 16.01 & 1.2 & 0.25 & -0.7 \\
\hline chr8:54-85 & significant & WL & 18.17 & 2.9 & 0.82 & -1.94 \\
\hline chr8:52-84 & suggestive & WL & 19.84 & 1.76 & 0.73 & -1.09 \\
\hline chr8:52-82 & significant & WL & 15.46 & 1.56 & 0.44 & -2.11 \\
\hline chr8:52-77 & significant & WL & 20.28 & 0.3 & 0.35 & -0.41 \\
\hline chr8:50-79 & suggestive & WL & 21.06 & 0.03 & 0.48 & -0.15 \\
\hline chr8:53-77 & suggestive & WL & 18.31 & 0.67 & 0.15 & -0.73 \\
\hline chr8:53-79 & suggestive & WL & 17.87 & 0.65 & 0.24 & -1.54 \\
\hline chr8:43-98 & suggestive & WL & 12.28 & 1.48 & 0.37 & 0.21 \\
\hline chr8:49-85 & suggestive & WL & 15.23 & 0.91 & 0.29 & -1.53 \\
\hline chr8:48-115 & suggestive & WL & 13.64 & 0.86 & 0.25 & -1.06 \\
\hline chr8:53-96 & suggestive & WL & 13.37 & 0.53 & 0.14 & -0.47 \\
\hline chr8:51-95 & suggestive & RJF & 13.02 & -28.32 & 6.24 & 6.41 \\
\hline chr8:53-94 & significant & RJF & 15.27 & -42.54 & 9.12 & 2.71 \\
\hline chr8:51-94 & significant & RJF & 14.3 & -38.15 & 8.59 & 0.62 \\
\hline
\end{tabular}




\begin{tabular}{|c|c|c|c|c|c|c|}
\hline chr8:51-94 & significant & RJF & 14.18 & -34.95 & 8.05 & -1.04 \\
\hline chr8:52-95 & suggestive & RJF & 13.63 & -43.39 & 10.15 & -0.95 \\
\hline chr8:53-94 & significant & RJF & 16.14 & -40.3 & 8.74 & -2.49 \\
\hline chr8:54-94 & significant & RJF & 15.99 & -47.76 & 9.97 & 2.57 \\
\hline chr8:53-94 & significant & RJF & 15.65 & -41.01 & 8.82 & 0.65 \\
\hline chr8:53-94 & significant & RJF & 15.51 & -37.73 & 8.13 & 0.53 \\
\hline chr8:51-94 & suggestive & RJF & 13.2 & -31.67 & 7.62 & -2.54 \\
\hline chr8:54-94 & significant & RJF & 15.14 & -41.53 & 9.2 & -1 \\
\hline chr8:55-94 & significant & RJF & 15.84 & -36.26 & 7.63 & 3.33 \\
\hline chr8:52-94 & significant & RJF & 16.04 & -53.36 & 11.59 & -3.08 \\
\hline chr8:62-122 & significant & WL & 19.53 & 1.61 & 0.63 & 2.19 \\
\hline chr8:51-94 & suggestive & RJF & 13.31 & -43.03 & 10.38 & -2.55 \\
\hline chr8:53-94 & suggestive & RJF & 14.03 & -44.93 & 10.41 & -0.32 \\
\hline chr8:52-104 & significant & WL & 16.07 & 3.86 & 1 & 1.41 \\
\hline chr8:55-94 & significant & RJF & 15.98 & -45.61 & 10.2 & -5.07 \\
\hline chr8:56-94 & significant & RJF & 15.84 & -36.35 & 7.88 & -0.49 \\
\hline chr8:55-94 & significant & RJF & 16.37 & -40.6 & 8.75 & -2.34 \\
\hline chr8:47-94 & suggestive & WL & 14.83 & 3.1 & 1.12 & 1.84 \\
\hline chr8:57-94 & significant & RJF & 16.37 & -33.74 & 7.4 & -2.28 \\
\hline chr8:52-94 & suggestive & RJF & 13.13 & -47.11 & 11.74 & -5.92 \\
\hline chr8:63-94 & significant & RJF & 18.7 & -41.12 & 8.05 & 2.1 \\
\hline chr8:63-93 & significant & RJF & 20.8 & -51.31 & 9.52 & -0.07 \\
\hline chr8:61-93 & significant & RJF & 19.63 & -55.02 & 10.82 & -4.89 \\
\hline chr8:51-94 & suggestive & RJF & 12.86 & -48.01 & 12.44 & -10.18 \\
\hline chr8:53-94 & significant & RJF & 14.1 & -57.92 & 14.18 & -11.56 \\
\hline chr8:60-93 & significant & RJF & 17.04 & -52.65 & 11.21 & -2.33 \\
\hline chr8:58-94 & significant & RJF & 14.77 & -38.53 & 9.31 & -6.36 \\
\hline chr8:71-99 & significant & WL & 30.41 & 3.49 & 0.5 & -1.28 \\
\hline chr8:52-95 & suggestive & RJF & 14.24 & -1.48 & 0.36 & -0.03 \\
\hline chr8:78-96 & suggestive & WL & 22.81 & 1.18 & 0.49 & 2.6 \\
\hline chr8:75-94 & suggestive & WL & 17.07 & 0.34 & 0.49 & 0.17 \\
\hline chr8:80-90 & significant & WL & 29.56 & 5.03 & 0.97 & 2.63 \\
\hline chr8:78-91 & significant & WL & 31.3 & 12.82 & 2.13 & 1.9 \\
\hline chr8:77-91 & significant & WL & 32.24 & 2.94 & 0.47 & 0.94 \\
\hline chr8:72-97 & suggestive & WL & 18.08 & 0.64 & 0.65 & 0.51 \\
\hline chr8:76-97 & significant & WL & 17.98 & 1.54 & 0.34 & 0.33 \\
\hline chr8:74-135 & suggestive & RJF & 14.08 & -1.93 & 0.54 & -0.75 \\
\hline chr8:67-94 & suggestive & WL & 13.25 & 1.77 & 0.43 & -0.37 \\
\hline chr8:62-95 & significant & RJF & 16.25 & -3.38 & 0.79 & -0.74 \\
\hline chr8:68-99 & significant & WL & 13.4 & 1.8 & 0.39 & -0.74 \\
\hline chr8:71-99 & significant & WL & 13.91 & 1.58 & 0.38 & -0.12 \\
\hline chr8:84-90 & significant & WL & 49.94 & 6.3 & 0.7 & 2.27 \\
\hline chr8:80-91 & significant & WL & 37.49 & 2.27 & 0.28 & 0.08 \\
\hline
\end{tabular}




\begin{tabular}{|c|c|c|c|c|c|c|}
\hline chr8:71-95 & suggestive & WL & 12.03 & 1.33 & 0.39 & 0.41 \\
\hline chr8:59-97 & suggestive & WL & 12.1 & 1.08 & 0.35 & 0.63 \\
\hline chr8:72-103 & significant & WL & 13.81 & 1.67 & 0.41 & -0.16 \\
\hline chr8:72-135 & significant & WL & 12.95 & 3.71 & 0.9 & -0.93 \\
\hline chr8:75-97 & significant & WL & 21.48 & 1.56 & 0.3 & 0.01 \\
\hline chr8:73-122 & significant & WL & 14.52 & 2.63 & 0.68 & 0.55 \\
\hline chr8:76-98 & significant & WL & 20.97 & 1.42 & 0.28 & 0.12 \\
\hline chr8:43-131 & significant & WL & 14.9 & 1.89 & 0.42 & -0.56 \\
\hline chr8:70-122 & significant & WL & 14.43 & 1.66 & 0.48 & 0.87 \\
\hline chr8:71-92 & suggestive & WL & 20.9 & 3.53 & 0.86 & -0.08 \\
\hline chr8:64-93 & suggestive & WL & 20.58 & 4.32 & 1.13 & -0.08 \\
\hline chr8:84-96 & significant & RJF & 26.15 & -2.28 & 0.39 & -0.11 \\
\hline chr8:73-132 & significant & WL & 18 & 3.59 & 0.81 & -0.55 \\
\hline chr8:53-98 & suggestive & WL & 13.13 & 1.23 & 0.31 & 0.02 \\
\hline chr8:64-96 & suggestive & WL & 10.53 & 1.38 & 0.54 & 1.12 \\
\hline chr8:76-158 & suggestive & WL & 11.24 & 1.16 & 0.38 & 0.56 \\
\hline chr8:67-132 & significant & WL & 14.56 & 1.49 & 0.36 & 0.04 \\
\hline chr8:83-94 & significant & WL & 31.65 & 1.98 & 0.28 & -0.37 \\
\hline chr8:73-99 & significant & WL & 16.09 & 1.61 & 0.36 & -0.04 \\
\hline chr8:91-92 & significant & WL & 35.55 & 2.04 & 0.29 & 0.55 \\
\hline chr8:76-96 & significant & WL & 24.03 & 3.94 & 0.67 & -0.17 \\
\hline chr8:78-99 & suggestive & RJF & 15.57 & -1.66 & 0.38 & 0.09 \\
\hline chr8:77-98 & suggestive & WL & 12.14 & 1.62 & 0.55 & 1.06 \\
\hline chr8:75-98 & suggestive & WL & 11.26 & 2.68 & 0.77 & 0.15 \\
\hline chr8:73-98 & suggestive & WL & 12.45 & 2.57 & 0.77 & 0.84 \\
\hline chr8:75-98 & suggestive & WL & 12.46 & 2 & 0.5 & -0.5 \\
\hline chr8:77-96 & suggestive & WL & 24.3 & 2.8 & 0.51 & -2.7 \\
\hline chr8:88-98 & significant & WL & 31.98 & 2.59 & 0.37 & 0.4 \\
\hline chr8:56-121 & significant & WL & 15.98 & 1.62 & 0.35 & -0.23 \\
\hline chr8:82-132 & significant & RJF & 22.73 & -3.87 & 0.66 & 1.41 \\
\hline chr8:75-131 & suggestive & RJF & 15.21 & -2.54 & 0.57 & 0.45 \\
\hline chr8:74-114 & significant & WL & 17.82 & 1.06 & 0.22 & -0.04 \\
\hline chr8:80-124 & significant & RJF & 16.97 & -1.92 & 0.4 & 0.6 \\
\hline chr8:81-127 & significant & RJF & 17.11 & -1.95 & 0.41 & 0.19 \\
\hline chr8:75-133 & suggestive & RJF & 13.93 & -3.28 & 0.78 & 0.19 \\
\hline chr8:80-122 & suggestive & WL & 14.58 & 1.78 & 0.4 & -1.54 \\
\hline chr8:94-113 & significant & WL & 28.74 & 8.57 & 1.39 & 1.31 \\
\hline chr8:90-104 & significant & RJF & 18.9 & -1.63 & 0.37 & -0.48 \\
\hline chr8:95-132 & significant & RJF & 19.45 & -0.95 & 0.91 & -0.41 \\
\hline chr8:96-104 & suggestive & WL & 19.89 & 0.1 & 0.45 & 0.06 \\
\hline chr8:95-103 & significant & RJF & 21.08 & -2.04 & 0.39 & -0.12 \\
\hline chr8:96-132 & significant & RJF & 19.37 & -2.94 & 0.59 & 0.12 \\
\hline chr8:94-117 & significant & RJF & 17.95 & -3.49 & 0.75 & -0.74 \\
\hline
\end{tabular}




\begin{tabular}{|c|c|c|c|c|c|c|}
\hline chr8:95-126 & significant & WL & 14.94 & 3.91 & 0.89 & -0.38 \\
\hline chr8:94-131 & significant & WL & 20.81 & 3.52 & 0.71 & 0.62 \\
\hline chr8:97-103 & significant & WL & 29.23 & 4.9 & 0.78 & 0.85 \\
\hline chr8:95-157 & suggestive & RJF & 14.69 & -2.43 & 0.6 & -0.37 \\
\hline chr8:92-120 & suggestive & RJF & 14.68 & -3.59 & 0.81 & 0.73 \\
\hline chr8:97-104 & suggestive & RJF & 15.21 & -1.73 & 0.4 & 0.13 \\
\hline chr8:96-119 & significant & RJF & 17.6 & -2.83 & 0.6 & 0.12 \\
\hline chr8:95-111 & significant & RJF & 17.5 & -2.16 & 0.46 & 0.23 \\
\hline chr8:95-113 & significant & RJF & 25.34 & -2.98 & 0.53 & -0.42 \\
\hline chr8:96-119 & significant & RJF & 22.51 & -2.27 & 0.44 & -0.32 \\
\hline chr8:96-133 & significant & RJF & 16.42 & -1.69 & 0.36 & 0.44 \\
\hline chr8:45-122 & suggestive & WL & 13.7 & 1.73 & 0.44 & 0.08 \\
\hline chr8:96-140 & significant & RJF & 16.6 & -2.38 & 0.53 & -0.06 \\
\hline chr8:83-118 & significant & WL & 20.89 & 13.93 & 3.2 & 7.65 \\
\hline chr8:81-121 & significant & WL & 23.29 & 15.24 & 2.96 & 3.48 \\
\hline chr8:92-128 & suggestive & WL & 13.29 & 3.68 & 0.83 & -1.74 \\
\hline chr8:89-123 & significant & WL & 17.14 & 2.31 & 0.48 & -0.21 \\
\hline chr8:93-133 & significant & WL & 15.22 & 2.49 & 0.54 & -0.54 \\
\hline chr8:97-132 & significant & RJF & 20.09 & -3.21 & 0.74 & -1.67 \\
\hline chr8:95-114 & significant & WL & 37.21 & 1.86 & 0.22 & -0.16 \\
\hline chr8:97-113 & significant & WL & 48.97 & 2.23 & 0.21 & 0.13 \\
\hline chr8:102-110 & significant & WL & 65.59 & 5.28 & 0.31 & -0.59 \\
\hline chr8:95-128 & significant & RJF & 16.88 & -1.37 & 0.38 & -0.94 \\
\hline chr8:94-142 & suggestive & RJF & 14.55 & -2 & 0.46 & 0.08 \\
\hline chr8:54-129 & significant & WL & 16.21 & 1.37 & 0.29 & -1.12 \\
\hline chr8:84-137 & suggestive & RJF & 13.98 & -2 & 0.51 & -0.53 \\
\hline chr8:85-138 & suggestive & RJF & 14.55 & -1.51 & 0.33 & 0.76 \\
\hline chr8:79-130 & significant & WL & 17.35 & 2.06 & 0.4 & -0.85 \\
\hline chr8:81-121 & significant & WL & 18.44 & 9.61 & 2.51 & 6.77 \\
\hline chr8:103-111 & significant & WL & 67.18 & 6.22 & 0.37 & 0.27 \\
\hline chr8:103-109 & significant & WL & 67.34 & 5.99 & 0.4 & 1.67 \\
\hline chr8:83-125 & significant & WL & 16.44 & 1.95 & 0.4 & -0.53 \\
\hline chr8:99-134 & significant & RJF & 22.98 & -2.51 & 0.61 & -2.16 \\
\hline chr8:48-125 & suggestive & WL & 14.13 & 3.2 & 0.73 & -2.36 \\
\hline chr8:71-124 & significant & WL & 15.23 & 1.97 & 0.41 & -1.09 \\
\hline chr8:97-136 & significant & RJF & 16.52 & -1.34 & 0.4 & -1.13 \\
\hline chr8:96-134 & significant & RJF & 15.89 & -3.28 & 0.73 & -0.19 \\
\hline chr8:96-119 & suggestive & WL & 20.59 & 0.23 & 0.4 & -0.41 \\
\hline chr8:98-128 & significant & RJF & 16.02 & -1.43 & 0.48 & -1.63 \\
\hline chr8:99-134 & significant & RJF & 22.32 & -3.23 & 0.63 & -0.7 \\
\hline chr8:101-133 & significant & RJF & 17.67 & -3.33 & 0.74 & -0.75 \\
\hline chr8:98-132 & significant & RJF & 17.01 & -3.51 & 0.73 & 0.54 \\
\hline chr8:97-125 & suggestive & WL & 16.49 & 0.84 & 0.67 & -0.76 \\
\hline
\end{tabular}




\begin{tabular}{|c|c|c|c|c|c|}
\hline chr8:101-130 suggestive & WL & 16.38 & 0.6 & 0.47 & 0.21 \\
\hline chr8:103-114 significant & WL & 51.8 & 3.1 & 0.25 & -0.59 \\
\hline chr8:92-129 suggestive & WL & 17.01 & 1.65 & 0.33 & -0.85 \\
\hline chr8:61-165 suggestive & WL & 15.82 & 3.01 & 0.7 & 0.07 \\
\hline chr8:77-132 significant & RJF & 16.12 & -2.21 & 0.48 & 0.26 \\
\hline chr8:97-133 suggestive & RJF & 15.24 & -1.03 & 0.3 & -0.67 \\
\hline chr8:100-134 significant & WL & 14.13 & 1.38 & 0.35 & 0.33 \\
\hline chr8:99-131 significant & RJF & 22.4 & -1.36 & 0.86 & -0.77 \\
\hline chr8:97-133 significant & RJF & 16.73 & -2.26 & 0.49 & -0.18 \\
\hline chr8:74-134 suggestive & RJF & 13.94 & -1.74 & 0.45 & -0.58 \\
\hline chr8:98-132 suggestive & RJF & 19.02 & -0.28 & 0.24 & -0.39 \\
\hline chr8:91-130 significant & WL & 17.39 & 2.14 & 0.43 & -1.93 \\
\hline chr8:92-129 significant & WL & 13.42 & 1.39 & 0.34 & -1.41 \\
\hline chr8:101-134 significant & WL & 18.39 & 3.67 & 0.78 & 0.81 \\
\hline chr8:97-135 significant & RJF & 15.3 & -2.34 & 0.56 & -0.52 \\
\hline chr8:95-134 suggestive & RJF & 13.58 & -1.26 & 0.5 & -1.77 \\
\hline chr8:101-136 significant & RJF & 16.15 & -2.06 & 0.53 & -1.16 \\
\hline chr8:92-138 significant & RJF & 15.89 & -1.32 & 0.34 & -0.66 \\
\hline chr8:98-154 significant & RJF & 13.31 & -1.21 & 0.52 & -1.56 \\
\hline chr8:98-148 suggestive & RJF & 13.95 & -1.49 & 0.59 & -1.7 \\
\hline chr8:122-152 suggestive & WL & 16.32 & 0.79 & 0.93 & -4.3 \\
\hline chr8:118-147 significant & RJF & 18.38 & -4 & 1.08 & -2.42 \\
\hline chr8:115-147 significant & RJF & 17.84 & -5.01 & 1.33 & -2.75 \\
\hline chr8:99-153 suggestive & RJF & 13.8 & -1.07 & 0.48 & -1.51 \\
\hline chr8:113-148 significant & RJF & 15.8 & -3.37 & 0.98 & -1.92 \\
\hline chr8:122-151 suggestive & RJF & 16.81 & -0.12 & 0.47 & -0.74 \\
\hline chr8:118-148 significant & RJF & 18.18 & -3.76 & 1.03 & -2.33 \\
\hline chr8:116-148 significant & RJF & 16.49 & -3.37 & 0.95 & -1.82 \\
\hline chr8:117-147 significant & RJF & 16.07 & -2.33 & 0.68 & -1.42 \\
\hline chr8:123-156 suggestive & WL & 17.61 & 1.02 & 0.65 & -3.06 \\
\hline chr8:124-164 suggestive & WL & 13.71 & 0.72 & 0.46 & -2.65 \\
\hline chr8:124-153 significant & WL & 15.36 & 0.95 & 0.45 & -2.82 \\
\hline chr8:124-157 significant & RJF & 13.04 & -1.84 & 0.83 & -2.49 \\
\hline chr8:122-151 suggestive & WL & 11.71 & 0.68 & 1.11 & -5.65 \\
\hline chr8:126-149 suggestive & RJF & 17.72 & -0.57 & 0.47 & -2.46 \\
\hline chr8:127-151 suggestive & WL & 19.86 & 0.76 & 0.34 & -2.54 \\
\hline chr8:125-160 suggestive & WL & 18.33 & 3.03 & 0.68 & -3.68 \\
\hline chr8:128-153 significant & RJF & 18.12 & -1.77 & 0.51 & -1.39 \\
\hline chr8:130-150 significant & WL & 9.54 & 0.54 & 0.48 & -3.21 \\
\hline chr8:128-152 suggestive & RJF & 17.05 & -0.26 & 0.48 & -2.7 \\
\hline chr8:129-150 suggestive & RJF & 17.42 & -2.07 & 0.66 & -1.85 \\
\hline chr8:130-149 suggestive & RJF & 19.22 & -0.99 & 0.37 & -1.49 \\
\hline chr8:125-170 suggestive & RJF & 12.71 & -0.73 & 0.45 & -1.67 \\
\hline
\end{tabular}




\begin{tabular}{|c|c|c|c|c|c|}
\hline chr8:130-163 suggestive & WL & 18.18 & 0.43 & 0.8 & -5.22 \\
\hline chr8:129-161 significant & RJF & 15.61 & -0.72 & 0.27 & -0.87 \\
\hline chr8:122-168 significant & RJF & 14.16 & -4.11 & 1.12 & -0.94 \\
\hline chr8:131-163 suggestive & WL & 18.52 & 1.2 & 0.3 & -1.88 \\
\hline chr8:128-163 suggestive & WL & 18.58 & 2.17 & 0.41 & -1.55 \\
\hline chr8:105-166 suggestive & WL & 15.16 & 3.18 & 0.74 & -2.34 \\
\hline chr8:128-179 suggestive & WL & 16 & 1.43 & 0.45 & -2.77 \\
\hline chr8:157-180 significant & WL & 19.76 & 3.53 & 0.71 & -3.38 \\
\hline chr8:159-182 significant & WL & 23.51 & 2.39 & 0.46 & -2.2 \\
\hline chr8:160-184 suggestive & WL & 20.12 & 2.18 & 0.51 & -2.95 \\
\hline chr8:156-190 suggestive & WL & 18.72 & 2.22 & 0.43 & -1.58 \\
\hline chr8:161-179 significant & WL & 22.39 & 2.54 & 0.55 & -3.4 \\
\hline chr8:158-181 significant & WL & 19.07 & 3.52 & 0.82 & -3.29 \\
\hline chr8:159-186 significant & WL & 18.38 & 1.68 & 0.33 & -1.45 \\
\hline chr8:161-183 suggestive & WL & 19.49 & 2.23 & 0.51 & -2.75 \\
\hline chr8:161-180 suggestive & WL & 18.57 & 3.31 & 0.71 & -3.61 \\
\hline chr8:165-186 significant & WL & 19.87 & 1.25 & 0.25 & -1.06 \\
\hline chr8:163-183 suggestive & WL & 18.12 & 3.51 & 0.78 & -3.58 \\
\hline chr8:165-182 suggestive & WL & 19.89 & 4.57 & 0.96 & -5.26 \\
\hline chr8:163-181 significant & WL & 22.27 & 2.21 & 0.52 & -3.01 \\
\hline chr8:165-183 significant & WL & 23.1 & 2.24 & 0.41 & -2.2 \\
\hline chr8:165-181 significant & WL & 21.41 & 2.9 & 0.51 & -2.36 \\
\hline chr8:164-182 suggestive & WL & 19.31 & 3.85 & 0.76 & -3.31 \\
\hline chr8:166-181 suggestive & WL & 19.42 & 2.75 & 0.54 & -2.36 \\
\hline chr8:163-182 significant & WL & 20.49 & 1.83 & 0.52 & -2.35 \\
\hline chr8:159-185 suggestive & WL & 14.75 & 1.49 & 0.43 & -2.29 \\
\hline chr8:164-185 suggestive & WL & 14.76 & 1.79 & 0.4 & -1.52 \\
\hline chr8:165-180 suggestive & WL & 24.76 & 2.21 & 0.49 & -3.44 \\
\hline chr8:165-182 suggestive & WL & 22.53 & 2.26 & 0.43 & -2.44 \\
\hline chr8:165-179 significant & WL & 29.08 & 3.01 & 0.48 & -3.12 \\
\hline chr8:167-182 suggestive & WL & 23.72 & 1.43 & 0.26 & -1.42 \\
\hline chr8:164-184 suggestive & WL & 24.41 & 2.83 & 0.56 & -3.38 \\
\hline chr8:164-177 suggestive & WL & 26.59 & 2.45 & 0.44 & -2.65 \\
\hline chr8:162-183 suggestive & WL & 20.59 & 0.18 & 0.04 & -0.19 \\
\hline chr8:161-182 suggestive & WL & 19.69 & 1.64 & 0.32 & -1.52 \\
\hline chr8:163-183 suggestive & WL & 19.19 & 1.49 & 0.3 & -1.53 \\
\hline chr8:164-185 significant & WL & 32.56 & 4 & 0.53 & -1.26 \\
\hline chr8:164-186 suggestive & WL & 21.01 & 0.17 & 0.03 & -0.18 \\
\hline chr8:162-188 suggestive & WL & 14.57 & 1.1 & 0.24 & -1.47 \\
\hline chr8:159-192 suggestive & WL & 14.9 & 2.08 & 0.4 & -1.96 \\
\hline chr8:160-194 significant & WL & 20.34 & 1.85 & 0.34 & -0.97 \\
\hline chr8:225-244 significant & WL & 15.18 & 0.97 & 0.87 & 1.89 \\
\hline chr8:222-244 significant & RJF & 16.19 & -1.49 & 0.31 & -0.12 \\
\hline
\end{tabular}




\begin{tabular}{|c|c|c|c|c|c|c|}
\hline \multicolumn{2}{|c|}{ chr8:226-244 significant } & RJF & 23.01 & -1.4 & 0.24 & 0.04 \\
\hline \multicolumn{2}{|c|}{ chr8:229-244 significant } & RJF & 18.91 & -1.8 & 0.33 & 0.89 \\
\hline \multicolumn{2}{|c|}{ chr8:231-244 significant } & RJF & 23.2 & -2.07 & 0.35 & -0.08 \\
\hline \multicolumn{2}{|c|}{ chr8:231-244 suggestive } & RJF & 18.98 & -3.06 & 0.75 & -0.3 \\
\hline chr9:0-14 & significant & RJF & 12.76 & -1.2 & 0.33 & 1.09 \\
\hline chr9:0-20 & significant & RJF & 19.33 & -1.83 & 0.34 & 0.2 \\
\hline chr9:0-7 & suggestive & WL & 26.46 & 0.15 & 0.54 & 4.89 \\
\hline chr9:0-20 & significant & RJF & 16.45 & -1.34 & 0.28 & 0.08 \\
\hline chr9:0-17 & significant & RJF & 17.36 & -1.25 & 0.25 & -0.42 \\
\hline chr9:0-24 & significant & RJF & 13.24 & -1.14 & 0.28 & -0.64 \\
\hline chr9:0-20 & significant & RJF & 16.72 & -2.23 & 0.46 & -0.59 \\
\hline chr9:0-25 & significant & RJF & 17.73 & -1.19 & 0.24 & 0.42 \\
\hline chr9:0-19 & significant & RJF & 24.31 & -1.06 & 0.17 & 0.03 \\
\hline chr9:0-19 & suggestive & RJF & 17.53 & -1.99 & 0.78 & 3.43 \\
\hline chr9:0-20 & suggestive & RJF & 16.73 & -0.52 & 0.58 & 2.54 \\
\hline chr9:0-20 & significant & WL & 21.62 & 2.27 & 0.4 & -0.48 \\
\hline chr9:0-20 & significant & RJF & 20.37 & -1.64 & 0.3 & 0.18 \\
\hline chr9:0-17 & significant & RJF & 17.29 & -1.57 & 0.3 & -0.09 \\
\hline chr9:0-25 & suggestive & RJF & 12.95 & -1.69 & 0.4 & 0.42 \\
\hline chr9:0-29 & suggestive & RJF & 11.21 & -1.46 & 0.37 & 0.19 \\
\hline chr9:0-18 & significant & RJF & 14.78 & -1.21 & 0.26 & -0.28 \\
\hline chr9:0-39 & significant & WL & 15.1 & 3.69 & 0.84 & 2.08 \\
\hline chr9:0-42 & significant & RJF & 22.05 & -2.33 & 0.4 & -0.27 \\
\hline chr9:0-37 & significant & RJF & 30.34 & -4.2 & 0.59 & -1.33 \\
\hline chr9:0-103 & significant & RJF & 10.33 & -2.23 & 0.61 & -0.26 \\
\hline chr9:0-47 & suggestive & RJF & 10.48 & -1.62 & 0.53 & 0.75 \\
\hline chr9:25-41 & significant & WL & 35.8 & 2.47 & 0.31 & 0.07 \\
\hline chr9:22-49 & significant & WL & 16.9 & 1.97 & 0.4 & 0.54 \\
\hline chr9:38-73 & significant & WL & 18.25 & 0.47 & 0.49 & -2.57 \\
\hline chr9:44-68 & significant & WL & 21.12 & 5.15 & 0.88 & 0.37 \\
\hline chr9:44-73 & significant & WL & 20.42 & 2.06 & 0.84 & -3.42 \\
\hline chr9:46-79 & significant & RJF & 17.46 & -1.67 & 0.34 & 0.1 \\
\hline chr9:21-96 & significant & RJF & 13.5 & -1.78 & 0.44 & 0.46 \\
\hline chr9:45-103 & significant & WL & 18.75 & 4.22 & 1.31 & -5.97 \\
\hline chr9:46-103 & significant & WL & 17.45 & 2.23 & 0.44 & -1.46 \\
\hline chr9:48-103 & significant & WL & 16.73 & 1.77 & 0.38 & -1.77 \\
\hline chr9:42-95 & significant & RJF & 16.87 & -1.88 & 0.39 & -0.11 \\
\hline chr9:47-104 & significant & WL & 16.74 & 1.88 & 0.41 & -1.58 \\
\hline chr9:49-103 & significant & WL & 16.04 & 1.89 & 0.39 & -0.56 \\
\hline chr9:48-95 & suggestive & WL & 14.55 & 1.72 & 0.38 & -0.66 \\
\hline chr9:40-103 & suggestive & WL & 14.25 & 2.52 & 0.56 & -0.71 \\
\hline chr9:48-104 & significant & WL & 15.01 & 2.3 & 0.5 & -0.77 \\
\hline chr9:49-105 & significant & WL & 16.11 & 2.43 & 0.5 & -0.68 \\
\hline
\end{tabular}




\begin{tabular}{|c|c|c|c|c|c|c|}
\hline chr9:57-102 & significant & WL & 23.68 & 3.96 & 0.64 & -1.91 \\
\hline chr9:50-103 & significant & RJF & 16.48 & -1.3 & 0.28 & 0.98 \\
\hline chr9:58-102 & significant & WL & 24.98 & 3.19 & 0.51 & -1.48 \\
\hline chr9:56-104 & significant & WL & 17.29 & 3.46 & 0.69 & -1.46 \\
\hline chr9:52-96 & significant & WL & 14.82 & 2.13 & 0.46 & -1.05 \\
\hline chr9:66-94 & significant & WL & 37.41 & 1.83 & 0.22 & -0.24 \\
\hline chr9:57-101 & significant & WL & 17.77 & 1.97 & 0.4 & -1.42 \\
\hline chr9:58-128 & significant & RJF & 16.51 & -1.34 & 0.28 & 0.27 \\
\hline chr9:52-95 & suggestive & WL & 12.96 & 1.96 & 0.47 & -1.09 \\
\hline chr9:59-95 & significant & WL & 16.09 & 2.19 & 0.46 & -0.14 \\
\hline chr9:57-124 & significant & RJF & 15.23 & -2.19 & 0.47 & 1.22 \\
\hline chr9:62-102 & significant & WL & 20.62 & 2.99 & 0.53 & -1.51 \\
\hline chr9:70-96 & significant & WL & 39.13 & 3.47 & 0.39 & -0.7 \\
\hline chr9:61-113 & significant & RJF & 15.15 & -1.62 & 0.36 & 1.15 \\
\hline chr9:67-92 & significant & RJF & 17.77 & -2.64 & 0.52 & 1.38 \\
\hline chr9:77-92 & significant & RJF & 35.34 & -41.32 & 5.19 & 1.48 \\
\hline chr9:77-92 & significant & RJF & 35.93 & -64.7 & 8.03 & 1.49 \\
\hline chr9:77-92 & significant & RJF & 34.96 & -81.8 & 10.34 & 4.83 \\
\hline chr9:77-92 & significant & RJF & 34.74 & -66.95 & 8.52 & 2.35 \\
\hline chr9:76-93 & significant & RJF & 33.02 & -58.8 & 7.77 & 2.94 \\
\hline chr9:77-92 & significant & RJF & 33.69 & -59.19 & 7.72 & 1.33 \\
\hline chr9:77-92 & significant & RJF & 35.82 & -71.96 & 8.92 & 4.15 \\
\hline chr9:76-92 & significant & RJF & 35.03 & -62.88 & 7.94 & 3.34 \\
\hline chr9:77-92 & significant & RJF & 33.84 & -60.3 & 7.81 & 3.24 \\
\hline chr9:77-92 & significant & RJF & 35.42 & -37.15 & 4.65 & 2.11 \\
\hline chr9:77-98 & significant & RJF & 27.68 & -2.46 & 0.36 & 0.75 \\
\hline chr9:69-100 & significant & RJF & 23.68 & -3.69 & 0.59 & 1.35 \\
\hline chr9:71-97 & significant & RJF & 18.93 & -2.74 & 0.54 & -0.37 \\
\hline chr9:72-93 & significant & WL & 23.98 & 1.64 & 0.28 & 0.39 \\
\hline chr9:68-120 & significant & RJF & 16.28 & -2.27 & 0.53 & -1.09 \\
\hline chr9:65-100 & significant & WL & 18.33 & 2.48 & 0.48 & -1.46 \\
\hline chr9:68-91 & significant & WL & 28.16 & 3.07 & 0.45 & -1.7 \\
\hline chr9:71-94 & significant & WL & 27.63 & 2.43 & 0.35 & -0.82 \\
\hline chr9:69-113 & significant & RJF & 17.94 & -1.96 & 0.4 & -0.39 \\
\hline chr9:76-94 & significant & RJF & 34.59 & -4.68 & 0.65 & -2.06 \\
\hline chr9:63-95 & significant & RJF & 17.84 & -2.15 & 0.42 & 0.55 \\
\hline chr9:61-104 & significant & WL & 14.95 & 2.03 & 0.45 & -1.38 \\
\hline chr9:68-136 & significant & RJF & 13.19 & -2.34 & 0.55 & 0.54 \\
\hline chr9:57-96 & suggestive & RJF & 12.85 & -3.08 & 0.74 & 0.13 \\
\hline chr9:64-104 & suggestive & RJF & 13.27 & -1.21 & 0.42 & -1.64 \\
\hline chr9:69-96 & suggestive & RJF & 12.89 & -1.09 & 0.34 & -1.04 \\
\hline chr9:66-124 & suggestive & RJF & 13.04 & -1.13 & 0.27 & 0.7 \\
\hline chr9:63-95 & significant & WL & 15.69 & 1.13 & 0.24 & -0.47 \\
\hline
\end{tabular}




\begin{tabular}{|c|c|c|c|c|c|c|}
\hline chr9:61-104 & suggestive & RJF & 14.38 & -1.98 & 0.48 & 1.87 \\
\hline chr9:70-93 & significant & RJF & 22.87 & -3.37 & 0.55 & 0.58 \\
\hline chr9:77-92 & significant & RJF & 40.63 & -4.39 & 0.49 & 0.25 \\
\hline chr9:78-90 & significant & WL & 58.51 & 4.28 & 0.33 & -0.95 \\
\hline chr9:73-117 & significant & RJF & 15.07 & -2.13 & 0.45 & 0.56 \\
\hline chr9:67-100 & significant & RJF & 15.54 & -1.59 & 0.35 & -0.04 \\
\hline chr9:55-96 & significant & RJF & 17.97 & -1.85 & 0.4 & 1.91 \\
\hline chr9:62-96 & significant & RJF & 19.11 & -1.02 & 0.36 & 0.55 \\
\hline chr9:75-95 & significant & RJF & 25.66 & -1.64 & 0.26 & -0.08 \\
\hline chr9:74-93 & significant & RJF & 18.8 & -2.13 & 0.4 & 0.31 \\
\hline chr9:56-104 & suggestive & WL & 13.75 & 2.68 & 0.62 & -1.42 \\
\hline chr9:63-95 & significant & RJF & 14.98 & -2.36 & 0.53 & -0.2 \\
\hline chr9:70-92 & suggestive & RJF & 24.93 & -0.66 & 0.62 & 1.42 \\
\hline chr9:80-91 & significant & RJF & 49.56 & -4.54 & 0.43 & -0.53 \\
\hline chr9:73-96 & significant & RJF & 22.23 & -2.05 & 0.36 & -0.36 \\
\hline chr9:68-95 & significant & WL & 25.59 & 3.09 & 0.48 & -1.37 \\
\hline chr9:82-91 & significant & WL & 70.71 & 6.14 & 0.35 & -1.19 \\
\hline chr9:64-103 & significant & RJF & 14.46 & -1.34 & 0.31 & -0.27 \\
\hline chr9:62-96 & significant & WL & 22.47 & 1.25 & 0.72 & -0.96 \\
\hline chr9:68-101 & suggestive & RJF & 14.14 & -1.41 & 0.34 & -0.26 \\
\hline chr9:72-135 & significant & RJF & 16.12 & -1.37 & 0.28 & 0.05 \\
\hline chr9:51-102 & significant & WL & 14.68 & 3.22 & 0.73 & -2.41 \\
\hline chr9:66-132 & significant & RJF & 17.73 & -1.58 & 0.33 & 1.36 \\
\hline chr9:66-102 & significant & WL & 26.76 & 2.97 & 0.45 & -0.34 \\
\hline chr9:65-101 & suggestive & RJF & 13.24 & -1.67 & 0.49 & -1.42 \\
\hline chr9:74-96 & significant & RJF & 26.05 & -2.62 & 0.43 & -0.81 \\
\hline chr9:61-102 & significant & WL & 14.41 & 2.48 & 0.58 & -2 \\
\hline chr9:58-119 & significant & RJF & 19 & -1.95 & 0.37 & -0.02 \\
\hline chr9:63-101 & significant & RJF & 16.72 & -3.52 & 0.89 & -2.05 \\
\hline chr9:63-104 & significant & RJF & 17.66 & -2.67 & 0.55 & -0.56 \\
\hline chr9:66-102 & significant & RJF & 15.05 & -2.07 & 0.53 & -1.36 \\
\hline chr9:56-103 & significant & WL & 17.08 & 3.91 & 0.8 & -2.73 \\
\hline chr9:57-119 & suggestive & RJF & 12.34 & -2.32 & 0.59 & 1.59 \\
\hline chr9:57-102 & significant & WL & 18.34 & 3.64 & 0.66 & -1.58 \\
\hline chr9:60-103 & significant & WL & 24.08 & 5.05 & 0.81 & -2.29 \\
\hline chr9:75-119 & significant & RJF & 16.83 & -1.58 & 0.33 & -0.28 \\
\hline chr9:59-102 & significant & RJF & 16.57 & -1.84 & 0.48 & -1.55 \\
\hline chr9:57-127 & significant & WL & 15.86 & 1.87 & 0.49 & 0.95 \\
\hline chr9:63-125 & significant & RJF & 14.22 & -1.27 & 0.3 & -0.32 \\
\hline chr9:69-116 & significant & RJF & 14.75 & -2.37 & 0.57 & -0.88 \\
\hline chr9:68-104 & suggestive & RJF & 18.18 & -1.02 & 0.34 & -1.89 \\
\hline chr9:66-101 & significant & RJF & 15.95 & -2.42 & 0.53 & -0.38 \\
\hline chr9:63-122 & significant & RJF & 15.21 & -1.79 & 0.39 & 0.09 \\
\hline
\end{tabular}




\begin{tabular}{|c|c|c|c|c|c|c|}
\hline chr9:65-124 & significant & RJF & 16.25 & -1.52 & 0.32 & 0.71 \\
\hline chr9:77-100 & significant & RJF & 28.31 & -2.2 & 0.38 & -1.42 \\
\hline chr9:44-104 & suggestive & WL & 12.69 & 3.24 & 0.8 & -2.71 \\
\hline chr9:73-118 & significant & RJF & 14.28 & -1.53 & 0.45 & -1.46 \\
\hline chr9:54-104 & significant & WL & 14.2 & 1.92 & 0.43 & -1.35 \\
\hline chr9:66-114 & suggestive & RJF & 13.58 & -2.16 & 0.63 & -1.78 \\
\hline chr9:63-127 & suggestive & RJF & 13.02 & -2.21 & 0.56 & -0.77 \\
\hline chr9:70-117 & significant & RJF & 17.82 & -1.77 & 0.42 & -1.19 \\
\hline chr9:73-118 & significant & RJF & 23.75 & -1.17 & 0.19 & 0.15 \\
\hline chr9:66-102 & significant & RJF & 18.61 & -2.88 & 0.57 & -0.47 \\
\hline chr9:77-112 & significant & RJF & 25.81 & -3.01 & 0.5 & -0.91 \\
\hline chr9:65-111 & significant & RJF & 16.26 & -2.66 & 0.62 & -1.16 \\
\hline chr9:73-116 & significant & RJF & 14.21 & -1.59 & 0.41 & -0.92 \\
\hline chr9:43-104 & significant & WL & 13.84 & 2.38 & 0.57 & -1.82 \\
\hline chr9:67-129 & suggestive & RJF & 12.55 & -2.17 & 0.58 & -0.86 \\
\hline chr9:52-107 & suggestive & WL & 13.73 & 2.58 & 0.6 & -0.51 \\
\hline chr9:48-126 & suggestive & WL & 13.86 & 2.92 & 0.66 & -1.57 \\
\hline chr9:53-104 & significant & WL & 16.15 & 2.48 & 0.54 & -2.19 \\
\hline chr9:59-103 & significant & RJF & 22.52 & -2.07 & 0.35 & 0.4 \\
\hline chr9:67-111 & suggestive & RJF & 13.05 & -1.44 & 0.39 & -0.8 \\
\hline chr9:71-118 & significant & RJF & 13.78 & -2.48 & 0.7 & -1.93 \\
\hline chr9:68-118 & suggestive & RJF & 13.19 & -1.89 & 0.59 & -1.47 \\
\hline chr9:68-102 & significant & WL & 19.46 & 4.01 & 0.75 & -2.41 \\
\hline chr9:53-102 & significant & WL & 19.6 & 4.59 & 0.86 & -3.22 \\
\hline chr9:62-104 & significant & WL & 18.19 & 4.37 & 0.85 & -2.72 \\
\hline chr9:67-113 & significant & WL & 17.3 & 1.84 & 0.37 & -1.02 \\
\hline chr9:77-115 & significant & RJF & 15.65 & -0.88 & 0.27 & -0.92 \\
\hline chr9:65-103 & suggestive & WL & 16.84 & 2.42 & 0.76 & -5.06 \\
\hline chr9:87-103 & suggestive & WL & 21.19 & 1.47 & 0.41 & -3.17 \\
\hline chr9:86-110 & significant & RJF & 22.78 & -1.56 & 0.35 & -1.14 \\
\hline chr9:64-102 & significant & WL & 21.54 & 3.17 & 0.59 & -3.48 \\
\hline chr9:64-103 & significant & WL & 23.11 & 2.21 & 0.77 & -3.39 \\
\hline chr9:89-105 & significant & RJF & 25.39 & -0.74 & 0.32 & 0.49 \\
\hline chr9:87-112 & significant & RJF & 20.54 & -3.12 & 0.82 & -3.09 \\
\hline chr9:64-118 & suggestive & RJF & 14.13 & -1.35 & 0.41 & -1.16 \\
\hline chr9:69-120 & suggestive & RJF & 14.21 & -1.08 & 0.35 & -1.03 \\
\hline chr9:72-120 & significant & RJF & 16.63 & -1.71 & 0.38 & -0.29 \\
\hline chr9:87-112 & significant & RJF & 18.3 & -1.77 & 0.57 & -2.42 \\
\hline chr9:85-115 & significant & RJF & 17.69 & -1.94 & 0.52 & -1.55 \\
\hline chr9:57-116 & significant & WL & 12.97 & 2.13 & 0.49 & -0.99 \\
\hline chr9:88-101 & suggestive & WL & 22.04 & 1.86 & 0.41 & -2.91 \\
\hline chr9:87-102 & significant & WL & 20.36 & 2.58 & 0.49 & -2.4 \\
\hline chr9:51-103 & significant & WL & 18.63 & 4.32 & 0.88 & -4.5 \\
\hline
\end{tabular}




\begin{tabular}{|c|c|c|c|c|c|c|}
\hline chr9:87-119 & significant & RJF & 21.96 & -1.71 & 0.32 & -0.43 \\
\hline chr9:90-103 & significant & WL & 33.06 & 3.88 & 0.5 & -2.02 \\
\hline chr9:87-104 & significant & WL & 16.75 & 2.16 & 0.5 & -2.73 \\
\hline chr9:81-119 & suggestive & RJF & 15.13 & -1.49 & 0.55 & -2.12 \\
\hline chr9:50-104 & suggestive & WL & 14.75 & 1.97 & 0.47 & -2.05 \\
\hline chr9:90-103 & significant & WL & 17.97 & 1.24 & 0.4 & -2.92 \\
\hline chr9:88-109 & significant & RJF & 17.66 & -1.92 & 0.45 & -0.74 \\
\hline chr9:89-103 & significant & WL & 19.92 & 1.68 & 0.35 & -2.08 \\
\hline chr9:90-102 & significant & WL & 25.85 & 1.99 & 0.67 & -3.27 \\
\hline chr9:92-101 & significant & RJF & 30.61 & -1.46 & 0.26 & -0.96 \\
\hline chr9:91-102 & significant & RJF & 18.54 & -1.1 & 0.29 & -0.93 \\
\hline chr9:90-102 & significant & WL & 24.11 & 1.41 & 0.23 & -0.74 \\
\hline chr9:93-105 & significant & RJF & 33.03 & -3.38 & 0.5 & -1.02 \\
\hline chr9:92-110 & significant & RJF & 28.96 & -2.98 & 0.61 & -2.75 \\
\hline chr9:87-103 & suggestive & WL & 18.62 & 5.44 & 1.18 & -4.02 \\
\hline chr9:90-111 & significant & RJF & 19.59 & -1.63 & 0.48 & -1.96 \\
\hline chr9:90-110 & significant & RJF & 19.72 & -1.65 & 0.39 & -1.12 \\
\hline chr9:92-103 & significant & WL & 22.86 & 2.56 & 0.48 & -2.13 \\
\hline chr9:89-102 & significant & WL & 24.5 & 3.94 & 0.72 & -2.36 \\
\hline chr9:90-110 & significant & RJF & 25.86 & -2.22 & 0.44 & -1.35 \\
\hline chr9:89-103 & suggestive & WL & 16.35 & 2.38 & 0.6 & -3.38 \\
\hline chr9:91-112 & significant & RJF & 17.06 & -1.16 & 0.41 & -1.78 \\
\hline chr9:87-104 & significant & WL & 17.14 & 2.44 & 0.52 & -2.28 \\
\hline chr9:89-124 & significant & RJF & 14.07 & -1.74 & 0.4 & 0.37 \\
\hline chr9:89-104 & significant & RJF & 16.04 & -1.24 & 0.4 & -1.4 \\
\hline chr9:89-104 & significant & WL & 18.14 & 2.06 & 0.42 & -1.9 \\
\hline chr9:90-113 & suggestive & RJF & 15.17 & -1.27 & 0.44 & -1.59 \\
\hline chr9:91-113 & significant & RJF & 17.19 & -1.69 & 0.57 & -2.3 \\
\hline chr9:91-108 & significant & RJF & 17.36 & -1.62 & 0.51 & -1.96 \\
\hline chr9:90-126 & suggestive & RJF & 15.2 & -1.01 & 0.35 & -1.23 \\
\hline chr9:90-114 & suggestive & RJF & 15.72 & -1.24 & 0.37 & -1.06 \\
\hline chr9:90-112 & suggestive & RJF & 16.46 & -0.96 & 0.63 & -3.48 \\
\hline chr9:89-106 & suggestive & RJF & 16.49 & -1.46 & 0.61 & -2.78 \\
\hline chr9:94-102 & suggestive & WL & 24.88 & 1.84 & 0.65 & -5.34 \\
\hline chr9:90-103 & significant & WL & 23.6 & 5.54 & 0.93 & -4.6 \\
\hline chr9:92-109 & significant & RJF & 30.97 & -2.22 & 0.39 & -1.56 \\
\hline chr9:90-113 & significant & RJF & 25.78 & -2.01 & 0.39 & -1.07 \\
\hline chr9:90-103 & significant & WL & 26.44 & 5.19 & 0.79 & -2.96 \\
\hline chr9:90-103 & significant & WL & 21.29 & 2.83 & 0.67 & -4.79 \\
\hline chr9:90-122 & significant & RJF & 17.54 & -1.22 & 0.3 & -0.75 \\
\hline chr9:89-119 & suggestive & WL & 13.94 & 3.24 & 0.73 & -2.22 \\
\hline chr9:87-104 & significant & WL & 16.67 & 3.27 & 0.7 & -3.12 \\
\hline chr9:52-104 & significant & WL & 16.86 & 3.24 & 0.7 & -3.19 \\
\hline
\end{tabular}




\begin{tabular}{|c|c|c|c|c|c|c|}
\hline chr9:90-112 & suggestive & RJF & 16.67 & -1.31 & 0.59 & -2.91 \\
\hline chr9:67-112 & significant & WL & 15 & 3.09 & 0.68 & -2.1 \\
\hline chr9:91-102 & significant & WL & 20.05 & 3.98 & 0.73 & -3.03 \\
\hline chr9:64-119 & significant & RJF & 17.57 & -2.16 & 0.5 & -0.74 \\
\hline chr9:89-102 & significant & WL & 23.13 & 2.62 & 0.87 & -3.47 \\
\hline chr9:87-102 & significant & WL & 18.91 & 3.29 & 0.64 & -2.71 \\
\hline chr9:89-105 & significant & WL & 17.21 & 2.78 & 0.55 & -1.55 \\
\hline chr9:50-102 & significant & WL & 16.18 & 3.88 & 0.82 & -3.48 \\
\hline chr9:71-134 & suggestive & WL & 15.03 & 2.94 & 0.66 & -2.51 \\
\hline chr9:71-102 & significant & WL & 17.12 & 3.12 & 0.74 & -4.22 \\
\hline chr9:88-133 & suggestive & WL & 14.89 & 2.43 & 0.54 & -1.66 \\
\hline chr9:91-102 & suggestive & WL & 15.94 & 1.5 & 0.38 & -2.1 \\
\hline chr9:78-128 & significant & WL & 17.25 & 2.88 & 0.58 & -2.04 \\
\hline chr9:70-102 & significant & WL & 18.38 & 3.76 & 0.89 & -5.57 \\
\hline chr9:77-111 & significant & WL & 20.87 & 1.22 & 1.08 & -2.12 \\
\hline chr9:71-103 & significant & WL & 24.3 & 3.26 & 0.55 & -3.23 \\
\hline chr9:91-103 & significant & WL & 28.98 & 3.97 & 0.6 & -3.96 \\
\hline chr9:89-105 & significant & WL & 24.06 & 3.65 & 0.59 & -1.02 \\
\hline chr9:90-113 & significant & RJF & 21.4 & -0.63 & 0.88 & -4.08 \\
\hline chr9:90-102 & significant & WL & 21.42 & 3.08 & 0.59 & -3.42 \\
\hline chr9:91-102 & suggestive & WL & 19.1 & 4.25 & 0.99 & -5.32 \\
\hline chr9:90-102 & significant & WL & 16.03 & 2.51 & 0.6 & -3.41 \\
\hline chr9:57-102 & significant & WL & 16.18 & 2.93 & 0.66 & -3 \\
\hline chr9:88-102 & suggestive & WL & 16.06 & 3.24 & 0.79 & -4.25 \\
\hline chr9:51-123 & significant & WL & 16.4 & 3.39 & 0.73 & -3.13 \\
\hline chr9:88-102 & significant & WL & 17.64 & 2.55 & 0.53 & -2.38 \\
\hline chr9:62-130 & significant & WL & 15.95 & 4.96 & 1.03 & -3.2 \\
\hline chr9:92-102 & suggestive & WL & 20.12 & 3.07 & 0.72 & -4.88 \\
\hline chr9:92-103 & suggestive & WL & 19.64 & 1.93 & 0.49 & -3.48 \\
\hline chr9:60-131 & significant & WL & 13.02 & 4.37 & 1.03 & -3.07 \\
\hline chr9:61-102 & significant & WL & 16.95 & 3.3 & 0.67 & -2.64 \\
\hline chr9:87-120 & significant & RJF & 17.43 & -1.74 & 0.36 & 0.14 \\
\hline chr9:91-105 & significant & WL & 20.82 & 2.73 & 0.49 & -1.52 \\
\hline chr9:49-115 & significant & WL & 14.84 & 3.89 & 0.84 & -2.84 \\
\hline chr9:89-102 & significant & WL & 19.01 & 4.48 & 0.88 & -4.01 \\
\hline chr9:94-103 & significant & WL & 35.01 & 5.15 & 0.63 & -3.78 \\
\hline chr9:60-118 & significant & WL & 15.74 & 3.51 & 0.73 & -1.64 \\
\hline chr9:96-102 & significant & WL & 32.82 & 6.36 & 0.83 & -3.81 \\
\hline chr9:92-105 & significant & WL & 21 & 4.39 & 0.79 & -3.25 \\
\hline chr9:95-103 & significant & WL & 25.52 & 2.69 & 0.65 & -3.41 \\
\hline chr9:91-122 & significant & WL & 19.19 & 2.43 & 0.45 & -1.17 \\
\hline chr9:90-120 & significant & WL & 16.6 & 4.11 & 0.84 & -2.32 \\
\hline chr9:91-120 & significant & WL & 14.92 & 2.94 & 0.63 & -1.36 \\
\hline
\end{tabular}




\begin{tabular}{|c|c|c|c|c|c|c|}
\hline chr9:93-111 & significant & WL & 22.25 & 3.51 & 0.61 & -2.73 \\
\hline chr9:94-117 & significant & WL & 18.99 & 3.03 & 0.55 & -1.73 \\
\hline chr9:45-105 & suggestive & WL & 15.33 & 3.84 & 0.93 & -4.53 \\
\hline chr9:51-104 & suggestive & WL & 15.65 & 2.92 & 0.71 & -3.69 \\
\hline chr9:78-102 & suggestive & WL & 15.22 & 3.11 & 0.73 & -3.32 \\
\hline chr9:87-126 & suggestive & WL & 15.16 & 2.85 & 0.65 & -2.93 \\
\hline chr9:92-102 & significant & WL & 16.52 & 2.18 & 0.45 & -1.45 \\
\hline chr9:90-102 & suggestive & WL & 15.72 & 3.27 & 0.74 & -3.44 \\
\hline chr9:92-113 & suggestive & WL & 16.3 & 3.68 & 0.88 & -4.64 \\
\hline chr9:89-114 & suggestive & WL & 13.82 & 2.54 & 0.58 & -0.85 \\
\hline chr9:90-110 & suggestive & WL & 14.03 & 2.07 & 0.48 & -1.58 \\
\hline chr9:90-116 & suggestive & WL & 15.51 & 2.6 & 0.63 & -3.23 \\
\hline chr9:66-135 & significant & WL & 14.03 & 2.52 & 0.57 & -1.66 \\
\hline chr9:91-107 & suggestive & WL & 14.8 & 2.76 & 0.65 & -2.86 \\
\hline chr9:90-105 & suggestive & WL & 15.48 & 2.1 & 0.48 & -2.2 \\
\hline chr9:93-108 & suggestive & RJF & 13.19 & -0.24 & 0.5 & 0 \\
\hline chr9:89-109 & suggestive & RJF & 18.6 & -0.19 & 0.5 & 0.04 \\
\hline chr9:55-140 & significant & WL & 12.52 & 5.44 & 1.3 & -2.8 \\
\hline chr9:52-136 & suggestive & WL & 11.7 & 2.55 & 0.65 & -0.59 \\
\hline chr9:84-119 & significant & RJF & 14.94 & -2.11 & 0.48 & 0.03 \\
\hline chr9:98-118 & suggestive & RJF & 18.11 & -0.81 & 0.27 & 1.99 \\
\hline chr9:93-114 & significant & RJF & 30.88 & -3.43 & 0.52 & -0.82 \\
\hline chr9:89-127 & significant & RJF & 14.73 & -2.15 & 0.47 & 0.47 \\
\hline chr9:93-119 & significant & RJF & 26.73 & -3.56 & 0.55 & 0.32 \\
\hline chr9:69-132 & suggestive & RJF & 14.81 & -1.66 & 0.41 & -0.45 \\
\hline chr9:93-129 & significant & RJF & 14.01 & -2.35 & 0.68 & -1.27 \\
\hline chr9:68-129 & suggestive & RJF & 14.99 & -2.4 & 0.56 & -0.14 \\
\hline chr9:92-122 & significant & RJF & 20.57 & -2.94 & 0.52 & 0.99 \\
\hline chr9:93-127 & significant & RJF & 17.03 & -3.32 & 0.7 & 0.11 \\
\hline chr9:108-124 & significant & WL & 33.62 & 1.1 & 0.16 & 0.28 \\
\hline chr9:109-120 & significant & WL & 48.23 & 2.33 & 0.23 & 0.35 \\
\hline chr9:108-122 & significant & WL & 45.63 & 2.41 & 0.25 & -0.14 \\
\hline chr9:109-124 & significant & WL & 38.63 & 3.08 & 0.38 & 0.58 \\
\hline chr9:111-120 & significant & WL & 41.26 & 0.91 & 0.12 & 0.42 \\
\hline chr9:74-133 & significant & WL & 14.33 & 1.66 & 0.41 & 0.21 \\
\hline chr9:112-125 & significant & WL & 37.38 & 2.42 & 0.29 & 0.05 \\
\hline chr9:90-131 & significant & RJF & 13.86 & -2.36 & 0.53 & 1.19 \\
\hline chr9:48-125 & suggestive & RJF & 14.12 & -1.67 & 0.37 & 0.54 \\
\hline chr9:107-127 & significant & RJF & 22.56 & -0.56 & 0.38 & 0.98 \\
\hline chr9:114-132 & significant & WL & 19.23 & 1.12 & 0.48 & 2.27 \\
\hline chr9:112-128 & significant & WL & 22 & 3.16 & 0.55 & -0.42 \\
\hline chr9:90-133 & significant & WL & 12.87 & 2.17 & 0.52 & -0.62 \\
\hline chr9:61-137 & suggestive & WL & 11.54 & 3.86 & 1.02 & -0.19 \\
\hline
\end{tabular}




\begin{tabular}{|c|c|c|c|c|c|}
\hline \multirow{2}{*}{ chr9:96-131 suggestive } & RJF & 13.94 & -0.68 & 0.69 & -0.52 \\
\hline & RJF & 20.01 & -0.17 & 0.7 & -0.21 \\
\hline chr9:112-134 suggestive & RJF & 24.47 & -0.96 & 0.62 & 0.37 \\
\hline chr9:115-136 suggestive & RJF & 21.08 & -0.61 & 0.54 & 2.23 \\
\hline chr9:108-207 suggestive & WL & 15.96 & 0.8 & 0.96 & 0.04 \\
\hline chr9:101-171 suggestive & WL & 18.82 & 0.35 & 0.24 & -0.19 \\
\hline \multirow{2}{*}{$\begin{array}{l}\text { chr9:89-180 suggestive } \\
\text { chr9:125-175 significant }\end{array}$} & RJF & 12.04 & -0.46 & 0.24 & -1.05 \\
\hline & WL & 23.34 & 0.42 & 0.61 & 0.23 \\
\hline chr9:90-183 suggestive & RJF & 12.08 & -1.85 & 0.52 & -0.87 \\
\hline chr9:91-175 significant & RJF & 11.87 & -1.66 & 0.56 & -1.62 \\
\hline chr9:135-167 significant & RJF & 22.13 & -2.47 & 0.46 & -0.63 \\
\hline chr9:136-180 suggestive & WL & 14.09 & 1.47 & 0.33 & -0.67 \\
\hline chr9:137-184 significant & WL & 16.46 & 1.21 & 0.25 & -0.81 \\
\hline chr9:127-182 significant & WL & 16.77 & 2.72 & 0.57 & 0.03 \\
\hline chr9:126-207 suggestive & WL & 12.6 & 1.87 & 0.49 & -0.69 \\
\hline chr9:160-207 significant & WL & 15.6 & 2.47 & 0.52 & 0.94 \\
\hline chr9:165-207 significant & WL & 12.89 & 1.88 & 0.44 & 0.25 \\
\hline chr9:168-207 significant & RJF & 18.44 & -3.77 & 0.85 & 1.48 \\
\hline chr9:175-207 significant & WL & 20.54 & 1.83 & 0.33 & 0.12 \\
\hline chr9:169-207 significant & WL & 14.62 & 2.97 & 0.67 & -0.44 \\
\hline chr9:174-207 significant & WL & 16.46 & 2.62 & 0.54 & 0.98 \\
\hline chr9:148-207 suggestive & WL & 11.48 & 1.88 & 0.48 & 0.6 \\
\hline chr9:172-207 suggestive & WL & 12.07 & 2.17 & 0.52 & 0.61 \\
\hline chr9:183-207 significant & WL & 22.26 & 1.35 & 0.23 & 0.6 \\
\hline chr9:184-207 significant & WL & 20.17 & 2.4 & 0.43 & 0.61 \\
\hline chr9:190-207 suggestive & WL & 13.71 & 1.4 & 0.33 & 0.93 \\
\hline chr9:177-207 significant & WL & 15.39 & 1.5 & 0.32 & 0.41 \\
\hline chr9:186-207 suggestive & WL & 14.02 & 1.62 & 0.35 & 0.71 \\
\hline chr10:0-25 significant & RJF & 18.19 & -2.16 & 0.42 & 0.07 \\
\hline chr10:0-25 significant & RJF & 17.84 & -2.63 & 0.52 & -0.2 \\
\hline chr10:0-24 & RJF & 18.93 & -2.71 & 0.52 & -0.22 \\
\hline chr10:0-30 & RJF & 15.76 & -3.01 & 0.67 & -0.82 \\
\hline suggestive & RJF & 13.54 & -3.3 & 0.79 & -0.66 \\
\hline chr10:0-34 & RJF & 17.33 & -2.32 & 0.49 & -0.63 \\
\hline suggestive & RJF & 14.49 & -2 & 0.47 & -0.46 \\
\hline suggestive & RJF & 14.3 & -1.51 & 0.37 & -0.57 \\
\hline suggestive & RJF & 15.2 & -2.09 & 0.48 & -0.52 \\
\hline chr10:0-40 & RJF & 15.52 & -2.17 & 0.49 & -0.56 \\
\hline chr10:0-41 suggestive & RJF & 13.97 & -1.5 & 0.36 & -0.45 \\
\hline chr10:72-107 suggestive & RJF & 20.91 & -1.77 & 0.31 & -0.01 \\
\hline chr10:71-110 suggestive & RJF & 14.97 & -1.99 & 0.44 & 0.26 \\
\hline chr10:78-109 significant & RJF & 17.63 & -2.53 & 0.5 & 0.48 \\
\hline chr10:79-124 significant & RJF & 17.38 & -2.23 & 0.44 & -0.28 \\
\hline
\end{tabular}




\begin{tabular}{|c|c|c|c|c|c|}
\hline chr10:73-106 significant & RJF & 16.52 & -2.26 & 0.47 & 0.46 \\
\hline chr10:85-102 significant & RJF & 30.23 & -3.63 & 0.53 & 1.11 \\
\hline chr10:88-108 significant & RJF & 30.1 & -3.64 & 0.52 & 0.65 \\
\hline chr10:80-105 significant & RJF & 24.69 & -3.63 & 0.59 & 0.45 \\
\hline chr10:83-108 significant & RJF & 20.68 & -1.95 & 0.35 & 0.24 \\
\hline chr10:83-112 suggestive & RJF & 15.59 & -1.72 & 0.37 & 0.2 \\
\hline chr10:79-107 significant & RJF & 16.07 & -1.69 & 0.35 & -0.42 \\
\hline chr10:76-109 suggestive & RJF & 15.32 & -1.27 & 0.27 & 0.1 \\
\hline chr10:96-108 suggestive & RJF & 20.97 & -1.84 & 0.33 & -0.89 \\
\hline chr10:78-110 significant & RJF & 19.24 & -1.76 & 0.33 & -0.11 \\
\hline chr10:91-105 significant & RJF & 23.91 & -1.94 & 0.32 & -0.08 \\
\hline chr10:96-110 significant & RJF & 22.37 & -1.66 & 0.28 & 0.19 \\
\hline chr10:97-108 suggestive & RJF & 23.28 & -3.17 & 0.81 & 2.77 \\
\hline chr10:89-105 significant & RJF & 25.7 & -4.64 & 0.78 & 2.66 \\
\hline chr10:88-109 suggestive & RJF & 15.82 & -1.43 & 0.3 & 0.03 \\
\hline chr10:81-107 suggestive & RJF & 15.27 & -1.72 & 0.4 & 0.72 \\
\hline chr10:80-109 suggestive & RJF & 15.53 & -1.89 & 0.48 & 1.37 \\
\hline chr10:73-109 suggestive & RJF & 16.22 & -2.91 & 0.61 & 0.08 \\
\hline chr10:84-110 significant & RJF & 18.23 & -2.39 & 0.46 & -0.06 \\
\hline chr10:71-106 suggestive & RJF & 15.25 & -1.52 & 0.33 & -0.11 \\
\hline chr10:95-109 suggestive & RJF & 19.63 & -1.95 & 0.35 & -0.23 \\
\hline chr10:98-105 significant & RJF & 58.77 & -4.61 & 0.35 & -0.9 \\
\hline chr10:96-114 significant & RJF & 17.44 & -1.74 & 0.35 & -0.63 \\
\hline chr10:75-112 suggestive & RJF & 21.13 & -2.53 & 0.44 & 0.41 \\
\hline chr10:76-117 significant & WL & 13.07 & 1.77 & 0.42 & 0.48 \\
\hline chr10:95-117 significant & RJF & 18.52 & -2.57 & 0.49 & 0.15 \\
\hline chr10:96-114 suggestive & RJF & 20.97 & -0.36 & 0.17 & -0.04 \\
\hline chr10:83-115 significant & RJF & 20.64 & -3.44 & 0.62 & 0.59 \\
\hline chr10:97-109 significant & RJF & 64.64 & -6.04 & 0.4 & -0.26 \\
\hline chr10:96-116 significant & RJF & 20.35 & -2.48 & 0.45 & -0.74 \\
\hline chr10:96-127 significant & RJF & 17.6 & -3.34 & 0.65 & -0.19 \\
\hline chr10:82-127 significant & RJF & 23.3 & -3.14 & 0.52 & 0.42 \\
\hline chr10:96-126 significant & RJF & 18.15 & -3.95 & 0.77 & -0.66 \\
\hline chr10:96-126 significant & RJF & 16.18 & -1.81 & 0.38 & -1.03 \\
\hline chr10:77-135 significant & WL & 11.03 & 2.04 & 0.53 & 0.48 \\
\hline chr10:85-135 significant & RJF & 18.09 & -4.89 & 0.96 & -1.15 \\
\hline chr10:78-145 significant & RJF & 15.25 & -2.65 & 0.58 & -0.69 \\
\hline chr10:98-129 significant & RJF & 20.92 & -2.86 & 0.51 & -1.46 \\
\hline chr10:97-130 significant & RJF & 25.4 & -8.18 & 1.35 & -2.47 \\
\hline chr10:100-12 suggestive & RJF & 14.99 & -0.83 & 0.68 & -0.66 \\
\hline chr10:101-13 significant & RJF & 15.9 & -2.92 & 0.68 & -2.84 \\
\hline chr10:105-12 suggestive & RJF & 23.29 & -0.81 & 0.61 & -0.49 \\
\hline chr10:104-13 significant & RJF & 16.08 & -3.43 & 0.77 & -3.11 \\
\hline
\end{tabular}




\begin{tabular}{|c|c|c|c|c|c|}
\hline chr10:106-12 suggestive & RJF & 19.17 & -2.02 & 0.44 & -2.26 \\
\hline chr10:109-12 suggestive & WL & 24.5 & 0.03 & 0.02 & -0.03 \\
\hline chr10:109-13 significant & WL & 13.33 & 2.98 & 0.88 & -1.45 \\
\hline chr10:112-13 suggestive & WL & 13.35 & 1.31 & 0.49 & -1.35 \\
\hline chr10:112-12 suggestive & RJF & 22.28 & -2.39 & 0.43 & -2.03 \\
\hline chr10:111-12 suggestive & RJF & 23.87 & -0.22 & 0.41 & -0.74 \\
\hline chr10:105-13 suggestive & WL & 11 & 1.97 & 0.58 & -0.46 \\
\hline chr10:111-12 suggestive & WL & 23.3 & 2.14 & 0.42 & -1.37 \\
\hline chr10:106-13 significant & RJF & 18.25 & -2.83 & 0.56 & -2.42 \\
\hline chr10:108-13 suggestive & RJF & 19.51 & -3.08 & 0.6 & -2.77 \\
\hline chr10:95-134 significant & RJF & 17.35 & -4 & 0.81 & -2.88 \\
\hline chr10:103-14 significant & RJF & 17.27 & -2.86 & 0.56 & -1.65 \\
\hline chr10:125-14 suggestive & RJF & 20.11 & -1.18 & 0.28 & -1.7 \\
\hline chr10:121-20 significant & RJF & 15.33 & -2.42 & 0.52 & -0.34 \\
\hline chr10:130-14 significant & RJF & 20.08 & -0.49 & 0.52 & -3.4 \\
\hline chr10:141-15 significant & WL & 27.7 & 1.65 & 0.65 & -3.56 \\
\hline chr10:141-15 significant & WL & 28.14 & 1.23 & 0.51 & -2.59 \\
\hline chr10:143-15 suggestive & WL & 22.56 & 1.28 & 0.41 & -1.39 \\
\hline chr10:144-15 suggestive & WL & 23.71 & 1.22 & 0.42 & -1.59 \\
\hline chr10:142-16 suggestive & WL & 22.35 & 1.53 & 0.76 & -3.85 \\
\hline chr10:143-16 suggestive & WL & 23.98 & 1.38 & 0.49 & -2.05 \\
\hline chr10:125-18 significant & RJF & 14.01 & -2.69 & 0.63 & -0.54 \\
\hline chr10:109-17 significant & RJF & 15.71 & -3.56 & 0.91 & -4.81 \\
\hline chr10:128-18 suggestive & RJF & 20.55 & -2.42 & 0.49 & -2.41 \\
\hline chr10:152-18 significant & RJF & 16.19 & -1.4 & 0.44 & -2.68 \\
\hline chr10:156-18 suggestive & RJF & 14.87 & -0.31 & 0.56 & 0.87 \\
\hline chr10:99-189 suggestive & RJF & 12.61 & -3.44 & 0.84 & -1.18 \\
\hline chr10:150-18 suggestive & RJF & 14.78 & -2.59 & 0.57 & -1.88 \\
\hline chr10:156-18 suggestive & WL & 14.28 & 0.73 & 0.36 & -1.79 \\
\hline chr10:160-18 suggestive & RJF & 25.77 & -1.1 & 0.66 & -0.75 \\
\hline chr10:161-18 suggestive & RJF & 26.02 & -1.68 & 0.73 & -0.4 \\
\hline chr10:164-17 significant & RJF & 34.51 & -4.48 & 0.56 & -2.31 \\
\hline chr10:160-18 suggestive & RJF & 23.85 & -0.8 & 0.44 & 0.22 \\
\hline chr10:154-19 significant & RJF & 16.99 & -2.71 & 0.54 & -1.16 \\
\hline chr10:163-19 significant & RJF & 51.76 & -1.91 & 0.15 & -0.23 \\
\hline chr10:155-20 significant & RJF & 16.89 & -5.22 & 0.99 & -2.43 \\
\hline chr10:165-19 significant & RJF & 37.75 & -3.56 & 0.39 & -1.19 \\
\hline chr10:151-20 suggestive & RJF & 13.37 & -1.42 & 0.34 & -0.94 \\
\hline chr10:177-19 significant & RJF & 67.26 & -6.46 & 0.39 & -0.13 \\
\hline chr10:178-19 significant & RJF & 70.47 & -7.98 & 0.4 & -0.65 \\
\hline chr10:176-19 significant & RJF & 51.15 & -2.35 & 0.2 & -0.13 \\
\hline chr10:150-20 significant & RJF & 16.69 & -1.95 & 0.4 & -0.54 \\
\hline chr10:170-19 significant & RJF & 34.52 & -6.83 & 0.79 & -2.8 \\
\hline
\end{tabular}




\begin{tabular}{|c|c|c|c|c|c|c|}
\hline \multirow{2}{*}{\multicolumn{2}{|c|}{ chr10:167-19 significant }} & RJF & 29.34 & -7.07 & 0.95 & -2.53 \\
\hline & & RJF & 68.55 & -4.05 & 0.24 & 0.08 \\
\hline \multicolumn{2}{|c|}{ chr10:180-19 significant } & RJF & 67.78 & -3.04 & 0.18 & -0.07 \\
\hline \multicolumn{2}{|c|}{$\begin{array}{l}\text { chr10:179-19 significant } \\
\text { chr10:178-18 significant }\end{array}$} & RJF & 59.24 & -4.59 & 0.35 & 0.79 \\
\hline \multirow{2}{*}{\multicolumn{2}{|c|}{$\begin{array}{l}\text { chr10:163-19 significant } \\
\text { chr10:175-19 significant }\end{array}$}} & RJF & 22.6 & -9.82 & 1.58 & -3.79 \\
\hline & & RJF & 36.71 & -4.31 & 0.48 & -1.3 \\
\hline \multicolumn{2}{|c|}{ chr10:174-19 significant } & RJF & 30.56 & -3.91 & 0.53 & -1.28 \\
\hline \multirow{2}{*}{\multicolumn{2}{|c|}{$\begin{array}{l}\text { chr10:179-19 significant } \\
\text { chr10:177-19 significant }\end{array}$}} & RJF & 53.79 & -2.5 & 0.19 & -0.28 \\
\hline & & RJF & 50.94 & -3.3 & 0.28 & 0.38 \\
\hline \multirow{2}{*}{\multicolumn{2}{|c|}{$\begin{array}{l}\text { chr10:161-21 suggestive } \\
\text { chr10:154-21 suggestive }\end{array}$}} & WL & 12.58 & 1.77 & 0.42 & 0.46 \\
\hline & & RJF & 12.18 & -2.55 & 0.59 & -0.83 \\
\hline \multicolumn{2}{|c|}{ chr10:178-20 suggestive } & RJF & 25.92 & -0.03 & 0.44 & 1.78 \\
\hline \multicolumn{2}{|c|}{ chr10:173-20 suggestive } & RJF & 22.72 & -0.13 & 0.12 & 0.17 \\
\hline \multicolumn{2}{|c|}{ chr10:190-23 suggestive } & RJF & 19.65 & -0.55 & 0.83 & 0.5 \\
\hline \multicolumn{2}{|c|}{ chr10:192-23 suggestive } & RJF & 24.13 & -0.66 & 0.69 & 0.81 \\
\hline \multicolumn{2}{|c|}{ chr10:198-23 suggestive } & WL & 21.39 & 0.43 & 0.75 & 0.89 \\
\hline \multicolumn{2}{|c|}{ chr10:198-23 significant } & WL & 24.23 & 0.19 & 0.64 & 2.63 \\
\hline \multicolumn{2}{|c|}{ chr10:199-23 significant } & RJF & 22.01 & -0.59 & 0.73 & 1.43 \\
\hline \multirow{2}{*}{\multicolumn{2}{|c|}{$\begin{array}{l}\text { chr10:199-23 significant } \\
\text { chr10:201-23 suggestive }\end{array}$}} & RJF & 21.41 & -2.99 & 1.51 & 5.05 \\
\hline & & RJF & 13.43 & -0.94 & 0.35 & -1.89 \\
\hline \multicolumn{2}{|c|}{ chr10:203-23 suggestive } & RJF & 20.51 & -0.06 & 1.19 & 4.24 \\
\hline \multicolumn{2}{|c|}{ chr10:204-23 significant } & WL & 23.02 & 0.51 & 0.63 & 3.01 \\
\hline \multicolumn{2}{|c|}{ chr10:194-23 suggestive } & RJF & 15.08 & -0.36 & 0.08 & -0.29 \\
\hline \multicolumn{2}{|c|}{ chr10:203-23 significant } & WL & 28.01 & 2.73 & 1 & 2.46 \\
\hline chr11:0-27 & suggestive & WL & 19.45 & 0.29 & 0.08 & -0.41 \\
\hline chr11:1-32 & suggestive & WL & 18.99 & 0.73 & 0.21 & -1.12 \\
\hline chr11:0-32 & significant & WL & 16.74 & 1.53 & 0.31 & -0.03 \\
\hline chr11:0-32 & suggestive & WL & 13.78 & 1.66 & 0.45 & -1.29 \\
\hline chr11:3-28 & suggestive & RJF & 15.46 & -0.37 & 0.44 & -0.43 \\
\hline chr11:7-23 & significant & WL & 19.77 & 1.44 & 0.48 & 1.19 \\
\hline chr11:0-35 & suggestive & WL & 11.78 & 3.34 & 0.82 & 0.51 \\
\hline chr11:0-29 & suggestive & WL & 13.91 & 1.01 & 0.23 & -0.02 \\
\hline chr11:5-43 & suggestive & WL & 14.01 & 1.13 & 0.3 & -0.81 \\
\hline chr11:0-32 & suggestive & RJF & 12.36 & -45.8 & 12.36 & 22.6 \\
\hline chr11:3-29 & significant & RJF & 15.83 & -57.59 & 13.19 & 24.4 \\
\hline chr11:5-28 & significant & RJF & 16.39 & -50.24 & 11 & 17.08 \\
\hline chr11:5-35 & significant & WL & 13.86 & 1.44 & 0.32 & 0.11 \\
\hline chr11:2-34 & suggestive & WL & 13.83 & 1.46 & 0.36 & -0.6 \\
\hline chr11:4-61 & suggestive & WL & 13.27 & 2.58 & 0.62 & -0.21 \\
\hline chr11:8-35 & suggestive & WL & 14.09 & 0.54 & 0.12 & -0.09 \\
\hline chr11:10-25 & suggestive & RJF & 21.93 & -3.15 & 0.62 & -2.94 \\
\hline chr11:9-24 & suggestive & RJF & 22.97 & -2.39 & 0.54 & -2.72 \\
\hline chr11:2-33 & suggestive & WL & 13.81 & 1.1 & 0.32 & -1.1 \\
\hline
\end{tabular}




\begin{tabular}{|c|c|c|c|c|c|c|}
\hline chr11:9-31 & suggestive & WL & 21.38 & 0.99 & 0.19 & -0.55 \\
\hline chr11:8-29 & suggestive & WL & 21.19 & 0.87 & 0.22 & -1.18 \\
\hline chr11:7-63 & suggestive & WL & 13.91 & 1.59 & 0.37 & -0.13 \\
\hline chr11:0-38 & significant & RJF & 13.36 & -1.5 & 0.35 & -0.42 \\
\hline chr11:6-33 & suggestive & WL & 18.05 & 0.88 & 0.18 & -0.2 \\
\hline chr11:0-50 & suggestive & WL & 13.18 & 3.06 & 0.77 & -1.12 \\
\hline chr11:1-41 & significant & WL & 14.72 & 1.9 & 0.41 & 0.22 \\
\hline chr11:0-38 & significant & RJF & 15.2 & -2.83 & 0.62 & -0.13 \\
\hline chr11:8-37 & suggestive & WL & 18.57 & 0.18 & 0.05 & -0.2 \\
\hline chr11:9-35 & suggestive & WL & 19.6 & 1.6 & 0.39 & -1.67 \\
\hline chr11:0-54 & suggestive & WL & 13.03 & 3.14 & 0.73 & 0.44 \\
\hline chr11:3-35 & suggestive & WL & 17.7 & 0.21 & 0.07 & -0.37 \\
\hline chr11:0-51 & suggestive & WL & 13.93 & 1.4 & 0.31 & -0.04 \\
\hline chr11:3-50 & significant & WL & 16.85 & 0.62 & 0.17 & -0.72 \\
\hline chr11:7-58 & significant & WL & 16.95 & 1.66 & 0.38 & -0.88 \\
\hline chr11:5-55 & suggestive & WL & 14.24 & 0.43 & 0.11 & -0.33 \\
\hline chr11:6-71 & suggestive & WL & 13.83 & 1.35 & 0.34 & -0.73 \\
\hline chr11:3-47 & significant & WL & 22.28 & 0.42 & 0.08 & -0.3 \\
\hline chr11:9-58 & suggestive & WL & 14.27 & 0.77 & 0.21 & -0.69 \\
\hline chr11:10-55 & significant & RJF & 21.15 & -4.09 & 0.76 & -2.2 \\
\hline chr11:17-47 & suggestive & WL & 20.14 & 0.38 & 0.25 & -1.44 \\
\hline chr11:0-47 & significant & RJF & 18.68 & -1.64 & 0.76 & -3.52 \\
\hline chr11:6-50 & significant & RJF & 13.95 & -2.97 & 0.68 & -0.52 \\
\hline chr11:14-49 & significant & WL & 16.41 & 1.58 & 0.37 & -0.27 \\
\hline chr11:18-56 & suggestive & RJF & 12.97 & -1.41 & 0.37 & 0.25 \\
\hline chr11:23-55 & suggestive & RJF & 13.66 & -2.09 & 0.49 & -0.21 \\
\hline chr11:24-48 & significant & RJF & 14.22 & -2.54 & 0.58 & -1.81 \\
\hline chr11:26-54 & suggestive & WL & 18.14 & 0.26 & 0.08 & -0.27 \\
\hline chr11:56-85 & suggestive & WL & 15.9 & 1.89 & 0.4 & -0.52 \\
\hline chr11:60-88 & significant & WL & 16.05 & 4.24 & 0.89 & -0.11 \\
\hline chr11:76-99 & significant & RJF & 17.12 & -2.65 & 1.2 & -4.34 \\
\hline chr11:81-111 & suggestive & RJF & 10.57 & -1.64 & 0.56 & -1.27 \\
\hline chr11:86-96 & suggestive & WL & 25.67 & 0.93 & 0.16 & -0.74 \\
\hline chr11:84-92 & suggestive & WL & 21.27 & 0.77 & 0.95 & -4.75 \\
\hline chr11:72-110 & significant & RJF & 12.04 & -1.47 & 0.38 & -0.18 \\
\hline chr11:80-100 & suggestive & WL & 19.1 & 0.7 & 0.33 & -2.29 \\
\hline chr11:55-162 & significant & WL & 12.31 & 1.94 & 0.48 & -0.41 \\
\hline chr11:80-114 & significant & RJF & 19.53 & -1.83 & 0.34 & 0.16 \\
\hline chr11:75-132 & significant & RJF & 19.63 & -1.91 & 0.36 & -0.36 \\
\hline chr11:89-117 & significant & RJF & 24.46 & -3.45 & 0.55 & 0.35 \\
\hline chr11:77-110 & significant & RJF & 16.38 & -1.59 & 0.35 & -0.89 \\
\hline chr11:78-119 & suggestive & RJF & 13.74 & -2.52 & 0.56 & -0.12 \\
\hline chr11:78-152 & significant & WL & 15.49 & 1.77 & 0.39 & -0.57 \\
\hline
\end{tabular}




\begin{tabular}{|c|c|c|c|c|c|}
\hline chr11:92-115 suggestive & RJF & 19.22 & -0.97 & 0.4 & -2.81 \\
\hline chr11:95-107 significant & WL & 57.28 & 3.62 & 0.28 & -0.94 \\
\hline chr11:62-148 significant & WL & 13.76 & 2.03 & 0.53 & -1.56 \\
\hline chr11:96-108 significant & WL & 62.93 & 5.18 & 0.36 & -0.83 \\
\hline chr11:62-155 significant & WL & 15.13 & 2.09 & 0.45 & -0.08 \\
\hline chr11:90-114 significant & RJF & 17.61 & -2.72 & 0.54 & -0.62 \\
\hline chr11:93-115 significant & RJF & 14.97 & -1.85 & 0.43 & -1.07 \\
\hline chr11:92-155 significant & WL & 15.07 & 2.05 & 0.45 & -0.19 \\
\hline chr11:85-124 significant & RJF & 13.74 & -2.17 & 0.49 & 0.36 \\
\hline chr11:95-121 significant & WL & 19.41 & 0.82 & 0.17 & -0.66 \\
\hline chr11:92-114 significant & RJF & 16.3 & -2.49 & 0.52 & -0.43 \\
\hline chr11:93-118 significant & RJF & 32.03 & -2.85 & 0.38 & 0.04 \\
\hline chr11:95-130 significant & RJF & 13.52 & -1.93 & 0.44 & 0.12 \\
\hline chr11:94-122 significant & RJF & 28.63 & -4.06 & 0.58 & -0.37 \\
\hline chr11:98-121 significant & RJF & 19.94 & -1.94 & 0.36 & -0.81 \\
\hline chr11:98-118 suggestive & WL & 20.78 & 0.09 & 0.46 & -3.85 \\
\hline chr11:90-129 significant & RJF & 22.33 & -2.15 & 0.37 & 0.22 \\
\hline chr11:92-155 suggestive & WL & 13.41 & 1.45 & 0.4 & -1.45 \\
\hline chr11:91-133 significant & RJF & 19.21 & -1.66 & 0.32 & 0.52 \\
\hline chr11:92-153 significant & WL & 15.01 & 2.23 & 0.49 & 0.03 \\
\hline chr11:90-135 significant & RJF & 20.65 & -3.17 & 0.59 & 1.19 \\
\hline chr11:99-148 significant & WL & 25.54 & 2.96 & 0.48 & -1.08 \\
\hline chr11:75-157 significant & WL & 12.77 & 2.7 & 0.66 & -0.67 \\
\hline chr11:98-130 significant & RJF & 17.6 & -2.57 & 0.51 & 0.83 \\
\hline chr11:106-12 suggestive & RJF & 21.52 & -0.13 & 0.39 & -0.04 \\
\hline chr11:119-13 significant & WL & 24.89 & 2.01 & 0.35 & 0.65 \\
\hline chr11:113-13 significant & WL & 22.19 & 4.27 & 0.87 & 2.55 \\
\hline chr11:112-13 significant & WL & 18.82 & 6.09 & 1.15 & 0.63 \\
\hline chr11:90-136 suggestive & WL & 14.42 & 1.58 & 0.35 & -0.46 \\
\hline chr11:113-13 suggestive & WL & 13.84 & 1.69 & 0.47 & 1.27 \\
\hline chr11:115-13 suggestive & WL & 18.91 & 1.73 & 0.35 & -1.43 \\
\hline chr11:115-13 significant & WL & 19.82 & 1.57 & 0.28 & -0.31 \\
\hline chr11:117-13 suggestive & WL & 23.46 & 0.14 & 0.54 & -2.14 \\
\hline chr11:115-13 significant & WL & 31.76 & 4.75 & 0.64 & -0.7 \\
\hline chr11:115-13 significant & WL & 45.29 & 4.67 & 0.47 & -1.93 \\
\hline chr11:111-13 significant & WL & 23.21 & 2.36 & 0.4 & -0.02 \\
\hline chr11:111-13 significant & WL & 23.27 & 5.1 & 0.85 & 0.45 \\
\hline chr11:111-13 significant & WL & 16.22 & 1.46 & 0.3 & -0.63 \\
\hline chr11:108-13 suggestive & WL & 23.48 & 0.53 & 0.31 & 0.44 \\
\hline chr11:112-13 significant & WL & 28.65 & 5.78 & 0.84 & -2.96 \\
\hline chr11:112-13 significant & WL & 42.97 & 3.39 & 0.35 & -1.45 \\
\hline chr11:113-15 significant & WL & 20.73 & 1.92 & 0.38 & 0.62 \\
\hline chr11:110-13 suggestive & RJF & 14.07 & -2.36 & 0.55 & 1.57 \\
\hline
\end{tabular}




\begin{tabular}{|c|c|c|c|c|c|}
\hline chr11:98-156 significant & WL & 14.61 & 1.2 & 0.25 & -0.5 \\
\hline chr11:110-15 suggestive & WL & 14 & 1.86 & 0.42 & -0.22 \\
\hline chr11:74-157 suggestive & WL & 12.21 & 1.63 & 0.41 & -0.63 \\
\hline chr11:118-13 significant & WL & 30.13 & 2.16 & 1.11 & 1.54 \\
\hline chr11:113-13 significant & WL & 23.32 & 2.69 & 1.15 & -0.17 \\
\hline chr11:90-136 significant & RJF & 16.85 & -2.09 & 0.46 & 1.92 \\
\hline chr11:97-161 suggestive & WL & 14.49 & 1.93 & 0.43 & -0.39 \\
\hline chr11:109-15 suggestive & WL & 12.16 & 4.76 & 1.18 & 0.93 \\
\hline chr11:110-15 significant & WL & 16.48 & 1.44 & 0.29 & -0.24 \\
\hline chr11:115-14 suggestive & WL & 18.88 & 1.41 & 0.73 & 0.1 \\
\hline chr11:27-155 suggestive & WL & 12.6 & 1.42 & 0.35 & -0.34 \\
\hline chr11:93-165 significant & RJF & 19.65 & -3.76 & 0.7 & 2.31 \\
\hline chr11:108-15 significant & WL & 16.21 & 2.17 & 0.46 & -1.28 \\
\hline chr11:77-156 significant & WL & 14.15 & 1.7 & 0.39 & -0.86 \\
\hline chr11:113-15 significant & WL & 18.92 & 1.37 & 0.96 & -0.72 \\
\hline chr11:115-15 significant & WL & 20.93 & 2.33 & 0.46 & 0.8 \\
\hline chr11:55-159 significant & WL & 14.06 & 1.91 & 0.43 & -0.36 \\
\hline chr11:111-16 suggestive & WL & 14.14 & 0.21 & 0.4 & 0.39 \\
\hline chr11:113-15 suggestive & WL & 17.46 & 1.96 & 0.38 & -0.41 \\
\hline chr11:98-160 significant & WL & 16.58 & 1.88 & 0.4 & -1.39 \\
\hline chr11:91-157 significant & WL & 13.28 & 3.41 & 0.8 & -0.49 \\
\hline chr11:111-15 significant & WL & 17.71 & 1.77 & 0.38 & 0.56 \\
\hline chr11:95-175 significant & RJF & 19.01 & -2.26 & 0.43 & 1.31 \\
\hline chr11:113-15 significant & WL & 20.17 & 2.04 & 0.39 & -1.85 \\
\hline chr11:92-157 suggestive & WL & 14.89 & 2.29 & 0.53 & 0.47 \\
\hline chr11:97-158 suggestive & WL & 20.08 & 1.83 & 0.33 & -0.87 \\
\hline chr11:78-159 significant & WL & 14.23 & 1.78 & 0.41 & -0.59 \\
\hline chr11:99-153 significant & WL & 18.49 & 2.4 & 0.49 & 0.54 \\
\hline chr11:126-16 significant & WL & 18.39 & 3.31 & 0.64 & -1.5 \\
\hline chr11:120-15 significant & WL & 22.74 & 3.42 & 0.8 & 1.62 \\
\hline chr11:115-15 significant & WL & 18.07 & 2.02 & 0.39 & -0.51 \\
\hline chr11:103-16 significant & WL & 16.01 & 1.48 & 0.34 & -1.39 \\
\hline chr11:127-15 significant & WL & 15.27 & 2.07 & 0.45 & -1.49 \\
\hline chr11:129-15 suggestive & WL & 16.54 & 0.83 & 0.7 & 0.36 \\
\hline chr11:128-15 suggestive & WL & 24.09 & 2.41 & 0.45 & -0.53 \\
\hline chr11:99-167 suggestive & WL & 14.42 & 2.21 & 0.49 & -0.63 \\
\hline chr11:119-16 significant & WL & 15.97 & 1.02 & 0.22 & -0.09 \\
\hline chr11:119-15 significant & WL & 17.81 & 2.1 & 0.42 & 0.01 \\
\hline chr11:127-16 significant & WL & 16.82 & 1.96 & 0.4 & -0.94 \\
\hline chr11:127-16 significant & WL & 13.34 & 1.79 & 0.41 & -1.12 \\
\hline chr11:128-15 suggestive & WL & 19.14 & 1.95 & 0.36 & -0.33 \\
\hline chr11:129-15 significant & WL & 21.06 & 2.29 & 0.41 & -0.95 \\
\hline chr11:130-15 significant & WL & 22.6 & 1.98 & 0.34 & -0.89 \\
\hline
\end{tabular}




\begin{tabular}{|c|c|c|c|c|c|}
\hline chr11:128-15 significant & WL & 16.51 & 1.64 & 0.34 & -0.84 \\
\hline chr11:116-15 significant & WL & 17.05 & 2.07 & 0.51 & 1.48 \\
\hline chr11:120-15 suggestive & WL & 14.55 & 1.43 & 0.33 & 0.13 \\
\hline chr11:124-15 significant & WL & 17.98 & 2.83 & 0.6 & 0.66 \\
\hline chr11:119-15 suggestive & WL & 15.19 & 1.48 & 0.35 & 0.43 \\
\hline chr11:119-16 significant & WL & 15.59 & 1.61 & 0.34 & -0.45 \\
\hline chr11:126-15 suggestive & WL & 17.74 & 1.65 & 0.33 & -0.01 \\
\hline chr11:130-15 suggestive & WL & 20.53 & 0.09 & 0.02 & -0.12 \\
\hline chr11:124-15 suggestive & WL & 19.01 & 1.46 & 0.32 & 0.8 \\
\hline chr11:129-15 significant & WL & 18.59 & 1.5 & 0.29 & -0.24 \\
\hline chr11:129-15 significant & WL & 18.62 & 2.43 & 0.48 & 0.33 \\
\hline chr11:128-15 significant & WL & 18.98 & 3.56 & 0.69 & 0.03 \\
\hline chr11:131-15 significant & WL & 18.68 & 1.9 & 0.37 & -1.04 \\
\hline chr11:132-16 suggestive & WL & 18.41 & 0.45 & 0.13 & -0.59 \\
\hline chr11:131-15 significant & WL & 20.66 & 3.24 & 0.58 & -0.45 \\
\hline chr11:131-15 significant & WL & 19.49 & 2.29 & 0.42 & -0.67 \\
\hline chr11:129-15 significant & WL & 18.46 & 1.66 & 0.33 & 0.04 \\
\hline chr11:132-15 significant & WL & 16.04 & 1.2 & 0.29 & -1.42 \\
\hline chr11:131-14 significant & WL & 22.64 & 2.81 & 0.57 & 1.68 \\
\hline chr11:127-15 significant & WL & 17.47 & 2.49 & 0.54 & 0.81 \\
\hline chr11:129-15 significant & WL & 15.28 & 1.35 & 0.29 & 0.09 \\
\hline chr11:127-15 significant & WL & 16.34 & 2.51 & 0.53 & -0.08 \\
\hline chr11:126-16 suggestive & WL & 15.72 & 2.15 & 0.48 & 0.28 \\
\hline chr11:130-16 suggestive & WL & 14.24 & 1.92 & 0.45 & -1.34 \\
\hline chr11:130-15 suggestive & WL & 15.29 & 1.87 & 0.64 & 2.67 \\
\hline chr11:128-15 significant & WL & 17.49 & 1.8 & 0.36 & -0.26 \\
\hline chr11:126-15 suggestive & WL & 14.28 & 3.36 & 0.78 & 0.12 \\
\hline chr11:128-15 suggestive & WL & 15.37 & 2.22 & 0.48 & -0.79 \\
\hline chr11:133-14 suggestive & WL & 9.37 & 1.58 & 0.26 & -1.16 \\
\hline chr11:131-16 suggestive & WL & 12.26 & 1.56 & 0.28 & -0.49 \\
\hline chr11:133-15 suggestive & WL & 21.8 & 1.53 & 0.26 & -0.5 \\
\hline chr11:132-15 suggestive & WL & 19.9 & 1.57 & 0.29 & -0.46 \\
\hline chr11:129-15 suggestive & WL & 21.39 & 1.55 & 0.27 & -0.55 \\
\hline chr11:130-15 suggestive & WL & 19.52 & 1.33 & 0.27 & 0.36 \\
\hline chr11:133-16 suggestive & WL & 18.84 & 2.2 & 0.44 & -1.74 \\
\hline chr11:133-15 suggestive & WL & 20.22 & 0.56 & 0.11 & -0.55 \\
\hline chr11:132-16 suggestive & WL & 12.31 & 1.79 & 0.36 & -1.19 \\
\hline chr11:131-15 suggestive & WL & 12.78 & 1.27 & 0.24 & -0.63 \\
\hline chr11:131-15 suggestive & WL & 18.83 & 1.65 & 0.31 & -0.31 \\
\hline chr11:131-15 suggestive & WL & 19.37 & 1.28 & 0.24 & -0.53 \\
\hline chr11:131-15 suggestive & WL & 19.24 & 2.47 & 0.49 & -2.26 \\
\hline chr11:129-16 suggestive & WL & 18.54 & 1.48 & 0.28 & -0.28 \\
\hline chr11:95-166 significant & RJF & 14.16 & -1.57 & 0.36 & -0.18 \\
\hline
\end{tabular}




\begin{tabular}{|c|c|c|c|c|c|c|}
\hline chr11:128-16 & 5 significant & WL & 16.5 & 2.28 & 0.47 & -0.75 \\
\hline chr11:130-16 & suggestive & WL & 25.8 & 2.11 & 0.43 & -2.47 \\
\hline chr11:177-18 & 3 suggestive & RJF & 18.73 & -2.54 & 0.54 & -1.64 \\
\hline chr12:0-21 & significant & WL & 12.19 & 1.67 & 0.46 & -0.41 \\
\hline chr12:0-9 & significant & WL & 13.52 & 2.23 & 0.53 & 0.44 \\
\hline chr12:0-18 & significant & WL & 9.42 & 1.63 & 0.53 & -0.47 \\
\hline chr12:0-9 & significant & RJF & 17.12 & -34.69 & 7.27 & -7.37 \\
\hline chr12:0-9 & significant & RJF & 17.11 & -35.52 & 7.5 & -6.39 \\
\hline chr12:0-9 & suggestive & RJF & 15.92 & -30.45 & 6.68 & -6.05 \\
\hline chr12:0-10 & suggestive & RJF & 15.81 & -31.38 & 6.84 & -7.11 \\
\hline chr12:0-10 & suggestive & RJF & 15.73 & -29.17 & 6.39 & -6.75 \\
\hline chr12:10-32 & suggestive & RJF & 18.87 & -1.93 & 0.37 & -1.07 \\
\hline chr12:17-32 & significant & WL & 28.52 & 6.75 & 1.11 & 0.34 \\
\hline chr12:18-31 & significant & WL & 34.44 & 7.11 & 1 & 0.85 \\
\hline chr12:21-31 & significant & WL & 34.19 & 12.58 & 1.7 & 2.73 \\
\hline chr12:18-31 & significant & WL & 30.48 & 5.23 & 0.77 & 1.14 \\
\hline chr12:22-31 & significant & WL & 41.3 & 11.74 & 1.34 & 3.25 \\
\hline chr12:12-42 & suggestive & RJF & 14.53 & -1.57 & 0.4 & -0.02 \\
\hline chr12:0-242 & significant & RJF & 7.15 & -0.35 & 0.27 & -0.28 \\
\hline chr12:13-39 & suggestive & RJF & 15.51 & -0.83 & 0.22 & -1.28 \\
\hline chr12:14-46 & significant & RJF & 25.7 & -13.75 & 2.06 & -5.62 \\
\hline chr12:12-51 & significant & RJF & 19.32 & -10.31 & 1.9 & -3.43 \\
\hline chr12:12-47 & significant & RJF & 24.06 & -13.23 & 2.07 & -4.34 \\
\hline chr12:20-46 & significant & RJF & 29.72 & -1.5 & 0.23 & -0.16 \\
\hline chr12:12-48 & significant & RJF & 19.82 & -11.1 & 1.97 & -3.9 \\
\hline chr12:13-55 & suggestive & RJF & 13.61 & -4.73 & 1.09 & -0.97 \\
\hline chr12:12-55 & significant & RJF & 16.3 & -8.8 & 1.79 & -2.39 \\
\hline chr12:13-48 & significant & RJF & 21.7 & -12.75 & 2.14 & -3.97 \\
\hline chr12:30-46 & suggestive & RJF & 17.37 & -1.71 & 0.35 & -0.25 \\
\hline chr12:17-59 & suggestive & RJF & 12.83 & -2.17 & 0.63 & 0.6 \\
\hline chr12:30-53 & suggestive & WL & 17.38 & 0.31 & 0.67 & -2.98 \\
\hline chr12:29-55 & suggestive & RJF & 19.04 & -0.5 & 0.09 & -0.27 \\
\hline chr12:16-57 & significant & RJF & 19.12 & -7.88 & 1.47 & -1.88 \\
\hline chr12:16-67 & significant & RJF & 15.75 & -4.38 & 1.02 & -0.17 \\
\hline chr12:27-68 & significant & RJF & 13.94 & -2.4 & 0.57 & -0.48 \\
\hline chr12:34-72 & suggestive & RJF & 14.21 & -1.45 & 0.33 & -0.66 \\
\hline chr12:44-66 & significant & RJF & 17.32 & -1.36 & 0.28 & -1.35 \\
\hline chr12:36-67 & suggestive & RJF & 17.21 & -1.24 & 0.25 & -0.92 \\
\hline chr12:49-66 & significant & RJF & 46.9 & -3.28 & 0.32 & -1.38 \\
\hline chr12:48-66 & significant & RJF & 30.01 & -2.71 & 0.38 & -1.29 \\
\hline chr12:47-66 & significant & RJF & 18.82 & -0.84 & 0.16 & -0.59 \\
\hline chr12:48-67 & significant & RJF & 20.86 & -2.04 & 0.38 & -2.01 \\
\hline chr12:47-67 & significant & RJF & 21.61 & -3.44 & 0.6 & -2.31 \\
\hline
\end{tabular}




\begin{tabular}{|c|c|c|c|c|c|}
\hline chr12:53-61 suggestive & RJF & 25.88 & -0.86 & 0.14 & -0.75 \\
\hline chr12:43-74 suggestive & RJF & 15.49 & -2.19 & 0.47 & -1.06 \\
\hline chr12:48-69 significant & RJF & 25.66 & -0.38 & 0.06 & -0.24 \\
\hline chr12:47-70 suggestive & RJF & 19.43 & -1.83 & 0.34 & -1.44 \\
\hline chr12:51-71 significant & RJF & 17.66 & -1.13 & 0.23 & -1.15 \\
\hline chr12:53-70 significant & WL & 20.87 & 0.22 & 0.9 & -0.99 \\
\hline chr12:53-71 significant & RJF & 21.97 & -1.06 & 0.8 & -0.16 \\
\hline chr12:51-69 suggestive & RJF & 26.25 & -0.1 & 0.52 & 0.74 \\
\hline chr12:131-17 suggestive & WL & 18.84 & 0.52 & 0.12 & -0.68 \\
\hline chr12:139-17 suggestive & WL & 20.07 & 0.35 & 0.11 & -0.7 \\
\hline chr12:128-18 significant & WL & 14.83 & 2.27 & 0.5 & -0.16 \\
\hline chr12:136-16 suggestive & WL & 20.78 & 1.41 & 0.26 & -0.82 \\
\hline chr12:137-21 suggestive & WL & 14.21 & 1.4 & 0.37 & -1.58 \\
\hline chr12:143-16 suggestive & WL & 21.24 & 0.02 & 0.1 & -0.12 \\
\hline chr12:138-16 suggestive & WL & 19.08 & 0.3 & 0.07 & -0.4 \\
\hline chr12:130-20 significant & WL & 15.08 & 0.73 & 0.19 & -0.84 \\
\hline chr12:151-18 suggestive & WL & 15.46 & 0.39 & 0.08 & -0.56 \\
\hline chr12:152-18 suggestive & WL & 16.48 & 0.14 & 0.1 & -0.84 \\
\hline chr12:136-18 suggestive & WL & 15.87 & 0.17 & 0.43 & -1.22 \\
\hline chr12:152-17 significant & WL & 25.94 & 0.85 & 0.16 & -1.14 \\
\hline chr12:136-18 significant & WL & 14.88 & 1.99 & 0.44 & -0.82 \\
\hline chr12:152-18 suggestive & WL & 25.97 & 1.57 & 0.39 & -2.92 \\
\hline chr12:162-18 suggestive & WL & 23.04 & 0.38 & 0.08 & -0.45 \\
\hline chr12:157-18 suggestive & WL & 21.4 & 0.44 & 0.11 & -0.76 \\
\hline chr12:151-18 significant & WL & 17.42 & 0.78 & 0.13 & -0.93 \\
\hline chr12:152-18 suggestive & WL & 18.16 & 0.13 & 0.36 & -0.34 \\
\hline chr12:159-19 suggestive & WL & 20.96 & 1.21 & 0.27 & -1.7 \\
\hline chr12:178-22 significant & RJF & 19.09 & -2.87 & 0.5 & -1.39 \\
\hline chr12:190-21 suggestive & RJF & 15.87 & -3.74 & 0.86 & -3.61 \\
\hline chr12:204-24 significant & RJF & 16.51 & -4.33 & 0.89 & -3.37 \\
\hline chr12:223-24 suggestive & RJF & 22.54 & -0.52 & 0.1 & -0.62 \\
\hline chr12:224-23 suggestive & RJF & 13.24 & -1.69 & 0.58 & -3.28 \\
\hline chr12:220-24 suggestive & RJF & 13.11 & -0.88 & 0.33 & -1.86 \\
\hline chr12:220-24 suggestive & RJF & 18.53 & -1.06 & 0.21 & -1.25 \\
\hline chr12:222-24 significant & RJF & 23.22 & -3.63 & 0.6 & -2.31 \\
\hline chr12:228-24 suggestive & RJF & 15.69 & -2 & 0.42 & -1.57 \\
\hline chr12:219-24 suggestive & RJF & 19.03 & -2.57 & 0.51 & -2.94 \\
\hline chr12:215-24 suggestive & RJF & 17.95 & -2.75 & 0.55 & -3.02 \\
\hline chr12:234-24 significant & RJF & 33.13 & -5.08 & 0.68 & -1.74 \\
\hline chr12:213-24 significant & RJF & 23.01 & -6.25 & 1.04 & -3.63 \\
\hline chr12:215-24 suggestive & RJF & 13.72 & -3.17 & 0.71 & -2.9 \\
\hline chr12:233-24 significant & RJF & 39.24 & -3.95 & 0.48 & -0.89 \\
\hline chr12:217-24 significant & RJF & 16.64 & -3.68 & 0.75 & -3 \\
\hline
\end{tabular}




\begin{tabular}{|c|c|c|c|c|c|}
\hline chr12:206-24 suggestive & RJF & 14.3 & -4.18 & 0.93 & -1.73 \\
\hline chr12:211-24 significant & RJF & 15.72 & -2.99 & 0.63 & -2.04 \\
\hline chr12:213-24 suggestive & RJF & 14.69 & -5.12 & 1.1 & -3.39 \\
\hline chr12:226-24 suggestive & RJF & 19.9 & -3.17 & 0.66 & -2.73 \\
\hline chr12:221-24 suggestive & RJF & 18.22 & -1.35 & 0.7 & -1.35 \\
\hline chr12:234-24 significant & RJF & 19.01 & -2.98 & 0.62 & 0.4 \\
\hline chr12:232-24 significant & RJF & 19.02 & -2.69 & 0.51 & -1.55 \\
\hline chr12:234-24 significant & WL & 14.74 & 2.02 & 0.47 & 0.21 \\
\hline chr12:233-24 significant & RJF & 21.46 & -2.04 & 0.47 & -2.16 \\
\hline chr13:0-16 significant & RJF & 23.22 & -11.31 & 2.58 & 6.77 \\
\hline chr13:0-17 suggestive & RJF & 22.56 & -8.82 & 2.12 & 4.45 \\
\hline chr13:0-17 suggestive & RJF & 20.47 & -7.11 & 2.31 & 2.28 \\
\hline chr13:0-16 significant & RJF & 22.27 & -3.58 & 1.34 & 1.33 \\
\hline chr13:0-10 & RJF & 34.32 & -3.45 & 0.94 & -0.06 \\
\hline chr13:0-16 & RJF & 17.13 & -6.99 & 1.53 & 1.38 \\
\hline chr13:0-17 & RJF & 19.93 & -6.68 & 1.51 & -0.65 \\
\hline chr13:0-17 & RJF & 20.86 & -6.86 & 1.46 & -0.18 \\
\hline chr13:0-18 & RJF & 22.19 & -1.23 & 0.29 & -0.37 \\
\hline chr13:0-10 & RJF & 34.45 & -2.51 & 0.38 & -0.04 \\
\hline significant & RJF & 39.2 & -3.38 & 0.45 & 0.18 \\
\hline chr13:0-17 & RJF & 19.72 & -8.64 & 1.7 & 2.04 \\
\hline chr13:0-14 & RJF & 35.72 & -1.92 & 0.54 & 0.56 \\
\hline chr13:0-17 & RJF & 22.86 & -9.22 & 1.74 & 1.2 \\
\hline chr13:0-20 & WL & 16.87 & 2.6 & 0.62 & -0.09 \\
\hline chr13:0-19 & RJF & 19.65 & -5.66 & 1.28 & -0.36 \\
\hline chr13:0-20 & RJF & 21.4 & -6.04 & 1.82 & 3.88 \\
\hline chr13:0-19 & RJF & 22.76 & -4.01 & 1.11 & 2.85 \\
\hline chr13:0-19 & RJF & 21.04 & -8.53 & 1.96 & 3.97 \\
\hline chr13:0-22 & RJF & 20.14 & -5.54 & 1.66 & 3.24 \\
\hline chr13:0-20 & RJF & 17.94 & -5.07 & 1.14 & 0.28 \\
\hline chr13:0-18 & RJF & 24.72 & -9.77 & 1.66 & 2.42 \\
\hline chr13:0-19 & RJF & 19.54 & -5.84 & 1.15 & 1.4 \\
\hline chr13:0-22 & RJF & 15.54 & -6.1 & 1.37 & 1.57 \\
\hline chr13:0-16 & WL & 49.78 & 6.57 & 0.7 & -0.6 \\
\hline chr13:0-18 & WL & 31.16 & 5.07 & 0.72 & -1.73 \\
\hline chr13:0-18 & WL & 27.05 & 4.79 & 1.3 & -0.66 \\
\hline chr13:0-29 & WL & 10.69 & 5.01 & 1.39 & -1.64 \\
\hline chr13:0-19 & WL & 30.55 & 7.23 & 1.83 & -0.96 \\
\hline chr13:0-24 significant & WL & 15.18 & 4.64 & 1.07 & -1.37 \\
\hline chr13:40-109 significant & WL & 16.51 & 2.53 & 0.53 & -1.64 \\
\hline chr13:58-85 significant & RJF & 22.12 & -2.05 & 0.64 & 0.94 \\
\hline chr13:56-104 significant & RJF & 13.72 & -4.19 & 0.97 & 0.72 \\
\hline chr13:53-87 suggestive & RJF & 13.99 & -1.95 & 0.48 & -0.42 \\
\hline
\end{tabular}




\begin{tabular}{|c|c|c|c|c|c|}
\hline chr13:53-114 significant & WL & 22.05 & 2.64 & 0.47 & -0.05 \\
\hline chr13:54-116 suggestive & WL & 15.95 & 2.62 & 0.69 & -1.96 \\
\hline chr13:55-110 significant & RJF & 13.25 & -2.11 & 0.6 & -0.45 \\
\hline chr13:47-100 suggestive & RJF & 10.04 & -1.93 & 0.89 & -2.07 \\
\hline chr13:75-96 suggestive & RJF & 25.96 & -2.1 & 0.67 & -2.65 \\
\hline chr13:80-128 significant & WL & 18.41 & 1.97 & 0.39 & -0.5 \\
\hline chr13:81-107 significant & WL & 17.37 & 1.31 & 0.26 & -0.69 \\
\hline chr13:80-108 suggestive & WL & 14.94 & 1.75 & 0.4 & -0.38 \\
\hline chr13:54-131 significant & WL & 16.87 & 2.81 & 0.6 & -0.62 \\
\hline chr13:81-108 significant & WL & 17.38 & 2.49 & 0.49 & -1.87 \\
\hline chr13:59-107 suggestive & WL & 15.44 & 2.82 & 0.6 & -1.86 \\
\hline chr13:57-121 significant & WL & 17.94 & 1.64 & 0.32 & -0.77 \\
\hline chr13:82-110 significant & WL & 21.01 & 3.35 & 0.62 & -0.95 \\
\hline chr13:80-109 suggestive & WL & 19.4 & 1.22 & 0.21 & -0.81 \\
\hline chr13:82-105 significant & WL & 24.18 & 2.31 & 0.38 & -1.95 \\
\hline chr13:82-108 significant & WL & 22.65 & 4.56 & 0.85 & -0.36 \\
\hline chr13:81-153 suggestive & WL & 15.49 & 2.49 & 0.54 & -1.02 \\
\hline chr13:57-124 significant & WL & 21.31 & 4.84 & 0.87 & -1.9 \\
\hline chr13:49-132 suggestive & WL & 13.62 & 1.41 & 0.32 & -0.54 \\
\hline chr13:82-154 suggestive & WL & 15.19 & 2.38 & 0.54 & -0.56 \\
\hline chr13:80-110 suggestive & WL & 21.04 & 0.08 & 0.02 & -0.09 \\
\hline chr13:81-124 suggestive & WL & 19.71 & 1.79 & 0.4 & -2.59 \\
\hline chr13:84-130 significant & WL & 17.69 & 2.01 & 0.42 & -0.39 \\
\hline chr13:83-110 suggestive & WL & 26.51 & 1.62 & 0.59 & -4.42 \\
\hline chr13:83-113 significant & WL & 31.92 & 2.77 & 0.57 & -4.46 \\
\hline chr13:85-139 significant & WL & 17.18 & 3.53 & 0.71 & -1.84 \\
\hline chr13:93-128 significant & RJF & 14.48 & -2.13 & 0.55 & -0.5 \\
\hline chr13:81-121 significant & WL & 20.35 & 2.42 & 0.47 & -0.16 \\
\hline chr13:102-11 significant & WL & 37.97 & 5.17 & 0.77 & 2.06 \\
\hline chr13:88-123 significant & WL & 18.63 & 1.69 & 0.34 & -0.25 \\
\hline chr13:100-11 significant & WL & 18.33 & 2.6 & 0.59 & 0.63 \\
\hline chr13:81-120 significant & WL & 17.97 & 2.3 & 0.47 & -0.42 \\
\hline chr13:101-11 suggestive & RJF & 20.07 & -1.08 & 0.54 & 0.36 \\
\hline chr13:98-115 suggestive & RJF & 22.74 & -0.56 & 0.61 & 2.18 \\
\hline chr13:87-128 significant & WL & 18.01 & 3.07 & 0.62 & -0.66 \\
\hline chr13:37-152 significant & WL & 14.47 & 1.77 & 0.51 & 0.65 \\
\hline chr13:84-165 suggestive & WL & 13.66 & 2.54 & 0.68 & 0.22 \\
\hline chr13:95-171 suggestive & WL & 14.98 & 1.59 & 0.34 & -1.31 \\
\hline chr13:113-17 significant & WL & 18.27 & 4.37 & 0.86 & -1.4 \\
\hline chr13:90-171 suggestive & WL & 15.49 & 1.8 & 0.4 & -0.55 \\
\hline chr13:114-16 suggestive & WL & 18.1 & 2.58 & 0.88 & -0.93 \\
\hline chr13:115-15 significant & WL & 17.74 & 2.57 & 0.61 & 0.33 \\
\hline chr13:115-16 significant & WL & 17.94 & 2.31 & 0.47 & -0.42 \\
\hline
\end{tabular}




\begin{tabular}{|c|c|c|c|c|c|}
\hline chr13:120-16 significant & WL & 25.34 & 3.75 & 0.61 & -0.85 \\
\hline chr13:115-15 significant & WL & 15.78 & 1.93 & 0.52 & 0.49 \\
\hline chr13:119-16 significant & WL & 21.62 & 1.38 & 0.57 & -1.16 \\
\hline chr13:119-15 significant & WL & 19.49 & 3.38 & 0.75 & 0.42 \\
\hline chr13:121-15 significant & WL & 17.07 & 3.71 & 0.78 & -0.88 \\
\hline chr13:118-15 suggestive & WL & 14.28 & 2.37 & 0.56 & -0.62 \\
\hline chr13:126-16 suggestive & WL & 15.23 & 2.69 & 0.59 & -1.29 \\
\hline chr13:122-16 significant & WL & 15.64 & 3.44 & 0.78 & -0.76 \\
\hline chr13:118-15 suggestive & WL & 13.16 & 2.45 & 0.6 & -0.63 \\
\hline chr13:123-17 significant & WL & 15.46 & 2.64 & 0.59 & -0.85 \\
\hline chr13:120-16 significant & WL & 16.11 & 2.19 & 0.46 & -0.72 \\
\hline chr13:122-17 suggestive & WL & 14.16 & 2.53 & 0.59 & -0.82 \\
\hline chr13:121-15 suggestive & WL & 14.39 & 2.04 & 0.57 & 0.46 \\
\hline chr13:126-16 significant & WL & 22.69 & 1.98 & 0.35 & -0.59 \\
\hline chr13:123-16 suggestive & WL & 19.23 & 1.88 & 0.36 & -0.63 \\
\hline chr13:119-15 suggestive & WL & 15.21 & 1.73 & 0.32 & -0.62 \\
\hline chr13:121-15 suggestive & WL & 19.82 & 2.35 & 0.44 & -0.9 \\
\hline chr13:121-15 suggestive & WL & 17.76 & 1.13 & 0.36 & 0.99 \\
\hline chr13:123-15 suggestive & WL & 19.42 & 1.91 & 0.44 & 0.33 \\
\hline chr13:125-15 suggestive & WL & 19.61 & 1.76 & 0.34 & -0.52 \\
\hline chr13:122-17 suggestive & WL & 14.42 & 2.95 & 0.8 & 0.49 \\
\hline chr13:123-16 suggestive & WL & 18.52 & 1.78 & 0.36 & -0.49 \\
\hline chr13:127-16 suggestive & WL & 19.42 & 2.64 & 0.55 & -0.18 \\
\hline chr13:119-17 suggestive & WL & 14.54 & 2.45 & 0.57 & -0.74 \\
\hline chr13:119-16 significant & WL & 14.73 & 1.12 & 0.34 & 0.5 \\
\hline chr13:124-15 suggestive & WL & 19.97 & 1.82 & 0.49 & 1.24 \\
\hline chr13:121-16 significant & WL & 16.41 & 1.43 & 0.38 & 0.46 \\
\hline chr13:119-17 significant & WL & 15.37 & 2.15 & 0.48 & -0.52 \\
\hline chr13:120-17 significant & WL & 16.21 & 2.42 & 0.51 & -1.43 \\
\hline chr13:118-17 suggestive & WL & 13.31 & 2.03 & 0.61 & 0.82 \\
\hline chr13:141-18 significant & RJF & 18.76 & -5.28 & 1.32 & -2.14 \\
\hline chr13:120-18 suggestive & WL & 15.06 & 3.43 & 0.76 & -4.07 \\
\hline chr13:151-17 suggestive & WL & 24.43 & 0.21 & 0.04 & -0.41 \\
\hline chr13:154-19 significant & WL & 19.64 & 3.15 & 1.54 & -5.29 \\
\hline chr13:165-17 significant & WL & 35.68 & 0.3 & 0.04 & -0.41 \\
\hline chr13:155-17 suggestive & WL & 20.41 & 0.03 & 0.01 & -0.06 \\
\hline chr13:156-18 significant & WL & 22.3 & 2.48 & 0.55 & -4.28 \\
\hline chr13:157-18 suggestive & WL & 24.2 & 2.82 & 0.68 & -5.97 \\
\hline chr13:154-19 significant & WL & 24.49 & 4.07 & 0.66 & -2.66 \\
\hline chr13:162-18 suggestive & WL & 17.49 & 1.58 & 0.37 & -3.59 \\
\hline chr13:150-19 suggestive & WL & 24.44 & 1.66 & 0.69 & -6.07 \\
\hline chr13:163-19 suggestive & RJF & 8.36 & -0.93 & 0.41 & 4 \\
\hline chr13:152-21 significant & RJF & 13.88 & -5.08 & 1.36 & -0.94 \\
\hline
\end{tabular}




\begin{tabular}{|c|c|c|c|c|c|c|}
\hline \multicolumn{2}{|c|}{ chr13:161-21 significant } & WL & 19.89 & 1.4 & 0.33 & 0.59 \\
\hline \multicolumn{2}{|c|}{ chr13:170-21 significant } & WL & 18.22 & 0.81 & 0.9 & 3.19 \\
\hline \multicolumn{2}{|c|}{ chr13:182-20 suggestive } & WL & 23.92 & 1.39 & 0.23 & -0.87 \\
\hline \multicolumn{2}{|c|}{ chr13:177-21 suggestive } & WL & 24.05 & 1.56 & 0.41 & -2.21 \\
\hline \multicolumn{2}{|c|}{ chr13:179-21 suggestive } & RJF & 14.17 & -1.81 & 0.43 & -0.14 \\
\hline \multirow{2}{*}{\multicolumn{2}{|c|}{$\begin{array}{l}\text { chr13:161-21 significant } \\
\text { chr13:199-20 suggestive }\end{array}$}} & RJF & 15.69 & -2.14 & 0.65 & -2.06 \\
\hline & & WL & 23.89 & 3.39 & 0.69 & -3.53 \\
\hline \multicolumn{2}{|c|}{ chr13:200-20 suggestive } & WL & 23.52 & 3.42 & 0.72 & -3.5 \\
\hline \multicolumn{2}{|c|}{ chr13:169-21 significant } & WL & 20.03 & 2.97 & 0.56 & -0.15 \\
\hline \multicolumn{2}{|c|}{ chr13:203-20 suggestive } & WL & 23.42 & 3.68 & 0.77 & -4.12 \\
\hline \multicolumn{2}{|c|}{ chr13:202-21 significant } & WL & 23.67 & 2.81 & 0.61 & -2.89 \\
\hline \multicolumn{2}{|c|}{ chr13:200-21 suggestive } & WL & 22.72 & 3.15 & 0.56 & -1.88 \\
\hline chr14:0-16 & suggestive & WL & 16.38 & 0.85 & 0.18 & -0.26 \\
\hline chr14:0-13 & suggestive & WL & 9.58 & 0.67 & 0.17 & -0.94 \\
\hline chr14:0-22 & significant & RJF & 28.36 & -3.22 & 0.49 & -0.4 \\
\hline chr14:0-34 & significant & RJF & 13.12 & -1.51 & 0.38 & -0.42 \\
\hline chr14:8-24 & significant & WL & 29.22 & 3.44 & 0.53 & -0.95 \\
\hline chr14:11-22 & significant & WL & 43.19 & 4.57 & 0.49 & -0.43 \\
\hline chr14:12-22 & significant & WL & 49.88 & 8.11 & 0.75 & -0.01 \\
\hline chr14:13-22 & significant & WL & 49.6 & 6.83 & 0.62 & 0.31 \\
\hline chr14:15-22 & significant & WL & 54.87 & 8.26 & 0.68 & 0.46 \\
\hline chr14:12-34 & suggestive & RJF & 12.98 & -1.8 & 0.43 & -1.18 \\
\hline chr14:3-78 & suggestive & RJF & 10.83 & -2.21 & 0.57 & -0.4 \\
\hline chr14:6-58 & suggestive & RJF & 10.39 & -3.7 & 0.99 & -1.55 \\
\hline chr14:12-49 & significant & RJF & 14.54 & -2.02 & 0.46 & -1.34 \\
\hline chr14:14-31 & suggestive & WL & 20.16 & 0.33 & 0.32 & -2.34 \\
\hline chr14:0-35 & significant & RJF & 11.88 & -2.26 & 0.55 & 0.35 \\
\hline chr14:13-31 & significant & RJF & 14.73 & -3.2 & 0.7 & -0.45 \\
\hline chr14:13-33 & significant & RJF & 14.07 & -1.95 & 0.45 & -0.96 \\
\hline chr14:22-33 & significant & RJF & 13.94 & -2.85 & 0.69 & -2.34 \\
\hline chr14:0-32 & suggestive & RJF & 11.34 & -4.41 & 1.12 & -0.56 \\
\hline chr14:12-46 & suggestive & RJF & 12.63 & -2.11 & 0.5 & -0.87 \\
\hline chr14:21-34 & significant & RJF & 15.7 & -2.94 & 0.62 & -0.89 \\
\hline chr14:17-33 & significant & RJF & 12.6 & -1.82 & 0.43 & -0.66 \\
\hline chr14:11-51 & suggestive & RJF & 9.16 & -2.23 & 0.63 & -0.24 \\
\hline chr14:23-29 & suggestive & WL & 20.83 & 0.24 & 0.25 & -1.89 \\
\hline chr14:24-30 & suggestive & RJF & 21.43 & -1.22 & 0.27 & -1.69 \\
\hline chr14:23-31 & suggestive & WL & 19.79 & 0.17 & 0.22 & -1.67 \\
\hline chr14:8-50 & significant & RJF & 12.55 & -2.15 & 0.53 & -1.41 \\
\hline chr14:0-60 & suggestive & RJF & 11.98 & -3.44 & 0.87 & -2.42 \\
\hline chr14:16-51 & suggestive & RJF & 13.95 & -2.58 & 0.62 & -1.93 \\
\hline chr14:0-65 & significant & RJF & 8.7 & -2.7 & 0.79 & 0.18 \\
\hline chr14:23-56 & significant & RJF & 14.53 & -2.64 & 0.58 & -2.29 \\
\hline
\end{tabular}




\begin{tabular}{|c|c|c|c|c|c|}
\hline chr14:24-56 suggestive & RJF & 20.53 & -2.89 & 0.54 & -3.05 \\
\hline chr14:0-67 suggestive & RJF & 12.72 & -3.06 & 0.74 & -2.59 \\
\hline chr14:5-68 suggestive & RJF & 9.44 & -3.82 & 1.08 & -2.09 \\
\hline chr14:30-45 significant & RJF & 51.88 & -5.77 & 0.57 & -0.93 \\
\hline chr14:26-51 suggestive & RJF & 14.31 & -1.8 & 0.42 & -1.95 \\
\hline chr14:30-56 significant & RJF & 20.77 & -2.55 & 0.46 & -1.14 \\
\hline chr14:0-68 significant & RJF & 11.44 & -1.64 & 0.58 & 0.68 \\
\hline chr14:32-46 significant & RJF & 41.19 & -8.2 & 0.91 & -7.52 \\
\hline chr14:32-46 significant & RJF & 39.8 & -10.59 & 1.21 & -9.79 \\
\hline chr14:30-74 significant & RJF & 17.32 & -2.27 & 0.44 & -1.12 \\
\hline chr14:32-70 suggestive & RJF & 13.35 & -0.07 & 0.43 & 2.12 \\
\hline chr14:36-108 significant & RJF & 11.97 & -2.21 & 0.55 & 0.67 \\
\hline chr14:42-115 significant & RJF & 15.89 & -2.11 & 0.44 & -0.2 \\
\hline chr14:58-109 significant & WL & 17.67 & 1.61 & 0.33 & -0.75 \\
\hline chr14:73-99 suggestive & RJF & 21.2 & -0.82 & 0.27 & 1.83 \\
\hline chr14:70-123 suggestive & WL & 17.45 & 3.18 & 0.64 & -0.6 \\
\hline chr14:73-118 suggestive & WL & 17.08 & 3.33 & 0.68 & -0.82 \\
\hline chr14:73-165 suggestive & RJF & 11.42 & -2.22 & 0.55 & 0.75 \\
\hline chr14:110-15 significant & RJF & 16.88 & -1.89 & 0.4 & -0.16 \\
\hline chr14:112-16 significant & WL & 17.8 & 1.41 & 0.29 & 0.11 \\
\hline chr14:108-16 significant & RJF & 14.66 & -3.43 & 0.74 & 0.32 \\
\hline chr14:118-16 significant & WL & 22.09 & 2.7 & 0.51 & 0.98 \\
\hline chr14:119-16 significant & WL & 24.93 & 3.91 & 0.67 & 1.13 \\
\hline chr14:117-16 suggestive & WL & 19.85 & 2.59 & 0.94 & 3.4 \\
\hline chr14:121-16 significant & WL & 25.41 & 3.76 & 0.61 & 0.47 \\
\hline chr14:120-16 suggestive & WL & 16.78 & 2.87 & 0.94 & 3.24 \\
\hline chr14:118-16 significant & WL & 17.98 & 3.87 & 0.78 & 0.26 \\
\hline chr14:122-16 significant & WL & 25.05 & 4.21 & 0.7 & 1.04 \\
\hline chr14:118-16 suggestive & WL & 15.9 & 3.56 & 0.87 & 1.76 \\
\hline chr14:120-16 significant & WL & 17.48 & 1.79 & 0.38 & 0.4 \\
\hline chr14:114-17 suggestive & WL & 14.63 & 2.61 & 0.58 & -0.11 \\
\hline chr14:122-16 significant & WL & 25.69 & 3.91 & 0.64 & 0.66 \\
\hline chr14:118-17 suggestive & WL & 13.95 & 1.7 & 0.37 & 0 \\
\hline chr14:119-17 significant & WL & 17.1 & 2.05 & 0.4 & -0.42 \\
\hline chr14:126-17 significant & WL & 22.5 & 0.57 & 0.82 & 5.74 \\
\hline chr14:117-19 suggestive & WL & 17.49 & 2.24 & 0.57 & 0.98 \\
\hline chr14:166-26 significant & WL & 21.61 & 1.06 & 0.57 & -1.13 \\
\hline chr14:202-26 significant & WL & 15.63 & 2 & 0.47 & -1.85 \\
\hline chr14:203-26 significant & WL & 12.24 & 2.11 & 0.48 & -0.34 \\
\hline chr14:200-25 significant & WL & 18.39 & 2.01 & 0.46 & -2.33 \\
\hline chr14:210-26 suggestive & WL & 19.24 & 2.22 & 0.44 & -1.86 \\
\hline chr14:215-25 significant & WL & 19.16 & 1.52 & 0.29 & -0.86 \\
\hline chr14:219-25 suggestive & WL & 13.82 & 0.42 & 0.14 & -0.82 \\
\hline
\end{tabular}




\begin{tabular}{|c|c|c|c|c|c|}
\hline chr14:217-26 suggestive & WL & 14.31 & 1.25 & 0.3 & -1.13 \\
\hline chr14:225-26 significant & RJF & 22.43 & -1.97 & 0.41 & -1.7 \\
\hline chr14:226-26 significant & WL & 22.1 & 4.37 & 0.79 & -3.14 \\
\hline chr14:247-26 suggestive & WL & 20.39 & 1.39 & 0.93 & 1 \\
\hline chr14:222-26 significant & WL & 22.16 & 1.81 & 0.31 & -1.28 \\
\hline chr14:246-26 suggestive & RJF & 22.74 & -0.66 & 0.61 & -1.57 \\
\hline chr14:243-26 suggestive & WL & 19.48 & 0.36 & 0.29 & -0.74 \\
\hline chr14:215-26 suggestive & WL & 18.8 & 0.26 & 0.29 & -0.8 \\
\hline chr14:243-26 significant & RJF & 15.16 & -6.3 & 1.41 & -0.76 \\
\hline chr14:245-26 suggestive & WL & 23.88 & 0.66 & 0.47 & -1.47 \\
\hline chr14:247-26 suggestive & RJF & 24.64 & -0.46 & 0.52 & -0.47 \\
\hline chr14:247-26 suggestive & RJF & 20.65 & -0.22 & 0.25 & -0.32 \\
\hline chr14:245-26 suggestive & WL & 24.9 & 0.06 & 0.41 & -1.27 \\
\hline chr14:248-26 suggestive & RJF & 18.26 & -0.28 & 0.31 & 0.2 \\
\hline chr14:250-26 suggestive & RJF & 18.62 & -0.27 & 0.21 & 0.19 \\
\hline chr14:250-26 suggestive & RJF & 25.75 & -0.72 & 0.72 & -2.08 \\
\hline chr14:249-26 suggestive & WL & 25.11 & 0.32 & 0.47 & -1.5 \\
\hline chr14:247-26 suggestive & WL & 16.33 & 0.09 & 0.42 & -0.88 \\
\hline chr14:237-26 suggestive & WL & 18.56 & 0.18 & 0.04 & -0.26 \\
\hline chr14:230-26 suggestive & WL & 18.97 & 0.58 & 0.11 & -0.2 \\
\hline chr14:244-26 significant & WL & 16.28 & 2.09 & 0.44 & -0.33 \\
\hline chr14:251-26 suggestive & WL & 24.24 & 0.19 & 0.67 & -3.04 \\
\hline chr14:250-26 suggestive & WL & 20.21 & 0.22 & 0.36 & -0.57 \\
\hline chr14:252-26 suggestive & RJF & 27.07 & -0.82 & 0.56 & -1.66 \\
\hline chr14:248-26 suggestive & RJF & 25.66 & -0.37 & 0.24 & -0.46 \\
\hline chr14:250-26 suggestive & RJF & 23.77 & -0.41 & 0.4 & -1.18 \\
\hline chr14:253-26 suggestive & WL & 21.7 & 0.04 & 0.3 & -0.83 \\
\hline chr14:251-26 suggestive & RJF & 19.94 & -0.77 & 0.58 & -0.18 \\
\hline chr14:250-26 suggestive & RJF & 23.46 & -0.21 & 0.7 & -2.15 \\
\hline chr14:252-26 suggestive & RJF & 25.1 & -0.98 & 0.54 & -1.5 \\
\hline chr14:251-26 suggestive & RJF & 23.77 & -0.45 & 0.6 & -1.43 \\
\hline chr14:251-26 suggestive & RJF & 23.41 & -0.29 & 0.6 & -2 \\
\hline chr14:253-26 suggestive & RJF & 12.99 & -0.32 & 0.42 & 0.17 \\
\hline chr14:249-26 suggestive & RJF & 21.89 & -0.18 & 0.34 & -0.45 \\
\hline chr14:252-26 suggestive & WL & 26.36 & 0.24 & 0.4 & -0.14 \\
\hline chr14:252-26 suggestive & RJF & 24.32 & -0.36 & 0.42 & -1.03 \\
\hline chr14:246-26 suggestive & WL & 19.98 & 2.98 & 0.54 & -1.36 \\
\hline chr14:244-26 suggestive & RJF & 12.5 & -15.09 & 3.57 & 2.02 \\
\hline chr14:252-26 suggestive & RJF & 26.36 & -0.26 & 0.23 & -0.6 \\
\hline chr14:248-26 suggestive & WL & 22.98 & 0.08 & 0.26 & 0.35 \\
\hline chr14:255-26 suggestive & WL & 18.46 & 0.07 & 0.45 & -0.34 \\
\hline chr14:251-26 suggestive & WL & 19.67 & 0.21 & 0.4 & 0.17 \\
\hline chr14:248-26 significant & WL & 19 & 2.02 & 0.38 & -0.7 \\
\hline
\end{tabular}




\begin{tabular}{|c|c|c|c|c|c|}
\hline chr14:251-26 significant & WL & 19.33 & 1.38 & 0.29 & 0.28 \\
\hline chr14:258-26 suggestive & RJF & 23.83 & -0.09 & 0.25 & -0.13 \\
\hline chr14:257-26 suggestive & RJF & 25.27 & -0.5 & 0.5 & 0.43 \\
\hline chr14:252-26 suggestive & RJF & 23.47 & -1.1 & 0.61 & -1.27 \\
\hline chr14:252-26 suggestive & WL & 23.12 & 0.06 & 0.31 & -0.81 \\
\hline chr14:256-26 suggestive & RJF & 24.08 & -0.81 & 0.62 & -1.89 \\
\hline chr14:254-26 suggestive & RJF & 23.65 & -0.14 & 0.23 & -0.59 \\
\hline chr14:253-26 suggestive & RJF & 18.31 & -0.16 & 0.38 & 0.41 \\
\hline chr14:255-26 suggestive & RJF & 22.36 & -0.12 & 0.23 & 0.03 \\
\hline chr14:255-26 suggestive & RJF & 18.51 & -0.22 & 0.32 & -0.62 \\
\hline chr14:254-26 suggestive & WL & 22.34 & 0.04 & 0.29 & -0.45 \\
\hline chr14:255-26 suggestive & RJF & 19.65 & -0.24 & 0.33 & -0.46 \\
\hline chr14:257-26 suggestive & RJF & 19.8 & -0.13 & 0.24 & 0.22 \\
\hline chr14:256-26 significant & RJF & 33.87 & -0.4 & 0.24 & 0.38 \\
\hline chr14:253-26 suggestive & RJF & 27.76 & -0.25 & 0.64 & -1.58 \\
\hline chr15:0-7 significant & WL & 21.55 & 3.72 & 0.66 & -0.19 \\
\hline significant & WL & 21.46 & 7.4 & 1.44 & -2.36 \\
\hline suggestive & WL & 17.23 & 5.98 & 1.35 & -0.71 \\
\hline suggestive & WL & 14.59 & 3.37 & 0.8 & 0.74 \\
\hline chr15:0-14 & WL & 12.47 & 2.78 & 0.71 & 0.53 \\
\hline suggestive & WL & 23.16 & 2.23 & 0.59 & -2.89 \\
\hline significant & WL & 17.95 & 1.83 & 0.36 & -0.69 \\
\hline suggestive & WL & 15.59 & 3.12 & 0.68 & -2.12 \\
\hline suggestive & WL & 23.68 & 3.58 & 0.68 & -3.64 \\
\hline suggestive & RJF & 13.32 & -1.71 & 0.45 & -0.64 \\
\hline chr15:0-17 & WL & 14.74 & 3.3 & 0.72 & -1.63 \\
\hline chr15:0-15 & RJF & 14.92 & -4.15 & 0.99 & -0.89 \\
\hline chr15:0-49 suggestive & RJF & 13.76 & -0.94 & 0.22 & 0 \\
\hline chr15:0-61 significant & WL & 13.36 & 2.4 & 0.57 & -0.82 \\
\hline chr15:0-37 significant & WL & 10.78 & 2.74 & 0.69 & 0.4 \\
\hline suggestive & RJF & 19.85 & -1.12 & 0.22 & 0.56 \\
\hline chr15:0-48 significant & WL & 16.19 & 4.28 & 0.88 & 0.08 \\
\hline chr15:13-44 suggestive & WL & 13.72 & 0.98 & 0.23 & 0.31 \\
\hline chr15:11-48 significant & WL & 15.16 & 1.47 & 0.33 & 0.55 \\
\hline chr15:0-58 significant & WL & 13.13 & 2.47 & 0.59 & -0.54 \\
\hline chr15:64-130 suggestive & RJF & 25.71 & -7.76 & 1.32 & -7.44 \\
\hline chr15:64-130 suggestive & RJF & 22.93 & -14.09 & 2.68 & -15.47 \\
\hline chr15:65-134 suggestive & RJF & 25.09 & -17.57 & 3.01 & -15 \\
\hline chr15:64-131 suggestive & RJF & 25.15 & -13.96 & 2.42 & -13.48 \\
\hline chr15:62-132 suggestive & RJF & 23.3 & -15.79 & 3 & -16.25 \\
\hline chr15:62-126 suggestive & RJF & 22.72 & -11.6 & 2.32 & -13.14 \\
\hline chr15:66-159 suggestive & RJF & 11.65 & -7.76 & 1.32 & -7.82 \\
\hline chr15:65-130 suggestive & RJF & 24.41 & -20.73 & 3.68 & -20.15 \\
\hline
\end{tabular}




\begin{tabular}{|c|c|c|c|c|c|}
\hline chr15:66-131 suggestive & RJF & 25.32 & -16.51 & 2.81 & -14.55 \\
\hline chr15:64-135 suggestive & RJF & 23.41 & -19.37 & 3.6 & -19.89 \\
\hline chr15:64-130 suggestive & RJF & 23.53 & -6.98 & 1.34 & -7 \\
\hline chr15:64-127 suggestive & RJF & 23.3 & -7.72 & 1.49 & -7.45 \\
\hline chr15:64-123 suggestive & RJF & 22.62 & -13.85 & 2.8 & -17.22 \\
\hline chr15:65-131 suggestive & RJF & 23.1 & -8.44 & 1.56 & -8.83 \\
\hline chr15:61-123 suggestive & RJF & 21.64 & -7.19 & 1.5 & -8.62 \\
\hline chr15:68-125 suggestive & RJF & 23.59 & -11.02 & 2.02 & -11.58 \\
\hline chr15:65-133 suggestive & RJF & 23.26 & -26.13 & 4.83 & -25.08 \\
\hline chr15:63-135 suggestive & RJF & 13.15 & -8.83 & 1.62 & -10.87 \\
\hline chr15:66-121 significant & RJF & 27.52 & -11.76 & 1.95 & -11.8 \\
\hline chr15:64-126 suggestive & RJF & 23.73 & -7.73 & 1.35 & -6.24 \\
\hline chr15:66-134 suggestive & RJF & 23.48 & -28.6 & 5.45 & -31.15 \\
\hline chr15:67-132 suggestive & RJF & 25.11 & -20.35 & 3.66 & -18.88 \\
\hline chr15:66-129 suggestive & RJF & 22.46 & -8.22 & 1.46 & -8.24 \\
\hline chr15:67-134 suggestive & RJF & 24.15 & -31.65 & 5.6 & -29.9 \\
\hline chr15:64-125 suggestive & RJF & 22.6 & -7.75 & 1.5 & -8.29 \\
\hline chr15:67-125 suggestive & RJF & 24.21 & -14.98 & 2.63 & -15.24 \\
\hline chr15:65-129 suggestive & RJF & 23.2 & -28.8 & 5.46 & -28.71 \\
\hline chr15:67-128 suggestive & RJF & 18.36 & -12.18 & 2.07 & -11.99 \\
\hline chr15:66-134 suggestive & RJF & 24.17 & -27.66 & 4.97 & -27.43 \\
\hline chr15:66-139 suggestive & RJF & 23.28 & -8.01 & 1.47 & -7.75 \\
\hline chr15:64-121 suggestive & RJF & 22.97 & -10.02 & 1.89 & -10.99 \\
\hline chr15:65-130 significant & RJF & 19.93 & -35.08 & 7.87 & -42.31 \\
\hline chr15:66-134 suggestive & RJF & 24.26 & -26.4 & 4.65 & -23.29 \\
\hline chr15:68-126 suggestive & RJF & 22.22 & -16.79 & 3.41 & -21.11 \\
\hline chr15:69-132 suggestive & RJF & 24.88 & -29.49 & 5.22 & -28.17 \\
\hline chr15:68-127 suggestive & RJF & 23.05 & -11.52 & 2.02 & -10.91 \\
\hline chr15:68-132 significant & RJF & 25.86 & -6.87 & 1.34 & -7.86 \\
\hline chr15:70-131 suggestive & RJF & 25.39 & -26.54 & 4.45 & -25.19 \\
\hline chr15:71-124 significant & RJF & 28.06 & -10.49 & 1.53 & -10.74 \\
\hline chr15:68-119 suggestive & RJF & 23.81 & -7.45 & 1.33 & -8.18 \\
\hline chr15:67-138 suggestive & RJF & 24.11 & -23.67 & 4.4 & -21.41 \\
\hline chr15:70-128 suggestive & RJF & 24.59 & -9.76 & 1.62 & -8.94 \\
\hline chr15:65-127 suggestive & RJF & 23.21 & -16.53 & 3.06 & -15.98 \\
\hline chr15:71-129 significant & RJF & 20.44 & -9.49 & 1.59 & -10.86 \\
\hline chr15:69-125 suggestive & RJF & 23.65 & -12.88 & 2.4 & -14.23 \\
\hline chr15:71-125 significant & RJF & 28.92 & -17.59 & 2.76 & -18.12 \\
\hline chr15:68-128 suggestive & RJF & 25.05 & -15.27 & 2.72 & -15.59 \\
\hline chr15:69-125 suggestive & RJF & 24.86 & -21.63 & 3.76 & -22.24 \\
\hline chr15:66-132 suggestive & RJF & 23.66 & -10.54 & 1.98 & -9.02 \\
\hline chr15:67-133 suggestive & RJF & 23.76 & -24.27 & 4.45 & -23.31 \\
\hline chr15:68-122 suggestive & RJF & 10.59 & -49.72 & 9.4 & -64.49 \\
\hline
\end{tabular}




\begin{tabular}{|c|c|c|c|c|c|}
\hline chr15:66-129 suggestive & RJF & 23.78 & -10.06 & 1.9 & -9.78 \\
\hline chr15:67-127 suggestive & RJF & 24.04 & -8.47 & 1.5 & -8.26 \\
\hline chr15:67-127 suggestive & RJF & 23.87 & -9.15 & 1.59 & -7.89 \\
\hline chr15:68-127 suggestive & RJF & 24.86 & -14.94 & 2.65 & -15.35 \\
\hline chr15:69-129 suggestive & RJF & 23.41 & -17.29 & 3.21 & -17.8 \\
\hline chr15:72-127 significant & RJF & 22.78 & -8.13 & 1.41 & -9.46 \\
\hline chr15:69-128 suggestive & RJF & 22.64 & -22.46 & 4.36 & -25.48 \\
\hline chr15:67-123 suggestive & RJF & 10.41 & -56.74 & 10.54 & -71.96 \\
\hline chr15:68-131 suggestive & RJF & 23.19 & -10.06 & 1.81 & -9.95 \\
\hline chr15:67-127 suggestive & RJF & 23.09 & -9.71 & 1.83 & -9.93 \\
\hline chr15:66-130 suggestive & RJF & 22.79 & -7.17 & 1.35 & -6.83 \\
\hline chr15:69-128 suggestive & RJF & 24.59 & -7.56 & 1.37 & -7.12 \\
\hline chr15:68-132 significant & RJF & 25.91 & -8.15 & 1.31 & -7.1 \\
\hline chr15:70-134 suggestive & RJF & 20.22 & -23.4 & 3.91 & -19.59 \\
\hline chr15:66-130 suggestive & RJF & 23.59 & -13.91 & 2.59 & -15.12 \\
\hline chr15:69-131 suggestive & RJF & 24.22 & -20.61 & 3.79 & -20.07 \\
\hline chr15:72-132 suggestive & RJF & 14.75 & -3.48 & 0.57 & -3.65 \\
\hline chr15:72-123 suggestive & RJF & 25.15 & -7.07 & 1.2 & -7.68 \\
\hline chr15:69-126 suggestive & RJF & 24.16 & -14.34 & 2.55 & -14.55 \\
\hline chr15:73-127 significant & RJF & 29.31 & -10.39 & 1.58 & -10.62 \\
\hline chr15:72-123 suggestive & RJF & 24.53 & -12.84 & 2.27 & -14.18 \\
\hline chr15:71-128 suggestive & RJF & 23.24 & -12.25 & 2.27 & -12.55 \\
\hline chr15:70-126 suggestive & RJF & 25.34 & -13.01 & 2.27 & -13.48 \\
\hline chr15:72-128 suggestive & RJF & 25.01 & -27.92 & 4.8 & -27.91 \\
\hline chr15:71-122 suggestive & RJF & 23.71 & -7.52 & 1.37 & -8.06 \\
\hline chr15:70-126 suggestive & RJF & 23.22 & -6.42 & 1.31 & -7.67 \\
\hline chr15:71-133 suggestive & RJF & 23.78 & -36 & 6.45 & -35.71 \\
\hline chr15:69-130 suggestive & RJF & 23.99 & -18.92 & 3.45 & -19.35 \\
\hline chr15:71-130 significant & RJF & 26.38 & -31.12 & 5.14 & -29.66 \\
\hline chr15:72-127 significant & RJF & 26.42 & -18.94 & 3.14 & -19.12 \\
\hline chr15:71-134 suggestive & RJF & 23.37 & -32.9 & 5.86 & -33.05 \\
\hline chr15:72-122 suggestive & RJF & 23.81 & -7.25 & 1.35 & -8.6 \\
\hline chr15:70-129 suggestive & RJF & 22.75 & -11 & 2.25 & -12.95 \\
\hline chr15:71-128 significant & RJF & 27.07 & -20.52 & 3.27 & -20.48 \\
\hline chr15:72-117 suggestive & RJF & 23.11 & -8.15 & 1.4 & -7.89 \\
\hline chr15:71-121 significant & RJF & 26.62 & -13.74 & 2.24 & -13.81 \\
\hline chr15:69-133 suggestive & RJF & 23.73 & -13.73 & 2.5 & -12.18 \\
\hline chr15:70-127 suggestive & RJF & 23.5 & -8.8 & 1.58 & -7.4 \\
\hline chr15:69-127 suggestive & RJF & 23.04 & -7.25 & 1.34 & -7.64 \\
\hline chr15:72-121 significant & RJF & 29.75 & -10.44 & 1.45 & -10.47 \\
\hline chr15:69-123 suggestive & RJF & 23.71 & -7.67 & 1.37 & -7.21 \\
\hline chr15:68-129 suggestive & RJF & 23.22 & -8.08 & 1.43 & -6.6 \\
\hline chr15:71-129 suggestive & RJF & 23.16 & -18.33 & 3.36 & -17.62 \\
\hline
\end{tabular}




\begin{tabular}{|c|c|c|c|c|c|}
\hline chr15:65-144 significant & RJF & 17.27 & -3.03 & 0.61 & -1.52 \\
\hline chr15:72-121 suggestive & RJF & 24.18 & -4.51 & 0.8 & -4.35 \\
\hline chr15:72-129 suggestive & RJF & 23.67 & -9.39 & 1.79 & -9.97 \\
\hline chr15:74-123 significant & RJF & 28.26 & -2.31 & 0.38 & -2.71 \\
\hline chr15:74-123 significant & RJF & 17.07 & -3.07 & 0.36 & -2.53 \\
\hline chr15:73-125 suggestive & RJF & 23.79 & -12.73 & 2.41 & -14.71 \\
\hline chr15:72-120 suggestive & RJF & 25.18 & -8.47 & 1.47 & -9.28 \\
\hline chr15:73-128 suggestive & RJF & 24.14 & -7.45 & 1.48 & -9.54 \\
\hline chr15:72-123 suggestive & RJF & 22.96 & -12.08 & 2.36 & -14.29 \\
\hline chr15:72-123 suggestive & RJF & 24.21 & -12.99 & 2.4 & -15.03 \\
\hline chr15:73-125 significant & RJF & 26.38 & -8.7 & 1.55 & -8.52 \\
\hline chr15:75-116 significant & RJF & 20.99 & -9.7 & 1.34 & -10.6 \\
\hline chr15:73-127 suggestive & RJF & 24.47 & -11.83 & 2.22 & -14.09 \\
\hline chr15:69-122 suggestive & RJF & 23.16 & -10.24 & 1.95 & -10.03 \\
\hline chr15:72-122 suggestive & RJF & 23.35 & -12.79 & 2.34 & -13.73 \\
\hline chr15:72-131 suggestive & RJF & 24.57 & -10.97 & 1.93 & -10.59 \\
\hline chr15:72-117 suggestive & RJF & 25.53 & -7.95 & 1.43 & -9.06 \\
\hline chr15:72-130 suggestive & RJF & 23.17 & -17.11 & 3.18 & -15.53 \\
\hline chr15:73-128 suggestive & RJF & 25.39 & -19.31 & 3.4 & -21.44 \\
\hline chr15:71-128 suggestive & RJF & 23.25 & -9.2 & 1.69 & -8.36 \\
\hline chr15:72-126 significant & RJF & 26.02 & -21.69 & 3.72 & -21.47 \\
\hline chr15:72-121 suggestive & RJF & 25.43 & -11.08 & 1.96 & -12.14 \\
\hline chr15:73-126 suggestive & RJF & 23.8 & -25.67 & 4.66 & -27.61 \\
\hline chr15:74-125 significant & RJF & 14.37 & -14.39 & 1.86 & -13.26 \\
\hline chr15:72-123 significant & RJF & 25.8 & -15.76 & 2.82 & -17.73 \\
\hline chr15:72-126 suggestive & RJF & 23.24 & -17.55 & 3.4 & -20.71 \\
\hline chr15:72-132 suggestive & RJF & 23.49 & -16.39 & 2.89 & -16.38 \\
\hline chr15:72-127 suggestive & RJF & 25.34 & -27.87 & 4.7 & -25.92 \\
\hline chr15:72-124 suggestive & RJF & 22.96 & -9.13 & 1.78 & -10.3 \\
\hline chr15:73-125 suggestive & RJF & 23.01 & -8.5 & 1.59 & -9.48 \\
\hline chr15:73-125 suggestive & RJF & 24.1 & -7.88 & 1.37 & -8.32 \\
\hline chr15:73-124 suggestive & RJF & 25.38 & -15.5 & 2.71 & -16.14 \\
\hline chr15:73-121 suggestive & RJF & 24.69 & -9.06 & 1.73 & -10.56 \\
\hline chr15:74-123 suggestive & RJF & 26.22 & -5.29 & 0.86 & -5.26 \\
\hline chr15:74-118 suggestive & RJF & 24.51 & -4.55 & 0.84 & -5.56 \\
\hline chr15:75-117 significant & RJF & 28.08 & -7.44 & 1.09 & -6.54 \\
\hline chr15:73-124 suggestive & RJF & 24.07 & -8.37 & 1.54 & -8.76 \\
\hline chr15:74-127 significant & RJF & 26.05 & -9.75 & 1.71 & -10.7 \\
\hline chr15:73-127 suggestive & RJF & 22.94 & -18.79 & 3.47 & -20.08 \\
\hline chr15:73-126 suggestive & RJF & 23.72 & -9.92 & 1.96 & -11.68 \\
\hline chr15:74-127 significant & RJF & 26.25 & -16.21 & 2.8 & -17.97 \\
\hline chr15:73-127 suggestive & RJF & 24.33 & -35.82 & 6.36 & -36.57 \\
\hline chr15:74-127 suggestive & RJF & 25.03 & -23.85 & 4.11 & -23.67 \\
\hline
\end{tabular}




\begin{tabular}{|c|c|c|c|c|c|}
\hline chr15:73-126 significant & RJF & 26.52 & -19.05 & 3.13 & -18.62 \\
\hline chr15:73-130 suggestive & RJF & 24.02 & -15.51 & 2.76 & -16.7 \\
\hline chr15:72-122 suggestive & RJF & 22.7 & -10.33 & 1.96 & -11.76 \\
\hline chr15:72-124 suggestive & RJF & 24.46 & -20.68 & 3.69 & -20.8 \\
\hline chr15:73-122 suggestive & RJF & 24.37 & -15.35 & 2.82 & -16.52 \\
\hline chr15:73-130 suggestive & RJF & 23.61 & -8.63 & 1.56 & -9.34 \\
\hline chr15:74-127 significant & RJF & 26.41 & -21.81 & 3.57 & -21.45 \\
\hline chr15:73-130 suggestive & RJF & 23.86 & -28.29 & 5.28 & -30.33 \\
\hline chr15:72-128 suggestive & RJF & 22.72 & -13.29 & 2.64 & -14.96 \\
\hline chr15:74-122 significant & RJF & 28.42 & -20.97 & 3.32 & -22.56 \\
\hline chr15:74-128 significant & RJF & 23.99 & -16.21 & 2.47 & -15.01 \\
\hline chr15:73-125 suggestive & RJF & 24.56 & -17.89 & 3.14 & -17.33 \\
\hline chr15:73-123 suggestive & RJF & 23.24 & -20.28 & 3.99 & -23.49 \\
\hline chr15:74-126 suggestive & RJF & 25.72 & -22.66 & 3.88 & -22.91 \\
\hline chr15:74-127 suggestive & RJF & 23.28 & -8.79 & 1.57 & -9.12 \\
\hline chr15:75-122 significant & RJF & 31.07 & -10.01 & 1.46 & -10.04 \\
\hline chr15:74-124 suggestive & RJF & 23.39 & -5.58 & 1.01 & -4.74 \\
\hline chr15:74-125 significant & RJF & 26.74 & -21.37 & 3.48 & -21.32 \\
\hline chr15:73-126 suggestive & RJF & 24.45 & -14.8 & 2.61 & -15.58 \\
\hline chr15:74-123 significant & RJF & 26.57 & -9.99 & 1.73 & -10.51 \\
\hline chr15:74-130 suggestive & RJF & 23.77 & -27.22 & 5 & -29.34 \\
\hline chr15:74-127 suggestive & RJF & 24.83 & -23.65 & 4.21 & -25.62 \\
\hline chr15:73-130 suggestive & RJF & 23.67 & -14.91 & 2.76 & -16.53 \\
\hline chr15:73-127 suggestive & RJF & 23.98 & -21.27 & 3.81 & -20.8 \\
\hline chr15:73-128 suggestive & RJF & 22.7 & -15.74 & 3 & -16.8 \\
\hline chr15:75-115 significant & RJF & 28.51 & -11.14 & 1.79 & -11.83 \\
\hline chr15:73-127 suggestive & RJF & 22.82 & -22.9 & 4.21 & -23.62 \\
\hline chr15:74-128 suggestive & RJF & 22.11 & -12.18 & 2.44 & -15.13 \\
\hline chr15:74-120 suggestive & RJF & 23.51 & -6.86 & 1.45 & -8.36 \\
\hline chr15:73-129 suggestive & RJF & 23.84 & -33.22 & 5.94 & -33.05 \\
\hline chr15:73-125 suggestive & RJF & 22.85 & -11.98 & 2.26 & -12.42 \\
\hline chr15:73-127 suggestive & RJF & 23.5 & -36.19 & 6.5 & -36.16 \\
\hline chr15:76-120 significant & RJF & 21.85 & -10.32 & 1.72 & -12.34 \\
\hline chr15:74-125 significant & RJF & 28.22 & -21.27 & 3.33 & -20.79 \\
\hline chr15:74-123 significant & RJF & 26.62 & -15.99 & 2.7 & -16.59 \\
\hline chr15:73-126 suggestive & RJF & 25.17 & -12.72 & 2.16 & -11.84 \\
\hline chr15:74-124 suggestive & RJF & 23.78 & -9.83 & 1.78 & -10.04 \\
\hline chr15:74-126 suggestive & RJF & 23.7 & -8.48 & 1.5 & -8.32 \\
\hline chr15:74-128 suggestive & RJF & 25.56 & -21.38 & 3.8 & -23.2 \\
\hline chr15:74-127 suggestive & RJF & 25.04 & -32.12 & 5.6 & -32.78 \\
\hline chr15:74-125 suggestive & RJF & 21.87 & -8.74 & 1.74 & -9.94 \\
\hline chr15:74-125 suggestive & RJF & 23.14 & -11.27 & 1.94 & -10.64 \\
\hline chr15:74-119 suggestive & RJF & 23.48 & -7.91 & 1.38 & -7.5 \\
\hline
\end{tabular}




\begin{tabular}{|c|c|c|c|c|c|}
\hline chr15:74-127 suggestive & RJF & 24.65 & -33.71 & 5.92 & -34.89 \\
\hline chr15:75-118 suggestive & RJF & 24.16 & -7.2 & 1.35 & -7.8 \\
\hline chr15:74-121 significant & RJF & 27.22 & -12.38 & 1.99 & -11.09 \\
\hline chr15:74-118 suggestive & RJF & 24.07 & -8.88 & 1.82 & -9.87 \\
\hline chr15:74-127 suggestive & RJF & 22.44 & -42.3 & 6.78 & -34.71 \\
\hline chr15:73-128 suggestive & RJF & 23.07 & -35.6 & 6.54 & -34.67 \\
\hline chr15:74-124 suggestive & RJF & 24.79 & -12.06 & 2.21 & -12.28 \\
\hline chr15:73-120 suggestive & RJF & 23.17 & -19.63 & 3.74 & -22.02 \\
\hline chr15:74-123 suggestive & RJF & 23.07 & -6.92 & 1.35 & -7.41 \\
\hline chr15:76-118 suggestive & RJF & 5.89 & -3.93 & 0.68 & -4.28 \\
\hline chr15:74-128 suggestive & RJF & 25.01 & -22.23 & 3.99 & -22.85 \\
\hline chr15:74-128 suggestive & RJF & 23.57 & -33.71 & 6.08 & -34.31 \\
\hline chr15:75-125 significant & RJF & 26.19 & -13.59 & 2.38 & -15.12 \\
\hline chr15:75-121 suggestive & RJF & 23.32 & -14.32 & 2.97 & -17.77 \\
\hline chr15:74-123 suggestive & RJF & 23.31 & -11.05 & 1.9 & -9.32 \\
\hline chr15:75-122 significant & RJF & 27.15 & -11.41 & 1.86 & -10.37 \\
\hline chr15:75-122 suggestive & RJF & 24.34 & -15.65 & 2.99 & -18.68 \\
\hline chr15:75-121 suggestive & RJF & 24.18 & -7.68 & 1.32 & -7.51 \\
\hline chr15:76-120 significant & RJF & 25.86 & -11.17 & 1.9 & -10.73 \\
\hline chr15:74-125 suggestive & RJF & 24.28 & -32.92 & 5.9 & -33.27 \\
\hline chr15:73-125 suggestive & RJF & 24.28 & -29.41 & 5.02 & -26.4 \\
\hline chr15:77-119 significant & RJF & 19.13 & -3.42 & 0.51 & -3.52 \\
\hline chr15:75-123 suggestive & RJF & 23.9 & -12.44 & 2.23 & -11.69 \\
\hline chr15:75-126 suggestive & RJF & 24.43 & -15 & 2.71 & -13.76 \\
\hline chr15:75-120 suggestive & RJF & 24.17 & -10.84 & 2.11 & -11.63 \\
\hline chr15:75-121 suggestive & RJF & 24.59 & -7.56 & 1.31 & -7.16 \\
\hline chr15:77-117 significant & RJF & 27.32 & -7.64 & 1.32 & -8.17 \\
\hline chr15:74-119 suggestive & RJF & 24.04 & -12.63 & 2.37 & -13.54 \\
\hline chr15:75-126 suggestive & RJF & 23.35 & -20.15 & 3.75 & -21.46 \\
\hline chr15:75-121 suggestive & RJF & 24.24 & -11.94 & 2.25 & -12.41 \\
\hline chr15:75-128 suggestive & RJF & 22.91 & -14.02 & 2.57 & -13.36 \\
\hline chr15:76-122 suggestive & RJF & 26.11 & -15.04 & 2.59 & -15.89 \\
\hline chr15:79-115 significant & RJF & 19.89 & -5.2 & 0.84 & -6.01 \\
\hline chr15:78-122 suggestive & RJF & 24.8 & -3.51 & 0.64 & -3.26 \\
\hline chr15:75-121 suggestive & RJF & 22.89 & -9.35 & 1.77 & -9.31 \\
\hline chr15:76-118 suggestive & RJF & 25.32 & -9.21 & 1.7 & -9.68 \\
\hline chr15:77-121 suggestive & RJF & 23.72 & -7.97 & 1.49 & -8.32 \\
\hline chr15:76-119 suggestive & RJF & 23.84 & -8.8 & 1.53 & -8.61 \\
\hline chr15:76-114 significant & RJF & 30.36 & -9 & 1.33 & -8.81 \\
\hline chr15:75-116 suggestive & RJF & 23.59 & -8.53 & 1.63 & -9.39 \\
\hline chr15:76-115 suggestive & RJF & 20.76 & -3.51 & 0.64 & -2.9 \\
\hline chr15:78-120 significant & RJF & 26.77 & -14.5 & 2.56 & -16.25 \\
\hline chr15:76-118 suggestive & RJF & 24.22 & -12.62 & 2.28 & -12.55 \\
\hline
\end{tabular}




\begin{tabular}{|c|c|c|c|c|c|}
\hline chr15:76-121 suggestive & RJF & 24.3 & -10.63 & 1.93 & -10.46 \\
\hline chr15:76-118 suggestive & RJF & 22.89 & -11.92 & 2.42 & -12.1 \\
\hline chr15:77-120 suggestive & RJF & 22.94 & -6.77 & 1.32 & -7.29 \\
\hline chr15:73-118 suggestive & RJF & 25.37 & -8.22 & 1.4 & -7.36 \\
\hline chr15:78-126 suggestive & RJF & 18.75 & -12.64 & 2.85 & -15.17 \\
\hline chr15:80-112 significant & RJF & 31.71 & -4 & 0.57 & -3.64 \\
\hline chr15:78-120 suggestive & RJF & 23.84 & -3.24 & 0.58 & -2.76 \\
\hline chr15:80-111 suggestive & RJF & 9.3 & -1.53 & 0.24 & -1.24 \\
\hline chr15:81-116 suggestive & RJF & 23.45 & -8.71 & 1.73 & -9.2 \\
\hline chr15:77-117 suggestive & RJF & 23.5 & -5.53 & 0.96 & -5.34 \\
\hline chr15:82-113 suggestive & RJF & 18.72 & -0.51 & 0.22 & 0.75 \\
\hline chr15:78-113 suggestive & RJF & 23.89 & -6.79 & 1.36 & -7.4 \\
\hline chr15:77-120 suggestive & RJF & 23.64 & -11.23 & 2.1 & -11.24 \\
\hline chr15:81-116 suggestive & RJF & 23.99 & -8.91 & 1.55 & -8.95 \\
\hline chr15:76-121 suggestive & RJF & 23.32 & -10.27 & 1.87 & -9.14 \\
\hline chr15:79-120 suggestive & RJF & 24.02 & -12.72 & 2.3 & -11.4 \\
\hline chr15:78-117 suggestive & RJF & 23.35 & -8.36 & 1.62 & -7.44 \\
\hline chr15:75-121 suggestive & RJF & 23.53 & -10.58 & 2 & -10.94 \\
\hline chr15:81-117 suggestive & RJF & 25.44 & -10.74 & 1.91 & -9.71 \\
\hline chr15:74-119 suggestive & RJF & 23.45 & -11.7 & 2.1 & -9.83 \\
\hline chr15:77-117 suggestive & RJF & 23.38 & -7.07 & 1.46 & -8.09 \\
\hline chr15:76-118 suggestive & RJF & 24.2 & -8.78 & 1.54 & -7.72 \\
\hline chr15:79-121 suggestive & RJF & 23.26 & -15.46 & 2.96 & -15.68 \\
\hline chr15:78-119 significant & RJF & 28.03 & -11.51 & 1.85 & -11.4 \\
\hline chr15:76-123 suggestive & RJF & 23.89 & -20.34 & 3.74 & -19.85 \\
\hline chr15:76-122 significant & RJF & 25.74 & -7.77 & 1.31 & -8.09 \\
\hline chr15:80-121 suggestive & RJF & 23.72 & -10.8 & 1.98 & -10.28 \\
\hline chr15:76-123 suggestive & RJF & 24.06 & -6.31 & 1.15 & -5.39 \\
\hline chr15:77-122 significant & RJF & 25.67 & -16.92 & 2.75 & -14.8 \\
\hline chr15:77-121 suggestive & RJF & 22.9 & -14.58 & 2.67 & -14.71 \\
\hline chr15:77-123 suggestive & RJF & 23.3 & -7.86 & 1.41 & -6.64 \\
\hline chr15:79-121 suggestive & RJF & 23.82 & -8.44 & 1.54 & -8.17 \\
\hline chr15:76-120 suggestive & RJF & 25.09 & -10.87 & 1.91 & -11.13 \\
\hline chr15:76-121 suggestive & RJF & 23.41 & -12.51 & 2.26 & -12.35 \\
\hline chr15:76-125 suggestive & RJF & 23.03 & -12.09 & 2.24 & -12.14 \\
\hline chr15:78-121 suggestive & RJF & 25.3 & -10.67 & 1.81 & -10.9 \\
\hline chr15:75-123 suggestive & RJF & 25.14 & -7.19 & 1.26 & -6.8 \\
\hline chr15:78-122 suggestive & RJF & 23.78 & -7.98 & 1.44 & -7.53 \\
\hline chr15:75-121 suggestive & RJF & 24.08 & -9.83 & 1.83 & -9.35 \\
\hline chr15:77-122 suggestive & RJF & 24.79 & -12.29 & 2.1 & -10.88 \\
\hline chr15:78-126 suggestive & RJF & 23.11 & -7.39 & 1.33 & -6.12 \\
\hline chr15:77-123 suggestive & RJF & 24.23 & -13.23 & 2.37 & -13.09 \\
\hline chr15:77-121 suggestive & RJF & 25.88 & -9.24 & 1.53 & -8.2 \\
\hline
\end{tabular}




\begin{tabular}{|c|c|c|c|c|c|}
\hline chr15:78-128 significant & RJF & 20.76 & -6.16 & 1.3 & -4.07 \\
\hline chr15:81-123 suggestive & RJF & 23.45 & -9.26 & 1.73 & -8.97 \\
\hline chr15:80-120 significant & RJF & 28.05 & -8.79 & 1.33 & -7.54 \\
\hline chr15:83-125 suggestive & RJF & 24.75 & -4.11 & 0.73 & -2.05 \\
\hline chr15:79-125 suggestive & RJF & 21.84 & -3.65 & 0.67 & -4.01 \\
\hline chr15:74-123 suggestive & RJF & 22.98 & -7.67 & 1.46 & -7.58 \\
\hline chr15:78-127 suggestive & RJF & 24.53 & -14.65 & 2.53 & -12.18 \\
\hline chr15:77-122 suggestive & RJF & 24.93 & -10.6 & 1.81 & -9.8 \\
\hline chr15:76-124 significant & RJF & 26.24 & -17.62 & 2.94 & -16.81 \\
\hline chr15:74-125 suggestive & RJF & 23.28 & -27.97 & 5.1 & -26.81 \\
\hline chr15:76-121 suggestive & RJF & 23.5 & -10.55 & 2.14 & -12.81 \\
\hline chr15:75-125 suggestive & RJF & 23.27 & -9.78 & 1.71 & -8.27 \\
\hline chr15:77-120 significant & RJF & 30.96 & -6.56 & 0.98 & -6.01 \\
\hline chr15:77-122 suggestive & RJF & 24.05 & -8.96 & 1.73 & -10.05 \\
\hline chr15:78-122 significant & RJF & 25.98 & -13.77 & 2.36 & -13.85 \\
\hline chr15:78-123 significant & RJF & 27.5 & -13.34 & 2.3 & -14.58 \\
\hline chr15:76-126 suggestive & RJF & 23.23 & -11.56 & 1.99 & -10.28 \\
\hline chr15:76-125 suggestive & RJF & 23.79 & -20.43 & 3.65 & -19.25 \\
\hline chr15:78-124 significant & RJF & 25.89 & -13.16 & 2.21 & -12.87 \\
\hline chr15:77-122 suggestive & RJF & 25.56 & -15 & 2.59 & -14.61 \\
\hline chr15:77-121 significant & RJF & 27.7 & -10.13 & 1.75 & -12.03 \\
\hline chr15:77-123 significant & RJF & 26.65 & -12.71 & 2.05 & -11.02 \\
\hline chr15:77-120 significant & RJF & 28.23 & -5.07 & 0.81 & -5.54 \\
\hline chr15:79-125 suggestive & RJF & 24.26 & -8.83 & 1.6 & -6.67 \\
\hline chr15:77-125 suggestive & RJF & 24.2 & -7.96 & 1.4 & -6.98 \\
\hline chr15:76-121 significant & RJF & 27.58 & -11.84 & 1.93 & -11.53 \\
\hline chr15:72-125 suggestive & RJF & 23.54 & -9.39 & 1.72 & -8.87 \\
\hline chr15:76-124 significant & RJF & 26.4 & -15.71 & 2.66 & -16.54 \\
\hline chr15:77-126 suggestive & RJF & 18.14 & -7.93 & 1.53 & -8.71 \\
\hline chr15:75-127 suggestive & RJF & 24.71 & -39.05 & 6.81 & -37.53 \\
\hline chr15:75-127 suggestive & RJF & 23.21 & -31.99 & 5.82 & -30.02 \\
\hline chr15:75-125 suggestive & RJF & 24.69 & -21.26 & 3.72 & -20.32 \\
\hline chr15:73-126 suggestive & RJF & 22.63 & -10.75 & 2.11 & -12.1 \\
\hline chr15:75-123 suggestive & RJF & 25.27 & -12.87 & 2.13 & -11.18 \\
\hline chr15:77-126 suggestive & RJF & 24.72 & -16.09 & 2.93 & -17.58 \\
\hline chr15:74-126 suggestive & RJF & 22.93 & -17.75 & 3.36 & -19.85 \\
\hline chr15:78-125 significant & RJF & 24.02 & -17.78 & 2.9 & -16.18 \\
\hline chr15:78-125 suggestive & RJF & 22.09 & -11.79 & 2.05 & -9.76 \\
\hline chr15:74-124 suggestive & RJF & 23.39 & -10.19 & 1.86 & -9.94 \\
\hline chr15:75-124 suggestive & RJF & 24.38 & -9.32 & 1.75 & -10.52 \\
\hline chr15:77-126 suggestive & RJF & 23.88 & -22.06 & 3.95 & -22.42 \\
\hline chr15:81-124 significant & RJF & 28.19 & -8.92 & 1.39 & -7.22 \\
\hline chr15:74-127 suggestive & RJF & 25.16 & -10 & 1.73 & -9.29 \\
\hline
\end{tabular}




\begin{tabular}{|c|c|c|c|c|c|}
\hline chr15:76-127 suggestive & RJF & 24 & -20.77 & 3.8 & -20.28 \\
\hline chr15:78-127 suggestive & RJF & 23.08 & -13.24 & 2.6 & -11.94 \\
\hline chr15:75-123 significant & RJF & 26.11 & -11.69 & 1.97 & -11.57 \\
\hline chr15:77-126 suggestive & RJF & 23.57 & -22.87 & 4.08 & -22.67 \\
\hline chr15:76-126 suggestive & RJF & 23.81 & -8.81 & 1.6 & -8.13 \\
\hline chr15:77-125 suggestive & RJF & 24.34 & -8.09 & 1.39 & -6.99 \\
\hline chr15:76-129 suggestive & RJF & 23.24 & -8.07 & 1.45 & -7.48 \\
\hline chr15:78-125 significant & RJF & 26.89 & -9.48 & 1.6 & -9.57 \\
\hline chr15:76-122 significant & RJF & 29.32 & -12.63 & 1.96 & -13.22 \\
\hline chr15:74-128 suggestive & RJF & 23.49 & -36.2 & 6.6 & -36.45 \\
\hline chr15:78-123 significant & RJF & 27.13 & -10.54 & 1.67 & -10.07 \\
\hline chr15:76-125 suggestive & RJF & 24.31 & -9.71 & 1.69 & -9.11 \\
\hline chr15:76-126 suggestive & RJF & 23.77 & -8.49 & 1.63 & -8.93 \\
\hline chr15:74-127 suggestive & RJF & 23.9 & -13.81 & 2.53 & -14.64 \\
\hline chr15:76-129 suggestive & RJF & 23.2 & -13.17 & 2.43 & -10.4 \\
\hline chr15:75-124 suggestive & RJF & 24.87 & -23.38 & 4.07 & -23.31 \\
\hline chr15:75-129 suggestive & RJF & 22.76 & -23.95 & 4.19 & -20.04 \\
\hline chr15:77-125 significant & RJF & 26.69 & -10.02 & 1.63 & -8.58 \\
\hline chr15:78-127 suggestive & RJF & 23.31 & -11.88 & 2.19 & -10.91 \\
\hline chr15:76-124 suggestive & RJF & 24.34 & -9.66 & 1.67 & -9.16 \\
\hline chr15:76-128 suggestive & RJF & 23.07 & -13.83 & 2.65 & -14.11 \\
\hline chr15:81-133 significant & RJF & 18.37 & -2.28 & 0.43 & -1.17 \\
\hline chr15:78-125 suggestive & RJF & 27.41 & -5.01 & 0.79 & -4.4 \\
\hline chr15:79-127 suggestive & RJF & 18.55 & -4.07 & 0.81 & -3.58 \\
\hline chr15:74-128 suggestive & RJF & 24.68 & -24.1 & 4.26 & -23.86 \\
\hline chr15:74-128 suggestive & RJF & 23.97 & -29.46 & 5.26 & -27.43 \\
\hline chr15:78-126 suggestive & RJF & 24.65 & -11.32 & 1.91 & -8.69 \\
\hline chr15:81-119 significant & RJF & 19.35 & -8.1 & 0.85 & -7.6 \\
\hline chr15:74-127 suggestive & RJF & 24.73 & -39.62 & 6.83 & -37.31 \\
\hline chr15:76-128 suggestive & RJF & 17.84 & -8.01 & 1.55 & -8.61 \\
\hline chr15:75-126 suggestive & RJF & 24.69 & -19.27 & 3.46 & -18.64 \\
\hline chr15:74-125 suggestive & RJF & 23.58 & -11.31 & 2.15 & -12.18 \\
\hline chr15:75-126 suggestive & RJF & 24.89 & -9.96 & 1.71 & -8.45 \\
\hline chr15:79-127 suggestive & RJF & 23.27 & -20.76 & 3.8 & -17.39 \\
\hline chr15:74-129 suggestive & RJF & 23.34 & -23.14 & 4.12 & -20.89 \\
\hline chr15:76-129 suggestive & RJF & 24.06 & -20.08 & 3.51 & -18.8 \\
\hline chr15:79-123 significant & RJF & 31.82 & -12.46 & 1.71 & -9.58 \\
\hline chr15:75-124 suggestive & RJF & 25.25 & -8.42 & 1.41 & -8.35 \\
\hline chr15:74-129 suggestive & RJF & 22.04 & -9.2 & 1.68 & -9.17 \\
\hline chr15:74-126 suggestive & RJF & 23.82 & -7.48 & 1.34 & -6.62 \\
\hline chr15:83-131 suggestive & RJF & 19.4 & -2.89 & 0.54 & -1.38 \\
\hline chr15:78-128 suggestive & RJF & 22.98 & -22.44 & 4.02 & -21.57 \\
\hline chr15:77-128 suggestive & RJF & 23.56 & -23.05 & 4.13 & -22.82 \\
\hline
\end{tabular}




\begin{tabular}{|c|c|c|c|c|c|}
\hline chr15:74-127 suggestive & RJF & 24.7 & -34.91 & 6.06 & -35.64 \\
\hline chr15:78-129 suggestive & RJF & 23.88 & -7.38 & 1.46 & -9.07 \\
\hline chr15:74-128 suggestive & RJF & 22.98 & -8.5 & 1.45 & -7.17 \\
\hline chr15:75-126 significant & RJF & 27.85 & -20.88 & 3.33 & -19.96 \\
\hline chr15:81-127 suggestive & RJF & 25.13 & -7.82 & 1.35 & -6.73 \\
\hline chr15:73-128 suggestive & RJF & 23.42 & -14.16 & 2.53 & -13.85 \\
\hline chr15:80-126 significant & RJF & 26.61 & -7.43 & 1.28 & -5.83 \\
\hline chr15:78-126 significant & RJF & 28.67 & -12.17 & 1.89 & -11.54 \\
\hline chr15:76-130 suggestive & RJF & 23.66 & -10.59 & 1.9 & -10.05 \\
\hline chr15:79-129 significant & RJF & 18.65 & -7.09 & 1.1 & -2.52 \\
\hline chr15:75-127 suggestive & RJF & 24.1 & -33.21 & 5.92 & -33.15 \\
\hline chr15:76-130 suggestive & RJF & 24.05 & -9.59 & 1.67 & -7.76 \\
\hline chr15:74-130 suggestive & RJF & 23.64 & -8.3 & 1.51 & -8.23 \\
\hline chr15:74-129 suggestive & RJF & 23.26 & -31.9 & 5.92 & -33.08 \\
\hline chr15:75-128 suggestive & RJF & 22.79 & -14.02 & 2.55 & -13.17 \\
\hline chr15:77-131 suggestive & RJF & 24.38 & -7.3 & 1.3 & -7.44 \\
\hline chr15:77-126 suggestive & RJF & 25.44 & -4.33 & 0.74 & -3.4 \\
\hline chr15:76-129 suggestive & RJF & 24.78 & -35.37 & 6.05 & -32.13 \\
\hline chr15:74-129 suggestive & RJF & 25.52 & -15.44 & 2.59 & -13.3 \\
\hline chr15:76-128 significant & RJF & 26.34 & -21.01 & 3.44 & -19.11 \\
\hline chr15:78-127 suggestive & RJF & 23.75 & -8.84 & 1.54 & -6.35 \\
\hline chr15:74-129 suggestive & RJF & 25.77 & -47.52 & 7.87 & -43.21 \\
\hline chr15:74-130 suggestive & RJF & 23.68 & -17.55 & 3.11 & -14.99 \\
\hline chr15:74-129 suggestive & RJF & 23.3 & -40.17 & 7.46 & -41.81 \\
\hline chr15:75-127 suggestive & RJF & 23.4 & -8.29 & 1.51 & -8.33 \\
\hline chr15:74-129 suggestive & RJF & 23.75 & -19.77 & 3.58 & -18.45 \\
\hline chr15:74-130 suggestive & RJF & 23.35 & -34.94 & 6.28 & -33.59 \\
\hline chr15:76-128 suggestive & RJF & 24.25 & -19.2 & 3.43 & -19.29 \\
\hline chr15:75-128 suggestive & RJF & 23.78 & -13.32 & 2.51 & -14.79 \\
\hline chr15:81-129 significant & RJF & 11.16 & -7.99 & 1.25 & -3.31 \\
\hline chr15:74-129 suggestive & RJF & 24.38 & -42.36 & 7.41 & -40.31 \\
\hline chr15:74-131 suggestive & RJF & 23.32 & -43.99 & 7.98 & -40.68 \\
\hline chr15:74-127 suggestive & RJF & 24.09 & -9.44 & 1.58 & -8.34 \\
\hline chr15:74-128 suggestive & RJF & 23.58 & -34.27 & 6.19 & -33.9 \\
\hline chr15:74-130 suggestive & RJF & 24.31 & -24.68 & 4.44 & -25.16 \\
\hline chr15:75-126 significant & RJF & 27.01 & -32.97 & 5.23 & -32.21 \\
\hline chr15:77-121 significant & RJF & 18.23 & -4.8 & 0.57 & -4.9 \\
\hline chr15:74-129 suggestive & RJF & 25.72 & -19.13 & 3.24 & -19.27 \\
\hline chr15:75-131 suggestive & RJF & 23.42 & -33.75 & 6.26 & -34.35 \\
\hline chr15:74-128 suggestive & RJF & 24.69 & -21.12 & 3.7 & -21.64 \\
\hline chr15:74-131 suggestive & RJF & 24.47 & -55.71 & 9.67 & -50.62 \\
\hline chr15:79-129 suggestive & RJF & 25.77 & -9.83 & 1.59 & -8.1 \\
\hline chr15:74-130 suggestive & RJF & 24.27 & -36.69 & 6.52 & -36.88 \\
\hline
\end{tabular}




\begin{tabular}{|c|c|c|c|c|c|}
\hline chr15:74-129 suggestive & RJF & 25.71 & -26.48 & 4.5 & -25.46 \\
\hline chr15:73-130 suggestive & RJF & 23.31 & -8.75 & 1.64 & -8.83 \\
\hline chr15:75-129 suggestive & RJF & 25.15 & -27.41 & 4.68 & -25.49 \\
\hline chr15:75-130 suggestive & RJF & 23.12 & -11.35 & 2.08 & -10.16 \\
\hline chr15:78-124 significant & RJF & 15.47 & -6.73 & 0.82 & -6.93 \\
\hline chr15:73-129 suggestive & RJF & 23.75 & -9.22 & 1.75 & -10 \\
\hline chr15:77-131 suggestive & RJF & 23.99 & -24.87 & 4.35 & -21.51 \\
\hline chr15:75-131 suggestive & RJF & 23.99 & -24.84 & 4.45 & -23.26 \\
\hline chr15:79-137 suggestive & RJF & 18.62 & -5.87 & 1.21 & -5.35 \\
\hline chr15:74-130 suggestive & RJF & 22.93 & -4.81 & 0.81 & -3.86 \\
\hline chr15:75-131 suggestive & RJF & 25.14 & -3.13 & 0.51 & -2.22 \\
\hline chr15:74-131 suggestive & RJF & 24.71 & -15.14 & 2.71 & -14.67 \\
\hline chr15:74-129 suggestive & RJF & 22.98 & -8.9 & 1.64 & -9 \\
\hline chr15:75-132 suggestive & RJF & 23.64 & -65.93 & 11.52 & -58.29 \\
\hline chr15:82-130 suggestive & RJF & 25.54 & -9.79 & 1.63 & -9.02 \\
\hline chr15:74-130 suggestive & RJF & 25.75 & -20.48 & 3.59 & -22.19 \\
\hline chr15:79-129 suggestive & RJF & 24.41 & -14.93 & 2.52 & -14.22 \\
\hline chr15:75-129 significant & RJF & 26.66 & -16.64 & 2.81 & -17.57 \\
\hline chr15:74-130 suggestive & RJF & 24.57 & -33.39 & 5.79 & -31.55 \\
\hline chr15:74-128 suggestive & RJF & 23.28 & -7.67 & 1.39 & -7.89 \\
\hline chr15:75-130 suggestive & RJF & 24.64 & -28.84 & 5.13 & -29.42 \\
\hline chr15:74-130 suggestive & RJF & 22.13 & -10.63 & 1.95 & -10.11 \\
\hline chr15:75-131 suggestive & RJF & 24.93 & -48.76 & 8.2 & -43.14 \\
\hline chr15:74-131 suggestive & RJF & 24.64 & -40.18 & 6.97 & -38.51 \\
\hline chr15:80-129 suggestive & RJF & 23.93 & -10.04 & 1.75 & -9.19 \\
\hline chr15:75-133 suggestive & RJF & 23.47 & -49.34 & 8.76 & -43.8 \\
\hline chr15:82-133 suggestive & RJF & 16.61 & -9.86 & 1.62 & -5.51 \\
\hline chr15:74-127 suggestive & RJF & 25.61 & -5.55 & 0.97 & -5.77 \\
\hline chr15:75-130 suggestive & RJF & 23.1 & -28.19 & 5.17 & -28.05 \\
\hline chr15:75-131 suggestive & RJF & 23.28 & -17.92 & 3.23 & -17.14 \\
\hline chr15:76-136 suggestive & RJF & 23.68 & -7.16 & 1.36 & -6.85 \\
\hline chr15:75-134 suggestive & RJF & 23.35 & -10 & 1.75 & -8.46 \\
\hline chr15:73-132 suggestive & RJF & 25.12 & -44.73 & 7.69 & -41.53 \\
\hline chr15:79-132 suggestive & RJF & 25.31 & -13.23 & 2.17 & -9.92 \\
\hline chr15:74-130 suggestive & RJF & 24.22 & -17.93 & 3.07 & -14.41 \\
\hline chr15:74-129 suggestive & RJF & 23.54 & -14.29 & 2.51 & -13.8 \\
\hline chr15:75-132 significant & RJF & 22.79 & -18.66 & 2.99 & -14.59 \\
\hline chr15:72-132 suggestive & RJF & 22.5 & -16.35 & 3.12 & -17.26 \\
\hline chr15:74-131 suggestive & RJF & 25.3 & -29.54 & 5.07 & -29.02 \\
\hline chr15:74-131 suggestive & RJF & 25.05 & -12.77 & 2.22 & -11.83 \\
\hline chr15:72-133 suggestive & RJF & 24.43 & -46.92 & 8.08 & -42.36 \\
\hline chr15:76-131 suggestive & RJF & 23.45 & -10.41 & 1.89 & -10.24 \\
\hline chr15:74-131 significant & RJF & 26.31 & -18.88 & 3.19 & -19.05 \\
\hline
\end{tabular}




\begin{tabular}{|c|c|c|c|c|c|}
\hline chr15:78-130 suggestive & RJF & 27.25 & -6.16 & 0.95 & -4.36 \\
\hline chr15:73-133 suggestive & RJF & 24.39 & -42.41 & 7.35 & -36.83 \\
\hline chr15:81-133 significant & RJF & 15.1 & -10.04 & 1.55 & -4.98 \\
\hline chr15:77-136 suggestive & RJF & 23.97 & -7.88 & 1.39 & -7.62 \\
\hline chr15:77-131 suggestive & RJF & 23.3 & -23.31 & 4.2 & -23.95 \\
\hline chr15:74-135 suggestive & RJF & 18.81 & -9.44 & 1.67 & -6.9 \\
\hline chr15:75-133 suggestive & RJF & 23.15 & -12.9 & 2.35 & -11.02 \\
\hline chr15:74-134 suggestive & RJF & 23.88 & -58.45 & 10.17 & -54.74 \\
\hline chr15:78-131 significant & RJF & 14.38 & -16.22 & 2.43 & -13.53 \\
\hline chr15:75-133 suggestive & RJF & 24.79 & -44.19 & 7.3 & -37.93 \\
\hline chr15:73-134 suggestive & RJF & 23.6 & -28.88 & 5.12 & -27.47 \\
\hline chr15:75-133 suggestive & RJF & 24 & -52.58 & 9.1 & -44.92 \\
\hline chr15:73-134 suggestive & RJF & 23.9 & -28.31 & 4.93 & -23.87 \\
\hline chr15:74-133 suggestive & RJF & 22.85 & -11.06 & 2.01 & -10.64 \\
\hline chr15:75-132 significant & RJF & 25.83 & -21.33 & 3.63 & -21.67 \\
\hline chr15:80-131 suggestive & RJF & 25.81 & -6.42 & 1.12 & -6.47 \\
\hline chr15:72-133 suggestive & RJF & 24 & -14.28 & 2.53 & -13.65 \\
\hline chr15:73-133 suggestive & RJF & 25.22 & -16.29 & 2.82 & -15.4 \\
\hline chr15:75-131 suggestive & RJF & 23.75 & -7.07 & 1.35 & -8.16 \\
\hline chr15:71-135 suggestive & RJF & 23.99 & -20 & 3.46 & -18.26 \\
\hline chr15:77-137 suggestive & RJF & 22.2 & -19.48 & 3.32 & -13.64 \\
\hline chr15:78-136 suggestive & RJF & 23.11 & -60 & 10.63 & -46.93 \\
\hline chr15:83-130 significant & RJF & 20.44 & -11.32 & 1.61 & -6.64 \\
\hline chr15:73-134 suggestive & RJF & 23.82 & -17.97 & 3.13 & -16 \\
\hline chr15:76-134 suggestive & RJF & 24.45 & -41.92 & 6.95 & -36.39 \\
\hline chr15:75-136 suggestive & RJF & 22.99 & -47.96 & 8.48 & -42.02 \\
\hline chr15:76-136 suggestive & RJF & 22.59 & -40.11 & 6.9 & -33.8 \\
\hline chr15:77-135 suggestive & RJF & 23.58 & -72.31 & 12.64 & -56.99 \\
\hline chr15:75-135 suggestive & RJF & 23.67 & -13.23 & 2.27 & -9.12 \\
\hline chr15:73-137 suggestive & RJF & 23.86 & -11.46 & 2.26 & -9.4 \\
\hline chr15:76-132 significant & RJF & 21.3 & -30.23 & 4.57 & -21.71 \\
\hline chr15:73-136 significant & RJF & 24.24 & -4.47 & 0.84 & -3.41 \\
\hline chr15:83-135 suggestive & RJF & 24.79 & -3.74 & 0.62 & -2.82 \\
\hline chr15:78-131 significant & RJF & 24.55 & -5.23 & 0.8 & -4.4 \\
\hline chr15:76-133 suggestive & RJF & 26.86 & -13.7 & 2.21 & -12.19 \\
\hline chr15:79-137 suggestive & RJF & 24.07 & -3.96 & 0.72 & -3.81 \\
\hline chr15:75-135 suggestive & RJF & 22.27 & -35.36 & 5.97 & -26.24 \\
\hline chr15:72-135 suggestive & RJF & 23.95 & -20.81 & 3.85 & -20.56 \\
\hline chr15:73-136 suggestive & RJF & 22.94 & -7.31 & 1.35 & -6.88 \\
\hline chr15:78-135 significant & RJF & 13.59 & -10.37 & 1.59 & -6.73 \\
\hline chr15:70-134 suggestive & RJF & 19.84 & -11.43 & 1.93 & -8.97 \\
\hline chr15:74-136 suggestive & RJF & 23.25 & -7.47 & 1.46 & -7.09 \\
\hline chr15:73-133 suggestive & RJF & 24.47 & -9.85 & 1.67 & -8.34 \\
\hline
\end{tabular}




\begin{tabular}{|c|c|c|c|c|c|}
\hline chr15:78-134 significant & RJF & 13.4 & -10.1 & 1.56 & -8.16 \\
\hline chr15:72-134 suggestive & RJF & 22.68 & -9.63 & 1.74 & -9.27 \\
\hline chr15:74-133 suggestive & RJF & 23.86 & -22.62 & 3.88 & -20.58 \\
\hline chr15:81-138 suggestive & RJF & 19.01 & -13 & 2.26 & -6.51 \\
\hline chr15:84-143 significant & RJF & 27.32 & -3.6 & 0.78 & 1.48 \\
\hline chr15:74-133 suggestive & RJF & 27.28 & -9.96 & 1.58 & -8.4 \\
\hline chr15:74-135 suggestive & RJF & 25.89 & -27.87 & 4.64 & -26.5 \\
\hline chr15:73-137 suggestive & RJF & 23.69 & -29.05 & 5.12 & -25.02 \\
\hline chr15:78-137 significant & RJF & 11.61 & -8.71 & 1.34 & -3.61 \\
\hline chr15:76-138 suggestive & RJF & 18.39 & -21.62 & 3.83 & -13.71 \\
\hline chr15:73-137 suggestive & RJF & 22.95 & -24.09 & 4.41 & -24.3 \\
\hline chr15:81-137 suggestive & RJF & 11.22 & -5.92 & 1 & -1.83 \\
\hline chr15:80-132 significant & RJF & 27.74 & -9.88 & 1.5 & -8.21 \\
\hline chr15:70-137 suggestive & RJF & 24.11 & -16.1 & 2.97 & -14.66 \\
\hline chr15:73-133 suggestive & RJF & 24.35 & -10.19 & 1.81 & -10.87 \\
\hline chr15:76-136 suggestive & RJF & 24.73 & -26.6 & 4.52 & -23.6 \\
\hline chr15:77-136 suggestive & RJF & 23.22 & -44.4 & 7.27 & -31.97 \\
\hline chr15:82-132 significant & RJF & 16.67 & -7.04 & 0.84 & -4.21 \\
\hline chr15:76-132 significant & RJF & 23.56 & -8.27 & 1.25 & -7.85 \\
\hline chr15:68-135 suggestive & RJF & 25.65 & -16.48 & 2.83 & -12.91 \\
\hline chr15:76-132 significant & RJF & 28.63 & -16.83 & 2.39 & -14.44 \\
\hline chr15:73-137 suggestive & RJF & 22.85 & -14.81 & 2.64 & -11.92 \\
\hline chr15:80-135 suggestive & RJF & 24.8 & -4.99 & 0.82 & -4.12 \\
\hline chr15:79-134 significant & RJF & 15.82 & -9.57 & 1.3 & -7.33 \\
\hline chr15:72-141 suggestive & RJF & 20.13 & -13.51 & 2.27 & -8.25 \\
\hline chr15:69-137 suggestive & RJF & 23.29 & -14.49 & 2.62 & -14.39 \\
\hline chr15:72-137 suggestive & RJF & 20.18 & -7.91 & 1.37 & -7.25 \\
\hline chr15:77-138 significant & RJF & 18.67 & -7.31 & 1.06 & -4.37 \\
\hline chr15:75-135 suggestive & RJF & 12.43 & -6.29 & 1.02 & -4.66 \\
\hline chr15:80-137 significant & RJF & 24.16 & -22.75 & 3.42 & -15.87 \\
\hline chr15:76-140 suggestive & RJF & 23.52 & -20.48 & 3.68 & -17.67 \\
\hline chr15:76-141 suggestive & RJF & 21.91 & -16.79 & 2.77 & -11.08 \\
\hline chr15:73-141 suggestive & RJF & 22.81 & -10.03 & 1.75 & -8.47 \\
\hline chr15:78-141 suggestive & RJF & 24.59 & -11.52 & 2.07 & -7.79 \\
\hline chr15:76-140 suggestive & RJF & 23.46 & -36.93 & 6.33 & -31.99 \\
\hline chr15:72-140 suggestive & RJF & 18.17 & -46.48 & 8.18 & -37.23 \\
\hline chr15:74-137 significant & RJF & 26.52 & -9.95 & 1.81 & -9.48 \\
\hline chr15:75-139 suggestive & RJF & 22.37 & -37.55 & 6.24 & -27.48 \\
\hline chr15:73-140 suggestive & RJF & 16.9 & -9.75 & 1.6 & -5.07 \\
\hline chr15:66-142 suggestive & RJF & 20.89 & -5.43 & 0.94 & -4.59 \\
\hline chr15:77-142 suggestive & RJF & 18.59 & -20.16 & 3.48 & -17.71 \\
\hline chr15:79-142 suggestive & RJF & 20.34 & -7.48 & 1.24 & -5.59 \\
\hline chr15:76-142 suggestive & RJF & 22.96 & -49.14 & 8.61 & -40.16 \\
\hline
\end{tabular}




\begin{tabular}{|c|c|c|c|c|c|}
\hline chr15:80-142 significant & RJF & 22.65 & -18.81 & 2.98 & -11.69 \\
\hline chr15:75-141 suggestive & RJF & 22.09 & -43.93 & 7.48 & -32.68 \\
\hline chr15:78-141 suggestive & RJF & 24.6 & -31.74 & 5.1 & -21.51 \\
\hline chr15:82-139 suggestive & RJF & 14.84 & -5.91 & 0.9 & -2.71 \\
\hline chr15:81-141 suggestive & RJF & 27.13 & -5.48 & 0.86 & -4.09 \\
\hline chr15:77-143 suggestive & RJF & 22.48 & -12.15 & 2 & -7.12 \\
\hline chr15:72-140 significant & RJF & 28.67 & -16.04 & 2.53 & -13.63 \\
\hline chr15:73-144 suggestive & RJF & 21.67 & -12.72 & 2.28 & -11.27 \\
\hline chr15:78-143 significant & RJF & 13.27 & -11.03 & 1.71 & -7.42 \\
\hline chr15:68-141 significant & RJF & 26.5 & -10.59 & 1.71 & -8.35 \\
\hline chr15:74-141 suggestive & RJF & 21.8 & -48.07 & 8.01 & -36.92 \\
\hline chr15:74-142 suggestive & RJF & 18.07 & -8 & 1.36 & -6.04 \\
\hline chr15:71-143 suggestive & RJF & 23.04 & -21.44 & 3.67 & -16.38 \\
\hline chr15:73-144 suggestive & RJF & 21.5 & -23.49 & 3.91 & -15.8 \\
\hline chr15:81-146 suggestive & RJF & 23.42 & -8.72 & 1.42 & -5.7 \\
\hline chr15:72-142 suggestive & RJF & 17.56 & -15.2 & 2.55 & -11.02 \\
\hline chr15:78-140 significant & RJF & 10.86 & -12.17 & 1.58 & -7.54 \\
\hline chr15:72-146 suggestive & RJF & 16.45 & -44.39 & 7.53 & -32.25 \\
\hline chr15:78-142 significant & RJF & 21.88 & -16.31 & 2.43 & -11.58 \\
\hline chr15:77-146 suggestive & RJF & 23.86 & -14.25 & 2.47 & -10.57 \\
\hline chr15:68-144 suggestive & RJF & 15.78 & -40.1 & 6.42 & -29.18 \\
\hline chr15:77-146 suggestive & RJF & 15.17 & -9.89 & 1.63 & -4.97 \\
\hline chr15:83-143 significant & RJF & 20.2 & -8.2 & 1.28 & -5.67 \\
\hline chr15:79-165 significant & RJF & 17.94 & -2.29 & 0.51 & -0.26 \\
\hline chr15:77-147 suggestive & RJF & 17.23 & -21.17 & 3.57 & -13.57 \\
\hline chr15:78-146 suggestive & RJF & 20.96 & -25.78 & 4.28 & -16.49 \\
\hline chr15:77-145 suggestive & RJF & 18.46 & -27.14 & 4.51 & -18.14 \\
\hline chr15:73-150 suggestive & RJF & 12.81 & -11.38 & 1.79 & -8.62 \\
\hline chr15:75-143 significant & RJF & 16.89 & -7.59 & 1.19 & -6.07 \\
\hline chr15:82-145 significant & RJF & 18.25 & -16.45 & 2.37 & -4.58 \\
\hline chr15:81-143 significant & RJF & 16.41 & -5.02 & 0.63 & -3.76 \\
\hline chr15:72-150 suggestive & RJF & 16.24 & -12.35 & 2.03 & -9.71 \\
\hline chr15:79-146 significant & RJF & 19.57 & -21.33 & 3.26 & -12.9 \\
\hline chr15:70-155 suggestive & RJF & 22.87 & -16.37 & 2.93 & -13.6 \\
\hline chr15:81-147 suggestive & RJF & 18.9 & -13.81 & 2.19 & -6.34 \\
\hline chr15:79-154 suggestive & RJF & 18.47 & -15.39 & 2.6 & -6.79 \\
\hline chr15:79-150 significant & RJF & 9.2 & -9.79 & 1.52 & -3.62 \\
\hline chr15:78-151 significant & RJF & 9.17 & -9.89 & 1.49 & -3.92 \\
\hline chr15:81-156 suggestive & RJF & 14.7 & -13.99 & 2.28 & -6.33 \\
\hline chr15:76-155 suggestive & RJF & 7.02 & -5.87 & 1.02 & -1.62 \\
\hline chr15:82-156 suggestive & RJF & 15.94 & -20.01 & 3.44 & -8.08 \\
\hline chr15:75-165 suggestive & RJF & 20.48 & -10.81 & 1.92 & -6.71 \\
\hline chr15:81-165 suggestive & RJF & 15.56 & -14.77 & 2.43 & -4.88 \\
\hline
\end{tabular}




\begin{tabular}{|c|c|c|c|c|c|c|}
\hline \multicolumn{2}{|c|}{ chr15:79-164 significant } & RJF & 15.86 & -11.15 & 1.73 & -3.76 \\
\hline \multicolumn{2}{|c|}{ chr15:78-167 suggestive } & RJF & 22.62 & -15.5 & 2.65 & -9.75 \\
\hline \multicolumn{2}{|c|}{ chr15:80-170 suggestive } & RJF & 18.75 & -7.77 & 1.29 & -4.7 \\
\hline \multicolumn{2}{|c|}{ chr15:77-166 suggestive } & RJF & 10.7 & -6.97 & 1.14 & -3.72 \\
\hline \multicolumn{2}{|c|}{ chr15:83-170 suggestive } & RJF & 18.99 & -6.7 & 1.17 & -3.6 \\
\hline \multicolumn{2}{|c|}{ chr15:61-176 significant } & RJF & 17.91 & -1.4 & 0.28 & -0.18 \\
\hline \multicolumn{2}{|c|}{ chr15:87-168 suggestive } & RJF & 23.21 & -7.73 & 1.54 & -7.5 \\
\hline \multicolumn{2}{|c|}{ chr15:85-176 significant } & RJF & 18.03 & -1.1 & 0.22 & -0.44 \\
\hline \multicolumn{2}{|c|}{ chr15:104-16 suggestive } & RJF & 9.32 & -3.05 & 0.54 & -2.51 \\
\hline \multicolumn{2}{|c|}{ chr15:100-18 significant } & RJF & 23.52 & -2.27 & 0.42 & -0.73 \\
\hline \multicolumn{2}{|c|}{ chr15:112-16 suggestive } & RJF & 5.18 & -3.93 & 0.77 & -3.39 \\
\hline \multicolumn{2}{|c|}{ chr15:108-17 suggestive } & RJF & 19.4 & -1.61 & 0.31 & -1.11 \\
\hline \multicolumn{2}{|c|}{ chr15:116-16 suggestive } & RJF & 5.75 & -3.88 & 0.76 & -3.83 \\
\hline \multicolumn{2}{|c|}{ chr15:106-17 suggestive } & RJF & 17.28 & -2.17 & 0.46 & -1.03 \\
\hline \multicolumn{2}{|c|}{ chr15:98-177 significant } & RJF & 14.17 & -1.5 & 0.38 & -1.05 \\
\hline \multicolumn{2}{|c|}{ chr15:121-17 suggestive } & RJF & 18.61 & -1.53 & 0.29 & -0.01 \\
\hline \multicolumn{2}{|c|}{ chr15:121-17 suggestive } & RJF & 6.18 & -1.24 & 0.21 & -0.57 \\
\hline \multicolumn{2}{|c|}{ chr15:172-20 significant } & RJF & 17.83 & -2.77 & 0.55 & 0.94 \\
\hline \multicolumn{2}{|c|}{ chr15:181-19 suggestive } & RJF & 20.31 & -0.19 & 0.34 & 2.77 \\
\hline \multicolumn{2}{|c|}{ chr15:171-20 suggestive } & RJF & 12.87 & -1.45 & 0.38 & -1.15 \\
\hline \multicolumn{2}{|c|}{ chr15:197-20 significant } & WL & 25.27 & 1.07 & 0.49 & -1.31 \\
\hline chr16:0-0 & suggestive & WL & 14.88 & 0.5 & 0.59 & -0.11 \\
\hline chr16:0-0 & suggestive & RJF & 17.52 & -4.66 & 2.03 & 4.52 \\
\hline chr16:0-0 & suggestive & WL & 17.37 & 1.03 & 0.69 & -0.9 \\
\hline chr16:0-0 & significant & RJF & 15.47 & -9.71 & 2.15 & -2.31 \\
\hline chr16:0-0 & suggestive & WL & 10.76 & 3.58 & 0.95 & -1.26 \\
\hline chr16:0-0 & suggestive & WL & 10.33 & 3.68 & 1 & -1.18 \\
\hline chr16:0-0 & significant & RJF & 16.71 & -2.73 & 0.56 & -0.11 \\
\hline chr16:0-0 & suggestive & WL & 15.75 & 0.07 & 0.12 & -0.99 \\
\hline chr16:0-0 & suggestive & WL & 23.42 & 1.13 & 0.27 & -1.22 \\
\hline chr16:0-0 & suggestive & WL & 18.26 & 0.8 & 0.17 & -0.69 \\
\hline chr16:0-0 & suggestive & WL & 14.7 & 1.05 & 0.23 & -0.8 \\
\hline chr16:0-0 & suggestive & WL & 15.22 & 1.15 & 0.22 & -0.35 \\
\hline chr16:0-0 & suggestive & WL & 17.82 & 2.12 & 0.44 & -1.25 \\
\hline chr16:0-0 & suggestive & WL & 20.67 & 1.87 & 0.35 & -1.05 \\
\hline chr16:0-0 & suggestive & WL & 18.28 & 1.21 & 0.23 & -0.49 \\
\hline chr16:0-1 & suggestive & RJF & 14.39 & -11.52 & 2.71 & -3.61 \\
\hline chr16:0-1 & significant & RJF & 16.26 & -8.83 & 1.88 & -1.5 \\
\hline chr16:0-1 & significant & RJF & 15.04 & -7.82 & 1.73 & -0.91 \\
\hline chr16:0-1 & suggestive & RJF & 13.99 & -10.96 & 2.62 & -3.35 \\
\hline chr16:0-1 & suggestive & WL & 22.97 & 0.06 & 0.46 & -2.81 \\
\hline chr16:0-1 & suggestive & WL & 19.73 & 0.88 & 0.16 & -0.35 \\
\hline chr16:0-1 & suggestive & WL & 19.82 & 1.32 & 0.24 & -0.44 \\
\hline
\end{tabular}




\begin{tabular}{|c|c|c|c|c|c|c|}
\hline chr16:0-1 & suggestive & WL & 18.88 & 1.46 & 0.29 & -0.77 \\
\hline chr17:0-0 & significant & WL & 33.94 & 1.33 & 0.18 & -1.08 \\
\hline chr17:0-2 & suggestive & WL & 18.61 & 1.86 & 0.4 & -1.34 \\
\hline chr17:0-2 & suggestive & WL & 23.26 & 3.06 & 0.62 & -3.29 \\
\hline chr17:0-0 & significant & WL & 27.92 & 2.62 & 0.41 & -2.19 \\
\hline chr17:0-3 & suggestive & WL & 15.01 & 0.83 & 0.22 & -1.22 \\
\hline chr17:0-3 & suggestive & RJF & 18.82 & -1.08 & 0.31 & 2.04 \\
\hline chr17:0-4 & significant & WL & 14.48 & 1.5 & 0.37 & -1.86 \\
\hline chr17:0-2 & suggestive & WL & 23.78 & 1.96 & 0.36 & -1.8 \\
\hline chr17:0-12 & significant & RJF & 20.76 & -2.24 & 0.4 & 1.53 \\
\hline chr17:0-2 & significant & WL & 26.97 & 3.38 & 0.54 & -2.58 \\
\hline chr17:0-2 & suggestive & WL & 24.03 & 0.78 & 0.16 & -0.98 \\
\hline chr17:0-2 & significant & WL & 27.05 & 6.6 & 1.09 & -5.86 \\
\hline chr17:0-3 & suggestive & WL & 24.98 & 2.45 & 0.41 & -1.64 \\
\hline chr17:0-3 & suggestive & WL & 22.15 & 3.14 & 0.58 & -2.61 \\
\hline chr17:0-6 & suggestive & RJF & 18.05 & -1.75 & 0.39 & 2.25 \\
\hline chr17:0-3 & suggestive & RJF & 22.66 & -1 & 0.44 & 3.06 \\
\hline chr17:0-2 & suggestive & WL & 24.79 & 3.08 & 0.53 & -2.92 \\
\hline chr17:0-2 & suggestive & WL & 24.43 & 1.55 & 0.28 & -1.47 \\
\hline chr17:0-4 & suggestive & WL & 25.72 & 1.83 & 0.33 & -2.05 \\
\hline chr17:0-3 & suggestive & WL & 24.14 & 1.76 & 0.33 & -1.91 \\
\hline chr17:18-73 & significant & RJF & 19.12 & -1.65 & 0.38 & -0.59 \\
\hline chr17:23-72 & suggestive & RJF & 24.1 & -2.16 & 0.37 & -0.02 \\
\hline chr17:36-66 & suggestive & WL & 27.86 & 0.85 & 0.14 & -0.9 \\
\hline chr17:39-66 & significant & WL & 34.22 & 2.87 & 0.42 & -3.09 \\
\hline chr17:35-68 & suggestive & RJF & 22.55 & -3.55 & 0.62 & 3.29 \\
\hline chr17:34-68 & suggestive & RJF & 22.92 & -3.37 & 0.56 & 1.9 \\
\hline chr17:39-69 & suggestive & RJF & 29.1 & -3.06 & 1.22 & 5.69 \\
\hline chr17:41-65 & suggestive & RJF & 29.25 & -2.61 & 0.84 & 5.11 \\
\hline chr17:38-65 & suggestive & RJF & 19.35 & -0.56 & 0.4 & 0.43 \\
\hline chr17:41-66 & suggestive & RJF & 27.25 & -1.54 & 0.82 & 5.11 \\
\hline chr17:38-67 & suggestive & RJF & 17.65 & -0.65 & 0.78 & 1.88 \\
\hline chr17:37-69 & suggestive & RJF & 24.59 & -3.16 & 0.51 & 2.43 \\
\hline chr17:41-66 & suggestive & RJF & 23.46 & -2.57 & 0.5 & 3.5 \\
\hline chr17:30-70 & suggestive & WL & 17.89 & 3.56 & 0.71 & -0.92 \\
\hline chr17:42-66 & suggestive & RJF & 22.06 & -1.82 & 0.85 & 4.35 \\
\hline chr17:40-71 & significant & RJF & 23.23 & -1.18 & 0.58 & 1.32 \\
\hline chr17:46-67 & suggestive & RJF & 14.76 & -0.96 & 0.59 & 0.84 \\
\hline chr17:45-68 & suggestive & RJF & 19.64 & -0.35 & 0.47 & 0.4 \\
\hline chr17:42-70 & suggestive & RJF & 18.64 & -0.41 & 0.43 & 1.12 \\
\hline chr17:43-68 & suggestive & RJF & 22.39 & -1.96 & 0.35 & 1.88 \\
\hline chr17:37-80 & suggestive & RJF & 18.86 & -2.34 & 0.46 & 0.51 \\
\hline chr17:29-76 & suggestive & RJF & 18.72 & -2.79 & 0.57 & 0.14 \\
\hline
\end{tabular}


chr17:38-77 suggestive RJF chr17:40-74 suggestive RJF chr17:39-87 significant RJF chr17:32-85 suggestive RJF chr17:27-83 significant RJF chr17:41-85 significant RJF chr17:78-104 suggestive WL chr17:83-103 suggestive WL chr17:82-102 suggestive WL chr17:84-99 significant chr17:82-101 suggestive chr17:85-101 suggestive chr17:85-101 suggestive chr17:85-101 suggestive chr17:84-101 suggestive chr17:83-103 suggestive chr17:89-104 suggestive chr17:125-15 suggestive chr17:97-158 significant chr17:119-15 significant chr17:128-14 suggestive chr17:143-16 suggestive chr17:168-17 suggestive chr17:167-17 suggestive chr18:1-58 suggestive chr18:0-65 suggestive chr18:6-66 suggestive chr18:18-60 suggestive chr18:34-59 significant chr18:28-64 significant chr18:22-63 significant chr18:25-64 suggestive chr18:26-59 suggestive chr18:34-60 suggestive chr18:8-71 suggestive chr18:18-66 suggestive chr18:17-64 suggestive chr18:1-71 suggestive chr18:25-64 suggestive chr18:18-71 significant chr18:26-64 significant chr18:97-118 suggestive chr18:102-12 suggestive
22.59

22.95

26.77

17.35

18.7

31.51

18.9

24.8

19.94

23.36

18.41

19.08

18.83

12.45

19.67

18.64

25.06

15.24

12.59

19.44

15.68

21.72

15.06

14.06

22.92

21.45

13.94

19.84

19.07

13.84

14.05

12.72

23.08

23.7

12.3

21.8

19.29

18.04

22.93

14.95

24.17

24.81

16.59
$-2.42$

$-1.84$

$-2.6$

$-2.93$

$-3.48$

$-3.38$

0.63

0.12

1.03

1.53

2.23

0.66

1.26

0.32

0.07

0.4

1.04

$-1.61$

$-2.34$

1.54

1

$-1.1$

2.65

2.23

0

$-0.07$

$-0.09$

$-0.74$

$-1.89$

$-3.23$

$-2.84$

$-2.36$

$-1.41$

$-3.59$

$-1.52$

0.25

$-0.78$

1.15

$-1.35$

$-2.56$

$-0.44$

$-2$

$-0.85$
0.4

0.32

0.45

0.58

0.67

0.51

0.89

0.49

0.63

0.35

0.54

0.48

0.58

0.85

0.01

0.08

0.2

0.47

0.81

0.29

0.31

0.52

0.78

0.51

0.01

0.12

0.53

0.63

0.4

0.74

0.64

0.56

0.29

0.65

0.37

0.76

0.19

0.72

0.28

0.52

0.07

0.37

0.15
1.52

1.69

$-0.46$

1.21

0.96

$-0.45$

$-5.06$

$-3.14$

$-0.91$

$-2.68$

$-3.58$

$-3.11$

$-3.88$

$-0.44$

$-0.07$

$-0.5$

$-1.32$

$-1.46$

$-2.81$

$-0.77$

$-1.79$

3.08

$-3.2$

$-0.6$

0

$-0.13$

$-1.52$

$-4.33$

$-1.49$

$-0.2$

$-0.52$

$-0.79$

$-1.3$

$-3.19$

$-0.66$

$-3.4$

$-1.1$

$-1.69$

$-1.62$

$-0.77$

$-0.42$

$-1.04$

$-0.99$ 


\begin{tabular}{|c|c|c|c|c|c|c|}
\hline chr18:103-12 & suggestive & RJF & 25.03 & -0.98 & 0.23 & -1.02 \\
\hline chr19:0-14 & suggestive & RJF & 14.57 & -1.39 & 0.37 & 1.39 \\
\hline chr19:0-26 & significant & WL & 15.56 & 3.94 & 0.83 & -1.34 \\
\hline chr19:0-41 & suggestive & RJF & 14.85 & -1.65 & 0.39 & -0.3 \\
\hline chr19:1-25 & significant & RJF & 17.18 & -2.23 & 0.57 & -1.51 \\
\hline chr19:6-25 & suggestive & WL & 19.42 & 3.15 & 1.17 & -5.03 \\
\hline chr19:15-36 & significant & WL & 21.54 & 0.27 & 0.05 & -0.28 \\
\hline chr19:52-75 & significant & RJF & 22.57 & -0.17 & 0.36 & 0.03 \\
\hline chr19:50-72 & suggestive & WL & 23.74 & 2.44 & 0.57 & -1.18 \\
\hline chr19:47-77 & suggestive & WL & 16.27 & 0.45 & 0.31 & -1.96 \\
\hline chr19:49-77 & suggestive & WL & 27.07 & 3.35 & 0.76 & -2.04 \\
\hline chr19:46-77 & suggestive & WL & 25.48 & 1.77 & 0.44 & -1.95 \\
\hline chr19:44-77 & suggestive & WL & 12.98 & 2.6 & 0.61 & -0.46 \\
\hline chr19:64-77 & significant & WL & 22.1 & 2.39 & 0.72 & -2.22 \\
\hline chr19:66-77 & significant & WL & 23.25 & 21.2 & 4.58 & -9.28 \\
\hline chr19:65-77 & significant & WL & 23.09 & 15.69 & 3.34 & -6.39 \\
\hline chr19:67-77 & suggestive & WL & 26.69 & 4.88 & 0.89 & 0.1 \\
\hline chr19:67-77 & suggestive & RJF & 24.06 & -0.45 & 0.08 & -0.65 \\
\hline chr19:68-77 & suggestive & WL & 26.34 & 4.32 & 0.71 & -0.13 \\
\hline chr19:68-77 & suggestive & WL & 28.27 & 4.84 & 0.84 & -0.83 \\
\hline chr19:67-77 & suggestive & WL & 20.4 & 1.61 & 0.35 & -0.35 \\
\hline chr19:70-77 & suggestive & WL & 29.4 & 5.41 & 0.98 & -1.66 \\
\hline chr19:69-77 & significant & WL & 32.56 & 3.63 & 0.72 & -1.51 \\
\hline chr19:69-77 & suggestive & WL & 28.08 & 5.17 & 0.98 & -0.92 \\
\hline chr19:72-77 & suggestive & WL & 21.33 & 3.01 & 0.7 & -1.43 \\
\hline chr19:70-77 & suggestive & WL & 23.37 & 2.93 & 0.62 & 0.58 \\
\hline chr19:72-77 & significant & WL & 24.99 & 4.41 & 0.78 & 0.97 \\
\hline chr19:69-77 & suggestive & WL & 20.06 & 2.3 & 0.63 & -1.51 \\
\hline chr19:72-77 & significant & WL & 25.85 & 3.14 & 0.62 & -0.86 \\
\hline chr19:70-77 & significant & WL & 24.3 & 3.23 & 0.76 & -2.05 \\
\hline chr19:70-77 & significant & WL & 22.37 & 2.79 & 0.76 & -1.86 \\
\hline chr19:69-77 & suggestive & WL & 22.09 & 4.19 & 0.87 & -0.37 \\
\hline chr19:71-77 & significant & WL & 22.26 & 2.51 & 0.54 & -0.69 \\
\hline chr19:71-77 & suggestive & WL & 20.7 & 2.29 & 0.53 & -1.01 \\
\hline chr19:70-77 & significant & WL & 22.13 & 2.67 & 0.63 & -1.41 \\
\hline chr19:65-77 & significant & WL & 18.57 & 2.18 & 0.44 & -0.24 \\
\hline chr19:71-77 & significant & WL & 27.32 & 5.6 & 0.92 & 0.33 \\
\hline chr19:70-77 & suggestive & WL & 20.98 & 4.71 & 1.04 & -0.95 \\
\hline chr19:70-77 & suggestive & WL & 22.16 & 3.81 & 0.87 & -0.88 \\
\hline chr19:69-77 & suggestive & WL & 21.2 & 2.17 & 0.54 & -1.21 \\
\hline chr19:71-77 & suggestive & WL & 21.95 & 3.92 & 0.82 & -0.5 \\
\hline chr19:71-77 & suggestive & WL & 21.09 & 2.21 & 0.56 & -0.86 \\
\hline chr19:68-77 & suggestive & WL & 22.06 & 4.61 & 0.98 & -0.78 \\
\hline
\end{tabular}




\begin{tabular}{|c|c|c|c|c|c|c|}
\hline chr19:72-77 & significant & WL & 23.15 & 4.06 & 0.93 & -1.41 \\
\hline chr19:68-77 & suggestive & WL & 23.73 & 4.03 & 0.83 & 0.11 \\
\hline chr19:70-77 & significant & WL & 25.76 & 5.62 & 1.06 & 1.82 \\
\hline chr19:70-77 & significant & WL & 21.69 & 3.56 & 0.81 & -1.62 \\
\hline chr19:72-77 & suggestive & WL & 22.62 & 2.72 & 0.61 & -1.1 \\
\hline chr19:71-77 & suggestive & WL & 21.92 & 2.36 & 0.48 & 0.01 \\
\hline chr19:71-77 & suggestive & WL & 20.62 & 1.32 & 0.37 & -0.9 \\
\hline chr19:68-77 & suggestive & WL & 13.84 & 0.94 & 0.29 & -0.84 \\
\hline chr19:68-77 & suggestive & WL & 14.47 & 0.39 & 0.33 & -1.81 \\
\hline chr19:74-77 & suggestive & WL & 25.47 & 1.6 & 0.49 & -1.66 \\
\hline chr19:70-77 & suggestive & WL & 25.08 & 2.51 & 0.64 & -1.38 \\
\hline chr19:73-77 & suggestive & WL & 28.71 & 4.55 & 0.83 & -1.18 \\
\hline chr19:75-77 & suggestive & WL & 24.69 & 2.43 & 0.86 & -4.19 \\
\hline chr19:72-77 & suggestive & WL & 27.2 & 2.1 & 0.47 & -0.84 \\
\hline chr19:71-77 & suggestive & WL & 27.37 & 4.85 & 0.9 & -0.9 \\
\hline chr19:72-77 & suggestive & WL & 24.09 & 2 & 0.55 & -1.95 \\
\hline chr19:71-77 & suggestive & WL & 26.09 & 3.23 & 0.58 & -0.65 \\
\hline chr19:71-77 & suggestive & WL & 24.76 & 2.37 & 0.55 & -1.33 \\
\hline chr19:71-77 & suggestive & WL & 26.52 & 2.71 & 0.64 & -2.02 \\
\hline chr19:73-77 & suggestive & WL & 26.08 & 1.9 & 0.52 & -1.44 \\
\hline chr19:70-77 & suggestive & WL & 27.58 & 2.21 & 0.59 & -2.25 \\
\hline chr19:71-77 & suggestive & WL & 25.83 & 2.67 & 0.67 & -1.97 \\
\hline chr19:73-77 & suggestive & WL & 25.63 & 3.07 & 0.72 & -2.05 \\
\hline chr19:69-77 & suggestive & WL & 28.21 & 2.93 & 0.63 & -1.6 \\
\hline chr19:71-77 & suggestive & WL & 25.11 & 3.7 & 0.79 & -2.12 \\
\hline chr19:70-77 & significant & WL & 32.24 & 2.58 & 0.43 & -0.47 \\
\hline chr19:70-77 & suggestive & WL & 25.51 & 2.04 & 0.4 & -0.74 \\
\hline chr19:72-77 & suggestive & WL & 29.61 & 3.03 & 0.49 & 0.15 \\
\hline chr19:71-77 & suggestive & WL & 28.96 & 2.94 & 0.56 & -1.01 \\
\hline chr19:72-77 & significant & WL & 29.9 & 2.98 & 0.51 & -0.89 \\
\hline chr19:71-77 & suggestive & WL & 25.29 & 2.27 & 0.58 & -2.09 \\
\hline chr19:69-77 & suggestive & RJF & 21.26 & -2.39 & 0.51 & -3.11 \\
\hline chr19:72-77 & suggestive & WL & 26.67 & 78.76 & 18.33 & -54.84 \\
\hline chr19:72-77 & suggestive & WL & 26.79 & 77.89 & 17.6 & -50.44 \\
\hline chr19:72-77 & suggestive & WL & 27.25 & 74.24 & 16.85 & -49.77 \\
\hline chr19:72-77 & suggestive & WL & 26.49 & 81.79 & 18.67 & -52.67 \\
\hline chr19:72-77 & suggestive & WL & 26.19 & 76.25 & 17.36 & -47.6 \\
\hline chr19:72-77 & suggestive & WL & 26.78 & 85.86 & 19.25 & -55.75 \\
\hline chr19:72-77 & suggestive & WL & 26.68 & 76.66 & 17.18 & -47.48 \\
\hline chr19:72-77 & suggestive & WL & 27.49 & 2.59 & 0.5 & -0.77 \\
\hline chr19:71-77 & suggestive & WL & 19.47 & 1.47 & 0.43 & -1.97 \\
\hline chr19:68-77 & suggestive & WL & 19.4 & 2.24 & 0.52 & -1.54 \\
\hline chr20:0-30 & suggestive & WL & 12.95 & 0.62 & 0.52 & -0.16 \\
\hline
\end{tabular}




\begin{tabular}{|c|c|c|c|c|c|c|}
\hline chr20:2-38 & significant & RJF & 15.81 & -3.99 & 0.82 & -0.68 \\
\hline chr20:1-28 & significant & RJF & 16.01 & -1.51 & 0.3 & -0.11 \\
\hline chr20:8-34 & significant & RJF & 29.6 & -2.88 & 0.39 & -0.7 \\
\hline chr20:4-31 & suggestive & WL & 18.17 & 1.65 & 0.31 & 0.44 \\
\hline chr20:6-28 & significant & RJF & 17.73 & -2.16 & 0.44 & 0.47 \\
\hline chr20:9-29 & significant & RJF & 20.76 & -14.59 & 2.51 & 0.89 \\
\hline chr20:8-27 & suggestive & RJF & 17.23 & -6.29 & 1.92 & 3.33 \\
\hline chr20:9-29 & significant & RJF & 17.89 & -2.3 & 0.45 & -1.4 \\
\hline chr20:8-30 & significant & RJF & 15.32 & -2.62 & 0.54 & -0.5 \\
\hline chr20:6-37 & suggestive & RJF & 14.73 & -1.26 & 0.27 & -0.13 \\
\hline chr20:8-32 & suggestive & RJF & 15.08 & -6.57 & 1.41 & 0.94 \\
\hline chr20:8-34 & suggestive & RJF & 14.49 & -14.5 & 3.13 & 0.02 \\
\hline chr20:5-36 & suggestive & RJF & 14.21 & -2.36 & 0.53 & -1.02 \\
\hline chr20:6-41 & suggestive & WL & 16.36 & 1.55 & 0.35 & -0.57 \\
\hline chr20:6-42 & suggestive & RJF & 14.4 & -3.46 & 0.75 & -1.56 \\
\hline chr20:3-58 & significant & WL & 11.85 & 1.4 & 0.36 & -0.11 \\
\hline chr20:8-41 & suggestive & RJF & 18.12 & -3.44 & 0.66 & -1.26 \\
\hline chr20:8-45 & significant & RJF & 17.13 & -3.46 & 0.68 & -0.28 \\
\hline chr20:8-41 & suggestive & RJF & 16.98 & -2.14 & 0.47 & -2.08 \\
\hline chr20:2-52 & significant & WL & 14.27 & 1.51 & 0.38 & -0.77 \\
\hline chr20:3-51 & suggestive & WL & 12.37 & 0.67 & 0.17 & -0.17 \\
\hline chr20:7-46 & significant & RJF & 20.02 & -1.68 & 0.31 & -0.5 \\
\hline chr20:2-48 & significant & WL & 15.57 & 0.86 & 0.2 & -0.33 \\
\hline chr20:10-59 & significant & RJF & 18.91 & -3.43 & 0.62 & -0.54 \\
\hline chr20:8-51 & significant & RJF & 16.72 & -3.72 & 0.78 & -2.31 \\
\hline chr20:6-46 & significant & RJF & 16.35 & -1.49 & 0.37 & -1.94 \\
\hline chr20:5-51 & significant & WL & 21.88 & 10.28 & 1.69 & 4.52 \\
\hline chr20:6-54 & significant & WL & 19.79 & 7.87 & 1.42 & -0.75 \\
\hline chr20:3-55 & significant & WL & 13.31 & 3.23 & 0.72 & -0.31 \\
\hline chr20:9-66 & suggestive & RJF & 14.88 & -3.76 & 0.84 & -2.12 \\
\hline chr20:5-56 & significant & WL & 15.28 & 10.86 & 2.25 & -0.72 \\
\hline chr20:4-56 & significant & WL & 15.85 & 12.94 & 2.68 & -2.41 \\
\hline chr20:10-58 & suggestive & RJF & 14.72 & -2.66 & 0.64 & -2.25 \\
\hline chr20:14-55 & significant & RJF & 22.24 & -2.59 & 0.45 & -0.96 \\
\hline chr20:5-57 & significant & WL & 14.04 & 11.34 & 2.49 & -1.68 \\
\hline chr20:5-56 & significant & WL & 14.12 & 11.29 & 2.46 & -1.68 \\
\hline chr20:18-59 & suggestive & RJF & 12.5 & -1.15 & 0.59 & -3.27 \\
\hline chr20:8-59 & significant & WL & 15.98 & 3.57 & 1.66 & 1.41 \\
\hline chr20:23-57 & significant & RJF & 20.84 & -4.15 & 0.78 & -3.04 \\
\hline chr20:20-83 & suggestive & RJF & 14.74 & -2.11 & 0.53 & -2.13 \\
\hline chr20:27-58 & suggestive & RJF & 19.79 & -2.33 & 0.61 & -3.88 \\
\hline chr20:5-56 & significant & WL & 12.41 & 1.31 & 0.32 & -0.23 \\
\hline chr20:26-105 & suggestive & RJF & 14.12 & -2.23 & 0.6 & -2.53 \\
\hline
\end{tabular}




\begin{tabular}{|c|c|c|c|c|c|}
\hline chr20:29-55 suggestive & RJF & 14.56 & -1.2 & 0.41 & -2.29 \\
\hline chr20:22-56 suggestive & WL & 13.34 & 1.3 & 0.34 & -0.74 \\
\hline chr20:32-54 significant & WL & 17.4 & 1.01 & 0.26 & -0.96 \\
\hline chr20:34-54 significant & RJF & 21.85 & -2.85 & 0.64 & -3.87 \\
\hline chr20:23-110 suggestive & WL & 8.44 & 1.76 & 0.58 & -0.98 \\
\hline chr20:27-101 suggestive & RJF & 13.8 & -2.06 & 0.54 & -2.1 \\
\hline chr20:34-60 significant & WL & 14.7 & 1.85 & 0.41 & 0.04 \\
\hline chr20:25-108 significant & WL & 10.38 & 1.23 & 0.33 & -0.16 \\
\hline chr20:11-100 suggestive & RJF & 14.84 & -3.51 & 0.77 & -1.46 \\
\hline chr20:29-77 suggestive & RJF & 20.69 & -3.59 & 0.65 & -1.52 \\
\hline chr20:52-75 suggestive & RJF & 22.41 & -0.88 & 0.58 & 4.6 \\
\hline chr20:30-80 suggestive & RJF & 18.55 & -2.42 & 0.47 & -1.16 \\
\hline chr20:41-83 significant & RJF & 21.78 & -1.79 & 0.33 & -1.39 \\
\hline chr20:30-89 significant & RJF & 15.89 & -1.64 & 0.4 & -1.75 \\
\hline chr20:53-70 suggestive & RJF & 24.29 & -0.84 & 0.51 & 2.53 \\
\hline chr20:50-77 suggestive & RJF & 15.06 & -2.3 & 0.5 & -0.89 \\
\hline chr20:50-80 significant & RJF & 15.64 & -1.98 & 0.41 & -0.34 \\
\hline chr20:54-78 suggestive & RJF & 23.91 & -1 & 0.34 & 2.31 \\
\hline chr20:41-81 significant & RJF & 19.79 & -2.66 & 0.49 & -0.52 \\
\hline chr20:55-78 significant & RJF & 18.14 & -1.39 & 0.28 & 0.27 \\
\hline chr20:52-79 significant & RJF & 17.2 & -1.44 & 0.37 & 1.35 \\
\hline chr20:55-80 significant & RJF & 16.49 & -1.86 & 0.4 & 0.59 \\
\hline chr20:32-82 significant & RJF & 16.68 & -2.25 & 0.46 & -0.66 \\
\hline chr20:56-78 suggestive & RJF & 23.3 & -1.22 & 0.49 & 3.49 \\
\hline chr20:55-71 suggestive & RJF & 21.89 & -1.02 & 0.44 & 3.05 \\
\hline chr20:53-79 suggestive & RJF & 23.86 & -1.38 & 0.45 & 1.17 \\
\hline chr20:52-80 suggestive & RJF & 21.68 & -1.55 & 0.45 & 2.77 \\
\hline chr20:39-83 significant & RJF & 21.46 & -2.48 & 0.44 & -0.15 \\
\hline chr20:31-95 significant & RJF & 16.14 & -2 & 0.43 & -1.21 \\
\hline chr20:30-103 significant & RJF & 16.11 & -1.91 & 0.46 & -1.93 \\
\hline chr20:31-105 suggestive & RJF & 15.17 & -1.9 & 0.41 & -0.51 \\
\hline chr20:26-107 significant & RJF & 15.59 & -3.59 & 0.84 & -3.19 \\
\hline chr20:32-148 significant & RJF & 11.68 & -1.45 & 0.41 & -0.5 \\
\hline chr20:44-144 significant & RJF & 12.2 & -2.68 & 0.69 & -0.01 \\
\hline chr20:40-114 suggestive & WL & 10.65 & 1.71 & 0.46 & 0.19 \\
\hline chr20:11-125 significant & RJF & 14.39 & -1.89 & 0.47 & -0.43 \\
\hline chr20:60-147 significant & RJF & 15.55 & -2.2 & 0.5 & -0.07 \\
\hline chr20:13-143 significant & RJF & 15.09 & -3.44 & 0.84 & -1.16 \\
\hline chr20:14-147 suggestive & RJF & 10.5 & -1.83 & 0.55 & -0.74 \\
\hline chr20:13-142 suggestive & RJF & 10.73 & -2.35 & 0.62 & 0.63 \\
\hline chr20:35-127 significant & WL & 11.8 & 2.15 & 0.54 & -0.17 \\
\hline chr20:28-144 suggestive & RJF & 13.05 & -3.38 & 0.82 & 0.31 \\
\hline chr20:8-139 significant & RJF & 14.51 & -1.57 & 0.39 & -0.51 \\
\hline
\end{tabular}




\begin{tabular}{|c|c|c|c|c|c|}
\hline chr20:66-120 significant & RJF & 20.11 & -3.27 & 0.61 & 0.09 \\
\hline chr20:66-137 significant & RJF & 18.86 & -0.69 & 0.36 & -0.36 \\
\hline chr20:73-155 significant & RJF & 18.5 & -3.05 & 0.6 & 0.36 \\
\hline chr20:67-131 significant & RJF & 12.42 & -2.13 & 0.51 & 0.38 \\
\hline chr20:92-112 significant & WL & 25.31 & 3.29 & 0.54 & -0.05 \\
\hline chr20:99-113 significant & WL & 22.32 & 2.81 & 0.51 & 0.3 \\
\hline chr20:92-113 significant & WL & 21.87 & 1.72 & 0.34 & 0.63 \\
\hline chr20:98-111 suggestive & WL & 15.48 & 1.05 & 0.18 & -1.08 \\
\hline chr20:86-131 significant & WL & 15.66 & 2.23 & 0.49 & -0.28 \\
\hline chr20:73-130 suggestive & WL & 14.37 & 1.14 & 0.36 & 1.08 \\
\hline chr20:87-133 significant & RJF & 24.96 & -3 & 0.51 & -0.1 \\
\hline chr20:111-13 significant & RJF & 19.86 & -0.61 & 0.37 & 0.43 \\
\hline chr20:111-13 significant & RJF & 20.71 & -1.61 & 0.55 & 0.92 \\
\hline chr20:112-13 significant & RJF & 26.84 & -2.45 & 0.37 & 0.41 \\
\hline chr20:111-13 significant & RJF & 25.06 & -3.5 & 0.58 & 0.21 \\
\hline chr20:109-13 significant & WL & 23.56 & 4.75 & 0.8 & -0.84 \\
\hline chr20:75-141 significant & RJF & 12.81 & -2.73 & 0.67 & -0.29 \\
\hline chr20:109-13 significant & WL & 34.08 & 3.26 & 0.43 & -0.42 \\
\hline chr20:91-170 significant & WL & 14.69 & 3.01 & 0.69 & -0.06 \\
\hline chr20:74-146 significant & RJF & 21.06 & -2.1 & 0.41 & -0.4 \\
\hline chr20:110-16 significant & RJF & 20.05 & -1.78 & 0.35 & -0.06 \\
\hline chr20:74-152 significant & RJF & 21.25 & -2.21 & 0.41 & -0.14 \\
\hline chr20:70-166 suggestive & RJF & 12.55 & -1.47 & 0.39 & -0.41 \\
\hline chr20:111-16 suggestive & RJF & 19.66 & -1.82 & 0.76 & 1.86 \\
\hline chr20:111-15 significant & RJF & 22.16 & -1.81 & 0.34 & -0.34 \\
\hline chr20:89-157 suggestive & RJF & 18.48 & -0.15 & 0.56 & -0.45 \\
\hline chr20:67-164 significant & RJF & 17.61 & -3.09 & 0.62 & -3.25 \\
\hline chr20:112-15 significant & RJF & 16.82 & -2.74 & 0.54 & -2.43 \\
\hline chr20:128-15 suggestive & RJF & 18.52 & -0.71 & 0.99 & -0.62 \\
\hline chr20:128-15 suggestive & WL & 19.12 & 0.84 & 0.8 & -1.31 \\
\hline chr20:133-15 suggestive & RJF & 19.43 & -0.56 & 0.67 & -3.06 \\
\hline chr20:136-15 suggestive & WL & 18.59 & 0.43 & 1.06 & -2.83 \\
\hline chr20:135-15 significant & RJF & 20.66 & -0.38 & 0.61 & -2.89 \\
\hline chr20:136-15 suggestive & WL & 19.9 & 0.29 & 0.84 & -3.32 \\
\hline chr20:132-16 suggestive & RJF & 18.42 & -1.24 & 1.09 & -2.56 \\
\hline chr20:212-25 significant & RJF & 16.47 & -3.74 & 0.78 & -2.7 \\
\hline chr20:219-25 significant & RJF & 15.66 & -1.31 & 0.28 & -0.41 \\
\hline chr20:206-25 suggestive & WL & 13.08 & 1.64 & 0.52 & -1.09 \\
\hline chr20:208-25 significant & RJF & 15.06 & -2.89 & 0.64 & -1.3 \\
\hline chr20:209-25 significant & RJF & 16.65 & -1.45 & 0.4 & -2.17 \\
\hline chr20:219-25 significant & WL & 16.83 & 2.02 & 0.41 & 0.57 \\
\hline chr20:220-25 suggestive & RJF & 19.17 & -3.24 & 0.83 & -4.5 \\
\hline chr20:229-25 suggestive & RJF & 19.29 & -1.34 & 0.47 & -3.18 \\
\hline
\end{tabular}




\begin{tabular}{|c|c|c|c|c|c|c|}
\hline \multicolumn{2}{|c|}{ chr20:239-25 significant } & WL & 23.87 & 2.02 & 0.33 & 0.55 \\
\hline \multicolumn{2}{|c|}{ chr20:241-25 significant } & WL & 17.59 & 1.66 & 0.4 & -0.76 \\
\hline \multicolumn{2}{|c|}{ chr20:240-25 suggestive } & WL & 14.08 & 2.24 & 0.51 & 0.16 \\
\hline \multicolumn{2}{|c|}{ chr20:243-25 suggestive } & RJF & 25.95 & -2.98 & 0.59 & -3.91 \\
\hline \multicolumn{2}{|c|}{ chr20:242-25 suggestive } & RJF & 19.2 & -2.3 & 0.57 & -3.27 \\
\hline \multicolumn{2}{|c|}{ chr20:239-25 significant } & RJF & 22.36 & -1.47 & 0.49 & -3.87 \\
\hline \multicolumn{2}{|c|}{ chr20:221-25 significant } & RJF & 17.93 & -1.88 & 0.35 & -0.72 \\
\hline \multicolumn{2}{|c|}{ chr20:225-25 significant } & RJF & 15.84 & -1.88 & 0.7 & -3.92 \\
\hline \multicolumn{2}{|c|}{ chr20:238-25 suggestive } & RJF & 20.21 & -1.03 & 0.42 & -2.93 \\
\hline \multicolumn{2}{|c|}{ chr20:219-25 significant } & RJF & 23.48 & -6.59 & 1.12 & -3.78 \\
\hline \multicolumn{2}{|c|}{ chr20:242-25 suggestive } & RJF & 19.66 & -1.83 & 0.5 & -2.99 \\
\hline \multicolumn{2}{|c|}{ chr20:246-25 suggestive } & RJF & 19.78 & -1.61 & 0.52 & -3.42 \\
\hline \multicolumn{2}{|c|}{ chr20:239-25 suggestive } & RJF & 18.85 & -3.8 & 0.91 & -4.5 \\
\hline \multicolumn{2}{|c|}{ chr20:237-25 significant } & WL & 18.82 & 2.38 & 0.44 & 0.65 \\
\hline \multicolumn{2}{|c|}{ chr20:244-25 suggestive } & RJF & 8.95 & -35.32 & 9.06 & -65.33 \\
\hline \multicolumn{2}{|c|}{ chr20:244-25 suggestive } & RJF & 8.2 & -45.9 & 11.48 & -81.8 \\
\hline chr21:0-13 & suggestive & RJF & 18.15 & -0.21 & 0.31 & -0.41 \\
\hline chr21:0-18 & suggestive & RJF & 18.97 & -1.03 & 0.2 & -0.44 \\
\hline chr21:0-15 & suggestive & RJF & 14.29 & -0.56 & 0.29 & -1.64 \\
\hline chr21:0-15 & suggestive & RJF & 19.5 & -1.78 & 0.36 & -1.49 \\
\hline chr21:3-12 & significant & RJF & 23.97 & -0.64 & 0.39 & -1.07 \\
\hline chr21:3-13 & significant & RJF & 17.17 & -0.89 & 0.54 & -3.57 \\
\hline chr21:3-49 & significant & RJF & 18.48 & -1.67 & 0.34 & -0.96 \\
\hline chr21:2-18 & suggestive & RJF & 15.72 & -1.02 & 0.22 & -0.55 \\
\hline chr21:3-46 & significant & RJF & 16.39 & -1.32 & 0.3 & -1.26 \\
\hline chr21:5-16 & suggestive & RJF & 24.62 & -1.27 & 0.44 & 0.49 \\
\hline chr21:5-13 & suggestive & RJF & 16.57 & -0.58 & 0.49 & -0.47 \\
\hline chr21:2-13 & suggestive & RJF & 16.47 & -0.41 & 0.33 & -0.47 \\
\hline chr21:3-15 & suggestive & RJF & 22.76 & -0.55 & 0.45 & -0.01 \\
\hline chr21:3-18 & suggestive & RJF & 19.89 & -1.03 & 0.19 & -0.51 \\
\hline chr21:3-18 & suggestive & RJF & 19.46 & -1.87 & 0.37 & -1.41 \\
\hline chr21:3-44 & suggestive & RJF & 19.76 & -0.98 & 0.19 & -0.61 \\
\hline chr21:2-41 & suggestive & RJF & 14.86 & -2.33 & 0.52 & -0.99 \\
\hline chr21:3-48 & suggestive & RJF & 20.21 & -1.46 & 0.27 & -0.76 \\
\hline chr21:3-32 & suggestive & RJF & 20.36 & -0.63 & 0.45 & -3.27 \\
\hline chr21:5-31 & suggestive & RJF & 19.73 & -1.04 & 0.2 & -0.58 \\
\hline chr21:1-19 & suggestive & RJF & 19.28 & -1.59 & 0.29 & -0.44 \\
\hline chr21:3-50 & suggestive & RJF & 14.96 & -1.53 & 0.36 & -1.02 \\
\hline chr21:0-47 & suggestive & RJF & 13.7 & -1.79 & 0.45 & -1.03 \\
\hline chr21:1-55 & suggestive & RJF & 13.67 & -2.75 & 0.65 & -0.99 \\
\hline chr21:4-56 & suggestive & RJF & 19.6 & -1.51 & 0.33 & -1.27 \\
\hline chr21:0-52 & significant & RJF & 18.73 & -1.95 & 0.52 & -2.45 \\
\hline chr21:6-48 & suggestive & RJF & 21.77 & -0.93 & 0.26 & -1.5 \\
\hline
\end{tabular}




\begin{tabular}{|c|c|c|c|c|c|c|}
\hline chr21:10-48 & suggestive & RJF & 19.05 & -0.87 & 0.23 & -1.14 \\
\hline chr21:4-49 & suggestive & RJF & 18.26 & -1.65 & 0.32 & -0.45 \\
\hline chr21:11-36 & suggestive & RJF & 17.36 & -0.08 & 0.4 & -0.08 \\
\hline chr21:5-50 & significant & RJF & 15.51 & -1.78 & 0.56 & -2.2 \\
\hline chr21:10-40 & significant & RJF & 23.03 & -0.23 & 0.35 & -2.7 \\
\hline chr21:11-74 & suggestive & WL & 11.64 & 1.72 & 0.49 & 0.95 \\
\hline chr21:7-43 & suggestive & RJF & 15.67 & -0.57 & 0.27 & -1.75 \\
\hline chr21:10-44 & suggestive & RJF & 20.2 & -0.11 & 0.47 & -3.31 \\
\hline chr21:4-62 & significant & RJF & 15.42 & -1.69 & 0.7 & -3.41 \\
\hline chr21:7-55 & significant & RJF & 15.49 & -1.45 & 0.61 & -2.97 \\
\hline chr21:3-59 & suggestive & RJF & 20.22 & -1.49 & 0.32 & -1.46 \\
\hline chr21:5-56 & significant & RJF & 17.62 & -0.61 & 0.4 & -2.41 \\
\hline chr21:1-57 & suggestive & RJF & 18.06 & -2.13 & 0.45 & -1.16 \\
\hline chr21:6-69 & suggestive & RJF & 15.54 & -0.77 & 0.26 & -1.22 \\
\hline chr21:3-59 & suggestive & RJF & 20.29 & -1.53 & 0.33 & -1.26 \\
\hline chr21:3-91 & significant & RJF & 16.51 & -2.43 & 0.55 & -1.98 \\
\hline chr21:0-106 & significant & RJF & 15.45 & -1.69 & 0.38 & -0.35 \\
\hline chr21:23-68 & significant & RJF & 18.44 & -3.2 & 0.63 & -2.81 \\
\hline chr21:7-75 & suggestive & RJF & 19.57 & -1.9 & 0.37 & -2.1 \\
\hline chr21:26-91 & significant & RJF & 20.81 & -1.81 & 0.34 & -0.24 \\
\hline chr21:31-87 & significant & RJF & 23.9 & -3.34 & 0.54 & -1.75 \\
\hline chr21:27-75 & significant & RJF & 17.48 & -2.58 & 0.52 & -1.99 \\
\hline chr21:32-74 & suggestive & RJF & 20.15 & -2.19 & 0.42 & -2.33 \\
\hline chr21:36-72 & significant & RJF & 25.15 & -1.77 & 0.29 & -0.22 \\
\hline chr21:39-71 & suggestive & RJF & 21.16 & -1.33 & 0.24 & -1.31 \\
\hline chr21:41-69 & suggestive & RJF & 20.56 & -1.67 & 0.33 & -2.35 \\
\hline chr21:44-66 & suggestive & RJF & 19.67 & -0.03 & 0.23 & 1.79 \\
\hline chr21:38-72 & significant & WL & 12.14 & 1.77 & 0.5 & -0.44 \\
\hline chr21:45-67 & significant & RJF & 16.33 & -1.41 & 0.38 & -2.59 \\
\hline chr21:44-71 & suggestive & RJF & 19.66 & -2.64 & 0.5 & -2.59 \\
\hline chr21:41-70 & suggestive & RJF & 23.61 & -1.53 & 0.28 & -1.77 \\
\hline chr21:38-72 & suggestive & RJF & 21.75 & -1.93 & 0.38 & -2.66 \\
\hline chr21:41-72 & significant & RJF & 27.79 & -2.31 & 0.47 & -1.84 \\
\hline chr21:41-74 & suggestive & RJF & 14.48 & -0.35 & 0.43 & 2.58 \\
\hline chr21:46-83 & suggestive & RJF & 19.35 & -1.29 & 0.24 & -0.83 \\
\hline chr21:30-82 & significant & RJF & 24.37 & -3.11 & 0.68 & -2.93 \\
\hline chr21:38-82 & suggestive & RJF & 19.3 & -1.1 & 0.22 & -1.27 \\
\hline chr21:64-85 & suggestive & RJF & 24.25 & -3.1 & 0.54 & -2.94 \\
\hline chr21:65-83 & suggestive & RJF & 25.13 & -3.76 & 0.79 & -3.85 \\
\hline chr21:65-93 & suggestive & RJF & 23.09 & -0.12 & 0.43 & -0.43 \\
\hline chr21:44-96 & suggestive & RJF & 22.21 & -0.31 & 0.32 & 0.27 \\
\hline chr21:67-88 & suggestive & RJF & 19.32 & -1.76 & 0.54 & -1.47 \\
\hline chr21:67-102 & suggestive & RJF & 22.84 & -0.04 & 0.36 & -0.06 \\
\hline
\end{tabular}




\begin{tabular}{|c|c|c|c|c|c|c|}
\hline chr21:68-95 & suggestive & RJF & 26.17 & -0.23 & 0.04 & -0.24 \\
\hline chr21:74-93 & significant & RJF & 53.26 & -0.88 & 0.08 & -0.9 \\
\hline chr21:74-98 & suggestive & RJF & 24.7 & -0.46 & 0.08 & -0.41 \\
\hline chr21:89-121 & suggestive & RJF & 19.27 & -1.26 & 0.23 & -0.35 \\
\hline chr23:0-9 & suggestive & WL & 18.39 & 4.78 & 0.89 & 3.26 \\
\hline chr23:0-4 & suggestive & RJF & 17.24 & -2.3 & 0.56 & 0.56 \\
\hline chr23:0-4 & suggestive & RJF & 15.74 & -3.55 & 0.9 & 0.79 \\
\hline chr23:0-28 & significant & RJF & 13.1 & -1.53 & 0.42 & -0.01 \\
\hline chr23:3-15 & significant & WL & 16.51 & 1.49 & 0.46 & -0.77 \\
\hline chr23:0-13 & suggestive & WL & 21.35 & 0.37 & 0.67 & -1.04 \\
\hline chr23:7-13 & suggestive & WL & 8.1 & 4.89 & 1.1 & -3.05 \\
\hline chr23:8-13 & suggestive & WL & 17.12 & 7.31 & 1.51 & -4.25 \\
\hline chr23:7-15 & suggestive & WL & 17.82 & 2.66 & 0.55 & -1.09 \\
\hline chr23:7-14 & suggestive & WL & 6.64 & 4.75 & 0.98 & -2.48 \\
\hline chr23:7-15 & significant & WL & 7.34 & 4.07 & 0.65 & -0.19 \\
\hline chr23:6-18 & significant & WL & 14.02 & 1.14 & 0.32 & -1.73 \\
\hline chr23:7-15 & suggestive & WL & 6.29 & 3.86 & 0.79 & -1.95 \\
\hline chr23:6-15 & suggestive & WL & 11.75 & 0.35 & 0.16 & -0.97 \\
\hline chr23:6-29 & significant & WL & 23.19 & 2.63 & 0.66 & -1.73 \\
\hline chr23:7-21 & suggestive & WL & 9.76 & 11.51 & 2.18 & -2.98 \\
\hline chr23:8-27 & suggestive & WL & 9.96 & 10.68 & 1.99 & 1.79 \\
\hline chr23:11-35 & suggestive & RJF & 19.71 & -2.02 & 0.45 & -2.89 \\
\hline chr23:10-32 & suggestive & RJF & 19.28 & -0.73 & 0.36 & -2.66 \\
\hline chr23:14-29 & significant & WL & 27.9 & 0.01 & 0.05 & -0.01 \\
\hline chr23:13-31 & suggestive & RJF & 22.95 & -2.92 & 0.58 & -2.97 \\
\hline chr23:13-33 & suggestive & RJF & 19.33 & -1.84 & 0.36 & -1.84 \\
\hline chr23:31-89 & significant & WL & 16.25 & 1.49 & 0.39 & -1.15 \\
\hline chr24:0-12 & significant & WL & 20.44 & 1.28 & 0.23 & 0.04 \\
\hline chr24:0-19 & significant & WL & 17.99 & 1.81 & 0.35 & -0.3 \\
\hline chr24:0-13 & suggestive & WL & 24.14 & 2.34 & 0.42 & -2.25 \\
\hline chr24:0-9 & significant & RJF & 24.11 & -1 & 0.55 & -1.99 \\
\hline chr24:0-39 & suggestive & WL & 23.77 & 2.43 & 0.45 & -1.94 \\
\hline chr24:0-42 & suggestive & RJF & 16.98 & -2.72 & 0.64 & -0.97 \\
\hline chr24:0-19 & suggestive & RJF & 14.13 & -2.88 & 0.81 & -0.45 \\
\hline chr24:0-36 & suggestive & WL & 25.05 & 2.34 & 0.4 & -1.53 \\
\hline chr24:0-9 & suggestive & WL & 25.38 & 1.23 & 0.23 & -1.21 \\
\hline chr24:0-38 & suggestive & WL & 20.35 & 1.64 & 0.38 & -2.25 \\
\hline chr24:0-9 & suggestive & RJF & 24.73 & -0.94 & 0.53 & 0.65 \\
\hline chr24:0-41 & suggestive & WL & 16.48 & 2.05 & 0.42 & -0.09 \\
\hline chr24:0-36 & suggestive & WL & 13.62 & 1.2 & 0.49 & -2.73 \\
\hline chr24:0-36 & suggestive & WL & 25.37 & 0.72 & 0.13 & -0.71 \\
\hline chr24:2-33 & suggestive & WL & 23.69 & 1.45 & 0.47 & -3.49 \\
\hline chr24:0-33 & significant & WL & 21.76 & 0.85 & 0.29 & -1.8 \\
\hline
\end{tabular}




\begin{tabular}{|c|c|c|c|c|c|}
\hline chr24:0-49 & RJF & 8.78 & -2.19 & 0.61 & -0.08 \\
\hline chr24:0-41 & RJF & 8.66 & -1.72 & 0.51 & 0.98 \\
\hline chr24:3-33 & WL & 26.52 & 2.24 & 0.35 & -1.1 \\
\hline chr24:0-42 & WL & 13.88 & 0.83 & 0.34 & -1.97 \\
\hline chr24:9-34 & WL & 23.47 & 0.99 & 0.18 & -0.87 \\
\hline chr24:17-29 suggestive & WL & 8.6 & 1.16 & 0.26 & -2.29 \\
\hline chr24:0-42 significant & WL & 24.91 & 1.24 & 0.23 & -1.33 \\
\hline chr24:0-49 & WL & 20.09 & 0.48 & 0.11 & -0.57 \\
\hline chr25:0-18 & RJF & 31.32 & -0.31 & 0.05 & -0.4 \\
\hline chr25:0-36 & RJF & 15.58 & -1.86 & 0.5 & 0.23 \\
\hline chr25:0-35 significant & RJF & 26.18 & -0.19 & 0.03 & -0.19 \\
\hline chr25:49-151 significant & RJF & 29.1 & -5 & 0.82 & -0.44 \\
\hline chr25:62-135 suggestive & RJF & 17.4 & -0.76 & 0.65 & 4.39 \\
\hline chr25:62-140 significant & RJF & 34.08 & -3.72 & 0.51 & 0.29 \\
\hline chr25:72-129 suggestive & RJF & 14.47 & -0.99 & 0.66 & 4.7 \\
\hline chr25:71-125 significant & RJF & 18.78 & -1.34 & 0.9 & 7.64 \\
\hline chr25:64-132 significant & RJF & 11.57 & -0.97 & 0.74 & 4.02 \\
\hline chr25:58-140 suggestive & RJF & 8.05 & -0.64 & 0.63 & 2.79 \\
\hline chr25:66-134 significant & RJF & 10.17 & -0.29 & 0.39 & 1.92 \\
\hline chr25:66-133 suggestive & RJF & 12.45 & -0.8 & 0.79 & 4.44 \\
\hline chr25:67-128 suggestive & RJF & 14.82 & -0.53 & 0.5 & 3.06 \\
\hline chr25:67-130 suggestive & RJF & 16.67 & -0.34 & 0.38 & 2.49 \\
\hline chr25:64-133 suggestive & RJF & 12.51 & -1.35 & 0.87 & 4.98 \\
\hline chr25:60-136 suggestive & RJF & 10.14 & -0.13 & 0.22 & 1.07 \\
\hline chr25:65-131 suggestive & RJF & 11.21 & -0.7 & 0.52 & 2.78 \\
\hline chr25:52-191 suggestive & RJF & 8.18 & -0.55 & 0.99 & 4.18 \\
\hline chr25:71-126 suggestive & RJF & 19.35 & -0.04 & 0.12 & 0.94 \\
\hline chr25:73-126 significant & RJF & 55.62 & -6.22 & 0.57 & -0.06 \\
\hline chr25:75-123 significant & RJF & 59.66 & -11.63 & 0.97 & 0.29 \\
\hline chr25:73-124 significant & RJF & 48.61 & -10.57 & 1.04 & 2.02 \\
\hline chr25:65-137 significant & RJF & 36.15 & -3.95 & 0.52 & 0.25 \\
\hline chr25:69-131 significant & RJF & 43.36 & -5.04 & 0.57 & 0.47 \\
\hline chr25:72-124 significant & RJF & 52.1 & -13.63 & 1.26 & 2.28 \\
\hline chr25:74-123 significant & RJF & 59.54 & -10.55 & 0.89 & -0.02 \\
\hline chr25:72-127 significant & RJF & 55.29 & -6.8 & 0.62 & 0.08 \\
\hline chr25:61-134 suggestive & WL & 18.24 & 0.21 & 0.2 & 1.41 \\
\hline chr25:60-136 significant & RJF & 9.57 & -0.4 & 0.33 & 1.58 \\
\hline chr25:58-153 suggestive & RJF & 11.56 & -0.59 & 0.78 & 4.23 \\
\hline chr25:58-167 significant & RJF & 12.23 & -2.85 & 0.77 & 0.02 \\
\hline chr25:51-169 significant & RJF & 14.79 & -4.21 & 0.95 & 1.28 \\
\hline chr25:45-168 significant & RJF & 22.88 & -4.25 & 0.85 & -0.79 \\
\hline chr25:52-168 significant & RJF & 14.69 & -4.3 & 0.97 & 1.42 \\
\hline chr25:48-186 significant & RJF & 15.7 & -3.93 & 0.9 & 0.31 \\
\hline
\end{tabular}




\begin{tabular}{|c|c|c|c|c|c|c|}
\hline \multicolumn{2}{|c|}{ chr25:154-19 significant } & WL & 20.92 & 1.22 & 0.38 & -1.27 \\
\hline \multicolumn{2}{|c|}{ chr25:159-19 suggestive } & RJF & 23.59 & -0.76 & 0.17 & -0.05 \\
\hline \multicolumn{2}{|c|}{ chr25:168-19 significant } & RJF & 22.3 & -0.37 & 0.14 & -0.65 \\
\hline \multicolumn{2}{|c|}{ chr25:160-19 suggestive } & RJF & 21.48 & -0.97 & 0.29 & 1.76 \\
\hline \multicolumn{2}{|c|}{ chr25:156-19 suggestive } & RJF & 11.36 & -1.47 & 0.31 & -0.98 \\
\hline chr26:0-14 & suggestive & RJF & 12.69 & -1.04 & 0.25 & -0.02 \\
\hline chr26:0-13 & significant & RJF & 14.79 & -0.81 & 0.18 & -0.23 \\
\hline chr26:0-8 & significant & RJF & 12.46 & -1.51 & 0.41 & 1.16 \\
\hline chr26:0-65 & suggestive & RJF & 11.07 & -0.8 & 0.22 & 0.34 \\
\hline chr26:0-12 & suggestive & RJF & 18.08 & -13.22 & 2.55 & -0.4 \\
\hline chr26:0-13 & significant & RJF & 19.79 & -2.81 & 0.52 & -0.28 \\
\hline chr26:0-13 & suggestive & WL & 23.51 & 1.86 & 0.45 & -2.23 \\
\hline chr26:0-42 & significant & RJF & 12.2 & -3.94 & 1.03 & -2.33 \\
\hline chr26:6-27 & suggestive & RJF & 23.62 & -11.61 & 3.21 & -19.28 \\
\hline chr26:6-30 & significant & RJF & 6.14 & -5.7 & 1.16 & -8.14 \\
\hline chr26:0-39 & suggestive & WL & 12.72 & 0.38 & 0.39 & 2.34 \\
\hline chr26:5-33 & suggestive & WL & 21.39 & 1.16 & 0.62 & 3.99 \\
\hline chr26:0-37 & suggestive & RJF & 20.35 & -14.94 & 3.8 & -15.68 \\
\hline chr26:6-11 & suggestive & RJF & 24.29 & -1.55 & 0.37 & -1.82 \\
\hline chr26:6-17 & suggestive & WL & 10.4 & 0.57 & 0.59 & -4.84 \\
\hline chr26:6-17 & suggestive & RJF & 6.25 & -1.77 & 0.44 & -2.89 \\
\hline chr26:6-15 & significant & RJF & 11.17 & -0.91 & 0.18 & -1.04 \\
\hline chr26:6-18 & suggestive & RJF & 3.85 & -2.32 & 0.68 & -4.98 \\
\hline chr26:8-28 & suggestive & RJF & 12.52 & -3.13 & 0.93 & -5.63 \\
\hline chr26:6-28 & suggestive & WL & 21.03 & 1.42 & 0.73 & 4.49 \\
\hline chr26:13-26 & suggestive & RJF & 23.39 & -0.16 & 0.29 & 2.13 \\
\hline chr26:131-15 & 5 suggestive & RJF & 15.36 & -0.39 & 0.3 & 2.07 \\
\hline chr27:0-9 & significant & RJF & 30.92 & -6.86 & 1.07 & 0.15 \\
\hline chr27:0-7 & significant & RJF & 26.1 & -5.45 & 0.97 & -0.07 \\
\hline chr27:0-7 & significant & RJF & 29.43 & -9.72 & 1.58 & 1.29 \\
\hline $\operatorname{chr} 27: 0-7$ & significant & RJF & 34.81 & -7.96 & 1.18 & 1.1 \\
\hline chr27:0-9 & significant & RJF & 27.23 & -5.56 & 0.93 & -0.18 \\
\hline chr27:0-8 & significant & RJF & 35.89 & -8.01 & 1.1 & -0.74 \\
\hline chr27:0-4 & significant & RJF & 32.72 & -3.97 & 0.55 & -1.26 \\
\hline chr27:0-8 & significant & RJF & 41.69 & -8.07 & 0.99 & 0.44 \\
\hline chr27:0-4 & significant & RJF & 43.42 & -7.33 & 0.87 & 0.53 \\
\hline chr27:0-10 & significant & RJF & 19.01 & -5.85 & 1.33 & 0.62 \\
\hline chr27:0-5 & significant & RJF & 28.79 & -3.76 & 0.58 & -1.15 \\
\hline chr27:0-4 & significant & RJF & 42.91 & -5.7 & 0.7 & -0.06 \\
\hline chr27:0-4 & significant & RJF & 41.83 & -5.53 & 0.69 & -0.14 \\
\hline chr27:0-5 & significant & RJF & 38.02 & -9.42 & 1.2 & -1.98 \\
\hline chr27:0-11 & significant & RJF & 20.72 & -6.45 & 1.17 & -3.24 \\
\hline chr27:0-8 & significant & RJF & 23.86 & -7.96 & 1.37 & -2.69 \\
\hline
\end{tabular}




\begin{tabular}{|c|c|c|c|c|c|c|}
\hline chr27:0-7 & significant & RJF & 24.94 & -4.68 & 0.85 & -0.33 \\
\hline chr27:0-8 & significant & RJF & 25.9 & -7.83 & 1.28 & -2.51 \\
\hline chr27:0-7 & significant & RJF & 17.9 & -0.83 & 0.19 & 0.05 \\
\hline chr27:0-11 & significant & RJF & 19.39 & -6.49 & 1.29 & -1.54 \\
\hline chr27:0-10 & significant & RJF & 25.46 & -7.22 & 1.19 & -2.05 \\
\hline chr27:0-11 & significant & RJF & 28.12 & -0.09 & 0.1 & 0.06 \\
\hline chr27:0-15 & significant & RJF & 17.15 & -0.27 & 0.06 & -0.08 \\
\hline chr27:0-15 & significant & RJF & 16.12 & -5.71 & 1.44 & 0.25 \\
\hline chr27:0-11 & significant & RJF & 18.2 & -4.39 & 0.94 & -0.73 \\
\hline chr27:0-13 & suggestive & RJF & 14.38 & -5.96 & 1.63 & 1.17 \\
\hline chr27:0-12 & suggestive & RJF & 14.16 & -6.07 & 1.77 & 1.99 \\
\hline chr27:0-12 & suggestive & RJF & 14.8 & -6.65 & 1.67 & -0.54 \\
\hline chr27:0-15 & suggestive & RJF & 15.19 & -4.89 & 1.28 & 0.18 \\
\hline chr27:0-9 & suggestive & RJF & 14.61 & -1.13 & 0.29 & -0.05 \\
\hline chr27:0-13 & significant & WL & 15.59 & 7.49 & 1.84 & 0.78 \\
\hline chr27:0-10 & suggestive & RJF & 15.93 & -7.63 & 1.72 & -2.02 \\
\hline chr27:0-8 & suggestive & RJF & 15.49 & -4.32 & 1.07 & -0.28 \\
\hline chr27:0-14 & suggestive & WL & 12.66 & 5.93 & 1.99 & -2.04 \\
\hline chr27:0-11 & significant & RJF & 16.83 & -5.35 & 1.12 & -2.05 \\
\hline chr27:0-12 & suggestive & RJF & 15.02 & -4.13 & 0.9 & -2.25 \\
\hline chr27:0-13 & significant & RJF & 15.84 & -5.75 & 1.42 & 0.02 \\
\hline chr27:0-10 & significant & RJF & 19.62 & -5.87 & 1.32 & 0.75 \\
\hline chr27:0-10 & significant & RJF & 20.67 & -8.61 & 1.77 & -0.56 \\
\hline chr27:0-9 & significant & RJF & 34.58 & -10.98 & 1.59 & -0.85 \\
\hline chr27:0-10 & significant & RJF & 23.38 & -9.49 & 1.78 & -0.88 \\
\hline chr27:0-10 & significant & RJF & 27.46 & -6.74 & 1.27 & 1.01 \\
\hline chr27:0-9 & significant & RJF & 36.99 & -12.14 & 1.67 & -0.79 \\
\hline chr27:0-11 & significant & RJF & 29.82 & -11.35 & 1.81 & -1.14 \\
\hline chr27:0-8 & significant & RJF & 23.84 & -3.02 & 0.58 & -0.18 \\
\hline chr27:0-9 & suggestive & RJF & 17.99 & -2.1 & 0.58 & 0.49 \\
\hline chr27:0-9 & significant & RJF & 30.73 & -5.96 & 0.93 & 0.43 \\
\hline chr27:0-8 & significant & RJF & 24.32 & -3.35 & 0.63 & -0.26 \\
\hline chr27:0-6 & significant & RJF & 33.36 & -9.75 & 1.37 & -2.22 \\
\hline chr27:0-5 & significant & RJF & 35.46 & -3.27 & 0.48 & 0.15 \\
\hline chr27:0-5 & significant & RJF & 39.46 & -9.61 & 1.19 & -2.17 \\
\hline chr27:0-13 & significant & RJF & 17.98 & -5.39 & 1.07 & -2.35 \\
\hline chr27:0-11 & significant & RJF & 17.67 & -2.37 & 0.51 & -0.45 \\
\hline chr27:0-11 & significant & RJF & 24.11 & -4.93 & 0.87 & -0.82 \\
\hline chr27:0-11 & significant & RJF & 21.4 & -4.42 & 0.9 & -0.23 \\
\hline chr27:0-13 & significant & RJF & 17.33 & -5.5 & 1.11 & -2.85 \\
\hline chr27:0-13 & significant & RJF & 16.16 & -4.97 & 1.08 & -1.8 \\
\hline chr27:0-12 & suggestive & RJF & 15.01 & -4.27 & 0.93 & -2.82 \\
\hline chr27:0-11 & suggestive & RJF & 15.9 & -6.09 & 1.31 & -2.53 \\
\hline
\end{tabular}




\begin{tabular}{|c|c|c|c|c|c|c|}
\hline chr27:0-12 & significant & RJF & 17.96 & -2.48 & 0.53 & -0.46 \\
\hline chr27:0-12 & suggestive & RJF & 14.73 & -5.65 & 1.55 & 0.89 \\
\hline chr27:0-13 & significant & RJF & 16.9 & -3 & 0.68 & -0.36 \\
\hline chr27:0-11 & significant & RJF & 18.46 & -4.99 & 1.2 & 0.8 \\
\hline chr27:0-14 & suggestive & RJF & 16.11 & -4.53 & 1.03 & -0.97 \\
\hline chr27:0-9 & significant & RJF & 23.69 & -1.67 & 0.29 & -1.84 \\
\hline chr27:0-5 & significant & RJF & 30.8 & 0 & 0.01 & 0 \\
\hline chr27:0-7 & significant & RJF & 31.2 & 0 & 0.02 & -0.04 \\
\hline chr27:0-10 & suggestive & RJF & 15.11 & -0.44 & 0.08 & -0.42 \\
\hline chr27:0-12 & significant & RJF & 17.92 & -0.82 & 0.13 & -0.55 \\
\hline chr27:0-11 & suggestive & RJF & 23.91 & -0.72 & 0.57 & -0.72 \\
\hline chr27:0-11 & suggestive & RJF & 28.13 & -0.02 & 0.02 & -0.02 \\
\hline chr27:0-13 & suggestive & RJF & 24.3 & -0.09 & 0.1 & 0.11 \\
\hline chr27:0-13 & significant & RJF & 24.12 & -1.27 & 0.62 & 0.63 \\
\hline chr27:0-18 & significant & RJF & 17.65 & -1.21 & 0.24 & -0.59 \\
\hline chr27:0-21 & suggestive & RJF & 15.57 & -0.79 & 0.17 & -0.41 \\
\hline chr27:0-23 & suggestive & WL & 19.17 & 0.97 & 0.32 & -0.83 \\
\hline chr27:0-26 & significant & WL & 12.36 & 1.69 & 0.58 & -0.67 \\
\hline chr27:0-17 & suggestive & RJF & 24.01 & -0.05 & 0.04 & -0.05 \\
\hline chr27:0-18 & suggestive & WL & 24.55 & 0.01 & 0.01 & -0.01 \\
\hline chr27:0-19 & suggestive & RJF & 18.23 & -1.46 & 0.29 & -1.51 \\
\hline chr28:0-12 & suggestive & WL & 18.16 & 1.52 & 0.29 & -0.87 \\
\hline chr28:15-38 & suggestive & WL & 10.95 & 1.11 & 0.19 & -0.51 \\
\hline chr28:18-38 & suggestive & WL & 23.53 & 0.57 & 0.54 & 3.96 \\
\hline chr28:16-46 & suggestive & WL & 17.94 & 1.05 & 0.2 & -0.45 \\
\hline chr28:45-77 & suggestive & RJF & 19.05 & -0.02 & 0.27 & 0.02 \\
\hline chr28:54-79 & suggestive & RJF & 21.81 & -2.74 & 0.54 & -2.67 \\
\hline chr28:43-81 & suggestive & WL & 20.54 & 0.93 & 0.29 & -1.92 \\
\hline chr28:48-81 & significant & RJF & 22.86 & -0.24 & 0.29 & -0.48 \\
\hline chr28:44-85 & suggestive & WL & 26.73 & 1 & 0.46 & -0.23 \\
\hline chr28:43-84 & suggestive & WL & 18.04 & 0.18 & 0.24 & -0.22 \\
\hline chr28:83-114 & suggestive & WL & 21.57 & 0.33 & 0.46 & -0.07 \\
\hline chr28:85-116 & suggestive & WL & 21.86 & 2.07 & 0.37 & -2.02 \\
\hline chr28:88-116 & suggestive & WL & 24.86 & 2.54 & 0.42 & -1.93 \\
\hline chr28:95-114 & significant & WL & 15.2 & 1.86 & 0.21 & -1.59 \\
\hline chr28:92-119 & suggestive & WL & 23.61 & 2.17 & 0.39 & -2.06 \\
\hline chr28:93-123 & suggestive & WL & 20.58 & 1.16 & 0.2 & -0.92 \\
\hline chr28:122-13 & suggestive & WL & 21.26 & 1.74 & 0.35 & -2.15 \\
\hline
\end{tabular}


ce intervals are provided.

\begin{tabular}{|c|c|c|c|c|c|}
\hline $1.57 \mathrm{NA}$ & NA & NA & NA & rbl449 & X1_406616 \\
\hline $1.43 \mathrm{NA}$ & NA & NA & NA & rbl449 & X1_406616 \\
\hline $1.31 \mathrm{NA}$ & NA & NA & NA & rbl449 & X1_406616 \\
\hline $1.84 \mathrm{NA}$ & NA & NA & NA & rbl449 & X1_406616 \\
\hline $0.8 \mathrm{NA}$ & NA & NA & NA & rbl449 & X1_406616 \\
\hline $0.92 \mathrm{NA}$ & NA & NA & NA & rbl449 & X1_406616 \\
\hline $1.82 \mathrm{NA}$ & NA & NA & NA & rbl449 & X1_406616 \\
\hline $1.42 \mathrm{NA}$ & NA & NA & NA & rbl449 & X1_406616 \\
\hline $1.26 \mathrm{NA}$ & NA & NA & NA & rbl449 & X1_406616 \\
\hline $1.06 \mathrm{NA}$ & NA & NA & NA & rbl449 & X1_406616 \\
\hline $1.67 \mathrm{NA}$ & NA & NA & NA & rbl449 & X1_406616 \\
\hline $1.08 \mathrm{NA}$ & NA & NA & NA & rbl449 & X1_406616 \\
\hline $1.83 \mathrm{NA}$ & NA & NA & NA & rbl449 & X1_406616 \\
\hline $2.13 \mathrm{NA}$ & NA & NA & NA & rbl449 & X1_406616 \\
\hline $2.08 \mathrm{NA}$ & NA & NA & NA & rbl449 & X1_406616 \\
\hline $2.32 \mathrm{NA}$ & NA & NA & NA & rbl449 & X1_406616 \\
\hline $0.16 \mathrm{NA}$ & NA & NA & NA & rbl449 & X1_406616 \\
\hline $0.42 \mathrm{NA}$ & NA & NA & NA & rbl449 & X1_406616 \\
\hline $0.43 \mathrm{NA}$ & NA & NA & NA & rbl449 & X1_406616 \\
\hline $0.91 \mathrm{NA}$ & NA & NA & NA & rbl449 & X1_406616 \\
\hline $0.5 \mathrm{NA}$ & NA & NA & NA & rbl449 & X1_406616 \\
\hline $0.51 \mathrm{NA}$ & NA & NA & NA & rbl449 & X1_406616 \\
\hline $0.56 \mathrm{NA}$ & NA & NA & NA & rbl449 & X1_406616 \\
\hline 0.67 & -1.9 & 0.66 & -1.71 & 0.9 rbl449 & X1_406616 \\
\hline $0.86 \mathrm{NA}$ & NA & NA & NA & X1_366532 & Gg_rs138324 \\
\hline $1.23 \mathrm{NA}$ & NA & NA & NA & X1_366532 & Gg_rs138261 \\
\hline $2.59 \mathrm{NA}$ & NA & NA & NA & X1_366532 & Gg_rs140803 \\
\hline $4.33 \mathrm{NA}$ & NA & NA & NA & X1_366532 & Gg_rs140803 \\
\hline $4.5 \mathrm{NA}$ & NA & NA & NA & X1_366532 & Gg_rs140803 \\
\hline $0.79 \mathrm{NA}$ & NA & NA & NA & X1_366532 & Gg_rs140803 \\
\hline $1.56 \mathrm{NA}$ & NA & NA & NA & X1_366532 & Gg_rs147885 \\
\hline $0.83 \mathrm{NA}$ & NA & NA & NA & X1_366532 & Gg_rs138261 \\
\hline $7.89 \mathrm{NA}$ & NA & NA & NA & X1_366532 & Gg_rs140803 \\
\hline $0.88 \mathrm{NA}$ & NA & NA & NA & X1_366532 & Gg_rs140803 \\
\hline $0.78 \mathrm{NA}$ & NA & NA & NA & X1_366532 & Gg_rs151948 \\
\hline $3.72 \mathrm{NA}$ & NA & NA & NA & X1_366532 & Gg_rs140803 \\
\hline $2.58 \mathrm{NA}$ & NA & NA & NA & X1_366532 & Gg_rs138261 \\
\hline $0.97 \mathrm{NA}$ & NA & NA & NA & X1_366532 & Gg_rs138261 \\
\hline $0.91 \mathrm{NA}$ & NA & NA & NA & X1_366532 & chr1.929557C \\
\hline $0.84 \mathrm{NA}$ & NA & NA & NA & X1_366532 & Gg_rs138261 \\
\hline $0.55 \mathrm{NA}$ & NA & NA & NA & X1_366532 & chr1.929557C \\
\hline
\end{tabular}




\begin{tabular}{|c|c|c|c|c|c|c|}
\hline $0.45 \mathrm{NA}$ & NA & NA & NA & & X1_366532 & Gg_rs138261 \\
\hline 0.71 & 2.04 & 0.58 & 0 & 0.97 & X1_366532 & RBL4976 \\
\hline 0.37 & 1.04 & 0.3 & -0.62 & 0.5 & X1_406616 & Gg_rs151869 \\
\hline 1.1 & 2.54 & 0.9 & -0.3 & 1.51 & X1_366532 & Gg_rs138261 \\
\hline 0.66 NA & NA & NA & NA & & X1_406616 & Gg_rs138261 \\
\hline $0.29 \mathrm{NA}$ & NA & NA & NA & & X1_366532 & chr1.929557C \\
\hline $0.41 \mathrm{NA}$ & NA & NA & NA & & X1_366532 & Gg_rs138261 \\
\hline 0.42 NA & NA & NA & NA & & X1_366532 & Gg_rs138261 \\
\hline $0.64 \mathrm{NA}$ & NA & NA & NA & & X1_366532 & chr1.929557C \\
\hline 0.38 NA & NA & NA & NA & & X1_366532 & Gg_rs138261 \\
\hline 0.76 NA & NA & NA & NA & & rbl449 & hrm_chr1.29l \\
\hline $0.74 \mathrm{NA}$ & NA & NA & NA & & X1_366532 & chr1.929557C \\
\hline $0.43 \mathrm{NA}$ & NA & NA & NA & & X1_406616 & Gg_rs138324 \\
\hline $0.42 \mathrm{NA}$ & NA & NA & NA & & X1_406616 & Gg_rs138261 \\
\hline $0.41 \mathrm{NA}$ & NA & NA & NA & & Gg_rs140803 & 3 Gg_rs147885 \\
\hline 0.37 NA & NA & NA & NA & & X1_406616 & Gg_rs138261 \\
\hline 0.55 & 1.42 & 0.45 & 0.18 & 0.75 & X1_406616 & Gg_rs138261 \\
\hline 0.68 NA & NA & NA & NA & & X1_366532 & Gg_rs147937 \\
\hline $0.51 \mathrm{NA}$ & NA & NA & NA & & X1_406616 & chr1.929557C \\
\hline $0.67 \mathrm{NA}$ & NA & NA & NA & & X1_366532 & Gg_rs151948 \\
\hline $0.29 \mathrm{NA}$ & NA & NA & NA & & X1_366532 & Gg_rs147937 \\
\hline 0.59 NA & NA & NA & NA & & X1_366532 & Gg_rs138324 \\
\hline $0.5 \mathrm{NA}$ & NA & NA & NA & & X1_406616 & chr1.929557C \\
\hline $2.72 \mathrm{NA}$ & NA & NA & NA & & Gg_rs140803 & 3 Gg_rs138324 \\
\hline $0.51 \mathrm{NA}$ & NA & NA & NA & & X1_406616 & chr1.929557C \\
\hline $2.04 \mathrm{NA}$ & NA & NA & NA & & Gg_rs140803 & 3 Gg_rs138261 \\
\hline $0.74 \mathrm{NA}$ & NA & NA & NA & & X1_406616 & Gg_rs138324 \\
\hline $0.69 \mathrm{NA}$ & NA & NA & NA & & X1_406616 & Gg_rs138324 \\
\hline 1 & 1.5 & 0.82 & -5.87 & 1.36 & Gg_rs140803 & 3 Gg_rs138261 \\
\hline $2.14 \mathrm{NA}$ & NA & NA & NA & & Gg_rs140803 & 3 Gg_rs138324 \\
\hline $1.93 \mathrm{NA}$ & NA & NA & NA & & X1_406616 & Gg_rs138283 \\
\hline $1.08 \mathrm{NA}$ & NA & NA & NA & & X1_366532 & chr1.929557C \\
\hline $0.7 \mathrm{NA}$ & NA & NA & NA & & X1_406616 & Gg_rs138324 \\
\hline $0.6 \mathrm{NA}$ & NA & NA & NA & & X1_366532 & Gg_rs138324 \\
\hline $0.5 \mathrm{NA}$ & NA & NA & NA & & X1_406616 & Gg_rs151948 \\
\hline $1.52 \mathrm{NA}$ & NA & NA & NA & & Gg_rs14080 & 3 Gg_rs138261 \\
\hline $0.92 \mathrm{NA}$ & NA & NA & NA & & Gg_rs14080 & 3 Gg_rs138261 \\
\hline $2.37 \mathrm{NA}$ & NA & NA & NA & & Gg_rs14080 & 3 Gg_rs138261 \\
\hline $1.62 \mathrm{NA}$ & NA & NA & NA & & Gg_rs14080 & 3 Gg_rs138261 \\
\hline $2.52 \mathrm{NA}$ & NA & NA & NA & & Gg_rs14080 & 3 Gg_rs138261 \\
\hline $1.4 \mathrm{NA}$ & NA & NA & NA & & Gg_rs14080 & 3 Gg_rs138324 \\
\hline $1.88 \mathrm{NA}$ & NA & NA & NA & & X1_406616 & Gg_rs147981 \\
\hline $0.28 \mathrm{NA}$ & NA & NA & NA & & Gg_rs14080 & 3 Gg_rs147885 \\
\hline
\end{tabular}




\begin{tabular}{|c|c|c|c|c|c|c|c|c|}
\hline $1.79 \mathrm{NA}$ & & NA & & NA & NA & & \multicolumn{2}{|c|}{ Gg_rs140803 Gg_rs138324 } \\
\hline $1.48 \mathrm{NA}$ & & NA & & NA & NA & & \multicolumn{2}{|c|}{ Gg_rs140803 Gg_rs138261 } \\
\hline $1.63 \mathrm{NA}$ & & NA & & NA & NA & & \multicolumn{2}{|c|}{ Gg_rs140803 Gg_rs138261 } \\
\hline $1.63 \mathrm{NA}$ & & NA & & NA & NA & & \multicolumn{2}{|c|}{ Gg_rs140803 Gg_rs147937 } \\
\hline $2.04 \mathrm{NA}$ & & NA & & NA & NA & & X1_406616 & Gg_rs147981 \\
\hline $1.8 \mathrm{NA}$ & & NA & & NA & NA & & \multicolumn{2}{|c|}{ Gg_rs140803 Gg_rs138324 } \\
\hline $1.92 \mathrm{NA}$ & & NA & & NA & NA & & \multicolumn{2}{|c|}{ Gg_rs140803 Gg_rs138324 } \\
\hline $1.26 \mathrm{NA}$ & & NA & & NA & NA & & \multicolumn{2}{|c|}{ Gg_rs140803 Gg_rs138324 } \\
\hline 3.29 & 6.75 & & 2.65 & & -3.1 & 4.47 & \multicolumn{2}{|c|}{ Gg_rs140803 Gg_rs138261 } \\
\hline $2.3 \mathrm{NA}$ & & NA & & NA & NA & & \multicolumn{2}{|c|}{ Gg_rs140803 Gg_rs138261 } \\
\hline $1.32 \mathrm{NA}$ & & NA & & NA & NA & & \multicolumn{2}{|c|}{ Gg_rs140803 Gg_rs138261 } \\
\hline $2.26 \mathrm{NA}$ & & NA & & NA & NA & & \multicolumn{2}{|c|}{ Gg_rs140803 Gg_rs138261 } \\
\hline $2.21 \mathrm{NA}$ & & NA & & NA & NA & & \multicolumn{2}{|c|}{ Gg_rs140803 Gg_rs147937 } \\
\hline $1.41 \mathrm{NA}$ & & NA & & NA & NA & & \multicolumn{2}{|c|}{ Gg_rs140803 Gg_rs138261 } \\
\hline 2.99 & 8.15 & & 2.41 & & -2.38 & 4.06 & \multicolumn{2}{|c|}{ Gg_rs140803 Gg_rs138261 } \\
\hline $1.77 \mathrm{NA}$ & & NA & & NA & NA & & \multicolumn{2}{|c|}{ Gg_rs140803 Gg_rs138324 } \\
\hline $1.89 \mathrm{NA}$ & & NA & & NA & NA & & \multicolumn{2}{|c|}{ Gg_rs140803 Gg_rs138324 } \\
\hline $1.18 \mathrm{NA}$ & & NA & & NA & NA & & \multicolumn{2}{|c|}{ Gg_rs140803 Gg_rs138324 } \\
\hline 0.6 & 0.38 & & 0.48 & & -1.88 & 0.82 & X1_366532 & Gg_rs138324 \\
\hline $1.42 \mathrm{NA}$ & & NA & & NA & NA & & X1_406616 & Gg_rs151948 \\
\hline $1.47 \mathrm{NA}$ & & NA & & NA & NA & & \multicolumn{2}{|c|}{ Gg_rs140803 Gg_rs151948. } \\
\hline $0.95 \mathrm{NA}$ & & NA & & NA & NA & & \multicolumn{2}{|c|}{ Gg_rs140803 Gg_rs138324 } \\
\hline 0.31 & 0.36 & & 0.25 & & -1.98 & 0.42 & \multicolumn{2}{|c|}{ Gg_rs140803 Gg_rs138261 } \\
\hline $1.57 \mathrm{NA}$ & & NA & & NA & NA & & \multicolumn{2}{|c|}{ Gg_rs140803 Gg_rs138261 } \\
\hline $1.31 \mathrm{NA}$ & & NA & & NA & NA & & \multicolumn{2}{|c|}{ Gg_rs140803 Gg_rs138261 } \\
\hline 0.55 & -1.04 & & 0.46 & & -2.18 & 0.75 & X1_406616 & Gg_rs138261 \\
\hline $0.52 \mathrm{NA}$ & & NA & & NA & NA & & X1_406616 & chr1.929557C \\
\hline 0.52 NA & & NA & & NA & NA & & X1_406616 & Gg_rs138261 \\
\hline $1.13 \mathrm{NA}$ & & NA & & NA & NA & & X1_366532 & chr1.929557C \\
\hline $0.31 \mathrm{NA}$ & & NA & & NA & NA & & Gg_rs140803 & 3 Gg_rs138261 \\
\hline $0.79 \mathrm{NA}$ & & NA & & NA & NA & & X1_406616 & Gg_rs138261 \\
\hline $0.61 \mathrm{NA}$ & & NA & & NA & NA & & RBL4976 & Gg_rs138261 \\
\hline $0.83 \mathrm{NA}$ & & NA & & NA & NA & & X1_406616 & Gg_rs151948. \\
\hline $0.56 \mathrm{NA}$ & & NA & & NA & NA & & X1_366532 & chr1.929557C \\
\hline 0.46 NA & & NA & & NA & NA & & Gg_rs140803 & chr1.929557C \\
\hline 1.81 & 5.43 & & 1.53 & & -0.37 & 2.48 & Gg_rs140803 & 3 Gg_rs138261 \\
\hline $1.35 \mathrm{NA}$ & & NA & & NA & NA & & Gg_rs140803 & 3 Gg_rs138324 \\
\hline $0.8 \mathrm{NA}$ & & NA & & NA & NA & & X1_366532 & Gg_rs138324 \\
\hline $2.54 \mathrm{NA}$ & & NA & & NA & NA & & Gg_rs140803 & 3 Gg_rs138324 \\
\hline $0.61 \mathrm{NA}$ & & NA & & NA & NA & & X1_366532 & Gg_rs147937 \\
\hline 0.87 NA & & NA & & NA & NA & & X1_366532 & Gg_rs151948. \\
\hline $0.38 \mathrm{NA}$ & & NA & & NA & NA & & X1_406616 & Gg_rs138324 \\
\hline $2.91 \mathrm{NA}$ & & NA & & NA & NA & & Gg_rs140803 & 3 Gg_rs138324 \\
\hline
\end{tabular}




\begin{tabular}{|c|c|c|c|c|c|c|}
\hline $2.92 \mathrm{NA}$ & NA & NA & NA & & \multicolumn{2}{|c|}{ Gg_rs140803 Gg_rs138324 } \\
\hline $2.11 \mathrm{NA}$ & NA & NA & NA & & \multicolumn{2}{|c|}{ Gg_rs140803 Gg_rs138324 } \\
\hline $0.78 \mathrm{NA}$ & NA & NA & NA & & \multicolumn{2}{|c|}{ Gg_rs140803 Gg_rs138324 } \\
\hline $1.03 \mathrm{NA}$ & NA & NA & NA & & X1_406616 & Gg_rs138324 \\
\hline $0.58 \mathrm{NA}$ & NA & NA & NA & & X1_366532 & Gg_rs138324 \\
\hline $0.53 \mathrm{NA}$ & NA & NA & NA & & X1_366532 & Gg_rs151948 \\
\hline $0.54 \mathrm{NA}$ & NA & NA & NA & & X1_406616 & Gg_rs147937 \\
\hline $0.56 \mathrm{NA}$ & NA & NA & NA & & X1_366532 & Gg_rs138324 \\
\hline $0.82 \mathrm{NA}$ & NA & NA & NA & & X1_406616 & Gg_rs138324 \\
\hline 0.44 NA & NA & NA & NA & & X1_406616 & Gg_rs138324 \\
\hline $0.65 \mathrm{NA}$ & NA & NA & NA & & \multicolumn{2}{|c|}{ Gg_rs140803 Gg_rs138324 } \\
\hline 0.86 NA & NA & NA & NA & & X1_406616 & Gg_rs138324 \\
\hline 0.84 NA & NA & NA & NA & & X1_366532 & Gg_rs138283 \\
\hline $0.66 \mathrm{NA}$ & NA & NA & NA & & \multicolumn{2}{|c|}{ Gg_rs140803 Gg_rs138324 } \\
\hline $0.47 \mathrm{NA}$ & NA & NA & NA & & X1_406616 & Gg_rs138324 \\
\hline $0.61 \mathrm{NA}$ & NA & NA & NA & & X1_406616 & Gg_rs151948 \\
\hline 0.87 NA & NA & NA & NA & & X1_366532 & Gg_rs138324 \\
\hline $0.52 \mathrm{NA}$ & NA & NA & NA & & X1_366532 & Gg_rs147937 \\
\hline $0.85 \mathrm{NA}$ & NA & NA & NA & & X1_406616 & Gg_rs147937 \\
\hline $0.68 \mathrm{NA}$ & NA & NA & NA & & X1_406616 & Gg_rs138324 \\
\hline $0.44 \mathrm{NA}$ & NA & NA & NA & & RBL4976 & Gg_rs138324 \\
\hline $1.19 \mathrm{NA}$ & NA & NA & NA & & X1_366532 & Gg_rs151948 \\
\hline $0.67 \mathrm{NA}$ & NA & NA & NA & & X1_406616 & Gg_rs138324 \\
\hline 0.43 NA & NA & NA & NA & & X1_406616 & Gg_rs151948 \\
\hline $0.56 \mathrm{NA}$ & NA & NA & NA & & X1_366532 & chr1.929557C \\
\hline $0.67 \mathrm{NA}$ & NA & NA & NA & & \multicolumn{2}{|c|}{ Gg_rs140803 Gg_rs138324 } \\
\hline $0.61 \mathrm{NA}$ & NA & NA & NA & & X1_406616 & Gg_rs138324 \\
\hline $0.69 \mathrm{NA}$ & NA & NA & NA & & X1_366532 & Gg_rs138324 \\
\hline $0.61 \mathrm{NA}$ & NA & NA & NA & & \multicolumn{2}{|c|}{ Gg_rs140803 Gg_rs138324 } \\
\hline $0.57 \mathrm{NA}$ & NA & NA & NA & & \multicolumn{2}{|c|}{ Gg_rs140803 Gg_rs151948. } \\
\hline $0.3 \mathrm{NA}$ & NA & NA & NA & & \multicolumn{2}{|c|}{ Gg_rs147885 Gg_rs138324 } \\
\hline $0.61 \mathrm{NA}$ & NA & NA & NA & & \multicolumn{2}{|c|}{ Gg_rs147885 Gg_rs138324 } \\
\hline 0.53 NA & NA & NA & NA & & X1_366532 & Gg_rs151948 \\
\hline $0.62 \mathrm{NA}$ & NA & NA & NA & & X1_366532 & Gg_rs138324 \\
\hline 0.48 NA & NA & NA & NA & & \multicolumn{2}{|c|}{ Gg_rs140803 Gg_rs138324 } \\
\hline $1 \mathrm{NA}$ & NA & NA & NA & & \multicolumn{2}{|c|}{ Gg_rs140803 Gg_rs138324 } \\
\hline $0.5 \mathrm{NA}$ & NA & NA & NA & & X1_406616 & Gg_rs138324 \\
\hline $0.51 \mathrm{NA}$ & NA & NA & NA & & \multicolumn{2}{|c|}{ Gg_rs151869 Gg_rs138324 } \\
\hline 1.35 & 2 & 1.14 & 3.1 & 1.85 & X1_366532 & Gg_rs138324 \\
\hline $0.48 \mathrm{NA}$ & NA & NA & NA & & \multicolumn{2}{|c|}{ Gg_rs147885 Gg_rs138324 } \\
\hline $0.52 \mathrm{NA}$ & NA & NA & NA & & \multicolumn{2}{|c|}{ Gg_rs147885 Gg_rs138324 } \\
\hline $0.86 \mathrm{NA}$ & NA & NA & NA & & \multicolumn{2}{|c|}{ Gg_rs140803 Gg_rs138324 } \\
\hline $0.64 \mathrm{NA}$ & NA & NA & NA & & X1_406616 & Gg_rs138283 \\
\hline
\end{tabular}




\begin{tabular}{|c|c|c|c|c|c|}
\hline $0.36 \mathrm{NA}$ & NA & NA & NA & & Gg_rs151869Gg_rs147937 \\
\hline $0.55 \mathrm{NA}$ & NA & NA & NA & & Gg_rs140803 Gg_rs138324 \\
\hline $1.13 \mathrm{NA}$ & NA & NA & NA & & Gg_rs147885 Gg_rs138324 \\
\hline $1.32 \mathrm{NA}$ & NA & NA & NA & & Gg_rs147885 Gg_rs138324 \\
\hline $3.55 \mathrm{NA}$ & NA & NA & NA & & Gg_rs147885 Gg_rs138324 \\
\hline $2.2 \mathrm{NA}$ & NA & NA & NA & & Gg_rs147885 Gg_rs138324 \\
\hline $2.47 \mathrm{NA}$ & NA & NA & NA & & Gg_rs147885 Gg_rs147937 \\
\hline $1.67 \mathrm{NA}$ & NA & NA & NA & & Gg_rs140803 Gg_rs138324 \\
\hline $3.5 \mathrm{NA}$ & NA & NA & NA & & Gg_rs147885 Gg_rs138324 \\
\hline $1.57 \mathrm{NA}$ & NA & NA & NA & & Gg_rs147885 Gg_rs138324 \\
\hline $3.51 \mathrm{NA}$ & NA & NA & NA & & Gg_rs147885 Gg_rs138324 \\
\hline $1.6 \mathrm{NA}$ & NA & NA & NA & & Gg_rs147885 Gg_rs138324 \\
\hline $0.77 \mathrm{NA}$ & NA & NA & NA & & Gg_rs147885 Gg_rs138324 \\
\hline $1.54 \mathrm{NA}$ & NA & NA & NA & & Gg_rs140803 Gg_rs147937 \\
\hline $1.55 \mathrm{NA}$ & NA & NA & NA & & Gg_rs147885 Gg_rs138324 \\
\hline $1.48 \mathrm{NA}$ & NA & NA & NA & & Gg_rs140803 Gg_rs147937 \\
\hline $1.33 \mathrm{NA}$ & NA & NA & NA & & Gg_rs147885 Gg_rs147937 \\
\hline $1.58 \mathrm{NA}$ & NA & NA & NA & & RBL4976 Gg_rs138324 \\
\hline $3 \mathrm{NA}$ & NA & NA & NA & & Gg_rs147885 Gg_rs138324 \\
\hline $2.38 \mathrm{NA}$ & NA & NA & NA & & X1_366532 Gg_rs138324 \\
\hline $0.69 \mathrm{NA}$ & NA & NA & NA & & Gg_rs140803 Gg_rs138324 \\
\hline $0.61 \mathrm{NA}$ & NA & NA & NA & & Gg_rs147885 Gg_rs138324 \\
\hline $1.74 \mathrm{NA}$ & NA & NA & NA & & Gg_rs140803 Gg_rs138324 \\
\hline $1.78 \mathrm{NA}$ & NA & NA & NA & & Gg_rs140803 Gg_rs147937 \\
\hline $0.96 \mathrm{NA}$ & NA & NA & NA & & Gg_rs140803 Gg_rs147937 \\
\hline $1.31 \mathrm{NA}$ & NA & NA & NA & & Gg_rs147885 Gg_rs147937 \\
\hline $2.71 \mathrm{NA}$ & NA & NA & NA & & Gg_rs140803 Gg_rs138324 \\
\hline $2.75 \mathrm{NA}$ & NA & NA & NA & & Gg_rs140803 Gg_rs138324 \\
\hline $2.8 \mathrm{NA}$ & NA & NA & NA & & Gg_rs140803 Gg_rs138324 \\
\hline $2.75 \mathrm{NA}$ & NA & NA & NA & & Gg_rs140803 Gg_rs138324 \\
\hline $0.75 \mathrm{NA}$ & NA & NA & NA & & Gg_rs147885 Gg_rs138324 \\
\hline $1.52 \mathrm{NA}$ & NA & NA & NA & & Gg_rs140803 Gg_rs147937 \\
\hline $1.65 \mathrm{NA}$ & NA & NA & NA & & Gg_rs147885 Gg_rs147937 \\
\hline $1.14 \mathrm{NA}$ & NA & NA & NA & & Gg_rs147885 Gg_rs147937 \\
\hline $0.53 \mathrm{NA}$ & NA & NA & NA & & Gg_rs147885 Gg_rs147937 \\
\hline 1.1 & 0.09 & 0.92 & 3.32 & 1.49 & Gg_rs147885 Gg_rs151948. \\
\hline $0.85 \mathrm{NA}$ & NA & NA & NA & & X1_406616 Gg_rs138283 \\
\hline $1.77 \mathrm{NA}$ & NA & NA & NA & & Gg_rs140803 Gg_rs147937 \\
\hline 0.56 NA & NA & NA & NA & & Gg_rs147885 Gg_rs147937 \\
\hline $0.47 \mathrm{NA}$ & NA & NA & NA & & Gg_rs147885 Gg_rs147937 \\
\hline $1.61 \mathrm{NA}$ & NA & NA & NA & & Gg_rs147885 Gg_rs147937 \\
\hline $1.3 \mathrm{NA}$ & NA & NA & NA & & Gg_rs140803 Gg_rs147937 \\
\hline $2.25 \mathrm{NA}$ & NA & NA & NA & & Gg_rs140803 Gg_rs147937 \\
\hline
\end{tabular}




\begin{tabular}{|c|c|c|c|c|c|c|c|}
\hline $1.62 \mathrm{NA}$ & NA & & NA & & NA & & Gg_rs147885 Gg_rs147937 \\
\hline 0.82 NA & NA & & NA & & NA & & Gg_rs140803 Gg_rs147937 \\
\hline 1.02 & 2.66 & 0.81 & & 2.5 & & 1.36 & X1_1130108EGg_rs151948. \\
\hline $0.32 \mathrm{NA}$ & NA & & NA & & NA & & X1_1130108EGg_rs151948. \\
\hline $2.11 \mathrm{NA}$ & NA & & NA & & NA & & Gg_rs140803 Gg_rs151948. \\
\hline $1.15 \mathrm{NA}$ & NA & & NA & & NA & & Gg_rs140803 Gg_rs147937 \\
\hline $0.48 \mathrm{NA}$ & NA & & NA & & NA & & Gg_rs140803 Gg_rs147937 \\
\hline $0.32 \mathrm{NA}$ & NA & & NA & & NA & & Gg_rs147885 Gg_rs147937 \\
\hline $0.41 \mathrm{NA}$ & NA & & NA & & NA & & Gg_rs140803 Gg_rs151948. \\
\hline $0.58 \mathrm{NA}$ & NA & & NA & & NA & & Gg_rs140803 Gg_rs151948. \\
\hline $1.12 \mathrm{NA}$ & NA & & NA & & NA & & Gg_rs147885 Gg_rs147937 \\
\hline 0.69 NA & NA & & NA & & NA & & Gg_rs147885 Gg_rs151948. \\
\hline $0.81 \mathrm{NA}$ & NA & & NA & & NA & & X1_406616 Gg_rs151948. \\
\hline $1.15 \mathrm{NA}$ & NA & & NA & & NA & & Gg_rs140803 Gg_rs147937 \\
\hline $0.33 \mathrm{NA}$ & NA & & NA & & NA & & X1_1130108EGg_rs147937 \\
\hline 0.73 & 0.54 & 0.58 & & 1.88 & & 0.98 & X1_1130108EGg_rs147937 \\
\hline $1.63 \mathrm{NA}$ & NA & & NA & & NA & & Gg_rs140803 Gg_rs147937 \\
\hline $0.3 \mathrm{NA}$ & NA & & NA & & NA & & X1_1130108EGg_rs147937 \\
\hline 0.88 NA & NA & & NA & & NA & & X1_366532 Gg_rs151948. \\
\hline $0.57 \mathrm{NA}$ & NA & & NA & & NA & & X1_1130108EGg_rs151948. \\
\hline $0.34 \mathrm{NA}$ & NA & & NA & & NA & & X1_1130108EGg_rs147937 \\
\hline $0.75 \mathrm{NA}$ & NA & & NA & & NA & & X1_1130108EGg_rs147937 \\
\hline 1.37 & 3.07 & 1.08 & & -1.75 & & 1.82 & X1_1130108EGg_rs147937 \\
\hline 1.05 & 1.69 & 0.83 & & -0.49 & & 1.4 & X1_1130108EGg_rs147937 \\
\hline $0.54 \mathrm{NA}$ & NA & & NA & & NA & & X1_1130108EGg_rs147937 \\
\hline 0.3 & 0.45 & 0.23 & & 0.8 & & 0.39 & X1_1130108EGg_rs147937 \\
\hline $0.46 \mathrm{NA}$ & NA & & NA & & NA & & X1_1130108EGg_rs151948. \\
\hline 0.99 & 2.23 & 0.78 & & 6.02 & & 1.31 & X1_1130108EGg_rs147937 \\
\hline $0.84 \mathrm{NA}$ & NA & & NA & & NA & & X1_1130108EGg_rs147937 \\
\hline 0.47 & 0.57 & 0.37 & & 2.51 & & 0.62 & X1_1130108EGg_rs147937 \\
\hline 0.74 & 0.67 & 0.59 & & -3.81 & & 0.98 & X1_1130108EGg_rs147937 \\
\hline 0.98 & 0.68 & 0.78 & & -6.29 & & 1.31 & X1_1130108EGg_rs147937 \\
\hline $0.46 \mathrm{NA}$ & NA & & NA & & NA & & X1_1130108EGg_rs147937 \\
\hline $0.26 \mathrm{NA}$ & NA & & NA & & NA & & X1_1130108EGg_rs147937 \\
\hline $0.35 \mathrm{NA}$ & NA & & NA & & NA & & X1_1130108EGg_rs151948. \\
\hline 0.54 NA & NA & & NA & & NA & & X1_1130108EGg_rs147981 \\
\hline 0.64 NA & NA & & NA & & NA & & X1_1130108EGg_rs147937 \\
\hline $0.31 \mathrm{NA}$ & NA & & NA & & NA & & X1_1130108EGg_rs147937 \\
\hline 1.69 & 1.49 & 1.34 & & -4.81 & & 2.25 & X1_1130108EGg_rs147937 \\
\hline 0.17 & -0.3 & 0.14 & & -0.97 & & 0.23 & X1_1130108EGg_rs147937 \\
\hline 0.69 NA & NA & & NA & & NA & & X1_1130108EGg_rs151948. \\
\hline $0.5 \mathrm{NA}$ & NA & & NA & & NA & & X1_1130108EGg_rs137990. \\
\hline $0.85 \mathrm{NA}$ & NA & & NA & & NA & & X1_1130108EGg_rs151948. \\
\hline
\end{tabular}




\begin{tabular}{|c|c|c|c|c|c|}
\hline 0.36 & 0.21 & 0.28 & -2.17 & 0.48 & X1_1130108EGg_rs147937 \\
\hline 0.89 & -0.15 & 0.7 & -3.33 & 1.18 & X1_1130108EGg_rs147937 \\
\hline $0.63 \mathrm{NA}$ & NA & NA & NA & & X1_1130108EGg_rs138283 \\
\hline 0.78 NA & NA & NA & NA & & Gg_rs140803 Gg_rs138283 \\
\hline $0.54 \mathrm{NA}$ & NA & NA & NA & & X1_1130108EGg_rs151948. \\
\hline $0.72 \mathrm{NA}$ & NA & NA & NA & & X1_1130108EGg_rs151948. \\
\hline $0.43 \mathrm{NA}$ & NA & NA & NA & & X1_1130108EGg_rs147981 \\
\hline $0.63 \mathrm{NA}$ & NA & NA & NA & & X1_1130108EGg_rs151948. \\
\hline 1.54 & 2.57 & 1.22 & 1.6 & 2.05 & X1_1130108EGg_rs151948. \\
\hline 0.94 NA & NA & NA & NA & & X1_1130108EGg_rs151948. \\
\hline $0.67 \mathrm{NA}$ & NA & NA & NA & & X1_1130108EGg_rs151948. \\
\hline $0.63 \mathrm{NA}$ & NA & NA & NA & & X1_1130108EGg_rs138283 \\
\hline $0.41 \mathrm{NA}$ & NA & NA & NA & & X1_1130108EGg_rs151948. \\
\hline $0.71 \mathrm{NA}$ & NA & NA & NA & & X1_1130108EGg_rs138283 \\
\hline 0.98 & -0.2 & 0.79 & -2.4 & 1.31 & X1_1130108EGg_rs151948. \\
\hline 0.65 NA & NA & NA & NA & & Gg_rs138324Gg_rs151948. \\
\hline $0.53 \mathrm{NA}$ & NA & NA & NA & & Gg_rs138324Gg_rs151948. \\
\hline 1.56 & 4.78 & 1.26 & 0.22 & 2.1 & Gg_rs138324Gg_rs151948. \\
\hline $0.61 \mathrm{NA}$ & NA & NA & NA & & X1_1130108EGg_rs151948. \\
\hline $0.59 \mathrm{NA}$ & NA & NA & NA & & X1_1130108EGg_rs151948. \\
\hline 0.79 NA & NA & NA & NA & & Gg_rs138324 Gg_rs151948. \\
\hline $1.08 \mathrm{NA}$ & NA & NA & NA & & Gg_rs138324 Gg_rs151948. \\
\hline 0.86 NA & NA & NA & NA & & Gg_rs138324Gg_rs151948. \\
\hline 0.62 NA & NA & NA & NA & & Gg_rs138324 Gg_rs151948. \\
\hline 1.48 & 3.33 & 1.19 & -0.03 & 1.98 & Gg_rs138324Gg_rs151948. \\
\hline 1.14 & 2.49 & 0.92 & -0.04 & 1.53 & Gg_rs138324 Gg_rs151948. \\
\hline 0.63 NA & NA & NA & NA & & Gg_rs138324 Gg_rs151948. \\
\hline $0.54 \mathrm{NA}$ & NA & NA & NA & & Gg_rs138324 Gg_rs151948. \\
\hline $1.04 \mathrm{NA}$ & NA & NA & NA & & Gg_rs138324 Gg_rs151948. \\
\hline $0.6 \mathrm{NA}$ & NA & NA & NA & & Gg_rs138324Gg_rs151948. \\
\hline 1.68 & 0.83 & 1.35 & -3.99 & 2.25 & Gg_rs138324 Gg_rs151948. \\
\hline 0.83 & -0.04 & 0.67 & -2.39 & 1.11 & Gg_rs138324 Gg_rs151948. \\
\hline $0.5 \mathrm{NA}$ & NA & NA & NA & & Gg_rs138324Gg_rs151948. \\
\hline 0.84 & 0.28 & 0.68 & -2.23 & 1.13 & Gg_rs138324 Gg_rs151948. \\
\hline $0.41 \mathrm{NA}$ & NA & NA & NA & & Gg_rs138324Gg_rs151948. \\
\hline 0.74 & 1.43 & 0.59 & -0.41 & 0.99 & Gg_rs138324Gg_rs151948. \\
\hline $0.54 \mathrm{NA}$ & NA & NA & NA & & Gg_rs138324 Gg_rs151948. \\
\hline $0.79 \mathrm{NA}$ & NA & NA & NA & & Gg_rs138324Gg_rs151948. \\
\hline $1.63 \mathrm{NA}$ & NA & NA & NA & & Gg_rs138324 Gg_rs151948. \\
\hline 0.64 NA & NA & NA & NA & & Gg_rs138324 Gg_rs151948. \\
\hline 0.63 NA & NA & NA & NA & & Gg_rs138324 Gg_rs151948. \\
\hline $0.62 \mathrm{NA}$ & NA & NA & NA & & Gg_rs138324 Gg_rs151948. \\
\hline $1.02 \mathrm{NA}$ & NA & NA & NA & & Gg_rs138324 Gg_rs151948 \\
\hline
\end{tabular}




\begin{tabular}{|c|c|c|c|c|c|}
\hline $0.72 \mathrm{NA}$ & NA & NA & NA & & Gg_rs138324 Gg_rs151948. \\
\hline $0.91 \mathrm{NA}$ & NA & NA & NA & & Gg_rs138324 Gg_rs151948. \\
\hline $0.35 \mathrm{NA}$ & NA & NA & NA & & Gg_rs138324 Gg_rs138283 \\
\hline $0.84 \mathrm{NA}$ & NA & NA & NA & & Gg_rs138324 Gg_rs151948. \\
\hline $0.67 \mathrm{NA}$ & NA & NA & NA & & Gg_rs138324Gg_rs151948. \\
\hline $0.5 \mathrm{NA}$ & NA & NA & NA & & Gg_rs138324Gg_rs151948. \\
\hline $0.59 \mathrm{NA}$ & NA & NA & NA & & Gg_rs138324 Gg_rs151948. \\
\hline $0.5 \mathrm{NA}$ & NA & NA & NA & & Gg_rs138324 Gg_rs151948. \\
\hline $0.59 \mathrm{NA}$ & NA & NA & NA & & Gg_rs138324 Gg_rs151948. \\
\hline $0.72 \mathrm{NA}$ & NA & NA & NA & & Gg_rs138324 Gg_rs151948. \\
\hline $0.64 \mathrm{NA}$ & NA & NA & NA & & Gg_rs138324 Gg_rs151948. \\
\hline $2.74 \mathrm{NA}$ & NA & NA & NA & & Gg_rs138324 Gg_rs151948. \\
\hline $0.5 \mathrm{NA}$ & NA & NA & NA & & Gg_rs138324 Gg_rs138283 \\
\hline $0.56 \mathrm{NA}$ & NA & NA & NA & & X1_1130108EGg_rs151948. \\
\hline $0.48 \mathrm{NA}$ & NA & NA & NA & & X1_1130108EGg_rs151948. \\
\hline $0.75 \mathrm{NA}$ & NA & NA & NA & & Gg_rs138324 Gg_rs151948. \\
\hline $0.73 \mathrm{NA}$ & NA & NA & NA & & Gg_rs138324 Gg_rs138283 \\
\hline $0.67 \mathrm{NA}$ & NA & NA & NA & & Gg_rs138324 Gg_rs138283 \\
\hline $0.52 \mathrm{NA}$ & NA & NA & NA & & Gg_rs138324 Gg_rs151948. \\
\hline $0.62 \mathrm{NA}$ & NA & NA & NA & & Gg_rs138324 Gg_rs138283 \\
\hline $0.61 \mathrm{NA}$ & NA & NA & NA & & Gg_rs138324 Gg_rs138283 \\
\hline $0.55 \mathrm{NA}$ & NA & NA & NA & & X1_1130108EGg_rs138283 \\
\hline $0.34 \mathrm{NA}$ & NA & NA & NA & & Gg_rs138324 Gg_rs151948. \\
\hline $0.42 \mathrm{NA}$ & NA & NA & NA & & Gg_rs138324 Gg_rs151948. \\
\hline $0.81 \mathrm{NA}$ & NA & NA & NA & & Gg_rs138324 Gg_rs151948. \\
\hline $0.89 \mathrm{NA}$ & NA & NA & NA & & Gg_rs138324 Gg_rs151948. \\
\hline $0.56 \mathrm{NA}$ & NA & NA & NA & & Gg_rs138324 Gg_rs151948. \\
\hline $0.55 \mathrm{NA}$ & NA & NA & NA & & Gg_rs138324 Gg_rs151948. \\
\hline $0.49 \mathrm{NA}$ & NA & NA & NA & & Gg_rs138324 Gg_rs151948. \\
\hline $0.94 \mathrm{NA}$ & NA & NA & NA & & Gg_rs138324Gg_rs151948. \\
\hline 0.94 & -0.4 & 0.76 & -3 & 1.26 & Gg_rs138324 Gg_rs151948. \\
\hline 0.99 & 3.45 & 0.8 & 0.16 & 1.32 & X1_11301086Gg_rs151948. \\
\hline 1 & 2.08 & 0.81 & -0.93 & 1.35 & Gg_rs138324Gg_rs151948. \\
\hline $0.6 \mathrm{NA}$ & NA & NA & NA & & Gg_rs138324Gg_rs151948 \\
\hline 0.84 & 0.13 & 0.68 & -3.4 & 1.13 & Gg_rs138324Gg_rs151948 \\
\hline 1.15 & 1.94 & 0.93 & -3.16 & 1.54 & Gg_rs138324Gg_rs151948 \\
\hline 0.72 & 1.06 & 0.58 & -1.06 & 0.96 & Gg_rs138324 Gg_rs151948 \\
\hline 1.27 & 3.1 & 1.03 & -1.31 & 1.71 & Gg_rs138324Gg_rs151948 \\
\hline 0.97 & 1.72 & 0.79 & -2.14 & 1.31 & Gg_rs138324Gg_rs151948 \\
\hline $0.45 \mathrm{NA}$ & NA & NA & NA & & Gg_rs138324Gg_rs151948 \\
\hline $0.56 \mathrm{NA}$ & NA & NA & NA & & Gg_rs138324 Gg_rs151948 \\
\hline $0.82 \mathrm{NA}$ & NA & NA & NA & & Gg_rs138324 Gg_rs151948 \\
\hline $0.53 \mathrm{NA}$ & NA & NA & NA & & Gg_rs138324 Gg_rs151948 \\
\hline
\end{tabular}




\begin{tabular}{|c|c|c|c|c|c|}
\hline $0.62 \mathrm{NA}$ & NA & NA & NA & & Gg_rs138324Gg_rs151948 \\
\hline $0.55 \mathrm{NA}$ & NA & NA & NA & & Gg_rs138324 Gg_rs151948 \\
\hline 0.59 NA & NA & NA & NA & & Gg_rs138324 Gg_rs151948 \\
\hline $0.95 \mathrm{NA}$ & NA & NA & NA & & Gg_rs138324 Gg_rs151948 \\
\hline $0.55 \mathrm{NA}$ & NA & NA & NA & & Gg_rs138324 Gg_rs151948 \\
\hline $0.58 \mathrm{NA}$ & NA & NA & NA & & Gg_rs138324 Gg_rs151948 \\
\hline $0.5 \mathrm{NA}$ & NA & NA & NA & & Gg_rs138324 Gg_rs151948 \\
\hline $0.38 \mathrm{NA}$ & NA & NA & NA & & Gg_rs138324Gg_rs151948 \\
\hline $0.7 \mathrm{NA}$ & NA & NA & NA & & Gg_rs138324 Gg_rs151948 \\
\hline $0.74 \mathrm{NA}$ & NA & NA & NA & & Gg_rs138324Gg_rs151948 \\
\hline $0.92 \mathrm{NA}$ & NA & NA & NA & & Gg_rs138324 Gg_rs151948 \\
\hline $0.6 \mathrm{NA}$ & NA & NA & NA & & Gg_rs138324Gg_rs151948 \\
\hline $0.44 \mathrm{NA}$ & NA & NA & NA & & Gg_rs138324Gg_rs151948 \\
\hline $0.57 \mathrm{NA}$ & NA & NA & NA & & Gg_rs138324 Gg_rs151948 \\
\hline $0.75 \mathrm{NA}$ & NA & NA & NA & & Gg_rs138324 Gg_rs151948 \\
\hline $0.45 \mathrm{NA}$ & NA & NA & NA & & Gg_rs138324Gg_rs151948 \\
\hline $0.51 \mathrm{NA}$ & NA & NA & NA & & Gg_rs138324 Gg_rs151948 \\
\hline $0.98 \mathrm{NA}$ & NA & NA & NA & & Gg_rs138324 Gg_rs151948 \\
\hline $0.51 \mathrm{NA}$ & NA & NA & NA & & Gg_rs138324 Gg_rs151948 \\
\hline $0.74 \mathrm{NA}$ & NA & NA & NA & & Gg_rs138324 Gg_rs151948 \\
\hline 0.49 NA & NA & NA & NA & & Gg_rs138324 Gg_rs151948 \\
\hline $1.04 \mathrm{NA}$ & NA & NA & NA & & Gg_rs138324Gg_rs151948 \\
\hline $0.67 \mathrm{NA}$ & NA & NA & NA & & Gg_rs138324 Gg_rs151948 \\
\hline $0.91 \mathrm{NA}$ & NA & NA & NA & & Gg_rs138324 Gg_rs151948 \\
\hline $0.47 \mathrm{NA}$ & NA & NA & NA & & Gg_rs138324 Gg_rs151948 \\
\hline $0.57 \mathrm{NA}$ & NA & NA & NA & & Gg_rs138324 Gg_rs151948 \\
\hline 1.11 & 1.28 & 0.9 & -2.76 & 1.49 & Gg_rs138324 Gg_rs151948 \\
\hline $0.91 \mathrm{NA}$ & NA & NA & NA & & Gg_rs138324 Gg_rs151948 \\
\hline $0.66 \mathrm{NA}$ & NA & NA & NA & & Gg_rs138324Gg_rs151948 \\
\hline $0.71 \mathrm{NA}$ & NA & NA & NA & & Gg_rs138324Gg_rs151948 \\
\hline $0.78 \mathrm{NA}$ & NA & NA & NA & & Gg_rs138324 Gg_rs151948 \\
\hline $0.89 \mathrm{NA}$ & NA & NA & NA & & Gg_rs138324Gg_rs151948 \\
\hline 1.39 & 2.85 & 1.12 & -0.96 & 1.86 & Gg_rs138324Gg_rs138283 \\
\hline $0.76 \mathrm{NA}$ & NA & NA & NA & & X1_1130108EGg_rs138283 \\
\hline $0.78 \mathrm{NA}$ & NA & NA & NA & & Gg_rs138324 Gg_rs138283 \\
\hline $0.38 \mathrm{NA}$ & NA & NA & NA & & Gg_rs138324 Gg_rs151948 \\
\hline $0.82 \mathrm{NA}$ & NA & NA & NA & & Gg_rs138324 Gg_rs138283 \\
\hline $0.53 \mathrm{NA}$ & NA & NA & NA & & Gg_rs138324 Gg_rs151948 \\
\hline $0.66 \mathrm{NA}$ & NA & NA & NA & & Gg_rs138324Gg_rs151948 \\
\hline $0.81 \mathrm{NA}$ & NA & NA & NA & & Gg_rs138324 Gg_rs151948 \\
\hline $0.7 \mathrm{NA}$ & NA & NA & NA & & Gg_rs138324 Gg_rs151948 \\
\hline $0.72 \mathrm{NA}$ & NA & NA & NA & & Gg_rs138324 Gg_rs138283 \\
\hline $0.7 \mathrm{NA}$ & NA & NA & NA & & Gg_rs138324 Gg_rs138283 \\
\hline
\end{tabular}




\begin{tabular}{|c|c|c|c|c|c|}
\hline $0.79 \mathrm{NA}$ & NA & NA & NA & & Gg_rs138324Gg_rs138283 \\
\hline $0.76 \mathrm{NA}$ & NA & NA & NA & & Gg_rs138324Gg_rs138283 \\
\hline $0.83 \mathrm{NA}$ & NA & NA & NA & & Gg_rs138324Gg_rs138283 \\
\hline $0.9 \mathrm{NA}$ & NA & NA & NA & & Gg_rs138324 Gg_rs138283 \\
\hline $0.47 \mathrm{NA}$ & NA & NA & NA & & Gg_rs138324 Gg_rs151948. \\
\hline $0.8 \mathrm{NA}$ & NA & NA & NA & & Gg_rs138324 Gg_rs151948. \\
\hline 1.38 & 2.01 & 1.11 & -0.27 & 1.85 & X1_1130108EGg_rs138283 \\
\hline $0.45 \mathrm{NA}$ & NA & NA & NA & & Gg_rs138324Gg_rs138283 \\
\hline $0.59 \mathrm{NA}$ & NA & NA & NA & & Gg_rs138324Gg_rs138283 \\
\hline $0.5 \mathrm{NA}$ & NA & NA & NA & & Gg_rs138324Gg_rs138283 \\
\hline 1.26 & 2.51 & 1.02 & -0.56 & 1.69 & X1_1130108EGg_rs138283 \\
\hline $0.32 \mathrm{NA}$ & NA & NA & NA & & X1_1130108EGg_rs147981 \\
\hline $1.08 \mathrm{NA}$ & NA & NA & NA & & X1_1130108EGg_rs138283 \\
\hline $0.67 \mathrm{NA}$ & NA & NA & NA & & X1_1130108EGg_rs138283 \\
\hline $0.64 \mathrm{NA}$ & NA & NA & NA & & Gg_rs138324Gg_rs138283 \\
\hline $0.47 \mathrm{NA}$ & NA & NA & NA & & Gg_rs138324Gg_rs138283 \\
\hline 0.97 & 0.67 & 0.84 & -1.23 & 1.3 & Gg_rs138324Gg_rs138283 \\
\hline 0.91 & 3.13 & 0.79 & -0.05 & 1.21 & Gg_rs138324Gg_rs138283 \\
\hline $0.82 \mathrm{NA}$ & NA & NA & NA & & Gg_rs138324Gg_rs138283 \\
\hline 0.54 & 0.79 & 0.47 & -1.18 & 0.72 & Gg_rs138324Gg_rs138283 \\
\hline $0.65 \mathrm{NA}$ & NA & NA & NA & & Gg_rs147937 Gg_rs138283 \\
\hline $1.08 \mathrm{NA}$ & NA & NA & NA & & Gg_rs138324Gg_rs138283 \\
\hline $0.72 \mathrm{NA}$ & NA & NA & NA & & Gg_rs147937 Gg_rs138283 \\
\hline $0.38 \mathrm{NA}$ & NA & NA & NA & & Gg_rs138324 Gg_rs138283 \\
\hline $0.34 \mathrm{NA}$ & NA & NA & NA & & Gg_rs147937 Gg_rs138283 \\
\hline $0.3 \mathrm{NA}$ & NA & NA & NA & & Gg_rs147937 Gg_rs138283 \\
\hline $0.85 \mathrm{NA}$ & NA & NA & NA & & Gg_rs138324Gg_rs137990. \\
\hline $0.56 \mathrm{NA}$ & NA & NA & NA & & Gg_rs138324Gg_rs138283 \\
\hline $0.57 \mathrm{NA}$ & NA & NA & NA & & Gg_rs138324Gg_rs138283 \\
\hline $0.84 \mathrm{NA}$ & NA & NA & NA & & Gg_rs138324 Gg_rs147981 \\
\hline $0.47 \mathrm{NA}$ & NA & NA & NA & & Gg_rs138324X1_2401256€ \\
\hline $0.39 \mathrm{NA}$ & NA & NA & NA & & Gg_rs138324Gg_rs147981 \\
\hline $0.18 \mathrm{NA}$ & NA & NA & NA & & Gg_rs151948 Gg_rs147981 \\
\hline $0.1 \mathrm{NA}$ & NA & NA & NA & & Gg_rs151948 Gg_rs137990. \\
\hline $0.68 \mathrm{NA}$ & NA & NA & NA & & Gg_rs151948 X1_2401256€ \\
\hline $0.61 \mathrm{NA}$ & NA & NA & NA & & Gg_rs151948 X1_2401256€ \\
\hline $0.47 \mathrm{NA}$ & NA & NA & NA & & Gg_rs151948 Gg_rs147981 \\
\hline $0.77 \mathrm{NA}$ & NA & NA & NA & & Gg_rs151948 Gg_rs147981 \\
\hline $0.36 \mathrm{NA}$ & NA & NA & NA & & Gg_rs151948Gg_rs147981 \\
\hline $0.44 \mathrm{NA}$ & NA & NA & NA & & Gg_rs151948X1_2401256€ \\
\hline $0.78 \mathrm{NA}$ & NA & NA & NA & & Gg_rs151948X1_2401256€ \\
\hline $0.5 \mathrm{NA}$ & NA & NA & NA & & Gg_rs151948 Gg_rs147998. \\
\hline $0.47 \mathrm{NA}$ & NA & NA & NA & & Gg_rs138283X1_2401256€ \\
\hline
\end{tabular}




\begin{tabular}{|c|c|c|c|c|c|}
\hline $0.4 \mathrm{NA}$ & NA & NA & NA & & Gg_rs137990 X1_2401256€ \\
\hline $0.66 \mathrm{NA}$ & NA & NA & NA & & Gg_rs137990 X1_2401256€ \\
\hline 0.71 & 1.93 & 0.72 & 2.13 & 0.94 & Gg_rs137990 X1_2401256€ \\
\hline $0.62 \mathrm{NA}$ & NA & NA & NA & & Gg_rs151948 Gg_rs147998. \\
\hline $0.56 \mathrm{NA}$ & NA & NA & NA & & Gg_rs137990 Gg_rs147998. \\
\hline $0.51 \mathrm{NA}$ & NA & NA & NA & & Gg_rs137990 Gg_rs147998. \\
\hline 0.75 & 1.57 & 0.67 & 0.9 & 0.98 & Gg_rs137990 Gg_rs147998. \\
\hline 1.04 & 2.11 & 0.93 & 0.6 & 1.37 & Gg_rs147981 Gg_rs147998. \\
\hline $0.89 \mathrm{NA}$ & NA & NA & NA & & Gg_rs151948 Gg_rs147998. \\
\hline $0.77 \mathrm{NA}$ & NA & NA & NA & & Gg_rs147981 Gg_rs147998. \\
\hline $0.59 \mathrm{NA}$ & NA & NA & NA & & Gg_rs147981 Gg_rs147998. \\
\hline 1.08 & -3.1 & 0.96 & -5.74 & 1.42 & Gg_rs147981X1_27115232 \\
\hline $0.72 \mathrm{NA}$ & NA & NA & NA & & Gg_rs147981 Gg_rs147998. \\
\hline $0.32 \mathrm{NA}$ & NA & NA & NA & & Gg_rs147981 Gg_rs147998. \\
\hline $0.36 \mathrm{NA}$ & NA & NA & NA & & Gg_rs147981 Gg_rs147998. \\
\hline 0.69 NA & NA & NA & NA & & Gg_rs147981 Gg_rs147998. \\
\hline 1.35 & 2.02 & 1.21 & 4.06 & 1.78 & Gg_rs147981 Gg_rs147998. \\
\hline 0.46 NA & NA & NA & NA & & Gg_rs147981 Gg_rs147998. \\
\hline $0.91 \mathrm{NA}$ & NA & NA & NA & & Gg_rs147981 Gg_rs147998. \\
\hline $0.45 \mathrm{NA}$ & NA & NA & NA & & Gg_rs147981 Gg_rs147998. \\
\hline $0.59 \mathrm{NA}$ & NA & NA & NA & & Gg_rs147981 Gg_rs147998. \\
\hline $0.3 \mathrm{NA}$ & NA & NA & NA & & Gg_rs147981 Gg_rs147998. \\
\hline 0.67 NA & NA & NA & NA & & Gg_rs147981X1_27115232 \\
\hline $0.71 \mathrm{NA}$ & NA & NA & NA & & Gg_rs147981X1_27115232 \\
\hline $0.41 \mathrm{NA}$ & NA & NA & NA & & Gg_rs147981X1_27115232 \\
\hline 0.48 NA & NA & NA & NA & & Gg_rs147981X1_27115232 \\
\hline $0.42 \mathrm{NA}$ & NA & NA & NA & & Gg_rs147981 hrm_chr1.29l \\
\hline $1.02 \mathrm{NA}$ & NA & NA & NA & & X1_2401256€X1_27115232 \\
\hline $0.4 \mathrm{NA}$ & NA & NA & NA & & Gg_rs147981X1_27115232 \\
\hline 0.45 & -0.91 & 0.56 & -0.68 & 0.66 & X1_2401256EX1_27115232 \\
\hline $0.68 \mathrm{NA}$ & NA & NA & NA & & Gg_rs147998hrm_chr1.29I \\
\hline $1.08 \mathrm{NA}$ & NA & NA & NA & & X1_2401256€hrm_chr1.29I \\
\hline $0.83 \mathrm{NA}$ & NA & NA & NA & & Gg_rs147998hrm_chr1.29I \\
\hline $0.61 \mathrm{NA}$ & NA & NA & NA & & X1_2401256€X32_1 \\
\hline 0.46 NA & NA & NA & NA & & Gg_rs147998 X32_1 \\
\hline $0.61 \mathrm{NA}$ & NA & NA & NA & & Gg_rs147998 X32_1 \\
\hline 0.62 NA & NA & NA & NA & & Gg_rs147998 X33_2 \\
\hline 0.62 NA & NA & NA & NA & & X1_27115232X32_1 \\
\hline $0.45 \mathrm{NA}$ & NA & NA & NA & & X1_27115232X1_30454963 \\
\hline $0.54 \mathrm{NA}$ & NA & NA & NA & & X1_27115232X33_2 \\
\hline $0.69 \mathrm{NA}$ & NA & NA & NA & & Gg_rs147998 X32_1 \\
\hline $0.51 \mathrm{NA}$ & NA & NA & NA & & X1_27115232X32_1 \\
\hline $0.46 \mathrm{NA}$ & NA & NA & NA & & X1_27115232X32_1 \\
\hline
\end{tabular}




\begin{tabular}{|c|c|c|c|c|c|c|}
\hline $0.47 \mathrm{NA}$ & NA & NA & NA & \multicolumn{3}{|c|}{ X1_27115232X32_1 } \\
\hline $0.69 \mathrm{NA}$ & NA & NA & NA & \multicolumn{3}{|c|}{ Gg_rs147998 X32_1 } \\
\hline $0.78 \mathrm{NA}$ & NA & NA & NA & \multicolumn{3}{|c|}{ chr1_310000।X33_2 } \\
\hline $0.91 \mathrm{NA}$ & NA & NA & NA & \multicolumn{3}{|c|}{ chr1_3100001X33_2 } \\
\hline $0.55 \mathrm{NA}$ & NA & NA & NA & \multicolumn{3}{|c|}{ chr1_310000।X33_2 } \\
\hline $0.5 \mathrm{NA}$ & NA & NA & NA & \multicolumn{3}{|c|}{ chr1_310000।X33_2 } \\
\hline 0.76 & 0.85 & 0.73 & 1.92 & \multicolumn{3}{|c|}{1.03 chr1_310000।X33_2 } \\
\hline $0.5 \mathrm{NA}$ & NA & NA & NA & \multicolumn{3}{|c|}{ chr1_310000।X33_2 } \\
\hline $0.41 \mathrm{NA}$ & NA & NA & NA & \multicolumn{3}{|c|}{ chr1_310000।X33_2 } \\
\hline $0.53 \mathrm{NA}$ & NA & NA & NA & \multicolumn{3}{|c|}{ chr1_310000।X33_2 } \\
\hline $0.91 \mathrm{NA}$ & NA & NA & NA & \multicolumn{3}{|c|}{ chr1_310000।X33_2 } \\
\hline $0.67 \mathrm{NA}$ & NA & NA & NA & \multicolumn{3}{|c|}{ chr1_310000।X33_2 } \\
\hline 0.43 NA & NA & NA & NA & & X32_1 & $\mathrm{X} 35.5 \mathrm{Mb}$ \\
\hline $0.77 \mathrm{NA}$ & NA & NA & NA & & X32_1 & X36.15Mb \\
\hline 0.39 NA & NA & NA & NA & & X32_1 & $\mathrm{X} 36.15 \mathrm{Mb}$ \\
\hline 0.06 & -0.33 & 0.06 & 0.25 & 0.08 & X33_2 & $\mathrm{X} 36.15 \mathrm{Mb}$ \\
\hline $0.61 \mathrm{NA}$ & NA & NA & NA & & X33_2 & $\mathrm{X} 36.15 \mathrm{Mb}$ \\
\hline $0.9 \mathrm{NA}$ & NA & NA & NA & & X33_2 & X36.15Mb \\
\hline $0.44 \mathrm{NA}$ & NA & NA & NA & & X33_2 & X36.15Mb \\
\hline $1.11 \mathrm{NA}$ & NA & NA & NA & & X33_2 & $\mathrm{X} 36.15 \mathrm{Mb}$ \\
\hline 0.11 & 0.67 & 0.12 & -0.82 & 0.16 & X35.5Mb & X1_36652477 \\
\hline 0.08 & 0.45 & 0.09 & -0.56 & 0.12 & $\mathrm{X} 35.5 \mathrm{Mb}$ & X1_36652477 \\
\hline 0.07 & 0.36 & 0.07 & -0.47 & 0.09 & X35.5Mb & X1_36652477 \\
\hline 0.09 & 0.47 & 0.1 & -0.64 & 0.13 & $\mathrm{X} 35.5 \mathrm{Mb}$ & X1_36652477 \\
\hline 0.11 & 0.4 & 0.12 & -0.68 & 0.15 & $\mathrm{X} 35.5 \mathrm{Mb}$ & X1_36652477 \\
\hline $0.59 \mathrm{NA}$ & NA & NA & NA & & X35.5Mb & X1_36652477 \\
\hline $0.6 \mathrm{NA}$ & NA & NA & NA & & X35.5Mb & X36_9 \\
\hline 0.42 NA & NA & NA & NA & & X35.5Mb & X1_37164711 \\
\hline 0.02 NA & NA & NA & NA & & X36_45 & X1_37164711 \\
\hline 0.07 NA & NA & NA & NA & & X1_36652 & 7X1_37164711 \\
\hline 0.89 & -3.26 & 0.87 & 6.31 & 1.16 & X1_36652 & 7X1_37164711 \\
\hline 0.43 NA & NA & NA & NA & & X36_9 & Gg_rs137516 \\
\hline $0.68 \mathrm{NA}$ & NA & NA & NA & & X36_9 & Gg_rs148147. \\
\hline $0.56 \mathrm{NA}$ & NA & NA & NA & & X36_9 & Gg_rs148147. \\
\hline 0.86 NA & NA & NA & NA & & X1_36652 & 7Gg_rs148208 \\
\hline $0.72 \mathrm{NA}$ & NA & NA & NA & & X35.5Mb & Gg_rs148147 \\
\hline 0.65 NA & NA & NA & NA & & X36_9 & Gg_rs138586 \\
\hline $0.59 \mathrm{NA}$ & NA & NA & NA & & X32_1 & Gg_rs148208 \\
\hline 0.66 & 1.68 & 0.64 & -0.4 & 0.91 & Gg_rs148 & 5 Gg_rs148147 \\
\hline $0.4 \mathrm{NA}$ & NA & NA & NA & & Gg_rs148 & 5 Gg_rs148147. \\
\hline $1.18 \mathrm{NA}$ & NA & NA & NA & & Gg_rs148 & 5 Gg_rs148147. \\
\hline $0.45 \mathrm{NA}$ & NA & NA & NA & & X36_9 & Gg_rs148159 \\
\hline 0.49 NA & NA & NA & NA & & Gg_rs148 & 5 Gg_rs148147. \\
\hline
\end{tabular}




\begin{tabular}{|c|c|c|c|c|c|}
\hline $0.46 \mathrm{NA}$ & NA & NA & NA & & Gg_rs148105 Gg_rs148147. \\
\hline $1.19 \mathrm{NA}$ & NA & NA & NA & & Gg_rs148105 Gg_rs148147. \\
\hline $0.48 \mathrm{NA}$ & NA & NA & NA & & Gg_rs148105 Gg_rs148147. \\
\hline 0.49 NA & NA & NA & NA & & Gg_rs148105 Gg_rs148147. \\
\hline $0.75 \mathrm{NA}$ & NA & NA & NA & & Gg_rs148105 Gg_rs148147. \\
\hline $0.38 \mathrm{NA}$ & NA & NA & NA & & Gg_rs148105 Gg_rs148147. \\
\hline $0.35 \mathrm{NA}$ & NA & NA & NA & & Gg_rs148105 Gg_rs148147. \\
\hline $1.08 \mathrm{NA}$ & NA & NA & NA & & Gg_rs148105 Gg_rs148147. \\
\hline $0.56 \mathrm{NA}$ & NA & NA & NA & & Gg_rs148105 Gg_rs148147. \\
\hline $0.48 \mathrm{NA}$ & NA & NA & NA & & Gg_rs148105 Gg_rs148147. \\
\hline 1.6 & -1.48 & 1.55 & -4.67 & 2.19 & Gg_rs148105 Gg_rs148147. \\
\hline $0.95 \mathrm{NA}$ & NA & NA & NA & & Gg_rs148105 Gg_rs148147. \\
\hline $0.6 \mathrm{NA}$ & NA & NA & NA & & Gg_rs148105 Gg_rs148147. \\
\hline 0.49 NA & NA & NA & NA & & Gg_rs148105 Gg_rs148147. \\
\hline 0.99 NA & NA & NA & NA & & Gg_rs148105 Gg_rs148147. \\
\hline $0.33 \mathrm{NA}$ & NA & NA & NA & & Gg_rs148105 Gg_rs148159 \\
\hline $0.6 \mathrm{NA}$ & NA & NA & NA & & Gg_rs148105 Gg_rs148147. \\
\hline 0.77 NA & NA & NA & NA & & Gg_rs148105 Gg_rs148147. \\
\hline 0.72 & -1.06 & 0.7 & -2.64 & 0.98 & Gg_rs148105 Gg_rs148147. \\
\hline $0.65 \mathrm{NA}$ & NA & NA & NA & & Gg_rs148105 Gg_rs148147. \\
\hline 0.45 NA & NA & NA & NA & & Gg_rs148105 Gg_rs148147. \\
\hline $0.84 \mathrm{NA}$ & NA & NA & NA & & Gg_rs148105 Gg_rs148147. \\
\hline $0.57 \mathrm{NA}$ & NA & NA & NA & & X1_36652477Gg_rs148159 \\
\hline $0.7 \mathrm{NA}$ & NA & NA & NA & & Gg_rs148105 Gg_rs148147. \\
\hline $0.41 \mathrm{NA}$ & NA & NA & NA & & Gg_rs148105 Gg_rs138586 \\
\hline $0.64 \mathrm{NA}$ & NA & NA & NA & & Gg_rs148105 Gg_rs148147. \\
\hline $1.03 \mathrm{NA}$ & NA & NA & NA & & X35.5Mb Gg_rs148159 \\
\hline $0.7 \mathrm{NA}$ & NA & NA & NA & & Gg_rs148105 Gg_rs138586 \\
\hline $0.64 \mathrm{NA}$ & NA & NA & NA & & Gg_rs148105 Gg_rs138586 \\
\hline $0.52 \mathrm{NA}$ & NA & NA & NA & & Gg_rs148105 Gg_rs148159 \\
\hline $0.36 \mathrm{NA}$ & NA & NA & NA & & Gg_rs148105 Gg_rs148147. \\
\hline $0.45 \mathrm{NA}$ & NA & NA & NA & & Gg_rs148105 Gg_rs148159 \\
\hline $0.54 \mathrm{NA}$ & NA & NA & NA & & Gg_rs148105 Gg_rs148159 \\
\hline $0.4 \mathrm{NA}$ & NA & NA & NA & & Gg_rs148105 Gg_rs138586 \\
\hline 0.56 NA & NA & NA & NA & & Gg_rs148105 Gg_rs138586 \\
\hline 0.44 NA & NA & NA & NA & & Gg_rs148105 Gg_rs138586 \\
\hline 0.67 NA & NA & NA & NA & & Gg_rs148105 Gg_rs138586 \\
\hline $0.31 \mathrm{NA}$ & NA & NA & NA & & Gg_rs148105 Gg_rs138586 \\
\hline 0.74 NA & NA & NA & NA & & Gg_rs148105 Gg_rs148159 \\
\hline $0.63 \mathrm{NA}$ & NA & NA & NA & & Gg_rs148105 Gg_rs138586 \\
\hline $0.68 \mathrm{NA}$ & NA & NA & NA & & Gg_rs148105 Gg_rs138586 \\
\hline $0.85 \mathrm{NA}$ & NA & NA & NA & & Gg_rs148105 Gg_rs138586 \\
\hline $0.56 \mathrm{NA}$ & NA & NA & NA & & Gg_rs152393 Gg_rs138586 \\
\hline
\end{tabular}




\begin{tabular}{|c|c|c|c|c|c|}
\hline $0.48 \mathrm{NA}$ & NA & NA & NA & & Gg_rs148105 Gg_rs138586 \\
\hline $0.87 \mathrm{NA}$ & NA & NA & NA & & Gg_rs148105 Gg_rs138586 \\
\hline $0.8 \mathrm{NA}$ & NA & NA & NA & & Gg_rs148105 Gg_rs138586 \\
\hline $0.47 \mathrm{NA}$ & NA & NA & NA & & Gg_rs152393 Gg_rs148159 \\
\hline $0.45 \mathrm{NA}$ & NA & NA & NA & & Gg_rs152393 Gg_rs138586 \\
\hline $0.43 \mathrm{NA}$ & NA & NA & NA & & Gg_rs148105 Gg_rs148159 \\
\hline $0.36 \mathrm{NA}$ & NA & NA & NA & & Gg_rs148105 Gg_rs148208 \\
\hline $0.37 \mathrm{NA}$ & NA & NA & NA & & Gg_rs148105 Gg_rs138586 \\
\hline $0.55 \mathrm{NA}$ & NA & NA & NA & & Gg_rs152393 Gg_rs148159 \\
\hline $0.42 \mathrm{NA}$ & NA & NA & NA & & Gg_rs152393 Gg_rs138586 \\
\hline $0.38 \mathrm{NA}$ & NA & NA & NA & & Gg_rs148105 Gg_rs138586 \\
\hline $0.38 \mathrm{NA}$ & NA & NA & NA & & Gg_rs152393 Gg_rs138586 \\
\hline $0.42 \mathrm{NA}$ & NA & NA & NA & & Gg_rs152393 Gg_rs148159 \\
\hline $0.7 \mathrm{NA}$ & NA & NA & NA & & Gg_rs148105 Gg_rs148186 \\
\hline $0.5 \mathrm{NA}$ & NA & NA & NA & & Gg_rs152393 Gg_rs138586 \\
\hline $0.53 \mathrm{NA}$ & NA & NA & NA & & Gg_rs152393 Gg_rs148159 \\
\hline $0.77 \mathrm{NA}$ & NA & NA & NA & & Gg_rs152393 Gg_rs138586 \\
\hline $0.49 \mathrm{NA}$ & NA & NA & NA & & Gg_rs152393 Gg_rs148186 \\
\hline $0.96 \mathrm{NA}$ & NA & NA & NA & & Gg_rs152393 Gg_rs138586 \\
\hline $0.36 \mathrm{NA}$ & NA & NA & NA & & Gg_rs152393 Gg_rs148186 \\
\hline $0.73 \mathrm{NA}$ & NA & NA & NA & & X1_37164711rbl1791 \\
\hline 0.32 & -0.33 & 0.28 & 1.99 & 0.42 & Gg_rs152393 Gg_rs148159 \\
\hline $0.48 \mathrm{NA}$ & NA & NA & NA & & Gg_rs152393 Gg_rs138586 \\
\hline $0.71 \mathrm{NA}$ & NA & NA & NA & & Gg_rs152393 Gg_rs138586 \\
\hline $0.68 \mathrm{NA}$ & NA & NA & NA & & Gg_rs152393 Gg_rs138586 \\
\hline $0.35 \mathrm{NA}$ & NA & NA & NA & & Gg_rs152393 Gg_rs138586 \\
\hline $0.64 \mathrm{NA}$ & NA & NA & NA & & Gg_rs152393 Gg_rs138586 \\
\hline $0.51 \mathrm{NA}$ & NA & NA & NA & & Gg_rs152393 Gg_rs138586 \\
\hline $0.7 \mathrm{NA}$ & NA & NA & NA & & X36_9 Gg_rs138586 \\
\hline $0.5 \mathrm{NA}$ & NA & NA & NA & & Gg_rs148105 Gg_rs138586 \\
\hline $0.37 \mathrm{NA}$ & NA & NA & NA & & Gg_rs137516Gg_rs138586 \\
\hline $0.72 \mathrm{NA}$ & NA & NA & NA & & Gg_rs152393 Gg_rs138586 \\
\hline $0.47 \mathrm{NA}$ & NA & NA & NA & & Gg_rs152393 Gg_rs138586 \\
\hline $0.39 \mathrm{NA}$ & NA & NA & NA & & Gg_rs152393 Gg_rs138586 \\
\hline 0.74 & 0.3 & 0.69 & 2.78 & 1.01 & Gg_rs152393 Gg_rs138586 \\
\hline $0.48 \mathrm{NA}$ & NA & NA & NA & & Gg_rs152393 Gg_rs138586 \\
\hline $0.35 \mathrm{NA}$ & NA & NA & NA & & Gg_rs148147 Gg_rs138586 \\
\hline $0.6 \mathrm{NA}$ & NA & NA & NA & & Gg_rs152393 Gg_rs138586 \\
\hline $0.23 \mathrm{NA}$ & NA & NA & NA & & Gg_rs148147 Gg_rs138586 \\
\hline $0.45 \mathrm{NA}$ & NA & NA & NA & & Gg_rs148147 Gg_rs148159 \\
\hline $0.43 \mathrm{NA}$ & NA & NA & NA & & Gg_rs148147 Gg_rs138586 \\
\hline $0.43 \mathrm{NA}$ & NA & NA & NA & & Gg_rs148147 Gg_rs138586 \\
\hline $0.39 \mathrm{NA}$ & NA & NA & NA & & Gg_rs148147 Gg_rs138586 \\
\hline
\end{tabular}




\begin{tabular}{|c|c|c|c|c|c|c|c|}
\hline $0.38 \mathrm{NA}$ & & NA & & NA & & & Gg_rs148147 Gg_rs138586 \\
\hline $0.67 \mathrm{NA}$ & & NA & & NA & & & Gg_rs148147 Gg_rs138586 \\
\hline $1 \mathrm{NA}$ & & NA & & NA & & & Gg_rs148186 \\
\hline $0.81 \mathrm{NA}$ & & NA & & NA & & & Calle_cGLA1 \\
\hline $0.52 \mathrm{NA}$ & & NA & & NA & & & Gg_rs152393 Gg_rs148186 \\
\hline $0.39 \mathrm{NA}$ & & NA & & NA & & & Gg_rs152393 Gg_rs148186 \\
\hline $0.38 \mathrm{NA}$ & & NA & & NA & & & Gg_rs148147 Gg_rs148186 \\
\hline $0.35 \mathrm{NA}$ & & NA & & NA & & & Gg_rs148147 Gg_rs148186 \\
\hline $0.72 \mathrm{NA}$ & & NA & & NA & & & Gg_rs152393 Gg_rs138586 \\
\hline $0.53 \mathrm{NA}$ & & NA & & NA & & & Gg_rs148105 Gg_rs138586 \\
\hline $0.87 \mathrm{NA}$ & & NA & & NA & & & X36_9 Gg_rs148186 \\
\hline $0.45 \mathrm{NA}$ & & NA & & NA & & & Gg_rs148147 Gg_rs138586 \\
\hline 0.74 & -2.16 & & 0.69 & & 3.74 & 1.01 & Gg_rs148147 Gg_rs138586 \\
\hline $0.21 \mathrm{NA}$ & & NA & & NA & & & Gg_rs148147 Gg_rs138586 \\
\hline $0.42 \mathrm{NA}$ & & NA & & NA & & & Gg_rs152393 Gg_rs138586 \\
\hline $0.61 \mathrm{NA}$ & & NA & & NA & & & Gg_rs148147 Gg_rs138586 \\
\hline $0.69 \mathrm{NA}$ & & NA & & NA & & & Gg_rs152393 Gg_rs148186 \\
\hline $0.5 \mathrm{NA}$ & & NA & & NA & & & Gg_rs148147 Gg_rs138586 \\
\hline 0.52 & 1.76 & & 0.48 & & 0.7 & 0.71 & Gg_rs152393 Gg_rs148186 \\
\hline $0.41 \mathrm{NA}$ & & NA & & NA & & & Gg_rs148147 Gg_rs138586 \\
\hline $0.6 \mathrm{NA}$ & & NA & & NA & & & Gg_rs152393 Gg_rs148186 \\
\hline $0.42 \mathrm{NA}$ & & NA & & NA & & & Gg_rs148147 Gg_rs138586 \\
\hline $0.46 \mathrm{NA}$ & & NA & & NA & & & Gg_rs152393 Gg_rs148186 \\
\hline $0.9 \mathrm{NA}$ & & NA & & NA & & & Gg_rs152393 Gg_rs148186 \\
\hline 1.33 & -4.38 & & 1.3 & & 3.12 & 1.76 & Gg_rs152393 Gg_rs148186 \\
\hline $0.44 \mathrm{NA}$ & & NA & & NA & & & Gg_rs148147 Gg_rs148186 \\
\hline 0.79 & -2.81 & & 0.78 & & 4.1 & 1.05 & Gg_rs148159 Gg_rs148186 \\
\hline $0.61 \mathrm{NA}$ & & NA & & NA & & & Gg_rs148159 Gg_rs148186 \\
\hline 0.88 & -3.72 & & 0.87 & & 2.17 & 1.17 & Gg_rs148159 Gg_rs148186 \\
\hline $0.16 \mathrm{NA}$ & & NA & & NA & & & Gg_rs148159 Gg_rs148186 \\
\hline $0.81 \mathrm{NA}$ & & NA & & NA & & & Gg_rs152393 Gg_rs148186 \\
\hline 1.95 & -8.64 & & 1.92 & & 5.21 & 2.6 & Gg_rs148159 Gg_rs148186 \\
\hline $0.41 \mathrm{NA}$ & & NA & & NA & & & Gg_rs148159 Gg_rs148186 \\
\hline $0.55 \mathrm{NA}$ & & NA & & NA & & & Gg_rs148159 Gg_rs148186 \\
\hline 1.4 & -5.92 & & 1.38 & & 3.49 & 1.86 & Gg_rs148159 Gg_rs148186 \\
\hline 1.72 & -5.98 & & 1.69 & & 3.47 & 2.29 & Gg_rs148159 Gg_rs148186 \\
\hline 1.75 & -7.88 & & 1.72 & & 6.53 & 2.32 & Gg_rs148159 Gg_rs148186 \\
\hline 1.59 & -7.2 & & 1.56 & & 4.83 & 2.12 & Gg_rs148159 Gg_rs148186 \\
\hline 1.79 & -7.6 & & 1.76 & & 8.45 & 2.38 & Gg_rs148159 Gg_rs148186 \\
\hline 1.22 & -5.67 & & 1.2 & & 5.15 & 1.62 & Gg_rs148159 Gg_rs148186 \\
\hline 0.75 & -1.29 & & 0.74 & & 1.08 & 1 & Gg_rs148159 Gg_rs148186 \\
\hline 1.23 & -4.2 & & 1.21 & & 4.4 & 1.63 & Gg_rs148159 Gg_rs148186 \\
\hline $0.2 \mathrm{NA}$ & & NA & & NA & & & Gg_rs148159 Gg_rs148186 \\
\hline
\end{tabular}




\begin{tabular}{|c|c|c|c|c|c|}
\hline 0.35 & -0.52 & 0.34 & 2.07 & 0.46 & Gg_rs148159 Gg_rs148186. \\
\hline $0.02 \mathrm{NA}$ & NA & NA & NA & & Gg_rs148159 Gg_rs148186. \\
\hline $0.45 \mathrm{NA}$ & NA & NA & NA & & Gg_rs148159 Gg_rs148186 \\
\hline $0.87 \mathrm{NA}$ & NA & NA & NA & & Gg_rs148159 Calle_cGLA1 \\
\hline $0.6 \mathrm{NA}$ & NA & NA & NA & & Gg_rs148159 Calle_cGLA1 \\
\hline $0.93 \mathrm{NA}$ & NA & NA & NA & & Gg_rs138586 Calle_cGLA1 \\
\hline $1.59 \mathrm{NA}$ & NA & NA & NA & & Gg_rs138586 Calle_cGLA1 \\
\hline $1.92 \mathrm{NA}$ & NA & NA & NA & & Gg_rs138586 Calle_cGLA1 \\
\hline $2.25 \mathrm{NA}$ & NA & NA & NA & & Gg_rs138586 Calle_cGLA1 \\
\hline $1.07 \mathrm{NA}$ & NA & NA & NA & & Gg_rs138586 Calle_cGLA1 \\
\hline $1.45 \mathrm{NA}$ & NA & NA & NA & & Gg_rs138586 Calle_cGLA1 \\
\hline $1.22 \mathrm{NA}$ & NA & NA & NA & & Gg_rs148159 Calle_cGLA1 \\
\hline $3.18 \mathrm{NA}$ & NA & NA & NA & & Gg_rs138586 Calle_cGLA1 \\
\hline $1.35 \mathrm{NA}$ & NA & NA & NA & & Gg_rs138586 Calle_cGLA1 \\
\hline $2.34 \mathrm{NA}$ & NA & NA & NA & & Gg_rs138586 Calle_cGLA1 \\
\hline $0.99 \mathrm{NA}$ & NA & NA & NA & & Gg_rs138586 Calle_cGLA1 \\
\hline $1.24 \mathrm{NA}$ & NA & NA & NA & & Gg_rs138586 Calle_cGLA1 \\
\hline $3.32 \mathrm{NA}$ & NA & NA & NA & & Gg_rs138586 Calle_cGLA1 \\
\hline $1.25 \mathrm{NA}$ & NA & NA & NA & & Gg_rs138586 Gg_rs148208. \\
\hline $0.63 \mathrm{NA}$ & NA & NA & NA & & Gg_rs138586 Calle_cGLA1 \\
\hline $1.36 \mathrm{NA}$ & NA & NA & NA & & Gg_rs138586 Calle_cGLA1 \\
\hline $0.48 \mathrm{NA}$ & NA & NA & NA & & Gg_rs138586 Calle_cGLA1 \\
\hline 1.33 & -1.46 & 0.99 & -4.76 & 1.8 & Gg_rs138586 Calle_cGLA1 \\
\hline $1.04 \mathrm{NA}$ & NA & NA & NA & & Gg_rs138586 Calle_cGLA1 \\
\hline $0.99 \mathrm{NA}$ & NA & NA & NA & & Gg_rs138586 Calle_cGLA1 \\
\hline $1.22 \mathrm{NA}$ & NA & NA & NA & & Gg_rs138586 Gg_rs148208. \\
\hline $1.06 \mathrm{NA}$ & NA & NA & NA & & Gg_rs138586 Gg_rs148208. \\
\hline $1.07 \mathrm{NA}$ & NA & NA & NA & & Gg_rs138586 Gg_rs148208. \\
\hline $0.91 \mathrm{NA}$ & NA & NA & NA & & Gg_rs138586Gg_rs148208. \\
\hline $1.25 \mathrm{NA}$ & NA & NA & NA & & Gg_rs138586 Gg_rs148208. \\
\hline $1.13 \mathrm{NA}$ & NA & NA & NA & & Gg_rs138586 Gg_rs148208. \\
\hline $1.09 \mathrm{NA}$ & NA & NA & NA & & Gg_rs138586 Gg_rs148208. \\
\hline $1.08 \mathrm{NA}$ & NA & NA & NA & & Gg_rs148147 Gg_rs148208. \\
\hline $0.64 \mathrm{NA}$ & NA & NA & NA & & Gg_rs148159 Gg_rs148208. \\
\hline $0.5 \mathrm{NA}$ & NA & NA & NA & & Gg_rs138586 Gg_rs148208. \\
\hline $0.89 \mathrm{NA}$ & NA & NA & NA & & Gg_rs138586 rbl1791 \\
\hline $0.91 \mathrm{NA}$ & NA & NA & NA & & Gg_rs138586 Gg_rs148208 \\
\hline 0.29 & 1 & 0.29 & 0.5 & 0.37 & Gg_rs152393 Gg_rs148208. \\
\hline 0.4 NA & NA & NA & NA & & Gg_rs138586 Gg_rs148208. \\
\hline $0.35 \mathrm{NA}$ & NA & NA & NA & & Gg_rs138586 Gg_rs148208. \\
\hline $0.65 \mathrm{NA}$ & NA & NA & NA & & Gg_rs148159 rbl1791 \\
\hline $0.62 \mathrm{NA}$ & NA & NA & NA & & Gg_rs148186 Gg_rs148208. \\
\hline $0.26 \mathrm{NA}$ & NA & NA & NA & & Gg_rs148186 Gg_rs148208 \\
\hline
\end{tabular}




\begin{tabular}{|c|c|c|c|c|c|}
\hline 1.28 & -5.5 & 1.25 & 5.99 & 1.61 & Gg_rs148186 Gg_rs148208. \\
\hline $0.5 \mathrm{NA}$ & NA & NA & NA & & Gg_rs138586 Gg_rs148208. \\
\hline $0.5 \mathrm{NA}$ & NA & NA & NA & & Gg_rs148186 Gg_rs148208. \\
\hline 0.96 & 3.64 & 0.94 & -0.09 & 1.21 & Gg_rs148186 rbl1791 \\
\hline 0.88 & 2.01 & 0.86 & -2.46 & 1.11 & Gg_rs148186 rbl1791 \\
\hline $0.56 \mathrm{NA}$ & NA & NA & NA & & Gg_rs148186 rbl1791 \\
\hline $0.67 \mathrm{NA}$ & NA & NA & NA & & Gg_rs148186 rbl1791 \\
\hline $0.58 \mathrm{NA}$ & NA & NA & NA & & Calle_cGLA1 Gg_rs138635 \\
\hline $0.6 \mathrm{NA}$ & NA & NA & NA & & Gg_rs148186Gg_rs138635 \\
\hline $0.59 \mathrm{NA}$ & NA & NA & NA & & Calle_cGLA1 rbl1791 \\
\hline $0.25 \mathrm{NA}$ & NA & NA & NA & & Gg_rs148186 rbl1791 \\
\hline $0.53 \mathrm{NA}$ & NA & NA & NA & & Gg_rs148186 rbl1791 \\
\hline $0.73 \mathrm{NA}$ & NA & NA & NA & & Gg_rs148208 rbl1791 \\
\hline $0.41 \mathrm{NA}$ & NA & NA & NA & & Gg_rs148208 rbl1791 \\
\hline $0.81 \mathrm{NA}$ & NA & NA & NA & & Gg_rs138635 Gg_rs136528 \\
\hline $0.27 \mathrm{NA}$ & NA & NA & NA & & Gg_rs138635 Gg_rs136528 \\
\hline 0.74 & 1.65 & 0.7 & -2.68 & 0.98 & Gg_rs138635 Gg_rs136528 \\
\hline $0.29 \mathrm{NA}$ & NA & NA & NA & & Gg_rs138635 Gg_rs136528 \\
\hline $0.3 \mathrm{NA}$ & NA & NA & NA & & Gg_rs138635 Gg_rs136528 \\
\hline $1.19 \mathrm{NA}$ & NA & NA & NA & & Gg_rs138635 Gg_rs136528 \\
\hline 0.73 NA & NA & NA & NA & & Gg_rs138635 Gg_rs136528 \\
\hline $0.56 \mathrm{NA}$ & NA & NA & NA & & Gg_rs138635 Gg_rs136528 \\
\hline 0.59 NA & NA & NA & NA & & Gg_rs138635 Gg_rs136528 \\
\hline 0.49 NA & NA & NA & NA & & Gg_rs138635 Gg_rs136528 \\
\hline $0.43 \mathrm{NA}$ & NA & NA & NA & & rbl1791 Gg_rs148323. \\
\hline $0.61 \mathrm{NA}$ & NA & NA & NA & & Gg_rs148323 \\
\hline $0.54 \mathrm{NA}$ & NA & NA & NA & & Gg_rs148323 \\
\hline $0.55 \mathrm{NA}$ & NA & NA & NA & & Gg_rs148323 \\
\hline $1.14 \mathrm{NA}$ & NA & NA & NA & & Gg_rs148323 \\
\hline $0.67 \mathrm{NA}$ & NA & NA & NA & & Gg_rs148323 \\
\hline $0.44 \mathrm{NA}$ & NA & NA & NA & & Gg_rs148323 \\
\hline $0.84 \mathrm{NA}$ & NA & NA & NA & & rbl1791 Gg_rs148323. \\
\hline $1.02 \mathrm{NA}$ & NA & NA & NA & & Gg_rs138635 Gg_rs148323. \\
\hline $1.6 \mathrm{NA}$ & NA & NA & NA & & Gg_rs148323 \\
\hline $1.2 \mathrm{NA}$ & NA & NA & NA & & Gg_rs148323 \\
\hline $1 \mathrm{NA}$ & NA & NA & NA & & Gg_rs148323. \\
\hline $0.57 \mathrm{NA}$ & NA & NA & NA & & rbl1791 Gg_rs148323. \\
\hline $0.6 \mathrm{NA}$ & NA & NA & NA & & Gg_rs136528Gg_rs148323. \\
\hline $0.55 \mathrm{NA}$ & NA & NA & NA & & Gg_rs138635 Gg_rs148323. \\
\hline $0.41 \mathrm{NA}$ & NA & NA & NA & & Gg_rs136528 Gg_rs148323. \\
\hline $1.47 \mathrm{NA}$ & NA & NA & NA & & rbl1791 Gg_rs148323. \\
\hline 1.06 & -0.88 & 0.89 & 3.86 & 1.39 & rbl1791 Gg_rs148323. \\
\hline 0.59 NA & NA & NA & NA & & Gg_rs136528 Gg_rs148323. \\
\hline
\end{tabular}




\begin{tabular}{|c|c|c|c|c|c|}
\hline $0.58 \mathrm{NA}$ & NA & NA & NA & & rbl1791 Gg_rs148323. \\
\hline $1.31 \mathrm{NA}$ & NA & NA & NA & & Gg_rs138635 Gg_rs148323. \\
\hline 1.47 NA & NA & NA & NA & & Gg_rs148323 \\
\hline $0.61 \mathrm{NA}$ & NA & NA & NA & & Gg_rs136528 Gg_rs148323. \\
\hline $1.16 \mathrm{NA}$ & NA & NA & NA & & rbl1791 Gg_rs148323. \\
\hline $0.75 \mathrm{NA}$ & NA & NA & NA & & Gg_rs148323 \\
\hline $0.71 \mathrm{NA}$ & NA & NA & NA & & Gg_rs148323 \\
\hline $0.95 \mathrm{NA}$ & NA & NA & NA & & rbl1791 Gg_rs148323. \\
\hline $0.63 \mathrm{NA}$ & NA & NA & NA & & Gg_rs136528 Gg_rs148323. \\
\hline $0.9 \mathrm{NA}$ & NA & NA & NA & & rbl1791 Gg_rs148323 \\
\hline $0.52 \mathrm{NA}$ & NA & NA & NA & & X1_5881184\&Gg_rs138749. \\
\hline $0.05 \mathrm{NA}$ & NA & NA & NA & & X1_58811848Gg_rs138749. \\
\hline $0.34 \mathrm{NA}$ & NA & NA & NA & & X1_58811848Gg_rs138749. \\
\hline 0.69 & -1.27 & 0.81 & 1.75 & 1.02 & X1_58811848Gg_rs138749. \\
\hline $0.52 \mathrm{NA}$ & NA & NA & NA & & X1_58811848Gg_rs138749. \\
\hline $0.77 \mathrm{NA}$ & NA & NA & NA & & X1_5881184\&Gg_rs138749. \\
\hline 0.9 & 4.31 & 1.07 & -0.6 & 1.33 & X1_58811848Gg_rs138749. \\
\hline 0.48 NA & NA & NA & NA & & X1_58811848Gg_rs138749. \\
\hline 0.31 & -0.74 & 0.3 & 1.36 & 0.41 & Gg_rs138749Gg_rs148351 \\
\hline $0.55 \mathrm{NA}$ & NA & NA & NA & & Gg_rs138749Gg_rs148351 \\
\hline $0.56 \mathrm{NA}$ & NA & NA & NA & & Gg_rs138749Gg_rs148351 \\
\hline 0.65 & -1.33 & 0.62 & -1.9 & 0.86 & Gg_rs138749Gg_rs148351 \\
\hline 0.62 NA & NA & NA & NA & & Gg_rs138749 Gg_rs148351 \\
\hline 0.48 NA & NA & NA & NA & & Gg_rs138749 Gg_rs148351 \\
\hline 0.64 & -2.51 & 0.62 & 0.58 & 0.85 & Gg_rs138749 Gg_rs148351 \\
\hline $0.48 \mathrm{NA}$ & NA & NA & NA & & Gg_rs138749 Gg_rs148351 \\
\hline $0.38 \mathrm{NA}$ & NA & NA & NA & & Gg_rs138749 Gg_rs148351 \\
\hline $0.63 \mathrm{NA}$ & NA & NA & NA & & Gg_rs138749 Gg_rs148363 \\
\hline $0.7 \mathrm{NA}$ & NA & NA & NA & & Gg_rs138749X1_71708614 \\
\hline $0.4 \mathrm{NA}$ & NA & NA & NA & & Gg_rs138763 Gg_rs148363 \\
\hline $0.75 \mathrm{NA}$ & NA & NA & NA & & Gg_rs138763 Gg_rs148372 \\
\hline $0.53 \mathrm{NA}$ & NA & NA & NA & & Gg_rs138763 Gg_rs148363 \\
\hline $0.42 \mathrm{NA}$ & NA & NA & NA & & Gg_rs138763 X1_71708614 \\
\hline $1.1 \mathrm{NA}$ & NA & NA & NA & & Gg_rs138763 X1_6802129C \\
\hline 0.97 NA & NA & NA & NA & & Gg_rs138763 Gg_rs148363 \\
\hline 1.43 NA & NA & NA & NA & & Gg_rs138763 Gg_rs148372 \\
\hline 0.88 NA & NA & NA & NA & & Gg_rs138763 X1_6802129C \\
\hline 0.95 NA & NA & NA & NA & & Gg_rs138763 Gg_rs148372 \\
\hline $0.47 \mathrm{NA}$ & NA & NA & NA & & Gg_rs138763 X1_71708614 \\
\hline $0.82 \mathrm{NA}$ & NA & NA & NA & & Gg_rs138763 X1_71708614 \\
\hline $0.64 \mathrm{NA}$ & NA & NA & NA & & Gg_rs138763 X1_6802129C \\
\hline 0.49 NA & NA & NA & NA & & Gg_rs138763 Gg_rs148372 \\
\hline $0.84 \mathrm{NA}$ & NA & NA & NA & & Gg_rs138763 Gg_rs148372 \\
\hline
\end{tabular}




\begin{tabular}{|c|c|c|c|c|c|c|c|}
\hline $0.4 \mathrm{NA}$ & NA & & NA & & NA & & Gg_rs138763 Gg_rs148372 \\
\hline 0.89 & 0.86 & 0.88 & & -2.75 & & 1.15 & Gg_rs148351 Gg_rs148372 \\
\hline 0.48 & -0.93 & 0.48 & & 1.01 & & 0.62 & Gg_rs148351Gg_rs148372 \\
\hline $0.65 \mathrm{NA}$ & NA & & NA & & NA & & Gg_rs148351Gg_rs148372 \\
\hline $0.63 \mathrm{NA}$ & NA & & NA & & NA & & Gg_rs148351Gg_rs148372 \\
\hline $0.39 \mathrm{NA}$ & NA & & NA & & NA & & Gg_rs148351Gg_rs148372 \\
\hline $0.59 \mathrm{NA}$ & NA & & NA & & NA & & Gg_rs138763 X1_71708614 \\
\hline $0.55 \mathrm{NA}$ & NA & & NA & & NA & & Gg_rs148351Gg_rs148372 \\
\hline $0.45 \mathrm{NA}$ & NA & & NA & & NA & & Gg_rs148351X1_71708614 \\
\hline $0.88 \mathrm{NA}$ & NA & & NA & & NA & & Gg_rs138749 X1_71708614 \\
\hline $0.82 \mathrm{NA}$ & NA & & NA & & NA & & Gg_rs138749 X1_71708614 \\
\hline 0.28 & -0.44 & 0.3 & & -1.05 & & 0.37 & Gg_rs148363X1_6802129C \\
\hline $0.76 \mathrm{NA}$ & NA & & NA & & NA & & Gg_rs148351X1_71708614 \\
\hline $0.62 \mathrm{NA}$ & NA & & NA & & NA & & Gg_rs148363 X1_71708614 \\
\hline 0.79 NA & NA & & NA & & NA & & Gg_rs148363 X1_71708614 \\
\hline 0.92 & 2.21 & 0.97 & & 0.28 & & 1.22 & Gg_rs148363 X1_71708614 \\
\hline $0.65 \mathrm{NA}$ & NA & & NA & & NA & & Gg_rs148363 X1_71708614 \\
\hline $0.38 \mathrm{NA}$ & NA & & NA & & NA & & Gg_rs148363 X1_71708614 \\
\hline $0.72 \mathrm{NA}$ & NA & & NA & & NA & & Gg_rs148363 X1_71708614 \\
\hline 0.79 NA & NA & & NA & & NA & & Gg_rs148363 X1_71708614 \\
\hline 0.46 NA & NA & & NA & & NA & & Gg_rs148363 X1_71708614 \\
\hline 1.2 & -2.99 & 1.12 & & 1.51 & & 1.6 & Gg_rs148363 Gg_rs138195 \\
\hline 0.97 NA & NA & & NA & & NA & & Gg_rs148363 X1_71708614 \\
\hline $1.11 \mathrm{NA}$ & NA & & NA & & NA & & Gg_rs148363 Gg_rs138195. \\
\hline $0.77 \mathrm{NA}$ & NA & & NA & & NA & & Gg_rs148363 Gg_rs138195. \\
\hline $2.32 \mathrm{NA}$ & NA & & NA & & NA & & Gg_rs148363 X1_71708614 \\
\hline $2.41 \mathrm{NA}$ & NA & & NA & & NA & & Gg_rs148363 X1_71708614 \\
\hline $2.02 \mathrm{NA}$ & NA & & NA & & NA & & Gg_rs148363 X1_71708614 \\
\hline $2.25 \mathrm{NA}$ & NA & & NA & & NA & & Gg_rs148363 X1_71708614 \\
\hline 0.99 NA & NA & & NA & & NA & & Gg_rs148363 X1_71708614 \\
\hline $3.02 \mathrm{NA}$ & NA & & NA & & NA & & Gg_rs148363 X1_71708614 \\
\hline 0.99 NA & NA & & NA & & NA & & Gg_rs148363 X1_71708614 \\
\hline $1.53 \mathrm{NA}$ & NA & & NA & & NA & & Gg_rs148363 X1_71708614 \\
\hline $1.55 \mathrm{NA}$ & NA & & NA & & NA & & Gg_rs148363 Gg_rs138195. \\
\hline $3.24 \mathrm{NA}$ & NA & & NA & & NA & & X1_6802129CGg_rs138195. \\
\hline 3.11 & -5.14 & 1.77 & & -1.17 & & 3.61 & X1_6802129CGg_rs138195 \\
\hline 3.39 & 5.71 & 1.93 & & -14.45 & & 3.94 & X1_6802129CGg_rs137259 \\
\hline 2.86 & 5.43 & 1.63 & & -7.35 & & 3.33 & X1_6802129CGg_rs138195 \\
\hline $2.54 \mathrm{NA}$ & NA & & NA & & NA & & X1_6802129CGg_rs138195. \\
\hline $1.82 \mathrm{NA}$ & NA & & NA & & NA & & X1_6802129CGg_rs138195. \\
\hline 0.49 NA & NA & & NA & & NA & & X1_82323161X1_88934871 \\
\hline 0.09 & -0.31 & 0.09 & & 0.55 & & 0.12 & X1_82323161X1_88934871 \\
\hline $0.37 \mathrm{NA}$ & NA & & NA & & NA & & X1_82323161X1_8893487 \\
\hline
\end{tabular}




\begin{tabular}{|c|c|c|c|c|}
\hline $0.74 \mathrm{NA}$ & NA & NA & NA & X1_82323161X1_88934871 \\
\hline $0.5 \mathrm{NA}$ & NA & NA & NA & X1_82323161X1_88934871 \\
\hline $0.37 \mathrm{NA}$ & NA & NA & NA & X1_82323161X1_88934871 \\
\hline $0.56 \mathrm{NA}$ & NA & NA & NA & X1_82323161Gg_rs139109. \\
\hline $0.39 \mathrm{NA}$ & NA & NA & NA & Gg_rs148495 Gg_rs139109. \\
\hline $0.42 \mathrm{NA}$ & NA & NA & NA & X1_82323161Gg_rs139109. \\
\hline $0.54 \mathrm{NA}$ & NA & NA & NA & X1_82323161Gg_rs139109. \\
\hline $0.58 \mathrm{NA}$ & NA & NA & NA & Gg_rs148495 Gg_rs139109. \\
\hline 0.79 NA & NA & NA & NA & X1_82323161Gg_rs139109. \\
\hline $0.56 \mathrm{NA}$ & NA & NA & NA & Gg_rs148495 Gg_rs139109. \\
\hline $0.3 \mathrm{NA}$ & NA & NA & NA & Gg_rs148495 Gg_rs139018 \\
\hline $0.55 \mathrm{NA}$ & NA & NA & NA & Gg_rs148495 Gg_rs139018 \\
\hline $0.75 \mathrm{NA}$ & NA & NA & NA & Gg_rs148495 Gg_rs139018 \\
\hline $0.51 \mathrm{NA}$ & NA & NA & NA & Gg_rs148495 Gg_rs139109. \\
\hline $0.6 \mathrm{NA}$ & NA & NA & NA & Gg_rs148495 Gg_rs139018 \\
\hline $0.26 \mathrm{NA}$ & NA & NA & NA & Gg_rs148495 Gg_rs139018 \\
\hline $0.32 \mathrm{NA}$ & NA & NA & NA & Gg_rs148495 Gg_rs139018 \\
\hline $1.17 \mathrm{NA}$ & NA & NA & NA & Gg_rs148495 Gg_rs139018 \\
\hline $0.57 \mathrm{NA}$ & NA & NA & NA & Gg_rs148495 Gg_rs139109. \\
\hline $0.37 \mathrm{NA}$ & NA & NA & NA & Gg_rs148495 Gg_rs148584. \\
\hline $0.29 \mathrm{NA}$ & NA & NA & NA & Gg_rs148495 Gg_rs139018 \\
\hline $0.61 \mathrm{NA}$ & NA & NA & NA & Gg_rs148495 Gg_rs139109. \\
\hline $0.53 \mathrm{NA}$ & NA & NA & NA & Gg_rs148495 Gg_rs139109. \\
\hline $0.38 \mathrm{NA}$ & NA & NA & NA & Gg_rs148495 Gg_rs139109. \\
\hline $0.5 \mathrm{NA}$ & NA & NA & NA & Gg_rs148495 Gg_rs148584. \\
\hline $1.68 \mathrm{NA}$ & NA & NA & NA & Gg_rs148495 Gg_rs148584. \\
\hline $0.53 \mathrm{NA}$ & NA & NA & NA & Gg_rs148495 Gg_rs139109. \\
\hline $0.63 \mathrm{NA}$ & NA & NA & NA & Gg_rs148495 Gg_rs139109. \\
\hline 0.46 NA & NA & NA & NA & Gg_rs148495 Gg_rs139109. \\
\hline $0.38 \mathrm{NA}$ & NA & NA & NA & Gg_rs136480 Gg_rs139109. \\
\hline $0.25 \mathrm{NA}$ & NA & NA & NA & Gg_rs136480 Gg_rs148589. \\
\hline $0.23 \mathrm{NA}$ & NA & NA & NA & Gg_rs136480 Gg_rs148589. \\
\hline $0.47 \mathrm{NA}$ & NA & NA & NA & Gg_rs148495 Gg_rs139109. \\
\hline $0.41 \mathrm{NA}$ & NA & NA & NA & Gg_rs136480 Gg_rs139109. \\
\hline $0.54 \mathrm{NA}$ & NA & NA & NA & Gg_rs148495 Gg_rs139109. \\
\hline $0.5 \mathrm{NA}$ & NA & NA & NA & Gg_rs136480 Gg_rs148589. \\
\hline $0.7 \mathrm{NA}$ & NA & NA & NA & Gg_rs136480 Gg_rs148589. \\
\hline $0.66 \mathrm{NA}$ & NA & NA & NA & Gg_rs136480 Gg_rs139109. \\
\hline 0.52 NA & NA & NA & NA & Gg_rs136480 Gg_rs139109. \\
\hline $0.33 \mathrm{NA}$ & NA & NA & NA & Gg_rs136480 Gg_rs148589. \\
\hline $0.33 \mathrm{NA}$ & NA & NA & NA & Gg_rs148495 Gg_rs139109. \\
\hline $0.89 \mathrm{NA}$ & NA & NA & NA & Gg_rs136480 Gg_rs139109. \\
\hline $0.57 \mathrm{NA}$ & NA & NA & NA & Gg_rs136480 Gg_rs139109. \\
\hline
\end{tabular}




\begin{tabular}{|c|c|c|c|c|c|}
\hline $0.55 \mathrm{NA}$ & NA & NA & NA & & Gg_rs148495 Gg_rs139109. \\
\hline 0.43 & -0.86 & 0.45 & -0.94 & 0.58 & Gg_rs136480 Gg_rs148589. \\
\hline 0.57 & -1.46 & 0.59 & -1.82 & 0.76 & Gg_rs139018 Gg_rs148589. \\
\hline 0.55 & -0.64 & 0.57 & -1.73 & 0.74 & Gg_rs139018Gg_rs139109. \\
\hline $0.41 \mathrm{NA}$ & NA & NA & NA & & Gg_rs136480Gg_rs139109. \\
\hline $0.51 \mathrm{NA}$ & NA & NA & NA & & Gg_rs136480 Gg_rs148589. \\
\hline $0.58 \mathrm{NA}$ & NA & NA & NA & & Gg_rs136480 Gg_rs139109. \\
\hline 0.7 & 2.25 & 0.72 & 0.56 & 0.93 & Gg_rs136480Gg_rs139109. \\
\hline 0.46 NA & NA & NA & NA & & Gg_rs136480Gg_rs139109. \\
\hline $0.35 \mathrm{NA}$ & NA & NA & NA & & Gg_rs136480 Gg_rs139109. \\
\hline $0.49 \mathrm{NA}$ & NA & NA & NA & & Gg_rs136480 Gg_rs139109. \\
\hline $0.47 \mathrm{NA}$ & NA & NA & NA & & Gg_rs136480Gg_rs148589. \\
\hline $0.5 \mathrm{NA}$ & NA & NA & NA & & Gg_rs136480 Gg_rs139109. \\
\hline $0.38 \mathrm{NA}$ & NA & NA & NA & & Gg_rs136480 Gg_rs148589. \\
\hline $0.65 \mathrm{NA}$ & NA & NA & NA & & Gg_rs136480 Gg_rs139109. \\
\hline $0.29 \mathrm{NA}$ & NA & NA & NA & & Gg_rs136480 Gg_rs148589. \\
\hline $0.53 \mathrm{NA}$ & NA & NA & NA & & Gg_rs148495 Gg_rs139109. \\
\hline $0.38 \mathrm{NA}$ & NA & NA & NA & & Gg_rs148495 Gg_rs139109. \\
\hline 0.54 & -1.86 & 0.55 & -1.19 & 0.72 & Gg_rs136480 Gg_rs148589. \\
\hline $0.38 \mathrm{NA}$ & NA & NA & NA & & Gg_rs139018Gg_rs148589. \\
\hline 0.56 & -2.08 & 0.57 & -2.43 & 0.74 & Gg_rs139018Gg_rs148589. \\
\hline 0.78 & -2.24 & 0.81 & -3.77 & 1.04 & Gg_rs139018Gg_rs148589. \\
\hline $0.28 \mathrm{NA}$ & NA & NA & NA & & Gg_rs136480Gg_rs139109. \\
\hline 0.42 NA & NA & NA & NA & & Gg_rs136480 Gg_rs139109. \\
\hline $0.24 \mathrm{NA}$ & NA & NA & NA & & Gg_rs136480 Gg_rs148589. \\
\hline $0.45 \mathrm{NA}$ & NA & NA & NA & & Gg_rs148495 Gg_rs139109. \\
\hline $0.6 \mathrm{NA}$ & NA & NA & NA & & Gg_rs136480 Gg_rs139109. \\
\hline $0.58 \mathrm{NA}$ & NA & NA & NA & & Gg_rs136480 Gg_rs139109. \\
\hline $0.38 \mathrm{NA}$ & NA & NA & NA & & Gg_rs136480 Gg_rs139109. \\
\hline $0.35 \mathrm{NA}$ & NA & NA & NA & & Gg_rs136480 Gg_rs139109. \\
\hline $0.43 \mathrm{NA}$ & NA & NA & NA & & Gg_rs148495 Gg_rs139109. \\
\hline $0.65 \mathrm{NA}$ & NA & NA & NA & & Gg_rs136480 Gg_rs139109. \\
\hline $0.74 \mathrm{NA}$ & NA & NA & NA & & Gg_rs136480 Gg_rs139109. \\
\hline $0.45 \mathrm{NA}$ & NA & NA & NA & & Gg_rs136480 Gg_rs139109. \\
\hline $0.55 \mathrm{NA}$ & NA & NA & NA & & Gg_rs136480 Gg_rs139109. \\
\hline $0.35 \mathrm{NA}$ & NA & NA & NA & & Gg_rs148495 Gg_rs139109. \\
\hline $0.52 \mathrm{NA}$ & NA & NA & NA & & Gg_rs136480 Gg_rs139109. \\
\hline 0.58 & -0.44 & 0.56 & -1.77 & 0.77 & Gg_rs139018Gg_rs139109. \\
\hline 0.36 NA & NA & NA & NA & & Gg_rs136480 Gg_rs139109. \\
\hline $1.21 \mathrm{NA}$ & NA & NA & NA & & Gg_rs148495 Gg_rs139109. \\
\hline $0.24 \mathrm{NA}$ & NA & NA & NA & & Gg_rs148495 Gg_rs139109. \\
\hline $0.5 \mathrm{NA}$ & NA & NA & NA & & Gg_rs148584 Gg_rs139109. \\
\hline $0.33 \mathrm{NA}$ & NA & NA & NA & & Gg_rs148584 Gg_rs139109. \\
\hline
\end{tabular}




\begin{tabular}{|c|c|c|c|c|c|}
\hline $0.4 \mathrm{NA}$ & NA & NA & NA & & Gg_rs148584 Gg_rs139109. \\
\hline 0.61 & 2.03 & 0.59 & 0.81 & 0.82 & Gg_rs148584 Gg_rs139109. \\
\hline $0.39 \mathrm{NA}$ & NA & NA & NA & & Gg_rs139018Gg_rs139109. \\
\hline $0.4 \mathrm{NA}$ & NA & NA & NA & & Gg_rs139018 Gg_rs139109. \\
\hline $0.52 \mathrm{NA}$ & NA & NA & NA & & Gg_rs136480 Gg_rs139109. \\
\hline $0.35 \mathrm{NA}$ & NA & NA & NA & & Gg_rs139018Gg_rs139109. \\
\hline $0.39 \mathrm{NA}$ & NA & NA & NA & & Gg_rs139018 Gg_rs139109. \\
\hline $0.58 \mathrm{NA}$ & NA & NA & NA & & Gg_rs148495 Gg_rs139109. \\
\hline $0.41 \mathrm{NA}$ & NA & NA & NA & & Gg_rs136480 Gg_rs139109. \\
\hline 0.56 & -1.1 & 0.55 & -0.8 & 0.75 & Gg_rs139018 Gg_rs139109. \\
\hline $0.22 \mathrm{NA}$ & NA & NA & NA & & Gg_rs139018 Gg_rs139109. \\
\hline $0.37 \mathrm{NA}$ & NA & NA & NA & & Gg_rs139018Gg_rs139109. \\
\hline $0.56 \mathrm{NA}$ & NA & NA & NA & & Gg_rs136480Gg_rs139109. \\
\hline $0.39 \mathrm{NA}$ & NA & NA & NA & & Gg_rs136480 Gg_rs139109. \\
\hline $0.31 \mathrm{NA}$ & NA & NA & NA & & Gg_rs139018Gg_rs139109. \\
\hline $0.55 \mathrm{NA}$ & NA & NA & NA & & Gg_rs148495 snp.26.313.2 \\
\hline 0.46 NA & NA & NA & NA & & Gg_rs148495 Gg_rs139109. \\
\hline $0.53 \mathrm{NA}$ & NA & NA & NA & & Gg_rs148495 Gg_rs139109. \\
\hline $0.43 \mathrm{NA}$ & NA & NA & NA & & Gg_rs136480 Gg_rs139109. \\
\hline $0.34 \mathrm{NA}$ & NA & NA & NA & & Gg_rs139018Gg_rs139109. \\
\hline $0.44 \mathrm{NA}$ & NA & NA & NA & & Gg_rs136480 Gg_rs139109. \\
\hline $0.36 \mathrm{NA}$ & NA & NA & NA & & Gg_rs136480 Gg_rs139109. \\
\hline $0.52 \mathrm{NA}$ & NA & NA & NA & & Gg_rs136480 Gg_rs139109. \\
\hline $0.64 \mathrm{NA}$ & NA & NA & NA & & Gg_rs136480 Gg_rs139109. \\
\hline $0.45 \mathrm{NA}$ & NA & NA & NA & & Gg_rs136480Gg_rs139109. \\
\hline $0.36 \mathrm{NA}$ & NA & NA & NA & & Gg_rs136480 snp.26.313.2 \\
\hline $0.27 \mathrm{NA}$ & NA & NA & NA & & Gg_rs136480 snp.26.313.2 \\
\hline 0.49 NA & NA & NA & NA & & Gg_rs136480 snp.26.313.2 \\
\hline $0.48 \mathrm{NA}$ & NA & NA & NA & & Gg_rs136480 snp.26.313.2 \\
\hline $0.85 \mathrm{NA}$ & NA & NA & NA & & Gg_rs148584 snp.26.313.2 \\
\hline $0.73 \mathrm{NA}$ & NA & NA & NA & & Gg_rs136480 Gg_rs139109. \\
\hline 0.47 NA & NA & NA & NA & & Gg_rs148589 X1_1115558C \\
\hline $0.44 \mathrm{NA}$ & NA & NA & NA & & Gg_rs148589 X1_1167836؟ \\
\hline $0.32 \mathrm{NA}$ & NA & NA & NA & & Gg_rs148584 X1_1167836؟ \\
\hline $0.4 \mathrm{NA}$ & NA & NA & NA & & Gg_rs148589 X1_1115558C \\
\hline $0.57 \mathrm{NA}$ & NA & NA & NA & & Gg_rs148589 X1_1167836C \\
\hline $0.51 \mathrm{NA}$ & NA & NA & NA & & Gg_rs148589 X1_1167836C \\
\hline $0.56 \mathrm{NA}$ & NA & NA & NA & & Gg_rs148589 snp.26.313.2 \\
\hline $0.33 \mathrm{NA}$ & NA & NA & NA & & Gg_rs148589 snp.26.313.2 \\
\hline $0.44 \mathrm{NA}$ & NA & NA & NA & & Gg_rs148589 X1_1115558C \\
\hline $0.46 \mathrm{NA}$ & NA & NA & NA & & Gg_rs148589 X1_1115558C \\
\hline $0.31 \mathrm{NA}$ & NA & NA & NA & & Gg_rs136480 snp.26.313.2 \\
\hline $0.38 \mathrm{NA}$ & NA & NA & NA & & Gg_rs148589 X1_1167836C \\
\hline
\end{tabular}




\begin{tabular}{|c|c|c|c|c|c|}
\hline $0.44 \mathrm{NA}$ & NA & NA & NA & & Gg_rs148589 X1_1167836C \\
\hline 0.63 NA & NA & NA & NA & & Gg_rs148589 X1_1167836C \\
\hline $0.55 \mathrm{NA}$ & NA & NA & NA & & Gg_rs148589 X1_1115558C \\
\hline $0.36 \mathrm{NA}$ & NA & NA & NA & & Gg_rs148589 X1_1115558C \\
\hline $0.61 \mathrm{NA}$ & NA & NA & NA & & Gg_rs148589 X1_1167836؟ \\
\hline $0.51 \mathrm{NA}$ & NA & NA & NA & & Gg_rs148589 X1_1115558C \\
\hline $0.55 \mathrm{NA}$ & NA & NA & NA & & Gg_rs148589 snp.26.313.2 \\
\hline $0.29 \mathrm{NA}$ & NA & NA & NA & & Gg_rs148589 X1_1115558C \\
\hline $0.5 \mathrm{NA}$ & NA & NA & NA & & Gg_rs148589 snp.26.313.2 \\
\hline $0.42 \mathrm{NA}$ & NA & NA & NA & & Gg_rs148589 X1_1115558C \\
\hline 0.68 & -0.07 & 0.66 & -2.81 & 0.91 & Gg_rs148589 X1_1115558C \\
\hline 0.53 & 1.03 & 0.51 & 1.14 & 0.71 & Gg_rs148589 X1_1167836؟ \\
\hline $0.51 \mathrm{NA}$ & NA & NA & NA & & Gg_rs148589 X1_1167836؟ \\
\hline $0.55 \mathrm{NA}$ & NA & NA & NA & & Gg_rs148589 X1_1115558C \\
\hline $0.64 \mathrm{NA}$ & NA & NA & NA & & Gg_rs148589 X1_1167836C \\
\hline $0.53 \mathrm{NA}$ & NA & NA & NA & & Gg_rs148589 X1_1115558C \\
\hline 0.51 & -0.34 & 0.49 & -1.56 & 0.69 & Gg_rs148589 X1_1115558C \\
\hline $0.65 \mathrm{NA}$ & NA & NA & NA & & Gg_rs148589 X1_1115558C \\
\hline $0.52 \mathrm{NA}$ & NA & NA & NA & & Gg_rs148589 X1_1115558C \\
\hline 0.57 & 0.72 & 0.54 & -1.96 & 0.76 & Gg_rs148589 X1_1115558C \\
\hline 0.63 NA & NA & NA & NA & & Gg_rs148589 X1_1167836C \\
\hline $0.75 \mathrm{NA}$ & NA & NA & NA & & Gg_rs148589 X1_1167836C \\
\hline $0.71 \mathrm{NA}$ & NA & NA & NA & & Gg_rs148589 X1_1167836؟ \\
\hline 0.62 NA & NA & NA & NA & & Gg_rs148589 X1_1115558C \\
\hline $0.47 \mathrm{NA}$ & NA & NA & NA & & Gg_rs148589 X1_1167836؟ \\
\hline $0.6 \mathrm{NA}$ & NA & NA & NA & & Gg_rs148589 X1_1167836؟ \\
\hline 0.8 & -0.08 & 0.75 & -3.02 & 1.07 & Gg_rs148589 X1_1115558C \\
\hline $0.62 \mathrm{NA}$ & NA & NA & NA & & Gg_rs148589 X1_1115558C \\
\hline $0.3 \mathrm{NA}$ & NA & NA & NA & & Gg_rs148589 X1_1167836C \\
\hline $0.72 \mathrm{NA}$ & NA & NA & NA & & Gg_rs148589 X1_1115558C \\
\hline 0.65 NA & NA & NA & NA & & Gg_rs148589 X1_1115558C \\
\hline $0.59 \mathrm{NA}$ & NA & NA & NA & & Gg_rs148589 X1_1115558C \\
\hline 1.58 & -2.84 & 1.49 & -3.4 & 2.11 & Gg_rs148589 X1_1115558C \\
\hline 1.24 & 2.93 & 1.17 & -1.56 & 1.66 & Gg_rs139109 X1_1115558C \\
\hline $0.85 \mathrm{NA}$ & NA & NA & NA & & Gg_rs139109 X1_1115558C \\
\hline $0.63 \mathrm{NA}$ & NA & NA & NA & & Gg_rs148589 X1_1115558C \\
\hline $0.91 \mathrm{NA}$ & NA & NA & NA & & Gg_rs148589 X1_1115558C \\
\hline $0.56 \mathrm{NA}$ & NA & NA & NA & & Gg_rs139109 X1_1115558C \\
\hline $0.4 \mathrm{NA}$ & NA & NA & NA & & Gg_rs139109 X1_1115558C \\
\hline $0.79 \mathrm{NA}$ & NA & NA & NA & & Gg_rs139109 X1_1167836C \\
\hline $0.47 \mathrm{NA}$ & NA & NA & NA & & Gg_rs139109 X1_1115558C \\
\hline $0.78 \mathrm{NA}$ & NA & NA & NA & & Gg_rs148589 X1_1115558C \\
\hline 0.63 NA & NA & NA & NA & & Gg_rs148589 X1_1167836C \\
\hline
\end{tabular}




\begin{tabular}{|c|c|c|c|c|c|}
\hline $0.54 \mathrm{NA}$ & NA & NA & NA & & Gg_rs139109 X1_1167836؟ \\
\hline $0.8 \mathrm{NA}$ & NA & NA & NA & & Gg_rs139109 X1_1115558C \\
\hline $0.67 \mathrm{NA}$ & NA & NA & NA & & Gg_rs139109 X1_1115558C \\
\hline 0.39 NA & NA & NA & NA & & Gg_rs139109 X1_1115558C \\
\hline $0.97 \mathrm{NA}$ & NA & NA & NA & & Gg_rs148589 X1_1167836C \\
\hline 0.79 & -0.24 & 0.75 & -2.22 & 1.06 & Gg_rs148589 X1_1167836C \\
\hline $0.57 \mathrm{NA}$ & NA & NA & NA & & Gg_rs148589 X1_1167836C \\
\hline $0.51 \mathrm{NA}$ & NA & NA & NA & & Gg_rs139109 X1_1167836؟ \\
\hline 0.79 & 0.5 & 0.75 & -3.59 & 1.06 & Gg_rs139109 X1_1167836 \\
\hline $0.5 \mathrm{NA}$ & NA & NA & NA & & Gg_rs148589 X1_1167836 \\
\hline $0.56 \mathrm{NA}$ & NA & NA & NA & & Gg_rs139109 X1_1167836C \\
\hline $0.63 \mathrm{NA}$ & NA & NA & NA & & Gg_rs148589 X1_1167836C \\
\hline 0.69 NA & NA & NA & NA & & Gg_rs148589 X1_1167836C \\
\hline $0.6 \mathrm{NA}$ & NA & NA & NA & & Gg_rs139109 X1_1167836؟ \\
\hline $0.86 \mathrm{NA}$ & NA & NA & NA & & Gg_rs148589 X1_1167836 \\
\hline 1.16 & -0.96 & 1.01 & -3.49 & 1.5 & Gg_rs139109 X1_1167836؟ \\
\hline $0.57 \mathrm{NA}$ & NA & NA & NA & & Gg_rs148589 X1_1167836C \\
\hline $0.7 \mathrm{NA}$ & NA & NA & NA & & Gg_rs148589 X1_1167836C \\
\hline 0.13 & -0.64 & 0.12 & -0.14 & 0.17 & Gg_rs148589 X1_1167836؟ \\
\hline $0.75 \mathrm{NA}$ & NA & NA & NA & & Gg_rs139109 X1_1167836 \\
\hline $0.7 \mathrm{NA}$ & NA & NA & NA & & Gg_rs139109 X1_1167836؟ \\
\hline $0.51 \mathrm{NA}$ & NA & NA & NA & & Gg_rs148589 X1_1167836؟ \\
\hline $0.33 \mathrm{NA}$ & NA & NA & NA & & snp.26.313.2‘X1_1167836؟ \\
\hline $0.68 \mathrm{NA}$ & NA & NA & NA & & snp.26.313.2‘X1_1167836؟ \\
\hline 0.87 & -0.58 & 0.76 & -0.45 & 1.13 & snp.26.313.2‘X1_1167836؟ \\
\hline $0.55 \mathrm{NA}$ & NA & NA & NA & & snp.26.313.2‘X1_1167836؟ \\
\hline $0.71 \mathrm{NA}$ & NA & NA & NA & & snp.26.313.2‘X1_1167836؟ \\
\hline 0.99 NA & NA & NA & NA & & snp.26.313.2‘X1_1167836؟ \\
\hline $0.82 \mathrm{NA}$ & NA & NA & NA & & snp.26.313.2‘X1_1167836؟ \\
\hline $0.72 \mathrm{NA}$ & NA & NA & NA & & snp.26.313.2‘X1_1167836؟ \\
\hline $0.45 \mathrm{NA}$ & NA & NA & NA & & snp.26.313.2‘X1_1167836؟ \\
\hline $0.39 \mathrm{NA}$ & NA & NA & NA & & snp.26.313.2 ¿X1_1167836؟ \\
\hline $0.46 \mathrm{NA}$ & NA & NA & NA & & Gg_rs139109 X1_1167836؟ \\
\hline $0.58 \mathrm{NA}$ & NA & NA & NA & & snp.26.313.2‘X1_1167836؟ \\
\hline 1.26 & 3.82 & 1.1 & -0.87 & 1.63 & snp.26.313.2‘X1_1167836؟ \\
\hline $1.31 \mathrm{NA}$ & NA & NA & NA & & snp.26.313.2‘X1_1167836؟ \\
\hline 0.82 NA & NA & NA & NA & & snp.26.313.2‘X1_1167836؟ \\
\hline $0.58 \mathrm{NA}$ & NA & NA & NA & & snp.26.313.2‘X1_1167836؟ \\
\hline $1.4 \mathrm{NA}$ & NA & NA & NA & & snp.26.313.2‘X1_1167836؟ \\
\hline 1.3 & -3.38 & 1.13 & -4.84 & 1.68 & snp.26.313.2‘X1_1167836؟ \\
\hline $0.98 \mathrm{NA}$ & NA & NA & NA & & Gg_rs139109 X1_1167836؟ \\
\hline $0.76 \mathrm{NA}$ & NA & NA & NA & & snp.26.313.2‘X1_1167836؟ \\
\hline 0.95 & 0.86 & 0.83 & -2.13 & 1.23 & Gg_rs139109 X1_1167836 \\
\hline
\end{tabular}




\begin{tabular}{|c|c|c|c|c|c|}
\hline 0.34 & -0.83 & 0.29 & -1.02 & 0.44 & snp.26.313.2‘X1_1167836؛ \\
\hline $0.59 \mathrm{NA}$ & NA & NA & NA & & snp.26.313.2‘X1_1167836؛ \\
\hline $0.72 \mathrm{NA}$ & NA & NA & NA & & snp.26.313.2‘X1_1182262C \\
\hline $0.42 \mathrm{NA}$ & NA & NA & NA & & snp.26.313.2‘X1_1182262C \\
\hline $0.55 \mathrm{NA}$ & NA & NA & NA & & X1_1115558CX1_1182262C \\
\hline 0.49 NA & NA & NA & NA & & X1_1115558CX1_1182262C \\
\hline $0.32 \mathrm{NA}$ & NA & NA & NA & & X1_1115558CX1_1182262C \\
\hline 0.75 & -0.78 & 0.71 & 2.31 & 1 & X1_1115558CX1_1182262C \\
\hline 0.66 & 1.54 & 0.62 & 3.95 & 0.87 & X1_1115558CX1_1182262C \\
\hline $0.55 \mathrm{NA}$ & NA & NA & NA & & X1_1115558CGg_rs139298 \\
\hline 0.64 NA & NA & NA & NA & & X1_1115558CGg_rs139298. \\
\hline $0.41 \mathrm{NA}$ & NA & NA & NA & & X1_1115558CGg_rs139298 \\
\hline $0.39 \mathrm{NA}$ & NA & NA & NA & & X1_1115558CGg_rs139298. \\
\hline $0.61 \mathrm{NA}$ & NA & NA & NA & & X1_1115558Csnp.146.19.5! \\
\hline 0.81 & -0.74 & 0.71 & 3.48 & 1.07 & X1_1115558CGg_rs139298 \\
\hline 0.48 NA & NA & NA & NA & & X1_1115558Csnp.146.19.5! \\
\hline 0.68 NA & NA & NA & NA & & X1_11700171Gg_rs139298. \\
\hline $0.47 \mathrm{NA}$ & NA & NA & NA & & X1_1115558Csnp.146.19.5! \\
\hline 0.52 & -1.11 & 0.49 & 0.81 & 0.69 & X1_1115558CGg_rs139298 \\
\hline $0.28 \mathrm{NA}$ & NA & NA & NA & & X1_1115558Csnp.146.19.5! \\
\hline $0.85 \mathrm{NA}$ & NA & NA & NA & & X1_1115558CGg_rs139298. \\
\hline $0.38 \mathrm{NA}$ & NA & NA & NA & & X1_11700171Gg_rs139298. \\
\hline $0.39 \mathrm{NA}$ & NA & NA & NA & & X1_11700171Gg_rs139298. \\
\hline $0.58 \mathrm{NA}$ & NA & NA & NA & & X1_11700171Gg_rs139298. \\
\hline 0.81 & -1.88 & 0.7 & 2.63 & 1.06 & X1_11700171Gg_rs139298 \\
\hline $0.47 \mathrm{NA}$ & NA & NA & NA & & X1_11700171Gg_rs139298. \\
\hline $0.79 \mathrm{NA}$ & NA & NA & NA & & X1_11700171Gg_rs139298. \\
\hline $0.33 \mathrm{NA}$ & NA & NA & NA & & X1_11700171Gg_rs139298 \\
\hline 1.08 & 0.05 & 0.94 & 3.06 & 1.42 & X1_11700171Gg_rs139298 \\
\hline $0.71 \mathrm{NA}$ & NA & NA & NA & & X1_11700171Gg_rs139298 \\
\hline 0.96 NA & NA & NA & NA & & X1_11700171Gg_rs139298. \\
\hline $0.53 \mathrm{NA}$ & NA & NA & NA & & X1_1167836కGg_rs139298 \\
\hline 0.71 & -0.83 & 0.62 & 2.14 & 0.93 & X1_11700171Gg_rs139298 \\
\hline 0.71 & 0.27 & 0.62 & 1.77 & 0.94 & X1_11700171snp.146.19.5! \\
\hline 0.84 NA & NA & NA & NA & & X1_11700171Gg_rs139298. \\
\hline $0.95 \mathrm{NA}$ & NA & NA & NA & & X1_11700171Gg_rs139298. \\
\hline $0.58 \mathrm{NA}$ & NA & NA & NA & & X1_11700171snp.146.19.5! \\
\hline 1.06 & -0.8 & 0.93 & 3.51 & 1.4 & X1_11700171Gg_rs139298 \\
\hline 0.66 & 2.34 & 0.58 & -0.15 & 0.87 & X1_11700171snp.146.19.5! \\
\hline $0.53 \mathrm{NA}$ & NA & NA & NA & & X1_11700171snp.146.19.5! \\
\hline $1.07 \mathrm{NA}$ & NA & NA & NA & & X1_11700171snp.146.19.5! \\
\hline $1.13 \mathrm{NA}$ & NA & NA & NA & & X1_11700171snp.146.19.5! \\
\hline $0.37 \mathrm{NA}$ & NA & NA & NA & & X1_11700171X1_12837468 \\
\hline
\end{tabular}




\begin{tabular}{|c|c|c|c|c|c|}
\hline 0.45 & 2.18 & 0.47 & -2.66 & 0.69 & Gg_rs148779 X1_12837468 \\
\hline $0.38 \mathrm{NA}$ & NA & NA & NA & & X1_1115558CX1_1341656C \\
\hline $0.26 \mathrm{NA}$ & NA & NA & NA & & Gg_rs148779 X1_12837468 \\
\hline $0.8 \mathrm{NA}$ & NA & NA & NA & & Gg_rs148779 X1_12837468 \\
\hline $0.54 \mathrm{NA}$ & NA & NA & NA & & X1_12585307X1_12837468 \\
\hline $0.46 \mathrm{NA}$ & NA & NA & NA & & X1_12585307X1_12837468 \\
\hline $0.73 \mathrm{NA}$ & NA & NA & NA & & Gg_rs148779 X1_12837468 \\
\hline $0.6 \mathrm{NA}$ & NA & NA & NA & & X1_12585307X1_12837468 \\
\hline $0.41 \mathrm{NA}$ & NA & NA & NA & & Gg_rs148779 X1_12837468 \\
\hline 0.66 & -0.42 & 0.69 & 3.34 & 1.01 & X1_12585307X1_12837468 \\
\hline $0.54 \mathrm{NA}$ & NA & NA & NA & & X1_12585307X1_12837468 \\
\hline $0.82 \mathrm{NA}$ & NA & NA & NA & & X1_12585307X1_12837468 \\
\hline 0.73 & -1.23 & 0.76 & 1.37 & 1.11 & X1_12585307X1_12837468 \\
\hline 0.65 & -1.33 & 0.68 & 2.67 & 0.99 & X1_12585307X1_12837468 \\
\hline 0.77 & 1.48 & 0.8 & -4.05 & 1.17 & X1_12585307X1_12837468 \\
\hline $0.36 \mathrm{NA}$ & NA & NA & NA & & X1_12585307X1_12837468 \\
\hline $0.34 \mathrm{NA}$ & NA & NA & NA & & X1_12585307X1_12837468 \\
\hline $0.39 \mathrm{NA}$ & NA & NA & NA & & X1_12585307X1_12837468 \\
\hline 0.69 NA & NA & NA & NA & & X1_12585307X1_12837468 \\
\hline $0.68 \mathrm{NA}$ & NA & NA & NA & & X1_12585307X1_12837468 \\
\hline $0.67 \mathrm{NA}$ & NA & NA & NA & & X1_12585307X1_12837468 \\
\hline 0.19 & 0.23 & 0.2 & 0.81 & 0.28 & X1_12585307X1_12837468 \\
\hline 0.01 & -0.04 & 0.01 & 0.04 & 0.01 & X1_12585307X1_12837468 \\
\hline $0.45 \mathrm{NA}$ & NA & NA & NA & & X1_12585307X1_12837468 \\
\hline $0.31 \mathrm{NA}$ & NA & NA & NA & & X1_12585307X1_12837468 \\
\hline $0.06 \mathrm{NA}$ & NA & NA & NA & & X1_12585307X1_12837468 \\
\hline $0.43 \mathrm{NA}$ & NA & NA & NA & & X1_12585307X1_12837468 \\
\hline $0.3 \mathrm{NA}$ & NA & NA & NA & & X1_12585307X1_12837468 \\
\hline $0.11 \mathrm{NA}$ & NA & NA & NA & & X1_12585307X1_12837468 \\
\hline 0.12 & -0.25 & 0.12 & 0.63 & 0.18 & X1_12585307X1_12837468 \\
\hline $0.57 \mathrm{NA}$ & NA & NA & NA & & X1_12585307X1_12837468 \\
\hline $0.04 \mathrm{NA}$ & NA & NA & NA & & Gg_rs139298 X1_12837468 \\
\hline $0.53 \mathrm{NA}$ & NA & NA & NA & & snp.146.19.5!Gg_rs139396 \\
\hline 0.44 NA & NA & NA & NA & & X1_12585307X1_1341656؟ \\
\hline $0.4 \mathrm{NA}$ & NA & NA & NA & & snp.146.19.5!X1_1341656؟ \\
\hline $0.57 \mathrm{NA}$ & NA & NA & NA & & snp.146.19.5!X1_1341656؟ \\
\hline $0.51 \mathrm{NA}$ & NA & NA & NA & & snp.146.19.5!X1_1341656؟ \\
\hline 0.64 NA & NA & NA & NA & & snp.146.19.5!X1_1341656؟ \\
\hline $0.33 \mathrm{NA}$ & NA & NA & NA & & snp.146.19.5!X1_1341656؟ \\
\hline $0.58 \mathrm{NA}$ & NA & NA & NA & & snp.146.19.5!X1_1341656؟ \\
\hline 0.46 NA & NA & NA & NA & & snp.146.19.5!X1_1341656؟ \\
\hline $0.43 \mathrm{NA}$ & NA & NA & NA & & snp.146.19.5!X1_1341656؟ \\
\hline $0.62 \mathrm{NA}$ & NA & NA & NA & & snp.146.19.5!X1_1341656؟ \\
\hline
\end{tabular}




\begin{tabular}{|c|c|c|c|c|c|c|c|}
\hline $0.53 \mathrm{NA}$ & NA & & NA & & NA & & snp.146.19.5!X1_1341656؟ \\
\hline $0.4 \mathrm{NA}$ & NA & & NA & & NA & & snp.146.19.5!X1_1341656؟ \\
\hline $0.37 \mathrm{NA}$ & NA & & NA & & NA & & snp.146.19.5!X1_1341656؟ \\
\hline 0.86 NA & NA & & NA & & NA & & snp.146.19.5!X1_1341656؟ \\
\hline $0.59 \mathrm{NA}$ & NA & & NA & & NA & & snp.146.19.5!X1_1341656؟ \\
\hline $0.48 \mathrm{NA}$ & NA & & NA & & NA & & snp.146.19.5!Gg_rs139396 \\
\hline $0.57 \mathrm{NA}$ & NA & & NA & & NA & & snp.146.19.5!Gg_rs139396 \\
\hline $0.58 \mathrm{NA}$ & NA & & NA & & NA & & snp.146.19.5!X1_1341656؟ \\
\hline 0.35 & -1.21 & 0.29 & & 1.15 & & 0.46 & snp.146.19.5!X1_1341656 \\
\hline $0.43 \mathrm{NA}$ & NA & & NA & & NA & & snp.146.19.5!Gg_rs139396 \\
\hline 0.84 & 0.46 & 0.68 & & 2.55 & & 1.12 & snp.146.19.5!Gg_rs154270 \\
\hline $1.01 \mathrm{NA}$ & NA & & NA & & NA & & snp.146.19.5!Gg_rs139396 \\
\hline $0.73 \mathrm{NA}$ & NA & & NA & & NA & & snp.146.19.5!Gg_rs148910 \\
\hline $0.54 \mathrm{NA}$ & NA & & NA & & NA & & Gg_rs148853 Gg_rs139396 \\
\hline 0.58 & -1.27 & 0.54 & & 2.09 & & 0.78 & X1_1341656કGg_rs148910 \\
\hline $0.6 \mathrm{NA}$ & NA & & NA & & NA & & X1_1341656؟Gg_rs148934 \\
\hline $0.4 \mathrm{NA}$ & NA & & NA & & NA & & X1_1341656CGg_rs148910. \\
\hline $0.61 \mathrm{NA}$ & NA & & NA & & NA & & Gg_rs148853:Gg_rs148910. \\
\hline $0.58 \mathrm{NA}$ & NA & & NA & & NA & & Gg_rs148853.Gg_rs148910. \\
\hline 0.65 & -1.97 & 0.6 & & 3.47 & & 0.87 & X1_1341656વGg_rs148910. \\
\hline 0.46 NA & NA & & NA & & NA & & Gg_rs148853.Gg_rs148910. \\
\hline $0.34 \mathrm{NA}$ & NA & & NA & & NA & & X1_1341656ভGg_rs148934 \\
\hline 0.98 & 1.16 & 0.91 & & 3.83 & & 1.32 & X1_1341656కGg_rs148934 \\
\hline $1.43 \mathrm{NA}$ & NA & & NA & & NA & & X1_1341656ฐGg_rs148934 \\
\hline $0.4 \mathrm{NA}$ & NA & & NA & & NA & & Gg_rs148853:Gg_rs148934 \\
\hline $0.84 \mathrm{NA}$ & NA & & NA & & NA & & Gg_rs148853.Gg_rs148934 \\
\hline 0.04 & 0.17 & 0.04 & & 0.15 & & 0.06 & Gg_rs154270 Gg_rs148934 \\
\hline 0.98 & 3.81 & 1.07 & & -0.04 & & 1.38 & Gg_rs139396 Gg_rs148934 \\
\hline $0.77 \mathrm{NA}$ & NA & & NA & & NA & & X1_1130108EGg_rs149396 \\
\hline $0.55 \mathrm{NA}$ & NA & & NA & & NA & & X1_1341656@Gg_rs148934 \\
\hline 0.3 & -1.43 & 0.33 & & 0.77 & & 0.42 & Gg_rs154270 Gg_rs148934 \\
\hline $0.55 \mathrm{NA}$ & NA & & NA & & NA & & Gg_rs154270 Gg_rs148934 \\
\hline $0.58 \mathrm{NA}$ & NA & & NA & & NA & & Gg_rs154270 Gg_rs148934 \\
\hline $1.25 \mathrm{NA}$ & NA & & NA & & NA & & X1_1341656CGg_rs148934 \\
\hline 0.96 NA & NA & & NA & & NA & & Gg_rs139396Gg_rs148934 \\
\hline $0.58 \mathrm{NA}$ & NA & & NA & & NA & & Gg_rs154270 Gg_rs148934 \\
\hline $0.68 \mathrm{NA}$ & NA & & NA & & NA & & Gg_rs154270 Gg_rs148934 \\
\hline $0.43 \mathrm{NA}$ & NA & & NA & & NA & & Gg_rs148853.Gg_rs148934 \\
\hline $0.62 \mathrm{NA}$ & NA & & NA & & NA & & Gg_rs154270 Gg_rs148954. \\
\hline 0.58 & -1.86 & 0.63 & & 1.33 & & 0.82 & Gg_rs154270Gg_rs148934 \\
\hline $0.6 \mathrm{NA}$ & NA & & NA & & NA & & X1_1341656ฐGg_rs148954 \\
\hline 1.42 & 1.74 & 1.55 & & -1.95 & & 2 & X1_1341656ฐGg_rs148954 \\
\hline 0.59 NA & NA & & NA & & NA & & Gg_rs154270Gg_rs148954 \\
\hline
\end{tabular}




\begin{tabular}{|c|c|c|c|c|}
\hline $0.29 \mathrm{NA}$ & NA & NA & NA & Gg_rs154270 Gg_rs148973 \\
\hline $0.54 \mathrm{NA}$ & NA & NA & NA & Gg_rs154270 Gg_rs148954 \\
\hline 0.63 NA & NA & NA & NA & Gg_rs148853.Gg_rs148973 \\
\hline $0.62 \mathrm{NA}$ & NA & NA & NA & Gg_rs154270 Gg_rs148954 \\
\hline $0.48 \mathrm{NA}$ & NA & NA & NA & Gg_rs148853 Gg_rs148954 \\
\hline $0.91 \mathrm{NA}$ & NA & NA & NA & Gg_rs148910Gg_rs148954 \\
\hline 0.93 NA & NA & NA & NA & Gg_rs154270Gg_rs148954 \\
\hline 1.39 & -3.01 & 1.41 & 1.77 & 1.86 Gg_rs148910Gg_rs148954 \\
\hline $0.97 \mathrm{NA}$ & NA & NA & NA & Gg_rs148910.Gg_rs148954 \\
\hline $0.75 \mathrm{NA}$ & NA & NA & NA & Gg_rs148910Gg_rs148973 \\
\hline $0.85 \mathrm{NA}$ & NA & NA & NA & Gg_rs148910Gg_rs148954 \\
\hline $1.02 \mathrm{NA}$ & NA & NA & NA & Gg_rs148910Gg_rs148954 \\
\hline $0.97 \mathrm{NA}$ & NA & NA & NA & Gg_rs148910Gg_rs148954 \\
\hline $0.72 \mathrm{NA}$ & NA & NA & NA & X1_1341656ভGg_rs148954 \\
\hline 0.49 NA & NA & NA & NA & Gg_rs148910Gg_rs148954 \\
\hline $0.94 \mathrm{NA}$ & NA & NA & NA & Gg_rs148910Gg_rs148954 \\
\hline $1.06 \mathrm{NA}$ & NA & NA & NA & Gg_rs148910Gg_rs148954 \\
\hline $0.86 \mathrm{NA}$ & NA & NA & NA & Gg_rs148910.Gg_rs148954 \\
\hline $0.62 \mathrm{NA}$ & NA & NA & NA & Gg_rs148910Gg_rs148954 \\
\hline $3 N A$ & NA & NA & NA & Gg_rs148910.Gg_rs148954 \\
\hline $3.09 \mathrm{NA}$ & NA & NA & NA & Gg_rs148910Gg_rs148954 \\
\hline $3.95 \mathrm{NA}$ & NA & NA & NA & Gg_rs148910Gg_rs148954 \\
\hline $13.04 \mathrm{NA}$ & NA & NA & NA & Gg_rs148910Gg_rs148954 \\
\hline $3.88 \mathrm{NA}$ & NA & NA & NA & Gg_rs148910Gg_rs148954 \\
\hline $3.46 \mathrm{NA}$ & NA & NA & NA & Gg_rs148910.Gg_rs148954 \\
\hline $2.07 \mathrm{NA}$ & NA & NA & NA & Gg_rs148910.Gg_rs148954 \\
\hline $14.38 \mathrm{NA}$ & NA & NA & NA & Gg_rs148910.Gg_rs148954 \\
\hline $14.36 \mathrm{NA}$ & NA & NA & NA & Gg_rs148910Gg_rs148954 \\
\hline 13.47 NA & NA & NA & NA & Gg_rs148910Gg_rs148954 \\
\hline $3.44 \mathrm{NA}$ & NA & NA & NA & Gg_rs148910Gg_rs148954 \\
\hline $15.15 \mathrm{NA}$ & NA & NA & NA & Gg_rs148910Gg_rs148954 \\
\hline $11.77 \mathrm{NA}$ & NA & NA & NA & Gg_rs148910Gg_rs148954 \\
\hline $3.32 \mathrm{NA}$ & NA & NA & NA & Gg_rs148910Gg_rs148954 \\
\hline $12.35 \mathrm{NA}$ & NA & NA & NA & Gg_rs148910Gg_rs148954 \\
\hline $12.8 \mathrm{NA}$ & NA & NA & NA & Gg_rs148910Gg_rs148954 \\
\hline $12.49 \mathrm{NA}$ & NA & NA & NA & Gg_rs148910.Gg_rs148954 \\
\hline $13.28 \mathrm{NA}$ & NA & NA & NA & Gg_rs148910.Gg_rs148954 \\
\hline $12.64 \mathrm{NA}$ & NA & NA & NA & Gg_rs148910Gg_rs148954 \\
\hline $10.6 \mathrm{NA}$ & NA & NA & NA & Gg_rs148910.Gg_rs148954 \\
\hline $12.99 \mathrm{NA}$ & NA & NA & NA & Gg_rs148910.Gg_rs148954 \\
\hline $4.19 \mathrm{NA}$ & NA & NA & NA & Gg_rs148910Gg_rs148954 \\
\hline $15.04 \mathrm{NA}$ & NA & NA & NA & Gg_rs148910.Gg_rs148954 \\
\hline $13.98 \mathrm{NA}$ & NA & NA & NA & Gg_rs148910Gg_rs148954 \\
\hline
\end{tabular}




\begin{tabular}{|c|c|c|c|c|}
\hline $9.97 \mathrm{NA}$ & NA & NA & NA & Gg_rs148910.Gg_rs148954 \\
\hline $15 \mathrm{NA}$ & NA & NA & NA & Gg_rs148910.Gg_rs148954 \\
\hline $12.11 \mathrm{NA}$ & NA & NA & NA & Gg_rs148910.Gg_rs148954 \\
\hline $10.82 \mathrm{NA}$ & NA & NA & NA & Gg_rs148910.Gg_rs148954 \\
\hline $3.76 \mathrm{NA}$ & NA & NA & NA & Gg_rs148910.Gg_rs148954 \\
\hline $12.11 \mathrm{NA}$ & NA & NA & NA & Gg_rs148910.Gg_rs148954 \\
\hline 14.23 NA & NA & NA & NA & Gg_rs148910.Gg_rs148954 \\
\hline $3.23 \mathrm{NA}$ & NA & NA & NA & Gg_rs148910.Gg_rs148954 \\
\hline $0.34 \mathrm{NA}$ & NA & NA & NA & Gg_rs148910.Gg_rs148954 \\
\hline $0.57 \mathrm{NA}$ & NA & NA & NA & Gg_rs148910.Gg_rs148973 \\
\hline $5.4 \mathrm{NA}$ & NA & NA & NA & Gg_rs148910.Gg_rs148954 \\
\hline $6.79 \mathrm{NA}$ & NA & NA & NA & Gg_rs148910.Gg_rs148954 \\
\hline $8.2 \mathrm{NA}$ & NA & NA & NA & Gg_rs148910.Gg_rs148954 \\
\hline $7.21 \mathrm{NA}$ & NA & NA & NA & Gg_rs148910.Gg_rs148954 \\
\hline $7.14 \mathrm{NA}$ & NA & NA & NA & Gg_rs148910.Gg_rs148954 \\
\hline 4.36 NA & NA & NA & NA & Gg_rs148910Gg_rs148954 \\
\hline 4.7 NA & NA & NA & NA & Gg_rs148910Gg_rs148954 \\
\hline $5.16 \mathrm{NA}$ & NA & NA & NA & Gg_rs148910Gg_rs148954 \\
\hline $7.94 \mathrm{NA}$ & NA & NA & NA & Gg_rs148910.Gg_rs148954 \\
\hline $12.09 \mathrm{NA}$ & NA & NA & NA & Gg_rs148910.Gg_rs148954 \\
\hline $6.95 \mathrm{NA}$ & NA & NA & NA & Gg_rs148910.Gg_rs148954 \\
\hline $10.02 \mathrm{NA}$ & NA & NA & NA & Gg_rs148910.Gg_rs148954 \\
\hline $7.05 \mathrm{NA}$ & NA & NA & NA & Gg_rs148910.Gg_rs148954 \\
\hline $5.32 \mathrm{NA}$ & NA & NA & NA & Gg_rs148910.Gg_rs148954 \\
\hline 4.49 NA & NA & NA & NA & Gg_rs148910.Gg_rs148954 \\
\hline $1.69 \mathrm{NA}$ & NA & NA & NA & Gg_rs148910.Gg_rs148973 \\
\hline $5.79 \mathrm{NA}$ & NA & NA & NA & Gg_rs148910.Gg_rs148954 \\
\hline $11.44 \mathrm{NA}$ & NA & NA & NA & Gg_rs148910.Gg_rs148954 \\
\hline $6.18 \mathrm{NA}$ & NA & NA & NA & Gg_rs148910.Gg_rs148954 \\
\hline $6.28 \mathrm{NA}$ & NA & NA & NA & Gg_rs148910.Gg_rs148954 \\
\hline 4.16 NA & NA & NA & NA & Gg_rs148910Gg_rs148954 \\
\hline $4.54 \mathrm{NA}$ & NA & NA & NA & Gg_rs148910.Gg_rs148954 \\
\hline $1.69 \mathrm{NA}$ & NA & NA & NA & Gg_rs148910.Gg_rs148954 \\
\hline $5.36 \mathrm{NA}$ & NA & NA & NA & Gg_rs148910.Gg_rs148954 \\
\hline $7.64 \mathrm{NA}$ & NA & NA & NA & Gg_rs148910Gg_rs148954 \\
\hline $6.09 \mathrm{NA}$ & NA & NA & NA & Gg_rs148910.Gg_rs148954 \\
\hline $12.77 \mathrm{NA}$ & NA & NA & NA & Gg_rs148910Gg_rs148954 \\
\hline $10.3 \mathrm{NA}$ & NA & NA & NA & Gg_rs148910.Gg_rs148954 \\
\hline $10.86 \mathrm{NA}$ & NA & NA & NA & Gg_rs148910.Gg_rs148954 \\
\hline $5.92 \mathrm{NA}$ & NA & NA & NA & Gg_rs148910.Gg_rs148954 \\
\hline $6.37 \mathrm{NA}$ & NA & NA & NA & Gg_rs148910.Gg_rs148954 \\
\hline $3.85 \mathrm{NA}$ & NA & NA & NA & Gg_rs148910.Gg_rs148954 \\
\hline $1.59 \mathrm{NA}$ & NA & NA & NA & Gg_rs148910.Gg_rs148954 \\
\hline
\end{tabular}




\begin{tabular}{|c|c|c|c|c|}
\hline $7.54 \mathrm{NA}$ & NA & NA & NA & Gg_rs148910Gg_rs148954 \\
\hline $6.57 \mathrm{NA}$ & NA & NA & NA & Gg_rs148910Gg_rs148954 \\
\hline $5.72 \mathrm{NA}$ & NA & NA & NA & Gg_rs148910Gg_rs148954 \\
\hline 4.59 NA & NA & NA & NA & Gg_rs148910Gg_rs148954 \\
\hline $1.8 \mathrm{NA}$ & NA & NA & NA & Gg_rs148910Gg_rs148954 \\
\hline $7.7 \mathrm{NA}$ & NA & NA & NA & Gg_rs148910.Gg_rs148954 \\
\hline $6.54 \mathrm{NA}$ & NA & NA & NA & Gg_rs148910Gg_rs148954 \\
\hline $13.84 \mathrm{NA}$ & NA & NA & NA & Gg_rs148910Gg_rs148954 \\
\hline $9.75 \mathrm{NA}$ & NA & NA & NA & Gg_rs148910Gg_rs148954 \\
\hline $13.23 \mathrm{NA}$ & NA & NA & NA & Gg_rs148910Gg_rs148954 \\
\hline $9.45 \mathrm{NA}$ & NA & NA & NA & Gg_rs148910Gg_rs148954 \\
\hline 8.12 NA & NA & NA & NA & Gg_rs148910Gg_rs148954 \\
\hline $6.97 \mathrm{NA}$ & NA & NA & NA & Gg_rs148910Gg_rs148954 \\
\hline 4.77 NA & NA & NA & NA & Gg_rs148910Gg_rs148954 \\
\hline 4.89 NA & NA & NA & NA & Gg_rs148910Gg_rs148954 \\
\hline 4.54 NA & NA & NA & NA & Gg_rs148910Gg_rs148954 \\
\hline $3.77 \mathrm{NA}$ & NA & NA & NA & Gg_rs148910Gg_rs148954 \\
\hline $0.53 \mathrm{NA}$ & NA & NA & NA & Gg_rs148910Gg_rs148954 \\
\hline $0.41 \mathrm{NA}$ & NA & NA & NA & Gg_rs148910Gg_rs148954 \\
\hline 1.83 NA & NA & NA & NA & Gg_rs148910.Gg_rs148973 \\
\hline 0.59 NA & NA & NA & NA & Gg_rs148910Gg_rs148954 \\
\hline 7.43 NA & NA & NA & NA & Gg_rs148910Gg_rs148954 \\
\hline $7.82 \mathrm{NA}$ & NA & NA & NA & Gg_rs148910Gg_rs148954 \\
\hline $5.68 \mathrm{NA}$ & NA & NA & NA & Gg_rs148910Gg_rs148954 \\
\hline $1.82 \mathrm{NA}$ & NA & NA & NA & Gg_rs148910Gg_rs148954 \\
\hline 7.13 NA & NA & NA & NA & Gg_rs148910.Gg_rs148954 \\
\hline $7.93 \mathrm{NA}$ & NA & NA & NA & Gg_rs148910.Gg_rs148954 \\
\hline $7.96 \mathrm{NA}$ & NA & NA & NA & Gg_rs148910Gg_rs148954 \\
\hline $5.57 \mathrm{NA}$ & NA & NA & NA & Gg_rs148910Gg_rs148954 \\
\hline 4.72 NA & NA & NA & NA & Gg_rs148910Gg_rs148954 \\
\hline $4.26 \mathrm{NA}$ & NA & NA & NA & Gg_rs148910Gg_rs148954 \\
\hline $2.04 \mathrm{NA}$ & NA & NA & NA & Gg_rs148910Gg_rs148954 \\
\hline $6.7 \mathrm{NA}$ & NA & NA & NA & Gg_rs148910Gg_rs148954 \\
\hline 4.09 NA & NA & NA & NA & Gg_rs148910Gg_rs148954 \\
\hline $4.93 \mathrm{NA}$ & NA & NA & NA & Gg_rs148910Gg_rs148954 \\
\hline $6.74 \mathrm{NA}$ & NA & NA & NA & Gg_rs148910Gg_rs148954 \\
\hline $7.77 \mathrm{NA}$ & NA & NA & NA & Gg_rs148910.Gg_rs148954 \\
\hline $8.25 \mathrm{NA}$ & NA & NA & NA & Gg_rs148910Gg_rs148954 \\
\hline $5.74 \mathrm{NA}$ & NA & NA & NA & Gg_rs148910.Gg_rs148954 \\
\hline $4.86 \mathrm{NA}$ & NA & NA & NA & Gg_rs148910Gg_rs148954 \\
\hline $5.82 \mathrm{NA}$ & NA & NA & NA & Gg_rs148910Gg_rs148954 \\
\hline $4.1 \mathrm{NA}$ & NA & NA & NA & Gg_rs148910Gg_rs148954 \\
\hline $5.08 \mathrm{NA}$ & NA & NA & NA & Gg_rs148910Gg_rs148954 \\
\hline
\end{tabular}




\begin{tabular}{|c|c|c|c|c|}
\hline $8 \mathrm{NA}$ & NA & NA & NA & Gg_rs148910.Gg_rs148954 \\
\hline $8.41 \mathrm{NA}$ & NA & NA & NA & Gg_rs148910Gg_rs148954 \\
\hline 5.47 NA & NA & NA & NA & Gg_rs148910.Gg_rs148954 \\
\hline $5.37 \mathrm{NA}$ & NA & NA & NA & Gg_rs148910:Gg_rs148973 \\
\hline $5.72 \mathrm{NA}$ & NA & NA & NA & Gg_rs148910Gg_rs148954 \\
\hline $7.55 \mathrm{NA}$ & NA & NA & NA & Gg_rs148910Gg_rs148954 \\
\hline $6.87 \mathrm{NA}$ & NA & NA & NA & Gg_rs148910Gg_rs148954 \\
\hline $0.35 \mathrm{NA}$ & NA & NA & NA & Gg_rs148910.Gg_rs148973 \\
\hline $0.52 \mathrm{NA}$ & NA & NA & NA & Gg_rs148910.Gg_rs148954 \\
\hline $2.18 \mathrm{NA}$ & NA & NA & NA & Gg_rs148910Gg_rs148973 \\
\hline $5.06 \mathrm{NA}$ & NA & NA & NA & Gg_rs148910Gg_rs148954 \\
\hline $5.85 \mathrm{NA}$ & NA & NA & NA & Gg_rs148910Gg_rs148954 \\
\hline $3.78 \mathrm{NA}$ & NA & NA & NA & Gg_rs148910Gg_rs148954 \\
\hline $7.42 \mathrm{NA}$ & NA & NA & NA & Gg_rs148910Gg_rs148954 \\
\hline $6.08 \mathrm{NA}$ & NA & NA & NA & Gg_rs148910Gg_rs148954 \\
\hline $8.05 \mathrm{NA}$ & NA & NA & NA & Gg_rs148910.Gg_rs148954 \\
\hline $5.88 \mathrm{NA}$ & NA & NA & NA & Gg_rs148910Gg_rs148954 \\
\hline $5.46 \mathrm{NA}$ & NA & NA & NA & Gg_rs148910Gg_rs148954 \\
\hline $5.17 \mathrm{NA}$ & NA & NA & NA & Gg_rs148910Gg_rs148954 \\
\hline $3.55 \mathrm{NA}$ & NA & NA & NA & Gg_rs148910.Gg_rs148954 \\
\hline $7.63 \mathrm{NA}$ & NA & NA & NA & Gg_rs148910Gg_rs148954 \\
\hline $7.71 \mathrm{NA}$ & NA & NA & NA & Gg_rs148910.Gg_rs148954 \\
\hline $4.58 \mathrm{NA}$ & NA & NA & NA & Gg_rs148910.Gg_rs148954 \\
\hline $5.95 \mathrm{NA}$ & NA & NA & NA & Gg_rs148910.Gg_rs148954 \\
\hline 7.98 NA & NA & NA & NA & Gg_rs148910Gg_rs148954 \\
\hline $5.54 \mathrm{NA}$ & NA & NA & NA & Gg_rs148910Gg_rs148954 \\
\hline $6.1 \mathrm{NA}$ & NA & NA & NA & Gg_rs148910Gg_rs148954 \\
\hline $7.95 \mathrm{NA}$ & NA & NA & NA & Gg_rs148910.Gg_rs148954 \\
\hline $7.9 \mathrm{NA}$ & NA & NA & NA & Gg_rs148910.Gg_rs148954 \\
\hline 8.07 NA & NA & NA & NA & Gg_rs148910Gg_rs148954 \\
\hline $8.12 \mathrm{NA}$ & NA & NA & NA & Gg_rs148910Gg_rs148954 \\
\hline $7.47 \mathrm{NA}$ & NA & NA & NA & Gg_rs148910Gg_rs148954 \\
\hline $6.12 \mathrm{NA}$ & NA & NA & NA & Gg_rs148910Gg_rs148954 \\
\hline 5.7 NA & NA & NA & NA & Gg_rs148910Gg_rs148954 \\
\hline $3.96 \mathrm{NA}$ & NA & NA & NA & Gg_rs148910.Gg_rs148954 \\
\hline $2.9 \mathrm{NA}$ & NA & NA & NA & Gg_rs148910.Gg_rs148954 \\
\hline $7.67 \mathrm{NA}$ & NA & NA & NA & Gg_rs148910Gg_rs148954 \\
\hline $5.28 \mathrm{NA}$ & NA & NA & NA & Gg_rs148910.Gg_rs148954 \\
\hline $5.02 \mathrm{NA}$ & NA & NA & NA & Gg_rs148910Gg_rs148954 \\
\hline $5.88 \mathrm{NA}$ & NA & NA & NA & Gg_rs148910Gg_rs148954 \\
\hline 2.43 NA & NA & NA & NA & Gg_rs148910.Gg_rs148954 \\
\hline $7.79 \mathrm{NA}$ & NA & NA & NA & Gg_rs148910Gg_rs148954 \\
\hline $6.61 \mathrm{NA}$ & NA & NA & NA & Gg_rs148910Gg_rs148954 \\
\hline
\end{tabular}




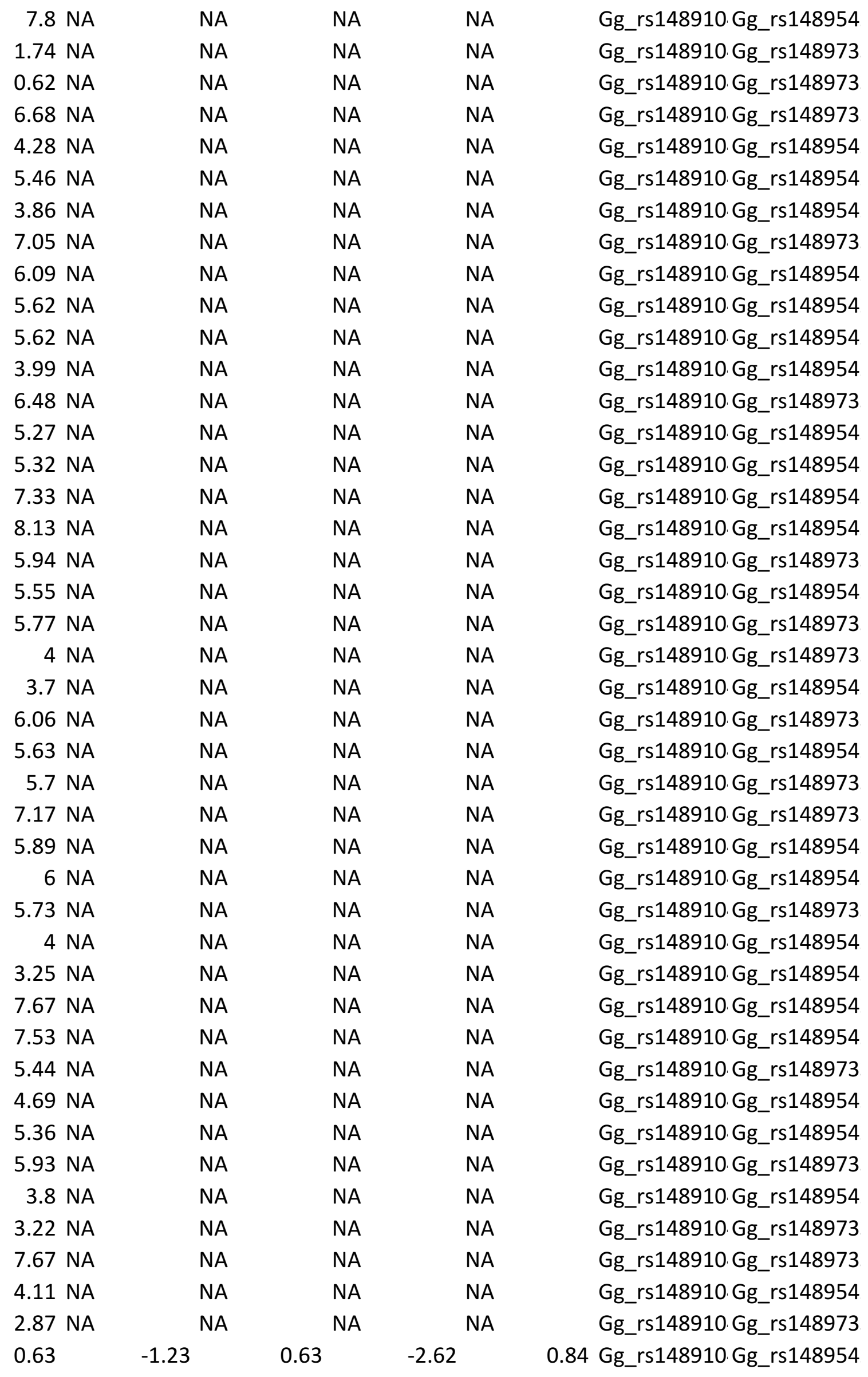




\begin{tabular}{|c|c|c|c|c|c|}
\hline $0.38 \mathrm{NA}$ & NA & NA & NA & & Gg_rs148910Gg_rs148973 \\
\hline $0.51 \mathrm{NA}$ & NA & NA & NA & & Gg_rs148910.Gg_rs148973 \\
\hline $0.66 \mathrm{NA}$ & NA & NA & NA & & Gg_rs148910Gg_rs148973 \\
\hline $1 \mathrm{NA}$ & NA & NA & NA & & Gg_rs148910Gg_rs148973 \\
\hline $6.32 \mathrm{NA}$ & NA & NA & NA & & Gg_rs148910Gg_rs148973 \\
\hline 8.08 NA & NA & NA & NA & & Gg_rs148910Gg_rs148973 \\
\hline $7.75 \mathrm{NA}$ & NA & NA & NA & & Gg_rs148910.Gg_rs148973 \\
\hline 1.8 NA & NA & NA & NA & & Gg_rs148910Gg_rs148973 \\
\hline $2.67 \mathrm{NA}$ & NA & NA & NA & & Gg_rs148910Gg_rs148973 \\
\hline $5.33 \mathrm{NA}$ & NA & NA & NA & & Gg_rs148910Gg_rs148973 \\
\hline $5.27 \mathrm{NA}$ & NA & NA & NA & & Gg_rs148910Gg_rs148973 \\
\hline $5.91 \mathrm{NA}$ & NA & NA & NA & & Gg_rs148910Gg_rs148973 \\
\hline $7.27 \mathrm{NA}$ & NA & NA & NA & & Gg_rs148910.Gg_rs148973 \\
\hline $6.1 \mathrm{NA}$ & NA & NA & NA & & Gg_rs148910Gg_rs148973 \\
\hline $0.52 \mathrm{NA}$ & NA & NA & NA & & Gg_rs154270Gg_rs148973 \\
\hline $4.87 \mathrm{NA}$ & NA & NA & NA & & Gg_rs148910Gg_rs148973 \\
\hline 0.72 & -1.79 & 0.73 & 0.65 & 0.96 & Gg_rs148910Gg_rs148973 \\
\hline 4.76 NA & NA & NA & NA & & Gg_rs148910.Gg_rs148973 \\
\hline $4.92 \mathrm{NA}$ & NA & NA & NA & & Gg_rs148910.Gg_rs148973 \\
\hline $7.03 \mathrm{NA}$ & NA & NA & NA & & Gg_rs148910.Gg_rs148973 \\
\hline $4.38 \mathrm{NA}$ & NA & NA & NA & & Gg_rs148910.Gg_rs148973 \\
\hline $0.73 \mathrm{NA}$ & NA & NA & NA & & Gg_rs148910Gg_rs148973 \\
\hline 5.4 NA & NA & NA & NA & & Gg_rs148910.Gg_rs148973 \\
\hline $5.45 \mathrm{NA}$ & NA & NA & NA & & Gg_rs148910Gg_rs148973 \\
\hline 5.47 NA & NA & NA & NA & & Gg_rs148910.Gg_rs148973 \\
\hline $6.79 \mathrm{NA}$ & NA & NA & NA & & Gg_rs148910.Gg_rs148973 \\
\hline $0.48 \mathrm{NA}$ & NA & NA & NA & & Gg_rs148910.Gg_rs148973 \\
\hline $0.37 \mathrm{NA}$ & NA & NA & NA & & Gg_rs148910.Gg_rs148973 \\
\hline $0.56 \mathrm{NA}$ & NA & NA & NA & & Gg_rs154270 Gg_rs148973 \\
\hline $0.57 \mathrm{NA}$ & NA & NA & NA & & Gg_rs148910Gg_rs148973 \\
\hline 0.71 & -2.21 & 0.73 & 0 & 0.96 & Gg_rs148910.Gg_rs148973 \\
\hline 0.03 & -0.12 & 0.03 & -0.1 & 0.04 & Gg_rs148934 Gg_rs148973 \\
\hline $0.41 \mathrm{NA}$ & NA & NA & NA & & Gg_rs148934 Gg_rs148973 \\
\hline 0.7 & -1.88 & 0.77 & -1.43 & 0.97 & Gg_rs148934 Gg_rs148973 \\
\hline $0.42 \mathrm{NA}$ & NA & NA & NA & & Gg_rs148934 Gg_rs148973 \\
\hline $3.04 \mathrm{NA}$ & NA & NA & NA & & Gg_rs148910.Gg_rs148984 \\
\hline $0.56 \mathrm{NA}$ & NA & NA & NA & & Gg_rs148934 Gg_rs148973 \\
\hline 1.03 & 0.42 & 1.13 & 4.07 & 1.43 & Gg_rs148934 Gg_rs148973 \\
\hline 0.63 & -1.48 & 0.69 & 0.89 & 0.88 & Gg_rs148910.Gg_rs148973 \\
\hline $2.95 \mathrm{NA}$ & NA & NA & NA & & Gg_rs148910.Gg_rs139535 \\
\hline $3.04 \mathrm{NA}$ & NA & NA & NA & & Gg_rs148910Gg_rs148984 \\
\hline $3.52 \mathrm{NA}$ & NA & NA & NA & & Gg_rs148910Gg_rs139535 \\
\hline $2.62 \mathrm{NA}$ & NA & NA & NA & & Gg_rs148910Gg_rs139535 \\
\hline
\end{tabular}




\begin{tabular}{|c|c|c|c|c|}
\hline $0.75 \mathrm{NA}$ & NA & NA & NA & Gg_rs148934 Gg_rs139535 \\
\hline $0.14 \mathrm{NA}$ & NA & NA & NA & Gg_rs148954 Gg_rs107286 \\
\hline $0.66 \mathrm{NA}$ & NA & NA & NA & Gg_rs148954 Gg_rs139535 \\
\hline $0.42 \mathrm{NA}$ & NA & NA & NA & Gg_rs148954 Gg_rs107286 \\
\hline $0.48 \mathrm{NA}$ & NA & NA & NA & Gg_rs148954 Gg_rs139535 \\
\hline $0.42 \mathrm{NA}$ & NA & NA & NA & Gg_rs148954 Gg_rs139535 \\
\hline $1.04 \mathrm{NA}$ & NA & NA & NA & Gg_rs148954 Gg_rs107286. \\
\hline $1.46 \mathrm{NA}$ & NA & NA & NA & Gg_rs148954 Gg_rs107286 \\
\hline $1.11 \mathrm{NA}$ & NA & NA & NA & Gg_rs148954 Gg_rs139535 \\
\hline $1.37 \mathrm{NA}$ & NA & NA & NA & Gg_rs148954 Gg_rs139535 \\
\hline $0.44 \mathrm{NA}$ & NA & NA & NA & Gg_rs148954 Gg_rs148984 \\
\hline $1.05 \mathrm{NA}$ & NA & NA & NA & Gg_rs148954 Gg_rs107286 \\
\hline $1.55 \mathrm{NA}$ & NA & NA & NA & Gg_rs148954 Gg_rs139535 \\
\hline $1.18 \mathrm{NA}$ & NA & NA & NA & Gg_rs148954 snp.23.342.1\{ \\
\hline $0.53 \mathrm{NA}$ & NA & NA & NA & Gg_rs148954 Gg_rs139535 \\
\hline $0.87 \mathrm{NA}$ & NA & NA & NA & Gg_rs148954 snp.23.342.1§ \\
\hline $1.79 \mathrm{NA}$ & NA & NA & NA & Gg_rs148954 Gg_rs148984 \\
\hline $1.8 \mathrm{NA}$ & NA & NA & NA & Gg_rs148954 Gg_rs148984 \\
\hline $1.9 \mathrm{NA}$ & NA & NA & NA & Gg_rs148954 Gg_rs139535 \\
\hline $1.98 \mathrm{NA}$ & NA & NA & NA & Gg_rs148954 Gg_rs139535 \\
\hline $0.5 \mathrm{NA}$ & NA & NA & NA & Gg_rs148954 Gg_rs148984 \\
\hline 0.74 & -1.5 & 0.71 & -0.35 & 1 Gg_rs148954 Gg_rs139535 \\
\hline $0.55 \mathrm{NA}$ & NA & NA & NA & Gg_rs148954 Gg_rs139535 \\
\hline $2.05 \mathrm{NA}$ & NA & NA & NA & Gg_rs148954 Gg_rs139535 \\
\hline $0.5 \mathrm{NA}$ & NA & NA & NA & Gg_rs148954 Gg_rs107286. \\
\hline $2.9 \mathrm{NA}$ & NA & NA & NA & Gg_rs148954 Gg_rs139535 \\
\hline 4.13 NA & NA & NA & NA & Gg_rs148954 Gg_rs139535 \\
\hline $3.85 \mathrm{NA}$ & NA & NA & NA & Gg_rs148954 Gg_rs139535 \\
\hline 0.86 NA & NA & NA & NA & Gg_rs148954 Gg_rs148984 \\
\hline $3.7 \mathrm{NA}$ & NA & NA & NA & Gg_rs148954 Gg_rs139535 \\
\hline $1.25 \mathrm{NA}$ & NA & NA & NA & Gg_rs148954 Gg_rs107286 \\
\hline $0.66 \mathrm{NA}$ & NA & NA & NA & Gg_rs148954 Gg_rs139535 \\
\hline $1.82 \mathrm{NA}$ & NA & NA & NA & Gg_rs148954 Gg_rs139535 \\
\hline $2.35 \mathrm{NA}$ & NA & NA & NA & Gg_rs148954 Gg_rs139535 \\
\hline $1.43 \mathrm{NA}$ & NA & NA & NA & Gg_rs148954 Gg_rs139535 \\
\hline $2.5 \mathrm{NA}$ & NA & NA & NA & Gg_rs148954 Gg_rs139535 \\
\hline $2.44 \mathrm{NA}$ & NA & NA & NA & Gg_rs148954 Gg_rs107286. \\
\hline 4.96 NA & NA & NA & NA & Gg_rs148954 Gg_rs139535 \\
\hline 3.79 NA & NA & NA & NA & Gg_rs148954 Gg_rs139535 \\
\hline $2.03 \mathrm{NA}$ & NA & NA & NA & Gg_rs148954 Gg_rs139535 \\
\hline $1.31 \mathrm{NA}$ & NA & NA & NA & Gg_rs148954 Gg_rs148984 \\
\hline $1.08 \mathrm{NA}$ & NA & NA & NA & Gg_rs148954 Gg_rs139535 \\
\hline $1.14 \mathrm{NA}$ & NA & NA & NA & Gg_rs148954 Gg_rs139535 \\
\hline
\end{tabular}




\begin{tabular}{|c|c|c|c|c|}
\hline $1.04 \mathrm{NA}$ & NA & NA & NA & Gg_rs148954 Gg_rs107286. \\
\hline $2.13 \mathrm{NA}$ & NA & NA & NA & Gg_rs148954 Gg_rs139535 \\
\hline $3.92 \mathrm{NA}$ & NA & NA & NA & Gg_rs148954 Gg_rs107286، \\
\hline $3.78 \mathrm{NA}$ & NA & NA & NA & Gg_rs148954 Gg_rs139535 \\
\hline $5.01 \mathrm{NA}$ & NA & NA & NA & Gg_rs148954 Gg_rs139535 \\
\hline $3.43 \mathrm{NA}$ & NA & NA & NA & Gg_rs148954 Gg_rs139535 \\
\hline $4.13 \mathrm{NA}$ & NA & NA & NA & Gg_rs148954 Gg_rs139535 \\
\hline $1.33 \mathrm{NA}$ & NA & NA & NA & Gg_rs148954 Gg_rs139535 \\
\hline $2.03 \mathrm{NA}$ & NA & NA & NA & Gg_rs148954 Gg_rs148984 \\
\hline $2.97 \mathrm{NA}$ & NA & NA & NA & Gg_rs148954 Gg_rs139535 \\
\hline $4.45 \mathrm{NA}$ & NA & NA & NA & Gg_rs148954 Gg_rs139535 \\
\hline $3.03 \mathrm{NA}$ & NA & NA & NA & Gg_rs148954 Gg_rs139535 \\
\hline $1.63 \mathrm{NA}$ & NA & NA & NA & Gg_rs148954 Gg_rs139535 \\
\hline $1.44 \mathrm{NA}$ & NA & NA & NA & Gg_rs148954 Gg_rs139535 \\
\hline $1.45 \mathrm{NA}$ & NA & NA & NA & Gg_rs148954 Gg_rs139535 \\
\hline $1.09 \mathrm{NA}$ & NA & NA & NA & Gg_rs148954 Gg_rs139535 \\
\hline $1.24 \mathrm{NA}$ & NA & NA & NA & Gg_rs148954 Gg_rs148984 \\
\hline $1.16 \mathrm{NA}$ & NA & NA & NA & Gg_rs148954 Gg_rs107286 \\
\hline $1.04 \mathrm{NA}$ & NA & NA & NA & Gg_rs148954 Gg_rs139535 \\
\hline $2.45 \mathrm{NA}$ & NA & NA & NA & Gg_rs148954 Gg_rs139535 \\
\hline $4.51 \mathrm{NA}$ & NA & NA & NA & Gg_rs148954 Gg_rs139535 \\
\hline $3.34 \mathrm{NA}$ & NA & NA & NA & Gg_rs148954 Gg_rs139535 \\
\hline $1.58 \mathrm{NA}$ & NA & NA & NA & Gg_rs148954 Gg_rs139535 \\
\hline $2.1 \mathrm{NA}$ & NA & NA & NA & Gg_rs148954 Gg_rs139535 \\
\hline $1.51 \mathrm{NA}$ & NA & NA & NA & Gg_rs148954 Gg_rs139535 \\
\hline $1.39 \mathrm{NA}$ & NA & NA & NA & Gg_rs148954 Gg_rs139535 \\
\hline $1.69 \mathrm{NA}$ & NA & NA & NA & Gg_rs148954 Gg_rs139535 \\
\hline $1.91 \mathrm{NA}$ & NA & NA & NA & Gg_rs148954 Gg_rs139535 \\
\hline $1.4 \mathrm{NA}$ & NA & NA & NA & Gg_rs148954 Gg_rs107286. \\
\hline $0.78 \mathrm{NA}$ & NA & NA & NA & Gg_rs148954 Gg_rs139535 \\
\hline $1.4 \mathrm{NA}$ & NA & NA & NA & Gg_rs148954 Gg_rs107286 \\
\hline 0.99 NA & NA & NA & NA & Gg_rs148954 Gg_rs107286. \\
\hline $0.7 \mathrm{NA}$ & NA & NA & NA & Gg_rs148954 Gg_rs107286 \\
\hline $1.41 \mathrm{NA}$ & NA & NA & NA & Gg_rs148954 Gg_rs139535 \\
\hline $1.22 \mathrm{NA}$ & NA & NA & NA & Gg_rs148954 Gg_rs139535 \\
\hline $2.28 \mathrm{NA}$ & NA & NA & NA & Gg_rs148954 Gg_rs139535 \\
\hline $0.31 \mathrm{NA}$ & NA & NA & NA & Gg_rs148954 Gg_rs139535 \\
\hline $2.1 \mathrm{NA}$ & NA & NA & NA & Gg_rs148954 Gg_rs139535 \\
\hline $1.38 \mathrm{NA}$ & NA & NA & NA & Gg_rs148954 Gg_rs139535 \\
\hline $0.97 \mathrm{NA}$ & NA & NA & NA & Gg_rs148954 Gg_rs139535 \\
\hline $2.18 \mathrm{NA}$ & NA & NA & NA & Gg_rs148954 Gg_rs139535 \\
\hline $0.87 \mathrm{NA}$ & NA & NA & NA & Gg_rs148954 Gg_rs139535 \\
\hline $2.39 \mathrm{NA}$ & NA & NA & NA & Gg_rs148954 Gg_rs139535 \\
\hline
\end{tabular}




\begin{tabular}{|c|c|c|c|c|c|}
\hline $2.17 \mathrm{NA}$ & NA & NA & NA & & Gg_rs148954 Gg_rs139535 \\
\hline $1.44 \mathrm{NA}$ & NA & NA & NA & & Gg_rs148954 Gg_rs139535 \\
\hline $0.32 \mathrm{NA}$ & NA & NA & NA & & Gg_rs148954 Gg_rs107286. \\
\hline $1.36 \mathrm{NA}$ & NA & NA & NA & & Gg_rs148954 Gg_rs107286. \\
\hline $1.22 \mathrm{NA}$ & NA & NA & NA & & Gg_rs148954 Gg_rs107286 \\
\hline $3.39 \mathrm{NA}$ & NA & NA & NA & & Gg_rs148954 Gg_rs139535 \\
\hline $3.78 \mathrm{NA}$ & NA & NA & NA & & Gg_rs148954 Gg_rs139535 \\
\hline $1.42 \mathrm{NA}$ & NA & NA & NA & & Gg_rs148954 Gg_rs107286. \\
\hline $1.28 \mathrm{NA}$ & NA & NA & NA & & Gg_rs148954 Gg_rs107286. \\
\hline $0.47 \mathrm{NA}$ & NA & NA & NA & & Gg_rs148954 Gg_rs139535 \\
\hline $1 \mathrm{NA}$ & NA & NA & NA & & Gg_rs148954 Gg_rs139535 \\
\hline $1.02 \mathrm{NA}$ & NA & NA & NA & & Gg_rs148954 Gg_rs139535 \\
\hline 3.24 & -5.96 & 3.14 & 2.23 & 4.38 & Gg_rs148954 Gg_rs139535 \\
\hline $0.42 \mathrm{NA}$ & NA & NA & NA & & Gg_rs148954 Gg_rs139535 \\
\hline $0.4 \mathrm{NA}$ & NA & NA & NA & & Gg_rs148954 Gg_rs107286. \\
\hline 0.49 NA & NA & NA & NA & & Gg_rs148954 Gg_rs107286 \\
\hline 0.68 NA & NA & NA & NA & & Gg_rs148954 Gg_rs139535 \\
\hline $0.62 \mathrm{NA}$ & NA & NA & NA & & Gg_rs148954 Gg_rs139535 \\
\hline $0.78 \mathrm{NA}$ & NA & NA & NA & & Gg_rs148954 Gg_rs139535 \\
\hline $0.68 \mathrm{NA}$ & NA & NA & NA & & Gg_rs148954 Gg_rs139535 \\
\hline $0.56 \mathrm{NA}$ & NA & NA & NA & & Gg_rs148954 Gg_rs139535 \\
\hline $0.22 \mathrm{NA}$ & NA & NA & NA & & Gg_rs148954 Gg_rs139535 \\
\hline 1.79 NA & NA & NA & NA & & Gg_rs148954 Gg_rs139535 \\
\hline $0.5 \mathrm{NA}$ & NA & NA & NA & & Gg_rs148954 Gg_rs139535 \\
\hline $2.33 \mathrm{NA}$ & NA & NA & NA & & Gg_rs148954 Gg_rs139535 \\
\hline $1.52 \mathrm{NA}$ & NA & NA & NA & & Gg_rs148954 Gg_rs139535 \\
\hline $1.95 \mathrm{NA}$ & NA & NA & NA & & Gg_rs148954 Gg_rs139535 \\
\hline $1.97 \mathrm{NA}$ & NA & NA & NA & & Gg_rs148954 Gg_rs139535 \\
\hline 0.89 NA & NA & NA & NA & & Gg_rs148954 Gg_rs139535 \\
\hline $1.71 \mathrm{NA}$ & NA & NA & NA & & Gg_rs148954 Gg_rs139535 \\
\hline $1.61 \mathrm{NA}$ & NA & NA & NA & & Gg_rs148984 Gg_rs139535 \\
\hline $2 \mathrm{NA}$ & NA & NA & NA & & Gg_rs148954 Gg_rs139535 \\
\hline $1.57 \mathrm{NA}$ & NA & NA & NA & & Gg_rs148984 Gg_rs139535 \\
\hline $1.41 \mathrm{NA}$ & NA & NA & NA & & Gg_rs148984 Gg_rs139535 \\
\hline $0.52 \mathrm{NA}$ & NA & NA & NA & & Gg_rs148984 Gg_rs139535 \\
\hline $1.75 \mathrm{NA}$ & NA & NA & NA & & Gg_rs148984 Gg_rs139535 \\
\hline $1.71 \mathrm{NA}$ & NA & NA & NA & & Gg_rs148984 Gg_rs139535 \\
\hline $1.75 \mathrm{NA}$ & NA & NA & NA & & Gg_rs148984 Gg_rs139535 \\
\hline $0.41 \mathrm{NA}$ & NA & NA & NA & & Gg_rs148954 Gg_rs139535 \\
\hline $1.03 \mathrm{NA}$ & NA & NA & NA & & Gg_rs148954 Gg_rs139535 \\
\hline $0.48 \mathrm{NA}$ & NA & NA & NA & & Gg_rs148984 Gg_rs139535 \\
\hline $0.68 \mathrm{NA}$ & NA & NA & NA & & Gg_rs148984 Gg_rs139535 \\
\hline $0.38 \mathrm{NA}$ & NA & NA & NA & & Gg_rs148954 Gg_rs139535 \\
\hline
\end{tabular}




\begin{tabular}{|c|c|c|c|c|c|}
\hline $0.53 \mathrm{NA}$ & NA & NA & NA & & Gg_rs148973 Gg_rs139535 \\
\hline 0.87 & -3.63 & 0.86 & -2.4 & 1.2 & Gg_rs107286.snp.23.342.1尺 \\
\hline 1.01 & 4.13 & 0.99 & -0.03 & 1.38 & Gg_rs107286.snp.23.342.1§ \\
\hline $0.58 \mathrm{NA}$ & NA & NA & NA & & Gg_rs107286.Gg_rs149092 \\
\hline 0.8 & 1.54 & 0.79 & -0.59 & 1.1 & Gg_rs107286.snp.23.342.1§ \\
\hline $0.58 \mathrm{NA}$ & NA & NA & NA & & Gg_rs107286.Gg_rs149092 \\
\hline 0.62 NA & NA & NA & NA & & Gg_rs107286.Gg_rs149092 \\
\hline 1.35 & 0.18 & 1.33 & -3.78 & 1.85 & Gg_rs107286.snp.23.342.18 \\
\hline $0.42 \mathrm{NA}$ & NA & NA & NA & & Gg_rs107286.Gg_rs137793 \\
\hline $1.05 \mathrm{NA}$ & NA & NA & NA & & Gg_rs107286.Gg_rs139567 \\
\hline $0.67 \mathrm{NA}$ & NA & NA & NA & & Gg_rs107286.snp.23.342.1尺 \\
\hline $0.79 \mathrm{NA}$ & NA & NA & NA & & Gg_rs107286.Gg_rs137793 \\
\hline 0.77 NA & NA & NA & NA & & Gg_rs107286.snp.23.342.1§ \\
\hline 0.97 & 0.1 & 0.96 & -3.31 & 1.33 & Gg_rs107286.snp.23.342.1§ \\
\hline $1.07 \mathrm{NA}$ & NA & NA & NA & & Gg_rs107286.snp.23.342.18 \\
\hline $1.32 \mathrm{NA}$ & NA & NA & NA & & Gg_rs107286.snp.23.342.1§ \\
\hline 0.88 & -3.3 & 0.87 & -3.03 & 1.2 & Gg_rs107286.snp.23.342.1§ \\
\hline $0.65 \mathrm{NA}$ & NA & NA & NA & & Gg_rs107286.Gg_rs137793 \\
\hline 0.61 & -1.71 & 0.6 & -1.46 & 0.84 & Gg_rs107286.snp.23.342.1尺 \\
\hline 0.89 NA & NA & NA & NA & & Gg_rs107286.Gg_rs139567 \\
\hline $0.72 \mathrm{NA}$ & NA & NA & NA & & Gg_rs107286.Gg_rs137793 \\
\hline 0.65 & -2.53 & 0.64 & -3.87 & 0.9 & Gg_rs107286.Gg_rs137793 \\
\hline $0.43 \mathrm{NA}$ & NA & NA & NA & & Gg_rs139535 Gg_rs149092 \\
\hline $0.33 \mathrm{NA}$ & NA & NA & NA & & Gg_rs107286.Gg_rs149092 \\
\hline $0.32 \mathrm{NA}$ & NA & NA & NA & & Gg_rs107286.Gg_rs137793 \\
\hline $0.45 \mathrm{NA}$ & NA & NA & NA & & Gg_rs107286.Gg_rs149092 \\
\hline 0.88 & 2.82 & 0.85 & 5.09 & 1.18 & Gg_rs139535 Gg_rs137793 \\
\hline 0.81 & 2.28 & 0.78 & 4.78 & 1.08 & Gg_rs139535 Gg_rs139567 \\
\hline 0.98 & 2.1 & 0.95 & 5.4 & 1.31 & Gg_rs139535 Gg_rs137793 \\
\hline 0.44 NA & NA & NA & NA & & Gg_rs139535 Gg_rs149092 \\
\hline $0.65 \mathrm{NA}$ & NA & NA & NA & & Gg_rs139535 Gg_rs137793 \\
\hline 0.48 NA & NA & NA & NA & & Gg_rs139535 Gg_rs149092 \\
\hline 0.74 NA & NA & NA & NA & & Gg_rs107286.Gg_rs149092 \\
\hline $0.35 \mathrm{NA}$ & NA & NA & NA & & Gg_rs139535 Gg_rs137793 \\
\hline $0.55 \mathrm{NA}$ & NA & NA & NA & & Gg_rs139535 Gg_rs149092 \\
\hline 0.31 & -1.13 & 0.3 & 1.04 & 0.41 & Gg_rs139535 Gg_rs149092 \\
\hline $0.35 \mathrm{NA}$ & NA & NA & NA & & Gg_rs139535 Gg_rs149092 \\
\hline $1.38 \mathrm{NA}$ & NA & NA & NA & & Gg_rs139535 Gg_rs149092 \\
\hline 0.83 NA & NA & NA & NA & & Gg_rs139567 Gg_rs149092 \\
\hline $0.95 \mathrm{NA}$ & NA & NA & NA & & Gg_rs139535 Gg_rs149092 \\
\hline $0.56 \mathrm{NA}$ & NA & NA & NA & & Gg_rs139535 Gg_rs149092 \\
\hline $0.85 \mathrm{NA}$ & NA & NA & NA & & Gg_rs139535 X1_16196114 \\
\hline $0.53 \mathrm{NA}$ & NA & NA & NA & & Gg_rs139567 Gg_rs139652. \\
\hline
\end{tabular}




\begin{tabular}{|c|c|c|c|c|c|c|}
\hline $0.71 \mathrm{NA}$ & NA & & NA & NA & & Gg_rs139567 X1_16196114 \\
\hline $0.57 \mathrm{NA}$ & NA & & NA & NA & & Gg_rs139535 Gg_rs154918. \\
\hline $0.34 \mathrm{NA}$ & NA & & NA & NA & & Gg_rs139535 Gg_rs154918. \\
\hline 1.46 & -2.58 & 1.5 & & -1.43 & 1.87 & Gg_rs139535 Gg_rs139703. \\
\hline $0.34 \mathrm{NA}$ & NA & & NA & NA & & Gg_rs139535 Gg_rs139652. \\
\hline 0.37 & 1.82 & 0.38 & & 1.83 & 0.47 & Gg_rs139567 Gg_rs154918. \\
\hline $0.31 \mathrm{NA}$ & NA & & NA & NA & & Gg_rs139567 Gg_rs154918. \\
\hline $1.39 \mathrm{NA}$ & NA & & NA & NA & & Gg_rs137793 Gg_rs154918. \\
\hline 0.38 & 2.15 & 0.39 & & 1.61 & 0.47 & Gg_rs137793 Gg_rs154918. \\
\hline 0.65 & 3.71 & 0.69 & & 2.58 & 0.86 & Gg_rs137793 Gg_rs154918. \\
\hline $1.52 \mathrm{NA}$ & NA & & NA & NA & & Gg_rs137793 Gg_rs154918. \\
\hline 1.21 & 2.31 & 1.3 & & 2.42 & 1.6 & Gg_rs137969 Gg_rs154918. \\
\hline $0.38 \mathrm{NA}$ & NA & & NA & NA & & Gg_rs137793 Gg_rs154918. \\
\hline $0.7 \mathrm{NA}$ & NA & & NA & NA & & Gg_rs137969 Gg_rs154918. \\
\hline 1.12 & 3.78 & 1.2 & & 4.38 & 1.49 & Gg_rs139652 Gg_rs154918. \\
\hline $0.5 \mathrm{NA}$ & NA & & NA & NA & & Gg_rs139567 Gg_rs139703. \\
\hline 0.46 NA & NA & & NA & NA & & Gg_rs137793 Gg_rs139703. \\
\hline 0.66 NA & NA & & NA & NA & & Gg_rs139567 Gg_rs139703. \\
\hline $0.6 \mathrm{NA}$ & NA & & NA & NA & & Gg_rs139652 Gg_rs139703. \\
\hline $0.59 \mathrm{NA}$ & NA & & NA & NA & & Gg_rs139652 Gg_rs139703. \\
\hline $0.79 \mathrm{NA}$ & NA & & NA & NA & & Gg_rs139652 Gg_rs139703. \\
\hline $0.56 \mathrm{NA}$ & NA & & NA & NA & & Gg_rs139652 Gg_rs139703. \\
\hline $2.41 \mathrm{NA}$ & NA & & NA & NA & & Gg_rs139703 Gg_rs139760 \\
\hline $0.71 \mathrm{NA}$ & NA & & NA & NA & & Gg_rs154918snp.4.489.26 \\
\hline $0.45 \mathrm{NA}$ & NA & & NA & NA & & X1_17441654Gg_rs135559: \\
\hline $0.7 \mathrm{NA}$ & NA & & NA & NA & & Gg_rs139703 X1_18389794 \\
\hline $0.62 \mathrm{NA}$ & NA & & NA & NA & & Gg_rs154918.Gg_rs135559: \\
\hline $0.32 \mathrm{NA}$ & NA & & NA & NA & & X1_17441654Gg_rs135559: \\
\hline $0.53 \mathrm{NA}$ & NA & & NA & NA & & X1_17441654snp.4.489.26 \\
\hline 0.46 NA & NA & & NA & NA & & X1_17441654Gg_rs135559: \\
\hline $0.43 \mathrm{NA}$ & NA & & NA & NA & & X1_17441654Gg_rs135559: \\
\hline $0.45 \mathrm{NA}$ & NA & & NA & NA & & X1_17441654snp.4.489.26 \\
\hline $0.49 \mathrm{NA}$ & NA & & NA & NA & & X1_17441654Gg_rs135559: \\
\hline $0.38 \mathrm{NA}$ & NA & & NA & NA & & X1_17441654Gg_rs135559: \\
\hline $0.84 \mathrm{NA}$ & NA & & NA & NA & & X1_17441654X1_19033467 \\
\hline $0.66 \mathrm{NA}$ & NA & & NA & NA & & X1_17441654Gg_rs135559: \\
\hline $0.52 \mathrm{NA}$ & NA & & NA & NA & & X1_17441654Gg_rs135559: \\
\hline $0.75 \mathrm{NA}$ & NA & & NA & NA & & X1_17441654Gg_rs135559: \\
\hline 0.63 & 0.6 & 0.58 & & 3.16 & 0.84 & X1_17441654Gg_rs135559: \\
\hline $0.16 \mathrm{NA}$ & NA & & NA & NA & & X1_17441654Gg_rs135559: \\
\hline 0.97 & 0.66 & 0.85 & & 4.71 & 1.28 & X1_17441654Gg_rs135559: \\
\hline $0.34 \mathrm{NA}$ & NA & & NA & NA & & X1_17441654Gg_rs135559: \\
\hline $0.56 \mathrm{NA}$ & NA & & NA & NA & & X1_17441654Gg_rs135559: \\
\hline
\end{tabular}




\begin{tabular}{|c|c|c|c|c|c|c|c|}
\hline $0.48 \mathrm{NA}$ & NA & & NA & & NA & & X1_17441654Gg_rs135559: \\
\hline $1.12 \mathrm{NA}$ & NA & & NA & & NA & & X1_17441654snp.4.489.26 \\
\hline $0.51 \mathrm{NA}$ & NA & & NA & & NA & & Gg_rs139703 snp.4.489.26 \\
\hline $0.55 \mathrm{NA}$ & NA & & NA & & NA & & X1_17441654X1_19033467 \\
\hline 0.49 NA & NA & & NA & & NA & & X1_17441654snp.4.489.26 \\
\hline $0.77 \mathrm{NA}$ & NA & & NA & & NA & & X1_17441654snp.4.489.26 \\
\hline $0.73 \mathrm{NA}$ & NA & & NA & & NA & & X1_17441654snp.4.489.26 \\
\hline $0.61 \mathrm{NA}$ & NA & & NA & & NA & & Gg_rs139760X1_18389794 \\
\hline $0.61 \mathrm{NA}$ & NA & & NA & & NA & & Gg_rs139703 snp.4.489.26 \\
\hline 1.2 & 1.54 & 1.17 & & 5.65 & & 1.53 & X1_1785128@snp.4.489.26 \\
\hline 0.03 & -0.14 & 0.03 & & 0.16 & & 0.04 & X1_1785128SGg_rs135559: \\
\hline 0.64 & 0.52 & 0.62 & & 1.93 & & 0.81 & X1_1785128@snp.4.489.26 \\
\hline 0.97 & 2.82 & 0.94 & & 1.16 & & 1.23 & X1_1785128ভsnp.4.489.26 \\
\hline $0.86 \mathrm{NA}$ & NA & & NA & & NA & & X1_1785128@snp.4.489.26 \\
\hline 0.27 & -1.41 & 0.26 & & 1.42 & & 0.35 & X1_1785128@snp.4.489.26، \\
\hline 0.81 & 2.17 & 0.78 & & 3.67 & & 1.02 & X1_1785128@snp.4.489.26 \\
\hline $0.3 \mathrm{NA}$ & NA & & NA & & NA & & X1_1785128@X1_18389794 \\
\hline $0.43 \mathrm{NA}$ & NA & & NA & & NA & & Gg_rs139703 X1_19033467 \\
\hline 0.98 & 2.37 & 0.98 & & -2.05 & & 1.2 & X1_1785128@X1_19033467 \\
\hline 0.04 & -0.24 & 0.04 & & 0.24 & & 0.05 & X1_1785128@X1_18389794 \\
\hline $0.33 \mathrm{NA}$ & NA & & NA & & NA & & Gg_rs135559X1_18448663 \\
\hline $0.52 \mathrm{NA}$ & NA & & NA & & NA & & Gg_rs135559 X1_19033467 \\
\hline 0.09 & -0.56 & 0.09 & & 0.67 & & 0.1 & Gg_rs135559 X1_18389794 \\
\hline 0.52 & 1.68 & 0.52 & & -1.23 & & 0.63 & Gg_rs135559 X1_18389794 \\
\hline $0.58 \mathrm{NA}$ & NA & & NA & & NA & & Gg_rs135559 X1_18448663 \\
\hline $0.94 \mathrm{NA}$ & NA & & NA & & NA & & Gg_rs135559 X1_19527164 \\
\hline $0.36 \mathrm{NA}$ & NA & & NA & & NA & & Gg_rs135559X1_19033467 \\
\hline $0.51 \mathrm{NA}$ & NA & & NA & & NA & & Gg_rs135559X1_19033467 \\
\hline $0.32 \mathrm{NA}$ & NA & & NA & & NA & & Gg_rs135559X1_19033467 \\
\hline $0.6 \mathrm{NA}$ & NA & & NA & & NA & & Gg_rs135559X1_19033467 \\
\hline $0.39 \mathrm{NA}$ & NA & & NA & & NA & & Gg_rs135559 X1_19033467 \\
\hline $0.52 \mathrm{NA}$ & NA & & NA & & NA & & Gg_rs135559X1_19033467 \\
\hline $0.57 \mathrm{NA}$ & NA & & NA & & NA & & Gg_rs135559X1_19033467 \\
\hline $0.38 \mathrm{NA}$ & NA & & NA & & NA & & Gg_rs135559X1_19033467 \\
\hline $0.36 \mathrm{NA}$ & NA & & NA & & NA & & Gg_rs135559 X1_19033467 \\
\hline 1.3 & -1.5 & 1.26 & & 3.78 & & 1.66 & snp.4.489.26‘X1_19033467 \\
\hline $0.64 \mathrm{NA}$ & NA & & NA & & NA & & Gg_rs135559 X1_19033467 \\
\hline $0.54 \mathrm{NA}$ & NA & & NA & & NA & & Gg_rs135559X1_19033467 \\
\hline $0.35 \mathrm{NA}$ & NA & & NA & & NA & & snp.4.489.26<X1_19033467 \\
\hline 0.04 & -0.16 & 0.04 & & 0.17 & & 0.05 & snp.4.489.26<X1_19033467 \\
\hline 0.27 & 0.79 & 0.26 & & -0.93 & & 0.34 & snp.4.489.26<X1_19033467 \\
\hline $0.32 \mathrm{NA}$ & NA & & NA & & NA & & snp.4.489.26<X1_19033467 \\
\hline $0.17 \mathrm{NA}$ & NA & & NA & & NA & & snp.4.489.26<X1_1903346ד \\
\hline
\end{tabular}




\begin{tabular}{|c|c|c|c|c|c|}
\hline $0.37 \mathrm{NA}$ & NA & NA & NA & & Gg_rs135559X1_19033467 \\
\hline $0.68 \mathrm{NA}$ & NA & NA & NA & & Gg_rs135559X1_19033467 \\
\hline $0.27 \mathrm{NA}$ & NA & NA & NA & & Gg_rs135559X1_19033467 \\
\hline $0.45 \mathrm{NA}$ & NA & NA & NA & & snp.4.489.26<X1_19033467 \\
\hline $0.48 \mathrm{NA}$ & NA & NA & NA & & snp.4.489.26<X1_19033467 \\
\hline $0.47 \mathrm{NA}$ & NA & NA & NA & & snp.4.489.26<X1_19033467 \\
\hline $0.62 \mathrm{NA}$ & NA & NA & NA & & snp.4.489.26<X1_19033467 \\
\hline $0.67 \mathrm{NA}$ & NA & NA & NA & & snp.4.489.26<X1_19033467 \\
\hline $0.47 \mathrm{NA}$ & NA & NA & NA & & snp.4.489.26‘X1_1903346† \\
\hline $0.38 \mathrm{NA}$ & NA & NA & NA & & snp.4.489.26<X1_1903346ד \\
\hline $0.55 \mathrm{NA}$ & NA & NA & NA & & snp.4.489.26<X1_19033467 \\
\hline $0.8 \mathrm{NA}$ & NA & NA & NA & & snp.4.489.26<X1_1903346ד \\
\hline 0.49 NA & NA & NA & NA & & Gg_rs135559X1_19033467 \\
\hline $0.41 \mathrm{NA}$ & NA & NA & NA & & snp.4.489.26<X1_19033467 \\
\hline $0.38 \mathrm{NA}$ & NA & NA & NA & & snp.4.489.26<X1_1903346ד \\
\hline $0.4 \mathrm{NA}$ & NA & NA & NA & & snp.4.489.26<X1_1903346ד \\
\hline $0.43 \mathrm{NA}$ & NA & NA & NA & & snp.4.489.26<X1_1903346ד \\
\hline $0.45 \mathrm{NA}$ & NA & NA & NA & & Gg_rs135559 X1_19033467 \\
\hline $0.51 \mathrm{NA}$ & NA & NA & NA & & Gg_rs135559X1_19033467 \\
\hline $0.93 \mathrm{NA}$ & NA & NA & NA & & Gg_rs135559 X1_19033467 \\
\hline 0.48 NA & NA & NA & NA & & snp.4.489.26<X1_19033467 \\
\hline $0.4 \mathrm{NA}$ & NA & NA & NA & & snp.4.489.26<X1_19033467 \\
\hline 0.7 & -2.03 & 0.65 & 3.12 & 0.9 & X1_18389794X1_19033467 \\
\hline $0.26 \mathrm{NA}$ & NA & NA & NA & & Gg_rs135559X1_19033467 \\
\hline $0.45 \mathrm{NA}$ & NA & NA & NA & & snp.4.489.26<X1_19033467 \\
\hline $0.2 \mathrm{NA}$ & NA & NA & NA & & snp.4.489.26<X1_19033467 \\
\hline 0.34 & 0.67 & 0.32 & -0.79 & 0.44 & Gg_rs135559 X1_19033467 \\
\hline $0.51 \mathrm{NA}$ & NA & NA & NA & & X1_18389794X1_19033467 \\
\hline $0.71 \mathrm{NA}$ & NA & NA & NA & & Gg_rs135559 X1_19033467 \\
\hline 0.73 & 0.52 & 0.68 & -2.08 & 0.94 & Gg_rs135559 X1_19033467 \\
\hline $0.41 \mathrm{NA}$ & NA & NA & NA & & Gg_rs135559 X1_19033467 \\
\hline 0.72 & 2.17 & 0.67 & -3.27 & 0.93 & snp.4.489.26‘X1_19033467 \\
\hline $0.59 \mathrm{NA}$ & NA & NA & NA & & Gg_rs135559X1_19033467 \\
\hline $0.75 \mathrm{NA}$ & NA & NA & NA & & Gg_rs135559X1_19033467 \\
\hline $0.49 \mathrm{NA}$ & NA & NA & NA & & Gg_rs135559X1_19033467 \\
\hline $0.41 \mathrm{NA}$ & NA & NA & NA & & Gg_rs135559 X1_19033467 \\
\hline 0.65 & 0.54 & 0.63 & -3.4 & 0.83 & snp.4.489.26‘X1_19033467 \\
\hline $0.5 \mathrm{NA}$ & NA & NA & NA & & Gg_rs135559X1_19527164 \\
\hline $0.4 \mathrm{NA}$ & NA & NA & NA & & Gg_rs135559 X1_19527164 \\
\hline $0.39 \mathrm{NA}$ & NA & NA & NA & & Gg_rs135559X1_19527164 \\
\hline 0.88 NA & NA & NA & NA & & Gg_rs135559X1_19527164 \\
\hline $0.46 \mathrm{NA}$ & NA & NA & NA & & X1_17441654X1_19527164 \\
\hline $1.9 \mathrm{NA}$ & NA & NA & NA & & X1_18598868X1_19527164 \\
\hline
\end{tabular}




\begin{tabular}{|c|c|c|c|c|c|}
\hline 0.31 & 0.47 & 0.22 & -1.42 & 0.4 & X1_18598868X1_19527164 \\
\hline 0.75 & -2.09 & 0.54 & 4.16 & 0.97 & X1_18598868X1_19527164 \\
\hline 0.07 & -0.13 & 0.05 & 0.23 & 0.08 & X1_18598868X1_19527164 \\
\hline $1.09 \mathrm{NA}$ & NA & NA & NA & & X1_18598868X1_19527164 \\
\hline 0.18 & -0.7 & 0.13 & 0.89 & 0.24 & X1_18598868X1_19527164 \\
\hline $0.89 \mathrm{NA}$ & NA & NA & NA & & X1_18598868X1_19527164 \\
\hline $0.73 \mathrm{NA}$ & NA & NA & NA & & Gg_rs135559 X1_19527164 \\
\hline $0.39 \mathrm{NA}$ & NA & NA & NA & & X1_18598868X1_19527164 \\
\hline $1.42 \mathrm{NA}$ & NA & NA & NA & & X1_18598868X1_2004005€ \\
\hline $1.08 \mathrm{NA}$ & NA & NA & NA & & X1_18598868X1_2004005€ \\
\hline $0.88 \mathrm{NA}$ & NA & NA & NA & & X1_18598868X1_2004005€ \\
\hline $1.16 \mathrm{NA}$ & NA & NA & NA & & X1_18598868X1_2004005€ \\
\hline $0.58 \mathrm{NA}$ & NA & NA & NA & & X1_18598868X1_2004005€ \\
\hline $0.52 \mathrm{NA}$ & NA & NA & NA & & X1_18598868X1_2004005€ \\
\hline $1 \mathrm{NA}$ & NA & NA & NA & & X1_18598868X1_2004005€ \\
\hline $0.61 \mathrm{NA}$ & NA & NA & NA & & X1_19202981X1_2004005€ \\
\hline $3.27 \mathrm{NA}$ & NA & NA & NA & & X1_19202981X1_2004005€ \\
\hline 0.59 NA & NA & NA & NA & & X1_19202981X1_2004005€ \\
\hline $0.57 \mathrm{NA}$ & NA & NA & NA & & X1_19202981X1_2004005€ \\
\hline $0.54 \mathrm{NA}$ & NA & NA & NA & & X1_19202981X1_2004005€ \\
\hline 0.64 NA & NA & NA & NA & & X1_19202981X1_2004005€ \\
\hline 0.97 & -0.95 & 0.84 & -2.63 & 1.32 & X1_19202981X1_2004005€ \\
\hline 0.47 NA & NA & NA & NA & & X1_19202981X1_2004005€ \\
\hline $0.45 \mathrm{NA}$ & NA & NA & NA & & X1_19202981X1_2004005€ \\
\hline $0.8 \mathrm{NA}$ & NA & NA & NA & & X1_19202981X1_2004005€ \\
\hline $0.56 \mathrm{NA}$ & NA & NA & NA & & X1_19202981X1_2004005€ \\
\hline $0.41 \mathrm{NA}$ & NA & NA & NA & & X1_19202981X1_2004005€ \\
\hline $0.34 \mathrm{NA}$ & NA & NA & NA & & X1_19202981X1_2004005€ \\
\hline $3.94 \mathrm{NA}$ & NA & NA & NA & & X1_19202981X1_2004005€ \\
\hline $0.7 \mathrm{NA}$ & NA & NA & NA & & X1_19202981X1_2004005€ \\
\hline $0.59 \mathrm{NA}$ & NA & NA & NA & & X1_19202981X1_2004005€ \\
\hline 0.86 NA & NA & NA & NA & & X1_19202981X1_2004005€ \\
\hline $0.63 \mathrm{NA}$ & NA & NA & NA & & X1_19202981X1_2004005€ \\
\hline $0.5 \mathrm{NA}$ & NA & NA & NA & & X1_19202981X1_2004005€ \\
\hline $3.37 \mathrm{NA}$ & NA & NA & NA & & X1_19202981X1_2004005€ \\
\hline $0.48 \mathrm{NA}$ & NA & NA & NA & & X1_19202981X1_2004005€ \\
\hline $0.51 \mathrm{NA}$ & NA & NA & NA & & X1_19202981X1_2004005€ \\
\hline $0.91 \mathrm{NA}$ & NA & NA & NA & & X1_19202981X1_2004005€ \\
\hline $0.56 \mathrm{NA}$ & NA & NA & NA & & X1_19202981X1_2004005€ \\
\hline $0.58 \mathrm{NA}$ & NA & NA & NA & & X1_19202981X1_2004005€ \\
\hline $3.81 \mathrm{NA}$ & NA & NA & NA & & X1_19202981X1_2004005€ \\
\hline $0.31 \mathrm{NA}$ & NA & NA & NA & & X1_19202981X1_2004005€ \\
\hline $0.51 \mathrm{NA}$ & NA & NA & NA & & X1_19202981X1_2004005€ \\
\hline
\end{tabular}




\begin{tabular}{|c|c|c|c|c|c|}
\hline $0.68 \mathrm{NA}$ & NA & NA & NA & & X1_19202981X1_2004005€ \\
\hline 4.09 NA & NA & NA & NA & & X1_19202981X1_2004005€ \\
\hline 4.26 NA & NA & NA & NA & & X1_19202981X1_2004005€ \\
\hline $5.1 \mathrm{NA}$ & NA & NA & NA & & X1_19202981X1_2004005€ \\
\hline $3.87 \mathrm{NA}$ & NA & NA & NA & & X1_19202981X1_2004005€ \\
\hline 3.7 NA & NA & NA & NA & & X1_19202981X1_2004005€ \\
\hline $5.15 \mathrm{NA}$ & NA & NA & NA & & X1_19202981X1_2004005€ \\
\hline 4.22 NA & NA & NA & NA & & X1_19202981X1_2004005€ \\
\hline 4.11 NA & NA & NA & NA & & X1_19202981X1_2004005€ \\
\hline $4.41 \mathrm{NA}$ & NA & NA & NA & & X1_19202981X1_2004005€ \\
\hline 4.69 NA & NA & NA & NA & & X1_19202981X1_2004005€ \\
\hline 4.18 NA & NA & NA & NA & & X1_19202981X1_2004005€ \\
\hline 4.74 NA & NA & NA & NA & & X1_19202981X1_2004005€ \\
\hline $0.72 \mathrm{NA}$ & NA & NA & NA & & X1_19202981X1_2004005€ \\
\hline $0.9 \mathrm{NA}$ & NA & NA & NA & & X1_19202981X1_2004005€ \\
\hline $0.57 \mathrm{NA}$ & NA & NA & NA & & X1_19202981X1_2004005€ \\
\hline $1.12 \mathrm{NA}$ & NA & NA & NA & & X1_19202981X1_2004005€ \\
\hline $0.53 \mathrm{NA}$ & NA & NA & NA & & X1_19202981X1_2004005€ \\
\hline $0.6 \mathrm{NA}$ & NA & NA & NA & & X1_19202981X1_2004005€ \\
\hline $0.86 \mathrm{NA}$ & NA & NA & NA & & X1_19202981X1_2004005€ \\
\hline $0.9 \mathrm{NA}$ & NA & NA & NA & & X1_19202981X1_2004005€ \\
\hline 0.64 & -1.4 & 0.56 & -2.12 & 0.88 & X1_19202981X1_2004005€ \\
\hline $0.55 \mathrm{NA}$ & NA & NA & NA & & X1_19202981X1_2004005€ \\
\hline 0.73 NA & NA & NA & NA & & X1_19202981X1_2004005€ \\
\hline $0.38 \mathrm{NA}$ & NA & NA & NA & & X1_19202981X1_2004005€ \\
\hline $0.48 \mathrm{NA}$ & NA & NA & NA & & X1_19202981X1_2004005€ \\
\hline 0.46 & -1.05 & 0.4 & 0.2 & 0.62 & X1_19202981X1_2004005€ \\
\hline $2.67 \mathrm{NA}$ & NA & NA & NA & & X1_19202981X1_2004005€ \\
\hline $2.64 \mathrm{NA}$ & NA & NA & NA & & X1_19202981X1_2004005€ \\
\hline $0.51 \mathrm{NA}$ & NA & NA & NA & & X1_19202981X1_2004005€ \\
\hline $2.6 \mathrm{NA}$ & NA & NA & NA & & X1_19202981X1_2004005€ \\
\hline 0.46 NA & NA & NA & NA & & snp.317.20.1:Gg_rs149396 \\
\hline $0.77 \mathrm{NA}$ & NA & NA & NA & & snp.317.20.1:Gg_rs149396 \\
\hline $1.94 \mathrm{NA}$ & NA & NA & NA & & snp.317.20.1:Gg_rs149396 \\
\hline $0.42 \mathrm{NA}$ & NA & NA & NA & & snp.317.20.1:Gg_rs149396 \\
\hline $1.65 \mathrm{NA}$ & NA & NA & NA & & snp.317.20.1:Gg_rs149396 \\
\hline $1.79 \mathrm{NA}$ & NA & NA & NA & & snp.317.20.1:Gg_rs149396 \\
\hline $1.3 \mathrm{NA}$ & NA & NA & NA & & snp.317.20.1:Gg_rs149396 \\
\hline $0.35 \mathrm{NA}$ & NA & NA & NA & & snp.317.20.1:Gg_rs149396 \\
\hline $1.52 \mathrm{NA}$ & NA & NA & NA & & snp.317.20.1:Gg_rs149396 \\
\hline $1.15 \mathrm{NA}$ & NA & NA & NA & & snp.317.20.1:Gg_rs149396 \\
\hline $2.03 \mathrm{NA}$ & NA & NA & NA & & X1_19202981Gg_rs149396 \\
\hline 0.78 NA & NA & NA & NA & & snp.317.20.1:Gg_rs14939 \\
\hline
\end{tabular}




\begin{tabular}{|c|c|c|c|c|c|c|c|}
\hline $0.89 \mathrm{NA}$ & NA & & NA & NA & & \multicolumn{2}{|c|}{ snp.317.20.1:Gg_rs149396 } \\
\hline $1.14 \mathrm{NA}$ & NA & & NA & NA & & rbl1360 & Gg_rs149396: \\
\hline $0.52 \mathrm{NA}$ & NA & & NA & NA & & rbl1360 & Gg_rs149396: \\
\hline $1.15 \mathrm{NA}$ & NA & & NA & NA & & rbl1360 & Gg_rs149396 \\
\hline 0.87 & -2.53 & 0.84 & & 2.42 & 1.11 & rbl1360 & Gg_rs149396 \\
\hline 0.55 & -1.63 & 0.53 & & 1.25 & 0.71 & rbl1360 & Gg_rs149396 \\
\hline 0.8 & -3.16 & 0.77 & & 3.17 & 1.02 & rbl1360 & Gg_rs149396 \\
\hline 1.29 & -4.77 & 1.25 & & 1.14 & 1.65 & rbl1360 & Gg_rs149396 \\
\hline $0.61 \mathrm{NA}$ & NA & & NA & NA & & rbl1360 & Gg_rs149396 \\
\hline $0.58 \mathrm{NA}$ & NA & & NA & NA & & X2_3527379 & Gg_rs141323: \\
\hline $0.88 \mathrm{NA}$ & NA & & NA & NA & & X2_3527379 & Gg_rs141323 \\
\hline $0.61 \mathrm{NA}$ & NA & & NA & NA & & X2_3527379 & Gg_rs141323 \\
\hline 1.39 & -4.23 & 1.42 & & 5.16 & 1.86 & X2_3527379 & Gg_rs141323 \\
\hline $11.5 \mathrm{NA}$ & NA & & NA & NA & & X2_3527379 & Gg_rs141323 \\
\hline 8.48 NA & NA & & NA & NA & & X2_3527379 & Gg_rs141323 \\
\hline $2.04 \mathrm{NA}$ & NA & & NA & NA & & X2_3527379 & Gg_rs141323 \\
\hline $6.83 \mathrm{NA}$ & NA & & NA & NA & & X2_3527379 & Gg_rs141323 \\
\hline $3.36 \mathrm{NA}$ & NA & & NA & NA & & X2_3527379 & Gg_rs141323 \\
\hline 1.07 & -2.75 & 1.11 & & 2.38 & 1.43 & X2_3527379 & Gg_rs141323 \\
\hline 1.29 & -2.37 & 1.35 & & -1.65 & 1.73 & X2_3527379 & Gg_rs141339: \\
\hline $0.4 \mathrm{NA}$ & NA & & NA & NA & & X2_3527379 & Gg_rs150605 \\
\hline 0.78 NA & NA & & NA & NA & & Gg_rs141315 & 5 Gg_rs141339 \\
\hline 0.02 NA & NA & & NA & NA & & Gg_rs141315 & 5 Gg_rs141339 \\
\hline 0.53 NA & NA & & NA & NA & & Gg_rs141315 & 5 Gg_rs141339: \\
\hline $0.73 \mathrm{NA}$ & NA & & NA & NA & & Gg_rs141315 & 5 Gg_rs141339; \\
\hline 1.15 & -2.47 & 1.21 & & -2.1 & 1.64 & Gg_rs141315 & 5 Gg_rs141339 \\
\hline $0.45 \mathrm{NA}$ & NA & & NA & NA & & Gg_rs141315 & 5 Gg_rs141339 \\
\hline $0.59 \mathrm{NA}$ & NA & & NA & NA & & Gg_rs141315 & 5 Gg_rs141339 \\
\hline $0.36 \mathrm{NA}$ & NA & & NA & NA & & Gg_rs141315 & 5 Gg_rs141339 \\
\hline $0.3 \mathrm{NA}$ & NA & & NA & NA & & Gg_rs141315 & 5 Gg_rs141339; \\
\hline $0.84 \mathrm{NA}$ & NA & & NA & NA & & X2_3527379 & Gg_rs150605 \\
\hline $0.51 \mathrm{NA}$ & NA & & NA & NA & & Gg_rs141315 & 5 Gg_rs150605 \\
\hline 0.54 & 1.95 & 0.56 & & 2.09 & 0.74 & Gg_rs141323 & 3 Gg_rs141391. \\
\hline 0.42 NA & NA & & NA & NA & & Gg_rs141315 & 5 Gg_rs141391. \\
\hline 0.49 NA & NA & & NA & NA & & Gg_rs141323 & 3 Gg_rs141391. \\
\hline $0.75 \mathrm{NA}$ & NA & & NA & NA & & Gg_rs141315 & 5 Gg_rs141391. \\
\hline $0.52 \mathrm{NA}$ & NA & & NA & NA & & Gg_rs141323 & 3 Gg_rs141391 \\
\hline 0.59 NA & NA & & NA & NA & & Gg_rs141323 & 3 Gg_rs141391. \\
\hline $0.42 \mathrm{NA}$ & NA & & NA & NA & & Gg_rs141323 & 3 Gg_rs141391. \\
\hline $0.56 \mathrm{NA}$ & NA & & NA & NA & & Gg_rs141323 & 3 Gg_rs150605 \\
\hline 0.46 NA & NA & & NA & NA & & Gg_rs141323 & 3 Gg_rs141391. \\
\hline $0.55 \mathrm{NA}$ & NA & & NA & NA & & Gg_rs141323 & 3 Gg_rs141391. \\
\hline $0.35 \mathrm{NA}$ & NA & & NA & NA & & Gg_rs141323 & 3 Gg_rs150605 \\
\hline
\end{tabular}




\begin{tabular}{|c|c|c|c|c|c|}
\hline $0.47 \mathrm{NA}$ & NA & NA & NA & & Gg_rs141323 Gg_rs141391 \\
\hline $0.63 \mathrm{NA}$ & NA & NA & NA & & Gg_rs141323 Gg_rs141391 \\
\hline $0.61 \mathrm{NA}$ & NA & NA & NA & & Gg_rs141323 Gg_rs141391 \\
\hline $0.42 \mathrm{NA}$ & NA & NA & NA & & Gg_rs141323 Gg_rs141391 \\
\hline $0.55 \mathrm{NA}$ & NA & NA & NA & & Gg_rs141323 Gg_rs150605 \\
\hline $0.41 \mathrm{NA}$ & NA & NA & NA & & Gg_rs141323 Gg_rs141391 \\
\hline $0.51 \mathrm{NA}$ & NA & NA & NA & & Gg_rs141323 Gg_rs150605 \\
\hline 0.46 NA & NA & NA & NA & & Gg_rs141323 Gg_rs150605 \\
\hline $0.45 \mathrm{NA}$ & NA & NA & NA & & Gg_rs141323 Gg_rs150605 \\
\hline $0.68 \mathrm{NA}$ & NA & NA & NA & & Gg_rs141323 Gg_rs141391 \\
\hline $0.43 \mathrm{NA}$ & NA & NA & NA & & Gg_rs141323 Gg_rs141391 \\
\hline $0.46 \mathrm{NA}$ & NA & NA & NA & & Gg_rs141323 Gg_rs150605 \\
\hline $0.32 \mathrm{NA}$ & NA & NA & NA & & Gg_rs141323 Gg_rs141391 \\
\hline $0.36 \mathrm{NA}$ & NA & NA & NA & & Gg_rs141323 Gg_rs141391 \\
\hline $0.44 \mathrm{NA}$ & NA & NA & NA & & Gg_rs141323 Gg_rs150605 \\
\hline $0.53 \mathrm{NA}$ & NA & NA & NA & & Gg_rs141323 Gg_rs141391 \\
\hline $0.44 \mathrm{NA}$ & NA & NA & NA & & Gg_rs141323 Gg_rs150605 \\
\hline $0.36 \mathrm{NA}$ & NA & NA & NA & & Gg_rs141323 Gg_rs150605 \\
\hline $0.64 \mathrm{NA}$ & NA & NA & NA & & Gg_rs141323 Gg_rs150605 \\
\hline $0.54 \mathrm{NA}$ & NA & NA & NA & & Gg_rs141323 Gg_rs141391 \\
\hline 0.43 NA & NA & NA & NA & & Gg_rs141323 Gg_rs150605 \\
\hline $1.01 \mathrm{NA}$ & NA & NA & NA & & Gg_rs141323 Gg_rs150605 \\
\hline $0.61 \mathrm{NA}$ & NA & NA & NA & & Gg_rs141323 Gg_rs141391 \\
\hline $0.59 \mathrm{NA}$ & NA & NA & NA & & Gg_rs141323 Gg_rs150605 \\
\hline $0.61 \mathrm{NA}$ & NA & NA & NA & & Gg_rs141323 Gg_rs150605 \\
\hline $0.37 \mathrm{NA}$ & NA & NA & NA & & Gg_rs141323 Gg_rs150605 \\
\hline 0.49 NA & NA & NA & NA & & Gg_rs141323 Gg_rs150605 \\
\hline $0.69 \mathrm{NA}$ & NA & NA & NA & & Gg_rs141323 Gg_rs150605 \\
\hline $0.53 \mathrm{NA}$ & NA & NA & NA & & Gg_rs141323 Gg_rs150605 \\
\hline $0.66 \mathrm{NA}$ & NA & NA & NA & & Gg_rs141323 Gg_rs150605 \\
\hline 0.63 & 1.86 & 0.66 & 0.32 & 0.89 & Gg_rs141323 Gg_rs150605 \\
\hline $0.29 \mathrm{NA}$ & NA & NA & NA & & Gg_rs141323 Gg_rs150605 \\
\hline $0.43 \mathrm{NA}$ & NA & NA & NA & & Gg_rs141323 Gg_rs150605 \\
\hline $0.45 \mathrm{NA}$ & NA & NA & NA & & Gg_rs141323 Gg_rs150605 \\
\hline $1.09 \mathrm{NA}$ & NA & NA & NA & & Gg_rs141323 Gg_rs141391 \\
\hline $0.5 \mathrm{NA}$ & NA & NA & NA & & Gg_rs141323 Gg_rs150605 \\
\hline 0.79 & -0.71 & 0.83 & -3.15 & 1.11 & Gg_rs141323 Gg_rs150605 \\
\hline 0.69 & -2.7 & 0.72 & -2.97 & 0.97 & Gg_rs141323 Gg_rs150605 \\
\hline $0.77 \mathrm{NA}$ & NA & NA & NA & & Gg_rs141323 Gg_rs150605 \\
\hline 0.79 NA & NA & NA & NA & & Gg_rs141323 Gg_rs141391 \\
\hline $0.45 \mathrm{NA}$ & NA & NA & NA & & Gg_rs141323 Gg_rs141391 \\
\hline $0.55 \mathrm{NA}$ & NA & NA & NA & & Gg_rs141323 Gg_rs141391 \\
\hline $0.68 \mathrm{NA}$ & NA & NA & NA & & Gg_rs141323 Gg_rs150605 \\
\hline
\end{tabular}




\begin{tabular}{|c|c|c|c|c|c|}
\hline 0.47 NA & NA & NA & NA & & Gg_rs141323 Gg_rs150605 \\
\hline $0.63 \mathrm{NA}$ & NA & NA & NA & & Gg_rs141323 Gg_rs141391. \\
\hline 0.48 NA & NA & NA & NA & & Gg_rs141323 Gg_rs150605 \\
\hline $0.73 \mathrm{NA}$ & NA & NA & NA & & Gg_rs141323 Gg_rs141391 \\
\hline $0.35 \mathrm{NA}$ & NA & NA & NA & & Gg_rs141323 Gg_rs150605 \\
\hline $0.58 \mathrm{NA}$ & NA & NA & NA & & Gg_rs141323 Gg_rs141391. \\
\hline $0.38 \mathrm{NA}$ & NA & NA & NA & & Gg_rs141323 Gg_rs150605 \\
\hline $0.37 \mathrm{NA}$ & NA & NA & NA & & Gg_rs141323 Gg_rs150605 \\
\hline 0.56 & -0.35 & 0.58 & -2.95 & 0.79 & Gg_rs141323 Gg_rs150605 \\
\hline 0.53 & 1.83 & 0.56 & -0.09 & 0.75 & Gg_rs141323 Gg_rs150605 \\
\hline 0.66 NA & NA & NA & NA & & Gg_rs141323 Gg_rs150605 \\
\hline 0.86 & -1.28 & 0.9 & -4.81 & 1.21 & Gg_rs141323 Gg_rs150605 \\
\hline $0.53 \mathrm{NA}$ & NA & NA & NA & & Gg_rs141323 Gg_rs150605 \\
\hline $0.57 \mathrm{NA}$ & NA & NA & NA & & Gg_rs141323 Gg_rs150605 \\
\hline $0.45 \mathrm{NA}$ & NA & NA & NA & & Gg_rs141323 Gg_rs150605 \\
\hline 0.69 NA & NA & NA & NA & & Gg_rs141323 Gg_rs150605 \\
\hline $0.73 \mathrm{NA}$ & NA & NA & NA & & Gg_rs141323 Gg_rs150605 \\
\hline $0.59 \mathrm{NA}$ & NA & NA & NA & & Gg_rs141323 Gg_rs150605 \\
\hline 0.49 NA & NA & NA & NA & & Gg_rs141323 Gg_rs141391. \\
\hline $0.39 \mathrm{NA}$ & NA & NA & NA & & Gg_rs141323 Gg_rs150605 \\
\hline 0.48 NA & NA & NA & NA & & Gg_rs141323 Gg_rs150605 \\
\hline $0.14 \mathrm{NA}$ & NA & NA & NA & & Gg_rs141323 Gg_rs150605 \\
\hline 0.74 NA & NA & NA & NA & & Gg_rs141323 Gg_rs150605 \\
\hline $0.36 \mathrm{NA}$ & NA & NA & NA & & Gg_rs141323 Gg_rs150605 \\
\hline $0.9 \mathrm{NA}$ & NA & NA & NA & & Gg_rs141323 Gg_rs150605 \\
\hline $0.38 \mathrm{NA}$ & NA & NA & NA & & Gg_rs141323 Gg_rs150605 \\
\hline $0.58 \mathrm{NA}$ & NA & NA & NA & & Gg_rs141323 Gg_rs150605 \\
\hline $0.69 \mathrm{NA}$ & NA & NA & NA & & Gg_rs141323 Gg_rs150605 \\
\hline $0.45 \mathrm{NA}$ & NA & NA & NA & & Gg_rs141323 Gg_rs150605 \\
\hline 0.59 NA & NA & NA & NA & & Gg_rs141323 Gg_rs141391. \\
\hline $0.55 \mathrm{NA}$ & NA & NA & NA & & Gg_rs141323 Gg_rs141391. \\
\hline $0.6 \mathrm{NA}$ & NA & NA & NA & & Gg_rs141323 Gg_rs141391 \\
\hline $0.54 \mathrm{NA}$ & NA & NA & NA & & Gg_rs141323 Gg_rs150605 \\
\hline 0.64 & -1.21 & 0.67 & -1.8 & 0.9 & Gg_rs141323 Gg_rs150605 \\
\hline 1 & 1.62 & 1.04 & -1.88 & 1.41 & Gg_rs141323 Gg_rs150605 \\
\hline 0.47 & 0.4 & 0.49 & -2.19 & 0.66 & Gg_rs141323 Gg_rs150605 \\
\hline $0.41 \mathrm{NA}$ & NA & NA & NA & & Gg_rs141323 Gg_rs141391. \\
\hline $0.55 \mathrm{NA}$ & NA & NA & NA & & Gg_rs141323 Gg_rs141391. \\
\hline $0.23 \mathrm{NA}$ & NA & NA & NA & & Gg_rs141323 Gg_rs150605 \\
\hline $0.52 \mathrm{NA}$ & NA & NA & NA & & Gg_rs141323 Gg_rs141391. \\
\hline $0.58 \mathrm{NA}$ & NA & NA & NA & & Gg_rs141323 Gg_rs141391. \\
\hline $0.52 \mathrm{NA}$ & NA & NA & NA & & Gg_rs141323 Gg_rs141391. \\
\hline 0.79 NA & NA & NA & NA & & Gg_rs141323 Gg_rs150605 \\
\hline
\end{tabular}




\begin{tabular}{|c|c|c|c|c|}
\hline $0.8 \mathrm{NA}$ & NA & NA & NA & Gg_rs141323 Gg_rs141391 \\
\hline $0.29 \mathrm{NA}$ & NA & NA & NA & Gg_rs141323 Gg_rs150605 \\
\hline $0.47 \mathrm{NA}$ & NA & NA & NA & Gg_rs141323 Gg_rs141391 \\
\hline $0.6 \mathrm{NA}$ & NA & NA & NA & Gg_rs141323 Gg_rs141391 \\
\hline $0.78 \mathrm{NA}$ & NA & NA & NA & Gg_rs141323 Gg_rs141391 \\
\hline $0.5 \mathrm{NA}$ & NA & NA & NA & Gg_rs141323 Gg_rs141391 \\
\hline $0.39 \mathrm{NA}$ & NA & NA & NA & Gg_rs141323 Gg_rs141391 \\
\hline $0.4 \mathrm{NA}$ & NA & NA & NA & Gg_rs141323 Gg_rs141391 \\
\hline $0.48 \mathrm{NA}$ & NA & NA & NA & Gg_rs141323 Gg_rs141391 \\
\hline $0.23 \mathrm{NA}$ & NA & NA & NA & Gg_rs141323 Gg_rs141391 \\
\hline 0.44 NA & NA & NA & NA & Gg_rs141323 Gg_rs141391 \\
\hline 0.69 NA & NA & NA & NA & Gg_rs141323 Gg_rs141391 \\
\hline $0.6 \mathrm{NA}$ & NA & NA & NA & Gg_rs141323 Gg_rs141391 \\
\hline $0.44 \mathrm{NA}$ & NA & NA & NA & Gg_rs141323 Gg_rs141391 \\
\hline $0.68 \mathrm{NA}$ & NA & NA & NA & Gg_rs141323 Gg_rs141391 \\
\hline $0.4 \mathrm{NA}$ & NA & NA & NA & Gg_rs141323 Gg_rs141391 \\
\hline $0.48 \mathrm{NA}$ & NA & NA & NA & Gg_rs141323 Gg_rs141391 \\
\hline 0.48 NA & NA & NA & NA & Gg_rs141323 Gg_rs141391 \\
\hline $0.75 \mathrm{NA}$ & NA & NA & NA & Gg_rs141323 Gg_rs141391 \\
\hline $0.58 \mathrm{NA}$ & NA & NA & NA & Gg_rs141323 Gg_rs141391 \\
\hline $0.59 \mathrm{NA}$ & NA & NA & NA & Gg_rs141323 Gg_rs141391 \\
\hline 0.36 NA & NA & NA & NA & Gg_rs141323 Gg_rs141391 \\
\hline $0.57 \mathrm{NA}$ & NA & NA & NA & Gg_rs141323 Gg_rs141391 \\
\hline $0.47 \mathrm{NA}$ & NA & NA & NA & Gg_rs141323 Gg_rs141391 \\
\hline $0.58 \mathrm{NA}$ & NA & NA & NA & Gg_rs141323 Gg_rs141391 \\
\hline $0.49 \mathrm{NA}$ & NA & NA & NA & Gg_rs141323 Gg_rs141391 \\
\hline 0.48 NA & NA & NA & NA & Gg_rs141323 Gg_rs141391 \\
\hline $0.61 \mathrm{NA}$ & NA & NA & NA & Gg_rs141323 Gg_rs141391 \\
\hline 0.63 NA & NA & NA & NA & Gg_rs141323 Gg_rs141391 \\
\hline $0.54 \mathrm{NA}$ & NA & NA & NA & Gg_rs141323 Gg_rs141391 \\
\hline $0.55 \mathrm{NA}$ & NA & NA & NA & Gg_rs141323 Gg_rs141391 \\
\hline 0.53 NA & NA & NA & NA & Gg_rs141323 Gg_rs141391 \\
\hline $0.45 \mathrm{NA}$ & NA & NA & NA & Gg_rs141323 Gg_rs141391 \\
\hline $0.54 \mathrm{NA}$ & NA & NA & NA & Gg_rs141323 Gg_rs141391 \\
\hline $0.53 \mathrm{NA}$ & NA & NA & NA & Gg_rs141323 Gg_rs141391 \\
\hline $0.66 \mathrm{NA}$ & NA & NA & NA & Gg_rs141323 Gg_rs141391 \\
\hline 0.66 NA & NA & NA & NA & Gg_rs141323 Gg_rs141391 \\
\hline $0.51 \mathrm{NA}$ & NA & NA & NA & Gg_rs141323 Gg_rs141391 \\
\hline $0.35 \mathrm{NA}$ & NA & NA & NA & Gg_rs141323 Gg_rs141391 \\
\hline $0.37 \mathrm{NA}$ & NA & NA & NA & Gg_rs141323 Gg_rs141391 \\
\hline $0.83 \mathrm{NA}$ & NA & NA & NA & Gg_rs141323 Gg_rs141391 \\
\hline $0.73 \mathrm{NA}$ & NA & NA & NA & Gg_rs141323 Gg_rs141391 \\
\hline $0.58 \mathrm{NA}$ & NA & NA & NA & Gg_rs141323 Gg_rs141391 \\
\hline
\end{tabular}




\begin{tabular}{|c|c|c|c|c|c|}
\hline $0.39 \mathrm{NA}$ & NA & NA & NA & & Gg_rs141323 Gg_rs141391 \\
\hline $0.52 \mathrm{NA}$ & NA & NA & NA & & Gg_rs141323 Gg_rs141391 \\
\hline 0.75 & -0.33 & 0.78 & -2.6 & 1.03 & Gg_rs141323 Gg_rs141391 \\
\hline $0.62 \mathrm{NA}$ & NA & NA & NA & & Gg_rs141323 Gg_rs141391 \\
\hline $0.5 \mathrm{NA}$ & NA & NA & NA & & Gg_rs141323 Gg_rs141391 \\
\hline $0.58 \mathrm{NA}$ & NA & NA & NA & & Gg_rs141323 Gg_rs141391 \\
\hline $0.57 \mathrm{NA}$ & NA & NA & NA & & Gg_rs141323 Gg_rs141391 \\
\hline $0.61 \mathrm{NA}$ & NA & NA & NA & & Gg_rs141323 Gg_rs141391 \\
\hline $0.59 \mathrm{NA}$ & NA & NA & NA & & Gg_rs141323 Gg_rs141391 \\
\hline $0.83 \mathrm{NA}$ & NA & NA & NA & & Gg_rs141323 Gg_rs141391 \\
\hline $0.47 \mathrm{NA}$ & NA & NA & NA & & Gg_rs141323 Gg_rs141391 \\
\hline $0.67 \mathrm{NA}$ & NA & NA & NA & & Gg_rs141323 Gg_rs141391 \\
\hline $0.55 \mathrm{NA}$ & NA & NA & NA & & Gg_rs141323 Gg_rs141391 \\
\hline $0.72 \mathrm{NA}$ & NA & NA & NA & & Gg_rs141323 Gg_rs141391 \\
\hline $0.7 \mathrm{NA}$ & NA & NA & NA & & Gg_rs141323 Gg_rs141391 \\
\hline 0.49 NA & NA & NA & NA & & Gg_rs141323 Gg_rs141391 \\
\hline 0.86 & -3.01 & 0.9 & -3.01 & 1.21 & Gg_rs141323 Gg_rs141391 \\
\hline $0.56 \mathrm{NA}$ & NA & NA & NA & & Gg_rs141323 Gg_rs141391 \\
\hline $0.84 \mathrm{NA}$ & NA & NA & NA & & Gg_rs141323 Gg_rs141391 \\
\hline $0.57 \mathrm{NA}$ & NA & NA & NA & & Gg_rs141323 Gg_rs141391 \\
\hline $0.54 \mathrm{NA}$ & NA & NA & NA & & Gg_rs141323 Gg_rs141391 \\
\hline $0.57 \mathrm{NA}$ & NA & NA & NA & & Gg_rs141323 Gg_rs141391 \\
\hline $0.24 \mathrm{NA}$ & NA & NA & NA & & Gg_rs141323 Gg_rs141391 \\
\hline $0.45 \mathrm{NA}$ & NA & NA & NA & & Gg_rs141315 Gg_rs141391 \\
\hline $0.7 \mathrm{NA}$ & NA & NA & NA & & Gg_rs141323 Gg_rs141391 \\
\hline $0.3 \mathrm{NA}$ & NA & NA & NA & & Gg_rs141323 Gg_rs141391 \\
\hline $0.43 \mathrm{NA}$ & NA & NA & NA & & Gg_rs141323 Gg_rs141391 \\
\hline 0.46 NA & NA & NA & NA & & Gg_rs141323 Gg_rs141391 \\
\hline $0.77 \mathrm{NA}$ & NA & NA & NA & & Gg_rs141323 Gg_rs141391 \\
\hline $0.57 \mathrm{NA}$ & NA & NA & NA & & Gg_rs141323 Gg_rs141391 \\
\hline $0.65 \mathrm{NA}$ & NA & NA & NA & & Gg_rs141323 Gg_rs141391 \\
\hline 0.87 & -1.76 & 0.91 & -1.26 & 1.19 & Gg_rs141323 Gg_rs141391 \\
\hline $0.78 \mathrm{NA}$ & NA & NA & NA & & Gg_rs141323 Gg_rs141391 \\
\hline $0.36 \mathrm{NA}$ & NA & NA & NA & & Gg_rs141323 Gg_rs141391 \\
\hline $0.83 \mathrm{NA}$ & NA & NA & NA & & Gg_rs141323 Gg_rs141391 \\
\hline $0.47 \mathrm{NA}$ & NA & NA & NA & & Gg_rs141323 Gg_rs141391 \\
\hline $0.58 \mathrm{NA}$ & NA & NA & NA & & Gg_rs141323 Gg_rs141391 \\
\hline $0.67 \mathrm{NA}$ & NA & NA & NA & & Gg_rs141323 Gg_rs141391 \\
\hline $0.56 \mathrm{NA}$ & NA & NA & NA & & Gg_rs141323 Gg_rs141391 \\
\hline $0.68 \mathrm{NA}$ & NA & NA & NA & & Gg_rs141323 Gg_rs141391 \\
\hline $0.52 \mathrm{NA}$ & NA & NA & NA & & Gg_rs141323 Gg_rs141391 \\
\hline $0.52 \mathrm{NA}$ & NA & NA & NA & & Gg_rs141323 Gg_rs141391 \\
\hline $0.41 \mathrm{NA}$ & NA & NA & NA & & Gg_rs141323 Gg_rs141391 \\
\hline
\end{tabular}




\begin{tabular}{|c|c|c|c|c|c|}
\hline $0.9 \mathrm{NA}$ & NA & NA & NA & & Gg_rs141323 Gg_rs141391. \\
\hline $0.64 \mathrm{NA}$ & NA & NA & NA & & Gg_rs141323 Gg_rs141391. \\
\hline $0.68 \mathrm{NA}$ & NA & NA & NA & & Gg_rs141323 Gg_rs141391. \\
\hline $0.68 \mathrm{NA}$ & NA & NA & NA & & Gg_rs141323 Gg_rs141391. \\
\hline $1.15 \mathrm{NA}$ & NA & NA & NA & & Gg_rs141323 Gg_rs141391. \\
\hline $0.71 \mathrm{NA}$ & NA & NA & NA & & Gg_rs141323 Gg_rs141391. \\
\hline $0.56 \mathrm{NA}$ & NA & NA & NA & & Gg_rs141323 Gg_rs141391. \\
\hline $0.48 \mathrm{NA}$ & NA & NA & NA & & Gg_rs141323 Gg_rs141391. \\
\hline $0.72 \mathrm{NA}$ & NA & NA & NA & & Gg_rs141323 Gg_rs141391. \\
\hline $0.83 \mathrm{NA}$ & NA & NA & NA & & Gg_rs141323 Gg_rs141391. \\
\hline $0.28 \mathrm{NA}$ & NA & NA & NA & & Gg_rs141323 Gg_rs141391. \\
\hline $0.52 \mathrm{NA}$ & NA & NA & NA & & Gg_rs141323 Gg_rs141391. \\
\hline $0.55 \mathrm{NA}$ & NA & NA & NA & & Gg_rs141323 Gg_rs141391. \\
\hline $0.66 \mathrm{NA}$ & NA & NA & NA & & Gg_rs141323 Gg_rs141391. \\
\hline $0.55 \mathrm{NA}$ & NA & NA & NA & & Gg_rs141323 Gg_rs141391 \\
\hline $0.52 \mathrm{NA}$ & NA & NA & NA & & Gg_rs141323 Gg_rs141391. \\
\hline $0.45 \mathrm{NA}$ & NA & NA & NA & & Gg_rs141323 Gg_rs141391. \\
\hline $0.74 \mathrm{NA}$ & NA & NA & NA & & Gg_rs141323 Gg_rs141391. \\
\hline $0.79 \mathrm{NA}$ & NA & NA & NA & & Gg_rs141323 Gg_rs141391. \\
\hline $0.55 \mathrm{NA}$ & NA & NA & NA & & Gg_rs141323 Gg_rs141391. \\
\hline $0.37 \mathrm{NA}$ & NA & NA & NA & & Gg_rs141323 Gg_rs141391. \\
\hline $0.41 \mathrm{NA}$ & NA & NA & NA & & Gg_rs141323 Gg_rs141391. \\
\hline $1.67 \mathrm{NA}$ & NA & NA & NA & & Gg_rs141323 Gg_rs141391. \\
\hline 0.73 & 2.48 & 0.75 & -0.15 & 0.99 & Gg_rs141323 Gg_rs141391. \\
\hline 0.97 & 0.11 & 1 & -2.23 & 1.32 & Gg_rs141323 Gg_rs141391 \\
\hline $0.58 \mathrm{NA}$ & NA & NA & NA & & Gg_rs141323 Gg_rs141391. \\
\hline $0.95 \mathrm{NA}$ & NA & NA & NA & & Gg_rs141323 Gg_rs141391. \\
\hline $0.38 \mathrm{NA}$ & NA & NA & NA & & Gg_rs141323 Gg_rs141391. \\
\hline $0.37 \mathrm{NA}$ & NA & NA & NA & & Gg_rs141323 Gg_rs141391. \\
\hline $0.84 \mathrm{NA}$ & NA & NA & NA & & Gg_rs141323 Gg_rs141391. \\
\hline $0.3 \mathrm{NA}$ & NA & NA & NA & & Gg_rs141323 Gg_rs141391. \\
\hline 0.75 & -1.67 & 0.78 & -2.2 & 1.03 & Gg_rs141323 Gg_rs141391 \\
\hline $0.43 \mathrm{NA}$ & NA & NA & NA & & Gg_rs141323 Gg_rs141391. \\
\hline $0.5 \mathrm{NA}$ & NA & NA & NA & & Gg_rs141323 Gg_rs141391 \\
\hline $0.57 \mathrm{NA}$ & NA & NA & NA & & Gg_rs141323 Gg_rs141391. \\
\hline $0.35 \mathrm{NA}$ & NA & NA & NA & & Gg_rs141323 Gg_rs141391. \\
\hline 1.41 & -0.62 & 1.47 & -3.42 & 1.93 & Gg_rs141323 Gg_rs141391 \\
\hline $0.48 \mathrm{NA}$ & NA & NA & NA & & Gg_rs141323 Gg_rs141391 \\
\hline $0.42 \mathrm{NA}$ & NA & NA & NA & & Gg_rs141323 Gg_rs141391. \\
\hline $0.48 \mathrm{NA}$ & NA & NA & NA & & Gg_rs141323 Gg_rs141391. \\
\hline $0.74 \mathrm{NA}$ & NA & NA & NA & & Gg_rs141323 Gg_rs141391. \\
\hline $0.64 \mathrm{NA}$ & NA & NA & NA & & Gg_rs141323 Gg_rs141391. \\
\hline $0.47 \mathrm{NA}$ & NA & NA & NA & & Gg_rs141323 Gg_rs141391 \\
\hline
\end{tabular}




\begin{tabular}{|c|c|c|c|c|}
\hline $0.73 \mathrm{NA}$ & NA & NA & NA & Gg_rs141323 Gg_rs141391. \\
\hline $0.59 \mathrm{NA}$ & NA & NA & NA & Gg_rs141323 Gg_rs141391. \\
\hline $0.64 \mathrm{NA}$ & NA & NA & NA & Gg_rs141323 Gg_rs141391. \\
\hline $0.55 \mathrm{NA}$ & NA & NA & NA & Gg_rs141323 Gg_rs141391. \\
\hline $0.48 \mathrm{NA}$ & NA & NA & NA & Gg_rs141323 Gg_rs141391. \\
\hline 0.49 NA & NA & NA & NA & Gg_rs141323 Gg_rs141391. \\
\hline $0.71 \mathrm{NA}$ & NA & NA & NA & Gg_rs141323 Gg_rs141391. \\
\hline $0.52 \mathrm{NA}$ & NA & NA & NA & Gg_rs141323 Gg_rs141391. \\
\hline $0.29 \mathrm{NA}$ & NA & NA & NA & Gg_rs141323 Gg_rs141391. \\
\hline $0.48 \mathrm{NA}$ & NA & NA & NA & Gg_rs141323 Gg_rs141391. \\
\hline 0.69 NA & NA & NA & NA & Gg_rs141323 Gg_rs141391. \\
\hline $0.38 \mathrm{NA}$ & NA & NA & NA & Gg_rs141323 Gg_rs141391. \\
\hline $0.52 \mathrm{NA}$ & NA & NA & NA & Gg_rs141323 Gg_rs141391. \\
\hline $0.43 \mathrm{NA}$ & NA & NA & NA & Gg_rs141323 Gg_rs141391. \\
\hline $0.65 \mathrm{NA}$ & NA & NA & NA & Gg_rs141323 Gg_rs141391. \\
\hline $0.6 \mathrm{NA}$ & NA & NA & NA & Gg_rs141323 Gg_rs141391. \\
\hline $0.66 \mathrm{NA}$ & NA & NA & NA & Gg_rs141323 Gg_rs141391. \\
\hline $0.51 \mathrm{NA}$ & NA & NA & NA & Gg_rs141323 Gg_rs141391. \\
\hline $0.41 \mathrm{NA}$ & NA & NA & NA & Gg_rs141323 Gg_rs141391. \\
\hline 0.68 NA & NA & NA & NA & Gg_rs141323 Gg_rs141391. \\
\hline $0.71 \mathrm{NA}$ & NA & NA & NA & Gg_rs141323 Gg_rs141391. \\
\hline $0.64 \mathrm{NA}$ & NA & NA & NA & Gg_rs141323 Gg_rs141391. \\
\hline $0.55 \mathrm{NA}$ & NA & NA & NA & Gg_rs141323 Gg_rs141391. \\
\hline $0.56 \mathrm{NA}$ & NA & NA & NA & Gg_rs141323 Gg_rs141391. \\
\hline 0.96 NA & NA & NA & NA & Gg_rs141323 Gg_rs141391. \\
\hline $0.38 \mathrm{NA}$ & NA & NA & NA & Gg_rs141323 Gg_rs141391. \\
\hline $0.58 \mathrm{NA}$ & NA & NA & NA & Gg_rs141323 Gg_rs141391. \\
\hline $0.67 \mathrm{NA}$ & NA & NA & NA & Gg_rs141323 Gg_rs141391. \\
\hline $0.72 \mathrm{NA}$ & NA & NA & NA & Gg_rs141323 Gg_rs141391. \\
\hline $0.43 \mathrm{NA}$ & NA & NA & NA & Gg_rs141323 Gg_rs141391. \\
\hline $0.57 \mathrm{NA}$ & NA & NA & NA & Gg_rs141323 Gg_rs141391. \\
\hline $0.51 \mathrm{NA}$ & NA & NA & NA & Gg_rs141323 Gg_rs141391. \\
\hline $0.57 \mathrm{NA}$ & NA & NA & NA & Gg_rs141323 Gg_rs141391. \\
\hline $0.58 \mathrm{NA}$ & NA & NA & NA & Gg_rs141323 Gg_rs141391. \\
\hline $0.61 \mathrm{NA}$ & NA & NA & NA & Gg_rs141323 Gg_rs141391. \\
\hline 0.49 NA & NA & NA & NA & Gg_rs141323 Gg_rs141391. \\
\hline 0.89 NA & NA & NA & NA & Gg_rs141323 Gg_rs141391. \\
\hline $0.75 \mathrm{NA}$ & NA & NA & NA & Gg_rs141323 Gg_rs141391. \\
\hline $0.45 \mathrm{NA}$ & NA & NA & NA & Gg_rs141323 Gg_rs141391. \\
\hline $0.56 \mathrm{NA}$ & NA & NA & NA & Gg_rs141323 Gg_rs141391. \\
\hline $0.75 \mathrm{NA}$ & NA & NA & NA & Gg_rs141323 Gg_rs141391. \\
\hline $0.22 \mathrm{NA}$ & NA & NA & NA & Gg_rs141323 Gg_rs141391. \\
\hline $0.49 \mathrm{NA}$ & NA & NA & NA & Gg_rs141323 Gg_rs141391 \\
\hline
\end{tabular}




\begin{tabular}{|c|c|c|c|c|c|}
\hline $0.44 \mathrm{NA}$ & NA & NA & NA & & Gg_rs141323 Gg_rs141391. \\
\hline $0.66 \mathrm{NA}$ & NA & NA & NA & & Gg_rs141323 Gg_rs141391. \\
\hline $0.61 \mathrm{NA}$ & NA & NA & NA & & Gg_rs141323 Gg_rs141391. \\
\hline 0.64 NA & NA & NA & NA & & Gg_rs141323 Gg_rs141391. \\
\hline $0.42 \mathrm{NA}$ & NA & NA & NA & & Gg_rs141323 Gg_rs141391. \\
\hline $0.46 \mathrm{NA}$ & NA & NA & NA & & Gg_rs141323 Gg_rs141391. \\
\hline $0.54 \mathrm{NA}$ & NA & NA & NA & & Gg_rs141323 Gg_rs141391. \\
\hline $0.65 \mathrm{NA}$ & NA & NA & NA & & Gg_rs141323 Gg_rs141391. \\
\hline $0.4 \mathrm{NA}$ & NA & NA & NA & & Gg_rs141339 Gg_rs141391. \\
\hline $0.51 \mathrm{NA}$ & NA & NA & NA & & Gg_rs141323 Gg_rs141391. \\
\hline 0.77 & -1.54 & 0.8 & -0.9 & 1.06 & Gg_rs141323 Gg_rs141391. \\
\hline $0.53 \mathrm{NA}$ & NA & NA & NA & & Gg_rs141323 Gg_rs141391. \\
\hline $0.71 \mathrm{NA}$ & NA & NA & NA & & Gg_rs141323 Gg_rs141391. \\
\hline $0.59 \mathrm{NA}$ & NA & NA & NA & & Gg_rs141339 Gg_rs141391. \\
\hline $0.77 \mathrm{NA}$ & NA & NA & NA & & Gg_rs141323 Gg_rs141391. \\
\hline 0.49 NA & NA & NA & NA & & Gg_rs141323 Gg_rs141391. \\
\hline 0.43 & 0.68 & 0.45 & -1.56 & 0.59 & Gg_rs141339Gg_rs141391. \\
\hline $0.39 \mathrm{NA}$ & NA & NA & NA & & Gg_rs141323 Gg_rs141391. \\
\hline $0.7 \mathrm{NA}$ & NA & NA & NA & & Gg_rs141323 Gg_rs141391. \\
\hline $0.39 \mathrm{NA}$ & NA & NA & NA & & Gg_rs141339 Gg_rs141391. \\
\hline $0.67 \mathrm{NA}$ & NA & NA & NA & & Gg_rs141323 Gg_rs141391. \\
\hline $0.55 \mathrm{NA}$ & NA & NA & NA & & Gg_rs141323 Gg_rs141391. \\
\hline $0.52 \mathrm{NA}$ & NA & NA & NA & & Gg_rs141323 Gg_rs141391. \\
\hline $0.41 \mathrm{NA}$ & NA & NA & NA & & Gg_rs141323 Gg_rs141391. \\
\hline $0.58 \mathrm{NA}$ & NA & NA & NA & & Gg_rs141323 Gg_rs141391. \\
\hline $0.82 \mathrm{NA}$ & NA & NA & NA & & Gg_rs141323 Gg_rs141391. \\
\hline $0.67 \mathrm{NA}$ & NA & NA & NA & & Gg_rs141323 Gg_rs141391. \\
\hline $0.77 \mathrm{NA}$ & NA & NA & NA & & Gg_rs141323 Gg_rs141391. \\
\hline 0.49 NA & NA & NA & NA & & Gg_rs141323 Gg_rs141391. \\
\hline $0.56 \mathrm{NA}$ & NA & NA & NA & & Gg_rs141323 Gg_rs141391. \\
\hline $0.74 \mathrm{NA}$ & NA & NA & NA & & Gg_rs141323 Gg_rs141391. \\
\hline $0.63 \mathrm{NA}$ & NA & NA & NA & & Gg_rs141323 Gg_rs141391. \\
\hline $0.66 \mathrm{NA}$ & NA & NA & NA & & Gg_rs141323 Gg_rs141391. \\
\hline $1.12 \mathrm{NA}$ & NA & NA & NA & & Gg_rs141323 Gg_rs141391. \\
\hline $0.57 \mathrm{NA}$ & NA & NA & NA & & Gg_rs141323 Gg_rs141391. \\
\hline $0.71 \mathrm{NA}$ & NA & NA & NA & & Gg_rs141323 Gg_rs141391. \\
\hline $0.61 \mathrm{NA}$ & NA & NA & NA & & Gg_rs141323 Gg_rs141391. \\
\hline $0.56 \mathrm{NA}$ & NA & NA & NA & & Gg_rs141323 Gg_rs141391. \\
\hline $0.57 \mathrm{NA}$ & NA & NA & NA & & Gg_rs141323 Gg_rs141391. \\
\hline $0.31 \mathrm{NA}$ & NA & NA & NA & & Gg_rs141323 Gg_rs141391. \\
\hline $0.8 \mathrm{NA}$ & NA & NA & NA & & Gg_rs141323 Gg_rs141391. \\
\hline $1.06 \mathrm{NA}$ & NA & NA & NA & & Gg_rs141315 Gg_rs141391. \\
\hline $0.6 \mathrm{NA}$ & NA & NA & NA & & Gg_rs141339 Gg_rs14139 \\
\hline
\end{tabular}




\begin{tabular}{|c|c|c|c|c|c|}
\hline 0.44 NA & NA & NA & NA & & Gg_rs141323 Gg_rs141391. \\
\hline $0.8 \mathrm{NA}$ & NA & NA & NA & & Gg_rs141323 Gg_rs141391. \\
\hline 0.99 NA & NA & NA & NA & & Gg_rs141323 Gg_rs141391. \\
\hline $0.42 \mathrm{NA}$ & NA & NA & NA & & Gg_rs141323 Gg_rs141391. \\
\hline $0.56 \mathrm{NA}$ & NA & NA & NA & & Gg_rs141323 Gg_rs141391. \\
\hline 0.89 NA & NA & NA & NA & & Gg_rs141323 Gg_rs141391. \\
\hline $0.61 \mathrm{NA}$ & NA & NA & NA & & Gg_rs141339 Gg_rs141391. \\
\hline 0.52 NA & NA & NA & NA & & Gg_rs141323 Gg_rs141391. \\
\hline $0.55 \mathrm{NA}$ & NA & NA & NA & & Gg_rs141323 Gg_rs141391. \\
\hline $0.34 \mathrm{NA}$ & NA & NA & NA & & Gg_rs141323 Gg_rs141391. \\
\hline $0.77 \mathrm{NA}$ & NA & NA & NA & & Gg_rs141323 Gg_rs141391. \\
\hline 0.49 NA & NA & NA & NA & & Gg_rs141323 Gg_rs141391. \\
\hline $0.67 \mathrm{NA}$ & NA & NA & NA & & Gg_rs141323 Gg_rs141391. \\
\hline $0.71 \mathrm{NA}$ & NA & NA & NA & & Gg_rs141323 Gg_rs141391. \\
\hline $0.76 \mathrm{NA}$ & NA & NA & NA & & Gg_rs141323 Gg_rs141391. \\
\hline $0.46 \mathrm{NA}$ & NA & NA & NA & & Gg_rs141323 Gg_rs141391. \\
\hline 0.79 NA & NA & NA & NA & & Gg_rs141323 Gg_rs141391. \\
\hline $0.62 \mathrm{NA}$ & NA & NA & NA & & Gg_rs141323 Gg_rs141391. \\
\hline 0.83 NA & NA & NA & NA & & Gg_rs141323 Gg_rs141391. \\
\hline 0.89 NA & NA & NA & NA & & Gg_rs141323 Gg_rs141391. \\
\hline $0.71 \mathrm{NA}$ & NA & NA & NA & & Gg_rs141323 Gg_rs141391. \\
\hline $0.66 \mathrm{NA}$ & NA & NA & NA & & Gg_rs141323 Gg_rs141391. \\
\hline $0.5 \mathrm{NA}$ & NA & NA & NA & & Gg_rs141339 Gg_rs141391. \\
\hline 0.62 NA & NA & NA & NA & & Gg_rs141323 Gg_rs141391. \\
\hline $0.56 \mathrm{NA}$ & NA & NA & NA & & Gg_rs141339 Gg_rs141391. \\
\hline $0.66 \mathrm{NA}$ & NA & NA & NA & & Gg_rs141339Gg_rs141391. \\
\hline $0.71 \mathrm{NA}$ & NA & NA & NA & & Gg_rs141323 Gg_rs141391. \\
\hline $0.57 \mathrm{NA}$ & NA & NA & NA & & Gg_rs141339Gg_rs141391. \\
\hline 0.64 NA & NA & NA & NA & & Gg_rs141339 Gg_rs141391. \\
\hline $0.71 \mathrm{NA}$ & NA & NA & NA & & Gg_rs141323 Gg_rs141391. \\
\hline $0.61 \mathrm{NA}$ & NA & NA & NA & & Gg_rs141323 Gg_rs141391. \\
\hline 0.33 NA & NA & NA & NA & & Gg_rs141339Gg_rs141391. \\
\hline $0.52 \mathrm{NA}$ & NA & NA & NA & & Gg_rs141323 Gg_rs150652 \\
\hline $1.13 \mathrm{NA}$ & NA & NA & NA & & Gg_rs141323 Gg_rs141391. \\
\hline $0.35 \mathrm{NA}$ & NA & NA & NA & & Gg_rs141339 Gg_rs141391. \\
\hline $0.92 \mathrm{NA}$ & NA & NA & NA & & Gg_rs141339Gg_rs141391. \\
\hline 0.97 NA & NA & NA & NA & & Gg_rs141339 Gg_rs141391. \\
\hline $0.55 \mathrm{NA}$ & NA & NA & NA & & Gg_rs141339Gg_rs141391. \\
\hline 0.45 & 1.51 & 0.46 & -0.62 & 0.61 & Gg_rs141339 Gg_rs141391. \\
\hline 0.6 & -0.84 & 0.63 & -1 & 0.83 & Gg_rs141339Gg_rs141391. \\
\hline $0.47 \mathrm{NA}$ & NA & NA & NA & & Gg_rs141339 Gg_rs141391. \\
\hline $0.9 \mathrm{NA}$ & NA & NA & NA & & Gg_rs141339Gg_rs141391. \\
\hline 0.77 & 1.4 & 0.8 & 3.8 & 1.05 & Gg_rs141323 Gg_rs141391 \\
\hline
\end{tabular}




\begin{tabular}{|c|c|c|c|c|}
\hline $0.17 \mathrm{NA}$ & NA & NA & NA & Gg_rs141323 Gg_rs141391. \\
\hline $0.56 \mathrm{NA}$ & NA & NA & NA & Gg_rs141339 Gg_rs141391. \\
\hline $0.55 \mathrm{NA}$ & NA & NA & NA & Gg_rs141339 Gg_rs141391. \\
\hline $0.76 \mathrm{NA}$ & NA & NA & NA & Gg_rs141323 Gg_rs141391. \\
\hline $0.56 \mathrm{NA}$ & NA & NA & NA & Gg_rs141339 Gg_rs141391. \\
\hline 0.49 NA & NA & NA & NA & Gg_rs141339 Gg_rs141391. \\
\hline $0.5 \mathrm{NA}$ & NA & NA & NA & Gg_rs141339 Gg_rs141391. \\
\hline $0.33 \mathrm{NA}$ & NA & NA & NA & Gg_rs141339 Gg_rs141391. \\
\hline $0.64 \mathrm{NA}$ & NA & NA & NA & Gg_rs141323 Gg_rs141391. \\
\hline $0.81 \mathrm{NA}$ & NA & NA & NA & Gg_rs141323 Gg_rs141391. \\
\hline $0.41 \mathrm{NA}$ & NA & NA & NA & Gg_rs141339 Gg_rs141391. \\
\hline 0.89 NA & NA & NA & NA & Gg_rs141323 Gg_rs141391. \\
\hline 0.83 NA & NA & NA & NA & Gg_rs141323 Gg_rs141391. \\
\hline $0.44 \mathrm{NA}$ & NA & NA & NA & Gg_rs141323 Gg_rs141391. \\
\hline $0.92 \mathrm{NA}$ & NA & NA & NA & Gg_rs141323 Gg_rs141391. \\
\hline $0.11 \mathrm{NA}$ & NA & NA & NA & Gg_rs141339 Gg_rs141391. \\
\hline $0.53 \mathrm{NA}$ & NA & NA & NA & Gg_rs141339 Gg_rs141391. \\
\hline 0.63 NA & NA & NA & NA & Gg_rs141339 Gg_rs141391. \\
\hline $0.52 \mathrm{NA}$ & NA & NA & NA & Gg_rs141339 Gg_rs141391. \\
\hline $0.58 \mathrm{NA}$ & NA & NA & NA & Gg_rs141339 Gg_rs141391. \\
\hline $0.56 \mathrm{NA}$ & NA & NA & NA & Gg_rs141323 Gg_rs141391. \\
\hline $0.59 \mathrm{NA}$ & NA & NA & NA & Gg_rs141323 Gg_rs141391. \\
\hline $0.62 \mathrm{NA}$ & NA & NA & NA & Gg_rs141339 Gg_rs141391. \\
\hline $0.36 \mathrm{NA}$ & NA & NA & NA & Gg_rs141339 Gg_rs141391. \\
\hline $0.71 \mathrm{NA}$ & NA & NA & NA & Gg_rs141339 Gg_rs141391. \\
\hline $1.1 \mathrm{NA}$ & NA & NA & NA & Gg_rs141339 Gg_rs141391. \\
\hline $1.13 \mathrm{NA}$ & NA & NA & NA & Gg_rs141323 Gg_rs141391. \\
\hline $0.39 \mathrm{NA}$ & NA & NA & NA & Gg_rs141339Gg_rs141391. \\
\hline $0.55 \mathrm{NA}$ & NA & NA & NA & Gg_rs141323 Gg_rs141391. \\
\hline $0.75 \mathrm{NA}$ & NA & NA & NA & Gg_rs141323 Gg_rs141391. \\
\hline 0.93 NA & NA & NA & NA & Gg_rs141339 Gg_rs141391. \\
\hline 0.07 NA & NA & NA & NA & Gg_rs141339 Gg_rs141391. \\
\hline $0.7 \mathrm{NA}$ & NA & NA & NA & Gg_rs141323 Gg_rs141391. \\
\hline $1.02 \mathrm{NA}$ & NA & NA & NA & Gg_rs141339 Gg_rs141391. \\
\hline $0.5 \mathrm{NA}$ & NA & NA & NA & Gg_rs141339 Gg_rs141391. \\
\hline $0.54 \mathrm{NA}$ & NA & NA & NA & Gg_rs141339 Gg_rs141391. \\
\hline $0.32 \mathrm{NA}$ & NA & NA & NA & Gg_rs141339 Gg_rs141391. \\
\hline 0.63 NA & NA & NA & NA & Gg_rs141339 Gg_rs141391. \\
\hline $0.43 \mathrm{NA}$ & NA & NA & NA & Gg_rs141323 Gg_rs141391. \\
\hline $0.52 \mathrm{NA}$ & NA & NA & NA & Gg_rs141339 Gg_rs141391. \\
\hline $0.52 \mathrm{NA}$ & NA & NA & NA & Gg_rs141339 Gg_rs141391. \\
\hline 0.49 NA & NA & NA & NA & Gg_rs141339 Gg_rs141391. \\
\hline $0.37 \mathrm{NA}$ & NA & NA & NA & Gg_rs141323 Gg_rs141391 \\
\hline
\end{tabular}




\begin{tabular}{|c|c|c|c|c|}
\hline $0.4 \mathrm{NA}$ & NA & NA & NA & Gg_rs141339Gg_rs141391. \\
\hline $0.31 \mathrm{NA}$ & NA & NA & NA & Gg_rs141339Gg_rs141391. \\
\hline 0.49 NA & NA & NA & NA & Gg_rs141339Gg_rs141391. \\
\hline $0.29 \mathrm{NA}$ & NA & NA & NA & Gg_rs141339Gg_rs141391. \\
\hline 0.66 NA & NA & NA & NA & Gg_rs141339Gg_rs141391. \\
\hline $0.34 \mathrm{NA}$ & NA & NA & NA & Gg_rs141339Gg_rs141391. \\
\hline $0.28 \mathrm{NA}$ & NA & NA & NA & Gg_rs141339Gg_rs141391. \\
\hline $1.23 \mathrm{NA}$ & NA & NA & NA & Gg_rs141339Gg_rs141391. \\
\hline $0.75 \mathrm{NA}$ & NA & NA & NA & Gg_rs141339Gg_rs141391. \\
\hline $0.56 \mathrm{NA}$ & NA & NA & NA & Gg_rs141339Gg_rs141391. \\
\hline $0.45 \mathrm{NA}$ & NA & NA & NA & Gg_rs141339Gg_rs141391. \\
\hline $0.37 \mathrm{NA}$ & NA & NA & NA & Gg_rs141339Gg_rs141391. \\
\hline 0.63 NA & NA & NA & NA & Gg_rs141339Gg_rs141391. \\
\hline $0.63 \mathrm{NA}$ & NA & NA & NA & Gg_rs141339Gg_rs141391. \\
\hline $0.71 \mathrm{NA}$ & NA & NA & NA & Gg_rs141323 Gg_rs141391 \\
\hline $0.68 \mathrm{NA}$ & NA & NA & NA & Gg_rs141339Gg_rs141391. \\
\hline $0.79 \mathrm{NA}$ & NA & NA & NA & Gg_rs141339Gg_rs141391. \\
\hline $0.52 \mathrm{NA}$ & NA & NA & NA & Gg_rs141339Gg_rs141391. \\
\hline $0.71 \mathrm{NA}$ & NA & NA & NA & Gg_rs141339Gg_rs141391. \\
\hline $0.74 \mathrm{NA}$ & NA & NA & NA & Gg_rs141339Gg_rs141391. \\
\hline $0.69 \mathrm{NA}$ & NA & NA & NA & Gg_rs141339Gg_rs141391. \\
\hline $0.55 \mathrm{NA}$ & NA & NA & NA & Gg_rs141323 Gg_rs141391. \\
\hline $0.49 \mathrm{NA}$ & NA & NA & NA & Gg_rs141323 Gg_rs141391. \\
\hline 0.46 NA & NA & NA & NA & Gg_rs141339Gg_rs141391. \\
\hline $0.91 \mathrm{NA}$ & NA & NA & NA & Gg_rs141339Gg_rs141391. \\
\hline $0.5 \mathrm{NA}$ & NA & NA & NA & Gg_rs141339Gg_rs141391. \\
\hline $0.27 \mathrm{NA}$ & NA & NA & NA & Gg_rs141323 Gg_rs141391 \\
\hline $0.35 \mathrm{NA}$ & NA & NA & NA & Gg_rs141339Gg_rs141391. \\
\hline $0.62 \mathrm{NA}$ & NA & NA & NA & Gg_rs141339Gg_rs141391. \\
\hline $0.55 \mathrm{NA}$ & NA & NA & NA & Gg_rs141339Gg_rs141391. \\
\hline $0.32 \mathrm{NA}$ & NA & NA & NA & Gg_rs141339Gg_rs141391. \\
\hline 0.45 NA & NA & NA & NA & Gg_rs141339Gg_rs141391. \\
\hline $0.4 \mathrm{NA}$ & NA & NA & NA & Gg_rs141339Gg_rs141391. \\
\hline $0.44 \mathrm{NA}$ & NA & NA & NA & Gg_rs141339Gg_rs141391 \\
\hline $0.96 \mathrm{NA}$ & NA & NA & NA & Gg_rs141323 Gg_rs141391. \\
\hline $0.4 \mathrm{NA}$ & NA & NA & NA & Gg_rs141323 Gg_rs141391. \\
\hline $0.87 \mathrm{NA}$ & NA & NA & NA & Gg_rs141339 Gg_rs141391 \\
\hline $0.59 \mathrm{NA}$ & NA & NA & NA & Gg_rs141323 Gg_rs141391. \\
\hline $0.53 \mathrm{NA}$ & NA & NA & NA & Gg_rs141323 Gg_rs141391 \\
\hline $0.61 \mathrm{NA}$ & NA & NA & NA & Gg_rs141339Gg_rs141391. \\
\hline $0.39 \mathrm{NA}$ & NA & NA & NA & Gg_rs141339Gg_rs141391. \\
\hline $0.62 \mathrm{NA}$ & NA & NA & NA & Gg_rs141339Gg_rs141391. \\
\hline $0.48 \mathrm{NA}$ & NA & NA & NA & Gg_rs141339 Gg_rs141391 \\
\hline
\end{tabular}




\begin{tabular}{|c|c|c|c|c|c|}
\hline $0.4 \mathrm{NA}$ & NA & NA & NA & & Gg_rs141339 Gg_rs141391. \\
\hline $0.87 \mathrm{NA}$ & NA & NA & NA & & Gg_rs141339Gg_rs141391. \\
\hline $0.55 \mathrm{NA}$ & NA & NA & NA & & Gg_rs141339 Gg_rs141391. \\
\hline $0.5 \mathrm{NA}$ & NA & NA & NA & & Gg_rs141339 Gg_rs141391. \\
\hline $0.75 \mathrm{NA}$ & NA & NA & NA & & Gg_rs141323 Gg_rs141391 \\
\hline $0.84 \mathrm{NA}$ & NA & NA & NA & & Gg_rs141339Gg_rs141391. \\
\hline $1.46 \mathrm{NA}$ & NA & NA & NA & & Gg_rs141323 Gg_rs141391. \\
\hline $0.78 \mathrm{NA}$ & NA & NA & NA & & Gg_rs141339Gg_rs141391. \\
\hline $0.73 \mathrm{NA}$ & NA & NA & NA & & Gg_rs141323 Gg_rs141391. \\
\hline $0.63 \mathrm{NA}$ & NA & NA & NA & & Gg_rs141339 Gg_rs141391. \\
\hline $0.73 \mathrm{NA}$ & NA & NA & NA & & Gg_rs141323 Gg_rs141391. \\
\hline $0.57 \mathrm{NA}$ & NA & NA & NA & & Gg_rs141339 Gg_rs141391 \\
\hline 0.85 & 3.36 & 0.88 & 4.35 & 1.16 & Gg_rs141323 Gg_rs141391. \\
\hline $0.44 \mathrm{NA}$ & NA & NA & NA & & Gg_rs141323 Gg_rs141391 \\
\hline $0.74 \mathrm{NA}$ & NA & NA & NA & & Gg_rs141323 Gg_rs141391. \\
\hline $0.66 \mathrm{NA}$ & NA & NA & NA & & Gg_rs141323 Gg_rs141391. \\
\hline $0.32 \mathrm{NA}$ & NA & NA & NA & & Gg_rs141339 Gg_rs141391 \\
\hline $0.73 \mathrm{NA}$ & NA & NA & NA & & Gg_rs141339 Gg_rs141391 \\
\hline $0.66 \mathrm{NA}$ & NA & NA & NA & & Gg_rs141339 Gg_rs141391 \\
\hline $0.59 \mathrm{NA}$ & NA & NA & NA & & Gg_rs141323 Gg_rs141391. \\
\hline $0.36 \mathrm{NA}$ & NA & NA & NA & & Gg_rs141323 Gg_rs141391. \\
\hline $0.54 \mathrm{NA}$ & NA & NA & NA & & Gg_rs141339 Gg_rs141391. \\
\hline $0.57 \mathrm{NA}$ & NA & NA & NA & & Gg_rs141323 Gg_rs141391. \\
\hline $0.93 \mathrm{NA}$ & NA & NA & NA & & Gg_rs141339 Gg_rs141391. \\
\hline $0.59 \mathrm{NA}$ & NA & NA & NA & & Gg_rs141323 Gg_rs141391. \\
\hline $0.84 \mathrm{NA}$ & NA & NA & NA & & Gg_rs141339Gg_rs141391. \\
\hline $0.57 \mathrm{NA}$ & NA & NA & NA & & Gg_rs141339Gg_rs141391. \\
\hline $0.96 \mathrm{NA}$ & NA & NA & NA & & Gg_rs141339 Gg_rs141391. \\
\hline $0.46 \mathrm{NA}$ & NA & NA & NA & & Gg_rs141339 Gg_rs141391. \\
\hline $0.68 \mathrm{NA}$ & NA & NA & NA & & Gg_rs141339Gg_rs141391. \\
\hline $0.45 \mathrm{NA}$ & NA & NA & NA & & Gg_rs141339Gg_rs141391. \\
\hline $0.67 \mathrm{NA}$ & NA & NA & NA & & Gg_rs141339Gg_rs141391. \\
\hline $0.5 \mathrm{NA}$ & NA & NA & NA & & Gg_rs141323 Gg_rs141391. \\
\hline $0.46 \mathrm{NA}$ & NA & NA & NA & & Gg_rs141339 Gg_rs141391. \\
\hline $0.92 \mathrm{NA}$ & NA & NA & NA & & Gg_rs141339 Gg_rs141391. \\
\hline 0.91 & -2.59 & 0.94 & -0.24 & 1.24 & Gg_rs141323 Gg_rs141391. \\
\hline $0.61 \mathrm{NA}$ & NA & NA & NA & & Gg_rs141323 Gg_rs141391. \\
\hline $1.48 \mathrm{NA}$ & NA & NA & NA & & Gg_rs141323 Gg_rs141391. \\
\hline $0.59 \mathrm{NA}$ & NA & NA & NA & & Gg_rs141339 Gg_rs141391. \\
\hline $0.7 \mathrm{NA}$ & NA & NA & NA & & Gg_rs141323 Gg_rs141391. \\
\hline $0.36 \mathrm{NA}$ & NA & NA & NA & & Gg_rs141323 Gg_rs141391. \\
\hline $0.68 \mathrm{NA}$ & NA & NA & NA & & Gg_rs141339Gg_rs141391. \\
\hline $0.42 \mathrm{NA}$ & NA & NA & NA & & Gg_rs141339 Gg_rs141391 \\
\hline
\end{tabular}




\begin{tabular}{|c|c|c|c|c|c|}
\hline $0.52 \mathrm{NA}$ & NA & NA & NA & & Gg_rs141339 Gg_rs141391 \\
\hline $1 \mathrm{NA}$ & NA & NA & NA & & Gg_rs141323 Gg_rs141391 \\
\hline $0.5 \mathrm{NA}$ & NA & NA & NA & & Gg_rs141323 Gg_rs141391 \\
\hline $0.54 \mathrm{NA}$ & NA & NA & NA & & Gg_rs141339 Gg_rs141391 \\
\hline $0.34 \mathrm{NA}$ & NA & NA & NA & & Gg_rs141323 Gg_rs150652 \\
\hline $1.41 \mathrm{NA}$ & NA & NA & NA & & Gg_rs141339 Gg_rs141391 \\
\hline 0.98 & -0.73 & 1.02 & 3.01 & 1.33 & Gg_rs141323 Gg_rs141391 \\
\hline 0.29 & 0.95 & 0.3 & 1.14 & 0.39 & Gg_rs141323 Gg_rs141391 \\
\hline 0.44 NA & NA & NA & NA & & Gg_rs141339 Gg_rs141391 \\
\hline $0.16 \mathrm{NA}$ & NA & NA & NA & & Gg_rs141323 Gg_rs141391 \\
\hline $0.61 \mathrm{NA}$ & NA & NA & NA & & Gg_rs141339 Gg_rs141391 \\
\hline $0.44 \mathrm{NA}$ & NA & NA & NA & & Gg_rs141323 Gg_rs141391 \\
\hline $0.57 \mathrm{NA}$ & NA & NA & NA & & Gg_rs141323 Gg_rs141391 \\
\hline $0.55 \mathrm{NA}$ & NA & NA & NA & & Gg_rs141323 Gg_rs141391 \\
\hline $0.33 \mathrm{NA}$ & NA & NA & NA & & Gg_rs141323 Gg_rs141391 \\
\hline $1 \mathrm{NA}$ & NA & NA & NA & & Gg_rs141339 Gg_rs141391 \\
\hline $0.55 \mathrm{NA}$ & NA & NA & NA & & Gg_rs141323 Gg_rs141391 \\
\hline 0.89 & 1.26 & 0.93 & 4.41 & 1.22 & Gg_rs141323 Gg_rs141391 \\
\hline $0.62 \mathrm{NA}$ & NA & NA & NA & & Gg_rs141323 Gg_rs141391 \\
\hline 0.66 NA & NA & NA & NA & & Gg_rs141339 Gg_rs141391 \\
\hline $0.59 \mathrm{NA}$ & NA & NA & NA & & Gg_rs141323 Gg_rs141391 \\
\hline $0.78 \mathrm{NA}$ & NA & NA & NA & & Gg_rs141339 Gg_rs141391 \\
\hline $0.31 \mathrm{NA}$ & NA & NA & NA & & Gg_rs141323 Gg_rs141391 \\
\hline $0.61 \mathrm{NA}$ & NA & NA & NA & & Gg_rs141323 Gg_rs141391 \\
\hline 1.08 & -2.58 & 1.13 & -1.73 & 1.48 & Gg_rs141339 Gg_rs141391 \\
\hline $0.54 \mathrm{NA}$ & NA & NA & NA & & Gg_rs141323 Gg_rs141391 \\
\hline $0.48 \mathrm{NA}$ & NA & NA & NA & & Gg_rs141323 Gg_rs141391 \\
\hline $0.95 \mathrm{NA}$ & NA & NA & NA & & Gg_rs141339 Gg_rs141391 \\
\hline $0.39 \mathrm{NA}$ & NA & NA & NA & & Gg_rs141323 Gg_rs141391 \\
\hline $0.5 \mathrm{NA}$ & NA & NA & NA & & Gg_rs141323 Gg_rs141391 \\
\hline $0.68 \mathrm{NA}$ & NA & NA & NA & & Gg_rs141339Gg_rs141391 \\
\hline $0.45 \mathrm{NA}$ & NA & NA & NA & & Gg_rs141323 Gg_rs141391 \\
\hline 0.68 & -1.44 & 0.71 & 0.35 & 0.93 & Gg_rs141323 Gg_rs141391 \\
\hline $0.59 \mathrm{NA}$ & NA & NA & NA & & Gg_rs141323 Gg_rs141391 \\
\hline $0.47 \mathrm{NA}$ & NA & NA & NA & & Gg_rs141323 Gg_rs141391 \\
\hline $0.47 \mathrm{NA}$ & NA & NA & NA & & Gg_rs141339 Gg_rs141391 \\
\hline $0.78 \mathrm{NA}$ & NA & NA & NA & & Gg_rs141339 Gg_rs141391 \\
\hline $0.58 \mathrm{NA}$ & NA & NA & NA & & Gg_rs141323 Gg_rs141391 \\
\hline $0.4 \mathrm{NA}$ & NA & NA & NA & & Gg_rs141339 Gg_rs150652 \\
\hline $0.48 \mathrm{NA}$ & NA & NA & NA & & Gg_rs150605 Gg_rs150652 \\
\hline $0.27 \mathrm{NA}$ & NA & NA & NA & & Gg_rs150605 Gg_rs150692 \\
\hline $0.6 \mathrm{NA}$ & NA & NA & NA & & Gg_rs150605 Gg_rs150652 \\
\hline $0.89 \mathrm{NA}$ & NA & NA & NA & & Gg_rs150605 Gg_rs150652 \\
\hline
\end{tabular}




\begin{tabular}{|c|c|c|c|c|c|c|c|}
\hline $0.63 \mathrm{NA}$ & NA & & NA & & NA & & Gg_rs150605 Gg_rs150652 \\
\hline 1.93 & 3.59 & 1.85 & & 2.7 & & 2.62 & Gg_rs150605 Gg_rs150652 \\
\hline $1.17 \mathrm{NA}$ & NA & & NA & & NA & & Gg_rs150605 Gg_rs150652 \\
\hline $0.59 \mathrm{NA}$ & NA & & NA & & NA & & Gg_rs150605 Gg_rs150676. \\
\hline $0.6 \mathrm{NA}$ & NA & & NA & & NA & & Gg_rs150605 Gg_rs150652 \\
\hline 0.43 NA & NA & & NA & & NA & & Gg_rs150605 Gg_rs150652 \\
\hline 1.09 & -1.21 & 1.04 & & 5.29 & & 1.47 & Gg_rs150605 Gg_rs150652 \\
\hline 0.58 & -2.04 & 0.55 & & 3.52 & & 0.78 & Gg_rs150605 Gg_rs150652 \\
\hline $0.77 \mathrm{NA}$ & NA & & NA & & NA & & Gg_rs150605 Gg_rs150652 \\
\hline $0.37 \mathrm{NA}$ & NA & & NA & & NA & & Gg_rs150605 Gg_rs150652 \\
\hline 1.23 & 1.46 & 1.17 & & 3.04 & & 1.66 & Gg_rs150605 Gg_rs150652 \\
\hline $1.13 \mathrm{NA}$ & NA & & NA & & NA & & Gg_rs150605 Gg_rs150652 \\
\hline 0.98 NA & NA & & NA & & NA & & Gg_rs150605 Gg_rs150638. \\
\hline $0.92 \mathrm{NA}$ & NA & & NA & & NA & & Gg_rs150605 Gg_rs150652 \\
\hline $0.87 \mathrm{NA}$ & NA & & NA & & NA & & Gg_rs150605 Gg_rs150652 \\
\hline 0.75 & 1.84 & 0.72 & & -1.07 & & 1.02 & Gg_rs150605 Gg_rs150638. \\
\hline $0.55 \mathrm{NA}$ & NA & & NA & & NA & & Gg_rs150605 Gg_rs150652 \\
\hline $0.65 \mathrm{NA}$ & NA & & NA & & NA & & Gg_rs150605 Gg_rs150652 \\
\hline 0.63 NA & NA & & NA & & NA & & Gg_rs150605 Gg_rs150652 \\
\hline 1.31 & 3.24 & 1.25 & & -1.67 & & 1.77 & Gg_rs150605 Gg_rs150652 \\
\hline 0.63 & 1.52 & 0.6 & & -1.61 & & 0.85 & Gg_rs150605 Gg_rs150676. \\
\hline 0.65 & 1 & 0.62 & & -1.65 & & 0.88 & Gg_rs150605 Gg_rs150692 \\
\hline $0.61 \mathrm{NA}$ & NA & & NA & & NA & & Gg_rs150605 Gg_rs150652 \\
\hline $0.71 \mathrm{NA}$ & NA & & NA & & NA & & Gg_rs150605 Gg_rs150652 \\
\hline $0.62 \mathrm{NA}$ & NA & & NA & & NA & & Gg_rs150605 Gg_rs150692 \\
\hline 0.78 NA & NA & & NA & & NA & & Gg_rs150605 Gg_rs150676. \\
\hline $0.61 \mathrm{NA}$ & NA & & NA & & NA & & Gg_rs150605 Gg_rs150652 \\
\hline $0.88 \mathrm{NA}$ & NA & & NA & & NA & & Gg_rs150605 Gg_rs150676. \\
\hline $0.59 \mathrm{NA}$ & NA & & NA & & NA & & Gg_rs150605 Gg_rs150652 \\
\hline 0.5 & 1.21 & 0.47 & & 0.32 & & 0.67 & Gg_rs150605 Gg_rs150652 \\
\hline $0.5 \mathrm{NA}$ & NA & & NA & & NA & & Gg_rs150605 Gg_rs150652 \\
\hline $0.51 \mathrm{NA}$ & NA & & NA & & NA & & Gg_rs150605 Gg_rs150652 \\
\hline 0.94 NA & NA & & NA & & NA & & Gg_rs150605 Gg_rs150676. \\
\hline $0.56 \mathrm{NA}$ & NA & & NA & & NA & & Gg_rs150605 Gg_rs150652 \\
\hline 0.73 & 1.79 & 0.7 & & -3.02 & & 0.99 & Gg_rs150605 Gg_rs141392. \\
\hline 0.65 NA & NA & & NA & & NA & & Gg_rs150605 Gg_rs150652 \\
\hline $0.74 \mathrm{NA}$ & NA & & NA & & NA & & Gg_rs150605 Gg_rs150652 \\
\hline $0.41 \mathrm{NA}$ & NA & & NA & & NA & & Gg_rs150605 Gg_rs150652 \\
\hline 1.43 & 3.85 & 1.37 & & 0.66 & & 1.93 & Gg_rs150605 Gg_rs150652 \\
\hline $0.94 \mathrm{NA}$ & NA & & NA & & NA & & Gg_rs150605 Gg_rs150652 \\
\hline $0.65 \mathrm{NA}$ & NA & & NA & & NA & & Gg_rs150605 Gg_rs150652 \\
\hline 0.55 & -1.1 & 0.53 & & 0.45 & & 0.74 & Gg_rs150605 Gg_rs150652 \\
\hline $0.55 \mathrm{NA}$ & NA & & NA & & NA & & Gg_rs150605 Gg_rs150652 \\
\hline
\end{tabular}




\begin{tabular}{|c|c|c|c|c|c|}
\hline $0.59 \mathrm{NA}$ & NA & NA & NA & & Gg_rs150605 Gg_rs150652 \\
\hline $0.52 \mathrm{NA}$ & NA & NA & NA & & Gg_rs150605 Gg_rs150652 \\
\hline $0.84 \mathrm{NA}$ & NA & NA & NA & & Gg_rs141339Gg_rs150676. \\
\hline $0.62 \mathrm{NA}$ & NA & NA & NA & & Gg_rs150605 Gg_rs150652 \\
\hline $0.43 \mathrm{NA}$ & NA & NA & NA & & Gg_rs150605 Gg_rs150652 \\
\hline $0.41 \mathrm{NA}$ & NA & NA & NA & & Gg_rs141392.Gg_rs150652 \\
\hline 0.78 & -0.7 & 0.74 & 3.07 & 1.04 & Gg_rs150605 Gg_rs150652 \\
\hline $0.57 \mathrm{NA}$ & NA & NA & NA & & Gg_rs150605 Gg_rs150652 \\
\hline $0.79 \mathrm{NA}$ & NA & NA & NA & & Gg_rs150605 Gg_rs150676. \\
\hline $0.62 \mathrm{NA}$ & NA & NA & NA & & Gg_rs150605 Gg_rs150652 \\
\hline $0.69 \mathrm{NA}$ & NA & NA & NA & & Gg_rs150605 Gg_rs150676. \\
\hline $0.87 \mathrm{NA}$ & NA & NA & NA & & Gg_rs150605 Gg_rs150652 \\
\hline $0.84 \mathrm{NA}$ & NA & NA & NA & & Gg_rs150605 Gg_rs150676. \\
\hline $0.77 \mathrm{NA}$ & NA & NA & NA & & Gg_rs150605 Gg_rs150676. \\
\hline $0.48 \mathrm{NA}$ & NA & NA & NA & & Gg_rs150605 Gg_rs150692 \\
\hline 0.36 & -1.78 & 0.34 & 0.12 & 0.48 & Gg_rs150605 Gg_rs150676. \\
\hline $0.55 \mathrm{NA}$ & NA & NA & NA & & Gg_rs150605 Gg_rs150676. \\
\hline $0.42 \mathrm{NA}$ & NA & NA & NA & & Gg_rs150605 Gg_rs150692 \\
\hline $0.61 \mathrm{NA}$ & NA & NA & NA & & Gg_rs150605 Gg_rs150676. \\
\hline 0.92 & -1.84 & 0.87 & 4.81 & 1.23 & Gg_rs150605 Gg_rs150676. \\
\hline $0.49 \mathrm{NA}$ & NA & NA & NA & & Gg_rs141323 Gg_rs150692 \\
\hline $0.48 \mathrm{NA}$ & NA & NA & NA & & Gg_rs150605 Gg_rs150692 \\
\hline 0.76 & -2.02 & 0.73 & 3.15 & 1.03 & Gg_rs150605 Gg_rs150676. \\
\hline $0.42 \mathrm{NA}$ & NA & NA & NA & & Gg_rs150605 Gg_rs150676. \\
\hline $0.28 \mathrm{NA}$ & NA & NA & NA & & Gg_rs150605 Gg_rs150692 \\
\hline $0.59 \mathrm{NA}$ & NA & NA & NA & & Gg_rs150605 Gg_rs150692 \\
\hline 0.78 & -3.02 & 0.74 & 4.43 & 1.05 & Gg_rs150605 Gg_rs150676. \\
\hline 1.35 & 2.84 & 1.23 & -3.88 & 1.82 & Gg_rs141339 Gg_rs150692 \\
\hline $0.47 \mathrm{NA}$ & NA & NA & NA & & Gg_rs150605 Gg_rs150692 \\
\hline $0.53 \mathrm{NA}$ & NA & NA & NA & & Gg_rs150605 snp.2.314.16! \\
\hline $0.49 \mathrm{NA}$ & NA & NA & NA & & Gg_rs150605 Gg_rs150692 \\
\hline 0.9 & -3.09 & 0.82 & 5.24 & 1.22 & Gg_rs141392.Gg_rs150676. \\
\hline $0.71 \mathrm{NA}$ & NA & NA & NA & & Gg_rs150638.Gg_rs150676. \\
\hline $0.99 \mathrm{NA}$ & NA & NA & NA & & Gg_rs150605 Gg_rs150692 \\
\hline $0.65 \mathrm{NA}$ & NA & NA & NA & & Gg_rs141392:Gg_rs150676. \\
\hline $0.5 \mathrm{NA}$ & NA & NA & NA & & Gg_rs150638.Gg_rs150676. \\
\hline $0.7 \mathrm{NA}$ & NA & NA & NA & & Gg_rs150638.Gg_rs150676. \\
\hline $0.47 \mathrm{NA}$ & NA & NA & NA & & Gg_rs141392.Gg_rs150676. \\
\hline 0.66 & -2.74 & 0.6 & 3.55 & 0.89 & Gg_rs150605 Gg_rs150676. \\
\hline $0.66 \mathrm{NA}$ & NA & NA & NA & & Gg_rs150605 Gg_rs150676. \\
\hline 0.64 & 0.61 & 0.58 & -1.05 & 0.86 & Gg_rs150605 Gg_rs150676. \\
\hline $0.6 \mathrm{NA}$ & NA & NA & NA & & Gg_rs150638.Gg_rs150692 \\
\hline $0.41 \mathrm{NA}$ & NA & NA & NA & & Gg_rs150638.Gg_rs150676. \\
\hline
\end{tabular}




\begin{tabular}{|c|c|c|c|c|c|}
\hline $0.27 \mathrm{NA}$ & NA & NA & NA & & Gg_rs150638.Gg_rs150676. \\
\hline 0.59 & -0.49 & 0.54 & -3.27 & 0.8 & Gg_rs150638.Gg_rs150676. \\
\hline 0.35 & -0.78 & 0.32 & 1.17 & 0.47 & Gg_rs150638.Gg_rs150676. \\
\hline $0.36 \mathrm{NA}$ & NA & NA & NA & & Gg_rs150638.Gg_rs150676. \\
\hline $0.44 \mathrm{NA}$ & NA & NA & NA & & Gg_rs150638.Gg_rs150676. \\
\hline 0.7 & -0.41 & 0.64 & -2.48 & 0.94 & Gg_rs150638snp.2.314.16! \\
\hline $0.37 \mathrm{NA}$ & NA & NA & NA & & Gg_rs150638.Gg_rs150692 \\
\hline $0.32 \mathrm{NA}$ & NA & NA & NA & & Gg_rs150638.Gg_rs150692 \\
\hline $0.41 \mathrm{NA}$ & NA & NA & NA & & Gg_rs150638.Gg_rs150692 \\
\hline $0.34 \mathrm{NA}$ & NA & NA & NA & & Gg_rs150638.Gg_rs150692 \\
\hline 0.69 NA & NA & NA & NA & & Gg_rs150638.Gg_rs150692 \\
\hline $1.38 \mathrm{NA}$ & NA & NA & NA & & Gg_rs150638snp.2.314.16! \\
\hline 0.55 & -1.12 & 0.5 & 2.95 & 0.74 & Gg_rs150638.Gg_rs150676. \\
\hline $0.34 \mathrm{NA}$ & NA & NA & NA & & Gg_rs150638.Gg_rs150692 \\
\hline $0.57 \mathrm{NA}$ & NA & NA & NA & & Gg_rs150638.Gg_rs150692 \\
\hline 0.89 NA & NA & NA & NA & & Gg_rs150638.Gg_rs150692 \\
\hline $0.53 \mathrm{NA}$ & NA & NA & NA & & Gg_rs150638.Gg_rs150692 \\
\hline 0.64 & -1.18 & 0.58 & 4.11 & 0.86 & Gg_rs150638.Gg_rs150676. \\
\hline $0.6 \mathrm{NA}$ & NA & NA & NA & & Gg_rs150638.Gg_rs150692 \\
\hline $0.42 \mathrm{NA}$ & NA & NA & NA & & Gg_rs150638.Gg_rs150692 \\
\hline $0.67 \mathrm{NA}$ & NA & NA & NA & & Gg_rs150638.Gg_rs150692 \\
\hline 0.44 NA & NA & NA & NA & & Gg_rs150638.Gg_rs150692 \\
\hline 1.37 & -2.66 & 1.25 & 3.35 & 1.84 & Gg_rs150638.Gg_rs150692 \\
\hline 0.46 NA & NA & NA & NA & & Gg_rs150638.Gg_rs150692 \\
\hline $0.56 \mathrm{NA}$ & NA & NA & NA & & Gg_rs150638.Gg_rs150692 \\
\hline $0.58 \mathrm{NA}$ & NA & NA & NA & & Gg_rs150638.Gg_rs150692 \\
\hline $0.65 \mathrm{NA}$ & NA & NA & NA & & Gg_rs150638.Gg_rs150692 \\
\hline 0.63 & -1.5 & 0.53 & 2.27 & 0.8 & Gg_rs150605 Gg_rs150692 \\
\hline 0.86 & -1.41 & 0.74 & 1.85 & 1.1 & Gg_rs150638.Gg_rs150692 \\
\hline 0.64 NA & NA & NA & NA & & Gg_rs150638.Gg_rs150692 \\
\hline $0.39 \mathrm{NA}$ & NA & NA & NA & & Gg_rs150638.Gg_rs150692 \\
\hline 0.61 & -1.34 & 0.52 & 2.95 & 0.79 & Gg_rs150638.Gg_rs150692 \\
\hline $0.84 \mathrm{NA}$ & NA & NA & NA & & Gg_rs150638.Gg_rs150692 \\
\hline $0.39 \mathrm{NA}$ & NA & NA & NA & & Gg_rs150638.Gg_rs150692 \\
\hline $0.25 \mathrm{NA}$ & NA & NA & NA & & Gg_rs150638.Gg_rs150692 \\
\hline $0.53 \mathrm{NA}$ & NA & NA & NA & & Gg_rs150638.Gg_rs150692 \\
\hline 0.79 & -0.37 & 0.68 & 3.33 & 1.02 & Gg_rs150652 Gg_rs150692 \\
\hline 0.64 NA & NA & NA & NA & & Gg_rs150638.Gg_rs150692 \\
\hline 1.22 & 2.02 & 1.04 & 2.91 & 1.56 & Gg_rs150638.Gg_rs150692 \\
\hline $0.71 \mathrm{NA}$ & NA & NA & NA & & Gg_rs150638.Gg_rs150692 \\
\hline 0.42 NA & NA & NA & NA & & Gg_rs150638.Gg_rs150692 \\
\hline 0.83 NA & NA & NA & NA & & Gg_rs150652 Gg_rs150692 \\
\hline 0.44 NA & NA & NA & NA & & Gg_rs150638.Gg_rs150692 \\
\hline
\end{tabular}




\begin{tabular}{|c|c|c|c|c|c|}
\hline $0.61 \mathrm{NA}$ & NA & NA & NA & & Gg_rs150652 Gg_rs150692 \\
\hline $0.39 \mathrm{NA}$ & NA & NA & NA & & Gg_rs150638.Gg_rs150692 \\
\hline $1.1 \mathrm{NA}$ & NA & NA & NA & & Gg_rs150638.Gg_rs150692 \\
\hline $0.48 \mathrm{NA}$ & NA & NA & NA & & Gg_rs150652 Gg_rs150692 \\
\hline $0.94 \mathrm{NA}$ & NA & NA & NA & & Gg_rs150638.Gg_rs150692 \\
\hline $0.28 \mathrm{NA}$ & NA & NA & NA & & Gg_rs150638.Gg_rs150692 \\
\hline 1.73 & 3.19 & 1.47 & 2.58 & 2.21 & Gg_rs150638X2_23979784 \\
\hline $0.83 \mathrm{NA}$ & NA & NA & NA & & Gg_rs150652 snp.2.314.16! \\
\hline 0.64 & 1.37 & 0.55 & 1.63 & 0.82 & Gg_rs150652 Gg_rs150692 \\
\hline $0.35 \mathrm{NA}$ & NA & NA & NA & & Gg_rs150652 Gg_rs150692 \\
\hline $0.58 \mathrm{NA}$ & NA & NA & NA & & Gg_rs150638.Gg_rs150692 \\
\hline 0.73 NA & NA & NA & NA & & Gg_rs150652 Gg_rs150692 \\
\hline 0.59 & 0.32 & 0.51 & -1.6 & 0.75 & Gg_rs150652 Gg_rs150692 \\
\hline $0.51 \mathrm{NA}$ & NA & NA & NA & & Gg_rs150652 Gg_rs150692 \\
\hline $0.7 \mathrm{NA}$ & NA & NA & NA & & Gg_rs150638X2_23979784 \\
\hline $0.51 \mathrm{NA}$ & NA & NA & NA & & Gg_rs150652 snp.2.314.16! \\
\hline 0.96 & 1.29 & 0.93 & 0.07 & 1.24 & Gg_rs150652 snp.2.314.16! \\
\hline $0.76 \mathrm{NA}$ & NA & NA & NA & & Gg_rs150638X2_23979784 \\
\hline 0.66 NA & NA & NA & NA & & Gg_rs150676 snp.2.314.16! \\
\hline 0.85 NA & NA & NA & NA & & Gg_rs150676 snp.2.314.16! \\
\hline 1.32 & 2.3 & 1.28 & 1.71 & 1.71 & Gg_rs150676 snp.2.314.16! \\
\hline $0.73 \mathrm{NA}$ & NA & NA & NA & & Gg_rs150676 X2_23979784 \\
\hline 0.07 & 0.33 & 0.07 & 0.33 & 0.09 & Gg_rs150676 snp.2.314.16! \\
\hline 0.05 & 0.25 & 0.05 & 0.31 & 0.07 & Gg_rs150676 snp.2.314.16! \\
\hline 0.31 & 1.65 & 0.3 & 1.21 & 0.4 & Gg_rs150676 snp.2.314.16! \\
\hline 0.46 NA & NA & NA & NA & & Gg_rs150676 snp.2.314.16! \\
\hline $0.3 \mathrm{NA}$ & NA & NA & NA & & Gg_rs150676 snp.2.314.16! \\
\hline 0.44 & 1.9 & 0.44 & 1.7 & 0.57 & Gg_rs150676 snp.2.314.16! \\
\hline $1.44 \mathrm{NA}$ & NA & NA & NA & & Gg_rs150676 snp.2.314.16! \\
\hline 0.7 & -0.94 & 0.68 & -1.9 & 0.9 & Gg_rs150676 snp.2.314.16! \\
\hline $0.73 \mathrm{NA}$ & NA & NA & NA & & Gg_rs150676 X2_23979784 \\
\hline $0.9 \mathrm{NA}$ & NA & NA & NA & & Gg_rs150676 snp.2.314.16! \\
\hline 2.65 & 1.16 & 2.61 & 5.83 & 3.44 & Gg_rs150652 snp.2.314.16! \\
\hline $0.96 \mathrm{NA}$ & NA & NA & NA & & Gg_rs150676X2_23979784 \\
\hline $0.97 \mathrm{NA}$ & NA & NA & NA & & Gg_rs150652 X2_23979784 \\
\hline $0.47 \mathrm{NA}$ & NA & NA & NA & & Gg_rs150676 X2_23979784 \\
\hline $0.7 \mathrm{NA}$ & NA & NA & NA & & Gg_rs150638X2_23979784 \\
\hline $0.57 \mathrm{NA}$ & NA & NA & NA & & Gg_rs150638X2_23979784 \\
\hline $0.71 \mathrm{NA}$ & NA & NA & NA & & Gg_rs150652 X2_23979784 \\
\hline 0.31 & -0.46 & 0.28 & -2.19 & 0.4 & Gg_rs150700X2_23979784 \\
\hline $0.9 \mathrm{NA}$ & NA & NA & NA & & Gg_rs150700X2_23979784 \\
\hline $0.66 \mathrm{NA}$ & NA & NA & NA & & Gg_rs150700X2_23979784 \\
\hline 0.96 & 0.58 & 0.86 & 1 & 1.24 & Gg_rs150700X2_23979784 \\
\hline
\end{tabular}




\begin{tabular}{|c|c|c|c|c|c|}
\hline 0.62 & 1.72 & 0.56 & 3.78 & 0.8 & Gg_rs150700X2_23979784 \\
\hline 0.61 & -0.77 & 0.55 & -3.97 & 0.79 & Gg_rs150700X2_23979784 \\
\hline 1.29 & 1.28 & 1.16 & 3.91 & 1.67 & Gg_rs150700X2_23979784 \\
\hline $0.67 \mathrm{NA}$ & NA & NA & NA & & Gg_rs150700X2_23979784 \\
\hline $0.72 \mathrm{NA}$ & NA & NA & NA & & Gg_rs150700X2_23979784 \\
\hline 1.11 & -2.09 & 1 & -6.54 & 1.45 & Gg_rs150700X2_23979784 \\
\hline $0.82 \mathrm{NA}$ & NA & NA & NA & & Gg_rs150700X2_23979784 \\
\hline $0.41 \mathrm{NA}$ & NA & NA & NA & & Gg_rs150700X2_23979784 \\
\hline $0.63 \mathrm{NA}$ & NA & NA & NA & & Gg_rs150700X2_23979784 \\
\hline 0.73 NA & NA & NA & NA & & Gg_rs150700.snp.5.86.557 \\
\hline $0.63 \mathrm{NA}$ & NA & NA & NA & & Gg_rs150700.snp.5.86.557 \\
\hline $0.68 \mathrm{NA}$ & NA & NA & NA & & snp.2.314.16!snp.5.86.557£ \\
\hline $0.86 \mathrm{NA}$ & NA & NA & NA & & snp.2.314.16!snp.5.86.557 \\
\hline $0.72 \mathrm{NA}$ & NA & NA & NA & & snp.2.314.16!snp.5.86.557£ \\
\hline $1.29 \mathrm{NA}$ & NA & NA & NA & & Gg_rs150700.snp.5.86.557£ \\
\hline $1.38 \mathrm{NA}$ & NA & NA & NA & & snp.2.314.16!snp.5.86.557£ \\
\hline $0.47 \mathrm{NA}$ & NA & NA & NA & & snp.2.314.16!snp.5.86.557£ \\
\hline $0.63 \mathrm{NA}$ & NA & NA & NA & & snp.2.314.16!snp.5.86.557£ \\
\hline $1.82 \mathrm{NA}$ & NA & NA & NA & & Gg_rs150700.snp.5.86.557^ \\
\hline $0.45 \mathrm{NA}$ & NA & NA & NA & & snp.2.314.16!snp.5.86.557 \\
\hline $0.54 \mathrm{NA}$ & NA & NA & NA & & snp.2.314.16!snp.5.86.557£ \\
\hline $0.87 \mathrm{NA}$ & NA & NA & NA & & snp.2.314.16!Gg_rs150782 \\
\hline $1.26 \mathrm{NA}$ & NA & NA & NA & & snp.2.314.16!snp.5.86.557£ \\
\hline $3.37 \mathrm{NA}$ & NA & NA & NA & & snp.2.314.16!snp.5.86.557£ \\
\hline $2.31 \mathrm{NA}$ & NA & NA & NA & & snp.2.314.16!snp.5.86.557 \\
\hline $1.44 \mathrm{NA}$ & NA & NA & NA & & snp.2.314.16!snp.5.86.557 \\
\hline $1.85 \mathrm{NA}$ & NA & NA & NA & & Gg_rs150700.snp.5.86.557 \\
\hline $1.05 \mathrm{NA}$ & NA & NA & NA & & snp.2.314.16!snp.5.86.557£ \\
\hline $1.35 \mathrm{NA}$ & NA & NA & NA & & snp.2.314.16!snp.5.86.557£ \\
\hline $0.86 \mathrm{NA}$ & NA & NA & NA & & snp.2.314.16!snp.5.86.557؛ \\
\hline $0.17 \mathrm{NA}$ & NA & NA & NA & & snp.2.314.16!Gg_rs150782 \\
\hline 0.75 & 0.91 & 0.65 & -2.16 & 0.97 & snp.2.314.16!snp.5.86.557 \\
\hline $2.97 \mathrm{NA}$ & NA & NA & NA & & snp.2.314.16!snp.5.86.557£ \\
\hline $2.51 \mathrm{NA}$ & NA & NA & NA & & snp.2.314.16!snp.5.86.557£ \\
\hline $1.68 \mathrm{NA}$ & NA & NA & NA & & snp.2.314.16!snp.5.86.557 \\
\hline $2.02 \mathrm{NA}$ & NA & NA & NA & & snp.2.314.16!snp.5.86.557£ \\
\hline $0.44 \mathrm{NA}$ & NA & NA & NA & & snp.2.314.16!snp.5.86.557 \\
\hline $1.3 \mathrm{NA}$ & NA & NA & NA & & snp.2.314.16!snp.5.86.557£ \\
\hline $1.98 \mathrm{NA}$ & NA & NA & NA & & Gg_rs150700.snp.5.86.557 \\
\hline $2.91 \mathrm{NA}$ & NA & NA & NA & & snp.2.314.16!snp.5.86.557 \\
\hline $2.13 \mathrm{NA}$ & NA & NA & NA & & Gg_rs150700.snp.5.86.557£ \\
\hline $0.45 \mathrm{NA}$ & NA & NA & NA & & snp.2.314.16!snp.5.86.557 \\
\hline $2.79 \mathrm{NA}$ & NA & NA & NA & & snp.2.314.16!snp.5.86.557? \\
\hline
\end{tabular}




\begin{tabular}{|c|c|c|c|c|c|}
\hline 2.48 NA & NA & NA & NA & & snp.2.314.16!snp.5.86.557 \\
\hline $1.99 \mathrm{NA}$ & NA & NA & NA & & Gg_rs150700.snp.5.86.557^ \\
\hline $2.15 \mathrm{NA}$ & NA & NA & NA & & snp.2.314.16!snp.5.86.557£ \\
\hline $2.88 \mathrm{NA}$ & NA & NA & NA & & snp.2.314.16!snp.5.86.557^ \\
\hline $1.75 \mathrm{NA}$ & NA & NA & NA & & snp.2.314.16!snp.5.86.557£ \\
\hline $1.81 \mathrm{NA}$ & NA & NA & NA & & snp.2.314.16!snp.5.86.557£ \\
\hline $0.56 \mathrm{NA}$ & NA & NA & NA & & snp.2.314.16!snp.5.86.557£ \\
\hline $2.53 \mathrm{NA}$ & NA & NA & NA & & snp.2.314.16!snp.5.86.557^ \\
\hline $1.8 \mathrm{NA}$ & NA & NA & NA & & snp.2.314.16!snp.5.86.557^ \\
\hline $1.64 \mathrm{NA}$ & NA & NA & NA & & Gg_rs150700.snp.5.86.557 \\
\hline $2.16 \mathrm{NA}$ & NA & NA & NA & & Gg_rs150700.snp.5.86.557 \\
\hline $1.22 \mathrm{NA}$ & NA & NA & NA & & Gg_rs150700 snp.5.86.557 \\
\hline $1.56 \mathrm{NA}$ & NA & NA & NA & & snp.2.314.16!snp.5.86.557 \\
\hline $1.51 \mathrm{NA}$ & NA & NA & NA & & snp.2.314.16!snp.5.86.557£ \\
\hline $2.14 \mathrm{NA}$ & NA & NA & NA & & Gg_rs150700snp.5.86.557 \\
\hline $1.57 \mathrm{NA}$ & NA & NA & NA & & Gg_rs150700.snp.5.86.557^ \\
\hline $1.8 \mathrm{NA}$ & NA & NA & NA & & snp.2.314.16!snp.5.86.557£ \\
\hline $2.73 \mathrm{NA}$ & NA & NA & NA & & snp.2.314.16!snp.5.86.557 \\
\hline $0.11 \mathrm{NA}$ & NA & NA & NA & & snp.2.314.16!snp.5.86.557£ \\
\hline $2.05 \mathrm{NA}$ & NA & NA & NA & & snp.2.314.16!snp.5.86.557£ \\
\hline $2.09 \mathrm{NA}$ & NA & NA & NA & & Gg_rs150700.snp.5.86.557^ \\
\hline $2.18 \mathrm{NA}$ & NA & NA & NA & & Gg_rs150700.snp.5.86.557 \\
\hline $1.99 \mathrm{NA}$ & NA & NA & NA & & Gg_rs150700 snp.5.86.557^ \\
\hline 1.99 NA & NA & NA & NA & & Gg_rs150700.snp.5.86.557 \\
\hline $2.05 \mathrm{NA}$ & NA & NA & NA & & Gg_rs150700.snp.5.86.557 \\
\hline $0.36 \mathrm{NA}$ & NA & NA & NA & & snp.2.314.16!snp.5.86.557^ \\
\hline $0.38 \mathrm{NA}$ & NA & NA & NA & & snp.2.314.16!snp.5.86.557^ \\
\hline $0.47 \mathrm{NA}$ & NA & NA & NA & & snp.2.314.16!snp.5.86.557^ \\
\hline 0.52 NA & NA & NA & NA & & snp.2.314.16!snp.5.86.557 \\
\hline $0.32 \mathrm{NA}$ & NA & NA & NA & & snp.2.314.16!snp.5.86.557 \\
\hline 0.86 NA & NA & NA & NA & & snp.2.314.16!snp.5.86.557 \\
\hline 0.73 NA & NA & NA & NA & & snp.2.314.16!snp.5.86.557 \\
\hline 0.85 & -1.3 & 0.78 & -2.2 & 1.11 & Gg_rs150700 snp.5.242.10 \\
\hline $0.31 \mathrm{NA}$ & NA & NA & NA & & snp.2.314.16!snp.5.86.557 \\
\hline $1.32 \mathrm{NA}$ & NA & NA & NA & & snp.2.314.16!snp.5.86.557^ \\
\hline $1.07 \mathrm{NA}$ & NA & NA & NA & & snp.2.314.16!snp.5.86.557 \\
\hline $0.81 \mathrm{NA}$ & NA & NA & NA & & snp.2.314.16!snp.5.86.557 \\
\hline $1.09 \mathrm{NA}$ & NA & NA & NA & & snp.2.314.16!snp.5.86.557 \\
\hline $0.61 \mathrm{NA}$ & NA & NA & NA & & X2_23979784snp.5.86.557 \\
\hline $0.62 \mathrm{NA}$ & NA & NA & NA & & snp.2.314.16!snp.5.86.557^ \\
\hline $1.07 \mathrm{NA}$ & NA & NA & NA & & snp.2.314.16!snp.5.86.557^ \\
\hline $0.56 \mathrm{NA}$ & NA & NA & NA & & snp.2.314.16!snp.5.86.557^ \\
\hline 0.9 & 2.15 & 0.83 & -3.25 & 1.18 & snp.2.314.16!snp.5.86.557? \\
\hline
\end{tabular}




\begin{tabular}{|c|c|c|c|c|c|c|}
\hline $1.23 \mathrm{NA}$ & NA & & NA & NA & & snp.2.314.16!snp.5.86.557 \\
\hline $1 \mathrm{NA}$ & NA & & NA & NA & & snp.2.314.16!snp.5.86.557^ \\
\hline 0.48 NA & NA & & NA & NA & & snp.2.314.16!snp.5.86.557 \\
\hline 0.68 NA & NA & & NA & NA & & snp.2.314.16!snp.5.86.557 \\
\hline $0.61 \mathrm{NA}$ & NA & & NA & NA & & X2_23979784snp.5.86.557^ \\
\hline $0.5 \mathrm{NA}$ & NA & & NA & NA & & snp.2.314.16!snp.5.86.557 \\
\hline $0.52 \mathrm{NA}$ & NA & & NA & NA & & snp.2.314.16!snp.5.86.557 \\
\hline 0.92 NA & NA & & NA & NA & & X2_23979784snp.5.86.557 \\
\hline $0.79 \mathrm{NA}$ & NA & & NA & NA & & snp.2.314.16!snp.5.86.557^ \\
\hline $0.81 \mathrm{NA}$ & NA & & NA & NA & & snp.2.314.16!snp.5.86.557 \\
\hline $0.75 \mathrm{NA}$ & NA & & NA & NA & & snp.2.314.16!snp.5.86.557 \\
\hline $0.59 \mathrm{NA}$ & NA & & NA & NA & & snp.2.314.16!snp.5.86.557 \\
\hline $0.55 \mathrm{NA}$ & NA & & NA & NA & & snp.2.314.16!snp.5.86.557 \\
\hline $1.06 \mathrm{NA}$ & NA & & NA & NA & & snp.2.314.16!snp.5.86.557^ \\
\hline $1.35 \mathrm{NA}$ & NA & & NA & NA & & snp.2.314.16!snp.5.86.557 \\
\hline $0.81 \mathrm{NA}$ & NA & & NA & NA & & snp.2.314.16!snp.5.86.557 \\
\hline $0.82 \mathrm{NA}$ & NA & & NA & NA & & snp.2.314.16!snp.5.86.557 \\
\hline $0.59 \mathrm{NA}$ & NA & & NA & NA & & snp.2.314.16!snp.5.86.557 \\
\hline $0.43 \mathrm{NA}$ & NA & & NA & NA & & snp.2.314.16!snp.5.86.557^ \\
\hline $0.73 \mathrm{NA}$ & NA & & NA & NA & & snp.2.314.16!snp.5.86.557 \\
\hline $0.52 \mathrm{NA}$ & NA & & NA & NA & & snp.2.314.16!snp.5.86.557^ \\
\hline $0.75 \mathrm{NA}$ & NA & & NA & NA & & X2_23979784snp.5.86.557 \\
\hline $0.66 \mathrm{NA}$ & NA & & NA & NA & & snp.2.314.16!snp.5.86.557 \\
\hline 1.08 & 2.45 & 0.99 & & 1.02 & 1.41 & snp.2.314.16!snp.5.86.557 \\
\hline $0.38 \mathrm{NA}$ & NA & & NA & NA & & snp.2.314.16!snp.5.86.557 \\
\hline $0.77 \mathrm{NA}$ & NA & & NA & NA & & snp.2.314.16!snp.5.242.10t \\
\hline $0.47 \mathrm{NA}$ & NA & & NA & NA & & snp.2.314.16!snp.5.86.557 \\
\hline $0.49 \mathrm{NA}$ & NA & & NA & NA & & snp.2.314.16!snp.5.86.557 \\
\hline $0.38 \mathrm{NA}$ & NA & & NA & NA & & snp.2.314.16!snp.5.86.557 \\
\hline $0.81 \mathrm{NA}$ & NA & & NA & NA & & snp.2.314.16!snp.5.242.10 \\
\hline 0.13 & -0.57 & 0.13 & & 0.59 & 0.16 & Gg_rs150782 snp.5.242.10 \\
\hline $0.53 \mathrm{NA}$ & NA & & NA & NA & & Gg_rs150782 snp.5.242.10t \\
\hline $0.68 \mathrm{NA}$ & NA & & NA & NA & & Gg_rs150782 snp.5.242.10t \\
\hline 0.82 & -0.3 & 0.79 & & 2.26 & 0.96 & Gg_rs150782 snp.5.242.10 \\
\hline $0.5 \mathrm{NA}$ & NA & & NA & NA & & Gg_rs150782 snp.5.242.10t \\
\hline 0.27 & -1.42 & 0.26 & & 1.58 & 0.32 & Gg_rs150782 snp.5.242.10 \\
\hline 0.23 & -1.41 & 0.22 & & 1.55 & 0.27 & Gg_rs150782 snp.5.242.10t \\
\hline 0.27 & -1.41 & 0.25 & & 0.99 & 0.31 & Gg_rs150782 snp.5.242.10 \\
\hline $0.67 \mathrm{NA}$ & NA & & NA & NA & & Gg_rs150782 snp.5.242.10t \\
\hline 0.33 & -1.8 & 0.32 & & 1.21 & 0.39 & Gg_rs150782 snp.5.242.10t \\
\hline 0.44 & -2.06 & 0.43 & & 1.05 & 0.52 & Gg_rs150782 snp.5.242.10 \\
\hline $0.95 \mathrm{NA}$ & NA & & NA & NA & & Gg_rs150782 snp.5.242.10t \\
\hline $0.62 \mathrm{NA}$ & NA & & NA & NA & & Gg_rs150782 snp.5.242.10 \\
\hline
\end{tabular}




\begin{tabular}{|c|c|c|c|c|}
\hline 0.98 & 1.85 & 0.94 & 2.3 & 1.16 Gg_rs150782 snp.5.242.10 \\
\hline $0.64 \mathrm{NA}$ & NA & NA & NA & Gg_rs150782 snp.5.242.10 \\
\hline $0.81 \mathrm{NA}$ & NA & NA & NA & Gg_rs150782.snp.5.242.10t \\
\hline $0.77 \mathrm{NA}$ & NA & NA & NA & Gg_rs150782 snp.5.242.10t \\
\hline $0.58 \mathrm{NA}$ & NA & NA & NA & Gg_rs150782 snp.5.242.10t \\
\hline $0.88 \mathrm{NA}$ & NA & NA & NA & Gg_rs150782.snp.5.242.10t \\
\hline $0.7 \mathrm{NA}$ & NA & NA & NA & Gg_rs150782.snp.5.242.10t \\
\hline $0.31 \mathrm{NA}$ & NA & NA & NA & Gg_rs150782 snp.5.242.10t \\
\hline $0.49 \mathrm{NA}$ & NA & NA & NA & Gg_rs150782 snp.5.242.10t \\
\hline $0.6 \mathrm{NA}$ & NA & NA & NA & Gg_rs150782 snp.5.242.10t \\
\hline 0.18 & -1.13 & 0.18 & 1 & 0.21 Gg_rs150782 snp.5.242.10 \\
\hline $0.52 \mathrm{NA}$ & NA & NA & NA & Gg_rs150782 snp.5.242.10t \\
\hline $0.5 \mathrm{NA}$ & NA & NA & NA & Gg_rs150782 snp.5.242.10t \\
\hline 1.2 & 2.41 & 1.15 & 0.77 & 1.41 Gg_rs150782 snp.5.242.10 \\
\hline $0.71 \mathrm{NA}$ & NA & NA & NA & Gg_rs150782 snp.5.242.10 \\
\hline $1 \mathrm{NA}$ & NA & NA & NA & Gg_rs150782.snp.5.242.10t \\
\hline $0.68 \mathrm{NA}$ & NA & NA & NA & Gg_rs150782.snp.5.242.10t \\
\hline 0.2 & -1.16 & 0.2 & 0.61 & 0.24 Gg_rs150782 snp.5.242.10 \\
\hline $0.69 \mathrm{NA}$ & NA & NA & NA & Gg_rs150782.snp.5.242.10t \\
\hline 0.68 NA & NA & NA & NA & Gg_rs150782.snp.5.242.10t \\
\hline $1 \mathrm{NA}$ & NA & NA & NA & Gg_rs150782 snp.5.242.10t \\
\hline 0.17 & -1.08 & 0.17 & 0.84 & 0.2 Gg_rs150782 snp.5.242.10t \\
\hline 0.19 & -0.97 & 0.19 & 0.79 & 0.23 Gg_rs150782 snp.5.242.10t \\
\hline 1.52 & -0.2 & 1.47 & -0.85 & 1.8 Gg_rs150782X2_40263015 \\
\hline 0.35 & -1.35 & 0.34 & 1.3 & 0.41 Gg_rs150782 snp.5.242.10 \\
\hline $0.5 \mathrm{NA}$ & NA & NA & NA & snp.5.86.557iX2_40263015 \\
\hline $1.51 \mathrm{NA}$ & NA & NA & NA & snp.5.86.557^Gg_rs150944. \\
\hline $0.65 \mathrm{NA}$ & NA & NA & NA & snp.5.86.557^Gg_rs150910. \\
\hline $0.94 \mathrm{NA}$ & NA & NA & NA & snp.5.242.10tGg_rs150944. \\
\hline 1 & 2.59 & 0.84 & 0.62 & 1.34 snp.5.242.10łGg_rs150944. \\
\hline $0.61 \mathrm{NA}$ & NA & NA & NA & snp.5.242.10tGg_rs150944. \\
\hline $3.52 \mathrm{NA}$ & NA & NA & NA & snp.5.86.557^Gg_rs150944. \\
\hline $3.25 \mathrm{NA}$ & NA & NA & NA & snp.5.86.557^Gg_rs150944. \\
\hline $1.75 \mathrm{NA}$ & NA & NA & NA & snp.5.242.10tGg_rs150910. \\
\hline $1.57 \mathrm{NA}$ & NA & NA & NA & snp.5.242.10tGg_rs150910. \\
\hline 4.18 NA & NA & NA & NA & snp.5.242.10tGg_rs150944. \\
\hline $1.66 \mathrm{NA}$ & NA & NA & NA & snp.5.242.10tGg_rs150910 \\
\hline $1.67 \mathrm{NA}$ & NA & NA & NA & snp.5.242.10tGg_rs150910. \\
\hline $2.3 \mathrm{NA}$ & NA & NA & NA & snp.5.242.10tGg_rs150910. \\
\hline $1.6 \mathrm{NA}$ & NA & NA & NA & snp.5.242.10tGg_rs150910. \\
\hline $1.4 \mathrm{NA}$ & NA & NA & NA & snp.5.242.10tGg_rs150910، \\
\hline $1.54 \mathrm{NA}$ & NA & NA & NA & X2_40263015Gg_rs150910. \\
\hline 0.92 & -0.12 & 0.82 & 5.79 & 1.22 snp.5.242.10tGg_rs150910. \\
\hline
\end{tabular}




\begin{tabular}{|c|c|c|c|c|c|}
\hline $2.24 \mathrm{NA}$ & NA & NA & NA & & X2_40263015Gg_rs150910. \\
\hline 1.26 & 0.98 & 1.13 & 5.32 & 1.68 & snp.5.242.10łGg_rs150944 \\
\hline $1.37 \mathrm{NA}$ & NA & NA & NA & & X2_40263015Gg_rs150910 \\
\hline 3 NA & NA & NA & NA & & snp.5.242.10tGg_rs150910 \\
\hline 1.2 & -1.07 & 1.07 & 4.07 & 1.6 & snp.5.242.10łGg_rs150944 \\
\hline $2.98 \mathrm{NA}$ & NA & NA & NA & & X2_40263015Gg_rs150910. \\
\hline $1.48 \mathrm{NA}$ & NA & NA & NA & & X2_40263015Gg_rs150910. \\
\hline 1.61 & 1.13 & 1.44 & 4.39 & 2.14 & X2_40263015Gg_rs150944. \\
\hline $0.69 \mathrm{NA}$ & NA & NA & NA & & X2_40263015Gg_rs150910 \\
\hline 1.39 & -2.55 & 1.24 & 5 & 1.85 & X2_40263015Gg_rs150910 \\
\hline 1.06 & -2.37 & 0.95 & 2.75 & 1.41 & X2_40263015Gg_rs150910. \\
\hline 1.43 & 1.32 & 1.27 & 6.48 & 1.9 & X2_40263015Gg_rs150944 \\
\hline $2.46 \mathrm{NA}$ & NA & NA & NA & & snp.5.242.10łGg_rs150910 \\
\hline $0.73 \mathrm{NA}$ & NA & NA & NA & & X2_40263015Gg_rs150944. \\
\hline $0.62 \mathrm{NA}$ & NA & NA & NA & & snp.5.242.10tGg_rs150944. \\
\hline $1.41 \mathrm{NA}$ & NA & NA & NA & & snp.5.242.10tGg_rs150944. \\
\hline $2.54 \mathrm{NA}$ & NA & NA & NA & & snp.5.242.10tGg_rs150944. \\
\hline $1.84 \mathrm{NA}$ & NA & NA & NA & & snp.5.242.10tGg_rs150944. \\
\hline $0.9 \mathrm{NA}$ & NA & NA & NA & & X2_40263015Gg_rs150944. \\
\hline $2.28 \mathrm{NA}$ & NA & NA & NA & & snp.5.242.10tGg_rs150910. \\
\hline $0.78 \mathrm{NA}$ & NA & NA & NA & & snp.5.242.10tGg_rs150944. \\
\hline $0.58 \mathrm{NA}$ & NA & NA & NA & & snp.5.242.10tGg_rs150944. \\
\hline 0.86 NA & NA & NA & NA & & snp.5.242.10tGg_rs150944. \\
\hline $0.4 \mathrm{NA}$ & NA & NA & NA & & snp.5.242.10tGg_rs150944. \\
\hline $0.36 \mathrm{NA}$ & NA & NA & NA & & X2_40263015Gg_rs150944. \\
\hline $0.29 \mathrm{NA}$ & NA & NA & NA & & snp.5.242.10tGg_rs150944. \\
\hline $0.46 \mathrm{NA}$ & NA & NA & NA & & X2_40263015Gg_rs150944. \\
\hline $0.35 \mathrm{NA}$ & NA & NA & NA & & X2_40263015Gg_rs150944. \\
\hline $0.59 \mathrm{NA}$ & NA & NA & NA & & X2_41000405Gg_rs150944. \\
\hline $0.41 \mathrm{NA}$ & NA & NA & NA & & X2_41000405Gg_rs150944. \\
\hline $0.55 \mathrm{NA}$ & NA & NA & NA & & X2_40263015Gg_rs150944. \\
\hline $0.61 \mathrm{NA}$ & NA & NA & NA & & X2_41000405Gg_rs150944. \\
\hline $0.47 \mathrm{NA}$ & NA & NA & NA & & X2_40263015Gg_rs150944. \\
\hline $0.31 \mathrm{NA}$ & NA & NA & NA & & X2_40263015Gg_rs150944. \\
\hline $0.42 \mathrm{NA}$ & NA & NA & NA & & X2_40263015Gg_rs150944. \\
\hline $0.5 \mathrm{NA}$ & NA & NA & NA & & X2_41000405snp.5.524.14: \\
\hline $0.31 \mathrm{NA}$ & NA & NA & NA & & snp.5.242.10tsnp.5.524.14! \\
\hline $0.57 \mathrm{NA}$ & NA & NA & NA & & X2_40263015snp.5.524.14: \\
\hline $0.56 \mathrm{NA}$ & NA & NA & NA & & X2_40263015snp.5.524.14: \\
\hline $0.48 \mathrm{NA}$ & NA & NA & NA & & X2_41000405snp.5.524.14: \\
\hline $0.62 \mathrm{NA}$ & NA & NA & NA & & snp.5.242.10tGg_rs150944. \\
\hline $0.58 \mathrm{NA}$ & NA & NA & NA & & X2_40263015GG_rs150996 \\
\hline $0.58 \mathrm{NA}$ & NA & NA & NA & & snp.5.242.10tsnp.5.524.14: \\
\hline
\end{tabular}




\begin{tabular}{|c|c|c|c|c|c|c|}
\hline $0.45 \mathrm{NA}$ & NA & & NA & NA & & X2_41000405Gg_rs150944. \\
\hline 0.78 & 0.45 & 0.7 & & 1.95 & 1.04 & X2_40263015Gg_rs150944. \\
\hline $0.7 \mathrm{NA}$ & NA & & NA & NA & & X2_40263015snp.5.524.14! \\
\hline 1.05 & 1.86 & 0.88 & & -0.11 & 1.38 & X2_41000405snp.5.524.14! \\
\hline $0.82 \mathrm{NA}$ & NA & & NA & NA & & snp.5.86.557^GG_rs150996 \\
\hline $0.37 \mathrm{NA}$ & NA & & NA & NA & & X2_41000405snp.5.524.14! \\
\hline $0.78 \mathrm{NA}$ & NA & & NA & NA & & X2_40263015snp.5.524.14: \\
\hline 0.64 NA & NA & & NA & NA & & X2_41000405snp.5.524.14: \\
\hline $0.88 \mathrm{NA}$ & NA & & NA & NA & & Gg_rs150910.snp.5.524.14: \\
\hline $0.75 \mathrm{NA}$ & NA & & NA & NA & & X2_41000405snp.5.524.14: \\
\hline $0.7 \mathrm{NA}$ & NA & & NA & NA & & X2_41000405GG_rs150996 \\
\hline $1.23 \mathrm{NA}$ & NA & & NA & NA & & X2_41000405snp.5.524.14: \\
\hline $0.73 \mathrm{NA}$ & NA & & NA & NA & & X2_41000405snp.5.524.14! \\
\hline $0.72 \mathrm{NA}$ & NA & & NA & NA & & X2_41000405snp.5.524.14: \\
\hline $0.32 \mathrm{NA}$ & NA & & NA & NA & & Gg_rs150910.snp.5.524.14: \\
\hline $0.64 \mathrm{NA}$ & NA & & NA & NA & & Gg_rs150910.snp.5.524.14: \\
\hline $0.65 \mathrm{NA}$ & NA & & NA & NA & & Gg_rs150910 snp.5.524.14: \\
\hline 0.79 NA & NA & & NA & NA & & Gg_rs150910 snp.5.524.14: \\
\hline $0.86 \mathrm{NA}$ & NA & & NA & NA & & Gg_rs150910.snp.5.524.14: \\
\hline $1.2 \mathrm{NA}$ & NA & & NA & NA & & Gg_rs150910.snp.5.524.14: \\
\hline 0.66 & 1.36 & 0.63 & & -2.57 & 0.87 & Gg_rs150910 snp.5.524.14: \\
\hline 0.64 NA & NA & & NA & NA & & Gg_rs150910 snp.5.524.14: \\
\hline $0.66 \mathrm{NA}$ & NA & & NA & NA & & Gg_rs150910 snp.5.524.14: \\
\hline $0.62 \mathrm{NA}$ & NA & & NA & NA & & Gg_rs150910.snp.5.524.14: \\
\hline $0.58 \mathrm{NA}$ & NA & & NA & NA & & Gg_rs150910.snp.5.524.14: \\
\hline $1.32 \mathrm{NA}$ & NA & & NA & NA & & Gg_rs150910.snp.5.524.14: \\
\hline 0.56 NA & NA & & NA & NA & & Gg_rs150910.snp.5.524.14: \\
\hline $0.67 \mathrm{NA}$ & NA & & NA & NA & & Gg_rs150910.snp.5.524.14: \\
\hline 0.66 NA & NA & & NA & NA & & Gg_rs150910.snp.5.524.14: \\
\hline $0.51 \mathrm{NA}$ & NA & & NA & NA & & Gg_rs150910.snp.5.524.14: \\
\hline $0.77 \mathrm{NA}$ & NA & & NA & NA & & Gg_rs150910.snp.5.524.14: \\
\hline $0.8 \mathrm{NA}$ & NA & & NA & NA & & Gg_rs150910.snp.5.524.14: \\
\hline $0.45 \mathrm{NA}$ & NA & & NA & NA & & Gg_rs150910.snp.5.524.14: \\
\hline 0.8 & 3.16 & 0.76 & & -2.4 & 1.06 & Gg_rs150910 snp.5.524.14: \\
\hline 1.33 & 4.92 & 1.26 & & -2.31 & 1.76 & Gg_rs150910 snp.5.524.14: \\
\hline 1.44 & 6.55 & 1.37 & & -5.38 & 1.91 & Gg_rs150910 snp.5.524.14: \\
\hline 0.8 & 3.08 & 0.76 & & -4.64 & 1.06 & Gg_rs150910 snp.5.524.14: \\
\hline 0.68 & 1.16 & 0.65 & & 0.84 & 0.9 & Gg_rs150910 snp.5.524.14: \\
\hline 0.97 NA & NA & & NA & NA & & Gg_rs150910.snp.5.524.14: \\
\hline 0.68 NA & NA & & NA & NA & & Gg_rs150910GG_rs150996 \\
\hline $0.85 \mathrm{NA}$ & NA & & NA & NA & & Gg_rs150910.snp.5.524.14: \\
\hline $0.54 \mathrm{NA}$ & NA & & NA & NA & & Gg_rs150910GG_rs150996 \\
\hline 0.78 & 3.03 & 0.74 & & -3.62 & 1.03 & Gg_rs150910 snp.5.524.14: \\
\hline
\end{tabular}




\begin{tabular}{|c|c|c|c|c|c|}
\hline $0.57 \mathrm{NA}$ & NA & NA & NA & & Gg_rs150910.snp.5.524.14: \\
\hline $0.51 \mathrm{NA}$ & NA & NA & NA & & X2_41000405GG_rs150996 \\
\hline 0.67 & 2.37 & 0.63 & -2.69 & 0.88 & Gg_rs150910GG_rs150996 \\
\hline $0.35 \mathrm{NA}$ & NA & NA & NA & & Gg_rs150910GG_rs150996 \\
\hline 0.95 & 2.39 & 0.9 & -1.66 & 1.26 & Gg_rs150910GG_rs150996 \\
\hline $0.56 \mathrm{NA}$ & NA & NA & NA & & Gg_rs150944 snp.20.234.4: \\
\hline $0.59 \mathrm{NA}$ & NA & NA & NA & & Gg_rs150910.snp.20.234.4; \\
\hline $0.56 \mathrm{NA}$ & NA & NA & NA & & Gg_rs150944 snp.20.234.4: \\
\hline $0.5 \mathrm{NA}$ & NA & NA & NA & & Gg_rs150975 snp.20.234.4: \\
\hline $0.62 \mathrm{NA}$ & NA & NA & NA & & Gg_rs150910.snp.20.141.7( \\
\hline $0.24 \mathrm{NA}$ & NA & NA & NA & & Gg_rs150975 snp.20.360.5: \\
\hline 0.73 NA & NA & NA & NA & & Gg_rs150944 Gg_rs151076. \\
\hline $0.54 \mathrm{NA}$ & NA & NA & NA & & Gg_rs150975 snp.20.360.5: \\
\hline $0.66 \mathrm{NA}$ & NA & NA & NA & & Gg_rs150975 snp.20.141.7( \\
\hline $0.61 \mathrm{NA}$ & NA & NA & NA & & Gg_rs150975 snp.20.234.4; \\
\hline 1.33 & 1.79 & 1.31 & -4.05 & 1.64 & Gg_rs150975 snp.20.234.4: \\
\hline $0.62 \mathrm{NA}$ & NA & NA & NA & & Gg_rs150975 snp.20.234.4: \\
\hline 0.62 & -2.17 & 0.61 & 1.44 & 0.77 & Gg_rs150975 snp.20.234.4: \\
\hline $0.84 \mathrm{NA}$ & NA & NA & NA & & Gg_rs150975 snp.20.234.4: \\
\hline $0.75 \mathrm{NA}$ & NA & NA & NA & & Gg_rs150975 snp.20.234.4; \\
\hline $0.74 \mathrm{NA}$ & NA & NA & NA & & Gg_rs150975 snp.20.234.4: \\
\hline $0.97 \mathrm{NA}$ & NA & NA & NA & & Gg_rs150975 snp.20.360.5: \\
\hline $0.21 \mathrm{NA}$ & NA & NA & NA & & Gg_rs150975 snp.20.234.4: \\
\hline $0.26 \mathrm{NA}$ & NA & NA & NA & & Gg_rs150975 snp.20.234.4: \\
\hline $0.99 \mathrm{NA}$ & NA & NA & NA & & Gg_rs150975 snp.20.360.5: \\
\hline $0.55 \mathrm{NA}$ & NA & NA & NA & & Gg_rs150975 snp.20.141.7( \\
\hline 0.03 & -0.13 & 0.03 & 0.14 & 0.03 & Gg_rs150975 snp.20.360.5: \\
\hline 0.95 & 1.25 & 0.94 & -4.54 & 1.18 & Gg_rs150975 snp.20.234.4; \\
\hline $0.43 \mathrm{NA}$ & NA & NA & NA & & Gg_rs150975 snp.20.234.4: \\
\hline $0.42 \mathrm{NA}$ & NA & NA & NA & & Gg_rs150975 snp.20.234.4; \\
\hline $0.82 \mathrm{NA}$ & NA & NA & NA & & Gg_rs150975 snp.20.234.4" \\
\hline $0.59 \mathrm{NA}$ & NA & NA & NA & & Gg_rs150975 snp.20.234.4: \\
\hline $0.63 \mathrm{NA}$ & NA & NA & NA & & Gg_rs150975 snp.20.234.4; \\
\hline $0.73 \mathrm{NA}$ & NA & NA & NA & & Gg_rs150975 snp.20.234.4; \\
\hline $0.94 \mathrm{NA}$ & NA & NA & NA & & Gg_rs150975 snp.20.234.4: \\
\hline $0.69 \mathrm{NA}$ & NA & NA & NA & & Gg_rs150944 snp.20.234.4: \\
\hline 0.66 NA & NA & NA & NA & & Gg_rs150944 Gg_rs141909. \\
\hline $0.23 \mathrm{NA}$ & NA & NA & NA & & Gg_rs150975 snp.20.234.4: \\
\hline $0.8 \mathrm{NA}$ & NA & NA & NA & & Gg_rs150975 snp.20.234.4; \\
\hline $0.8 \mathrm{NA}$ & NA & NA & NA & & Gg_rs150975 snp.20.234.4; \\
\hline $0.01 \mathrm{NA}$ & NA & NA & NA & & Gg_rs150944 snp.20.234.4: \\
\hline 0.42 & -1.71 & 0.4 & 2.23 & 0.54 & Gg_rs141832 snp.20.234.4: \\
\hline $0.71 \mathrm{NA}$ & NA & NA & NA & & Gg_rs150944 snp.20.234.4; \\
\hline
\end{tabular}




\begin{tabular}{|c|c|c|c|c|c|c|c|}
\hline $0.52 \mathrm{NA}$ & NA & & NA & & NA & & Gg_rs150975 snp.20.234.4; \\
\hline $1 \mathrm{NA}$ & NA & & NA & & NA & & Gg_rs150975 snp.20.234.4; \\
\hline 1.67 & -1.33 & 1.58 & & 4.28 & & 2.14 & Gg_rs150975 snp.20.234.4: \\
\hline $0.54 \mathrm{NA}$ & NA & & NA & & NA & & Gg_rs150975 snp.20.234.4; \\
\hline 0.75 & 1.62 & 0.71 & & 0.4 & & 0.96 & Gg_rs150975 snp.20.234.4: \\
\hline 0.64 & -0.92 & 0.61 & & -1.69 & & 0.82 & Gg_rs150975 snp.20.234.4: \\
\hline 0.8 & -2.52 & 0.76 & & 4.4 & & 1.02 & Gg_rs141832 snp.20.234.4; \\
\hline 1.24 & -3.27 & 1.18 & & 2.29 & & 1.59 & Gg_rs150975 snp.20.234.4: \\
\hline $0.56 \mathrm{NA}$ & NA & & NA & & NA & & Gg_rs150975 Gg_rs151076. \\
\hline $0.23 \mathrm{NA}$ & NA & & NA & & NA & & Gg_rs150975 snp.20.234.4: \\
\hline $0.37 \mathrm{NA}$ & NA & & NA & & NA & & Gg_rs141832 snp.20.234.4; \\
\hline $0.64 \mathrm{NA}$ & NA & & NA & & NA & & Gg_rs150975 snp.20.234.4: \\
\hline $0.58 \mathrm{NA}$ & NA & & NA & & NA & & Gg_rs150975 snp.20.234.4; \\
\hline $0.83 \mathrm{NA}$ & NA & & NA & & NA & & Gg_rs150975 snp.20.234.4; \\
\hline $0.67 \mathrm{NA}$ & NA & & NA & & NA & & Gg_rs141832 snp.20.234.4; \\
\hline $0.82 \mathrm{NA}$ & NA & & NA & & NA & & Gg_rs150975 Gg_rs151076. \\
\hline $0.53 \mathrm{NA}$ & NA & & NA & & NA & & Gg_rs150944 Gg_rs141909. \\
\hline $0.35 \mathrm{NA}$ & NA & & NA & & NA & & Gg_rs150975 snp.20.141.7( \\
\hline $0.36 \mathrm{NA}$ & NA & & NA & & NA & & Gg_rs150975 Gg_rs151076. \\
\hline $0.7 \mathrm{NA}$ & NA & & NA & & NA & & Gg_rs150975 Gg_rs151076. \\
\hline $0.66 \mathrm{NA}$ & NA & & NA & & NA & & Gg_rs150975 Gg_rs151076. \\
\hline 0.91 & -1.35 & 0.86 & & 4.91 & & 1.17 & Gg_rs141832 Gg_rs141871. \\
\hline 0.92 & -0.42 & 0.87 & & -3.08 & & 1.18 & Gg_rs150975 snp.20.141.7( \\
\hline $0.76 \mathrm{NA}$ & NA & & NA & & NA & & Gg_rs150975 Gg_rs151076. \\
\hline 1.09 & -1.39 & 1.03 & & 5.67 & & 1.4 & Gg_rs150975 Gg_rs141871. \\
\hline 1.14 & -1.63 & 1.08 & & 6.52 & & 1.46 & Gg_rs141832 snp.20.234.4; \\
\hline 0.59 & -0.8 & 0.56 & & 4.12 & & 0.75 & Gg_rs141832 snp.20.234.4; \\
\hline $0.76 \mathrm{NA}$ & NA & & NA & & NA & & Gg_rs150975 Gg_rs151076. \\
\hline 1.09 & -0.43 & 1.03 & & 2.84 & & 1.39 & Gg_rs150975 Gg_rs141909. \\
\hline $0.25 \mathrm{NA}$ & NA & & NA & & NA & & Gg_rs141832 Gg_rs151076. \\
\hline 0.46 NA & NA & & NA & & NA & & snp.20.360.5:Gg_rs151076. \\
\hline 0.44 NA & NA & & NA & & NA & & Gg_rs150975 Gg_rs151076. \\
\hline 0.49 NA & NA & & NA & & NA & & Gg_rs141832 Gg_rs151076. \\
\hline 1.29 & 2 & 1.25 & & -4.22 & & 1.62 & Gg_rs150975 Gg_rs151076. \\
\hline $0.73 \mathrm{NA}$ & NA & & NA & & NA & & Gg_rs150975 Gg_rs151076. \\
\hline 0.88 & 2.28 & 0.86 & & -1.69 & & 1.11 & Gg_rs141871 Gg_rs151076. \\
\hline 0.46 & -1.53 & 0.45 & & 2.13 & & 0.58 & Gg_rs141871 Gg_rs141909. \\
\hline 0.94 & 1.41 & 0.91 & & -2.58 & & 1.18 & Gg_rs141871 Gg_rs141909. \\
\hline 0.37 & -2.28 & 0.36 & & 1.88 & & 0.46 & Gg_rs141871 Gg_rs141909. \\
\hline 0.38 & -1.47 & 0.36 & & 1.51 & & 0.47 & Gg_rs141871 Gg_rs141909. \\
\hline 0.04 & -0.28 & 0.04 & & 0.23 & & 0.05 & Gg_rs141871 Gg_rs141909. \\
\hline 0.6 & -2.47 & 0.58 & & 2.68 & & 0.75 & Gg_rs141871 Gg_rs141909. \\
\hline 0.34 & -1.61 & 0.33 & & 1.6 & & 0.42 & Gg_rs141871 Gg_rs141909. \\
\hline
\end{tabular}




\begin{tabular}{|c|c|c|c|c|}
\hline 0.17 & -0.87 & 0.17 & 0.7 & 0.22 Gg_rs141871 Gg_rs141909. \\
\hline 1.12 & 4.81 & 1.09 & -5.48 & 1.41 Gg_rs141871 Gg_rs141909. \\
\hline 0.86 & 3.59 & 0.83 & -3.57 & 1.08 Gg_rs141871 Gg_rs141909. \\
\hline 0.95 & 4.19 & 0.93 & -3.17 & 1.2 Gg_rs141871 Gg_rs141909. \\
\hline 0.63 & 1.13 & 0.61 & -3.58 & 0.79 Gg_rs141871 Gg_rs141909. \\
\hline 1.37 & 6.04 & 1.33 & -5.53 & 1.72 Gg_rs141871 Gg_rs141909. \\
\hline $1.07 \mathrm{NA}$ & NA & NA & NA & Gg_rs150944 Gg_rs151076 \\
\hline $0.72 \mathrm{NA}$ & NA & NA & NA & Gg_rs141871 Gg_rs151076. \\
\hline 0.83 & -3.32 & 0.81 & 2.87 & 1.05 Gg_rs141871 Gg_rs151076. \\
\hline 4.26 & 15.96 & 4.14 & -10.39 & 5.36 snp.20.360.5:Gg_rs151076 \\
\hline 4.84 & 17.64 & 4.71 & -16 & 6.09 Gg_rs141871 Gg_rs151076. \\
\hline $0.85 \mathrm{NA}$ & NA & NA & NA & Gg_rs141871 Gg_rs151076. \\
\hline 1.06 & 3.43 & 1.04 & -6.34 & 1.34 Gg_rs141871 Gg_rs141909. \\
\hline $0.33 \mathrm{NA}$ & NA & NA & NA & snp.20.360.5:Gg_rs151076 \\
\hline 0.26 & -1.28 & 0.25 & 1.43 & 0.33 Gg_rs141871 Gg_rs141909. \\
\hline 0.9 & 2.92 & 0.87 & -4.05 & 1.13 Gg_rs141871 Gg_rs151076. \\
\hline $0.57 \mathrm{NA}$ & NA & NA & NA & Gg_rs141832 Gg_rs151076. \\
\hline $0.6 \mathrm{NA}$ & NA & NA & NA & Gg_rs141871 Gg_rs151076. \\
\hline 0.07 & -0.29 & 0.07 & 0.38 & 0.09 Gg_rs141871 Gg_rs151076. \\
\hline 0.83 NA & NA & NA & NA & Gg_rs151104 Gg_rs151154 \\
\hline $0.64 \mathrm{NA}$ & NA & NA & NA & Gg_rs151104 Gg_rs142004 \\
\hline $0.55 \mathrm{NA}$ & NA & NA & NA & Gg_rs151104 Gg_rs151154 \\
\hline $0.56 \mathrm{NA}$ & NA & NA & NA & Gg_rs151104 Gg_rs142082 \\
\hline $0.56 \mathrm{NA}$ & NA & NA & NA & Gg_rs151076 Gg_rs142061. \\
\hline $0.47 \mathrm{NA}$ & NA & NA & NA & Gg_rs151104 Gg_rs142082 \\
\hline 1.07 & -0.41 & 1.07 & 5.84 & 1.42 Gg_rs151120 Gg_rs151154 \\
\hline $0.5 \mathrm{NA}$ & NA & NA & NA & Gg_rs151120Gg_rs151154 \\
\hline $0.34 \mathrm{NA}$ & NA & NA & NA & Gg_rs151120 Gg_rs142082 \\
\hline $0.6 \mathrm{NA}$ & NA & NA & NA & Gg_rs151120Gg_rs142061. \\
\hline 1.17 & -1.33 & 1.17 & -5.25 & 1.55 Gg_rs151120 Gg_rs142061. \\
\hline $0.22 \mathrm{NA}$ & NA & NA & NA & Gg_rs151120Gg_rs142061. \\
\hline $0.39 \mathrm{NA}$ & NA & NA & NA & Gg_rs151104 Gg_rs142061. \\
\hline $0.28 \mathrm{NA}$ & NA & NA & NA & Gg_rs151120 Gg_rs142082 \\
\hline 0.69 NA & NA & NA & NA & Gg_rs151120Gg_rs142061. \\
\hline 1.15 & -0.54 & 1.15 & -4.5 & 1.52 Gg_rs151120 Gg_rs142061. \\
\hline $0.64 \mathrm{NA}$ & NA & NA & NA & Gg_rs151120Gg_rs142061. \\
\hline $0.65 \mathrm{NA}$ & NA & NA & NA & Gg_rs151120Gg_rs142061. \\
\hline $0.21 \mathrm{NA}$ & NA & NA & NA & Gg_rs151120Gg_rs142061. \\
\hline $0.52 \mathrm{NA}$ & NA & NA & NA & Gg_rs151104 Gg_rs142061. \\
\hline $0.16 \mathrm{NA}$ & NA & NA & NA & Gg_rs151120 Gg_rs142061. \\
\hline $0.43 \mathrm{NA}$ & NA & NA & NA & Gg_rs151120 Gg_rs142061. \\
\hline $0.29 \mathrm{NA}$ & NA & NA & NA & Gg_rs151120 Gg_rs142061. \\
\hline $0.5 \mathrm{NA}$ & NA & NA & NA & Gg_rs151120Gg_rs142061. \\
\hline
\end{tabular}




\begin{tabular}{|c|c|c|c|c|c|}
\hline 0.7 & 1.42 & 0.7 & 3.34 & 0.92 & Gg_rs142004 Gg_rs142061. \\
\hline 0.7 & -2.11 & 0.7 & -0.6 & 0.92 & Gg_rs151076 Gg_rs142061. \\
\hline 0.49 & -1.32 & 0.48 & -0.01 & 0.64 & Gg_rs151104 Gg_rs142061 \\
\hline $0.36 \mathrm{NA}$ & NA & NA & NA & & Gg_rs142004 Gg_rs142061 \\
\hline $0.18 \mathrm{NA}$ & NA & NA & NA & & Gg_rs142004 Gg_rs142061. \\
\hline $0.44 \mathrm{NA}$ & NA & NA & NA & & Gg_rs151120Gg_rs142061. \\
\hline $0.25 \mathrm{NA}$ & NA & NA & NA & & Gg_rs142004 Gg_rs142061. \\
\hline $0.21 \mathrm{NA}$ & NA & NA & NA & & Gg_rs142004 Gg_rs142061. \\
\hline $0.48 \mathrm{NA}$ & NA & NA & NA & & Gg_rs151120Gg_rs142061. \\
\hline 0.44 NA & NA & NA & NA & & Gg_rs151120Gg_rs142061 \\
\hline $0.43 \mathrm{NA}$ & NA & NA & NA & & Gg_rs151120Gg_rs142061 \\
\hline $0.41 \mathrm{NA}$ & NA & NA & NA & & Gg_rs151120Gg_rs142061. \\
\hline $0.37 \mathrm{NA}$ & NA & NA & NA & & Gg_rs151120Gg_rs142082 \\
\hline $0.22 \mathrm{NA}$ & NA & NA & NA & & Gg_rs151120Gg_rs142061. \\
\hline $0.35 \mathrm{NA}$ & NA & NA & NA & & Gg_rs151120Gg_rs142061. \\
\hline $0.28 \mathrm{NA}$ & NA & NA & NA & & Gg_rs151120 Gg_rs142061. \\
\hline $0.61 \mathrm{NA}$ & NA & NA & NA & & Gg_rs151120Gg_rs142061. \\
\hline $0.46 \mathrm{NA}$ & NA & NA & NA & & Gg_rs151120 Gg_rs142061. \\
\hline $0.24 \mathrm{NA}$ & NA & NA & NA & & Gg_rs142004 Gg_rs142061. \\
\hline 0.04 & 0.19 & 0.04 & 0.04 & 0.05 & Gg_rs142004 Gg_rs142061. \\
\hline $0.69 \mathrm{NA}$ & NA & NA & NA & & Gg_rs151120Gg_rs142061. \\
\hline $0.61 \mathrm{NA}$ & NA & NA & NA & & Gg_rs151120 Gg_rs142061. \\
\hline $0.18 \mathrm{NA}$ & NA & NA & NA & & Gg_rs151120 Gg_rs142061. \\
\hline $0.45 \mathrm{NA}$ & NA & NA & NA & & Gg_rs151120Gg_rs142061. \\
\hline $0.37 \mathrm{NA}$ & NA & NA & NA & & Gg_rs142004 Gg_rs142061. \\
\hline 0.76 & 0.22 & 0.75 & 2.33 & 0.99 & Gg_rs142004 Gg_rs142061. \\
\hline 0.57 & 2.42 & 0.56 & 1.69 & 0.74 & Gg_rs142004 Gg_rs142061 \\
\hline $0.46 \mathrm{NA}$ & NA & NA & NA & & Gg_rs151120Gg_rs142061 \\
\hline $0.5 \mathrm{NA}$ & NA & NA & NA & & Gg_rs151120 Gg_rs142061. \\
\hline $0.58 \mathrm{NA}$ & NA & NA & NA & & Gg_rs151120Gg_rs142061. \\
\hline 0.49 NA & NA & NA & NA & & Gg_rs151120Gg_rs142082 \\
\hline $0.52 \mathrm{NA}$ & NA & NA & NA & & Gg_rs142004 Gg_rs142061. \\
\hline 0.49 NA & NA & NA & NA & & Gg_rs151104 Gg_rs142082 \\
\hline $0.47 \mathrm{NA}$ & NA & NA & NA & & Gg_rs151120 Gg_rs142082 \\
\hline $0.74 \mathrm{NA}$ & NA & NA & NA & & Gg_rs151120Gg_rs142082 \\
\hline $0.26 \mathrm{NA}$ & NA & NA & NA & & Gg_rs151120Gg_rs142061. \\
\hline $0.71 \mathrm{NA}$ & NA & NA & NA & & Gg_rs142004 Gg_rs142082 \\
\hline 0.61 & 0.71 & 0.6 & 1.45 & 0.79 & Gg_rs151120Gg_rs142061. \\
\hline $0.33 \mathrm{NA}$ & NA & NA & NA & & Gg_rs151120Gg_rs142061. \\
\hline $0.78 \mathrm{NA}$ & NA & NA & NA & & Gg_rs151120 Gg_rs142061. \\
\hline $0.63 \mathrm{NA}$ & NA & NA & NA & & Gg_rs151120 Gg_rs142061. \\
\hline $0.84 \mathrm{NA}$ & NA & NA & NA & & Gg_rs151104 Gg_rs142082 \\
\hline $0.45 \mathrm{NA}$ & NA & NA & NA & & Gg_rs151120 chr2.846684c \\
\hline
\end{tabular}




\begin{tabular}{|c|c|c|c|c|c|}
\hline $0.66 \mathrm{NA}$ & NA & NA & NA & & Gg_rs151120 Gg_rs142082 \\
\hline $0.62 \mathrm{NA}$ & NA & NA & NA & & Gg_rs151120Gg_rs151251 \\
\hline $0.74 \mathrm{NA}$ & NA & NA & NA & & Gg_rs151104 chr2.846684C \\
\hline $0.56 \mathrm{NA}$ & NA & NA & NA & & Gg_rs151120 Gg_rs142082 \\
\hline $0.67 \mathrm{NA}$ & NA & NA & NA & & Gg_rs151120 Gg_rs142082 \\
\hline $0.37 \mathrm{NA}$ & NA & NA & NA & & Gg_rs151120Gg_rs142082 \\
\hline $0.29 \mathrm{NA}$ & NA & NA & NA & & Gg_rs151120Gg_rs142082 \\
\hline $0.37 \mathrm{NA}$ & NA & NA & NA & & Gg_rs151120 Gg_rs142082 \\
\hline $0.48 \mathrm{NA}$ & NA & NA & NA & & Gg_rs151120Gg_rs142082 \\
\hline $0.47 \mathrm{NA}$ & NA & NA & NA & & Gg_rs151120Gg_rs151251 \\
\hline 0.76 NA & NA & NA & NA & & Gg_rs142004 Gg_rs142082 \\
\hline $0.43 \mathrm{NA}$ & NA & NA & NA & & Gg_rs142004 Gg_rs142082 \\
\hline 0.59 NA & NA & NA & NA & & Gg_rs151104 chr2.846684c \\
\hline $0.48 \mathrm{NA}$ & NA & NA & NA & & Gg_rs151104 Gg_rs151225. \\
\hline $0.51 \mathrm{NA}$ & NA & NA & NA & & Gg_rs151076 Gg_rs151251 \\
\hline $0.46 \mathrm{NA}$ & NA & NA & NA & & Gg_rs151120 chr2.846684c \\
\hline $0.51 \mathrm{NA}$ & NA & NA & NA & & Gg_rs151104 Gg_rs142082 \\
\hline $0.59 \mathrm{NA}$ & NA & NA & NA & & Gg_rs151120 chr2.846684C \\
\hline $0.41 \mathrm{NA}$ & NA & NA & NA & & Gg_rs151120 Gg_rs142082 \\
\hline $0.57 \mathrm{NA}$ & NA & NA & NA & & Gg_rs151120 chr2.846684c \\
\hline $0.74 \mathrm{NA}$ & NA & NA & NA & & Gg_rs142004 Gg_rs151251 \\
\hline 0.52 NA & NA & NA & NA & & Gg_rs151104 Gg_rs151225. \\
\hline 1.25 & 2.78 & 1.23 & 3.04 & 1.67 & Gg_rs151162 Gg_rs142082 \\
\hline 0.39 NA & NA & NA & NA & & Gg_rs151104 Gg_rs142082 \\
\hline 0.79 NA & NA & NA & NA & & Gg_rs151162 Gg_rs142082 \\
\hline $0.39 \mathrm{NA}$ & NA & NA & NA & & Gg_rs142004 chr2.846684C \\
\hline $0.97 \mathrm{NA}$ & NA & NA & NA & & Gg_rs151162 Gg_rs142082 \\
\hline $0.37 \mathrm{NA}$ & NA & NA & NA & & Gg_rs151162 Gg_rs142189. \\
\hline $0.41 \mathrm{NA}$ & NA & NA & NA & & Gg_rs151162 Gg_rs151265 \\
\hline 0.66 NA & NA & NA & NA & & Gg_rs151162 Gg_rs142082 \\
\hline $0.35 \mathrm{NA}$ & NA & NA & NA & & Gg_rs151162 Gg_rs142082 \\
\hline 0.49 NA & NA & NA & NA & & Gg_rs151162 Gg_rs151251 \\
\hline 0.64 & 2.1 & 0.63 & -0.27 & 0.85 & Gg_rs151162 Gg_rs142082 \\
\hline 0.48 & -0.8 & 0.47 & 1.54 & 0.64 & Gg_rs151162 Gg_rs142082 \\
\hline $0.26 \mathrm{NA}$ & NA & NA & NA & & Gg_rs151162 Gg_rs142082 \\
\hline $0.31 \mathrm{NA}$ & NA & NA & NA & & Gg_rs151162 Gg_rs142082 \\
\hline $0.36 \mathrm{NA}$ & NA & NA & NA & & Gg_rs151162 chr2.846684C \\
\hline $0.45 \mathrm{NA}$ & NA & NA & NA & & Gg_rs151162 Gg_rs142082 \\
\hline $0.38 \mathrm{NA}$ & NA & NA & NA & & Gg_rs151162 Gg_rs142082 \\
\hline $0.35 \mathrm{NA}$ & NA & NA & NA & & Gg_rs151162 Gg_rs151251 \\
\hline $0.45 \mathrm{NA}$ & NA & NA & NA & & Gg_rs151162 Gg_rs142082 \\
\hline $0.46 \mathrm{NA}$ & NA & NA & NA & & Gg_rs151162 Gg_rs142082 \\
\hline $0.49 \mathrm{NA}$ & NA & NA & NA & & Gg_rs151162 Gg_rs142082 \\
\hline
\end{tabular}




\begin{tabular}{|c|c|c|c|c|}
\hline $0.3 \mathrm{NA}$ & NA & NA & NA & Gg_rs151162 Gg_rs142082 \\
\hline $0.33 \mathrm{NA}$ & NA & NA & NA & Gg_rs151162 Gg_rs142082 \\
\hline $0.34 \mathrm{NA}$ & NA & NA & NA & Gg_rs151162 chr2.846684c \\
\hline $0.55 \mathrm{NA}$ & NA & NA & NA & Gg_rs151162 Gg_rs151251 \\
\hline $0.51 \mathrm{NA}$ & NA & NA & NA & Gg_rs151162 Gg_rs142082 \\
\hline $0.53 \mathrm{NA}$ & NA & NA & NA & Gg_rs151162 Gg_rs142082 \\
\hline $0.55 \mathrm{NA}$ & NA & NA & NA & Gg_rs151162 Gg_rs142082 \\
\hline $0.52 \mathrm{NA}$ & NA & NA & NA & Gg_rs151162 Gg_rs151231. \\
\hline $0.36 \mathrm{NA}$ & NA & NA & NA & Gg_rs151162 Gg_rs151251 \\
\hline $0.58 \mathrm{NA}$ & NA & NA & NA & Gg_rs151162 chr2.846684c \\
\hline $0.4 \mathrm{NA}$ & NA & NA & NA & Gg_rs151162 Gg_rs151251 \\
\hline $0.38 \mathrm{NA}$ & NA & NA & NA & Gg_rs151162 Gg_rs142082 \\
\hline $0.42 \mathrm{NA}$ & NA & NA & NA & Gg_rs151162 Gg_rs142189. \\
\hline $0.52 \mathrm{NA}$ & NA & NA & NA & Gg_rs151162 Gg_rs151251 \\
\hline $0.47 \mathrm{NA}$ & NA & NA & NA & Gg_rs151162 chr2.846684c \\
\hline $0.58 \mathrm{NA}$ & NA & NA & NA & Gg_rs151162 chr2.846684c \\
\hline $0.59 \mathrm{NA}$ & NA & NA & NA & Gg_rs151162 Gg_rs151251 \\
\hline $0.53 \mathrm{NA}$ & NA & NA & NA & Gg_rs151162 chr2.846684c \\
\hline $0.45 \mathrm{NA}$ & NA & NA & NA & Gg_rs151162 chr2.846684c \\
\hline $0.32 \mathrm{NA}$ & NA & NA & NA & Gg_rs151162 Gg_rs151265 \\
\hline 1.89 & 1.53 & 1.86 & 6.67 & 2.51 Gg_rs151162 chr2.846684c \\
\hline 0.45 NA & NA & NA & NA & Gg_rs151162 Gg_rs151251 \\
\hline $0.29 \mathrm{NA}$ & NA & NA & NA & Gg_rs151162 Gg_rs151251 \\
\hline $0.31 \mathrm{NA}$ & NA & NA & NA & Gg_rs151162 Gg_rs151225. \\
\hline $0.33 \mathrm{NA}$ & NA & NA & NA & Gg_rs151162 Gg_rs151251 \\
\hline $0.31 \mathrm{NA}$ & NA & NA & NA & Gg_rs151162 Gg_rs151251 \\
\hline $0.46 \mathrm{NA}$ & NA & NA & NA & Gg_rs151162 Gg_rs151251 \\
\hline $0.32 \mathrm{NA}$ & NA & NA & NA & Gg_rs151162 Gg_rs151265 \\
\hline $0.57 \mathrm{NA}$ & NA & NA & NA & Gg_rs151162 Gg_rs151251 \\
\hline $0.78 \mathrm{NA}$ & NA & NA & NA & Gg_rs151154 Gg_rs151231. \\
\hline $0.32 \mathrm{NA}$ & NA & NA & NA & Gg_rs151162 Gg_rs151251 \\
\hline $0.49 \mathrm{NA}$ & NA & NA & NA & Gg_rs151162 Gg_rs151251 \\
\hline $0.4 \mathrm{NA}$ & NA & NA & NA & Gg_rs151162 Gg_rs151251 \\
\hline $0.51 \mathrm{NA}$ & NA & NA & NA & Gg_rs151162 Gg_rs151251 \\
\hline $0.41 \mathrm{NA}$ & NA & NA & NA & Gg_rs151162 Gg_rs151251 \\
\hline $0.27 \mathrm{NA}$ & NA & NA & NA & Gg_rs151120Gg_rs151231. \\
\hline 0.43 NA & NA & NA & NA & Gg_rs151162 Gg_rs151251 \\
\hline 0.47 NA & NA & NA & NA & Gg_rs151162 Gg_rs142189. \\
\hline $0.55 \mathrm{NA}$ & NA & NA & NA & Gg_rs151162 Gg_rs151251 \\
\hline $0.37 \mathrm{NA}$ & NA & NA & NA & Gg_rs151162 Gg_rs151251 \\
\hline $0.58 \mathrm{NA}$ & NA & NA & NA & Gg_rs151162 Gg_rs151251 \\
\hline 0.49 NA & NA & NA & NA & Gg_rs151162 Gg_rs151251 \\
\hline $0.42 \mathrm{NA}$ & NA & NA & NA & Gg_rs151162 Gg_rs151251 \\
\hline
\end{tabular}




\begin{tabular}{|c|c|c|c|c|c|}
\hline 0.68 NA & NA & NA & NA & & Gg_rs151162 Gg_rs142189. \\
\hline $0.54 \mathrm{NA}$ & NA & NA & NA & & Gg_rs151162 Gg_rs151251 \\
\hline $0.46 \mathrm{NA}$ & NA & NA & NA & & Gg_rs151162 Gg_rs151251 \\
\hline $0.43 \mathrm{NA}$ & NA & NA & NA & & Gg_rs151162 Gg_rs151251 \\
\hline 0.47 NA & NA & NA & NA & & Gg_rs151162 Gg_rs151251 \\
\hline $0.35 \mathrm{NA}$ & NA & NA & NA & & Gg_rs142004 Gg_rs142189. \\
\hline $0.29 \mathrm{NA}$ & NA & NA & NA & & Gg_rs151120Gg_rs151251 \\
\hline $0.31 \mathrm{NA}$ & NA & NA & NA & & Gg_rs151162 Gg_rs142189. \\
\hline $0.45 \mathrm{NA}$ & NA & NA & NA & & Gg_rs151162 Gg_rs151251 \\
\hline $0.5 \mathrm{NA}$ & NA & NA & NA & & Gg_rs151162 Gg_rs151251 \\
\hline $0.42 \mathrm{NA}$ & NA & NA & NA & & Gg_rs151162 Gg_rs151251 \\
\hline $0.72 \mathrm{NA}$ & NA & NA & NA & & Gg_rs151162 Gg_rs151251 \\
\hline 0.64 NA & NA & NA & NA & & Gg_rs151162 Gg_rs151251 \\
\hline $0.62 \mathrm{NA}$ & NA & NA & NA & & Gg_rs151162 Gg_rs151251 \\
\hline $0.47 \mathrm{NA}$ & NA & NA & NA & & Gg_rs151162 chr2.846684C \\
\hline $0.6 \mathrm{NA}$ & NA & NA & NA & & Gg_rs151162 Gg_rs151251 \\
\hline $0.45 \mathrm{NA}$ & NA & NA & NA & & Gg_rs151162 Gg_rs151251 \\
\hline $0.16 \mathrm{NA}$ & NA & NA & NA & & Gg_rs151162 Gg_rs151251 \\
\hline $0.34 \mathrm{NA}$ & NA & NA & NA & & Gg_rs151162 Gg_rs151251 \\
\hline $0.41 \mathrm{NA}$ & NA & NA & NA & & Gg_rs151120Gg_rs151251 \\
\hline 0.71 & 1.3 & 0.65 & 1.8 & 0.95 & Gg_rs151162 Gg_rs151251 \\
\hline $0.33 \mathrm{NA}$ & NA & NA & NA & & Gg_rs151162 Gg_rs151251 \\
\hline $0.46 \mathrm{NA}$ & NA & NA & NA & & Gg_rs151162 Gg_rs151251 \\
\hline $0.41 \mathrm{NA}$ & NA & NA & NA & & Gg_rs151162 Gg_rs151251 \\
\hline $0.51 \mathrm{NA}$ & NA & NA & NA & & Gg_rs151120Gg_rs151265 \\
\hline $0.31 \mathrm{NA}$ & NA & NA & NA & & Gg_rs151162 Gg_rs151251 \\
\hline $0.55 \mathrm{NA}$ & NA & NA & NA & & Gg_rs151162 Gg_rs151251 \\
\hline $0.61 \mathrm{NA}$ & NA & NA & NA & & Gg_rs151162 Gg_rs151265 \\
\hline $0.31 \mathrm{NA}$ & NA & NA & NA & & Gg_rs151162 Gg_rs151251 \\
\hline $0.5 \mathrm{NA}$ & NA & NA & NA & & Gg_rs151162 chr2.846684C \\
\hline 0.73 & -1.25 & 0.68 & 1.54 & 0.98 & Gg_rs151183 chr2.846684C \\
\hline $0.86 \mathrm{NA}$ & NA & NA & NA & & Gg_rs151162 Gg_rs151265 \\
\hline 0.63 NA & NA & NA & NA & & Gg_rs151162 Gg_rs151251 \\
\hline $0.41 \mathrm{NA}$ & NA & NA & NA & & Gg_rs142004 Gg_rs151251 \\
\hline $0.47 \mathrm{NA}$ & NA & NA & NA & & Gg_rs151162 Gg_rs151251 \\
\hline $0.47 \mathrm{NA}$ & NA & NA & NA & & Gg_rs151162 Gg_rs151251 \\
\hline 0.83 NA & NA & NA & NA & & Gg_rs151162 Gg_rs142189. \\
\hline $0.6 \mathrm{NA}$ & NA & NA & NA & & Gg_rs151162 Gg_rs142189. \\
\hline $0.5 \mathrm{NA}$ & NA & NA & NA & & Gg_rs151162 Gg_rs151251 \\
\hline $0.35 \mathrm{NA}$ & NA & NA & NA & & Gg_rs151183 Gg_rs151251 \\
\hline $0.4 \mathrm{NA}$ & NA & NA & NA & & Gg_rs151162 Gg_rs151225. \\
\hline $0.52 \mathrm{NA}$ & NA & NA & NA & & Gg_rs142061 Gg_rs151251 \\
\hline $0.33 \mathrm{NA}$ & NA & NA & NA & & Gg_rs151162 Gg_rs151251 \\
\hline
\end{tabular}




\begin{tabular}{|c|c|c|c|c|c|}
\hline $0.44 \mathrm{NA}$ & NA & NA & NA & & Gg_rs151162 Gg_rs151251 \\
\hline $0.38 \mathrm{NA}$ & NA & NA & NA & & Gg_rs151162 Gg_rs142189. \\
\hline $0.42 \mathrm{NA}$ & NA & NA & NA & & Gg_rs151162 Gg_rs151251 \\
\hline 0.52 & -0.58 & 0.48 & 1.81 & 0.7 & Gg_rs151183 chr2.846684C \\
\hline $0.52 \mathrm{NA}$ & NA & NA & NA & & Gg_rs151183 Gg_rs151251 \\
\hline 0.43 NA & NA & NA & NA & & Gg_rs151162 Gg_rs151251 \\
\hline $0.36 \mathrm{NA}$ & NA & NA & NA & & Gg_rs151183 Gg_rs151251 \\
\hline 0.99 & 0.5 & 0.91 & 3.68 & 1.32 & Gg_rs151162 chr2.846684c \\
\hline 0.46 NA & NA & NA & NA & & Gg_rs151162 Gg_rs142189. \\
\hline $0.5 \mathrm{NA}$ & NA & NA & NA & & Gg_rs151162 Gg_rs151251 \\
\hline $0.38 \mathrm{NA}$ & NA & NA & NA & & Gg_rs151162 Gg_rs142189. \\
\hline $0.38 \mathrm{NA}$ & NA & NA & NA & & Gg_rs142004 Gg_rs151251 \\
\hline $0.27 \mathrm{NA}$ & NA & NA & NA & & Gg_rs151183 Gg_rs151251 \\
\hline $0.3 \mathrm{NA}$ & NA & NA & NA & & Gg_rs151183 Gg_rs151251 \\
\hline $0.46 \mathrm{NA}$ & NA & NA & NA & & Gg_rs142061 Gg_rs151251 \\
\hline $0.28 \mathrm{NA}$ & NA & NA & NA & & Gg_rs151183 chr2.846684c \\
\hline $0.35 \mathrm{NA}$ & NA & NA & NA & & Gg_rs151183 chr2.846684c \\
\hline 0.48 & 0.48 & 0.45 & 1.69 & 0.65 & Gg_rs151183 chr2.846684c \\
\hline 0.49 & 0 & 0.45 & 2.09 & 0.65 & Gg_rs151162 chr2.846684c \\
\hline 0.59 NA & NA & NA & NA & & Gg_rs151183 Gg_rs151251 \\
\hline $0.66 \mathrm{NA}$ & NA & NA & NA & & Gg_rs151162 Gg_rs142189. \\
\hline $0.38 \mathrm{NA}$ & NA & NA & NA & & Gg_rs151183 Gg_rs151251 \\
\hline $0.79 \mathrm{NA}$ & NA & NA & NA & & Gg_rs151183 Gg_rs151251 \\
\hline $0.4 \mathrm{NA}$ & NA & NA & NA & & Gg_rs151162 Gg_rs151225. \\
\hline $0.33 \mathrm{NA}$ & NA & NA & NA & & Gg_rs151183 chr2.846684C \\
\hline 0.63 & 0.18 & 0.58 & 2.31 & 0.84 & Gg_rs151183 Gg_rs142082 \\
\hline $0.39 \mathrm{NA}$ & NA & NA & NA & & Gg_rs151183 Gg_rs151251 \\
\hline $0.42 \mathrm{NA}$ & NA & NA & NA & & Gg_rs151183 Gg_rs151251 \\
\hline $0.46 \mathrm{NA}$ & NA & NA & NA & & Gg_rs151183 Gg_rs151251 \\
\hline $0.39 \mathrm{NA}$ & NA & NA & NA & & Gg_rs151183 Gg_rs151251 \\
\hline $0.52 \mathrm{NA}$ & NA & NA & NA & & Gg_rs151162 Gg_rs142189. \\
\hline $0.65 \mathrm{NA}$ & NA & NA & NA & & Gg_rs151183 Gg_rs151231. \\
\hline $1.03 \mathrm{NA}$ & NA & NA & NA & & Gg_rs151183 Gg_rs151251 \\
\hline $0.64 \mathrm{NA}$ & NA & NA & NA & & Gg_rs151183 chr2.846684C \\
\hline $3.46 \mathrm{NA}$ & NA & NA & NA & & Gg_rs151183Gg_rs151265 \\
\hline $3.86 \mathrm{NA}$ & NA & NA & NA & & Gg_rs151183Gg_rs151265 \\
\hline $2.73 \mathrm{NA}$ & NA & NA & NA & & Gg_rs151183 chr2.846684c \\
\hline 4.02 NA & NA & NA & NA & & Gg_rs151183 Gg_rs151251 \\
\hline $3.03 \mathrm{NA}$ & NA & NA & NA & & Gg_rs151183 Gg_rs151251 \\
\hline $3.69 \mathrm{NA}$ & NA & NA & NA & & Gg_rs151183 Gg_rs151251 \\
\hline $1.78 \mathrm{NA}$ & NA & NA & NA & & Gg_rs151183Gg_rs142189. \\
\hline $0.9 \mathrm{NA}$ & NA & NA & NA & & Gg_rs151183 Gg_rs151231. \\
\hline $1.02 \mathrm{NA}$ & NA & NA & NA & & Gg_rs151183 Gg_rs151265 \\
\hline
\end{tabular}




\begin{tabular}{|c|c|c|c|c|c|}
\hline $0.41 \mathrm{NA}$ & NA & NA & NA & & Gg_rs151183 Gg_rs142189 \\
\hline 0.51 & 0.55 & 0.47 & 1.94 & 0.68 & Gg_rs151183 chr2.8466849 \\
\hline $0.4 \mathrm{NA}$ & NA & NA & NA & & Gg_rs151183 Gg_rs151251 \\
\hline $0.57 \mathrm{NA}$ & NA & NA & NA & & Gg_rs151183 Gg_rs151251 \\
\hline 0.99 NA & NA & NA & NA & & Gg_rs151183 Gg_rs151251 \\
\hline $0.54 \mathrm{NA}$ & NA & NA & NA & & Gg_rs151183 Gg_rs151225 \\
\hline $0.47 \mathrm{NA}$ & NA & NA & NA & & Gg_rs142061 Gg_rs151251 \\
\hline $0.61 \mathrm{NA}$ & NA & NA & NA & & Gg_rs151183 Gg_rs151251 \\
\hline $0.36 \mathrm{NA}$ & NA & NA & NA & & Gg_rs151183 Gg_rs142189 \\
\hline $0.62 \mathrm{NA}$ & NA & NA & NA & & Gg_rs151183 Gg_rs151251 \\
\hline $0.66 \mathrm{NA}$ & NA & NA & NA & & Gg_rs151183 Gg_rs142189 \\
\hline $0.38 \mathrm{NA}$ & NA & NA & NA & & Gg_rs151183 Gg_rs151251 \\
\hline $0.63 \mathrm{NA}$ & NA & NA & NA & & Gg_rs151183 Gg_rs151251 \\
\hline $0.83 \mathrm{NA}$ & NA & NA & NA & & Gg_rs151162 Gg_rs151251 \\
\hline 0.87 & 0.98 & 0.8 & 3.06 & 1.16 & Gg_rs151183 chr2.8466849 \\
\hline $0.55 \mathrm{NA}$ & NA & NA & NA & & Gg_rs151183 Gg_rs151225 \\
\hline $0.34 \mathrm{NA}$ & NA & NA & NA & & Gg_rs151183 chr2.8466849 \\
\hline $0.5 \mathrm{NA}$ & NA & NA & NA & & Gg_rs151183 chr2.8466849 \\
\hline 0.82 & 0.77 & 0.76 & 3.49 & 1.1 & Gg_rs151183 chr2.8466849 \\
\hline 0.59 NA & NA & NA & NA & & Gg_rs151183 chr2.8466849 \\
\hline 0.64 & 1.45 & 0.59 & 2.71 & 0.86 & Gg_rs151183 chr2.8466849 \\
\hline 0.52 & -0.47 & 0.48 & 2.43 & 0.7 & Gg_rs151183 chr2.8466849 \\
\hline $0.38 \mathrm{NA}$ & NA & NA & NA & & Gg_rs151183 Gg_rs151251 \\
\hline $0.5 \mathrm{NA}$ & NA & NA & NA & & Gg_rs151183 chr2.8466849 \\
\hline $0.57 \mathrm{NA}$ & NA & NA & NA & & Gg_rs151183 Gg_rs151251 \\
\hline $0.36 \mathrm{NA}$ & NA & NA & NA & & Gg_rs151183 chr2.8466849 \\
\hline $0.45 \mathrm{NA}$ & NA & NA & NA & & Gg_rs151183 chr2.8466849 \\
\hline $0.55 \mathrm{NA}$ & NA & NA & NA & & Gg_rs151183 Gg_rs142189 \\
\hline 0.96 & -2.08 & 0.88 & 3.43 & 1.28 & Gg_rs151183 chr2.8466849 \\
\hline $0.28 \mathrm{NA}$ & NA & NA & NA & & Gg_rs151183 Gg_rs151251 \\
\hline 0.46 NA & NA & NA & NA & & Gg_rs151183 Gg_rs151251 \\
\hline 0.98 & 0.94 & 0.9 & 1.99 & 1.31 & Gg_rs151183 Gg_rs151231 \\
\hline $0.41 \mathrm{NA}$ & NA & NA & NA & & Gg_rs151183 Gg_rs151251 \\
\hline 0.87 & -1.13 & 0.8 & 3.52 & 1.16 & Gg_rs151183 Gg_rs151251 \\
\hline $0.41 \mathrm{NA}$ & NA & NA & NA & & Gg_rs151183 Gg_rs151251 \\
\hline 0.89 NA & NA & NA & NA & & Gg_rs151183 Gg_rs151251 \\
\hline $0.65 \mathrm{NA}$ & NA & NA & NA & & Gg_rs151183 Gg_rs151251 \\
\hline $0.31 \mathrm{NA}$ & NA & NA & NA & & Gg_rs151183 Gg_rs151251 \\
\hline 0.8 & -0.17 & 0.74 & 3.49 & 1.07 & Gg_rs151183 chr2.8466849 \\
\hline 0.63 & 0.21 & 0.58 & 2.01 & 0.84 & Gg_rs151183 Gg_rs151231 \\
\hline $0.57 \mathrm{NA}$ & NA & NA & NA & & Gg_rs151183 Gg_rs151251 \\
\hline $0.42 \mathrm{NA}$ & NA & NA & NA & & Gg_rs151183 Gg_rs151251 \\
\hline $0.61 \mathrm{NA}$ & NA & NA & NA & & Gg_rs151183 Gg_rs151251 \\
\hline
\end{tabular}




\begin{tabular}{|c|c|c|c|c|c|}
\hline $0.55 \mathrm{NA}$ & NA & NA & NA & & Gg_rs151183 Gg_rs151251 \\
\hline $0.4 \mathrm{NA}$ & NA & NA & NA & & Gg_rs151183 Gg_rs151251 \\
\hline $0.51 \mathrm{NA}$ & NA & NA & NA & & Gg_rs151183 Gg_rs151225. \\
\hline $0.53 \mathrm{NA}$ & NA & NA & NA & & Gg_rs151183 Gg_rs151251 \\
\hline 0.68 & -0.64 & 0.63 & 2.31 & 0.91 & Gg_rs151183 Gg_rs151225. \\
\hline $0.4 \mathrm{NA}$ & NA & NA & NA & & Gg_rs151183 Gg_rs151251 \\
\hline $0.48 \mathrm{NA}$ & NA & NA & NA & & Gg_rs151183 Gg_rs151251 \\
\hline $0.62 \mathrm{NA}$ & NA & NA & NA & & Gg_rs151183 Gg_rs151265 \\
\hline 0.46 & 0.82 & 0.43 & 1.67 & 0.62 & Gg_rs151183Gg_rs151265 \\
\hline 0.46 NA & NA & NA & NA & & Gg_rs151162 Gg_rs151251 \\
\hline $0.6 \mathrm{NA}$ & NA & NA & NA & & Gg_rs151162 Gg_rs151251 \\
\hline $0.53 \mathrm{NA}$ & NA & NA & NA & & Gg_rs151183 Gg_rs142189. \\
\hline $0.54 \mathrm{NA}$ & NA & NA & NA & & Gg_rs151183 Gg_rs151251 \\
\hline $0.73 \mathrm{NA}$ & NA & NA & NA & & Gg_rs151183 Gg_rs142189. \\
\hline $0.82 \mathrm{NA}$ & NA & NA & NA & & Gg_rs151183 Gg_rs151251 \\
\hline 0.32 & -1.12 & 0.3 & 0.87 & 0.43 & Gg_rs151183 Gg_rs151225. \\
\hline 0.22 & -0.46 & 0.21 & 1.11 & 0.3 & Gg_rs151183 chr2.846684C \\
\hline 0.92 & 1.39 & 0.85 & 1.98 & 1.23 & Gg_rs151183 Gg_rs151251 \\
\hline $0.38 \mathrm{NA}$ & NA & NA & NA & & Gg_rs151183 Gg_rs151251 \\
\hline $0.66 \mathrm{NA}$ & NA & NA & NA & & Gg_rs151183 Gg_rs151251 \\
\hline $0.56 \mathrm{NA}$ & NA & NA & NA & & Gg_rs151183 Gg_rs151251 \\
\hline $0.25 \mathrm{NA}$ & NA & NA & NA & & Gg_rs151183 Gg_rs151251 \\
\hline 0.63 NA & NA & NA & NA & & Gg_rs151183 Gg_rs142189. \\
\hline $0.37 \mathrm{NA}$ & NA & NA & NA & & Gg_rs151162 Gg_rs151251 \\
\hline 0.61 & 0.42 & 0.57 & 1.85 & 0.82 & Gg_rs142004 Gg_rs151251 \\
\hline 0.59 & -0.11 & 0.55 & 2.49 & 0.79 & Gg_rs151183 Gg_rs151251 \\
\hline 0.98 & -0.93 & 0.91 & 3.12 & 1.32 & Gg_rs151183 Gg_rs151251 \\
\hline 0.96 & -0.74 & 0.89 & 3.5 & 1.29 & Gg_rs151183 Gg_rs151251 \\
\hline $0.3 \mathrm{NA}$ & NA & NA & NA & & Gg_rs151183 Gg_rs151251 \\
\hline $0.38 \mathrm{NA}$ & NA & NA & NA & & Gg_rs151162 Gg_rs151251 \\
\hline $0.44 \mathrm{NA}$ & NA & NA & NA & & Gg_rs151162 Gg_rs151251 \\
\hline $0.53 \mathrm{NA}$ & NA & NA & NA & & Gg_rs151162 Gg_rs142189. \\
\hline $0.39 \mathrm{NA}$ & NA & NA & NA & & Gg_rs151183 Gg_rs142189. \\
\hline $0.6 \mathrm{NA}$ & NA & NA & NA & & Gg_rs151183 Gg_rs151251 \\
\hline $0.5 \mathrm{NA}$ & NA & NA & NA & & Gg_rs151162 Gg_rs151251 \\
\hline $0.42 \mathrm{NA}$ & NA & NA & NA & & Gg_rs151183 Gg_rs151251 \\
\hline $0.34 \mathrm{NA}$ & NA & NA & NA & & Gg_rs151183 Gg_rs142189. \\
\hline $0.48 \mathrm{NA}$ & NA & NA & NA & & Gg_rs151183 Gg_rs151251 \\
\hline $0.37 \mathrm{NA}$ & NA & NA & NA & & Gg_rs142061 Gg_rs151251 \\
\hline $0.44 \mathrm{NA}$ & NA & NA & NA & & Gg_rs151183 Gg_rs151251 \\
\hline 0.49 NA & NA & NA & NA & & Gg_rs151183 Gg_rs151251 \\
\hline 0.43 NA & NA & NA & NA & & Gg_rs151162 Gg_rs142189. \\
\hline $0.42 \mathrm{NA}$ & NA & NA & NA & & Gg_rs151162 Gg_rs151251 \\
\hline
\end{tabular}




\begin{tabular}{|c|c|c|c|c|c|}
\hline 0.52 & 1.19 & 0.48 & 0.34 & 0.7 & Gg_rs151183 Gg_rs151251 \\
\hline 0.91 & -0.3 & 0.83 & 3.45 & 1.22 & Gg_rs151183 Gg_rs151251 \\
\hline $0.58 \mathrm{NA}$ & NA & NA & NA & & Gg_rs142061 Gg_rs151251 \\
\hline $0.31 \mathrm{NA}$ & NA & NA & NA & & Gg_rs151183 Gg_rs151251 \\
\hline $0.56 \mathrm{NA}$ & NA & NA & NA & & Gg_rs151183 Gg_rs151251 \\
\hline $0.41 \mathrm{NA}$ & NA & NA & NA & & Gg_rs151183 Gg_rs151251 \\
\hline $0.32 \mathrm{NA}$ & NA & NA & NA & & Gg_rs151183 Gg_rs151251 \\
\hline $0.45 \mathrm{NA}$ & NA & NA & NA & & Gg_rs151183 Gg_rs151251 \\
\hline $0.58 \mathrm{NA}$ & NA & NA & NA & & Gg_rs151183 Gg_rs151251 \\
\hline $0.39 \mathrm{NA}$ & NA & NA & NA & & Gg_rs151183 Gg_rs151251 \\
\hline $0.39 \mathrm{NA}$ & NA & NA & NA & & Gg_rs151183 Gg_rs151251 \\
\hline $0.34 \mathrm{NA}$ & NA & NA & NA & & Gg_rs151183 Gg_rs151251 \\
\hline $0.45 \mathrm{NA}$ & NA & NA & NA & & Gg_rs151183 Gg_rs151251 \\
\hline $0.35 \mathrm{NA}$ & NA & NA & NA & & Gg_rs151183 Gg_rs151251 \\
\hline $0.4 \mathrm{NA}$ & NA & NA & NA & & Gg_rs151162 Gg_rs151251 \\
\hline $0.42 \mathrm{NA}$ & NA & NA & NA & & Gg_rs151183 Gg_rs151251 \\
\hline $0.39 \mathrm{NA}$ & NA & NA & NA & & Gg_rs151183 Gg_rs151251 \\
\hline $0.42 \mathrm{NA}$ & NA & NA & NA & & Gg_rs151162 Gg_rs151251 \\
\hline 0.49 NA & NA & NA & NA & & Gg_rs151183 Gg_rs151251 \\
\hline 0.48 NA & NA & NA & NA & & Gg_rs151183 Gg_rs151265 \\
\hline $0.4 \mathrm{NA}$ & NA & NA & NA & & Gg_rs151183 Gg_rs151265 \\
\hline $0.36 \mathrm{NA}$ & NA & NA & NA & & Gg_rs151183 Gg_rs151251 \\
\hline $0.2 \mathrm{NA}$ & NA & NA & NA & & Gg_rs151162 Gg_rs151251 \\
\hline $0.31 \mathrm{NA}$ & NA & NA & NA & & Gg_rs151162 chr2.846684C \\
\hline $0.55 \mathrm{NA}$ & NA & NA & NA & & Gg_rs151183 Gg_rs151251 \\
\hline $0.5 \mathrm{NA}$ & NA & NA & NA & & Gg_rs151183 Gg_rs151251 \\
\hline $0.52 \mathrm{NA}$ & NA & NA & NA & & Gg_rs151183 Gg_rs142189. \\
\hline $0.76 \mathrm{NA}$ & NA & NA & NA & & Gg_rs151162 Gg_rs151251 \\
\hline $0.47 \mathrm{NA}$ & NA & NA & NA & & Gg_rs151183 Gg_rs151251 \\
\hline 0.79 & 1.82 & 0.72 & -0.2 & 1.06 & Gg_rs151183 Gg_rs151251 \\
\hline $0.29 \mathrm{NA}$ & NA & NA & NA & & Gg_rs151183Gg_rs151265 \\
\hline $0.58 \mathrm{NA}$ & NA & NA & NA & & Gg_rs142082 Gg_rs151251 \\
\hline 0.44 NA & NA & NA & NA & & Gg_rs151183 Gg_rs151251 \\
\hline $0.47 \mathrm{NA}$ & NA & NA & NA & & Gg_rs151183 Gg_rs151251 \\
\hline $0.48 \mathrm{NA}$ & NA & NA & NA & & Gg_rs151183 Gg_rs151251 \\
\hline $0.67 \mathrm{NA}$ & NA & NA & NA & & Gg_rs151183 Gg_rs151251 \\
\hline $0.57 \mathrm{NA}$ & NA & NA & NA & & Gg_rs151183 Gg_rs151231. \\
\hline $0.38 \mathrm{NA}$ & NA & NA & NA & & Gg_rs151183 Gg_rs151225. \\
\hline 0.39 NA & NA & NA & NA & & Gg_rs151183 Gg_rs151251 \\
\hline $0.42 \mathrm{NA}$ & NA & NA & NA & & Gg_rs151183 Gg_rs151251 \\
\hline $0.37 \mathrm{NA}$ & NA & NA & NA & & Gg_rs151183 Gg_rs151251 \\
\hline $0.51 \mathrm{NA}$ & NA & NA & NA & & Gg_rs151183 Gg_rs151251 \\
\hline $0.63 \mathrm{NA}$ & NA & NA & NA & & Gg_rs151183 Gg_rs151251 \\
\hline
\end{tabular}




\begin{tabular}{|c|c|c|c|c|}
\hline $0.48 \mathrm{NA}$ & NA & NA & NA & Gg_rs142082 Gg_rs151251 \\
\hline $0.54 \mathrm{NA}$ & NA & NA & NA & Gg_rs142082 Gg_rs151251 \\
\hline $0.51 \mathrm{NA}$ & NA & NA & NA & Gg_rs151162 Gg_rs151251 \\
\hline $0.29 \mathrm{NA}$ & NA & NA & NA & Gg_rs151183 Gg_rs151251 \\
\hline $0.44 \mathrm{NA}$ & NA & NA & NA & Gg_rs151162 Gg_rs151251 \\
\hline $0.67 \mathrm{NA}$ & NA & NA & NA & Gg_rs151162 Gg_rs142189 \\
\hline $0.37 \mathrm{NA}$ & NA & NA & NA & Gg_rs151183 Gg_rs151251 \\
\hline 0.44 NA & NA & NA & NA & Gg_rs151183 Gg_rs151265 \\
\hline $0.82 \mathrm{NA}$ & NA & NA & NA & Gg_rs151183 Gg_rs151251 \\
\hline $0.27 \mathrm{NA}$ & NA & NA & NA & Gg_rs142082 Gg_rs151251 \\
\hline $0.45 \mathrm{NA}$ & NA & NA & NA & Gg_rs151162 Gg_rs151251 \\
\hline $0.44 \mathrm{NA}$ & NA & NA & NA & Gg_rs151183 Gg_rs151251 \\
\hline $0.3 \mathrm{NA}$ & NA & NA & NA & Gg_rs142082 chr2.8466849 \\
\hline $0.43 \mathrm{NA}$ & NA & NA & NA & Gg_rs151183 Gg_rs151251 \\
\hline $0.57 \mathrm{NA}$ & NA & NA & NA & Gg_rs142082 Gg_rs151251 \\
\hline $0.35 \mathrm{NA}$ & NA & NA & NA & Gg_rs151183 Gg_rs151251 \\
\hline $0.43 \mathrm{NA}$ & NA & NA & NA & Gg_rs151183 Gg_rs151265 \\
\hline $0.29 \mathrm{NA}$ & NA & NA & NA & Gg_rs151183 Gg_rs151251 \\
\hline $0.36 \mathrm{NA}$ & NA & NA & NA & Gg_rs151183 Gg_rs151251 \\
\hline $0.38 \mathrm{NA}$ & NA & NA & NA & Gg_rs151162 Gg_rs142189 \\
\hline $0.54 \mathrm{NA}$ & NA & NA & NA & Gg_rs151183 Gg_rs151265 \\
\hline $0.48 \mathrm{NA}$ & NA & NA & NA & Gg_rs151183 Gg_rs151251 \\
\hline $0.31 \mathrm{NA}$ & NA & NA & NA & Gg_rs151183 Gg_rs151251 \\
\hline 0.42 NA & NA & NA & NA & Gg_rs151183 Gg_rs151251 \\
\hline $0.29 \mathrm{NA}$ & NA & NA & NA & Gg_rs142061 Gg_rs151251 \\
\hline $0.22 \mathrm{NA}$ & NA & NA & NA & Gg_rs151183 Gg_rs151251 \\
\hline $0.46 \mathrm{NA}$ & NA & NA & NA & Gg_rs151183 Gg_rs151251 \\
\hline $0.83 \mathrm{NA}$ & NA & NA & NA & Gg_rs151183 Gg_rs142189 \\
\hline $0.29 \mathrm{NA}$ & NA & NA & NA & Gg_rs151183 Gg_rs151251 \\
\hline $0.41 \mathrm{NA}$ & NA & NA & NA & Gg_rs151183 Gg_rs151251 \\
\hline $0.45 \mathrm{NA}$ & NA & NA & NA & Gg_rs151162 Gg_rs151251 \\
\hline $0.54 \mathrm{NA}$ & NA & NA & NA & Gg_rs151162 Gg_rs151251 \\
\hline $0.67 \mathrm{NA}$ & NA & NA & NA & Gg_rs151162 Gg_rs151265 \\
\hline $0.37 \mathrm{NA}$ & NA & NA & NA & Gg_rs151183 Gg_rs151251 \\
\hline $0.91 \mathrm{NA}$ & NA & NA & NA & Gg_rs151183 Gg_rs151251 \\
\hline $0.52 \mathrm{NA}$ & NA & NA & NA & Gg_rs151162 Gg_rs151251 \\
\hline $0.36 \mathrm{NA}$ & NA & NA & NA & Gg_rs151162 Gg_rs142189 \\
\hline $0.72 \mathrm{NA}$ & NA & NA & NA & Gg_rs151183 Gg_rs151251 \\
\hline $0.45 \mathrm{NA}$ & NA & NA & NA & Gg_rs151162 Gg_rs151251 \\
\hline $0.42 \mathrm{NA}$ & NA & NA & NA & Gg_rs151183 Gg_rs151251 \\
\hline $0.48 \mathrm{NA}$ & NA & NA & NA & Gg_rs151162 Gg_rs151251 \\
\hline $0.49 \mathrm{NA}$ & NA & NA & NA & Gg_rs151183 Gg_rs151251 \\
\hline $0.53 \mathrm{NA}$ & NA & NA & NA & Gg_rs151162 Gg_rs151251 \\
\hline
\end{tabular}




\begin{tabular}{|c|c|c|c|c|c|}
\hline $0.83 \mathrm{NA}$ & NA & NA & NA & & Gg_rs151162 Gg_rs151251 \\
\hline $0.68 \mathrm{NA}$ & NA & NA & NA & & Gg_rs151162 Gg_rs151251 \\
\hline $0.58 \mathrm{NA}$ & NA & NA & NA & & Gg_rs151183 Gg_rs151251 \\
\hline $0.37 \mathrm{NA}$ & NA & NA & NA & & Gg_rs151183 Gg_rs151251 \\
\hline $0.36 \mathrm{NA}$ & NA & NA & NA & & Gg_rs151162 Gg_rs151251 \\
\hline $0.4 \mathrm{NA}$ & NA & NA & NA & & Gg_rs151162 Gg_rs151251 \\
\hline $0.36 \mathrm{NA}$ & NA & NA & NA & & Gg_rs151183 Gg_rs151265 \\
\hline $0.37 \mathrm{NA}$ & NA & NA & NA & & Gg_rs151183 Gg_rs151251 \\
\hline $0.52 \mathrm{NA}$ & NA & NA & NA & & Gg_rs151162 Gg_rs151265 \\
\hline $0.54 \mathrm{NA}$ & NA & NA & NA & & Gg_rs151162 Gg_rs142189. \\
\hline 0.63 NA & NA & NA & NA & & Gg_rs151162 Gg_rs151251 \\
\hline $0.39 \mathrm{NA}$ & NA & NA & NA & & Gg_rs151162 Gg_rs142189. \\
\hline $0.39 \mathrm{NA}$ & NA & NA & NA & & Gg_rs151183 Gg_rs151251 \\
\hline 0.69 NA & NA & NA & NA & & Gg_rs151183Gg_rs151265 \\
\hline 0.82 & 1.3 & 0.77 & 2.18 & 1.09 & Gg_rs151183 Gg_rs151251 \\
\hline $0.39 \mathrm{NA}$ & NA & NA & NA & & Gg_rs142082Gg_rs151251 \\
\hline $0.5 \mathrm{NA}$ & NA & NA & NA & & snp.12.170.1:Gg_rs151251 \\
\hline $0.77 \mathrm{NA}$ & NA & NA & NA & & Gg_rs151183 Gg_rs142189. \\
\hline $0.54 \mathrm{NA}$ & NA & NA & NA & & Gg_rs151183 Gg_rs151265 \\
\hline $0.53 \mathrm{NA}$ & NA & NA & NA & & Gg_rs151183 Gg_rs142189. \\
\hline $0.35 \mathrm{NA}$ & NA & NA & NA & & Gg_rs151183 Gg_rs151265 \\
\hline 0.46 NA & NA & NA & NA & & Gg_rs151183 Gg_rs142189. \\
\hline $0.47 \mathrm{NA}$ & NA & NA & NA & & Gg_rs151183 Gg_rs151265 \\
\hline 0.42 NA & NA & NA & NA & & Gg_rs151183 Gg_rs142189. \\
\hline $0.57 \mathrm{NA}$ & NA & NA & NA & & Gg_rs151183 Gg_rs142189. \\
\hline 0.61 & 1.82 & 0.58 & 1.21 & 0.81 & Gg_rs142082 Gg_rs151251 \\
\hline $0.33 \mathrm{NA}$ & NA & NA & NA & & Gg_rs151183 Gg_rs151251 \\
\hline $0.87 \mathrm{NA}$ & NA & NA & NA & & Gg_rs151183 Gg_rs151265 \\
\hline $0.61 \mathrm{NA}$ & NA & NA & NA & & Gg_rs151183 Gg_rs151265 \\
\hline $0.58 \mathrm{NA}$ & NA & NA & NA & & Gg_rs151162 Gg_rs142189. \\
\hline 0.72 & 0.34 & 0.68 & 2.17 & 0.96 & Gg_rs151183 Gg_rs142189. \\
\hline $0.56 \mathrm{NA}$ & NA & NA & NA & & snp.12.170.1:Gg_rs151251 \\
\hline $0.26 \mathrm{NA}$ & NA & NA & NA & & Gg_rs151162 Gg_rs142189. \\
\hline $0.42 \mathrm{NA}$ & NA & NA & NA & & Gg_rs151183 Gg_rs142189. \\
\hline $0.39 \mathrm{NA}$ & NA & NA & NA & & Gg_rs151162 Gg_rs142189. \\
\hline $0.49 \mathrm{NA}$ & NA & NA & NA & & Gg_rs151183 Gg_rs142189. \\
\hline $0.64 \mathrm{NA}$ & NA & NA & NA & & Gg_rs151162 Gg_rs142189. \\
\hline $0.39 \mathrm{NA}$ & NA & NA & NA & & Gg_rs151183 Gg_rs142189. \\
\hline $0.5 \mathrm{NA}$ & NA & NA & NA & & Gg_rs151183 Gg_rs142189. \\
\hline $0.53 \mathrm{NA}$ & NA & NA & NA & & Gg_rs142082 Gg_rs142189. \\
\hline $0.75 \mathrm{NA}$ & NA & NA & NA & & Gg_rs151183 Gg_rs142189. \\
\hline 0.48 NA & NA & NA & NA & & Gg_rs151183 Gg_rs142189. \\
\hline $0.5 \mathrm{NA}$ & NA & NA & NA & & Gg_rs151183 Gg_rs142189 \\
\hline
\end{tabular}




\begin{tabular}{|c|c|c|c|c|c|}
\hline $0.51 \mathrm{NA}$ & NA & NA & NA & & Gg_rs151162 Gg_rs142189. \\
\hline $0.49 \mathrm{NA}$ & NA & NA & NA & & Gg_rs151162 Gg_rs142189. \\
\hline $0.72 \mathrm{NA}$ & NA & NA & NA & & Gg_rs151183 Gg_rs142189. \\
\hline $0.4 \mathrm{NA}$ & NA & NA & NA & & Gg_rs151183 Gg_rs142189. \\
\hline $0.34 \mathrm{NA}$ & NA & NA & NA & & Gg_rs151162 Gg_rs142209: \\
\hline $0.46 \mathrm{NA}$ & NA & NA & NA & & Gg_rs151183 Gg_rs142189. \\
\hline $0.58 \mathrm{NA}$ & NA & NA & NA & & Gg_rs151183 Gg_rs142189. \\
\hline $0.49 \mathrm{NA}$ & NA & NA & NA & & Gg_rs151162 Gg_rs142189. \\
\hline $0.79 \mathrm{NA}$ & NA & NA & NA & & Gg_rs151183 Gg_rs142189. \\
\hline $0.34 \mathrm{NA}$ & NA & NA & NA & & Gg_rs151183Gg_rs151265 \\
\hline $0.44 \mathrm{NA}$ & NA & NA & NA & & Gg_rs151183 Gg_rs142189. \\
\hline $0.65 \mathrm{NA}$ & NA & NA & NA & & Gg_rs151225 Gg_rs142189. \\
\hline $0.71 \mathrm{NA}$ & NA & NA & NA & & Gg_rs151162 Gg_rs142189. \\
\hline $0.42 \mathrm{NA}$ & NA & NA & NA & & Gg_rs151183 Gg_rs142189. \\
\hline $0.57 \mathrm{NA}$ & NA & NA & NA & & Gg_rs151162 Gg_rs142189. \\
\hline $0.86 \mathrm{NA}$ & NA & NA & NA & & Gg_rs142082 Gg_rs142189. \\
\hline $0.44 \mathrm{NA}$ & NA & NA & NA & & Gg_rs151183 Gg_rs142209: \\
\hline $0.79 \mathrm{NA}$ & NA & NA & NA & & Gg_rs151225 Gg_rs142189. \\
\hline $0.91 \mathrm{NA}$ & NA & NA & NA & & Gg_rs151162 Gg_rs142189. \\
\hline $0.57 \mathrm{NA}$ & NA & NA & NA & & Gg_rs142004 snp.397.7.57! \\
\hline $0.75 \mathrm{NA}$ & NA & NA & NA & & Gg_rs151231 Gg_rs142189. \\
\hline $0.45 \mathrm{NA}$ & NA & NA & NA & & Gg_rs151225 Gg_rs142189. \\
\hline $0.61 \mathrm{NA}$ & NA & NA & NA & & Gg_rs151225 Gg_rs142189. \\
\hline $0.46 \mathrm{NA}$ & NA & NA & NA & & Gg_rs151231 Gg_rs142189. \\
\hline $0.73 \mathrm{NA}$ & NA & NA & NA & & snp.12.170.1:Gg_rs142189. \\
\hline $0.54 \mathrm{NA}$ & NA & NA & NA & & Gg_rs151231 Gg_rs142189. \\
\hline $0.65 \mathrm{NA}$ & NA & NA & NA & & Gg_rs142082 Gg_rs142189. \\
\hline $0.49 \mathrm{NA}$ & NA & NA & NA & & Gg_rs151225 Gg_rs142189. \\
\hline $0.75 \mathrm{NA}$ & NA & NA & NA & & Gg_rs142082 Gg_rs142189. \\
\hline $0.56 \mathrm{NA}$ & NA & NA & NA & & Gg_rs151231 Gg_rs142189. \\
\hline $0.44 \mathrm{NA}$ & NA & NA & NA & & Gg_rs151183 Gg_rs142189. \\
\hline $0.44 \mathrm{NA}$ & NA & NA & NA & & Gg_rs151231 Gg_rs142189. \\
\hline $0.44 \mathrm{NA}$ & NA & NA & NA & & Gg_rs151231 Gg_rs142189. \\
\hline $0.59 \mathrm{NA}$ & NA & NA & NA & & Gg_rs151231 Gg_rs142189. \\
\hline $0.46 \mathrm{NA}$ & NA & NA & NA & & Gg_rs151183 Gg_rs142189. \\
\hline 0.51 & -0.43 & 0.46 & 1.39 & 0.67 & snp.12.170.1:Gg_rs142189. \\
\hline $0.66 \mathrm{NA}$ & NA & NA & NA & & Gg_rs151231 Gg_rs142189. \\
\hline 0.82 & 1.97 & 0.74 & 2.05 & 1.08 & Gg_rs151231 Gg_rs142189. \\
\hline $0.71 \mathrm{NA}$ & NA & NA & NA & & Gg_rs151231 Gg_rs142189. \\
\hline $0.7 \mathrm{NA}$ & NA & NA & NA & & Gg_rs151231 Gg_rs142189. \\
\hline $0.78 \mathrm{NA}$ & NA & NA & NA & & Gg_rs151231 Gg_rs142189. \\
\hline $0.59 \mathrm{NA}$ & NA & NA & NA & & Gg_rs151225 Gg_rs142189. \\
\hline $1 \mathrm{NA}$ & NA & NA & NA & & Gg_rs151231 Gg_rs142189. \\
\hline
\end{tabular}




\begin{tabular}{|c|c|c|c|c|c|c|}
\hline $0.63 \mathrm{NA}$ & NA & & NA & NA & & Gg_rs151225 Gg_rs142189. \\
\hline 0.68 & 1.77 & 0.62 & & -0.03 & 0.9 & Gg_rs151231 Gg_rs142189. \\
\hline 1.12 & 1.71 & 1.01 & & 1.35 & 1.48 & Gg_rs151162 Gg_rs142209: \\
\hline $0.82 \mathrm{NA}$ & NA & & NA & NA & & Gg_rs151231 Gg_rs142189. \\
\hline $0.74 \mathrm{NA}$ & NA & & NA & NA & & Gg_rs151251 Gg_rs142189. \\
\hline $0.54 \mathrm{NA}$ & NA & & NA & NA & & Gg_rs151183 Gg_rs142189. \\
\hline 0.62 NA & NA & & NA & NA & & Gg_rs151183 Gg_rs142189. \\
\hline $0.5 \mathrm{NA}$ & NA & & NA & NA & & Gg_rs151225 Gg_rs142189. \\
\hline $0.46 \mathrm{NA}$ & NA & & NA & NA & & Gg_rs151183 Gg_rs142189. \\
\hline $0.71 \mathrm{NA}$ & NA & & NA & NA & & Gg_rs151183 Gg_rs142189. \\
\hline 0.69 NA & NA & & NA & NA & & Gg_rs151231 Gg_rs142189. \\
\hline $0.4 \mathrm{NA}$ & NA & & NA & NA & & Gg_rs151225 Gg_rs142189. \\
\hline $1.1 \mathrm{NA}$ & NA & & NA & NA & & Gg_rs151225 Gg_rs142189. \\
\hline $0.5 \mathrm{NA}$ & NA & & NA & NA & & Gg_rs151231 Gg_rs142189. \\
\hline $0.5 \mathrm{NA}$ & NA & & NA & NA & & Gg_rs151231 Gg_rs142189. \\
\hline 0.71 & 1.95 & 0.65 & & -0.52 & 0.94 & Gg_rs142082 Gg_rs142189. \\
\hline $0.62 \mathrm{NA}$ & NA & & NA & NA & & Gg_rs151231 Gg_rs142189. \\
\hline $0.67 \mathrm{NA}$ & NA & & NA & NA & & Gg_rs151231 Gg_rs142209: \\
\hline $0.62 \mathrm{NA}$ & NA & & NA & NA & & snp.12.170.1:Gg_rs142189. \\
\hline 0.65 & 1.22 & 0.59 & & 2.99 & 0.86 & Gg_rs151231 Gg_rs142189. \\
\hline 1.06 & 1.59 & 0.96 & & 1.02 & 1.39 & Gg_rs151231 Gg_rs142209: \\
\hline $0.65 \mathrm{NA}$ & NA & & NA & NA & & Gg_rs151231 Gg_rs142189. \\
\hline $0.41 \mathrm{NA}$ & NA & & NA & NA & & Gg_rs151231 Gg_rs142189. \\
\hline $0.67 \mathrm{NA}$ & NA & & NA & NA & & Gg_rs151231 Gg_rs142189. \\
\hline $0.68 \mathrm{NA}$ & NA & & NA & NA & & Gg_rs151231 Gg_rs142189. \\
\hline 0.39 NA & NA & & NA & NA & & Gg_rs151231 Gg_rs142223 \\
\hline 0.76 & 0.74 & 0.68 & & -2.22 & 1.03 & Gg_rs151225 snp.397.7.57! \\
\hline $0.28 \mathrm{NA}$ & NA & & NA & NA & & Gg_rs151162 Gg_rs142209: \\
\hline $0.65 \mathrm{NA}$ & NA & & NA & NA & & Gg_rs151231 Gg_rs142189. \\
\hline 0.83 NA & NA & & NA & NA & & Gg_rs151231 Gg_rs142209: \\
\hline 1.01 & 1.83 & 0.92 & & 1.9 & 1.33 & Gg_rs151231 Gg_rs142223 \\
\hline 0.87 NA & NA & & NA & NA & & Gg_rs151231 snp.397.7.57! \\
\hline 0.63 & 0.96 & 0.57 & & 1.77 & 0.83 & Gg_rs151225 Gg_rs107232. \\
\hline $1.11 \mathrm{NA}$ & NA & & NA & NA & & Gg_rs151183 snp.397.7.57! \\
\hline $0.56 \mathrm{NA}$ & NA & & NA & NA & & Gg_rs151251 Gg_rs107232. \\
\hline $0.78 \mathrm{NA}$ & NA & & NA & NA & & Gg_rs151231 Gg_rs142209: \\
\hline 0.63 NA & NA & & NA & NA & & Gg_rs151265 Gg_rs142209: \\
\hline $0.4 \mathrm{NA}$ & NA & & NA & NA & & Gg_rs151225 Gg_rs107232. \\
\hline $1.07 \mathrm{NA}$ & NA & & NA & NA & & Gg_rs151251 Gg_rs107232. \\
\hline $1.52 \mathrm{NA}$ & NA & & NA & NA & & Gg_rs151225 snp.397.7.57! \\
\hline $0.39 \mathrm{NA}$ & NA & & NA & NA & & Gg_rs151231 Gg_rs142209: \\
\hline $1.67 \mathrm{NA}$ & NA & & NA & NA & & Gg_rs151183 Gg_rs142209: \\
\hline $0.98 \mathrm{NA}$ & NA & & NA & NA & & Gg_rs151265 Gg_rs142209: \\
\hline
\end{tabular}




\begin{tabular}{|c|c|c|c|c|c|c|}
\hline $0.55 \mathrm{NA}$ & NA & & NA & NA & & Gg_rs151225 Gg_rs142209: \\
\hline $0.78 \mathrm{NA}$ & NA & & NA & NA & & Gg_rs151231 snp.397.7.57! \\
\hline $0.82 \mathrm{NA}$ & NA & & NA & NA & & Gg_rs151231 snp.397.7.57! \\
\hline $1.41 \mathrm{NA}$ & NA & & NA & NA & & Gg_rs151265 Gg_rs142209: \\
\hline $0.79 \mathrm{NA}$ & NA & & NA & NA & & Gg_rs151265 Gg_rs142209: \\
\hline $0.78 \mathrm{NA}$ & NA & & NA & NA & & Gg_rs151251 Gg_rs142209: \\
\hline $0.62 \mathrm{NA}$ & NA & & NA & NA & & Gg_rs151231 snp.397.7.57! \\
\hline 1.68 & 2.72 & 1.49 & & -0.54 & 2.26 & Gg_rs151265 snp.397.7.57! \\
\hline $0.86 \mathrm{NA}$ & NA & & NA & NA & & Gg_rs151265 Gg_rs142209: \\
\hline 0.86 NA & NA & & NA & NA & & Gg_rs142061 Gg_rs142209: \\
\hline 0.65 & 2.29 & 0.55 & & 0.19 & 0.88 & Gg_rs151265 Gg_rs142209: \\
\hline $0.53 \mathrm{NA}$ & NA & & NA & NA & & Gg_rs151265 Gg_rs142209: \\
\hline 0.99 & 2.47 & 0.83 & & 1.11 & 1.34 & Gg_rs151265 Gg_rs142209: \\
\hline $0.56 \mathrm{NA}$ & NA & & NA & NA & & Gg_rs151265 Gg_rs107232 \\
\hline 1.2 & 3.37 & 1.01 & & -0.18 & 1.63 & Gg_rs151265 Gg_rs142209: \\
\hline 0.85 & 1.93 & 0.72 & & 2.63 & 1.16 & Gg_rs151265 Gg_rs142209: \\
\hline $0.45 \mathrm{NA}$ & NA & & NA & NA & & Gg_rs151265 Gg_rs142209: \\
\hline $0.52 \mathrm{NA}$ & NA & & NA & NA & & Gg_rs151265 Gg_rs142209: \\
\hline 0.7 & -1.22 & 0.58 & & -2.38 & 0.94 & Gg_rs151265 Gg_rs142209: \\
\hline 0.81 & -0.54 & 0.68 & & 2.59 & 1.1 & Gg_rs151265 Gg_rs142209: \\
\hline 1.35 & 3.93 & 1.13 & & -0.55 & 1.83 & Gg_rs151265 Gg_rs142209: \\
\hline 0.99 & 3.26 & 0.84 & & -1.69 & 1.35 & Gg_rs151265 Gg_rs142209: \\
\hline 1.02 & 4.21 & 0.85 & & 0.37 & 1.38 & Gg_rs151265 Gg_rs142209: \\
\hline 1.16 & 2.07 & 0.97 & & 1.98 & 1.57 & Gg_rs151265 Gg_rs142209: \\
\hline $0.41 \mathrm{NA}$ & NA & & NA & NA & & Gg_rs151265 Gg_rs142209: \\
\hline 0.56 & 2.28 & 0.47 & & 1.48 & 0.76 & Gg_rs151265 Gg_rs142209: \\
\hline 0.86 & 1.57 & 0.72 & & 0.62 & 1.17 & Gg_rs151265 snp.397.7.57! \\
\hline $0.54 \mathrm{NA}$ & NA & & NA & NA & & Gg_rs151265 Gg_rs142223 \\
\hline $0.57 \mathrm{NA}$ & NA & & NA & NA & & Gg_rs151265 Gg_rs142209: \\
\hline 1.47 & 1.72 & 1.33 & & -1.84 & 1.96 & Gg_rs151251 snp.397.7.57! \\
\hline 1.43 & 2.49 & 1.2 & & 2.34 & 1.95 & Gg_rs151265 Gg_rs142223 \\
\hline $0.32 \mathrm{NA}$ & NA & & NA & NA & & Gg_rs151265 snp.397.7.57! \\
\hline $0.51 \mathrm{NA}$ & NA & & NA & NA & & Gg_rs151265 Gg_rs142223 \\
\hline $0.57 \mathrm{NA}$ & NA & & NA & NA & & Gg_rs151265 Gg_rs107232 \\
\hline 0.79 NA & NA & & NA & NA & & Gg_rs151265 Gg_rs107232. \\
\hline 1.19 & 1.52 & 1.08 & & -1.48 & 1.58 & Gg_rs151251 snp.397.7.57! \\
\hline $0.64 \mathrm{NA}$ & NA & & NA & NA & & Gg_rs151265 snp.397.7.57! \\
\hline $0.54 \mathrm{NA}$ & NA & & NA & NA & & Gg_rs151265 snp.397.7.57! \\
\hline $0.35 \mathrm{NA}$ & NA & & NA & NA & & Gg_rs151231 snp.397.7.57! \\
\hline $0.69 \mathrm{NA}$ & NA & & NA & NA & & Gg_rs151265 snp.397.7.57! \\
\hline 0.96 & 1.11 & 0.85 & & -1.26 & 1.3 & Gg_rs151231 snp.397.7.57! \\
\hline 0.44 NA & NA & & NA & NA & & Gg_rs151265 Gg_rs107232. \\
\hline $0.71 \mathrm{NA}$ & NA & & NA & NA & & Gg_rs151162 Gg_rs142253 \\
\hline
\end{tabular}




\begin{tabular}{|c|c|c|c|c|c|}
\hline $0.43 \mathrm{NA}$ & NA & NA & NA & & Gg_rs151231 Gg_rs142329 \\
\hline $0.43 \mathrm{NA}$ & NA & NA & NA & & Gg_rs151265 snp.397.7.57! \\
\hline 0.42 NA & NA & NA & NA & & Gg_rs151251 snp.397.7.57! \\
\hline $0.4 \mathrm{NA}$ & NA & NA & NA & & Gg_rs151265 snp.397.7.57! \\
\hline $0.59 \mathrm{NA}$ & NA & NA & NA & & Gg_rs151265 snp.397.7.57! \\
\hline $0.51 \mathrm{NA}$ & NA & NA & NA & & Gg_rs142189 snp.397.7.57! \\
\hline $0.57 \mathrm{NA}$ & NA & NA & NA & & Gg_rs142189 Gg_rs107232. \\
\hline $0.71 \mathrm{NA}$ & NA & NA & NA & & Gg_rs151265 snp.397.7.57! \\
\hline $0.64 \mathrm{NA}$ & NA & NA & NA & & Gg_rs151265 snp.397.7.57! \\
\hline $0.76 \mathrm{NA}$ & NA & NA & NA & & Gg_rs151265 Gg_rs107232. \\
\hline $0.55 \mathrm{NA}$ & NA & NA & NA & & Gg_rs151265 snp.397.7.57! \\
\hline $0.66 \mathrm{NA}$ & NA & NA & NA & & Gg_rs142189 Gg_rs107232 \\
\hline $0.5 \mathrm{NA}$ & NA & NA & NA & & Gg_rs142189 Gg_rs107232. \\
\hline $0.92 \mathrm{NA}$ & NA & NA & NA & & Gg_rs142189 Gg_rs107232. \\
\hline $0.52 \mathrm{NA}$ & NA & NA & NA & & Gg_rs142189 snp.397.7.57! \\
\hline 0.66 & 1.76 & 0.58 & 2.48 & 0.89 & Gg_rs142189 snp.397.7.57! \\
\hline 4.71 NA & NA & NA & NA & & Gg_rs142189 snp.397.7.57! \\
\hline $7.11 \mathrm{NA}$ & NA & NA & NA & & Gg_rs142189 snp.397.7.57! \\
\hline $6.71 \mathrm{NA}$ & NA & NA & NA & & Gg_rs142189 snp.397.7.57! \\
\hline 4.52 NA & NA & NA & NA & & Gg_rs142189 snp.397.7.57! \\
\hline 6.44 NA & NA & NA & NA & & Gg_rs142189 snp.397.7.57! \\
\hline $7.63 \mathrm{NA}$ & NA & NA & NA & & Gg_rs142189 snp.397.7.57! \\
\hline $6.79 \mathrm{NA}$ & NA & NA & NA & & Gg_rs142189 snp.397.7.57! \\
\hline 0.73 NA & NA & NA & NA & & Gg_rs142189 Gg_rs107232. \\
\hline $0.84 \mathrm{NA}$ & NA & NA & NA & & Gg_rs142189 snp.397.7.57! \\
\hline 8.58 NA & NA & NA & NA & & Gg_rs142189 snp.397.7.57! \\
\hline $6.14 \mathrm{NA}$ & NA & NA & NA & & Gg_rs142189 snp.397.7.57! \\
\hline $6.28 \mathrm{NA}$ & NA & NA & NA & & Gg_rs142189 snp.397.7.57! \\
\hline 7.74 NA & NA & NA & NA & & Gg_rs142189 snp.397.7.57! \\
\hline 4.63 NA & NA & NA & NA & & Gg_rs142189 snp.397.7.57! \\
\hline $3.21 \mathrm{NA}$ & NA & NA & NA & & Gg_rs142189 Gg_rs107232. \\
\hline 8.05 NA & NA & NA & NA & & Gg_rs142189 snp.397.7.57! \\
\hline $6.81 \mathrm{NA}$ & NA & NA & NA & & Gg_rs142189 snp.397.7.57! \\
\hline $7.29 \mathrm{NA}$ & NA & NA & NA & & Gg_rs142189 snp.397.7.57! \\
\hline $8.34 \mathrm{NA}$ & NA & NA & NA & & Gg_rs142189 snp.397.7.57! \\
\hline $5.78 \mathrm{NA}$ & NA & NA & NA & & Gg_rs142189 snp.397.7.57! \\
\hline $5.03 \mathrm{NA}$ & NA & NA & NA & & Gg_rs142189 snp.397.7.57! \\
\hline $2.05 \mathrm{NA}$ & NA & NA & NA & & Gg_rs142189 snp.397.7.57! \\
\hline $6.7 \mathrm{NA}$ & NA & NA & NA & & Gg_rs142189 snp.397.7.57! \\
\hline $7.96 \mathrm{NA}$ & NA & NA & NA & & Gg_rs142189 snp.397.7.57! \\
\hline 7.98 NA & NA & NA & NA & & Gg_rs142189 snp.397.7.57! \\
\hline $5.3 \mathrm{NA}$ & NA & NA & NA & & Gg_rs142189 snp.397.7.57! \\
\hline $0.47 \mathrm{NA}$ & NA & NA & NA & & Gg_rs142189 snp.397.7.57! \\
\hline
\end{tabular}




\begin{tabular}{|c|c|c|c|c|c|}
\hline $6.38 \mathrm{NA}$ & NA & NA & NA & & Gg_rs142189 Gg_rs142329 \\
\hline $7.76 \mathrm{NA}$ & NA & NA & NA & & Gg_rs142189 Gg_rs142329 \\
\hline 7.05 NA & NA & NA & NA & & Gg_rs142189 Gg_rs142329 \\
\hline $7.08 \mathrm{NA}$ & NA & NA & NA & & Gg_rs142189 Gg_rs142329 \\
\hline $7.17 \mathrm{NA}$ & NA & NA & NA & & Gg_rs142189 snp.397.7.57! \\
\hline 4.91 NA & NA & NA & NA & & Gg_rs142189 snp.397.7.57! \\
\hline $0.5 \mathrm{NA}$ & NA & NA & NA & & Gg_rs142209 Gg_rs107232. \\
\hline $0.77 \mathrm{NA}$ & NA & NA & NA & & Gg_rs142189 Gg_rs107232. \\
\hline $0.39 \mathrm{NA}$ & NA & NA & NA & & Gg_rs142189 Gg_rs107232 \\
\hline $0.28 \mathrm{NA}$ & NA & NA & NA & & Gg_rs142189 Gg_rs107232. \\
\hline $0.32 \mathrm{NA}$ & NA & NA & NA & & Gg_rs142189 snp.397.7.57! \\
\hline 0.28 & -0.27 & 0.25 & 0.9 & 0.38 & Gg_rs142189 Gg_rs107232 \\
\hline $1.06 \mathrm{NA}$ & NA & NA & NA & & Gg_rs142189 snp.397.7.57! \\
\hline $0.72 \mathrm{NA}$ & NA & NA & NA & & Gg_rs142189 Gg_rs107232. \\
\hline $0.74 \mathrm{NA}$ & NA & NA & NA & & Gg_rs142189 snp.397.7.57! \\
\hline $0.85 \mathrm{NA}$ & NA & NA & NA & & Gg_rs142189 Gg_rs107232. \\
\hline $0.46 \mathrm{NA}$ & NA & NA & NA & & Gg_rs142189 Gg_rs107232. \\
\hline $0.24 \mathrm{NA}$ & NA & NA & NA & & Gg_rs142189 Gg_rs107232. \\
\hline $0.45 \mathrm{NA}$ & NA & NA & NA & & Gg_rs142189 snp.397.7.57! \\
\hline $0.41 \mathrm{NA}$ & NA & NA & NA & & Gg_rs142189 Gg_rs107232. \\
\hline $0.16 \mathrm{NA}$ & NA & NA & NA & & Gg_rs142189 Gg_rs107232. \\
\hline 0.44 NA & NA & NA & NA & & Gg_rs142189 snp.397.7.57! \\
\hline 0.43 NA & NA & NA & NA & & Gg_rs151265 snp.397.7.57! \\
\hline $0.32 \mathrm{NA}$ & NA & NA & NA & & Gg_rs142209 Gg_rs107232. \\
\hline $1.28 \mathrm{NA}$ & NA & NA & NA & & Gg_rs151265 snp.397.7.57! \\
\hline $0.5 \mathrm{NA}$ & NA & NA & NA & & Gg_rs142189 snp.397.7.57! \\
\hline $0.32 \mathrm{NA}$ & NA & NA & NA & & Gg_rs142189 snp.397.7.57! \\
\hline $0.38 \mathrm{NA}$ & NA & NA & NA & & Gg_rs151265 snp.397.7.57! \\
\hline $0.35 \mathrm{NA}$ & NA & NA & NA & & Gg_rs142189 snp.397.7.57! \\
\hline $0.54 \mathrm{NA}$ & NA & NA & NA & & Gg_rs151265 snp.397.7.57! \\
\hline 0.32 & 1.17 & 0.28 & 1.25 & 0.43 & Gg_rs142209 snp.397.7.57! \\
\hline $0.5 \mathrm{NA}$ & NA & NA & NA & & Gg_rs142209 Gg_rs107232. \\
\hline $0.37 \mathrm{NA}$ & NA & NA & NA & & Gg_rs142189 snp.397.7.57! \\
\hline $0.88 \mathrm{NA}$ & NA & NA & NA & & Gg_rs142189 snp.397.7.57! \\
\hline $0.46 \mathrm{NA}$ & NA & NA & NA & & Gg_rs151265 snp.397.7.57! \\
\hline $0.48 \mathrm{NA}$ & NA & NA & NA & & Gg_rs151265 Gg_rs142329 \\
\hline $0.31 \mathrm{NA}$ & NA & NA & NA & & Gg_rs151265 snp.397.7.57! \\
\hline $0.35 \mathrm{NA}$ & NA & NA & NA & & Gg_rs142189 snp.397.7.57! \\
\hline $0.31 \mathrm{NA}$ & NA & NA & NA & & Gg_rs142189 snp.397.7.57! \\
\hline $0.56 \mathrm{NA}$ & NA & NA & NA & & Gg_rs151265 snp.397.7.57! \\
\hline $0.31 \mathrm{NA}$ & NA & NA & NA & & Gg_rs142189 snp.397.7.57! \\
\hline $0.38 \mathrm{NA}$ & NA & NA & NA & & Gg_rs142189 snp.397.7.57! \\
\hline $0.72 \mathrm{NA}$ & NA & NA & NA & & Gg_rs142189 snp.397.7.57! \\
\hline
\end{tabular}




\begin{tabular}{|c|c|c|c|c|c|}
\hline $0.16 \mathrm{NA}$ & NA & NA & NA & & Gg_rs142189 snp.397.7.57! \\
\hline 0.44 NA & NA & NA & NA & & Gg_rs142189 snp.397.7.57! \\
\hline 0.42 NA & NA & NA & NA & & Gg_rs142189 snp.397.7.57! \\
\hline $0.38 \mathrm{NA}$ & NA & NA & NA & & Gg_rs142189 snp.397.7.57! \\
\hline $0.3 \mathrm{NA}$ & NA & NA & NA & & Gg_rs142189 snp.397.7.57! \\
\hline $0.48 \mathrm{NA}$ & NA & NA & NA & & Gg_rs142189 snp.397.7.57! \\
\hline $0.31 \mathrm{NA}$ & NA & NA & NA & & Gg_rs151265 snp.397.7.57! \\
\hline $0.38 \mathrm{NA}$ & NA & NA & NA & & Gg_rs142189Gg_rs142329 \\
\hline $0.49 \mathrm{NA}$ & NA & NA & NA & & Gg_rs142189 snp.397.7.57! \\
\hline $0.58 \mathrm{NA}$ & NA & NA & NA & & Gg_rs142189 snp.397.7.57! \\
\hline $0.48 \mathrm{NA}$ & NA & NA & NA & & Gg_rs142189 snp.397.7.57! \\
\hline $0.44 \mathrm{NA}$ & NA & NA & NA & & Gg_rs142189 snp.397.7.57! \\
\hline $0.45 \mathrm{NA}$ & NA & NA & NA & & Gg_rs151265 snp.397.7.57! \\
\hline $0.38 \mathrm{NA}$ & NA & NA & NA & & Gg_rs142189 snp.397.7.57! \\
\hline 0.65 & 1.45 & 0.59 & 0.8 & 0.87 & Gg_rs142189 snp.397.7.57! \\
\hline 0.64 NA & NA & NA & NA & & Gg_rs142189 snp.397.7.57! \\
\hline $0.43 \mathrm{NA}$ & NA & NA & NA & & Gg_rs142189 Gg_rs142329 \\
\hline $0.38 \mathrm{NA}$ & NA & NA & NA & & Gg_rs142189 Gg_rs142329 \\
\hline $0.65 \mathrm{NA}$ & NA & NA & NA & & Gg_rs142189 snp.397.7.57! \\
\hline $0.35 \mathrm{NA}$ & NA & NA & NA & & Gg_rs142189 snp.397.7.57! \\
\hline 0.48 NA & NA & NA & NA & & Gg_rs151231 snp.397.7.57! \\
\hline $0.6 \mathrm{NA}$ & NA & NA & NA & & Gg_rs142189 snp.397.7.57! \\
\hline $0.41 \mathrm{NA}$ & NA & NA & NA & & Gg_rs142189 snp.397.7.57! \\
\hline $0.36 \mathrm{NA}$ & NA & NA & NA & & Gg_rs151265 snp.397.7.57! \\
\hline 0.52 & 1.02 & 0.47 & 1.45 & 0.69 & Gg_rs142223 snp.397.7.57! \\
\hline $0.45 \mathrm{NA}$ & NA & NA & NA & & Gg_rs142209 snp.397.7.57! \\
\hline $0.54 \mathrm{NA}$ & NA & NA & NA & & Gg_rs142189 snp.397.7.57! \\
\hline $0.47 \mathrm{NA}$ & NA & NA & NA & & Gg_rs142223 snp.397.7.57! \\
\hline 0.69 NA & NA & NA & NA & & Gg_rs142189 snp.397.7.57! \\
\hline $0.39 \mathrm{NA}$ & NA & NA & NA & & Gg_rs142223 snp.397.7.57! \\
\hline $0.51 \mathrm{NA}$ & NA & NA & NA & & Gg_rs151265 snp.397.7.57! \\
\hline $0.34 \mathrm{NA}$ & NA & NA & NA & & Gg_rs142189 Gg_rs142253 \\
\hline $0.36 \mathrm{NA}$ & NA & NA & NA & & Gg_rs142189 snp.397.7.57! \\
\hline $0.5 \mathrm{NA}$ & NA & NA & NA & & Gg_rs142189 snp.397.7.57! \\
\hline $0.35 \mathrm{NA}$ & NA & NA & NA & & Gg_rs142189 snp.397.7.57! \\
\hline $0.61 \mathrm{NA}$ & NA & NA & NA & & Gg_rs142209 snp.397.7.57! \\
\hline $0.42 \mathrm{NA}$ & NA & NA & NA & & Gg_rs142223 snp.397.7.57! \\
\hline $0.38 \mathrm{NA}$ & NA & NA & NA & & Gg_rs142189 snp.397.7.57! \\
\hline $0.67 \mathrm{NA}$ & NA & NA & NA & & Gg_rs142189 Gg_rs142253 \\
\hline $0.47 \mathrm{NA}$ & NA & NA & NA & & Gg_rs142189 snp.397.7.57! \\
\hline $0.36 \mathrm{NA}$ & NA & NA & NA & & Gg_rs142189 snp.397.7.57! \\
\hline $0.59 \mathrm{NA}$ & NA & NA & NA & & Gg_rs142223 snp.397.7.57! \\
\hline 1.38 & 1.74 & 1.26 & -2.31 & 1.84 & Gg_rs142209 snp.397.7.57! \\
\hline
\end{tabular}




\begin{tabular}{|c|c|c|c|c|}
\hline $0.44 \mathrm{NA}$ & NA & NA & NA & Gg_rs142189 X2_10635231 \\
\hline $0.45 \mathrm{NA}$ & NA & NA & NA & Gg_rs142223 Gg_rs142253 \\
\hline $0.58 \mathrm{NA}$ & NA & NA & NA & Gg_rs142189 Gg_rs142329 \\
\hline $0.45 \mathrm{NA}$ & NA & NA & NA & Gg_rs142189 snp.397.7.57! \\
\hline 0.65 NA & NA & NA & NA & Gg_rs142209 Gg_rs142329 \\
\hline $0.54 \mathrm{NA}$ & NA & NA & NA & Gg_rs142223 X2_10700517 \\
\hline $0.96 \mathrm{NA}$ & NA & NA & NA & Gg_rs151251Gg_rs142329 \\
\hline $0.97 \mathrm{NA}$ & NA & NA & NA & Gg_rs142209 Gg_rs142329 \\
\hline $0.44 \mathrm{NA}$ & NA & NA & NA & Gg_rs151265 Gg_rs142329 \\
\hline $0.59 \mathrm{NA}$ & NA & NA & NA & Gg_rs151265 Gg_rs142329 \\
\hline $0.57 \mathrm{NA}$ & NA & NA & NA & Gg_rs142223 Gg_rs142329 \\
\hline $0.25 \mathrm{NA}$ & NA & NA & NA & Gg_rs142223 Gg_rs142329 \\
\hline $0.41 \mathrm{NA}$ & NA & NA & NA & Gg_rs142189 Gg_rs142329 \\
\hline $0.56 \mathrm{NA}$ & NA & NA & NA & Gg_rs151265 Gg_rs142329 \\
\hline $0.63 \mathrm{NA}$ & NA & NA & NA & Gg_rs142223 Gg_rs142329 \\
\hline $0.44 \mathrm{NA}$ & NA & NA & NA & Gg_rs107232 Gg_rs142253 \\
\hline 0.69 NA & NA & NA & NA & Gg_rs142209 X2_10635231 \\
\hline $0.71 \mathrm{NA}$ & NA & NA & NA & Gg_rs107232 X2_10700517 \\
\hline $0.72 \mathrm{NA}$ & NA & NA & NA & Gg_rs142209 Gg_rs142329 \\
\hline $0.72 \mathrm{NA}$ & NA & NA & NA & Gg_rs107232 X2_10635231 \\
\hline $0.71 \mathrm{NA}$ & NA & NA & NA & Gg_rs107232 Gg_rs142253 \\
\hline $0.58 \mathrm{NA}$ & NA & NA & NA & Gg_rs107232 Gg_rs142329 \\
\hline $0.48 \mathrm{NA}$ & NA & NA & NA & Gg_rs107232 Gg_rs142329 \\
\hline 0.43 NA & NA & NA & NA & Gg_rs107232 X2_10700517 \\
\hline $0.8 \mathrm{NA}$ & NA & NA & NA & Gg_rs142209 Gg_rs142329 \\
\hline $0.52 \mathrm{NA}$ & NA & NA & NA & Gg_rs107232 Gg_rs142329 \\
\hline $0.48 \mathrm{NA}$ & NA & NA & NA & Gg_rs107232 X2_10700517 \\
\hline $1.33 \mathrm{NA}$ & NA & NA & NA & Gg_rs107232 Gg_rs142329 \\
\hline $1.03 \mathrm{NA}$ & NA & NA & NA & Gg_rs142223 Gg_rs142329 \\
\hline 0.86 NA & NA & NA & NA & Gg_rs107232 Gg_rs142329 \\
\hline 0.46 NA & NA & NA & NA & Gg_rs107232 Gg_rs142329 \\
\hline 0.73 NA & NA & NA & NA & Gg_rs107232 Gg_rs142329 \\
\hline $0.34 \mathrm{NA}$ & NA & NA & NA & Gg_rs107232 Gg_rs142329 \\
\hline $0.57 \mathrm{NA}$ & NA & NA & NA & Gg_rs107232 Gg_rs142329 \\
\hline $0.57 \mathrm{NA}$ & NA & NA & NA & Gg_rs107232 Gg_rs142329 \\
\hline $0.57 \mathrm{NA}$ & NA & NA & NA & Gg_rs142189 Gg_rs142329 \\
\hline $0.4 \mathrm{NA}$ & NA & NA & NA & Gg_rs107232 Gg_rs142329 \\
\hline 0.69 NA & NA & NA & NA & Gg_rs142209 Gg_rs142329 \\
\hline $0.57 \mathrm{NA}$ & NA & NA & NA & Gg_rs107232 Gg_rs142329 \\
\hline $0.46 \mathrm{NA}$ & NA & NA & NA & Gg_rs107232 Gg_rs142329 \\
\hline $0.59 \mathrm{NA}$ & NA & NA & NA & Gg_rs142223 Gg_rs142329 \\
\hline $0.53 \mathrm{NA}$ & NA & NA & NA & Gg_rs107232 Gg_rs142329 \\
\hline $0.57 \mathrm{NA}$ & NA & NA & NA & Gg_rs142209 Gg_rs142329 \\
\hline
\end{tabular}




\begin{tabular}{|c|c|c|c|c|c|}
\hline $1.1 \mathrm{NA}$ & NA & NA & NA & & Gg_rs107232 Gg_rs142329 \\
\hline $0.5 \mathrm{NA}$ & NA & NA & NA & & Gg_rs107232 Gg_rs142329 \\
\hline $0.62 \mathrm{NA}$ & NA & NA & NA & & Gg_rs107232 Gg_rs142329 \\
\hline $0.85 \mathrm{NA}$ & NA & NA & NA & & Gg_rs107232 Gg_rs142329 \\
\hline $0.71 \mathrm{NA}$ & NA & NA & NA & & Gg_rs142223 Gg_rs142329 \\
\hline 0.61 & 1.37 & 0.58 & -0.75 & 0.82 & Gg_rs107232 Gg_rs142329 \\
\hline $0.61 \mathrm{NA}$ & NA & NA & NA & & Gg_rs107232 Gg_rs142329 \\
\hline 0.66 NA & NA & NA & NA & & Gg_rs142223 Gg_rs142329 \\
\hline $0.46 \mathrm{NA}$ & NA & NA & NA & & snp.397.7.57!Gg_rs142329 \\
\hline $0.51 \mathrm{NA}$ & NA & NA & NA & & Gg_rs107232 Gg_rs142329 \\
\hline $0.44 \mathrm{NA}$ & NA & NA & NA & & snp.397.7.57!Gg_rs142329 \\
\hline $0.68 \mathrm{NA}$ & NA & NA & NA & & snp.397.7.57!Gg_rs142329 \\
\hline 0.6 & 1.19 & 0.56 & -1.9 & 0.8 & snp.397.7.57!Gg_rs142329 \\
\hline $0.31 \mathrm{NA}$ & NA & NA & NA & & snp.397.7.57!Gg_rs142329 \\
\hline $0.42 \mathrm{NA}$ & NA & NA & NA & & snp.397.7.57!Gg_rs142329 \\
\hline $0.65 \mathrm{NA}$ & NA & NA & NA & & snp.397.7.57!Gg_rs142329 \\
\hline $0.43 \mathrm{NA}$ & NA & NA & NA & & Gg_rs107232 X2_10700517 \\
\hline $0.56 \mathrm{NA}$ & NA & NA & NA & & Gg_rs107232 Gg_rs142329 \\
\hline $0.54 \mathrm{NA}$ & NA & NA & NA & & Gg_rs142209 Gg_rs142329 \\
\hline $0.31 \mathrm{NA}$ & NA & NA & NA & & Gg_rs107232 Gg_rs142329 \\
\hline $0.36 \mathrm{NA}$ & NA & NA & NA & & snp.397.7.57!Gg_rs142329 \\
\hline $0.41 \mathrm{NA}$ & NA & NA & NA & & Gg_rs142223 Gg_rs142329 \\
\hline $0.37 \mathrm{NA}$ & NA & NA & NA & & Gg_rs142223 Gg_rs142329 \\
\hline $0.37 \mathrm{NA}$ & NA & NA & NA & & Gg_rs142223 Gg_rs142329 \\
\hline 1.39 & 3.97 & 1.09 & -2.8 & 1.96 & snp.397.7.57!Gg_rs142329 \\
\hline $0.36 \mathrm{NA}$ & NA & NA & NA & & Gg_rs142189 Gg_rs142329 \\
\hline 0.46 NA & NA & NA & NA & & Gg_rs142223 Gg_rs142329 \\
\hline $0.49 \mathrm{NA}$ & NA & NA & NA & & snp.397.7.57!Gg_rs142329 \\
\hline 0.82 NA & NA & NA & NA & & snp.397.7.57!Gg_rs142329 \\
\hline $0.29 \mathrm{NA}$ & NA & NA & NA & & Gg_rs107232 Gg_rs142329 \\
\hline 0.83 NA & NA & NA & NA & & Gg_rs142223 Gg_rs142329 \\
\hline $0.54 \mathrm{NA}$ & NA & NA & NA & & Gg_rs142223 Gg_rs142329 \\
\hline 0.86 NA & NA & NA & NA & & snp.397.7.57!Gg_rs142329 \\
\hline $0.74 \mathrm{NA}$ & NA & NA & NA & & snp.397.7.57!Gg_rs142329 \\
\hline $0.64 \mathrm{NA}$ & NA & NA & NA & & Gg_rs107232 Gg_rs142329 \\
\hline 0.85 & 0.89 & 0.7 & 2.11 & 1.17 & Gg_rs107232 Gg_rs142329 \\
\hline 0.79 NA & NA & NA & NA & & Gg_rs107232 Gg_rs142329 \\
\hline $0.76 \mathrm{NA}$ & NA & NA & NA & & Gg_rs142209 Gg_rs142329 \\
\hline $0.58 \mathrm{NA}$ & NA & NA & NA & & Gg_rs107232 Gg_rs142329 \\
\hline $0.79 \mathrm{NA}$ & NA & NA & NA & & snp.397.7.57!Gg_rs142329 \\
\hline 0.79 NA & NA & NA & NA & & Gg_rs107232 Gg_rs142329 \\
\hline $1.42 \mathrm{NA}$ & NA & NA & NA & & snp.397.7.57!Gg_rs142329 \\
\hline $0.86 \mathrm{NA}$ & NA & NA & NA & & snp.397.7.57!Gg_rs142329 \\
\hline
\end{tabular}




\begin{tabular}{|c|c|c|c|c|c|}
\hline $0.59 \mathrm{NA}$ & NA & NA & NA & & snp.397.7.57!Gg_rs142329 \\
\hline $0.72 \mathrm{NA}$ & NA & NA & NA & & Gg_rs107232 Gg_rs142329 \\
\hline $0.54 \mathrm{NA}$ & NA & NA & NA & & Gg_rs107232 Gg_rs142329 \\
\hline $0.7 \mathrm{NA}$ & NA & NA & NA & & snp.397.7.57!Gg_rs142329 \\
\hline $0.6 \mathrm{NA}$ & NA & NA & NA & & snp.397.7.57!Gg_rs142329 \\
\hline $0.65 \mathrm{NA}$ & NA & NA & NA & & Gg_rs107232 Gg_rs142329 \\
\hline $0.71 \mathrm{NA}$ & NA & NA & NA & & Gg_rs142223 Gg_rs142329 \\
\hline $0.5 \mathrm{NA}$ & NA & NA & NA & & snp.397.7.57!Gg_rs142329 \\
\hline $0.6 \mathrm{NA}$ & NA & NA & NA & & Gg_rs107232 Gg_rs142329 \\
\hline $1.28 \mathrm{NA}$ & NA & NA & NA & & Gg_rs107232 Gg_rs142329 \\
\hline $0.69 \mathrm{NA}$ & NA & NA & NA & & Gg_rs142189 Gg_rs142329 \\
\hline $0.84 \mathrm{NA}$ & NA & NA & NA & & snp.397.7.57!Gg_rs142329 \\
\hline $0.5 \mathrm{NA}$ & NA & NA & NA & & Gg_rs142223 Gg_rs142329 \\
\hline $0.6 \mathrm{NA}$ & NA & NA & NA & & snp.397.7.57!Gg_rs142329 \\
\hline $0.7 \mathrm{NA}$ & NA & NA & NA & & snp.397.7.57!Gg_rs142329 \\
\hline $1.39 \mathrm{NA}$ & NA & NA & NA & & Gg_rs107232 Gg_rs142329 \\
\hline $1.36 \mathrm{NA}$ & NA & NA & NA & & snp.397.7.57!Gg_rs142329 \\
\hline 1.52 & 4.85 & 1.2 & -4.88 & 2.16 & snp.397.7.57!Gg_rs142329 \\
\hline $1.17 \mathrm{NA}$ & NA & NA & NA & & snp.397.7.57!Gg_rs142329 \\
\hline $0.94 \mathrm{NA}$ & NA & NA & NA & & Gg_rs107232 Gg_rs142329 \\
\hline $1.56 \mathrm{NA}$ & NA & NA & NA & & snp.397.7.57!Gg_rs142329 \\
\hline $1.18 \mathrm{NA}$ & NA & NA & NA & & snp.397.7.57!Gg_rs142329 \\
\hline $0.72 \mathrm{NA}$ & NA & NA & NA & & Gg_rs107232 Gg_rs142329 \\
\hline $1.27 \mathrm{NA}$ & NA & NA & NA & & Gg_rs107232 Gg_rs142329 \\
\hline $1.07 \mathrm{NA}$ & NA & NA & NA & & Gg_rs142209 Gg_rs142329 \\
\hline $1.32 \mathrm{NA}$ & NA & NA & NA & & Gg_rs142223 Gg_rs142329 \\
\hline $1.31 \mathrm{NA}$ & NA & NA & NA & & Gg_rs142223 Gg_rs142329 \\
\hline $2 \mathrm{NA}$ & NA & NA & NA & & snp.397.7.57!Gg_rs142329 \\
\hline $0.97 \mathrm{NA}$ & NA & NA & NA & & snp.397.7.57!Gg_rs142329 \\
\hline $1.4 \mathrm{NA}$ & NA & NA & NA & & snp.397.7.57!Gg_rs142329 \\
\hline $0.9 \mathrm{NA}$ & NA & NA & NA & & Gg_rs142223 Gg_rs142329 \\
\hline $0.94 \mathrm{NA}$ & NA & NA & NA & & Gg_rs142223 Gg_rs142329 \\
\hline $0.98 \mathrm{NA}$ & NA & NA & NA & & snp.397.7.57!Gg_rs142329 \\
\hline $1 \mathrm{NA}$ & NA & NA & NA & & Gg_rs142253 Gg_rs142329 \\
\hline $1.29 \mathrm{NA}$ & NA & NA & NA & & Gg_rs107232 Gg_rs142329 \\
\hline $0.68 \mathrm{NA}$ & NA & NA & NA & & Gg_rs142223 Gg_rs142329 \\
\hline $1.06 \mathrm{NA}$ & NA & NA & NA & & snp.397.7.57!Gg_rs142329 \\
\hline $0.86 \mathrm{NA}$ & NA & NA & NA & & snp.397.7.57!Gg_rs142329 \\
\hline $0.63 \mathrm{NA}$ & NA & NA & NA & & Gg_rs142223 Gg_rs142329 \\
\hline $1.59 \mathrm{NA}$ & NA & NA & NA & & Gg_rs107232 Gg_rs142329 \\
\hline 0.89 NA & NA & NA & NA & & Gg_rs107232 Gg_rs142329 \\
\hline $1.73 \mathrm{NA}$ & NA & NA & NA & & Gg_rs107232 Gg_rs142329 \\
\hline $1.96 \mathrm{NA}$ & NA & NA & NA & & Gg_rs142223 Gg_rs142329 \\
\hline
\end{tabular}




\begin{tabular}{|c|c|c|c|c|c|}
\hline $0.65 \mathrm{NA}$ & NA & NA & NA & & X2_10635231Gg_rs151445 \\
\hline $0.57 \mathrm{NA}$ & NA & NA & NA & & Gg_rs151265 Gg_rs151429: \\
\hline 0.62 & -0.34 & 0.6 & 2.47 & 0.82 & X2_10700517Gg_rs151429: \\
\hline $0.58 \mathrm{NA}$ & NA & NA & NA & & X2_10700517Gg_rs151445 \\
\hline $0.41 \mathrm{NA}$ & NA & NA & NA & & X2_10700517Gg_rs151429: \\
\hline $0.37 \mathrm{NA}$ & NA & NA & NA & & X2_10700517RBL1120 \\
\hline $0.52 \mathrm{NA}$ & NA & NA & NA & & X2_10700517RBL1120 \\
\hline 0.73 NA & NA & NA & NA & & X2_10700517RBL1120 \\
\hline $0.64 \mathrm{NA}$ & NA & NA & NA & & X2_10700517Gg_rs151429: \\
\hline $0.9 \mathrm{NA}$ & NA & NA & NA & & X2_10700517Gg_rs151429: \\
\hline $0.64 \mathrm{NA}$ & NA & NA & NA & & X2_10700517Gg_rs151429: \\
\hline 0.89 & -1.3 & 0.86 & 2.77 & 1.17 & X2_10700517Gg_rs151429: \\
\hline $0.53 \mathrm{NA}$ & NA & NA & NA & & X2_10700517Gg_rs151429: \\
\hline $0.65 \mathrm{NA}$ & NA & NA & NA & & X2_10700517RBL1120 \\
\hline $0.39 \mathrm{NA}$ & NA & NA & NA & & X2_10700517Gg_rs151429: \\
\hline 0.79 & 0.29 & 0.77 & 3.59 & 1.05 & X2_10700517Gg_rs151429: \\
\hline $0.8 \mathrm{NA}$ & NA & NA & NA & & X2_10700517rbl2524 \\
\hline $0.46 \mathrm{NA}$ & NA & NA & NA & & Gg_rs142329 Gg_rs151424 \\
\hline $0.61 \mathrm{NA}$ & NA & NA & NA & & X2_10700517Gg_rs151424 \\
\hline 0.95 & 0.82 & 0.92 & 6.95 & 1.25 & X2_10700517Gg_rs151429: \\
\hline $0.28 \mathrm{NA}$ & NA & NA & NA & & Gg_rs142329Gg_rs151424 \\
\hline $0.35 \mathrm{NA}$ & NA & NA & NA & & X2_10700517RBL1120 \\
\hline 0.48 NA & NA & NA & NA & & X2_10700517RBL1120 \\
\hline 0.48 NA & NA & NA & NA & & X2_10700517RBL1120 \\
\hline $0.4 \mathrm{NA}$ & NA & NA & NA & & X2_10700517Gg_rs151445 \\
\hline $0.6 \mathrm{NA}$ & NA & NA & NA & & X2_10700517Gg_rs151445 \\
\hline $0.39 \mathrm{NA}$ & NA & NA & NA & & X2_10700517RBL1120 \\
\hline $0.64 \mathrm{NA}$ & NA & NA & NA & & X2_10700517Gg_rs151445 \\
\hline $0.65 \mathrm{NA}$ & NA & NA & NA & & X2_10700517rbl2524 \\
\hline $0.75 \mathrm{NA}$ & NA & NA & NA & & X2_10700517RBL1120 \\
\hline $0.48 \mathrm{NA}$ & NA & NA & NA & & X2_10700517Gg_rs151465. \\
\hline $0.88 \mathrm{NA}$ & NA & NA & NA & & X2_10700517Gg_rs151465. \\
\hline $0.42 \mathrm{NA}$ & NA & NA & NA & & Gg_rs142329 RBL1120 \\
\hline $0.73 \mathrm{NA}$ & NA & NA & NA & & X2_10700517RBL1120 \\
\hline $1.09 \mathrm{NA}$ & NA & NA & NA & & X2_10700517Gg_rs151465. \\
\hline $0.57 \mathrm{NA}$ & NA & NA & NA & & X2_10700517RBL1120 \\
\hline $0.66 \mathrm{NA}$ & NA & NA & NA & & X2_10700517RBL1120 \\
\hline $0.43 \mathrm{NA}$ & NA & NA & NA & & Gg_rs142329 Gg_rs151445 \\
\hline 0.89 NA & NA & NA & NA & & X2_10700517Gg_rs151465. \\
\hline $0.31 \mathrm{NA}$ & NA & NA & NA & & X2_10700517rbl2524 \\
\hline 0.44 NA & NA & NA & NA & & X2_10700517RBL1120 \\
\hline 0.79 NA & NA & NA & NA & & X2_10700517Gg_rs151465. \\
\hline 0.48 NA & NA & NA & NA & & Gg_rs151424 RBL1120 \\
\hline
\end{tabular}




\begin{tabular}{|c|c|c|c|c|c|c|c|c|}
\hline $0.63 \mathrm{NA}$ & & NA & & NA & & NA & & Gg_rs151424 RBL1120 \\
\hline 0.49 NA & & NA & & NA & & NA & & Gg_rs151424 RBL1120 \\
\hline $1.02 \mathrm{NA}$ & & NA & & NA & & NA & & Gg_rs142329 RBL1120 \\
\hline $0.83 \mathrm{NA}$ & & NA & & NA & & NA & & Gg_rs151424 RBL1120 \\
\hline $0.32 \mathrm{NA}$ & & NA & & NA & & NA & & X2_10700517Gg_rs142424. \\
\hline $0.52 \mathrm{NA}$ & & NA & & NA & & NA & & X2_10700517Gg_rs151465. \\
\hline $1.02 \mathrm{NA}$ & & NA & & NA & & NA & & X2_10700517Gg_rs151445 \\
\hline 0.49 NA & & NA & & NA & & NA & & Gg_rs151424 RBL1120 \\
\hline $0.51 \mathrm{NA}$ & & NA & & NA & & NA & & Gg_rs151424 RBL1120 \\
\hline $0.72 \mathrm{NA}$ & & NA & & NA & & NA & & Gg_rs151424 RBL1120 \\
\hline $0.65 \mathrm{NA}$ & & NA & & NA & & NA & & Gg_rs151424 RBL1120 \\
\hline $0.54 \mathrm{NA}$ & & NA & & NA & & NA & & Gg_rs151424 RBL1120 \\
\hline 0.35 & 1.11 & & 0.33 & & 0.01 & & 0.47 & Gg_rs151424 Gg_rs151465. \\
\hline 0.67 & -2.32 & & 0.63 & & 0.08 & & 0.9 & X2_10700517RBL1120 \\
\hline $0.47 \mathrm{NA}$ & & NA & & NA & & NA & & Gg_rs142329 rbl2524 \\
\hline $0.61 \mathrm{NA}$ & & NA & & NA & & NA & & Gg_rs151424 Gg_rs151445 \\
\hline $0.39 \mathrm{NA}$ & & NA & & NA & & NA & & X2_10700517rbl2524 \\
\hline $0.52 \mathrm{NA}$ & & NA & & NA & & NA & & Gg_rs151424 Gg_rs151445 \\
\hline 0.79 NA & & NA & & NA & & NA & & X2_10700517Gg_rs151465. \\
\hline $0.35 \mathrm{NA}$ & & NA & & NA & & NA & & Gg_rs151424 Gg_rs151445 \\
\hline 1.11 & -2.09 & & 1.03 & & -2.21 & & 1.48 & Gg_rs151424 rbl2524 \\
\hline $0.38 \mathrm{NA}$ & & NA & & NA & & NA & & X2_10700517Gg_rs151445 \\
\hline $0.53 \mathrm{NA}$ & & NA & & NA & & NA & & X2_10700517Gg_rs151465. \\
\hline $0.53 \mathrm{NA}$ & & NA & & NA & & NA & & X2_10700517Gg_rs151445 \\
\hline 0.66 & -1.87 & & 0.61 & & -1.78 & & 0.88 & Gg_rs151429Gg_rs151445 \\
\hline $0.34 \mathrm{NA}$ & & NA & & NA & & NA & & Gg_rs151429 rbl2524 \\
\hline $0.73 \mathrm{NA}$ & & NA & & NA & & NA & & X2_10700517Gg_rs151465. \\
\hline $0.62 \mathrm{NA}$ & & NA & & NA & & NA & & X2_10700517Gg_rs151465. \\
\hline 0.75 & 3.62 & & 0.77 & & -2.78 & & 1.15 & X2_10700517rbl2524 \\
\hline 0.51 & 2.54 & & 0.51 & & -2.17 & & 0.77 & X2_10700517rbl2524 \\
\hline 0.35 & 1.9 & & 0.36 & & -1.62 & & 0.54 & Gg_rs142329 rbl2524 \\
\hline 0.48 NA & & NA & & NA & & NA & & Gg_rs151424 rbl2524 \\
\hline 1.39 & -2.92 & & 1.43 & & 2.88 & & 2.14 & X2_10700517Gg_rs151465. \\
\hline $0.71 \mathrm{NA}$ & & NA & & NA & & NA & & RBL1120 rbl2524 \\
\hline 0.35 & -0.8 & & 0.36 & & 1.61 & & 0.54 & RBL1120 \\
\hline $0.36 \mathrm{NA}$ & & NA & & NA & & NA & & RBL1120 Gg_rs151465. \\
\hline $0.55 \mathrm{NA}$ & & NA & & NA & & NA & & Gg_rs151429 rbl2524 \\
\hline $0.58 \mathrm{NA}$ & & NA & & NA & & NA & & X2_10700517Gg_rs151465. \\
\hline 0.99 NA & & NA & & NA & & NA & & X2_10700517Gg_rs151465. \\
\hline 0.87 & -1.18 & & 0.89 & & 3.27 & & 1.33 & Gg_rs151429Gg_rs151465. \\
\hline 0.8 & 0.01 & & 0.82 & & 2.72 & & 1.22 & RBL1120 Gg_rs151465. \\
\hline $0.46 \mathrm{NA}$ & & NA & & NA & & NA & & Gg_rs151465 \\
\hline $0.76 \mathrm{NA}$ & & NA & & NA & & NA & & Gg_rs151465 \\
\hline
\end{tabular}




\begin{tabular}{|c|c|c|c|c|c|c|}
\hline 0.46 & 0.36 & 0.47 & -1.81 & 0.71 & RBL1120 & Gg_rs151465 \\
\hline $0.66 \mathrm{NA}$ & NA & NA & NA & & Gg_rs151424 & 4 Gg_rs151465 \\
\hline $0.58 \mathrm{NA}$ & NA & NA & NA & & RBL1120 & Gg_rs151465 \\
\hline 0.94 & -3.67 & 1.13 & -0.8 & 1.39 & RBL1120 & Gg_rs151465 \\
\hline $0.47 \mathrm{NA}$ & NA & NA & NA & & RBL1120 & Gg_rs151465 \\
\hline 0.31 & 1.47 & 0.38 & -1.23 & 0.46 & Gg_rs151445 & 5 Gg_rs151465 \\
\hline $0.6 \mathrm{NA}$ & NA & NA & NA & & RBL1120 & Gg_rs151465 \\
\hline 0.67 NA & NA & NA & NA & & RBL1120 & Gg_rs151526 \\
\hline 0.45 & 2.14 & 0.58 & -3.9 & 0.7 & Gg_rs151445 & 5 Gg_rs142424 \\
\hline $0.57 \mathrm{NA}$ & NA & NA & NA & & Gg_rs151445 & 5 Gg_rs142424 \\
\hline $0.21 \mathrm{NA}$ & NA & NA & NA & & rbl2524 & Gg_rs151526 \\
\hline $0.41 \mathrm{NA}$ & NA & NA & NA & & rbl2524 & Gg_rs142424 \\
\hline $0.56 \mathrm{NA}$ & NA & NA & NA & & rbl2524 & Gg_rs142424 \\
\hline 0.49 NA & NA & NA & NA & & rbl2524 & Gg_rs151526 \\
\hline $0.41 \mathrm{NA}$ & NA & NA & NA & & rbl2524 & Gg_rs151526 \\
\hline $0.25 \mathrm{NA}$ & NA & NA & NA & & rbl2524 & Gg_rs142487 \\
\hline 0.72 NA & NA & NA & NA & & rbl2524 & Gg_rs151546 \\
\hline 0.64 & 1.94 & 0.76 & -4.31 & 0.96 & Gg_rs151465 & 5 Gg_rs151526 \\
\hline $0.36 \mathrm{NA}$ & NA & NA & NA & & rbl2524 & Gg_rs151526 \\
\hline $1.45 \mathrm{NA}$ & NA & NA & NA & & rbl2524 & Gg_rs151526 \\
\hline 0.47 NA & NA & NA & NA & & rbl2524 & Gg_rs151526 \\
\hline 0.66 NA & NA & NA & NA & & Gg_rs151465 & 5 Gg_rs151526 \\
\hline $0.4 \mathrm{NA}$ & NA & NA & NA & & Gg_rs151465 & 5 Gg_rs151526 \\
\hline $0.5 \mathrm{NA}$ & NA & NA & NA & & Gg_rs151465 & 5 Gg_rs151526 \\
\hline 0.57 & 0.96 & 0.68 & -3.07 & 0.86 & Gg_rs151465 & 5 Gg_rs151526 \\
\hline 0.52 NA & NA & NA & NA & & Gg_rs151465 & 5 Gg_rs151526 \\
\hline 0.95 NA & NA & NA & NA & & Gg_rs151465 & 5 Gg_rs151526 \\
\hline $0.64 \mathrm{NA}$ & NA & NA & NA & & Gg_rs151465 & 5 Gg_rs151526 \\
\hline 0.82 & 1.36 & 0.99 & -2.69 & 1.24 & Gg_rs151465 & 5 Gg_rs151526 \\
\hline $0.72 \mathrm{NA}$ & NA & NA & NA & & Gg_rs151465 & 5 Gg_rs151526 \\
\hline 0.55 NA & NA & NA & NA & & Gg_rs151465 & 5 Gg_rs151526 \\
\hline $0.63 \mathrm{NA}$ & NA & NA & NA & & Gg_rs151445 & 5 Gg_rs151546 \\
\hline $0.35 \mathrm{NA}$ & NA & NA & NA & & Gg_rs151465 & 5 Gg_rs151526 \\
\hline $0.67 \mathrm{NA}$ & NA & NA & NA & & Gg_rs151465 & 5 Gg_rs151526 \\
\hline $0.38 \mathrm{NA}$ & NA & NA & NA & & Gg_rs151465 & 5 Gg_rs151546 \\
\hline $0.54 \mathrm{NA}$ & NA & NA & NA & & Gg_rs151465 & 5 Gg_rs151526 \\
\hline $0.5 \mathrm{NA}$ & NA & NA & NA & & rbl2524 & Gg_rs151546 \\
\hline $0.59 \mathrm{NA}$ & NA & NA & NA & & RBL1120 & Gg_rs151526 \\
\hline $0.48 \mathrm{NA}$ & NA & NA & NA & & Gg_rs151465 & 5 Gg_rs151546 \\
\hline $0.4 \mathrm{NA}$ & NA & NA & NA & & Gg_rs151465 & 5 Gg_rs151546 \\
\hline $0.6 \mathrm{NA}$ & NA & NA & NA & & Gg_rs151465 & 5 Gg_rs151546 \\
\hline $0.53 \mathrm{NA}$ & NA & NA & NA & & Gg_rs151465 & 5 Gg_rs151546 \\
\hline $0.63 \mathrm{NA}$ & NA & NA & NA & & Gg_rs151465 & 5 Gg_rs151546 \\
\hline
\end{tabular}




\begin{tabular}{|c|c|c|c|c|}
\hline 0.5 & 0.55 & 0.46 & 2.31 & 0.66 snp.21.121.2:Gg_rs151546 \\
\hline $0.29 \mathrm{NA}$ & NA & NA & NA & snp.21.121.2:Gg_rs151546 \\
\hline $0.51 \mathrm{NA}$ & NA & NA & NA & snp.21.121.2:Gg_rs151546 \\
\hline $0.98 \mathrm{NA}$ & NA & NA & NA & Gg_rs151465 Gg_rs151546 \\
\hline $1.02 \mathrm{NA}$ & NA & NA & NA & snp.21.121.2:Gg_rs151546 \\
\hline $0.61 \mathrm{NA}$ & NA & NA & NA & snp.21.121.2:Gg_rs151546 \\
\hline $0.92 \mathrm{NA}$ & NA & NA & NA & snp.21.121.2:Gg_rs151546 \\
\hline $0.45 \mathrm{NA}$ & NA & NA & NA & snp.21.121.2:Gg_rs151546 \\
\hline $0.25 \mathrm{NA}$ & NA & NA & NA & snp.21.121.2:Gg_rs151546 \\
\hline $0.59 \mathrm{NA}$ & NA & NA & NA & snp.21.121.2:Gg_rs151546 \\
\hline $0.62 \mathrm{NA}$ & NA & NA & NA & snp.21.121.2:Gg_rs151546 \\
\hline 0.89 & -1.98 & 0.82 & 0.02 & 1.17 snp.21.121.2:Gg_rs151546 \\
\hline 0.53 & -0.17 & 0.49 & 2.45 & 0.7 snp.21.121.2:Gg_rs151546 \\
\hline $0.55 \mathrm{NA}$ & NA & NA & NA & snp.21.121.2:Gg_rs151546 \\
\hline 0.72 & -0.64 & 0.66 & 2.69 & 0.94 snp.21.121.2:Gg_rs151546 \\
\hline $0.58 \mathrm{NA}$ & NA & NA & NA & snp.21.121.2:Gg_rs151546 \\
\hline $0.66 \mathrm{NA}$ & NA & NA & NA & snp.21.121.2:Gg_rs151546 \\
\hline $0.6 \mathrm{NA}$ & NA & NA & NA & snp.21.121.2:Gg_rs151546 \\
\hline $0.64 \mathrm{NA}$ & NA & NA & NA & snp.21.121.2irbl2639 \\
\hline $0.67 \mathrm{NA}$ & NA & NA & NA & snp.21.121.2:Gg_rs151546 \\
\hline $0.38 \mathrm{NA}$ & NA & NA & NA & snp.21.121.2:Gg_rs151546 \\
\hline $0.73 \mathrm{NA}$ & NA & NA & NA & snp.21.121.2:Gg_rs151546 \\
\hline $0.54 \mathrm{NA}$ & NA & NA & NA & Gg_rs151465 Gg_rs151546 \\
\hline $0.77 \mathrm{NA}$ & NA & NA & NA & snp.21.121.2:Gg_rs151546 \\
\hline $0.52 \mathrm{NA}$ & NA & NA & NA & snp.21.121.2:Gg_rs151546 \\
\hline $0.47 \mathrm{NA}$ & NA & NA & NA & snp.21.121.2:Gg_rs151546 \\
\hline $0.6 \mathrm{NA}$ & NA & NA & NA & snp.21.121.2:Gg_rs151546 \\
\hline 0.68 NA & NA & NA & NA & snp.21.121.2:Gg_rs151546 \\
\hline 1.04 & 0.62 & 0.96 & 5.38 & 1.37 snp.21.121.2:Gg_rs151546 \\
\hline $0.43 \mathrm{NA}$ & NA & NA & NA & snp.21.121.2،Gg_rs151546 \\
\hline $0.56 \mathrm{NA}$ & NA & NA & NA & snp.21.121.2:Gg_rs151546 \\
\hline $0.37 \mathrm{NA}$ & NA & NA & NA & snp.21.121.2:Gg_rs151546 \\
\hline $0.41 \mathrm{NA}$ & NA & NA & NA & Gg_rs151445 Gg_rs151546 \\
\hline $0.69 \mathrm{NA}$ & NA & NA & NA & snp.21.121.2:Gg_rs151546 \\
\hline $0.35 \mathrm{NA}$ & NA & NA & NA & snp.21.121.2:Gg_rs151546 \\
\hline $0.28 \mathrm{NA}$ & NA & NA & NA & snp.21.121.2:Gg_rs151546 \\
\hline $0.27 \mathrm{NA}$ & NA & NA & NA & snp.21.121.2:Gg_rs151546 \\
\hline $0.47 \mathrm{NA}$ & NA & NA & NA & Gg_rs142424 Gg_rs151546 \\
\hline $0.45 \mathrm{NA}$ & NA & NA & NA & snp.21.121.2:rbl2639 \\
\hline $0.33 \mathrm{NA}$ & NA & NA & NA & snp.21.121.2:Gg_rs151546 \\
\hline $0.72 \mathrm{NA}$ & NA & NA & NA & rbl2524 Gg_rs151546 \\
\hline $0.55 \mathrm{NA}$ & NA & NA & NA & snp.21.121.2:Gg_rs151546 \\
\hline 0.47 NA & NA & NA & NA & Gg_rs151465 Gg_rs151546 \\
\hline
\end{tabular}




\begin{tabular}{|c|c|c|c|c|c|}
\hline $0.36 \mathrm{NA}$ & NA & NA & NA & & snp.21.121.2:Gg_rs151546 \\
\hline 0.98 & -2.05 & 0.9 & 1.75 & 1.29 & snp.21.121.2:Gg_rs151546 \\
\hline $0.35 \mathrm{NA}$ & NA & NA & NA & & snp.21.121.2‘rbl2639 \\
\hline $0.59 \mathrm{NA}$ & NA & NA & NA & & snp.21.121.2:Gg_rs151546 \\
\hline $0.35 \mathrm{NA}$ & NA & NA & NA & & Gg_rs151465 rbl2639 \\
\hline $0.95 \mathrm{NA}$ & NA & NA & NA & & snp.21.121.2:rbl2639 \\
\hline $0.4 \mathrm{NA}$ & NA & NA & NA & & Gg_rs151465 rbl2639 \\
\hline $2.73 \mathrm{NA}$ & NA & NA & NA & & snp.21.121.2:rbl2639 \\
\hline $2.67 \mathrm{NA}$ & NA & NA & NA & & snp.21.121.2:rbl2639 \\
\hline $1.1 \mathrm{NA}$ & NA & NA & NA & & Gg_rs142487 rbl2639 \\
\hline $0.76 \mathrm{NA}$ & NA & NA & NA & & Gg_rs142487 rbl2639 \\
\hline $0.13 \mathrm{NA}$ & NA & NA & NA & & Gg_rs142487 rbl2639 \\
\hline 0.61 & -2.04 & 0.59 & 1.92 & 0.77 & Gg_rs142487 rbl2639 \\
\hline $0.16 \mathrm{NA}$ & NA & NA & NA & & Gg_rs142487 rbl2639 \\
\hline $0.32 \mathrm{NA}$ & NA & NA & NA & & Gg_rs142487 rbl2639 \\
\hline $1.23 \mathrm{NA}$ & NA & NA & NA & & Gg_rs142487 rbl2639 \\
\hline $0.6 \mathrm{NA}$ & NA & NA & NA & & Gg_rs142487 rbl2639 \\
\hline $0.72 \mathrm{NA}$ & NA & NA & NA & & Gg_rs142487 rbl2639 \\
\hline $0.86 \mathrm{NA}$ & NA & NA & NA & & Gg_rs142487 rbl2639 \\
\hline 0.37 & 0.06 & 0.36 & -1.3 & 0.46 & Gg_rs142487 rbl2639 \\
\hline 0.46 & 0.09 & 0.44 & -1.48 & 0.58 & Gg_rs142487 rbl2639 \\
\hline $1.05 \mathrm{NA}$ & NA & NA & NA & & Gg_rs142487 rbl2639 \\
\hline $0.52 \mathrm{NA}$ & NA & NA & NA & & Gg_rs142487 rbl2639 \\
\hline $0.59 \mathrm{NA}$ & NA & NA & NA & & Gg_rs142487 rbl2639 \\
\hline $0.42 \mathrm{NA}$ & NA & NA & NA & & Gg_rs142487 rbl2639 \\
\hline 0.05 NA & NA & NA & NA & & Gg_rs142487 rbl2639 \\
\hline $0.33 \mathrm{NA}$ & NA & NA & NA & & Gg_rs142487 rbl2639 \\
\hline $0.23 \mathrm{NA}$ & NA & NA & NA & & Gg_rs142487 rbl2639 \\
\hline $0.15 \mathrm{NA}$ & NA & NA & NA & & Gg_rs142487 rbl2639 \\
\hline $0.62 \mathrm{NA}$ & NA & NA & NA & & Gg_rs142487 rbl2639 \\
\hline 0.49 NA & NA & NA & NA & & Gg_rs142487 rbl2639 \\
\hline 0.64 NA & NA & NA & NA & & Gg_rs142487 rbl2639 \\
\hline 0.76 & -1.46 & 0.73 & 0.9 & 0.96 & Gg_rs142487 rbl2639 \\
\hline $0.71 \mathrm{NA}$ & NA & NA & NA & & Gg_rs151526 rbl2639 \\
\hline $0.65 \mathrm{NA}$ & NA & NA & NA & & snp.21.121.2:rbl2639 \\
\hline $0.17 \mathrm{NA}$ & NA & NA & NA & & Gg_rs151546Gg_rs142536 \\
\hline $0.4 \mathrm{NA}$ & NA & NA & NA & & Gg_rs151546Gg_rs142536 \\
\hline $0.6 \mathrm{NA}$ & NA & NA & NA & & snp.21.121.2:Gg_rs151670 \\
\hline $0.5 \mathrm{NA}$ & NA & NA & NA & & Gg_rs151546Gg_rs142536 \\
\hline $0.52 \mathrm{NA}$ & NA & NA & NA & & Gg_rs151546Gg_rs142536 \\
\hline $0.49 \mathrm{NA}$ & NA & NA & NA & & Gg_rs151546Gg_rs142536 \\
\hline $0.47 \mathrm{NA}$ & NA & NA & NA & & Gg_rs151546Gg_rs142536 \\
\hline 0.79 NA & NA & NA & NA & & Gg_rs151546 rbl4207 \\
\hline
\end{tabular}




\begin{tabular}{|c|c|c|c|c|c|c|}
\hline $0.81 \mathrm{NA}$ & NA & & NA & NA & & Gg_rs151546 Gg_rs151670 \\
\hline 0.73 & 1.49 & 0.73 & & -0.71 & 0.97 & Gg_rs151546 Gg_rs142536 \\
\hline $0.51 \mathrm{NA}$ & NA & & NA & NA & & Gg_rs151546 Gg_rs142536 \\
\hline $0.63 \mathrm{NA}$ & NA & & NA & NA & & Gg_rs151546 Gg_rs137949 \\
\hline $0.85 \mathrm{NA}$ & NA & & NA & NA & & Gg_rs151546 Gg_rs161553 \\
\hline $0.71 \mathrm{NA}$ & NA & & NA & NA & & Gg_rs151546 Gg_rs151559 \\
\hline $0.47 \mathrm{NA}$ & NA & & NA & NA & & Gg_rs151546 Gg_rs137949 \\
\hline $0.97 \mathrm{NA}$ & NA & & NA & NA & & Gg_rs151546 Gg_rs142536 \\
\hline $0.52 \mathrm{NA}$ & NA & & NA & NA & & Gg_rs151546 Gg_rs142536 \\
\hline $0.89 \mathrm{NA}$ & NA & & NA & NA & & Gg_rs151546 Gg_rs142536 \\
\hline $0.21 \mathrm{NA}$ & NA & & NA & NA & & Gg_rs151546 Gg_rs137949 \\
\hline $0.89 \mathrm{NA}$ & NA & & NA & NA & & Gg_rs151546 Gg_rs161553 \\
\hline $0.33 \mathrm{NA}$ & NA & & NA & NA & & Gg_rs151546 Gg_rs142536 \\
\hline $0.9 \mathrm{NA}$ & NA & & NA & NA & & Gg_rs151546 Gg_rs151670 \\
\hline $0.86 \mathrm{NA}$ & NA & & NA & NA & & Gg_rs151546 Gg_rs142536 \\
\hline $0.82 \mathrm{NA}$ & NA & & NA & NA & & Gg_rs151546 Gg_rs142536 \\
\hline $0.3 \mathrm{NA}$ & NA & & NA & NA & & Gg_rs151546 Gg_rs142536 \\
\hline $0.94 \mathrm{NA}$ & NA & & NA & NA & & Gg_rs151546 Gg_rs142536 \\
\hline 0.86 & 0.79 & 0.82 & & -3.06 & 1.17 & Gg_rs151546 Gg_rs142536 \\
\hline $0.75 \mathrm{NA}$ & NA & & NA & NA & & Gg_rs151546 Gg_rs142536 \\
\hline $0.56 \mathrm{NA}$ & NA & & NA & NA & & Gg_rs151546 Gg_rs142536 \\
\hline $0.53 \mathrm{NA}$ & NA & & NA & NA & & Gg_rs151546 Gg_rs161553 \\
\hline $0.39 \mathrm{NA}$ & NA & & NA & NA & & Gg_rs151546 Gg_rs142536 \\
\hline $0.72 \mathrm{NA}$ & NA & & NA & NA & & Gg_rs151546 Gg_rs142536 \\
\hline $0.76 \mathrm{NA}$ & NA & & NA & NA & & rbl2639 Gg_rs142536 \\
\hline 0.97 & 2.31 & 0.92 & & 0.59 & 1.3 & Gg_rs151559.Gg_rs142536 \\
\hline $0.73 \mathrm{NA}$ & NA & & NA & NA & & Gg_rs151546 Gg_rs161553 \\
\hline $0.71 \mathrm{NA}$ & NA & & NA & NA & & rbl2639 Gg_rs161553 \\
\hline 0.24 & 0.96 & 0.22 & & 1.08 & 0.32 & Gg_rs151559.Gg_rs142536 \\
\hline $0.76 \mathrm{NA}$ & NA & & NA & NA & & Gg_rs151559.Gg_rs161553 \\
\hline 0.62 & 1.86 & 0.59 & & -0.68 & 0.83 & rbl2639 Gg_rs161553 \\
\hline $0.5 \mathrm{NA}$ & NA & & NA & NA & & Gg_rs151546 Gg_rs151670 \\
\hline $0.55 \mathrm{NA}$ & NA & & NA & NA & & Gg_rs151559.Gg_rs161553 \\
\hline $0.4 \mathrm{NA}$ & NA & & NA & NA & & Gg_rs151559.Gg_rs161553 \\
\hline $0.58 \mathrm{NA}$ & NA & & NA & NA & & Gg_rs151546 Gg_rs161553 \\
\hline $0.97 \mathrm{NA}$ & NA & & NA & NA & & Gg_rs151559.Gg_rs161553 \\
\hline 0.52 & -0.49 & 0.51 & & 3.26 & 0.71 & Gg_rs137949 Gg_rs161553 \\
\hline $0.37 \mathrm{NA}$ & NA & & NA & NA & & Gg_rs151559.Gg_rs161553 \\
\hline 1.23 & -3.75 & 1.19 & & 6.63 & 1.66 & Gg_rs137949 Gg_rs161553 \\
\hline 0.9 & 1.49 & 0.87 & & 1.64 & 1.21 & Gg_rs151559.Gg_rs161553 \\
\hline $0.83 \mathrm{NA}$ & NA & & NA & NA & & Gg_rs137949 Gg_rs151670 \\
\hline $0.87 \mathrm{NA}$ & NA & & NA & NA & & Gg_rs137949Gg_rs161553 \\
\hline $0.55 \mathrm{NA}$ & NA & & NA & NA & & rbl2639 Gg_rs161553 \\
\hline
\end{tabular}




\begin{tabular}{|c|c|c|c|c|c|}
\hline 1.05 & -3.19 & 1.02 & 5.83 & 1.42 & Gg_rs137949Gg_rs161553 \\
\hline $0.61 \mathrm{NA}$ & NA & NA & NA & & Gg_rs137949Gg_rs161553 \\
\hline $0.45 \mathrm{NA}$ & NA & NA & NA & & Gg_rs137949Gg_rs161553 \\
\hline $0.76 \mathrm{NA}$ & NA & NA & NA & & Gg_rs137949Gg_rs161553 \\
\hline $0.59 \mathrm{NA}$ & NA & NA & NA & & Gg_rs151546Gg_rs137615. \\
\hline $0.41 \mathrm{NA}$ & NA & NA & NA & & Gg_rs137949Gg_rs151670 \\
\hline $0.65 \mathrm{NA}$ & NA & NA & NA & & Gg_rs137949 rbl4207 \\
\hline $0.52 \mathrm{NA}$ & NA & NA & NA & & Gg_rs151546 Gg_rs137615 \\
\hline $0.51 \mathrm{NA}$ & NA & NA & NA & & Gg_rs137949Gg_rs151670 \\
\hline $0.55 \mathrm{NA}$ & NA & NA & NA & & Gg_rs151546Gg_rs151590 \\
\hline 1.5 & -2.13 & 1.45 & -1 & 2.01 & Gg_rs137949Gg_rs151670 \\
\hline 1.24 & 1.96 & 1.2 & -2.67 & 1.67 & Gg_rs151546 Gg_rs142580. \\
\hline $0.41 \mathrm{NA}$ & NA & NA & NA & & Gg_rs151559.Gg_rs151670 \\
\hline 1.26 & -2.14 & 1.22 & -2.02 & 1.69 & Gg_rs151559.Gg_rs151670 \\
\hline $0.59 \mathrm{NA}$ & NA & NA & NA & & Gg_rs137949Gg_rs151670 \\
\hline $0.45 \mathrm{NA}$ & NA & NA & NA & & Gg_rs137949Gg_rs151670 \\
\hline $0.7 \mathrm{NA}$ & NA & NA & NA & & Gg_rs137949Gg_rs137615. \\
\hline $0.38 \mathrm{NA}$ & NA & NA & NA & & Gg_rs137949 rbl4207 \\
\hline $0.37 \mathrm{NA}$ & NA & NA & NA & & Gg_rs137949Gg_rs151670 \\
\hline $0.53 \mathrm{NA}$ & NA & NA & NA & & Gg_rs137949Gg_rs151670 \\
\hline $1.21 \mathrm{NA}$ & NA & NA & NA & & Gg_rs142536Gg_rs151670 \\
\hline $0.61 \mathrm{NA}$ & NA & NA & NA & & Gg_rs137949Gg_rs151670 \\
\hline $0.8 \mathrm{NA}$ & NA & NA & NA & & Gg_rs137949Gg_rs151590 \\
\hline 0.59 NA & NA & NA & NA & & Gg_rs142536Gg_rs151670 \\
\hline $0.42 \mathrm{NA}$ & NA & NA & NA & & Gg_rs142536 Gg_rs151670 \\
\hline 0.76 NA & NA & NA & NA & & Gg_rs142536 Gg_rs151670 \\
\hline 0.52 NA & NA & NA & NA & & Gg_rs142536 Gg_rs151670 \\
\hline $0.78 \mathrm{NA}$ & NA & NA & NA & & Gg_rs142536Gg_rs142580. \\
\hline $0.48 \mathrm{NA}$ & NA & NA & NA & & Gg_rs142536 Gg_rs142580. \\
\hline 0.56 & 0.11 & 0.55 & -1.96 & 0.76 & Gg_rs142536Gg_rs151670 \\
\hline 0.53 NA & NA & NA & NA & & Gg_rs142536 rbl4207 \\
\hline $0.78 \mathrm{NA}$ & NA & NA & NA & & Gg_rs137949 rbl4207 \\
\hline $0.61 \mathrm{NA}$ & NA & NA & NA & & Gg_rs151546 rbl4207 \\
\hline $0.62 \mathrm{NA}$ & NA & NA & NA & & Gg_rs137949 rbl4207 \\
\hline $0.24 \mathrm{NA}$ & NA & NA & NA & & Gg_rs142536Gg_rs151670 \\
\hline $1.17 \mathrm{NA}$ & NA & NA & NA & & Gg_rs142536 rbl4207 \\
\hline $0.42 \mathrm{NA}$ & NA & NA & NA & & Gg_rs137949 rbl4207 \\
\hline 0.75 & 0.84 & 0.69 & -2.45 & 1.01 & Gg_rs142536 rbl4207 \\
\hline 0.43 NA & NA & NA & NA & & Gg_rs142536 Gg_rs142580. \\
\hline $0.4 \mathrm{NA}$ & NA & NA & NA & & Gg_rs161553 Gg_rs137615. \\
\hline 1.04 & 1.87 & 0.87 & -2.06 & 1.35 & Gg_rs161553 Gg_rs137615. \\
\hline 0.08 & 0.22 & 0.07 & 0.37 & 0.1 & Gg_rs161553 Gg_rs137615. \\
\hline 0.07 & 0.23 & 0.06 & 0.21 & 0.1 & Gg_rs142536 rbl4207 \\
\hline
\end{tabular}




\begin{tabular}{|c|c|c|c|c|c|}
\hline 0.06 & 0.2 & 0.05 & 0.19 & 0.08 & Gg_rs142536 Gg_rs137615. \\
\hline 0.6 & 0.58 & 0.5 & -2.05 & 0.78 & Gg_rs161553 Gg_rs142580. \\
\hline 0.71 & 0.57 & 0.6 & -3.33 & 0.92 & Gg_rs161553 rbl4207 \\
\hline $0.43 \mathrm{NA}$ & NA & NA & NA & & Gg_rs161553 rbl4207 \\
\hline $0.36 \mathrm{NA}$ & NA & NA & NA & & Gg_rs161553 rbl4207 \\
\hline $0.66 \mathrm{NA}$ & NA & NA & NA & & Gg_rs161553 rbl4207 \\
\hline 0.86 & 0.15 & 0.78 & -3.11 & 1.14 & Gg_rs161553 rbl4207 \\
\hline $0.43 \mathrm{NA}$ & NA & NA & NA & & Gg_rs161553 rbl4207 \\
\hline $0.62 \mathrm{NA}$ & NA & NA & NA & & Gg_rs161553 rbl4207 \\
\hline $0.51 \mathrm{NA}$ & NA & NA & NA & & Gg_rs161553 rbl4207 \\
\hline 0.53 & 2.27 & 0.48 & 0.75 & 0.71 & Gg_rs142536 rbl4207 \\
\hline 0.53 & -0.81 & 0.48 & -1.39 & 0.7 & Gg_rs161553 rbl4207 \\
\hline $0.36 \mathrm{NA}$ & NA & NA & NA & & Gg_rs161553 rbl4207 \\
\hline $0.53 \mathrm{NA}$ & NA & NA & NA & & Gg_rs161553 rbl4207 \\
\hline 0.76 & 0.64 & 0.68 & -2.52 & 1 & Gg_rs161553 rbl4207 \\
\hline 0.51 & 0.57 & 0.46 & -1.42 & 0.67 & Gg_rs161553 rbl4207 \\
\hline $0.41 \mathrm{NA}$ & NA & NA & NA & & Gg_rs142536 rbl4207 \\
\hline $0.44 \mathrm{NA}$ & NA & NA & NA & & Gg_rs142536 rbl4207 \\
\hline $0.38 \mathrm{NA}$ & NA & NA & NA & & Gg_rs161553 rbl4207 \\
\hline $0.27 \mathrm{NA}$ & NA & NA & NA & & Gg_rs151670 rbl4207 \\
\hline 0.79 NA & NA & NA & NA & & Gg_rs151670 rbl4207 \\
\hline $0.48 \mathrm{NA}$ & NA & NA & NA & & Gg_rs151670 rbl4207 \\
\hline $0.58 \mathrm{NA}$ & NA & NA & NA & & Gg_rs151670 rbl4207 \\
\hline $0.53 \mathrm{NA}$ & NA & NA & NA & & Gg_rs151670 rbl4207 \\
\hline $0.64 \mathrm{NA}$ & NA & NA & NA & & Gg_rs151670 rbl4207 \\
\hline $0.2 \mathrm{NA}$ & NA & NA & NA & & Gg_rs151670 rbl4207 \\
\hline 0.5 & -0.86 & 0.45 & -1.43 & 0.66 & Gg_rs151670 rbl4207 \\
\hline $0.36 \mathrm{NA}$ & NA & NA & NA & & Gg_rs151670 rbl4207 \\
\hline $0.5 \mathrm{NA}$ & NA & NA & NA & & Gg_rs161553 rbl4207 \\
\hline 0.43 & 0.7 & 0.39 & -1.71 & 0.58 & Gg_rs137949 rbl4207 \\
\hline $0.87 \mathrm{NA}$ & NA & NA & NA & & Gg_rs151670 rbl4207 \\
\hline $0.59 \mathrm{NA}$ & NA & NA & NA & & Gg_rs161553 rbl4207 \\
\hline $0.52 \mathrm{NA}$ & NA & NA & NA & & Gg_rs151670 rbl4207 \\
\hline 0.86 & 1.37 & 0.77 & -1.33 & 1.14 & Gg_rs161553 rbl4207 \\
\hline $0.58 \mathrm{NA}$ & NA & NA & NA & & Gg_rs151670 rbl4207 \\
\hline $0.45 \mathrm{NA}$ & NA & NA & NA & & Gg_rs161553 rbl4207 \\
\hline $0.47 \mathrm{NA}$ & NA & NA & NA & & Gg_rs161553 rbl4207 \\
\hline $0.38 \mathrm{NA}$ & NA & NA & NA & & Gg_rs137949 rbl4207 \\
\hline 0.58 NA & NA & NA & NA & & Gg_rs161553 rbl4207 \\
\hline 0.69 NA & NA & NA & NA & & Gg_rs161553 rbl4207 \\
\hline $0.42 \mathrm{NA}$ & NA & NA & NA & & Gg_rs142536 rbl4207 \\
\hline $0.54 \mathrm{NA}$ & NA & NA & NA & & Gg_rs151670 rbl4207 \\
\hline $0.82 \mathrm{NA}$ & NA & NA & NA & & Gg_rs161553 rbl4207 \\
\hline
\end{tabular}




\begin{tabular}{|c|c|c|c|c|c|}
\hline 0.29 & 0.33 & 0.26 & -1.53 & 0.38 & Gg_rs151670 rb|4207 \\
\hline 0.37 & -0.82 & 0.33 & -1.93 & 0.49 & Gg_rs151670 rbl4207 \\
\hline $0.5 \mathrm{NA}$ & NA & NA & NA & & Gg_rs151670 rbl4207 \\
\hline 1.5 & 3.97 & 1.37 & 0.82 & 2.01 & Gg_rs161553 rbl4207 \\
\hline $0.59 \mathrm{NA}$ & NA & NA & NA & & Gg_rs151670rb|4207 \\
\hline $0.39 \mathrm{NA}$ & NA & NA & NA & & Gg_rs142536rbl4207 \\
\hline 0.39 & 1.57 & 0.35 & -0.43 & 0.51 & Gg_rs151670 rbl4207 \\
\hline $0.53 \mathrm{NA}$ & NA & NA & NA & & Gg_rs151670 rb|4207 \\
\hline $0.52 \mathrm{NA}$ & NA & NA & NA & & Gg_rs151670 rb|4207 \\
\hline $0.36 \mathrm{NA}$ & NA & NA & NA & & Gg_rs151670 rb|4207 \\
\hline $0.37 \mathrm{NA}$ & NA & NA & NA & & Gg_rs151670 rbl4207 \\
\hline $1.31 \mathrm{NA}$ & NA & NA & NA & & Gg_rs161553 rbl4207 \\
\hline $0.42 \mathrm{NA}$ & NA & NA & NA & & Gg_rs137615 rb|4207 \\
\hline $0.48 \mathrm{NA}$ & NA & NA & NA & & Gg_rs151670 rb|4207 \\
\hline 0.47 & 0.91 & 0.43 & 0.23 & 0.63 & Gg_rs142536rb|4207 \\
\hline $1.01 \mathrm{NA}$ & NA & NA & NA & & Gg_rs161553 rbl4207 \\
\hline $0.69 \mathrm{NA}$ & NA & NA & NA & & Gg_rs151670 rb|4207 \\
\hline $0.47 \mathrm{NA}$ & NA & NA & NA & & Gg_rs151670 rb|4207 \\
\hline $0.28 \mathrm{NA}$ & NA & NA & NA & & Gg_rs137615 rb|4207 \\
\hline $0.39 \mathrm{NA}$ & NA & NA & NA & & Gg_rs137949 rbl4207 \\
\hline $0.77 \mathrm{NA}$ & NA & NA & NA & & Gg_rs151670 rbl4207 \\
\hline $2.05 \mathrm{NA}$ & NA & NA & NA & & rbl2639 rbl4207 \\
\hline $0.46 \mathrm{NA}$ & NA & NA & NA & & Gg_rs151670 rb|4207 \\
\hline 0.35 & 1.32 & 0.32 & -0.29 & 0.47 & Gg_rs151670rb|4207 \\
\hline 0.02 & -0.07 & 0.02 & -0.07 & 0.02 & Gg_rs151670rb|4207 \\
\hline $1.25 \mathrm{NA}$ & NA & NA & NA & & Gg_rs151670 rb|4207 \\
\hline 0.59 & -1 & 0.53 & -1.98 & 0.78 & Gg_rs151670 rbl4207 \\
\hline $0.22 \mathrm{NA}$ & NA & NA & NA & & Gg_rs137615 rbl4207 \\
\hline $0.33 \mathrm{NA}$ & NA & NA & NA & & Gg_rs161553 rbl4207 \\
\hline $0.94 \mathrm{NA}$ & NA & NA & NA & & Gg_rs161553 rbl4207 \\
\hline $1.97 \mathrm{NA}$ & NA & NA & NA & & Gg_rs161553 rbl4207 \\
\hline $0.67 \mathrm{NA}$ & NA & NA & NA & & Gg_rs151670 rb|4207 \\
\hline $0.26 \mathrm{NA}$ & NA & NA & NA & & Gg_rs151670 rb|4207 \\
\hline $0.55 \mathrm{NA}$ & NA & NA & NA & & Gg_rs151670 rbl4207 \\
\hline $0.41 \mathrm{NA}$ & NA & NA & NA & & Gg_rs151670 rb|4207 \\
\hline $1.37 \mathrm{NA}$ & NA & NA & NA & & Gg_rs151670 rb|4207 \\
\hline $0.93 \mathrm{NA}$ & NA & NA & NA & & Gg_rs137615 rb|4207 \\
\hline $1.17 \mathrm{NA}$ & NA & NA & NA & & Gg_rs161553 rbl4207 \\
\hline $0.51 \mathrm{NA}$ & NA & NA & NA & & Gg_rs151670 rb|4207 \\
\hline $0.49 \mathrm{NA}$ & NA & NA & NA & & Gg_rs151670 rb|4207 \\
\hline $0.57 \mathrm{NA}$ & NA & NA & NA & & Gg_rs151670 rbl4207 \\
\hline $1.11 \mathrm{NA}$ & NA & NA & NA & & Gg_rs151670 rb|4207 \\
\hline $2.12 \mathrm{NA}$ & NA & NA & NA & & Gg_rs161553 rb|4207 \\
\hline
\end{tabular}




\begin{tabular}{|c|c|c|c|c|c|c|}
\hline $1.51 \mathrm{NA}$ & NA & & NA & NA & & Gg_rs151670 rbl4207 \\
\hline $0.28 \mathrm{NA}$ & NA & & NA & NA & & Gg_rs151670 rbl4207 \\
\hline $0.54 \mathrm{NA}$ & NA & & NA & NA & & Gg_rs151670 rbl4207 \\
\hline $0.95 \mathrm{NA}$ & NA & & NA & NA & & Gg_rs151670 rbl4207 \\
\hline 0.46 NA & NA & & NA & NA & & Gg_rs151670 rbl4207 \\
\hline $0.52 \mathrm{NA}$ & NA & & NA & NA & & Gg_rs151670 rbl4207 \\
\hline 0.77 & 0.98 & 0.7 & & -2.01 & 1.03 & Gg_rs151670 rbl4207 \\
\hline $0.49 \mathrm{NA}$ & NA & & NA & NA & & Gg_rs161553 rbl4207 \\
\hline $0.39 \mathrm{NA}$ & NA & & NA & NA & & Gg_rs151670 rbl4207 \\
\hline 0.74 & 0.31 & 0.67 & & -2.89 & 0.99 & Gg_rs151670 rbl4207 \\
\hline 1.04 & -0.59 & 0.95 & & -4.08 & 1.39 & Gg_rs151670 rbl4207 \\
\hline $0.51 \mathrm{NA}$ & NA & & NA & NA & & Gg_rs151670 rbl4207 \\
\hline 0.49 NA & NA & & NA & NA & & Gg_rs161553 rbl4207 \\
\hline 0.52 & -0.81 & 0.47 & & -2.8 & 0.69 & Gg_rs151670 rbl4207 \\
\hline 0.36 & -1.05 & 0.33 & & -1.53 & 0.49 & Gg_rs151670 rbl4207 \\
\hline $0.32 \mathrm{NA}$ & NA & & NA & NA & & Gg_rs161553 rbl4207 \\
\hline 0.72 & 0.55 & 0.65 & & -2.32 & 0.96 & Gg_rs161553 rbl4207 \\
\hline $0.67 \mathrm{NA}$ & NA & & NA & NA & & Gg_rs151670 rbl4207 \\
\hline 0.74 & 0.01 & 0.68 & & -2.37 & 1 & Gg_rs161553 rbl4207 \\
\hline 0.89 NA & NA & & NA & NA & & Gg_rs151670 rbl4207 \\
\hline $0.59 \mathrm{NA}$ & NA & & NA & NA & & Gg_rs151670 rbl4207 \\
\hline $0.52 \mathrm{NA}$ & NA & & NA & NA & & Gg_rs151670 rbl4207 \\
\hline $0.97 \mathrm{NA}$ & NA & & NA & NA & & Gg_rs161553 rbl4207 \\
\hline 0.64 NA & NA & & NA & NA & & Gg_rs161553 rbl4207 \\
\hline $0.57 \mathrm{NA}$ & NA & & NA & NA & & Gg_rs151670 rbl4207 \\
\hline $0.7 \mathrm{NA}$ & NA & & NA & NA & & Gg_rs142536 rbl4207 \\
\hline $0.48 \mathrm{NA}$ & NA & & NA & NA & & Gg_rs151670 rbl4207 \\
\hline 0.49 & -0.92 & 0.44 & & -2.71 & 0.65 & Gg_rs151670 rbl4207 \\
\hline $1.1 \mathrm{NA}$ & NA & & NA & NA & & Gg_rs161553 rbl4207 \\
\hline $0.48 \mathrm{NA}$ & NA & & NA & NA & & Gg_rs151670 rbl4207 \\
\hline $0.48 \mathrm{NA}$ & NA & & NA & NA & & Gg_rs151670 rbl4207 \\
\hline 0.79 & 0.63 & 0.72 & & -4.34 & 1.06 & Gg_rs161553 rbl4207 \\
\hline $0.51 \mathrm{NA}$ & NA & & NA & NA & & Gg_rs151670 rbl4207 \\
\hline 0.06 & -0.27 & 0.06 & & -0.3 & 0.08 & Gg_rs151670 rbl4207 \\
\hline $0.58 \mathrm{NA}$ & NA & & NA & NA & & Gg_rs161553 rbl4207 \\
\hline $1.13 \mathrm{NA}$ & NA & & NA & NA & & Gg_rs151670 rbl4207 \\
\hline 1.23 & 0.71 & 1.02 & & -4.35 & 1.59 & Gg_rs161553 rbl4207 \\
\hline $0.76 \mathrm{NA}$ & NA & & NA & NA & & Gg_rs161553 rbl4207 \\
\hline 4.35 & 14.49 & 3.36 & & 17 & 5.69 & X3_4521634 X3_6300742 \\
\hline 2.28 & 6.37 & 2.22 & & -5.68 & 2.99 & X3_1651995€X3_2199277C \\
\hline 2.07 & 8.63 & 2.01 & & -6.82 & 2.71 & X3_1651995EX3_2199277C \\
\hline 1.84 & 5.62 & 1.79 & & -4.52 & 2.41 & X3_1651995EX3_2199277C \\
\hline 1.86 & 8.23 & 1.81 & & -5.52 & 2.44 & X3_1651995€X3_2199277C \\
\hline
\end{tabular}




\begin{tabular}{|c|c|c|c|c|}
\hline 1.58 & 6.96 & 1.53 & -3.42 & 2.07 X3_1651995€X3_2199277C \\
\hline 1.44 & 5.06 & 1.4 & -2.68 & 1.88 X3_1651995€X3_2199277C \\
\hline 2.3 & 8.73 & 2.23 & -5.11 & 3.02 X3_1651995€X3_2199277C \\
\hline 1.48 & 5.45 & 1.44 & -3.45 & 1.95 X3_1651995€X3_2199277C \\
\hline 2.29 & 8.68 & 2.23 & -3.49 & 3.01 X3_1651995€X3_2199277C \\
\hline 1.51 & 6.15 & 1.47 & -6.03 & 1.99 X3_1651995€X3_2199277C \\
\hline $0.83 \mathrm{NA}$ & NA & NA & NA & X3_1651995€X3_2199277C \\
\hline 1.27 & 4.74 & 1.24 & -2.81 & 1.67 X3_1651995€X3_2199277C \\
\hline 1.68 & 5.56 & 1.64 & -2.7 & 2.21 X3_1651995€X3_2199277C \\
\hline 1.9 & 6.85 & 1.85 & -5.18 & 2.49 X3_1651995€X3_2199277C \\
\hline 1.66 & 6.01 & 1.61 & -6.61 & 2.18 X3_1651995€X3_2199277C \\
\hline $1.53 \mathrm{NA}$ & NA & NA & NA & X3_1651995EX3_2199277C \\
\hline 1.63 & 6.14 & 1.58 & -4.67 & 2.14 X3_1651995€X3_2199277C \\
\hline 3.08 & 12.51 & 2.99 & -7.92 & 4.04 X3_1651995€X3_2199277C \\
\hline $1.03 \mathrm{NA}$ & NA & NA & NA & X3_1651995€X3_2199277C \\
\hline 1.81 & 6.7 & 1.76 & -6.14 & 2.38 X3_1651995€X3_2199277C \\
\hline 2.37 & 8.07 & 2.3 & -7.95 & 3.11 X3_1651995€X3_2199277C \\
\hline 1.93 & 4.98 & 1.88 & -5.3 & 2.54 X3_1651995€X3_2199277C \\
\hline 1.8 & 6.7 & 1.75 & -5.76 & 2.36 X3_1651995€X3_2199277C \\
\hline 1.79 & 5.31 & 1.74 & -4.97 & 2.35 X3_1651995€X3_2199277C \\
\hline 1.6 & 4.1 & 1.56 & -5.11 & 2.1 X3_1651995€X3_2199277C \\
\hline $1.55 \mathrm{NA}$ & NA & NA & NA & X3_1651995€X3_2199277C \\
\hline 1.52 & 3.97 & 1.48 & -4.85 & 1.99 X3_1651995€X3_2199277C \\
\hline 2.03 & 6.65 & 1.97 & -7.3 & 2.66 X3_1651995€X3_2199277C \\
\hline 2.46 & 9.42 & 2.39 & -4.81 & 3.23 X3_1651995€X3_2199277C \\
\hline 1.24 & 4.14 & 1.21 & -3.04 & 1.63 X3_1651995€X3_2199277C \\
\hline $1.04 \mathrm{NA}$ & NA & NA & NA & X3_1651995€X3_2199277C \\
\hline 1.94 & 6.57 & 1.88 & -4.63 & 2.54 X3_1651995€X3_2199277C \\
\hline 1.55 & 5.22 & 1.5 & -2.3 & 2.03 X3_1651995€X3_2199277C \\
\hline 1.53 & 6.33 & 1.49 & -4.85 & 2.01 X3_1651995€X3_2199277C \\
\hline 2.55 & 10.23 & 2.48 & -7.26 & 3.35 X3_1651995€X3_2199277C \\
\hline 2.47 & 6.47 & 2.4 & -4.64 & 3.24 X3_1651995€X3_2199277C \\
\hline 1.55 & 6.57 & 1.5 & -5.35 & 2.03 X3_1651995€X3_2199277C \\
\hline 2.22 & 8.59 & 2.16 & -5.14 & 2.91 X3_1651995€X3_2199277C \\
\hline 1.5 & 5.02 & 1.45 & -6.02 & 1.96 X3_1651995€X3_2199277C \\
\hline $1.51 \mathrm{NA}$ & NA & NA & NA & X3_1651995EX3_2199277C \\
\hline 1.57 & 6.33 & 1.52 & -5.92 & 2.06 X3_1651995€X3_2199277C \\
\hline 1.69 & 6.18 & 1.64 & -4.26 & 2.22 X3_1651995€X3_2199277C \\
\hline 1.47 & 5.39 & 1.43 & -4.68 & 1.93 X3_1651995€X3_2199277C \\
\hline 1.61 & 7.64 & 1.57 & -3.96 & 2.11 X3_1651995€X3_2199277C \\
\hline 3.1 & 10.58 & 3.01 & -9.6 & 4.07 X3_1651995€X3_2199277C \\
\hline 1.81 & 4.41 & 1.76 & -4.51 & 2.38 X3_1651995€X3_2199277C \\
\hline 1.65 & 7.01 & 1.6 & -6.84 & 2.16 X3_1651995€X3_2199277C \\
\hline
\end{tabular}




\begin{tabular}{|c|c|c|c|c|c|}
\hline 2.01 & 6.36 & 1.95 & -2.95 & 2.63 & X3_1651995EX3_2199277C \\
\hline $1.02 \mathrm{NA}$ & NA & NA & NA & & X3_1651995€X3_2199277C \\
\hline 2.27 & 7.3 & 2.21 & -2.27 & 2.98 & X3_1651995EX3_2199277C \\
\hline 1.72 & 6.23 & 1.67 & -4.51 & 2.25 & X3_1651995EX3_2199277C \\
\hline 1.73 & 6.21 & 1.68 & -6.58 & 2.27 & X3_1651995EX3_2199277C \\
\hline 1.71 & 5.23 & 1.66 & -5.71 & 2.24 & X3_1651995€X3_2199277C \\
\hline 1.87 & 7.07 & 1.82 & -5.98 & 2.45 & X3_1651995EX3_2199277C \\
\hline $1.03 \mathrm{NA}$ & NA & NA & NA & & X3_1651995EX3_2199277C \\
\hline 1.65 & 6.09 & 1.6 & -4.79 & 2.17 & X3_1651995€X3_2199277C \\
\hline $1.42 \mathrm{NA}$ & NA & NA & NA & & X3_1651995EX3_2199277C \\
\hline 1.88 & 7.83 & 1.82 & -4.74 & 2.46 & X3_1651995€X3_2199277C \\
\hline 1.5 & 3.03 & 1.45 & 1.15 & 1.97 & X3_1651995EX3_2199277C \\
\hline 2.07 & 6.99 & 2.02 & -4.49 & 2.72 & X3_1651995EX3_2199277C \\
\hline 2.3 & 7.92 & 2.23 & -5.74 & 3.01 & X3_1651995€X3_2199277C \\
\hline 1.48 & 4.89 & 1.43 & -3.21 & 1.94 & X3_1651995EX3_2199277C \\
\hline 3.23 & 11.57 & 3.14 & -9.76 & 4.25 & X3_1651995EX3_2199277C \\
\hline 1.5 & 4.26 & 1.46 & -4.32 & 1.97 & X3_1651995EX3_2199277C \\
\hline 2.51 & 9.71 & 2.44 & -7.26 & 3.3 & X3_1651995€X3_2199277C \\
\hline 1.93 & 7.74 & 1.88 & -7.35 & 2.54 & X3_1651995€X3_2199277C \\
\hline 2.34 & 5.71 & 2.28 & -4.56 & 3.08 & X3_1651995EX3_2199277C \\
\hline $1 \mathrm{NA}$ & NA & NA & NA & & X3_1651995EX3_2199277C \\
\hline 2.11 & 7.52 & 2.05 & -6.55 & 2.77 & X3_1651995€X3_2199277C \\
\hline 2.01 & 7.87 & 1.96 & -6.1 & 2.64 & X3_1651995€X3_2199277C \\
\hline $1.01 \mathrm{NA}$ & NA & NA & NA & & X3_1651995EX3_2199277C \\
\hline 1.83 & 5.71 & 1.78 & -7.21 & 2.4 & X3_1651995€X3_2199277C \\
\hline 2.03 & 5.76 & 1.97 & -7.17 & 2.66 & X3_1651995EX3_2199277C \\
\hline 1.78 & 6.12 & 1.73 & -6.9 & 2.34 & X3_1651995EX3_2199277C \\
\hline 1.66 & 5.46 & 1.61 & -6.17 & 2.18 & X3_1651995€X3_2199277C \\
\hline 2.06 & 6.67 & 2 & -4.94 & 2.7 & X3_1651995EX3_2199277C \\
\hline 1.8 & 6.14 & 1.75 & -5.42 & 2.36 & X3_1651995€X3_2199277C \\
\hline 1.93 & 6.44 & 1.87 & -4.87 & 2.53 & X3_1651995EX3_2199277C \\
\hline 1.64 & 5.86 & 1.59 & -5.36 & 2.15 & X3_1651995EX3_2199277C \\
\hline $1.58 \mathrm{NA}$ & NA & NA & NA & & X3_1651995EX3_2199277C \\
\hline 2.03 & 6.74 & 1.97 & -5.02 & 2.67 & X3_1651995EX3_2199277C \\
\hline 1.29 & 5.51 & 1.25 & -4.81 & 1.69 & X3_1651995EX3_2199277C \\
\hline 2.43 & 8.01 & 2.36 & -5.68 & 3.19 & X3_1651995€X3_2199277C \\
\hline 2.46 & 9.36 & 2.39 & -7.05 & 3.23 & X3_1651995€X3_2199277C \\
\hline 1.82 & 4.46 & 1.77 & 0.77 & 2.39 & X3_1651995€X3_2199277C \\
\hline 1.36 & 5.35 & 1.33 & -3.92 & 1.79 & X3_1651995EX3_2199277C \\
\hline 1.82 & 5.36 & 1.77 & -5.05 & 2.39 & X3_1651995€X3_2199277C \\
\hline 1.95 & 6.05 & 1.89 & -5.29 & 2.56 & X3_1651995€X3_2199277C \\
\hline 1.78 & 5.57 & 1.73 & -3.11 & 2.34 & X3_1651995€X3_2199277C \\
\hline 1.58 & 5.32 & 1.53 & -4.02 & 2.07 & X3_1651995EX3_2199277C \\
\hline
\end{tabular}




\begin{tabular}{|c|c|c|c|c|c|}
\hline 2.68 & 10.3 & 2.61 & -5.77 & 3.52 & X3_1651995EX3_2199277C \\
\hline 1.49 & 4.63 & 1.44 & -3.7 & 1.95 & X3_1651995EX3_2199277C \\
\hline 1.4 & 5.33 & 1.36 & -4.64 & 1.84 & X3_1651995EX3_2199277C \\
\hline 1.78 & 7.17 & 1.73 & -4.68 & 2.34 & X3_1651995EX3_2199277C \\
\hline 1.65 & 4.81 & 1.6 & -6.06 & 2.16 & X3_1651995€X3_2199277C \\
\hline $1.07 \mathrm{NA}$ & NA & NA & NA & & X3_1651995EX3_2199277C \\
\hline 1.54 & 5.04 & 1.49 & -4.06 & 2.02 & X3_1651995EX3_2199277C \\
\hline 1.9 & 6.1 & 1.85 & -5.39 & 2.5 & X3_1651995EX3_2199277C \\
\hline $1.16 \mathrm{NA}$ & NA & NA & NA & & X3_1651995EX3_2199277C \\
\hline 2.46 & 9.07 & 2.39 & -7.15 & 3.23 & X3_1651995€X3_2199277C \\
\hline $1.76 \mathrm{NA}$ & NA & NA & NA & & X3_1651995EX3_2199277C \\
\hline 1.86 & 8.39 & 1.81 & -5.55 & 2.45 & X3_1651995EX3_2199277C \\
\hline 2.03 & 7.82 & 1.97 & -5.59 & 2.66 & X3_1651995EX3_2199277C \\
\hline 1.38 & 3.67 & 1.34 & -0.93 & 1.81 & X3_1651995EX3_2199277C \\
\hline 1.82 & 7.24 & 1.77 & -6.92 & 2.39 & X3_1651995€X3_2199277C \\
\hline $1.19 \mathrm{NA}$ & NA & NA & NA & & X3_1651995EX3_2199277C \\
\hline 1.91 & 7.09 & 1.85 & -5.63 & 2.5 & X3_1651995€X3_2199277C \\
\hline 1.98 & 5.97 & 1.92 & -2.17 & 2.59 & X3_1651995€X3_2199277C \\
\hline 1.63 & 6.63 & 1.58 & -5.26 & 2.14 & X3_1651995EX3_2199277C \\
\hline 1.5 & 5.33 & 1.46 & -2.79 & 1.97 & X3_1651995€X3_2199277C \\
\hline $1.27 \mathrm{NA}$ & NA & NA & NA & & X3_1651995EX3_2199277C \\
\hline 1.52 & 5.98 & 1.47 & -6.02 & 1.99 & X3_1651995€X3_2199277C \\
\hline 2.51 & 7.62 & 2.44 & -6.06 & 3.29 & X3_1651995EX3_2199277C \\
\hline 2.08 & 7.32 & 2.02 & -6.47 & 2.73 & X3_1651995EX3_2199277C \\
\hline $1.02 \mathrm{NA}$ & NA & NA & NA & & X3_1651995EX3_2199277C \\
\hline 2.17 & 7.86 & 2.11 & -5.95 & 2.85 & X3_1651995EX3_2199277C \\
\hline 1.73 & 6.51 & 1.68 & -7.38 & 2.27 & X3_1651995€X3_2199277C \\
\hline 2 & 6.84 & 1.94 & -4.21 & 2.62 & X3_1651995EX3_2199277C \\
\hline 1.83 & 5.8 & 1.77 & -3.48 & 2.4 & X3_1651995€X3_2199277C \\
\hline 1.31 & 4.29 & 1.28 & -3.67 & 1.72 & X3_1651995EX3_2199277C \\
\hline 1.55 & 6.76 & 1.5 & -4.55 & 2.03 & X3_1651995€X3_2199277C \\
\hline 2.17 & 7.35 & 2.11 & -5.5 & 2.85 & X3_1651995EX3_2199277C \\
\hline 2.58 & 9.8 & 2.5 & -6.63 & 3.38 & X3_1651995EX3_2199277C \\
\hline 1.49 & 5.85 & 1.45 & -4.28 & 1.95 & X3_1651995EX3_2199277C \\
\hline 1.51 & 4.85 & 1.47 & -3.15 & 1.98 & X3_1651995EX3_2199277C \\
\hline $1.02 \mathrm{NA}$ & NA & NA & NA & & X3_1651995€X3_2199277C \\
\hline 1.66 & 3.58 & 1.61 & -4.12 & 2.17 & X3_1651995€X3_2199277C \\
\hline 2 & 6.12 & 1.94 & -4.31 & 2.62 & X3_1651995€X3_2199277C \\
\hline 2.67 & 9.98 & 2.6 & -6.64 & 3.51 & X3_1651995EX3_2199277C \\
\hline 2.27 & 9.13 & 2.2 & -7.12 & 2.98 & X3_1651995EX3_2199277C \\
\hline $1.24 \mathrm{NA}$ & NA & NA & NA & & X3_1651995€X3_2199277C \\
\hline 1.49 & 4.17 & 1.44 & -2.91 & 1.95 & X3_1651995€X3_2199277C \\
\hline $1.21 \mathrm{NA}$ & NA & NA & NA & & X3_1651995€X3_2199277C \\
\hline
\end{tabular}




\begin{tabular}{|c|c|c|c|c|c|c|}
\hline $1.24 \mathrm{NA}$ & NA & NA & NA & \multicolumn{3}{|c|}{ X3_1651995€X3_2199277C } \\
\hline $1.11 \mathrm{NA}$ & NA & NA & NA & \multicolumn{3}{|c|}{ X3_1651995€X3_2199277C } \\
\hline $1 \mathrm{NA}$ & NA & NA & NA & \multicolumn{3}{|c|}{ X3_1651995€X3_2199277C } \\
\hline 0.95 NA & NA & NA & NA & \multicolumn{3}{|c|}{ X3_1651995€X3_2199277C } \\
\hline $1.42 \mathrm{NA}$ & NA & NA & NA & \multicolumn{3}{|c|}{ X3_1651995EX3_2199277C } \\
\hline $1.04 \mathrm{NA}$ & NA & NA & NA & \multicolumn{3}{|c|}{ X3_1651995€X3_2199277C } \\
\hline $1.03 \mathrm{NA}$ & NA & NA & NA & \multicolumn{3}{|c|}{ X3_1651995€X3_2199277C } \\
\hline $1.12 \mathrm{NA}$ & NA & NA & NA & \multicolumn{3}{|c|}{ X3_1651995€X3_2199277C } \\
\hline $1.28 \mathrm{NA}$ & NA & NA & NA & \multicolumn{3}{|c|}{ X3_1651995€X3_2199277C } \\
\hline 2.09 & 6.37 & 2.03 & -5.85 & 2.75 & \multirow{2}{*}{\multicolumn{2}{|c|}{$\begin{array}{l}\text { X3_1651995€X3_2199277C } \\
\text { X3_1651995€X3_2199277C }\end{array}$}} \\
\hline $2.03 \mathrm{NA}$ & NA & NA & NA & & & \\
\hline $1.06 \mathrm{NA}$ & NA & NA & NA & & \multicolumn{2}{|c|}{ X3_1651995EX3_2199277C } \\
\hline $1.27 \mathrm{NA}$ & NA & NA & NA & & \multicolumn{2}{|c|}{ X3_1651995EX3_2199277C } \\
\hline $1.32 \mathrm{NA}$ & NA & NA & NA & & \multicolumn{2}{|c|}{ X3_1651995EX3_2199277C } \\
\hline $1.07 \mathrm{NA}$ & NA & NA & NA & & \multicolumn{2}{|c|}{ X3_1651995€X3_2199277C } \\
\hline $1.01 \mathrm{NA}$ & NA & NA & NA & & \multicolumn{2}{|c|}{ X3_1651995€X3_2199277C } \\
\hline $1.98 \mathrm{NA}$ & NA & NA & NA & & \multicolumn{2}{|c|}{ X3_1651995EX3_2199277C } \\
\hline 0.47 & -1.55 & 0.49 & 2.18 & 0.63 & rbl1438 & Gg_rs155771 \\
\hline 0.29 & -0.74 & 0.3 & 0.72 & 0.39 & rbl1438 & Gg_rs155771 \\
\hline 0.19 & -0.95 & 0.2 & 0.48 & 0.26 & rbl1438 & Gg_rs155771 \\
\hline 0.66 & -2.92 & 0.69 & 1.9 & 0.89 & rbl1438 & Gg_rs155771 \\
\hline 0.36 & -1.23 & 0.38 & 0.99 & 0.49 & rbl1438 & Gg_rs155771 \\
\hline 0.35 & -0.93 & 0.37 & 0.65 & 0.48 & rbl1438 & Gg_rs155771 \\
\hline 0.59 & -2.08 & 0.62 & 1.37 & 0.8 & rbl1438 & Gg_rs155771 \\
\hline 0.46 & -0.39 & 0.48 & 1.89 & 0.62 & rbl1438 & Gg_rs155771 \\
\hline 0.53 & -1.5 & 0.55 & 1.69 & 0.71 & rbl1438 & Gg_rs155771 \\
\hline 0.53 & -1.43 & 0.55 & 2.24 & 0.71 & rbl1438 & Gg_rs155771 \\
\hline 0.68 & -2.23 & 0.71 & 3.1 & 0.92 & rbl1438 & Gg_rs155771 \\
\hline 0.88 & -3.07 & 0.92 & 3.65 & 1.19 & rbl1438 & Gg_rs155771 \\
\hline 0.48 & -1.32 & 0.5 & 0.62 & 0.65 & rbl1438 & Gg_rs155771 \\
\hline 0.49 & -1.73 & 0.52 & 2.06 & 0.66 & rbl1438 & Gg_rs155771 \\
\hline 0.43 NA & NA & NA & NA & & rbl1438 & Gg_rs155771 \\
\hline $0.36 \mathrm{NA}$ & NA & NA & NA & & rbl1438 & Gg_rs155771 \\
\hline $0.38 \mathrm{NA}$ & NA & NA & NA & & rbl1438 & Gg_rs155771 \\
\hline 0.45 NA & NA & NA & NA & & rbl1438 & Gg_rs155771 \\
\hline 0.37 NA & NA & NA & NA & & rbl1438 & Gg_rs155771 \\
\hline $0.34 \mathrm{NA}$ & NA & NA & NA & & rbl1438 & Gg_rs155771 \\
\hline 0.18 & -0.67 & 0.19 & 0.67 & 0.24 & rbl1438 & Gg_rs155771 \\
\hline 0.55 & -1.37 & 0.58 & 1.26 & 0.74 & rbl1438 & Gg_rs155771 \\
\hline 0.62 NA & NA & NA & NA & & rbl1438 & Gg_rs155771 \\
\hline $0.32 \mathrm{NA}$ & NA & NA & NA & & rbl1438 & Gg_rs155771 \\
\hline $0.22 \mathrm{NA}$ & NA & NA & NA & & rbl1438 & Gg_rs155771 \\
\hline $0.36 \mathrm{NA}$ & NA & NA & NA & & rbl1438 & Gg_rs155771 \\
\hline
\end{tabular}




\begin{tabular}{|c|c|c|c|c|c|c|c|}
\hline $0.53 \mathrm{NA}$ & NA & & NA & NA & & rbl1438 & Gg_rs155771 \\
\hline 0.46 NA & NA & & NA & NA & & rbl1438 & Gg_rs155771 \\
\hline $0.25 \mathrm{NA}$ & NA & & NA & NA & & rbl1438 & Gg_rs155771 \\
\hline $0.32 \mathrm{NA}$ & NA & & NA & NA & & rbl1438 & Gg_rs155771 \\
\hline 0.59 & -2.33 & 0.62 & & 2.84 & 0.79 & rbl1438 & Gg_rs155771 \\
\hline $0.3 \mathrm{NA}$ & NA & & NA & NA & & rbl1438 & Gg_rs155771 \\
\hline 0.64 & -2.2 & 0.68 & & 2.41 & 0.87 & rbl1438 & Gg_rs155771 \\
\hline 0.42 & -1.28 & 0.44 & & 1.97 & 0.57 & rbl1438 & Gg_rs155771 \\
\hline $0.35 \mathrm{NA}$ & NA & & NA & NA & & rbl1438 & Gg_rs155771 \\
\hline $0.43 \mathrm{NA}$ & NA & & NA & NA & & rbl1438 & Gg_rs155771 \\
\hline 0.47 & -1.42 & 0.49 & & 1.67 & 0.63 & rbl1438 & Gg_rs155771 \\
\hline $0.3 \mathrm{NA}$ & NA & & NA & NA & & rbl1438 & Gg_rs155771 \\
\hline $0.26 \mathrm{NA}$ & NA & & NA & NA & & rbl1438 & Gg_rs155771 \\
\hline $0.75 \mathrm{NA}$ & NA & & NA & NA & & rbl1438 & Gg_rs155771 \\
\hline 0.43 NA & NA & & NA & NA & & rbl1438 & Gg_rs155771 \\
\hline $0.39 \mathrm{NA}$ & NA & & NA & NA & & rbl1438 & Gg_rs155771 \\
\hline 0.33 & -1.43 & 0.34 & & 0.95 & 0.44 & rbl1438 & Gg_rs155771 \\
\hline $0.24 \mathrm{NA}$ & NA & & NA & NA & & rbl1438 & Gg_rs155771 \\
\hline 0.15 & 0.28 & 0.15 & & -0.51 & 0.19 & Gg_rs156C & 5.Gg_rs144874 \\
\hline 0.74 NA & NA & & NA & NA & & X4_57822 & 53Gg_rs144874 \\
\hline 0.56 & -1.73 & 0.59 & & -2.87 & 0.75 & Gg_rs156C & 5.Gg_rs156084 \\
\hline $0.03 \mathrm{NA}$ & NA & & NA & NA & & Gg_rs156C & 5.Gg_rs156084 \\
\hline 1.03 & -1.32 & 1.07 & & -0.97 & 1.37 & Gg_rs156C & 5. chr4.7183334 \\
\hline $1.07 \mathrm{NA}$ & NA & & NA & NA & & MTUS_1 & chr4.7183334 \\
\hline $0.36 \mathrm{NA}$ & NA & & NA & NA & & Gg_rs156c & 5. chr4.7183334 \\
\hline 0.98 NA & NA & & NA & NA & & Gg_rs1447 & $2 \mathrm{chr} 4.7183334$ \\
\hline $0.38 \mathrm{NA}$ & NA & & NA & NA & & Gg_rs156c & 5. chr4.7183334 \\
\hline $0.5 \mathrm{NA}$ & NA & & NA & NA & & Gg_rs156C & 5. chr4.7183334 \\
\hline 0.79 NA & NA & & NA & NA & & Gg_rs156C & 5. chr4.7183334 \\
\hline 0.44 NA & NA & & NA & NA & & Gg_rs156c & 4 chr4.7183334 \\
\hline 0.96 & -1.87 & 0.95 & & -3.55 & 1.24 & Gg_rs156c & 4 chr4.7183334 \\
\hline 0.58 NA & NA & & NA & NA & & Gg_rs156C & 5. chr4.7183334 \\
\hline $0.52 \mathrm{NA}$ & NA & & NA & NA & & MTUS_1 & chr4.7183334 \\
\hline 0.7 & -1.31 & 0.69 & & -2.98 & 0.9 & Gg_rs156c & 4 chr4.7183334 \\
\hline $0.78 \mathrm{NA}$ & NA & & NA & NA & & Gg_rs156C & 5 chr4.7183334 \\
\hline 1.21 & -1.38 & 1.19 & & -4.24 & 1.56 & Gg_rs156C & 4 chr4.7183334 \\
\hline 0.77 NA & NA & & NA & NA & & Gg_rs156C & 4 chr4.7183334 \\
\hline 1.24 & -5.57 & 1.22 & & -2.95 & 1.61 & Gg_rs156 & 4 chr4.7183334 \\
\hline 0.75 NA & NA & & NA & NA & & Gg_rs156C & 4 chr4.7183334 \\
\hline $0.6 \mathrm{NA}$ & NA & & NA & NA & & Gg_rs156C & 4 chr4.7183334 \\
\hline $0.53 \mathrm{NA}$ & NA & & NA & NA & & Gg_rs156C & 4 chr4.7183334 \\
\hline $0.63 \mathrm{NA}$ & NA & & NA & NA & & Gg_rs156C & 4 chr4.7183334 \\
\hline $0.41 \mathrm{NA}$ & NA & & NA & NA & & Gg_rs156c & 4 chr4.7183334 \\
\hline
\end{tabular}




\begin{tabular}{|c|c|c|c|c|c|}
\hline 0.8 & -3.04 & 0.78 & -2.94 & 1.03 & Gg_rs156084 chr4.7183334 \\
\hline $0.51 \mathrm{NA}$ & NA & NA & NA & & Gg_rs156084 chr4.7183334 \\
\hline $0.27 \mathrm{NA}$ & NA & NA & NA & & Gg_rs156084 Gg_rs144887، \\
\hline $0.54 \mathrm{NA}$ & NA & NA & NA & & Gg_rs156084 chr4.7183334 \\
\hline $0.64 \mathrm{NA}$ & NA & NA & NA & & Gg_rs156035.Gg_rs144887. \\
\hline $1.17 \mathrm{NA}$ & NA & NA & NA & & Gg_rs144762 Gg_rs144966 \\
\hline $1.17 \mathrm{NA}$ & NA & NA & NA & & Gg_rs156035.Gg_rs144966 \\
\hline $0.68 \mathrm{NA}$ & NA & NA & NA & & Gg_rs144762 Gg_rs145002 \\
\hline 1.28 & -0.45 & 1.12 & -3.75 & 1.68 & Gg_rs156084 snp.131.95.1( \\
\hline $0.47 \mathrm{NA}$ & NA & NA & NA & & Gg_rs156084 snp.131.95.1( \\
\hline $0.57 \mathrm{NA}$ & NA & NA & NA & & Gg_rs156084 snp.131.95.1( \\
\hline $0.73 \mathrm{NA}$ & NA & NA & NA & & Gg_rs156084 snp.131.95.1( \\
\hline $0.65 \mathrm{NA}$ & NA & NA & NA & & Gg_rs156084 snp.131.95.1( \\
\hline $0.4 \mathrm{NA}$ & NA & NA & NA & & Gg_rs156084 snp.131.95.1( \\
\hline 0.52 & -1.84 & 0.46 & 0.16 & 0.68 & Gg_rs144874 snp.131.95.1( \\
\hline $0.72 \mathrm{NA}$ & NA & NA & NA & & Gg_rs156084 snp.131.95.1( \\
\hline 0.68 NA & NA & NA & NA & & Gg_rs156084 snp.131.95.1 \\
\hline $0.72 \mathrm{NA}$ & NA & NA & NA & & Gg_rs156084 snp.131.95.1( \\
\hline $0.95 \mathrm{NA}$ & NA & NA & NA & & Gg_rs156084 snp.131.95.1( \\
\hline $0.57 \mathrm{NA}$ & NA & NA & NA & & Gg_rs156084 snp.131.95.1( \\
\hline $0.56 \mathrm{NA}$ & NA & NA & NA & & Gg_rs144874 snp.131.95.1( \\
\hline $0.59 \mathrm{NA}$ & NA & NA & NA & & Gg_rs144874 Gg_rs156277 \\
\hline $1.03 \mathrm{NA}$ & NA & NA & NA & & Gg_rs156084 snp.131.95.1( \\
\hline 0.96 NA & NA & NA & NA & & Gg_rs144874 snp.131.95.1( \\
\hline $0.54 \mathrm{NA}$ & NA & NA & NA & & Gg_rs144874 snp.131.95.1( \\
\hline $0.74 \mathrm{NA}$ & NA & NA & NA & & Gg_rs144874 snp.131.95.1( \\
\hline $0.96 \mathrm{NA}$ & NA & NA & NA & & Gg_rs144874 snp.131.95.1( \\
\hline $0.42 \mathrm{NA}$ & NA & NA & NA & & Gg_rs144874 snp.131.95.1( \\
\hline $0.82 \mathrm{NA}$ & NA & NA & NA & & Gg_rs144874 snp.131.95.1( \\
\hline $1.31 \mathrm{NA}$ & NA & NA & NA & & Gg_rs144874 snp.131.95.1 \\
\hline 0.48 NA & NA & NA & NA & & Gg_rs144874 snp.131.95.1 \\
\hline $1.04 \mathrm{NA}$ & NA & NA & NA & & Gg_rs144874 Gg_rs144966 \\
\hline $0.58 \mathrm{NA}$ & NA & NA & NA & & Gg_rs144874 Gg_rs156277 \\
\hline $1.35 \mathrm{NA}$ & NA & NA & NA & & Gg_rs144874 snp.131.95.1( \\
\hline $0.37 \mathrm{NA}$ & NA & NA & NA & & Gg_rs144874 snp.131.95.1( \\
\hline 0.99 NA & NA & NA & NA & & Gg_rs144874 snp.131.95.1( \\
\hline $0.71 \mathrm{NA}$ & NA & NA & NA & & Gg_rs144874 snp.131.95.1 \\
\hline $0.74 \mathrm{NA}$ & NA & NA & NA & & Gg_rs144874 snp.131.95.1( \\
\hline $0.33 \mathrm{NA}$ & NA & NA & NA & & Gg_rs144874 Gg_rs156277 \\
\hline $0.49 \mathrm{NA}$ & NA & NA & NA & & Gg_rs144874 snp.131.95.1( \\
\hline $0.4 \mathrm{NA}$ & NA & NA & NA & & Gg_rs144874 snp.131.95.1( \\
\hline $1.08 \mathrm{NA}$ & NA & NA & NA & & Gg_rs144874 snp.131.95.1 \\
\hline $0.43 \mathrm{NA}$ & NA & NA & NA & & Gg_rs144874 snp.131.95. \\
\hline
\end{tabular}




\begin{tabular}{|c|c|c|c|c|c|c|}
\hline $0.54 \mathrm{NA}$ & NA & & NA & $\mathrm{N}$ & & Gg_rs144874Gg_rs156277 \\
\hline $0.38 \mathrm{NA}$ & NA & & NA & $\mathrm{N}$ & & Gg_rs144874 snp.131.95.1( \\
\hline 0.77 NA & NA & & NA & $\mathrm{N}$ & & Gg_rs144874 Gg_rs156277 \\
\hline 1.19 & -0.27 & 1.04 & & -4.34 & 1.56 & Gg_rs144874 Gg_rs156277 \\
\hline $0.4 \mathrm{NA}$ & NA & & NA & $\mathrm{N}$ & & Gg_rs144874Gg_rs156277 \\
\hline $0.68 \mathrm{NA}$ & NA & & NA & $\mathrm{N}$ & & Gg_rs144874 Gg_rs156277 \\
\hline $0.3 \mathrm{NA}$ & NA & & NA & $\mathrm{N}$ & & Gg_rs144874 snp.131.95.1( \\
\hline $0.51 \mathrm{NA}$ & NA & & NA & $\mathrm{N}$ & & Gg_rs144874 Gg_rs144966. \\
\hline $0.72 \mathrm{NA}$ & NA & & NA & $\mathrm{N}$ & & Gg_rs144874 Gg_rs156277 \\
\hline $1.18 \mathrm{NA}$ & NA & & NA & $\mathrm{N}$ & & Gg_rs144874 Gg_rs144966. \\
\hline $0.89 \mathrm{NA}$ & NA & & NA & $\mathrm{N}$ & & Gg_rs144874Gg_rs145002 \\
\hline $0.71 \mathrm{NA}$ & NA & & NA & $\mathrm{N}$ & & Gg_rs144874Gg_rs156277 \\
\hline $0.82 \mathrm{NA}$ & NA & & NA & $\mathrm{N}$ & & Gg_rs144874 Gg_rs144966. \\
\hline 0.11 & 0.52 & 0.11 & & 0.38 & 0.14 & Gg_rs144887:Gg_rs156277 \\
\hline 0.45 & 1.58 & 0.4 & & 2.39 & 0.56 & Gg_rs144887.Gg_rs156277 \\
\hline 0.77 NA & NA & & NA & $\mathrm{N}$ & & Gg_rs144874Gg_rs144966. \\
\hline 0.63 & -0.35 & 0.57 & & -4.21 & 0.79 & Gg_rs144887.snp.454.6.31؛ \\
\hline $0.55 \mathrm{NA}$ & NA & & NA & $\mathrm{N}$ & & Gg_rs144874Gg_rs156277 \\
\hline 1.12 & -2.38 & 1.02 & & -3.51 & 1.41 & Gg_rs144887:Gg_rs156277 \\
\hline 0.77 & 0.05 & 0.7 & & -3.37 & 0.97 & Gg_rs144887.snp.454.6.31 \\
\hline 0.69 & 0.17 & 0.62 & & -2.55 & 0.86 & Gg_rs144887.Gg_rs156277 \\
\hline $0.75 \mathrm{NA}$ & NA & & NA & $\mathrm{N}$ & & Gg_rs144887.snp.454.6.31؛ \\
\hline 0.85 & 0.27 & 0.77 & & -3.04 & 1.07 & Gg_rs144887.snp.454.6.31 \\
\hline 0.87 & -0.95 & 0.79 & & -4.21 & 1.1 & Gg_rs144887.snp.454.6.31؛ \\
\hline 0.75 & 0.39 & 0.68 & & -3.84 & 0.94 & Gg_rs144887.snp.454.6.31 \\
\hline 0.87 & -0.6 & 0.79 & & -4.32 & 1.1 & Gg_rs144887.snp.454.6.31 \\
\hline 0.77 & 0.03 & 0.7 & & -3.65 & 0.97 & Gg_rs144887.snp.454.6.31 \\
\hline 0.7 & -0.71 & 0.64 & & -3.47 & 0.88 & Gg_rs144887.snp.454.6.31 \\
\hline $0.62 \mathrm{NA}$ & NA & & NA & $\mathrm{N}$ & & Gg_rs144887.Gg_rs156277 \\
\hline $0.51 \mathrm{NA}$ & NA & & NA & $\mathrm{N}$ & & Gg_rs144887:Gg_rs156277 \\
\hline 0.59 NA & NA & & NA & $\mathrm{N}$ & & Gg_rs144887:Gg_rs156277 \\
\hline $0.69 \mathrm{NA}$ & NA & & NA & $\mathrm{N}$ & & Gg_rs144887.snp.454.6.31 \\
\hline $0.75 \mathrm{NA}$ & NA & & NA & $\mathrm{N}$ & & Gg_rs144887:Gg_rs156277 \\
\hline $0.68 \mathrm{NA}$ & NA & & NA & $\mathrm{N}$ & & Gg_rs144887.snp.454.6.31 \\
\hline $0.54 \mathrm{NA}$ & NA & & NA & $\mathrm{N}$ & & Gg_rs144887.Gg_rs156277 \\
\hline 0.81 & -0.92 & 0.74 & & -4.69 & 1.02 & Gg_rs144887.snp.454.6.31 \\
\hline 0.86 & -0.28 & 0.78 & & -5.09 & 1.08 & Gg_rs144887.snp.454.6.31 \\
\hline 0.15 & 0.7 & 0.13 & & 0.91 & 0.18 & Gg_rs144887.Gg_rs156277 \\
\hline 0.29 & 1.6 & 0.26 & & 2.01 & 0.36 & Gg_rs144887.Gg_rs156277 \\
\hline 0.61 & 0.11 & 0.55 & & -3.12 & 0.76 & Gg_rs144887.snp.454.6.31 \\
\hline 1 & -1.31 & 0.91 & & -4.63 & 1.26 & Gg_rs144887.snp.454.6.31؛ \\
\hline $0.48 \mathrm{NA}$ & NA & & NA & $\mathrm{N}$ & & Gg_rs144887.snp.454.6.31 \\
\hline 0.39 NA & NA & & NA & $\mathrm{N}$ & & Gg_rs144887.snp.454.6.31؛ \\
\hline
\end{tabular}




\begin{tabular}{|c|c|c|c|c|c|}
\hline 0.55 NA & NA & NA & NA & & Gg_rs144887.snp.454.6.31 \\
\hline $0.54 \mathrm{NA}$ & NA & NA & NA & & Gg_rs144887:Gg_rs156277 \\
\hline $0.64 \mathrm{NA}$ & NA & NA & NA & & Gg_rs144887.snp.454.6.31\{ \\
\hline $0.56 \mathrm{NA}$ & NA & NA & NA & & Gg_rs144887.snp.454.6.31\{ \\
\hline $0.51 \mathrm{NA}$ & NA & NA & NA & & Gg_rs144887.snp.454.6.31 \\
\hline $0.32 \mathrm{NA}$ & NA & NA & NA & & Gg_rs144887.snp.454.6.31 \\
\hline 0.85 & -0.26 & 0.77 & -4.49 & 1.07 & Gg_rs144887.snp.454.6.31؛ \\
\hline $0.81 \mathrm{NA}$ & NA & NA & NA & & Gg_rs144874 Gg_rs156277 \\
\hline $0.4 \mathrm{NA}$ & NA & NA & NA & & Gg_rs144874Gg_rs145002 \\
\hline $0.59 \mathrm{NA}$ & NA & NA & NA & & Gg_rs144887.Gg_rs156277 \\
\hline $0.62 \mathrm{NA}$ & NA & NA & NA & & Gg_rs144887:Gg_rs156277 \\
\hline 0.46 NA & NA & NA & NA & & chr4.7183334Gg_rs156277 \\
\hline 0.46 NA & NA & NA & NA & & Gg_rs144887.Gg_rs144966. \\
\hline $0.63 \mathrm{NA}$ & NA & NA & NA & & Gg_rs144874Gg_rs156277 \\
\hline $0.51 \mathrm{NA}$ & NA & NA & NA & & Gg_rs144887:Gg_rs156277 \\
\hline $0.41 \mathrm{NA}$ & NA & NA & NA & & Gg_rs144887:Gg_rs156277 \\
\hline 0.87 NA & NA & NA & NA & & Gg_rs144887.Gg_rs156277 \\
\hline 0.85 & -1.39 & 0.8 & 0.77 & 1.08 & snp.131.95.1(Gg_rs156277 \\
\hline $1.14 \mathrm{NA}$ & NA & NA & NA & & snp.131.95.1(Gg_rs156277 \\
\hline 0.48 NA & NA & NA & NA & & snp.131.95.1(Gg_rs156277 \\
\hline 0.49 NA & NA & NA & NA & & snp.131.95.1(Gg_rs156277 \\
\hline $0.77 \mathrm{NA}$ & NA & NA & NA & & snp.131.95.1(Gg_rs156277 \\
\hline 0.76 & -1.63 & 0.71 & 0.06 & 0.96 & snp.131.95.1(Gg_rs156277 \\
\hline 0.39 NA & NA & NA & NA & & chr4.7183334Gg_rs156277 \\
\hline 0.63 NA & NA & NA & NA & & Gg_rs144887.Gg_rs156277 \\
\hline $0.52 \mathrm{NA}$ & NA & NA & NA & & Gg_rs144874 Gg_rs156277 \\
\hline 0.15 & 0.55 & 0.14 & 0.22 & 0.19 & Gg_rs144887.Gg_rs156277 \\
\hline 1.02 & -2.09 & 0.96 & -3.05 & 1.29 & snp.131.95.1(Gg_rs156277 \\
\hline 0.52 NA & NA & NA & NA & & Gg_rs144874Gg_rs144966 \\
\hline $0.56 \mathrm{NA}$ & NA & NA & NA & & Gg_rs144887:Gg_rs156277 \\
\hline 0.43 NA & NA & NA & NA & & snp.131.95.1(Gg_rs156277 \\
\hline 0.67 NA & NA & NA & NA & & snp.131.95.1(Gg_rs156277 \\
\hline $0.31 \mathrm{NA}$ & NA & NA & NA & & snp.131.95.1(Gg_rs156277 \\
\hline $0.38 \mathrm{NA}$ & NA & NA & NA & & Gg_rs144874 Gg_rs144966. \\
\hline $0.37 \mathrm{NA}$ & NA & NA & NA & & snp.131.95.1(Gg_rs144966 \\
\hline $0.35 \mathrm{NA}$ & NA & NA & NA & & snp.131.95.1(Gg_rs144966 \\
\hline $0.64 \mathrm{NA}$ & NA & NA & NA & & Gg_rs144874 Gg_rs144966. \\
\hline $0.48 \mathrm{NA}$ & NA & NA & NA & & Gg_rs138210.Gg_rs144966 \\
\hline $0.55 \mathrm{NA}$ & NA & NA & NA & & snp.131.95.1(Gg_rs144966. \\
\hline $0.41 \mathrm{NA}$ & NA & NA & NA & & Gg_rs138210.Gg_rs144966 \\
\hline $0.85 \mathrm{NA}$ & NA & NA & NA & & Gg_rs144887:Gg_rs144966 \\
\hline $0.56 \mathrm{NA}$ & NA & NA & NA & & Gg_rs144887.Gg_rs144966. \\
\hline $0.81 \mathrm{NA}$ & NA & NA & NA & & Gg_rs144887.Gg_rs144966. \\
\hline
\end{tabular}




\begin{tabular}{|c|c|c|c|c|c|c|}
\hline $0.77 \mathrm{NA}$ & NA & & NA & NA & & snp.131.95.1(Gg_rs144966 \\
\hline 0.73 NA & NA & & NA & NA & & Gg_rs138210.Gg_rs144966 \\
\hline 0.67 NA & NA & & NA & NA & & Gg_rs156277 Gg_rs145002 \\
\hline $0.99 \mathrm{NA}$ & NA & & NA & NA & & Gg_rs156277 Gg_rs145002 \\
\hline 0.53 & 2.67 & 0.55 & & 2.89 & 0.62 & Gg_rs156277 Gg_rs145002 \\
\hline 0.08 & 0.48 & 0.09 & & 0.42 & 0.1 & Gg_rs156277 Gg_rs145002 \\
\hline 0.42 & 1.5 & 0.43 & & 1.85 & 0.49 & Gg_rs156277 Gg_rs145002 \\
\hline 0.97 NA & NA & & NA & NA & & Gg_rs156277 Gg_rs145002 \\
\hline 1.04 & 2.28 & 1.01 & & 5.61 & 1.37 & Gg_rs156310.Gg_rs145002 \\
\hline 0.69 NA & NA & & NA & NA & & Gg_rs156310Gg_rs145002 \\
\hline 0.56 & 0.18 & 0.55 & & 3.62 & 0.74 & Gg_rs156310Gg_rs145002 \\
\hline 0.4 & 1.05 & 0.39 & & 1.4 & 0.53 & Gg_rs156310Gg_rs145002 \\
\hline 0.57 & 0.38 & 0.55 & & 3.42 & 0.75 & Gg_rs156310.Gg_rs145002 \\
\hline 0.58 & 2.05 & 0.57 & & 2.77 & 0.77 & Gg_rs156310.Gg_rs145002 \\
\hline 0.55 & 0.44 & 0.54 & & 3.33 & 0.73 & Gg_rs156310.Gg_rs145002 \\
\hline $0.54 \mathrm{NA}$ & NA & & NA & NA & & Gg_rs156310Gg_rs145002 \\
\hline $0.32 \mathrm{NA}$ & NA & & NA & NA & & Gg_rs156310Gg_rs145002 \\
\hline 0.77 & 0.2 & 0.75 & & 4.37 & 1.01 & Gg_rs156310Gg_rs145002 \\
\hline $0.72 \mathrm{NA}$ & NA & & NA & NA & & Gg_rs156310Gg_rs145002 \\
\hline 0.44 & 0.32 & 0.42 & & 2.92 & 0.58 & Gg_rs156310.Gg_rs145002 \\
\hline 0.48 NA & NA & & NA & NA & & Gg_rs156310.Gg_rs145002 \\
\hline 0.49 NA & NA & & NA & NA & & Gg_rs156310.Gg_rs145002 \\
\hline 0.64 & 0.31 & 0.62 & & 3.72 & 0.84 & Gg_rs156310.Gg_rs145002 \\
\hline 0.37 & -0.5 & 0.36 & & 1.2 & 0.48 & Gg_rs156310Gg_rs145002 \\
\hline 0.81 & 2.75 & 0.79 & & 2.75 & 1.07 & Gg_rs156310.Gg_rs145002 \\
\hline $0.39 \mathrm{NA}$ & NA & & NA & NA & & Gg_rs156310.Gg_rs145002 \\
\hline 0.57 & 0.08 & 0.56 & & 3.23 & 0.75 & Gg_rs156310.Gg_rs145002 \\
\hline $1.68 \mathrm{NA}$ & NA & & NA & NA & & Gg_rs156310.Gg_rs145002 \\
\hline $2.11 \mathrm{NA}$ & NA & & NA & NA & & Gg_rs156310.Gg_rs145002 \\
\hline $2.45 \mathrm{NA}$ & NA & & NA & NA & & Gg_rs156310Gg_rs145002 \\
\hline $2.33 \mathrm{NA}$ & NA & & NA & NA & & Gg_rs156310.Gg_rs145002 \\
\hline $1.81 \mathrm{NA}$ & NA & & NA & NA & & Gg_rs156310.Gg_rs145002 \\
\hline $2.58 \mathrm{NA}$ & NA & & NA & NA & & Gg_rs156310.Gg_rs145002 \\
\hline $1.55 \mathrm{NA}$ & NA & & NA & NA & & Gg_rs156310.Gg_rs145002 \\
\hline $2.05 \mathrm{NA}$ & NA & & NA & NA & & Gg_rs156310Gg_rs145002 \\
\hline $1.59 \mathrm{NA}$ & NA & & NA & NA & & Gg_rs156310.Gg_rs145002 \\
\hline $2.28 \mathrm{NA}$ & NA & & NA & NA & & Gg_rs156310.Gg_rs145002 \\
\hline $1.71 \mathrm{NA}$ & NA & & NA & NA & & Gg_rs156310.Gg_rs145002 \\
\hline $2.83 \mathrm{NA}$ & NA & & NA & NA & & Gg_rs156310.Gg_rs145002 \\
\hline $0.86 \mathrm{NA}$ & NA & & NA & NA & & Gg_rs156310.Gg_rs145002 \\
\hline $1.47 \mathrm{NA}$ & NA & & NA & NA & & Gg_rs156310.Gg_rs145002 \\
\hline $2.03 \mathrm{NA}$ & NA & & NA & NA & & Gg_rs156310.Gg_rs145002 \\
\hline $1.46 \mathrm{NA}$ & NA & & NA & NA & & Gg_rs156310 Gg_rs145002 \\
\hline
\end{tabular}




\begin{tabular}{|c|c|c|c|c|c|}
\hline $1.58 \mathrm{NA}$ & NA & NA & NA & & Gg_rs156310.Gg_rs145002 \\
\hline $1.45 \mathrm{NA}$ & NA & NA & NA & & Gg_rs156310.Gg_rs145002 \\
\hline $1.77 \mathrm{NA}$ & NA & NA & NA & & Gg_rs156310Gg_rs145002 \\
\hline $2.16 \mathrm{NA}$ & NA & NA & NA & & Gg_rs156310Gg_rs145002 \\
\hline $2.76 \mathrm{NA}$ & NA & NA & NA & & Gg_rs156310Gg_rs145002 \\
\hline $1.16 \mathrm{NA}$ & NA & NA & NA & & Gg_rs156310Gg_rs145002 \\
\hline $2.16 \mathrm{NA}$ & NA & NA & NA & & Gg_rs156310Gg_rs145002 \\
\hline $2.23 \mathrm{NA}$ & NA & NA & NA & & Gg_rs156310.Gg_rs145002 \\
\hline $1.79 \mathrm{NA}$ & NA & NA & NA & & Gg_rs156310.Gg_rs145002 \\
\hline $2.66 \mathrm{NA}$ & NA & NA & NA & & Gg_rs156310 Gg_rs145002 \\
\hline $2.56 \mathrm{NA}$ & NA & NA & NA & & Gg_rs156310Gg_rs145002 \\
\hline $1.97 \mathrm{NA}$ & NA & NA & NA & & Gg_rs156310.Gg_rs145002 \\
\hline $2.57 \mathrm{NA}$ & NA & NA & NA & & Gg_rs156310.Gg_rs145002 \\
\hline 1.37 & -2.46 & 1.33 & -0.71 & 1.81 & Gg_rs156310.Gg_rs145002 \\
\hline $1.68 \mathrm{NA}$ & NA & NA & NA & & Gg_rs156310.Gg_rs145002 \\
\hline $1.87 \mathrm{NA}$ & NA & NA & NA & & Gg_rs156310 Gg_rs145002 \\
\hline $2.2 \mathrm{NA}$ & NA & NA & NA & & Gg_rs156310Gg_rs145002 \\
\hline $2.9 \mathrm{NA}$ & NA & NA & NA & & Gg_rs156310.Gg_rs145002 \\
\hline $2.13 \mathrm{NA}$ & NA & NA & NA & & Gg_rs156310Gg_rs145002 \\
\hline $2.85 \mathrm{NA}$ & NA & NA & NA & & Gg_rs156310Gg_rs145002 \\
\hline $1.69 \mathrm{NA}$ & NA & NA & NA & & Gg_rs156310.Gg_rs145002 \\
\hline $1.18 \mathrm{NA}$ & NA & NA & NA & & Gg_rs156310.Gg_rs145002 \\
\hline $1.36 \mathrm{NA}$ & NA & NA & NA & & Gg_rs156310.Gg_rs145002 \\
\hline $1.96 \mathrm{NA}$ & NA & NA & NA & & Gg_rs156310 Gg_rs145002 \\
\hline $1.5 \mathrm{NA}$ & NA & NA & NA & & Gg_rs156310Gg_rs145002 \\
\hline $1.45 \mathrm{NA}$ & NA & NA & NA & & Gg_rs156310Gg_rs145002 \\
\hline $1.91 \mathrm{NA}$ & NA & NA & NA & & Gg_rs156310.Gg_rs145002 \\
\hline 0.85 & 1.2 & 0.83 & 2.36 & 1.13 & Gg_rs156310.Gg_rs145002 \\
\hline $0.39 \mathrm{NA}$ & NA & NA & NA & & Gg_rs156310Gg_rs145002 \\
\hline $0.52 \mathrm{NA}$ & NA & NA & NA & & Gg_rs156310Gg_rs145002 \\
\hline $0.52 \mathrm{NA}$ & NA & NA & NA & & Gg_rs156310.Gg_rs145002 \\
\hline $0.47 \mathrm{NA}$ & NA & NA & NA & & Gg_rs156310.Gg_rs145002 \\
\hline 0.95 & 2.96 & 0.93 & -0.33 & 1.26 & Gg_rs156310Gg_rs145002 \\
\hline $0.88 \mathrm{NA}$ & NA & NA & NA & & Gg_rs156310.Gg_rs145002 \\
\hline $0.77 \mathrm{NA}$ & NA & NA & NA & & Gg_rs156310Gg_rs145002 \\
\hline $0.92 \mathrm{NA}$ & NA & NA & NA & & Gg_rs156310.Gg_rs145002 \\
\hline $0.64 \mathrm{NA}$ & NA & NA & NA & & Gg_rs156310.Gg_rs145002 \\
\hline 0.64 & 1.52 & 0.63 & 1.52 & 0.85 & Gg_rs156310Gg_rs145002 \\
\hline $0.64 \mathrm{NA}$ & NA & NA & NA & & Gg_rs156310.Gg_rs145002 \\
\hline $0.57 \mathrm{NA}$ & NA & NA & NA & & Gg_rs156310Gg_rs145002 \\
\hline $0.46 \mathrm{NA}$ & NA & NA & NA & & Gg_rs156310.Gg_rs145002 \\
\hline $0.42 \mathrm{NA}$ & NA & NA & NA & & Gg_rs156310.Gg_rs145002 \\
\hline 0.85 & 1.8 & 0.83 & -0.03 & 1.13 & Gg_rs156310Gg_rs145002 \\
\hline
\end{tabular}




\begin{tabular}{|c|c|c|c|c|c|c|c|c|}
\hline $0.51 \mathrm{NA}$ & & NA & & NA & & NA & & Gg_rs156310.Gg_rs145002 \\
\hline $0.59 \mathrm{NA}$ & & NA & & NA & & NA & & Gg_rs156310.Gg_rs145002 \\
\hline 0.57 & 1.2 & & 0.56 & & 1.2 & & 0.76 & Gg_rs156310.Gg_rs145002 \\
\hline 0.61 & 1.44 & & 0.59 & & 0.34 & & 0.8 & Gg_rs156310Gg_rs145002 \\
\hline 0.74 & 1.73 & & 0.72 & & 1.05 & & 0.98 & Gg_rs156310.Gg_rs145002 \\
\hline 0.78 & 1.67 & & 0.76 & & 1.53 & & 1.03 & Gg_rs156310.Gg_rs145002 \\
\hline 0.54 & 1.02 & & 0.52 & & 1.72 & & 0.71 & Gg_rs156310.Gg_rs145002 \\
\hline 0.42 & 0.71 & & 0.41 & & 0.85 & & 0.56 & Gg_rs156310Gg_rs145002 \\
\hline 0.82 & 2.64 & & 0.8 & & 2.62 & & 1.09 & Gg_rs156310Gg_rs145002 \\
\hline 1.22 & 2.44 & & 1.18 & & 2.85 & & 1.61 & Gg_rs156310.Gg_rs145002 \\
\hline 0.8 & 1.73 & & 0.78 & & 1.37 & & 1.06 & Gg_rs156310.Gg_rs145002 \\
\hline 0.51 & 0.68 & & 0.5 & & 1.36 & & 0.67 & Gg_rs156310Gg_rs145002 \\
\hline 0.56 & 1.05 & & 0.55 & & 1.25 & & 0.74 & Gg_rs156310Gg_rs145002 \\
\hline $0.41 \mathrm{NA}$ & & NA & & NA & & NA & & Gg_rs156310.Gg_rs145002 \\
\hline 0.45 & 1.23 & & 0.44 & & 1.74 & & 0.59 & Gg_rs156310Gg_rs145002 \\
\hline $0.63 \mathrm{NA}$ & & NA & & NA & & NA & & Gg_rs156310Gg_rs145002 \\
\hline $1.17 \mathrm{NA}$ & & NA & & NA & & NA & & Gg_rs156310.Gg_rs145002 \\
\hline $2.11 \mathrm{NA}$ & & NA & & NA & & NA & & Gg_rs156310.Gg_rs145002 \\
\hline $2.13 \mathrm{NA}$ & & NA & & NA & & NA & & Gg_rs156310 Gg_rs145002 \\
\hline $1.53 \mathrm{NA}$ & & NA & & NA & & NA & & Gg_rs156310.Gg_rs145002 \\
\hline $3.05 \mathrm{NA}$ & & NA & & NA & & NA & & Gg_rs156310.Gg_rs145002 \\
\hline $1.17 \mathrm{NA}$ & & NA & & NA & & NA & & Gg_rs156310Gg_rs145002 \\
\hline $2.47 \mathrm{NA}$ & & NA & & NA & & NA & & Gg_rs156310Gg_rs145002 \\
\hline $1.77 \mathrm{NA}$ & & NA & & NA & & NA & & Gg_rs156310 Gg_rs145002 \\
\hline $2.59 \mathrm{NA}$ & & NA & & NA & & NA & & Gg_rs156310.Gg_rs145002 \\
\hline $2.28 \mathrm{NA}$ & & NA & & NA & & NA & & Gg_rs156310 Gg_rs145002 \\
\hline $2.35 \mathrm{NA}$ & & NA & & NA & & NA & & Gg_rs156310Gg_rs145002 \\
\hline $1.92 \mathrm{NA}$ & & NA & & NA & & NA & & Gg_rs156310Gg_rs145002 \\
\hline $1.89 \mathrm{NA}$ & & NA & & NA & & NA & & Gg_rs156310 Gg_rs145002 \\
\hline 2.09 NA & & NA & & NA & & NA & & Gg_rs156310.Gg_rs145002 \\
\hline $2.77 \mathrm{NA}$ & & NA & & NA & & NA & & Gg_rs156310.Gg_rs145002 \\
\hline $2.27 \mathrm{NA}$ & & NA & & NA & & NA & & Gg_rs156310 Gg_rs145002 \\
\hline $2.92 \mathrm{NA}$ & & NA & & NA & & NA & & Gg_rs156310 Gg_rs145002 \\
\hline $1.77 \mathrm{NA}$ & & NA & & NA & & NA & & Gg_rs156310 Gg_rs145002 \\
\hline $1.08 \mathrm{NA}$ & & NA & & NA & & NA & & Gg_rs156310Gg_rs145002 \\
\hline $2.25 \mathrm{NA}$ & & NA & & NA & & NA & & Gg_rs156310Gg_rs145002 \\
\hline $2.14 \mathrm{NA}$ & & NA & & NA & & NA & & Gg_rs156310.Gg_rs145002 \\
\hline $1.27 \mathrm{NA}$ & & NA & & NA & & NA & & Gg_rs156310.Gg_rs145002 \\
\hline $1.79 \mathrm{NA}$ & & NA & & NA & & NA & & Gg_rs156310.Gg_rs145002 \\
\hline $1.02 \mathrm{NA}$ & & NA & & NA & & NA & & Gg_rs156310Gg_rs145002 \\
\hline $1.76 \mathrm{NA}$ & & NA & & NA & & NA & & Gg_rs156310Gg_rs145002 \\
\hline $1.34 \mathrm{NA}$ & & NA & & NA & & NA & & Gg_rs156310Gg_rs145002 \\
\hline $2.34 \mathrm{NA}$ & & NA & & NA & & NA & & Gg_rs156310 Gg_rs145002 \\
\hline
\end{tabular}




\begin{tabular}{|c|c|c|c|c|c|}
\hline $1.92 \mathrm{NA}$ & NA & NA & NA & & Gg_rs156310.Gg_rs145002 \\
\hline $2.31 \mathrm{NA}$ & NA & NA & NA & & Gg_rs156310.Gg_rs145002 \\
\hline $0.52 \mathrm{NA}$ & NA & NA & NA & & Gg_rs156310.Gg_rs145002 \\
\hline $0.51 \mathrm{NA}$ & NA & NA & NA & & Gg_rs156310Gg_rs145002 \\
\hline 0.52 & -0.45 & 0.5 & 3.49 & 0.68 & Gg_rs156310.Gg_rs145002 \\
\hline $0.04 \mathrm{NA}$ & NA & NA & NA & & Gg_rs156490 Gg_rs145097. \\
\hline $1.08 \mathrm{NA}$ & NA & NA & NA & & Gg_rs156490 Gg_rs143531 \\
\hline $0.64 \mathrm{NA}$ & NA & NA & NA & & Gg_rs156490Gg_rs143531 \\
\hline 0.96 & -1.95 & 1.02 & -1.85 & 1.3 & Gg_rs156490 Gg_rs143531 \\
\hline $0.43 \mathrm{NA}$ & NA & NA & NA & & X5_5571125 Gg_rs145189. \\
\hline $0.31 \mathrm{NA}$ & NA & NA & NA & & X5_5571125 Gg_rs145189. \\
\hline 0.77 & -1.95 & 0.65 & -1.95 & 1.01 & X5_5571125 Gg_rs156661 \\
\hline $0.42 \mathrm{NA}$ & NA & NA & NA & & X5_5571125 Gg_rs145189. \\
\hline 1.02 & -2.94 & 0.86 & 0.11 & 1.34 & X5_5571125 Gg_rs145189. \\
\hline $0.64 \mathrm{NA}$ & NA & NA & NA & & X5_5571125 Gg_rs145189. \\
\hline $1.06 \mathrm{NA}$ & NA & NA & NA & & X5_5571125 Gg_rs145189. \\
\hline $0.38 \mathrm{NA}$ & NA & NA & NA & & X5_5571125 Gg_rs145189. \\
\hline $1.17 \mathrm{NA}$ & NA & NA & NA & & X5_5571125 snp.98.79.91( \\
\hline $0.28 \mathrm{NA}$ & NA & NA & NA & & X5_5571125 Gg_rs145189. \\
\hline $0.9 \mathrm{NA}$ & NA & NA & NA & & X5_5571125 Gg_rs145189. \\
\hline 1.53 & -3.57 & 1.29 & -1.42 & 2.01 & X5_5571125 Gg_rs145189. \\
\hline $1.1 \mathrm{NA}$ & NA & NA & NA & & X5_5571125 X5_21008191 \\
\hline $0.52 \mathrm{NA}$ & NA & NA & NA & & X5_5571125 Gg_rs145189. \\
\hline $1.41 \mathrm{NA}$ & NA & NA & NA & & X5_5571125 Gg_rs145189. \\
\hline $0.54 \mathrm{NA}$ & NA & NA & NA & & X5_5571125 Gg_rs156661 \\
\hline $0.39 \mathrm{NA}$ & NA & NA & NA & & X5_5571125 Gg_rs145189. \\
\hline $0.67 \mathrm{NA}$ & NA & NA & NA & & X5_5571125 X5_21008191 \\
\hline $0.38 \mathrm{NA}$ & NA & NA & NA & & X5_5571125 Gg_rs145189. \\
\hline 1.33 & -1.95 & 1.15 & -2.05 & 1.76 & X5_5571125 snp.98.79.91( \\
\hline $1.02 \mathrm{NA}$ & NA & NA & NA & & X5_5571125 Gg_rs145189. \\
\hline $0.54 \mathrm{NA}$ & NA & NA & NA & & X5_5571125 Gg_rs145189. \\
\hline 0.48 NA & NA & NA & NA & & X5_5571125 Gg_rs145189. \\
\hline $0.48 \mathrm{NA}$ & NA & NA & NA & & X5_5571125 Gg_rs145189. \\
\hline 0.44 NA & NA & NA & NA & & X5_5571125 Gg_rs145189. \\
\hline 0.49 NA & NA & NA & NA & & X5_5571125 Gg_rs145189. \\
\hline $0.54 \mathrm{NA}$ & NA & NA & NA & & X5_5571125 Gg_rs145189. \\
\hline $0.47 \mathrm{NA}$ & NA & NA & NA & & X5_5571125 Gg_rs145189. \\
\hline $0.66 \mathrm{NA}$ & NA & NA & NA & & X5_5571125 Gg_rs145189. \\
\hline $0.36 \mathrm{NA}$ & NA & NA & NA & & X5_5571125 Gg_rs145189. \\
\hline $0.74 \mathrm{NA}$ & NA & NA & NA & & X5_5571125 Gg_rs145189. \\
\hline 0.57 & -1 & 0.5 & 0.59 & 0.76 & X5_5571125 Gg_rs145189. \\
\hline $0.75 \mathrm{NA}$ & NA & NA & NA & & X5_5571125 Gg_rs145189. \\
\hline $0.55 \mathrm{NA}$ & NA & NA & NA & & X5_5571125 Gg_rs145189. \\
\hline
\end{tabular}




\begin{tabular}{|c|c|c|c|c|c|}
\hline $0.43 \mathrm{NA}$ & NA & NA & NA & & X5_5571125 Gg_rs145189. \\
\hline $0.93 \mathrm{NA}$ & NA & NA & NA & & X5_5571125 Gg_rs145189. \\
\hline 0.89 NA & NA & NA & NA & & X5_5571125 Gg_rs145189. \\
\hline $0.54 \mathrm{NA}$ & NA & NA & NA & & X5_5571125 Gg_rs145189. \\
\hline $0.81 \mathrm{NA}$ & NA & NA & NA & & X5_5571125 Gg_rs145189. \\
\hline $0.54 \mathrm{NA}$ & NA & NA & NA & & X5_5571125 Gg_rs145189. \\
\hline $0.6 \mathrm{NA}$ & NA & NA & NA & & X5_5571125 Gg_rs145189. \\
\hline $0.18 \mathrm{NA}$ & NA & NA & NA & & Gg_rs145155 Gg_rs145189. \\
\hline $0.66 \mathrm{NA}$ & NA & NA & NA & & X5_5571125 Gg_rs145189. \\
\hline 0.67 & 0.07 & 0.58 & -2.96 & 0.89 & X5_5571125 snp.98.79.91( \\
\hline $1.59 \mathrm{NA}$ & NA & NA & NA & & X5_5571125 snp.98.79.91( \\
\hline $0.41 \mathrm{NA}$ & NA & NA & NA & & X5_5571125 snp.98.79.91( \\
\hline $0.56 \mathrm{NA}$ & NA & NA & NA & & X5_5571125 Gg_rs145189. \\
\hline $0.64 \mathrm{NA}$ & NA & NA & NA & & X5_5571125 snp.98.79.91( \\
\hline $0.37 \mathrm{NA}$ & NA & NA & NA & & X5_5571125 X5_21008191 \\
\hline $0.5 \mathrm{NA}$ & NA & NA & NA & & X5_5571125 snp.98.79.91( \\
\hline $0.05 \mathrm{NA}$ & NA & NA & NA & & X5_5571125 snp.98.79.91( \\
\hline $0.42 \mathrm{NA}$ & NA & NA & NA & & X5_5571125 Gg_rs145189. \\
\hline $0.38 \mathrm{NA}$ & NA & NA & NA & & X5_5571125 Gg_rs145189. \\
\hline $0.35 \mathrm{NA}$ & NA & NA & NA & & X5_5571125 Gg_rs145189. \\
\hline $1.04 \mathrm{NA}$ & NA & NA & NA & & X5_5571125 snp.98.79.91( \\
\hline $0.48 \mathrm{NA}$ & NA & NA & NA & & X5_5571125 X5_21008191 \\
\hline $0.89 \mathrm{NA}$ & NA & NA & NA & & X5_5571125 X5_21008191 \\
\hline $0.81 \mathrm{NA}$ & NA & NA & NA & & X5_5571125 X5_21008191 \\
\hline 0.43 NA & NA & NA & NA & & X5_5571125 snp.98.79.91( \\
\hline $0.84 \mathrm{NA}$ & NA & NA & NA & & X5_5571125 X5_21008191 \\
\hline $0.72 \mathrm{NA}$ & NA & NA & NA & & X5_5571125 X5_21008191 \\
\hline 0.93 & -0.63 & 0.79 & -3.38 & 1.22 & X5_5571125 snp.98.79.91( \\
\hline $0.69 \mathrm{NA}$ & NA & NA & NA & & X5_5571125 X5_21008191 \\
\hline $0.75 \mathrm{NA}$ & NA & NA & NA & & X5_5571125 X5_21008191 \\
\hline $1.05 \mathrm{NA}$ & NA & NA & NA & & X5_5571125 snp.98.79.91( \\
\hline 0.83 NA & NA & NA & NA & & X5_5571125 X5_21008191 \\
\hline 1.3 & -1.81 & 1.1 & -1.96 & 1.71 & X5_5571125 X5_21008191 \\
\hline 0.85 & -1.39 & 0.84 & -1.71 & 1.12 & X5_5571125 X5_21008191 \\
\hline $0.42 \mathrm{NA}$ & NA & NA & NA & & X5_5571125 X5_21008191 \\
\hline $1.33 \mathrm{NA}$ & NA & NA & NA & & X5_5571125 X5_21008191 \\
\hline 0.73 NA & NA & NA & NA & & X5_5571125 X5_21008191 \\
\hline $0.59 \mathrm{NA}$ & NA & NA & NA & & Gg_rs156661 snp.98.79.91( \\
\hline $0.57 \mathrm{NA}$ & NA & NA & NA & & Gg_rs156661X5_21008191 \\
\hline $0.79 \mathrm{NA}$ & NA & NA & NA & & Gg_rs145155 snp.98.79.91( \\
\hline 1.69 & -2.68 & 1.43 & -5.24 & 2.18 & Gg_rs156661X5_21008191 \\
\hline 0.32 & 1.1 & 0.27 & 1.95 & 0.42 & Gg_rs156661X5_21008191 \\
\hline 0.46 & 1.7 & 0.38 & 0.81 & 0.59 & Gg_rs156661X5_21008191 \\
\hline
\end{tabular}




\begin{tabular}{|c|c|c|c|c|c|}
\hline 0.47 & 1.84 & 0.4 & 2.42 & 0.61 & Gg_rs156661 X5_21008191 \\
\hline $1.39 \mathrm{NA}$ & NA & NA & NA & & Gg_rs156661X5_21008191 \\
\hline 1.36 & -0.16 & 1.14 & -6.04 & 1.75 & Gg_rs156661X5_21008191 \\
\hline $0.45 \mathrm{NA}$ & NA & NA & NA & & Gg_rs156661 snp.98.79.91( \\
\hline 2.4 & 0.82 & 2.03 & -8.95 & 3.09 & Gg_rs156661X5_21008191 \\
\hline 1.28 & -1.6 & 1.08 & -3.92 & 1.65 & Gg_rs156661 snp.98.79.91( \\
\hline $0.7 \mathrm{NA}$ & NA & NA & NA & & Gg_rs156661X5_21008191 \\
\hline 2.11 & -1.67 & 1.78 & -6.91 & 2.71 & Gg_rs156661X5_21008191 \\
\hline 2.2 & -7.41 & 1.86 & 4.51 & 2.84 & Gg_rs156661X5_21008191 \\
\hline 6.98 & -23.82 & 5.89 & -5.8 & 8.99 & Gg_rs156661X5_21008191 \\
\hline 3.76 & -14.86 & 3.17 & 1.26 & 4.84 & Gg_rs156661X5_21008191 \\
\hline 0.69 NA & NA & NA & NA & & Gg_rs156661 X5_21008191 \\
\hline $1.18 \mathrm{NA}$ & NA & NA & NA & & Gg_rs156661X5_21008191 \\
\hline $1.03 \mathrm{NA}$ & NA & NA & NA & & Gg_rs156661 X5_21008191 \\
\hline $0.42 \mathrm{NA}$ & NA & NA & NA & & Gg_rs156661X5_21008191 \\
\hline $1.3 \mathrm{NA}$ & NA & NA & NA & & Gg_rs156661X5_21008191 \\
\hline $1.5 \mathrm{NA}$ & NA & NA & NA & & Gg_rs156661X5_21008191 \\
\hline 0.99 NA & NA & NA & NA & & Gg_rs156661X5_21008191 \\
\hline 1.79 & 1.46 & 1.51 & -8.91 & 2.3 & Gg_rs156661X5_21008191 \\
\hline $1.09 \mathrm{NA}$ & NA & NA & NA & & Gg_rs145155 X5_21008191 \\
\hline $1.24 \mathrm{NA}$ & NA & NA & NA & & Gg_rs156661X5_21008191 \\
\hline $1.03 \mathrm{NA}$ & NA & NA & NA & & Gg_rs156661X5_21008191 \\
\hline 0.78 NA & NA & NA & NA & & Gg_rs156661X5_21008191 \\
\hline 0.75 & -2.26 & 0.74 & -0.8 & 0.98 & Gg_rs156661X5_21008191 \\
\hline 0.68 & -0.16 & 0.67 & 3.69 & 0.89 & Gg_rs156661X5_21008191 \\
\hline $0.46 \mathrm{NA}$ & NA & NA & NA & & X5_5571125 X5_21008191 \\
\hline 0.6 & 1.72 & 0.59 & 3.97 & 0.78 & Gg_rs156661X5_21008191 \\
\hline $0.71 \mathrm{NA}$ & NA & NA & NA & & X5_5571125 X5_21008191 \\
\hline 1.73 & -4.92 & 1.71 & -7.38 & 2.28 & Gg_rs156661X5_21008191 \\
\hline 1.5 & -4.44 & 1.48 & -5.52 & 1.97 & X5_5571125 X5_21008191 \\
\hline 2.16 & -3.98 & 2.13 & -9.59 & 2.84 & Gg_rs156661X5_21008191 \\
\hline 0.06 & 0.36 & 0.06 & 0.08 & 0.08 & Gg_rs156661X5_21008191 \\
\hline 0.49 NA & NA & NA & NA & & Gg_rs156661X5_21008191 \\
\hline $1.39 \mathrm{NA}$ & NA & NA & NA & & Gg_rs156661X5_21008191 \\
\hline $0.6 \mathrm{NA}$ & NA & NA & NA & & Gg_rs156661X5_21008191 \\
\hline 0.38 & 1.05 & 0.37 & 1.14 & 0.49 & Gg_rs156661X5_21008191 \\
\hline $0.55 \mathrm{NA}$ & NA & NA & NA & & snp.98.79.91(Gg_rs156859. \\
\hline 0.75 & -1.14 & 0.77 & -0.92 & 0.97 & snp.98.79.91(Gg_rs156859. \\
\hline $0.55 \mathrm{NA}$ & NA & NA & NA & & snp.98.79.91(RBL3041 \\
\hline $0.3 \mathrm{NA}$ & NA & NA & NA & & snp.98.79.91(Gg_rs156859. \\
\hline $0.52 \mathrm{NA}$ & NA & NA & NA & & snp.98.79.91(Gg_rs156859. \\
\hline $0.37 \mathrm{NA}$ & NA & NA & NA & & snp.98.79.91(Gg_rs156859. \\
\hline $0.51 \mathrm{NA}$ & NA & NA & NA & & snp.98.79.91(Gg_rs156859. \\
\hline
\end{tabular}




\begin{tabular}{|c|c|c|c|c|c|c|c|c|}
\hline $0.52 \mathrm{NA}$ & & NA & & NA & & NA & & snp.98.79.91(Gg_rs156859. \\
\hline 0.48 NA & & NA & & NA & & NA & & X5_21008191Gg_rs156859. \\
\hline $0.27 \mathrm{NA}$ & & NA & & NA & & NA & & snp.98.79.91(Gg_rs156859. \\
\hline 0.56 NA & & NA & & NA & & NA & & snp.98.79.91(Gg_rs156859. \\
\hline 0.51 & 2.29 & & 0.52 & & 3.36 & & 0.65 & X5_21008191Gg_rs156859. \\
\hline $0.78 \mathrm{NA}$ & & NA & & NA & & NA & & snp.98.79.91(Gg_rs156859. \\
\hline $0.2 \mathrm{NA}$ & & NA & & NA & & NA & & X5_21008191Gg_rs156859. \\
\hline $0.35 \mathrm{NA}$ & & NA & & NA & & NA & & X5_21008191Gg_rs156859. \\
\hline $0.69 \mathrm{NA}$ & & NA & & NA & & NA & & snp.98.79.91(Gg_rs156859. \\
\hline $0.79 \mathrm{NA}$ & & NA & & NA & & NA & & X5_21008191Gg_rs156859. \\
\hline $0.39 \mathrm{NA}$ & & NA & & NA & & NA & & X5_21008191Gg_rs135852 \\
\hline 0.12 & 0.71 & & 0.12 & & 0.76 & & 0.15 & X5_21008191Gg_rs156859. \\
\hline 0.48 & 1.37 & & 0.49 & & 2.11 & & 0.62 & X5_21008191Gg_rs156859. \\
\hline 0.31 & 1.52 & & 0.32 & & 1.62 & & 0.4 & X5_21008191Gg_rs156859. \\
\hline 0.38 & 1.56 & & 0.39 & & 2.59 & & 0.49 & X5_21008191Gg_rs156859. \\
\hline $0.42 \mathrm{NA}$ & & NA & & NA & & NA & & snp.98.79.91(Gg_rs135852 \\
\hline 0.6 & 2.99 & & 0.61 & & 3 & & 0.77 & X5_21008191RBL3041 \\
\hline 0.01 & 0.08 & & 0.01 & & 0.09 & & 0.02 & X5_21008191Gg_rs156859. \\
\hline 0.47 NA & & NA & & NA & & NA & & snp.98.79.91(Gg_rs156859. \\
\hline 0.18 & 0.94 & & 0.19 & & 1.02 & & 0.24 & X5_21008191RBL3041 \\
\hline 0.37 & 2.05 & & 0.37 & & 1.77 & & 0.47 & X5_21008191RBL3041 \\
\hline 0.52 & 2.01 & & 0.53 & & 2.61 & & 0.67 & X5_21008191RBL3041 \\
\hline 0.22 & 0.86 & & 0.23 & & 0.76 & & 0.29 & X5_21008191Gg_rs135852 \\
\hline 0.63 NA & & NA & & NA & & NA & & X5_21008191Gg_rs135852 \\
\hline 0.96 NA & & NA & & NA & & NA & & snp.98.79.91(GG_rs157021 \\
\hline 0.26 & 1.1 & & 0.26 & & 0.88 & & 0.33 & snp.98.79.91(Gg_rs135852 \\
\hline 0.09 & 0.52 & & 0.09 & & 0.43 & & 0.11 & X5_21008191RBL3041 \\
\hline 0.3 & 1.34 & & 0.31 & & 0.97 & & 0.4 & snp.98.79.91(RBL3041 \\
\hline $0.46 \mathrm{NA}$ & & NA & & NA & & NA & & X5_21008191RBL3041 \\
\hline 0.13 & 0.6 & & 0.14 & & 0.58 & & 0.18 & Gg_rs156795 RBL3041 \\
\hline 0.57 & 1.66 & & 0.6 & & 2.23 & & 0.77 & Gg_rs156795 RBL3041 \\
\hline 0.71 & 2.87 & & 0.75 & & 2.83 & & 0.96 & Gg_rs156795 RBL3041 \\
\hline 0.43 & 1.95 & & 0.46 & & 2.13 & & 0.59 & Gg_rs156795 RBL3041 \\
\hline 0.81 & 4.25 & & 0.86 & & 4.73 & & 1.11 & Gg_rs156795 RBL3041 \\
\hline 0.32 & 1.21 & & 0.34 & & 0.96 & & 0.43 & Gg_rs156795 RBL3041 \\
\hline $0.17 \mathrm{NA}$ & & NA & & NA & & NA & & X5_21008191RBL3041 \\
\hline 0.71 & 3.45 & & 0.75 & & 2.85 & & 0.96 & Gg_rs156795 RBL3041 \\
\hline $0.3 \mathrm{NA}$ & & NA & & NA & & NA & & Gg_rs156795 RBL3041 \\
\hline 0.77 & 2.79 & & 0.81 & & 3.19 & & 1.05 & Gg_rs156795 RBL3041 \\
\hline 1.08 & 4.31 & & 1.14 & & 5.82 & & 1.47 & Gg_rs156795 RBL3041 \\
\hline 0.3 & 1.36 & & 0.32 & & 1.88 & & 0.41 & Gg_rs156795 RBL3041 \\
\hline 0.47 & 1.35 & & 0.5 & & 1.69 & & 0.65 & Gg_rs156795 RBL3041 \\
\hline $0.18 \mathrm{NA}$ & & NA & & NA & & NA & & Gg_rs156795 RBL3041 \\
\hline
\end{tabular}




\begin{tabular}{|c|c|c|c|c|c|c|c|c|}
\hline $0.12 \mathrm{NA}$ & & NA & & NA & & NA & & Gg_rs156795 RBL3041 \\
\hline $0.41 \mathrm{NA}$ & & NA & & NA & & NA & & Gg_rs156795 RBL3041 \\
\hline 0.47 & 2.21 & & 0.5 & & 2.42 & & 0.64 & Gg_rs156795 RBL3041 \\
\hline 0.43 & 1.63 & & 0.45 & & 1.64 & & 0.58 & Gg_rs156795 RBL3041 \\
\hline 0.36 & 1.93 & & 0.38 & & 1.73 & & 0.49 & Gg_rs156795 RBL3041 \\
\hline 0.54 & 2.47 & & 0.57 & & 1.28 & & 0.73 & Gg_rs156795 RBL3041 \\
\hline 0.72 & 2.61 & & 0.76 & & 1.94 & & 0.98 & Gg_rs156795 RBL3041 \\
\hline 0.35 & 1.84 & & 0.36 & & 1.73 & & 0.47 & Gg_rs156795 RBL3041 \\
\hline 0.55 & 2.09 & & 0.58 & & 1.26 & & 0.75 & Gg_rs156795 RBL3041 \\
\hline 0.59 & 1.55 & & 0.62 & & 0.91 & & 0.8 & Gg_rs156795 RBL3041 \\
\hline 0.14 & 0.58 & & 0.15 & & 0.73 & & 0.19 & Gg_rs156795 RBL3041 \\
\hline 0.03 & 0.16 & & 0.03 & & 0.21 & & 0.04 & Gg_rs156795 RBL3041 \\
\hline 0.72 & 2.49 & & 0.76 & & 3.02 & & 0.98 & Gg_rs156795 RBL3041 \\
\hline $0.25 \mathrm{NA}$ & & NA & & NA & & NA & & Gg_rs156795 RBL3041 \\
\hline $1.02 \mathrm{NA}$ & & NA & & NA & & NA & & Gg_rs156795 RBL3041 \\
\hline $1.27 \mathrm{NA}$ & & NA & & NA & & NA & & Gg_rs156795 RBL3041 \\
\hline 0.6 & 3.02 & & 0.63 & & 2.94 & & 0.82 & Gg_rs156795 RBL3041 \\
\hline 0.83 & 5.5 & & 0.87 & & 5.59 & & 1.13 & Gg_rs156795 RBL3041 \\
\hline 0.65 & 3.39 & & 0.68 & & 3.94 & & 0.88 & Gg_rs156795 RBL3041 \\
\hline 0.53 & 2.24 & & 0.56 & & 1.84 & & 0.72 & Gg_rs156795 RBL3041 \\
\hline $0.35 \mathrm{NA}$ & & NA & & NA & & NA & & Gg_rs156795 RBL3041 \\
\hline 0.27 & 1.17 & & 0.29 & & 1.79 & & 0.37 & Gg_rs156795 RBL3041 \\
\hline 0.5 & 2.9 & & 0.53 & & 2.27 & & 0.68 & Gg_rs156795 RBL3041 \\
\hline 0.73 & 4.21 & & 0.77 & & 4.17 & & 1 & Gg_rs156795 RBL3041 \\
\hline 1.23 & 3.09 & & 1.3 & & 3.15 & & 1.67 & Gg_rs156795 RBL3041 \\
\hline 0.8 & 2.78 & & 0.84 & & 3.83 & & 1.08 & Gg_rs156795 RBL3041 \\
\hline 0.92 & 3.87 & & 0.97 & & 3.67 & & 1.25 & Gg_rs156795 RBL3041 \\
\hline 0.5 & 2.85 & & 0.52 & & 2.69 & & 0.67 & Gg_rs156795 RBL3041 \\
\hline 1.31 & 6.22 & & 1.38 & & 5.54 & & 1.78 & Gg_rs156795 RBL3041 \\
\hline 0.89 & 3.1 & & 0.94 & & 3.63 & & 1.22 & Gg_rs156795 RBL3041 \\
\hline $0.5 \mathrm{NA}$ & & NA & & NA & & NA & & Gg_rs156795 RBL3041 \\
\hline $0.31 \mathrm{NA}$ & & NA & & NA & & NA & & Gg_rs156795 RBL3041 \\
\hline $3.68 \mathrm{NA}$ & & NA & & NA & & NA & & Gg_rs156795 RBL3041 \\
\hline 0.38 & 1.72 & & 0.4 & & 1.62 & & 0.51 & Gg_rs156795 RBL3041 \\
\hline 1.03 & 4.74 & & 1.08 & & 5.68 & & 1.4 & Gg_rs156795 RBL3041 \\
\hline 0.8 & 3.77 & & 0.84 & & 4.37 & & 1.09 & Gg_rs156795 RBL3041 \\
\hline 0.76 & 4.08 & & 0.8 & & 3.99 & & 1.03 & Gg_rs156795 RBL3041 \\
\hline 1.11 & 4.11 & & 1.17 & & 2.31 & & 1.51 & Gg_rs156795 RBL3041 \\
\hline 0.72 & 3.82 & & 0.76 & & 3.69 & & 0.98 & Gg_rs156795 RBL3041 \\
\hline 0.99 & 2.75 & & 1.04 & & 4.01 & & 1.34 & Gg_rs156795 RBL3041 \\
\hline 1.08 & 3.43 & & 1.14 & & 3.61 & & 1.46 & Gg_rs156795 RBL3041 \\
\hline 0.49 & 2.2 & & 0.52 & & 2.14 & & 0.67 & Gg_rs156795 RBL3041 \\
\hline 0.39 & 1.06 & & 0.41 & & 1.54 & & 0.53 & Gg_rs156795 RBL3041 \\
\hline
\end{tabular}




\begin{tabular}{|c|c|c|c|c|}
\hline 0.63 & 4.09 & 0.66 & 3.37 & 0.85 Gg_rs156795 RBL3041 \\
\hline 0.78 & 3.4 & 0.82 & 4.93 & 1.06 Gg_rs156795 RBL3041 \\
\hline 0.39 & 2.43 & 0.41 & 1.99 & 0.53 Gg_rs156795 RBL3041 \\
\hline 0.26 & 1.2 & 0.28 & 0.76 & 0.36 Gg_rs156795 RBL3041 \\
\hline 1.37 & 5.03 & 1.44 & 4.88 & 1.86 Gg_rs156795 RBL3041 \\
\hline 0.33 & 1.09 & 0.35 & 1.5 & 0.45 Gg_rs156795 RBL3041 \\
\hline 1.07 & 5.28 & 1.13 & 4.52 & 1.46 Gg_rs156795 RBL3041 \\
\hline 0.39 & 1.89 & 0.41 & 2.09 & 0.53 Gg_rs156795 RBL3041 \\
\hline 0.68 & 2.47 & 0.72 & 3.53 & 0.93 Gg_rs156795 RBL3041 \\
\hline 0.53 & 3.84 & 0.56 & 3.39 & 0.72 Gg_rs156795 RBL3041 \\
\hline $0.46 \mathrm{NA}$ & NA & NA & NA & Gg_rs156795 RBL3041 \\
\hline 0.15 & 0.82 & 0.16 & 0.69 & 0.21 Gg_rs156795 RBL3041 \\
\hline 0.37 & 1.69 & 0.4 & 1.99 & 0.51 Gg_rs156795 RBL3041 \\
\hline 0.44 & 2.01 & 0.46 & 1.36 & 0.59 Gg_rs156795 RBL3041 \\
\hline 0.81 & 4.68 & 0.85 & 4.05 & 1.1 Gg_rs156795 RBL3041 \\
\hline 0.45 & 2.11 & 0.47 & 2.45 & 0.61 Gg_rs156795 RBL3041 \\
\hline 0.79 & 2.82 & 0.83 & 3.12 & 1.08 Gg_rs156795 RBL3041 \\
\hline 0.42 & 1.46 & 0.45 & 1.58 & 0.57 Gg_rs156795 RBL3041 \\
\hline 0.57 & 2.14 & 0.6 & 2.1 & 0.77 Gg_rs156795 RBL3041 \\
\hline 0.28 & 1.09 & 0.29 & 1.37 & 0.37 Gg_rs156795 RBL3041 \\
\hline 0.15 & 0.82 & 0.16 & 0.58 & 0.2 Gg_rs156795 RBL3041 \\
\hline 0.46 & 2.13 & 0.49 & 2.89 & 0.63 Gg_rs156795 RBL3041 \\
\hline 0.91 & 5.21 & 0.96 & 3.93 & 1.24 Gg_rs156795 RBL3041 \\
\hline 0.81 & 4.24 & 0.86 & 5.38 & 1.1 Gg_rs156795 RBL3041 \\
\hline 0.83 & 4.32 & 0.88 & 4.78 & 1.13 Gg_rs156795 RBL3041 \\
\hline 0.28 & 1.82 & 0.29 & 1.34 & 0.38 Gg_rs156795 RBL3041 \\
\hline 0.5 & 2.65 & 0.53 & 2.16 & 0.69 Gg_rs156795 RBL3041 \\
\hline 0.81 & 3.58 & 0.85 & 4.74 & 1.09 Gg_rs156795 Gg_rs135852 \\
\hline 0.39 & 1.63 & 0.41 & 1.52 & 0.52 Gg_rs156795 RBL3041 \\
\hline 0.58 & 2.33 & 0.61 & 2.83 & 0.78 Gg_rs156795 Gg_rs135852 \\
\hline 0.13 & 0.59 & 0.14 & 0.7 & 0.18 Gg_rs156795 Gg_rs135852 \\
\hline 0.35 & 1.6 & 0.37 & 1.85 & 0.48 Gg_rs156795 Gg_rs135852 \\
\hline 0.5 & 2.4 & 0.53 & 2.96 & 0.68 Gg_rs156795 Gg_rs135852 \\
\hline $0.46 \mathrm{NA}$ & NA & NA & NA & Gg_rs156795 X5_36025717 \\
\hline 0.48 NA & NA & NA & NA & Gg_rs156795 Gg_rs135852 \\
\hline 0.57 & 2.92 & 0.6 & 1.8 & 0.78 Gg_rs156795 Gg_rs135852 \\
\hline 0.94 & 4.48 & 1 & 4.6 & 1.28 Gg_rs156795 Gg_rs135852 \\
\hline 0.25 & 1.4 & 0.26 & 1.03 & 0.33 Gg_rs156795 Gg_rs135852 \\
\hline 0.36 & 1.17 & 0.38 & 1.09 & 0.48 X5_21008191Gg_rs135852 \\
\hline $0.42 \mathrm{NA}$ & NA & NA & NA & Gg_rs156795 Gg_rs135852 \\
\hline $0.65 \mathrm{NA}$ & NA & NA & NA & Gg_rs156795 Gg_rs135852 \\
\hline 1.01 & 3.34 & 0.99 & 4.03 & 1.38 Gg_rs156795 Gg_rs135852 \\
\hline 1.03 & 3.92 & 1.01 & 4.99 & 1.41 Gg_rs156795 Gg_rs135852 \\
\hline
\end{tabular}




\begin{tabular}{|c|c|c|c|c|}
\hline 0.72 & 3.24 & 0.71 & 3.78 & 0.99 Gg_rs156795 Gg_rs135852 \\
\hline 0.52 & 1.96 & 0.51 & 3.17 & 0.71 Gg_rs156795 Gg_rs135852 \\
\hline 0.84 & 2.61 & 0.82 & 2.45 & 1.14 Gg_rs156795 Gg_rs135852 \\
\hline 1.06 & 3.76 & 1.04 & 6.41 & 1.45 Gg_rs156795 Gg_rs135852 \\
\hline 0.65 & 3.08 & 0.64 & 3 & 0.89 Gg_rs156795 Gg_rs135852 \\
\hline 0.27 & 0.89 & 0.27 & 1.07 & 0.37 Gg_rs156795 Gg_rs135852 \\
\hline 0.53 & 2.17 & 0.52 & 2.29 & 0.72 Gg_rs156795 Gg_rs135852 \\
\hline 0.34 & 1.59 & 0.34 & 1.06 & 0.47 Gg_rs156795 Gg_rs135852 \\
\hline 0.36 & 1.49 & 0.36 & 1.66 & 0.5 Gg_rs156795 Gg_rs135852 \\
\hline 0.45 & 1.48 & 0.44 & 1.67 & 0.62 X5_21008191Gg_rs135852 \\
\hline 0.42 & 1.9 & 0.41 & 1.19 & 0.57 Gg_rs156795 Gg_rs135852 \\
\hline 0.61 & 2.98 & 0.6 & 2.68 & 0.84 Gg_rs156795 Gg_rs135852 \\
\hline 0.65 & 2.95 & 0.64 & 3.2 & 0.89 Gg_rs156795 Gg_rs135852 \\
\hline 0.61 & 2.25 & 0.6 & 2.87 & 0.83 Gg_rs156795 Gg_rs135852 \\
\hline $0.51 \mathrm{NA}$ & NA & NA & NA & X5_21008191Gg_rs135852 \\
\hline 0.77 & 2.56 & 0.75 & 3.77 & 1.05 Gg_rs156795 Gg_rs135852 \\
\hline 0.29 & 1.17 & 0.28 & 1.03 & 0.4 Gg_rs156795 Gg_rs135852 \\
\hline 0.39 & 1.92 & 0.39 & 1.17 & 0.54 Gg_rs156795 Gg_rs135852 \\
\hline 0.27 & 0.95 & 0.27 & 0.94 & 0.37 Gg_rs156795 Gg_rs135852 \\
\hline 0.59 & 2.24 & 0.58 & 2.63 & 0.81 Gg_rs156795 Gg_rs135852 \\
\hline 0.31 & 1.24 & 0.3 & 1.63 & 0.42 Gg_rs156795 Gg_rs135852 \\
\hline 0.46 & 1.4 & 0.45 & 1.87 & 0.63 Gg_rs156795 Gg_rs135852 \\
\hline 0.66 & 3.29 & 0.64 & 2.9 & 0.9 Gg_rs156795 Gg_rs135852 \\
\hline 1.16 & 3.99 & 1.14 & 6.48 & 1.59 Gg_rs156795 Gg_rs135852 \\
\hline $0.4 \mathrm{NA}$ & NA & NA & NA & Gg_rs156795 Gg_rs135852 \\
\hline 0.43 & 1.94 & 0.42 & 1.7 & 0.58 Gg_rs156795 Gg_rs135852 \\
\hline 0.39 & 1.55 & 0.38 & 1.48 & 0.54 Gg_rs156859 Gg_rs135852 \\
\hline 0.18 & 0.68 & 0.17 & 0.65 & 0.24 Gg_rs156795 Gg_rs135852 \\
\hline 0.46 & 1.55 & 0.45 & 2.49 & 0.63 Gg_rs156859 Gg_rs135852 \\
\hline 0.37 & 1.5 & 0.37 & 1.82 & 0.51 Gg_rs156859 Gg_rs135852 \\
\hline 0.46 & 1.41 & 0.45 & 2.86 & 0.63 Gg_rs156795 Gg_rs135852 \\
\hline 0.38 & 1.76 & 0.37 & 1.61 & 0.52 Gg_rs156859 Gg_rs135852 \\
\hline 1.28 & 5.96 & 1.26 & 7.52 & 1.75 Gg_rs156859 Gg_rs135852 \\
\hline 0.36 & 1.42 & 0.35 & 2.04 & 0.49 Gg_rs156859 Gg_rs135852 \\
\hline 0.56 & 2.32 & 0.55 & 2.68 & 0.76 Gg_rs156859 Gg_rs135852 \\
\hline 0.07 & 0.34 & 0.07 & 0.4 & 0.09 Gg_rs156859 Gg_rs135852 \\
\hline 0.4 & 1.46 & 0.39 & 2.56 & 0.54 Gg_rs156859 Gg_rs135852 \\
\hline 1.74 & 4.74 & 1.71 & 8.68 & 2.38 Gg_rs156859 Gg_rs135852 \\
\hline 0.68 & 2.6 & 0.67 & 3.78 & 0.93 Gg_rs156859 Gg_rs135852 \\
\hline 0.46 & 1.77 & 0.45 & 2.41 & 0.62 Gg_rs156859 Gg_rs135852 \\
\hline 0.66 & 2.85 & 0.65 & 3.65 & 0.9 Gg_rs156859 Gg_rs135852 \\
\hline 0.63 & 3.02 & 0.62 & 2.59 & 0.86 Gg_rs156859 Gg_rs135852 \\
\hline 0.3 & 1.19 & 0.29 & 1.09 & 0.4 Gg_rs156859 Gg_rs135852 \\
\hline
\end{tabular}




\begin{tabular}{|c|c|c|c|c|c|c|}
\hline 0.63 & 2.4 & 0.62 & 2.99 & 0.86 & \multicolumn{2}{|c|}{ Gg_rs156859 Gg_rs135852 } \\
\hline 0.48 & 1.45 & 0.47 & 2.55 & 0.66 & Gg_rs156 & 9 Gg_rs135852 \\
\hline 0.98 & 3.93 & 0.96 & 3.15 & 1.33 & Gg_rs156 & 9 Gg_rs135852 \\
\hline 0.18 & 0.76 & 0.17 & 1.07 & 0.24 & Gg_rs156 & 9 Gg_rs135852 \\
\hline 0.73 & 3.08 & 0.72 & 3.9 & 1 & Gg_rs156 & 9 Gg_rs135852 \\
\hline 0.48 NA & NA & NA & NA & & Gg_rs156 & 9 Gg_rs135852 \\
\hline 0.04 & 0.21 & 0.04 & 0.17 & 0.06 & Gg_rs156 & 9 Gg_rs135852 \\
\hline 0.74 & 1.95 & 0.72 & 4.12 & 1.01 & Gg_rs156 & 9 Gg_rs135852 \\
\hline 0.21 & 0.7 & 0.21 & 0.94 & 0.29 & Gg_rs156 & 9 Gg_rs135852 \\
\hline $0.46 \mathrm{NA}$ & NA & NA & NA & & Gg_rs156 & 9 Gg_rs135852 \\
\hline $0.38 \mathrm{NA}$ & NA & NA & NA & & Gg_rs156 & 9 Gg_rs135852 \\
\hline 0.43 & 1.25 & 0.43 & 2.03 & 0.59 & Gg_rs156 & 9 Gg_rs135852 \\
\hline 1.28 & 5.58 & 1.26 & 5.98 & 1.75 & Gg_rs156 & 9 Gg_rs135852 \\
\hline 0.49 & 0.14 & 0.48 & 1.91 & 0.67 & Gg_rs156 & 9 Gg_rs135852 \\
\hline 0.55 & 1.56 & 0.54 & 1.87 & 0.76 & Gg_rs156 & 9 Gg_rs135852 \\
\hline 1.15 & 3.45 & 1.13 & 7.74 & 1.57 & Gg_rs156 & 9 Gg_rs135852 \\
\hline 0.71 & 2.84 & 0.7 & 2.92 & 0.98 & Gg_rs156 & 9 Gg_rs135852 \\
\hline 0.14 NA & NA & NA & NA & & Gg_rs156 & 9 Gg_rs135852 \\
\hline 0.53 & 1.4 & 0.52 & 1.15 & 0.72 & Gg_rs156 & 9 Gg_rs135852 \\
\hline 0.85 & 3.81 & 0.84 & 2.93 & 1.17 & Gg_rs156 & 9 Gg_rs135852 \\
\hline $0.58 \mathrm{NA}$ & NA & NA & NA & & Gg_rs156 & 5 Gg_rs135852 \\
\hline $0.38 \mathrm{NA}$ & NA & NA & NA & & Gg_rs156 & 5 Gg_rs135852 \\
\hline 0.69 & 3.08 & 0.68 & 3.26 & 0.94 & Gg_rs156 & 9 Gg_rs135852 \\
\hline 0.45 & 1.24 & 0.44 & 2.27 & 0.61 & Gg_rs156 & 9 Gg_rs135852 \\
\hline 0.13 & 0.61 & 0.13 & 0.49 & 0.18 & Gg_rs156 & 9 Gg_rs135852 \\
\hline 1 & 4.57 & 0.98 & 5.51 & 1.36 & Gg_rs156 & 9 Gg_rs135852 \\
\hline 0.63 & 1.93 & 0.62 & 3.85 & 0.86 & Gg_rs156 & 9 Gg_rs135852 \\
\hline $0.55 \mathrm{NA}$ & NA & NA & NA & & RBL3041 & GG_rs157021 \\
\hline $0.34 \mathrm{NA}$ & NA & NA & NA & & RBL3041 & GG_rs157021 \\
\hline 0.67 & -1.74 & 0.63 & 1.57 & 0.89 & RBL3041 & Gg_rs135852 \\
\hline $1.52 \mathrm{NA}$ & NA & NA & NA & & RBL3041 & GG_rs157021 \\
\hline 0.89 & -2.03 & 0.84 & -0.25 & 1.19 & RBL3041 & Gg_rs135852 \\
\hline $1.2 \mathrm{NA}$ & NA & NA & NA & & RBL3041 & X5_36025717 \\
\hline 0.59 NA & NA & NA & NA & & RBL3041 & GG_rs157021 \\
\hline $0.43 \mathrm{NA}$ & NA & NA & NA & & RBL3041 & GG_rs156965 \\
\hline $0.72 \mathrm{NA}$ & NA & NA & NA & & RBL3041 & GG_rs157021 \\
\hline 0.74 NA & NA & NA & NA & & RBL3041 & GG_rs156965 \\
\hline 0.72 NA & NA & NA & NA & & RBL3041 & GG_rs157021 \\
\hline 0.79 NA & NA & NA & NA & & RBL3041 & GG_rs157021 \\
\hline $0.95 \mathrm{NA}$ & NA & NA & NA & & RBL3041 & GG_rs157021 \\
\hline $0.74 \mathrm{NA}$ & NA & NA & NA & & RBL3041 & GG_rs157021 \\
\hline $0.52 \mathrm{NA}$ & NA & NA & NA & & RBL3041 & GG_rs157021 \\
\hline $0.32 \mathrm{NA}$ & NA & NA & NA & & RBL3041 & GG_rs157021 \\
\hline
\end{tabular}




\begin{tabular}{|c|c|c|c|c|c|c|c|}
\hline $0.58 \mathrm{NA}$ & NA & & NA & NA & & RBL3041 & GG_rs157021 \\
\hline 0.31 & 1.24 & 0.3 & & 1.16 & 0.42 & RBL3041 & GG_rs157021 \\
\hline $0.22 \mathrm{NA}$ & NA & & NA & NA & & RBL3041 & GG_rs157021 \\
\hline $0.17 \mathrm{NA}$ & NA & & NA & NA & & RBL3041 & GG_rs157021 \\
\hline $0.39 \mathrm{NA}$ & NA & & NA & NA & & \multicolumn{2}{|c|}{ Gg_rs135852 GG_rs157021 } \\
\hline $1.16 \mathrm{NA}$ & NA & & NA & NA & & \multicolumn{2}{|c|}{ Gg_rs135852GG_rs157021 } \\
\hline 0.58 & 2.2 & 0.57 & & 1.61 & 0.78 & \multicolumn{2}{|c|}{ Gg_rs135852 GG_rs157021 } \\
\hline 0.13 & 0.45 & 0.13 & & 0.38 & 0.18 & \multicolumn{2}{|c|}{ Gg_rs135852 GG_rs157021 } \\
\hline 0.73 & 2.29 & 0.71 & & 1.13 & 0.98 & \multicolumn{2}{|c|}{ Gg_rs135852 GG_rs157021 } \\
\hline $0.44 \mathrm{NA}$ & NA & & NA & NA & & RBL3041 & GG_rs157021 \\
\hline $0.47 \mathrm{NA}$ & NA & & NA & NA & & \multicolumn{2}{|c|}{ Gg_rs135852 GG_rs157021 } \\
\hline 0.47 NA & NA & & NA & NA & & \multicolumn{2}{|c|}{ Gg_rs135852 GG_rs157021 } \\
\hline $0.19 \mathrm{NA}$ & NA & & NA & NA & & \multicolumn{2}{|c|}{ Gg_rs135852 GG_rs157021 } \\
\hline $0.85 \mathrm{NA}$ & NA & & NA & NA & & RBL3041 & GG_rs157021 \\
\hline $0.33 \mathrm{NA}$ & NA & & NA & NA & & \multicolumn{2}{|c|}{ snp.98.79.91(GG_rs157021 } \\
\hline $0.4 \mathrm{NA}$ & NA & & NA & NA & & RBL3041 & GG_rs157021 \\
\hline $0.41 \mathrm{NA}$ & NA & & NA & NA & & RBL3041 & GG_rs157021 \\
\hline 0.82 & -2.73 & 0.8 & & 3.33 & 1.1 & \multicolumn{2}{|c|}{ Gg_rs135852 GG_rs157021 } \\
\hline 0.46 & 0.58 & 0.45 & & 2.64 & 0.62 & \multicolumn{2}{|c|}{ Gg_rs135852 GG_rs157021 } \\
\hline 0.77 NA & NA & & NA & NA & & RBL3041 & GG_rs157021 \\
\hline 0.77 & -1.36 & 0.72 & & -0.65 & 1.04 & RBL3041 & GG_rs157021 \\
\hline $0.6 \mathrm{NA}$ & NA & & NA & NA & & RBL3041 & GG_rs157021 \\
\hline $0.83 \mathrm{NA}$ & NA & & NA & NA & & RBL3041 & GG_rs157021 \\
\hline $0.45 \mathrm{NA}$ & NA & & NA & NA & & RBL3041 & GG_rs157021 \\
\hline $0.75 \mathrm{NA}$ & NA & & NA & NA & & RBL3041 & GG_rs157021 \\
\hline 0.46 NA & NA & & NA & NA & & RBL3041 & GG_rs157021 \\
\hline $0.65 \mathrm{NA}$ & NA & & NA & NA & & RBL3041 & GG_rs157021 \\
\hline $0.62 \mathrm{NA}$ & NA & & NA & NA & & RBL3041 & GG_rs157021 \\
\hline $0.45 \mathrm{NA}$ & NA & & NA & NA & & RBL3041 & Gg_rs157060 \\
\hline 0.02 & 0.06 & 0.02 & & 0.07 & 0.03 & \multicolumn{2}{|c|}{ X5_36025717GG_rs157021 } \\
\hline 0.38 & 0.99 & 0.36 & & 2.74 & 0.51 & \multicolumn{2}{|c|}{ GG_rs156965GG_rs157021 } \\
\hline 0.68 & 0.42 & 0.64 & & 4.32 & 0.92 & \multicolumn{2}{|c|}{ GG_rs156965GG_rs157021 } \\
\hline 0.67 & -2.65 & 0.63 & & 2.67 & 0.9 & \multicolumn{2}{|c|}{ Gg_rs135852 GG_rs157021 } \\
\hline $0.63 \mathrm{NA}$ & NA & & NA & NA & & \multicolumn{2}{|c|}{ Gg_rs135852 GG_rs157021 } \\
\hline $0.47 \mathrm{NA}$ & NA & & NA & NA & & \multicolumn{2}{|c|}{ Gg_rs135852GG_rs157021 } \\
\hline $0.4 \mathrm{NA}$ & NA & & NA & NA & & RBL3041 & GG_rs157021 \\
\hline $0.61 \mathrm{NA}$ & NA & & NA & NA & & RBL3041 & GG_rs157021 \\
\hline 0.66 & 0.11 & 0.62 & & 3.83 & 0.88 & \multicolumn{2}{|c|}{ GG_rs156965GG_rs157021 } \\
\hline 0.25 & 0.44 & 0.23 & & 1.33 & 0.33 & \multicolumn{2}{|c|}{ GG_rs156965GG_rs157021 } \\
\hline $0.46 \mathrm{NA}$ & NA & & NA & NA & & RBL3041 & Gg_rs157060. \\
\hline $0.45 \mathrm{NA}$ & NA & & NA & NA & & RBL3041 & Gg_rs157060. \\
\hline $0.4 \mathrm{NA}$ & NA & & NA & NA & & \multicolumn{2}{|c|}{ Gg_rs135852 GG_rs157021 } \\
\hline $0.43 \mathrm{NA}$ & NA & & NA & NA & & RBL3041 & Gg_rs157060. \\
\hline
\end{tabular}




\begin{tabular}{|c|c|c|c|c|c|}
\hline 0.67 & 0.23 & 0.63 & 2.7 & 0.9 & RBL3041 Gg_rs157060 \\
\hline 0.62 & -2.15 & 0.58 & 3.03 & 0.84 & Gg_rs135852Gg_rs157060. \\
\hline $0.29 \mathrm{NA}$ & NA & NA & NA & & Gg_rs156859Gg_rs157060. \\
\hline 0.62 & -1.08 & 0.56 & 0.31 & 0.8 & RBL3041 Gg_rs157060 \\
\hline $0.4 \mathrm{NA}$ & NA & NA & NA & & Gg_rs135852Gg_rs157060. \\
\hline $0.68 \mathrm{NA}$ & NA & NA & NA & & X5_3804386CGg_rs157060. \\
\hline $0.59 \mathrm{NA}$ & NA & NA & NA & & X5_3804386CGg_rs157060. \\
\hline $0.61 \mathrm{NA}$ & NA & NA & NA & & X5_3804386CGg_rs157060، \\
\hline $1.33 \mathrm{NA}$ & NA & NA & NA & & Gg_rs135852Gg_rs157060 \\
\hline 0.84 & 1.31 & 0.77 & 3.89 & 1.09 & X5_3804386@Gg_rs157060. \\
\hline $0.5 \mathrm{NA}$ & NA & NA & NA & & X5_3804386@Gg_rs157060. \\
\hline $0.07 \mathrm{NA}$ & NA & NA & NA & & X5_3804386CGg_rs157060. \\
\hline $0.45 \mathrm{NA}$ & NA & NA & NA & & Gg_rs157184 \\
\hline 0.61 & 0 & 0.57 & 2 & 0.81 & GG_rs156965Gg_rs157269 \\
\hline $0.51 \mathrm{NA}$ & NA & NA & NA & & X5_3804386@Gg_rs157269 \\
\hline $0.34 \mathrm{NA}$ & NA & NA & NA & & GG_rs157021Gg_rs157184 \\
\hline 0.89 NA & NA & NA & NA & & GG_rs157021Gg_rs157269 \\
\hline $0.71 \mathrm{NA}$ & NA & NA & NA & & GG_rs157021Gg_rs157184 \\
\hline $0.64 \mathrm{NA}$ & NA & NA & NA & & GG_rs157021Gg_rs157184 \\
\hline $0.77 \mathrm{NA}$ & NA & NA & NA & & GG_rs157021Gg_rs157184 \\
\hline $0.9 \mathrm{NA}$ & NA & NA & NA & & GG_rs157021Gg_rs157269 \\
\hline $0.62 \mathrm{NA}$ & NA & NA & NA & & GG_rs157021Gg_rs157269 \\
\hline 0.64 NA & NA & NA & NA & & GG_rs157021Gg_rs157269 \\
\hline $0.55 \mathrm{NA}$ & NA & NA & NA & & GG_rs157021Gg_rs157184 \\
\hline $0.59 \mathrm{NA}$ & NA & NA & NA & & GG_rs157021Gg_rs157269 \\
\hline $0.41 \mathrm{NA}$ & NA & NA & NA & & GG_rs157021Gg_rs157184 \\
\hline $0.79 \mathrm{NA}$ & NA & NA & NA & & GG_rs157021Gg_rs157269 \\
\hline $0.8 \mathrm{NA}$ & NA & NA & NA & & GG_rs157021Gg_rs157184 \\
\hline 0.94 NA & NA & NA & NA & & GG_rs157021Gg_rs157269 \\
\hline 0.49 NA & NA & NA & NA & & GG_rs157021Gg_rs157184 \\
\hline $0.61 \mathrm{NA}$ & NA & NA & NA & & GG_rs157021Gg_rs157184 \\
\hline $0.51 \mathrm{NA}$ & NA & NA & NA & & GG_rs157021Gg_rs157184 \\
\hline $0.61 \mathrm{NA}$ & NA & NA & NA & & GG_rs157021Gg_rs157184 \\
\hline $0.87 \mathrm{NA}$ & NA & NA & NA & & GG_rs157021Gg_rs157269 \\
\hline 0.9 & -3.3 & 0.85 & 3.42 & 1.2 & GG_rs157021Gg_rs157184 \\
\hline $0.57 \mathrm{NA}$ & NA & NA & NA & & GG_rs157021Gg_rs157184 \\
\hline $0.79 \mathrm{NA}$ & NA & NA & NA & & GG_rs157021Gg_rs157269 \\
\hline 0.48 NA & NA & NA & NA & & Gg_rs157060.Gg_rs157184 \\
\hline 0.99 & 0.6 & 0.93 & 3.58 & 1.31 & Gg_rs157060.Gg_rs157184 \\
\hline 0.71 & -1.37 & 0.67 & 3.29 & 0.94 & Gg_rs157060.Gg_rs157184 \\
\hline 0.74 NA & NA & NA & NA & & GG_rs157021Gg_rs157184 \\
\hline $0.36 \mathrm{NA}$ & NA & NA & NA & & GG_rs157021Gg_rs157269 \\
\hline $0.31 \mathrm{NA}$ & NA & NA & NA & & GG_rs157021Gg_rs157184 \\
\hline
\end{tabular}




\begin{tabular}{|c|c|c|c|c|c|c|c|}
\hline $0.54 \mathrm{NA}$ & NA & & NA & & NA & & GG_rs157021Gg_rs157269 \\
\hline 0.74 NA & NA & & NA & & NA & & GG_rs157021Gg_rs157184 \\
\hline $0.66 \mathrm{NA}$ & NA & & NA & & NA & & GG_rs157021Gg_rs157184 \\
\hline 0.54 NA & NA & & NA & & NA & & GG_rs157021Gg_rs157269 \\
\hline 1.32 & 0.51 & 1.25 & & 4.29 & & 1.76 & Gg_rs157060.Gg_rs157184 \\
\hline $0.74 \mathrm{NA}$ & NA & & NA & & NA & & GG_rs157021Gg_rs157269 \\
\hline 0.59 NA & NA & & NA & & NA & & GG_rs157021Gg_rs157269 \\
\hline $0.91 \mathrm{NA}$ & NA & & NA & & NA & & GG_rs157021Gg_rs157269 \\
\hline $0.56 \mathrm{NA}$ & NA & & NA & & NA & & GG_rs157021Gg_rs157269 \\
\hline $0.34 \mathrm{NA}$ & NA & & NA & & NA & & GG_rs157021Gg_rs157269 \\
\hline $0.82 \mathrm{NA}$ & NA & & NA & & NA & & GG_rs157021Gg_rs157269 \\
\hline 0.72 & 1.41 & 0.68 & & 0.97 & & 0.96 & GG_rs157021Gg_rs157269 \\
\hline $0.6 \mathrm{NA}$ & NA & & NA & & NA & & GG_rs157021Gg_rs157269 \\
\hline $0.5 \mathrm{NA}$ & NA & & NA & & NA & & GG_rs157021Gg_rs157269 \\
\hline 0.49 NA & NA & & NA & & NA & & GG_rs157021Gg_rs157269 \\
\hline $0.61 \mathrm{NA}$ & NA & & NA & & NA & & GG_rs157021Gg_rs157269 \\
\hline $0.75 \mathrm{NA}$ & NA & & NA & & NA & & Gg_rs157060.Gg_rs157269 \\
\hline 0.49 NA & NA & & NA & & NA & & GG_rs157021Gg_rs157269 \\
\hline 0.86 NA & NA & & NA & & NA & & GG_rs157021Gg_rs157269 \\
\hline $0.61 \mathrm{NA}$ & NA & & NA & & NA & & GG_rs157021Gg_rs157269 \\
\hline $0.9 \mathrm{NA}$ & NA & & NA & & NA & & GG_rs157021Gg_rs157269 \\
\hline 0.72 & 1.32 & 0.68 & & 2.73 & & 0.96 & GG_rs157021Gg_rs157269 \\
\hline 1 & 0.49 & 0.94 & & 3.32 & & 1.33 & GG_rs157021Gg_rs157269 \\
\hline 0.42 NA & NA & & NA & & NA & & GG_rs157021Gg_rs157269 \\
\hline $1.52 \mathrm{NA}$ & NA & & NA & & NA & & GG_rs157021Gg_rs157269 \\
\hline 1.25 & -1.56 & 1.18 & & 1.72 & & 1.67 & GG_rs157021Gg_rs157269 \\
\hline $0.75 \mathrm{NA}$ & NA & & NA & & NA & & GG_rs157021Gg_rs157269 \\
\hline $0.65 \mathrm{NA}$ & NA & & NA & & NA & & GG_rs157021Gg_rs157269 \\
\hline $0.32 \mathrm{NA}$ & NA & & NA & & NA & & GG_rs157021Gg_rs157269 \\
\hline 1.04 & 1.26 & 0.98 & & 6.26 & & 1.39 & Gg_rs157135 Gg_rs157269 \\
\hline 0.94 & 0.93 & 0.89 & & 3.39 & & 1.25 & Gg_rs157135 Gg_rs157269 \\
\hline 0.79 & 0.65 & 0.74 & & 2.71 & & 1.05 & Gg_rs157135 Gg_rs157269 \\
\hline 0.83 & 1.09 & 0.78 & & 3.84 & & 1.11 & Gg_rs157135 Gg_rs157269 \\
\hline 1.36 & 1.02 & 1.28 & & 6.22 & & 1.82 & Gg_rs157135 Gg_rs157269 \\
\hline 1.31 & 0.5 & 1.24 & & 6.12 & & 1.75 & Gg_rs157135 Gg_rs157269 \\
\hline 0.88 & -0.51 & 0.83 & & 4.82 & & 1.17 & Gg_rs157135 Gg_rs157269 \\
\hline 0.72 NA & NA & & NA & & NA & & Gg_rs157135 Gg_rs157269 \\
\hline 0.68 NA & NA & & NA & & NA & & chr5.4325034Gg_rs157269 \\
\hline $0.83 \mathrm{NA}$ & NA & & NA & & NA & & Gg_rs157060.Gg_rs157269 \\
\hline 0.66 & 1 & 0.62 & & 3.96 & & 0.88 & Gg_rs157135 Gg_rs157269 \\
\hline 0.85 & 2.33 & 0.8 & & 4.57 & & 1.14 & Gg_rs157135 Gg_rs157269 \\
\hline $0.51 \mathrm{NA}$ & NA & & NA & & NA & & Gg_rs157135 Gg_rs157269 \\
\hline $0.53 \mathrm{NA}$ & NA & & NA & & NA & & Gg_rs157135 Gg_rs157269 \\
\hline
\end{tabular}




\begin{tabular}{|c|c|c|c|c|c|}
\hline 0.89 & 0.7 & 0.84 & 3.36 & 1.19 & Gg_rs157060.Gg_rs157269 \\
\hline $0.41 \mathrm{NA}$ & NA & NA & NA & & Gg_rs157135 Gg_rs157269 \\
\hline 1.3 & 1.25 & 1.23 & 5.35 & 1.74 & Gg_rs157060.Gg_rs157269 \\
\hline 0.82 & -1.58 & 0.77 & 1.07 & 1.09 & Gg_rs157135 Gg_rs157269 \\
\hline 0.66 & -0.08 & 0.62 & 2.5 & 0.88 & Gg_rs157135 Gg_rs157269 \\
\hline 0.69 & -0.95 & 0.65 & 0.9 & 0.91 & GG_rs157021Gg_rs157269 \\
\hline 0.78 & -1.08 & 0.74 & 1.61 & 1.04 & Gg_rs157135 Gg_rs157269 \\
\hline 0.2 & -0.77 & 0.19 & 0.5 & 0.27 & Gg_rs157135 Gg_rs157269 \\
\hline $0.57 \mathrm{NA}$ & NA & NA & NA & & GG_rs157021Gg_rs135913. \\
\hline $0.6 \mathrm{NA}$ & NA & NA & NA & & GG_rs157021Gg_rs157269 \\
\hline $0.42 \mathrm{NA}$ & NA & NA & NA & & GG_rs157021Gg_rs157269 \\
\hline $0.24 \mathrm{NA}$ & NA & NA & NA & & GG_rs157021Gg_rs157269 \\
\hline $0.52 \mathrm{NA}$ & NA & NA & NA & & GG_rs157021Gg_rs135913. \\
\hline $0.71 \mathrm{NA}$ & NA & NA & NA & & GG_rs157021Gg_rs135913. \\
\hline $0.64 \mathrm{NA}$ & NA & NA & NA & & GG_rs157021Gg_rs135913. \\
\hline 0.78 NA & NA & NA & NA & & Gg_rs157184 Gg_rs135913. \\
\hline 0.49 NA & NA & NA & NA & & Gg_rs157184 Gg_rs135913. \\
\hline $0.78 \mathrm{NA}$ & NA & NA & NA & & Gg_rs157184 Gg_rs135913. \\
\hline $0.59 \mathrm{NA}$ & NA & NA & NA & & Gg_rs157184 Gg_rs135913. \\
\hline $0.92 \mathrm{NA}$ & NA & NA & NA & & Gg_rs157184 Gg_rs135913. \\
\hline $0.78 \mathrm{NA}$ & NA & NA & NA & & Gg_rs157184 Gg_rs135913. \\
\hline $2.11 \mathrm{NA}$ & NA & NA & NA & & Gg_rs157184 Gg_rs135913. \\
\hline 0.63 NA & NA & NA & NA & & Gg_rs157184 Gg_rs135913. \\
\hline $0.45 \mathrm{NA}$ & NA & NA & NA & & Gg_rs157184X5_56411157 \\
\hline $0.78 \mathrm{NA}$ & NA & NA & NA & & Gg_rs157184 Gg_rs135913. \\
\hline $1.44 \mathrm{NA}$ & NA & NA & NA & & Gg_rs157184 Gg_rs135913. \\
\hline $0.85 \mathrm{NA}$ & NA & NA & NA & & Gg_rs157184 Gg_rs135913. \\
\hline 0.79 NA & NA & NA & NA & & Gg_rs157184 Gg_rs135913. \\
\hline 0.73 NA & NA & NA & NA & & Gg_rs157184 Gg_rs135913. \\
\hline $0.66 \mathrm{NA}$ & NA & NA & NA & & Gg_rs157184 Gg_rs135913. \\
\hline $0.59 \mathrm{NA}$ & NA & NA & NA & & Gg_rs157184 Gg_rs135913 \\
\hline $0.38 \mathrm{NA}$ & NA & NA & NA & & Gg_rs157184 Gg_rs135913 \\
\hline $0.3 \mathrm{NA}$ & NA & NA & NA & & Gg_rs157184 GG_rs145495 \\
\hline $0.43 \mathrm{NA}$ & NA & NA & NA & & Gg_rs157184 GG_rs145495 \\
\hline $0.34 \mathrm{NA}$ & NA & NA & NA & & Gg_rs157184 GG_rs145495 \\
\hline $0.45 \mathrm{NA}$ & NA & NA & NA & & Gg_rs157184X5_56411157 \\
\hline $0.97 \mathrm{NA}$ & NA & NA & NA & & Gg_rs157184 GG_rs145495 \\
\hline $0.46 \mathrm{NA}$ & NA & NA & NA & & Gg_rs157184 GG_rs145495 \\
\hline 0.65 NA & NA & NA & NA & & Gg_rs157184 GG_rs145495 \\
\hline 0.75 NA & NA & NA & NA & & Gg_rs157269 GG_rs145495 \\
\hline $0.75 \mathrm{NA}$ & NA & NA & NA & & Gg_rs157269 GG_rs145495 \\
\hline 0.62 & -1.74 & 0.57 & -2.61 & 0.83 & Gg_rs157269 GG_rs145495 \\
\hline $0.56 \mathrm{NA}$ & NA & NA & NA & & Gg_rs157269 GG_rs145495 \\
\hline
\end{tabular}




\begin{tabular}{|c|c|c|c|c|c|}
\hline 0.85 NA & NA & NA & NA & & Gg_rs157269 GG_rs145495 \\
\hline $0.61 \mathrm{NA}$ & NA & NA & NA & & Gg_rs157269 GG_rs145495 \\
\hline $0.66 \mathrm{NA}$ & NA & NA & NA & & Gg_rs157269GG_rs145495 \\
\hline $0.61 \mathrm{NA}$ & NA & NA & NA & & Gg_rs157269GG_rs145495 \\
\hline $1.01 \mathrm{NA}$ & NA & NA & NA & & Gg_rs157269 GG_rs145495 \\
\hline 0.44 NA & NA & NA & NA & & Gg_rs157269 GG_rs145495 \\
\hline $0.8 \mathrm{NA}$ & NA & NA & NA & & Gg_rs157269X5_56411157 \\
\hline $0.64 \mathrm{NA}$ & NA & NA & NA & & Gg_rs157269X5_56411157 \\
\hline $0.53 \mathrm{NA}$ & NA & NA & NA & & Gg_rs157269 GG_rs145495 \\
\hline $0.66 \mathrm{NA}$ & NA & NA & NA & & Gg_rs157269X5_56411157 \\
\hline $0.42 \mathrm{NA}$ & NA & NA & NA & & Gg_rs157269X5_56411157 \\
\hline $0.7 \mathrm{NA}$ & NA & NA & NA & & Gg_rs157269X5_56411157 \\
\hline 0.82 NA & NA & NA & NA & & Gg_rs157269X5_56411157 \\
\hline $0.8 \mathrm{NA}$ & NA & NA & NA & & Gg_rs157184X5_56411157 \\
\hline $0.74 \mathrm{NA}$ & NA & NA & NA & & Gg_rs157184X5_56411157 \\
\hline $0.37 \mathrm{NA}$ & NA & NA & NA & & Gg_rs157269X5_56411157 \\
\hline $0.53 \mathrm{NA}$ & NA & NA & NA & & Gg_rs157269X5_56411157 \\
\hline $0.71 \mathrm{NA}$ & NA & NA & NA & & Gg_rs157269X5_56411157 \\
\hline $0.39 \mathrm{NA}$ & NA & NA & NA & & Gg_rs157269X5_56411157 \\
\hline $0.33 \mathrm{NA}$ & NA & NA & NA & & Gg_rs157184X5_56411157 \\
\hline 0.5 & -2.17 & 0.49 & -2.27 & 0.69 & Gg_rs157269X5_56411157 \\
\hline 0.49 NA & NA & NA & NA & & Gg_rs157184 X5_56411157 \\
\hline $1.03 \mathrm{NA}$ & NA & NA & NA & & Gg_rs157269X5_56411157 \\
\hline 0.73 & -1.7 & 0.72 & -1.21 & 1.01 & Gg_rs157269X5_56411157 \\
\hline $0.4 \mathrm{NA}$ & NA & NA & NA & & Gg_rs135913 X5_56411157 \\
\hline $0.39 \mathrm{NA}$ & NA & NA & NA & & Gg_rs135913 X5_56411157 \\
\hline $0.47 \mathrm{NA}$ & NA & NA & NA & & Gg_rs135913 X5_56411157 \\
\hline $0.35 \mathrm{NA}$ & NA & NA & NA & & Gg_rs135913 X5_56411157 \\
\hline 0.51 & -2.27 & 0.5 & -2.41 & 0.7 & Gg_rs135913 X5_56411157 \\
\hline 0.62 & -2.3 & 0.62 & -2.51 & 0.87 & Gg_rs135913 X5_56411157 \\
\hline $0.6 \mathrm{NA}$ & NA & NA & NA & & Gg_rs135913 X5_56411157 \\
\hline $0.47 \mathrm{NA}$ & NA & NA & NA & & Gg_rs135913 X5_56411157 \\
\hline $0.71 \mathrm{NA}$ & NA & NA & NA & & Gg_rs135913 Gg_rs157434 \\
\hline 1.05 & 3.15 & 0.96 & 4.24 & 1.4 & GG_rs145495Gg_rs157434 \\
\hline 0.74 & 2.54 & 0.67 & 4.05 & 0.98 & GG_rs145495Gg_rs157434 \\
\hline 0.34 & 0.98 & 0.31 & 1.05 & 0.45 & GG_rs145495Gg_rs157434 \\
\hline 0.57 & 1.08 & 0.52 & 3.19 & 0.76 & GG_rs145495Gg_rs157434 \\
\hline 0.56 & 1.93 & 0.5 & 1.76 & 0.74 & GG_rs145495Gg_rs157434 \\
\hline $3.62 \mathrm{NA}$ & NA & NA & NA & & GG_rs145495Gg_rs157434 \\
\hline $0.55 \mathrm{NA}$ & NA & NA & NA & & GG_rs145495Gg_rs157434 \\
\hline $5.38 \mathrm{NA}$ & NA & NA & NA & & GG_rs145495Gg_rs157434 \\
\hline $5.29 \mathrm{NA}$ & NA & NA & NA & & GG_rs145495Gg_rs157434 \\
\hline $0.56 \mathrm{NA}$ & NA & NA & NA & & GG_rs145495Gg_rs157434 \\
\hline
\end{tabular}




\begin{tabular}{|c|c|c|c|c|c|}
\hline $7.92 \mathrm{NA}$ & NA & NA & NA & & GG_rs145495Gg_rs157434 \\
\hline 0.29 & 0.9 & 0.26 & 1.39 & 0.38 & GG_rs145495Gg_rs157434 \\
\hline 0.07 & 0.29 & 0.07 & 0.37 & 0.1 & GG_rs145495Gg_rs157434 \\
\hline 0.79 & 3.12 & 0.71 & 2.83 & 1.04 & GG_rs145495Gg_rs157434 \\
\hline 0.57 & 2.48 & 0.52 & 1.62 & 0.76 & GG_rs145495Gg_rs157434 \\
\hline 0.44 & 1.99 & 0.4 & 1.96 & 0.58 & GG_rs145495Gg_rs157434 \\
\hline 0.4 & 2.13 & 0.38 & 1.75 & 0.51 & Gg_rs157408Gg_rs157434 \\
\hline 0.52 & 2.53 & 0.49 & 2.67 & 0.67 & Gg_rs157408Gg_rs157434 \\
\hline 0.2 & 0.8 & 0.19 & 1.02 & 0.26 & Gg_rs157408Gg_rs157434 \\
\hline 0.21 & 1.09 & 0.2 & 0.99 & 0.27 & Gg_rs157408Gg_rs157434 \\
\hline 0.56 & 2.84 & 0.54 & 2.64 & 0.73 & Gg_rs157408 Gg_rs157434 \\
\hline 0.18 & 0.91 & 0.17 & 1.17 & 0.23 & Gg_rs157408Gg_rs157434 \\
\hline 0.23 & 0.9 & 0.22 & 0.84 & 0.29 & Gg_rs157408Gg_rs157434 \\
\hline 1.19 & -4.09 & 1.13 & 0.09 & 1.54 & Gg_rs157408Gg_rs157434 \\
\hline 0.61 & -1.56 & 0.58 & -1.1 & 0.79 & Gg_rs157408Gg_rs157434 \\
\hline $0.13 \mathrm{NA}$ & NA & NA & NA & & GG_rs145495Gg_rs157434 \\
\hline $0.48 \mathrm{NA}$ & NA & NA & NA & & Gg_rs157408Gg_rs157434 \\
\hline 0.03 & -0.1 & 0.03 & -0.12 & 0.04 & Gg_rs157408Gg_rs157434 \\
\hline $3.1 \mathrm{NA}$ & NA & NA & NA & & Gg_rs157502 X6_2930562 \\
\hline $2.47 \mathrm{NA}$ & NA & NA & NA & & Gg_rs157502 X6_2930562 \\
\hline $0.47 \mathrm{NA}$ & NA & NA & NA & & Gg_rs157502 X6_2930562 \\
\hline $1.75 \mathrm{NA}$ & NA & NA & NA & & Gg_rs157502 Gg_rs157568. \\
\hline $0.35 \mathrm{NA}$ & NA & NA & NA & & Gg_rs157502 Gg_rs157568. \\
\hline $1.19 \mathrm{NA}$ & NA & NA & NA & & Gg_rs157502 Gg_rs157568. \\
\hline $1.62 \mathrm{NA}$ & NA & NA & NA & & Gg_rs157502 Gg_rs157568. \\
\hline $0.33 \mathrm{NA}$ & NA & NA & NA & & Gg_rs157502 X6_2930562 \\
\hline $0.33 \mathrm{NA}$ & NA & NA & NA & & Gg_rs157502 Gg_rs157568 \\
\hline $0.46 \mathrm{NA}$ & NA & NA & NA & & Gg_rs157502 X6_2930562 \\
\hline $0.81 \mathrm{NA}$ & NA & NA & NA & & Gg_rs157502 X6_2930562 \\
\hline $0.76 \mathrm{NA}$ & NA & NA & NA & & Gg_rs157502 X6_2930562 \\
\hline 0.88 & -0.91 & 0.86 & 5.03 & 1.18 & Gg_rs157502 X6_2930562 \\
\hline $2.13 \mathrm{NA}$ & NA & NA & NA & & Gg_rs157502 X6_2930562 \\
\hline $2.71 \mathrm{NA}$ & NA & NA & NA & & Gg_rs157502 X6_2930562 \\
\hline $0.47 \mathrm{NA}$ & NA & NA & NA & & Gg_rs157502 X6_2930562 \\
\hline $2.3 \mathrm{NA}$ & NA & NA & NA & & Gg_rs157502 X6_2930562 \\
\hline $2.19 \mathrm{NA}$ & NA & NA & NA & & Gg_rs157502 X6_2930562 \\
\hline $2.38 \mathrm{NA}$ & NA & NA & NA & & Gg_rs157502 X6_2930562 \\
\hline $2.16 \mathrm{NA}$ & NA & NA & NA & & Gg_rs157502 X6_2930562 \\
\hline $3.28 \mathrm{NA}$ & NA & NA & NA & & Gg_rs157502 X6_2930562 \\
\hline $2.31 \mathrm{NA}$ & NA & NA & NA & & Gg_rs157502 X6_2930562 \\
\hline $2.51 \mathrm{NA}$ & NA & NA & NA & & Gg_rs157502 X6_2930562 \\
\hline $3.29 \mathrm{NA}$ & NA & NA & NA & & Gg_rs157502 Gg_rs157568. \\
\hline $2.26 \mathrm{NA}$ & NA & NA & NA & & Gg_rs157502 Gg_rs157568 \\
\hline
\end{tabular}




\begin{tabular}{|c|c|c|c|c|c|}
\hline $4.48 \mathrm{NA}$ & NA & NA & NA & & Gg_rs157502 Gg_rs157568. \\
\hline $1.55 \mathrm{NA}$ & NA & NA & NA & & Gg_rs157502 Gg_rs157568. \\
\hline $0.53 \mathrm{NA}$ & NA & NA & NA & & Gg_rs157502 Gg_rs157568. \\
\hline $5.19 \mathrm{NA}$ & NA & NA & NA & & Gg_rs157502 Gg_rs157568 \\
\hline $0.62 \mathrm{NA}$ & NA & NA & NA & & Gg_rs157502 Gg_rs157568. \\
\hline $4.28 \mathrm{NA}$ & NA & NA & NA & & Gg_rs157502 Gg_rs157568. \\
\hline $2.21 \mathrm{NA}$ & NA & NA & NA & & Gg_rs157502 Gg_rs157568. \\
\hline $5.09 \mathrm{NA}$ & NA & NA & NA & & Gg_rs157502 Gg_rs157568. \\
\hline $1.17 \mathrm{NA}$ & NA & NA & NA & & Gg_rs157502 Gg_rs157568 \\
\hline 4.69 NA & NA & NA & NA & & Gg_rs157502 Gg_rs157568. \\
\hline $0.4 \mathrm{NA}$ & NA & NA & NA & & Gg_rs157502 Gg_rs157568. \\
\hline $2.65 \mathrm{NA}$ & NA & NA & NA & & Gg_rs157502 Gg_rs157568 \\
\hline $5.03 \mathrm{NA}$ & NA & NA & NA & & Gg_rs157502 Gg_rs157568 \\
\hline $2.39 \mathrm{NA}$ & NA & NA & NA & & Gg_rs157502 Gg_rs157568. \\
\hline $4.33 \mathrm{NA}$ & NA & NA & NA & & Gg_rs157502 Gg_rs157568. \\
\hline $4.85 \mathrm{NA}$ & NA & NA & NA & & Gg_rs157502 Gg_rs157568. \\
\hline 0.09 & 0.26 & 0.08 & -0.35 & 0.12 & Gg_rs157520 Gg_rs157568. \\
\hline 4.68 NA & NA & NA & NA & & Gg_rs157502 Gg_rs157568. \\
\hline $0.66 \mathrm{NA}$ & NA & NA & NA & & Gg_rs157520 Gg_rs157568. \\
\hline $1.73 \mathrm{NA}$ & NA & NA & NA & & Gg_rs157520 Gg_rs157568. \\
\hline 0.99 NA & NA & NA & NA & & Gg_rs157520Gg_rs157591. \\
\hline $0.13 \mathrm{NA}$ & NA & NA & NA & & Gg_rs157520Gg_rs157568 \\
\hline $0.77 \mathrm{NA}$ & NA & NA & NA & & X6_2930562 Gg_rs157654 \\
\hline $1.2 \mathrm{NA}$ & NA & NA & NA & & X6_2930562 Gg_rs157654 \\
\hline 1.09 & 0.71 & 1.15 & -4.32 & 1.5 & X6_2930562 Gg_rs157654 \\
\hline $0.65 \mathrm{NA}$ & NA & NA & NA & & X6_2930562 Gg_rs157596. \\
\hline $0.27 \mathrm{NA}$ & NA & NA & NA & & X6_2930562 Gg_rs157654 \\
\hline 2.15 & -4.17 & 2.29 & 3.04 & 2.94 & X6_2930562 Gg_rs157654 \\
\hline $0.5 \mathrm{NA}$ & NA & NA & NA & & X6_2930562 Gg_rs157596 \\
\hline $1.05 \mathrm{NA}$ & NA & NA & NA & & X6_2930562 Gg_rs157654 \\
\hline $0.5 \mathrm{NA}$ & NA & NA & NA & & X6_2930562 Gg_rs157654 \\
\hline $0.29 \mathrm{NA}$ & NA & NA & NA & & X6_2930562 Gg_rs157654 \\
\hline $0.68 \mathrm{NA}$ & NA & NA & NA & & X6_2930562 Gg_rs157654 \\
\hline $0.61 \mathrm{NA}$ & NA & NA & NA & & X6_2930562 Gg_rs157654 \\
\hline 0.49 & -1.09 & 0.52 & 1.28 & 0.66 & X6_2930562 Gg_rs157654 \\
\hline $0.58 \mathrm{NA}$ & NA & NA & NA & & Gg_rs157568 Gg_rs157654 \\
\hline $0.6 \mathrm{NA}$ & NA & NA & NA & & Gg_rs157568 Gg_rs145688 \\
\hline $0.57 \mathrm{NA}$ & NA & NA & NA & & Gg_rs157520 Gg_rs157730. \\
\hline $1.14 \mathrm{NA}$ & NA & NA & NA & & Gg_rs157596 Gg_rs157730. \\
\hline 0.03 & 0.1 & 0.03 & -0.11 & 0.04 & Gg_rs157596 Gg_rs145688. \\
\hline $0.91 \mathrm{NA}$ & NA & NA & NA & & X6_2930562 Gg_rs157730. \\
\hline 0.75 & -1.19 & 0.81 & 3.65 & 1.05 & Gg_rs157596Gg_rs145688 \\
\hline $4.53 \mathrm{NA}$ & NA & NA & NA & & Gg_rs157596 Gg_rs145688 \\
\hline
\end{tabular}




\begin{tabular}{|c|c|c|c|c|c|}
\hline $0.53 \mathrm{NA}$ & NA & NA & NA & & Gg_rs157596 Gg_rs145688 \\
\hline 4.61 NA & NA & NA & NA & & Gg_rs157596 Gg_rs145688 \\
\hline $0.55 \mathrm{NA}$ & NA & NA & NA & & Gg_rs157596 Gg_rs145688 \\
\hline $2.66 \mathrm{NA}$ & NA & NA & NA & & Gg_rs157596 Gg_rs145688 \\
\hline $1.39 \mathrm{NA}$ & NA & NA & NA & & Gg_rs157596 Gg_rs145688 \\
\hline $0.52 \mathrm{NA}$ & NA & NA & NA & & Gg_rs157596 Gg_rs145688 \\
\hline $0.74 \mathrm{NA}$ & NA & NA & NA & & Gg_rs157596 Gg_rs145688 \\
\hline $2.92 \mathrm{NA}$ & NA & NA & NA & & Gg_rs157596 Gg_rs145688 \\
\hline $2.39 \mathrm{NA}$ & NA & NA & NA & & Gg_rs157596 Gg_rs145688 \\
\hline $1.82 \mathrm{NA}$ & NA & NA & NA & & Gg_rs157596 Gg_rs145688 \\
\hline $3.02 \mathrm{NA}$ & NA & NA & NA & & Gg_rs157596 Gg_rs145688 \\
\hline $0.8 \mathrm{NA}$ & NA & NA & NA & & Gg_rs157596 Gg_rs145688 \\
\hline $0.84 \mathrm{NA}$ & NA & NA & NA & & Gg_rs157596 Gg_rs145688 \\
\hline $2.46 \mathrm{NA}$ & NA & NA & NA & & Gg_rs157596 Gg_rs145688 \\
\hline $0.65 \mathrm{NA}$ & NA & NA & NA & & Gg_rs157596 Gg_rs145688 \\
\hline $0.56 \mathrm{NA}$ & NA & NA & NA & & Gg_rs157596 Gg_rs145688 \\
\hline $0.82 \mathrm{NA}$ & NA & NA & NA & & Gg_rs157596 Gg_rs145688 \\
\hline $0.57 \mathrm{NA}$ & NA & NA & NA & & Gg_rs157596Gg_rs145688 \\
\hline $0.61 \mathrm{NA}$ & NA & NA & NA & & Gg_rs157596 Gg_rs145688 \\
\hline $0.68 \mathrm{NA}$ & NA & NA & NA & & Gg_rs157596 Gg_rs145688 \\
\hline $0.45 \mathrm{NA}$ & NA & NA & NA & & Gg_rs157596 Gg_rs145688 \\
\hline $2.78 \mathrm{NA}$ & NA & NA & NA & & Gg_rs157596 Gg_rs145688 \\
\hline 0.32 & -1 & 0.34 & 0.95 & 0.45 & Gg_rs157596 Gg_rs145688 \\
\hline $0.75 \mathrm{NA}$ & NA & NA & NA & & Gg_rs157596Gg_rs145688 \\
\hline $0.74 \mathrm{NA}$ & NA & NA & NA & & Gg_rs157596 Gg_rs157730. \\
\hline $0.37 \mathrm{NA}$ & NA & NA & NA & & Gg_rs157596 Gg_rs157816. \\
\hline 0.74 & -0.16 & 0.8 & 2.11 & 1.04 & Gg_rs157596 Gg_rs157730. \\
\hline $0.35 \mathrm{NA}$ & NA & NA & NA & & Gg_rs157596 Gg_rs157730. \\
\hline $0.45 \mathrm{NA}$ & NA & NA & NA & & Gg_rs157596 Gg_rs157770 \\
\hline $0.58 \mathrm{NA}$ & NA & NA & NA & & Gg_rs157596 Gg_rs157730. \\
\hline 1.22 & 4.74 & 1.32 & -3.08 & 1.72 & Gg_rs157596 Gg_rs157770 \\
\hline 0.4 & 0.19 & 0.38 & 1.2 & 0.52 & Gg_rs157596 Gg_rs157730. \\
\hline $0.75 \mathrm{NA}$ & NA & NA & NA & & Gg_rs157596 Gg_rs157770 \\
\hline $0.52 \mathrm{NA}$ & NA & NA & NA & & Gg_rs157596 Gg_rs157770 \\
\hline $0.81 \mathrm{NA}$ & NA & NA & NA & & Gg_rs157596 Gg_rs157730. \\
\hline $0.52 \mathrm{NA}$ & NA & NA & NA & & Gg_rs157568 Gg_rs157730. \\
\hline $0.52 \mathrm{NA}$ & NA & NA & NA & & Gg_rs157596 Gg_rs157770 \\
\hline 0.88 & 3.14 & 0.83 & -1.08 & 1.16 & Gg_rs157596 Gg_rs157770 \\
\hline $0.46 \mathrm{NA}$ & NA & NA & NA & & Gg_rs157654 Gg_rs157730. \\
\hline $0.47 \mathrm{NA}$ & NA & NA & NA & & Gg_rs157596 Gg_rs157730. \\
\hline 0.99 NA & NA & NA & NA & & Gg_rs157520Gg_rs157730. \\
\hline $0.59 \mathrm{NA}$ & NA & NA & NA & & Gg_rs157596 Gg_rs157730، \\
\hline $0.41 \mathrm{NA}$ & NA & NA & NA & & Gg_rs157654 Gg_rs157730. \\
\hline
\end{tabular}




\begin{tabular}{|c|c|c|c|c|c|c|c|c|}
\hline 0.49 NA & & NA & & NA & & NA & & Gg_rs157596 Gg_rs157770 \\
\hline $2.47 \mathrm{NA}$ & & NA & & NA & & NA & & Gg_rs157654 Gg_rs157770 \\
\hline $0.49 \mathrm{NA}$ & & NA & & NA & & NA & & Gg_rs157654 Gg_rs157730. \\
\hline $0.37 \mathrm{NA}$ & & NA & & NA & & NA & & Gg_rs157654 Gg_rs157730. \\
\hline 1.14 & 2.37 & & 1.07 & & 3.22 & & 1.5 & Gg_rs157596Gg_rs157770 \\
\hline 0.34 & 1.05 & & 0.32 & & -1.32 & & 0.45 & Gg_rs157654 Gg_rs157730. \\
\hline 1.32 & 1.67 & & 1.25 & & 3.2 & & 1.74 & Gg_rs157654 Gg_rs157770 \\
\hline 1.25 & 3.13 & & 1.18 & & 2.67 & & 1.65 & Gg_rs157654 Gg_rs157770 \\
\hline 0.95 & 2.65 & & 0.9 & & 1.55 & & 1.25 & Gg_rs157654 Gg_rs157770 \\
\hline 0.77 & 2.22 & & 0.72 & & 2.34 & & 1.01 & Gg_rs157654 Gg_rs157730. \\
\hline 0.69 & 2.92 & & 0.65 & & 0.76 & & 0.91 & Gg_rs157654 Gg_rs157730. \\
\hline 0.66 & 1.62 & & 0.62 & & -1.77 & & 0.87 & Gg_rs157654 Gg_rs157730. \\
\hline 0.63 & -1.43 & & 0.6 & & -4.32 & & 0.83 & Gg_rs157654 Gg_rs157730. \\
\hline 0.59 & 2.36 & & 0.56 & & -0.16 & & 0.77 & Gg_rs157654 Gg_rs157730. \\
\hline 0.51 & 1.38 & & 0.48 & & -0.53 & & 0.67 & Gg_rs157654 Gg_rs157730. \\
\hline 0.57 & 1.99 & & 0.53 & & -1.27 & & 0.74 & Gg_rs157654 Gg_rs157730. \\
\hline 0.62 & 1.99 & & 0.59 & & -0.75 & & 0.82 & Gg_rs157654 Gg_rs157730. \\
\hline 0.56 & 2.57 & & 0.53 & & 0.13 & & 0.74 & Gg_rs157654 Gg_rs157730. \\
\hline 0.46 & 1.88 & & 0.44 & & -0.53 & & 0.61 & Gg_rs157654 Gg_rs157730. \\
\hline 0.64 & 2.03 & & 0.6 & & -0.77 & & 0.84 & Gg_rs157654 Gg_rs157730. \\
\hline $0.35 \mathrm{NA}$ & & NA & & NA & & NA & & Gg_rs157654 Gg_rs157730. \\
\hline $0.35 \mathrm{NA}$ & & NA & & NA & & NA & & Gg_rs157596 Gg_rs157770 \\
\hline $0.32 \mathrm{NA}$ & & NA & & NA & & NA & & Gg_rs157654 Gg_rs157730. \\
\hline $0.47 \mathrm{NA}$ & & NA & & NA & & NA & & Gg_rs157654 Gg_rs157770 \\
\hline 0.44 NA & & NA & & NA & & NA & & Gg_rs157654 Gg_rs157770 \\
\hline 0.56 & -0.39 & & 0.53 & & -3.5 & & 0.74 & Gg_rs157654 Gg_rs157730. \\
\hline 0.35 & 0.78 & & 0.33 & & -1.76 & & 0.46 & Gg_rs157654 Gg_rs157770 \\
\hline 0.48 & 1.59 & & 0.46 & & -1.05 & & 0.64 & Gg_rs157654 Gg_rs157770 \\
\hline 0.6 & 2.6 & & 0.57 & & 0.53 & & 0.79 & Gg_rs157654 Gg_rs157770 \\
\hline $0.34 \mathrm{NA}$ & & NA & & NA & & NA & & Gg_rs157654 Gg_rs157770 \\
\hline 0.63 NA & & NA & & NA & & NA & & Gg_rs157654 Gg_rs157770 \\
\hline $1.07 \mathrm{NA}$ & & NA & & NA & & NA & & Gg_rs157596 Gg_rs157770 \\
\hline $1.08 \mathrm{NA}$ & & NA & & NA & & NA & & Gg_rs157654 Gg_rs157816. \\
\hline $0.64 \mathrm{NA}$ & & NA & & NA & & NA & & Gg_rs157654 Gg_rs157770 \\
\hline $0.59 \mathrm{NA}$ & & NA & & NA & & NA & & Gg_rs157596 Gg_rs157770 \\
\hline $0.47 \mathrm{NA}$ & & NA & & NA & & NA & & Gg_rs157654 Gg_rs157770 \\
\hline $0.33 \mathrm{NA}$ & & NA & & NA & & NA & & Gg_rs157654 Gg_rs157816. \\
\hline 0.56 & 2.28 & & 0.53 & & -0.99 & & 0.74 & Gg_rs157654 Gg_rs157816. \\
\hline 0.61 & 2.18 & & 0.58 & & 1.28 & & 0.8 & Gg_rs157654 Gg_rs157770 \\
\hline 0.89 & 2.81 & & 0.84 & & 2.94 & & 1.18 & Gg_rs157654 Gg_rs157816. \\
\hline $0.66 \mathrm{NA}$ & & NA & & NA & & NA & & Gg_rs157596 Gg_rs157770 \\
\hline $0.63 \mathrm{NA}$ & & NA & & NA & & NA & & Gg_rs157654 Gg_rs157770 \\
\hline $0.47 \mathrm{NA}$ & & NA & & NA & & NA & & Gg_rs157654 Gg_rs157770 \\
\hline
\end{tabular}




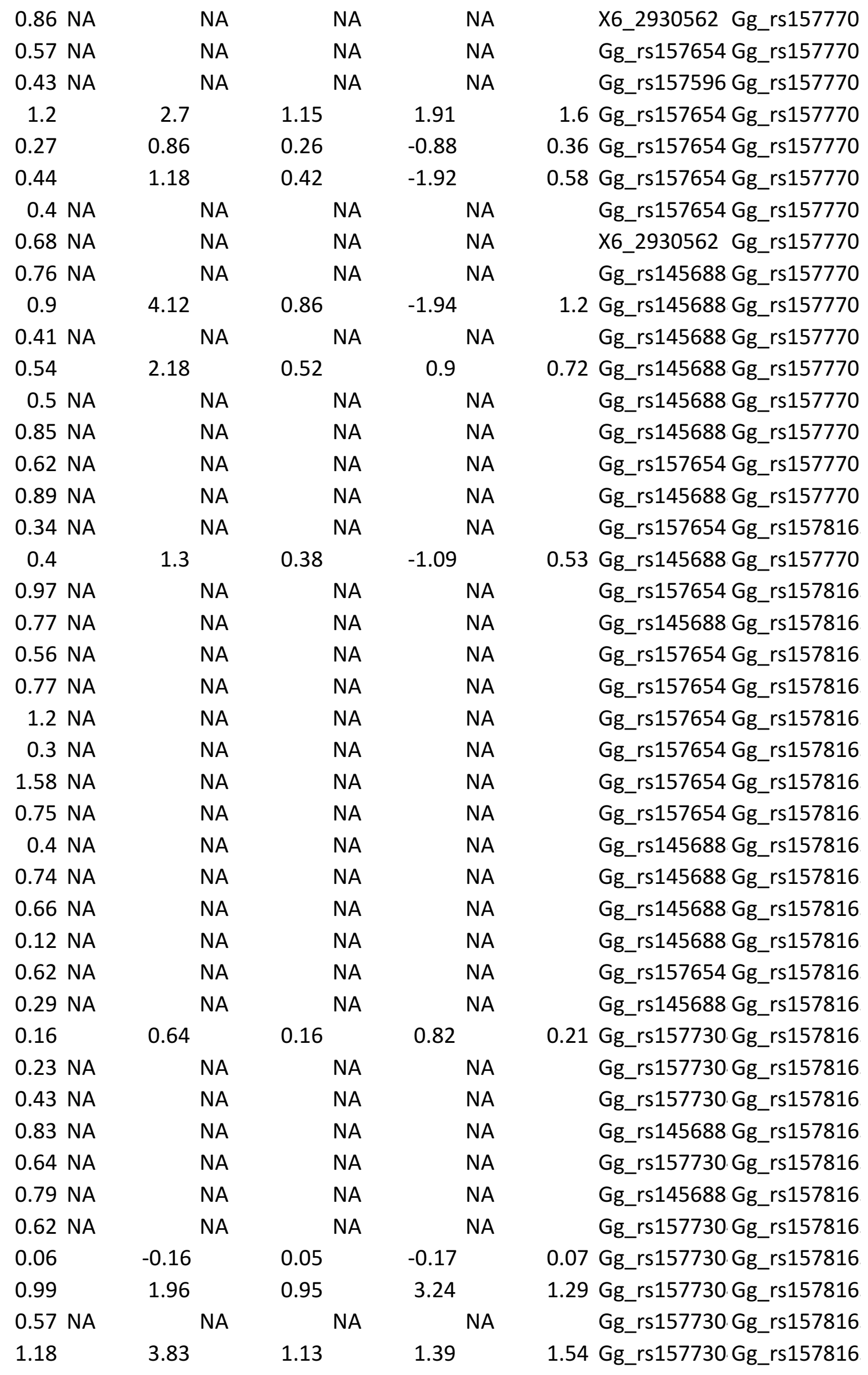




\begin{tabular}{|c|c|c|c|c|c|}
\hline 1.18 & 3.63 & 1.14 & 2.04 & 1.54 & Gg_rs157730.Gg_rs157816 \\
\hline 0.45 & 1.23 & 0.44 & 0.76 & 0.59 & Gg_rs157730.Gg_rs157816 \\
\hline $0.54 \mathrm{NA}$ & NA & NA & NA & & Gg_rs157730.Gg_rs157816 \\
\hline $0.62 \mathrm{NA}$ & NA & NA & NA & & Gg_rs157730.Gg_rs157816 \\
\hline $1.3 \mathrm{NA}$ & NA & NA & NA & & Gg_rs157730.Gg_rs157816 \\
\hline $0.49 \mathrm{NA}$ & NA & NA & NA & & Gg_rs157730.Gg_rs157816 \\
\hline $0.84 \mathrm{NA}$ & NA & NA & NA & & Gg_rs157730.Gg_rs157816 \\
\hline $0.78 \mathrm{NA}$ & NA & NA & NA & & Gg_rs157730.Gg_rs157816 \\
\hline $0.61 \mathrm{NA}$ & NA & NA & NA & & Gg_rs157730.Gg_rs157816 \\
\hline 0.06 & 0.3 & 0.06 & 0.3 & 0.08 & Gg_rs157730.Gg_rs157816 \\
\hline $0.53 \mathrm{NA}$ & NA & NA & NA & & Gg_rs157730.Gg_rs157816 \\
\hline 1.81 & 5.04 & 1.75 & 3.9 & 2.37 & Gg_rs157730Gg_rs157816 \\
\hline 1.96 & 5.72 & 1.89 & 2.46 & 2.56 & Gg_rs157730.Gg_rs157816 \\
\hline 1.96 & 5.39 & 1.89 & 2.77 & 2.57 & Gg_rs157730Gg_rs157816 \\
\hline $1.85 \mathrm{NA}$ & NA & NA & NA & & Gg_rs157730.Gg_rs157816 \\
\hline 0.01 & 0.07 & 0.01 & 0.08 & 0.02 & Gg_rs157730.Gg_rs157816 \\
\hline $1.16 \mathrm{NA}$ & NA & NA & NA & & Gg_rs157730.Gg_rs157816 \\
\hline $2.9 \mathrm{NA}$ & NA & NA & NA & & Gg_rs157730.Gg_rs157816 \\
\hline $1.25 \mathrm{NA}$ & NA & NA & NA & & Gg_rs157730.Gg_rs157816 \\
\hline $3.29 \mathrm{NA}$ & NA & NA & NA & & Gg_rs157730.Gg_rs157816 \\
\hline $1.73 \mathrm{NA}$ & NA & NA & NA & & Gg_rs157730 Gg_rs157816 \\
\hline $1.43 \mathrm{NA}$ & NA & NA & NA & & Gg_rs157730.Gg_rs157816 \\
\hline $2.6 \mathrm{NA}$ & NA & NA & NA & & Gg_rs157730.Gg_rs157816 \\
\hline $2.18 \mathrm{NA}$ & NA & NA & NA & & Gg_rs157730 Gg_rs157816 \\
\hline $2.53 \mathrm{NA}$ & NA & NA & NA & & Gg_rs157730.Gg_rs157816 \\
\hline $1.35 \mathrm{NA}$ & NA & NA & NA & & Gg_rs157730 Gg_rs157816 \\
\hline $2.17 \mathrm{NA}$ & NA & NA & NA & & Gg_rs157730Gg_rs157816 \\
\hline $1.56 \mathrm{NA}$ & NA & NA & NA & & Gg_rs157730.Gg_rs157816 \\
\hline 0.93 NA & NA & NA & NA & & Gg_rs157730 Gg_rs157816 \\
\hline $1.05 \mathrm{NA}$ & NA & NA & NA & & Gg_rs157730.Gg_rs157816 \\
\hline $1.17 \mathrm{NA}$ & NA & NA & NA & & Gg_rs157730.Gg_rs157816 \\
\hline $2.35 \mathrm{NA}$ & NA & NA & NA & & Gg_rs157730 Gg_rs157816 \\
\hline $2.53 \mathrm{NA}$ & NA & NA & NA & & Gg_rs157730.Gg_rs157816 \\
\hline $2.24 \mathrm{NA}$ & NA & NA & NA & & Gg_rs157730 Gg_rs157816 \\
\hline $1.04 \mathrm{NA}$ & NA & NA & NA & & Gg_rs157730 Gg_rs157816 \\
\hline $1.31 \mathrm{NA}$ & NA & NA & NA & & Gg_rs157730.Gg_rs157816 \\
\hline $1.37 \mathrm{NA}$ & NA & NA & NA & & Gg_rs157730.Gg_rs157816 \\
\hline $2.23 \mathrm{NA}$ & NA & NA & NA & & Gg_rs157730.Gg_rs157816 \\
\hline $2.36 \mathrm{NA}$ & NA & NA & NA & & Gg_rs157730.Gg_rs157816 \\
\hline $2.11 \mathrm{NA}$ & NA & NA & NA & & Gg_rs157730 Gg_rs157816 \\
\hline $1.57 \mathrm{NA}$ & NA & NA & NA & & Gg_rs157730 Gg_rs157816 \\
\hline $2.08 \mathrm{NA}$ & NA & NA & NA & & Gg_rs157730.Gg_rs157830 \\
\hline $0.95 \mathrm{NA}$ & NA & NA & NA & & Gg_rs157730.Gg_rs157874 \\
\hline
\end{tabular}




\begin{tabular}{|c|c|c|c|c|}
\hline $1.9 \mathrm{NA}$ & NA & NA & NA & Gg_rs157730.Gg_rs157830 \\
\hline $1.92 \mathrm{NA}$ & NA & NA & NA & Gg_rs157730.Gg_rs157874 \\
\hline $1.87 \mathrm{NA}$ & NA & NA & NA & Gg_rs157730 Gg_rs157830 \\
\hline $1.85 \mathrm{NA}$ & NA & NA & NA & Gg_rs157730 Gg_rs157874 \\
\hline $3.1 \mathrm{NA}$ & NA & NA & NA & Gg_rs157730 Gg_rs157874 \\
\hline $2.58 \mathrm{NA}$ & NA & NA & NA & Gg_rs157730 Gg_rs145756 \\
\hline $3.21 \mathrm{NA}$ & NA & NA & NA & Gg_rs157730.Gg_rs157816 \\
\hline 3.54 NA & NA & NA & NA & Gg_rs157730.Gg_rs145756 \\
\hline $1.71 \mathrm{NA}$ & NA & NA & NA & Gg_rs157730.Gg_rs157874 \\
\hline 0.98 NA & NA & NA & NA & Gg_rs157730.Gg_rs157874 \\
\hline 5.09 NA & NA & NA & NA & Gg_rs157730 Gg_rs157830 \\
\hline $1.34 \mathrm{NA}$ & NA & NA & NA & Gg_rs157730 Gg_rs157874 \\
\hline $4.91 \mathrm{NA}$ & NA & NA & NA & Gg_rs157730Gg_rs145756 \\
\hline $1.14 \mathrm{NA}$ & NA & NA & NA & Gg_rs157730 Gg_rs157830 \\
\hline $2.51 \mathrm{NA}$ & NA & NA & NA & Gg_rs157730 Gg_rs157816 \\
\hline $3.85 \mathrm{NA}$ & NA & NA & NA & Gg_rs157730.Gg_rs157874 \\
\hline $2.91 \mathrm{NA}$ & NA & NA & NA & Gg_rs157730 Gg_rs157874 \\
\hline $1.86 \mathrm{NA}$ & NA & NA & NA & Gg_rs157730.Gg_rs157874 \\
\hline $2.9 \mathrm{NA}$ & NA & NA & NA & Gg_rs157730 Gg_rs157874 \\
\hline $1.9 \mathrm{NA}$ & NA & NA & NA & Gg_rs157730.Gg_rs157874 \\
\hline $2.68 \mathrm{NA}$ & NA & NA & NA & Gg_rs157770 Gg_rs145756 \\
\hline 4.79 NA & NA & NA & NA & Gg_rs157730.Gg_rs157830 \\
\hline $3.23 \mathrm{NA}$ & NA & NA & NA & Gg_rs157730.Gg_rs157874 \\
\hline $3.4 \mathrm{NA}$ & NA & NA & NA & Gg_rs157730.Gg_rs157874 \\
\hline $2.75 \mathrm{NA}$ & NA & NA & NA & Gg_rs157730.Gg_rs157874 \\
\hline $1.85 \mathrm{NA}$ & NA & NA & NA & Gg_rs157730.Gg_rs157874 \\
\hline $1.88 \mathrm{NA}$ & NA & NA & NA & Gg_rs157770 Gg_rs145756 \\
\hline $2.02 \mathrm{NA}$ & NA & NA & NA & Gg_rs157770 Gg_rs157816 \\
\hline $2.55 \mathrm{NA}$ & NA & NA & NA & Gg_rs157770 Gg_rs157830 \\
\hline $2.85 \mathrm{NA}$ & NA & NA & NA & Gg_rs157770 Gg_rs157816 \\
\hline 3.67 NA & NA & NA & NA & Gg_rs157770 Gg_rs157874 \\
\hline $2.08 \mathrm{NA}$ & NA & NA & NA & Gg_rs157770 Gg_rs157816 \\
\hline 4.8 NA & NA & NA & NA & Gg_rs157770 Gg_rs157830 \\
\hline 4.04 NA & NA & NA & NA & Gg_rs157770 Gg_rs157874 \\
\hline $2.78 \mathrm{NA}$ & NA & NA & NA & Gg_rs157770 Gg_rs157874 \\
\hline $2.72 \mathrm{NA}$ & NA & NA & NA & Gg_rs157770Gg_rs157874 \\
\hline $3.07 \mathrm{NA}$ & NA & NA & NA & Gg_rs157770 Gg_rs157816 \\
\hline 4.36 NA & NA & NA & NA & Gg_rs157770 Gg_rs157874 \\
\hline $2.73 \mathrm{NA}$ & NA & NA & NA & Gg_rs157770 Gg_rs157874 \\
\hline $1.44 \mathrm{NA}$ & NA & NA & NA & Gg_rs157770 Gg_rs157874 \\
\hline 0.54 NA & NA & NA & NA & Gg_rs157770 Gg_rs157874 \\
\hline $1.69 \mathrm{NA}$ & NA & NA & NA & Gg_rs157770 Gg_rs157874 \\
\hline $2.43 \mathrm{NA}$ & NA & NA & NA & Gg_rs157770 Gg_rs157874 \\
\hline
\end{tabular}




\begin{tabular}{|c|c|c|c|c|c|}
\hline 4.48 NA & NA & NA & NA & & Gg_rs157770Gg_rs157874 \\
\hline $3.8 \mathrm{NA}$ & NA & NA & NA & & Gg_rs157770 Gg_rs157874 \\
\hline $2.11 \mathrm{NA}$ & NA & NA & NA & & Gg_rs157770 Gg_rs157874 \\
\hline $3.96 \mathrm{NA}$ & NA & NA & NA & & Gg_rs157770 Gg_rs157874 \\
\hline 5.95 & 11.24 & 6.99 & 3.71 & 8.9 & Gg_rs157770 Gg_rs157830. \\
\hline 5.7 NA & NA & NA & NA & & Gg_rs157770Gg_rs157874 \\
\hline $5.21 \mathrm{NA}$ & NA & NA & NA & & Gg_rs157770 Gg_rs157874 \\
\hline $5.82 \mathrm{NA}$ & NA & NA & NA & & Gg_rs157770Gg_rs157874 \\
\hline $2.46 \mathrm{NA}$ & NA & NA & NA & & Gg_rs157770 Gg_rs145756: \\
\hline 3.3 & 3.33 & 3.87 & 5.59 & 4.93 & Gg_rs157770 Gg_rs157830 \\
\hline $2.46 \mathrm{NA}$ & NA & NA & NA & & Gg_rs157770Gg_rs157874 \\
\hline $2.47 \mathrm{NA}$ & NA & NA & NA & & Gg_rs157770 Gg_rs157874 \\
\hline $1.95 \mathrm{NA}$ & NA & NA & NA & & Gg_rs157770 Gg_rs157874 \\
\hline 4.59 & 9.65 & 5.39 & 2.56 & 6.87 & Gg_rs157770 Gg_rs157874 \\
\hline $6.82 \mathrm{NA}$ & NA & NA & NA & & Gg_rs157770Gg_rs157874 \\
\hline $5.47 \mathrm{NA}$ & NA & NA & NA & & Gg_rs157770 Gg_rs157874 \\
\hline $0.69 \mathrm{NA}$ & NA & NA & NA & & Gg_rs157730X6_18893102 \\
\hline $1.61 \mathrm{NA}$ & NA & NA & NA & & Gg_rs157770 Gg_rs157830. \\
\hline $6.09 \mathrm{NA}$ & NA & NA & NA & & Gg_rs157770 Gg_rs157874 \\
\hline $7.09 \mathrm{NA}$ & NA & NA & NA & & Gg_rs157770 Gg_rs157874 \\
\hline $6.6 \mathrm{NA}$ & NA & NA & NA & & Gg_rs157770 Gg_rs157874 \\
\hline $6.26 \mathrm{NA}$ & NA & NA & NA & & Gg_rs157770 Gg_rs157874 \\
\hline $5.64 \mathrm{NA}$ & NA & NA & NA & & Gg_rs157770 Gg_rs157874 \\
\hline $6.81 \mathrm{NA}$ & NA & NA & NA & & Gg_rs157770 Gg_rs157874 \\
\hline $6.3 \mathrm{NA}$ & NA & NA & NA & & Gg_rs157770Gg_rs157874 \\
\hline $5.04 \mathrm{NA}$ & NA & NA & NA & & Gg_rs157770 Gg_rs157874 \\
\hline 4.69 NA & NA & NA & NA & & Gg_rs157770 Gg_rs157874 \\
\hline $2.39 \mathrm{NA}$ & NA & NA & NA & & Gg_rs157770 Gg_rs157874 \\
\hline $6.44 \mathrm{NA}$ & NA & NA & NA & & Gg_rs157770Gg_rs157874 \\
\hline $3.32 \mathrm{NA}$ & NA & NA & NA & & Gg_rs157770Gg_rs157874 \\
\hline $0.5 \mathrm{NA}$ & NA & NA & NA & & Gg_rs157770 Gg_rs157874 \\
\hline $2.51 \mathrm{NA}$ & NA & NA & NA & & Gg_rs157770 Gg_rs157874 \\
\hline $2.76 \mathrm{NA}$ & NA & NA & NA & & Gg_rs157770 Gg_rs157874 \\
\hline $1.11 \mathrm{NA}$ & NA & NA & NA & & Gg_rs157770Gg_rs157874 \\
\hline 3.48 & 4.78 & 4.08 & 5.6 & 5.2 & Gg_rs157770 Gg_rs145756 \\
\hline 1.39 & -0.33 & 1.63 & -4.31 & 2.08 & Gg_rs157770 Gg_rs157874 \\
\hline $1.89 \mathrm{NA}$ & NA & NA & NA & & Gg_rs157770 Gg_rs157874 \\
\hline $0.91 \mathrm{NA}$ & NA & NA & NA & & Gg_rs157770 Gg_rs157874 \\
\hline $0.8 \mathrm{NA}$ & NA & NA & NA & & Gg_rs157770 Gg_rs157874 \\
\hline $1.43 \mathrm{NA}$ & NA & NA & NA & & Gg_rs157770 Gg_rs145756: \\
\hline $1.26 \mathrm{NA}$ & NA & NA & NA & & Gg_rs157770 Gg_rs157874 \\
\hline $0.7 \mathrm{NA}$ & NA & NA & NA & & Gg_rs157770 X6_18893102 \\
\hline $2.58 \mathrm{NA}$ & NA & NA & NA & & Gg_rs157770 Gg_rs157874 \\
\hline
\end{tabular}




\begin{tabular}{|c|c|c|c|c|c|c|}
\hline $0.91 \mathrm{NA}$ & NA & & NA & NA & & Gg_rs157770 X6_18893102 \\
\hline $0.6 \mathrm{NA}$ & NA & & NA & NA & & Gg_rs157770 X6_18893102 \\
\hline 1.25 & -3.18 & 1.47 & & -1.63 & 1.87 & Gg_rs157770 Gg_rs157874 \\
\hline $0.61 \mathrm{NA}$ & NA & & NA & NA & & Gg_rs157770 Gg_rs157874 \\
\hline 1.23 & -1.25 & 1.45 & & -2.48 & 1.84 & Gg_rs157770 X6_18893102 \\
\hline 0.69 & -2.03 & 0.81 & & 0.38 & 1.03 & Gg_rs157770 X6_18893102 \\
\hline 1.53 & 2.36 & 1.8 & & -5.04 & 2.29 & Gg_rs157770Gg_rs157874 \\
\hline $0.72 \mathrm{NA}$ & NA & & NA & NA & & Gg_rs157770 X6_18893102 \\
\hline 1.39 & -0.63 & 1.64 & & -4.04 & 2.08 & Gg_rs157770 X6_18893102 \\
\hline 1.41 & -3.28 & 1.65 & & -1.15 & 2.1 & Gg_rs157770 X6_18893102 \\
\hline 0.91 & -2.05 & 1.06 & & -0.75 & 1.35 & Gg_rs157770 X6_18893102 \\
\hline $0.73 \mathrm{NA}$ & NA & & NA & NA & & Gg_rs157770 X6_18893102 \\
\hline $1.57 \mathrm{NA}$ & NA & & NA & NA & & Gg_rs157770 Gg_rs157874 \\
\hline $1.52 \mathrm{NA}$ & NA & & NA & NA & & Gg_rs157770 X6_18893102 \\
\hline $0.71 \mathrm{NA}$ & NA & & NA & NA & & Gg_rs157770X6_18893102 \\
\hline $0.93 \mathrm{NA}$ & NA & & NA & NA & & Gg_rs157770 X6_18893102 \\
\hline $0.7 \mathrm{NA}$ & NA & & NA & NA & & Gg_rs157770 X6_18893102 \\
\hline $0.74 \mathrm{NA}$ & NA & & NA & NA & & Gg_rs157770X6_18893102 \\
\hline $0.72 \mathrm{NA}$ & NA & & NA & NA & & Gg_rs157770X6_18893102 \\
\hline 0.84 & -3.38 & 0.98 & & 0.58 & 1.25 & Gg_rs157770Gg_rs157830. \\
\hline $0.01 \mathrm{NA}$ & NA & & NA & NA & & Gg_rs157770 Gg_rs157874 \\
\hline 1.03 & -3.14 & 1.2 & & 0.47 & 1.53 & Gg_rs157770 X6_18893102 \\
\hline $5.21 \mathrm{NA}$ & NA & & NA & NA & & Gg_rs157770 Gg_rs157874 \\
\hline 0.79 NA & NA & & NA & NA & & Gg_rs157770 Gg_rs157874 \\
\hline 2.35 & -4.15 & 2.76 & & 1.92 & 3.51 & Gg_rs157770 X6_18893102 \\
\hline 1 & -2.54 & 1.17 & & 2.35 & 1.49 & Gg_rs157770 X6_18893102 \\
\hline $2.7 \mathrm{NA}$ & NA & & NA & NA & & Gg_rs157770 Gg_rs157874 \\
\hline $0.63 \mathrm{NA}$ & NA & & NA & NA & & Gg_rs157770 Gg_rs157874 \\
\hline $0.83 \mathrm{NA}$ & NA & & NA & NA & & Gg_rs157770X6_18893102 \\
\hline $0.97 \mathrm{NA}$ & NA & & NA & NA & & Gg_rs157770 X6_18893102 \\
\hline $1.15 \mathrm{NA}$ & NA & & NA & NA & & Gg_rs157770 X6_18893102 \\
\hline $0.75 \mathrm{NA}$ & NA & & NA & NA & & Gg_rs157770X6_18893102 \\
\hline 1.18 & -0.27 & 1.39 & & -3.42 & 1.76 & Gg_rs157770 X6_18893102 \\
\hline $0.81 \mathrm{NA}$ & NA & & NA & NA & & Gg_rs157770 X6_18893102 \\
\hline 1.29 & -1.2 & 1.39 & & -2.5 & 1.83 & Gg_rs157770 X6_18893102 \\
\hline 1.11 & 0.47 & 1.19 & & -3.69 & 1.58 & Gg_rs157770 Gg_rs157874 \\
\hline $0.67 \mathrm{NA}$ & NA & & NA & NA & & Gg_rs157770 X6_18893102 \\
\hline 0.93 NA & NA & & NA & NA & & Gg_rs157770 X6_18893102 \\
\hline 6.89 NA & NA & & NA & NA & & Gg_rs157770 X6_18893102 \\
\hline 4.14 NA & NA & & NA & NA & & Gg_rs157770 Gg_rs157874 \\
\hline $0.73 \mathrm{NA}$ & NA & & NA & NA & & Gg_rs157770 Gg_rs157874 \\
\hline $0.91 \mathrm{NA}$ & NA & & NA & NA & & Gg_rs157770 Gg_rs157874 \\
\hline $3.26 \mathrm{NA}$ & NA & & NA & NA & & Gg_rs157770X6_18893102 \\
\hline
\end{tabular}




\begin{tabular}{|c|c|c|c|c|c|}
\hline $4.48 \mathrm{NA}$ & NA & NA & NA & & Gg_rs157770 X6_18893102 \\
\hline $3.72 \mathrm{NA}$ & NA & NA & NA & & Gg_rs157770 Gg_rs157874 \\
\hline $0.74 \mathrm{NA}$ & NA & NA & NA & & Gg_rs157770 Gg_rs157874 \\
\hline 0.69 & -0.72 & 0.74 & -1.17 & 0.98 & Gg_rs157770 X6_18893102 \\
\hline $0.54 \mathrm{NA}$ & NA & NA & NA & & Gg_rs157770 X6_18893102 \\
\hline $0.78 \mathrm{NA}$ & NA & NA & NA & & Gg_rs157770 X6_18893102 \\
\hline $1.06 \mathrm{NA}$ & NA & NA & NA & & Gg_rs157770 X6_18893102 \\
\hline $0.62 \mathrm{NA}$ & NA & NA & NA & & Gg_rs157816Gg_rs157874 \\
\hline 0.73 & -0.51 & 0.79 & -2.5 & 1.04 & Gg_rs157770X6_18893102 \\
\hline $1.05 \mathrm{NA}$ & NA & NA & NA & & Gg_rs157770 X6_18893102 \\
\hline $0.7 \mathrm{NA}$ & NA & NA & NA & & Gg_rs157770 X6_18893102 \\
\hline $0.56 \mathrm{NA}$ & NA & NA & NA & & Gg_rs157770X6_18893102 \\
\hline $0.7 \mathrm{NA}$ & NA & NA & NA & & Gg_rs157770X6_18893102 \\
\hline $0.8 \mathrm{NA}$ & NA & NA & NA & & Gg_rs157770 Gg_rs157874 \\
\hline $0.89 \mathrm{NA}$ & NA & NA & NA & & Gg_rs157770 X6_18893102 \\
\hline $0.61 \mathrm{NA}$ & NA & NA & NA & & Gg_rs157770 X6_18893102 \\
\hline $0.84 \mathrm{NA}$ & NA & NA & NA & & Gg_rs157770 X6_18893102 \\
\hline $0.63 \mathrm{NA}$ & NA & NA & NA & & Gg_rs157816 X6_18893102 \\
\hline $0.82 \mathrm{NA}$ & NA & NA & NA & & Gg_rs157770 X6_18893102 \\
\hline $0.96 \mathrm{NA}$ & NA & NA & NA & & Gg_rs157770 X6_18893102 \\
\hline $0.62 \mathrm{NA}$ & NA & NA & NA & & Gg_rs157770X6_25762392 \\
\hline $0.95 \mathrm{NA}$ & NA & NA & NA & & Gg_rs157770 X6_18893102 \\
\hline 0.95 & -3.45 & 1.02 & -0.58 & 1.35 & Gg_rs157770Gg_rs135785 \\
\hline $0.73 \mathrm{NA}$ & NA & NA & NA & & Gg_rs157770 X6_18893102 \\
\hline $0.52 \mathrm{NA}$ & NA & NA & NA & & Gg_rs157770 X6_18893102 \\
\hline $0.58 \mathrm{NA}$ & NA & NA & NA & & Gg_rs157770X6_25762392 \\
\hline $0.81 \mathrm{NA}$ & NA & NA & NA & & Gg_rs157770 X6_18893102 \\
\hline $1.08 \mathrm{NA}$ & NA & NA & NA & & Gg_rs157770X6_18893102 \\
\hline 1.03 & -2.37 & 1.21 & 1.38 & 1.54 & Gg_rs157770X6_25762392 \\
\hline $1.05 \mathrm{NA}$ & NA & NA & NA & & Gg_rs157770 X6_18893102 \\
\hline $0.99 \mathrm{NA}$ & NA & NA & NA & & Gg_rs157770 X6_18893102 \\
\hline $0.72 \mathrm{NA}$ & NA & NA & NA & & Gg_rs157770 X6_18893102 \\
\hline $0.64 \mathrm{NA}$ & NA & NA & NA & & Gg_rs157770X6_25762392 \\
\hline $0.65 \mathrm{NA}$ & NA & NA & NA & & Gg_rs157770X6_25762392 \\
\hline $0.63 \mathrm{NA}$ & NA & NA & NA & & Gg_rs157770 X6_18893102 \\
\hline $0.63 \mathrm{NA}$ & NA & NA & NA & & Gg_rs157770 X6_18893102 \\
\hline 0.95 & -1.77 & 0.95 & -0.56 & 1.3 & Gg_rs157770 X6_18893102 \\
\hline $0.78 \mathrm{NA}$ & NA & NA & NA & & Gg_rs157816 X6_18893102 \\
\hline $0.52 \mathrm{NA}$ & NA & NA & NA & & Gg_rs157770 X6_18893102 \\
\hline $0.65 \mathrm{NA}$ & NA & NA & NA & & Gg_rs157816 X6_18893102 \\
\hline $0.82 \mathrm{NA}$ & NA & NA & NA & & Gg_rs157770 X6_18893102 \\
\hline $0.43 \mathrm{NA}$ & NA & NA & NA & & Gg_rs157770 X6_18893102 \\
\hline 0.6 & -1.94 & 0.59 & -0.91 & 0.81 & Gg_rs157816 X6_1889310 \\
\hline
\end{tabular}




\begin{tabular}{|c|c|c|c|c|c|}
\hline 0.63 & -2.22 & 0.63 & -0.67 & 0.86 & Gg_rs145756 X6_18893102 \\
\hline 0.83 & -1.9 & 0.82 & 0.15 & 1.12 & Gg_rs145756 X6_18893102 \\
\hline $0.62 \mathrm{NA}$ & NA & NA & NA & & Gg_rs145756 X6_18893102 \\
\hline $1.07 \mathrm{NA}$ & NA & NA & NA & & Gg_rs157770 X6_18893102 \\
\hline $0.65 \mathrm{NA}$ & NA & NA & NA & & Gg_rs145756 X6_18893102 \\
\hline 1.09 & -2.57 & 1.09 & 0.39 & 1.49 & Gg_rs145756 X6_18893102 \\
\hline $0.53 \mathrm{NA}$ & NA & NA & NA & & Gg_rs145756 X6_18893102 \\
\hline 0.93 & -2.27 & 0.93 & 2.65 & 1.26 & Gg_rs145756 X6_18893102 \\
\hline $0.84 \mathrm{NA}$ & NA & NA & NA & & Gg_rs145756 X6_18893102 \\
\hline $0.62 \mathrm{NA}$ & NA & NA & NA & & Gg_rs145756 X6_18893102 \\
\hline 1.04 & -2.19 & 1.04 & 2.86 & 1.42 & Gg_rs145756 X6_18893102 \\
\hline 0.73 & -1.39 & 0.73 & 1.3 & 0.99 & Gg_rs157770 X6_18893102 \\
\hline $0.64 \mathrm{NA}$ & NA & NA & NA & & Gg_rs157770 X6_18893102 \\
\hline 1.08 & -3.93 & 1.07 & 2.6 & 1.46 & Gg_rs145756 X6_18893102 \\
\hline $0.7 \mathrm{NA}$ & NA & NA & NA & & Gg_rs157770 X6_18893102 \\
\hline 0.53 NA & NA & NA & NA & & Gg_rs157816Gg_rs135785 \\
\hline $0.53 \mathrm{NA}$ & NA & NA & NA & & Gg_rs145756 X6_18893102 \\
\hline 0.65 NA & NA & NA & NA & & Gg_rs157770X6_18893102 \\
\hline 0.65 NA & NA & NA & NA & & Gg_rs157816 X6_18893102 \\
\hline 0.44 NA & NA & NA & NA & & Gg_rs157770 X6_18893102 \\
\hline $0.47 \mathrm{NA}$ & NA & NA & NA & & Gg_rs145756 X6_18893102 \\
\hline $0.68 \mathrm{NA}$ & NA & NA & NA & & Gg_rs157816 X6_18893102 \\
\hline 0.79 NA & NA & NA & NA & & Gg_rs157770 X6_18893102 \\
\hline 0.55 & -2.31 & 0.55 & -0.08 & 0.75 & Gg_rs145756 X6_18893102 \\
\hline $0.6 \mathrm{NA}$ & NA & NA & NA & & Gg_rs145756 X6_18893102 \\
\hline 1.15 & -2.52 & 1.14 & 3.19 & 1.56 & Gg_rs157770 X6_18893102 \\
\hline $0.67 \mathrm{NA}$ & NA & NA & NA & & Gg_rs145756 X6_18893102 \\
\hline $0.67 \mathrm{NA}$ & NA & NA & NA & & Gg_rs145756 X6_18893102 \\
\hline $0.85 \mathrm{NA}$ & NA & NA & NA & & Gg_rs145756 X6_25762392 \\
\hline $0.8 \mathrm{NA}$ & NA & NA & NA & & Gg_rs145756 X6_25762392 \\
\hline $0.58 \mathrm{NA}$ & NA & NA & NA & & Gg_rs145756 X6_18893102 \\
\hline $0.42 \mathrm{NA}$ & NA & NA & NA & & Gg_rs145756 X6_18893102 \\
\hline 0.96 NA & NA & NA & NA & & Gg_rs145756 X6_18893102 \\
\hline $0.74 \mathrm{NA}$ & NA & NA & NA & & Gg_rs145756 X6_18893102 \\
\hline 0.52 NA & NA & NA & NA & & Gg_rs145756 X6_18893102 \\
\hline 0.67 NA & NA & NA & NA & & Gg_rs145756 Gg_rs135785 \\
\hline $0.55 \mathrm{NA}$ & NA & NA & NA & & Gg_rs145756 X6_18893102 \\
\hline 0.96 NA & NA & NA & NA & & Gg_rs145756 X6_18893102 \\
\hline $0.5 \mathrm{NA}$ & NA & NA & NA & & Gg_rs145756 X6_18893102 \\
\hline 0.46 NA & NA & NA & NA & & Gg_rs145756 X6_18893102 \\
\hline 0.85 & -2.55 & 0.84 & 2.52 & 1.15 & Gg_rs145756 X6_18893102 \\
\hline 0.76 & -2.39 & 0.76 & 2.63 & 1.03 & Gg_rs145756 X6_18893102 \\
\hline $0.44 \mathrm{NA}$ & NA & NA & NA & & Gg_rs145756 X6_1889310 \\
\hline
\end{tabular}




\begin{tabular}{|c|c|c|c|c|c|c|}
\hline $0.66 \mathrm{NA}$ & NA & & NA & NA & & Gg_rs145756 X6_18893102 \\
\hline $0.66 \mathrm{NA}$ & NA & & NA & NA & & Gg_rs145756 X6_25762392 \\
\hline 1.15 & -3.05 & 1.14 & & -0.33 & 1.56 & Gg_rs157816 X6_25762392 \\
\hline 0.85 & -1.24 & 0.85 & & 1.77 & 1.15 & Gg_rs157816 X6_18893102 \\
\hline $0.59 \mathrm{NA}$ & NA & & NA & NA & & Gg_rs157770X6_25762392 \\
\hline $0.24 \mathrm{NA}$ & NA & & NA & NA & & Gg_rs145756 X6_25762392 \\
\hline 0.69 NA & NA & & NA & NA & & Gg_rs145756 Gg_rs135785 \\
\hline $0.7 \mathrm{NA}$ & NA & & NA & NA & & Gg_rs145756 X6_18893102 \\
\hline $1 \mathrm{NA}$ & NA & & NA & NA & & Gg_rs145756 X6_18893102 \\
\hline 0.81 & -2.41 & 0.81 & & 1.66 & 1.1 & Gg_rs145756 X6_18893102 \\
\hline $0.82 \mathrm{NA}$ & NA & & NA & NA & & Gg_rs145756 X6_25762392 \\
\hline 2.26 & -5.2 & 2.25 & & 2.56 & 3.07 & Gg_rs145756 X6_18893102 \\
\hline 0.84 NA & NA & & NA & NA & & Gg_rs157816 X6_18893102 \\
\hline $0.39 \mathrm{NA}$ & NA & & NA & NA & & Gg_rs145756 X6_25762392 \\
\hline $0.44 \mathrm{NA}$ & NA & & NA & NA & & Gg_rs157770X6_25762392 \\
\hline $0.82 \mathrm{NA}$ & NA & & NA & NA & & Gg_rs157770 X6_25762392 \\
\hline $0.46 \mathrm{NA}$ & NA & & NA & NA & & Gg_rs145756 Gg_rs158051 \\
\hline $0.56 \mathrm{NA}$ & NA & & NA & NA & & Gg_rs157770 X6_25762392 \\
\hline $0.74 \mathrm{NA}$ & NA & & NA & NA & & Gg_rs157770 X6_25762392 \\
\hline 0.92 & -1.22 & 0.91 & & -2.34 & 1.25 & Gg_rs145756 Gg_rs135785 \\
\hline $0.63 \mathrm{NA}$ & NA & & NA & NA & & Gg_rs145756 X6_25762392 \\
\hline 0.74 & -1.88 & 0.74 & & 0.33 & 1.01 & Gg_rs157770 Gg_rs135785 \\
\hline $0.77 \mathrm{NA}$ & NA & & NA & NA & & Gg_rs145756 X6_18893102 \\
\hline $0.56 \mathrm{NA}$ & NA & & NA & NA & & Gg_rs157816X6_18893102 \\
\hline 1.34 & -3.28 & 1.33 & & 3.39 & 1.82 & Gg_rs145756 Gg_rs135785 \\
\hline $1.09 \mathrm{NA}$ & NA & & NA & NA & & Gg_rs145756 Gg_rs135785 \\
\hline $0.73 \mathrm{NA}$ & NA & & NA & NA & & Gg_rs145756 X6_25762392 \\
\hline $0.91 \mathrm{NA}$ & NA & & NA & NA & & Gg_rs145756 X6_25762392 \\
\hline $0.88 \mathrm{NA}$ & NA & & NA & NA & & Gg_rs157770 Gg_rs135785 \\
\hline $0.58 \mathrm{NA}$ & NA & & NA & NA & & Gg_rs157770 X6_25762392 \\
\hline $0.64 \mathrm{NA}$ & NA & & NA & NA & & Gg_rs145756 X6_25762392 \\
\hline $0.58 \mathrm{NA}$ & NA & & NA & NA & & Gg_rs145756X6_25762392 \\
\hline $0.76 \mathrm{NA}$ & NA & & NA & NA & & Gg_rs145756 X6_18893102 \\
\hline 0.97 & -2.02 & 0.96 & & 0.89 & 1.32 & Gg_rs157816Gg_rs135785 \\
\hline $0.76 \mathrm{NA}$ & NA & & NA & NA & & Gg_rs145756 X6_25762392 \\
\hline 0.8 & -1.53 & 0.8 & & -0.82 & 1.08 & Gg_rs157816Gg_rs135785 \\
\hline $0.92 \mathrm{NA}$ & NA & & NA & NA & & Gg_rs145756 X6_25762392 \\
\hline 1.39 & 1.22 & 1.38 & & -5.37 & 1.89 & Gg_rs145756 Gg_rs145824 \\
\hline $0.58 \mathrm{NA}$ & NA & & NA & NA & & Gg_rs145756 X6_25762392 \\
\hline $0.68 \mathrm{NA}$ & NA & & NA & NA & & Gg_rs157770 X6_25762392 \\
\hline $1.78 \mathrm{NA}$ & NA & & NA & NA & & Gg_rs145756 X6_25762392 \\
\hline $1.25 \mathrm{NA}$ & NA & & NA & NA & & Gg_rs145756 X6_25762392 \\
\hline $0.57 \mathrm{NA}$ & NA & & NA & NA & & Gg_rs157830 Gg_rs135785 \\
\hline
\end{tabular}




\begin{tabular}{|c|c|c|c|c|c|c|}
\hline $0.82 \mathrm{NA}$ & NA & & NA & NA & & Gg_rs145756 X6_25762392 \\
\hline $0.83 \mathrm{NA}$ & NA & & NA & NA & & Gg_rs145756 X6_25762392 \\
\hline $0.46 \mathrm{NA}$ & NA & & NA & NA & & Gg_rs145756 X6_25762392 \\
\hline $0.91 \mathrm{NA}$ & NA & & NA & NA & & Gg_rs145756 X6_25762392 \\
\hline $0.85 \mathrm{NA}$ & NA & & NA & NA & & Gg_rs145756 X6_25762392 \\
\hline $0.41 \mathrm{NA}$ & NA & & NA & NA & & Gg_rs157830X6_25762392 \\
\hline $0.87 \mathrm{NA}$ & NA & & NA & NA & & Gg_rs145756 X6_25762392 \\
\hline $0.87 \mathrm{NA}$ & NA & & NA & NA & & Gg_rs145756 X6_25762392 \\
\hline $0.42 \mathrm{NA}$ & NA & & NA & NA & & Gg_rs145756 X6_25762392 \\
\hline $0.54 \mathrm{NA}$ & NA & & NA & NA & & Gg_rs145756 X6_25762392 \\
\hline 0.96 NA & NA & & NA & NA & & Gg_rs145756 X6_25762392 \\
\hline 0.66 NA & NA & & NA & NA & & Gg_rs157816X6_25762392 \\
\hline $0.3 \mathrm{NA}$ & NA & & NA & NA & & Gg_rs145756 Gg_rs135785 \\
\hline $0.48 \mathrm{NA}$ & NA & & NA & NA & & Gg_rs145756 X6_25762392 \\
\hline $0.53 \mathrm{NA}$ & NA & & NA & NA & & Gg_rs145756 X6_25762392 \\
\hline $0.49 \mathrm{NA}$ & NA & & NA & NA & & Gg_rs145756 Gg_rs135785 \\
\hline $0.29 \mathrm{NA}$ & NA & & NA & NA & & Gg_rs157874 X6_25762392 \\
\hline $0.57 \mathrm{NA}$ & NA & & NA & NA & & Gg_rs157816X6_25762392 \\
\hline $0.71 \mathrm{NA}$ & NA & & NA & NA & & Gg_rs157874 Gg_rs135785 \\
\hline $0.54 \mathrm{NA}$ & NA & & NA & NA & & Gg_rs157770 X6_25762392 \\
\hline 0.82 & -1.79 & 0.8 & & 0.85 & 1.05 & Gg_rs157874 X6_25762392 \\
\hline $0.81 \mathrm{NA}$ & NA & & NA & NA & & Gg_rs145756 X6_25762392 \\
\hline 0.43 NA & NA & & NA & NA & & Gg_rs145756 X6_25762392 \\
\hline $0.56 \mathrm{NA}$ & NA & & NA & NA & & Gg_rs157874 X6_25762392 \\
\hline 1.44 & -5 & 1.41 & & 0.89 & 1.84 & Gg_rs157770Gg_rs135785 \\
\hline 0.02 & -0.1 & 0.02 & & 0.1 & 0.03 & Gg_rs157874 Gg_rs135785 \\
\hline 0.75 & -2.32 & 0.73 & & 2.52 & 0.95 & Gg_rs157874 X6_25762392 \\
\hline 1.47 & 3.46 & 1.43 & & -4.28 & 1.87 & Gg_rs157874 X6_25762392 \\
\hline 0.75 NA & NA & & NA & NA & & Gg_rs157874 X6_25762392 \\
\hline 1.53 & -1.26 & 1.49 & & -5.13 & 1.95 & Gg_rs157874 Gg_rs135785 \\
\hline 1.17 & -2.64 & 1.14 & & -3.03 & 1.49 & Gg_rs157874 Gg_rs135785 \\
\hline $0.41 \mathrm{NA}$ & NA & & NA & NA & & Gg_rs157874 Gg_rs135785 \\
\hline 1.81 & -1.9 & 1.77 & & -3.82 & 2.3 & Gg_rs157874 Gg_rs135785 \\
\hline $0.65 \mathrm{NA}$ & NA & & NA & NA & & Gg_rs157874 Gg_rs135785 \\
\hline 0.49 NA & NA & & NA & NA & & Gg_rs145756 X6_25762392 \\
\hline 0.76 & -1.67 & 0.75 & & 0.4 & 0.97 & Gg_rs157874 X6_25762392 \\
\hline $0.63 \mathrm{NA}$ & NA & & NA & NA & & Gg_rs157874 X6_25762392 \\
\hline $0.74 \mathrm{NA}$ & NA & & NA & NA & & Gg_rs157874 X6_25762392 \\
\hline $0.66 \mathrm{NA}$ & NA & & NA & NA & & Gg_rs157874 X6_25762392 \\
\hline $0.61 \mathrm{NA}$ & NA & & NA & NA & & Gg_rs145756 X6_25762392 \\
\hline $0.58 \mathrm{NA}$ & NA & & NA & NA & & Gg_rs157874 X6_25762392 \\
\hline $0.62 \mathrm{NA}$ & NA & & NA & NA & & Gg_rs157874 X6_25762392 \\
\hline $1.27 \mathrm{NA}$ & NA & & NA & NA & & Gg_rs145756 X6_25762392 \\
\hline
\end{tabular}




\begin{tabular}{|c|c|c|c|c|}
\hline $0.5 \mathrm{NA}$ & NA & NA & NA & Gg_rs157816X6_25762392 \\
\hline $0.43 \mathrm{NA}$ & NA & NA & NA & Gg_rs157874 X6_25762392 \\
\hline $0.72 \mathrm{NA}$ & NA & NA & NA & Gg_rs145756 X6_25762392 \\
\hline $0.65 \mathrm{NA}$ & NA & NA & NA & Gg_rs157770X6_25762392 \\
\hline $0.55 \mathrm{NA}$ & NA & NA & NA & Gg_rs145756 X6_25762392 \\
\hline $0.31 \mathrm{NA}$ & NA & NA & NA & Gg_rs145824 X6_25762392 \\
\hline $0.6 \mathrm{NA}$ & NA & NA & NA & Gg_rs145824 X6_25762392 \\
\hline $0.68 \mathrm{NA}$ & NA & NA & NA & Gg_rs145824 X6_25762392 \\
\hline $0.83 \mathrm{NA}$ & NA & NA & NA & Gg_rs145824 X6_25762392 \\
\hline $0.65 \mathrm{NA}$ & NA & NA & NA & Gg_rs157874 X6_25762392 \\
\hline $0.61 \mathrm{NA}$ & NA & NA & NA & Gg_rs145824 X6_25762392 \\
\hline $0.91 \mathrm{NA}$ & NA & NA & NA & Gg_rs145824 X6_25762392 \\
\hline $0.9 \mathrm{NA}$ & NA & NA & NA & Gg_rs145824 X6_25762392 \\
\hline $0.7 \mathrm{NA}$ & NA & NA & NA & Gg_rs145824 X6_25762392 \\
\hline $0.88 \mathrm{NA}$ & NA & NA & NA & Gg_rs145756 X6_25762392 \\
\hline $0.85 \mathrm{NA}$ & NA & NA & NA & Gg_rs145756 X6_25762392 \\
\hline 0.68 NA & NA & NA & NA & Gg_rs157874 Gg_rs158051 \\
\hline $0.75 \mathrm{NA}$ & NA & NA & NA & Gg_rs145756 Gg_rs158051 \\
\hline 0.99 NA & NA & NA & NA & Gg_rs157830 Gg_rs145922. \\
\hline $1.31 \mathrm{NA}$ & NA & NA & NA & Gg_rs135785 Gg_rs158051 \\
\hline $0.78 \mathrm{NA}$ & NA & NA & NA & Gg_rs135785 Gg_rs158051 \\
\hline $0.76 \mathrm{NA}$ & NA & NA & NA & Gg_rs135785 Gg_rs158077 \\
\hline $1.05 \mathrm{NA}$ & NA & NA & NA & Gg_rs135785 Gg_rs158051 \\
\hline $1 \mathrm{NA}$ & NA & NA & NA & Gg_rs135785 Gg_rs145922. \\
\hline $0.76 \mathrm{NA}$ & NA & NA & NA & Gg_rs145824 Gg_rs145922. \\
\hline $0.82 \mathrm{NA}$ & NA & NA & NA & Gg_rs145824 Gg_rs158051 \\
\hline $0.79 \mathrm{NA}$ & NA & NA & NA & Gg_rs145824 Gg_rs158051 \\
\hline $0.71 \mathrm{NA}$ & NA & NA & NA & Gg_rs145824 Gg_rs145922 \\
\hline $0.55 \mathrm{NA}$ & NA & NA & NA & Gg_rs135785 Gg_rs158051 \\
\hline $1.14 \mathrm{NA}$ & NA & NA & NA & Gg_rs135785 Gg_rs158051 \\
\hline 0.95 NA & NA & NA & NA & Gg_rs135785 Gg_rs158051 \\
\hline $0.75 \mathrm{NA}$ & NA & NA & NA & Gg_rs145824 Gg_rs145922 \\
\hline $0.51 \mathrm{NA}$ & NA & NA & NA & Gg_rs135785 Gg_rs158051 \\
\hline $0.73 \mathrm{NA}$ & NA & NA & NA & Gg_rs135785 Gg_rs145922. \\
\hline $0.64 \mathrm{NA}$ & NA & NA & NA & Gg_rs135785 Gg_rs158051 \\
\hline $1.29 \mathrm{NA}$ & NA & NA & NA & Gg_rs135785 Gg_rs158051 \\
\hline $0.82 \mathrm{NA}$ & NA & NA & NA & Gg_rs135785 Gg_rs158051 \\
\hline $1.04 \mathrm{NA}$ & NA & NA & NA & Gg_rs135785 Gg_rs158051 \\
\hline $0.81 \mathrm{NA}$ & NA & NA & NA & Gg_rs135785 Gg_rs158077 \\
\hline $0.61 \mathrm{NA}$ & NA & NA & NA & Gg_rs135785 Gg_rs145922. \\
\hline $0.63 \mathrm{NA}$ & NA & NA & NA & Gg_rs135785 Gg_rs158051 \\
\hline $1.67 \mathrm{NA}$ & NA & NA & NA & Gg_rs135785 Gg_rs158051 \\
\hline $0.66 \mathrm{NA}$ & NA & NA & NA & Gg_rs135785 Gg_rs158051 \\
\hline
\end{tabular}




\begin{tabular}{|c|c|c|c|c|c|}
\hline $0.54 \mathrm{NA}$ & NA & NA & NA & & Gg_rs135785 Gg_rs158051 \\
\hline $0.58 \mathrm{NA}$ & NA & NA & NA & & Gg_rs135785 Gg_rs158051 \\
\hline $0.96 \mathrm{NA}$ & NA & NA & NA & & Gg_rs135785 Gg_rs158077 \\
\hline $0.7 \mathrm{NA}$ & NA & NA & NA & & Gg_rs135785 Gg_rs158051 \\
\hline $0.9 \mathrm{NA}$ & NA & NA & NA & & Gg_rs135785 Gg_rs158051 \\
\hline $0.54 \mathrm{NA}$ & NA & NA & NA & & Gg_rs135785 Gg_rs145922. \\
\hline $0.55 \mathrm{NA}$ & NA & NA & NA & & Gg_rs135785 Gg_rs158051 \\
\hline $0.59 \mathrm{NA}$ & NA & NA & NA & & Gg_rs135785 Gg_rs158051 \\
\hline $0.7 \mathrm{NA}$ & NA & NA & NA & & Gg_rs135785 Gg_rs158077 \\
\hline $0.52 \mathrm{NA}$ & NA & NA & NA & & Gg_rs135785 Gg_rs158051 \\
\hline $0.73 \mathrm{NA}$ & NA & NA & NA & & Gg_rs135785 Gg_rs158051 \\
\hline $1.04 \mathrm{NA}$ & NA & NA & NA & & Gg_rs135785 Gg_rs158051 \\
\hline $0.55 \mathrm{NA}$ & NA & NA & NA & & Gg_rs135785 Gg_rs158051 \\
\hline $0.81 \mathrm{NA}$ & NA & NA & NA & & Gg_rs135785 Gg_rs158077 \\
\hline $1.08 \mathrm{NA}$ & NA & NA & NA & & Gg_rs135785 Gg_rs158051 \\
\hline $0.92 \mathrm{NA}$ & NA & NA & NA & & Gg_rs135785 Gg_rs158051 \\
\hline $0.92 \mathrm{NA}$ & NA & NA & NA & & Gg_rs135785 Gg_rs158051 \\
\hline $1.15 \mathrm{NA}$ & NA & NA & NA & & Gg_rs135785 Gg_rs158051 \\
\hline $0.75 \mathrm{NA}$ & NA & NA & NA & & Gg_rs135785 Gg_rs158051 \\
\hline $1.16 \mathrm{NA}$ & NA & NA & NA & & Gg_rs135785 Gg_rs158051 \\
\hline $0.51 \mathrm{NA}$ & NA & NA & NA & & Gg_rs135785 Gg_rs158051 \\
\hline $0.61 \mathrm{NA}$ & NA & NA & NA & & Gg_rs135785 Gg_rs158051 \\
\hline $0.8 \mathrm{NA}$ & NA & NA & NA & & Gg_rs135785 Gg_rs145922. \\
\hline 0.94 NA & NA & NA & NA & & Gg_rs135785 Gg_rs145922. \\
\hline $0.91 \mathrm{NA}$ & NA & NA & NA & & Gg_rs135785 Gg_rs158077 \\
\hline $0.82 \mathrm{NA}$ & NA & NA & NA & & Gg_rs135785 Gg_rs145922. \\
\hline $0.52 \mathrm{NA}$ & NA & NA & NA & & Gg_rs135785 Gg_rs145922. \\
\hline $0.86 \mathrm{NA}$ & NA & NA & NA & & Gg_rs135785 Gg_rs145922. \\
\hline $0.74 \mathrm{NA}$ & NA & NA & NA & & Gg_rs135785 Gg_rs145922. \\
\hline 0.96 NA & NA & NA & NA & & Gg_rs135785 Gg_rs145922. \\
\hline 0.64 NA & NA & NA & NA & & Gg_rs135785 Gg_rs145922. \\
\hline 0.69 NA & NA & NA & NA & & Gg_rs135785 Gg_rs158177. \\
\hline $0.91 \mathrm{NA}$ & NA & NA & NA & & Gg_rs135785 Gg_rs145922. \\
\hline $0.7 \mathrm{NA}$ & NA & NA & NA & & Gg_rs145824 Gg_rs145922. \\
\hline 1.28 & 2.23 & 1.22 & 3.65 & 1.66 & Gg_rs135785 Gg_rs145922. \\
\hline $0.63 \mathrm{NA}$ & NA & NA & NA & & Gg_rs135785 Gg_rs145922. \\
\hline 0.84 NA & NA & NA & NA & & Gg_rs135785 Gg_rs145922. \\
\hline $1.51 \mathrm{NA}$ & NA & NA & NA & & Gg_rs135785 Gg_rs145922. \\
\hline $0.63 \mathrm{NA}$ & NA & NA & NA & & Gg_rs135785 Gg_rs145922. \\
\hline $0.46 \mathrm{NA}$ & NA & NA & NA & & Gg_rs135785 Gg_rs145922. \\
\hline $0.75 \mathrm{NA}$ & NA & NA & NA & & Gg_rs135785 Gg_rs145922. \\
\hline $0.62 \mathrm{NA}$ & NA & NA & NA & & Gg_rs135785 Gg_rs145922. \\
\hline $0.6 \mathrm{NA}$ & NA & NA & NA & & Gg_rs135785 Gg_rs145922. \\
\hline
\end{tabular}




\begin{tabular}{|c|c|c|c|c|c|}
\hline $0.89 \mathrm{NA}$ & NA & NA & NA & & Gg_rs135785 Gg_rs145922. \\
\hline $0.62 \mathrm{NA}$ & NA & NA & NA & & Gg_rs135785 Gg_rs145922. \\
\hline $0.58 \mathrm{NA}$ & NA & NA & NA & & Gg_rs135785 Gg_rs158177. \\
\hline $0.78 \mathrm{NA}$ & NA & NA & NA & & X6_25762392Gg_rs145922. \\
\hline 0.46 NA & NA & NA & NA & & Gg_rs135785 Gg_rs145922. \\
\hline 0.39 NA & NA & NA & NA & & X6_25762392Gg_rs145922. \\
\hline $0.34 \mathrm{NA}$ & NA & NA & NA & & X6_25762392Gg_rs145922. \\
\hline $0.58 \mathrm{NA}$ & NA & NA & NA & & Gg_rs135785 Gg_rs145922. \\
\hline 0.95 & 0.94 & 0.96 & 4.29 & 1.27 & X6_25762392Gg_rs145922. \\
\hline $0.37 \mathrm{NA}$ & NA & NA & NA & & X6_25762392Gg_rs145922. \\
\hline $0.71 \mathrm{NA}$ & NA & NA & NA & & X6_25762392Gg_rs145922. \\
\hline $0.56 \mathrm{NA}$ & NA & NA & NA & & X6_25762392Gg_rs145922. \\
\hline 0.5 & -1.44 & 0.5 & -1.08 & 0.66 & X6_25762392Gg_rs145922. \\
\hline $0.61 \mathrm{NA}$ & NA & NA & NA & & Gg_rs135785 Gg_rs145922. \\
\hline $0.66 \mathrm{NA}$ & NA & NA & NA & & X6_25762392Gg_rs145922. \\
\hline $0.89 \mathrm{NA}$ & NA & NA & NA & & X6_25762392Gg_rs145922. \\
\hline $0.75 \mathrm{NA}$ & NA & NA & NA & & X6_25762392Gg_rs145922. \\
\hline 0.92 & 2.76 & 0.93 & 3.35 & 1.22 & X6_25762392Gg_rs145922 \\
\hline $0.29 \mathrm{NA}$ & NA & NA & NA & & X6_25762392Gg_rs145922. \\
\hline $0.71 \mathrm{NA}$ & NA & NA & NA & & X6_25762392Gg_rs145922. \\
\hline $0.46 \mathrm{NA}$ & NA & NA & NA & & X6_25762392Gg_rs145922. \\
\hline $0.56 \mathrm{NA}$ & NA & NA & NA & & Gg_rs135785 Gg_rs145922. \\
\hline $1.03 \mathrm{NA}$ & NA & NA & NA & & X6_25762392Gg_rs145922 \\
\hline $0.55 \mathrm{NA}$ & NA & NA & NA & & X6_25762392Gg_rs145922 \\
\hline $0.95 \mathrm{NA}$ & NA & NA & NA & & X6_25762392Gg_rs145922 \\
\hline $0.69 \mathrm{NA}$ & NA & NA & NA & & Gg_rs135785 Gg_rs145922 \\
\hline $0.72 \mathrm{NA}$ & NA & NA & NA & & X6_25762392Gg_rs145922 \\
\hline 0.91 & -0.64 & 0.92 & -2.33 & 1.22 & X6_25762392Gg_rs158177 \\
\hline $0.95 \mathrm{NA}$ & NA & NA & NA & & X6_25762392Gg_rs158177 \\
\hline $0.38 \mathrm{NA}$ & NA & NA & NA & & X6_25762392Gg_rs158177 \\
\hline $0.59 \mathrm{NA}$ & NA & NA & NA & & X6_25762392Gg_rs158177 \\
\hline $0.78 \mathrm{NA}$ & NA & NA & NA & & X6_25762392Gg_rs158177 \\
\hline $0.59 \mathrm{NA}$ & NA & NA & NA & & Gg_rs158077 Gg_rs158177 \\
\hline 0.76 & -1.21 & 0.73 & 1.56 & 1.03 & X6_25762392Gg_rs158177 \\
\hline $0.67 \mathrm{NA}$ & NA & NA & NA & & Gg_rs158077 Gg_rs158177 \\
\hline $0.61 \mathrm{NA}$ & NA & NA & NA & & Gg_rs158077 Gg_rs158177 \\
\hline $0.47 \mathrm{NA}$ & NA & NA & NA & & Gg_rs158077 Gg_rs158177 \\
\hline $0.79 \mathrm{NA}$ & NA & NA & NA & & Gg_rs158077 Gg_rs158177 \\
\hline $0.68 \mathrm{NA}$ & NA & NA & NA & & Gg_rs158077 Gg_rs158177 \\
\hline 0.97 & 0.79 & 0.93 & 3.23 & 1.31 & Gg_rs158077 Gg_rs158177 \\
\hline $0.81 \mathrm{NA}$ & NA & NA & NA & & Gg_rs158077 Gg_rs158177 \\
\hline $0.71 \mathrm{NA}$ & NA & NA & NA & & Gg_rs158077 Gg_rs158177 \\
\hline $0.57 \mathrm{NA}$ & NA & NA & NA & & Gg_rs135785 Gg_rs158177 \\
\hline
\end{tabular}




\begin{tabular}{|c|c|c|c|c|c|c|}
\hline $0.57 \mathrm{NA}$ & NA & & NA & NA & & Gg_rs158077 Gg_rs158177. \\
\hline $0.67 \mathrm{NA}$ & NA & & NA & NA & & Gg_rs158077 Gg_rs158177. \\
\hline $0.61 \mathrm{NA}$ & NA & & NA & NA & & Gg_rs158077 Gg_rs158177. \\
\hline $0.57 \mathrm{NA}$ & NA & & NA & NA & & Gg_rs158077 Gg_rs158177. \\
\hline $0.7 \mathrm{NA}$ & NA & & NA & NA & & Gg_rs158077 Gg_rs158177. \\
\hline $0.54 \mathrm{NA}$ & NA & & NA & NA & & Gg_rs135785 Gg_rs158177. \\
\hline $0.6 \mathrm{NA}$ & NA & & NA & NA & & Gg_rs158077 Gg_rs158177. \\
\hline 0.43 NA & NA & & NA & NA & & Gg_rs145922 Gg_rs158177. \\
\hline $1.38 \mathrm{NA}$ & NA & & NA & NA & & Gg_rs145922 Gg_rs158177. \\
\hline 0.13 & -0.69 & 0.13 & & 0.51 & 0.17 & Gg_rs145922 Gg_rs158177. \\
\hline $1.23 \mathrm{NA}$ & NA & & NA & NA & & Gg_rs145922 Gg_rs158177. \\
\hline $0.3 \mathrm{NA}$ & NA & & NA & NA & & Gg_rs158261Gg_rs158284 \\
\hline $0.9 \mathrm{NA}$ & NA & & NA & NA & & Gg_rs158261Gg_rs158284 \\
\hline 0.22 & -0.87 & 0.2 & & 0.79 & 0.27 & Gg_rs158261 Gg_rs158284 \\
\hline 0.09 & -0.45 & 0.09 & & 0.47 & 0.11 & Gg_rs158261Gg_rs158284 \\
\hline 0.06 & -0.53 & 0.06 & & 0.41 & 0.08 & Gg_rs158261Gg_rs158284 \\
\hline $0.35 \mathrm{NA}$ & NA & & NA & NA & & Gg_rs158261 Gg_rs158284 \\
\hline 0.62 & -2.42 & 0.58 & & 3.1 & 0.78 & Gg_rs158261Gg_rs158284 \\
\hline 0.19 & -1.03 & 0.18 & & 0.87 & 0.24 & Gg_rs158261 Gg_rs158284 \\
\hline $0.75 \mathrm{NA}$ & NA & & NA & NA & & Gg_rs158261Gg_rs165755. \\
\hline 0.73 & -3.66 & 0.69 & & 3.37 & 0.93 & Gg_rs158261Gg_rs165755. \\
\hline 0.7 & -1.58 & 0.65 & & 2.95 & 0.88 & Gg_rs158261Gg_rs158284 \\
\hline $1.04 \mathrm{NA}$ & NA & & NA & NA & & Gg_rs158261 Gg_rs165755. \\
\hline 0.64 NA & NA & & NA & NA & & Gg_rs158261Gg_rs165755. \\
\hline $0.55 \mathrm{NA}$ & NA & & NA & NA & & Gg_rs158261Gg_rs165755. \\
\hline $0.47 \mathrm{NA}$ & NA & & NA & NA & & Gg_rs158261Gg_rs165755. \\
\hline $0.64 \mathrm{NA}$ & NA & & NA & NA & & Gg_rs158261Gg_rs165755. \\
\hline $0.57 \mathrm{NA}$ & NA & & NA & NA & & Gg_rs158261Gg_rs165755. \\
\hline $0.47 \mathrm{NA}$ & NA & & NA & NA & & Gg_rs158261Gg_rs165755. \\
\hline 0.5 & -2.02 & 0.47 & & 3.1 & 0.66 & Gg_rs158261 Gg_rs165755. \\
\hline $0.35 \mathrm{NA}$ & NA & & NA & NA & & Gg_rs158261 Gg_rs165755. \\
\hline $1.06 \mathrm{NA}$ & NA & & NA & NA & & Gg_rs158261Gg_rs165755. \\
\hline $0.49 \mathrm{NA}$ & NA & & NA & NA & & Gg_rs158261Gg_rs165755. \\
\hline $0.67 \mathrm{NA}$ & NA & & NA & NA & & Gg_rs158261Gg_rs165755. \\
\hline $0.8 \mathrm{NA}$ & NA & & NA & NA & & Gg_rs158261 Gg_rs165755. \\
\hline 0.41 & -1.63 & 0.38 & & 2.7 & 0.54 & Gg_rs158261 Gg_rs165755. \\
\hline 0.18 & -0.53 & 0.17 & & 0.83 & 0.23 & Gg_rs158261Gg_rs165755. \\
\hline $0.51 \mathrm{NA}$ & NA & & NA & NA & & Gg_rs158261 Gg_rs165755. \\
\hline $0.73 \mathrm{NA}$ & NA & & NA & NA & & Gg_rs158261Gg_rs165755. \\
\hline 1.12 & 0.7 & 1.05 & & 3.45 & 1.46 & Gg_rs158261Gg_rs165755. \\
\hline 0.75 & 1.68 & 0.7 & & 1.11 & 0.98 & Gg_rs158261 Gg_rs165755. \\
\hline $1.01 \mathrm{NA}$ & NA & & NA & NA & & Gg_rs158261 Gg_rs165755. \\
\hline $0.82 \mathrm{NA}$ & NA & & NA & NA & & Gg_rs158261Gg_rs165755. \\
\hline
\end{tabular}




\begin{tabular}{|c|c|c|c|c|c|}
\hline $0.44 \mathrm{NA}$ & NA & NA & NA & & Gg_rs158261Gg_rs165755 \\
\hline 0.55 & 2.15 & 0.51 & 0.87 & 0.71 & Gg_rs158261Gg_rs165755 \\
\hline $0.72 \mathrm{NA}$ & NA & NA & NA & & Gg_rs158261Gg_rs165755 \\
\hline $0.59 \mathrm{NA}$ & NA & NA & NA & & Gg_rs158261Gg_rs165755 \\
\hline $0.83 \mathrm{NA}$ & NA & NA & NA & & Gg_rs158261 Gg_rs165755 \\
\hline $0.3 \mathrm{NA}$ & NA & NA & NA & & Gg_rs158261Gg_rs165755 \\
\hline $0.47 \mathrm{NA}$ & NA & NA & NA & & Gg_rs158261Gg_rs165755 \\
\hline $0.47 \mathrm{NA}$ & NA & NA & NA & & Gg_rs158261Gg_rs165755 \\
\hline $0.92 \mathrm{NA}$ & NA & NA & NA & & Gg_rs158261Gg_rs165755 \\
\hline $0.26 \mathrm{NA}$ & NA & NA & NA & & Gg_rs158261 Gg_rs165755 \\
\hline $0.37 \mathrm{NA}$ & NA & NA & NA & & Gg_rs158261 Gg_rs165755 \\
\hline $0.5 \mathrm{NA}$ & NA & NA & NA & & Gg_rs158261Gg_rs165755 \\
\hline $0.58 \mathrm{NA}$ & NA & NA & NA & & Gg_rs158261Gg_rs165755 \\
\hline $0.52 \mathrm{NA}$ & NA & NA & NA & & Gg_rs158261Gg_rs165755 \\
\hline $0.66 \mathrm{NA}$ & NA & NA & NA & & Gg_rs158261 Gg_rs165755 \\
\hline $0.73 \mathrm{NA}$ & NA & NA & NA & & Gg_rs158261 Gg_rs165755 \\
\hline $0.47 \mathrm{NA}$ & NA & NA & NA & & Gg_rs158261Gg_rs165755 \\
\hline $0.76 \mathrm{NA}$ & NA & NA & NA & & Gg_rs158261Gg_rs165755 \\
\hline $0.43 \mathrm{NA}$ & NA & NA & NA & & Gg_rs158261Gg_rs165755 \\
\hline $0.48 \mathrm{NA}$ & NA & NA & NA & & Gg_rs158261Gg_rs165755 \\
\hline $0.37 \mathrm{NA}$ & NA & NA & NA & & Gg_rs158261 Gg_rs165755 \\
\hline $0.4 \mathrm{NA}$ & NA & NA & NA & & Gg_rs158261Gg_rs165755 \\
\hline $0.51 \mathrm{NA}$ & NA & NA & NA & & Gg_rs158261Gg_rs165755 \\
\hline $0.3 \mathrm{NA}$ & NA & NA & NA & & Gg_rs158261Gg_rs165755 \\
\hline 0.4 & -1.75 & 0.37 & 1.31 & 0.52 & Gg_rs158261Gg_rs165755 \\
\hline 0.78 & 1.72 & 0.73 & 1.08 & 1.01 & Gg_rs158261Gg_rs165755 \\
\hline 0.36 & -1.73 & 0.34 & 1.87 & 0.47 & Gg_rs158261Gg_rs165755 \\
\hline 0.82 & 2.5 & 0.77 & 1.37 & 1.07 & Gg_rs158261Gg_rs165755 \\
\hline $0.34 \mathrm{NA}$ & NA & NA & NA & & Gg_rs158261Gg_rs165755 \\
\hline 0.25 & -1.05 & 0.23 & 1.05 & 0.33 & Gg_rs158261Gg_rs165755 \\
\hline 0.67 & -3.57 & 0.62 & 4.1 & 0.87 & Gg_rs158261Gg_rs165755 \\
\hline 0.19 & -0.81 & 0.18 & 0.82 & 0.25 & Gg_rs158261Gg_rs165755 \\
\hline 0.15 & -0.73 & 0.14 & 0.88 & 0.2 & Gg_rs158261Gg_rs165755 \\
\hline 0.24 & -1.18 & 0.22 & 0.86 & 0.31 & Gg_rs158261Gg_rs165755 \\
\hline $0.41 \mathrm{NA}$ & NA & NA & NA & & Gg_rs158261Gg_rs165755 \\
\hline $0.49 \mathrm{NA}$ & NA & NA & NA & & Gg_rs158261Gg_rs165755 \\
\hline $0.45 \mathrm{NA}$ & NA & NA & NA & & Gg_rs158261Gg_rs165755 \\
\hline $0.49 \mathrm{NA}$ & NA & NA & NA & & Gg_rs158261Gg_rs165755 \\
\hline $0.26 \mathrm{NA}$ & NA & NA & NA & & Gg_rs158261Gg_rs165755 \\
\hline $0.35 \mathrm{NA}$ & NA & NA & NA & & Gg_rs158261Gg_rs165755 \\
\hline $0.41 \mathrm{NA}$ & NA & NA & NA & & Gg_rs158261Gg_rs165755 \\
\hline $0.55 \mathrm{NA}$ & NA & NA & NA & & Gg_rs158261Gg_rs165755 \\
\hline $0.52 \mathrm{NA}$ & NA & NA & NA & & Gg_rs158261Gg_rs165755 \\
\hline
\end{tabular}




\begin{tabular}{|c|c|c|c|c|c|}
\hline $0.44 \mathrm{NA}$ & NA & NA & NA & & Gg_rs158261 Gg_rs165755. \\
\hline $0.45 \mathrm{NA}$ & NA & NA & NA & & Gg_rs158261 Gg_rs165755. \\
\hline $0.38 \mathrm{NA}$ & NA & NA & NA & & Gg_rs158261 Gg_rs165755. \\
\hline $0.35 \mathrm{NA}$ & NA & NA & NA & & Gg_rs158261 Gg_rs165755. \\
\hline $0.43 \mathrm{NA}$ & NA & NA & NA & & Gg_rs158261 Gg_rs165755. \\
\hline $0.49 \mathrm{NA}$ & NA & NA & NA & & Gg_rs158261 Gg_rs165755. \\
\hline $0.48 \mathrm{NA}$ & NA & NA & NA & & Gg_rs158261 Gg_rs165755. \\
\hline $0.42 \mathrm{NA}$ & NA & NA & NA & & Gg_rs158261 Gg_rs165755. \\
\hline $0.37 \mathrm{NA}$ & NA & NA & NA & & Gg_rs158261 Gg_rs165755. \\
\hline $0.4 \mathrm{NA}$ & NA & NA & NA & & Gg_rs158261 Gg_rs165755. \\
\hline $0.51 \mathrm{NA}$ & NA & NA & NA & & Gg_rs158261 Gg_rs165755. \\
\hline $0.36 \mathrm{NA}$ & NA & NA & NA & & Gg_rs158261 Gg_rs165755. \\
\hline $0.27 \mathrm{NA}$ & NA & NA & NA & & Gg_rs158261 Gg_rs165755. \\
\hline $0.51 \mathrm{NA}$ & NA & NA & NA & & Gg_rs158261 Gg_rs165755. \\
\hline $0.63 \mathrm{NA}$ & NA & NA & NA & & Gg_rs158261 Gg_rs165755. \\
\hline $0.55 \mathrm{NA}$ & NA & NA & NA & & Gg_rs158261 Gg_rs165755. \\
\hline $0.49 \mathrm{NA}$ & NA & NA & NA & & Gg_rs158261 Gg_rs165755. \\
\hline $0.53 \mathrm{NA}$ & NA & NA & NA & & Gg_rs158261 Gg_rs165755. \\
\hline $0.53 \mathrm{NA}$ & NA & NA & NA & & Gg_rs158261 Gg_rs165755. \\
\hline $0.39 \mathrm{NA}$ & NA & NA & NA & & Gg_rs158261 Gg_rs165755. \\
\hline $0.58 \mathrm{NA}$ & NA & NA & NA & & Gg_rs158261 Gg_rs165755. \\
\hline $0.37 \mathrm{NA}$ & NA & NA & NA & & Gg_rs158261 Gg_rs165755. \\
\hline $0.58 \mathrm{NA}$ & NA & NA & NA & & Gg_rs158261 Gg_rs165755. \\
\hline $0.99 \mathrm{NA}$ & NA & NA & NA & & Gg_rs158261 Gg_rs165755. \\
\hline $0.46 \mathrm{NA}$ & NA & NA & NA & & Gg_rs158261 Gg_rs165755. \\
\hline $0.84 \mathrm{NA}$ & NA & NA & NA & & Gg_rs158261 Gg_rs165755. \\
\hline $0.39 \mathrm{NA}$ & NA & NA & NA & & Gg_rs158261 Gg_rs165755. \\
\hline $0.64 \mathrm{NA}$ & NA & NA & NA & & Gg_rs158261 Gg_rs165755. \\
\hline $0.45 \mathrm{NA}$ & NA & NA & NA & & Gg_rs158261 Gg_rs165755. \\
\hline $0.44 \mathrm{NA}$ & NA & NA & NA & & Gg_rs158261 Gg_rs165755. \\
\hline $0.49 \mathrm{NA}$ & NA & NA & NA & & Gg_rs158261 Gg_rs165755. \\
\hline $0.44 \mathrm{NA}$ & NA & NA & NA & & Gg_rs158261 Gg_rs165755. \\
\hline $0.69 \mathrm{NA}$ & NA & NA & NA & & Gg_rs158284 Gg_rs158345 \\
\hline $0.49 \mathrm{NA}$ & NA & NA & NA & & Gg_rs158284 Gg_rs158345 \\
\hline $0.51 \mathrm{NA}$ & NA & NA & NA & & Gg_rs158284 Gg_rs158345 \\
\hline $0.84 \mathrm{NA}$ & NA & NA & NA & & Gg_rs158284 Gg_rs158345 \\
\hline 1.06 & -1.77 & 0.97 & 1.2 & 1.41 & Gg_rs158284 Gg_rs158345 \\
\hline $0.72 \mathrm{NA}$ & NA & NA & NA & & Gg_rs158284 Gg_rs158345 \\
\hline $0.47 \mathrm{NA}$ & NA & NA & NA & & Gg_rs158284 Gg_rs158345 \\
\hline $1.2 \mathrm{NA}$ & NA & NA & NA & & Gg_rs158284 Gg_rs158345 \\
\hline $0.65 \mathrm{NA}$ & NA & NA & NA & & Gg_rs158284 Gg_rs158345 \\
\hline $0.4 \mathrm{NA}$ & NA & NA & NA & & Gg_rs158284 Gg_rs158345 \\
\hline $0.98 \mathrm{NA}$ & NA & NA & NA & & Gg_rs158284 Gg_rs158345 \\
\hline
\end{tabular}




\begin{tabular}{|c|c|c|c|c|c|c|c|}
\hline $0.8 \mathrm{NA}$ & NA & & NA & & NA & & Gg_rs158284 Gg_rs158345 \\
\hline $0.33 \mathrm{NA}$ & NA & & NA & & NA & & Gg_rs158284 Gg_rs158345 \\
\hline $0.65 \mathrm{NA}$ & NA & & NA & & NA & & Gg_rs158284 Gg_rs158345 \\
\hline $0.29 \mathrm{NA}$ & NA & & NA & & NA & & Gg_rs158284 Gg_rs158345 \\
\hline 0.3 & -1.12 & 0.27 & & 1.05 & & 0.4 & Gg_rs158284 Gg_rs158345 \\
\hline $0.57 \mathrm{NA}$ & NA & & NA & & NA & & Gg_rs158284 Gg_rs158353، \\
\hline 0.68 NA & NA & & NA & & NA & & Gg_rs158322 Gg_rs158353. \\
\hline 0.45 & -2.06 & 0.39 & & 0.52 & & 0.59 & Gg_rs158322 Gg_rs158353، \\
\hline 0.72 & -2.55 & 0.74 & & 0.19 & & 0.99 & Gg_rs158322 Gg_rs158453، \\
\hline $1 \mathrm{NA}$ & NA & & NA & & NA & & Gg_rs158322 Gg_rs146071. \\
\hline 0.66 & -2.35 & 0.68 & & 1.5 & & 0.91 & Gg_rs158345 Gg_rs146071. \\
\hline $0.57 \mathrm{NA}$ & NA & & NA & & NA & & Gg_rs158345 Gg_rs158387. \\
\hline $0.55 \mathrm{NA}$ & NA & & NA & & NA & & Gg_rs158345 Gg_rs137409. \\
\hline 0.87 & -2.43 & 0.9 & & 0.21 & & 1.2 & Gg_rs158345 Gg_rs146071. \\
\hline 0.79 NA & NA & & NA & & NA & & Gg_rs158322 Gg_rs146071. \\
\hline $1.62 \mathrm{NA}$ & NA & & NA & & NA & & Gg_rs158345 Gg_rs158453، \\
\hline $1.43 \mathrm{NA}$ & NA & & NA & & NA & & Gg_rs158345 Gg_rs158453. \\
\hline $0.63 \mathrm{NA}$ & NA & & NA & & NA & & Gg_rs158353.Gg_rs158453. \\
\hline $1.35 \mathrm{NA}$ & NA & & NA & & NA & & Gg_rs158345 Gg_rs158453، \\
\hline $0.7 \mathrm{NA}$ & NA & & NA & & NA & & Gg_rs158353.Gg_rs158453، \\
\hline $2.26 \mathrm{NA}$ & NA & & NA & & NA & & Gg_rs158345 Gg_rs158453، \\
\hline $2.98 \mathrm{NA}$ & NA & & NA & & NA & & Gg_rs158353.Gg_rs158453، \\
\hline $2.31 \mathrm{NA}$ & NA & & NA & & NA & & Gg_rs158345 Gg_rs158453. \\
\hline $0.73 \mathrm{NA}$ & NA & & NA & & NA & & Gg_rs158353.Gg_rs158453. \\
\hline 0.03 & 0.18 & 0.04 & & -0.14 & & 0.05 & Gg_rs158387.Gg_rs158453، \\
\hline 0.29 & -1.13 & 0.35 & & 0.66 & & 0.45 & Gg_rs158345 Gg_rs158453. \\
\hline 0.26 & 1.55 & 0.32 & & -0.59 & & 0.4 & Gg_rs137409Gg_rs158453. \\
\hline 0.69 NA & NA & & NA & & NA & & Gg_rs158387.Gg_rs158453، \\
\hline $1.11 \mathrm{NA}$ & NA & & NA & & NA & & Gg_rs158387.Gg_rs158453، \\
\hline $0.56 \mathrm{NA}$ & NA & & NA & & NA & & Gg_rs158353.Gg_rs158453. \\
\hline $1.34 \mathrm{NA}$ & NA & & NA & & NA & & Gg_rs158387.Gg_rs158453، \\
\hline 0.03 & 0.19 & 0.03 & & -0.17 & & 0.04 & Gg_rs158387.Gg_rs158453. \\
\hline $0.9 \mathrm{NA}$ & NA & & NA & & NA & & Gg_rs158387.Gg_rs158453. \\
\hline 0.93 & -5.08 & 1.13 & & 3.28 & & 1.44 & Gg_rs158387.Gg_rs158453، \\
\hline 0.19 & 1.31 & 0.23 & & -0.89 & & 0.3 & Gg_rs158387:GG_rs158464 \\
\hline 0.43 & 1.87 & 0.45 & & -1.68 & & 0.59 & Gg_rs158537Gg_rs146144 \\
\hline $1.02 \mathrm{NA}$ & NA & & NA & & NA & & X7_24150587 \\
\hline 1.33 & -2.38 & 1.41 & & 2.37 & & 1.84 & X7_24150587 \\
\hline 2.57 & -2.72 & 2.65 & & 7.12 & & 3.59 & Gg_rs158507 X7_24150587 \\
\hline $1.77 \mathrm{NA}$ & NA & & NA & & NA & & Gg_rs158548X7_24150587 \\
\hline $2 \mathrm{NA}$ & NA & & NA & & NA & & Gg_rs158548X7_24150587 \\
\hline $2.33 \mathrm{NA}$ & NA & & NA & & NA & & Gg_rs158548X7_24150587 \\
\hline $1.37 \mathrm{NA}$ & NA & & NA & & NA & & Gg_rs158548X7_24150587 \\
\hline
\end{tabular}




\begin{tabular}{|c|c|c|c|c|c|c|}
\hline $2.13 \mathrm{NA}$ & NA & NA & NA & & RBL4833 & X7_24150587 \\
\hline $2.6 \mathrm{NA}$ & NA & NA & NA & & \multicolumn{2}{|c|}{ Gg_rs158548X7_2415058ד } \\
\hline $1.95 \mathrm{NA}$ & NA & NA & NA & & \multicolumn{2}{|c|}{ Gg_rs158548X7_2415058ד } \\
\hline $1.06 \mathrm{NA}$ & NA & NA & NA & & RBL4833 & X7_24150587 \\
\hline $0.66 \mathrm{NA}$ & NA & NA & NA & & RBL4833 & X7_24150587 \\
\hline $0.98 \mathrm{NA}$ & NA & NA & NA & & \multicolumn{2}{|c|}{ Gg_rs158548X7_24150587 } \\
\hline $1.67 \mathrm{NA}$ & NA & NA & NA & & \multicolumn{2}{|c|}{ Gg_rs158548X7_24150587 } \\
\hline 0.97 NA & NA & NA & NA & & \multicolumn{2}{|c|}{ Gg_rs158548 X7_2415058ד } \\
\hline $2.25 \mathrm{NA}$ & NA & NA & NA & & \multicolumn{2}{|c|}{ Gg_rs158548 X7_24150587 } \\
\hline $1.43 \mathrm{NA}$ & NA & NA & NA & & \multicolumn{2}{|c|}{ Gg_rs158548 X7_24150587 } \\
\hline $1.29 \mathrm{NA}$ & NA & NA & NA & & \multicolumn{2}{|c|}{ Gg_rs158548X7_24150587 } \\
\hline $0.81 \mathrm{NA}$ & NA & NA & NA & & \multicolumn{2}{|c|}{ Gg_rs158548X7_24150587 } \\
\hline $2.39 \mathrm{NA}$ & NA & NA & NA & & RBL4833 & X7_24150587 \\
\hline $1.69 \mathrm{NA}$ & NA & NA & NA & & \multicolumn{2}{|c|}{ Gg_rs158548X7_24150587 } \\
\hline $1.45 \mathrm{NA}$ & NA & NA & NA & & \multicolumn{2}{|c|}{ Gg_rs158548X7_24150587 } \\
\hline $1.79 \mathrm{NA}$ & NA & NA & NA & & RBL4833 & X7_24150587 \\
\hline $2.39 \mathrm{NA}$ & NA & NA & NA & & RBL4833 & X7_24150587 \\
\hline $1.25 \mathrm{NA}$ & NA & NA & NA & & RBL4833 & X7_24150587 \\
\hline $2.06 \mathrm{NA}$ & NA & NA & NA & & RBL4833 & X7_24150587 \\
\hline $1.07 \mathrm{NA}$ & NA & NA & NA & & \multicolumn{2}{|c|}{ Gg_rs158548 X7_24150587 } \\
\hline $1.54 \mathrm{NA}$ & NA & NA & NA & & RBL4833 & X7_24150587 \\
\hline $1.46 \mathrm{NA}$ & NA & NA & NA & & \multicolumn{2}{|c|}{ Gg_rs158548X7_24150587 } \\
\hline 0.77 NA & NA & NA & NA & & RBL4833 & X7_24150587 \\
\hline 0.79 NA & NA & NA & NA & & \multicolumn{2}{|c|}{ Gg_rs158507 X7_24150587 } \\
\hline $2.52 \mathrm{NA}$ & NA & NA & NA & & RBL4833 & X7_24150587 \\
\hline 2.61 & -2.66 & 2.69 & 7.65 & 3.64 & \multicolumn{2}{|c|}{ Gg_rs158507 X7_24150587 } \\
\hline $2.12 \mathrm{NA}$ & NA & NA & NA & & \multicolumn{2}{|c|}{ Gg_rs158548 X7_24150587 } \\
\hline $2.55 \mathrm{NA}$ & NA & NA & NA & & \multicolumn{2}{|c|}{ Gg_rs158548 X7_24150587 } \\
\hline $2.08 \mathrm{NA}$ & NA & NA & NA & & RBL4833 & X7_24150587 \\
\hline $1.43 \mathrm{NA}$ & NA & NA & NA & & \multicolumn{2}{|c|}{ Gg_rs158548X7_2415058ד } \\
\hline 1.77 NA & NA & NA & NA & & RBL4833 & X7_24150587 \\
\hline $2.54 \mathrm{NA}$ & NA & NA & NA & & RBL4833 & X7_24150587 \\
\hline $2.02 \mathrm{NA}$ & NA & NA & NA & & \multicolumn{2}{|c|}{ Gg_rs158548X7_24150587 } \\
\hline 2.82 & -2.81 & 2.91 & 8.14 & 3.94 & & \\
\hline $1.59 \mathrm{NA}$ & NA & NA & NA & & \multicolumn{2}{|c|}{ Gg_rs158548 X7_2415058 } \\
\hline $1.49 \mathrm{NA}$ & NA & NA & NA & & RBL4833 & X7_24150587 \\
\hline $1.08 \mathrm{NA}$ & NA & NA & NA & & RBL4833 & X7_24150587 \\
\hline $1.99 \mathrm{NA}$ & NA & NA & NA & & \multicolumn{2}{|c|}{ Gg_rs158548X7_24150587 } \\
\hline $2.59 \mathrm{NA}$ & NA & NA & NA & & \multicolumn{2}{|c|}{ Gg_rs158548 X7_24150587 } \\
\hline 2.55 & -2.65 & 2.63 & 7.01 & 3.56 & \multicolumn{2}{|c|}{ Gg_rs158507 X7_24150587 } \\
\hline $2 \mathrm{NA}$ & NA & NA & NA & & \multicolumn{2}{|c|}{ Gg_rs158548X7_24150587 } \\
\hline $2.62 \mathrm{NA}$ & NA & NA & NA & & Gg_rs1585 & $8 \times 7 \_24150587$ \\
\hline $2.47 \mathrm{NA}$ & NA & NA & NA & & Gg_rs1585 & $8 \times 7 \_24150587$ \\
\hline
\end{tabular}




\begin{tabular}{|c|c|c|c|c|c|}
\hline $2.12 \mathrm{NA}$ & NA & NA & NA & RBL4833 & X7_24150587 \\
\hline $2.1 \mathrm{NA}$ & NA & NA & NA & RBL4833 & X7_24150587 \\
\hline $1.06 \mathrm{NA}$ & NA & NA & NA & \multicolumn{2}{|c|}{ Gg_rs158548X7_2415058ד } \\
\hline $2.64 \mathrm{NA}$ & NA & NA & NA & \multicolumn{2}{|c|}{ Gg_rs158548 X7_2415058ד } \\
\hline $2.18 \mathrm{NA}$ & NA & NA & NA & RBL4833 & X7_24150587 \\
\hline $2.13 \mathrm{NA}$ & NA & NA & NA & RBL4833 & X7_24150587 \\
\hline $2.37 \mathrm{NA}$ & NA & NA & NA & RBL4833 & X7_24150587 \\
\hline $2.3 \mathrm{NA}$ & NA & NA & NA & RBL4833 & X7_24150587 \\
\hline $1.33 \mathrm{NA}$ & NA & NA & NA & \multicolumn{2}{|c|}{ Gg_rs158548X7_24150587 } \\
\hline $1.13 \mathrm{NA}$ & NA & NA & NA & RBL4833 & X7_24150587 \\
\hline $2.17 \mathrm{NA}$ & NA & NA & NA & RBL4833 & X7_24150587 \\
\hline $1.08 \mathrm{NA}$ & NA & NA & NA & \multicolumn{2}{|c|}{ Gg_rs158548X7_24150587 } \\
\hline $1.72 \mathrm{NA}$ & NA & NA & NA & \multicolumn{2}{|c|}{ Gg_rs158548X7_24150587 } \\
\hline $1.86 \mathrm{NA}$ & NA & NA & NA & \multicolumn{2}{|c|}{ Gg_rs158548X7_24150587 } \\
\hline $2.39 \mathrm{NA}$ & NA & NA & NA & \multicolumn{2}{|c|}{ Gg_rs158548X7_24150587 } \\
\hline 0.99 NA & NA & NA & NA & \multicolumn{2}{|c|}{ Gg_rs158548X7_24150587 } \\
\hline $1.46 \mathrm{NA}$ & NA & NA & NA & RBL4833 & X7_24150587 \\
\hline $2.35 \mathrm{NA}$ & NA & NA & NA & RBL4833 & X7_24150587 \\
\hline $2.43 \mathrm{NA}$ & NA & NA & NA & RBL4833 & X7_24150587 \\
\hline $3.36 \mathrm{NA}$ & NA & NA & NA & \multicolumn{2}{|c|}{ Gg_rs158548X7_24150587 } \\
\hline $3.71 \mathrm{NA}$ & NA & NA & NA & \multicolumn{2}{|c|}{ Gg_rs158548X7_24150587 } \\
\hline $3.01 \mathrm{NA}$ & NA & NA & NA & \multicolumn{2}{|c|}{ Gg_rs158548X7_24150587 } \\
\hline $3.48 \mathrm{NA}$ & NA & NA & NA & \multicolumn{2}{|c|}{ Gg_rs158548X7_24150587 } \\
\hline $3.36 \mathrm{NA}$ & NA & NA & NA & \multicolumn{2}{|c|}{ Gg_rs158548X7_24150587 } \\
\hline 4.24 NA & NA & NA & NA & \multicolumn{2}{|c|}{ Gg_rs158548X7_24150587 } \\
\hline $3.66 \mathrm{NA}$ & NA & NA & NA & \multicolumn{2}{|c|}{ Gg_rs158548X7_24150587 } \\
\hline $2.95 \mathrm{NA}$ & NA & NA & NA & \multicolumn{2}{|c|}{ Gg_rs158548X7_24150587 } \\
\hline $3.33 \mathrm{NA}$ & NA & NA & NA & \multicolumn{2}{|c|}{ Gg_rs158548X7_24150587 } \\
\hline $3.79 \mathrm{NA}$ & NA & NA & NA & \multicolumn{2}{|c|}{ Gg_rs158548X7_24150587 } \\
\hline $3.42 \mathrm{NA}$ & NA & NA & NA & \multicolumn{2}{|c|}{ Gg_rs158548X7_24150587 } \\
\hline $2.41 \mathrm{NA}$ & NA & NA & NA & \multicolumn{2}{|c|}{ Gg_rs158548X7_24150587 } \\
\hline $2.97 \mathrm{NA}$ & NA & NA & NA & \multicolumn{2}{|c|}{ Gg_rs158548X7_24150587 } \\
\hline $2.43 \mathrm{NA}$ & NA & NA & NA & \multicolumn{2}{|c|}{ Gg_rs158548X7_24150587 } \\
\hline $3.7 \mathrm{NA}$ & NA & NA & NA & \multicolumn{2}{|c|}{ Gg_rs158548X7_24150587 } \\
\hline $3.72 \mathrm{NA}$ & NA & NA & NA & \multicolumn{2}{|c|}{ Gg_rs146144X7_24150587 } \\
\hline 3.44 NA & NA & NA & NA & \multicolumn{2}{|c|}{ Gg_rs158548X7_24150587 } \\
\hline 4.04 NA & NA & NA & NA & Gg_rs1585 & $8 \times 7 \_24150587$ \\
\hline 3 NA & NA & NA & NA & Gg_rs1585 & $8 \times 7 \_24150587$ \\
\hline $3.42 \mathrm{NA}$ & NA & NA & NA & Gg_rs1585 & 8 X7_24150587 \\
\hline 4.12 NA & NA & NA & NA & Gg_rs1585 & 8 X7_24150587 \\
\hline $3.62 \mathrm{NA}$ & NA & NA & NA & Gg_rs1585 & 8 X7_24150587 \\
\hline $2.74 \mathrm{NA}$ & NA & NA & NA & Gg_rs1585 & 8 X7_24150587 \\
\hline $4.03 \mathrm{NA}$ & NA & NA & NA & Gg_rs1585 & 8X7_24150587 \\
\hline
\end{tabular}




\begin{tabular}{|c|c|c|c|c|c|}
\hline $3.95 \mathrm{NA}$ & NA & NA & NA & & Gg_rs146144 X7_24150587 \\
\hline $3.59 \mathrm{NA}$ & NA & NA & NA & & Gg_rs146144X7_24150587 \\
\hline $2.91 \mathrm{NA}$ & NA & NA & NA & & Gg_rs158548X7_24150587 \\
\hline 4.17 NA & NA & NA & NA & & Gg_rs158548X7_24150587 \\
\hline $2.83 \mathrm{NA}$ & NA & NA & NA & & Gg_rs158548X7_24150587 \\
\hline $2.04 \mathrm{NA}$ & NA & NA & NA & & Gg_rs158548X7_24150587 \\
\hline $2.35 \mathrm{NA}$ & NA & NA & NA & & Gg_rs158548 X7_24150587 \\
\hline $1.48 \mathrm{NA}$ & NA & NA & NA & & Gg_rs158548 X7_24150587 \\
\hline $1.17 \mathrm{NA}$ & NA & NA & NA & & Gg_rs158548X7_24150587 \\
\hline $2.83 \mathrm{NA}$ & NA & NA & NA & & Gg_rs158548X7_24150587 \\
\hline 0.66 & 3.63 & 0.68 & -2.38 & 0.92 & Gg_rs146144 X7_24150587 \\
\hline $1.24 \mathrm{NA}$ & NA & NA & NA & & Gg_rs158548X7_24150587 \\
\hline $1.9 \mathrm{NA}$ & NA & NA & NA & & RBL4833 X7_24150587 \\
\hline $1.43 \mathrm{NA}$ & NA & NA & NA & & Gg_rs158548X7_24150587 \\
\hline $1.88 \mathrm{NA}$ & NA & NA & NA & & Gg_rs158548X7_24150587 \\
\hline $2.67 \mathrm{NA}$ & NA & NA & NA & & RBL4833 X7_24150587 \\
\hline $1.38 \mathrm{NA}$ & NA & NA & NA & & X7_24150587 \\
\hline $1.9 \mathrm{NA}$ & NA & NA & NA & & Gg_rs158548 X7_24150587 \\
\hline $1.96 \mathrm{NA}$ & NA & NA & NA & & RBL4833 X7_24150587 \\
\hline $3.61 \mathrm{NA}$ & NA & NA & NA & & RBL4833 X7_24150587 \\
\hline $1.38 \mathrm{NA}$ & NA & NA & NA & & Gg_rs158548X7_24150587 \\
\hline $1.93 \mathrm{NA}$ & NA & NA & NA & & RBL4833 X7_24150587 \\
\hline $1.34 \mathrm{NA}$ & NA & NA & NA & & X7_24150587 \\
\hline $1.25 \mathrm{NA}$ & NA & NA & NA & & Gg_rs158548X7_24150587 \\
\hline $1.85 \mathrm{NA}$ & NA & NA & NA & & Gg_rs158548X7_24150587 \\
\hline $2.41 \mathrm{NA}$ & NA & NA & NA & & Gg_rs158548 X7_24150587 \\
\hline $2.42 \mathrm{NA}$ & NA & NA & NA & & RBL4833 X7_2415058ד \\
\hline $2.22 \mathrm{NA}$ & NA & NA & NA & & Gg_rs158548 X7_24150587 \\
\hline $2.58 \mathrm{NA}$ & NA & NA & NA & & RBL4833 X7_2415058ד \\
\hline $1.98 \mathrm{NA}$ & NA & NA & NA & & X7_24150587 \\
\hline $0.96 \mathrm{NA}$ & NA & NA & NA & & X7_24150587 \\
\hline $1.36 \mathrm{NA}$ & NA & NA & NA & & X7_24150587 \\
\hline $1.95 \mathrm{NA}$ & NA & NA & NA & & X7_24150587 \\
\hline $2.14 \mathrm{NA}$ & NA & NA & NA & & Gg_rs158548 X7_24150587 \\
\hline $1.53 \mathrm{NA}$ & NA & NA & NA & & X7_24150587 \\
\hline $1.53 \mathrm{NA}$ & NA & NA & NA & & RBL4833 X7_24150587 \\
\hline 4.36 NA & NA & NA & NA & & Gg_rs158507 X7_24150587 \\
\hline 4.17 NA & NA & NA & NA & & Gg_rs158548 X7_24150587 \\
\hline $2.92 \mathrm{NA}$ & NA & NA & NA & & RBL4833 X7_24150587 \\
\hline $0.42 \mathrm{NA}$ & NA & NA & NA & & RBL4833 X7_24150587 \\
\hline 0.48 NA & NA & NA & NA & & Gg_rs158548X7_24150587 \\
\hline 4.76 NA & NA & NA & NA & & Gg_rs158548X7_24150587 \\
\hline $1.74 \mathrm{NA}$ & NA & NA & NA & & X7_24150587 \\
\hline
\end{tabular}




\begin{tabular}{|c|c|c|c|c|c|}
\hline $2.69 \mathrm{NA}$ & NA & NA & NA & RBL4833 & X7_24150587 \\
\hline $1.44 \mathrm{NA}$ & NA & NA & NA & \multicolumn{2}{|c|}{ Gg_rs158548 X7_24150587 } \\
\hline $1.85 \mathrm{NA}$ & NA & NA & NA & RBL4833 & X7_24150587 \\
\hline $1.63 \mathrm{NA}$ & NA & NA & NA & RBL4833 & X7_24150587 \\
\hline $2.32 \mathrm{NA}$ & NA & NA & NA & RBL4833 & X7_24150587 \\
\hline $1.87 \mathrm{NA}$ & NA & NA & NA & RBL4833 & X7_24150587 \\
\hline $2.97 \mathrm{NA}$ & NA & NA & NA & RBL4833 & X7_24150587 \\
\hline $1.91 \mathrm{NA}$ & NA & NA & NA & RBL4833 & X7_24150587 \\
\hline $3.59 \mathrm{NA}$ & NA & NA & NA & \multicolumn{2}{|c|}{ Gg_rs158548X7_2415058ד } \\
\hline $1.46 \mathrm{NA}$ & NA & NA & NA & \multicolumn{2}{|c|}{ Gg_rs158548 X7_2415058ד } \\
\hline $3.47 \mathrm{NA}$ & NA & NA & NA & RBL4833 & X7_24150587 \\
\hline $1.55 \mathrm{NA}$ & NA & NA & NA & \multicolumn{2}{|c|}{ Gg_rs158507 X7_24150587 } \\
\hline $1.95 \mathrm{NA}$ & NA & NA & NA & \multicolumn{2}{|c|}{ Gg_rs158548 X7_24150587 } \\
\hline $1.66 \mathrm{NA}$ & NA & NA & NA & \multicolumn{2}{|c|}{ Gg_rs158548X7_24150587 } \\
\hline $1.33 \mathrm{NA}$ & NA & NA & NA & RBL4833 & X7_24150587 \\
\hline $1.15 \mathrm{NA}$ & NA & NA & NA & RBL4833 & X7_24150587 \\
\hline $1.45 \mathrm{NA}$ & NA & NA & NA & RBL4833 & X7_24150587 \\
\hline $1.77 \mathrm{NA}$ & NA & NA & NA & RBL4833 & X7_24150587 \\
\hline $3.01 \mathrm{NA}$ & NA & NA & NA & \multicolumn{2}{|c|}{ Gg_rs158548 X7_24150587 } \\
\hline $0.07 \mathrm{NA}$ & NA & NA & NA & \multicolumn{2}{|c|}{ Gg_rs146144X7_24150587 } \\
\hline $0.83 \mathrm{NA}$ & NA & NA & NA & \multicolumn{2}{|c|}{ Gg_rs158507 X7_24150587 } \\
\hline $0.96 \mathrm{NA}$ & NA & NA & NA & RBL4833 & X7_24150587 \\
\hline 4.17 NA & NA & NA & NA & \multicolumn{2}{|c|}{ Gg_rs158548X7_2415058ד } \\
\hline $2.14 \mathrm{NA}$ & NA & NA & NA & \multicolumn{2}{|c|}{ Gg_rs158548 X7_24150587 } \\
\hline $1.33 \mathrm{NA}$ & NA & NA & NA & RBL4833 & X7_24150587 \\
\hline $2.1 \mathrm{NA}$ & NA & NA & NA & RBL4833 & X7_24150587 \\
\hline $1.38 \mathrm{NA}$ & NA & NA & NA & \multicolumn{2}{|c|}{ Gg_rs158548X7_24150587 } \\
\hline $1.84 \mathrm{NA}$ & NA & NA & NA & \multicolumn{2}{|c|}{ Gg_rs158548X7_24150587 } \\
\hline $1.65 \mathrm{NA}$ & NA & NA & NA & \multicolumn{2}{|c|}{ Gg_rs158548X7_24150587 } \\
\hline $2.59 \mathrm{NA}$ & NA & NA & NA & RBL4833 & X7_24150587 \\
\hline $2.34 \mathrm{NA}$ & NA & NA & NA & RBL4833 & X7_24150587 \\
\hline $1.45 \mathrm{NA}$ & NA & NA & NA & RBL4833 & X7_24150587 \\
\hline $0.6 \mathrm{NA}$ & NA & NA & NA & \multicolumn{2}{|c|}{ Gg_rs158507 X7_24150587 } \\
\hline $2.21 \mathrm{NA}$ & NA & NA & NA & \multicolumn{2}{|c|}{ Gg_rs158548X7_24150587 } \\
\hline $2.33 \mathrm{NA}$ & NA & NA & NA & RBL4833 & X7_24150587 \\
\hline $2.31 \mathrm{NA}$ & NA & NA & NA & RBL4833 & X7_24150587 \\
\hline $2.74 \mathrm{NA}$ & NA & NA & NA & RBL4833 & X7_24150587 \\
\hline $0.92 \mathrm{NA}$ & NA & NA & NA & \multicolumn{2}{|c|}{ Gg_rs158548 X7_2415058ד } \\
\hline $1.66 \mathrm{NA}$ & NA & NA & NA & RBL4833 & X7_24150587 \\
\hline $1.02 \mathrm{NA}$ & NA & NA & NA & RBL4833 & X7_24150587 \\
\hline $2.2 \mathrm{NA}$ & NA & NA & NA & \multicolumn{2}{|c|}{ Gg_rs158548 X7_24150587 } \\
\hline $0.81 \mathrm{NA}$ & NA & NA & NA & RBL4833 & X7_24150587 \\
\hline $1.24 \mathrm{NA}$ & NA & NA & NA & \multicolumn{2}{|c|}{ Gg_rs158548X7_24150587 } \\
\hline
\end{tabular}




\begin{tabular}{|c|c|c|c|c|c|}
\hline $0.96 \mathrm{NA}$ & NA & NA & NA & RBL4833 & X7_24150587 \\
\hline $2.33 \mathrm{NA}$ & NA & NA & NA & RBL4833 & X7_24150587 \\
\hline $1.87 \mathrm{NA}$ & NA & NA & NA & \multicolumn{2}{|c|}{ Gg_rs158548X7_24150587 } \\
\hline $1.05 \mathrm{NA}$ & NA & NA & NA & \multicolumn{2}{|c|}{ Gg_rs158548 X7_2415058ד } \\
\hline $1.93 \mathrm{NA}$ & NA & NA & NA & RBL4833 & X7_24150587 \\
\hline $1.76 \mathrm{NA}$ & NA & NA & NA & \multicolumn{2}{|c|}{ Gg_rs158548X7_24150587 } \\
\hline $0.94 \mathrm{NA}$ & NA & NA & NA & RBL4833 & X7_24150587 \\
\hline $3.47 \mathrm{NA}$ & NA & NA & NA & \multicolumn{2}{|c|}{ Gg_rs158548 X7_2415058ד } \\
\hline 4.97 NA & NA & NA & NA & \multicolumn{2}{|c|}{ Gg_rs158548X7_24150587 } \\
\hline $2.42 \mathrm{NA}$ & NA & NA & NA & \multicolumn{2}{|c|}{ Gg_rs158548X7_24150587 } \\
\hline $3.82 \mathrm{NA}$ & NA & NA & NA & \multicolumn{2}{|c|}{ Gg_rs158548X7_24150587 } \\
\hline $4.91 \mathrm{NA}$ & NA & NA & NA & \multicolumn{2}{|c|}{ Gg_rs158548X7_24150587 } \\
\hline $3.82 \mathrm{NA}$ & NA & NA & NA & \multicolumn{2}{|c|}{ Gg_rs158548X7_24150587 } \\
\hline $0.91 \mathrm{NA}$ & NA & NA & NA & \multicolumn{2}{|c|}{ Gg_rs158548X7_24150587 } \\
\hline $1.54 \mathrm{NA}$ & NA & NA & NA & \multicolumn{2}{|c|}{ Gg_rs158548X7_24150587 } \\
\hline $1.92 \mathrm{NA}$ & NA & NA & NA & RBL4833 & X7_24150587 \\
\hline $2.31 \mathrm{NA}$ & NA & NA & NA & RBL4833 & X7_24150587 \\
\hline $2.07 \mathrm{NA}$ & NA & NA & NA & RBL4833 & X7_24150587 \\
\hline $3.31 \mathrm{NA}$ & NA & NA & NA & \multicolumn{2}{|c|}{ Gg_rs158548 X7_2415058ד } \\
\hline $1.9 \mathrm{NA}$ & NA & NA & NA & \multicolumn{2}{|c|}{ Gg_rs158548X7_24150587 } \\
\hline $1.88 \mathrm{NA}$ & NA & NA & NA & \multicolumn{2}{|c|}{ Gg_rs158548X7_24150587 } \\
\hline $1.98 \mathrm{NA}$ & NA & NA & NA & RBL4833 & X7_24150587 \\
\hline $2.09 \mathrm{NA}$ & NA & NA & NA & \multicolumn{2}{|c|}{ Gg_rs158548X7_24150587 } \\
\hline $1.59 \mathrm{NA}$ & NA & NA & NA & RBL4833 & X7_24150587 \\
\hline 2.09 NA & NA & NA & NA & RBL4833 & X7_24150587 \\
\hline $1.98 \mathrm{NA}$ & NA & NA & NA & \multicolumn{2}{|c|}{ Gg_rs158548X7_24150587 } \\
\hline $1.4 \mathrm{NA}$ & NA & NA & NA & RBL4833 & X7_24150587 \\
\hline $0.93 \mathrm{NA}$ & NA & NA & NA & RBL4833 & X7_24150587 \\
\hline 4.71 NA & NA & NA & NA & \multicolumn{2}{|c|}{ Gg_rs158548 X7_24150587 } \\
\hline $2.83 \mathrm{NA}$ & NA & NA & NA & \multicolumn{2}{|c|}{ Gg_rs158548X7_24150587 } \\
\hline $3.03 \mathrm{NA}$ & NA & NA & NA & \multicolumn{2}{|c|}{ Gg_rs158548X7_24150587 } \\
\hline $2.72 \mathrm{NA}$ & NA & NA & NA & \multicolumn{2}{|c|}{ Gg_rs158548X7_24150587 } \\
\hline $3.91 \mathrm{NA}$ & NA & NA & NA & \multicolumn{2}{|c|}{ Gg_rs158548X7_24150587 } \\
\hline $3.63 \mathrm{NA}$ & NA & NA & NA & \multicolumn{2}{|c|}{ Gg_rs158548X7_24150587 } \\
\hline $2.97 \mathrm{NA}$ & NA & NA & NA & \multicolumn{2}{|c|}{ Gg_rs158548X7_24150587 } \\
\hline $2.82 \mathrm{NA}$ & NA & NA & NA & \multicolumn{2}{|c|}{ Gg_rs158548X7_24150587 } \\
\hline $4.03 \mathrm{NA}$ & NA & NA & NA & \multicolumn{2}{|c|}{ Gg_rs158548X7_24150587 } \\
\hline 3.69 NA & NA & NA & NA & Gg_rs1585 & $8 \times 7 \_24150587$ \\
\hline $2.48 \mathrm{NA}$ & NA & NA & NA & Gg_rs1585 & 8 X7_24150587 \\
\hline $1.87 \mathrm{NA}$ & NA & NA & NA & Gg_rs1585 & 8 X7_24150587 \\
\hline 3.79 NA & NA & NA & NA & Gg_rs1585 & 8 X7_24150587 \\
\hline $2.74 \mathrm{NA}$ & NA & NA & NA & Gg_rs1585 & 8 X7_24150587 \\
\hline $4.86 \mathrm{NA}$ & NA & NA & NA & Gg_rs15854 & 8X7_24150587 \\
\hline
\end{tabular}




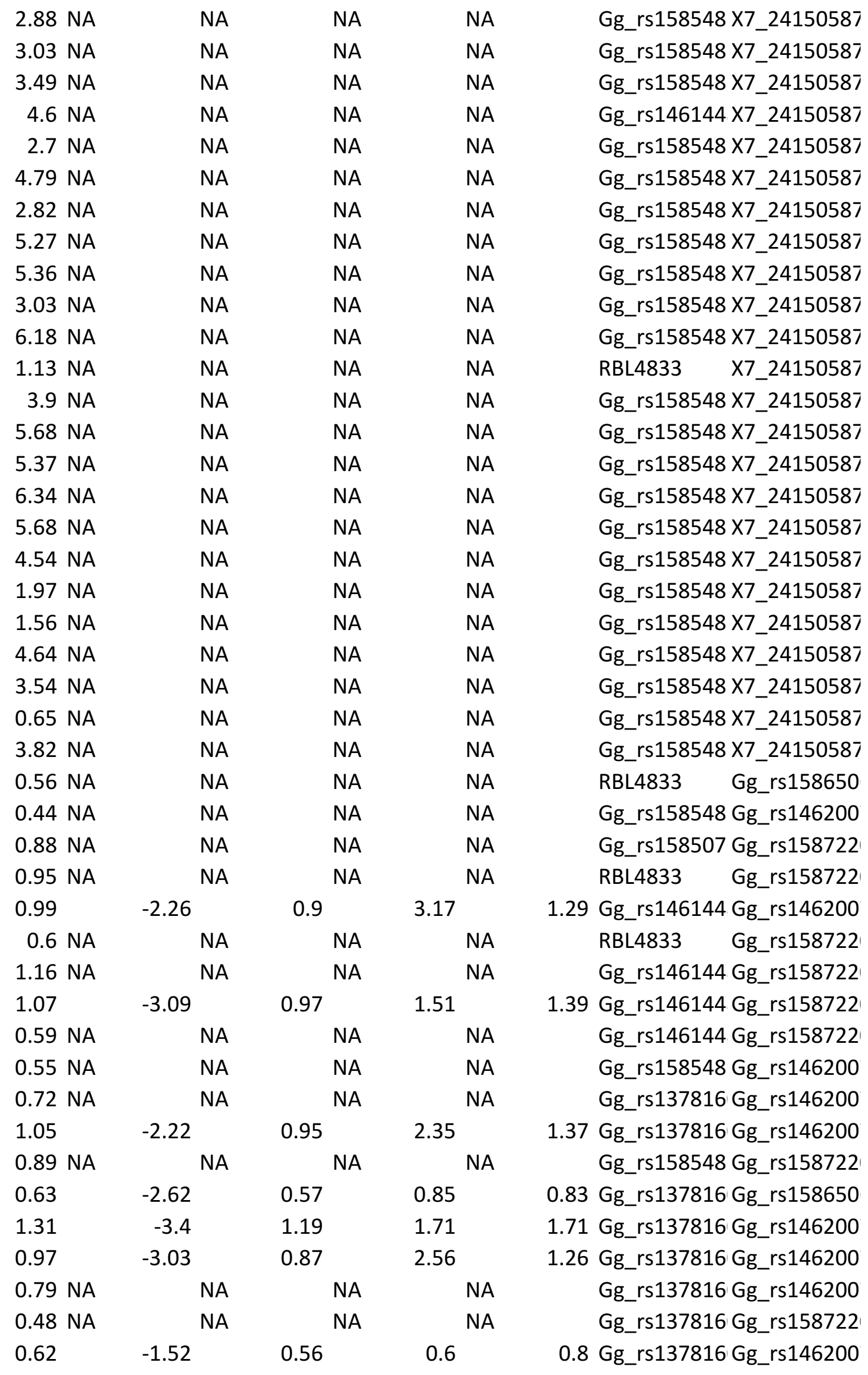




\begin{tabular}{|c|c|c|c|c|c|}
\hline $0.58 \mathrm{NA}$ & NA & NA & NA & & Gg_rs137816 Gg_rs146200 \\
\hline $0.82 \mathrm{NA}$ & NA & NA & NA & & Gg_rs137816Gg_rs158722 \\
\hline $0.56 \mathrm{NA}$ & NA & NA & NA & & Gg_rs137816 Gg_rs146200 \\
\hline $0.74 \mathrm{NA}$ & NA & NA & NA & & Gg_rs137816Gg_rs146225 \\
\hline $0.85 \mathrm{NA}$ & NA & NA & NA & & Gg_rs146144 Gg_rs146225 \\
\hline $0.52 \mathrm{NA}$ & NA & NA & NA & & Gg_rs137816Gg_rs158674 \\
\hline $0.87 \mathrm{NA}$ & NA & NA & NA & & Gg_rs137816Gg_rs158722 \\
\hline $0.5 \mathrm{NA}$ & NA & NA & NA & & Gg_rs137816Gg_rs158722 \\
\hline $0.77 \mathrm{NA}$ & NA & NA & NA & & Gg_rs137816Gg_rs158650 \\
\hline 0.79 NA & NA & NA & NA & & Gg_rs137816 Gg_rs146200 \\
\hline 0.74 & -1.72 & 0.72 & -0.55 & 0.98 & Gg_rs137816 Gg_rs146200 \\
\hline $1.12 \mathrm{NA}$ & NA & NA & NA & & Gg_rs137816Gg_rs158722 \\
\hline 0.94 NA & NA & NA & NA & & Gg_rs137816Gg_rs158722 \\
\hline 0.69 NA & NA & NA & NA & & Gg_rs137816 Gg_rs158722 \\
\hline 0.94 & -3.55 & 0.85 & 2.94 & 1.23 & Gg_rs137816 Gg_rs146200 \\
\hline 0.74 & -2.55 & 0.67 & 1.04 & 0.96 & Gg_rs137816 Gg_rs146200 \\
\hline $0.65 \mathrm{NA}$ & NA & NA & NA & & Gg_rs137816 Gg_rs146200 \\
\hline 0.9 & -1.28 & 0.81 & 4.25 & 1.17 & Gg_rs137816 Gg_rs146200 \\
\hline 0.77 & -2.28 & 0.75 & 3.1 & 1.02 & Gg_rs137816 Gg_rs146200 \\
\hline $0.55 \mathrm{NA}$ & NA & NA & NA & & Gg_rs137816 Gg_rs146200 \\
\hline 0.69 & -2.75 & 0.67 & 1.2 & 0.91 & Gg_rs137816 Gg_rs158722 \\
\hline $0.46 \mathrm{NA}$ & NA & NA & NA & & Gg_rs137816 Gg_rs146200 \\
\hline 0.86 & -2.7 & 0.84 & 2.84 & 1.15 & Gg_rs137816 Gg_rs146200 \\
\hline 0.44 NA & NA & NA & NA & & Gg_rs137816 Gg_rs146200 \\
\hline $0.51 \mathrm{NA}$ & NA & NA & NA & & Gg_rs137816Gg_rs158722 \\
\hline $0.38 \mathrm{NA}$ & NA & NA & NA & & Gg_rs137816 Gg_rs146200 \\
\hline $0.58 \mathrm{NA}$ & NA & NA & NA & & Gg_rs137816 Gg_rs146200 \\
\hline $0.74 \mathrm{NA}$ & NA & NA & NA & & Gg_rs137816Gg_rs158722 \\
\hline $0.6 \mathrm{NA}$ & NA & NA & NA & & Gg_rs137816 Gg_rs146200 \\
\hline 0.86 & -2.99 & 0.84 & 3.91 & 1.14 & Gg_rs137816 Gg_rs146200 \\
\hline 1.26 & -3.34 & 1.23 & 5.46 & 1.68 & X7_24150587Gg_rs146200 \\
\hline $0.67 \mathrm{NA}$ & NA & NA & NA & & Gg_rs137816 Gg_rs146200 \\
\hline $0.4 \mathrm{NA}$ & NA & NA & NA & & Gg_rs137816 Gg_rs146200 \\
\hline $0.79 \mathrm{NA}$ & NA & NA & NA & & Gg_rs137816 Gg_rs146200 \\
\hline $0.47 \mathrm{NA}$ & NA & NA & NA & & Gg_rs137816 Gg_rs146200 \\
\hline $0.7 \mathrm{NA}$ & NA & NA & NA & & Gg_rs137816 Gg_rs146200 \\
\hline $0.42 \mathrm{NA}$ & NA & NA & NA & & Gg_rs137816 Gg_rs146200 \\
\hline $0.65 \mathrm{NA}$ & NA & NA & NA & & Gg_rs137816Gg_rs158722 \\
\hline 0.89 NA & NA & NA & NA & & Gg_rs137816 Gg_rs146200 \\
\hline $0.4 \mathrm{NA}$ & NA & NA & NA & & Gg_rs137816 Gg_rs146200 \\
\hline $0.64 \mathrm{NA}$ & NA & NA & NA & & Gg_rs137816 Gg_rs146200 \\
\hline $0.29 \mathrm{NA}$ & NA & NA & NA & & Gg_rs137816 Gg_rs146200 \\
\hline $0.55 \mathrm{NA}$ & NA & NA & NA & & Gg_rs137816 Gg_rs146200 \\
\hline
\end{tabular}




\begin{tabular}{|c|c|c|c|c|c|}
\hline $0.6 \mathrm{NA}$ & NA & NA & NA & & Gg_rs137816 Gg_rs146200 \\
\hline $0.62 \mathrm{NA}$ & NA & NA & NA & & Gg_rs137816 Gg_rs146200 \\
\hline 0.8 & -2.14 & 0.77 & 1.65 & 1.06 & Gg_rs137816 Gg_rs158722 \\
\hline $0.53 \mathrm{NA}$ & NA & NA & NA & & Gg_rs137816 Gg_rs146200 \\
\hline 0.66 & -1.92 & 0.64 & -1.19 & 0.88 & Gg_rs137816 Gg_rs146200 \\
\hline 0.98 NA & NA & NA & NA & & Gg_rs137816 Gg_rs146200 \\
\hline $0.98 \mathrm{NA}$ & NA & NA & NA & & Gg_rs137816 Gg_rs146200 \\
\hline $0.48 \mathrm{NA}$ & NA & NA & NA & & Gg_rs137816 Gg_rs146200 \\
\hline $0.7 \mathrm{NA}$ & NA & NA & NA & & Gg_rs137816 Gg_rs146200 \\
\hline $0.33 \mathrm{NA}$ & NA & NA & NA & & Gg_rs137816 Gg_rs146200 \\
\hline $0.36 \mathrm{NA}$ & NA & NA & NA & & Gg_rs137816Gg_rs158722 \\
\hline $0.33 \mathrm{NA}$ & NA & NA & NA & & Gg_rs137816 Gg_rs146200 \\
\hline $1 \mathrm{NA}$ & NA & NA & NA & & Gg_rs137816 Gg_rs146200 \\
\hline $0.76 \mathrm{NA}$ & NA & NA & NA & & Gg_rs137816 Gg_rs146200 \\
\hline $0.77 \mathrm{NA}$ & NA & NA & NA & & Gg_rs137816 Gg_rs146200 \\
\hline $1.19 \mathrm{NA}$ & NA & NA & NA & & Gg_rs137816Gg_rs158722 \\
\hline $0.52 \mathrm{NA}$ & NA & NA & NA & & Gg_rs137816 Gg_rs146200 \\
\hline $0.78 \mathrm{NA}$ & NA & NA & NA & & Gg_rs137816 Gg_rs146200 \\
\hline $0.87 \mathrm{NA}$ & NA & NA & NA & & Gg_rs137816 Gg_rs146200 \\
\hline 0.64 & -1.95 & 0.62 & -0.55 & 0.84 & Gg_rs137816 Gg_rs146200 \\
\hline $0.98 \mathrm{NA}$ & NA & NA & NA & & Gg_rs137816 Gg_rs146200 \\
\hline $0.68 \mathrm{NA}$ & NA & NA & NA & & Gg_rs158650 Gg_rs146200 \\
\hline $0.59 \mathrm{NA}$ & NA & NA & NA & & Gg_rs137816Gg_rs158722 \\
\hline $1.17 \mathrm{NA}$ & NA & NA & NA & & Gg_rs137816 Gg_rs146200 \\
\hline $0.84 \mathrm{NA}$ & NA & NA & NA & & Gg_rs137816Gg_rs158722 \\
\hline $0.68 \mathrm{NA}$ & NA & NA & NA & & Gg_rs137816 Gg_rs146200 \\
\hline 0.64 NA & NA & NA & NA & & Gg_rs137816 Gg_rs146200 \\
\hline 2.21 & -1.87 & 2.14 & 7.88 & 2.94 & Gg_rs137816 Gg_rs146200 \\
\hline 1.01 & -1.23 & 0.98 & 3.89 & 1.34 & Gg_rs137816 Gg_rs146200 \\
\hline 0.79 NA & NA & NA & NA & & Gg_rs137816 Gg_rs146200 \\
\hline $0.48 \mathrm{NA}$ & NA & NA & NA & & Gg_rs137816Gg_rs158722 \\
\hline 0.83 NA & NA & NA & NA & & Gg_rs137816Gg_rs158722 \\
\hline 0.48 NA & NA & NA & NA & & Gg_rs137816Gg_rs158722 \\
\hline $0.81 \mathrm{NA}$ & NA & NA & NA & & Gg_rs137816Gg_rs158722 \\
\hline $1.04 \mathrm{NA}$ & NA & NA & NA & & Gg_rs137816Gg_rs158722 \\
\hline $0.85 \mathrm{NA}$ & NA & NA & NA & & Gg_rs137816Gg_rs158722 \\
\hline 0.79 NA & NA & NA & NA & & Gg_rs137816Gg_rs158722 \\
\hline 0.44 NA & NA & NA & NA & & Gg_rs137816 Gg_rs146200 \\
\hline $0.8 \mathrm{NA}$ & NA & NA & NA & & Gg_rs137816 Gg_rs146200 \\
\hline 0.67 NA & NA & NA & NA & & Gg_rs137816Gg_rs158722 \\
\hline $0.68 \mathrm{NA}$ & NA & NA & NA & & X7_24150587Gg_rs146200 \\
\hline $1.08 \mathrm{NA}$ & NA & NA & NA & & Gg_rs137816Gg_rs158722 \\
\hline $0.85 \mathrm{NA}$ & NA & NA & NA & & Gg_rs137816 Gg_rs146200 \\
\hline
\end{tabular}




\begin{tabular}{|c|c|c|c|c|c|}
\hline $1.12 \mathrm{NA}$ & NA & NA & NA & & Gg_rs137816Gg_rs158722 \\
\hline $1.01 \mathrm{NA}$ & NA & NA & NA & & Gg_rs137816Gg_rs158722 \\
\hline 1.41 & -3.44 & 1.28 & 3.94 & 1.84 & Gg_rs137816 Gg_rs158722 \\
\hline $0.74 \mathrm{NA}$ & NA & NA & NA & & Gg_rs137816 Gg_rs158722 \\
\hline $1 \mathrm{NA}$ & NA & NA & NA & & Gg_rs137816Gg_rs158722 \\
\hline $0.7 \mathrm{NA}$ & NA & NA & NA & & Gg_rs137816Gg_rs146225 \\
\hline $0.78 \mathrm{NA}$ & NA & NA & NA & & Gg_rs137816Gg_rs146225 \\
\hline $1.11 \mathrm{NA}$ & NA & NA & NA & & Gg_rs137816Gg_rs158722 \\
\hline $0.64 \mathrm{NA}$ & NA & NA & NA & & Gg_rs137816Gg_rs158722 \\
\hline 0.86 NA & NA & NA & NA & & Gg_rs137816Gg_rs158722 \\
\hline 0.49 NA & NA & NA & NA & & Gg_rs137816Gg_rs146225 \\
\hline $0.61 \mathrm{NA}$ & NA & NA & NA & & Gg_rs137816Gg_rs158722 \\
\hline $0.6 \mathrm{NA}$ & NA & NA & NA & & Gg_rs137816Gg_rs158783. \\
\hline $0.52 \mathrm{NA}$ & NA & NA & NA & & Gg_rs137816Gg_rs158722 \\
\hline $0.78 \mathrm{NA}$ & NA & NA & NA & & Gg_rs137816Gg_rs158722 \\
\hline $0.81 \mathrm{NA}$ & NA & NA & NA & & Gg_rs137816Gg_rs146225 \\
\hline $0.63 \mathrm{NA}$ & NA & NA & NA & & Gg_rs137816Gg_rs158722 \\
\hline $0.41 \mathrm{NA}$ & NA & NA & NA & & Gg_rs137816Gg_rs158783. \\
\hline $0.81 \mathrm{NA}$ & NA & NA & NA & & Gg_rs137816 Gg_rs158722 \\
\hline $1.16 \mathrm{NA}$ & NA & NA & NA & & Gg_rs137816Gg_rs158722 \\
\hline $0.5 \mathrm{NA}$ & NA & NA & NA & & Gg_rs137816 Gg_rs158722 \\
\hline $1.07 \mathrm{NA}$ & NA & NA & NA & & Gg_rs137816Gg_rs158722 \\
\hline $0.76 \mathrm{NA}$ & NA & NA & NA & & Gg_rs137816Gg_rs146225 \\
\hline $0.85 \mathrm{NA}$ & NA & NA & NA & & Gg_rs137816Gg_rs146225 \\
\hline $0.91 \mathrm{NA}$ & NA & NA & NA & & Gg_rs137816Gg_rs158722 \\
\hline $0.75 \mathrm{NA}$ & NA & NA & NA & & Gg_rs137816Gg_rs146225 \\
\hline $1.16 \mathrm{NA}$ & NA & NA & NA & & Gg_rs137816Gg_rs146225 \\
\hline $0.58 \mathrm{NA}$ & NA & NA & NA & & Gg_rs137816Gg_rs158722 \\
\hline 0.56 NA & NA & NA & NA & & Gg_rs137816Gg_rs158722 \\
\hline 0.64 NA & NA & NA & NA & & Gg_rs137816Gg_rs146225 \\
\hline 0.83 NA & NA & NA & NA & & Gg_rs137816Gg_rs158722 \\
\hline $1.1 \mathrm{NA}$ & NA & NA & NA & & Gg_rs137816Gg_rs158783. \\
\hline 0.68 NA & NA & NA & NA & & Gg_rs137816Gg_rs146225 \\
\hline $0.91 \mathrm{NA}$ & NA & NA & NA & & Gg_rs137816Gg_rs146225 \\
\hline $1.21 \mathrm{NA}$ & NA & NA & NA & & Gg_rs137816Gg_rs158722 \\
\hline $0.56 \mathrm{NA}$ & NA & NA & NA & & Gg_rs137816Gg_rs158722 \\
\hline $0.55 \mathrm{NA}$ & NA & NA & NA & & Gg_rs137816Gg_rs158722 \\
\hline $1.48 \mathrm{NA}$ & NA & NA & NA & & Gg_rs137816Gg_rs146225 \\
\hline 0.83 NA & NA & NA & NA & & Gg_rs137816Gg_rs146225 \\
\hline $1.11 \mathrm{NA}$ & NA & NA & NA & & Gg_rs137816Gg_rs146225 \\
\hline 0.54 NA & NA & NA & NA & & Gg_rs137816Gg_rs158722 \\
\hline $0.95 \mathrm{NA}$ & NA & NA & NA & & Gg_rs137816Gg_rs146225 \\
\hline $1 \mathrm{NA}$ & NA & NA & NA & & Gg_rs137816 Gg_rs158783. \\
\hline
\end{tabular}




\begin{tabular}{|c|c|c|c|c|c|}
\hline $1.13 \mathrm{NA}$ & NA & NA & NA & & Gg_rs137816Gg_rs158783. \\
\hline 1.28 & -0.13 & 1.21 & 3.36 & 1.71 & Gg_rs137816Gg_rs158722 \\
\hline $0.57 \mathrm{NA}$ & NA & NA & NA & & Gg_rs137816 Gg_rs158722 \\
\hline $0.54 \mathrm{NA}$ & NA & NA & NA & & Gg_rs137816 Gg_rs158722 \\
\hline $0.9 \mathrm{NA}$ & NA & NA & NA & & Gg_rs137816Gg_rs146225 \\
\hline $0.53 \mathrm{NA}$ & NA & NA & NA & & Gg_rs137816Gg_rs158722 \\
\hline 0.79 NA & NA & NA & NA & & Gg_rs158674 Gg_rs158722 \\
\hline $0.51 \mathrm{NA}$ & NA & NA & NA & & Gg_rs158674 Gg_rs158722 \\
\hline $0.53 \mathrm{NA}$ & NA & NA & NA & & Gg_rs158674 Gg_rs158722 \\
\hline $0.5 \mathrm{NA}$ & NA & NA & NA & & Gg_rs158674 Gg_rs158722 \\
\hline $0.52 \mathrm{NA}$ & NA & NA & NA & & Gg_rs146144 Gg_rs158722 \\
\hline $0.67 \mathrm{NA}$ & NA & NA & NA & & Gg_rs137816Gg_rs158722 \\
\hline $0.4 \mathrm{NA}$ & NA & NA & NA & & Gg_rs137816Gg_rs158722 \\
\hline $0.54 \mathrm{NA}$ & NA & NA & NA & & Gg_rs158674 Gg_rs158722 \\
\hline $0.66 \mathrm{NA}$ & NA & NA & NA & & Gg_rs158674 Gg_rs158722 \\
\hline $0.5 \mathrm{NA}$ & NA & NA & NA & & Gg_rs158674 Gg_rs158722 \\
\hline $0.53 \mathrm{NA}$ & NA & NA & NA & & Gg_rs158674 Gg_rs158722 \\
\hline 0.64 & -2.09 & 0.6 & -2.79 & 0.85 & Gg_rs158674 Gg_rs158722 \\
\hline $0.35 \mathrm{NA}$ & NA & NA & NA & & Gg_rs158674 Gg_rs146225 \\
\hline $1 \mathrm{NA}$ & NA & NA & NA & & Gg_rs158674 Gg_rs146225 \\
\hline 0.39 NA & NA & NA & NA & & Gg_rs158674 Gg_rs146225 \\
\hline 0.76 & -1.21 & 0.71 & -1.99 & 1.01 & Gg_rs137816 Gg_rs158783. \\
\hline 0.48 NA & NA & NA & NA & & Gg_rs158674 Gg_rs158783. \\
\hline $0.72 \mathrm{NA}$ & NA & NA & NA & & Gg_rs158674 Gg_rs146225 \\
\hline $0.79 \mathrm{NA}$ & NA & NA & NA & & Gg_rs158674 Gg_rs146225 \\
\hline 0.68 NA & NA & NA & NA & & Gg_rs158674 Gg_rs158783. \\
\hline $0.62 \mathrm{NA}$ & NA & NA & NA & & Gg_rs137816Gg_rs158783. \\
\hline 0.95 NA & NA & NA & NA & & Gg_rs158674 Gg_rs146225 \\
\hline $0.55 \mathrm{NA}$ & NA & NA & NA & & Gg_rs158674 Gg_rs158783. \\
\hline 1.39 & -0.21 & 1.16 & 3.62 & 1.81 & Gg_rs158674 Gg_rs146225 \\
\hline 0.56 NA & NA & NA & NA & & Gg_rs158674 Gg_rs146225 \\
\hline $0.79 \mathrm{NA}$ & NA & NA & NA & & Gg_rs137816Gg_rs158783. \\
\hline $0.87 \mathrm{NA}$ & NA & NA & NA & & Gg_rs137816Gg_rs146225 \\
\hline $0.82 \mathrm{NA}$ & NA & NA & NA & & Gg_rs158674 Gg_rs158783. \\
\hline 0.64 NA & NA & NA & NA & & Gg_rs158674 Gg_rs146225 \\
\hline $0.71 \mathrm{NA}$ & NA & NA & NA & & Gg_rs158674 Gg_rs158808. \\
\hline $0.43 \mathrm{NA}$ & NA & NA & NA & & Gg_rs158674 Gg_rs146225 \\
\hline $0.48 \mathrm{NA}$ & NA & NA & NA & & Gg_rs158674 Gg_rs146225 \\
\hline 0.99 NA & NA & NA & NA & & Gg_rs158674 Gg_rs146225 \\
\hline 0.39 NA & NA & NA & NA & & Gg_rs158674 Gg_rs146225 \\
\hline $1.16 \mathrm{NA}$ & NA & NA & NA & & Gg_rs158674 Gg_rs146225 \\
\hline $0.7 \mathrm{NA}$ & NA & NA & NA & & Gg_rs146200 Gg_rs146225 \\
\hline $0.5 \mathrm{NA}$ & NA & NA & NA & & Gg_rs158674 Gg_rs146225 \\
\hline
\end{tabular}




\begin{tabular}{|c|c|c|c|c|c|}
\hline $0.59 \mathrm{NA}$ & NA & NA & NA & & Gg_rs158674 Gg_rs146225 \\
\hline $0.9 \mathrm{NA}$ & NA & NA & NA & & Gg_rs158674 Gg_rs146225 \\
\hline $0.54 \mathrm{NA}$ & NA & NA & NA & & Gg_rs158674 Gg_rs146225 \\
\hline $0.74 \mathrm{NA}$ & NA & NA & NA & & Gg_rs158674 Gg_rs146225 \\
\hline 0.99 NA & NA & NA & NA & & Gg_rs158674 Gg_rs146225 \\
\hline 0.96 NA & NA & NA & NA & & Gg_rs158674 Gg_rs158783 \\
\hline 0.46 NA & NA & NA & NA & & Gg_rs158674 Gg_rs146225 \\
\hline 0.42 & -0.03 & 0.35 & -2.27 & 0.54 & Gg_rs146200 Gg_rs146225 \\
\hline $0.64 \mathrm{NA}$ & NA & NA & NA & & Gg_rs146200 Gg_rs146225 \\
\hline $0.88 \mathrm{NA}$ & NA & NA & NA & & Gg_rs158674 Gg_rs146225 \\
\hline $1.14 \mathrm{NA}$ & NA & NA & NA & & Gg_rs146200 Gg_rs146225 \\
\hline $1.02 \mathrm{NA}$ & NA & NA & NA & & Gg_rs158674 Gg_rs146225 \\
\hline 0.18 & -0.14 & 0.15 & -1.23 & 0.23 & Gg_rs146200 Gg_rs146225 \\
\hline $0.58 \mathrm{NA}$ & NA & NA & NA & & Gg_rs146200 Gg_rs146225 \\
\hline 0.83 NA & NA & NA & NA & & Gg_rs146200 Gg_rs158783 \\
\hline $0.6 \mathrm{NA}$ & NA & NA & NA & & Gg_rs146200 Gg_rs146225 \\
\hline $0.71 \mathrm{NA}$ & NA & NA & NA & & Gg_rs146200 Gg_rs146225 \\
\hline 0.93 NA & NA & NA & NA & & Gg_rs146200 Gg_rs158783 \\
\hline $0.42 \mathrm{NA}$ & NA & NA & NA & & Gg_rs146200 Gg_rs158783 \\
\hline $0.63 \mathrm{NA}$ & NA & NA & NA & & Gg_rs146200 Gg_rs146225 \\
\hline $0.95 \mathrm{NA}$ & NA & NA & NA & & Gg_rs146200 Gg_rs158783 \\
\hline $0.66 \mathrm{NA}$ & NA & NA & NA & & Gg_rs158674 Gg_rs158783 \\
\hline $1.01 \mathrm{NA}$ & NA & NA & NA & & Gg_rs146200 Gg_rs158808 \\
\hline $0.82 \mathrm{NA}$ & NA & NA & NA & & Gg_rs158674 Gg_rs158783 \\
\hline 1.83 & -2 & 1.52 & 4.17 & 2.37 & Gg_rs158674 Gg_rs158808 \\
\hline $0.96 \mathrm{NA}$ & NA & NA & NA & & Gg_rs146200 Gg_rs158808 \\
\hline $0.7 \mathrm{NA}$ & NA & NA & NA & & Gg_rs158674 Gg_rs158808 \\
\hline 1.34 & -0.63 & 1.12 & 5.06 & 1.74 & Gg_rs158674 Gg_rs158783 \\
\hline $0.87 \mathrm{NA}$ & NA & NA & NA & & Gg_rs137816Gg_rs158783 \\
\hline $0.72 \mathrm{NA}$ & NA & NA & NA & & Gg_rs146200 Gg_rs158783 \\
\hline $0.64 \mathrm{NA}$ & NA & NA & NA & & Gg_rs158674 Gg_rs158783 \\
\hline $0.88 \mathrm{NA}$ & NA & NA & NA & & Gg_rs146200 Gg_rs158783 \\
\hline $0.81 \mathrm{NA}$ & NA & NA & NA & & Gg_rs146200 Gg_rs158808 \\
\hline $0.43 \mathrm{NA}$ & NA & NA & NA & & Gg_rs146200 Gg_rs158783 \\
\hline 0.69 NA & NA & NA & NA & & Gg_rs158674 Gg_rs158808 \\
\hline $0.44 \mathrm{NA}$ & NA & NA & NA & & Gg_rs146200 Gg_rs158783 \\
\hline $0.55 \mathrm{NA}$ & NA & NA & NA & & Gg_rs146200 Gg_rs158783 \\
\hline $0.47 \mathrm{NA}$ & NA & NA & NA & & Gg_rs146200 Gg_rs158783 \\
\hline 0.86 NA & NA & NA & NA & & Gg_rs158674 Gg_rs158783 \\
\hline 0.99 & -0.71 & 0.86 & 3.42 & 1.32 & Gg_rs158722 Gg_rs158808 \\
\hline 0.86 & -0.43 & 0.74 & 3.25 & 1.15 & Gg_rs158722 Gg_rs158783 \\
\hline $0.26 \mathrm{NA}$ & NA & NA & NA & & Gg_rs158722 Gg_rs158783 \\
\hline $0.67 \mathrm{NA}$ & NA & NA & NA & & Gg_rs158722 Gg_rs158783 \\
\hline
\end{tabular}




\begin{tabular}{|c|c|c|c|c|c|}
\hline $0.37 \mathrm{NA}$ & NA & NA & NA & & Gg_rs158722 Gg_rs158783 \\
\hline $0.35 \mathrm{NA}$ & NA & NA & NA & & Gg_rs158722 Gg_rs158783 \\
\hline $0.35 \mathrm{NA}$ & NA & NA & NA & & Gg_rs158722 Gg_rs158783 \\
\hline $0.49 \mathrm{NA}$ & NA & NA & NA & & Gg_rs146200 Gg_rs158783 \\
\hline 0.59 & 0.11 & 0.51 & -3.74 & 0.79 & Gg_rs158722 Gg_rs158783 \\
\hline $0.36 \mathrm{NA}$ & NA & NA & NA & & Gg_rs158722 Gg_rs158783 \\
\hline $0.52 \mathrm{NA}$ & NA & NA & NA & & Gg_rs158722 Gg_rs158783 \\
\hline $0.56 \mathrm{NA}$ & NA & NA & NA & & Gg_rs158722 Gg_rs158783 \\
\hline $0.61 \mathrm{NA}$ & NA & NA & NA & & Gg_rs158722 Gg_rs158783 \\
\hline 0.64 NA & NA & NA & NA & & Gg_rs158722 Gg_rs158783 \\
\hline $0.91 \mathrm{NA}$ & NA & NA & NA & & Gg_rs146200 Gg_rs136003 \\
\hline $0.39 \mathrm{NA}$ & NA & NA & NA & & Gg_rs146200 Gg_rs158808 \\
\hline 0.74 & -0.9 & 0.65 & 2.97 & 0.99 & Gg_rs158722 Gg_rs158808 \\
\hline $0.4 \mathrm{NA}$ & NA & NA & NA & & Gg_rs146200 Gg_rs136003 \\
\hline $0.5 \mathrm{NA}$ & NA & NA & NA & & Gg_rs158722 Gg_rs158808 \\
\hline $0.58 \mathrm{NA}$ & NA & NA & NA & & Gg_rs146200 Gg_rs158808 \\
\hline $0.55 \mathrm{NA}$ & NA & NA & NA & & Gg_rs146200 Gg_rs158808 \\
\hline 1.55 & -0.99 & 1.41 & 6.62 & 2.04 & Gg_rs158722 Gg_rs158808 \\
\hline $0.54 \mathrm{NA}$ & NA & NA & NA & & Gg_rs158722 Gg_rs158808 \\
\hline $0.76 \mathrm{NA}$ & NA & NA & NA & & Gg_rs158722 Gg_rs158808 \\
\hline 0.94 NA & NA & NA & NA & & Gg_rs146225 Gg_rs158808 \\
\hline $0.76 \mathrm{NA}$ & NA & NA & NA & & Gg_rs146200 Gg_rs158808 \\
\hline 0.73 NA & NA & NA & NA & & Gg_rs146225 Gg_rs158808 \\
\hline $0.54 \mathrm{NA}$ & NA & NA & NA & & Gg_rs146225 Gg_rs158808 \\
\hline $0.54 \mathrm{NA}$ & NA & NA & NA & & Gg_rs146225 Gg_rs158808 \\
\hline $0.74 \mathrm{NA}$ & NA & NA & NA & & Gg_rs146225 Gg_rs158808 \\
\hline $1.12 \mathrm{NA}$ & NA & NA & NA & & Gg_rs146225 Gg_rs158808 \\
\hline $0.4 \mathrm{NA}$ & NA & NA & NA & & Gg_rs146225 Gg_rs158808 \\
\hline $0.32 \mathrm{NA}$ & NA & NA & NA & & Gg_rs146225 Gg_rs158808 \\
\hline 1.4 & -2.67 & 1.27 & 5 & 1.85 & Gg_rs146225 Gg_rs136003 \\
\hline $0.75 \mathrm{NA}$ & NA & NA & NA & & Gg_rs146225 Gg_rs158808 \\
\hline $0.56 \mathrm{NA}$ & NA & NA & NA & & Gg_rs146225 Gg_rs158808 \\
\hline 0.84 & 0.11 & 0.77 & 2.61 & 1.11 & Gg_rs146225 Gg_rs158808 \\
\hline $0.72 \mathrm{NA}$ & NA & NA & NA & & Gg_rs146225 Gg_rs136003 \\
\hline $0.56 \mathrm{NA}$ & NA & NA & NA & & Gg_rs146225 Gg_rs136003 \\
\hline $1.13 \mathrm{NA}$ & NA & NA & NA & & Gg_rs146225 Gg_rs158808 \\
\hline $1.12 \mathrm{NA}$ & NA & NA & NA & & Gg_rs146225 Gg_rs136003 \\
\hline $0.45 \mathrm{NA}$ & NA & NA & NA & & Gg_rs146225 Gg_rs136003 \\
\hline $0.61 \mathrm{NA}$ & NA & NA & NA & & Gg_rs146225 Gg_rs158808 \\
\hline $0.67 \mathrm{NA}$ & NA & NA & NA & & Gg_rs146225 Gg_rs136003 \\
\hline $0.61 \mathrm{NA}$ & NA & NA & NA & & Gg_rs146225 Gg_rs136003 \\
\hline $0.76 \mathrm{NA}$ & NA & NA & NA & & Gg_rs146225 Gg_rs136003 \\
\hline $1.34 \mathrm{NA}$ & NA & NA & NA & & Gg_rs146225 Gg_rs136003 \\
\hline
\end{tabular}




\begin{tabular}{|c|c|c|c|c|c|}
\hline $0.7 \mathrm{NA}$ & NA & NA & NA & & Gg_rs146225 Gg_rs136003 \\
\hline $0.47 \mathrm{NA}$ & NA & NA & NA & & Gg_rs146200 Gg_rs136003 \\
\hline $0.49 \mathrm{NA}$ & NA & NA & NA & & Gg_rs146225 Gg_rs136003 \\
\hline $0.46 \mathrm{NA}$ & NA & NA & NA & & Gg_rs158722 Gg_rs136003 \\
\hline $0.7 \mathrm{NA}$ & NA & NA & NA & & Gg_rs146225 Gg_rs136003 \\
\hline $0.58 \mathrm{NA}$ & NA & NA & NA & & Gg_rs146225 Gg_rs136003 \\
\hline 1.24 & -4.08 & 1.14 & -3.06 & 1.6 & Gg_rs146225 Gg_rs136003 \\
\hline $0.56 \mathrm{NA}$ & NA & NA & NA & & Gg_rs158722 Gg_rs136003 \\
\hline $0.81 \mathrm{NA}$ & NA & NA & NA & & Gg_rs158783 Gg_rs136003 \\
\hline $0.74 \mathrm{NA}$ & NA & NA & NA & & Gg_rs158783 Gg_rs136003 \\
\hline $0.54 \mathrm{NA}$ & NA & NA & NA & & Gg_rs158722 Gg_rs136003 \\
\hline $0.83 \mathrm{NA}$ & NA & NA & NA & & Gg_rs146225 Gg_rs136003 \\
\hline $0.43 \mathrm{NA}$ & NA & NA & NA & & Gg_rs146225 Gg_rs136003 \\
\hline 0.58 & -1.57 & 0.53 & -0.82 & 0.74 & Gg_rs146225 Gg_rs136003 \\
\hline $0.55 \mathrm{NA}$ & NA & NA & NA & & Gg_rs158783 Gg_rs136003 \\
\hline $0.43 \mathrm{NA}$ & NA & NA & NA & & Gg_rs158783 Gg_rs136003 \\
\hline $0.86 \mathrm{NA}$ & NA & NA & NA & & Gg_rs146225 Gg_rs136003 \\
\hline 1.42 & -5.16 & 1.31 & -0.75 & 1.83 & Gg_rs158783 Gg_rs136003 \\
\hline $0.86 \mathrm{NA}$ & NA & NA & NA & & Gg_rs158783 Gg_rs136003 \\
\hline $0.89 \mathrm{NA}$ & NA & NA & NA & & Gg_rs158783 Gg_rs136003 \\
\hline $0.31 \mathrm{NA}$ & NA & NA & NA & & Gg_rs158783 Gg_rs136003 \\
\hline $0.71 \mathrm{NA}$ & NA & NA & NA & & Gg_rs158783 Gg_rs136003 \\
\hline $0.7 \mathrm{NA}$ & NA & NA & NA & & Gg_rs158783 Gg_rs136003 \\
\hline $0.88 \mathrm{NA}$ & NA & NA & NA & & Gg_rs158783 Gg_rs136003 \\
\hline $0.84 \mathrm{NA}$ & NA & NA & NA & & Gg_rs158783 Gg_rs136003 \\
\hline $0.74 \mathrm{NA}$ & NA & NA & NA & & Gg_rs158783 Gg_rs136003 \\
\hline 0.88 & -3.42 & 0.81 & -3.29 & 1.13 & Gg_rs158783 Gg_rs136003 \\
\hline 1.1 & -3.74 & 1.01 & -2.98 & 1.42 & Gg_rs158783 Gg_rs136003 \\
\hline $0.48 \mathrm{NA}$ & NA & NA & NA & & Gg_rs158783 Gg_rs136003 \\
\hline $0.83 \mathrm{NA}$ & NA & NA & NA & & Gg_rs158783 Gg_rs136003 \\
\hline $0.81 \mathrm{NA}$ & NA & NA & NA & & Gg_rs158783 Gg_rs136003 \\
\hline $0.8 \mathrm{NA}$ & NA & NA & NA & & Gg_rs158783 Gg_rs136003 \\
\hline $0.97 \mathrm{NA}$ & NA & NA & NA & & Gg_rs158783 Gg_rs136003 \\
\hline $0.52 \mathrm{NA}$ & NA & NA & NA & & Gg_rs158783 Gg_rs136003 \\
\hline $0.49 \mathrm{NA}$ & NA & NA & NA & & Gg_rs158783 Gg_rs136003 \\
\hline $0.91 \mathrm{NA}$ & NA & NA & NA & & Gg_rs158783 Gg_rs136003 \\
\hline $0.54 \mathrm{NA}$ & NA & NA & NA & & Gg_rs158783 Gg_rs136003 \\
\hline $0.56 \mathrm{NA}$ & NA & NA & NA & & Gg_rs158783 Gg_rs136003 \\
\hline $0.41 \mathrm{NA}$ & NA & NA & NA & & Gg_rs158783 Gg_rs136003 \\
\hline $0.49 \mathrm{NA}$ & NA & NA & NA & & Gg_rs158783 Gg_rs136003 \\
\hline $0.5 \mathrm{NA}$ & NA & NA & NA & & Gg_rs158783 Gg_rs136003 \\
\hline $0.52 \mathrm{NA}$ & NA & NA & NA & & Gg_rs158783 Gg_rs136003 \\
\hline $0.64 \mathrm{NA}$ & NA & NA & NA & & Gg_rs158783 Gg_rs136003 \\
\hline
\end{tabular}




\begin{tabular}{|c|c|c|c|c|c|c|}
\hline 0.73 & -0.49 & 0.67 & 1.86 & 0.94 & \multirow{2}{*}{\multicolumn{2}{|c|}{$\begin{array}{l}\text { Gg_rs158783 Gg_rs136003. } \\
\text { Gg_rs158783 Gg_rs136003. }\end{array}$}} \\
\hline $0.75 \mathrm{NA}$ & NA & NA & NA & & & \\
\hline $0.32 \mathrm{NA}$ & NA & NA & NA & & \multicolumn{2}{|c|}{ Gg_rs158783 Gg_rs136003. } \\
\hline $1.02 \mathrm{NA}$ & NA & NA & NA & & \multicolumn{2}{|c|}{ Gg_rs158783 Gg_rs136003 } \\
\hline $0.76 \mathrm{NA}$ & NA & NA & NA & & \multicolumn{2}{|c|}{ Gg_rs158783 Gg_rs136003 } \\
\hline $0.61 \mathrm{NA}$ & NA & NA & NA & & \multicolumn{2}{|c|}{ Gg_rs158783 Gg_rs136003 } \\
\hline $1.05 \mathrm{NA}$ & NA & NA & NA & & \multicolumn{2}{|c|}{ Gg_rs146225 Gg_rs158905 } \\
\hline $0.77 \mathrm{NA}$ & NA & NA & NA & & \multicolumn{2}{|c|}{ Gg_rs158783 Gg_rs158905 } \\
\hline $0.78 \mathrm{NA}$ & NA & NA & NA & & \multicolumn{2}{|c|}{ Gg_rs158783 Gg_rs158905 } \\
\hline $0.54 \mathrm{NA}$ & NA & NA & NA & & \multicolumn{2}{|c|}{ Gg_rs158783 Gg_rs158905. } \\
\hline 0.99 & 2.16 & 1 & 1.79 & 1.25 & \multirow{2}{*}{\multicolumn{2}{|c|}{$\begin{array}{l}\text { Gg_rs158808.Gg_rs158905 } \\
\text { Gg_rs158808.Gg_rs158905 }\end{array}$}} \\
\hline 0.49 NA & NA & NA & NA & & & \\
\hline $0.88 \mathrm{NA}$ & NA & NA & NA & & \multicolumn{2}{|c|}{ Gg_rs158783 Gg_rs158905 } \\
\hline $0.59 \mathrm{NA}$ & NA & NA & NA & & \multicolumn{2}{|c|}{ Gg_rs158783 Gg_rs158905 } \\
\hline $0.52 \mathrm{NA}$ & NA & NA & NA & & \multicolumn{2}{|c|}{ Gg_rs158808.Gg_rs158905. } \\
\hline $0.52 \mathrm{NA}$ & NA & NA & NA & & \multicolumn{2}{|c|}{ Gg_rs158808.Gg_rs158905 } \\
\hline $0.8 \mathrm{NA}$ & NA & NA & NA & & \multicolumn{2}{|c|}{ Gg_rs158808.Gg_rs158905 } \\
\hline $1.05 \mathrm{NA}$ & NA & NA & NA & & \multicolumn{2}{|c|}{ Gg_rs158808.Gg_rs158905. } \\
\hline $1.21 \mathrm{NA}$ & NA & NA & NA & & \multicolumn{2}{|c|}{ Gg_rs158808.Gg_rs158905 } \\
\hline $0.81 \mathrm{NA}$ & NA & NA & NA & & \multicolumn{2}{|c|}{ Gg_rs158808.Gg_rs158905. } \\
\hline 0.66 NA & NA & NA & NA & & \multicolumn{2}{|c|}{ Gg_rs136003 Gg_rs158905. } \\
\hline 0.06 & 0.28 & 0.06 & 0.28 & 0.08 & \multicolumn{2}{|c|}{ Gg_rs136003 Gg_rs158905. } \\
\hline 0.13 & 0.76 & 0.13 & 0.29 & 0.18 & \multirow{2}{*}{\multicolumn{2}{|c|}{$\begin{array}{l}\text { Gg_rs136003 Gg_rs158905 } \\
\text { Gg_rs136003 Gg_rs158905 }\end{array}$}} \\
\hline 0.47 NA & NA & NA & NA & & & \\
\hline $0.52 \mathrm{NA}$ & NA & NA & NA & & \multicolumn{2}{|c|}{ Gg_rs158808.Gg_rs158905. } \\
\hline $0.14 \mathrm{NA}$ & NA & NA & NA & & \multicolumn{2}{|c|}{ Gg_rs136003 Gg_rs158905 } \\
\hline $0.63 \mathrm{NA}$ & NA & NA & NA & & \multicolumn{2}{|c|}{ Gg_rs158808.Gg_rs158905 } \\
\hline $0.41 \mathrm{NA}$ & NA & NA & NA & & Gg_rs1588 & 8Gg_rs158905 \\
\hline 0.46 NA & NA & NA & NA & & Gg_rs1588 & 3 Gg_rs158905 \\
\hline 0.98 & -0.43 & 0.91 & 2.65 & 1.27 & RBL4827 & Gg_rs136152 \\
\hline 0.57 NA & NA & NA & NA & & RBL4827 & Gg_rs136152 \\
\hline $0.54 \mathrm{NA}$ & NA & NA & NA & & RBL4827 & Gg_rs136152 \\
\hline $0.61 \mathrm{NA}$ & NA & NA & NA & & RBL4827 & Gg_rs136152 \\
\hline $0.54 \mathrm{NA}$ & NA & NA & NA & & RBL4827 & Gg_rs136152 \\
\hline 0.3 & -0.69 & 0.28 & 1.17 & 0.39 & RBL4827 & Gg_rs136152 \\
\hline 0.61 & -0.3 & 0.57 & 3.11 & 0.8 & RBL4827 & Gg_rs136152 \\
\hline 0.75 & -3.02 & 0.7 & 3.95 & 0.98 & RBL4827 & Gg_rs136152 \\
\hline 0.44 NA & NA & NA & NA & & RBL4827 & Gg_rs136152 \\
\hline 0.44 NA & NA & NA & NA & & RBL4827 & Gg_rs136152 \\
\hline $0.42 \mathrm{NA}$ & NA & NA & NA & & RBL4827 & Gg_rs136152 \\
\hline 0.39 NA & NA & NA & NA & & RBL4827 & Gg_rs136152 \\
\hline $0.5 \mathrm{NA}$ & NA & NA & NA & & RBL4827 & Gg_rs136152 \\
\hline 0.61 & -1.57 & 0.56 & 1.02 & 0.79 & RBL4827 & Gg_rs136152 \\
\hline
\end{tabular}




\begin{tabular}{|c|c|c|c|c|c|c|c|c|}
\hline $0.55 \mathrm{NA}$ & & NA & & NA & NA & & RBL4827 & Gg_rs136152. \\
\hline $0.28 \mathrm{NA}$ & & NA & & NA & NA & & RBL4827 & Gg_rs136152 \\
\hline $0.42 \mathrm{NA}$ & & NA & & NA & NA & & RBL4827 & Gg_rs136152 \\
\hline $0.43 \mathrm{NA}$ & & NA & & NA & NA & & RBL4827 & Gg_rs136152 \\
\hline $0.53 \mathrm{NA}$ & & NA & & NA & NA & & RBL4827 & Gg_rs136152 \\
\hline $0.25 \mathrm{NA}$ & & NA & & NA & NA & & RBL4827 & Gg_rs136152 \\
\hline $0.37 \mathrm{NA}$ & & NA & & NA & NA & & RBL4827 & Gg_rs136152 \\
\hline $0.57 \mathrm{NA}$ & & NA & & NA & NA & & RBL4827 & Gg_rs136152 \\
\hline $0.51 \mathrm{NA}$ & & NA & & NA & NA & & RBL4827 & Gg_rs146387 \\
\hline $0.54 \mathrm{NA}$ & & NA & & NA & NA & & RBL4827 & Gg_rs136152 \\
\hline $0.35 \mathrm{NA}$ & & NA & & NA & NA & & RBL4827 & Gg_rs146387 \\
\hline $0.63 \mathrm{NA}$ & & NA & & NA & NA & & RBL4827 & Gg_rs146387 \\
\hline $0.35 \mathrm{NA}$ & & NA & & NA & NA & & \multirow{2}{*}{\multicolumn{2}{|c|}{$\begin{array}{l}\text { Gg_rs136152 Gg_rs166258 } \\
\text { Gg_rs136152 Gg_rs166258 }\end{array}$}} \\
\hline $0.53 \mathrm{NA}$ & & NA & & NA & NA & & & \\
\hline 0.66 & 0.43 & & 0.63 & & -2.32 & 0.88 & \multicolumn{2}{|c|}{ Gg_rs136152 Gg_rs166258 } \\
\hline $0.46 \mathrm{NA}$ & & NA & & NA & NA & & \multicolumn{2}{|c|}{ Gg_rs136152X8_21627386 } \\
\hline 0.33 & 0.95 & & 0.31 & & -1.36 & 0.44 & \multicolumn{2}{|c|}{ Gg_rs136152 Gg_rs166258 } \\
\hline 0.88 & 0.42 & & 0.85 & & -2.88 & 1.19 & \multicolumn{2}{|c|}{ Gg_rs136152 Gg_rs166258 } \\
\hline 0.57 & 1.02 & & 0.55 & & -2.02 & 0.77 & \multicolumn{2}{|c|}{ Gg_rs136152 Gg_rs166258 } \\
\hline 0.7 & 1.07 & & 0.67 & & -2.93 & 0.94 & \multicolumn{2}{|c|}{ Gg_rs136152 Gg_rs166258 } \\
\hline 0.69 & 1.05 & & 0.66 & & -2.4 & 0.92 & \multicolumn{2}{|c|}{ Gg_rs136152 Gg_rs166258 } \\
\hline 0.66 & 1.89 & & 0.64 & & -2.2 & 0.89 & \multicolumn{2}{|c|}{ Gg_rs136152 Gg_rs166258 } \\
\hline 0.51 & 0.93 & & 0.49 & & -2.62 & 0.68 & \multicolumn{2}{|c|}{ Gg_rs136152 Gg_rs166258 } \\
\hline $0.62 \mathrm{NA}$ & & NA & & NA & NA & & \multicolumn{2}{|c|}{ Gg_rs136152X8_2162738€ } \\
\hline 0.92 & -2.07 & & 0.88 & & 0.86 & 1.23 & \multicolumn{2}{|c|}{ Gg_rs136152 Gg_rs166258 } \\
\hline 0.46 & 0.31 & & 0.44 & & -3 & 0.62 & \multicolumn{2}{|c|}{ Gg_rs136152 Gg_rs166258 } \\
\hline $0.53 \mathrm{NA}$ & & NA & & NA & NA & & \multicolumn{2}{|c|}{ Gg_rs136152 Gg_rs166258 } \\
\hline 0.58 & 1.65 & & 0.56 & & -2.64 & 0.78 & \multicolumn{2}{|c|}{ Gg_rs136152 Gg_rs166258 } \\
\hline $0.35 \mathrm{NA}$ & & NA & & NA & NA & & \multicolumn{2}{|c|}{ Gg_rs136152 Gg_rs159199، } \\
\hline 1.08 & -4.41 & & 1.04 & & 4.57 & 1.45 & \multicolumn{2}{|c|}{ Gg_rs136152 Gg_rs166258 } \\
\hline 0.96 & -3.9 & & 0.93 & & 3.97 & 1.29 & \multicolumn{2}{|c|}{ Gg_rs136152 Gg_rs166258 } \\
\hline $0.62 \mathrm{NA}$ & & NA & & NA & NA & & \multicolumn{2}{|c|}{ Gg_rs136152 Gg_rs166258 } \\
\hline 0.46 & 0.88 & & 0.44 & & -1.89 & 0.61 & \multicolumn{2}{|c|}{ Gg_rs136152 Gg_rs166258 } \\
\hline 0.63 & 1.67 & & 0.61 & & -2.75 & 0.85 & \multicolumn{2}{|c|}{ Gg_rs136152 Gg_rs166258 } \\
\hline $0.22 \mathrm{NA}$ & & NA & & NA & NA & & \multicolumn{2}{|c|}{ Gg_rs136152 Gg_rs166258 } \\
\hline $0.33 \mathrm{NA}$ & & NA & & NA & NA & & \multicolumn{2}{|c|}{ Gg_rs136152 Gg_rs166258 } \\
\hline $0.52 \mathrm{NA}$ & & NA & & NA & NA & & Gg_rs136 & 2 Gg_rs159199، \\
\hline $0.41 \mathrm{NA}$ & & NA & & NA & NA & & Gg_rs136 & 2 Gg_rs166258 \\
\hline $0.35 \mathrm{NA}$ & & NA & & NA & NA & & Gg_rs136 & 2 chr8_20.5 \\
\hline $0.19 \mathrm{NA}$ & & NA & & NA & NA & & Gg_rs136 & 2 Gg_rs159199. \\
\hline $8.85 \mathrm{NA}$ & & NA & & NA & NA & & Gg_rs136 & 2 Gg_rs159199، \\
\hline $12.89 \mathrm{NA}$ & & NA & & NA & NA & & Gg_rs136 & 2 Gg_rs159199، \\
\hline $12.13 \mathrm{NA}$ & & NA & & NA & NA & & Gg_rs136 & 2 Gg_rs159199. \\
\hline
\end{tabular}




\begin{tabular}{|c|c|c|c|c|c|}
\hline 11.37 NA & NA & NA & NA & & Gg_rs136152 Gg_rs159199 \\
\hline 14.35 NA & NA & NA & NA & & Gg_rs136152 Gg_rs159199 \\
\hline $12.34 \mathrm{NA}$ & NA & NA & NA & & Gg_rs136152 Gg_rs159199 \\
\hline 14.09 NA & NA & NA & NA & & Gg_rs136152 Gg_rs159199 \\
\hline $12.46 \mathrm{NA}$ & NA & NA & NA & & Gg_rs136152 Gg_rs159199 \\
\hline $11.49 \mathrm{NA}$ & NA & NA & NA & & Gg_rs136152 Gg_rs159199 \\
\hline 10.76 NA & NA & NA & NA & & Gg_rs136152 Gg_rs159199 \\
\hline $13 \mathrm{NA}$ & NA & NA & NA & & Gg_rs136152 Gg_rs159199 \\
\hline 10.77 NA & NA & NA & NA & & Gg_rs136152 Gg_rs159199 \\
\hline $16.38 \mathrm{NA}$ & NA & NA & NA & & Gg_rs136152 Gg_rs159199 \\
\hline 0.9 & -2.85 & 0.84 & -2.98 & 1.22 & Gg_rs136152 X8_21627386 \\
\hline 14.67 NA & NA & NA & NA & & Gg_rs136152 Gg_rs159199 \\
\hline $14.7 \mathrm{NA}$ & NA & NA & NA & & Gg_rs136152 Gg_rs159199 \\
\hline 1.41 & -2.65 & 1.33 & -2.28 & 1.92 & Gg_rs136152 Gg_rs146475 \\
\hline 14.41 NA & NA & NA & NA & & Gg_rs136152 Gg_rs159199 \\
\hline $11.13 \mathrm{NA}$ & NA & NA & NA & & Gg_rs136152 Gg_rs159199 \\
\hline $12.36 \mathrm{NA}$ & NA & NA & NA & & Gg_rs136152 Gg_rs159199 \\
\hline 1.59 & -5.14 & 1.49 & -3.54 & 2.15 & Gg_rs136152 Gg_rs159199 \\
\hline $10.45 \mathrm{NA}$ & NA & NA & NA & & Gg_rs136152 Gg_rs159199 \\
\hline $16.58 \mathrm{NA}$ & NA & NA & NA & & Gg_rs136152 Gg_rs159199 \\
\hline $11.38 \mathrm{NA}$ & NA & NA & NA & & Gg_rs136152 Gg_rs159199 \\
\hline $13.45 \mathrm{NA}$ & NA & NA & NA & & Gg_rs136152 Gg_rs159199 \\
\hline $15.28 \mathrm{NA}$ & NA & NA & NA & & Gg_rs136152 Gg_rs159199 \\
\hline $17.58 \mathrm{NA}$ & NA & NA & NA & & Gg_rs136152 Gg_rs159199 \\
\hline $20.04 N A$ & NA & NA & NA & & Gg_rs136152 Gg_rs159199 \\
\hline $15.83 \mathrm{NA}$ & NA & NA & NA & & Gg_rs136152 Gg_rs159199 \\
\hline 13.15 NA & NA & NA & NA & & Gg_rs136152 Gg_rs159199 \\
\hline 0.7 & -3.55 & 0.66 & 1.87 & 0.96 & Gg_rs146387 Gg_rs159199 \\
\hline $0.51 \mathrm{NA}$ & NA & NA & NA & & Gg_rs136152 Gg_rs159199 \\
\hline 0.7 & -1.6 & 0.65 & -3.57 & 0.95 & Gg_rs146387 Gg_rs159199 \\
\hline 0.69 & -1.73 & 0.65 & -2.19 & 0.94 & Gg_rs146387 Gg_rs159199 \\
\hline 1.37 & -2.78 & 1.28 & -3.95 & 1.86 & Gg_rs146387 Gg_rs159126 \\
\hline 3.01 & -7.32 & 2.82 & -3 & 4.1 & Gg_rs146387 Gg_rs159126 \\
\hline $0.66 \mathrm{NA}$ & NA & NA & NA & & Gg_rs146387 Gg_rs159126 \\
\hline 0.92 & 1.94 & 0.86 & -0.09 & 1.26 & Gg_rs146387 Gg_rs159199 \\
\hline $0.48 \mathrm{NA}$ & NA & NA & NA & & Gg_rs146387 Gg_rs159199 \\
\hline $0.77 \mathrm{NA}$ & NA & NA & NA & & Gg_rs146387 X8_21627386 \\
\hline $0.61 \mathrm{NA}$ & NA & NA & NA & & Gg_rs136152 Gg_rs159199 \\
\hline $1.11 \mathrm{NA}$ & NA & NA & NA & & Gg_rs136152 Gg_rs159199 \\
\hline $0.56 \mathrm{NA}$ & NA & NA & NA & & Gg_rs136152 Gg_rs159199 \\
\hline $0.54 \mathrm{NA}$ & NA & NA & NA & & Gg_rs146387 Gg_rs159199 \\
\hline $0.98 \mathrm{NA}$ & NA & NA & NA & & Gg_rs146387 Gg_rs159126 \\
\hline $0.4 \mathrm{NA}$ & NA & NA & NA & & Gg_rs146387 Gg_rs159126 \\
\hline
\end{tabular}




\begin{tabular}{|c|c|c|c|c|c|}
\hline $0.55 \mathrm{NA}$ & NA & NA & NA & & Gg_rs146387 Gg_rs159199، \\
\hline $0.5 \mathrm{NA}$ & NA & NA & NA & & Gg_rs136152 Gg_rs159199، \\
\hline $0.59 \mathrm{NA}$ & NA & NA & NA & & Gg_rs146387 Gg_rs146475 \\
\hline $1.29 \mathrm{NA}$ & NA & NA & NA & & Gg_rs146387 X8_2162738€ \\
\hline $0.42 \mathrm{NA}$ & NA & NA & NA & & Gg_rs146387 Gg_rs159199، \\
\hline $0.95 \mathrm{NA}$ & NA & NA & NA & & Gg_rs146387 X8_2162738€ \\
\hline 0.39 NA & NA & NA & NA & & Gg_rs146387 Gg_rs159199، \\
\hline $0.59 \mathrm{NA}$ & NA & NA & NA & & Gg_rs136152 X8_2162738€ \\
\hline $0.68 \mathrm{NA}$ & NA & NA & NA & & Gg_rs146387 X8_2162738€ \\
\hline 1.23 & -5.56 & 1.14 & -0.87 & 1.67 & Gg_rs146387 Gg_rs159199، \\
\hline 1.63 & -6.99 & 1.51 & -1.99 & 2.21 & Gg_rs136152 Gg_rs159199، \\
\hline $0.56 \mathrm{NA}$ & NA & NA & NA & & Gg_rs146387 Gg_rs159199، \\
\hline 1.15 & -2.18 & 1.04 & -0.55 & 1.53 & Gg_rs146387 X8_2162738€ \\
\hline $0.45 \mathrm{NA}$ & NA & NA & NA & & Gg_rs136152 Gg_rs159199، \\
\hline $0.78 \mathrm{NA}$ & NA & NA & NA & & Gg_rs136152 Gg_rs159199، \\
\hline $0.55 \mathrm{NA}$ & NA & NA & NA & & Gg_rs146387 Gg_rs146522 \\
\hline $0.52 \mathrm{NA}$ & NA & NA & NA & & Gg_rs136152 X8_2162738€ \\
\hline $0.4 \mathrm{NA}$ & NA & NA & NA & & Gg_rs146387 Gg_rs159199، \\
\hline $0.52 \mathrm{NA}$ & NA & NA & NA & & Gg_rs146387 Gg_rs159199، \\
\hline $0.41 \mathrm{NA}$ & NA & NA & NA & & Gg_rs146415 Gg_rs159126 \\
\hline $0.95 \mathrm{NA}$ & NA & NA & NA & & Gg_rs146387 Gg_rs159199، \\
\hline $0.55 \mathrm{NA}$ & NA & NA & NA & & Gg_rs146387 Gg_rs159199، \\
\hline $0.78 \mathrm{NA}$ & NA & NA & NA & & Gg_rs146387 Gg_rs159199، \\
\hline $1.1 \mathrm{NA}$ & NA & NA & NA & & Gg_rs146387 Gg_rs159199. \\
\hline $1.09 \mathrm{NA}$ & NA & NA & NA & & Gg_rs146387 Gg_rs159199، \\
\hline $0.71 \mathrm{NA}$ & NA & NA & NA & & Gg_rs146387 Gg_rs159199، \\
\hline 0.72 & -2.49 & 0.65 & 1.19 & 0.96 & Gg_rs146387 Gg_rs159199، \\
\hline $0.53 \mathrm{NA}$ & NA & NA & NA & & Gg_rs146387 Gg_rs159199. \\
\hline $0.5 \mathrm{NA}$ & NA & NA & NA & & Gg_rs136152 X8_2162738€ \\
\hline 0.94 NA & NA & NA & NA & & Gg_rs146387 X8_2162738€ \\
\hline 0.79 NA & NA & NA & NA & & Gg_rs146387 X8_2162738€ \\
\hline $0.31 \mathrm{NA}$ & NA & NA & NA & & Gg_rs146387 chr8_20.5 \\
\hline $0.53 \mathrm{NA}$ & NA & NA & NA & & Gg_rs146387 X8_2162738€ \\
\hline $0.55 \mathrm{NA}$ & NA & NA & NA & & Gg_rs146387 X8_2162738€ \\
\hline $1.06 \mathrm{NA}$ & NA & NA & NA & & Gg_rs146387 X8_2162738€ \\
\hline $0.54 \mathrm{NA}$ & NA & NA & NA & & Gg_rs146387 X8_2162738€ \\
\hline $1.87 \mathrm{NA}$ & NA & NA & NA & & Gg_rs159126chr8_20.5 \\
\hline $0.5 \mathrm{NA}$ & NA & NA & NA & & Gg_rs146415 Gg_rs146475 \\
\hline 1.24 & -2.26 & 1.15 & -1.15 & 1.61 & Gg_rs159126X8_2162738€ \\
\hline 0.61 & -1.76 & 0.57 & -1.46 & 0.79 & Gg_rs159126Gg_rs146475 \\
\hline $0.53 \mathrm{NA}$ & NA & NA & NA & & Gg_rs159126Gg_rs146475 \\
\hline $0.79 \mathrm{NA}$ & NA & NA & NA & & Gg_rs159126X8_2162738€ \\
\hline $1.01 \mathrm{NA}$ & NA & NA & NA & & Gg_rs159126chr8_20.5 \\
\hline
\end{tabular}




\begin{tabular}{|c|c|c|c|c|c|}
\hline $1.2 \mathrm{NA}$ & NA & NA & NA & & Gg_rs159126 X8_2162738€ \\
\hline $0.96 \mathrm{NA}$ & NA & NA & NA & & Gg_rs159126X8_2162738E \\
\hline $1.05 \mathrm{NA}$ & NA & NA & NA & & Gg_rs159126 Gg_rs146475 \\
\hline $0.81 \mathrm{NA}$ & NA & NA & NA & & Gg_rs159126 Gg_rs146522 \\
\hline 1.09 NA & NA & NA & NA & & Gg_rs159126chr8_20.5 \\
\hline $0.54 \mathrm{NA}$ & NA & NA & NA & & Gg_rs159126Gg_rs146475 \\
\hline $0.81 \mathrm{NA}$ & NA & NA & NA & & Gg_rs159126 chr8_20.5 \\
\hline $0.61 \mathrm{NA}$ & NA & NA & NA & & Gg_rs159126chr8_20.5 \\
\hline $0.72 \mathrm{NA}$ & NA & NA & NA & & Gg_rs159126chr8_20.5 \\
\hline $0.59 \mathrm{NA}$ & NA & NA & NA & & Gg_rs159126chr8_20.5 \\
\hline $0.48 \mathrm{NA}$ & NA & NA & NA & & Gg_rs159126X8_2162738€ \\
\hline 0.59 NA & NA & NA & NA & & Gg_rs136152 X8_2162738€ \\
\hline 0.72 NA & NA & NA & NA & & Gg_rs159126 Gg_rs146522 \\
\hline 4.31 NA & NA & NA & NA & & Gg_rs146387 chr8_20.5 \\
\hline $3.98 \mathrm{NA}$ & NA & NA & NA & & Gg_rs146387 X8_2162738€ \\
\hline $1.11 \mathrm{NA}$ & NA & NA & NA & & Gg_rs159126X8_2162738€ \\
\hline $0.65 \mathrm{NA}$ & NA & NA & NA & & GG_rs166276X8_2162738€ \\
\hline $0.72 \mathrm{NA}$ & NA & NA & NA & & Gg_rs159126X8_2162738€ \\
\hline $1 \mathrm{NA}$ & NA & NA & NA & & Gg_rs159126X8_2162738€ \\
\hline $0.3 \mathrm{NA}$ & NA & NA & NA & & Gg_rs159126chr8_20.5 \\
\hline $0.28 \mathrm{NA}$ & NA & NA & NA & & Gg_rs159126chr8_20.5 \\
\hline 0.43 NA & NA & NA & NA & & Gg_rs159199.chr8_20.5 \\
\hline $0.51 \mathrm{NA}$ & NA & NA & NA & & Gg_rs159126X8_2162738€ \\
\hline 0.63 NA & NA & NA & NA & & Gg_rs159126 Gg_rs146522 \\
\hline $0.4 \mathrm{NA}$ & NA & NA & NA & & Gg_rs136152 X8_2162738€ \\
\hline $0.72 \mathrm{NA}$ & NA & NA & NA & & Gg_rs146387 X8_2162738€ \\
\hline 0.46 NA & NA & NA & NA & & Gg_rs146387 X8_2162738€ \\
\hline $0.55 \mathrm{NA}$ & NA & NA & NA & & Gg_rs146387 X8_2162738€ \\
\hline $3.4 \mathrm{NA}$ & NA & NA & NA & & Gg_rs146387 X8_2162738€ \\
\hline $0.5 \mathrm{NA}$ & NA & NA & NA & & Gg_rs159199.chr8_20.5 \\
\hline $0.54 \mathrm{NA}$ & NA & NA & NA & & Gg_rs159199.chr8_20.5 \\
\hline $0.54 \mathrm{NA}$ & NA & NA & NA & & Gg_rs146387 X8_2162738€ \\
\hline 0.82 NA & NA & NA & NA & & Gg_rs159126X8_2162738€ \\
\hline $0.98 \mathrm{NA}$ & NA & NA & NA & & Gg_rs136152 X8_2162738€ \\
\hline $0.55 \mathrm{NA}$ & NA & NA & NA & & Gg_rs146387 X8_2162738€ \\
\hline $0.54 \mathrm{NA}$ & NA & NA & NA & & Gg_rs159126X8_2162738€ \\
\hline $0.98 \mathrm{NA}$ & NA & NA & NA & & Gg_rs159126X8_2162738€ \\
\hline 0.58 & 1.98 & 0.54 & -1.61 & 0.77 & Gg_rs159126 chr8_20.5 \\
\hline $0.66 \mathrm{NA}$ & NA & NA & NA & & Gg_rs159126X8_2162738€ \\
\hline $0.85 \mathrm{NA}$ & NA & NA & NA & & Gg_rs159126X8_2162738E \\
\hline $1.01 \mathrm{NA}$ & NA & NA & NA & & Gg_rs159199:X8_2162738E \\
\hline 0.99 NA & NA & NA & NA & & Gg_rs159126X8_2162738€ \\
\hline 0.98 & -4.5 & 0.91 & 0.99 & 1.3 & Gg_rs159126 X8_2162738 \\
\hline
\end{tabular}




\begin{tabular}{|c|c|c|c|c|c|c|}
\hline 0.69 & -2.28 & 0.64 & -2.07 & \multirow{2}{*}{\multicolumn{3}{|c|}{$\begin{array}{c}0.91 \text { Gg_rs159199 X8_21627386 } \\
\text { Gg_rs159199.chr8_20.5 }\end{array}$}} \\
\hline $0.34 \mathrm{NA}$ & NA & NA & NA & & & \\
\hline $0.45 \mathrm{NA}$ & NA & NA & NA & \multicolumn{3}{|c|}{ Gg_rs159126 X8_2162738€ } \\
\hline 1.01 & -3.16 & 0.94 & 0.99 & \multicolumn{3}{|c|}{1.34 Gg_rs136152 Gg_rs146522 } \\
\hline $0.65 \mathrm{NA}$ & NA & NA & NA & \multicolumn{3}{|c|}{ Gg_rs146387 X8_2162738€ } \\
\hline $0.4 \mathrm{NA}$ & NA & NA & NA & \multicolumn{3}{|c|}{ Gg_rs159126X8_2162738€ } \\
\hline $0.47 \mathrm{NA}$ & NA & NA & NA & \multicolumn{3}{|c|}{ Gg_rs159126X8_2162738€ } \\
\hline 1.25 & -2.69 & 1.16 & -0.04 & \multicolumn{3}{|c|}{1.65 Gg_rs159126 X8_2162738€ } \\
\hline $0.68 \mathrm{NA}$ & NA & NA & NA & \multicolumn{3}{|c|}{ Gg_rs159126X8_2162738€ } \\
\hline $0.63 \mathrm{NA}$ & NA & NA & NA & \multicolumn{3}{|c|}{ Gg_rs146387 X8_2162738€ } \\
\hline 0.35 & -0.9 & 0.32 & 0.32 & \multicolumn{3}{|c|}{0.47 Gg_rs159126 X8_2162738€ } \\
\hline $0.6 \mathrm{NA}$ & NA & NA & NA & \multicolumn{3}{|c|}{ Gg_rs146415X8_2162738€ } \\
\hline 0.48 NA & NA & NA & NA & \multicolumn{3}{|c|}{ Gg_rs159126X8_2162738€ } \\
\hline $1.1 \mathrm{NA}$ & NA & NA & NA & \multicolumn{3}{|c|}{ Gg_rs159199:X8_2162738€ } \\
\hline 0.79 NA & NA & NA & NA & \multicolumn{3}{|c|}{ Gg_rs159126X8_2162738€ } \\
\hline 0.69 NA & NA & NA & NA & \multicolumn{3}{|c|}{ Gg_rs159126X8_2162738€ } \\
\hline $0.75 \mathrm{NA}$ & NA & NA & NA & \multicolumn{3}{|c|}{ Gg_rs159199:X8_2162738€ } \\
\hline 0.48 NA & NA & NA & NA & \multicolumn{3}{|c|}{ Gg_rs159126X8_2162738€ } \\
\hline $0.7 \mathrm{NA}$ & NA & NA & NA & \multicolumn{3}{|c|}{ Gg_rs159126 Gg_rs146522 } \\
\hline $0.8 \mathrm{NA}$ & NA & NA & NA & \multicolumn{3}{|c|}{ Gg_rs159126 Gg_rs146522 } \\
\hline 1.42 & -3.32 & 1.48 & 2.63 & 1.99 & chr8_20.5 & Gg_rs146522 \\
\hline $1.44 \mathrm{NA}$ & NA & NA & NA & \multicolumn{3}{|c|}{ Gg_rs146475 Gg_rs146522 } \\
\hline $1.78 \mathrm{NA}$ & NA & NA & NA & \multicolumn{3}{|c|}{ Gg_rs146475 Gg_rs146522 } \\
\hline 0.64 NA & NA & NA & NA & \multicolumn{3}{|c|}{ Gg_rs159126 Gg_rs146522 } \\
\hline $1.31 \mathrm{NA}$ & NA & NA & NA & \multicolumn{3}{|c|}{ Gg_rs146475 Gg_rs146522 } \\
\hline 0.71 & -2.02 & 0.74 & -0.08 & & chr8_20.5 & Gg_rs146522 \\
\hline $1.38 \mathrm{NA}$ & NA & NA & NA & \multicolumn{3}{|c|}{ Gg_rs146475 Gg_rs146522 } \\
\hline $1.27 \mathrm{NA}$ & NA & NA & NA & & Gg_rs1464 & 5 Gg_rs146522 \\
\hline $0.92 \mathrm{NA}$ & NA & NA & NA & & Gg_rs1464 & 5 Gg_rs146522 \\
\hline 0.99 & -3.09 & 1.04 & 2.16 & 1.4 & chr8_20.5 & Gg_rs146522 \\
\hline $0.61 \mathrm{NA}$ & NA & NA & NA & & chr8_20.5 & Gg_rs146522 \\
\hline $0.61 \mathrm{NA}$ & NA & NA & NA & & chr8_20.5 & Gg_rs146522 \\
\hline $1.11 \mathrm{NA}$ & NA & NA & NA & & chr8_20.5 & Gg_rs146522 \\
\hline $1.48 \mathrm{NA}$ & NA & NA & NA & & chr8_20.5 & Gg_rs146522 \\
\hline $0.63 \mathrm{NA}$ & NA & NA & NA & & chr8_20.5 & Gg_rs146522 \\
\hline $0.46 \mathrm{NA}$ & NA & NA & NA & & chr8_20.5 & Gg_rs146522 \\
\hline 1.03 & -3.62 & 1.07 & 3.32 & 1.44 & chr8_20.5 & Gg_rs146522 \\
\hline 0.68 NA & NA & NA & NA & & chr8_20.5 & Gg_rs146522 \\
\hline 0.64 NA & NA & NA & NA & & chr8_20.5 & Gg_rs146522 \\
\hline $0.64 \mathrm{NA}$ & NA & NA & NA & & chr8_20.5 & Gg_rs146522 \\
\hline 0.88 NA & NA & NA & NA & & chr8_20.5 & Gg_rs146522 \\
\hline 0.49 NA & NA & NA & NA & & chr8_20.5 & Gg_rs146522 \\
\hline 0.6 NA & NA & NA & NA & & chr8_20.5 & Gg_rs146522 \\
\hline
\end{tabular}




\begin{tabular}{|c|c|c|c|c|c|c|}
\hline 1.21 & -1.4 & 1.26 & 3.55 & 1.7 & chr8_20.5 & Gg_rs146522 \\
\hline $0.36 \mathrm{NA}$ & NA & NA & NA & & chr8_20.5 & Gg_rs146522 \\
\hline $1.49 \mathrm{NA}$ & NA & NA & NA & & chr8_20.5 & Gg_rs146522 \\
\hline $0.4 \mathrm{NA}$ & NA & NA & NA & & chr8_20.5 & Gg_rs146522 \\
\hline $0.55 \mathrm{NA}$ & NA & NA & NA & & chr8_20.5 & Gg_rs146522 \\
\hline 1.13 & -2.43 & 1.17 & 0.66 & 1.58 & Gg_rs146475 & 5 Gg_rs146522 \\
\hline 0.68 & -1.4 & 0.71 & 1.88 & 0.95 & chr8_20.5 & X8_2551368E \\
\hline 0.89 & -2.79 & 0.92 & 2.69 & 1.19 & X8_21627386 & EX8_2551368E \\
\hline 0.58 & -1.96 & 0.6 & 3.13 & 0.78 & X8_21627386 & EX8_2551368E \\
\hline 0.64 & -1.57 & 0.66 & 2.79 & 0.86 & X8_21627386 & EX8_2551368E \\
\hline $0.54 \mathrm{NA}$ & NA & NA & NA & & X8_21627386 & EX8_2551368E \\
\hline 0.69 & -1.79 & 0.71 & 2.89 & 0.92 & X8_21627386 & EX8_2551368€ \\
\hline 1.02 & -2.17 & 1.05 & 0.88 & 1.37 & X8_21627386 & EX8_2551368E \\
\hline $0.42 \mathrm{NA}$ & NA & NA & NA & & X8_21627386 & EX8_2551368E \\
\hline 0.63 & -2.22 & 0.65 & 3.43 & 0.85 & X8_21627386 & EX8_2551368E \\
\hline 0.88 & -2.92 & 0.91 & 3.61 & 1.18 & X8_21627386 & EX8_2551368E \\
\hline 0.31 & -1.14 & 0.32 & 1.39 & 0.41 & X8_21627386 & EX8_2551368E \\
\hline 0.97 & -2.33 & 1 & 3.26 & 1.3 & X8_21627386 & EX8_2551368€ \\
\hline 1.2 & -4.17 & 1.24 & 5.27 & 1.62 & X8_21627386 & EX8_2551368E \\
\hline 0.64 & -2.12 & 0.66 & 4.09 & 0.86 & X8_21627386 & EX8_2551368E \\
\hline 0.51 & -2.13 & 0.53 & 2.38 & 0.69 & X8_21627386 & EX8_2551368E \\
\hline 0.64 NA & NA & NA & NA & & X8_21627386 & EX8_2551368€ \\
\hline 0.95 & -3.1 & 0.98 & 3 & 1.27 & X8_21627386 & EX8_2551368E \\
\hline 0.68 & -2.41 & 0.7 & 2.64 & 0.91 & X8_21627386 & EX8_2551368E \\
\hline 0.65 & -0.5 & 0.67 & 2.49 & 0.87 & X8_21627386 & EX8_2551368E \\
\hline $0.54 \mathrm{NA}$ & NA & NA & NA & & X8_21627386 & EX8_2551368E \\
\hline $0.5 \mathrm{NA}$ & NA & NA & NA & & X8_21627386 & EX8_2551368E \\
\hline 0.61 & -1.57 & 0.63 & 3.5 & 0.82 & X8_21627386 & EX8_2551368E \\
\hline 0.53 & -2.26 & 0.55 & 2.91 & 0.71 & X8_21627386 & EX8_2551368E \\
\hline 0.6 & -2.65 & 0.62 & 3.34 & 0.81 & X8_21627386 & EX8_2551368E \\
\hline 0.33 & -1.31 & 0.34 & 1.81 & 0.44 & X8_21627386 & EX8_2551368E \\
\hline 0.7 & -2.19 & 0.72 & 3.79 & 0.94 & X8_21627386 & EX8_2551368E \\
\hline 0.55 & -1.64 & 0.57 & 2.46 & 0.74 & X8_21627386 & EX8_2551368E \\
\hline 0.04 NA & NA & NA & NA & & X8_21627386 & EX8_2551368E \\
\hline $0.4 \mathrm{NA}$ & NA & NA & NA & & X8_21627386 & EX8_2551368E \\
\hline $0.38 \mathrm{NA}$ & NA & NA & NA & & X8_21627386 & EX8_2551368€ \\
\hline 0.66 NA & NA & NA & NA & & X8_21627386 & EX8_2551368€ \\
\hline 0.04 NA & NA & NA & NA & & X8_21627386 & EX8_2551368E \\
\hline $0.31 \mathrm{NA}$ & NA & NA & NA & & X8_21627386 & EX8_2551368E \\
\hline $0.51 \mathrm{NA}$ & NA & NA & NA & & X8_21627386 & EX8_2551368€ \\
\hline $0.42 \mathrm{NA}$ & NA & NA & NA & & X8_21627386 & EX8_2551368E \\
\hline 1.45 & 2.15 & 1.15 & -3.2 & 1.83 & Gg_rs159355 & 58_29856293 \\
\hline $0.5 \mathrm{NA}$ & NA & NA & NA & & Gg_rs159355 & X8_29856293 \\
\hline
\end{tabular}




\begin{tabular}{|c|c|c|c|c|c|}
\hline $0.38 \mathrm{NA}$ & NA & NA & NA & & Gg_rs159355 X8_29856293 \\
\hline $0.53 \mathrm{NA}$ & NA & NA & NA & & Gg_rs159355 X8_29856293 \\
\hline $0.55 \mathrm{NA}$ & NA & NA & NA & & Gg_rs159355 X8_29856293 \\
\hline 1.24 & 1.23 & 0.98 & 1.79 & 1.57 & Gg_rs159355 X8_29856293 \\
\hline $0.53 \mathrm{NA}$ & NA & NA & NA & & Gg_rs159444 Gg_rs159669 \\
\hline $0.55 \mathrm{NA}$ & NA & NA & NA & & Gg_rs159444Gg_rs159669 \\
\hline 0.9 & -1.81 & 0.73 & -5.61 & 1.18 & Gg_rs159444 Gg_rs146603. \\
\hline 0.44 NA & NA & NA & NA & & Gg_rs159444 Gg_rs159669 \\
\hline $0.4 \mathrm{NA}$ & NA & NA & NA & & Gg_rs159444Gg_rs159669 \\
\hline 0.44 NA & NA & NA & NA & & Gg_rs159444Gg_rs159669 \\
\hline 0.74 NA & NA & NA & NA & & Gg_rs159444Gg_rs159669 \\
\hline $0.39 \mathrm{NA}$ & NA & NA & NA & & Gg_rs159444Gg_rs159669 \\
\hline $0.28 \mathrm{NA}$ & NA & NA & NA & & Gg_rs159444 Gg_rs159669 \\
\hline 1.19 & 0.01 & 1.05 & -3.47 & 1.58 & Gg_rs159444 Gg_rs159669 \\
\hline 0.89 & -1.47 & 0.79 & -2.2 & 1.18 & Gg_rs159444 Gg_rs159669 \\
\hline $0.6 \mathrm{NA}$ & NA & NA & NA & & Gg_rs159444 Gg_rs159669 \\
\hline 0.44 NA & NA & NA & NA & & Gg_rs159444Gg_rs159669 \\
\hline $0.46 \mathrm{NA}$ & NA & NA & NA & & Gg_rs159444Gg_rs159669 \\
\hline $0.61 \mathrm{NA}$ & NA & NA & NA & & Gg_rs159444Gg_rs159669 \\
\hline $0.55 \mathrm{NA}$ & NA & NA & NA & & Gg_rs159444Gg_rs159669 \\
\hline $0.4 \mathrm{NA}$ & NA & NA & NA & & Gg_rs159444Gg_rs159669 \\
\hline $1.26 \mathrm{NA}$ & NA & NA & NA & & Gg_rs159444 Gg_rs137670 \\
\hline $0.6 \mathrm{NA}$ & NA & NA & NA & & Gg_rs159444 Gg_rs146695. \\
\hline 0.89 NA & NA & NA & NA & & Gg_rs159444 Gg_rs137670 \\
\hline $0.83 \mathrm{NA}$ & NA & NA & NA & & Gg_rs159444 Gg_rs166674 \\
\hline $0.71 \mathrm{NA}$ & NA & NA & NA & & Gg_rs159444 Gg_rs146695. \\
\hline $0.41 \mathrm{NA}$ & NA & NA & NA & & Gg_rs146603 Gg_rs146695. \\
\hline $0.54 \mathrm{NA}$ & NA & NA & NA & & Gg_rs146603 Gg_rs146695. \\
\hline 0.66 & 0.62 & 0.64 & 3.48 & 0.89 & Gg_rs159669Gg_rs159599 \\
\hline $1.23 \mathrm{NA}$ & NA & NA & NA & & Gg_rs137670Gg_rs159599 \\
\hline 1.14 & 0.38 & 1.1 & 4.46 & 1.53 & Gg_rs137670 Gg_rs159599 \\
\hline $0.47 \mathrm{NA}$ & NA & NA & NA & & Gg_rs137670 Gg_rs159599 \\
\hline $0.61 \mathrm{NA}$ & NA & NA & NA & & Gg_rs146603 Gg_rs137644 \\
\hline 2 & 0.22 & 1.75 & 5.78 & 2.54 & Gg_rs137670Gg_rs166674 \\
\hline 0.64 NA & NA & NA & NA & & Gg_rs137670Gg_rs166674 \\
\hline $0.55 \mathrm{NA}$ & NA & NA & NA & & Gg_rs137670Gg_rs166674 \\
\hline $0.58 \mathrm{NA}$ & NA & NA & NA & & Gg_rs137670 Gg_rs137644 \\
\hline $0.6 \mathrm{NA}$ & NA & NA & NA & & Gg_rs137670Gg_rs166674 \\
\hline 0.58 NA & NA & NA & NA & & Gg_rs137670 Gg_rs166674 \\
\hline $0.57 \mathrm{NA}$ & NA & NA & NA & & Gg_rs137670 Gg_rs137644 \\
\hline $0.85 \mathrm{NA}$ & NA & NA & NA & & Gg_rs159669 Gg_rs166674 \\
\hline $0.75 \mathrm{NA}$ & NA & NA & NA & & Gg_rs137670 Gg_rs166674 \\
\hline $0.74 \mathrm{NA}$ & NA & NA & NA & & Gg_rs137670Gg_rs166674 \\
\hline
\end{tabular}




\begin{tabular}{|c|c|c|c|c|}
\hline $0.9 \mathrm{NA}$ & NA & NA & NA & Gg_rs146695 Gg_rs146717 \\
\hline $0.42 \mathrm{NA}$ & NA & NA & NA & Gg_rs137670 Gg_rs166674 \\
\hline $0.76 \mathrm{NA}$ & NA & NA & NA & Gg_rs146695 Gg_rs146717 \\
\hline $1.04 \mathrm{NA}$ & NA & NA & NA & Gg_rs146695 Gg_rs166674 \\
\hline $0.7 \mathrm{NA}$ & NA & NA & NA & Gg_rs146695 Gg_rs137644 \\
\hline $0.32 \mathrm{NA}$ & NA & NA & NA & Gg_rs146695 Gg_rs137644 \\
\hline $0.61 \mathrm{NA}$ & NA & NA & NA & Gg_rs146695 Gg_rs146717 \\
\hline 0.42 NA & NA & NA & NA & Gg_rs146695 Gg_rs159781 \\
\hline $0.7 \mathrm{NA}$ & NA & NA & NA & Gg_rs146695 Gg_rs137644 \\
\hline 0.69 NA & NA & NA & NA & Gg_rs146695 Gg_rs137644 \\
\hline $0.71 \mathrm{NA}$ & NA & NA & NA & Gg_rs146695 Gg_rs159781 \\
\hline $0.8 \mathrm{NA}$ & NA & NA & NA & Gg_rs146695 Gg_rs146717 \\
\hline $0.59 \mathrm{NA}$ & NA & NA & NA & Gg_rs146695 Gg_rs137644 \\
\hline $0.54 \mathrm{NA}$ & NA & NA & NA & Gg_rs146695 Gg_rs159733 \\
\hline $0.79 \mathrm{NA}$ & NA & NA & NA & Gg_rs146695 Gg_rs137644 \\
\hline $7.8 \mathrm{NA}$ & NA & NA & NA & Gg_rs146695 Gg_rs137644 \\
\hline 12.07 NA & NA & NA & NA & Gg_rs146695 Gg_rs137644 \\
\hline $15.54 \mathrm{NA}$ & NA & NA & NA & Gg_rs146695 Gg_rs137644 \\
\hline $12.81 \mathrm{NA}$ & NA & NA & NA & Gg_rs146695 Gg_rs137644 \\
\hline $11.68 \mathrm{NA}$ & NA & NA & NA & Gg_rs146695 Gg_rs137644 \\
\hline $11.61 \mathrm{NA}$ & NA & NA & NA & Gg_rs146695 Gg_rs137644 \\
\hline $13.41 \mathrm{NA}$ & NA & NA & NA & Gg_rs146695 Gg_rs137644 \\
\hline $11.93 \mathrm{NA}$ & NA & NA & NA & Gg_rs146695 Gg_rs137644 \\
\hline $11.75 \mathrm{NA}$ & NA & NA & NA & Gg_rs146695 Gg_rs137644 \\
\hline $6.99 \mathrm{NA}$ & NA & NA & NA & Gg_rs146695 Gg_rs137644 \\
\hline $0.55 \mathrm{NA}$ & NA & NA & NA & Gg_rs146695 Gg_rs146717 \\
\hline 0.89 NA & NA & NA & NA & Gg_rs146695 Gg_rs146717 \\
\hline $0.81 \mathrm{NA}$ & NA & NA & NA & Gg_rs146695 Gg_rs146717 \\
\hline $0.42 \mathrm{NA}$ & NA & NA & NA & Gg_rs146695 Gg_rs137644 \\
\hline $0.8 \mathrm{NA}$ & NA & NA & NA & Gg_rs146695 Gg_rs136083 \\
\hline $0.72 \mathrm{NA}$ & NA & NA & NA & Gg_rs146695 Gg_rs146717 \\
\hline $0.68 \mathrm{NA}$ & NA & NA & NA & Gg_rs146695 Gg_rs137644 \\
\hline $0.53 \mathrm{NA}$ & NA & NA & NA & Gg_rs146695 Gg_rs137644 \\
\hline $0.6 \mathrm{NA}$ & NA & NA & NA & Gg_rs146695 Gg_rs159733 \\
\hline $0.98 \mathrm{NA}$ & NA & NA & NA & Gg_rs146695 Gg_rs137644 \\
\hline $0.63 \mathrm{NA}$ & NA & NA & NA & Gg_rs146695 Gg_rs137644 \\
\hline $0.68 \mathrm{NA}$ & NA & NA & NA & Gg_rs146695 Gg_rs166674 \\
\hline $0.83 \mathrm{NA}$ & NA & NA & NA & Gg_rs146695 Gg_rs159781 \\
\hline $1.11 \mathrm{NA}$ & NA & NA & NA & Gg_rs146695 Gg_rs137644 \\
\hline $0.64 \mathrm{NA}$ & NA & NA & NA & Gg_rs146695 Gg_rs166674 \\
\hline $0.5 \mathrm{NA}$ & NA & NA & NA & Gg_rs146695 Gg_rs137644 \\
\hline $0.4 \mathrm{NA}$ & NA & NA & NA & Gg_rs146695 Gg_rs159781 \\
\hline $0.36 \mathrm{NA}$ & NA & NA & NA & Gg_rs146695 Gg_rs137644 \\
\hline
\end{tabular}




\begin{tabular}{|c|c|c|c|c|c|}
\hline $0.72 \mathrm{NA}$ & NA & NA & NA & & Gg_rs146695 Gg_rs166674 \\
\hline $0.81 \mathrm{NA}$ & NA & NA & NA & & Gg_rs146695 Gg_rs137644 \\
\hline $0.72 \mathrm{NA}$ & NA & NA & NA & & Gg_rs146695 Gg_rs137644 \\
\hline $0.48 \mathrm{NA}$ & NA & NA & NA & & Gg_rs146695 Gg_rs137644 \\
\hline $0.66 \mathrm{NA}$ & NA & NA & NA & & Gg_rs146695 Gg_rs159733 \\
\hline $0.51 \mathrm{NA}$ & NA & NA & NA & & Gg_rs146695 Gg_rs146717 \\
\hline $0.59 \mathrm{NA}$ & NA & NA & NA & & Gg_rs146695 Gg_rs137644 \\
\hline 0.56 & -0.32 & 0.49 & -1.27 & 0.73 & Gg_rs146695 Gg_rs137644 \\
\hline $0.39 \mathrm{NA}$ & NA & NA & NA & & Gg_rs146695 Gg_rs137644 \\
\hline $0.59 \mathrm{NA}$ & NA & NA & NA & & Gg_rs146695 Gg_rs137644 \\
\hline $0.91 \mathrm{NA}$ & NA & NA & NA & & Gg_rs146695 Gg_rs166674 \\
\hline 0.78 NA & NA & NA & NA & & Gg_rs146695 Gg_rs137644 \\
\hline 0.95 & -2.42 & 0.84 & -2.71 & 1.24 & Gg_rs146695 Gg_rs137644 \\
\hline $0.65 \mathrm{NA}$ & NA & NA & NA & & Gg_rs146695 Gg_rs137644 \\
\hline $0.53 \mathrm{NA}$ & NA & NA & NA & & Gg_rs146695 Gg_rs137644 \\
\hline $0.71 \mathrm{NA}$ & NA & NA & NA & & Gg_rs146695 Gg_rs137644 \\
\hline $0.51 \mathrm{NA}$ & NA & NA & NA & & Gg_rs146695 Gg_rs137644 \\
\hline $0.45 \mathrm{NA}$ & NA & NA & NA & & Gg_rs146695 Gg_rs166674 \\
\hline 1.1 & 2.64 & 0.97 & -0.51 & 1.44 & Gg_rs146695 Gg_rs137644 \\
\hline 0.49 NA & NA & NA & NA & & Gg_rs146695 Gg_rs146717 \\
\hline $0.42 \mathrm{NA}$ & NA & NA & NA & & Gg_rs146695 Gg_rs159781 \\
\hline $1.07 \mathrm{NA}$ & NA & NA & NA & & Gg_rs137670 Gg_rs146717 \\
\hline $0.5 \mathrm{NA}$ & NA & NA & NA & & Gg_rs146695 Gg_rs159781 \\
\hline $0.67 \mathrm{NA}$ & NA & NA & NA & & Gg_rs146695 Gg_rs146717 \\
\hline $0.72 \mathrm{NA}$ & NA & NA & NA & & Gg_rs146695 Gg_rs146717 \\
\hline $0.63 \mathrm{NA}$ & NA & NA & NA & & Gg_rs146695 Gg_rs137644 \\
\hline $0.85 \mathrm{NA}$ & NA & NA & NA & & Gg_rs146695 Gg_rs146717 \\
\hline $0.56 \mathrm{NA}$ & NA & NA & NA & & Gg_rs146695 Gg_rs136083 \\
\hline 1.42 & 2.52 & 1.22 & 1.9 & 1.84 & Gg_rs146695 Gg_rs146717 \\
\hline $0.83 \mathrm{NA}$ & NA & NA & NA & & Gg_rs146695 Gg_rs166674 \\
\hline $0.8 \mathrm{NA}$ & NA & NA & NA & & Gg_rs146695 Gg_rs146717 \\
\hline $1.18 \mathrm{NA}$ & NA & NA & NA & & Gg_rs146695 Gg_rs166674 \\
\hline $0.86 \mathrm{NA}$ & NA & NA & NA & & Gg_rs146695 Gg_rs136083 \\
\hline $0.97 \mathrm{NA}$ & NA & NA & NA & & Gg_rs146695 Gg_rs146717 \\
\hline $1.18 \mathrm{NA}$ & NA & NA & NA & & Gg_rs146695 Gg_rs166674 \\
\hline 0.49 NA & NA & NA & NA & & Gg_rs146695 Gg_rs136083 \\
\hline $0.7 \mathrm{NA}$ & NA & NA & NA & & Gg_rs146695 Gg_rs146717 \\
\hline $0.68 \mathrm{NA}$ & NA & NA & NA & & Gg_rs146695 Gg_rs159781 \\
\hline $0.46 \mathrm{NA}$ & NA & NA & NA & & Gg_rs146695 Gg_rs159781 \\
\hline $0.85 \mathrm{NA}$ & NA & NA & NA & & Gg_rs146695 Gg_rs159733 \\
\hline $0.52 \mathrm{NA}$ & NA & NA & NA & & Gg_rs146695 Gg_rs166674 \\
\hline $0.78 \mathrm{NA}$ & NA & NA & NA & & Gg_rs146695 Gg_rs146717 \\
\hline $0.57 \mathrm{NA}$ & NA & NA & NA & & Gg_rs146695 Gg_rs159781 \\
\hline
\end{tabular}




\begin{tabular}{|c|c|c|c|c|c|}
\hline $0.47 \mathrm{NA}$ & NA & NA & NA & & Gg_rs146695 Gg_rs159781 \\
\hline $0.56 \mathrm{NA}$ & NA & NA & NA & & Gg_rs146695 Gg_rs146717 \\
\hline $1.17 \mathrm{NA}$ & NA & NA & NA & & Gg_rs137670Gg_rs166674 \\
\hline $0.67 \mathrm{NA}$ & NA & NA & NA & & Gg_rs146695 Gg_rs136083 \\
\hline $0.64 \mathrm{NA}$ & NA & NA & NA & & Gg_rs146695 Gg_rs166674 \\
\hline $0.94 \mathrm{NA}$ & NA & NA & NA & & Gg_rs146695 Gg_rs159733 \\
\hline $0.85 \mathrm{NA}$ & NA & NA & NA & & Gg_rs146695 Gg_rs159781 \\
\hline $0.61 \mathrm{NA}$ & NA & NA & NA & & Gg_rs146695 Gg_rs159733 \\
\hline $0.28 \mathrm{NA}$ & NA & NA & NA & & Gg_rs146695 Gg_rs159733 \\
\hline $0.84 \mathrm{NA}$ & NA & NA & NA & & Gg_rs146695 Gg_rs146717 \\
\hline $0.74 \mathrm{NA}$ & NA & NA & NA & & Gg_rs146695 Gg_rs159733 \\
\hline $0.91 \mathrm{NA}$ & NA & NA & NA & & Gg_rs146695 Gg_rs159733 \\
\hline $0.61 \mathrm{NA}$ & NA & NA & NA & & Gg_rs146695 Gg_rs159733 \\
\hline $0.84 \mathrm{NA}$ & NA & NA & NA & & Gg_rs137670 Gg_rs166674 \\
\hline $0.85 \mathrm{NA}$ & NA & NA & NA & & Gg_rs146695 Gg_rs159781 \\
\hline $0.86 \mathrm{NA}$ & NA & NA & NA & & Gg_rs146695 Gg_rs159733 \\
\hline $0.92 \mathrm{NA}$ & NA & NA & NA & & Gg_rs137670 Gg_rs159781 \\
\hline $0.81 \mathrm{NA}$ & NA & NA & NA & & Gg_rs146695 Gg_rs166674 \\
\hline $0.51 \mathrm{NA}$ & NA & NA & NA & & Gg_rs146695 Gg_rs166674 \\
\hline $0.58 \mathrm{NA}$ & NA & NA & NA & & Gg_rs146695 Gg_rs159733 \\
\hline $1.04 \mathrm{NA}$ & NA & NA & NA & & Gg_rs146695 Gg_rs136083 \\
\hline $0.85 \mathrm{NA}$ & NA & NA & NA & & Gg_rs146695 Gg_rs136083 \\
\hline $1.05 \mathrm{NA}$ & NA & NA & NA & & Gg_rs146695 Gg_rs146717 \\
\hline $1.2 \mathrm{NA}$ & NA & NA & NA & & Gg_rs146695 Gg_rs146717 \\
\hline $1.23 \mathrm{NA}$ & NA & NA & NA & & Gg_rs146695 Gg_rs166674 \\
\hline $0.53 \mathrm{NA}$ & NA & NA & NA & & Gg_rs146695 Gg_rs159733 \\
\hline 0.4 NA & NA & NA & NA & & Gg_rs146695 Gg_rs159733 \\
\hline $1.1 \mathrm{NA}$ & NA & NA & NA & & Gg_rs146695 Gg_rs166674 \\
\hline $0.59 \mathrm{NA}$ & NA & NA & NA & & Gg_rs146661 Gg_rs166674 \\
\hline $0.51 \mathrm{NA}$ & NA & NA & NA & & Gg_rs146695 Gg_rs159733 \\
\hline $0.85 \mathrm{NA}$ & NA & NA & NA & & Gg_rs146695 Gg_rs146717 \\
\hline 1.18 & 0.99 & 1.02 & 2.73 & 1.49 & Gg_rs146695 Gg_rs166674 \\
\hline 0.49 & -0.76 & 0.42 & -0.89 & 0.62 & GG_rs159573Gg_rs166674 \\
\hline $1.19 \mathrm{NA}$ & NA & NA & NA & & Gg_rs146661Gg_rs159733 \\
\hline $0.6 \mathrm{NA}$ & NA & NA & NA & & Gg_rs146695 Gg_rs136083 \\
\hline $0.5 \mathrm{NA}$ & NA & NA & NA & & Gg_rs146695 Gg_rs136083 \\
\hline $0.55 \mathrm{NA}$ & NA & NA & NA & & Gg_rs146695 Gg_rs136083 \\
\hline $0.83 \mathrm{NA}$ & NA & NA & NA & & Gg_rs146661Gg_rs159733 \\
\hline $0.75 \mathrm{NA}$ & NA & NA & NA & & Gg_rs146695 Gg_rs159733 \\
\hline $0.71 \mathrm{NA}$ & NA & NA & NA & & Gg_rs146695 Gg_rs159733 \\
\hline $0.59 \mathrm{NA}$ & NA & NA & NA & & GG_rs159573Gg_rs146717 \\
\hline 0.74 & -2.31 & 0.65 & 2.25 & 0.94 & GG_rs159573Gg_rs146717 \\
\hline $1.26 \mathrm{NA}$ & NA & NA & NA & & Gg_rs137670Gg_rs166674 \\
\hline
\end{tabular}




\begin{tabular}{|c|c|c|c|c|c|}
\hline $0.47 \mathrm{NA}$ & NA & NA & NA & & Gg_rs146661Gg_rs136083 \\
\hline $0.72 \mathrm{NA}$ & NA & NA & NA & & GG_rs159573Gg_rs166674 \\
\hline $0.73 \mathrm{NA}$ & NA & NA & NA & & Gg_rs146661Gg_rs166674 \\
\hline $0.79 \mathrm{NA}$ & NA & NA & NA & & Gg_rs146695 Gg_rs136083 \\
\hline $0.68 \mathrm{NA}$ & NA & NA & NA & & Gg_rs137670 Gg_rs166674 \\
\hline $0.58 \mathrm{NA}$ & NA & NA & NA & & GG_rs159573Gg_rs166674 \\
\hline $0.65 \mathrm{NA}$ & NA & NA & NA & & GG_rs159573Gg_rs159733 \\
\hline $0.51 \mathrm{NA}$ & NA & NA & NA & & GG_rs159573Gg_rs166674 \\
\hline 1.02 & 1.01 & 0.89 & 2.39 & 1.29 & GG_rs159573Gg_rs146717 \\
\hline $0.37 \mathrm{NA}$ & NA & NA & NA & & GG_rs159573Gg_rs146717 \\
\hline $0.42 \mathrm{NA}$ & NA & NA & NA & & GG_rs159573Gg_rs146717 \\
\hline $0.33 \mathrm{NA}$ & NA & NA & NA & & GG_rs159573Gg_rs146717 \\
\hline $0.73 \mathrm{NA}$ & NA & NA & NA & & GG_rs159573Gg_rs166674 \\
\hline $0.88 \mathrm{NA}$ & NA & NA & NA & & GG_rs159573Gg_rs159733 \\
\hline 1.81 & -3.1 & 1.58 & 3.94 & 2.29 & GG_rs159573Gg_rs166674 \\
\hline $0.69 \mathrm{NA}$ & NA & NA & NA & & GG_rs159573Gg_rs159733 \\
\hline $0.57 \mathrm{NA}$ & NA & NA & NA & & GG_rs159573Gg_rs159733 \\
\hline 0.74 & -1.5 & 0.64 & 1.96 & 0.93 & GG_rs159573Gg_rs166674 \\
\hline 1.1 & -2.67 & 0.96 & 3.56 & 1.4 & GG_rs159573Gg_rs146717 \\
\hline $0.63 \mathrm{NA}$ & NA & NA & NA & & GG_rs159573Gg_rs159733 \\
\hline $0.86 \mathrm{NA}$ & NA & NA & NA & & GG_rs159573Gg_rs166674 \\
\hline $0.59 \mathrm{NA}$ & NA & NA & NA & & GG_rs159573Gg_rs159733 \\
\hline $0.75 \mathrm{NA}$ & NA & NA & NA & & GG_rs159573Gg_rs166674 \\
\hline $0.58 \mathrm{NA}$ & NA & NA & NA & & GG_rs159573Gg_rs159781 \\
\hline $0.57 \mathrm{NA}$ & NA & NA & NA & & GG_rs159573Gg_rs166674 \\
\hline $0.61 \mathrm{NA}$ & NA & NA & NA & & GG_rs159573Gg_rs166674 \\
\hline $0.64 \mathrm{NA}$ & NA & NA & NA & & GG_rs159573Gg_rs159733 \\
\hline $0.82 \mathrm{NA}$ & NA & NA & NA & & GG_rs159573Gg_rs159733 \\
\hline $0.74 \mathrm{NA}$ & NA & NA & NA & & GG_rs159573Gg_rs159733 \\
\hline $0.5 \mathrm{NA}$ & NA & NA & NA & & GG_rs159573Gg_rs159781 \\
\hline $0.53 \mathrm{NA}$ & NA & NA & NA & & GG_rs159573Gg_rs159733 \\
\hline $0.91 \mathrm{NA}$ & NA & NA & NA & & GG_rs159573Gg_rs159733 \\
\hline $0.88 \mathrm{NA}$ & NA & NA & NA & & GG_rs159573Gg_rs159733 \\
\hline 1 & -3.59 & 0.87 & 5.51 & 1.26 & GG_rs159573Gg_rs146717 \\
\hline $1.34 \mathrm{NA}$ & NA & NA & NA & & GG_rs159573Gg_rs166674 \\
\hline $0.57 \mathrm{NA}$ & NA & NA & NA & & GG_rs159573Gg_rs159733 \\
\hline $0.56 \mathrm{NA}$ & NA & NA & NA & & GG_rs159573Gg_rs159733 \\
\hline $1.14 \mathrm{NA}$ & NA & NA & NA & & GG_rs159573Gg_rs166674 \\
\hline $0.96 \mathrm{NA}$ & NA & NA & NA & & GG_rs159573Gg_rs166674 \\
\hline $0.44 \mathrm{NA}$ & NA & NA & NA & & GG_rs159573Gg_rs159781 \\
\hline $1.06 \mathrm{NA}$ & NA & NA & NA & & GG_rs159573Gg_rs136083 \\
\hline $1.01 \mathrm{NA}$ & NA & NA & NA & & GG_rs159573Gg_rs166674 \\
\hline $1.01 \mathrm{NA}$ & NA & NA & NA & & Gg_rs146695 Gg_rs166674 \\
\hline
\end{tabular}




\begin{tabular}{|c|c|c|c|c|c|}
\hline 0.86 NA & NA & NA & NA & & GG_rs159573Gg_rs159733 \\
\hline $0.95 \mathrm{NA}$ & NA & NA & NA & & Gg_rs146695 Gg_rs159733 \\
\hline $1.02 \mathrm{NA}$ & NA & NA & NA & & GG_rs159573Gg_rs146717 \\
\hline $0.71 \mathrm{NA}$ & NA & NA & NA & & Gg_rs146695 Gg_rs136083 \\
\hline 1.24 & 0.88 & 1.14 & 3.14 & 1.57 & GG_rs159573Gg_rs146717 \\
\hline 0.89 NA & NA & NA & NA & & GG_rs159573Gg_rs146717 \\
\hline $0.78 \mathrm{NA}$ & NA & NA & NA & & GG_rs159573Gg_rs166674 \\
\hline $1.15 \mathrm{NA}$ & NA & NA & NA & & Gg_rs137670 Gg_rs146717 \\
\hline $0.92 \mathrm{NA}$ & NA & NA & NA & & Gg_rs146695 Gg_rs159781. \\
\hline $1.04 \mathrm{NA}$ & NA & NA & NA & & Gg_rs146695 Gg_rs146717 \\
\hline $0.75 \mathrm{NA}$ & NA & NA & NA & & GG_rs159573Gg_rs159781. \\
\hline $0.53 \mathrm{NA}$ & NA & NA & NA & & GG_rs159573Gg_rs146717 \\
\hline $0.81 \mathrm{NA}$ & NA & NA & NA & & Gg_rs146695 Gg_rs159781. \\
\hline $1.25 \mathrm{NA}$ & NA & NA & NA & & Gg_rs146695 Gg_rs146717 \\
\hline 1.54 & 3.37 & 1.41 & -2.08 & 1.96 & Gg_rs146695 Gg_rs159733 \\
\hline 0.78 NA & NA & NA & NA & & Gg_rs146695 Gg_rs166674 \\
\hline $0.84 \mathrm{NA}$ & NA & NA & NA & & GG_rs159573Gg_rs166674 \\
\hline $0.82 \mathrm{NA}$ & NA & NA & NA & & GG_rs159573Gg_rs166674 \\
\hline 1.26 & -1.99 & 1.15 & 3.23 & 1.6 & GG_rs159573Gg_rs159733 \\
\hline $0.83 \mathrm{NA}$ & NA & NA & NA & & GG_rs159573Gg_rs146717 \\
\hline 1.42 & -2.15 & 1.3 & 4.04 & 1.8 & GG_rs159573Gg_rs146717 \\
\hline 0.84 NA & NA & NA & NA & & GG_rs159573Gg_rs146717 \\
\hline 0.92 NA & NA & NA & NA & & Gg_rs146695 Gg_rs146717 \\
\hline $1.11 \mathrm{NA}$ & NA & NA & NA & & GG_rs159573Gg_rs146717 \\
\hline $1.02 \mathrm{NA}$ & NA & NA & NA & & Gg_rs137670 Gg_rs159781. \\
\hline $0.73 \mathrm{NA}$ & NA & NA & NA & & GG_rs159573Gg_rs146717 \\
\hline $1.44 \mathrm{NA}$ & NA & NA & NA & & Gg_rs146695 Gg_rs159781. \\
\hline $1.01 \mathrm{NA}$ & NA & NA & NA & & GG_rs159573Gg_rs146717 \\
\hline $0.68 \mathrm{NA}$ & NA & NA & NA & & GG_rs159573Gg_rs166674 \\
\hline $1.4 \mathrm{NA}$ & NA & NA & NA & & Gg_rs146695 Gg_rs159781. \\
\hline $0.94 \mathrm{NA}$ & NA & NA & NA & & Gg_rs146695 Gg_rs146717 \\
\hline $0.5 \mathrm{NA}$ & NA & NA & NA & & GG_rs159573Gg_rs136083 \\
\hline $0.68 \mathrm{NA}$ & NA & NA & NA & & GG_rs159573Gg_rs166674 \\
\hline $1.18 \mathrm{NA}$ & NA & NA & NA & & Gg_rs137670 Gg_rs159733 \\
\hline $1.23 \mathrm{NA}$ & NA & NA & NA & & GG_rs159573Gg_rs146717 \\
\hline $0.88 \mathrm{NA}$ & NA & NA & NA & & GG_rs159573Gg_rs166674 \\
\hline $1.02 \mathrm{NA}$ & NA & NA & NA & & Gg_rs146695 Gg_rs136083 \\
\hline $1.15 \mathrm{NA}$ & NA & NA & NA & & GG_rs159573Gg_rs146717 \\
\hline $1.1 \mathrm{NA}$ & NA & NA & NA & & GG_rs159573Gg_rs166674 \\
\hline 0.94 & -0.29 & 0.86 & 3.39 & 1.19 & GG_rs159573Gg_rs166674 \\
\hline 0.63 NA & NA & NA & NA & & GG_rs159573Gg_rs159781. \\
\hline $1.18 \mathrm{NA}$ & NA & NA & NA & & GG_rs159573Gg_rs136083 \\
\hline 0.88 NA & NA & NA & NA & & GG_rs159573Gg_rs136083 \\
\hline
\end{tabular}




\begin{tabular}{|c|c|c|c|c|c|}
\hline $0.85 \mathrm{NA}$ & NA & NA & NA & & GG_rs159573Gg_rs159733 \\
\hline $0.77 \mathrm{NA}$ & NA & NA & NA & & GG_rs159573Gg_rs159733 \\
\hline $1.3 \mathrm{NA}$ & NA & NA & NA & & Gg_rs137670 Gg_rs166674 \\
\hline $0.99 \mathrm{NA}$ & NA & NA & NA & & Gg_rs137670 Gg_rs166674 \\
\hline $1.02 \mathrm{NA}$ & NA & NA & NA & & Gg_rs146695 Gg_rs146717 \\
\hline $0.91 \mathrm{NA}$ & NA & NA & NA & & GG_rs159573Gg_rs159781. \\
\hline $0.63 \mathrm{NA}$ & NA & NA & NA & & GG_rs159573Gg_rs146717 \\
\hline $1.04 \mathrm{NA}$ & NA & NA & NA & & GG_rs159573Gg_rs146717 \\
\hline $1.23 \mathrm{NA}$ & NA & NA & NA & & GG_rs159573Gg_rs159733 \\
\hline $0.82 \mathrm{NA}$ & NA & NA & NA & & GG_rs159573Gg_rs159733 \\
\hline $0.67 \mathrm{NA}$ & NA & NA & NA & & GG_rs159573Gg_rs159733 \\
\hline $0.88 \mathrm{NA}$ & NA & NA & NA & & GG_rs159573Gg_rs159733 \\
\hline $0.79 \mathrm{NA}$ & NA & NA & NA & & Gg_rs146695 Gg_rs159781. \\
\hline $0.91 \mathrm{NA}$ & NA & NA & NA & & GG_rs159573Gg_rs159733 \\
\hline $0.67 \mathrm{NA}$ & NA & NA & NA & & GG_rs159573Gg_rs166674 \\
\hline 0.72 & -0.22 & 0.66 & 2.86 & 0.91 & GG_rs159573Gg_rs159733 \\
\hline 0.72 & -1.57 & 0.66 & -1.73 & 0.91 & GG_rs159573Gg_rs159733 \\
\hline $1.76 \mathrm{NA}$ & NA & NA & NA & & Gg_rs146695 Gg_rs159781. \\
\hline $0.88 \mathrm{NA}$ & NA & NA & NA & & Gg_rs146695 Gg_rs159781. \\
\hline $0.7 \mathrm{NA}$ & NA & NA & NA & & Gg_rs146695 Gg_rs136083 \\
\hline $0.4 \mathrm{NA}$ & NA & NA & NA & & Gg_rs137644 Gg_rs159733 \\
\hline $0.76 \mathrm{NA}$ & NA & NA & NA & & GG_rs159573Gg_rs159733 \\
\hline $0.68 \mathrm{NA}$ & NA & NA & NA & & GG_rs159573Gg_rs159781. \\
\hline $0.8 \mathrm{NA}$ & NA & NA & NA & & GG_rs159573Gg_rs136083 \\
\hline $0.59 \mathrm{NA}$ & NA & NA & NA & & Gg_rs146695 Gg_rs159781. \\
\hline $0.94 \mathrm{NA}$ & NA & NA & NA & & GG_rs159573Gg_rs159781. \\
\hline $0.81 \mathrm{NA}$ & NA & NA & NA & & Gg_rs146695 Gg_rs159781. \\
\hline $0.73 \mathrm{NA}$ & NA & NA & NA & & GG_rs159573Gg_rs159781. \\
\hline $0.97 \mathrm{NA}$ & NA & NA & NA & & GG_rs159573Gg_rs159781. \\
\hline $0.22 \mathrm{NA}$ & NA & NA & NA & & Gg_rs166674 Gg_rs159781. \\
\hline $0.32 \mathrm{NA}$ & NA & NA & NA & & Gg_rs166674 Gg_rs136083 \\
\hline $0.34 \mathrm{NA}$ & NA & NA & NA & & Gg_rs166674 Gg_rs159781. \\
\hline $0.53 \mathrm{NA}$ & NA & NA & NA & & Gg_rs166674 Gg_rs159781. \\
\hline $0.16 \mathrm{NA}$ & NA & NA & NA & & Gg_rs166674 Gg_rs136083 \\
\hline $0.57 \mathrm{NA}$ & NA & NA & NA & & Gg_rs146695 Gg_rs159781 \\
\hline $0.41 \mathrm{NA}$ & NA & NA & NA & & Gg_rs166674 Gg_rs159781 \\
\hline $0.73 \mathrm{NA}$ & NA & NA & NA & & GG_rs159573Gg_rs159781 \\
\hline $0.51 \mathrm{NA}$ & NA & NA & NA & & Gg_rs137670 Gg_rs159781 \\
\hline 0.54 & 2.81 & 0.55 & -1.94 & 0.72 & Gg_rs166674 Gg_rs159781 \\
\hline $0.65 \mathrm{NA}$ & NA & NA & NA & & Gg_rs166674 Gg_rs159781 \\
\hline $0.75 \mathrm{NA}$ & NA & NA & NA & & Gg_rs166674 Gg_rs159781 \\
\hline $0.71 \mathrm{NA}$ & NA & NA & NA & & GG_rs159573Gg_rs159781 \\
\hline $1.39 \mathrm{NA}$ & NA & NA & NA & & Gg_rs146695 Gg_rs159781 \\
\hline
\end{tabular}




\begin{tabular}{|c|c|c|c|c|}
\hline 0.98 & 4.62 & 0.99 & -0.89 & 1.29 GG_rs159573Gg_rs159781. \\
\hline 1 & 4.06 & 1.01 & -1.26 & 1.32 Gg_rs166674 Gg_rs159781. \\
\hline 0.88 & 4.73 & 0.89 & -3.13 & 1.16 Gg_rs166674 Gg_rs159781. \\
\hline 0.76 & 3.19 & 0.77 & -4.32 & 1 Gg_rs166674 Gg_rs159781. \\
\hline 1.37 & 3.73 & 1.38 & -2.24 & 1.81 Gg_rs166674 Gg_rs146817. \\
\hline 0.37 & 0.82 & 0.33 & 0.01 & 0.5 Gg_rs137644 X9_20745674 \\
\hline $0.35 \mathrm{NA}$ & NA & NA & NA & GG_rs159573X9_20745674 \\
\hline 0.93 & 2.96 & 0.85 & -0.46 & 1.26 Gg_rs136083 X9_20745674 \\
\hline $0.78 \mathrm{NA}$ & NA & NA & NA & GG_rs159573X9_20745674 \\
\hline $0.84 \mathrm{NA}$ & NA & NA & NA & GG_rs159573X9_20745674 \\
\hline $0.68 \mathrm{NA}$ & NA & NA & NA & Gg_rs136083 X9_20745674 \\
\hline 0.49 NA & NA & NA & NA & Gg_rs136083 X9_20745674 \\
\hline $0.38 \mathrm{NA}$ & NA & NA & NA & Gg_rs136083 X9_20745674 \\
\hline $0.85 \mathrm{NA}$ & NA & NA & NA & Gg_rs136083 X9_20745674 \\
\hline $0.88 \mathrm{NA}$ & NA & NA & NA & Gg_rs136083 Gg_rs146817. \\
\hline 0.94 NA & NA & NA & NA & Gg_rs159781 Gg_rs146817. \\
\hline $0.68 \mathrm{NA}$ & NA & NA & NA & Gg_rs159781 Gg_rs146817. \\
\hline 1.49 & 2.31 & 1.11 & -1.59 & 1.99 Gg_rs159781 Gg_rs146817. \\
\hline $0.6 \mathrm{NA}$ & NA & NA & NA & Gg_rs159781 Gg_rs146817 \\
\hline $1.21 \mathrm{NA}$ & NA & NA & NA & Gg_rs159781 Gg_rs146817. \\
\hline $0.97 \mathrm{NA}$ & NA & NA & NA & Gg_rs159781 Gg_rs146817. \\
\hline 0.74 NA & NA & NA & NA & Gg_rs159781 Gg_rs146817. \\
\hline $0.81 \mathrm{NA}$ & NA & NA & NA & Gg_rs159781 Gg_rs146817. \\
\hline $0.36 \mathrm{NA}$ & NA & NA & NA & Gg_rs159781 Gg_rs146817. \\
\hline $0.67 \mathrm{NA}$ & NA & NA & NA & Gg_rs159781 Gg_rs146817. \\
\hline 0.52 NA & NA & NA & NA & Gg_rs159781 Gg_rs146817. \\
\hline $0.5 \mathrm{NA}$ & NA & NA & NA & Gg_rs159781 Gg_rs146817. \\
\hline $0.55 \mathrm{NA}$ & NA & NA & NA & Gg_rs159781 Gg_rs146817. \\
\hline $0.58 \mathrm{NA}$ & NA & NA & NA & Gg_rs149412 Gg_rs149416. \\
\hline $0.72 \mathrm{NA}$ & NA & NA & NA & Gg_rs149412 Gg_rs149416. \\
\hline 0.72 NA & NA & NA & NA & Gg_rs149412 Gg_rs149416. \\
\hline $0.92 \mathrm{NA}$ & NA & NA & NA & Gg_rs149412 Gg_rs149416. \\
\hline $1.09 \mathrm{NA}$ & NA & NA & NA & Gg_rs149412 Gg_rs149416. \\
\hline $0.67 \mathrm{NA}$ & NA & NA & NA & Gg_rs149412 Gg_rs149416. \\
\hline $0.64 \mathrm{NA}$ & NA & NA & NA & Gg_rs149412 Gg_rs149416. \\
\hline $0.5 \mathrm{NA}$ & NA & NA & NA & Gg_rs149412 Gg_rs149416. \\
\hline $0.65 \mathrm{NA}$ & NA & NA & NA & Gg_rs149412 Gg_rs149416. \\
\hline $0.67 \mathrm{NA}$ & NA & NA & NA & Gg_rs149412 Gg_rs149416. \\
\hline $0.5 \mathrm{NA}$ & NA & NA & NA & Gg_rs149412 Gg_rs149416. \\
\hline 0.42 NA & NA & NA & NA & Gg_rs149416 Gg_rs140031. \\
\hline 0.59 NA & NA & NA & NA & Gg_rs149416Gg_rs140031. \\
\hline $0.69 \mathrm{NA}$ & NA & NA & NA & Gg_rs149416Gg_rs140031. \\
\hline $0.6 \mathrm{NA}$ & NA & NA & NA & Gg_rs149416 Gg_rs140060 \\
\hline
\end{tabular}




\begin{tabular}{|c|c|c|c|c|c|}
\hline $0.64 \mathrm{NA}$ & NA & NA & NA & & Gg_rs149416Gg_rs140031 \\
\hline $0.68 \mathrm{NA}$ & NA & NA & NA & & Gg_rs149416Gg_rs140031 \\
\hline $0.66 \mathrm{NA}$ & NA & NA & NA & & Gg_rs149416Gg_rs140031 \\
\hline $0.75 \mathrm{NA}$ & NA & NA & NA & & Gg_rs149416Gg_rs140031 \\
\hline $0.44 \mathrm{NA}$ & NA & NA & NA & & Gg_rs149416 Gg_rs140031 \\
\hline $0.48 \mathrm{NA}$ & NA & NA & NA & & Gg_rs149416 Gg_rs140031 \\
\hline $0.45 \mathrm{NA}$ & NA & NA & NA & & Gg_rs149416Gg_rs140031 \\
\hline $0.35 \mathrm{NA}$ & NA & NA & NA & & Gg_rs149416Gg_rs140031 \\
\hline $0.42 \mathrm{NA}$ & NA & NA & NA & & Gg_rs140016Gg_rs140031 \\
\hline $0.42 \mathrm{NA}$ & NA & NA & NA & & Gg_rs149416Gg_rs140031 \\
\hline $0.41 \mathrm{NA}$ & NA & NA & NA & & Gg_rs149416 Gg_rs140031 \\
\hline $0.36 \mathrm{NA}$ & NA & NA & NA & & Gg_rs140016 Gg_rs140031 \\
\hline 1.03 & 0.48 & 1.1 & -4.38 & 1.4 & Gg_rs140016 Gg_rs140031 \\
\hline 1 & 4.09 & 1.06 & -3.25 & 1.35 & Gg_rs149416 Gg_rs140031 \\
\hline $0.39 \mathrm{NA}$ & NA & NA & NA & & Gg_rs149416Gg_rs140031 \\
\hline $0.51 \mathrm{NA}$ & NA & NA & NA & & Gg_rs149416Gg_rs140031 \\
\hline $0.61 \mathrm{NA}$ & NA & NA & NA & & Gg_rs149416Gg_rs140031 \\
\hline $0.78 \mathrm{NA}$ & NA & NA & NA & & Gg_rs149416Gg_rs140031 \\
\hline $0.6 \mathrm{NA}$ & NA & NA & NA & & Gg_rs149416 Gg_rs140031 \\
\hline $0.42 \mathrm{NA}$ & NA & NA & NA & & Gg_rs149416Gg_rs140031 \\
\hline $0.45 \mathrm{NA}$ & NA & NA & NA & & Gg_rs140016Gg_rs140031 \\
\hline $0.45 \mathrm{NA}$ & NA & NA & NA & & Gg_rs140016Gg_rs140031 \\
\hline $0.45 \mathrm{NA}$ & NA & NA & NA & & Gg_rs140016Gg_rs140031 \\
\hline $0.56 \mathrm{NA}$ & NA & NA & NA & & Gg_rs149416Gg_rs140031 \\
\hline $0.54 \mathrm{NA}$ & NA & NA & NA & & Gg_rs149416 Gg_rs140031 \\
\hline $0.63 \mathrm{NA}$ & NA & NA & NA & & Gg_rs140016Gg_rs140031 \\
\hline 0.22 & -0.4 & 0.23 & -0.43 & 0.3 & Gg_rs140016Gg_rs140031 \\
\hline $0.79 \mathrm{NA}$ & NA & NA & NA & & Gg_rs149416Gg_rs140031 \\
\hline $0.52 \mathrm{NA}$ & NA & NA & NA & & Gg_rs140016Gg_rs140031 \\
\hline $0.57 \mathrm{NA}$ & NA & NA & NA & & Gg_rs140016 Gg_rs140031 \\
\hline $0.83 \mathrm{NA}$ & NA & NA & NA & & Gg_rs140016 Gg_rs140060 \\
\hline $0.66 \mathrm{NA}$ & NA & NA & NA & & Gg_rs149416 Gg_rs140060 \\
\hline $0.98 \mathrm{NA}$ & NA & NA & NA & & Gg_rs140016 Gg_rs140060 \\
\hline $0.49 \mathrm{NA}$ & NA & NA & NA & & Gg_rs140016Gg_rs140060 \\
\hline $0.67 \mathrm{NA}$ & NA & NA & NA & & Gg_rs149416Gg_rs140060 \\
\hline $1.21 \mathrm{NA}$ & NA & NA & NA & & Gg_rs149416Gg_rs140060 \\
\hline $0.79 \mathrm{NA}$ & NA & NA & NA & & Gg_rs149416 Gg_rs149477 \\
\hline $0.64 \mathrm{NA}$ & NA & NA & NA & & Gg_rs140016Gg_rs140060 \\
\hline 1.79 & 6.17 & 2.08 & 2.59 & 2.61 & Gg_rs140016Gg_rs140060 \\
\hline 0.9 & -3.78 & 1.05 & -3.3 & 1.31 & Gg_rs140020Gg_rs140060 \\
\hline $0.85 \mathrm{NA}$ & NA & NA & NA & & Gg_rs140020 Gg_rs140060 \\
\hline 0.8 & -3.34 & 0.93 & -2.86 & 1.17 & Gg_rs140020 Gg_rs140060 \\
\hline $0.97 \mathrm{NA}$ & NA & NA & NA & & Gg_rs140020Gg_rs140060 \\
\hline
\end{tabular}




\begin{tabular}{|c|c|c|c|c|c|}
\hline $0.55 \mathrm{NA}$ & NA & NA & NA & & Gg_rs140020X10_798626€ \\
\hline 0.02 & -0.15 & 0.03 & -0.08 & 0.04 & Gg_rs140020 Gg_rs140060. \\
\hline $1.11 \mathrm{NA}$ & NA & NA & NA & & Gg_rs140020 Gg_rs140060. \\
\hline $0.61 \mathrm{NA}$ & NA & NA & NA & & Gg_rs140020 Gg_rs140060. \\
\hline $0.54 \mathrm{NA}$ & NA & NA & NA & & Gg_rs140020 Gg_rs140060. \\
\hline 0.54 & -2.51 & 0.63 & -1.82 & 0.79 & Gg_rs140020Gg_rs140060. \\
\hline 0.73 NA & NA & NA & NA & & Gg_rs140020Gg_rs140060. \\
\hline 0.55 & -2.28 & 0.64 & 1.53 & 0.8 & Gg_rs140020 Gg_rs140060. \\
\hline $0.72 \mathrm{NA}$ & NA & NA & NA & & Gg_rs140020 Gg_rs140060. \\
\hline $0.77 \mathrm{NA}$ & NA & NA & NA & & Gg_rs140020 Gg_rs140060. \\
\hline $1.05 \mathrm{NA}$ & NA & NA & NA & & Gg_rs140016 Gg_rs140060. \\
\hline 0.73 NA & NA & NA & NA & & Gg_rs140020Gg_rs149477 \\
\hline $0.36 \mathrm{NA}$ & NA & NA & NA & & X10_952577વGg_rs149477 \\
\hline $0.72 \mathrm{NA}$ & NA & NA & NA & & Gg_rs140031 GG_rs149515 \\
\hline 0.63 & 0.4 & 0.67 & 3.87 & 0.85 & X10_952577વGg_rs149477 \\
\hline 0.8 & -1.79 & 0.83 & 4.53 & 1.05 & Gg_rs140060 Gg_rs140082. \\
\hline 0.63 & -2.38 & 0.65 & 2.45 & 0.83 & Gg_rs140060 Gg_rs140082. \\
\hline 0.51 & -1.17 & 0.53 & 2.26 & 0.67 & Gg_rs140060 Gg_rs140082. \\
\hline 0.51 & -1.07 & 0.53 & 2.49 & 0.68 & Gg_rs140060 Gg_rs140082. \\
\hline 0.94 & -1.97 & 0.97 & 3.39 & 1.24 & Gg_rs140060 Gg_rs140082. \\
\hline 0.61 & -1.13 & 0.63 & 3.04 & 0.8 & Gg_rs140060 Gg_rs140082. \\
\hline $0.81 \mathrm{NA}$ & NA & NA & NA & & X10_952577૬Gg_rs149498. \\
\hline $1.17 \mathrm{NA}$ & NA & NA & NA & & Gg_rs140020 Gg_rs149498. \\
\hline $0.67 \mathrm{NA}$ & NA & NA & NA & & X10_952577વGG_rs149515 \\
\hline $0.6 \mathrm{NA}$ & NA & NA & NA & & Gg_rs149477 Gg_rs149498. \\
\hline 0.82 & 1.22 & 0.77 & -4.99 & 1.11 & Gg_rs149477 Gg_rs149498. \\
\hline $1.07 \mathrm{NA}$ & NA & NA & NA & & Gg_rs140020GG_rs149515 \\
\hline 0.79 NA & NA & NA & NA & & Gg_rs149477 GG_rs149515 \\
\hline 0.49 NA & NA & NA & NA & & Gg_rs149477 Gg_rs149498. \\
\hline 0.97 & 2.82 & 0.91 & -3.46 & 1.3 & Gg_rs149477 Gg_rs149498. \\
\hline 1.08 & 2.64 & 1.02 & -4.94 & 1.46 & Gg_rs149477 Gg_rs149498. \\
\hline 0.77 NA & NA & NA & NA & & Gg_rs149477 Gg_rs149498. \\
\hline 0.65 & 1.08 & 0.61 & -3.6 & 0.87 & Gg_rs149477 Gg_rs149498. \\
\hline $0.75 \mathrm{NA}$ & NA & NA & NA & & Gg_rs149477 GG_rs149515 \\
\hline $0.21 \mathrm{NA}$ & NA & NA & NA & & Gg_rs149477 GG_rs149515 \\
\hline $1.37 \mathrm{NA}$ & NA & NA & NA & & Gg_rs149477 GG_rs149515 \\
\hline $0.55 \mathrm{NA}$ & NA & NA & NA & & Gg_rs149477 GG_rs149515 \\
\hline 0.47 NA & NA & NA & NA & & Gg_rs149477 GG_rs149515 \\
\hline $0.54 \mathrm{NA}$ & NA & NA & NA & & Gg_rs140082 GG_rs149515 \\
\hline $0.55 \mathrm{NA}$ & NA & NA & NA & & Gg_rs140082 GG_rs149515 \\
\hline $0.27 \mathrm{NA}$ & NA & NA & NA & & Gg_rs140082 GG_rs149515 \\
\hline $0.55 \mathrm{NA}$ & NA & NA & NA & & Gg_rs149477 GG_rs149515 \\
\hline $1.1 \mathrm{NA}$ & NA & NA & NA & & Gg_rs149477 GG_rs149515 \\
\hline
\end{tabular}




\begin{tabular}{|c|c|c|c|c|c|c|c|c|}
\hline $1.32 \mathrm{NA}$ & & NA & & NA & & NA & & Gg_rs149477 GG_rs149515 \\
\hline $0.33 \mathrm{NA}$ & & NA & & NA & & NA & & Gg_rs140082 GG_rs149515 \\
\hline $0.25 \mathrm{NA}$ & & NA & & NA & & NA & & Gg_rs140082 GG_rs149515 \\
\hline $0.48 \mathrm{NA}$ & & NA & & NA & & NA & & Gg_rs140082 GG_rs149515 \\
\hline $2.19 \mathrm{NA}$ & & NA & & NA & & NA & & Gg_rs149477 GG_rs149515 \\
\hline $0.67 \mathrm{NA}$ & & NA & & NA & & NA & & Gg_rs140082 GG_rs149515 \\
\hline $0.74 \mathrm{NA}$ & & NA & & NA & & NA & & Gg_rs140082 GG_rs149515 \\
\hline $0.26 \mathrm{NA}$ & & NA & & NA & & NA & & Gg_rs140082 GG_rs149515 \\
\hline $0.39 \mathrm{NA}$ & & NA & & NA & & NA & & Gg_rs140082 GG_rs149515 \\
\hline $0.58 \mathrm{NA}$ & & NA & & NA & & NA & & Gg_rs149477 GG_rs149515 \\
\hline $0.82 \mathrm{NA}$ & & NA & & NA & & NA & & Gg_rs149477 GG_rs149515 \\
\hline 0.7 & 0.23 & & 0.65 & & -5.24 & & 0.95 & Gg_rs140082 GG_rs149515 \\
\hline 0.19 & 0.46 & & 0.18 & & -0.96 & & 0.26 & Gg_rs140082 GG_rs149515 \\
\hline 1.13 & 1.22 & & 1.18 & & -4.87 & & 1.54 & Gg_rs149498 Gg_rs149523 \\
\hline 0.94 & 1.61 & & 0.98 & & -4.63 & & 1.28 & Gg_rs149498 Gg_rs149523 \\
\hline 1.02 & 0.76 & & 1.06 & & -4.62 & & 1.39 & Gg_rs149498 Gg_rs149523. \\
\hline 0.87 & 0.94 & & 0.91 & & -5.41 & & 1.18 & Gg_rs149498 Gg_rs149523. \\
\hline 0.99 & -0.25 & & 1.03 & & -6.26 & & 1.35 & Gg_rs149498 Gg_rs149523. \\
\hline 2.06 & -3.08 & & 2.15 & & -8.92 & & 2.81 & Gg_rs149498 Gg_rs149523 \\
\hline $0.44 \mathrm{NA}$ & & NA & & NA & & NA & & Gg_rs149498 Gg_rs149523. \\
\hline 1.62 & 1.23 & & 1.69 & & -9.76 & & 2.21 & Gg_rs149498 Gg_rs149523. \\
\hline 0.86 & 0.21 & & 0.89 & & -5.79 & & 1.17 & Gg_rs149498 Gg_rs149523. \\
\hline $0.1 \mathrm{NA}$ & & NA & & NA & & NA & & Gg_rs149498 Gg_rs149523. \\
\hline 1.36 & 0.9 & & 1.42 & & -5.94 & & 1.86 & Gg_rs149498 Gg_rs149523. \\
\hline $0.13 \mathrm{NA}$ & & NA & & NA & & NA & & Gg_rs149579 X11_6450003 \\
\hline $0.34 \mathrm{NA}$ & & NA & & NA & & NA & & Gg_rs149579 X11_6450003 \\
\hline $0.48 \mathrm{NA}$ & & NA & & NA & & NA & & Gg_rs149579 X11_6450003 \\
\hline $0.68 \mathrm{NA}$ & & NA & & NA & & NA & & Gg_rs149579 X11_6450003 \\
\hline 0.65 & 2.34 & & 0.57 & & -0.79 & & 0.86 & Gg_rs149579 X11_6450003 \\
\hline 0.71 & -0.04 & & 0.63 & & -2.7 & & 0.95 & Gg_rs149579 X11_6450003 \\
\hline $1.23 \mathrm{NA}$ & & NA & & NA & & NA & & Gg_rs149579 Gg_rs149618. \\
\hline $0.35 \mathrm{NA}$ & & NA & & NA & & NA & & Gg_rs149579 X11_6450003 \\
\hline $0.45 \mathrm{NA}$ & & NA & & NA & & NA & & Gg_rs149579Gg_rs149630 \\
\hline $18.7 \mathrm{NA}$ & & NA & & NA & & NA & & Gg_rs149579 X11_6450003 \\
\hline $19.96 \mathrm{NA}$ & & NA & & NA & & NA & & Gg_rs149579 X11_6450003 \\
\hline $16.65 \mathrm{NA}$ & & NA & & NA & & NA & & Gg_rs149579 X11_6450003 \\
\hline $0.49 \mathrm{NA}$ & & NA & & NA & & NA & & Gg_rs149579 Gg_rs149618. \\
\hline $0.55 \mathrm{NA}$ & & NA & & NA & & NA & & Gg_rs149579 X11_6450003 \\
\hline $0.93 \mathrm{NA}$ & & NA & & NA & & NA & & Gg_rs149579 Gg_rs149630 \\
\hline $0.19 \mathrm{NA}$ & & NA & & NA & & NA & & Gg_rs149579 Gg_rs149618. \\
\hline 0.92 & 4.11 & & 0.82 & & 3.61 & & 1.23 & X11_4341055X11_6450003 \\
\hline 0.8 & 3.22 & & 0.71 & & 1.12 & & 1.06 & X11_4341055X11_6450003 \\
\hline $0.49 \mathrm{NA}$ & & NA & & NA & & NA & & Gg_rs149579 X11_6450003 \\
\hline
\end{tabular}




\begin{tabular}{|c|c|c|c|c|c|}
\hline $0.29 \mathrm{NA}$ & NA & NA & NA & & X11_4341055X11_6450003 \\
\hline $0.33 \mathrm{NA}$ & NA & NA & NA & & Gg_rs149579 X11_6450003 \\
\hline $0.56 \mathrm{NA}$ & NA & NA & NA & & Gg_rs149579 Gg_rs149630 \\
\hline $0.53 \mathrm{NA}$ & NA & NA & NA & & Gg_rs149579 Gg_rs149630 \\
\hline $0.27 \mathrm{NA}$ & NA & NA & NA & & Gg_rs149579 X11_6450003 \\
\hline $1.16 \mathrm{NA}$ & NA & NA & NA & & Gg_rs149579 Gg_rs149630 \\
\hline $0.62 \mathrm{NA}$ & NA & NA & NA & & Gg_rs149579 Gg_rs149630 \\
\hline 0.93 NA & NA & NA & NA & & Gg_rs149579 Gg_rs149630 \\
\hline $0.07 \mathrm{NA}$ & NA & NA & NA & & Gg_rs149579 Gg_rs149630 \\
\hline $0.58 \mathrm{NA}$ & NA & NA & NA & & X11_4341055Gg_rs149618. \\
\hline $1.11 \mathrm{NA}$ & NA & NA & NA & & Gg_rs149579 Gg_rs149630 \\
\hline $0.1 \mathrm{NA}$ & NA & NA & NA & & Gg_rs149579 Gg_rs149618. \\
\hline 0.48 NA & NA & NA & NA & & Gg_rs149579 Gg_rs149630 \\
\hline $0.26 \mathrm{NA}$ & NA & NA & NA & & Gg_rs149579 Gg_rs149630 \\
\hline $0.57 \mathrm{NA}$ & NA & NA & NA & & Gg_rs149579 Gg_rs149630 \\
\hline $0.17 \mathrm{NA}$ & NA & NA & NA & & Gg_rs149579 Gg_rs149630 \\
\hline $0.51 \mathrm{NA}$ & NA & NA & NA & & Gg_rs149579 Gg_rs140250' \\
\hline $0.13 \mathrm{NA}$ & NA & NA & NA & & Gg_rs149579 Gg_rs149630 \\
\hline $0.32 \mathrm{NA}$ & NA & NA & NA & & X11_4341055Gg_rs149630 \\
\hline 1 & 4.26 & 1.03 & 3.7 & 1.35 & X11_4341055Gg_rs149630 \\
\hline $0.33 \mathrm{NA}$ & NA & NA & NA & & Gg_rs140198 Gg_rs149630 \\
\hline 1 & 3.93 & 1.03 & 3.23 & 1.34 & Gg_rs149579 Gg_rs149630 \\
\hline $0.89 \mathrm{NA}$ & NA & NA & NA & & Gg_rs149579 Gg_rs149630 \\
\hline 0.48 NA & NA & NA & NA & & X11_4341055Gg_rs149630 \\
\hline 0.48 NA & NA & NA & NA & & Gg_rs140198 Gg_rs149630 \\
\hline $0.64 \mathrm{NA}$ & NA & NA & NA & & Gg_rs140198 Gg_rs149630 \\
\hline $0.75 \mathrm{NA}$ & NA & NA & NA & & Gg_rs140198 Gg_rs149630 \\
\hline $0.1 \mathrm{NA}$ & NA & NA & NA & & Gg_rs140198 Gg_rs149630 \\
\hline $0.55 \mathrm{NA}$ & NA & NA & NA & & Gg_rs149618 Gg_rs140250' \\
\hline $1.24 \mathrm{NA}$ & NA & NA & NA & & Gg_rs149618Gg_rs149653. \\
\hline 1.61 & 1.16 & 1.53 & 5.42 & 2.04 & Gg_rs149630 Gg_rs149653. \\
\hline $0.75 \mathrm{NA}$ & NA & NA & NA & & Gg_rs149630 RBL3647 \\
\hline 0.21 & -1.08 & 0.2 & 1.04 & 0.27 & Gg_rs149630 Gg_rs149653. \\
\hline 1.28 & 0.5 & 1.22 & 9.01 & 1.62 & Gg_rs149630 Gg_rs149653. \\
\hline $0.51 \mathrm{NA}$ & NA & NA & NA & & Gg_rs149630 RBL3647 \\
\hline 0.44 NA & NA & NA & NA & & Gg_rs149630 Gg_rs149653. \\
\hline $0.7 \mathrm{NA}$ & NA & NA & NA & & Gg_rs149618 RBL2392 \\
\hline $0.45 \mathrm{NA}$ & NA & NA & NA & & Gg_rs149630 RBL3647 \\
\hline $0.52 \mathrm{NA}$ & NA & NA & NA & & Gg_rs149630 Gg_rs149669 \\
\hline $0.8 \mathrm{NA}$ & NA & NA & NA & & Gg_rs140250 RBL3647 \\
\hline $0.5 \mathrm{NA}$ & NA & NA & NA & & Gg_rs149630 RBL3647 \\
\hline $0.82 \mathrm{NA}$ & NA & NA & NA & & Gg_rs149630 RBL3647 \\
\hline $0.56 \mathrm{NA}$ & NA & NA & NA & & Gg_rs149630 RBL2392 \\
\hline
\end{tabular}




\begin{tabular}{|c|c|c|c|c|c|}
\hline $0.58 \mathrm{NA}$ & NA & NA & NA & & Gg_rs140250 RBL3647 \\
\hline $0.41 \mathrm{NA}$ & NA & NA & NA & & Gg_rs140250 RBL3647 \\
\hline $0.77 \mathrm{NA}$ & NA & NA & NA & & Gg_rs149618 RBL2392 \\
\hline $0.53 \mathrm{NA}$ & NA & NA & NA & & Gg_rs140250 RBL3647 \\
\hline $0.66 \mathrm{NA}$ & NA & NA & NA & & Gg_rs149618 RBL2392 \\
\hline $0.79 \mathrm{NA}$ & NA & NA & NA & & Gg_rs140250 RBL3647 \\
\hline $0.62 \mathrm{NA}$ & NA & NA & NA & & Gg_rs140250 RBL3647 \\
\hline $0.65 \mathrm{NA}$ & NA & NA & NA & & Gg_rs140250 RBL2392 \\
\hline $0.71 \mathrm{NA}$ & NA & NA & NA & & Gg_rs149630 RBL3647 \\
\hline $0.25 \mathrm{NA}$ & NA & NA & NA & & Gg_rs140250 RBL3647 \\
\hline $0.76 \mathrm{NA}$ & NA & NA & NA & & Gg_rs140250 RBL3647 \\
\hline $0.55 \mathrm{NA}$ & NA & NA & NA & & Gg_rs140250 RBL3647 \\
\hline $0.64 \mathrm{NA}$ & NA & NA & NA & & Gg_rs140250 Gg_rs149669 \\
\hline $0.85 \mathrm{NA}$ & NA & NA & NA & & Gg_rs140250 RBL3647 \\
\hline $0.53 \mathrm{NA}$ & NA & NA & NA & & Gg_rs140250 RBL3647 \\
\hline $0.67 \mathrm{NA}$ & NA & NA & NA & & Gg_rs140250 RBL3647 \\
\hline $0.53 \mathrm{NA}$ & NA & NA & NA & & Gg_rs140250 Gg_rs149669 \\
\hline $0.59 \mathrm{NA}$ & NA & NA & NA & & Gg_rs140250 RBL2392 \\
\hline $0.46 \mathrm{NA}$ & NA & NA & NA & & Gg_rs140250 Gg_rs149669 \\
\hline $0.71 \mathrm{NA}$ & NA & NA & NA & & Gg_rs140250 RBL2392 \\
\hline $0.85 \mathrm{NA}$ & NA & NA & NA & & Gg_rs140250 Gg_rs149669 \\
\hline $0.7 \mathrm{NA}$ & NA & NA & NA & & Gg_rs140250 RBL2392 \\
\hline $0.96 \mathrm{NA}$ & NA & NA & NA & & Gg_rs149630 RBL2392 \\
\hline $0.74 \mathrm{NA}$ & NA & NA & NA & & Gg_rs140250 Gg_rs149669 \\
\hline 0.52 & 2.03 & 0.5 & -1.03 & 0.7 & Gg_rs140262 Gg_rs149669 \\
\hline $0.51 \mathrm{NA}$ & NA & NA & NA & & Gg_rs140262 Gg_rs149669 \\
\hline $1.27 \mathrm{NA}$ & NA & NA & NA & & Gg_rs140262 Gg_rs149669 \\
\hline $1.68 \mathrm{NA}$ & NA & NA & NA & & Gg_rs140262 Gg_rs149669 \\
\hline $0.51 \mathrm{NA}$ & NA & NA & NA & & Gg_rs140250 Gg_rs149669 \\
\hline $0.69 \mathrm{NA}$ & NA & NA & NA & & Gg_rs140262 Gg_rs149669 \\
\hline $0.51 \mathrm{NA}$ & NA & NA & NA & & Gg_rs140262 Gg_rs149669 \\
\hline $0.41 \mathrm{NA}$ & NA & NA & NA & & Gg_rs140262 Gg_rs149669 \\
\hline 0.73 & 1.84 & 0.69 & 3.16 & 0.98 & Gg_rs140262 Gg_rs149669 \\
\hline 0.94 NA & NA & NA & NA & & Gg_rs140262 Gg_rs149669 \\
\hline $0.69 \mathrm{NA}$ & NA & NA & NA & & Gg_rs140262 Gg_rs149669 \\
\hline $0.58 \mathrm{NA}$ & NA & NA & NA & & Gg_rs140262 Gg_rs149669 \\
\hline $1.24 \mathrm{NA}$ & NA & NA & NA & & Gg_rs140262 Gg_rs149669 \\
\hline $0.43 \mathrm{NA}$ & NA & NA & NA & & Gg_rs140262 Gg_rs149669 \\
\hline 0.42 & 0.84 & 0.4 & -1.29 & 0.56 & Gg_rs140262 Gg_rs149669 \\
\hline $1.23 \mathrm{NA}$ & NA & NA & NA & & Gg_rs140262 Gg_rs149669 \\
\hline $0.52 \mathrm{NA}$ & NA & NA & NA & & Gg_rs140262 Gg_rs149669 \\
\hline $0.55 \mathrm{NA}$ & NA & NA & NA & & Gg_rs140262 RBL2392 \\
\hline $0.8 \mathrm{NA}$ & NA & NA & NA & & Gg_rs140262 Gg_rs149669 \\
\hline
\end{tabular}




\begin{tabular}{|c|c|c|c|c|c|c|}
\hline $0.37 \mathrm{NA}$ & NA & & NA & NA & & Gg_rs140250 RBL2392 \\
\hline $0.62 \mathrm{NA}$ & NA & & NA & NA & & Gg_rs140262 RBL2392 \\
\hline $0.6 \mathrm{NA}$ & NA & & NA & NA & & Gg_rs149630 RBL2392 \\
\hline 1.49 & 3.53 & 1.41 & & -5.09 & 2 & Gg_rs140262 Gg_rs149669 \\
\hline 1.55 & 2.08 & 1.46 & & -3.47 & 2.08 & Gg_rs140262 Gg_rs149669 \\
\hline $0.67 \mathrm{NA}$ & NA & & NA & NA & & Gg_rs140250 Gg_rs149669 \\
\hline 0.63 NA & NA & & NA & NA & & Gg_rs140250 RBL2392 \\
\hline $1.72 \mathrm{NA}$ & NA & & NA & NA & & Gg_rs140262 RBL2392 \\
\hline $0.43 \mathrm{NA}$ & NA & & NA & NA & & Gg_rs140262 RBL2392 \\
\hline 0.98 & 1.35 & 0.93 & & -0.92 & 1.32 & Gg_rs140262 RBL2392 \\
\hline $0.51 \mathrm{NA}$ & NA & & NA & NA & & Gg_rs140198 RBL2392 \\
\hline $1.03 \mathrm{NA}$ & NA & & NA & NA & & Gg_rs140250 RBL2392 \\
\hline $0.67 \mathrm{NA}$ & NA & & NA & NA & & Gg_rs140262 RBL2392 \\
\hline $0.57 \mathrm{NA}$ & NA & & NA & NA & & Gg_rs149630 RBL2392 \\
\hline 1.3 & 2.48 & 1.22 & & -0.18 & 1.74 & Gg_rs140262 RBL2392 \\
\hline $0.67 \mathrm{NA}$ & NA & & NA & NA & & Gg_rs140262 RBL2392 \\
\hline $0.63 \mathrm{NA}$ & NA & & NA & NA & & Gg_rs149618 RBL2392 \\
\hline 0.54 & 1.6 & 0.51 & & -0.74 & 0.72 & Gg_rs140262 RBL2392 \\
\hline $0.55 \mathrm{NA}$ & NA & & NA & NA & & Gg_rs140262 RBL2392 \\
\hline $0.57 \mathrm{NA}$ & NA & & NA & NA & & Gg_rs140250 RBL2392 \\
\hline $1.17 \mathrm{NA}$ & NA & & NA & NA & & Gg_rs140250 RBL2392 \\
\hline $0.54 \mathrm{NA}$ & NA & & NA & NA & & Gg_rs140262 RBL2392 \\
\hline $0.62 \mathrm{NA}$ & NA & & NA & NA & & Gg_rs140250 RBL2392 \\
\hline $0.56 \mathrm{NA}$ & NA & & NA & NA & & Gg_rs140262 RBL2392 \\
\hline $0.76 \mathrm{NA}$ & NA & & NA & NA & & Gg_rs140250 RBL2392 \\
\hline 0.47 NA & NA & & NA & NA & & Gg_rs140250 RBL2392 \\
\hline $0.6 \mathrm{NA}$ & NA & & NA & NA & & Gg_rs149630 RBL2392 \\
\hline $0.7 \mathrm{NA}$ & NA & & NA & NA & & Gg_rs140250 RBL2392 \\
\hline $0.91 \mathrm{NA}$ & NA & & NA & NA & & RBL3647 RBL2392 \\
\hline 1.1 & -1.24 & 1.03 & & -2.35 & 1.47 & Gg_rs140262 RBL2392 \\
\hline $0.56 \mathrm{NA}$ & NA & & NA & NA & & Gg_rs140262 RBL2392 \\
\hline 0.48 NA & NA & & NA & NA & & Gg_rs140250 RBL2392 \\
\hline 0.65 NA & NA & & NA & NA & & RBL3647 RBL2392 \\
\hline 0.96 & 2.54 & 0.9 & & -2.69 & 1.28 & RBL3647 \\
\hline 0.63 & -1.37 & 0.59 & & -0.56 & 0.83 & RBL3647 \\
\hline $0.7 \mathrm{NA}$ & NA & & NA & NA & & Gg_rs140250 RBL2392 \\
\hline $0.31 \mathrm{NA}$ & NA & & NA & NA & & Gg_rs140262 RBL2392 \\
\hline $0.6 \mathrm{NA}$ & NA & & NA & NA & & Gg_rs140262 RBL2392 \\
\hline $0.57 \mathrm{NA}$ & NA & & NA & NA & & RBL3647 RBL2392 \\
\hline 0.59 NA & NA & & NA & NA & & RBL2392 \\
\hline $0.51 \mathrm{NA}$ & NA & & NA & NA & & RBL2392 \\
\hline $0.58 \mathrm{NA}$ & NA & & NA & NA & & RBL2392 \\
\hline 0.48 NA & NA & & NA & NA & & RBL3647 \\
\hline
\end{tabular}




\begin{tabular}{|c|c|c|c|c|c|c|}
\hline 0.48 NA & NA & NA & NA & & RBL3647 & RBL2392 \\
\hline $0.73 \mathrm{NA}$ & NA & NA & NA & & Gg_rs14026 & 2 RBL2392 \\
\hline 0.47 NA & NA & NA & NA & & Gg_rs14026 & 2 RBL2392 \\
\hline $0.85 \mathrm{NA}$ & NA & NA & NA & & Gg_rs14026 & 2 RBL2392 \\
\hline 0.49 NA & NA & NA & NA & & Gg_rs14026 & 2 RBL2392 \\
\hline 0.49 NA & NA & NA & NA & & Gg_rs14026 & 2 RBL2392 \\
\hline 0.47 NA & NA & NA & NA & & RBL3647 & RBL2392 \\
\hline 0.03 NA & NA & NA & NA & & RBL3647 & RBL2392 \\
\hline $0.46 \mathrm{NA}$ & NA & NA & NA & & Gg_rs14026 & 2 RBL2392 \\
\hline $0.41 \mathrm{NA}$ & NA & NA & NA & & RBL3647 & RBL2392 \\
\hline 0.69 NA & NA & NA & NA & & RBL3647 & RBL2392 \\
\hline 0.99 NA & NA & NA & NA & & RBL3647 & RBL2392 \\
\hline $0.52 \mathrm{NA}$ & NA & NA & NA & & RBL3647 & RBL2392 \\
\hline 0.18 & -0.4 & 0.17 & 0.13 & 0.24 & RBL3647 & RBL2392 \\
\hline 0.83 NA & NA & NA & NA & & RBL3647 & RBL2392 \\
\hline $0.61 \mathrm{NA}$ & NA & NA & NA & & RBL3647 & RBL2392 \\
\hline 0.47 NA & NA & NA & NA & & RBL3647 & RBL2392 \\
\hline $0.42 \mathrm{NA}$ & NA & NA & NA & & RBL3647 & RBL2392 \\
\hline $0.81 \mathrm{NA}$ & NA & NA & NA & & RBL3647 & RBL2392 \\
\hline $0.78 \mathrm{NA}$ & NA & NA & NA & & RBL3647 & RBL2392 \\
\hline 0.42 NA & NA & NA & NA & & RBL3647 & RBL2392 \\
\hline $0.76 \mathrm{NA}$ & NA & NA & NA & & RBL3647 & RBL2392 \\
\hline $0.68 \mathrm{NA}$ & NA & NA & NA & & RBL3647 & RBL2392 \\
\hline 0.64 NA & NA & NA & NA & & RBL3647 & RBL2392 \\
\hline 0.92 NA & NA & NA & NA & & RBL3647 & RBL2392 \\
\hline $0.52 \mathrm{NA}$ & NA & NA & NA & & RBL3647 & RBL2392 \\
\hline $1.11 \mathrm{NA}$ & NA & NA & NA & & RBL3647 & RBL2392 \\
\hline 0.68 NA & NA & NA & NA & & RBL3647 & RBL2392 \\
\hline 0.36 & -1.61 & 0.34 & 1.17 & 0.48 & RBL3647 & RBL2392 \\
\hline 0.39 NA & NA & NA & NA & & RBL3647 & RBL2392 \\
\hline 0.38 NA & NA & NA & NA & & RBL3647 & RBL2392 \\
\hline 0.41 NA & NA & NA & NA & & RBL3647 & RBL2392 \\
\hline 0.38 NA & NA & NA & NA & & RBL3647 & RBL2392 \\
\hline $0.38 \mathrm{NA}$ & NA & NA & NA & & RBL3647 & RBL2392 \\
\hline 0.63 NA & NA & NA & NA & & RBL3647 & RBL2392 \\
\hline 0.16 NA & NA & NA & NA & & RBL3647 & RBL2392 \\
\hline $0.51 \mathrm{NA}$ & NA & NA & NA & & RBL3647 & RBL2392 \\
\hline 0.34 NA & NA & NA & NA & & RBL3647 & RBL2392 \\
\hline 0.44 NA & NA & NA & NA & & RBL3647 & RBL2392 \\
\hline $0.34 \mathrm{NA}$ & NA & NA & NA & & RBL3647 & RBL2392 \\
\hline $0.7 \mathrm{NA}$ & NA & NA & NA & & RBL3647 & RBL2392 \\
\hline $0.4 \mathrm{NA}$ & NA & NA & NA & & RBL3647 & RBL2392 \\
\hline $0.52 \mathrm{NA}$ & NA & NA & NA & & Gg_rs14025 & 0 RBL2392 \\
\hline
\end{tabular}




\begin{tabular}{|c|c|c|c|c|c|c|c|}
\hline 0.67 NA & NA & & NA & NA & & RBL3647 & RBL2392 \\
\hline 0.6 & -1.78 & 0.56 & & 1.05 & 0.79 & RBL3647 & RBL2392 \\
\hline 0.81 & 2.88 & 0.82 & & 0.91 & 1.11 & Gg_rs14966 & 69 RBL2392 \\
\hline $0.6 \mathrm{NA}$ & NA & & NA & NA & & Gg_rs14031 & 12 Gg_rs149711 \\
\hline $0.68 \mathrm{NA}$ & NA & & NA & NA & & Gg_rs14031 & 12 Gg_rs149711 \\
\hline 0.69 NA & NA & & NA & NA & & Gg_rs14031 & 12 Gg_rs149711 \\
\hline $9.27 \mathrm{NA}$ & NA & & NA & NA & & Gg_rs14031 & 12 Gg_rs149711 \\
\hline $9.58 \mathrm{NA}$ & NA & & NA & NA & & Gg_rs14031 & 12 Gg_rs149711 \\
\hline $8.53 \mathrm{NA}$ & NA & & NA & NA & & Gg_rs14031 & 12 Gg_rs149711 \\
\hline 8.73 NA & NA & & NA & NA & & Gg_rs14031 & 12 Gg_rs149711 \\
\hline 8.16 NA & NA & & NA & NA & & Gg_rs14031 & 12 Gg_rs149711 \\
\hline 0.48 NA & NA & & NA & NA & & Gg_rs1497c & 06 Gg_rs136214 \\
\hline $1.45 \mathrm{NA}$ & NA & & NA & NA & & Gg_rs1497c & 06 Gg_rs136214 \\
\hline $1.3 \mathrm{NA}$ & NA & & NA & NA & & Gg_rs1497C & 06 Gg_rs136214 \\
\hline $2.22 \mathrm{NA}$ & NA & & NA & NA & & Gg_rs1497C & 06 Gg_rs136214 \\
\hline $1 \mathrm{NA}$ & NA & & NA & NA & & Gg_rs1497C & 06 Gg_rs136214 \\
\hline $1.75 \mathrm{NA}$ & NA & & NA & NA & & Gg_rs1497C & 06 Gg_rs136214 \\
\hline $0.53 \mathrm{NA}$ & NA & & NA & NA & & Gg_rs1497C & 06 Gg_rs136094 \\
\hline 0.35 & -0.16 & 0.35 & & -0.37 & 0.45 & Gg_rs14031 & 12 Gg_rs149849 \\
\hline $0.29 \mathrm{NA}$ & NA & & NA & NA & & Gg_rs1497C & 06 Gg_rs136094 \\
\hline $2.69 \mathrm{NA}$ & NA & & NA & NA & & Gg_rs1497C & 06 Gg_rs136094 \\
\hline $2.48 \mathrm{NA}$ & NA & & NA & NA & & Gg_rs1497C & 06 Gg_rs136094 \\
\hline $2.83 \mathrm{NA}$ & NA & & NA & NA & & Gg_rs1497C & 06 Gg_rs136094 \\
\hline $0.31 \mathrm{NA}$ & NA & & NA & NA & & Gg_rs1497C & 06 Gg_rs136094 \\
\hline $2.69 \mathrm{NA}$ & NA & & NA & NA & & Gg_rs1497C & 06 Gg_rs136094 \\
\hline $1.48 \mathrm{NA}$ & NA & & NA & NA & & Gg_rs1497c & 06 Gg_rs136094 \\
\hline $2.44 \mathrm{NA}$ & NA & & NA & NA & & Gg_rs1497C & 06 Gg_rs136094 \\
\hline $2.92 \mathrm{NA}$ & NA & & NA & NA & & Gg_rs1497C & 06 Gg_rs136094 \\
\hline 0.48 NA & NA & & NA & NA & & Gg_rs14971 & 11 Gg_rs136094 \\
\hline 0.86 NA & NA & & NA & NA & & Gg_rs1497C & 06 Gg_rs149745 \\
\hline 0.92 & 1.23 & 0.87 & & 3.2 & 1.18 & Gg_rs14971 & 11 Gg_rs136094 \\
\hline $0.13 \mathrm{NA}$ & NA & & NA & NA & & Gg_rs14971 & 11 Gg_rs136094 \\
\hline $2.01 \mathrm{NA}$ & NA & & NA & NA & & Gg_rs1497c & 06 Gg_rs136094 \\
\hline $1.4 \mathrm{NA}$ & NA & & NA & NA & & Gg_rs1497c & 06 Gg_rs149745 \\
\hline $0.75 \mathrm{NA}$ & NA & & NA & NA & & Gg_rs1497: & 11 Gg_rs149745 \\
\hline $0.44 \mathrm{NA}$ & NA & & NA & NA & & Gg_rs1497: & 11 Gg_rs149745 \\
\hline 0.36 NA & NA & & NA & NA & & Gg_rs1362: & 14 Gg_rs149745 \\
\hline $0.32 \mathrm{NA}$ & NA & & NA & NA & & Gg_rs1362: & 14 Gg_rs149745 \\
\hline 0.42 NA & NA & & NA & NA & & Gg_rs1362: & 14 Gg_rs149745 \\
\hline $0.5 \mathrm{NA}$ & NA & & NA & NA & & Gg_rs1362: & 14 Gg_rs149745 \\
\hline $0.21 \mathrm{NA}$ & NA & & NA & NA & & Gg_rs1362: & 14 Gg_rs149745 \\
\hline 0.49 NA & NA & & NA & NA & & Gg_rs1362: & 14 Gg_rs149745 \\
\hline $0.78 \mathrm{NA}$ & NA & & NA & NA & & Gg_rs1362: & 14 Gg_rs149745 \\
\hline
\end{tabular}




\begin{tabular}{|c|c|c|c|c|c|}
\hline 0.19 & 0.68 & 0.19 & 0.51 & 0.25 & Gg_rs136214 Gg_rs149745 \\
\hline $0.62 \mathrm{NA}$ & NA & NA & NA & & Gg_rs136214 Gg_rs149745 \\
\hline $0.08 \mathrm{NA}$ & NA & NA & NA & & Gg_rs136214 Gg_rs149745 \\
\hline $0.45 \mathrm{NA}$ & NA & NA & NA & & Gg_rs136214 Gg_rs149745. \\
\hline $0.31 \mathrm{NA}$ & NA & NA & NA & & Gg_rs136214Gg_rs149745. \\
\hline 1.19 & -4.28 & 1.21 & -3.99 & 1.59 & Gg_rs136214 Gg_rs149745. \\
\hline 1.06 & -3.03 & 1.08 & -3.4 & 1.41 & Gg_rs136214 Gg_rs149745 \\
\hline 0.69 & -2.83 & 0.7 & -3.62 & 0.92 & Gg_rs136214 Gg_rs149745. \\
\hline $0.16 \mathrm{NA}$ & NA & NA & NA & & Gg_rs140384 X12_140511€ \\
\hline $0.14 \mathrm{NA}$ & NA & NA & NA & & Gg_rs140384 X12_140511€ \\
\hline $0.67 \mathrm{NA}$ & NA & NA & NA & & Gg_rs140384 X12_140511€ \\
\hline $0.33 \mathrm{NA}$ & NA & NA & NA & & Gg_rs140384 Gg_rs149803. \\
\hline $0.5 \mathrm{NA}$ & NA & NA & NA & & Gg_rs140384 Gg_rs156655. \\
\hline 0.14 & 0.41 & 0.13 & -0.38 & 0.18 & Gg_rs140384 Gg_rs149803. \\
\hline $0.1 \mathrm{NA}$ & NA & NA & NA & & Gg_rs140384 Gg_rs149803. \\
\hline $0.26 \mathrm{NA}$ & NA & NA & NA & & Gg_rs140384 X12_140511€ \\
\hline 0.11 & -0.42 & 0.11 & 0.6 & 0.15 & Gg_rs149791 X12_140511€ \\
\hline 0.14 & -0.19 & 0.14 & 0.94 & 0.19 & Gg_rs149791 X12_140511€ \\
\hline 0.64 & 1.74 & 0.62 & 2.27 & 0.86 & Gg_rs140384 X12_140511€ \\
\hline $0.22 \mathrm{NA}$ & NA & NA & NA & & Gg_rs140408 X12_140511€ \\
\hline $0.6 \mathrm{NA}$ & NA & NA & NA & & Gg_rs140384 X12_140511€ \\
\hline 0.58 & -0.5 & 0.56 & 2.14 & 0.78 & Gg_rs149791 X12_140511€ \\
\hline 0.11 & -0.23 & 0.11 & 0.42 & 0.15 & Gg_rs140408 X12_140511€ \\
\hline $0.15 \mathrm{NA}$ & NA & NA & NA & & Gg_rs140408 X12_140511€ \\
\hline 0.19 & -0.73 & 0.19 & 0.97 & 0.26 & Gg_rs149791 X12_140511€ \\
\hline 0.54 & 2.12 & 0.52 & -1.36 & 0.72 & Gg_rs140408 X12_140511€ \\
\hline $0.37 \mathrm{NA}$ & NA & NA & NA & & Gg_rs140408 X12_140511€ \\
\hline $0.68 \mathrm{NA}$ & NA & NA & NA & & Gg_rs149803 Gg_rs156655. \\
\hline $1.15 \mathrm{NA}$ & NA & NA & NA & & Gg_rs149803 Gg_rs156655. \\
\hline $1.26 \mathrm{NA}$ & NA & NA & NA & & X12_140511EGg_rs149849: \\
\hline $0.13 \mathrm{NA}$ & NA & NA & NA & & X12_140511EGg_rs149849: \\
\hline $0.77 \mathrm{NA}$ & NA & NA & NA & & X12_140511EGg_rs149849: \\
\hline 0.44 NA & NA & NA & NA & & X12_140511EGg_rs149849: \\
\hline $0.3 \mathrm{NA}$ & NA & NA & NA & & X12_140511EGg_rs149849: \\
\hline $0.85 \mathrm{NA}$ & NA & NA & NA & & X12_140511EGg_rs149849: \\
\hline $0.6 \mathrm{NA}$ & NA & NA & NA & & X12_140511EGg_rs149849: \\
\hline $0.72 \mathrm{NA}$ & NA & NA & NA & & X12_140511EGg_rs149849: \\
\hline $0.77 \mathrm{NA}$ & NA & NA & NA & & X12_140511EGg_rs149849: \\
\hline $0.97 \mathrm{NA}$ & NA & NA & NA & & Gg_rs156655 Gg_rs149849: \\
\hline $1.47 \mathrm{NA}$ & NA & NA & NA & & X12_140511EGg_rs149849: \\
\hline $1 \mathrm{NA}$ & NA & NA & NA & & X12_140511EGg_rs149849: \\
\hline $0.67 \mathrm{NA}$ & NA & NA & NA & & Gg_rs156655 Gg_rs149849: \\
\hline $1.05 \mathrm{NA}$ & NA & NA & NA & & X12_140511EGg_rs149849 \\
\hline
\end{tabular}




\begin{tabular}{|c|c|c|c|c|c|c|c|}
\hline $1.32 \mathrm{NA}$ & NA & & NA & & NA & & X12_140511EGg_rs149849: \\
\hline 0.89 NA & NA & & NA & & NA & & X12_140511EGg_rs149849: \\
\hline $1.55 \mathrm{NA}$ & NA & & NA & & NA & & X12_140511EGg_rs149849: \\
\hline 1.02 & 2 & 0.88 & & 1.15 & & 1.29 & X12_140511EGg_rs149849: \\
\hline 1.08 & -1.47 & 0.92 & & -0.67 & & 1.36 & X12_140511EGg_rs149849: \\
\hline $0.9 \mathrm{NA}$ & NA & & NA & & NA & & Gg_rs156655 Gg_rs149849: \\
\hline $0.74 \mathrm{NA}$ & NA & & NA & & NA & & Gg_rs156655 Gg_rs149849: \\
\hline 0.69 NA & NA & & NA & & NA & & Gg_rs156655 Gg_rs149849: \\
\hline 0.73 & 1.53 & 0.63 & & 3.62 & & 0.92 & Gg_rs156655 Gg_rs149849: \\
\hline 3.32 & 3.45 & 3.49 & & -6.89 & & 4.31 & Gg_rs149872 Gg_rs140568 \\
\hline 2.73 & 2.93 & 2.87 & & -5.29 & & 3.54 & Gg_rs149872 Gg_rs140568 \\
\hline 2.97 & 0.47 & 3.12 & & -4.3 & & 3.85 & Gg_rs149872 Gg_rs140568 \\
\hline 1.73 & -0.73 & 1.81 & & -2.81 & & 2.24 & Gg_rs149872 Gg_rs140568 \\
\hline 1.21 & -0.84 & 1.28 & & -0.77 & & 1.57 & Gg_rs149872 Gg_rs140568 \\
\hline $1.9 \mathrm{NA}$ & NA & & NA & & NA & & Gg_rs149872 Gg_rs140568 \\
\hline $1.87 \mathrm{NA}$ & NA & & NA & & NA & & Gg_rs149872 Gg_rs140568 \\
\hline $1.81 \mathrm{NA}$ & NA & & NA & & NA & & Gg_rs149872 Gg_rs140568 \\
\hline $0.36 \mathrm{NA}$ & NA & & NA & & NA & & Gg_rs149872 Gg_rs140568 \\
\hline $0.47 \mathrm{NA}$ & NA & & NA & & NA & & Gg_rs149872 Gg_rs140568 \\
\hline 0.56 NA & NA & & NA & & NA & & Gg_rs149872 Gg_rs140568 \\
\hline $2.11 \mathrm{NA}$ & NA & & NA & & NA & & Gg_rs149872 Gg_rs140568 \\
\hline 0.7 & -0.74 & 0.73 & & -0.94 & & 0.9 & Gg_rs149872 Gg_rs140568 \\
\hline $2.16 \mathrm{NA}$ & NA & & NA & & NA & & Gg_rs149872 Gg_rs140568 \\
\hline $0.77 \mathrm{NA}$ & NA & & NA & & NA & & Gg_rs149872 Gg_rs140568 \\
\hline $1.58 \mathrm{NA}$ & NA & & NA & & NA & & Gg_rs149872 Gg_rs140568 \\
\hline 2.34 & 1.05 & 2.46 & & -6.1 & & 3.04 & Gg_rs149872 Gg_rs140568 \\
\hline 1.43 & 1.13 & 1.5 & & -4.66 & & 1.85 & Gg_rs149872 Gg_rs140568 \\
\hline 2.52 & 3.27 & 2.65 & & -3.81 & & 3.27 & Gg_rs149872 Gg_rs140568 \\
\hline 2.13 & 1.4 & 2.24 & & -5.29 & & 2.76 & Gg_rs149872 Gg_rs140568 \\
\hline $1.41 \mathrm{NA}$ & NA & & NA & & NA & & Gg_rs149872 Gg_rs140568 \\
\hline $2.06 \mathrm{NA}$ & NA & & NA & & NA & & Gg_rs149872 Gg_rs140568 \\
\hline $1.43 \mathrm{NA}$ & NA & & NA & & NA & & Gg_rs149872 Gg_rs140568 \\
\hline $1.7 \mathrm{NA}$ & NA & & NA & & NA & & Gg_rs149872 Gg_rs140568 \\
\hline $0.86 \mathrm{NA}$ & NA & & NA & & NA & & Gg_rs149872 Gg_rs140568 \\
\hline $0.9 \mathrm{NA}$ & NA & & NA & & NA & & Gg_rs149872 Gg_rs140568 \\
\hline 1.67 & 1.01 & 1.76 & & -2.33 & & 2.17 & Gg_rs149872 Gg_rs140568 \\
\hline $1.72 \mathrm{NA}$ & NA & & NA & & NA & & Gg_rs149872 Gg_rs140568 \\
\hline 2.36 & 1.47 & 2.48 & & -2.13 & & 3.05 & Gg_rs149872 Gg_rs140568 \\
\hline $1.32 \mathrm{NA}$ & NA & & NA & & NA & & Gg_rs149872 Gg_rs140568 \\
\hline $0.68 \mathrm{NA}$ & NA & & NA & & NA & & Gg_rs140568Gg_rs140586 \\
\hline 0.82 & 4.69 & 0.86 & & -0.62 & & 1.07 & Gg_rs140568X13_555545c \\
\hline $1.24 \mathrm{NA}$ & NA & & NA & & NA & & Gg_rs140568 X13_906174C \\
\hline $0.62 \mathrm{NA}$ & NA & & NA & & NA & & Gg_rs140568 X13_5555459 \\
\hline
\end{tabular}




\begin{tabular}{|c|c|c|c|c|c|}
\hline $0.6 \mathrm{NA}$ & NA & NA & NA & & Gg_rs140568 Gg_rs149956 \\
\hline 0.89 & -1.26 & 0.92 & 2.67 & 1.15 & Gg_rs140568 Gg_rs149956 \\
\hline $0.82 \mathrm{NA}$ & NA & NA & NA & & Gg_rs140568 Gg_rs140586 \\
\hline $1.2 \mathrm{NA}$ & NA & NA & NA & & Gg_rs140568X13_8702725 \\
\hline 0.89 & 4.01 & 0.91 & 0.98 & 1.2 & Gg_rs140568X13_8702725 \\
\hline $0.53 \mathrm{NA}$ & NA & NA & NA & & Gg_rs149910Gg_rs149956. \\
\hline $0.36 \mathrm{NA}$ & NA & NA & NA & & Gg_rs149910 Gg_rs140586 \\
\hline $0.55 \mathrm{NA}$ & NA & NA & NA & & Gg_rs149910Gg_rs140586 \\
\hline $0.81 \mathrm{NA}$ & NA & NA & NA & & Gg_rs140568 Gg_rs149956 \\
\hline $0.67 \mathrm{NA}$ & NA & NA & NA & & Gg_rs149910Gg_rs140586 \\
\hline $0.81 \mathrm{NA}$ & NA & NA & NA & & Gg_rs140568 Gg_rs140586 \\
\hline 0.44 NA & NA & NA & NA & & Gg_rs140568 Gg_rs149956. \\
\hline 0.84 NA & NA & NA & NA & & Gg_rs149910Gg_rs140586 \\
\hline $0.29 \mathrm{NA}$ & NA & NA & NA & & Gg_rs149910Gg_rs140586 \\
\hline $0.51 \mathrm{NA}$ & NA & NA & NA & & Gg_rs149910Gg_rs140586 \\
\hline $1.15 \mathrm{NA}$ & NA & NA & NA & & Gg_rs149910 Gg_rs140586 \\
\hline 0.73 NA & NA & NA & NA & & Gg_rs149910X13_129794€ \\
\hline $1.18 \mathrm{NA}$ & NA & NA & NA & & Gg_rs140568 Gg_rs149956. \\
\hline $0.41 \mathrm{NA}$ & NA & NA & NA & & Gg_rs140568 Gg_rs149956 \\
\hline 0.73 NA & NA & NA & NA & & Gg_rs149910X13_129794€ \\
\hline 0.02 NA & NA & NA & NA & & Gg_rs149910Gg_rs140586 \\
\hline $0.54 \mathrm{NA}$ & NA & NA & NA & & Gg_rs149910Gg_rs149956. \\
\hline $0.57 \mathrm{NA}$ & NA & NA & NA & & Gg_rs149910Gg_rs149956. \\
\hline 0.79 & -0.08 & 0.81 & 4.59 & 1.07 & 'Gg_rs149910Gg_rs140586 \\
\hline 0.79 & -1.45 & 0.86 & 5.12 & 1.16 & Gg_rs149910Gg_rs149956 \\
\hline 0.97 NA & NA & NA & NA & & Gg_rs149910X13_129794€ \\
\hline 0.72 NA & NA & NA & NA & & X13_555545CGg_rs149956. \\
\hline $0.64 \mathrm{NA}$ & NA & NA & NA & & Gg_rs149910Gg_rs149956. \\
\hline $1 \mathrm{NA}$ & NA & NA & NA & & X13_555545@Gg_rs149956 \\
\hline 0.44 NA & NA & NA & NA & & X13_555545CGg_rs149956. \\
\hline $0.77 \mathrm{NA}$ & NA & NA & NA & & X13_555545CGg_rs149956. \\
\hline $0.61 \mathrm{NA}$ & NA & NA & NA & & Gg_rs149910Gg_rs149956. \\
\hline 0.72 & 4.29 & 0.83 & -4.42 & 1.06 & X13_555545કGg_rs149956 \\
\hline 0.81 & 4.21 & 0.93 & -6.2 & 1.2 & X13_555545@Gg_rs149956 \\
\hline $0.81 \mathrm{NA}$ & NA & NA & NA & & Gg_rs149910Gg_rs149956. \\
\hline $0.69 \mathrm{NA}$ & NA & NA & NA & & Gg_rs140568X13_129794€ \\
\hline $0.91 \mathrm{NA}$ & NA & NA & NA & & Gg_rs149910X13_129794€ \\
\hline 0.46 NA & NA & NA & NA & & X13_555545CGg_rs140628. \\
\hline $1.14 \mathrm{NA}$ & NA & NA & NA & & Gg_rs140586Gg_rs140628. \\
\hline $0.54 \mathrm{NA}$ & NA & NA & NA & & X13_555545ऽGg_rs140628 \\
\hline 1.23 & -0.84 & 1.31 & 3.41 & 1.75 & Gg_rs140586 X13_129794€ \\
\hline $0.81 \mathrm{NA}$ & NA & NA & NA & & Gg_rs140586 X13_129794€ \\
\hline $0.63 \mathrm{NA}$ & NA & NA & NA & & Gg_rs140586 X13_129794€ \\
\hline
\end{tabular}




\begin{tabular}{|c|c|c|c|c|c|c|c|}
\hline $0.82 \mathrm{NA}$ & NA & & NA & & NA & & Gg_rs140586 X13_1297946 \\
\hline 0.69 NA & NA & & NA & & NA & & Gg_rs140586 X13_1297946 \\
\hline 0.79 & 0.83 & 0.85 & & 2.03 & & 1.14 & Gg_rs140586 X13_1297946 \\
\hline $1.01 \mathrm{NA}$ & NA & & NA & & NA & & Gg_rs140586X13_1297946 \\
\hline $1.05 \mathrm{NA}$ & NA & & NA & & NA & & Gg_rs140586 X13_1297946 \\
\hline $0.74 \mathrm{NA}$ & NA & & NA & & NA & & Gg_rs140586 X13_1297946 \\
\hline $0.78 \mathrm{NA}$ & NA & & NA & & NA & & Gg_rs140586 X13_1297946 \\
\hline $1.04 \mathrm{NA}$ & NA & & NA & & NA & & Gg_rs140586 X13_1297946 \\
\hline $0.8 \mathrm{NA}$ & NA & & NA & & NA & & Gg_rs140586 X13_1297946 \\
\hline $0.78 \mathrm{NA}$ & NA & & NA & & NA & & Gg_rs140586 Gg_rs140628 \\
\hline $0.62 \mathrm{NA}$ & NA & & NA & & NA & & Gg_rs140586 X13_1297946 \\
\hline $0.79 \mathrm{NA}$ & NA & & NA & & NA & & Gg_rs140586 Gg_rs140628 \\
\hline $0.77 \mathrm{NA}$ & NA & & NA & & NA & & Gg_rs140586 X13_1297946 \\
\hline $0.47 \mathrm{NA}$ & NA & & NA & & NA & & Gg_rs140586 X13_1297946 \\
\hline 0.49 NA & NA & & NA & & NA & & Gg_rs140586 X13_1297946 \\
\hline 0.43 NA & NA & & NA & & NA & & Gg_rs140586 X13_1297946 \\
\hline $0.59 \mathrm{NA}$ & NA & & NA & & NA & & Gg_rs140586 X13_1297946 \\
\hline $0.48 \mathrm{NA}$ & NA & & NA & & NA & & Gg_rs140586 X13_1297946 \\
\hline $0.58 \mathrm{NA}$ & NA & & NA & & NA & & Gg_rs140586 X13_1297946 \\
\hline $0.46 \mathrm{NA}$ & NA & & NA & & NA & & Gg_rs140586 X13_1297946 \\
\hline $1.08 \mathrm{NA}$ & NA & & NA & & NA & & Gg_rs140586 Gg_rs140628 \\
\hline 0.48 NA & NA & & NA & & NA & & Gg_rs140586 X13_1297946 \\
\hline $0.73 \mathrm{NA}$ & NA & & NA & & NA & & Gg_rs140586 X13_1297946 \\
\hline 0.76 NA & NA & & NA & & NA & & Gg_rs140586Gg_rs140628 \\
\hline $0.45 \mathrm{NA}$ & NA & & NA & & NA & & Gg_rs140586 X13_1297946 \\
\hline $0.65 \mathrm{NA}$ & NA & & NA & & NA & & Gg_rs140586 X13_1297946 \\
\hline $0.51 \mathrm{NA}$ & NA & & NA & & NA & & Gg_rs140586 X13_1297946 \\
\hline $0.64 \mathrm{NA}$ & NA & & NA & & NA & & Gg_rs140586 Gg_rs140628 \\
\hline $0.68 \mathrm{NA}$ & NA & & NA & & NA & & Gg_rs140586Gg_rs140628 \\
\hline $1.05 \mathrm{NA}$ & NA & & NA & & NA & & Gg_rs140586 Gg_rs140628 \\
\hline $2.26 \mathrm{NA}$ & NA & & NA & & NA & & Gg_rs149956 Gg_rs140628 \\
\hline $1.3 \mathrm{NA}$ & NA & & NA & & NA & & Gg_rs140586 Gg_rs140628 \\
\hline 0.07 & -0.24 & 0.07 & & 0.46 & & 0.11 & Gg_rs149956:Gg_rs140628 \\
\hline 2.57 & 3.17 & 2.41 & & 5.94 & & 4.11 & Gg_rs149956.Gg_rs140628 \\
\hline 0.07 & -0.31 & 0.06 & & 0.46 & & 0.11 & Gg_rs149956.Gg_rs140628 \\
\hline $0.01 \mathrm{NA}$ & NA & & NA & & NA & & Gg_rs149956.Gg_rs140628 \\
\hline 0.91 & -1.83 & 0.85 & & 4.33 & & 1.46 & Gg_rs149956.Gg_rs140628 \\
\hline 1.13 & -3.24 & 1.06 & & 8.41 & & 1.81 & Gg_rs149956:Gg_rs140628 \\
\hline $1.13 \mathrm{NA}$ & NA & & NA & & NA & & Gg_rs149956.Gg_rs140628 \\
\hline 0.62 & -1.61 & 0.58 & & 4.47 & & 0.99 & Gg_rs149956.Gg_rs140628 \\
\hline 1.15 & -0.43 & 1.08 & & 7.6 & & 1.84 & Gg_rs149956:Gg_rs140628 \\
\hline 0.68 & 0.94 & 0.64 & & -4.09 & & 1.09 & Gg_rs149956.Gg_rs140640 \\
\hline $2.34 \mathrm{NA}$ & NA & & NA & & NA & & Gg_rs149956.Gg_rs135057 \\
\hline
\end{tabular}




\begin{tabular}{|c|c|c|c|c|c|}
\hline $0.41 \mathrm{NA}$ & NA & NA & NA & & Gg_rs149956.Gg_rs135057. \\
\hline 1.09 & 2.15 & 1.19 & -4.85 & 1.47 & X13_129794EGg_rs135057. \\
\hline 0.28 & -1.39 & 0.31 & 0.94 & 0.38 & X13_129794EGg_rs135057. \\
\hline 0.51 & -2.71 & 0.54 & 2.74 & 0.69 & X13_129794EGg_rs135057. \\
\hline $0.56 \mathrm{NA}$ & NA & NA & NA & & X13_129794EGg_rs135057. \\
\hline $0.84 \mathrm{NA}$ & NA & NA & NA & & Gg_rs149956.Gg_rs135057. \\
\hline 0.86 & -4.53 & 0.9 & 4.25 & 1.15 & Gg_rs140628 Gg_rs135057. \\
\hline 0.89 & -4.76 & 0.94 & 4.6 & 1.19 & Gg_rs140628 Gg_rs135057. \\
\hline $0.72 \mathrm{NA}$ & NA & NA & NA & & X13_129794EGg_rs135057. \\
\hline 0.96 & -4.81 & 1.01 & 5.11 & 1.28 & Gg_rs140628 Gg_rs140640. \\
\hline 0.76 & -4.28 & 0.8 & 3.48 & 1.02 & Gg_rs140628 Gg_rs135057. \\
\hline 0.69 & -3.65 & 0.72 & 2.61 & 0.92 & Gg_rs140628 Gg_rs135057. \\
\hline $0.23 \mathrm{NA}$ & NA & NA & NA & & Gg_rs135301 Gg_rs135309. \\
\hline $0.22 \mathrm{NA}$ & NA & NA & NA & & Gg_rs135301 Gg_rs135309. \\
\hline $0.64 \mathrm{NA}$ & NA & NA & NA & & Gg_rs135301Gg_rs135311. \\
\hline 0.49 NA & NA & NA & NA & & Gg_rs135301 Gg_rs140717 \\
\hline $0.76 \mathrm{NA}$ & NA & NA & NA & & Gg_rs135304.Gg_rs135311. \\
\hline $0.7 \mathrm{NA}$ & NA & NA & NA & & Gg_rs135304.Gg_rs135309. \\
\hline $1.07 \mathrm{NA}$ & NA & NA & NA & & Gg_rs135304.Gg_rs135309. \\
\hline 0.89 NA & NA & NA & NA & & Gg_rs135304.Gg_rs135309. \\
\hline $0.97 \mathrm{NA}$ & NA & NA & NA & & Gg_rs135304:Gg_rs135311. \\
\hline $0.62 \mathrm{NA}$ & NA & NA & NA & & Gg_rs135304:Gg_rs140717 \\
\hline $0.82 \mathrm{NA}$ & NA & NA & NA & & Gg_rs135301 Gg_rs135319. \\
\hline $1.42 \mathrm{NA}$ & NA & NA & NA & & Gg_rs135304.Gg_rs135319. \\
\hline $0.66 \mathrm{NA}$ & NA & NA & NA & & Gg_rs135304.Gg_rs135319. \\
\hline $0.45 \mathrm{NA}$ & NA & NA & NA & & Gg_rs135304:Gg_rs140717 \\
\hline $0.72 \mathrm{NA}$ & NA & NA & NA & & Gg_rs135301 Gg_rs140717 \\
\hline $1 \mathrm{NA}$ & NA & NA & NA & & Gg_rs135304:Gg_rs140717 \\
\hline $0.64 \mathrm{NA}$ & NA & NA & NA & & Gg_rs135304.Gg_rs140717 \\
\hline 0.99 NA & NA & NA & NA & & Gg_rs135309 Gg_rs140717 \\
\hline $1.59 \mathrm{NA}$ & NA & NA & NA & & Gg_rs135301 Gg_rs140717 \\
\hline $0.71 \mathrm{NA}$ & NA & NA & NA & & Gg_rs135304.Gg_rs135319. \\
\hline 0.89 NA & NA & NA & NA & & Gg_rs135304.Gg_rs140717 \\
\hline $0.61 \mathrm{NA}$ & NA & NA & NA & & Gg_rs135304.Gg_rs140717 \\
\hline $0.9 \mathrm{NA}$ & NA & NA & NA & & Gg_rs135304:Gg_rs135319. \\
\hline $0.36 \mathrm{NA}$ & NA & NA & NA & & Gg_rs135309 Gg_rs140717 \\
\hline $0.38 \mathrm{NA}$ & NA & NA & NA & & Gg_rs135309 Gg_rs140717 \\
\hline $0.32 \mathrm{NA}$ & NA & NA & NA & & Gg_rs135309 Gg_rs140717 \\
\hline $0.75 \mathrm{NA}$ & NA & NA & NA & & Gg_rs135304.Gg_rs135319. \\
\hline $1.24 \mathrm{NA}$ & NA & NA & NA & & Gg_rs135301 Gg_rs135319. \\
\hline $0.88 \mathrm{NA}$ & NA & NA & NA & & Gg_rs135304.Gg_rs135319. \\
\hline $1.03 \mathrm{NA}$ & NA & NA & NA & & Gg_rs135301Gg_rs135319. \\
\hline $0.78 \mathrm{NA}$ & NA & NA & NA & & Gg_rs135309 Gg_rs135319. \\
\hline
\end{tabular}




\begin{tabular}{|c|c|c|c|c|c|}
\hline $0.72 \mathrm{NA}$ & NA & NA & NA & & Gg_rs135309 Gg_rs135319. \\
\hline $0.99 \mathrm{NA}$ & NA & NA & NA & & Gg_rs135301 Gg_rs135319. \\
\hline $1.45 \mathrm{NA}$ & NA & NA & NA & & Gg_rs135304.Gg_rs135319. \\
\hline $0.76 \mathrm{NA}$ & NA & NA & NA & & Gg_rs135311 Gg_rs135319. \\
\hline $0.57 \mathrm{NA}$ & NA & NA & NA & & Gg_rs135311 Gg_rs135319. \\
\hline $0.62 \mathrm{NA}$ & NA & NA & NA & & Gg_rs135311 Gg_rs135319. \\
\hline $0.78 \mathrm{NA}$ & NA & NA & NA & & Gg_rs135301 Gg_rs135319. \\
\hline 1.29 & 8.82 & 1.34 & 7.76 & 1.79 & Gg_rs135311 Gg_rs135319. \\
\hline 1.72 & 11.42 & 1.79 & 10.37 & 2.39 & Gg_rs135311 Gg_rs135319. \\
\hline $0.59 \mathrm{NA}$ & NA & NA & NA & & Gg_rs135311 Gg_rs135319. \\
\hline $0.57 \mathrm{NA}$ & NA & NA & NA & & Gg_rs135311Gg_rs135319. \\
\hline $0.77 \mathrm{NA}$ & NA & NA & NA & & Gg_rs135311 Gg_rs140765. \\
\hline $0.61 \mathrm{NA}$ & NA & NA & NA & & Gg_rs140717Gg_rs140765. \\
\hline $0.46 \mathrm{NA}$ & NA & NA & NA & & Gg_rs140717Gg_rs140765. \\
\hline $0.37 \mathrm{NA}$ & NA & NA & NA & & Gg_rs140717Gg_rs135322 \\
\hline $0.88 \mathrm{NA}$ & NA & NA & NA & & Gg_rs140717Gg_rs140765. \\
\hline $0.94 \mathrm{NA}$ & NA & NA & NA & & Gg_rs140717Gg_rs140765. \\
\hline $0.73 \mathrm{NA}$ & NA & NA & NA & & Gg_rs140717X14_101724C \\
\hline $0.53 \mathrm{NA}$ & NA & NA & NA & & Gg_rs135322 X14_101724C \\
\hline $0.38 \mathrm{NA}$ & NA & NA & NA & & Gg_rs135322 X14_101724C \\
\hline 0.97 NA & NA & NA & NA & & Gg_rs135322 X14_101724C \\
\hline $0.68 \mathrm{NA}$ & NA & NA & NA & & Gg_rs135322 X14_101724C \\
\hline 0.89 NA & NA & NA & NA & & Gg_rs135322 X14_101724C \\
\hline 1.34 & 1.44 & 1.4 & -4.18 & 1.89 & Gg_rs135322 X14_101724C \\
\hline $0.81 \mathrm{NA}$ & NA & NA & NA & & Gg_rs135322 X14_101724C \\
\hline 1.35 & 0.49 & 1.41 & -5.19 & 1.9 & Gg_rs135322 X14_101724C \\
\hline $1.03 \mathrm{NA}$ & NA & NA & NA & & Gg_rs135322 X14_101724C \\
\hline 0.93 NA & NA & NA & NA & & Gg_rs135322 X14_101724C \\
\hline $1.15 \mathrm{NA}$ & NA & NA & NA & & Gg_rs135322 X14_101724C \\
\hline $0.51 \mathrm{NA}$ & NA & NA & NA & & Gg_rs135322 X14_101724C \\
\hline 0.77 NA & NA & NA & NA & & Gg_rs135322 X14_101724C \\
\hline $0.84 \mathrm{NA}$ & NA & NA & NA & & Gg_rs135322 X14_101724C \\
\hline $0.5 \mathrm{NA}$ & NA & NA & NA & & Gg_rs135322 X14_101724C \\
\hline $0.52 \mathrm{NA}$ & NA & NA & NA & & Gg_rs135322 X14_101724C \\
\hline 1.17 & 2.29 & 1.22 & -6.41 & 1.64 & Gg_rs135322 X14_101724C \\
\hline 0.81 & -0.4 & 0.85 & -2.59 & 1.14 & Gg_rs135322 X14_101724C \\
\hline 0.89 & 1.53 & 0.78 & -1.51 & 1.17 & Gg_rs140765 rbl1871 \\
\hline $0.71 \mathrm{NA}$ & NA & NA & NA & & rbl1499 rbl1871 \\
\hline $0.72 \mathrm{NA}$ & NA & NA & NA & & rbl1499 \\
\hline $0.69 \mathrm{NA}$ & NA & NA & NA & & rbl1871 \\
\hline 0.66 NA & NA & NA & NA & & rbl1499 \\
\hline $0.44 \mathrm{NA}$ & NA & NA & NA & & rbl1499 \\
\hline $0.21 \mathrm{NA}$ & NA & NA & NA & & rbl1499 \\
\hline
\end{tabular}




\begin{tabular}{|c|c|c|c|c|c|c|c|}
\hline $0.46 \mathrm{NA}$ & & NA & & NA & & & rbl1499 \\
\hline $0.61 \mathrm{NA}$ & & NA & & NA & & & rbl1499 \\
\hline $1.18 \mathrm{NA}$ & & NA & & NA & & & rbl1499 \\
\hline 1.66 & -5.05 & & 1.38 & & -4.1 & 2.15 & Gg_rs150026 rbl1871 \\
\hline $0.48 \mathrm{NA}$ & & NA & & NA & & & rbl1499 rbl1871 \\
\hline 1.09 & 3.91 & & 0.91 & & 2.46 & 1.41 & Gg_rs150026 rbl1871 \\
\hline 0.53 & 1.68 & & 0.44 & & -0.54 & 0.68 & Gg_rs150026 rbl1871 \\
\hline 0.51 & 1.5 & & 0.42 & & 0.36 & 0.66 & rbl1499 \\
\hline $2.19 \mathrm{NA}$ & & NA & & NA & & & Gg_rs150026rbl1871 \\
\hline 0.83 & 2.09 & & 0.69 & & 1.62 & 1.08 & Gg_rs150026rbl1871 \\
\hline 0.92 & 3.84 & & 0.77 & & -0.05 & 1.2 & Gg_rs150026rbl1871 \\
\hline 0.45 & 1.9 & & 0.37 & & -0.12 & 0.58 & Gg_rs150026 rbl1871 \\
\hline 0.74 & 2.68 & & 0.61 & & 0.55 & 0.95 & Gg_rs150026rbl1871 \\
\hline 0.55 & 1.78 & & 0.46 & & 0.66 & 0.71 & Gg_rs150026 rbl1871 \\
\hline 0.37 & 1.02 & & 0.3 & & 0.82 & 0.47 & Gg_rs150026 rbl1871 \\
\hline 1.29 & 5.03 & & 1.07 & & 2.75 & 1.67 & Gg_rs150026 rbl1871 \\
\hline 0.83 & 2.54 & & 0.69 & & 1.78 & 1.08 & Gg_rs150026 rbl1871 \\
\hline 0.75 & 2.47 & & 0.62 & & 0.89 & 0.97 & Gg_rs150026 rbl1871 \\
\hline $0.06 \mathrm{NA}$ & & NA & & NA & & & rbl1499 rbl1871 \\
\hline $0.17 \mathrm{NA}$ & & NA & & NA & & & rbl1499 rbl1871 \\
\hline $0.68 \mathrm{NA}$ & & NA & & NA & & & Gg_rs150026rbl1871 \\
\hline 1.2 & 3.65 & & 1 & & 3.12 & 1.55 & Gg_rs150026 rbl1871 \\
\hline 0.65 & 2 & & 0.54 & & 0.8 & 0.84 & Gg_rs150026 rbl1871 \\
\hline 1 & 4.42 & & 0.83 & & 1.52 & 1.29 & Gg_rs150026 rbl1871 \\
\hline 0.43 & 1.98 & & 0.35 & & -0.58 & 0.55 & Gg_rs150026 rbl1871 \\
\hline 0.71 & 2.88 & & 0.59 & & 0.41 & 0.92 & Gg_rs150026 rbl1871 \\
\hline 0.53 & 1.52 & & 0.44 & & 1.79 & 0.68 & Gg_rs150026rbl1871 \\
\hline 1.04 & 4.51 & & 0.86 & & -0.23 & 1.34 & Gg_rs150026rbl1871 \\
\hline 1.26 & 4.5 & & 1.05 & & 1.6 & 1.63 & Gg_rs150026rbl1871 \\
\hline 0.96 & 3.97 & & 0.8 & & 1.9 & 1.24 & Gg_rs150026 rbl1871 \\
\hline 1.07 & 3.81 & & 0.89 & & 2.17 & 1.39 & Gg_rs150026 rbl1871 \\
\hline 1.07 & 3.56 & & 0.89 & & 2.59 & 1.38 & Gg_rs150026rbl1871 \\
\hline 0.75 & 2.16 & & 0.62 & & 1.46 & 0.97 & Gg_rs150026 rbl1871 \\
\hline 0.61 & 2.47 & & 0.51 & & -0.81 & 0.79 & Gg_rs150026 rbl1871 \\
\hline 0.71 & 2.43 & & 0.59 & & 0.02 & 0.92 & Gg_rs150026 rbl1871 \\
\hline 0.76 & 2.88 & & 0.63 & & 1.16 & 0.98 & Gg_rs150026 rbl1871 \\
\hline $0.84 \mathrm{NA}$ & & NA & & NA & & & Gg_rs150026rbl1871 \\
\hline $5.54 \mathrm{NA}$ & & NA & & NA & & & Gg_rs150026 rbl1871 \\
\hline 0.41 & 1.84 & & 0.34 & & -0.21 & 0.53 & Gg_rs150026rbl1871 \\
\hline 0.47 & 1.68 & & 0.39 & & -1.14 & 0.61 & Gg_rs150026rbl1871 \\
\hline 0.8 & 1.51 & & 0.66 & & 3.12 & 1.03 & Gg_rs150026rbl1871 \\
\hline 0.71 & 2.25 & & 0.59 & & -0.29 & 0.92 & Gg_rs150026rbl1871 \\
\hline $0.59 \mathrm{NA}$ & & NA & & NA & & & Gg_rs150026 rbl1871 \\
\hline
\end{tabular}




\begin{tabular}{|c|c|c|c|c|c|}
\hline $0.45 \mathrm{NA}$ & NA & NA & NA & & Gg_rs150026 rbl1871 \\
\hline 0.44 & 1.76 & 0.37 & -0.45 & 0.57 & Gg_rs150026rbl1871 \\
\hline 0.89 & 3.04 & 0.74 & 1.1 & 1.15 & Gg_rs150026 rbl1871 \\
\hline 1.1 & 4.89 & 0.91 & -0.87 & 1.42 & Gg_rs150026 rbl1871 \\
\hline 0.56 & 1.88 & 0.47 & 0.47 & 0.73 & Gg_rs150026 rbl1871 \\
\hline 1.1 & 3.87 & 0.92 & 3.2 & 1.43 & Gg_rs150026 rbl1871 \\
\hline 0.42 & 1.56 & 0.34 & 0.64 & 0.54 & Gg_rs150026 rbl1871 \\
\hline 0.69 & 2.43 & 0.57 & 0.06 & 0.89 & Gg_rs150026 rbl1871 \\
\hline 0.41 & 1.5 & 0.34 & 0.13 & 0.53 & Gg_rs150026 rbl1871 \\
\hline 0.57 & 1.74 & 0.48 & 1.46 & 0.74 & Gg_rs150026 rbl1871 \\
\hline 0.52 & 1.98 & 0.43 & -0.16 & 0.68 & Gg_rs150026 rbl1871 \\
\hline 0.59 & 2.39 & 0.49 & 0.02 & 0.76 & Gg_rs150026 rbl1871 \\
\hline 0.44 & 1.6 & 0.36 & -0.18 & 0.56 & Gg_rs150026 rbl1871 \\
\hline 0.43 & 2.45 & 0.35 & -0.93 & 0.55 & Gg_rs150026 rbl1871 \\
\hline 1.14 & 4.76 & 0.95 & 0.51 & 1.47 & Gg_rs150026 rbl1871 \\
\hline $0.95 \mathrm{NA}$ & NA & NA & NA & & Gg_rs157609 Gg_rs157630 \\
\hline 2.22 & -5.55 & 2.04 & 5.77 & 2.98 & Gg_rs157609 Gg_rs157630 \\
\hline 2.1 & -4.67 & 1.93 & 3.9 & 2.8 & Gg_rs157609 Gg_rs157630 \\
\hline $1.16 \mathrm{NA}$ & NA & NA & NA & & Gg_rs157609 Gg_rs157630 \\
\hline $1.03 \mathrm{NA}$ & NA & NA & NA & & Gg_rs157609 Gg_rs150194 \\
\hline 0.92 & -4.56 & 0.84 & 3.26 & 1.23 & Gg_rs157609 Gg_rs157630 \\
\hline $0.52 \mathrm{NA}$ & NA & NA & NA & & Gg_rs157609 Gg_rs157630 \\
\hline $0.98 \mathrm{NA}$ & NA & NA & NA & & Gg_rs157609 Gg_rs157630 \\
\hline 1.05 & -4.82 & 0.96 & 3.53 & 1.4 & Gg_rs157609 Gg_rs157630 \\
\hline $0.66 \mathrm{NA}$ & NA & NA & NA & & Gg_rs157609 Gg_rs150194 \\
\hline $1.04 \mathrm{NA}$ & NA & NA & NA & & Gg_rs157609 Gg_rs150194 \\
\hline $1.42 \mathrm{NA}$ & NA & NA & NA & & Gg_rs157609 Gg_rs150194 \\
\hline $0.32 \mathrm{NA}$ & NA & NA & NA & & Gg_rs157609 X15_6012728 \\
\hline $0.79 \mathrm{NA}$ & NA & NA & NA & & Gg_rs157609 X15_6012728 \\
\hline $0.97 \mathrm{NA}$ & NA & NA & NA & & Gg_rs157609 X15_6012728 \\
\hline $0.3 \mathrm{NA}$ & NA & NA & NA & & Gg_rs140871 X15_6012728 \\
\hline $1.22 \mathrm{NA}$ & NA & NA & NA & & Gg_rs157609 X15_6012728 \\
\hline $0.32 \mathrm{NA}$ & NA & NA & NA & & Gg_rs157637 X15_6012728 \\
\hline $0.46 \mathrm{NA}$ & NA & NA & NA & & Gg_rs157630X15_6012728 \\
\hline $0.82 \mathrm{NA}$ & NA & NA & NA & & Gg_rs157609 X15_6012728 \\
\hline 1.65 & 5.73 & 1.72 & 7.25 & 2.16 & Gg_rs150194 Gg_rs140951 \\
\hline 3.33 & 13.62 & 3.47 & 19.92 & 4.38 & Gg_rs150194 Gg_rs140951 \\
\hline 3.75 & 13.33 & 3.91 & 16.28 & 4.92 & Gg_rs150194 Gg_rs140951 \\
\hline 3.01 & 11.7 & 3.14 & 15.97 & 3.95 & Gg_rs150194 Gg_rs140951 \\
\hline 3.73 & 12.7 & 3.89 & 19.46 & 4.9 & Gg_rs150194 Gg_rs140951 \\
\hline 2.89 & 9.82 & 3.01 & 16.76 & 3.79 & Gg_rs150194 Gg_rs140951 \\
\hline 1.64 & 7.17 & 1.71 & 7.58 & 2.16 & Gg_rs150194 Gg_rs140951 \\
\hline 4.59 & 16.37 & 4.78 & 22.56 & 6.02 & Gg_rs150194 Gg_rs140951 \\
\hline
\end{tabular}




\begin{tabular}{|c|c|c|c|c|}
\hline 3.5 & 12.74 & 3.64 & 15.46 & 4.59 Gg_rs150194 Gg_rs140951 \\
\hline 4.49 & 16.11 & 4.67 & 23.57 & 5.89 Gg_rs150194 Gg_rs140951 \\
\hline 1.67 & 4.27 & 1.74 & 6.86 & 2.19 Gg_rs150194 Gg_rs140951 \\
\hline 1.86 & 5.06 & 1.93 & 8.1 & 2.44 Gg_rs150194 Gg_rs140951 \\
\hline 3.48 & 11.75 & 3.63 & 20.73 & 4.57 Gg_rs150194 Gg_rs140951 \\
\hline 1.94 & 6.91 & 2.02 & 10.14 & 2.55 Gg_rs150194 Gg_rs140951 \\
\hline 1.87 & 5.71 & 1.94 & 10.1 & 2.45 Gg_rs150194 Gg_rs140951 \\
\hline 2.52 & 8.61 & 2.62 & 12.22 & 3.3 Gg_rs150194 Gg_rs140951 \\
\hline 6.02 & 19.57 & 6.27 & 27.42 & 7.9 Gg_rs150194 Gg_rs140951 \\
\hline 2.02 & 8.07 & 2.11 & 10.9 & 2.65 Gg_rs150194 Gg_rs140951 \\
\hline 2.42 & 8.98 & 2.53 & 13.28 & 3.18 Gg_rs150194 Gg_rs140951 \\
\hline 1.69 & 5.83 & 1.76 & 6.24 & 2.22 Gg_rs150194 Gg_rs140951 \\
\hline 6.79 & 23.22 & 7.07 & 37.14 & 8.91 Gg_rs150194 Gg_rs140951 \\
\hline 4.56 & 14.22 & 4.75 & 20.71 & 5.99 Gg_rs150194 Gg_rs140951 \\
\hline 1.82 & 6.38 & 1.9 & 8.65 & 2.39 Gg_rs150194 Gg_rs140951 \\
\hline 6.97 & 26.83 & 7.27 & 35.4 & 9.15 Gg_rs150194 Gg_rs140951 \\
\hline 1.87 & 6.79 & 1.95 & 10.54 & 2.45 Gg_rs150194 Gg_rs140951 \\
\hline 3.28 & 13.47 & 3.41 & 17.85 & 4.3 Gg_rs150194 Gg_rs140951 \\
\hline 6.81 & 24.36 & 7.09 & 36.6 & 8.94 Gg_rs150194 Gg_rs140951 \\
\hline 2.58 & 10.2 & 2.69 & 13.24 & 3.39 Gg_rs150194 Gg_rs140951 \\
\hline 6.2 & 20.79 & 6.45 & 28.94 & 8.13 Gg_rs150194 Gg_rs140951 \\
\hline 1.83 & 6.23 & 1.91 & 8.77 & 2.4 Gg_rs150194 Gg_rs140951 \\
\hline 2.36 & 8.58 & 2.46 & 13.1 & 3.09 Gg_rs150194 Gg_rs140951 \\
\hline 9.81 & 30.39 & 10.22 & 54.39 & 12.88 Gg_rs150194 Gg_rs140951 \\
\hline 5.79 & 19.76 & 6.03 & 25.51 & 7.6 Gg_rs150194 Gg_rs140951 \\
\hline 4.25 & 15.11 & 4.43 & 26.21 & 5.58 Gg_rs150194 Gg_rs140951 \\
\hline 6.5 & 22.66 & 6.77 & 32.01 & 8.53 Gg_rs150194 Gg_rs140951 \\
\hline 2.52 & 10.07 & 2.62 & 11.96 & 3.31 Gg_rs150194 Gg_rs140951 \\
\hline 1.67 & 5.19 & 1.74 & 10.49 & 2.19 Gg_rs150194 Gg_rs140951 \\
\hline 5.55 & 23.28 & 5.78 & 29.04 & 7.28 Gg_rs150194 Gg_rs140951 \\
\hline 1.91 & 8.31 & 1.99 & 11.01 & 2.5 Gg_rs150194 Gg_rs140951 \\
\hline 1.66 & 7.17 & 1.73 & 9.39 & 2.18 Gg_rs150194 Gg_rs140951 \\
\hline 5.49 & 15.98 & 5.71 & 23.46 & 7.2 Gg_rs150194 Gg_rs140951 \\
\hline 2.02 & 8.53 & 2.11 & 9.94 & 2.66 Gg_rs150194 Gg_rs140951 \\
\hline 3.81 & 14.61 & 3.97 & 20.61 & 5 Gg_rs150194 Gg_rs140951 \\
\hline 1.99 & 9.25 & 2.07 & 12.49 & 2.61 Gg_rs150194 Gg_rs140951 \\
\hline 2.98 & 11.53 & 3.11 & 17.1 & 3.92 Gg_rs150194 Gg_rs140951 \\
\hline 3.44 & 15.22 & 3.58 & 21.04 & 4.52 Gg_rs150194 Gg_rs140951 \\
\hline 3.38 & 13.46 & 3.52 & 19.95 & 4.44 Gg_rs150194 Gg_rs140951 \\
\hline 4.69 & 19.88 & 4.88 & 26.61 & 6.15 Gg_rs150194 Gg_rs140951 \\
\hline 2.47 & 7.34 & 2.57 & 10.37 & 3.24 Gg_rs150194 Gg_rs140951 \\
\hline 5.54 & 20.12 & 5.77 & 28.66 & 7.27 Gg_rs150194 Gg_rs140951 \\
\hline 11.71 & 49.67 & 12.2 & 64.44 & 15.37 Gg_rs150194 Gg_rs140951 \\
\hline
\end{tabular}




\begin{tabular}{|c|c|c|c|c|}
\hline 2.37 & 9.51 & 2.47 & 13.99 & 3.11 Gg_rs150194 Gg_rs140951 \\
\hline 1.87 & 7.7 & 1.95 & 10.42 & 2.46 Gg_rs150194 Gg_rs140951 \\
\hline 1.98 & 8.1 & 2.07 & 9.52 & 2.6 Gg_rs150194 Gg_rs140951 \\
\hline 3.3 & 12.41 & 3.44 & 18.09 & 4.34 Gg_rs150194 Gg_rs140951 \\
\hline 4 & 15.41 & 4.17 & 22.68 & 5.25 Gg_rs150194 Gg_rs140951 \\
\hline 1.76 & 7.9 & 1.83 & 11.23 & 2.31 X15_601272عGg_rs140951 \\
\hline 5.43 & 19.3 & 5.66 & 30.57 & 7.13 Gg_rs150194 Gg_rs140951 \\
\hline 13.13 & 56.35 & 13.68 & 71.84 & 17.24 Gg_rs150194 Gg_rs140951 \\
\hline 2.25 & 8.58 & 2.35 & 11.02 & 2.96 Gg_rs150194 Gg_rs140951 \\
\hline 2.28 & 8.2 & 2.37 & 12.54 & 2.99 Gg_rs150194 Gg_rs140951 \\
\hline 1.69 & 4.74 & 1.76 & 6.66 & 2.21 Gg_rs150194 Gg_rs140951 \\
\hline 1.71 & 5.92 & 1.78 & 8.67 & 2.24 Gg_rs150194 Gg_rs140951 \\
\hline 1.64 & 5.87 & 1.71 & 6.44 & 2.15 Gg_rs150194 Gg_rs140951 \\
\hline 4.87 & 20.93 & 5.08 & 23.45 & 6.4 Gg_rs150194 Gg_rs140951 \\
\hline 3.23 & 11.82 & 3.36 & 18.2 & 4.23 Gg_rs150194 Gg_rs140951 \\
\hline 4.73 & 15.58 & 4.92 & 23.59 & 6.2 Gg_rs150194 Gg_rs140951 \\
\hline 0.71 & 3.52 & 0.74 & 3.72 & 0.94 Gg_rs150230 Gg_rs140951 \\
\hline 1.5 & 6.95 & 1.56 & 9.69 & 1.96 X15_6012728Gg_rs140951 \\
\hline 3.18 & 13.56 & 3.31 & 18.01 & 4.17 Gg_rs150194 Gg_rs140951 \\
\hline 1.97 & 9.68 & 2.05 & 12.21 & 2.59 Gg_rs150230 Gg_rs140951 \\
\hline 2.83 & 10.73 & 2.95 & 14.9 & 3.72 Gg_rs150230 Gg_rs140951 \\
\hline 2.83 & 10.78 & 2.95 & 15.54 & 3.72 Gg_rs150194 Gg_rs140951 \\
\hline 2.83 & 11.02 & 2.95 & 15.63 & 3.71 Gg_rs150194 Gg_rs140951 \\
\hline 5.98 & 25.2 & 6.23 & 33.37 & 7.85 X15_601272عGg_rs140951 \\
\hline 1.71 & 6.67 & 1.78 & 9.77 & 2.25 Gg_rs150194 Gg_rs140951 \\
\hline 1.63 & 6.24 & 1.7 & 10.73 & 2.15 Gg_rs150194 Gg_rs140951 \\
\hline 8.04 & 33.45 & 8.37 & 44.55 & 10.55 Gg_rs150194 Gg_rs140951 \\
\hline 4.29 & 15.3 & 4.47 & 22.23 & 5.63 Gg_rs150194 Gg_rs140951 \\
\hline 6.4 & 28.52 & 6.67 & 36.26 & 8.4 Gg_rs150194 Gg_rs140951 \\
\hline 3.91 & 16.65 & 4.08 & 22.68 & 5.14 X15_601272\&Gg_rs140951 \\
\hline 7.3 & 30.93 & 7.61 & 39.06 & 9.59 Gg_rs150194 Gg_rs140951 \\
\hline 1.68 & 7.37 & 1.75 & 10.39 & 2.21 Gg_rs150230 Gg_rs140951 \\
\hline 2.8 & 9.05 & 2.92 & 16.43 & 3.68 Gg_rs150194 Gg_rs140951 \\
\hline 4.08 & 18.57 & 4.25 & 22.12 & 5.35 Gg_rs150194 Gg_rs140951 \\
\hline 1.75 & 7.29 & 1.82 & 8.55 & 2.29 X15_601272عGg_rs140951 \\
\hline 2.8 & 10.91 & 2.91 & 11.66 & 3.67 Gg_rs150194 Gg_rs140951 \\
\hline 3.11 & 11.06 & 3.24 & 14.74 & 4.08 Gg_rs150194 Gg_rs140951 \\
\hline 1.97 & 7.2 & 2.05 & 9.05 & 2.59 Gg_rs150194 Gg_rs140951 \\
\hline 1.67 & 6.17 & 1.74 & 8.75 & 2.19 Gg_rs150194 Gg_rs140951 \\
\hline 1.81 & 8.26 & 1.89 & 10.75 & 2.38 Gg_rs150230 Gg_rs140951 \\
\hline 1.7 & 5.39 & 1.77 & 6.46 & 2.23 Gg_rs150194 Gg_rs140951 \\
\hline 1.78 & 7.61 & 1.85 & 8.7 & 2.33 Gg_rs150194 Gg_rs140951 \\
\hline 4.19 & 15.96 & 4.36 & 21.99 & 5.5 Gg_rs150194 Gg_rs140951 \\
\hline
\end{tabular}




\begin{tabular}{|c|c|c|c|c|}
\hline $0.78 \mathrm{NA}$ & NA & NA & NA & Gg_rs150194 Gg_rs140951 \\
\hline 0.99 & 3.47 & 1.03 & 4.37 & 1.3 Gg_rs150230 Gg_rs140951 \\
\hline 2.23 & 8.1 & 2.32 & 12.73 & 2.93 Gg_rs150230 Gg_rs140951 \\
\hline 0.48 & 1.94 & 0.5 & 2.86 & 0.62 Gg_rs150230 Gg_rs140951 \\
\hline 0.45 & 3.01 & 0.47 & 2.59 & 0.59 Gg_rs150230 Gg_rs140951 \\
\hline 3.01 & 12.34 & 3.13 & 19.17 & 3.95 Gg_rs150230 Gg_rs140951 \\
\hline 1.83 & 7.61 & 1.9 & 10.87 & 2.4 X15_601272عGg_rs140951 \\
\hline 1.84 & 7.11 & 1.92 & 12.23 & 2.42 Gg_rs150230 Gg_rs140951 \\
\hline 2.95 & 11.22 & 3.07 & 18.06 & 3.87 X15_601272عGg_rs140951 \\
\hline 2.99 & 12.04 & 3.11 & 18.27 & 3.92 Gg_rs150230 Gg_rs140951 \\
\hline 1.93 & 7.85 & 2.01 & 11.86 & 2.53 Gg_rs150230 Gg_rs140951 \\
\hline 1.67 & 9.53 & 1.74 & 11.23 & 2.19 Gg_rs150230 Gg_rs140951 \\
\hline 2.77 & 10.16 & 2.88 & 16.74 & 3.63 Gg_rs150230 Gg_rs140951 \\
\hline 2.43 & 9.47 & 2.54 & 14.02 & 3.19 Gg_rs150194 Gg_rs140951 \\
\hline 2.91 & 11.71 & 3.04 & 16.12 & 3.83 Gg_rs150230 Gg_rs140951 \\
\hline 2.41 & 8.78 & 2.51 & 11.83 & 3.16 X15_601272عGg_rs140951 \\
\hline 1.79 & 6.78 & 1.86 & 10.99 & 2.34 Gg_rs150230 Gg_rs140951 \\
\hline 3.96 & 12.68 & 4.13 & 17.54 & 5.2 Gg_rs150230 Gg_rs140951 \\
\hline 4.24 & 18.04 & 4.41 & 26.12 & 5.56 Gg_rs150230 Gg_rs140951 \\
\hline 2.1 & 7.65 & 2.19 & 10.3 & 2.76 Gg_rs150194 Gg_rs140951 \\
\hline 4.64 & 18.82 & 4.83 & 26.53 & 6.08 Gg_rs150230 Gg_rs140951 \\
\hline 2.44 & 9.55 & 2.54 & 14.64 & 3.2 Gg_rs150230 Gg_rs140951 \\
\hline 5.8 & 23.68 & 6.04 & 33.44 & 7.62 Gg_rs150230 Gg_rs140951 \\
\hline 2.32 & 14.22 & 2.42 & 12.57 & 3.05 Gg_rs150230 Gg_rs140951 \\
\hline 3.51 & 13.78 & 3.66 & 21.96 & 4.61 Gg_rs150230 Gg_rs140951 \\
\hline 4.24 & 14.85 & 4.41 & 24.09 & 5.56 Gg_rs150230 Gg_rs140951 \\
\hline 3.6 & 15.26 & 3.75 & 18.94 & 4.72 Gg_rs150230 Gg_rs140951 \\
\hline 5.86 & 25.71 & 6.1 & 32.26 & 7.69 Gg_rs150230 Gg_rs140951 \\
\hline 2.22 & 7.82 & 2.31 & 12.66 & 2.91 Gg_rs150230 Gg_rs140951 \\
\hline 1.99 & 6.16 & 2.07 & 8.82 & 2.61 Gg_rs150230 Gg_rs140951 \\
\hline 1.71 & 6.97 & 1.78 & 8.26 & 2.24 Gg_rs150230 Gg_rs140951 \\
\hline 3.37 & 15.01 & 3.51 & 20.81 & 4.43 Gg_rs150230 Gg_rs140951 \\
\hline 2.16 & 8.58 & 2.25 & 14.07 & 2.83 Gg_rs150230 Gg_rs140951 \\
\hline 1.07 & 4.55 & 1.12 & 6.04 & 1.41 Gg_rs150230 Gg_rs140951 \\
\hline 1.04 & 4.13 & 1.09 & 6.5 & 1.37 Gg_rs150230 Gg_rs140951 \\
\hline 1.35 & 5.92 & 1.41 & 5.39 & 1.78 Gg_rs150230 Gg_rs140951 \\
\hline 1.92 & 7.09 & 2 & 10.76 & 2.52 Gg_rs150230 Gg_rs140951 \\
\hline 2.13 & 7.69 & 2.22 & 11.92 & 2.79 Gg_rs150230 Gg_rs140951 \\
\hline 4.33 & 17.5 & 4.51 & 24.48 & 5.68 Gg_rs150230 Gg_rs140951 \\
\hline 2.44 & 9.36 & 2.54 & 15.59 & 3.2 Gg_rs150230 Gg_rs140951 \\
\hline 3.49 & 15.78 & 3.64 & 22.41 & 4.59 Gg_rs150230 Gg_rs140951 \\
\hline 7.92 & 34.32 & 8.26 & 46.37 & 10.4 Gg_rs150230 Gg_rs140951 \\
\hline 5.12 & 21.98 & 5.34 & 28.93 & 6.72 Gg_rs150230 Gg_rs140951 \\
\hline
\end{tabular}




\begin{tabular}{|c|c|c|c|c|}
\hline 3.9 & 18.67 & 4.06 & 23.96 & 5.11 Gg_rs150230 Gg_rs140951 \\
\hline 3.44 & 15.32 & 3.58 & 20.32 & 4.51 Gg_rs150230 Gg_rs140951 \\
\hline 2.44 & 9.28 & 2.54 & 13.73 & 3.21 Gg_rs150230 Gg_rs140951 \\
\hline 4.59 & 18.84 & 4.78 & 26.07 & 6.03 Gg_rs150230 Gg_rs140951 \\
\hline 3.52 & 13.53 & 3.67 & 21.03 & 4.62 Gg_rs150230 Gg_rs140951 \\
\hline 1.95 & 8.67 & 2.03 & 11.81 & 2.56 Gg_rs150230 Gg_rs140951 \\
\hline 4.45 & 20.34 & 4.63 & 25.7 & 5.84 Gg_rs150230 Gg_rs140951 \\
\hline 6.58 & 23.24 & 6.86 & 36.14 & 8.64 Gg_rs150230 Gg_rs140951 \\
\hline 3.29 & 11.91 & 3.42 & 19.55 & 4.31 Gg_rs150230 Gg_rs140951 \\
\hline 4.13 & 19.8 & 4.31 & 26.23 & 5.43 Gg_rs150230 Gg_rs140951 \\
\hline 3.08 & 13.75 & 3.21 & 16.73 & 4.04 Gg_rs150230 Gg_rs140951 \\
\hline 3.91 & 15.71 & 4.07 & 21.28 & 5.13 Gg_rs150230 Gg_rs140951 \\
\hline 4.97 & 18.77 & 5.18 & 30.85 & 6.52 Gg_rs150230 Gg_rs140951 \\
\hline 4.84 & 20.61 & 5.04 & 28.46 & 6.35 Gg_rs150230 Gg_rs140951 \\
\hline 1.95 & 7.41 & 2.04 & 7.95 & 2.57 Gg_rs150230 Gg_rs140951 \\
\hline 1.82 & 8.54 & 1.9 & 11.33 & 2.39 Gg_rs150230 Gg_rs140951 \\
\hline 1.26 & 3.69 & 1.32 & 3.93 & 1.66 Gg_rs150230 Gg_rs140951 \\
\hline 4.34 & 19.36 & 4.52 & 25.41 & 5.7 Gg_rs150230 Gg_rs140951 \\
\hline 3.25 & 13.93 & 3.38 & 18.58 & 4.26 Gg_rs150230 Gg_rs140951 \\
\hline 2.15 & 7.77 & 2.24 & 11.87 & 2.82 Gg_rs150230 Gg_rs140951 \\
\hline 6.23 & 24.84 & 6.49 & 35.78 & 8.18 Gg_rs150230 Gg_rs140951 \\
\hline 5.24 & 22.07 & 5.46 & 31.51 & 6.88 Gg_rs150230 Gg_rs140951 \\
\hline 3.44 & 13.88 & 3.58 & 20.47 & 4.51 Gg_rs150230 Gg_rs140951 \\
\hline 4.74 & 19.93 & 4.94 & 26.92 & 6.22 Gg_rs150230 Gg_rs140951 \\
\hline 3.74 & 13.64 & 3.89 & 20.61 & 4.9 Gg_rs150230 Gg_rs140951 \\
\hline 2.23 & 10.61 & 2.32 & 14.79 & 2.92 Gg_rs150230 Gg_rs140951 \\
\hline 5.25 & 20.66 & 5.47 & 28.58 & 6.89 Gg_rs150230 Gg_rs140951 \\
\hline 3.04 & 10.98 & 3.17 & 18.44 & 3.99 Gg_rs150230 Gg_rs140951 \\
\hline 1.81 & 5.86 & 1.88 & 11.38 & 2.37 Gg_rs150230 Gg_rs140951 \\
\hline 7.4 & 30.85 & 7.7 & 40.9 & 9.71 Gg_rs150230 Gg_rs140951 \\
\hline 2.81 & 9.68 & 2.93 & 14.85 & 3.69 Gg_rs150230 Gg_rs140951 \\
\hline 8.1 & 34.65 & 8.44 & 44.88 & 10.63 Gg_rs150230 Gg_rs140951 \\
\hline 2.14 & 10.33 & 2.23 & 15.48 & 2.81 Gg_rs150230 Gg_rs140951 \\
\hline 4.15 & 19.13 & 4.33 & 24.25 & 5.45 Gg_rs150230 Gg_rs140951 \\
\hline 3.36 & 14.5 & 3.5 & 20.55 & 4.41 Gg_rs150230 Gg_rs140951 \\
\hline 2.7 & 12.69 & 2.81 & 16.32 & 3.54 Gg_rs150230 Gg_rs140951 \\
\hline 2.22 & 9.7 & 2.31 & 13.17 & 2.91 Gg_rs150230 Gg_rs140951 \\
\hline 1.87 & 7.93 & 1.95 & 10.16 & 2.46 Gg_rs150230 Gg_rs140951 \\
\hline 4.74 & 19.18 & 4.94 & 28.99 & 6.22 Gg_rs150230 Gg_rs140951 \\
\hline 6.98 & 29.97 & 7.27 & 41.31 & 9.16 Gg_rs150230 Gg_rs140951 \\
\hline 2.17 & 7.7 & 2.26 & 12.55 & 2.85 Gg_rs150230 Gg_rs140951 \\
\hline 2.41 & 10.85 & 2.51 & 12.66 & 3.17 Gg_rs150230 Gg_rs140951 \\
\hline 1.72 & 7.8 & 1.8 & 8.97 & 2.26 Gg_rs150230 Gg_rs140951 \\
\hline
\end{tabular}




\begin{tabular}{|c|c|c|c|c|}
\hline 7.38 & 32.86 & 7.69 & 43.51 & 9.68 Gg_rs150230 Gg_rs140951 \\
\hline 1.68 & 7.01 & 1.75 & 10.84 & 2.21 Gg_rs150230 Gg_rs140951 \\
\hline 2.47 & 10.99 & 2.58 & 13.68 & 3.25 Gg_rs150230 Gg_rs140951 \\
\hline 2.27 & 8.5 & 2.36 & 14.84 & 2.97 Gg_rs150230 Gg_rs140951 \\
\hline 8.45 & 39.02 & 8.8 & 41.2 & 11.09 Gg_rs150230 Gg_rs140951 \\
\hline 8.15 & 33.17 & 8.5 & 44.31 & 10.7 Gg_rs150230 Gg_rs140951 \\
\hline 2.75 & 10.55 & 2.87 & 15.95 & 3.61 Gg_rs150230 Gg_rs140951 \\
\hline 4.66 & 19 & 4.85 & 28.37 & 6.11 Gg_rs150230 Gg_rs140951 \\
\hline 1.68 & 5.41 & 1.75 & 9.34 & 2.2 Gg_rs150230 Gg_rs140951 \\
\hline 0.85 & 4.23 & 0.88 & 4.65 & 1.11 Gg_rs150230 Gg_rs140951 \\
\hline 4.98 & 20.1 & 5.18 & 29.58 & 6.53 Gg_rs150230 Gg_rs140951 \\
\hline 7.58 & 31.87 & 7.9 & 42.24 & 9.95 Gg_rs150230 Gg_rs140951 \\
\hline 2.96 & 11.8 & 3.09 & 18.16 & 3.89 Gg_rs150230 Gg_rs140951 \\
\hline 3.7 & 14.07 & 3.85 & 24.93 & 4.85 Gg_rs150230 Gg_rs140951 \\
\hline 2.37 & 10.49 & 2.47 & 11.07 & 3.11 Gg_rs150230 Gg_rs140951 \\
\hline 2.32 & 11.14 & 2.41 & 13.96 & 3.04 Gg_rs150230 Gg_rs140951 \\
\hline 3.73 & 14.72 & 3.89 & 24.38 & 4.9 Gg_rs150230 Gg_rs140951 \\
\hline 1.64 & 6.56 & 1.71 & 8.66 & 2.15 Gg_rs150230 Gg_rs140951 \\
\hline 2.37 & 10.72 & 2.47 & 14.46 & 3.11 Gg_rs150230 Gg_rs140951 \\
\hline 7.35 & 30.71 & 7.66 & 42.48 & 9.65 Gg_rs150230 Gg_rs140951 \\
\hline 6.25 & 28.1 & 6.51 & 33.17 & 8.2 Gg_rs150230 Gg_rs140951 \\
\hline 0.63 & 3.52 & 0.66 & 4.09 & 0.83 Gg_rs150230 Gg_rs140951 \\
\hline 2.78 & 11.92 & 2.89 & 15.78 & 3.64 Gg_rs150230 Gg_rs140951 \\
\hline 3.38 & 14.19 & 3.52 & 19.33 & 4.43 Gg_rs150230 Gg_rs140951 \\
\hline 2.63 & 9.91 & 2.74 & 16.57 & 3.46 Gg_rs150230 Gg_rs140951 \\
\hline 1.64 & 7.19 & 1.71 & 9.41 & 2.15 Gg_rs150230 Gg_rs140951 \\
\hline 1.64 & 7.37 & 1.71 & 11.27 & 2.15 Gg_rs150230 Gg_rs140951 \\
\hline 2.95 & 11.42 & 3.08 & 17.58 & 3.88 Gg_rs150230 Gg_rs140951 \\
\hline 4.68 & 20.03 & 4.87 & 27.96 & 6.14 Gg_rs150230 Gg_rs140951 \\
\hline 2.8 & 11.04 & 2.91 & 17.07 & 3.67 Gg_rs150230 Gg_rs140951 \\
\hline 3.21 & 13.41 & 3.34 & 17.63 & 4.21 Gg_rs150230 Gg_rs140951 \\
\hline 3.23 & 14.34 & 3.37 & 20.16 & 4.24 Gg_rs150230 Gg_rs140951 \\
\hline 1.05 & 5.4 & 1.09 & 7.49 & 1.37 Gg_rs150230 Gg_rs140951 \\
\hline 0.8 & 3.04 & 0.83 & 4.54 & 1.05 Gg_rs150230 Gg_rs140951 \\
\hline 2.21 & 8.89 & 2.3 & 12.56 & 2.9 Gg_rs150230 Gg_rs140951 \\
\hline 2.12 & 8.88 & 2.2 & 13.71 & 2.78 Gg_rs150230 Gg_rs140951 \\
\hline 1.86 & 7.28 & 1.94 & 10.83 & 2.44 Gg_rs150230 Gg_rs140951 \\
\hline 1.9 & 8.62 & 1.98 & 10.7 & 2.5 Gg_rs150230 Gg_rs140951 \\
\hline 1.66 & 8.49 & 1.73 & 10.48 & 2.18 Gg_rs150230 Gg_rs140951 \\
\hline 2.04 & 8.41 & 2.12 & 12.69 & 2.67 Gg_rs150230 Gg_rs140951 \\
\hline 0.8 & 3.96 & 0.83 & 3.04 & 1.05 Gg_rs150230 Gg_rs140951 \\
\hline 3.19 & 12.84 & 3.33 & 20.46 & 4.19 Gg_rs150230 Gg_rs140951 \\
\hline 2.84 & 12.54 & 2.96 & 16.92 & 3.73 Gg_rs150230 Gg_rs140951 \\
\hline
\end{tabular}




\begin{tabular}{|c|c|c|c|c|}
\hline 2.4 & 9.35 & 2.5 & 13.35 & 3.15 Gg_rs150230 Gg_rs140951 \\
\hline 3.02 & 11.98 & 3.14 & 18.75 & 3.96 Gg_rs150230 Gg_rs140951 \\
\hline 1.65 & 6.51 & 1.72 & 10.17 & 2.16 Gg_rs150230 Gg_rs140951 \\
\hline 1.74 & 7.41 & 1.82 & 9.3 & 2.29 Gg_rs150230 Gg_rs140951 \\
\hline 3.56 & 13.24 & 3.7 & 20.3 & 4.67 Gg_rs150230 Gg_rs140951 \\
\hline 0.71 & 3.13 & 0.73 & 3.63 & 0.93 Gg_rs150230 Gg_rs140951 \\
\hline 0.72 & 2.66 & 0.75 & 3.35 & 0.94 Gg_rs150230 Gg_rs140951 \\
\hline 0.3 & 1.38 & 0.32 & 1.28 & 0.4 Gg_rs150230 Gg_rs140951 \\
\hline 2.16 & 7.87 & 2.25 & 13.43 & 2.83 Gg_rs150230 Gg_rs140951 \\
\hline 1.2 & 4.89 & 1.25 & 5.98 & 1.58 Gg_rs150230 Gg_rs140951 \\
\hline $0.28 \mathrm{NA}$ & NA & NA & NA & Gg_rs150230 Gg_rs140951 \\
\hline 1.69 & 6.59 & 1.76 & 10.95 & 2.22 Gg_rs150230 Gg_rs140951 \\
\hline 2.62 & 10.81 & 2.73 & 15.36 & 3.44 Gg_rs150230 Gg_rs140951 \\
\hline 1.93 & 8.76 & 2.02 & 10.81 & 2.54 Gg_rs150230 Gg_rs140951 \\
\hline 2.33 & 9.37 & 2.43 & 12.07 & 3.06 Gg_rs150230 Gg_rs140951 \\
\hline 2.87 & 11.95 & 2.99 & 15.69 & 3.76 Gg_rs150230 Gg_rs140951 \\
\hline 2.02 & 8.27 & 2.11 & 11.67 & 2.66 Gg_rs150230 Gg_rs140951 \\
\hline 2.49 & 9.39 & 2.6 & 14.08 & 3.27 Gg_rs150230 Gg_rs140951 \\
\hline 2.38 & 10.31 & 2.48 & 14.06 & 3.12 Gg_rs150230 Gg_rs140951 \\
\hline 2.62 & 11.39 & 2.73 & 13.67 & 3.44 Gg_rs150230 Gg_rs140951 \\
\hline 1.81 & 5.9 & 1.89 & 10.95 & 2.38 Gg_rs150230 Gg_rs140951 \\
\hline 1.92 & 7.7 & 2 & 9.56 & 2.52 Gg_rs150230 Gg_rs140951 \\
\hline 3.69 & 15.82 & 3.85 & 22.45 & 4.84 Gg_rs150230 Gg_rs140951 \\
\hline 2.31 & 10.78 & 2.4 & 14.39 & 3.03 Gg_rs150230 Gg_rs140951 \\
\hline 4.65 & 18.27 & 4.85 & 26.23 & 6.11 Gg_rs150230 Gg_rs140951 \\
\hline 1.63 & 7.45 & 1.7 & 10.16 & 2.14 Gg_rs150230 Gg_rs140951 \\
\hline 2.46 & 10.21 & 2.56 & 13.82 & 3.23 Gg_rs150230 Gg_rs140951 \\
\hline 1.44 & 4.34 & 1.5 & 5.97 & 1.89 Gg_rs150230 Gg_rs140951 \\
\hline 3.42 & 15.49 & 3.57 & 17.34 & 4.49 Gg_rs150230 Gg_rs140951 \\
\hline 3.33 & 13.5 & 3.46 & 18.65 & 4.36 Gg_rs150230 Gg_rs140951 \\
\hline 1.76 & 7.62 & 1.83 & 9.34 & 2.3 Gg_rs150230 Gg_rs140951 \\
\hline 1.92 & 8.35 & 2 & 11.21 & 2.52 Gg_rs150230 Gg_rs140951 \\
\hline 2.38 & 9.76 & 2.48 & 13.58 & 3.12 Gg_rs150230 Gg_rs140951 \\
\hline 2.82 & 12.35 & 2.93 & 15.84 & 3.69 Gg_rs150230 Gg_rs140951 \\
\hline 2.79 & 11.33 & 2.91 & 15.65 & 3.66 Gg_rs150230 Gg_rs140951 \\
\hline 2.25 & 10.73 & 2.34 & 13.29 & 2.95 Gg_rs150230 Gg_rs140951 \\
\hline 1.57 & 5.97 & 1.63 & 8.32 & 2.06 Gg_rs150230 Gg_rs140951 \\
\hline 1.79 & 7.99 & 1.87 & 10.23 & 2.35 Gg_rs150230 Gg_rs140951 \\
\hline 2.28 & 7.68 & 2.38 & 11.61 & 3 Gg_rs150230 Gg_rs140951 \\
\hline 2.61 & 11.23 & 2.72 & 13.46 & 3.43 Gg_rs150230 Gg_rs140951 \\
\hline 1.66 & 6.35 & 1.73 & 7.6 & 2.17 Gg_rs150230 Gg_rs140951 \\
\hline 2.95 & 12.93 & 3.08 & 17.4 & 3.88 Gg_rs150230 Gg_rs140951 \\
\hline 1.9 & 9.16 & 1.98 & 10.77 & 2.5 Gg_rs150230 Gg_rs140951 \\
\hline
\end{tabular}




\begin{tabular}{|c|c|c|c|c|}
\hline 1.62 & 5.55 & 1.68 & 6.99 & 2.12 Gg_rs150230 Gg_rs140951 \\
\hline 2.15 & 8.5 & 2.24 & 12.03 & 2.83 Gg_rs150230 Gg_rs140951 \\
\hline 1.66 & 8.81 & 1.73 & 9 & 2.18 Gg_rs150230 Gg_rs140951 \\
\hline 0.91 & 3.02 & 0.95 & 2.45 & 1.2 Gg_rs150230 Gg_rs140951 \\
\hline 0.83 & 3.85 & 0.86 & 4.84 & 1.09 Gg_rs150230 Gg_rs140951 \\
\hline 1.81 & 7.31 & 1.89 & 10.82 & 2.38 Gg_rs150230 Gg_rs140951 \\
\hline 3.16 & 11.44 & 3.29 & 14.38 & 4.14 Gg_rs150230 Gg_rs140951 \\
\hline 2.26 & 10.08 & 2.36 & 12.85 & 2.97 Gg_rs150230 Gg_rs140951 \\
\hline 3.66 & 16.13 & 3.82 & 21.23 & 4.81 Gg_rs150230 Gg_rs140951 \\
\hline 6.35 & 26.9 & 6.62 & 35.47 & 8.34 Gg_rs150230 Gg_rs140951 \\
\hline 2.66 & 9.27 & 2.78 & 16.49 & 3.5 Gg_rs150230 Gg_rs140951 \\
\hline 2.14 & 9.67 & 2.22 & 11.05 & 2.8 Gg_rs150230 Gg_rs140951 \\
\hline 1.22 & 5.41 & 1.27 & 7.11 & 1.6 Gg_rs150230 Gg_rs140951 \\
\hline 2.15 & 7.95 & 2.24 & 13.1 & 2.82 Gg_rs150230 Gg_rs140951 \\
\hline 2.94 & 12.82 & 3.06 & 17.6 & 3.86 Gg_rs150230 Gg_rs140951 \\
\hline 2.87 & 12.24 & 2.99 & 19.22 & 3.76 Gg_rs150230 Gg_rs140951 \\
\hline 2.47 & 10.55 & 2.58 & 11.41 & 3.25 Gg_rs150230 Gg_rs140951 \\
\hline 4.55 & 19.94 & 4.74 & 26.09 & 5.97 Gg_rs150230 Gg_rs140951 \\
\hline 2.75 & 12.22 & 2.87 & 16.13 & 3.61 Gg_rs150230 Gg_rs140951 \\
\hline 3.23 & 13.98 & 3.37 & 18.89 & 4.24 Gg_rs150230 Gg_rs140951 \\
\hline 2.17 & 9.09 & 2.27 & 14.38 & 2.85 Gg_rs150230 Gg_rs140951 \\
\hline 2.56 & 11.31 & 2.67 & 13.39 & 3.36 Gg_rs150230 Gg_rs140951 \\
\hline 1.01 & 3.95 & 1.05 & 5.05 & 1.32 Gg_rs150230 Gg_rs140951 \\
\hline 2 & 9.51 & 2.08 & 10.92 & 2.62 Gg_rs150230 Gg_rs140951 \\
\hline 1.75 & 7.68 & 1.82 & 9.59 & 2.3 Gg_rs150230 Gg_rs140951 \\
\hline 2.4 & 9.69 & 2.5 & 13.33 & 3.15 Gg_rs150230 Gg_rs140951 \\
\hline 2.14 & 7.64 & 2.23 & 10.45 & 2.81 Gg_rs150230 Gg_rs140951 \\
\hline 3.31 & 13.79 & 3.45 & 19.34 & 4.35 Gg_rs150230 Gg_rs140951 \\
\hline 1.91 & 7 & 1.99 & 11.21 & 2.5 Gg_rs150230 Gg_rs140951 \\
\hline 8.49 & 36.29 & 8.84 & 47.56 & 11.14 Gg_rs150230 Gg_rs140951 \\
\hline 7.25 & 32.65 & 7.55 & 40.66 & 9.52 Gg_rs150230 Gg_rs140951 \\
\hline 4.63 & 20.79 & 4.83 & 27.03 & 6.08 Gg_rs150230 Gg_rs140951 \\
\hline 2.63 & 9.42 & 2.74 & 14.99 & 3.45 Gg_rs150230 Gg_rs140951 \\
\hline 2.66 & 12.22 & 2.77 & 13.85 & 3.49 Gg_rs150230 Gg_rs140951 \\
\hline 3.66 & 14.89 & 3.81 & 22.2 & 4.8 Gg_rs150230 Gg_rs140951 \\
\hline 4.18 & 17.03 & 4.36 & 24.66 & 5.49 Gg_rs150230 Gg_rs140951 \\
\hline 3.61 & 16.5 & 3.76 & 20.31 & 4.74 Gg_rs150230 Gg_rs140951 \\
\hline 2.55 & 11.1 & 2.66 & 12.92 & 3.35 Gg_rs150230 Gg_rs140951 \\
\hline 2.32 & 9.04 & 2.42 & 12.28 & 3.04 Gg_rs150230 Gg_rs140951 \\
\hline 2.18 & 9.06 & 2.27 & 13.9 & 2.86 Gg_rs150230 Gg_rs140951 \\
\hline 4.92 & 20.64 & 5.13 & 27.4 & 6.46 Gg_rs150230 Gg_rs140951 \\
\hline 1.74 & 8.38 & 1.81 & 9.55 & 2.28 Gg_rs150230 Gg_rs140951 \\
\hline 2.16 & 8.86 & 2.25 & 11.79 & 2.83 Gg_rs150230 Gg_rs140951 \\
\hline
\end{tabular}




\begin{tabular}{|c|c|c|c|c|}
\hline 4.74 & 20.3 & 4.94 & 28.05 & 6.22 Gg_rs150230 Gg_rs140951 \\
\hline 3.24 & 10.91 & 3.37 & 16.71 & 4.25 Gg_rs150230 Gg_rs140951 \\
\hline 2.45 & 10.8 & 2.56 & 14.52 & 3.22 Gg_rs150230 Gg_rs140951 \\
\hline 5.09 & 21.78 & 5.3 & 27.71 & 6.68 Gg_rs150230 Gg_rs140951 \\
\hline 1.99 & 9.13 & 2.07 & 11.69 & 2.61 Gg_rs150230 Gg_rs140951 \\
\hline 1.74 & 7.27 & 1.81 & 8.71 & 2.28 Gg_rs150230 Gg_rs140951 \\
\hline 1.81 & 7.75 & 1.88 & 9.64 & 2.37 Gg_rs150230 Gg_rs140951 \\
\hline 1.99 & 8.66 & 2.08 & 12.19 & 2.62 Gg_rs150230 Gg_rs140951 \\
\hline 2.45 & 12.61 & 2.55 & 16.63 & 3.21 Gg_rs150230 Gg_rs140951 \\
\hline 8.23 & 35.23 & 8.57 & 47.28 & 10.8 Gg_rs150230 Gg_rs140951 \\
\hline 2.08 & 10.54 & 2.17 & 12.25 & 2.73 Gg_rs150230 Gg_rs140951 \\
\hline 2.1 & 8.7 & 2.19 & 10.85 & 2.76 Gg_rs150230 Gg_rs140951 \\
\hline 2.03 & 6.79 & 2.11 & 11.01 & 2.66 Gg_rs150230 Gg_rs140951 \\
\hline 3.16 & 12.67 & 3.29 & 18.49 & 4.14 Gg_rs150230 Gg_rs140951 \\
\hline 3.02 & 9.97 & 3.15 & 12.36 & 3.97 Gg_rs150230 Gg_rs140951 \\
\hline 5.07 & 19.01 & 5.28 & 26.97 & 6.65 Gg_rs150230 Gg_rs140951 \\
\hline 5.22 & 23.81 & 5.44 & 26.33 & 6.86 Gg_rs150230 Gg_rs140951 \\
\hline 2.03 & 10.12 & 2.11 & 11.56 & 2.66 Gg_rs150230 Gg_rs140951 \\
\hline 2.73 & 11.52 & 2.85 & 15.18 & 3.59 Gg_rs150230 Gg_rs140951 \\
\hline 2.09 & 8.05 & 2.17 & 9.92 & 2.74 Gg_rs150230 Gg_rs140951 \\
\hline 3.3 & 13.27 & 3.43 & 19.34 & 4.33 Gg_rs150230 Gg_rs140951 \\
\hline $0.55 \mathrm{NA}$ & NA & NA & NA & Gg_rs150230 Gg_rs140951 \\
\hline 0.99 & 4.35 & 1.03 & 5.14 & 1.3 Gg_rs150230 Gg_rs140951 \\
\hline 1 & 3.46 & 1.05 & 5.39 & 1.32 Gg_rs150230 Gg_rs140951 \\
\hline 5.31 & 21.81 & 5.53 & 30.06 & 6.97 Gg_rs150230 Gg_rs140951 \\
\hline 6.55 & 27.32 & 6.82 & 36.03 & 8.6 Gg_rs150230 Gg_rs140951 \\
\hline 2.38 & 10.15 & 2.47 & 10.8 & 3.12 Gg_rs150230 Gg_rs140951 \\
\hline 1.06 & 7.98 & 1.1 & 7.83 & 1.39 Gg_rs150230 Gg_rs140951 \\
\hline 8.51 & 37.54 & 8.86 & 46.93 & 11.17 Gg_rs150230 Gg_rs140951 \\
\hline 1.93 & 7.09 & 2.02 & 11.11 & 2.54 Gg_rs150230 Gg_rs140951 \\
\hline 4.32 & 16.4 & 4.5 & 23.48 & 5.66 Gg_rs150230 Gg_rs140951 \\
\hline 2.68 & 10.29 & 2.79 & 16.14 & 3.52 Gg_rs150230 Gg_rs140951 \\
\hline 2.13 & 9.54 & 2.22 & 11.4 & 2.8 Gg_rs150230 Gg_rs140951 \\
\hline 4.74 & 19.45 & 4.93 & 24.78 & 6.22 Gg_rs150230 Gg_rs140951 \\
\hline 5.14 & 21.98 & 5.35 & 26.63 & 6.74 Gg_rs150230 Gg_rs140951 \\
\hline 4.37 & 19.06 & 4.55 & 23.79 & 5.74 Gg_rs150230 Gg_rs140951 \\
\hline 2.13 & 11.02 & 2.21 & 10.65 & 2.79 Gg_rs150230 Gg_rs140951 \\
\hline 1.76 & 8.07 & 1.84 & 9.74 & 2.31 Gg_rs150230 Gg_rs140951 \\
\hline 2.09 & 8.51 & 2.18 & 11.5 & 2.75 Gg_rs150230 Gg_rs140951 \\
\hline 1.67 & 6.32 & 1.74 & 8.11 & 2.19 Gg_rs150230 Gg_rs140951 \\
\hline 0.68 & 2.77 & 0.7 & 1.53 & 0.89 Gg_rs150230 Gg_rs140951 \\
\hline 5.01 & 22.2 & 5.22 & 26.61 & 6.57 Gg_rs150230 Gg_rs140951 \\
\hline 5.15 & 21.58 & 5.36 & 27.79 & 6.75 Gg_rs150230 Gg_rs140951 \\
\hline
\end{tabular}




\begin{tabular}{|c|c|c|c|c|}
\hline 7.56 & 32.57 & 7.87 & 42.59 & 9.92 Gg_rs150230 Gg_rs140951 \\
\hline 1.83 & 6.19 & 1.9 & 10.95 & 2.4 Gg_rs150230 Gg_rs140951 \\
\hline 1.81 & 7.94 & 1.88 & 8.38 & 2.37 Gg_rs150230 Gg_rs140951 \\
\hline 4.14 & 18.82 & 4.32 & 24.39 & 5.44 Gg_rs150230 Gg_rs140951 \\
\hline 1.68 & 7.71 & 1.75 & 9.38 & 2.2 Gg_rs150230 Gg_rs140951 \\
\hline 3.15 & 13.36 & 3.28 & 16.75 & 4.13 Gg_rs150230 Gg_rs140951 \\
\hline 1.6 & 6.75 & 1.67 & 8.88 & 2.1 Gg_rs150230 Gg_rs140951 \\
\hline 2.36 & 10.74 & 2.45 & 13.49 & 3.09 Gg_rs150230 Gg_rs140951 \\
\hline 2.37 & 10.41 & 2.47 & 13.51 & 3.11 Gg_rs150230 Gg_rs140951 \\
\hline 1.37 & 6.83 & 1.43 & 3.07 & 1.8 Gg_rs150230 Gg_rs140951 \\
\hline 7.37 & 29.83 & 7.68 & 41.45 & 9.67 Gg_rs150230 Gg_rs140951 \\
\hline 2.08 & 8.85 & 2.16 & 10.03 & 2.72 Gg_rs150230 Gg_rs140951 \\
\hline 1.88 & 7.06 & 1.96 & 9.81 & 2.47 Gg_rs150230 Gg_rs140951 \\
\hline 7.38 & 31.31 & 7.69 & 43.05 & 9.68 Gg_rs150230 Gg_rs140951 \\
\hline 3.18 & 11.96 & 3.31 & 15.98 & 4.17 Gg_rs150230 Gg_rs140951 \\
\hline 1.63 & 6.85 & 1.69 & 9.46 & 2.13 Gg_rs150230 Gg_rs140951 \\
\hline 0.92 & 3.9 & 0.96 & 4.71 & 1.21 Gg_rs150230 Gg_rs140951 \\
\hline 7.54 & 32.95 & 7.85 & 40.47 & 9.89 Gg_rs150230 Gg_rs140951 \\
\hline 3.23 & 14.24 & 3.36 & 17.35 & 4.24 Gg_rs150230 Gg_rs140951 \\
\hline 4.29 & 20.33 & 4.46 & 25.3 & 5.62 Gg_rs150230 Gg_rs140951 \\
\hline 1.92 & 6.52 & 2 & 4.01 & 2.51 Gg_rs150230 Gg_rs140951 \\
\hline 9.8 & 45.3 & 10.21 & 54.45 & 12.86 Gg_rs150230 Gg_rs140951 \\
\hline 3.88 & 15.78 & 4.04 & 19.45 & 5.09 Gg_rs150230 Gg_rs140951 \\
\hline 9.3 & 37.18 & 9.69 & 52.65 & 12.2 Gg_rs150230 Gg_rs140951 \\
\hline 1.88 & 8.27 & 1.96 & 10.79 & 2.47 Gg_rs150230 Gg_rs140951 \\
\hline 4.46 & 18.1 & 4.65 & 24.14 & 5.85 Gg_rs150230 Gg_rs140951 \\
\hline 7.82 & 34.09 & 8.15 & 42.94 & 10.26 Gg_rs150230 Gg_rs140951 \\
\hline 4.27 & 17.37 & 4.45 & 24.42 & 5.6 Gg_rs150230 Gg_rs140951 \\
\hline 3.13 & 12.53 & 3.26 & 19.22 & 4.1 Gg_rs150230 Gg_rs140951 \\
\hline 1.56 & 8.07 & 1.62 & 4.74 & 2.04 Gg_rs150230 Gg_rs140951 \\
\hline 9.23 & 41.38 & 9.61 & 52.26 & 12.11 Gg_rs150230 Gg_rs140951 \\
\hline 9.94 & 42.1 & 10.36 & 53.77 & 13.05 Gg_rs150230 Gg_rs140951 \\
\hline 1.97 & 9.25 & 2.05 & 9.21 & 2.58 Gg_rs150230 Gg_rs140951 \\
\hline 7.72 & 29.82 & 8.04 & 41.75 & 10.13 Gg_rs150230 Gg_rs140951 \\
\hline 5.54 & 22.67 & 5.77 & 31.89 & 7.27 Gg_rs150230 Gg_rs140951 \\
\hline 6.52 & 31.62 & 6.79 & 39.49 & 8.56 Gg_rs150230 Gg_rs140951 \\
\hline 0.71 & 4.52 & 0.74 & 4.8 & 0.94 Gg_rs150230 Gg_rs140951 \\
\hline 4.03 & 17.33 & 4.2 & 23.27 & 5.29 Gg_rs150230 Gg_rs140951 \\
\hline 7.8 & 31.18 & 8.12 & 44.22 & 10.24 Gg_rs150230 Gg_rs140951 \\
\hline 4.62 & 17.37 & 4.81 & 25.14 & 6.06 Gg_rs150230 Gg_rs140951 \\
\hline 12.05 & 52.31 & 12.55 & 65.14 & 15.81 Gg_rs150230 Gg_rs140951 \\
\hline 1.98 & 9.37 & 2.06 & 9.88 & 2.6 Gg_rs150230 Gg_rs140951 \\
\hline 8.12 & 34.23 & 8.46 & 46.2 & 10.66 Gg_rs150230 Gg_rs140951 \\
\hline
\end{tabular}




\begin{tabular}{|c|c|c|c|c|}
\hline 5.61 & 24.48 & 5.84 & 32.12 & 7.36 Gg_rs150230 Gg_rs140951 \\
\hline 2.05 & 7.39 & 2.14 & 11.39 & 2.69 Gg_rs150230 Gg_rs140951 \\
\hline 5.83 & 23.75 & 6.08 & 30.9 & 7.65 Gg_rs150230 Gg_rs140951 \\
\hline 2.59 & 10.76 & 2.7 & 13.82 & 3.4 Gg_rs150230 Gg_rs140951 \\
\hline 1.02 & 6.56 & 1.06 & 7.14 & 1.34 Gg_rs150230 Gg_rs140951 \\
\hline 2.18 & 9.03 & 2.27 & 13.68 & 2.86 Gg_rs150230 Gg_rs140951 \\
\hline 5.42 & 22.87 & 5.64 & 27.97 & 7.11 Gg_rs150230 Gg_rs140951 \\
\hline 5.54 & 21.83 & 5.77 & 29.56 & 7.27 Gg_rs150230 Gg_rs140951 \\
\hline 1.51 & 6.14 & 1.57 & 7.31 & 1.98 Gg_rs150230 Gg_rs140951 \\
\hline 1.01 & 4.05 & 1.06 & 4.38 & 1.33 Gg_rs150230 Gg_rs140951 \\
\hline 0.63 & 2.71 & 0.66 & 2.3 & 0.83 Gg_rs150230 Gg_rs140951 \\
\hline 3.38 & 13.1 & 3.52 & 18.87 & 4.44 Gg_rs150230 Gg_rs140951 \\
\hline 2.05 & 7.78 & 2.13 & 10.8 & 2.69 Gg_rs150230 Gg_rs140951 \\
\hline 14.36 & 63.28 & 14.96 & 73.8 & 18.84 Gg_rs150230 Gg_rs140951 \\
\hline 2.03 & 9.63 & 2.12 & 11.43 & 2.67 Gg_rs150230 Gg_rs140951 \\
\hline 4.47 & 19.04 & 4.66 & 27.82 & 5.87 Gg_rs150230 Gg_rs140951 \\
\hline 3.14 & 13.67 & 3.27 & 16.32 & 4.12 Gg_rs150230 Gg_rs140951 \\
\hline 3.5 & 15.69 & 3.64 & 22.01 & 4.59 Gg_rs150230 Gg_rs140951 \\
\hline 7.22 & 32.06 & 7.52 & 40.28 & 9.47 Gg_rs150230 Gg_rs140951 \\
\hline 1.73 & 6.61 & 1.8 & 8.88 & 2.27 Gg_rs150230 Gg_rs140951 \\
\hline 6.39 & 26.73 & 6.65 & 37.64 & 8.38 Gg_rs150230 Gg_rs140951 \\
\hline 2.43 & 8.97 & 2.53 & 12.04 & 3.19 Gg_rs150230 Gg_rs140951 \\
\hline 10.22 & 47.37 & 10.64 & 54.72 & 13.41 Gg_rs150230 Gg_rs140951 \\
\hline 8.69 & 38.39 & 9.05 & 48.95 & 11.4 Gg_rs150230 Gg_rs140951 \\
\hline 2.18 & 9.52 & 2.27 & 11.46 & 2.86 Gg_rs150230 Gg_rs140951 \\
\hline 10.92 & 45.21 & 11.38 & 55.36 & 14.33 Gg_rs150230 Gg_rs140951 \\
\hline 2.02 & 9.77 & 2.1 & 7.71 & 2.65 Gg_rs150230 Gg_rs140951 \\
\hline 1.2 & 4.37 & 1.25 & 7.27 & 1.58 Gg_rs150230 Gg_rs140951 \\
\hline 6.44 & 23.97 & 6.71 & 33.53 & 8.45 Gg_rs150230 Gg_rs140951 \\
\hline 4.02 & 16.31 & 4.19 & 21.6 & 5.28 Gg_rs150230 Gg_rs140951 \\
\hline 1.69 & 5.68 & 1.76 & 8.68 & 2.22 Gg_rs150230 Gg_rs140951 \\
\hline 2.18 & 9.62 & 2.27 & 10.75 & 2.87 Gg_rs150230 Gg_rs140951 \\
\hline 9.59 & 42.45 & 9.99 & 54.06 & 12.58 Gg_rs150230 Gg_rs140951 \\
\hline 2.7 & 11.25 & 2.81 & 10.73 & 3.54 Gg_rs150230 Gg_rs140951 \\
\hline 3.82 & 14.95 & 3.98 & 15.97 & 5.01 Gg_rs150230 Gg_rs140951 \\
\hline 3.13 & 11.33 & 3.26 & 14.02 & 4.11 Gg_rs150230 Gg_rs140951 \\
\hline 3.73 & 17.52 & 3.88 & 19.58 & 4.89 Gg_rs150230 Gg_rs140951 \\
\hline 3.89 & 13.81 & 4.05 & 21.15 & 5.11 X15_601272عGg_rs140951 \\
\hline 6.32 & 28.65 & 6.58 & 37.28 & 8.29 Gg_rs150230 Gg_rs140951 \\
\hline 2.76 & 11.33 & 2.88 & 15.15 & 3.63 Gg_rs150230 Gg_rs140951 \\
\hline 10.07 & 43.63 & 10.49 & 53.15 & 13.21 Gg_rs150230 Gg_rs140951 \\
\hline 2.35 & 7.39 & 2.45 & 9.41 & 3.09 Gg_rs150230 Gg_rs140951 \\
\hline 3.97 & 17.38 & 4.14 & 23.96 & 5.21 Gg_rs150230 Gg_rs140951 \\
\hline
\end{tabular}




\begin{tabular}{|c|c|c|c|c|}
\hline 1.18 & 5.03 & 1.23 & 3.18 & 1.55 Gg_rs150230 Gg_rs140951 \\
\hline 9.16 & 39.21 & 9.54 & 48.1 & 12.02 Gg_rs150230 Gg_rs140951 \\
\hline 1.93 & 8.72 & 2.01 & 4.15 & 2.54 Gg_rs150230 Gg_rs140951 \\
\hline 1.73 & 7.11 & 1.8 & 8.99 & 2.27 Gg_rs150230 Gg_rs140951 \\
\hline 5.23 & 20.75 & 5.45 & 27.72 & 6.87 Gg_rs150230 Gg_rs140951 \\
\hline 2.08 & 7.89 & 2.16 & 8.4 & 2.73 Gg_rs150230 Gg_rs140951 \\
\hline 2.93 & 11.01 & 3.05 & 13.99 & 3.84 Gg_rs150230 Gg_rs140951 \\
\hline 12.68 & 55.52 & 13.2 & 67.49 & 16.64 Gg_rs150230 Gg_rs140951 \\
\hline 3.03 & 15.34 & 3.16 & 15.87 & 3.98 Gg_rs150230 Gg_rs140951 \\
\hline 9.1 & 42.29 & 9.48 & 47.72 & 11.94 Gg_rs150230 Gg_rs140951 \\
\hline 6.38 & 28.37 & 6.64 & 35.1 & 8.37 Gg_rs150230 Gg_rs140951 \\
\hline 11.34 & 50.78 & 11.81 & 58.86 & 14.88 Gg_rs150230 Gg_rs140951 \\
\hline 6.15 & 26.17 & 6.41 & 31.04 & 8.07 Gg_rs150230 Gg_rs140951 \\
\hline 2.5 & 9.64 & 2.6 & 12.23 & 3.28 Gg_rs150230 Gg_rs140951 \\
\hline 4.53 & 18.89 & 4.72 & 26.19 & 5.94 Gg_rs150230 Gg_rs140951 \\
\hline 1.4 & 5.78 & 1.46 & 8.3 & 1.84 Gg_rs150230 Gg_rs140951 \\
\hline 3.15 & 13.01 & 3.28 & 17.17 & 4.13 Gg_rs150230 Gg_rs140951 \\
\hline 3.51 & 11.95 & 3.66 & 15.44 & 4.61 Gg_rs150230 Gg_rs140951 \\
\hline 1.69 & 5.82 & 1.76 & 10.09 & 2.21 Gg_rs150230 Gg_rs140951 \\
\hline 4.31 & 18.67 & 4.49 & 22.27 & 5.66 Gg_rs150194 Gg_rs140951 \\
\hline 4.14 & 17.81 & 4.31 & 17.43 & 5.43 Gg_rs150230 Gg_rs140951 \\
\hline 13.25 & 56.84 & 13.8 & 63.22 & 17.39 Gg_rs150230 Gg_rs140951 \\
\hline 2.01 & 11.27 & 2.09 & 8.91 & 2.64 Gg_rs150230 Gg_rs140951 \\
\hline 3.89 & 16.44 & 4.06 & 19.5 & 5.11 Gg_rs150230 Gg_rs140951 \\
\hline 8.66 & 40.64 & 9.02 & 42.49 & 11.36 Gg_rs150230 Gg_rs140951 \\
\hline 10.57 & 45.76 & 11.01 & 52.92 & 13.87 Gg_rs150230 Gg_rs140951 \\
\hline 8.59 & 35.41 & 8.95 & 38.54 & 11.28 Gg_rs150230 Gg_rs140951 \\
\hline 15.75 & 68.52 & 16.41 & 76.79 & 20.67 Gg_rs150230 Gg_rs140951 \\
\hline 2.82 & 13 & 2.94 & 12.38 & 3.7 Gg_rs150230 Gg_rs140951 \\
\hline 2.82 & 7.01 & 2.94 & 11 & 3.7 Gg_rs150230 Gg_rs140951 \\
\hline 5.69 & 26.75 & 5.93 & 24.98 & 7.47 Gg_rs150230 Gg_rs140951 \\
\hline 1.04 & 2.36 & 1.09 & 2.2 & 1.37 Gg_rs150230 Gg_rs140951 \\
\hline 0.77 & 3.09 & 0.8 & 2.57 & 1.01 Gg_rs150230 Gg_rs140951 \\
\hline 0.99 & 4.13 & 1.03 & 4.8 & 1.3 Gg_rs150230 Gg_rs140951 \\
\hline 2.75 & 12.44 & 2.86 & 15.01 & 3.61 Gg_rs150230 Gg_rs140951 \\
\hline 0.9 & 3.53 & 0.94 & 5.01 & 1.18 Gg_rs150230 Gg_rs140951 \\
\hline 7.44 & 31.12 & 7.75 & 31.91 & 9.77 Gg_rs150230 Gg_rs140951 \\
\hline 4.8 & 15.84 & 5 & 23.74 & 6.3 Gg_rs150230 Gg_rs140951 \\
\hline 1.68 & 5.32 & 1.75 & 7.68 & 2.2 Gg_rs150230 Gg_rs140951 \\
\hline 1.98 & 9.71 & 2.07 & 7.68 & 2.6 Gg_rs150230 Gg_rs140951 \\
\hline 2.4 & 11.23 & 2.5 & 12.18 & 3.15 Gg_rs150194 Gg_rs140951 \\
\hline 1.82 & 5.69 & 1.9 & 9.1 & 2.39 Gg_rs150230 Gg_rs140951 \\
\hline 2.08 & 7.94 & 2.17 & 8.88 & 2.73 Gg_rs150230 Gg_rs140951 \\
\hline
\end{tabular}




\begin{tabular}{|c|c|c|c|c|}
\hline 1.94 & 9.49 & 2.02 & 8.79 & 2.55 Gg_rs150230 Gg_rs140951 \\
\hline 2.17 & 8.4 & 2.26 & 10.92 & 2.85 Gg_rs150230 Gg_rs140951 \\
\hline 4.84 & 18.72 & 5.04 & 19.52 & 6.35 Gg_rs150230 Gg_rs140951 \\
\hline 2.81 & 12.64 & 2.93 & 10.19 & 3.69 Gg_rs150230 Gg_rs140951 \\
\hline $0.98 \mathrm{NA}$ & NA & NA & NA & Gg_rs150230 Gg_rs140951 \\
\hline 1.97 & 7.34 & 2.05 & 7.1 & 2.58 Gg_rs150230 Gg_rs140951 \\
\hline 5.78 & 25.6 & 6.02 & 32.13 & 7.59 Gg_rs150230 Gg_rs140951 \\
\hline 6.38 & 24.87 & 6.65 & 30.25 & 8.38 Gg_rs150230 Gg_rs140951 \\
\hline 1.67 & 8.05 & 1.74 & 2.79 & 2.19 Gg_rs150230 Gg_rs140951 \\
\hline 4.77 & 19.02 & 4.97 & 18.88 & 6.26 Gg_rs150230 Gg_rs140951 \\
\hline 5.5 & 20.2 & 5.73 & 28.87 & 7.22 Gg_rs150230 Gg_rs140951 \\
\hline 1.25 & 5.28 & 1.3 & 2.87 & 1.64 Gg_rs150230 Gg_rs140951 \\
\hline 1.87 & 8.31 & 1.95 & 7.53 & 2.46 Gg_rs150230 Gg_rs140951 \\
\hline 3.7 & 11.16 & 3.85 & 16.07 & 4.86 Gg_rs150194 Gg_rs140951 \\
\hline 2.25 & 8.41 & 2.34 & 11.37 & 2.95 Gg_rs150230 Gg_rs140951 \\
\hline 5.63 & 23.14 & 5.86 & 27.94 & 7.39 Gg_rs150230 Gg_rs140951 \\
\hline 9.06 & 41.73 & 9.44 & 40.81 & 11.89 Gg_rs150230 Gg_rs140951 \\
\hline 1.05 & 6.95 & 1.1 & 4.14 & 1.38 Gg_rs150230 Gg_rs140951 \\
\hline 1.55 & 8.26 & 1.62 & 9.83 & 2.04 Gg_rs150230 Gg_rs140951 \\
\hline 3.53 & 11.95 & 3.67 & 14.06 & 4.63 Gg_rs150194 Gg_rs140951 \\
\hline 2.98 & 14.83 & 3.11 & 16.56 & 3.91 Gg_rs150230 Gg_rs140951 \\
\hline 3.29 & 12.43 & 3.42 & 14.18 & 4.31 Gg_rs150230 Gg_rs140951 \\
\hline 1.02 & 4.34 & 1.06 & 3.92 & 1.34 Gg_rs150230 Gg_rs140951 \\
\hline 1.61 & 9.08 & 1.68 & 7.89 & 2.12 Gg_rs150230 Gg_rs140951 \\
\hline 2.83 & 10.93 & 2.94 & 8.19 & 3.71 Gg_rs150230 Gg_rs140951 \\
\hline 3.27 & 12.16 & 3.4 & 15.99 & 4.29 Gg_rs150194 Gg_rs140951 \\
\hline 1.71 & 7.31 & 1.78 & 9.08 & 2.25 X15_601272عGg_rs140951 \\
\hline 1.33 & 7.06 & 1.38 & 4.42 & 1.74 Gg_rs150230 Gg_rs140951 \\
\hline 1.27 & 5.53 & 1.32 & 5.67 & 1.67 Gg_rs150230 Gg_rs140951 \\
\hline 4.26 & 20.2 & 4.44 & 17.73 & 5.59 Gg_rs150230 Gg_rs140951 \\
\hline 4.59 & 17.17 & 4.78 & 22 & 6.02 Gg_rs150230 Gg_rs140951 \\
\hline 3.45 & 15.72 & 3.59 & 13.59 & 4.52 Gg_rs150230 Gg_rs140951 \\
\hline 2.18 & 7.94 & 2.27 & 10.22 & 2.86 Gg_rs150230 Gg_rs140951 \\
\hline 2.58 & 8.53 & 2.69 & 9.83 & 3.38 Gg_rs150230 Gg_rs140951 \\
\hline 7.88 & 34.09 & 8.21 & 39.69 & 10.35 Gg_rs150230 Gg_rs140951 \\
\hline 10.19 & 39.32 & 10.61 & 43.53 & 13.37 X15_6012728Gg_rs140951 \\
\hline 2.25 & 7.35 & 2.35 & 12.15 & 2.96 Gg_rs150230 Gg_rs140951 \\
\hline 7.77 & 33.44 & 8.09 & 33.5 & 10.2 Gg_rs150230 Gg_rs140951 \\
\hline 2 & 8.51 & 2.08 & 5.53 & 2.62 Gg_rs150230 Gg_rs140951 \\
\hline 1.17 & 4.78 & 1.22 & 5.41 & 1.54 Gg_rs150194 Gg_rs140951 \\
\hline 4.34 & 17.03 & 4.52 & 19.41 & 5.69 Gg_rs150230 Gg_rs140951 \\
\hline 1.54 & 6.79 & 1.6 & 7.25 & 2.02 Gg_rs150230 Gg_rs140951 \\
\hline 10.73 & 45.78 & 11.18 & 51.14 & 14.08 Gg_rs150230 Gg_rs140951 \\
\hline
\end{tabular}




\begin{tabular}{|c|c|c|c|c|}
\hline 3.72 & 15.8 & 3.87 & 13.03 & 4.88 Gg_rs150230 Gg_rs140951 \\
\hline 9.33 & 40.96 & 9.72 & 43.54 & 12.24 Gg_rs150230 Gg_rs140951 \\
\hline 6.36 & 26.51 & 6.62 & 23.07 & 8.34 Gg_rs150230 Gg_rs140951 \\
\hline 1.12 & 5.4 & 1.16 & 2.12 & 1.46 Gg_rs150230 Gg_rs140951 \\
\hline 1.07 & 4.63 & 1.12 & 4.56 & 1.41 Gg_rs150230 Gg_rs140951 \\
\hline 2.49 & 10.67 & 2.59 & 7.58 & 3.26 Gg_rs150230 Gg_rs140951 \\
\hline 3.15 & 12.73 & 3.28 & 15.84 & 4.13 Gg_rs150230 Gg_rs140951 \\
\hline 2.84 & 10.89 & 2.95 & 12.99 & 3.72 Gg_rs150230 Gg_rs140951 \\
\hline 2.13 & 10 & 2.22 & 8.33 & 2.8 Gg_rs150230 Gg_rs140951 \\
\hline 2.13 & 8.52 & 2.21 & 8.9 & 2.79 Gg_rs150194 Gg_rs140951 \\
\hline 9.97 & 40.25 & 10.39 & 42.32 & 13.09 Gg_rs150230 Gg_rs140951 \\
\hline 1.69 & 7.35 & 1.76 & 7.29 & 2.22 Gg_rs150230 Gg_rs140951 \\
\hline 4.58 & 17.03 & 4.77 & 16.84 & 6.01 Gg_rs150194 Gg_rs140951 \\
\hline 4.87 & 20.7 & 5.07 & 16.65 & 6.39 Gg_rs150230 Gg_rs140951 \\
\hline 1.77 & 8.31 & 1.85 & 8.43 & 2.33 Gg_rs150230 Gg_rs140951 \\
\hline 3.18 & 13.7 & 3.32 & 12.93 & 4.18 Gg_rs150230 Gg_rs140951 \\
\hline 1.97 & 11.75 & 2.06 & 7.11 & 2.59 Gg_rs150230 Gg_rs140951 \\
\hline 9.38 & 36.66 & 9.77 & 37.45 & 12.31 X15_6012728Gg_rs140951 \\
\hline 3.02 & 14.46 & 3.15 & 12.89 & 3.97 Gg_rs150230 Gg_rs140951 \\
\hline 3.08 & 10.47 & 3.21 & 10.27 & 4.04 Gg_rs150230 Gg_rs140951 \\
\hline 7.99 & 34.9 & 8.33 & 33.07 & 10.49 Gg_rs150194 Gg_rs140951 \\
\hline 2.03 & 8.83 & 2.12 & 4.16 & 2.67 Gg_rs150230 Gg_rs140951 \\
\hline 1.59 & 7.27 & 1.66 & 7.55 & 2.09 Gg_rs150230 Gg_rs140951 \\
\hline $0.64 \mathrm{NA}$ & NA & NA & NA & Gg_rs150230 Gg_rs150257 \\
\hline 4.44 & 18.68 & 4.63 & 16.22 & 5.83 Gg_rs150230 Gg_rs140951 \\
\hline 5.33 & 22.62 & 5.55 & 18.83 & 7 Gg_rs150230 Gg_rs140951 \\
\hline 5.63 & 23.24 & 5.86 & 21.08 & 7.38 Gg_rs150230 Gg_rs140951 \\
\hline 2.23 & 10.83 & 2.33 & 8.54 & 2.93 Gg_rs150230 Gg_rs140951 \\
\hline 1.48 & 7.51 & 1.54 & 7.3 & 1.94 Gg_rs150230 Gg_rs140951 \\
\hline 2.96 & 15.27 & 3.08 & 4.79 & 3.88 Gg_rs150230 Gg_rs140951 \\
\hline 0.79 & 4.76 & 0.82 & 4.1 & 1.03 Gg_rs150230 Gg_rs140951 \\
\hline 2.53 & 11.1 & 2.64 & 9.82 & 3.32 X15_6012728Gg_rs140951 \\
\hline 4.06 & 19.77 & 4.23 & 14.25 & 5.33 Gg_rs150230 Gg_rs140951 \\
\hline 3.65 & 11.82 & 3.8 & 13.45 & 4.79 Gg_rs150194 Gg_rs140951 \\
\hline 2.72 & 11.31 & 2.84 & 5.1 & 3.57 Gg_rs150230 Gg_rs140951 \\
\hline 3.24 & 13.56 & 3.38 & 7.43 & 4.25 Gg_rs150230 Gg_rs140951 \\
\hline 1.9 & 9.47 & 1.98 & 3.14 & 2.49 Gg_rs150230 Gg_rs140951 \\
\hline 1.85 & 9.44 & 1.93 & 3.39 & 2.43 Gg_rs150230 Gg_rs140951 \\
\hline 2.84 & 13 & 2.96 & 5.34 & 3.73 Gg_rs150230 Gg_rs140951 \\
\hline 1.27 & 5.87 & 1.32 & 1.72 & 1.66 Gg_rs150230 Gg_rs140951 \\
\hline 4.28 & 18.86 & 4.46 & 10.78 & 5.62 Gg_rs150230 Gg_rs140951 \\
\hline 2.39 & 8.11 & 2.49 & 7.48 & 3.14 Gg_rs150230 Gg_rs150257 \\
\hline 3.03 & 12.45 & 3.16 & 4.6 & 3.98 Gg_rs150230 Gg_rs150257 \\
\hline
\end{tabular}




\begin{tabular}{|c|c|c|c|c|c|}
\hline 2.16 & 9.88 & 2.25 & 2.77 & 2.83 & Gg_rs150230 Gg_rs140951 \\
\hline 3.31 & 11.3 & 3.44 & 10.28 & 4.34 & Gg_rs150230 Gg_rs150257. \\
\hline 1.61 & 6.96 & 1.68 & 5.08 & 2.11 & Gg_rs150230 Gg_rs150257. \\
\hline 1.42 & 6.38 & 1.48 & 4.08 & 1.86 & Gg_rs150230 Gg_rs150257. \\
\hline 1.7 & 4.82 & 1.56 & 3.73 & 2.23 & Gg_rs150230 Gg_rs150257. \\
\hline $0.41 \mathrm{NA}$ & NA & NA & NA & & Gg_rs150194 Gg_rs150257. \\
\hline 2.24 & 5.83 & 2.06 & 7.6 & 2.94 & Gg_rs150230 Gg_rs150257. \\
\hline $0.33 \mathrm{NA}$ & NA & NA & NA & & Gg_rs150230 Gg_rs150257. \\
\hline 0.79 & 2.8 & 0.72 & 2.71 & 1.03 & Gg_rs150238 Gg_rs150257. \\
\hline 0.61 & 1.55 & 0.56 & 0.9 & 0.8 & Gg_rs150238 Gg_rs150257. \\
\hline 1.12 & 3.94 & 1.03 & 3.35 & 1.47 & Gg_rs150238 Gg_rs150257. \\
\hline $0.46 \mathrm{NA}$ & NA & NA & NA & & Gg_rs150238 Gg_rs150257. \\
\hline 1.1 & 3.88 & 1.01 & 3.71 & 1.44 & Gg_rs150238 Gg_rs150257. \\
\hline $0.68 \mathrm{NA}$ & NA & NA & NA & & Gg_rs150238 Gg_rs150257. \\
\hline $0.56 \mathrm{NA}$ & NA & NA & NA & & Gg_rs150238 Gg_rs150257. \\
\hline $0.43 \mathrm{NA}$ & NA & NA & NA & & Gg_rs150238 Gg_rs150257. \\
\hline 0.31 & 1.29 & 0.29 & 0.78 & 0.41 & Gg_rs150238 Gg_rs150257. \\
\hline $0.83 \mathrm{NA}$ & NA & NA & NA & & Gg_rs140951 Gg_rs150262 \\
\hline $0.5 \mathrm{NA}$ & NA & NA & NA & & Gg_rs140951 Gg_rs150262 \\
\hline $0.57 \mathrm{NA}$ & NA & NA & NA & & Gg_rs140951Gg_rs150262 \\
\hline 0.86 & 1.75 & 0.71 & 1.22 & 1.12 & Gg_rs140959 Gg_rs150262 \\
\hline 0.78 & 1.42 & 0.77 & -1.74 & 1.04 & Gg_rs150267 Gg_rs150267 \\
\hline 2.68 & -2.15 & 2.64 & -6.21 & 3.56 & Gg_rs150267 Gg_rs150267 \\
\hline 0.92 & 1.62 & 0.9 & 0.74 & 1.22 & Gg_rs150267 Gg_rs150267 \\
\hline $2.96 \mathrm{NA}$ & NA & NA & NA & & Gg_rs150267 Gg_rs150267 \\
\hline $1.3 \mathrm{NA}$ & NA & NA & NA & & Gg_rs150267 Gg_rs150267 \\
\hline $1.37 \mathrm{NA}$ & NA & NA & NA & & Gg_rs150267 Gg_rs150267 \\
\hline $0.77 \mathrm{NA}$ & NA & NA & NA & & Gg_rs150267 Gg_rs150267 \\
\hline 0.16 & 0 & 0.16 & 1 & 0.22 & Gg_rs150267 Gg_rs150267 \\
\hline 0.36 & -0.55 & 0.35 & 1.37 & 0.48 & Gg_rs150267 Gg_rs150267 \\
\hline $0.24 \mathrm{NA}$ & NA & NA & NA & & Gg_rs150267 Gg_rs150267 \\
\hline $0.31 \mathrm{NA}$ & NA & NA & NA & & Gg_rs150267 Gg_rs150267 \\
\hline $0.3 \mathrm{NA}$ & NA & NA & NA & & Gg_rs150267 Gg_rs150267 \\
\hline $0.61 \mathrm{NA}$ & NA & NA & NA & & Gg_rs150267 Gg_rs150267 \\
\hline $0.48 \mathrm{NA}$ & NA & NA & NA & & Gg_rs150267 Gg_rs150267 \\
\hline $0.32 \mathrm{NA}$ & NA & NA & NA & & Gg_rs150267 Gg_rs150267 \\
\hline 3.7 NA & NA & NA & NA & & Gg_rs150267 Gg_rs140966: \\
\hline $2.56 \mathrm{NA}$ & NA & NA & NA & & Gg_rs150267 Gg_rs140966: \\
\hline $2.36 \mathrm{NA}$ & NA & NA & NA & & Gg_rs150267 Gg_rs140966: \\
\hline $3.57 \mathrm{NA}$ & NA & NA & NA & & Gg_rs150267 Gg_rs140966: \\
\hline 0.6 & -0.06 & 0.59 & 4.65 & 0.79 & Gg_rs150267 Gg_rs140966: \\
\hline $0.22 \mathrm{NA}$ & NA & NA & NA & & Gg_rs150267 Gg_rs140966: \\
\hline $0.33 \mathrm{NA}$ & NA & NA & NA & & Gg_rs150267 Gg_rs140966: \\
\hline
\end{tabular}




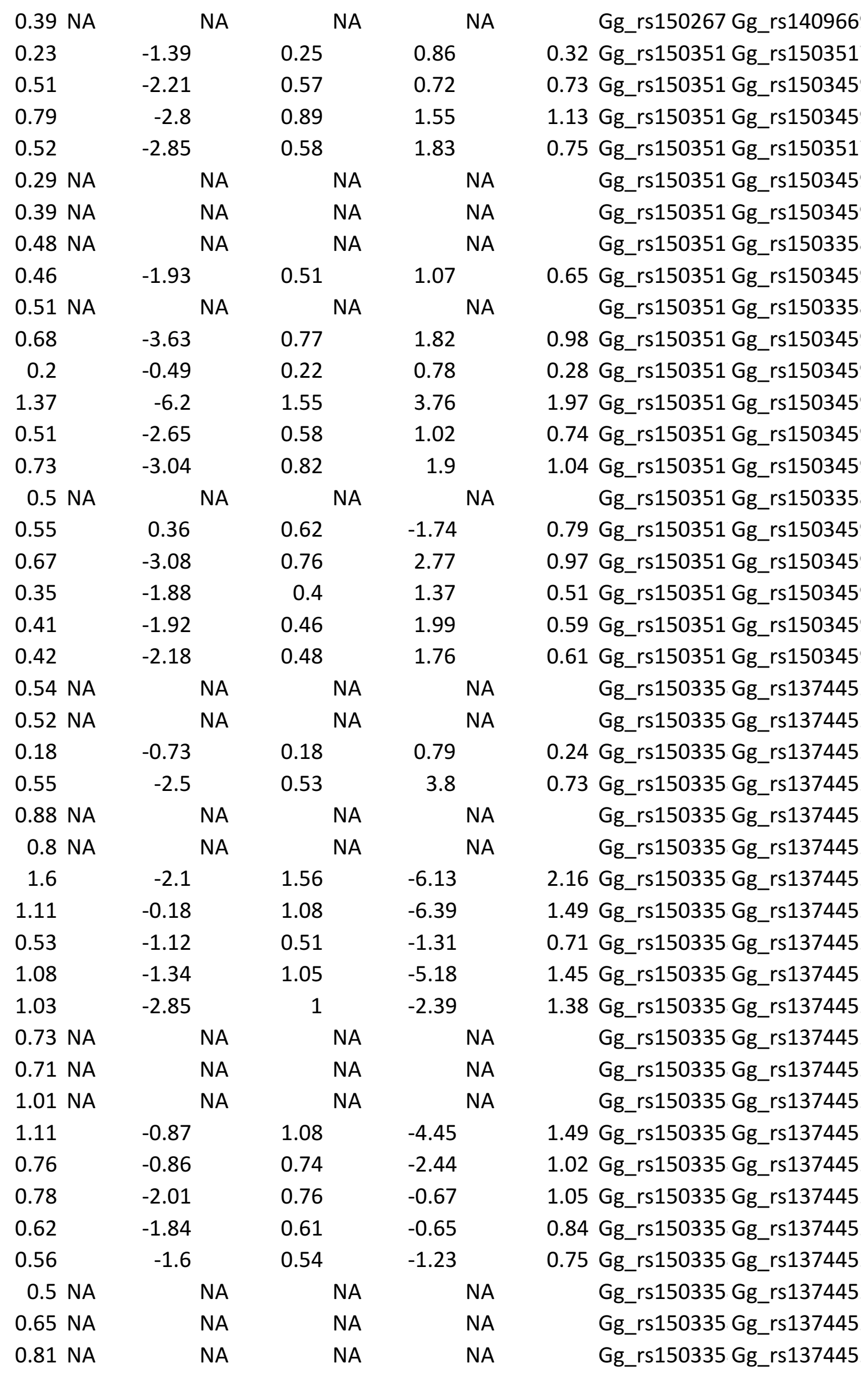




\begin{tabular}{|c|c|c|c|c|c|c|c|}
\hline $0.58 \mathrm{NA}$ & & NA & & NA & & & Gg_rs150335 Gg_rs137445 \\
\hline $0.46 \mathrm{NA}$ & & NA & & NA & & & Gg_rs150335 Gg_rs137445 \\
\hline $0.64 \mathrm{NA}$ & & NA & & NA & & & Gg_rs150335 rbl4379 \\
\hline $0.83 \mathrm{NA}$ & & NA & & NA & & & Gg_rs150335 rbl4379 \\
\hline 0.95 NA & & NA & & NA & & & Gg_rs150335 rbl4379 \\
\hline $0.73 \mathrm{NA}$ & & NA & & NA & & & Gg_rs150335 rbl4379 \\
\hline 1.13 & -1.37 & & 1.13 & & 4.68 & 1.46 & Gg_rs137449 Gg_rs150304 \\
\hline 0.62 & -0.17 & & 0.62 & & 3.7 & 0.8 & Gg_rs137445 Gg_rs150304 \\
\hline 0.8 & 2.02 & & 0.8 & & -2.55 & 1.03 & Gg_rs137445 Gg_rs150304 \\
\hline $0.45 \mathrm{NA}$ & & NA & & NA & & & Gg_rs137445 Gg_rs150304 \\
\hline $0.7 \mathrm{NA}$ & & NA & & NA & & & Gg_rs137445 Gg_rs150304 \\
\hline $0.63 \mathrm{NA}$ & & NA & & NA & & & Gg_rs137445 Gg_rs150304 \\
\hline 0.75 NA & & NA & & NA & & & Gg_rs137445 Gg_rs150304 \\
\hline 1.08 & 2.32 & & 1.09 & & -4.56 & 1.4 & Gg_rs137445 Gg_rs150304 \\
\hline $0.02 \mathrm{NA}$ & & NA & & NA & & & Gg_rs137445 Gg_rs150304 \\
\hline $0.11 \mathrm{NA}$ & & NA & & NA & & & Gg_rs137445 Gg_rs150304 \\
\hline 0.25 & -1.23 & & 0.26 & & 1.81 & 0.33 & Gg_rs137445 Gg_rs150304 \\
\hline $0.64 \mathrm{NA}$ & & NA & & NA & & & Gg_rs140998 Gg_rs140981 \\
\hline $1.07 \mathrm{NA}$ & & NA & & NA & & & rb|4379 Gg_rs140981 \\
\hline $0.39 \mathrm{NA}$ & & NA & & NA & & & Gg_rs150304 Gg_rs140981 \\
\hline $0.42 \mathrm{NA}$ & & NA & & NA & & & RBL3555 Gg_rs140981 \\
\hline 0.93 & 2.24 & & 0.78 & & -5.56 & 1.22 & Gg_rs140992 Gg_rs140972 \\
\hline 1.14 & -1.9 & & 1.08 & & 3.01 & 1.53 & Gg_rs140981 Gg_rs140972 \\
\hline 0.73 NA & & NA & & NA & & & Gg_rs140981 Gg_rs140972 \\
\hline 0.02 & -0.07 & & 0.02 & & -0.08 & 0.03 & X18_3045583Gg_rs135077 \\
\hline 0.16 & -0.51 & & 0.16 & & -0.42 & 0.22 & Gg_rs141057 Gg_rs135077 \\
\hline 0.71 & -2.38 & & 0.73 & & 1.13 & 0.97 & X18_3045583Gg_rs135077 \\
\hline 0.84 & -0.1 & & 0.87 & & 4.73 & 1.16 & X18_3045583Gg_rs135077 \\
\hline 0.53 & 2.32 & & 0.54 & & 1.5 & 0.72 & X18_3045583Gg_rs135077 \\
\hline $0.97 \mathrm{NA}$ & & NA & & NA & & & X18_3045583Gg_rs135077 \\
\hline $0.85 \mathrm{NA}$ & & NA & & NA & & & X18_3045583Gg_rs135077 \\
\hline 0.74 NA & & NA & & NA & & & X18_3045583Gg_rs135077 \\
\hline 0.39 & 2.13 & & 0.4 & & 1.52 & 0.53 & X18_3045583Gg_rs135077 \\
\hline 0.87 & 3.38 & & 0.89 & & 3.47 & 1.2 & X18_3045583Gg_rs135077 \\
\hline $0.49 \mathrm{NA}$ & & NA & & NA & & & X18_3045583Gg_rs135077 \\
\hline 1.01 & -3.37 & & 1.04 & & 2.56 & 1.39 & X18_3045583Gg_rs135077 \\
\hline $0.25 \mathrm{NA}$ & & NA & & NA & & & X18_3045583Gg_rs135077 \\
\hline 0.96 & -3.92 & & 0.99 & & 1.42 & 1.32 & X18_3045583Gg_rs135077 \\
\hline 0.37 & 1.56 & & 0.38 & & 1.78 & 0.51 & X18_3045583Gg_rs135077 \\
\hline 0.69 NA & & NA & & NA & & & X18_3045583Gg_rs135077 \\
\hline 0.09 & 0.45 & & 0.1 & & 0.55 & 0.13 & X18_3045583Gg_rs135077 \\
\hline 0.53 & 2.13 & & 0.5 & & -0.23 & 0.73 & Gg_rs150404 rbl11793 \\
\hline 0.22 & 0.88 & & 0.2 & & 1.03 & 0.29 & Gg_rs150404 rbl11793 \\
\hline
\end{tabular}




\begin{tabular}{|c|c|c|c|c|c|}
\hline 0.33 & 1.47 & 0.31 & 0.4 & 0.45 & Gg_rs150404 rbl11793 \\
\hline $0.52 \mathrm{NA}$ & NA & NA & NA & & Gg_rs141150Gg_rs150444. \\
\hline $1.07 \mathrm{NA}$ & NA & NA & NA & & Gg_rs141150 Gg_rs141168 \\
\hline $0.51 \mathrm{NA}$ & NA & NA & NA & & Gg_rs141150 Gg_rs141191 \\
\hline $0.74 \mathrm{NA}$ & NA & NA & NA & & Gg_rs141150 Gg_rs141168 \\
\hline 1.38 & -0.22 & 1.41 & 4.89 & 1.76 & Gg_rs141150 Gg_rs141168 \\
\hline 0.06 & -0.29 & 0.06 & 0.23 & 0.07 & Gg_rs141150Gg_rs141191 \\
\hline 0.47 & 1.76 & 0.45 & -1.63 & 0.62 & Gg_rs141168Gg_rs150470 \\
\hline 0.74 & -3.63 & 0.72 & -0.84 & 0.98 & Gg_rs141168Gg_rs150470 \\
\hline $0.43 \mathrm{NA}$ & NA & NA & NA & & Gg_rs141168 Gg_rs150470 \\
\hline 0.99 & -5.69 & 0.96 & -0.02 & 1.31 & Gg_rs141168Gg_rs150470 \\
\hline 0.57 & -2.69 & 0.55 & 0.74 & 0.75 & Gg_rs141168Gg_rs150470 \\
\hline $0.85 \mathrm{NA}$ & NA & NA & NA & & Gg_rs141168Gg_rs150470 \\
\hline 0.92 & -3.01 & 0.87 & 0.39 & 1.2 & Gg_rs141195 Gg_rs150470 \\
\hline 5.84 & -21.98 & 5.52 & 5.74 & 7.58 & Gg_rs141195 Gg_rs150470 \\
\hline 4.26 & -15.84 & 4.02 & 4.94 & 5.52 & Gg_rs141195 Gg_rs150470 \\
\hline 1.14 & -5.94 & 1.07 & -2.75 & 1.47 & Gg_rs141195 Gg_rs150470 \\
\hline 0.11 & 0.47 & 0.1 & 0.6 & 0.14 & Gg_rs141195 Gg_rs150470 \\
\hline 0.9 & -5.11 & 0.86 & -0.34 & 1.17 & Gg_rs141195 Gg_rs150470 \\
\hline 1.07 & -5.1 & 1.01 & -0.57 & 1.39 & Gg_rs141195 Gg_rs150470 \\
\hline 0.44 & -1.31 & 0.42 & 0.15 & 0.57 & Gg_rs141195 Gg_rs150470 \\
\hline 1.25 & -6.02 & 1.18 & -0.48 & 1.62 & Gg_rs141195 Gg_rs150470 \\
\hline 0.91 & -4.88 & 0.86 & -0.95 & 1.18 & Gg_rs141195 Gg_rs150470 \\
\hline 1.25 & -5.61 & 1.18 & -2.09 & 1.62 & Gg_rs141195 Gg_rs150470 \\
\hline 0.9 & -2.9 & 0.85 & 1.17 & 1.16 & Gg_rs141195 Gg_rs150470 \\
\hline 0.79 & -4.17 & 0.75 & -2.05 & 1.02 & Gg_rs141195 Gg_rs150470 \\
\hline 1 & -4.75 & 0.94 & -2.9 & 1.29 & Gg_rs141195 Gg_rs150470 \\
\hline 0.8 & -2.71 & 0.76 & 0.28 & 1.04 & Gg_rs141195 Gg_rs150470 \\
\hline 0.78 & -3.84 & 0.74 & -0.03 & 1.02 & Gg_rs141195 Gg_rs150470 \\
\hline 0.97 & -2.84 & 0.92 & 0.77 & 1.26 & Gg_rs141195 Gg_rs150470 \\
\hline 0.97 & -3.21 & 0.92 & -0.13 & 1.26 & Gg_rs141195 Gg_rs150470 \\
\hline 1.11 & -4.87 & 1.05 & -1.8 & 1.44 & Gg_rs141195 Gg_rs150470 \\
\hline 0.69 & -2.27 & 0.66 & -0.26 & 0.9 & Gg_rs141195 Gg_rs150470 \\
\hline 0.67 & -2.4 & 0.64 & 0.4 & 0.87 & Gg_rs141195 Gg_rs150470 \\
\hline 0.81 & -2.76 & 0.76 & 0.22 & 1.05 & Gg_rs141195 Gg_rs150470 \\
\hline $0.62 \mathrm{NA}$ & NA & NA & NA & & Gg_rs141195 Gg_rs150470 \\
\hline 1.18 & -5.25 & 1.11 & -1.25 & 1.53 & Gg_rs141195 Gg_rs150470 \\
\hline 1.32 & -3.54 & 1.25 & 1.72 & 1.72 & Gg_rs141195 Gg_rs150470 \\
\hline 1.11 & -4.63 & 1.05 & -1.38 & 1.44 & Gg_rs141195 Gg_rs150470 \\
\hline 0.68 & -2.49 & 0.65 & 0.58 & 0.89 & Gg_rs141195 Gg_rs150470 \\
\hline 1.05 & -4.28 & 0.99 & -1.36 & 1.36 & Gg_rs141195 Gg_rs150470 \\
\hline 0.72 & -2.75 & 0.68 & -0.51 & 0.93 & Gg_rs141195 Gg_rs150470 \\
\hline 1.25 & -5.3 & 1.18 & -1.05 & 1.62 & Gg_rs141195 Gg_rs150470 \\
\hline
\end{tabular}




\begin{tabular}{|c|c|c|c|c|}
\hline 1.19 & -5.03 & 1.13 & -0.8 & 1.55 Gg_rs141195 Gg_rs150470 \\
\hline 1.05 & -5.5 & 1 & -1.76 & 1.37 Gg_rs141195 Gg_rs150470 \\
\hline 1.35 & -7.64 & 1.28 & -4.83 & 1.75 Gg_rs141195 Gg_rs150470 \\
\hline 1.03 & -4.29 & 0.98 & 0.89 & 1.34 Gg_rs141195 Gg_rs150470 \\
\hline 0.77 & -3.1 & 0.73 & 0.37 & 1 Gg_rs141195 Gg_rs150470 \\
\hline 0.61 & -2.29 & 0.58 & -1 & 0.79 Gg_rs141195 Gg_rs150470 \\
\hline 0.47 & -1.52 & 0.44 & 0.18 & 0.61 Gg_rs141195 Gg_rs150470 \\
\hline $0.41 \mathrm{NA}$ & NA & NA & NA & Gg_rs141195 Gg_rs150470 \\
\hline 0.46 NA & NA & NA & NA & Gg_rs141195 Gg_rs150470 \\
\hline 0.63 & -2.42 & 0.59 & 0.36 & 0.81 Gg_rs141195 Gg_rs150470 \\
\hline 0.82 & -3.7 & 0.77 & -0.95 & 1.06 Gg_rs141195 Gg_rs150470 \\
\hline 1.06 & -4.83 & 1 & -0.58 & 1.37 Gg_rs141195 Gg_rs150470 \\
\hline 1.09 & -2.71 & 1.03 & 3.39 & 1.42 Gg_rs141195 Gg_rs150470 \\
\hline 0.6 & -3.01 & 0.57 & -0.15 & 0.78 Gg_rs141195 Gg_rs150470 \\
\hline 1.14 & -6.01 & 1.08 & -0.62 & 1.48 Gg_rs141195 Gg_rs150470 \\
\hline 0.7 & -2.73 & 0.67 & 1.02 & 0.91 Gg_rs141195 Gg_rs150470 \\
\hline 0.75 & -3.37 & 0.7 & 1.06 & 0.97 Gg_rs141195 Gg_rs150470 \\
\hline 0.71 & -3.35 & 0.67 & 0.48 & 0.92 Gg_rs141195 Gg_rs150470 \\
\hline 0.82 & -3.66 & 0.78 & 1.7 & 1.07 Gg_rs141195 Gg_rs150470 \\
\hline 0.67 & -3.06 & 0.63 & 0.43 & 0.87 Gg_rs141195 Gg_rs150470 \\
\hline 0.75 & -3.23 & 0.71 & 0.75 & 0.97 Gg_rs141195 Gg_rs150470 \\
\hline 0.85 & -3.72 & 0.8 & 1.08 & 1.1 Gg_rs141195 Gg_rs150470 \\
\hline 0.92 & -3.56 & 0.87 & 0.32 & 1.19 Gg_rs141195 Gg_rs150470 \\
\hline 0.81 & -3.09 & 0.76 & -0.32 & 1.04 Gg_rs141195 Gg_rs150470 \\
\hline 1.01 & -3.53 & 0.96 & 1.14 & 1.32 Gg_rs141195 Gg_rs150470 \\
\hline 0.55 & -3.43 & 0.52 & -0.95 & 0.71 Gg_rs141195 Gg_rs150470 \\
\hline 0.51 & -1.95 & 0.48 & 0.28 & 0.66 Gg_rs141195 Gg_rs150470 \\
\hline 0.6 & -3.31 & 0.58 & -0.8 & 0.77 Gg_rs141195 Gg_rs150470 \\
\hline 0.71 & -3.56 & 0.68 & -0.19 & 0.93 Gg_rs141195 Gg_rs150470 \\
\hline 0.65 & -3.62 & 0.61 & 0.25 & 0.84 Gg_rs141195 Gg_rs150470 \\
\hline 0.74 & -2.72 & 0.7 & 1.75 & 0.96 Gg_rs141195 Gg_rs150470 \\
\hline 0.65 & 2.74 & 0.62 & 2.95 & 0.85 Gg_rs141195 Gg_rs150470 \\
\hline 23.37 & -102.12 & 22.09 & 31.32 & 30.33 Gg_rs141195 Gg_rs150470 \\
\hline 22.44 & -99.75 & 21.21 & 29.58 & 29.11 Gg_rs141195 Gg_rs150470 \\
\hline 21.48 & -96.25 & 20.31 & 27.42 & 27.87 Gg_rs141195 Gg_rs150470 \\
\hline 23.8 & -105.22 & 22.5 & 29.38 & 30.88 Gg_rs141195 Gg_rs150470 \\
\hline 22.14 & -97.6 & 20.92 & 25.86 & 28.72 Gg_rs141195 Gg_rs150470 \\
\hline 24.55 & -107.55 & 23.2 & 32.92 & 31.84 Gg_rs141195 Gg_rs150470 \\
\hline 21.9 & -98.28 & 20.7 & 25.27 & 28.42 Gg_rs141195 Gg_rs150470 \\
\hline 0.64 & -3.51 & 0.61 & -0.09 & 0.83 Gg_rs141195 Gg_rs150470 \\
\hline $0.61 \mathrm{NA}$ & NA & NA & NA & Gg_rs141195 Gg_rs150470 \\
\hline $0.75 \mathrm{NA}$ & NA & NA & NA & Gg_rs141195 Gg_rs150470 \\
\hline 0.7 & 1.33 & 0.63 & -0.4 & 0.94 Gg_rs142677 Gg_rs142708 \\
\hline
\end{tabular}




\begin{tabular}{|c|c|c|c|c|c|}
\hline $1.26 \mathrm{NA}$ & NA & NA & NA & & Gg_rs136307 Gg_rs142708 \\
\hline $0.46 \mathrm{NA}$ & NA & NA & NA & & Gg_rs136307 Gg_rs142708 \\
\hline 0.59 NA & NA & NA & NA & & Gg_rs136307 Gg_rs142708 \\
\hline $0.48 \mathrm{NA}$ & NA & NA & NA & & Gg_rs136307 Gg_rs142708 \\
\hline $0.67 \mathrm{NA}$ & NA & NA & NA & & Gg_rs136307 Gg_rs142708 \\
\hline 3.84 NA & NA & NA & NA & & Gg_rs136307 Gg_rs142708 \\
\hline 2.57 & 2.08 & 2.32 & -4.86 & 3.43 & Gg_rs136307 Gg_rs142708 \\
\hline 0.68 NA & NA & NA & NA & & Gg_rs136307 Gg_rs142708 \\
\hline $0.83 \mathrm{NA}$ & NA & NA & NA & & Gg_rs136307 Gg_rs142708 \\
\hline 0.42 NA & NA & NA & NA & & Gg_rs136307 Gg_rs142708 \\
\hline $2.15 \mathrm{NA}$ & NA & NA & NA & & Gg_rs136307 Gg_rs142708 \\
\hline 4.79 NA & NA & NA & NA & & Gg_rs136307 Gg_rs142708 \\
\hline $0.81 \mathrm{NA}$ & NA & NA & NA & & Gg_rs136307 Gg_rs142708 \\
\hline $0.53 \mathrm{NA}$ & NA & NA & NA & & Gg_rs136307 Gg_rs142708 \\
\hline $1.15 \mathrm{NA}$ & NA & NA & NA & & Gg_rs136307 Gg_rs142708 \\
\hline $0.55 \mathrm{NA}$ & NA & NA & NA & & Gg_rs136307 Gg_rs151715 \\
\hline $1.01 \mathrm{NA}$ & NA & NA & NA & & Gg_rs136307 Gg_rs142708 \\
\hline $1.04 \mathrm{NA}$ & NA & NA & NA & & Gg_rs136307 Gg_rs142708 \\
\hline $0.72 \mathrm{NA}$ & NA & NA & NA & & Gg_rs136307 Gg_rs142708 \\
\hline $0.58 \mathrm{NA}$ & NA & NA & NA & & Gg_rs136307 Gg_rs151715. \\
\hline $0.26 \mathrm{NA}$ & NA & NA & NA & & Gg_rs136307 Gg_rs151715. \\
\hline 0.47 NA & NA & NA & NA & & Gg_rs136307 Gg_rs142708 \\
\hline $0.31 \mathrm{NA}$ & NA & NA & NA & & Gg_rs136307 Gg_rs151715. \\
\hline 0.94 NA & NA & NA & NA & & Gg_rs136307 GG_rs151718 \\
\hline $1.19 \mathrm{NA}$ & NA & NA & NA & & Gg_rs136307 Gg_rs151715 \\
\hline $0.57 \mathrm{NA}$ & NA & NA & NA & & Gg_rs136307 Gg_rs142708 \\
\hline $2.59 \mathrm{NA}$ & NA & NA & NA & & Gg_rs136307 Gg_rs151715. \\
\hline $2.03 \mathrm{NA}$ & NA & NA & NA & & Gg_rs136307 Gg_rs151715. \\
\hline $1.04 \mathrm{NA}$ & NA & NA & NA & & Gg_rs136307 Gg_rs151715. \\
\hline $1.2 \mathrm{NA}$ & NA & NA & NA & & Gg_rs136307 Gg_rs151737. \\
\hline $3.23 \mathrm{NA}$ & NA & NA & NA & & Gg_rs136307 Gg_rs151715 \\
\hline $3.85 \mathrm{NA}$ & NA & NA & NA & & Gg_rs136307 Gg_rs151715. \\
\hline 0.92 NA & NA & NA & NA & & Gg_rs136307 Gg_rs151715 \\
\hline $0.64 \mathrm{NA}$ & NA & NA & NA & & Gg_rs136307 Gg_rs151715. \\
\hline $3.57 \mathrm{NA}$ & NA & NA & NA & & Gg_rs136307 Gg_rs151715 \\
\hline $3.54 \mathrm{NA}$ & NA & NA & NA & & Gg_rs136307 Gg_rs151715 \\
\hline $0.84 \mathrm{NA}$ & NA & NA & NA & & Gg_rs151697 GG_rs151718 \\
\hline 2.55 & 3.26 & 2.34 & -4.4 & 3.37 & Gg_rs136307 Gg_rs151715 \\
\hline $1.11 \mathrm{NA}$ & NA & NA & NA & & Gg_rs151697 Gg_rs151715 \\
\hline $0.75 \mathrm{NA}$ & NA & NA & NA & & Gg_rs151697 Gg_rs151737. \\
\hline $0.88 \mathrm{NA}$ & NA & NA & NA & & Gg_rs151697 Gg_rs151715. \\
\hline $0.46 \mathrm{NA}$ & NA & NA & NA & & Gg_rs136307 Gg_rs151715. \\
\hline $0.86 \mathrm{NA}$ & NA & NA & NA & & Gg_rs151697 Gg_rs151737. \\
\hline
\end{tabular}




\begin{tabular}{|c|c|c|c|c|c|}
\hline $0.6 \mathrm{NA}$ & NA & NA & NA & & Gg_rs151697 Gg_rs151715. \\
\hline $0.49 \mathrm{NA}$ & NA & NA & NA & & Gg_rs151697 Gg_rs151715. \\
\hline $0.37 \mathrm{NA}$ & NA & NA & NA & & Gg_rs151697 Gg_rs151715 \\
\hline $0.91 \mathrm{NA}$ & NA & NA & NA & & Gg_rs151697 Gg_rs151715. \\
\hline 0.83 NA & NA & NA & NA & & Gg_rs151697 Gg_rs151744 \\
\hline $0.77 \mathrm{NA}$ & NA & NA & NA & & Gg_rs151697 Gg_rs151737. \\
\hline $0.59 \mathrm{NA}$ & NA & NA & NA & & Gg_rs151697 GG_rs151718 \\
\hline 0.47 NA & NA & NA & NA & & Gg_rs151697 Gg_rs151737. \\
\hline $1.11 \mathrm{NA}$ & NA & NA & NA & & Gg_rs136307 Gg_rs151737. \\
\hline 0.97 NA & NA & NA & NA & & Gg_rs151697 Gg_rs151737. \\
\hline 0.96 & -0.26 & 0.83 & -6.97 & 1.25 & Gg_rs142708 Gg_rs151737. \\
\hline $0.7 \mathrm{NA}$ & NA & NA & NA & & Gg_rs151697 Gg_rs151737. \\
\hline $0.48 \mathrm{NA}$ & NA & NA & NA & & Gg_rs151697 Gg_rs151737. \\
\hline $0.59 \mathrm{NA}$ & NA & NA & NA & & Gg_rs151697 Gg_rs151737. \\
\hline 0.85 & -1.55 & 0.74 & -4.76 & 1.12 & Gg_rs142708 Gg_rs151737. \\
\hline $0.75 \mathrm{NA}$ & NA & NA & NA & & Gg_rs142708 Gg_rs151737. \\
\hline $0.61 \mathrm{NA}$ & NA & NA & NA & & Gg_rs142708 Gg_rs151737. \\
\hline 0.57 & -0.08 & 0.5 & -3.1 & 0.75 & Gg_rs142708 Gg_rs151737. \\
\hline $0.72 \mathrm{NA}$ & NA & NA & NA & & Gg_rs151697 Gg_rs151737 \\
\hline $0.41 \mathrm{NA}$ & NA & NA & NA & & Gg_rs142708 Gg_rs151737. \\
\hline $0.54 \mathrm{NA}$ & NA & NA & NA & & Gg_rs142708 Gg_rs151737. \\
\hline 0.59 NA & NA & NA & NA & & Gg_rs142708 Gg_rs151737. \\
\hline $0.67 \mathrm{NA}$ & NA & NA & NA & & Gg_rs151697 Gg_rs151737. \\
\hline 0.82 & -0.33 & 0.73 & -4.3 & 1.07 & Gg_rs142708 Gg_rs151737. \\
\hline 0.73 & 1.16 & 0.64 & -1.15 & 0.95 & Gg_rs142708 Gg_rs151737. \\
\hline 0.75 & -0.81 & 0.66 & -3.1 & 0.98 & Gg_rs142708 Gg_rs151737. \\
\hline 0.75 & 0.07 & 0.67 & -3 & 0.99 & Gg_rs142708 Gg_rs151737. \\
\hline $0.64 \mathrm{NA}$ & NA & NA & NA & & Gg_rs151697 Gg_rs151737. \\
\hline 0.63 NA & NA & NA & NA & & Gg_rs151697 Gg_rs151737. \\
\hline 0.66 NA & NA & NA & NA & & Gg_rs151697 Gg_rs151737. \\
\hline $0.6 \mathrm{NA}$ & NA & NA & NA & & Gg_rs151697 Gg_rs151737. \\
\hline $1.21 \mathrm{NA}$ & NA & NA & NA & & Gg_rs151697 Gg_rs151737. \\
\hline 0.59 NA & NA & NA & NA & & Gg_rs151697 Gg_rs151769 \\
\hline $0.97 \mathrm{NA}$ & NA & NA & NA & & Gg_rs151697 Gg_rs151751 \\
\hline $0.67 \mathrm{NA}$ & NA & NA & NA & & Gg_rs151697 Gg_rs151744 \\
\hline $0.67 \mathrm{NA}$ & NA & NA & NA & & Gg_rs136307 Gg_rs151751 \\
\hline $0.7 \mathrm{NA}$ & NA & NA & NA & & Gg_rs151715 Gg_rs151769 \\
\hline $1.2 \mathrm{NA}$ & NA & NA & NA & & Gg_rs136307 Gg_rs151751 \\
\hline $0.79 \mathrm{NA}$ & NA & NA & NA & & Gg_rs136307 Gg_rs151769 \\
\hline $0.88 \mathrm{NA}$ & NA & NA & NA & & Gg_rs136307 Gg_rs151751 \\
\hline $0.78 \mathrm{NA}$ & NA & NA & NA & & Gg_rs151697 Gg_rs151751 \\
\hline $1.16 \mathrm{NA}$ & NA & NA & NA & & Gg_rs151697 Gg_rs151751 \\
\hline $0.56 \mathrm{NA}$ & NA & NA & NA & & Gg_rs136307 Gg_rs151751 \\
\hline
\end{tabular}




\begin{tabular}{|c|c|c|c|c|c|c|c|}
\hline $0.87 \mathrm{NA}$ & NA & & NA & & NA & & GG_rs151718Gg_rs151751 \\
\hline 0.55 & -1.03 & 0.52 & & 0.65 & & 0.75 & GG_rs151718Gg_rs151751 \\
\hline $0.84 \mathrm{NA}$ & NA & & NA & & NA & & GG_rs151718Gg_rs151769 \\
\hline $0.73 \mathrm{NA}$ & NA & & NA & & NA & & GG_rs151718Gg_rs151751 \\
\hline $0.77 \mathrm{NA}$ & NA & & NA & & NA & & GG_rs151718Gg_rs151744 \\
\hline $0.74 \mathrm{NA}$ & NA & & NA & & NA & & GG_rs151718Gg_rs151744 \\
\hline $0.49 \mathrm{NA}$ & NA & & NA & & NA & & GG_rs151718Gg_rs151744 \\
\hline 0.27 & -1.19 & 0.26 & & 1.15 & & 0.37 & GG_rs151718Gg_rs151744 \\
\hline $0.69 \mathrm{NA}$ & NA & & NA & & NA & & GG_rs151718Gg_rs151751 \\
\hline $0.51 \mathrm{NA}$ & NA & & NA & & NA & & GG_rs151718Gg_rs151751 \\
\hline $0.71 \mathrm{NA}$ & NA & & NA & & NA & & GG_rs151718Gg_rs151751 \\
\hline 0.55 & -1.24 & 0.54 & & -0.45 & & 0.76 & Gg_rs151737 Gg_rs151751 \\
\hline 0.82 & 0.49 & 0.81 & & -3.34 & & 1.13 & Gg_rs151737 Gg_rs151751 \\
\hline $0.53 \mathrm{NA}$ & NA & & NA & & NA & & Gg_rs151737 Gg_rs151751 \\
\hline $0.81 \mathrm{NA}$ & NA & & NA & & NA & & Gg_rs151737 Gg_rs151751 \\
\hline $1.12 \mathrm{NA}$ & NA & & NA & & NA & & Gg_rs151737 Gg_rs151751 \\
\hline $0.95 \mathrm{NA}$ & NA & & NA & & NA & & GG_rs151718Gg_rs151751 \\
\hline $0.6 \mathrm{NA}$ & NA & & NA & & NA & & Gg_rs151737 Gg_rs151751 \\
\hline $0.98 \mathrm{NA}$ & NA & & NA & & NA & & GG_rs151718Gg_rs151769 \\
\hline $0.58 \mathrm{NA}$ & NA & & NA & & NA & & GG_rs151718Gg_rs151769 \\
\hline $0.49 \mathrm{NA}$ & NA & & NA & & NA & & Gg_rs151737 Gg_rs151769 \\
\hline $0.58 \mathrm{NA}$ & NA & & NA & & NA & & GG_rs151718Gg_rs151769 \\
\hline $0.56 \mathrm{NA}$ & NA & & NA & & NA & & GG_rs151718Gg_rs151769 \\
\hline 1.14 & -1.19 & 1.12 & & -3.09 & & 1.57 & Gg_rs151737 Gg_rs151769 \\
\hline $0.48 \mathrm{NA}$ & NA & & NA & & NA & & Gg_rs151737 Gg_rs151769 \\
\hline 1.1 & -3.17 & 0.88 & & -3.39 & & 1.42 & GG_rs151718Gg_rs151769 \\
\hline $1 \mathrm{NA}$ & NA & & NA & & NA & & GG_rs151718Gg_rs151769 \\
\hline $0.88 \mathrm{NA}$ & NA & & NA & & NA & & Gg_rs151737 Gg_rs151769 \\
\hline 1.97 & -5.33 & 1.56 & & -5.05 & & 2.53 & Gg_rs151744 Gg_rs151769 \\
\hline 1.59 & -5.63 & 1.26 & & -3.01 & & 2.05 & Gg_rs151744 Gg_rs151769 \\
\hline 1.34 & -3.28 & 1.06 & & -0.9 & & 1.72 & Gg_rs151744 Gg_rs151769 \\
\hline 2.11 & -6.79 & 1.67 & & -3.11 & & 2.71 & Gg_rs151744 Gg_rs151769 \\
\hline 1.22 & -3.4 & 0.97 & & -0.67 & & 1.57 & Gg_rs151744 Gg_rs151769 \\
\hline 1.66 & -5.17 & 1.32 & & -1.22 & & 2.14 & Gg_rs151744 Gg_rs151769 \\
\hline 2.17 & -5.37 & 1.72 & & -2.87 & & 2.8 & Gg_rs151744 Gg_rs151769 \\
\hline $1.07 \mathrm{NA}$ & NA & & NA & & NA & & Gg_rs151769 Gg_rs142808 \\
\hline $0.38 \mathrm{NA}$ & NA & & NA & & NA & & Gg_rs151769 Gg_rs142808 \\
\hline $0.71 \mathrm{NA}$ & NA & & NA & & NA & & Gg_rs151769 Gg_rs142808 \\
\hline $0.84 \mathrm{NA}$ & NA & & NA & & NA & & Gg_rs151769 Gg_rs142808 \\
\hline $0.55 \mathrm{NA}$ & NA & & NA & & NA & & Gg_rs151769 Gg_rs142808 \\
\hline $0.54 \mathrm{NA}$ & NA & & NA & & NA & & Gg_rs151769 Gg_rs142808 \\
\hline $1.09 \mathrm{NA}$ & NA & & NA & & NA & & Gg_rs151769 Gg_rs142808 \\
\hline $0.64 \mathrm{NA}$ & NA & & NA & & NA & & Gg_rs151769 Gg_rs142808 \\
\hline
\end{tabular}




\begin{tabular}{|c|c|c|c|c|c|c|c|}
\hline $0.45 \mathrm{NA}$ & NA & & NA & & NA & & Gg_rs151779 Gg_rs142808 \\
\hline 0.53 & -1.8 & 0.52 & & 1.09 & & 0.7 & Gg_rs151779 Gg_rs142808 \\
\hline $0.7 \mathrm{NA}$ & NA & & NA & & NA & & Gg_rs151779 Gg_rs142808 \\
\hline 0.78 & 2.85 & 0.76 & & 2.87 & & 1.03 & Gg_rs151779 Gg_rs142808 \\
\hline $0.78 \mathrm{NA}$ & NA & & NA & & NA & & Gg_rs151779 Gg_rs142808 \\
\hline $0.67 \mathrm{NA}$ & NA & & NA & & NA & & Gg_rs151779 Gg_rs142808 \\
\hline $0.45 \mathrm{NA}$ & NA & & NA & & NA & & Gg_rs151769 Gg_rs142808 \\
\hline 0.92 NA & NA & & NA & & NA & & Gg_rs151769 Gg_rs142808 \\
\hline $0.55 \mathrm{NA}$ & NA & & NA & & NA & & Gg_rs151779 Gg_rs142808 \\
\hline $1.47 \mathrm{NA}$ & NA & & NA & & NA & & Gg_rs151769 Gg_rs142808 \\
\hline $0.65 \mathrm{NA}$ & NA & & NA & & NA & & Gg_rs151779 Gg_rs142808 \\
\hline $0.69 \mathrm{NA}$ & NA & & NA & & NA & & Gg_rs151779 Gg_rs142808 \\
\hline $1.19 \mathrm{NA}$ & NA & & NA & & NA & & Gg_rs151779 Gg_rs142808 \\
\hline $0.57 \mathrm{NA}$ & NA & & NA & & NA & & Gg_rs151779 Gg_rs142808 \\
\hline 12.12 & 35.36 & 12.14 & & 65.11 & & 16.09 & Gg_rs151779 Gg_rs142808 \\
\hline 15.37 & 45.81 & 15.38 & & 81.39 & & 20.39 & Gg_rs151779 Gg_rs142808 \\
\hline 0.44 & -1.11 & 0.44 & & -1.35 & & 0.59 & Gg_rs151802 Gg_rs151816 \\
\hline $0.26 \mathrm{NA}$ & NA & & NA & & NA & & Gg_rs151802 Gg_rs151821 \\
\hline $0.38 \mathrm{NA}$ & NA & & NA & & NA & & Gg_rs151802 Gg_rs151816 \\
\hline $0.49 \mathrm{NA}$ & NA & & NA & & NA & & Gg_rs151802 Gg_rs151816 \\
\hline 0.55 & -1.55 & 0.54 & & -0.95 & & 0.73 & Gg_rs151802 Gg_rs151816 \\
\hline $0.73 \mathrm{NA}$ & NA & & NA & & NA & & Gg_rs151802 Gg_rs151816 \\
\hline $0.45 \mathrm{NA}$ & NA & & NA & & NA & & Gg_rs151802 Gg_rs151839 \\
\hline $0.3 \mathrm{NA}$ & NA & & NA & & NA & & Gg_rs151802 Gg_rs151821 \\
\hline $0.41 \mathrm{NA}$ & NA & & NA & & NA & & Gg_rs151802 Gg_rs151839 \\
\hline 0.61 & -0.71 & 0.61 & & -2.25 & & 0.83 & Gg_rs151802 Gg_rs151816 \\
\hline 0.7 & -1.86 & 0.69 & & -2.37 & & 0.93 & Gg_rs151802 Gg_rs151816 \\
\hline 0.46 & -0.72 & 0.46 & & -1.53 & & 0.62 & Gg_rs151802 Gg_rs151816 \\
\hline 0.63 & -1.58 & 0.63 & & -2.1 & & 0.85 & Gg_rs151802 Gg_rs151816 \\
\hline $0.25 \mathrm{NA}$ & NA & & NA & & NA & & Gg_rs151802 Gg_rs151821 \\
\hline $0.49 \mathrm{NA}$ & NA & & NA & & NA & & Gg_rs151802 Gg_rs151821 \\
\hline $0.25 \mathrm{NA}$ & NA & & NA & & NA & & Gg_rs151802 Gg_rs151839 \\
\hline $0.7 \mathrm{NA}$ & NA & & NA & & NA & & Gg_rs151802 Gg_rs151839 \\
\hline $0.37 \mathrm{NA}$ & NA & & NA & & NA & & Gg_rs151802 Gg_rs151839 \\
\hline $0.6 \mathrm{NA}$ & NA & & NA & & NA & & Gg_rs151802 Gg_rs151839 \\
\hline $0.26 \mathrm{NA}$ & NA & & NA & & NA & & Gg_rs151802 Gg_rs151839 \\
\hline $0.39 \mathrm{NA}$ & NA & & NA & & NA & & Gg_rs151802 Gg_rs151821 \\
\hline $0.48 \mathrm{NA}$ & NA & & NA & & NA & & Gg_rs151802 Gg_rs151839 \\
\hline $0.61 \mathrm{NA}$ & NA & & NA & & NA & & Gg_rs151802 Gg_rs151839 \\
\hline $0.87 \mathrm{NA}$ & NA & & NA & & NA & & Gg_rs151802 Gg_rs151839 \\
\hline $0.44 \mathrm{NA}$ & NA & & NA & & NA & & Gg_rs151802 Gg_rs151839 \\
\hline $0.69 \mathrm{NA}$ & NA & & NA & & NA & & Gg_rs151802 Gg_rs151839 \\
\hline $0.35 \mathrm{NA}$ & NA & & NA & & NA & & Gg_rs151802 Gg_rs151839 \\
\hline
\end{tabular}




\begin{tabular}{|c|c|c|c|c|c|c|c|}
\hline $0.31 \mathrm{NA}$ & NA & & NA & & NA & & GG_rs151812Gg_rs151839 \\
\hline $0.43 \mathrm{NA}$ & NA & & NA & & NA & & Gg_rs151802 Gg_rs151839 \\
\hline 0.53 & 1.21 & 0.56 & & -2.74 & & 0.72 & GG_rs151812Gg_rs151839 \\
\hline $0.73 \mathrm{NA}$ & NA & & NA & & NA & & Gg_rs151802 Gg_rs151839 \\
\hline $0.45 \mathrm{NA}$ & NA & & NA & & NA & & GG_rs151812Gg_rs151839 \\
\hline $0.64 \mathrm{NA}$ & NA & & NA & & NA & & GG_rs151812Gg_rs151842 \\
\hline $0.36 \mathrm{NA}$ & NA & & NA & & NA & & Gg_rs151802 Gg_rs151839 \\
\hline $0.61 \mathrm{NA}$ & NA & & NA & & NA & & GG_rs151812Gg_rs151839 \\
\hline $0.92 \mathrm{NA}$ & NA & & NA & & NA & & Gg_rs151802 Gg_rs151842 \\
\hline $0.79 \mathrm{NA}$ & NA & & NA & & NA & & Gg_rs151802 Gg_rs151839 \\
\hline $0.42 \mathrm{NA}$ & NA & & NA & & NA & & Gg_rs151802 Gg_rs151839 \\
\hline $0.52 \mathrm{NA}$ & NA & & NA & & NA & & Gg_rs151802 Gg_rs151839 \\
\hline $0.6 \mathrm{NA}$ & NA & & NA & & NA & & Gg_rs151802 Gg_rs151839 \\
\hline $0.35 \mathrm{NA}$ & NA & & NA & & NA & & Gg_rs151802 Gg_rs151842 \\
\hline $0.45 \mathrm{NA}$ & NA & & NA & & NA & & Gg_rs151802 Gg_rs151839 \\
\hline $0.74 \mathrm{NA}$ & NA & & NA & & NA & & Gg_rs151802 GG_rs161833 \\
\hline $0.63 \mathrm{NA}$ & NA & & NA & & NA & & Gg_rs151802 GG_rs161833 \\
\hline $0.98 \mathrm{NA}$ & NA & & NA & & NA & & Gg_rs151821Gg_rs151842 \\
\hline $0.57 \mathrm{NA}$ & NA & & NA & & NA & & Gg_rs151802 Gg_rs151842 \\
\hline $0.53 \mathrm{NA}$ & NA & & NA & & NA & & Gg_rs151821GG_rs161833 \\
\hline $0.84 \mathrm{NA}$ & NA & & NA & & NA & & Gg_rs151821GG_rs161833 \\
\hline $0.81 \mathrm{NA}$ & NA & & NA & & NA & & Gg_rs151821Gg_rs151842 \\
\hline $0.65 \mathrm{NA}$ & NA & & NA & & NA & & Gg_rs151821 Gg_rs151842 \\
\hline $0.45 \mathrm{NA}$ & NA & & NA & & NA & & Gg_rs151821Gg_rs151842 \\
\hline $0.38 \mathrm{NA}$ & NA & & NA & & NA & & Gg_rs151821 Gg_rs151842 \\
\hline $0.52 \mathrm{NA}$ & NA & & NA & & NA & & Gg_rs151821Gg_rs151842 \\
\hline $0.36 \mathrm{NA}$ & NA & & NA & & NA & & Gg_rs151821Gg_rs151842 \\
\hline $0.78 \mathrm{NA}$ & NA & & NA & & NA & & Gg_rs151821Gg_rs151842 \\
\hline $0.6 \mathrm{NA}$ & NA & & NA & & NA & & Gg_rs151821Gg_rs151842 \\
\hline $0.78 \mathrm{NA}$ & NA & & NA & & NA & & Gg_rs151821 Gg_rs151842 \\
\hline 0.4 & 1.33 & 0.35 & & 2.05 & & 0.51 & Gg_rs151821 Gg_rs151842 \\
\hline $0.6 \mathrm{NA}$ & NA & & NA & & NA & & Gg_rs151821Gg_rs151842 \\
\hline 0.66 & 1.03 & 0.57 & & 2.51 & & 0.85 & Gg_rs151821 Gg_rs151842 \\
\hline $0.67 \mathrm{NA}$ & NA & & NA & & NA & & Gg_rs151821 Gg_rs151842 \\
\hline $0.38 \mathrm{NA}$ & NA & & NA & & NA & & Gg_rs151821 GG_rs161833 \\
\hline 0.96 & 1.23 & 0.84 & & 2.99 & & 1.24 & Gg_rs151821 GG_rs161833 \\
\hline $0.34 \mathrm{NA}$ & NA & & NA & & NA & & Gg_rs151821 GG_rs161833 \\
\hline 0.71 & 2.84 & 0.61 & & 3.84 & & 0.9 & Gg_rs151839 GG_rs161833 \\
\hline 1.04 & 2.9 & 0.9 & & 6.13 & & 1.32 & Gg_rs151839 GG_rs161833 \\
\hline 0.57 & -0.21 & 0.49 & & 2.72 & & 0.72 & Gg_rs151839GG_rs161833 \\
\hline 0.42 & -0.28 & 0.37 & & 0.97 & & 0.54 & Gg_rs151821 GG_rs161833 \\
\hline 0.71 & 1.12 & 0.61 & & 3.64 & & 0.9 & Gg_rs151839 GG_rs161833 \\
\hline 0.47 & -0.84 & 0.4 & & 1.2 & & 0.6 & Gg_rs151839 GG_rs161833 \\
\hline
\end{tabular}




\begin{tabular}{|c|c|c|c|c|c|}
\hline 0.05 & 0.23 & 0.04 & 0.23 & 0.06 & Gg_rs151839 GG_rs161833 \\
\hline 0.1 & 0.75 & 0.09 & 0.86 & 0.13 & Gg_rs151839 GG_rs161833 \\
\hline 0.1 & 0.46 & 0.09 & 0.6 & 0.13 & Gg_rs151839 GG_rs161833 \\
\hline $0.35 \mathrm{NA}$ & NA & NA & NA & & Gg_rs151842 rbl2878 \\
\hline $1.22 \mathrm{NA}$ & NA & NA & NA & & Gg_rs142875 Gg_rs151940 \\
\hline $0.77 \mathrm{NA}$ & NA & NA & NA & & Gg_rs142875 Gg_rs151926، \\
\hline $1.23 \mathrm{NA}$ & NA & NA & NA & & Gg_rs142875 Gg_rs151926. \\
\hline $0.56 \mathrm{NA}$ & NA & NA & NA & & Gg_rs142875 RBL4501 \\
\hline $0.61 \mathrm{NA}$ & NA & NA & NA & & Gg_rs142875 Gg_rs151940 \\
\hline 0.96 & -4.55 & 1.03 & -3.43 & 1.37 & Gg_rs142875 Gg_rs151940 \\
\hline 1.57 & -4.88 & 1.69 & 3.15 & 2.24 & Gg_rs142875 Gg_rs151940 \\
\hline 2.16 & -6.47 & 2.32 & 5.59 & 3.06 & Gg_rs142875 Gg_rs151940 \\
\hline 0.79 & -1.88 & 0.85 & 1.84 & 1.12 & Gg_rs142875 Gg_rs151940 \\
\hline 1.41 & -4.92 & 1.51 & 1.99 & 2 & Gg_rs142875 Gg_rs151940 \\
\hline 0.93 & -4.23 & 1 & 0.14 & 1.32 & Gg_rs142875 Gg_rs151940 \\
\hline 0.47 & -1.33 & 0.5 & 1.4 & 0.66 & Gg_rs142875 RBL4501 \\
\hline 1.14 & -3.95 & 1.22 & 1.85 & 1.62 & Gg_rs142875 Gg_rs151940 \\
\hline 0.23 & -0.3 & 0.25 & 0.98 & 0.33 & Gg_rs142875 Gg_rs151940 \\
\hline 0.95 & -1.6 & 1.02 & 1.51 & 1.35 & Gg_rs142875 RBL4501 \\
\hline 3.13 & -10.34 & 3.36 & 4.88 & 4.44 & Gg_rs142875 RBL4501 \\
\hline 2.54 & -11.63 & 2.5 & -0.4 & 3.36 & Gg_rs142875 RBL4501 \\
\hline $0.62 \mathrm{NA}$ & NA & NA & NA & & Gg_rs151926 RBL4501 \\
\hline $0.5 \mathrm{NA}$ & NA & NA & NA & & Gg_rs151926 RBL4501 \\
\hline 0.06 & -0.26 & 0.06 & -0.24 & 0.09 & Gg_rs151926 RBL4501 \\
\hline 0.74 & 3.42 & 0.73 & 1.95 & 0.98 & Gg_rs151926 RBL4501 \\
\hline $0.5 \mathrm{NA}$ & NA & NA & NA & & Gg_rs151926 RBL4501 \\
\hline $0.51 \mathrm{NA}$ & NA & NA & NA & & Gg_rs151940Gg_rs152020. \\
\hline $0.34 \mathrm{NA}$ & NA & NA & NA & & Gg_rs142928 Gg_rs142931 \\
\hline $0.52 \mathrm{NA}$ & NA & NA & NA & & Gg_rs142928GG_rs161944 \\
\hline 0.64 & -2.02 & 0.56 & 2.52 & 0.85 & Gg_rs142928Gg_rs142931 \\
\hline 0.86 & 2.36 & 0.74 & -1.32 & 1.12 & Gg_rs142928 Gg_rs142931 \\
\hline 0.69 & -2.41 & 0.59 & 2.86 & 0.9 & Gg_rs142928Gg_rs136047. \\
\hline 0.99 & 2.17 & 0.86 & 2.56 & 1.3 & Gg_rs142928Gg_rs136047. \\
\hline 1.24 & 3.39 & 1.08 & -2 & 1.63 & Gg_rs142928GG_rs161944 \\
\hline 0.61 & -2.19 & 0.53 & 0.83 & 0.81 & Gg_rs142928 Gg_rs136047. \\
\hline 0.35 & -1.17 & 0.3 & 1.63 & 0.46 & Gg_rs142928Gg_rs142931 \\
\hline $0.57 \mathrm{NA}$ & NA & NA & NA & & Gg_rs142928Gg_rs136047. \\
\hline 0.82 & 2.44 & 0.71 & -4.2 & 1.07 & Gg_rs142928Gg_rs142931 \\
\hline 0.63 NA & NA & NA & NA & & Gg_rs142928 Gg_rs136047. \\
\hline $0.73 \mathrm{NA}$ & NA & NA & NA & & Gg_rs142928 Gg_rs136047. \\
\hline 0.2 & -0.7 & 0.17 & 0.74 & 0.26 & Gg_rs142928 Gg_rs136047. \\
\hline 0.69 & -1.52 & 0.68 & 2.52 & 0.93 & Gg_rs142928Gg_rs136047. \\
\hline $0.39 \mathrm{NA}$ & NA & NA & NA & & Gg_rs142928 Gg_rs136047. \\
\hline
\end{tabular}




\begin{tabular}{|c|c|c|c|c|c|}
\hline $0.91 \mathrm{NA}$ & NA & NA & NA & & Gg_rs142928 Gg_rs136047. \\
\hline $0.76 \mathrm{NA}$ & NA & NA & NA & & Gg_rs142928Gg_rs136047. \\
\hline 0.55 & -2.1 & 0.47 & 1.03 & 0.72 & Gg_rs142928 Gg_rs136047. \\
\hline $0.51 \mathrm{NA}$ & NA & NA & NA & & Gg_rs142928 Gg_rs136047. \\
\hline 0.29 & -0.94 & 0.25 & 0.37 & 0.38 & Gg_rs142928 Gg_rs136047. \\
\hline 0.41 & -1.14 & 0.35 & 2.37 & 0.54 & Gg_rs142931 Gg_rs136047. \\
\hline 0.37 & -1.09 & 0.32 & 0.92 & 0.48 & Gg_rs142928Gg_rs136047. \\
\hline $0.16 \mathrm{NA}$ & NA & NA & NA & & Gg_rs142928Gg_rs136047. \\
\hline 0.06 & 0.34 & 0.07 & 0.4 & 0.09 & GG_rs167237Gg_rs166878 \\
\hline $0.63 \mathrm{NA}$ & NA & NA & NA & & GG_rs167237Gg_rs166878 \\
\hline $0.04 \mathrm{NA}$ & NA & NA & NA & & GG_rs167237Gg_rs166878 \\
\hline $1.1 \mathrm{NA}$ & NA & NA & NA & & GG_rs167237Gg_rs166823 \\
\hline 0.89 NA & NA & NA & NA & & GG_rs167237Gg_rs166823 \\
\hline 0.69 NA & NA & NA & NA & & GG_rs167237Gg_rs166823 \\
\hline 1.08 & 0.94 & 1.09 & -3.85 & 1.5 & GG_rs167237Gg_rs166823 \\
\hline 1.46 & 1.76 & 1.48 & -7.78 & 2.03 & GG_rs167237Gg_rs166823 \\
\hline $1.02 \mathrm{NA}$ & NA & NA & NA & & GG_rs167237Gg_rs166823 \\
\hline 0.86 NA & NA & NA & NA & & GG_rs167237Gg_rs166823 \\
\hline $0.53 \mathrm{NA}$ & NA & NA & NA & & GG_rs167237Gg_rs166823 \\
\hline 1.09 NA & NA & NA & NA & & GG_rs167237Gg_rs166823 \\
\hline $0.68 \mathrm{NA}$ & NA & NA & NA & & GG_rs167237Gg_rs166823 \\
\hline $0.52 \mathrm{NA}$ & NA & NA & NA & & GG_rs167237Gg_rs166823 \\
\hline $1.2 \mathrm{NA}$ & NA & NA & NA & & GG_rs167237Gg_rs166823 \\
\hline $0.3 \mathrm{NA}$ & NA & NA & NA & & GG_rs167237Gg_rs166823 \\
\hline $0.72 \mathrm{NA}$ & NA & NA & NA & & GG_rs167237Gg_rs166823 \\
\hline $1.34 \mathrm{NA}$ & NA & NA & NA & & GG_rs167237Gg_rs166823 \\
\hline 0.19 & -0.11 & 0.19 & -0.86 & 0.26 & GG_rs167237Gg_rs166823 \\
\hline $0.77 \mathrm{NA}$ & NA & NA & NA & & GG_rs167237Gg_rs166823 \\
\hline $1.31 \mathrm{NA}$ & NA & NA & NA & & GG_rs167237Gg_rs166823 \\
\hline $1.41 \mathrm{NA}$ & NA & NA & NA & & GG_rs167237Gg_rs166823 \\
\hline $0.71 \mathrm{NA}$ & NA & NA & NA & & GG_rs167237Gg_rs166823 \\
\hline $0.77 \mathrm{NA}$ & NA & NA & NA & & GG_rs167237Gg_rs166823 \\
\hline $1.7 \mathrm{NA}$ & NA & NA & NA & & GG_rs167237Gg_rs166823 \\
\hline $1.2 \mathrm{NA}$ & NA & NA & NA & & GG_rs167237Gg_rs166823 \\
\hline $0.83 \mathrm{NA}$ & NA & NA & NA & & GG_rs167237Gg_rs166823 \\
\hline 0.32 & -0.15 & 0.33 & -1.45 & 0.45 & GG_rs167237Gg_rs166823 \\
\hline 0.44 NA & NA & NA & NA & & GG_rs167237Gg_rs166823 \\
\hline $1.08 \mathrm{NA}$ & NA & NA & NA & & GG_rs167237Gg_rs166823 \\
\hline $1.04 \mathrm{NA}$ & NA & NA & NA & & GG_rs167237Gg_rs166823 \\
\hline $1.29 \mathrm{NA}$ & NA & NA & NA & & GG_rs167237Gg_rs166823 \\
\hline $1.14 \mathrm{NA}$ & NA & NA & NA & & GG_rs167237Gg_rs166823 \\
\hline $1.31 \mathrm{NA}$ & NA & NA & NA & & GG_rs167237Gg_rs166823 \\
\hline $1.21 \mathrm{NA}$ & NA & NA & NA & & GG_rs167237Gg_rs166823 \\
\hline
\end{tabular}




\begin{tabular}{|c|c|c|c|c|c|c|}
\hline 0.46 & -1.03 & 0.45 & 0.5 & \multicolumn{3}{|c|}{0.6 snp.2347.3.31Gg_rs166823 } \\
\hline 0.21 & 0.63 & 0.2 & -0.57 & \multicolumn{3}{|c|}{0.27 snp. $2347.3 .3(G g$ grs 166823} \\
\hline 0.17 & 0.69 & 0.17 & 0.35 & 0.22 & \multirow{2}{*}{\multicolumn{2}{|c|}{$\begin{array}{l}\text { snp.2347.3.31Gg_rs166823 } \\
\text { snp.2347.3.31Gg_rs166823 }\end{array}$}} \\
\hline $0.41 \mathrm{NA}$ & NA & NA & NA & & & \\
\hline $0.44 \mathrm{NA}$ & NA & NA & NA & & \multicolumn{2}{|c|}{ snp.2347.3.3(Gg_rs166823 } \\
\hline $0.38 \mathrm{NA}$ & NA & NA & NA & & \multicolumn{2}{|c|}{ Gg_rs137248 Gg_rs136057. } \\
\hline $0.27 \mathrm{NA}$ & NA & NA & NA & & \multicolumn{2}{|c|}{ Gg_rs137248 Gg_rs136057. } \\
\hline $0.63 \mathrm{NA}$ & NA & NA & NA & & \multicolumn{2}{|c|}{ Gg_rs137248 Gg_rs154660' } \\
\hline $0.33 \mathrm{NA}$ & NA & NA & NA & & \multicolumn{2}{|c|}{ Gg_rs137248 Gg_rs152336. } \\
\hline $3.91 \mathrm{NA}$ & NA & NA & NA & & \multicolumn{2}{|c|}{ Gg_rs137248 Gg_rs136057. } \\
\hline $0.8 \mathrm{NA}$ & NA & NA & NA & & \multicolumn{2}{|c|}{ Gg_rs137248 Gg_rs136057. } \\
\hline 0.61 & -2.68 & 0.57 & 1.14 & 0.81 & \multicolumn{2}{|c|}{ Gg_rs137248 Gg_rs136057. } \\
\hline $1.48 \mathrm{NA}$ & NA & NA & NA & & \multicolumn{2}{|c|}{ Gg_rs137248 Gg_rs152309. } \\
\hline 4.4 & 9.98 & 4.14 & 14.99 & 5.86 & \multicolumn{2}{|c|}{ Gg_rs137246.Gg_rs152309. } \\
\hline 1.59 & 5.77 & 1.5 & 8.83 & 2.12 & \multicolumn{2}{|c|}{ Gg_rs137246.Gg_rs152309. } \\
\hline $0.57 \mathrm{NA}$ & NA & NA & NA & & \multicolumn{2}{|c|}{ Gg_rs137248 Gg_rs152309. } \\
\hline 0.85 & -2.17 & 0.8 & -3.75 & 1.13 & \multicolumn{2}{|c|}{ Gg_rs137246.Gg_rs152309. } \\
\hline 5.21 & 8.44 & 4.9 & 15.78 & 6.93 & \multicolumn{2}{|c|}{ Gg_rs137248 Gg_rs152309. } \\
\hline 0.5 & 1.35 & 0.47 & 1.17 & 0.67 & \multicolumn{2}{|c|}{ Gg_rs137246.Gg_rs154660 } \\
\hline 0.8 & -0.78 & 0.76 & 5.27 & 1.07 & \multicolumn{2}{|c|}{ Gg_rs137246.X26_1308001 } \\
\hline 0.6 & 1.79 & 0.56 & 3.22 & 0.8 & \multicolumn{2}{|c|}{ Gg_rs137246.X26_1308001 } \\
\hline 0.25 & 0.82 & 0.23 & 1.23 & 0.33 & \multicolumn{2}{|c|}{ Gg_rs137246.Gg_rs136057. } \\
\hline 0.93 & 2.41 & 0.87 & 5.45 & 1.23 & \multicolumn{2}{|c|}{ Gg_rs137246:X26_1308001 } \\
\hline 1.27 & 2.99 & 1.19 & 4.82 & 1.69 & \multicolumn{2}{|c|}{ Gg_rs137246.Gg_rs152309. } \\
\hline 0.99 & -2.66 & 0.93 & -4.5 & 1.32 & Gg_rs137 & Gg_rs152309. \\
\hline 0.41 & -0.3 & 0.38 & -3.13 & 0.55 & Gg_rs15 & Gg_rs152309. \\
\hline $0.39 \mathrm{NA}$ & NA & NA & NA & & Gg_rs136 & 5 Gg_rs143013 \\
\hline $1.41 \mathrm{NA}$ & NA & NA & NA & & RBL280 & Gg_rs152385 \\
\hline $1.28 \mathrm{NA}$ & NA & NA & NA & & RBL280 & Gg_rs152385 \\
\hline $2.09 \mathrm{NA}$ & NA & NA & NA & & RBL280 & Gg_rs152385 \\
\hline $1.56 \mathrm{NA}$ & NA & NA & NA & & RBL280 & Gg_rs152385 \\
\hline $1.23 \mathrm{NA}$ & NA & NA & NA & & RBL280 & Gg_rs152385 \\
\hline $1.45 \mathrm{NA}$ & NA & NA & NA & & RBL280 & Gg_rs152385 \\
\hline 0.73 NA & NA & NA & NA & & RBL280 & Gg_rs152385 \\
\hline $1.31 \mathrm{NA}$ & NA & NA & NA & & RBL280 & Gg_rs152385 \\
\hline $1.15 \mathrm{NA}$ & NA & NA & NA & & RBL280 & Gg_rs152385 \\
\hline $1.76 \mathrm{NA}$ & NA & NA & NA & & RBL280 & Gg_rs152385 \\
\hline 0.76 NA & NA & NA & NA & & RBL280 & Gg_rs152385 \\
\hline 0.93 NA & NA & NA & NA & & RBL280 & Gg_rs152385 \\
\hline $0.91 \mathrm{NA}$ & NA & NA & NA & & RBL280 & Gg_rs152385 \\
\hline $1.58 \mathrm{NA}$ & NA & NA & NA & & RBL280 & Gg_rs152385 \\
\hline $1.54 \mathrm{NA}$ & NA & NA & NA & & RBL280 & Gg_rs152385 \\
\hline $1.8 \mathrm{NA}$ & NA & NA & NA & & RBL280 & Gg_rs152385 \\
\hline
\end{tabular}




\begin{tabular}{|c|c|c|c|c|c|}
\hline $1.13 \mathrm{NA}$ & NA & NA & NA & RBL280 & Gg_rs152385 \\
\hline $1.69 \mathrm{NA}$ & NA & NA & NA & RBL280 & Gg_rs152385 \\
\hline $0.26 \mathrm{NA}$ & NA & NA & NA & RBL280 & Gg_rs152385 \\
\hline $1.7 \mathrm{NA}$ & NA & NA & NA & RBL280 & Gg_rs152385 \\
\hline $1.57 \mathrm{NA}$ & NA & NA & NA & RBL280 & Gg_rs152385 \\
\hline 0.14 & -0.61 & 0.17 & -0.18 & 0.21 RBL280 & Gg_rs152385 \\
\hline $0.08 \mathrm{NA}$ & NA & NA & NA & RBL280 & Gg_rs152385 \\
\hline $1.9 \mathrm{NA}$ & NA & NA & NA & RBL280 & Gg_rs152385 \\
\hline $1.24 \mathrm{NA}$ & NA & NA & NA & RBL280 & Gg_rs152385 \\
\hline $2.16 \mathrm{NA}$ & NA & NA & NA & RBL280 & Gg_rs152385 \\
\hline $2.34 \mathrm{NA}$ & NA & NA & NA & RBL280 & Gg_rs152385 \\
\hline $2.21 \mathrm{NA}$ & NA & NA & NA & RBL280 & Gg_rs152385 \\
\hline $1.69 \mathrm{NA}$ & NA & NA & NA & RBL280 & Gg_rs152385 \\
\hline $0.38 \mathrm{NA}$ & NA & NA & NA & RBL280 & Gg_rs152385 \\
\hline $2.43 \mathrm{NA}$ & NA & NA & NA & RBL280 & Gg_rs152385 \\
\hline $2.27 \mathrm{NA}$ & NA & NA & NA & RBL280 & Gg_rs152385 \\
\hline $1.42 \mathrm{NA}$ & NA & NA & NA & RBL280 & Gg_rs152385 \\
\hline $2.63 \mathrm{NA}$ & NA & NA & NA & RBL280 & Gg_rs152385 \\
\hline $1.48 \mathrm{NA}$ & NA & NA & NA & RBL280 & Gg_rs152385 \\
\hline $1.18 \mathrm{NA}$ & NA & NA & NA & RBL280 & Gg_rs152385 \\
\hline $1.87 \mathrm{NA}$ & NA & NA & NA & RBL280 & Gg_rs152385 \\
\hline $1.75 \mathrm{NA}$ & NA & NA & NA & RBL280 & Gg_rs152385 \\
\hline $2.35 \mathrm{NA}$ & NA & NA & NA & RBL280 & Gg_rs152385 \\
\hline $2.11 \mathrm{NA}$ & NA & NA & NA & RBL280 & Gg_rs152385 \\
\hline $2.37 \mathrm{NA}$ & NA & NA & NA & RBL280 & Gg_rs152385 \\
\hline $1.68 \mathrm{NA}$ & NA & NA & NA & RBL280 & Gg_rs152385 \\
\hline $2.22 \mathrm{NA}$ & NA & NA & NA & RBL280 & Gg_rs152385 \\
\hline $2.41 \mathrm{NA}$ & NA & NA & NA & RBL280 & Gg_rs152385 \\
\hline $0.76 \mathrm{NA}$ & NA & NA & NA & RBL280 & Gg_rs152385 \\
\hline 0.84 & -0.33 & 1.02 & -2.53 & 1.3 RBL280 & Gg_rs152385 \\
\hline $1.24 \mathrm{NA}$ & NA & NA & NA & RBL280 & Gg_rs152385 \\
\hline $0.83 \mathrm{NA}$ & NA & NA & NA & RBL280 & Gg_rs152385 \\
\hline $1.81 \mathrm{NA}$ & NA & NA & NA & RBL280 & Gg_rs152385 \\
\hline $0.64 \mathrm{NA}$ & NA & NA & NA & RBL280 & Gg_rs152385 \\
\hline $1.57 \mathrm{NA}$ & NA & NA & NA & RBL280 & Gg_rs152385 \\
\hline $1.42 \mathrm{NA}$ & NA & NA & NA & RBL280 & Gg_rs152385 \\
\hline $0.67 \mathrm{NA}$ & NA & NA & NA & RBL280 & Gg_rs152385 \\
\hline $1.16 \mathrm{NA}$ & NA & NA & NA & RBL280 & Gg_rs152385 \\
\hline $1.19 \mathrm{NA}$ & NA & NA & NA & RBL280 & Gg_rs152385 \\
\hline $1.48 \mathrm{NA}$ & NA & NA & NA & RBL280 & Gg_rs152385 \\
\hline $1.43 \mathrm{NA}$ & NA & NA & NA & RBL280 & Gg_rs152385 \\
\hline $1.23 \mathrm{NA}$ & NA & NA & NA & RBL280 & Gg_rs152385 \\
\hline $1.74 \mathrm{NA}$ & NA & NA & NA & RBL280 & Gg_rs152385 \\
\hline
\end{tabular}




\begin{tabular}{|c|c|c|c|c|c|c|c|}
\hline $0.7 \mathrm{NA}$ & NA & & NA & & & RBL280 & Gg_rs152385 \\
\hline $2.05 \mathrm{NA}$ & NA & & NA & & & RBL280 & Gg_rs152385 \\
\hline $0.91 \mathrm{NA}$ & NA & & NA & & & RBL280 & Gg_rs152385 \\
\hline $1.6 \mathrm{NA}$ & NA & & NA & & & RBL280 & Gg_rs152385 \\
\hline $1.37 \mathrm{NA}$ & NA & & NA & & & RBL280 & Gg_rs152385 \\
\hline $0.38 \mathrm{NA}$ & NA & & NA & & & RBL280 & Gg_rs152385 \\
\hline 0.01 & -0.05 & 0.01 & & -0.05 & 0.01 & RBL280 & Gg_rs152385 \\
\hline 0.03 & -0.21 & 0.04 & & -0.17 & 0.05 & RBL280 & Gg_rs152385 \\
\hline $0.11 \mathrm{NA}$ & NA & & NA & & & RBL280 & Gg_rs152385 \\
\hline $0.17 \mathrm{NA}$ & NA & & NA & & & RBL280 & Gg_rs152385 \\
\hline 0.81 & -3.69 & 0.99 & & -4.58 & 1.26 & RBL280 & Gg_rs152385 \\
\hline 0.03 & -0.16 & 0.03 & & -0.12 & 0.04 & RBL280 & Gg_rs152385 \\
\hline 0.15 & -0.78 & 0.18 & & -0.91 & 0.23 & RBL280 & Gg_rs152385 \\
\hline 0.89 & -4.54 & 1.08 & & -6.29 & 1.37 & RBL280 & Gg_rs152385 \\
\hline $0.32 \mathrm{NA}$ & NA & & NA & & & RBL280 & Gg_rs152385 \\
\hline $0.23 \mathrm{NA}$ & NA & & NA & & & RBL280 & Gg_rs152385 \\
\hline $0.42 \mathrm{NA}$ & NA & & NA & & & RBL280 & Gg_rs152385 \\
\hline $0.77 \mathrm{NA}$ & NA & & NA & & & RBL280 & Gg_rs152385 \\
\hline 0.06 & -0.29 & 0.07 & & -0.16 & 0.09 & RBL280 & Gg_rs152385 \\
\hline 0.02 & -0.12 & 0.02 & & -0.09 & 0.03 & RBL280 & Gg_rs152385 \\
\hline $0.38 \mathrm{NA}$ & NA & & NA & & & RBL280 & Gg_rs152385 \\
\hline $0.36 \mathrm{NA}$ & NA & & NA & & & \multirow{2}{*}{\multicolumn{2}{|c|}{$\begin{array}{l}\text { Gg_rs152462 X28_1608048 } \\
\text { Gg_rs152462 Gg_rs152476 }\end{array}$}} \\
\hline 0.27 & -1.2 & 0.25 & & 0.62 & 0.36 & & \\
\hline 0.77 & 0.71 & 0.73 & & -4.26 & 1.05 & \multicolumn{2}{|c|}{ Gg_rs152462 Gg_rs152476 } \\
\hline $0.29 \mathrm{NA}$ & NA & & NA & & & \multicolumn{2}{|c|}{ Gg_rs152462 Gg_rs152476 } \\
\hline 0.43 & 1.33 & 0.36 & & -1.16 & 0.55 & \multicolumn{2}{|c|}{ X28_1608048Gg_rs143072 } \\
\hline 0.91 & 1.8 & 0.74 & & 3 & 1.17 & \multirow{2}{*}{\multicolumn{2}{|c|}{$\begin{array}{l}\text { Gg_rs152476 Gg_rs } 143072 \\
\text { X28_1608048Gg_rs } 143072\end{array}$}} \\
\hline $0.45 \mathrm{NA}$ & NA & & NA & & & & \\
\hline 0.5 & 1.95 & 0.4 & & -0.46 & 0.63 & \multicolumn{2}{|c|}{ X28_1608048Gg_rs143072 } \\
\hline 0.73 & 1.51 & 0.62 & & -0.2 & 0.95 & \multicolumn{2}{|c|}{ X28_160804\&Gg_rs143072 } \\
\hline 0.39 & 1.03 & 0.33 & & -0.73 & 0.5 & \multicolumn{2}{|c|}{ X28_1608048Gg_rs143072 } \\
\hline 0.61 & 2.4 & 0.64 & & -2 & 0.84 & \multicolumn{2}{|c|}{ Gg_rs143061 X28_3803332 } \\
\hline $0.49 \mathrm{NA}$ & NA & & NA & & & \multicolumn{2}{|c|}{ Gg_rs143061 X28_3803332 } \\
\hline 0.55 & -2.02 & 0.58 & & 1.5 & 0.76 & \multicolumn{2}{|c|}{ Gg_rs143061X28_3803332 } \\
\hline 0.28 & -1.97 & 0.29 & & 1.56 & 0.38 & \multicolumn{2}{|c|}{ Gg_rs143061 X28_3803332 } \\
\hline 0.52 & -2.76 & 0.54 & & 1.84 & 0.71 & \multicolumn{2}{|c|}{ Gg_rs143061X28_3803332 } \\
\hline 0.26 & -1.3 & 0.27 & & 0.96 & 0.36 & \multirow{2}{*}{\multicolumn{2}{|c|}{$\begin{array}{l}\text { Gg_rs143061 X28_3803332 } \\
\text { Gg_rs143072 GG_rs152528 }\end{array}$}} \\
\hline $0.45 \mathrm{NA}$ & NA & & NA & & & & \\
\hline
\end{tabular}


lower_marke upper_mark€Cl_lower_po: $\mathrm{Cl}$ _upper_po: $\mathrm{Cl}$ _size

\begin{tabular}{|c|c|c|c|c|}
\hline 479025 & 1293096 & 50 & 115 & 65 \\
\hline 479025 & 1293096 & 50 & 110 & 60 \\
\hline 479025 & 1293096 & 50 & 102 & 52 \\
\hline 479025 & 1293096 & 50 & 114 & 64 \\
\hline 479025 & 1293096 & 50 & 113 & 63 \\
\hline 479025 & 1293096 & 50 & 107 & 57 \\
\hline 479025 & 1293096 & 50 & 113 & 63 \\
\hline 479025 & 1293096 & 50 & 109 & 59 \\
\hline 479025 & 1293096 & 50 & 107 & 57 \\
\hline 479025 & 1293096 & 50 & 108 & 58 \\
\hline 479025 & 1293096 & 50 & 114 & 64 \\
\hline 479025 & 1293096 & 50 & 105 & 55 \\
\hline 479025 & 1293096 & 50 & 122 & 72 \\
\hline 479025 & 1293096 & 50 & 121 & 71 \\
\hline 479025 & 1293096 & 50 & 123 & 73 \\
\hline 479025 & 1293096 & 50 & 121 & 71 \\
\hline 479025 & 1293096 & 50 & 119 & 69 \\
\hline 479025 & 1293096 & 50 & 124 & 74 \\
\hline 479025 & 1293096 & 50 & 127 & 77 \\
\hline 479025 & 1293096 & 50 & 116 & 66 \\
\hline 479025 & 1293096 & 50 & 125 & 75 \\
\hline 479025 & 1293096 & 50 & 129 & 79 \\
\hline 479025 & 1293096 & 50 & 129 & 79 \\
\hline 479025 & 1293096 & 50 & 126 & 76 \\
\hline 648718 & 12488579 & 116 & 268 & 152 \\
\hline 648718 & 7683739 & 127 & 245 & 118 \\
\hline 648718 & 1440585 & 133 & 228 & 95 \\
\hline 648718 & 1440585 & 135 & 228 & 93 \\
\hline 648718 & 1440585 & 134 & 228 & 94 \\
\hline 648718 & 1440585 & 134 & 228 & 94 \\
\hline 648718 & 4080964 & 130 & 242 & 112 \\
\hline 648718 & 7683739 & 142 & 246 & 104 \\
\hline 648718 & 1440585 & 144 & 233 & 89 \\
\hline 648718 & 1440585 & 169 & 228 & 59 \\
\hline 648718 & 14847187 & 157 & 287 & 130 \\
\hline 648718 & 1440585 & 149 & 232 & 83 \\
\hline 648718 & 7683739 & 141 & 245 & 104 \\
\hline 648718 & 7683739 & 139 & 246 & 107 \\
\hline 648718 & 9217865 & 136 & 253 & 117 \\
\hline 648718 & 7683739 & 147 & 246 & 99 \\
\hline 648718 & 9217865 & 136 & 251 & 115 \\
\hline
\end{tabular}




\begin{tabular}{|c|c|c|c|}
\hline 648718 & 7683739 & 171 & 244 \\
\hline 648718 & 3678621 & 187 & 242 \\
\hline 1293096 & 2923116 & 220 & 237 \\
\hline 648718 & 7683739 & 139 & 246 \\
\hline 1293096 & 7683739 & 222 & 248 \\
\hline 648718 & 9217865 & 175 & 253 \\
\hline 648718 & 7683739 & 150 & 248 \\
\hline 648718 & 7683739 & 162 & 244 \\
\hline 648718 & 9217865 & 155 & 251 \\
\hline 648718 & 7683739 & 147 & 249 \\
\hline 479025 & 27333617 & 50 & 424 \\
\hline 648718 & 9217865 & 163 & 253 \\
\hline 1293096 & 12488579 & 220 & 265 \\
\hline 1293096 & 7683739 & 223 & 249 \\
\hline 1440585 & 4080964 & 236 & 242 \\
\hline 1293096 & 7683739 & 224 & 248 \\
\hline 1293096 & 7683739 & 223 & 248 \\
\hline 648718 & 13847380 & 182 & 277 \\
\hline 1293096 & 9217865 & 226 & 251 \\
\hline 648718 & 14847187 & 171 & 287 \\
\hline 648718 & 13847380 & 167 & 274 \\
\hline 648718 & 12488579 & 182 & 266 \\
\hline 1293096 & 9217865 & 221 & 253 \\
\hline 1440585 & 12488579 & 236 & 265 \\
\hline 1293096 & 9217865 & 229 & 251 \\
\hline 1440585 & 7683739 & 237 & 248 \\
\hline 1293096 & 12488579 & 220 & 261 \\
\hline 1293096 & 12488579 & 228 & 257 \\
\hline 1440585 & 7683739 & 236 & 246 \\
\hline 1440585 & 12488579 & 235 & 268 \\
\hline 1293096 & 16419237 & 226 & 302 \\
\hline 648718 & 9217865 & 179 & 254 \\
\hline 1293096 & 12488579 & 218 & 268 \\
\hline 648718 & 12488579 & 162 & 266 \\
\hline 1293096 & 14847187 & 224 & 282 \\
\hline 1440585 & 7683739 & 236 & 248 \\
\hline 1440585 & 7683739 & 237 & 246 \\
\hline 1440585 & 7683739 & 237 & 247 \\
\hline 1440585 & 7683739 & 236 & 248 \\
\hline 1440585 & 7683739 & 237 & 247 \\
\hline 1440585 & 12488579 & 236 & 268 \\
\hline 1293096 & 20892359 & 223 & 342 \\
\hline 1440585 & 4080964 & 235 & 242 \\
\hline
\end{tabular}




\begin{tabular}{|c|c|c|c|}
\hline 1440585 & 12488579 & 236 & 265 \\
\hline 1440585 & 7683739 & 236 & 248 \\
\hline 1440585 & 7683739 & 236 & 249 \\
\hline 1440585 & 13847380 & 236 & 273 \\
\hline 1293096 & 20892359 & 230 & 341 \\
\hline 1440585 & 12488579 & 236 & 265 \\
\hline 1440585 & 12488579 & 236 & 264 \\
\hline 1440585 & 12488579 & 236 & 266 \\
\hline 1440585 & 7683739 & 235 & 248 \\
\hline 1440585 & 7683739 & 238 & 247 \\
\hline 1440585 & 7683739 & 237 & 249 \\
\hline 1440585 & 7683739 & 238 & 246 \\
\hline 1440585 & 13847380 & 236 & 276 \\
\hline 1440585 & 7683739 & 237 & 248 \\
\hline 1440585 & 7683739 & 236 & 245 \\
\hline 1440585 & 12488579 & 237 & 264 \\
\hline 1440585 & 12488579 & 237 & 265 \\
\hline 1440585 & 12488579 & 235 & 255 \\
\hline 648718 & 12488579 & 156 & 268 \\
\hline 1293096 & 14847187 & 227 & 291 \\
\hline 1440585 & 14847187 & 235 & 289 \\
\hline 1440585 & 12488579 & 235 & 265 \\
\hline 1440585 & 7683739 & 237 & 247 \\
\hline 1440585 & 7683739 & 238 & 247 \\
\hline 1440585 & 7683739 & 238 & 247 \\
\hline 1293096 & 7683739 & 219 & 248 \\
\hline 1293096 & 9217865 & 228 & 250 \\
\hline 1293096 & 7683739 & 232 & 249 \\
\hline 648718 & 9217865 & 156 & 253 \\
\hline 1440585 & 7683739 & 236 & 247 \\
\hline 1293096 & 7683739 & 219 & 249 \\
\hline 3678621 & 7683739 & 242 & 248 \\
\hline 1293096 & 14847187 & 225 & 295 \\
\hline 648718 & 9217865 & 174 & 252 \\
\hline 1440585 & 9217865 & 235 & 252 \\
\hline 1440585 & 7683739 & 237 & 246 \\
\hline 1440585 & 12488579 & 235 & 266 \\
\hline 648718 & 12488579 & 152 & 263 \\
\hline 1440585 & 12488579 & 236 & 264 \\
\hline 648718 & 13847380 & 176 & 277 \\
\hline 648718 & 14847187 & 194 & 294 \\
\hline 1293096 & 12488579 & 233 & 255 \\
\hline 1440585 & 12488579 & 237 & 262 \\
\hline
\end{tabular}




\begin{tabular}{|c|c|c|c|}
\hline 1440585 & 12488579 & 237 & 262 \\
\hline 1440585 & 12488579 & 236 & 265 \\
\hline 1440585 & 12488579 & 237 & 266 \\
\hline 1293096 & 12488579 & 223 & 268 \\
\hline 648718 & 12488579 & 197 & 264 \\
\hline 648718 & 14847187 & 171 & 289 \\
\hline 1293096 & 13847380 & 222 & 279 \\
\hline 648718 & 12488579 & 180 & 264 \\
\hline 1293096 & 12488579 & 222 & 261 \\
\hline 1293096 & 12488579 & 218 & 264 \\
\hline 1440585 & 12488579 & 236 & 266 \\
\hline 1293096 & 12488579 & 222 & 264 \\
\hline 648718 & 16419237 & 206 & 316 \\
\hline 1440585 & 12488579 & 238 & 260 \\
\hline 1293096 & 12488579 & 221 & 262 \\
\hline 1293096 & 14847187 & 226 & 287 \\
\hline 648718 & 12488579 & 139 & 267 \\
\hline 648718 & 13847380 & 136 & 271 \\
\hline 1293096 & 13847380 & 220 & 279 \\
\hline 1293096 & 12488579 & 234 & 265 \\
\hline 3678621 & 12488579 & 242 & 263 \\
\hline 648718 & 14847187 & 152 & 289 \\
\hline 1293096 & 12488579 & 228 & 267 \\
\hline 1293096 & 14847187 & 227 & 285 \\
\hline 648718 & 9217865 & 205 & 254 \\
\hline 1440585 & 12488579 & 236 & 264 \\
\hline 1293096 & 12488579 & 224 & 259 \\
\hline 648718 & 12488579 & 169 & 258 \\
\hline 1440585 & 12488579 & 237 & 262 \\
\hline 1440585 & 14847187 & 238 & 289 \\
\hline 4080964 & 12488579 & 246 & 265 \\
\hline 4080964 & 12488579 & 245 & 259 \\
\hline 648718 & 14847187 & 169 & 289 \\
\hline 648718 & 12488579 & 140 & 262 \\
\hline 1440585 & 12488579 & 237 & 262 \\
\hline 1440585 & 12488579 & 236 & 268 \\
\hline 1293096 & 12488579 & 226 & 263 \\
\hline 2923116 & 12488579 & 241 & 261 \\
\hline 648718 & 12488579 & 191 & 269 \\
\hline 4080964 & 12488579 & 246 & 270 \\
\hline 4080964 & 12488579 & 247 & 264 \\
\hline 1440585 & 12488579 & 237 & 268 \\
\hline 1293096 & 16419237 & 218 & 314 \\
\hline
\end{tabular}




\begin{tabular}{|c|c|c|c|}
\hline 2923116 & 13847380 & 240 & 273 \\
\hline 1440585 & 12488579 & 237 & 268 \\
\hline 4080964 & 12488579 & 245 & 267 \\
\hline 4080964 & 12488579 & 243 & 267 \\
\hline 4080964 & 12488579 & 243 & 266 \\
\hline 4080964 & 12488579 & 244 & 265 \\
\hline 4080964 & 13847380 & 245 & 270 \\
\hline 1440585 & 12488579 & 237 & 265 \\
\hline 4080964 & 12488579 & 243 & 267 \\
\hline 4080964 & 12488579 & 245 & 269 \\
\hline 4080964 & 12488579 & 244 & 267 \\
\hline 4080964 & 12488579 & 244 & 265 \\
\hline 4080964 & 12488579 & 244 & 269 \\
\hline 1440585 & 13847380 & 236 & 271 \\
\hline 4080964 & 12488579 & 244 & 268 \\
\hline 1440585 & 13847380 & 238 & 272 \\
\hline 4080964 & 13847380 & 245 & 270 \\
\hline 3678621 & 12488579 & 242 & 268 \\
\hline 4080964 & 12488579 & 244 & 267 \\
\hline 648718 & 12488579 & 168 & 270 \\
\hline 1440585 & 12488579 & 239 & 267 \\
\hline 4080964 & 12488579 & 245 & 267 \\
\hline 1440585 & 12488579 & 236 & 266 \\
\hline 1440585 & 13847380 & 239 & 271 \\
\hline 1440585 & 13847380 & 236 & 272 \\
\hline 4080964 & 13847380 & 243 & 273 \\
\hline 1440585 & 12488579 & 237 & 265 \\
\hline 1440585 & 12488579 & 237 & 265 \\
\hline 1440585 & 12488579 & 237 & 266 \\
\hline 1440585 & 12488579 & 237 & 266 \\
\hline 4080964 & 12488579 & 244 & 269 \\
\hline 1440585 & 13847380 & 239 & 273 \\
\hline 4080964 & 13847380 & 244 & 275 \\
\hline 4080964 & 13847380 & 245 & 274 \\
\hline 4080964 & 13847380 & 246 & 275 \\
\hline 4080964 & 14847187 & 247 & 286 \\
\hline 1293096 & 16419237 & 219 & 313 \\
\hline 1440585 & 13847380 & 236 & 275 \\
\hline 4080964 & 13847380 & 244 & 276 \\
\hline 4080964 & 13847380 & 246 & 279 \\
\hline 4080964 & 13847380 & 245 & 274 \\
\hline 1440585 & 13847380 & 238 & 275 \\
\hline 1440585 & 13847380 & 236 & 273 \\
\hline
\end{tabular}




\begin{tabular}{|c|c|c|c|c|}
\hline 4080964 & 13847380 & 244 & 274 & 30 \\
\hline 1440585 & 13847380 & 235 & 278 & 43 \\
\hline 10671331 & 14847187 & 257 & 289 & 32 \\
\hline 10671331 & 14847187 & 257 & 292 & 35 \\
\hline 1440585 & 14847187 & 235 & 288 & 53 \\
\hline 1440585 & 13847380 & 236 & 274 & 38 \\
\hline 1440585 & 13847380 & 239 & 275 & 36 \\
\hline 4080964 & 13847380 & 247 & 279 & 32 \\
\hline 1440585 & 14847187 & 237 & 295 & 58 \\
\hline 1440585 & 14847187 & 238 & 291 & 53 \\
\hline 4080964 & 13847380 & 246 & 276 & 30 \\
\hline 4080964 & 14847187 & 245 & 285 & 40 \\
\hline 1293096 & 14847187 & 233 & 290 & 57 \\
\hline 1440585 & 13847380 & 239 & 277 & 38 \\
\hline 10671331 & 13847380 & 257 & 276 & 19 \\
\hline 10671331 & 13847380 & 257 & 279 & 22 \\
\hline 1440585 & 13847380 & 236 & 277 & 41 \\
\hline 10671331 & 13847380 & 257 & 279 & 22 \\
\hline 648718 & 14847187 & 200 & 296 & 96 \\
\hline 10671331 & 14847187 & 257 & 288 & 31 \\
\hline 10671331 & 13847380 & 257 & 279 & 22 \\
\hline 10671331 & 13847380 & 257 & 279 & 22 \\
\hline 10671331 & 13847380 & 257 & 280 & 23 \\
\hline 10671331 & 13847380 & 257 & 278 & 21 \\
\hline 10671331 & 13847380 & 257 & 280 & 23 \\
\hline 10671331 & 13847380 & 257 & 279 & 22 \\
\hline 10671331 & 14847187 & 257 & 293 & 36 \\
\hline 10671331 & 13847380 & 258 & 273 & 15 \\
\hline 10671331 & 13847380 & 257 & 277 & 20 \\
\hline 10671331 & 13847380 & 262 & 272 & 10 \\
\hline 10671331 & 13847380 & 263 & 276 & 13 \\
\hline 10671331 & 13847380 & 262 & 275 & 13 \\
\hline 10671331 & 13847380 & 260 & 279 & 19 \\
\hline 10671331 & 13847380 & 261 & 278 & 17 \\
\hline 10671331 & 14847187 & 257 & 286 & 29 \\
\hline 10671331 & 20892359 & 257 & 349 & 92 \\
\hline 10671331 & 13847380 & 257 & 280 & 23 \\
\hline 10671331 & 13847380 & 259 & 280 & 21 \\
\hline 10671331 & 13847380 & 257 & 275 & 18 \\
\hline 10671331 & 13847380 & 264 & 274 & 10 \\
\hline 10671331 & 14847187 & 261 & 282 & 21 \\
\hline 10671331 & 19755536 & 257 & 338 & 81 \\
\hline 10671331 & 14847187 & 257 & 295 & 38 \\
\hline
\end{tabular}




\begin{tabular}{|c|c|c|c|c|}
\hline 10671331 & 13847380 & 264 & 276 & 12 \\
\hline 10671331 & 13847380 & 258 & 281 & 23 \\
\hline 10671331 & 16419237 & 257 & 310 & 53 \\
\hline 1440585 & 16419237 & 239 & 308 & 69 \\
\hline 10671331 & 14847187 & 258 & 292 & 34 \\
\hline 10671331 & 14847187 & 258 & 288 & 30 \\
\hline 10671331 & 20892359 & 257 & 341 & 84 \\
\hline 10671331 & 14847187 & 257 & 293 & 36 \\
\hline 10671331 & 14847187 & 257 & 293 & 36 \\
\hline 10671331 & 14847187 & 257 & 295 & 38 \\
\hline 10671331 & 14847187 & 260 & 295 & 35 \\
\hline 10671331 & 16419237 & 258 & 313 & 55 \\
\hline 10671331 & 14847187 & 257 & 294 & 37 \\
\hline 10671331 & 16419237 & 262 & 306 & 44 \\
\hline 10671331 & 14847187 & 262 & 294 & 32 \\
\hline 12488579 & 14847187 & 271 & 295 & 24 \\
\hline 12488579 & 14847187 & 270 & 293 & 23 \\
\hline 12488579 & 14847187 & 270 & 291 & 21 \\
\hline 10671331 & 14847187 & 257 & 289 & 32 \\
\hline 10671331 & 14847187 & 257 & 293 & 36 \\
\hline 12488579 & 14847187 & 274 & 292 & 18 \\
\hline 12488579 & 14847187 & 275 & 291 & 16 \\
\hline 12488579 & 14847187 & 277 & 290 & 13 \\
\hline 12488579 & 14847187 & 278 & 290 & 12 \\
\hline 12488579 & 14847187 & 275 & 293 & 18 \\
\hline 12488579 & 14847187 & 277 & 292 & 15 \\
\hline 12488579 & 14847187 & 278 & 289 & 11 \\
\hline 12488579 & 14847187 & 278 & 288 & 10 \\
\hline 12488579 & 14847187 & 278 & 290 & 12 \\
\hline 12488579 & 14847187 & 277 & 290 & 13 \\
\hline 12488579 & 14847187 & 278 & 286 & 8 \\
\hline 12488579 & 14847187 & 277 & 291 & 14 \\
\hline 12488579 & 14847187 & 275 & 290 & 15 \\
\hline 12488579 & 14847187 & 273 & 291 & 18 \\
\hline 12488579 & 14847187 & 274 & 289 & 15 \\
\hline 12488579 & 14847187 & 277 & 292 & 15 \\
\hline 12488579 & 14847187 & 279 & 290 & 11 \\
\hline 12488579 & 14847187 & 278 & 292 & 14 \\
\hline 12488579 & 14847187 & 276 & 290 & 14 \\
\hline 12488579 & 14847187 & 277 & 293 & 16 \\
\hline 12488579 & 14847187 & 275 & 291 & 16 \\
\hline 12488579 & 14847187 & 278 & 290 & 12 \\
\hline 12488579 & 14847187 & 278 & 290 & 12 \\
\hline
\end{tabular}




\begin{tabular}{|c|c|c|c|c|}
\hline 12488579 & 14847187 & 277 & 289 & 12 \\
\hline 12488579 & 14847187 & 277 & 289 & 12 \\
\hline 12488579 & 16419237 & 271 & 310 & 39 \\
\hline 12488579 & 14847187 & 273 & 294 & 21 \\
\hline 12488579 & 14847187 & 274 & 288 & 14 \\
\hline 12488579 & 14847187 & 277 & 290 & 13 \\
\hline 12488579 & 14847187 & 277 & 292 & 15 \\
\hline 12488579 & 14847187 & 277 & 290 & 13 \\
\hline 12488579 & 14847187 & 277 & 292 & 15 \\
\hline 12488579 & 14847187 & 277 & 289 & 12 \\
\hline 12488579 & 14847187 & 276 & 288 & 12 \\
\hline 12488579 & 14847187 & 277 & 288 & 11 \\
\hline 12488579 & 16419237 & 273 & 301 & 28 \\
\hline 10671331 & 14847187 & 267 & 292 & 25 \\
\hline 10671331 & 14847187 & 257 & 291 & 34 \\
\hline 12488579 & 14847187 & 276 & 292 & 16 \\
\hline 12488579 & 16419237 & 270 & 311 & 41 \\
\hline 12488579 & 16419237 & 271 & 305 & 34 \\
\hline 12488579 & 14847187 & 277 & 293 & 16 \\
\hline 12488579 & 16419237 & 273 & 319 & 46 \\
\hline 12488579 & 16419237 & 273 & 306 & 33 \\
\hline 10671331 & 16419237 & 257 & 303 & 46 \\
\hline 12488579 & 14847187 & 276 & 290 & 14 \\
\hline 12488579 & 14847187 & 277 & 289 & 12 \\
\hline 12488579 & 14847187 & 277 & 290 & 13 \\
\hline 12488579 & 14847187 & 277 & 292 & 15 \\
\hline 12488579 & 14847187 & 277 & 291 & 14 \\
\hline 12488579 & 14847187 & 278 & 292 & 14 \\
\hline 12488579 & 14847187 & 278 & 292 & 14 \\
\hline 12488579 & 14847187 & 277 & 286 & 9 \\
\hline 12488579 & 14847187 & 278 & 287 & 9 \\
\hline 10671331 & 14847187 & 266 & 294 & 28 \\
\hline 12488579 & 14847187 & 277 & 290 & 13 \\
\hline 12488579 & 14847187 & 278 & 286 & 8 \\
\hline 12488579 & 14847187 & 278 & 286 & 8 \\
\hline 12488579 & 14847187 & 276 & 287 & 11 \\
\hline 12488579 & 14847187 & 277 & 289 & 12 \\
\hline 12488579 & 14847187 & 275 & 294 & 19 \\
\hline 12488579 & 14847187 & 278 & 288 & 10 \\
\hline 12488579 & 14847187 & 277 & 291 & 14 \\
\hline 12488579 & 14847187 & 276 & 291 & 15 \\
\hline 12488579 & 14847187 & 276 & 289 & 13 \\
\hline 12488579 & 14847187 & 278 & 290 & 12 \\
\hline
\end{tabular}




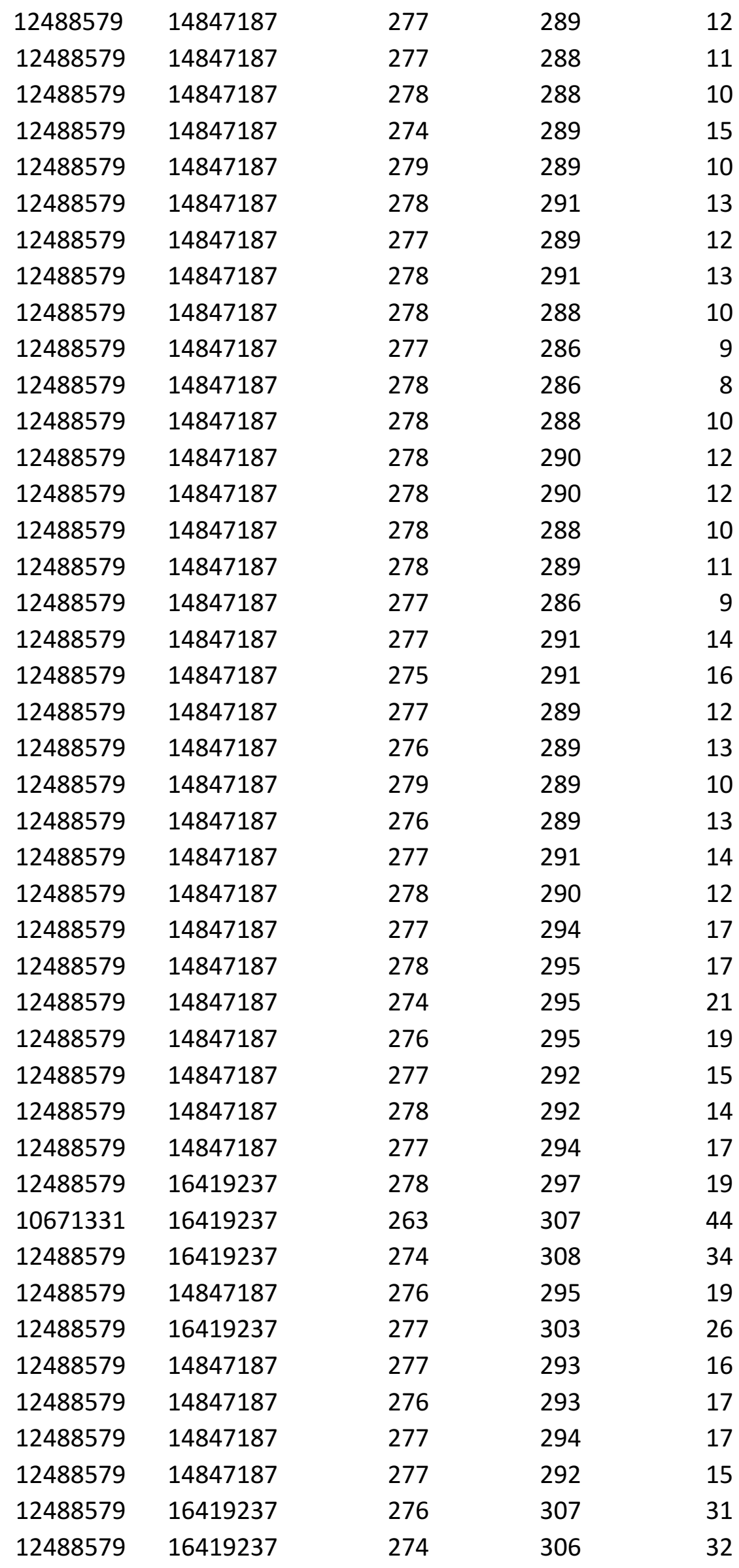




\begin{tabular}{|c|c|c|c|}
\hline 12488579 & 16419237 & 270 & 311 \\
\hline 12488579 & 16419237 & 272 & 306 \\
\hline 12488579 & 16419237 & 276 & 304 \\
\hline 12488579 & 16419237 & 276 & 299 \\
\hline 12488579 & 14847187 & 275 & 294 \\
\hline 12488579 & 14847187 & 277 & 294 \\
\hline 10671331 & 16419237 & 264 & 309 \\
\hline 12488579 & 16419237 & 276 & 305 \\
\hline 12488579 & 16419237 & 276 & 305 \\
\hline 12488579 & 16419237 & 277 & 300 \\
\hline 10671331 & 16419237 & 261 & 311 \\
\hline 10671331 & 20892359 & 256 & 344 \\
\hline 10671331 & 16419237 & 263 & 312 \\
\hline 10671331 & 16419237 & 257 & 322 \\
\hline 12488579 & 16419237 & 274 & 311 \\
\hline 12488579 & 16419237 & 272 & 320 \\
\hline 12488579 & 16419237 & 272 & 320 \\
\hline 12488579 & 16419237 & 275 & 310 \\
\hline 12488579 & 16419237 & 272 & 313 \\
\hline 12488579 & 16419237 & 281 & 313 \\
\hline 13847380 & 16419237 & 291 & 311 \\
\hline 12488579 & 16419237 & 276 & 317 \\
\hline 13847380 & 16419237 & 287 & 316 \\
\hline 12488579 & 16419237 & 273 & 325 \\
\hline 13847380 & 16419237 & 284 & 325 \\
\hline 13847380 & 16419237 & 283 & 324 \\
\hline 12488579 & 19755536 & 277 & 333 \\
\hline 12488579 & 16419237 & 275 & 323 \\
\hline 12488579 & 16419237 & 272 & 328 \\
\hline 12488579 & 20892359 & 270 & 342 \\
\hline 12488579 & 22379284 & 273 & 356 \\
\hline 12488579 & 20892359 & 271 & 346 \\
\hline 14847187 & 20892359 & 308 & 343 \\
\hline 14847187 & 19755536 & 314 & 339 \\
\hline 14847187 & 22379284 & 310 & 355 \\
\hline 14847187 & 22379284 & 316 & 357 \\
\hline 14847187 & 20892359 & 317 & 350 \\
\hline 14847187 & 20892359 & 302 & 345 \\
\hline 14847187 & 20892359 & 302 & 351 \\
\hline 14847187 & 22379284 & 321 & 355 \\
\hline 14847187 & 22379284 & 320 & 359 \\
\hline 14847187 & 23646445 & 321 & 372 \\
\hline 16419237 & 22379284 & 334 & 370 \\
\hline
\end{tabular}




\begin{tabular}{|c|c|c|c|}
\hline 19755536 & 22379284 & 344 & 363 \\
\hline 19755536 & 22379284 & 345 & 364 \\
\hline 19755536 & 22379284 & 346 & 370 \\
\hline 14847187 & 23646445 & 319 & 380 \\
\hline 19755536 & 23646445 & 346 & 373 \\
\hline 19755536 & 23646445 & 349 & 383 \\
\hline 19755536 & 23646445 & 350 & 381 \\
\hline 20892359 & 23646445 & 358 & 384 \\
\hline 14847187 & 23646445 & 327 & 386 \\
\hline 20892359 & 23646445 & 359 & 385 \\
\hline 20892359 & 23646445 & 359 & 387 \\
\hline 20892359 & 25461613 & 363 & 396 \\
\hline 20892359 & 23646445 & 360 & 380 \\
\hline 20892359 & 23646445 & 355 & 386 \\
\hline 20892359 & 23646445 & 363 & 388 \\
\hline 20892359 & 23646445 & 366 & 385 \\
\hline 20892359 & 23646445 & 367 & 382 \\
\hline 20892359 & 23646445 & 361 & 386 \\
\hline 20892359 & 23646445 & 358 & 389 \\
\hline 20892359 & 23646445 & 366 & 389 \\
\hline 20892359 & 23646445 & 363 & 390 \\
\hline 20892359 & 23646445 & 359 & 387 \\
\hline 20892359 & 25461613 & 364 & 396 \\
\hline 20892359 & 25461613 & 362 & 400 \\
\hline 20892359 & 25461613 & 366 & 397 \\
\hline 20892359 & 25461613 & 363 & 397 \\
\hline 20892359 & 27333617 & 368 & 428 \\
\hline 22379284 & 25461613 & 373 & 405 \\
\hline 20892359 & 25461613 & 371 & 407 \\
\hline 22379284 & 25461613 & 383 & 403 \\
\hline 23646445 & 27333617 & 404 & 425 \\
\hline 22379284 & 27333617 & 384 & 436 \\
\hline 23646445 & 27333617 & 400 & 427 \\
\hline 22379284 & 30335134 & 383 & 461 \\
\hline 23646445 & 30335134 & 397 & 495 \\
\hline 23646445 & 30335134 & 399 & 486 \\
\hline 23646445 & 31325838 & 408 & 536 \\
\hline 25461613 & 30335134 & 424 & 475 \\
\hline 25461613 & 28801838 & 424 & 444 \\
\hline 25461613 & 31325838 & 421 & 529 \\
\hline 23646445 & 30335134 & 413 & 465 \\
\hline 25461613 & 30335134 & 434 & 456 \\
\hline 25461613 & 30335134 & 422 & 463 \\
\hline
\end{tabular}




\begin{tabular}{|c|c|c|c|c|}
\hline 25461613 & 30335134 & 418 & 477 & 59 \\
\hline 23646445 & 30335134 & 397 & 501 & 104 \\
\hline 29344086 & 31325838 & 468 & 533 & 65 \\
\hline 29344086 & 31325838 & 468 & 536 & 68 \\
\hline 29344086 & 31325838 & 470 & 531 & 61 \\
\hline 29344086 & 31325838 & 472 & 537 & 65 \\
\hline 29344086 & 31325838 & 476 & 528 & 52 \\
\hline 29344086 & 31325838 & 484 & 529 & 45 \\
\hline 29344086 & 31325838 & 480 & 529 & 49 \\
\hline 29344086 & 31325838 & 483 & 528 & 45 \\
\hline 29344086 & 31325838 & 471 & 541 & 70 \\
\hline 29344086 & 31325838 & 477 & 538 & 61 \\
\hline 30335134 & 33757240 & 546 & 593 & 47 \\
\hline 30335134 & 34401823 & 515 & 677 & 162 \\
\hline 30335134 & 34401823 & 549 & 666 & 117 \\
\hline 31325838 & 34401823 & 606 & 639 & 33 \\
\hline 31325838 & 34401823 & 584 & 662 & 78 \\
\hline 31325838 & 34401823 & 588 & 666 & 78 \\
\hline 31325838 & 34401823 & 592 & 663 & 71 \\
\hline 31325838 & 34401823 & 592 & 667 & 75 \\
\hline 33757240 & 34901278 & 688 & 710 & 22 \\
\hline 33757240 & 34901278 & 686 & 709 & 23 \\
\hline 33757240 & 34901278 & 688 & 712 & 24 \\
\hline 33757240 & 34901278 & 684 & 709 & 25 \\
\hline 33757240 & 34901278 & 680 & 713 & 33 \\
\hline 33757240 & 34901278 & 687 & 708 & 21 \\
\hline 33757240 & 35149051 & 661 & 719 & 58 \\
\hline 33757240 & 35411442 & 653 & 745 & 92 \\
\hline 34700598 & 35411442 & 713 & 729 & 16 \\
\hline 34901278 & 35411442 & 719 & 730 & 11 \\
\hline 34901278 & 35411442 & 719 & 731 & 12 \\
\hline 35149051 & 36845644 & 734 & 756 & 22 \\
\hline 35149051 & 40160048 & 732 & 773 & 41 \\
\hline 35149051 & 40160048 & 734 & 772 & 38 \\
\hline 34901278 & 48075656 & 719 & 885 & 166 \\
\hline 33757240 & 40160048 & 648 & 771 & 123 \\
\hline 35149051 & 44237442 & 730 & 796 & 66 \\
\hline 30335134 & 48075656 & 549 & 885 & 336 \\
\hline 35549849 & 40160048 & 750 & 775 & 25 \\
\hline 35549849 & 40160048 & 750 & 776 & 26 \\
\hline 35549849 & 40160048 & 750 & 771 & 21 \\
\hline 35149051 & 41584336 & 728 & 786 & 58 \\
\hline 35549849 & 40160048 & 751 & 771 & 20 \\
\hline
\end{tabular}




\begin{tabular}{|c|c|c|c|c|}
\hline 35549849 & 40160048 & 751 & 775 & 24 \\
\hline 35549849 & 40160048 & 753 & 769 & 16 \\
\hline 35549849 & 40160048 & 752 & 772 & 20 \\
\hline 35549849 & 40160048 & 757 & 770 & 13 \\
\hline 35549849 & 40160048 & 755 & 778 & 23 \\
\hline 35549849 & 40160048 & 751 & 774 & 23 \\
\hline 35549849 & 40160048 & 751 & 777 & 26 \\
\hline 35549849 & 40160048 & 753 & 772 & 19 \\
\hline 35549849 & 40160048 & 755 & 769 & 14 \\
\hline 35549849 & 40160048 & 755 & 772 & 17 \\
\hline 35549849 & 40160048 & 753 & 776 & 23 \\
\hline 35549849 & 40160048 & 753 & 774 & 21 \\
\hline 35549849 & 40160048 & 754 & 774 & 20 \\
\hline 35549849 & 40160048 & 752 & 777 & 25 \\
\hline 35549849 & 40160048 & 751 & 780 & 29 \\
\hline 35549849 & 41584336 & 755 & 787 & 32 \\
\hline 35549849 & 40160048 & 752 & 776 & 24 \\
\hline 35549849 & 40160048 & 752 & 776 & 24 \\
\hline 35549849 & 40160048 & 755 & 775 & 20 \\
\hline 35549849 & 40160048 & 755 & 780 & 25 \\
\hline 35549849 & 40160048 & 754 & 772 & 18 \\
\hline 35549849 & 40160048 & 754 & 777 & 23 \\
\hline 34901278 & 41584336 & 718 & 787 & 69 \\
\hline 35549849 & 40160048 & 754 & 780 & 26 \\
\hline 35549849 & 44237442 & 752 & 793 & 41 \\
\hline 35549849 & 40160048 & 754 & 781 & 27 \\
\hline 33757240 & 41584336 & 645 & 786 & 141 \\
\hline 35549849 & 44237442 & 755 & 799 & 44 \\
\hline 35549849 & 44237442 & 754 & 792 & 38 \\
\hline 35549849 & 41584336 & 754 & 787 & 33 \\
\hline 35549849 & 40160048 & 753 & 782 & 29 \\
\hline 35549849 & 41584336 & 751 & 788 & 37 \\
\hline 35549849 & 41584336 & 750 & 788 & 38 \\
\hline 35549849 & 44237442 & 755 & 791 & 36 \\
\hline 35549849 & 44237442 & 752 & 796 & 44 \\
\hline 35549849 & 44237442 & 756 & 794 & 38 \\
\hline 35549849 & 44237442 & 754 & 796 & 42 \\
\hline 35549849 & 44237442 & 752 & 794 & 42 \\
\hline 35549849 & 41584336 & 754 & 788 & 34 \\
\hline 35549849 & 44237442 & 756 & 796 & 40 \\
\hline 35549849 & 44237442 & 755 & 800 & 45 \\
\hline 35549849 & 44237442 & 757 & 796 & 39 \\
\hline 37887738 & 44237442 & 765 & 798 & 33 \\
\hline
\end{tabular}




\begin{tabular}{|c|c|c|c|c|}
\hline 35549849 & 44237442 & 758 & 800 & 42 \\
\hline 35549849 & 44237442 & 754 & 797 & 43 \\
\hline 35549849 & 44237442 & 757 & 796 & 39 \\
\hline 37887738 & 41584336 & 766 & 787 & 21 \\
\hline 37887738 & 44237442 & 764 & 798 & 34 \\
\hline 35549849 & 41584336 & 751 & 788 & 37 \\
\hline 35549849 & 48075656 & 756 & 884 & 128 \\
\hline 35549849 & 44237442 & 755 & 795 & 40 \\
\hline 37887738 & 41584336 & 766 & 786 & 20 \\
\hline 37887738 & 44237442 & 764 & 796 & 32 \\
\hline 35549849 & 44237442 & 759 & 796 & 37 \\
\hline 37887738 & 44237442 & 764 & 797 & 33 \\
\hline 37887738 & 41584336 & 772 & 787 & 15 \\
\hline 35549849 & 45892486 & 755 & 831 & 76 \\
\hline 37887738 & 44237442 & 774 & 793 & 19 \\
\hline 37887738 & 41584336 & 774 & 785 & 11 \\
\hline 37887738 & 44237442 & 767 & 793 & 26 \\
\hline 37887738 & 45892486 & 768 & 832 & 64 \\
\hline 37887738 & 44237442 & 767 & 790 & 23 \\
\hline 37887738 & 45892486 & 765 & 819 & 54 \\
\hline 35411442 & 50771270 & 748 & 921 & 173 \\
\hline 37887738 & 41584336 & 774 & 787 & 13 \\
\hline 37887738 & 44237442 & 765 & 795 & 30 \\
\hline 37887738 & 44237442 & 766 & 798 & 32 \\
\hline 37887738 & 44237442 & 770 & 795 & 25 \\
\hline 37887738 & 44237442 & 774 & 800 & 26 \\
\hline 37887738 & 44237442 & 770 & 795 & 25 \\
\hline 37887738 & 44237442 & 775 & 797 & 22 \\
\hline 35149051 & 44237442 & 729 & 799 & 70 \\
\hline 35549849 & 44237442 & 751 & 798 & 47 \\
\hline 36845644 & 44237442 & 760 & 798 & 38 \\
\hline 37887738 & 44237442 & 767 & 800 & 33 \\
\hline 37887738 & 44237442 & 769 & 796 & 27 \\
\hline 37887738 & 44237442 & 771 & 800 & 29 \\
\hline 37887738 & 44237442 & 775 & 794 & 19 \\
\hline 37887738 & 44237442 & 776 & 796 & 20 \\
\hline 40160048 & 44237442 & 784 & 792 & 8 \\
\hline 37887738 & 44237442 & 768 & 797 & 29 \\
\hline 40160048 & 44237442 & 785 & 795 & 10 \\
\hline 40160048 & 41584336 & 787 & 789 & 2 \\
\hline 40160048 & 44237442 & 787 & 791 & 4 \\
\hline 40160048 & 44237442 & 787 & 792 & 5 \\
\hline 40160048 & 44237442 & 786 & 794 & 8 \\
\hline
\end{tabular}




\begin{tabular}{|c|c|c|c|c|}
\hline 40160048 & 44237442 & 786 & 796 & 10 \\
\hline 40160048 & 44237442 & 787 & 795 & 8 \\
\hline 35149051 & 45892486 & 722 & 827 & 105 \\
\hline 35149051 & 48033947 & 721 & 869 & 148 \\
\hline 37887738 & 45892486 & 767 & 831 & 64 \\
\hline 37887738 & 45892486 & 780 & 806 & 26 \\
\hline 40160048 & 45892486 & 785 & 825 & 40 \\
\hline 40160048 & 45892486 & 786 & 825 & 39 \\
\hline 37887738 & 44237442 & 774 & 797 & 23 \\
\hline 35549849 & 44237442 & 756 & 799 & 43 \\
\hline 35149051 & 45892486 & 730 & 828 & 98 \\
\hline 40160048 & 44237442 & 784 & 797 & 13 \\
\hline 40160048 & 44237442 & 786 & 794 & 8 \\
\hline 40160048 & 44237442 & 786 & 795 & 9 \\
\hline 37887738 & 44237442 & 778 & 797 & 19 \\
\hline 40160048 & 44237442 & 785 & 799 & 14 \\
\hline 37887738 & 45892486 & 767 & 809 & 42 \\
\hline 40160048 & 44237442 & 785 & 799 & 14 \\
\hline 37887738 & 45892486 & 779 & 824 & 45 \\
\hline 40160048 & 44237442 & 784 & 799 & 15 \\
\hline 37887738 & 45892486 & 781 & 827 & 46 \\
\hline 40160048 & 44237442 & 785 & 799 & 14 \\
\hline 37887738 & 45892486 & 768 & 835 & 67 \\
\hline 37887738 & 45892486 & 774 & 826 & 52 \\
\hline 37887738 & 45892486 & 781 & 823 & 42 \\
\hline 40160048 & 45892486 & 784 & 833 & 49 \\
\hline 41584336 & 45892486 & 794 & 817 & 23 \\
\hline 41584336 & 45892486 & 792 & 822 & 30 \\
\hline 41584336 & 45892486 & 794 & 820 & 26 \\
\hline 41584336 & 45892486 & 791 & 824 & 33 \\
\hline 37887738 & 45892486 & 782 & 831 & 49 \\
\hline 41584336 & 45892486 & 794 & 824 & 30 \\
\hline 41584336 & 45892486 & 793 & 824 & 31 \\
\hline 41584336 & 45892486 & 792 & 830 & 38 \\
\hline 41584336 & 45892486 & 795 & 827 & 32 \\
\hline 41584336 & 45892486 & 797 & 826 & 29 \\
\hline 41584336 & 45892486 & 797 & 817 & 20 \\
\hline 41584336 & 45892486 & 796 & 817 & 21 \\
\hline 41584336 & 45892486 & 799 & 821 & 22 \\
\hline 41584336 & 45892486 & 796 & 823 & 27 \\
\hline 41584336 & 45892486 & 794 & 839 & 45 \\
\hline 41584336 & 45892486 & 799 & 829 & 30 \\
\hline 41584336 & 45892486 & 793 & 832 & 39 \\
\hline
\end{tabular}




\begin{tabular}{|c|c|c|c|c|}
\hline 41584336 & 45892486 & 798 & 824 & 26 \\
\hline 41584336 & 45892486 & 796 & 828 & 32 \\
\hline 41584336 & 45892486 & 791 & 839 & 48 \\
\hline 41584336 & 48033947 & 792 & 868 & 76 \\
\hline 41584336 & 48033947 & 795 & 880 & 85 \\
\hline 44237442 & 48033947 & 832 & 857 & 25 \\
\hline 44237442 & 48033947 & 826 & 856 & 30 \\
\hline 44237442 & 48033947 & 826 & 860 & 34 \\
\hline 44237442 & 48033947 & 831 & 859 & 28 \\
\hline 44237442 & 48033947 & 832 & 870 & 38 \\
\hline 44237442 & 48033947 & 817 & 872 & 55 \\
\hline 41584336 & 48033947 & 799 & 870 & 71 \\
\hline 44237442 & 48033947 & 822 & 869 & 47 \\
\hline 44237442 & 48033947 & 823 & 871 & 48 \\
\hline 44237442 & 48033947 & 819 & 876 & 57 \\
\hline 44237442 & 48033947 & 835 & 863 & 28 \\
\hline 44237442 & 48033947 & 828 & 866 & 38 \\
\hline 44237442 & 48033947 & 823 & 872 & 49 \\
\hline 44237442 & 48075656 & 822 & 888 & 66 \\
\hline 44237442 & 48033947 & 832 & 872 & 40 \\
\hline 44237442 & 48033947 & 834 & 866 & 32 \\
\hline 44237442 & 48033947 & 824 & 865 & 41 \\
\hline 44237442 & 48033947 & 830 & 867 & 37 \\
\hline 44237442 & 48033947 & 832 & 871 & 39 \\
\hline 44237442 & 48033947 & 819 & 878 & 59 \\
\hline 44237442 & 48075656 & 827 & 884 & 57 \\
\hline 44237442 & 48075656 & 822 & 885 & 63 \\
\hline 44237442 & 48075656 & 824 & 888 & 64 \\
\hline 44237442 & 48075656 & 829 & 885 & 56 \\
\hline 44237442 & 48075656 & 823 & 886 & 63 \\
\hline 44237442 & 48075656 & 819 & 886 & 67 \\
\hline 44237442 & 48075656 & 817 & 889 & 72 \\
\hline 40160048 & 48075656 & 787 & 885 & 98 \\
\hline 41584336 & 48075656 & 793 & 889 & 96 \\
\hline 44237442 & 48075656 & 816 & 886 & 70 \\
\hline 44237442 & 50771270 & 810 & 924 & 114 \\
\hline 44237442 & 48075656 & 813 & 887 & 74 \\
\hline 37887738 & 48075656 & 781 & 888 & 107 \\
\hline 44237442 & 48075656 & 833 & 886 & 53 \\
\hline 44237442 & 48075656 & 822 & 888 & 66 \\
\hline 41584336 & 50771270 & 793 & 921 & 128 \\
\hline 45892486 & 48075656 & 846 & 889 & 43 \\
\hline 45892486 & 48075656 & 846 & 890 & 44 \\
\hline
\end{tabular}




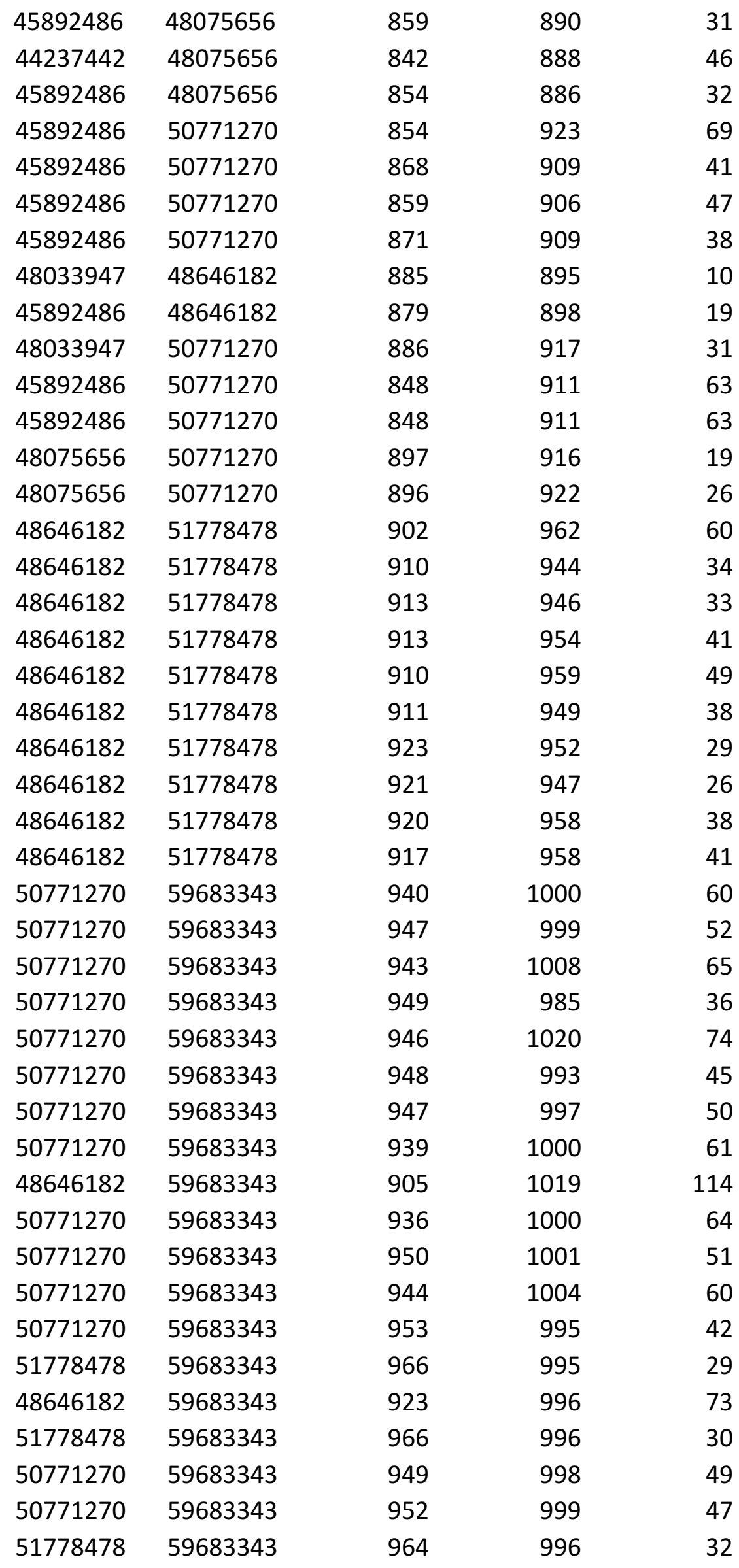




\begin{tabular}{|c|c|c|c|c|}
\hline 50771270 & 59683343 & 948 & 1004 & 56 \\
\hline 48646182 & 59683343 & 909 & 1012 & 103 \\
\hline 50771270 & 59683343 & 961 & 1010 & 49 \\
\hline 51778478 & 59683343 & 968 & 1002 & 34 \\
\hline 50771270 & 59683343 & 951 & 1010 & 59 \\
\hline 50771270 & 59683343 & 960 & 1013 & 53 \\
\hline 50771270 & 59683343 & 946 & 1014 & 68 \\
\hline 50771270 & 59683343 & 953 & 1021 & 68 \\
\hline 51778478 & 59683343 & 966 & 1017 & 51 \\
\hline 50771270 & 59683343 & 959 & 1026 & 67 \\
\hline 56746212 & 60080719 & 996 & 1038 & 42 \\
\hline 56746212 & 60080719 & 1000 & 1037 & 37 \\
\hline 56746212 & 60080719 & 1010 & 1037 & 27 \\
\hline 56746212 & 60080719 & 1004 & 1039 & 35 \\
\hline 56746212 & 60080719 & 1003 & 1041 & 38 \\
\hline 56746212 & 60080719 & 1003 & 1043 & 40 \\
\hline 56746212 & 60080719 & 1011 & 1040 & 29 \\
\hline 56746212 & 60080719 & 1002 & 1049 & 47 \\
\hline 60080719 & 62186792 & 1060 & 1089 & 29 \\
\hline 60080719 & 62186792 & 1058 & 1093 & 35 \\
\hline 60080719 & 62186792 & 1061 & 1094 & 33 \\
\hline 60080719 & 62186792 & 1064 & 1096 & 32 \\
\hline 60080719 & 62186792 & 1070 & 1090 & 20 \\
\hline 60080719 & 62186792 & 1057 & 1092 & 35 \\
\hline 60080719 & 62186792 & 1069 & 1091 & 22 \\
\hline 60080719 & 62186792 & 1066 & 1099 & 33 \\
\hline 60080719 & 62186792 & 1071 & 1096 & 25 \\
\hline 60080719 & 63385690 & 1065 & 1109 & 44 \\
\hline 60080719 & 69525397 & 1066 & 1166 & 100 \\
\hline 61428536 & 63385690 & 1086 & 1106 & 20 \\
\hline 61428536 & 64551845 & 1094 & 1123 & 29 \\
\hline 61428536 & 63385690 & 1095 & 1106 & 11 \\
\hline 61428536 & 69525397 & 1092 & 1173 & 81 \\
\hline 61428536 & 65862504 & 1093 & 1126 & 33 \\
\hline 61428536 & 63385690 & 1094 & 1110 & 16 \\
\hline 61428536 & 64551845 & 1094 & 1120 & 26 \\
\hline 61428536 & 65862504 & 1093 & 1130 & 37 \\
\hline 61428536 & 64551845 & 1094 & 1123 & 29 \\
\hline 61428536 & 69525397 & 1094 & 1171 & 77 \\
\hline 61428536 & 69525397 & 1091 & 1163 & 72 \\
\hline 61428536 & 65862504 & 1096 & 1130 & 34 \\
\hline 61428536 & 64551845 & 1089 & 1121 & 32 \\
\hline 61428536 & 64551845 & 1091 & 1124 & 33 \\
\hline
\end{tabular}




\begin{tabular}{|c|c|c|c|c|}
\hline 61428536 & 64551845 & 1096 & 1123 & 27 \\
\hline 62186792 & 64551845 & 1105 & 1118 & 13 \\
\hline 62186792 & 64551845 & 1105 & 1121 & 16 \\
\hline 62186792 & 64551845 & 1104 & 1120 & 16 \\
\hline 62186792 & 64551845 & 1106 & 1122 & 16 \\
\hline 62186792 & 64551845 & 1102 & 1126 & 24 \\
\hline 61428536 & 69525397 & 1094 & 1172 & 78 \\
\hline 62186792 & 64551845 & 1105 & 1122 & 17 \\
\hline 62186792 & 69525397 & 1105 & 1196 & 91 \\
\hline 60080719 & 69525397 & 1072 & 1181 & 109 \\
\hline 60080719 & 69525397 & 1060 & 1169 & 109 \\
\hline 63385690 & 65862504 & 1112 & 1132 & 20 \\
\hline 62186792 & 69525397 & 1108 & 1189 & 81 \\
\hline 63385690 & 69525397 & 1116 & 1160 & 44 \\
\hline 63385690 & 69525397 & 1119 & 1163 & 44 \\
\hline 63385690 & 69525397 & 1117 & 1184 & 67 \\
\hline 63385690 & 69525397 & 1118 & 1186 & 68 \\
\hline 63385690 & 69525397 & 1114 & 1188 & 74 \\
\hline 63385690 & 69525397 & 1114 & 1174 & 60 \\
\hline 63385690 & 69525397 & 1117 & 1181 & 64 \\
\hline 63385690 & 69525397 & 1117 & 1186 & 69 \\
\hline 63385690 & 71659908 & 1121 & 1222 & 101 \\
\hline 63385690 & 69525397 & 1121 & 1182 & 61 \\
\hline 63385690 & 71659908 & 1116 & 1211 & 95 \\
\hline 63385690 & 71659908 & 1115 & 1205 & 90 \\
\hline 63385690 & 69525397 & 1120 & 1188 & 68 \\
\hline 63385690 & 69525397 & 1120 & 1189 & 69 \\
\hline 63385690 & 69525397 & 1123 & 1192 & 69 \\
\hline 63385690 & 69525397 & 1124 & 1192 & 68 \\
\hline 63385690 & 69525397 & 1119 & 1193 & 74 \\
\hline 63385690 & 69525397 & 1117 & 1196 & 79 \\
\hline 63385690 & 69525397 & 1123 & 1190 & 67 \\
\hline 63385690 & 69525397 & 1114 & 1205 & 91 \\
\hline 63385690 & 71659908 & 1114 & 1215 & 101 \\
\hline 65862504 & 71659908 & 1147 & 1231 & 84 \\
\hline 65862504 & 71659908 & 1168 & 1235 & 67 \\
\hline 65862504 & 73244382 & 1172 & 1265 & 93 \\
\hline 65862504 & 71659908 & 1192 & 1233 & 41 \\
\hline 65862504 & 71659908 & 1155 & 1257 & 102 \\
\hline 65862504 & 71659908 & 1181 & 1223 & 42 \\
\hline 79907247 & 86711726 & 1304 & 1360 & 56 \\
\hline 79907247 & 86711726 & 1312 & 1343 & 31 \\
\hline 79907247 & 86711726 & 1308 & 1355 & 47 \\
\hline
\end{tabular}




\begin{tabular}{|c|c|c|c|c|}
\hline 79907247 & 86711726 & 1303 & 1352 & 49 \\
\hline 79907247 & 86711726 & 1307 & 1351 & 44 \\
\hline 79907247 & 86711726 & 1297 & 1381 & 84 \\
\hline 79907247 & 101131756 & 1306 & 1470 & 164 \\
\hline 82590932 & 101131756 & 1329 & 1470 & 141 \\
\hline 79907247 & 101131756 & 1302 & 1441 & 139 \\
\hline 79907247 & 101131756 & 1314 & 1445 & 131 \\
\hline 82590932 & 101131756 & 1342 & 1445 & 103 \\
\hline 79907247 & 101131756 & 1319 & 1473 & 154 \\
\hline 82590932 & 101131756 & 1338 & 1459 & 121 \\
\hline 82590932 & 91200315 & 1345 & 1407 & 62 \\
\hline 82590932 & 91200315 & 1347 & 1400 & 53 \\
\hline 82590932 & 91200315 & 1349 & 1402 & 53 \\
\hline 82590932 & 101131756 & 1363 & 1474 & 111 \\
\hline 82590932 & 91200315 & 1366 & 1403 & 37 \\
\hline 82590932 & 91200315 & 1371 & 1401 & 30 \\
\hline 82590932 & 91200315 & 1368 & 1402 & 34 \\
\hline 82590932 & 91200315 & 1360 & 1403 & 43 \\
\hline 82590932 & 101131756 & 1364 & 1459 & 95 \\
\hline 82590932 & 92741754 & 1363 & 1408 & 45 \\
\hline 82590932 & 91200315 & 1372 & 1403 & 31 \\
\hline 82590932 & 101131756 & 1366 & 1442 & 76 \\
\hline 82590932 & 101131756 & 1349 & 1466 & 117 \\
\hline 82590932 & 101131756 & 1366 & 1448 & 82 \\
\hline 82590932 & 92741754 & 1364 & 1408 & 44 \\
\hline 82590932 & 92741754 & 1357 & 1409 & 52 \\
\hline 82590932 & 101131756 & 1369 & 1453 & 84 \\
\hline 82590932 & 101131756 & 1356 & 1435 & 79 \\
\hline 82590932 & 101131756 & 1358 & 1455 & 97 \\
\hline 87784709 & 101131756 & 1394 & 1460 & 66 \\
\hline 87784709 & 93318656 & 1395 & 1416 & 21 \\
\hline 87784709 & 93318656 & 1395 & 1416 & 21 \\
\hline 82590932 & 101131756 & 1368 & 1490 & 122 \\
\hline 87784709 & 101131756 & 1396 & 1487 & 91 \\
\hline 82590932 & 101131756 & 1375 & 1471 & 96 \\
\hline 87784709 & 93318656 & 1400 & 1412 & 12 \\
\hline 87784709 & 93318656 & 1403 & 1414 & 11 \\
\hline 87784709 & 101131756 & 1398 & 1458 & 60 \\
\hline 87784709 & 101131756 & 1398 & 1464 & 66 \\
\hline 87784709 & 93318656 & 1402 & 1414 & 12 \\
\hline 82590932 & 101131756 & 1386 & 1465 & 79 \\
\hline 87784709 & 101131756 & 1396 & 1443 & 47 \\
\hline 87784709 & 101131756 & 1396 & 1463 & 67 \\
\hline
\end{tabular}




\begin{tabular}{|c|c|c|c|c|}
\hline 82590932 & 101131756 & 1338 & 1448 & 110 \\
\hline 87784709 & 93318656 & 1407 & 1416 & 9 \\
\hline 91200315 & 93318656 & 1408 & 1415 & 7 \\
\hline 91200315 & 101131756 & 1408 & 1436 & 28 \\
\hline 87784709 & 101131756 & 1397 & 1449 & 52 \\
\hline 87784709 & 93318656 & 1401 & 1416 & 15 \\
\hline 87784709 & 101131756 & 1402 & 1456 & 54 \\
\hline 87784709 & 101131756 & 1403 & 1464 & 61 \\
\hline 87784709 & 101131756 & 1396 & 1436 & 40 \\
\hline 87784709 & 101131756 & 1399 & 1462 & 63 \\
\hline 87784709 & 101131756 & 1400 & 1452 & 52 \\
\hline 87784709 & 93318656 & 1402 & 1414 & 12 \\
\hline 87784709 & 101131756 & 1399 & 1462 & 63 \\
\hline 87784709 & 93318656 & 1399 & 1416 & 17 \\
\hline 87784709 & 101131756 & 1396 & 1428 & 32 \\
\hline 87784709 & 93318656 & 1402 & 1414 & 12 \\
\hline 82590932 & 101131756 & 1359 & 1440 & 81 \\
\hline 82590932 & 101131756 & 1351 & 1459 & 108 \\
\hline 87784709 & 93318656 & 1404 & 1413 & 9 \\
\hline 91200315 & 93318656 & 1408 & 1417 & 9 \\
\hline 91200315 & 93318656 & 1409 & 1414 & 5 \\
\hline 91200315 & 93318656 & 1409 & 1413 & 4 \\
\hline 87784709 & 101131756 & 1402 & 1435 & 33 \\
\hline 87784709 & 101131756 & 1403 & 1448 & 45 \\
\hline 87784709 & 93318656 & 1405 & 1416 & 11 \\
\hline 82590932 & 101131756 & 1359 & 1467 & 108 \\
\hline 87784709 & 101131756 & 1396 & 1454 & 58 \\
\hline 87784709 & 101131756 & 1401 & 1460 & 59 \\
\hline 87784709 & 101131756 & 1404 & 1465 & 61 \\
\hline 87784709 & 101131756 & 1403 & 1470 & 67 \\
\hline 82590932 & 101131756 & 1383 & 1454 & 71 \\
\hline 87784709 & 101131756 & 1401 & 1471 & 70 \\
\hline 87784709 & 101131756 & 1398 & 1451 & 53 \\
\hline 87784709 & 101131756 & 1394 & 1451 & 57 \\
\hline 87784709 & 101131756 & 1402 & 1454 & 52 \\
\hline 82590932 & 101131756 & 1386 & 1458 & 72 \\
\hline 87784709 & 101131756 & 1402 & 1455 & 53 \\
\hline 91200315 & 101131756 & 1409 & 1460 & 51 \\
\hline 87784709 & 101131756 & 1398 & 1468 & 70 \\
\hline 82590932 & 101131756 & 1369 & 1452 & 83 \\
\hline 82590932 & 101131756 & 1349 & 1456 & 107 \\
\hline 92741754 & 101131756 & 1412 & 1455 & 43 \\
\hline 92741754 & 101131756 & 1412 & 1446 & 34 \\
\hline
\end{tabular}




\begin{tabular}{|c|c|c|c|c|}
\hline 92741754 & 101131756 & 1411 & 1458 & 47 \\
\hline 92741754 & 101131756 & 1413 & 1467 & 54 \\
\hline 91200315 & 101131756 & 1409 & 1458 & 49 \\
\hline 91200315 & 101131756 & 1410 & 1454 & 44 \\
\hline 87784709 & 101131756 & 1405 & 1464 & 59 \\
\hline 91200315 & 101131756 & 1409 & 1459 & 50 \\
\hline 91200315 & 101131756 & 1409 & 1463 & 54 \\
\hline 82590932 & 101131756 & 1360 & 1481 & 121 \\
\hline 87784709 & 101131756 & 1402 & 1476 & 74 \\
\hline 91200315 & 101131756 & 1410 & 1461 & 51 \\
\hline 91200315 & 101131756 & 1410 & 1464 & 54 \\
\hline 91200315 & 101131756 & 1409 & 1475 & 66 \\
\hline 87784709 & 101131756 & 1401 & 1467 & 66 \\
\hline 87784709 & 101131756 & 1394 & 1478 & 84 \\
\hline 91200315 & 101131756 & 1409 & 1473 & 64 \\
\hline 82590932 & 102649167 & 1343 & 1504 & 161 \\
\hline 82590932 & 101131756 & 1379 & 1493 & 114 \\
\hline 82590932 & 101131756 & 1363 & 1477 & 114 \\
\hline 87784709 & 101131756 & 1403 & 1476 & 73 \\
\hline 91200315 & 101131756 & 1409 & 1477 & 68 \\
\hline 87784709 & 101131756 & 1404 & 1477 & 73 \\
\hline 87784709 & 101131756 & 1404 & 1472 & 68 \\
\hline 87784709 & 101131756 & 1397 & 1482 & 85 \\
\hline 87784709 & 101131756 & 1402 & 1492 & 90 \\
\hline 87784709 & 101131756 & 1399 & 1491 & 92 \\
\hline 87784709 & 102649167 & 1395 & 1501 & 106 \\
\hline 87784709 & 102649167 & 1396 & 1501 & 105 \\
\hline 87784709 & 102649167 & 1398 & 1501 & 103 \\
\hline 87784709 & 102649167 & 1399 & 1506 & 107 \\
\hline 92741754 & 102649167 & 1413 & 1506 & 93 \\
\hline 87784709 & 101131756 & 1401 & 1494 & 93 \\
\hline 93318656 & 109433438 & 1420 & 1510 & 90 \\
\hline 93318656 & 114520237 & 1440 & 1540 & 100 \\
\hline 92741754 & 114520237 & 1411 & 1536 & 125 \\
\hline 93318656 & 109433438 & 1427 & 1517 & 90 \\
\hline 93318656 & 114520237 & 1443 & 1533 & 90 \\
\hline 93318656 & 114520237 & 1435 & 1532 & 97 \\
\hline 93318656 & 102649167 & 1430 & 1508 & 78 \\
\hline 93318656 & 102649167 & 1443 & 1507 & 64 \\
\hline 93318656 & 109433438 & 1441 & 1511 & 70 \\
\hline 93318656 & 109433438 & 1444 & 1514 & 70 \\
\hline 87784709 & 102649167 & 1400 & 1504 & 104 \\
\hline 93318656 & 114520237 & 1446 & 1538 & 92 \\
\hline
\end{tabular}




\begin{tabular}{|c|c|c|c|c|}
\hline 93318656 & 114520237 & 1450 & 1536 & 86 \\
\hline 93318656 & 114520237 & 1449 & 1524 & 75 \\
\hline 93318656 & 109433438 & 1445 & 1517 & 72 \\
\hline 93318656 & 109433438 & 1441 & 1518 & 77 \\
\hline 93318656 & 114520237 & 1449 & 1571 & 122 \\
\hline 93318656 & 109433438 & 1454 & 1518 & 64 \\
\hline 93318656 & 102649167 & 1439 & 1508 & 69 \\
\hline 93318656 & 109433438 & 1451 & 1513 & 62 \\
\hline 93318656 & 102649167 & 1472 & 1507 & 35 \\
\hline 93318656 & 109433438 & 1471 & 1519 & 48 \\
\hline 93318656 & 109433438 & 1466 & 1511 & 45 \\
\hline 93318656 & 114520237 & 1450 & 1557 & 107 \\
\hline 93318656 & 114520237 & 1453 & 1540 & 87 \\
\hline 93318656 & 109433438 & 1459 & 1515 & 56 \\
\hline 93318656 & 114520237 & 1467 & 1544 & 77 \\
\hline 93318656 & 109433438 & 1463 & 1517 & 54 \\
\hline 93318656 & 109433438 & 1465 & 1517 & 52 \\
\hline 93318656 & 109433438 & 1465 & 1520 & 55 \\
\hline 93318656 & 109433438 & 1468 & 1518 & 50 \\
\hline 93318656 & 109433438 & 1459 & 1517 & 58 \\
\hline 93318656 & 114520237 & 1458 & 1534 & 76 \\
\hline 93318656 & 114520237 & 1468 & 1535 & 67 \\
\hline 93318656 & 114520237 & 1459 & 1533 & 74 \\
\hline 93318656 & 109433438 & 1474 & 1518 & 44 \\
\hline 93318656 & 114520237 & 1476 & 1526 & 50 \\
\hline 93318656 & 114520237 & 1471 & 1521 & 50 \\
\hline 93318656 & 109433438 & 1476 & 1518 & 42 \\
\hline 93318656 & 109433438 & 1486 & 1516 & 30 \\
\hline 93318656 & 114520237 & 1463 & 1536 & 73 \\
\hline 93318656 & 109433438 & 1468 & 1516 & 48 \\
\hline 93318656 & 109433438 & 1480 & 1517 & 37 \\
\hline 93318656 & 109433438 & 1473 & 1516 & 43 \\
\hline 93318656 & 109433438 & 1480 & 1517 & 37 \\
\hline 101131756 & 109433438 & 1504 & 1515 & 11 \\
\hline 101131756 & 109433438 & 1504 & 1518 & 14 \\
\hline 93318656 & 109433438 & 1485 & 1519 & 34 \\
\hline 93318656 & 109433438 & 1481 & 1516 & 35 \\
\hline 101131756 & 109433438 & 1502 & 1519 & 17 \\
\hline 101131756 & 109433438 & 1503 & 1516 & 13 \\
\hline 101131756 & 114520237 & 1505 & 1550 & 45 \\
\hline 101131756 & 109433438 & 1502 & 1519 & 17 \\
\hline 93318656 & 109433438 & 1465 & 1519 & 54 \\
\hline 93318656 & 114520237 & 1490 & 1531 & 41 \\
\hline
\end{tabular}




\begin{tabular}{|c|c|c|c|}
\hline 101131756 & 114520237 & 1505 & 1523 \\
\hline 101131756 & 109433438 & 1503 & 1517 \\
\hline 101131756 & 109433438 & 1503 & 1515 \\
\hline 101131756 & 109433438 & 1504 & 1518 \\
\hline 93318656 & 114520237 & 1481 & 1554 \\
\hline 93318656 & 114520237 & 1482 & 1568 \\
\hline 93318656 & 114520237 & 1483 & 1553 \\
\hline 101131756 & 114520237 & 1503 & 1540 \\
\hline 101131756 & 114520237 & 1502 & 1544 \\
\hline 93318656 & 114520237 & 1481 & 1537 \\
\hline 101131756 & 114520237 & 1504 & 1552 \\
\hline 93318656 & 114520237 & 1470 & 1545 \\
\hline 93318656 & 114520237 & 1458 & 1542 \\
\hline 101131756 & 114520237 & 1504 & 1567 \\
\hline 93318656 & 114520237 & 1454 & 1549 \\
\hline 101131756 & 114520237 & 1505 & 1537 \\
\hline 93318656 & 114520237 & 1456 & 1544 \\
\hline 93318656 & 114520237 & 1493 & 1553 \\
\hline 93318656 & 114520237 & 1477 & 1537 \\
\hline 101131756 & 114520237 & 1505 & 1555 \\
\hline 101131756 & 114520237 & 1504 & 1543 \\
\hline 93318656 & 114520237 & 1453 & 1549 \\
\hline 102649167 & 114520237 & 1510 & 1553 \\
\hline 102649167 & 114520237 & 1511 & 1543 \\
\hline 102649167 & 114520237 & 1516 & 1534 \\
\hline 102649167 & 114520237 & 1514 & 1538 \\
\hline 102649167 & 114520237 & 1514 & 1538 \\
\hline 102649167 & 114520237 & 1513 & 1548 \\
\hline 102649167 & 114520237 & 1517 & 1541 \\
\hline 102649167 & 114520237 & 1517 & 1535 \\
\hline 102649167 & 114520237 & 1516 & 1548 \\
\hline 102649167 & 114520237 & 1516 & 1541 \\
\hline 101131756 & 114520237 & 1504 & 1559 \\
\hline 102649167 & 114520237 & 1511 & 1551 \\
\hline 102649167 & 114520237 & 1512 & 1540 \\
\hline 102649167 & 114520237 & 1512 & 1553 \\
\hline 102649167 & 114520237 & 1517 & 1545 \\
\hline 102649167 & 114520237 & 1515 & 1548 \\
\hline 102649167 & 114520237 & 1518 & 1546 \\
\hline 102649167 & 114520237 & 1518 & 1548 \\
\hline 101131756 & 114520237 & 1505 & 1565 \\
\hline 102649167 & 114520237 & 1514 & 1567 \\
\hline 101131756 & 114520237 & 1505 & 1570 \\
\hline
\end{tabular}




\begin{tabular}{|c|c|c|c|c|}
\hline 102649167 & 114520237 & 1513 & 1553 & 40 \\
\hline 102649167 & 114520237 & 1510 & 1572 & 62 \\
\hline 102649167 & 117032829 & 1513 & 1610 & 97 \\
\hline 102649167 & 117032829 & 1518 & 1623 & 105 \\
\hline 109433438 & 117032829 & 1557 & 1607 & 50 \\
\hline 109433438 & 117032829 & 1575 & 1605 & 30 \\
\hline 109433438 & 117032829 & 1563 & 1614 & 51 \\
\hline 109433438 & 117032829 & 1569 & 1616 & 47 \\
\hline 109433438 & 117032829 & 1560 & 1613 & 53 \\
\hline 109433438 & 121263709 & 1565 & 1632 & 67 \\
\hline 109433438 & 121263709 & 1559 & 1647 & 88 \\
\hline 109433438 & 121263709 & 1545 & 1636 & 91 \\
\hline 109433438 & 121263709 & 1545 & 1644 & 99 \\
\hline 109433438 & 125004874 & 1572 & 1681 & 109 \\
\hline 109433438 & 121263709 & 1573 & 1633 & 60 \\
\hline 109433438 & 125004874 & 1535 & 1654 & 119 \\
\hline 114832053 & 121263709 & 1598 & 1642 & 44 \\
\hline 109433438 & 125004874 & 1548 & 1660 & 112 \\
\hline 109433438 & 121263709 & 1546 & 1639 & 93 \\
\hline 109433438 & 125004874 & 1565 & 1653 & 88 \\
\hline 109433438 & 121263709 & 1582 & 1647 & 65 \\
\hline 114832053 & 121263709 & 1596 & 1649 & 53 \\
\hline 114832053 & 121263709 & 1604 & 1640 & 36 \\
\hline 114832053 & 121263709 & 1603 & 1646 & 43 \\
\hline 114832053 & 121263709 & 1601 & 1642 & 41 \\
\hline 114832053 & 121263709 & 1602 & 1646 & 44 \\
\hline 114832053 & 121263709 & 1604 & 1642 & 38 \\
\hline 114832053 & 121263709 & 1608 & 1647 & 39 \\
\hline 114832053 & 121263709 & 1604 & 1634 & 30 \\
\hline 114832053 & 121263709 & 1595 & 1647 & 52 \\
\hline 114832053 & 121263709 & 1601 & 1649 & 48 \\
\hline 114520237 & 121263709 & 1595 & 1650 & 55 \\
\hline 114832053 & 121263709 & 1613 & 1642 & 29 \\
\hline 114832053 & 125004874 & 1602 & 1653 & 51 \\
\hline 114832053 & 121263709 & 1603 & 1649 & 46 \\
\hline 114832053 & 121263709 & 1615 & 1642 & 27 \\
\hline 114832053 & 125004874 & 1607 & 1659 & 52 \\
\hline 114832053 & 121263709 & 1614 & 1647 & 33 \\
\hline 114832053 & 125004874 & 1606 & 1665 & 59 \\
\hline 114832053 & 125004874 & 1599 & 1669 & 70 \\
\hline 114832053 & 125004874 & 1614 & 1668 & 54 \\
\hline 114832053 & 125004874 & 1607 & 1671 & 64 \\
\hline 114832053 & 126047891 & 1612 & 1704 & \\
\hline
\end{tabular}




\begin{tabular}{|c|c|c|c|c|}
\hline 117882924 & 126047891 & 1638 & 1707 & 69 \\
\hline 109433438 & 131350907 & 1564 & 1731 & 167 \\
\hline 117882924 & 126047891 & 1650 & 1708 & 58 \\
\hline 117882924 & 126047891 & 1650 & 1706 & 56 \\
\hline 123796210 & 126047891 & 1652 & 1720 & 68 \\
\hline 123796210 & 126047891 & 1653 & 1704 & 51 \\
\hline 117882924 & 126047891 & 1647 & 1708 & 61 \\
\hline 123796210 & 126047891 & 1659 & 1706 & 47 \\
\hline 117882924 & 126047891 & 1647 & 1717 & 70 \\
\hline 123796210 & 126047891 & 1669 & 1697 & 28 \\
\hline 123796210 & 126047891 & 1667 & 1701 & 34 \\
\hline 123796210 & 126047891 & 1660 & 1705 & 45 \\
\hline 123796210 & 126047891 & 1669 & 1708 & 39 \\
\hline 123796210 & 126047891 & 1673 & 1698 & 25 \\
\hline 123796210 & 126047891 & 1671 & 1702 & 31 \\
\hline 123796210 & 126047891 & 1666 & 1710 & 44 \\
\hline 123796210 & 126047891 & 1669 & 1702 & 33 \\
\hline 123796210 & 126047891 & 1668 & 1700 & 32 \\
\hline 123796210 & 126047891 & 1673 & 1699 & 26 \\
\hline 123796210 & 126047891 & 1668 & 1704 & 36 \\
\hline 123796210 & 126047891 & 1663 & 1704 & 41 \\
\hline 123796210 & 126047891 & 1676 & 1699 & 23 \\
\hline 123796210 & 126047891 & 1674 & 1700 & 26 \\
\hline 123796210 & 126047891 & 1676 & 1695 & 19 \\
\hline 123796210 & 126047891 & 1673 & 1696 & 23 \\
\hline 123796210 & 126047891 & 1664 & 1703 & 39 \\
\hline 123796210 & 126047891 & 1670 & 1709 & 39 \\
\hline 123796210 & 126047891 & 1662 & 1716 & 54 \\
\hline 123796210 & 126047891 & 1669 & 1706 & 37 \\
\hline 123796210 & 126047891 & 1673 & 1705 & 32 \\
\hline 123796210 & 126047891 & 1656 & 1715 & 59 \\
\hline 121263709 & 126047891 & 1651 & 1713 & 62 \\
\hline 125004874 & 132460300 & 1689 & 1752 & 63 \\
\hline 123796210 & 131350907 & 1669 & 1739 & 70 \\
\hline 125004874 & 131350907 & 1696 & 1742 & 46 \\
\hline 125004874 & 131350907 & 1697 & 1743 & 46 \\
\hline 125004874 & 131350907 & 1705 & 1733 & 28 \\
\hline 125004874 & 131350907 & 1705 & 1744 & 39 \\
\hline 125004874 & 131350907 & 1712 & 1745 & 33 \\
\hline 125004874 & 131350907 & 1708 & 1744 & 36 \\
\hline 125004874 & 131350907 & 1716 & 1741 & 25 \\
\hline 125004874 & 131350907 & 1717 & 1737 & 20 \\
\hline 125004874 & 131350907 & 1708 & 1739 & 31 \\
\hline
\end{tabular}




\begin{tabular}{|c|c|c|c|c|}
\hline 125004874 & 131350907 & 1709 & 1741 & 32 \\
\hline 125004874 & 131350907 & 1702 & 1737 & 35 \\
\hline 125004874 & 131350907 & 1704 & 1741 & 37 \\
\hline 125004874 & 131350907 & 1710 & 1740 & 30 \\
\hline 125004874 & 131350907 & 1708 & 1741 & 33 \\
\hline 125004874 & 132460300 & 1697 & 1751 & 54 \\
\hline 125004874 & 132460300 & 1709 & 1748 & 39 \\
\hline 125004874 & 131350907 & 1713 & 1743 & 30 \\
\hline 125004874 & 131350907 & 1718 & 1743 & 25 \\
\hline 125004874 & 132460300 & 1703 & 1751 & 48 \\
\hline 125004874 & 133337850 & 1713 & 1758 & 45 \\
\hline 125004874 & 132460300 & 1720 & 1752 & 32 \\
\hline 125004874 & 135194339 & 1712 & 1766 & 54 \\
\hline 128977630 & 132460300 & 1730 & 1754 & 24 \\
\hline 131350907 & 135194339 & 1753 & 1772 & 19 \\
\hline 131350907 & 138065689 & 1749 & 1779 & 30 \\
\hline 131350907 & 135194339 & 1750 & 1770 & 20 \\
\hline 128977630 & 135194339 & 1735 & 1771 & 36 \\
\hline 128977630 & 135194339 & 1743 & 1771 & 28 \\
\hline 131350907 & 135194339 & 1752 & 1770 & 18 \\
\hline 128977630 & 135194339 & 1737 & 1774 & 37 \\
\hline 131350907 & 138065689 & 1746 & 1780 & 34 \\
\hline 131350907 & 138065689 & 1750 & 1793 & 43 \\
\hline 131350907 & 138065689 & 1748 & 1782 & 34 \\
\hline 128977630 & 138065689 & 1739 & 1784 & 45 \\
\hline 128977630 & 138065689 & 1732 & 1787 & 55 \\
\hline 133337850 & 138065689 & 1763 & 1779 & 16 \\
\hline 132460300 & 138065689 & 1759 & 1784 & 25 \\
\hline 10671331 & 197455851 & 257 & 2352 & 2095 \\
\hline 131350907 & 138065689 & 1755 & 1782 & 27 \\
\hline 133337850 & 138065689 & 1766 & 1785 & 19 \\
\hline 133337850 & 138065689 & 1768 & 1786 & 18 \\
\hline 133337850 & 138065689 & 1768 & 1787 & 19 \\
\hline 131350907 & 138065689 & 1751 & 1790 & 39 \\
\hline 132460300 & 138065689 & 1758 & 1789 & 31 \\
\hline 133337850 & 138065689 & 1763 & 1788 & 25 \\
\hline 133337850 & 138065689 & 1762 & 1786 & 24 \\
\hline 128977630 & 138065689 & 1733 & 1792 & \\
\hline 133337850 & 139705107 & 1763 & 1794 & 31 \\
\hline 133337850 & 138065689 & 1763 & 1792 & 29 \\
\hline 131350907 & 139705107 & 1749 & 1805 & \\
\hline 131350907 & 139705107 & 1749 & 1797 & \\
\hline 133337850 & 139705107 & 1765 & 1804 & \\
\hline
\end{tabular}




\begin{tabular}{|c|c|c|c|}
\hline 133337850 & 142746882 & 1771 & 1830 \\
\hline 133337850 & 139705107 & 1772 & 1801 \\
\hline 128977630 & 142746882 & 1737 & 1825 \\
\hline 133337850 & 139705107 & 1774 & 1800 \\
\hline 128977630 & 139705107 & 1733 & 1805 \\
\hline 135194339 & 139705107 & 1780 & 1802 \\
\hline 133337850 & 139705107 & 1772 & 1804 \\
\hline 135194339 & 139705107 & 1782 & 1802 \\
\hline 135194339 & 139705107 & 1781 & 1804 \\
\hline 135194339 & 142746882 & 1779 & 1812 \\
\hline 135194339 & 139705107 & 1781 & 1806 \\
\hline 135194339 & 139705107 & 1782 & 1802 \\
\hline 135194339 & 139705107 & 1780 & 1804 \\
\hline 131350907 & 139705107 & 1756 & 1805 \\
\hline 135194339 & 139705107 & 1783 & 1799 \\
\hline 135194339 & 139705107 & 1781 & 1806 \\
\hline 135194339 & 139705107 & 1783 & 1804 \\
\hline 135194339 & 139705107 & 1780 & 1807 \\
\hline 135194339 & 139705107 & 1781 & 1809 \\
\hline 135194339 & 139705107 & 1785 & 1805 \\
\hline 135194339 & 139705107 & 1788 & 1801 \\
\hline 135194339 & 139705107 & 1786 & 1803 \\
\hline 135194339 & 139705107 & 1787 & 1803 \\
\hline 135194339 & 139705107 & 1785 & 1803 \\
\hline 135194339 & 139705107 & 1786 & 1802 \\
\hline 135194339 & 139705107 & 1784 & 1805 \\
\hline 135194339 & 139705107 & 1787 & 1805 \\
\hline 135194339 & 139705107 & 1787 & 1804 \\
\hline 135194339 & 139705107 & 1786 & 1805 \\
\hline 135194339 & 139705107 & 1790 & 1801 \\
\hline 135194339 & 139705107 & 1787 & 1804 \\
\hline 135194339 & 139705107 & 1787 & 1804 \\
\hline 135194339 & 139705107 & 1788 & 1804 \\
\hline 135194339 & 139705107 & 1787 & 1804 \\
\hline 135194339 & 139705107 & 1787 & 1804 \\
\hline 135194339 & 139705107 & 1787 & 1804 \\
\hline 135194339 & 139705107 & 1787 & 1804 \\
\hline 135194339 & 139705107 & 1786 & 1805 \\
\hline 135194339 & 139705107 & 1787 & 1804 \\
\hline 135194339 & 139705107 & 1787 & 1804 \\
\hline 135194339 & 139705107 & 1786 & 1804 \\
\hline 135194339 & 139705107 & 1787 & 1805 \\
\hline 135194339 & 139705107 & 1787 & 1804 \\
\hline
\end{tabular}




\begin{tabular}{|c|c|c|c|c|}
\hline 135194339 & 139705107 & 1788 & 1804 & 16 \\
\hline 135194339 & 139705107 & 1787 & 1804 & 17 \\
\hline 135194339 & 139705107 & 1787 & 1804 & 17 \\
\hline 135194339 & 139705107 & 1787 & 1804 & 17 \\
\hline 135194339 & 139705107 & 1786 & 1804 & 18 \\
\hline 135194339 & 139705107 & 1787 & 1804 & 17 \\
\hline 135194339 & 139705107 & 1786 & 1804 & 18 \\
\hline 135194339 & 139705107 & 1789 & 1802 & 13 \\
\hline 135194339 & 139705107 & 1781 & 1808 & 27 \\
\hline 135194339 & 142746882 & 1782 & 1816 & 34 \\
\hline 135194339 & 139705107 & 1787 & 1804 & 17 \\
\hline 135194339 & 139705107 & 1785 & 1807 & 22 \\
\hline 135194339 & 139705107 & 1788 & 1805 & 17 \\
\hline 135194339 & 139705107 & 1787 & 1805 & 18 \\
\hline 135194339 & 139705107 & 1789 & 1804 & 15 \\
\hline 135194339 & 139705107 & 1787 & 1804 & 17 \\
\hline 135194339 & 139705107 & 1789 & 1804 & 15 \\
\hline 135194339 & 139705107 & 1787 & 1805 & 18 \\
\hline 135194339 & 139705107 & 1784 & 1808 & 24 \\
\hline 135194339 & 139705107 & 1786 & 1805 & 19 \\
\hline 135194339 & 139705107 & 1788 & 1804 & 16 \\
\hline 135194339 & 139705107 & 1787 & 1805 & 18 \\
\hline 135194339 & 139705107 & 1788 & 1805 & 17 \\
\hline 135194339 & 139705107 & 1788 & 1804 & 16 \\
\hline 135194339 & 139705107 & 1790 & 1803 & 13 \\
\hline 135194339 & 142746882 & 1783 & 1815 & 32 \\
\hline 135194339 & 139705107 & 1788 & 1803 & 15 \\
\hline 135194339 & 139705107 & 1787 & 1804 & 17 \\
\hline 135194339 & 139705107 & 1788 & 1804 & 16 \\
\hline 135194339 & 139705107 & 1789 & 1804 & 15 \\
\hline 135194339 & 139705107 & 1790 & 1803 & 13 \\
\hline 135194339 & 139705107 & 1789 & 1804 & 15 \\
\hline 135194339 & 139705107 & 1784 & 1807 & 23 \\
\hline 135194339 & 139705107 & 1787 & 1804 & 17 \\
\hline 135194339 & 139705107 & 1785 & 1807 & 22 \\
\hline 135194339 & 139705107 & 1788 & 1803 & 15 \\
\hline 135194339 & 139705107 & 1787 & 1805 & 18 \\
\hline 135194339 & 139705107 & 1787 & 1805 & 18 \\
\hline 135194339 & 139705107 & 1787 & 1805 & 18 \\
\hline 135194339 & 139705107 & 1788 & 1804 & 16 \\
\hline 135194339 & 139705107 & 1789 & 1804 & 15 \\
\hline 135194339 & 139705107 & 1790 & 1802 & 12 \\
\hline 135194339 & 139705107 & 1784 & 1808 & 24 \\
\hline
\end{tabular}




\begin{tabular}{|c|c|c|c|}
\hline 135194339 & 139705107 & 1785 & 1807 \\
\hline 135194339 & 139705107 & 1788 & 1805 \\
\hline 135194339 & 139705107 & 1788 & 1804 \\
\hline 135194339 & 139705107 & 1790 & 1803 \\
\hline 135194339 & 139705107 & 1784 & 1808 \\
\hline 135194339 & 139705107 & 1785 & 1807 \\
\hline 135194339 & 139705107 & 1787 & 1806 \\
\hline 135194339 & 139705107 & 1787 & 1805 \\
\hline 135194339 & 139705107 & 1787 & 1805 \\
\hline 135194339 & 139705107 & 1786 & 1805 \\
\hline 135194339 & 139705107 & 1787 & 1805 \\
\hline 135194339 & 139705107 & 1787 & 1805 \\
\hline 135194339 & 139705107 & 1788 & 1804 \\
\hline 135194339 & 139705107 & 1788 & 1804 \\
\hline 135194339 & 139705107 & 1790 & 1804 \\
\hline 135194339 & 139705107 & 1787 & 1805 \\
\hline 135194339 & 139705107 & 1788 & 1805 \\
\hline 135194339 & 139705107 & 1787 & 1805 \\
\hline 135194339 & 139705107 & 1787 & 1808 \\
\hline 135194339 & 142746882 & 1784 & 1836 \\
\hline 135194339 & 139705107 & 1786 & 1805 \\
\hline 135194339 & 139705107 & 1785 & 1807 \\
\hline 135194339 & 139705107 & 1787 & 1806 \\
\hline 135194339 & 139705107 & 1788 & 1806 \\
\hline 135194339 & 139705107 & 1786 & 1808 \\
\hline 135194339 & 139705107 & 1786 & 1807 \\
\hline 135194339 & 139705107 & 1789 & 1805 \\
\hline 135194339 & 139705107 & 1787 & 1806 \\
\hline 135194339 & 139705107 & 1788 & 1805 \\
\hline 135194339 & 139705107 & 1789 & 1805 \\
\hline 135194339 & 139705107 & 1789 & 1804 \\
\hline 135194339 & 139705107 & 1787 & 1806 \\
\hline 135194339 & 139705107 & 1786 & 1808 \\
\hline 135194339 & 139705107 & 1789 & 1805 \\
\hline 135194339 & 139705107 & 1787 & 1806 \\
\hline 135194339 & 139705107 & 1787 & 1807 \\
\hline 135194339 & 139705107 & 1789 & 1806 \\
\hline 135194339 & 139705107 & 1787 & 1805 \\
\hline 135194339 & 139705107 & 1788 & 1806 \\
\hline 135194339 & 139705107 & 1788 & 1805 \\
\hline 135194339 & 139705107 & 1788 & 1806 \\
\hline 135194339 & 139705107 & 1790 & 1804 \\
\hline 135194339 & 139705107 & 1787 & 1806 \\
\hline
\end{tabular}




\begin{tabular}{|c|c|c|c|c|}
\hline 135194339 & 139705107 & 1789 & 1805 & 16 \\
\hline 135194339 & 139705107 & 1787 & 1805 & 18 \\
\hline 135194339 & 139705107 & 1789 & 1805 & 16 \\
\hline 135194339 & 142746882 & 1784 & 1818 & 34 \\
\hline 135194339 & 139705107 & 1785 & 1808 & 23 \\
\hline 135194339 & 139705107 & 1787 & 1805 & 18 \\
\hline 135194339 & 139705107 & 1786 & 1806 & 20 \\
\hline 135194339 & 142746882 & 1781 & 1821 & 40 \\
\hline 135194339 & 139705107 & 1788 & 1808 & 20 \\
\hline 135194339 & 142746882 & 1787 & 1815 & 28 \\
\hline 135194339 & 139705107 & 1789 & 1807 & 18 \\
\hline 135194339 & 139705107 & 1788 & 1807 & 19 \\
\hline 135194339 & 139705107 & 1788 & 1807 & 19 \\
\hline 135194339 & 139705107 & 1788 & 1807 & 19 \\
\hline 135194339 & 139705107 & 1789 & 1807 & 18 \\
\hline 135194339 & 139705107 & 1789 & 1808 & 19 \\
\hline 135194339 & 139705107 & 1788 & 1807 & 19 \\
\hline 135194339 & 139705107 & 1789 & 1807 & 18 \\
\hline 135194339 & 139705107 & 1789 & 1808 & 19 \\
\hline 135194339 & 139705107 & 1789 & 1808 & 19 \\
\hline 135194339 & 139705107 & 1788 & 1808 & 20 \\
\hline 135194339 & 139705107 & 1788 & 1808 & 20 \\
\hline 135194339 & 139705107 & 1789 & 1807 & 18 \\
\hline 135194339 & 139705107 & 1788 & 1807 & 19 \\
\hline 135194339 & 139705107 & 1788 & 1807 & 19 \\
\hline 135194339 & 139705107 & 1789 & 1808 & 19 \\
\hline 135194339 & 139705107 & 1790 & 1808 & 18 \\
\hline 135194339 & 139705107 & 1788 & 1809 & 21 \\
\hline 135194339 & 139705107 & 1789 & 1806 & 17 \\
\hline 135194339 & 139705107 & 1789 & 1807 & 18 \\
\hline 135194339 & 139705107 & 1787 & 1809 & 22 \\
\hline 135194339 & 139705107 & 1787 & 1808 & 21 \\
\hline 135194339 & 139705107 & 1790 & 1808 & 18 \\
\hline 135194339 & 139705107 & 1789 & 1807 & 18 \\
\hline 135194339 & 139705107 & 1789 & 1807 & 18 \\
\hline 135194339 & 139705107 & 1788 & 1808 & 20 \\
\hline 135194339 & 139705107 & 1789 & 1808 & 19 \\
\hline 135194339 & 139705107 & 1789 & 1807 & 18 \\
\hline 135194339 & 139705107 & 1789 & 1807 & 18 \\
\hline 135194339 & 139705107 & 1789 & 1807 & 18 \\
\hline 135194339 & 139705107 & 1787 & 1808 & 21 \\
\hline 135194339 & 139705107 & 1788 & 1809 & 21 \\
\hline 135194339 & 139705107 & 1789 & 1806 & 17 \\
\hline
\end{tabular}




\begin{tabular}{|c|c|c|c|c|}
\hline 135194339 & 139705107 & 1789 & 1808 & 19 \\
\hline 135194339 & 142746882 & 1786 & 1822 & 36 \\
\hline 135194339 & 142746882 & 1783 & 1819 & 36 \\
\hline 135194339 & 142746882 & 1786 & 1836 & 50 \\
\hline 135194339 & 139705107 & 1789 & 1806 & 17 \\
\hline 135194339 & 139705107 & 1789 & 1808 & 19 \\
\hline 135194339 & 139705107 & 1789 & 1808 & 19 \\
\hline 135194339 & 142746882 & 1787 & 1816 & 29 \\
\hline 135194339 & 139705107 & 1789 & 1808 & 19 \\
\hline 135194339 & 139705107 & 1790 & 1808 & 18 \\
\hline 135194339 & 139705107 & 1789 & 1810 & 21 \\
\hline 135194339 & 139705107 & 1789 & 1808 & 19 \\
\hline 135194339 & 142746882 & 1789 & 1813 & 24 \\
\hline 135194339 & 139705107 & 1789 & 1808 & 19 \\
\hline 135194339 & 139705107 & 1790 & 1809 & 19 \\
\hline 135194339 & 139705107 & 1789 & 1809 & 20 \\
\hline 135194339 & 139705107 & 1787 & 1809 & 22 \\
\hline 135194339 & 142746882 & 1788 & 1815 & 27 \\
\hline 135194339 & 139705107 & 1789 & 1808 & 19 \\
\hline 135194339 & 142746882 & 1789 & 1812 & 23 \\
\hline 135194339 & 142746882 & 1789 & 1812 & 23 \\
\hline 135194339 & 139705107 & 1789 & 1808 & 19 \\
\hline 135194339 & 142746882 & 1789 & 1813 & 24 \\
\hline 135194339 & 139705107 & 1789 & 1809 & 20 \\
\hline 135194339 & 142746882 & 1789 & 1814 & 25 \\
\hline 135194339 & 142746882 & 1787 & 1814 & 27 \\
\hline 135194339 & 139705107 & 1789 & 1809 & 20 \\
\hline 135194339 & 139705107 & 1789 & 1808 & 19 \\
\hline 135194339 & 142746882 & 1790 & 1812 & 22 \\
\hline 135194339 & 139705107 & 1789 & 1809 & 20 \\
\hline 135194339 & 139705107 & 1789 & 1808 & 19 \\
\hline 135194339 & 139705107 & 1789 & 1809 & 20 \\
\hline 135194339 & 139705107 & 1788 & 1808 & 20 \\
\hline 135194339 & 142746882 & 1789 & 1812 & 23 \\
\hline 135194339 & 139705107 & 1789 & 1808 & 19 \\
\hline 135194339 & 139705107 & 1790 & 1808 & 18 \\
\hline 135194339 & 142746882 & 1790 & 1810 & 20 \\
\hline 135194339 & 139705107 & 1789 & 1808 & 19 \\
\hline 135194339 & 142746882 & 1788 & 1813 & 25 \\
\hline 135194339 & 142746882 & 1787 & 1817 & 30 \\
\hline 135194339 & 139705107 & 1788 & 1808 & 20 \\
\hline 135194339 & 142746882 & 1786 & 1828 & 42 \\
\hline 135194339 & 139705107 & 1789 & 1808 & 19 \\
\hline
\end{tabular}




\begin{tabular}{|c|c|c|c|}
\hline 135194339 & 142746882 & 1780 & 1832 \\
\hline 135194339 & 142746882 & 1782 & 1829 \\
\hline 135194339 & 142746882 & 1779 & 1823 \\
\hline 135194339 & 142746882 & 1788 & 1822 \\
\hline 135194339 & 142746882 & 1788 & 1818 \\
\hline 135194339 & 142746882 & 1788 & 1820 \\
\hline 135194339 & 142746882 & 1788 & 1819 \\
\hline 135194339 & 142746882 & 1787 & 1820 \\
\hline 135194339 & 142746882 & 1787 & 1822 \\
\hline 135194339 & 142746882 & 1786 & 1841 \\
\hline 135194339 & 142746882 & 1786 & 1841 \\
\hline 135194339 & 142746882 & 1786 & 1829 \\
\hline 135194339 & 142746882 & 1788 & 1823 \\
\hline 135194339 & 142746882 & 1787 & 1831 \\
\hline 133337850 & 142746882 & 1771 & 1830 \\
\hline 135194339 & 142746882 & 1786 & 1828 \\
\hline 135194339 & 142746882 & 1785 & 1831 \\
\hline 135194339 & 142746882 & 1786 & 1828 \\
\hline 135194339 & 142746882 & 1786 & 1828 \\
\hline 135194339 & 142746882 & 1783 & 1836 \\
\hline 135194339 & 142746882 & 1785 & 1831 \\
\hline 135194339 & 142746882 & 1786 & 1829 \\
\hline 135194339 & 142746882 & 1786 & 1833 \\
\hline 135194339 & 142746882 & 1787 & 1831 \\
\hline 135194339 & 142746882 & 1786 & 1830 \\
\hline 135194339 & 142746882 & 1785 & 1836 \\
\hline 135194339 & 142746882 & 1786 & 1838 \\
\hline 135194339 & 142746882 & 1784 & 1833 \\
\hline 133337850 & 142746882 & 1770 & 1824 \\
\hline 135194339 & 142746882 & 1787 & 1834 \\
\hline 135194339 & 142746882 & 1785 & 1834 \\
\hline 138065689 & 142746882 & 1800 & 1814 \\
\hline 138065689 & 142746882 & 1795 & 1828 \\
\hline 138065689 & 142746882 & 1799 & 1826 \\
\hline 138065689 & 142746882 & 1800 & 1827 \\
\hline 135194339 & 144855155 & 1787 & 1844 \\
\hline 138065689 & 142746882 & 1795 & 1840 \\
\hline 138065689 & 142746882 & 1806 & 1837 \\
\hline 135194339 & 142746882 & 1790 & 1841 \\
\hline 135194339 & 146988746 & 1783 & 1859 \\
\hline 135194339 & 144855155 & 1787 & 1844 \\
\hline 135194339 & 146988746 & 1786 & 1861 \\
\hline 135194339 & 146988746 & 1786 & 1864 \\
\hline
\end{tabular}




\begin{tabular}{|c|c|c|c|c|}
\hline 138065689 & 146988746 & 1802 & 1866 & 64 \\
\hline 139705107 & 145518682 & 1814 & 1849 & 35 \\
\hline 139705107 & 146988746 & 1814 & 1864 & 50 \\
\hline 139705107 & 145518682 & 1813 & 1849 & 36 \\
\hline 139705107 & 146988746 & 1811 & 1868 & 57 \\
\hline 139705107 & 146988746 & 1820 & 1858 & 38 \\
\hline 139705107 & 145518682 & 1823 & 1847 & 24 \\
\hline 139705107 & 145518682 & 1821 & 1848 & 27 \\
\hline 139705107 & 146988746 & 1823 & 1855 & 32 \\
\hline 139705107 & 146988746 & 1821 & 1867 & 46 \\
\hline 139705107 & 144855155 & 1820 & 1846 & 26 \\
\hline 139705107 & 145518682 & 1825 & 1849 & 24 \\
\hline 139705107 & 146988746 & 1820 & 1855 & 35 \\
\hline 139705107 & 148698440 & 1820 & 1902 & 82 \\
\hline 139705107 & 146988746 & 1821 & 1856 & 35 \\
\hline 139705107 & 148698440 & 1814 & 1901 & 87 \\
\hline 139705107 & 144855155 & 1824 & 1846 & 22 \\
\hline 139705107 & 144855155 & 1825 & 1845 & 20 \\
\hline 139705107 & 146988746 & 1824 & 1850 & 26 \\
\hline 139705107 & 146988746 & 1825 & 1850 & 25 \\
\hline 139705107 & 144855155 & 1827 & 1845 & 18 \\
\hline 139705107 & 146988746 & 1822 & 1861 & 39 \\
\hline 139705107 & 146988746 & 1820 & 1854 & 34 \\
\hline 139705107 & 146988746 & 1823 & 1853 & 30 \\
\hline 139705107 & 145518682 & 1825 & 1848 & 23 \\
\hline 139705107 & 146988746 & 1823 & 1858 & 35 \\
\hline 139705107 & 146988746 & 1824 & 1858 & 34 \\
\hline 139705107 & 146988746 & 1823 & 1857 & 34 \\
\hline 139705107 & 144855155 & 1827 & 1846 & 19 \\
\hline 139705107 & 146988746 & 1824 & 1856 & 32 \\
\hline 139705107 & 145518682 & 1823 & 1848 & 25 \\
\hline 139705107 & 146988746 & 1817 & 1851 & 34 \\
\hline 139705107 & 146988746 & 1821 & 1864 & 43 \\
\hline 139705107 & 146988746 & 1829 & 1867 & 38 \\
\hline 139705107 & 146988746 & 1834 & 1850 & 16 \\
\hline 139705107 & 146988746 & 1831 & 1863 & 32 \\
\hline 139705107 & 145518682 & 1830 & 1848 & 18 \\
\hline 139705107 & 146988746 & 1828 & 1873 & 45 \\
\hline 139705107 & 146988746 & 1828 & 1860 & 32 \\
\hline 139705107 & 146988746 & 1829 & 1861 & 32 \\
\hline 139705107 & 144855155 & 1832 & 1845 & 13 \\
\hline 139705107 & 146988746 & 1832 & 1852 & 20 \\
\hline 139705107 & 146988746 & 1831 & 1853 & 22 \\
\hline
\end{tabular}




\begin{tabular}{|c|c|c|c|}
\hline 139705107 & 145518682 & 1829 & 1847 \\
\hline 139705107 & 146988746 & 1827 & 1861 \\
\hline 139705107 & 145518682 & 1831 & 1849 \\
\hline 139705107 & 146988746 & 1829 & 1861 \\
\hline 139705107 & 146988746 & 1830 & 1870 \\
\hline 139705107 & 146988746 & 1827 & 1854 \\
\hline 139705107 & 146988746 & 1828 & 1857 \\
\hline 139705107 & 146988746 & 1830 & 1862 \\
\hline 139705107 & 144855155 & 1833 & 1846 \\
\hline 139705107 & 146988746 & 1828 & 1862 \\
\hline 139705107 & 146988746 & 1831 & 1858 \\
\hline 139705107 & 146988746 & 1829 & 1855 \\
\hline 139705107 & 146988746 & 1827 & 1850 \\
\hline 139705107 & 146988746 & 1830 & 1861 \\
\hline 139705107 & 146988746 & 1831 & 1861 \\
\hline 139705107 & 146988746 & 1830 & 1864 \\
\hline 139705107 & 144855155 & 1832 & 1845 \\
\hline 139705107 & 145518682 & 1833 & 1850 \\
\hline 139705107 & 146988746 & 1831 & 1854 \\
\hline 139705107 & 146988746 & 1826 & 1857 \\
\hline 139705107 & 146988746 & 1831 & 1863 \\
\hline 139705107 & 146988746 & 1828 & 1856 \\
\hline 139705107 & 146988746 & 1826 & 1850 \\
\hline 139705107 & 146988746 & 1828 & 1853 \\
\hline 139705107 & 146988746 & 1831 & 1861 \\
\hline 139705107 & 146988746 & 1828 & 1857 \\
\hline 139705107 & 146988746 & 1828 & 1861 \\
\hline 139705107 & 146988746 & 1823 & 1861 \\
\hline 139705107 & 145518682 & 1832 & 1848 \\
\hline 139705107 & 146988746 & 1822 & 1857 \\
\hline 139705107 & 145518682 & 1831 & 1848 \\
\hline 139705107 & 145518682 & 1834 & 1849 \\
\hline 139705107 & 145518682 & 1815 & 1849 \\
\hline 139705107 & 146988746 & 1828 & 1863 \\
\hline 139705107 & 146988746 & 1830 & 1865 \\
\hline 139705107 & 146988746 & 1826 & 1865 \\
\hline 139705107 & 146988746 & 1824 & 1862 \\
\hline 139705107 & 146988746 & 1825 & 1862 \\
\hline 139705107 & 146988746 & 1829 & 1866 \\
\hline 139705107 & 146988746 & 1828 & 1866 \\
\hline 139705107 & 146988746 & 1824 & 1861 \\
\hline 139705107 & 146988746 & 1829 & 1859 \\
\hline 139705107 & 146988746 & 1824 & 1861 \\
\hline
\end{tabular}




\begin{tabular}{|c|c|c|c|}
\hline 139705107 & 146988746 & 1825 & 1864 \\
\hline 139705107 & 146988746 & 1826 & 1854 \\
\hline 139705107 & 145518682 & 1829 & 1848 \\
\hline 139705107 & 145518682 & 1829 & 1848 \\
\hline 139705107 & 145518682 & 1829 & 1850 \\
\hline 139705107 & 146988746 & 1828 & 1868 \\
\hline 139705107 & 146988746 & 1828 & 1878 \\
\hline 139705107 & 145518682 & 1829 & 1848 \\
\hline 139705107 & 145518682 & 1831 & 1849 \\
\hline 139705107 & 146988746 & 1826 & 1868 \\
\hline 139705107 & 146988746 & 1830 & 1860 \\
\hline 139705107 & 146988746 & 1826 & 1862 \\
\hline 139705107 & 146988746 & 1829 & 1863 \\
\hline 139705107 & 146988746 & 1832 & 1856 \\
\hline 139705107 & 145518682 & 1825 & 1849 \\
\hline 139705107 & 145518682 & 1825 & 1849 \\
\hline 139705107 & 146988746 & 1832 & 1884 \\
\hline 139705107 & 146988746 & 1840 & 1859 \\
\hline 139705107 & 146988746 & 1825 & 1873 \\
\hline 139705107 & 146988746 & 1830 & 1870 \\
\hline 139705107 & 146988746 & 1822 & 1871 \\
\hline 139705107 & 146988746 & 1818 & 1863 \\
\hline 139705107 & 146988746 & 1831 & 1863 \\
\hline 139705107 & 146988746 & 1822 & 1880 \\
\hline 139705107 & 146988746 & 1831 & 1861 \\
\hline 139705107 & 146988746 & 1831 & 1863 \\
\hline 139705107 & 146988746 & 1826 & 1865 \\
\hline 139705107 & 146988746 & 1827 & 1865 \\
\hline 139705107 & 146988746 & 1829 & 1866 \\
\hline 139705107 & 146988746 & 1835 & 1861 \\
\hline 144855155 & 146988746 & 1847 & 1862 \\
\hline 139705107 & 146988746 & 1831 & 1861 \\
\hline 144855155 & 146988746 & 1847 & 1860 \\
\hline 144855155 & 146988746 & 1848 & 1861 \\
\hline 144855155 & 146988746 & 1847 & 1866 \\
\hline 144855155 & 146988746 & 1849 & 1869 \\
\hline 144855155 & 146988746 & 1848 & 1866 \\
\hline 144855155 & 146988746 & 1849 & 1869 \\
\hline 139705107 & 146988746 & 1823 & 1867 \\
\hline 139705107 & 146988746 & 1824 & 1873 \\
\hline 144855155 & 146988746 & 1848 & 1873 \\
\hline 144855155 & 146988746 & 1848 & 1875 \\
\hline 139705107 & 146988746 & 1829 & 1878 \\
\hline
\end{tabular}




\begin{tabular}{|c|c|c|c|c|}
\hline 142746882 & 146988746 & 1843 & 1887 & 44 \\
\hline 145518682 & 148698440 & 1853 & 1903 & 50 \\
\hline 145518682 & 148698440 & 1854 & 1897 & 43 \\
\hline 145518682 & 157333643 & 1854 & 1926 & 72 \\
\hline 145518682 & 148698440 & 1852 & 1901 & 49 \\
\hline 145518682 & 157333643 & 1850 & 1926 & 76 \\
\hline 145518682 & 157333643 & 1850 & 1922 & 72 \\
\hline 145518682 & 148698440 & 1861 & 1907 & 46 \\
\hline 145518682 & 155510340 & 1860 & 1908 & 48 \\
\hline 145518682 & 150960511 & 1865 & 1907 & 42 \\
\hline 145518682 & 148698440 & 1866 & 1899 & 33 \\
\hline 145518682 & 155510340 & 1870 & 1912 & 42 \\
\hline 145518682 & 148698440 & 1875 & 1900 & 25 \\
\hline 145518682 & 148698440 & 1873 & 1899 & 26 \\
\hline 145518682 & 148698440 & 1868 & 1903 & 35 \\
\hline 145518682 & 148698440 & 1870 & 1903 & 33 \\
\hline 145518682 & 148698440 & 1866 & 1902 & 36 \\
\hline 145518682 & 155510340 & 1871 & 1908 & 37 \\
\hline 145518682 & 148698440 & 1864 & 1904 & 40 \\
\hline 145518682 & 150960511 & 1861 & 1907 & 46 \\
\hline 145518682 & 155510340 & 1869 & 1909 & 40 \\
\hline 145518682 & 155510340 & 1866 & 1910 & 44 \\
\hline 146988746 & 157333643 & 1890 & 1933 & 43 \\
\hline 145518682 & 157333643 & 1882 & 1931 & 49 \\
\hline 145518682 & 155510340 & 1881 & 1911 & 30 \\
\hline 145518682 & 157333643 & 1864 & 1915 & 51 \\
\hline 146988746 & 155510340 & 1898 & 1912 & 14 \\
\hline 146988746 & 150960511 & 1897 & 1907 & 10 \\
\hline 146988746 & 155510340 & 1897 & 1909 & 12 \\
\hline 146988746 & 157333643 & 1895 & 1915 & 20 \\
\hline 146988746 & 155510340 & 1905 & 1912 & 7 \\
\hline 146988746 & 157333643 & 1889 & 1918 & 29 \\
\hline 145518682 & 157333643 & 1873 & 1919 & 46 \\
\hline 146988746 & 155510340 & 1898 & 1912 & 14 \\
\hline 146988746 & 157333643 & 1892 & 1926 & 34 \\
\hline 146988746 & 157333643 & 1905 & 1920 & 15 \\
\hline 146988746 & 157333643 & 1891 & 1925 & 34 \\
\hline 146988746 & 157333643 & 1903 & 1930 & 27 \\
\hline 150960511 & 157333643 & 1908 & 1925 & 17 \\
\hline 146988746 & 157333643 & 1901 & 1930 & 29 \\
\hline 146988746 & 157333643 & 1898 & 1932 & 34 \\
\hline 146988746 & 159222917 & 1906 & 1935 & 29 \\
\hline 150960511 & 163985765 & 1910 & 1940 & 30 \\
\hline
\end{tabular}




\begin{tabular}{|c|c|c|c|}
\hline 150960511 & 159222917 & 1911 & 1937 \\
\hline 146988746 & 168505773 & 1891 & 1967 \\
\hline 146988746 & 168505773 & 1900 & 1957 \\
\hline 146988746 & 169510679 & 1896 & 1971 \\
\hline 146988746 & 163985765 & 1893 & 1941 \\
\hline 150960511 & 168505773 & 1912 & 1964 \\
\hline 150960511 & 168505773 & 1910 & 1955 \\
\hline 155510340 & 168505773 & 1924 & 1953 \\
\hline 155510340 & 168505773 & 1924 & 1948 \\
\hline 155510340 & 168505773 & 1924 & 1955 \\
\hline 155510340 & 168505773 & 1929 & 1955 \\
\hline 163259020 & 168505773 & 1940 & 1959 \\
\hline 155510340 & 168505773 & 1920 & 1961 \\
\hline 163259020 & 168505773 & 1941 & 1967 \\
\hline 163985765 & 168505773 & 1942 & 1965 \\
\hline 150960511 & 169510679 & 1911 & 1974 \\
\hline 155510340 & 169510679 & 1923 & 1974 \\
\hline 150960511 & 169510679 & 1913 & 1976 \\
\hline 163985765 & 169510679 & 1953 & 1979 \\
\hline 163985765 & 169510679 & 1956 & 1977 \\
\hline 163985765 & 169510679 & 1952 & 1978 \\
\hline 163985765 & 169510679 & 1951 & 1976 \\
\hline 169510679 & 174277216 & 1988 & 2002 \\
\hline 168505773 & 179059657 & 1987 & 2077 \\
\hline 171463002 & 177451389 & 2000 & 2064 \\
\hline 169510679 & 180918951 & 1991 & 2086 \\
\hline 168505773 & 177451389 & 1985 & 2053 \\
\hline 171463002 & 177451389 & 2007 & 2052 \\
\hline 171463002 & 179059657 & 2000 & 2068 \\
\hline 171463002 & 177451389 & 2000 & 2055 \\
\hline 171463002 & 177451389 & 2014 & 2056 \\
\hline 171463002 & 179059657 & 2005 & 2070 \\
\hline 171463002 & 177451389 & 2006 & 2051 \\
\hline 171463002 & 177451389 & 2014 & 2050 \\
\hline 171463002 & 187348973 & 2014 & 2124 \\
\hline 171463002 & 177451389 & 2013 & 2055 \\
\hline 171463002 & 177451389 & 2010 & 2058 \\
\hline 171463002 & 177451389 & 2013 & 2053 \\
\hline 171463002 & 177451389 & 2022 & 2051 \\
\hline 171463002 & 177451389 & 2021 & 2053 \\
\hline 171463002 & 177451389 & 2016 & 2056 \\
\hline 171463002 & 177451389 & 2007 & 2062 \\
\hline 171463002 & 177451389 & 2006 & 2062 \\
\hline
\end{tabular}




\begin{tabular}{|c|c|c|c|c|}
\hline 171463002 & 177451389 & 2012 & 2061 & 49 \\
\hline 171463002 & 179059657 & 2002 & 2070 & 68 \\
\hline 169510679 & 179059657 & 1994 & 2075 & 81 \\
\hline 171463002 & 187348973 & 2012 & 2122 & 110 \\
\hline 171463002 & 179059657 & 2014 & 2076 & 62 \\
\hline 171463002 & 179059657 & 2008 & 2073 & 65 \\
\hline 171463002 & 179059657 & 2033 & 2076 & 43 \\
\hline 174277216 & 180918951 & 2043 & 2080 & 37 \\
\hline 169510679 & 179059657 & 1996 & 2072 & 76 \\
\hline 175577246 & 179059657 & 2054 & 2069 & 15 \\
\hline 175577246 & 177451389 & 2056 & 2066 & 10 \\
\hline 175577246 & 179059657 & 2050 & 2070 & 20 \\
\hline 175577246 & 179059657 & 2049 & 2070 & 21 \\
\hline 175577246 & 179059657 & 2052 & 2077 & 25 \\
\hline 175577246 & 179059657 & 2057 & 2068 & 11 \\
\hline 175577246 & 179059657 & 2055 & 2072 & 17 \\
\hline 175577246 & 180918951 & 2044 & 2080 & 36 \\
\hline 169510679 & 187348973 & 1995 & 2106 & 111 \\
\hline 175577246 & 187348973 & 2048 & 2104 & 56 \\
\hline 175577246 & 180918951 & 2053 & 2083 & 30 \\
\hline 177451389 & 181505126 & 2073 & 2090 & 17 \\
\hline 177451389 & 187348973 & 2068 & 2130 & 62 \\
\hline 177451389 & 180918951 & 2072 & 2081 & 9 \\
\hline 177451389 & 180918951 & 2073 & 2085 & 12 \\
\hline 177451389 & 181505126 & 2068 & 2093 & 25 \\
\hline 177451389 & 191937702 & 2071 & 2141 & 70 \\
\hline 177451389 & 187348973 & 2070 & 2120 & 50 \\
\hline 177451389 & 187348973 & 2070 & 2126 & 56 \\
\hline 177451389 & 187348973 & 2069 & 2101 & 32 \\
\hline 177451389 & 187348973 & 2070 & 2134 & 64 \\
\hline 177451389 & 187348973 & 2069 & 2121 & 52 \\
\hline 177451389 & 187348973 & 2068 & 2130 & 62 \\
\hline 177451389 & 187348973 & 2071 & 2121 & 50 \\
\hline 177451389 & 187348973 & 2068 & 2128 & 60 \\
\hline 177451389 & 187348973 & 2070 & 2126 & 56 \\
\hline 179059657 & 187348973 & 2082 & 2122 & 40 \\
\hline 177451389 & 187348973 & 2077 & 2117 & 40 \\
\hline 177451389 & 187348973 & 2073 & 2111 & 38 \\
\hline 179059657 & 187348973 & 2083 & 2118 & 35 \\
\hline 179059657 & 187348973 & 2086 & 2101 & 15 \\
\hline 179059657 & 187348973 & 2084 & 2109 & 25 \\
\hline 179059657 & 187348973 & 2083 & 2107 & 24 \\
\hline 179059657 & 187348973 & 2083 & 2111 & \\
\hline
\end{tabular}




\begin{tabular}{|c|c|c|c|c|}
\hline 177451389 & 187348973 & 2077 & 2108 & 31 \\
\hline 177451389 & 187348973 & 2072 & 2114 & 42 \\
\hline 177451389 & 187348973 & 2075 & 2114 & 39 \\
\hline 179059657 & 187348973 & 2081 & 2108 & 27 \\
\hline 179059657 & 187348973 & 2078 & 2118 & 40 \\
\hline 179059657 & 187348973 & 2083 & 2115 & 32 \\
\hline 179059657 & 187348973 & 2081 & 2131 & 50 \\
\hline 179059657 & 187348973 & 2080 & 2112 & 32 \\
\hline 179059657 & 187348973 & 2079 & 2116 & 37 \\
\hline 179059657 & 187348973 & 2081 & 2119 & 38 \\
\hline 179059657 & 187348973 & 2080 & 2123 & 43 \\
\hline 179059657 & 187348973 & 2084 & 2118 & 34 \\
\hline 177451389 & 187348973 & 2074 & 2120 & 46 \\
\hline 179059657 & 187348973 & 2080 & 2125 & 45 \\
\hline 179059657 & 187348973 & 2085 & 2110 & 25 \\
\hline 179059657 & 187348973 & 2083 & 2114 & 31 \\
\hline 179059657 & 187348973 & 2080 & 2130 & 50 \\
\hline 177451389 & 187348973 & 2072 & 2129 & 57 \\
\hline 177451389 & 187348973 & 2070 & 2121 & 51 \\
\hline 177451389 & 187348973 & 2071 & 2121 & 50 \\
\hline 179059657 & 187348973 & 2082 & 2127 & 45 \\
\hline 179059657 & 187348973 & 2079 & 2115 & 36 \\
\hline 180918951 & 187348973 & 2092 & 2107 & 15 \\
\hline 177451389 & 187348973 & 2072 & 2119 & 47 \\
\hline 179059657 & 187348973 & 2080 & 2113 & 33 \\
\hline 179059657 & 187348973 & 2082 & 2119 & 37 \\
\hline 177451389 & 187348973 & 2073 & 2118 & 45 \\
\hline 180918951 & 187348973 & 2090 & 2114 & 24 \\
\hline 177451389 & 187348973 & 2075 & 2125 & 50 \\
\hline 177451389 & 187348973 & 2073 & 2118 & 45 \\
\hline 177451389 & 187348973 & 2069 & 2127 & 58 \\
\hline 179059657 & 187348973 & 2081 & 2119 & 38 \\
\hline 177451389 & 187348973 & 2067 & 2135 & 68 \\
\hline 177451389 & 187348973 & 2076 & 2128 & 52 \\
\hline 177451389 & 187348973 & 2066 & 2134 & 68 \\
\hline 177451389 & 187348973 & 2068 & 2131 & 63 \\
\hline 179059657 & 187348973 & 2080 & 2129 & 49 \\
\hline 177451389 & 191937702 & 2074 & 2155 & 81 \\
\hline 177451389 & 191937702 & 2066 & 2147 & 81 \\
\hline 177451389 & 191937702 & 2071 & 2161 & 90 \\
\hline 177451389 & 191937702 & 2067 & 2159 & 92 \\
\hline 171463002 & 191937702 & 2005 & 2161 & 156 \\
\hline 182987407 & 191937702 & 2100 & 2165 & 65 \\
\hline
\end{tabular}




\begin{tabular}{|c|c|c|c|c|}
\hline 182987407 & 191937702 & 2113 & 2157 & 44 \\
\hline 182987407 & 191937702 & 2097 & 2162 & 65 \\
\hline 182987407 & 191937702 & 2116 & 2156 & 40 \\
\hline 182987407 & 191937702 & 2102 & 2163 & 61 \\
\hline 182987407 & 191937702 & 2119 & 2159 & 40 \\
\hline 182987407 & 191937702 & 2111 & 2155 & 44 \\
\hline 177451389 & 191937702 & 2069 & 2179 & 110 \\
\hline 182987407 & 191937702 & 2131 & 2177 & 46 \\
\hline 182987407 & 196261195 & 2121 & 2251 & 130 \\
\hline 182987407 & 196261195 & 2123 & 2252 & 129 \\
\hline 182987407 & 196261195 & 2130 & 2231 & 101 \\
\hline 182987407 & 196261195 & 2132 & 2249 & 117 \\
\hline 182987407 & 196261195 & 2131 & 2264 & 133 \\
\hline 182987407 & 196261195 & 2121 & 2269 & 148 \\
\hline 182987407 & 196261195 & 2132 & 2261 & 129 \\
\hline 189047467 & 196261195 & 2151 & 2256 & 105 \\
\hline 189047467 & 196261195 & 2142 & 2277 & 135 \\
\hline 189047467 & 196261195 & 2144 & 2268 & 124 \\
\hline 189047467 & 196261195 & 2151 & 2259 & 108 \\
\hline 189047467 & 196261195 & 2143 & 2279 & 136 \\
\hline 189047467 & 196261195 & 2150 & 2253 & 103 \\
\hline 189047467 & 196261195 & 2153 & 2259 & 106 \\
\hline 189047467 & 196261195 & 2150 & 2271 & 121 \\
\hline 189047467 & 196261195 & 2147 & 2261 & 114 \\
\hline 189047467 & 196261195 & 2156 & 2259 & 103 \\
\hline 189047467 & 196261195 & 2160 & 2251 & 91 \\
\hline 189047467 & 196261195 & 2147 & 2279 & 132 \\
\hline 189047467 & 196261195 & 2158 & 2250 & 92 \\
\hline 189047467 & 196261195 & 2150 & 2271 & 121 \\
\hline 189047467 & 196261195 & 2148 & 2253 & 105 \\
\hline 189047467 & 196261195 & 2155 & 2257 & 102 \\
\hline 189047467 & 196261195 & 2155 & 2260 & 105 \\
\hline 189047467 & 196261195 & 2158 & 2248 & 90 \\
\hline 189047467 & 196261195 & 2151 & 2265 & 114 \\
\hline 189047467 & 196261195 & 2155 & 2270 & 115 \\
\hline 189047467 & 196261195 & 2152 & 2265 & 113 \\
\hline 189047467 & 196261195 & 2159 & 2266 & 107 \\
\hline 189047467 & 196261195 & 2160 & 2259 & 99 \\
\hline 189047467 & 196261195 & 2157 & 2262 & 105 \\
\hline 189047467 & 196261195 & 2158 & 2266 & 108 \\
\hline 189047467 & 196261195 & 2155 & 2271 & 116 \\
\hline 189047467 & 196261195 & 2148 & 2261 & 113 \\
\hline 189047467 & 196261195 & 2161 & 2263 & 102 \\
\hline
\end{tabular}




\begin{tabular}{|c|c|c|c|c|}
\hline 189047467 & 196261195 & 2158 & 2266 & 108 \\
\hline 189047467 & 196261195 & 2163 & 2274 & 111 \\
\hline 189047467 & 196261195 & 2159 & 2274 & 115 \\
\hline 189047467 & 196261195 & 2163 & 2276 & 113 \\
\hline 189047467 & 196261195 & 2157 & 2274 & 117 \\
\hline 189047467 & 196261195 & 2158 & 2273 & 115 \\
\hline 189047467 & 196261195 & 2163 & 2275 & 112 \\
\hline 189047467 & 196261195 & 2157 & 2274 & 117 \\
\hline 189047467 & 196261195 & 2157 & 2273 & 116 \\
\hline 189047467 & 196261195 & 2159 & 2271 & 112 \\
\hline 189047467 & 196261195 & 2164 & 2273 & 109 \\
\hline 189047467 & 196261195 & 2162 & 2273 & 111 \\
\hline 189047467 & 196261195 & 2161 & 2274 & 113 \\
\hline 189047467 & 196261195 & 2167 & 2248 & 81 \\
\hline 189047467 & 196261195 & 2175 & 2241 & 66 \\
\hline 189047467 & 196261195 & 2175 & 2251 & 76 \\
\hline 189047467 & 196261195 & 2147 & 2270 & 123 \\
\hline 189047467 & 196261195 & 2158 & 2260 & 102 \\
\hline 189047467 & 196261195 & 2158 & 2270 & 112 \\
\hline 189047467 & 196261195 & 2158 & 2257 & 99 \\
\hline 189047467 & 196261195 & 2171 & 2250 & 79 \\
\hline 189047467 & 196261195 & 2170 & 2255 & 85 \\
\hline 189047467 & 196261195 & 2172 & 2262 & 90 \\
\hline 189047467 & 196261195 & 2166 & 2259 & 93 \\
\hline 189047467 & 196261195 & 2163 & 2274 & 111 \\
\hline 189047467 & 196261195 & 2158 & 2280 & 122 \\
\hline 189047467 & 196261195 & 2142 & 2272 & 130 \\
\hline 189047467 & 196261195 & 2163 & 2292 & 129 \\
\hline 189047467 & 196261195 & 2162 & 2290 & 128 \\
\hline 189047467 & 196261195 & 2170 & 2288 & 118 \\
\hline 189047467 & 196261195 & 2158 & 2300 & 142 \\
\hline 194020914 & 197455851 & 2255 & 2350 & 95 \\
\hline 194020914 & 197455851 & 2279 & 2350 & 71 \\
\hline 194020914 & 197455851 & 2274 & 2352 & 78 \\
\hline 194020914 & 197455851 & 2283 & 2348 & 65 \\
\hline 194020914 & 197455851 & 2273 & 2352 & 79 \\
\hline 194020914 & 197455851 & 2277 & 2352 & 75 \\
\hline 194020914 & 197455851 & 2257 & 2352 & 95 \\
\hline 194020914 & 197455851 & 2277 & 2348 & 71 \\
\hline 194020914 & 197455851 & 2267 & 2352 & 85 \\
\hline 194020914 & 197455851 & 2268 & 2352 & 84 \\
\hline 189047467 & 197455851 & 2187 & 2352 & 165 \\
\hline 194020914 & 197455851 & 2298 & 2352 & 54 \\
\hline
\end{tabular}




\begin{tabular}{|c|c|c|c|}
\hline 194020914 & 197455851 & 2282 & 2352 \\
\hline 196530962 & 197455851 & 2337 & 2352 \\
\hline 196530962 & 197455851 & 2335 & 2352 \\
\hline 196530962 & 197455851 & 2336 & 2352 \\
\hline 196530962 & 197455851 & 2339 & 2352 \\
\hline 196530962 & 197455851 & 2342 & 2352 \\
\hline 196530962 & 197455851 & 2344 & 2352 \\
\hline 196530962 & 197455851 & 2345 & 2352 \\
\hline 196530962 & 197455851 & 2341 & 2352 \\
\hline 3690634 & 5843745 & 0 & 25 \\
\hline 3690634 & 5843745 & 0 & 26 \\
\hline 3690634 & 5843745 & 0 & 32 \\
\hline 3690634 & 5843745 & 0 & 27 \\
\hline 3690634 & 5843745 & 0 & 28 \\
\hline 3690634 & 5843745 & 0 & 30 \\
\hline 3690634 & 5843745 & 0 & 34 \\
\hline 3690634 & 5843745 & 0 & 30 \\
\hline 3690634 & 5843745 & 0 & 32 \\
\hline 3690634 & 5843745 & 0 & 35 \\
\hline 3690634 & 7017142 & 0 & 74 \\
\hline 3690634 & 8387159 & 0 & 97 \\
\hline 5056555 & 7017142 & 3 & 67 \\
\hline 5056555 & 7017142 & 17 & 58 \\
\hline 5056555 & 7017142 & 17 & 64 \\
\hline 5056555 & 7017142 & 20 & 59 \\
\hline 5056555 & 7017142 & 14 & 60 \\
\hline 5056555 & 7017142 & 23 & 60 \\
\hline 5056555 & 7017142 & 10 & 65 \\
\hline 5056555 & 7017142 & 19 & 63 \\
\hline 5056555 & 7017142 & 14 & 85 \\
\hline 3690634 & 8387159 & 0 & 95 \\
\hline 5056555 & 8387159 & 11 & 96 \\
\hline 5843745 & 11697701 & 50 & 110 \\
\hline 5056555 & 11697701 & 31 & 103 \\
\hline 5843745 & 11697701 & 55 & 108 \\
\hline 5056555 & 11697701 & 3 & 115 \\
\hline 5843745 & 11697701 & 51 & 117 \\
\hline 5843745 & 11697701 & 51 & 122 \\
\hline 5843745 & 11697701 & 47 & 111 \\
\hline 5843745 & 8387159 & 57 & 97 \\
\hline 5843745 & 11697701 & 56 & 107 \\
\hline 5843745 & 11697701 & 50 & 117 \\
\hline 5843745 & 8387159 & 57 & 97 \\
\hline
\end{tabular}




\begin{tabular}{|c|c|c|c|}
\hline 5843745 & 11697701 & 57 & 99 \\
\hline 5843745 & 11697701 & 57 & 112 \\
\hline 5843745 & 11697701 & 57 & 104 \\
\hline 5843745 & 11697701 & 54 & 110 \\
\hline 5843745 & 8387159 & 62 & 96 \\
\hline 5843745 & 11697701 & 62 & 100 \\
\hline 5843745 & 8387159 & 57 & 98 \\
\hline 5843745 & 8387159 & 63 & 95 \\
\hline 5843745 & 8387159 & 63 & 97 \\
\hline 5843745 & 11697701 & 62 & 106 \\
\hline 5843745 & 11697701 & 60 & 116 \\
\hline 5843745 & 8387159 & 58 & 97 \\
\hline 5843745 & 11697701 & 60 & 102 \\
\hline 5843745 & 11697701 & 63 & 111 \\
\hline 5843745 & 8387159 & 60 & 98 \\
\hline 5843745 & 11697701 & 58 & 107 \\
\hline 5843745 & 8387159 & 56 & 97 \\
\hline 5843745 & 8387159 & 66 & 97 \\
\hline 5843745 & 8387159 & 63 & 96 \\
\hline 5843745 & 11697701 & 62 & 118 \\
\hline 5843745 & 8387159 & 68 & 94 \\
\hline 5843745 & 8387159 & 62 & 97 \\
\hline 5843745 & 11697701 & 65 & 103 \\
\hline 5843745 & 8387159 & 64 & 98 \\
\hline 5843745 & 8387159 & 66 & 96 \\
\hline 5843745 & 8387159 & 68 & 94 \\
\hline 5843745 & 8387159 & 68 & 97 \\
\hline 5843745 & 8387159 & 68 & 98 \\
\hline 5843745 & 8387159 & 69 & 94 \\
\hline 5843745 & 8387159 & 69 & 96 \\
\hline 5843745 & 8387159 & 64 & 98 \\
\hline 5843745 & 8387159 & 69 & 97 \\
\hline 5843745 & 8387159 & 70 & 96 \\
\hline 5843745 & 8387159 & 72 & 98 \\
\hline 5843745 & 11697701 & 75 & 103 \\
\hline 5843745 & 8387159 & 73 & 96 \\
\hline 5843745 & 8387159 & 70 & 96 \\
\hline 5843745 & 8387159 & 66 & 96 \\
\hline 5843745 & 8387159 & 72 & 97 \\
\hline 5843745 & 11697701 & 71 & 106 \\
\hline 5843745 & 11697701 & 68 & 114 \\
\hline 5843745 & 11697701 & 71 & 99 \\
\hline 5843745 & 8387159 & 66 & 97 \\
\hline
\end{tabular}




\begin{tabular}{|c|c|c|c|}
\hline 5843745 & 8387159 & 69 & 96 \\
\hline 5843745 & 11697701 & 66 & 110 \\
\hline 5843745 & 8387159 & 62 & 96 \\
\hline 5843745 & 11697701 & 72 & 114 \\
\hline 5843745 & 8387159 & 74 & 96 \\
\hline 5843745 & 11697701 & 69 & 121 \\
\hline 5843745 & 8387159 & 71 & 96 \\
\hline 5843745 & 8387159 & 71 & 97 \\
\hline 5843745 & 8387159 & 69 & 93 \\
\hline 5843745 & 8387159 & 79 & 96 \\
\hline 5843745 & 8387159 & 70 & 96 \\
\hline 5843745 & 8387159 & 73 & 95 \\
\hline 5843745 & 8387159 & 75 & 97 \\
\hline 5843745 & 8387159 & 70 & 95 \\
\hline 5843745 & 8387159 & 59 & 96 \\
\hline 5843745 & 8387159 & 73 & 97 \\
\hline 5843745 & 8387159 & 75 & 97 \\
\hline 5843745 & 8387159 & 66 & 97 \\
\hline 5843745 & 11697701 & 75 & 106 \\
\hline 5843745 & 8387159 & 69 & 96 \\
\hline 5843745 & 8387159 & 75 & 97 \\
\hline 5843745 & 8387159 & 70 & 95 \\
\hline 5843745 & 8387159 & 67 & 95 \\
\hline 5843745 & 8387159 & 80 & 96 \\
\hline 5843745 & 8387159 & 72 & 95 \\
\hline 5843745 & 8387159 & 72 & 97 \\
\hline 5843745 & 8387159 & 74 & 96 \\
\hline 5843745 & 8387159 & 73 & 96 \\
\hline 5843745 & 8387159 & 77 & 95 \\
\hline 5843745 & 11697701 & 68 & 110 \\
\hline 5843745 & 11697701 & 73 & 126 \\
\hline 5843745 & 11697701 & 67 & 110 \\
\hline 5843745 & 8387159 & 68 & 98 \\
\hline 5843745 & 8387159 & 67 & 97 \\
\hline 5843745 & 8387159 & 76 & 97 \\
\hline 5843745 & 8387159 & 76 & 96 \\
\hline 5843745 & 11697701 & 70 & 106 \\
\hline 5843745 & 11697701 & 62 & 101 \\
\hline 5843745 & 8387159 & 70 & 98 \\
\hline 5843745 & 11697701 & 72 & 108 \\
\hline 5843745 & 11697701 & 63 & 103 \\
\hline 5843745 & 11697701 & 72 & 103 \\
\hline 5843745 & 8387159 & 77 & 98 \\
\hline
\end{tabular}




\begin{tabular}{|c|c|c|c|}
\hline 5843745 & 11697701 & 79 & 122 \\
\hline 5843745 & 8387159 & 70 & 98 \\
\hline 5843745 & 11697701 & 76 & 108 \\
\hline 5843745 & 11697701 & 77 & 105 \\
\hline 5843745 & 11697701 & 64 & 108 \\
\hline 5843745 & 11697701 & 63 & 114 \\
\hline 5843745 & 11697701 & 73 & 104 \\
\hline 5843745 & 11697701 & 73 & 120 \\
\hline 5843745 & 11697701 & 66 & 120 \\
\hline 5843745 & 11697701 & 70 & 116 \\
\hline 5843745 & 11697701 & 61 & 134 \\
\hline 5843745 & 11697701 & 63 & 117 \\
\hline 5843745 & 11697701 & 65 & 114 \\
\hline 5843745 & 11697701 & 70 & 105 \\
\hline 5843745 & 11697701 & 77 & 106 \\
\hline 5843745 & 11697701 & 66 & 108 \\
\hline 5843745 & 11697701 & 72 & 112 \\
\hline 5843745 & 11697701 & 72 & 104 \\
\hline 5843745 & 11697701 & 64 & 105 \\
\hline 5843745 & 11697701 & 73 & 114 \\
\hline 5843745 & 11697701 & 70 & 111 \\
\hline 5843745 & 11697701 & 68 & 105 \\
\hline 5843745 & 11697701 & 74 & 114 \\
\hline 5843745 & 11697701 & 58 & 129 \\
\hline 5843745 & 11697701 & 54 & 122 \\
\hline 5843745 & 11697701 & 65 & 115 \\
\hline 5843745 & 11697701 & 58 & 121 \\
\hline 5843745 & 11697701 & 69 & 117 \\
\hline 5843745 & 11697701 & 60 & 123 \\
\hline 5843745 & 11697701 & 74 & 117 \\
\hline 5843745 & 11697701 & 69 & 114 \\
\hline 5843745 & 11697701 & 69 & 119 \\
\hline 5843745 & 11697701 & 71 & 118 \\
\hline 5843745 & 11697701 & 73 & 123 \\
\hline 5843745 & 11697701 & 68 & 117 \\
\hline 5843745 & 11697701 & 78 & 117 \\
\hline 5843745 & 11697701 & 76 & 116 \\
\hline 5843745 & 11697701 & 76 & 111 \\
\hline 5843745 & 11697701 & 78 & 111 \\
\hline 5843745 & 11697701 & 71 & 114 \\
\hline 5843745 & 11697701 & 64 & 123 \\
\hline 5843745 & 11697701 & 66 & 123 \\
\hline 5843745 & 11697701 & 44 & 117 \\
\hline
\end{tabular}




\begin{tabular}{|c|c|c|c|}
\hline 5843745 & 11697701 & 61 & 118 \\
\hline 5843745 & 11697701 & 71 & 116 \\
\hline 5843745 & 11697701 & 44 & 115 \\
\hline 5843745 & 11697701 & 63 & 120 \\
\hline 5843745 & 11697701 & 62 & 108 \\
\hline 5843745 & 11697701 & 69 & 123 \\
\hline 5843745 & 11697701 & 62 & 119 \\
\hline 5843745 & 11697701 & 58 & 112 \\
\hline 5843745 & 11697701 & 60 & 118 \\
\hline 5843745 & 11697701 & 63 & 116 \\
\hline 5843745 & 11697701 & 67 & 116 \\
\hline 5843745 & 11697701 & 63 & 126 \\
\hline 5843745 & 11697701 & 59 & 125 \\
\hline 5843745 & 11697701 & 56 & 122 \\
\hline 5843745 & 11697701 & 60 & 120 \\
\hline 5843745 & 11697701 & 63 & 122 \\
\hline 5843745 & 11697701 & 62 & 124 \\
\hline 5843745 & 11697701 & 75 & 120 \\
\hline 5843745 & 11697701 & 64 & 114 \\
\hline 5843745 & 11697701 & 55 & 111 \\
\hline 5843745 & 11697701 & 58 & 119 \\
\hline 5843745 & 11697701 & 71 & 119 \\
\hline 5843745 & 11697701 & 76 & 112 \\
\hline 5056555 & 11697701 & 27 & 114 \\
\hline 5843745 & 11697701 & 64 & 121 \\
\hline 5843745 & 11697701 & 71 & 118 \\
\hline 5843745 & 11697701 & 58 & 121 \\
\hline 5843745 & 11697701 & 66 & 114 \\
\hline 5843745 & 11697701 & 67 & 122 \\
\hline 5843745 & 11697701 & 71 & 116 \\
\hline 5843745 & 11697701 & 71 & 113 \\
\hline 5843745 & 11697701 & 58 & 122 \\
\hline 5843745 & 11697701 & 67 & 118 \\
\hline 5843745 & 11697701 & 75 & 122 \\
\hline 5843745 & 11697701 & 62 & 119 \\
\hline 5843745 & 11697701 & 67 & 115 \\
\hline 5843745 & 11697701 & 67 & 117 \\
\hline 5843745 & 11697701 & 67 & 115 \\
\hline 5843745 & 11697701 & 72 & 115 \\
\hline 5843745 & 11697701 & 47 & 114 \\
\hline 5843745 & 11697701 & 50 & 119 \\
\hline 5843745 & 11697701 & 71 & 118 \\
\hline 5843745 & 11697701 & 67 & 113 \\
\hline
\end{tabular}




\begin{tabular}{|c|c|c|c|}
\hline 5843745 & 11697701 & 70 & 122 \\
\hline 5843745 & 11697701 & 51 & 114 \\
\hline 5843745 & 11697701 & 63 & 117 \\
\hline 5843745 & 11697701 & 67 & 118 \\
\hline 5843745 & 11697701 & 72 & 114 \\
\hline 5843745 & 11697701 & 65 & 119 \\
\hline 5843745 & 11697701 & 76 & 120 \\
\hline 5843745 & 11697701 & 73 & 119 \\
\hline 5843745 & 11697701 & 70 & 115 \\
\hline 5843745 & 11697701 & 76 & 119 \\
\hline 5843745 & 11697701 & 77 & 126 \\
\hline 5843745 & 11697701 & 74 & 121 \\
\hline 5843745 & 11697701 & 78 & 117 \\
\hline 5843745 & 11697701 & 75 & 118 \\
\hline 5843745 & 11697701 & 66 & 116 \\
\hline 5843745 & 11697701 & 70 & 121 \\
\hline 5843745 & 11697701 & 62 & 110 \\
\hline 5843745 & 11697701 & 73 & 124 \\
\hline 5843745 & 11697701 & 69 & 115 \\
\hline 5843745 & 11697701 & 70 & 119 \\
\hline 5843745 & 11697701 & 76 & 115 \\
\hline 5843745 & 11697701 & 71 & 132 \\
\hline 5843745 & 11697701 & 70 & 125 \\
\hline 5843745 & 11697701 & 81 & 132 \\
\hline 5843745 & 11697701 & 80 & 120 \\
\hline 5843745 & 11697701 & 80 & 121 \\
\hline 5843745 & 11697701 & 74 & 119 \\
\hline 5843745 & 11697701 & 67 & 113 \\
\hline 5843745 & 11697701 & 74 & 120 \\
\hline 5843745 & 11697701 & 72 & 120 \\
\hline 5843745 & 11697701 & 85 & 120 \\
\hline 5843745 & 11697701 & 47 & 114 \\
\hline 5843745 & 11697701 & 79 & 122 \\
\hline 5843745 & 11697701 & 87 & 109 \\
\hline 5843745 & 11697701 & 84 & 116 \\
\hline 5843745 & 11697701 & 85 & 117 \\
\hline 5843745 & 11697701 & 82 & 121 \\
\hline 5843745 & 11697701 & 76 & 113 \\
\hline 5843745 & 11697701 & 75 & 111 \\
\hline 5843745 & 11697701 & 79 & 124 \\
\hline 5843745 & 11697701 & 83 & 116 \\
\hline 5843745 & 11697701 & 71 & 117 \\
\hline 5843745 & 11697701 & 81 & 116 \\
\hline
\end{tabular}




\begin{tabular}{|c|c|c|c|}
\hline 5843745 & 11697701 & 68 & 118 \\
\hline 5843745 & 11697701 & 75 & 117 \\
\hline 5843745 & 11697701 & 77 & 117 \\
\hline 5843745 & 11697701 & 83 & 114 \\
\hline 5843745 & 11697701 & 59 & 114 \\
\hline 5843745 & 11697701 & 73 & 116 \\
\hline 5843745 & 11697701 & 60 & 113 \\
\hline 5843745 & 11697701 & 62 & 116 \\
\hline 5843745 & 11697701 & 63 & 113 \\
\hline 5843745 & 11697701 & 69 & 112 \\
\hline 5843745 & 11697701 & 85 & 116 \\
\hline 5843745 & 11697701 & 80 & 118 \\
\hline 5843745 & 11697701 & 81 & 118 \\
\hline 5843745 & 11697701 & 76 & 122 \\
\hline 5843745 & 11697701 & 72 & 127 \\
\hline 5843745 & 11697701 & 66 & 115 \\
\hline 5843745 & 11697701 & 73 & 116 \\
\hline 5843745 & 11697701 & 81 & 116 \\
\hline 5843745 & 11697701 & 81 & 115 \\
\hline 5843745 & 11697701 & 72 & 121 \\
\hline 5843745 & 11697701 & 80 & 118 \\
\hline 5843745 & 11697701 & 65 & 117 \\
\hline 5843745 & 11697701 & 76 & 120 \\
\hline 5843745 & 11697701 & 71 & 112 \\
\hline 5843745 & 11697701 & 78 & 124 \\
\hline 5843745 & 11697701 & 82 & 112 \\
\hline 5843745 & 11697701 & 69 & 113 \\
\hline 5843745 & 11697701 & 78 & 116 \\
\hline 5843745 & 11697701 & 71 & 115 \\
\hline 5843745 & 11697701 & 79 & 119 \\
\hline 5843745 & 11697701 & 73 & 116 \\
\hline 5843745 & 11697701 & 77 & 114 \\
\hline 5843745 & 11697701 & 76 & 115 \\
\hline 5843745 & 11697701 & 63 & 124 \\
\hline 5843745 & 11697701 & 83 & 116 \\
\hline 5843745 & 11697701 & 82 & 127 \\
\hline 5843745 & 11697701 & 72 & 119 \\
\hline 5843745 & 11697701 & 68 & 118 \\
\hline 5843745 & 11697701 & 74 & 116 \\
\hline 5843745 & 11697701 & 67 & 119 \\
\hline 5843745 & 11697701 & 73 & 116 \\
\hline 5843745 & 11697701 & 65 & 115 \\
\hline 5843745 & 11697701 & 73 & 124 \\
\hline
\end{tabular}




\begin{tabular}{|c|c|c|c|}
\hline 5843745 & 11697701 & 76 & 116 \\
\hline 5843745 & 11697701 & 77 & 117 \\
\hline 5843745 & 11697701 & 65 & 122 \\
\hline 5843745 & 11697701 & 75 & 125 \\
\hline 5843745 & 11697701 & 81 & 120 \\
\hline 5843745 & 11697701 & 68 & 116 \\
\hline 5843745 & 11697701 & 70 & 117 \\
\hline 5843745 & 11697701 & 90 & 111 \\
\hline 7017142 & 11697701 & 92 & 116 \\
\hline 5843745 & 11697701 & 89 & 116 \\
\hline 5843745 & 11697701 & 85 & 117 \\
\hline 5843745 & 11697701 & 85 & 118 \\
\hline 5843745 & 11697701 & 86 & 113 \\
\hline 7017142 & 11697701 & 92 & 115 \\
\hline 5843745 & 11697701 & 84 & 115 \\
\hline 5843745 & 11697701 & 83 & 117 \\
\hline 7017142 & 11697701 & 92 & 110 \\
\hline 5843745 & 11697701 & 69 & 117 \\
\hline 5843745 & 11697701 & 89 & 118 \\
\hline 7017142 & 11697701 & 92 & 114 \\
\hline 5843745 & 11697701 & 81 & 119 \\
\hline 5843745 & 11697701 & 68 & 116 \\
\hline 5843745 & 11697701 & 76 & 113 \\
\hline 5843745 & 11697701 & 90 & 117 \\
\hline 5843745 & 11697701 & 73 & 124 \\
\hline 5843745 & 11697701 & 81 & 120 \\
\hline 5843745 & 11697701 & 76 & 115 \\
\hline 5843745 & 11697701 & 79 & 120 \\
\hline 5843745 & 11697701 & 82 & 122 \\
\hline 5843745 & 11697701 & 76 & 117 \\
\hline 5843745 & 11697701 & 79 & 113 \\
\hline 5843745 & 11697701 & 84 & 115 \\
\hline 5843745 & 11697701 & 80 & 116 \\
\hline 5843745 & 11697701 & 74 & 118 \\
\hline 5843745 & 11697701 & 76 & 119 \\
\hline 5843745 & 11697701 & 65 & 116 \\
\hline 5843745 & 11697701 & 76 & 121 \\
\hline 5843745 & 11697701 & 85 & 117 \\
\hline 5843745 & 11697701 & 71 & 118 \\
\hline 5843745 & 11697701 & 88 & 119 \\
\hline 5843745 & 11697701 & 77 & 116 \\
\hline 5056555 & 11697701 & 12 & 120 \\
\hline 7017142 & 11697701 & 92 & 112 \\
\hline
\end{tabular}




\begin{tabular}{|c|c|c|c|}
\hline 5843745 & 11697701 & 77 & 118 \\
\hline 5843745 & 11697701 & 78 & 122 \\
\hline 5843745 & 11697701 & 70 & 116 \\
\hline 5843745 & 11697701 & 87 & 116 \\
\hline 5843745 & 11697701 & 87 & 118 \\
\hline 5843745 & 11697701 & 80 & 111 \\
\hline 7017142 & 11697701 & 91 & 115 \\
\hline 5843745 & 11697701 & 68 & 121 \\
\hline 5843745 & 11697701 & 76 & 113 \\
\hline 5843745 & 11697701 & 86 & 116 \\
\hline 5843745 & 11697701 & 72 & 121 \\
\hline 5843745 & 11697701 & 73 & 117 \\
\hline 5843745 & 11697701 & 88 & 116 \\
\hline 5843745 & 11697701 & 75 & 118 \\
\hline 5843745 & 11697701 & 80 & 119 \\
\hline 5843745 & 11697701 & 72 & 119 \\
\hline 5843745 & 11697701 & 78 & 117 \\
\hline 5843745 & 11697701 & 65 & 116 \\
\hline 5843745 & 11697701 & 72 & 119 \\
\hline 5843745 & 11697701 & 87 & 115 \\
\hline 5843745 & 11697701 & 64 & 122 \\
\hline 5843745 & 11697701 & 86 & 119 \\
\hline 7017142 & 11697701 & 93 & 118 \\
\hline 5843745 & 11697701 & 80 & 119 \\
\hline 7017142 & 11697701 & 92 & 117 \\
\hline 7017142 & 11697701 & 92 & 117 \\
\hline 5843745 & 11697701 & 68 & 119 \\
\hline 7017142 & 11697701 & 94 & 115 \\
\hline 7017142 & 11697701 & 93 & 120 \\
\hline 5843745 & 11697701 & 73 & 123 \\
\hline 5843745 & 11697701 & 70 & 119 \\
\hline 7017142 & 11697701 & 92 & 123 \\
\hline 5843745 & 14607591 & 80 & 152 \\
\hline 5843745 & 11697701 & 60 & 117 \\
\hline 7017142 & 11697701 & 95 & 109 \\
\hline 7017142 & 11697701 & 92 & 118 \\
\hline 7017142 & 11697701 & 93 & 115 \\
\hline 7017142 & 11697701 & 94 & 114 \\
\hline 7017142 & 11697701 & 95 & 115 \\
\hline 7017142 & 11697701 & 92 & 118 \\
\hline 7017142 & 11697701 & 92 & 115 \\
\hline 7017142 & 11697701 & 92 & 113 \\
\hline 5843745 & 11697701 & 67 & 110 \\
\hline
\end{tabular}




\begin{tabular}{|c|c|c|c|}
\hline 5843745 & 11697701 & 71 & 110 \\
\hline 7017142 & 11697701 & 91 & 120 \\
\hline 7017142 & 11697701 & 93 & 118 \\
\hline 5843745 & 11697701 & 90 & 115 \\
\hline 7017142 & 11697701 & 92 & 119 \\
\hline 7017142 & 11697701 & 93 & 114 \\
\hline 7017142 & 11697701 & 95 & 116 \\
\hline 7017142 & 11697701 & 93 & 117 \\
\hline 5843745 & 11697701 & 89 & 117 \\
\hline 5843745 & 11697701 & 71 & 117 \\
\hline 7017142 & 11697701 & 92 & 116 \\
\hline 5843745 & 11697701 & 89 & 116 \\
\hline 5843745 & 11697701 & 64 & 115 \\
\hline 5843745 & 11697701 & 73 & 116 \\
\hline 5843745 & 11697701 & 67 & 118 \\
\hline 7017142 & 11697701 & 92 & 117 \\
\hline 7017142 & 11697701 & 93 & 119 \\
\hline 7017142 & 11697701 & 93 & 114 \\
\hline 7017142 & 11697701 & 92 & 115 \\
\hline 7017142 & 11697701 & 93 & 117 \\
\hline 5843745 & 11697701 & 50 & 118 \\
\hline 5843745 & 11697701 & 84 & 122 \\
\hline 7017142 & 11697701 & 94 & 113 \\
\hline 7017142 & 11697701 & 91 & 112 \\
\hline 7017142 & 11697701 & 93 & 113 \\
\hline 7017142 & 11697701 & 92 & 116 \\
\hline 5843745 & 11697701 & 71 & 117 \\
\hline 7017142 & 11697701 & 93 & 115 \\
\hline 5843745 & 11697701 & 89 & 117 \\
\hline 5843745 & 11697701 & 78 & 133 \\
\hline 7017142 & 11697701 & 93 & 117 \\
\hline 7017142 & 11697701 & 92 & 111 \\
\hline 5843745 & 11697701 & 85 & 118 \\
\hline 7017142 & 11697701 & 94 & 113 \\
\hline 7017142 & 11697701 & 93 & 114 \\
\hline 7017142 & 11697701 & 93 & 115 \\
\hline 7017142 & 11697701 & 91 & 122 \\
\hline 7017142 & 11697701 & 94 & 113 \\
\hline 5843745 & 11697701 & 86 & 112 \\
\hline 7017142 & 11697701 & 93 & 121 \\
\hline 7017142 & 11697701 & 93 & 120 \\
\hline 7017142 & 11697701 & 93 & 120 \\
\hline 5843745 & 11697701 & 89 & 120 \\
\hline
\end{tabular}




\begin{tabular}{|c|c|c|c|}
\hline 7017142 & 11697701 & 93 & 115 \\
\hline 7017142 & 11697701 & 94 & 115 \\
\hline 7017142 & 11697701 & 93 & 114 \\
\hline 7017142 & 11697701 & 93 & 112 \\
\hline 7017142 & 11697701 & 93 & 120 \\
\hline 7017142 & 11697701 & 92 & 118 \\
\hline 7017142 & 11697701 & 93 & 115 \\
\hline 7017142 & 11697701 & 93 & 114 \\
\hline 7017142 & 11697701 & 91 & 116 \\
\hline 7017142 & 11697701 & 94 & 116 \\
\hline 7017142 & 11697701 & 92 & 117 \\
\hline 7017142 & 11697701 & 94 & 120 \\
\hline 7017142 & 11697701 & 93 & 119 \\
\hline 7017142 & 11697701 & 94 & 115 \\
\hline 5843745 & 11697701 & 87 & 112 \\
\hline 7017142 & 11697701 & 94 & 114 \\
\hline 7017142 & 11697701 & 92 & 117 \\
\hline 7017142 & 11697701 & 94 & 113 \\
\hline 7017142 & 11697701 & 92 & 116 \\
\hline 7017142 & 11697701 & 92 & 117 \\
\hline 7017142 & 11697701 & 92 & 119 \\
\hline 5843745 & 11697701 & 86 & 119 \\
\hline 5843745 & 11697701 & 83 & 123 \\
\hline 7017142 & 11697701 & 93 & 117 \\
\hline 7017142 & 11697701 & 93 & 120 \\
\hline 7017142 & 11697701 & 92 & 118 \\
\hline 5843745 & 11697701 & 89 & 121 \\
\hline 7017142 & 11697701 & 92 & 118 \\
\hline 7017142 & 11697701 & 92 & 118 \\
\hline 7017142 & 11697701 & 92 & 119 \\
\hline 7017142 & 11697701 & 94 & 118 \\
\hline 7017142 & 11697701 & 92 & 120 \\
\hline 7017142 & 11697701 & 92 & 126 \\
\hline 7017142 & 11697701 & 92 & 119 \\
\hline 5843745 & 11697701 & 72 & 124 \\
\hline 5843745 & 11697701 & 64 & 124 \\
\hline 7017142 & 11697701 & 92 & 118 \\
\hline 5843745 & 11697701 & 64 & 117 \\
\hline 5843745 & 11697701 & 81 & 118 \\
\hline 7017142 & 11697701 & 91 & 120 \\
\hline 7017142 & 11697701 & 92 & 120 \\
\hline 7017142 & 11697701 & 93 & 120 \\
\hline 7017142 & 11697701 & 94 & 119 \\
\hline
\end{tabular}




\begin{tabular}{|c|c|c|c|}
\hline 7017142 & 11697701 & 92 & 119 \\
\hline 7017142 & 11697701 & 93 & 119 \\
\hline 7017142 & 11697701 & 93 & 120 \\
\hline 7017142 & 11697701 & 91 & 140 \\
\hline 5843745 & 11697701 & 76 & 124 \\
\hline 7017142 & 11697701 & 93 & 119 \\
\hline 5843745 & 11697701 & 60 & 122 \\
\hline 7017142 & 11697701 & 93 & 119 \\
\hline 5843745 & 11697701 & 86 & 119 \\
\hline 7017142 & 11697701 & 93 & 120 \\
\hline 5843745 & 11697701 & 79 & 122 \\
\hline 7017142 & 11697701 & 94 & 118 \\
\hline 5843745 & 11697701 & 69 & 119 \\
\hline 5843745 & 11697701 & 88 & 123 \\
\hline 5843745 & 11697701 & 73 & 118 \\
\hline 5843745 & 11697701 & 61 & 121 \\
\hline 7017142 & 11697701 & 94 & 123 \\
\hline 7017142 & 11697701 & 92 & 119 \\
\hline 7017142 & 11697701 & 92 & 119 \\
\hline 5843745 & 11697701 & 86 & 120 \\
\hline 5843745 & 11697701 & 80 & 124 \\
\hline 7017142 & 11697701 & 94 & 126 \\
\hline 5843745 & 11697701 & 87 & 127 \\
\hline 7017142 & 11697701 & 92 & 120 \\
\hline 5843745 & 11697701 & 82 & 119 \\
\hline 7017142 & 11697701 & 92 & 123 \\
\hline 7017142 & 11697701 & 92 & 122 \\
\hline 7017142 & 11697701 & 93 & 125 \\
\hline 7017142 & 11697701 & 93 & 123 \\
\hline 7017142 & 11697701 & 93 & 120 \\
\hline 7017142 & 11697701 & 93 & 120 \\
\hline 7017142 & 11697701 & 91 & 120 \\
\hline 5843745 & 11697701 & 86 & 120 \\
\hline 7017142 & 11697701 & 92 & 125 \\
\hline 7017142 & 11697701 & 92 & 120 \\
\hline 5843745 & 11697701 & 85 & 121 \\
\hline 5843745 & 11697701 & 68 & 130 \\
\hline 5843745 & 11697701 & 76 & 126 \\
\hline 7017142 & 11697701 & 93 & 127 \\
\hline 5843745 & 11697701 & 62 & 122 \\
\hline 5843745 & 11697701 & 77 & 121 \\
\hline 7017142 & 11697701 & 93 & 123 \\
\hline 7017142 & 11697701 & 93 & 121 \\
\hline
\end{tabular}




\begin{tabular}{|c|c|c|c|}
\hline 7017142 & 11697701 & 93 & 122 \\
\hline 5843745 & 11697701 & 70 & 125 \\
\hline 5843745 & 11697701 & 80 & 124 \\
\hline 7017142 & 11697701 & 94 & 125 \\
\hline 5843745 & 14607591 & 86 & 157 \\
\hline 7017142 & 11697701 & 91 & 123 \\
\hline 5843745 & 11697701 & 72 & 121 \\
\hline 5843745 & 11697701 & 87 & 126 \\
\hline 7017142 & 11697701 & 93 & 121 \\
\hline 5843745 & 11697701 & 73 & 124 \\
\hline 7017142 & 11697701 & 92 & 124 \\
\hline 5843745 & 11697701 & 79 & 125 \\
\hline 5843745 & 11697701 & 70 & 122 \\
\hline 5843745 & 11697701 & 72 & 123 \\
\hline 5843745 & 11697701 & 87 & 129 \\
\hline 7017142 & 11697701 & 92 & 124 \\
\hline 5843745 & 11697701 & 54 & 129 \\
\hline 5843745 & 11697701 & 74 & 125 \\
\hline 5843745 & 11697701 & 75 & 124 \\
\hline 7017142 & 11697701 & 91 & 123 \\
\hline 5843745 & 11697701 & 78 & 125 \\
\hline 7017142 & 11697701 & 92 & 122 \\
\hline 5843745 & 11697701 & 82 & 122 \\
\hline 5843745 & 11697701 & 61 & 124 \\
\hline 7017142 & 11697701 & 93 & 124 \\
\hline 5843745 & 11697701 & 74 & 129 \\
\hline 5843745 & 11697701 & 72 & 127 \\
\hline 7017142 & 11697701 & 92 & 124 \\
\hline 5843745 & 11697701 & 72 & 125 \\
\hline 5843745 & 11697701 & 78 & 123 \\
\hline 7017142 & 11697701 & 92 & 123 \\
\hline 5843745 & 11697701 & 68 & 130 \\
\hline 5843745 & 11697701 & 84 & 124 \\
\hline 5843745 & 11697701 & 75 & 128 \\
\hline 5843745 & 11697701 & 87 & 134 \\
\hline 7017142 & 11697701 & 91 & 133 \\
\hline 7017142 & 11697701 & 92 & 134 \\
\hline 5843745 & 11697701 & 77 & 139 \\
\hline 7017142 & 14607591 & 92 & 156 \\
\hline 8387159 & 14607591 & 110 & 153 \\
\hline 8387159 & 18471725 & 101 & 190 \\
\hline 8387159 & 14607591 & 107 & 157 \\
\hline 8387159 & 14607591 & 110 & 158 \\
\hline
\end{tabular}




\begin{tabular}{|c|c|c|c|}
\hline 8387159 & 14607591 & 117 & 156 \\
\hline 8387159 & 14607591 & 113 & 153 \\
\hline 8387159 & 14607591 & 116 & 155 \\
\hline 8387159 & 16771127 & 118 & 164 \\
\hline 8387159 & 14607591 & 123 & 159 \\
\hline 8387159 & 14607591 & 118 & 161 \\
\hline 8387159 & 14607591 & 120 & 158 \\
\hline 8387159 & 14607591 & 128 & 161 \\
\hline 8387159 & 14607591 & 121 & 154 \\
\hline 8387159 & 14607591 & 122 & 158 \\
\hline 8387159 & 14607591 & 113 & 154 \\
\hline 8387159 & 14607591 & 124 & 152 \\
\hline 8387159 & 12861882 & 136 & 147 \\
\hline 8387159 & 14607591 & 129 & 149 \\
\hline 8387159 & 14607591 & 118 & 160 \\
\hline 8387159 & 12861882 & 133 & 147 \\
\hline 8387159 & 14607591 & 127 & 152 \\
\hline 8387159 & 14607591 & 122 & 151 \\
\hline 8387159 & 14607591 & 122 & 154 \\
\hline 8387159 & 14607591 & 118 & 154 \\
\hline 8387159 & 16771127 & 129 & 166 \\
\hline 8387159 & 18471725 & 118 & 201 \\
\hline 8387159 & 14607591 & 129 & 154 \\
\hline 8387159 & 14607591 & 125 & 153 \\
\hline 8387159 & 18471725 & 121 & 197 \\
\hline 8387159 & 16771127 & 123 & 167 \\
\hline 8387159 & 14607591 & 126 & 157 \\
\hline 8387159 & 16771127 & 125 & 173 \\
\hline 8387159 & 14607591 & 128 & 153 \\
\hline 8387159 & 14607591 & 127 & 160 \\
\hline 8387159 & 14607591 & 125 & 157 \\
\hline 8387159 & 14607591 & 128 & 155 \\
\hline 8387159 & 16771127 & 121 & 176 \\
\hline 8387159 & 14607591 & 136 & 151 \\
\hline 8387159 & 11934340 & 136 & 146 \\
\hline 8387159 & 14607591 & 127 & 160 \\
\hline 8387159 & 14607591 & 120 & 161 \\
\hline 8387159 & 14607591 & 129 & 160 \\
\hline 8387159 & 14607591 & 121 & 155 \\
\hline 8387159 & 14607591 & 126 & 156 \\
\hline 8387159 & 14607591 & 129 & 157 \\
\hline 8387159 & 14607591 & 126 & 154 \\
\hline 8387159 & 14607591 & 123 & 153 \\
\hline
\end{tabular}




\begin{tabular}{|c|c|c|c|c|}
\hline 8387159 & 14607591 & 127 & 155 & 28 \\
\hline 8387159 & 14607591 & 101 & 156 & 55 \\
\hline 7017142 & 16771127 & 93 & 168 & 75 \\
\hline 8387159 & 14607591 & 128 & 158 & 30 \\
\hline 8387159 & 14607591 & 129 & 159 & 30 \\
\hline 11934340 & 14607591 & 147 & 156 & 9 \\
\hline 8387159 & 14607591 & 134 & 160 & 26 \\
\hline 8387159 & 14607591 & 120 & 162 & 42 \\
\hline 8387159 & 16771127 & 101 & 172 & 71 \\
\hline 8387159 & 14607591 & 133 & 159 & 26 \\
\hline 8387159 & 16771127 & 131 & 172 & 41 \\
\hline 8387159 & 14607591 & 129 & 159 & 30 \\
\hline 8387159 & 16771127 & 123 & 171 & 48 \\
\hline 8387159 & 16771127 & 107 & 172 & 65 \\
\hline 8387159 & 18471725 & 131 & 193 & 62 \\
\hline 8387159 & 16771127 & 132 & 166 & 34 \\
\hline 8387159 & 16771127 & 126 & 168 & 42 \\
\hline 8387159 & 18471725 & 116 & 198 & 82 \\
\hline 8387159 & 16771127 & 128 & 172 & 44 \\
\hline 8387159 & 16771127 & 133 & 168 & 35 \\
\hline 5843745 & 18471725 & 74 & 194 & 120 \\
\hline 8387159 & 18471725 & 127 & 201 & 74 \\
\hline 8387159 & 16771127 & 126 & 172 & 46 \\
\hline 8387159 & 16771127 & 121 & 173 & 52 \\
\hline 8387159 & 18471725 & 118 & 192 & 74 \\
\hline 8387159 & 18471725 & 125 & 197 & 72 \\
\hline 8387159 & 16771127 & 124 & 168 & 44 \\
\hline 7017142 & 18471725 & 95 & 196 & 101 \\
\hline 8387159 & 18471725 & 112 & 193 & 81 \\
\hline 8387159 & 21311378 & 114 & 206 & 92 \\
\hline 8387159 & 18471725 & 126 & 187 & 61 \\
\hline 11934340 & 16771127 & 148 & 177 & 29 \\
\hline 12861882 & 16771127 & 150 & 171 & 21 \\
\hline 8387159 & 18471725 & 125 & 187 & 62 \\
\hline 11934340 & 16771127 & 148 & 177 & 29 \\
\hline 12861882 & 16771127 & 151 & 177 & 26 \\
\hline 12861882 & 16771127 & 150 & 172 & 22 \\
\hline 11934340 & 16771127 & 147 & 176 & 29 \\
\hline 8387159 & 16771127 & 133 & 170 & 37 \\
\hline 8387159 & 16771127 & 135 & 176 & 41 \\
\hline 8387159 & 16771127 & 125 & 175 & 50 \\
\hline 12861882 & 18471725 & 152 & 187 & 35 \\
\hline 12861882 & 16771127 & 154 & 179 & 25 \\
\hline
\end{tabular}




\begin{tabular}{|c|c|c|c|}
\hline 12861882 & 16771127 & 151 & 175 \\
\hline 12861882 & 16771127 & 157 & 171 \\
\hline 12861882 & 16771127 & 153 & 171 \\
\hline 12861882 & 16771127 & 154 & 179 \\
\hline 12861882 & 16771127 & 153 & 174 \\
\hline 12861882 & 21311378 & 156 & 217 \\
\hline 12861882 & 18471725 & 152 & 200 \\
\hline 12861882 & 18471725 & 153 & 183 \\
\hline 12861882 & 18471725 & 153 & 184 \\
\hline 12861882 & 18471725 & 151 & 194 \\
\hline 12861882 & 18471725 & 150 & 190 \\
\hline 12861882 & 21311378 & 153 & 215 \\
\hline 12861882 & 16771127 & 153 & 172 \\
\hline 12861882 & 18471725 & 151 & 198 \\
\hline 12861882 & 18471725 & 157 & 186 \\
\hline 12861882 & 18471725 & 157 & 184 \\
\hline 12861882 & 18471725 & 154 & 191 \\
\hline 12861882 & 16771127 & 148 & 175 \\
\hline 12861882 & 18471725 & 156 & 191 \\
\hline 12861882 & 18471725 & 157 & 192 \\
\hline 12861882 & 18471725 & 156 & 196 \\
\hline 12861882 & 18471725 & 156 & 193 \\
\hline 12861882 & 18471725 & 156 & 189 \\
\hline 12861882 & 18471725 & 157 & 190 \\
\hline 12861882 & 18471725 & 155 & 199 \\
\hline 12861882 & 18471725 & 151 & 194 \\
\hline 12861882 & 18471725 & 153 & 200 \\
\hline 8387159 & 18471725 & 123 & 189 \\
\hline 12861882 & 18471725 & 158 & 193 \\
\hline 12861882 & 18471725 & 155 & 193 \\
\hline 12861882 & 18471725 & 156 & 194 \\
\hline 12861882 & 18471725 & 160 & 186 \\
\hline 12861882 & 18471725 & 152 & 199 \\
\hline 12861882 & 18471725 & 156 & 196 \\
\hline 12861882 & 18471725 & 162 & 190 \\
\hline 12861882 & 18471725 & 155 & 198 \\
\hline 14607591 & 18471725 & 165 & 192 \\
\hline 12861882 & 18471725 & 160 & 194 \\
\hline 12861882 & 18471725 & 156 & 192 \\
\hline 12861882 & 18471725 & 156 & 198 \\
\hline 12861882 & 18471725 & 159 & 191 \\
\hline 14607591 & 18471725 & 164 & 190 \\
\hline 12861882 & 18471725 & 157 & 202 \\
\hline
\end{tabular}




\begin{tabular}{|c|c|c|c|}
\hline 14607591 & 18471725 & 164 & 194 \\
\hline 12861882 & 18471725 & 154 & 193 \\
\hline 12861882 & 18471725 & 154 & 199 \\
\hline 14607591 & 18471725 & 168 & 192 \\
\hline 12861882 & 18471725 & 155 & 200 \\
\hline 12861882 & 18471725 & 160 & 199 \\
\hline 12861882 & 24018632 & 158 & 234 \\
\hline 14607591 & 21311378 & 167 & 208 \\
\hline 14607591 & 18471725 & 168 & 190 \\
\hline 14607591 & 18471725 & 169 & 193 \\
\hline 12861882 & 18471725 & 159 & 202 \\
\hline 14607591 & 18471725 & 170 & 195 \\
\hline 14607591 & 18471725 & 171 & 193 \\
\hline 14607591 & 18471725 & 167 & 201 \\
\hline 12861882 & 24018632 & 157 & 247 \\
\hline 14607591 & 21311378 & 164 & 208 \\
\hline 14607591 & 21311378 & 169 & 221 \\
\hline 12861882 & 24018632 & 155 & 233 \\
\hline 16771127 & 21311378 & 199 & 217 \\
\hline 16771127 & 21311378 & 198 & 216 \\
\hline 16771127 & 21311378 & 192 & 213 \\
\hline 16771127 & 24018632 & 185 & 238 \\
\hline 16771127 & 21311378 & 194 & 224 \\
\hline 16771127 & 21311378 & 193 & 221 \\
\hline 16771127 & 21311378 & 194 & 217 \\
\hline 16771127 & 21311378 & 190 & 213 \\
\hline 16771127 & 21311378 & 192 & 222 \\
\hline 16771127 & 21311378 & 194 & 217 \\
\hline 16771127 & 21311378 & 191 & 215 \\
\hline 16771127 & 21311378 & 191 & 222 \\
\hline 16771127 & 24018632 & 198 & 241 \\
\hline 16771127 & 21311378 & 195 & 222 \\
\hline 14607591 & 21311378 & 176 & 221 \\
\hline 16771127 & 24018632 & 186 & 250 \\
\hline 14607591 & 24018632 & 166 & 246 \\
\hline 16771127 & 24018632 & 183 & 243 \\
\hline 12861882 & 24018632 & 157 & 267 \\
\hline 12861882 & 24018632 & 157 & 253 \\
\hline 14607591 & 24018632 & 170 & 261 \\
\hline 19445503 & 24018632 & 211 & 249 \\
\hline 19445503 & 24018632 & 210 & 250 \\
\hline 19445503 & 24018632 & 204 & 249 \\
\hline 19445503 & 24018632 & 210 & 259 \\
\hline
\end{tabular}




\begin{tabular}{|c|c|c|c|c|}
\hline 19445503 & 24018632 & 219 & 246 & 27 \\
\hline 19445503 & 24018632 & 219 & 245 & 26 \\
\hline 19445503 & 24018632 & 215 & 274 & 59 \\
\hline 19445503 & 24018632 & 218 & 247 & 29 \\
\hline 19445503 & 24018632 & 213 & 259 & 46 \\
\hline 19445503 & 24018632 & 220 & 248 & 28 \\
\hline 19445503 & 24018632 & 215 & 266 & 51 \\
\hline 19445503 & 24018632 & 206 & 256 & 50 \\
\hline 19445503 & 24018632 & 210 & 277 & 67 \\
\hline 19445503 & 33411211 & 211 & 301 & 90 \\
\hline 19445503 & 33411211 & 218 & 327 & 109 \\
\hline 21311378 & 33411211 & 233 & 328 & 95 \\
\hline 21311378 & 33411211 & 227 & 307 & 80 \\
\hline 21311378 & 33411211 & 239 & 322 & 83 \\
\hline 19445503 & 33411211 & 221 & 332 & 111 \\
\hline 21311378 & 33411211 & 234 & 326 & 92 \\
\hline 21311378 & 33411211 & 227 & 306 & 79 \\
\hline 21311378 & 33411211 & 235 & 325 & 90 \\
\hline 19445503 & 33411211 & 214 & 325 & 111 \\
\hline 21311378 & 33411211 & 239 & 323 & 84 \\
\hline 21311378 & 33411211 & 228 & 315 & 87 \\
\hline 21311378 & 28852740 & 245 & 286 & 41 \\
\hline 21311378 & 33411211 & 239 & 320 & 81 \\
\hline 21311378 & 33411211 & 232 & 320 & 88 \\
\hline 21311378 & 33411211 & 241 & 324 & 83 \\
\hline 21311378 & 33411211 & 236 & 324 & 88 \\
\hline 19445503 & 33411211 & 216 & 329 & 113 \\
\hline 21311378 & 33411211 & 234 & 321 & 87 \\
\hline 21311378 & 33411211 & 236 & 326 & 90 \\
\hline 21311378 & 33411211 & 246 & 328 & 82 \\
\hline 21311378 & 28852740 & 254 & 287 & 33 \\
\hline 21311378 & 33411211 & 246 & 302 & 56 \\
\hline 21311378 & 33411211 & 234 & 326 & 92 \\
\hline 21311378 & 33411211 & 229 & 329 & 100 \\
\hline 21311378 & 33411211 & 235 & 324 & 89 \\
\hline 21311378 & 33411211 & 249 & 320 & 71 \\
\hline 21311378 & 33411211 & 244 & 323 & 79 \\
\hline 21311378 & 33411211 & 247 & 327 & 80 \\
\hline 19445503 & 33411211 & 217 & 321 & 104 \\
\hline 21311378 & 33411211 & 241 & 324 & 83 \\
\hline 19445503 & 33411211 & 221 & 322 & 101 \\
\hline 21311378 & 33411211 & 261 & 327 & 66 \\
\hline 21311378 & 33411211 & 252 & 318 & 66 \\
\hline
\end{tabular}




\begin{tabular}{|c|c|c|c|c|}
\hline 21311378 & 33411211 & 248 & 317 & 69 \\
\hline 19445503 & 33411211 & 215 & 323 & 108 \\
\hline 21311378 & 33411211 & 250 & 321 & 71 \\
\hline 21311378 & 33411211 & 248 & 318 & 70 \\
\hline 21311378 & 33411211 & 240 & 320 & 80 \\
\hline 21311378 & 33411211 & 257 & 315 & 58 \\
\hline 21311378 & 33411211 & 229 & 334 & 105 \\
\hline 21311378 & 33411211 & 231 & 330 & 99 \\
\hline 21311378 & 33411211 & 241 & 323 & 82 \\
\hline 19445503 & 33411211 & 213 & 327 & 114 \\
\hline 19445503 & 33411211 & 220 & 321 & 101 \\
\hline 19445503 & 33411211 & 213 & 333 & 120 \\
\hline 21311378 & 33411211 & 244 & 323 & 79 \\
\hline 21311378 & 33411211 & 237 & 326 & 89 \\
\hline 19445503 & 33411211 & 215 & 329 & 114 \\
\hline 19445503 & 33411211 & 216 & 327 & 111 \\
\hline 21311378 & 33411211 & 244 & 321 & 77 \\
\hline 21311378 & 33411211 & 247 & 317 & 70 \\
\hline 21311378 & 33411211 & 257 & 301 & 44 \\
\hline 21311378 & 33411211 & 249 & 317 & 68 \\
\hline 19445503 & 33411211 & 214 & 327 & 113 \\
\hline 19445503 & 33411211 & 214 & 330 & 116 \\
\hline 19445503 & 33411211 & 214 & 330 & 116 \\
\hline 19445503 & 33411211 & 214 & 329 & 115 \\
\hline 19445503 & 33411211 & 214 & 334 & 120 \\
\hline 21311378 & 33411211 & 253 & 318 & 65 \\
\hline 21311378 & 33411211 & 263 & 328 & 65 \\
\hline 21311378 & 33411211 & 255 & 329 & 74 \\
\hline 21311378 & 33411211 & 257 & 319 & 62 \\
\hline 21311378 & 33411211 & 241 & 318 & 77 \\
\hline 21311378 & 33411211 & 246 & 319 & 73 \\
\hline 21311378 & 33411211 & 259 & 308 & 49 \\
\hline 19445503 & 38433535 & 213 & 364 & 151 \\
\hline 21311378 & 33411211 & 240 & 311 & 71 \\
\hline 21311378 & 33411211 & 238 & 320 & 82 \\
\hline 21311378 & 33411211 & 261 & 318 & 57 \\
\hline 21311378 & 33411211 & 228 & 316 & 88 \\
\hline 21311378 & 33411211 & 265 & 316 & 51 \\
\hline 24018632 & 33411211 & 281 & 316 & 35 \\
\hline 21311378 & 33411211 & 237 & 313 & 76 \\
\hline 21311378 & 33411211 & 251 & 321 & 70 \\
\hline 21311378 & 33411211 & 257 & 308 & 51 \\
\hline 21311378 & 33411211 & 262 & 309 & 47 \\
\hline
\end{tabular}




\begin{tabular}{|c|c|c|c|c|}
\hline 21311378 & 33411211 & 250 & 326 & 76 \\
\hline 21311378 & 33411211 & 250 & 327 & 77 \\
\hline 21311378 & 33411211 & 270 & 319 & 49 \\
\hline 21311378 & 33411211 & 261 & 318 & 57 \\
\hline 24018632 & 33411211 & 281 & 324 & 43 \\
\hline 21311378 & 33411211 & 261 & 315 & 54 \\
\hline 21311378 & 33411211 & 254 & 319 & 65 \\
\hline 24018632 & 33411211 & 283 & 316 & 33 \\
\hline 21311378 & 33411211 & 243 & 326 & 83 \\
\hline 21311378 & 33411211 & 251 & 327 & 76 \\
\hline 21311378 & 33411211 & 251 & 324 & 73 \\
\hline 21311378 & 33411211 & 247 & 327 & 80 \\
\hline 21311378 & 33411211 & 257 & 314 & 57 \\
\hline 21311378 & 33411211 & 261 & 324 & 63 \\
\hline 21311378 & 33411211 & 243 & 327 & 84 \\
\hline 21311378 & 33411211 & 247 & 325 & 78 \\
\hline 21311378 & 33411211 & 262 & 318 & 56 \\
\hline 21311378 & 33411211 & 266 & 325 & 59 \\
\hline 21311378 & 33411211 & 251 & 322 & 71 \\
\hline 21311378 & 33411211 & 260 & 329 & 69 \\
\hline 21311378 & 33411211 & 260 & 324 & 64 \\
\hline 24018632 & 33411211 & 281 & 323 & 42 \\
\hline 21311378 & 33411211 & 252 & 330 & 78 \\
\hline 21311378 & 33411211 & 264 & 331 & 67 \\
\hline 21311378 & 33411211 & 259 & 337 & 78 \\
\hline 21311378 & 38433535 & 251 & 360 & 109 \\
\hline 21311378 & 33411211 & 247 & 338 & 91 \\
\hline 21311378 & 33411211 & 254 & 342 & 88 \\
\hline 21311378 & 33411211 & 261 & 339 & 78 \\
\hline 21311378 & 38433535 & 280 & 379 & 99 \\
\hline 28852740 & 38433535 & 315 & 374 & 59 \\
\hline 28852740 & 38433535 & 305 & 375 & 70 \\
\hline 28852740 & 38433535 & 297 & 372 & 75 \\
\hline 28852740 & 38433535 & 315 & 371 & 56 \\
\hline 28852740 & 38433535 & 314 & 366 & 52 \\
\hline 28852740 & 38433535 & 328 & 377 & 49 \\
\hline 28852740 & 38433535 & 329 & 373 & 44 \\
\hline 28852740 & 38433535 & 328 & 374 & 46 \\
\hline 28852740 & 38433535 & 315 & 368 & 53 \\
\hline 28852740 & 38433535 & 330 & 380 & 50 \\
\hline 28852740 & 38433535 & 329 & 375 & 46 \\
\hline 28852740 & 38433535 & 309 & 378 & 69 \\
\hline 28852740 & 38433535 & 324 & 370 & 46 \\
\hline
\end{tabular}




\begin{tabular}{|c|c|c|c|c|}
\hline 28852740 & 38433535 & 327 & 367 & 40 \\
\hline 28852740 & 38433535 & 329 & 376 & 47 \\
\hline 28852740 & 38433535 & 323 & 375 & 52 \\
\hline 28852740 & 38433535 & 321 & 383 & 62 \\
\hline 28852740 & 38433535 & 320 & 378 & 58 \\
\hline 28852740 & 38433535 & 326 & 381 & 55 \\
\hline 28852740 & 38433535 & 326 & 386 & 60 \\
\hline 28852740 & 38433535 & 325 & 373 & 48 \\
\hline 28852740 & 38433535 & 322 & 380 & 58 \\
\hline 28852740 & 38433535 & 328 & 379 & 51 \\
\hline 28852740 & 38433535 & 336 & 371 & 35 \\
\hline 28852740 & 38433535 & 331 & 363 & 32 \\
\hline 28852740 & 38433535 & 325 & 377 & 52 \\
\hline 28852740 & 38433535 & 324 & 383 & 59 \\
\hline 28852740 & 38433535 & 322 & 389 & 67 \\
\hline 28852740 & 38433535 & 325 & 392 & 67 \\
\hline 28852740 & 38433535 & 325 & 390 & 65 \\
\hline 28852740 & 38433535 & 330 & 381 & 51 \\
\hline 28852740 & 38433535 & 327 & 396 & 69 \\
\hline 28852740 & 38433535 & 304 & 392 & 88 \\
\hline 28852740 & 38433535 & 321 & 401 & 80 \\
\hline 28852740 & 38433535 & 331 & 385 & 54 \\
\hline 28852740 & 38433535 & 332 & 391 & 59 \\
\hline 28852740 & 40194958 & 321 & 422 & 101 \\
\hline 28852740 & 38433535 & 328 & 394 & 66 \\
\hline 33411211 & 40194958 & 374 & 427 & 53 \\
\hline 33411211 & 45384469 & 376 & 510 & 134 \\
\hline 33411211 & 42781548 & 399 & 454 & 55 \\
\hline 38433535 & 45384469 & 421 & 500 & 79 \\
\hline 38433535 & 45384469 & 416 & 513 & 97 \\
\hline 38433535 & 45384469 & 419 & 505 & 86 \\
\hline 33411211 & 45384469 & 403 & 478 & 75 \\
\hline 33411211 & 45384469 & 409 & 476 & 67 \\
\hline 38433535 & 42781548 & 417 & 448 & 31 \\
\hline 38433535 & 42781548 & 418 & 454 & 36 \\
\hline 38433535 & 45384469 & 413 & 472 & 59 \\
\hline 38433535 & 42781548 & 422 & 454 & 32 \\
\hline 38433535 & 42781548 & 421 & 450 & 29 \\
\hline 38433535 & 42781548 & 422 & 453 & 31 \\
\hline 38433535 & 42781548 & 428 & 447 & 19 \\
\hline 38433535 & 42781548 & 428 & 448 & 20 \\
\hline 40194958 & 42781548 & 432 & 445 & 13 \\
\hline 38433535 & 42781548 & 427 & 447 & 20 \\
\hline
\end{tabular}




\begin{tabular}{|c|c|c|c|}
\hline 40194958 & 42781548 & 432 & 445 \\
\hline 38433535 & 45384469 & 415 & 467 \\
\hline 40194958 & 42781548 & 434 & 446 \\
\hline 38433535 & 42781548 & 430 & 449 \\
\hline 38433535 & 45384469 & 427 & 463 \\
\hline 40194958 & 42781548 & 432 & 449 \\
\hline 40194958 & 42781548 & 433 & 450 \\
\hline 40194958 & 45384469 & 432 & 465 \\
\hline 40194958 & 42781548 & 436 & 446 \\
\hline 40194958 & 42781548 & 432 & 452 \\
\hline 40194958 & 42781548 & 433 & 454 \\
\hline 40194958 & 45384469 & 433 & 490 \\
\hline 38433535 & 42781548 & 430 & 451 \\
\hline 40194958 & 45384469 & 431 & 493 \\
\hline 38433535 & 45384469 & 423 & 470 \\
\hline 38433535 & 45384469 & 426 & 462 \\
\hline 38433535 & 45384469 & 425 & 460 \\
\hline 38433535 & 45384469 & 425 & 469 \\
\hline 40194958 & 45384469 & 431 & 481 \\
\hline 38433535 & 42781548 & 429 & 453 \\
\hline 38433535 & 45384469 & 420 & 501 \\
\hline 38433535 & 45384469 & 414 & 488 \\
\hline 38433535 & 45384469 & 427 & 491 \\
\hline 38433535 & 45384469 & 426 & 488 \\
\hline 40194958 & 45384469 & 433 & 493 \\
\hline 38433535 & 45384469 & 425 & 499 \\
\hline 40194958 & 45384469 & 437 & 495 \\
\hline 40194958 & 45384469 & 435 & 489 \\
\hline 40928880 & 45384469 & 444 & 494 \\
\hline 40928880 & 45384469 & 449 & 479 \\
\hline 40194958 & 45384469 & 434 & 503 \\
\hline 40928880 & 45384469 & 447 & 513 \\
\hline 40194958 & 45384469 & 433 & 515 \\
\hline 40194958 & 45384469 & 435 & 510 \\
\hline 40194958 & 45384469 & 432 & 518 \\
\hline 40928880 & 47589124 & 445 & 528 \\
\hline 38433535 & 47589124 & 420 & 538 \\
\hline 40194958 & 47589124 & 437 & 546 \\
\hline 40194958 & 47589124 & 437 & 528 \\
\hline 40928880 & 47589124 & 447 & 537 \\
\hline 38433535 & 45384469 & 425 & 518 \\
\hline 40194958 & 51756699 & 436 & 552 \\
\hline 38433535 & 47589124 & 418 & 547 \\
\hline
\end{tabular}




\begin{tabular}{|c|c|c|c|c|}
\hline 40928880 & 45384469 & 447 & 516 & 69 \\
\hline 40194958 & 45384469 & 433 & 510 & 77 \\
\hline 40194958 & 47589124 & 436 & 527 & 91 \\
\hline 40928880 & 47589124 & 445 & 539 & 94 \\
\hline 33411211 & 51756699 & 365 & 554 & 189 \\
\hline 40928880 & 47589124 & 445 & 534 & 89 \\
\hline 40194958 & 47589124 & 435 & 541 & 106 \\
\hline 40928880 & 47589124 & 449 & 542 & 93 \\
\hline 42781548 & 47589124 & 456 & 540 & 84 \\
\hline 40928880 & 47589124 & 449 & 532 & 83 \\
\hline 40928880 & 51756699 & 447 & 554 & 107 \\
\hline 40928880 & 47589124 & 443 & 534 & 91 \\
\hline 40928880 & 47589124 & 446 & 543 & 97 \\
\hline 40928880 & 47589124 & 451 & 536 & 85 \\
\hline 42781548 & 47589124 & 480 & 545 & 65 \\
\hline 42781548 & 47589124 & 467 & 539 & 72 \\
\hline 42781548 & 47589124 & 462 & 538 & 76 \\
\hline 42781548 & 47589124 & 463 & 545 & 82 \\
\hline 42781548 & 47589124 & 465 & 538 & 73 \\
\hline 42781548 & 47589124 & 468 & 546 & 78 \\
\hline 42781548 & 47589124 & 486 & 529 & 43 \\
\hline 42781548 & 47589124 & 473 & 548 & 75 \\
\hline 42781548 & 47589124 & 466 & 545 & 79 \\
\hline 42781548 & 47589124 & 464 & 547 & 83 \\
\hline 42781548 & 47589124 & 475 & 543 & 68 \\
\hline 42781548 & 47589124 & 463 & 541 & 78 \\
\hline 42781548 & 47589124 & 479 & 538 & 59 \\
\hline 42781548 & 47589124 & 481 & 537 & 56 \\
\hline 42781548 & 47589124 & 490 & 532 & 42 \\
\hline 42781548 & 47589124 & 502 & 531 & 29 \\
\hline 42781548 & 47589124 & 488 & 529 & 41 \\
\hline 42781548 & 47589124 & 499 & 529 & 30 \\
\hline 42781548 & 47589124 & 498 & 534 & 36 \\
\hline 42781548 & 47589124 & 497 & 536 & 39 \\
\hline 42781548 & 47589124 & 498 & 529 & 31 \\
\hline 42781548 & 47589124 & 494 & 530 & 36 \\
\hline 42781548 & 47589124 & 498 & 531 & 33 \\
\hline 42781548 & 47589124 & 485 & 535 & 50 \\
\hline 42781548 & 47589124 & 487 & 539 & 52 \\
\hline 42781548 & 51756699 & 486 & 551 & 65 \\
\hline 42781548 & 47589124 & 494 & 540 & 46 \\
\hline 42781548 & 51756699 & 492 & 552 & 60 \\
\hline 42781548 & 47589124 & 496 & 547 & 51 \\
\hline
\end{tabular}




\begin{tabular}{|c|c|c|c|c|}
\hline 42781548 & 47589124 & 477 & 542 & 65 \\
\hline 40928880 & 51756699 & 454 & 554 & 100 \\
\hline 42781548 & 51756699 & 495 & 552 & 57 \\
\hline 42781548 & 51756699 & 484 & 555 & 71 \\
\hline 42781548 & 51756699 & 494 & 556 & 62 \\
\hline 45384469 & 56870978 & 535 & 570 & 35 \\
\hline 42781548 & 56870978 & 478 & 570 & 92 \\
\hline 45384469 & 56870978 & 538 & 570 & 32 \\
\hline 48157811 & 56870978 & 550 & 565 & 15 \\
\hline 42781548 & 59154844 & 474 & 579 & 105 \\
\hline 48157811 & 54401974 & 552 & 561 & 9 \\
\hline 45384469 & 62058258 & 541 & 605 & 64 \\
\hline 48157811 & 54401974 & 553 & 561 & 8 \\
\hline 48157811 & 59154844 & 552 & 582 & 30 \\
\hline 48157811 & 56870978 & 554 & 565 & 11 \\
\hline 48157811 & 56870978 & 555 & 572 & 17 \\
\hline 48157811 & 56870978 & 553 & 563 & 10 \\
\hline 48157811 & 56870978 & 550 & 566 & 16 \\
\hline 48157811 & 56870978 & 556 & 562 & 6 \\
\hline 48157811 & 56870978 & 556 & 562 & 6 \\
\hline 48157811 & 56870978 & 555 & 569 & 14 \\
\hline 48157811 & 54401974 & 556 & 561 & 5 \\
\hline 48157811 & 56870978 & 555 & 571 & 16 \\
\hline 48157811 & 56870978 & 553 & 573 & 20 \\
\hline 48157811 & 54401974 & 556 & 561 & 5 \\
\hline 48157811 & 59154844 & 555 & 581 & 26 \\
\hline 48157811 & 54401974 & 558 & 560 & 2 \\
\hline 48157811 & 56870978 & 554 & 566 & 12 \\
\hline 48157811 & 56870978 & 550 & 567 & 17 \\
\hline 48157811 & 56870978 & 555 & 569 & 14 \\
\hline 48157811 & 56870978 & 553 & 569 & 16 \\
\hline 48157811 & 56870978 & 552 & 570 & 18 \\
\hline 48157811 & 56870978 & 554 & 571 & 17 \\
\hline 48157811 & 56870978 & 552 & 567 & 15 \\
\hline 48157811 & 56870978 & 553 & 570 & 17 \\
\hline 45384469 & 56870978 & 537 & 574 & 37 \\
\hline 45384469 & 60161629 & 546 & 589 & 43 \\
\hline 48157811 & 56870978 & 553 & 571 & 18 \\
\hline 48157811 & 56870978 & 551 & 572 & 21 \\
\hline 48157811 & 56870978 & 552 & 569 & 17 \\
\hline 45384469 & 56870978 & 544 & 566 & 22 \\
\hline 53171670 & 56870978 & 560 & 568 & 8 \\
\hline 45384469 & 56870978 & 526 & 572 & 46 \\
\hline
\end{tabular}




\begin{tabular}{|c|c|c|c|c|}
\hline 48157811 & 56870978 & 553 & 569 & 16 \\
\hline 48157811 & 56870978 & 551 & 569 & 18 \\
\hline 48157811 & 56870978 & 554 & 571 & 17 \\
\hline 48157811 & 56870978 & 554 & 571 & 17 \\
\hline 48157811 & 56870978 & 553 & 570 & 17 \\
\hline 48157811 & 56870978 & 556 & 571 & 15 \\
\hline 53171670 & 56870978 & 560 & 571 & 11 \\
\hline 48157811 & 56870978 & 554 & 571 & 17 \\
\hline 48157811 & 62058258 & 554 & 604 & 50 \\
\hline 48157811 & 56870978 & 553 & 572 & 19 \\
\hline 53171670 & 56870978 & 560 & 571 & 11 \\
\hline 48157811 & 56870978 & 559 & 570 & 11 \\
\hline 48157811 & 56870978 & 554 & 572 & 18 \\
\hline 48157811 & 56870978 & 550 & 572 & 22 \\
\hline 53171670 & 56870978 & 561 & 572 & 11 \\
\hline 48157811 & 62058258 & 554 & 601 & 47 \\
\hline 45384469 & 60161629 & 542 & 588 & 46 \\
\hline 48157811 & 59154844 & 554 & 580 & 26 \\
\hline 48157811 & 62058258 & 553 & 611 & 58 \\
\hline 48157811 & 62058258 & 553 & 597 & 44 \\
\hline 48157811 & 62058258 & 552 & 600 & 48 \\
\hline 53171670 & 57172051 & 560 & 574 & 14 \\
\hline 48157811 & 59154844 & 556 & 583 & 27 \\
\hline 48157811 & 62058258 & 557 & 598 & 41 \\
\hline 48157811 & 57172051 & 553 & 574 & 21 \\
\hline 53171670 & 56870978 & 560 & 572 & 12 \\
\hline 53171670 & 56870978 & 561 & 573 & 12 \\
\hline 48157811 & 62058258 & 550 & 599 & 49 \\
\hline 48157811 & 60161629 & 555 & 588 & 33 \\
\hline 53171670 & 62058258 & 561 & 597 & 36 \\
\hline 54401974 & 62058258 & 569 & 601 & 32 \\
\hline 48157811 & 62058258 & 556 & 595 & 39 \\
\hline 53171670 & 62058258 & 561 & 607 & 46 \\
\hline 48157811 & 62058258 & 551 & 598 & 47 \\
\hline 48157811 & 62058258 & 550 & 603 & 53 \\
\hline 57172051 & 62058258 & 577 & 605 & 28 \\
\hline 57172051 & 60161629 & 582 & 589 & 7 \\
\hline 57172051 & 60161629 & 578 & 590 & 12 \\
\hline 57172051 & 60161629 & 582 & 587 & 5 \\
\hline 57172051 & 60161629 & 582 & 588 & 6 \\
\hline 57172051 & 60161629 & 582 & 585 & 3 \\
\hline 57172051 & 60161629 & 582 & 588 & 6 \\
\hline 57172051 & 60161629 & 581 & 588 & 7 \\
\hline
\end{tabular}




\begin{tabular}{|c|c|c|c|}
\hline 57172051 & 60161629 & 583 & 587 \\
\hline 57172051 & 60161629 & 580 & 588 \\
\hline 57172051 & 60161629 & 580 & 588 \\
\hline 57172051 & 60161629 & 578 & 589 \\
\hline 57172051 & 60161629 & 580 & 590 \\
\hline 57172051 & 60161629 & 576 & 590 \\
\hline 45384469 & 62058258 & 537 & 606 \\
\hline 57172051 & 62058258 & 576 & 598 \\
\hline 57172051 & 62058258 & 581 & 594 \\
\hline 54401974 & 62058258 & 573 & 606 \\
\hline 57172051 & 62058258 & 576 & 605 \\
\hline 57172051 & 62058258 & 578 & 592 \\
\hline 57172051 & 60161629 & 578 & 590 \\
\hline 54401974 & 62058258 & 564 & 608 \\
\hline 57172051 & 60161629 & 581 & 590 \\
\hline 57172051 & 62058258 & 577 & 599 \\
\hline 53171670 & 62058258 & 561 & 602 \\
\hline 57172051 & 62058258 & 577 & 600 \\
\hline 57172051 & 62058258 & 581 & 598 \\
\hline 64998570 & 69290382 & 655 & 689 \\
\hline 64998570 & 67680565 & 657 & 686 \\
\hline 64998570 & 69290382 & 658 & 689 \\
\hline 64998570 & 79034049 & 651 & 717 \\
\hline 62058258 & 76137316 & 622 & 703 \\
\hline 64998570 & 79034049 & 651 & 722 \\
\hline 66361941 & 69290382 & 676 & 691 \\
\hline 66361941 & 69290382 & 674 & 693 \\
\hline 66361941 & 79034049 & 676 & 717 \\
\hline 66361941 & 76137316 & 673 & 707 \\
\hline 66361941 & 76137316 & 677 & 699 \\
\hline 66361941 & 76137316 & 675 & 704 \\
\hline 64998570 & 76137316 & 663 & 701 \\
\hline 66361941 & 79034049 & 676 & 722 \\
\hline 66361941 & 76137316 & 674 & 701 \\
\hline 66361941 & 76137316 & 678 & 700 \\
\hline 66361941 & 76137316 & 674 & 705 \\
\hline 66361941 & 76137316 & 677 & 706 \\
\hline 66361941 & 76137316 & 676 & 712 \\
\hline 64998570 & 76137316 & 669 & 703 \\
\hline 66361941 & 76137316 & 685 & 697 \\
\hline 66361941 & 76137316 & 678 & 704 \\
\hline 66361941 & 76137316 & 675 & 708 \\
\hline 66361941 & 76137316 & 679 & 704 \\
\hline
\end{tabular}




\begin{tabular}{|c|c|c|c|}
\hline 67680565 & 76137316 & 689 & 703 \\
\hline 62058258 & 76137316 & 643 & 706 \\
\hline 64998570 & 76137316 & 655 & 707 \\
\hline 67680565 & 76137316 & 688 & 698 \\
\hline 67680565 & 76137316 & 690 & 701 \\
\hline 66361941 & 76137316 & 686 & 699 \\
\hline 67680565 & 76137316 & 690 & 698 \\
\hline 67680565 & 76137316 & 691 & 698 \\
\hline 66361941 & 76137316 & 683 & 701 \\
\hline 66361941 & 76137316 & 686 & 705 \\
\hline 66361941 & 76137316 & 681 & 705 \\
\hline 66361941 & 76137316 & 685 & 707 \\
\hline 66361941 & 79034049 & 678 & 718 \\
\hline 66361941 & 76137316 & 678 & 701 \\
\hline 66361941 & 76137316 & 684 & 704 \\
\hline 66361941 & 76137316 & 686 & 703 \\
\hline 66361941 & 76137316 & 679 & 709 \\
\hline 66361941 & 76137316 & 673 & 708 \\
\hline 67680565 & 76137316 & 689 & 701 \\
\hline 67680565 & 76137316 & 690 & 706 \\
\hline 66361941 & 76137316 & 680 & 707 \\
\hline 66361941 & 76137316 & 683 & 708 \\
\hline 66361941 & 76137316 & 680 & 711 \\
\hline 66361941 & 76137316 & 677 & 708 \\
\hline 67680565 & 76137316 & 689 & 708 \\
\hline 67680565 & 76137316 & 688 & 709 \\
\hline 67680565 & 76137316 & 688 & 709 \\
\hline 66361941 & 76137316 & 678 & 709 \\
\hline 66361941 & 76137316 & 677 & 709 \\
\hline 66361941 & 76137316 & 682 & 712 \\
\hline 66361941 & 79034049 & 672 & 717 \\
\hline 67680565 & 76137316 & 688 & 707 \\
\hline 64998570 & 79034049 & 663 & 714 \\
\hline 66361941 & 79034049 & 671 & 721 \\
\hline 66361941 & 79034049 & 676 & 716 \\
\hline 66361941 & 76137316 & 677 & 708 \\
\hline 67680565 & 79034049 & 688 & 713 \\
\hline 66361941 & 76137316 & 677 & 711 \\
\hline 66361941 & 76137316 & 677 & 712 \\
\hline 66361941 & 76137316 & 675 & 709 \\
\hline 66361941 & 76137316 & 685 & 709 \\
\hline 64998570 & 79034049 & 660 & 715 \\
\hline 66361941 & 82401512 & 679 & 727 \\
\hline
\end{tabular}




\begin{tabular}{|c|c|c|c|c|}
\hline 66361941 & 79034049 & 682 & 717 & 35 \\
\hline 66361941 & 86276688 & 678 & 736 & 58 \\
\hline 64998570 & 82401512 & 656 & 727 & 71 \\
\hline 66361941 & 79034049 & 675 & 723 & 48 \\
\hline 66361941 & 79034049 & 683 & 719 & 36 \\
\hline 66361941 & 79034049 & 679 & 720 & 41 \\
\hline 66361941 & 79034049 & 677 & 721 & 44 \\
\hline 66361941 & 79034049 & 674 & 716 & 42 \\
\hline 66361941 & 79034049 & 678 & 719 & 41 \\
\hline 66361941 & 86276688 & 674 & 737 & 63 \\
\hline 67680565 & 79034049 & 689 & 719 & 30 \\
\hline 67680565 & 79034049 & 688 & 714 & 26 \\
\hline 64998570 & 82401512 & 661 & 726 & 65 \\
\hline 64998570 & 84367585 & 659 & 731 & 72 \\
\hline 62058258 & 86276688 & 638 & 736 & 98 \\
\hline 66361941 & 82401512 & 675 & 729 & 54 \\
\hline 64998570 & 79034049 & 658 & 723 & 65 \\
\hline 66361941 & 82401512 & 682 & 728 & 46 \\
\hline 66361941 & 79034049 & 683 & 722 & 39 \\
\hline 66361941 & 82401512 & 681 & 729 & 48 \\
\hline 67680565 & 86276688 & 691 & 737 & 46 \\
\hline 64998570 & 84367585 & 662 & 731 & 69 \\
\hline 72176743 & 79034049 & 699 & 719 & 20 \\
\hline 64998570 & 79034049 & 655 & 720 & 65 \\
\hline 72176743 & 79034049 & 703 & 719 & 16 \\
\hline 67680565 & 82401512 & 688 & 725 & 37 \\
\hline 72176743 & 79034049 & 705 & 718 & 13 \\
\hline 72176743 & 90682837 & 699 & 749 & 50 \\
\hline 72176743 & 88754535 & 701 & 741 & 40 \\
\hline 72176743 & 79034049 & 700 & 721 & 21 \\
\hline 72176743 & 79034049 & 701 & 720 & 19 \\
\hline 72176743 & 86276688 & 706 & 735 & 29 \\
\hline 72176743 & 79034049 & 700 & 722 & 22 \\
\hline 72176743 & 79034049 & 706 & 717 & 11 \\
\hline 72176743 & 79034049 & 705 & 721 & 16 \\
\hline 72176743 & 79034049 & 704 & 722 & 18 \\
\hline 72176743 & 82401512 & 701 & 729 & 28 \\
\hline 72176743 & 79034049 & 703 & 722 & 19 \\
\hline 72176743 & 79034049 & 697 & 723 & 26 \\
\hline 72176743 & 86276688 & 702 & 736 & 34 \\
\hline 72176743 & 79034049 & 698 & 722 & 24 \\
\hline 72176743 & 79034049 & 703 & 721 & 18 \\
\hline 72176743 & 79034049 & 704 & 721 & 17 \\
\hline
\end{tabular}




\begin{tabular}{|c|c|c|c|c|}
\hline 72176743 & 79034049 & 706 & 720 & 14 \\
\hline 72176743 & 79034049 & 707 & 721 & 14 \\
\hline 72176743 & 82401512 & 704 & 727 & 23 \\
\hline 72176743 & 86276688 & 699 & 737 & 38 \\
\hline 72176743 & 79034049 & 706 & 724 & 18 \\
\hline 72176743 & 79034049 & 708 & 723 & 15 \\
\hline 72176743 & 79034049 & 709 & 722 & 13 \\
\hline 72176743 & 84825964 & 696 & 733 & 37 \\
\hline 72176743 & 86276688 & 701 & 737 & 36 \\
\hline 72176743 & 82401512 & 701 & 728 & 27 \\
\hline 72176743 & 86276688 & 706 & 738 & 32 \\
\hline 72176743 & 79034049 & 705 & 724 & 19 \\
\hline 72176743 & 90682837 & 704 & 749 & 45 \\
\hline 72176743 & 86276688 & 701 & 737 & 36 \\
\hline 72176743 & 82401512 & 704 & 729 & 25 \\
\hline 72176743 & 82401512 & 698 & 729 & 31 \\
\hline 72176743 & 86276688 & 703 & 738 & 35 \\
\hline 72176743 & 82401512 & 705 & 728 & 23 \\
\hline 72176743 & 82401512 & 705 & 726 & 21 \\
\hline 72176743 & 88754535 & 700 & 745 & 45 \\
\hline 72176743 & 82401512 & 699 & 729 & 30 \\
\hline 72176743 & 86276688 & 699 & 736 & 37 \\
\hline 72176743 & 86276688 & 704 & 734 & 30 \\
\hline 72176743 & 84367585 & 704 & 731 & 27 \\
\hline 72176743 & 86276688 & 700 & 737 & 37 \\
\hline 72176743 & 86276688 & 697 & 739 & 42 \\
\hline 72176743 & 86276688 & 701 & 735 & 34 \\
\hline 72176743 & 88754535 & 700 & 745 & 45 \\
\hline 72176743 & 86276688 & 702 & 736 & 34 \\
\hline 69290382 & 84825964 & 694 & 733 & 39 \\
\hline 72176743 & 86276688 & 702 & 736 & 34 \\
\hline 72176743 & 86276688 & 696 & 739 & 43 \\
\hline 72176743 & 86276688 & 697 & 737 & 40 \\
\hline 72176743 & 86276688 & 698 & 738 & 40 \\
\hline 72176743 & 86276688 & 697 & 738 & 41 \\
\hline 66361941 & 84825964 & 683 & 733 & 50 \\
\hline 72176743 & 86276688 & 703 & 738 & 35 \\
\hline 72176743 & 90682837 & 705 & 754 & 49 \\
\hline 72176743 & 86276688 & 707 & 739 & 32 \\
\hline 72176743 & 86276688 & 701 & 739 & 38 \\
\hline 72176743 & 86276688 & 705 & 736 & 31 \\
\hline 72176743 & 86276688 & 707 & 737 & 30 \\
\hline 72176743 & 86276688 & 705 & 738 & 33 \\
\hline
\end{tabular}




\begin{tabular}{|c|c|c|c|c|}
\hline 72176743 & 90682837 & 709 & 761 & 52 \\
\hline 72176743 & 86276688 & 708 & 737 & 29 \\
\hline 72176743 & 86276688 & 702 & 734 & 32 \\
\hline 72176743 & 86276688 & 705 & 740 & 35 \\
\hline 72176743 & 86276688 & 700 & 739 & 39 \\
\hline 67680565 & 90682837 & 692 & 748 & 56 \\
\hline 66361941 & 86276688 & 686 & 738 & 52 \\
\hline 72176743 & 90682837 & 703 & 759 & 56 \\
\hline 72176743 & 86276688 & 708 & 736 & 28 \\
\hline 72176743 & 86276688 & 710 & 737 & 27 \\
\hline 72176743 & 86276688 & 705 & 738 & 33 \\
\hline 72176743 & 86276688 & 709 & 739 & 30 \\
\hline 72176743 & 86276688 & 707 & 736 & 29 \\
\hline 72176743 & 86276688 & 712 & 739 & 27 \\
\hline 72176743 & 82401512 & 707 & 729 & 22 \\
\hline 72176743 & 86276688 & 708 & 737 & 29 \\
\hline 72176743 & 86276688 & 707 & 739 & 32 \\
\hline 72176743 & 86276688 & 702 & 737 & 35 \\
\hline 72176743 & 86276688 & 701 & 739 & 38 \\
\hline 66361941 & 86276688 & 683 & 737 & 54 \\
\hline 72176743 & 86276688 & 710 & 734 & 24 \\
\hline 72176743 & 86276688 & 706 & 735 & 29 \\
\hline 72176743 & 86276688 & 697 & 734 & 37 \\
\hline 72176743 & 86276688 & 707 & 735 & 28 \\
\hline 66361941 & 88754535 & 683 & 741 & 58 \\
\hline 72176743 & 86276688 & 697 & 738 & 41 \\
\hline 72176743 & 86276688 & 708 & 737 & 29 \\
\hline 72176743 & 88754535 & 701 & 742 & 41 \\
\hline 72176743 & 86276688 & 705 & 738 & 33 \\
\hline 72176743 & 82401512 & 712 & 729 & 17 \\
\hline 77434720 & 82401512 & 715 & 730 & 15 \\
\hline 72176743 & 88754535 & 711 & 742 & 31 \\
\hline 72176743 & 86276688 & 709 & 737 & 28 \\
\hline 67680565 & 86276688 & 689 & 739 & 50 \\
\hline 72176743 & 86276688 & 710 & 737 & 27 \\
\hline 72176743 & 86276688 & 705 & 737 & 32 \\
\hline 72176743 & 90682837 & 704 & 755 & 51 \\
\hline 72176743 & 90682837 & 704 & 753 & 49 \\
\hline 72176743 & 86276688 & 707 & 738 & 31 \\
\hline 77434720 & 86276688 & 713 & 734 & 21 \\
\hline 72176743 & 84367585 & 711 & 731 & 20 \\
\hline 76137316 & 86276688 & 713 & 737 & 24 \\
\hline 72176743 & 86276688 & 709 & 735 & 26 \\
\hline
\end{tabular}




\begin{tabular}{|c|c|c|c|c|}
\hline 72176743 & 86276688 & 696 & 735 & 39 \\
\hline 72176743 & 90682837 & 704 & 755 & 51 \\
\hline 72176743 & 86276688 & 710 & 740 & 30 \\
\hline 77434720 & 82401512 & 715 & 730 & 15 \\
\hline 77434720 & 86276688 & 714 & 736 & 22 \\
\hline 72176743 & 86276688 & 706 & 738 & 32 \\
\hline 77434720 & 86276688 & 714 & 735 & 21 \\
\hline 72176743 & 82401512 & 696 & 729 & 33 \\
\hline 72176743 & 90682837 & 705 & 746 & 41 \\
\hline 72176743 & 86276688 & 706 & 737 & 31 \\
\hline 72176743 & 90682837 & 703 & 754 & 51 \\
\hline 67680565 & 86276688 & 692 & 738 & 46 \\
\hline 77434720 & 86276688 & 714 & 737 & 23 \\
\hline 77434720 & 86276688 & 714 & 738 & 24 \\
\hline 76137316 & 86276688 & 713 & 740 & 27 \\
\hline 77434720 & 82401512 & 715 & 729 & 14 \\
\hline 77434720 & 82401512 & 716 & 729 & 13 \\
\hline 77434720 & 82401512 & 716 & 729 & 13 \\
\hline 72176743 & 82401512 & 705 & 729 & 24 \\
\hline 77434720 & 86276688 & 714 & 738 & 24 \\
\hline 72176743 & 90682837 & 703 & 755 & 52 \\
\hline 77434720 & 86276688 & 715 & 734 & 19 \\
\hline 77434720 & 86276688 & 714 & 735 & 21 \\
\hline 72176743 & 84367585 & 711 & 731 & 20 \\
\hline 77434720 & 82401512 & 716 & 728 & 12 \\
\hline 77434720 & 79034049 & 717 & 724 & 7 \\
\hline 77434720 & 86276688 & 716 & 735 & 19 \\
\hline 77434720 & 86276688 & 715 & 738 & 23 \\
\hline 77434720 & 86276688 & 716 & 734 & 18 \\
\hline 77434720 & 86276688 & 715 & 735 & 20 \\
\hline 72176743 & 90682837 & 704 & 748 & 44 \\
\hline 77434720 & 84825964 & 716 & 733 & 17 \\
\hline 77434720 & 86276688 & 716 & 739 & 23 \\
\hline 77434720 & 82401512 & 717 & 728 & 11 \\
\hline 77434720 & 88754535 & 716 & 743 & 27 \\
\hline 77434720 & 88754535 & 716 & 744 & 28 \\
\hline 77434720 & 82401512 & 718 & 729 & 11 \\
\hline 77434720 & 86276688 & 716 & 740 & 24 \\
\hline 77434720 & 86276688 & 718 & 739 & 21 \\
\hline 77434720 & 86276688 & 717 & 739 & 22 \\
\hline 77434720 & 90682837 & 715 & 767 & 52 \\
\hline 77434720 & 84825964 & 713 & 733 & 20 \\
\hline 77434720 & 88754535 & 715 & 743 & 28 \\
\hline
\end{tabular}




\begin{tabular}{|c|c|c|c|c|}
\hline 77434720 & 90682837 & 713 & 753 & 40 \\
\hline 77434720 & 82401512 & 718 & 729 & 11 \\
\hline 77434720 & 86276688 & 714 & 735 & 21 \\
\hline 77434720 & 86276688 & 714 & 740 & 26 \\
\hline 77434720 & 86276688 & 716 & 740 & 24 \\
\hline 77434720 & 84367585 & 713 & 731 & 18 \\
\hline 76137316 & 86276688 & 713 & 740 & 27 \\
\hline 77434720 & 86276688 & 717 & 738 & 21 \\
\hline 77434720 & 90682837 & 717 & 751 & 34 \\
\hline 77434720 & 86276688 & 716 & 739 & 23 \\
\hline 77434720 & 90682837 & 716 & 753 & 37 \\
\hline 77434720 & 86276688 & 716 & 737 & 21 \\
\hline 77434720 & 86276688 & 717 & 740 & 23 \\
\hline 72176743 & 86276688 & 698 & 738 & 40 \\
\hline 77434720 & 82401512 & 717 & 729 & 12 \\
\hline 77434720 & 84367585 & 717 & 731 & 14 \\
\hline 77434720 & 82401512 & 718 & 728 & 10 \\
\hline 77434720 & 82401512 & 719 & 729 & 10 \\
\hline 77434720 & 82401512 & 717 & 729 & 12 \\
\hline 77434720 & 82401512 & 717 & 729 & 12 \\
\hline 77434720 & 82401512 & 718 & 729 & 11 \\
\hline 77434720 & 82401512 & 718 & 728 & 10 \\
\hline 77434720 & 86276688 & 717 & 736 & 19 \\
\hline 77434720 & 82401512 & 717 & 729 & 12 \\
\hline 77434720 & 86276688 & 716 & 740 & 24 \\
\hline 77434720 & 82401512 & 720 & 728 & 8 \\
\hline 77434720 & 82401512 & 718 & 729 & 11 \\
\hline 77434720 & 90682837 & 716 & 752 & 36 \\
\hline 77434720 & 82401512 & 718 & 729 & 11 \\
\hline 77434720 & 86276688 & 716 & 736 & 20 \\
\hline 77434720 & 86276688 & 718 & 739 & 21 \\
\hline 77434720 & 84825964 & 717 & 733 & 16 \\
\hline 77434720 & 86276688 & 717 & 737 & 20 \\
\hline 77434720 & 86276688 & 719 & 736 & 17 \\
\hline 77434720 & 86276688 & 716 & 736 & 20 \\
\hline 77434720 & 86276688 & 714 & 736 & 22 \\
\hline 77434720 & 86276688 & 714 & 738 & 24 \\
\hline 77434720 & 86276688 & 718 & 736 & 18 \\
\hline 77434720 & 82401512 & 719 & 729 & 10 \\
\hline 77434720 & 84825964 & 717 & 733 & 16 \\
\hline 77434720 & 86276688 & 719 & 737 & 18 \\
\hline 77434720 & 86276688 & 717 & 736 & 19 \\
\hline 77434720 & 86276688 & 716 & 740 & 24 \\
\hline
\end{tabular}




\begin{tabular}{|c|c|c|c|c|}
\hline 77434720 & 86276688 & 718 & 738 & 20 \\
\hline 77434720 & 86276688 & 716 & 738 & 22 \\
\hline 77434720 & 84367585 & 717 & 731 & 14 \\
\hline 77434720 & 86276688 & 717 & 734 & 17 \\
\hline 77434720 & 84367585 & 719 & 731 & 12 \\
\hline 77434720 & 86276688 & 718 & 736 & 18 \\
\hline 77434720 & 86276688 & 717 & 740 & 23 \\
\hline 77434720 & 88754535 & 715 & 745 & 30 \\
\hline 77434720 & 88754535 & 718 & 745 & 27 \\
\hline 72176743 & 86276688 & 704 & 737 & 33 \\
\hline 72176743 & 86276688 & 709 & 739 & 30 \\
\hline 77434720 & 90682837 & 715 & 752 & 37 \\
\hline 77434720 & 86276688 & 714 & 739 & 25 \\
\hline 77434720 & 90682837 & 713 & 751 & 38 \\
\hline 77434720 & 86276688 & 717 & 739 & 22 \\
\hline 77434720 & 84367585 & 720 & 731 & 11 \\
\hline 77434720 & 82401512 & 720 & 730 & 10 \\
\hline 77434720 & 86276688 & 716 & 736 & 20 \\
\hline 77434720 & 86276688 & 715 & 736 & 21 \\
\hline 77434720 & 86276688 & 717 & 735 & 18 \\
\hline 77434720 & 86276688 & 716 & 736 & 20 \\
\hline 77434720 & 86276688 & 716 & 734 & 18 \\
\hline 77434720 & 90682837 & 715 & 750 & 35 \\
\hline 72176743 & 86276688 & 705 & 739 & 34 \\
\hline 67680565 & 86276688 & 691 & 737 & 46 \\
\hline 77434720 & 86276688 & 717 & 738 & 21 \\
\hline 77434720 & 86276688 & 715 & 734 & 19 \\
\hline 77434720 & 86276688 & 715 & 739 & 24 \\
\hline 77434720 & 86276688 & 716 & 737 & 21 \\
\hline 72176743 & 86276688 & 701 & 738 & 37 \\
\hline 72176743 & 86276688 & 706 & 736 & 30 \\
\hline 72176743 & 90682837 & 698 & 750 & 52 \\
\hline 77434720 & 90682837 & 716 & 746 & 30 \\
\hline 77434720 & 86276688 & 714 & 735 & 21 \\
\hline 72176743 & 86276688 & 708 & 738 & 30 \\
\hline 77434720 & 86276688 & 719 & 737 & 18 \\
\hline 77434720 & 90682837 & 715 & 747 & 32 \\
\hline 77434720 & 86276688 & 718 & 736 & 18 \\
\hline 76137316 & 86276688 & 713 & 738 & 25 \\
\hline 77434720 & 86276688 & 715 & 736 & 21 \\
\hline 77434720 & 86276688 & 714 & 735 & 21 \\
\hline 72176743 & 90682837 & 710 & 757 & 47 \\
\hline 72176743 & 86276688 & 700 & 736 & 36 \\
\hline
\end{tabular}




\begin{tabular}{|c|c|c|c|c|}
\hline 77434720 & 86276688 & 716 & 734 & 18 \\
\hline 77434720 & 86276688 & 720 & 735 & 15 \\
\hline 76137316 & 86276688 & 713 & 738 & 25 \\
\hline 77434720 & 86276688 & 716 & 736 & 20 \\
\hline 77434720 & 86276688 & 717 & 736 & 19 \\
\hline 77434720 & 86276688 & 717 & 738 & 21 \\
\hline 77434720 & 86276688 & 718 & 736 & 18 \\
\hline 77434720 & 86276688 & 717 & 739 & 22 \\
\hline 77434720 & 86276688 & 714 & 739 & 25 \\
\hline 77434720 & 86276688 & 714 & 735 & 21 \\
\hline 77434720 & 86276688 & 715 & 736 & 21 \\
\hline 77434720 & 86276688 & 717 & 737 & 20 \\
\hline 77434720 & 86276688 & 714 & 737 & 23 \\
\hline 77434720 & 86276688 & 714 & 738 & 24 \\
\hline 72176743 & 86276688 & 699 & 738 & 39 \\
\hline 77434720 & 86276688 & 714 & 737 & 23 \\
\hline 77434720 & 86276688 & 718 & 737 & 19 \\
\hline 72176743 & 86276688 & 702 & 736 & 34 \\
\hline 77434720 & 86276688 & 715 & 734 & 19 \\
\hline 77434720 & 88754535 & 717 & 744 & 27 \\
\hline 77434720 & 88754535 & 716 & 741 & 25 \\
\hline 77434720 & 86276688 & 716 & 739 & 23 \\
\hline 72176743 & 86276688 & 711 & 739 & 28 \\
\hline 72176743 & 82401512 & 709 & 730 & 21 \\
\hline 77434720 & 86276688 & 716 & 736 & 20 \\
\hline 77434720 & 86276688 & 716 & 739 & 23 \\
\hline 77434720 & 90682837 & 717 & 750 & 33 \\
\hline 72176743 & 86276688 & 704 & 737 & 33 \\
\hline 77434720 & 86276688 & 718 & 735 & 17 \\
\hline 77434720 & 86276688 & 715 & 739 & 24 \\
\hline 77434720 & 88754535 & 717 & 741 & 24 \\
\hline 79034049 & 86276688 & 726 & 735 & 9 \\
\hline 77434720 & 86276688 & 715 & 738 & 23 \\
\hline 77434720 & 86276688 & 715 & 738 & 23 \\
\hline 77434720 & 86276688 & 721 & 737 & 16 \\
\hline 77434720 & 86276688 & 719 & 735 & 16 \\
\hline 77434720 & 84825964 & 717 & 732 & 15 \\
\hline 77434720 & 84367585 & 723 & 731 & 8 \\
\hline 77434720 & 86276688 & 716 & 734 & 18 \\
\hline 77434720 & 86276688 & 721 & 738 & 17 \\
\hline 77434720 & 86276688 & 716 & 735 & 19 \\
\hline 77434720 & 86276688 & 719 & 738 & 19 \\
\hline 77434720 & 86276688 & 720 & 738 & 18 \\
\hline
\end{tabular}




\begin{tabular}{|c|c|c|c|c|}
\hline 79034049 & 86276688 & 725 & 735 & 10 \\
\hline 79034049 & 86276688 & 725 & 734 & 9 \\
\hline 72176743 & 86276688 & 708 & 739 & 31 \\
\hline 77434720 & 86276688 & 724 & 737 & 13 \\
\hline 72176743 & 86276688 & 708 & 738 & 30 \\
\hline 72176743 & 90682837 & 710 & 761 & 51 \\
\hline 77434720 & 86276688 & 719 & 737 & 18 \\
\hline 77434720 & 88754535 & 716 & 744 & 28 \\
\hline 77434720 & 86276688 & 715 & 739 & 24 \\
\hline 79034049 & 86276688 & 726 & 736 & 10 \\
\hline 72176743 & 86276688 & 710 & 740 & 30 \\
\hline 77434720 & 86276688 & 713 & 736 & 23 \\
\hline 79034049 & 82401512 & 725 & 730 & 5 \\
\hline 77434720 & 86276688 & 714 & 736 & 22 \\
\hline 79034049 & 86276688 & 725 & 734 & 9 \\
\hline 77434720 & 86276688 & 714 & 735 & 21 \\
\hline 77434720 & 88754535 & 718 & 741 & 23 \\
\hline 77434720 & 86276688 & 721 & 739 & 18 \\
\hline 77434720 & 86276688 & 715 & 737 & 22 \\
\hline 72176743 & 90682837 & 707 & 754 & 47 \\
\hline 77434720 & 88754535 & 718 & 741 & 23 \\
\hline 77434720 & 86276688 & 720 & 735 & 15 \\
\hline 77434720 & 86276688 & 716 & 739 & 23 \\
\hline 77434720 & 86276688 & 718 & 737 & 19 \\
\hline 76137316 & 86276688 & 713 & 739 & 26 \\
\hline 77434720 & 86276688 & 716 & 737 & 21 \\
\hline 77434720 & 86276688 & 715 & 740 & 25 \\
\hline 77434720 & 90682837 & 721 & 757 & 36 \\
\hline 77434720 & 86276688 & 715 & 738 & 23 \\
\hline 77434720 & 86276688 & 723 & 736 & 13 \\
\hline 72176743 & 86276688 & 701 & 736 & 35 \\
\hline 72176743 & 86276688 & 711 & 738 & 27 \\
\hline 72176743 & 88754535 & 711 & 743 & 32 \\
\hline 77434720 & 86276688 & 717 & 739 & 22 \\
\hline 77434720 & 86276688 & 713 & 738 & 25 \\
\hline 72176743 & 86276688 & 711 & 737 & 26 \\
\hline 72176743 & 90682837 & 706 & 766 & 60 \\
\hline 77434720 & 86276688 & 719 & 738 & 19 \\
\hline 72176743 & 86276688 & 710 & 740 & 30 \\
\hline 77434720 & 86276688 & 716 & 739 & 23 \\
\hline 72176743 & 86276688 & 712 & 739 & 27 \\
\hline 77434720 & 86276688 & 713 & 738 & 25 \\
\hline 72176743 & 86276688 & 707 & 740 & 33 \\
\hline
\end{tabular}




\begin{tabular}{|c|c|c|c|}
\hline 72176743 & 86276688 & 704 & 737 \\
\hline 72176743 & 86276688 & 710 & 740 \\
\hline 77434720 & 86276688 & 716 & 739 \\
\hline 77434720 & 86276688 & 719 & 739 \\
\hline 72176743 & 86276688 & 706 & 740 \\
\hline 72176743 & 86276688 & 707 & 739 \\
\hline 77434720 & 88754535 & 716 & 743 \\
\hline 77434720 & 86276688 & 715 & 739 \\
\hline 72176743 & 88754535 & 709 & 743 \\
\hline 72176743 & 90682837 & 708 & 750 \\
\hline 72176743 & 86276688 & 708 & 738 \\
\hline 72176743 & 90682837 & 710 & 746 \\
\hline 77434720 & 86276688 & 718 & 738 \\
\hline 77434720 & 88754535 & 715 & 744 \\
\hline 77434720 & 86276688 & 720 & 740 \\
\hline 79034049 & 86276688 & 726 & 739 \\
\hline 83317202 & 86276688 & 731 & 738 \\
\hline 77434720 & 90682837 & 718 & 748 \\
\hline 77434720 & 88754535 & 717 & 741 \\
\hline 77434720 & 90682837 & 714 & 746 \\
\hline 77434720 & 88754535 & 715 & 743 \\
\hline 77434720 & 90682837 & 718 & 758 \\
\hline 77434720 & 88754535 & 716 & 744 \\
\hline 77434720 & 90682837 & 720 & 747 \\
\hline 77434720 & 90682837 & 713 & 751 \\
\hline 79034049 & 86276688 & 727 & 739 \\
\hline 77434720 & 86276688 & 720 & 740 \\
\hline 77434720 & 88754535 & 715 & 743 \\
\hline 77434720 & 88754535 & 718 & 743 \\
\hline 72176743 & 90682837 & 709 & 749 \\
\hline 77434720 & 90682837 & 717 & 752 \\
\hline 83317202 & 86276688 & 731 & 739 \\
\hline 72176743 & 90682837 & 700 & 751 \\
\hline 77434720 & 90682837 & 717 & 750 \\
\hline 72176743 & 90682837 & 706 & 770 \\
\hline 77434720 & 90682837 & 719 & 751 \\
\hline 72176743 & 90682837 & 706 & 752 \\
\hline 77434720 & 90682837 & 714 & 755 \\
\hline 77434720 & 90682837 & 713 & 757 \\
\hline 79034049 & 90682837 & 726 & 755 \\
\hline 77434720 & 90682837 & 714 & 761 \\
\hline 77434720 & 90682837 & 718 & 747 \\
\hline 77434720 & 90682837 & 713 & 752 \\
\hline
\end{tabular}




\begin{tabular}{|c|c|c|c|c|}
\hline 72176743 & 90682837 & 709 & 754 & 45 \\
\hline 72176743 & 90682837 & 711 & 754 & 43 \\
\hline 77434720 & 90682837 & 721 & 754 & 33 \\
\hline 77434720 & 90682837 & 716 & 751 & 35 \\
\hline 72176743 & 93266458 & 711 & 774 & 63 \\
\hline 77434720 & 90682837 & 724 & 754 & 30 \\
\hline 77434720 & 90682837 & 720 & 756 & 36 \\
\hline 72176743 & 90682837 & 703 & 758 & 55 \\
\hline 77434720 & 90682837 & 716 & 755 & 39 \\
\hline 77434720 & 88754535 & 716 & 744 & 28 \\
\hline 77434720 & 90682837 & 721 & 752 & 31 \\
\hline 84367585 & 90682837 & 732 & 748 & 16 \\
\hline 72176743 & 90682837 & 703 & 753 & 50 \\
\hline 77434720 & 90682837 & 721 & 758 & 37 \\
\hline 72176743 & 90682837 & 706 & 755 & 49 \\
\hline 79034049 & 90682837 & 726 & 749 & 23 \\
\hline 77434720 & 93266458 & 720 & 775 & 55 \\
\hline 84367585 & 90682837 & 732 & 768 & 36 \\
\hline 72176743 & 90682837 & 711 & 758 & 47 \\
\hline 67680565 & 98535208 & 693 & 806 & 113 \\
\hline 84825964 & 90682837 & 737 & 750 & 13 \\
\hline 84367585 & 90682837 & 732 & 764 & 32 \\
\hline 84367585 & 90682837 & 733 & 756 & 23 \\
\hline 84825964 & 90682837 & 736 & 759 & 23 \\
\hline 83317202 & 90682837 & 731 & 761 & 30 \\
\hline 84825964 & 90682837 & 735 & 757 & 22 \\
\hline 79034049 & 90682837 & 726 & 762 & 36 \\
\hline 84367585 & 90682837 & 732 & 753 & 21 \\
\hline 79034049 & 90682837 & 729 & 761 & 32 \\
\hline 84825964 & 90682837 & 734 & 758 & 24 \\
\hline 77434720 & 90682837 & 715 & 755 & 40 \\
\hline 84825964 & 90682837 & 734 & 754 & 20 \\
\hline 84825964 & 90682837 & 735 & 751 & 16 \\
\hline 84825964 & 90682837 & 735 & 754 & 19 \\
\hline 77434720 & 90682837 & 723 & 755 & 32 \\
\hline 83317202 & 90682837 & 731 & 756 & 25 \\
\hline 84825964 & 90682837 & 736 & 755 & 19 \\
\hline 84825964 & 90682837 & 736 & 758 & 22 \\
\hline 84825964 & 90682837 & 736 & 760 & 24 \\
\hline 84825964 & 90682837 & 735 & 758 & 23 \\
\hline 84825964 & 90682837 & 737 & 755 & 18 \\
\hline 84367585 & 90682837 & 732 & 754 & 22 \\
\hline 84825964 & 90682837 & 737 & 758 & 21 \\
\hline
\end{tabular}




\begin{tabular}{|c|c|c|c|c|}
\hline 84367585 & 90682837 & 732 & 754 & 22 \\
\hline 84825964 & 90682837 & 737 & 760 & 23 \\
\hline 72176743 & 93266458 & 712 & 774 & 62 \\
\hline 84825964 & 90682837 & 734 & 754 & 20 \\
\hline 86276688 & 90682837 & 741 & 754 & 13 \\
\hline 77434720 & 90682837 & 715 & 766 & 51 \\
\hline 77434720 & 90682837 & 723 & 757 & 34 \\
\hline 84367585 & 90682837 & 732 & 756 & 24 \\
\hline 77434720 & 90682837 & 724 & 754 & 30 \\
\hline 77434720 & 90682837 & 718 & 755 & 37 \\
\hline 84825964 & 90682837 & 738 & 756 & 18 \\
\hline 84367585 & 90682837 & 732 & 759 & 27 \\
\hline 84367585 & 90682837 & 732 & 753 & 21 \\
\hline 84825964 & 90682837 & 735 & 757 & 22 \\
\hline 84825964 & 90682837 & 735 & 758 & 23 \\
\hline 79034049 & 90682837 & 725 & 759 & 34 \\
\hline 84825964 & 90682837 & 738 & 759 & 21 \\
\hline 84825964 & 93266458 & 740 & 773 & 33 \\
\hline 83317202 & 90682837 & 731 & 760 & 29 \\
\hline 84825964 & 90682837 & 739 & 761 & 22 \\
\hline 84825964 & 93266458 & 736 & 776 & 40 \\
\hline 84825964 & 90682837 & 736 & 764 & 28 \\
\hline 84825964 & 90682837 & 736 & 769 & 33 \\
\hline 84825964 & 90682837 & 736 & 769 & 33 \\
\hline 84825964 & 90682837 & 740 & 769 & 29 \\
\hline 84825964 & 95593899 & 738 & 786 & 48 \\
\hline 84367585 & 98535208 & 732 & 798 & 66 \\
\hline 72176743 & 93266458 & 712 & 779 & 67 \\
\hline 84825964 & 90682837 & 737 & 770 & 33 \\
\hline 84825964 & 93266458 & 737 & 782 & 45 \\
\hline 84825964 & 95593899 & 740 & 787 & 47 \\
\hline 84825964 & 98535208 & 737 & 809 & 72 \\
\hline 84367585 & 97040983 & 732 & 793 & 61 \\
\hline 77434720 & 98535208 & 718 & 804 & 86 \\
\hline 86276688 & 97040983 & 741 & 797 & 56 \\
\hline 84825964 & 93266458 & 738 & 781 & 43 \\
\hline 88754535 & 93266458 & 747 & 779 & 32 \\
\hline 84367585 & 97040983 & 732 & 791 & 59 \\
\hline 86276688 & 97040983 & 745 & 796 & 51 \\
\hline 84367585 & 98535208 & 732 & 804 & 72 \\
\hline 84825964 & 93266458 & 736 & 778 & 42 \\
\hline 77434720 & 93266458 & 714 & 779 & 65 \\
\hline 88754535 & 93266458 & 753 & 776 & 23 \\
\hline
\end{tabular}




\begin{tabular}{|c|c|c|c|c|}
\hline 84367585 & 93266458 & 732 & 781 & 49 \\
\hline 84825964 & 98535208 & 739 & 802 & 63 \\
\hline 84825964 & 98535208 & 738 & 809 & 71 \\
\hline 88754535 & 93266458 & 749 & 778 & 29 \\
\hline 88754535 & 93266458 & 749 & 780 & 31 \\
\hline 86276688 & 93266458 & 741 & 781 & 40 \\
\hline 84825964 & 98535208 & 737 & 807 & 70 \\
\hline 88754535 & 98535208 & 746 & 804 & 58 \\
\hline 88754535 & 93266458 & 747 & 784 & 37 \\
\hline 76137316 & 93266458 & 713 & 784 & 71 \\
\hline 88754535 & 93266458 & 751 & 782 & 31 \\
\hline 88754535 & 93266458 & 749 & 783 & 34 \\
\hline 88754535 & 93266458 & 750 & 779 & 29 \\
\hline 88754535 & 97040983 & 754 & 796 & 42 \\
\hline 88754535 & 93266458 & 748 & 781 & 33 \\
\hline 88754535 & 93266458 & 759 & 781 & 22 \\
\hline 88754535 & 93266458 & 754 & 778 & 24 \\
\hline 88754535 & 93266458 & 748 & 778 & 30 \\
\hline 88754535 & 93266458 & 760 & 777 & 17 \\
\hline 88754535 & 93266458 & 754 & 781 & 27 \\
\hline 88754535 & 93266458 & 753 & 780 & 27 \\
\hline 88754535 & 93266458 & 750 & 784 & 34 \\
\hline 88754535 & 93266458 & 759 & 778 & 19 \\
\hline 88754535 & 93266458 & 761 & 781 & 20 \\
\hline 88754535 & 93266458 & 757 & 783 & 26 \\
\hline 88754535 & 93266458 & 759 & 779 & 20 \\
\hline 88754535 & 98535208 & 759 & 800 & 41 \\
\hline 88754535 & 95593899 & 762 & 785 & 23 \\
\hline 88754535 & 93266458 & 762 & 783 & 21 \\
\hline 86276688 & 98535208 & 744 & 807 & 63 \\
\hline 88754535 & 95593899 & 765 & 786 & 21 \\
\hline 88754535 & 98535208 & 748 & 801 & 53 \\
\hline 88754535 & 95593899 & 751 & 787 & 36 \\
\hline 88754535 & 97040983 & 751 & 795 & 44 \\
\hline 88754535 & 97040983 & 755 & 795 & 40 \\
\hline 86276688 & 98535208 & 741 & 809 & 68 \\
\hline 88754535 & 98535208 & 754 & 804 & 50 \\
\hline 88754535 & 98535208 & 747 & 803 & 56 \\
\hline 84825964 & 98535208 & 736 & 803 & 67 \\
\hline 88754535 & 98535208 & 762 & 802 & 40 \\
\hline 84825964 & 98535208 & 738 & 801 & 63 \\
\hline 88754535 & 97040983 & 754 & 795 & 41 \\
\hline 72176743 & 100283237 & 709 & 815 & 106 \\
\hline
\end{tabular}




\begin{tabular}{|c|c|c|c|c|}
\hline 84825964 & 109567246 & 738 & 839 & 101 \\
\hline 88754535 & 98535208 & 766 & 810 & 44 \\
\hline 86276688 & 98535208 & 742 & 803 & 61 \\
\hline 88754535 & 98535208 & 765 & 802 & 37 \\
\hline 88754535 & 98535208 & 763 & 808 & 45 \\
\hline 90682837 & 98535208 & 773 & 803 & 30 \\
\hline 90682837 & 97040983 & 772 & 793 & 21 \\
\hline 88754535 & 98535208 & 763 & 803 & 40 \\
\hline 88754535 & 98535208 & 769 & 804 & 35 \\
\hline 88754535 & 97040983 & 767 & 795 & 28 \\
\hline 88754535 & 98535208 & 770 & 804 & 34 \\
\hline 90682837 & 97040983 & 773 & 793 & 20 \\
\hline 90682837 & 97040983 & 777 & 790 & 13 \\
\hline 90682837 & 97040983 & 779 & 790 & 11 \\
\hline 90682837 & 98535208 & 779 & 802 & 23 \\
\hline 90682837 & 98535208 & 779 & 804 & 25 \\
\hline 90682837 & 98535208 & 778 & 805 & 27 \\
\hline 90682837 & 98535208 & 778 & 807 & 29 \\
\hline 90682837 & 98535208 & 778 & 807 & 29 \\
\hline 90682837 & 98535208 & 778 & 807 & 29 \\
\hline 90682837 & 98535208 & 777 & 808 & 31 \\
\hline 90682837 & 98535208 & 778 & 807 & 29 \\
\hline 90682837 & 98535208 & 777 & 808 & 31 \\
\hline 90682837 & 97040983 & 780 & 795 & 15 \\
\hline 90682837 & 98535208 & 774 & 803 & 29 \\
\hline 90682837 & 98535208 & 776 & 809 & 33 \\
\hline 90682837 & 98535208 & 777 & 809 & 32 \\
\hline 90682837 & 98535208 & 777 & 808 & 31 \\
\hline 90682837 & 98535208 & 777 & 810 & 33 \\
\hline 90682837 & 98535208 & 776 & 810 & 34 \\
\hline 90682837 & 97040983 & 776 & 796 & 20 \\
\hline 90682837 & 98535208 & 776 & 809 & 33 \\
\hline 90682837 & 98535208 & 777 & 810 & 33 \\
\hline 90682837 & 98535208 & 777 & 809 & 32 \\
\hline 90682837 & 98535208 & 777 & 808 & 31 \\
\hline 90682837 & 98535208 & 777 & 809 & 32 \\
\hline 90682837 & 98535208 & 777 & 809 & 32 \\
\hline 90682837 & 98535208 & 779 & 806 & 27 \\
\hline 90682837 & 98535208 & 776 & 810 & 34 \\
\hline 90682837 & 98535208 & 777 & 809 & 32 \\
\hline 90682837 & 98535208 & 777 & 808 & 31 \\
\hline 90682837 & 98535208 & 778 & 806 & 28 \\
\hline 90682837 & 98535208 & 773 & 810 & 37 \\
\hline
\end{tabular}




\begin{tabular}{|c|c|c|c|}
\hline 90682837 & 109567246 & 775 & 870 \\
\hline 90682837 & 109567246 & 777 & 870 \\
\hline 90682837 & 109567246 & 776 & 873 \\
\hline 90682837 & 109567246 & 776 & 874 \\
\hline 90682837 & 98535208 & 776 & 810 \\
\hline 90682837 & 98535208 & 777 & 810 \\
\hline 93266458 & 97040983 & 785 & 793 \\
\hline 90682837 & 97040983 & 783 & 794 \\
\hline 90682837 & 97040983 & 780 & 793 \\
\hline 90682837 & 97040983 & 773 & 796 \\
\hline 90682837 & 98535208 & 776 & 800 \\
\hline 90682837 & 97040983 & 775 & 795 \\
\hline 90682837 & 98535208 & 774 & 804 \\
\hline 90682837 & 97040983 & 780 & 796 \\
\hline 90682837 & 98535208 & 781 & 801 \\
\hline 90682837 & 97040983 & 774 & 796 \\
\hline 90682837 & 97040983 & 778 & 795 \\
\hline 90682837 & 97040983 & 777 & 793 \\
\hline 90682837 & 98535208 & 777 & 799 \\
\hline 90682837 & 97040983 & 781 & 796 \\
\hline 90682837 & 97040983 & 777 & 794 \\
\hline 90682837 & 98535208 & 774 & 804 \\
\hline 88754535 & 98535208 & 764 & 809 \\
\hline 93266458 & 97040983 & 786 & 792 \\
\hline 88754535 & 98535208 & 755 & 798 \\
\hline 90682837 & 98535208 & 775 & 805 \\
\hline 90682837 & 98535208 & 776 & 809 \\
\hline 88754535 & 98535208 & 767 & 807 \\
\hline 90682837 & 98535208 & 778 & 805 \\
\hline 88754535 & 98535208 & 766 & 810 \\
\hline 93266458 & 98535208 & 786 & 802 \\
\hline 93266458 & 97040983 & 786 & 797 \\
\hline 90682837 & 98535208 & 776 & 809 \\
\hline 90682837 & 98535208 & 775 & 809 \\
\hline 88754535 & 98535208 & 758 & 807 \\
\hline 88754535 & 109567246 & 752 & 866 \\
\hline 88754535 & 98535208 & 762 & 802 \\
\hline 90682837 & 98535208 & 772 & 806 \\
\hline 90682837 & 98535208 & 777 & 801 \\
\hline 88754535 & 98535208 & 745 & 810 \\
\hline 90682837 & 98535208 & 774 & 806 \\
\hline 90682837 & 98535208 & 776 & 803 \\
\hline 90682837 & 98535208 & 781 & 809 \\
\hline
\end{tabular}




\begin{tabular}{|c|c|c|c|}
\hline 90682837 & 98535208 & 782 & 807 \\
\hline 90682837 & 98535208 & 779 & 805 \\
\hline 90682837 & 98535208 & 775 & 808 \\
\hline 90682837 & 98535208 & 777 & 807 \\
\hline 90682837 & 98535208 & 780 & 808 \\
\hline 90682837 & 98535208 & 779 & 807 \\
\hline 88754535 & 98535208 & 758 & 808 \\
\hline 90682837 & 109567246 & 780 & 863 \\
\hline 90682837 & 98535208 & 777 & 806 \\
\hline 90682837 & 98535208 & 778 & 810 \\
\hline 90682837 & 98535208 & 773 & 808 \\
\hline 90682837 & 98535208 & 776 & 805 \\
\hline 88754535 & 98535208 & 757 & 808 \\
\hline 90682837 & 98535208 & 779 & 808 \\
\hline 90682837 & 98535208 & 783 & 806 \\
\hline 90682837 & 98535208 & 778 & 809 \\
\hline 90682837 & 109567246 & 772 & 844 \\
\hline 90682837 & 109567246 & 781 & 859 \\
\hline 90682837 & 98535208 & 778 & 806 \\
\hline 90682837 & 98535208 & 772 & 809 \\
\hline 84825964 & 98535208 & 737 & 805 \\
\hline 90682837 & 98535208 & 779 & 806 \\
\hline 90682837 & 98535208 & 782 & 806 \\
\hline 88754535 & 98535208 & 768 & 804 \\
\hline 95593899 & 98535208 & 790 & 801 \\
\hline 93266458 & 98535208 & 787 & 807 \\
\hline 90682837 & 98535208 & 778 & 806 \\
\hline 95593899 & 98535208 & 790 & 806 \\
\hline 90682837 & 98535208 & 773 & 807 \\
\hline 95593899 & 98535208 & 791 & 807 \\
\hline 88754535 & 98535208 & 763 & 806 \\
\hline 90682837 & 100283237 & 775 & 815 \\
\hline 90682837 & 98535208 & 783 & 808 \\
\hline 90682837 & 98535208 & 781 & 807 \\
\hline 90682837 & 98535208 & 776 & 810 \\
\hline 93266458 & 98535208 & 786 & 808 \\
\hline 95593899 & 98535208 & 789 & 808 \\
\hline 90682837 & 98535208 & 782 & 810 \\
\hline 90682837 & 100283237 & 776 & 816 \\
\hline 90682837 & 98535208 & 771 & 810 \\
\hline 90682837 & 98535208 & 783 & 810 \\
\hline 95593899 & 98535208 & 792 & 808 \\
\hline 93266458 & 98535208 & 786 & 809 \\
\hline
\end{tabular}




\begin{tabular}{|c|c|c|c|c|}
\hline 90682837 & 103195604 & 779 & 826 & 47 \\
\hline 95593899 & 100283237 & 790 & 813 & 23 \\
\hline 90682837 & 109567246 & 774 & 859 & 85 \\
\hline 90682837 & 98535208 & 783 & 810 & 27 \\
\hline 93266458 & 109567246 & 787 & 851 & 64 \\
\hline 95593899 & 103850588 & 789 & 836 & 47 \\
\hline 86276688 & 109567246 & 745 & 849 & 104 \\
\hline 93266458 & 109567246 & 785 & 864 & 79 \\
\hline 88754535 & 109567246 & 756 & 848 & 92 \\
\hline 88754535 & 109567246 & 752 & 875 & 123 \\
\hline 95593899 & 109567246 & 790 & 862 & 72 \\
\hline 95593899 & 109567246 & 788 & 854 & 66 \\
\hline 90682837 & 109567246 & 776 & 865 & 89 \\
\hline 88754535 & 109567246 & 747 & 867 & 120 \\
\hline 95593899 & 109567246 & 791 & 855 & 64 \\
\hline 97040983 & 100283237 & 802 & 814 & 12 \\
\hline 93266458 & 103195604 & 785 & 821 & 36 \\
\hline 97040983 & 103850588 & 800 & 835 & 35 \\
\hline 93266458 & 109567246 & 786 & 847 & 61 \\
\hline 97040983 & 103195604 & 806 & 826 & 20 \\
\hline 97040983 & 100283237 & 801 & 818 & 17 \\
\hline 97040983 & 109567246 & 800 & 858 & 58 \\
\hline 97040983 & 109567246 & 801 & 848 & 47 \\
\hline 97040983 & 103850588 & 801 & 836 & 35 \\
\hline 93266458 & 109567246 & 787 & 863 & 76 \\
\hline 97040983 & 109567246 & 800 & 861 & 61 \\
\hline 97040983 & 103850588 & 801 & 831 & 30 \\
\hline 97040983 & 109567246 & 802 & 851 & 49 \\
\hline 95593899 & 109567246 & 791 & 866 & 75 \\
\hline 97040983 & 109567246 & 802 & 847 & 45 \\
\hline 97040983 & 109567246 & 801 & 861 & 60 \\
\hline 97040983 & 109567246 & 800 & 861 & 61 \\
\hline 97040983 & 109567246 & 800 & 862 & 62 \\
\hline 97040983 & 109567246 & 803 & 859 & 56 \\
\hline 97040983 & 109567246 & 800 & 863 & 63 \\
\hline 90682837 & 109567246 & 779 & 862 & 83 \\
\hline 97040983 & 109567246 & 801 & 851 & 50 \\
\hline 93266458 & 109567246 & 787 & 871 & 84 \\
\hline 97040983 & 109567246 & 801 & 870 & 69 \\
\hline 97040983 & 109567246 & 801 & 844 & 43 \\
\hline 95593899 & 109567246 & 795 & 870 & 75 \\
\hline 97040983 & 109567246 & 800 & 859 & 59 \\
\hline 93266458 & 109567246 & 787 & 856 & 69 \\
\hline
\end{tabular}




\begin{tabular}{|c|c|c|c|c|}
\hline 97040983 & 109567246 & 802 & 870 & 68 \\
\hline 97040983 & 109567246 & 805 & 851 & 46 \\
\hline 97040983 & 109567246 & 806 & 848 & 42 \\
\hline 97040983 & 109567246 & 811 & 855 & 44 \\
\hline 95593899 & 109567246 & 792 & 847 & 55 \\
\hline 97040983 & 109567246 & 808 & 855 & 47 \\
\hline 97040983 & 109567246 & 803 & 860 & 57 \\
\hline 95593899 & 109567246 & 792 & 850 & 58 \\
\hline 98535208 & 109567246 & 814 & 853 & 39 \\
\hline 97040983 & 109567246 & 807 & 853 & 46 \\
\hline 98535208 & 109567246 & 813 & 865 & 52 \\
\hline 98535208 & 109567246 & 812 & 852 & 40 \\
\hline 98535208 & 109567246 & 813 & 855 & 42 \\
\hline 98535208 & 109567246 & 814 & 858 & 44 \\
\hline 98535208 & 109567246 & 812 & 841 & 29 \\
\hline 98535208 & 109567246 & 813 & 851 & 38 \\
\hline 97040983 & 103850588 & 804 & 835 & 31 \\
\hline 97040983 & 109567246 & 805 & 849 & 44 \\
\hline 93266458 & 109567246 & 787 & 859 & 72 \\
\hline 97040983 & 109567246 & 809 & 856 & 47 \\
\hline 98535208 & 109567246 & 813 & 857 & 44 \\
\hline 95593899 & 109567246 & 791 & 868 & 77 \\
\hline 95593899 & 109567246 & 794 & 862 & 68 \\
\hline 95593899 & 109567246 & 789 & 862 & 73 \\
\hline 98535208 & 109567246 & 813 & 862 & 49 \\
\hline 90682837 & 109567246 & 781 & 862 & 81 \\
\hline 95593899 & 109567246 & 793 & 858 & 65 \\
\hline 98535208 & 109567246 & 813 & 855 & 42 \\
\hline 98535208 & 109567246 & 814 & 854 & 40 \\
\hline 97040983 & 109567246 & 805 & 862 & 57 \\
\hline 95593899 & 109567246 & 795 & 859 & 64 \\
\hline 95593899 & 109567246 & 792 & 861 & 69 \\
\hline 98535208 & 109567246 & 815 & 864 & 49 \\
\hline 98535208 & 109567246 & 816 & 847 & 31 \\
\hline 97040983 & 109567246 & 802 & 866 & 64 \\
\hline 97040983 & 109567246 & 810 & 861 & 51 \\
\hline 97040983 & 109567246 & 801 & 872 & 71 \\
\hline 93266458 & 109567246 & 787 & 869 & 82 \\
\hline 97040983 & 109567246 & 807 & 860 & 53 \\
\hline 98535208 & 109567246 & 814 & 875 & 61 \\
\hline 97040983 & 109567246 & 804 & 863 & 59 \\
\hline 98535208 & 109567246 & 814 & 857 & 43 \\
\hline 98535208 & 109567246 & 816 & 867 & 51 \\
\hline
\end{tabular}




\begin{tabular}{|c|c|c|c|c|}
\hline 98535208 & 109567246 & 813 & 856 & 43 \\
\hline 97040983 & 109567246 & 807 & 864 & 57 \\
\hline 97040983 & 109567246 & 806 & 863 & 57 \\
\hline 98535208 & 109567246 & 814 & 865 & 51 \\
\hline 98535208 & 109567246 & 816 & 860 & 44 \\
\hline 97040983 & 109567246 & 802 & 862 & 60 \\
\hline 95593899 & 109567246 & 796 & 862 & 66 \\
\hline 98535208 & 109567246 & 815 & 871 & 56 \\
\hline 97040983 & 109567246 & 804 & 865 & 61 \\
\hline 97040983 & 109567246 & 801 & 860 & 59 \\
\hline 90682837 & 109567246 & 778 & 875 & 97 \\
\hline 98535208 & 109567246 & 813 & 865 & 52 \\
\hline 95593899 & 109567246 & 796 & 871 & 75 \\
\hline 98535208 & 109567246 & 815 & 865 & 50 \\
\hline 98535208 & 109567246 & 815 & 870 & 55 \\
\hline 97040983 & 109567246 & 800 & 858 & 58 \\
\hline 98535208 & 109567246 & 816 & 857 & 41 \\
\hline 98535208 & 109567246 & 814 & 859 & 45 \\
\hline 98535208 & 109567246 & 814 & 866 & 52 \\
\hline 97040983 & 109567246 & 805 & 865 & 60 \\
\hline 98535208 & 109567246 & 814 & 864 & 50 \\
\hline 98535208 & 109567246 & 815 & 860 & 45 \\
\hline 97040983 & 109567246 & 800 & 871 & 71 \\
\hline 97040983 & 109567246 & 802 & 864 & 62 \\
\hline 93266458 & 109567246 & 787 & 865 & 78 \\
\hline 95593899 & 109567246 & 795 & 875 & 80 \\
\hline 95593899 & 109567246 & 790 & 873 & 83 \\
\hline 98535208 & 109567246 & 815 & 869 & 54 \\
\hline 98535208 & 109567246 & 814 & 872 & 58 \\
\hline 98535208 & 109567246 & 813 & 868 & 55 \\
\hline 95593899 & 109567246 & 792 & 877 & 85 \\
\hline 95593899 & 109567246 & 795 & 874 & 79 \\
\hline 98535208 & 109567246 & 814 & 876 & 62 \\
\hline 100283237 & 109567246 & 825 & 866 & 41 \\
\hline 97040983 & 109567246 & 801 & 877 & 76 \\
\hline 95593899 & 109567246 & 788 & 874 & 86 \\
\hline 98535208 & 109567246 & 813 & 873 & 60 \\
\hline 98535208 & 109567246 & 815 & 878 & 63 \\
\hline 95593899 & 109567246 & 797 & 879 & 82 \\
\hline 97040983 & 109567246 & 811 & 877 & 66 \\
\hline 97040983 & 109567246 & 804 & 876 & 72 \\
\hline 97040983 & 109567246 & 807 & 882 & 75 \\
\hline 95593899 & 109567246 & 790 & 894 & 104 \\
\hline
\end{tabular}




\begin{tabular}{|c|c|c|c|c|}
\hline 103195604 & 114337754 & 834 & 947 & 113 \\
\hline 88754535 & 111656920 & 761 & 929 & 168 \\
\hline 103850588 & 111656920 & 858 & 928 & 70 \\
\hline 103850588 & 114337754 & 844 & 945 & 101 \\
\hline 103850588 & 111656920 & 855 & 929 & 74 \\
\hline 103850588 & 112709273 & 861 & 936 & 75 \\
\hline 103850588 & 112709273 & 867 & 941 & 74 \\
\hline 103850588 & 112709273 & 890 & 933 & 43 \\
\hline 103850588 & 111656920 & 893 & 930 & 37 \\
\hline 103850588 & 111656920 & 882 & 927 & 45 \\
\hline 103850588 & 111656920 & 883 & 928 & 45 \\
\hline 103850588 & 111656920 & 884 & 928 & 44 \\
\hline 103850588 & 111656920 & 881 & 929 & 48 \\
\hline 103850588 & 112709273 & 884 & 938 & 54 \\
\hline 103850588 & 111656920 & 888 & 930 & 42 \\
\hline 103850588 & 111656920 & 888 & 929 & 41 \\
\hline 103850588 & 114798111 & 875 & 956 & 81 \\
\hline 109567246 & 110592265 & 921 & 926 & 5 \\
\hline 103850588 & 110592265 & 911 & 926 & 15 \\
\hline 103850588 & 111656920 & 878 & 927 & 49 \\
\hline 109567246 & 110592265 & 923 & 926 & 3 \\
\hline 103850588 & 112709273 & 877 & 937 & 60 \\
\hline 103850588 & 112709273 & 884 & 937 & 53 \\
\hline 103850588 & 112709273 & 887 & 939 & 52 \\
\hline 103850588 & 114337754 & 886 & 950 & 64 \\
\hline 103850588 & 114337754 & 895 & 948 & 53 \\
\hline 103850588 & 112709273 & 881 & 937 & 56 \\
\hline 103850588 & 114337754 & 857 & 952 & 95 \\
\hline 103850588 & 114798111 & 884 & 956 & 72 \\
\hline 103850588 & 112709273 & 897 & 939 & 42 \\
\hline 103850588 & 117236337 & 894 & 962 & 68 \\
\hline 103850588 & 117236337 & 872 & 964 & 92 \\
\hline 109567246 & 112709273 & 921 & 941 & 20 \\
\hline 103850588 & 112709273 & 905 & 938 & 33 \\
\hline 103850588 & 117236337 & 903 & 967 & 64 \\
\hline 103850588 & 112709273 & 895 & 941 & 46 \\
\hline 103850588 & 112709273 & 899 & 940 & 41 \\
\hline 109567246 & 114337754 & 922 & 950 & 28 \\
\hline 103850588 & 117236337 & 897 & 970 & 73 \\
\hline 103850588 & 114798111 & 894 & 958 & 64 \\
\hline 103850588 & 112709273 & 885 & 940 & 55 \\
\hline 103850588 & 117236337 & 875 & 968 & 93 \\
\hline 110592265 & 112709273 & 927 & 938 & \\
\hline
\end{tabular}




\begin{tabular}{|c|c|c|c|}
\hline 110592265 & 112709273 & 927 & 938 \\
\hline 110592265 & 112709273 & 927 & 937 \\
\hline 109567246 & 112709273 & 925 & 941 \\
\hline 110592265 & 112709273 & 928 & 939 \\
\hline 103850588 & 120193154 & 889 & 989 \\
\hline 103850588 & 117236337 & 893 & 970 \\
\hline 103850588 & 114337754 & 886 & 953 \\
\hline 110592265 & 112709273 & 928 & 938 \\
\hline 110592265 & 112709273 & 929 & 940 \\
\hline 110592265 & 112709273 & 928 & 937 \\
\hline 110592265 & 112709273 & 927 & 938 \\
\hline 110592265 & 112709273 & 928 & 937 \\
\hline 110592265 & 117236337 & 929 & 970 \\
\hline 103850588 & 112709273 & 903 & 942 \\
\hline 109567246 & 114798111 & 923 & 961 \\
\hline 110592265 & 114337754 & 927 & 945 \\
\hline 103850588 & 114798111 & 889 & 960 \\
\hline 110592265 & 114337754 & 927 & 954 \\
\hline 103850588 & 117236337 & 890 & 963 \\
\hline 110592265 & 114337754 & 928 & 949 \\
\hline 110592265 & 114798111 & 927 & 955 \\
\hline 103850588 & 114337754 & 853 & 952 \\
\hline 103850588 & 117236337 & 888 & 965 \\
\hline 103850588 & 114337754 & 877 & 954 \\
\hline 111656920 & 114337754 & 932 & 953 \\
\hline 111656920 & 114798111 & 933 & 956 \\
\hline 103850588 & 117236337 & 881 & 967 \\
\hline 103850588 & 117236337 & 884 & 965 \\
\hline 103850588 & 114798111 & 879 & 959 \\
\hline 103850588 & 114798111 & 895 & 959 \\
\hline 109567246 & 114798111 & 923 & 959 \\
\hline 110592265 & 114798111 & 928 & 959 \\
\hline 103850588 & 117236337 & 880 & 969 \\
\hline 112709273 & 114798111 & 947 & 959 \\
\hline 112709273 & 114798111 & 947 & 957 \\
\hline 112709273 & 117236337 & 948 & 963 \\
\hline 111656920 & 114798111 & 932 & 960 \\
\hline 103850588 & 117236337 & 893 & 972 \\
\hline 103850588 & 117236337 & 899 & 975 \\
\hline 111656920 & 117236337 & 939 & 974 \\
\hline 112709273 & 117236337 & 946 & 974 \\
\hline 112709273 & 117236337 & 944 & 968 \\
\hline 112709273 & 117236337 & 944 & 968 \\
\hline
\end{tabular}




\begin{tabular}{|c|c|c|c|}
\hline 112709273 & 117236337 & 947 & 978 \\
\hline 110592265 & 117236337 & 929 & 972 \\
\hline 112709273 & 117236337 & 947 & 970 \\
\hline 112709273 & 117236337 & 947 & 971 \\
\hline 112709273 & 117236337 & 950 & 971 \\
\hline 114337754 & 117236337 & 955 & 976 \\
\hline 112709273 & 117236337 & 947 & 975 \\
\hline 112709273 & 124905306 & 950 & 999 \\
\hline 114337754 & 120193154 & 959 & 991 \\
\hline 114337754 & 120193154 & 961 & 991 \\
\hline 114798111 & 124905306 & 970 & 1003 \\
\hline 114798111 & 120193154 & 970 & 990 \\
\hline 114798111 & 120193154 & 968 & 991 \\
\hline 114798111 & 124905306 & 967 & 1005 \\
\hline 114798111 & 124905306 & 962 & 1020 \\
\hline 114798111 & 125665077 & 966 & 1032 \\
\hline 114798111 & 127004900 & 963 & 1043 \\
\hline 117236337 & 124905306 & 982 & 1003 \\
\hline 114798111 & 124905306 & 978 & 1015 \\
\hline 114798111 & 124905306 & 969 & 1011 \\
\hline 114798111 & 124905306 & 965 & 1016 \\
\hline 117236337 & 124905306 & 985 & 1009 \\
\hline 117236337 & 124905306 & 986 & 1013 \\
\hline 117236337 & 124905306 & 987 & 1019 \\
\hline 117236337 & 124905306 & 985 & 1011 \\
\hline 117236337 & 124905306 & 984 & 1013 \\
\hline 117236337 & 124905306 & 985 & 1024 \\
\hline 117236337 & 124905306 & 988 & 1017 \\
\hline 117236337 & 124905306 & 984 & 1028 \\
\hline 117236337 & 124905306 & 982 & 1023 \\
\hline 117236337 & 124905306 & 988 & 1017 \\
\hline 114337754 & 127004900 & 958 & 1041 \\
\hline 117236337 & 124905306 & 989 & 1027 \\
\hline 117236337 & 124905306 & 985 & 1025 \\
\hline 117236337 & 127004900 & 984 & 1041 \\
\hline 117236337 & 124905306 & 987 & 1029 \\
\hline 114798111 & 127004900 & 966 & 1043 \\
\hline 112709273 & 124905306 & 945 & 1030 \\
\hline 117236337 & 127004900 & 984 & 1040 \\
\hline 117236337 & 127004900 & 987 & 1043 \\
\hline 117236337 & 127004900 & 990 & 1039 \\
\hline 117236337 & 127004900 & 990 & 1049 \\
\hline 117236337 & 127004900 & 992 & 1041 \\
\hline
\end{tabular}




\begin{tabular}{|c|c|c|c|c|}
\hline 120303305 & 127004900 & 1002 & 1048 & 46 \\
\hline 120303305 & 127004900 & 996 & 1044 & 48 \\
\hline 120303305 & 127004900 & 997 & 1042 & 45 \\
\hline 117236337 & 127004900 & 985 & 1050 & 65 \\
\hline 120303305 & 127004900 & 1002 & 1046 & 44 \\
\hline 120303305 & 127004900 & 1011 & 1044 & 33 \\
\hline 120303305 & 127004900 & 1014 & 1040 & 26 \\
\hline 120303305 & 127004900 & 1005 & 1042 & 37 \\
\hline 120303305 & 127004900 & 1009 & 1042 & 33 \\
\hline 120303305 & 127004900 & 1015 & 1040 & 25 \\
\hline 120303305 & 127004900 & 1015 & 1043 & 28 \\
\hline 120303305 & 127004900 & 1021 & 1043 & 22 \\
\hline 120303305 & 127004900 & 1025 & 1042 & 17 \\
\hline 120303305 & 127004900 & 1022 & 1041 & 19 \\
\hline 120303305 & 127004900 & 1014 & 1041 & 27 \\
\hline 120303305 & 127004900 & 1012 & 1044 & 32 \\
\hline 120303305 & 127004900 & 1018 & 1046 & 28 \\
\hline 120303305 & 127004900 & 1016 & 1044 & 28 \\
\hline 120303305 & 129145419 & 1004 & 1056 & 52 \\
\hline 120303305 & 127004900 & 1002 & 1047 & 45 \\
\hline 120303305 & 127004900 & 1013 & 1045 & 32 \\
\hline 120303305 & 127004900 & 1015 & 1043 & 28 \\
\hline 117236337 & 127004900 & 987 & 1041 & 54 \\
\hline 120303305 & 127004900 & 1012 & 1042 & 30 \\
\hline 120303305 & 127004900 & 1003 & 1047 & 44 \\
\hline 120303305 & 127004900 & 1004 & 1042 & 38 \\
\hline 120303305 & 127004900 & 1008 & 1048 & 40 \\
\hline 120303305 & 127004900 & 1015 & 1049 & 34 \\
\hline 120303305 & 127004900 & 1017 & 1043 & 26 \\
\hline 120303305 & 127004900 & 1007 & 1042 & 35 \\
\hline 120303305 & 127004900 & 1017 & 1046 & 29 \\
\hline 120303305 & 127004900 & 1004 & 1047 & 43 \\
\hline 114337754 & 127004900 & 959 & 1047 & 88 \\
\hline 120303305 & 127004900 & 1013 & 1048 & 35 \\
\hline 120303305 & 127004900 & 1014 & 1042 & 28 \\
\hline 120303305 & 127004900 & 1006 & 1047 & 41 \\
\hline 120303305 & 127004900 & 1017 & 1045 & 28 \\
\hline 120193154 & 127004900 & 994 & 1049 & 55 \\
\hline 120303305 & 129145419 & 1002 & 1095 & 93 \\
\hline 120303305 & 127004900 & 1007 & 1050 & 43 \\
\hline 114798111 & 127004900 & 970 & 1049 & 79 \\
\hline 120303305 & 127004900 & 1000 & 1050 & 50 \\
\hline 117236337 & 127004900 & 994 & 1046 & 52 \\
\hline
\end{tabular}




\begin{tabular}{|c|c|c|c|c|}
\hline 120303305 & 127004900 & 1008 & 1050 & 42 \\
\hline 120303305 & 127004900 & 1014 & 1048 & 34 \\
\hline 120303305 & 129145419 & 1006 & 1097 & 91 \\
\hline 120303305 & 127004900 & 1014 & 1049 & 35 \\
\hline 117236337 & 129145419 & 991 & 1111 & 120 \\
\hline 120303305 & 129145419 & 1005 & 1088 & 83 \\
\hline 117236337 & 129145419 & 990 & 1075 & 85 \\
\hline 120303305 & 129145419 & 998 & 1084 & 86 \\
\hline 120303305 & 129145419 & 1002 & 1080 & 78 \\
\hline 125665077 & 129145419 & 1032 & 1098 & 66 \\
\hline 125665077 & 129145419 & 1033 & 1089 & 56 \\
\hline 125665077 & 129145419 & 1042 & 1075 & 33 \\
\hline 125665077 & 129145419 & 1039 & 1083 & 44 \\
\hline 125665077 & 129145419 & 1040 & 1080 & 40 \\
\hline 125665077 & 129145419 & 1041 & 1071 & 30 \\
\hline 125665077 & 129145419 & 1041 & 1078 & 37 \\
\hline 125665077 & 129145419 & 1048 & 1062 & 14 \\
\hline 125665077 & 129145419 & 1044 & 1071 & 27 \\
\hline 125665077 & 129145419 & 1044 & 1075 & 31 \\
\hline 125665077 & 129145419 & 1045 & 1068 & 23 \\
\hline 125665077 & 129145419 & 1043 & 1077 & 34 \\
\hline 125665077 & 129145419 & 1040 & 1083 & 43 \\
\hline 125665077 & 129145419 & 1045 & 1074 & 29 \\
\hline 125665077 & 129145419 & 1045 & 1078 & 33 \\
\hline 125665077 & 129145419 & 1041 & 1078 & 37 \\
\hline 125665077 & 129145419 & 1042 & 1071 & 29 \\
\hline 125665077 & 129145419 & 1045 & 1071 & 26 \\
\hline 125665077 & 129145419 & 1039 & 1079 & 40 \\
\hline 125665077 & 129145419 & 1044 & 1077 & 33 \\
\hline 125665077 & 129145419 & 1039 & 1083 & 44 \\
\hline 125665077 & 129145419 & 1045 & 1085 & 40 \\
\hline 125665077 & 129145419 & 1040 & 1088 & 48 \\
\hline 125665077 & 129145419 & 1040 & 1089 & 49 \\
\hline 124905306 & 129145419 & 1032 & 1092 & 60 \\
\hline 120303305 & 129145419 & 999 & 1109 & 110 \\
\hline 127004900 & 136129932 & 1063 & 1137 & 74 \\
\hline 127004900 & 136129932 & 1057 & 1136 & 79 \\
\hline 120303305 & 138605748 & 1027 & 1172 & 145 \\
\hline 127004900 & 136129932 & 1064 & 1137 & 73 \\
\hline 127004900 & 136129932 & 1084 & 1132 & 48 \\
\hline 127004900 & 136129932 & 1069 & 1133 & 64 \\
\hline 127004900 & 136129932 & 1075 & 1132 & 57 \\
\hline 127004900 & 149310477 & 1051 & 1234 & 183 \\
\hline
\end{tabular}




\begin{tabular}{|c|c|c|c|c|}
\hline 127004900 & 138605748 & 1079 & 1183 & 104 \\
\hline 127004900 & 136129932 & 1091 & 1135 & 44 \\
\hline 127004900 & 136129932 & 1094 & 1135 & 41 \\
\hline 127004900 & 133441175 & 1090 & 1128 & 38 \\
\hline 127004900 & 138238126 & 1084 & 1145 & 61 \\
\hline 127004900 & 130486897 & 1089 & 1124 & 35 \\
\hline 127004900 & 133441175 & 1099 & 1128 & 29 \\
\hline 127004900 & 136129932 & 1090 & 1134 & 44 \\
\hline 127004900 & 136129932 & 1096 & 1135 & 39 \\
\hline 127004900 & 136129932 & 1088 & 1137 & 49 \\
\hline 127004900 & 133441175 & 1104 & 1130 & 26 \\
\hline 127004900 & 138238126 & 1084 & 1142 & 58 \\
\hline 127004900 & 136129932 & 1096 & 1136 & 40 \\
\hline 127004900 & 138605748 & 1084 & 1179 & 95 \\
\hline 127004900 & 136129932 & 1092 & 1135 & 43 \\
\hline 127004900 & 136129932 & 1092 & 1136 & 44 \\
\hline 127004900 & 136129932 & 1087 & 1138 & 51 \\
\hline 127004900 & 136129932 & 1096 & 1134 & 38 \\
\hline 127004900 & 136129932 & 1097 & 1135 & 38 \\
\hline 127004900 & 136129932 & 1093 & 1136 & 43 \\
\hline 127004900 & 136129932 & 1085 & 1136 & 51 \\
\hline 127004900 & 138238126 & 1083 & 1148 & 65 \\
\hline 127004900 & 136129932 & 1091 & 1136 & 45 \\
\hline 127004900 & 136129932 & 1094 & 1136 & 42 \\
\hline 129145419 & 136129932 & 1122 & 1136 & 14 \\
\hline 130486897 & 136129932 & 1126 & 1136 & 10 \\
\hline 127004900 & 138238126 & 1078 & 1148 & 70 \\
\hline 129145419 & 138238126 & 1120 & 1154 & 34 \\
\hline 130486897 & 136129932 & 1127 & 1138 & 11 \\
\hline 130486897 & 138238126 & 1127 & 1155 & 28 \\
\hline 129145419 & 138238126 & 1123 & 1153 & 30 \\
\hline 127004900 & 138605748 & 1091 & 1180 & 89 \\
\hline 130486897 & 138238126 & 1127 & 1154 & 27 \\
\hline 130486897 & 138238126 & 1126 & 1149 & 23 \\
\hline 127004900 & 138238126 & 1083 & 1147 & 64 \\
\hline 130486897 & 138238126 & 1126 & 1156 & 30 \\
\hline 133441175 & 138238126 & 1134 & 1144 & 10 \\
\hline 130486897 & 138238126 & 1128 & 1158 & 30 \\
\hline 133441175 & 138238126 & 1134 & 1144 & 10 \\
\hline 130486897 & 138238126 & 1129 & 1152 & 23 \\
\hline 133441175 & 138605748 & 1134 & 1168 & 34 \\
\hline 133441175 & 138238126 & 1133 & 1158 & 25 \\
\hline 129145419 & 138238126 & 1121 & 1157 & 36 \\
\hline
\end{tabular}




\begin{tabular}{|c|c|c|c|c|}
\hline 133441175 & 138238126 & 1136 & 1145 & 9 \\
\hline 133441175 & 138238126 & 1136 & 1149 & 13 \\
\hline 133441175 & 138238126 & 1134 & 1154 & 20 \\
\hline 133441175 & 138238126 & 1133 & 1162 & 29 \\
\hline 127004900 & 139780488 & 1080 & 1186 & 106 \\
\hline 133441175 & 138605748 & 1131 & 1168 & 37 \\
\hline 133441175 & 149310477 & 1131 & 1241 & 110 \\
\hline 127004900 & 139780488 & 1098 & 1185 & 87 \\
\hline 133441175 & 138605748 & 1133 & 1173 & 40 \\
\hline 127004900 & 148354542 & 1087 & 1193 & 106 \\
\hline 133441175 & 138605748 & 1132 & 1172 & 40 \\
\hline 127004900 & 145964815 & 1094 & 1193 & 99 \\
\hline 130486897 & 138605748 & 1128 & 1175 & 47 \\
\hline 130486897 & 138605748 & 1130 & 1177 & 47 \\
\hline 133441175 & 138605748 & 1135 & 1175 & 40 \\
\hline 133441175 & 138605748 & 1138 & 1179 & 41 \\
\hline 133441175 & 139780488 & 1134 & 1188 & 54 \\
\hline 133441175 & 149310477 & 1135 & 1229 & 94 \\
\hline 133441175 & 138605748 & 1136 & 1177 & 41 \\
\hline 133441175 & 138605748 & 1137 & 1175 & 38 \\
\hline 136129932 & 138605748 & 1142 & 1176 & 34 \\
\hline 133441175 & 138605748 & 1139 & 1183 & 44 \\
\hline 133441175 & 148354542 & 1136 & 1193 & 57 \\
\hline 136129932 & 138605748 & 1144 & 1182 & 38 \\
\hline 136129932 & 138605748 & 1146 & 1179 & 33 \\
\hline 136129932 & 138605748 & 1140 & 1179 & 39 \\
\hline 136129932 & 138605748 & 1140 & 1183 & 43 \\
\hline 136129932 & 145964815 & 1139 & 1192 & 53 \\
\hline 136129932 & 145964815 & 1145 & 1192 & 47 \\
\hline 136129932 & 138605748 & 1148 & 1180 & 32 \\
\hline 136129932 & 149310477 & 1141 & 1237 & 96 \\
\hline 133441175 & 149310477 & 1139 & 1244 & 105 \\
\hline 127004900 & 149310477 & 1111 & 1206 & 95 \\
\hline 133441175 & 149310477 & 1139 & 1234 & 95 \\
\hline 136129932 & 138605748 & 1144 & 1184 & 40 \\
\hline 136129932 & 149310477 & 1144 & 1240 & 96 \\
\hline 133441175 & 149310477 & 1138 & 1234 & 96 \\
\hline 136129932 & 149310477 & 1156 & 1229 & 73 \\
\hline 136129932 & 145964815 & 1150 & 1190 & 40 \\
\hline 138238126 & 139780488 & 1167 & 1188 & 21 \\
\hline 138238126 & 139780488 & 1171 & 1189 & 18 \\
\hline 138238126 & 139780488 & 1168 & 1188 & 20 \\
\hline 136129932 & 149310477 & 1154 & 1220 & 66 \\
\hline
\end{tabular}




\begin{tabular}{|c|c|c|c|c|}
\hline 136129932 & 139780488 & 1149 & 1187 & 38 \\
\hline 138238126 & 145964815 & 1173 & 1192 & 19 \\
\hline 138238126 & 149310477 & 1173 & 1232 & 59 \\
\hline 138238126 & 149310477 & 1174 & 1233 & 59 \\
\hline 138238126 & 149310477 & 1166 & 1233 & 67 \\
\hline 138238126 & 149310477 & 1172 & 1239 & 67 \\
\hline 138238126 & 149310477 & 1176 & 1230 & 54 \\
\hline 138238126 & 149310477 & 1167 & 1219 & 52 \\
\hline 138238126 & 149310477 & 1172 & 1232 & 60 \\
\hline 138238126 & 149310477 & 1169 & 1225 & 56 \\
\hline 136129932 & 149310477 & 1143 & 1218 & 75 \\
\hline 138238126 & 149310477 & 1177 & 1218 & 41 \\
\hline 138238126 & 149310477 & 1170 & 1230 & 60 \\
\hline 138238126 & 149310477 & 1173 & 1222 & 49 \\
\hline 138238126 & 149310477 & 1169 & 1229 & 60 \\
\hline 138238126 & 149310477 & 1174 & 1229 & 55 \\
\hline 136129932 & 149310477 & 1151 & 1237 & 86 \\
\hline 136129932 & 149310477 & 1152 & 1234 & 82 \\
\hline 138238126 & 149310477 & 1167 & 1225 & 58 \\
\hline 138605748 & 149310477 & 1187 & 1222 & 35 \\
\hline 138605748 & 149310477 & 1186 & 1224 & 38 \\
\hline 138605748 & 149310477 & 1187 & 1215 & 28 \\
\hline 138605748 & 149310477 & 1187 & 1226 & 39 \\
\hline 138605748 & 149310477 & 1186 & 1234 & 48 \\
\hline 138605748 & 149310477 & 1187 & 1213 & 26 \\
\hline 138605748 & 149310477 & 1188 & 1214 & 26 \\
\hline 138605748 & 149310477 & 1188 & 1229 & 41 \\
\hline 138605748 & 149310477 & 1185 & 1234 & 49 \\
\hline 138238126 & 149310477 & 1175 & 1234 & 59 \\
\hline 133441175 & 149310477 & 1135 & 1233 & 98 \\
\hline 138605748 & 149310477 & 1187 & 1236 & 49 \\
\hline 138238126 & 149310477 & 1173 & 1232 & 59 \\
\hline 138605748 & 149310477 & 1187 & 1218 & 31 \\
\hline 138238126 & 149310477 & 1182 & 1241 & 59 \\
\hline 138605748 & 149310477 & 1188 & 1220 & 32 \\
\hline 138238126 & 149310477 & 1177 & 1236 & 59 \\
\hline 138238126 & 149310477 & 1167 & 1219 & 52 \\
\hline 133441175 & 149310477 & 1134 & 1231 & 97 \\
\hline 138238126 & 149310477 & 1177 & 1231 & 54 \\
\hline 138238126 & 149310477 & 1169 & 1245 & 76 \\
\hline 136129932 & 149310477 & 1152 & 1239 & 87 \\
\hline 138605748 & 149310477 & 1186 & 1232 & 46 \\
\hline 138238126 & 149310477 & 1176 & 1236 & 60 \\
\hline
\end{tabular}




\begin{tabular}{|c|c|c|c|}
\hline 138605748 & 149310477 & 1188 & 1223 \\
\hline 138605748 & 149310477 & 1188 & 1217 \\
\hline 138605748 & 149310477 & 1187 & 1237 \\
\hline 138238126 & 149310477 & 1177 & 1223 \\
\hline 138605748 & 149310477 & 1186 & 1235 \\
\hline 136129932 & 149310477 & 1151 & 1231 \\
\hline 138605748 & 149310477 & 1185 & 1222 \\
\hline 138605748 & 149310477 & 1188 & 1228 \\
\hline 138605748 & 149310477 & 1188 & 1234 \\
\hline 138605748 & 149310477 & 1186 & 1225 \\
\hline 138605748 & 149310477 & 1189 & 1229 \\
\hline 138238126 & 149310477 & 1170 & 1227 \\
\hline 139780488 & 149310477 & 1190 & 1224 \\
\hline 138605748 & 149310477 & 1188 & 1219 \\
\hline 136129932 & 149310477 & 1153 & 1223 \\
\hline 138238126 & 149310477 & 1178 & 1220 \\
\hline 138605748 & 149310477 & 1187 & 1230 \\
\hline 138605748 & 149310477 & 1185 & 1222 \\
\hline 139780488 & 149310477 & 1190 & 1227 \\
\hline 133441175 & 149310477 & 1131 & 1233 \\
\hline 138605748 & 149310477 & 1186 & 1232 \\
\hline 129145419 & 149310477 & 1120 & 1238 \\
\hline 138605748 & 149310477 & 1188 & 1232 \\
\hline 138605748 & 149310477 & 1187 & 1231 \\
\hline 138605748 & 149310477 & 1188 & 1219 \\
\hline 138605748 & 149310477 & 1187 & 1220 \\
\hline 138605748 & 149310477 & 1188 & 1221 \\
\hline 139780488 & 149310477 & 1190 & 1226 \\
\hline 138238126 & 149310477 & 1166 & 1229 \\
\hline 138238126 & 149310477 & 1165 & 1233 \\
\hline 138238126 & 149310477 & 1168 & 1230 \\
\hline 138605748 & 149310477 & 1186 & 1225 \\
\hline 138605748 & 149310477 & 1187 & 1221 \\
\hline 138605748 & 149310477 & 1186 & 1227 \\
\hline 138605748 & 149310477 & 1189 & 1217 \\
\hline 138605748 & 149310477 & 1186 & 1222 \\
\hline 139780488 & 149310477 & 1190 & 1216 \\
\hline 138238126 & 149310477 & 1184 & 1223 \\
\hline 138605748 & 149310477 & 1186 & 1228 \\
\hline 138605748 & 149310477 & 1187 & 1230 \\
\hline 138605748 & 149310477 & 1188 & 1224 \\
\hline 138605748 & 149310477 & 1186 & 1224 \\
\hline 138238126 & 149310477 & 1182 & 1229 \\
\hline
\end{tabular}




\begin{tabular}{|c|c|c|c|c|}
\hline 138605748 & 149310477 & 1185 & 1228 & 43 \\
\hline 138605748 & 149310477 & 1189 & 1220 & 31 \\
\hline 138605748 & 149310477 & 1187 & 1231 & 44 \\
\hline 138605748 & 149310477 & 1186 & 1232 & 46 \\
\hline 138605748 & 149310477 & 1188 & 1225 & 37 \\
\hline 138605748 & 149310477 & 1187 & 1234 & 47 \\
\hline 138605748 & 149310477 & 1187 & 1230 & 43 \\
\hline 138238126 & 149310477 & 1167 & 1237 & 70 \\
\hline 138605748 & 149310477 & 1188 & 1229 & 41 \\
\hline 138605748 & 149310477 & 1188 & 1230 & 42 \\
\hline 138605748 & 149310477 & 1187 & 1230 & 43 \\
\hline 138605748 & 149310477 & 1187 & 1231 & 44 \\
\hline 138238126 & 149310477 & 1169 & 1238 & 69 \\
\hline 138605748 & 149310477 & 1188 & 1229 & 41 \\
\hline 138605748 & 149310477 & 1188 & 1230 & 42 \\
\hline 138238126 & 149310477 & 1165 & 1235 & 70 \\
\hline 138238126 & 149310477 & 1170 & 1235 & 65 \\
\hline 138605748 & 149310477 & 1186 & 1243 & 57 \\
\hline 138238126 & 149310477 & 1182 & 1234 & 52 \\
\hline 138605748 & 149310477 & 1188 & 1233 & 45 \\
\hline 138605748 & 149310477 & 1187 & 1236 & 49 \\
\hline 138605748 & 149310477 & 1186 & 1242 & 56 \\
\hline 138238126 & 149310477 & 1169 & 1241 & 72 \\
\hline 138238126 & 149310477 & 1167 & 1238 & 71 \\
\hline 138605748 & 149310477 & 1186 & 1239 & 53 \\
\hline 136129932 & 149310477 & 1150 & 1245 & 95 \\
\hline 138605748 & 149310477 & 1187 & 1244 & 57 \\
\hline 138605748 & 149310477 & 1188 & 1234 & 46 \\
\hline 138238126 & 149310477 & 1181 & 1243 & 62 \\
\hline 138605748 & 149310477 & 1187 & 1239 & 52 \\
\hline 138605748 & 149310477 & 1187 & 1238 & 51 \\
\hline 138238126 & 149310477 & 1178 & 1240 & 62 \\
\hline 138605748 & 149310477 & 1186 & 1244 & 58 \\
\hline 138605748 & 149310477 & 1189 & 1235 & 46 \\
\hline 138238126 & 149310477 & 1171 & 1252 & 81 \\
\hline 138605748 & 149310477 & 1186 & 1251 & 65 \\
\hline 138238126 & 149310477 & 1174 & 1247 & 73 \\
\hline 138238126 & 149310477 & 1166 & 1256 & 90 \\
\hline 4610016 & 7019129 & 0 & 13 & 13 \\
\hline 15705386 & 20949420 & 197 & 231 & 34 \\
\hline 15705386 & 20949420 & 201 & 232 & 31 \\
\hline 15705386 & 20949420 & 197 & 239 & 42 \\
\hline 15705386 & 20949420 & 202 & 229 & 27 \\
\hline
\end{tabular}




\begin{tabular}{|c|c|c|c|}
\hline 15705386 & 20949420 & 198 & 234 \\
\hline 15705386 & 20949420 & 197 & 233 \\
\hline 15705386 & 20949420 & 200 & 231 \\
\hline 15705386 & 20949420 & 201 & 235 \\
\hline 15705386 & 20949420 & 184 & 231 \\
\hline 15705386 & 20949420 & 203 & 236 \\
\hline 15705386 & 20949420 & 194 & 244 \\
\hline 15705386 & 20949420 & 201 & 237 \\
\hline 15705386 & 20949420 & 202 & 230 \\
\hline 15705386 & 20949420 & 203 & 237 \\
\hline 15705386 & 20949420 & 206 & 236 \\
\hline 15705386 & 20949420 & 203 & 252 \\
\hline 15705386 & 20949420 & 203 & 234 \\
\hline 15705386 & 20949420 & 207 & 241 \\
\hline 15705386 & 20949420 & 202 & 245 \\
\hline 15705386 & 20949420 & 202 & 233 \\
\hline 15705386 & 20949420 & 206 & 233 \\
\hline 15705386 & 20949420 & 206 & 235 \\
\hline 15705386 & 20949420 & 207 & 236 \\
\hline 15705386 & 20949420 & 205 & 231 \\
\hline 15705386 & 20949420 & 209 & 233 \\
\hline 15705386 & 20949420 & 203 & 241 \\
\hline 15705386 & 20949420 & 205 & 230 \\
\hline 15705386 & 20949420 & 205 & 234 \\
\hline 15705386 & 20949420 & 203 & 237 \\
\hline 15705386 & 20949420 & 206 & 229 \\
\hline 15705386 & 20949420 & 203 & 246 \\
\hline 15705386 & 20949420 & 202 & 235 \\
\hline 15705386 & 20949420 & 204 & 236 \\
\hline 15705386 & 20949420 & 203 & 238 \\
\hline 15705386 & 20949420 & 205 & 238 \\
\hline 15705386 & 20949420 & 202 & 234 \\
\hline 15705386 & 20949420 & 204 & 238 \\
\hline 15705386 & 20949420 & 204 & 235 \\
\hline 15705386 & 20949420 & 207 & 232 \\
\hline 15705386 & 20949420 & 202 & 245 \\
\hline 15705386 & 20949420 & 211 & 232 \\
\hline 15705386 & 20949420 & 207 & 234 \\
\hline 15705386 & 20949420 & 205 & 231 \\
\hline 15705386 & 20949420 & 208 & 231 \\
\hline 15705386 & 20949420 & 209 & 239 \\
\hline 15705386 & 20949420 & 209 & 234 \\
\hline 15705386 & 20949420 & 212 & 234 \\
\hline
\end{tabular}




\begin{tabular}{|c|c|c|c|c|}
\hline 15705386 & 20949420 & 207 & 235 & 28 \\
\hline 15705386 & 20949420 & 205 & 236 & 31 \\
\hline 15705386 & 20949420 & 203 & 240 & 37 \\
\hline 15705386 & 20949420 & 204 & 232 & 28 \\
\hline 15705386 & 20949420 & 209 & 233 & 24 \\
\hline 15705386 & 20949420 & 205 & 228 & 23 \\
\hline 15705386 & 20949420 & 206 & 237 & 31 \\
\hline 15705386 & 20949420 & 204 & 241 & 37 \\
\hline 15705386 & 20949420 & 205 & 231 & 26 \\
\hline 15705386 & 20949420 & 204 & 246 & 42 \\
\hline 15705386 & 20949420 & 202 & 238 & 36 \\
\hline 15705386 & 20949420 & 201 & 237 & 36 \\
\hline 15705386 & 20949420 & 206 & 233 & 27 \\
\hline 15705386 & 20949420 & 206 & 236 & 30 \\
\hline 15705386 & 20949420 & 201 & 232 & 31 \\
\hline 15705386 & 20949420 & 208 & 232 & 24 \\
\hline 15705386 & 20949420 & 205 & 233 & 28 \\
\hline 15705386 & 20949420 & 205 & 231 & 26 \\
\hline 15705386 & 20949420 & 207 & 234 & 27 \\
\hline 15705386 & 20949420 & 206 & 236 & 30 \\
\hline 15705386 & 20949420 & 199 & 242 & 43 \\
\hline 15705386 & 20949420 & 203 & 239 & 36 \\
\hline 15705386 & 20949420 & 208 & 237 & 29 \\
\hline 15705386 & 20949420 & 203 & 241 & 38 \\
\hline 15705386 & 20949420 & 205 & 235 & 30 \\
\hline 15705386 & 20949420 & 207 & 233 & 26 \\
\hline 15705386 & 20949420 & 209 & 235 & 26 \\
\hline 15705386 & 20949420 & 206 & 240 & 34 \\
\hline 15705386 & 20949420 & 205 & 238 & 33 \\
\hline 15705386 & 20949420 & 205 & 232 & 27 \\
\hline 15705386 & 20949420 & 204 & 231 & 27 \\
\hline 15705386 & 20949420 & 207 & 235 & 28 \\
\hline 15705386 & 20949420 & 208 & 240 & 32 \\
\hline 15705386 & 20949420 & 206 & 238 & 32 \\
\hline 15705386 & 20949420 & 208 & 231 & 23 \\
\hline 15705386 & 20949420 & 203 & 232 & 29 \\
\hline 15705386 & 20949420 & 207 & 233 & 26 \\
\hline 15705386 & 20949420 & 205 & 237 & 32 \\
\hline 15705386 & 20949420 & 205 & 235 & 30 \\
\hline 15705386 & 20949420 & 207 & 235 & 28 \\
\hline 15705386 & 20949420 & 204 & 235 & 31 \\
\hline 15705386 & 20949420 & 208 & 241 & 33 \\
\hline 15705386 & 20949420 & 205 & 232 & 27 \\
\hline
\end{tabular}




\begin{tabular}{|c|c|c|c|c|}
\hline 15705386 & 20949420 & 203 & 236 & 33 \\
\hline 15705386 & 20949420 & 207 & 234 & 27 \\
\hline 15705386 & 20949420 & 210 & 232 & 22 \\
\hline 15705386 & 20949420 & 208 & 231 & 23 \\
\hline 15705386 & 20949420 & 205 & 230 & 25 \\
\hline 15705386 & 20949420 & 204 & 246 & 42 \\
\hline 15705386 & 20949420 & 206 & 240 & 34 \\
\hline 15705386 & 20949420 & 206 & 233 & 27 \\
\hline 15705386 & 20949420 & 204 & 239 & 35 \\
\hline 15705386 & 20949420 & 205 & 236 & 31 \\
\hline 15705386 & 20949420 & 208 & 240 & 32 \\
\hline 15705386 & 20949420 & 204 & 238 & 34 \\
\hline 15705386 & 20949420 & 203 & 233 & 30 \\
\hline 15705386 & 20949420 & 199 & 240 & 41 \\
\hline 15705386 & 20949420 & 206 & 235 & 29 \\
\hline 15705386 & 20949420 & 201 & 240 & 39 \\
\hline 15705386 & 20949420 & 203 & 232 & 29 \\
\hline 15705386 & 20949420 & 201 & 237 & 36 \\
\hline 15705386 & 20949420 & 208 & 235 & 27 \\
\hline 15705386 & 20949420 & 195 & 236 & 41 \\
\hline 15705386 & 20949420 & 206 & 240 & 34 \\
\hline 15705386 & 20949420 & 208 & 230 & 22 \\
\hline 15705386 & 20949420 & 206 & 233 & 27 \\
\hline 15705386 & 20949420 & 205 & 230 & 25 \\
\hline 15705386 & 20949420 & 206 & 244 & 38 \\
\hline 15705386 & 20949420 & 204 & 237 & 33 \\
\hline 15705386 & 20949420 & 208 & 234 & 26 \\
\hline 15705386 & 20949420 & 205 & 235 & 30 \\
\hline 15705386 & 20949420 & 208 & 232 & 24 \\
\hline 15705386 & 20949420 & 204 & 231 & 27 \\
\hline 15705386 & 20949420 & 207 & 233 & 26 \\
\hline 15705386 & 20949420 & 204 & 236 & 32 \\
\hline 15705386 & 20949420 & 203 & 235 & 32 \\
\hline 15705386 & 20949420 & 207 & 232 & 25 \\
\hline 15705386 & 20949420 & 200 & 236 & 36 \\
\hline 15705386 & 20949420 & 207 & 240 & 33 \\
\hline 15705386 & 20949420 & 209 & 232 & 23 \\
\hline 15705386 & 20949420 & 207 & 239 & 32 \\
\hline 15705386 & 20949420 & 206 & 237 & 31 \\
\hline 15705386 & 20949420 & 203 & 234 & 31 \\
\hline 15705386 & 20949420 & 208 & 243 & 35 \\
\hline 15705386 & 20949420 & 203 & 241 & 38 \\
\hline 15705386 & 20949420 & 202 & 248 & 46 \\
\hline
\end{tabular}




\begin{tabular}{|c|c|c|c|c|}
\hline 15705386 & 20949420 & 203 & 249 & 46 \\
\hline 15705386 & 20949420 & 202 & 248 & 46 \\
\hline 15705386 & 20949420 & 205 & 247 & 42 \\
\hline 15705386 & 20949420 & 206 & 245 & 39 \\
\hline 15705386 & 20949420 & 203 & 249 & 46 \\
\hline 15705386 & 20949420 & 207 & 249 & 42 \\
\hline 15705386 & 20949420 & 206 & 244 & 38 \\
\hline 15705386 & 20949420 & 197 & 247 & 50 \\
\hline 15705386 & 20949420 & 204 & 247 & 43 \\
\hline 15705386 & 20949420 & 210 & 247 & 37 \\
\hline 15705386 & 20949420 & 205 & 251 & 46 \\
\hline 15705386 & 20949420 & 200 & 250 & 50 \\
\hline 15705386 & 20949420 & 203 & 251 & 48 \\
\hline 15705386 & 20949420 & 206 & 249 & 43 \\
\hline 15705386 & 20949420 & 206 & 258 & 52 \\
\hline 15705386 & 20949420 & 200 & 259 & 59 \\
\hline 15705386 & 20949420 & 206 & 264 & 58 \\
\hline 37834638 & 51483475 & 299 & 342 & 43 \\
\hline 37834638 & 51483475 & 300 & 339 & 39 \\
\hline 37834638 & 51483475 & 301 & 349 & 48 \\
\hline 37834638 & 51483475 & 300 & 347 & 47 \\
\hline 37834638 & 51483475 & 300 & 339 & 39 \\
\hline 37834638 & 51483475 & 298 & 351 & 53 \\
\hline 37834638 & 51483475 & 300 & 346 & 46 \\
\hline 37834638 & 51483475 & 304 & 342 & 38 \\
\hline 37834638 & 51483475 & 302 & 350 & 48 \\
\hline 37834638 & 51483475 & 302 & 343 & 41 \\
\hline 37834638 & 51483475 & 302 & 345 & 43 \\
\hline 37834638 & 51483475 & 305 & 343 & 38 \\
\hline 37834638 & 51483475 & 302 & 339 & 37 \\
\hline 37834638 & 51483475 & 305 & 344 & 39 \\
\hline 37834638 & 51483475 & 303 & 348 & 45 \\
\hline 37834638 & 51483475 & 302 & 344 & 42 \\
\hline 37834638 & 51483475 & 303 & 343 & 40 \\
\hline 37834638 & 51483475 & 304 & 348 & 44 \\
\hline 37834638 & 51483475 & 306 & 340 & 34 \\
\hline 37834638 & 51483475 & 306 & 343 & 37 \\
\hline 37834638 & 51483475 & 310 & 338 & 28 \\
\hline 37834638 & 51483475 & 309 & 338 & 29 \\
\hline 37834638 & 51483475 & 307 & 344 & 37 \\
\hline 37834638 & 51483475 & 310 & 344 & 34 \\
\hline 37834638 & 51483475 & 310 & 345 & 35 \\
\hline 37834638 & 51483475 & 306 & 348 & 42 \\
\hline
\end{tabular}




\begin{tabular}{|c|c|c|c|c|}
\hline 37834638 & 51483475 & 309 & 347 & 38 \\
\hline 37834638 & 51483475 & 305 & 346 & 41 \\
\hline 37834638 & 51483475 & 307 & 346 & 39 \\
\hline 37834638 & 51483475 & 305 & 347 & 42 \\
\hline 37834638 & 51483475 & 309 & 345 & 36 \\
\hline 37834638 & 51483475 & 305 & 346 & 41 \\
\hline 37834638 & 51483475 & 305 & 346 & 41 \\
\hline 37834638 & 51483475 & 308 & 346 & 38 \\
\hline 37834638 & 51483475 & 308 & 349 & 41 \\
\hline 37834638 & 51483475 & 305 & 352 & 47 \\
\hline 37834638 & 51483475 & 300 & 350 & 50 \\
\hline 37834638 & 51483475 & 307 & 351 & 44 \\
\hline 37834638 & 51483475 & 301 & 354 & 53 \\
\hline 37834638 & 51483475 & 309 & 355 & 46 \\
\hline 37834638 & 51483475 & 305 & 356 & 51 \\
\hline 37834638 & 51483475 & 310 & 356 & 46 \\
\hline 37834638 & 51483475 & 313 & 359 & 46 \\
\hline 37834638 & 51483475 & 302 & 366 & 64 \\
\hline 64852372 & 68738722 & 422 & 441 & 19 \\
\hline 56258958 & 68738722 & 398 & 446 & 48 \\
\hline 64852372 & 66455327 & 426 & 433 & 7 \\
\hline 64852372 & 66455327 & 431 & 433 & 2 \\
\hline 64852372 & 69744119 & 424 & 448 & 24 \\
\hline 62926903 & 69744119 & 421 & 451 & 30 \\
\hline 64852372 & 69744119 & 425 & 454 & 29 \\
\hline 57770912 & 69744119 & 406 & 454 & 48 \\
\hline 64852372 & 69744119 & 425 & 461 & 36 \\
\hline 64852372 & 69744119 & 423 & 462 & 39 \\
\hline 64852372 & 69744119 & 424 & 460 & 36 \\
\hline 66455327 & 69744119 & 435 & 456 & 21 \\
\hline 66455327 & 69744119 & 435 & 461 & 26 \\
\hline 64852372 & 69744119 & 422 & 458 & 36 \\
\hline 62926903 & 69744119 & 419 & 461 & 42 \\
\hline 66455327 & 69744119 & 435 & 464 & 29 \\
\hline 64852372 & 69744119 & 430 & 461 & 31 \\
\hline 66455327 & 69744119 & 438 & 462 & 24 \\
\hline 66455327 & 69744119 & 436 & 462 & 26 \\
\hline 66455327 & 69744119 & 438 & 462 & 24 \\
\hline 66455327 & 69744119 & 436 & 465 & 29 \\
\hline 66455327 & 69744119 & 438 & 464 & 26 \\
\hline 66455327 & 69744119 & 437 & 465 & 28 \\
\hline 66455327 & 69744119 & 438 & 461 & 23 \\
\hline 66455327 & 69744119 & 440 & 462 & 22 \\
\hline
\end{tabular}




\begin{tabular}{|c|c|c|c|c|}
\hline 66455327 & 69744119 & 444 & 460 & 16 \\
\hline 66455327 & 69744119 & 435 & 465 & 30 \\
\hline 66455327 & 70910344 & 434 & 472 & 38 \\
\hline 66455327 & 69744119 & 440 & 463 & 23 \\
\hline 64852372 & 70910344 & 427 & 471 & 44 \\
\hline 57770912 & 80976548 & 407 & 526 & 119 \\
\hline 64852372 & 80976548 & 422 & 540 & 118 \\
\hline 57770912 & 84154671 & 407 & 565 & 158 \\
\hline 66455327 & 74199336 & 439 & 482 & 43 \\
\hline 66455327 & 74199336 & 439 & 480 & 41 \\
\hline 66455327 & 74199336 & 443 & 481 & 38 \\
\hline 66455327 & 74199336 & 439 & 480 & 41 \\
\hline 66455327 & 74199336 & 445 & 480 & 35 \\
\hline 66455327 & 74199336 & 436 & 480 & 44 \\
\hline 68738722 & 74199336 & 451 & 483 & 32 \\
\hline 66455327 & 74199336 & 445 & 480 & 35 \\
\hline 66455327 & 74199336 & 435 & 482 & 47 \\
\hline 66455327 & 74199336 & 440 & 480 & 40 \\
\hline 66455327 & 74199336 & 439 & 482 & 43 \\
\hline 66455327 & 74199336 & 441 & 484 & 43 \\
\hline 68738722 & 74199336 & 450 & 480 & 30 \\
\hline 68738722 & 79685620 & 452 & 509 & 57 \\
\hline 66455327 & 74199336 & 443 & 483 & 40 \\
\hline 68738722 & 74199336 & 456 & 483 & 27 \\
\hline 68738722 & 74199336 & 455 & 485 & 30 \\
\hline 68738722 & 74199336 & 453 & 480 & 27 \\
\hline 68738722 & 74199336 & 452 & 483 & 31 \\
\hline 68738722 & 74199336 & 454 & 483 & 29 \\
\hline 68738722 & 74199336 & 460 & 482 & 22 \\
\hline 68738722 & 74199336 & 456 & 484 & 28 \\
\hline 68738722 & 74199336 & 458 & 486 & 28 \\
\hline 68738722 & 80976548 & 453 & 528 & 75 \\
\hline 68738722 & 79685620 & 459 & 504 & 45 \\
\hline 68738722 & 74199336 & 457 & 480 & 23 \\
\hline 68738722 & 74199336 & 459 & 484 & 25 \\
\hline 68738722 & 74199336 & 460 & 483 & 23 \\
\hline 68738722 & 74199336 & 461 & 481 & 20 \\
\hline 68738722 & 74199336 & 462 & 482 & 20 \\
\hline 68738722 & 79685620 & 459 & 509 & 50 \\
\hline 68738722 & 74199336 & 462 & 483 & 21 \\
\hline 68738722 & 74199336 & 465 & 484 & 19 \\
\hline 68738722 & 74199336 & 460 & 485 & 25 \\
\hline 68738722 & 74199336 & 466 & 480 & 14 \\
\hline
\end{tabular}




\begin{tabular}{|c|c|c|c|c|}
\hline 68738722 & 79685620 & 464 & 502 & 38 \\
\hline 68738722 & 74199336 & 468 & 482 & 14 \\
\hline 68738722 & 79685620 & 458 & 510 & 52 \\
\hline 68738722 & 79685620 & 462 & 496 & 34 \\
\hline 68738722 & 79685620 & 456 & 508 & 52 \\
\hline 68738722 & 79685620 & 458 & 506 & 48 \\
\hline 68738722 & 74199336 & 466 & 485 & 19 \\
\hline 68738722 & 80976548 & 450 & 526 & 76 \\
\hline 68738722 & 79685620 & 463 & 505 & 42 \\
\hline 68738722 & 80976548 & 459 & 513 & 54 \\
\hline 68738722 & 84154671 & 454 & 569 & 115 \\
\hline 68738722 & 79685620 & 464 & 508 & 44 \\
\hline 68738722 & 80976548 & 458 & 529 & 71 \\
\hline 70910344 & 79685620 & 477 & 502 & 25 \\
\hline 70910344 & 79685620 & 480 & 500 & 20 \\
\hline 68738722 & 80976548 & 467 & 528 & 61 \\
\hline 70910344 & 75904188 & 480 & 490 & 10 \\
\hline 68738722 & 79685620 & 465 & 503 & 38 \\
\hline 70910344 & 79685620 & 483 & 501 & 18 \\
\hline 70910344 & 75904188 & 479 & 491 & 12 \\
\hline 70910344 & 79685620 & 482 & 496 & 14 \\
\hline 70910344 & 75904188 & 477 & 491 & 14 \\
\hline 70910344 & 75904188 & 483 & 491 & 8 \\
\hline 70910344 & 75904188 & 482 & 490 & 8 \\
\hline 70910344 & 75904188 & 482 & 491 & 9 \\
\hline 70910344 & 75904188 & 483 & 491 & 8 \\
\hline 70910344 & 75904188 & 481 & 491 & 10 \\
\hline 70910344 & 75904188 & 481 & 491 & 10 \\
\hline 70910344 & 79685620 & 478 & 500 & 22 \\
\hline 70910344 & 79685620 & 479 & 504 & 25 \\
\hline 70910344 & 79685620 & 481 & 497 & 16 \\
\hline 70910344 & 75904188 & 479 & 491 & 12 \\
\hline 70910344 & 79685620 & 482 & 505 & 23 \\
\hline 70910344 & 75904188 & 479 & 491 & 12 \\
\hline 70910344 & 79685620 & 482 & 511 & 29 \\
\hline 70910344 & 75904188 & 483 & 490 & 7 \\
\hline 70910344 & 75904188 & 483 & 489 & 6 \\
\hline 70910344 & 79685620 & 482 & 500 & 18 \\
\hline 70910344 & 79685620 & 483 & 499 & 16 \\
\hline 70910344 & 75904188 & 483 & 490 & 7 \\
\hline 70910344 & 75904188 & 481 & 491 & 10 \\
\hline 70910344 & 75904188 & 481 & 490 & 9 \\
\hline 70910344 & 75904188 & 481 & 489 & 8 \\
\hline
\end{tabular}




\begin{tabular}{|c|c|c|c|c|}
\hline 70910344 & 75904188 & 479 & 491 & 12 \\
\hline 70910344 & 79685620 & 484 & 494 & 10 \\
\hline 70910344 & 75904188 & 482 & 490 & 8 \\
\hline 70910344 & 75904188 & 482 & 491 & 9 \\
\hline 70910344 & 75904188 & 480 & 489 & 9 \\
\hline 70910344 & 75904188 & 481 & 490 & 9 \\
\hline 70910344 & 75904188 & 482 & 492 & 10 \\
\hline 68738722 & 79685620 & 461 & 510 & 49 \\
\hline 68738722 & 84154671 & 464 & 552 & 88 \\
\hline 70910344 & 79685620 & 476 & 506 & 30 \\
\hline 70910344 & 79685620 & 480 & 506 & 26 \\
\hline 69744119 & 79685620 & 471 & 503 & 32 \\
\hline 70910344 & 80976548 & 477 & 533 & 56 \\
\hline 68738722 & 79685620 & 465 & 508 & 43 \\
\hline 70910344 & 79685620 & 481 & 503 & 22 \\
\hline 70910344 & 79685620 & 476 & 500 & 24 \\
\hline 70910344 & 79685620 & 476 & 505 & 29 \\
\hline 74199336 & 79685620 & 489 & 502 & 13 \\
\hline 74199336 & 79685620 & 490 & 498 & 8 \\
\hline 74199336 & 79685620 & 492 & 502 & 10 \\
\hline 74199336 & 79685620 & 489 & 502 & 13 \\
\hline 74199336 & 79685620 & 489 & 506 & 17 \\
\hline 74199336 & 79685620 & 489 & 505 & 16 \\
\hline 69744119 & 79685620 & 472 & 505 & 33 \\
\hline 70910344 & 79685620 & 478 & 506 & 28 \\
\hline 68738722 & 79685620 & 470 & 509 & 39 \\
\hline 70910344 & 79685620 & 479 & 502 & 23 \\
\hline 74199336 & 79685620 & 488 & 507 & 19 \\
\hline 68738722 & 80976548 & 469 & 515 & 46 \\
\hline 70910344 & 79685620 & 480 & 510 & 30 \\
\hline 74199336 & 79685620 & 489 & 510 & 21 \\
\hline 74199336 & 79685620 & 490 & 510 & 20 \\
\hline 74199336 & 79685620 & 489 & 507 & 18 \\
\hline 68738722 & 80976548 & 460 & 528 & 68 \\
\hline 74199336 & 80976548 & 489 & 523 & 34 \\
\hline 74199336 & 80976548 & 489 & 524 & 35 \\
\hline 68738722 & 80976548 & 467 & 526 & 59 \\
\hline 76162624 & 80976548 & 496 & 518 & 22 \\
\hline 74199336 & 80976548 & 488 & 528 & 40 \\
\hline 76162624 & 80976548 & 497 & 527 & 30 \\
\hline 70910344 & 80976548 & 482 & 533 & 51 \\
\hline 70910344 & 80976548 & 486 & 531 & 45 \\
\hline 70910344 & 80976548 & 485 & 527 & 42 \\
\hline
\end{tabular}




\begin{tabular}{|c|c|c|c|}
\hline 74199336 & 80976548 & 491 & 537 \\
\hline 76162624 & 80976548 & 499 & 534 \\
\hline 79685620 & 84154671 & 528 & 557 \\
\hline 79685620 & 84154671 & 529 & 555 \\
\hline 79685620 & 84154671 & 530 & 552 \\
\hline 79685620 & 84154671 & 529 & 550 \\
\hline 79685620 & 84154671 & 532 & 555 \\
\hline 79685620 & 84154671 & 526 & 569 \\
\hline 81046751 & 84154671 & 549 & 569 \\
\hline 81046751 & 84154671 & 547 & 569 \\
\hline 81046751 & 84154671 & 553 & 569 \\
\hline 81046751 & 84154671 & 553 & 569 \\
\hline 81046751 & 84154671 & 554 & 569 \\
\hline 81046751 & 84154671 & 554 & 569 \\
\hline 81046751 & 84154671 & 553 & 569 \\
\hline 81046751 & 84154671 & 551 & 569 \\
\hline 81046751 & 84154671 & 549 & 569 \\
\hline 81046751 & 84154671 & 555 & 569 \\
\hline 81046751 & 84154671 & 549 & 569 \\
\hline 81046751 & 84154671 & 556 & 569 \\
\hline 81046751 & 84154671 & 553 & 569 \\
\hline 81046751 & 84154671 & 547 & 569 \\
\hline 81046751 & 84154671 & 556 & 569 \\
\hline 81046751 & 84154671 & 559 & 569 \\
\hline 81046751 & 84154671 & 554 & 569 \\
\hline 81046751 & 84154671 & 557 & 569 \\
\hline 81046751 & 84154671 & 558 & 569 \\
\hline 81046751 & 84154671 & 556 & 569 \\
\hline 81046751 & 84154671 & 556 & 569 \\
\hline 81046751 & 84154671 & 554 & 569 \\
\hline 81046751 & 84154671 & 556 & 569 \\
\hline 81046751 & 84154671 & 561 & 569 \\
\hline 81046751 & 84154671 & 559 & 569 \\
\hline 81046751 & 84154671 & 560 & 569 \\
\hline 81046751 & 84154671 & 563 & 569 \\
\hline 81046751 & 84154671 & 555 & 569 \\
\hline 81046751 & 84154671 & 557 & 569 \\
\hline 81046751 & 84154671 & 558 & 569 \\
\hline 81046751 & 84154671 & 557 & 569 \\
\hline 81046751 & 84154671 & 556 & 569 \\
\hline 81046751 & 84154671 & 561 & 569 \\
\hline 81046751 & 84154671 & 563 & 569 \\
\hline 81046751 & 84154671 & 553 & 569 \\
\hline
\end{tabular}




\begin{tabular}{|c|c|c|c|}
\hline 81046751 & 84154671 & 559 & 569 \\
\hline 81046751 & 84154671 & 555 & 569 \\
\hline 81046751 & 84154671 & 556 & 569 \\
\hline 81046751 & 84154671 & 553 & 569 \\
\hline 81046751 & 84154671 & 555 & 569 \\
\hline 81046751 & 84154671 & 556 & 569 \\
\hline 81046751 & 84154671 & 555 & 569 \\
\hline 81046751 & 84154671 & 557 & 569 \\
\hline 81046751 & 84154671 & 562 & 569 \\
\hline 81046751 & 84154671 & 559 & 569 \\
\hline 81046751 & 84154671 & 559 & 569 \\
\hline 81046751 & 84154671 & 561 & 569 \\
\hline 81046751 & 84154671 & 557 & 569 \\
\hline 81046751 & 84154671 & 549 & 569 \\
\hline 81046751 & 84154671 & 557 & 569 \\
\hline 81046751 & 84154671 & 562 & 569 \\
\hline 81046751 & 84154671 & 560 & 569 \\
\hline 81046751 & 84154671 & 559 & 569 \\
\hline 81046751 & 84154671 & 554 & 569 \\
\hline 81046751 & 84154671 & 558 & 569 \\
\hline 81046751 & 84154671 & 557 & 569 \\
\hline 81046751 & 84154671 & 557 & 569 \\
\hline 81046751 & 84154671 & 558 & 569 \\
\hline 81046751 & 84154671 & 556 & 569 \\
\hline 81046751 & 84154671 & 556 & 569 \\
\hline 81046751 & 84154671 & 556 & 569 \\
\hline 81046751 & 84154671 & 556 & 569 \\
\hline 81046751 & 84154671 & 560 & 569 \\
\hline 81046751 & 84154671 & 564 & 569 \\
\hline 81046751 & 84154671 & 563 & 569 \\
\hline 81046751 & 84154671 & 565 & 569 \\
\hline 81046751 & 84154671 & 561 & 569 \\
\hline 81046751 & 84154671 & 560 & 569 \\
\hline 81046751 & 84154671 & 564 & 569 \\
\hline 81046751 & 84154671 & 562 & 569 \\
\hline 81046751 & 84154671 & 562 & 569 \\
\hline 81046751 & 84154671 & 564 & 569 \\
\hline 81046751 & 84154671 & 561 & 569 \\
\hline 81046751 & 84154671 & 562 & 569 \\
\hline 81046751 & 84154671 & 563 & 569 \\
\hline 81046751 & 84154671 & 562 & 569 \\
\hline 81046751 & 84154671 & 557 & 569 \\
\hline 81046751 & 84154671 & 559 & 569 \\
\hline
\end{tabular}




\begin{tabular}{|c|c|c|c|c|}
\hline 81046751 & 84154671 & 563 & 569 & 6 \\
\hline 81046751 & 84154671 & 561 & 569 & 8 \\
\hline 81046751 & 84154671 & 558 & 569 & 11 \\
\hline 81046751 & 84154671 & 561 & 569 & 8 \\
\hline 81046751 & 84154671 & 564 & 569 & 5 \\
\hline 81046751 & 84154671 & 558 & 569 & 11 \\
\hline 81046751 & 84154671 & 556 & 569 & 13 \\
\hline 81046751 & 84154671 & 557 & 569 & 12 \\
\hline 81046751 & 84154671 & 562 & 569 & 7 \\
\hline 81046751 & 84154671 & 563 & 569 & 6 \\
\hline 81046751 & 84154671 & 561 & 569 & 8 \\
\hline 81046751 & 84154671 & 561 & 569 & 8 \\
\hline 81046751 & 84154671 & 557 & 569 & 12 \\
\hline 81046751 & 84154671 & 561 & 569 & 8 \\
\hline 81046751 & 84154671 & 555 & 569 & 14 \\
\hline 81046751 & 84154671 & 550 & 569 & 19 \\
\hline 81046751 & 84154671 & 551 & 569 & 18 \\
\hline 81046751 & 84154671 & 553 & 569 & 16 \\
\hline 81046751 & 84154671 & 556 & 569 & 13 \\
\hline 81046751 & 84154671 & 552 & 569 & 17 \\
\hline 81046751 & 84154671 & 553 & 569 & 16 \\
\hline 81046751 & 84154671 & 555 & 569 & 14 \\
\hline 81046751 & 84154671 & 554 & 569 & 15 \\
\hline 81046751 & 84154671 & 554 & 569 & 15 \\
\hline 81046751 & 84154671 & 550 & 569 & 19 \\
\hline 81046751 & 84154671 & 553 & 569 & 16 \\
\hline 81046751 & 84154671 & 548 & 569 & 21 \\
\hline 81046751 & 84154671 & 551 & 569 & 18 \\
\hline 81046751 & 84154671 & 553 & 569 & 16 \\
\hline 81046751 & 84154671 & 555 & 569 & 14 \\
\hline 81046751 & 84154671 & 554 & 569 & 15 \\
\hline 81046751 & 84154671 & 548 & 569 & 21 \\
\hline 81046751 & 84154671 & 555 & 569 & 14 \\
\hline 81046751 & 84154671 & 554 & 569 & 15 \\
\hline 81046751 & 84154671 & 551 & 569 & 18 \\
\hline 81046751 & 84154671 & 555 & 569 & 14 \\
\hline 81046751 & 84154671 & 553 & 569 & 16 \\
\hline 81046751 & 84154671 & 555 & 569 & 14 \\
\hline 81046751 & 84154671 & 551 & 569 & 18 \\
\hline 81046751 & 84154671 & 554 & 569 & 15 \\
\hline 81046751 & 84154671 & 555 & 569 & 14 \\
\hline 81046751 & 84154671 & 554 & 569 & 15 \\
\hline 81046751 & 84154671 & 554 & 569 & 15 \\
\hline
\end{tabular}




\begin{tabular}{|c|c|c|c|}
\hline 81046751 & 84154671 & 554 & 569 \\
\hline 81046751 & 84154671 & 552 & 569 \\
\hline 81046751 & 84154671 & 555 & 569 \\
\hline 81046751 & 84154671 & 558 & 569 \\
\hline 81046751 & 84154671 & 559 & 569 \\
\hline 542497 & 1061606 & 0 & 7 \\
\hline 542497 & 4074625 & 0 & 17 \\
\hline 542497 & 4074625 & 6 & 27 \\
\hline 542497 & 4074625 & 0 & 30 \\
\hline 5752853 & 15461425 & 46 & 118 \\
\hline 5752853 & 15461425 & 61 & 103 \\
\hline 5752853 & 13243005 & 58 & 95 \\
\hline 5752853 & 15461425 & 61 & 110 \\
\hline 5752853 & 15461425 & 61 & 120 \\
\hline 5752853 & 15461425 & 60 & 118 \\
\hline 5752853 & 15461425 & 61 & 99 \\
\hline 5752853 & 15461425 & 64 & 108 \\
\hline 5752853 & 17614557 & 58 & 126 \\
\hline 5752853 & 15461425 & 65 & 100 \\
\hline 5752853 & 15461425 & 72 & 96 \\
\hline 5752853 & 15461425 & 68 & 98 \\
\hline 5752853 & 19572670 & 61 & 136 \\
\hline 5752853 & 15461425 & 65 & 118 \\
\hline 5752853 & 15461425 & 65 & 103 \\
\hline 5752853 & 13243005 & 69 & 95 \\
\hline 5752853 & 15461425 & 64 & 109 \\
\hline 5752853 & 19572670 & 57 & 156 \\
\hline 5752853 & 15461425 & 64 & 119 \\
\hline 5752853 & 17614557 & 64 & 131 \\
\hline 5752853 & 15461425 & 67 & 109 \\
\hline 5752853 & 15461425 & 69 & 117 \\
\hline 5752853 & 15461425 & 65 & 115 \\
\hline 5752853 & 15461425 & 62 & 115 \\
\hline 5752853 & 15461425 & 68 & 109 \\
\hline 5752853 & 15461425 & 64 & 115 \\
\hline 5752853 & 15461425 & 59 & 117 \\
\hline 5752853 & 15461425 & 65 & 113 \\
\hline 5752853 & 15461425 & 70 & 111 \\
\hline 5752853 & 15461425 & 78 & 110 \\
\hline 5752853 & 15461425 & 80 & 102 \\
\hline 5752853 & 15461425 & 67 & 109 \\
\hline 5752853 & 15461425 & 72 & 107 \\
\hline 5752853 & 15461425 & 68 & 115 \\
\hline
\end{tabular}




\begin{tabular}{|c|c|c|c|}
\hline 5752853 & 15461425 & 63 & 116 \\
\hline 5752853 & 15461425 & 71 & 110 \\
\hline 5752853 & 15461425 & 73 & 109 \\
\hline 5752853 & 15461425 & 69 & 114 \\
\hline 5752853 & 15461425 & 73 & 107 \\
\hline 5752853 & 15461425 & 78 & 113 \\
\hline 5752853 & 15461425 & 64 & 119 \\
\hline 12264317 & 15461425 & 94 & 109 \\
\hline 5752853 & 15461425 & 61 & 114 \\
\hline 5752853 & 17614557 & 74 & 121 \\
\hline 5752853 & 17614557 & 75 & 127 \\
\hline 5752853 & 17614557 & 72 & 121 \\
\hline 5752853 & 15461425 & 90 & 116 \\
\hline 5752853 & 17614557 & 75 & 121 \\
\hline 5752853 & 19572670 & 65 & 133 \\
\hline 5752853 & 17614557 & 70 & 131 \\
\hline 5752853 & 17614557 & 62 & 127 \\
\hline 5752853 & 15461425 & 75 & 119 \\
\hline 5752853 & 15461425 & 69 & 120 \\
\hline 5752853 & 15461425 & 69 & 118 \\
\hline 5752853 & 17614557 & 64 & 126 \\
\hline 5752853 & 19572670 & 56 & 143 \\
\hline 5752853 & 19572670 & 62 & 136 \\
\hline 5752853 & 19572670 & 65 & 136 \\
\hline 5752853 & 17614557 & 73 & 124 \\
\hline 5752853 & 19572670 & 70 & 138 \\
\hline 5752853 & 19572670 & 66 & 154 \\
\hline 5752853 & 17614557 & 59 & 129 \\
\hline 5752853 & 19572670 & 64 & 148 \\
\hline 5752853 & 19572670 & 75 & 144 \\
\hline 5752853 & 17614557 & 68 & 127 \\
\hline 5752853 & 19572670 & 58 & 145 \\
\hline 5752853 & 19572670 & 64 & 148 \\
\hline 5752853 & 19572670 & 78 & 149 \\
\hline 5752853 & 19572670 & 64 & 140 \\
\hline 5752853 & 19572670 & 60 & 150 \\
\hline 5752853 & 19572670 & 50 & 159 \\
\hline 13243005 & 17614557 & 100 & 128 \\
\hline 13243005 & 19572670 & 100 & 143 \\
\hline 12264317 & 17614557 & 95 & 131 \\
\hline 13243005 & 19572670 & 100 & 144 \\
\hline 13243005 & 19572670 & 108 & 147 \\
\hline 13243005 & 19572670 & 104 & 138 \\
\hline
\end{tabular}




\begin{tabular}{|c|c|c|c|c|}
\hline 13243005 & 19572670 & 108 & 141 & 33 \\
\hline 13243005 & 19572670 & 97 & 146 & 49 \\
\hline 13243005 & 19572670 & 108 & 142 & 34 \\
\hline 13243005 & 17614557 & 111 & 126 & 15 \\
\hline 13243005 & 19572670 & 109 & 137 & 28 \\
\hline 13243005 & 17614557 & 110 & 128 & 18 \\
\hline 13243005 & 19572670 & 110 & 168 & 58 \\
\hline 13243005 & 19572670 & 111 & 144 & 33 \\
\hline 13243005 & 19572670 & 110 & 143 & 33 \\
\hline 13243005 & 19572670 & 103 & 150 & 47 \\
\hline 13243005 & 19572670 & 108 & 145 & 37 \\
\hline 13243005 & 19572670 & 110 & 136 & 26 \\
\hline 13243005 & 19572670 & 111 & 157 & 46 \\
\hline 13243005 & 19572670 & 110 & 150 & 40 \\
\hline 13243005 & 19572670 & 102 & 160 & 58 \\
\hline 13243005 & 19572670 & 110 & 145 & 35 \\
\hline 13243005 & 19572670 & 110 & 151 & 41 \\
\hline 13243005 & 19572670 & 108 & 149 & 41 \\
\hline 13243005 & 19572670 & 113 & 146 & 33 \\
\hline 12264317 & 19572670 & 94 & 152 & 58 \\
\hline 13243005 & 19572670 & 108 & 161 & 53 \\
\hline 13243005 & 19572670 & 111 & 158 & 47 \\
\hline 13243005 & 19572670 & 96 & 158 & 62 \\
\hline 13243005 & 19572670 & 110 & 147 & 37 \\
\hline 13243005 & 19572670 & 109 & 145 & 36 \\
\hline 5752853 & 19572670 & 51 & 166 & 115 \\
\hline 13243005 & 19572670 & 109 & 146 & 37 \\
\hline 5752853 & 19572670 & 82 & 160 & 78 \\
\hline 13243005 & 19572670 & 108 & 155 & 47 \\
\hline 5752853 & 19572670 & 83 & 152 & 69 \\
\hline 13243005 & 19572670 & 113 & 152 & 39 \\
\hline 13243005 & 19572670 & 117 & 143 & 26 \\
\hline 13243005 & 19572670 & 113 & 148 & 35 \\
\hline 13243005 & 19572670 & 115 & 155 & 40 \\
\hline 13243005 & 19572670 & 106 & 166 & 60 \\
\hline 13243005 & 19572670 & 118 & 157 & 39 \\
\hline 17614557 & 25665093 & 161 & 195 & 34 \\
\hline 17614557 & 25665093 & 163 & 198 & 35 \\
\hline 17614557 & 27552588 & 160 & 203 & 43 \\
\hline 17614557 & 25665093 & 158 & 190 & 32 \\
\hline 17614557 & 25665093 & 169 & 193 & 24 \\
\hline 17614557 & 25665093 & 146 & 198 & 52 \\
\hline 17614557 & 25665093 & 170 & 192 & 22 \\
\hline
\end{tabular}




\begin{tabular}{|c|c|c|c|c|}
\hline 17614557 & 25665093 & 167 & 195 & 28 \\
\hline 19572670 & 25665093 & 174 & 197 & 23 \\
\hline 17614557 & 25665093 & 169 & 197 & 28 \\
\hline 17614557 & 25665093 & 166 & 197 & 31 \\
\hline 19572670 & 25665093 & 176 & 192 & 16 \\
\hline 17614557 & 25665093 & 164 & 190 & 26 \\
\hline 19572670 & 25665093 & 175 & 191 & 16 \\
\hline 19572670 & 25665093 & 176 & 193 & 17 \\
\hline 17614557 & 25665093 & 167 & 199 & 32 \\
\hline 19572670 & 25665093 & 175 & 199 & 24 \\
\hline 19572670 & 30607808 & 177 & 230 & 53 \\
\hline 19572670 & 25665093 & 180 & 187 & 7 \\
\hline 19572670 & 25665093 & 179 & 193 & 14 \\
\hline 19572670 & 25665093 & 178 & 193 & 15 \\
\hline 19572670 & 25665093 & 177 & 196 & 19 \\
\hline 17614557 & 30607808 & 169 & 223 & 54 \\
\hline 19572670 & 27552588 & 178 & 210 & 32 \\
\hline 19572670 & 25665093 & 177 & 198 & 21 \\
\hline 17614557 & 25665093 & 172 & 199 & 27 \\
\hline 19572670 & 27552588 & 178 & 203 & 25 \\
\hline 19572670 & 27552588 & 177 & 208 & 31 \\
\hline 19572670 & 27552588 & 179 & 207 & 28 \\
\hline 19572670 & 30607808 & 176 & 225 & 49 \\
\hline 19572670 & 30607808 & 174 & 229 & 55 \\
\hline 17614557 & 37011684 & 171 & 252 & 81 \\
\hline 17614557 & 30607808 & 169 & 226 & 57 \\
\hline 19572670 & 27552588 & 177 & 203 & 26 \\
\hline 17614557 & 27552588 & 173 & 206 & 33 \\
\hline 19572670 & 27552588 & 177 & 207 & 30 \\
\hline 22362570 & 27552588 & 183 & 206 & 23 \\
\hline 22362570 & 27552588 & 184 & 207 & 23 \\
\hline 22362570 & 27552588 & 183 & 207 & 24 \\
\hline 22362570 & 27552588 & 184 & 208 & 24 \\
\hline 22362570 & 27552588 & 185 & 208 & 23 \\
\hline 22362570 & 27552588 & 186 & 209 & 23 \\
\hline 19572670 & 27552588 & 180 & 206 & 26 \\
\hline 22362570 & 27552588 & 186 & 208 & 22 \\
\hline 22362570 & 27552588 & 184 & 207 & 23 \\
\hline 22362570 & 27552588 & 186 & 207 & 21 \\
\hline 22362570 & 27552588 & 187 & 207 & 20 \\
\hline 22362570 & 27552588 & 187 & 206 & 19 \\
\hline 22362570 & 27552588 & 190 & 205 & 15 \\
\hline 22362570 & 27552588 & 186 & 206 & 20 \\
\hline
\end{tabular}




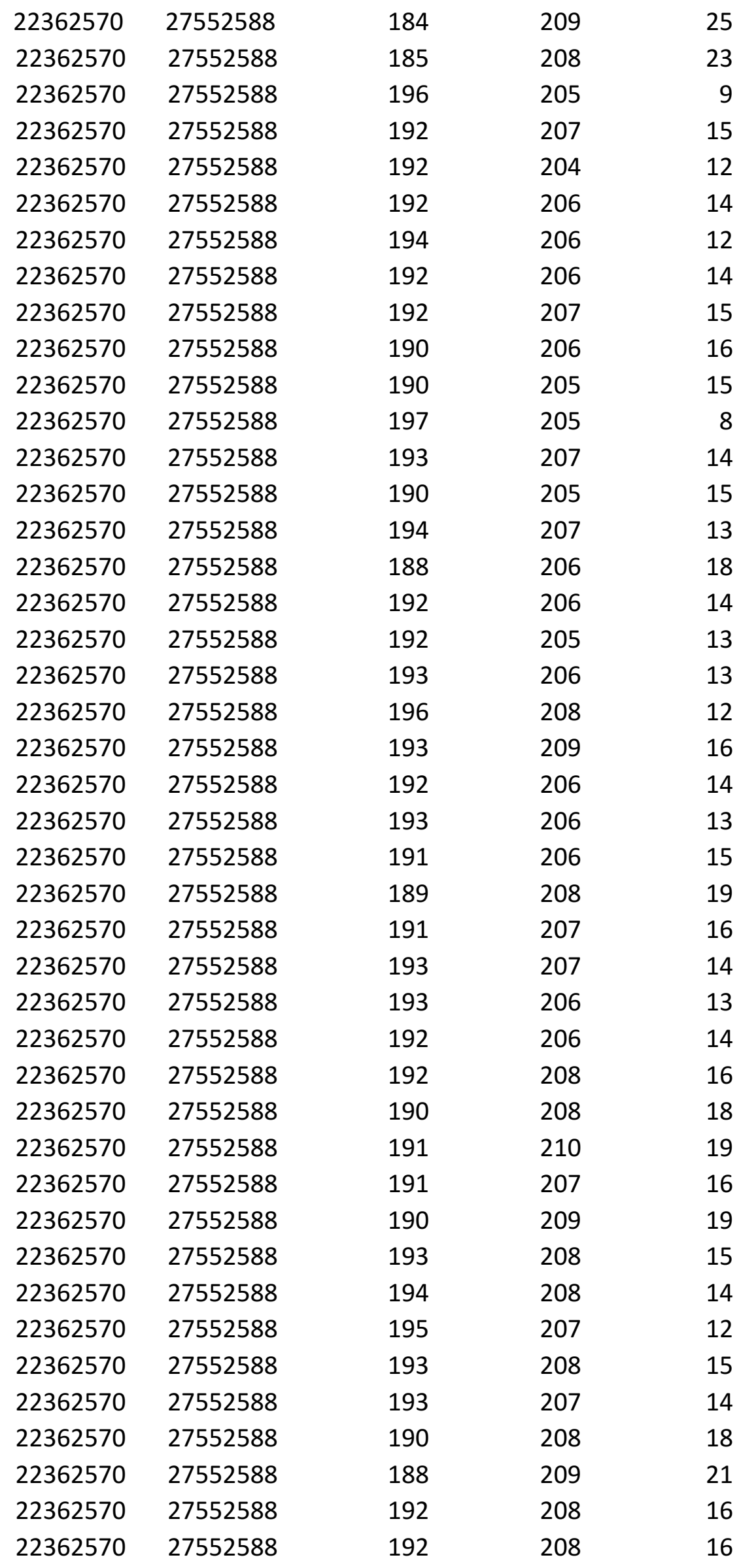




\begin{tabular}{|c|c|c|c|}
\hline 22362570 & 27552588 & 194 & 207 \\
\hline 22362570 & 27552588 & 194 & 207 \\
\hline 22362570 & 27552588 & 196 & 206 \\
\hline 22362570 & 27552588 & 189 & 209 \\
\hline 22362570 & 27552588 & 192 & 208 \\
\hline 22362570 & 27552588 & 185 & 210 \\
\hline 22362570 & 27552588 & 188 & 208 \\
\hline 22362570 & 27552588 & 189 & 207 \\
\hline 22362570 & 27552588 & 187 & 207 \\
\hline 22362570 & 27552588 & 194 & 206 \\
\hline 22362570 & 27552588 & 188 & 209 \\
\hline 22362570 & 27552588 & 190 & 210 \\
\hline 22362570 & 27552588 & 187 & 210 \\
\hline 22362570 & 27552588 & 194 & 208 \\
\hline 22362570 & 27552588 & 191 & 208 \\
\hline 22362570 & 27552588 & 189 & 209 \\
\hline 22362570 & 27552588 & 190 & 209 \\
\hline 22362570 & 27552588 & 189 & 210 \\
\hline 22362570 & 27552588 & 190 & 209 \\
\hline 22362570 & 27552588 & 189 & 210 \\
\hline 22362570 & 27552588 & 187 & 208 \\
\hline 22362570 & 27552588 & 191 & 209 \\
\hline 22362570 & 27552588 & 191 & 209 \\
\hline 22362570 & 27552588 & 190 & 209 \\
\hline 22362570 & 27552588 & 195 & 208 \\
\hline 22362570 & 27552588 & 193 & 210 \\
\hline 22362570 & 27552588 & 190 & 211 \\
\hline 22362570 & 30607808 & 193 & 219 \\
\hline 22362570 & 27552588 & 192 & 211 \\
\hline 22362570 & 30607808 & 191 & 221 \\
\hline 22362570 & 30607808 & 192 & 221 \\
\hline 22362570 & 30607808 & 189 & 219 \\
\hline 22362570 & 30607808 & 192 & 223 \\
\hline 22362570 & 33875344 & 192 & 236 \\
\hline 22362570 & 30607808 & 191 & 218 \\
\hline 22362570 & 30607808 & 192 & 224 \\
\hline 22362570 & 30607808 & 192 & 220 \\
\hline 22362570 & 30607808 & 190 & 219 \\
\hline 19572670 & 30607808 & 181 & 220 \\
\hline 22362570 & 30607808 & 192 & 226 \\
\hline 22362570 & 30607808 & 186 & 225 \\
\hline 22362570 & 30607808 & 185 & 224 \\
\hline 22362570 & 30607808 & 192 & 222 \\
\hline
\end{tabular}




\begin{tabular}{|c|c|c|c|c|}
\hline 22362570 & 30607808 & 193 & 225 & 32 \\
\hline 22362570 & 30607808 & 193 & 223 & 30 \\
\hline 22362570 & 30607808 & 190 & 222 & 32 \\
\hline 22362570 & 30607808 & 192 & 221 & 29 \\
\hline 22362570 & 30607808 & 188 & 223 & 35 \\
\hline 22362570 & 30607808 & 191 & 221 & 30 \\
\hline 22362570 & 30607808 & 197 & 222 & 25 \\
\hline 22362570 & 30607808 & 184 & 223 & 39 \\
\hline 22362570 & 30607808 & 186 & 225 & 39 \\
\hline 19572670 & 30607808 & 180 & 223 & 43 \\
\hline 22362570 & 30607808 & 193 & 222 & 29 \\
\hline 22362570 & 30607808 & 189 & 223 & 34 \\
\hline 22362570 & 30607808 & 188 & 224 & 36 \\
\hline 22362570 & 30607808 & 195 & 219 & 24 \\
\hline 19572670 & 30607808 & 180 & 224 & 44 \\
\hline 22362570 & 30607808 & 196 & 222 & 26 \\
\hline 22362570 & 30607808 & 191 & 224 & 33 \\
\hline 22362570 & 30607808 & 198 & 223 & 25 \\
\hline 22362570 & 30607808 & 187 & 225 & 38 \\
\hline 22362570 & 30607808 & 185 & 224 & 39 \\
\hline 22362570 & 30607808 & 194 & 220 & 26 \\
\hline 22362570 & 30607808 & 186 & 223 & 37 \\
\hline 22362570 & 30607808 & 197 & 224 & 27 \\
\hline 22362570 & 30607808 & 191 & 223 & 32 \\
\hline 22362570 & 30607808 & 198 & 225 & 27 \\
\hline 22362570 & 30607808 & 191 & 219 & 28 \\
\hline 25665093 & 30607808 & 201 & 223 & 22 \\
\hline 22362570 & 30607808 & 187 & 224 & 37 \\
\hline 25665093 & 30607808 & 202 & 219 & 17 \\
\hline 25665093 & 30607808 & 202 & 220 & 18 \\
\hline 22362570 & 30607808 & 200 & 221 & 21 \\
\hline 25665093 & 30607808 & 201 & 222 & 21 \\
\hline 25665093 & 30607808 & 202 & 221 & 19 \\
\hline 25665093 & 30607808 & 201 & 219 & 18 \\
\hline 25665093 & 30607808 & 202 & 221 & 19 \\
\hline 25665093 & 30607808 & 205 & 219 & 14 \\
\hline 25665093 & 30607808 & 203 & 223 & 20 \\
\hline 25665093 & 30607808 & 203 & 221 & 18 \\
\hline 25665093 & 30607808 & 203 & 223 & 20 \\
\hline 25665093 & 30607808 & 202 & 221 & 19 \\
\hline 25665093 & 30607808 & 204 & 220 & 16 \\
\hline 25665093 & 30607808 & 203 & 220 & 17 \\
\hline 25665093 & 30607808 & 203 & 220 & 17 \\
\hline
\end{tabular}




\begin{tabular}{|c|c|c|c|}
\hline 25665093 & 30607808 & 203 & 220 \\
\hline 25665093 & 30607808 & 203 & 221 \\
\hline 25665093 & 30607808 & 204 & 220 \\
\hline 25665093 & 30607808 & 205 & 220 \\
\hline 25665093 & 30607808 & 205 & 218 \\
\hline 25665093 & 30607808 & 203 & 221 \\
\hline 25665093 & 30607808 & 210 & 217 \\
\hline 25665093 & 30607808 & 205 & 219 \\
\hline 25665093 & 30607808 & 206 & 219 \\
\hline 25665093 & 30607808 & 204 & 221 \\
\hline 25665093 & 30607808 & 204 & 221 \\
\hline 25665093 & 30607808 & 204 & 222 \\
\hline 25665093 & 30607808 & 205 & 222 \\
\hline 25665093 & 30607808 & 206 & 222 \\
\hline 25665093 & 30607808 & 204 & 223 \\
\hline 25665093 & 30607808 & 203 & 223 \\
\hline 25665093 & 30607808 & 202 & 225 \\
\hline 25665093 & 30607808 & 204 & 225 \\
\hline 25665093 & 30607808 & 201 & 228 \\
\hline 25665093 & 30607808 & 202 & 227 \\
\hline 22362570 & 30607808 & 185 & 230 \\
\hline 22362570 & 30607808 & 184 & 229 \\
\hline 25665093 & 30607808 & 203 & 225 \\
\hline 25665093 & 30607808 & 203 & 227 \\
\hline 25665093 & 30607808 & 202 & 226 \\
\hline 25665093 & 30607808 & 204 & 225 \\
\hline 25665093 & 30607808 & 202 & 229 \\
\hline 27552588 & 37011684 & 214 & 243 \\
\hline 27552588 & 37011684 & 215 & 245 \\
\hline 27552588 & 30607808 & 220 & 232 \\
\hline 27552588 & 37011684 & 215 & 245 \\
\hline 27552588 & 30607808 & 223 & 232 \\
\hline 27552588 & 33875344 & 223 & 236 \\
\hline 27552588 & 37011684 & 223 & 247 \\
\hline 27552588 & 32817273 & 225 & 235 \\
\hline 27552588 & 37011684 & 221 & 245 \\
\hline 27552588 & 32817273 & 221 & 235 \\
\hline 27552588 & 37011684 & 222 & 248 \\
\hline 27552588 & 37011684 & 220 & 244 \\
\hline 27552588 & 37011684 & 216 & 248 \\
\hline 27552588 & 37011684 & 223 & 255 \\
\hline 27552588 & 37011684 & 219 & 238 \\
\hline 27552588 & 37011684 & 213 & 244 \\
\hline
\end{tabular}




\begin{tabular}{|c|c|c|c|c|}
\hline 27552588 & 37011684 & 217 & 251 & 34 \\
\hline 27552588 & 37011684 & 220 & 242 & 22 \\
\hline 27552588 & 37011684 & 218 & 247 & 29 \\
\hline 27552588 & 37011684 & 214 & 248 & 34 \\
\hline 30607808 & 37011684 & 233 & 255 & 22 \\
\hline 30607808 & 37011684 & 233 & 242 & 9 \\
\hline 30607808 & 37011684 & 234 & 244 & 10 \\
\hline 30607808 & 37011684 & 234 & 246 & 12 \\
\hline 30607808 & 37011684 & 234 & 238 & 4 \\
\hline 27552588 & 37011684 & 224 & 245 & 21 \\
\hline 30607808 & 37011684 & 233 & 237 & 4 \\
\hline 30607808 & 37011684 & 233 & 238 & 5 \\
\hline 30607808 & 37011684 & 234 & 249 & 15 \\
\hline 27552588 & 37011684 & 225 & 253 & 28 \\
\hline 17614557 & 37011684 & 146 & 256 & 110 \\
\hline 27552588 & 37011684 & 221 & 253 & 32 \\
\hline 27552588 & 37011684 & 215 & 243 & 28 \\
\hline 30607808 & 37011684 & 234 & 244 & 10 \\
\hline 30607808 & 37011684 & 234 & 243 & 9 \\
\hline 27552588 & 37011684 & 215 & 253 & 38 \\
\hline 27552588 & 37011684 & 221 & 258 & 37 \\
\hline 27552588 & 37011684 & 223 & 250 & 27 \\
\hline 27552588 & 37011684 & 216 & 258 & 42 \\
\hline 27552588 & 37011684 & 227 & 257 & 30 \\
\hline 27552588 & 37011684 & 220 & 252 & 32 \\
\hline 27552588 & 37011684 & 220 & 253 & 33 \\
\hline 27552588 & 37011684 & 216 & 252 & 36 \\
\hline 27552588 & 37011684 & 217 & 247 & 30 \\
\hline 27552588 & 38849288 & 214 & 269 & 55 \\
\hline 33875344 & 37011684 & 237 & 246 & 9 \\
\hline 32817273 & 37011684 & 236 & 242 & 6 \\
\hline 32817273 & 37011684 & 236 & 250 & 14 \\
\hline 30607808 & 37011684 & 233 & 246 & 13 \\
\hline 30607808 & 37011684 & 233 & 247 & 14 \\
\hline 30607808 & 37011684 & 234 & 254 & 20 \\
\hline 27552588 & 37011684 & 226 & 253 & 27 \\
\hline 27552588 & 37011684 & 226 & 258 & 32 \\
\hline 32817273 & 37011684 & 236 & 254 & 18 \\
\hline 32817273 & 37011684 & 236 & 253 & 17 \\
\hline 27552588 & 38849288 & 231 & 289 & 58 \\
\hline 27552588 & 38849288 & 214 & 277 & 63 \\
\hline 30607808 & 37011684 & 235 & 257 & 22 \\
\hline 27552588 & 38849288 & 218 & 289 & 71 \\
\hline
\end{tabular}




\begin{tabular}{|c|c|c|c|c|}
\hline 27552588 & 38849288 & 231 & 287 & 56 \\
\hline 30607808 & 38849288 & 233 & 290 & 57 \\
\hline 25665093 & 38849288 & 203 & 287 & 84 \\
\hline 27552588 & 38849288 & 232 & 282 & 50 \\
\hline 30607808 & 38849288 & 233 & 283 & 50 \\
\hline 35879487 & 38849288 & 240 & 278 & 38 \\
\hline 35879487 & 38849288 & 239 & 291 & 52 \\
\hline 35879487 & 38849288 & 241 & 285 & 44 \\
\hline 30607808 & 38849288 & 235 & 279 & 44 \\
\hline 35879487 & 38849288 & 248 & 275 & 27 \\
\hline 35879487 & 38849288 & 245 & 279 & 34 \\
\hline 35879487 & 38849288 & 248 & 281 & 33 \\
\hline 27552588 & 45643182 & 216 & 317 & 101 \\
\hline 32817273 & 50092059 & 236 & 343 & 107 \\
\hline 35879487 & 50092059 & 259 & 323 & 64 \\
\hline 37011684 & 45643182 & 265 & 316 & 51 \\
\hline 37011684 & 50092059 & 262 & 346 & 84 \\
\hline 37011684 & 45643182 & 277 & 314 & 37 \\
\hline 37011684 & 45643182 & 279 & 315 & 36 \\
\hline 37011684 & 45643182 & 275 & 317 & 42 \\
\hline 37011684 & 50092059 & 281 & 344 & 63 \\
\hline 37011684 & 50092059 & 273 & 335 & 62 \\
\hline 37011684 & 50092059 & 277 & 339 & 62 \\
\hline 37011684 & 45643182 & 287 & 313 & 26 \\
\hline 37011684 & 50092059 & 297 & 332 & 35 \\
\hline 37011684 & 45643182 & 300 & 315 & 15 \\
\hline 37011684 & 50092059 & 289 & 338 & 49 \\
\hline 37011684 & 45643182 & 283 & 317 & 34 \\
\hline 37011684 & 50092059 & 287 & 340 & 53 \\
\hline 37011684 & 45643182 & 294 & 315 & 21 \\
\hline 37011684 & 45643182 & 283 & 316 & 33 \\
\hline 37011684 & 45643182 & 290 & 315 & 25 \\
\hline 37011684 & 45643182 & 278 & 314 & 36 \\
\hline 37011684 & 50092059 & 283 & 338 & 55 \\
\hline 37011684 & 45643182 & 289 & 313 & 24 \\
\hline 37011684 & 45643182 & 284 & 315 & 31 \\
\hline 37011684 & 50092059 & 276 & 319 & 43 \\
\hline 38849288 & 45643182 & 306 & 315 & 9 \\
\hline 38849288 & 45643182 & 306 & 317 & 11 \\
\hline 38849288 & 45643182 & 305 & 314 & 9 \\
\hline 37011684 & 45643182 & 301 & 317 & 16 \\
\hline 37011684 & 50092059 & 295 & 320 & 25 \\
\hline 37011684 & 45643182 & 293 & 317 & 24 \\
\hline
\end{tabular}




\begin{tabular}{|c|c|c|c|c|}
\hline 37011684 & 50092059 & 289 & 328 & 39 \\
\hline 37011684 & 45643182 & 304 & 318 & 14 \\
\hline 37011684 & 45643182 & 296 & 316 & 20 \\
\hline 37011684 & 50092059 & 296 & 327 & 31 \\
\hline 38849288 & 45643182 & 306 & 317 & 11 \\
\hline 37011684 & 50092059 & 296 & 347 & 51 \\
\hline 37011684 & 50092059 & 286 & 344 & 58 \\
\hline 37011684 & 50092059 & 299 & 335 & 36 \\
\hline 37011684 & 50092059 & 279 & 343 & 64 \\
\hline 37011684 & 50092059 & 276 & 340 & 64 \\
\hline 37011684 & 50092059 & 287 & 354 & 67 \\
\hline 37011684 & 50092059 & 295 & 328 & 33 \\
\hline 37011684 & 50092059 & 287 & 329 & 42 \\
\hline 37011684 & 50092059 & 286 & 344 & 58 \\
\hline 37011684 & 50092059 & 288 & 349 & 61 \\
\hline 37011684 & 50092059 & 292 & 341 & 49 \\
\hline 38849288 & 50092059 & 306 & 328 & 22 \\
\hline 37011684 & 50092059 & 295 & 338 & 43 \\
\hline 37011684 & 50092059 & 275 & 350 & 75 \\
\hline 37011684 & 50092059 & 285 & 341 & 56 \\
\hline 37011684 & 50092059 & 263 & 346 & 83 \\
\hline 37011684 & 50092059 & 288 & 341 & 53 \\
\hline 37011684 & 50092059 & 286 & 356 & 70 \\
\hline 37011684 & 50092059 & 285 & 337 & 52 \\
\hline 37011684 & 50092059 & 290 & 347 & 57 \\
\hline 37011684 & 50092059 & 268 & 340 & 72 \\
\hline 37011684 & 50092059 & 293 & 351 & 58 \\
\hline 37011684 & 50092059 & 279 & 335 & 56 \\
\hline 37011684 & 50092059 & 288 & 340 & 52 \\
\hline 43649536 & 50092059 & 313 & 333 & 20 \\
\hline 43649536 & 50092059 & 313 & 336 & 23 \\
\hline 43649536 & 50092059 & 312 & 339 & 27 \\
\hline 43649536 & 50092059 & 314 & 338 & 24 \\
\hline 43649536 & 50092059 & 313 & 337 & 24 \\
\hline 43649536 & 50092059 & 313 & 335 & 22 \\
\hline 43649536 & 50092059 & 315 & 333 & 18 \\
\hline 43649536 & 50092059 & 313 & 338 & 25 \\
\hline 41020238 & 50092059 & 309 & 335 & 26 \\
\hline 38849288 & 50092059 & 307 & 340 & 33 \\
\hline 43649536 & 50092059 & 315 & 335 & 20 \\
\hline 43649536 & 50092059 & 312 & 332 & 20 \\
\hline 43649536 & 50092059 & 314 & 331 & 17 \\
\hline 43649536 & 50092059 & 314 & 337 & 23 \\
\hline
\end{tabular}




\begin{tabular}{|c|c|c|c|c|}
\hline 38849288 & 50092059 & 306 & 340 & 34 \\
\hline 43649536 & 50092059 & 313 & 340 & 27 \\
\hline 38849288 & 50092059 & 306 & 342 & 36 \\
\hline 43649536 & 50092059 & 313 & 339 & 26 \\
\hline 43649536 & 50092059 & 312 & 343 & 31 \\
\hline 37011684 & 50092059 & 274 & 344 & 70 \\
\hline 43649536 & 50092059 & 313 & 340 & 27 \\
\hline 43649536 & 50092059 & 316 & 338 & 22 \\
\hline 37011684 & 50986558 & 271 & 362 & 91 \\
\hline 37011684 & 50092059 & 294 & 349 & 55 \\
\hline 37011684 & 50092059 & 282 & 357 & 75 \\
\hline 37011684 & 50092059 & 294 & 358 & 64 \\
\hline 37011684 & 50986558 & 272 & 361 & 89 \\
\hline 37011684 & 50986558 & 271 & 359 & 88 \\
\hline 37011684 & 50986558 & 274 & 364 & 90 \\
\hline 45643182 & 50986558 & 326 & 368 & 42 \\
\hline 45643182 & 50986558 & 325 & 365 & 40 \\
\hline 45643182 & 50986558 & 329 & 364 & 35 \\
\hline 45643182 & 50986558 & 329 & 364 & 35 \\
\hline 45643182 & 50986558 & 330 & 366 & 36 \\
\hline 45643182 & 50986558 & 331 & 366 & 35 \\
\hline 45643182 & 50986558 & 334 & 364 & 30 \\
\hline 45643182 & 50986558 & 333 & 365 & 32 \\
\hline 45643182 & 54052573 & 336 & 403 & 67 \\
\hline 45643182 & 50986558 & 340 & 364 & 24 \\
\hline 45643182 & 50986558 & 341 & 362 & 21 \\
\hline 45643182 & 50986558 & 347 & 365 & 18 \\
\hline 45643182 & 50986558 & 345 & 363 & 18 \\
\hline 45643182 & 50986558 & 338 & 368 & 30 \\
\hline 45643182 & 50986558 & 338 & 366 & 28 \\
\hline 45643182 & 50986558 & 344 & 367 & 23 \\
\hline 45643182 & 50986558 & 344 & 366 & 22 \\
\hline 45643182 & 52411403 & 335 & 371 & 36 \\
\hline 45643182 & 52411403 & 337 & 378 & 41 \\
\hline 45643182 & 52411403 & 339 & 378 & 39 \\
\hline 45643182 & 54052573 & 335 & 406 & 71 \\
\hline 45643182 & 52411403 & 339 & 376 & 37 \\
\hline 45643182 & 52411403 & 346 & 376 & 30 \\
\hline 45643182 & 52411403 & 343 & 378 & 35 \\
\hline 50092059 & 52411403 & 361 & 374 & 13 \\
\hline 50092059 & 52411403 & 358 & 375 & 17 \\
\hline 50092059 & 52411403 & 362 & 376 & 14 \\
\hline 50092059 & 52411403 & 361 & 376 & 15 \\
\hline
\end{tabular}




\begin{tabular}{|c|c|c|c|}
\hline 50092059 & 52411403 & 361 & 377 \\
\hline 50092059 & 52411403 & 362 & 377 \\
\hline 50092059 & 52411403 & 361 & 377 \\
\hline 50092059 & 52411403 & 360 & 376 \\
\hline 50092059 & 52411403 & 359 & 377 \\
\hline 50092059 & 52411403 & 362 & 376 \\
\hline 50092059 & 54052573 & 359 & 382 \\
\hline 50092059 & 54052573 & 361 & 392 \\
\hline 50092059 & 52411403 & 365 & 378 \\
\hline 50092059 & 54052573 & 360 & 397 \\
\hline 50092059 & 54052573 & 361 & 393 \\
\hline 50092059 & 54052573 & 363 & 390 \\
\hline 50092059 & 54052573 & 360 & 395 \\
\hline 45643182 & 54052573 & 357 & 395 \\
\hline 45643182 & 54052573 & 355 & 410 \\
\hline 50092059 & 54052573 & 364 & 395 \\
\hline 50092059 & 54052573 & 360 & 416 \\
\hline 50092059 & 54052573 & 360 & 411 \\
\hline 50092059 & 54052573 & 363 & 392 \\
\hline 45643182 & 54052573 & 346 & 398 \\
\hline 50092059 & 54052573 & 359 & 393 \\
\hline 45643182 & 54052573 & 353 & 397 \\
\hline 50092059 & 54052573 & 361 & 408 \\
\hline 50092059 & 54052573 & 365 & 396 \\
\hline 50986558 & 54052573 & 371 & 401 \\
\hline 50986558 & 54052573 & 371 & 393 \\
\hline 50986558 & 54052573 & 375 & 397 \\
\hline 50986558 & 54052573 & 373 & 406 \\
\hline 50986558 & 54052573 & 377 & 402 \\
\hline 50986558 & 54052573 & 372 & 405 \\
\hline 50986558 & 54052573 & 374 & 414 \\
\hline 50986558 & 54052573 & 374 & 417 \\
\hline 50986558 & 56925084 & 371 & 436 \\
\hline 52411403 & 56925084 & 394 & 439 \\
\hline 52411403 & 56925084 & 397 & 444 \\
\hline 52411403 & 56925084 & 398 & 440 \\
\hline 52411403 & 56925084 & 400 & 441 \\
\hline 52411403 & 56925084 & 397 & 439 \\
\hline 52411403 & 56925084 & 396 & 436 \\
\hline 52411403 & 56925084 & 408 & 449 \\
\hline 52411403 & 56925084 & 399 & 436 \\
\hline 52411403 & 56925084 & 400 & 436 \\
\hline 52411403 & 56925084 & 400 & 438 \\
\hline
\end{tabular}




\begin{tabular}{|c|c|c|c|}
\hline 52411403 & 56925084 & 406 & 437 \\
\hline 52411403 & 56925084 & 403 & 441 \\
\hline 52411403 & 56925084 & 407 & 443 \\
\hline 52411403 & 56925084 & 407 & 451 \\
\hline 52411403 & 56925084 & 401 & 452 \\
\hline 52411403 & 56925084 & 404 & 452 \\
\hline 55787716 & 56925084 & 431 & 452 \\
\hline 55787716 & 56925084 & 430 & 452 \\
\hline 55787716 & 56925084 & 432 & 452 \\
\hline 55787716 & 56925084 & 428 & 452 \\
\hline 55787716 & 56925084 & 433 & 452 \\
\hline 55787716 & 56925084 & 435 & 452 \\
\hline 55787716 & 56925084 & 435 & 452 \\
\hline 55787716 & 56925084 & 435 & 452 \\
\hline 55787716 & 56925084 & 445 & 452 \\
\hline 52411403 & 56925084 & 401 & 452 \\
\hline 55787716 & 56925084 & 440 & 452 \\
\hline 55787716 & 56925084 & 446 & 452 \\
\hline 1284139 & 2658552 & 0 & 28 \\
\hline 1284139 & 2658552 & 0 & 27 \\
\hline 1284139 & 2658552 & 0 & 22 \\
\hline 1284139 & 4193290 & 0 & 41 \\
\hline 1284139 & 4193290 & 0 & 41 \\
\hline 1284139 & 4193290 & 0 & 42 \\
\hline 1284139 & 4193290 & 0 & 42 \\
\hline 1284139 & 2658552 & 0 & 21 \\
\hline 1284139 & 4193290 & 0 & 42 \\
\hline 1284139 & 2658552 & 0 & 20 \\
\hline 1284139 & 2658552 & 0 & 20 \\
\hline 1284139 & 2658552 & 0 & 20 \\
\hline 1284139 & 2658552 & 0 & 21 \\
\hline 1284139 & 2658552 & 0 & 34 \\
\hline 1284139 & 2658552 & 0 & 31 \\
\hline 1284139 & 2658552 & 0 & 33 \\
\hline 1284139 & 2658552 & 0 & 28 \\
\hline 1284139 & 2658552 & 0 & 28 \\
\hline 1284139 & 2658552 & 0 & 32 \\
\hline 1284139 & 2658552 & 0 & 32 \\
\hline 1284139 & 2658552 & 0 & 31 \\
\hline 1284139 & 2658552 & 0 & 33 \\
\hline 1284139 & 2658552 & 0 & 32 \\
\hline 1284139 & 4193290 & 0 & 38 \\
\hline 1284139 & 4193290 & 0 & 49 \\
\hline
\end{tabular}




\begin{tabular}{|c|c|c|c|}
\hline 1284139 & 4193290 & 0 & 40 \\
\hline 1284139 & 4193290 & 0 & 42 \\
\hline 1284139 & 4193290 & 0 & 59 \\
\hline 1284139 & 4193290 & 0 & 45 \\
\hline 1284139 & 4193290 & 0 & 50 \\
\hline 1284139 & 4193290 & 0 & 45 \\
\hline 1284139 & 4193290 & 0 & 50 \\
\hline 1284139 & 4193290 & 0 & 45 \\
\hline 1284139 & 4193290 & 0 & 52 \\
\hline 1284139 & 4193290 & 0 & 46 \\
\hline 1284139 & 4193290 & 0 & 44 \\
\hline 1284139 & 4193290 & 0 & 52 \\
\hline 1284139 & 4193290 & 0 & 51 \\
\hline 1284139 & 4193290 & 0 & 52 \\
\hline 1284139 & 4193290 & 0 & 51 \\
\hline 1284139 & 4193290 & 0 & 56 \\
\hline 2058639 & 4193290 & 17 & 46 \\
\hline 1284139 & 4193290 & 0 & 54 \\
\hline 2058639 & 4193290 & 2 & 59 \\
\hline 2058639 & 4193290 & 26 & 62 \\
\hline 2058639 & 5014708 & 7 & 65 \\
\hline 2058639 & 4193290 & 21 & 56 \\
\hline 2658552 & 6568227 & 41 & 82 \\
\hline 2658552 & 6568227 & 45 & 74 \\
\hline 2658552 & 6568227 & 50 & 85 \\
\hline 2658552 & 5175596 & 52 & 69 \\
\hline 2658552 & 6568227 & 47 & 81 \\
\hline 2658552 & 6568227 & 42 & 79 \\
\hline 2658552 & 5175596 & 52 & 69 \\
\hline 2658552 & 6568227 & 47 & 76 \\
\hline 2658552 & 6568227 & 51 & 74 \\
\hline 2658552 & 6568227 & 47 & 85 \\
\hline 2658552 & 6568227 & 49 & 79 \\
\hline 2658552 & 6568227 & 52 & 77 \\
\hline 2658552 & 6568227 & 39 & 80 \\
\hline 4193290 & 6568227 & 65 & 81 \\
\hline 4193290 & 7742744 & 66 & 103 \\
\hline 2058639 & 8866392 & 31 & 109 \\
\hline 5175596 & 8866392 & 72 & 111 \\
\hline 5175596 & 7742744 & 71 & 95 \\
\hline 2658552 & 8866392 & 56 & 106 \\
\hline 5175596 & 7742744 & 73 & 94 \\
\hline 5175596 & 7742744 & 73 & 102 \\
\hline
\end{tabular}




\begin{tabular}{|c|c|c|c|}
\hline 5175596 & 7742744 & 72 & 100 \\
\hline 5175596 & 7742744 & 76 & 99 \\
\hline 5175596 & 7742744 & 72 & 102 \\
\hline 5175596 & 7742744 & 78 & 97 \\
\hline 5175596 & 7742744 & 79 & 100 \\
\hline 5175596 & 7742744 & 82 & 90 \\
\hline 5175596 & 7742744 & 79 & 95 \\
\hline 5175596 & 7742744 & 78 & 95 \\
\hline 5175596 & 7742744 & 80 & 93 \\
\hline 5175596 & 7742744 & 79 & 94 \\
\hline 5175596 & 7742744 & 77 & 96 \\
\hline 5175596 & 7742744 & 80 & 93 \\
\hline 5175596 & 7742744 & 74 & 100 \\
\hline 5175596 & 7742744 & 79 & 100 \\
\hline 5175596 & 7742744 & 75 & 96 \\
\hline 5175596 & 7742744 & 73 & 100 \\
\hline 5175596 & 7742744 & 76 & 95 \\
\hline 5175596 & 7742744 & 76 & 101 \\
\hline 5175596 & 7742744 & 75 & 102 \\
\hline 5175596 & 7742744 & 72 & 103 \\
\hline 5175596 & 7742744 & 74 & 99 \\
\hline 5175596 & 7742744 & 75 & 102 \\
\hline 5175596 & 7742744 & 75 & 98 \\
\hline 5175596 & 7742744 & 76 & 101 \\
\hline 5175596 & 8866392 & 72 & 107 \\
\hline 5175596 & 13220219 & 76 & 139 \\
\hline 5175596 & 8866392 & 73 & 109 \\
\hline 5175596 & 8866392 & 77 & 110 \\
\hline 5175596 & 10633493 & 74 & 119 \\
\hline 5175596 & 8866392 & 70 & 108 \\
\hline 5175596 & 10633493 & 74 & 116 \\
\hline 5175596 & 8866392 & 75 & 110 \\
\hline 5175596 & 10633493 & 75 & 114 \\
\hline 5175596 & 10633493 & 69 & 121 \\
\hline 5175596 & 8866392 & 69 & 111 \\
\hline 4193290 & 8866392 & 68 & 109 \\
\hline 5175596 & 10633493 & 70 & 116 \\
\hline 5175596 & 10633493 & 75 & 114 \\
\hline 6568227 & 8866392 & 89 & 108 \\
\hline 5175596 & 8866392 & 77 & 111 \\
\hline 2058639 & 8866392 & 19 & 111 \\
\hline 5175596 & 8866392 & 78 & 112 \\
\hline 6568227 & 8866392 & 89 & 111 \\
\hline
\end{tabular}




\begin{tabular}{|c|c|c|c|}
\hline 5175596 & 10633493 & 72 & 115 \\
\hline 6568227 & 10633493 & 89 & 113 \\
\hline 6568227 & 8866392 & 92 & 110 \\
\hline 6568227 & 8866392 & 90 & 110 \\
\hline 5175596 & 10633493 & 78 & 124 \\
\hline 6568227 & 8866392 & 99 & 111 \\
\hline 6568227 & 10633493 & 95 & 121 \\
\hline 6568227 & 10633493 & 90 & 117 \\
\hline 6568227 & 10633493 & 98 & 119 \\
\hline 6568227 & 8866392 & 93 & 109 \\
\hline 6568227 & 8866392 & 96 & 111 \\
\hline 6568227 & 8866392 & 96 & 111 \\
\hline 6568227 & 8866392 & 97 & 108 \\
\hline 6568227 & 8866392 & 98 & 112 \\
\hline 6568227 & 8866392 & 100 & 111 \\
\hline 6568227 & 8866392 & 97 & 111 \\
\hline 6568227 & 8866392 & 100 & 111 \\
\hline 6568227 & 8866392 & 100 & 110 \\
\hline 6568227 & 8866392 & 100 & 109 \\
\hline 6568227 & 8866392 & 98 & 111 \\
\hline 6568227 & 8866392 & 94 & 110 \\
\hline 5175596 & 10633493 & 75 & 117 \\
\hline 6568227 & 8866392 & 99 & 109 \\
\hline 6568227 & 10633493 & 90 & 116 \\
\hline 6568227 & 10633493 & 91 & 113 \\
\hline 6568227 & 8866392 & 98 & 111 \\
\hline 6568227 & 10633493 & 93 & 121 \\
\hline 6568227 & 10633493 & 97 & 119 \\
\hline 6568227 & 10633493 & 100 & 117 \\
\hline 6568227 & 10633493 & 92 & 118 \\
\hline 6568227 & 10633493 & 88 & 117 \\
\hline 5175596 & 10633493 & 72 & 120 \\
\hline 6568227 & 13220219 & 92 & 132 \\
\hline 6568227 & 10633493 & 91 & 124 \\
\hline 5175596 & 10633493 & 77 & 127 \\
\hline 6568227 & 10633493 & 95 & 119 \\
\hline 6568227 & 13220219 & 91 & 142 \\
\hline 6568227 & 13220219 & 95 & 141 \\
\hline 6568227 & 10633493 & 92 & 122 \\
\hline 6568227 & 13220219 & 95 & 130 \\
\hline 5175596 & 10633493 & 72 & 122 \\
\hline 6568227 & 10633493 & 89 & 124 \\
\hline 6568227 & 10633493 & 101 & 122 \\
\hline
\end{tabular}




\begin{tabular}{|c|c|c|c|}
\hline 2658552 & 10633493 & 46 & 123 \\
\hline 6568227 & 10633493 & 92 & 122 \\
\hline 5175596 & 10633493 & 76 & 118 \\
\hline 6568227 & 10633493 & 90 & 121 \\
\hline 6568227 & 10633493 & 105 & 119 \\
\hline 6568227 & 10633493 & 102 & 119 \\
\hline 6568227 & 10633493 & 102 & 120 \\
\hline 2658552 & 10633493 & 46 & 124 \\
\hline 7742744 & 10633493 & 106 & 126 \\
\hline 7742744 & 10633493 & 106 & 120 \\
\hline 7742744 & 10633493 & 110 & 117 \\
\hline 7742744 & 10633493 & 107 & 120 \\
\hline 7742744 & 10633493 & 109 & 121 \\
\hline 7742744 & 10633493 & 108 & 121 \\
\hline 6568227 & 10633493 & 100 & 123 \\
\hline 7742744 & 10633493 & 108 & 124 \\
\hline 6568227 & 13220219 & 95 & 132 \\
\hline 7742744 & 10633493 & 108 & 122 \\
\hline 6568227 & 13220219 & 96 & 135 \\
\hline 7742744 & 13220219 & 107 & 139 \\
\hline 6568227 & 13220219 & 97 & 143 \\
\hline 6568227 & 13220219 & 89 & 140 \\
\hline 6568227 & 13220219 & 88 & 145 \\
\hline 6568227 & 13220219 & 90 & 141 \\
\hline 6568227 & 13220219 & 105 & 151 \\
\hline 6568227 & 13220219 & 92 & 146 \\
\hline 7742744 & 13220219 & 108 & 156 \\
\hline 7742744 & 13220219 & 109 & 146 \\
\hline 7742744 & 13220219 & 106 & 152 \\
\hline 7742744 & 13220219 & 107 & 141 \\
\hline 6568227 & 13220219 & 105 & 147 \\
\hline 7742744 & 13220219 & 110 & 144 \\
\hline 8866392 & 13220219 & 116 & 136 \\
\hline 8866392 & 13220219 & 115 & 144 \\
\hline 8866392 & 13220219 & 112 & 147 \\
\hline 7742744 & 13220219 & 107 & 149 \\
\hline 8866392 & 13220219 & 114 & 150 \\
\hline 7742744 & 13220219 & 108 & 152 \\
\hline 8866392 & 13220219 & 116 & 147 \\
\hline 8866392 & 13220219 & 119 & 142 \\
\hline 8866392 & 13220219 & 119 & 149 \\
\hline 8866392 & 13220219 & 118 & 147 \\
\hline 8866392 & 13220219 & 121 & 149 \\
\hline
\end{tabular}




\begin{tabular}{|c|c|c|c|}
\hline 8866392 & 13220219 & 120 & 148 \\
\hline 8866392 & 13220219 & 120 & 145 \\
\hline 8866392 & 13220219 & 119 & 151 \\
\hline 8866392 & 13220219 & 117 & 147 \\
\hline 8866392 & 13220219 & 113 & 158 \\
\hline 8866392 & 13220219 & 119 & 151 \\
\hline 8866392 & 13220219 & 117 & 149 \\
\hline 8866392 & 13220219 & 118 & 148 \\
\hline 8866392 & 13220219 & 119 & 152 \\
\hline 8866392 & 13220219 & 125 & 146 \\
\hline 8866392 & 13220219 & 123 & 154 \\
\hline 8866392 & 13220219 & 123 & 160 \\
\hline 8866392 & 13220219 & 123 & 162 \\
\hline 8866392 & 13220219 & 123 & 159 \\
\hline 8866392 & 13220219 & 118 & 165 \\
\hline 8866392 & 13220219 & 125 & 153 \\
\hline 8866392 & 13220219 & 120 & 163 \\
\hline 8866392 & 13220219 & 119 & 169 \\
\hline 8866392 & 13220219 & 123 & 161 \\
\hline 8866392 & 13220219 & 120 & 166 \\
\hline 8866392 & 13220219 & 123 & 161 \\
\hline 8866392 & 13220219 & 122 & 164 \\
\hline 8866392 & 13220219 & 121 & 165 \\
\hline 8866392 & 13220219 & 122 & 163 \\
\hline 8866392 & 13220219 & 124 & 161 \\
\hline 8866392 & 13220219 & 119 & 170 \\
\hline 8866392 & 13220219 & 121 & 172 \\
\hline 8866392 & 13220219 & 123 & 162 \\
\hline 8866392 & 13220219 & 124 & 162 \\
\hline 8866392 & 13220219 & 123 & 167 \\
\hline 8866392 & 13220219 & 123 & 166 \\
\hline 8866392 & 13220219 & 122 & 171 \\
\hline 8866392 & 13220219 & 123 & 167 \\
\hline 8866392 & 13220219 & 121 & 174 \\
\hline 8866392 & 13220219 & 122 & 169 \\
\hline 8866392 & 13220219 & 121 & 171 \\
\hline 8866392 & 13220219 & 122 & 171 \\
\hline 8866392 & 13220219 & 120 & 180 \\
\hline 8866392 & 13220219 & 123 & 172 \\
\hline 8866392 & 13220219 & 122 & 174 \\
\hline 8866392 & 13220219 & 122 & 178 \\
\hline 8866392 & 14544747 & 123 & 180 \\
\hline 8866392 & 17327995 & 122 & 184 \\
\hline
\end{tabular}




\begin{tabular}{|c|c|c|c|c|}
\hline 8866392 & 14544747 & 123 & 181 & 58 \\
\hline 8866392 & 17327995 & 121 & 183 & 62 \\
\hline 8866392 & 14544747 & 122 & 182 & 60 \\
\hline 8866392 & 17327995 & 121 & 183 & 62 \\
\hline 8866392 & 17327995 & 123 & 184 & 61 \\
\hline 8866392 & 15083839 & 122 & 182 & 60 \\
\hline 8866392 & 13220219 & 124 & 180 & 56 \\
\hline 8866392 & 15083839 & 124 & 182 & 58 \\
\hline 8866392 & 17327995 & 123 & 183 & 60 \\
\hline 8866392 & 17327995 & 125 & 187 & 62 \\
\hline 8866392 & 14544747 & 125 & 182 & 57 \\
\hline 8866392 & 17327995 & 125 & 184 & 59 \\
\hline 8866392 & 15083839 & 125 & 182 & 57 \\
\hline 8866392 & 14544747 & 126 & 182 & 56 \\
\hline 8866392 & 13220219 & 124 & 180 & 56 \\
\hline 8866392 & 17327995 & 124 & 183 & 59 \\
\hline 8866392 & 17327995 & 124 & 183 & 59 \\
\hline 8866392 & 17327995 & 125 & 183 & 58 \\
\hline 8866392 & 17327995 & 123 & 183 & 60 \\
\hline 8866392 & 17327995 & 125 & 183 & 58 \\
\hline 10633493 & 15083839 & 127 & 182 & 55 \\
\hline 8866392 & 14544747 & 125 & 182 & 57 \\
\hline 8866392 & 17327995 & 124 & 185 & 61 \\
\hline 8866392 & 17327995 & 123 & 183 & 60 \\
\hline 8866392 & 17327995 & 126 & 184 & 58 \\
\hline 8866392 & 17327995 & 126 & 187 & 61 \\
\hline 10633493 & 15083839 & 128 & 182 & 54 \\
\hline 10633493 & 13220219 & 129 & 180 & 51 \\
\hline 10633493 & 14544747 & 127 & 182 & 55 \\
\hline 10633493 & 13220219 & 135 & 180 & 45 \\
\hline 10633493 & 17327995 & 131 & 184 & 53 \\
\hline 10633493 & 13220219 & 135 & 180 & 45 \\
\hline 10633493 & 14544747 & 132 & 182 & 50 \\
\hline 10633493 & 17327995 & 135 & 183 & 48 \\
\hline 10633493 & 17327995 & 132 & 184 & 52 \\
\hline 10633493 & 17327995 & 132 & 185 & 53 \\
\hline 10633493 & 13220219 & 138 & 180 & 42 \\
\hline 10633493 & 17327995 & 138 & 184 & 46 \\
\hline 10633493 & 17327995 & 132 & 184 & 52 \\
\hline 10633493 & 17327995 & 132 & 186 & 54 \\
\hline 10633493 & 17327995 & 134 & 188 & 54 \\
\hline 10633493 & 17327995 & 131 & 183 & 52 \\
\hline 10633493 & 17327995 & 137 & 185 & 48 \\
\hline
\end{tabular}




\begin{tabular}{|c|c|c|c|c|}
\hline 10633493 & 17327995 & 142 & 186 & 44 \\
\hline 10633493 & 17327995 & 138 & 184 & 46 \\
\hline 10633493 & 17327995 & 141 & 187 & 46 \\
\hline 10633493 & 17327995 & 137 & 186 & 49 \\
\hline 10633493 & 14544747 & 147 & 182 & 35 \\
\hline 10633493 & 17327995 & 143 & 187 & 44 \\
\hline 10633493 & 17327995 & 143 & 186 & 43 \\
\hline 10633493 & 17327995 & 146 & 185 & 39 \\
\hline 10633493 & 15083839 & 144 & 182 & 38 \\
\hline 10633493 & 14544747 & 145 & 182 & 37 \\
\hline 10633493 & 17327995 & 137 & 184 & 47 \\
\hline 10633493 & 17327995 & 139 & 185 & 46 \\
\hline 10633493 & 17327995 & 136 & 185 & 49 \\
\hline 10633493 & 17327995 & 148 & 183 & 35 \\
\hline 10633493 & 17327995 & 145 & 187 & 42 \\
\hline 10633493 & 17327995 & 144 & 185 & 41 \\
\hline 8866392 & 18645658 & 126 & 194 & 68 \\
\hline 10633493 & 14544747 & 145 & 182 & 37 \\
\hline 10633493 & 17327995 & 146 & 186 & 40 \\
\hline 10633493 & 17327995 & 146 & 187 & 41 \\
\hline 10633493 & 17327995 & 146 & 187 & 41 \\
\hline 10633493 & 17327995 & 146 & 186 & 40 \\
\hline 10633493 & 17327995 & 145 & 186 & 41 \\
\hline 10633493 & 17327995 & 148 & 187 & 39 \\
\hline 10633493 & 17327995 & 148 & 187 & 39 \\
\hline 10633493 & 17327995 & 147 & 187 & 40 \\
\hline 10633493 & 17327995 & 148 & 186 & 38 \\
\hline 10633493 & 17327995 & 145 & 184 & 39 \\
\hline 10633493 & 17327995 & 148 & 186 & 38 \\
\hline 10633493 & 17327995 & 147 & 188 & 41 \\
\hline 10633493 & 17327995 & 150 & 189 & 39 \\
\hline 10633493 & 17327995 & 143 & 184 & 41 \\
\hline 10633493 & 17327995 & 145 & 184 & 39 \\
\hline 10633493 & 17327995 & 141 & 186 & 45 \\
\hline 10633493 & 15083839 & 153 & 182 & 29 \\
\hline 10633493 & 17327995 & 153 & 186 & 33 \\
\hline 10633493 & 17327995 & 148 & 186 & 38 \\
\hline 10633493 & 17327995 & 145 & 185 & 40 \\
\hline 10633493 & 17327995 & 152 & 189 & 37 \\
\hline 10633493 & 15083839 & 151 & 182 & 31 \\
\hline 10633493 & 17327995 & 148 & 188 & 40 \\
\hline 10633493 & 18645658 & 155 & 191 & 36 \\
\hline 10633493 & 17327995 & 153 & 186 & 33 \\
\hline
\end{tabular}




\begin{tabular}{|c|c|c|c|c|}
\hline 10633493 & 18645658 & 162 & 197 & 35 \\
\hline 10633493 & 18645658 & 163 & 199 & 36 \\
\hline 10633493 & 17327995 & 167 & 189 & 22 \\
\hline 10633493 & 17327995 & 159 & 188 & 29 \\
\hline 10633493 & 18645658 & 162 & 195 & 33 \\
\hline 10633493 & 18645658 & 164 & 195 & 31 \\
\hline 10633493 & 17327995 & 165 & 189 & 24 \\
\hline 10633493 & 18645658 & 165 & 192 & 27 \\
\hline 10633493 & 18645658 & 165 & 199 & 34 \\
\hline 10633493 & 18645658 & 164 & 195 & 31 \\
\hline 10633493 & 18645658 & 164 & 202 & 38 \\
\hline 10633493 & 18645658 & 163 & 197 & 34 \\
\hline 10633493 & 17327995 & 151 & 186 & 35 \\
\hline 10633493 & 18645658 & 156 & 203 & 47 \\
\hline 10633493 & 18645658 & 153 & 198 & 45 \\
\hline 10633493 & 18645658 & 154 & 193 & 39 \\
\hline 10633493 & 18645658 & 158 & 191 & 33 \\
\hline 10633493 & 18645658 & 153 & 197 & 44 \\
\hline 10633493 & 18645658 & 156 & 199 & 43 \\
\hline 10633493 & 14544747 & 163 & 182 & 19 \\
\hline 10633493 & 17327995 & 163 & 186 & 23 \\
\hline 10633493 & 18645658 & 163 & 194 & 31 \\
\hline 10633493 & 17327995 & 150 & 188 & 38 \\
\hline 10633493 & 17327995 & 167 & 188 & 21 \\
\hline 10633493 & 18645658 & 159 & 199 & 40 \\
\hline 10633493 & 18645658 & 157 & 201 & 44 \\
\hline 10633493 & 17327995 & 148 & 189 & 41 \\
\hline 10633493 & 17327995 & 151 & 189 & 38 \\
\hline 10633493 & 18645658 & 156 & 196 & 40 \\
\hline 10633493 & 18645658 & 151 & 193 & 42 \\
\hline 10633493 & 18645658 & 152 & 197 & 45 \\
\hline 10633493 & 18645658 & 152 & 194 & 42 \\
\hline 10633493 & 18645658 & 166 & 195 & 29 \\
\hline 10633493 & 18645658 & 162 & 191 & 29 \\
\hline 10633493 & 18645658 & 167 & 202 & 35 \\
\hline 10633493 & 17327995 & 166 & 187 & 21 \\
\hline 10633493 & 18645658 & 165 & 190 & 25 \\
\hline 10633493 & 18645658 & 154 & 195 & 41 \\
\hline 10633493 & 18645658 & 153 & 194 & 41 \\
\hline 10633493 & 17327995 & 155 & 188 & 33 \\
\hline 10633493 & 17327995 & 165 & 186 & 21 \\
\hline 10633493 & 17327995 & 157 & 189 & 32 \\
\hline 10633493 & 18645658 & 153 & 190 & 37 \\
\hline
\end{tabular}




\begin{tabular}{|c|c|c|c|c|}
\hline 10633493 & 18645658 & 154 & 191 & 37 \\
\hline 10633493 & 17327995 & 154 & 189 & 35 \\
\hline 10633493 & 17327995 & 161 & 188 & 27 \\
\hline 10633493 & 18645658 & 163 & 203 & 40 \\
\hline 10633493 & 18645658 & 159 & 190 & 31 \\
\hline 10633493 & 18645658 & 157 & 191 & 34 \\
\hline 10633493 & 18645658 & 176 & 190 & 14 \\
\hline 13220219 & 17327995 & 181 & 186 & 5 \\
\hline 10633493 & 18645658 & 177 & 192 & 15 \\
\hline 10633493 & 18645658 & 167 & 191 & 24 \\
\hline 10633493 & 18645658 & 173 & 205 & 32 \\
\hline 10633493 & 18645658 & 164 & 196 & 32 \\
\hline 10633493 & 18645658 & 171 & 192 & 21 \\
\hline 10633493 & 17327995 & 175 & 188 & 13 \\
\hline 10633493 & 18645658 & 168 & 202 & 34 \\
\hline 10633493 & 18645658 & 170 & 194 & 24 \\
\hline 10633493 & 18645658 & 176 & 191 & 15 \\
\hline 13220219 & 18645658 & 180 & 197 & 17 \\
\hline 10633493 & 18645658 & 163 & 200 & 37 \\
\hline 10633493 & 18645658 & 166 & 195 & 29 \\
\hline 10633493 & 25275062 & 159 & 222 & 63 \\
\hline 10633493 & 18645658 & 160 & 196 & 36 \\
\hline 10633493 & 23044232 & 168 & 214 & 46 \\
\hline 10633493 & 18645658 & 177 & 196 & 19 \\
\hline 10633493 & 18645658 & 166 & 199 & 33 \\
\hline 10633493 & 25275062 & 169 & 217 & 48 \\
\hline 10633493 & 18645658 & 166 & 201 & 35 \\
\hline 10633493 & 18645658 & 160 & 205 & 45 \\
\hline 10633493 & 25275062 & 164 & 228 & 64 \\
\hline 10633493 & 18645658 & 165 & 200 & 35 \\
\hline 10633493 & 18645658 & 175 & 204 & 29 \\
\hline 10633493 & 18645658 & 159 & 204 & 45 \\
\hline 10633493 & 25275062 & 172 & 235 & 63 \\
\hline 10633493 & 25275062 & 158 & 215 & 57 \\
\hline 10633493 & 18645658 & 171 & 202 & 31 \\
\hline 10633493 & 18645658 & 170 & 204 & 34 \\
\hline 10633493 & 18645658 & 171 & 202 & 31 \\
\hline 13220219 & 18645658 & 180 & 204 & 24 \\
\hline 10633493 & 18645658 & 178 & 205 & 27 \\
\hline 13220219 & 18645658 & 181 & 197 & 16 \\
\hline 10633493 & 18645658 & 169 & 200 & 31 \\
\hline 10633493 & 18645658 & 169 & 204 & 35 \\
\hline 13220219 & 18645658 & 182 & 197 & 15 \\
\hline
\end{tabular}




\begin{tabular}{|c|c|c|c|}
\hline 15083839 & 18645658 & 183 & 198 \\
\hline 15083839 & 18645658 & 184 & 197 \\
\hline 15083839 & 18645658 & 184 & 199 \\
\hline 10633493 & 18645658 & 176 & 201 \\
\hline 15083839 & 18645658 & 184 & 199 \\
\hline 15083839 & 18645658 & 185 & 198 \\
\hline 15083839 & 18645658 & 183 & 202 \\
\hline 15083839 & 18645658 & 185 & 201 \\
\hline 15083839 & 18645658 & 185 & 198 \\
\hline 15083839 & 18645658 & 185 & 199 \\
\hline 15083839 & 18645658 & 186 & 203 \\
\hline 10633493 & 18645658 & 177 & 201 \\
\hline 10633493 & 18645658 & 168 & 200 \\
\hline 15083839 & 18645658 & 185 & 199 \\
\hline 10633493 & 18645658 & 173 & 202 \\
\hline 13220219 & 23044232 & 182 & 207 \\
\hline 15083839 & 18645658 & 184 & 199 \\
\hline 10633493 & 18645658 & 162 & 202 \\
\hline 13220219 & 18645658 & 180 & 204 \\
\hline 10633493 & 18645658 & 174 & 201 \\
\hline 15083839 & 18645658 & 183 & 202 \\
\hline 13220219 & 18645658 & 181 & 200 \\
\hline 10633493 & 18645658 & 174 & 198 \\
\hline 15083839 & 18645658 & 184 & 200 \\
\hline 15083839 & 18645658 & 184 & 201 \\
\hline 10633493 & 18645658 & 172 & 205 \\
\hline 15083839 & 18645658 & 183 & 202 \\
\hline 15083839 & 18645658 & 184 & 204 \\
\hline 15083839 & 25275062 & 184 & 236 \\
\hline 15083839 & 25275062 & 183 & 227 \\
\hline 15083839 & 18645658 & 184 & 205 \\
\hline 15083839 & 18645658 & 185 & 201 \\
\hline 15083839 & 18645658 & 186 & 204 \\
\hline 15083839 & 18645658 & 184 & 204 \\
\hline 15083839 & 18645658 & 184 & 205 \\
\hline 15083839 & 23044232 & 183 & 211 \\
\hline 15083839 & 18645658 & 184 & 203 \\
\hline 15083839 & 18645658 & 186 & 200 \\
\hline 15083839 & 18645658 & 187 & 199 \\
\hline 15083839 & 18645658 & 185 & 204 \\
\hline 15083839 & 18645658 & 187 & 202 \\
\hline 15083839 & 18645658 & 185 & 203 \\
\hline 15083839 & 18645658 & 185 & 205 \\
\hline
\end{tabular}




\begin{tabular}{|c|c|c|c|c|}
\hline 15083839 & 18645658 & 186 & 205 & 19 \\
\hline 15083839 & 25275062 & 185 & 221 & 36 \\
\hline 13220219 & 25275062 & 181 & 225 & 44 \\
\hline 13220219 & 18645658 & 181 & 204 & 23 \\
\hline 10633493 & 25275062 & 169 & 223 & 54 \\
\hline 15083839 & 25275062 & 184 & 231 & 47 \\
\hline 15083839 & 23044232 & 185 & 211 & 26 \\
\hline 15083839 & 18645658 & 183 & 204 & 21 \\
\hline 15083839 & 18645658 & 187 & 204 & 17 \\
\hline 15083839 & 18645658 & 187 & 203 & 16 \\
\hline 15083839 & 25275062 & 185 & 227 & 42 \\
\hline 15083839 & 18645658 & 185 & 205 & 20 \\
\hline 13220219 & 18645658 & 181 & 204 & 23 \\
\hline 15083839 & 25275062 & 184 & 220 & 36 \\
\hline 10633493 & 25275062 & 174 & 234 & 60 \\
\hline 10633493 & 25275062 & 162 & 239 & 77 \\
\hline 15083839 & 26868425 & 185 & 243 & 58 \\
\hline 10633493 & 25275062 & 167 & 224 & 57 \\
\hline 10633493 & 25275062 & 166 & 220 & 54 \\
\hline 15083839 & 23044232 & 186 & 210 & 24 \\
\hline 15083839 & 25275062 & 185 & 226 & 41 \\
\hline 10633493 & 23044232 & 176 & 214 & 38 \\
\hline 15083839 & 18645658 & 184 & 205 & 21 \\
\hline 13220219 & 18645658 & 182 & 205 & 23 \\
\hline 15083839 & 23044232 & 185 & 209 & 24 \\
\hline 15083839 & 23044232 & 185 & 214 & 29 \\
\hline 15083839 & 25275062 & 186 & 228 & 42 \\
\hline 15083839 & 25275062 & 184 & 233 & 49 \\
\hline 10633493 & 23044232 & 171 & 212 & 41 \\
\hline 10633493 & 25275062 & 173 & 235 & 62 \\
\hline 15083839 & 25275062 & 187 & 232 & 45 \\
\hline 15083839 & 25275062 & 184 & 232 & 48 \\
\hline 15083839 & 18645658 & 188 & 205 & 17 \\
\hline 13220219 & 23044232 & 182 & 212 & 30 \\
\hline 15083839 & 25275062 & 188 & 227 & 39 \\
\hline 13220219 & 23044232 & 180 & 214 & 34 \\
\hline 15083839 & 25275062 & 185 & 228 & 43 \\
\hline 15083839 & 22394452 & 185 & 206 & 21 \\
\hline 15083839 & 25275062 & 185 & 229 & 44 \\
\hline 10633493 & 25275062 & 166 & 230 & 64 \\
\hline 15083839 & 25275062 & 183 & 232 & 49 \\
\hline 15083839 & 25275062 & 186 & 229 & 43 \\
\hline 14544747 & 23044232 & 182 & 214 & 32 \\
\hline
\end{tabular}




\begin{tabular}{|c|c|c|c|c|}
\hline 15083839 & 25275062 & 184 & 229 & 45 \\
\hline 15083839 & 25275062 & 185 & 235 & 50 \\
\hline 15083839 & 25275062 & 185 & 231 & 46 \\
\hline 15083839 & 25275062 & 184 & 236 & 52 \\
\hline 15083839 & 25275062 & 183 & 234 & 51 \\
\hline 14544747 & 25275062 & 182 & 234 & 52 \\
\hline 15083839 & 25275062 & 184 & 233 & 49 \\
\hline 15083839 & 25275062 & 185 & 224 & 39 \\
\hline 15083839 & 25275062 & 188 & 225 & 37 \\
\hline 15083839 & 25275062 & 184 & 236 & 52 \\
\hline 15083839 & 25275062 & 186 & 232 & 46 \\
\hline 13220219 & 25275062 & 182 & 235 & 53 \\
\hline 15083839 & 23044232 & 186 & 214 & 28 \\
\hline 15083839 & 25275062 & 183 & 227 & 44 \\
\hline 15083839 & 25275062 & 184 & 231 & 47 \\
\hline 15083839 & 23044232 & 183 & 214 & 31 \\
\hline 17327995 & 25275062 & 190 & 223 & 33 \\
\hline 13220219 & 25275062 & 181 & 230 & 49 \\
\hline 17327995 & 23044232 & 192 & 214 & 22 \\
\hline 10633493 & 25275062 & 166 & 241 & 75 \\
\hline 17327995 & 25275062 & 190 & 219 & 29 \\
\hline 15083839 & 25275062 & 188 & 232 & 44 \\
\hline 15083839 & 25275062 & 184 & 231 & 47 \\
\hline 17327995 & 25275062 & 192 & 225 & 33 \\
\hline 10633493 & 23044232 & 176 & 214 & 38 \\
\hline 17327995 & 23044232 & 197 & 210 & 13 \\
\hline 17327995 & 25275062 & 191 & 217 & 26 \\
\hline 17327995 & 25275062 & 193 & 232 & 39 \\
\hline 17327995 & 25275062 & 194 & 240 & 46 \\
\hline 17327995 & 23044232 & 194 & 214 & 20 \\
\hline 17327995 & 23044232 & 194 & 213 & 19 \\
\hline 17327995 & 23044232 & 195 & 214 & 19 \\
\hline 17327995 & 23044232 & 197 & 211 & 14 \\
\hline 17327995 & 23044232 & 203 & 209 & 6 \\
\hline 15083839 & 25275062 & 189 & 226 & 37 \\
\hline 17327995 & 25275062 & 197 & 222 & 25 \\
\hline 17327995 & 25275062 & 192 & 229 & 37 \\
\hline 17327995 & 25275062 & 196 & 225 & 29 \\
\hline 17327995 & 25275062 & 195 & 242 & 47 \\
\hline 15083839 & 25275062 & 188 & 232 & 44 \\
\hline 17327995 & 25275062 & 190 & 235 & 45 \\
\hline 17327995 & 25275062 & 195 & 232 & 37 \\
\hline 15083839 & 25275062 & 185 & 235 & 50 \\
\hline
\end{tabular}




\begin{tabular}{|c|c|c|c|c|}
\hline 13220219 & 25275062 & 181 & 224 & 43 \\
\hline 17327995 & 25275062 & 193 & 227 & 34 \\
\hline 15083839 & 25275062 & 186 & 233 & 47 \\
\hline 10633493 & 25275062 & 146 & 233 & 87 \\
\hline 15083839 & 25275062 & 183 & 234 & 51 \\
\hline 22394452 & 25275062 & 209 & 226 & 17 \\
\hline 22394452 & 25275062 & 208 & 228 & 20 \\
\hline 22394452 & 25275062 & 211 & 230 & 19 \\
\hline 22394452 & 25275062 & 209 & 228 & 19 \\
\hline 17327995 & 25275062 & 191 & 237 & 46 \\
\hline 22394452 & 25275062 & 208 & 227 & 19 \\
\hline 22394452 & 25275062 & 209 & 235 & 26 \\
\hline 22394452 & 25275062 & 208 & 236 & 28 \\
\hline 22394452 & 25275062 & 211 & 236 & 25 \\
\hline 15083839 & 25275062 & 189 & 233 & 44 \\
\hline 15083839 & 25275062 & 188 & 236 & 48 \\
\hline 17327995 & 26868425 & 193 & 250 & 57 \\
\hline 15083839 & 26868425 & 184 & 246 & 62 \\
\hline 14544747 & 31360627 & 182 & 277 & 95 \\
\hline 23044232 & 26868425 & 215 & 248 & 33 \\
\hline 23044232 & 26868425 & 222 & 247 & 25 \\
\hline 23044232 & 28016896 & 221 & 256 & 35 \\
\hline 23044232 & 26868425 & 220 & 248 & 28 \\
\hline 23044232 & 31360627 & 215 & 281 & 66 \\
\hline 22394452 & 31360627 & 210 & 272 & 62 \\
\hline 22394452 & 26868425 & 214 & 252 & 38 \\
\hline 22394452 & 26868425 & 214 & 254 & 40 \\
\hline 22394452 & 31360627 & 210 & 298 & 88 \\
\hline 23044232 & 26868425 & 217 & 251 & 34 \\
\hline 23044232 & 26868425 & 218 & 251 & 33 \\
\hline 23044232 & 26868425 & 220 & 250 & 30 \\
\hline 22394452 & 31360627 & 212 & 261 & 49 \\
\hline 23044232 & 26868425 & 223 & 249 & 26 \\
\hline 23044232 & 31360627 & 221 & 266 & 45 \\
\hline 23044232 & 26868425 & 217 & 251 & 34 \\
\hline 23044232 & 26868425 & 218 & 251 & 33 \\
\hline 23044232 & 26868425 & 225 & 250 & 25 \\
\hline 23044232 & 26868425 & 226 & 247 & 21 \\
\hline 23044232 & 28016896 & 221 & 257 & 36 \\
\hline 23044232 & 31360627 & 220 & 259 & 39 \\
\hline 23044232 & 26868425 & 221 & 250 & 29 \\
\hline 23044232 & 26868425 & 221 & 250 & 29 \\
\hline 23044232 & 26868425 & 222 & 251 & 29 \\
\hline
\end{tabular}




\begin{tabular}{|c|c|c|c|c|}
\hline 23044232 & 26868425 & 221 & 253 & 32 \\
\hline 23044232 & 26868425 & 223 & 250 & 27 \\
\hline 23044232 & 28016896 & 224 & 256 & 32 \\
\hline 23044232 & 26868425 & 228 & 250 & 22 \\
\hline 23044232 & 26868425 & 231 & 250 & 19 \\
\hline 23044232 & 31360627 & 222 & 283 & 61 \\
\hline 23044232 & 26868425 & 228 & 253 & 25 \\
\hline 23044232 & 26868425 & 227 & 252 & 25 \\
\hline 23044232 & 28016896 & 227 & 255 & 28 \\
\hline 23044232 & 26868425 & 230 & 252 & 22 \\
\hline 23044232 & 26868425 & 230 & 249 & 19 \\
\hline 23044232 & 26868425 & 236 & 251 & 15 \\
\hline 23044232 & 26868425 & 230 & 251 & 21 \\
\hline 23044232 & 28016896 & 228 & 256 & 28 \\
\hline 23044232 & 26868425 & 229 & 251 & 22 \\
\hline 23044232 & 26868425 & 230 & 251 & 21 \\
\hline 23044232 & 26868425 & 225 & 250 & 25 \\
\hline 23044232 & 26868425 & 224 & 253 & 29 \\
\hline 23044232 & 26868425 & 224 & 251 & 27 \\
\hline 23044232 & 26868425 & 227 & 254 & 27 \\
\hline 23044232 & 26868425 & 234 & 254 & 20 \\
\hline 23044232 & 26868425 & 235 & 252 & 17 \\
\hline 23044232 & 31360627 & 227 & 266 & 39 \\
\hline 23044232 & 31360627 & 236 & 272 & 36 \\
\hline 23044232 & 28016896 & 227 & 256 & 29 \\
\hline 23044232 & 31360627 & 233 & 280 & 47 \\
\hline 23044232 & 31360627 & 237 & 269 & 32 \\
\hline 23044232 & 31360627 & 233 & 283 & 50 \\
\hline 23044232 & 31360627 & 235 & 270 & 35 \\
\hline 23044232 & 31360627 & 226 & 297 & 71 \\
\hline 23044232 & 31360627 & 221 & 281 & 60 \\
\hline 23044232 & 32743333 & 220 & 309 & 89 \\
\hline 23044232 & 31360627 & 223 & 283 & 60 \\
\hline 22394452 & 31360627 & 209 & 281 & 72 \\
\hline 23044232 & 31360627 & 234 & 288 & 54 \\
\hline 23044232 & 31360627 & 229 & 286 & 57 \\
\hline 23044232 & 31360627 & 236 & 283 & 47 \\
\hline 23044232 & 31360627 & 224 & 293 & 69 \\
\hline 23044232 & 31360627 & 233 & 278 & 45 \\
\hline 23044232 & 31360627 & 228 & 294 & 66 \\
\hline 23044232 & 31360627 & 235 & 286 & 51 \\
\hline 23044232 & 31360627 & 235 & 291 & 56 \\
\hline 23044232 & 31360627 & 237 & 283 & 46 \\
\hline
\end{tabular}




\begin{tabular}{|c|c|c|c|c|}
\hline 23044232 & 31360627 & 236 & 283 & 47 \\
\hline 23044232 & 31360627 & 238 & 276 & 38 \\
\hline 23044232 & 32743333 & 220 & 317 & 97 \\
\hline 25275062 & 31360627 & 245 & 281 & 36 \\
\hline 23044232 & 31360627 & 240 & 285 & 45 \\
\hline 25275062 & 31360627 & 243 & 275 & 32 \\
\hline 25275062 & 31360627 & 244 & 273 & 29 \\
\hline 23044232 & 31360627 & 237 & 278 & 41 \\
\hline 25275062 & 31360627 & 245 & 289 & 44 \\
\hline 25275062 & 31360627 & 247 & 277 & 30 \\
\hline 25275062 & 31360627 & 246 & 283 & 37 \\
\hline 25275062 & 31360627 & 247 & 285 & 38 \\
\hline 25275062 & 31360627 & 247 & 284 & 37 \\
\hline 23044232 & 31360627 & 242 & 281 & 39 \\
\hline 25275062 & 31360627 & 250 & 293 & 43 \\
\hline 25275062 & 31360627 & 246 & 286 & 40 \\
\hline 25275062 & 31360627 & 243 & 282 & 39 \\
\hline 25275062 & 31360627 & 247 & 281 & 34 \\
\hline 25275062 & 31360627 & 245 & 289 & 44 \\
\hline 25275062 & 31360627 & 253 & 278 & 25 \\
\hline 25275062 & 31360627 & 245 & 280 & 35 \\
\hline 23044232 & 31360627 & 239 & 282 & 43 \\
\hline 25275062 & 31360627 & 242 & 290 & 48 \\
\hline 25275062 & 31360627 & 252 & 280 & 28 \\
\hline 25275062 & 31360627 & 243 & 292 & 49 \\
\hline 23044232 & 31360627 & 235 & 292 & 57 \\
\hline 25275062 & 31360627 & 244 & 291 & 47 \\
\hline 25275062 & 32743333 & 248 & 307 & 59 \\
\hline 25275062 & 32743333 & 245 & 307 & 62 \\
\hline 25275062 & 32743333 & 244 & 311 & 67 \\
\hline 25275062 & 32743333 & 248 & 309 & 61 \\
\hline 25275062 & 32743333 & 249 & 313 & 64 \\
\hline 28016896 & 32743333 & 267 & 316 & 49 \\
\hline 25275062 & 32743333 & 254 & 311 & 57 \\
\hline 28016896 & 32743333 & 270 & 308 & 38 \\
\hline 28016896 & 32743333 & 271 & 314 & 43 \\
\hline 28016896 & 32743333 & 280 & 308 & 28 \\
\hline 28016896 & 32743333 & 277 & 312 & 35 \\
\hline 28016896 & 32743333 & 276 & 309 & 33 \\
\hline 28016896 & 32743333 & 277 & 306 & 29 \\
\hline 28016896 & 32743333 & 280 & 308 & 28 \\
\hline 28016896 & 32743333 & 277 & 324 & 47 \\
\hline 23044232 & 32743333 & 223 & 315 & 92 \\
\hline
\end{tabular}




\begin{tabular}{|c|c|c|c|c|}
\hline 28016896 & 32743333 & 283 & 310 & 27 \\
\hline 28016896 & 32743333 & 276 & 311 & 35 \\
\hline 28016896 & 32743333 & 277 & 314 & 37 \\
\hline 28016896 & 32743333 & 280 & 317 & 37 \\
\hline 28016896 & 32743333 & 287 & 323 & 36 \\
\hline 23044232 & 32743333 & 219 & 325 & 106 \\
\hline 28016896 & 32743333 & 280 & 325 & 45 \\
\hline 31360627 & 32743333 & 307 & 325 & 18 \\
\hline 31360627 & 32743333 & 309 & 325 & 16 \\
\hline 31360627 & 32743333 & 314 & 325 & 11 \\
\hline 31360627 & 32743333 & 314 & 325 & 11 \\
\hline 1680380 & 2469584 & 0 & 10 & 10 \\
\hline 1680380 & 2469584 & 0 & 7 & 7 \\
\hline 1680380 & 2469584 & 0 & 5 & 5 \\
\hline 1680380 & 2469584 & 0 & 6 & 6 \\
\hline 1680380 & 2469584 & 0 & 3 & 3 \\
\hline 1680380 & 2469584 & 0 & 6 & 6 \\
\hline 1680380 & 2469584 & 0 & 8 & 8 \\
\hline 1680380 & 2469584 & 0 & 7 & 7 \\
\hline 1680380 & 3673817 & 0 & 24 & 24 \\
\hline 1680380 & 3673817 & 0 & 28 & 28 \\
\hline 1680380 & 2469584 & 0 & 9 & 9 \\
\hline 1680380 & 3673817 & 0 & 36 & 36 \\
\hline 1680380 & 3673817 & 0 & 35 & 35 \\
\hline 1680380 & 3673817 & 0 & 24 & 24 \\
\hline 1680380 & 3673817 & 0 & 31 & 31 \\
\hline 1680380 & 3673817 & 0 & 35 & 35 \\
\hline 1680380 & 3673817 & 0 & 34 & 34 \\
\hline 1680380 & 3673817 & 0 & 35 & 35 \\
\hline 1680380 & 3673817 & 2 & 28 & 26 \\
\hline 1680380 & 3673817 & 1 & 29 & 28 \\
\hline 1680380 & 3673817 & 0 & 29 & 29 \\
\hline 1680380 & 3673817 & 1 & 24 & 23 \\
\hline 1680380 & 3673817 & 0 & 26 & 26 \\
\hline 1680380 & 3673817 & 1 & 30 & 29 \\
\hline 1680380 & 3673817 & 4 & 23 & 19 \\
\hline 1680380 & 3673817 & 1 & 29 & 28 \\
\hline 1680380 & 3673817 & 3 & 24 & 21 \\
\hline 1680380 & 3673817 & 2 & 25 & 23 \\
\hline 1680380 & 3673817 & 3 & 28 & 25 \\
\hline 1680380 & 3673817 & 5 & 21 & 16 \\
\hline 1680380 & 3673817 & 4 & 27 & 23 \\
\hline 1680380 & 3673817 & 2 & 31 & 29 \\
\hline
\end{tabular}




\begin{tabular}{|c|c|c|c|}
\hline 1680380 & 3673817 & 5 & 27 \\
\hline 1680380 & 3673817 & 7 & 25 \\
\hline 1680380 & 3673817 & 4 & 25 \\
\hline 1680380 & 3673817 & 3 & 26 \\
\hline 1680380 & 3673817 & 3 & 29 \\
\hline 1680380 & 3673817 & 3 & 29 \\
\hline 1680380 & 3673817 & 4 & 28 \\
\hline 1680380 & 3673817 & 5 & 23 \\
\hline 1680380 & 3673817 & 2 & 27 \\
\hline 1680380 & 3673817 & 6 & 28 \\
\hline 1680380 & 3673817 & 2 & 35 \\
\hline 1680380 & 3673817 & 0 & 26 \\
\hline 1680380 & 3673817 & 0 & 26 \\
\hline 1680380 & 3673817 & 2 & 27 \\
\hline 1680380 & 3673817 & 2 & 26 \\
\hline 1680380 & 3673817 & 3 & 31 \\
\hline 1680380 & 3673817 & 5 & 24 \\
\hline 1680380 & 3673817 & 3 & 31 \\
\hline 1680380 & 3673817 & 3 & 25 \\
\hline 1680380 & 3673817 & 0 & 35 \\
\hline 1680380 & 3673817 & 3 & 24 \\
\hline 1680380 & 3673817 & 3 & 32 \\
\hline 1680380 & 3673817 & 6 & 30 \\
\hline 1680380 & 3673817 & 5 & 29 \\
\hline 1680380 & 3673817 & 7 & 20 \\
\hline 1680380 & 3673817 & 6 & 23 \\
\hline 1680380 & 3673817 & 7 & 18 \\
\hline 1680380 & 3673817 & 6 & 23 \\
\hline 1680380 & 3673817 & 6 & 27 \\
\hline 1680380 & 3673817 & 4 & 25 \\
\hline 1680380 & 3673817 & 7 & 18 \\
\hline 1680380 & 3673817 & 7 & 20 \\
\hline 1680380 & 3673817 & 7 & 18 \\
\hline 1680380 & 3673817 & 8 & 21 \\
\hline 1680380 & 3673817 & 5 & 24 \\
\hline 1680380 & 3673817 & 4 & 27 \\
\hline 1680380 & 3673817 & 6 & 21 \\
\hline 1680380 & 3673817 & 6 & 26 \\
\hline 1680380 & 3673817 & 6 & 23 \\
\hline 1680380 & 3673817 & 6 & 25 \\
\hline 1680380 & 3673817 & 7 & 27 \\
\hline 1680380 & 3673817 & 5 & 25 \\
\hline 1680380 & 3673817 & 6 & 22 \\
\hline
\end{tabular}




\begin{tabular}{|c|c|c|c|}
\hline 1680380 & 3673817 & 6 & 23 \\
\hline 1680380 & 3673817 & 6 & 22 \\
\hline 1680380 & 3673817 & 7 & 22 \\
\hline 1680380 & 3673817 & 4 & 28 \\
\hline 1680380 & 3673817 & 5 & 27 \\
\hline 1680380 & 3673817 & 7 & 27 \\
\hline 1680380 & 3673817 & 6 & 24 \\
\hline 1680380 & 3673817 & 7 & 25 \\
\hline 1680380 & 3673817 & 4 & 28 \\
\hline 1680380 & 3673817 & 5 & 28 \\
\hline 1680380 & 3673817 & 6 & 25 \\
\hline 1680380 & 3673817 & 6 & 27 \\
\hline 1680380 & 3673817 & 3 & 24 \\
\hline 1680380 & 3673817 & 6 & 20 \\
\hline 1680380 & 3673817 & 5 & 25 \\
\hline 1680380 & 3673817 & 5 & 23 \\
\hline 1680380 & 3673817 & 5 & 23 \\
\hline 1680380 & 3673817 & 6 & 30 \\
\hline 1680380 & 3673817 & 2 & 31 \\
\hline 1680380 & 3673817 & 3 & 36 \\
\hline 1680380 & 3673817 & 3 & 31 \\
\hline 1680380 & 3673817 & 7 & 32 \\
\hline 1680380 & 3673817 & 6 & 31 \\
\hline 1680380 & 3673817 & 0 & 36 \\
\hline 1680380 & 3673817 & 5 & 28 \\
\hline 1680380 & 3673817 & 2 & 39 \\
\hline 1680380 & 3673817 & 0 & 32 \\
\hline 1680380 & 3673817 & 4 & 35 \\
\hline 1680380 & 3673817 & 6 & 32 \\
\hline 1680380 & 3673817 & 5 & 33 \\
\hline 1680380 & 3673817 & 5 & 36 \\
\hline 1680380 & 3673817 & 0 & 55 \\
\hline 2469584 & 5228531 & 25 & 76 \\
\hline 2469584 & 5228531 & 25 & 76 \\
\hline 2469584 & 5228531 & 32 & 70 \\
\hline 2469584 & 5228531 & 17 & 76 \\
\hline 2469584 & 5228531 & 39 & 73 \\
\hline 2469584 & 5228531 & 47 & 67 \\
\hline 2469584 & 5228531 & 54 & 63 \\
\hline 2469584 & 5228531 & 33 & 76 \\
\hline 2469584 & 5228531 & 42 & 75 \\
\hline 2469584 & 5228531 & 39 & 72 \\
\hline 2469584 & 5228531 & 33 & 78 \\
\hline
\end{tabular}




\begin{tabular}{|c|c|c|c|c|}
\hline 2469584 & 5228531 & 41 & 77 & 36 \\
\hline 2469584 & 5228531 & 44 & 70 & 26 \\
\hline 2469584 & 5228531 & 36 & 77 & 41 \\
\hline 2469584 & 5228531 & 43 & 79 & 36 \\
\hline 2469584 & 5228531 & 41 & 80 & 39 \\
\hline 2469584 & 5615323 & 21 & 90 & 69 \\
\hline 3989157 & 5615323 & 73 & 89 & 16 \\
\hline 3989157 & 5615323 & 69 & 91 & 22 \\
\hline 3989157 & 12509488 & 69 & 109 & 40 \\
\hline 3989157 & 11260790 & 72 & 106 & 34 \\
\hline 5228531 & 11260790 & 85 & 105 & 20 \\
\hline 5228531 & 9065738 & 84 & 102 & 18 \\
\hline 5228531 & 6873621 & 87 & 100 & 13 \\
\hline 5228531 & 11260790 & 84 & 105 & 21 \\
\hline 3989157 & 11260790 & 73 & 106 & 33 \\
\hline 5228531 & 12509488 & 86 & 116 & 30 \\
\hline 5228531 & 12509488 & 85 & 117 & 32 \\
\hline 5615323 & 12509488 & 95 & 118 & 23 \\
\hline 5228531 & 12509488 & 83 & 116 & 33 \\
\hline 5615323 & 12509488 & 95 & 112 & 17 \\
\hline 5228531 & 12509488 & 88 & 114 & 26 \\
\hline 5615323 & 12509488 & 94 & 114 & 20 \\
\hline 5228531 & 12509488 & 85 & 115 & 30 \\
\hline 5615323 & 12509488 & 98 & 119 & 21 \\
\hline 9065738 & 12509488 & 103 & 118 & 15 \\
\hline 5228531 & 12509488 & 88 & 114 & 26 \\
\hline 6873621 & 12509488 & 102 & 114 & 12 \\
\hline 9065738 & 12509488 & 104 & 117 & 13 \\
\hline 9065738 & 12509488 & 104 & 117 & 13 \\
\hline 5615323 & 12509488 & 95 & 117 & 22 \\
\hline 9065738 & 12509488 & 104 & 119 & 15 \\
\hline 9065738 & 12509488 & 104 & 118 & 14 \\
\hline 9065738 & 12509488 & 104 & 122 & 18 \\
\hline 9065738 & 12509488 & 106 & 122 & 16 \\
\hline 9065738 & 13386491 & 105 & 125 & 20 \\
\hline 16751130 & 20658484 & 163 & 174 & 11 \\
\hline 16961743 & 22616032 & 164 & 178 & 14 \\
\hline 16961743 & 22616032 & 166 & 189 & 23 \\
\hline 15205783 & 22616032 & 155 & 186 & 31 \\
\hline 18209201 & 22616032 & 174 & 192 & 18 \\
\hline 18209201 & 22616032 & 174 & 188 & 14 \\
\hline 18209201 & 22616032 & 174 & 188 & 14 \\
\hline 18209201 & 22616032 & 173 & 190 & 17 \\
\hline
\end{tabular}




\begin{tabular}{|c|c|c|c|}
\hline 16961743 & 22616032 & 171 & 185 \\
\hline 18209201 & 22616032 & 173 & 188 \\
\hline 18209201 & 22616032 & 174 & 189 \\
\hline 16961743 & 22616032 & 170 & 190 \\
\hline 16961743 & 22616032 & 171 & 186 \\
\hline 18209201 & 22616032 & 173 & 187 \\
\hline 18209201 & 22616032 & 174 & 188 \\
\hline 18209201 & 22616032 & 173 & 190 \\
\hline 18209201 & 22616032 & 174 & 189 \\
\hline 18209201 & 22616032 & 173 & 190 \\
\hline 18209201 & 22616032 & 174 & 190 \\
\hline 18209201 & 22616032 & 174 & 187 \\
\hline 16961743 & 22616032 & 169 & 186 \\
\hline 18209201 & 22616032 & 173 & 190 \\
\hline 18209201 & 22616032 & 174 & 188 \\
\hline 16961743 & 22616032 & 169 & 187 \\
\hline 16961743 & 22616032 & 171 & 186 \\
\hline 16961743 & 22616032 & 166 & 187 \\
\hline 16961743 & 22616032 & 169 & 189 \\
\hline 18209201 & 22616032 & 174 & 186 \\
\hline 16961743 & 22616032 & 170 & 187 \\
\hline 18209201 & 22616032 & 173 & 187 \\
\hline 16961743 & 22616032 & 169 & 188 \\
\hline 15205783 & 22616032 & 156 & 190 \\
\hline 16961743 & 22616032 & 169 & 186 \\
\hline 15205783 & 22616032 & 155 & 187 \\
\hline 18209201 & 22616032 & 174 & 188 \\
\hline 18209201 & 22616032 & 174 & 189 \\
\hline 16961743 & 22616032 & 168 & 188 \\
\hline 18209201 & 22616032 & 174 & 188 \\
\hline 16961743 & 22616032 & 168 & 187 \\
\hline 16961743 & 22616032 & 170 & 186 \\
\hline 18209201 & 22616032 & 173 & 188 \\
\hline 18209201 & 22616032 & 174 & 186 \\
\hline 18209201 & 22616032 & 173 & 188 \\
\hline 16961743 & 22616032 & 170 & 185 \\
\hline 16961743 & 22616032 & 167 & 189 \\
\hline 18209201 & 22616032 & 173 & 188 \\
\hline 18209201 & 22616032 & 174 & 189 \\
\hline 15205783 & 22616032 & 154 & 186 \\
\hline 18209201 & 22616032 & 173 & 188 \\
\hline 18209201 & 22616032 & 174 & 190 \\
\hline 18209201 & 22616032 & 173 & 188 \\
\hline
\end{tabular}




\begin{tabular}{|c|c|c|c|}
\hline 16961743 & 22616032 & 169 & 187 \\
\hline 16961743 & 22616032 & 168 & 186 \\
\hline 18209201 & 22616032 & 173 & 188 \\
\hline 18209201 & 22616032 & 173 & 189 \\
\hline 16961743 & 22616032 & 169 & 187 \\
\hline 16961743 & 22616032 & 167 & 188 \\
\hline 16961743 & 22616032 & 168 & 187 \\
\hline 16961743 & 22616032 & 170 & 186 \\
\hline 18209201 & 22616032 & 173 & 188 \\
\hline 16961743 & 22616032 & 164 & 188 \\
\hline 16961743 & 22616032 & 170 & 186 \\
\hline 18209201 & 22616032 & 173 & 187 \\
\hline 18209201 & 22616032 & 173 & 189 \\
\hline 18209201 & 22616032 & 173 & 189 \\
\hline 18209201 & 22616032 & 174 & 189 \\
\hline 18209201 & 22616032 & 173 & 189 \\
\hline 16961743 & 22616032 & 167 & 187 \\
\hline 16961743 & 22616032 & 169 & 185 \\
\hline 16961743 & 22616032 & 166 & 190 \\
\hline 18209201 & 22616032 & 174 & 189 \\
\hline 18209201 & 22616032 & 175 & 190 \\
\hline 18209201 & 22616032 & 175 & 190 \\
\hline 18209201 & 22616032 & 175 & 188 \\
\hline 18209201 & 22616032 & 174 & 190 \\
\hline 18209201 & 22616032 & 174 & 192 \\
\hline 18209201 & 22616032 & 175 & 190 \\
\hline 18209201 & 22616032 & 174 & 190 \\
\hline 18209201 & 22616032 & 175 & 188 \\
\hline 18209201 & 22616032 & 173 & 189 \\
\hline 18209201 & 22616032 & 175 & 190 \\
\hline 18209201 & 22616032 & 175 & 189 \\
\hline 18209201 & 22616032 & 175 & 187 \\
\hline 18209201 & 22616032 & 174 & 190 \\
\hline 18209201 & 22616032 & 174 & 192 \\
\hline 20658484 & 22616032 & 176 & 189 \\
\hline 18209201 & 22616032 & 174 & 189 \\
\hline 18209201 & 22616032 & 175 & 191 \\
\hline 18209201 & 22616032 & 175 & 190 \\
\hline 18209201 & 22616032 & 174 & 189 \\
\hline 18209201 & 22616032 & 175 & 190 \\
\hline 18209201 & 22616032 & 175 & 188 \\
\hline 18209201 & 22616032 & 173 & 190 \\
\hline 18209201 & 22616032 & 173 & 191 \\
\hline
\end{tabular}




\begin{tabular}{|c|c|c|c|}
\hline 20658484 & 22616032 & 176 & 189 \\
\hline 20658484 & 22616032 & 176 & 187 \\
\hline 18209201 & 22616032 & 174 & 189 \\
\hline 18209201 & 22616032 & 175 & 190 \\
\hline 18209201 & 22616032 & 174 & 187 \\
\hline 18209201 & 22616032 & 174 & 188 \\
\hline 18209201 & 22616032 & 173 & 187 \\
\hline 18209201 & 22616032 & 174 & 190 \\
\hline 18209201 & 22616032 & 173 & 187 \\
\hline 18209201 & 22616032 & 175 & 187 \\
\hline 20658484 & 22616032 & 176 & 190 \\
\hline 18209201 & 22616032 & 173 & 189 \\
\hline 16961743 & 22616032 & 170 & 188 \\
\hline 18209201 & 22616032 & 173 & 190 \\
\hline 18209201 & 22616032 & 174 & 190 \\
\hline 16961743 & 22616032 & 167 & 191 \\
\hline 16961743 & 22616032 & 167 & 190 \\
\hline 18209201 & 22616032 & 172 & 191 \\
\hline 16961743 & 22616032 & 167 & 188 \\
\hline 16961743 & 22616032 & 165 & 188 \\
\hline 18209201 & 22616032 & 173 & 190 \\
\hline 16961743 & 22616032 & 165 & 190 \\
\hline 16961743 & 22616032 & 168 & 189 \\
\hline 18209201 & 22616032 & 174 & 189 \\
\hline 18209201 & 22616032 & 173 & 190 \\
\hline 18209201 & 22616032 & 173 & 190 \\
\hline 16961743 & 22616032 & 167 & 191 \\
\hline 18209201 & 22616032 & 173 & 191 \\
\hline 16961743 & 22616032 & 167 & 190 \\
\hline 16961743 & 22616032 & 166 & 194 \\
\hline 16961743 & 22616032 & 166 & 191 \\
\hline 16961743 & 22616032 & 168 & 188 \\
\hline 16961743 & 22616032 & 167 & 191 \\
\hline 18209201 & 22616032 & 173 & 189 \\
\hline 16961743 & 22616032 & 166 & 188 \\
\hline 16961743 & 22616032 & 166 & 190 \\
\hline 15205783 & 22616032 & 150 & 191 \\
\hline 18209201 & 22616032 & 174 & 188 \\
\hline 16961743 & 22616032 & 167 & 191 \\
\hline 16961743 & 22616032 & 165 & 190 \\
\hline 18209201 & 22616032 & 174 & 191 \\
\hline 18209201 & 22616032 & 174 & 192 \\
\hline 16961743 & 22616032 & 165 & 191 \\
\hline
\end{tabular}




\begin{tabular}{|c|c|c|c|}
\hline 16961743 & 22616032 & 168 & 191 \\
\hline 18209201 & 22616032 & 174 & 190 \\
\hline 16961743 & 22616032 & 166 & 187 \\
\hline 16961743 & 22616032 & 169 & 190 \\
\hline 16961743 & 22616032 & 167 & 190 \\
\hline 16961743 & 22616032 & 166 & 193 \\
\hline 16961743 & 22616032 & 165 & 191 \\
\hline 16961743 & 22616032 & 166 & 189 \\
\hline 18209201 & 22616032 & 174 & 191 \\
\hline 18209201 & 22616032 & 173 & 190 \\
\hline 16961743 & 22616032 & 166 & 190 \\
\hline 15205783 & 22616032 & 155 & 193 \\
\hline 18209201 & 22616032 & 173 & 192 \\
\hline 18209201 & 22616032 & 173 & 190 \\
\hline 16961743 & 22616032 & 166 & 186 \\
\hline 16961743 & 22616032 & 165 & 190 \\
\hline 16961743 & 22616032 & 167 & 191 \\
\hline 16961743 & 22616032 & 168 & 190 \\
\hline 18209201 & 22616032 & 173 & 189 \\
\hline 20658484 & 22616032 & 176 & 190 \\
\hline 15205783 & 22616032 & 161 & 188 \\
\hline 16961743 & 22616032 & 171 & 190 \\
\hline 18209201 & 22616032 & 174 & 188 \\
\hline 18209201 & 22616032 & 173 & 188 \\
\hline 16961743 & 22616032 & 167 & 189 \\
\hline 16961743 & 22616032 & 169 & 196 \\
\hline 18209201 & 22616032 & 173 & 188 \\
\hline 18209201 & 22616032 & 174 & 191 \\
\hline 18209201 & 22616032 & 173 & 189 \\
\hline 16961743 & 22616032 & 165 & 191 \\
\hline 16961743 & 22616032 & 170 & 190 \\
\hline 16961743 & 22616032 & 168 & 188 \\
\hline 15205783 & 22616032 & 156 & 189 \\
\hline 18209201 & 22616032 & 173 & 189 \\
\hline 16961743 & 22616032 & 165 & 191 \\
\hline 16961743 & 22616032 & 167 & 189 \\
\hline 16961743 & 22616032 & 167 & 192 \\
\hline 18209201 & 22616032 & 173 & 189 \\
\hline 16961743 & 22616032 & 167 & 190 \\
\hline 16961743 & 22616032 & 167 & 188 \\
\hline 18209201 & 22616032 & 173 & 190 \\
\hline 16961743 & 22616032 & 166 & 190 \\
\hline 18209201 & 22616032 & 174 & 190 \\
\hline
\end{tabular}




\begin{tabular}{|c|c|c|c|c|}
\hline 16961743 & 22616032 & 171 & 190 & 19 \\
\hline 16961743 & 22616032 & 168 & 191 & 23 \\
\hline 18209201 & 22616032 & 173 & 192 & 19 \\
\hline 18209201 & 22616032 & 173 & 192 & 19 \\
\hline 16961743 & 22616032 & 166 & 191 & 25 \\
\hline 18209201 & 22616032 & 174 & 189 & 15 \\
\hline 16961743 & 22616032 & 164 & 189 & 25 \\
\hline 18209201 & 22616032 & 173 & 193 & 20 \\
\hline 18209201 & 22616032 & 174 & 192 & 18 \\
\hline 18209201 & 22616032 & 174 & 192 & 18 \\
\hline 18209201 & 22616032 & 174 & 193 & 19 \\
\hline 18209201 & 22616032 & 174 & 192 & 18 \\
\hline 18209201 & 22616032 & 173 & 193 & 20 \\
\hline 18209201 & 22616032 & 174 & 190 & 16 \\
\hline 18209201 & 22616032 & 172 & 189 & 17 \\
\hline 16961743 & 22616032 & 168 & 191 & 23 \\
\hline 16961743 & 22616032 & 165 & 192 & 27 \\
\hline 16961743 & 22616032 & 164 & 189 & 25 \\
\hline 18209201 & 22616032 & 174 & 192 & 18 \\
\hline 18209201 & 22616032 & 174 & 190 & 16 \\
\hline 18209201 & 22616032 & 173 & 190 & 17 \\
\hline 16961743 & 22616032 & 169 & 187 & 18 \\
\hline 18209201 & 22616032 & 174 & 188 & 14 \\
\hline 16961743 & 22616032 & 171 & 188 & 17 \\
\hline 16961743 & 22616032 & 165 & 191 & 26 \\
\hline 18209201 & 22616032 & 173 & 191 & 18 \\
\hline 16961743 & 22616032 & 167 & 189 & 22 \\
\hline 16961743 & 22616032 & 166 & 189 & 23 \\
\hline 18209201 & 22616032 & 174 & 191 & 17 \\
\hline 18209201 & 22616032 & 174 & 191 & 17 \\
\hline 18209201 & 22616032 & 173 & 191 & 18 \\
\hline 18209201 & 22616032 & 175 & 192 & 17 \\
\hline 18209201 & 22616032 & 175 & 191 & 16 \\
\hline 18209201 & 22616032 & 175 & 191 & 16 \\
\hline 18209201 & 22616032 & 174 & 191 & 17 \\
\hline 18209201 & 22616032 & 175 & 190 & 15 \\
\hline 18209201 & 22616032 & 173 & 192 & 19 \\
\hline 18209201 & 22616032 & 175 & 190 & 15 \\
\hline 18209201 & 22616032 & 174 & 193 & 19 \\
\hline 18209201 & 22616032 & 174 & 194 & 20 \\
\hline 18209201 & 22616032 & 175 & 191 & 16 \\
\hline 18209201 & 22616032 & 175 & 192 & 17 \\
\hline 18209201 & 22616032 & 175 & 192 & 17 \\
\hline
\end{tabular}




\begin{tabular}{|c|c|c|c|c|}
\hline 18209201 & 22616032 & 174 & 192 & 18 \\
\hline 18209201 & 22616032 & 174 & 191 & 17 \\
\hline 18209201 & 22616032 & 175 & 190 & 15 \\
\hline 20658484 & 22616032 & 176 & 191 & 15 \\
\hline 18209201 & 22616032 & 175 & 190 & 15 \\
\hline 18209201 & 22616032 & 175 & 194 & 19 \\
\hline 18209201 & 22616032 & 174 & 191 & 17 \\
\hline 18209201 & 22616032 & 175 & 193 & 18 \\
\hline 18209201 & 22616032 & 175 & 193 & 18 \\
\hline 18209201 & 22616032 & 175 & 193 & 18 \\
\hline 18209201 & 22616032 & 175 & 194 & 19 \\
\hline 16961743 & 22616032 & 167 & 202 & 35 \\
\hline 18209201 & 22616032 & 175 & 194 & 19 \\
\hline 18209201 & 22616032 & 175 & 195 & 20 \\
\hline 18209201 & 22616032 & 175 & 196 & 21 \\
\hline 18209201 & 22616032 & 175 & 194 & 19 \\
\hline 18209201 & 22616032 & 174 & 195 & 21 \\
\hline 18209201 & 22616032 & 174 & 196 & 22 \\
\hline 18209201 & 22616032 & 174 & 196 & 22 \\
\hline 18209201 & 22616032 & 174 & 197 & 23 \\
\hline 18209201 & 22616032 & 174 & 197 & 23 \\
\hline 18209201 & 22616032 & 175 & 197 & 22 \\
\hline 18209201 & 22616032 & 173 & 202 & 29 \\
\hline 18209201 & 22616032 & 174 & 199 & 25 \\
\hline 16961743 & 23840020 & 169 & 211 & 42 \\
\hline 18209201 & 26299134 & 174 & 222 & 48 \\
\hline 15205783 & 27027570 & 156 & 231 & 75 \\
\hline 16961743 & 27027570 & 168 & 231 & 63 \\
\hline 20658484 & 26299134 & 176 & 225 & 49 \\
\hline 16961743 & 27027570 & 169 & 232 & 63 \\
\hline 20658484 & 27027570 & 176 & 227 & 51 \\
\hline 20658484 & 27027570 & 176 & 230 & 54 \\
\hline 20658484 & 27027570 & 176 & 230 & 54 \\
\hline 18209201 & 26299134 & 175 & 224 & 49 \\
\hline 21856047 & 26299134 & 176 & 222 & 46 \\
\hline 21856047 & 26299134 & 176 & 225 & 49 \\
\hline 18209201 & 27027570 & 174 & 230 & 56 \\
\hline 21856047 & 23840020 & 178 & 212 & 34 \\
\hline 21856047 & 26299134 & 176 & 223 & 47 \\
\hline 21856047 & 26299134 & 176 & 220 & 44 \\
\hline 21856047 & 26299134 & 179 & 221 & 42 \\
\hline 21856047 & 27027570 & 179 & 226 & 47 \\
\hline 21856047 & 26299134 & 176 & 222 & 46 \\
\hline
\end{tabular}




\begin{tabular}{|c|c|c|c|}
\hline 21856047 & 26299134 & 178 & 223 \\
\hline 21856047 & 27027570 & 179 & 234 \\
\hline 21856047 & 26299134 & 187 & 225 \\
\hline 21856047 & 28250540 & 179 & 237 \\
\hline 20658484 & 28250540 & 176 & 240 \\
\hline 21856047 & 24882677 & 179 & 213 \\
\hline 21856047 & 27027570 & 180 & 233 \\
\hline 21856047 & 27027570 & 185 & 227 \\
\hline 21856047 & 23840020 & 188 & 212 \\
\hline 21856047 & 26299134 & 188 & 222 \\
\hline 21856047 & 26299134 & 185 & 222 \\
\hline 21856047 & 27027570 & 183 & 228 \\
\hline 21856047 & 27027570 & 182 & 229 \\
\hline 21856047 & 27027570 & 192 & 226 \\
\hline 21856047 & 26299134 & 194 & 219 \\
\hline 21856047 & 26299134 & 195 & 221 \\
\hline 21856047 & 26299134 & 185 & 224 \\
\hline 21856047 & 26299134 & 178 & 220 \\
\hline 21856047 & 26299134 & 195 & 219 \\
\hline 21856047 & 26299134 & 190 & 220 \\
\hline 21856047 & 27027570 & 193 & 231 \\
\hline 21856047 & 26299134 & 197 & 220 \\
\hline 21856047 & 26299134 & 192 & 218 \\
\hline 21856047 & 26299134 & 200 & 221 \\
\hline 21856047 & 27027570 & 177 & 227 \\
\hline 21856047 & 26299134 & 190 & 223 \\
\hline 21856047 & 26299134 & 189 & 223 \\
\hline 21856047 & 27027570 & 189 & 226 \\
\hline 21856047 & 26299134 & 201 & 219 \\
\hline 21856047 & 26299134 & 201 & 222 \\
\hline 22616032 & 26299134 & 209 & 219 \\
\hline 21856047 & 26299134 & 189 & 223 \\
\hline 21856047 & 26299134 & 198 & 222 \\
\hline 21856047 & 26299134 & 203 & 220 \\
\hline 21856047 & 26299134 & 191 & 222 \\
\hline 21856047 & 26299134 & 206 & 222 \\
\hline 21856047 & 26299134 & 206 & 217 \\
\hline 21856047 & 27027570 & 200 & 226 \\
\hline 21856047 & 26299134 & 190 & 223 \\
\hline 21856047 & 26299134 & 203 & 220 \\
\hline 21856047 & 26299134 & 194 & 222 \\
\hline 21856047 & 26299134 & 201 & 219 \\
\hline 21856047 & 26299134 & 195 & 224 \\
\hline
\end{tabular}




\begin{tabular}{|c|c|c|c|}
\hline 21856047 & 26299134 & 204 & 220 \\
\hline 21856047 & 26299134 & 206 & 219 \\
\hline 21856047 & 27027570 & 197 & 228 \\
\hline 21856047 & 26299134 & 193 & 220 \\
\hline 21856047 & 26299134 & 199 & 222 \\
\hline 21856047 & 26299134 & 200 & 222 \\
\hline 21856047 & 26299134 & 197 & 221 \\
\hline 21856047 & 26299134 & 188 & 223 \\
\hline 21856047 & 26299134 & 197 & 222 \\
\hline 21856047 & 26299134 & 199 & 221 \\
\hline 21856047 & 27027570 & 190 & 228 \\
\hline 21856047 & 26299134 & 190 & 224 \\
\hline 21856047 & 26299134 & 183 & 224 \\
\hline 21856047 & 26299134 & 197 & 221 \\
\hline 21856047 & 26299134 & 189 & 223 \\
\hline 21856047 & 27027570 & 176 & 229 \\
\hline 21856047 & 26299134 & 189 & 222 \\
\hline 21856047 & 26299134 & 188 & 223 \\
\hline 21856047 & 26299134 & 194 & 224 \\
\hline 21856047 & 26299134 & 199 & 223 \\
\hline 21856047 & 26299134 & 203 & 222 \\
\hline 23840020 & 26299134 & 213 & 218 \\
\hline 21856047 & 27027570 & 201 & 235 \\
\hline 21856047 & 26299134 & 193 & 224 \\
\hline 21856047 & 27027570 & 195 & 233 \\
\hline 21856047 & 26299134 & 186 & 225 \\
\hline 21856047 & 26299134 & 195 & 222 \\
\hline 21856047 & 26299134 & 192 & 224 \\
\hline 21856047 & 26299134 & 203 & 224 \\
\hline 21856047 & 26299134 & 197 & 222 \\
\hline 21856047 & 27027570 & 195 & 233 \\
\hline 21856047 & 27027570 & 187 & 227 \\
\hline 21856047 & 27027570 & 200 & 234 \\
\hline 21856047 & 27027570 & 187 & 228 \\
\hline 21856047 & 27027570 & 188 & 226 \\
\hline 21856047 & 27027570 & 192 & 229 \\
\hline 21856047 & 27027570 & 195 & 231 \\
\hline 21856047 & 26299134 & 198 & 222 \\
\hline 21856047 & 26299134 & 190 & 224 \\
\hline 21856047 & 27027570 & 193 & 230 \\
\hline 22616032 & 26299134 & 209 & 222 \\
\hline 21856047 & 27027570 & 199 & 229 \\
\hline 21856047 & 26299134 & 194 & 225 \\
\hline
\end{tabular}




\begin{tabular}{|c|c|c|c|c|}
\hline 21856047 & 27027570 & 200 & 231 & 31 \\
\hline 21856047 & 27027570 & 176 & 234 & 58 \\
\hline 21856047 & 27027570 & 177 & 228 & 51 \\
\hline 21856047 & 27027570 & 192 & 231 & 39 \\
\hline 21856047 & 27027570 & 191 & 234 & 43 \\
\hline 21856047 & 28250540 & 182 & 239 & 57 \\
\hline 21856047 & 28250540 & 185 & 237 & 52 \\
\hline 21856047 & 27027570 & 192 & 232 & 40 \\
\hline 21856047 & 27027570 & 197 & 229 & 32 \\
\hline 21856047 & 27027570 & 176 & 230 & 54 \\
\hline 21856047 & 28250540 & 196 & 238 & 42 \\
\hline 21856047 & 27027570 & 183 & 231 & 48 \\
\hline 21856047 & 29525593 & 181 & 248 & 67 \\
\hline 21856047 & 27027570 & 186 & 231 & 45 \\
\hline 21856047 & 27027570 & 188 & 234 & 46 \\
\hline 21856047 & 28250540 & 183 & 240 & 57 \\
\hline 21856047 & 27027570 & 194 & 230 & 36 \\
\hline 21856047 & 29525593 & 194 & 248 & 54 \\
\hline 21856047 & 27027570 & 189 & 234 & 45 \\
\hline 21856047 & 27027570 & 194 & 230 & 36 \\
\hline 21856047 & 27027570 & 187 & 234 & 47 \\
\hline 21856047 & 27027570 & 193 & 234 & 41 \\
\hline 21856047 & 28250540 & 184 & 246 & 62 \\
\hline 21856047 & 28250540 & 183 & 239 & 56 \\
\hline 21856047 & 27027570 & 181 & 235 & 54 \\
\hline 21856047 & 28250540 & 192 & 242 & 50 \\
\hline 21856047 & 28250540 & 186 & 245 & 59 \\
\hline 21856047 & 27027570 & 182 & 233 & 51 \\
\hline 21856047 & 27027570 & 176 & 230 & 54 \\
\hline 21856047 & 28250540 & 190 & 240 & 50 \\
\hline 21856047 & 27027570 & 182 & 234 & 52 \\
\hline 21856047 & 29525593 & 185 & 254 & 69 \\
\hline 21856047 & 28250540 & 184 & 247 & 63 \\
\hline 21856047 & 28250540 & 190 & 243 & 53 \\
\hline 21856047 & 27027570 & 193 & 233 & 40 \\
\hline 21856047 & 27027570 & 192 & 232 & 40 \\
\hline 21856047 & 27027570 & 186 & 232 & 46 \\
\hline 21856047 & 28250540 & 186 & 242 & 56 \\
\hline 21856047 & 28250540 & 183 & 243 & 60 \\
\hline 21856047 & 28250540 & 180 & 239 & 59 \\
\hline 21856047 & 27027570 & 186 & 234 & 48 \\
\hline 21856047 & 28250540 & 199 & 245 & 46 \\
\hline 21856047 & 29525593 & 184 & 255 & 71 \\
\hline
\end{tabular}




\begin{tabular}{|c|c|c|c|c|}
\hline 21856047 & 29525593 & 187 & 249 & 62 \\
\hline 21856047 & 27027570 & 207 & 235 & 28 \\
\hline 21856047 & 27027570 & 201 & 231 & 30 \\
\hline 21856047 & 27027570 & 190 & 232 & 42 \\
\hline 21856047 & 28250540 & 197 & 244 & 47 \\
\hline 21856047 & 27027570 & 185 & 233 & 48 \\
\hline 24882677 & 27027570 & 216 & 235 & 19 \\
\hline 24882677 & 27027570 & 216 & 231 & 15 \\
\hline 24882677 & 27027570 & 216 & 231 & 15 \\
\hline 24882677 & 27027570 & 217 & 233 & 16 \\
\hline 20658484 & 27027570 & 176 & 231 & 55 \\
\hline 21856047 & 27027570 & 199 & 233 & 34 \\
\hline 21856047 & 27027570 & 207 & 232 & 25 \\
\hline 24882677 & 27027570 & 217 & 232 & 15 \\
\hline 24882677 & 27027570 & 217 & 235 & 18 \\
\hline 24882677 & 27027570 & 217 & 235 & 18 \\
\hline 24882677 & 27027570 & 220 & 232 & 12 \\
\hline 24882677 & 27027570 & 220 & 231 & 11 \\
\hline 24882677 & 28250540 & 219 & 241 & 22 \\
\hline 24882677 & 28250540 & 217 & 241 & 24 \\
\hline 24882677 & 28250540 & 216 & 247 & 31 \\
\hline 21856047 & 29525593 & 206 & 257 & 51 \\
\hline 24882677 & 29525593 & 221 & 251 & 30 \\
\hline 24882677 & 28250540 & 218 & 247 & 29 \\
\hline 24882677 & 28250540 & 216 & 245 & 29 \\
\hline 24882677 & 29525593 & 217 & 252 & 35 \\
\hline 21856047 & 29525593 & 192 & 249 & 57 \\
\hline 24882677 & 28250540 & 216 & 244 & 28 \\
\hline 24882677 & 29525593 & 218 & 253 & 35 \\
\hline 24882677 & 28250540 & 220 & 245 & 25 \\
\hline 24882677 & 28250540 & 221 & 247 & 26 \\
\hline 21856047 & 29525593 & 177 & 254 & 77 \\
\hline 21856047 & 28250540 & 195 & 245 & 50 \\
\hline 24882677 & 29525593 & 221 & 259 & 38 \\
\hline 24882677 & 28250540 & 221 & 247 & 26 \\
\hline 24882677 & 30852447 & 220 & 269 & 49 \\
\hline 24882677 & 28250540 & 221 & 245 & 24 \\
\hline 24882677 & 28250540 & 224 & 247 & 23 \\
\hline 24882677 & 28250540 & 220 & 245 & 25 \\
\hline 24882677 & 28250540 & 222 & 246 & 24 \\
\hline 24882677 & 28250540 & 223 & 243 & 20 \\
\hline 26299134 & 28250540 & 226 & 244 & 18 \\
\hline 24882677 & 28250540 & 223 & 247 & 24 \\
\hline
\end{tabular}




\begin{tabular}{|c|c|c|c|c|}
\hline 24882677 & 28250540 & 220 & 246 & 26 \\
\hline 24882677 & 28250540 & 220 & 242 & 22 \\
\hline 24882677 & 28250540 & 222 & 246 & 24 \\
\hline 24882677 & 28250540 & 223 & 244 & 21 \\
\hline 24882677 & 28250540 & 223 & 244 & 21 \\
\hline 24882677 & 29525593 & 224 & 253 & 29 \\
\hline 24882677 & 28250540 & 224 & 245 & 21 \\
\hline 26299134 & 28250540 & 227 & 243 & 16 \\
\hline 26299134 & 28250540 & 227 & 242 & 15 \\
\hline 24882677 & 28250540 & 224 & 242 & 18 \\
\hline 26299134 & 28250540 & 226 & 242 & 16 \\
\hline 24882677 & 28250540 & 224 & 243 & 19 \\
\hline 26299134 & 28250540 & 230 & 239 & 9 \\
\hline 26299134 & 28250540 & 227 & 241 & 14 \\
\hline 26299134 & 29525593 & 229 & 262 & 33 \\
\hline 26299134 & 28250540 & 230 & 247 & 17 \\
\hline 26299134 & 28250540 & 226 & 244 & 18 \\
\hline 26299134 & 29525593 & 226 & 254 & 28 \\
\hline 26299134 & 29525593 & 228 & 251 & 23 \\
\hline 26299134 & 28250540 & 229 & 246 & 17 \\
\hline 26299134 & 29525593 & 230 & 259 & 29 \\
\hline 24882677 & 29525593 & 224 & 261 & 37 \\
\hline 26299134 & 30852447 & 227 & 275 & 48 \\
\hline 24882677 & 29525593 & 222 & 251 & 29 \\
\hline 24882677 & 30852447 & 218 & 265 & 47 \\
\hline 26299134 & 30852447 & 228 & 276 & 48 \\
\hline 24882677 & 30852447 & 225 & 273 & 48 \\
\hline 24882677 & 29525593 & 224 & 254 & 30 \\
\hline 21856047 & 29525593 & 195 & 255 & 60 \\
\hline 26299134 & 29525593 & 230 & 259 & 29 \\
\hline 24882677 & 29525593 & 223 & 253 & 30 \\
\hline 26299134 & 29525593 & 227 & 254 & 27 \\
\hline 26299134 & 30852447 & 227 & 268 & 41 \\
\hline 26299134 & 29525593 & 232 & 258 & 26 \\
\hline 24882677 & 30852447 & 220 & 269 & 49 \\
\hline 26299134 & 29525593 & 231 & 256 & 25 \\
\hline 26299134 & 29525593 & 232 & 261 & 29 \\
\hline 26299134 & 29525593 & 229 & 255 & 26 \\
\hline 24882677 & 29525593 & 225 & 258 & 33 \\
\hline 27027570 & 30852447 & 238 & 268 & 30 \\
\hline 27027570 & 29525593 & 237 & 258 & 21 \\
\hline 27027570 & 29525593 & 238 & 255 & 17 \\
\hline 27027570 & 29525593 & 237 & 258 & 21 \\
\hline
\end{tabular}




\begin{tabular}{|c|c|c|c|c|}
\hline 27027570 & 29525593 & 240 & 253 & 13 \\
\hline 27027570 & 29525593 & 240 & 255 & 15 \\
\hline 27027570 & 29525593 & 239 & 257 & 18 \\
\hline 26299134 & 29525593 & 227 & 255 & 28 \\
\hline 27027570 & 29525593 & 243 & 253 & 10 \\
\hline 27027570 & 29525593 & 240 & 256 & 16 \\
\hline 27027570 & 29525593 & 239 & 258 & 19 \\
\hline 27027570 & 29525593 & 244 & 252 & 8 \\
\hline 27027570 & 29525593 & 240 & 254 & 14 \\
\hline 27027570 & 29525593 & 238 & 258 & 20 \\
\hline 26299134 & 33485260 & 230 & 296 & 66 \\
\hline 26299134 & 30852447 & 231 & 268 & 37 \\
\hline 27027570 & 30852447 & 239 & 270 & 31 \\
\hline 26299134 & 33485260 & 231 & 289 & 58 \\
\hline 27027570 & 30852447 & 237 & 274 & 37 \\
\hline 26299134 & 30852447 & 232 & 271 & 39 \\
\hline 26299134 & 30852447 & 226 & 277 & 51 \\
\hline 27027570 & 30852447 & 238 & 270 & 32 \\
\hline 27027570 & 30852447 & 239 & 273 & 34 \\
\hline 27027570 & 30852447 & 242 & 272 & 30 \\
\hline 28250540 & 30852447 & 250 & 277 & 27 \\
\hline 26299134 & 30852447 & 229 & 277 & 48 \\
\hline 28250540 & 30852447 & 251 & 271 & 20 \\
\hline 28250540 & 30852447 & 252 & 269 & 17 \\
\hline 28250540 & 30852447 & 256 & 274 & 18 \\
\hline 28250540 & 30852447 & 256 & 271 & 15 \\
\hline 28250540 & 30852447 & 252 & 270 & 18 \\
\hline 28250540 & 30852447 & 252 & 273 & 21 \\
\hline 28250540 & 30852447 & 253 & 275 & 22 \\
\hline 28250540 & 33485260 & 252 & 279 & 27 \\
\hline 28250540 & 30852447 & 251 & 277 & 26 \\
\hline 28250540 & 30852447 & 252 & 275 & 23 \\
\hline 28250540 & 30852447 & 255 & 277 & 22 \\
\hline 28250540 & 33485260 & 254 & 293 & 39 \\
\hline 28250540 & 33485260 & 255 & 292 & 37 \\
\hline 28250540 & 30852447 & 253 & 276 & 23 \\
\hline 28250540 & 33485260 & 253 & 286 & 33 \\
\hline 28250540 & 33485260 & 253 & 280 & 27 \\
\hline 28250540 & 30852447 & 252 & 277 & 25 \\
\hline 28250540 & 33485260 & 255 & 292 & 37 \\
\hline 28250540 & 33485260 & 255 & 288 & 33 \\
\hline 28250540 & 33485260 & 254 & 286 & 32 \\
\hline 28250540 & 33485260 & 253 & 291 & 38 \\
\hline
\end{tabular}




\begin{tabular}{|c|c|c|c|c|}
\hline 28250540 & 33485260 & 254 & 285 & 31 \\
\hline 26299134 & 33485260 & 231 & 300 & 69 \\
\hline 28250540 & 33485260 & 254 & 284 & 30 \\
\hline 27027570 & 33485260 & 242 & 303 & 61 \\
\hline 28250540 & 33485260 & 252 & 290 & 38 \\
\hline 28250540 & 33485260 & 255 & 291 & 36 \\
\hline 28250540 & 33485260 & 260 & 291 & 31 \\
\hline 27027570 & 33485260 & 247 & 293 & 46 \\
\hline 29525593 & 33485260 & 264 & 290 & 26 \\
\hline 29525593 & 33485260 & 264 & 293 & 29 \\
\hline 27027570 & 33485260 & 246 & 302 & 56 \\
\hline 28250540 & 33485260 & 254 & 294 & 40 \\
\hline 28250540 & 33485260 & 260 & 290 & 30 \\
\hline 28250540 & 33485260 & 254 & 292 & 38 \\
\hline 29525593 & 33485260 & 265 & 299 & 34 \\
\hline 29525593 & 33485260 & 268 & 295 & 27 \\
\hline 28250540 & 33485260 & 259 & 297 & 38 \\
\hline 29525593 & 33485260 & 264 & 289 & 25 \\
\hline 29525593 & 33485260 & 265 & 293 & 28 \\
\hline 29525593 & 33485260 & 267 & 291 & 24 \\
\hline 29525593 & 33485260 & 268 & 292 & 24 \\
\hline 29525593 & 33485260 & 265 & 291 & 26 \\
\hline 29525593 & 33485260 & 267 & 290 & 23 \\
\hline 29525593 & 33485260 & 262 & 292 & 30 \\
\hline 29525593 & 33485260 & 264 & 296 & 32 \\
\hline 29525593 & 33485260 & 270 & 291 & 21 \\
\hline 29525593 & 33485260 & 269 & 291 & 22 \\
\hline 29525593 & 33485260 & 274 & 287 & 13 \\
\hline 29525593 & 33485260 & 270 & 293 & 23 \\
\hline 29525593 & 33485260 & 264 & 295 & 31 \\
\hline 29525593 & 33485260 & 263 & 296 & 33 \\
\hline 29525593 & 33485260 & 265 & 297 & 32 \\
\hline 29525593 & 33485260 & 269 & 292 & 23 \\
\hline 29525593 & 33485260 & 264 & 296 & 32 \\
\hline 29525593 & 33485260 & 268 & 299 & 31 \\
\hline 29525593 & 33485260 & 268 & 298 & 30 \\
\hline 29525593 & 33485260 & 268 & 296 & 28 \\
\hline 29525593 & 33485260 & 269 & 292 & 23 \\
\hline 29525593 & 33485260 & 270 & 293 & 23 \\
\hline 29525593 & 33485260 & 265 & 301 & 36 \\
\hline 29525593 & 33485260 & 272 & 293 & 21 \\
\hline 29525593 & 33485260 & 270 & 297 & 27 \\
\hline 29525593 & 33485260 & 269 & 298 & 29 \\
\hline
\end{tabular}




\begin{tabular}{|c|c|c|c|c|}
\hline 29525593 & 33485260 & 264 & 301 & 37 \\
\hline 29525593 & 33485260 & 270 & 296 & 26 \\
\hline 29525593 & 33485260 & 267 & 298 & 31 \\
\hline 29525593 & 33485260 & 269 & 299 & 30 \\
\hline 29525593 & 33485260 & 268 & 300 & 32 \\
\hline 29525593 & 33485260 & 267 & 304 & 37 \\
\hline 28250540 & 35682862 & 257 & 330 & 73 \\
\hline 29525593 & 35682862 & 270 & 332 & 62 \\
\hline 29525593 & 35682862 & 272 & 336 & 64 \\
\hline 29525593 & 35682862 & 268 & 336 & 68 \\
\hline 30852447 & 35682862 & 282 & 323 & 41 \\
\hline 30852447 & 35682862 & 281 & 325 & 44 \\
\hline 29525593 & 35682862 & 269 & 334 & 65 \\
\hline 29525593 & 35682862 & 275 & 332 & 57 \\
\hline 30852447 & 35682862 & 289 & 328 & 39 \\
\hline 30852447 & 35682862 & 291 & 332 & 41 \\
\hline 30852447 & 35682862 & 291 & 329 & 38 \\
\hline 30852447 & 35682862 & 292 & 322 & 30 \\
\hline 30852447 & 35682862 & 293 & 322 & 29 \\
\hline 30852447 & 35682862 & 294 & 330 & 36 \\
\hline 33485260 & 35682862 & 316 & 333 & 17 \\
\hline 33485260 & 35682862 & 317 & 327 & 10 \\
\hline 33485260 & 35682862 & 317 & 326 & 9 \\
\hline 33485260 & 35682862 & 314 & 332 & 18 \\
\hline 30852447 & 35682862 & 302 & 336 & 34 \\
\hline 33485260 & 35682862 & 316 & 336 & 20 \\
\hline 30852447 & 35682862 & 292 & 336 & 44 \\
\hline 30852447 & 35682862 & 297 & 336 & 39 \\
\hline 34731340 & 35682862 & 322 & 336 & 14 \\
\hline 2738111 & 4487570 & 0 & 9 & 9 \\
\hline 2738111 & 4487570 & 0 & 9 & 9 \\
\hline 2738111 & 4487570 & 0 & 7 & 7 \\
\hline 2738111 & 4487570 & 0 & 8 & 8 \\
\hline 2738111 & 4487570 & 0 & 12 & 12 \\
\hline 2738111 & 4487570 & 0 & 9 & 9 \\
\hline 2738111 & 4487570 & 0 & 8 & 8 \\
\hline 2738111 & 4487570 & 0 & 6 & 6 \\
\hline 2738111 & 4487570 & 0 & 12 & 12 \\
\hline 2738111 & 4487570 & 0 & 14 & 14 \\
\hline 2738111 & 4487570 & 0 & 9 & 9 \\
\hline 2738111 & 4487570 & 0 & 12 & 12 \\
\hline 2738111 & 4487570 & 0 & 14 & 14 \\
\hline 2738111 & 4487570 & 0 & 16 & 16 \\
\hline
\end{tabular}




\begin{tabular}{|c|c|c|c|}
\hline 2738111 & 4487570 & 0 & 12 \\
\hline 2738111 & 4487570 & 0 & 13 \\
\hline 2738111 & 4487570 & 0 & 13 \\
\hline 2738111 & 4487570 & 0 & 15 \\
\hline 2738111 & 4487570 & 0 & 17 \\
\hline 2738111 & 4487570 & 0 & 18 \\
\hline 2738111 & 4487570 & 0 & 19 \\
\hline 2738111 & 4487570 & 0 & 19 \\
\hline 2738111 & 7613280 & 0 & 36 \\
\hline 2738111 & 4487570 & 0 & 19 \\
\hline 2738111 & 7613280 & 0 & 46 \\
\hline 2738111 & 7613280 & 0 & 51 \\
\hline 4487570 & 8426011 & 34 & 78 \\
\hline 4487570 & 8426011 & 38 & 80 \\
\hline 4487570 & 8426011 & 42 & 80 \\
\hline 4487570 & 21275999 & 41 & 122 \\
\hline 4487570 & 8426011 & 45 & 82 \\
\hline 4487570 & 8426011 & 45 & 78 \\
\hline 4487570 & 8426011 & 45 & 74 \\
\hline 4487570 & 8426011 & 45 & 76 \\
\hline 4487570 & 8426011 & 46 & 75 \\
\hline 4487570 & 8426011 & 46 & 80 \\
\hline 4487570 & 8426011 & 48 & 76 \\
\hline 4487570 & 21275999 & 39 & 132 \\
\hline 4487570 & 8426011 & 43 & 80 \\
\hline 4487570 & 8426011 & 49 & 76 \\
\hline 4487570 & 8426011 & 48 & 79 \\
\hline 4487570 & 8426011 & 50 & 78 \\
\hline 4487570 & 17962738 & 48 & 93 \\
\hline 4487570 & 8426011 & 54 & 85 \\
\hline 4487570 & 8426011 & 52 & 84 \\
\hline 4487570 & 8426011 & 52 & 82 \\
\hline 4487570 & 8426011 & 52 & 77 \\
\hline 4487570 & 8426011 & 50 & 79 \\
\hline 4487570 & 8426011 & 53 & 77 \\
\hline 4487570 & 8426011 & 53 & 79 \\
\hline 4487570 & 17962738 & 43 & 98 \\
\hline 4487570 & 8426011 & 49 & 85 \\
\hline 4487570 & 20145093 & 48 & 115 \\
\hline 4487570 & 17962738 & 53 & 96 \\
\hline 4487570 & 17962738 & 51 & 95 \\
\hline 4487570 & 17962738 & 53 & 94 \\
\hline 4487570 & 17962738 & 51 & 94 \\
\hline
\end{tabular}




\begin{tabular}{|c|c|c|c|}
\hline 4487570 & 17962738 & 51 & 94 \\
\hline 4487570 & 17962738 & 52 & 95 \\
\hline 4487570 & 17962738 & 53 & 94 \\
\hline 4487570 & 17962738 & 54 & 94 \\
\hline 4487570 & 17962738 & 53 & 94 \\
\hline 4487570 & 17962738 & 53 & 94 \\
\hline 4487570 & 17962738 & 51 & 94 \\
\hline 4487570 & 17962738 & 54 & 94 \\
\hline 4487570 & 17962738 & 55 & 94 \\
\hline 4487570 & 17962738 & 52 & 94 \\
\hline 4487570 & 21275999 & 62 & 122 \\
\hline 4487570 & 17962738 & 51 & 94 \\
\hline 4487570 & 17962738 & 53 & 94 \\
\hline 4487570 & 19253244 & 52 & 104 \\
\hline 4487570 & 17962738 & 55 & 94 \\
\hline 4487570 & 17962738 & 56 & 94 \\
\hline 4487570 & 17962738 & 55 & 94 \\
\hline 4487570 & 17962738 & 47 & 94 \\
\hline 4487570 & 17962738 & 57 & 94 \\
\hline 4487570 & 17962738 & 52 & 94 \\
\hline 4487570 & 17962738 & 63 & 94 \\
\hline 4487570 & 17962738 & 63 & 93 \\
\hline 4487570 & 17962738 & 61 & 93 \\
\hline 4487570 & 17962738 & 51 & 94 \\
\hline 4487570 & 17962738 & 53 & 94 \\
\hline 4487570 & 17962738 & 60 & 93 \\
\hline 4487570 & 17962738 & 58 & 94 \\
\hline 7613280 & 17962738 & 71 & 99 \\
\hline 4487570 & 17962738 & 52 & 95 \\
\hline 7613280 & 17962738 & 78 & 96 \\
\hline 7613280 & 17962738 & 75 & 94 \\
\hline 7613280 & 14100831 & 80 & 90 \\
\hline 7613280 & 14100831 & 78 & 91 \\
\hline 7613280 & 14100831 & 77 & 91 \\
\hline 7613280 & 17962738 & 72 & 97 \\
\hline 7613280 & 17962738 & 76 & 97 \\
\hline 7613280 & 21275999 & 74 & 135 \\
\hline 4487570 & 17962738 & 67 & 94 \\
\hline 4487570 & 17962738 & 62 & 95 \\
\hline 4487570 & 17962738 & 68 & 99 \\
\hline 7613280 & 17962738 & 71 & 99 \\
\hline 7613280 & 14100831 & 84 & 90 \\
\hline 7613280 & 14100831 & 80 & 91 \\
\hline
\end{tabular}




\begin{tabular}{|c|c|c|c|}
\hline 7613280 & 17962738 & 71 & 95 \\
\hline 4487570 & 17962738 & 59 & 97 \\
\hline 7613280 & 19253244 & 72 & 103 \\
\hline 7613280 & 21275999 & 72 & 135 \\
\hline 7613280 & 17962738 & 75 & 97 \\
\hline 7613280 & 21275999 & 73 & 122 \\
\hline 7613280 & 17962738 & 76 & 98 \\
\hline 4487570 & 21275999 & 43 & 131 \\
\hline 7613280 & 21275999 & 70 & 122 \\
\hline 7613280 & 17962738 & 71 & 92 \\
\hline 4487570 & 17962738 & 64 & 93 \\
\hline 7613280 & 17962738 & 84 & 96 \\
\hline 7613280 & 21275999 & 73 & 132 \\
\hline 4487570 & 17962738 & 53 & 98 \\
\hline 4487570 & 17962738 & 64 & 96 \\
\hline 7613280 & 23555825 & 76 & 158 \\
\hline 4487570 & 21275999 & 67 & 132 \\
\hline 7613280 & 17962738 & 83 & 94 \\
\hline 7613280 & 17962738 & 73 & 99 \\
\hline 12568541 & 14100831 & 91 & 92 \\
\hline 7613280 & 17962738 & 76 & 96 \\
\hline 7613280 & 17962738 & 78 & 99 \\
\hline 7613280 & 17962738 & 77 & 98 \\
\hline 7613280 & 17962738 & 75 & 98 \\
\hline 7613280 & 17962738 & 73 & 98 \\
\hline 7613280 & 17962738 & 75 & 98 \\
\hline 7613280 & 17962738 & 77 & 96 \\
\hline 7613280 & 17962738 & 88 & 98 \\
\hline 4487570 & 21275999 & 56 & 121 \\
\hline 7613280 & 21275999 & 82 & 132 \\
\hline 7613280 & 21275999 & 75 & 131 \\
\hline 7613280 & 20145093 & 74 & 114 \\
\hline 7613280 & 21275999 & 80 & 124 \\
\hline 7613280 & 21275999 & 81 & 127 \\
\hline 7613280 & 21275999 & 75 & 133 \\
\hline 7613280 & 21275999 & 80 & 122 \\
\hline 14100831 & 20145093 & 94 & 113 \\
\hline 12568541 & 19253244 & 90 & 104 \\
\hline 14100831 & 21275999 & 95 & 132 \\
\hline 14100831 & 19253244 & 96 & 104 \\
\hline 14100831 & 19253244 & 95 & 103 \\
\hline 14100831 & 21275999 & 96 & 132 \\
\hline 14100831 & 20145093 & 94 & 117 \\
\hline
\end{tabular}




\begin{tabular}{|c|c|c|c|}
\hline 14100831 & 21275999 & 95 & 126 \\
\hline 14100831 & 21275999 & 94 & 131 \\
\hline 14100831 & 19253244 & 97 & 103 \\
\hline 14100831 & 23555825 & 95 & 157 \\
\hline 14100831 & 20145093 & 92 & 120 \\
\hline 14100831 & 19253244 & 97 & 104 \\
\hline 14100831 & 20145093 & 96 & 119 \\
\hline 14100831 & 20145093 & 95 & 111 \\
\hline 14100831 & 20145093 & 95 & 113 \\
\hline 14100831 & 20145093 & 96 & 119 \\
\hline 14100831 & 21275999 & 96 & 133 \\
\hline 4487570 & 21275999 & 45 & 122 \\
\hline 14100831 & 23555825 & 96 & 140 \\
\hline 7613280 & 20145093 & 83 & 118 \\
\hline 7613280 & 21275999 & 81 & 121 \\
\hline 14100831 & 21275999 & 92 & 128 \\
\hline 10378748 & 21275999 & 89 & 123 \\
\hline 14100831 & 21275999 & 93 & 133 \\
\hline 14100831 & 21275999 & 97 & 132 \\
\hline 14100831 & 20145093 & 95 & 114 \\
\hline 14100831 & 20145093 & 97 & 113 \\
\hline 17962738 & 20145093 & 102 & 110 \\
\hline 14100831 & 21275999 & 95 & 128 \\
\hline 14100831 & 23555825 & 94 & 142 \\
\hline 4487570 & 21275999 & 54 & 129 \\
\hline 7613280 & 21275999 & 84 & 137 \\
\hline 7613280 & 21275999 & 85 & 138 \\
\hline 7613280 & 21275999 & 79 & 130 \\
\hline 7613280 & 21275999 & 81 & 121 \\
\hline 17962738 & 20145093 & 103 & 111 \\
\hline 17962738 & 20145093 & 103 & 109 \\
\hline 7613280 & 21275999 & 83 & 125 \\
\hline 14100831 & 21275999 & 99 & 134 \\
\hline 4487570 & 21275999 & 48 & 125 \\
\hline 7613280 & 21275999 & 71 & 124 \\
\hline 14100831 & 21275999 & 97 & 136 \\
\hline 14100831 & 21275999 & 96 & 134 \\
\hline 14100831 & 20145093 & 96 & 119 \\
\hline 14100831 & 21275999 & 98 & 128 \\
\hline 14100831 & 21275999 & 99 & 134 \\
\hline 17962738 & 21275999 & 101 & 133 \\
\hline 14100831 & 21275999 & 98 & 132 \\
\hline 14100831 & 21275999 & 97 & 125 \\
\hline
\end{tabular}




\begin{tabular}{|c|c|c|c|c|}
\hline 17962738 & 21275999 & 101 & 130 & 29 \\
\hline 17962738 & 20145093 & 103 & 114 & 11 \\
\hline 14100831 & 21275999 & 92 & 129 & 37 \\
\hline 4487570 & 23555825 & 61 & 165 & 104 \\
\hline 7613280 & 21275999 & 77 & 132 & 55 \\
\hline 14100831 & 21275999 & 97 & 133 & 36 \\
\hline 14100831 & 21275999 & 100 & 134 & 34 \\
\hline 14100831 & 21275999 & 99 & 131 & 32 \\
\hline 14100831 & 21275999 & 97 & 133 & 36 \\
\hline 7613280 & 21275999 & 74 & 134 & 60 \\
\hline 14100831 & 21275999 & 98 & 132 & 34 \\
\hline 12568541 & 21275999 & 91 & 130 & 39 \\
\hline 14100831 & 21275999 & 92 & 129 & 37 \\
\hline 17962738 & 21275999 & 101 & 134 & 33 \\
\hline 14100831 & 21275999 & 97 & 135 & 38 \\
\hline 14100831 & 21275999 & 95 & 134 & 39 \\
\hline 17962738 & 21275999 & 101 & 136 & 35 \\
\hline 14100831 & 21275999 & 92 & 138 & 46 \\
\hline 14100831 & 23555825 & 98 & 154 & 56 \\
\hline 14100831 & 23555825 & 98 & 148 & 50 \\
\hline 20145093 & 23555825 & 122 & 152 & 30 \\
\hline 19253244 & 23555825 & 118 & 147 & 29 \\
\hline 19253244 & 23555825 & 115 & 147 & 32 \\
\hline 14100831 & 23555825 & 99 & 153 & 54 \\
\hline 19253244 & 23555825 & 113 & 148 & 35 \\
\hline 20145093 & 23555825 & 122 & 151 & 29 \\
\hline 19253244 & 23555825 & 118 & 148 & 30 \\
\hline 19253244 & 23555825 & 116 & 148 & 32 \\
\hline 19253244 & 23555825 & 117 & 147 & 30 \\
\hline 20145093 & 23555825 & 123 & 156 & 33 \\
\hline 20145093 & 23555825 & 124 & 164 & 40 \\
\hline 20145093 & 23555825 & 124 & 153 & 29 \\
\hline 20145093 & 23555825 & 124 & 157 & 33 \\
\hline 20145093 & 23555825 & 122 & 151 & 29 \\
\hline 20145093 & 23555825 & 126 & 149 & 23 \\
\hline 20145093 & 23555825 & 127 & 151 & 24 \\
\hline 20145093 & 23555825 & 125 & 160 & 35 \\
\hline 20145093 & 23555825 & 128 & 153 & 25 \\
\hline 20145093 & 23555825 & 130 & 150 & 20 \\
\hline 20145093 & 23555825 & 128 & 152 & 24 \\
\hline 20145093 & 23555825 & 129 & 150 & 21 \\
\hline 20145093 & 23555825 & 130 & 149 & 19 \\
\hline 20145093 & 23555825 & 125 & 170 & 45 \\
\hline
\end{tabular}




\begin{tabular}{|c|c|c|c|c|}
\hline 20145093 & 23555825 & 130 & 163 & 33 \\
\hline 20145093 & 23555825 & 129 & 161 & 32 \\
\hline 20145093 & 23555825 & 122 & 168 & 46 \\
\hline 20145093 & 23555825 & 131 & 163 & 32 \\
\hline 20145093 & 23555825 & 128 & 163 & 35 \\
\hline 19253244 & 23555825 & 105 & 166 & 61 \\
\hline 20145093 & 25117014 & 128 & 179 & 51 \\
\hline 21275999 & 25117014 & 157 & 180 & 23 \\
\hline 21275999 & 25117014 & 159 & 182 & 23 \\
\hline 21275999 & 25117014 & 160 & 184 & 24 \\
\hline 21275999 & 25117014 & 156 & 190 & 34 \\
\hline 21275999 & 25117014 & 161 & 179 & 18 \\
\hline 21275999 & 25117014 & 158 & 181 & 23 \\
\hline 21275999 & 25117014 & 159 & 186 & 27 \\
\hline 21275999 & 25117014 & 161 & 183 & 22 \\
\hline 21275999 & 25117014 & 161 & 180 & 19 \\
\hline 21275999 & 25117014 & 165 & 186 & 21 \\
\hline 21275999 & 25117014 & 163 & 183 & 20 \\
\hline 21275999 & 25117014 & 165 & 182 & 17 \\
\hline 21275999 & 25117014 & 163 & 181 & 18 \\
\hline 21275999 & 25117014 & 165 & 183 & 18 \\
\hline 21275999 & 25117014 & 165 & 181 & 16 \\
\hline 21275999 & 25117014 & 164 & 182 & 18 \\
\hline 21275999 & 25117014 & 166 & 181 & 15 \\
\hline 21275999 & 25117014 & 163 & 182 & 19 \\
\hline 21275999 & 25117014 & 159 & 185 & 26 \\
\hline 21275999 & 25117014 & 164 & 185 & 21 \\
\hline 21275999 & 25117014 & 165 & 180 & 15 \\
\hline 21275999 & 25117014 & 165 & 182 & 17 \\
\hline 21275999 & 25117014 & 165 & 179 & 14 \\
\hline 21275999 & 25117014 & 167 & 182 & 15 \\
\hline 21275999 & 25117014 & 164 & 184 & 20 \\
\hline 21275999 & 25117014 & 164 & 177 & 13 \\
\hline 21275999 & 25117014 & 162 & 183 & 21 \\
\hline 21275999 & 25117014 & 161 & 182 & 21 \\
\hline 21275999 & 25117014 & 163 & 183 & 20 \\
\hline 21275999 & 25117014 & 164 & 185 & 21 \\
\hline 21275999 & 25117014 & 164 & 186 & 22 \\
\hline 21275999 & 25117014 & 162 & 188 & 26 \\
\hline 21275999 & 25117014 & 159 & 192 & 33 \\
\hline 21275999 & 25117014 & 160 & 194 & 34 \\
\hline 26226321 & 29410049 & 225 & 244 & 19 \\
\hline 26226321 & 29410049 & 222 & 244 & 22 \\
\hline
\end{tabular}




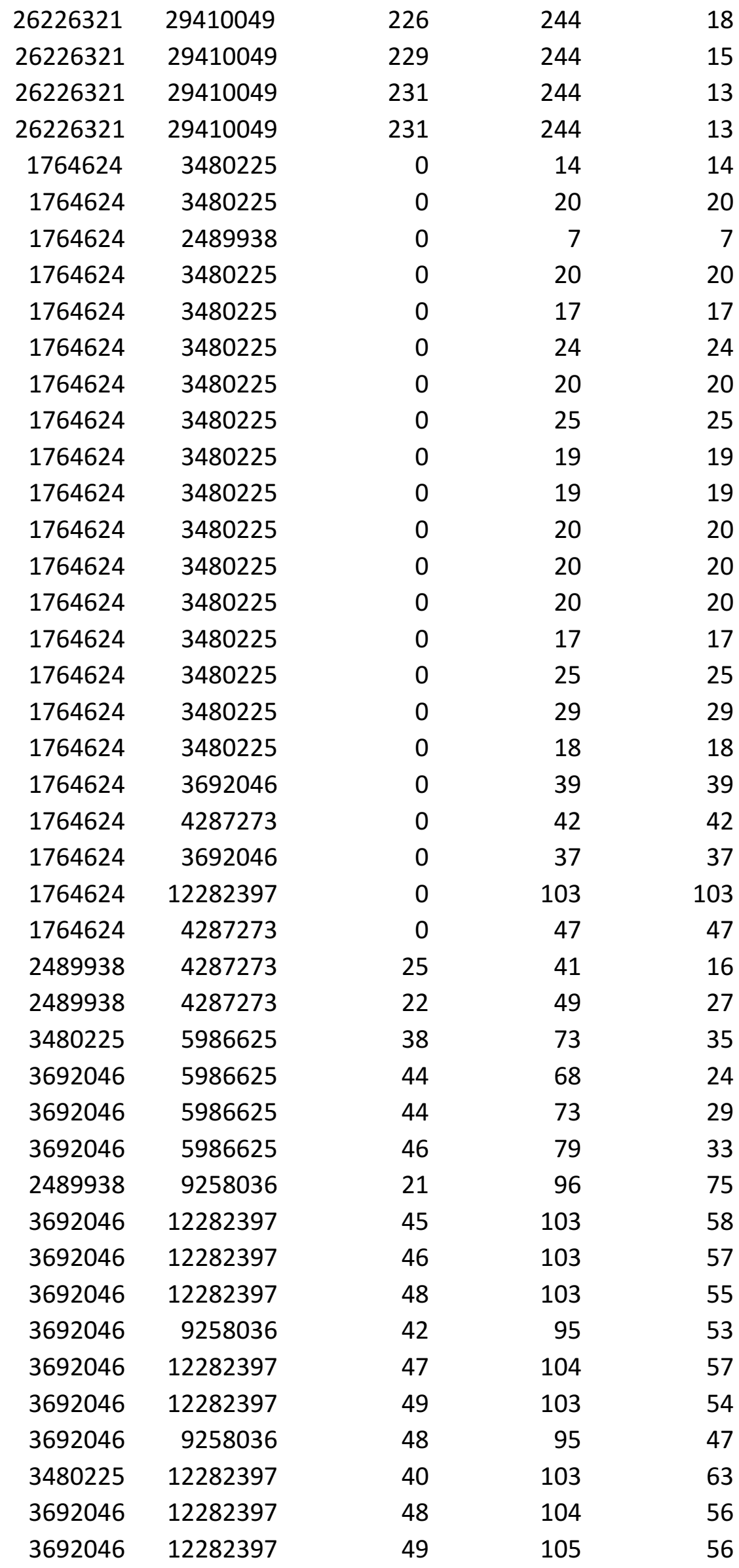




\begin{tabular}{|c|c|c|c|}
\hline 4287273 & 11818418 & 57 & 102 \\
\hline 3692046 & 12282397 & 50 & 103 \\
\hline 4287273 & 11818418 & 58 & 102 \\
\hline 4287273 & 12282397 & 56 & 104 \\
\hline 4287273 & 9258036 & 52 & 96 \\
\hline 4287273 & 9258036 & 66 & 94 \\
\hline 4287273 & 11818418 & 57 & 101 \\
\hline 4287273 & 16688383 & 58 & 128 \\
\hline 4287273 & 9258036 & 52 & 95 \\
\hline 4287273 & 9258036 & 59 & 95 \\
\hline 4287273 & 16688383 & 57 & 124 \\
\hline 4287273 & 11818418 & 62 & 102 \\
\hline 4287273 & 9258036 & 70 & 96 \\
\hline 4287273 & 13912221 & 61 & 113 \\
\hline 4287273 & 9258036 & 67 & 92 \\
\hline 4287273 & 9258036 & 77 & 92 \\
\hline 4287273 & 9258036 & 77 & 92 \\
\hline 4287273 & 9258036 & 77 & 92 \\
\hline 4287273 & 9258036 & 77 & 92 \\
\hline 4287273 & 9258036 & 76 & 93 \\
\hline 4287273 & 9258036 & 77 & 92 \\
\hline 4287273 & 9258036 & 77 & 92 \\
\hline 4287273 & 9258036 & 76 & 92 \\
\hline 4287273 & 9258036 & 77 & 92 \\
\hline 4287273 & 9258036 & 77 & 92 \\
\hline 4287273 & 11818418 & 77 & 98 \\
\hline 4287273 & 11818418 & 69 & 100 \\
\hline 4287273 & 11818418 & 71 & 97 \\
\hline 4287273 & 9258036 & 72 & 93 \\
\hline 4287273 & 14731322 & 68 & 120 \\
\hline 4287273 & 11818418 & 65 & 100 \\
\hline 4287273 & 9258036 & 68 & 91 \\
\hline 4287273 & 9258036 & 71 & 94 \\
\hline 4287273 & 13912221 & 69 & 113 \\
\hline 4287273 & 9258036 & 76 & 94 \\
\hline 4287273 & 9258036 & 63 & 95 \\
\hline 4287273 & 12282397 & 61 & 104 \\
\hline 4287273 & 16688383 & 68 & 136 \\
\hline 4287273 & 9258036 & 57 & 96 \\
\hline 4287273 & 12282397 & 64 & 104 \\
\hline 4287273 & 9258036 & 69 & 96 \\
\hline 4287273 & 16688383 & 66 & 124 \\
\hline 4287273 & 9258036 & 63 & 95 \\
\hline
\end{tabular}




\begin{tabular}{|c|c|c|c|}
\hline 4287273 & 12282397 & 61 & 104 \\
\hline 4287273 & 9258036 & 70 & 93 \\
\hline 4287273 & 9258036 & 77 & 92 \\
\hline 4287273 & 9258036 & 78 & 90 \\
\hline 4287273 & 13912221 & 73 & 117 \\
\hline 4287273 & 11818418 & 67 & 100 \\
\hline 4287273 & 9258036 & 55 & 96 \\
\hline 4287273 & 9258036 & 62 & 96 \\
\hline 4287273 & 9258036 & 75 & 95 \\
\hline 4287273 & 9258036 & 74 & 93 \\
\hline 4287273 & 12282397 & 56 & 104 \\
\hline 4287273 & 9258036 & 63 & 95 \\
\hline 4287273 & 9258036 & 70 & 92 \\
\hline 4287273 & 9258036 & 80 & 91 \\
\hline 4287273 & 9258036 & 73 & 96 \\
\hline 4287273 & 9258036 & 68 & 95 \\
\hline 4287273 & 9258036 & 82 & 91 \\
\hline 4287273 & 12282397 & 64 & 103 \\
\hline 4287273 & 9258036 & 62 & 96 \\
\hline 4287273 & 11818418 & 68 & 101 \\
\hline 4287273 & 16688383 & 72 & 135 \\
\hline 3692046 & 11818418 & 51 & 102 \\
\hline 4287273 & 16688383 & 66 & 132 \\
\hline 4287273 & 11818418 & 66 & 102 \\
\hline 4287273 & 11818418 & 65 & 101 \\
\hline 4287273 & 9258036 & 74 & 96 \\
\hline 4287273 & 11818418 & 61 & 102 \\
\hline 4287273 & 14731322 & 58 & 119 \\
\hline 4287273 & 11818418 & 63 & 101 \\
\hline 4287273 & 12282397 & 63 & 104 \\
\hline 4287273 & 11818418 & 66 & 102 \\
\hline 4287273 & 12282397 & 56 & 103 \\
\hline 4287273 & 14731322 & 57 & 119 \\
\hline 4287273 & 11818418 & 57 & 102 \\
\hline 4287273 & 12282397 & 60 & 103 \\
\hline 4287273 & 14731322 & 75 & 119 \\
\hline 4287273 & 11818418 & 59 & 102 \\
\hline 4287273 & 16688383 & 57 & 127 \\
\hline 4287273 & 16688383 & 63 & 125 \\
\hline 4287273 & 13912221 & 69 & 116 \\
\hline 4287273 & 12282397 & 68 & 104 \\
\hline 4287273 & 11818418 & 66 & 101 \\
\hline 4287273 & 16688383 & 63 & 122 \\
\hline
\end{tabular}




\begin{tabular}{|c|c|c|c|}
\hline 4287273 & 16688383 & 65 & 124 \\
\hline 4287273 & 11818418 & 77 & 100 \\
\hline 3692046 & 12282397 & 44 & 104 \\
\hline 4287273 & 14731322 & 73 & 118 \\
\hline 4287273 & 12282397 & 54 & 104 \\
\hline 4287273 & 13912221 & 66 & 114 \\
\hline 4287273 & 16688383 & 63 & 127 \\
\hline 4287273 & 13912221 & 70 & 117 \\
\hline 4287273 & 13912221 & 73 & 118 \\
\hline 4287273 & 11818418 & 66 & 102 \\
\hline 4287273 & 13912221 & 77 & 112 \\
\hline 4287273 & 13912221 & 65 & 111 \\
\hline 4287273 & 13912221 & 73 & 116 \\
\hline 3692046 & 12282397 & 43 & 104 \\
\hline 4287273 & 16688383 & 67 & 129 \\
\hline 4287273 & 13912221 & 52 & 107 \\
\hline 3692046 & 16688383 & 48 & 126 \\
\hline 4287273 & 12282397 & 53 & 104 \\
\hline 4287273 & 12282397 & 59 & 103 \\
\hline 4287273 & 13912221 & 67 & 111 \\
\hline 4287273 & 14731322 & 71 & 118 \\
\hline 4287273 & 14731322 & 68 & 118 \\
\hline 4287273 & 11818418 & 68 & 102 \\
\hline 4287273 & 11818418 & 53 & 102 \\
\hline 4287273 & 12282397 & 62 & 104 \\
\hline 4287273 & 13912221 & 67 & 113 \\
\hline 4287273 & 13912221 & 77 & 115 \\
\hline 4287273 & 12282397 & 65 & 103 \\
\hline 7332602 & 12282397 & 87 & 103 \\
\hline 4287273 & 13912221 & 86 & 110 \\
\hline 4287273 & 11818418 & 64 & 102 \\
\hline 4287273 & 12282397 & 64 & 103 \\
\hline 7546777 & 12282397 & 89 & 105 \\
\hline 7332602 & 13912221 & 87 & 112 \\
\hline 4287273 & 14731322 & 64 & 118 \\
\hline 4287273 & 14731322 & 69 & 120 \\
\hline 4287273 & 14731322 & 72 & 120 \\
\hline 7332602 & 13912221 & 87 & 112 \\
\hline 4287273 & 13912221 & 85 & 115 \\
\hline 4287273 & 13912221 & 57 & 116 \\
\hline 7546777 & 11818418 & 88 & 101 \\
\hline 7546777 & 11818418 & 87 & 102 \\
\hline 3692046 & 12282397 & 51 & 103 \\
\hline
\end{tabular}




\begin{tabular}{|c|c|c|c|}
\hline 7332602 & 14731322 & 87 & 119 \\
\hline 7546777 & 12282397 & 90 & 103 \\
\hline 7332602 & 12282397 & 87 & 104 \\
\hline 4287273 & 14731322 & 81 & 119 \\
\hline 3692046 & 12282397 & 50 & 104 \\
\hline 7546777 & 12282397 & 90 & 103 \\
\hline 7546777 & 13912221 & 88 & 109 \\
\hline 7546777 & 12282397 & 89 & 103 \\
\hline 7546777 & 11818418 & 90 & 102 \\
\hline 7546777 & 11818418 & 92 & 101 \\
\hline 7546777 & 11818418 & 91 & 102 \\
\hline 7546777 & 11818418 & 90 & 102 \\
\hline 7546777 & 12282397 & 93 & 105 \\
\hline 7546777 & 13912221 & 92 & 110 \\
\hline 7546777 & 12282397 & 87 & 103 \\
\hline 7546777 & 13912221 & 90 & 111 \\
\hline 7546777 & 13912221 & 90 & 110 \\
\hline 7546777 & 12282397 & 92 & 103 \\
\hline 7546777 & 11818418 & 89 & 102 \\
\hline 7546777 & 13912221 & 90 & 110 \\
\hline 7546777 & 12282397 & 89 & 103 \\
\hline 7546777 & 13912221 & 91 & 112 \\
\hline 7546777 & 12282397 & 87 & 104 \\
\hline 7546777 & 16688383 & 89 & 124 \\
\hline 7546777 & 12282397 & 89 & 104 \\
\hline 7546777 & 12282397 & 89 & 104 \\
\hline 7546777 & 13912221 & 90 & 113 \\
\hline 7546777 & 13912221 & 91 & 113 \\
\hline 7546777 & 13912221 & 91 & 108 \\
\hline 7546777 & 16688383 & 90 & 126 \\
\hline 7546777 & 13912221 & 90 & 114 \\
\hline 7546777 & 13912221 & 90 & 112 \\
\hline 7546777 & 13912221 & 89 & 106 \\
\hline 7546777 & 11818418 & 94 & 102 \\
\hline 7546777 & 12282397 & 90 & 103 \\
\hline 7546777 & 13912221 & 92 & 109 \\
\hline 7546777 & 13912221 & 90 & 113 \\
\hline 7546777 & 12282397 & 90 & 103 \\
\hline 7546777 & 12282397 & 90 & 103 \\
\hline 7546777 & 16688383 & 90 & 122 \\
\hline 7546777 & 14731322 & 89 & 119 \\
\hline 7546777 & 12282397 & 87 & 104 \\
\hline 4287273 & 12282397 & 52 & 104 \\
\hline
\end{tabular}




\begin{tabular}{|c|c|c|c|}
\hline 7546777 & 13912221 & 90 & 112 \\
\hline 4287273 & 13912221 & 67 & 112 \\
\hline 7546777 & 11818418 & 91 & 102 \\
\hline 4287273 & 14731322 & 64 & 119 \\
\hline 7546777 & 11818418 & 89 & 102 \\
\hline 7546777 & 11818418 & 87 & 102 \\
\hline 7546777 & 12282397 & 89 & 105 \\
\hline 3692046 & 11818418 & 50 & 102 \\
\hline 4287273 & 16688383 & 71 & 134 \\
\hline 4287273 & 11818418 & 71 & 102 \\
\hline 7546777 & 16688383 & 88 & 133 \\
\hline 7546777 & 11818418 & 91 & 102 \\
\hline 4287273 & 16688383 & 78 & 128 \\
\hline 4287273 & 11818418 & 70 & 102 \\
\hline 4287273 & 13912221 & 77 & 111 \\
\hline 4287273 & 12282397 & 71 & 103 \\
\hline 7546777 & 12282397 & 91 & 103 \\
\hline 7546777 & 12282397 & 89 & 105 \\
\hline 7546777 & 13912221 & 90 & 113 \\
\hline 7546777 & 11818418 & 90 & 102 \\
\hline 7546777 & 11818418 & 91 & 102 \\
\hline 7546777 & 11818418 & 90 & 102 \\
\hline 4287273 & 11818418 & 57 & 102 \\
\hline 7546777 & 11818418 & 88 & 102 \\
\hline 3692046 & 16688383 & 51 & 123 \\
\hline 7546777 & 11818418 & 88 & 102 \\
\hline 4287273 & 16688383 & 62 & 130 \\
\hline 7546777 & 11818418 & 92 & 102 \\
\hline 7546777 & 12282397 & 92 & 103 \\
\hline 4287273 & 16688383 & 60 & 131 \\
\hline 4287273 & 11818418 & 61 & 102 \\
\hline 7546777 & 14731322 & 87 & 120 \\
\hline 7546777 & 12282397 & 91 & 105 \\
\hline 3692046 & 13912221 & 49 & 115 \\
\hline 7546777 & 11818418 & 89 & 102 \\
\hline 7546777 & 12282397 & 94 & 103 \\
\hline 4287273 & 14731322 & 60 & 118 \\
\hline 7546777 & 11818418 & 96 & 102 \\
\hline 7546777 & 12282397 & 92 & 105 \\
\hline 7546777 & 12282397 & 95 & 103 \\
\hline 7546777 & 16688383 & 91 & 122 \\
\hline 7546777 & 14731322 & 90 & 120 \\
\hline 7546777 & 14731322 & 91 & 120 \\
\hline
\end{tabular}




\begin{tabular}{|c|c|c|c|}
\hline 7546777 & 13912221 & 93 & 111 \\
\hline 7546777 & 13912221 & 94 & 117 \\
\hline 3692046 & 12282397 & 45 & 105 \\
\hline 3692046 & 12282397 & 51 & 104 \\
\hline 4287273 & 11818418 & 78 & 102 \\
\hline 7546777 & 16688383 & 87 & 126 \\
\hline 7546777 & 11818418 & 92 & 102 \\
\hline 7546777 & 11818418 & 90 & 102 \\
\hline 7546777 & 13912221 & 92 & 113 \\
\hline 7546777 & 13912221 & 89 & 114 \\
\hline 7546777 & 13912221 & 90 & 110 \\
\hline 7546777 & 13912221 & 90 & 116 \\
\hline 4287273 & 16688383 & 66 & 135 \\
\hline 7546777 & 13912221 & 91 & 107 \\
\hline 7546777 & 12282397 & 90 & 105 \\
\hline 7546777 & 13912221 & 93 & 108 \\
\hline 7546777 & 13912221 & 89 & 109 \\
\hline 4287273 & 16688383 & 55 & 140 \\
\hline 4287273 & 16688383 & 52 & 136 \\
\hline 4287273 & 14731322 & 84 & 119 \\
\hline 9258036 & 13912221 & 98 & 118 \\
\hline 7546777 & 13912221 & 93 & 114 \\
\hline 7546777 & 16688383 & 89 & 127 \\
\hline 7546777 & 14731322 & 93 & 119 \\
\hline 4287273 & 16688383 & 69 & 132 \\
\hline 7546777 & 16688383 & 93 & 129 \\
\hline 4287273 & 16688383 & 68 & 129 \\
\hline 7546777 & 16688383 & 92 & 122 \\
\hline 7546777 & 16688383 & 93 & 127 \\
\hline 12282397 & 16688383 & 108 & 124 \\
\hline 12282397 & 14731322 & 109 & 120 \\
\hline 12282397 & 16688383 & 108 & 122 \\
\hline 12282397 & 16688383 & 109 & 124 \\
\hline 12282397 & 14731322 & 111 & 120 \\
\hline 4287273 & 16688383 & 74 & 133 \\
\hline 12282397 & 16688383 & 112 & 125 \\
\hline 7546777 & 16688383 & 90 & 131 \\
\hline 3692046 & 16688383 & 48 & 125 \\
\hline 12282397 & 16688383 & 107 & 127 \\
\hline 12282397 & 16688383 & 114 & 132 \\
\hline 12282397 & 16688383 & 112 & 128 \\
\hline 7546777 & 16688383 & 90 & 133 \\
\hline 4287273 & 16688383 & 61 & 137 \\
\hline
\end{tabular}




\begin{tabular}{|c|c|c|c|c|}
\hline 7546777 & 16688383 & 96 & 131 & 35 \\
\hline 12282397 & 16688383 & 110 & 133 & 23 \\
\hline 12282397 & 16688383 & 112 & 134 & 22 \\
\hline 12282397 & 16688383 & 115 & 136 & 21 \\
\hline 12282397 & 21849192 & 108 & 207 & 99 \\
\hline 9258036 & 19356938 & 101 & 171 & 70 \\
\hline 7546777 & 19356938 & 89 & 180 & 91 \\
\hline 14731322 & 19356938 & 125 & 175 & 50 \\
\hline 7546777 & 19356938 & 90 & 183 & 93 \\
\hline 7546777 & 19356938 & 91 & 175 & 84 \\
\hline 14731322 & 19356938 & 135 & 167 & 32 \\
\hline 14731322 & 19356938 & 136 & 180 & 44 \\
\hline 14731322 & 19356938 & 137 & 184 & 47 \\
\hline 14731322 & 19356938 & 127 & 182 & 55 \\
\hline 14731322 & 21849192 & 126 & 207 & 81 \\
\hline 16688383 & 21849192 & 160 & 207 & 47 \\
\hline 16688383 & 21849192 & 165 & 207 & 42 \\
\hline 16688383 & 21849192 & 168 & 207 & 39 \\
\hline 16688383 & 21849192 & 175 & 207 & 32 \\
\hline 16688383 & 21849192 & 169 & 207 & 38 \\
\hline 16688383 & 21849192 & 174 & 207 & 33 \\
\hline 16688383 & 21849192 & 148 & 207 & 59 \\
\hline 16688383 & 21849192 & 172 & 207 & 35 \\
\hline 16688383 & 21849192 & 183 & 207 & 24 \\
\hline 16688383 & 21849192 & 184 & 207 & 23 \\
\hline 16688383 & 21849192 & 190 & 207 & 17 \\
\hline 16688383 & 21849192 & 177 & 207 & 30 \\
\hline 16688383 & 21849192 & 186 & 207 & 21 \\
\hline 1847396 & 3202353 & 0 & 25 & 25 \\
\hline 1847396 & 3202353 & 0 & 25 & 25 \\
\hline 1847396 & 3202353 & 0 & 24 & 24 \\
\hline 1847396 & 3202353 & 0 & 30 & 30 \\
\hline 1847396 & 3202353 & 0 & 32 & 32 \\
\hline 1847396 & 3202353 & 0 & 34 & 34 \\
\hline 1847396 & 3202353 & 0 & 39 & 39 \\
\hline 1847396 & 3202353 & 0 & 40 & 40 \\
\hline 1847396 & 3202353 & 0 & 39 & 39 \\
\hline 1847396 & 3202353 & 0 & 40 & 40 \\
\hline 1847396 & 3202353 & 0 & 41 & 41 \\
\hline 3202353 & 6317387 & 72 & 107 & 35 \\
\hline 3202353 & 6317387 & 71 & 110 & 39 \\
\hline 3202353 & 6317387 & 78 & 109 & 31 \\
\hline 3202353 & 11362934 & 79 & 124 & 45 \\
\hline
\end{tabular}




\begin{tabular}{|c|c|c|c|c|}
\hline 3202353 & 6317387 & 73 & 106 & 33 \\
\hline 3202353 & 6317387 & 85 & 102 & 17 \\
\hline 3202353 & 6317387 & 88 & 108 & 20 \\
\hline 3202353 & 6317387 & 80 & 105 & 25 \\
\hline 3202353 & 6317387 & 83 & 108 & 25 \\
\hline 3202353 & 6317387 & 83 & 112 & 29 \\
\hline 3202353 & 6317387 & 79 & 107 & 28 \\
\hline 3202353 & 6317387 & 76 & 109 & 33 \\
\hline 3853777 & 6317387 & 96 & 108 & 12 \\
\hline 3202353 & 6317387 & 78 & 110 & 32 \\
\hline 3202353 & 6317387 & 91 & 105 & 14 \\
\hline 3853777 & 6317387 & 96 & 110 & 14 \\
\hline 3853777 & 6317387 & 97 & 108 & 11 \\
\hline 3202353 & 6317387 & 89 & 105 & 16 \\
\hline 3202353 & 6317387 & 88 & 109 & 21 \\
\hline 3202353 & 6317387 & 81 & 107 & 26 \\
\hline 3202353 & 6317387 & 80 & 109 & 29 \\
\hline 3202353 & 6317387 & 73 & 109 & 36 \\
\hline 3202353 & 6317387 & 84 & 110 & 26 \\
\hline 3202353 & 6317387 & 71 & 106 & 35 \\
\hline 3853777 & 6317387 & 95 & 109 & 14 \\
\hline 3853777 & 6317387 & 98 & 105 & 7 \\
\hline 3853777 & 6317387 & 96 & 114 & 18 \\
\hline 3202353 & 6317387 & 75 & 112 & 37 \\
\hline 3202353 & 6317387 & 76 & 117 & 41 \\
\hline 3853777 & 6317387 & 95 & 117 & 22 \\
\hline 3853777 & 6317387 & 96 & 114 & 18 \\
\hline 3202353 & 6317387 & 83 & 115 & 32 \\
\hline 3853777 & 6317387 & 97 & 109 & 12 \\
\hline 3853777 & 6317387 & 96 & 116 & 20 \\
\hline 3853777 & 11362934 & 96 & 127 & 31 \\
\hline 3202353 & 11362934 & 82 & 127 & 45 \\
\hline 3853777 & 11362934 & 96 & 126 & 30 \\
\hline 3853777 & 11362934 & 96 & 126 & 30 \\
\hline 3202353 & 11362934 & 77 & 135 & 58 \\
\hline 3202353 & 11362934 & 85 & 135 & 50 \\
\hline 3202353 & 12071751 & 78 & 145 & 67 \\
\hline 3853777 & 11362934 & 98 & 129 & 31 \\
\hline 3853777 & 11362934 & 97 & 130 & 33 \\
\hline 4252654 & 11362934 & 100 & 124 & 24 \\
\hline 4252654 & 11362934 & 101 & 137 & 36 \\
\hline 4252654 & 11362934 & 105 & 128 & 23 \\
\hline 4252654 & 11362934 & 104 & 136 & 32 \\
\hline
\end{tabular}




\begin{tabular}{|c|c|c|c|c|}
\hline 4252654 & 7461703 & 106 & 122 & 16 \\
\hline 4252654 & 11362934 & 109 & 123 & 14 \\
\hline 4252654 & 11362934 & 109 & 132 & 23 \\
\hline 4252654 & 11362934 & 112 & 138 & 26 \\
\hline 4252654 & 11362934 & 112 & 126 & 14 \\
\hline 4252654 & 11362934 & 111 & 127 & 16 \\
\hline 4252654 & 11362934 & 105 & 133 & 28 \\
\hline 4252654 & 11362934 & 111 & 127 & 16 \\
\hline 4252654 & 11362934 & 106 & 135 & 29 \\
\hline 4252654 & 11362934 & 108 & 132 & 24 \\
\hline 3853777 & 11362934 & 95 & 134 & 39 \\
\hline 4252654 & 12071751 & 103 & 140 & 37 \\
\hline 8783670 & 12071751 & 125 & 143 & 18 \\
\hline 6317387 & 16035116 & 121 & 200 & 79 \\
\hline 8783670 & 12071751 & 130 & 147 & 17 \\
\hline 11362934 & 13146665 & 141 & 153 & 12 \\
\hline 11362934 & 13146665 & 141 & 153 & 12 \\
\hline 11362934 & 13146665 & 143 & 153 & 10 \\
\hline 11362934 & 13146665 & 144 & 153 & 9 \\
\hline 11362934 & 13146665 & 142 & 160 & 18 \\
\hline 11362934 & 13146665 & 143 & 160 & 17 \\
\hline 8783670 & 13793654 & 125 & 185 & 60 \\
\hline 4252654 & 13793654 & 109 & 178 & 69 \\
\hline 8783670 & 16035116 & 128 & 187 & 59 \\
\hline 12071751 & 13793654 & 152 & 180 & 28 \\
\hline 12071751 & 13793654 & 156 & 182 & 26 \\
\hline 4252654 & 16035116 & 99 & 189 & 90 \\
\hline 12071751 & 16035116 & 150 & 186 & 36 \\
\hline 12071751 & 13793654 & 156 & 181 & 25 \\
\hline 12071751 & 13793654 & 160 & 180 & 20 \\
\hline 12071751 & 13793654 & 161 & 180 & 19 \\
\hline 12071751 & 13793654 & 164 & 179 & 15 \\
\hline 12071751 & 13793654 & 160 & 184 & 24 \\
\hline 12071751 & 16035116 & 154 & 199 & 45 \\
\hline 12071751 & 16035116 & 163 & 190 & 27 \\
\hline 12071751 & 16035116 & 155 & 203 & 48 \\
\hline 12071751 & 16035116 & 165 & 191 & 26 \\
\hline 12071751 & 16035116 & 151 & 204 & 53 \\
\hline 13146665 & 16035116 & 177 & 191 & 14 \\
\hline 13146665 & 16035116 & 178 & 190 & 12 \\
\hline 13146665 & 16035116 & 176 & 196 & 20 \\
\hline 12071751 & 16035116 & 150 & 202 & 52 \\
\hline 12071751 & 16035116 & 170 & 194 & 24 \\
\hline
\end{tabular}




\begin{tabular}{|c|c|c|c|c|}
\hline 12071751 & 16035116 & 167 & 196 & 29 \\
\hline 13146665 & 16035116 & 180 & 190 & 10 \\
\hline 13146665 & 16035116 & 179 & 191 & 12 \\
\hline 13146665 & 16035116 & 178 & 189 & 11 \\
\hline 12071751 & 16035116 & 163 & 196 & 33 \\
\hline 13146665 & 16035116 & 175 & 195 & 20 \\
\hline 13146665 & 16035116 & 174 & 197 & 23 \\
\hline 13146665 & 16035116 & 179 & 196 & 17 \\
\hline 13146665 & 16035116 & 177 & 197 & 20 \\
\hline 12071751 & 16035116 & 161 & 212 & 51 \\
\hline 12071751 & 16035116 & 154 & 211 & 57 \\
\hline 13146665 & 16035116 & 178 & 202 & 24 \\
\hline 13146665 & 16035116 & 173 & 207 & 34 \\
\hline 13793654 & 17409527 & 190 & 233 & 43 \\
\hline 13793654 & 17409527 & 192 & 232 & 40 \\
\hline 13793654 & 17409527 & 198 & 233 & 35 \\
\hline 13793654 & 17409527 & 198 & 232 & 34 \\
\hline 13793654 & 17409527 & 199 & 231 & 32 \\
\hline 13793654 & 17409527 & 199 & 233 & 34 \\
\hline 13793654 & 17409527 & 201 & 232 & 31 \\
\hline 13793654 & 17409527 & 203 & 235 & 32 \\
\hline 13793654 & 17409527 & 204 & 236 & 32 \\
\hline 13793654 & 17409527 & 194 & 237 & 43 \\
\hline 13793654 & 17409527 & 203 & 239 & 36 \\
\hline 1326452 & 6145288 & 0 & 27 & 27 \\
\hline 1326452 & 6145288 & 1 & 32 & 31 \\
\hline 1326452 & 6145288 & 0 & 32 & 32 \\
\hline 1326452 & 6145288 & 0 & 32 & 32 \\
\hline 1326452 & 6145288 & 3 & 28 & 25 \\
\hline 1326452 & 6145288 & 7 & 23 & 16 \\
\hline 1326452 & 7933354 & 0 & 35 & 35 \\
\hline 1326452 & 6145288 & 0 & 29 & 29 \\
\hline 1326452 & 9233556 & 5 & 43 & 38 \\
\hline 1326452 & 6145288 & 0 & 32 & 32 \\
\hline 1326452 & 6145288 & 3 & 29 & 26 \\
\hline 1326452 & 6145288 & 5 & 28 & 23 \\
\hline 1326452 & 7933354 & 5 & 35 & 30 \\
\hline 1326452 & 6145288 & 2 & 34 & 32 \\
\hline 1326452 & 9233556 & 4 & 61 & 57 \\
\hline 1326452 & 7933354 & 8 & 35 & 27 \\
\hline 4361902 & 6145288 & 10 & 25 & 15 \\
\hline 4361902 & 6145288 & 9 & 24 & 15 \\
\hline 1326452 & 6145288 & 2 & 33 & 31 \\
\hline
\end{tabular}




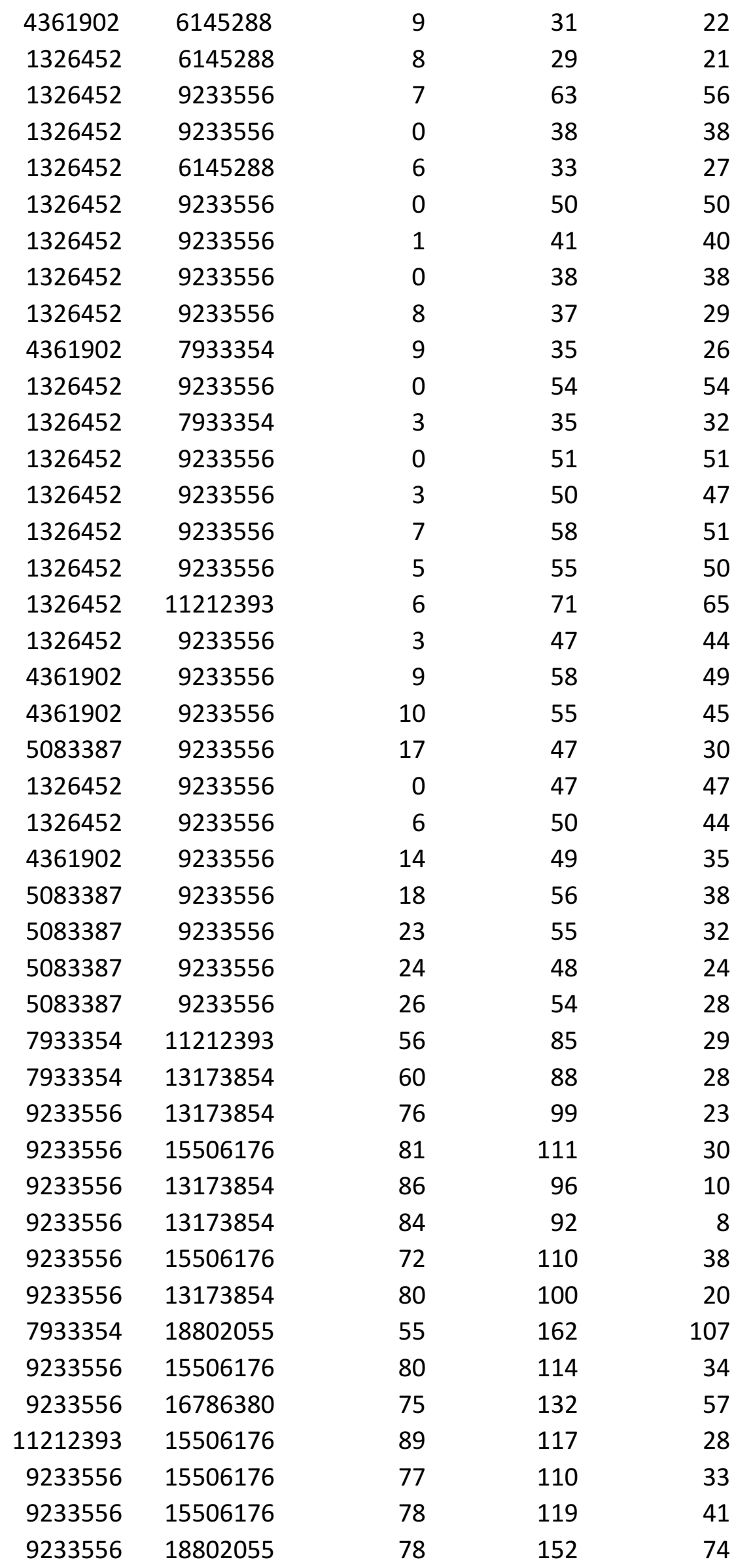




\begin{tabular}{|c|c|c|c|}
\hline 11212393 & 15506176 & 92 & 115 \\
\hline 11212393 & 15506176 & 95 & 107 \\
\hline 7933354 & 18802055 & 62 & 148 \\
\hline 11212393 & 15506176 & 96 & 108 \\
\hline 7933354 & 18802055 & 62 & 155 \\
\hline 11212393 & 15506176 & 90 & 114 \\
\hline 11212393 & 15506176 & 93 & 115 \\
\hline 11212393 & 18802055 & 92 & 155 \\
\hline 9233556 & 15506176 & 85 & 124 \\
\hline 11212393 & 15506176 & 95 & 121 \\
\hline 11212393 & 15506176 & 92 & 114 \\
\hline 11212393 & 15506176 & 93 & 118 \\
\hline 11212393 & 16786380 & 95 & 130 \\
\hline 11212393 & 15506176 & 94 & 122 \\
\hline 11212393 & 15506176 & 98 & 121 \\
\hline 11212393 & 15506176 & 98 & 118 \\
\hline 11212393 & 16786380 & 90 & 129 \\
\hline 11212393 & 18802055 & 92 & 155 \\
\hline 11212393 & 16786380 & 91 & 133 \\
\hline 11212393 & 18802055 & 92 & 153 \\
\hline 11212393 & 16786380 & 90 & 135 \\
\hline 11212393 & 18802055 & 99 & 148 \\
\hline 9233556 & 18802055 & 75 & 157 \\
\hline 11212393 & 16786380 & 98 & 130 \\
\hline 13835372 & 16786380 & 106 & 129 \\
\hline 13835372 & 16786380 & 119 & 134 \\
\hline 13835372 & 16786380 & 113 & 134 \\
\hline 13835372 & 16786380 & 112 & 135 \\
\hline 11212393 & 16786380 & 90 & 136 \\
\hline 13835372 & 16786380 & 113 & 135 \\
\hline 13835372 & 16786380 & 115 & 130 \\
\hline 13835372 & 16786380 & 115 & 134 \\
\hline 13835372 & 16786380 & 117 & 136 \\
\hline 13835372 & 16786380 & 115 & 133 \\
\hline 13835372 & 16786380 & 115 & 131 \\
\hline 13835372 & 16786380 & 111 & 135 \\
\hline 13835372 & 16786380 & 111 & 135 \\
\hline 13835372 & 16786380 & 111 & 135 \\
\hline 13835372 & 16786380 & 108 & 135 \\
\hline 13835372 & 16786380 & 112 & 133 \\
\hline 13835372 & 16786380 & 112 & 132 \\
\hline 13835372 & 18802055 & 113 & 153 \\
\hline 13835372 & 16786380 & 110 & 136 \\
\hline
\end{tabular}




\begin{tabular}{|c|c|c|c|c|}
\hline 11212393 & 18802055 & 98 & 156 & 58 \\
\hline 13835372 & 18802055 & 110 & 155 & 45 \\
\hline 9233556 & 18802055 & 74 & 157 & 83 \\
\hline 13835372 & 16786380 & 118 & 134 & 16 \\
\hline 13835372 & 16786380 & 113 & 135 & 22 \\
\hline 11212393 & 16786380 & 90 & 136 & 46 \\
\hline 11212393 & 18802055 & 97 & 161 & 64 \\
\hline 13835372 & 18802055 & 109 & 156 & 47 \\
\hline 13835372 & 18802055 & 110 & 154 & 44 \\
\hline 13835372 & 18802055 & 115 & 144 & 29 \\
\hline 5083387 & 18802055 & 27 & 155 & 128 \\
\hline 11212393 & 18802055 & 93 & 165 & 72 \\
\hline 13835372 & 18802055 & 108 & 150 & 42 \\
\hline 9233556 & 18802055 & 77 & 156 & 79 \\
\hline 13835372 & 18802055 & 113 & 153 & 40 \\
\hline 13835372 & 18802055 & 115 & 152 & 37 \\
\hline 7933354 & 18802055 & 55 & 159 & 104 \\
\hline 13835372 & 18802055 & 111 & 166 & 55 \\
\hline 13835372 & 18802055 & 113 & 159 & 46 \\
\hline 11212393 & 18802055 & 98 & 160 & 62 \\
\hline 11212393 & 18802055 & 91 & 157 & 66 \\
\hline 13835372 & 18802055 & 111 & 155 & 44 \\
\hline 11212393 & 18802055 & 95 & 175 & 80 \\
\hline 13835372 & 18802055 & 113 & 157 & 44 \\
\hline 11212393 & 18802055 & 92 & 157 & 65 \\
\hline 11212393 & 18802055 & 97 & 158 & 61 \\
\hline 9233556 & 18802055 & 78 & 159 & 81 \\
\hline 11212393 & 18802055 & 99 & 153 & 54 \\
\hline 15506176 & 18802055 & 126 & 166 & 40 \\
\hline 13835372 & 18802055 & 120 & 150 & 30 \\
\hline 13835372 & 18802055 & 115 & 153 & 38 \\
\hline 11212393 & 18802055 & 103 & 167 & 64 \\
\hline 15506176 & 18802055 & 127 & 159 & 32 \\
\hline 15506176 & 18802055 & 129 & 156 & 27 \\
\hline 15506176 & 18802055 & 128 & 156 & 28 \\
\hline 11212393 & 18802055 & 99 & 167 & 68 \\
\hline 13835372 & 18802055 & 119 & 163 & 44 \\
\hline 13835372 & 18802055 & 119 & 151 & 32 \\
\hline 15506176 & 18802055 & 127 & 160 & 33 \\
\hline 15506176 & 18802055 & 127 & 160 & 33 \\
\hline 15506176 & 18802055 & 128 & 152 & 24 \\
\hline 15506176 & 18802055 & 129 & 153 & 24 \\
\hline 15506176 & 18802055 & 130 & 154 & 24 \\
\hline
\end{tabular}




\begin{tabular}{|c|c|c|c|c|}
\hline 15506176 & 18802055 & 128 & 158 & 30 \\
\hline 13835372 & 18802055 & 116 & 154 & 38 \\
\hline 13835372 & 18802055 & 120 & 157 & 37 \\
\hline 13835372 & 18802055 & 124 & 154 & 30 \\
\hline 13835372 & 18802055 & 119 & 157 & 38 \\
\hline 13835372 & 18802055 & 119 & 166 & 47 \\
\hline 15506176 & 18802055 & 126 & 159 & 33 \\
\hline 15506176 & 18802055 & 130 & 156 & 26 \\
\hline 13835372 & 18802055 & 124 & 153 & 29 \\
\hline 15506176 & 18802055 & 129 & 152 & 23 \\
\hline 15506176 & 18802055 & 129 & 157 & 28 \\
\hline 15506176 & 18802055 & 128 & 152 & 24 \\
\hline 15506176 & 18802055 & 131 & 156 & 25 \\
\hline 15506176 & 18802055 & 132 & 160 & 28 \\
\hline 15506176 & 18802055 & 131 & 153 & 22 \\
\hline 15506176 & 18802055 & 131 & 153 & 22 \\
\hline 15506176 & 18802055 & 129 & 152 & 23 \\
\hline 15506176 & 18802055 & 132 & 159 & 27 \\
\hline 15506176 & 18802055 & 131 & 149 & 18 \\
\hline 15506176 & 18802055 & 127 & 159 & 32 \\
\hline 15506176 & 18802055 & 129 & 154 & 25 \\
\hline 15506176 & 18802055 & 127 & 155 & 28 \\
\hline 15506176 & 18802055 & 126 & 160 & 34 \\
\hline 15506176 & 18802055 & 130 & 162 & 32 \\
\hline 15506176 & 18802055 & 130 & 152 & 22 \\
\hline 15506176 & 18802055 & 128 & 152 & 24 \\
\hline 15506176 & 18802055 & 126 & 157 & 31 \\
\hline 15506176 & 18802055 & 128 & 151 & 23 \\
\hline 15506176 & 18802055 & 133 & 149 & 16 \\
\hline 15506176 & 18802055 & 131 & 161 & 30 \\
\hline 15506176 & 18802055 & 133 & 156 & 23 \\
\hline 15506176 & 18802055 & 132 & 158 & 26 \\
\hline 15506176 & 18802055 & 129 & 158 & 29 \\
\hline 15506176 & 18802055 & 130 & 157 & 27 \\
\hline 15506176 & 18802055 & 133 & 162 & 29 \\
\hline 15506176 & 18802055 & 133 & 156 & 23 \\
\hline 15506176 & 18802055 & 132 & 162 & 30 \\
\hline 15506176 & 18802055 & 131 & 159 & 28 \\
\hline 15506176 & 18802055 & 131 & 156 & 25 \\
\hline 15506176 & 18802055 & 131 & 153 & 22 \\
\hline 15506176 & 18802055 & 131 & 156 & 25 \\
\hline 15506176 & 18802055 & 129 & 162 & 33 \\
\hline 11212393 & 18802055 & 95 & 166 & 71 \\
\hline
\end{tabular}




\begin{tabular}{|c|c|c|c|c|}
\hline 15506176 & 18802055 & 128 & 168 & 40 \\
\hline 15506176 & 18802055 & 130 & 163 & 33 \\
\hline 16786380 & 18802055 & 177 & 189 & 12 \\
\hline 2036593 & 2766168 & 0 & 21 & 21 \\
\hline 2036593 & 2766168 & 0 & 9 & 9 \\
\hline 2036593 & 2766168 & 0 & 18 & 18 \\
\hline 2036593 & 2766168 & 0 & 9 & 9 \\
\hline 2036593 & 2766168 & 0 & 9 & 9 \\
\hline 2036593 & 2766168 & 0 & 9 & 9 \\
\hline 2036593 & 2766168 & 0 & 10 & 10 \\
\hline 2036593 & 2766168 & 0 & 10 & 10 \\
\hline 2176134 & 3076405 & 10 & 32 & 22 \\
\hline 2176134 & 3076405 & 17 & 32 & 15 \\
\hline 2176134 & 3076405 & 18 & 31 & 13 \\
\hline 2176134 & 3076405 & 21 & 31 & 10 \\
\hline 2176134 & 3076405 & 18 & 31 & 13 \\
\hline 2176134 & 3076405 & 22 & 31 & 9 \\
\hline 2176134 & 5538321 & 12 & 42 & 30 \\
\hline 2036593 & 17812125 & 0 & 242 & 242 \\
\hline 2176134 & 5538321 & 13 & 39 & 26 \\
\hline 2176134 & 5538321 & 14 & 46 & 32 \\
\hline 2176134 & 5538321 & 12 & 51 & 39 \\
\hline 2176134 & 5538321 & 12 & 47 & 35 \\
\hline 2176134 & 5538321 & 20 & 46 & 26 \\
\hline 2176134 & 5538321 & 12 & 48 & 36 \\
\hline 2176134 & 5538321 & 13 & 55 & 42 \\
\hline 2176134 & 5538321 & 12 & 55 & 43 \\
\hline 2176134 & 5538321 & 13 & 48 & 35 \\
\hline 2766168 & 5538321 & 30 & 46 & 16 \\
\hline 2176134 & 6304262 & 17 & 59 & 42 \\
\hline 2766168 & 5538321 & 30 & 53 & 23 \\
\hline 2766168 & 5538321 & 29 & 55 & 26 \\
\hline 2176134 & 5538321 & 16 & 57 & 41 \\
\hline 2176134 & 6304262 & 16 & 67 & 51 \\
\hline 2766168 & 6304262 & 27 & 68 & 41 \\
\hline 2766168 & 6304262 & 34 & 72 & 38 \\
\hline 3076405 & 6304262 & 44 & 66 & 22 \\
\hline 3076405 & 6304262 & 36 & 67 & 31 \\
\hline 3076405 & 6304262 & 49 & 66 & 17 \\
\hline 3076405 & 6304262 & 48 & 66 & 18 \\
\hline 3076405 & 6304262 & 47 & 66 & 19 \\
\hline 3076405 & 6304262 & 48 & 67 & 19 \\
\hline 3076405 & 6304262 & 47 & 67 & 20 \\
\hline
\end{tabular}




\begin{tabular}{|c|c|c|c|c|}
\hline 3076405 & 6304262 & 53 & 61 & 8 \\
\hline 3076405 & 6304262 & 43 & 74 & 31 \\
\hline 3076405 & 6304262 & 48 & 69 & 21 \\
\hline 3076405 & 6304262 & 47 & 70 & 23 \\
\hline 3076405 & 6304262 & 51 & 71 & 20 \\
\hline 3076405 & 6304262 & 53 & 70 & 17 \\
\hline 3076405 & 6304262 & 53 & 71 & 18 \\
\hline 3076405 & 6304262 & 51 & 69 & 18 \\
\hline 8569364 & 14150069 & 131 & 179 & 48 \\
\hline 8569364 & 14150069 & 139 & 174 & 35 \\
\hline 8569364 & 14150069 & 128 & 184 & 56 \\
\hline 8569364 & 12167140 & 136 & 164 & 28 \\
\hline 8569364 & 16678176 & 137 & 210 & 73 \\
\hline 8569364 & 12167140 & 143 & 160 & 17 \\
\hline 8569364 & 12167140 & 138 & 162 & 24 \\
\hline 8569364 & 14150069 & 130 & 200 & 70 \\
\hline 10740553 & 14150069 & 151 & 183 & 32 \\
\hline 10740553 & 14150069 & 152 & 186 & 34 \\
\hline 8569364 & 14150069 & 136 & 180 & 44 \\
\hline 11125456 & 14150069 & 152 & 178 & 26 \\
\hline 8569364 & 14150069 & 136 & 184 & 48 \\
\hline 10740553 & 14150069 & 152 & 180 & 28 \\
\hline 11125456 & 14150069 & 162 & 181 & 19 \\
\hline 11125456 & 14150069 & 157 & 185 & 28 \\
\hline 10740553 & 14150069 & 151 & 182 & 31 \\
\hline 11125456 & 14150069 & 152 & 188 & 36 \\
\hline 11125456 & 14150069 & 159 & 191 & 32 \\
\hline 12167140 & 16678176 & 178 & 221 & 43 \\
\hline 12167140 & 16678176 & 190 & 218 & 28 \\
\hline 14150069 & 17812125 & 204 & 242 & 38 \\
\hline 14150069 & 17812125 & 223 & 241 & 18 \\
\hline 14150069 & 17812125 & 224 & 239 & 15 \\
\hline 14150069 & 17812125 & 220 & 242 & 22 \\
\hline 14150069 & 17812125 & 220 & 241 & 21 \\
\hline 14150069 & 17812125 & 222 & 242 & 20 \\
\hline 14150069 & 17812125 & 228 & 242 & 14 \\
\hline 14150069 & 17812125 & 219 & 240 & 21 \\
\hline 14150069 & 17812125 & 215 & 241 & 26 \\
\hline 16678176 & 17812125 & 234 & 242 & 8 \\
\hline 14150069 & 17812125 & 213 & 242 & 29 \\
\hline 14150069 & 17812125 & 215 & 242 & 27 \\
\hline 16678176 & 17812125 & 233 & 242 & 9 \\
\hline 14150069 & 17812125 & 217 & 242 & 25 \\
\hline
\end{tabular}




\begin{tabular}{|c|c|c|c|c|}
\hline 14150069 & 17812125 & 206 & 242 & 36 \\
\hline 14150069 & 17812125 & 211 & 242 & 31 \\
\hline 14150069 & 17812125 & 213 & 242 & 29 \\
\hline 14150069 & 17812125 & 226 & 242 & 16 \\
\hline 14150069 & 17812125 & 221 & 242 & 21 \\
\hline 16678176 & 17812125 & 234 & 242 & 8 \\
\hline 16678176 & 17812125 & 232 & 242 & 10 \\
\hline 16678176 & 17812125 & 234 & 242 & 8 \\
\hline 16678176 & 17812125 & 233 & 242 & 9 \\
\hline 1624738 & 3424796 & 0 & 16 & 16 \\
\hline 1624738 & 3424796 & 0 & 17 & 17 \\
\hline 1624738 & 3424796 & 0 & 17 & 17 \\
\hline 1624738 & 3424796 & 0 & 16 & 16 \\
\hline 1624738 & 3424796 & 0 & 10 & 10 \\
\hline 1624738 & 3424796 & 0 & 16 & 16 \\
\hline 1624738 & 3424796 & 0 & 17 & 17 \\
\hline 1624738 & 3424796 & 0 & 17 & 17 \\
\hline 1624738 & 3424796 & 0 & 18 & 18 \\
\hline 1624738 & 3424796 & 0 & 10 & 10 \\
\hline 1624738 & 3424796 & 0 & 8 & 8 \\
\hline 1624738 & 3424796 & 0 & 17 & 17 \\
\hline 1624738 & 3424796 & 0 & 14 & 14 \\
\hline 1624738 & 3424796 & 0 & 17 & 17 \\
\hline 1624738 & 3424796 & 0 & 20 & 20 \\
\hline 1624738 & 3424796 & 0 & 19 & 19 \\
\hline 1624738 & 3424796 & 0 & 20 & 20 \\
\hline 1624738 & 3424796 & 0 & 19 & 19 \\
\hline 1624738 & 3424796 & 0 & 19 & 19 \\
\hline 1624738 & 3424796 & 0 & 22 & 22 \\
\hline 1624738 & 3424796 & 0 & 20 & 20 \\
\hline 1624738 & 3424796 & 0 & 18 & 18 \\
\hline 1624738 & 3424796 & 0 & 19 & 19 \\
\hline 1624738 & 3424796 & 0 & 22 & 22 \\
\hline 1624738 & 3424796 & 0 & 16 & 16 \\
\hline 1624738 & 3424796 & 0 & 18 & 18 \\
\hline 1624738 & 3424796 & 0 & 18 & 18 \\
\hline 1624738 & 3424796 & 0 & 29 & 29 \\
\hline 1624738 & 3424796 & 0 & 19 & 19 \\
\hline 1624738 & 3424796 & 0 & 24 & 24 \\
\hline 3424796 & 10742939 & 40 & 109 & 69 \\
\hline 3424796 & 6538686 & 58 & 85 & 27 \\
\hline 3424796 & 9849448 & 56 & 104 & 48 \\
\hline 3424796 & 6538686 & 53 & 87 & 34 \\
\hline
\end{tabular}




\begin{tabular}{|c|c|c|c|c|}
\hline 3424796 & 11735757 & 53 & 114 & 61 \\
\hline 3424796 & 11735757 & 54 & 116 & 62 \\
\hline 3424796 & 10742939 & 55 & 110 & 55 \\
\hline 3424796 & 9546962 & 47 & 100 & 53 \\
\hline 3424796 & 9546962 & 75 & 96 & 21 \\
\hline 5102045 & 11735757 & 80 & 128 & 48 \\
\hline 5102045 & 10742939 & 81 & 107 & 26 \\
\hline 5102045 & 10742939 & 80 & 108 & 28 \\
\hline 3424796 & 11735757 & 54 & 131 & 77 \\
\hline 5102045 & 10742939 & 81 & 108 & 27 \\
\hline 3424796 & 10742939 & 59 & 107 & 48 \\
\hline 3424796 & 11735757 & 57 & 121 & 64 \\
\hline 5102045 & 10742939 & 82 & 110 & 28 \\
\hline 5102045 & 10742939 & 80 & 109 & 29 \\
\hline 5102045 & 10742939 & 82 & 105 & 23 \\
\hline 5102045 & 10742939 & 82 & 108 & 26 \\
\hline 5102045 & 13610537 & 81 & 153 & 72 \\
\hline 3424796 & 11735757 & 57 & 124 & 67 \\
\hline 3424796 & 11735757 & 49 & 132 & 83 \\
\hline 5102045 & 13610537 & 82 & 154 & 72 \\
\hline 5102045 & 10742939 & 80 & 110 & 30 \\
\hline 5102045 & 11735757 & 81 & 124 & 43 \\
\hline 5102045 & 11735757 & 84 & 130 & 46 \\
\hline 5102045 & 10742939 & 83 & 110 & 27 \\
\hline 5102045 & 11735757 & 83 & 113 & 30 \\
\hline 5102045 & 13610537 & 85 & 139 & 54 \\
\hline 6538686 & 11735757 & 93 & 128 & 35 \\
\hline 5102045 & 11735757 & 81 & 121 & 40 \\
\hline 6538686 & 11735757 & 102 & 113 & 11 \\
\hline 6538686 & 11735757 & 88 & 123 & 35 \\
\hline 6538686 & 11735757 & 100 & 119 & 19 \\
\hline 5102045 & 11735757 & 81 & 120 & 39 \\
\hline 6538686 & 11735757 & 101 & 117 & 16 \\
\hline 6538686 & 11735757 & 98 & 115 & 17 \\
\hline 5102045 & 11735757 & 87 & 128 & 41 \\
\hline 3424796 & 13610537 & 37 & 152 & 115 \\
\hline 5102045 & 13610537 & 84 & 165 & 81 \\
\hline 6538686 & 15507714 & 95 & 171 & 76 \\
\hline 10742939 & 15507714 & 113 & 172 & 59 \\
\hline 6538686 & 15507714 & 90 & 171 & 81 \\
\hline 10742939 & 13610537 & 114 & 160 & 46 \\
\hline 10742939 & 13610537 & 115 & 154 & 39 \\
\hline 10742939 & 13610537 & 115 & 166 & 51 \\
\hline
\end{tabular}




\begin{tabular}{|c|c|c|c|}
\hline 10742939 & 13610537 & 120 & 161 \\
\hline 10742939 & 13610537 & 115 & 153 \\
\hline 10742939 & 13610537 & 119 & 160 \\
\hline 10742939 & 13610537 & 119 & 152 \\
\hline 10742939 & 13610537 & 121 & 155 \\
\hline 10742939 & 13610537 & 118 & 157 \\
\hline 10742939 & 13610537 & 126 & 160 \\
\hline 10742939 & 13610537 & 122 & 164 \\
\hline 10742939 & 13610537 & 118 & 157 \\
\hline 10742939 & 15507714 & 123 & 173 \\
\hline 10742939 & 13610537 & 120 & 162 \\
\hline 10742939 & 15507714 & 122 & 178 \\
\hline 10742939 & 13610537 & 121 & 159 \\
\hline 10742939 & 13610537 & 126 & 161 \\
\hline 10742939 & 13610537 & 123 & 162 \\
\hline 10742939 & 13610537 & 119 & 154 \\
\hline 10742939 & 13610537 & 121 & 152 \\
\hline 10742939 & 13610537 & 121 & 150 \\
\hline 10742939 & 13610537 & 123 & 154 \\
\hline 10742939 & 13610537 & 125 & 159 \\
\hline 10742939 & 15507714 & 122 & 176 \\
\hline 10742939 & 13610537 & 123 & 163 \\
\hline 10742939 & 13610537 & 127 & 162 \\
\hline 10742939 & 15507714 & 119 & 176 \\
\hline 10742939 & 13610537 & 119 & 164 \\
\hline 10742939 & 13610537 & 124 & 159 \\
\hline 10742939 & 13610537 & 121 & 163 \\
\hline 10742939 & 15507714 & 119 & 172 \\
\hline 10742939 & 15507714 & 120 & 176 \\
\hline 10742939 & 15507714 & 118 & 178 \\
\hline 11735757 & 15507714 & 141 & 180 \\
\hline 10742939 & 15507714 & 120 & 184 \\
\hline 11735757 & 15507714 & 151 & 174 \\
\hline 11735757 & 15507714 & 154 & 192 \\
\hline 11735757 & 15507714 & 165 & 173 \\
\hline 11735757 & 15507714 & 155 & 172 \\
\hline 11735757 & 15507714 & 156 & 185 \\
\hline 11735757 & 15507714 & 157 & 187 \\
\hline 11735757 & 15507714 & 154 & 194 \\
\hline 11735757 & 15507714 & 162 & 189 \\
\hline 11735757 & 15507714 & 150 & 191 \\
\hline 11735757 & 16667620 & 163 & 199 \\
\hline 11735757 & 18032473 & 152 & 213 \\
\hline
\end{tabular}




\begin{tabular}{|c|c|c|c|}
\hline 11735757 & 18032473 & 161 & 213 \\
\hline 13610537 & 18032473 & 170 & 212 \\
\hline 13610537 & 18032473 & 182 & 208 \\
\hline 13610537 & 18032473 & 177 & 210 \\
\hline 13610537 & 18032473 & 179 & 213 \\
\hline 11735757 & 18032473 & 161 & 212 \\
\hline 15507714 & 18032473 & 199 & 209 \\
\hline 15507714 & 18032473 & 200 & 208 \\
\hline 13610537 & 18032473 & 169 & 211 \\
\hline 15507714 & 16667620 & 203 & 205 \\
\hline 15507714 & 18032473 & 202 & 210 \\
\hline 15507714 & 18032473 & 200 & 211 \\
\hline 1150805 & 3086769 & 0 & 16 \\
\hline 1150805 & 3086769 & 0 & 13 \\
\hline 1150805 & 3497335 & 0 & 22 \\
\hline 1150805 & 3985497 & 0 & 34 \\
\hline 2047947 & 3497335 & 8 & 24 \\
\hline 2047947 & 3086769 & 11 & 22 \\
\hline 2047947 & 3086769 & 12 & 22 \\
\hline 2047947 & 3086769 & 13 & 22 \\
\hline 2047947 & 3497335 & 15 & 22 \\
\hline 2047947 & 3985497 & 12 & 34 \\
\hline 1150805 & 5487660 & 3 & 78 \\
\hline 2047947 & 5487660 & 6 & 58 \\
\hline 2047947 & 5487660 & 12 & 49 \\
\hline 2047947 & 3985497 & 14 & 31 \\
\hline 1150805 & 3985497 & 0 & 35 \\
\hline 2047947 & 3985497 & 13 & 31 \\
\hline 2047947 & 3985497 & 13 & 33 \\
\hline 3086769 & 3985497 & 22 & 33 \\
\hline 1150805 & 3985497 & 0 & 32 \\
\hline 2047947 & 5487660 & 12 & 46 \\
\hline 2047947 & 3985497 & 21 & 34 \\
\hline 2047947 & 3985497 & 17 & 33 \\
\hline 2047947 & 5487660 & 11 & 51 \\
\hline 3086769 & 3985497 & 23 & 29 \\
\hline 3086769 & 3985497 & 24 & 30 \\
\hline 3086769 & 3985497 & 23 & 31 \\
\hline 2047947 & 5487660 & 8 & 50 \\
\hline 1150805 & 5487660 & 0 & 60 \\
\hline 2047947 & 5487660 & 16 & 51 \\
\hline 1150805 & 5487660 & 0 & 65 \\
\hline 3086769 & 5487660 & 23 & 56 \\
\hline
\end{tabular}




\begin{tabular}{|c|c|c|c|}
\hline 3086769 & 5487660 & 24 & 56 \\
\hline 1150805 & 5487660 & 0 & 67 \\
\hline 2047947 & 5487660 & 5 & 68 \\
\hline 3497335 & 5487660 & 30 & 45 \\
\hline 3497335 & 5487660 & 26 & 51 \\
\hline 3497335 & 5487660 & 30 & 56 \\
\hline 1150805 & 5487660 & 0 & 68 \\
\hline 3497335 & 5487660 & 32 & 46 \\
\hline 3497335 & 5487660 & 32 & 46 \\
\hline 3497335 & 5487660 & 30 & 74 \\
\hline 3497335 & 5487660 & 32 & 70 \\
\hline 3497335 & 8901874 & 36 & 108 \\
\hline 3985497 & 8901874 & 42 & 115 \\
\hline 3985497 & 8901874 & 58 & 109 \\
\hline 3985497 & 6092798 & 73 & 99 \\
\hline 3985497 & 8901874 & 70 & 123 \\
\hline 3985497 & 8901874 & 73 & 118 \\
\hline 3985497 & 10348340 & 73 & 165 \\
\hline 6092798 & 10348340 & 110 & 154 \\
\hline 6092798 & 10348340 & 112 & 166 \\
\hline 6092798 & 10348340 & 108 & 163 \\
\hline 6092798 & 10348340 & 118 & 165 \\
\hline 6092798 & 10348340 & 119 & 165 \\
\hline 6092798 & 10348340 & 117 & 164 \\
\hline 6092798 & 10348340 & 121 & 160 \\
\hline 6092798 & 10348340 & 120 & 166 \\
\hline 6092798 & 10348340 & 118 & 167 \\
\hline 6092798 & 10348340 & 122 & 161 \\
\hline 6092798 & 10348340 & 118 & 166 \\
\hline 6092798 & 10348340 & 120 & 163 \\
\hline 6092798 & 10348340 & 114 & 174 \\
\hline 6092798 & 10348340 & 122 & 167 \\
\hline 6092798 & 10348340 & 118 & 178 \\
\hline 6092798 & 10348340 & 119 & 179 \\
\hline 6092798 & 10348340 & 126 & 171 \\
\hline 6092798 & 10348340 & 117 & 194 \\
\hline 8901874 & 15000631 & 166 & 261 \\
\hline 12066832 & 15000631 & 202 & 260 \\
\hline 12066832 & 15000631 & 203 & 266 \\
\hline 12066832 & 15000631 & 200 & 259 \\
\hline 12066832 & 15000631 & 210 & 262 \\
\hline 12066832 & 15000631 & 215 & 253 \\
\hline 12066832 & 15000631 & 219 & 259 \\
\hline
\end{tabular}




\begin{tabular}{|c|c|c|c|c|}
\hline 12066832 & 15000631 & 217 & 266 & 49 \\
\hline 12066832 & 15000631 & 225 & 261 & 36 \\
\hline 12066832 & 15000631 & 226 & 266 & 40 \\
\hline 13628710 & 15000631 & 247 & 266 & 19 \\
\hline 12066832 & 15000631 & 222 & 266 & 44 \\
\hline 13628710 & 15000631 & 246 & 266 & 20 \\
\hline 13628710 & 15000631 & 243 & 266 & 23 \\
\hline 12066832 & 15000631 & 215 & 266 & 51 \\
\hline 13628710 & 15000631 & 243 & 266 & 23 \\
\hline 13628710 & 15000631 & 245 & 266 & 21 \\
\hline 13628710 & 15000631 & 247 & 266 & 19 \\
\hline 13628710 & 15000631 & 247 & 266 & 19 \\
\hline 13628710 & 15000631 & 245 & 266 & 21 \\
\hline 13628710 & 15000631 & 248 & 266 & 18 \\
\hline 13628710 & 15000631 & 250 & 266 & 16 \\
\hline 13628710 & 15000631 & 250 & 266 & 16 \\
\hline 13628710 & 15000631 & 249 & 266 & 17 \\
\hline 13628710 & 15000631 & 247 & 266 & 19 \\
\hline 12066832 & 15000631 & 237 & 266 & 29 \\
\hline 12066832 & 15000631 & 230 & 266 & 36 \\
\hline 13628710 & 15000631 & 244 & 266 & 22 \\
\hline 13628710 & 15000631 & 251 & 266 & 15 \\
\hline 13628710 & 15000631 & 250 & 266 & 16 \\
\hline 13628710 & 15000631 & 252 & 266 & 14 \\
\hline 13628710 & 15000631 & 248 & 266 & 18 \\
\hline 13628710 & 15000631 & 250 & 266 & 16 \\
\hline 13628710 & 15000631 & 253 & 266 & 13 \\
\hline 13628710 & 15000631 & 251 & 266 & 15 \\
\hline 13628710 & 15000631 & 250 & 266 & 16 \\
\hline 13628710 & 15000631 & 252 & 266 & 14 \\
\hline 13628710 & 15000631 & 251 & 266 & 15 \\
\hline 13628710 & 15000631 & 251 & 266 & 15 \\
\hline 13628710 & 15000631 & 253 & 266 & 13 \\
\hline 13628710 & 15000631 & 249 & 266 & 17 \\
\hline 13628710 & 15000631 & 252 & 266 & 14 \\
\hline 13628710 & 15000631 & 252 & 266 & 14 \\
\hline 13628710 & 15000631 & 246 & 266 & 20 \\
\hline 13628710 & 15000631 & 244 & 266 & 22 \\
\hline 13628710 & 15000631 & 252 & 266 & 14 \\
\hline 13628710 & 15000631 & 248 & 266 & 18 \\
\hline 13628710 & 15000631 & 255 & 266 & 11 \\
\hline 13628710 & 15000631 & 251 & 266 & 15 \\
\hline 13628710 & 15000631 & 248 & 266 & 18 \\
\hline
\end{tabular}




\begin{tabular}{|c|c|c|c|}
\hline 13628710 & 15000631 & 251 & 266 \\
\hline 13628710 & 15000631 & 258 & 266 \\
\hline 13628710 & 15000631 & 257 & 266 \\
\hline 13628710 & 15000631 & 252 & 266 \\
\hline 13628710 & 15000631 & 252 & 266 \\
\hline 13628710 & 15000631 & 256 & 266 \\
\hline 13628710 & 15000631 & 254 & 266 \\
\hline 13628710 & 15000631 & 253 & 266 \\
\hline 13628710 & 15000631 & 255 & 266 \\
\hline 13628710 & 15000631 & 255 & 266 \\
\hline 13628710 & 15000631 & 254 & 266 \\
\hline 13628710 & 15000631 & 255 & 266 \\
\hline 13628710 & 15000631 & 257 & 266 \\
\hline 13628710 & 15000631 & 256 & 266 \\
\hline 13628710 & 15000631 & 253 & 266 \\
\hline 403059 & 1872721 & 0 & 7 \\
\hline 403059 & 1872721 & 0 & 7 \\
\hline 403059 & 1872721 & 0 & 5 \\
\hline 403059 & 1872721 & 0 & 8 \\
\hline 403059 & 4308352 & 0 & 14 \\
\hline 403059 & 1872721 & 0 & 5 \\
\hline 403059 & 1872721 & 0 & 7 \\
\hline 403059 & 1872721 & 0 & 9 \\
\hline 403059 & 1872721 & 0 & 6 \\
\hline 403059 & 4308352 & 0 & 15 \\
\hline 403059 & 4308352 & 0 & 17 \\
\hline 403059 & 4308352 & 0 & 15 \\
\hline 403059 & 6258358 & 0 & 49 \\
\hline 403059 & 6258358 & 0 & 61 \\
\hline 403059 & 6258358 & 0 & 37 \\
\hline 1697236 & 6258358 & 5 & 40 \\
\hline 403059 & 6258358 & 0 & 48 \\
\hline 2230582 & 6258358 & 13 & 44 \\
\hline 1872721 & 6258358 & 11 & 48 \\
\hline 403059 & 6258358 & 0 & 58 \\
\hline 4308352 & 10707964 & 64 & 130 \\
\hline 4308352 & 10707964 & 64 & 130 \\
\hline 4308352 & 10707964 & 65 & 134 \\
\hline 4308352 & 10707964 & 64 & 131 \\
\hline 4308352 & 10707964 & 62 & 132 \\
\hline 4308352 & 10707964 & 62 & 126 \\
\hline 4308352 & 10707964 & 66 & 159 \\
\hline 4308352 & 10707964 & 65 & 130 \\
\hline
\end{tabular}




\begin{tabular}{|c|c|c|c|}
\hline 4308352 & 10707964 & 66 & 131 \\
\hline 4308352 & 10707964 & 64 & 135 \\
\hline 4308352 & 10707964 & 64 & 130 \\
\hline 4308352 & 10707964 & 64 & 127 \\
\hline 4308352 & 10707964 & 64 & 123 \\
\hline 4308352 & 10707964 & 65 & 131 \\
\hline 4308352 & 10707964 & 61 & 123 \\
\hline 4308352 & 10707964 & 68 & 125 \\
\hline 4308352 & 10707964 & 65 & 133 \\
\hline 4308352 & 10707964 & 63 & 135 \\
\hline 4308352 & 10707964 & 66 & 121 \\
\hline 4308352 & 10707964 & 64 & 126 \\
\hline 4308352 & 10707964 & 66 & 134 \\
\hline 4308352 & 10707964 & 67 & 132 \\
\hline 4308352 & 10707964 & 66 & 129 \\
\hline 4308352 & 10707964 & 67 & 134 \\
\hline 4308352 & 10707964 & 64 & 125 \\
\hline 4308352 & 10707964 & 67 & 125 \\
\hline 4308352 & 10707964 & 65 & 129 \\
\hline 4308352 & 10707964 & 67 & 128 \\
\hline 4308352 & 10707964 & 66 & 134 \\
\hline 4308352 & 10707964 & 66 & 139 \\
\hline 4308352 & 10707964 & 64 & 121 \\
\hline 4308352 & 10707964 & 65 & 130 \\
\hline 4308352 & 10707964 & 66 & 134 \\
\hline 4308352 & 10707964 & 68 & 126 \\
\hline 4308352 & 10707964 & 69 & 132 \\
\hline 4308352 & 10707964 & 68 & 127 \\
\hline 4308352 & 10707964 & 68 & 132 \\
\hline 4308352 & 10707964 & 70 & 131 \\
\hline 4308352 & 10707964 & 71 & 124 \\
\hline 4308352 & 10707964 & 68 & 119 \\
\hline 4308352 & 10707964 & 67 & 138 \\
\hline 4308352 & 10707964 & 70 & 128 \\
\hline 4308352 & 10707964 & 65 & 127 \\
\hline 4308352 & 10707964 & 71 & 129 \\
\hline 4308352 & 10707964 & 69 & 125 \\
\hline 4308352 & 10707964 & 71 & 125 \\
\hline 4308352 & 10707964 & 68 & 128 \\
\hline 4308352 & 10707964 & 69 & 125 \\
\hline 4308352 & 10707964 & 66 & 132 \\
\hline 4308352 & 10707964 & 67 & 133 \\
\hline 4308352 & 10707964 & 68 & 122 \\
\hline
\end{tabular}




\begin{tabular}{|c|c|c|c|}
\hline 4308352 & 10707964 & 66 & 129 \\
\hline 4308352 & 10707964 & 67 & 127 \\
\hline 4308352 & 10707964 & 67 & 127 \\
\hline 4308352 & 10707964 & 68 & 127 \\
\hline 4308352 & 10707964 & 69 & 129 \\
\hline 6258358 & 10707964 & 72 & 127 \\
\hline 4308352 & 10707964 & 69 & 128 \\
\hline 4308352 & 10707964 & 67 & 123 \\
\hline 4308352 & 10707964 & 68 & 131 \\
\hline 4308352 & 10707964 & 67 & 127 \\
\hline 4308352 & 10707964 & 66 & 130 \\
\hline 4308352 & 10707964 & 69 & 128 \\
\hline 4308352 & 10707964 & 68 & 132 \\
\hline 4308352 & 10707964 & 70 & 134 \\
\hline 4308352 & 10707964 & 66 & 130 \\
\hline 4308352 & 10707964 & 69 & 131 \\
\hline 8486347 & 10707964 & 72 & 132 \\
\hline 6258358 & 10707964 & 72 & 123 \\
\hline 4308352 & 10707964 & 69 & 126 \\
\hline 8486347 & 10707964 & 73 & 127 \\
\hline 8486347 & 10707964 & 72 & 123 \\
\hline 4308352 & 10707964 & 71 & 128 \\
\hline 4308352 & 10707964 & 70 & 126 \\
\hline 6258358 & 10707964 & 72 & 128 \\
\hline 4308352 & 10707964 & 71 & 122 \\
\hline 4308352 & 10707964 & 70 & 126 \\
\hline 4308352 & 10707964 & 71 & 133 \\
\hline 4308352 & 10707964 & 69 & 130 \\
\hline 4308352 & 10707964 & 71 & 130 \\
\hline 6258358 & 10707964 & 72 & 127 \\
\hline 4308352 & 10707964 & 71 & 134 \\
\hline 8486347 & 10707964 & 72 & 122 \\
\hline 4308352 & 10707964 & 70 & 129 \\
\hline 4308352 & 10707964 & 71 & 128 \\
\hline 6258358 & 10707964 & 72 & 117 \\
\hline 4308352 & 10707964 & 71 & 121 \\
\hline 4308352 & 10707964 & 69 & 133 \\
\hline 4308352 & 10707964 & 70 & 127 \\
\hline 4308352 & 10707964 & 69 & 127 \\
\hline 8486347 & 10707964 & 72 & 121 \\
\hline 4308352 & 10707964 & 69 & 123 \\
\hline 4308352 & 10707964 & 68 & 129 \\
\hline 4308352 & 10707964 & 71 & 129 \\
\hline
\end{tabular}




\begin{tabular}{|c|c|c|c|c|}
\hline 4308352 & 10707964 & 65 & 144 & 79 \\
\hline 8486347 & 10707964 & 72 & 121 & 49 \\
\hline 8486347 & 10707964 & 72 & 129 & 57 \\
\hline 8486347 & 10707964 & 74 & 123 & 49 \\
\hline 8486347 & 10707964 & 74 & 123 & 49 \\
\hline 8486347 & 10707964 & 73 & 125 & 52 \\
\hline 6258358 & 10707964 & 72 & 120 & 48 \\
\hline 8486347 & 10707964 & 73 & 128 & 55 \\
\hline 6258358 & 10707964 & 72 & 123 & 51 \\
\hline 8486347 & 10707964 & 72 & 123 & 51 \\
\hline 8486347 & 10707964 & 73 & 125 & 52 \\
\hline 8486347 & 10707964 & 75 & 116 & 41 \\
\hline 8486347 & 10707964 & 73 & 127 & 54 \\
\hline 4308352 & 10707964 & 69 & 122 & 53 \\
\hline 8486347 & 10707964 & 72 & 122 & 50 \\
\hline 6258358 & 10707964 & 72 & 131 & 59 \\
\hline 8486347 & 10707964 & 72 & 117 & 45 \\
\hline 8486347 & 10707964 & 72 & 130 & 58 \\
\hline 8486347 & 10707964 & 73 & 128 & 55 \\
\hline 4308352 & 10707964 & 71 & 128 & 57 \\
\hline 8486347 & 10707964 & 72 & 126 & 54 \\
\hline 8486347 & 10707964 & 72 & 121 & 49 \\
\hline 8486347 & 10707964 & 73 & 126 & 53 \\
\hline 8486347 & 10707964 & 74 & 125 & 51 \\
\hline 8486347 & 10707964 & 72 & 123 & 51 \\
\hline 8486347 & 10707964 & 72 & 126 & 54 \\
\hline 8486347 & 10707964 & 72 & 132 & 60 \\
\hline 8486347 & 10707964 & 72 & 127 & 55 \\
\hline 8486347 & 10707964 & 72 & 124 & 52 \\
\hline 8486347 & 10707964 & 73 & 125 & 52 \\
\hline 8486347 & 10707964 & 73 & 125 & 52 \\
\hline 8486347 & 10707964 & 73 & 124 & 51 \\
\hline 8486347 & 10707964 & 73 & 121 & 48 \\
\hline 8486347 & 10707964 & 74 & 123 & 49 \\
\hline 8486347 & 10707964 & 74 & 118 & 44 \\
\hline 8486347 & 10707964 & 75 & 117 & 42 \\
\hline 8486347 & 10707964 & 73 & 124 & 51 \\
\hline 8486347 & 10707964 & 74 & 127 & 53 \\
\hline 8486347 & 10707964 & 73 & 127 & 54 \\
\hline 8486347 & 10707964 & 73 & 126 & 53 \\
\hline 8486347 & 10707964 & 74 & 127 & 53 \\
\hline 8486347 & 10707964 & 73 & 127 & 54 \\
\hline 8486347 & 10707964 & 74 & 127 & 53 \\
\hline
\end{tabular}




\begin{tabular}{|c|c|c|c|}
\hline 8486347 & 10707964 & 73 & 126 \\
\hline 8486347 & 10707964 & 73 & 130 \\
\hline 8486347 & 10707964 & 72 & 122 \\
\hline 8486347 & 10707964 & 72 & 124 \\
\hline 8486347 & 10707964 & 73 & 122 \\
\hline 8486347 & 10707964 & 73 & 130 \\
\hline 8486347 & 10707964 & 74 & 127 \\
\hline 8486347 & 10707964 & 73 & 130 \\
\hline 8486347 & 10707964 & 72 & 128 \\
\hline 8486347 & 10707964 & 74 & 122 \\
\hline 8486347 & 10707964 & 74 & 128 \\
\hline 8486347 & 10707964 & 73 & 125 \\
\hline 8486347 & 10707964 & 73 & 123 \\
\hline 8486347 & 10707964 & 74 & 126 \\
\hline 8486347 & 10707964 & 74 & 127 \\
\hline 8486347 & 10707964 & 75 & 122 \\
\hline 8486347 & 10707964 & 74 & 124 \\
\hline 8486347 & 10707964 & 74 & 125 \\
\hline 8486347 & 10707964 & 73 & 126 \\
\hline 8486347 & 10707964 & 74 & 123 \\
\hline 8486347 & 10707964 & 74 & 130 \\
\hline 8486347 & 10707964 & 74 & 127 \\
\hline 8486347 & 10707964 & 73 & 130 \\
\hline 8486347 & 10707964 & 73 & 127 \\
\hline 8486347 & 10707964 & 73 & 128 \\
\hline 8486347 & 10707964 & 75 & 115 \\
\hline 8486347 & 10707964 & 73 & 127 \\
\hline 8486347 & 10707964 & 74 & 128 \\
\hline 8486347 & 10707964 & 74 & 120 \\
\hline 8486347 & 10707964 & 73 & 129 \\
\hline 8486347 & 10707964 & 73 & 125 \\
\hline 8486347 & 10707964 & 73 & 127 \\
\hline 8486347 & 10707964 & 76 & 120 \\
\hline 8486347 & 10707964 & 74 & 125 \\
\hline 8486347 & 10707964 & 74 & 123 \\
\hline 8486347 & 10707964 & 73 & 126 \\
\hline 8486347 & 10707964 & 74 & 124 \\
\hline 8486347 & 10707964 & 74 & 126 \\
\hline 8486347 & 10707964 & 74 & 128 \\
\hline 8486347 & 10707964 & 74 & 127 \\
\hline 8486347 & 10707964 & 74 & 125 \\
\hline 8486347 & 10707964 & 74 & 125 \\
\hline 8486347 & 10707964 & 74 & 119 \\
\hline
\end{tabular}




\begin{tabular}{|c|c|c|c|}
\hline 8486347 & 10707964 & 74 & 127 \\
\hline 8486347 & 10707964 & 75 & 118 \\
\hline 8486347 & 10707964 & 74 & 121 \\
\hline 8486347 & 10707964 & 74 & 118 \\
\hline 8486347 & 10707964 & 74 & 127 \\
\hline 8486347 & 10707964 & 73 & 128 \\
\hline 8486347 & 10707964 & 74 & 124 \\
\hline 8486347 & 10707964 & 73 & 120 \\
\hline 8486347 & 10707964 & 74 & 123 \\
\hline 8486347 & 10707964 & 76 & 118 \\
\hline 8486347 & 10707964 & 74 & 128 \\
\hline 8486347 & 10707964 & 74 & 128 \\
\hline 8486347 & 10707964 & 75 & 125 \\
\hline 8486347 & 10707964 & 75 & 121 \\
\hline 8486347 & 10707964 & 74 & 123 \\
\hline 8486347 & 10707964 & 75 & 122 \\
\hline 8486347 & 10707964 & 75 & 122 \\
\hline 8486347 & 10707964 & 75 & 121 \\
\hline 8486347 & 10707964 & 76 & 120 \\
\hline 8486347 & 10707964 & 74 & 125 \\
\hline 8486347 & 10707964 & 73 & 125 \\
\hline 8486347 & 10707964 & 77 & 119 \\
\hline 8486347 & 10707964 & 75 & 123 \\
\hline 8486347 & 10707964 & 75 & 126 \\
\hline 8486347 & 10707964 & 75 & 120 \\
\hline 8486347 & 10707964 & 75 & 121 \\
\hline 8486347 & 10707964 & 77 & 117 \\
\hline 8486347 & 10707964 & 74 & 119 \\
\hline 8486347 & 10707964 & 75 & 126 \\
\hline 8486347 & 10707964 & 75 & 121 \\
\hline 8486347 & 10707964 & 75 & 128 \\
\hline 8486347 & 10707964 & 76 & 122 \\
\hline 8486347 & 10707964 & 79 & 115 \\
\hline 8486347 & 10707964 & 78 & 122 \\
\hline 8486347 & 10707964 & 75 & 121 \\
\hline 8486347 & 10707964 & 76 & 118 \\
\hline 8486347 & 10707964 & 77 & 121 \\
\hline 8486347 & 10707964 & 76 & 119 \\
\hline 8486347 & 10707964 & 76 & 114 \\
\hline 8486347 & 10707964 & 75 & 116 \\
\hline 8486347 & 10707964 & 76 & 115 \\
\hline 8486347 & 10707964 & 78 & 120 \\
\hline 8486347 & 10707964 & 76 & 118 \\
\hline
\end{tabular}




\begin{tabular}{|c|c|c|c|}
\hline 8486347 & 10707964 & 76 & 121 \\
\hline 8486347 & 10707964 & 76 & 118 \\
\hline 8486347 & 10707964 & 77 & 120 \\
\hline 8486347 & 10707964 & 73 & 118 \\
\hline 8486347 & 10707964 & 78 & 126 \\
\hline 8486347 & 10707964 & 80 & 112 \\
\hline 8486347 & 10707964 & 78 & 120 \\
\hline 8486347 & 10707964 & 80 & 111 \\
\hline 8486347 & 10707964 & 81 & 116 \\
\hline 8486347 & 10707964 & 77 & 117 \\
\hline 8486347 & 10707964 & 82 & 113 \\
\hline 8486347 & 10707964 & 78 & 113 \\
\hline 8486347 & 10707964 & 77 & 120 \\
\hline 8486347 & 10707964 & 81 & 116 \\
\hline 8486347 & 10707964 & 76 & 121 \\
\hline 8486347 & 10707964 & 79 & 120 \\
\hline 8486347 & 10707964 & 78 & 117 \\
\hline 8486347 & 10707964 & 75 & 121 \\
\hline 8486347 & 10707964 & 81 & 117 \\
\hline 8486347 & 10707964 & 74 & 119 \\
\hline 8486347 & 10707964 & 77 & 117 \\
\hline 8486347 & 10707964 & 76 & 118 \\
\hline 8486347 & 10707964 & 79 & 121 \\
\hline 8486347 & 10707964 & 78 & 119 \\
\hline 8486347 & 10707964 & 76 & 123 \\
\hline 8486347 & 10707964 & 76 & 122 \\
\hline 8486347 & 10707964 & 80 & 121 \\
\hline 8486347 & 10707964 & 76 & 123 \\
\hline 8486347 & 10707964 & 77 & 122 \\
\hline 8486347 & 10707964 & 77 & 121 \\
\hline 8486347 & 10707964 & 77 & 123 \\
\hline 8486347 & 10707964 & 79 & 121 \\
\hline 8486347 & 10707964 & 76 & 120 \\
\hline 8486347 & 10707964 & 76 & 121 \\
\hline 8486347 & 10707964 & 76 & 125 \\
\hline 8486347 & 10707964 & 78 & 121 \\
\hline 8486347 & 10707964 & 75 & 123 \\
\hline 8486347 & 10707964 & 78 & 122 \\
\hline 8486347 & 10707964 & 75 & 121 \\
\hline 8486347 & 10707964 & 77 & 122 \\
\hline 8486347 & 10707964 & 78 & 126 \\
\hline 8486347 & 10707964 & 77 & 123 \\
\hline 8486347 & 10707964 & 77 & 121 \\
\hline
\end{tabular}




\begin{tabular}{|c|c|c|c|}
\hline 8486347 & 10707964 & 78 & 128 \\
\hline 8486347 & 10707964 & 81 & 123 \\
\hline 8486347 & 10707964 & 80 & 120 \\
\hline 8486347 & 10707964 & 83 & 125 \\
\hline 8486347 & 10707964 & 79 & 125 \\
\hline 8486347 & 10707964 & 74 & 123 \\
\hline 8486347 & 10707964 & 78 & 127 \\
\hline 8486347 & 10707964 & 77 & 122 \\
\hline 8486347 & 10707964 & 76 & 124 \\
\hline 8486347 & 10707964 & 74 & 125 \\
\hline 8486347 & 10707964 & 76 & 121 \\
\hline 8486347 & 10707964 & 75 & 125 \\
\hline 8486347 & 10707964 & 77 & 120 \\
\hline 8486347 & 10707964 & 77 & 122 \\
\hline 8486347 & 10707964 & 78 & 122 \\
\hline 8486347 & 10707964 & 78 & 123 \\
\hline 8486347 & 10707964 & 76 & 126 \\
\hline 8486347 & 10707964 & 76 & 125 \\
\hline 8486347 & 10707964 & 78 & 124 \\
\hline 8486347 & 10707964 & 77 & 122 \\
\hline 8486347 & 10707964 & 77 & 121 \\
\hline 8486347 & 10707964 & 77 & 123 \\
\hline 8486347 & 10707964 & 77 & 120 \\
\hline 8486347 & 10707964 & 79 & 125 \\
\hline 8486347 & 10707964 & 77 & 125 \\
\hline 8486347 & 10707964 & 76 & 121 \\
\hline 8486347 & 10707964 & 72 & 125 \\
\hline 8486347 & 10707964 & 76 & 124 \\
\hline 8486347 & 10707964 & 77 & 126 \\
\hline 8486347 & 10707964 & 75 & 127 \\
\hline 8486347 & 10707964 & 75 & 127 \\
\hline 8486347 & 10707964 & 75 & 125 \\
\hline 8486347 & 10707964 & 73 & 126 \\
\hline 8486347 & 10707964 & 75 & 123 \\
\hline 8486347 & 10707964 & 77 & 126 \\
\hline 8486347 & 10707964 & 74 & 126 \\
\hline 8486347 & 10707964 & 78 & 125 \\
\hline 8486347 & 10707964 & 78 & 125 \\
\hline 8486347 & 10707964 & 74 & 124 \\
\hline 8486347 & 10707964 & 75 & 124 \\
\hline 8486347 & 10707964 & 77 & 126 \\
\hline 8486347 & 10707964 & 81 & 124 \\
\hline 8486347 & 10707964 & 74 & 127 \\
\hline
\end{tabular}




\begin{tabular}{|c|c|c|c|}
\hline 8486347 & 10707964 & 76 & 127 \\
\hline 8486347 & 10707964 & 78 & 127 \\
\hline 8486347 & 10707964 & 75 & 123 \\
\hline 8486347 & 10707964 & 77 & 126 \\
\hline 8486347 & 10707964 & 76 & 126 \\
\hline 8486347 & 10707964 & 77 & 125 \\
\hline 8486347 & 10707964 & 76 & 129 \\
\hline 8486347 & 10707964 & 78 & 125 \\
\hline 8486347 & 10707964 & 76 & 122 \\
\hline 8486347 & 10707964 & 74 & 128 \\
\hline 8486347 & 10707964 & 78 & 123 \\
\hline 8486347 & 10707964 & 76 & 125 \\
\hline 8486347 & 10707964 & 76 & 126 \\
\hline 8486347 & 10707964 & 74 & 127 \\
\hline 8486347 & 10707964 & 76 & 129 \\
\hline 8486347 & 10707964 & 75 & 124 \\
\hline 8486347 & 10707964 & 75 & 129 \\
\hline 8486347 & 10707964 & 77 & 125 \\
\hline 8486347 & 10707964 & 78 & 127 \\
\hline 8486347 & 10707964 & 76 & 124 \\
\hline 8486347 & 10707964 & 76 & 128 \\
\hline 8486347 & 10707964 & 81 & 133 \\
\hline 8486347 & 10707964 & 78 & 125 \\
\hline 8486347 & 10707964 & 79 & 127 \\
\hline 8486347 & 10707964 & 74 & 128 \\
\hline 8486347 & 10707964 & 74 & 128 \\
\hline 8486347 & 10707964 & 78 & 126 \\
\hline 8486347 & 10707964 & 81 & 119 \\
\hline 8486347 & 10707964 & 74 & 127 \\
\hline 8486347 & 10707964 & 76 & 128 \\
\hline 8486347 & 10707964 & 75 & 126 \\
\hline 8486347 & 10707964 & 74 & 125 \\
\hline 8486347 & 10707964 & 75 & 126 \\
\hline 8486347 & 10707964 & 79 & 127 \\
\hline 8486347 & 10707964 & 74 & 129 \\
\hline 8486347 & 10707964 & 76 & 129 \\
\hline 8486347 & 10707964 & 79 & 123 \\
\hline 8486347 & 10707964 & 75 & 124 \\
\hline 8486347 & 10707964 & 74 & 129 \\
\hline 8486347 & 10707964 & 74 & 126 \\
\hline 8486347 & 10707964 & 83 & 131 \\
\hline 8486347 & 10707964 & 78 & 128 \\
\hline 8486347 & 10707964 & 77 & 128 \\
\hline
\end{tabular}




\begin{tabular}{|c|c|c|c|}
\hline 8486347 & 10707964 & 74 & 127 \\
\hline 8486347 & 10707964 & 78 & 129 \\
\hline 8486347 & 10707964 & 74 & 128 \\
\hline 8486347 & 10707964 & 75 & 126 \\
\hline 8486347 & 10707964 & 81 & 127 \\
\hline 8486347 & 10707964 & 73 & 128 \\
\hline 8486347 & 10707964 & 80 & 126 \\
\hline 8486347 & 10707964 & 78 & 126 \\
\hline 8486347 & 10707964 & 76 & 130 \\
\hline 8486347 & 10707964 & 79 & 129 \\
\hline 8486347 & 10707964 & 75 & 127 \\
\hline 8486347 & 10707964 & 76 & 130 \\
\hline 8486347 & 10707964 & 74 & 130 \\
\hline 8486347 & 10707964 & 74 & 129 \\
\hline 8486347 & 10707964 & 75 & 128 \\
\hline 8486347 & 10707964 & 77 & 131 \\
\hline 8486347 & 10707964 & 77 & 126 \\
\hline 8486347 & 10707964 & 76 & 129 \\
\hline 8486347 & 10707964 & 74 & 129 \\
\hline 8486347 & 10707964 & 76 & 128 \\
\hline 8486347 & 10707964 & 78 & 127 \\
\hline 8486347 & 10707964 & 74 & 129 \\
\hline 8486347 & 10707964 & 74 & 130 \\
\hline 8486347 & 10707964 & 74 & 129 \\
\hline 8486347 & 10707964 & 75 & 127 \\
\hline 8486347 & 10707964 & 74 & 129 \\
\hline 8486347 & 10707964 & 74 & 130 \\
\hline 8486347 & 10707964 & 76 & 128 \\
\hline 8486347 & 10707964 & 75 & 128 \\
\hline 8486347 & 10707964 & 81 & 129 \\
\hline 8486347 & 10707964 & 74 & 129 \\
\hline 8486347 & 10707964 & 74 & 131 \\
\hline 8486347 & 10707964 & 74 & 127 \\
\hline 8486347 & 10707964 & 74 & 128 \\
\hline 8486347 & 10707964 & 74 & 130 \\
\hline 8486347 & 10707964 & 75 & 126 \\
\hline 8486347 & 10707964 & 77 & 121 \\
\hline 8486347 & 10707964 & 74 & 129 \\
\hline 8486347 & 10707964 & 75 & 131 \\
\hline 8486347 & 10707964 & 74 & 128 \\
\hline 8486347 & 10707964 & 74 & 131 \\
\hline 8486347 & 10707964 & 79 & 129 \\
\hline 8486347 & 10707964 & 74 & 130 \\
\hline
\end{tabular}




\begin{tabular}{|c|c|c|c|}
\hline 8486347 & 10707964 & 74 & 129 \\
\hline 8486347 & 10707964 & 73 & 130 \\
\hline 8486347 & 10707964 & 75 & 129 \\
\hline 8486347 & 10707964 & 75 & 130 \\
\hline 8486347 & 10707964 & 78 & 124 \\
\hline 8486347 & 10707964 & 73 & 129 \\
\hline 8486347 & 10707964 & 77 & 131 \\
\hline 8486347 & 10707964 & 75 & 131 \\
\hline 8486347 & 10707964 & 79 & 137 \\
\hline 8486347 & 10707964 & 74 & 130 \\
\hline 8486347 & 10707964 & 75 & 131 \\
\hline 8486347 & 10707964 & 74 & 131 \\
\hline 8486347 & 10707964 & 74 & 129 \\
\hline 8486347 & 10707964 & 75 & 132 \\
\hline 8486347 & 10707964 & 82 & 130 \\
\hline 8486347 & 10707964 & 74 & 130 \\
\hline 8486347 & 10707964 & 79 & 129 \\
\hline 8486347 & 10707964 & 75 & 129 \\
\hline 8486347 & 10707964 & 74 & 130 \\
\hline 8486347 & 10707964 & 74 & 128 \\
\hline 8486347 & 10707964 & 75 & 130 \\
\hline 8486347 & 10707964 & 74 & 130 \\
\hline 8486347 & 10707964 & 75 & 131 \\
\hline 8486347 & 10707964 & 74 & 131 \\
\hline 8486347 & 10707964 & 80 & 129 \\
\hline 8486347 & 10707964 & 75 & 133 \\
\hline 8486347 & 10707964 & 82 & 133 \\
\hline 8486347 & 10707964 & 74 & 127 \\
\hline 8486347 & 10707964 & 75 & 130 \\
\hline 8486347 & 10707964 & 75 & 131 \\
\hline 8486347 & 10707964 & 76 & 136 \\
\hline 8486347 & 10707964 & 75 & 134 \\
\hline 8486347 & 10707964 & 73 & 132 \\
\hline 8486347 & 10707964 & 79 & 132 \\
\hline 8486347 & 10707964 & 74 & 130 \\
\hline 8486347 & 10707964 & 74 & 129 \\
\hline 8486347 & 10707964 & 75 & 132 \\
\hline 6258358 & 10707964 & 72 & 132 \\
\hline 8486347 & 10707964 & 74 & 131 \\
\hline 8486347 & 10707964 & 74 & 131 \\
\hline 8486347 & 10707964 & 72 & 133 \\
\hline 8486347 & 10707964 & 76 & 131 \\
\hline 8486347 & 10707964 & 74 & 131 \\
\hline
\end{tabular}




\begin{tabular}{|c|c|c|c|}
\hline 8486347 & 10707964 & 78 & 130 \\
\hline 8486347 & 10707964 & 73 & 133 \\
\hline 8486347 & 10707964 & 81 & 133 \\
\hline 8486347 & 10707964 & 77 & 136 \\
\hline 8486347 & 10707964 & 77 & 131 \\
\hline 8486347 & 10707964 & 74 & 135 \\
\hline 8486347 & 10707964 & 75 & 133 \\
\hline 8486347 & 10707964 & 74 & 134 \\
\hline 8486347 & 10707964 & 78 & 131 \\
\hline 8486347 & 10707964 & 75 & 133 \\
\hline 8486347 & 10707964 & 73 & 134 \\
\hline 8486347 & 10707964 & 75 & 133 \\
\hline 8486347 & 10707964 & 73 & 134 \\
\hline 8486347 & 10707964 & 74 & 133 \\
\hline 8486347 & 10707964 & 75 & 132 \\
\hline 8486347 & 10707964 & 80 & 131 \\
\hline 8486347 & 10707964 & 72 & 133 \\
\hline 8486347 & 10707964 & 73 & 133 \\
\hline 8486347 & 10707964 & 75 & 131 \\
\hline 4308352 & 10707964 & 71 & 135 \\
\hline 8486347 & 10707964 & 77 & 137 \\
\hline 8486347 & 10707964 & 78 & 136 \\
\hline 8486347 & 10707964 & 83 & 130 \\
\hline 8486347 & 10707964 & 73 & 134 \\
\hline 8486347 & 10707964 & 76 & 134 \\
\hline 8486347 & 10707964 & 75 & 136 \\
\hline 8486347 & 10707964 & 76 & 136 \\
\hline 8486347 & 10707964 & 77 & 135 \\
\hline 8486347 & 10707964 & 75 & 135 \\
\hline 8486347 & 10707964 & 73 & 137 \\
\hline 8486347 & 10707964 & 76 & 132 \\
\hline 8486347 & 10707964 & 73 & 136 \\
\hline 8486347 & 10707964 & 83 & 135 \\
\hline 8486347 & 10707964 & 78 & 131 \\
\hline 8486347 & 10707964 & 76 & 133 \\
\hline 8486347 & 10707964 & 79 & 137 \\
\hline 8486347 & 10707964 & 75 & 135 \\
\hline 8486347 & 10707964 & 72 & 135 \\
\hline 8486347 & 10707964 & 73 & 136 \\
\hline 8486347 & 10707964 & 78 & 135 \\
\hline 4308352 & 10707964 & 70 & 134 \\
\hline 8486347 & 10707964 & 74 & 136 \\
\hline 8486347 & 10707964 & 73 & 133 \\
\hline
\end{tabular}




\begin{tabular}{|c|c|c|c|}
\hline 8486347 & 10707964 & 78 & 134 \\
\hline 8486347 & 10707964 & 72 & 134 \\
\hline 8486347 & 10707964 & 74 & 133 \\
\hline 8486347 & 10707964 & 81 & 138 \\
\hline 8486347 & 10707964 & 84 & 143 \\
\hline 8486347 & 10707964 & 74 & 133 \\
\hline 8486347 & 10707964 & 74 & 135 \\
\hline 8486347 & 10707964 & 73 & 137 \\
\hline 8486347 & 10707964 & 78 & 137 \\
\hline 8486347 & 10707964 & 76 & 138 \\
\hline 8486347 & 10707964 & 73 & 137 \\
\hline 8486347 & 10707964 & 81 & 137 \\
\hline 8486347 & 10707964 & 80 & 132 \\
\hline 4308352 & 10707964 & 70 & 137 \\
\hline 8486347 & 10707964 & 73 & 133 \\
\hline 8486347 & 10707964 & 76 & 136 \\
\hline 8486347 & 10707964 & 77 & 136 \\
\hline 8486347 & 10707964 & 82 & 132 \\
\hline 8486347 & 10707964 & 76 & 132 \\
\hline 4308352 & 10707964 & 68 & 135 \\
\hline 8486347 & 10707964 & 76 & 132 \\
\hline 8486347 & 10707964 & 73 & 137 \\
\hline 8486347 & 10707964 & 80 & 135 \\
\hline 8486347 & 10707964 & 79 & 134 \\
\hline 8486347 & 10707964 & 72 & 141 \\
\hline 4308352 & 10707964 & 69 & 137 \\
\hline 6258358 & 10707964 & 72 & 137 \\
\hline 8486347 & 10707964 & 77 & 138 \\
\hline 8486347 & 10707964 & 75 & 135 \\
\hline 8486347 & 10707964 & 80 & 137 \\
\hline 8486347 & 10707964 & 76 & 140 \\
\hline 8486347 & 10707964 & 76 & 141 \\
\hline 8486347 & 10707964 & 73 & 141 \\
\hline 8486347 & 10707964 & 78 & 141 \\
\hline 8486347 & 10707964 & 76 & 140 \\
\hline 6258358 & 10707964 & 72 & 140 \\
\hline 8486347 & 10707964 & 74 & 137 \\
\hline 8486347 & 10707964 & 75 & 139 \\
\hline 8486347 & 10707964 & 73 & 140 \\
\hline 4308352 & 10707964 & 66 & 142 \\
\hline 8486347 & 10707964 & 77 & 142 \\
\hline 8486347 & 10707964 & 79 & 142 \\
\hline 8486347 & 10707964 & 76 & 142 \\
\hline
\end{tabular}




\begin{tabular}{|c|c|c|c|}
\hline 8486347 & 10707964 & 80 & 142 \\
\hline 8486347 & 10707964 & 75 & 141 \\
\hline 8486347 & 10707964 & 78 & 141 \\
\hline 8486347 & 10707964 & 82 & 139 \\
\hline 8486347 & 10707964 & 81 & 141 \\
\hline 8486347 & 10707964 & 77 & 143 \\
\hline 8486347 & 10707964 & 72 & 140 \\
\hline 8486347 & 10707964 & 73 & 144 \\
\hline 8486347 & 10707964 & 78 & 143 \\
\hline 4308352 & 10707964 & 68 & 141 \\
\hline 8486347 & 10707964 & 74 & 141 \\
\hline 8486347 & 10707964 & 74 & 142 \\
\hline 4308352 & 10707964 & 71 & 143 \\
\hline 8486347 & 10707964 & 73 & 144 \\
\hline 8486347 & 10707964 & 81 & 146 \\
\hline 8486347 & 10707964 & 72 & 142 \\
\hline 8486347 & 10707964 & 78 & 140 \\
\hline 6258358 & 10707964 & 72 & 146 \\
\hline 8486347 & 10707964 & 78 & 142 \\
\hline 8486347 & 10707964 & 77 & 146 \\
\hline 4308352 & 10707964 & 68 & 144 \\
\hline 8486347 & 10707964 & 77 & 146 \\
\hline 8486347 & 10707964 & 83 & 143 \\
\hline 8486347 & 11401597 & 79 & 165 \\
\hline 8486347 & 10707964 & 77 & 147 \\
\hline 8486347 & 10707964 & 78 & 146 \\
\hline 8486347 & 10707964 & 77 & 145 \\
\hline 8486347 & 10707964 & 73 & 150 \\
\hline 8486347 & 10707964 & 75 & 143 \\
\hline 8486347 & 10707964 & 82 & 145 \\
\hline 8486347 & 10707964 & 81 & 143 \\
\hline 6258358 & 10707964 & 72 & 150 \\
\hline 8486347 & 10707964 & 79 & 146 \\
\hline 4308352 & 10707964 & 70 & 155 \\
\hline 8486347 & 10707964 & 81 & 147 \\
\hline 8486347 & 10707964 & 79 & 154 \\
\hline 8486347 & 10707964 & 79 & 150 \\
\hline 8486347 & 10707964 & 78 & 151 \\
\hline 8486347 & 10707964 & 81 & 156 \\
\hline 8486347 & 10707964 & 76 & 155 \\
\hline 8486347 & 10707964 & 82 & 156 \\
\hline 8486347 & 11401597 & 75 & 165 \\
\hline 8486347 & 11401597 & 81 & 165 \\
\hline
\end{tabular}




\begin{tabular}{|c|c|c|c|c|}
\hline 8486347 & 10707964 & 79 & 164 & 85 \\
\hline 8486347 & 11401597 & 78 & 167 & 89 \\
\hline 8486347 & 11401597 & 80 & 170 & 90 \\
\hline 8486347 & 11401597 & 77 & 166 & 89 \\
\hline 8486347 & 11401597 & 83 & 170 & 87 \\
\hline 4308352 & 11401597 & 61 & 176 & 115 \\
\hline 8486347 & 11401597 & 87 & 168 & 81 \\
\hline 8486347 & 11401597 & 85 & 176 & 91 \\
\hline 9414707 & 11401597 & 104 & 168 & 64 \\
\hline 9414707 & 11401597 & 100 & 180 & 80 \\
\hline 9414707 & 11401597 & 112 & 169 & 57 \\
\hline 9414707 & 11401597 & 108 & 170 & 62 \\
\hline 9414707 & 11401597 & 116 & 168 & 52 \\
\hline 9414707 & 11401597 & 106 & 172 & 66 \\
\hline 9414707 & 11401597 & 98 & 177 & 79 \\
\hline 9414707 & 11401597 & 121 & 179 & 58 \\
\hline 9414707 & 11401597 & 121 & 178 & 57 \\
\hline 10707964 & 12084299 & 172 & 201 & 29 \\
\hline 10707964 & 12084299 & 181 & 195 & 14 \\
\hline 10707964 & 12084299 & 171 & 202 & 31 \\
\hline 11708108 & 12084299 & 197 & 202 & 5 \\
\hline 2546359 & 2546359 & 0 & 0 & 0 \\
\hline 2546359 & 2546359 & 0 & 0 & 0 \\
\hline 2546359 & 2546359 & 0 & 0 & 0 \\
\hline 2546359 & 2546359 & 0 & 0 & 0 \\
\hline 2546359 & 2546359 & 0 & 0 & 0 \\
\hline 2546359 & 2546359 & 0 & 0 & 0 \\
\hline 2546359 & 2546359 & 0 & 0 & 0 \\
\hline 2546359 & 2546359 & 0 & 0 & 0 \\
\hline 2546359 & 2546359 & 0 & 0 & 0 \\
\hline 2546359 & 2546359 & 0 & 0 & 0 \\
\hline 2546359 & 2546359 & 0 & 0 & 0 \\
\hline 2546359 & 2546359 & 0 & 0 & 0 \\
\hline 2546359 & 2546359 & 0 & 0 & 0 \\
\hline 2546359 & 2546359 & 0 & 0 & 0 \\
\hline 2546359 & 2546359 & 0 & 0 & 0 \\
\hline 2546359 & 2480204 & 0 & 1 & 1 \\
\hline 2546359 & 2480204 & 0 & 1 & 1 \\
\hline 2546359 & 2480204 & 0 & 1 & 1 \\
\hline 2546359 & 2480204 & 0 & 1 & 1 \\
\hline 2546359 & 2480204 & 0 & 1 & 1 \\
\hline 2546359 & 2480204 & 0 & 1 & 1 \\
\hline 2546359 & 2480204 & 0 & 1 & 1 \\
\hline
\end{tabular}




\begin{tabular}{|c|c|c|c|}
\hline 2546359 & 2480204 & 0 & 1 \\
\hline 969239 & 969239 & 0 & 0 \\
\hline 969239 & 1473750 & 0 & 2 \\
\hline 969239 & 1473750 & 0 & 2 \\
\hline 969239 & 969239 & 0 & 0 \\
\hline 969239 & 1473750 & 0 & 3 \\
\hline 969239 & 1473750 & 0 & 3 \\
\hline 969239 & 2473253 & 0 & 4 \\
\hline 969239 & 1473750 & 0 & 2 \\
\hline 969239 & 2473253 & 0 & 12 \\
\hline 969239 & 1473750 & 0 & 2 \\
\hline 969239 & 1473750 & 0 & 2 \\
\hline 969239 & 1473750 & 0 & 2 \\
\hline 969239 & 1473750 & 0 & 3 \\
\hline 969239 & 1473750 & 0 & 3 \\
\hline 969239 & 2473253 & 0 & 6 \\
\hline 969239 & 1473750 & 0 & 3 \\
\hline 969239 & 1473750 & 0 & 2 \\
\hline 969239 & 1473750 & 0 & 2 \\
\hline 969239 & 1473750 & 0 & 4 \\
\hline 969239 & 1473750 & 0 & 3 \\
\hline 2473253 & 5465495 & 18 & 73 \\
\hline 2473253 & 5465495 & 23 & 72 \\
\hline 2473253 & 5465495 & 36 & 66 \\
\hline 2473253 & 5465495 & 39 & 66 \\
\hline 2473253 & 5465495 & 35 & 68 \\
\hline 2473253 & 5465495 & 34 & 68 \\
\hline 2473253 & 5465495 & 39 & 69 \\
\hline 2473253 & 5465495 & 41 & 65 \\
\hline 2473253 & 5465495 & 38 & 65 \\
\hline 2473253 & 5465495 & 41 & 66 \\
\hline 2473253 & 5465495 & 38 & 67 \\
\hline 2473253 & 5465495 & 37 & 69 \\
\hline 2473253 & 5465495 & 41 & 66 \\
\hline 2473253 & 5465495 & 30 & 70 \\
\hline 2473253 & 5465495 & 42 & 66 \\
\hline 2473253 & 5465495 & 40 & 71 \\
\hline 2473253 & 5465495 & 46 & 67 \\
\hline 2473253 & 5465495 & 45 & 68 \\
\hline 2473253 & 5465495 & 42 & 70 \\
\hline 2473253 & 5465495 & 43 & 68 \\
\hline 2473253 & 5465495 & 37 & 80 \\
\hline 2473253 & 5465495 & 29 & 76 \\
\hline
\end{tabular}




\begin{tabular}{|c|c|c|c|}
\hline 2473253 & 5465495 & 38 & 77 \\
\hline 2473253 & 5465495 & 40 & 74 \\
\hline 2473253 & 5817334 & 39 & 87 \\
\hline 2473253 & 5817334 & 32 & 85 \\
\hline 2473253 & 5817334 & 27 & 83 \\
\hline 2473253 & 5817334 & 41 & 85 \\
\hline 3944254 & 6408779 & 78 & 104 \\
\hline 5465495 & 6408779 & 83 & 103 \\
\hline 5465495 & 6408779 & 82 & 102 \\
\hline 5465495 & 6408779 & 84 & 99 \\
\hline 5465495 & 6408779 & 82 & 101 \\
\hline 5465495 & 6408779 & 85 & 101 \\
\hline 5465495 & 6408779 & 85 & 101 \\
\hline 5465495 & 6408779 & 85 & 101 \\
\hline 5465495 & 6408779 & 84 & 101 \\
\hline 5465495 & 6408779 & 83 & 103 \\
\hline 5465495 & 6408779 & 89 & 104 \\
\hline 6728614 & 8396399 & 125 & 151 \\
\hline 5817334 & 8396399 & 97 & 158 \\
\hline 6408779 & 8396399 & 119 & 150 \\
\hline 6957395 & 8396399 & 128 & 149 \\
\hline 7470995 & 9796419 & 143 & 162 \\
\hline 8396399 & 9796419 & 168 & 178 \\
\hline 8396399 & 9796419 & 167 & 178 \\
\hline 3073300 & 4931562 & 1 & 58 \\
\hline 553274 & 4931562 & 0 & 65 \\
\hline 3073300 & 4931562 & 6 & 66 \\
\hline 3073300 & 4931562 & 18 & 60 \\
\hline 3073300 & 4931562 & 34 & 59 \\
\hline 3073300 & 4931562 & 28 & 64 \\
\hline 3073300 & 4931562 & 22 & 63 \\
\hline 3073300 & 4931562 & 25 & 64 \\
\hline 3073300 & 4931562 & 26 & 59 \\
\hline 3073300 & 4931562 & 34 & 60 \\
\hline 3073300 & 4931562 & 8 & 71 \\
\hline 3073300 & 4931562 & 18 & 66 \\
\hline 3073300 & 4931562 & 17 & 64 \\
\hline 3073300 & 4931562 & 1 & 71 \\
\hline 3073300 & 4931562 & 25 & 64 \\
\hline 3073300 & 4931562 & 18 & 71 \\
\hline 3073300 & 4931562 & 26 & 64 \\
\hline 6195698 & 9437678 & 97 & 118 \\
\hline 6195698 & 9437678 & 102 & 125 \\
\hline
\end{tabular}




\begin{tabular}{|c|c|c|c|}
\hline 6195698 & 9437678 & 103 & 128 \\
\hline 400229 & 1720582 & 0 & 14 \\
\hline 400229 & 2028198 & 0 & 26 \\
\hline 400229 & 3827260 & 0 & 41 \\
\hline 400229 & 2028198 & 1 & 25 \\
\hline 400229 & 2028198 & 6 & 25 \\
\hline 400229 & 3827260 & 15 & 36 \\
\hline 2028198 & 4331777 & 52 & 75 \\
\hline 2028198 & 4331777 & 50 & 72 \\
\hline 2028198 & 4331777 & 47 & 77 \\
\hline 2028198 & 4331777 & 49 & 77 \\
\hline 2028198 & 4331777 & 46 & 77 \\
\hline 2028198 & 4331777 & 44 & 77 \\
\hline 4153676 & 4331777 & 64 & 77 \\
\hline 4153676 & 4331777 & 66 & 77 \\
\hline 4153676 & 4331777 & 65 & 77 \\
\hline 4153676 & 4331777 & 67 & 77 \\
\hline 4153676 & 4331777 & 67 & 77 \\
\hline 4153676 & 4331777 & 68 & 77 \\
\hline 4153676 & 4331777 & 68 & 77 \\
\hline 4153676 & 4331777 & 67 & 77 \\
\hline 4153676 & 4331777 & 70 & 77 \\
\hline 4153676 & 4331777 & 69 & 77 \\
\hline 4153676 & 4331777 & 69 & 77 \\
\hline 4153676 & 4331777 & 72 & 77 \\
\hline 4153676 & 4331777 & 70 & 77 \\
\hline 4153676 & 4331777 & 72 & 77 \\
\hline 4153676 & 4331777 & 69 & 77 \\
\hline 4153676 & 4331777 & 72 & 77 \\
\hline 4153676 & 4331777 & 70 & 77 \\
\hline 4153676 & 4331777 & 70 & 77 \\
\hline 4153676 & 4331777 & 69 & 77 \\
\hline 4153676 & 4331777 & 71 & 77 \\
\hline 4153676 & 4331777 & 71 & 77 \\
\hline 4153676 & 4331777 & 70 & 77 \\
\hline 4153676 & 4331777 & 65 & 77 \\
\hline 4153676 & 4331777 & 71 & 77 \\
\hline 4153676 & 4331777 & 70 & 77 \\
\hline 4153676 & 4331777 & 70 & 77 \\
\hline 4153676 & 4331777 & 69 & 77 \\
\hline 4153676 & 4331777 & 71 & 77 \\
\hline 4153676 & 4331777 & 71 & 77 \\
\hline 4153676 & 4331777 & 68 & 77 \\
\hline
\end{tabular}




\begin{tabular}{|c|c|c|c|}
\hline 4153676 & 4331777 & 72 & 77 \\
\hline 4153676 & 4331777 & 68 & 77 \\
\hline 4153676 & 4331777 & 70 & 77 \\
\hline 4153676 & 4331777 & 70 & 77 \\
\hline 4153676 & 4331777 & 72 & 77 \\
\hline 4153676 & 4331777 & 71 & 77 \\
\hline 4153676 & 4331777 & 71 & 77 \\
\hline 4153676 & 4331777 & 68 & 77 \\
\hline 4153676 & 4331777 & 68 & 77 \\
\hline 4153676 & 4331777 & 74 & 77 \\
\hline 4153676 & 4331777 & 70 & 77 \\
\hline 4153676 & 4331777 & 73 & 77 \\
\hline 4153676 & 4331777 & 75 & 77 \\
\hline 4153676 & 4331777 & 72 & 77 \\
\hline 4153676 & 4331777 & 71 & 77 \\
\hline 4153676 & 4331777 & 72 & 77 \\
\hline 4153676 & 4331777 & 71 & 77 \\
\hline 4153676 & 4331777 & 71 & 77 \\
\hline 4153676 & 4331777 & 71 & 77 \\
\hline 4153676 & 4331777 & 73 & 77 \\
\hline 4153676 & 4331777 & 70 & 77 \\
\hline 4153676 & 4331777 & 71 & 77 \\
\hline 4153676 & 4331777 & 73 & 77 \\
\hline 4153676 & 4331777 & 69 & 77 \\
\hline 4153676 & 4331777 & 71 & 77 \\
\hline 4153676 & 4331777 & 70 & 77 \\
\hline 4153676 & 4331777 & 70 & 77 \\
\hline 4153676 & 4331777 & 72 & 77 \\
\hline 4153676 & 4331777 & 71 & 77 \\
\hline 4153676 & 4331777 & 72 & 77 \\
\hline 4153676 & 4331777 & 71 & 77 \\
\hline 4153676 & 4331777 & 69 & 77 \\
\hline 4153676 & 4331777 & 72 & 77 \\
\hline 4153676 & 4331777 & 72 & 77 \\
\hline 4153676 & 4331777 & 72 & 77 \\
\hline 4153676 & 4331777 & 72 & 77 \\
\hline 4153676 & 4331777 & 72 & 77 \\
\hline 4153676 & 4331777 & 72 & 77 \\
\hline 4153676 & 4331777 & 72 & 77 \\
\hline 4153676 & 4331777 & 72 & 77 \\
\hline 4153676 & 4331777 & 71 & 77 \\
\hline 4153676 & 4331777 & 68 & 77 \\
\hline 625045 & 3157160 & 0 & 30 \\
\hline
\end{tabular}




\begin{tabular}{|c|c|c|c|}
\hline 1222389 & 3157160 & 2 & 38 \\
\hline 1222389 & 3157160 & 1 & 28 \\
\hline 1222389 & 3157160 & 8 & 34 \\
\hline 1222389 & 3157160 & 4 & 31 \\
\hline 1222389 & 3157160 & 6 & 28 \\
\hline 1222389 & 3157160 & 9 & 29 \\
\hline 1222389 & 3157160 & 8 & 27 \\
\hline 1222389 & 3157160 & 9 & 29 \\
\hline 1222389 & 3157160 & 8 & 30 \\
\hline 1222389 & 3157160 & 6 & 37 \\
\hline 1222389 & 3157160 & 8 & 32 \\
\hline 1222389 & 3157160 & 8 & 34 \\
\hline 1222389 & 3157160 & 5 & 36 \\
\hline 1222389 & 3157160 & 6 & 41 \\
\hline 1222389 & 3157160 & 6 & 42 \\
\hline 1222389 & 3825225 & 3 & 58 \\
\hline 1222389 & 3157160 & 8 & 41 \\
\hline 1222389 & 3157160 & 8 & 45 \\
\hline 1222389 & 3157160 & 8 & 41 \\
\hline 1222389 & 3825225 & 2 & 52 \\
\hline 1222389 & 3825225 & 3 & 51 \\
\hline 1222389 & 3157160 & 7 & 46 \\
\hline 1222389 & 3825225 & 2 & 48 \\
\hline 1222389 & 4177657 & 10 & 59 \\
\hline 1222389 & 3825225 & 8 & 51 \\
\hline 1222389 & 3157160 & 6 & 46 \\
\hline 1222389 & 3825225 & 5 & 51 \\
\hline 1222389 & 3825225 & 6 & 54 \\
\hline 1222389 & 3825225 & 3 & 55 \\
\hline 1222389 & 6216604 & 9 & 66 \\
\hline 1222389 & 3825225 & 5 & 56 \\
\hline 1222389 & 3825225 & 4 & 56 \\
\hline 1222389 & 3825225 & 10 & 58 \\
\hline 1222389 & 3825225 & 14 & 55 \\
\hline 1222389 & 3825225 & 5 & 57 \\
\hline 1222389 & 3825225 & 5 & 56 \\
\hline 1566484 & 4177657 & 18 & 59 \\
\hline 1222389 & 3825225 & 8 & 59 \\
\hline 1566484 & 3825225 & 23 & 57 \\
\hline 1566484 & 6216604 & 20 & 83 \\
\hline 1566484 & 3825225 & 27 & 58 \\
\hline 1222389 & 3825225 & 5 & 56 \\
\hline 1566484 & 6216604 & 26 & 105 \\
\hline
\end{tabular}




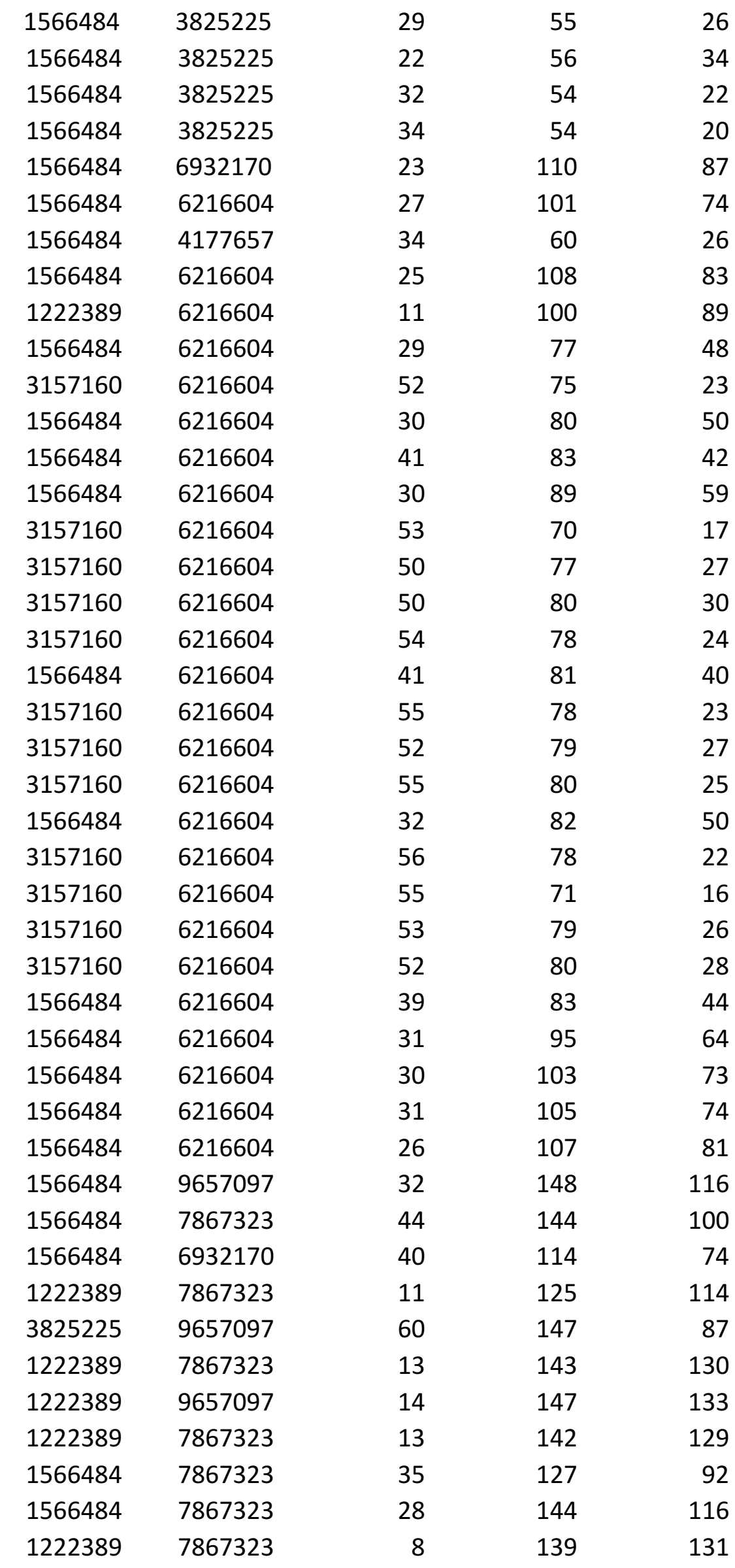




\begin{tabular}{|c|c|c|c|}
\hline 4177657 & 7867323 & 66 & 120 \\
\hline 4177657 & 7867323 & 66 & 137 \\
\hline 4177657 & 9657097 & 73 & 155 \\
\hline 4177657 & 7867323 & 67 & 131 \\
\hline 4177657 & 6932170 & 92 & 112 \\
\hline 4177657 & 6932170 & 99 & 113 \\
\hline 4177657 & 6932170 & 92 & 113 \\
\hline 4177657 & 6932170 & 98 & 111 \\
\hline 4177657 & 7867323 & 86 & 131 \\
\hline 4177657 & 7867323 & 73 & 130 \\
\hline 4177657 & 7867323 & 87 & 133 \\
\hline 6216604 & 7867323 & 111 & 134 \\
\hline 6216604 & 7867323 & 111 & 133 \\
\hline 6216604 & 7867323 & 112 & 133 \\
\hline 6216604 & 7867323 & 111 & 135 \\
\hline 6216604 & 7867323 & 109 & 134 \\
\hline 4177657 & 7867323 & 75 & 141 \\
\hline 6216604 & 7867323 & 109 & 136 \\
\hline 4177657 & 9657097 & 91 & 170 \\
\hline 4177657 & 9657097 & 74 & 146 \\
\hline 6216604 & 9657097 & 110 & 166 \\
\hline 4177657 & 9657097 & 74 & 152 \\
\hline 4177657 & 9657097 & 70 & 166 \\
\hline 6216604 & 9657097 & 111 & 160 \\
\hline 6216604 & 9657097 & 111 & 152 \\
\hline 4177657 & 9657097 & 89 & 157 \\
\hline 4177657 & 9657097 & 67 & 164 \\
\hline 6216604 & 9657097 & 112 & 157 \\
\hline 6932170 & 9657097 & 128 & 159 \\
\hline 6932170 & 9657097 & 128 & 157 \\
\hline 6932170 & 9657097 & 133 & 158 \\
\hline 6932170 & 9657097 & 136 & 157 \\
\hline 6932170 & 9657097 & 135 & 159 \\
\hline 6932170 & 9657097 & 136 & 159 \\
\hline 6932170 & 9657097 & 132 & 163 \\
\hline 9657097 & 13149304 & 212 & 251 \\
\hline 9657097 & 13149304 & 219 & 252 \\
\hline 9657097 & 13149304 & 206 & 252 \\
\hline 9657097 & 13149304 & 208 & 252 \\
\hline 9657097 & 13149304 & 209 & 252 \\
\hline 9657097 & 13149304 & 219 & 252 \\
\hline 9657097 & 13149304 & 220 & 252 \\
\hline 9657097 & 13149304 & 229 & 252 \\
\hline
\end{tabular}




\begin{tabular}{|c|c|c|c|}
\hline 10729434 & 13149304 & 239 & 252 \\
\hline 10729434 & 13149304 & 241 & 252 \\
\hline 10729434 & 13149304 & 240 & 252 \\
\hline 10729434 & 13149304 & 243 & 252 \\
\hline 10729434 & 13149304 & 242 & 252 \\
\hline 10729434 & 13149304 & 239 & 252 \\
\hline 9657097 & 13149304 & 221 & 252 \\
\hline 9657097 & 13149304 & 225 & 252 \\
\hline 10729434 & 13149304 & 238 & 252 \\
\hline 9657097 & 13149304 & 219 & 252 \\
\hline 10729434 & 13149304 & 242 & 252 \\
\hline 10729434 & 13149304 & 246 & 252 \\
\hline 10729434 & 13149304 & 239 & 252 \\
\hline 10729434 & 13149304 & 237 & 252 \\
\hline 10729434 & 13149304 & 244 & 252 \\
\hline 10729434 & 13149304 & 244 & 252 \\
\hline 673999 & 2194516 & 0 & 13 \\
\hline 673999 & 2702007 & 0 & 18 \\
\hline 673999 & 2194516 & 0 & 15 \\
\hline 673999 & 2194516 & 0 & 15 \\
\hline 673999 & 2194516 & 3 & 12 \\
\hline 673999 & 2194516 & 3 & 13 \\
\hline 673999 & 4620849 & 3 & 49 \\
\hline 673999 & 2702007 & 2 & 18 \\
\hline 673999 & 4620849 & 3 & 46 \\
\hline 673999 & 2194516 & 5 & 16 \\
\hline 673999 & 2194516 & 5 & 13 \\
\hline 673999 & 2194516 & 2 & 13 \\
\hline 673999 & 2194516 & 3 & 15 \\
\hline 673999 & 2702007 & 3 & 18 \\
\hline 673999 & 2702007 & 3 & 18 \\
\hline 673999 & 4620849 & 3 & 44 \\
\hline 673999 & 4620849 & 2 & 41 \\
\hline 673999 & 4620849 & 3 & 48 \\
\hline 673999 & 4620849 & 3 & 32 \\
\hline 673999 & 4620849 & 5 & 31 \\
\hline 673999 & 2702007 & 1 & 19 \\
\hline 673999 & 4620849 & 3 & 50 \\
\hline 673999 & 4620849 & 0 & 47 \\
\hline 673999 & 4620849 & 1 & 55 \\
\hline 673999 & 4620849 & 4 & 56 \\
\hline 673999 & 4620849 & 0 & 52 \\
\hline 673999 & 4620849 & 6 & 48 \\
\hline
\end{tabular}




\begin{tabular}{|c|c|c|c|}
\hline 1808590 & 4620849 & 10 & 48 \\
\hline 673999 & 4620849 & 4 & 49 \\
\hline 1808590 & 4620849 & 11 & 36 \\
\hline 673999 & 4620849 & 5 & 50 \\
\hline 1808590 & 4620849 & 10 & 40 \\
\hline 1808590 & 5376623 & 11 & 74 \\
\hline 673999 & 4620849 & 7 & 43 \\
\hline 1808590 & 4620849 & 10 & 44 \\
\hline 673999 & 5376623 & 4 & 62 \\
\hline 673999 & 4620849 & 7 & 55 \\
\hline 673999 & 4620849 & 3 & 59 \\
\hline 673999 & 4620849 & 5 & 56 \\
\hline 673999 & 4620849 & 1 & 57 \\
\hline 673999 & 5376623 & 6 & 69 \\
\hline 673999 & 4620849 & 3 & 59 \\
\hline 673999 & 6494925 & 3 & 91 \\
\hline 673999 & 6494925 & 0 & 106 \\
\hline 2702007 & 5376623 & 23 & 68 \\
\hline 673999 & 5376623 & 7 & 75 \\
\hline 2702007 & 6494925 & 26 & 91 \\
\hline 2702007 & 6494925 & 31 & 87 \\
\hline 2702007 & 5376623 & 27 & 75 \\
\hline 2702007 & 5376623 & 32 & 74 \\
\hline 2702007 & 5376623 & 36 & 72 \\
\hline 2702007 & 5376623 & 39 & 71 \\
\hline 2702007 & 5376623 & 41 & 69 \\
\hline 2702007 & 5376623 & 44 & 66 \\
\hline 2702007 & 5376623 & 38 & 72 \\
\hline 2702007 & 5376623 & 45 & 67 \\
\hline 2702007 & 5376623 & 44 & 71 \\
\hline 2702007 & 5376623 & 41 & 70 \\
\hline 2702007 & 5376623 & 38 & 72 \\
\hline 2702007 & 5376623 & 41 & 72 \\
\hline 2702007 & 5376623 & 41 & 74 \\
\hline 2702007 & 6494925 & 46 & 83 \\
\hline 2702007 & 6494925 & 30 & 82 \\
\hline 2702007 & 6494925 & 38 & 82 \\
\hline 4620849 & 6494925 & 64 & 85 \\
\hline 4620849 & 6494925 & 65 & 83 \\
\hline 4620849 & 6494925 & 65 & 93 \\
\hline 2702007 & 6494925 & 44 & 96 \\
\hline 4620849 & 6494925 & 67 & 88 \\
\hline 4620849 & 6494925 & 67 & 102 \\
\hline
\end{tabular}




\begin{tabular}{|c|c|c|c|}
\hline 4620849 & 6494925 & 68 & 95 \\
\hline 4620849 & 6494925 & 74 & 93 \\
\hline 4620849 & 6494925 & 74 & 98 \\
\hline 5376623 & 6560677 & 89 & 121 \\
\hline 318416 & 1163038 & 0 & 9 \\
\hline 318416 & 752333 & 0 & 4 \\
\hline 318416 & 752333 & 0 & 4 \\
\hline 318416 & 2275005 & 0 & 28 \\
\hline 318416 & 1163038 & 3 & 15 \\
\hline 318416 & 1163038 & 0 & 13 \\
\hline 318416 & 1163038 & 7 & 13 \\
\hline 318416 & 1163038 & 8 & 13 \\
\hline 318416 & 1163038 & 7 & 15 \\
\hline 318416 & 1163038 & 7 & 14 \\
\hline 318416 & 1163038 & 7 & 15 \\
\hline 318416 & 2275005 & 6 & 18 \\
\hline 318416 & 1163038 & 7 & 15 \\
\hline 318416 & 1163038 & 6 & 15 \\
\hline 318416 & 2275005 & 6 & 29 \\
\hline 318416 & 2275005 & 7 & 21 \\
\hline 318416 & 2275005 & 8 & 27 \\
\hline 752333 & 2275005 & 11 & 35 \\
\hline 752333 & 2275005 & 10 & 32 \\
\hline 752333 & 2275005 & 14 & 29 \\
\hline 752333 & 2275005 & 13 & 31 \\
\hline 752333 & 2275005 & 13 & 33 \\
\hline 1163038 & 3689556 & 31 & 89 \\
\hline 1143074 & 1506870 & 0 & 12 \\
\hline 1143074 & 1812195 & 0 & 19 \\
\hline 1143074 & 1506870 & 0 & 13 \\
\hline 1143074 & 1506870 & 0 & 9 \\
\hline 1143074 & 2601122 & 0 & 39 \\
\hline 1143074 & 2601122 & 0 & 42 \\
\hline 1143074 & 1812195 & 0 & 19 \\
\hline 1143074 & 2601122 & 0 & 36 \\
\hline 1143074 & 1506870 & 0 & 9 \\
\hline 1143074 & 2601122 & 0 & 38 \\
\hline 1143074 & 1506870 & 0 & 9 \\
\hline 1143074 & 2601122 & 0 & 41 \\
\hline 1143074 & 2601122 & 0 & 36 \\
\hline 1143074 & 2601122 & 0 & 36 \\
\hline 1143074 & 2601122 & 2 & 33 \\
\hline 1143074 & 2601122 & 0 & 33 \\
\hline
\end{tabular}




\begin{tabular}{|c|c|c|c|}
\hline 1143074 & 2601122 & 0 & 49 \\
\hline 1143074 & 2601122 & 0 & 41 \\
\hline 1143074 & 2601122 & 3 & 33 \\
\hline 1143074 & 2601122 & 0 & 42 \\
\hline 1143074 & 2601122 & 9 & 34 \\
\hline 1506870 & 2601122 & 17 & 29 \\
\hline 1143074 & 2601122 & 0 & 42 \\
\hline 1143074 & 2601122 & 0 & 49 \\
\hline 177009 & 2338142 & 0 & 18 \\
\hline 177009 & 2338142 & 0 & 36 \\
\hline 177009 & 2338142 & 0 & 35 \\
\hline 177009 & 3687372 & 49 & 151 \\
\hline 177009 & 3687372 & 62 & 135 \\
\hline 177009 & 3687372 & 62 & 140 \\
\hline 177009 & 3687372 & 72 & 129 \\
\hline 177009 & 3687372 & 71 & 125 \\
\hline 177009 & 3687372 & 64 & 132 \\
\hline 177009 & 3687372 & 58 & 140 \\
\hline 177009 & 3687372 & 66 & 134 \\
\hline 177009 & 3687372 & 66 & 133 \\
\hline 177009 & 3687372 & 67 & 128 \\
\hline 177009 & 3687372 & 67 & 130 \\
\hline 177009 & 3687372 & 64 & 133 \\
\hline 177009 & 3687372 & 60 & 136 \\
\hline 177009 & 3687372 & 65 & 131 \\
\hline 177009 & 3687372 & 52 & 191 \\
\hline 177009 & 3687372 & 71 & 126 \\
\hline 177009 & 3687372 & 73 & 126 \\
\hline 177009 & 3687372 & 75 & 123 \\
\hline 177009 & 3687372 & 73 & 124 \\
\hline 177009 & 3687372 & 65 & 137 \\
\hline 177009 & 3687372 & 69 & 131 \\
\hline 177009 & 3687372 & 72 & 124 \\
\hline 177009 & 3687372 & 74 & 123 \\
\hline 177009 & 3687372 & 72 & 127 \\
\hline 177009 & 3687372 & 61 & 134 \\
\hline 177009 & 3687372 & 60 & 136 \\
\hline 177009 & 3687372 & 58 & 153 \\
\hline 177009 & 3687372 & 58 & 167 \\
\hline 177009 & 3687372 & 51 & 169 \\
\hline 177009 & 3687372 & 45 & 168 \\
\hline 177009 & 3687372 & 52 & 168 \\
\hline 177009 & 3687372 & 48 & 186 \\
\hline
\end{tabular}




\begin{tabular}{|c|c|c|c|}
\hline 2464112 & 3687372 & 154 & 191 \\
\hline 2464112 & 3687372 & 159 & 191 \\
\hline 2464112 & 3687372 & 168 & 191 \\
\hline 2464112 & 3687372 & 160 & 191 \\
\hline 2464112 & 3687372 & 156 & 191 \\
\hline 227502 & 1437975 & 0 & 14 \\
\hline 227502 & 1437975 & 0 & 13 \\
\hline 227502 & 1110806 & 0 & 8 \\
\hline 227502 & 3224844 & 0 & 65 \\
\hline 227502 & 1437975 & 0 & 12 \\
\hline 227502 & 1437975 & 0 & 13 \\
\hline 227502 & 1437975 & 0 & 13 \\
\hline 227502 & 2511713 & 0 & 42 \\
\hline 877972 & 2511713 & 6 & 27 \\
\hline 877972 & 2511713 & 6 & 30 \\
\hline 227502 & 2511713 & 0 & 39 \\
\hline 877972 & 2511713 & 5 & 33 \\
\hline 227502 & 2511713 & 0 & 37 \\
\hline 877972 & 1110806 & 6 & 11 \\
\hline 877972 & 1511882 & 6 & 17 \\
\hline 877972 & 1511882 & 6 & 17 \\
\hline 877972 & 1437975 & 6 & 15 \\
\hline 877972 & 1511882 & 6 & 18 \\
\hline 877972 & 2511713 & 8 & 28 \\
\hline 877972 & 2511713 & 6 & 28 \\
\hline 1110806 & 2511713 & 13 & 26 \\
\hline 3991875 & 4995520 & 131 & 150 \\
\hline 2635524 & 4372230 & 0 & 9 \\
\hline 2635524 & 4372230 & 0 & 7 \\
\hline 2635524 & 4372230 & 0 & 7 \\
\hline 2635524 & 4372230 & 0 & 7 \\
\hline 2635524 & 4372230 & 0 & 9 \\
\hline 2635524 & 4372230 & 0 & 8 \\
\hline 2635524 & 4372230 & 0 & 4 \\
\hline 2635524 & 4372230 & 0 & 8 \\
\hline 2635524 & 4372230 & 0 & 4 \\
\hline 2635524 & 4372230 & 0 & 10 \\
\hline 2635524 & 4372230 & 0 & 5 \\
\hline 2635524 & 4372230 & 0 & 4 \\
\hline 2635524 & 4372230 & 0 & 4 \\
\hline 2635524 & 4372230 & 0 & 5 \\
\hline 2635524 & 4372230 & 0 & 11 \\
\hline 2635524 & 4372230 & 0 & 8 \\
\hline
\end{tabular}




\begin{tabular}{|c|c|c|c|}
\hline 2635524 & 4372230 & 0 & 7 \\
\hline 2635524 & 4372230 & 0 & 8 \\
\hline 2635524 & 4372230 & 0 & 7 \\
\hline 2635524 & 4372230 & 0 & 11 \\
\hline 2635524 & 4372230 & 0 & 10 \\
\hline 2635524 & 4372230 & 0 & 11 \\
\hline 2635524 & 4372230 & 0 & 15 \\
\hline 2635524 & 4372230 & 0 & 15 \\
\hline 2635524 & 4372230 & 0 & 11 \\
\hline 2635524 & 4372230 & 0 & 13 \\
\hline 2635524 & 4372230 & 0 & 12 \\
\hline 2635524 & 4372230 & 0 & 12 \\
\hline 2635524 & 4372230 & 0 & 15 \\
\hline 2635524 & 4372230 & 0 & 9 \\
\hline 2635524 & 4372230 & 0 & 13 \\
\hline 2635524 & 4372230 & 0 & 10 \\
\hline 2635524 & 4372230 & 0 & 8 \\
\hline 2635524 & 4372230 & 0 & 14 \\
\hline 2635524 & 4372230 & 0 & 11 \\
\hline 2635524 & 4372230 & 0 & 12 \\
\hline 2635524 & 4372230 & 0 & 13 \\
\hline 2635524 & 4372230 & 0 & 10 \\
\hline 2635524 & 4372230 & 0 & 10 \\
\hline 2635524 & 4372230 & 0 & 9 \\
\hline 2635524 & 4372230 & 0 & 10 \\
\hline 2635524 & 4372230 & 0 & 10 \\
\hline 2635524 & 4372230 & 0 & 9 \\
\hline 2635524 & 4372230 & 0 & 11 \\
\hline 2635524 & 4372230 & 0 & 8 \\
\hline 2635524 & 4372230 & 0 & 9 \\
\hline 2635524 & 4372230 & 0 & 9 \\
\hline 2635524 & 4372230 & 0 & 8 \\
\hline 2635524 & 4372230 & 0 & 6 \\
\hline 2635524 & 4372230 & 0 & 5 \\
\hline 2635524 & 4372230 & 0 & 5 \\
\hline 2635524 & 4372230 & 0 & 13 \\
\hline 2635524 & 4372230 & 0 & 11 \\
\hline 2635524 & 4372230 & 0 & 11 \\
\hline 2635524 & 4372230 & 0 & 11 \\
\hline 2635524 & 4372230 & 0 & 13 \\
\hline 2635524 & 4372230 & 0 & 13 \\
\hline 2635524 & 4372230 & 0 & 12 \\
\hline 2635524 & 4372230 & 0 & 11 \\
\hline
\end{tabular}




\begin{tabular}{|c|c|c|c|}
\hline 2635524 & 4372230 & 0 & 12 \\
\hline 2635524 & 4372230 & 0 & 12 \\
\hline 2635524 & 4372230 & 0 & 13 \\
\hline 2635524 & 4372230 & 0 & 11 \\
\hline 2635524 & 4372230 & 0 & 14 \\
\hline 2635524 & 4372230 & 0 & 9 \\
\hline 2635524 & 4372230 & 0 & 5 \\
\hline 2635524 & 4372230 & 0 & 7 \\
\hline 2635524 & 4372230 & 0 & 10 \\
\hline 2635524 & 4372230 & 0 & 12 \\
\hline 2635524 & 4372230 & 0 & 11 \\
\hline 2635524 & 4372230 & 0 & 11 \\
\hline 2635524 & 4372230 & 0 & 13 \\
\hline 2635524 & 4372230 & 0 & 13 \\
\hline 2635524 & 4372230 & 0 & 18 \\
\hline 2635524 & 4372230 & 0 & 21 \\
\hline 2635524 & 4372230 & 0 & 23 \\
\hline 2635524 & 4372230 & 0 & 26 \\
\hline 2635524 & 4372230 & 0 & 17 \\
\hline 2635524 & 4372230 & 0 & 18 \\
\hline 2635524 & 4372230 & 0 & 19 \\
\hline 1119248 & 2115727 & 0 & 12 \\
\hline 1119248 & 2582768 & 15 & 38 \\
\hline 1119248 & 2582768 & 18 & 38 \\
\hline 1119248 & 2582768 & 16 & 46 \\
\hline 2115727 & 3814681 & 45 & 77 \\
\hline 2582768 & 3814681 & 54 & 79 \\
\hline 2115727 & 3814681 & 43 & 81 \\
\hline 2115727 & 3814681 & 48 & 81 \\
\hline 2115727 & 3814681 & 44 & 85 \\
\hline 2115727 & 3814681 & 43 & 84 \\
\hline 2746445 & 4349920 & 83 & 114 \\
\hline 2746445 & 4349920 & 85 & 116 \\
\hline 2746445 & 4349920 & 88 & 116 \\
\hline 2746445 & 4349920 & 95 & 114 \\
\hline 2746445 & 4349920 & 92 & 119 \\
\hline 2746445 & 4349920 & 93 & 123 \\
\hline 3814681 & 4757842 & 122 & 131 \\
\hline
\end{tabular}




\begin{tabular}{|c|c|c|c|c|c|c|c|}
\hline meth_block\# & methylation_window_start & methylation_window_end & \#_continous_methQTL & methQTL_interval (cM) & mqtl_direction & block_direction & avg_additive_effect \\
\hline 1 & chr1_2638000 & chr1_2652000 & 6 & chr1:235-289 & $6 \mathrm{WL} \mid \mathrm{RJF} 0$ & WL & 6.19 \\
\hline 2 & chr1_3421000 & chr1_3455000 & 27 & chr1:227-341 & $27 \mathrm{WL} \mid \mathrm{RJF} 0$ & WL & 7.61 \\
\hline 3 & chr1_13859000 & chr1_13864000 & 4 & chr1:262-311 & $0 \mathrm{WL} \mid \mathrm{RJF} 4$ & RJF & -1.87 \\
\hline 4 & chr1_41177000 & chr1_41180000 & 4 & chr1:785-795 & 0 WL | RJF 4 & RJF & -2.56 \\
\hline 5 & chr1_46163000 & chr1_46179000 & 14 & chr1:823-888 & $0 \mathrm{WL} \mid \mathrm{RJF} 14$ & RJF & -4.01 \\
\hline 6 & chr1_62140000 & chr1_62152000 & 9 & chr1:1060-1169 & 0 WL | RJF 9 & RJF & -2.63 \\
\hline 7 & chr1_111231000 & chr1_111238000 & 8 & chr1:1513-1548 & $0 \mathrm{WL} \mid \mathrm{RJF} 8$ & RJF & -4 \\
\hline 8 & chr1_137574000 & chr1_137633000 & 58 & chr1:1784-1830 & 0 WL | RJF 58 & RJF & -38 \\
\hline 9 & chr1_137641000 & chr1_137758000 & 118 & chr1:1784-1822 & $0 \mathrm{WL} \mid \mathrm{RJF} 118$ & RJF & -39.83 \\
\hline 10 & chr1_137767000 & chr1_137778000 & 10 & chr1:1780-1836 & $0 \mathrm{WL} \mid \mathrm{RJF} 10$ & RJF & -27.43 \\
\hline 11 & chr1_137785000 & chr1_137790000 & 4 & chr1:1780-1828 & $1 \mathrm{WL} \mid \mathrm{RJF} 3$ & - & -12.39 \\
\hline 12 & chr1_138281000 & chr1_138288000 & 4 & chr1:1733-1806 & $4 \mathrm{WL} \mid \mathrm{RJF} 0$ & WL & 2.86 \\
\hline 13 & chr1_141258000 & chr1_141272000 & 7 & chr1:1826-1869 & 0 WL | RJF 7 & RJF & -7.69 \\
\hline 14 & chr1_141286000 & chr1_141301000 & 10 & chr1:1823-1873 & $0 \mathrm{WL} \mid \mathrm{RJF} 10$ & RJF & -10.37 \\
\hline 15 & chr1_141317000 & chr1_141337000 & 12 & chr1:1826-1866 & $0 \mathrm{WL} \mid \mathrm{RJF} 12$ & RJF & -6.87 \\
\hline 16 & chr1_141352000 & chr1_141376000 & 15 & chr1:1814-1878 & $0 \mathrm{WL} \mid \mathrm{RJF} 15$ & RJF & -11.58 \\
\hline 17 & chr1_141384000 & chr1_141391000 & 6 & chr1:1820-1862 & $0 \mathrm{WL} \mid \mathrm{RJF} 6$ & RJF & -12.47 \\
\hline 18 & chr1_141480000 & chr1_141488000 & 7 & chr1:1829-1869 & $0 \mathrm{WL} \mid \mathrm{RJF} 7$ & RJF & -5.51 \\
\hline 19 & chr1_141503000 & chr1_141511000 & 7 & chr1:1824-1863 & $0 \mathrm{WL} \mid \mathrm{RJF} 7$ & RJF & -12.03 \\
\hline 20 & chr1_141556000 & chr1_141562000 & 4 & chr1:1786-1902 & $0 \mathrm{WL} \mid \mathrm{RJF} 4$ & RJF & -6.77 \\
\hline 21 & chr1_194066000 & chr1_194071000 & 4 & chr1:2157-2276 & $4 \mathrm{WL} \mid \mathrm{RJF} 0$ & WL & 11.98 \\
\hline 22 & chr1_194235000 & chr1_194239000 & 4 & chr1:2142-2277 & $4 \mathrm{WL} \mid \mathrm{RJF} 0$ & WL & 11.68 \\
\hline 23 & chr2_9639000 & chr2_9645000 & 6 & chr2:121-156 & $6 \mathrm{WL} \mid \mathrm{RJF} 0$ & WL & 3.76 \\
\hline 24 & chr2_27959000 & chr2_27966000 & 4 & chr2:246-329 & $0 \mathrm{WL} \mid \mathrm{RJF} 4$ & RJF & -2.24 \\
\hline 25 & chr2_31012000 & chr2_31017000 & 6 & chr2:214-330 & $0 \mathrm{WL} \mid \mathrm{RJF} 6$ & RJF & -6.41 \\
\hline 26 & chr2_31032000 & chr2_31039000 & 6 & chr2:216-329 & $0 \mathrm{WL} \mid \mathrm{RJF} 6$ & RJF & -6.3 \\
\hline 27 & chr2_31063000 & chr2_31070000 & 8 & chr2:213-334 & $0 \mathrm{WL} \mid \mathrm{RJF} 8$ & RJF & -6.06 \\
\hline 28 & chr2_31087000 & chr2_31095000 & 7 & chr2:214-330 & $0 \mathrm{WL} \mid \mathrm{RJF} 7$ & RJF & -6.45 \\
\hline 29 & chr2_31120000 & chr2_31123000 & 4 & chr2:214-330 & $0 \mathrm{WL} \mid \mathrm{RJF} 4$ & RJF & -5.59 \\
\hline 30 & chr2_40582000 & chr2_40610000 & 20 & chr2:403-478 & $20 \mathrm{WL} \mid \mathrm{RJF} 0$ & WL & 11.17 \\
\hline 31 & chr2_52699000 & chr2_52715000 & 11 & chr2:552-573 & $0 \mathrm{WL} \mid \mathrm{RJF} 11$ & RJF & -4.41 \\
\hline 32 & chr2_54992000 & chr2_54997000 & 5 & chr2:550-599 & $0 \mathrm{WL} \mid \mathrm{RJF} 5$ & RJF & -2.92 \\
\hline 33 & chr2_71857000 & chr2_71878000 & 14 & chr2:662-737 & $14 \mathrm{WL} \mid \mathrm{RJF} 0$ & WL & 2.31 \\
\hline 34 & chr2_74470000 & chr2_74491000 & 10 & chr2:677-709 & $0 \mathrm{WL} \mid \mathrm{RJF} 10$ & RJF & -1.48 \\
\hline 35 & chr2_74763000 & chr2_74770000 & 4 & chr2:677-721 & $4 \mathrm{WL} \mid \mathrm{RJF} 0$ & WL & 1.84 \\
\hline 36 & chr2_76152000 & chr2_76159000 & 5 & chr2:694-733 & $0 \mathrm{WL} \mid \mathrm{RJF} 5$ & RJF & -3.78 \\
\hline 37 & chr2_80348000 & chr2_80354000 & 6 & chr2:716-744 & $6 \mathrm{WL} \mid \mathrm{RJF} 0$ & WL & 15.7 \\
\hline 38 & chr2_94415000 & chr2_94420000 & 5 & chr2:773-803 & $5 \mathrm{WL} \mid \mathrm{RJF} 0$ & WL & 1.74 \\
\hline 39 & chr2_94486000 & chr2_94491000 & 4 & chr2:757-810 & $0 \mathrm{WL} \mid \mathrm{RJF} 4$ & RJF & -1.34 \\
\hline 40 & chr2_96310000 & chr2_96319000 & 6 & chr2:775-870 & $0 \mathrm{WL} \mid \mathrm{RJF} 6$ & RJF & -20.09 \\
\hline 41 & chr2_96348000 & chr2_96356000 & 5 & chr2:776-810 & $0 \mathrm{WL} \mid \mathrm{RJF} 5$ & RJF & -18.77 \\
\hline 42 & chr2_96373000 & chr2_96393000 & 11 & chr2:776-873 & $0 \mathrm{WL} \mid \mathrm{RJF} 11$ & RJF & -18.8 \\
\hline 43 & chr2_111729000 & chr2_111745000 & 14 & chr2:884-970 & $0 \mathrm{WL}$ | RJF 14 & RJF & -2.71 \\
\hline 44 & chr2_111752000 & chr2_111764000 & 6 & chr2:885-961 & $0 \mathrm{WL} \mid \mathrm{RJF} 6$ & RJF & -2.67 \\
\hline 45 & chr4_85662000 & chr4_85672000 & 5 & chr4:552-569 & $0 \mathrm{WL} \mid \mathrm{RJF} 5$ & RJF & -6.78 \\
\hline 46 & chr4_85682000 & chr4_85705000 & 20 & chr4:550-569 & $0 \mathrm{WL} \mid \mathrm{RJF} 20$ & RJF & -8.22 \\
\hline 47 & chr4_85735000 & chr4_85739000 & 5 & chr4:555-569 & $0 \mathrm{WL} \mid \mathrm{RJF} 5$ & RJF & -8.74 \\
\hline 48 & chr4_85753000 & chr4_85758000 & 5 & chr4:551-569 & $0 \mathrm{WL} \mid \mathrm{RJF} 5$ & RJF & -7.83 \\
\hline 49 & chr4_85783000 & chr4_85798000 & 9 & chr4:551-569 & 0 WL | RJF 9 & RJF & -8.6 \\
\hline 50 & chr4_86423000 & chr4_86461000 & 29 & chr4:556-569 & 0 WL | RJF 29 & RJF & -2.75 \\
\hline 51 & chr5_49458000 & chr5_49468000 & 4 & chr5:336-403 & $0 \mathrm{WL} \mid \mathrm{RJF} 4$ & RJF & -3.08 \\
\hline 52 & chr6_1016000 & chr6_1024000 & 4 & chr6:0-52 & $4 \mathrm{WL} \mid \mathrm{RJF} 0$ & WL & 13.1 \\
\hline 53 & chr6_1046000 & chr6_1060000 & 9 & chr6:0-52 & $9 \mathrm{WL} \mid \mathrm{RJF} 0$ & WL & 13.69 \\
\hline 54 & chr6_4433000 & chr6_4450000 & 8 & chr6:0-97 & $8 \mathrm{WL} \mid \mathrm{RJF} 0$ & WL & 12.6 \\
\hline 55 & chr6_6717000 & chr6_6727000 & 7 & chr6:73-102 & $7 \mathrm{WL} \mid \mathrm{RJF} 0$ & WL & 12.08 \\
\hline 56 & chr6_6930000 & chr6_6935000 & 5 & chr6:46-135 & $0 \mathrm{WL} \mid \mathrm{RJF} 5$ & RJF & -3.41 \\
\hline 57 & chr6_11027000 & chr6_11070000 & 34 & chr6:119-185 & $0 \mathrm{WL}$ | RJF 34 & RJF & -7.2 \\
\hline 58 & chr6_11078000 & chr6_11103000 & 22 & chr6:118-187 & 0 WL | RJF 22 & RJF & -12.02 \\
\hline 59 & chr6_111111000 & chr6_11121000 & 10 & chr6:120-186 & $0 \mathrm{WL} \mid \mathrm{RJF} 10$ & RJF & -9.94 \\
\hline 60 & chr6_11761000 & chr6_11787000 & 20 & chr6:121-188 & $0 \mathrm{WL} \mid \mathrm{RJF} 20$ & RJF & -7.01 \\
\hline 61 & chr6_11827000 & chr6_11830000 & 4 & chr6:123-186 & $0 \mathrm{WL} \mid \mathrm{RJF} 4$ & RJF & -6.35 \\
\hline 62 & chr6_12798000 & chr6_12810000 & 4 & chr6:120-191 & 0 WL | RJF 4 & RJF & -8.16 \\
\hline 63 & chr6_21469000 & chr6_21476000 & 4 & chr6:166-241 & $4 \mathrm{WL} \mid \mathrm{RJF} 0$ & WL & 2.29 \\
\hline 64 & chr7_24131000 & chr7_24143000 & 5 & chr7:188-231 & $3 \mathrm{WL} \mid \mathrm{RJF} 2$ & - & 0.5 \\
\hline 65 & chr7_32069000 & chr7_32077000 & 9 & chr7:264-304 & $9 \mathrm{WL} \mid \mathrm{RJF} 0$ & WL & 3.25 \\
\hline 66 & chr7_32856000 & chr7_32865000 & 10 & chr7:268-336 & $10 \mathrm{WL} \mid \mathrm{RJF} 0$ & WL & 2.62 \\
\hline 67 & chr8_11310000 & chr8_11331000 & 12 & chr8:51-95 & $0 \mathrm{WL} \mid \mathrm{RJF} 12$ & RJF & -39.38 \\
\hline 68 & chr8_11356000 & chr8_11364000 & 5 & chr8:51-94 & $0 \mathrm{WL} \mid \mathrm{RJF} 5$ & RJF & -50.1 \\
\hline 69 & chr8_18832000 & chr8_18839000 & 7 & chr8:95-166 & $7 \mathrm{WL} \mid$ RJF 0 & WL & 3.75 \\
\hline 70 & chr8_21115000 & chr8_21119000 & 5 & chr8:113-148 & $0 \mathrm{WL} \mid \mathrm{RJF} 5$ & RJF & -3.9 \\
\hline 71 & chr9_5457000 & chr9_5465000 & 9 & chr9:76-93 & $0 \mathrm{WL} \mid \mathrm{RJF} 9$ & RJF & -63.1 \\
\hline 72 & chr9_10810000 & chr9_10815000 & 4 & chr9:47-104 & 4 WL | RJF 0 & WL & 3.9 \\
\hline 73 & chr9_11415000 & chr9_11423000 & 4 & chr9:90-114 & $1 \mathrm{WL} \mid \mathrm{RJF} 3$ & - & -1.08 \\
\hline 74 & chr9_13425000 & chr9_13431000 & 5 & chr9:108-124 & $5 \mathrm{WL} \mid \mathrm{RJF} 0$ & WL & 1.97 \\
\hline 75 & chr10_13769000 & chr10_13776000 & 8 & chr10:163-197 & $0 \mathrm{WL} \mid \mathrm{RJF} 8$ & RJF & -3.95 \\
\hline 76 & chr11_11864000 & chr11_11868000 & 5 & chr11:112-135 & $5 \mathrm{WL} \mid \mathrm{RJF} 0$ & WL & 4.01 \\
\hline 77 & chr11_13592000 & chr11_13604000 & 9 & chr11:75-175 & $0 \mathrm{WL} \mid \mathrm{RJF} 9$ & RJF & -2.59 \\
\hline 78 & chr11_15650000 & chr11_15663000 & 7 & chr11:109-156 & $7 \mathrm{WL} \mid \mathrm{RJF} 0$ & WL & 3.69 \\
\hline 79 & chr12_478000 & chr12_485000 & 4 & chr12:0-10 & $0 \mathrm{WL} \mid \mathrm{RJF} 4$ & RJF & -31.42 \\
\hline 80 & chr12_2717000 & chr12_2731000 & 8 & chr12:12-51 & 4 WL | RJF 4 & - & -2.3 \\
\hline 81 & chr14_2647000 & chr14_2650000 & 4 & chr14:11-22 & $4 \mathrm{WL} \mid \mathrm{RJF} 0$ & WL & 6.94 \\
\hline 82 & chr25_3183000 & chr25_3197000 & 14 & chr25:45-186 & $0 \mathrm{WL} \mid \mathrm{RJF} 14$ & RJF & -6.6 \\
\hline 83 & chr27_2222000 & chr27_2235000 & 5 & chr27:0-13 & $0 \mathrm{WL} \mid \mathrm{RJF} 5$ & RJF & -9.06 \\
\hline 84 & chr27_2245000 & chr27_2255000 & 8 & chr27:0-15 & $0 \mathrm{WL} \mid \mathrm{RJF} 8$ & RJF & -6.89 \\
\hline 85 & chr27_2324000 & chr27_2343000 & 10 & chr27:0-10 & $0 \mathrm{WL} \mid \mathrm{RJF} 10$ & RJF & -4.93 \\
\hline 86 & chr27_2423000 & chr27_2430000 & 5 & chr27:0-8 & O WL | RJF 5 & RJF & -6.43 \\
\hline
\end{tabular}


Supplementary Table 3. Trans methQTL hotpsots. Locations of hotspots, number of meth phenotypes per hotspot, number with positive additive effects (WL genotype methylated), number with negative additive effects (RFF genotype methylated) for all hotspots with ten or more ph eqt__ene_na \#_significant \#__suggestive
me _epistatic_nt_epistatic_int

\begin{tabular}{|c|c|c|c|c|c|c|c|c|c|c|c|c|}
\hline hotspotID & num_qtl & chr & QTL_dir_rif & QTL_dir_wI & avg_pos & lower_max & upper_min & lower_bp & $\begin{array}{l}\text { per_bp } \quad \text { me } \\
\text { PATZ1 LIMK2 }\end{array}$ & eractions & $\begin{array}{l}\text { Significant GO_terms } \\
\text { SM01375:SM01375; } \\
\text { IPR001372:Dynin light chain, }\end{array}$ & 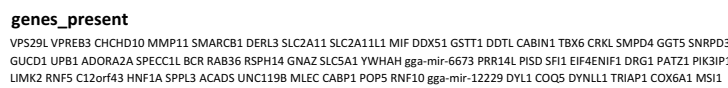 \\
\hline & 561 Gg_rs150238 & 15 & 561 & 0 & 95.61 & 83 & 111 & 8187241 & 9781703 & 0 & 36 type $1 / 2$ & 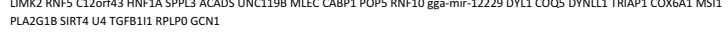 \\
\hline & 324 Gg_rs150605 & 2 & 39 & 285 & 98.43 & 95 & 108 & 7800010 & 9076859 & 1 & 18 & INSIG1 EN2 RBM33 SHH RNF32 LMBR1 NOM1 MNX1 UBE3C DNAJB6 gga-mir-153 \\
\hline & 242 Gg_rs137816 & 7 & 1 & 241 & 176.39 & 176 & 185 & 20658484 & 21209041 & 0 & 5 & $\begin{array}{l}\text { KCNH7 IFIH1 FAP GCG DPP4 } \\
\text { CNIH3 WDR26 CNIH4 NVL DEGS1 FBXO28 TP53BP2 CAPN2 CAPN8 SUSD4 TLR5 DISP1 BROX }\end{array}$ \\
\hline & 150 Gg_rs152823. & 3 & 66 & 84 & 219.85 & 212 & 228 & 17541260 & 18540332 & 1 & 24 & $\begin{array}{l}\text { AIDA MIA3 TAF1A HHIPL2 DUSP10 } \\
\text { AXL MGA gga-mir-6685 MAPKBP1 JMJD7 EHD4 PLA2G4F VPS39 TMEM87A GANC CAPN3 }\end{array}$ \\
\hline & 98 Gg_rs156859 & 5 & 98 & 0 & 200.87 & 197 & 204 & 25114680 & $26294257^{\mathrm{N} 3 \mathrm{ACOT1L}} \mathrm{G}$ & 12 & 14 & ZNF106 SNAP23 LRRC57 HAUS2 TTBK2 ROM1 UBR1 TMEM62 \\
\hline & 69 Gg_rs141339. & 2 & 37 & 32 & 90.26 & 80 & 93 & 6758997 & 7408576 & 0 & 4 & $\begin{array}{l}\text { gga-mir-6554 XRCC2 } \\
\text { (g) }\end{array}$ \\
\hline & 65 RBL3041 & 5 & 65 & 0 & 209.45 & 210 & 217 & 27238003 & 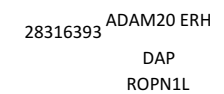 & 0 & 6 & $\begin{array}{l}\text { PCNX1 MAP3K9 TTC9 MED6 ADAM20 SYNJ2BP COX16 SLC8A3 SMOC1 SRSF5 gga-mir-1710 } \\
\text { SUSD6 PLEKHD1 SLC39A9 ERH GALNT16 }\end{array}$ \\
\hline & 52 Gg_rs142082. & 2 & 3 & 49 & 723.81 & 720 & 724 & 77980689 & 79034049 & 0 & 4 & DAP ANKRD33B SNRNP48 ROPN1L MARCH6 SBK2 CCT5 ATPSCKMT SNORD123 SEMA5A \\
\hline & 50 Gg_rs147937 & 1 & 48 & 2 & 282.34 & 279 & 286 & 13507676 & 14113996 & 1 & 1 & LHFPL3 KMT2E SRPK2 \\
\hline & 50 rbl1871 & 14 & 30 & 20 & 263 & 258 & 266 & 14561619 & 15000631 & 0 & 3 & CCNF gga-mir-1715 gga-mir-7471 MAPK8IP3 MRPS34 GLYR1 ROGDI MCHR2 ACSM5 GPR139 \\
\hline 11 & 47 Gg_rs158284 & 7 & 36 & 11 & 10.36 & 8 & 18 & 2311740 & 2670288 & 0 & $\begin{array}{l}1 \\
\text { IPR015497:Epidermal growth } \\
\text { factor receptor ligand; } \\
\text { GO:0005154 epidermal growth }\end{array}$ & FRZB MAP2 \\
\hline 12 & 44 Gg_rs144576. & 4 & 0 & 44 & 321.7 & 313 & 338 & 40216634 & 45018205 & 0 & 2 factor receptor binding & 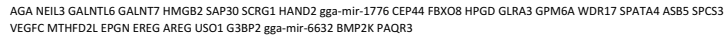 \\
\hline 13 & 40 Gg_rs150470 & 19 & 2 & 38 & 76.67 & 75 & 77 & 4306340 & 4331777 & 1 & 1 & OLR2J RASAAB UPK3B \\
\hline
\end{tabular}




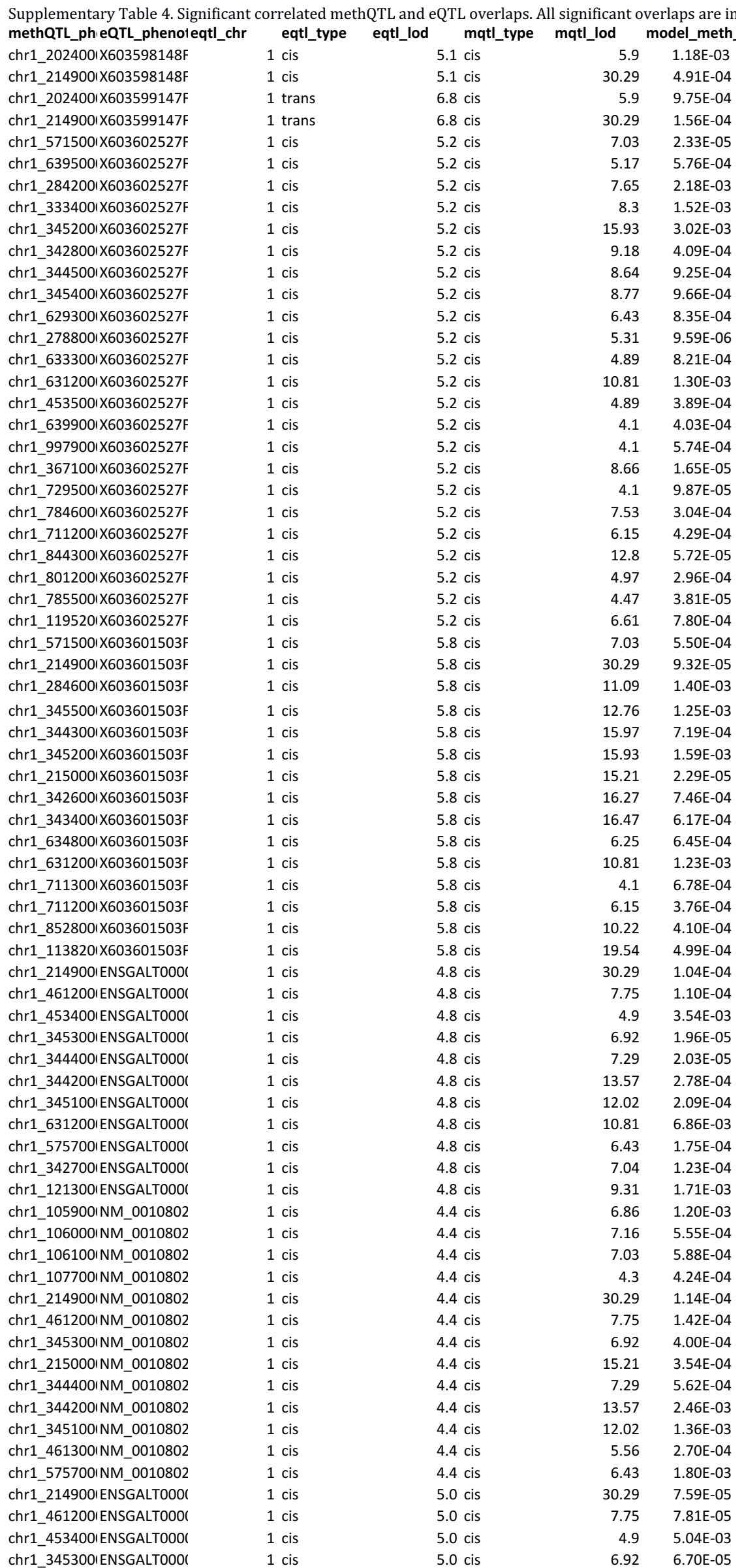




\begin{tabular}{|c|c|c|c|c|}
\hline chr1_215000IENSGALT000( & 1 cis & 5.0 cis & 15.21 & $5.90 \mathrm{E}-04$ \\
\hline chr1_344400IENSGALTO00 & 1 cis & 5.0 cis & 7.29 & $7.28 \mathrm{E}-05$ \\
\hline chr1_344200IENSGALT000( & 1 cis & 5.0 cis & 13.57 & $5.23 \mathrm{E}-04$ \\
\hline chr1_345100IENSGALT000( & $1 \mathrm{cis}$ & 5.0 cis & 12.02 & $3.95 \mathrm{E}-04$ \\
\hline chr1_631200IENSGALT000( & 1 cis & 5.0 cis & 10.81 & $5.42 \mathrm{E}-03$ \\
\hline chr1_575700IENSGALT000( & $1 \mathrm{cis}$ & 5.0 cis & 6.43 & 2.67E-04 \\
\hline chr1_342700IENSGALTO00( & 1 cis & 5.0 cis & 7.04 & $3.84 \mathrm{E}-04$ \\
\hline chr1_132300INM_204242_ & $1 \mathrm{cis}$ & $4.3 \mathrm{cis}$ & 7.78 & 4.55E-04 \\
\hline chr1_461200INM_204242_ & 1 cis & $4.3 \mathrm{cis}$ & 7.75 & $1.09 \mathrm{E}-05$ \\
\hline chr1_711100INM_204242_ & $1 \mathrm{cis}$ & $4.3 \mathrm{cis}$ & 4.1 & $1.53 \mathrm{E}-03$ \\
\hline chr1_552100INM_204242_ & 1 cis & $4.3 \mathrm{cis}$ & 5.62 & $1.71 \mathrm{E}-04$ \\
\hline chr1_344200INM_204242_ & $1 \mathrm{cis}$ & 4.3 cis & 13.57 & $3.78 \mathrm{E}-03$ \\
\hline chr1_345100INM_204242_ & 1 cis & $4.3 \mathrm{cis}$ & 12.02 & $2.34 \mathrm{E}-03$ \\
\hline chr1_631200INM_204242_ & $1 \mathrm{cis}$ & $4.3 \mathrm{cis}$ & 10.81 & $1.60 \mathrm{E}-03$ \\
\hline chr1_488900INM_204242_ & $1 \mathrm{cis}$ & $4.3 \mathrm{cis}$ & 4.1 & $9.39 \mathrm{E}-05$ \\
\hline chr1_575700INM_204242_ & $1 \mathrm{cis}$ & $4.3 \mathrm{cis}$ & 6.43 & $1.50 \mathrm{E}-04$ \\
\hline chr1_265200INM_204242_ & $1 \mathrm{cis}$ & $4.3 \mathrm{cis}$ & 7.26 & $1.61 \mathrm{E}-02$ \\
\hline chr1_113820INM_204242_ & $1 \mathrm{cis}$ & $4.3 \mathrm{cis}$ & 19.54 & 4.40E-04 \\
\hline chr1_121300INM_204242_ & 1 cis & $4.3 \mathrm{cis}$ & 9.31 & 4.42E-05 \\
\hline chr1_130810IENSGALT000 & $1 \mathrm{cis}$ & $5.2 \mathrm{cis}$ & 5.88 & $1.96 \mathrm{E}-04$ \\
\hline chr1_130240IENSGALT000( & $1 \mathrm{cis}$ & 5.2 cis & 7.86 & $3.62 \mathrm{E}-04$ \\
\hline chr1_115810।ENSGALT000( & $1 \mathrm{cis}$ & $5.2 \mathrm{cis}$ & 8.44 & 8.57E-04 \\
\hline chr1_117050IENSGALT000( & $1 \mathrm{cis}$ & $5.2 \mathrm{cis}$ & 8.23 & $1.37 \mathrm{E}-03$ \\
\hline chr1_132620।X603868019F & $1 \mathrm{cis}$ & $14.4 \mathrm{cis}$ & 6.42 & $1.11 \mathrm{E}-06$ \\
\hline chr1_126710।X603868019F & 1 cis & 14.4 cis & 4.1 & 9.37E-05 \\
\hline chr1_132250!X603868019F & $1 \mathrm{cis}$ & $14.4 \mathrm{cis}$ & 8.46 & $6.51 \mathrm{E}-05$ \\
\hline chr1_130810।X603868019F & $1 \mathrm{cis}$ & $14.4 \mathrm{cis}$ & 5.88 & $1.11 \mathrm{E}-04$ \\
\hline chr1_117720।X603868019F & $1 \mathrm{cis}$ & $14.4 \mathrm{cis}$ & 5.98 & $3.45 \mathrm{E}-04$ \\
\hline chr1_130240।X603868019F & $1 \mathrm{cis}$ & $14.4 \mathrm{cis}$ & 7.86 & $3.44 \mathrm{E}-06$ \\
\hline chr1_129820।X603868019F & $1 \mathrm{cis}$ & $14.4 \mathrm{cis}$ & 5.49 & 1.37E-04 \\
\hline chr1_1138201X603868019F & 1 cis & 14.4 cis & 19.54 & 7.30E-09 \\
\hline chr1_113830!X603868019F & $1 \mathrm{cis}$ & $14.4 \mathrm{cis}$ & 11.23 & 4.26E-05 \\
\hline chr1_131560।X603868019F & $1 \mathrm{cis}$ & $14.4 \mathrm{cis}$ & 4.93 & $1.72 \mathrm{E}-04$ \\
\hline chr1_113810।X603868019F & 1 cis & 14.4 cis & 7.73 & 7.79E-06 \\
\hline chr1_121300ıX03868019F & $1 \mathrm{cis}$ & 14.4 cis & 9.31 & $7.44 \mathrm{E}-06$ \\
\hline chr1_128320।X603868019F & $1 \mathrm{cis}$ & $14.4 \mathrm{cis}$ & 5.54 & 5.69E-04 \\
\hline chr1_1322201X603868019F & 1 cis & 14.4 cis & 8.65 & $2.82 \mathrm{E}-05$ \\
\hline chr1_117050)X603868019F & $1 \mathrm{cis}$ & $14.4 \mathrm{cis}$ & 8.23 & $1.40 \mathrm{E}-04$ \\
\hline chr1_134450।X603868019F & $1 \mathrm{cis}$ & $14.4 \mathrm{cis}$ & 5.24 & 8.56E-05 \\
\hline chr1_134640।X603868019F & 1 cis & 14.4 cis & 8.05 & $1.44 \mathrm{E}-04$ \\
\hline chr1_126700(X603868019F & 1 cis & 14.4 cis & 6.5 & 7.63E-07 \\
\hline chr1_119520।X603868019F & $1 \mathrm{cis}$ & $14.4 \mathrm{cis}$ & 6.61 & 4.64E-05 \\
\hline chr1_1324101X603868019F & 1 cis & 14.4 cis & 7.82 & $2.28 \mathrm{E}-04$ \\
\hline chr1_132630।X603868019F & $1 \mathrm{cis}$ & $14.4 \mathrm{cis}$ & 4.26 & $1.26 \mathrm{E}-04$ \\
\hline chr1_135070)X603868019F & $1 \mathrm{cis}$ & $14.4 \mathrm{cis}$ & 4.72 & $4.92 \mathrm{E}-04$ \\
\hline chr1_134640।X603596753F & 1 cis & 4.4 cis & 8.05 & $1.95 \mathrm{E}-04$ \\
\hline chr1_130810IENSGALT000( & 1 cis & 4.9 cis & 5.88 & $2.18 \mathrm{E}-04$ \\
\hline chr1_130240IENSGALT000( & $1 \mathrm{cis}$ & 4.9 cis & 7.86 & 8.14E-05 \\
\hline chr1_113820IENSGALTO00( & $1 \mathrm{cis}$ & 4.9 cis & 19.54 & 3.39E-03 \\
\hline chr1_115810IENSGALT000 & $1 \mathrm{cis}$ & $4.9 \mathrm{cis}$ & 8.44 & $1.32 \mathrm{E}-03$ \\
\hline chr1_147980IENSGALT000( & 1 cis & 4.9 cis & 7.98 & $5.62 \mathrm{E}-04$ \\
\hline chr1_147860IENSGALT000( & $1 \mathrm{cis}$ & 4.9 cis & 4.25 & $1.43 \mathrm{E}-03$ \\
\hline chr1_1321301X603143689F & 1 cis & 11.5 cis & 4.19 & $1.61 \mathrm{E}-05$ \\
\hline chr1_126710'X603143689F & $1 \mathrm{cis}$ & $11.5 \mathrm{cis}$ & 4.1 & $2.56 \mathrm{E}-05$ \\
\hline chr1_132250।X603143689F & $1 \mathrm{cis}$ & 11.5 cis & 8.46 & $2.55 \mathrm{E}-04$ \\
\hline chr1_128110।X603143689F & $1 \mathrm{cis}$ & $11.5 \mathrm{cis}$ & 5.54 & 7.70E-05 \\
\hline chr1_142310।X603143689F & $1 \mathrm{cis}$ & $11.5 \mathrm{cis}$ & 6.08 & $6.34 \mathrm{E}-09$ \\
\hline chr1_129820।X603143689F & $1 \mathrm{cis}$ & $11.5 \mathrm{cis}$ & 5.49 & $6.43 E-06$ \\
\hline chr1_128320।X603143689F & 1 cis & 11.5 cis & 5.54 & $1.60 \mathrm{E}-03$ \\
\hline chr1_124170)X603143689F & $1 \mathrm{cis}$ & $11.5 \mathrm{cis}$ & 8.92 & $6.15 \mathrm{E}-08$ \\
\hline chr1_1322201X603143689F & $1 \mathrm{cis}$ & 11.5 cis & 8.65 & $1.89 \mathrm{E}-07$ \\
\hline chr1_132380।X603143689F & $1 \mathrm{cis}$ & $11.5 \mathrm{cis}$ & 7.92 & $1.70 \mathrm{E}-06$ \\
\hline chr1_134640।X603143689F & 1 cis & 11.5 cis & 8.05 & 3.53E-04 \\
\hline chr1_126700।X603143689F & $1 \mathrm{cis}$ & $11.5 \mathrm{cis}$ & 6.5 & 4.51E-06 \\
\hline chr1_135460।X603143689F & $1 \mathrm{cis}$ & 11.5 cis & 11.12 & 7.07E-10 \\
\hline chr1_132410।X603143689F & $1 \mathrm{cis}$ & $11.5 \mathrm{cis}$ & 7.82 & $4.32 \mathrm{E}-04$ \\
\hline chr1_136280ı603143689F & $1 \mathrm{cis}$ & 11.5 cis & 5.54 & 3.83E-05 \\
\hline chr1_132390।X603143689F & $1 \mathrm{cis}$ & $11.5 \mathrm{cis}$ & 4.78 & $3.90 \mathrm{E}-06$ \\
\hline chr1_128950।X603143689F & 1 cis & 11.5 cis & 4.31 & $3.21 \mathrm{E}-05$ \\
\hline chr1_138640।X603143689F & $1 \mathrm{cis}$ & $11.5 \mathrm{cis}$ & 7.13 & $1.32 \mathrm{E}-06$ \\
\hline chr1_138630ıX603143689F & 1 cis & 11.5 cis & 5.83 & $5.02 \mathrm{E}-08$ \\
\hline chr1_132630।X603143689F & $1 \mathrm{cis}$ & $11.5 \mathrm{cis}$ & 4.26 & $1.28 \mathrm{E}-04$ \\
\hline
\end{tabular}




\begin{tabular}{|c|c|c|c|c|}
\hline chr1_1386501X603143689F & 1 cis & $11.5 \mathrm{cis}$ & 5.22 & $5.46 \mathrm{E}-05$ \\
\hline chr1_1350701X603143689F & $1 \mathrm{cis}$ & 11.5 cis & 4.72 & 3.99E-04 \\
\hline chr1_137750।X603143689F & 1 cis & 11.5 cis & 4.54 & $2.80 \mathrm{E}-07$ \\
\hline chr1_1423201X603143689F & 1 cis & 11.5 cis & 7.06 & 7.47E-06 \\
\hline chr1_142240ıX603143689F & 1 cis & 11.5 cis & 6.51 & $9.55 \mathrm{E}-05$ \\
\hline chr1_142810।X603143689F & 1 cis & 11.5 cis & 8.19 & $1.25 \mathrm{E}-06$ \\
\hline chr1_147990।X603862742F & $1 \mathrm{cis}$ & 4.9 cis & 11.08 & $6.52 \mathrm{E}-04$ \\
\hline chr1_220340IENSGALT000( & $1 \mathrm{cis}$ & 4.1 cis & 8.05 & $1.75 \mathrm{E}-04$ \\
\hline chr1_2203401X603598591F & 1 cis & 4.0 cis & 8.05 & $6.48 \mathrm{E}-04$ \\
\hline chr1_1658101X603598591F & 1 cis & 4.0 cis & 4.1 & $3.20 \mathrm{E}-03$ \\
\hline chr1_2203401X603597317F & 1 cis & $9.6 \mathrm{cis}$ & 8.05 & $2.18 \mathrm{E}-04$ \\
\hline chr1_2139101X603597317F & 1 cis & 9.6 trans & 6.79 & $6.76 \mathrm{E}-04$ \\
\hline chr1_220340IENSGALT000( & $1 \mathrm{cis}$ & $4.2 \mathrm{cis}$ & 8.05 & $1.13 \mathrm{E}-04$ \\
\hline chr1_220340IENSGALT000( & $1 \mathrm{cis}$ & 5.4 cis & 8.05 & 7.06E-04 \\
\hline chr1_202020IENSGALT000( & 1 cis & 5.4 cis & 5.48 & 3.34E-04 \\
\hline chr1_213910IENSGALT000( & 1 cis & 5.4 trans & 6.79 & 2.07E-03 \\
\hline chr1_2556001X603866079F & 1 cis & $3.7 \mathrm{cis}$ & 4.31 & 4.66E-05 \\
\hline chr1_2556001X603601949F & $1 \mathrm{cis}$ & $4.3 \mathrm{cis}$ & 4.31 & $6.35 \mathrm{E}-04$ \\
\hline chr1_4103501X603598966F & $1 \mathrm{cis}$ & 4.7 cis & 4.1 & $9.64 \mathrm{E}-05$ \\
\hline chr1_3602501X603598966F & $1 \mathrm{cis}$ & 4.7 cis & 4.1 & $1.31 \mathrm{E}-05$ \\
\hline chr1_3999401X603598966F & 1 cis & 4.7 cis & 5.54 & 2.02E-04 \\
\hline chr1_3643501X603598966F & 1 cis & 4.7 cis & 4.1 & $1.48 \mathrm{E}-04$ \\
\hline chr1_3638601X603598966F & 1 cis & 4.7 cis & 4.1 & 3.13E-07 \\
\hline chr1_358770IENSGALTO00( & $1 \mathrm{cis}$ & 4.9 cis & 6.17 & $1.63 \mathrm{E}-03$ \\
\hline chr1_377310IENSGALT000( & 1 cis & 4.9 cis & 7.06 & $1.42 \mathrm{E}-03$ \\
\hline chr1_378430IENSGALT000( & $1 \mathrm{cis}$ & 4.9 cis & 4.1 & $8.01 \mathrm{E}-04$ \\
\hline chr1_396230IENSGALT000( & 1 cis & 4.9 cis & 10.1 & $1.34 \mathrm{E}-05$ \\
\hline chr1_409150IENSGALT000( & 1 cis & 4.9 cis & 4.1 & 8.23E-04 \\
\hline chr1_409500IENSGALT000( & 1 cis & 4.9 cis & 5.09 & $6.41 \mathrm{E}-04$ \\
\hline chr1_363860IENSGALT000( & $1 \mathrm{cis}$ & 4.9 cis & 4.1 & $6.80 \mathrm{E}-04$ \\
\hline chr1_396230INM_0010307 & $1 \mathrm{cis}$ & $4.3 \mathrm{cis}$ & 10.1 & $6.63 \mathrm{E}-05$ \\
\hline chr1_409500।NM_0010307 & $1 \mathrm{cis}$ & 4.3 cis & 5.09 & $6.14 \mathrm{E}-04$ \\
\hline chr1_396230।NM_205208_ & 1 cis & 4.0 cis & 10.1 & $1.84 \mathrm{E}-04$ \\
\hline chr1_405250।NM_205208_ & $1 \mathrm{cis}$ & 4.0 cis & 4.66 & $5.41 E-04$ \\
\hline chr1_402400INM_205208_ & 1 cis & 4.0 cis & 7.49 & $1.28 \mathrm{E}-03$ \\
\hline chr1_3636101X603867954F & $1 \mathrm{cis}$ & 4.2 cis & 4.1 & $1.79 \mathrm{E}-03$ \\
\hline chr1_3587701X603867954F & $1 \mathrm{cis}$ & $4.2 \mathrm{cis}$ & 6.17 & 8.83E-04 \\
\hline chr1_3962301X603867954F & $1 \mathrm{cis}$ & 4.2 cis & 10.1 & 8.27E-04 \\
\hline chr1_4040401X603867954F & 1 cis & 4.2 cis & 4.55 & $3.64 \mathrm{E}-04$ \\
\hline chr1_4091501X603867954F & $1 \mathrm{cis}$ & 4.2 cis & 4.1 & $9.22 \mathrm{E}-04$ \\
\hline chr1_3961701X603867954F & 1 cis & 4.2 cis & 4.1 & $2.96 \mathrm{E}-03$ \\
\hline chr1_411810iX603867954F & $1 \mathrm{cis}$ & 4.2 cis & 10.05 & $8.98 \mathrm{E}-04$ \\
\hline chr1_382120IENSGALT000( & 1 cis & 4.5 cis & 5.59 & $2.59 \mathrm{E}-03$ \\
\hline chr1_409150IENSGALT000( & $1 \mathrm{cis}$ & 4.5 cis & 4.1 & $2.12 \mathrm{E}-04$ \\
\hline chr1_410350IENSGALT000( & 1 cis & 4.5 cis & 4.1 & $1.88 \mathrm{E}-03$ \\
\hline chr1_3962301X603595948F & $1 \mathrm{cis}$ & 3.9 cis & 10.1 & $6.63 \mathrm{E}-04$ \\
\hline chr1_4052501X603595948F & 1 cis & 3.9 cis & 4.66 & $1.30 \mathrm{E}-03$ \\
\hline chr1_4024001X603595948F & $1 \mathrm{cis}$ & 3.9 cis & 7.49 & 2.09E-03 \\
\hline chr1_411160।X603595948F & $1 \mathrm{cis}$ & 3.9 cis & 5.17 & $1.28 \mathrm{E}-03$ \\
\hline chr1_4019001X603599019F & $1 \mathrm{cis}$ & 4.5 cis & 7.04 & $5.80 \mathrm{E}-03$ \\
\hline chr1_4061001X603599019F & 1 cis & 4.5 cis & 11.07 & $6.81 \mathrm{E}-04$ \\
\hline chr1_4034901X603599019F & $1 \mathrm{cis}$ & 4.5 cis & 5.89 & $1.31 \mathrm{E}-05$ \\
\hline chr1_4123501X603599019F & $1 \mathrm{cis}$ & 4.5 cis & 9.9 & $1.62 \mathrm{E}-03$ \\
\hline chr1_4061101X603599019F & $1 \mathrm{cis}$ & 4.5 cis & 13.78 & 2.37E-04 \\
\hline chr1_3553701X603599269F & 1 cis & $4.3 \mathrm{cis}$ & 11.8 & $2.98 \mathrm{E}-03$ \\
\hline chr1_3602501X603599269F & $1 \mathrm{cis}$ & 4.3 cis & 4.1 & $1.85 \mathrm{E}-04$ \\
\hline chr1_3998601X603599269F & 1 cis & $4.3 \mathrm{cis}$ & 4.1 & $1.54 \mathrm{E}-03$ \\
\hline chr1_3999401X603599269F & $1 \mathrm{cis}$ & 4.3 cis & 5.54 & 8.47E-04 \\
\hline chr1_3643501X603599269F & 1 cis & $4.3 \mathrm{cis}$ & 4.1 & 2.13E-04 \\
\hline chr1_3638601X603599269F & $1 \mathrm{cis}$ & $4.3 \mathrm{cis}$ & 4.1 & 7.44E-04 \\
\hline chr1_461740IENSGALT000( & $1 \mathrm{cis}$ & 7.9 cis & 9.89 & $2.95 \mathrm{E}-03$ \\
\hline chr1_461690IENSGALTO00( & $1 \mathrm{cis}$ & 7.9 cis & 5.76 & 5.59E-04 \\
\hline chr1_490940IENSGALT000( & 1 cis & 4.2 cis & 11.92 & $2.44 \mathrm{E}-03$ \\
\hline chr1_514790IENSGALTO00( & $1 \mathrm{cis}$ & $4.1 \mathrm{cis}$ & 4.43 & 8.12E-04 \\
\hline chr1_5075001X603600031F & 1 cis & $7.2 \mathrm{cis}$ & 6.33 & $1.06 \mathrm{E}-04$ \\
\hline chr1_514790IENSGALTO00( & $1 \mathrm{cis}$ & $3.7 \mathrm{cis}$ & 4.43 & $5.45 \mathrm{E}-04$ \\
\hline chr1_544980IENSGALT000( & $1 \mathrm{cis}$ & $3.7 \mathrm{cis}$ & 4.1 & $2.36 \mathrm{E}-04$ \\
\hline chr1_5376901X603143082F & $1 \mathrm{cis}$ & 5.3 cis & 4.6 & 4.47E-04 \\
\hline chr1_5382001X603143082F & 1 cis & $5.3 \mathrm{cis}$ & 4.93 & $2.54 \mathrm{E}-04$ \\
\hline chr1_5369901X603143082F & $1 \mathrm{cis}$ & 5.3 cis & 9.88 & $2.21 \mathrm{E}-04$ \\
\hline chr1_5372301X603143082F & 1 cis & $5.3 \mathrm{cis}$ & 4.2 & 8.77E-06 \\
\hline chr1_5436401X603143082F & $1 \mathrm{cis}$ & 5.3 cis & 5.12 & $3.52 \mathrm{E}-06$ \\
\hline
\end{tabular}




\begin{tabular}{|c|c|c|c|c|}
\hline chr1_536040(X603143082F & 1 cis & $5.3 \mathrm{cis}$ & 6.47 & $3.64 \mathrm{E}-05$ \\
\hline chr1_537230ıx603866558F & $1 \mathrm{cis}$ & 3.7 cis & 4.2 & $6.16 \mathrm{E}-04$ \\
\hline chr1_543640ıX603866558F & 1 cis & 3.7 cis & 5.12 & 7.54E-04 \\
\hline chr1_536040ıX603866558F & $1 \mathrm{cis}$ & 3.7 cis & 6.47 & 1.07E-04 \\
\hline chr1_536040ıNM_0010128 & 1 cis & $5.8 \mathrm{cis}$ & 6.47 & $6.01 \mathrm{E}-04$ \\
\hline chr1_532720ıX603599470F & $1 \mathrm{cis}$ & 4.9 cis & 4.64 & $8.08 \mathrm{E}-04$ \\
\hline chr1_536040ıX603599470F & $1 \mathrm{cis}$ & 4.9 cis & 6.47 & $1.21 \mathrm{E}-03$ \\
\hline chr1_525000IENSGALT000( & $1 \mathrm{cis}$ & 5.9 cis & 4.1 & $2.15 \mathrm{E}-04$ \\
\hline chr1_527980IENSGALT000( & 1 cis & 5.9 cis & 4.1 & 2.97E-03 \\
\hline chr1_536990IENSGALTO00( & $1 \mathrm{cis}$ & 5.9 cis & 9.88 & $3.16 \mathrm{E}-04$ \\
\hline chr1_537230IENSGALTO00( & 1 cis & 5.9 cis & 4.2 & $4.36 \mathrm{E}-04$ \\
\hline chr1_543640IENSGALTO00( & 1 cis & 5.9 cis & 5.12 & $1.40 \mathrm{E}-03$ \\
\hline chr1_536040IENSGALT000( & $1 \mathrm{cis}$ & 5.9 cis & 6.47 & $2.11 \mathrm{E}-04$ \\
\hline chr1_536990ıX603599379F & $1 \mathrm{cis}$ & 4.8 cis & 9.88 & 6.37E-05 \\
\hline chr1_537230ıX03599379F & $1 \mathrm{cis}$ & $4.8 \mathrm{cis}$ & 4.2 & $4.55 \mathrm{E}-04$ \\
\hline chr1_536040ıX603599379F & $1 \mathrm{cis}$ & $4.8 \mathrm{cis}$ & 6.47 & $2.11 \mathrm{E}-06$ \\
\hline chr1_515260ıNM_0011307 & $1 \mathrm{cis}$ & 4.4 cis & 5.99 & $1.58 \mathrm{E}-03$ \\
\hline chr1_532820ıNM_0011307 & 1 cis & 4.4 cis & 7.83 & 2.09E-04 \\
\hline chr1_537690IENSGALTO00( & $1 \mathrm{cis}$ & $7.2 \mathrm{cis}$ & 4.6 & $1.32 \mathrm{E}-03$ \\
\hline chr1_530420IENSGALT000( & $1 \mathrm{cis}$ & 7.2 cis & 4.1 & $1.54 \mathrm{E}-03$ \\
\hline chr1_536810ıENSGALT000( & $1 \mathrm{cis}$ & 7.2 cis & 4.1 & $1.39 \mathrm{E}-02$ \\
\hline chr1_527980IENSGALT000( & $1 \mathrm{cis}$ & $7.2 \mathrm{cis}$ & 4.1 & $1.45 \mathrm{E}-03$ \\
\hline chr1_529370IENSGALTO00( & $1 \mathrm{cis}$ & 7.2 cis & 5.21 & 7.01E-04 \\
\hline chr1_536040IENSGALT000( & $1 \mathrm{cis}$ & 7.2 cis & 6.47 & $2.50 \mathrm{E}-04$ \\
\hline chr1_532830IENSGALTO00( & 1 trans & 6.5 cis & 5.64 & 8.99E-04 \\
\hline chr1_532820IENSGALTO00( & 1 trans & 6.5 cis & 7.83 & $1.94 \mathrm{E}-05$ \\
\hline chr1_536810ıNM_205344_ & $1 \mathrm{cis}$ & 6.3 cis & 4.1 & $3.28 \mathrm{E}-02$ \\
\hline chr1_527980ıNM_205344_ & $1 \mathrm{cis}$ & $6.3 \mathrm{cis}$ & 4.1 & $1.18 \mathrm{E}-02$ \\
\hline chr1_536040।NM_205344_ & $1 \mathrm{cis}$ & $6.3 \mathrm{cis}$ & 6.47 & $9.44 \mathrm{E}-04$ \\
\hline chr1_536990IENSGALTO00 & 1 cis & 5.7 cis & 9.88 & 4.97E-03 \\
\hline chr1_536040IENSGALT000( & $1 \mathrm{cis}$ & $5.7 \mathrm{cis}$ & 6.47 & 3.70E-03 \\
\hline chr1_537230ıX603601419F & $1 \mathrm{cis}$ & $9.1 \mathrm{cis}$ & 4.2 & 1.77E-03 \\
\hline chr1_543640ıX03601419F & $1 \mathrm{cis}$ & $9.1 \mathrm{cis}$ & 5.12 & $2.22 \mathrm{E}-03$ \\
\hline chr1_566040ıX603601419F & $1 \mathrm{cis}$ & $9.1 \mathrm{cis}$ & 6.5 & $6.29 E-05$ \\
\hline chr1_589810ıX03601419F & $1 \mathrm{cis}$ & $9.1 \mathrm{cis}$ & 6.57 & $3.85 \mathrm{E}-04$ \\
\hline chr1_621510ıNM_0010307 & 1 cis & 5.0 cis & 4.64 & $2.92 \mathrm{E}-05$ \\
\hline chr1_621420ıNM_0010307 & $1 \mathrm{cis}$ & $5.0 \mathrm{cis}$ & 7.74 & $9.06 \mathrm{E}-04$ \\
\hline chr1_621530ıNM_0010307 & $1 \mathrm{cis}$ & $5.0 \mathrm{cis}$ & 6.84 & $9.38 \mathrm{E}-04$ \\
\hline chr1_621410ıNM_0010307 & $1 \mathrm{cis}$ & 5.0 cis & 5.72 & $3.32 \mathrm{E}-03$ \\
\hline chr1_621400ıNM_0010307 & $1 \mathrm{cis}$ & $5.0 \mathrm{cis}$ & 4.39 & $1.11 \mathrm{E}-03$ \\
\hline chr1_606470ıNM_0010307 & $1 \mathrm{cis}$ & 5.0 trans & 7.85 & $2.04 \mathrm{E}-03$ \\
\hline chr1_927920IENSGALTO00( & 1 cis & $4.6 \mathrm{cis}$ & 4.29 & $2.25 \mathrm{E}-03$ \\
\hline chr1_938830IENSGALT000( & $1 \mathrm{cis}$ & $4.6 \mathrm{cis}$ & 4.8 & $4.29 E-04$ \\
\hline chr1_927060IENSGALT000( & $1 \mathrm{cis}$ & $4.6 \mathrm{cis}$ & 4.1 & $6.84 \mathrm{E}-04$ \\
\hline chr1_878250IENSGALTO00( & $1 \mathrm{cis}$ & $5.7 \mathrm{cis}$ & 8.86 & $3.26 \mathrm{E}-05$ \\
\hline chr1_895130IENSGALT000( & $1 \mathrm{cis}$ & $5.7 \mathrm{cis}$ & 7.35 & $2.45 \mathrm{E}-04$ \\
\hline chr1_873100IENSGALT000( & $1 \mathrm{cis}$ & $5.7 \mathrm{cis}$ & 6.97 & $1.05 \mathrm{E}-03$ \\
\hline chr1_898200IENSGALT000( & 1 cis & 5.7 cis & 4.59 & $2.23 \mathrm{E}-04$ \\
\hline chr1_878250IENSGALTOOO & $1 \mathrm{cis}$ & $5.3 \mathrm{cis}$ & 8.86 & $2.44 \mathrm{E}-04$ \\
\hline chr1_898200IENSGALT000( & $1 \mathrm{cis}$ & $5.3 \mathrm{cis}$ & 4.59 & $4.82 \mathrm{E}-04$ \\
\hline chr1_926110ıENSGALT000( & $1 \mathrm{cis}$ & $4.7 \mathrm{cis}$ & 6.2 & $6.66 \mathrm{E}-03$ \\
\hline chr1_898040IENSGALT000( & $1 \mathrm{cis}$ & $4.7 \mathrm{cis}$ & 5.1 & $3.38 \mathrm{E}-03$ \\
\hline chr1_933150IENSGALT000( & $1 \mathrm{cis}$ & $4.7 \mathrm{cis}$ & 5.6 & $7.44 \mathrm{E}-03$ \\
\hline chr1_116981।ENSGALT000( & 1 cis & 5.4 cis & 5.18 & $3.50 \mathrm{E}-03$ \\
\hline chr1_119144IENSGALT000( & $1 \mathrm{cis}$ & $5.4 \mathrm{cis}$ & 10.07 & $1.39 \mathrm{E}-03$ \\
\hline chr1_118205ıNM_0010128 & $1 \mathrm{cis}$ & $4.3 \mathrm{cis}$ & 5.6 & $9.40 \mathrm{E}-04$ \\
\hline chr1_110954ıX603596950F & $1 \mathrm{cis}$ & $3.2 \mathrm{cis}$ & 4.68 & $8.46 \mathrm{E}-05$ \\
\hline chr1_116981।X603596950F & $1 \mathrm{cis}$ & $3.2 \mathrm{cis}$ & 5.18 & $6.33 \mathrm{E}-05$ \\
\hline chr1_117180ıX603596950F & $1 \mathrm{cis}$ & $3.2 \mathrm{cis}$ & 5.82 & $3.76 \mathrm{E}-03$ \\
\hline chr1_119144ıX603596950F & 1 cis & $3.2 \mathrm{cis}$ & 10.07 & $1.39 \mathrm{E}-04$ \\
\hline chr1_123394IENSGALT000( & $1 \mathrm{cis}$ & $5.0 \mathrm{cis}$ & 4.1 & $1.03 \mathrm{E}-03$ \\
\hline chr1_126629ıENSGALT000( & $1 \mathrm{cis}$ & 10.9 cis & 4.42 & $1.38 \mathrm{E}-05$ \\
\hline chr1_127636IENSGALT000( & $1 \mathrm{cis}$ & 10.9 cis & 4.73 & $1.30 \mathrm{E}-03$ \\
\hline chr1_128935IENSGALT000( & $1 \mathrm{cis}$ & $10.9 \mathrm{cis}$ & 12.14 & $4.56 \mathrm{E}-04$ \\
\hline chr1_130171'ENSGALT000( & $1 \mathrm{cis}$ & 4.8 cis & 5.59 & $7.98 \mathrm{E}-04$ \\
\hline chr1_130174IENSGALT000( & 1 cis & $4.8 \mathrm{cis}$ & 4.32 & $5.53 \mathrm{E}-04$ \\
\hline chr1_129633ıX03598304F & $1 \mathrm{cis}$ & $10.0 \mathrm{cis}$ & 4.1 & $8.30 \mathrm{E}-04$ \\
\hline chr1_131263ıX603598304F & $1 \mathrm{cis}$ & $10.0 \mathrm{cis}$ & 6.95 & 2.07E-06 \\
\hline chr1_133244।X603598304F & $1 \mathrm{cis}$ & $10.0 \mathrm{cis}$ & 7.74 & 1.15E-06 \\
\hline chr1_131968ıX603598304F & $1 \mathrm{cis}$ & $10.0 \mathrm{cis}$ & 4.1 & $2.39 \mathrm{E}-04$ \\
\hline chr1_137201'NM_0010303 & $1 \mathrm{cis}$ & $4.5 \mathrm{cis}$ & 6.7 & $4.32 \mathrm{E}-04$ \\
\hline chr1_133943ıENSGALT000( & $1 \mathrm{cis}$ & $13.5 \mathrm{cis}$ & 4.4 & $6.72 \mathrm{E}-04$ \\
\hline
\end{tabular}




\begin{tabular}{|c|c|c|c|c|}
\hline chr1_135072।ENSGALT000( & 1 cis & $13.5 \mathrm{cis}$ & 6.11 & 4.77E-04 \\
\hline chr1_133995IENSGALTO00( & $1 \mathrm{cis}$ & $13.5 \mathrm{cis}$ & 6.99 & 2.51E-04 \\
\hline chr1_133896IENSGALT000( & 1 cis & $13.5 \mathrm{cis}$ & 4.99 & $1.35 \mathrm{E}-05$ \\
\hline chr1_136389IENSGALTO00( & $1 \mathrm{cis}$ & 13.5 cis & 4.91 & 5.43E-04 \\
\hline chr1_136991IENSGALT000( & 1 cis & $13.5 \mathrm{cis}$ & 4.73 & 4.93E-04 \\
\hline chr1_137382IENSGALT000( & $1 \mathrm{cis}$ & $13.5 \mathrm{cis}$ & 6.07 & 2.63E-07 \\
\hline chr1_136500IENSGALT000( & $1 \mathrm{cis}$ & $13.5 \mathrm{cis}$ & 7.17 & 2.59E-06 \\
\hline chr1_137610IENSGALT000( & 1 cis & 13.5 cis & 8.68 & 5.05E-09 \\
\hline chr1_137629IENSGALT000( & $1 \mathrm{cis}$ & $13.5 \mathrm{cis}$ & 19.85 & $1.06 \mathrm{E}-11$ \\
\hline chr1_137656IENSGALT000( & $1 \mathrm{cis}$ & 13.5 cis & 11.5 & 1.47E-10 \\
\hline chr1_137682IENSGALT000( & 1 cis & $13.5 \mathrm{cis}$ & 11.7 & 7.21E-11 \\
\hline chr1_137686IENSGALT000( & $1 \mathrm{cis}$ & 13.5 cis & 11.2 & 8.59E-11 \\
\hline chr1_137581IENSGALT000( & $1 \mathrm{cis}$ & $13.5 \mathrm{cis}$ & 7.77 & $3.46 \mathrm{E}-09$ \\
\hline chr1_137594IENSGALT000( & $1 \mathrm{cis}$ & 13.5 cis & 10.53 & $5.65 \mathrm{E}-10$ \\
\hline chr1_137596IENSGALT000( & $1 \mathrm{cis}$ & $13.5 \mathrm{cis}$ & 11.39 & $2.31 \mathrm{E}-10$ \\
\hline chr1_137597IENSGALT000( & $1 \mathrm{cis}$ & 13.5 cis & 10.26 & 3.69E-10 \\
\hline chr1_137601।ENSGALT000( & $1 \mathrm{cis}$ & $13.5 \mathrm{cis}$ & 21.57 & $4.09 \mathrm{E}-10$ \\
\hline chr1_137624IENSGALTO00( & $1 \mathrm{cis}$ & 13.5 cis & 11.53 & $2.08 \mathrm{E}-10$ \\
\hline chr1_137625IENSGALT000( & $1 \mathrm{cis}$ & $13.5 \mathrm{cis}$ & 11.15 & $2.18 \mathrm{E}-10$ \\
\hline chr1_137641।ENSGALT000( & $1 \mathrm{cis}$ & $13.5 \mathrm{cis}$ & 11.45 & 1.64E-09 \\
\hline chr1_137648IENSGALT000( & $1 \mathrm{cis}$ & 13.5 cis & 11.67 & 3.72E-11 \\
\hline chr1_137649IENSGALT000( & $1 \mathrm{cis}$ & $13.5 \mathrm{cis}$ & 11.23 & $4.72 \mathrm{E}-10$ \\
\hline chr1_137650IENSGALT000( & $1 \mathrm{cis}$ & $13.5 \mathrm{cis}$ & 10.88 & $3.11 E-10$ \\
\hline chr1_137652IENSGALTO00( & $1 \mathrm{cis}$ & $13.5 \mathrm{cis}$ & 11.68 & $2.25 \mathrm{E}-10$ \\
\hline chr1_137653IENSGALT000( & $1 \mathrm{cis}$ & $13.5 \mathrm{cis}$ & 10.35 & $2.58 \mathrm{E}-10$ \\
\hline chr1_137678IENSGALT000( & $1 \mathrm{cis}$ & $13.5 \mathrm{cis}$ & 12.09 & 5.91E-11 \\
\hline chr1_137680IENSGALT000( & $1 \mathrm{cis}$ & 13.5 cis & 11.65 & $1.77 \mathrm{E}-10$ \\
\hline chr1_137696IENSGALT000( & $1 \mathrm{cis}$ & $13.5 \mathrm{cis}$ & 10.03 & $6.31 E-09$ \\
\hline chr1_137582IENSGALT000( & $1 \mathrm{cis}$ & $13.5 \mathrm{cis}$ & 12.1 & 4.37E-09 \\
\hline chr1_137583IENSGALTO00( & $1 \mathrm{cis}$ & $13.5 \mathrm{cis}$ & 7.53 & $2.45 \mathrm{E}-08$ \\
\hline chr1_137595IENSGALT000( & $1 \mathrm{cis}$ & $13.5 \mathrm{cis}$ & 12.69 & 2.61E-09 \\
\hline chr1_137598IENSGALT000( & $1 \mathrm{cis}$ & $13.5 \mathrm{cis}$ & 11.89 & $1.60 \mathrm{E}-10$ \\
\hline chr1_137599IENSGALT000( & $1 \mathrm{cis}$ & $13.5 \mathrm{cis}$ & 14.05 & 1.36E-09 \\
\hline chr1_137600IENSGALT000( & $1 \mathrm{cis}$ & $13.5 \mathrm{cis}$ & 14.13 & $7.88 \mathrm{E}-11$ \\
\hline chr1_137602IENSGALT000( & $1 \mathrm{cis}$ & $13.5 \mathrm{cis}$ & 18.72 & 5.33E-10 \\
\hline chr1_137611।ENSGALTO00( & $1 \mathrm{cis}$ & $13.5 \mathrm{cis}$ & 11.58 & $1.46 \mathrm{E}-08$ \\
\hline chr1_137612IENSGALTO00( & $1 \mathrm{cis}$ & $13.5 \mathrm{cis}$ & 6.53 & 2.87E-08 \\
\hline chr1_137622IENSGALT000( & $1 \mathrm{cis}$ & $13.5 \mathrm{cis}$ & 10.07 & $5.22 \mathrm{E}-10$ \\
\hline chr1_137623IENSGALT000( & $1 \mathrm{cis}$ & $13.5 \mathrm{cis}$ & 13.09 & 1.16E-09 \\
\hline chr1_137626IENSGALTO00( & $1 \mathrm{cis}$ & $13.5 \mathrm{cis}$ & 11.6 & $6.53 E-11$ \\
\hline chr1_137627IENSGALTO00( & $1 \mathrm{cis}$ & $13.5 \mathrm{cis}$ & 12.04 & $1.22 \mathrm{E}-09$ \\
\hline chr1_137628IENSGALTO00( & $1 \mathrm{cis}$ & $13.5 \mathrm{cis}$ & 14.6 & $9.71 E-11$ \\
\hline chr1_137630IENSGALT000( & $1 \mathrm{cis}$ & $13.5 \mathrm{cis}$ & 20.13 & $1.04 \mathrm{E}-08$ \\
\hline chr1_137634IENSGALT000( & $1 \mathrm{cis}$ & $13.5 \mathrm{cis}$ & 5.22 & $1.01 \mathrm{E}-05$ \\
\hline chr1_137644IENSGALT000( & $1 \mathrm{cis}$ & $13.5 \mathrm{cis}$ & 17.4 & $3.41 E-10$ \\
\hline chr1_137651।ENSGALT000( & $1 \mathrm{cis}$ & $13.5 \mathrm{cis}$ & 11.52 & $1.83 \mathrm{E}-10$ \\
\hline chr1_137654IENSGALTO00( & 1 cis & $13.5 \mathrm{cis}$ & 12 & 4.55E-10 \\
\hline chr1_137655IENSGALTO00( & $1 \mathrm{cis}$ & $13.5 \mathrm{cis}$ & 14.8 & $2.45 \mathrm{E}-09$ \\
\hline chr1_137657IENSGALT000( & $1 \mathrm{cis}$ & $13.5 \mathrm{cis}$ & 20.05 & $8.20 \mathrm{E}-08$ \\
\hline chr1_137661।ENSGALT000( & $1 \mathrm{cis}$ & $13.5 \mathrm{cis}$ & 14.44 & $3.76 \mathrm{E}-09$ \\
\hline chr1_137666IENSGALT000( & $1 \mathrm{cis}$ & $13.5 \mathrm{cis}$ & 8.69 & $5.79 E-10$ \\
\hline chr1_137667IENSGALTO00( & $1 \mathrm{cis}$ & $13.5 \mathrm{cis}$ & 12.12 & $1.42 \mathrm{E}-09$ \\
\hline chr1_137669IENSGALTO00( & $1 \mathrm{cis}$ & $13.5 \mathrm{cis}$ & 7.63 & $1.99 \mathrm{E}-08$ \\
\hline chr1_137674IENSGALTO00( & $1 \mathrm{cis}$ & $13.5 \mathrm{cis}$ & 17.46 & 3.26E-10 \\
\hline chr1_137679IENSGALT000( & $1 \mathrm{cis}$ & $13.5 \mathrm{cis}$ & 11.19 & 4.41E-10 \\
\hline chr1_137681IENSGALT000( & $1 \mathrm{cis}$ & $13.5 \mathrm{cis}$ & 11.75 & 3.14E-10 \\
\hline chr1_137683IENSGALTO00( & $1 \mathrm{cis}$ & $13.5 \mathrm{cis}$ & 10.91 & $1.84 \mathrm{E}-10$ \\
\hline chr1_137684IENSGALTO00( & $1 \mathrm{cis}$ & $13.5 \mathrm{cis}$ & 13.1 & $1.78 \mathrm{E}-10$ \\
\hline chr1_137685IENSGALT000( & $1 \mathrm{cis}$ & $13.5 \mathrm{cis}$ & 15.45 & $1.83 \mathrm{E}-09$ \\
\hline chr1_137687IENSGALTO00( & $1 \mathrm{cis}$ & $13.5 \mathrm{cis}$ & 20.63 & $5.20 \mathrm{E}-08$ \\
\hline chr1_137695IENSGALTO00( & $1 \mathrm{cis}$ & $13.5 \mathrm{cis}$ & 5.8 & $1.55 \mathrm{E}-06$ \\
\hline chr1_137698IENSGALT000( & $1 \mathrm{cis}$ & $13.5 \mathrm{cis}$ & 7.13 & $1.32 \mathrm{E}-08$ \\
\hline chr1_137584IENSGALT000( & $1 \mathrm{cis}$ & $13.5 \mathrm{cis}$ & 7.86 & $1.18 \mathrm{E}-08$ \\
\hline chr1_137589IENSGALTO00( & $1 \mathrm{cis}$ & $13.5 \mathrm{cis}$ & 13.65 & $7.02 E-09$ \\
\hline chr1_137605IENSGALTO00( & $1 \mathrm{cis}$ & $13.5 \mathrm{cis}$ & 12.38 & $3.11 E-08$ \\
\hline chr1_137609IENSGALTO00( & $1 \mathrm{cis}$ & $13.5 \mathrm{cis}$ & 7.31 & $2.72 \mathrm{E}-07$ \\
\hline chr1_137613IENSGALTO00( & $1 \mathrm{cis}$ & $13.5 \mathrm{cis}$ & 8.76 & $1.82 \mathrm{E}-08$ \\
\hline chr1_137615IENSGALT000( & $1 \mathrm{cis}$ & $13.5 \mathrm{cis}$ & 15.89 & $1.25 \mathrm{E}-08$ \\
\hline chr1_137617IENSGALTO00( & $1 \mathrm{cis}$ & $13.5 \mathrm{cis}$ & 14.17 & 3.80E-09 \\
\hline chr1_137618IENSGALTO00( & $1 \mathrm{cis}$ & $13.5 \mathrm{cis}$ & 16.44 & 8.25E-10 \\
\hline chr1_137631IENSGALT000( & $1 \mathrm{cis}$ & $13.5 \mathrm{cis}$ & 14.54 & 3.27E-08 \\
\hline chr1_137658IENSGALT000( & $1 \mathrm{cis}$ & 13.5 cis & 17.96 & $4.52 \mathrm{E}-11$ \\
\hline
\end{tabular}




\begin{tabular}{|c|c|c|c|c|}
\hline chr1_137665IENSGALTO0OC & 1 cis & $13.5 \mathrm{cis}$ & 10 & $1.98 \mathrm{E}-08$ \\
\hline chr1_137668IENSGALTO0OI & 1 cis & 13.5 cis & 7.29 & $1.25 \mathrm{E}-07$ \\
\hline chr1_137688IENSGALTO0OC & 1 cis & 13.5 cis & 17.84 & $1.01 \mathrm{E}-10$ \\
\hline chr1_137697IENSGALTO0OC & 1 cis & 13.5 cis & 10.75 & $2.57 \mathrm{E}-08$ \\
\hline chr1_137699IENSGALTO0Or & 1 cis & 13.5 cis & 9.84 & $1.64 \mathrm{E}-07$ \\
\hline chr1_137701IENSGALTO0OC & 1 cis & 13.5 cis & 14.71 & 2.27E-08 \\
\hline chr1_137703IENSGALTO0OC & 1 cis & 13.5 cis & 14.78 & $1.96 \mathrm{E}-09$ \\
\hline chr1_137574IENSGALTO0OC & 1 cis & 13.5 cis & 13.87 & $1.21 \mathrm{E}-07$ \\
\hline chr1_137576IENSGALTO0OC & 1 cis & 13.5 cis & 11.94 & $1.55 \mathrm{E}-08$ \\
\hline chr1_137579IENSGALTO0OC & 1 cis & 13.5 cis & 12.49 & $3.73 \mathrm{E}-06$ \\
\hline chr1_137585IENSGALTO0OC & 1 cis & 13.5 cis & 11 & $9.24 \mathrm{E}-06$ \\
\hline chr1_137586IENSGALTO0OC & 1 cis & 13.5 cis & 13.87 & $3.47 \mathrm{E}-07$ \\
\hline chr1_137587IENSGALTO0OC & 1 cis & 13.5 cis & 11.77 & $1.35 \mathrm{E}-07$ \\
\hline chr1_137590IENSGALTO0OC & 1 cis & 13.5 cis & 13.24 & $1.49 \mathrm{E}-09$ \\
\hline chr1_137593IENSGALTO0OC & 1 cis & 13.5 cis & 13.66 & $6.65 \mathrm{E}-08$ \\
\hline chr1_137603IENSGALTO00( & 1 cis & 13.5 cis & 13.07 & $1.82 \mathrm{E}-06$ \\
\hline chr1_137608IENSGALTO0OO & 1 cis & 13.5 cis & 12.22 & $4.11 \mathrm{E}-06$ \\
\hline chr1_137614IENSGALTO0OC & 1 cis & 13.5 cis & 10.31 & $4.82 \mathrm{E}-05$ \\
\hline chr1_137619iENSGALTO0OC & 1 cis & 13.5 cis & 12.43 & $2.71 \mathrm{E}-09$ \\
\hline chr1_137621IENSGALTO00 & 1 cis & 13.5 cis & 13.68 & $1.65 \mathrm{E}-06$ \\
\hline chr1_137633IENSGALTO0OC & 1 cis & 13.5 cis & 11.92 & $1.09 \mathrm{E}-08$ \\
\hline chr1_137643IENSGALTO0OC & 1 cis & 13.5 cis & 13.72 & $6.19 \mathrm{E}-09$ \\
\hline chr1_137647IENSGALTO0OC & 1 cis & 13.5 cis & 12.23 & $3.55 \mathrm{E}-06$ \\
\hline chr1_137659IENSGALTO0OC & 1 cis & 13.5 cis & 13.41 & $5.24 \mathrm{E}-05$ \\
\hline chr1_137670IENSGALTO0OI & 1 cis & 13.5 cis & 9.41 & $3.62 \mathrm{E}-05$ \\
\hline chr1_137671IENSGALTO0OC & 1 cis & $13.5 \mathrm{cis}$ & 15.22 & $5.85 \mathrm{E}-08$ \\
\hline chr1_137672IENSGALTO0OC & 1 cis & 13.5 cis & 12.01 & $2.20 \mathrm{E}-07$ \\
\hline chr1_137673IENSGALTO0OC & 1 cis & 13.5 cis & 11.1 & $3.91 \mathrm{E}-08$ \\
\hline chr1_137675IENSGALTO0OC & 1 cis & 13.5 cis & 11.47 & $1.64 \mathrm{E}-09$ \\
\hline chr1_137689IENSGALTO0OC & 1 cis & 13.5 cis & 13.29 & $6.99 \mathrm{E}-05$ \\
\hline chr1_137691|ENSGALTO0OC & 1 cis & 13.5 cis & 13.54 & 2.99E-07 \\
\hline chr1_137693IENSGALTO0OC & 1 cis & 13.5 cis & 15.25 & 7.09E-06 \\
\hline chr1_137694IENSGALTO0OC & 1 cis & 13.5 cis & 10.96 & $1.20 \mathrm{E}-07$ \\
\hline chr1_137700IENSGALTO0OC & 1 cis & 13.5 cis & 12.24 & $2.84 \mathrm{E}-05$ \\
\hline chr1_137575IENSGALTO0OC & 1 cis & 13.5 cis & 14.57 & 3.40E-06 \\
\hline chr1_137578IENSGALTO0OC & 1 cis & 13.5 cis & 14.89 & $1.12 \mathrm{E}-05$ \\
\hline chr1_137591|ENSGALTO0O & 1 cis & 13.5 cis & 11.3 & $2.51 \mathrm{E}-08$ \\
\hline chr1_137592IENSGALTO0OC & 1 cis & 13.5 cis & 11.45 & $1.93 \mathrm{E}-05$ \\
\hline chr1_137604IENSGALTO0OC & 1 cis & 13.5 cis & 14.51 & $1.20 \mathrm{E}-06$ \\
\hline chr1_137606IENSGALTO0OC & 1 cis & 13.5 cis & 13.67 & $1.85 \mathrm{E}-05$ \\
\hline chr1_137607IENSGALTO0OC & 1 cis & 13.5 cis & 14.26 & $1.74 \mathrm{E}-05$ \\
\hline chr1_137616IENSGALTO0OC & 1 cis & 13.5 cis & 12.35 & 3.44E-07 \\
\hline chr1_137620IENSGALTO0OI & 1 cis & 13.5 cis & 12.52 & $3.01 \mathrm{E}-06$ \\
\hline chr1_137632IENSGALTO0O & 1 cis & 13.5 cis & 14.85 & $3.56 \mathrm{E}-06$ \\
\hline chr1_137642IENSGALTO0OC & 1 cis & 13.5 cis & 11.5 & $3.72 \mathrm{E}-07$ \\
\hline chr1_137645IENSGALTO0OC & 1 cis & 13.5 cis & 11.49 & $3.19 \mathrm{E}-09$ \\
\hline chr1_137646IENSGALTO0OC & 1 cis & 13.5 cis & 11.33 & 3.56E-06 \\
\hline chr1_137660IENSGALTO0OC & 1 cis & 13.5 cis & 13.64 & $5.26 \mathrm{E}-08$ \\
\hline chr1_137662IENSGALTO0OC & 1 cis & 13.5 cis & 13.75 & $8.01 \mathrm{E}-06$ \\
\hline chr1_137663IENSGALTO0OI & 1 cis & 13.5 cis & 13.79 & $5.19 \mathrm{E}-05$ \\
\hline chr1_137664IENSGALTO0OC & 1 cis & 13.5 cis & 12.5 & $3.60 \mathrm{E}-06$ \\
\hline chr1_137677IENSGALTO0OC & 1 cis & 13.5 cis & 11.72 & $9.64 \mathrm{E}-06$ \\
\hline chr1_137690IENSGALTO0OC & 1 cis & 13.5 cis & 13.19 & $5.39 \mathrm{E}-08$ \\
\hline chr1_137692IENSGALTO0OC & 1 cis & 13.5 cis & 13.15 & 4.01E-05 \\
\hline chr1_137676IENSGALTO0OC & 1 cis & 13.5 cis & 10.73 & $2.31 \mathrm{E}-06$ \\
\hline chr1_137702IENSGALTO0Or & 1 cis & 13.5 cis & 10.02 & $1.76 \mathrm{E}-06$ \\
\hline chr1_137588IENSGALTO0OC & 1 cis & 13.5 cis & 6.55 & $7.01 E-05$ \\
\hline chr1_1338961X603142577F & 1 cis & 10.6 cis & 4.99 & $1.10 \mathrm{E}-04$ \\
\hline chr1_1382091X603142577F & 1 cis & 10.6 cis & 8.03 & $7.01 \mathrm{E}-06$ \\
\hline chr1_137382।X603142577F & 1 cis & 10.6 cis & 6.07 & 7.07E-06 \\
\hline chr1_138835IX603142577F & 1 cis & 10.6 cis & 7.82 & $1.26 \mathrm{E}-03$ \\
\hline chr1_1365001X603142577F & 1 cis & 10.6 cis & 7.17 & $1.23 \mathrm{E}-04$ \\
\hline chr1_137768IX603142577F & 1 cis & 10.6 cis & 4.39 & $6.02 \mathrm{E}-05$ \\
\hline chr1_1376101X603142577F & 1 cis & 10.6 cis & 8.68 & $1.86 \mathrm{E}-06$ \\
\hline chr1_1376291X603142577F & 1 cis & 10.6 cis & 19.85 & $5.66 \mathrm{E}-09$ \\
\hline chr1_137656IX603142577F & 1 cis & 10.6 cis & 11.5 & 4.66E-08 \\
\hline chr1_137682।X603142577F & 1 cis & 10.6 cis & 11.7 & $1.87 \mathrm{E}-08$ \\
\hline chr1_1376861X603142577F & 1 cis & 10.6 cis & 11.2 & $3.15 \mathrm{E}-08$ \\
\hline chr1_1377161X603142577F & 1 cis & 10.6 cis & 14.66 & $2.72 \mathrm{E}-08$ \\
\hline chr1_137581|X603142577F & 1 cis & 10.6 cis & 7.77 & $1.41 \mathrm{E}-06$ \\
\hline chr1_137594ıX603142577F & 1 cis & 10.6 cis & 10.53 & $9.49 \mathrm{E}-08$ \\
\hline chr1_137596iX603142577F & $1 \mathrm{cis}$ & $10.6 \mathrm{cis}$ & 11.39 & $4.68 \mathrm{E}-08$ \\
\hline
\end{tabular}




\begin{tabular}{|c|c|c|c|c|}
\hline chr1_137597।X603142577F & 1 cis & $10.6 \mathrm{cis}$ & 10.26 & $6.34 \mathrm{E}-08$ \\
\hline chr1_137601।X603142577F & 1 cis & 10.6 cis & 21.57 & $6.29 E-08$ \\
\hline chr1_137624ıX603142577F & 1 cis & 10.6 cis & 11.53 & $4.41 \mathrm{E}-08$ \\
\hline chr1_137625ıX603142577F & 1 cis & 10.6 cis & 11.15 & 4.00E-08 \\
\hline chr1_137641।X603142577F & 1 cis & 10.6 cis & 11.45 & $1.64 \mathrm{E}-07$ \\
\hline chr1_137648ıX603142577F & 1 cis & 10.6 cis & 11.67 & $2.03 E-08$ \\
\hline chr1_137649ıX603142577F & 1 cis & $10.6 \mathrm{cis}$ & 11.23 & $8.84 \mathrm{E}-08$ \\
\hline chr1_137650ıX603142577F & 1 cis & 10.6 cis & 10.88 & $5.31 \mathrm{E}-08$ \\
\hline chr1_137652ıX03142577F & 1 cis & 10.6 cis & 11.68 & 4.00E-08 \\
\hline chr1_137653ıX03142577F & 1 cis & 10.6 cis & 10.35 & $5.85 \mathrm{E}-08$ \\
\hline chr1_137678ıX603142577F & 1 cis & 10.6 cis & 12.09 & $2.66 \mathrm{E}-08$ \\
\hline chr1_137680ıX603142577F & 1 cis & 10.6 cis & 11.65 & $3.88 \mathrm{E}-08$ \\
\hline chr1_137696।X603142577F & 1 cis & $10.6 \mathrm{cis}$ & 10.03 & $1.98 \mathrm{E}-06$ \\
\hline chr1_137708ıX603142577F & 1 cis & 10.6 cis & 10.55 & $4.94 \mathrm{E}-08$ \\
\hline chr1_137709ıX603142577F & 1 cis & $10.6 \mathrm{cis}$ & 11.02 & $5.82 \mathrm{E}-08$ \\
\hline chr1_137710ıX603142577F & 1 cis & 10.6 cis & 12.66 & $4.32 \mathrm{E}-08$ \\
\hline chr1_137711।X603142577F & 1 cis & 10.6 cis & 11.03 & $5.70 \mathrm{E}-08$ \\
\hline chr1_137712।X603142577F & 1 cis & $10.6 \mathrm{cis}$ & 11.4 & $6.77 \mathrm{E}-08$ \\
\hline chr1_137713ıX603142577F & 1 cis & 10.6 cis & 11.54 & $1.38 \mathrm{E}-08$ \\
\hline chr1_137726ıX603142577F & 1 cis & 10.6 cis & 9.81 & $1.48 \mathrm{E}-06$ \\
\hline chr1_137739ıX603142577F & 1 cis & $10.6 \mathrm{cis}$ & 11.17 & $8.21 \mathrm{E}-08$ \\
\hline chr1_137743ıX603142577F & 1 cis & 10.6 cis & 11.15 & $3.22 \mathrm{E}-08$ \\
\hline chr1_137747।X603142577F & 1 cis & 10.6 cis & 18.69 & $2.91 \mathrm{E}-08$ \\
\hline chr1_137582।X603142577F & 1 cis & $10.6 \mathrm{cis}$ & 12.1 & $7.41 \mathrm{E}-07$ \\
\hline chr1_137583ıX603142577F & 1 cis & 10.6 cis & 7.53 & $2.08 \mathrm{E}-06$ \\
\hline chr1_137595ıX603142577F & 1 cis & 10.6 cis & 12.69 & $3.58 \mathrm{E}-07$ \\
\hline chr1_137598ıX603142577F & 1 cis & $10.6 \mathrm{cis}$ & 11.89 & $3.30 \mathrm{E}-08$ \\
\hline chr1_137599IX603142577F & $1 \mathrm{cis}$ & $10.6 \mathrm{cis}$ & 14.05 & $2.55 \mathrm{E}-07$ \\
\hline chr1_137600ıX03142577F & 1 cis & 10.6 cis & 14.13 & $1.75 \mathrm{E}-08$ \\
\hline chr1_137602ı603142577F & 1 cis & $10.6 \mathrm{cis}$ & 18.72 & $7.56 \mathrm{E}-08$ \\
\hline chr1_137611'X603142577F & 1 cis & 10.6 cis & 11.58 & $1.92 \mathrm{E}-06$ \\
\hline chr1_137612।X603142577F & 1 cis & 10.6 cis & 6.53 & $2.05 \mathrm{E}-06$ \\
\hline chr1_137622।X603142577F & 1 cis & 10.6 cis & 10.07 & $1.18 \mathrm{E}-07$ \\
\hline chr1_137623ıX603142577F & $1 \mathrm{cis}$ & 10.6 cis & 13.09 & $1.53 \mathrm{E}-07$ \\
\hline chr1_137626ıX603142577F & 1 cis & 10.6 cis & 11.6 & $1.86 \mathrm{E}-08$ \\
\hline chr1_137627ıX603142577F & 1 cis & $10.6 \mathrm{cis}$ & 12.04 & $2.80 \mathrm{E}-07$ \\
\hline chr1_1376281X603142577F & 1 cis & 10.6 cis & 14.6 & $2.16 \mathrm{E}-08$ \\
\hline chr1_137630ıX603142577F & 1 cis & 10.6 cis & 20.13 & $6.53 \mathrm{E}-07$ \\
\hline chr1_137634।X603142577F & 1 cis & 10.6 cis & 5.22 & $1.92 \mathrm{E}-05$ \\
\hline chr1_137644ıX603142577F & $1 \mathrm{cis}$ & 10.6 cis & 17.4 & $3.03 E-08$ \\
\hline chr1_137651।X603142577F & 1 cis & 10.6 cis & 11.52 & $5.90 \mathrm{E}-08$ \\
\hline chr1_137654ıX03142577F & 1 cis & $10.6 \mathrm{cis}$ & 12 & $1.43 \mathrm{E}-07$ \\
\hline chr1_137655ıX603142577F & 1 cis & 10.6 cis & 14.8 & 2.93E-07 \\
\hline chr1_137657।X603142577F & $1 \mathrm{cis}$ & $10.6 \mathrm{cis}$ & 20.05 & 3.10E-06 \\
\hline chr1_137661।X603142577F & 1 cis & 10.6 cis & 14.44 & 3.53E-07 \\
\hline chr1_137666।X603142577F & $1 \mathrm{cis}$ & 10.6 cis & 8.69 & 5.06E-07 \\
\hline chr1_137667।X603142577F & 1 cis & 10.6 cis & 12.12 & $3.15 \mathrm{E}-07$ \\
\hline chr1_137669।X603142577F & 1 cis & $10.6 \mathrm{cis}$ & 7.63 & $6.22 \mathrm{E}-07$ \\
\hline chr1_137674ıX603142577F & 1 cis & $10.6 \mathrm{cis}$ & 17.46 & $3.85 \mathrm{E}-08$ \\
\hline chr1_137679ıX603142577F & $1 \mathrm{cis}$ & 10.6 cis & 11.19 & $9.07 \mathrm{E}-08$ \\
\hline chr1_137681।X603142577F & 1 cis & 10.6 cis & 11.75 & $1.04 \mathrm{E}-07$ \\
\hline chr1_137683ı603142577F & $1 \mathrm{cis}$ & 10.6 cis & 10.91 & $2.96 \mathrm{E}-08$ \\
\hline chr1_137684IX603142577F & 1 cis & 10.6 cis & 13.1 & $8.24 \mathrm{E}-08$ \\
\hline chr1_137685ıX603142577F & 1 cis & $10.6 \mathrm{cis}$ & 15.45 & $2.94 \mathrm{E}-07$ \\
\hline chr1_137687।X603142577F & 1 cis & $10.6 \mathrm{cis}$ & 20.63 & $2.71 \mathrm{E}-06$ \\
\hline chr1_137695ıX03142577F & $1 \mathrm{cis}$ & 10.6 cis & 5.8 & $2.10 \mathrm{E}-06$ \\
\hline chr1_1376981X603142577F & 1 cis & 10.6 cis & 7.13 & $7.46 \mathrm{E}-07$ \\
\hline chr1_137714ıX603142577F & $1 \mathrm{cis}$ & 10.6 cis & 12.06 & 2.93E-07 \\
\hline chr1_137715ıX603142577F & 1 cis & 10.6 cis & 14.88 & $8.69 \mathrm{E}-08$ \\
\hline chr1_137717ıX603142577F & 1 cis & $10.6 \mathrm{cis}$ & 19.34 & $2.06 \mathrm{E}-06$ \\
\hline chr1_137725ıX603142577F & 1 cis & $10.6 \mathrm{cis}$ & 6.06 & $1.26 \mathrm{E}-06$ \\
\hline chr1_137728ıX603142577F & $1 \mathrm{cis}$ & 10.6 cis & 7.61 & 5.76E-07 \\
\hline chr1_137729ıX603142577F & 1 cis & 10.6 cis & 9.84 & $1.75 \mathrm{E}-06$ \\
\hline chr1_137738ıx603142577F & $1 \mathrm{cis}$ & 10.6 cis & 10.68 & $4.21 \mathrm{E}-08$ \\
\hline chr1_137740(X603142577F & 1 cis & 10.6 cis & 11.54 & $3.02 \mathrm{E}-08$ \\
\hline chr1_137741।X603142577F & $1 \mathrm{cis}$ & 10.6 cis & 11.01 & $2.92 \mathrm{E}-08$ \\
\hline chr1_137742।X603142577F & 1 cis & $10.6 \mathrm{cis}$ & 11.49 & $3.62 \mathrm{E}-08$ \\
\hline chr1_137744ı X603142577F & $1 \mathrm{cis}$ & 10.6 cis & 11.31 & $4.51 \mathrm{E}-08$ \\
\hline chr1_137745ıX603142577F & 1 cis & 10.6 cis & 13.04 & $1.65 \mathrm{E}-07$ \\
\hline chr1_1377461X603142577F & $1 \mathrm{cis}$ & 10.6 cis & 15.17 & $3.05 \mathrm{E}-08$ \\
\hline chr1_137748ıX603142577F & 1 cis & 10.6 cis & 18.61 & $2.05 \mathrm{E}-07$ \\
\hline chr1_137769ıX603142577F & $1 \mathrm{cis}$ & 10.6 cis & 10.67 & $2.88 \mathrm{E}-06$ \\
\hline
\end{tabular}




\begin{tabular}{|c|c|c|c|c|}
\hline chr1_137791ıX603142577F & 1 cis & $10.6 \mathrm{cis}$ & 14.07 & 4.79E-08 \\
\hline chr1_139238ıX603142577F & 1 cis & 10.6 cis & 11.53 & $5.78 \mathrm{E}-06$ \\
\hline chr1_137767ıX603142577F & 1 cis & 10.6 cis & 5.11 & $1.46 \mathrm{E}-06$ \\
\hline chr1_137584ıX03142577F & 1 cis & 10.6 cis & 7.86 & $5.20 \mathrm{E}-07$ \\
\hline chr1_137589ıX603142577F & 1 cis & 10.6 cis & 13.65 & $3.92 \mathrm{E}-07$ \\
\hline chr1_137605ıX603142577F & 1 cis & 10.6 cis & 12.38 & 7.07E-07 \\
\hline chr1_137609ıX603142577F & 1 cis & $10.6 \mathrm{cis}$ & 7.31 & $5.93 \mathrm{E}-07$ \\
\hline chr1_137613ıX603142577F & 1 cis & 10.6 cis & 8.76 & $9.07 \mathrm{E}-07$ \\
\hline chr1_137615ıX603142577F & 1 cis & 10.6 cis & 15.89 & $9.21 \mathrm{E}-07$ \\
\hline chr1_137617।X603142577F & 1 cis & 10.6 cis & 14.17 & $1.60 \mathrm{E}-07$ \\
\hline chr1_137618ıX603142577F & 1 cis & 10.6 cis & 16.44 & $6.98 \mathrm{E}-08$ \\
\hline chr1_137631।X603142577F & 1 cis & 10.6 cis & 14.54 & $4.38 \mathrm{E}-07$ \\
\hline chr1_137658ıX603142577F & 1 cis & $10.6 \mathrm{cis}$ & 17.96 & 3.37E-09 \\
\hline chr1_137665ıX603142577F & 1 cis & 10.6 cis & 10 & $5.44 \mathrm{E}-08$ \\
\hline chr1_137668ıX603142577F & 1 cis & $10.6 \mathrm{cis}$ & 7.29 & $9.01 \mathrm{E}-06$ \\
\hline chr1_137688ıX603142577F & 1 cis & 10.6 cis & 17.84 & 7.11E-09 \\
\hline chr1_137697।X603142577F & 1 cis & 10.6 cis & 10.75 & $2.98 \mathrm{E}-06$ \\
\hline chr1_137699ıX603142577F & 1 cis & $10.6 \mathrm{cis}$ & 9.84 & $6.27 \mathrm{E}-06$ \\
\hline chr1_137701ıX603142577F & 1 cis & 10.6 cis & 14.71 & $1.72 \mathrm{E}-06$ \\
\hline chr1_137703ıX603142577F & 1 cis & 10.6 cis & 14.78 & $1.75 \mathrm{E}-07$ \\
\hline chr1_137704ıX03142577F & 1 cis & $10.6 \mathrm{cis}$ & 14.85 & $5.80 \mathrm{E}-08$ \\
\hline chr1_137718ıX603142577F & 1 cis & 10.6 cis & 14.8 & 1.57E-07 \\
\hline chr1_137720ıX603142577F & 1 cis & 10.6 cis & 12.94 & $2.51 \mathrm{E}-07$ \\
\hline chr1_137721।X603142577F & 1 cis & $10.6 \mathrm{cis}$ & 17.19 & 7.71E-07 \\
\hline chr1_137727ıX603142577F & 1 cis & 10.6 cis & 10.77 & $5.24 \mathrm{E}-06$ \\
\hline chr1_137731।X603142577F & 1 cis & 10.6 cis & 15.52 & $1.14 \mathrm{E}-06$ \\
\hline chr1_137733ıX03142577F & 1 cis & $10.6 \mathrm{cis}$ & 14.23 & $1.42 \mathrm{E}-07$ \\
\hline chr1_137734ıX603142577F & $1 \mathrm{cis}$ & $10.6 \mathrm{cis}$ & 15.49 & $9.22 \mathrm{E}-08$ \\
\hline chr1_137770ıX603142577F & 1 cis & 10.6 cis & 6.25 & $1.46 \mathrm{E}-06$ \\
\hline chr1_137771।X603142577F & 1 cis & $10.6 \mathrm{cis}$ & 6.84 & $4.52 \mathrm{E}-06$ \\
\hline chr1_137777ıX603142577F & 1 cis & 10.6 cis & 13.14 & 2.91E-07 \\
\hline chr1_137790ıX603142577F & 1 cis & 10.6 cis & 9.73 & $1.18 \mathrm{E}-07$ \\
\hline chr1_137574ıX603142577F & 1 cis & 10.6 cis & 13.87 & $1.02 \mathrm{E}-06$ \\
\hline chr1_1375761X603142577F & $1 \mathrm{cis}$ & 10.6 cis & 11.94 & $5.78 \mathrm{E}-07$ \\
\hline chr1_137579ıX603142577F & 1 cis & 10.6 cis & 12.49 & $2.89 \mathrm{E}-05$ \\
\hline chr1_137585ıX603142577F & 1 cis & $10.6 \mathrm{cis}$ & 11 & $1.12 \mathrm{E}-04$ \\
\hline chr1_137586ıX603142577F & 1 cis & 10.6 cis & 13.87 & $1.08 \mathrm{E}-05$ \\
\hline chr1_137587ıX603142577F & 1 cis & 10.6 cis & 11.77 & 5.77E-06 \\
\hline chr1_137590।X603142577F & 1 cis & 10.6 cis & 13.24 & $1.09 \mathrm{E}-07$ \\
\hline chr1_137593ıX03142577F & $1 \mathrm{cis}$ & 10.6 cis & 13.66 & $2.16 \mathrm{E}-06$ \\
\hline chr1_137603ıX603142577F & 1 cis & 10.6 cis & 13.07 & $1.19 \mathrm{E}-05$ \\
\hline chr1_137608ıX603142577F & 1 cis & $10.6 \mathrm{cis}$ & 12.22 & $2.71 E-05$ \\
\hline chr1_137614ıX603142577F & 1 cis & $10.6 \mathrm{cis}$ & 10.31 & 4.46E-04 \\
\hline chr1_137619ıX603142577F & $1 \mathrm{cis}$ & $10.6 \mathrm{cis}$ & 12.43 & $1.30 \mathrm{E}-07$ \\
\hline chr1_137621।X603142577F & 1 cis & 10.6 cis & 13.68 & $9.90 \mathrm{E}-06$ \\
\hline chr1_137633ı603142577F & $1 \mathrm{cis}$ & 10.6 cis & 11.92 & 4.37E-07 \\
\hline chr1_137643ıX603142577F & 1 cis & 10.6 cis & 13.72 & $2.31 \mathrm{E}-07$ \\
\hline chr1_137647।X603142577F & 1 cis & $10.6 \mathrm{cis}$ & 12.23 & $1.54 \mathrm{E}-05$ \\
\hline chr1_1376591X603142577F & 1 cis & 10.6 cis & 13.41 & $1.41 \mathrm{E}-04$ \\
\hline chr1_137670ıX03142577F & $1 \mathrm{cis}$ & 10.6 cis & 9.41 & 3.59E-04 \\
\hline chr1_137671।X603142577F & 1 cis & 10.6 cis & 15.22 & 3.50E-06 \\
\hline chr1_137672।X603142577F & $1 \mathrm{cis}$ & 10.6 cis & 12.01 & $7.80 \mathrm{E}-06$ \\
\hline chr1_137673ıX603142577F & 1 cis & 10.6 cis & 11.1 & 1.19E-06 \\
\hline chr1_137675ıX603142577F & 1 cis & $10.6 \mathrm{cis}$ & 11.47 & $6.09 \mathrm{E}-08$ \\
\hline chr1_137689ıX603142577F & 1 cis & $10.6 \mathrm{cis}$ & 13.29 & $1.59 \mathrm{E}-04$ \\
\hline chr1_137691।X603142577F & $1 \mathrm{cis}$ & 10.6 cis & 13.54 & 2.47E-06 \\
\hline chr1_137693ıX603142577F & 1 cis & 10.6 cis & 15.25 & $5.04 \mathrm{E}-05$ \\
\hline chr1_137694ıX603142577F & $1 \mathrm{cis}$ & 10.6 cis & 10.96 & $1.29 \mathrm{E}-06$ \\
\hline chr1_137700।X603142577F & 1 cis & 10.6 cis & 12.24 & $2.62 \mathrm{E}-04$ \\
\hline chr1_137707ıX603142577F & 1 cis & $10.6 \mathrm{cis}$ & 14.34 & $1.73 \mathrm{E}-06$ \\
\hline chr1_137749ıX603142577F & 1 cis & $10.6 \mathrm{cis}$ & 14.29 & $7.34 \mathrm{E}-07$ \\
\hline chr1_137751।X603142577F & $1 \mathrm{cis}$ & 10.6 cis & 12.18 & 2.49E-07 \\
\hline chr1_137756।X603142577F & 1 cis & 10.6 cis & 11.17 & $1.65 \mathrm{E}-06$ \\
\hline chr1_137772।X603142577F & $1 \mathrm{cis}$ & 10.6 cis & 9.71 & 7.71E-04 \\
\hline chr1_137773ıX603142577F & 1 cis & 10.6 cis & 14.08 & 2.99E-06 \\
\hline chr1_137774ı X603142577F & $1 \mathrm{cis}$ & 10.6 cis & 11.46 & $9.79 \mathrm{E}-06$ \\
\hline chr1_137786ıX603142577F & 1 cis & $10.6 \mathrm{cis}$ & 6.34 & 4.16E-04 \\
\hline chr1_137575ıX603142577F & $1 \mathrm{cis}$ & 10.6 cis & 14.57 & $1.91 \mathrm{E}-05$ \\
\hline chr1_137578ıX603142577F & 1 cis & 10.6 cis & 14.89 & $7.62 \mathrm{E}-05$ \\
\hline chr1_137591।X603142577F & $1 \mathrm{cis}$ & 10.6 cis & 11.3 & $6.42 \mathrm{E}-07$ \\
\hline chr1_137592ıX603142577F & 1 cis & 10.6 cis & 11.45 & $6.56 \mathrm{E}-05$ \\
\hline chr1_137604ıX603142577F & $1 \mathrm{cis}$ & 10.6 cis & 14.51 & $9.75 \mathrm{E}-06$ \\
\hline
\end{tabular}




\begin{tabular}{|c|c|c|c|c|}
\hline chr1_137606।X603142577F & 1 cis & $10.6 \mathrm{cis}$ & 13.67 & $1.01 \mathrm{E}-04$ \\
\hline chr1_137607ıX603142577F & 1 cis & 10.6 cis & 14.26 & 8.30E-05 \\
\hline chr1_137616।X603142577F & 1 cis & 10.6 cis & 12.35 & $9.13 \mathrm{E}-06$ \\
\hline chr1_137620ıX603142577F & 1 cis & 10.6 cis & 12.52 & $2.29 \mathrm{E}-05$ \\
\hline chr1_137632।X603142577F & 1 cis & 10.6 cis & 14.85 & $2.64 \mathrm{E}-05$ \\
\hline chr1_137642ıX03142577F & 1 cis & 10.6 cis & 11.5 & $8.86 \mathrm{E}-06$ \\
\hline chr1_137645ıX603142577F & 1 cis & $10.6 \mathrm{cis}$ & 11.49 & $1.20 \mathrm{E}-07$ \\
\hline chr1_137646ıX603142577F & 1 cis & 10.6 cis & 11.33 & $2.51 \mathrm{E}-05$ \\
\hline chr1_137660।X603142577F & 1 cis & 10.6 cis & 13.64 & $1.09 \mathrm{E}-06$ \\
\hline chr1_137662।X603142577F & 1 cis & 10.6 cis & 13.75 & 5.29E-05 \\
\hline chr1_137663ıX03142577F & 1 cis & 10.6 cis & 13.79 & $2.78 \mathrm{E}-04$ \\
\hline chr1_137664ıX603142577F & 1 cis & 10.6 cis & 12.5 & 2.94E-05 \\
\hline chr1_137677।X603142577F & 1 cis & $10.6 \mathrm{cis}$ & 11.72 & $3.18 \mathrm{E}-05$ \\
\hline chr1_137690ıX603142577F & 1 cis & 10.6 cis & 13.19 & $1.08 \mathrm{E}-06$ \\
\hline chr1_137692ı603142577F & 1 cis & $10.6 \mathrm{cis}$ & 13.15 & 1.69E-04 \\
\hline chr1_137705ıX603142577F & 1 cis & 10.6 cis & 11.7 & $3.78 \mathrm{E}-07$ \\
\hline chr1_137706ıX603142577F & 1 cis & 10.6 cis & 11.74 & 4.26E-05 \\
\hline chr1_137719ıX603142577F & 1 cis & $10.6 \mathrm{cis}$ & 13.47 & $8.89 \mathrm{E}-05$ \\
\hline chr1_137722ıX03142577F & 1 cis & 10.6 cis & 13.95 & $7.12 \mathrm{E}-05$ \\
\hline chr1_137723ıX603142577F & 1 cis & 10.6 cis & 15.21 & 4.12E-05 \\
\hline chr1_137724ıX603142577F & 1 cis & $10.6 \mathrm{cis}$ & 12.58 & $1.44 \mathrm{E}-05$ \\
\hline chr1_137730ıX603142577F & 1 cis & 10.6 cis & 10.98 & $5.26 \mathrm{E}-04$ \\
\hline chr1_137735ıX603142577F & 1 cis & 10.6 cis & 12.23 & $2.43 \mathrm{E}-07$ \\
\hline chr1_137736ıX603142577F & 1 cis & $10.6 \mathrm{cis}$ & 11.26 & 4.75E-05 \\
\hline chr1_137737ıX603142577F & 1 cis & 10.6 cis & 12.9 & $1.07 \mathrm{E}-05$ \\
\hline chr1_137750ıX603142577F & 1 cis & 10.6 cis & 14.69 & $1.66 \mathrm{E}-05$ \\
\hline chr1_137752।X603142577F & 1 cis & $10.6 \mathrm{cis}$ & 13.9 & $6.21 \mathrm{E}-05$ \\
\hline chr1_137753ıX603142577F & $1 \mathrm{cis}$ & $10.6 \mathrm{cis}$ & 14.86 & $7.41 \mathrm{E}-05$ \\
\hline chr1_137754ı603142577F & 1 cis & 10.6 cis & 11.23 & $5.25 \mathrm{E}-05$ \\
\hline chr1_137757।X603142577F & 1 cis & $10.6 \mathrm{cis}$ & 10.92 & $2.29 \mathrm{E}-07$ \\
\hline chr1_137778ıX603142577F & 1 cis & 10.6 cis & 13.73 & 4.03E-08 \\
\hline chr1_137676ıX603142577F & 1 cis & 10.6 cis & 10.73 & 2.17E-05 \\
\hline chr1_137702।X603142577F & 1 cis & 10.6 cis & 10.02 & $2.62 \mathrm{E}-05$ \\
\hline chr1_137732।X603142577F & $1 \mathrm{cis}$ & 10.6 cis & 10.16 & 2.77E-05 \\
\hline chr1_137755ıX603142577F & 1 cis & 10.6 cis & 10.63 & 2.37E-04 \\
\hline chr1_137758ıX603142577F & 1 cis & $10.6 \mathrm{cis}$ & 8.15 & $1.43 \mathrm{E}-04$ \\
\hline chr1_139815ıX603142577F & 1 cis & $10.6 \mathrm{cis}$ & 4.1 & $3.81 \mathrm{E}-04$ \\
\hline chr1_137779ıX603142577F & 1 cis & 10.6 cis & 8.81 & 7.01E-06 \\
\hline chr1_137588ıX603142577F & 1 cis & 10.6 cis & 6.55 & 4.09E-04 \\
\hline chr1_134539IENSGALT000( & $1 \mathrm{cis}$ & 9.3 cis & 5.34 & $2.67 \mathrm{E}-04$ \\
\hline chr1_134200IENSGALT000( & 1 cis & $9.3 \mathrm{cis}$ & 4.51 & 4.54E-04 \\
\hline chr1_133896IENSGALT000( & 1 cis & $9.3 \mathrm{cis}$ & 4.99 & $2.23 \mathrm{E}-06$ \\
\hline chr1_138209IENSGALT000( & 1 cis & 9.3 cis & 8.03 & 1.13E-05 \\
\hline chr1_136991।ENSGALTO00( & $1 \mathrm{cis}$ & 9.3 cis & 4.73 & $2.02 \mathrm{E}-04$ \\
\hline chr1_137382।ENSGALTO00( & 1 cis & 9.3 cis & 6.07 & $6.38 \mathrm{E}-08$ \\
\hline chr1_138835IENSGALT000( & $1 \mathrm{cis}$ & $9.3 \mathrm{cis}$ & 7.82 & $1.01 \mathrm{E}-04$ \\
\hline chr1_136500IENSGALT000( & 1 cis & 9.3 cis & 7.17 & $5.90 \mathrm{E}-06$ \\
\hline chr1_137768ıENSGALT000( & 1 cis & $9.3 \mathrm{cis}$ & 4.39 & 3.19E-05 \\
\hline chr1_137610IENSGALT000( & 1 cis & 9.3 cis & 8.68 & 4.13E-07 \\
\hline chr1_137629IENSGALT000( & $1 \mathrm{cis}$ & $9.3 \mathrm{cis}$ & 19.85 & $1.79 \mathrm{E}-08$ \\
\hline chr1_137656IENSGALT000( & 1 cis & 9.3 cis & 11.5 & $1.52 \mathrm{E}-08$ \\
\hline chr1_137682।ENSGALT000( & $1 \mathrm{cis}$ & $9.3 \mathrm{cis}$ & 11.7 & $3.48 \mathrm{E}-08$ \\
\hline chr1_137686IENSGALT000( & 1 cis & $9.3 \mathrm{cis}$ & 11.2 & $1.38 \mathrm{E}-08$ \\
\hline chr1_137716।ENSGALT000( & 1 cis & $9.3 \mathrm{cis}$ & 14.66 & $3.39 \mathrm{E}-08$ \\
\hline chr1_137581।ENSGALTO00( & 1 cis & $9.3 \mathrm{cis}$ & 7.77 & $1.73 \mathrm{E}-07$ \\
\hline chr1_137594IENSGALT000( & $1 \mathrm{cis}$ & $9.3 \mathrm{cis}$ & 10.53 & $1.02 \mathrm{E}-07$ \\
\hline chr1_137596IENSGALT000( & 1 cis & 9.3 cis & 11.39 & $4.80 \mathrm{E}-08$ \\
\hline chr1_137597IENSGALT000( & $1 \mathrm{cis}$ & $9.3 \mathrm{cis}$ & 10.26 & $1.02 \mathrm{E}-07$ \\
\hline chr1_137601।ENSGALT000( & 1 cis & $9.3 \mathrm{cis}$ & 21.57 & $3.61 \mathrm{E}-07$ \\
\hline chr1_137624IENSGALT000( & 1 cis & $9.3 \mathrm{cis}$ & 11.53 & $3.75 \mathrm{E}-08$ \\
\hline chr1_137625।ENSGALTO00( & 1 cis & $9.3 \mathrm{cis}$ & 11.15 & 4.03E-08 \\
\hline chr1_137641।ENSGALT000( & $1 \mathrm{cis}$ & $9.3 \mathrm{cis}$ & 11.45 & 5.99E-08 \\
\hline chr1_137648IENSGALT000( & 1 cis & 9.3 cis & 11.67 & 7.17E-09 \\
\hline chr1_137649IENSGALT000( & $1 \mathrm{cis}$ & $9.3 \mathrm{cis}$ & 11.23 & $1.41 \mathrm{E}-07$ \\
\hline chr1_137650IENSGALT000( & 1 cis & $9.3 \mathrm{cis}$ & 10.88 & $1.01 \mathrm{E}-07$ \\
\hline chr1_137652।ENSGALT000( & $1 \mathrm{cis}$ & $9.3 \mathrm{cis}$ & 11.68 & $9.42 \mathrm{E}-08$ \\
\hline chr1_137653IENSGALT000 & 1 cis & 9.3 cis & 10.35 & 4.00E-08 \\
\hline chr1_137678IENSGALT000( & $1 \mathrm{cis}$ & $9.3 \mathrm{cis}$ & 12.09 & $1.06 \mathrm{E}-08$ \\
\hline chr1_137680IENSGALT000( & 1 cis & $9.3 \mathrm{cis}$ & 11.65 & $4.26 \mathrm{E}-08$ \\
\hline chr1_137696IENSGALT000( & $1 \mathrm{cis}$ & $9.3 \mathrm{cis}$ & 10.03 & $6.33 \mathrm{E}-07$ \\
\hline chr1_137708IENSGALT000( & 1 cis & $9.3 \mathrm{cis}$ & 10.55 & $4.66 \mathrm{E}-08$ \\
\hline chr1_137709ıENSGALT000( & $1 \mathrm{cis}$ & $9.3 \mathrm{cis}$ & 11.02 & $6.70 \mathrm{E}-08$ \\
\hline
\end{tabular}




\begin{tabular}{|c|c|c|c|c|}
\hline chr1_137710IENSGALT000( & 1 cis & 9.3 cis & 12.66 & $1.37 \mathrm{E}-07$ \\
\hline chr1_137711।ENSGALT000( & 1 cis & $9.3 \mathrm{cis}$ & 11.03 & $5.08 \mathrm{E}-08$ \\
\hline chr1_137712।ENSGALT000( & 1 cis & $9.3 \mathrm{cis}$ & 11.4 & 7.15E-08 \\
\hline chr1_137713ıENSGALT000( & 1 cis & $9.3 \mathrm{cis}$ & 11.54 & 4.00E-08 \\
\hline chr1_137726IENSGALT000( & 1 cis & $9.3 \mathrm{cis}$ & 9.81 & $5.82 \mathrm{E}-07$ \\
\hline chr1_137739IENSGALTO00( & 1 cis & $9.3 \mathrm{cis}$ & 11.17 & $1.43 \mathrm{E}-07$ \\
\hline chr1_137743ıENSGALT000( & 1 cis & 9.3 cis & 11.15 & 4.29E-08 \\
\hline chr1_137747IENSGALT000( & 1 cis & $9.3 \mathrm{cis}$ & 18.69 & $1.42 \mathrm{E}-07$ \\
\hline chr1_137582।ENSGALT000( & 1 cis & $9.3 \mathrm{cis}$ & 12.1 & $5.98 \mathrm{E}-07$ \\
\hline chr1_137583ıENSGALT000( & 1 cis & $9.3 \mathrm{cis}$ & 7.53 & 5.90E-07 \\
\hline chr1_137595ıENSGALT000( & 1 cis & $9.3 \mathrm{cis}$ & 12.69 & 3.27E-07 \\
\hline chr1_137598IENSGALTO00( & 1 cis & 9.3 cis & 11.89 & 2.37E-08 \\
\hline chr1_137599IENSGALT000( & 1 cis & 9.3 cis & 14.05 & $1.33 \mathrm{E}-07$ \\
\hline chr1_137600IENSGALT000( & 1 cis & $9.3 \mathrm{cis}$ & 14.13 & $1.79 \mathrm{E}-08$ \\
\hline chr1_137602।ENSGALT000( & 1 cis & $9.3 \mathrm{cis}$ & 18.72 & 3.36E-07 \\
\hline chr1_137611।ENSGALT000( & 1 cis & $9.3 \mathrm{cis}$ & 11.58 & $1.96 \mathrm{E}-06$ \\
\hline chr1_137612।ENSGALT000( & 1 cis & $9.3 \mathrm{cis}$ & 6.53 & 4.23E-07 \\
\hline chr1_137622।ENSGALT000( & 1 cis & $9.3 \mathrm{cis}$ & 10.07 & $6.81 \mathrm{E}-08$ \\
\hline chr1_137623IENSGALT000( & 1 cis & 9.3 cis & 13.09 & $1.18 \mathrm{E}-07$ \\
\hline chr1_137626IENSGALT000( & 1 cis & $9.3 \mathrm{cis}$ & 11.6 & 3.19E-08 \\
\hline chr1_137627IENSGALT000( & 1 cis & $9.3 \mathrm{cis}$ & 12.04 & 4.99E-08 \\
\hline chr1_137628IENSGALT000( & 1 cis & $9.3 \mathrm{cis}$ & 14.6 & $2.24 \mathrm{E}-08$ \\
\hline chr1_137630IENSGALT000( & 1 cis & $9.3 \mathrm{cis}$ & 20.13 & $5.82 \mathrm{E}-06$ \\
\hline chr1_137634IENSGALT000( & 1 cis & $9.3 \mathrm{cis}$ & 5.22 & $1.24 \mathrm{E}-04$ \\
\hline chr1_137644IENSGALT000( & 1 cis & 9.3 cis & 17.4 & 2.19E-06 \\
\hline chr1_137651।ENSGALT000( & 1 cis & $9.3 \mathrm{cis}$ & 11.52 & $1.63 \mathrm{E}-08$ \\
\hline chr1_137654IENSGALT000( & 1 cis & $9.3 \mathrm{cis}$ & 12 & $1.82 \mathrm{E}-08$ \\
\hline chr1_137655IENSGALTO00( & $1 \mathrm{cis}$ & 9.3 cis & 14.8 & $2.69 \mathrm{E}-07$ \\
\hline chr1_137657IENSGALT000( & 1 cis & $9.3 \mathrm{cis}$ & 20.05 & 3.15E-05 \\
\hline chr1_137661।ENSGALT000( & 1 cis & $9.3 \mathrm{cis}$ & 14.44 & $9.31 \mathrm{E}-08$ \\
\hline chr1_137666ıENSGALT000( & 1 cis & 9.3 cis & 8.69 & 4.83E-08 \\
\hline chr1_137667IENSGALT000( & 1 cis & $9.3 \mathrm{cis}$ & 12.12 & $2.33 \mathrm{E}-07$ \\
\hline chr1_137669IENSGALT000( & 1 cis & 9.3 cis & 7.63 & $2.92 \mathrm{E}-07$ \\
\hline chr1_137674IENSGALT000( & $1 \mathrm{cis}$ & $9.3 \mathrm{cis}$ & 17.46 & $1.27 \mathrm{E}-06$ \\
\hline chr1_137679IENSGALT000( & 1 cis & $9.3 \mathrm{cis}$ & 11.19 & $1.14 \mathrm{E}-07$ \\
\hline chr1_137681।ENSGALT000( & 1 cis & $9.3 \mathrm{cis}$ & 11.75 & $3.25 \mathrm{E}-08$ \\
\hline chr1_137683!ENSGALTO00( & 1 cis & $9.3 \mathrm{cis}$ & 10.91 & $2.80 \mathrm{E}-08$ \\
\hline chr1_137684IENSGALT000( & 1 cis & $9.3 \mathrm{cis}$ & 13.1 & $6.39 \mathrm{E}-09$ \\
\hline chr1_137685ıENSGALT000( & 1 cis & 9.3 cis & 15.45 & $1.93 \mathrm{E}-07$ \\
\hline chr1_137687IENSGALT000( & $1 \mathrm{cis}$ & $9.3 \mathrm{cis}$ & 20.63 & 3.16E-05 \\
\hline chr1_137695ıENSGALT000( & 1 cis & $9.3 \mathrm{cis}$ & 5.8 & 5.13E-06 \\
\hline chr1_137698IENSGALT000( & 1 cis & $9.3 \mathrm{cis}$ & 7.13 & $1.84 \mathrm{E}-07$ \\
\hline chr1_137714ıENSGALT000( & 1 cis & $9.3 \mathrm{cis}$ & 12.06 & 4.47E-08 \\
\hline chr1_137715ıENSGALTO00( & $1 \mathrm{cis}$ & 9.3 cis & 14.88 & $6.76 \mathrm{E}-08$ \\
\hline chr1_137717।ENSGALTO00( & 1 cis & 9.3 cis & 19.34 & $2.32 \mathrm{E}-05$ \\
\hline chr1_137725ıENSGALT000( & $1 \mathrm{cis}$ & $9.3 \mathrm{cis}$ & 6.06 & $1.05 \mathrm{E}-06$ \\
\hline chr1_137728IENSGALT000( & 1 cis & 9.3 cis & 7.61 & $1.60 \mathrm{E}-07$ \\
\hline chr1_137729IENSGALT000( & 1 cis & $9.3 \mathrm{cis}$ & 9.84 & $1.79 \mathrm{E}-06$ \\
\hline chr1_137738IENSGALTO00( & 1 cis & $9.3 \mathrm{cis}$ & 10.68 & $5.15 \mathrm{E}-08$ \\
\hline chr1_137740ıENSGALT000( & $1 \mathrm{cis}$ & $9.3 \mathrm{cis}$ & 11.54 & 1.57E-07 \\
\hline chr1_137741।ENSGALT000( & 1 cis & 9.3 cis & 11.01 & $1.96 \mathrm{E}-08$ \\
\hline chr1_137742।ENSGALT000( & $1 \mathrm{cis}$ & $9.3 \mathrm{cis}$ & 11.49 & $2.45 \mathrm{E}-07$ \\
\hline chr1_137744ıENSGALTO00( & 1 cis & $9.3 \mathrm{cis}$ & 11.31 & $7.22 \mathrm{E}-08$ \\
\hline chr1_137745ıENSGALT000( & 1 cis & $9.3 \mathrm{cis}$ & 13.04 & 4.53E-08 \\
\hline chr1_137746IENSGALTO00( & 1 cis & $9.3 \mathrm{cis}$ & 15.17 & 2.37E-08 \\
\hline chr1_137748IENSGALT000( & $1 \mathrm{cis}$ & $9.3 \mathrm{cis}$ & 18.61 & 1.47E-06 \\
\hline chr1_137769IENSGALT000( & 1 cis & 9.3 cis & 10.67 & $3.01 \mathrm{E}-06$ \\
\hline chr1_137791।ENSGALT000( & $1 \mathrm{cis}$ & $9.3 \mathrm{cis}$ & 14.07 & $6.84 \mathrm{E}-08$ \\
\hline chr1_139238IENSGALT000( & 1 cis & $9.3 \mathrm{cis}$ & 11.53 & $1.84 \mathrm{E}-07$ \\
\hline chr1_137767IENSGALT000( & 1 cis & $9.3 \mathrm{cis}$ & 5.11 & 5.55E-06 \\
\hline chr1_137584IENSGALTO00( & 1 cis & $9.3 \mathrm{cis}$ & 7.86 & $2.18 \mathrm{E}-07$ \\
\hline chr1_137589IENSGALT000( & $1 \mathrm{cis}$ & $9.3 \mathrm{cis}$ & 13.65 & $9.93 \mathrm{E}-06$ \\
\hline chr1_137605ıENSGALT000( & 1 cis & 9.3 cis & 12.38 & $9.12 \mathrm{E}-07$ \\
\hline chr1_137609IENSGALT000( & $1 \mathrm{cis}$ & $9.3 \mathrm{cis}$ & 7.31 & 8.36E-07 \\
\hline chr1_137613ıENSGALT000( & $1 \mathrm{cis}$ & $9.3 \mathrm{cis}$ & 8.76 & $4.58 \mathrm{E}-07$ \\
\hline chr1_137615ıENSGALT000( & $1 \mathrm{cis}$ & $9.3 \mathrm{cis}$ & 15.89 & $1.44 \mathrm{E}-06$ \\
\hline chr1_137617IENSGALT000 & 1 cis & 9.3 cis & 14.17 & $3.82 \mathrm{E}-06$ \\
\hline chr1_137618IENSGALT000( & $1 \mathrm{cis}$ & $9.3 \mathrm{cis}$ & 16.44 & 2.79E-06 \\
\hline chr1_137631।ENSGALT000( & 1 cis & 9.3 cis & 14.54 & 5.55E-06 \\
\hline chr1_137658IENSGALT000( & $1 \mathrm{cis}$ & $9.3 \mathrm{cis}$ & 17.96 & $3.49 \mathrm{E}-08$ \\
\hline chr1_137665ıENSGALT000( & 1 cis & $9.3 \mathrm{cis}$ & 10 & 3.67E-07 \\
\hline chr1_137668ıENSGALT000( & $1 \mathrm{cis}$ & $9.3 \mathrm{cis}$ & 7.29 & $2.72 \mathrm{E}-06$ \\
\hline
\end{tabular}




\begin{tabular}{|c|c|c|c|c|}
\hline chr1_137688IENSGALT000( & 1 cis & 9.3 cis & 17.84 & $9.23 \mathrm{E}-08$ \\
\hline chr1_137697IENSGALT000( & 1 cis & $9.3 \mathrm{cis}$ & 10.75 & $2.32 \mathrm{E}-06$ \\
\hline chr1_137699IENSGALT000( & 1 cis & $9.3 \mathrm{cis}$ & 9.84 & $5.50 \mathrm{E}-06$ \\
\hline chr1_137701।ENSGALT000( & 1 cis & $9.3 \mathrm{cis}$ & 14.71 & $1.49 \mathrm{E}-06$ \\
\hline chr1_137703ıENSGALT000( & 1 cis & $9.3 \mathrm{cis}$ & 14.78 & $3.38 \mathrm{E}-06$ \\
\hline chr1_137704IENSGALT000( & 1 cis & $9.3 \mathrm{cis}$ & 14.85 & $1.44 \mathrm{E}-06$ \\
\hline chr1_137718IENSGALT000( & 1 cis & 9.3 cis & 14.8 & $3.00 \mathrm{E}-06$ \\
\hline chr1_137720IENSGALT000( & 1 cis & $9.3 \mathrm{cis}$ & 12.94 & 1.07E-07 \\
\hline chr1_137721।ENSGALT000( & 1 cis & $9.3 \mathrm{cis}$ & 17.19 & $7.55 \mathrm{E}-07$ \\
\hline chr1_137727IENSGALT000( & 1 cis & $9.3 \mathrm{cis}$ & 10.77 & $1.01 \mathrm{E}-05$ \\
\hline chr1_137731।ENSGALT000( & 1 cis & $9.3 \mathrm{cis}$ & 15.52 & $1.46 \mathrm{E}-06$ \\
\hline chr1_137733ıENSGALTO00( & 1 cis & 9.3 cis & 14.23 & 3.75E-06 \\
\hline chr1_137734!ENSGALT000( & 1 cis & 9.3 cis & 15.49 & $4.18 \mathrm{E}-06$ \\
\hline chr1_137770IENSGALT000( & 1 cis & $9.3 \mathrm{cis}$ & 6.25 & 5.89E-07 \\
\hline chr1_137771।ENSGALT000( & 1 cis & $9.3 \mathrm{cis}$ & 6.84 & $1.52 \mathrm{E}-06$ \\
\hline chr1_137777|ENSGALT000( & 1 cis & $9.3 \mathrm{cis}$ & 13.14 & 4.96E-06 \\
\hline chr1_137790IENSGALT000( & 1 cis & $9.3 \mathrm{cis}$ & 9.73 & $6.72 \mathrm{E}-08$ \\
\hline chr1_137574IENSGALT000( & 1 cis & $9.3 \mathrm{cis}$ & 13.87 & $1.49 \mathrm{E}-05$ \\
\hline chr1_137576ıENSGALT000( & 1 cis & 9.3 cis & 11.94 & $6.12 \mathrm{E}-07$ \\
\hline chr1_137579IENSGALT000( & 1 cis & $9.3 \mathrm{cis}$ & 12.49 & 3.59E-04 \\
\hline chr1_137585ıENSGALT000( & 1 cis & $9.3 \mathrm{cis}$ & 11 & $2.78 \mathrm{E}-04$ \\
\hline chr1_137586IENSGALT000( & 1 cis & $9.3 \mathrm{cis}$ & 13.87 & $2.90 \mathrm{E}-05$ \\
\hline chr1_137587IENSGALT000( & 1 cis & $9.3 \mathrm{cis}$ & 11.77 & $4.88 \mathrm{E}-06$ \\
\hline chr1_137590IENSGALT000( & 1 cis & $9.3 \mathrm{cis}$ & 13.24 & $3.24 \mathrm{E}-06$ \\
\hline chr1_137593ıENSGALT000( & 1 cis & $9.3 \mathrm{cis}$ & 13.66 & 2.67E-05 \\
\hline chr1_137603ıENSGALT000( & 1 cis & $9.3 \mathrm{cis}$ & 13.07 & $1.12 \mathrm{E}-04$ \\
\hline chr1_137608ıENSGALT000( & 1 cis & $9.3 \mathrm{cis}$ & 12.22 & $4.85 \mathrm{E}-04$ \\
\hline chr1_137614IENSGALTO00( & $1 \mathrm{cis}$ & 9.3 cis & 10.31 & $1.48 \mathrm{E}-03$ \\
\hline chr1_137619IENSGALT000( & 1 cis & $9.3 \mathrm{cis}$ & 12.43 & $1.30 \mathrm{E}-06$ \\
\hline chr1_137621।ENSGALT000( & 1 cis & $9.3 \mathrm{cis}$ & 13.68 & $7.60 \mathrm{E}-04$ \\
\hline chr1_137633ıENSGALT000( & 1 cis & $9.3 \mathrm{cis}$ & 11.92 & $3.05 \mathrm{E}-07$ \\
\hline chr1_137643ıENSGALT000( & 1 cis & $9.3 \mathrm{cis}$ & 13.72 & 5.91E-06 \\
\hline chr1_137647IENSGALTO00( & 1 cis & 9.3 cis & 12.23 & $9.29 \mathrm{E}-04$ \\
\hline chr1_137659IENSGALT000( & $1 \mathrm{cis}$ & $9.3 \mathrm{cis}$ & 13.41 & $2.12 \mathrm{E}-03$ \\
\hline chr1_137670IENSGALT000( & 1 cis & $9.3 \mathrm{cis}$ & 9.41 & $6.61 \mathrm{E}-04$ \\
\hline chr1_137671।ENSGALT000( & 1 cis & $9.3 \mathrm{cis}$ & 15.22 & 4.63E-06 \\
\hline chr1_137672।ENSGALT000( & 1 cis & $9.3 \mathrm{cis}$ & 12.01 & $6.63 \mathrm{E}-06$ \\
\hline chr1_137673ıENSGALT000( & 1 cis & $9.3 \mathrm{cis}$ & 11.1 & $1.21 \mathrm{E}-05$ \\
\hline chr1_137675ıENSGALT000( & 1 cis & 9.3 cis & 11.47 & $1.22 \mathrm{E}-06$ \\
\hline chr1_137689IENSGALT000( & $1 \mathrm{cis}$ & $9.3 \mathrm{cis}$ & 13.29 & $2.88 \mathrm{E}-03$ \\
\hline chr1_137691।ENSGALT000( & 1 cis & $9.3 \mathrm{cis}$ & 13.54 & $1.08 \mathrm{E}-05$ \\
\hline chr1_137693ıENSGALT000( & 1 cis & $9.3 \mathrm{cis}$ & 15.25 & $9.52 \mathrm{E}-04$ \\
\hline chr1_137694IENSGALT000( & 1 cis & $9.3 \mathrm{cis}$ & 10.96 & $2.06 \mathrm{E}-05$ \\
\hline chr1_137700IENSGALTO00( & $1 \mathrm{cis}$ & 9.3 cis & 12.24 & $1.16 \mathrm{E}-03$ \\
\hline chr1_137707IENSGALT000( & 1 cis & 9.3 cis & 14.34 & $6.12 \mathrm{E}-05$ \\
\hline chr1_137749IENSGALT000( & $1 \mathrm{cis}$ & $9.3 \mathrm{cis}$ & 14.29 & $7.58 \mathrm{E}-06$ \\
\hline chr1_137751।ENSGALT000( & 1 cis & 9.3 cis & 12.18 & 2.67E-07 \\
\hline chr1_137756IENSGALT000( & 1 cis & $9.3 \mathrm{cis}$ & 11.17 & $1.72 \mathrm{E}-05$ \\
\hline chr1_137772।ENSGALTO00( & 1 cis & $9.3 \mathrm{cis}$ & 9.71 & $1.93 \mathrm{E}-03$ \\
\hline chr1_137773ıENSGALT000( & $1 \mathrm{cis}$ & $9.3 \mathrm{cis}$ & 14.08 & $2.41 \mathrm{E}-06$ \\
\hline chr1_137774!ENSGALT000( & 1 cis & 9.3 cis & 11.46 & $4.68 \mathrm{E}-06$ \\
\hline chr1_137786IENSGALT000( & $1 \mathrm{cis}$ & $9.3 \mathrm{cis}$ & 6.34 & $5.36 \mathrm{E}-04$ \\
\hline chr1_137575ıENSGALT000( & 1 cis & $9.3 \mathrm{cis}$ & 14.57 & $2.95 \mathrm{E}-04$ \\
\hline chr1_137578ıENSGALT000( & 1 cis & $9.3 \mathrm{cis}$ & 14.89 & $1.99 \mathrm{E}-03$ \\
\hline chr1_137591।ENSGALT000( & 1 cis & $9.3 \mathrm{cis}$ & 11.3 & $6.91 \mathrm{E}-06$ \\
\hline chr1_137592।ENSGALT000( & $1 \mathrm{cis}$ & $9.3 \mathrm{cis}$ & 11.45 & $1.20 \mathrm{E}-03$ \\
\hline chr1_137604IENSGALT000( & 1 cis & 9.3 cis & 14.51 & $1.31 \mathrm{E}-04$ \\
\hline chr1_137606IENSGALT000( & $1 \mathrm{cis}$ & $9.3 \mathrm{cis}$ & 13.67 & $1.22 \mathrm{E}-03$ \\
\hline chr1_137607IENSGALT000( & 1 cis & $9.3 \mathrm{cis}$ & 14.26 & $2.43 \mathrm{E}-03$ \\
\hline chr1_137616।ENSGALT000( & 1 cis & $9.3 \mathrm{cis}$ & 12.35 & $1.64 \mathrm{E}-05$ \\
\hline chr1_137620IENSGALTO00( & 1 cis & $9.3 \mathrm{cis}$ & 12.52 & $1.73 \mathrm{E}-04$ \\
\hline chr1_137632।ENSGALT000( & $1 \mathrm{cis}$ & $9.3 \mathrm{cis}$ & 14.85 & $2.80 \mathrm{E}-04$ \\
\hline chr1_137642।ENSGALT000( & 1 cis & 9.3 cis & 11.5 & $1.42 \mathrm{E}-05$ \\
\hline chr1_137645ıENSGALT000( & $1 \mathrm{cis}$ & $9.3 \mathrm{cis}$ & 11.49 & $1.78 \mathrm{E}-06$ \\
\hline chr1_137646IENSGALT000( & 1 cis & $9.3 \mathrm{cis}$ & 11.33 & $2.46 \mathrm{E}-04$ \\
\hline chr1_137660IENSGALT000( & $1 \mathrm{cis}$ & $9.3 \mathrm{cis}$ & 13.64 & 4.54E-06 \\
\hline chr1_137662।ENSGALT000 & 1 cis & 9.3 cis & 13.75 & 4.37E-04 \\
\hline chr1_137664IENSGALT000( & $1 \mathrm{cis}$ & $9.3 \mathrm{cis}$ & 12.5 & 5.93E-04 \\
\hline chr1_137677IENSGALTO00( & 1 cis & 9.3 cis & 11.72 & $1.43 \mathrm{E}-03$ \\
\hline chr1_137690IENSGALT000( & $1 \mathrm{cis}$ & $9.3 \mathrm{cis}$ & 13.19 & 4.37E-06 \\
\hline chr1_137692।ENSGALT000( & 1 cis & $9.3 \mathrm{cis}$ & 13.15 & $3.29 \mathrm{E}-03$ \\
\hline chr1_137705ıENSGALT000( & $1 \mathrm{cis}$ & $9.3 \mathrm{cis}$ & 11.7 & 3.99E-06 \\
\hline
\end{tabular}




\begin{tabular}{|c|c|c|c|c|}
\hline chr1_137706ıENSGALT000( & 1 cis & $9.3 \mathrm{cis}$ & 11.74 & 7.32E-04 \\
\hline chr1_137719IENSGALTO00( & $1 \mathrm{cis}$ & $9.3 \mathrm{cis}$ & 13.47 & $1.39 \mathrm{E}-03$ \\
\hline chr1_137722IENSGALT000( & $1 \mathrm{cis}$ & 9.3 cis & 13.95 & $1.18 \mathrm{E}-03$ \\
\hline chr1_137723IENSGALT000( & $1 \mathrm{cis}$ & 9.3 cis & 15.21 & $1.03 \mathrm{E}-03$ \\
\hline chr1_137724IENSGALT000( & $1 \mathrm{cis}$ & $9.3 \mathrm{cis}$ & 12.58 & 1.67E-04 \\
\hline chr1_137730IENSGALTO00( & $1 \mathrm{cis}$ & $9.3 \mathrm{cis}$ & 10.98 & $2.44 \mathrm{E}-03$ \\
\hline chr1_137735IENSGALT000( & $1 \mathrm{cis}$ & 9.3 cis & 12.23 & 2.77E-06 \\
\hline chr1_137736IENSGALT000( & $1 \mathrm{cis}$ & 9.3 cis & 11.26 & $6.72 \mathrm{E}-04$ \\
\hline chr1_137737IENSGALT000( & $1 \mathrm{cis}$ & $9.3 \mathrm{cis}$ & 12.9 & 7.09E-04 \\
\hline chr1_137750IENSGALTO00( & $1 \mathrm{cis}$ & 9.3 cis & 14.69 & 2.19E-04 \\
\hline chr1_137752IENSGALT000( & $1 \mathrm{cis}$ & $9.3 \mathrm{cis}$ & 13.9 & $6.43 \mathrm{E}-04$ \\
\hline chr1_137753IENSGALT000( & $1 \mathrm{cis}$ & 9.3 cis & 14.86 & $2.10 \mathrm{E}-03$ \\
\hline chr1_137754IENSGALT000( & $1 \mathrm{cis}$ & $9.3 \mathrm{cis}$ & 11.23 & 7.65E-04 \\
\hline chr1_137757IENSGALT000( & $1 \mathrm{cis}$ & 9.3 cis & 10.92 & $2.26 \mathrm{E}-06$ \\
\hline chr1_137778IENSGALT000( & $1 \mathrm{cis}$ & $9.3 \mathrm{cis}$ & 13.73 & $1.41 \mathrm{E}-06$ \\
\hline chr1_137785IENSGALT000( & $1 \mathrm{cis}$ & $9.3 \mathrm{cis}$ & 4.97 & 7.24E-04 \\
\hline chr1_137676IENSGALT000( & $1 \mathrm{cis}$ & $9.3 \mathrm{cis}$ & 10.73 & 1.64E-04 \\
\hline chr1_137702IENSGALTO00( & $1 \mathrm{cis}$ & $9.3 \mathrm{cis}$ & 10.02 & $6.90 \mathrm{E}-05$ \\
\hline chr1_137732IENSGALT000( & $1 \mathrm{cis}$ & $9.3 \mathrm{cis}$ & 10.16 & 5.21E-05 \\
\hline chr1_137755IENSGALT000( & $1 \mathrm{cis}$ & $9.3 \mathrm{cis}$ & 10.63 & 1.19E-03 \\
\hline chr1_137758IENSGALT000( & $1 \mathrm{cis}$ & $9.3 \mathrm{cis}$ & 8.15 & 3.84E-04 \\
\hline chr1_137779IENSGALT000( & $1 \mathrm{cis}$ & $9.3 \mathrm{cis}$ & 8.81 & 3.44E-05 \\
\hline chr1_137760IENSGALT000( & $1 \mathrm{cis}$ & $9.3 \mathrm{cis}$ & 5.32 & $3.95 \mathrm{E}-03$ \\
\hline chr1_137588IENSGALTO00( & $1 \mathrm{cis}$ & $9.3 \mathrm{cis}$ & 6.55 & $1.83 \mathrm{E}-03$ \\
\hline chr1_139940IENSGALT000( & $1 \mathrm{cis}$ & $9.3 \mathrm{cis}$ & 4.44 & 8.00E-06 \\
\hline chr1_140937INM_0010086 & $1 \mathrm{cis}$ & $4.6 \mathrm{cis}$ & 5.35 & $6.48 \mathrm{E}-05$ \\
\hline chr1_142072ıNM_0010086 & $1 \mathrm{cis}$ & $4.6 \mathrm{cis}$ & 5.33 & 4.29E-04 \\
\hline chr1_144697ıNM_0010086 & $1 \mathrm{cis}$ & $4.6 \mathrm{cis}$ & 7.58 & 1.37E-03 \\
\hline chr1_141190IENSGALT000( & $1 \mathrm{cis}$ & 7.7 cis & 8.96 & $1.04 \mathrm{E}-03$ \\
\hline chr1_144171।ENSGALTO00( & $1 \mathrm{cis}$ & $7.7 \mathrm{cis}$ & 10.37 & $2.04 \mathrm{E}-03$ \\
\hline chr1_141287IENSGALT000( & $1 \mathrm{cis}$ & 7.7 cis & 8.59 & 2.98E-04 \\
\hline chr1_141332IENSGALT000( & $1 \mathrm{cis}$ & $7.7 \mathrm{cis}$ & 10.16 & $5.32 \mathrm{E}-03$ \\
\hline chr1_141333IENSGALT000( & $1 \mathrm{cis}$ & $7.7 \mathrm{cis}$ & 9.1 & 7.89E-04 \\
\hline chr1_141404IENSGALT000( & $1 \mathrm{cis}$ & $7.7 \mathrm{cis}$ & 6.07 & 8.65E-04 \\
\hline chr1_141472IENSGALT000( & $1 \mathrm{cis}$ & $7.7 \mathrm{cis}$ & 6.27 & 3.86E-04 \\
\hline chr1_141484IENSGALTO00( & $1 \mathrm{cis}$ & $7.7 \mathrm{cis}$ & 11.08 & $1.21 \mathrm{E}-04$ \\
\hline chr1_141523IENSGALT000( & $1 \mathrm{cis}$ & $7.7 \mathrm{cis}$ & 6.57 & 2.27E-03 \\
\hline chr1_141524IENSGALT000( & $1 \mathrm{cis}$ & $7.7 \mathrm{cis}$ & 5.85 & 9.35E-04 \\
\hline chr1_141629IENSGALT000( & $1 \mathrm{cis}$ & $7.7 \mathrm{cis}$ & 8.07 & 1.19E-03 \\
\hline chr1_141634IENSGALTO00( & $1 \mathrm{cis}$ & 7.7 cis & 11.6 & $1.34 \mathrm{E}-04$ \\
\hline chr1_141286IENSGALT000( & $1 \mathrm{cis}$ & $7.7 \mathrm{cis}$ & 5.41 & 4.16E-04 \\
\hline chr1_141489IENSGALTO00( & $1 \mathrm{cis}$ & $7.7 \mathrm{cis}$ & 8.32 & $9.33 \mathrm{E}-04$ \\
\hline chr1_141265IENSGALT000( & $1 \mathrm{cis}$ & 7.7 cis & 8.87 & 5.59E-03 \\
\hline chr1_141264IENSGALT000( & $1 \mathrm{cis}$ & $7.7 \mathrm{cis}$ & 10.72 & 3.99E-04 \\
\hline chr1_141324IENSGALT000( & $1 \mathrm{cis}$ & $7.7 \mathrm{cis}$ & 11.38 & $4.50 \mathrm{E}-04$ \\
\hline chr1_141259IENSGALTO00( & $1 \mathrm{cis}$ & 7.7 cis & 8.23 & $2.96 \mathrm{E}-03$ \\
\hline chr1_141329IENSGALT000( & $1 \mathrm{cis}$ & 7.7 cis & 9.17 & $1.03 \mathrm{E}-03$ \\
\hline chr1_141480IENSGALTO00( & $1 \mathrm{cis}$ & $7.7 \mathrm{cis}$ & 8.28 & $1.56 \mathrm{E}-03$ \\
\hline chr1_141330IENSGALTO00( & $1 \mathrm{cis}$ & $7.7 \mathrm{cis}$ & 6.06 & $1.64 \mathrm{E}-02$ \\
\hline chr1_141481IENSGALT000( & $1 \mathrm{cis}$ & $7.7 \mathrm{cis}$ & 5.79 & $1.98 \mathrm{E}-02$ \\
\hline chr1_1409281X603602468F & $1 \mathrm{cis}$ & $4.0 \mathrm{cis}$ & 7.86 & 2.40E-04 \\
\hline chr1_150681ıX603865265F & $1 \mathrm{cis}$ & 6.1 trans & 7.51 & $1.91 \mathrm{E}-03$ \\
\hline chr1_151794ıX603865265F & $1 \mathrm{cis}$ & 6.1 trans & 7.61 & 5.03E-04 \\
\hline chr1_146186ıX603865265F & $1 \mathrm{cis}$ & 6.1 trans & 7.28 & $2.24 \mathrm{E}-03$ \\
\hline chr1_150711ıX603865265F & $1 \mathrm{cis}$ & 6.1 trans & 9.11 & $1.32 \mathrm{E}-03$ \\
\hline chr1_151275ıX603865265F & $1 \mathrm{cis}$ & 6.1 trans & 7.8 & 1.13E-03 \\
\hline chr1_148777ıX603002887F & $1 \mathrm{cis}$ & 5.6 cis & 4.1 & 7.49E-04 \\
\hline chr1_148807ıX603002887F & $1 \mathrm{cis}$ & $5.6 \mathrm{cis}$ & 4.92 & $1.68 \mathrm{E}-04$ \\
\hline chr1_1478661X603002887F & $1 \mathrm{cis}$ & $5.6 \mathrm{cis}$ & 5.28 & 4.81E-04 \\
\hline chr1_1480501X603002887F & $1 \mathrm{cis}$ & $5.6 \mathrm{cis}$ & 6.77 & 7.71E-04 \\
\hline chr1_148800।X603002887F & $1 \mathrm{cis}$ & 5.6 trans & 6.56 & $5.46 \mathrm{E}-04$ \\
\hline chr1_151962।NM_0010308 & $1 \mathrm{cis}$ & $11.6 \mathrm{cis}$ & 5.99 & 1.47E-04 \\
\hline chr1_155715ıENSGALT000( & 1 trans & $7.7 \mathrm{cis}$ & 5.54 & 1.49E-04 \\
\hline chr1_155760IENSGALTO00( & $1 \mathrm{cis}$ & $5.6 \mathrm{cis}$ & 5.19 & $1.09 \mathrm{E}-03$ \\
\hline chr1_156059IENSGALTO00( & $1 \mathrm{cis}$ & 5.6 trans & 8.86 & 2.15E-04 \\
\hline chr1_145493IENSGALTO00( & $1 \mathrm{cis}$ & 4.9 cis & 5.01 & 5.50E-03 \\
\hline chr1_157382IENSGALT000( & $1 \mathrm{cis}$ & 4.9 cis & 5.52 & 7.35E-03 \\
\hline chr1_159670IENSGALT000( & $1 \mathrm{cis}$ & 4.9 cis & 4.85 & $3.78 \mathrm{E}-03$ \\
\hline chr1_164280IENSGALTO00( & $1 \mathrm{cis}$ & 4.9 trans & 8.51 & 4.69E-03 \\
\hline chr1_160219IENSGALTO00( & $1 \mathrm{cis}$ & 4.9 trans & 9.24 & $1.62 \mathrm{E}-03$ \\
\hline chr1_153661IENSGALTO00( & $1 \mathrm{cis}$ & 4.9 trans & 8.23 & $1.89 \mathrm{E}-03$ \\
\hline chr1_167468IENSGALT000( & $1 \mathrm{cis}$ & 4.9 trans & 9.34 & 4.63E-03 \\
\hline
\end{tabular}




\begin{tabular}{|c|c|c|c|c|}
\hline chr1_151228IENSGALT000( & 1 cis & 4.9 trans & 8.69 & $3.31 \mathrm{E}-03$ \\
\hline chr1_153310ıENSGALTO00( & 1 cis & 4.9 trans & 8.34 & $1.60 \mathrm{E}-03$ \\
\hline chr1_164414!ENSGALT000( & 1 cis & 4.9 trans & 8.57 & $2.21 \mathrm{E}-03$ \\
\hline chr1_150541IENSGALTO00( & 1 cis & 4.9 trans & 8.58 & $1.05 \mathrm{E}-03$ \\
\hline chr1_153057IENSGALT000( & 1 cis & 4.9 trans & 8.02 & $8.60 \mathrm{E}-04$ \\
\hline chr1_151794IENSGALTO00( & $1 \mathrm{cis}$ & 4.9 trans & 7.61 & $6.27 \mathrm{E}-04$ \\
\hline chr1_165612।ENSGALT000( & $1 \mathrm{cis}$ & 4.9 trans & 7.96 & $2.00 \mathrm{E}-03$ \\
\hline chr1_161386IENSGALTO00( & $1 \mathrm{cis}$ & 4.9 trans & 7.72 & $1.39 \mathrm{E}-03$ \\
\hline chr1_156059IENSGALTO00( & 1 cis & 4.9 trans & 8.86 & $3.59 \mathrm{E}-03$ \\
\hline chr1_160066IENSGALTO00( & 1 cis & 4.9 trans & 7.28 & $1.50 \mathrm{E}-03$ \\
\hline chr1_150711।ENSGALTO00( & 1 cis & 4.9 trans & 9.11 & $2.51 \mathrm{E}-03$ \\
\hline chr1_162429IENSGALTO00( & 1 cis & 4.9 trans & 8.71 & 4.76E-03 \\
\hline chr1_162758IENSGALT000( & 1 cis & 4.9 trans & 7.53 & 2.77E-03 \\
\hline chr1_152131IENSGALTO00( & $1 \mathrm{cis}$ & 4.9 trans & 7.32 & $1.18 \mathrm{E}-03$ \\
\hline chr1_152927IENSGALTO00( & 1 cis & 4.9 trans & 7.76 & 4.74E-04 \\
\hline chr1_151275IENSGALTO00( & 1 cis & 4.9 trans & 7.8 & $9.13 \mathrm{E}-04$ \\
\hline chr1_162438IENSGALTO00( & 1 cis & 4.9 trans & 8.19 & $9.90 \mathrm{E}-04$ \\
\hline chr1_164415IENSGALTO00( & 1 cis & 4.9 trans & 7.64 & $1.07 \mathrm{E}-03$ \\
\hline chr1_162106IENSGALTO00( & 1 cis & 4.9 trans & 8 & $4.68 \mathrm{E}-05$ \\
\hline chr1_154452IENSGALTOOO( & $1 \mathrm{cis}$ & 4.9 trans & 8.86 & $1.47 \mathrm{E}-03$ \\
\hline chr1_163615।X603603033F & 1 cis & 7.0 cis & 4.57 & $1.65 \mathrm{E}-03$ \\
\hline chr1_167035IENSGALTO00( & 1 cis & $5.2 \mathrm{cis}$ & 4.97 & $1.95 \mathrm{E}-04$ \\
\hline chr1_163487IENSGALTOOO( & 1 cis & 5.2 cis & 4.17 & $1.01 \mathrm{E}-03$ \\
\hline chr1_162106IENSGALTO00( & 1 cis & 5.2 trans & 8 & $3.08 \mathrm{E}-03$ \\
\hline chr1_173226IENSGALTO00( & 1 cis & $4.8 \mathrm{cis}$ & 4.35 & $2.35 \mathrm{E}-04$ \\
\hline chr1_173486IENSGALTO00( & $1 \mathrm{cis}$ & $4.8 \mathrm{cis}$ & 14.19 & $8.82 \mathrm{E}-04$ \\
\hline chr1_175527IENSGALTOOO( & 1 cis & $4.8 \mathrm{cis}$ & 4.44 & $4.34 \mathrm{E}-03$ \\
\hline chr1_172387IENSGALTO00( & $1 \mathrm{cis}$ & $4.8 \mathrm{cis}$ & 7.57 & $6.76 \mathrm{E}-04$ \\
\hline chr1_183280IENSGALTO00( & $1 \mathrm{cis}$ & $4.8 \mathrm{cis}$ & 5.7 & $2.18 \mathrm{E}-04$ \\
\hline chr1_183279IENSGALTO00( & 1 cis & $4.8 \mathrm{cis}$ & 4.79 & $5.44 \mathrm{E}-04$ \\
\hline chr1_182304IENSGALTO00( & 1 cis & $4.8 \mathrm{cis}$ & 16.7 & $1.02 \mathrm{E}-05$ \\
\hline chr1_1732261X603865290F & $1 \mathrm{cis}$ & $6.2 \mathrm{cis}$ & 4.35 & $4.82 \mathrm{E}-04$ \\
\hline chr1_172387ıX603865290F & $1 \mathrm{cis}$ & $6.2 \mathrm{cis}$ & 7.57 & 4.06E-05 \\
\hline chr1_174482!X603865290F & $1 \mathrm{cis}$ & $6.2 \mathrm{cis}$ & 4.28 & $5.71 \mathrm{E}-04$ \\
\hline chr1_175527IENSGALTOOO( & $1 \mathrm{cis}$ & $4.3 \mathrm{cis}$ & 4.44 & $2.15 \mathrm{E}-03$ \\
\hline chr1_183293IENSGALTO00( & 1 cis & $4.3 \mathrm{cis}$ & 5.44 & $1.20 \mathrm{E}-03$ \\
\hline chr1_175527ıX603863736F & 1 cis & 4.7 cis & 4.44 & $3.04 \mathrm{E}-04$ \\
\hline chr1_183280ıX603863736F & $1 \mathrm{cis}$ & 4.7 cis & 5.7 & 5.56E-04 \\
\hline chr1_183279ıX603863736F & 1 cis & 4.7 cis & 4.79 & $2.18 \mathrm{E}-03$ \\
\hline chr1_182304ıX603863736F & $1 \mathrm{cis}$ & $4.7 \mathrm{cis}$ & 16.7 & $2.83 E-05$ \\
\hline chr1_183504IX603598770F & 1 trans & 10.0 cis & 5.36 & $7.65 \mathrm{E}-05$ \\
\hline chr1_182304IX603598770F & 1 trans & $10.0 \mathrm{cis}$ & 16.7 & $2.03 E-03$ \\
\hline chr1_175647IENSGALTO00( & 1 cis & 5.2 trans & 8.64 & 1.17E-04 \\
\hline chr1_182166IENSGALTO00 & $1 \mathrm{cis}$ & $5.2 \mathrm{cis}$ & 5.9 & $7.32 \mathrm{E}-04$ \\
\hline chr1_182304IENSGALTO00( & 1 cis & 5.2 cis & 16.7 & $1.90 \mathrm{E}-04$ \\
\hline chr1_187951।X603862514F & $1 \mathrm{cis}$ & 4.4 trans & 7.69 & $2.03 E-03$ \\
\hline chr1_177071।ENSGALT000( & 1 cis & 4.2 cis & 5.97 & $1.99 \mathrm{E}-03$ \\
\hline chr1_186038IENSGALTO00( & $1 \mathrm{cis}$ & $4.6 \mathrm{cis}$ & 4.1 & $1.15 \mathrm{E}-02$ \\
\hline chr1_181868ıX603867858F & 1 cis & 4.4 trans & 8.22 & $1.60 \mathrm{E}-03$ \\
\hline chr1_186681।X603863843F & $1 \mathrm{cis}$ & 4.6 trans & 6.33 & $1.03 \mathrm{E}-03$ \\
\hline chr1_1818681X603863843F & 1 cis & 4.6 trans & 8.22 & $1.06 \mathrm{E}-03$ \\
\hline chr1_182457IENSGALTO00( & $1 \mathrm{cis}$ & 4.4 trans & 6.14 & $8.13 E-04$ \\
\hline chr1_186681।ENSGALTO00( & 1 cis & 6.1 trans & 6.33 & $1.12 \mathrm{E}-03$ \\
\hline chr1_189679IX603595547F & $1 \mathrm{cis}$ & $4.3 \mathrm{cis}$ & 4.64 & $3.95 \mathrm{E}-03$ \\
\hline chr1_191153INM_0010308 & 1 cis & $6.1 \mathrm{cis}$ & 6.84 & $3.74 \mathrm{E}-03$ \\
\hline chr1_190661IENSGALATO00( & $1 \mathrm{cis}$ & $4.8 \mathrm{cis}$ & 6.53 & $7.56 \mathrm{E}-04$ \\
\hline chr1_188617IENSGALTO00( & 1 cis & $4.8 \mathrm{cis}$ & 4.47 & $9.00 \mathrm{E}-04$ \\
\hline chr1_191153IENSGALTO00( & 1 cis & $4.8 \mathrm{cis}$ & 6.84 & $3.34 \mathrm{E}-03$ \\
\hline chr10_480901X603862632F & 10 cis & $3.8 \mathrm{cis}$ & 30.99 & $2.29 \mathrm{E}-04$ \\
\hline chr10_70600।X603862632F & 10 cis & $3.8 \mathrm{cis}$ & 6.44 & 4.36E-04 \\
\hline chr10_58590ıX603862632F & 10 cis & $3.8 \mathrm{cis}$ & 4.1 & $3.02 \mathrm{E}-03$ \\
\hline chr10_867901X603862632F & $10 \mathrm{cis}$ & $3.8 \mathrm{cis}$ & 4.1 & $1.02 \mathrm{E}-03$ \\
\hline chr10_703801X603862632F & 10 cis & $3.8 \mathrm{cis}$ & 5.14 & $8.18 \mathrm{E}-05$ \\
\hline chr10_42000IX603863210F & $10 \mathrm{cis}$ & $4.4 \mathrm{cis}$ & 4.1 & $1.80 \mathrm{E}-03$ \\
\hline chr10_48090INM_204261_ & 10 cis & $4.0 \mathrm{cis}$ & 30.99 & 1.16E-03 \\
\hline chr10_54810ı603862030̄ & $10 \mathrm{cis}$ & 3.5 trans & 7.26 & $1.22 \mathrm{E}-03$ \\
\hline chr10_12817ıX603600179F & 10 cis & 7.7 cis & 11.79 & $6.68 \mathrm{E}-05$ \\
\hline chr10_13771IENSGALTO00( & $10 \mathrm{cis}$ & $4.8 \mathrm{cis}$ & 33.97 & $3.93 E-04$ \\
\hline chr10_13772।ENSGALTO00( & 10 cis & $4.8 \mathrm{cis}$ & 41.62 & 5.97E-05 \\
\hline chr10_13770IENSGALTO00( & $10 \mathrm{cis}$ & $4.8 \mathrm{cis}$ & 35.24 & $7.45 \mathrm{E}-05$ \\
\hline chr10_13773IENSGALTO00( & $10 \mathrm{cis}$ & $4.8 \mathrm{cis}$ & 34.13 & $2.47 \mathrm{E}-04$ \\
\hline chr10_12504INM_0010246 & $10 \mathrm{cis}$ & $7.6 \mathrm{cis}$ & 4.75 & 5.33E-04 \\
\hline
\end{tabular}




\begin{tabular}{|c|c|c|c|c|}
\hline chr10_12817INM_0010246 & $10 \mathrm{cis}$ & 7.6 cis & 11.79 & 3.00E-05 \\
\hline chr10_14254INM_0010246 & $10 \mathrm{cis}$ & 7.6 cis & 5.43 & $2.30 \mathrm{E}-05$ \\
\hline chr10_13771ıNM_0010246 & 10 cis & 7.6 cis & 33.97 & 4.10E-05 \\
\hline chr10_13772।NM_0010246 & 10 cis & 7.6 cis & 41.62 & $9.92 \mathrm{E}-07$ \\
\hline chr10_13775ıNM_0010246 & $10 \mathrm{cis}$ & 7.6 cis & 22.26 & $2.80 \mathrm{E}-05$ \\
\hline chr10_14172ıNM_0010246 & 10 cis & $7.6 \mathrm{cis}$ & 13.35 & $4.80 \mathrm{E}-07$ \\
\hline chr10_14174INM_0010246 & $10 \mathrm{cis}$ & 7.6 cis & 10.44 & $2.44 \mathrm{E}-06$ \\
\hline chr10_13770ıNM_0010246 & 10 cis & 7.6 cis & 35.24 & 4.23E-06 \\
\hline chr10_14173ıNM_0010246 & 10 cis & 7.6 cis & 7.61 & $1.59 \mathrm{E}-05$ \\
\hline chr10_14252।NM_0010246 & $10 \mathrm{cis}$ & 7.6 cis & 13.88 & 7.59E-09 \\
\hline chr10_14250।NM_0010246 & 10 cis & 7.6 cis & 10.11 & $3.45 \mathrm{E}-06$ \\
\hline chr10_13774INM_0010246 & 10 cis & 7.6 cis & 25.54 & 1.13E-05 \\
\hline chr10_13769INM_0010246 & $10 \mathrm{cis}$ & 7.6 cis & 22.7 & $3.76 \mathrm{E}-06$ \\
\hline chr10_15866INM_0010246 & 10 cis & 7.6 cis & 7 & $1.66 \mathrm{E}-06$ \\
\hline chr11_64120IENSGALT000( & 11 cis & $4.8 \mathrm{cis}$ & 4.23 & $8.45 \mathrm{E}-04$ \\
\hline chr11_79620IENSGALT000( & 11 cis & 4.8 cis & 4.15 & $6.63 \mathrm{E}-04$ \\
\hline chr11_58070ıX603864355F & 11 cis & 10.1 cis & 4.64 & 2.17E-05 \\
\hline chr11_76600ıX03864355F & 11 cis & 10.1 cis & 4.5 & $1.72 \mathrm{E}-05$ \\
\hline chr11_72030ıX603864355F & 11 cis & 10.1 cis & 4.87 & $6.49 \mathrm{E}-06$ \\
\hline chr11_58070ıX603603027F & 11 cis & 11.3 cis & 4.64 & $2.35 \mathrm{E}-05$ \\
\hline chr11_76600ıX03603027F & 11 cis & 11.3 cis & 4.5 & 8.27E-06 \\
\hline chr11_72030ıX603603027F & 11 cis & 11.3 cis & 4.87 & 4.37E-05 \\
\hline chr11_59610ıNM_0010058 & 11 cis & 6.6 cis & 4.51 & $1.25 \mathrm{E}-03$ \\
\hline chr11_13605ıNM_0010017 & 11 cis & 7.2 cis & 9.45 & $2.65 \mathrm{E}-04$ \\
\hline chr11_15433ıNM_0010017 & 11 cis & 7.2 cis & 5.49 & $3.87 \mathrm{E}-03$ \\
\hline chr11_13604INM_0010017 & 11 cis & 7.2 cis & 7.13 & $2.04 \mathrm{E}-03$ \\
\hline chr12_36210IENSGALT000( & 12 trans & 9.6 cis & 5.54 & 1.77E-05 \\
\hline chr12_27210IENSGALTO00( & 12 trans & 9.6 cis & 9.53 & 5.39E-04 \\
\hline chr12_27290IENSGALT000( & 12 trans & 9.6 cis & 6.94 & 8.59E-04 \\
\hline chr12_27280IENSGALT000( & 12 trans & 9.6 cis & 9.42 & $1.98 \mathrm{E}-03$ \\
\hline chr12_27580IENSGALT000( & 12 trans & 9.6 cis & 4.76 & $2.90 \mathrm{E}-03$ \\
\hline chr12_49540ıNM_0010128 & 12 trans & 8.5 trans & 7.28 & $3.41 \mathrm{E}-04$ \\
\hline chr12_36210ıNM_0010128 & 12 trans & 8.5 cis & 5.54 & $2.28 \mathrm{E}-06$ \\
\hline chr12_27210ıNM_0010128 & 12 trans & $8.5 \mathrm{cis}$ & 9.53 & $6.77 \mathrm{E}-03$ \\
\hline chr12_27210IENSGALT000( & 12 trans & 8.0 cis & 9.53 & $3.81 \mathrm{E}-03$ \\
\hline chr12_27280IENSGALT000( & 12 trans & 8.0 cis & 9.42 & $8.44 \mathrm{E}-03$ \\
\hline chr12_27390IENSGALT000( & 12 trans & 8.0 cis & 5.75 & 4.19E-03 \\
\hline chr12_49540IENSGALT000( & 12 trans & 7.7 trans & 7.28 & $8.84 \mathrm{E}-04$ \\
\hline chr12_36210IENSGALT000( & 12 trans & 7.7 cis & 5.54 & 3.39E-04 \\
\hline chr12_27210IENSGALT000( & 12 trans & 7.0 cis & 9.53 & $1.06 \mathrm{E}-02$ \\
\hline chr12_27390IENSGALT000( & 12 trans & 7.0 cis & 5.75 & $3.41 \mathrm{E}-03$ \\
\hline chr12_49540ıX603144566F & 12 cis & 5.2 trans & 7.28 & $3.26 \mathrm{E}-04$ \\
\hline chr12_36210।X603144566F & 12 cis & $5.2 \mathrm{cis}$ & 5.54 & $3.44 \mathrm{E}-04$ \\
\hline chr12_27390ıX603144566F & $12 \mathrm{cis}$ & 5.2 cis & 5.75 & 7.95E-04 \\
\hline chr12_36210।X603142827F & 12 trans & 6.6 cis & 5.54 & $4.62 \mathrm{E}-03$ \\
\hline chr12_45840IENSGALT000( & 12 cis & $4.2 \mathrm{cis}$ & 17.62 & $3.28 \mathrm{E}-03$ \\
\hline chr12_43740ıX603603373F & 12 cis & 4.6 cis & 5.15 & $1.84 \mathrm{E}-04$ \\
\hline chr12_45840।X603603373F & 12 cis & 4.6 cis & 17.62 & $4.18 \mathrm{E}-04$ \\
\hline chr12_52780।X603603373F & 12 cis & 4.6 cis & 6.56 & $3.20 \mathrm{E}-04$ \\
\hline chr12_45840IENSGALT000( & 12 cis & $4.4 \mathrm{cis}$ & 17.62 & $5.30 \mathrm{E}-04$ \\
\hline chr12_52780IENSGALT000( & 12 cis & 4.4 cis & 6.56 & $1.13 \mathrm{E}-03$ \\
\hline chr12_36210IENSGALT000( & 12 cis & $5.0 \mathrm{cis}$ & 5.54 & $1.20 \mathrm{E}-03$ \\
\hline chr12_45840IENSGALT000( & 12 cis & $5.0 \mathrm{cis}$ & 17.62 & $2.66 \mathrm{E}-03$ \\
\hline chr12_11001।ENSGALT000( & 12 cis & $5.8 \mathrm{cis}$ & 4.63 & $4.32 \mathrm{E}-04$ \\
\hline chr12_16909IENSGALT000( & 12 cis & 4.6 cis & 6.03 & 4.77E-03 \\
\hline chr12_16909ıX603862915F & 12 cis & $5.2 \mathrm{cis}$ & 6.03 & 4.36E-04 \\
\hline chr12_16909ıX603864922F & 12 trans & 6.5 cis & 6.03 & $1.14 \mathrm{E}-03$ \\
\hline chr13_14230ıX03568189F & 13 cis & $3.5 \mathrm{cis}$ & 11.56 & $1.30 \mathrm{E}-04$ \\
\hline chr13_10545ıX03144253F & 13 cis & 5.9 trans & 6.56 & $8.66 \mathrm{E}-04$ \\
\hline chr13_52600।X603144253F & 13 cis & 5.9 cis & 7.09 & 5.37E-05 \\
\hline chr13_890201X603144253F & 13 cis & 5.9 cis & 6.44 & $1.28 \mathrm{E}-03$ \\
\hline chr13_51540ıX603144253F & 13 cis & 5.9 cis & 6.71 & $3.29 E-04$ \\
\hline chr13_10205ıX603144253F & 13 cis & 5.9 cis & 11.76 & $2.14 \mathrm{E}-03$ \\
\hline chr13_65710।X603144253F & 13 cis & 5.9 cis & 5.89 & 5.29E-04 \\
\hline chr13_66020।X603144253F & 13 cis & 5.9 cis & 5.19 & 1.10E-04 \\
\hline chr13_52600IENSGALT000( & 13 cis & $7.6 \mathrm{cis}$ & 7.09 & $4.51 \mathrm{E}-04$ \\
\hline chr13_79470IENSGALT000 & $13 \mathrm{cis}$ & $7.6 \mathrm{cis}$ & 4.7 & $9.58 \mathrm{E}-06$ \\
\hline chr13_77670ıENSGALT000( & 13 cis & $7.6 \mathrm{cis}$ & 4.83 & $6.04 \mathrm{E}-04$ \\
\hline chr13_78180IENSGALT000( & 13 cis & 7.6 cis & 6.84 & 4.56E-04 \\
\hline chr13_81220IENSGALT000( & 13 cis & $7.6 \mathrm{cis}$ & 4.27 & $7.58 \mathrm{E}-04$ \\
\hline chr13_65210ı603863557F & 13 cis & 4.5 cis & 5.02 & $1.46 \mathrm{E}-04$ \\
\hline chr13_60230ıX603863557F & $13 \mathrm{cis}$ & 4.5 cis & 5.4 & $9.80 \mathrm{E}-05$ \\
\hline
\end{tabular}




\begin{tabular}{|c|c|c|c|c|}
\hline chr13_89020IENSGALTO00 & 13 cis & $4.8 \mathrm{cis}$ & 6.44 & $1.59 \mathrm{E}-04$ \\
\hline chr13_80900IENSGALTO00 & 13 cis & 4.8 cis & 5.63 & $6.66 \mathrm{E}-04$ \\
\hline chr13_65710IENSGALTO00 & 13 cis & 4.8 cis & 5.89 & $2.67 \mathrm{E}-05$ \\
\hline chr13_52600IENSGALTO00 & 13 cis & 6.3 cis & 7.09 & $1.77 \mathrm{E}-03$ \\
\hline chr13_89020IENSGALTO00 & 13 cis & 6.3 cis & 6.44 & $4.84 \mathrm{E}-04$ \\
\hline chr13_79470IENSGALTO0O & 13 cis & 6.3 cis & 4.7 & $7.61 \mathrm{E}-05$ \\
\hline chr13_82350IENSGALTO0O & 13 cis & 6.3 cis & 5.44 & $1.15 \mathrm{E}-05$ \\
\hline chr13_83310IENSGALTO00 & 13 cis & 6.3 cis & 5.42 & $5.26 \mathrm{E}-04$ \\
\hline chr13_85940IENSGALTO0O & 13 cis & 6.3 cis & 4.75 & $2.02 \mathrm{E}-04$ \\
\hline chr13_78180IENSGALTO0O & 13 cis & 6.3 cis & 6.84 & $1.40 \mathrm{E}-03$ \\
\hline chr13_65710IENSGALTO0O & 13 cis & 6.3 cis & 5.89 & $2.21 \mathrm{E}-03$ \\
\hline chr13_660201ENSGALTO00 & 13 cis & 6.3 cis & 5.19 & $4.21 \mathrm{E}-04$ \\
\hline chr13_81220IENSGALTO00 & 13 cis & 6.3 cis & 4.27 & $3.53 \mathrm{E}-04$ \\
\hline chr13_52600(X603598354F & 13 cis & 8.3 cis & 7.09 & $4.84 \mathrm{E}-04$ \\
\hline chr13_870701X603598354F & 13 cis & 8.3 cis & 5.2 & $1.21 \mathrm{E}-03$ \\
\hline chr13_890201X603598354F & 13 cis & 8.3 cis & 6.44 & $1.26 \mathrm{E}-04$ \\
\hline chr13_794701X603598354F & 13 cis & 8.3 cis & 4.7 & $1.78 \mathrm{E}-05$ \\
\hline chr13_80510(X603598354F & 13 cis & 8.3 cis & 5.69 & $1.31 \mathrm{E}-03$ \\
\hline chr13_82350।X603598354F & 13 cis & 8.3 cis & 5.44 & $3.12 \mathrm{E}-06$ \\
\hline chr13_83310।X603598354F & 13 cis & 8.3 cis & 5.42 & $9.58 \mathrm{E}-05$ \\
\hline chr13_85940)X603598354F & 13 cis & 8.3 cis & 4.75 & $5.97 \mathrm{E}-05$ \\
\hline chr13_781801X603598354F & 13 cis & $8.3 \mathrm{cis}$ & 6.84 & 2.17E-04 \\
\hline chr13_660201X603598354F & 13 cis & 8.3 cis & 5.19 & $1.04 \mathrm{E}-04$ \\
\hline chr13_52600IENSGALTO00 & 13 cis & 4.9 cis & 7.09 & $2.93 \mathrm{E}-03$ \\
\hline chr13_63370IENSGALTO00 & 13 cis & 4.9 cis & 5.71 & $2.73 \mathrm{E}-03$ \\
\hline chr13_79470IENSGALTOOO & 13 cis & 4.9 cis & 4.7 & $1.19 \mathrm{E}-03$ \\
\hline chr13_82350IENSGALTO00 & 13 cis & 4.9 cis & 5.44 & $4.01 \mathrm{E}-03$ \\
\hline chr13_52600(X603866983F & 13 cis & 9.1 cis & 7.09 & $3.09 \mathrm{E}-04$ \\
\hline chr13_870701X603866983F & 13 cis & $9.1 \mathrm{cis}$ & 5.2 & $6.09 \mathrm{E}-04$ \\
\hline chr13_890201X603866983F & 13 cis & 9.1 cis & 6.44 & $3.73 \mathrm{E}-05$ \\
\hline chr13_794701X603866983F & 13 cis & 9.1 cis & 4.7 & $4.46 \mathrm{E}-06$ \\
\hline chr13_80510(X603866983F & 13 cis & $9.1 \mathrm{cis}$ & 5.69 & $1.05 \mathrm{E}-03$ \\
\hline chr13_82350।X603866983F & 13 cis & 9.1 cis & 5.44 & $8.81 \mathrm{E}-07$ \\
\hline chr13_83310(X603866983F & 13 cis & 9.1 cis & 5.42 & $2.53 \mathrm{E}-05$ \\
\hline chr13_776701X603866983F & 13 cis & 9.1 cis & 4.83 & $6.18 \mathrm{E}-04$ \\
\hline chr13_85940।X603866983F & 13 cis & 9.1 cis & 4.75 & $8.27 \mathrm{E}-06$ \\
\hline chr13_78180।X603866983F & 13 cis & $9.1 \mathrm{cis}$ & 6.84 & $9.02 \mathrm{E}-05$ \\
\hline chr13_660201X603866983F & 13 cis & $9.1 \mathrm{cis}$ & 5.19 & 5.97E-05 \\
\hline chr13_52600(X603597807F & 13 cis & $5.8 \mathrm{cis}$ & 7.09 & $1.09 \mathrm{E}-03$ \\
\hline chr13_633701X603597807F & 13 cis & 5.8 cis & 5.71 & $3.84 \mathrm{E}-03$ \\
\hline chr13_794701X603597807F & 13 cis & $5.8 \mathrm{cis}$ & 4.7 & $1.06 \mathrm{E}-03$ \\
\hline chr13_52600IENSGALTO00 & 13 cis & $7.7 \mathrm{cis}$ & 7.09 & $1.26 \mathrm{E}-04$ \\
\hline chr13_89020IENSGALTO0O & 13 cis & $7.7 \mathrm{cis}$ & 6.44 & $3.82 \mathrm{E}-06$ \\
\hline chr13_83310IENSGALTOOO & 13 cis & $7.7 \mathrm{cis}$ & 5.42 & $1.59 \mathrm{E}-05$ \\
\hline chr13_85940IENSGALTO00 & 13 cis & $7.7 \mathrm{cis}$ & 4.75 & $1.23 \mathrm{E}-06$ \\
\hline chr13_11573।X603598203F & 13 cis & 4.6 cis & 8.36 & $4.51 \mathrm{E}-03$ \\
\hline chr14_26480(X603863190F & 14 cis & 4.2 cis & 16.35 & $9.70 \mathrm{E}-04$ \\
\hline chr14_264901X603863190F & 14 cis & 4.2 cis & 19.89 & 4.07E-03 \\
\hline chr14_264701X603863190F & 14 cis & 4.2 cis & 19.66 & $2.40 \mathrm{E}-03$ \\
\hline chr14_26500।X603863190F & 14 cis & $4.2 \mathrm{cis}$ & 22.44 & $3.54 \mathrm{E}-03$ \\
\hline chr14_26490IENSGALTO0O & 14 cis & $4.1 \mathrm{cis}$ & 19.89 & $1.29 \mathrm{E}-02$ \\
\hline chr14_82030INM_0010061 & 14 cis & $4.6 \mathrm{cis}$ & 4.1 & $8.59 \mathrm{E}-06$ \\
\hline chr14_82020INM_0010061 & 14 cis & $4.6 \mathrm{cis}$ & 4.68 & $1.37 \mathrm{E}-05$ \\
\hline chr14_820301X603863473F & 14 cis & $8.5 \mathrm{cis}$ & 4.1 & $1.60 \mathrm{E}-03$ \\
\hline chr14_820201X603863473F & $14 \mathrm{cis}$ & $8.5 \mathrm{cis}$ & 4.68 & $6.71 \mathrm{E}-04$ \\
\hline chr14_56710(X603596248F & 14 cis & $8.8 \mathrm{cis}$ & 5.2 & $1.44 \mathrm{E}-05$ \\
\hline chr14_58640(X603596248F & 14 cis & $8.8 \mathrm{cis}$ & 5.05 & $2.87 \mathrm{E}-05$ \\
\hline chr14_820301X603596248F & 14 cis & $8.8 \mathrm{cis}$ & 4.1 & $4.84 \mathrm{E}-04$ \\
\hline chr14_794001X603596248F & 14 cis & $8.8 \mathrm{cis}$ & 8.04 & $8.27 \mathrm{E}-06$ \\
\hline chr14_90010)X603596248F & 14 cis & $8.8 \mathrm{cis}$ & 8.12 & $2.22 \mathrm{E}-06$ \\
\hline chr14_974101X603596248F & 14 cis & $8.8 \mathrm{cis}$ & 5.22 & $7.46 \mathrm{E}-06$ \\
\hline chr14_96310।X603596248F & 14 cis & $8.8 \mathrm{cis}$ & 8.35 & $7.39 \mathrm{E}-06$ \\
\hline chr14_82030IENSGALT000 & 14 cis & 4.5 cis & 4.1 & $2.71 \mathrm{E}-04$ \\
\hline chr14_82020IENSGALTO00 & 14 cis & $4.5 \mathrm{cis}$ & 4.68 & $7.62 \mathrm{E}-05$ \\
\hline chr14_97410IENSGALTO0O & 14 cis & $5.0 \mathrm{cis}$ & 5.22 & $1.05 \mathrm{E}-04$ \\
\hline chr14_87840।X603599183F & 14 cis & 5.9 cis & 6 & $3.80 \mathrm{E}-04$ \\
\hline chr14_969701X603599183F & 14 cis & 5.9 cis & 8.26 & $1.72 \mathrm{E}-04$ \\
\hline chr14_899001X603599183F & 14 cis & 5.9 cis & 4.8 & $1.67 \mathrm{E}-04$ \\
\hline chr14_974101X603599183F & 14 cis & 5.9 cis & 5.22 & $4.72 \mathrm{E}-05$ \\
\hline chr14_820301X603603312F & 14 cis & $10.5 \mathrm{cis}$ & 4.1 & $2.09 \mathrm{E}-05$ \\
\hline chr14_82020(X603603312F & 14 cis & $10.5 \mathrm{cis}$ & 4.68 & $1.14 \mathrm{E}-06$ \\
\hline chr14_794001X603603312F & 14 cis & 10.5 cis & 8.04 & $1.50 \mathrm{E}-\mathrm{C}$ \\
\hline
\end{tabular}




\begin{tabular}{|c|c|c|c|c|}
\hline chr14_878401X603603312F & 14 cis & 10.5 cis & 6 & $1.37 \mathrm{E}-04$ \\
\hline chr14_900101X603603312F & 14 cis & 10.5 cis & 8.12 & $1.25 \mathrm{E}-04$ \\
\hline chr14_969701X603603312F & 14 cis & 10.5 cis & 8.26 & $4.53 \mathrm{E}-05$ \\
\hline chr14_974101X603603312F & 14 cis & 10.5 cis & 5.22 & $9.49 \mathrm{E}-06$ \\
\hline chr14_96310।X603603312F & 14 cis & 10.5 cis & 8.35 & $5.33 \mathrm{E}-06$ \\
\hline chr14_94450INM_0010061 & 14 cis & 9.1 cis & 7.11 & $4.52 \mathrm{E}-05$ \\
\hline chr14_79400INM_0010061 & 14 cis & 9.1 cis & 8.04 & $8.70 \mathrm{E}-07$ \\
\hline chr14_87840INM_0010061 & 14 cis & 9.1 cis & 6 & $3.55 \mathrm{E}-06$ \\
\hline chr14_90010INM_0010061 & 14 cis & 9.1 cis & 8.12 & $4.19 \mathrm{E}-06$ \\
\hline chr14_94250INM_0010061 & 14 cis & 9.1 cis & 5.44 & $1.40 \mathrm{E}-04$ \\
\hline chr14_96970INM_0010061 & 14 cis & 9.1 cis & 8.26 & $1.51 \mathrm{E}-06$ \\
\hline chr14_89900INM_0010061 & 14 cis & 9.1 cis & 4.8 & $2.40 \mathrm{E}-05$ \\
\hline chr14_97410INM_0010061 & 14 cis & 9.1 cis & 5.22 & $5.89 \mathrm{E}-05$ \\
\hline chr14_96310INM_0010061 & 14 cis & 9.1 cis & 8.35 & $2.86 \mathrm{E}-05$ \\
\hline chr14_83340INM_0010061 & 14 cis & 9.1 cis & 5.7 & $3.33 \mathrm{E}-04$ \\
\hline chr14_820301X603595988F & 14 cis & 4.5 cis & 4.1 & $1.43 \mathrm{E}-04$ \\
\hline chr14_820201X603595988F & 14 cis & 4.5 cis & 4.68 & $4.58 \mathrm{E}-05$ \\
\hline chr14_974101X603595988F & 14 cis & 4.5 cis & 5.22 & $3.22 \mathrm{E}-04$ \\
\hline chr14_97410IENSGALT000 & 14 trans & 7.0 cis & 5.22 & $1.28 \mathrm{E}-06$ \\
\hline chr14_820301X603863911F & $14 \mathrm{cis}$ & 8.6 cis & 4.1 & $2.11 \mathrm{E}-05$ \\
\hline chr14_820201X603863911F & 14 cis & 8.6 cis & 4.68 & $1.42 \mathrm{E}-06$ \\
\hline chr14_794001X603863911F & 14 cis & 8.6 cis & 8.04 & 4.86E-05 \\
\hline chr14_87840IX603863911F & 14 cis & 8.6 cis & 6 & $1.83 \mathrm{E}-04$ \\
\hline chr14_900101X603863911F & 14 cis & 8.6 cis & 8.12 & $2.61 \mathrm{E}-04$ \\
\hline chr14_974101X603863911F & 14 cis & 8.6 cis & 5.22 & $1.39 \mathrm{E}-05$ \\
\hline chr14_96310।X603863911F & 14 cis & 8.6 cis & 8.35 & $8.65 \mathrm{E}-06$ \\
\hline chr14_97410IENSGALT000 & 14 cis & $5.0 \mathrm{cis}$ & 5.22 & $1.60 \mathrm{E}-04$ \\
\hline chr14_820201X603597475F & 14 cis & 14.4 cis & 4.68 & $2.34 \mathrm{E}-03$ \\
\hline chr14_94450IX603597475F & 14 cis & 14.4 cis & 7.11 & 5.10E-06 \\
\hline chr14_794001X603597475F & 14 cis & 14.4 cis & 8.04 & $1.29 \mathrm{E}-05$ \\
\hline chr14_900101X603597475F & 14 cis & 14.4 cis & 8.12 & $1.49 \mathrm{E}-05$ \\
\hline chr14_96970।X603597475F & 14 cis & 14.4 cis & 8.26 & $2.84 \mathrm{E}-07$ \\
\hline chr14_974101X603597475F & 14 cis & 14.4 cis & 5.22 & $1.19 \mathrm{E}-07$ \\
\hline chr14_86860।X603597475F & 14 cis & 14.4 cis & 4.4 & 7.73E-06 \\
\hline chr14_96310।X603597475F & 14 cis & 14.4 cis & 8.35 & $5.33 \mathrm{E}-07$ \\
\hline chr14_86970IX603597475F & 14 cis & 14.4 cis & 4.58 & $2.13 \mathrm{E}-06$ \\
\hline chr14_820201ENSGALT000( & 14 cis & $7.6 \mathrm{cis}$ & 4.68 & $3.26 \mathrm{E}-04$ \\
\hline chr14_79400IENSGALT000( & 14 cis & 7.6 cis & 8.04 & $1.21 \mathrm{E}-03$ \\
\hline chr14_87840IENSGALT000 & 14 cis & 7.6 cis & 6 & $1.34 \mathrm{E}-04$ \\
\hline chr14_96970IENSGALT000 & 14 cis & 7.6 cis & 8.26 & $5.89 \mathrm{E}-04$ \\
\hline chr14_97410IENSGALT000( & 14 cis & 7.6 cis & 5.22 & $3.92 \mathrm{E}-04$ \\
\hline chr14_820201ENSGALT000( & 14 cis & 9.8 cis & 4.68 & $5.32 \mathrm{E}-04$ \\
\hline chr14_87840IENSGALT000 & 14 cis & 9.8 cis & 6 & $1.25 \mathrm{E}-04$ \\
\hline chr14_97410IENSGALT000( & 14 cis & 9.8 cis & 5.22 & $4.59 \mathrm{E}-06$ \\
\hline chr14_878401X603868033F & 14 cis & 8.5 cis & 6 & $8.73 \mathrm{E}-04$ \\
\hline chr14_974101X603868033F & 14 cis & 8.5 cis & 5.22 & 2.97E-04 \\
\hline chr14_96970।X603867683F & 14 cis & 5.4 cis & 8.26 & $9.42 \mathrm{E}-04$ \\
\hline chr14_13772IENSGALTO00( & 14 cis & 5.5 cis & 4.48 & $2.53 \mathrm{E}-02$ \\
\hline chr15_15350।X603598221F & 15 cis & 7.0 cis & 5.33 & $3.56 \mathrm{E}-05$ \\
\hline chr15_23710IENSGALTO0OO & 15 cis & 4.1 cis & 4.1 & $1.54 \mathrm{E}-02$ \\
\hline chr15_359701X603603039F & 15 cis & 7.4 cis & 4.94 & $3.50 \mathrm{E}-04$ \\
\hline chr15_235201X603603039F & 15 cis & 7.4 cis & 4.1 & $5.33 \mathrm{E}-04$ \\
\hline chr15_89990IENSGALT000 & 15 cis & 4.3 cis & 6.56 & $3.75 \mathrm{E}-03$ \\
\hline chr15_91910।X603599406F & 15 cis & 5.3 cis & 9.27 & $9.29 \mathrm{E}-04$ \\
\hline chr17_46780IENSGALTO00( & 17 cis & 6.4 cis & 13.07 & $3.70 \mathrm{E}-04$ \\
\hline chr17_863801X603602420F & 17 cis & 7.6 cis & 4.11 & $2.86 \mathrm{E}-04$ \\
\hline chr18_40090INM_205240_ & $18 \mathrm{cis}$ & 7.4 cis & 4.24 & $2.42 \mathrm{E}-03$ \\
\hline chr18_40070INM_205240_ & 18 cis & 7.4 cis & 4.1 & $2.04 \mathrm{E}-03$ \\
\hline chr2_7056001X603866028F & 2 cis & 4.8 cis & 5.9 & $7.77 \mathrm{E}-04$ \\
\hline chr2_7055001X603866028F & 2 cis & 4.8 cis & 7.9 & 4.97E-03 \\
\hline chr2_8335001X603866028F & 2 cis & 4.8 cis & 14.61 & $3.63 \mathrm{E}-04$ \\
\hline chr2_962900।X603867141F & 2 cis & 5.3 cis & 5.57 & $7.41 \mathrm{E}-04$ \\
\hline chr2_103450।X603867141F & 2 cis & 5.3 cis & 7.52 & $4.80 \mathrm{E}-03$ \\
\hline chr2_9902001X603867141F & 2 cis & 5.3 cis & 10.15 & $1.90 \mathrm{E}-04$ \\
\hline chr2_9530001X603867141F & 2 cis & 5.3 cis & 5.46 & $4.81 \mathrm{E}-04$ \\
\hline chr2_964300।X603867141F & 2 cis & 5.3 cis & 18.73 & $1.16 \mathrm{E}-03$ \\
\hline chr2_103910।X603867141F & 2 cis & 5.3 cis & 6.41 & $4.08 \mathrm{E}-03$ \\
\hline chr2_118230।X603867141F & 2 cis & 5.3 cis & 6.77 & $4.48 \mathrm{E}-04$ \\
\hline chr2_9400001X603867141F & 2 cis & 5.3 cis & 7.42 & $2.45 \mathrm{E}-05$ \\
\hline chr2_113450IX603867141F & 2 cis & 5.3 cis & 4.1 & $9.24 \mathrm{E}-04$ \\
\hline chr2_964100।X603867141F & 2 cis & 5.3 cis & 12.83 & $1.74 \mathrm{E}-04$ \\
\hline chr2 9642001X603867141F & 2 cis & 5.3 cis & 19.56 & $3.60 \mathrm{E}-$ \\
\hline
\end{tabular}




\begin{tabular}{|c|c|c|c|c|}
\hline chr2_9168001X603867141F & 2 cis & $5.3 \mathrm{cis}$ & 8.75 & $3.60 \mathrm{E}-03$ \\
\hline chr2_960700ıx603867141F & 2 cis & 5.3 cis & 9.36 & 4.77E-04 \\
\hline chr2_128490ıX603867141F & 2 cis & 5.3 cis & 10.42 & $2.16 \mathrm{E}-03$ \\
\hline chr2_9618001X603867141F & 2 cis & 5.3 cis & 6.57 & $1.41 \mathrm{E}-05$ \\
\hline chr2_114220ıX603867141F & 2 cis & 5.3 cis & 11.32 & 8.50E-04 \\
\hline chr2_9432001X603867141F & 2 cis & 5.3 cis & 6.83 & $6.95 \mathrm{E}-03$ \\
\hline chr2_111190ıX603867141F & 2 cis & 5.3 cis & 6.13 & $1.18 \mathrm{E}-04$ \\
\hline chr2_142070ıX603867141F & 2 cis & 5.3 cis & 11.69 & $3.78 \mathrm{E}-04$ \\
\hline chr2_142060ıX603867141F & 2 cis & 5.3 cis & 8.3 & $2.78 \mathrm{E}-03$ \\
\hline chr2_103910IENSGALT000( & 2 cis & 4.3 cis & 6.41 & 9.97E-04 \\
\hline chr2_157440IENSGALT000( & 2 cis & 4.3 cis & 4.1 & $7.26 \mathrm{E}-04$ \\
\hline chr2_142070IENSGALTO00( & 2 cis & $4.4 \mathrm{cis}$ & 11.69 & $5.56 \mathrm{E}-03$ \\
\hline chr2_142060IENSGALT000( & 2 cis & $4.4 \mathrm{cis}$ & 8.3 & $4.68 \mathrm{E}-03$ \\
\hline chr2_144670IENSGALT000( & 2 cis & 4.4 cis & 5.29 & $1.44 \mathrm{E}-04$ \\
\hline chr2_171640IENSGALT000( & 2 cis & 4.4 cis & 6.49 & $1.51 \mathrm{E}-02$ \\
\hline chr2_156270IENSGALT000( & 2 cis & $4.4 \mathrm{cis}$ & 6.42 & 1.67E-02 \\
\hline chr2_156280IENSGALTO00( & 2 cis & 4.4 cis & 7.04 & $4.83 \mathrm{E}-03$ \\
\hline chr2_171640INM_0010063 & 2 cis & 4.5 cis & 6.49 & 4.65E-03 \\
\hline chr2_163370ıNM_0010063 & 2 cis & 4.5 cis & 6.65 & $3.76 \mathrm{E}-03$ \\
\hline chr2_163980ıX603866182F & 2 cis & 4.6 cis & 6.3 & 9.97E-04 \\
\hline chr2_157440ıX603866182F & 2 cis & 4.6 cis & 4.1 & $7.85 \mathrm{E}-04$ \\
\hline chr2_193810IENSGALTO00( & 2 cis & $11.6 \mathrm{cis}$ & 4.69 & 9.07E-04 \\
\hline chr2_211380IENSGALTO00( & 2 cis & $11.6 \mathrm{cis}$ & 4.66 & $1.60 \mathrm{E}-03$ \\
\hline chr2_199980IENSGALTO00( & 2 cis & $11.6 \mathrm{cis}$ & 4.36 & $9.55 \mathrm{E}-05$ \\
\hline chr2_216030IENSGALTO00( & 2 cis & $11.6 \mathrm{cis}$ & 8.75 & $2.54 \mathrm{E}-04$ \\
\hline chr2_210640IENSGALTO00( & 2 cis & $11.6 \mathrm{cis}$ & 13.76 & $1.06 \mathrm{E}-04$ \\
\hline chr2_214470IENSGALT000( & 2 cis & $11.6 \mathrm{cis}$ & 4.73 & $8.15 E-05$ \\
\hline chr2_219660IENSGALT000( & 2 cis & $11.6 \mathrm{cis}$ & 4.1 & 4.40E-04 \\
\hline chr2_193810ıNM_204894_ & 2 cis & $12.5 \mathrm{cis}$ & 4.69 & $9.56 \mathrm{E}-04$ \\
\hline chr2_220690INM_204894_ & 2 cis & $12.5 \mathrm{cis}$ & 6.32 & 8.77E-04 \\
\hline chr2_211380!NM_204894_ & 2 cis & $12.5 \mathrm{cis}$ & 4.66 & 8.33E-04 \\
\hline chr2_199980ıNM_204894_ & 2 cis & $12.5 \mathrm{cis}$ & 4.36 & $2.58 \mathrm{E}-04$ \\
\hline chr2_216030INM_204894_ & 2 cis & $12.5 \mathrm{cis}$ & 8.75 & $5.31 \mathrm{E}-05$ \\
\hline chr2_210640ıNM_204894_ & 2 cis & $12.5 \mathrm{cis}$ & 13.76 & 3.57E-07 \\
\hline chr2_212710ıNM_204894_ & 2 cis & $12.5 \mathrm{cis}$ & 4.91 & $5.40 \mathrm{E}-04$ \\
\hline chr2_240120INM_204894_ & 2 cis & $12.5 \mathrm{cis}$ & 5.93 & $5.54 \mathrm{E}-03$ \\
\hline chr2_214470INM_204894_ & 2 cis & $12.5 \mathrm{cis}$ & 4.73 & $6.07 E-07$ \\
\hline chr2_210640ıX603595549F & 2 cis & 5.1 cis & 13.76 & $6.55 \mathrm{E}-04$ \\
\hline chr2_212710ıX603595549F & 2 cis & $5.1 \mathrm{cis}$ & 4.91 & $8.44 \mathrm{E}-05$ \\
\hline chr2_225640ı603595549F & 2 cis & $5.1 \mathrm{cis}$ & 4.38 & 3.97E-04 \\
\hline chr2_199980IENSGALTO00( & 2 cis & $9.6 \mathrm{cis}$ & 4.36 & $1.71 \mathrm{E}-04$ \\
\hline chr2_210640IENSGALTO00( & 2 cis & 9.6 cis & 13.76 & $1.86 \mathrm{E}-05$ \\
\hline chr2_219660IENSGALTO00( & 2 cis & $9.6 \mathrm{cis}$ & 4.1 & $5.24 \mathrm{E}-05$ \\
\hline chr2_212710IENSGALTO00( & 2 cis & 4.0 cis & 4.91 & $1.55 \mathrm{E}-03$ \\
\hline chr2_269600IENSGALT000( & 2 cis & 4.0 cis & 5.49 & 6.07E-04 \\
\hline chr2_284500IENSGALTO00( & 2 cis & $4.0 \mathrm{cis}$ & 9.02 & 3.37E-03 \\
\hline chr2_214470IENSGALTO00( & 2 trans & 7.6 cis & 4.73 & 3.17E-03 \\
\hline chr2_219740ıX03598188F & 2 cis & $11.3 \mathrm{cis}$ & 5.54 & $6.11 \mathrm{E}-05$ \\
\hline chr2_215230ıX603598188F & 2 cis & $11.3 \mathrm{cis}$ & 6.19 & $1.20 \mathrm{E}-06$ \\
\hline chr2_239330ıX603598188F & 2 cis & $11.3 \mathrm{cis}$ & 5.82 & $1.43 \mathrm{E}-05$ \\
\hline chr2_242450ıX603598188F & 2 cis & $11.3 \mathrm{cis}$ & 4.98 & 7.16E-07 \\
\hline chr2_2352801X603598188F & 2 cis & $11.3 \mathrm{cis}$ & 4.39 & 7.33E-05 \\
\hline chr2_231930ı603598188F & 2 cis & $11.3 \mathrm{cis}$ & 5.67 & $1.34 \mathrm{E}-05$ \\
\hline chr2_272180ı603598188F & 2 cis & $11.3 \mathrm{cis}$ & 4.36 & $1.74 \mathrm{E}-03$ \\
\hline chr2 2649401X603598188F & 2 cis & $11.3 \mathrm{cis}$ & 5.32 & $3.24 \mathrm{E}-04$ \\
\hline chr2_2384201X603598188F & 2 cis & $11.3 \mathrm{cis}$ & 5.08 & 7.17E-06 \\
\hline chr2_239740ıX603598188F & 2 cis & $11.3 \mathrm{cis}$ & 4.63 & 1.87E-05 \\
\hline chr2_244940ıX603598188F & 2 cis & $11.3 \mathrm{cis}$ & 4.13 & $5.72 \mathrm{E}-05$ \\
\hline chr2_285010ıX603598188F & 2 cis & $11.3 \mathrm{cis}$ & 4.23 & $2.80 \mathrm{E}-06$ \\
\hline chr2_240450ıX603598188F & 2 cis & $11.3 \mathrm{cis}$ & 4.1 & $1.57 \mathrm{E}-05$ \\
\hline chr2_241480ıX603598188F & 2 cis & $11.3 \mathrm{cis}$ & 5.15 & $1.51 \mathrm{E}-05$ \\
\hline chr2_284500ı603598188F & 2 cis & $11.3 \mathrm{cis}$ & 9.02 & $1.53 \mathrm{E}-06$ \\
\hline chr2_280490ıX603598188F & 2 cis & $11.3 \mathrm{cis}$ & 9.33 & $2.22 \mathrm{E}-04$ \\
\hline chr2_2390201X603598188F & 2 cis & 11.3 trans & 6.91 & 2.67E-02 \\
\hline chr2_216030ı603600947F & 2 cis & $17.7 \mathrm{cis}$ & 8.75 & $4.90 \mathrm{E}-05$ \\
\hline chr2_212710ıx603600947F & 2 cis & $17.7 \mathrm{cis}$ & 4.91 & 4.69E-05 \\
\hline chr2_2152301X603600947F & 2 cis & $17.7 \mathrm{cis}$ & 6.19 & 3.31E-06 \\
\hline chr2_239330ıx603600947F & 2 cis & $17.7 \mathrm{cis}$ & 5.82 & $1.46 \mathrm{E}-06$ \\
\hline chr2_242450ıX603600947F & 2 cis & $17.7 \mathrm{cis}$ & 4.98 & $2.43 E-07$ \\
\hline chr2_2352801X603600947F & 2 cis & $17.7 \mathrm{cis}$ & 4.39 & $1.83 \mathrm{E}-08$ \\
\hline chr2_239010ı603600947F & 2 cis & $17.7 \mathrm{cis}$ & 4.56 & 5.07E-05 \\
\hline chr2_2239201X603600947F & 2 cis & $17.7 \mathrm{cis}$ & 5.14 & $9.85 \mathrm{E}-05$ \\
\hline
\end{tabular}




\begin{tabular}{|c|c|c|c|c|}
\hline chr2_2319301X603600947F & 2 cis & $17.7 \mathrm{cis}$ & 5.67 & $1.40 \mathrm{E}-05$ \\
\hline chr2_2721801X603600947F & 2 cis & $17.7 \mathrm{cis}$ & 4.36 & $3.35 \mathrm{E}-06$ \\
\hline chr2_2649401X603600947F & 2 cis & 17.7 cis & 5.32 & $1.52 \mathrm{E}-07$ \\
\hline chr2_2384201X603600947F & 2 cis & 17.7 cis & 5.08 & 2.01E-06 \\
\hline chr2_2696001X603600947F & 2 cis & 17.7 cis & 5.49 & 3.47E-05 \\
\hline chr2_2368901X603600947F & 2 cis & $17.7 \mathrm{cis}$ & 4.1 & $6.79 \mathrm{E}-05$ \\
\hline chr2_2397401X603600947F & 2 cis & $17.7 \mathrm{cis}$ & 4.63 & 8.10E-05 \\
\hline chr2_2449401X603600947F & 2 cis & $17.7 \mathrm{cis}$ & 4.13 & 7.74E-05 \\
\hline chr2_2850101X603600947F & 2 cis & 17.7 cis & 4.23 & $2.45 \mathrm{E}-06$ \\
\hline chr2_2854101X603600947F & 2 cis & 17.7 cis & 7.78 & 4.51E-04 \\
\hline chr2_2404501X603600947F & 2 cis & 17.7 cis & 4.1 & 4.75E-05 \\
\hline chr2_2547301X603600947F & 2 cis & $17.7 \mathrm{cis}$ & 13.47 & $1.10 \mathrm{E}-04$ \\
\hline chr2_2414801X603600947F & 2 cis & $17.7 \mathrm{cis}$ & 5.15 & 1.13E-05 \\
\hline chr2_2845001X603600947F & 2 cis & $17.7 \mathrm{cis}$ & 9.02 & 3.05E-09 \\
\hline chr2_2804901X603600947F & 2 cis & 17.7 cis & 9.33 & 5.52E-06 \\
\hline chr2_2390201X603600947F & 2 cis & 17.7 trans & 6.91 & 5.64E-04 \\
\hline chr2_212710IENSGALTO00( & 2 cis & $4.8 \mathrm{cis}$ & 4.91 & 3.74E-03 \\
\hline chr2_284500IENSGALTO00( & 2 cis & $4.8 \mathrm{cis}$ & 9.02 & 2.07E-03 \\
\hline chr2_2914901X603865064F & 2 cis & $8.8 \mathrm{cis}$ & 4.6 & 4.80E-04 \\
\hline chr2_3043901X603865064F & 2 cis & $8.8 \mathrm{cis}$ & 6.68 & 2.29E-04 \\
\hline chr2_2602001X603865064F & 2 cis & 8.8 cis & 6.7 & 6.93E-06 \\
\hline chr2_3137101X603865064F & 2 cis & $8.8 \mathrm{cis}$ & 5.08 & 3.87E-04 \\
\hline chr2_2790801X603865064F & 2 cis & $8.8 \mathrm{cis}$ & 4.1 & 8.11E-05 \\
\hline chr2_3137901X603865064F & 2 cis & $8.8 \mathrm{cis}$ & 7.33 & $1.93 \mathrm{E}-04$ \\
\hline chr2_3072501X603865064F & 2 cis & $8.8 \mathrm{cis}$ & 8.61 & $1.69 \mathrm{E}-04$ \\
\hline chr2_2881201X603865064F & 2 cis & $8.8 \mathrm{cis}$ & 4.1 & 1.16E-03 \\
\hline chr2_2973601X603865064F & 2 cis & 8.8 cis & 7.52 & $5.73 \mathrm{E}-05$ \\
\hline chr2_2855901X603865064F & 2 cis & $8.8 \mathrm{cis}$ & 7.73 & $7.82 \mathrm{E}-06$ \\
\hline chr2_212710ıX603865923F & 2 cis & $10.3 \mathrm{cis}$ & 4.91 & $9.18 \mathrm{E}-05$ \\
\hline chr2_2152301X603865923F & 2 cis & $10.3 \mathrm{cis}$ & 6.19 & $1.20 \mathrm{E}-04$ \\
\hline chr2_2144701X603865923F & 2 cis & $10.3 \mathrm{cis}$ & 4.73 & 5.91E-04 \\
\hline chr2_3109601X603865923F & 2 cis & $10.3 \mathrm{cis}$ & 4.59 & 2.68E-05 \\
\hline chr2_3101201X603865923F & 2 cis & $10.3 \mathrm{cis}$ & 5.49 & 4.73E-06 \\
\hline chr2_2352801X603865923F & 2 cis & $10.3 \mathrm{cis}$ & 4.39 & $6.25 \mathrm{E}-05$ \\
\hline chr2_2390101X603865923F & 2 cis & $10.3 \mathrm{cis}$ & 4.56 & 2.84E-05 \\
\hline chr2_3104001X603865923F & 2 cis & $10.3 \mathrm{cis}$ & 4.1 & $9.95 \mathrm{E}-04$ \\
\hline chr2_2239201X603865923F & 2 cis & $10.3 \mathrm{cis}$ & 5.14 & $6.88 \mathrm{E}-05$ \\
\hline chr2_2914901X603865923F & 2 cis & $10.3 \mathrm{cis}$ & 4.6 & $1.94 \mathrm{E}-05$ \\
\hline chr2_3107001X603865923F & 2 cis & $10.3 \mathrm{cis}$ & 6.2 & $1.22 \mathrm{E}-06$ \\
\hline chr2_3106301X603865923F & 2 cis & $10.3 \mathrm{cis}$ & 4.1 & $1.38 \mathrm{E}-02$ \\
\hline chr2_3103801X603865923F & 2 cis & $10.3 \mathrm{cis}$ & 6.24 & 3.77E-04 \\
\hline chr2_3103501X603865923F & 2 cis & $10.3 \mathrm{cis}$ & 5.1 & 1.97E-05 \\
\hline chr2_3103901X603865923F & 2 cis & $10.3 \mathrm{cis}$ & 4.1 & $2.08 \mathrm{E}-03$ \\
\hline chr2_3107201X603865923F & 2 cis & $10.3 \mathrm{cis}$ & 4.55 & 5.86E-04 \\
\hline chr2_3103201X603865923F & 2 cis & $10.3 \mathrm{cis}$ & 4.1 & 7.15E-04 \\
\hline chr2_3112401X603865923F & 2 cis & $10.3 \mathrm{cis}$ & 7.45 & $1.78 \mathrm{E}-04$ \\
\hline chr2_3106901X603865923F & 2 cis & $10.3 \mathrm{cis}$ & 4.1 & 3.83E-03 \\
\hline chr2_3101301X603865923F & 2 cis & $10.3 \mathrm{cis}$ & 4.1 & 3.59E-03 \\
\hline chr2_3043901X603865923F & 2 cis & $10.3 \mathrm{cis}$ & 6.68 & $6.70 \mathrm{E}-05$ \\
\hline chr2_3101501X603865923F & 2 cis & $10.3 \mathrm{cis}$ & 6.48 & 2.12E-03 \\
\hline chr2_3106701X603865923F & 2 cis & $10.3 \mathrm{cis}$ & 6.3 & $1.38 \mathrm{E}-03$ \\
\hline chr2_3109001X603865923F & 2 cis & $10.3 \mathrm{cis}$ & 4.1 & $2.50 \mathrm{E}-04$ \\
\hline chr2_3109201X603865923F & 2 cis & $10.3 \mathrm{cis}$ & 5.09 & $1.35 \mathrm{E}-03$ \\
\hline chr2_3109301X603865923F & 2 cis & $10.3 \mathrm{cis}$ & 6.2 & 2.01E-03 \\
\hline chr2_3109501X603865923F & 2 cis & $10.3 \mathrm{cis}$ & 4.45 & 8.69E-04 \\
\hline chr2_3112301X603865923F & 2 cis & $10.3 \mathrm{cis}$ & 7.28 & $1.02 \mathrm{E}-04$ \\
\hline chr2_3012901X603865923F & 2 cis & $10.3 \mathrm{cis}$ & 4.1 & $3.49 \mathrm{E}-04$ \\
\hline chr2_3101601X603865923F & 2 cis & $10.3 \mathrm{cis}$ & 4.1 & $4.58 \mathrm{E}-03$ \\
\hline chr2_3101801X603865923F & 2 cis & $10.3 \mathrm{cis}$ & 4.1 & $5.43 \mathrm{E}-04$ \\
\hline chr2_3103601X603865923F & 2 cis & $10.3 \mathrm{cis}$ & 4.1 & 4.73E-03 \\
\hline chr2_3106401X603865923F & 2 cis & $10.3 \mathrm{cis}$ & 4.1 & 1.07E-03 \\
\hline chr2_3106601X603865923F & 2 cis & $10.3 \mathrm{cis}$ & 4.1 & 3.68E-03 \\
\hline chr2_3108801X603865923F & 2 cis & $10.3 \mathrm{cis}$ & 4.1 & 4.59E-03 \\
\hline chr2_3112001X603865923F & 2 cis & $10.3 \mathrm{cis}$ & 4.1 & $2.41 \mathrm{E}-04$ \\
\hline chr2_3112201X603865923F & 2 cis & $10.3 \mathrm{cis}$ & 4.1 & $3.24 \mathrm{E}-03$ \\
\hline chr2_3099201X603865923F & 2 cis & $10.3 \mathrm{cis}$ & 4.1 & $6.83 \mathrm{E}-04$ \\
\hline chr2_3108701X603865923F & 2 cis & $10.3 \mathrm{cis}$ & 4.1 & $8.21 \mathrm{E}-04$ \\
\hline chr2_3112101X603865923F & 2 cis & $10.3 \mathrm{cis}$ & 4.1 & 2.49E-03 \\
\hline chr2_3101701X603865923F & 2 cis & $10.3 \mathrm{cis}$ & 4.1 & $2.32 \mathrm{E}-03$ \\
\hline chr2_3106501X603865923F & 2 cis & $10.3 \mathrm{cis}$ & 4.1 & $1.82 \mathrm{E}-03$ \\
\hline chr2_2721801X603865923F & 2 cis & $10.3 \mathrm{cis}$ & 4.36 & $1.85 \mathrm{E}-04$ \\
\hline chr2_304960ı603865923F & 2 cis & 10.3 cis & 4.32 & $1.48 \mathrm{E}-04$ \\
\hline
\end{tabular}




\begin{tabular}{|c|c|c|c|c|}
\hline chr2_2696001X603865923F & 2 cis & $10.3 \mathrm{cis}$ & 5.49 & $1.51 \mathrm{E}-05$ \\
\hline chr2_3096401X603865923F & 2 cis & 10.3 cis & 4.61 & $1.06 \mathrm{E}-08$ \\
\hline chr2_3137101X603865923F & 2 cis & 10.3 cis & 5.08 & $2.83 \mathrm{E}-04$ \\
\hline chr2_3137901X603865923F & 2 cis & 10.3 cis & 7.33 & $2.21 \mathrm{E}-07$ \\
\hline chr2_3125501X603865923F & 2 cis & 10.3 cis & 4.48 & $1.26 \mathrm{E}-04$ \\
\hline chr2_2854101X603865923F & 2 cis & 10.3 cis & 7.78 & 4.29E-04 \\
\hline chr2_2893601X603865923F & 2 cis & 10.3 cis & 5.26 & $2.29 \mathrm{E}-03$ \\
\hline chr2_3072501X603865923F & 2 cis & 10.3 cis & 8.61 & $9.60 \mathrm{E}-08$ \\
\hline chr2_3072601X603865923F & 2 cis & $10.3 \mathrm{cis}$ & 4.17 & $1.76 \mathrm{E}-05$ \\
\hline chr2_2547301X603865923F & 2 cis & 10.3 cis & 13.47 & $2.61 \mathrm{E}-06$ \\
\hline chr2_2414801X603865923F & 2 cis & 10.3 cis & 5.15 & 4.60E-05 \\
\hline chr2_2919601X603865923F & 2 cis & 10.3 cis & 4.43 & $1.35 \mathrm{E}-04$ \\
\hline chr2_2796801X603865923F & 2 cis & 10.3 cis & 4.1 & $1.36 \mathrm{E}-04$ \\
\hline chr2_2845001X603865923F & 2 cis & 10.3 cis & 9.02 & $2.61 \mathrm{E}-08$ \\
\hline chr2_2805001X603865923F & 2 cis & 10.3 cis & 5.87 & $1.38 \mathrm{E}-05$ \\
\hline chr2_2973601X603865923F & 2 cis & 10.3 cis & 7.52 & 5.79E-04 \\
\hline chr2_2796601X603865923F & 2 cis & 10.3 cis & 4.52 & 2.19E-03 \\
\hline chr2_2855901X603865923F & 2 cis & 10.3 cis & 7.73 & $1.39 \mathrm{E}-03$ \\
\hline chr2_2804901X603865923F & 2 cis & 10.3 cis & 9.33 & $3.23 \mathrm{E}-07$ \\
\hline chr2_2698801X603865923F & 2 cis & 10.3 cis & 5.66 & $1.84 \mathrm{E}-06$ \\
\hline chr2_3162901X603865923F & 2 cis & 10.3 cis & 6.7 & 3.57E-05 \\
\hline chr2_2805101X603865923F & 2 cis & 10.3 cis & 5.85 & $1.11 \mathrm{E}-04$ \\
\hline chr2_2883201X603865923F & 2 cis & 10.3 cis & 4.1 & 7.65E-04 \\
\hline chr2_2931901X603865923F & 2 cis & 10.3 cis & 8.11 & 7.10E-08 \\
\hline chr2_318010।X603865923F & 2 cis & $10.3 \mathrm{cis}$ & 6.52 & $1.29 \mathrm{E}-04$ \\
\hline chr2_2932001X603865923F & 2 cis & $10.3 \mathrm{cis}$ & 5.91 & $6.79 \mathrm{E}-04$ \\
\hline chr2_2564901X603865923F & 2 cis & 10.3 trans & 7.86 & 7.63E-04 \\
\hline chr2_2390201X603865923F & 2 cis & 10.3 trans & 6.91 & $9.55 \mathrm{E}-04$ \\
\hline chr2_2144701X603868123F & 2 cis & 10.2 cis & 4.73 & $1.21 \mathrm{E}-03$ \\
\hline chr2_3109601X603868123F & 2 cis & 10.2 cis & 4.59 & 4.95E-04 \\
\hline chr2_3101201X603868123F & 2 cis & 10.2 cis & 5.49 & $5.08 \mathrm{E}-04$ \\
\hline chr2_2390101X603868123F & 2 cis & 10.2 cis & 4.56 & 8.57E-04 \\
\hline chr2_3104001X603868123F & 2 cis & 10.2 cis & 4.1 & $6.86 \mathrm{E}-04$ \\
\hline chr2_2239201X603868123F & 2 cis & $10.2 \mathrm{cis}$ & 5.14 & 3.39E-04 \\
\hline chr2_3107001X603868123F & 2 cis & 10.2 cis & 6.2 & $1.64 \mathrm{E}-04$ \\
\hline chr2_3103501X603868123F & 2 cis & 10.2 cis & 5.1 & $1.26 \mathrm{E}-03$ \\
\hline chr2_2796401X603868123F & 2 cis & 10.2 cis & 4.64 & $6.74 \mathrm{E}-04$ \\
\hline chr2_3103201X603868123F & 2 cis & 10.2 cis & 4.1 & $3.82 \mathrm{E}-04$ \\
\hline chr2_3043901X603868123F & 2 cis & 10.2 cis & 6.68 & $1.39 \mathrm{E}-04$ \\
\hline chr2_3112301X603868123F & 2 cis & $10.2 \mathrm{cis}$ & 7.28 & $1.09 \mathrm{E}-03$ \\
\hline chr2_3012901X603868123F & 2 cis & 10.2 cis & 4.1 & $9.89 \mathrm{E}-04$ \\
\hline chr2_3049601X603868123F & 2 cis & 10.2 cis & 4.32 & $1.06 \mathrm{E}-03$ \\
\hline chr2_269600।X603868123F & 2 cis & 10.2 cis & 5.49 & $5.46 \mathrm{E}-05$ \\
\hline chr2_2790801X603868123F & 2 cis & 10.2 cis & 4.1 & $1.15 \mathrm{E}-03$ \\
\hline chr2_3137901X603868123F & 2 cis & 10.2 cis & 7.33 & 2.04E-04 \\
\hline chr2_2854101X603868123F & 2 cis & $10.2 \mathrm{cis}$ & 7.78 & $1.86 \mathrm{E}-04$ \\
\hline chr2_2893601X603868123F & 2 cis & 10.2 cis & 5.26 & 4.56E-04 \\
\hline chr2_2796501X603868123F & 2 cis & 10.2 cis & 5.42 & 7.05E-04 \\
\hline chr2_2547301X603868123F & 2 cis & 10.2 cis & 13.47 & $2.32 \mathrm{E}-05$ \\
\hline chr2_2796801X603868123F & 2 cis & 10.2 cis & 4.1 & $1.61 \mathrm{E}-04$ \\
\hline chr2_2845001X603868123F & 2 cis & 10.2 cis & 9.02 & $2.94 \mathrm{E}-06$ \\
\hline chr2_2805001X603868123F & 2 cis & $10.2 \mathrm{cis}$ & 5.87 & $1.04 \mathrm{E}-04$ \\
\hline chr2_2973601X603868123F & 2 cis & $10.2 \mathrm{cis}$ & 7.52 & 2.63E-06 \\
\hline chr2_2796601X603868123F & 2 cis & 10.2 cis & 4.52 & 3.83E-06 \\
\hline chr2_2855901X603868123F & 2 cis & 10.2 cis & 7.73 & $1.50 \mathrm{E}-04$ \\
\hline chr2_280490ıX603868123F & 2 cis & 10.2 cis & 9.33 & 1.97E-06 \\
\hline chr2_2698801X603868123F & 2 cis & 10.2 cis & 5.66 & $3.66 \mathrm{E}-06$ \\
\hline chr2_2883201X603868123F & 2 cis & $10.2 \mathrm{cis}$ & 4.1 & $9.20 \mathrm{E}-05$ \\
\hline chr2_2720901X603868123F & 2 cis & $10.2 \mathrm{cis}$ & 6.41 & $1.25 \mathrm{E}-04$ \\
\hline chr2_2931901X603868123F & 2 cis & 10.2 cis & 8.11 & $2.12 \mathrm{E}-05$ \\
\hline chr2_318010।X603868123F & 2 cis & $10.2 \mathrm{cis}$ & 6.52 & $1.80 \mathrm{E}-04$ \\
\hline chr2_2390201X603868123F & 2 cis & 10.2 trans & 6.91 & $1.10 \mathrm{E}-03$ \\
\hline chr2_330510IENSGALT000( & 2 cis & 4.1 cis & 4.61 & $2.25 \mathrm{E}-04$ \\
\hline chr2_335620IENSGALTO00( & 2 cis & $4.1 \mathrm{cis}$ & 4.27 & 4.40E-03 \\
\hline chr2_340780IENSGALTO00( & 2 cis & 4.9 cis & 4.82 & $9.11 E-03$ \\
\hline chr2_4061201X603596713F & 2 cis & 7.3 cis & 7.98 & 5.31E-06 \\
\hline chr2_405980IX603596713F & 2 cis & 7.3 cis & 5.76 & $5.75 \mathrm{E}-04$ \\
\hline chr2_4059501X603596713F & 2 cis & 7.3 cis & 10.88 & 4.89E-05 \\
\hline chr2_4059201X603596713F & 2 cis & 7.3 cis & 9.63 & $2.46 \mathrm{E}-04$ \\
\hline chr2_4059401X603596713F & 2 cis & 7.3 cis & 10.54 & $1.60 \mathrm{E}-04$ \\
\hline chr2_406040ıX603596713F & 2 cis & $7.3 \mathrm{cis}$ & 19.77 & $1.86 \mathrm{E}-05$ \\
\hline chr2_4059901X603596713F & 2 cis & 7.3 cis & 13.5 & $2.18 \mathrm{E}-05$ \\
\hline
\end{tabular}




\begin{tabular}{|c|c|c|c|c|}
\hline chr2_406020ıX603596713F & $2 \mathrm{cis}$ & 7.3 cis & 26.04 & $1.08 \mathrm{E}-06$ \\
\hline chr2_4060301X603596713F & 2 cis & $7.3 \mathrm{cis}$ & 25.16 & $1.92 \mathrm{E}-05$ \\
\hline chr2_406010।X603596713F & 2 cis & 7.3 cis & 24.19 & 4.91E-04 \\
\hline chr2_406090।X603596713F & 2 cis & 7.3 cis & 14.14 & 4.87E-05 \\
\hline chr2_406000।X603596713F & 2 cis & 7.3 cis & 15.1 & $6.85 \mathrm{E}-05$ \\
\hline chr2_406080IX603596713F & 2 cis & $7.3 \mathrm{cis}$ & 14.51 & 4.59E-05 \\
\hline chr2_4097001X603596713F & 2 cis & 7.3 cis & 19.16 & 8.03E-04 \\
\hline chr2_405910।X603596713F & 2 cis & $7.3 \mathrm{cis}$ & 12.88 & $1.08 \mathrm{E}-05$ \\
\hline chr2_405870ıX603596713F & 2 cis & 7.3 cis & 7.6 & $1.22 \mathrm{E}-03$ \\
\hline chr2_406100IX603596713F & 2 cis & 7.3 cis & 7.23 & $3.68 \mathrm{E}-03$ \\
\hline chr2_405860ıX603596713F & 2 cis & 7.3 cis & 11.59 & $6.72 \mathrm{E}-05$ \\
\hline chr2_413540।X603596713F & 2 cis & $7.3 \mathrm{cis}$ & 5.32 & $3.40 \mathrm{E}-04$ \\
\hline chr2_419360।X603868164F & 2 cis & $4.1 \mathrm{cis}$ & 4.1 & 7.94E-02 \\
\hline chr2_405920IENSGALT000 & 2 cis & $4.7 \mathrm{cis}$ & 9.63 & $1.24 \mathrm{E}-03$ \\
\hline chr2_405910IENSGALT000( & 2 cis & $4.7 \mathrm{cis}$ & 12.88 & $7.64 \mathrm{E}-04$ \\
\hline chr2_405860IENSGALT000( & 2 cis & $4.7 \mathrm{cis}$ & 11.59 & $9.94 \mathrm{E}-04$ \\
\hline chr2_459710।ENSGALTO00( & 2 cis & 4.5 cis & 5.25 & $6.21 \mathrm{E}-04$ \\
\hline chr2_450630IENSGALTO00 & 2 cis & $4.5 \mathrm{cis}$ & 6.93 & $1.08 \mathrm{E}-03$ \\
\hline chr2_451530ı603866511F & 2 cis & 5.4 cis & 5.45 & 8.35E-05 \\
\hline chr2_459550।X603866511F & 2 cis & 5.4 cis & 9.43 & $1.05 \mathrm{E}-03$ \\
\hline chr2_459710।X603866511F & 2 cis & 5.4 cis & 5.25 & $8.50 \mathrm{E}-04$ \\
\hline chr2_4506301X603866511F & 2 cis & 5.4 cis & 6.93 & $6.28 \mathrm{E}-05$ \\
\hline chr2_456690।X603866511F & 2 cis & 5.4 cis & 13.78 & $2.42 \mathrm{E}-05$ \\
\hline chr2_4451001X603866511F & 2 cis & 5.4 cis & 8.68 & $1.58 \mathrm{E}-03$ \\
\hline chr2_445090।X603866511F & 2 cis & 5.4 cis & 9.04 & $5.76 \mathrm{E}-04$ \\
\hline chr2_549920IENSGALTO00( & 2 cis & $4.8 \mathrm{cis}$ & 6.84 & $2.07 \mathrm{E}-03$ \\
\hline chr2_526990।ENSGALT000( & 2 cis & $4.8 \mathrm{cis}$ & 11.99 & $1.62 \mathrm{E}-03$ \\
\hline chr2_549950IENSGALTO00 & 2 cis & $4.8 \mathrm{cis}$ & 9.13 & $3.78 \mathrm{E}-03$ \\
\hline chr2_549920INM_001007C & 2 cis & $5.5 \mathrm{cis}$ & 6.84 & $7.88 \mathrm{E}-04$ \\
\hline chr2_549930ıNM_001007C & 2 cis & $5.5 \mathrm{cis}$ & 7.06 & 2.07E-04 \\
\hline chr2_549950INM_001007C & 2 cis & $5.5 \mathrm{cis}$ & 9.13 & $6.20 \mathrm{E}-06$ \\
\hline chr2_558570ıNM_001007C & 2 cis & 5.5 cis & 4.92 & $6.76 \mathrm{E}-04$ \\
\hline chr2_510790ıNM_001007C & 2 cis & $5.5 \mathrm{cis}$ & 5.73 & $6.05 \mathrm{E}-05$ \\
\hline chr2_490700INM_001007C & 2 cis & $5.5 \mathrm{cis}$ & 5.54 & $2.80 \mathrm{E}-04$ \\
\hline chr2_491080INM_001007C & 2 cis & $5.5 \mathrm{cis}$ & 5.54 & $2.75 \mathrm{E}-04$ \\
\hline chr2_527010।X603864267F & 2 cis & $4.7 \mathrm{cis}$ & 23.35 & $2.46 \mathrm{E}-03$ \\
\hline chr2_5499201X603864267F & 2 cis & 4.7 cis & 6.84 & $4.80 \mathrm{E}-04$ \\
\hline chr2_5499301X603864267F & 2 cis & $4.7 \mathrm{cis}$ & 7.06 & $4.89 \mathrm{E}-04$ \\
\hline chr2_549950।X603864267F & 2 cis & $4.7 \mathrm{cis}$ & 9.13 & $6.82 \mathrm{E}-04$ \\
\hline chr2_515700IENSGALT000( & 2 cis & $8.2 \mathrm{cis}$ & 4.8 & $3.53 \mathrm{E}-04$ \\
\hline chr2_527010IENSGALT000( & 2 cis & 8.2 cis & 23.35 & $3.08 \mathrm{E}-07$ \\
\hline chr2_527020IENSGALTO00 & 2 cis & $8.2 \mathrm{cis}$ & 16.48 & $1.53 \mathrm{E}-05$ \\
\hline chr2_527040IENSGALT000( & 2 cis & 8.2 cis & 27.63 & $4.84 \mathrm{E}-06$ \\
\hline chr2_527150IENSGALTO00 & 2 cis & 8.2 cis & 28.95 & $6.99 \mathrm{E}-06$ \\
\hline chr2_488040IENSGALT000( & 2 cis & $8.2 \mathrm{cis}$ & 6.78 & 4.79E-05 \\
\hline chr2_527140।ENSGALTO00( & 2 cis & $8.2 \mathrm{cis}$ & 14.34 & $4.78 \mathrm{E}-06$ \\
\hline chr2_527050IENSGALT000( & 2 cis & 8.2 cis & 11.15 & 2.57E-05 \\
\hline chr2_534080IENSGALTO00 & 2 cis & $8.2 \mathrm{cis}$ & 8.53 & 3.37E-05 \\
\hline chr2_480300IENSGALT000( & 2 cis & 8.2 cis & 4.9 & 6.37E-04 \\
\hline chr2_496120IENSGALTO00( & 2 cis & $8.2 \mathrm{cis}$ & 5.7 & $1.62 \mathrm{E}-05$ \\
\hline chr2_496630IENSGALT000( & 2 cis & $8.2 \mathrm{cis}$ & 6.07 & 7.63E-04 \\
\hline chr2_549300IENSGALT000( & 2 cis & $8.2 \mathrm{cis}$ & 5.76 & $1.67 \mathrm{E}-04$ \\
\hline chr2_526990IENSGALT000( & 2 cis & 8.2 cis & 11.99 & $3.08 \mathrm{E}-05$ \\
\hline chr2_549950IENSGALTO00 & 2 cis & $8.2 \mathrm{cis}$ & 9.13 & $8.71 \mathrm{E}-04$ \\
\hline chr2_532200IENSGALTO00( & 2 cis & 8.2 cis & 4.62 & $3.67 \mathrm{E}-03$ \\
\hline chr2_543590IENSGALTO00( & 2 cis & $8.2 \mathrm{cis}$ & 6.29 & $3.41 \mathrm{E}-04$ \\
\hline chr2_515020IENSGALT000( & 2 cis & 8.2 cis & 6.94 & $1.14 \mathrm{E}-04$ \\
\hline chr2_528870IENSGALT000( & 2 cis & 8.2 trans & 8.27 & $2.29 \mathrm{E}-03$ \\
\hline chr2_5499201X603866284F & 2 cis & 4.4 cis & 6.84 & $6.04 \mathrm{E}-04$ \\
\hline chr2_5499301X603866284F & 2 cis & $4.4 \mathrm{cis}$ & 7.06 & $5.70 \mathrm{E}-04$ \\
\hline chr2_5499501X603866284F & 2 cis & 4.4 cis & 9.13 & 7.53E-04 \\
\hline chr2_480310।X603142604F & 2 cis & 5.9 cis & 8.77 & $1.78 \mathrm{E}-04$ \\
\hline chr2_5317701X603142604F & 2 cis & 5.9 cis & 7.13 & $1.35 \mathrm{E}-04$ \\
\hline chr2_527010।X603142604F & 2 cis & 5.9 cis & 23.35 & 7.01E-04 \\
\hline chr2_527020।X603142604F & 2 cis & $5.9 \mathrm{cis}$ & 16.48 & $6.70 \mathrm{E}-04$ \\
\hline chr2_5270301X603142604F & 2 cis & 5.9 cis & 12.36 & $1.31 \mathrm{E}-04$ \\
\hline chr2_527040।X603142604F & 2 cis & 5.9 cis & 27.63 & 2.37E-04 \\
\hline chr2_527150।X603142604F & 2 cis & $5.9 \mathrm{cis}$ & 28.95 & $8.79 \mathrm{E}-05$ \\
\hline chr2_527140(X603142604F & 2 cis & 5.9 cis & 14.34 & $1.56 \mathrm{E}-03$ \\
\hline chr2_5499201X603142604F & 2 cis & 5.9 cis & 6.84 & $5.80 \mathrm{E}-04$ \\
\hline chr2_549930(X603142604F & 2 cis & 5.9 cis & 7.06 & $2.47 \mathrm{E}-03$ \\
\hline chr2_488050।X603142604F & 2 cis & 5.9 cis & 7.24 & 4.94E-05 \\
\hline
\end{tabular}




\begin{tabular}{|c|c|c|c|c|}
\hline chr2_4966301X603142604F & 2 cis & 5.9 cis & 6.07 & $9.85 \mathrm{E}-04$ \\
\hline chr2_5498201X603142604F & 2 cis & 5.9 cis & 6.23 & $1.25 \mathrm{E}-03$ \\
\hline chr2_5499801X603142604F & 2 cis & 5.9 cis & 4.48 & $1.25 \mathrm{E}-03$ \\
\hline chr2_5499501X603867382F & 2 cis & $5.0 \mathrm{cis}$ & 9.13 & 3.69E-04 \\
\hline chr2_661920IENSGALT000( & 2 cis & 4.7 trans & 6.32 & 1.10E-03 \\
\hline chr2_658880INM_0010336 & 2 trans & 6.6 trans & 6.08 & $1.10 \mathrm{E}-03$ \\
\hline chr2_7477001X603862212F & 2 cis & $4.8 \mathrm{cis}$ & 18.45 & $6.58 \mathrm{E}-04$ \\
\hline chr2_7186301X603596980F & 2 cis & 4.9 cis & 7.31 & 3.57E-03 \\
\hline chr2_716230INM_204134_ & 2 cis & 4.5 trans & 5.69 & $6.14 \mathrm{E}-04$ \\
\hline chr2_842800INM_204134_ & 2 cis & 4.5 cis & 11.63 & $1.85 \mathrm{E}-04$ \\
\hline chr2_859150INM_204134_ & 2 cis & 4.5 cis & 6.11 & $6.25 \mathrm{E}-04$ \\
\hline chr2_738880INM_0010044 & 2 cis & 4.5 cis & 6.39 & $6.83 \mathrm{E}-05$ \\
\hline chr2_744590IENSGALTO00( & 2 cis & $4.3 \mathrm{cis}$ & 6.22 & $1.56 \mathrm{E}-04$ \\
\hline chr2_724870IENSGALTO00( & 2 cis & 4.3 cis & 5.32 & $1.91 \mathrm{E}-04$ \\
\hline chr2_745270IENSGALTO00( & 2 cis & 4.3 cis & 4.81 & $1.46 \mathrm{E}-03$ \\
\hline chr2_787900IENSGALT000( & 2 cis & $4.3 \mathrm{cis}$ & 6.26 & $6.33 \mathrm{E}-03$ \\
\hline chr2_805810IENSGALT000( & 2 cis & 4.3 cis & 7.37 & $2.24 \mathrm{E}-03$ \\
\hline chr2_820760IENSGALTO00( & 2 cis & $4.3 \mathrm{cis}$ & 6.61 & $1.57 \mathrm{E}-03$ \\
\hline chr2_722570IENSGALTO00( & 2 cis & $10.4 \mathrm{cis}$ & 4.54 & 4.19E-04 \\
\hline chr2_747720IENSGALTO00( & 2 cis & $10.4 \mathrm{cis}$ & 7.52 & 7.15E-04 \\
\hline chr2_747700IENSGALTO00( & 2 cis & 10.4 cis & 18.45 & 2.72E-04 \\
\hline chr2_747650IENSGALT000( & 2 cis & $10.4 \mathrm{cis}$ & 11.24 & 2.64E-04 \\
\hline chr2_741810IENSGALTO00( & 2 cis & $10.4 \mathrm{cis}$ & 6.19 & 1.57E-04 \\
\hline chr2_744580IENSGALTO00( & 2 cis & 10.4 cis & 12.65 & $8.52 \mathrm{E}-04$ \\
\hline chr2_752370IENSGALTO00( & 2 cis & $10.4 \mathrm{cis}$ & 11.75 & $2.36 \mathrm{E}-04$ \\
\hline chr2_747630IENSGALTO00( & 2 cis & $10.4 \mathrm{cis}$ & 13.56 & 1.49E-04 \\
\hline chr2_744590IENSGALTO00( & 2 cis & 10.4 cis & 6.22 & $1.03 \mathrm{E}-04$ \\
\hline chr2_761590IENSGALTO00( & 2 cis & $10.4 \mathrm{cis}$ & 12.47 & $6.43 E-04$ \\
\hline chr2_775090IENSGALT000( & 2 cis & 10.4 cis & 4.77 & $6.12 \mathrm{E}-04$ \\
\hline chr2_775020IENSGALTO00( & 2 cis & $10.4 \mathrm{cis}$ & 9.97 & 8.79E-05 \\
\hline chr2_730580IENSGALTO00( & 2 cis & $10.4 \mathrm{cis}$ & 4.38 & 2.80E-04 \\
\hline chr2_789610IENSGALT000( & 2 cis & $10.4 \mathrm{cis}$ & 8.34 & 2.07E-04 \\
\hline chr2_787380IENSGALTO00( & 2 cis & $10.4 \mathrm{cis}$ & 4.25 & 7.38E-05 \\
\hline chr2_781080IENSGALT000( & 2 cis & $10.4 \mathrm{cis}$ & 4.38 & $3.08 \mathrm{E}-04$ \\
\hline chr2_746750IENSGALTO00( & 2 cis & 10.4 cis & 4.21 & 7.80E-05 \\
\hline chr2_741220IENSGALTO00( & 2 cis & $10.4 \mathrm{cis}$ & 4.67 & $8.88 \mathrm{E}-04$ \\
\hline chr2_749210IENSGALTO00( & 2 cis & $10.4 \mathrm{cis}$ & 4.97 & 7.81E-06 \\
\hline chr2_744590IENSGALTO00( & 2 cis & 4.4 cis & 6.22 & $9.08 \mathrm{E}-04$ \\
\hline chr2_765830IENSGALTO00( & 2 cis & 4.4 cis & 5.34 & $3.45 \mathrm{E}-04$ \\
\hline chr2_842800IENSGALT000( & 2 cis & $4.4 \mathrm{cis}$ & 11.63 & $1.54 \mathrm{E}-03$ \\
\hline chr2_850080IENSGALT000( & 2 cis & $4.4 \mathrm{cis}$ & 17.23 & 2.37E-05 \\
\hline chr2_8334501X603144121F & 2 cis & 4.5 cis & 7.94 & 2.47E-04 \\
\hline chr2_8480801X603144121F & 2 cis & $4.5 \mathrm{cis}$ & 11.8 & 8.66E-04 \\
\hline chr2_8500801X603144121F & 2 cis & $4.5 \mathrm{cis}$ & 17.23 & 1.07E-04 \\
\hline chr2_8500701X603144121F & 2 cis & $4.5 \mathrm{cis}$ & 7.3 & 2.67E-05 \\
\hline chr2_813900IENSGALT000( & 2 cis & $4.5 \mathrm{cis}$ & 5.77 & 3.74E-04 \\
\hline chr2_833450IENSGALT000( & 2 cis & 4.5 cis & 7.94 & $1.95 \mathrm{E}-03$ \\
\hline chr2_850080IENSGALTO00( & 2 cis & $4.5 \mathrm{cis}$ & 17.23 & $4.68 \mathrm{E}-06$ \\
\hline chr2_853760IENSGALTO00 & 2 cis & 4.5 cis & 4.92 & 2.09E-04 \\
\hline chr2_862670IENSGALT000( & 2 cis & $4.5 \mathrm{cis}$ & 5 & $3.82 \mathrm{E}-04$ \\
\hline chr2_854420IENSGALT000( & 2 cis & $4.5 \mathrm{cis}$ & 4.17 & 3.01E-04 \\
\hline chr2_868300।NM_001031C & 2 cis & 7.2 cis & 5.42 & $2.38 \mathrm{E}-03$ \\
\hline chr2_842800।NM_001031C & 2 cis & 7.2 cis & 11.63 & $3.24 \mathrm{E}-05$ \\
\hline chr2_798150INM_001031C & 2 cis & 7.2 cis & 6.32 & $3.84 \mathrm{E}-04$ \\
\hline chr2_803480INM_001031C & 2 cis & 7.2 cis & 7.23 & 4.74E-03 \\
\hline chr2_803500।NM_001031C & 2 cis & $7.2 \mathrm{cis}$ & 7.2 & $6.70 \mathrm{E}-03$ \\
\hline chr2_803510।NM_001031C & 2 cis & 7.2 cis & 10.87 & 7.99E-04 \\
\hline chr2_803520।NM_001031C & 2 cis & 7.2 cis & 7.75 & 4.76E-03 \\
\hline chr2_803530INM_001031C & 2 cis & 7.2 cis & 10.05 & $1.72 \mathrm{E}-03$ \\
\hline chr2_803540INM_001031C & 2 cis & $7.2 \mathrm{cis}$ & 8.45 & 3.86E-03 \\
\hline chr2_803550।NM_001031C & 2 cis & $7.2 \mathrm{cis}$ & 6.34 & $2.88 \mathrm{E}-03$ \\
\hline chr2_850080INM_001031C & 2 cis & $7.2 \mathrm{cis}$ & 17.23 & 4.84E-04 \\
\hline chr2_857800।NM_001031C & 2 cis & $7.2 \mathrm{cis}$ & 6.43 & $2.06 \mathrm{E}-03$ \\
\hline chr2_859680INM_001031C & 2 cis & 7.2 cis & 13.39 & $1.40 \mathrm{E}-03$ \\
\hline chr2_842800IENSGALTO00( & 2 cis & $4.5 \mathrm{cis}$ & 11.63 & $1.61 \mathrm{E}-03$ \\
\hline chr2_803480IENSGALTO00( & 2 cis & 4.5 cis & 7.23 & 2.13E-02 \\
\hline chr2_803510IENSGALTO00 & 2 cis & $4.5 \mathrm{cis}$ & 10.87 & $1.01 \mathrm{E}-02$ \\
\hline chr2_803520IENSGALT000( & 2 cis & 4.5 cis & 7.75 & $2.42 \mathrm{E}-02$ \\
\hline chr2_803540IENSGALT000( & 2 cis & 4.5 cis & 8.45 & $1.93 \mathrm{E}-02$ \\
\hline chr2_803550IENSGALT000( & 2 cis & 4.5 cis & 6.34 & $1.51 \mathrm{E}-02$ \\
\hline chr2_850080IENSGALT000( & 2 cis & $4.5 \mathrm{cis}$ & 17.23 & $1.22 \mathrm{E}-02$ \\
\hline chr2_859870IENSGALT000( & 2 cis & $4.5 \mathrm{cis}$ & 6.01 & $1.24 \mathrm{E}-02$ \\
\hline
\end{tabular}




\begin{tabular}{|c|c|c|c|c|}
\hline chr2_859150IENSGALT000( & 2 cis & 4.5 cis & 6.11 & 4.46E-02 \\
\hline chr2_888410IENSGALTOOO & 2 cis & $4.5 \mathrm{cis}$ & 7.12 & 3.16E-02 \\
\hline chr2_858400ıX603866808F & 2 cis & 5.0 cis & 4.32 & $3.10 \mathrm{E}-03$ \\
\hline chr2_9068201X603866808F & 2 cis & $5.0 \mathrm{cis}$ & 4.49 & 4.81E-04 \\
\hline chr2_8540901X603866808F & 2 cis & 5.0 cis & 9.19 & $1.86 \mathrm{E}-03$ \\
\hline chr2_8626701X603866808F & 2 cis & 5.0 cis & 5 & $3.60 \mathrm{E}-03$ \\
\hline chr2_8748701X603866808F & 2 cis & $5.0 \mathrm{cis}$ & 5.06 & 3.66E-04 \\
\hline chr2_859640ıX603866808F & 2 cis & 5.0 cis & 10.6 & $9.28 \mathrm{E}-03$ \\
\hline chr2_8765201X603866808F & 2 cis & 5.0 cis & 4.51 & $1.16 \mathrm{E}-03$ \\
\hline chr2_8921301X603866808F & 2 cis & $5.0 \mathrm{cis}$ & 12.72 & $1.21 \mathrm{E}-03$ \\
\hline chr2_888410ıX603866808F & 2 cis & 5.0 cis & 7.12 & $1.85 \mathrm{E}-05$ \\
\hline chr2_889710ı603866808F & 2 cis & 5.0 cis & 5.75 & $1.43 \mathrm{E}-03$ \\
\hline chr2_891850ıX603866808F & 2 cis & $5.0 \mathrm{cis}$ & 5.56 & $2.15 \mathrm{E}-03$ \\
\hline chr2_892340ıX603866808F & 2 cis & $5.0 \mathrm{cis}$ & 8.14 & 4.90E-04 \\
\hline chr2_8775701X603866808F & 2 cis & 5.0 cis & 4.44 & $1.61 \mathrm{E}-03$ \\
\hline chr2_8835401X603866808F & 2 cis & $5.0 \mathrm{cis}$ & 5.38 & 2.09E-05 \\
\hline chr2_9062401X603866808F & 2 cis & $5.0 \mathrm{cis}$ & 7.69 & $1.33 \mathrm{E}-03$ \\
\hline chr2_912990ıX603866808F & 2 cis & $5.0 \mathrm{cis}$ & 4.14 & 3.79E-04 \\
\hline chr2_850080ıX603599695F & 2 cis & $8.7 \mathrm{cis}$ & 17.23 & $2.02 \mathrm{E}-05$ \\
\hline chr2_855360ıX603599695F & 2 cis & $8.7 \mathrm{cis}$ & 6.19 & $2.24 \mathrm{E}-05$ \\
\hline chr2_8880601X603599695F & 2 cis & $8.7 \mathrm{cis}$ & 9.18 & $1.86 \mathrm{E}-04$ \\
\hline chr2_850070ıX603599695F & 2 cis & $8.7 \mathrm{cis}$ & 7.3 & $1.71 \mathrm{E}-05$ \\
\hline chr2_855970ıX603599695F & 2 cis & $8.7 \mathrm{cis}$ & 8.12 & 1.09E-04 \\
\hline chr2_892920ıX603599695F & 2 cis & $8.7 \mathrm{cis}$ & 9.91 & $1.92 \mathrm{E}-04$ \\
\hline chr2_850850ıX603599695F & 2 cis & $8.7 \mathrm{cis}$ & 4.29 & 9.27E-05 \\
\hline chr2_8586901X603599695F & 2 cis & $8.7 \mathrm{cis}$ & 4.1 & 2.47E-04 \\
\hline chr2_8893501X603599695F & 2 cis & $8.7 \mathrm{cis}$ & 5.77 & 2.73E-04 \\
\hline chr2_891440ıX603599695F & 2 cis & $8.7 \mathrm{cis}$ & 6.96 & $2.65 \mathrm{E}-04$ \\
\hline chr2_8902301X603599695F & 2 cis & $8.7 \mathrm{cis}$ & 6.26 & $8.12 \mathrm{E}-05$ \\
\hline chr2_894000ıX03599695F & 2 cis & $8.7 \mathrm{cis}$ & 6.87 & 8.67E-05 \\
\hline chr2_889480ı603599695F & 2 cis & $8.7 \mathrm{cis}$ & 7.65 & $3.26 \mathrm{E}-04$ \\
\hline chr2_900140ıX603599695F & 2 cis & $8.7 \mathrm{cis}$ & 11.88 & 5.55E-05 \\
\hline chr2_9063601X603599695F & 2 cis & $8.7 \mathrm{cis}$ & 12.12 & $2.56 \mathrm{E}-04$ \\
\hline chr2_8874601X603599695F & 2 cis & $8.7 \mathrm{cis}$ & 4.1 & $1.50 \mathrm{E}-03$ \\
\hline chr2_906530ı603599695F & 2 cis & $8.7 \mathrm{cis}$ & 5.54 & $4.98 \mathrm{E}-03$ \\
\hline chr2_850080IENSGALTO00( & 2 cis & $9.4 \mathrm{cis}$ & 17.23 & $4.02 \mathrm{E}-04$ \\
\hline chr2_900610IENSGALT000( & 2 cis & $9.4 \mathrm{cis}$ & 8.43 & $3.71 E-05$ \\
\hline chr2_886960IENSGALT000( & 2 cis & $9.4 \mathrm{cis}$ & 8.42 & 1.60E-04 \\
\hline chr2_906870IENSGALT000( & 2 cis & 9.4 cis & 5.29 & $1.41 \mathrm{E}-03$ \\
\hline chr2_886970IENSGALT000( & 2 cis & $9.4 \mathrm{cis}$ & 5.31 & $3.73 E-05$ \\
\hline chr2_892130IENSGALT000( & 2 cis & $9.4 \mathrm{cis}$ & 12.72 & $1.98 \mathrm{E}-04$ \\
\hline chr2_850070IENSGALTO00( & 2 cis & $9.4 \mathrm{cis}$ & 7.3 & 5.87E-04 \\
\hline chr2_892920IENSGALT000( & 2 cis & $9.4 \mathrm{cis}$ & 9.91 & $2.78 \mathrm{E}-05$ \\
\hline chr2_889350IENSGALT000( & 2 cis & $9.4 \mathrm{cis}$ & 5.77 & 7.56E-04 \\
\hline chr2_894000IENSGALT000( & 2 cis & 9.4 cis & 6.87 & $2.60 \mathrm{E}-04$ \\
\hline chr2_900140IENSGALT000( & 2 cis & $9.4 \mathrm{cis}$ & 11.88 & $6.98 \mathrm{E}-05$ \\
\hline chr2_906360IENSGALT000( & 2 cis & $9.4 \mathrm{cis}$ & 12.12 & $2.51 \mathrm{E}-04$ \\
\hline chr2_887460IENSGALTOOO( & 2 cis & $9.4 \mathrm{cis}$ & 4.1 & $2.52 \mathrm{E}-04$ \\
\hline chr2_9096501X603602441F & 2 cis & 4.6 cis & 5.55 & $6.68 \mathrm{E}-04$ \\
\hline chr2_9141201X603602441F & 2 cis & $4.6 \mathrm{cis}$ & 6.44 & $8.50 \mathrm{E}-04$ \\
\hline chr2_8921301X603595629F & 2 cis & $15.2 \mathrm{cis}$ & 12.72 & 4.30E-05 \\
\hline chr2_9225601X603595629F & 2 cis & $15.2 \mathrm{cis}$ & 5.54 & 7.63E-05 \\
\hline chr2_8940001X603595629F & 2 cis & $15.2 \mathrm{cis}$ & 6.87 & $6.30 \mathrm{E}-06$ \\
\hline chr2_9001401X603595629F & 2 cis & $15.2 \mathrm{cis}$ & 11.88 & $1.46 \mathrm{E}-05$ \\
\hline chr2_9158501X603595629F & 2 cis & $15.2 \mathrm{cis}$ & 5.11 & $4.56 \mathrm{E}-06$ \\
\hline chr2_920540ıX603595629F & 2 cis & $15.2 \mathrm{cis}$ & 7.6 & $1.86 \mathrm{E}-05$ \\
\hline chr2_906360ı603595629F & 2 cis & $15.2 \mathrm{cis}$ & 12.12 & $9.95 \mathrm{E}-06$ \\
\hline chr2_916660ı603595629F & 2 cis & $15.2 \mathrm{cis}$ & 8.72 & $6.34 \mathrm{E}-07$ \\
\hline chr2_903650ıX603595629F & 2 cis & $15.2 \mathrm{cis}$ & 4.69 & $2.10 \mathrm{E}-04$ \\
\hline chr2_8980001X603595629F & 2 cis & $15.2 \mathrm{cis}$ & 6.53 & $1.40 \mathrm{E}-07$ \\
\hline chr2_907360ı X603595629F & 2 cis & $15.2 \mathrm{cis}$ & 5.21 & $1.79 \mathrm{E}-06$ \\
\hline chr2_906240ıX603595629F & 2 cis & $15.2 \mathrm{cis}$ & 7.69 & $2.48 \mathrm{E}-06$ \\
\hline chr2_9032701X603595629F & 2 cis & $15.2 \mathrm{cis}$ & 7.15 & $3.66 \mathrm{E}-07$ \\
\hline chr2_9065301X603595629F & 2 cis & $15.2 \mathrm{cis}$ & 5.54 & $8.48 \mathrm{E}-05$ \\
\hline chr2_898500ı603595629F & 2 cis & $15.2 \mathrm{cis}$ & 8.15 & $5.29 \mathrm{E}-06$ \\
\hline chr2_9047101X603595629F & 2 cis & $15.2 \mathrm{cis}$ & 9.31 & $9.18 \mathrm{E}-07$ \\
\hline chr2_915880ı603595629F & 2 cis & $15.2 \mathrm{cis}$ & 6.15 & 4.07E-05 \\
\hline chr2_9207901X603595629F & 2 cis & $15.2 \mathrm{cis}$ & 5.25 & $6.18 \mathrm{E}-05$ \\
\hline chr2_9222401X603595629F & 2 cis & $15.2 \mathrm{cis}$ & 6.17 & $1.74 \mathrm{E}-05$ \\
\hline chr2_908010ı603595629F & 2 cis & $15.2 \mathrm{cis}$ & 4.1 & $1.43 \mathrm{E}-05$ \\
\hline chr2_915420ıX603595629F & 2 cis & $15.2 \mathrm{cis}$ & 5.84 & $1.05 \mathrm{E}-04$ \\
\hline chr2_920530।X603595629F & 2 cis & $15.2 \mathrm{cis}$ & 8.55 & $5.38 \mathrm{E}-07$ \\
\hline
\end{tabular}




\begin{tabular}{|c|c|c|c|c|}
\hline chr2_918690।X603595629F & 2 cis & $15.2 \mathrm{cis}$ & 9.4 & 5.67E-06 \\
\hline chr2_9067101X603595629F & 2 cis & 15.2 cis & 4.27 & $2.35 \mathrm{E}-04$ \\
\hline chr2_907190ıX603595629F & 2 cis & 15.2 cis & 4.59 & $4.22 \mathrm{E}-04$ \\
\hline chr2_889350।X603597550F & 2 cis & 4.7 cis & 5.77 & $1.13 \mathrm{E}-03$ \\
\hline chr2_898500।X603597550F & 2 cis & 4.7 cis & 8.15 & $1.58 \mathrm{E}-03$ \\
\hline chr2_914120।X603597550F & 2 cis & $4.7 \mathrm{cis}$ & 6.44 & $1.24 \mathrm{E}-03$ \\
\hline chr2_9251601X603597550F & 2 cis & 4.7 cis & 6.2 & $1.06 \mathrm{E}-03$ \\
\hline chr2_890230IENSGALT000 & 2 cis & $4.4 \mathrm{cis}$ & 6.26 & $6.96 \mathrm{E}-04$ \\
\hline chr2_967770IENSGALT000( & 2 cis & $7.2 \mathrm{cis}$ & 5.45 & $2.91 \mathrm{E}-04$ \\
\hline chr2_892130IENSGALT000( & 2 cis & 7.2 cis & 12.72 & $2.52 \mathrm{E}-04$ \\
\hline chr2_891440IENSGALT000( & 2 cis & 7.2 cis & 6.96 & $2.80 \mathrm{E}-04$ \\
\hline chr2_922560IENSGALTO00 & 2 cis & $7.2 \mathrm{cis}$ & 5.54 & 4.15E-05 \\
\hline chr2_894000IENSGALT000( & 2 cis & 7.2 cis & 6.87 & $1.56 \mathrm{E}-08$ \\
\hline chr2_953190IENSGALT000( & 2 cis & 7.2 cis & 5.54 & $2.47 \mathrm{E}-05$ \\
\hline chr2_900140IENSGALT000( & 2 cis & $7.2 \mathrm{cis}$ & 11.88 & $9.96 \mathrm{E}-06$ \\
\hline chr2_915850IENSGALT000( & 2 cis & 7.2 cis & 5.11 & 4.64E-05 \\
\hline chr2_934080IENSGALT000( & 2 cis & $7.2 \mathrm{cis}$ & 7.62 & $2.32 \mathrm{E}-04$ \\
\hline chr2_965880IENSGALT000( & 2 cis & $7.2 \mathrm{cis}$ & 4.1 & $5.71 \mathrm{E}-04$ \\
\hline chr2_931240IENSGALT000( & 2 cis & $7.2 \mathrm{cis}$ & 7.8 & $3.23 \mathrm{E}-04$ \\
\hline chr2_920540IENSGALT000( & 2 cis & 7.2 cis & 7.6 & $6.41 \mathrm{E}-05$ \\
\hline chr2_906360।ENSGALT000( & 2 cis & $7.2 \mathrm{cis}$ & 12.12 & $2.35 \mathrm{E}-05$ \\
\hline chr2_916660IENSGALT000( & 2 cis & 7.2 cis & 8.72 & 4.11E-05 \\
\hline chr2_962840IENSGALT000( & 2 cis & $7.2 \mathrm{cis}$ & 5.2 & $1.22 \mathrm{E}-04$ \\
\hline chr2_919360IENSGALT000( & 2 cis & $7.2 \mathrm{cis}$ & 4.38 & $2.35 \mathrm{E}-04$ \\
\hline chr2_898000IENSGALT000( & 2 cis & $7.2 \mathrm{cis}$ & 6.53 & $7.00 \mathrm{E}-07$ \\
\hline chr2_966960IENSGALT000( & 2 cis & 7.2 cis & 5.73 & $1.65 \mathrm{E}-04$ \\
\hline chr2_906240IENSGALT000( & 2 cis & 7.2 cis & 7.69 & $1.98 \mathrm{E}-04$ \\
\hline chr2_903270IENSGALTO00 & 2 cis & 7.2 cis & 7.15 & $6.87 \mathrm{E}-05$ \\
\hline chr2_903540IENSGALT000( & 2 cis & $7.2 \mathrm{cis}$ & 6.64 & $3.01 \mathrm{E}-04$ \\
\hline chr2_915880IENSGALT000( & 2 cis & $7.2 \mathrm{cis}$ & 6.15 & $8.79 \mathrm{E}-03$ \\
\hline chr2_941850IENSGALT000( & 2 cis & $7.2 \mathrm{cis}$ & 4.53 & $1.95 \mathrm{E}-05$ \\
\hline chr2_920530IENSGALT000( & 2 cis & 7.2 cis & 8.55 & $7.12 \mathrm{E}-04$ \\
\hline chr2_929030IENSGALT000( & 2 cis & $7.2 \mathrm{cis}$ & 5.44 & $2.08 \mathrm{E}-04$ \\
\hline chr2_936500IENSGALT000( & 2 cis & 7.2 cis & 24.21 & $3.67 \mathrm{E}-03$ \\
\hline chr2_936060IENSGALT000( & 2 cis & $7.2 \mathrm{cis}$ & 5.37 & 2.79E-05 \\
\hline chr2_936070IENSGALT000( & 2 cis & $7.2 \mathrm{cis}$ & 9.9 & $8.39 \mathrm{E}-04$ \\
\hline chr2_941840IENSGALT000( & 2 cis & $7.2 \mathrm{cis}$ & 8.56 & $2.34 \mathrm{E}-05$ \\
\hline chr2_943600IENSGALT000( & 2 cis & $7.2 \mathrm{cis}$ & 13.55 & $1.68 \mathrm{E}-04$ \\
\hline chr2_944210।ENSGALT000( & 2 cis & $7.2 \mathrm{cis}$ & 7.38 & 2.37E-04 \\
\hline chr2_943500।ENSGALT000( & 2 cis & 7.2 cis & 4.75 & $8.70 \mathrm{E}-05$ \\
\hline chr2_965530IENSGALT000( & 2 cis & 7.2 cis & 8.89 & $9.67 \mathrm{E}-04$ \\
\hline chr2_859150IENSGALTO00( & 2 cis & $4.5 \mathrm{cis}$ & 6.11 & $8.41 \mathrm{E}-03$ \\
\hline chr2_962840IENSGALT000( & 2 cis & 4.5 cis & 5.2 & $6.59 \mathrm{E}-02$ \\
\hline chr2_944170IENSGALTO00 & 2 cis & 4.5 cis & 8.14 & $6.43 \mathrm{E}-04$ \\
\hline chr2_962780IENSGALT000( & 2 cis & $4.5 \mathrm{cis}$ & 7.84 & $1.03 \mathrm{E}-03$ \\
\hline chr2_941690IENSGALTO00( & 2 cis & $4.5 \mathrm{cis}$ & 7.4 & $4.66 \mathrm{E}-03$ \\
\hline chr2_962840।X603864386F & 2 cis & 4.4 cis & 5.2 & $1.25 \mathrm{E}-03$ \\
\hline chr2_942750।X603864386F & 2 cis & 4.4 cis & 10.26 & 1.17E-03 \\
\hline chr2_9415901X603864386F & 2 cis & 4.4 cis & 18.17 & 7.91E-04 \\
\hline chr2_962780!X603864386F & 2 cis & 4.4 cis & 7.84 & 7.43E-05 \\
\hline chr2_9605401X603864386F & 2 cis & 4.4 cis & 9.67 & $8.60 \mathrm{E}-04$ \\
\hline chr2_9496101X603864386F & 2 cis & $4.4 \mathrm{cis}$ & 5.12 & $7.48 \mathrm{E}-04$ \\
\hline chr2_941600।X603864386F & 2 cis & 4.4 cis & 7.89 & $2.65 \mathrm{E}-03$ \\
\hline chr2_9448701X603864386F & 2 cis & 4.4 cis & 4.4 & $2.99 \mathrm{E}-03$ \\
\hline chr2_9489201X603864386F & 2 cis & 4.4 cis & 4.9 & $1.96 \mathrm{E}-03$ \\
\hline chr2_9264701X603864386F & 2 cis & 4.4 cis & 6.34 & $2.96 \mathrm{E}-03$ \\
\hline chr2_941690।X603864386F & 2 cis & 4.4 cis & 7.4 & $3.50 \mathrm{E}-04$ \\
\hline chr2_971110।X603864386F & 2 cis & $4.4 \mathrm{cis}$ & 5.33 & 9.97E-04 \\
\hline chr2_960640।X603864386F & 2 cis & 4.4 cis & 4.49 & $1.69 \mathrm{E}-03$ \\
\hline chr2_943330!X603864386F & 2 cis & 4.4 cis & 5.06 & $4.91 \mathrm{E}-04$ \\
\hline chr2_9347301X603864386F & 2 cis & 4.4 cis & 4.41 & $7.60 \mathrm{E}-03$ \\
\hline chr2_959860।X603864386F & 2 cis & 4.4 trans & 8.01 & $1.73 \mathrm{E}-03$ \\
\hline chr2_898500IENSGALT000( & 2 cis & 4.0 cis & 8.15 & $6.13 \mathrm{E}-03$ \\
\hline chr2_915420IENSGALT000( & 2 cis & $4.0 \mathrm{cis}$ & 5.84 & $7.06 \mathrm{E}-03$ \\
\hline chr2_918690IENSGALT000( & 2 cis & 4.0 cis & 9.4 & $3.82 \mathrm{E}-03$ \\
\hline chr2_941520IENSGALT000( & 2 cis & $4.0 \mathrm{cis}$ & 10.38 & $2.31 \mathrm{E}-03$ \\
\hline chr2_943660IENSGALTO00( & 2 cis & 4.0 cis & 6.15 & $1.08 \mathrm{E}-03$ \\
\hline chr2_963190IENSGALT000( & 2 cis & $4.0 \mathrm{cis}$ & 6.65 & $3.93 \mathrm{E}-03$ \\
\hline chr2_963210IENSGALT000( & 2 cis & 4.0 cis & 5.87 & $3.67 \mathrm{E}-03$ \\
\hline chr2_963490IENSGALT000( & 2 cis & 4.0 cis & 6.48 & 4.79E-03 \\
\hline chr2_963820IENSGALT000( & 2 cis & 4.0 cis & 6.55 & $3.45 \mathrm{E}-03$ \\
\hline chr2_964030IENSGALT0001 & 2 cis & $4.0 \mathrm{cis}$ & 5.61 & $5.29 \mathrm{E}-03$ \\
\hline
\end{tabular}




\begin{tabular}{|c|c|c|c|c|}
\hline chr2_964080IENSGALTO00( & 2 cis & $4.0 \mathrm{cis}$ & 6.25 & $4.63 \mathrm{E}-03$ \\
\hline chr2_964100IENSGALTO00( & 2 cis & 4.0 cis & 5.34 & $9.18 \mathrm{E}-03$ \\
\hline chr2_963010IENSGALT000( & 2 cis & 4.0 cis & 4.51 & $7.75 \mathrm{E}-03$ \\
\hline chr2_963150IENSGALT000( & 2 cis & 4.0 cis & 4.78 & 8.99E-03 \\
\hline chr2_963480IENSGALT000( & 2 cis & 4.0 cis & 4.78 & 4.91E-03 \\
\hline chr2_963510IENSGALT000( & 2 cis & 4.0 cis & 4.78 & $9.75 \mathrm{E}-03$ \\
\hline chr2_963560IENSGALT000( & 2 cis & 4.0 cis & 4.28 & $8.45 E-03$ \\
\hline chr2_963790IENSGALT000( & 2 cis & 4.0 cis & 4.47 & $1.45 \mathrm{E}-02$ \\
\hline chr2_963840IENSGALT000( & 2 cis & 4.0 cis & 4.72 & $6.25 \mathrm{E}-03$ \\
\hline chr2_964010IENSGALT000( & 2 cis & 4.0 cis & 4.61 & 1.27E-02 \\
\hline chr2_963100IENSGALT000( & 2 cis & 4.0 cis & 5.58 & 7.47E-03 \\
\hline chr2_963110IENSGALT000( & 2 cis & 4.0 cis & 5.77 & 4.45E-03 \\
\hline chr2_963170IENSGALT000( & 2 cis & 4.0 cis & 5.11 & 1.16E-02 \\
\hline chr2_963530IENSGALT000( & 2 cis & 4.0 cis & 5.79 & 8.00E-03 \\
\hline chr2_963800IENSGALT000( & 2 cis & 4.0 cis & 7.4 & 4.26E-03 \\
\hline chr2_963870IENSGALT000( & 2 cis & 4.0 cis & 5.12 & 8.96E-03 \\
\hline chr2_963880IENSGALT000( & 2 cis & 4.0 cis & 5.63 & $4.08 \mathrm{E}-03$ \\
\hline chr2_963910IENSGALTO00( & 2 cis & 4.0 cis & 5.66 & $5.56 \mathrm{E}-03$ \\
\hline chr2_963940IENSGALT000( & 2 cis & 4.0 cis & 6.69 & 8.32E-03 \\
\hline chr2_963120IENSGALT000( & 2 cis & 4.0 cis & 4.33 & 4.96E-03 \\
\hline chr2_963730IENSGALT000( & 2 cis & 4.0 cis & 4.75 & $9.59 \mathrm{E}-03$ \\
\hline chr2_963830IENSGALT000( & 2 cis & 4.0 cis & 4.24 & 8.39E-03 \\
\hline chr2_963770IENSGALT000( & 2 cis & 4.0 cis & 4.89 & $6.18 \mathrm{E}-03$ \\
\hline chr2_963930IENSGALTO00( & 2 cis & 4.0 cis & 4.9 & $6.53 \mathrm{E}-03$ \\
\hline chr2_936500IENSGALT000( & 2 cis & 4.0 cis & 24.21 & 5.64E-04 \\
\hline chr2_936510IENSGALT000( & 2 cis & 4.0 cis & 26.95 & 4.07E-03 \\
\hline chr2_944880IENSGALT000( & 2 cis & 4.0 cis & 8.92 & $1.76 \mathrm{E}-03$ \\
\hline chr2_928690IENSGALT000( & 2 cis & 4.0 cis & 8.31 & 2.73E-03 \\
\hline chr2_944670IENSGALT000( & 2 cis & 4.0 cis & 6.67 & 5.27E-03 \\
\hline chr2_959190IENSGALTO00( & 2 cis & 4.0 cis & 6.45 & $5.35 \mathrm{E}-03$ \\
\hline chr2_970700IENSGALT000( & 2 cis & 4.0 cis & 8.53 & $6.70 \mathrm{E}-03$ \\
\hline chr2_917920IENSGALT000( & 2 cis & 4.0 cis & 4.22 & 1.19E-03 \\
\hline chr2_966960IENSGALT000( & 2 cis & $5.8 \mathrm{cis}$ & 5.73 & 2.97E-03 \\
\hline chr2_968260IENSGALT000( & 2 cis & $5.8 \mathrm{cis}$ & 9.21 & $2.40 \mathrm{E}-03$ \\
\hline chr2_936500IENSGALT000( & 2 cis & $5.8 \mathrm{cis}$ & 24.21 & $1.91 \mathrm{E}-04$ \\
\hline chr2_936510IENSGALTO00( & 2 cis & $5.8 \mathrm{cis}$ & 26.95 & 5.51E-05 \\
\hline chr2_936070IENSGALTO00 & 2 cis & $5.8 \mathrm{cis}$ & 9.9 & $5.72 \mathrm{E}-04$ \\
\hline chr2_959780IENSGALT000( & 2 cis & $5.8 \mathrm{cis}$ & 5.07 & 7.20E-05 \\
\hline chr2_960550IENSGALT000( & 2 cis & $5.8 \mathrm{cis}$ & 29.97 & 4.80E-04 \\
\hline chr2_975500IENSGALT000( & 2 cis & $5.8 \mathrm{cis}$ & 5.92 & $1.30 \mathrm{E}-03$ \\
\hline chr2_944190IENSGALT000( & 2 cis & $5.8 \mathrm{cis}$ & 8.76 & 8.27E-04 \\
\hline chr2_970700IENSGALTO00( & 2 cis & $5.8 \mathrm{cis}$ & 8.53 & $2.52 \mathrm{E}-03$ \\
\hline chr2_970900IENSGALT000( & 2 cis & $5.8 \mathrm{cis}$ & 5.98 & 6.19E-04 \\
\hline chr2_969570IENSGALT000( & 2 cis & $5.8 \mathrm{cis}$ & 8.96 & $1.91 \mathrm{E}-03$ \\
\hline chr2_972280IENSGALT000( & 2 cis & $5.8 \mathrm{cis}$ & 4.35 & 3.60E-05 \\
\hline chr2_990860IENSGALT000( & 2 cis & $5.8 \mathrm{cis}$ & 4.7 & 7.57E-04 \\
\hline chr2_984390IENSGALT000( & 2 cis & $5.8 \mathrm{cis}$ & 4.69 & 2.91E-03 \\
\hline chr2_984050IENSGALT000( & 2 cis & $5.8 \mathrm{cis}$ & 8.52 & 4.49E-05 \\
\hline chr2_996620IENSGALT000( & 2 cis & $5.8 \mathrm{cis}$ & 4.92 & $3.52 \mathrm{E}-06$ \\
\hline chr2_991300IENSGALT000( & 2 cis & $5.8 \mathrm{cis}$ & 11.29 & $1.95 \mathrm{E}-03$ \\
\hline chr2_101908IENSGALT000( & 2 cis & $5.8 \mathrm{cis}$ & 5.82 & 1.60E-03 \\
\hline chr2_993510IENSGALT000( & 2 cis & $5.8 \mathrm{cis}$ & 5.84 & $2.32 \mathrm{E}-04$ \\
\hline chr2_999520IENSGALT000( & 2 cis & $5.8 \mathrm{cis}$ & 5.32 & 8.70E-04 \\
\hline chr2_100049IENSGALTO00( & 2 cis & $5.8 \mathrm{cis}$ & 7.34 & 4.81E-05 \\
\hline chr2_100050IENSGALT000( & 2 cis & $5.8 \mathrm{cis}$ & 8.16 & $2.95 \mathrm{E}-04$ \\
\hline chr2_960420IENSGALT000( & 2 cis & $5.8 \mathrm{cis}$ & 5.15 & $1.32 \mathrm{E}-03$ \\
\hline chr2_981570IENSGALTO00( & 2 cis & $5.8 \mathrm{cis}$ & 6.24 & $1.35 \mathrm{E}-04$ \\
\hline chr2_986680IENSGALT000( & 2 cis & 5.8 cis & 5.68 & $9.72 \mathrm{E}-04$ \\
\hline chr2_9843901X603865192F & 2 cis & $12.0 \mathrm{cis}$ & 4.69 & $2.89 \mathrm{E}-04$ \\
\hline chr2_1008331X603865192F & 2 cis & $12.0 \mathrm{cis}$ & 5.14 & 1.65E-04 \\
\hline chr2_9966201X603865192F & 2 cis & $12.0 \mathrm{cis}$ & 4.92 & 7.50E-06 \\
\hline chr2_9913001X603865192F & 2 cis & $12.0 \mathrm{cis}$ & 11.29 & $9.75 \mathrm{E}-05$ \\
\hline chr2_1019081X603865192F & 2 cis & $12.0 \mathrm{cis}$ & 5.82 & 3.73E-04 \\
\hline chr2_9994601X603865192F & 2 cis & $12.0 \mathrm{cis}$ & 4.1 & $1.01 \mathrm{E}-05$ \\
\hline chr2_9981001X603865192F & 2 cis & $12.0 \mathrm{cis}$ & 9.19 & $2.49 \mathrm{E}-05$ \\
\hline chr2_100049ıX603865192F & 2 cis & $12.0 \mathrm{cis}$ & 7.34 & $1.38 \mathrm{E}-06$ \\
\hline chr2_100062।X603865192F & 2 cis & $12.0 \mathrm{cis}$ & 6.06 & $3.02 \mathrm{E}-07$ \\
\hline chr2_1000501X603865192F & 2 cis & $12.0 \mathrm{cis}$ & 8.16 & 1.17E-07 \\
\hline chr2_100939ıX603865192F & 2 cis & $12.0 \mathrm{cis}$ & 7.05 & $2.49 E-06$ \\
\hline chr2_101605।X603865192F & 2 cis & $12.0 \mathrm{cis}$ & 5.03 & $8.25 \mathrm{E}-05$ \\
\hline chr2_9994001X603865192F & 2 cis & $12.0 \mathrm{cis}$ & 12 & $1.55 \mathrm{E}-04$ \\
\hline chr2_102081ıX03865192F & 2 cis & $12.0 \mathrm{cis}$ & 4.79 & 7.89E-04 \\
\hline
\end{tabular}




\begin{tabular}{|c|c|c|c|c|}
\hline chr2_102593ıX603865192F & 2 cis & $12.0 \mathrm{cis}$ & 5.54 & 1.97E-03 \\
\hline chr2_987060ıX603865192F & 2 cis & $12.0 \mathrm{cis}$ & 5.63 & $1.12 \mathrm{E}-05$ \\
\hline chr2_999650।X603865192F & 2 cis & 12.0 cis & 7.54 & $2.35 \mathrm{E}-04$ \\
\hline chr2_102449ıX603865192F & 2 cis & 12.0 cis & 9.16 & $2.65 \mathrm{E}-04$ \\
\hline chr2_996620IENSGALT000( & 2 cis & 8.1 cis & 4.92 & $2.36 \mathrm{E}-07$ \\
\hline chr2_101677IENSGALTO00( & 2 cis & $8.1 \mathrm{cis}$ & 4.1 & $2.84 \mathrm{E}-05$ \\
\hline chr2_991300IENSGALT000( & 2 cis & $8.1 \mathrm{cis}$ & 11.29 & $6.94 \mathrm{E}-03$ \\
\hline chr2_101908IENSGALT000( & 2 cis & $8.1 \mathrm{cis}$ & 5.82 & $9.00 \mathrm{E}-03$ \\
\hline chr2_993510IENSGALT000( & 2 cis & $8.1 \mathrm{cis}$ & 5.84 & 8.23E-06 \\
\hline chr2_100144IENSGALT000( & 2 cis & $8.1 \mathrm{cis}$ & 6.07 & $2.82 \mathrm{E}-05$ \\
\hline chr2_999520IENSGALT000( & 2 cis & $8.1 \mathrm{cis}$ & 5.32 & $1.24 \mathrm{E}-05$ \\
\hline chr2_101099IENSGALT000( & 2 cis & 8.1 cis & 5.3 & 2.73E-04 \\
\hline chr2_999460IENSGALT000( & 2 cis & $8.1 \mathrm{cis}$ & 4.1 & $1.50 \mathrm{E}-07$ \\
\hline chr2_998100IENSGALT000( & 2 cis & $8.1 \mathrm{cis}$ & 9.19 & $5.50 \mathrm{E}-05$ \\
\hline chr2_100049IENSGALT000( & 2 cis & $8.1 \mathrm{cis}$ & 7.34 & $4.92 \mathrm{E}-07$ \\
\hline chr2_100062।ENSGALT000( & 2 cis & $8.1 \mathrm{cis}$ & 6.06 & $1.46 \mathrm{E}-05$ \\
\hline chr2_102958IENSGALT000( & 2 cis & $8.1 \mathrm{cis}$ & 5.67 & 1.10E-02 \\
\hline chr2_997610IENSGALT000( & 2 cis & $8.1 \mathrm{cis}$ & 4.5 & $5.22 \mathrm{E}-05$ \\
\hline chr2_990090IENSGALT000( & 2 cis & 8.1 cis & 4.12 & $3.95 \mathrm{E}-03$ \\
\hline chr2_100050IENSGALT000( & 2 cis & $8.1 \mathrm{cis}$ & 8.16 & $2.40 \mathrm{E}-05$ \\
\hline chr2_100939IENSGALT000( & 2 cis & $8.1 \mathrm{cis}$ & 7.05 & $4.80 \mathrm{E}-04$ \\
\hline chr2_989070IENSGALT000( & 2 cis & $8.1 \mathrm{cis}$ & 4.65 & $6.44 \mathrm{E}-04$ \\
\hline chr2_999270IENSGALT000( & 2 cis & $8.1 \mathrm{cis}$ & 7.75 & $3.46 \mathrm{E}-04$ \\
\hline chr2_986680IENSGALT000( & 2 cis & $8.1 \mathrm{cis}$ & 5.68 & $1.11 \mathrm{E}-03$ \\
\hline chr2_992010IENSGALT000( & 2 cis & 8.1 cis & 6.85 & 8.27E-04 \\
\hline chr2_970700ıNM_205341_ & 2 cis & 4.5 cis & 8.53 & $1.48 \mathrm{E}-03$ \\
\hline chr2_969570ıNM_205341_ & 2 cis & 4.5 cis & 8.96 & $4.28 \mathrm{E}-03$ \\
\hline chr2_978280INM_205341_ & 2 cis & 4.5 cis & 7.86 & $1.67 \mathrm{E}-03$ \\
\hline chr2_100939INM_205341_ & 2 cis & 4.5 cis & 7.05 & 7.47E-04 \\
\hline chr2_102081INM_205341_ & 2 cis & 4.5 cis & 4.79 & $2.10 \mathrm{E}-02$ \\
\hline chr2_101574IENSGALT000( & 2 cis & 8.6 cis & 6.36 & $4.94 \mathrm{E}-05$ \\
\hline chr2_984390IENSGALT000( & 2 cis & 8.6 cis & 4.69 & 3.47E-05 \\
\hline chr2_991300IENSGALT000( & 2 cis & 8.6 cis & 11.29 & $1.84 \mathrm{E}-07$ \\
\hline chr2_984870IENSGALT000( & 2 cis & 8.6 cis & 4.26 & $8.43 E-04$ \\
\hline chr2_990370IENSGALT000( & 2 cis & 8.6 cis & 4.97 & 4.96E-05 \\
\hline chr2_997970IENSGALT000( & 2 cis & 8.6 cis & 5.72 & $1.94 \mathrm{E}-04$ \\
\hline chr2_999810IENSGALT000( & 2 cis & 8.6 cis & 5.12 & $9.00 \mathrm{E}-05$ \\
\hline chr2_100055IENSGALT000( & 2 cis & 8.6 cis & 6.93 & $6.26 \mathrm{E}-05$ \\
\hline chr2_100905IENSGALT000( & 2 cis & 8.6 cis & 5.55 & $1.20 \mathrm{E}-04$ \\
\hline chr2_989070IENSGALT000( & 2 cis & 8.6 cis & 4.65 & $5.92 \mathrm{E}-04$ \\
\hline chr2_993190IENSGALT000( & 2 cis & 8.6 cis & 5.07 & 1.17E-04 \\
\hline chr2_999270IENSGALT000( & 2 cis & 8.6 cis & 7.75 & $1.09 \mathrm{E}-03$ \\
\hline chr2_991280IENSGALT000( & 2 cis & $8.6 \mathrm{cis}$ & 6.12 & $1.53 \mathrm{E}-05$ \\
\hline chr2_100507IENSGALTO00( & 2 cis & 8.6 cis & 5.78 & 3.97E-04 \\
\hline chr2_996030IENSGALT000( & 2 cis & 8.6 cis & 5.26 & $3.24 \mathrm{E}-04$ \\
\hline chr2_999400IENSGALT000( & 2 cis & 8.6 cis & 12 & $3.41 \mathrm{E}-06$ \\
\hline chr2_100297IENSGALTO00( & 2 cis & 8.6 cis & 4.1 & 4.64E-04 \\
\hline chr2_998400IENSGALT000( & 2 cis & 8.6 cis & 6.24 & $7.44 \mathrm{E}-04$ \\
\hline chr2_986770IENSGALTO00( & 2 cis & $8.6 \mathrm{cis}$ & 5.53 & $1.78 \mathrm{E}-04$ \\
\hline chr2_991560IENSGALT000( & 2 cis & $8.6 \mathrm{cis}$ & 5.34 & $2.76 \mathrm{E}-04$ \\
\hline chr2_994700IENSGALT000( & 2 cis & 8.6 cis & 6.29 & $2.01 \mathrm{E}-03$ \\
\hline chr2_999650IENSGALT000( & 2 cis & 8.6 cis & 7.54 & $6.08 \mathrm{E}-05$ \\
\hline chr2_100557IENSGALT000( & 2 cis & 8.6 cis & 5.42 & $4.78 \mathrm{E}-04$ \\
\hline chr2_100061।ENSGALT000( & 2 cis & 8.6 cis & 6.45 & $1.24 \mathrm{E}-05$ \\
\hline chr2_100912।ENSGALT000( & 2 cis & 8.6 cis & 4.74 & $7.08 \mathrm{E}-05$ \\
\hline chr2_101243IENSGALT000( & 2 cis & $8.6 \mathrm{cis}$ & 8.41 & $1.06 \mathrm{E}-05$ \\
\hline chr2_996430IENSGALT000( & 2 cis & 8.6 cis & 4.92 & 1.17E-03 \\
\hline chr2_988930IENSGALT000( & 2 cis & 8.6 cis & 4.8 & $1.26 \mathrm{E}-04$ \\
\hline chr2_997340IENSGALT000( & 2 cis & 8.6 cis & 4.1 & $1.62 \mathrm{E}-03$ \\
\hline chr2_101016IENSGALT000( & 2 cis & 8.6 cis & 4.1 & $2.23 \mathrm{E}-03$ \\
\hline chr2_987550IENSGALTO00( & 2 cis & $8.6 \mathrm{cis}$ & 4.72 & 4.06E-04 \\
\hline chr2_102449IENSGALT000( & 2 cis & $8.6 \mathrm{cis}$ & 9.16 & $4.12 \mathrm{E}-04$ \\
\hline chr2_101261।ENSGALT000( & 2 cis & 8.6 cis & 4.32 & $9.23 \mathrm{E}-04$ \\
\hline chr2_1155391X603597405F & 2 cis & 4.5 trans & 7.94 & $2.43 E-03$ \\
\hline chr2_110799ıX603865613F & 2 cis & $6.8 \mathrm{cis}$ & 14.22 & $2.91 \mathrm{E}-06$ \\
\hline chr2_110536।X603865613F & 2 cis & $6.8 \mathrm{cis}$ & 10.69 & $4.48 \mathrm{E}-04$ \\
\hline chr2_111731।X603865613F & 2 cis & $6.8 \mathrm{cis}$ & 8.55 & 3.49E-05 \\
\hline chr2_111764ıX603865613F & 2 cis & $6.8 \mathrm{cis}$ & 9.03 & $1.41 \mathrm{E}-05$ \\
\hline chr2_111743ıx603865613F & 2 cis & $6.8 \mathrm{cis}$ & 8.42 & $8.20 \mathrm{E}-04$ \\
\hline chr2_111744ıX603865613F & 2 cis & $6.8 \mathrm{cis}$ & 7.32 & $2.66 \mathrm{E}-04$ \\
\hline chr2_111752ıX603865613F & 2 cis & 6.8 cis & 9.5 & $2.36 \mathrm{E}-04$ \\
\hline chr2_111734ıX603865613F & 2 cis & 6.8 cis & 9.77 & $1.21 \mathrm{E}-07$ \\
\hline
\end{tabular}




\begin{tabular}{|c|c|c|c|c|}
\hline chr2_111745ıX603865613F & 2 cis & $6.8 \mathrm{cis}$ & 11.27 & $9.33 \mathrm{E}-04$ \\
\hline chr2_111755ıX603865613F & 2 cis & $6.8 \mathrm{cis}$ & 8.89 & $2.88 \mathrm{E}-05$ \\
\hline chr2_1117631X603865613F & 2 cis & $6.8 \mathrm{cis}$ & 7.8 & $5.50 \mathrm{E}-04$ \\
\hline chr2_1117531X603865613F & 2 cis & $6.8 \mathrm{cis}$ & 8.98 & 4.79E-05 \\
\hline chr2_111729ıX603865613F & 2 cis & $6.8 \mathrm{cis}$ & 9.75 & $2.25 \mathrm{E}-04$ \\
\hline chr2_111741।X603865613F & 2 cis & $6.8 \mathrm{cis}$ & 5.04 & $1.15 \mathrm{E}-04$ \\
\hline chr2_111740।X603865613F & 2 cis & $6.8 \mathrm{cis}$ & 4.7 & $3.65 \mathrm{E}-04$ \\
\hline chr2_112301।X603865613F & 2 cis & $6.8 \mathrm{cis}$ & 5.7 & 5.01E-05 \\
\hline chr2_114192।X603865613F & 2 cis & $6.8 \mathrm{cis}$ & 5.68 & $9.71 \mathrm{E}-05$ \\
\hline chr2_1107991X603863179F & 2 cis & 6.2 cis & 14.22 & 4.81E-07 \\
\hline chr2_111731ıX603863179F & 2 cis & $6.2 \mathrm{cis}$ & 8.55 & $8.45 \mathrm{E}-05$ \\
\hline chr2_111764!X603863179F & 2 cis & $6.2 \mathrm{cis}$ & 9.03 & 4.50E-05 \\
\hline chr2_111744ıX603863179F & 2 cis & 6.2 cis & 7.32 & $3.64 \mathrm{E}-04$ \\
\hline chr2_111734ıX603863179F & 2 cis & $6.2 \mathrm{cis}$ & 9.77 & $3.48 \mathrm{E}-07$ \\
\hline chr2_111755।X603863179F & 2 cis & 6.2 cis & 8.89 & 4.27E-05 \\
\hline chr2_1117531X603863179F & 2 cis & 6.2 cis & 8.98 & $9.47 \mathrm{E}-05$ \\
\hline chr2_111729ıX603863179F & 2 cis & $6.2 \mathrm{cis}$ & 9.75 & $3.44 \mathrm{E}-04$ \\
\hline chr2_111741ıX603863179F & 2 cis & $6.2 \mathrm{cis}$ & 5.04 & 2.19E-04 \\
\hline chr2_112301।X603863179F & 2 cis & 6.2 cis & 5.7 & $1.89 \mathrm{E}-05$ \\
\hline chr2_1157321X603863179F & 2 cis & $6.2 \mathrm{cis}$ & 4.13 & 2.86E-04 \\
\hline chr2_111764ıNM_0010393 & 2 cis & $4.3 \mathrm{cis}$ & 9.03 & 8.05E-05 \\
\hline chr2_111735ıNM_0010393 & 2 cis & $4.3 \mathrm{cis}$ & 5.45 & $2.42 \mathrm{E}-04$ \\
\hline chr2_111734ıNM_0010393 & 2 cis & $4.3 \mathrm{cis}$ & 9.77 & $5.21 \mathrm{E}-05$ \\
\hline chr2_1117291NM_0010393 & 2 cis & $4.3 \mathrm{cis}$ & 9.75 & $1.28 \mathrm{E}-03$ \\
\hline chr2_1141921X603866246F & 2 trans & 6.4 cis & 5.68 & $5.09 \mathrm{E}-03$ \\
\hline chr2_121857IENSGALT000( & 2 cis & $5.0 \mathrm{cis}$ & 7.68 & 4.93E-05 \\
\hline chr2_122966IENSGALT000( & 2 cis & 5.0 cis & 4.15 & $1.73 \mathrm{E}-04$ \\
\hline chr2_121300IENSGALTO00( & 2 cis & 5.0 cis & 4.33 & $7.49 \mathrm{E}-05$ \\
\hline chr2_121300।NM_0010063 & 2 cis & 4.5 cis & 4.33 & $4.02 \mathrm{E}-03$ \\
\hline chr2_121718IENSGALT000( & 2 cis & $5.4 \mathrm{cis}$ & 4.4 & $6.21 \mathrm{E}-04$ \\
\hline chr2_121273IENSGALTO00( & 2 cis & 5.4 cis & 7.75 & $2.36 \mathrm{E}-04$ \\
\hline chr2_121857IENSGALT000( & 2 cis & 5.4 cis & 7.68 & $2.55 \mathrm{E}-06$ \\
\hline chr2_121422IENSGALT000( & 2 cis & 5.4 cis & 7.45 & $3.89 \mathrm{E}-04$ \\
\hline chr2_121718ıNM_0010309 & 2 cis & $6.8 \mathrm{cis}$ & 4.4 & $2.12 \mathrm{E}-03$ \\
\hline chr2_121817ıNM_0010309 & 2 cis & $6.8 \mathrm{cis}$ & 4.53 & $9.06 \mathrm{E}-03$ \\
\hline chr2_118093ıNM_0010309 & 2 cis & $6.8 \mathrm{cis}$ & 5.6 & $9.36 \mathrm{E}-03$ \\
\hline chr2_121143ıNM_0010309 & 2 cis & $6.8 \mathrm{cis}$ & 6.86 & $1.78 \mathrm{E}-03$ \\
\hline chr2_120230।NM_0010309 & 2 cis & $6.8 \mathrm{cis}$ & 5.16 & $3.76 \mathrm{E}-03$ \\
\hline chr2_117471ıNM_0010309 & 2 cis & $6.8 \mathrm{cis}$ & 4.12 & 5.93E-03 \\
\hline chr2_120242ıNM_0010309 & 2 cis & $6.8 \mathrm{cis}$ & 4.67 & $5.02 \mathrm{E}-04$ \\
\hline chr2_121857ıNM_0010309 & 2 cis & $6.8 \mathrm{cis}$ & 7.68 & $2.65 \mathrm{E}-03$ \\
\hline chr2_123188ıNM_0010309 & 2 cis & $6.8 \mathrm{cis}$ & 8.99 & $2.82 \mathrm{E}-05$ \\
\hline chr2_1223591NM_0010309 & 2 cis & $6.8 \mathrm{cis}$ & 6.28 & $1.50 \mathrm{E}-03$ \\
\hline chr2_123014ıNM_0010309 & 2 cis & $6.8 \mathrm{cis}$ & 9.43 & $5.76 \mathrm{E}-03$ \\
\hline chr2_122665INM_0010309 & 2 cis & $6.8 \mathrm{cis}$ & 4.84 & $3.51 \mathrm{E}-03$ \\
\hline chr2_121422।NM_0010309 & 2 cis & $6.8 \mathrm{cis}$ & 7.45 & $1.12 \mathrm{E}-04$ \\
\hline chr2_124292iNM_0010309 & 2 cis & $6.8 \mathrm{cis}$ & 7.16 & $3.04 \mathrm{E}-03$ \\
\hline chr2_122557INM_0010309 & 2 cis & $6.8 \mathrm{cis}$ & 7.6 & $3.65 \mathrm{E}-04$ \\
\hline chr2_123189iNM_0010309 & 2 cis & $6.8 \mathrm{cis}$ & 10.12 & $1.26 \mathrm{E}-03$ \\
\hline chr2_125164ıNM_0010309 & 2 cis & $6.8 \mathrm{cis}$ & 4.63 & $7.32 \mathrm{E}-03$ \\
\hline chr2_124309ıNM_0010309 & 2 cis & $6.8 \mathrm{cis}$ & 4.1 & $3.36 \mathrm{E}-03$ \\
\hline chr2_125164INM_205513_ & 2 cis & $4.7 \mathrm{cis}$ & 4.63 & $4.25 E-04$ \\
\hline chr2_122799INM_205513_ & 2 cis & 4.7 cis & 4.1 & $6.78 \mathrm{E}-03$ \\
\hline chr2_123136INM_0010017 & 2 cis & 4.9 cis & 4.68 & $2.21 \mathrm{E}-03$ \\
\hline chr2_125133ıNM_0010017 & 2 cis & 4.9 cis & 4.86 & 4.75E-04 \\
\hline chr2_123189IENSGALT000( & 2 cis & $4.3 \mathrm{cis}$ & 10.12 & $6.25 \mathrm{E}-04$ \\
\hline chr2_125164IENSGALT000( & 2 cis & 4.3 cis & 4.63 & 4.80E-04 \\
\hline chr2_121422।NM_204290_ & 2 cis & 5.4 cis & 7.45 & $1.08 \mathrm{E}-03$ \\
\hline chr2_125870।NM_204290_ & 2 cis & 5.4 cis & 5.9 & 1.77E-04 \\
\hline chr2_122557INM_204290_ & 2 cis & $5.4 \mathrm{cis}$ & 7.6 & $6.12 \mathrm{E}-05$ \\
\hline chr2_122686INM_204290_ & 2 cis & 5.4 cis & 4.1 & 8.39E-06 \\
\hline chr2_125536INM_204290_ & 2 cis & 5.4 cis & 11.09 & $5.31 \mathrm{E}-04$ \\
\hline chr2_122799INM_204290_ & 2 cis & 5.4 cis & 4.1 & $4.85 \mathrm{E}-03$ \\
\hline chr2_121300INM_204290_ & 2 cis & 5.4 cis & 4.33 & $3.14 \mathrm{E}-05$ \\
\hline chr2_125870IENSGALTO00( & 2 cis & $4.3 \mathrm{cis}$ & 5.9 & $4.82 \mathrm{E}-04$ \\
\hline chr2_122557IENSGALTO00( & 2 cis & $4.3 \mathrm{cis}$ & 7.6 & $1.64 \mathrm{E}-03$ \\
\hline chr2_123362IENSGALT000( & 2 cis & 4.3 cis & 5.56 & $2.36 \mathrm{E}-03$ \\
\hline chr2_123074IENSGALT000( & 2 cis & $4.3 \mathrm{cis}$ & 4.16 & 4.24E-03 \\
\hline chr2_124356IENSGALTO00( & 2 cis & 4.3 cis & 4.1 & 4.39E-03 \\
\hline chr2_125137IENSGALTO00( & 2 cis & 4.3 cis & 4.1 & $3.80 \mathrm{E}-03$ \\
\hline chr2_126042IENSGALT000( & 2 cis & $4.3 \mathrm{cis}$ & 5.68 & $2.23 \mathrm{E}-03$ \\
\hline chr2_122686IENSGALTO00( & 2 cis & $4.3 \mathrm{cis}$ & 4.1 & 7.87E-03 \\
\hline
\end{tabular}




\begin{tabular}{|c|c|c|c|c|}
\hline chr2_125164!ENSGALT000( & 2 cis & 4.3 cis & 4.63 & $1.11 \mathrm{E}-03$ \\
\hline chr2_122799IENSGALTO00( & 2 cis & $4.3 \mathrm{cis}$ & 4.1 & $2.32 \mathrm{E}-02$ \\
\hline chr2_1217181X603595861F & 2 cis & $7.7 \mathrm{cis}$ & 4.4 & 2.17E-04 \\
\hline chr2_120205ıX603595861F & 2 cis & $7.7 \mathrm{cis}$ & 8.05 & 3.06E-05 \\
\hline chr2_121422।X603595861F & 2 cis & $7.7 \mathrm{cis}$ & 7.45 & $5.96 \mathrm{E}-06$ \\
\hline chr2_121717ıX603595861F & 2 cis & $7.7 \mathrm{cis}$ & 5.41 & 7.39E-05 \\
\hline chr2_125976ıX603595861F & 2 cis & $7.7 \mathrm{cis}$ & 5.37 & $1.32 \mathrm{E}-03$ \\
\hline chr2_126042।X603595861F & 2 cis & $7.7 \mathrm{cis}$ & 5.68 & 7.07E-04 \\
\hline chr2_125536ıX603595861F & 2 cis & $7.7 \mathrm{cis}$ & 11.09 & $9.03 \mathrm{E}-04$ \\
\hline chr2_121422।ENSGALT000( & 2 cis & $4.7 \mathrm{cis}$ & 7.45 & $1.64 \mathrm{E}-03$ \\
\hline chr2_125870IENSGALT000( & 2 cis & $4.7 \mathrm{cis}$ & 5.9 & 4.17E-03 \\
\hline chr2_121718ı X603864833F & 2 cis & 7.6 cis & 4.4 & $2.24 \mathrm{E}-03$ \\
\hline chr2_120205ıX603864833F & 2 cis & $7.6 \mathrm{cis}$ & 8.05 & $1.90 \mathrm{E}-03$ \\
\hline chr2_121422।X603864833F & 2 cis & $7.6 \mathrm{cis}$ & 7.45 & 7.31E-06 \\
\hline chr2_121717ıX603864833F & 2 cis & $7.6 \mathrm{cis}$ & 5.41 & $1.19 \mathrm{E}-03$ \\
\hline chr2_125870ı603864833F & 2 cis & $7.6 \mathrm{cis}$ & 5.9 & $6.25 \mathrm{E}-03$ \\
\hline chr2_126130ıX603864833F & 2 cis & $7.6 \mathrm{cis}$ & 4.8 & $8.44 \mathrm{E}-04$ \\
\hline chr2_123188INM_204337_ & 2 cis & $9.8 \mathrm{cis}$ & 8.99 & $1.58 \mathrm{E}-03$ \\
\hline chr2_124292!NM_204337_ & 2 cis & $9.8 \mathrm{cis}$ & 7.16 & $9.08 \mathrm{E}-04$ \\
\hline chr2_122557ıNM_204337_ & 2 cis & $9.8 \mathrm{cis}$ & 7.6 & 4.64E-04 \\
\hline chr2_123189!NM_204337_ & 2 cis & $9.8 \mathrm{cis}$ & 10.12 & 7.91E-05 \\
\hline chr2_125536ıNM_204337_ & 2 cis & $9.8 \mathrm{cis}$ & 11.09 & 2.90E-04 \\
\hline chr2_122799!NM_204337_ & 2 cis & $9.8 \mathrm{cis}$ & 4.1 & $7.46 \mathrm{E}-03$ \\
\hline chr2_121300INM_204337_ & 2 cis & $9.8 \mathrm{cis}$ & 4.33 & 3.66E-04 \\
\hline chr2_125870IENSGALTO00( & 2 cis & $4.3 \mathrm{cis}$ & 5.9 & $5.58 \mathrm{E}-04$ \\
\hline chr2_121300ıX603601310F & 2 cis & $4.7 \mathrm{cis}$ & 4.33 & 4.56E-04 \\
\hline chr2_126950ıX603865723F & 2 cis & $4.2 \mathrm{cis}$ & 21.9 & $1.38 \mathrm{E}-04$ \\
\hline chr2_127655ıX603865723F & 2 cis & $4.2 \mathrm{cis}$ & 4.19 & $8.41 \mathrm{E}-03$ \\
\hline chr2_132339IENSGALTO00( & 2 cis & $6.7 \mathrm{cis}$ & 13.6 & $2.14 \mathrm{E}-05$ \\
\hline chr2_132535IENSGALTO00( & 2 cis & $6.7 \mathrm{cis}$ & 7.39 & 2.09E-04 \\
\hline chr2_132338IENSGALTO00( & 2 cis & $6.7 \mathrm{cis}$ & 9.12 & $2.71 \mathrm{E}-04$ \\
\hline chr2_132339IENSGALTO00( & 2 cis & $5.1 \mathrm{cis}$ & 13.6 & $2.76 \mathrm{E}-03$ \\
\hline chr2_131519IENSGALTO00( & 2 cis & $5.1 \mathrm{cis}$ & 8.4 & 5.53E-04 \\
\hline chr2_132339IENSGALTO00( & 2 cis & $4.4 \mathrm{cis}$ & 13.6 & $2.70 \mathrm{E}-03$ \\
\hline chr2_131519IENSGALTO00( & 2 cis & $4.4 \mathrm{cis}$ & 8.4 & $1.49 \mathrm{E}-03$ \\
\hline chr2_146668IENSGALTO00( & 2 cis & $15.3 \mathrm{cis}$ & 4.36 & 2.37E-05 \\
\hline chr2_144673।ENSGALT000( & 2 cis & $15.3 \mathrm{cis}$ & 9.34 & $1.02 \mathrm{E}-04$ \\
\hline chr2_145473।ENSGALTO00( & 2 cis & $15.3 \mathrm{cis}$ & 6.64 & $3.42 \mathrm{E}-05$ \\
\hline chr2_139393।ENSGALT000( & 2 cis & $15.3 \mathrm{cis}$ & 6.61 & $2.53 \mathrm{E}-08$ \\
\hline chr2_148160IENSGALT000( & 2 cis & $15.3 \mathrm{cis}$ & 4.5 & $5.82 \mathrm{E}-07$ \\
\hline chr2_140107IENSGALT000( & 2 cis & $15.3 \mathrm{cis}$ & 6.39 & 1.69E-05 \\
\hline chr2_140435IENSGALTO00( & 2 cis & $15.3 \mathrm{cis}$ & 8.95 & $1.31 \mathrm{E}-04$ \\
\hline chr2_140436IENSGALT000( & 2 cis & $15.3 \mathrm{cis}$ & 13.67 & $6.45 \mathrm{E}-05$ \\
\hline chr2_140506IENSGALT000( & 2 cis & $15.3 \mathrm{cis}$ & 8.2 & 3.77E-04 \\
\hline chr2_145859IENSGALT000( & 2 cis & $15.3 \mathrm{cis}$ & 14.64 & $6.95 \mathrm{E}-08$ \\
\hline chr2_140458IENSGALT000( & 2 cis & $15.3 \mathrm{cis}$ & 4.27 & 8.59E-06 \\
\hline chr2_142376IENSGALT000( & 2 cis & $15.3 \mathrm{cis}$ & 9.28 & $6.14 \mathrm{E}-05$ \\
\hline chr2_140624IENSGALT000( & 2 cis & $15.3 \mathrm{cis}$ & 17.26 & 7.11E-08 \\
\hline chr2 141217IENSGALTO00 & 2 cis & $15.3 \mathrm{cis}$ & 6.18 & $3.74 \mathrm{E}-06$ \\
\hline chr2_144694IENSGALT000( & 2 cis & $15.3 \mathrm{cis}$ & 6.56 & $1.31 \mathrm{E}-04$ \\
\hline chr2_147470IENSGALT000( & 2 cis & $15.3 \mathrm{cis}$ & 4.75 & $1.58 \mathrm{E}-04$ \\
\hline chr2_146612।ENSGALT000( & 2 cis & $15.3 \mathrm{cis}$ & 6.79 & $1.21 \mathrm{E}-05$ \\
\hline chr2_148407IENSGALT000( & 2 cis & $15.3 \mathrm{cis}$ & 9.84 & $3.34 \mathrm{E}-06$ \\
\hline chr2_144680IENSGALT000( & 2 cis & $15.3 \mathrm{cis}$ & 5.06 & 7.36E-05 \\
\hline chr2 143184IENSGALTO00 & 2 cis & $15.3 \mathrm{cis}$ & 11.49 & $6.65 \mathrm{E}-06$ \\
\hline chr2_143699IENSGALT000( & 2 cis & $15.3 \mathrm{cis}$ & 6.47 & 2.31E-04 \\
\hline chr2_146352IENSGALT000( & 2 cis & $15.3 \mathrm{cis}$ & 9.24 & $6.71 \mathrm{E}-06$ \\
\hline chr2_143178IENSGALT000( & 2 cis & $15.3 \mathrm{cis}$ & 6.91 & 2.00E-04 \\
\hline chr2_143541।X603865519F & 2 cis & $9.3 \mathrm{cis}$ & 4.14 & $3.14 \mathrm{E}-04$ \\
\hline chr2_139393ıX603865519F & 2 cis & $9.3 \mathrm{cis}$ & 6.61 & 5.19E-06 \\
\hline chr2_140107ıX603865519F & 2 cis & $9.3 \mathrm{cis}$ & 6.39 & 7.95E-04 \\
\hline chr2_140436ıX603865519F & 2 cis & $9.3 \mathrm{cis}$ & 13.67 & 2.93E-04 \\
\hline chr2_145859!X603865519F & 2 cis & $9.3 \mathrm{cis}$ & 14.64 & $1.74 \mathrm{E}-05$ \\
\hline chr2_139695ıX603865519F & 2 cis & $9.3 \mathrm{cis}$ & 6 & 1.17E-04 \\
\hline chr2_142376ıX603865519F & 2 cis & $9.3 \mathrm{cis}$ & 9.28 & $6.94 \mathrm{E}-05$ \\
\hline chr2_140624ıX603865519F & 2 cis & $9.3 \mathrm{cis}$ & 17.26 & $6.39 \mathrm{E}-06$ \\
\hline chr2_141217ıX603865519F & 2 cis & $9.3 \mathrm{cis}$ & 6.18 & 4.33E-05 \\
\hline chr2_147681।X603865519F & 2 cis & $9.3 \mathrm{cis}$ & 5.48 & 3.69E-04 \\
\hline chr2_147470ıx603865519F & 2 cis & $9.3 \mathrm{cis}$ & 4.75 & $1.68 \mathrm{E}-04$ \\
\hline chr2_146612।X603865519F & 2 cis & $9.3 \mathrm{cis}$ & 6.79 & 5.89E-05 \\
\hline chr2_143184ıX603865519F & 2 cis & $9.3 \mathrm{cis}$ & 11.49 & $5.08 \mathrm{E}-05$ \\
\hline chr2_143699।X603865519F & 2 cis & $9.3 \mathrm{cis}$ & 6.47 & $2.25 \mathrm{E}-04$ \\
\hline
\end{tabular}




\begin{tabular}{|c|c|c|c|c|}
\hline chr2_143978ıX603865519F & 2 cis & 9.3 cis & 5.89 & 4.50E-05 \\
\hline chr2_142987।X603865519F & 2 cis & $9.3 \mathrm{cis}$ & 5.93 & $1.47 \mathrm{E}-04$ \\
\hline chr2_146352।X603865519F & 2 cis & 9.3 cis & 9.24 & $1.45 \mathrm{E}-04$ \\
\hline chr2_1435751X603865519F & 2 cis & $9.3 \mathrm{cis}$ & 5.55 & $6.48 \mathrm{E}-05$ \\
\hline chr2_145711'X603144230F & 2 cis & 8.5 cis & 4.53 & 2.90E-04 \\
\hline chr2_138671।X603144230F & 2 cis & 8.5 cis & 7.7 & $1.14 \mathrm{E}-04$ \\
\hline chr2_139393ıX603144230F & 2 cis & 8.5 cis & 6.61 & $1.23 \mathrm{E}-04$ \\
\hline chr2_1481881X603144230F & 2 cis & $8.5 \mathrm{cis}$ & 7.01 & $8.22 \mathrm{E}-04$ \\
\hline chr2_1458581X603144230F & 2 cis & 8.5 cis & 5.3 & 4.01E-04 \\
\hline chr2_139237।X603144230F & 2 cis & $8.5 \mathrm{cis}$ & 8.05 & $3.02 \mathrm{E}-05$ \\
\hline chr2_1405061X603144230F & 2 cis & $8.5 \mathrm{cis}$ & 8.2 & $9.62 \mathrm{E}-05$ \\
\hline chr2_145859।X603144230F & 2 cis & $8.5 \mathrm{cis}$ & 14.64 & 8.06E-06 \\
\hline chr2_148408ıX603144230F & 2 cis & $8.5 \mathrm{cis}$ & 6.32 & $4.84 \mathrm{E}-03$ \\
\hline chr2_1423761X603144230F & 2 cis & $8.5 \mathrm{cis}$ & 9.28 & $2.84 \mathrm{E}-04$ \\
\hline chr2_141217।X603144230F & 2 cis & $8.5 \mathrm{cis}$ & 6.18 & $6.86 \mathrm{E}-04$ \\
\hline chr2_147470)X603144230F & 2 cis & $8.5 \mathrm{cis}$ & 4.75 & $1.06 \mathrm{E}-03$ \\
\hline chr2_148407।X603144230F & 2 cis & $8.5 \mathrm{cis}$ & 9.84 & $7.68 \mathrm{E}-04$ \\
\hline chr2_138750!X603144230F & 2 cis & $8.5 \mathrm{cis}$ & 5.7 & $6.83 \mathrm{E}-04$ \\
\hline chr20_14550।X603598083F & 20 cis & $4.1 \mathrm{cis}$ & 7.03 & $7.48 \mathrm{E}-03$ \\
\hline chr20_28260।X603598083F & 20 cis & $4.1 \mathrm{cis}$ & 5.02 & $1.09 \mathrm{E}-03$ \\
\hline chr20_14550।X603597894F & 20 cis & $4.3 \mathrm{cis}$ & 7.03 & $1.43 \mathrm{E}-04$ \\
\hline chr20_14570)X603597894F & 20 cis & $4.3 \mathrm{cis}$ & 5.72 & $5.72 \mathrm{E}-03$ \\
\hline chr20_14480(X603597894F & 20 cis & $4.3 \mathrm{cis}$ & 4.85 & $5.61 \mathrm{E}-03$ \\
\hline chr20_14560।X603597894F & 20 cis & $4.3 \mathrm{cis}$ & 4.6 & $1.29 \mathrm{E}-03$ \\
\hline chr20_28260।X603597894F & 20 cis & $4.3 \mathrm{cis}$ & 5.02 & $1.29 \mathrm{E}-04$ \\
\hline chr20_29840INM_205318_ & 20 cis & 4.0 cis & 4.1 & 4.89E-04 \\
\hline chr20_384401X603600905F & 20 cis & $4.7 \mathrm{cis}$ & 6.01 & $9.06 \mathrm{E}-04$ \\
\hline chr20_58630।X603602359F & 20 cis & $4.7 \mathrm{cis}$ & 4.95 & 7.33E-04 \\
\hline chr20_58330!X603602359F & 20 cis & $4.7 \mathrm{cis}$ & 6.56 & $1.53 \mathrm{E}-04$ \\
\hline chr20_58320।X603602359F & 20 cis & $4.7 \mathrm{cis}$ & 7 & $2.47 \mathrm{E}-04$ \\
\hline chr20_76910।X603595816F & 20 cis & 5.0 cis & 5.87 & $1.11 \mathrm{E}-03$ \\
\hline chr20_61560)X603595816F & 20 cis & $5.0 \mathrm{cis}$ & 6.76 & $6.63 \mathrm{E}-04$ \\
\hline chr20_73830।X603595816F & 20 cis & 5.0 cis & 11.33 & $1.30 \mathrm{E}-03$ \\
\hline chr20_12060।X603865610F & 20 cis & $4.3 \mathrm{cis}$ & 4.7 & $7.24 \mathrm{E}-04$ \\
\hline chr20_12057।X603865610F & 20 cis & $4.3 \mathrm{cis}$ & 6.17 & $9.50 \mathrm{E}-04$ \\
\hline chr20_13141।X603862244F & 20 cis & $5.8 \mathrm{cis}$ & 6.08 & $1.15 \mathrm{E}-03$ \\
\hline chr21_95500।X603866715F & 21 cis & 4.3 cis & 4.36 & $3.89 \mathrm{E}-02$ \\
\hline chr21_95500ıX603867077F & 21 cis & $8.6 \mathrm{cis}$ & 4.36 & $1.52 \mathrm{E}-02$ \\
\hline chr21_47430!X603864620F & 21 cis & $6.3 \mathrm{cis}$ & 8.41 & $5.81 \mathrm{E}-04$ \\
\hline chr28_254301X603599785F & $28 \mathrm{cis}$ & 13.2 trans & 7.28 & $1.40 \mathrm{E}-05$ \\
\hline chr3_403720IENSGALTO00( & 3 cis & 5.3 trans & 7.63 & $2.41 \mathrm{E}-03$ \\
\hline chr3_393850IENSGALTO00( & 3 cis & 4.7 trans & 7.28 & $5.98 \mathrm{E}-03$ \\
\hline chr3_481550IENSGALTO00( & 3 cis & 4.2 trans & 7.64 & 4.34E-04 \\
\hline chr3_634930IENSGALTO00 & 3 cis & 3.9 trans & 7.28 & $1.44 \mathrm{E}-03$ \\
\hline chr3_618730IENSGALTO00( & 3 cis & 3.9 trans & 6.35 & $1.26 \mathrm{E}-03$ \\
\hline chr3_569860IENSGALTO00( & 3 cis & 5.3 trans & 6.05 & $1.53 \mathrm{E}-04$ \\
\hline chr3_106007IENSGALTO00 & 3 cis & 4.0 trans & 8.36 & $9.77 \mathrm{E}-04$ \\
\hline chr4_270300IENSGALTO00( & 4 cis & 4.3 trans & 6.6 & $2.31 \mathrm{E}-03$ \\
\hline chr4_136300ıX603863273F & 4 cis & 9.3 trans & 9.54 & 7.64E-05 \\
\hline chr4_164800।X603863273F & 4 cis & 9.3 trans & 8.11 & $4.60 \mathrm{E}-04$ \\
\hline chr4_129200ı603596676F & 4 cis & 10.2 trans & 7.86 & 8.79E-04 \\
\hline chr4_202030INM_0010125 & 4 cis & 4.8 trans & 7.88 & 4.29E-03 \\
\hline chr4_380270IENSGALTO00( & 4 cis & 6.4 trans & 8.5 & $3.12 \mathrm{E}-04$ \\
\hline chr4_487720IENSGALTOOO( & 4 cis & 6.4 trans & 7.28 & $1.27 \mathrm{E}-03$ \\
\hline chr4_581460IENSGALTO00( & 4 cis & 4.4 trans & 6.38 & $1.65 \mathrm{E}-03$ \\
\hline chr4_666250INM_0010044 & 4 cis & $4.0 \mathrm{cis}$ & 4.65 & 4.10E-03 \\
\hline chr4_659950IENSGALT000( & 4 cis & 7.6 cis & 4.1 & $2.70 \mathrm{E}-03$ \\
\hline chr4_666250IENSGALTO00( & 4 cis & $7.6 \mathrm{cis}$ & 4.65 & $1.34 \mathrm{E}-03$ \\
\hline chr4_6766301X603866410F & 4 cis & 5.4 cis & 6.35 & $1.04 \mathrm{E}-03$ \\
\hline chr4_666250ıX603866410F & 4 cis & $5.4 \mathrm{cis}$ & 4.65 & $6.09 \mathrm{E}-03$ \\
\hline chr4_6831601X603866410F & 4 cis & 5.4 cis & 6.42 & 3.00E-04 \\
\hline chr4_665660ıX603596285F & 4 cis & $5.3 \mathrm{cis}$ & 4.78 & $1.46 \mathrm{E}-02$ \\
\hline chr4_665820ıX603596285F & 4 cis & $5.3 \mathrm{cis}$ & 7.16 & 6.17E-04 \\
\hline chr4_689170ıX603596285F & 4 cis & $5.3 \mathrm{cis}$ & 4.83 & $2.56 \mathrm{E}-03$ \\
\hline chr4_680600।X603596285F & 4 cis & $5.3 \mathrm{cis}$ & 4.1 & 8.07E-04 \\
\hline chr4_665820IENSGALTO00( & 4 cis & $6.9 \mathrm{cis}$ & 7.16 & $1.71 \mathrm{E}-05$ \\
\hline chr4_685840IENSGALTO00( & 4 cis & 6.9 cis & 6.69 & $1.60 \mathrm{E}-04$ \\
\hline chr4_669740IENSGALTO00( & 4 cis & $6.9 \mathrm{cis}$ & 6.47 & $7.22 \mathrm{E}-04$ \\
\hline chr4_681060IENSGALTO00( & 4 cis & $6.9 \mathrm{cis}$ & 8.46 & $2.06 \mathrm{E}-04$ \\
\hline chr4_676630IENSGALTO00( & 4 cis & $6.9 \mathrm{cis}$ & 6.35 & $9.32 \mathrm{E}-05$ \\
\hline chr4_686410IENSGALTO00( & 4 cis & $6.9 \mathrm{cis}$ & 5.11 & $1.98 \mathrm{E}-03$ \\
\hline chr4_689170IENSGALT000( & $4 \mathrm{cis}$ & $6.9 \mathrm{cis}$ & 4.83 & 4.39E-04 \\
\hline
\end{tabular}




\begin{tabular}{|c|c|c|c|c|}
\hline chr4_685840IENSGALTO00( & 4 cis & $4.3 \mathrm{cis}$ & 6.69 & $1.36 \mathrm{E}-04$ \\
\hline chr4_676630IENSGALTO00( & 4 cis & $4.3 \mathrm{cis}$ & 6.35 & 4.70E-06 \\
\hline chr4_683160IENSGALT000( & 4 cis & $4.3 \mathrm{cis}$ & 6.42 & $9.43 \mathrm{E}-04$ \\
\hline chr4_6858401X603868314F & 4 cis & $5.7 \mathrm{cis}$ & 6.69 & 1.57E-03 \\
\hline chr4_6810601X603868314F & 4 cis & $5.7 \mathrm{cis}$ & 8.46 & $1.83 \mathrm{E}-04$ \\
\hline chr4_6766301X603868314F & 4 cis & $5.7 \mathrm{cis}$ & 6.35 & 2.57E-03 \\
\hline chr4_6864101X603868314F & 4 cis & 5.7 cis & 5.11 & $2.15 \mathrm{E}-03$ \\
\hline chr4_6917601X603868314F & 4 cis & $5.7 \mathrm{cis}$ & 6.98 & $1.89 \mathrm{E}-03$ \\
\hline chr4_6891701X603868314F & 4 cis & $5.7 \mathrm{cis}$ & 4.83 & 2.23E-04 \\
\hline chr4_6831601X603868314F & 4 cis & $5.7 \mathrm{cis}$ & 6.42 & 7.48E-05 \\
\hline chr4_7046901X603864570F & 4 cis & $5.8 \mathrm{cis}$ & 5.61 & 3.15E-04 \\
\hline chr4_7282201X603864570F & 4 cis & $5.8 \mathrm{cis}$ & 7.41 & $3.42 \mathrm{E}-04$ \\
\hline chr4_7316201X603864570F & 4 cis & $5.8 \mathrm{cis}$ & 5.8 & $1.73 \mathrm{E}-04$ \\
\hline chr4_7126201X603602358F & 4 cis & $11.5 \mathrm{cis}$ & 4.52 & $2.46 \mathrm{E}-03$ \\
\hline chr4_7064701X603602358F & 4 cis & 11.5 cis & 4.96 & $2.56 \mathrm{E}-03$ \\
\hline chr4_7371801X603602358F & 4 cis & $11.5 \mathrm{cis}$ & 4.1 & 1.09E-04 \\
\hline chr4_7374501X603602358F & 4 cis & $11.5 \mathrm{cis}$ & 4.78 & 4.57E-04 \\
\hline chr4_6970401X603602358F & 4 cis & $11.5 \mathrm{cis}$ & 4.4 & 3.63E-03 \\
\hline chr4_7323701X603602358F & 4 cis & $11.5 \mathrm{cis}$ & 8.75 & $1.42 \mathrm{E}-04$ \\
\hline chr4_7341101X603602358F & 4 cis & $11.5 \mathrm{cis}$ & 4.16 & $9.13 \mathrm{E}-06$ \\
\hline chr4_7312101X603602358F & 4 cis & 11.5 cis & 6.04 & 2.63E-04 \\
\hline chr4_7348701X603602358F & 4 cis & $11.5 \mathrm{cis}$ & 6.99 & 1.57E-06 \\
\hline chr4_7188301X603602358F & 4 cis & $11.5 \mathrm{cis}$ & 4.74 & 2.16E-05 \\
\hline chr4_7311201X603602358F & 4 cis & $11.5 \mathrm{cis}$ & 4.99 & $5.71 E-03$ \\
\hline chr4_7369301X603602358F & 4 cis & $11.5 \mathrm{cis}$ & 15.73 & $1.23 \mathrm{E}-07$ \\
\hline chr4_7369201X603602358F & 4 cis & $11.5 \mathrm{cis}$ & 16.4 & $9.85 E-09$ \\
\hline chr4_7126501X603602358F & 4 cis & 11.5 cis & 5.83 & $1.55 \mathrm{E}-06$ \\
\hline chr4_7141201X603602358F & 4 cis & $11.5 \mathrm{cis}$ & 6.42 & $2.05 E-04$ \\
\hline chr4_7127401X603599624F & 4 cis & 5.4 cis & 5.14 & $1.04 \mathrm{E}-03$ \\
\hline chr4_7282201X603599624F & 4 cis & $5.4 \mathrm{cis}$ & 7.41 & $1.79 \mathrm{E}-03$ \\
\hline chr4_7107201X603599624F & 4 cis & $5.4 \mathrm{cis}$ & 6.2 & 4.23E-04 \\
\hline chr4_7478601X603599624F & 4 cis & $5.4 \mathrm{cis}$ & 5.02 & 7.11E-04 \\
\hline chr4_7282201X603599417F & 4 cis & $6.7 \mathrm{cis}$ & 7.41 & 1.47E-03 \\
\hline chr4_7311201X603599417F & 4 cis & $6.7 \mathrm{cis}$ & 4.99 & $5.83 \mathrm{E}-03$ \\
\hline chr4_7369301X603599417F & 4 cis & $6.7 \mathrm{cis}$ & 15.73 & 3.60E-04 \\
\hline chr4_7369201X603599417F & 4 cis & $6.7 \mathrm{cis}$ & 16.4 & $8.89 \mathrm{E}-05$ \\
\hline chr4_7316201X603599417F & 4 cis & $6.7 \mathrm{cis}$ & 5.8 & $1.29 \mathrm{E}-03$ \\
\hline chr4_7126501X603599417F & 4 cis & $6.7 \mathrm{cis}$ & 5.83 & $1.52 \mathrm{E}-03$ \\
\hline chr4_7420401X603599417F & 4 cis & $6.7 \mathrm{cis}$ & 6.68 & 4.14E-04 \\
\hline chr4_7462701X603599417F & 4 cis & $6.7 \mathrm{cis}$ & 4.1 & 5.11E-04 \\
\hline chr4_7515001X603599417F & 4 cis & $6.7 \mathrm{cis}$ & 5.33 & $3.29 E-04$ \\
\hline chr4_7840901X603599417F & 4 cis & $6.7 \mathrm{cis}$ & 4.75 & 4.02E-05 \\
\hline chr4_7347201X603599417F & 4 cis & $6.7 \mathrm{cis}$ & 5.34 & $2.22 \mathrm{E}-03$ \\
\hline chr4_7484201X603599417F & 4 cis & $6.7 \mathrm{cis}$ & 9.45 & $2.06 \mathrm{E}-03$ \\
\hline chr4_7584701X603599417F & 4 cis & $6.7 \mathrm{cis}$ & 4.81 & 1.15E-03 \\
\hline chr4_7886301X603599417F & 4 cis & $6.7 \mathrm{cis}$ & 7.35 & $1.41 \mathrm{E}-04$ \\
\hline chr4_7883601X603599417F & 4 cis & $6.7 \mathrm{cis}$ & 8.71 & $9.22 \mathrm{E}-04$ \\
\hline chr4_7420401X603598729F & 4 cis & $16.6 \mathrm{cis}$ & 6.68 & $3.48 \mathrm{E}-05$ \\
\hline chr4_7462701X603598729F & 4 cis & $16.6 \mathrm{cis}$ & 4.1 & 4.81E-06 \\
\hline chr4_7515001X603598729F & 4 cis & $16.6 \mathrm{cis}$ & 5.33 & $2.21 \mathrm{E}-05$ \\
\hline chr4_7864201X603598729F & 4 cis & $16.6 \mathrm{cis}$ & 4.93 & 4.79E-05 \\
\hline chr4_7386401X603598729F & 4 cis & $16.6 \mathrm{cis}$ & 5.95 & $7.42 \mathrm{E}-05$ \\
\hline chr4_7483001X603598729F & 4 cis & $16.6 \mathrm{cis}$ & 7.67 & 2.81E-08 \\
\hline chr4_7840901X603598729F & 4 cis & $16.6 \mathrm{cis}$ & 4.75 & $3.18 \mathrm{E}-07$ \\
\hline chr4_7471701X603598729F & 4 cis & $16.6 \mathrm{cis}$ & 6.13 & 3.49E-07 \\
\hline chr4_7589801X603598729F & 4 cis & $16.6 \mathrm{cis}$ & 6.55 & $1.44 \mathrm{E}-04$ \\
\hline chr4_7557601X603598729F & 4 cis & $16.6 \mathrm{cis}$ & 17.03 & 7.06E-08 \\
\hline chr4_7484101X603598729F & 4 cis & $16.6 \mathrm{cis}$ & 11.82 & 2.54E-07 \\
\hline chr4_7484201X603598729F & 4 cis & $16.6 \mathrm{cis}$ & 9.45 & 3.07E-08 \\
\hline chr4_7589901X603598729F & 4 cis & $16.6 \mathrm{cis}$ & 7.52 & $9.95 \mathrm{E}-05$ \\
\hline chr4_7478601X603598729F & 4 cis & $16.6 \mathrm{cis}$ & 5.02 & $9.96 \mathrm{E}-07$ \\
\hline chr4_7584701X603598729F & 4 cis & $16.6 \mathrm{cis}$ & 4.81 & $1.93 \mathrm{E}-06$ \\
\hline chr4_7597001X603598729F & 4 cis & $16.6 \mathrm{cis}$ & 5 & 5.97E-06 \\
\hline chr4_7655601X603598729F & 4 cis & $16.6 \mathrm{cis}$ & 7.6 & $2.60 \mathrm{E}-06$ \\
\hline chr4_7444801X603598729F & 4 cis & $16.6 \mathrm{cis}$ & 6 & $8.78 \mathrm{E}-07$ \\
\hline chr4_7886301X603598729F & 4 cis & $16.6 \mathrm{cis}$ & 7.35 & 3.03E-05 \\
\hline chr4_7883601X603598729F & 4 cis & $16.6 \mathrm{cis}$ & 8.71 & 1.07E-06 \\
\hline chr4_7127401X603863112F & 4 cis & 7.3 cis & 5.14 & 1.97E-03 \\
\hline chr4_7117801X603863112F & 4 cis & 7.3 cis & 4.36 & $3.08 \mathrm{E}-04$ \\
\hline chr4_7282201X603863112F & 4 cis & 7.3 cis & 7.41 & $1.45 \mathrm{E}-04$ \\
\hline chr4_7107201X603863112F & 4 cis & $7.3 \mathrm{cis}$ & 6.2 & $3.75 \mathrm{E}-04$ \\
\hline chr4_7369201X603863112F & 4 cis & $7.3 \mathrm{cis}$ & 16.4 & $2.25 \mathrm{E}-05$ \\
\hline
\end{tabular}




\begin{tabular}{|c|c|c|c|c|}
\hline chr4_7141201X603863112F & 4 cis & $7.3 \mathrm{cis}$ & 6.42 & $2.10 \mathrm{E}-03$ \\
\hline chr4_7831901X603863112F & 4 cis & 7.3 cis & 4.1 & $2.57 \mathrm{E}-03$ \\
\hline chr4_7589901X603863112F & 4 cis & 7.3 cis & 7.52 & 3.74E-05 \\
\hline chr4_7678401X603863112F & 4 cis & 7.3 cis & 12.86 & 3.93E-05 \\
\hline chr4_7420401X603863258F & 4 cis & 24.1 cis & 6.68 & $4.36 \mathrm{E}-08$ \\
\hline chr4_7515001X603863258F & 4 cis & 24.1 cis & 5.33 & $1.55 \mathrm{E}-08$ \\
\hline chr4_7864201X603863258F & 4 cis & 24.1 cis & 4.93 & $1.93 \mathrm{E}-08$ \\
\hline chr4_7386401X603863258F & 4 cis & 24.1 cis & 5.95 & $2.16 \mathrm{E}-07$ \\
\hline chr4_7483001X603863258F & 4 cis & 24.1 cis & 7.67 & $1.02 \mathrm{E}-07$ \\
\hline chr4_7840901X603863258F & 4 cis & 24.1 cis & 4.75 & $2.02 \mathrm{E}-08$ \\
\hline chr4_7471701X603863258F & 4 cis & 24.1 cis & 6.13 & $1.74 \mathrm{E}-10$ \\
\hline chr4_7589801X603863258F & 4 cis & 24.1 cis & 6.55 & $2.28 \mathrm{E}-08$ \\
\hline chr4_7524101X603863258F & 4 cis & 24.1 cis & 6.12 & $1.31 \mathrm{E}-05$ \\
\hline chr4_7557601X603863258F & 4 cis & 24.1 cis & 17.03 & 8.73E-13 \\
\hline chr4_7484101X603863258F & 4 cis & 24.1 cis & 11.82 & $1.22 \mathrm{E}-09$ \\
\hline chr4_7484201X603863258F & 4 cis & 24.1 cis & 9.45 & $7.81 \mathrm{E}-12$ \\
\hline chr4_7589901X603863258F & 4 cis & 24.1 cis & 7.52 & $2.06 \mathrm{E}-08$ \\
\hline chr4_7478601X603863258F & 4 cis & 24.1 cis & 5.02 & $3.65 \mathrm{E}-08$ \\
\hline chr4_7584701X603863258F & 4 cis & 24.1 cis & 4.81 & $8.15 \mathrm{E}-10$ \\
\hline chr4_7589301X603863258F & 4 cis & 24.1 cis & 5.1 & $3.49 \mathrm{E}-06$ \\
\hline chr4_7597001X603863258F & 4 cis & 24.1 cis & 5 & $6.32 \mathrm{E}-09$ \\
\hline chr4_7655601X603863258F & 4 cis & 24.1 cis & 7.6 & $6.63 \mathrm{E}-08$ \\
\hline chr4_7678401X603863258F & 4 cis & 24.1 cis & 12.86 & $1.41 \mathrm{E}-09$ \\
\hline chr4_7444801X603863258F & 4 cis & 24.1 cis & 6 & 7.79E-09 \\
\hline chr4_7886301X603863258F & 4 cis & 24.1 cis & 7.35 & $1.81 \mathrm{E}-07$ \\
\hline chr4_7883601X603863258F & 4 cis & 24.1 cis & 8.71 & $6.83 \mathrm{E}-10$ \\
\hline chr4_7908201X603863258F & 4 cis & 24.1 cis & 5.12 & $9.09 \mathrm{E}-07$ \\
\hline chr4_7515001X603863527F & 4 cis & 5.8 cis & 5.33 & $2.71 E-03$ \\
\hline chr4_7864201X603863527F & 4 cis & $5.8 \mathrm{cis}$ & 4.93 & $4.86 \mathrm{E}-03$ \\
\hline chr4_7840901X603863527F & 4 cis & $5.8 \mathrm{cis}$ & 4.75 & 4.60E-04 \\
\hline chr4_7484201X603863527F & 4 cis & $5.8 \mathrm{cis}$ & 9.45 & $8.44 \mathrm{E}-04$ \\
\hline chr4_7584701X603863527F & 4 cis & 5.8 cis & 4.81 & 2.73E-03 \\
\hline chr4_7939101X603863527F & 4 cis & $5.8 \mathrm{cis}$ & 18.58 & $1.18 \mathrm{E}-03$ \\
\hline chr4_731210IENSGALTO00( & 4 cis & 4.7 cis & 6.04 & $3.91 \mathrm{E}-03$ \\
\hline chr4_731120IENSGALTO00( & 4 cis & 4.7 cis & 4.99 & $2.71 \mathrm{E}-03$ \\
\hline chr4_736920IENSGALTO00( & 4 cis & 4.7 cis & 16.4 & $5.55 \mathrm{E}-04$ \\
\hline chr4_714120IENSGALT000( & 4 cis & $4.7 \mathrm{cis}$ & 6.42 & $3.90 \mathrm{E}-04$ \\
\hline chr4_748420IENSGALTO00( & 4 cis & 4.7 cis & 9.45 & 5.54E-05 \\
\hline chr4_758990IENSGALTO00( & 4 cis & 4.7 cis & 7.52 & $1.95 \mathrm{E}-03$ \\
\hline chr4_744480IENSGALTO00( & 4 cis & 4.7 cis & 6 & $1.23 \mathrm{E}-04$ \\
\hline chr4_788360IENSGALTO00( & 4 cis & 4.7 cis & 8.71 & $6.64 \mathrm{E}-06$ \\
\hline chr4_793910IENSGALTO00( & 4 cis & 4.7 cis & 18.58 & $1.11 \mathrm{E}-04$ \\
\hline chr4_796860IENSGALT000( & 4 cis & $4.7 \mathrm{cis}$ & 5.83 & $2.62 \mathrm{E}-03$ \\
\hline chr4_734870IENSGALTO00( & 4 cis & 5.7 cis & 6.99 & $1.07 \mathrm{E}-03$ \\
\hline chr4_736920IENSGALT000( & 4 cis & $5.7 \mathrm{cis}$ & 16.4 & 2.69E-05 \\
\hline chr4_738640IENSGALTO00( & 4 cis & 5.7 cis & 5.95 & $3.24 \mathrm{E}-03$ \\
\hline chr4_748410IENSGALT000( & 4 cis & 5.7 cis & 11.82 & $2.55 \mathrm{E}-04$ \\
\hline chr4_748420IENSGALTO00( & 4 cis & 5.7 cis & 9.45 & $9.71 E-04$ \\
\hline chr4_765560IENSGALT000( & 4 cis & $5.7 \mathrm{cis}$ & 7.6 & $6.64 \mathrm{E}-04$ \\
\hline chr4_788360IENSGALTO00( & 4 cis & 5.7 cis & 8.71 & $1.33 \mathrm{E}-03$ \\
\hline chr4_793910IENSGALT000( & 4 cis & 5.7 cis & 18.58 & $1.14 \mathrm{E}-03$ \\
\hline chr4_738640IENSGALTO00( & 4 cis & $11.1 \mathrm{cis}$ & 5.95 & 4.96E-04 \\
\hline chr4_784090IENSGALTO00( & 4 cis & $11.1 \mathrm{cis}$ & 4.75 & $1.39 \mathrm{E}-04$ \\
\hline chr4_758980IENSGALTO00( & 4 cis & $11.1 \mathrm{cis}$ & 6.55 & 4.43E-05 \\
\hline chr4_755760IENSGALT000( & 4 cis & $11.1 \mathrm{cis}$ & 17.03 & 8.33E-06 \\
\hline chr4_748410IENSGALTO00( & 4 cis & $11.1 \mathrm{cis}$ & 11.82 & $6.68 \mathrm{E}-06$ \\
\hline chr4_748420IENSGALT000( & 4 cis & $11.1 \mathrm{cis}$ & 9.45 & $4.88 \mathrm{E}-07$ \\
\hline chr4_758990IENSGALTO00( & 4 cis & $11.1 \mathrm{cis}$ & 7.52 & $7.78 \mathrm{E}-04$ \\
\hline chr4_765560IENSGALTO00( & 4 cis & $11.1 \mathrm{cis}$ & 7.6 & $1.35 \mathrm{E}-05$ \\
\hline chr4_767840IENSGALTO00( & 4 cis & $11.1 \mathrm{cis}$ & 12.86 & 4.22E-04 \\
\hline chr4_744480IENSGALT000( & 4 cis & $11.1 \mathrm{cis}$ & 6 & $1.23 \mathrm{E}-04$ \\
\hline chr4_788630IENSGALTO00( & 4 cis & $11.1 \mathrm{cis}$ & 7.35 & $1.42 \mathrm{E}-04$ \\
\hline chr4_788360IENSGALT000( & 4 cis & $11.1 \mathrm{cis}$ & 8.71 & $3.20 \mathrm{E}-05$ \\
\hline chr4_793910IENSGALTO00( & 4 cis & $11.1 \mathrm{cis}$ & 18.58 & 3.70E-08 \\
\hline chr4_788360IENSGALTO00( & 4 cis & $4.2 \mathrm{cis}$ & 8.71 & 5.65E-04 \\
\hline chr4_836220INM_0010044 & 4 cis & 5.2 cis & 4.26 & $1.14 \mathrm{E}-03$ \\
\hline chr5_118420IENSGALTO00( & 5 cis & 4.7 cis & 9.95 & $3.24 \mathrm{E}-04$ \\
\hline chr5_118700IENSGALTO00( & 5 cis & 4.7 cis & 4.36 & 7.46E-08 \\
\hline chr5_134810IENSGALTO00( & 5 cis & 4.7 cis & 10.07 & $1.74 \mathrm{E}-04$ \\
\hline chr5_138110IENSGALTO00( & 5 cis & 4.7 cis & 5.93 & $1.82 \mathrm{E}-03$ \\
\hline chr5_138920IENSGALTO00( & 5 cis & $4.7 \mathrm{cis}$ & 8.2 & $2.80 \mathrm{E}-03$ \\
\hline chr5_794900ıX03596815F & 5 cis & 4.9 trans & 5.61 & 3.86E-04 \\
\hline
\end{tabular}




\begin{tabular}{|c|c|c|c|c|}
\hline chr5_129070।X603596815F & 5 cis & 4.9 cis & 4.32 & $9.28 \mathrm{E}-05$ \\
\hline chr5_122460ıX603596815F & 5 cis & 4.9 cis & 4.24 & $1.26 \mathrm{E}-04$ \\
\hline chr5_248560IENSGALT000( & 5 cis & $7.3 \mathrm{cis}$ & 7.42 & 6.17E-06 \\
\hline chr5_255610IENSGALTO00( & 5 cis & 7.3 cis & 5.6 & 1.07E-04 \\
\hline chr5_248500IENSGALT000( & 5 cis & $7.3 \mathrm{cis}$ & 4.53 & 1.14E-05 \\
\hline chr5_248570IENSGALTO00( & 5 cis & 7.3 trans & 8.13 & $3.35 \mathrm{E}-05$ \\
\hline chr5_244500ıNM_0010044 & 5 cis & 5.4 cis & 6.86 & $6.90 \mathrm{E}-05$ \\
\hline chr5_244490ıNM_0010044 & 5 cis & 5.4 cis & 11.51 & $3.88 \mathrm{E}-06$ \\
\hline chr5_248560ıNM_0010044 & 5 cis & 5.4 cis & 7.42 & $1.71 \mathrm{E}-04$ \\
\hline chr5_255610ıNM_0010044 & 5 cis & 5.4 cis & 5.6 & $2.29 \mathrm{E}-06$ \\
\hline chr5_283520ıNM_0010044 & 5 cis & $5.4 \mathrm{cis}$ & 5.85 & 4.37E-06 \\
\hline chr5_285550INM_0010044 & 5 cis & 5.4 trans & 8.21 & 4.97E-05 \\
\hline chr5_255610IENSGALT000( & 5 cis & $8.3 \mathrm{cis}$ & 5.6 & $4.24 \mathrm{E}-04$ \\
\hline chr5_285550IENSGALT000( & 5 cis & 8.3 trans & 8.21 & $5.20 \mathrm{E}-06$ \\
\hline chr5_306540ıX603601654F & 5 cis & 13.5 cis & 5.53 & $1.52 \mathrm{E}-04$ \\
\hline chr5_308300ıX603601654F & 5 cis & 13.5 cis & 4.31 & 6.57E-04 \\
\hline chr5_302650।X603601654F & 5 cis & 13.5 cis & 7.7 & 1.87E-04 \\
\hline chr5_308310ıX603601654F & 5 cis & 13.5 cis & 5.72 & 4.47E-05 \\
\hline chr5_295260ıX603601654F & 5 cis & 13.5 cis & 4.53 & $1.02 \mathrm{E}-04$ \\
\hline chr5_343050ıX603601654F & 5 cis & 13.5 cis & 5.49 & $2.86 \mathrm{E}-05$ \\
\hline chr5_343650।X603601654F & 5 cis & 13.5 cis & 6.23 & $1.65 \mathrm{E}-07$ \\
\hline chr5_343060ıX603601654F & 5 cis & 13.5 cis & 5.32 & $2.85 \mathrm{E}-04$ \\
\hline chr5_348780ıX603601654F & 5 cis & 13.5 cis & 6.44 & $1.65 \mathrm{E}-03$ \\
\hline chr5_331160ıX603601654F & 5 cis & 13.5 cis & 4.59 & 7.00E-04 \\
\hline chr5_287140ıX603865840F & 5 cis & 6.7 cis & 4.84 & $6.87 \mathrm{E}-03$ \\
\hline chr5_358470ıX603865840F & 5 cis & 6.7 cis & 5.25 & 3.97E-04 \\
\hline chr5_295640ıX603596306F & 5 cis & 5.6 cis & 4.95 & $2.21 \mathrm{E}-03$ \\
\hline chr5_368460ıX603144380F & 5 cis & 4.0 trans & 6.66 & $1.29 \mathrm{E}-03$ \\
\hline chr5_343650।X603868077F & 5 cis & $4.5 \mathrm{cis}$ & 6.23 & 1.57E-04 \\
\hline chr5_343650ıX603143975F & 5 cis & 15.3 cis & 6.23 & $7.42 \mathrm{E}-06$ \\
\hline chr5_343060ı603143975F & 5 cis & $15.3 \mathrm{cis}$ & 5.32 & 3.77E-06 \\
\hline chr5_348780ıX603143975F & 5 cis & 15.3 cis & 6.44 & $1.14 \mathrm{E}-04$ \\
\hline chr5_340270ıX603143975F & 5 cis & 15.3 cis & 4.34 & $8.46 \mathrm{E}-05$ \\
\hline chr5_358470ı603143975F & 5 cis & $15.3 \mathrm{cis}$ & 5.25 & $1.03 \mathrm{E}-04$ \\
\hline chr5_343650।NM_0010079 & 5 cis & 6.0 cis & 6.23 & $1.60 \mathrm{E}-04$ \\
\hline chr5_369750।NM_0010079 & 5 cis & 6.0 cis & 8.03 & 7.74E-05 \\
\hline chr5_369750IENSGALT000( & 5 cis & 7.9 cis & 8.03 & $1.29 \mathrm{E}-05$ \\
\hline chr5_377660IENSGALT000( & 5 cis & 7.9 cis & 4.13 & $1.53 \mathrm{E}-04$ \\
\hline chr5_363340ıNM_0010393 & 5 cis & 5.0 cis & 5.6 & 5.67E-03 \\
\hline chr5_369750ıNM_0010393 & 5 cis & $5.0 \mathrm{cis}$ & 8.03 & $3.18 \mathrm{E}-05$ \\
\hline chr5_413520IENSGALT000( & 5 trans & 7.5 cis & 7.42 & $4.22 \mathrm{E}-06$ \\
\hline chr5_413530IENSGALT000( & 5 trans & 7.5 cis & 6.59 & $1.00 \mathrm{E}-03$ \\
\hline chr5_3645101X603600251F & 5 cis & 6.6 cis & 4.1 & $2.88 \mathrm{E}-03$ \\
\hline chr5_413510ıX603600251F & 5 cis & 6.6 cis & 4.54 & $1.52 \mathrm{E}-04$ \\
\hline chr5_4135201X603600251F & 5 cis & 6.6 cis & 7.42 & $2.01 \mathrm{E}-07$ \\
\hline chr5_413530ıx603600251F & 5 cis & 6.6 cis & 6.59 & 4.10E-04 \\
\hline chr5_443540ıX603600251F & 5 cis & 6.6 trans & 6.63 & $1.01 \mathrm{E}-03$ \\
\hline chr5_441270IENSGALT000( & 5 cis & $4.7 \mathrm{cis}$ & 6.34 & $9.11 \mathrm{E}-05$ \\
\hline chr5_441810IENSGALTO00( & 5 cis & 4.7 cis & 4.73 & 8.97E-06 \\
\hline chr5_395970IENSGALT000( & 5 cis & 4.7 cis & 4.77 & $1.05 \mathrm{E}-06$ \\
\hline chr5_470740IX603868322F & 5 cis & 3.8 trans & 6.2 & $1.99 \mathrm{E}-03$ \\
\hline chr5_413510।X603868322F & 5 cis & $3.8 \mathrm{cis}$ & 4.54 & $8.43 E-05$ \\
\hline chr5_413530ı603868322F & 5 cis & $3.8 \mathrm{cis}$ & 6.59 & $1.08 \mathrm{E}-04$ \\
\hline chr5_440120ıX603868322F & 5 cis & $3.8 \mathrm{cis}$ & 4.1 & $7.18 \mathrm{E}-06$ \\
\hline chr5_4284301X603868322F & 5 cis & $3.8 \mathrm{cis}$ & 4.81 & $1.41 \mathrm{E}-04$ \\
\hline chr5_440490।X603868322F & 5 cis & $3.8 \mathrm{cis}$ & 6.17 & $1.72 \mathrm{E}-05$ \\
\hline chr5_461550IX603868322F & 5 cis & $3.8 \mathrm{cis}$ & 7.95 & $1.90 \mathrm{E}-04$ \\
\hline chr5_442290।X603868322F & 5 cis & $3.8 \mathrm{cis}$ & 4.1 & $1.03 \mathrm{E}-04$ \\
\hline chr5_462950।X603868322F & 5 cis & $3.8 \mathrm{cis}$ & 4.58 & $2.42 \mathrm{E}-04$ \\
\hline chr5_440510।X603868322F & 5 cis & $3.8 \mathrm{cis}$ & 5.22 & 2.19E-03 \\
\hline chr5_369750।X603864527F & 5 cis & $4.3 \mathrm{cis}$ & 8.03 & $1.11 \mathrm{E}-03$ \\
\hline chr5_397550ıX603864527F & 5 cis & $4.3 \mathrm{cis}$ & 6.32 & $1.04 \mathrm{E}-03$ \\
\hline chr5_461290IX603864527F & 5 cis & 4.3 cis & 5.73 & 4.03E-04 \\
\hline chr5_449670ı603867292F & 5 cis & 7.5 cis & 5.65 & $2.30 \mathrm{E}-03$ \\
\hline chr5_441800(X603867292F & 5 cis & 7.5 cis & 5.54 & 5.36E-03 \\
\hline chr5_451190।X603867292F & 5 cis & $7.5 \mathrm{cis}$ & 8.51 & $8.27 \mathrm{E}-06$ \\
\hline chr5_467450IX603867292F & 5 cis & 7.5 cis & 4.5 & 4.36E-03 \\
\hline chr5_485370ıx603867292F & 5 cis & 7.5 cis & 7.15 & $1.12 \mathrm{E}-03$ \\
\hline chr5_485990।X603867292F & 5 cis & 7.5 cis & 5.96 & $3.78 \mathrm{E}-03$ \\
\hline chr5_458900ı603598306F & 5 cis & $4.1 \mathrm{cis}$ & 5.68 & $1.27 \mathrm{E}-03$ \\
\hline chr5_494580ı603598306F & 5 cis & 4.1 cis & 8.96 & $1.40 \mathrm{E}-03$ \\
\hline chr5_526600।X603863866F & 5 cis & 6.5 cis & 4.1 & $1.98 \mathrm{E}-03$ \\
\hline
\end{tabular}




\begin{tabular}{|c|c|c|c|c|}
\hline chr5_5589601X603863866F & 5 cis & 6.5 cis & 5.92 & $1.05 \mathrm{E}-03$ \\
\hline chr5_5511301X603863866F & 5 cis & $6.5 \mathrm{cis}$ & 4.69 & $9.50 \mathrm{E}-05$ \\
\hline chr5_5511801X603863866F & 5 cis & 6.5 cis & 4.59 & $1.51 \mathrm{E}-04$ \\
\hline chr5_526600IENSGALT000( & 5 cis & $4.1 \mathrm{cis}$ & 4.1 & $1.86 \mathrm{E}-03$ \\
\hline chr5_558960IENSGALT000( & 5 cis & $4.1 \mathrm{cis}$ & 5.92 & $9.93 \mathrm{E}-04$ \\
\hline chr6_1862001X603598388F & 6 cis & $6.6 \mathrm{cis}$ & 9.15 & $1.06 \mathrm{E}-03$ \\
\hline chr6_2217001X603598388F & 6 cis & 6.6 cis & 6.77 & $1.75 \mathrm{E}-03$ \\
\hline chr6_186200IENSGALT000( & 6 cis & 6.5 cis & 9.15 & $1.43 \mathrm{E}-03$ \\
\hline chr6_4323001X603596512F & 6 cis & $5.2 \mathrm{cis}$ & 4.76 & $1.21 \mathrm{E}-03$ \\
\hline chr6_432300IENSGALTO00( & 6 cis & 4.6 cis & 4.76 & $1.20 \mathrm{E}-03$ \\
\hline chr6_452600IENSGALT000( & 6 cis & $4.6 \mathrm{cis}$ & 7.29 & $1.12 \mathrm{E}-04$ \\
\hline chr6_665400IENSGALT000( & 6 cis & 4.6 cis & 27.96 & $5.13 \mathrm{E}-04$ \\
\hline chr6_735800IENSGALT000( & $6 \mathrm{cis}$ & $4.6 \mathrm{cis}$ & 11.29 & $1.68 \mathrm{E}-05$ \\
\hline chr6_847600IENSGALT000( & 6 cis & $4.6 \mathrm{cis}$ & 5.62 & 3.71E-03 \\
\hline chr6_693400IENSGALT000( & 6 cis & $4.6 \mathrm{cis}$ & 6.65 & 5.29E-04 \\
\hline chr6_937200IENSGALT000( & 6 cis & 4.6 cis & 4.2 & 1.60E-04 \\
\hline chr6_558600IENSGALT000( & 6 cis & $5.0 \mathrm{cis}$ & 5.05 & 7.84E-04 \\
\hline chr6_672400IENSGALTO00( & 6 cis & $5.0 \mathrm{cis}$ & 7.28 & $1.34 \mathrm{E}-03$ \\
\hline chr6_777200IENSGALT000( & 6 cis & 5.0 cis & 4.8 & 2.83E-05 \\
\hline chr6_665400IENSGALT000( & 6 cis & $5.0 \mathrm{cis}$ & 27.96 & 1.83E-04 \\
\hline chr6_672200IENSGALT000( & 6 cis & $5.0 \mathrm{cis}$ & 9.79 & 5.87E-04 \\
\hline chr6_672300IENSGALT000( & 6 cis & $5.0 \mathrm{cis}$ & 14.97 & $3.44 \mathrm{E}-05$ \\
\hline chr6_671700IENSGALT000( & 6 cis & $5.0 \mathrm{cis}$ & 6.51 & 8.26E-04 \\
\hline chr6_921400IENSGALTO00( & 6 cis & $5.0 \mathrm{cis}$ & 7.85 & 3.68E-04 \\
\hline chr6_5233001X603862786F & 6 cis & $5.1 \mathrm{cis}$ & 4.66 & 9.37E-03 \\
\hline chr6_5586001X603865854F & $6 \mathrm{cis}$ & $7.6 \mathrm{cis}$ & 5.05 & 1.47E-03 \\
\hline chr6_7358001X603865854F & 6 cis & 7.6 cis & 11.29 & $2.76 \mathrm{E}-05$ \\
\hline chr6_5644001X603865854F & 6 cis & $7.6 \mathrm{cis}$ & 5.29 & 1.60E-04 \\
\hline chr6_7523001X603865854F & 6 cis & $7.6 \mathrm{cis}$ & 22.43 & 7.30E-04 \\
\hline chr6_635000IENSGALTO00( & 6 cis & $4.2 \mathrm{cis}$ & 6.08 & $1.14 \mathrm{E}-03$ \\
\hline chr6_564400IENSGALT000( & 6 cis & $4.2 \mathrm{cis}$ & 5.29 & $6.26 \mathrm{E}-04$ \\
\hline chr6_755800IENSGALT000( & 6 cis & $4.2 \mathrm{cis}$ & 9.32 & 5.87E-04 \\
\hline chr6_740200IENSGALT000( & 6 cis & $4.2 \mathrm{cis}$ & 8.05 & $2.80 \mathrm{E}-03$ \\
\hline chr6_7556001X603596093F & 6 cis & $5.0 \mathrm{cis}$ & 6.77 & $1.04 \mathrm{E}-02$ \\
\hline chr6_6934001X603596093F & 6 cis & $5.0 \mathrm{cis}$ & 6.65 & $1.16 \mathrm{E}-03$ \\
\hline chr6_693200IENSGALTO00( & 6 cis & $4.2 \mathrm{cis}$ & 7.27 & 3.56E-04 \\
\hline chr6_691500IENSGALTO00( & 6 cis & 4.2 cis & 4.53 & $1.10 \mathrm{E}-03$ \\
\hline chr6_652600IENSGALT000( & 6 cis & $4.2 \mathrm{cis}$ & 4.1 & $3.92 \mathrm{E}-03$ \\
\hline chr6_935100IENSGALT000( & 6 cis & $3.9 \mathrm{cis}$ & 18.79 & 9.27E-04 \\
\hline chr6_876100IENSGALT000( & 6 cis & 3.9 trans & 5.88 & $1.95 \mathrm{E}-03$ \\
\hline chr6_665400IENSGALT000( & 6 cis & $12.6 \mathrm{cis}$ & 27.96 & 2.99E-05 \\
\hline chr6_665900IENSGALTO00( & 6 cis & $12.6 \mathrm{cis}$ & 9.67 & 1.23E-04 \\
\hline chr6_672100IENSGALTO00( & 6 cis & $12.6 \mathrm{cis}$ & 9.64 & $1.74 \mathrm{E}-04$ \\
\hline chr6_678200IENSGALT000( & 6 cis & $12.6 \mathrm{cis}$ & 4.1 & 4.05E-03 \\
\hline chr6_946200IENSGALT000( & 6 cis & $12.6 \mathrm{cis}$ & 8.3 & $1.94 \mathrm{E}-04$ \\
\hline chr6_752300IENSGALTO00( & 6 cis & $12.6 \mathrm{cis}$ & 22.43 & 8.44E-07 \\
\hline chr6_691500IENSGALTO00( & 6 cis & $12.6 \mathrm{cis}$ & 4.53 & 8.97E-05 \\
\hline chr6_820900IENSGALT000( & 6 cis & $12.6 \mathrm{cis}$ & 4.62 & $2.12 \mathrm{E}-04$ \\
\hline chr6_935100IENSGALTO00 & 6 cis & $12.6 \mathrm{cis}$ & 18.79 & 7.05E-09 \\
\hline chr6_921400IENSGALT000( & 6 cis & $12.6 \mathrm{cis}$ & 7.85 & 5.33E-04 \\
\hline chr6_779600IENSGALTO00( & 6 cis & $12.6 \mathrm{cis}$ & 6.26 & 3.23E-04 \\
\hline chr6_780000IENSGALTO00( & 6 cis & $12.6 \mathrm{cis}$ & 8.49 & $2.38 \mathrm{E}-05$ \\
\hline chr6_779700IENSGALT000( & 6 cis & $12.6 \mathrm{cis}$ & 7.34 & $2.46 \mathrm{E}-04$ \\
\hline chr6_665400INM_0010312 & 6 cis & $11.4 \mathrm{cis}$ & 27.96 & $2.82 \mathrm{E}-05$ \\
\hline chr6_665900।NM_0010312 & 6 cis & $11.4 \mathrm{cis}$ & 9.67 & $1.26 \mathrm{E}-04$ \\
\hline chr6_672100INM_0010312 & 6 cis & $11.4 \mathrm{cis}$ & 9.64 & 5.01E-04 \\
\hline chr6_752300।NM_0010312 & 6 cis & $11.4 \mathrm{cis}$ & 22.43 & $1.20 \mathrm{E}-06$ \\
\hline chr6_691500।NM_0010312 & 6 cis & $11.4 \mathrm{cis}$ & 4.53 & 1.66E-04 \\
\hline chr6_820900।NM_0010312 & 6 cis & $11.4 \mathrm{cis}$ & 4.62 & $1.18 \mathrm{E}-04$ \\
\hline chr6_935100।NM_0010312 & 6 cis & $11.4 \mathrm{cis}$ & 18.79 & $2.25 \mathrm{E}-08$ \\
\hline chr6_921400।NM_0010312 & 6 cis & $11.4 \mathrm{cis}$ & 7.85 & 2.15E-03 \\
\hline chr6_780000।NM_0010312 & 6 cis & $11.4 \mathrm{cis}$ & 8.49 & 8.50E-05 \\
\hline chr6_6933001X603868348F & 6 cis & $5.7 \mathrm{cis}$ & 7.65 & 3.49E-04 \\
\hline chr6_6935001X603868348F & 6 cis & 5.7 cis & 5.27 & 2.77E-04 \\
\hline chr6_8902001X603868348F & 6 cis & $5.7 \mathrm{cis}$ & 4.97 & 7.05E-04 \\
\hline chr6_1046201X603868348F & 6 cis & $5.7 \mathrm{cis}$ & 7.53 & $1.20 \mathrm{E}-03$ \\
\hline chr6_107960।X603601830F & 6 cis & 4.9 cis & 5.54 & 6.99E-04 \\
\hline chr6_1104701X603601830F & 6 cis & 4.9 cis & 7.02 & 1.17E-03 \\
\hline chr6_1046201X603601830F & 6 cis & 4.9 cis & 7.53 & $1.88 \mathrm{E}-04$ \\
\hline chr6_1112101X603601830F & 6 cis & 4.9 cis & 7.13 & 6.07E-05 \\
\hline chr6_1104501X603601830F & 6 cis & 4.9 cis & 9.3 & 6.94E-05 \\
\hline chr6_111200ıX03601830F & 6 cis & 4.9 cis & 9.72 & 7.83E-05 \\
\hline
\end{tabular}




\begin{tabular}{|c|c|c|c|c|}
\hline chr6_110180IX603601830F & 6 cis & 4.9 cis & 4.91 & $1.54 \mathrm{E}-04$ \\
\hline chr6_110460ıX603601830F & 6 cis & 4.9 cis & 8.88 & 4.14E-05 \\
\hline chr6_110480ıX603601830F & 6 cis & 4.9 cis & 10.72 & 4.89E-04 \\
\hline chr6_110400ıX603601830F & 6 cis & 4.9 cis & 9.03 & $1.66 \mathrm{E}-04$ \\
\hline chr6_110790ıX603601830F & 6 cis & 4.9 cis & 9.14 & $4.98 \mathrm{E}-04$ \\
\hline chr6_110940ıX603601830F & 6 cis & 4.9 cis & 6.79 & $6.86 \mathrm{E}-05$ \\
\hline chr6_1111901X603601830F & 6 cis & 4.9 cis & 8.43 & $1.41 \mathrm{E}-05$ \\
\hline chr6_117650ıX603601830F & 6 cis & 4.9 cis & 5.22 & $5.02 \mathrm{E}-05$ \\
\hline chr6_1111201X603601830F & 6 cis & 4.9 cis & 8.1 & $3.32 \mathrm{E}-04$ \\
\hline chr6_118140ıX603601830F & 6 cis & 4.9 cis & 9.35 & $1.75 \mathrm{E}-05$ \\
\hline chr6_110690ıX603601830F & 6 cis & 4.9 cis & 9.3 & $8.32 \mathrm{E}-05$ \\
\hline chr6_110190ı603601830F & 6 cis & 4.9 cis & 10.28 & $2.34 \mathrm{E}-05$ \\
\hline chr6_1176601X603601830F & 6 cis & 4.9 cis & 9.09 & $2.84 \mathrm{E}-05$ \\
\hline chr6_110390ıX603601830F & 6 cis & 4.9 cis & 9.22 & $5.25 \mathrm{E}-04$ \\
\hline chr6_117740ıX603601830F & 6 cis & 4.9 cis & 6.08 & $6.58 \mathrm{E}-04$ \\
\hline chr6_110470ıX603865360F & 6 cis & 5.6 cis & 7.02 & 5.17E-04 \\
\hline chr6_110410ıX603865360F & 6 cis & 5.6 cis & 7.82 & 2.19E-04 \\
\hline chr6_110700ıX603865360F & 6 cis & 5.6 cis & 7.92 & 2.10E-04 \\
\hline chr6_1178801X603865360F & 6 cis & 5.6 cis & 9.08 & 3.13E-04 \\
\hline chr6_111210ıX603865360F & 6 cis & 5.6 cis & 7.13 & $2.52 \mathrm{E}-05$ \\
\hline chr6_1104501X603865360F & 6 cis & 5.6 cis & 9.3 & $6.58 \mathrm{E}-05$ \\
\hline chr6_111200ıX603865360F & 6 cis & 5.6 cis & 9.72 & $6.97 \mathrm{E}-05$ \\
\hline chr6_110460ıX603865360F & 6 cis & 5.6 cis & 8.88 & $3.95 \mathrm{E}-05$ \\
\hline chr6_1104001X603865360F & 6 cis & 5.6 cis & 9.03 & $1.38 \mathrm{E}-04$ \\
\hline chr6_110790ıX603865360F & 6 cis & 5.6 cis & 9.14 & $5.35 \mathrm{E}-05$ \\
\hline chr6_111190ıX603865360F & 6 cis & 5.6 cis & 8.43 & $2.02 \mathrm{E}-04$ \\
\hline chr6_1176501X603865360F & 6 cis & 5.6 cis & 5.22 & $3.20 \mathrm{E}-04$ \\
\hline chr6_1111201X603865360F & 6 cis & 5.6 cis & 8.1 & 7.75E-05 \\
\hline chr6_118140ıX603865360F & 6 cis & 5.6 cis & 9.35 & 2.79E-04 \\
\hline chr6_110690ıX603865360F & 6 cis & 5.6 cis & 9.3 & $1.92 \mathrm{E}-04$ \\
\hline chr6_1101901X603865360F & 6 cis & 5.6 cis & 10.28 & 2.69E-04 \\
\hline chr6_111000ıX603865360F & 6 cis & 5.6 cis & 7.96 & 3.30E-03 \\
\hline chr6_117660ıX603865360F & 6 cis & 5.6 cis & 9.09 & $1.41 \mathrm{E}-04$ \\
\hline chr6_117780ıX603865360F & 6 cis & $5.6 \mathrm{cis}$ & 5.67 & 4.35E-04 \\
\hline chr6_106090IENSGALT000( & 6 cis & 5.3 cis & 4.1 & $9.08 \mathrm{E}-03$ \\
\hline chr6_107820IENSGALTO00( & 6 cis & 5.3 cis & 4.1 & 4.99E-03 \\
\hline chr6_104620IENSGALT000( & 6 cis & 5.3 cis & 7.53 & $8.78 \mathrm{E}-06$ \\
\hline chr6_110450IENSGALTO00( & 6 cis & 5.3 cis & 9.3 & $1.05 \mathrm{E}-03$ \\
\hline chr6_111200IENSGALT000( & 6 cis & 5.3 cis & 9.72 & $1.01 \mathrm{E}-03$ \\
\hline chr6_110180IENSGALTO00( & 6 cis & $5.3 \mathrm{cis}$ & 4.91 & $1.12 \mathrm{E}-03$ \\
\hline chr6_110460IENSGALTO00( & 6 cis & 5.3 cis & 8.88 & $1.71 \mathrm{E}-03$ \\
\hline chr6_110480IENSGALTO00( & 6 cis & 5.3 cis & 10.72 & $1.67 \mathrm{E}-03$ \\
\hline chr6_110400IENSGALT000( & 6 cis & $5.3 \mathrm{cis}$ & 9.03 & $2.36 \mathrm{E}-04$ \\
\hline chr6_110940IENSGALT000( & 6 cis & 5.3 cis & 6.79 & $3.80 \mathrm{E}-04$ \\
\hline chr6_111190IENSGALT000( & 6 cis & 5.3 cis & 8.43 & $2.52 \mathrm{E}-04$ \\
\hline chr6_117650IENSGALTO00( & 6 cis & $5.3 \mathrm{cis}$ & 5.22 & $3.22 \mathrm{E}-04$ \\
\hline chr6_118140IENSGALT000( & 6 cis & 5.3 cis & 9.35 & $3.96 \mathrm{E}-05$ \\
\hline chr6_110690IENSGALTO00( & 6 cis & 5.3 cis & 9.3 & $2.20 \mathrm{E}-05$ \\
\hline chr6_110190IENSGALT000( & 6 cis & 5.3 cis & 10.28 & $3.34 \mathrm{E}-05$ \\
\hline chr6_117660IENSGALTO00( & 6 cis & $5.3 \mathrm{cis}$ & 9.09 & $3.65 \mathrm{E}-05$ \\
\hline chr6_110390IENSGALT000( & 6 cis & 5.3 cis & 9.22 & 2.87E-04 \\
\hline chr6_117860IENSGALTO00( & 6 cis & $5.3 \mathrm{cis}$ & 9.97 & $2.58 \mathrm{E}-04$ \\
\hline chr6_117740IENSGALT000( & 6 cis & 5.3 cis & 6.08 & $1.11 \mathrm{E}-03$ \\
\hline chr6_110570IENSGALTO00( & 6 cis & 5.3 cis & 7.27 & 4.19E-04 \\
\hline chr6_117750IENSGALT000( & 6 cis & $5.3 \mathrm{cis}$ & 6.55 & $2.31 \mathrm{E}-03$ \\
\hline chr6_117780IENSGALTO00( & 6 cis & $5.3 \mathrm{cis}$ & 5.67 & $7.12 \mathrm{E}-04$ \\
\hline chr6_110290IENSGALT000( & 6 cis & 5.3 cis & 7.21 & $4.80 \mathrm{E}-04$ \\
\hline chr6_117760IENSGALTO00( & 6 cis & $5.3 \mathrm{cis}$ & 7.15 & $3.74 \mathrm{E}-04$ \\
\hline chr6_110270IENSGALT000( & 6 cis & 5.3 cis & 6.2 & $1.44 \mathrm{E}-03$ \\
\hline chr6_110580IENSGALTO00( & 6 cis & 5.3 cis & 6.01 & 8.47E-04 \\
\hline chr6_110640IENSGALT000( & 6 cis & $5.3 \mathrm{cis}$ & 6.06 & $2.53 \mathrm{E}-04$ \\
\hline chr6_118210IENSGALTO00( & 6 cis & $5.3 \mathrm{cis}$ & 6 & $5.12 \mathrm{E}-04$ \\
\hline chr6_118270IENSGALT000( & 6 cis & 5.3 cis & 5.21 & 2.19E-03 \\
\hline chr6_118280IENSGALTO00( & 6 cis & $5.3 \mathrm{cis}$ & 5.41 & $3.63 \mathrm{E}-04$ \\
\hline chr6_118340IENSGALTO00( & 6 cis & $5.3 \mathrm{cis}$ & 5.83 & $1.11 \mathrm{E}-03$ \\
\hline chr6_110610IENSGALTO00( & 6 cis & $5.3 \mathrm{cis}$ & 7.12 & 5.59E-04 \\
\hline chr6_110340IENSGALT000( & 6 cis & $5.3 \mathrm{cis}$ & 7.46 & $7.80 \mathrm{E}-04$ \\
\hline chr6_110510IENSGALTO00( & 6 cis & $5.3 \mathrm{cis}$ & 7 & $6.34 \mathrm{E}-04$ \\
\hline chr6_110590IENSGALTO00( & 6 cis & $5.3 \mathrm{cis}$ & 6.28 & $9.23 \mathrm{E}-04$ \\
\hline chr6_110360IENSGALTO00( & 6 cis & $5.3 \mathrm{cis}$ & 5.7 & $3.29 E-03$ \\
\hline chr6_117810IENSGALT000( & 6 cis & 5.3 cis & 6.87 & $9.97 \mathrm{E}-04$ \\
\hline chr6_117850IENSGALT000( & 6 cis & 5.3 cis & 4.77 & $1.25 \mathrm{E}-03$ \\
\hline
\end{tabular}




\begin{tabular}{|c|c|c|c|c|}
\hline chr6_110920IENSGALT000( & 6 cis & $5.3 \mathrm{cis}$ & 4.56 & $2.36 \mathrm{E}-03$ \\
\hline chr6_110450IENSGALTO00( & 6 cis & 5.9 cis & 9.3 & $1.12 \mathrm{E}-04$ \\
\hline chr6_111200IENSGALT000( & 6 cis & 5.9 cis & 9.72 & $1.37 \mathrm{E}-04$ \\
\hline chr6_110400IENSGALTO00( & 6 cis & 5.9 cis & 9.03 & $6.79 \mathrm{E}-04$ \\
\hline chr6_118140IENSGALTO00( & 6 cis & 5.9 cis & 9.35 & $2.55 \mathrm{E}-04$ \\
\hline chr6_110690IENSGALT000( & 6 cis & 5.9 cis & 9.3 & $1.34 \mathrm{E}-04$ \\
\hline chr6_110190IENSGALTO00( & $6 \mathrm{cis}$ & 5.9 cis & 10.28 & 5.61E-05 \\
\hline chr6_117660IENSGALT000( & 6 cis & 5.9 cis & 9.09 & 2.31E-05 \\
\hline chr6_110390IENSGALT000( & 6 cis & 5.9 cis & 9.22 & 2.53E-05 \\
\hline chr6_117860IENSGALTO00( & 6 cis & 5.9 cis & 9.97 & $3.42 \mathrm{E}-05$ \\
\hline chr6_128110IENSGALTO00( & 6 cis & 5.9 cis & 6.27 & 1.57E-04 \\
\hline chr6_110490IENSGALT000( & 6 cis & 5.9 cis & 7.22 & 3.70E-04 \\
\hline chr6_117740IENSGALTO00( & $6 \mathrm{cis}$ & 5.9 cis & 6.08 & 1.09E-03 \\
\hline chr6_110570IENSGALTO00( & $6 \mathrm{cis}$ & 5.9 cis & 7.27 & 3.59E-04 \\
\hline chr6_117780IENSGALTO00( & 6 cis & 5.9 cis & 5.67 & 4.72E-04 \\
\hline chr6_110290IENSGALTO00( & 6 cis & 5.9 cis & 7.21 & 2.83E-04 \\
\hline chr6_110680IENSGALTO00( & 6 cis & 5.9 cis & 6.49 & $1.55 \mathrm{E}-05$ \\
\hline chr6_117760IENSGALTO00( & 6 cis & 5.9 cis & 7.15 & $2.16 \mathrm{E}-04$ \\
\hline chr6_110580IENSGALTO00( & $6 \mathrm{cis}$ & 5.9 cis & 6.01 & 4.66E-04 \\
\hline chr6_118210IENSGALTO00( & $6 \mathrm{cis}$ & 5.9 cis & 6 & 4.30E-04 \\
\hline chr6_127840IENSGALT000( & 6 cis & 5.9 cis & 5.73 & 1.19E-04 \\
\hline chr6_117680IENSGALTO00( & 6 cis & 5.9 cis & 7.39 & $1.82 \mathrm{E}-04$ \\
\hline chr6_118280IENSGALTO00( & 6 cis & 5.9 cis & 5.41 & 5.26E-04 \\
\hline chr6_110610IENSGALTO00( & 6 cis & 5.9 cis & 7.12 & $2.88 \mathrm{E}-04$ \\
\hline chr6_110340IENSGALTO00( & 6 cis & 5.9 cis & 7.46 & $1.59 \mathrm{E}-03$ \\
\hline chr6_110500IENSGALTO00( & $6 \mathrm{cis}$ & 5.9 cis & 6.4 & 1.27E-03 \\
\hline chr6_110510IENSGALT000( & 6 cis & 5.9 cis & 7 & 1.97E-04 \\
\hline chr6_110590IENSGALTO00( & 6 cis & 5.9 cis & 6.28 & $6.95 \mathrm{E}-04$ \\
\hline chr6_110660IENSGALTO00( & 6 cis & 5.9 cis & 6.94 & 1.60E-04 \\
\hline chr6_110360IENSGALT000( & 6 cis & 5.9 cis & 5.7 & 3.19E-04 \\
\hline chr6_117830IENSGALTO00( & 6 cis & 5.9 cis & 5.21 & 3.91E-04 \\
\hline chr6_117850IENSGALTO00( & $6 \mathrm{cis}$ & 5.9 cis & 4.77 & $1.25 \mathrm{E}-03$ \\
\hline chr6_111030IENSGALTO00( & 6 cis & 5.9 cis & 6.29 & 7.83E-03 \\
\hline chr6_118290IENSGALTO00( & 6 cis & 5.9 cis & 4.42 & 8.07E-04 \\
\hline chr6_110370IENSGALTO00( & 6 cis & 5.9 cis & 7.63 & $1.21 \mathrm{E}-03$ \\
\hline chr6_111150IENSGALTO00( & 6 cis & 5.9 cis & 8.37 & 2.33E-02 \\
\hline chr6_124070IENSGALTO00( & 6 cis & 5.9 cis & 4.1 & $4.78 \mathrm{E}-04$ \\
\hline chr6_110700।NM_205121_ & 6 cis & 4.7 cis & 7.92 & $3.94 \mathrm{E}-04$ \\
\hline chr6_117880।NM_205121_ & 6 cis & 4.7 cis & 9.08 & $2.54 \mathrm{E}-04$ \\
\hline chr6_110460INM_205121_ & 6 cis & $4.7 \mathrm{cis}$ & 8.88 & 8.44E-05 \\
\hline chr6_110400INM_205121_ & 6 cis & $4.7 \mathrm{cis}$ & 9.03 & 3.09E-04 \\
\hline chr6_110790INM_205121_ & 6 cis & $4.7 \mathrm{cis}$ & 9.14 & $2.21 \mathrm{E}-04$ \\
\hline chr6_111120।NM_205121_ & 6 cis & $4.7 \mathrm{cis}$ & 8.1 & $8.62 \mathrm{E}-05$ \\
\hline chr6_118140।NM_205121_ & 6 cis & 4.7 cis & 9.35 & $2.38 \mathrm{E}-04$ \\
\hline chr6_110690INM_205121_ & 6 cis & 4.7 cis & 9.3 & 1.67E-04 \\
\hline chr6_110190INM_205121_ & 6 cis & $4.7 \mathrm{cis}$ & 10.28 & 4.55E-05 \\
\hline chr6_111000INM_205121_ & 6 cis & $4.7 \mathrm{cis}$ & 7.96 & 2.01E-03 \\
\hline chr6_117660।NM_205121_ & 6 cis & $4.7 \mathrm{cis}$ & 9.09 & $8.21 \mathrm{E}-05$ \\
\hline chr6_110390।NM_205121_ & 6 cis & $4.7 \mathrm{cis}$ & 9.22 & 1.57E-04 \\
\hline chr6_117860।NM_205121_ & 6 cis & 4.7 cis & 9.97 & 3.01E-04 \\
\hline chr6_128110।NM_205121_ & 6 cis & 4.7 cis & 6.27 & $2.72 \mathrm{E}-04$ \\
\hline chr6_110490INM_205121_ & 6 cis & $4.7 \mathrm{cis}$ & 7.22 & 3.03E-04 \\
\hline chr6_117740INM_205121_ & 6 cis & $4.7 \mathrm{cis}$ & 6.08 & 4.41E-05 \\
\hline chr6_110570INM_205121_ & 6 cis & 4.7 cis & 7.27 & 5.47E-04 \\
\hline chr6_117780INM_205121_ & 6 cis & $4.7 \mathrm{cis}$ & 5.67 & $1.44 \mathrm{E}-04$ \\
\hline chr6_110680INM_205121_- & 6 cis & $4.7 \mathrm{cis}$ & 6.49 & 3.36E-04 \\
\hline chr6_110270INM_205121_ & 6 cis & 4.7 cis & 6.2 & 4.05E-05 \\
\hline chr6_118210।NM_205121_ & 6 cis & $4.7 \mathrm{cis}$ & 6 & $9.86 \mathrm{E}-04$ \\
\hline chr6_118270INM_205121_ & 6 cis & $4.7 \mathrm{cis}$ & 5.21 & 3.67E-04 \\
\hline chr6_127840INM_205121_ & 6 cis & 4.7 cis & 5.73 & $1.07 \mathrm{E}-03$ \\
\hline chr6_117680INM_205121_ & 6 cis & $4.7 \mathrm{cis}$ & 7.39 & 8.80E-04 \\
\hline chr6_117670।NM_205121_ & 6 cis & 4.7 cis & 5.15 & $6.05 \mathrm{E}-04$ \\
\hline chr6_110310INM_205121_ & 6 cis & $4.7 \mathrm{cis}$ & 6.48 & 4.20E-04 \\
\hline chr6_110500।NM_205121_ & 6 cis & $4.7 \mathrm{cis}$ & 6.4 & 8.10E-04 \\
\hline chr6_110510INM_205121_ & 6 cis & $4.7 \mathrm{cis}$ & 7 & 7.81E-04 \\
\hline chr6_117850।NM_205121_ & 6 cis & $4.7 \mathrm{cis}$ & 4.77 & $1.21 \mathrm{E}-03$ \\
\hline chr6_110300।NM_205121_ & 6 cis & $4.7 \mathrm{cis}$ & 5.3 & $8.52 \mathrm{E}-04$ \\
\hline chr6_111030।NM_205121_ & 6 cis & $4.7 \mathrm{cis}$ & 6.29 & $4.32 \mathrm{E}-03$ \\
\hline chr6_110370INM_205121_ & 6 cis & $4.7 \mathrm{cis}$ & 7.63 & $2.00 \mathrm{E}-03$ \\
\hline chr6_110600।NM_205121_ & 6 cis & $4.7 \mathrm{cis}$ & 4.65 & 3.05E-04 \\
\hline chr6_110560INM_205121_ & 6 cis & $4.7 \mathrm{cis}$ & 5.95 & $2.05 \mathrm{E}-04$ \\
\hline chr6_111170ıNM_205121_ & 6 cis & $4.7 \mathrm{cis}$ & 4.57 & $3.54 \mathrm{E}-04$ \\
\hline
\end{tabular}




\begin{tabular}{|c|c|c|c|c|}
\hline chr6_124070INM_205121_ & 6 cis & $4.7 \mathrm{cis}$ & 4.1 & 4.97E-04 \\
\hline chr6_117730INM_205121_ & 6 cis & 4.7 cis & 6.32 & $1.25 \mathrm{E}-03$ \\
\hline chr6_118300INM_205121_ & 6 cis & 4.7 cis & 4.29 & 2.19E-03 \\
\hline chr6_111040INM_205121_ & 6 cis & 4.7 cis & 5.86 & $1.03 \mathrm{E}-04$ \\
\hline chr6_111160INM_205121_ & 6 cis & 4.7 cis & 5.37 & $1.64 \mathrm{E}-04$ \\
\hline chr6_129860INM_205121_ & 6 cis & 4.7 cis & 7.22 & $3.82 \mathrm{E}-02$ \\
\hline chr6_117630।NM_205121_ & 6 cis & $4.7 \mathrm{cis}$ & 4.1 & 5.67E-04 \\
\hline chr6_110690ıX603600511F & 6 cis & 4.9 cis & 9.3 & 3.79E-04 \\
\hline chr6_110390ıX603600511F & 6 cis & 4.9 cis & 9.22 & 2.09E-04 \\
\hline chr6_117860ıX603600511F & 6 cis & 4.9 cis & 9.97 & $1.32 \mathrm{E}-04$ \\
\hline chr6_110570ıX603600511F & 6 cis & 4.9 cis & 7.27 & $1.52 \mathrm{E}-03$ \\
\hline chr6_117750ı603600511F & 6 cis & 4.9 cis & 6.55 & $9.68 \mathrm{E}-04$ \\
\hline chr6_1106801X603600511F & 6 cis & 4.9 cis & 6.49 & $1.12 \mathrm{E}-03$ \\
\hline chr6_110640ıX603600511F & 6 cis & 4.9 cis & 6.06 & $1.32 \mathrm{E}-03$ \\
\hline chr6_118210ıX603600511F & 6 cis & 4.9 cis & 6 & $9.81 \mathrm{E}-04$ \\
\hline chr6_118280ıX603600511F & 6 cis & 4.9 cis & 5.41 & $1.23 \mathrm{E}-03$ \\
\hline chr6_110340ıX603600511F & 6 cis & 4.9 cis & 7.46 & $1.32 \mathrm{E}-03$ \\
\hline chr6_110510ıX603600511F & 6 cis & 4.9 cis & 7 & $1.06 \mathrm{E}-03$ \\
\hline chr6_110660ıX603600511F & 6 cis & 4.9 cis & 6.94 & 2.97E-04 \\
\hline chr6_110360ıX603600511F & 6 cis & 4.9 cis & 5.7 & $1.92 \mathrm{E}-04$ \\
\hline chr6_117830ıX603600511F & 6 cis & 4.9 cis & 5.21 & $2.00 \mathrm{E}-04$ \\
\hline chr6_117810ıX603600511F & 6 cis & 4.9 cis & 6.87 & $9.48 \mathrm{E}-04$ \\
\hline chr6_111150ıX603600511F & 6 cis & 4.9 cis & 8.37 & 3.19E-03 \\
\hline chr6_117790ıX603600511F & 6 cis & 4.9 cis & 6.03 & $1.52 \mathrm{E}-03$ \\
\hline chr6_127350ıX603600511F & 6 cis & 4.9 cis & 4.32 & $1.16 \mathrm{E}-03$ \\
\hline chr6_1108201X603600511F & 6 cis & 4.9 cis & 7.29 & $3.75 \mathrm{E}-03$ \\
\hline chr6_110840ıX603600511F & 6 cis & 4.9 cis & 7.17 & $4.66 \mathrm{E}-03$ \\
\hline chr6_110860ı603600511F & 6 cis & 4.9 cis & 6.69 & 4.97E-03 \\
\hline chr6_110880ıX03600511F & 6 cis & 4.9 cis & 7.21 & $3.59 \mathrm{E}-03$ \\
\hline chr6_110890ıX603600511F & 6 cis & 4.9 cis & 7.1 & $4.41 \mathrm{E}-03$ \\
\hline chr6_1109001X603600511F & 6 cis & 4.9 cis & 7.39 & $3.16 \mathrm{E}-03$ \\
\hline chr6_110810ıX603600511F & 6 cis & 4.9 cis & 7.81 & $5.11 \mathrm{E}-03$ \\
\hline chr6_110920ıX603600511F & 6 cis & 4.9 cis & 4.56 & $1.31 \mathrm{E}-03$ \\
\hline chr6_127370ıX603600511F & 6 cis & 4.9 cis & 4.57 & $1.14 \mathrm{E}-03$ \\
\hline chr6_151590ıX603600511F & 6 cis & 4.9 cis & 5.78 & $4.88 \mathrm{E}-03$ \\
\hline chr6_162690ıX603600511F & 6 cis & 4.9 cis & 6.9 & $2.23 \mathrm{E}-03$ \\
\hline chr6_1697901X603600511F & 6 cis & 4.9 cis & 4.18 & 8.99E-04 \\
\hline chr6_117610ıX603600511F & 6 cis & 4.9 cis & 5.85 & $6.54 \mathrm{E}-03$ \\
\hline chr6_151580ıX603600511F & 6 cis & 4.9 cis & 11.02 & 4.96E-04 \\
\hline chr6_135730ıX603600511F & 6 cis & 4.9 cis & 5.24 & $2.62 \mathrm{E}-04$ \\
\hline chr6_152690ıX603600511F & 6 cis & 4.9 cis & 7.27 & $2.75 \mathrm{E}-04$ \\
\hline chr6_152680ıX603600511F & 6 cis & 4.9 cis & 7.2 & $6.53 \mathrm{E}-05$ \\
\hline chr6_153610ıX603600511F & 6 cis & 4.9 cis & 4.89 & $3.74 \mathrm{E}-03$ \\
\hline chr6_154410ıX603600511F & 6 cis & 4.9 cis & 18.34 & $4.58 \mathrm{E}-04$ \\
\hline chr6_1602501X603600511F & 6 cis & 4.9 cis & 4.44 & $1.23 \mathrm{E}-03$ \\
\hline chr6_170780ı603600511F & 6 cis & 4.9 cis & 6.95 & $8.30 \mathrm{E}-04$ \\
\hline chr6_111030ı603602109F & 6 cis & 4.5 cis & 6.29 & 8.07E-04 \\
\hline chr6_111150ıX603602109F & 6 cis & 4.5 cis & 8.37 & $3.22 \mathrm{E}-04$ \\
\hline chr6_136780ıX603602109F & 6 cis & 4.5 cis & 5.66 & $3.32 \mathrm{E}-05$ \\
\hline chr6_136770ıX603602109F & 6 cis & $4.5 \mathrm{cis}$ & 7.29 & $3.53 \mathrm{E}-04$ \\
\hline chr6_156990IX603602109F & 6 cis & 4.5 cis & 7.02 & $5.30 \mathrm{E}-05$ \\
\hline chr6_147020ıX603602109F & 6 cis & $4.5 \mathrm{cis}$ & 6.26 & 5.57E-04 \\
\hline chr6_152680ıX603602109F & 6 cis & $4.5 \mathrm{cis}$ & 7.2 & 5.16E-05 \\
\hline chr6_154410ıX603602109F & 6 cis & 4.5 cis & 18.34 & $1.60 \mathrm{E}-05$ \\
\hline chr6_160250ıX603602109F & 6 cis & 4.5 cis & 4.44 & 4.46E-04 \\
\hline chr6_151260ıX603602109F & 6 cis & $4.5 \mathrm{cis}$ & 6.27 & $6.47 \mathrm{E}-05$ \\
\hline chr6_151550IX603602109F & 6 cis & 4.5 cis & 7.6 & $1.41 \mathrm{E}-04$ \\
\hline chr6_179520ıX603595690F & 6 cis & $4.2 \mathrm{cis}$ & 4.71 & $6.23 \mathrm{E}-04$ \\
\hline chr6_169790ıX603866766F & 6 cis & $8.3 \mathrm{cis}$ & 4.18 & $2.49 \mathrm{E}-05$ \\
\hline chr6_151580ıX603866766F & 6 cis & $8.3 \mathrm{cis}$ & 11.02 & $8.59 \mathrm{E}-05$ \\
\hline chr6_155530ıX603866766F & 6 cis & $8.3 \mathrm{cis}$ & 5.54 & $6.47 \mathrm{E}-04$ \\
\hline chr6_148660ıX603866766F & 6 cis & $8.3 \mathrm{cis}$ & 4.86 & $6.25 \mathrm{E}-05$ \\
\hline chr6_1470201X603866766F & 6 cis & $8.3 \mathrm{cis}$ & 6.26 & $9.15 \mathrm{E}-05$ \\
\hline chr6_151250ıX603866766F & 6 cis & $8.3 \mathrm{cis}$ & 6.47 & $1.04 \mathrm{E}-04$ \\
\hline chr6_152690ıX603866766F & 6 cis & $8.3 \mathrm{cis}$ & 7.27 & $5.41 \mathrm{E}-05$ \\
\hline chr6_152680ıX603866766F & 6 cis & $8.3 \mathrm{cis}$ & 7.2 & $1.54 \mathrm{E}-04$ \\
\hline chr6_149550ıX603866766F & 6 cis & $8.3 \mathrm{cis}$ & 5.14 & 2.33E-04 \\
\hline chr6_149560ıX603866766F & 6 cis & $8.3 \mathrm{cis}$ & 4.51 & 4.10E-05 \\
\hline chr6_154410ıX603866766F & 6 cis & $8.3 \mathrm{cis}$ & 18.34 & $1.27 \mathrm{E}-07$ \\
\hline chr6_161480ıX603866766F & 6 cis & $8.3 \mathrm{cis}$ & 5.81 & $3.59 \mathrm{E}-04$ \\
\hline chr6_169760ı603866766F & 6 cis & 8.3 cis & 4.99 & $3.19 E-04$ \\
\hline chr6_140000ıX603866766F & 6 cis & $8.3 \mathrm{cis}$ & 12.78 & 4.47E-08 \\
\hline
\end{tabular}




\begin{tabular}{|c|c|c|c|c|}
\hline chr6_151550ıX603866766F & 6 cis & $8.3 \mathrm{cis}$ & 7.6 & $5.86 \mathrm{E}-05$ \\
\hline chr6_158680ıX603866766F & 6 cis & $8.3 \mathrm{cis}$ & 5.27 & $1.31 \mathrm{E}-04$ \\
\hline chr6_168630ıX603866766F & 6 cis & $8.3 \mathrm{cis}$ & 4.83 & 2.17E-04 \\
\hline chr6_170780ıX603866766F & 6 cis & $8.3 \mathrm{cis}$ & 6.95 & $1.94 \mathrm{E}-04$ \\
\hline chr6_155800ıX603866766F & 6 cis & $8.3 \mathrm{cis}$ & 9.36 & $1.26 \mathrm{E}-05$ \\
\hline chr6_167750ıX603866766F & 6 cis & $8.3 \mathrm{cis}$ & 4.38 & $3.12 \mathrm{E}-04$ \\
\hline chr6_157580ıX603866766F & 6 cis & $8.3 \mathrm{cis}$ & 5.8 & $1.02 \mathrm{E}-05$ \\
\hline chr6_172670ıX603866766F & 6 cis & $8.3 \mathrm{cis}$ & 5.57 & $1.24 \mathrm{E}-04$ \\
\hline chr6_138250ıX603866766F & 6 cis & $8.3 \mathrm{cis}$ & 4.17 & $2.04 \mathrm{E}-04$ \\
\hline chr6_171670ıX603866766F & 6 cis & $8.3 \mathrm{cis}$ & 5.35 & $6.43 \mathrm{E}-06$ \\
\hline chr6_172950ıX603866766F & 6 cis & $8.3 \mathrm{cis}$ & 7.13 & $1.67 \mathrm{E}-03$ \\
\hline chr6_154410IENSGALTO00( & 6 cis & 10.5 cis & 18.34 & $9.68 \mathrm{E}-04$ \\
\hline chr6_140000IENSGALT000( & 6 cis & $10.5 \mathrm{cis}$ & 12.78 & $9.32 \mathrm{E}-05$ \\
\hline chr6_165100IENSGALT000( & 6 cis & 10.5 cis & 4.75 & $2.96 \mathrm{E}-03$ \\
\hline chr6_150650IENSGALT000( & 6 cis & 10.5 cis & 9.04 & $7.82 \mathrm{E}-04$ \\
\hline chr6_172950IENSGALT000( & 6 cis & 10.5 cis & 7.13 & 3.64E-04 \\
\hline chr6_208330IENSGALT000( & 6 cis & 4.7 cis & 7.53 & 8.87E-05 \\
\hline chr6_208350IENSGALT000( & 6 cis & $4.7 \mathrm{cis}$ & 6.52 & $1.12 \mathrm{E}-04$ \\
\hline chr6_208320IENSGALT000( & 6 cis & $4.7 \mathrm{cis}$ & 4.26 & 2.93E-05 \\
\hline chr6_210210IENSGALT000( & 6 cis & $4.7 \mathrm{cis}$ & 6.19 & $3.22 \mathrm{E}-03$ \\
\hline chr6_169790IENSGALT000( & 6 cis & $4.7 \mathrm{cis}$ & 4.18 & $1.39 \mathrm{E}-05$ \\
\hline chr6_214500IENSGALT000( & 6 cis & $4.7 \mathrm{cis}$ & 4.45 & $5.55 \mathrm{E}-05$ \\
\hline chr6_170780IENSGALT000( & 6 cis & $4.7 \mathrm{cis}$ & 6.95 & 7.99E-05 \\
\hline chr6_167720IENSGALT000( & 6 cis & $4.7 \mathrm{cis}$ & 4.9 & $1.22 \mathrm{E}-03$ \\
\hline chr6_167750IENSGALT000( & 6 cis & $4.7 \mathrm{cis}$ & 4.38 & $5.04 \mathrm{E}-03$ \\
\hline chr6_216380IENSGALT000( & 6 cis & $4.7 \mathrm{cis}$ & 4.51 & $1.35 \mathrm{E}-04$ \\
\hline chr6_210190IENSGALT000( & 6 cis & $4.7 \mathrm{cis}$ & 5.94 & $5.36 \mathrm{E}-03$ \\
\hline chr6_167340IENSGALTO00( & 6 cis & 4.7 cis & 8.52 & 2.79E-03 \\
\hline chr6_183860IENSGALT000( & 6 cis & $4.7 \mathrm{cis}$ & 5.1 & $9.77 \mathrm{E}-04$ \\
\hline chr6_186820IENSGALT000( & 6 cis & $4.7 \mathrm{cis}$ & 7.43 & $2.42 \mathrm{E}-04$ \\
\hline chr6_189090IENSGALT000( & 6 cis & 4.7 cis & 7.03 & 8.10E-03 \\
\hline chr6_194480IENSGALT000( & 6 cis & $4.7 \mathrm{cis}$ & 8.37 & $1.21 \mathrm{E}-03$ \\
\hline chr6_205100IENSGALT000( & 6 cis & 4.7 cis & 4.91 & $1.90 \mathrm{E}-03$ \\
\hline chr6_169050IENSGALT000( & 6 cis & $4.7 \mathrm{cis}$ & 4.39 & $1.13 \mathrm{E}-03$ \\
\hline chr6_188980IENSGALT000( & 6 cis & $4.7 \mathrm{cis}$ & 16.05 & $9.39 \mathrm{E}-05$ \\
\hline chr6_190380IENSGALT000( & 6 cis & $4.7 \mathrm{cis}$ & 6.05 & $1.56 \mathrm{E}-03$ \\
\hline chr6_179170IENSGALT000 & 6 cis & $4.7 \mathrm{cis}$ & 5.52 & 8.67E-04 \\
\hline chr6_180620IENSGALT000( & 6 cis & $4.7 \mathrm{cis}$ & 8.61 & $1.68 \mathrm{E}-03$ \\
\hline chr6_193160IENSGALT000( & 6 cis & 4.7 cis & 9.19 & $2.20 \mathrm{E}-05$ \\
\hline chr6_207420IENSGALTO00( & 6 cis & $4.7 \mathrm{cis}$ & 6.11 & $2.82 \mathrm{E}-03$ \\
\hline chr6_207720IENSGALTO00( & 6 cis & $4.7 \mathrm{cis}$ & 5.58 & $1.38 \mathrm{E}-03$ \\
\hline chr6_183500IENSGALT000( & 6 cis & $4.7 \mathrm{cis}$ & 4.63 & $5.88 \mathrm{E}-04$ \\
\hline chr6_201760IENSGALTO00( & 6 cis & $4.7 \mathrm{cis}$ & 4.64 & $8.12 \mathrm{E}-04$ \\
\hline chr6_214750IENSGALTO00( & 6 cis & 4.7 cis & 4.43 & 1.49E-03 \\
\hline chr6_186340IENSGALT000( & 6 cis & 4.7 cis & 6.58 & $1.93 \mathrm{E}-05$ \\
\hline chr6_189870IENSGALT000( & 6 cis & $4.7 \mathrm{cis}$ & 6.58 & $2.05 \mathrm{E}-04$ \\
\hline chr6_195940IENSGALT000( & 6 cis & 4.7 cis & 6.98 & $3.72 \mathrm{E}-03$ \\
\hline chr6_211050IENSGALT000( & 6 cis & $4.7 \mathrm{cis}$ & 6.05 & $1.47 \mathrm{E}-03$ \\
\hline chr6_214710IENSGALTO00( & 6 cis & $4.7 \mathrm{cis}$ & 6.83 & 7.17E-04 \\
\hline chr6_217960IENSGALT000( & 6 cis & $4.7 \mathrm{cis}$ & 6.56 & $1.62 \mathrm{E}-05$ \\
\hline chr6_202390IENSGALTO00( & 6 cis & 4.7 cis & 4.79 & $7.51 \mathrm{E}-04$ \\
\hline chr6_190490IENSGALT000( & 6 cis & $4.7 \mathrm{cis}$ & 4.65 & $1.20 \mathrm{E}-03$ \\
\hline chr6_202570IENSGALT000( & 6 cis & 4.7 cis & 6.23 & $1.87 \mathrm{E}-03$ \\
\hline chr6_210530IENSGALT000( & 6 cis & $4.7 \mathrm{cis}$ & 8.06 & $3.63 \mathrm{E}-05$ \\
\hline chr6_215230IENSGALT000( & 6 cis & 4.7 cis & 9.03 & $1.07 \mathrm{E}-03$ \\
\hline chr6_224940IENSGALT000( & 6 cis & $4.7 \mathrm{cis}$ & 5.32 & $2.67 \mathrm{E}-04$ \\
\hline chr6_229190IENSGALT000( & 6 cis & 4.7 cis & 7.96 & 4.70E-03 \\
\hline chr6_199010ıENSGALT000( & 6 cis & $4.7 \mathrm{cis}$ & 5.82 & 4.47E-05 \\
\hline chr6_228220IENSGALT000( & 6 cis & $4.7 \mathrm{cis}$ & 6.13 & $6.70 \mathrm{E}-04$ \\
\hline chr6_197560IENSGALT000( & 6 cis & $4.7 \mathrm{cis}$ & 13.74 & 2.37E-05 \\
\hline chr6_214620IENSGALTO00( & 6 cis & $4.7 \mathrm{cis}$ & 10.74 & $8.29 \mathrm{E}-05$ \\
\hline chr6_222740IENSGALTO00( & 6 cis & $4.7 \mathrm{cis}$ & 7.8 & $1.89 \mathrm{E}-06$ \\
\hline chr6_227500IENSGALTO00( & 6 cis & 4.7 cis & 5.91 & $7.86 \mathrm{E}-04$ \\
\hline chr6_218600IENSGALT000( & 6 cis & $4.7 \mathrm{cis}$ & 5.54 & $3.84 \mathrm{E}-05$ \\
\hline chr6_228040IENSGALT000( & 6 cis & $4.7 \mathrm{cis}$ & 4.88 & 3.79E-04 \\
\hline chr6_225590IENSGALTO00( & 6 cis & $4.7 \mathrm{cis}$ & 6.04 & $2.82 \mathrm{E}-04$ \\
\hline chr6_201950IENSGALT000 & 6 cis & $4.7 \mathrm{cis}$ & 4.23 & $1.34 \mathrm{E}-03$ \\
\hline chr6_201560IENSGALT000( & 6 cis & $4.7 \mathrm{cis}$ & 9.19 & $2.48 \mathrm{E}-03$ \\
\hline chr6_210520IENSGALTO00( & 6 cis & 4.7 cis & 6.35 & $3.04 \mathrm{E}-06$ \\
\hline chr6_228610IENSGALT000( & 6 cis & $4.7 \mathrm{cis}$ & 4.32 & $6.58 \mathrm{E}-04$ \\
\hline chr6_235620IENSGALT000( & 6 cis & 4.7 cis & 4.41 & $3.90 \mathrm{E}-04$ \\
\hline chr6_235630IENSGALTO00( & 6 cis & $4.7 \mathrm{cis}$ & 4.74 & $1.72 \mathrm{E}-03$ \\
\hline
\end{tabular}




\begin{tabular}{|c|c|c|c|c|}
\hline chr6_200740IENSGALTO00 & $6 \mathrm{cis}$ & $4.7 \mathrm{cis}$ & 8.42 & $2.26 \mathrm{E}-05$ \\
\hline chr6_190020IENSGALTO00( & 6 cis & $4.7 \mathrm{cis}$ & 5.05 & 3.77E-04 \\
\hline chr6_182340ıENSGALTO00( & 6 cis & 4.7 trans & 10.77 & 5.13E-04 \\
\hline chr6_162690IENSGALT000( & 6 cis & 5.9 cis & 6.9 & $1.24 \mathrm{E}-06$ \\
\hline chr6_210210ıENSGALTO00( & 6 cis & 5.9 cis & 6.19 & $3.22 \mathrm{E}-05$ \\
\hline chr6_222130IENSGALTO00( & 6 cis & 5.9 cis & 6.62 & 4.34E-06 \\
\hline chr6_169790ıENSGALTO00( & 6 cis & 5.9 cis & 4.18 & $8.29 E-07$ \\
\hline chr6_214500ıENSGALTO00( & 6 cis & 5.9 cis & 4.45 & $1.74 \mathrm{E}-07$ \\
\hline chr6_161480ıENSGALT000( & 6 cis & 5.9 cis & 5.81 & $9.79 E-05$ \\
\hline chr6_165100ıENSGALT000( & 6 cis & 5.9 cis & 4.75 & 3.76E-04 \\
\hline chr6_170780ıENSGALT000( & 6 cis & 5.9 cis & 6.95 & $5.22 \mathrm{E}-06$ \\
\hline chr6_227430IENSGALTO00( & 6 cis & 5.9 cis & 4.42 & $9.16 \mathrm{E}-07$ \\
\hline chr6_167720ıENSGALTO00( & 6 cis & 5.9 cis & 4.9 & 9.17E-05 \\
\hline chr6_167750IENSGALTO00( & 6 cis & 5.9 cis & 4.38 & $2.62 \mathrm{E}-04$ \\
\hline chr6_216380ıENSGALTO00( & 6 cis & 5.9 cis & 4.51 & $2.66 \mathrm{E}-06$ \\
\hline chr6_217290ıENSGALTO00( & 6 cis & 5.9 cis & 4.54 & $2.18 \mathrm{E}-04$ \\
\hline chr6_223480ıENSGALTO00( & 6 cis & 5.9 cis & 4.84 & $1.48 \mathrm{E}-04$ \\
\hline chr6_189080IENSGALTO00( & 6 cis & 5.9 cis & 5.9 & $4.68 \mathrm{E}-06$ \\
\hline chr6_210190ıENSGALTO00( & 6 cis & 5.9 cis & 5.94 & $1.86 \mathrm{E}-04$ \\
\hline chr6_165310ıENSGALTO00( & 6 cis & 5.9 cis & 4.65 & $8.62 \mathrm{E}-04$ \\
\hline chr6_188360ıENSGALT000( & 6 cis & 5.9 cis & 5.16 & $2.24 \mathrm{E}-04$ \\
\hline chr6_172670ıENSGALT000( & 6 cis & 5.9 cis & 5.57 & $2.21 \mathrm{E}-06$ \\
\hline chr6_186820IENSGALT000( & 6 cis & 5.9 cis & 7.43 & 3.70E-05 \\
\hline chr6_189090IENSGALT000( & 6 cis & 5.9 cis & 7.03 & 4.74E-07 \\
\hline chr6_189880IENSGALTO00( & 6 cis & 5.9 cis & 5.54 & $8.12 \mathrm{E}-06$ \\
\hline chr6_194340ıENSGALTO00( & 6 cis & 5.9 cis & 5.65 & 2.87E-04 \\
\hline chr6_194480ıENSGALT000( & 6 cis & 5.9 cis & 8.37 & $9.46 \mathrm{E}-06$ \\
\hline chr6_196700ıENSGALTO00( & 6 cis & 5.9 cis & 6.37 & $6.31 \mathrm{E}-04$ \\
\hline chr6_226150IENSGALTO00( & 6 cis & 5.9 cis & 5.4 & $1.09 \mathrm{E}-04$ \\
\hline chr6_186910ıENSGALTO00( & 6 cis & 5.9 cis & 4.55 & $4.42 \mathrm{E}-05$ \\
\hline chr6_200040IENSGALTO00( & 6 cis & 5.9 cis & 4.34 & 4.10E-04 \\
\hline chr6_205100ıENSGALTO00( & 6 cis & 5.9 cis & 4.91 & 1.10E-05 \\
\hline chr6_193260ıENSGALTO00( & 6 cis & 5.9 cis & 6.76 & 3.57E-05 \\
\hline chr6_194380ıENSGALTO00( & 6 cis & 5.9 cis & 5.54 & $3.55 \mathrm{E}-04$ \\
\hline chr6_215040IENSGALTO00( & 6 cis & 5.9 cis & 6.16 & $6.05 \mathrm{E}-05$ \\
\hline chr6_169050IENSGALTO00( & 6 cis & 5.9 cis & 4.39 & $1.75 \mathrm{E}-04$ \\
\hline chr6_176860ıENSGALT000( & 6 cis & 5.9 cis & 4.25 & 7.16E-05 \\
\hline chr6_203170ıENSGALTO00( & 6 cis & 5.9 cis & 5.72 & $1.48 \mathrm{E}-05$ \\
\hline chr6_171670ıENSGALT000( & 6 cis & 5.9 cis & 5.35 & 4.71E-05 \\
\hline chr6_188980IENSGALTO00( & 6 cis & 5.9 cis & 16.05 & $2.19 \mathrm{E}-05$ \\
\hline chr6_190380IENSGALTO00( & 6 cis & 5.9 cis & 6.05 & $1.36 \mathrm{E}-05$ \\
\hline chr6_203190IENSGALTO00( & 6 cis & 5.9 cis & 5.01 & $2.20 \mathrm{E}-04$ \\
\hline chr6_203560IENSGALTO00( & 6 cis & 5.9 cis & 5.69 & $1.29 \mathrm{E}-04$ \\
\hline chr6_210950IENSGALTO00( & 6 cis & 5.9 cis & 5.63 & 7.02E-06 \\
\hline chr6_197570ıENSGALTO00( & 6 cis & 5.9 cis & 5.08 & $2.18 \mathrm{E}-04$ \\
\hline chr6_180620IENSGALT000( & 6 cis & 5.9 cis & 8.61 & 3.33E-05 \\
\hline chr6_193160IENSGALTO00( & 6 cis & 5.9 cis & 9.19 & 7.71E-06 \\
\hline chr6_194400IENSGALTO00( & 6 cis & 5.9 cis & 9.65 & $2.04 \mathrm{E}-04$ \\
\hline chr6_207420ıENSGALTO00( & 6 cis & 5.9 cis & 6.11 & $1.26 \mathrm{E}-06$ \\
\hline chr6_207720ıENSGALTO00( & 6 cis & 5.9 cis & 5.58 & $8.54 \mathrm{E}-05$ \\
\hline chr6_214570ıENSGALTO00( & 6 cis & 5.9 cis & 7.42 & $3.58 \mathrm{E}-05$ \\
\hline chr6_183500ıENSGALTO00 & 6 cis & 5.9 cis & 4.63 & 3.87E-04 \\
\hline chr6_201760ıENSGALTO00( & 6 cis & 5.9 cis & 4.64 & $3.98 \mathrm{E}-04$ \\
\hline chr6_202560IENSGALTO00( & 6 cis & 5.9 cis & 4.48 & $3.14 \mathrm{E}-03$ \\
\hline chr6_214750ıENSGALTO00( & 6 cis & 5.9 cis & 4.43 & 3.37E-07 \\
\hline chr6_221070ıENSGALTO00( & 6 cis & 5.9 cis & 4.57 & $3.44 \mathrm{E}-05$ \\
\hline chr6_186340ıENSGALTO00( & 6 cis & 5.9 cis & 6.58 & $1.33 \mathrm{E}-05$ \\
\hline chr6_189870ıENSGALT000 & 6 cis & 5.9 cis & 6.58 & $2.56 \mathrm{E}-06$ \\
\hline chr6_195940IENSGALTO00( & 6 cis & 5.9 cis & 6.98 & $1.70 \mathrm{E}-05$ \\
\hline chr6_211050IENSGALTO00( & 6 cis & 5.9 cis & 6.05 & $7.08 \mathrm{E}-06$ \\
\hline chr6_214710ıENSGALTO00( & 6 cis & 5.9 cis & 6.83 & 3.16E-06 \\
\hline chr6_217960ıENSGALTO00( & 6 cis & 5.9 cis & 6.56 & $6.26 \mathrm{E}-08$ \\
\hline chr6_202390ıENSGALTO00( & 6 cis & 5.9 cis & 4.79 & 7.95E-07 \\
\hline chr6_190490IENSGALTO00 & 6 cis & 5.9 cis & 4.65 & $5.29 \mathrm{E}-05$ \\
\hline chr6_187840IENSGALTO00( & 6 cis & 5.9 cis & 14.44 & 8.09E-05 \\
\hline chr6_193250IENSGALTO00 & 6 cis & 5.9 cis & 9.19 & $1.05 \mathrm{E}-04$ \\
\hline chr6_202570IENSGALTO00( & 6 cis & 5.9 cis & 6.23 & 7.27E-06 \\
\hline chr6_210530ıENSGALTO00( & 6 cis & 5.9 cis & 8.06 & $1.75 \mathrm{E}-05$ \\
\hline chr6_215230ıENSGALTO00( & 6 cis & 5.9 cis & 9.03 & 3.19E-05 \\
\hline chr6_224940IENSGALTO00( & 6 cis & 5.9 cis & 5.32 & $3.84 \mathrm{E}-07$ \\
\hline chr6_195950IENSGALTO00( & 6 cis & 5.9 cis & 5.49 & 7.17E-04 \\
\hline chr6_229190ıENSGALTO00( & 6 cis & 5.9 cis & 7.96 & $2.31 \mathrm{E}-05$ \\
\hline
\end{tabular}




\begin{tabular}{|c|c|c|c|c|}
\hline chr6_229310IENSGALTO00( & 6 cis & 5.9 cis & 6.86 & $9.91 \mathrm{E}-06$ \\
\hline chr6_199010IENSGALT000 & 6 cis & 5.9 cis & 5.82 & $1.34 \mathrm{E}-06$ \\
\hline chr6_214690IENSGALT000( & 6 cis & 5.9 cis & 5.19 & $8.02 \mathrm{E}-05$ \\
\hline chr6_228220IENSGALT000( & 6 cis & 5.9 cis & 6.13 & $1.29 \mathrm{E}-05$ \\
\hline chr6_199830IENSGALT000( & 6 cis & 5.9 cis & 5.65 & $2.78 \mathrm{E}-05$ \\
\hline chr6_227420IENSGALT000( & 6 cis & 5.9 cis & 8.07 & 7.17E-06 \\
\hline chr6_187550IENSGALT000( & 6 cis & 5.9 cis & 11.36 & $1.32 \mathrm{E}-04$ \\
\hline chr6_213320IENSGALTO00( & 6 cis & 5.9 cis & 6.8 & $1.02 \mathrm{E}-04$ \\
\hline chr6_228730IENSGALT000( & 6 cis & 5.9 cis & 5.43 & $1.91 \mathrm{E}-04$ \\
\hline chr6_197560IENSGALT000( & 6 cis & 5.9 cis & 13.74 & $6.67 \mathrm{E}-07$ \\
\hline chr6_201260IENSGALT000( & 6 cis & 5.9 cis & 4.92 & $1.04 \mathrm{E}-04$ \\
\hline chr6_214620IENSGALTO00( & 6 cis & 5.9 cis & 10.74 & $1.35 \mathrm{E}-07$ \\
\hline chr6_214760IENSGALT000( & $6 \mathrm{cis}$ & 5.9 cis & 4.47 & 4.36E-08 \\
\hline chr6_222740IENSGALT000( & 6 cis & 5.9 cis & 7.8 & $1.68 \mathrm{E}-05$ \\
\hline chr6_227500IENSGALT000( & 6 cis & 5.9 cis & 5.91 & $3.62 \mathrm{E}-08$ \\
\hline chr6_218600IENSGALT000( & 6 cis & 5.9 cis & 5.54 & 5.79E-08 \\
\hline chr6_222730IENSGALT000( & 6 cis & 5.9 cis & 8.71 & $7.02 \mathrm{E}-05$ \\
\hline chr6_222790IENSGALT000( & 6 cis & 5.9 cis & 6.5 & $6.46 \mathrm{E}-06$ \\
\hline chr6_214780IENSGALTO00 & 6 cis & 5.9 cis & 5.11 & $3.42 \mathrm{E}-07$ \\
\hline chr6_228040IENSGALT000( & 6 cis & 5.9 cis & 4.88 & $8.55 \mathrm{E}-06$ \\
\hline chr6_225590IENSGALT000( & 6 cis & 5.9 cis & 6.04 & $1.25 \mathrm{E}-04$ \\
\hline chr6_201750IENSGALT000( & 6 cis & 5.9 cis & 4.75 & $5.60 \mathrm{E}-04$ \\
\hline chr6_201560IENSGALT000( & 6 cis & 5.9 cis & 9.19 & $1.66 \mathrm{E}-04$ \\
\hline chr6_210520IENSGALT000( & 6 cis & 5.9 cis & 6.35 & 4.61E-06 \\
\hline chr6_236440IENSGALT000( & 6 cis & 5.9 cis & 6.19 & $1.21 \mathrm{E}-04$ \\
\hline chr6_238090IENSGALT000( & 6 cis & 5.9 cis & 5.74 & 1.37E-05 \\
\hline chr6_228610IENSGALT000( & 6 cis & 5.9 cis & 4.32 & $1.81 \mathrm{E}-04$ \\
\hline chr6_235630IENSGALTO00 & 6 cis & 5.9 cis & 4.74 & 7.79E-06 \\
\hline chr6_228050IENSGALT000( & 6 cis & 5.9 cis & 5.29 & $3.80 \mathrm{E}-05$ \\
\hline chr6_200740IENSGALT000( & 6 cis & 5.9 cis & 8.42 & $3.12 \mathrm{E}-06$ \\
\hline chr6_190020IENSGALT000( & 6 cis & 5.9 cis & 5.05 & $1.58 \mathrm{E}-05$ \\
\hline chr6_222130IENSGALTO00( & 6 cis & 5.4 cis & 6.62 & $1.88 \mathrm{E}-04$ \\
\hline chr6_214500IENSGALT000( & 6 cis & 5.4 cis & 4.45 & 5.49E-05 \\
\hline chr6_189090IENSGALT000( & 6 cis & 5.4 cis & 7.03 & $1.26 \mathrm{E}-04$ \\
\hline chr6_189880IENSGALT000( & 6 cis & 5.4 cis & 5.54 & $2.05 \mathrm{E}-05$ \\
\hline chr6_188980IENSGALT000( & 6 cis & 5.4 cis & 16.05 & $2.84 \mathrm{E}-07$ \\
\hline chr6_190380IENSGALT000( & 6 cis & 5.4 cis & 6.05 & 1.19E-04 \\
\hline chr6_197570IENSGALT000( & 6 cis & 5.4 cis & 5.08 & 4.47E-04 \\
\hline chr6_180620IENSGALT000( & 6 cis & 5.4 cis & 8.61 & 5.00E-06 \\
\hline chr6_221070IENSGALT000( & 6 cis & 5.4 cis & 4.57 & $8.61 \mathrm{E}-05$ \\
\hline chr6_214710IENSGALT000( & 6 cis & 5.4 cis & 6.83 & 5.19E-05 \\
\hline chr6_217960IENSGALT000( & 6 cis & 5.4 cis & 6.56 & $2.76 \mathrm{E}-04$ \\
\hline chr6_190490IENSGALT000( & 6 cis & 5.4 cis & 4.65 & 6.07E-04 \\
\hline chr6_187840IENSGALTOOOC & 6 cis & 5.4 cis & 14.44 & $1.34 \mathrm{E}-04$ \\
\hline chr6_193250IENSGALT000( & 6 cis & 5.4 cis & 9.19 & $3.48 \mathrm{E}-04$ \\
\hline chr6_210530IENSGALT000( & 6 cis & 5.4 cis & 8.06 & $1.26 \mathrm{E}-05$ \\
\hline chr6_203220IENSGALTO00 & 6 cis & 5.4 cis & 4.78 & $2.73 \mathrm{E}-05$ \\
\hline chr6_224940IENSGALT000( & 6 cis & 5.4 cis & 5.32 & $1.86 \mathrm{E}-04$ \\
\hline chr6_229310IENSGALTO00( & 6 cis & 5.4 cis & 6.86 & 8.19E-04 \\
\hline chr6_187550IENSGALT000 & 6 cis & 5.4 cis & 11.36 & $2.09 \mathrm{E}-05$ \\
\hline chr6_212390IENSGALT000( & 6 cis & 5.4 cis & 5.2 & 7.57E-05 \\
\hline chr6_197560IENSGALT000( & 6 cis & 5.4 cis & 13.74 & $2.78 \mathrm{E}-06$ \\
\hline chr6_201260IENSGALT000( & 6 cis & $5.4 \mathrm{cis}$ & 4.92 & $1.06 \mathrm{E}-05$ \\
\hline chr6_214620IENSGALT000( & 6 cis & 5.4 cis & 10.74 & $1.91 \mathrm{E}-06$ \\
\hline chr6_227500IENSGALT000( & $6 \mathrm{cis}$ & 5.4 cis & 5.91 & $2.85 \mathrm{E}-06$ \\
\hline chr6_222730IENSGALT000 & 6 cis & 5.4 cis & 8.71 & $6.43 E-05$ \\
\hline chr6_214780IENSGALT000( & 6 cis & 5.4 cis & 5.11 & $2.95 \mathrm{E}-04$ \\
\hline chr6_225590IENSGALT000( & 6 cis & 5.4 cis & 6.04 & $2.33 E-04$ \\
\hline chr6_201750IENSGALT000( & 6 cis & 5.4 cis & 4.75 & $3.70 \mathrm{E}-05$ \\
\hline chr6_201560IENSGALT000( & 6 cis & 5.4 cis & 9.19 & $3.08 \mathrm{E}-07$ \\
\hline chr6_210520IENSGALT000( & $6 \mathrm{cis}$ & 5.4 cis & 6.35 & $1.70 \mathrm{E}-04$ \\
\hline chr6_200740IENSGALT000( & 6 cis & 5.4 cis & 8.42 & $6.29 E-04$ \\
\hline chr6_205370IENSGALTO00 & 6 cis & $5.4 \mathrm{cis}$ & 4.1 & $1.22 \mathrm{E}-02$ \\
\hline chr6_235130IENSGALTO00( & 6 cis & 4.4 trans & 5.76 & $5.34 \mathrm{E}-04$ \\
\hline chr6_172950IENSGALTO00( & 6 cis & 4.4 cis & 7.13 & $1.40 \mathrm{E}-02$ \\
\hline chr6_214710IENSGALT000( & 6 cis & $4.4 \mathrm{cis}$ & 6.83 & $1.12 \mathrm{E}-04$ \\
\hline chr6_187840IENSGALT000( & $6 \mathrm{cis}$ & 4.4 cis & 14.44 & $3.35 \mathrm{E}-04$ \\
\hline chr6_203220IENSGALT000( & 6 cis & $4.4 \mathrm{cis}$ & 4.78 & $1.21 \mathrm{E}-05$ \\
\hline chr6_216810IENSGALT000( & 6 cis & 4.4 cis & 4.4 & $5.69 \mathrm{E}-04$ \\
\hline chr6_187550IENSGALT000( & 6 cis & $4.4 \mathrm{cis}$ & 11.36 & $2.60 \mathrm{E}-04$ \\
\hline chr6_236440IENSGALT000( & 6 cis & $4.4 \mathrm{cis}$ & 6.19 & $2.96 \mathrm{E}-04$ \\
\hline chr6_202310IENSGALTO00( & 6 cis & $4.4 \mathrm{cis}$ & 6.52 & 2.90E-04 \\
\hline
\end{tabular}




\begin{tabular}{|c|c|c|c|c|}
\hline chr6_2286101X603862991F & 6 cis & 4.5 cis & 4.32 & $1.59 \mathrm{E}-03$ \\
\hline chr6_200740IENSGALTO00( & 6 cis & 4.6 cis & 8.42 & 7.00E-05 \\
\hline chr6_2221301X603599830F & 6 cis & 6.4 cis & 6.62 & $1.18 \mathrm{E}-05$ \\
\hline chr6_2145001X603599830F & 6 cis & 6.4 cis & 4.45 & $5.56 \mathrm{E}-04$ \\
\hline chr6_2274301X603599830F & 6 cis & 6.4 cis & 4.42 & $5.26 \mathrm{E}-05$ \\
\hline chr6_2163801X603599830F & 6 cis & 6.4 cis & 4.51 & $1.07 \mathrm{E}-04$ \\
\hline chr6_2101901X603599830F & 6 cis & 6.4 cis & 5.94 & $1.90 \mathrm{E}-04$ \\
\hline chr6_2261501X603599830F & 6 cis & 6.4 cis & 5.4 & $9.15 \mathrm{E}-05$ \\
\hline chr6_2369401X603599830F & 6 cis & 6.4 cis & 4.46 & $8.45 \mathrm{E}-05$ \\
\hline chr6_2109501X603599830F & 6 cis & 6.4 cis & 5.63 & $5.01 \mathrm{E}-04$ \\
\hline chr6_2145701X603599830F & 6 cis & 6.4 cis & 7.42 & $8.88 \mathrm{E}-04$ \\
\hline chr6_2147501X603599830F & 6 cis & 6.4 cis & 4.43 & 4.35E-07 \\
\hline chr6_2210701X603599830F & 6 cis & 6.4 cis & 4.57 & $2.26 \mathrm{E}-03$ \\
\hline chr6_2147101X603599830F & 6 cis & 6.4 cis & 6.83 & $3.45 \mathrm{E}-04$ \\
\hline chr6_2179601X603599830F & 6 cis & 6.4 cis & 6.56 & 1.57E-05 \\
\hline chr6_2249401X603599830F & 6 cis & 6.4 cis & 5.32 & $1.59 \mathrm{E}-04$ \\
\hline chr6_2291901X603599830F & 6 cis & 6.4 cis & 7.96 & $6.23 \mathrm{E}-03$ \\
\hline chr6_2293101X603599830F & 6 cis & 6.4 cis & 6.86 & $5.76 \mathrm{E}-05$ \\
\hline chr6_2282201X603599830F & 6 cis & 6.4 cis & 6.13 & $1.21 \mathrm{E}-05$ \\
\hline chr6_2274201X603599830F & 6 cis & 6.4 cis & 8.07 & $9.04 \mathrm{E}-06$ \\
\hline chr6_2287301X603599830F & 6 cis & 6.4 cis & 5.43 & $2.55 \mathrm{E}-06$ \\
\hline chr6_2146201X603599830F & 6 cis & 6.4 cis & 10.74 & $5.56 \mathrm{E}-04$ \\
\hline chr6_2147601X603599830F & 6 cis & 6.4 cis & 4.47 & $6.08 \mathrm{E}-07$ \\
\hline chr6_2275001X603599830F & 6 cis & 6.4 cis & 5.91 & $1.22 \mathrm{E}-04$ \\
\hline chr6_2355501X603599830F & 6 cis & 6.4 cis & 6.98 & $2.05 \mathrm{E}-04$ \\
\hline chr6_2147801X603599830F & 6 cis & 6.4 cis & 5.11 & $1.05 \mathrm{E}-05$ \\
\hline chr6_2280401X603599830F & 6 cis & 6.4 cis & 4.88 & 5.91E-04 \\
\hline chr6_2255901X603599830F & 6 cis & 6.4 cis & 6.04 & $1.14 \mathrm{E}-03$ \\
\hline chr6_2105201X603599830F & 6 cis & 6.4 cis & 6.35 & 1.17E-04 \\
\hline chr6_2364401X603599830F & 6 cis & 6.4 cis & 6.19 & $3.62 \mathrm{E}-06$ \\
\hline chr6_2380901X603599830F & 6 cis & 6.4 cis & 5.74 & 9.00E-05 \\
\hline chr6_2286101X603599830F & 6 cis & 6.4 cis & 4.32 & 3.19E-05 \\
\hline chr6_2356201X603599830F & 6 cis & 6.4 cis & 4.41 & $2.89 \mathrm{E}-03$ \\
\hline chr6_2356301X603599830F & 6 cis & $6.4 \mathrm{cis}$ & 4.74 & $6.02 E-05$ \\
\hline chr6_2280501X603599830F & 6 cis & 6.4 cis & 5.29 & $3.61 \mathrm{E}-05$ \\
\hline chr6_228610IENSGALTO00( & 6 cis & $6.2 \mathrm{cis}$ & 4.32 & $2.51 \mathrm{E}-04$ \\
\hline chr6_2662701X603568048F & 6 cis & 5.1 trans & 6.8 & $5.38 \mathrm{E}-04$ \\
\hline chr6_2291901X603568048F & 6 cis & $5.1 \mathrm{cis}$ & 7.96 & $2.05 \mathrm{E}-03$ \\
\hline chr6_2280401X603568048F & 6 cis & 5.1 cis & 4.88 & $1.59 \mathrm{E}-04$ \\
\hline chr6_2456001X603568048F & 6 cis & $5.1 \mathrm{cis}$ & 5.6 & $1.22 \mathrm{E}-04$ \\
\hline chr6_2543401X603568048F & 6 cis & $5.1 \mathrm{cis}$ & 8.5 & 1.27E-04 \\
\hline chr6_2564301X603568048F & 6 cis & $5.1 \mathrm{cis}$ & 6.7 & 2.90E-04 \\
\hline chr6_2632601X603568048F & 6 cis & $5.1 \mathrm{cis}$ & 4.62 & 2.91E-04 \\
\hline chr6_2451501X603568048F & 6 cis & $5.1 \mathrm{cis}$ & 4.23 & 1.17E-05 \\
\hline chr6_2458501X603568048F & 6 cis & 5.1 cis & 5.12 & $9.19 \mathrm{E}-05$ \\
\hline chr6_2478101X603568048F & 6 cis & $5.1 \mathrm{cis}$ & 4.6 & $3.20 \mathrm{E}-04$ \\
\hline chr6_2512501X603568048F & 6 cis & 5.1 cis & 5.34 & $5.68 \mathrm{E}-05$ \\
\hline chr6_2551801X603568048F & 6 cis & $5.1 \mathrm{cis}$ & 4.1 & $6.46 \mathrm{E}-04$ \\
\hline chr6_2582201X603568048F & 6 cis & $5.1 \mathrm{cis}$ & 6.28 & $1.65 \mathrm{E}-04$ \\
\hline chr6_2581801X603568048F & 6 cis & $5.1 \mathrm{cis}$ & 4.11 & $1.36 \mathrm{E}-03$ \\
\hline chr6_254140।X603568048F & 6 cis & $5.1 \mathrm{cis}$ & 4.48 & $1.62 \mathrm{E}-04$ \\
\hline chr6_2620601X603568048F & 6 cis & $5.1 \mathrm{cis}$ & 4.18 & 3.34E-04 \\
\hline chr6_2545301X603568048F & 6 cis & $5.1 \mathrm{cis}$ & 4.85 & 4.93E-06 \\
\hline chr6_261310ıX603568048F & 6 cis & $5.1 \mathrm{cis}$ & 11.65 & $1.04 \mathrm{E}-04$ \\
\hline chr6_2407601X603568048F & 6 cis & $5.1 \mathrm{cis}$ & 5.61 & $1.34 \mathrm{E}-03$ \\
\hline chr6_2640301X603568048F & 6 cis & $5.1 \mathrm{cis}$ & 4.15 & $1.25 \mathrm{E}-05$ \\
\hline chr6_2594301X603568048F & 6 cis & $5.1 \mathrm{cis}$ & 5.65 & $3.70 \mathrm{E}-04$ \\
\hline chr6_2540001X603568048F & 6 cis & $5.1 \mathrm{cis}$ & 7.99 & $8.28 \mathrm{E}-03$ \\
\hline chr6_2820901X603568048F & 6 cis & $5.1 \mathrm{cis}$ & 5.49 & $4.02 \mathrm{E}-03$ \\
\hline chr6_2650401X603568048F & 6 cis & $5.1 \mathrm{cis}$ & 5.42 & $1.45 \mathrm{E}-03$ \\
\hline chr6_2696501X603568048F & 6 cis & $5.1 \mathrm{cis}$ & 4.1 & $2.69 \mathrm{E}-03$ \\
\hline chr6_2650201X603568048F & 6 cis & $5.1 \mathrm{cis}$ & 7.58 & 3.35E-04 \\
\hline chr6_2673601X603568048F & 6 cis & $5.1 \mathrm{cis}$ & 7.61 & $2.48 \mathrm{E}-05$ \\
\hline chr6_2484901X603568048F & 6 cis & $5.1 \mathrm{cis}$ & 5.64 & $1.70 \mathrm{E}-03$ \\
\hline chr6_2652701X603568048F & 6 cis & $5.1 \mathrm{cis}$ & 6.5 & 7.40E-04 \\
\hline chr6_2820801X603568048F & 6 cis & $5.1 \mathrm{cis}$ & 7.26 & $1.62 \mathrm{E}-03$ \\
\hline chr6_2647601X603568048F & 6 cis & $5.1 \mathrm{cis}$ & 4.42 & $8.12 \mathrm{E}-03$ \\
\hline chr6_3026201X603568048F & 6 cis & $5.1 \mathrm{cis}$ & 4.1 & $1.56 \mathrm{E}-04$ \\
\hline chr6_2465001X603862885F & 6 cis & 4.1 trans & 5.61 & $1.03 \mathrm{E}-04$ \\
\hline chr6_2249401X603862885F & 6 cis & $4.1 \mathrm{cis}$ & 5.32 & $1.94 \mathrm{E}-03$ \\
\hline chr6_2584501X603862885F & 6 cis & $4.1 \mathrm{cis}$ & 4.72 & $4.32 \mathrm{E}-03$ \\
\hline chr6_245600ıX603862885F & 6 cis & $4.1 \mathrm{cis}$ & 5.6 & $2.00 \mathrm{E}-03$ \\
\hline
\end{tabular}




\begin{tabular}{|c|c|c|c|c|}
\hline chr6_2515901X603862885F & 6 cis & $4.1 \mathrm{cis}$ & 5.18 & $1.85 \mathrm{E}-03$ \\
\hline chr6_2545301X603862885F & 6 cis & 4.1 cis & 4.85 & $3.66 \mathrm{E}-03$ \\
\hline chr6_2454201X603862885F & 6 cis & $4.1 \mathrm{cis}$ & 5.36 & $5.80 \mathrm{E}-04$ \\
\hline chr6_2650201X603862885F & 6 cis & $4.1 \mathrm{cis}$ & 7.58 & $2.42 \mathrm{E}-03$ \\
\hline chr6_265020IENSGALT000( & 6 cis & $4.3 \mathrm{cis}$ & 7.58 & $1.27 \mathrm{E}-03$ \\
\hline chr6_254000IX603601127F & 6 cis & 7.3 cis & 7.99 & $1.06 \mathrm{E}-03$ \\
\hline chr6_2650201X603601127F & 6 cis & 7.3 cis & 7.58 & $5.80 \mathrm{E}-04$ \\
\hline chr6_2673601X603601127F & 6 cis & 7.3 cis & 7.61 & 3.19E-04 \\
\hline chr6_2484901X603601127F & 6 cis & 7.3 cis & 5.64 & 4.01E-06 \\
\hline chr6_2652701X603601127F & 6 cis & 7.3 cis & 6.5 & 4.10E-04 \\
\hline chr6_2655301X603601127F & 6 cis & 7.3 cis & 5.47 & 2.77E-03 \\
\hline chr6_2659001X603601127F & 6 cis & 7.3 cis & 6.3 & $6.79 \mathrm{E}-04$ \\
\hline chr6_2956501X603601127F & 6 cis & 7.3 cis & 6.14 & $8.20 \mathrm{E}-06$ \\
\hline chr6_2867301X603601127F & 6 cis & 7.3 cis & 5.44 & 4.29E-03 \\
\hline chr6_2895001X603601127F & 6 cis & 7.3 cis & 10.88 & 3.07E-05 \\
\hline chr6_2649301X603601127F & 6 cis & 7.3 cis & 5.02 & $2.41 \mathrm{E}-03$ \\
\hline chr6_2767501X603601127F & 6 cis & 7.3 cis & 6.56 & 3.17E-03 \\
\hline chr6_2526701X603601127F & 6 cis & 7.3 cis & 6.25 & 4.15E-03 \\
\hline chr6_2647601X603601127F & 6 cis & 7.3 cis & 4.42 & 2.19E-05 \\
\hline chr6_2858101X603601127F & 6 cis & 7.3 cis & 5.54 & $5.78 \mathrm{E}-04$ \\
\hline chr6_254530IENSGALTO00( & 6 cis & $8.8 \mathrm{cis}$ & 4.85 & 3.90E-03 \\
\hline chr6_265020IENSGALT000( & 6 cis & $8.8 \mathrm{cis}$ & 7.58 & $1.29 \mathrm{E}-03$ \\
\hline chr6_265510IENSGALTO00( & 6 cis & $8.8 \mathrm{cis}$ & 4.99 & $1.03 \mathrm{E}-03$ \\
\hline chr6_289500IENSGALT000( & 6 cis & $8.8 \mathrm{cis}$ & 10.88 & $3.81 \mathrm{E}-05$ \\
\hline chr6_276750IENSGALTO00( & 6 cis & $8.8 \mathrm{cis}$ & 6.56 & $3.22 \mathrm{E}-04$ \\
\hline chr6_285810IENSGALTO00( & 6 cis & 8.8 cis & 5.54 & $5.54 \mathrm{E}-04$ \\
\hline chr6_267570IENSGALTO00( & 6 cis & 5.2 cis & 4.32 & $6.07 \mathrm{E}-03$ \\
\hline chr6_265020IENSGALT000( & 6 cis & 5.2 cis & 7.58 & $1.12 \mathrm{E}-03$ \\
\hline chr6_282080IENSGALT000( & 6 cis & 5.2 cis & 7.26 & $9.68 \mathrm{E}-03$ \\
\hline chr6_2613001X603596622F & 6 cis & 4.5 cis & 5.16 & $1.31 \mathrm{E}-03$ \\
\hline chr6_2540001X603596622F & $6 \mathrm{cis}$ & 4.5 cis & 7.99 & $5.46 \mathrm{E}-03$ \\
\hline chr6_2587901X603596622F & 6 cis & 4.5 cis & 5.33 & $3.85 \mathrm{E}-03$ \\
\hline chr6_2484901X603596622F & 6 cis & 4.5 cis & 5.64 & 2.67E-04 \\
\hline chr6_2652701X603596622F & 6 cis & 4.5 cis & 6.5 & $2.51 E-03$ \\
\hline chr6_2956501X603596622F & 6 cis & 4.5 cis & 6.14 & $1.66 \mathrm{E}-04$ \\
\hline chr6_2895001X603596622F & 6 cis & 4.5 cis & 10.88 & $1.32 \mathrm{E}-03$ \\
\hline chr6_2858101X603596622F & 6 cis & 4.5 cis & 5.54 & 2.07E-03 \\
\hline chr6_3087201X603596622F & 6 cis & 4.5 cis & 5.15 & 4.59E-04 \\
\hline chr6_248490IENSGALTO00( & 6 cis & 5.5 cis & 5.64 & 1.10E-04 \\
\hline chr6_265530IENSGALTO00( & 6 cis & 5.5 cis & 5.47 & 4.73E-03 \\
\hline chr6_295650IENSGALTO00( & 6 cis & 5.5 cis & 6.14 & $1.15 \mathrm{E}-04$ \\
\hline chr6_289500IENSGALT000( & 6 cis & 5.5 cis & 10.88 & $1.24 \mathrm{E}-06$ \\
\hline chr6_276750IENSGALTO00( & 6 cis & 5.5 cis & 6.56 & 4.68E-03 \\
\hline chr6_264760IENSGALT000( & 6 cis & 5.5 cis & 4.42 & 4.30E-04 \\
\hline chr6_308720IENSGALT000( & 6 cis & 5.5 cis & 5.15 & $9.84 \mathrm{E}-04$ \\
\hline chr6_307560IENSGALT000( & 6 cis & 5.5 cis & 4.45 & $1.04 \mathrm{E}-03$ \\
\hline chr6_307450IENSGALT000( & 6 cis & 5.5 cis & 8.84 & $6.04 \mathrm{E}-04$ \\
\hline chr6_2587901X603598822F & 6 cis & $5.3 \mathrm{cis}$ & 5.33 & $1.62 \mathrm{E}-03$ \\
\hline chr6_2655101X603598822F & 6 cis & $5.3 \mathrm{cis}$ & 4.99 & $9.49 \mathrm{E}-04$ \\
\hline chr6_2956501X603598822F & 6 cis & 5.3 cis & 6.14 & $2.60 \mathrm{E}-06$ \\
\hline chr6_2895001X603598822F & 6 cis & $5.3 \mathrm{cis}$ & 10.88 & $3.62 E-04$ \\
\hline chr6_2767501X603598822F & 6 cis & 5.3 cis & 6.56 & 3.27E-04 \\
\hline chr6_2647601X603598822F & 6 cis & $5.3 \mathrm{cis}$ & 4.42 & 8.17E-05 \\
\hline chr6_3087201X603598822F & 6 cis & $5.3 \mathrm{cis}$ & 5.15 & 7.59E-04 \\
\hline chr6_3075601X603598822F & 6 cis & $5.3 \mathrm{cis}$ & 4.45 & $1.70 \mathrm{E}-03$ \\
\hline chr6_3074501X603598822F & 6 cis & 5.3 cis & 8.84 & $1.84 \mathrm{E}-03$ \\
\hline chr6_258790IENSGALTO00( & 6 cis & $5.1 \mathrm{cis}$ & 5.33 & 7.55E-04 \\
\hline chr6_265510IENSGALTO00( & 6 cis & $5.1 \mathrm{cis}$ & 4.99 & $1.80 \mathrm{E}-04$ \\
\hline chr6_295650IENSGALTO00( & 6 cis & $5.1 \mathrm{cis}$ & 6.14 & $5.28 \mathrm{E}-05$ \\
\hline chr6_266160IENSGALTO00( & 6 cis & $5.1 \mathrm{cis}$ & 6.12 & $5.16 \mathrm{E}-04$ \\
\hline chr6_289500IENSGALTO00( & 6 cis & $5.1 \mathrm{cis}$ & 10.88 & 7.41E-04 \\
\hline chr6_308720IENSGALTO00( & 6 cis & $5.1 \mathrm{cis}$ & 5.15 & $9.92 \mathrm{E}-05$ \\
\hline chr6_307560IENSGALT000( & 6 cis & $5.1 \mathrm{cis}$ & 4.45 & $1.32 \mathrm{E}-04$ \\
\hline chr6_2956501X603599016F & 6 cis & 5.6 cis & 6.14 & $3.26 \mathrm{E}-05$ \\
\hline chr6_2661601X603599016F & 6 cis & 5.6 cis & 6.12 & $6.74 \mathrm{E}-04$ \\
\hline chr6_2895001X603599016F & 6 cis & 5.6 cis & 10.88 & 3.79E-04 \\
\hline chr6_2767501X603599016F & 6 cis & 5.6 cis & 6.56 & 9.77E-04 \\
\hline chr6_3087201X603599016F & 6 cis & 5.6 cis & 5.15 & 8.11E-05 \\
\hline chr6_3075601X603599016F & 6 cis & $5.6 \mathrm{cis}$ & 4.45 & $1.06 \mathrm{E}-03$ \\
\hline chr6_2956501X603863661F & 6 cis & $8.3 \mathrm{cis}$ & 6.14 & $3.81 E-06$ \\
\hline chr6_2661601X603863661F & 6 cis & $8.3 \mathrm{cis}$ & 6.12 & $1.70 \mathrm{E}-04$ \\
\hline chr6_2895001X603863661F & 6 cis & 8.3 cis & 10.88 & 1.60E-04 \\
\hline
\end{tabular}




\begin{tabular}{|c|c|c|c|c|}
\hline chr6_2767501X603863661F & 6 cis & $8.3 \mathrm{cis}$ & 6.56 & $1.14 \mathrm{E}-05$ \\
\hline chr6_285810ıX603863661F & 6 cis & 8.3 cis & 5.54 & $3.61 \mathrm{E}-04$ \\
\hline chr6_3087201X603863661F & 6 cis & $8.3 \mathrm{cis}$ & 5.15 & $3.41 \mathrm{E}-07$ \\
\hline chr6_3075601X603863661F & 6 cis & $8.3 \mathrm{cis}$ & 4.45 & $2.42 \mathrm{E}-07$ \\
\hline chr6_307450ıX603863661F & 6 cis & 8.3 cis & 8.84 & $5.98 \mathrm{E}-04$ \\
\hline chr6_295650IENSGALTO00( & 6 cis & 7.4 cis & 6.14 & $8.94 \mathrm{E}-06$ \\
\hline chr6_289500IENSGALT000( & 6 cis & 7.4 cis & 10.88 & $4.94 \mathrm{E}-04$ \\
\hline chr6_308720IENSGALTO00( & 6 cis & 7.4 cis & 5.15 & $1.90 \mathrm{E}-04$ \\
\hline chr6_307560IENSGALTO00( & 6 cis & 7.4 cis & 4.45 & $1.26 \mathrm{E}-05$ \\
\hline chr6_307450IENSGALTO00( & 6 cis & 7.4 cis & 8.84 & $3.22 \mathrm{E}-05$ \\
\hline chr7_217400IENSGALTO00( & 7 cis & 4.9 cis & 6.55 & 1.09E-04 \\
\hline chr7_220600IENSGALTO00( & 7 cis & 4.9 cis & 5.88 & $1.41 \mathrm{E}-04$ \\
\hline chr7_188200IENSGALT000( & 7 cis & 4.9 cis & 4.76 & $6.06 \mathrm{E}-04$ \\
\hline chr7_2320001X603601402F & 7 cis & $4.1 \mathrm{cis}$ & 7.71 & 4.40E-04 \\
\hline chr7_173400ıX603601402F & 7 cis & $4.1 \mathrm{cis}$ & 6.84 & $2.95 \mathrm{E}-03$ \\
\hline chr7_2323001X603601402F & 7 cis & $4.1 \mathrm{cis}$ & 4.1 & $9.21 \mathrm{E}-06$ \\
\hline chr7_400200IENSGALTO00( & 7 cis & 4.5 cis & 35.27 & $1.23 \mathrm{E}-03$ \\
\hline chr7_400200INM_205534_ & 7 cis & 4.6 cis & 35.27 & $2.91 \mathrm{E}-03$ \\
\hline chr7_111770ıX603868103F & 7 cis & 4.5 cis & 5.71 & $1.36 \mathrm{E}-03$ \\
\hline chr7_182110ıX603862432F & 7 cis & $6.3 \mathrm{cis}$ & 6.37 & $1.10 \mathrm{E}-02$ \\
\hline chr7_223990ıX603597339F & 7 cis & 5.2 trans & 9.64 & $6.23 \mathrm{E}-04$ \\
\hline chr7_182110ıX603597339F & 7 cis & 5.2 cis & 6.37 & 3.16E-04 \\
\hline chr7_220180ıX603597339F & 7 cis & 5.2 cis & 6.02 & $1.10 \mathrm{E}-03$ \\
\hline chr7_228590ıX603597339F & 7 cis & 5.2 cis & 5.06 & $1.00 \mathrm{E}-04$ \\
\hline chr7_2458101X603597339F & 7 cis & 5.2 cis & 5.25 & 4.15E-05 \\
\hline chr7_234870ıX603597339F & 7 cis & 5.2 cis & 7.17 & $8.56 \mathrm{E}-04$ \\
\hline chr7_253070ıX603597339F & 7 cis & 5.2 cis & 5.21 & $1.26 \mathrm{E}-03$ \\
\hline chr7_238860ıX603597339F & 7 cis & 5.2 cis & 6.11 & $1.76 \mathrm{E}-03$ \\
\hline chr7_250640ıX603597339F & 7 cis & 5.2 cis & 4.67 & $2.62 \mathrm{E}-03$ \\
\hline chr7_241370ıX603597339F & 7 cis & 5.2 cis & 4.39 & 4.39E-03 \\
\hline chr7_2420401X603597339F & 7 cis & 5.2 cis & 33.31 & $9.32 \mathrm{E}-04$ \\
\hline chr7_245830ıX603597339F & 7 cis & 5.2 cis & 5.83 & $1.35 \mathrm{E}-03$ \\
\hline chr7_241180ıX603597339F & 7 cis & 5.2 cis & 11.17 & $9.85 \mathrm{E}-04$ \\
\hline chr7_237140ıX603597339F & 7 cis & $5.2 \mathrm{cis}$ & 6.13 & $1.70 \mathrm{E}-03$ \\
\hline chr7_239030ıX603597339F & 7 cis & 5.2 cis & 5.52 & 4.57E-03 \\
\hline chr7_2424901X603597339F & 7 cis & 5.2 cis & 22.3 & $3.46 \mathrm{E}-03$ \\
\hline chr7_250950ıX603597339F & 7 cis & $5.2 \mathrm{cis}$ & 18.23 & $3.24 \mathrm{E}-04$ \\
\hline chr7_254140ıX603597339F & 7 cis & 5.2 cis & 9.91 & $1.83 \mathrm{E}-03$ \\
\hline chr7_2413901X603597339F & 7 cis & 5.2 cis & 5.56 & $5.46 \mathrm{E}-04$ \\
\hline chr7_240860ıX603597339F & 7 cis & $5.2 \mathrm{cis}$ & 7.95 & $3.44 \mathrm{E}-04$ \\
\hline chr7_240950ı603597339F & 7 cis & 5.2 cis & 9.13 & 5.75E-05 \\
\hline chr7_237270ıX603597339F & 7 cis & 5.2 cis & 6.93 & $1.70 \mathrm{E}-03$ \\
\hline chr7_2442501X603597339F & 7 cis & 5.2 cis & 7.51 & $1.68 \mathrm{E}-03$ \\
\hline chr7_2475401X603597339F & 7 cis & 5.2 cis & 6.34 & 5.10E-04 \\
\hline chr7_2506501X603597339F & 7 cis & 5.2 cis & 7.53 & $1.92 \mathrm{E}-03$ \\
\hline chr7_245070ıX603597339F & 7 cis & $5.2 \mathrm{cis}$ & 4.92 & $1.78 \mathrm{E}-05$ \\
\hline chr7_246630ı603597339F & 7 cis & 5.2 cis & 5.38 & $1.69 \mathrm{E}-03$ \\
\hline chr7_255230ıX603597339F & 7 cis & 5.2 cis & 6.51 & $1.60 \mathrm{E}-06$ \\
\hline chr7_237230ıX603597339F & 7 cis & $5.2 \mathrm{cis}$ & 4.61 & $1.82 \mathrm{E}-04$ \\
\hline chr7_237240ıX603597339F & 7 cis & $5.2 \mathrm{cis}$ & 4.82 & $2.84 \mathrm{E}-04$ \\
\hline chr7_2379701X603597339F & 7 cis & 5.2 cis & 7.92 & 7.83E-04 \\
\hline chr7_237290ıX603597339F & 7 cis & $5.2 \mathrm{cis}$ & 6.78 & $1.29 \mathrm{E}-03$ \\
\hline chr7_2267401X603597339F & 7 cis & 5.2 cis & 7.64 & 4.53E-05 \\
\hline chr7_231950ıX603597339F & 7 cis & 5.2 cis & 5.35 & $2.33 \mathrm{E}-03$ \\
\hline chr7_249190ıX603597339F & 7 cis & $5.2 \mathrm{cis}$ & 4.16 & 8.27E-04 \\
\hline chr7_182110ıX603143774F & 7 cis & 10.5 cis & 6.37 & $8.30 \mathrm{E}-03$ \\
\hline chr7_193900IX603868270F & 7 cis & 6.2 cis & 5.06 & $1.42 \mathrm{E}-03$ \\
\hline chr7_220180ıX603868270F & 7 cis & $6.2 \mathrm{cis}$ & 6.02 & $7.24 \mathrm{E}-04$ \\
\hline chr7_166640ıX603868270F & 7 cis & 6.2 trans & 5.78 & $3.71 \mathrm{E}-04$ \\
\hline chr7_182110ıX603002818F & 7 cis & 8.9 cis & 6.37 & $1.61 \mathrm{E}-02$ \\
\hline chr7_174460ıX603599149F & 7 cis & $8.0 \mathrm{cis}$ & 9.88 & 2.17E-05 \\
\hline chr7_251510IENSGALTOOO( & 7 cis & $11.3 \mathrm{cis}$ & 11.49 & $3.18 \mathrm{E}-04$ \\
\hline chr7_245070IENSGALTO00( & 7 cis & $11.3 \mathrm{cis}$ & 4.92 & $1.14 \mathrm{E}-02$ \\
\hline chr7_236500IENSGALTO00( & 7 cis & $11.3 \mathrm{cis}$ & 7.43 & $1.95 \mathrm{E}-02$ \\
\hline chr7_226740IENSGALTO00( & 7 cis & $11.3 \mathrm{cis}$ & 7.64 & $7.22 \mathrm{E}-02$ \\
\hline chr7_289400IENSGALTO00( & 7 cis & $11.3 \mathrm{cis}$ & 4.88 & $8.21 \mathrm{E}-03$ \\
\hline chr7_278600IENSGALTO00( & 7 cis & $11.3 \mathrm{cis}$ & 5.46 & 1.57E-02 \\
\hline chr7_273450IENSGALTOOO( & 7 cis & $11.3 \mathrm{cis}$ & 6.69 & 4.64E-02 \\
\hline chr7_302010IENSGALT000( & 7 cis & 11.3 cis & 4.56 & $9.49 \mathrm{E}-02$ \\
\hline chr7_282410IENSGALTOOO( & 7 cis & $11.3 \mathrm{cis}$ & 6.22 & 4.30E-02 \\
\hline chr7_281090IENSGALT000( & 7 cis & $11.3 \mathrm{cis}$ & 7.53 & $4.92 \mathrm{E}-02$ \\
\hline chr7_278360IENSGALTO00( & 7 cis & $11.3 \mathrm{cis}$ & 4.1 & $1.64 \mathrm{E}-04$ \\
\hline
\end{tabular}




\begin{tabular}{|c|c|c|c|c|}
\hline chr7_282210IENSGALTO00( & 7 cis & $11.3 \mathrm{cis}$ & 5.67 & $7.73 \mathrm{E}-03$ \\
\hline chr7_249190IENSGALTO00( & 7 cis & 11.3 cis & 4.16 & $1.68 \mathrm{E}-04$ \\
\hline chr7_283970IENSGALTO00( & 7 cis & $11.3 \mathrm{cis}$ & 8.06 & $1.72 \mathrm{E}-03$ \\
\hline chr7_293000IENSGALTO00( & 7 cis & $11.3 \mathrm{cis}$ & 7.59 & $1.03 \mathrm{E}-03$ \\
\hline chr7_289720IENSGALTO00( & 7 cis & $11.3 \mathrm{cis}$ & 6.65 & $1.89 \mathrm{E}-04$ \\
\hline chr7_291850IENSGALTO00( & 7 cis & 11.3 cis & 8.3 & $3.44 \mathrm{E}-02$ \\
\hline chr7_182110ıNM_0010125 & 7 cis & 10.0 cis & 6.37 & $1.10 \mathrm{E}-04$ \\
\hline chr7_220180INM_0010125 & 7 cis & 10.0 cis & 6.02 & 2.07E-04 \\
\hline chr7_182110IENSGALTO00( & 7 cis & 10.1 cis & 6.37 & $1.89 \mathrm{E}-04$ \\
\hline chr7_220180IENSGALTO00( & 7 cis & $10.1 \mathrm{cis}$ & 6.02 & $2.12 \mathrm{E}-04$ \\
\hline chr7_194370IENSGALTOOO( & 7 cis & 4.8 trans & 7.33 & $1.30 \mathrm{E}-03$ \\
\hline chr7_174460IENSGALTO00( & 7 cis & $4.8 \mathrm{cis}$ & 9.88 & 7.74E-04 \\
\hline chr7_2352001X603599760F & 7 cis & 10.6 cis & 10.37 & $1.02 \mathrm{E}-03$ \\
\hline chr7_253070ıX603599760F & 7 cis & 10.6 cis & 5.21 & $8.08 \mathrm{E}-03$ \\
\hline chr7_253130ıX603599760F & 7 cis & 10.6 cis & 5.01 & 4.70E-03 \\
\hline chr7_249960ıX603599760F & 7 cis & 10.6 cis & 8.04 & 4.76E-05 \\
\hline chr7_235580ıX603599760F & 7 cis & 10.6 cis & 4.92 & $7.20 \mathrm{E}-05$ \\
\hline chr7_251510ıX603599760F & 7 cis & 10.6 cis & 11.49 & $6.35 \mathrm{E}-04$ \\
\hline chr7_2492301X603599760F & 7 cis & 10.6 cis & 5.05 & $2.85 \mathrm{E}-04$ \\
\hline chr7_235750ıX603599760F & 7 cis & 10.6 cis & 8.21 & 7.03E-05 \\
\hline chr7_240170ıX603599760F & 7 cis & 10.6 cis & 11.96 & 5.07E-04 \\
\hline chr7_245620ıX603599760F & 7 cis & 10.6 cis & 8.92 & $1.13 \mathrm{E}-03$ \\
\hline chr7_242040ıX603599760F & 7 cis & 10.6 cis & 33.31 & $4.90 \mathrm{E}-04$ \\
\hline chr7_251960ıX03599760F & 7 cis & 10.6 cis & 14.27 & $1.22 \mathrm{E}-03$ \\
\hline chr7_2424901X603599760F & 7 cis & 10.6 cis & 22.3 & $6.12 \mathrm{E}-04$ \\
\hline chr7_250950ıX603599760F & 7 cis & 10.6 cis & 18.23 & $2.84 \mathrm{E}-04$ \\
\hline chr7_254900ı603599760F & 7 cis & 10.6 cis & 8.86 & 1.17E-03 \\
\hline chr7_254890ı603599760F & 7 cis & $10.6 \mathrm{cis}$ & 6.3 & $9.56 \mathrm{E}-04$ \\
\hline chr7_240950ıX603599760F & 7 cis & 10.6 cis & 9.13 & $7.08 \mathrm{E}-06$ \\
\hline chr7_243970ıX603599760F & 7 cis & 10.6 cis & 4.96 & $1.44 \mathrm{E}-03$ \\
\hline chr7_2393801X603599760F & 7 cis & 10.6 cis & 9.53 & $1.32 \mathrm{E}-03$ \\
\hline chr7_244250ıX603599760F & 7 cis & 10.6 cis & 7.51 & $2.59 \mathrm{E}-06$ \\
\hline chr7_245070ıX603599760F & 7 cis & 10.6 cis & 4.92 & $9.95 \mathrm{E}-04$ \\
\hline chr7_236500ıX603599760F & 7 cis & $10.6 \mathrm{cis}$ & 7.43 & $9.82 \mathrm{E}-04$ \\
\hline chr7_255230ıX603599760F & 7 cis & 10.6 cis & 6.51 & $2.30 \mathrm{E}-06$ \\
\hline chr7_254360ıX603599760F & 7 cis & 10.6 cis & 7.93 & $5.43 \mathrm{E}-03$ \\
\hline chr7_2372901X603599760F & 7 cis & 10.6 cis & 6.78 & $1.47 \mathrm{E}-03$ \\
\hline chr7_226740ıx603599760F & 7 cis & 10.6 cis & 7.64 & $2.88 \mathrm{E}-04$ \\
\hline chr7_233970ıX603599760F & 7 cis & 10.6 cis & 6.97 & $1.03 \mathrm{E}-03$ \\
\hline chr7_236300ıX603599760F & 7 cis & $10.6 \mathrm{cis}$ & 5.6 & $6.68 \mathrm{E}-04$ \\
\hline chr7_249190ıX603599760F & 7 cis & 10.6 cis & 4.16 & $1.24 \mathrm{E}-04$ \\
\hline chr7_233700IENSGALTOOO( & 7 cis & $10.1 \mathrm{cis}$ & 8.48 & $9.53 \mathrm{E}-04$ \\
\hline chr7_233880IENSGALTO00( & 7 cis & 10.1 cis & 4.9 & $6.91 \mathrm{E}-04$ \\
\hline chr7_228380IENSGALTO00 & 7 cis & $10.1 \mathrm{cis}$ & 5.88 & 7.73E-04 \\
\hline chr7_228730IENSGALTO00( & 7 cis & 10.1 cis & 5.75 & 5.77E-04 \\
\hline chr7_232900IENSGALTO00( & 7 cis & $10.1 \mathrm{cis}$ & 11.23 & 7.43E-04 \\
\hline chr7_235200IENSGALTO00( & 7 cis & 10.1 cis & 10.37 & $3.52 \mathrm{E}-04$ \\
\hline chr7_235580IENSGALTOOO( & 7 cis & $10.1 \mathrm{cis}$ & 4.92 & 2.47E-03 \\
\hline chr7_249110IENSGALTO00( & 7 cis & 10.1 cis & 5.69 & 3.63E-05 \\
\hline chr7_251510IENSGALTOOO & 7 cis & $10.1 \mathrm{cis}$ & 11.49 & $1.80 \mathrm{E}-04$ \\
\hline chr7_235750IENSGALTOOO( & 7 cis & 10.1 cis & 8.21 & $2.26 \mathrm{E}-05$ \\
\hline chr7_240170IENSGALTO00( & 7 cis & $10.1 \mathrm{cis}$ & 11.96 & 3.00E-05 \\
\hline chr7_245620IENSGALTO00( & 7 cis & 10.1 cis & 8.92 & $1.03 \mathrm{E}-05$ \\
\hline chr7_254910IENSGALTOOO( & 7 cis & $10.1 \mathrm{cis}$ & 7.92 & 3.03E-04 \\
\hline chr7_243540IENSGALTO00( & 7 cis & 10.1 cis & 9.31 & 4.46E-05 \\
\hline chr7_242040IENSGALTO00( & 7 cis & $10.1 \mathrm{cis}$ & 33.31 & $1.91 \mathrm{E}-04$ \\
\hline chr7_246010IENSGALTO00( & 7 cis & 10.1 cis & 4.9 & 2.19E-04 \\
\hline chr7_251960IENSGALTO00( & 7 cis & $10.1 \mathrm{cis}$ & 14.27 & $2.64 \mathrm{E}-05$ \\
\hline chr7_236790IENSGALTO00( & 7 cis & 10.1 cis & 6.79 & 4.46E-04 \\
\hline chr7_241180IENSGALTOOO( & 7 cis & $10.1 \mathrm{cis}$ & 11.17 & $5.15 \mathrm{E}-03$ \\
\hline chr7_242490IENSGALTO00( & 7 cis & 10.1 cis & 22.3 & $1.79 \mathrm{E}-04$ \\
\hline chr7_250950IENSGALTO00( & 7 cis & $10.1 \mathrm{cis}$ & 18.23 & $1.42 \mathrm{E}-03$ \\
\hline chr7_254140IENSGALTO00( & 7 cis & 10.1 cis & 9.91 & 2.91E-04 \\
\hline chr7_254900IENSGALTO00( & 7 cis & $10.1 \mathrm{cis}$ & 8.86 & $1.71 \mathrm{E}-04$ \\
\hline chr7_254890IENSGALTO00( & 7 cis & 10.1 cis & 6.3 & 2.09E-04 \\
\hline chr7_240950IENSGALTO00( & 7 cis & $10.1 \mathrm{cis}$ & 9.13 & $2.50 \mathrm{E}-04$ \\
\hline chr7_243970IENSGALTO00( & 7 cis & 10.1 cis & 4.96 & 2.39E-04 \\
\hline chr7_246440IENSGALTOOO( & 7 cis & $10.1 \mathrm{cis}$ & 5.52 & $1.00 \mathrm{E}-03$ \\
\hline chr7_254350IENSGALTO00( & 7 cis & 10.1 cis & 4.59 & $7.42 \mathrm{E}-04$ \\
\hline chr7_239380IENSGALTO00( & 7 cis & $10.1 \mathrm{cis}$ & 9.53 & $1.52 \mathrm{E}-04$ \\
\hline chr7_244250IENSGALTO00( & 7 cis & $10.1 \mathrm{cis}$ & 7.51 & $7.80 \mathrm{E}-04$ \\
\hline chr7_235030IENSGALTO00( & 7 cis & $10.1 \mathrm{cis}$ & 5.76 & $1.95 \mathrm{E}-03$ \\
\hline
\end{tabular}




\begin{tabular}{|c|c|c|c|c|}
\hline chr7_255230IENSGALTO00( & 7 cis & $10.1 \mathrm{cis}$ & 6.51 & $8.23 \mathrm{E}-04$ \\
\hline chr7_254360IENSGALTO00( & 7 cis & 10.1 cis & 7.93 & $6.14 \mathrm{E}-04$ \\
\hline chr7_239010।ENSGALT000( & 7 cis & 10.1 cis & 9.88 & 4.50E-03 \\
\hline chr7_236190ıENSGALTO00( & 7 cis & 10.1 cis & 5.48 & $1.14 \mathrm{E}-02$ \\
\hline chr7_233970ıENSGALTO00( & 7 cis & 10.1 cis & 6.97 & 4.16E-05 \\
\hline chr7_236300IENSGALTO00( & 7 cis & 10.1 cis & 5.6 & $2.66 \mathrm{E}-05$ \\
\hline chr7_231900ıENSGALTO00( & 7 cis & 10.1 cis & 4.84 & $9.36 \mathrm{E}-04$ \\
\hline chr7_231950ıENSGALTO00( & 7 cis & 10.1 cis & 5.35 & $1.36 \mathrm{E}-04$ \\
\hline chr7_257840ıENSGALTO00( & 7 cis & 10.1 cis & 5.67 & 1.10E-04 \\
\hline chr7_238750ıX603597508F & 7 cis & $5.1 \mathrm{cis}$ & 4.97 & $1.68 \mathrm{E}-03$ \\
\hline chr7_249110ıX603597508F & 7 cis & $5.1 \mathrm{cis}$ & 5.69 & 1.54E-04 \\
\hline chr7_249230ıX603597508F & 7 cis & $5.1 \mathrm{cis}$ & 5.05 & $1.20 \mathrm{E}-03$ \\
\hline chr7_240170ıX603597508F & 7 cis & $5.1 \mathrm{cis}$ & 11.96 & $5.58 \mathrm{E}-05$ \\
\hline chr7_245620ıX603597508F & 7 cis & $5.1 \mathrm{cis}$ & 8.92 & $2.65 \mathrm{E}-04$ \\
\hline chr7_250580ıX603597508F & 7 cis & $5.1 \mathrm{cis}$ & 5.54 & 5.80E-04 \\
\hline chr7_254910ıX603597508F & 7 cis & $5.1 \mathrm{cis}$ & 7.92 & $2.43 E-03$ \\
\hline chr7_238760ıX603597508F & 7 cis & $5.1 \mathrm{cis}$ & 6.62 & 5.97E-05 \\
\hline chr7_246010ı603597508F & 7 cis & $5.1 \mathrm{cis}$ & 4.9 & $5.55 \mathrm{E}-04$ \\
\hline chr7_254900ıX603597508F & 7 cis & $5.1 \mathrm{cis}$ & 8.86 & $3.36 \mathrm{E}-03$ \\
\hline chr7_240950ıX603597508F & 7 cis & $5.1 \mathrm{cis}$ & 9.13 & 5.59E-03 \\
\hline chr7_254350ıX603597508F & 7 cis & $5.1 \mathrm{cis}$ & 4.59 & 1.07E-02 \\
\hline chr7_236300ıX603597508F & 7 cis & $5.1 \mathrm{cis}$ & 5.6 & $2.63 \mathrm{E}-04$ \\
\hline chr7_249990ıX603597508F & 7 cis & $5.1 \mathrm{cis}$ & 4.34 & 1.77E-04 \\
\hline chr7_223990ı603597182F & 7 cis & 5.6 trans & 9.64 & $1.61 \mathrm{E}-03$ \\
\hline chr7_220180ıX603597182F & 7 cis & $5.6 \mathrm{cis}$ & 6.02 & $9.88 \mathrm{E}-04$ \\
\hline chr7_245690ıX603597182F & 7 cis & 5.6 cis & 5.44 & $5.76 \mathrm{E}-03$ \\
\hline chr7_228590ıX603597182F & 7 cis & 5.6 cis & 5.06 & $1.65 \mathrm{E}-04$ \\
\hline chr7_242980ıX603597182F & 7 cis & 5.6 cis & 6.34 & $1.96 \mathrm{E}-03$ \\
\hline chr7_251790ıX603597182F & 7 cis & 5.6 cis & 5.5 & 5.67E-04 \\
\hline chr7_245810ı603597182F & 7 cis & 5.6 cis & 5.25 & $7.86 \mathrm{E}-04$ \\
\hline chr7_228380ıX603597182F & 7 cis & 5.6 cis & 5.88 & $2.46 \mathrm{E}-04$ \\
\hline chr7_234870ıX603597182F & 7 cis & 5.6 cis & 7.17 & 3.70E-04 \\
\hline chr7_235200ıX603597182F & 7 cis & 5.6 cis & 10.37 & $2.33 \mathrm{E}-03$ \\
\hline chr7_253070ıX603597182F & 7 cis & 5.6 cis & 5.21 & $4.63 \mathrm{E}-03$ \\
\hline chr7_238860ıX603597182F & 7 cis & 5.6 cis & 6.11 & $2.90 \mathrm{E}-04$ \\
\hline chr7_250640ı603597182F & 7 cis & 5.6 cis & 4.67 & $3.88 \mathrm{E}-04$ \\
\hline chr7_244240ıX603597182F & 7 cis & 5.6 cis & 5.2 & 1.65E-04 \\
\hline chr7_239370ıX603597182F & 7 cis & 5.6 cis & 10.83 & $1.23 \mathrm{E}-03$ \\
\hline chr7_249960ıX603597182F & 7 cis & 5.6 cis & 8.04 & $1.52 \mathrm{E}-03$ \\
\hline chr7_248590ıX603597182F & 7 cis & 5.6 cis & 4.3 & $8.71 \mathrm{E}-04$ \\
\hline chr7_251130ıX603597182F & 7 cis & 5.6 cis & 4.73 & 4.14E-04 \\
\hline chr7_250740ı603597182F & 7 cis & 5.6 cis & 5.52 & $2.04 \mathrm{E}-03$ \\
\hline chr7_251510ıX603597182F & 7 cis & 5.6 cis & 11.49 & $1.20 \mathrm{E}-03$ \\
\hline chr7_241370ıX603597182F & 7 cis & 5.6 cis & 4.39 & $1.86 \mathrm{E}-04$ \\
\hline chr7_241430ıX603597182F & 7 cis & 5.6 cis & 8.17 & $7.25 \mathrm{E}-04$ \\
\hline chr7_242040ıX603597182F & 7 cis & 5.6 cis & 33.31 & $6.08 \mathrm{E}-05$ \\
\hline chr7_245830ıX603597182F & 7 cis & 5.6 cis & 5.83 & $3.93 \mathrm{E}-04$ \\
\hline chr7_251960ı603597182F & 7 cis & 5.6 cis & 14.27 & $1.26 \mathrm{E}-03$ \\
\hline chr7_241180ıX603597182F & 7 cis & $5.6 \mathrm{cis}$ & 11.17 & $3.78 \mathrm{E}-03$ \\
\hline chr7_237140ıX603597182F & 7 cis & 5.6 cis & 6.13 & $4.88 \mathrm{E}-04$ \\
\hline chr7_239030ı603597182F & 7 cis & 5.6 cis & 5.52 & $1.34 \mathrm{E}-03$ \\
\hline chr7_2420501X603597182F & 7 cis & $5.6 \mathrm{cis}$ & 8.87 & 4.10E-03 \\
\hline chr7_242490ıX603597182F & 7 cis & 5.6 cis & 22.3 & $5.82 \mathrm{E}-04$ \\
\hline chr7_250950ı603597182F & 7 cis & $5.6 \mathrm{cis}$ & 18.23 & $1.18 \mathrm{E}-04$ \\
\hline chr7_254140!X603597182F & 7 cis & $5.6 \mathrm{cis}$ & 9.91 & $6.85 \mathrm{E}-03$ \\
\hline chr7_241390ıX603597182F & 7 cis & 5.6 cis & 5.56 & 3.97E-04 \\
\hline chr7_237920ıX603597182F & 7 cis & 5.6 cis & 4.89 & $2.03 E-03$ \\
\hline chr7_2548901X603597182F & 7 cis & $5.6 \mathrm{cis}$ & 6.3 & $3.48 \mathrm{E}-03$ \\
\hline chr7_240860ıX603597182F & 7 cis & 5.6 cis & 7.95 & $3.40 \mathrm{E}-03$ \\
\hline chr7_240950!X603597182F & 7 cis & 5.6 cis & 9.13 & $3.34 \mathrm{E}-06$ \\
\hline chr7_2372701X603597182F & 7 cis & $5.6 \mathrm{cis}$ & 6.93 & 7.04E-05 \\
\hline chr7_246440ıX603597182F & 7 cis & 5.6 cis & 5.52 & $3.11 \mathrm{E}-03$ \\
\hline chr7_239380ıX603597182F & 7 cis & 5.6 cis & 9.53 & $1.28 \mathrm{E}-03$ \\
\hline chr7_2442501X603597182F & 7 cis & $5.6 \mathrm{cis}$ & 7.51 & $1.82 \mathrm{E}-06$ \\
\hline chr7_2464301X603597182F & 7 cis & $5.6 \mathrm{cis}$ & 5.76 & $2.13 \mathrm{E}-03$ \\
\hline chr7_2475401X603597182F & 7 cis & $5.6 \mathrm{cis}$ & 6.34 & $4.64 \mathrm{E}-03$ \\
\hline chr7_250650।X603597182F & 7 cis & $5.6 \mathrm{cis}$ & 7.53 & $2.30 \mathrm{E}-03$ \\
\hline chr7_245070ıX603597182F & 7 cis & $5.6 \mathrm{cis}$ & 4.92 & $6.66 \mathrm{E}-05$ \\
\hline chr7_2350301X603597182F & 7 cis & $5.6 \mathrm{cis}$ & 5.76 & $8.00 \mathrm{E}-03$ \\
\hline chr7_2365001X603597182F & 7 cis & $5.6 \mathrm{cis}$ & 7.43 & $2.27 \mathrm{E}-04$ \\
\hline chr7_255230ıX603597182F & 7 cis & $5.6 \mathrm{cis}$ & 6.51 & $4.90 \mathrm{E}-07$ \\
\hline chr7_237230ıX603597182F & 7 cis & 5.6 cis & 4.61 & $9.13 \mathrm{E}-06$ \\
\hline
\end{tabular}


chr7_2372401X603597182F

chr7_237970।X603597182F

chr7_2372901X603597182F

chr7_2446101X603597182F

chr7_2267401X603597182F

chr7_2576801X603597182F

chr7 2362401X603597182F

chr7_2319501X603597182F

chr7 2491901X603597182F

chr7_2405401X603597182F

chr7_2487401X603597890F

chr7_2333501X603597890F

chr7_2353001X603597890F

chr7_2329001X603597890F

chr7_2352001X603597890F

chr7 2554401X603597890F

chr7_2355801X603597890F

chr7 2491101X603597890F

chr7_2515101X603597890F

chr7 2357501X603597890F

chr7_243500।X603597890F

chr7 2456201X603597890F

chr7_2485601X603597890F

chr7_2435401X603597890F

chr7_2360401X603597890F

chr7_2420401X603597890F

chr7_2424901X603597890F

chr7_2509501X603597890F

chr7 2514901X603597890F

chr7_2359601X603597890F

chr7 2379801X603597890F

chr7_2460901X603597890F

chr7 2566101X603597890F

chr7_268380IENSGALTO0O(

chr7_292570IENSGALTO00(

chr7_269670IENSGALTO0O(

chr7_275200IENSGALTO0O(

chr7_295620IENSGALTO00(

chr7_290190IENSGALTO00(

chr7 267790IENSGALTO0O(

chr7_289110IENSGALTO0O(

chr7 292130IENSGALTOOO

chr7_285810IENSGALTOOO(

chr7 280610IENSGALTO00(

chr7_291760IENSGALTO0O(

chr7 286200IENSGALTOOO

chr7_289510IENSGALTO0O(

chr7_286210IENSGALTO0O(

chr7_288660IENSGALTOOO(

chr7_292560IENSGALTO0O(

chr7 288540IENSGALTO0O

chr7_291000IENSGALTO0O(

chr7 293370IENSGALTO00

chr7_288000IENSGALTO0O(

chr7_285990IENSGALTO00(

chr7_291700IENSGALTO00(

chr7 291710IENSGALTO0O(

chr7_291460IENSGALTO0O(

chr7_291850IENSGALTOOOC

chr7_2515101X603603407F

chr7_2450701X603603407F

chr7_2552301X603603407F

chr7_2460901X603603407F

chr7_2894001X603603407F

chr7_2820701X603603407F

chr7_2752001X603603407F

chr7_2708501X603603407F

chr7 2786001X603603407F

chr7_2677901X603603407F

chr7 2734501X603603407F

chr7_2815401X603603407F

chr7_2819301X603603407F
7 cis

7 cis

7 cis

7 cis

7 cis

7 cis

7 cis

7 cis

7 cis

7 cis

7 cis

7 cis

7 cis

7 cis

7 cis

7 cis

7 cis

7 cis

7 cis

7 cis

7 cis

7 cis

7 cis

7 cis

7 cis

7 cis

7 cis

7 cis

7 cis

7 cis

7 cis

7 cis

7 cis

$7 \mathrm{cis}$

7 cis

$7 \mathrm{cis}$

7 cis

7 cis

7 cis

7 cis

7 cis

7 cis

7 cis

7 cis

7 cis

7 cis

$7 \mathrm{cis}$

7 cis

7 cis

7 cis

7 cis

7 cis

7 cis

7 cis

7 cis

7 cis

7 cis

7 cis

7 cis

7 cis

7 cis

7 cis

7 cis

7 cis

7 cis

7 cis

7 cis

7 cis

7 cis

7 cis

7 cis

7 cis
$5.6 \mathrm{cis}$

5.6 cis

5.6 cis

$5.6 \mathrm{cis}$

$5.6 \mathrm{cis}$

5.6 cis

5.6 cis

$5.6 \mathrm{cis}$

5.6 cis

5.6 trans

$6.2 \mathrm{cis}$

6.2 cis

$6.2 \mathrm{cis}$

$6.2 \mathrm{cis}$

$6.2 \mathrm{cis}$

6.2 cis

6.2 cis

6.2 cis

6.2 cis

$6.2 \mathrm{cis}$

$6.2 \mathrm{cis}$

6.2 cis

$6.2 \mathrm{cis}$

6.2 cis

$6.2 \mathrm{cis}$

6.2 cis

6.2 cis

$6.2 \mathrm{cis}$

$6.2 \mathrm{cis}$

6.2 cis

6.2 cis

6.2 cis

6.2 cis

6.4 cis

6.4 cis

$6.4 \mathrm{cis}$

6.4 cis

6.4 cis

$6.4 \mathrm{cis}$

6.4 cis

$6.4 \mathrm{cis}$

$6.4 \mathrm{cis}$

6.4 cis

$6.4 \mathrm{cis}$

6.4 cis

6.4 cis

$6.4 \mathrm{cis}$

6.4 cis

6.4 cis

$6.4 \mathrm{cis}$

$6.4 \mathrm{cis}$

$6.4 \mathrm{cis}$

6.4 cis

$6.4 \mathrm{cis}$

6.4 cis

6.4 cis

6.4 cis

$6.4 \mathrm{cis}$

$6.4 \mathrm{cis}$

$6.3 \mathrm{cis}$

$6.3 \mathrm{cis}$

$6.3 \mathrm{cis}$

$6.3 \mathrm{cis}$

$6.3 \mathrm{cis}$

$6.3 \mathrm{cis}$

$6.3 \mathrm{cis}$

$6.3 \mathrm{cis}$

$6.3 \mathrm{cis}$

$6.3 \mathrm{cis}$

$6.3 \mathrm{cis}$

$6.3 \mathrm{cis}$

$6.3 \mathrm{cis}$
4.58E-04

9.89E-04

2.33E-03

8.69E-04

1.65E-04

3.86E-03

$1.65 \mathrm{E}-03$

8.83E-04

8.50E-05

5.04E-03

2.70E-04

1.31E-03

2.70E-04

1.25E-04

2.58E-04

7.38E-04

6.71E-05

5.29E-04

4.50E-04

$2.58 \mathrm{E}-05$

5.10E-05

$1.28 \mathrm{E}-03$

5.46E-04

1.90E-05

9.78E-04

1.14E-06

1.34E-06

4.65E-04

7.24E-04

6.70E-04

8.93E-04

3.13E-04

2.23E-03

2.58E-05

$1.01 \mathrm{E}-06$

5.42E-05

2.03E-05

5.97E-05

1.01E-05

3.41E-05

1.37E-05

6.09E-05

9.05E-06

4.03E-04

1.23E-05

3.97E-06

3.47E-06

1.07E-05

5.92E-05

$1.88 \mathrm{E}-05$

6.25E-06

5.15E-05

4.63E-05

1.78E-05

2.13E-08

1.77E-05

1.81E-05

7.37E-05

2.06E-05

1.45E-03

5.50E-04

1.76E-04

1.07E-03

6.90E-04

1.52E-04

5.92E-05

9.20E-04

2.92E-04

5.39E-04

1.23E-03

1.24E-03

4.07E-04 


\begin{tabular}{|c|c|c|c|c|}
\hline chr7_2831801X603603407F & 7 cis & $6.3 \mathrm{cis}$ & 7.67 & $3.44 \mathrm{E}-04$ \\
\hline chr7_289110ıX603603407F & 7 cis & 6.3 cis & 5.26 & $1.67 \mathrm{E}-03$ \\
\hline chr7_2735101X603603407F & 7 cis & 6.3 cis & 8.48 & 1.57E-04 \\
\hline chr7_286000ıX603603407F & 7 cis & 6.3 cis & 4.99 & $1.32 \mathrm{E}-04$ \\
\hline chr7_2825801X603603407F & 7 cis & 6.3 cis & 4.88 & $3.42 \mathrm{E}-04$ \\
\hline chr7_283970ı603603407F & 7 cis & $6.3 \mathrm{cis}$ & 8.06 & $8.88 \mathrm{E}-04$ \\
\hline chr7_2862001X603603407F & 7 cis & $6.3 \mathrm{cis}$ & 7.73 & 2.79E-04 \\
\hline chr7_288540ıX603603407F & 7 cis & 6.3 cis & 5.77 & 7.91E-05 \\
\hline chr7_2859901X603603407F & 7 cis & 6.3 cis & 10.24 & $3.85 \mathrm{E}-05$ \\
\hline chr7_2897201X603603407F & 7 cis & 6.3 cis & 6.65 & $1.23 \mathrm{E}-06$ \\
\hline chr7_280680ıX603864595F & 7 cis & 5.6 cis & 8.92 & 8.37E-04 \\
\hline chr7_273450ıX603864595F & 7 cis & 5.6 cis & 6.69 & 8.83E-04 \\
\hline chr7_2749301X603864595F & 7 cis & 5.6 cis & 5.54 & 2.57E-03 \\
\hline chr7_278360ıX603864595F & 7 cis & 5.6 cis & 4.1 & $2.10 \mathrm{E}-03$ \\
\hline chr7_280680IENSGALTO00( & 7 cis & 5.7 cis & 8.92 & 2.97E-03 \\
\hline chr7_273450IENSGALTO00( & 7 cis & 5.7 cis & 6.69 & $1.48 \mathrm{E}-03$ \\
\hline chr7_274930IENSGALTO00( & 7 cis & 5.7 cis & 5.54 & $1.18 \mathrm{E}-03$ \\
\hline chr7_278360IENSGALTO00( & 7 cis & 5.7 cis & 4.1 & 1.17E-03 \\
\hline chr7_2693701X603867770F & 7 cis & 4.8 trans & 6.99 & $9.82 \mathrm{E}-04$ \\
\hline chr7_245070ıX603867770F & 7 cis & $4.8 \mathrm{cis}$ & 4.92 & 4.17E-03 \\
\hline chr7_2460901X603867770F & 7 cis & $4.8 \mathrm{cis}$ & 6.98 & 8.13E-05 \\
\hline chr7_274930ıX603867770F & 7 cis & $4.8 \mathrm{cis}$ & 5.54 & $3.00 \mathrm{E}-06$ \\
\hline chr7_281090ıX03867770F & 7 cis & $4.8 \mathrm{cis}$ & 7.53 & $2.04 \mathrm{E}-02$ \\
\hline chr7_278360ıX603867770F & 7 cis & $4.8 \mathrm{cis}$ & 4.1 & $1.12 \mathrm{E}-05$ \\
\hline chr7_2860001X603867770F & 7 cis & $4.8 \mathrm{cis}$ & 4.99 & 2.93E-02 \\
\hline chr7_283970ıX603867770F & 7 cis & $4.8 \mathrm{cis}$ & 8.06 & $1.55 \mathrm{E}-06$ \\
\hline chr7_293010ıX603867770F & 7 cis & $4.8 \mathrm{cis}$ & 7.11 & 4.66E-04 \\
\hline chr7_2922601X603867770F & 7 cis & $4.8 \mathrm{cis}$ & 8.46 & $2.82 \mathrm{E}-03$ \\
\hline chr7_293000ıX603867770F & 7 cis & $4.8 \mathrm{cis}$ & 7.59 & 6.47E-04 \\
\hline chr7_289720ıX603867770F & 7 cis & $4.8 \mathrm{cis}$ & 6.65 & 4.97E-05 \\
\hline chr7_282730IENSGALTO00( & 7 cis & 5.1 trans & 6.62 & $1.15 \mathrm{E}-04$ \\
\hline chr7_289400IENSGALTO00( & 7 cis & 6.5 cis & 4.88 & $4.92 \mathrm{E}-04$ \\
\hline chr7_292570IENSGALTO00( & 7 cis & 6.5 cis & 4.88 & 7.51E-04 \\
\hline chr7_295620IENSGALTO00( & 7 cis & 6.5 cis & 5.35 & 1.67E-04 \\
\hline chr7_278600IENSGALTO00( & 7 cis & 6.5 cis & 5.46 & $4.98 \mathrm{E}-04$ \\
\hline chr7_303160IENSGALTO00( & 7 cis & 6.5 cis & 4.52 & $1.03 \mathrm{E}-04$ \\
\hline chr7_302010IENSGALT000( & 7 cis & 6.5 cis & 4.56 & $1.61 \mathrm{E}-05$ \\
\hline chr7_289110IENSGALTO00( & 7 cis & 6.5 cis & 5.26 & $1.51 \mathrm{E}-03$ \\
\hline chr7_282210IENSGALTO00( & 7 cis & 6.5 cis & 5.67 & $1.09 \mathrm{E}-05$ \\
\hline chr7_285810IENSGALTOOO( & 7 cis & 6.5 cis & 4.77 & $2.08 \mathrm{E}-03$ \\
\hline chr7_292890IENSGALTO00( & 7 cis & 6.5 cis & 5.39 & 2.77E-04 \\
\hline chr7_290950IENSGALTO00( & 7 cis & 6.5 cis & 5.54 & $2.24 \mathrm{E}-03$ \\
\hline chr7_280780IENSGALTO00( & 7 cis & 6.5 cis & 5.29 & $1.09 \mathrm{E}-03$ \\
\hline chr7_278450IENSGALTO00( & 7 cis & 6.5 cis & 4.33 & 7.04E-05 \\
\hline chr7_282580IENSGALTO00( & 7 cis & 6.5 cis & 4.88 & $2.28 \mathrm{E}-03$ \\
\hline chr7_291520IENSGALTO00( & 7 cis & 6.5 cis & 5.63 & $9.67 \mathrm{E}-05$ \\
\hline chr7_291760IENSGALTO00( & 7 cis & 6.5 cis & 5.45 & $1.62 \mathrm{E}-03$ \\
\hline chr7_294520IENSGALTO00( & 7 cis & 6.5 cis & 6.51 & $2.11 \mathrm{E}-03$ \\
\hline chr7_286200IENSGALTO00( & 7 cis & 6.5 cis & 7.73 & 4.29E-04 \\
\hline chr7_289510IENSGALTOOO( & 7 cis & $6.5 \mathrm{cis}$ & 7.92 & $1.60 \mathrm{E}-03$ \\
\hline chr7_296570IENSGALT000( & 7 cis & 6.5 cis & 5.26 & $1.84 \mathrm{E}-03$ \\
\hline chr7_288660IENSGALTO00( & 7 cis & 6.5 cis & 5.24 & $9.88 \mathrm{E}-05$ \\
\hline chr7_295100IENSGALT000( & 7 cis & 6.5 cis & 9.63 & $4.80 \mathrm{E}-05$ \\
\hline chr7_288540IENSGALTO00( & 7 cis & 6.5 cis & 5.77 & $1.87 \mathrm{E}-05$ \\
\hline chr7_293370IENSGALTO00( & 7 cis & 6.5 cis & 6.8 & $2.93 \mathrm{E}-08$ \\
\hline chr7_288000IENSGALTOOO( & 7 cis & $6.5 \mathrm{cis}$ & 4.27 & $3.29 \mathrm{E}-03$ \\
\hline chr7_285990IENSGALT000( & 7 cis & 6.5 cis & 10.24 & $9.26 \mathrm{E}-05$ \\
\hline chr7_291700IENSGALTO00( & 7 cis & 6.5 cis & 5.34 & $9.31 \mathrm{E}-04$ \\
\hline chr7_292260IENSGALTO00( & 7 cis & 6.5 cis & 8.46 & 7.49E-06 \\
\hline chr7_289720IENSGALTO00( & 7 cis & 6.5 cis & 6.65 & $1.25 \mathrm{E}-05$ \\
\hline chr7_291710IENSGALTO00( & 7 cis & 6.5 cis & 10.61 & 1.17E-07 \\
\hline chr7_291460IENSGALTOOO( & 7 cis & $6.5 \mathrm{cis}$ & 7.48 & 6.67E-07 \\
\hline chr7_291850IENSGALTO00( & 7 cis & $6.5 \mathrm{cis}$ & 8.3 & $9.94 \mathrm{E}-05$ \\
\hline chr7_292740IENSGALTOOO( & 7 cis & 6.5 cis & 6.59 & $3.30 \mathrm{E}-04$ \\
\hline chr7_302170IENSGALT000( & 7 cis & 6.5 cis & 8.85 & $1.36 \mathrm{E}-04$ \\
\hline chr7_294550IENSGALTOOOC & 7 cis & $6.5 \mathrm{cis}$ & 4.65 & 3.19E-03 \\
\hline chr7_289740IENSGALTO00I & 7 cis & $6.5 \mathrm{cis}$ & 5.92 & $7.88 \mathrm{E}-04$ \\
\hline chr7_295490IENSGALTOOOC & 7 cis & $6.5 \mathrm{cis}$ & 6.73 & $1.65 \mathrm{E}-03$ \\
\hline chr7_320750IENSGALTO00( & 7 cis & 6.5 cis & 7.65 & 3.77E-05 \\
\hline chr7_320770IENSGALTO00( & 7 cis & 6.5 cis & 9.7 & $5.85 \mathrm{E}-04$ \\
\hline chr7_302090IENSGALT000( & 7 cis & 6.5 cis & 4.29 & $1.65 \mathrm{E}-03$ \\
\hline chr7_304330IENSGALT000( & 7 cis & 6.5 cis & 4.1 & 8.19E-04 \\
\hline
\end{tabular}




\begin{tabular}{|c|c|c|c|c|}
\hline chr7_307540IENSGALT000( & 7 cis & 6.5 cis & 4.34 & $3.36 \mathrm{E}-03$ \\
\hline chr7_313470ıENSGALT000( & 7 cis & 6.5 cis & 4.1 & $1.59 \mathrm{E}-03$ \\
\hline chr7_320710।ENSGALT000( & 7 cis & 6.5 cis & 15.38 & 5.87E-04 \\
\hline chr7_320760IENSGALTO00( & 7 cis & 6.5 cis & 10.03 & $3.50 \mathrm{E}-05$ \\
\hline chr7_320690IENSGALT000( & 7 cis & 6.5 cis & 7.43 & $4.08 \mathrm{E}-05$ \\
\hline chr7_320700IENSGALT000( & 7 cis & 6.5 cis & 11.8 & 2.07E-05 \\
\hline chr7_320730IENSGALTO00( & 7 cis & 6.5 cis & 14.43 & $1.73 \mathrm{E}-06$ \\
\hline chr7_320740IENSGALT000( & 7 cis & 6.5 cis & 13.34 & $1.48 \mathrm{E}-06$ \\
\hline chr7_320720।ENSGALT000( & 7 cis & 6.5 cis & 12.08 & 7.19E-06 \\
\hline chr7_328580IENSGALTO00( & 7 cis & 6.5 cis & 8.53 & $3.24 \mathrm{E}-04$ \\
\hline chr7_328570IENSGALT000( & 7 cis & 6.5 cis & 7.78 & $2.49 \mathrm{E}-04$ \\
\hline chr7_328600IENSGALTO00( & 7 cis & 6.5 cis & 6.15 & $1.86 \mathrm{E}-05$ \\
\hline chr7_328610।ENSGALTO00( & 7 cis & 6.5 cis & 8.03 & 5.60E-05 \\
\hline chr7_328620IENSGALTO00( & 7 cis & 6.5 cis & 9.1 & $9.89 \mathrm{E}-04$ \\
\hline chr7_328630IENSGALTO00( & 7 cis & 6.5 cis & 6.78 & 4.01E-05 \\
\hline chr7_328640IENSGALT000( & 7 cis & 6.5 cis & 10.18 & 5.10E-05 \\
\hline chr7_328560IENSGALTO00( & 7 cis & 6.5 cis & 10.17 & $6.12 \mathrm{E}-06$ \\
\hline chr7_328590IENSGALT000( & 7 cis & 6.5 cis & 9.1 & $1.30 \mathrm{E}-03$ \\
\hline chr7_328660IENSGALTO00( & 7 cis & 6.5 cis & 11.58 & 8.77E-04 \\
\hline chr7_328650IENSGALTO00( & 7 cis & 6.5 cis & 10.6 & 1.39E-03 \\
\hline chr7_319530IENSGALTO00( & 7 cis & 4.1 trans & 7.55 & $3.32 \mathrm{E}-04$ \\
\hline chr8_808400।X603141831F & $8 \mathrm{cis}$ & $4.6 \mathrm{cis}$ & 5.54 & $9.64 \mathrm{E}-04$ \\
\hline chr8_899400।X603141831F & $8 \mathrm{cis}$ & 4.6 cis & 10.67 & $9.75 \mathrm{E}-04$ \\
\hline chr8_816400)X603141831F & $8 \mathrm{cis}$ & 4.6 cis & 4.1 & 4.61E-04 \\
\hline chr8_102330।X603141831F & $8 \mathrm{cis}$ & 4.6 cis & 13.69 & $2.01 \mathrm{E}-03$ \\
\hline chr8_136680!X603141831F & $8 \mathrm{cis}$ & 4.6 cis & 4.87 & $2.32 \mathrm{E}-03$ \\
\hline chr8_144480ıX603141831F & 8 cis & 4.6 cis & 13.02 & 1.76E-03 \\
\hline chr8_158970।X603141831F & $8 \mathrm{cis}$ & 4.6 cis & 4.1 & 1.64E-03 \\
\hline chr8_159960।X603141831F & $8 \mathrm{cis}$ & 4.6 cis & 4.1 & $1.87 \mathrm{E}-03$ \\
\hline chr8_766700।X603141830F & $8 \mathrm{cis}$ & $15.3 \mathrm{cis}$ & 4.1 & $1.07 \mathrm{E}-03$ \\
\hline chr8_808400।X603141830F & $8 \mathrm{cis}$ & $15.3 \mathrm{cis}$ & 5.54 & $1.35 \mathrm{E}-04$ \\
\hline chr8_751700।X603141830F & 8 cis & $15.3 \mathrm{cis}$ & 4.1 & 7.91E-06 \\
\hline chr8_113250।X603141830F & $8 \mathrm{cis}$ & $15.3 \mathrm{cis}$ & 4.85 & 8.97E-05 \\
\hline chr8_113200।X603141830F & $8 \mathrm{cis}$ & $15.3 \mathrm{cis}$ & 5.71 & $1.05 \mathrm{E}-04$ \\
\hline chr8_113100।X603141830F & $8 \mathrm{cis}$ & $15.3 \mathrm{cis}$ & 5.31 & $1.62 \mathrm{E}-04$ \\
\hline chr8_113110।X603141830F & $8 \mathrm{cis}$ & $15.3 \mathrm{cis}$ & 5.26 & $9.08 \mathrm{E}-05$ \\
\hline chr8_113130ıX603141830F & $8 \mathrm{cis}$ & $15.3 \mathrm{cis}$ & 5.1 & $1.05 \mathrm{E}-04$ \\
\hline chr8_113320।X603141830F & 8 cis & $15.3 \mathrm{cis}$ & 5.83 & 6.97E-05 \\
\hline chr8_113410।X603141830F & $8 \mathrm{cis}$ & $15.3 \mathrm{cis}$ & 5.82 & 1.67E-04 \\
\hline chr8_113910।X603141830F & $8 \mathrm{cis}$ & $15.3 \mathrm{cis}$ & 5.63 & 1.63E-04 \\
\hline chr8_113790।X603141830F & $8 \mathrm{cis}$ & $15.3 \mathrm{cis}$ & 5.71 & $8.40 \mathrm{E}-05$ \\
\hline chr8_113170)X603141830F & $8 \mathrm{cis}$ & $15.3 \mathrm{cis}$ & 4.88 & $2.24 \mathrm{E}-04$ \\
\hline chr8_113120।X603141830F & $8 \mathrm{cis}$ & $15.3 \mathrm{cis}$ & 5.36 & 9.91E-05 \\
\hline chr8_113260।X603141830F & 8 cis & $15.3 \mathrm{cis}$ & 5.57 & $1.08 \mathrm{E}-04$ \\
\hline chr8_113310।X603141830F & $8 \mathrm{cis}$ & $15.3 \mathrm{cis}$ & 5.66 & 1.15E-04 \\
\hline chr8_113220।X603141830F & 8 cis & $15.3 \mathrm{cis}$ & 5.47 & 7.17E-05 \\
\hline chr8_113400।X603141830F & $8 \mathrm{cis}$ & $15.3 \mathrm{cis}$ & 5.49 & $1.44 \mathrm{E}-04$ \\
\hline chr8_113620।X603141830F & $8 \mathrm{cis}$ & $15.3 \mathrm{cis}$ & 5.67 & 4.81E-05 \\
\hline chr8_113290।X603141830F & 8 cis & $15.3 \mathrm{cis}$ & 5.49 & $1.10 \mathrm{E}-04$ \\
\hline chr8_113420।X603141830F & 8 cis & $15.3 \mathrm{cis}$ & 6.2 & 7.65E-05 \\
\hline chr8_113560।X603141830F & $8 \mathrm{cis}$ & $15.3 \mathrm{cis}$ & 7.07 & 1.19E-05 \\
\hline chr8_113650।X603141830F & 8 cis & $15.3 \mathrm{cis}$ & 6.67 & 4.15E-05 \\
\hline chr8_811300।X603141830F & $8 \mathrm{cis}$ & $15.3 \mathrm{cis}$ & 4.31 & $2.44 \mathrm{E}-04$ \\
\hline chr8_846600।X603141830F & $8 \mathrm{cis}$ & $15.3 \mathrm{cis}$ & 10.44 & 3.36E-04 \\
\hline chr8_899400।X603141830F & 8 cis & $15.3 \mathrm{cis}$ & 10.67 & $3.26 \mathrm{E}-04$ \\
\hline chr8_899500।X603141830F & 8 cis & $15.3 \mathrm{cis}$ & 19.28 & $6.46 \mathrm{E}-05$ \\
\hline chr8_102330।X603141830F & 8 cis & $15.3 \mathrm{cis}$ & 13.69 & 4.59E-05 \\
\hline chr8_815100।X603141830F & 8 cis & $15.3 \mathrm{cis}$ & 6.64 & $9.15 E-05$ \\
\hline chr8_161970।X603141830F & $8 \mathrm{cis}$ & $15.3 \mathrm{cis}$ & 4.41 & 1.69E-04 \\
\hline chr8_131930)X603141830F & $8 \mathrm{cis}$ & $15.3 \mathrm{cis}$ & 10.34 & 3.65E-05 \\
\hline chr8_136680 X603141830F & 8 cis & $15.3 \mathrm{cis}$ & 4.87 & $1.18 \mathrm{E}-03$ \\
\hline chr8_144480।X603141830F & 8 cis & $15.3 \mathrm{cis}$ & 13.02 & $2.37 \mathrm{E}-07$ \\
\hline chr8_156350।X603141830F & 8 cis & $15.3 \mathrm{cis}$ & 11.84 & $3.84 \mathrm{E}-04$ \\
\hline chr8_146520।X603141830F & 8 cis & $15.3 \mathrm{cis}$ & 7.29 & $1.50 \mathrm{E}-04$ \\
\hline chr8_811300।X603142079F & $8 \mathrm{cis}$ & $8.6 \mathrm{cis}$ & 4.31 & 7.01E-05 \\
\hline chr8_846600।X603142079F & 8 cis & $8.6 \mathrm{cis}$ & 10.44 & $3.70 \mathrm{E}-03$ \\
\hline chr8_899500।X603142079F & 8 cis & $8.6 \mathrm{cis}$ & 19.28 & $1.28 \mathrm{E}-03$ \\
\hline chr8_102330।X603142079F & 8 cis & $8.6 \mathrm{cis}$ & 13.69 & $7.86 \mathrm{E}-04$ \\
\hline chr8_8997001X603142079F & 8 cis & $8.6 \mathrm{cis}$ & 4.1 & $2.26 \mathrm{E}-03$ \\
\hline chr8_128850।X603142079F & 8 cis & $8.6 \mathrm{cis}$ & 8.24 & $7.12 \mathrm{E}-06$ \\
\hline chr8_144480।X603142079F & $8 \mathrm{cis}$ & 8.6 cis & 13.02 & $1.20 \mathrm{E}-04$ \\
\hline chr8_751700ıNM_0010125 & $8 \mathrm{cis}$ & $16.1 \mathrm{cis}$ & 4.1 & $2.62 \mathrm{E}-04$ \\
\hline
\end{tabular}




\begin{tabular}{|c|c|c|c|c|}
\hline chr8_113200INM_0010125 & 8 cis & $16.1 \mathrm{cis}$ & 5.71 & $6.06 \mathrm{E}-05$ \\
\hline chr8_113110INM_0010125 & 8 cis & 16.1 cis & 5.26 & $8.87 \mathrm{E}-05$ \\
\hline chr8_113320INM_0010125 & 8 cis & 16.1 cis & 5.83 & $2.93 \mathrm{E}-05$ \\
\hline chr8_113410INM_0010125 & 8 cis & $16.1 \mathrm{cis}$ & 5.82 & $6.71 \mathrm{E}-05$ \\
\hline chr8_113910INM_0010125 & 8 cis & 16.1 cis & 5.63 & $6.37 \mathrm{E}-05$ \\
\hline chr8_113790।NM_0010125 & 8 cis & 16.1 cis & 5.71 & $7.28 \mathrm{E}-05$ \\
\hline chr8_113120।NM_0010125 & 8 cis & 16.1 cis & 5.36 & $6.49 \mathrm{E}-05$ \\
\hline chr8_113310INM_0010125 & 8 cis & $16.1 \mathrm{cis}$ & 5.66 & 3.63E-05 \\
\hline chr8_113220INM_0010125 & 8 cis & 16.1 cis & 5.47 & $2.70 \mathrm{E}-05$ \\
\hline chr8_113400INM_0010125 & 8 cis & 16.1 cis & 5.49 & 5.67E-05 \\
\hline chr8_113620INM_0010125 & 8 cis & 16.1 cis & 5.67 & $2.46 \mathrm{E}-05$ \\
\hline chr8_113290INM_0010125 & 8 cis & $16.1 \mathrm{cis}$ & 5.49 & $1.98 \mathrm{E}-05$ \\
\hline chr8_113420INM_0010125 & 8 cis & 16.1 cis & 6.2 & $6.12 \mathrm{E}-06$ \\
\hline chr8_113560।NM_0010125 & 8 cis & 16.1 cis & 7.07 & $1.70 \mathrm{E}-06$ \\
\hline chr8_113650।NM_0010125 & 8 cis & 16.1 cis & 6.67 & $1.96 \mathrm{E}-06$ \\
\hline chr8_113600INM_0010125 & 8 cis & 16.1 cis & 5.57 & $8.59 \mathrm{E}-06$ \\
\hline chr8_114080INM_0010125 & 8 cis & 16.1 cis & 4.75 & 3.06E-05 \\
\hline chr8_846500।NM_0010125 & 8 cis & 16.1 cis & 9.81 & $3.06 \mathrm{E}-04$ \\
\hline chr8_846600।NM_0010125 & 8 cis & 16.1 cis & 10.44 & $1.70 \mathrm{E}-05$ \\
\hline chr8_899400INM_0010125 & 8 cis & 16.1 cis & 10.67 & $1.06 \mathrm{E}-08$ \\
\hline chr8_816400।NM_0010125 & 8 cis & 16.1 cis & 4.1 & $3.86 \mathrm{E}-06$ \\
\hline chr8_836200।NM_0010125 & 8 cis & 16.1 cis & 5.16 & $1.21 \mathrm{E}-05$ \\
\hline chr8_859400।NM_0010125 & 8 cis & 16.1 cis & 4.43 & $9.41 \mathrm{E}-04$ \\
\hline chr8_899500।NM_0010125 & 8 cis & 16.1 cis & 19.28 & $1.25 \mathrm{E}-09$ \\
\hline chr8_102330INM_0010125 & 8 cis & 16.1 cis & 13.69 & 3.07E-10 \\
\hline chr8_899700।NM_0010125 & 8 cis & 16.1 cis & 4.1 & $9.08 \mathrm{E}-06$ \\
\hline chr8_110970INM_0010125 & 8 cis & 16.1 cis & 4.1 & $2.19 \mathrm{E}-04$ \\
\hline chr8_125800।NM_0010125 & 8 cis & $16.1 \mathrm{cis}$ & 4.17 & $2.30 \mathrm{E}-04$ \\
\hline chr8_815100।NM_0010125 & 8 cis & $16.1 \mathrm{cis}$ & 6.64 & $1.12 \mathrm{E}-05$ \\
\hline chr8_879200।NM_0010125 & 8 cis & $16.1 \mathrm{cis}$ & 6.61 & $5.65 \mathrm{E}-06$ \\
\hline chr8_878500।NM_0010125 & 8 cis & 16.1 cis & 4.1 & $2.39 \mathrm{E}-05$ \\
\hline chr8_982700।NM_0010125 & 8 cis & 16.1 cis & 9.64 & $8.79 \mathrm{E}-05$ \\
\hline chr8_998600।NM_0010125 & 8 cis & 16.1 cis & 4.99 & 2.49E-04 \\
\hline chr8_899400IENSGALTO00( & 8 cis & 4.6 cis & 10.67 & $1.42 \mathrm{E}-03$ \\
\hline chr8_816400|ENSGALTO0OC & 8 cis & 4.6 cis & 4.1 & $1.49 \mathrm{E}-03$ \\
\hline chr8_899500।ENSGALTO0OC & 8 cis & 4.6 cis & 19.28 & 1.37E-04 \\
\hline chr8_102330IENSGALTO0OI & 8 cis & 4.6 cis & 13.69 & $5.91 \mathrm{E}-03$ \\
\hline chr8_899700IENSGALTO0OI & 8 cis & 4.6 cis & 4.1 & $1.43 \mathrm{E}-02$ \\
\hline chr8_125800।ENSGALTO0OC & 8 cis & 4.6 cis & 4.17 & $1.19 \mathrm{E}-03$ \\
\hline chr8_879200IENSGALTO0OC & 8 cis & 4.6 cis & 6.61 & $1.11 \mathrm{E}-03$ \\
\hline chr8_158590|ENSGALTO0OC & 8 cis & 4.6 cis & 4.1 & $8.09 \mathrm{E}-03$ \\
\hline chr8_164260IENSGALTO0OC & 8 cis & 4.6 cis & 4.1 & $2.63 \mathrm{E}-03$ \\
\hline chr8_124050IENSGALTO0OC & 8 cis & 4.6 cis & 6.91 & $1.01 \mathrm{E}-02$ \\
\hline chr8_8997001X603601261F & 8 cis & 6.0 cis & 4.1 & $3.00 \mathrm{E}-03$ \\
\hline chr8_8994001X603868076F & 8 cis & 5.6 cis & 10.67 & $1.32 \mathrm{E}-04$ \\
\hline chr8_816400।X603868076F & 8 cis & 5.6 cis & 4.1 & $3.05 \mathrm{E}-04$ \\
\hline chr8_8362001X603868076F & 8 cis & 5.6 cis & 5.16 & $3.66 \mathrm{E}-03$ \\
\hline chr8_8995001X603868076F & 8 cis & 5.6 cis & 19.28 & $8.93 \mathrm{E}-06$ \\
\hline chr8_1023301X603868076F & 8 cis & 5.6 cis & 13.69 & $8.93 \mathrm{E}-04$ \\
\hline chr8_8997001X603868076F & 8 cis & 5.6 cis & 4.1 & $3.40 \mathrm{E}-03$ \\
\hline chr8_1258001X603868076F & 8 cis & 5.6 cis & 4.17 & $1.32 \mathrm{E}-04$ \\
\hline chr8_8792001X603868076F & 8 cis & 5.6 cis & 6.61 & 3.97E-04 \\
\hline chr8_149650।X603868076F & 8 cis & 5.6 cis & 4.1 & $2.27 \mathrm{E}-03$ \\
\hline chr8_158970ıX603868076F & 8 cis & 5.6 cis & 4.1 & $1.16 \mathrm{E}-03$ \\
\hline chr8_164260।X603868076F & 8 cis & 5.6 cis & 4.1 & $4.04 \mathrm{E}-04$ \\
\hline chr8_156350।X603868076F & 8 cis & 5.6 cis & 11.84 & $5.96 \mathrm{E}-04$ \\
\hline chr8_815100|ENSGALTO0OC & 8 cis & 4.2 cis & 6.64 & $1.61 \mathrm{E}-04$ \\
\hline chr8_128850|ENSGALTO0OC & 8 cis & 4.2 cis & 8.24 & $8.45 \mathrm{E}-04$ \\
\hline chr8_141180IENSGALTO0OC & 8 cis & 4.2 cis & 5.8 & $1.40 \mathrm{E}-04$ \\
\hline chr8_982800।ENSGALTO0OC & 8 cis & 4.2 cis & 7.56 & $2.10 \mathrm{E}-03$ \\
\hline chr8_188350|ENSGALTO0OC & 8 cis & 4.2 cis & 35.54 & $3.76 \mathrm{E}-04$ \\
\hline chr8_188370|ENSGALTO0OC & 8 cis & 4.2 cis & 34.59 & $8.20 \mathrm{E}-04$ \\
\hline chr8_7667001X603141556F & 8 cis & 4.5 cis & 4.1 & $1.59 \mathrm{E}-02$ \\
\hline chr8_899500।X603141556F & 8 cis & 4.5 cis & 19.28 & $7.06 \mathrm{E}-03$ \\
\hline chr8_125800।X603141556F & 8 cis & 4.5 cis & 4.17 & $7.33 \mathrm{E}-03$ \\
\hline chr8_1274201X603141556F & 8 cis & 4.5 cis & 4.29 & 3.27E-03 \\
\hline chr8_144760।X603141556F & 8 cis & 4.5 cis & 8.05 & $3.43 \mathrm{E}-03$ \\
\hline chr8_143830IENSGALTO00 & 8 cis & 4.8 cis & 5.22 & $1.65 \mathrm{E}-03$ \\
\hline chr8_144480IENSGALTO0OC & 8 cis & 4.8 cis & 13.02 & 2.93E-06 \\
\hline chr8_143830।X603866233F & 8 cis & 5.0 cis & 5.22 & $1.48 \mathrm{E}-04$ \\
\hline chr8_131930।X603866233F & 8 cis & 5.0 cis & 10.34 & $7.75 \mathrm{E}-06$ \\
\hline chr8_144480।X603866233F & 8 cis & 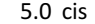 & 13.02 & 1.06E-06 \\
\hline
\end{tabular}




\begin{tabular}{|c|c|c|c|c|}
\hline chr8_1642601X603866233F & $8 \mathrm{cis}$ & $5.0 \mathrm{cis}$ & 4.1 & $1.39 \mathrm{E}-03$ \\
\hline chr8_156350।X603866233F & $8 \mathrm{cis}$ & 5.0 cis & 11.84 & $2.98 \mathrm{E}-04$ \\
\hline chr8_143830IENSGALTO0O & $8 \mathrm{cis}$ & $4.6 \mathrm{cis}$ & 5.22 & $5.08 \mathrm{E}-04$ \\
\hline chr8_144480IENSGALTO00 & $8 \mathrm{cis}$ & 4.6 cis & 13.02 & $1.25 \mathrm{E}-04$ \\
\hline chr8_195140IENSGALTO0OI & 8 cis & $4.3 \mathrm{cis}$ & 4.1 & $6.08 \mathrm{E}-03$ \\
\hline chr8_899500IENSGALTO0OC & 8 cis & 4.3 cis & 19.28 & $2.82 \mathrm{E}-03$ \\
\hline chr8_144480IENSGALTO0OI & 8 cis & 4.3 cis & 13.02 & $1.10 \mathrm{E}-03$ \\
\hline chr8_188380IENSGALTO0OI & $8 \mathrm{cis}$ & 4.3 cis & 13.84 & 4.87E-04 \\
\hline chr8_188390|ENSGALTO0OC & 8 cis & 4.3 cis & 21.62 & $6.00 \mathrm{E}-04$ \\
\hline chr8_188370IENSGALTO0OC & 8 cis & 4.3 cis & 34.59 & $2.56 \mathrm{E}-03$ \\
\hline chr8_188360IENSGALTO0O & 8 cis & 4.3 cis & 23.19 & $1.40 \mathrm{E}-03$ \\
\hline chr8_8464001X603599147F & 8 cis & 4.7 cis & 4.3 & $1.51 \mathrm{E}-02$ \\
\hline chr8_161970।X603599147F & 8 cis & 4.7 cis & 4.41 & $8.90 \mathrm{E}-03$ \\
\hline chr8_139860।X603599147F & 8 cis & 4.7 cis & 4.44 & $6.86 \mathrm{E}-05$ \\
\hline chr8_982700।X603599147F & 8 cis & 4.7 cis & 9.64 & $1.69 \mathrm{E}-03$ \\
\hline chr8_9301001X603599147F & 8 cis & 4.7 cis & 7.01 & $9.62 \mathrm{E}-03$ \\
\hline chr8_982800।X603599147F & 8 cis & 4.7 cis & 7.56 & $2.06 \mathrm{E}-04$ \\
\hline chr8_188400।X603599147F & 8 cis & 4.7 cis & 35.85 & $1.29 \mathrm{E}-04$ \\
\hline chr8_9300001X603599147F & 8 cis & 4.7 cis & 5.64 & $4.84 \mathrm{E}-04$ \\
\hline chr8_188350।X603599147F & 8 cis & 4.7 cis & 35.54 & $8.88 \mathrm{E}-04$ \\
\hline chr8_188370।X603599147F & 8 cis & 4.7 cis & 34.59 & $1.06 \mathrm{E}-03$ \\
\hline chr8_1953201X603599147F & $8 \mathrm{cis}$ & $4.7 \mathrm{cis}$ & 7.11 & $2.70 \mathrm{E}-05$ \\
\hline chr8_195380।X603599147F & 8 cis & 4.7 cis & 6.84 & $1.68 \mathrm{E}-04$ \\
\hline chr8_188360।X603599147F & 8 cis & 4.7 cis & 23.19 & $1.33 \mathrm{E}-04$ \\
\hline chr8_195380IENSGALTO0OC & 8 cis & 3.5 cis & 6.84 & $4.25 \mathrm{E}-04$ \\
\hline chr8_1619701X603599201F & $8 \mathrm{cis}$ & $6.1 \mathrm{cis}$ & 4.41 & $1.17 \mathrm{E}-02$ \\
\hline chr8_762800।X603599201F & 8 cis & 6.1 cis & 4.65 & $6.95 \mathrm{E}-05$ \\
\hline chr8_1884001X603599201F & 8 cis & $6.1 \mathrm{cis}$ & 35.85 & $5.62 \mathrm{E}-06$ \\
\hline chr8_188350।X603599201F & 8 cis & 6.1 cis & 35.54 & $5.40 \mathrm{E}-04$ \\
\hline chr8_1883701X603599201F & 8 cis & $6.1 \mathrm{cis}$ & 34.59 & $2.46 \mathrm{E}-04$ \\
\hline chr8_188360।X603599201F & 8 cis & $6.1 \mathrm{cis}$ & 23.19 & $4.02 \mathrm{E}-05$ \\
\hline chr8_195140।X603599396F & 8 cis & 7.3 cis & 4.1 & $4.29 \mathrm{E}-06$ \\
\hline chr8_195650।X603599396F & $8 \mathrm{cis}$ & 7.3 cis & 4.1 & $3.12 \mathrm{E}-04$ \\
\hline chr8_188380।X603599396F & 8 cis & 7.3 cis & 13.84 & $2.04 \mathrm{E}-04$ \\
\hline chr8_188390।X603599396F & 8 cis & 7.3 cis & 21.62 & $8.91 \mathrm{E}-06$ \\
\hline chr8_188400।X603599396F & 8 cis & 7.3 cis & 35.85 & $9.91 \mathrm{E}-05$ \\
\hline chr8_188350।X603599396F & 8 cis & 7.3 cis & 35.54 & $1.29 \mathrm{E}-05$ \\
\hline chr8_1883701X603599396F & 8 cis & 7.3 cis & 34.59 & $9.13 \mathrm{E}-05$ \\
\hline chr8_188360।X603599396F & 8 cis & 7.3 cis & 23.19 & $1.13 \mathrm{E}-06$ \\
\hline chr8_808400INM_204481_ & 8 cis & 6.0 cis & 5.54 & $7.92 \mathrm{E}-05$ \\
\hline chr8_846700INM_204481_ & 8 cis & 6.0 cis & 5.54 & $2.12 \mathrm{E}-07$ \\
\hline chr8_846500INM_204481_ & 8 cis & 6.0 cis & 9.81 & $2.56 \mathrm{E}-06$ \\
\hline chr8_846600INM_204481_ & $8 \mathrm{cis}$ & $6.0 \mathrm{cis}$ & 10.44 & $1.10 \mathrm{E}-05$ \\
\hline chr8_846400INM_204481_ & $8 \mathrm{cis}$ & $6.0 \mathrm{cis}$ & 4.3 & 7.97E-04 \\
\hline chr8_128850iNM_204481_ & 8 cis & 6.0 cis & 8.24 & $1.67 \mathrm{E}-03$ \\
\hline chr8_982700INM_204481_ & $8 \mathrm{cis}$ & 6.0 cis & 9.64 & $1.17 \mathrm{E}-03$ \\
\hline chr8_930100INM_204481_ & 8 cis & 6.0 cis & 7.01 & $1.10 \mathrm{E}-03$ \\
\hline chr8_982800INM_204481_ & 8 cis & 6.0 cis & 7.56 & $2.26 \mathrm{E}-03$ \\
\hline chr8_185870INM_204481_ & 8 cis & 6.0 cis & 6.44 & $1.70 \mathrm{E}-02$ \\
\hline chr8_188390INM_204481_ & $8 \mathrm{cis}$ & 6.0 cis & 21.62 & $2.11 \mathrm{E}-05$ \\
\hline chr8_188400INM_204481_ & 8 cis & 6.0 cis & 35.85 & $2.58 \mathrm{E}-05$ \\
\hline chr8_930000iNM_204481_ & 8 cis & 6.0 cis & 5.64 & $1.32 \mathrm{E}-03$ \\
\hline chr8_188350INM_204481_ & 8 cis & 6.0 cis & 35.54 & $9.70 \mathrm{E}-06$ \\
\hline chr8_188370INM_204481_ & 8 cis & 6.0 cis & 34.59 & $4.91 \mathrm{E}-05$ \\
\hline chr8_188360iNM_204481_ & 8 cis & 6.0 cis & 23.19 & $1.70 \mathrm{E}-06$ \\
\hline chr8_161970।X603602157F & 8 cis & 5.4 cis & 4.41 & $1.10 \mathrm{E}-02$ \\
\hline chr8_1884001X603602157F & 8 cis & 5.4 cis & 35.85 & $5.99 \mathrm{E}-04$ \\
\hline chr8_188360।X603602157F & 8 cis & 5.4 cis & 23.19 & $7.52 \mathrm{E}-04$ \\
\hline chr9_225000।X603598875F & 9 cis & 9.8 cis & 5.79 & $6.35 \mathrm{E}-04$ \\
\hline chr9_197700ıX603598875F & 9 cis & 9.8 cis & 4.86 & $1.45 \mathrm{E}-04$ \\
\hline chr9_195800।X603598875F & 9 cis & $9.8 \mathrm{cis}$ & 7.57 & $3.86 \mathrm{E}-04$ \\
\hline chr9_2707001X603598875F & 9 cis & 9.8 cis & 5.48 & $1.86 \mathrm{E}-03$ \\
\hline chr9_2654001X603598875F & 9 cis & 9.8 cis & 4.43 & $8.89 \mathrm{E}-04$ \\
\hline chr9_197700IENSGALTO0O & 9 cis & 7.6 cis & 4.86 & $1.75 \mathrm{E}-03$ \\
\hline chr9_270700IENSGALTO0OI & 9 cis & $7.6 \mathrm{cis}$ & 5.48 & $6.97 \mathrm{E}-04$ \\
\hline chr9_265400IENSGALTO0OC & 9 cis & 7.6 cis & 4.43 & $6.27 \mathrm{E}-04$ \\
\hline chr9_270700INM_204441_ & 9 cis & 4.4 cis & 5.48 & $5.95 \mathrm{E}-03$ \\
\hline chr9_285200INM_001168C & 9 cis & 4.5 cis & 8.95 & $2.19 \mathrm{E}-03$ \\
\hline chr9_285300INM_001168C & 9 cis & 4.5 cis & 14.49 & $6.41 \mathrm{E}-04$ \\
\hline chr9_8042001X603598638F & 9 cis & 7.0 cis & 5.4 & $2.46 \mathrm{E}-04$ \\
\hline chr9_546300।X603598638F & 9 cis & 7.0 cis & 11.98 & $2.70 \mathrm{E}-03$ \\
\hline chr9 5757001X603598638F & 9 cis & 7.0 cis & 8.83 & 8.82E-04 $>2 \Rightarrow$ \\
\hline
\end{tabular}




\begin{tabular}{|c|c|c|c|c|}
\hline chr9_5893001X603598638F & 9 cis & 7.0 cis & 7.69 & $1.84 \mathrm{E}-04$ \\
\hline chr9_634900।X603598638F & 9 cis & 7.0 cis & 8.98 & $3.26 \mathrm{E}-04$ \\
\hline chr9_657900।X603598638F & 9 cis & 7.0 cis & 11.59 & $8.08 \mathrm{E}-04$ \\
\hline chr9_6468001X603598638F & 9 cis & 7.0 cis & 14.24 & 4.10E-06 \\
\hline chr9_6787001X603598638F & 9 cis & 7.0 cis & 23.8 & $1.98 \mathrm{E}-04$ \\
\hline chr9_610500।X603598638F & 9 cis & 7.0 cis & 5.99 & $8.39 \mathrm{E}-04$ \\
\hline chr9_7205001X603598638F & 9 cis & 7.0 cis & 7.99 & $1.22 \mathrm{E}-03$ \\
\hline chr9_8351001X603598638F & 9 cis & 7.0 cis & 5.72 & $1.47 \mathrm{E}-02$ \\
\hline chr9_621300।X603598638F & 9 cis & 7.0 cis & 18.59 & $2.73 \mathrm{E}-04$ \\
\hline chr9_6785001X603598638F & 9 cis & 7.0 cis & 8.03 & $2.43 \mathrm{E}-04$ \\
\hline chr9_6786001X603598638F & 9 cis & 7.0 cis & 35.03 & $3.82 \mathrm{E}-06$ \\
\hline chr9_5503001X603598638F & 9 cis & 7.0 cis & 5.35 & $1.96 \mathrm{E}-03$ \\
\hline chr9_8373001X603598638F & 9 cis & 7.0 cis & 8.6 & $5.18 \mathrm{E}-05$ \\
\hline chr9_5708001X603598638F & 9 cis & 7.0 cis & 5.94 & $1.53 \mathrm{E}-04$ \\
\hline chr9_7356001X603598638F & 9 cis & 7.0 cis & 7.96 & $1.38 \mathrm{E}-04$ \\
\hline chr9_729800।X603598638F & 9 cis & 7.0 cis & 10.04 & $2.89 \mathrm{E}-03$ \\
\hline chr9_8160001X603598638F & 9 cis & 7.0 cis & 6.33 & $2.49 \mathrm{E}-03$ \\
\hline chr9_7344001X603598638F & 9 cis & 7.0 cis & 9.01 & 3.44E-04 \\
\hline chr9_7569001X603598638F & 9 cis & 7.0 cis & 4.83 & $6.52 \mathrm{E}-03$ \\
\hline chr9_8745001X603598638F & 9 cis & 7.0 cis & 4.33 & $7.21 \mathrm{E}-05$ \\
\hline chr9_8359001X603598638F & 9 cis & 7.0 cis & 8.06 & $1.78 \mathrm{E}-03$ \\
\hline chr9_5096001X603598638F & 9 cis & 7.0 cis & 7.03 & $8.90 \mathrm{E}-04$ \\
\hline chr9_7446001X603598638F & 9 cis & 7.0 cis & 4.42 & $1.46 \mathrm{E}-03$ \\
\hline chr9_7638001X603598638F & 9 cis & 7.0 cis & 4.27 & $2.61 \mathrm{E}-04$ \\
\hline chr9_1017201X603598638F & 9 cis & 7.0 cis & 5.17 & $2.60 \mathrm{E}-03$ \\
\hline chr9_1257301X603598638F & 9 cis & 7.0 cis & 5.54 & $1.86 \mathrm{E}-03$ \\
\hline chr9_666100।X603598638F & 9 cis & 7.0 cis & 4.52 & $6.57 \mathrm{E}-04$ \\
\hline chr9_6568001X603598638F & 9 cis & 7.0 cis & 5.21 & $1.03 \mathrm{E}-03$ \\
\hline chr9_105840।X603598638F & 9 cis & 7.0 cis & 9.32 & $2.40 \mathrm{E}-03$ \\
\hline chr9_122790।X603598638F & 9 cis & 7.0 cis & 8.06 & $3.90 \mathrm{E}-03$ \\
\hline chr9_1001001X603598638F & 9 cis & 7.0 cis & 5.43 & $2.21 \mathrm{E}-04$ \\
\hline chr9_114180ıX603598638F & 9 cis & 7.0 cis & 10.39 & $1.77 \mathrm{E}-03$ \\
\hline chr9_128580।X603598638F & 9 cis & 7.0 cis & 4.94 & $3.58 \mathrm{E}-04$ \\
\hline chr9_1141701X603598638F & 9 cis & 7.0 cis & 10.01 & $2.82 \mathrm{E}-03$ \\
\hline chr9_5757001X603144142F & 9 cis & 4.6 cis & 8.83 & 2.67E-03 \\
\hline chr9_5799001X603144142F & 9 cis & 4.6 cis & 5.66 & 4.15E-03 \\
\hline chr9_651500।X603144142F & 9 cis & 4.6 cis & 5.52 & $5.62 \mathrm{E}-04$ \\
\hline chr9_6468001X603144142F & 9 cis & 4.6 cis & 14.24 & $2.21 \mathrm{E}-04$ \\
\hline chr9_6213001X603144142F & 9 cis & 4.6 cis & 18.59 & $3.74 \mathrm{E}-03$ \\
\hline chr9_709300।X603144142F & 9 cis & 4.6 cis & 4.15 & $1.39 \mathrm{E}-03$ \\
\hline chr9_7417001X603144142F & 9 cis & 4.6 cis & 4.1 & $2.63 \mathrm{E}-04$ \\
\hline chr9_7152001X603144142F & 9 cis & 4.6 cis & 8.6 & $1.33 \mathrm{E}-03$ \\
\hline chr9_5708001X603144142F & 9 cis & 4.6 cis & 5.94 & 1.17E-03 \\
\hline chr9_619300।X603144142F & 9 cis & 4.6 cis & 5.37 & $1.58 \mathrm{E}-04$ \\
\hline chr9_6902001X603144142F & 9 cis & 4.6 cis & 4.81 & $1.91 \mathrm{E}-04$ \\
\hline chr9_729800।X603144142F & 9 cis & 4.6 cis & 10.04 & $5.50 \mathrm{E}-04$ \\
\hline chr9_647100(X603144142F & 9 cis & 4.6 cis & 4.27 & $1.74 \mathrm{E}-03$ \\
\hline chr9_816000।X603144142F & 9 cis & 4.6 cis & 6.33 & $7.98 \mathrm{E}-04$ \\
\hline chr9_7344001X603144142F & 9 cis & 4.6 cis & 9.01 & $6.32 \mathrm{E}-04$ \\
\hline chr9_756900।X603144142F & 9 cis & 4.6 cis & 4.83 & $5.43 \mathrm{E}-04$ \\
\hline chr9_835900।X603144142F & 9 cis & 4.6 cis & 8.06 & $3.80 \mathrm{E}-04$ \\
\hline chr9_7638001X603144142F & 9 cis & 4.6 cis & 4.27 & $6.31 \mathrm{E}-04$ \\
\hline chr9_656800।X603144142F & 9 cis & 4.6 cis & 5.21 & $1.57 \mathrm{E}-03$ \\
\hline chr9_646800IENSGALTO00 & 9 cis & $4.1 \mathrm{cis}$ & 14.24 & $1.06 \mathrm{E}-04$ \\
\hline chr9_835100IENSGALTO00 & 9 cis & 4.1 cis & 5.72 & 7.23E-04 \\
\hline chr9_678600।ENSGALTO00 & 9 cis & 4.1 cis & 35.03 & $5.50 \mathrm{E}-04$ \\
\hline chr9_5708001ENSGALTO00 & 9 cis & $4.1 \mathrm{cis}$ & 5.94 & $1.25 \mathrm{E}-03$ \\
\hline chr9_851600IENSGALT000 & 9 cis & $4.1 \mathrm{cis}$ & 5.67 & $5.21 \mathrm{E}-04$ \\
\hline chr9_734400iENSGALTO0OC & 9 cis & 4.1 cis & 9.01 & $2.54 \mathrm{E}-03$ \\
\hline chr9_744600।ENSGALTO0OC & 9 cis & 4.1 cis & 4.42 & $1.88 \mathrm{E}-04$ \\
\hline chr9_678700IENSGALTO00I & 9 cis & 4.3 cis & 23.8 & $1.24 \mathrm{E}-03$ \\
\hline chr9_678600IENSGALTO0OI & 9 cis & 4.3 cis & 35.03 & $2.72 \mathrm{E}-04$ \\
\hline chr9_744600iENSGALTO0OC & 9 cis & 4.3 cis & 4.42 & $1.77 \mathrm{E}-03$ \\
\hline chr9_919900IENSGALTO0OC & 9 cis & 4.3 cis & 7.16 & $1.72 \mathrm{E}-03$ \\
\hline chr9_927500IENSGALTO0OC & 9 cis & 4.3 cis & 10.91 & $2.21 \mathrm{E}-03$ \\
\hline chr9_118480iENSGALTO0OC & 9 cis & 4.3 cis & 8.46 & $4.20 \mathrm{E}-04$ \\
\hline chr9_933000IENSGALTO00 & 9 cis & 4.3 cis & 5.38 & $5.68 \mathrm{E}-03$ \\
\hline chr9_8677001X603596685F & 9 cis & 16.0 cis & 7.69 & $4.42 \mathrm{E}-05$ \\
\hline chr9_874500।X603596685F & 9 cis & $16.0 \mathrm{cis}$ & 4.33 & $8.82 \mathrm{E}-06$ \\
\hline chr9_103140।X603596685F & 9 cis & 16.0 cis & 8.28 & $9.66 \mathrm{E}-05$ \\
\hline chr9_106500।X603596685F & 9 cis & 16.0 cis & 6.44 & $6.04 \mathrm{E}-04$ \\
\hline chr9_123910।X603596685F & 9 cis & $16.0 \mathrm{cis}$ & 5.71 & $2.02 \mathrm{E}-\mathrm{C}$ \\
\hline
\end{tabular}




\begin{tabular}{|c|c|c|c|c|}
\hline chr9_125730ıX63596685F & 9 cis & $16.0 \mathrm{cis}$ & 5.54 & $5.16 \mathrm{E}-07$ \\
\hline chr9_919900ıX03596685F & 9 cis & 16.0 cis & 7.16 & $1.10 \mathrm{E}-07$ \\
\hline chr9_108990ıX603596685F & 9 cis & 16.0 cis & 11.04 & $2.89 \mathrm{E}-06$ \\
\hline chr9_903100ıX603596685F & 9 cis & 16.0 cis & 9.96 & $3.42 \mathrm{E}-06$ \\
\hline chr9_927500ı603596685F & 9 cis & 16.0 cis & 10.91 & $6.94 \mathrm{E}-07$ \\
\hline chr9_105840ıX603596685F & 9 cis & 16.0 cis & 9.32 & $8.96 \mathrm{E}-05$ \\
\hline chr9_106460।X603596685F & 9 cis & $16.0 \mathrm{cis}$ & 6.06 & 5.39E-05 \\
\hline chr9_122790ıX603596685F & 9 cis & 16.0 cis & 8.06 & $1.31 \mathrm{E}-06$ \\
\hline chr9_100100ıX603596685F & 9 cis & 16.0 cis & 5.43 & $1.21 \mathrm{E}-05$ \\
\hline chr9_114180ıX603596685F & 9 cis & 16.0 cis & 10.39 & $1.80 \mathrm{E}-05$ \\
\hline chr9_115430ıX603596685F & 9 cis & 16.0 cis & 8.15 & $1.39 \mathrm{E}-05$ \\
\hline chr9_118480ıx603596685F & 9 cis & $16.0 \mathrm{cis}$ & 8.46 & 2.14E-04 \\
\hline chr9_898100।X603596685F & 9 cis & $16.0 \mathrm{cis}$ & 5.46 & $6.25 \mathrm{E}-05$ \\
\hline chr9_110490ıX603596685F & 9 cis & 16.0 cis & 5.55 & $5.56 \mathrm{E}-05$ \\
\hline chr9_109610ıX603596685F & 9 cis & $16.0 \mathrm{cis}$ & 7.9 & 7.31E-06 \\
\hline chr9_114870ıX603596685F & 9 cis & 16.0 cis & 6.83 & $1.72 \mathrm{E}-04$ \\
\hline chr9_933000ıX03596685F & 9 cis & 16.0 cis & 5.38 & $2.34 \mathrm{E}-04$ \\
\hline chr9_993000ıX603596685F & 9 cis & $16.0 \mathrm{cis}$ & 6.32 & $1.31 \mathrm{E}-04$ \\
\hline chr9_108140ıX603596685F & 9 cis & 16.0 cis & 12.2 & $1.44 \mathrm{E}-05$ \\
\hline chr9_117450ıX603596685F & 9 cis & 16.0 cis & 4.8 & $1.75 \mathrm{E}-05$ \\
\hline chr9_126740ıX603596685F & 9 cis & $16.0 \mathrm{cis}$ & 10.94 & $6.39 \mathrm{E}-07$ \\
\hline chr9_128210ıX603596685F & 9 cis & 16.0 cis & 5.83 & $1.00 \mathrm{E}-06$ \\
\hline chr9_128580ıX603596685F & 9 cis & 16.0 cis & 4.94 & 3.99E-06 \\
\hline chr9_128640ıX603596685F & 9 cis & $16.0 \mathrm{cis}$ & 4.51 & 7.83E-06 \\
\hline chr9_129370ıX603596685F & 9 cis & 16.0 cis & 6.15 & $1.54 \mathrm{E}-06$ \\
\hline chr9_100110ıX603596685F & 9 cis & 16.0 cis & 4.96 & $1.18 \mathrm{E}-04$ \\
\hline chr9_102250ıX603596685F & 9 cis & $16.0 \mathrm{cis}$ & 4.66 & $1.41 \mathrm{E}-04$ \\
\hline chr9_123820ıX603596685F & 9 cis & $16.0 \mathrm{cis}$ & 4.1 & $1.22 \mathrm{E}-04$ \\
\hline chr9_924000ı603596685F & 9 cis & 16.0 cis & 4.38 & $6.41 \mathrm{E}-04$ \\
\hline chr9_114170ıX603596685F & 9 cis & $16.0 \mathrm{cis}$ & 10.01 & $3.84 \mathrm{E}-06$ \\
\hline chr9_110510ıX03596685F & 9 cis & 16.0 cis & 4.66 & $2.02 \mathrm{E}-04$ \\
\hline chr9_917000ıX603596685F & 9 cis & 16.0 cis & 9.24 & $6.56 \mathrm{E}-06$ \\
\hline chr9_888100।X603596685F & 9 cis & $16.0 \mathrm{cis}$ & 4.65 & $6.75 \mathrm{E}-05$ \\
\hline chr9_108190ıX603596685F & 9 cis & 16.0 cis & 5.54 & $5.35 \mathrm{E}-05$ \\
\hline chr9_131490ıX603596685F & 9 cis & 16.0 cis & 6.7 & $9.54 \mathrm{E}-07$ \\
\hline chr9_837300ıX603866863F & 9 cis & 4.9 cis & 8.6 & $5.78 \mathrm{E}-05$ \\
\hline chr9_735600(X603866863F & 9 cis & 4.9 cis & 7.96 & $9.61 \mathrm{E}-05$ \\
\hline chr9_874500ıX603866863F & 9 cis & $4.9 \mathrm{cis}$ & 4.33 & $6.48 \mathrm{E}-04$ \\
\hline chr9_125730ıX603866863F & 9 cis & 4.9 cis & 5.54 & $5.35 \mathrm{E}-03$ \\
\hline chr9_125730ı603599639F & 9 cis & $4.6 \mathrm{cis}$ & 5.54 & $1.51 \mathrm{E}-03$ \\
\hline chr9_804200IENSGALT000( & 9 cis & $5.3 \mathrm{cis}$ & 5.4 & $3.73 E-03$ \\
\hline chr9_837300IENSGALT000( & 9 cis & $5.3 \mathrm{cis}$ & 8.6 & $9.79 \mathrm{E}-04$ \\
\hline chr9_735600IENSGALT000( & 9 cis & $5.3 \mathrm{cis}$ & 7.96 & $3.82 \mathrm{E}-03$ \\
\hline chr9_885900IENSGALTO00( & 9 cis & $5.3 \mathrm{cis}$ & 8.08 & $1.54 \mathrm{E}-03$ \\
\hline chr9_100100IENSGALT000( & 9 cis & 5.3 cis & 5.43 & $2.75 \mathrm{E}-04$ \\
\hline chr9_103900IENSGALT000( & 9 cis & $5.3 \mathrm{cis}$ & 5.2 & $2.95 \mathrm{E}-03$ \\
\hline chr9_105980IENSGALTO00( & 9 cis & 5.3 cis & 4.45 & $4.78 \mathrm{E}-04$ \\
\hline chr9_132220IENSGALT000( & 9 cis & $5.3 \mathrm{cis}$ & 5.43 & 3.07E-03 \\
\hline chr9_100090IENSGALT000 & 9 cis & $5.3 \mathrm{cis}$ & 9.47 & $1.52 \mathrm{E}-03$ \\
\hline chr9_126740IENSGALT000( & 9 cis & $5.3 \mathrm{cis}$ & 10.94 & $6.47 \mathrm{E}-04$ \\
\hline chr9_126750IENSGALTO00( & 9 cis & 5.3 cis & 6.4 & 7.03E-04 \\
\hline chr9_130040IENSGALT000( & 9 cis & $5.3 \mathrm{cis}$ & 6.8 & $2.70 \mathrm{E}-03$ \\
\hline chr9_102250IENSGALT000( & 9 cis & $5.3 \mathrm{cis}$ & 4.66 & $3.99 E-04$ \\
\hline chr9_923000IENSGALT000( & 9 cis & $5.3 \mathrm{cis}$ & 4.95 & $1.06 \mathrm{E}-03$ \\
\hline chr9_646800।X603144063F & 9 cis & $4.3 \mathrm{cis}$ & 14.24 & $1.23 \mathrm{E}-04$ \\
\hline chr9_720500।X603144063F & 9 cis & $4.3 \mathrm{cis}$ & 7.99 & $2.52 \mathrm{E}-03$ \\
\hline chr9_621300।X603144063F & 9 cis & 4.3 cis & 18.59 & $2.81 \mathrm{E}-03$ \\
\hline chr9_678600।X603144063F & 9 cis & $4.3 \mathrm{cis}$ & 35.03 & $7.58 \mathrm{E}-05$ \\
\hline chr9_709300ı603144063F & 9 cis & $4.3 \mathrm{cis}$ & 4.15 & $8.45 \mathrm{E}-04$ \\
\hline chr9_570800ıX603144063F & 9 cis & $4.3 \mathrm{cis}$ & 5.94 & $2.12 \mathrm{E}-03$ \\
\hline chr9_851600।X603144063F & 9 cis & $4.3 \mathrm{cis}$ & 5.67 & $9.32 \mathrm{E}-06$ \\
\hline chr9_729800।X603144063F & 9 cis & $4.3 \mathrm{cis}$ & 10.04 & $3.61 \mathrm{E}-03$ \\
\hline chr9_816000IX603144063F & 9 cis & 4.3 cis & 6.33 & $9.49 \mathrm{E}-03$ \\
\hline chr9_734400ı603144063F & 9 cis & $4.3 \mathrm{cis}$ & 9.01 & $1.16 \mathrm{E}-03$ \\
\hline chr9_756900।X603144063F & 9 cis & $4.3 \mathrm{cis}$ & 4.83 & $1.27 \mathrm{E}-02$ \\
\hline chr9_874500।X603144063F & 9 cis & $4.3 \mathrm{cis}$ & 4.33 & 5.87E-04 \\
\hline chr9_728300।X603144063F & 9 cis & $4.3 \mathrm{cis}$ & 4.57 & $6.16 \mathrm{E}-03$ \\
\hline chr9_644300।X603144063F & 9 cis & $4.3 \mathrm{cis}$ & 6.05 & 7.77E-04 \\
\hline chr9_108100।X603144063F & 9 cis & $4.3 \mathrm{cis}$ & 6.36 & $2.65 \mathrm{E}-04$ \\
\hline chr9_751000ı603144063F & 9 cis & $4.3 \mathrm{cis}$ & 5.06 & $1.46 \mathrm{E}-03$ \\
\hline chr9_101720ıX603144063F & 9 cis & $4.3 \mathrm{cis}$ & 5.17 & $5.05 \mathrm{E}-03$ \\
\hline chr9_123910ıX03144063F & 9 cis & $4.3 \mathrm{cis}$ & 5.71 & $3.98 \mathrm{E}-03$ \\
\hline
\end{tabular}




\begin{tabular}{|c|c|c|c|c|}
\hline chr9_125730IX603144063F & 9 cis & $4.3 \mathrm{cis}$ & 5.54 & $2.23 \mathrm{E}-03$ \\
\hline chr9_122790ıX603144063F & 9 cis & 4.3 cis & 8.06 & $1.52 \mathrm{E}-03$ \\
\hline chr9_103140ıNM_0010065 & 9 cis & 6.2 cis & 8.28 & $1.98 \mathrm{E}-03$ \\
\hline chr9_108990ıNM_0010065 & 9 cis & 6.2 cis & 11.04 & $2.06 \mathrm{E}-03$ \\
\hline chr9_885900ıNM_0010065 & 9 cis & 6.2 cis & 8.08 & $1.67 \mathrm{E}-03$ \\
\hline chr9_100100ıNM_0010065 & 9 cis & 6.2 cis & 5.43 & 4.10E-04 \\
\hline chr9_100090INM_0010065 & 9 cis & $6.2 \mathrm{cis}$ & 9.47 & $2.42 \mathrm{E}-03$ \\
\hline chr9_126740ıNM_0010065 & 9 cis & 6.2 cis & 10.94 & $5.23 \mathrm{E}-03$ \\
\hline chr9_128210ıNM_0010065 & 9 cis & 6.2 cis & 5.83 & $1.42 \mathrm{E}-04$ \\
\hline chr9_128640ıNM_0010065 & 9 cis & 6.2 cis & 4.51 & $1.09 \mathrm{E}-03$ \\
\hline chr9_129370ıNM_0010065 & 9 cis & 6.2 cis & 6.15 & $4.75 \mathrm{E}-03$ \\
\hline chr9_123820INM_0010065 & 9 cis & 6.2 cis & 4.1 & $3.23 \mathrm{E}-03$ \\
\hline chr9_131490ıNM_0010065 & 9 cis & $6.2 \mathrm{cis}$ & 6.7 & $2.65 \mathrm{E}-03$ \\
\hline chr9_885900IENSGALT000( & 9 cis & 5.7 cis & 8.08 & 4.93E-03 \\
\hline chr9_100100IENSGALT000( & 9 cis & 5.7 cis & 5.43 & 7.41E-04 \\
\hline chr9_128210IENSGALT000( & 9 cis & 5.7 cis & 5.83 & $1.18 \mathrm{E}-03$ \\
\hline chr9_888100IENSGALT000( & 9 cis & 5.7 cis & 4.65 & 7.12E-04 \\
\hline chr9_720200ıX603862895F & 9 cis & 5.3 cis & 8.08 & $3.61 \mathrm{E}-04$ \\
\hline chr9_125730ıX603862895F & 9 cis & 5.3 cis & 5.54 & $4.26 \mathrm{E}-03$ \\
\hline chr9_905000ıX603862895F & 9 cis & 5.3 cis & 5.68 & $4.18 \mathrm{E}-03$ \\
\hline chr9_927500ı603862895F & 9 cis & 5.3 cis & 10.91 & $7.32 \mathrm{E}-03$ \\
\hline chr9_122790ıX603862895F & 9 cis & 5.3 cis & 8.06 & $3.58 \mathrm{E}-03$ \\
\hline chr9_903100ı603577149F & 9 cis & 4.4 cis & 9.96 & $3.32 \mathrm{E}-03$ \\
\hline chr9_100100ıX03577149F & 9 cis & 4.4 cis & 5.43 & $1.52 \mathrm{E}-03$ \\
\hline chr9_116870ıX603577149F & 9 cis & 4.4 cis & 4.17 & $1.09 \mathrm{E}-03$ \\
\hline chr9_100090ıX603577149F & 9 cis & 4.4 cis & 9.47 & $1.30 \mathrm{E}-03$ \\
\hline chr9_128210ıX603577149F & 9 cis & 4.4 cis & 5.83 & $6.37 \mathrm{E}-05$ \\
\hline chr9_128580ıX603577149F & 9 cis & 4.4 cis & 4.94 & 4.30E-04 \\
\hline chr9_128640ıX603577149F & 9 cis & 4.4 cis & 4.51 & $3.88 \mathrm{E}-04$ \\
\hline chr9_129370ıX603577149F & 9 cis & 4.4 cis & 6.15 & $5.05 \mathrm{E}-04$ \\
\hline chr9_135420ıX603577149F & 9 cis & 4.4 cis & 4.63 & $5.38 \mathrm{E}-04$ \\
\hline chr9_110510ıX603577149F & 9 cis & 4.4 cis & 4.66 & $1.89 \mathrm{E}-02$ \\
\hline chr9_903100ıX03867866F & 9 cis & 5.7 cis & 9.96 & $6.63 \mathrm{E}-04$ \\
\hline chr9_100100ı603867866F & 9 cis & 5.7 cis & 5.43 & $2.41 \mathrm{E}-04$ \\
\hline chr9_132220ıX603867866F & 9 cis & 5.7 cis & 5.43 & 1.17E-03 \\
\hline chr9_100090ıX603867866F & 9 cis & 5.7 cis & 9.47 & $6.99 \mathrm{E}-04$ \\
\hline chr9_126740ıX603867866F & 9 cis & 5.7 cis & 10.94 & $1.75 \mathrm{E}-04$ \\
\hline chr9_126750ıX603867866F & 9 cis & 5.7 cis & 6.4 & $1.57 \mathrm{E}-03$ \\
\hline chr9_128210।X603867866F & 9 cis & 5.7 cis & 5.83 & $9.75 \mathrm{E}-06$ \\
\hline chr9_128580ı603867866F & 9 cis & 5.7 cis & 4.94 & $2.74 \mathrm{E}-04$ \\
\hline chr9_128640ıX603867866F & 9 cis & 5.7 cis & 4.51 & $9.16 \mathrm{E}-05$ \\
\hline chr9_129370ıX603867866F & 9 cis & 5.7 cis & 6.15 & $2.29 \mathrm{E}-04$ \\
\hline chr9_135420ıX603867866F & 9 cis & 5.7 cis & 4.63 & $2.62 \mathrm{E}-04$ \\
\hline chr9_110510।X603867866F & 9 cis & 5.7 cis & 4.66 & $7.83 \mathrm{E}-03$ \\
\hline chr9_134300।X603867866F & 9 cis & 5.7 cis & 20.2 & $2.28 \mathrm{E}-04$ \\
\hline chr9_134680ı603867866F & 9 cis & 5.7 cis & 4.1 & 7.36E-04 \\
\hline chr9_100100IENSGALTO00( & 9 cis & 5.2 cis & 5.43 & 4.27E-05 \\
\hline chr9_135390IENSGALT000( & 9 cis & 5.2 cis & 4.88 & $9.87 \mathrm{E}-04$ \\
\hline chr9_132220IENSGALTO00( & 9 cis & $5.2 \mathrm{cis}$ & 5.43 & $1.41 \mathrm{E}-03$ \\
\hline chr9_100090IENSGALT000( & 9 cis & $5.2 \mathrm{cis}$ & 9.47 & $3.18 \mathrm{E}-05$ \\
\hline chr9_108140IENSGALT000( & 9 cis & 5.2 cis & 12.2 & $1.45 \mathrm{E}-03$ \\
\hline chr9_123820IENSGALT000( & 9 cis & $5.2 \mathrm{cis}$ & 4.1 & $1.98 \mathrm{E}-03$ \\
\hline chr9_134290IENSGALT000( & 9 cis & 5.2 cis & 12.09 & 4.25E-04 \\
\hline chr9_134300IENSGALT000( & 9 cis & 5.2 cis & 20.2 & $3.83 \mathrm{E}-05$ \\
\hline chr9_125730ı603866968F & 9 cis & 6.4 cis & 5.54 & 8.75E-05 \\
\hline chr9_134260ıx603866968F & 9 cis & $6.4 \mathrm{cis}$ & 15.8 & $1.39 \mathrm{E}-03$ \\
\hline chr9_134320।X603866968F & 9 cis & 6.4 cis & 13.75 & 4.70E-03 \\
\hline chr9_134290IENSGALT000( & 9 cis & 10.0 cis & 12.09 & $1.92 \mathrm{E}-05$ \\
\hline chr9_134300IENSGALT000( & 9 cis & 10.0 cis & 20.2 & 2.37E-07 \\
\hline chr9_134310IENSGALT000( & 9 cis & 10.0 cis & 17.39 & $7.18 \mathrm{E}-07$ \\
\hline chr9_134250IENSGALTO00( & 9 cis & 10.0 cis & 14.65 & 4.35E-07 \\
\hline chr9_134260IENSGALT000( & 9 cis & 10.0 cis & 15.8 & $2.95 \mathrm{E}-07$ \\
\hline chr9_134320IENSGALTO00( & 9 cis & 10.0 cis & 13.75 & $1.08 \mathrm{E}-05$ \\
\hline chr9_905000ıNM_0010393 & 9 cis & $5.1 \mathrm{cis}$ & 5.68 & 2.96E-04 \\
\hline chr9_122790ıNM_0010393 & 9 cis & $5.1 \mathrm{cis}$ & 8.06 & $1.90 \mathrm{E}-04$ \\
\hline chr9_128580ıNM_0010393 & 9 cis & $5.1 \mathrm{cis}$ & 4.94 & $9.07 \mathrm{E}-04$ \\
\hline chr9_125730।X603868153F & 9 cis & 4.9 cis & 5.54 & $3.20 \mathrm{E}-05$ \\
\hline chr9_132880।X603868153F & 9 cis & 4.9 cis & 5.34 & $5.41 \mathrm{E}-03$ \\
\hline chr9_134290।X603868153F & 9 cis & 4.9 cis & 12.09 & 2.73E-03 \\
\hline chr9_125730ı603866213F & 9 cis & $4.3 \mathrm{cis}$ & 5.54 & $4.52 \mathrm{E}-04$ \\
\hline chr9_132880(X603866213F & 9 cis & 4.3 cis & 5.34 & $1.27 \mathrm{E}-03$ \\
\hline chr9_134300।X603866213F & 9 cis & 4.3 cis & 20.2 & $1.08 \mathrm{E}-03$ \\
\hline
\end{tabular}




\begin{tabular}{|c|c|c|c|c|}
\hline chr9_134250ıX603866213F & 9 cis & 4.3 cis & 14.65 & $3.28 \mathrm{E}-04$ \\
\hline chr9_134260।X603866213F & 9 cis & $4.3 \mathrm{cis}$ & 15.8 & 5.27E-07 \\
\hline chr9_134320ıX603866213F & 9 cis & 4.3 cis & 13.75 & $6.34 \mathrm{E}-04$ \\
\hline chr9_125730IENSGALTO00 & 9 cis & 7.0 cis & 5.54 & $1.13 \mathrm{E}-06$ \\
\hline chr9_122360।ENSGALT000( & 9 cis & 7.0 cis & 4.86 & $1.46 \mathrm{E}-03$ \\
\hline chr9_132880IENSGALT000( & 9 cis & $7.0 \mathrm{cis}$ & 5.34 & $9.51 \mathrm{E}-04$ \\
\hline chr9_134300IENSGALT000( & 9 cis & 7.0 cis & 20.2 & $9.47 \mathrm{E}-04$ \\
\hline chr9_134310IENSGALTO00 & 9 cis & 7.0 cis & 17.39 & $3.26 \mathrm{E}-03$ \\
\hline chr9_134250IENSGALT000( & 9 cis & $7.0 \mathrm{cis}$ & 14.65 & $6.79 \mathrm{E}-03$ \\
\hline chr9_134260IENSGALTO00( & 9 cis & 7.0 cis & 15.8 & $3.32 \mathrm{E}-04$ \\
\hline chr9_134320IENSGALTO00( & 9 cis & 7.0 cis & 13.75 & $2.25 \mathrm{E}-02$ \\
\hline chr9_134300IENSGALTO00 & 9 cis & $5.0 \mathrm{cis}$ & 20.2 & $2.53 \mathrm{E}-05$ \\
\hline chr9_134310।ENSGALT000( & 9 cis & 5.0 cis & 17.39 & $8.58 \mathrm{E}-06$ \\
\hline chr9_134250IENSGALTO00 & 9 cis & 5.0 cis & 14.65 & $4.55 \mathrm{E}-08$ \\
\hline chr9_134260IENSGALTO00( & 9 cis & 5.0 cis & 15.8 & $8.23 \mathrm{E}-07$ \\
\hline chr9_137700।X603600966F & 9 cis & $8.3 \mathrm{cis}$ & 4.49 & $5.92 \mathrm{E}-05$ \\
\hline chr9_134250।X603600966F & 9 cis & $8.3 \mathrm{cis}$ & 14.65 & $1.23 \mathrm{E}-04$ \\
\hline chr9_169190।X603863036F & 9 cis & $4.1 \mathrm{cis}$ & 5.11 & $4.91 \mathrm{E}-04$ \\
\hline chr9_151250INM_204706_ & 9 cis & 4.9 trans & 5.61 & 6.17E-04 \\
\hline chr9_1257301NM_0010065 & 9 cis & 4.5 cis & 5.54 & $2.79 \mathrm{E}-03$ \\
\hline chr9_122790।NM_0010065 & 9 cis & 4.5 cis & 8.06 & $2.56 \mathrm{E}-03$ \\
\hline chr9_173060IENSGALTO00 & 9 trans & $7.8 \mathrm{cis}$ & 5.73 & $1.71 \mathrm{E}-03$ \\
\hline chr9_169150ıX603867781F & 9 cis & $4.7 \mathrm{cis}$ & 6.86 & 1.29E-02 \\
\hline chr9_183990।X603864983F & 9 cis & $15.7 \mathrm{cis}$ & 4.1 & $2.54 \mathrm{E}-03$ \\
\hline
\end{tabular}




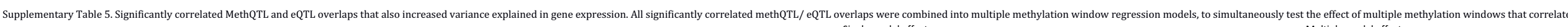

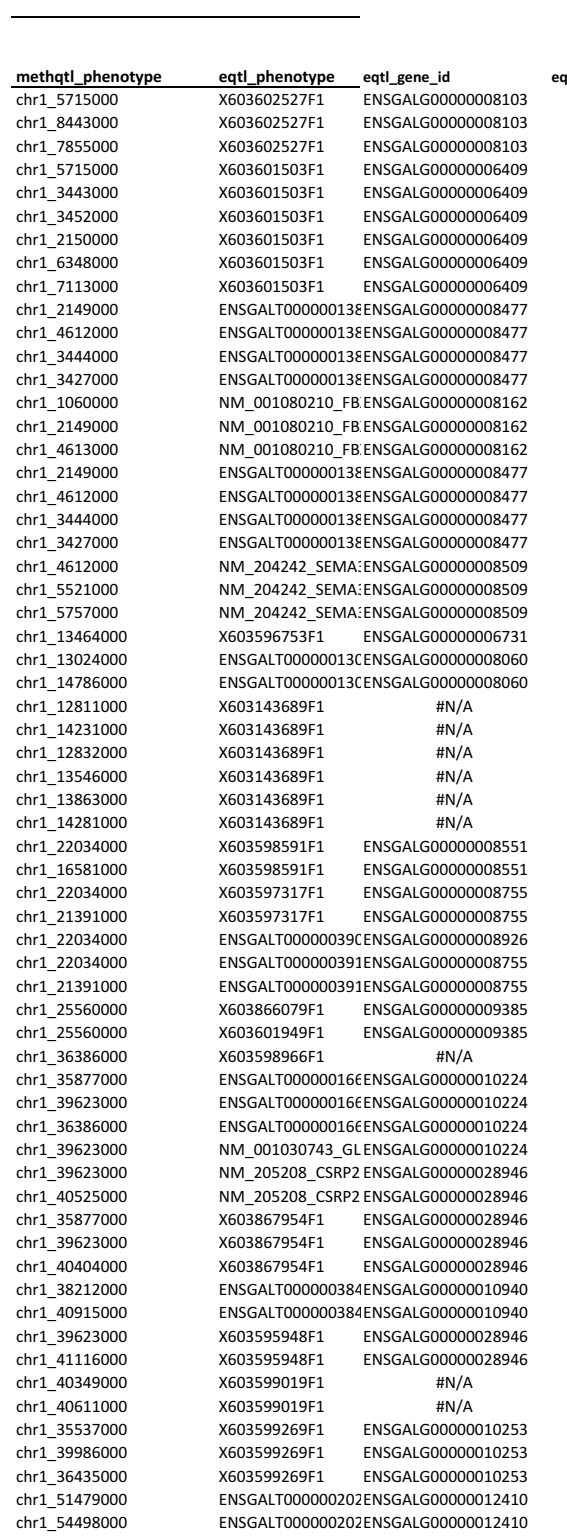

equt_type
1 cis
1 cis
1 cis
1 cis
1 cis
1 cis
1 cis
1 cis
1 cis
1 cis
1 cis
1 cis
1 cis
1 cis
1 cis
1 cis
1 cis
1 cis
1 cis
1 cis
1 cis
1 cis
1 cis
1 cis
1 cis
1 cis
1 cis
1 cis
1 cis
1 cis
1 cis
1 cis
1 cis
1 cis
1 cis
1 cis
1 cis
1 cis
1 cis
1 cis
1 cis
1 cis
1 cis
1 cis
1 cis
1 cis
1 cis
1 cis
1 cis
1 cis
1 cis
1 cis
1 cis
1 cis
1 cis
1 cis
1 cis
1 cis
1 cis
1 cis
1 cis
1 cis

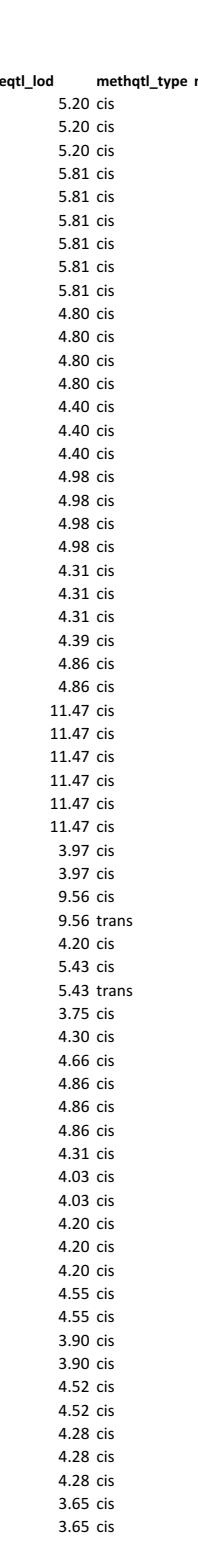

\begin{tabular}{|c|c|c|c|c|}
\hline & & $\begin{array}{l}\text { single_model_genoty } \\
\text { pe_pvalue_with_met }\end{array}$ & $\begin{array}{l}\text { Single.model.geno.pva } \\
\text { lue.without.meth.cov }\end{array}$ & \\
\hline & .pvalue & $\begin{array}{l}\text { h_covariate } \\
\text { nater }\end{array}$ & & with.meth.covariate \\
\hline & $2.3 \mathrm{E}-05$ & $4.3 \mathrm{E}-04$ & $\begin{array}{l}.3 E-07 \\
\end{array}$ & 0.20 \\
\hline & 5.7E-05 & 4.1E-04 & $8.3 E-07$ & \\
\hline & $3.8 E-05$ & $1.4 E-05$ & $8.3 E-07$ & 0.23 \\
\hline & $5.5 E-04$ & $1.3 E-04$ & $1.5 E-06$ & 0.19 \\
\hline 97 & $7.2 E-04$ & $3.7 E-04$ & $1.5 E-06$ & \\
\hline 93 & $1.6 E-03$ & $\begin{array}{l}1.8 E-04 \\
3 x=02\end{array}$ & $1.5 E-06$ & 0.17 \\
\hline .21 & $\begin{array}{l}2.3 E-05 \\
6.5-504\end{array}$ & $\begin{array}{l}3.5 E-03 \\
9.9-5.5 \\
9\end{array}$ & $\begin{array}{l}1.5 E-06 \\
1.5 E-06\end{array}$ & 0.19 \\
\hline 4.1 & $\begin{array}{l}6.5 E-04 \\
6.8--04\end{array}$ & $\begin{array}{l}9.9-9 E-05 \\
4.0 \mathrm{CE}-05\end{array}$ & $\begin{array}{l}1.5 E-06 \\
1.5 \mathrm{E}-06\end{array}$ & $\begin{array}{l}0.19 \\
0.20\end{array}$ \\
\hline 1.29 & $\begin{array}{l}1.0 E-04 \\
1.04\end{array}$ & $\begin{array}{l}4.0-0.03 \\
7.7-0 .-03\end{array}$ & $\begin{array}{l}1.5 E-06 \\
3.5 E-06\end{array}$ & 0.16 \\
\hline & $1.1 E-04$ & 5.2E-04 & $3.5 E-06$ & 0.19 \\
\hline & & & & \\
\hline & $1.2 E-04$ & $5.0 E-04$ & & \\
\hline & $\begin{array}{l}5.5 E-04 \\
115-0,\end{array}$ & $\begin{array}{l}2.9 E-04 \\
3.5-2 .\end{array}$ & & \\
\hline .296 & $\begin{array}{l}1.1 .1 E-04 \\
2.7-04\end{array}$ & $\begin{array}{l}3.1 E-02 \\
8.4-04\end{array}$ & & 0.15 \\
\hline 5.29 & $\begin{array}{l}2.7-2-04 \\
7.6 E-05\end{array}$ & $\begin{array}{l}8.4 E-04 \\
8.0-03\end{array}$ & $\begin{array}{l}1.4 E E-05 \\
2.8 E-06\end{array}$ & 0.18 \\
\hline & $\begin{array}{l}7.0 \mathrm{E}-\mathrm{-}-05 \\
7.85\end{array}$ & $\begin{array}{l}8.0=04 \\
4.7 Z-04\end{array}$ & $\begin{array}{l}2.8 \mathrm{E} E-06 \\
2.8 \mathrm{E}-06\end{array}$ & $\begin{array}{l}0.11 \\
0.19\end{array}$ \\
\hline .29 & $7.3 E-05$ & $5.2 E-04$ & $2.8 \mathrm{E}-06$ & 0.20 \\
\hline & $3.8 E-04$ & $2.2 E-04$ & $2.8 E-06$ & 0.19 \\
\hline & $1.11-05$ & $2.75-03$ & $1.0 E=-05$ & 0.22 \\
\hline & 1.7E-04 & $\begin{array}{l}8.7 E-04 \\
6750.4\end{array}$ & $\begin{array}{l}1.0 E-05 \\
1.0 E-05\end{array}$ & 0.22 \\
\hline & $\begin{array}{l}1.5 \mathrm{E}-04 \\
1.9 \mathrm{E}-04\end{array}$ & $\begin{array}{l}6.7 E-04 \\
45-5=0.1\end{array}$ & $\begin{array}{l}1.0 E-05 \\
28 E-02\end{array}$ & 0.21 \\
\hline & $\begin{array}{l}1.9 \mathrm{E}-04 \\
8.1 \mathrm{E}-05\end{array}$ & $\begin{array}{l}4.5 E-12 \\
8.6--04\end{array}$ & $\begin{array}{l}2.8 \mathrm{E}-02 \\
1.6 \mathrm{E}-05\end{array}$ & $\begin{array}{l}0.10 \\
0.18\end{array}$ \\
\hline & $1.4 \mathrm{E}-03$ & $1.9 \mathrm{E}-04$ & $\begin{array}{l}1.6 E-05 \\
1.6 E\end{array}$ & $\begin{array}{l}0.18 \\
0.18\end{array}$ \\
\hline & 7.7E-05 & 2.3E-10 & $1.8 \mathrm{E}-12$ & 0.33 \\
\hline & $6.3 \mathrm{E}-09$ & $3.7 \mathrm{E}-09$ & $1.8 \mathrm{E}-12$ & 0.38 \\
\hline & $1.6 \mathrm{E}-03$ & $1.3 E-11$ & $1.8 E-12$ & 0.34 \\
\hline .12 & 7.1E-10 & $8.2 E-07$ & $1.8 E-12$ & 0.36 \\
\hline & $\begin{array}{l}5.0 \mathrm{E}-08 \\
1.3 \mathrm{E}-06\end{array}$ & $\begin{array}{l}2.2 E-09 \\
83-5-9\end{array}$ & $\begin{array}{l}1.8 \mathrm{E}-12 \\
18 \mathrm{E}-12\end{array}$ & 0.37 \\
\hline & $\begin{array}{l}1.3 \mathrm{E}-06 \\
6.5 \mathrm{E}-04\end{array}$ & $\begin{array}{l}8.3 E-09 \\
4.11-01\end{array}$ & $\begin{array}{l}1.8 \mathrm{E}-12 \\
6 \mathrm{EE}-02\end{array}$ & $\begin{array}{l}0.33 \\
0.13\end{array}$ \\
\hline & $\begin{array}{l}0.5 \mathrm{E}-\mathrm{-}-4 \\
3.2 \mathrm{E}\end{array}$ & $\begin{array}{l}4.1 .6-11 \\
1.6 E-01\end{array}$ & $\begin{array}{l}6.6 \mathrm{E}-02 \\
6.6 \mathrm{E}-02\end{array}$ & $\begin{array}{l}0.13 \\
0.11\end{array}$ \\
\hline & 2.2E- -04 & 6.5E-06 & $1.3 E-07$ & 0.22 \\
\hline & $6.8 \mathrm{E}-04$ & 1.11E-06 & $1.3 E-07$ & 0.24 \\
\hline & 1.1.1E-04 & 4.9E- -01 & $6.6 \mathrm{E}-02$ & 0.09 \\
\hline & 7.1E-04 & $\begin{array}{l}2.3 E-04 \\
-5.509\end{array}$ & $8.9 E-06$ & 0.18 \\
\hline & $\begin{array}{l}2.1 E-03 \\
47 F-05\end{array}$ & 5.6E-05 & $8.9 E-06$ & 0.19 \\
\hline & $\begin{array}{l}4.7 E-05 \\
64 E-04\end{array}$ & $\begin{array}{l}6.0 \mathrm{E}-01 \\
24 \mathrm{E}-0.1\end{array}$ & $\begin{array}{l}8.9 E-02 \\
3.5 E-02\end{array}$ & $\begin{array}{l}0.14 \\
0.10\end{array}$ \\
\hline 4.1 & $\begin{array}{l}6.4 \mathrm{E}-04 \\
3.1 \mathrm{E}-07\end{array}$ & $\begin{array}{l}2.4-2-12 \\
7.95-02\end{array}$ & $\begin{array}{l}3.5 E E-02 \\
2.6 E-03\end{array}$ & $\begin{array}{l}0.10 \\
0.19\end{array}$ \\
\hline & $1.6 E-03$ & $1.8 \mathrm{E}-03$ & $\begin{array}{l}3.4 E-05 \\
\end{array}$ & 0.13 \\
\hline 0.1 & $1.3 \mathrm{E}-05$ & $8.8 \mathrm{E}-03$ & $3.4 E-05$ & 0.19 \\
\hline & $6.8 E-04$ & $4.0 \mathrm{E}-04$ & $3.4 E-05$ & 0.15 \\
\hline & $6.6 \mathrm{E}-05$ & $1.0 \mathrm{E}-01$ & $1.4 E-03$ & 0.13 \\
\hline & $1.8 \mathrm{E}-04$ & 4.6E-02 & $3.4 E-04$ & 0.12 \\
\hline & $5.4 E-04$ & $1.1 \mathrm{E}-02$ & 3.4E--04 & 0.12 \\
\hline & $8.8 E-04$ & $4.3 E-04$ & $1.9 \mathrm{E}-05$ & 0.17 \\
\hline & $8.3 \mathrm{E}-04$ & $2.2 E-03$ & $1.9 E-05$ & 0.15 \\
\hline .55 & 3.6E-04 & $1.4 E-03$ & $1.9 E-05$ & 0.16 \\
\hline 59 & $2.66-03$ & $2.2 E-03$ & $1.11 E-04$ & 0.13 \\
\hline & 2.11E-04 & 5.22E-03 & 1.1.1E-04 & 0.15 \\
\hline & 6.6-6E-04 & $\begin{array}{l}1.4-4 E-02 \\
\end{array}$ & $\begin{array}{l}1.4 E-04 \\
\text { 1 }\end{array}$ & 0.13 \\
\hline 89 & $\begin{array}{l}1.3 E-0.3 \\
13-505\end{array}$ & 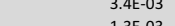 & $\begin{array}{l}1.4 E=-04 \\
1.30=0\end{array}$ & 0.13 \\
\hline 378 & $2.4 E-04$ & $\begin{array}{l}1.3 E=03 \\
13=-03\end{array}$ & $\begin{array}{l}3.9 E=-06 \\
3.9 E-06\end{array}$ & $\begin{array}{l}0.22 \\
0.20\end{array}$ \\
\hline 11.8 & $\begin{array}{l}2.4 E-44 \\
3.0 E-03\end{array}$ & $\begin{array}{l}1.3 E-U S \\
2.6 E-02\end{array}$ & $\begin{array}{l}3.9 E-00 \\
2.2 E-03\end{array}$ & $\begin{array}{l}0.20 \\
0.09\end{array}$ \\
\hline & $1.5 E-03$ & $\begin{array}{l}2.8 E-02 \\
2.8 E-02\end{array}$ & $\begin{array}{l}2.21-03 \\
2.2 E-03\end{array}$ & $\begin{array}{l}0.05 \\
0.10\end{array}$ \\
\hline 4. & 2.1E- -04 & 2.2E- -02 & $2.2 E-03$ & 0.12 \\
\hline & $5.4 E-04$ & $\begin{array}{l}5.8 E-04 \\
5.250 .0\end{array}$ & $\begin{array}{l}3.4 E-05 \\
3\end{array}$ & 0.18 \\
\hline & & & & \\
\hline
\end{tabular}
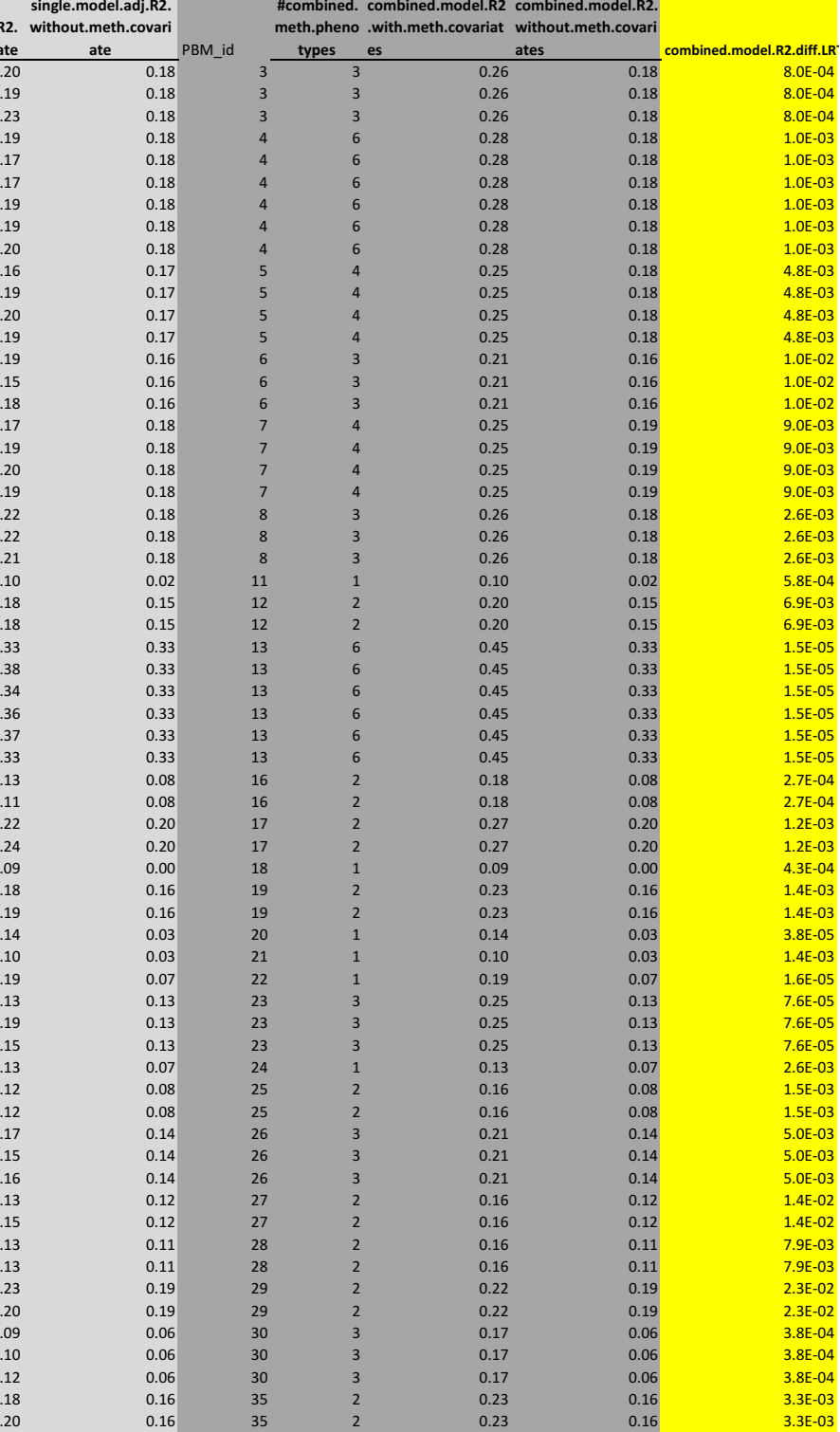


\begin{tabular}{|c|c|c|c|c|}
\hline chr1_53820000 & X603143082F1 & ENSGALG000000012870 & 1 cis & 5.35 cis \\
\hline chr1_53723000 & X603143082F1 & ENSGALG000000012870 & 1 cis & \\
\hline chr1_53604000 & X603866558F1 & ENSGALG00000012665 & 1 cis & 3.73 cis \\
\hline chr1_53604000 & NM_001012815_C_ & EYENSGALG000000012454 & 1 cis & 5.78 cis \\
\hline chr1553604000 & X603599379F1 & ENSGALG000000012718 & 1 cis & 4.79 cis \\
\hline chr1_51526000 & NM_001130738_ & DLENSGALG00000012281 & 1 cis & 4.43 cis \\
\hline chr1_53282000 & NM_001130738_' & DLENSGALG00000012281 & 1 cis & 4.43 cis \\
\hline chr1_53282000 & ENSGALT0000003 & 0\&ENSGALG00000015767 & 1 trans & 6.49 cis \\
\hline chr1_54364000 & X603601419F1 & ENSGALG00000012877 & 1 cis & 9.07 cis \\
\hline $\begin{array}{l}\text { chr1_56604000 } \\
\text { chr1 } 58980000\end{array}$ & $\begin{array}{l}\text { X603601419F1 } \\
\text { X66360141991 }\end{array}$ & ENSGALG600000012877 & 1 cis & $\begin{array}{l}9.07 \text { cis } \\
0.07 \text { cis }\end{array}$ \\
\hline $\begin{array}{l}\text { chrr_5598801000 } \\
\text { chr1_62151000 }\end{array}$ & NM_001030767_C & $\begin{array}{l}\text { ENSGGAGG0000000012877 } \\
\text { CE ENSGALG00000013031 }\end{array}$ & $\begin{array}{l}1 \text { cis } \\
1 \text { cis }\end{array}$ & $\begin{array}{l}9.07 \text { cis } \\
5.04 \text { cis }\end{array}$ \\
\hline chr1_62141000 & NM_001030767_- & EE ENSGALG00000013031 & 1 cis & 5.04 is \\
\hline chr1_62140000 & NM_001030767_- & & 1 cis & 5.04 is \\
\hline chr1_93883000 & ENSGALT0000003 & 6EENSGALG00000026184 & 1 cis & 4.58 cis \\
\hline chr1_92706000 & ENSGALTO000003 & & 1 cis & 4.58 cis \\
\hline chr1_89513000 & ENSGALTO000002 & 47ENSGALG00000015351 & 1 cis & 5.71 cis \\
\hline $\begin{array}{l}\text { chr1_89820000 } \\
\text { chr1 } 898200000\end{array}$ & $\begin{array}{l}\text { ENSGALTO0000002 } \\
\text { ENGGITOOPOP }\end{array}$ & 47ENSGALG00000015351 & 1 cis & $\begin{array}{l}5.71 \text { cis } \\
5.34 \text { cis }\end{array}$ \\
\hline $\begin{array}{l}\text { Chr1__898200000 } \\
\text { chr1_118205000 }\end{array}$ & $\begin{array}{l}\text { ENGGALTO0000002 } \\
\text { NM_010102823 }\end{array}$ & $\begin{array}{l}\text { 47ENENGALG0000000015347 } \\
\text { ACENGLAG00000016351 }\end{array}$ & $\begin{array}{l}1 \text { cis } \\
1 \text { cis }\end{array}$ & $\begin{array}{l}5.34 \text { cis } \\
4.28 \text { cis }\end{array}$ \\
\hline chr1_110954000 & X603596950F1 & $\# N / A$ & 1 cis & $\begin{array}{l}4.28 \mathrm{cls} \\
3.18 \mathrm{cis}\end{array}$ \\
\hline chr1_119144000 & X603596950F1 & $\# N / A$ & 1 cis & 3.18 is \\
\hline chr1_123394000 & ENSGALTO000002 & 6EENSGALG00000016518 & 1 cis & 4.98 cis \\
\hline chr1_126629000 & ENSGALTO000002 & 6EENSGALG00000016622 & 1 cis & 10.93 cis \\
\hline chr1_127636000 & ENSGALTO0000002 & 6EENSGALG00000016622 & 1 cis & 10.93 cis \\
\hline chr1_130171000 & ENSGALTO0000002 & 7CENSGALG00000016737 & 1 cis & 4.78 cis \\
\hline $\begin{array}{l}\text { chr1_13132630000 } \\
\text { chr1 133244000 }\end{array}$ & $\begin{array}{l}\text { X6035598300441 } \\
\times 603598304 F 1\end{array}$ & $\begin{array}{l}\text { ENSGALG600000025912 } \\
\text { ENSGAL60000025912 }\end{array}$ & $\begin{array}{l}\begin{array}{l}1 \text { cis } \\
1 \text { cis }\end{array} \\
.\end{array}$ & $\begin{array}{l}9.98 \text { cis } \\
9.98 \text { cis }\end{array}$ \\
\hline $\begin{array}{l}\text { chri1_13332444000 } \\
\text { chr1_137201000 }\end{array}$ & $\begin{array}{l}\text { X6603558803041 } \\
\text { NM_001030343_C }\end{array}$ & $\begin{array}{r}\text { ENSGGAGG0000000025912 } \\
\text { CHENSGALG0000016769 }\end{array}$ & $\begin{array}{l}1 \text { cis } \\
1 \text { cis }\end{array}$ & $\begin{array}{l}9.98 \text { cis } \\
4.54 \text { cis }\end{array}$ \\
\hline chr1_135072000 & ENSGALTTO000002 & 2EENSGALG00000016807 & 1 cis & 13.48 cis \\
\hline chr1_133995000 & ENSGALT00000002 & 2EENSGALG000000016807 & 1 cis & 13.48 cis \\
\hline chr1_133896000 & ENSGALTO000002 & 2EENSGALG00000016807 & 1 cis & 13.48 cis \\
\hline chr1_136991000 & ENSGALTO0000002 & 2EENSGALG00000016807 & 1 cis & 13.48 cis \\
\hline $\begin{array}{l}\text { chr1_1373828000 } \\
\text { chr1 } 137600000\end{array}$ & 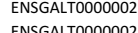 & 2EENSGALG00000016807 & $\begin{array}{l}1 \text { cis } \\
1 \text { cis }\end{array}$ & 13.48 is \\
\hline $\begin{array}{l}\text { chr1_ } 1376100000 \\
\text { chr1 } 137629000\end{array}$ & $\begin{array}{l}\text { ENGSALTO0000002 } \\
\text { ENGGATOO00002 }\end{array}$ & $\begin{array}{l}28 E N S G G A L G 000000016807 \\
\text { 2EENSGL G00000016807 }\end{array}$ & $\begin{array}{l}1 \text { cis } \\
1 \text { cis }\end{array}$ & 13.48 cis \\
\hline chr1_137656000 & ENSGALTO000002 & $2 \varepsilon E N S G A L G 00000016807$ & 1 cis & $\begin{array}{l}13.48 \mathrm{cis} \\
13.48 \mathrm{cis}\end{array}$ \\
\hline chr1_137682000 & ENSGALTO000002 & 2EENSGALG00000016807 & 1 cis & 13.48 cis \\
\hline chr1_137581000 & ENSGALTO000002 & 2EENSGALG00000016807 & 1 cis & 13.48 cis \\
\hline chr1_137594000 & ENSGALTO000002 & 2EENSGALG00000016807 & 1 cis & 13.48 cis \\
\hline chr1_137596000 & ENSGALTO0000002 & 2EENSGALG00000016807 & 1 cis & 13.48 cis \\
\hline chr1_137601000 & $\begin{array}{l}\text { ENSGALTO0000002 } \\
\text { ENGGITOPO }\end{array}$ & 28ENSGALG00000016807 & 1 cis & 13.48 cis \\
\hline $\begin{array}{l}\text { chr1__1376640000 } \\
\text { chr1_137625000 }\end{array}$ & $\begin{array}{l}\text { ENSGALTO0000002 } \\
\text { ENSGALOP0002 }\end{array}$ & $\begin{array}{l}\text { 2EENSGGLAG000000016807 } \\
\text { 2EENSGLG00000016807 }\end{array}$ & 1 cis & 13.48 cis \\
\hline 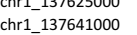 & ENSGALTO0000002 & 28ENSGALG00000016807 & $\begin{array}{l}1 \mathrm{cis} \\
1 \mathrm{cis}\end{array}$ & $\begin{array}{l}13.48 \mathrm{cis} \\
13.48\end{array}$ \\
\hline chr1_137648000 & ENSGALT0000002 & 2 2EENSGALG000000016807 & 1 cis & 13.48 cis \\
\hline chr1_1376499000 & ENSGALT00000002 & 2EENSGALG00000016807 & 1 cis & 13.48 cis \\
\hline chr1_137650000 & ENSGALT0000002 & 2EENSGALG00000016807 & 1 cis & 13.48 cis \\
\hline chr1_137652000 & ENSGALTO0000002 & 2EENSGALG00000016807 & 1 cis & 13.48 cis \\
\hline chr1_137653000 & ENSGALTO0000002 & 2EENSGALG00000016807 & 1 cis & 13.48 cis \\
\hline $\begin{array}{l}\text { chr1_13766780000 } \\
\text { chr1 137860000 }\end{array}$ & ENSGALTO000002 & 2EENSGALG00000016807 & 1 cis & 13.48 is \\
\hline $\begin{array}{l}\text { Chri_1313/8800000 } \\
\text { chr1_137696000 }\end{array}$ & ENSGALTO0000002 & $28 E N G G A L G 00000016807$ & $\begin{array}{l}1 \text { cls } \\
1 \text { cis }\end{array}$ & $\begin{array}{l}13.48 \mathrm{cis} \\
13.48 \mathrm{cis}\end{array}$ \\
\hline chr1_137582000 & ENSGALT0000002 & 28ENSGALG000000016807 & 1 cis & 13.48 cis \\
\hline chr1_1375883000 & ENSGALT0000002 & 2EENSGALG000000016807 & 1 cis & 13.48 cis \\
\hline chr1_1375988000 & ENSGALT0000002 & 28ENSGALG000000016807 & 1 cis & 13.48 cis \\
\hline chr1_137599000 & ENSGALT0000002 & 2EENSGALG00000016807 & 1 cis & 13.48 cis \\
\hline chr1_137600000 & ENSGALTO000002 & 2EENSGALG00000016807 & 1 cis & 13.48 is \\
\hline chr1_137602000 & ENSGALTO000002 & 2EENSGALG00000016807 & 1 cis & 13.48 cis \\
\hline $\begin{array}{l}\text { chr1__1376110000 } \\
\text { chr1_137612000 }\end{array}$ & $\begin{array}{l}\text { ENSGALOU00000 } \\
\text { ENSGALTO00002 }\end{array}$ & $28 E N G G A L G 00000016807$ & 1 cis & $\begin{array}{l}13.48 \mathrm{cis} \\
13.48 \mathrm{cis}\end{array}$ \\
\hline chr1_1376222000 & ENSGALT0000002 & 28ENSGALG000000016807 & 1 cis & 13.48 cis \\
\hline chr1_137623000 & ENSGALT0000002 & 2EENSGALG000000016807 & 1 cis & 13.48 cis \\
\hline chr1_137626000 & ENSGALT0000002 & & 1 cis & 13.48 cis $\mathrm{s}$. \\
\hline _137627000 & GALI0000002 & $28 E N S G A L G 00000016807$ & & \\
\hline chr1_137628000 & 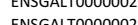 & 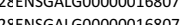 & 10 & 13.48 cis \\
\hline
\end{tabular}

\begin{tabular}{|c|c|c|c|c|c|c|c|c|c|c|}
\hline 4.93 & $2.5 \mathrm{E}-04$ & $9.3 E-04$ & $1.2 \mathrm{E}-05$ & 0.19 & 0.18 & 36 & 2 & 0.25 & 0.18 & 7.0E-04 \\
\hline 4.2 & $8.8 \mathrm{E}-06$ & $9.3 E-04$ & $1.2 E-05$ & 0.25 & 0.18 & 36 & 2 & 0.25 & 0.18 & 7.0E-04 \\
\hline 6.47 & 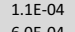 & $1.6 E-01$ & $9.9 E-03$ & 0.13 & 0.07 & 37 & 1 & 0.13 & 0.07 & $2.6 E-03$ \\
\hline $\begin{array}{l}6.47 \\
6.47\end{array}$ & $\begin{array}{l}6.0 E=-04 \\
2.1-06\end{array}$ & $\begin{array}{l}2.0 E-02 \\
5.7 E-03\end{array}$ & $\begin{array}{l}9.8 E-04 \\
46--05\end{array}$ & 0.18 & 0.15 & 38 & 1 & 0.18 & 0.15 & $1.8 E-02$ \\
\hline $\begin{array}{l}6.41 \\
5.99\end{array}$ & $\begin{array}{l}2.1-16-06 \\
1.6 E-03\end{array}$ & $\begin{array}{l}5.7 \mathrm{E}-03 \\
\text { 3.0E- }\end{array}$ & $\begin{array}{l}4.6 .6-505 \\
2.75-03\end{array}$ & $\begin{array}{l}0.24 \\
0.10\end{array}$ & $\begin{array}{l}0.19 \\
0.06\end{array}$ & $\begin{array}{l}41 \\
42\end{array}$ & 1 & $\begin{array}{l}0.24 \\
0.13\end{array}$ & $\begin{array}{l}0.19 \\
0.19\end{array}$ & $\begin{array}{l}2.9 E-03 \\
3.25-03\end{array}$ \\
\hline 7.83 & 2.1E-04 & $8.0 \mathrm{E}-02$ & 2.7E-03 & 0.10 & $\begin{array}{l}0.06 \\
0.06\end{array}$ & $\begin{array}{l}42 \\
42\end{array}$ & 2 & $\begin{array}{l}0.13 \\
0.13\end{array}$ & $\begin{array}{l}0.06 \\
0.06\end{array}$ & $\begin{array}{l}3.2 E-03 \\
3.2 E-03\end{array}$ \\
\hline 7.83 & $1.9 E-05$ & 3.6E-02 & $1.3 \mathrm{E}-04$ & 0.18 & 0.15 & 44 & 1 & 0.18 & $\begin{array}{l}0.00 \\
0.15\end{array}$ & $\begin{array}{l}3.2 E-03 \\
1.2 E-02\end{array}$ \\
\hline 5.12 & $2.2 E-03$ & $6.11-06$ & $\begin{array}{l}4.9 E-07 \\
\end{array}$ & 0.19 & 0.17 & 47 & 3 & 0.27 & 0.17 & $\begin{array}{l}1.2 L-02 \\
3.2 E-04\end{array}$ \\
\hline 6.5 & $6.3 E-05$ & $1.9 E-05$ & $4.9 E-07$ & 0.21 & 0.17 & 47 & 3 & 0.27 & 0.017 & $\begin{array}{l}3.2 L-04 \\
3.2 E-04\end{array}$ \\
\hline 6.57 & $3.9 E-04$ & $6.1 \mathrm{E}-05$ & $4.9 E-07$ & 0.18 & 0.17 & 47 & 3 & 0.27 & 0.17 & $\begin{array}{l}3.2 L-04 \\
3.2 E-04\end{array}$ \\
\hline 4.64 & $2.96-05$ & $9.1 \mathrm{E}-03$ & $4.5 \mathrm{E}-04$ & 0.17 & 0.09 & 48 & 3 & 0.20 & 0.09 & $3.55-04$ \\
\hline 5.72 & $3.3 E-03$ & $1.11-03$ & $4.5 \mathrm{E}-04$ & 0.16 & 0.09 & 48 & 3 & 0.20 & 0.09 & $3.55-04$ \\
\hline 4.39 & $1.1 \mathrm{E}-03$ & $2.3 \mathrm{E}-03$ & $4.5 \mathrm{E}-04$ & 0.14 & 0.09 & 48 & 3 & 0.20 & 0.09 & $3.5 \mathrm{E}-04$ \\
\hline 4.8 & $4.3 E-04$ & $4.0 \mathrm{E}-03$ & $1.2 E-04$ & 0.15 & 0.12 & 49 & 2 & 0.18 & 0.12 & $3.2 \mathrm{E}-03$ \\
\hline 4.1 & $6.8 E-04$ & $2.4 E-03$ & $1.2 \mathrm{E}-04$ & 0.14 & 0.12 & 49 & 2 & 0.18 & 0.12 & $3.2 \mathrm{E}-03$ \\
\hline 7.35 & $2.4 E-04$ & $4.9 E-06$ & $3.2 E-08$ & 0.25 & 0.24 & 50 & 2 & 0.28 & 0.24 & $1.3 \mathrm{E}-02$ \\
\hline 4.59 & $2.2 E-04$ & $7.8 E-07$ & $3.2 E-08$ & 0.26 & 0.24 & 50 & 2 & 0.28 & 0.24 & $1.3 \mathrm{E}-02$ \\
\hline 4.59 & $4.8 E-04$ & $1.2 E-04$ & $1.0 \mathrm{E}-05$ & 0.20 & 0.16 & 51 & 1 & 0.20 & 0.16 & $1.5 E-02$ \\
\hline 5.6 & $9.4 E-04$ & & 4.5E-04 & 0.12 & 0.09 & 54 & 1 & 0.12 & 0.09 & 3.7E-02 \\
\hline 4.68 & $8.5 E-05$ & $4.0 \mathrm{E}-02$ & $5.5 \mathrm{E}-03$ & 0.19 & 0.11 & 55 & 2 & 0.21 & 0.11 & 2.4E- 04 \\
\hline 10.07 & $1.4 E-04$ & $3.6 \mathrm{E}-01$ & $5.5 E-03$ & 0.15 & 0.11 & 55 & 2 & 0.21 & 0.11 & 2.4E-04 \\
\hline 4.1 & $\begin{array}{l}1.0 E-03 \\
0.0=02\end{array}$ & $1.8 E-04$ & $\begin{array}{r}7.2 E-06 \\
\end{array}$ & $\begin{array}{l}0.16 \\
0.09\end{array}$ & $\begin{array}{l}0.14 \\
0.26\end{array}$ & $\begin{array}{l}56 \\
57\end{array}$ & 1 & 0.16 & 0.14 & 4.9E-02 \\
\hline $\begin{array}{l}4.42 \\
473\end{array}$ & $1.4 E-05$ & $\begin{array}{l}1.4 E-07 \\
2.4 E-07\end{array}$ & $\begin{array}{l}4.4 E-10 \\
\end{array}$ & 0.29 & 0.26 & $\begin{array}{l}57 \\
57\end{array}$ & & 0.30 & $\begin{array}{l}0.26 \\
0.26\end{array}$ & $2.0 E-02$ \\
\hline $\begin{array}{l}4.153 \\
5.59\end{array}$ & $\begin{array}{l}1.3 E-03 \\
\end{array}$ & $\begin{array}{l}2.4 E=-08 \\
13 E-05\end{array}$ & $\begin{array}{l}\text { 4.4E-10 } \\
10-20\end{array}$ & $\begin{array}{l}.20 \\
0.24\end{array}$ & 0.00 & 58 & 1 & 0.30 & 0.20 & $2.0 E-02$ \\
\hline $\begin{array}{l}6.150 \\
695\end{array}$ & 作 & 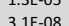 & $2,2=-10$ & 0.35 & 0.02 & 59 & 2 & 0.34 & 0.020 & $\begin{array}{l}1.1 E-02 \\
\end{array}$ \\
\hline 774 & $1.2 E-06$ & 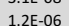 & $2.2 E-10$ & 0.030 & 0.29 & 59 & 2 & 0.0 .35 & 0.029 & $\begin{array}{l}1.55-03 \\
1.5-03\end{array}$ \\
\hline 6.7 & $4.3 E-04$ & $9.78-02$ & $9.9 E-03$ & 0.10 & 0.06 & 60 & 1 & 0.10 & 0.06 & $\begin{array}{l}1.5-5-0 \\
7.6 \mathrm{E}-0\end{array}$ \\
\hline 6.11 & $4.8 E-04$ & $1.76-10$ & $6.36-12$ & 0.32 & 0.31 & 61 & 106 & 0.99 & 0.31 & $0.0 E+0 C$ \\
\hline 6.99 & $2.5 E-04$ & $2.5 E-10$ & $6.3 \mathrm{E}-12$ & 0.33 & 0.31 & 61 & 106 & 0.99 & 0.31 & $0.0 \mathrm{E}+\mathrm{CC}$ \\
\hline 4.99 & $1.3 E-05$ & $3.3 E-10$ & $6.3 \mathrm{E}-12$ & 0.35 & 0.31 & 61 & 106 & 0.99 & 0.31 & $0.0 E+00$ \\
\hline 4.73 & $4.9 E-04$ & $4.8 E-10$ & $6.3 \mathrm{E}-12$ & 0.31 & 0.31 & 61 & 106 & 0.99 & 0.31 & $0.0 E+00$ \\
\hline 6.07 & $2.6 E-07$ & $1.9 E-08$ & 6.3E-12 & 0.34 & 0.31 & 61 & 106 & 0.99 & 0.31 & $0.0 E+0 C$ \\
\hline 8.68 & $5.0 \mathrm{E}-09$ & $5.5 \mathrm{E}-07$ & $6.3 \mathrm{E}-12$ & 0.34 & 0.31 & 61 & 106 & 0.99 & 0.31 & $0.0 E+O C$ \\
\hline 19.85 & 1.11E-11 & $8.2 E-04$ & 6.3E-12 & 0.34 & 0.31 & 61 & 106 & 0.99 & 0.31 & $0.0 E+00$ \\
\hline 11.5 & $1.5 E-10$ & $1.1 E-05$ & $6.3 \mathrm{E}-12$ & 0.35 & 0.31 & 61 & 106 & 0.99 & 0.31 & $0.0 E+00$ \\
\hline 11.7 & $7.2 E-11$ & $1.1 E-05$ & $6.3 \mathrm{E}-12$ & 0.36 & 0.31 & 61 & 106 & 0.99 & 0.31 & $0.0 E+0 C$ \\
\hline 7.77 & $3.5 E-09$ & $3.5 E-07$ & $6.3 E-12$ & 0.35 & 0.31 & 61 & 106 & 0.99 & 0.31 & $0.0 E+00$ \\
\hline 10.53 & $5.7 E-10$ & $2.5 E-06$ & 6.3EE-12 & 0.35 & 0.31 & 61 & 106 & 0.99 & 0.31 & $0.0 E+00$ \\
\hline 11.39 & $2.3 E-10$ & $5.88-06$ & 6.3EE-12 & 0.35 & 0.31 & 61 & 106 & 0.99 & 0.31 & $0.0 E+00$ \\
\hline 21.57 & 4.1E- -10 & $1.7 \mathrm{E}-04$ & $6.3 \mathrm{E}-12$ & 0.32 & 0.31 & & 106 & 0.99 & 0.31 & $0.0 E+00$ \\
\hline 11.53 & 2.1E-10 & $6.66-06$ & $6.3 E-12$ & 0.35 & 0.31 & 61 & 106 & 0.99 & 0.31 & $0.0 E+00$ \\
\hline 11.15 & $2.2 E-10$ & $5.38-06$ & $6.3 \mathrm{E}-12$ & 0.36 & 0.31 & 61 & 106 & 0.99 & 0.31 & $0.0 E+00$ \\
\hline 11.45 & $1.6 E-09$ & $2.78-06$ & $6.3 \mathrm{E}-12$ & 0.34 & 0.31 & 61 & 106 & 0.99 & 0.31 & $0.0 E+00$ \\
\hline 11.67 & $3.78-11$ & $1.3 E-05$ & $6.3 \mathrm{E}-12$ & 0.37 & 0.31 & 61 & 106 & 0.99 & & $0.0 E+0 C$ \\
\hline 11.23 & 4.7E- 10 & 4.1E- -06 & $6.3 \mathrm{E}-12$ & 0.35 & 0.31 & 61 & 106 & 0.99 & 0.31 & $0.0 \mathrm{E}+\mathrm{CC}$ \\
\hline 10.88 & 3.1E- 10 & $\begin{array}{l}4.0 \mathrm{E}-06 \\
\end{array}$ & 6.3EE-12 & 0.36 & 0.31 & 61 & 106 & 0.99 & 0.31 & $0.0 E+00$ \\
\hline 11.68 & $2.3 E-10$ & $6.7 \mathrm{E}-06$ & $6.3 \mathrm{E}-12$ & 0.35 & 0.31 & 61 & 106 & 0.99 & 0.31 & $0.0 E+00$ \\
\hline 10.35 & 2.6E-10 & 3.3E-06 & 6.3E-12 & 0.36 & 0.31 & 61 & 106 & 0.99 & 0.31 & $0.0 E+00$ \\
\hline 12.09 & $5.96-11$ & $1.3 E-05$ & $6.3 \mathrm{E}-12$ & 0.36 & 0.31 & 61 & 106 & 0.99 & 0.31 & $0.0 E+00$ \\
\hline 11.65 & $1.8 E-10$ & 7.5E-06 & 6.3E-12 & 0.36 & 0.31 & 61 & 106 & 0.99 & 0.31 & $0.0 E+00$ \\
\hline 10.03 & $6.3 E-09$ & $\begin{array}{l}9.5 E-07 \\
\end{array}$ & $6.3 E-12$ & 0.34 & 0.31 & 61 & 106 & 0.99 & 0.31 & $0.0 E+00$ \\
\hline $\begin{array}{l}12.1 \\
7.53\end{array}$ & $\begin{array}{l}4.4 E-09 \\
2.5 E-08\end{array}$ & $\begin{array}{l}2.4 E-06 \\
g .9 E-06\end{array}$ & $\begin{array}{l}6.3 \mathrm{~B}-12 \\
6 \mathrm{3}-\mathrm{e} 2\end{array}$ & $\begin{array}{l}0.33 \\
0.35\end{array}$ & $\begin{array}{l}0.31 \\
0.31\end{array}$ & 61 & 106 & 0.99 & $\begin{array}{l}0.31 \\
0.31\end{array}$ & $0.0 E+00$ \\
\hline $\begin{array}{r}1.53 \\
11.89\end{array}$ & $\begin{array}{l}2.5 E-08 \\
1.6-10\end{array}$ & $\begin{array}{l}9.9 E-08 \\
8.4 E-06\end{array}$ & $\begin{array}{l}6.3 .3-12 \\
63-12\end{array}$ & $\begin{array}{l}0.35 \\
0.35\end{array}$ & $\begin{array}{l}0.31 \\
0.31\end{array}$ & $\begin{array}{l}61 \\
61\end{array}$ & $\begin{array}{l}106 \\
106\end{array}$ & 0.99 & $\begin{array}{l}0.31 \\
0.31\end{array}$ & $0.0 E+00$ \\
\hline 14.05 & $\begin{array}{l}1.07 E-09 \\
1.45\end{array}$ & $\begin{array}{l}0.4 E-00 \\
9.1 E-06\end{array}$ & $6.3 \mathrm{E}-12$ & 0.33 & 0.31 & $\begin{array}{l}11 \\
61\end{array}$ & 106 & 0.99 & 0.31 & $\begin{array}{l}0.0 E++00 \\
0.0 E+00\end{array}$ \\
\hline 14.13 & 7.9E-11 & $4.2 E-05$ & 6.3E-12 & 0.35 & 0.31 & 61 & 106 & 0.99 & 0.31 & $0.0 E+00$ \\
\hline 18.72 & $5.3 E-10$ & $6.4 E-05$ & $6.3 \mathrm{E}-12$ & 0.33 & 0.31 & 61 & 106 & 0.99 & 0.31 & $0.0 E+00$ \\
\hline 11.58 & $1.5 E-08$ & $1.0 \mathrm{E}-06$ & $6.3 E-12$ & 0.33 & 0.31 & 61 & 106 & 0.99 & 0.31 & $0.0 E+00$ \\
\hline $\begin{array}{r}6.53 \\
0.07\end{array}$ & $2.9 E-08$ & $5.11-08$ & 6.3E-12 & 0.35 & 0.31 & 61 & 106 & 0.99 & 0.31 & $0.0 E+00$ \\
\hline $\begin{array}{l}10.07 \\
1307\end{array}$ & $5.2 E-10$ & $\begin{array}{l}2.0 E=-06 \\
56 E-06\end{array}$ & $\begin{array}{l}6.3 \mathrm{E}-12 \\
63-12\end{array}$ & 0.36 & 0.31 & 61 & 106 & 0.99 & 0.31 & $0.0 E+00$ \\
\hline $\begin{array}{r}13.09 \\
111.6\end{array}$ & $\begin{array}{l}1.2 E-09 \\
6.5-11\end{array}$ & $5.6 E-06$ & $\begin{array}{l}6.3 \mathrm{E}-12 \\
63-12\end{array}$ & $\begin{array}{l}0.34 \\
0.34\end{array}$ & 0.31 & 61 & $\begin{array}{l}106 \\
106\end{array}$ & 0.99 & $\begin{array}{l}0.31 \\
0.31\end{array}$ & $0.0 E+00$ \\
\hline 12.04 & $\begin{array}{l}0.2 E-09 \\
1.211\end{array}$ & $\begin{array}{l}1.16-05 \\
4.4 E-06\end{array}$ & $\begin{array}{l}.035-12 \\
6.3 E-12\end{array}$ & 0.34 & $\begin{array}{l}0.31 \\
0.31\end{array}$ & $\begin{array}{l}11 \\
61\end{array}$ & 106 & 0.99 & $\begin{array}{l}0.31 \\
0.31\end{array}$ & $\begin{array}{l}0.0 E++00 \\
0.0 E+00\end{array}$ \\
\hline 14.6 & $9.7 E-11$ & $4.3 E-05$ & $6.3 \mathrm{E}-12$ & 0.35 & 0.31 & 61 & 106 & 0.99 & 0.31 & $0.0 E+00$ \\
\hline & $1.0 E-08$ & $1.11-05$ & $6.3 E-12$ & 0.31 & 0.31 & 61 & 106 & 0.99 & 0.31 & $0.0 E+00$ \\
\hline
\end{tabular}




\begin{tabular}{|c|c|c|c|}
\hline chr1_137634000 & ENSGALTO0000022\&ENSGALG00000016807 & 1 cis & 13.48 cis \\
\hline chr1__137644000 & ENSGALTO00000022\&ENSGALG000000016807 & 1 cis & 13.48 cis \\
\hline chr1_137651000 & ENSGALTO0000022\&ENSGALG000000016807 & 1 cis & 13.48 cis \\
\hline chr1_137654000 & ENSGALTTO000000228ENSGALG000000016807 & 1 cis & 13.48 cis \\
\hline chr1_137655000 & ENSGALTO0000022\&ENSGALG00000016807 & 1 cis & 13.48 cis \\
\hline chr1_137657000 & ENSGALTO00000022EENSGALG00000016807 & 1 cis & 13.48 cis \\
\hline $\begin{array}{l}\text { chr1_137661000 } \\
\text { chr1 } 13766000\end{array}$ & ENSGALTO00000022\&ENSGALG00000016807 & $\begin{array}{l}1 \text { cis } \\
1 \text { cis }\end{array}$ & 13.48 cis \\
\hline $\begin{array}{l}\text { chr1_137666000 } \\
\text { chr1 } 137669000\end{array}$ & ENSGALTO000000222EENGALG000000016807 & $\begin{array}{l}1 \text { cis } \\
1 \text { cis }\end{array}$ & $\begin{array}{l}13.48 \text { cis } \\
13.48 \text { cis }\end{array}$ \\
\hline chr1_137674000 & ENSGALTO0000022EENSGALG00000016807 & 1 cis & $\begin{array}{l}13.48 \text { cis } \\
13.48 \text { cis }\end{array}$ \\
\hline chr1_137679000 & ENSGALTO00000228ENSGALG00000016807 & 1 cis & $\begin{array}{l}13.48 \text { cis } \\
13.48 \text { cis }\end{array}$ \\
\hline chr1_137681000 & ENSGALT00000022EENSGALG00000016807 & 1 cis & $\begin{array}{l}13.48 \mathrm{cis} \\
13.48 \mathrm{cis}\end{array}$ \\
\hline chr1_137683000 & ENSGALT000000022\&ENSGALG00000016807 & 1 cis & $\begin{array}{ll}13.48 \mathrm{cis} \\
13.4\end{array}$ \\
\hline chr11 137684000 & ENSGALTO00000022RENSGALG00000016807 & 1 cis & 13.48 cis \\
\hline hr1 137685000 & ENSGALT00000022\&ENSGALG00000016807 & 1 cis & $\begin{array}{l}13.4 \mathrm{cls} \\
13.48 \mathrm{cis}\end{array}$ \\
\hline$=$ =hr11 137687000 & ENSGALT00000022\&ENSGALG00000016807 & 1 cis & $13.48 \mathrm{cis}$ \\
\hline chr1 137695000 & ENSGALTO0000022\&ENSGALG000000016807 & 1 cis & 13.48 cis \\
\hline chr1_137589000 & ENSGALT00000022\&ENSGALG00000016807 & 1 cis & 13.48 cis \\
\hline chr1_137605000 & ENSGALT000000022EENSGALG00000016807 & 1 cis & 13.48 cis \\
\hline chr1_137613000 & ENSGALT00000022\&ENSGALG00000016807 & 1 cis & 13.48 cis \\
\hline chr1_137615000 & ENSGALT00000022\&ENSGALG00000016807 & 1 cis & 13.48 cis \\
\hline chr1_137617000 & ENGGALT00000022EENSGALG00000016807 & 1 cis & 13.48 cis \\
\hline chr1_137618000 & ENSGALT00000022\&ENSGALG00000016807 & 1 cis & 13.48 cis \\
\hline chr1_137631000 & ENGGALTO00000022EENSGALG000000016807 & $\begin{array}{l}1 \text { cis } \\
1 \text { cis }\end{array}$ & $\begin{array}{l}13.48 \text { cis } \\
13.48 \text { cis }\end{array}$ \\
\hline $\begin{array}{l}\text { chr1_1376685000 } \\
\text { chr1_137665000 }\end{array}$ & 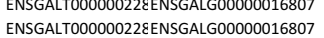 & $\begin{array}{l}1 \text { cis } \\
1 \text { cis }\end{array}$ & $\begin{array}{l}13.48 \text { cis } \\
13.48 \text { cis }\end{array}$ \\
\hline chr1_137668000 & ENSGALTO0000022EENSGALG00000016807 & 1 cis & $\begin{array}{l}13.48 \mathrm{cis} \\
13.48\end{array}$ \\
\hline chr1_137688000 & ENSGALTO0000022\&ENSGALG00000016807 & 1 cis & 13.48 cis \\
\hline chr1_137697000 & ENSGALT000000022EENSGALG00000016807 & 1 cis & 13.48 cis \\
\hline chr1_137699000 & ENSGALT00000022EENSGALG00000016807 & 1 cis & 13.48 cis \\
\hline chr1_137701000 & ENSGALTO00000022\&ENSGALG00000016807 & 1 cis & 13.48 cis \\
\hline chr1_137703000 & ENGGALTO000000228ENSGALG000000016807 & 1 cis & 13.48 cis \\
\hline $\begin{array}{l}\text { chr1_1375574000 } \\
\text { chr1_137576000 }\end{array}$ & 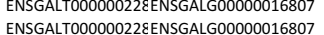 & $\begin{array}{l}1 \text { cis } \\
1 \text { cis }\end{array}$ & $\begin{array}{l}13.48 \text { cis } \\
13.48 \text { is }\end{array}$ \\
\hline $\begin{array}{l}\text { chrl_1_1375790000 } \\
\text { chros }\end{array}$ & ENSGALTO0000022EENSGALG00000016807 & 1 cis & $\begin{array}{l}13.48 \mathrm{cis} \\
13.48\end{array}$ \\
\hline chr1_137585000 & ENSGALT00000022\&ENSGALG00000016807 & 1 cis & 13.48 cis \\
\hline chr1_137586000 & ENSGALTO00000022EENSGALG00000016807 & 1 cis & 13.48 cis \\
\hline chr1_137587000 & ENSGALT00000022\&ENSGALG00000016807 & 1 cis & 13.48 cis \\
\hline chr1_137590000 & ENSGALTO0000022\&ENSGALG00000016807 & 1 cis & 13.48 cis \\
\hline $\begin{array}{l}\text { chr1_137593000 } \\
\text { chr1 } 1776600000\end{array}$ & ENGGALTO00000228ENSGALG000000016807 & 1 cis & 13.48 cis \\
\hline $\begin{array}{l}\text { chr1_1376030000 } \\
\text { chr1 } 137608000\end{array}$ & 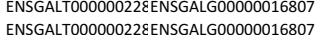 & $\begin{array}{l}1 \text { cis } \\
1 \text { cis }\end{array}$ & $\begin{array}{l}13.48 \text { cis } \\
13.48 \text { cis }\end{array}$ \\
\hline chr1_137614000 & ENSGALTO00000022EENSGALG00000016807 & 1 cis & $\begin{array}{l}13.48 \mathrm{cis} \\
13.48 \mathrm{cis}\end{array}$ \\
\hline chr1_137619000 & ENSGALTOO0000022\&ENSGALG00000016807 & 1 cis & 13.48 cis \\
\hline chr1_137621000 & ENSGALT00000022\&ENSGALG00000016807 & 1 cis & 13.48 cis \\
\hline chr1_137633000 & ENSGALT00000022\&ENSGALG00000016807 & 1 cis & 13.48 cis \\
\hline hr1_137643000 & ENGGALTO0000022\&ENSGALG00000016807 & 1 cis & 13.48 cis \\
\hline $\begin{array}{l}\text { chr1_137647000 } \\
\text { chr1 } 173659000\end{array}$ & ENGGALTO00000228ENSGALG00000016807 & 1 cis & 13.48 cis \\
\hline $\begin{array}{l}\text { Chr1_1376695000 } \\
\text { chr1_37671000 }\end{array}$ & 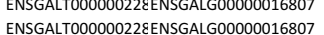 & $\begin{array}{l}1 \text { cis } \\
1 \text { cis }\end{array}$ & $\begin{array}{l}13.48 \text { cis } \\
13.48 \text { cis }\end{array}$ \\
\hline chr1_137672000 & ENSGALTOO0000022EENSGALG00000016807 & 1 cis & $\begin{array}{l}13.48 \mathrm{cis} \\
13.48 \mathrm{cis}\end{array}$ \\
\hline chr1_137673000 & ENSGALTO0000022\&ENSGALG00000016807 & 1 cis & 13.48 cis \\
\hline chr1_137675000 & ENSGALT00000022\&ENSGALG00000016807 & 1 cis & 13.48 cis \\
\hline chr1_137689000 & ENSGALT00000022\&ENSGALG00000016807 & 1 cis & 13.48 cis \\
\hline chr1_137691000 & ENSGALT00000022\&ENSGALG00000016807 & 1 cis & 13.48 cis \\
\hline Chr1_137693000 & ENSGALT00000022\&ENSGALG00000016807 & 1 cis & 13.48 cis \\
\hline $\begin{array}{l}\text { chr1_1376990000 } \\
\text { chr1 } 137700000\end{array}$ & ENGGALTO0000022\&ENSGALG00000016807 & 1 cis & 13.48 cis \\
\hline 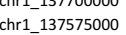 & 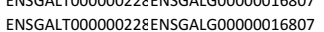 & 1 cis & $\begin{array}{l}13.48 \mathrm{cls} \\
13.48 \mathrm{cis}\end{array}$ \\
\hline chr1_137578000 & ENSGALT00000022\&ENSGALG00000016807 & 1 cis & 13.48 cis \\
\hline chr1_137591000 & ENSGALT00000022\&ENSGALG00000016807 & 1 cis & 13.48 cis \\
\hline chr1_137604000 & ENSGALT00000022\&ENSGALG00000016807 & 1 cis & 13.48 cis \\
\hline Chr1_137606000 & ENSGALT00000022\&ENSGALG00000016807 & 1 cis & 13.48 cis \\
\hline ri_1_137607000 & ENSGALTO00000022\&ENSGALG00000016807 & & 13.48 cis \\
\hline r1__137616000 & ENSGALTO00000022\&ENSGALG000000016807 & 1 cis & \\
\hline 137320000 & ENGSALLO000002028ENSGAGG000000016807 & 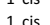 & $1308 e^{2}$ \\
\hline
\end{tabular}

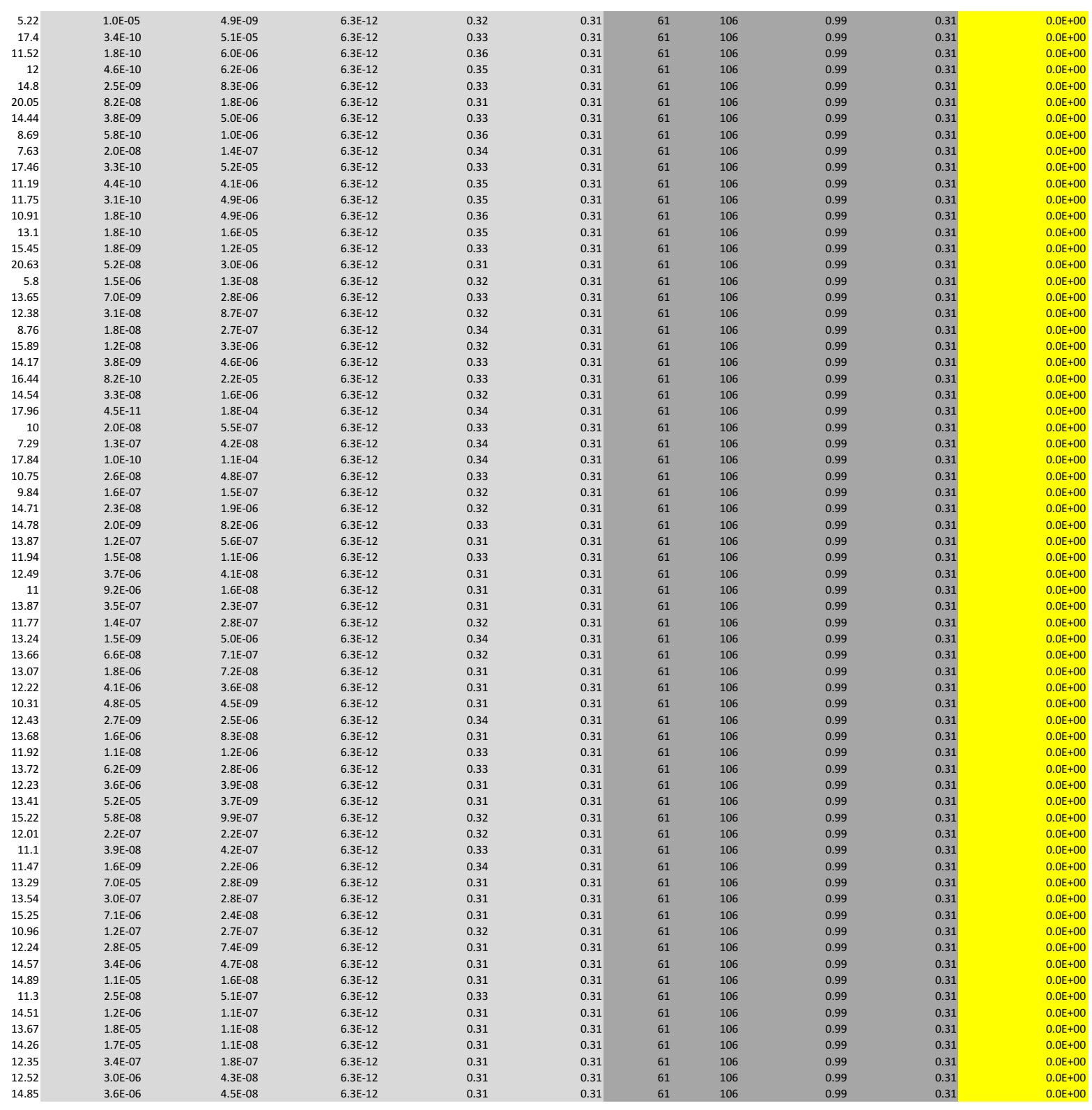




\begin{tabular}{|c|c|c|c|c|}
\hline chr1_137645000 & \multicolumn{2}{|c|}{ ENSGALTO00000022E ENSGALG00000016807 } & 1 cis & 13.48 cis \\
\hline chr1_138209000 & & ENSGALG00000016807 & 1 cis & 10.56 cis \\
\hline chr1_136500000 & X603142577F1 & ENSGALG00000016807 & 1 cis & 10.56 cis \\
\hline $\begin{array}{l}\text { chr1_1376629000 } \\
\text { chr1_37682000 }\end{array}$ & $\begin{array}{l}X 603142577771 \\
\times 60312577 F 1\end{array}$ & $\begin{array}{l}\text { ENSGALG000000016807 } \\
\text { ENSGA G0000016807 }\end{array}$ & $\begin{array}{l}1 \text { cis } \\
1 \text { cis }\end{array}$ & $\begin{array}{l}10.56 \text { cis } \\
105 \text { cis }\end{array}$ \\
\hline chr1_137716000 & $\begin{array}{l}\quad \times 00314253711 \\
\times 603142577 F 1\end{array}$ & $\begin{array}{l}\text { ENSGALG60000001680 } \\
\text { ENSGALO000001680 }\end{array}$ & $\begin{array}{l}1 \text { cis } \\
1 \text { cis }\end{array}$ & $\begin{array}{l}10.5 \text { c cis } \\
10.56 \text { cis }\end{array}$ \\
\hline chr1_137596000 & X603142577F1 & ENSGALG00000016807 & 1 cis & 10.56 cis \\
\hline chr1_137601000 & X603142577F1 1 & ENSGALG00000016807 & 1 cis & 10.56 cis \\
\hline chr1_137696000 & X603142577F1 & ENSGALG00000016807 & 1 cis & 10.56 cis \\
\hline chr1_137595000 & X6031425777F1 & ENSGALG000000016807 & 1 cis & 10.56 cis \\
\hline $\begin{array}{l}\text { chr1_1375980000 } \\
\text { chr1 } 137681000\end{array}$ & X603142577F1 & ENSGALG00000016807 & 1 cis & 10.56 cis \\
\hline $\begin{array}{l}\text { chr1_13778611000 } \\
\text { chr1_37684000 }\end{array}$ & $\begin{array}{l}X 603142577771 \\
X 60312577 F 1\end{array}$ & $\begin{array}{l}\text { ENSGALG000000016807 } \\
\text { ENGALG00000016877 }\end{array}$ & $\begin{array}{l}1 \text { cis } \\
1 \text { cis }\end{array}$ & 10.56 cis \\
\hline $\begin{array}{l}\text { chri_13737864000 } \\
\text { chr1_137728000 }\end{array}$ & $\begin{array}{l}\times 6031425 / F 1 \\
\times 603142577 F 1\end{array}$ & $\begin{array}{l}\text { ENGALG0000000016807 } \\
\text { ENSGALG0000016807 }\end{array}$ & $\begin{array}{l}1 \text { cis } \\
1 \text { cis }\end{array}$ & $\begin{array}{l}10.5 \text { c cis } \\
1.056 \text { cis }\end{array}$ \\
\hline chr1_137748000 & X603142577F1 & ENSGALG00000016807 & 1 cis & 10.56 cis \\
\hline chr1_137767000 & X603142577F1 & ENSGALG00000016807 & 1 cis & 10.56 cis \\
\hline chr1_137584000 & X603142577F1 & ENSGALG00000016807 & 1 cis & 10.56 cis \\
\hline chr1_134200000 & ENSGALTO000000271 & 1ENSGALG00000016807 & 1 cis & 9.28 cis \\
\hline $\begin{array}{l}\text { chr1_133896000 } \\
\text { chr1 } 138209000\end{array}$ & ENSGALTO000000271 & 1ENSGALG000000016807 & 1 cis & 9.28 cis \\
\hline $\begin{array}{l}\text { chr11 } 1382099000 \\
\text { chr1 } 136991000\end{array}$ & ENSGALTO000000271 & '1ENSGALGO00000016807 & 1 cis & 9.28 cis \\
\hline 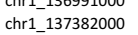 & $\begin{array}{l}\text { ENSGGLTT0000000271 } \\
\text { ENSGLTT00000271 }\end{array}$ & $\begin{array}{l}\text { 1ENSALGG0000000016807 } \\
\text { 1ENGALG0000016807 }\end{array}$ & $\begin{array}{l}1 \text { cis } \\
1 \text { cis }\end{array}$ & $\begin{array}{l}9.28 \text { cis } \\
9.28 \text { cis }\end{array}$ \\
\hline chr1_136500000 & ENSGALTO00000271 & 1ENSGALG00000016807 & 1 cis & $\begin{array}{l}9.28 \mathrm{cs} \\
9.28 \mathrm{cis}\end{array}$ \\
\hline chr1_137768000 & ENSGALTO00000271 & 1ENSGALG00000016807 & 1 cis & 9.28 cis \\
\hline chr1_137682000 & ENSGALTO00000271 & 1ENSGALG00000016807 & 1 cis & 9.28 cis \\
\hline chr1_137686000 & ENSGALT000000271 & 1ENSGALG00000016807 & 1 cis & 9.28 cis \\
\hline $\begin{array}{l}\text { chr1_137581000 } \\
\text { chr1 } 177594000\end{array}$ & ENSGALT0000000271 & 1ENSGALG00000016807 & 1 cis & 9.28 cis \\
\hline $\begin{array}{l}\text { chr1_13735540000 } \\
\text { chr1_137596000 }\end{array}$ & $\begin{array}{l}\text { ENSGALTT0000000271 } \\
\text { ENSGLTT000000271 }\end{array}$ & 1ENSGALGO0000016807 & $\begin{array}{l}1 \text { cis } \\
1 \text { cis }\end{array}$ & 9.28 cis \\
\hline $\begin{array}{l}\text { chrl1_13/3966000 } \\
\text { chr1_137597000 }\end{array}$ & $\begin{array}{l}\text { ENSGGLATO000000271 } \\
\text { ENSGLTO00000271 }\end{array}$ & 1ENSGALGG0000000001680 & $\begin{array}{l}1 \text { cis } \\
1 \text { cis }\end{array}$ & $\begin{array}{l}9.28 \text { cis } \\
9.28 \text { cis }\end{array}$ \\
\hline chr1_137601000 & ENSGALTO000000271 & 1ENSGALGO00000016807 & 1 cis & 9.28 cis \\
\hline chr1_137625000 & ENSGALTO00000271 & 1ENSGALG00000016807 & 1 cis & 9.28 cis \\
\hline chr1_137649000 & ENSGALTO00000271 & 1ENSGALG00000016807 & 1 cis & 9.28 cis \\
\hline chr1_137652000 & ENSGALT0000000271 & '1ENSGALG000000016807 & 1 cis & 9.28 cis \\
\hline chr1_137678000 & ENSGALTO000000271 & 1ENSGALG00000016807 & 1 cis & 9.28 cis \\
\hline $\begin{array}{l}\text { chr1_1376690000 } \\
\text { chr1 } 177709000\end{array}$ & ENSGALTO000000271 & 1ENSGALGO0000016807 & 1 cis & 9.28 cis \\
\hline $\begin{array}{l}\text { chr1_13777709000 } \\
\text { chr1_37711000 }\end{array}$ & $\begin{array}{l}\text { ENSGGLTT00000002777 } \\
\text { ENSGLITO000000271 }\end{array}$ & $\begin{array}{l}\text { 1ENSGALG000000016807 } \\
\text { 1ENSGALG000001680 }\end{array}$ & $\begin{array}{l}1 \text { cis } \\
1 \text { cis }\end{array}$ & $\begin{array}{l}9.28 \text { cis } \\
9.28 \text { cis }\end{array}$ \\
\hline $\begin{array}{l}\text { corri_1371111000 } \\
\text { chr1_137712000 }\end{array}$ & $\begin{array}{l}\text { ENSGGLATT0000000271 } \\
\text { ENSGLTT000000271 }\end{array}$ & 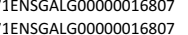 & $\begin{array}{l}1 \mathrm{cis} \\
1 \text { cis }\end{array}$ & $\begin{array}{l}9.28 \text { cis } \\
9.28 \text { cis }\end{array}$ \\
\hline chr1_137726000 & ENSGALTO00000271 & 1ENSGALGO0000016807 & 1 cis & 9.28 cis \\
\hline chr1_137743000 & ENSGALTO00000271 & 11ENSGALG00000016807 & 1 cis & 9.28 cis \\
\hline chr1_137582000 & ENSGALTO00000271 & 1ENSGALG00000016807 & 1 cis & 9.28 cis \\
\hline $\begin{array}{l}\text { chr1_1375853000 } \\
\text { chr1 } 137598000\end{array}$ & $\begin{array}{l}\text { ENSGGALTT0000000271 } \\
\text { ENSGLLTT00000271 }\end{array}$ & $\begin{array}{l}\text { 1'NESGALGG000000016807 } \\
\text { 1ENSGLGG0000016807 }\end{array}$ & $\begin{array}{l}1 \text { cis } \\
1 \text { cis }\end{array}$ & $\begin{array}{l}9.28 \text { cis } \\
9.28 \text { cis }\end{array}$ \\
\hline chr1_137599000 & ENSGALTO00000271 & 1ENSGALG00000016807 & 1 cis & $\begin{array}{l}9.28 \mathrm{cs} \\
9.28 \mathrm{cis}\end{array}$ \\
\hline chr1_137600000 & ENSGALT000000271 & '1ENSGALG00000016807 & 1 cis & 9.28 cis \\
\hline chr1_137611000 & ENSGALTO000000271 & '1ENSGALG00000016807 & 1 cis & 9.28 cis \\
\hline chr1_137612000 & ENSGALTO000000271 & '1ENSGALG00000016807 & 1 cis & 9.28 cis \\
\hline chr1_137623000 & ENSGALTO000000271] & 1'1ENSGALG00000016807 & 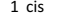 & 9.28 cis \\
\hline hr1_137627000 & ENSGALTO000000271 & 1ENSGALG000000016807 & 1 cis & 9.28 cis \\
\hline $\begin{array}{rl}r 11 & 137628000 \\
1\end{array}$ & ENSGALTO000000271 & 1ENSGALG000000016807 & 1 cis & 9.28 cis \\
\hline $\begin{array}{l}r_{1}^{1} \_137655000 \\
1137654000\end{array}$ & ENSGALTO000000271 & '1ENSGALG000000016807 & 1 cis & 9.28 cis \\
\hline $\begin{array}{l}\text { Chr1__1376544000 } \\
\text { chr1_137661000 }\end{array}$ & (1) & (II) & 1 cis & 9.28 cis \\
\hline chr1_137666000 & ENSGALTOO00000271 & ,ENGAG G000016007) & 1 cis & $\begin{array}{l}9.28 \mathrm{cls} \\
9.28 \text { cis }\end{array}$ \\
\hline chr1_137667000 & ENSGALTO00000271 & 1ENSGALGO0000016807 & 1 cis & 9.28 cis \\
\hline chr1_137674000 & ENSGALTO000000271 & 1ENSGALG00000016807 & 1 cis & 9.28 cis \\
\hline chr1_137679000 & ENSGALTO000000271 & '1ENSGALG000000016807 & 1 cis & 9.28 cis \\
\hline chr1_137681000 & ENSGALTO000000271 & '1ENSGALG000000016807 & 1 cis & 9.28 cis \\
\hline $\begin{array}{l}\text { Chr1_1376863000 } \\
\text { chr1 137685000 }\end{array}$ & ENSGALTO000000277 & 1ENSGALGO00000016807 & 1 cis & 9.28 cis \\
\hline 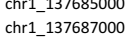 & ENSGALTO000000277 & 1ENSGALG00000016807 & 1 cis & 9.28 cis \\
\hline chr1_137695000 & ENSGALTOO00000271 & , & 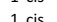 & $\begin{array}{l}9.28 \mathrm{cls} \\
0.28 \mathrm{csis}\end{array}$ \\
\hline chr1_137714000 & ENSGALTO00000271 & 1ENSGALG00000016807 & 1 cis & $\begin{array}{l}3.28 \\
9.28\end{array}$ \\
\hline hr1_137715000 & ENSGALTO000000271 & "1ENSGALG00000016807 & 1 cis & 9.28 cis \\
\hline _137725000 & ENSGALTO000000271 & 1ENSGALG00000016807 & 1 cis & 9.28 cis \\
\hline & & & & \\
\hline
\end{tabular}

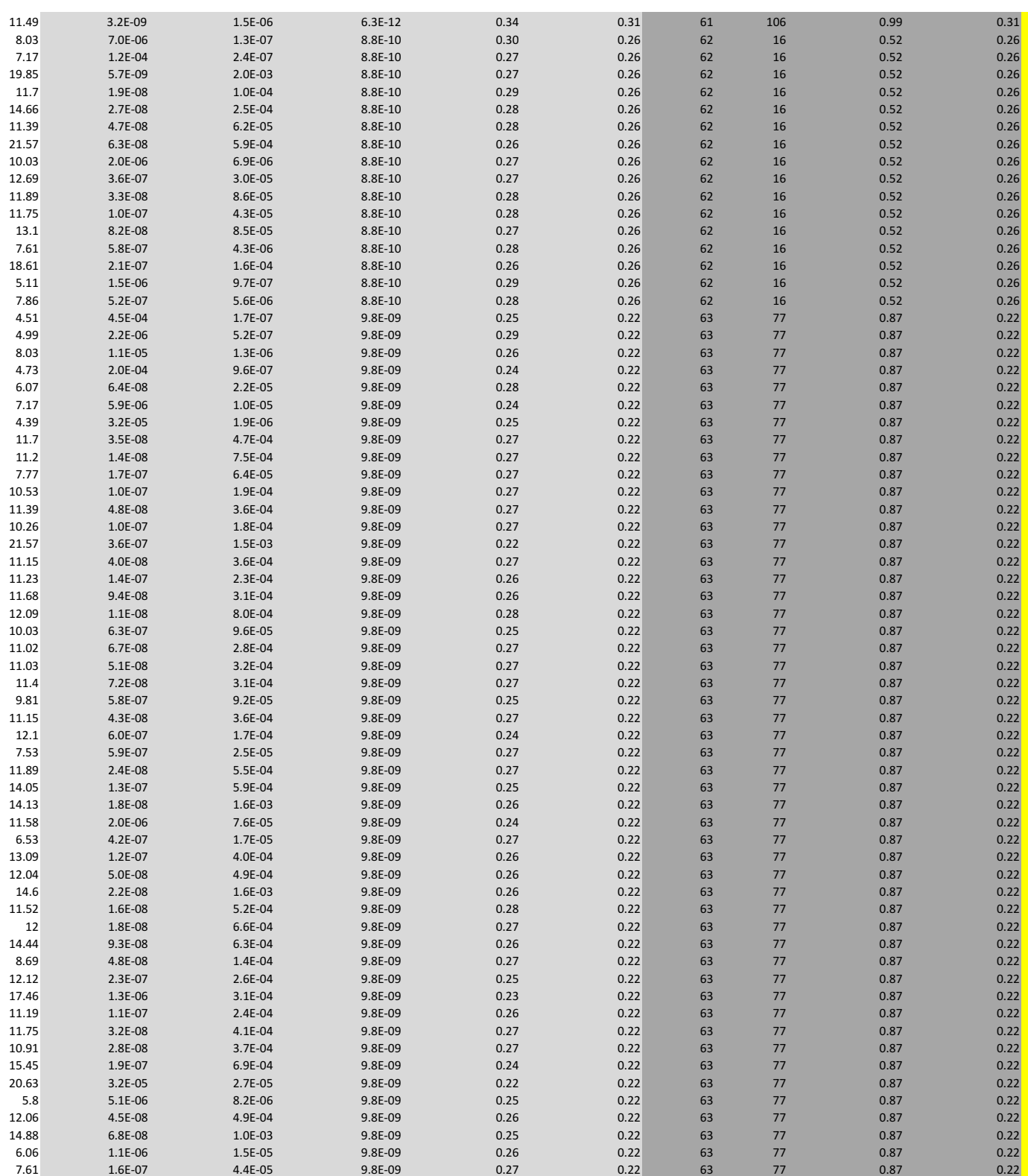




\begin{tabular}{|c|c|c|c|}
\hline chr1 137729000 & ENSGALTO00000271ENSGALG00000016807 & 1 cis & \\
\hline chr1_137738000 & ENSGALTO00000271ENSGALG00000016807 & 1 cis & 9.28 cis \\
\hline chr1_137740000 & ENSGALTO00000271ENSGALG00000016807 & 1 cis & 9.28 cis \\
\hline chr1_137741000 & ENSGALTO00000271ENSGALG00000016807 & 1 cis & 9.28 cis \\
\hline chr1_137745000 & ENSGALTO00000271ENSGALG00000016807 & 1 cis & 9.28 cis \\
\hline chr1_137746000 & ENSGALTO00000271ENSGALG00000016807 & 1 cis & 9.28 cis \\
\hline chr1_137748000 & ENSGALTT000000271ENSGALG000000016807 & 1 cis & 9.28 cis \\
\hline chr1_137769000 & ENSGALTO000000271ENSGALG000000016807 & 1 cis & 9.28 cis \\
\hline $\begin{array}{l}\text { chr1_193238000 } \\
\text { chr1 } 197584000\end{array}$ & 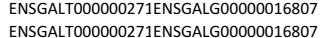 & $\begin{array}{l}1 \text { cis } \\
1 \text { cis }\end{array}$ & $\begin{array}{l}9.28 \text { cis } \\
9.28 \text { cis }\end{array}$ \\
\hline $\begin{array}{l}\text { chri1 } 1375840000 \\
\text { chr1 } 137605000\end{array}$ & $\begin{array}{l}\text { ENSGGLLTT0000000271ENGGAGG000000016807 } \\
\text { ENSGLLTTO0000271ESGGLG0000001687 }\end{array}$ & $\begin{array}{l}1 \text { cis } \\
1 \text { cis }\end{array}$ & $\begin{array}{l}9.28 \text { cis } \\
9.28 \text { cis }\end{array}$ \\
\hline $\begin{array}{l}\text { Chri_1 } 1370503000 \\
\text { chrl_137613000 }\end{array}$ & ENSGALTOO00000271ENSGALG00000016807 & $\begin{array}{l}1 \text { cis } \\
1 \text { cis }\end{array}$ & $\begin{array}{l}9.28 \mathrm{cis} \\
9.28 \mathrm{cis}\end{array}$ \\
\hline chr1_137615000 & ENSGALTO00000271ENSGALG00000016807 & 1 cis & 9.28 cis \\
\hline chr1_137618000 & ENSGALTO00000271ENSGALG00000016807 & 1 cis & 9.28 cis \\
\hline chr1_137631000 & ENSGALTO00000271ENSGALG00000016807 & 1 cis & 9.28 cis \\
\hline chr1_137658000 & ENSGALTO000000271ENSGALG00000016807 & 1 cis & 9.28 cis \\
\hline $\begin{array}{l}\text { chr1_137688000 } \\
\text { chr1 } 177697000\end{array}$ & ENSGALTO000000271ENSGALG00000016807 & 1 cis & 9.28 cis \\
\hline $\begin{array}{l}\text { chr11 } 1376697000 \\
\text { chrl_137699000 }\end{array}$ & 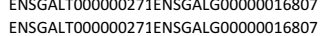 & $\begin{array}{l}1 \text { cis } \\
1 \text { cis }\end{array}$ & $\begin{array}{l}9.28 \text { cis } \\
9.28 \text { cis }\end{array}$ \\
\hline $\begin{array}{l}\text { Crri_1 } 1336599000 \\
\text { chr__137701000 }\end{array}$ & 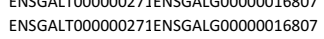 & $\begin{array}{l}1 \text { cis } \\
1 \text { cis }\end{array}$ & $\begin{array}{l}9.28 \text { cis } \\
9.28 \text { cis }\end{array}$ \\
\hline chr1_137718000 & ENSGALTO00000271ENSGALG00000016807 & 1 cis & 9.28 cis \\
\hline chr1_137721000 & ENSGALTO00000271ENSGALG00000016807 & 1 cis & 9.28 cis \\
\hline chr1_137727000 & ENSGALTO00000271ENSGALG00000016807 & 1 cis & 9.28 cis \\
\hline chr1_137731000 & ENSGALTO00000271ENSGALG00000016807 & 1 cis & 9.28 cis \\
\hline $\begin{array}{l}\text { chr1_137733000 } \\
\text { chr1 } 137734000\end{array}$ & ENSGALTO000000271ENSGALG00000016807 & 1 cis & 9.28 cis \\
\hline $\begin{array}{l}\text { chr1_1 } 1377734000 \\
\text { chr137770000 }\end{array}$ & 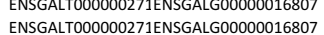 & $\begin{array}{l}1 \text { cis } \\
1 \text { cis }\end{array}$ & $\begin{array}{l}9.28 \text { cis } \\
9.28 \text { cis }\end{array}$ \\
\hline chr1_140937000 & NM_001008681_ABENSGALG00000016853 & 1 cis & 4.57 cis \\
\hline chr1_142072000 & NM_001008681_ABENSGALG00000016853 & 1 cis & 4.57 cis \\
\hline chr1_144697000 & NM_0010008681_ABENSGALG000000016853 & 1 cis & 4.57 cis \\
\hline chr1_140928000 & X603602468F1 \#N/A & 1 cis & 3.98 cis \\
\hline chr1_151794000 & X603865265F1 & 1 cis & 6.14 trans \\
\hline chr1_148807000 & 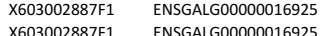 & $\begin{array}{ll}1 \text { cis } \\
1 \text { cis }\end{array}$ & 5.65 cis \\
\hline $\begin{array}{l}\text { chr11 } 1488800000 \\
\text { chrr_155715000 }\end{array}$ & 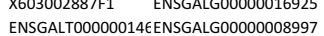 & $\begin{array}{l}1 \text { cis } \\
1 \text { trans }\end{array}$ & $\begin{array}{l}5.75 \text { trans } \\
7.74 \text { cis }\end{array}$ \\
\hline chr1_156059000 & ENSGALTO00000365ENSGALG00000016937 & 1 cis & 5.63 trans \\
\hline chr1_145493000 & ENSGALTO00000365ENSGALG00000016926 & 1 cis & 4.93 cis \\
\hline chr1_151275000 & ENSGALTO00000365ENSGALG00000016926 & 1 cis & 4.93 trans \\
\hline chr1_162106000 & ENSGALTO00000036EENSGALG000000016926 & 1 cis & 4.93 trans \\
\hline chr1_167035000 & ENSGALTO000000274ENSGALG00000016955 & 1 cis & 5.21 cis \\
\hline chr1_163487000 & ENSGALTO000000274ENSGALG00000016955 & 1 cis & 5.21 cis \\
\hline $\begin{array}{l}\text { chr1 } 1 \text { 1 } 1732260000 \\
\text { chr17287000 }\end{array}$ & 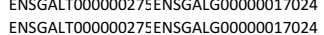 & $\begin{array}{l}1 \text { cis } \\
1 \text { cis }\end{array}$ & $\begin{array}{l}4.84 \text { cis } \\
4.84 \text { cis }\end{array}$ \\
\hline chr1_182304000 & ENSGALTO00000275ENSGALG00000017024 & 1 cis & $\begin{array}{l}4.84 \mathrm{cis} \\
4.84\end{array}$ \\
\hline chr1_173226000 & X603865290F1 $\quad$ \#N/A & 1 cis & 6.22 cis \\
\hline chr1_172387000 & X603865290F1 1 & 1 cis & 6.22 cis \\
\hline chr1_174482000 & X6038652900F 1 & 1 cis & 6.22 cis \\
\hline chr1_175527000 & 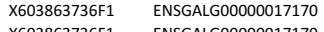 & 1 cis & 4.74 cis \\
\hline $\begin{array}{l}\text { chr1_1832880000 } \\
\text { chr1 } 182304000\end{array}$ & $\begin{array}{l}\text { ENSSAGG00000000177170 } \\
\text { ENGSAG G0000017770 }\end{array}$ & 1 cis & 4.74 cis \\
\hline $\begin{array}{l}\text { chr1_18283040000 } \\
\text { chr1_83504000 }\end{array}$ & $\begin{array}{ll}\text { ENSGALG000000017770 } \\
\text { HNA }\end{array}$ & 1 cis & $\begin{aligned} 4.74 \text { cis } \\
10.0 \text { cis }\end{aligned}$ \\
\hline $\begin{array}{l}\text { Chrl_1_18353040000 } \\
\text { chros }\end{array}$ & 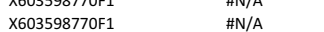 & 1 trans & $\begin{array}{l}10.01 \text { cis } \\
10.01 \text { cis }\end{array}$ \\
\hline chr1_175647000 & ENSGALTO00000364ENSGALG00000022715 & 1 cis & 5.19 trans \\
\hline chr1_182304000 & ENSGALTO00000364ENSGALG00000022715 & 1 cis & 5.19 cis \\
\hline chr1_177071000 & ENSGALTO00000277ENSGALG00000028658 & 1 cis & 4.24 cis \\
\hline chr1_186038000 & ENSGALTO00000277ENSGALG00000017181 & 1 cis & 4.56 cis \\
\hline $\begin{array}{l}\text { chr1_1818680000 } \\
\text { chr1 } 186681000\end{array}$ & 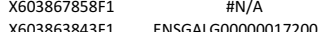 & 1 cis & 4.38 trans \\
\hline $\begin{array}{l}\text { chr1_186681000 } \\
\text { chr1_ } 182457000\end{array}$ & 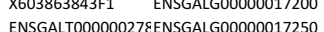 & 1 cis & $\begin{array}{l}4.64 \text { trans } \\
4.40 \text { trans }\end{array}$ \\
\hline chr1_191153000 & NM 010030832 CHENSGALG00000027409 & 1 cis & \\
\hline chr1_188617000 & ENSGALTO0000027EENSGALG00000027409 & 1 cis & 4.84 cis \\
\hline chr10_8679000 & X603862632F1 ENSGALG00000004212 & 10 cis & 3.80 cis \\
\hline chr10_7038000 & X603862632F1 ENSGALG00000004212 & 10 cis & 3.80 cis \\
\hline $\begin{array}{l}0.4809000 \\
-5498000\end{array}$ & NM_204261_ADAMENSGALG00000004212 & 10 cis & 3.98 cis \\
\hline & 6038620030F1 1 & 10 cis & \\
\hline 0 & M_-00010246964_R & 10 cis & $\begin{aligned} 1.62 \mathrm{cls} \\
1005 \mathrm{sis}\end{aligned}$ \\
\hline
\end{tabular}

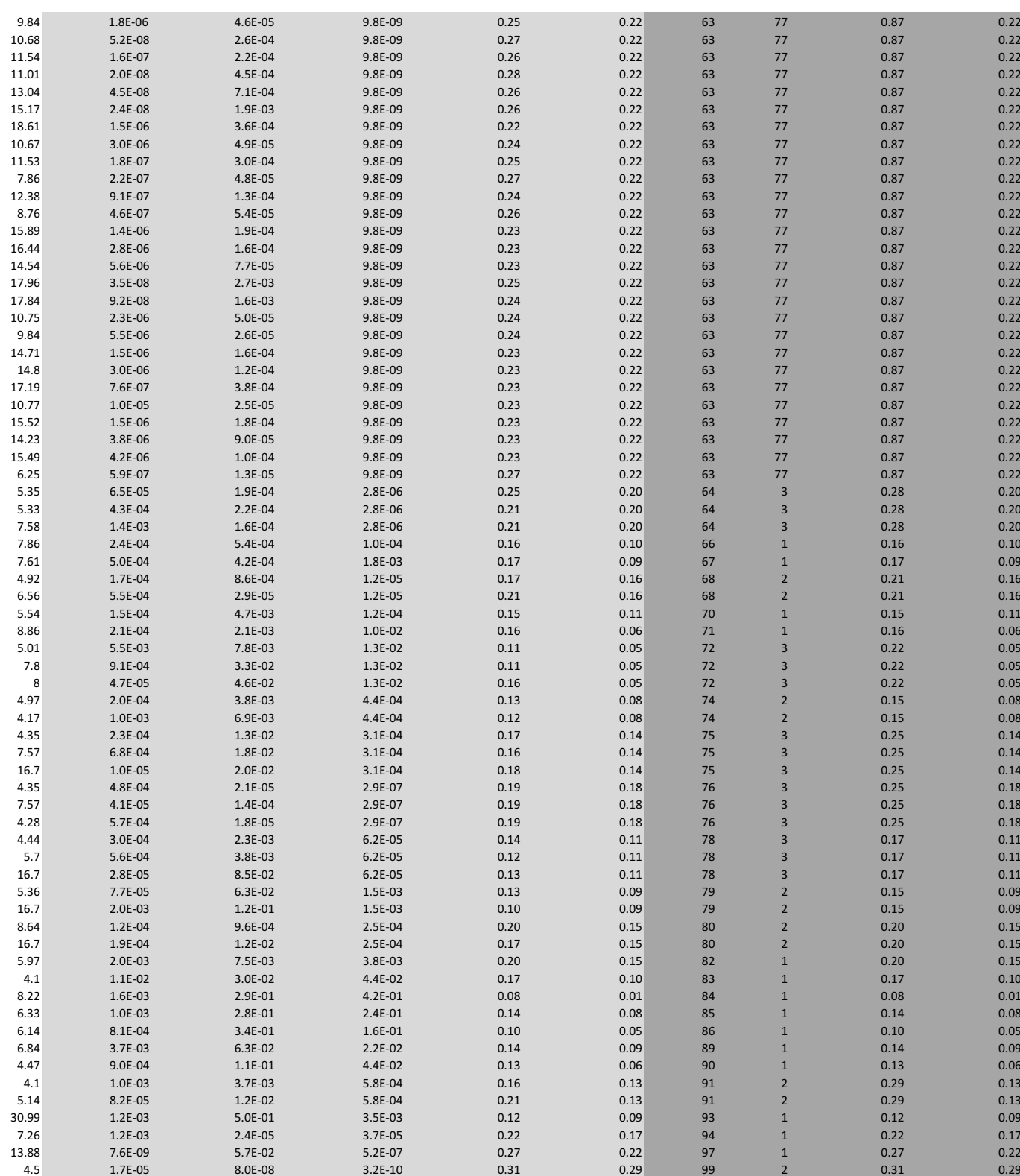




\begin{tabular}{|c|c|c|c|c|}
\hline chr11_7203000 & X603864355F1 & ENSGALG000000025819 & 11 cis & 10.05 cis \\
\hline chr11_5807000 & $\begin{array}{l}\times 603603027 F 1 \\
x_{6}\end{array}$ & $\begin{array}{l}\text { ENGGALG000000025819 } \\
\text { ENGSAGG00002519 }\end{array}$ & 11 cis & 11.30 cis \\
\hline $\begin{array}{l}\text { chr11_7660000 } \\
\text { chr11 } 5961000\end{array}$ & $\begin{array}{r}\times 603603027 F 1 \\
\end{array}$ & $\begin{array}{r}\text { ENGGALG000000025819 } \\
\text { IEFNGAG }\end{array}$ & $\begin{array}{ll}11 \text { cis } \\
11 \text { cis }\end{array}$ & $\begin{array}{ll}11.30 \text { cis } \\
660 \text { cis }\end{array}$ \\
\hline $\begin{array}{l}\text { chrr11_59610000 } \\
\text { chr12, 3620000 }\end{array}$ & $\begin{array}{l}\text { NM_0010055843- } \\
\text { ENSGALTO0000039 }\end{array}$ & $\begin{array}{l}\text { JEENGSAGG000000025819 } \\
\text { 92ENSGALG000002357 }\end{array}$ & $\begin{array}{l}11 \text { cis } \\
12 \text { trans }\end{array}$ & $\begin{array}{l}6.60 \text { cis } \\
9.63 \text { cis }\end{array}$ \\
\hline chr12_2721000 & ENSGALTO00000039 & 92ENSGALG00000023576 & 12 trans & $9.63 \mathrm{cis}$ \\
\hline chr12_3621000 & NM_001012842_B & SS ENSGALG00000003405 & 12 trans & 8.52 cis \\
\hline chr12_2721000 & NM_001012842_B & SS ENSGALG00000003405 & 12 trans & 8.52 cis \\
\hline chr12_4954000 & ENSGALT00000016 & 6 6EENSGALG00000010359 & 12 trans & 7.68 trans \\
\hline chr12_3621000 & ENSGALT00000016 & 68ENSGALG000000010359 & 12 trans & 7.68 cis \\
\hline chr12_2739000 & ENSGALT00000014 & 48ENSGALG00000008319 & 12 trans & 7.03 cis \\
\hline chr12_3621000 & X603144566F1 & ENSGALG00000002114 & 12 cis & 5.22 cis \\
\hline $\begin{array}{l}\text { chr12_2739000 } \\
\text { chr12 } 3621000\end{array}$ & Х603144566F1 & 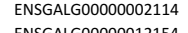 & 12 cis & 5.22 cis \\
\hline $\begin{array}{l}\text { chr12_362110000 } \\
\text { chr12 } 3621000\end{array}$ & $\begin{array}{l}\text { X6031428277F1 } \\
\text { ENSGATO0000005 }\end{array}$ & $\begin{array}{l}\text { ENSGALG000000012154 } \\
\text { S\&NSESGAG00000027060 }\end{array}$ & $\begin{array}{l}12 \text { trans } \\
12 \text { cis }\end{array}$ & $\begin{array}{l}6.57 \mathrm{cis} \\
5.01 \mathrm{cis}\end{array}$ \\
\hline $\begin{array}{l}\text { chr122-63261000 } \\
\text { chr12_4584000 }\end{array}$ & $\begin{array}{l}\text { ENSGALT000000005 } \\
\text { ENSGATO0000005 }\end{array}$ & 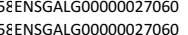 & $\begin{array}{l}12 \text { cis } \\
12 \text { cis }\end{array}$ & $\begin{array}{l}5.01 \text { cis } \\
5.01 \text { cis }\end{array}$ \\
\hline $\begin{array}{l}\text { Crr11_-4584000 } \\
\text { chr12_11001000 }\end{array}$ & ENSGALTO00000033 & $\begin{array}{l}\text { SEEENGAAGG000000027006 } \\
\text { 32ENSGALG0000020755 }\end{array}$ & $\begin{array}{l}12 \text { cis } \\
12 \text { cis }\end{array}$ & $\begin{array}{l}\text {.010 cis } \\
5.81 \text { cis }\end{array}$ \\
\hline chr12_16909000 & ENSGALT00000012 & 2 2EENSGALG00000027845 & 12 cis & 4.58 cis \\
\hline chr12_16909000 & X603862915F1 & ENSGALG00000008279 & 12 cis & 5.23 cis \\
\hline chr12_16909000 & X603864922F1 & ENSGALG000000006314 & 12 trans & 6.52 cis \\
\hline chr13_1423000 & Х603568189F1 & \#N/A & 13 cis & 3.55 cis \\
\hline $\begin{array}{l}\text { chr13_105450000 } \\
\text { chr13_5260000 }\end{array}$ & 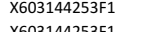 & 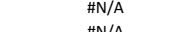 & 13 cis & 5.93 trans \\
\hline $\begin{array}{l}\text { chrr13__260000 } \\
\text { chr13_6571000 }\end{array}$ & $\begin{array}{l}X 603144253 F 1 \\
\times 603144253 F 1\end{array}$ & 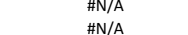 & $\begin{array}{l}13 \text { cis } \\
13 \text { cis }\end{array}$ & $\begin{array}{l}5.93 \text { cis } \\
5.93 \text { cis }\end{array}$ \\
\hline $\begin{array}{l}\operatorname{chr13} 33_{3} 65710000 \\
\operatorname{chr} 3947000\end{array}$ & 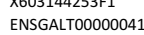 & $\begin{array}{c}\text { AN/A } \\
\text { 1CENSGALG0000001690 }\end{array}$ & $\begin{array}{l}13 \mathrm{cls} \\
13 \text { cis }\end{array}$ & $\begin{array}{l}5.93 \mathrm{cis} \\
7.59 \mathrm{cis}\end{array}$ \\
\hline chr13_6521000 & X603863557F1 & ENSGALG00000002665 & 13 cis & 4.50 cis \\
\hline chr13_6023000 & X603863557F1 & ENSGALG00000002665 & 13 cis & 4.50 cis \\
\hline chr13_8902000 & ENSGALTO00000034 & 47ENSGALG00000027809 & 13 cis & 4.79 cis \\
\hline $\begin{array}{l}\text { chr13_6571000 } \\
\text { chr13 } 79477000\end{array}$ & ENSGALT00000034 & 47ENSGALG00000027809 & 13 cis & 4.79 cis \\
\hline $\begin{array}{l}\text { chr13_79470000 } \\
\text { chr13 } 22350000\end{array}$ & $\begin{array}{l}\text { ENSGALTOO0000011 } \\
\text { ENGGATO000011 }\end{array}$ & 177NNSGALG000000001690 & 13 cis & 6.33 cis \\
\hline chr13_7947000 & $\begin{array}{l}\text { ENSAATL000000011 } \\
\text { X603598354F1 }\end{array}$ & $\begin{array}{l}\text { 17ENSGALG000000001690 } \\
\text { \#N/A }\end{array}$ & $\begin{array}{l}13 \text { cis } \\
13 \text { cis }\end{array}$ & $\begin{array}{l}6.32 \text { cis } \\
8.26 \text { cis }\end{array}$ \\
\hline chr13_8235000 & X603598354F1 & $\# \mathrm{~N} / \mathrm{A}$ & 13 cis & 8.26 cis \\
\hline chr13_7947000 & X603866983F1 & $\# N / A$ & 13 cis & 9.12 cis \\
\hline chr13_8235000 & X603866983F1 & \#N/A & 13 cis & 9.12 cis \\
\hline chr13_7947000 & X603597807F1 & ENSGALG00000002184 & 13 cis & 5.78 cis \\
\hline chr13_8902000 & ENSGALT00000004 & 45ENSGALG00000002863 & 13 cis & 7.65 cis \\
\hline $\begin{array}{l}\text { chr13_11573000 } \\
\text { crit_ } 8203000\end{array}$ & X603598203F1 & $\begin{array}{c}\text { \#N/A } \\
\text {. }\end{array}$ & 13 cis & 4.64 cis \\
\hline $\begin{array}{l}\text { chr14_88203000 } \\
\text { chr14820000 }\end{array}$ & $\begin{array}{l}\text { NM_0010006172_M } \\
\text { X60383637371 }\end{array}$ & MEENSGALG000000007044 & $\begin{array}{l}14 \text { cis } \\
14 \text { cis }\end{array}$ & $\begin{array}{l}4.59 \text { cis } \\
8.54 \text { cis }\end{array}$ \\
\hline $\begin{array}{l}\text { chr14_8 } 8020000 \\
\text { chr11_5864000 }\end{array}$ & $\begin{array}{l}\times 60383644 / 3 F 1 \\
\times 603596248 F 1\end{array}$ & ENSGALG0000003589 & $\begin{array}{l}14 \mathrm{cs} \\
14 \mathrm{cis}\end{array}$ & $\begin{array}{l}8.54 \mathrm{cls} \\
8.75 \text { cis }\end{array}$ \\
\hline chr14_8203000 & X603596248F1 & \#N/A & 14 cis & 8.75 cis \\
\hline chr14_9741000 & X603596248F1 & $\# N / A$ & 14 cis & 8.75 cis \\
\hline chr14_9741000 & ENSGALT00000039 & 9SENSGALG00000006889 & 14 cis & 5.02 cis \\
\hline chr14_9741000 & X603599183F1 & ENSGALG00000006307 & 14 cis & 5.90 cis \\
\hline $\begin{array}{l}\text { chr14_8202000 } \\
\text { ch14_ } 9679000\end{array}$ & X603603312F1 & ENGGALG000000007155 & 14 cis & 10.48 cis \\
\hline $\begin{array}{l}\text { chr14_96977000 } \\
\text { chr14-9740000 }\end{array}$ & $\begin{array}{l}\times 603603312 F 1 \\
\times 6003603312 F 1\end{array}$ & $\begin{array}{l}\text { ENGSAGG000000007755 } \\
\text { ENSGA G600000007155 }\end{array}$ & $\begin{array}{l}14 \text { cis } \\
14 \text { cis }\end{array}$ & 10.48 cis \\
\hline $\begin{array}{l}\text { Chr14-9941000 } \\
\text { chr14_7940000 }\end{array}$ & 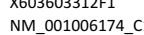 & $\begin{array}{l}\text { ENSGGAGG000000001755 } \\
\text { I ENSGALG0000007155 }\end{array}$ & $\begin{array}{l}14 \text { cis } \\
14 \text { cis }\end{array}$ & $\begin{array}{l}10.48 \text { cis } \\
9.11 \text { cis }\end{array}$ \\
\hline chr14_9741000 & NM_001006174_C & C1 ENSGALG00000007155 & 14 cis & 9.11 cis \\
\hline chr14_8202000 & Х6035959888F1 & ENSGALG000000007044 & 14 cis & 4.45 cis \\
\hline chr14_9741000 & X6035959888F1 & ENSGALG000000007044 & 14 cis & 4.45 cis \\
\hline chr14_9741000 & ENSGALTT000000006 & 67ENSGALG00000004251 & 14 trans & 7.04 cis \\
\hline $\begin{array}{l}\text { chr14_8202000 } \\
\text { ch14 8884000 }\end{array}$ & Х603863911F1 & ENSGALG00000007155 & 14 cis & 8.61 cis \\
\hline 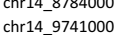 & $\begin{array}{l}\text { X66038663911F1 } \\
\text { X603863911F1 }\end{array}$ & $\begin{array}{l}\text { ENSGALG000000007155 } \\
\text { F }\end{array}$ & $\begin{array}{l}14 \text { cis } \\
14 \text { cis }\end{array}$ & 8.61 cis \\
\hline $\begin{array}{l}\text { Chr1_49-9471000 } \\
\text { chr14_9741000 }\end{array}$ & $\begin{array}{l}\text { DEUSB63511F1 } \\
\text { ENSGALTO0000010 }\end{array}$ & O3ENSGALG000000006418 & $\begin{array}{l}14 \mathrm{cls} \\
14 \mathrm{cis}\end{array}$ & $\begin{array}{l}8.61 \mathrm{cls} \\
4.96 \mathrm{cis}\end{array}$ \\
\hline chr14_8202000 & $\begin{array}{l}\text { X603597475F1 } \\
\end{array}$ & \#N/A & $14 \mathrm{cis}$ & 14.37 cis \\
\hline chr14_9697000 & X603597475F1 & $\# \mathrm{~N} / \mathrm{A}$ & 14 cis & 14.37 cis \\
\hline chr14_9741000 & X603597475F 1 & \#N/A & 14 cis & 14.37 cis \\
\hline chr14_8202000 & ENSGALTO00000011 & 15ENSGALG000000007155 & 14 cis & 7.64 cis \\
\hline chr14_8784000 & ENSGALT000000 & 15ENSGALG00000007155 & 14 cis & 7.64 cis \\
\hline $\begin{array}{l}\text { Chr1 } 14-96970000 \\
\text { hbl14 }\end{array}$ & ENSGALT00000011 & 15ENSGALG00000007155 & 14 cis & 7.64 cis \\
\hline 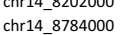 & $\begin{array}{l}\text { ENSGGATI0000011 } \\
\text { ENSGATO0000011 }\end{array}$ & 11ENSSALG0000000/7116 & $\begin{array}{l}14 \mathrm{cs} \\
14 \mathrm{cis}\end{array}$ & $\begin{array}{l}9.79 \text { c cls } \\
9.79 \text { cis }\end{array}$ \\
\hline & & & & \\
\hline 13772000 & ENSGALTO00000003 & $\begin{array}{l}1 \text { IENSGGAGG00000000/216 } \\
\text { 31ENSGALG000002026 }\end{array}$ & $\begin{array}{l}14 \mathrm{cs} \\
14\end{array}$ & 5.49 cis \\
\hline
\end{tabular}

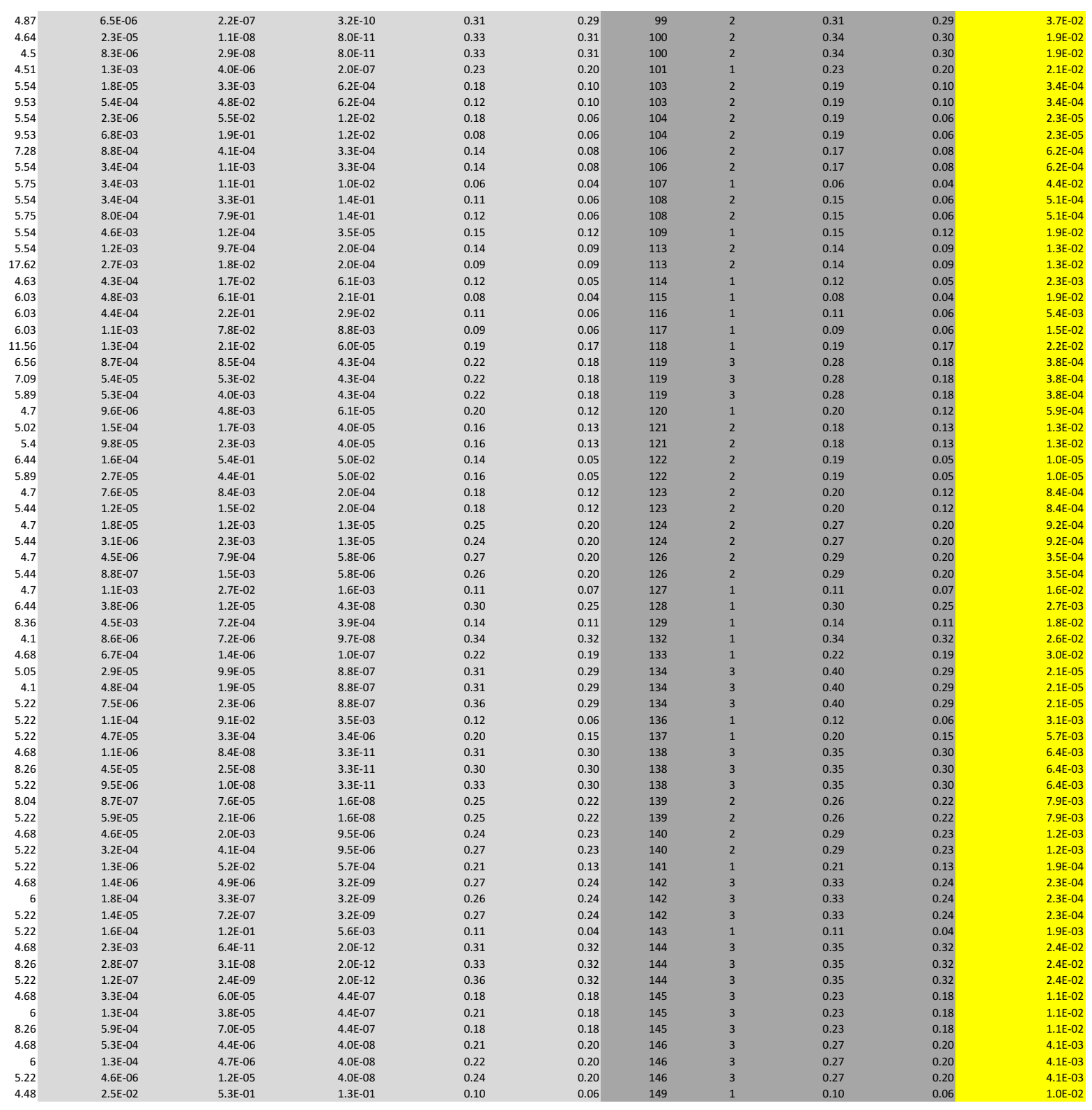




\begin{tabular}{|c|c|c|c|c|}
\hline chr15 15350000 & \multirow{2}{*}{\multicolumn{2}{|c|}{$\begin{array}{l}\text { X6603598221F1 } 1 \text { \#N/A } \\
\text { ENSGALO00000042ENSGALG00000002714 }\end{array}$}} & 15 cis & \\
\hline chr15_2371000 & & & 15 cis & 4.08 cis \\
\hline chr15_8999000 & \multicolumn{2}{|c|}{ ENSGALTO00000347ENSGALG00000002679 } & 15 cis & 4.29 cis \\
\hline chr2_96290000 & X603867141F1 & \#N/A & 2 cis & 5.30 cis \\
\hline chr2_11823000 & X603867141F1 & $\# N / A$ & 2 cis & 5.30 cis \\
\hline chr2_94000000 & X603867141F1 & \#N/A & 2 cis & 5.30 cis \\
\hline chr2_-11345000 & \multirow{2}{*}{\multicolumn{2}{|c|}{ ENSGALTO000000114ENSGALG000000007076 }} & 2 cis & 5.30 cis \\
\hline chr2_10391000 & & & 2 cis & 4.28 cis \\
\hline chr2_157444000 & \multirow{2}{*}{\multicolumn{2}{|c|}{$\begin{array}{l}\text { ENSGALTO00000114ENSGALG00000007076 } \\
\text { ENSGALTO0000011SENSGALG00000007417 }\end{array}$}} & 2 cis & 4.28 cis \\
\hline chr2_144677000 & & & 2 cis & 4.36 cis \\
\hline 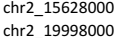 & \multicolumn{2}{|c|}{ ENSGALTO000000119ENSGALG00000007417 } & $\begin{array}{l}2 \text { cis } \\
2 \text { cis }\end{array}$ & $\begin{array}{r}4.36 \text { cis } \\
11.62 \text { cis }\end{array}$ \\
\hline 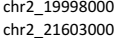 & \multicolumn{2}{|c|}{$\begin{array}{l}\text { ENSGALTO00000382ENSGALG00000008912 } \\
\text { ENSGALTO00000382ENSGALG00000008912 }\end{array}$} & $\begin{array}{l}2 \text { cis } \\
2 \text { cis }\end{array}$ & 11.62 cis \\
\hline chr__21447000 & \multicolumn{2}{|c|}{ ENSGALTOO00000382ENSGALG000000008912 } & 2 cis & $\begin{array}{l}11.62 \text { cis } \\
11.62 \text { cis }\end{array}$ \\
\hline chr2_-21966000 & \multicolumn{2}{|c|}{ ENSGALTO000000382ENSGALG000000088912 } & 2 cis & 11.62 cis \\
\hline chr2__19381000 & \multirow{2}{*}{\multicolumn{2}{|c|}{$\begin{array}{l}\text { NM_204894_ABCB1ENSGALG00000008912 } \\
\text { NM_204894_ABCB1ENSGALG00000008912 }\end{array}$}} & 2 cis & 12.47 cis \\
\hline chr2_210640000 & & & 2 cis & 12.47 cis \\
\hline chr2_24012000 & \multicolumn{2}{|c|}{ NM_204894_ABCB1ENSGALG000000089912 } & 2 cis & 12.47 cis \\
\hline $\begin{array}{l}\text { chrl_2 } 214477000 \\
\text { chr' } 21271000\end{array}$ & \multicolumn{2}{|c|}{ 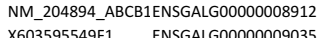 } & 2 cis & 12.47 cis \\
\hline 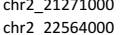 & \multirow{2}{*}{\multicolumn{2}{|c|}{ X603595549F1 ENSGALG000000009035 }} & $\begin{array}{l}2 \text { cis } \\
2 \text { cis }\end{array}$ & 5.06 cis \\
\hline $\begin{array}{ll}C h r 2 \\
\text { chr_e } 1999800000\end{array}$ & & & $\begin{array}{l}2 \text { cis } \\
2 \text { cis }\end{array}$ & $\begin{array}{l}5.06 \text { cis } \\
9.58 \text { cis }\end{array}$ \\
\hline chr___21064000 & \multirow{2}{*}{\multicolumn{2}{|c|}{$\begin{array}{l}\text { ENSGALTO0000014AENSGALG00000023271 } \\
\text { ENSGALTO0000014AENSGALG00000023271 }\end{array}$}} & 2 cis & 9.58 cis \\
\hline chr2_21966000 & & & 2 cis & 9.58 cis \\
\hline chr__26960000 & \multicolumn{2}{|c|}{$\begin{array}{l}\text { ENSGALTO00000144ENSGALG00000023271 } \\
\text { ENSGALTO0000014SENSGALG0000008963 }\end{array}$} & 2 cis & 4.02 cis \\
\hline chr2_28450000 & \multicolumn{2}{|c|}{ ENSGALTO000000145ENSGALG00000008963 } & 2 cis & 4.02 cis \\
\hline 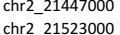 & \multicolumn{2}{|c|}{ ENSGALTO00000031SENSGALG000000020067 } & 2 trans & 7.56 cis \\
\hline 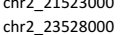 & $\begin{array}{l}\text { X60035988188F1 } \\
\text { X603598188F1 }\end{array}$ & $\begin{array}{l}\text { \#N//A } \\
\text { \#/A }\end{array}$ & 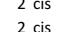 & $\begin{array}{l}11.26 \text { cis } \\
11.26 \text { cis }\end{array}$ \\
\hline chr__23193000 & X603598188F1 & AN/A & 2 cis & $\begin{array}{l}11.26 \text { c cs } \\
11.26 \text { is }\end{array}$ \\
\hline chr2_28450000 & X603598188F1 & \#N/A & 2 cis & 11.26 cis \\
\hline chr2_21603000 & X603600947F1 & \#N/A & 2 cis & 17.66 cis \\
\hline chr2_23901000 & X603600947F1 & \#N/A & 2 cis & 17.66 cis \\
\hline chr2_23193000 & X6036009977F1 & \#N/A & 2 cis & 17.66 cis \\
\hline chr2_264944000 & X603600947F1 & \#N/A & 2 cis & 17.66 cis \\
\hline $\begin{array}{ll}\text { chrt } 23238420000 \\
\text { chr' } 28450000\end{array}$ & $\begin{array}{l}\times 60360094771 \\
\times 6036094751\end{array}$ & 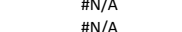 & $\begin{aligned} 2 \text { cis } \\
2 \text { cis }\end{aligned}$ & 17.66 cis \\
\hline $\begin{array}{ll}\text { Chri__2 } 284400000 \\
\text { chr } 29149000\end{array}$ & $\begin{array}{l}\text { X603600944F-1 } \\
\times 603865064 F 1\end{array}$ & 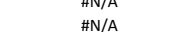 & $\begin{array}{l}2 \text { cis } \\
2 \text { cis }\end{array}$ & $\begin{array}{r}17.66 \text { cis } \\
8.82 \text { cis }\end{array}$ \\
\hline chr2_260200000 & X603865064F1 & \#N/A & 2 cis & 8.82 cis \\
\hline chr2_279080000 & X603865064F1 & \#N/A & 2 cis & 8.82 cis \\
\hline chr2_28812000 & X603865064F1 & \#N/A & 2 cis & 8.82 cis \\
\hline chr2_313032000 & X603865923F1 & \#N/A & 2 cis & 10.27 cis \\
\hline $\begin{array}{l}\text { chrl___310130000 } \\
\text { chr2 } 310900000\end{array}$ & X603865923F1 & \#N/A & 2 cis & 10.27 cis \\
\hline $\begin{array}{l}\text { Chr'_-313900000 } \\
\text { Chr2 } 31121000\end{array}$ & $\begin{array}{l}X 603865923 F 3 \\
\text { X603856923F1 }\end{array}$ & $\begin{array}{l}\# N / A \\
\# N / A \\
\# N A\end{array}$ & $\begin{array}{l}2 \text { cis } \\
2 \text { cis }\end{array}$ & 10.27 cis \\
\hline 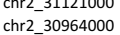 & $\begin{array}{r}\text { X6038656923F1 } \\
\text { X603865923F1 }\end{array}$ & $\begin{array}{l}\# N / A \\
\# N / A \\
\# / A\end{array}$ & 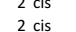 & $\begin{array}{l}10.27 \text { cis } \\
10.27 \text { cis }\end{array}$ \\
\hline chr___30725000 & х603865923F1 & 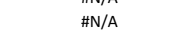 & 2 cis & $\begin{array}{l}10.27 \mathrm{cs} \\
10.27 \mathrm{cis}\end{array}$ \\
\hline chr2_28450000 & X603865923F1 & \#N/A & 2 cis & 10.27 cis \\
\hline chr2_26988000 & X603865923F1 & \#N/A & 2 cis & 10.27 cis \\
\hline chr2_313790000 & X603868123F1 & \#N/A & 2 cis & 10.18 cis \\
\hline chrr__28450000 & X603868123F1 & \#N/A & 2 cis & 10.18 cis \\
\hline 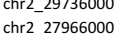 & $\begin{array}{l}\text { X60386812331 } \\
\text { X60386812351 }\end{array}$ & 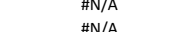 & $\begin{array}{l}2 \text { cis } \\
2 \text { cis }\end{array}$ & 10.18 cis \\
\hline 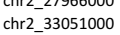 & ENSGALTO0000018 & IENSGALG00000011237 & 2 cis & $\begin{array}{r}10.18 \mathrm{cis} \\
4.09 \text { cis }\end{array}$ \\
\hline chr2_406002000 & X603596713F1 & $\# \mathrm{~N} / \mathrm{A}$ & 2 cis & 7.31 cis \\
\hline chr2_41936000 & X603868164F1 & \#N/A & 2 cis & 4.12 cis \\
\hline chr__-451530000 & X603866511F1 & ENSGALG0000000012066 & 2 cis & 5.38 cis \\
\hline $\begin{array}{l}\text { chr2__549920000 } \\
\text { che } 52509000\end{array}$ & ENSGALT00000020 & ENESGALG000000012666 & 2 cis & 4.84 cis \\
\hline 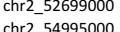 & ENSGALT000000020 & ENSGALG000000012666 & 2 cis & 4.84 cis \\
\hline $\begin{array}{l}\text { hr2_5 } 59995000 \\
\text { hr2 } 51079000\end{array}$ & NM_001007080_B & NENGGALG00000001212184 & 2 cis & $\begin{array}{l}5.50 \text { cis } \\
5.50 \text { cis }\end{array}$ \\
\hline 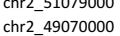 & NM_-001007080_B & 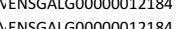 & 2 cis & $\begin{array}{l}5.50 \text { cis } \\
5.50 \text { cis }\end{array}$ \\
\hline chr___54992000 & X603864267F1 & ENSGALG00000012351 & 2 cis & 4.72 cis \\
\hline chr2_515700000 & ENSGALT00000020 & 1ENGGALG00000012337 & 2 cis & 8.23 cis \\
\hline chr2_52701000 & ENSGALT00000020 & 1ENGGALG000000012337 & 2 cis & 8.23 cis \\
\hline 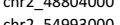 & 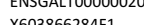 & (1) & 2 cis & $8.23 \mathrm{cls}$ \\
\hline
\end{tabular}

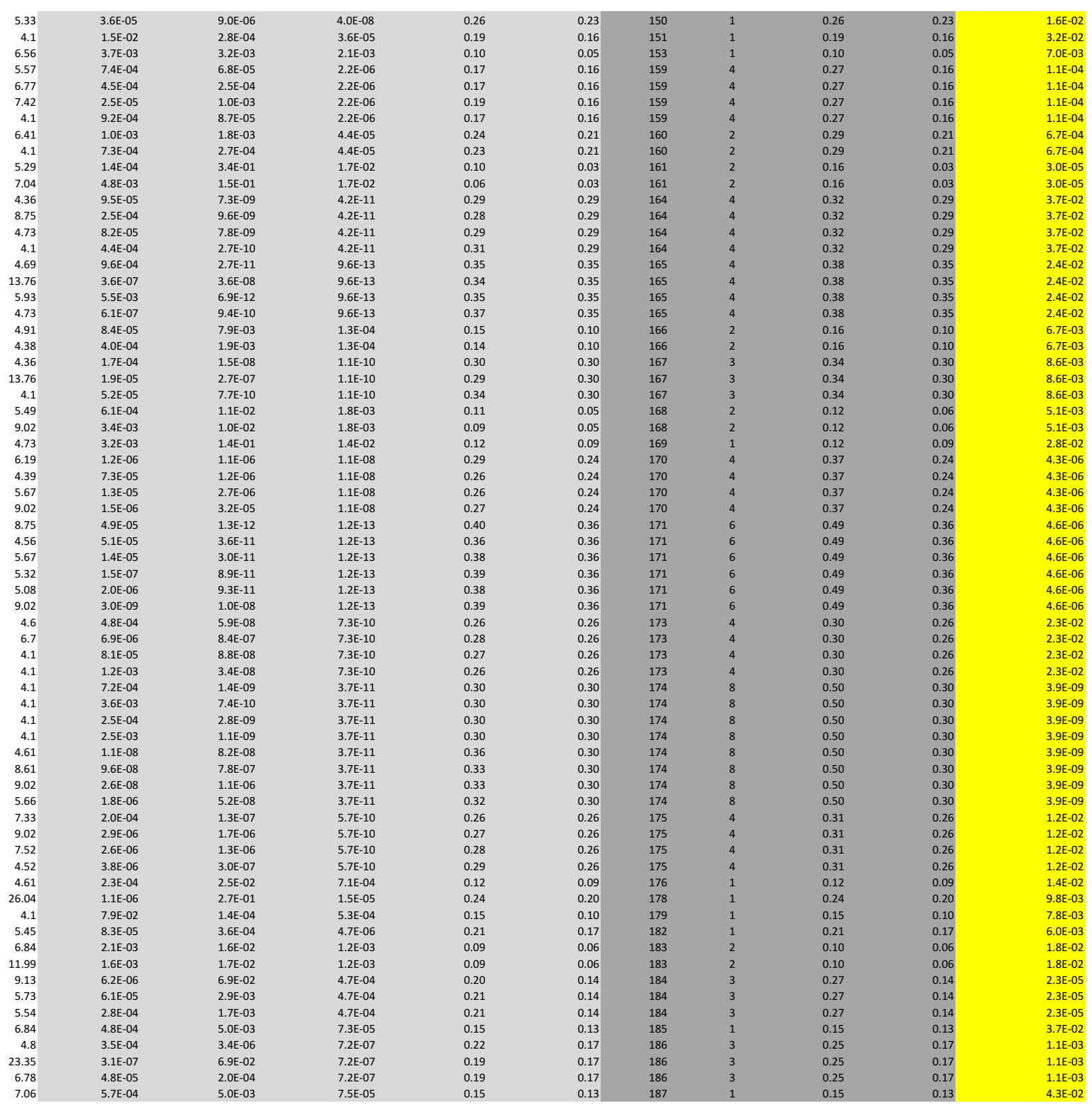




\begin{tabular}{|c|c|c|c|c|}
\hline $\operatorname{chr} 253177000$ & & & & \\
\hline $\begin{array}{ll}\mathrm{chr} 2-488050000 \\
-1\end{array}$ & X603142604F1 & 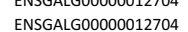 & $\begin{array}{l}2 \mathrm{cls} \\
2 \text { cis }\end{array}$ & \\
\hline chr2_54982000 & $\mathrm{X603142604F1}$ & ENSGALG000000012704 & 2 cis & \\
\hline chr2_54995000 & X603867382F1 & ENSGALG00000012732 & 2 cis & 5.02 cis \\
\hline chr2_66192000 & ENSGALT00000020 & OSENSGALG00000019555 & 2 cis & 4.75 trans \\
\hline chr2_-65888000 & NM_001033643_C & REENSGALG00000023950 & 2 trans & 6.56 trans \\
\hline chr2_71623000 & NM_204134_CDH2 & 2CENSGALG00000012906 & 2 cis & 4.46 trans \\
\hline chr2__84280000 & NM_204134_CDH2 & 2CENSGALG00000012906 & 2 cis & 4.46 cis \\
\hline chr2_73888000 & NM_001004400_T & UENSGALG00000012821 & 2 cis & \\
\hline chr2_-722487000 & ENSGALT00000021 & 13ENSGALG00000013053 & & 4.25 cis \\
\hline chr2_74527000 & ENSGALTO00000021 & 13ENSGALG000000013053 & 2 cis & 4.25 cis \\
\hline chr2_ 74459000 & ENSGALTO00000021 & 12ENSGALG00000013033 & 2 cis & 4.41 cis \\
\hline chr2_76583000 & ENSGALT00000021 & 12ENSGALG00000013033 & 2 cis & 4.41 cis \\
\hline chr2_-85008000 & ENSGALT00000037 & 7CENSGALG00000013183 & 2 cis & 4.46 cis \\
\hline chr2_85376000 & ENSGALT00000037 & 7CENSGALG00000013183 & 2 cis & 4.46 cis \\
\hline chr2_85442000 & ENSGALT00000037 & TCENSGALG000000013183 & 2 cis & 4.46 cis \\
\hline chr2_84280000 & NM_001031006_C & ECENSGALG00000013171 & & \\
\hline chr2_-80348000 & NM_001031006_C & CENSGALG00000013171 & 2 cis & 7.22 cis \\
\hline $\operatorname{chr} 280351000$ & NM $00100310006 \mathrm{C}$ & CENSGALG000000013171 & 2 cis & 7.22 cis \\
\hline chr2_80353000 & NM_001031006_C & CEENSGALG00000013171 & 2 cis & 7.22 cis \\
\hline chr2_80354000 & NM_001031006_C & CCENSGALG00000013171 & 2 cis & 7.22 cis \\
\hline chr2_85780000 & NM_001031006_C & CEENSGALG00000013171 & 2 cis & 7.22 cis \\
\hline chr2_-86267000 & X603866808F1 & \#N/A & 2 cis & 5.02 cis \\
\hline chr2_87757000 & X603866808F1 & \#N/A & 2 cis & 5.02 cis \\
\hline chr2_88354000 & Х603866808F1 & $\# \mathrm{~N} / \mathrm{A}$ & 2 cis & 5.02 cis \\
\hline $\begin{array}{l}\text { chr2_-85536000 } \\
\text { chr2 } 888060000\end{array}$ & Х603599695F1 & $\# \mathrm{~N} / \mathrm{A}$ & 2 cis & 8.75 cis \\
\hline $\begin{array}{l}\text { chr2_88806000 } \\
\text { chr2 } 85569000\end{array}$ & 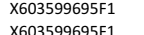 & $\begin{array}{ll}\# N / A \\
\# N A\end{array}$ & $\begin{array}{ll}2 \text { cis } \\
2 \text { cis }\end{array}$ & $\begin{array}{l}8.75 \text { cis } \\
8.75 \text { cis }\end{array}$ \\
\hline $\begin{array}{l}\text { chr___ } 858609000 \\
\text { chr2 } 89023000\end{array}$ & $\begin{array}{l}\text { X603599969551 } \\
\text { x600359969551 }\end{array}$ & $\begin{array}{l}\text { AN/A } \\
\text { AN/A } \\
\text { AN }\end{array}$ & $\begin{array}{l}2 \text { cis } \\
2 \text { cis }\end{array}$ & $\begin{array}{l}8.75 \text { cis } \\
8.75 \text { cis }\end{array}$ \\
\hline chr2_90653000 & $\begin{array}{l}\text { X0633999955)1 } \\
\times 6035996951\end{array}$ & $\begin{array}{l}\text { ANN/A } \\
\# N / A\end{array}$ & 2 cis & 8.75 cis \\
\hline chr2_90061000 & ENSGALT00000030 & OSENSGALG00000019522 & 2 cis & 9.36 cis \\
\hline chr2_88697000 & ENSGALT00000030 & OSENSGALG000000019522 & 2 cis & 9.36 cis \\
\hline chr2_90014000 & ENSGALT00000030 & OSENSGALG00000019522 & 2 cis & 9.36 cis \\
\hline $\begin{array}{l}\text { chr2_88746000 } \\
\text { chr2 } 909650000\end{array}$ & 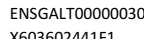 & OSENSGALG000000019522 & 2 cis & 9.36 cis \\
\hline $\begin{array}{l}\text { chr2_-900655000 } \\
\text { chr2 } 91585000\end{array}$ & $\begin{array}{l}\times 603602441 F 1 \\
\times 6605996251\end{array}$ & $\begin{array}{c}\text { \#N/A } \\
\text { ENSGA }\end{array}$ & 2 cis & $\begin{array}{l}4.58 \mathrm{cis} \\
1518 \text { sis }\end{array}$ \\
\hline $\begin{array}{l}\text { chr2_-91585000 } \\
\text { chr2_90365000 }\end{array}$ & $\begin{array}{l}\text { X6035956529F1 } \\
\times 6603599629 F 1\end{array}$ & $\begin{array}{l}\text { ENGSALG000000013637 } \\
\text { ENGGA G60000000163737 }\end{array}$ & $\begin{array}{l}2 \text { cis } \\
2 \text { cis }\end{array}$ & $\begin{array}{l}15.18 \text { cis } \\
15.15 \text { cis }\end{array}$ \\
\hline chr2_90736000 & $\begin{array}{r}1003539025911 \\
\times 603595629 F 1\end{array}$ & ENSGALGO00000013637 & 2 cis & 15.18 cis \\
\hline chr2_90653000 & X603595629F1 & ENSGALG000000013637 & 2 cis & 15.18 cis \\
\hline chr2_91588000 & X603595629F1 & ENSGALG000000013637 & 2 cis & 15.18 cis \\
\hline chr2_89850000 & X603597550F1 & ENGGALG000000013605 & 2 cis & 4.68 cis \\
\hline $\begin{array}{l}\text { chr2_914142000 } \\
\text { chr2 } 92516000\end{array}$ & $\begin{array}{l}\times 603597550 F 1 \\
\times\end{array}$ & ENSGALG00000013605 & 2 cis & 4.68 cis \\
\hline $\begin{array}{l}\text { chr2_-925160000 } \\
\text { chr2_ } 89023000\end{array}$ & $\begin{array}{l}\text { X60359755001 } \\
\text { ENGATT0000021 }\end{array}$ & $\begin{array}{l}\text { ENSGALG00000013605 } \\
\text { ent }\end{array}$ & $\begin{array}{l}2 \text { cis } \\
2 \text { cis }\end{array}$ & $\begin{array}{l}4.68 \text { cis } \\
4.43 \text { cis }\end{array}$ \\
\hline $\begin{array}{l}\text { Chri___ } 8092300000 \\
\text { chr_ } 89400000\end{array}$ & ENSGALTO00000022 & 21ENSGALG00000013637 & 2 cis & $\begin{array}{l}4.43 \mathrm{cls} \\
7.22 \mathrm{cis}\end{array}$ \\
\hline chr2_ 91585000 & ENSGALT00000022 & 21ENSGALG00000013637 & 2 cis & 7.22 cis \\
\hline chr2_-91588000 & ENSGALT00000022 & 21ENSGALG00000013637 & 2 cis & 7.22 cis \\
\hline chr2_94185000 & ENSGALT00000022 & 21ENSGALG00000013637 & 2 cis & 7.22 cis \\
\hline chr2_93650000 & ENSGALT00000022 & 21ENSGALG00000013637 & 2 cis & 7.22 cis \\
\hline $\begin{array}{l}\text { chr2_993060000 } \\
\text { chrr } 93007000\end{array}$ & ENSGALT00000022 & 21ENSGALG00000013637 & 2 cis & 7.22 cis \\
\hline $\begin{array}{l}\text { chr2_93607000 } \\
\text { chr2 } 85915000\end{array}$ & 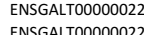 & 21ENSGALG000000013637 & 2 cis & 7.22 cis \\
\hline $\begin{array}{l}\text { chrr___89515000 } \\
\text { chr__94417000 }\end{array}$ & ENSGALTO00000022 & 22ENSENGALGG00000001361 & $\begin{array}{l}2 \text { cis } \\
2 \text { cis }\end{array}$ & $\begin{array}{l}\begin{array}{l}4.52 \mathrm{cls} \\
4.52 \mathrm{cis}\end{array} \\
4\end{array}$ \\
\hline chr2_94169000 & ENSGALT00000022 & 22ENSGALG00000013681 & 2 cis & 4.52 cis \\
\hline chr2_-96278000 & X603864386F1 & \#N/A & 2 cis & 4.42 cis \\
\hline chr2_94333000 & X603864386F1 1 & \#N/A & 2 cis & 4.42 cis \\
\hline chr2_959866000 & X603864386F1 & $\# N / A$ & 2 cis & 4.42 trans \\
\hline $\begin{array}{l}\text { chr2_-96321000 } \\
\text { cr.2 } 96612000\end{array}$ & ENSGALT00000022 & 25ENSGALG00000013880 & 2 cis & 3.96 cis \\
\hline $\begin{array}{l}\text { chr2_966322000 } \\
\text { chr2 } 96373000\end{array}$ & ENSGALT00000022 & 25ENSGALG00000013880 & 2 cis & 3.96 cis \\
\hline 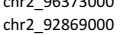 & ENSGALTO00000022 & 2EENSGALGG0000000138800 & $\begin{array}{l}2 \text { cis } \\
2 \text { cis }\end{array}$ & $\begin{array}{l}3.96 \mathrm{cls} \\
3.96 \mathrm{cis}\end{array}$ \\
\hline chr2_94419000 & ENSGALT00000022 & 2EENSGALG00000013954 & 2 cis & 5.81 cis \\
\hline chr2_ 98439000 & ENSGALT000000022 & 2EENSGALG000000013954 & 2 cis & 5.81 cis \\
\hline & ENSGALT000000022 & 2EENSGALG000000013954 & 2 cis & 5.81 cis \\
\hline hr2__100049000 & SGALT000000022 & 2EENSGALG000000013954 & 2 cis & \\
\hline hr2_98157000 & ENSGALT00000022 & 2EENSGALG00000013954 & 2 cis & \\
\hline & X603865192F1 & & & \\
\hline
\end{tabular}

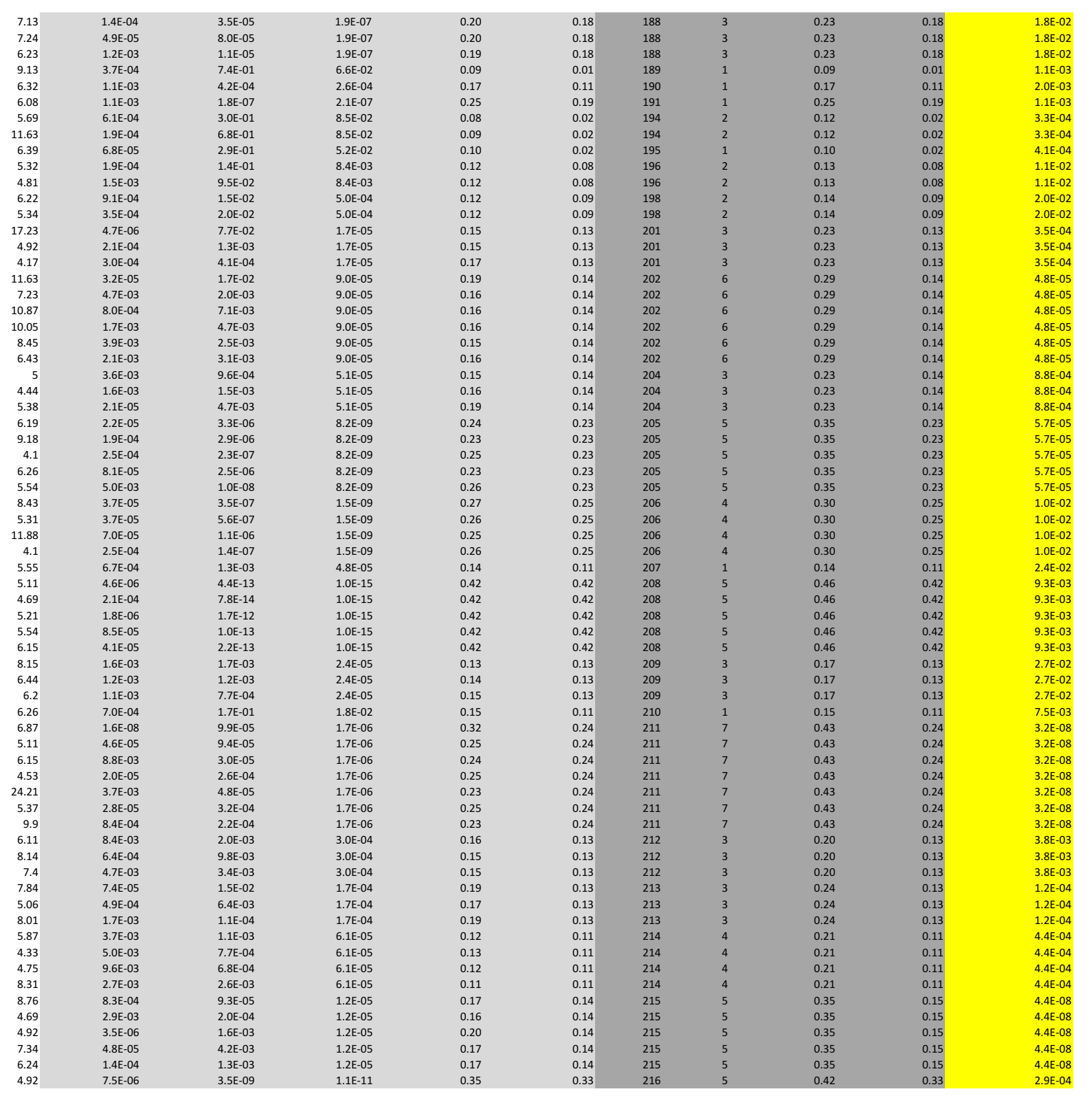




\begin{tabular}{|c|c|c|c|c|}
\hline chr2 100062000 & X603865192F1 & $\# N / A$ & 2 cis & 12.01 cis $\mathrm{Cl}$ \\
\hline chr2_100050000 & X603865192F1 & $\# N / A$ & & \\
\hline chr2_100939000 & X603865192F1 & $\# N / A$ & 2 cis & 12.01 cis \\
\hline chr2_987060000 & X603865192F1 & $\# N / A$ & 2 cis & \\
\hline chr2_99662000 & ENSGALT0000002 & 35ENSGALG000000014615 & 2 cis & 8.15 cis \\
\hline chr2_-99946000 & ENSGALTO000002 & 35ENSGALG00000014615 & 2 cis & 8.15 cis \\
\hline chr2_99009000 & ENSGALT0000002 & 35ENSGALG00000014615 & 2 cis & 8.15 cis \\
\hline chr2__99927000 & ENSGALT0000002 & 35ENSGALG00000014615 & 2 cis & 8.15 cis \\
\hline $\begin{array}{l}\text { chr2_999130000 } \\
\text { chr' } 100050000\end{array}$ & ENSGALT0000003 & 6EENSGALG00000014847 & 2 cis & 8.61 cis \\
\hline $\begin{array}{l}\text { chr2_-1000550000 } \\
\text { chr2 } 115539000\end{array}$ & 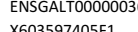 & 68ENSGALG000000014847 & 2 cis & $8.61 \mathrm{cis}$ \\
\hline $\begin{array}{l}\text { chr2 } 111155939000 \\
\text { chr2_ } 111734000\end{array}$ & $\begin{array}{l}\text { X60339577005F1 } \\
\text { X603856561F1 }\end{array}$ & $\begin{array}{l}\text { AN/A } \\
\text { NN/A }\end{array}$ & $\begin{array}{l}2 \text { cis } \\
2 \text { cis }\end{array}$ & $\begin{array}{l}4.50 \text { trans } \\
6.75 \text { cis }\end{array}$ \\
\hline chr2_ 112301000 & $\begin{array}{r}10303030513 F 1 \\
\times 6038613\end{array}$ & \#N/A & 2 cis & $\begin{array}{l}6.75 \text { cis } \\
6.75\end{array}$ \\
\hline chr2_-111734000 & X603863179F1 & $\# N / A$ & 2 cis & 6.21 cis \\
\hline chr2_112301000 & Х603863179F1 & \#N/A & 2 cis & 6.21 cis \\
\hline chr2_111734000 & NM_001039311_C & CEENSGALG00000014512 & 2 cis & 4.33 cis \\
\hline chr2_121857000 & ENSGALTO0000003 & OEENSGALG00000015767 & 2 cis & 4.96 cis \\
\hline $\begin{array}{l}\text { chr2_-12213000000 } \\
\text { chr2 } 121300000\end{array}$ & ENSGALTO0000003 & OEENSGALG000000015767 & 2 cis & 4.96 cis \\
\hline $\begin{array}{l}\text { chr2 } 12123000000 \\
\text { chr2-121718000 }\end{array}$ & $\begin{array}{l}\text { NM_001006345-F } \\
\text { ENGALTO0000003 }\end{array}$ & $\begin{array}{l}\text { RP RENSGAGG00000000015637 } \\
\text { 6ENSGALG00000015767 }\end{array}$ & $\begin{array}{l}2 \text { cis } \\
2 \text { cis }\end{array}$ & $\begin{array}{l}4.51 \text { cis } \\
5.39 \text { cis }\end{array}$ \\
\hline chr2_121857000 & ENSGALTO000003 & 6EENSGALG00000015767 & 2 cis & $\begin{array}{l}5.39 \text { cis } \\
5.39 \text { cis }\end{array}$ \\
\hline chr2_121422000 & ENSGALTO0000003 & 6EENSGALG00000015767 & 2 cis & $\begin{array}{l}5.39 \mathrm{cls} \\
5.39 \mathrm{cis}\end{array}$ \\
\hline chr2_120242000 & NM_001030943_F & l|FENSGALG00000015899 & 2 cis & 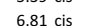 \\
\hline chr2_-122359000 & NM $001030943^{-}$ & SIFENSGALG00000015899 & 2 cis & 6.81 cis \\
\hline chr2 ${ }^{1212422000}$ & NM $001030943 \mathrm{~F}$ & IIFENSGALGO0000015899 & 2 cis & 6.81 cis \\
\hline chr2_123136000 & $\mathrm{NM}^{-0} 001001754{ }^{-\mathrm{L}}$ & OENSGALG00000015980 & 2 cis & $\begin{array}{l}0.91 \mathrm{cls} \\
4.93 \mathrm{cis}\end{array}$ \\
\hline chr2_125133000 & NM_001001754_L & OENSGALG00000015980 & 2 cis & $4.93 \mathrm{cis}$ \\
\hline chr2_121422000 & NM_204290_FAB! & PENSGALG000000015767 & 2 cis & 5.45 cis \\
\hline chr2_122557000 & NM_204290_FABE & P4ENSGALG000000015767 & 2 cis & 5.45 cis \\
\hline chr2_122686000 & NM_204290_FAB & 44ENSGALG00000015767 & 2 cis & 5.45 cis \\
\hline chr2_121300000 & NM_204290_FAB! & P4ENSGALG000000015767 & 2 cis & 5.45 cis \\
\hline chr2_125870000 & ENSGALT0000002 & 57ENSGALG00000015980 & 2 cis & 4.35 cis \\
\hline chr2_125164000 & ENSGALTO000002 & 57ENSGALG00000015980 & 2 cis & 4.35 cis \\
\hline $\begin{array}{l}\text { chr2_-1217118000 } \\
\text { chr2 } 121422000\end{array}$ & $\begin{array}{l}\text { X603595861F1 } \\
\text { x602595651 }\end{array}$ & $\begin{array}{l}\text { \#N/A } \\
\text { AN/A }\end{array}$ & 2 cis & 7.68 cis \\
\hline $\begin{array}{l}\text { chr' } 121214220000 \\
\text { chr2-125536000 }\end{array}$ & $\begin{array}{l}\text { X60035995856111 } \\
\text { X603595861F1 }\end{array}$ & $\begin{array}{l}\text { AN/A } \\
\text { AN/A }\end{array}$ & 2 cis & 7.68 cis \\
\hline 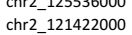 & 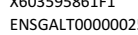 & 5EENSGALG000000015909 & 2 cis & $\begin{array}{l}7.68 \mathrm{cos} \\
4.67 \mathrm{cis}\end{array}$ \\
\hline chr2_ 125870000 & ENSGALTO000002 & SEENSGALG00000015909 & 2 cis & 4.67 cis \\
\hline chr2__121422000 & X6003864833F1 & ENSGALG000000015909 & 2 cis & 7.61 cis \\
\hline chr2_125870000 & X603864833F1 & ENSGALG00000015909 & 2 cis & 7.61 cis \\
\hline chr2_125870000 & ENSGALTO000002 & 5EENSGALG00000016066 & 2 cis & 4.30 cis \\
\hline $\begin{array}{l}\text { chr2_121300000 } \\
\text { cro20 } 14555000\end{array}$ & X603601310F1 & $\begin{array}{c}\# N / A \\
\end{array}$ & 2 cis & \\
\hline $\begin{array}{l}\text { chr20-14555000 } \\
\text { chr20_826000 }\end{array}$ & $\begin{array}{l}\text { X600359980883F1 } \\
\text { X603598083F1 }\end{array}$ & $\begin{array}{l}\text { ENSGALG0000000027126 } \\
\text { ENSGAGG00000027126 }\end{array}$ & $\begin{array}{ll}20 \text { cis } \\
20 \text { cis }\end{array}$ & 4.12 cis \\
\hline chr20_1455000 & $\begin{array}{l}\text { Xo335890883F1 } \\
\times 603597894 F 1\end{array}$ & ENSGALG00000003847 & 20 cis & $\begin{array}{l}4.12 \mathrm{cis} \\
4.31 \mathrm{cis}\end{array}$ \\
\hline chr20_2826000 & X603597894F1 & ENSGALG00000003847 & 20 cis & 4.31 cis \\
\hline chr20_2984000 & NM_205318_MYB & LLENSGALG000000003503 & 20 cis & 4.04 cis \\
\hline chr20_3844000 & X6036000905F1 & \#N/A & 20 cis & 4.68 cis \\
\hline $\begin{array}{l}\text { chr20_7691000 } \\
\text { chr20 } 6156000\end{array}$ & X603595816F1 & $\# N / A$ & 20 cis & 5.03 cis \\
\hline $\begin{array}{l}\text { chr20_6156000 } \\
\text { chr3 } 40372000\end{array}$ & X603595816F1 & $\begin{array}{c}\# N / A \\
\text { \# }\end{array}$ & 20 cis & 5.03 cis \\
\hline $\begin{array}{l}\text { chr3 } 4007322000 \\
\text { chr3 } 4815000\end{array}$ & $\begin{array}{l}\text { ENSGALTO0000002 } \\
\text { ESGITOP }\end{array}$ & 32ENSGALG000000014369 & 3 cis & 5.34 trans \\
\hline $\begin{array}{l}\text { crris3-48155000 } \\
\text { chr3_61873000 }\end{array}$ & ENSGALTO000002 & 25ENSGALG00000019967 & $\begin{array}{l}3 \text { cis } \\
3 \text { cis }\end{array}$ & $\begin{array}{l}4.19 \text { trans } \\
3.87 \text { trans }\end{array}$ \\
\hline chr3_56986000 & ENSGALT0000002 & 41ENSGALG00000014962 & 3 cis & 5.30 tran \\
\hline chr3_106007000 & ENSGALT0000003 & 15ENSGALG000000016707 & 3 cis & 4.04 tran \\
\hline chr4_2703000 & ENSGALTO0000000 & 95ENSGALG000000005964 & 4 cis & 4.34 trans $\mathrm{F}$. \\
\hline $\begin{array}{l}\text { chr4_1363000 } \\
\text { chr4 }\end{array}$ & X603863273F1 & $\# \mathrm{~N} / \mathrm{A}$ & 4 cis & 9.32 trans \\
\hline 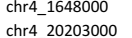 & X603863273F1 & $\begin{array}{c}\text { \#N/A } \\
\text { a }\end{array}$ & 4 cis & 9.32 trans \\
\hline $\begin{array}{l}\text { crrt__20203000 } \\
\text { chr4 } 38027000\end{array}$ & NM__010125/5_- & NENSGALG0000000/4443 & $\begin{array}{ll}4 \text { cis } \\
4 \text { sis }\end{array}$ & 4.81 trans \\
\hline chr4_48772000 & ENSGALTO000001 & 72ENSGALG00000010577 & $\begin{array}{l}4 \text { cis } \\
4 \text { cis }\end{array}$ & $\begin{array}{l}6.43 \text { trans } \\
6.43 \text { trans }\end{array}$ \\
\hline chr4_58146000 & ENSGALT0000002 & 01ENSGALG000000027860 & 4 cis & 4.44 trans \\
\hline chr4_66582000 & X603596285F1 & ENSGALG00000014261 & 4 cis & 5.32 cis \\
\hline chr4_680600000 & X603596285F1 & $\begin{array}{l}\text { ENSGALG00000014261 } \\
\end{array}$ & 4 cis & 5.32 cis \\
\hline $\begin{array}{l}-6 / 7663000 \\
69877000\end{array}$ & $\begin{array}{l}\text { INSGALI0000003 } \\
\text { ENSGALTO000003 }\end{array}$ & 7/EENSGALG00000014281 & $\begin{array}{ll}4 \text { cis } \\
4 \text { sis }\end{array}$ & $\begin{array}{ll}6.87 \mathrm{cs} \\
6.87 \mathrm{cs}\end{array}$ \\
\hline chr4_67663000 & ENSGALTO000003 & 75ENSGALG00000013684 & 4 cis & $\begin{array}{l}\begin{array}{l}.878 \mathrm{cs} \\
4.31 \mathrm{cis}\end{array} \\
\end{array}$ \\
\hline
\end{tabular}

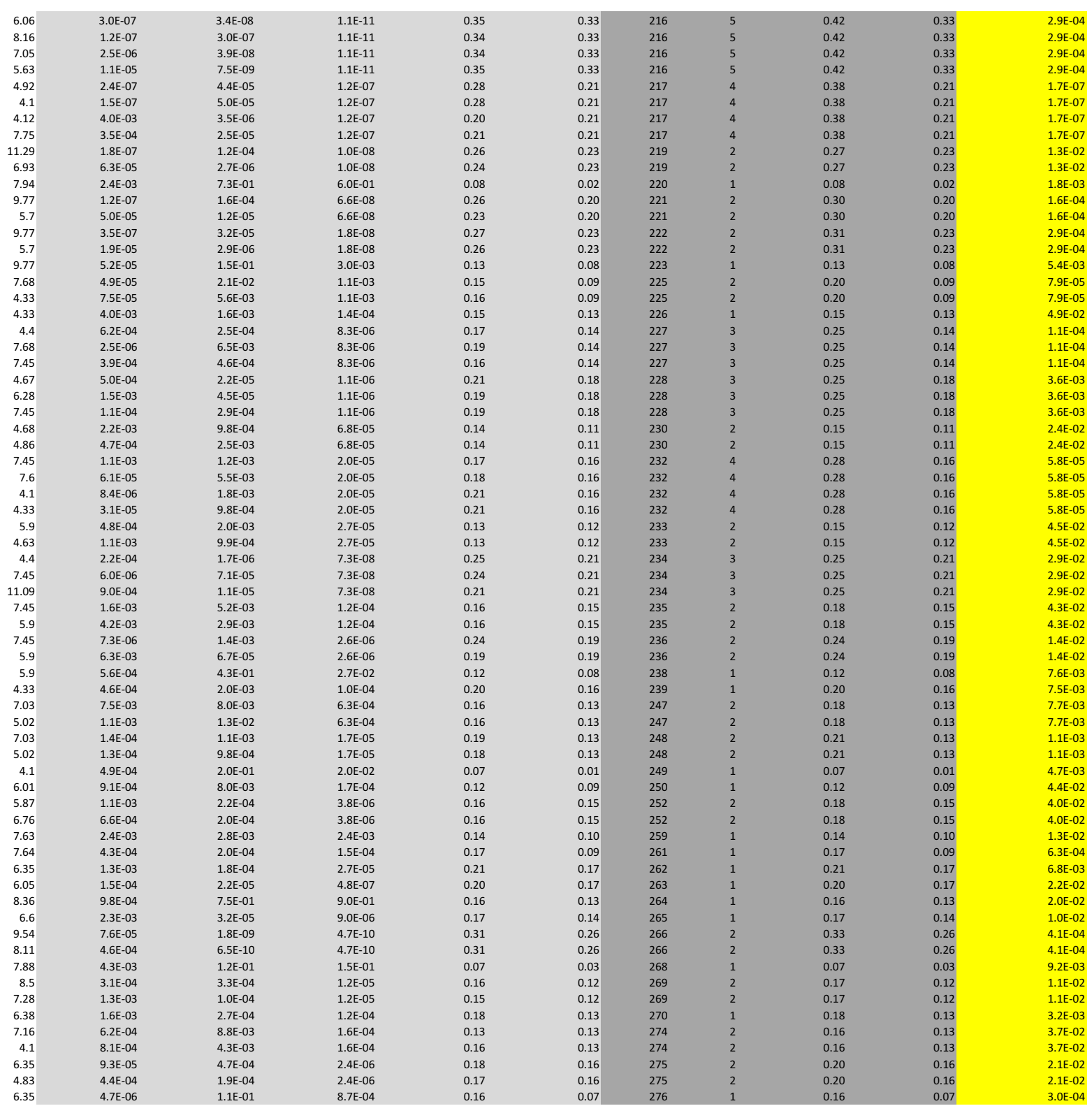




\begin{tabular}{|c|c|c|c|c|}
\hline chr4_67663000 & X603868314f1 & \#N/A & 4 cis & 5.66 cis \\
\hline chr4_68917000 & X603868314F1 & $\# N / A$ & 4 cis & 5.66 cis \\
\hline chr4-71274000 & X6035996244F1 & ENSGALG00000014379 & 4 cis & 5.44 cis \\
\hline chr4_73162000 & X603599417F1 & ENSGALG000000014514 & 4 cis & \\
\hline chr4_-74627000 & X6035994417F1 & ENSGALG00000014514 & 4 cis & 6.69 cis \\
\hline chr4_-784090000 & X603599417F1 & ENSGALG00000014514 & 4 cis & 6.69 cis \\
\hline chr4_74627000 & X603598729F1 & $\# N / A$ & 4 cis & 16.63 cis \\
\hline chr4_78642000 & X603598729F1 & \#N/A & 4 cis & 16.63 cis \\
\hline chr4_75576000 & X603598729F1 & $\# \mathrm{~N} / \mathrm{A}$ & 4 cis & 16.63 cis \\
\hline chr4_75847000 & $\begin{array}{r}\times 603598729 F 1 \\
\end{array}$ & \#N/A & 4 cis & 16.63 cis \\
\hline $\begin{array}{l}\text { chr4-76556000 } \\
\text { chr4 71072000 }\end{array}$ & $\begin{array}{l}\text { X6033998729191 } \\
\text { X60386312F1 }\end{array}$ & 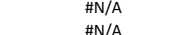 & $\begin{array}{l}4 \text { cis } \\
4 \text { cis }\end{array}$ & $\begin{array}{ll}16.63 \text { cis } \\
7.35 \text { cis }\end{array}$ \\
\hline $\begin{array}{l}\text { chr4-710720000 } \\
\text { chr4 78319000 }\end{array}$ & $\begin{array}{l}\times 603866311221 \\
\times 603863112 F 1\end{array}$ & $\begin{array}{l}\# N / A \\
\text { AN/A } \\
\text { ANA }\end{array}$ & $\begin{array}{l}4 \text { cis } \\
4 \text { cis }\end{array}$ & $\begin{array}{l}7.33 \text { cis } \\
7.35 \text { cis }\end{array}$ \\
\hline 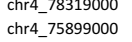 & $\begin{array}{l}X 6003863111212 \\
\times 60386312 F 1\end{array}$ & $\begin{array}{l}\# N / A \\
\# N / A\end{array}$ & $\begin{array}{l}4 \text { cis } \\
4 \text { cis }\end{array}$ & $\begin{array}{l}7.35 \text { cis } \\
7.35 \\
\text { cis }\end{array}$ \\
\hline $\mathrm{chr}_{4}$-78409000 & X603863527F1 & & 4 cis & 5.75 cis \\
\hline chr4_73112000 & \multirow{2}{*}{\multicolumn{2}{|c|}{$\begin{array}{l}\text { ENSGALTO000000242ENSGALG00000015012 } \\
\text { ENSGALTO00000242ENSGALG00000015012 }\end{array}$}} & 4 cis & 4.67 cis \\
\hline chr4_74448000 & & & 4 cis & 4.67 cis \\
\hline chr4_-78836000 & \multicolumn{2}{|c|}{ 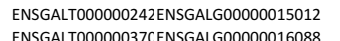 } & 4 cis & 4.67 cis \\
\hline $\begin{array}{l}\text { chra_-78886000 } \\
\text { chr5 } 11870000\end{array}$ & \multirow{2}{*}{\multicolumn{2}{|c|}{$\begin{array}{l}\text { ENSGALT00000037CENSGALG00000016088 } \\
\text { ENSGALTO0000010ENSGALG00000006608 }\end{array}$}} & 4 cis & 4.25 cis \\
\hline $\begin{array}{l}\text { Chr5 } 118700000 \\
\text { chr5 } 13892000\end{array}$ & & & $\begin{array}{l}5 \text { cis } \\
5 \text { cis }\end{array}$ & $\begin{array}{l}4.69 \text { cis } \\
4.69 \text { cis }\end{array}$ \\
\hline chr5_12907000 & \multicolumn{2}{|c|}{ 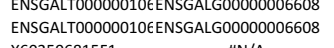 } & $\begin{array}{l}5 \text { cis } \\
5 \text { cis }\end{array}$ & $\begin{array}{l}4.69 \mathrm{cls} \\
4.92 \text { cis }\end{array}$ \\
\hline chrs_24856000 & \multicolumn{2}{|c|}{$\begin{array}{l}\text { X603596815F1 } \\
\text { ENSGALTO0000014CENSGALG00000008598 }\end{array}$} & 5 cis & $\begin{array}{l}4.92 \mathrm{cis} \\
7.26 \mathrm{cis}\end{array}$ \\
\hline chr5_25561000 & \multirow{2}{*}{\multicolumn{2}{|c|}{ ENSGALTOOOO00014CENSGALG000000008598 }} & 5 cis & 7.26 cis \\
\hline chr5_25561000 & & & 5 cis & 5.37 cis \\
\hline chr5_28352000 & \multicolumn{2}{|c|}{$\begin{array}{l}\text { NM_001004405_CAENSGALGG00000009050 } \\
\text { NM_001004405_CAENSGALG00000009050 }\end{array}$} & 5 cis & 5.37 cis \\
\hline chr___28555000 & \multicolumn{2}{|c|}{ NM_001004405_CAENSGALG000000009050 } & 5 cis & 5.37 trans \\
\hline chr5_25561000 & \multirow{2}{*}{\multicolumn{2}{|c|}{ ENSGALTO00000224ENSGALGG00000013838 }} & 5 cis & 8.32 cis \\
\hline chr5_-28555000 & & 24ENSGALG000000013838 & 5 cis & 8.32 trans \\
\hline chr5_-308300000 & $\begin{array}{l}\text { X603601654F1 } \\
\times 6 N / A\end{array}$ & $\# N / A$ & 5 cis & 13.45 cis \\
\hline chr5_29526000 & \multicolumn{2}{|l|}{ 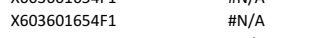 } & 5 cis & 13.45 cis \\
\hline chr5_34365000 & \multirow{2}{*}{\multicolumn{2}{|c|}{$\begin{array}{l}\text { X603601654F1 } \\
\text { X603144380F1 }\end{array}$}} & 5 cis & 13.45 cis \\
\hline chr5_368460000 & & & 5 cis & 4.01 trans $\mathrm{F}$ \\
\hline chr5_34365000 & \multicolumn{2}{|c|}{ X603868077F1 ENSGALG000000099801 } & 5 cis & 4.48 cis \\
\hline chr5_34365000 & \multirow{2}{*}{\multicolumn{2}{|c|}{$\begin{array}{l}\text { NM_001000935_BRENSGALG000000010093 } \\
\text { ENGGATT00000014ENSGALOCO00000089997 }\end{array}$}} & 5 cis & 5.97 cis \\
\hline $\begin{array}{l}\text { chr5_-4133252000 } \\
\text { chr5 } 36451000\end{array}$ & & & $\begin{array}{l}5 \text { trans } \\
5 \text { cis }\end{array}$ & 7.53 cis \\
\hline $\begin{array}{l}\text { chrs_366511000 } \\
\text { chr_41352000 }\end{array}$ & \multicolumn{2}{|c|}{$\begin{array}{ll}\times 6003600251 F 1 & \text { \#N/A } \\
\times 6603600251 F 1 & \text { \#N/A }\end{array}$} & $\begin{array}{l}5 \text { cis } \\
5 \text { cis }\end{array}$ & $\begin{array}{l}6.58 \text { cis } \\
6.58 \text { cis }\end{array}$ \\
\hline chr5_-4435340000 & \multicolumn{2}{|c|}{ X603600251F1 \#N/A } & 5 cis & $\begin{array}{l}6.58 \mathrm{cs} \\
6.58 \text { trans }\end{array}$ \\
\hline chr5_44181000 & ENSGALTO000001: & SEENSGALG00000009740 & 5 cis & 4.73 cis \\
\hline chr5_39597000 & ENSGALTO0000001: & 58ENSGALG0000000009740 & 5 cis & 4.73 cis \\
\hline chr5_41353000 & X603868322F1 & ENSGALG00000010677 & 5 cis & 3.77 cis \\
\hline chr5_44229000 & X603868322F1 & ENSGALG00000010677 & 5 cis & 3.77 cis \\
\hline $\begin{array}{l}\text { crr5_-440511000 } \\
\text { chr5 } 36975000\end{array}$ & 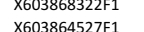 & ENSGALG00000010677 & $\begin{array}{l}5 \text { cis } \\
5 \text { cis }\end{array}$ & 3.77 cis \\
\hline $\begin{array}{l}\text { chrs_366755000 } \\
\text { chr5_39755000 }\end{array}$ & $\begin{array}{l}X 603864652 / F 1 \\
\times 603864527 F 1\end{array}$ & $\begin{array}{l}\# N / A \\
\# N / A\end{array}$ & $\begin{array}{l}5 \text { cis } \\
5 \text { cis }\end{array}$ & 4.29 cis \\
\hline chr5_46129000 & $\begin{array}{l}\mathrm{X} \\
\times 603864527 F 1\end{array}$ & 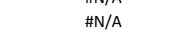 & 5 cis & 4.29 cis \\
\hline chr5_44180000 & X6038672922F1 & \#N/A & 5 cis & 7.50 cis \\
\hline chr5_451190000 & X603867292F1 & \#N/A & 5 cis & 7.50 cis \\
\hline chr5_52660000 & X603863866F1 & ENSGALG000000011855 & 5 cis & 6.48 cis \\
\hline chr5_55896000 & X603863866F1 & ENSGALG000000011855 & 5 cis & 6.48 cis \\
\hline $\begin{array}{l}\text { chr5_5551130000 } \\
\text { chr5 52660000 }\end{array}$ & X603863866F1 & $\begin{array}{l}\text { ENSGALG00000011855 } \\
\end{array}$ & $\begin{array}{l}5 \text { cis } \\
5 \text { cis }\end{array}$ & 6.48 cis \\
\hline chr5_55896000 & ENSGALTOO00001S & 3ENSGALG00000011855 & 5 cis & $\begin{array}{l}4.10 \mathrm{cis} \\
4.10 \text { cis }\end{array}$ \\
\hline chr6_-1862000 & X603598388F1 & ENSGALG00000002432 & 6 cis & 6.62 cis \\
\hline chr6_2217000 & X603598388F1 & ENSGALG000000002432 & 6 cis & 6.62 cis \\
\hline chr6_1862000 & ENSGALTO000000 & 42ENSGALG000000002701 & 6 cis & 6.45 cis \\
\hline chr6_4323000 & X603596512F1 & ENSGALG00000002421 & 6 cis & 5.19 cis \\
\hline chr6_7358000 & ENSGALT00000000: & 38ENSGALG00000002432 & 6 cis & 4.65 cis \\
\hline $\begin{array}{l}\text { chr6_693770000 } \\
\text { chr6 7772000 }\end{array}$ & ENSGALTO00000000 & BEENSGALG000000002432 & $\begin{array}{l}6 \mathrm{cis} \\
6\end{array}$ & 4.65 cis \\
\hline chr6 67230000 & $\begin{array}{l}\text { ENSGALTO0000000 } \\
\text { ENSGIOPO }\end{array}$ & 21ENSGALG000000002710 & 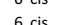 & 5.03 cis \\
\hline chr6_-9214000 & ENSGALTO000000 & H2ENSGALG00000002710 & 6 cis & 5.03 cis \\
\hline chr6__7358000 & X603865854F1 & & 6 cis & 7.57 cis \\
\hline chr6_6932000 & ENSGALTO0000000 & IfENSGALG000000002961 & 6 cis & 4.22 cis \\
\hline chr6_6915000 & ENSGALTT00000 & EENSGALG00000002961 & 6 cis & 4.22 cis \\
\hline chr6_9351000 & & & & \\
\hline
\end{tabular}

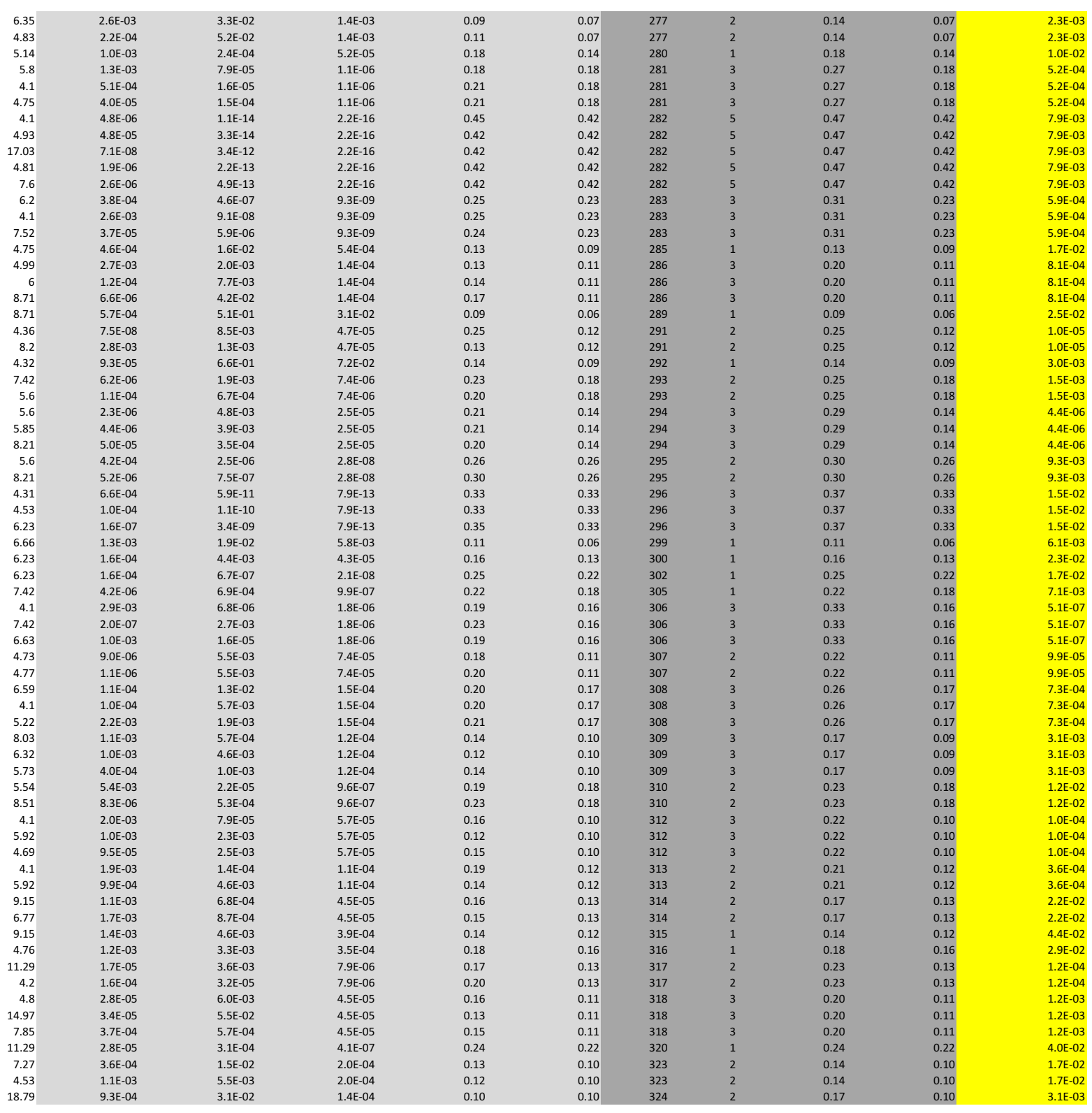




\begin{tabular}{|c|c|c|c|c|}
\hline chr6_87610000 & \multicolumn{2}{|c|}{ ENSGALT0000000047ENSGALG000000002980 } & 6 cis & 3.94 trans 4 - \\
\hline chr6_6933000 & X603868348F1 & ENSGALG00000002967 & 6 cis & 5.69 cis \\
\hline chr6_8902000 & X603868348F1 & ENSGALG00000002967 & 6 cis & 5.69 cis \\
\hline $\begin{array}{l}\text { chr6_10462000 } \\
\text { chr6 } 11766000\end{array}$ & X603868348F1 & ENSGALG00000002967 & 6 cis & 5.69 cis \\
\hline $\begin{array}{l}\text { chr6 } 111766000 \\
\text { chr6 } 11121000\end{array}$ & $\begin{array}{l}\text { X603601830F1 } \\
x_{66038653601}\end{array}$ & $\begin{array}{l}\text { ENSGALG000000003689 } \\
\text { ENGALG00000 }\end{array}$ & $\begin{array}{c}6 \text { cis } \\
6 \text { gis }\end{array}$ & 4.89 cis \\
\hline $\begin{array}{l}\text { chr6 } 1112111000 \\
\text { chr6 } 11079000\end{array}$ & $\begin{array}{l}\times 60038663660 F 1 \\
\times 603865360 F 1\end{array}$ & $\begin{array}{l}\text { ENSGALG6000000003689 } \\
\text { ENSGALG0000003689 }\end{array}$ & $\begin{array}{l}6 \text { cis } \\
6 \text { cis }\end{array}$ & $\begin{array}{l}5.62 \text { cis } \\
5.56 \text { cis }\end{array}$ \\
\hline chr6_10462000 & ENSGALTO000000 & 42ENSGALG00000002689 & 6 cis & 5.29 cis \\
\hline chr6_11069000 & ENSGALTO000000 & 42ENSGALG00000002686 & 6 cis & 5.29 cis \\
\hline chr6_11766000 & ENSGALTO000000 & SEENSGALG00000004167 & 6 cis & 5.89 cis \\
\hline chr6_11112000 & NM_205121_CHS & 3ENSGALG000000024056 & 6 cis & 4.73 cis \\
\hline chr6_11103000 & NM_205121_CHS & 3ENSGALG00000024056 & 6 cis & 4.73 cis \\
\hline $\begin{array}{l}\text { chr6 } 111116000 \\
\text { chr6 } 11783000\end{array}$ & NM_205121_CHS & 3 ENSGALG00000024056 & 6 cis & 4.73 cis \\
\hline $\begin{array}{l}\text { Chr6-_117830000 } \\
\text { chr6_15268000 }\end{array}$ & $\begin{array}{l}\times 60360051111 \\
\times 603605111\end{array}$ & $\begin{array}{l}\text { \#N/A } \\
\text { NN/A }\end{array}$ & $\begin{array}{l}6 \text { cis } \\
6 \text { cis }\end{array}$ & $\begin{array}{l}4.89 \text { cis } \\
4.89 \text { cis }\end{array}$ \\
\hline $\begin{array}{l}\text { Chr6__15688000 } \\
\text { chr6_17952000 }\end{array}$ & $\begin{array}{l}\times 60360051111 \\
\times 603595690 F 1\end{array}$ & $\begin{array}{c}\text { \#NNA } \\
\text { ENSGALG00000004769 }\end{array}$ & $\begin{array}{l}6 \text { cis } \\
6 \text { cis }\end{array}$ & $\begin{array}{l}4.89 \text { cis } \\
4.21 \text { cis }\end{array}$ \\
\hline chr6__4 14000000 & $\begin{array}{l}\mathrm{x} \\
\mathrm{x} 6003866766 \mathrm{~F} 1\end{array}$ & \#N/A & 6 cis & 8.33 cis \\
\hline chr6_15758000 & X603866766F1 & $\# N / A$ & 6 cis & 8.33 cis \\
\hline chr6_17295000 & X603866766FF1 & \#N/A & 6 cis & 8.33 cis \\
\hline chr6_20832000 & ENSGALTO0000004 & DCENSGALG0000000005978 & 6 cis & 4.75 cis \\
\hline $\begin{array}{l}\text { chr6_197566000 } \\
\text { chr6 22270000 }\end{array}$ & ENSGALTO000004 & OCENSGALG00000005978 & 6 cis & 4.75 cis \\
\hline $\begin{array}{l}\text { chr6-222744000 } \\
\text { chr6 22021000 }\end{array}$ & $\begin{array}{l}\text { ENGGALTO0000004 } \\
\text { ENGALTO00001 }\end{array}$ & $\begin{array}{l}\text { CEENGALG000000005978 } \\
\text { CEENGGALG0000027678 }\end{array}$ & $\begin{array}{l}6 \text { cis } \\
6 \text { cis }\end{array}$ & $\begin{array}{l}4.75 \text { cis } \\
5.54 \text { cis }\end{array}$ \\
\hline chr6_16979000 & ENSGALTO000001 & $\begin{array}{l}\text { DEENSGALGG00000027/6 } \\
\text { DEENSGLG0000027678 }\end{array}$ & $\begin{array}{l}6 \text { cis } \\
6 \text { cis }\end{array}$ & $\begin{array}{l}5.94 \text { cis } \\
5.94 \text { cis }\end{array}$ \\
\hline chr6_21638000 & ENSGALTO000001 & DEENSGALG00000027678 & 6 cis & $\begin{array}{l}5.94 \mathrm{cls} \\
5.94 \mathrm{cis}\end{array}$ \\
\hline chr6_17686000 & ENSGALTO000001 & DEENSGALG00000027678 & 6 cis & 5.94 cis \\
\hline chr6_19440000 & ENSGALT00000001 & DEENSGALG000000027678 & 6 cis & 5.94 cis \\
\hline chr6_21457000 & ENSGALTO000001 & DEENSGALG00000027678 & & 5.94 cis \\
\hline chr6_21053000 & ENSGALTO00000001 & JEENGGALG000000027678 & 6 cis & 5.94 cis \\
\hline chr6_19756000 & ENSGALTO0000001 & JEENSGALG000000027678 & 6 cis & 5.94 cis \\
\hline chr6_21462000 & ENSGALTO000001 & DEENSGALG000000027678 & 6 cis & 5.94 cis \\
\hline chr6_22750000 & ENSGALT0000001 & DEENSGALG000000027678 & 6 cis & 5.94 cis \\
\hline chr6_222790000 & ENSGALT0000001 & deENSGALG000000027678 & 6 cis & 5.94 cis \\
\hline chr6_20175000 & ENSGALT00000001 & DEENSGALG000000027678 & 6 cis & 5.94 cis \\
\hline chr6_23563000 & ENSGALT00000001 & DEENSGALG000000027678 & 6 cis & 5.94 cis \\
\hline chr6_18898000 & ENSGALTO0000001 & 13ENSGALG0000000006983 & 6 cis & 5.43 cis \\
\hline chr6_20156000 & ENSGALTO000001 & 13ENSGALG00000006983 & 6 cis & 5.43 cis \\
\hline chrb_23513000 & ENSGALTO000001 & $25 E N S G A L G 00000007715$ & 6 cis & 4.43 trans \\
\hline $\begin{array}{l}\text { Cnro_ } 1 / 29350000 \\
\text { chr6 } 20322000\end{array}$ & $\begin{array}{l}\text { ENGGALTT0000001 } \\
\text { ENSGALTO000001 }\end{array}$ & 25ENESGALGG000000007715 & 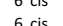 & $\begin{array}{l}4.43 \text { cis } \\
4.43 \text { cis }\end{array}$ \\
\hline chr6_20074000 & ENSGALTO000003 & 32ENSGALG00000020754 & 6 cis & $\begin{array}{l}\begin{array}{l}4.434 \mathrm{cis} \\
4.60 \mathrm{cis}\end{array}\end{array}$ \\
\hline chr6_21476000 & X603599830F1 & $\# N / A$ & 6 cis & 6.44 cis \\
\hline chr6_Z3555000 & X603599830F1 1 & $\# N / A$ & 6 cis & 6.44 cis \\
\hline chr6_23809000 & X6035998830F 1 & $\# N / A$ & 6 cis & 6.44 cis \\
\hline $\begin{array}{l}\text { chr6_22861000 } \\
\text { chr6 22861000 }\end{array}$ & X603599830F1 & & 6 cis & 6.44 cis \\
\hline $\begin{array}{l}\text { chr6-222661000 } \\
\text { chr6 } 26627000\end{array}$ & ENSGALTO000001 & DIENSGALG000000006302 & 6 cis & 6.19 cis \\
\hline $\begin{array}{l}\text { chr6__662677000 } \\
\text { chr6_24515000 }\end{array}$ & $\begin{array}{l}X 603566048881 \\
\times 6035680481\end{array}$ & $\begin{array}{l}\text { ENSGALGG00000027904 } \\
\text { ENSGALG0000027904 }\end{array}$ & $\begin{array}{l}6 \text { cis } \\
6 \text { cis }\end{array}$ & 5.09 trans \\
\hline $\begin{array}{l}\text { chr6_244155000 } \\
\text { chr6-25818000 }\end{array}$ & $\begin{array}{l}\times 603366048811 \\
\times 603568048 F 1\end{array}$ & $\begin{array}{l}\text { ESSGALGG00000297904 } \\
\text { ENSGALG00000027904 }\end{array}$ & $\begin{array}{l}6 \text { cis } \\
6 \text { cis }\end{array}$ & $\begin{array}{l}5.09 \text { cis } \\
5.59 \text { cis }\end{array}$ \\
\hline chr6_24849000 & X603568048F1 & ENSGALG00000027904 & 6 cis & 5.09 cis \\
\hline chr6_26527000 & X6035680048F1 & ENSGALG00000027904 & 6 cis & 5.09 cis \\
\hline chr6_30262000 & X603568048F1 & ENSGALG000000027904 & 6 cis & 5.09 cis \\
\hline chr6_24650000 & X603862885F1 & \#N/A & 6 cis & 4.07 trans \\
\hline $\begin{array}{l}\text { chr6_222444000 } \\
\text { chr6 25453000 }\end{array}$ & X603862885F1 & \#N/A & 6 cis & 4.07 cis \\
\hline $\begin{array}{l}\text { crr6-255453000 } \\
\text { chr6_24542000 }\end{array}$ & $\begin{array}{l}X 603862888551 \\
\text { X6038628851 }\end{array}$ & $\begin{array}{l}\text { AN/A } \\
\text { AN/A }\end{array}$ & 6 cis & $\begin{array}{l}4.07 \text { cis } \\
4.07 \text { cis }\end{array}$ \\
\hline chr6_26502000 & X603601127F1 & 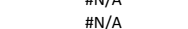 & 6 cis & $\begin{array}{l}4.74 \mathrm{cls} \\
7.25 \mathrm{cs}\end{array}$ \\
\hline chr6_29565000 & X603601127F1 & $\# N / A$ & 6 cis & 7.25 cis \\
\hline chr6_28673000 & X603601127F1 1 & $\# N / A$ & 6 cis & 7.25 cis \\
\hline chr6_26476000 & X603601127F1 & \#N/A & 6 cis & 7.25 cis \\
\hline chr6_26130000 & X6035966222F1 & $\# \mathrm{~N} / \mathrm{A}$ & 6 cis & 4.50 cis \\
\hline $\begin{array}{l}\text { Chr6__248490000 } \\
\text { chr6 } 380872000\end{array}$ & X6033596622F-1 & FN/A & 6 cis & $4.50 \mathrm{cis}$ \\
\hline 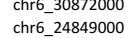 & & 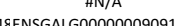 & 6 cis & 4.50 cis \\
\hline $\begin{array}{l}\text { chr6- } 248490000 \\
\text { Chr6 } 28950000\end{array}$ & N & (1) & 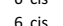 & $\begin{array}{l}5.45 \mathrm{cls} \\
5.45 \mathrm{cs}\end{array}$ \\
\hline chr6__30745000 & ENSGALTO000001 & EENSGALG00000009091 & 6 cis & $\begin{array}{l}5.45 \mathrm{cls} \\
5.45 \mathrm{cis}\end{array}$ \\
\hline & & & & \\
\hline
\end{tabular}

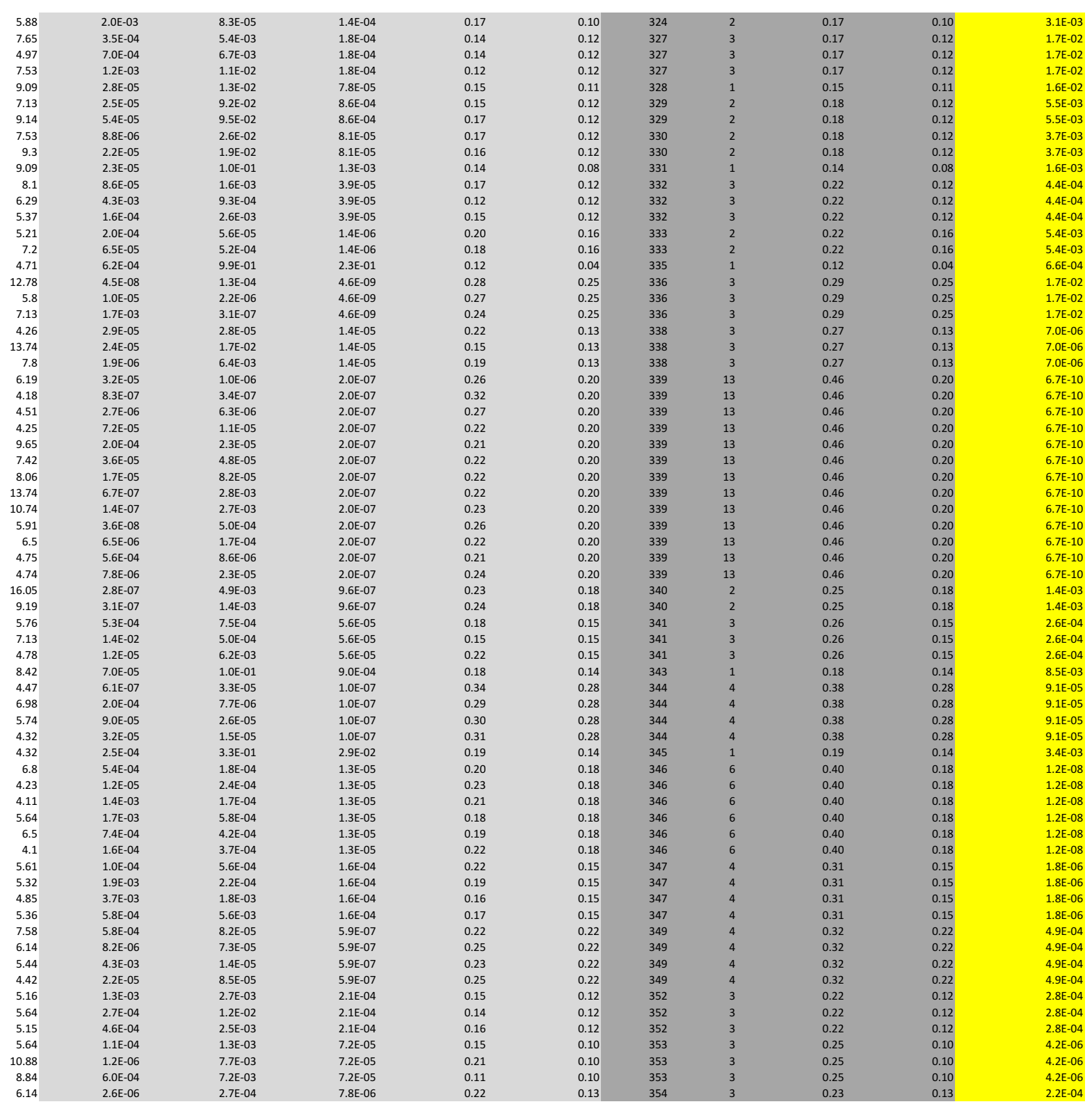




\begin{tabular}{|c|c|c|c|c|}
\hline chr6_26476000 & X603598822F1 & ENSGALG000000009152 & 6 cis & 5.30 cis \\
\hline chr6_30745000 & X603598822F1 & ENSGALG00000009152 & 6 cis & 5.30 cis \\
\hline chr6_29565000 & ENSGALTO000001 & 3ENSGALG000000009427 & 6 cis & 5.06 cis \\
\hline $\begin{array}{l}\text { chr6-30756000 } \\
\text { chr6 29565000 }\end{array}$ & $\begin{array}{l}\text { ESSGALTO0000001 } \\
\text { X603599016F1 }\end{array}$ & $\begin{array}{l}\text { IENSGALGG000000009427 } \\
\text { ENGALG0000009427 }\end{array}$ & $\begin{array}{l}6 \text { cis } \\
6 \text { cis }\end{array}$ & 5.06 cis \\
\hline $\begin{array}{l}\text { crrb_-25350000 } \\
\text { chr6_30872000 }\end{array}$ & $\begin{array}{l}\times 603599016 F 1 \\
\times 603599016 F 1\end{array}$ & $\begin{array}{l}\text { ENSGALG000000004427 } \\
\text { ENSGALO000009927 }\end{array}$ & $\begin{array}{l}6 \text { cis } \\
6 \text { cis }\end{array}$ & $\begin{array}{l}5.55 \text { cis } \\
5.55 \text { cis }\end{array}$ \\
\hline chr6_29565000 & X603863661F1 & $\mathrm{NN/A}$ & 6 cis & 8.30 cis \\
\hline chr6_30756000 & X603863661F1 & $\# N / A$ & 6 cis & 8.30 cis \\
\hline chr6_30745000 & \multirow{2}{*}{\multicolumn{2}{|c|}{$\begin{array}{l}\text { XE0038636661F1 } 1 \text { \#N/A } \\
\text { ENSGLLTO00000152ENSGALG00000009350 }\end{array}$}} & 6 cis & 8.30 cis \\
\hline chr6_29565000 & & & 6 cis & 7.37 cis \\
\hline $\begin{array}{l}\text { chr6_30745000 } \\
\text { chr7 27144000 }\end{array}$ & \multirow{2}{*}{\multicolumn{2}{|c|}{$\begin{array}{l}\text { ENSGALLT000000152ENSGALG00000009350 } \\
\text { ENSGALTO00000037FNSGALG00000028772 }\end{array}$}} & 6 cis & 7.37 cis \\
\hline $\begin{array}{l}\text { chrl_-2174000 } \\
\text { chr7 } 1734000\end{array}$ & & & $\begin{array}{l}7 \mathrm{cis} \\
7 \mathrm{cis}\end{array}$ & 4.90 cis \\
\hline 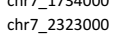 & 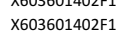 & $\begin{array}{l}\text { ENSGALG000000000509 } \\
\text { ENSGALO0000002509 }\end{array}$ & 7 cis & $\begin{array}{l}4.111 \mathrm{cls} \\
4.11 \text { cis }\end{array}$ \\
\hline chr7_18211000 & $\begin{array}{r}\times 1003862432 F 1 \\
\times 601\end{array}$ & 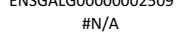 & 7 cis & $\begin{array}{l}6.33 \text { cis } \\
6.115\end{array}$ \\
\hline chr7_25523000 & X603597339F1 & & 7 cis & 5.23 cis \\
\hline chr7_16664000 & х603868270F1 & ENSGALG000000011153 & 7 cis & 6.17 trans \\
\hline chr7_17446000 & \multirow{2}{*}{\multicolumn{2}{|c|}{ 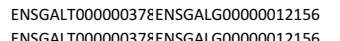 }} & 7 cis & 8.00 cis \\
\hline $\begin{array}{l}\text { chr7_24507000 } \\
\text { cr7 } 27836000\end{array}$ & & & 7 cis & 11.26 cis \\
\hline $\begin{array}{l}\text { chr772 } 283606000 \\
\text { chr7 } 24919000\end{array}$ & \multirow{2}{*}{\multicolumn{2}{|c|}{$\begin{array}{l}\text { ENSGALTO000000378ENSGALG00000012156 } \\
\text { ENSGALTO00000378ENSGALGO0000012156 }\end{array}$}} & $\begin{array}{l}7 \text { cis } \\
7 \text { cis }\end{array}$ & 11.26 cis \\
\hline $\begin{array}{l}\text { chrl_-249190000 } \\
\text { chr___9300000 }\end{array}$ & & & $\begin{array}{l}7 \mathrm{cs} \\
7 \mathrm{cis}\end{array}$ & $\begin{array}{l}11.22 \text { cis } \\
1.26 \text { cis }\end{array}$ \\
\hline chr7_-19437000 & \multicolumn{2}{|c|}{ 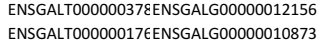 } & 7 cis & 4.82 trans \\
\hline chr7_23558000 & X603599760F1 $\quad$ \#N/A & \#N/A & 7 cis & 10.59 cis \\
\hline chr7_23575000 & X603599760F1 & \#N/A & 7 cis & 10.59 cis \\
\hline chr7_25523000 & \multirow{2}{*}{\multicolumn{2}{|c|}{ 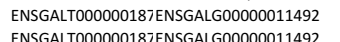 }} & 7 cis & 10.59 cis \\
\hline chr7_22873000 & & & 7 cis & 10.13 cis \\
\hline $\begin{array}{l}\text { chr77 } 24911000 \\
\text { chr7 24562000 }\end{array}$ & \multirow{2}{*}{\multicolumn{2}{|c|}{$\begin{array}{l}\text { ENSGALTO000000187ENSGALG00000011492 } \\
\text { ENSGALTO00000187ENSGALG00000011492 }\end{array}$}} & $\begin{array}{l}7 \text { cis } \\
7 \text { cis }\end{array}$ & $\begin{array}{l}10.13 \text { cis } \\
1013 \text { cis }\end{array}$ \\
\hline $\begin{array}{l}\text { Chrl_-24562000 } \\
\text { chr__24354000 }\end{array}$ & & & $\begin{array}{l}7 \text { cis } \\
7 \text { cis }\end{array}$ & $\begin{array}{l}10.13 \text { cis } \\
10.13 \text { cis }\end{array}$ \\
\hline chr7_24204000 & & 7 cis & 10.13 cis \\
\hline chr7_24601000 & \multicolumn{2}{|c|}{$\begin{array}{l}\text { ENSGALTO000000187ENSGALG00000011492 } \\
\text { ENSGALTO00000187ENSGALG00000011492 }\end{array}$} & 7 cis & 10.13 cis \\
\hline chr7_23679000 & \multicolumn{2}{|c|}{ ENSGALTOO000001878ENSGALG000000011492 } & 7 cis & 10.13 cis \\
\hline chr7_243997000 & \multicolumn{2}{|c|}{ 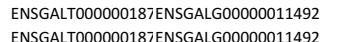 } & 7 cis & 10.13 cis \\
\hline chr7_24425000 & \multirow{2}{*}{\multicolumn{2}{|c|}{$\begin{array}{l}\text { ENSGALTO000000187ENSGALG00000011492 } \\
\text { ENSGALTO00000187ENSGALGO0000011492 }\end{array}$}} & 7 cis & 10.13 cis \\
\hline chr7_236190000 & & & $\begin{array}{l}7 \text { cis } \\
7 \text { cis }\end{array}$ & 10.13 cis \\
\hline $\begin{array}{l}\text { chrl_221995000 } \\
\text { chr_24911000 }\end{array}$ & \multicolumn{2}{|c|}{$\begin{array}{l}\text { ENSGALTO00000187ENSGALG00000011492 } \\
\text { ENSGALTO00000187ENSGALG00000011492 }\end{array}$} & $\begin{array}{l}7 \mathrm{cis} \\
7 \mathrm{cis}\end{array}$ & $\begin{array}{l}10.13 \text { cis } \\
5.07 \text { cis }\end{array}$ \\
\hline chr__24923000 & \multicolumn{2}{|r|}{$\begin{array}{l}\text { ENSGALG600000011493 } \\
\text { ENSGALG00000011493 }\end{array}$} & 7 cis & $\begin{array}{l}5.07 \text { cis } \\
5.07\end{array}$ \\
\hline chr7_23876000 & \multicolumn{2}{|r|}{ ENSGALG000000011493 } & 7 cis & 5.07 cis \\
\hline chr7_236300000 & \multirow{2}{*}{\multicolumn{2}{|c|}{ 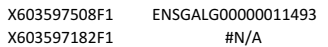 }} & 7 cis & 5.07 cis \\
\hline chr7_24424000 & & \#N/A & 7 cis & 5.61 cis \\
\hline chr7_25151000 & $\begin{array}{l}\text { X6035971822F1 } \\
\text { X653591821 }\end{array}$ & $\begin{array}{ll}\mathrm{HN} / \mathrm{A} \\
\mathrm{HN} / \mathrm{A}\end{array}$ & 7 cis & 5.61 cis \\
\hline chr7_25196000 & 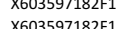 & FN/A & 7 cis & 5.61 cis \\
\hline $\begin{array}{l}\text { chrl_2 225523000 } \\
\text { chr__2674000 }\end{array}$ & $\begin{array}{l}X 6035597182 F 1 \\
X 603597182 F 1\end{array}$ & $\begin{array}{l}\# N / A \\
\# N / A\end{array}$ & $\begin{array}{l}7 \mathrm{cis} \\
7 \mathrm{cis}\end{array}$ & $\begin{array}{l}5.61 \mathrm{cls} \\
5.61 \mathrm{sis}\end{array}$ \\
\hline 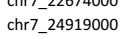 & 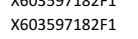 & $\begin{array}{l}\mathrm{H} \\
\mathrm{HNN} / \mathrm{A}\end{array}$ & 7 cis & $\begin{array}{l}5.61 \text { cis } \\
5.61\end{array}$ \\
\hline chr7_23558000 & X603597890F1 & \#N/A & 7 cis & 6.16 cis \\
\hline chr7_24911000 & X6035978900F1 & \#N/A & 7 cis & 6.16 cis \\
\hline chr7_243500000 & X6035978900F1 & \#N/A & 7 cis & 6.16 cis \\
\hline chr7_243540000 & $\begin{array}{l}\text { X603597890F1 } \\
\text { X6635978901 }\end{array}$ & \#N/A & 7 cis & 6.16 cis \\
\hline $\begin{array}{l}\text { chr7_23596000 } \\
\text { chr729100000 }\end{array}$ & $\begin{array}{l}\text { X6035978900F1 } \\
\text { ENSGATO000003 }\end{array}$ & $\begin{array}{c}\text { \#N/A } \\
\text { a }\end{array}$ & $\begin{array}{l}7 \text { cis } \\
7 \text { cis }\end{array}$ & 6.16 cis \\
\hline chr7_28599000 & ENSGALTO000003 & SENSGALGO0000012113 & 7 cis & $\begin{array}{l}0.39 \mathrm{cis} \\
6.39 \text { cis }\end{array}$ \\
\hline chr7_28193000 & X603603407F1 & \#N/A & 7 cis & 6.25 cis \\
\hline chr7_273551000 & X6003603407F1 & \#N/A & 7 cis & 6.25 cis \\
\hline chr7_28972000 & X6003603407F1 1 & $\# N / A$ & 7 cis & 6.25 cis \\
\hline chr7_28068000 & X6038864595F1 & ENSGALG600000011708 & 7 cis & 5.61 cis \\
\hline chr7_27345000 & X6038645955F1 & ENSGALG6000000011708 & 7 cis & 5.61 cis \\
\hline chr7_27836000 & X603864595F1 & ENSGALG000000011708 & $7 \mathrm{cis}$ & 5.61 cis \\
\hline chr7_27493000 & $\begin{array}{l}\text { ENGALTO0000003 } \\
\text { ENSGALTO000003 }\end{array}$ & SENSGALGO0000011708 & $7 \mathrm{cis}$ & $\begin{array}{l}5.72 \text { cis } \\
5.72 \text { cis }\end{array}$ \\
\hline chr7_24507000 & X603867770F1 & \#N/A & 7 cis & 4.81 cis \\
\hline chr7_246090000 & X603867770F1 & \#N/A & 7 cis & 4.81 cis \\
\hline chr7_27493000 & X603867770F1 & \#N/A & 7 cis & 4.81 cis \\
\hline chr7_27836000 & X603867770F1 & & 7 cis & 4.81 cis \\
\hline & & & & \\
\hline
\end{tabular}

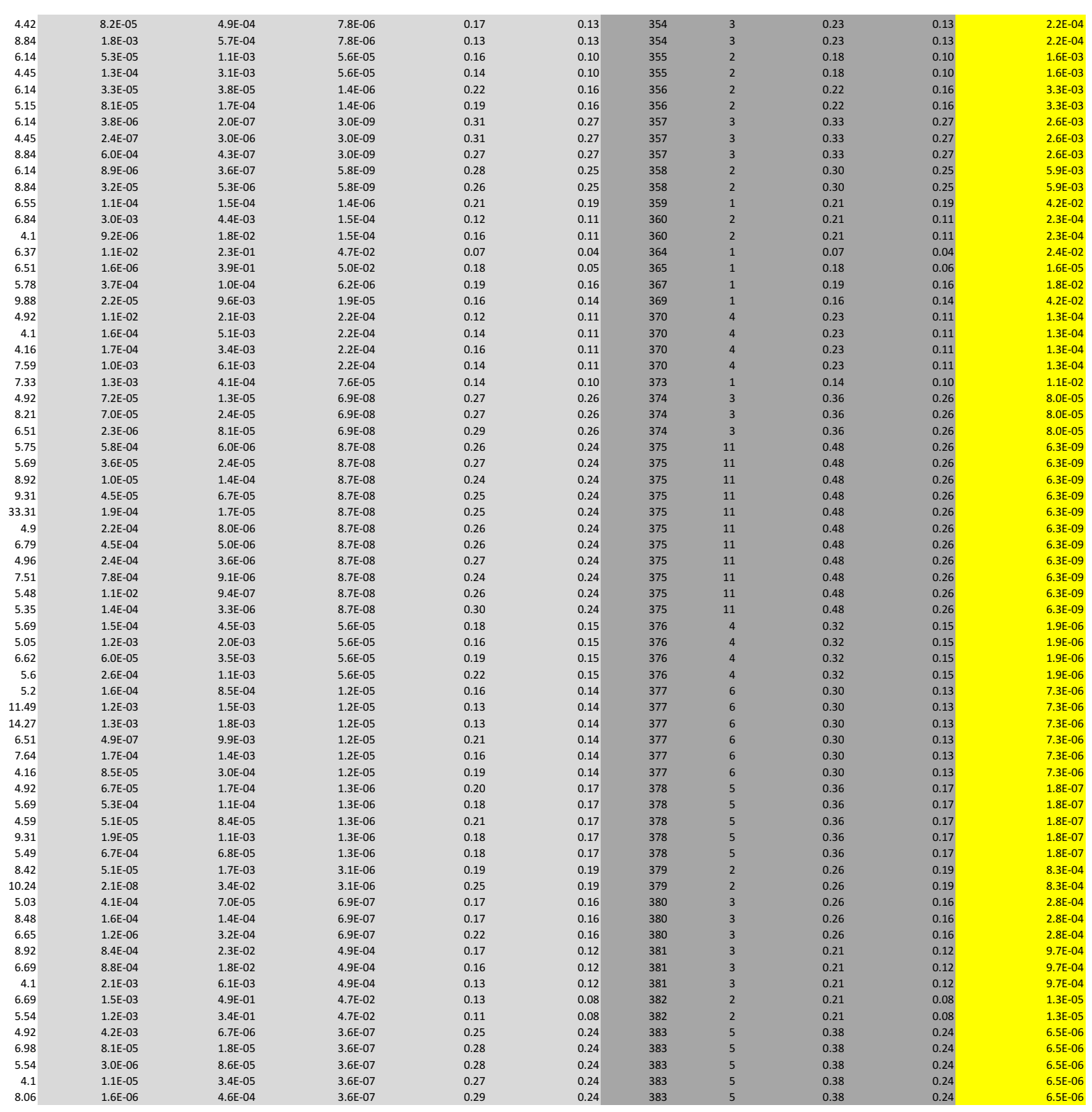




\begin{tabular}{|c|c|c|c|c|}
\hline chr7 28273000 & \multirow{2}{*}{\multicolumn{2}{|c|}{ ENSGALTT000000038ENSGALG000000002417 }} & 7 cis & \\
\hline chr7730201000 & & & 7 cis & 6.51 cis \\
\hline chr7_28221000 & \multicolumn{2}{|c|}{$\begin{array}{l}\text { ENSGALTO000000198ENSGALG00000012147 } \\
\text { ENSGALTO00000198ENSGALG00000012147 }\end{array}$} & 7 cis & 6.51 cis \\
\hline chr7_27845000 & \multicolumn{2}{|c|}{ ENSGALTOO00000198ENSGALG000000012147 } & 7 cis & 6.51 cis \\
\hline chr7730433000 & \multicolumn{2}{|c|}{ ENSGALTOO00000198ENSGALGO00000012147 } & 7 cis & 6.51 cis \\
\hline chr7_32857000 & \multirow{2}{*}{\multicolumn{2}{|c|}{$\begin{array}{l}\text { ENSGALTO000000198ENSGALG00000012147 } \\
\text { ENSGALLOO00000377ENSGALGO0000012511 }\end{array}$}} & 7 cis & 6.51 cis \\
\hline chr7_31953000 & & & 7 cis & 4.06 trans \\
\hline chr8_8084000 & & & 8 cis & 4.59 cis \\
\hline chr8_13668000 & X603141831F1 & $\# N / A$ & 8 cis & 4.59 cis \\
\hline chr8_14448000 & X603141831F1 & \#N/A & 8 cis & $\begin{array}{r}4.59 \mathrm{cis} \\
0.520\end{array}$ \\
\hline $\begin{array}{l}\text { chr8_80840000 } \\
\text { chr8 } 1325000\end{array}$ & $\begin{array}{l}\text { X603141830F1 } \\
\text { X603141830F1 }\end{array}$ & 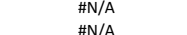 & $\begin{array}{l}8 \text { cis } \\
8 \text { cis }\end{array}$ & 15.28 cis \\
\hline $\begin{array}{l}\text { chrb_ } 113255000 \\
\text { chr8 } 11391000\end{array}$ & $\begin{array}{l}\text { X6031414830F1 } \\
\text { X6031430F1 }\end{array}$ & $\begin{array}{l}\mathrm{A} \text { \#N/A } \\
\text { \#N/A }\end{array}$ & $\begin{array}{l}8 \mathrm{cis} \\
8 \mathrm{cis}\end{array}$ & $\begin{array}{l}15.22 \text { cis } \\
1528 \text { cis }\end{array}$ \\
\hline $\begin{array}{l}\text { chro_-11391000 } \\
\text { chr__11322000 }\end{array}$ & 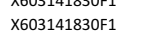 & 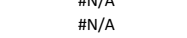 & $\begin{array}{l}8 \mathrm{cis} \\
8 \mathrm{cis}\end{array}$ & $\begin{array}{l}11.28 \mathrm{cis} \\
15.28 \mathrm{cis}\end{array}$ \\
\hline chr8_11329000 & X603141830F1 & $\# N / A$ & 8 cis & 15.28 cis \\
\hline chr8_8113000 & X603141830F1 & $\# \mathrm{~N} / \mathrm{A}$ & 8 cis & 15.28 cis \\
\hline chr8_10233000 & X603141830F1 & \#N/A & 8 cis & 15.28 cis \\
\hline chr8_14448000 & X603141830F1 & \#N/A & 8 cis & 15.28 cis \\
\hline $\begin{array}{l}\text { chr8_14652000 } \\
\text { chr8 } 8362000\end{array}$ & X603141830F1 & $\begin{array}{l}\text { \#N/A } \\
\text { ENSGA } \\
\text { EN6000005203 }\end{array}$ & 8 cis & 15.28 cis \\
\hline $\begin{array}{l}\text { chrb_88362000 } \\
\text { chr8 } 8792000\end{array}$ & $\begin{array}{l}\text { X603868076F1 } \\
\times 663860761\end{array}$ & $\begin{array}{l}\text { ENSGAAG6000000005203 } \\
\text { ENSGGLG600000005203 }\end{array}$ & 8 cis & $\begin{array}{l}5.56 \text { cis } \\
5.56 \text { cis }\end{array}$ \\
\hline $\begin{array}{l}\text { chrb_8 } 89202000 \\
\text { chr_15897000 }\end{array}$ & $\begin{array}{l}\text { X6038668076F1 } \\
\times 6038600651\end{array}$ & $\begin{array}{l}\text { ENSGAAGG000000000253 } \\
\text { ENSGALG00000005203 }\end{array}$ & $\begin{array}{l}8 \mathrm{cls} \\
8 \mathrm{cis}\end{array}$ & $\begin{array}{l}5.56 \text { cis } \\
5.56 \text { cis }\end{array}$ \\
\hline chr8_8151000 & ENSGALTO0000008C & EENSGALGO00000005571 & 8 cis & 4.24 cis \\
\hline chr8_12885000 & ENSGALT00000008s & EENSGALG00000005571 & 8 cis & 4.24 cis \\
\hline chr8_14118000 & ENSGALT00000008s & CENSGALG00000005571 & 8 cis & 4.24 cis \\
\hline chr8_14448000 & ENSGALT00000009 & AENSGALG00000005889 & 8 cis & 4.77 cis \\
\hline chr8_13193000 & х603866233F1 & \#N/A & 8 cis & 5.05 cis \\
\hline $\begin{array}{l}\text { Chr8_ } 14448000 \\
\text { chr8 } 16197000\end{array}$ & $\begin{array}{l}\text { X603866233F1 } \\
\times 630359914 F 1\end{array}$ & $\begin{array}{l}\mathrm{A} N \mathrm{~N} / \mathrm{A} \\
\mathrm{HN} / \mathrm{A}\end{array}$ & $\begin{array}{l}8 \text { cis } \\
8 \text { cis }\end{array}$ & $\begin{array}{l}5.05 \text { cis } \\
4.67 \text { cis }\end{array}$ \\
\hline $\begin{array}{l}\text { chr___16197000 } \\
\text { chr__9532000 }\end{array}$ & 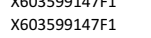 & 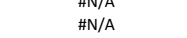 & 8 cis & $\begin{array}{l}4.67 \mathrm{cls} \\
4.67 \text { cis }\end{array}$ \\
\hline chr8_18836000 & X603599147F1 & $\# N / A$ & $8 \mathrm{cis}$ & 4.67 cis \\
\hline chr8_19538000 & ENSGALT000000168 & EENSGALG00000010360 & 8 cis & 3.51 cis \\
\hline chr8_7628000 & X603599201F1 & ENSGALG00000009988 & 8 cis & 6.11 cis \\
\hline chr8_18840000 & X603599201F1 & ENSGALG000000009988 & 8 cis & 6.11 cis \\
\hline chr8_19514000 & Х603599396F1 & ENSGALG00000028423 & 8 cis & 7.27 cis \\
\hline $\begin{array}{l}\text { chr8_18836000 } \\
\text { chr8 } 8467000\end{array}$ & $\begin{array}{l}\text { X603599396F1 } \\
\text { NM 204481 HS2ST }\end{array}$ & $\begin{array}{l}\text { ENSGALG000000028423 } \\
\text { ENSGALG Ge000028423 }\end{array}$ & $\begin{array}{l}8 \text { cis } \\
8 \text { cis }\end{array}$ & $\begin{array}{l}7.27 \text { cis } \\
5.96 \text { cis }\end{array}$ \\
\hline $\begin{array}{l}\text { chrb_84646000 } \\
\text { chr_12885000 }\end{array}$ & $\begin{array}{l}\text { NM_ 204481_HSSST } \\
\text { NM 204481 HS2ST }\end{array}$ & $\begin{array}{l}\text { ENSGAGG00000028423 } \\
\text { ENSGALGO000028423 }\end{array}$ & $\begin{array}{l}8 \text { cis } \\
8 \text { cis }\end{array}$ & $\begin{array}{l}5.96 \text { cis } \\
5.96 \text { cis }\end{array}$ \\
\hline chr___1883039000 & NM_204481_HS2ST & ENSGALGO00000028423 & $\begin{array}{l}8 \mathrm{cis} \\
8 \mathrm{cis}\end{array}$ & $\begin{array}{l}5.96 \mathrm{cis} \\
5.96\end{array}$ \\
\hline chr9_2853000 & NM_001168007_CE & ENSGALG00000006632 & 9 cis & 4.53 cis \\
\hline chr9_8373000 & X603598638F1 & $\# N / A$ & 9 cis & 6.96 cis \\
\hline chrr9-8745000 & 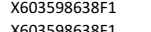 & \#N/A & 9 cis & 6.96 cis \\
\hline chr9,7638000 & X603598638F1 & $\# \mathrm{~N} / \mathrm{A}$ & 9 cis & 6.96 cis \\
\hline chr9-6661000 & $\begin{array}{l}X 603599683881 \\
\times 603596381\end{array}$ & $\begin{array}{l}A N / A \\
\# N / A\end{array}$ & $\begin{array}{l}9 \text { cis } \\
9 \text { cis }\end{array}$ & 6.96 cis \\
\hline $\begin{array}{l}\text { chrr91901010000 } \\
\text { chr97569000 }\end{array}$ & $\begin{array}{l}\text { X6035986638F1 } \\
\text { X603144142F1 }\end{array}$ & $\begin{array}{l}\text { AN/A } \\
\text { ENSGGL00000006298 }\end{array}$ & $\begin{array}{l}9 \text { cis } \\
9 \text { cis }\end{array}$ & $\begin{array}{l}6.96 \text { cis } \\
4.62 \text { cis }\end{array}$ \\
\hline $\begin{array}{l}\text { crrig-7569000 } \\
\text { chr9_7638000 }\end{array}$ & $\begin{array}{l}X 603144142 F 1 \\
\times 603144142 F 1\end{array}$ & ENSGALG000000006298 & $\begin{array}{l}9 \mathrm{cls} \\
9 \text { cis }\end{array}$ & $\begin{array}{l}4.62 \text { cis } \\
4.62 \text { cis }\end{array}$ \\
\hline chr9_8351000 & ENSGALT000000337 & JENSGALG00000020986 & 9 cis & 4.10 cis \\
\hline chr9__5516000 & ENSGALTT000000337 & TENSGALG000000020986 & 9 cis & 4.10 cis \\
\hline chr9_7446000 & ENSGALTO000000088 & EENSGALG00000005511 & 9 cis & 4.32 cis \\
\hline chr9_11848000 & ENSGALT000000088 & EENSGALG00000005511 & 9 cis & 4.32 cis \\
\hline chr9_87450000 & $\begin{array}{l}\mathrm{X} 603596668551 \\
\mathrm{X} 0635966851\end{array}$ & 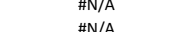 & $\begin{array}{l}9 \text { cis } \\
9 \text { cis }\end{array}$ & $\begin{array}{l}16.03 \text { cis } \\
10.03 \text { cis }\end{array}$ \\
\hline $\begin{array}{l}\text { chrig-10650000 } \\
\text { chr9 } 12573000\end{array}$ & $\begin{array}{l}\text { X60355666885F-1 } \\
\times 603596685 F 1\end{array}$ & $\begin{array}{l}\mathrm{AN} / \mathrm{A} \\
\# \mathrm{~N} / \mathrm{A}\end{array}$ & $\begin{array}{l}9 \text { cis } \\
9 \text { cis }\end{array}$ & $\begin{array}{ll}16.03 \text { cis } \\
1653 \text { cis }\end{array}$ \\
\hline chrg_9199000 & X603596685F1 & \#N/A & 9 cis & 16.03 cis \\
\hline chrg_100100000 & X603596685F1 & $\# N / A$ & 9 cis & 16.03 cis \\
\hline chrg_10814000 & X6035966855F1 & \#N/A & 9 cis & 16.03 cis \\
\hline chr9_10225000 & Х603596685F1 & \#N/A & 9 cis & 16.03 cis \\
\hline chr9_13149000 & X6035966685F1 & NN/A & 9 cis & 16.03 cis \\
\hline chr9_88373000 & 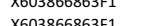 & $\begin{array}{l}\text { ENSGALG6000000005367 } \\
\text { ENSGGANG000005367 }\end{array}$ & 9 cis & 4.89 cis \\
\hline chr9_7356000 & 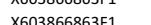 & 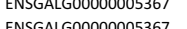 & 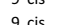 & $\begin{array}{l}4.89 \text { cis } \\
4.89 \text { cis }\end{array}$ \\
\hline $\begin{array}{l}\text { Crr9,125770000 } \\
\text { chrg_10010000 }\end{array}$ & ENSGALTO00000004] & 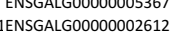 & 9 cis & $\begin{array}{l}4.89 \text { cis } \\
5.34 \text { cis }\end{array}$ \\
\hline chrg_9230000 & ENSGALTO0000004] & AENSGALG000000002612 & 9 cis & 5.34 cis \\
\hline chr9-7093000 & X603144063F1 & ENSGALG00000005809 & 9 cis & 4.27 cis \\
\hline chr9_7344000 & X603144063F1 & ENSGALG0000000058099 & 9 cis & 4.27 cis \\
\hline chr9_87450000 & & & & \\
\hline
\end{tabular}

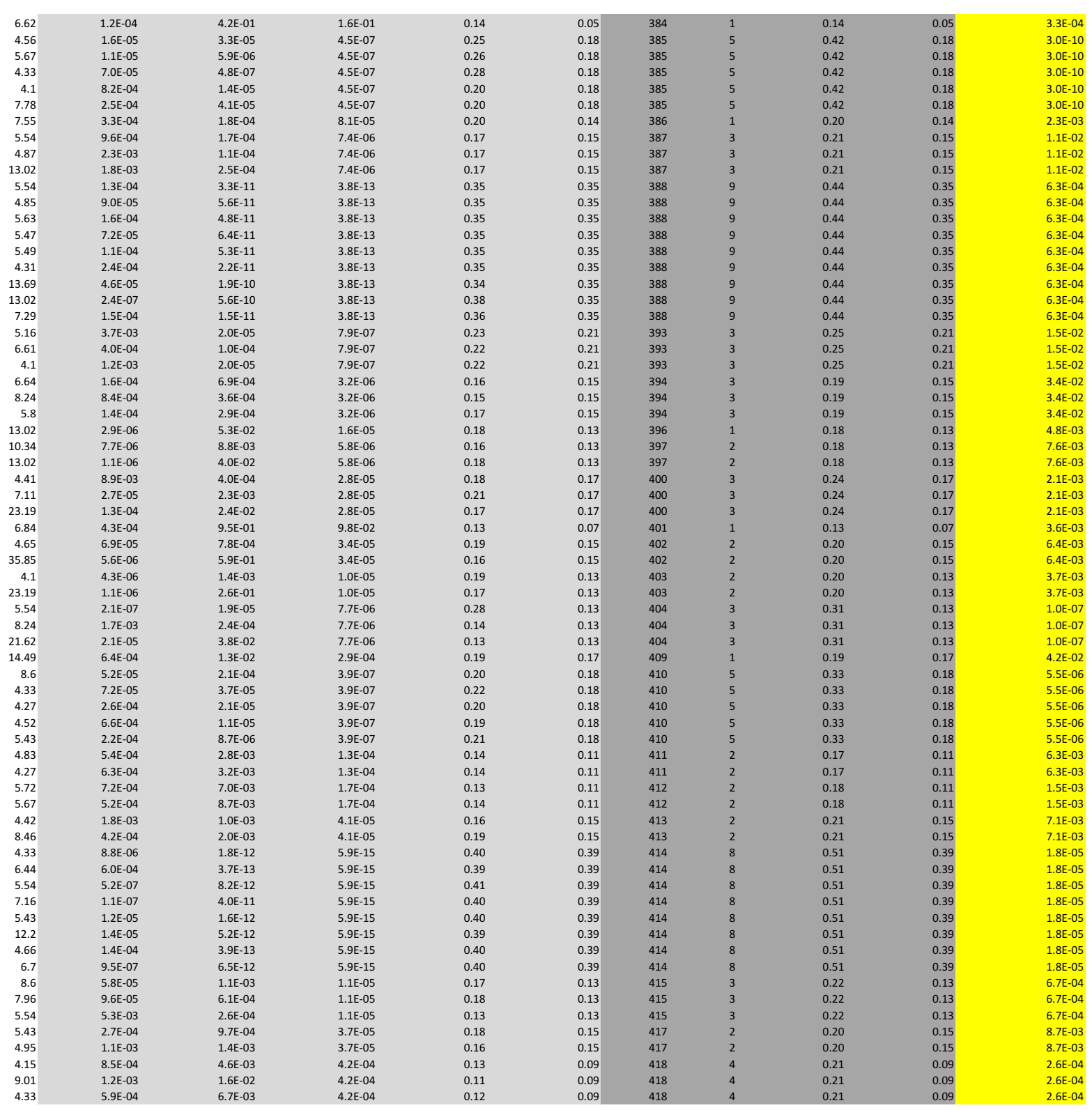




\begin{tabular}{|c|c|c|}
\hline & & \\
\hline chr9-6443000 & X603144063F1 & ENSGALG000000005809 \\
\hline $\begin{array}{l}\text { chr9__10314000 } \\
\text { chro } 100100000\end{array}$ & NM_001006547_ & DENSGAL6000000006947 \\
\hline $\begin{array}{l}\text { chrr9_101010000 } \\
\text { chrg } 131490000\end{array}$ & $\begin{array}{l}\text { NM_001006557_ } \\
\text { NM 001006547 }\end{array}$ & IDENSGAL 600000000069474 \\
\hline 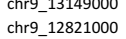 & $\begin{array}{l}\text { NM_001006557_ } \\
\text { ENGGALTO000001 }\end{array}$ & 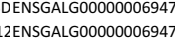 \\
\hline chrg_8881000 & ENSGALTOOOOOO1 & $12 \mathrm{ESSALG600000006947}$ \\
\hline chr9_9031000 & X603577149F1 & $\# N / A$ \\
\hline chr9_12821000 & X603577149F1 & \#N/A \\
\hline chr9_9031000 & X603867866F1 & \#N/A \\
\hline chrr9_12821000 & X603867866F1 & \#N/A \\
\hline $\begin{array}{l}\text { chrr9_1010100000 } \\
\text { chr9 } \\
134300000\end{array}$ & $\begin{array}{l}\text { ENSGALTO0000001 } \\
\text { ENGALTOP0001 }\end{array}$ & ICENSGALG000000006843 \\
\hline 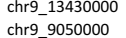 & $\begin{array}{l}\text { ENSGALTOT0000001 } \\
\text { NM_001039309 }\end{array}$ & 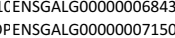 \\
\hline chr9__12858000 & NM_001039309_- & PENSGALG00000007150 \\
\hline chr9_12573000 & X603868153F1 & \#N/A \\
\hline chr9_13429000 & X603868153F1 & \#N/A \\
\hline chrr9_12573000 & X603866213F1 & ENSGALG000000007150 \\
\hline chrr9_13426000 & $\begin{array}{l}\text { X603866213F1 } \\
\text { F }\end{array}$ & ENSGALG000000007150 \\
\hline chrr9_12573000 & ENSGALTO000001 & \\
\hline 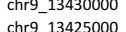 & $\begin{array}{l}\text { ENSGALTO000001 } \\
\text { ESSGT }\end{array}$ & 17 17NSGALGO000000269077 \\
\hline 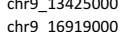 & $\begin{array}{l}\text { ENGGALTT000000 } \\
\text { X603863036F1 }\end{array}$ & ENSGALG000000007253 \\
\hline chri__15125000 & NM_204706_BOK & ENSGALG00000005772 \\
\hline Chr9_12573000 & NM_001006551_ & nENSGAL6000000008785 \\
\hline
\end{tabular}

$\begin{array}{ll}9 \text { cis } & 4.27 \text { cis } \\ 9 \text { cis } & 6.21 \text { cis } \\ 9 \text { cis } & 6.21 \text { cis } \\ 9 \text { cis } & 6.21 \text { cis } \\ 9 \text { cis } & 5.67 \text { cis } \\ 9 \text { cis } & 5.67 \text { cis } \\ 9 \text { cis } & 4.38 \text { cis } \\ 9 \text { cis } & 4.38 \text { cis } \\ 9 \text { cis } & 5.67 \text { cis } \\ 9 \text { cis } & 5.67 \text { cis } \\ 9 \text { cis } & 5.16 \text { cis } \\ 9 \text { cis } & 5.16 \text { cis } \\ 9 \text { cis } & 5.10 \text { cis } \\ 9 \text { cis } & 5.10 \text { cis } \\ 9 \text { cis } & 4.90 \text { cis } \\ 9 \text { cis } & 4.90 \text { cis } \\ 9 \text { cis } & 4.28 \text { cis } \\ 9 \text { cis } & 4.28 \text { cis } \\ 9 \text { cis } & 6.98 \text { cis } \\ 9 \text { cis } & 6.98 \text { cis } \\ 9 \text { cis } & 5.03 \text { cis } \\ 9 \text { cis } & 4.08 \text { cis } \\ 9 \text { cis } & 4.88 \text { trans } \\ 9 \text { cis } & 4.50 \text { cis }\end{array}$

\begin{tabular}{|c|c|}
\hline 6.05 & 7.8E-04 \\
\hline $\begin{array}{l}8.28 \\
5.43\end{array}$ & $2.0 E-03$ \\
\hline $\begin{array}{l}5.533 \\
6.7\end{array}$ & $\begin{array}{l}4.1 .1 E-04 \\
2.6 E-03\end{array}$ \\
\hline 5.83 & $1.2 \mathrm{E}-03$ \\
\hline 4.65 & 7.11E-04 \\
\hline 9.96 & $3.3 \mathrm{E}-03$ \\
\hline 5.83 & $6.4 \mathrm{E}-0$ \\
\hline 9.96 & $6.6 \mathrm{E}-04$ \\
\hline 5.83 & $\begin{array}{l}9.7 E-06 \\
\end{array}$ \\
\hline $\begin{array}{l}5.43 \\
.20 .2\end{array}$ & $\begin{array}{l}4.3 E-05 \\
0\end{array}$ \\
\hline 5.68 & $\begin{array}{l}3.8 E-05 \\
3.0 E-04\end{array}$ \\
\hline 4.94 & $\begin{array}{l}3.0 E-04 \\
9.1 .1-04\end{array}$ \\
\hline 5.54 & $\begin{array}{l}9.1 .1-2 E \\
3.2 E-05\end{array}$ \\
\hline 12.09 & $2.7 \mathrm{E}-03$ \\
\hline 5.54 & $4.5 E-04$ \\
\hline $\begin{array}{ll}15.8 \\
5.54\end{array}$ & $5.3 E-07$ \\
\hline $\begin{array}{l}5.54 \\
.20 .2\end{array}$ & $\begin{array}{l}1.1 E-06 \\
\text { SEE }\end{array}$ \\
\hline $\begin{array}{l}20.2 \\
4.65\end{array}$ & $\begin{array}{l}9.5 E-04 \\
4.6 E-04\end{array}$ \\
\hline 4.65 & $\begin{array}{l}4.6 E-08 \\
4\end{array}$ \\
\hline 5.61 & $\begin{array}{l}4.9-04 \\
6.2 \mathrm{E}-04\end{array}$ \\
\hline .54 & $2.8 \mathrm{E}-03$ \\
\hline
\end{tabular}

\begin{tabular}{|c|c|}
\hline $8.3 E-03$ & $4.2 \mathrm{E}-04$ \\
\hline $\begin{array}{l}9.2 E-05 \\
5.05\end{array}$ & 1.9E-06 \\
\hline $\begin{array}{l}5.8 \mathrm{E}-05 \\
4.8 \mathrm{E}-0\end{array}$ & $\begin{array}{l}1.9 \mathrm{E}-06 \\
1 \mathrm{~F}-06\end{array}$ \\
\hline $\begin{array}{l}4.8 \mathrm{E}-05 \\
1.6 \mathrm{-04}\end{array}$ & $\begin{array}{l}1.9 \mathrm{E}-06 \\
3.3 \mathrm{E}-06\end{array}$ \\
\hline $1.7 \mathrm{E}-04$ & 3.3E-06 \\
\hline $2.7 \mathrm{E}-03$ & $7.3 \mathrm{E}-0$. \\
\hline $6.4 \mathrm{E}-03$ & $7.3 \mathrm{E}-05$ \\
\hline $\begin{array}{l}3.0 \mathrm{E}-04 \\
\text { O-O4 }\end{array}$ & $2.8 \mathrm{E}-06$ \\
\hline $6.8 E-04$ & 2.8E-06 \\
\hline $\begin{array}{l}7.7 E-05 \\
3.5-5-03\end{array}$ & $1.6 \mathrm{E}-06$ \\
\hline $\begin{array}{l}3.5 \mathrm{E}-03 \\
6.6 \mathrm{E}-03\end{array}$ & $1.6 \mathrm{E}-06$ \\
\hline $\begin{array}{l}6.6-6-03 \\
5.2 \mathrm{E}-03\end{array}$ & $\begin{array}{l}4.6 \mathrm{E}-04 \\
4.6 \mathrm{E}-04\end{array}$ \\
\hline $\begin{array}{l}5.2-2 E-01 \\
1.2 E-01\end{array}$ & $\begin{array}{l}4.60=-0 \\
6.6 E-03\end{array}$ \\
\hline 2.3E-01 & $6.6 \mathrm{E}-03$ \\
\hline 2.2E-05 & $6.2 \mathrm{E}-07$ \\
\hline $\begin{array}{l}1.2 E-02 \\
2\end{array}$ & $\begin{array}{l}6.2 \mathrm{E}-07 \\
3\end{array}$ \\
\hline $\begin{array}{l}3.0 E-03 \\
0.0 E\end{array}$ & 3.9E-05 \\
\hline $\begin{array}{l}9.7 \mathrm{E}-03 \\
3.5 \mathrm{E}-02\end{array}$ & $\begin{array}{l}3.9 E-05 \\
5\end{array}$ \\
\hline $\begin{array}{l}3.5 E-02 \\
8.8 E-01\end{array}$ & $5.4 \mathrm{E}-07$ \\
\hline $\begin{array}{l}.8 Z E-O 1 \\
1.2 E-01\end{array}$ & \\
\hline $\begin{array}{l}4.4-02 \\
4\end{array}$ & $2.4 \mathrm{E}-04$ \\
\hline
\end{tabular}
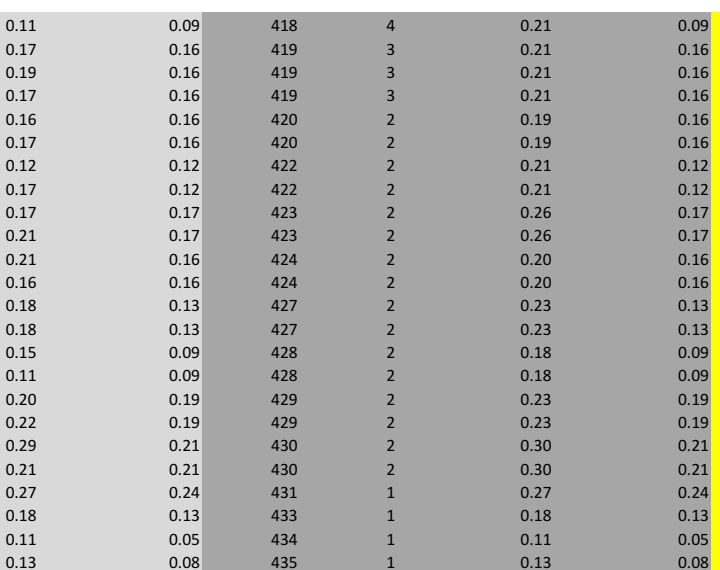

$2.6 E-04$
$2.0 \mathrm{E}-02$
2.02 
Supplementary Table 6. Significant causality NEO gene/methylation effects. NEO significance determined by LEO.nb.oca (significance $>0.3$, d

\section{probeset}

X603142577F1

ENSGALT00000027130_Q5ZL81_CHICK

X603596753F1

ENSGALT00000036508_KLF12

ENSGALT00000036508_KLF12

ENSGALT00000027404_KIAA0564

ENSGALT00000027507_NEK3

$\mathrm{X} 603863736 \mathrm{~F} 1$

X603143689F1

X603598770F1

ENSGALT00000036407_ENSGALG00000022715

ENSGALT00000036407_ENSGALG00000022715

ENSGALT00000027885_ANKRD42

X603862030F1

NM_001024694_RLBP1

X603603027F1

ENSGALT00000016866_LRRC41

X603143689F1

ENSGALT00000014848_ENSGALG00000009124

ENSGALT00000033255_ENSGALG00000020755

X603862915F1

$\mathrm{X} 603864922 \mathrm{~F} 1$

X603144253F1

ENSGALT00000034753_FAM196B

ENSGALT00000039948_TMC7_CHICK

ENSGALT00000006763_A4L9I7_CHICK

$\begin{array}{lllll}\text { meth_phenotype } & \text { edge_direction } & \text { LEO.NB.OCA } & \text { LEO.NB.CPA } & \text { Model.P.value.AtoB } \\ \text { chr1_138209000 } & \text { genexp -> meth } & 0.43 & 0.62 & 3.0 \mathrm{E}-11 \\ \text { chr1_137382000 } & \text { genexp -> meth } & 0.64 & 0.64 & 3.3 \mathrm{E}-03 \\ \text { chr1_13464000 } & \text { meth -> genexp } & 1.23 & 1.23 & 4.5 \mathrm{E}-01 \\ \text { chr1_145493000 } & \text { meth -> genexp } & 0.65 & 0.82 & 3.6 \mathrm{E}-02 \\ \text { chr1_162106000 } & \text { genexp -> meth } & 0.34 & 0.63 & 7.9 \mathrm{E}-01 \\ \text { chr1_167035000 } & \text { genexp -> meth } & 1.01 & 1.01 & 1.4 \mathrm{E}-01 \\ \text { chr1_182304000 } & \text { meth -> genexp } & 0.61 & 2.31 & 8.9 \mathrm{E}-08 \\ \text { chr1_182304000 } & \text { meth -> genexp } & 0.34 & 0.34 & 8.0 \mathrm{E}-02 \\ \text { chr1_14231000 } & \text { genexp -> meth } & 0.70 & 2.25 & 3.1 \mathrm{E}-31 \\ \text { chr1_183504000 } & \text { meth -> genexp } & 1.36 & 1.36 & 6.0 \mathrm{E}-02 \\ \text { chr1_175647000 } & \text { meth -> genexp } & 0.43 & 1.10 & 1.9 \mathrm{E}-01 \\ \text { chr1_182304000 } & \text { meth -> genexp } & 0.60 & 0.58 & 4.0 \mathrm{E}-09 \\ \text { chr1_182457000 } & \text { meth -> genexp } & 0.38 & 0.89 & 2.1 \mathrm{E}-01 \\ \text { chr10_5481000 } & \text { meth -> genexp } & 0.36 & 0.65 & 9.0 \mathrm{E}-01 \\ \text { chr10_14252000 } & \text { meth -> genexp } & 1.91 & 1.91 & 6.0 \mathrm{E}-02 \\ \text { chr11_7660000 } & \text { genexp -> meth } & 0.33 & 0.39 & 2.3 \mathrm{E}-19 \\ \text { chr12_3621000 } & \text { genexp -> meth } & 0.72 & 0.72 & 6.2 \mathrm{E}-01 \\ \text { chr1_13863000 } & \text { genexp -> meth } & 1.46 & 1.46 & 6.7 \mathrm{E}-02 \\ \text { chr12_2739000 } & \text { meth -> genexp } & 0.50 & 0.50 & 1.1 \mathrm{E}-01 \\ \text { chr12_11001000 } & \text { meth -> genexp } & 0.77 & 0.84 & 1.1 \mathrm{E}-02 \\ \text { chr12_16909000 } & \text { meth -> genexp } & 0.87 & 0.87 & 2.2 \mathrm{E}-01 \\ \text { chr12_16909000 } & \text { meth -> genexp } & 0.92 & 0.92 & 7.6 \mathrm{E}-02 \\ \text { chr13_5260000 } & \text { meth -> genexp } & 0.83 & 0.83 & 6.4 \mathrm{E}-02 \\ \text { chr13_8902000 } & \text { meth -> genexp } & 1.03 & 1.03 & 5.5 \mathrm{E}-01 \\ \text { chr14_9741000 } & \text { meth -> genexp } & 1.43 & 1.43 & 8.7 \mathrm{E}-02 \\ \text { chr14_9741000 } & \text { meth -> genexp } & 1.69 & 1.69 & 5.4 \mathrm{E}-02\end{array}$




\author{
ENSGALT00000010357_Q5F3U4_CHICK \\ X603597475F1 \\ X603597317F1 \\ ENSGALT00000003150_ENSGALG00000002026 \\ ENSGALT00000034720_Q5ZLV8_CHICK \\ ENSGALT00000039085_CADPS2 \\ ENSGALT00000011997_ARMC4 \\ X603595549F1 \\ ENSGALT00000039114_Q5ZKE1_CHICK \\ ENSGALT00000031934_VASH2 \\ X603866079F1 \\ $\mathrm{X} 603600947 \mathrm{~F} 1$ \\ $\mathrm{X} 603601949 \mathrm{~F} 1$ \\ X603865923F1 \\ ENSGALT00000018327_DPH3 \\ ENSGALT00000020160_MRPL32 \\ NM_001004400_TUBB2B \\ ENSGALT00000021306_MTRR \\ ENSGALT00000037043_Q2XT17_CHICK \\ NM_001030743_GLIPR1 \\ X603599695F1 \\ ENSGALT00000022184_TTRAP \\ ENSGALT00000023564_Q91429_CHICK \\ X603865613F1 \\ ENSGALT00000036651_Q6VYQ9_CHICK \\ NM_204290_FABP4 \\ NM_204290_FABP4 \\ X603595861F1
}

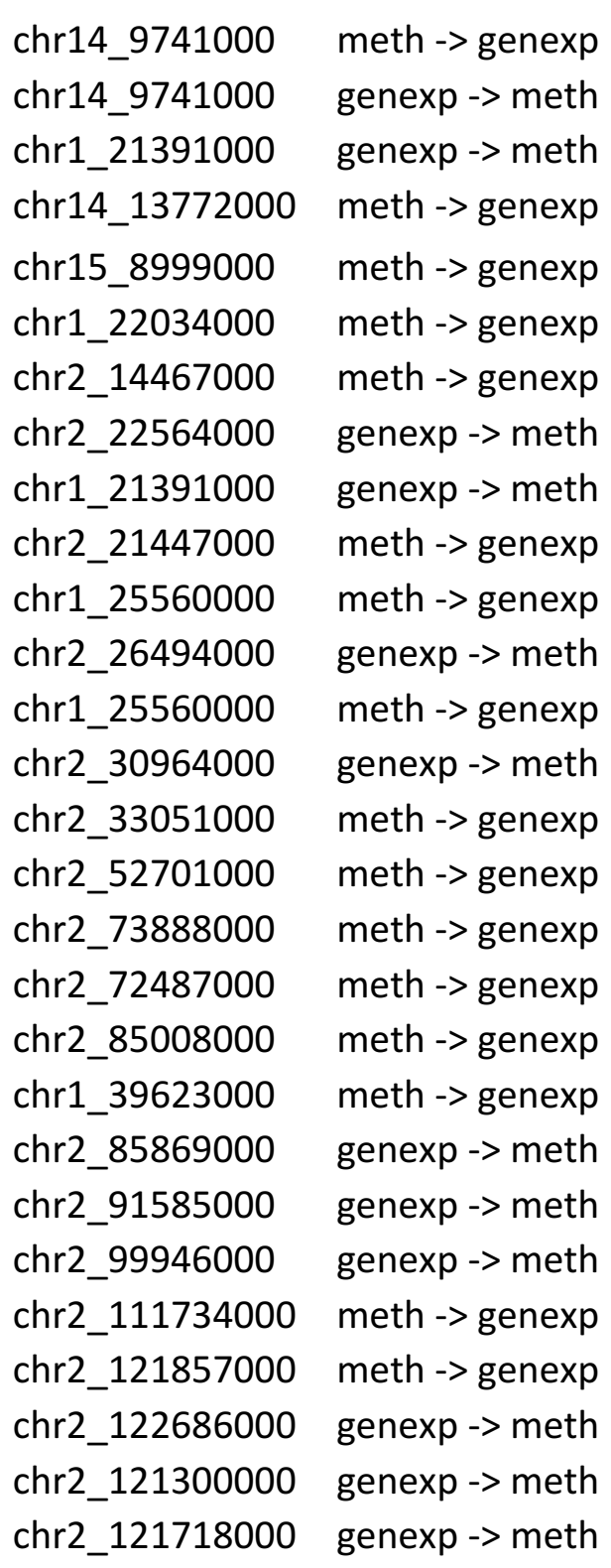

$\begin{array}{lll}1.34 & 1.34 & 1.1 \mathrm{E}-01 \\ 0.99 & 0.99 & 4.4 \mathrm{E}-02 \\ 0.32 & 1.13 & 1.1 \mathrm{E}-01 \\ 0.59 & 0.59 & 5.5 \mathrm{E}-01 \\ 0.32 & 0.44 & 3.0 \mathrm{E}-02 \\ 1.18 & 0.95 & 4.5 \mathrm{E}-12 \\ 1.33 & 1.33 & 3.3 \mathrm{E}-01 \\ 0.35 & 0.75 & 2.7 \mathrm{E}-06 \\ 0.48 & 0.74 & 1.8 \mathrm{E}-01 \\ 0.66 & 0.66 & 1.4 \mathrm{E}-01 \\ 0.83 & 0.83 & 6.0 \mathrm{E}-01 \\ 1.57 & 1.57 & 1.5 \mathrm{E}-01 \\ 0.84 & 0.84 & 2.4 \mathrm{E}-01 \\ 1.76 & 1.76 & 3.7 \mathrm{E}-02 \\ 0.45 & 0.45 & 2.3 \mathrm{E}-02 \\ 0.33 & 0.33 & 6.4 \mathrm{E}-02 \\ 0.60 & 1.41 & 3.5 \mathrm{E}-13 \\ 1.17 & 1.17 & 1.4 \mathrm{E}-01 \\ 0.58 & 0.58 & 7.2 \mathrm{E}-02 \\ 0.42 & 0.76 & 7.3 \mathrm{E}-21 \\ 0.61 & 0.61 & 1.1 \mathrm{E}-01 \\ 0.33 & 0.95 & 9.0 \mathrm{E}-05 \\ 2.05 & 2.05 & 2.8 \mathrm{E}-02 \\ 0.42 & 0.32 & 3.3 \mathrm{E}-18 \\ 0.32 & 0.32 & 5.6 \mathrm{E}-03 \\ 0.59 & 0.59 & 7.3 \mathrm{E}-03 \\ 0.54 & 0.54 & 1.2 \mathrm{E}-02 \\ 0.33 & 0.71 & 2.9 \mathrm{E}-08\end{array}$

1.1E-01

4.4E-02

5.5E-01

3. $0 \mathrm{E}-02$

3.3E-01

.7E-06

OE-01

$.5 \mathrm{E}-01$

$4 \mathrm{E}-01$

$3 \mathrm{E}-02$

.4E-02

$2 \mathrm{E}-02$

$3 \mathrm{E}-21$

OE-05

. $8 \mathrm{E}-02$

.3E-18

.6E-03

.2E-02

2.9E-08 
NM_205318_MYBL2

ENSGALT00000022584_FAM54A

ENSGALT00000009589_ENSGALG00000005964

X603863273F1

ENSGALT00000037559_SGCZ

X603868314F1

ENSGALT00000024208_STX18

X603596815F1

NM_001004405_CAPN3

ENSGALT00000022453_ENSGALG00000013838

ENSGALT00000015850_Q5F3V4_CHICK

X603601830F1

X603865360F1

X603865360F1

ENSGALT00000004232_ECD

ENSGALT00000004232_ECD

ENSGALT00000006627_SRGN

X603595690F1

ENSGALT00000040000_Q52P71_CHICK

ENSGALT00000040000_Q52P71_CHICK

ENSGALT00000010634_TMEM20

ENSGALT00000012505_FBXW4

ENSGALT00000012505_FBXW4

ENSGALT00000033256_ATP6V0E2

ENSGALT00000010189_ENSGALG00000006302

X603568048F1

$\mathrm{X} 603862885 \mathrm{~F} 1$

X603862885F1

\begin{abstract}
chr20_2984000
chr3_61873000

chr4_2703000

chr4_1363000

chr4_67663000

chr4_68917000

chr4_78836000

chr5_12907000

chr5_28555000

chr5_28555000

chr5_39597000

chr6_11766000

chr6_11121000

chr6_11079000

chr6_10462000

chr6_11069000

chr6_11766000

chr6_17952000

chr6_20832000

chr6_22274000

chr6_22750000

chr6_23513000

chr6_20322000

chr6_20074000

chr6_22861000

chr6_26627000

chr6_24650000

chr6_22494000

meth -> genexp

genexp $\rightarrow$ meth

genexp -> meth

genexp -> meth

meth $->$ genexp

meth $->$ genexp

meth -> genexp

meth $->$ genexp

genexp -> meth

genexp -> meth

meth $->$ genexp

meth -> genexp

meth -> genexp

meth -> genexp

meth $->$ genexp

meth -> genexp

meth -> genexp

meth $->$ genexp

meth -> genexp

meth $->$ genexp

genexp -> meth

genexp $->$ meth

meth -> genexp

meth -> genexp

meth $->$ genexp

genexp -> meth

genexp -> meth

meth -> genexp
\end{abstract}

\begin{tabular}{ll}
1.03 & 1.03 \\
0.31 & 0.89 \\
0.62 & 0.76 \\
0.38 & 0.81 \\
2.13 & 2.13 \\
0.80 & 0.80 \\
1.36 & 1.36 \\
0.92 & 0.92 \\
0.36 & 1.26 \\
0.40 & 1.49 \\
0.32 & 0.42 \\
0.37 & 0.37 \\
1.69 & 1.69 \\
1.20 & 1.20 \\
0.91 & 0.91 \\
0.48 & 0.48 \\
1.86 & 1.86 \\
0.62 & 0.62 \\
0.72 & 0.81 \\
0.67 & 0.67 \\
0.53 & 0.53 \\
0.83 & 0.75 \\
0.41 & 0.41 \\
1.12 & 1.12 \\
0.97 & 0.97 \\
0.76 & 0.70 \\
0.36 & 0.74 \\
0.43 & 0.88 \\
\hline &
\end{tabular}

1.9E-01

5.6E-02

1.9E-01

6.9E-01

1.0E-01

4.9E-02

4.0E-02

6.7E-01

8.8E-02

2.3E-01

1.4E-45

1.2E-02

9.1E-02

1.0E-01

2.4E-02

1.7E-02

1.0E-01

9.9E-01

1.1E-04

5.5E-03

1.3E-03

2.5E-01

7.3E-03

1.1E-01

3.5E-01

1.5E-01

9.5E-01

5.7E-01 


NM_001130738_PLA2G6
X603601127F1
ENSGALT00000014801_TRUB1
ENSGALT00000030858_Q6VYQ9_CHICK
X603863661F1
X603601402F1
X603597339F1
ENSGALT00000037879_DPP10
ENSGALT00000030858_Q6VYQ9_CHICK
X603597182F1
ENSGALT00000032941_LOC424274
ENSGALT00000037978_MYLK_CHICK
ENSGALT00000037978_MYLK_CHICK
X603867770F1
X603867770F1
ENSGALT00000003806_LOC769292
ENSGALT00000019841_DDX18
ENSGALT00000009468_CCDC18
X603866233F1
ENSGALT00000016868_NSUN4
X603599201F1
X603599396F1
X603868153F1
ENSGALT00000011741_IL1RAP
X603863036F1
NM_001006551_MCCC1
X603596950F1
NM_001030343_CHST10

$\begin{array}{lllll}\text { chr1_53282000 } & \text { meth -> genexp } & 0.33 & 0.60 & 2.7 \mathrm{E}-37 \\ \text { chr6_29565000 } & \text { genexp -> meth } & 1.01 & 1.01 & 2.7 \mathrm{E}-02 \\ \text { chr6_28950000 } & \text { meth -> genexp } & 0.71 & 1.92 & 3.2 \mathrm{E}-09 \\ \text { chr1_53282000 } & \text { meth -> genexp } & 0.80 & 0.80 & 3.8 \mathrm{E}-02 \\ \text { chr6_30756000 } & \text { genexp -> meth } & 0.96 & 0.96 & 1.5 \mathrm{E}-02 \\ \text { chr7_2323000 } & \text { meth -> genexp } & 1.18 & 1.18 & 1.6 \mathrm{E}-02 \\ \text { chr7_25523000 } & \text { meth -> genexp } & 1.11 & 2.02 & 7.8 \mathrm{E}-08 \\ \text { chr7_27836000 } & \text { genexp -> meth } & 0.55 & 0.55 & 1.6 \mathrm{E}-02 \\ \text { chr2_121300000 } & \text { meth -> genexp } & 0.49 & 1.07 & 1.2 \mathrm{E}-03 \\ \text { chr7_24919000 } & \text { genexp -> meth } & 0.45 & 1.36 & 1.4 \mathrm{E}-04 \\ \text { chr7_28599000 } & \text { meth -> genexp } & 2.25 & 2.25 & 3.4 \mathrm{E}-02 \\ \text { chr7_27345000 } & \text { meth -> genexp } & 0.99 & 0.99 & 5.1 \mathrm{E}-01 \\ \text { chr7_27493000 } & \text { meth -> genexp } & 0.83 & 0.83 & 3.5 \mathrm{E}-01 \\ \text { chr7_27493000 } & \text { genexp -> meth } & 0.76 & 0.76 & 1.3 \mathrm{E}-02 \\ \text { chr7_27836000 } & \text { genexp -> meth } & 1.30 & 1.30 & 5.5 \mathrm{E}-02 \\ \text { chr7_28273000 } & \text { meth -> genexp } & 0.66 & 0.47 & 3.2 \mathrm{E}-03 \\ \text { chr7_30201000 } & \text { genexp -> meth } & 0.56 & 0.58 & 1.5 \mathrm{E}-04 \\ \text { chr8_14448000 } & \text { meth -> genexp } & 0.66 & 0.66 & 5.1 \mathrm{E}-02 \\ \text { chr8_14448000 } & \text { meth -> genexp } & 0.67 & 0.67 & 3.6 \mathrm{E}-02 \\ \text { chr8_19538000 } & \text { meth -> genexp } & 0.96 & 0.96 & 9.5 \mathrm{E}-01 \\ \text { chr8_18840000 } & \text { meth -> genexp } & 1.07 & 1.07 & 6.0 \mathrm{E}-01 \\ \text { chr8_18836000 } & \text { meth -> genexp } & 1.14 & 1.14 & 2.5 \mathrm{E}-01 \\ \text { chr9_13429000 } & \text { meth -> genexp } & 0.42 & 0.42 & 2.4 \mathrm{E}-01 \\ \text { chr9_13425000 } & \text { meth -> genexp } & 1.10 & 1.10 & 3.9 \mathrm{E}-02 \\ \text { chr9_16919000 } & \text { meth -> genexp } & 0.78 & 0.78 & 8.9 \mathrm{E}-01 \\ \text { chr9_12573000 } & \text { meth -> genexp } & 0.38 & 0.90 & 4.0 \mathrm{E}-01 \\ \text { chr1_119144000 } & \text { meth -> genexp } & 1.74 & 1.74 & 3.7 \mathrm{E}-01 \\ \text { chr1_137201000 } & \text { meth -> genexp } & 0.98 & 0.98 & 9.4 \mathrm{E}-02 \\ & & & \end{array}$


lifferent SNP marker anchors for methylat

\section{eqtl_type mqtl_type model_type}

cis cis OCA_dual_mode

cis cis CPA_single_model

cis cis CPA_single_model

cis cis OCA_dual_model

cis trans OCA_dual_model

cis cis CPA_single_model

cis cis OCA_dual_model

cis cis CPA_single_model

cis cis OCA_dual_model

trans cis CPA_single_model

cis trans OCA_dual_model

cis cis OCA_dual_model

cis trans OCA_dual_model

cis trans OCA_dual_model

cis cis CPA_single_model

cis cis OCA_dual_model

trans cis CPA_single_model

cis cis CPA_single_model

trans cis CPA_single_model

cis cis OCA_dual_model

cis cis CPA_single_model

trans cis CPA_single_model

cis cis CPA_single_model

cis cis CPA_single_model

cis cis CPA_single_model

trans cis CPA_single_model 


$\begin{array}{lll}\text { cis } & \text { cis } & \text { CPA_single_model } \\ \text { cis } & \text { cis } & \text { CPA_single_model } \\ \text { cis } & \text { trans } & \text { OCA_dual_model } \\ \text { cis } & \text { cis } & \text { CPA_single_model } \\ \text { cis } & \text { cis } & \text { OCA_dual_model } \\ \text { cis } & \text { cis } & \text { OCA_dual_model } \\ \text { cis } & \text { cis } & \text { CPA_single_model } \\ \text { cis } & \text { cis } & \text { OCA_dual_model } \\ \text { cis } & \text { trans } & \text { OCA_dual_model } \\ \text { trans } & \text { cis } & \text { CPA_single_model } \\ \text { cis } & \text { cis } & \text { CPA_single_model } \\ \text { cis } & \text { cis } & \text { CPA_single_model } \\ \text { cis } & \text { cis } & \text { CPA_single_model } \\ \text { cis } & \text { cis } & \text { CPA_single_model } \\ \text { cis } & \text { cis } & \text { CPA_single_model } \\ \text { cis } & \text { cis } & \text { CPA_single_model } \\ \text { cis } & \text { cis } & \text { OCA_dual_model } \\ \text { cis } & \text { cis } & \text { CPA_single_model } \\ \text { cis } & \text { cis } & \text { CPA_single_model } \\ \text { cis } & \text { cis } & \text { OCA_dual_model } \\ \text { cis } & \text { cis } & \text { CPA_single_model } \\ \text { cis } & \text { cis } & \text { OCA_dual_model } \\ \text { cis } & \text { cis } & \text { CPA_single_model } \\ \text { cis } & \text { cis } & \text { OCA_dual_model } \\ \text { cis } & \text { cis } & \text { CPA_single_model } \\ \text { cis } & \text { cis } & \text { CPA_single_model } \\ \text { cis } & \text { cis } & \text { CPA_single_model } \\ \text { cis } & \text { cis } & \text { OCA_dual_model }\end{array}$




$\begin{array}{lll}\text { cis } & \text { cis } & \text { CPA_single_model } \\ \text { cis } & \text { trans } & \text { OCA_dual_model } \\ \text { cis } & \text { trans } & \text { OCA_dual_model } \\ \text { cis } & \text { trans } & \text { OCA_dual_model } \\ \text { cis } & \text { cis } & \text { CPA_single_model } \\ \text { cis } & \text { cis } & \text { CPA_single_model } \\ \text { cis } & \text { cis } & \text { CPA_single_model } \\ \text { cis } & \text { cis } & \text { CPA_single_model } \\ \text { cis } & \text { trans } & \text { OCA_dual_model } \\ \text { cis } & \text { trans } & \text { OCA_dual_model } \\ \text { cis } & \text { cis } & \text { OCA_dual_model } \\ \text { cis } & \text { cis } & \text { CPA_single_model } \\ \text { cis } & \text { cis } & \text { CPA_single_model } \\ \text { cis } & \text { cis } & \text { CPA_single_model } \\ \text { cis } & \text { cis } & \text { CPA_single_model } \\ \text { cis } & \text { cis } & \text { CPA_single_model } \\ \text { cis } & \text { cis } & \text { CPA_single_model } \\ \text { cis } & \text { cis } & \text { CPA_single_model } \\ \text { cis } & \text { cis } & \text { OCA_dual_model } \\ \text { cis } & \text { cis } & \text { CPA_single_model } \\ \text { cis } & \text { cis } & \text { CPA_single_model } \\ \text { cis } & \text { trans } & \text { OCA_dual_model } \\ \text { cis } & \text { cis } & \text { CPA_single_model } \\ \text { cis } & \text { cis } & \text { CPA_single_model } \\ \text { cis } & \text { cis } & \text { CPA_single_model } \\ \text { cis } & \text { trans } & \text { OCA_dual_model } \\ \text { cis } & \text { trans } & \text { OCA_dual_model } \\ \text { cis } & \text { cis } & \text { OCA_dual_model } \\ & & \end{array}$




$\begin{array}{lll}\text { cis } & \text { cis } & \text { OCA_dual_model } \\ \text { cis } & \text { cis } & \text { CPA_single_model } \\ \text { cis } & \text { cis } & \text { OCA_dual_model } \\ \text { trans } & \text { cis } & \text { CPA_single_model } \\ \text { cis } & \text { cis } & \text { CPA_single_model } \\ \text { cis } & \text { cis } & \text { CPA_single_model } \\ \text { cis } & \text { cis } & \text { OCA_dual_model } \\ \text { cis } & \text { cis } & \text { CPA_single_model } \\ \text { cis } & \text { cis } & \text { OCA_dual_model } \\ \text { cis } & \text { cis } & \text { OCA_dual_model } \\ \text { cis } & \text { cis } & \text { CPA_single_model } \\ \text { cis } & \text { cis } & \text { CPA_single_model } \\ \text { cis } & \text { cis } & \text { CPA_single_model } \\ \text { cis } & \text { cis } & \text { CPA_single_model } \\ \text { cis } & \text { cis } & \text { CPA_single_model } \\ \text { cis } & \text { trans } & \text { OCA_dual_model } \\ \text { cis } & \text { cis } & \text { OCA_dual_model } \\ \text { cis } & \text { cis } & \text { CPA_single_model } \\ \text { cis } & \text { cis } & \text { CPA_single_model } \\ \text { cis } & \text { cis } & \text { CPA_single_model } \\ \text { cis } & \text { cis } & \text { CPA_single_model } \\ \text { cis } & \text { cis } & \text { CPA_single_model } \\ \text { cis } & \text { cis } & \text { CPA_single_model } \\ \text { cis } & \text { cis } & \text { CPA_single_model } \\ \text { cis } & \text { cis } & \text { CPA_single_model } \\ \text { cis } & \text { cis } & \text { OCA_dual_model } \\ \text { cis } & \text { cis } & \text { CPA_single_model } \\ \text { cis } & \text { cis } & \text { CPA_single_model } \\ & & \end{array}$


Supplementary Table 7. SNP variants displaying allelic imbalance that fall within significant causal cis methQTL phenotype bins ide

\begin{tabular}{|c|c|c|c|c|c|c|}
\hline SNP_variant meth_phenot) & TTL_LOD probeset & ne_id & eQTL_lod & neo_dir & Deo_LE & _model_pl \\
\hline chr1.138626fchr1_138630। & $5.83 \times 603143689 \mathrm{~F}$ & $\# N / A$ & 11.5 & 5 genexp -> me 1.46 & 1.46 & $6.7 \mathrm{E}-02$ \\
\hline chr1.1191434chr1_119144। & $10.07 \times 603596950 F$ & $\# N / A$ & 3.2 & 2 meth $->$ gene 1.74 & 1.74 & 3.7E-01 \\
\hline chr1.182303Echr1_182304। & 16.7 ENSGALTOOOC & JSGALG000 & 4.8 & 8 meth $->$ gene 0.61 & 2.31 & 8.9E-08 \\
\hline chr1.182303Echr1_182304। & $16.7 \times 603863736 \mathrm{~F}$ & JSGALG000 & 4.7 & 7 meth $->$ gene 0.34 & 0.34 & 8.0E-02 \\
\hline chr1.182303Echr1_182304। & 16.7 ENSGALTOOO & JSGALG000 & 5.2 & 2 meth $->$ gene 0.60 & 0.58 & 4.0E-09 \\
\hline chr1.182303Echr1_182304। & 16.7 ENSGALTOOOC & JSGALG000 & 4.8 & 8 meth $->$ gene 0.61 & 2.31 & 8.9E-08 \\
\hline chr1.182303Echr1_182304। & $16.7 \times 603863736 \mathrm{~F}$ & JSGALG000 & 4.7 & 7 meth -> gene 0.34 & 0.34 & $8.0 \mathrm{E}-02$ \\
\hline chr1.182303Echr1_182304। & 16.7 ENSGALTOOOC & JSGALG000 & 5.2 & 2 meth $->$ gene 0.60 & 0.58 & 4.0E-09 \\
\hline chr12.27385\&chr12_27390। & 5.75 ENSGALTOOO & JSGALG000 & 7.0 & meth -> gene 0.5 & 0.5 & $1.1 \mathrm{E}-01$ \\
\hline chr12.27386ミchr12_27390। & 5.75 ENSGALTOOO & JSGALG000 & 7.0 & meth $->$ gene 0.5 & 0.5 & $1.1 \mathrm{E}-01$ \\
\hline chr13.89012Echr13_89020। & 6.44 ENSGALTO00( & JSGALG000 & 4.8 & 3 meth $->$ gene 1.03 & 1.03 & $5.5 \mathrm{E}-01$ \\
\hline chr2.3096312chr2_309640। & $4.61 \times 603865923 F$ & $\# N / A$ & 10.3 & 3 genexp -> me1.76 & 1.76 & 3.7E-02 \\
\hline chr2.3096351chr2_309640। & $4.61 \mathrm{X603865923F}$ & $\# N / A$ & 10.3 & 3 genexp -> me 1.76 & 1.76 & 3.7E-02 \\
\hline chr2.999450`ichr2_999460। & 4.1 ENSGALT000 & JSGALG000 & 8.1 & genexp -> me 2.05 & 2.05 & $2.8 \mathrm{E}-02$ \\
\hline chr2.999450؛chr2_999460। & 4.1 ENSGALTOOO & JSGALG000 & 8.1 & 1 genexp $->$ me 2.05 & 2.05 & $2.8 \mathrm{E}-02$ \\
\hline chr2.9994515chr2_999460। & 4.1 ENSGALTOOO & JSGALG000 & 8.1 & genexp $->$ me 2.05 & 2.05 & $2.8 \mathrm{E}-02$ \\
\hline chr2.9994515chr2_999460। & 4.1 ENSGALTOOO & JSGALG000 & 8.1 & genexp -> me 2.05 & 2.05 & $2.8 \mathrm{E}-02$ \\
\hline chr2.999452Cchr2_999460। & 4.1 ENSGALTOOO & JSGALG000 & 8.1 & 1 genexp $->$ me 2.05 & 2.05 & $2.8 \mathrm{E}-02$ \\
\hline chr2.999452عchr2_999460। & 4.1 ENSGALTO00 & JSGALG000 & 8.1 & 1 genexp -> me 2.05 & 2.05 & $2.8 \mathrm{E}-02$ \\
\hline chr6.2286021chr6_228610। & 4.32 ENSGALTOOO & JSGALG000 & 6.2 & 2 meth $\rightarrow$ gene 0.965 & 0.965 & $3.5 \mathrm{E}-01$ \\
\hline chr6.228602achr6_228610। & 4.32 ENSGALTOOO & JSGALG000 & 6.2 & meth $->$ gene 0.965 & 0.965 & $3.5 \mathrm{E}-01$ \\
\hline chr7.2552234chr7_255230। & $6.51 \times 603597339 \mathrm{~F}$ & $\# N / A$ & 5.2 & meth -> gene 1.11 & 2.02 & $7.8 \mathrm{E}-08$ \\
\hline chr7.255223Echr7_255230। & 6.51 X603597339F & $\# N / A$ & 5.2 & meth $->$ gene 1.11 & 2.02 & $7.8 \mathrm{E}-08$ \\
\hline chr7.255223ichr7_255230। & 6.51 X603597339F & $\# N / A$ & 5.2 & meth $->$ gene 1.11 & 2.02 & $7.8 \mathrm{E}-08$ \\
\hline chr7.255223ichr7_255230। & 6.51 X603597339F & $\# N / A$ & 5.2 & meth $->$ gene 1.11 & 2.02 & $7.8 \mathrm{E}-08$ \\
\hline chr7.255223\&chr7_255230। & 6.51 X603597339F & $\# N / A$ & 5.2 & meth $->$ gene 1.11 & 2.02 & $7.8 \mathrm{E}-08$ \\
\hline
\end{tabular}


chr7.255223chr7_255230 chr7.255225chr7_255230 chr7.273443ichr7_273450 chr7.278359Cchr7_278360। chr7.278359Cchr7_278360। chr7.2783591chr7_278360 chr7.2783591chr7_278360 chr7.278359 3 chr7_278360 chr7.278359 chr7_278360 chr7.2783595chr7_278360 chr7.2783595chr7_278360 chr7.278359€chr7_278360। chr7.278359€chr7_278360 chr7.2859881chr7_285990 chr7.285988zchr7_285990 chr7.285989Cchr7_285990 chr7.302007@chr7_302010 chr7.302009ichr7_302010 chr8.195372ichr8_195380।
$6.51 \times 603597339 \mathrm{~F} \quad \# \mathrm{~N} / \mathrm{A}$ $6.51 \times 603597339 \mathrm{~F} \quad \# \mathrm{~N} / \mathrm{A}$ 6.69 ENSGALTO00(ENSGALG000 4.1 ENSGALTO0O(ENSGALG000 4.1 X603867770F \#N/A 4.1 ENSGALTO0O(ENSGALG000 4.1 X603867770F \#N/A 4.1 ENSGALTO0OCENSGALG000 4.1 X603867770F \#N/A 4.1 ENSGALTO00(ENSGALG000 4.1 X603867770F \#N/A 4.1 ENSGALTO0O(ENSGALG000 4.1 X603867770F \#N/A 10.24 ENSGALTO0O(ENSGALG000 10.24 ENSGALTO0O(ENSGALG000 10.24 ENSGALTOOOCENSGALG000 4.56 ENSGALTOOO(ENSGALG000 4.56 ENSGALTOOO(ENSGALG000 6.84 ENSGALTOOO(ENSGALG000
5.2 meth $\rightarrow$ gene 1.11

5.2 meth $\rightarrow$ gene 1.11

5.7 meth $->$ gene 0.988

11.3 genexp $>$ me 0.55

4.8 genexp $\rightarrow$ me 1.30

11.3 genexp $->$ me 0.55

4.8 genexp $\rightarrow$ me 1.30

11.3 genexp $\rightarrow$ me 0.55

4.8 genexp $->$ me 1.30

11.3 genexp -> me 0.55

4.8 genexp $\rightarrow$ me 1.30

11.3 genexp $>$ me 0.55

4.8 genexp $>$ me 1.30

6.4 meth $\rightarrow$ gene 2.25

6.4 meth $\rightarrow$ gene 2.25

6.4 meth $->$ gene 2.25

6.5 genexp $>$ me 0.561

6.5 genexp -> me 0.561

3.5 meth -> gene 0.955

$\begin{array}{ll}2.02 & 7.8 \mathrm{E}-08 \\ 2.02 & 7.8 \mathrm{E}-08 \\ 0.988 & 5.1 \mathrm{E}-01 \\ 0.55 & 1.6 \mathrm{E}-02 \\ 1.30 & 5.5 \mathrm{E}-02 \\ 0.55 & 1.6 \mathrm{E}-02 \\ 1.30 & 5.5 \mathrm{E}-02 \\ 0.55 & 1.6 \mathrm{E}-02 \\ 1.30 & 5.5 \mathrm{E}-02 \\ 0.55 & 1.6 \mathrm{E}-02 \\ 1.30 & 5.5 \mathrm{E}-02 \\ 0.55 & 1.6 \mathrm{E}-02 \\ 1.30 & 5.5 \mathrm{E}-02 \\ 2.25 & 3.4 \mathrm{E}-02 \\ 2.25 & 3.4 \mathrm{E}-02 \\ 2.25 & 3.4 \mathrm{E}-02 \\ 0.581 & 1.5 \mathrm{E}-04 \\ 0.581 & 1.5 \mathrm{E}-04 \\ 0.955 & 9.5 \mathrm{E}-01\end{array}$

$7.8 \mathrm{E}-08$

$.8 \mathrm{E}-08$

.6E-02

$.5 \mathrm{E}-02$

$5.5 \mathrm{E}-02$

$6 \mathrm{E}-02$

.6E-02

$.5 \mathrm{E}-02$

$.4 \mathrm{E}-02$

$3.4 \mathrm{E}-02$

$9.5 \mathrm{E}-01$ 
!ntified using NEO causality analysis. Direction of effect, NEO score

\begin{tabular}{|c|c|c|c|}
\hline vcf_genotype_ & k_genotyp ai_var & ichisq_pvalue_ & q_pvalue_ \\
\hline 9.7E-03 & $4.9 \mathrm{E}-01 \mathrm{C} \mid \mathrm{T}$ & $3.7 \mathrm{E}-01$ & $5.4 \mathrm{E}-35$ \\
\hline $6.5 \mathrm{E}-03$ & 2.9E-03 C | G & $3.2 \mathrm{E}-02$ & $1.0 \mathrm{E}-18$ \\
\hline 1.3E-01 & 3.3E-03 A | T & $1.4 \mathrm{E}-04$ & $2.8 \mathrm{E}-30$ \\
\hline $1.3 \mathrm{E}-01$ & 3.3E-03 A | T & $1.4 \mathrm{E}-04$ & $2.8 \mathrm{E}-30$ \\
\hline 1.3E-01 & 3.3E-03 A / T & $1.4 \mathrm{E}-04$ & $2.8 \mathrm{E}-30$ \\
\hline $6.6 \mathrm{E}-04$ & $1.1 \mathrm{E}-03 \mathrm{~A} / \mathrm{T}$ & $2.0 \mathrm{E}-03$ & $2.8 \mathrm{E}-36$ \\
\hline $6.6 \mathrm{E}-04$ & 1.1E-03 A | T & 2.0E-03 & $2.8 \mathrm{E}-36$ \\
\hline $6.6 \mathrm{E}-04$ & 1.1E-03 A / T & $2.0 \mathrm{E}-03$ & $2.8 \mathrm{E}-36$ \\
\hline $9.8 \mathrm{E}-02$ & $1.1 \mathrm{E}-05 \mathrm{C} \mid \mathrm{T}$ & $6.5 \mathrm{E}-01$ & $6.1 \mathrm{E}-03$ \\
\hline $9.1 \mathrm{E}-01$ & $1.3 \mathrm{E}-05 \mathrm{~A} \mid \mathrm{G}$ & $5.5 \mathrm{E}-01$ & 1.6E-06 \\
\hline $2.8 \mathrm{E}-01$ & 6.9E-06 A | T & $1.9 \mathrm{E}-01$ & $1.3 \mathrm{E}-09$ \\
\hline $4.9 \mathrm{E}-04$ & $1.2 \mathrm{E}-02 \mathrm{~A} \mid \mathrm{G}$ & $3.9 \mathrm{E}-26$ & 1.7E-04 \\
\hline 7.9E-03 & $2.5 \mathrm{E}-05 \mathrm{C} \mid \mathrm{T}$ & $3.6 \mathrm{E}-01$ & $6.9 \mathrm{E}-15$ \\
\hline $3.4 \mathrm{E}-05$ & $2.4 \mathrm{E}-03 \mathrm{C} \mid \mathrm{T}$ & 7.3E-01 & $9.9 \mathrm{E}-11$ \\
\hline $1.1 \mathrm{E}-05$ & $1.1 \mathrm{E}-03 \mathrm{~A} \mid \mathrm{G}$ & $4.8 \mathrm{E}-01$ & $1.1 \mathrm{E}-11$ \\
\hline 7.1E-03 & 1.8E-03 A | T & $5.8 \mathrm{E}-01$ & $6.4 \mathrm{E}-12$ \\
\hline $1.6 \mathrm{E}-04$ & 7.5E-05 A | G & $7.2 \mathrm{E}-01$ & $4.9 \mathrm{E}-16$ \\
\hline 2.7E-04 & 8.6E-06 A | G & $3.1 \mathrm{E}-01$ & $1.9 \mathrm{E}-04$ \\
\hline 2.7E-02 & $3.8 \mathrm{E}-02 \mathrm{~A} \mid \mathrm{C}$ & n.s. & 1.7E-03 \\
\hline $6.3 E-02$ & $1.4 \mathrm{E}-06 \mathrm{~A} / \mathrm{G}$ & $5.3 \mathrm{E}-02$ & 1.3E-12 \\
\hline $5.4 \mathrm{E}-02$ & $5.4 \mathrm{E}-06 \mathrm{~A} \mid \mathrm{G}$ & 2.3E-01 & $1.9 \mathrm{E}-13$ \\
\hline $1.4 \mathrm{E}-02$ & $9.1 \mathrm{E}-06 \mathrm{C} \mid \mathrm{T}$ & n.s. & $1.3 \mathrm{E}-04$ \\
\hline $4.4 \mathrm{E}-03$ & $5.8 \mathrm{E}-06 \mathrm{C} \mid \mathrm{T}$ & $3.8 \mathrm{E}-01$ & $5.2 \mathrm{E}-03$ \\
\hline $2.0 \mathrm{E}-01$ & $1.5 \mathrm{E}-05 \mathrm{~A} \mid \mathrm{G}$ & $5.3 \mathrm{E}-01$ & $2.8 \mathrm{E}-03$ \\
\hline $1.0 \mathrm{E}-01$ & 1.6E-05 A | C & $5.1 \mathrm{E}-01$ & $3.5 \mathrm{E}-03$ \\
\hline $1.9 \mathrm{E}-04$ & 9.0E-06 C | G & $4.2 \mathrm{E}-01$ & $3.8 \mathrm{E}-03$ \\
\hline
\end{tabular}




\begin{tabular}{|c|c|c|c|}
\hline $1.2 \mathrm{E}-01$ & 3.8E-05 A | T & n.s. & $2.7 \mathrm{E}-03$ \\
\hline $9.1 \mathrm{E}-04$ & $3.5 \mathrm{E}-01 \mathrm{~A} \mid \mathrm{C}$ & $9.5 \mathrm{E}-01$ & $1.8 \mathrm{E}-02$ \\
\hline $6.8 \mathrm{E}-05$ & $2.4 \mathrm{E}-04 \mathrm{C} \mid \mathrm{T}$ & $6.9 \mathrm{E}-01$ & $1.2 \mathrm{E}-14$ \\
\hline $1.2 \mathrm{E}-02$ & $6.2 \mathrm{E}-03 \mathrm{CIT}$ & $4.1 \mathrm{E}-03$ & $1.1 \mathrm{E}-06$ \\
\hline $1.2 \mathrm{E}-02$ & $6.2 \mathrm{E}-03 \mathrm{CI} \mathrm{T}$ & $4.1 \mathrm{E}-03$ & $1.1 \mathrm{E}-06$ \\
\hline $6.3 \mathrm{E}-06$ & 4.6E-03 C। T & $1.0 \mathrm{E}-01$ & 2.1E-12 \\
\hline $6.3 \mathrm{E}-06$ & 4.6E-03 C। T & $1.0 \mathrm{E}-01$ & $2.1 \mathrm{E}-12$ \\
\hline $2.6 \mathrm{E}-01$ & $1.4 \mathrm{E}-02 \mathrm{~A} \mid \mathrm{C}$ & $1.4 \mathrm{E}-01$ & $1.5 \mathrm{E}-29$ \\
\hline $2.6 \mathrm{E}-01$ & $1.4 \mathrm{E}-02 \mathrm{~A} \mid \mathrm{C}$ & $1.4 \mathrm{E}-01$ & $1.5 \mathrm{E}-29$ \\
\hline 3.3E-01 & $1.4 \mathrm{E}-02 \mathrm{C} \mid \mathrm{T}$ & $5.6 \mathrm{E}-02$ & 2.1E-15 \\
\hline 3.3E-01 & $1.4 \mathrm{E}-02 \mathrm{C} \mid \mathrm{T}$ & $5.6 \mathrm{E}-02$ & $2.1 \mathrm{E}-15$ \\
\hline $5.3 \mathrm{E}-01$ & 5.0E-02 C | T & $1.7 \mathrm{E}-01$ & $2.4 \mathrm{E}-12$ \\
\hline $5.3 \mathrm{E}-01$ & $5.0 \mathrm{E}-02 \mathrm{C} \mid \mathrm{T}$ & $1.7 \mathrm{E}-01$ & $2.4 \mathrm{E}-12$ \\
\hline $1.9 \mathrm{E}-01$ & $5.8 \mathrm{E}-11 \mathrm{G} \mid \mathrm{T}$ & $4.3 \mathrm{E}-01$ & $1.5 \mathrm{E}-61$ \\
\hline $3.9 \mathrm{E}-05$ & $9.6 \mathrm{E}-11 \mathrm{C} \mid \mathrm{T}$ & 2.0E-06 & $2.9 \mathrm{E}-32$ \\
\hline $5.3 \mathrm{E}-01$ & $8.5 \mathrm{E}-08 \mathrm{~A} \mid \mathrm{G}$ & $3.5 \mathrm{E}-01$ & $6.5 \mathrm{E}-18$ \\
\hline $1.1 \mathrm{E}-01$ & $5.2 \mathrm{E}-06 \mathrm{C} \mid \mathrm{T}$ & $3.3 \mathrm{E}-01$ & 2.9E-05 \\
\hline 8.7E-02 & $2.4 \mathrm{E}-06 \mathrm{C} \mid \mathrm{T}$ & $5.4 \mathrm{E}-01$ & $9.3 \mathrm{E}-10$ \\
\hline $1.2 \mathrm{E}-03$ & $1.8 \mathrm{E}-07 \mathrm{~A} \mid \mathrm{G}$ & $3.0 \mathrm{E}-01$ & $3.1 \mathrm{E}-02$ \\
\hline
\end{tabular}


Supplementary Table 8. Significant behavioural correlations with methylation from the 16 probesets that were statistically causal to behaviour and tested against methylation directly.

\begin{tabular}{|c|c|c|c|c|c|c|c|c|}
\hline \multirow{2}{*}{$\begin{array}{l}\text { probeset gene } \\
\text { ENSGALTO00(TTRAP }\end{array}$} & \multirow{2}{*}{$\begin{array}{l}\text { methbin trait } \\
\text { chr2_915880ISocial_reinstatement_maximum_time_in_start_zone }\end{array}$} & \multirow{2}{*}{$\begin{array}{l}\text { adj.R2.full } \\
36.88 \%\end{array}$} & \multicolumn{6}{|c|}{ adj.R2.wo.ge adj.R2.wo.mı pvalue.meth. pvalue.meth. pvalue.genex pvalue.genex } \\
\hline & & & $34.03 \%$ & $35.39 \%$ & 0.05 & 0.01 & 0.01 & 0.00 \\
\hline ENSGALTOOOCTTRAP & chr2_965530|Social_reinstatement_average_time_in_start_zone & $46.69 \%$ & $43.59 \%$ & $45.93 \%$ & 0.10 & 0.01 & 0.01 & 0.00 \\
\hline ENSGALTOOO(TTRAP & chr2_965530|Social_reinstatement_average_latency_to_enter_stimulus_zone & $20.73 \%$ & $19.97 \%$ & $19.15 \%$ & 0.07 & 0.02 & 0.15 & 0.04 \\
\hline ENSGALTOOO(TTRAP & chr2_965530|Social_reinstatement_latency_to_enter_stimulus_zone_trial_2 & $18.55 \%$ & $17.1 \%$ & $17.23 \%$ & 0.09 & 0.02 & 0.08 & 0.02 \\
\hline ENSGALTOOO(TTRAP & chr2_9655301Social_reinstatement_minimum_time_in_start_zone & $44.07 \%$ & $42.24 \%$ & $43.49 \%$ & 0.14 & 0.03 & 0.03 & 0.01 \\
\hline ENSGALTOOO(TTRAP & chr2_965530|Social_reinstatement_maximum_time_in_start_zone & $35.85 \%$ & $32.8 \%$ & $35.39 \%$ & 0.18 & 0.03 & 0.01 & 0.00 \\
\hline ENSGALTOOO(TTRAP & chr2_920540|Social_reinstatement_minimum_time_in_start_zone & $43.85 \%$ & $41.98 \%$ & $43.49 \%$ & 0.19 & 0.04 & 0.03 & 0.01 \\
\hline ENSGALTOOOCTTRAP & chr2_8921301Social_reinstatement_average_latency_to_enter_stimulus_zone & $20.01 \%$ & $19.05 \%$ & $19.15 \%$ & 0.13 & 0.04 & 0.12 & 0.04 \\
\hline ENSGALT000(ITGBL1 & chr1_141629|Predictability_time_spent_in_tonic_immobility & $14.55 \%$ & $11.04 \%$ & $9.41 \%$ & 0.01 & 0.00 & 0.02 & 0.00 \\
\hline ENSGALT000(ITGBL1 & chr1_141265ımaximum_time_spent_in_tonic_immobility & $12.57 \%$ & $9.54 \%$ & $7.65 \%$ & 0.01 & 0.00 & 0.03 & 0.00 \\
\hline ENSGALT000(ITGBL1 & chr1_141265।Predictability_time_spent_in_tonic_immobility & $14.03 \%$ & $10.59 \%$ & $9.41 \%$ & 0.01 & 0.00 & 0.02 & 0.00 \\
\hline ENSGALT000(ITGBL1 & chr1_141264IPredictability_time_spent_in_tonic_immobility & $12.65 \%$ & $9.67 \%$ & $9.41 \%$ & 0.02 & 0.00 & 0.03 & 0.00 \\
\hline ENSGALTO00(ITGBL1 & chr1_141287|Predictability_time_spent_in_tonic_immobility & $12.58 \%$ & $9.41 \%$ & $9.41 \%$ & 0.02 & 0.00 & 0.02 & 0.00 \\
\hline ENSGALT000(ITGBL1 & chr1_141324IPredictability_time_spent_in_tonic_immobility & $12.47 \%$ & $9.41 \%$ & $9.41 \%$ & 0.03 & 0.00 & 0.03 & 0.00 \\
\hline ENSGALT000(ITGBL1 & chr1_141629ımaximum_time_spent_in_tonic_immobility & $11.41 \%$ & $8.07 \%$ & $7.65 \%$ & 0.02 & 0.00 & 0.02 & 0.00 \\
\hline ENSGALT000(ITGBL1 & chr1_141404lPredictability_time_spent_in_tonic_immobility & $12.27 \%$ & $8.76 \%$ & $9.41 \%$ & 0.03 & 0.00 & 0.02 & 0.00 \\
\hline ENSGALT000(ITGBL1 & chr1_141472।Predictability_time_spent_in_tonic_immobility & $12.14 \%$ & $8.74 \%$ & $9.41 \%$ & 0.03 & 0.00 & 0.02 & 0.00 \\
\hline ENSGALT000(ITGBL1 & chr1_141333iPredictability_time_spent_in_tonic_immobility & $12.54 \%$ & $8.7 \%$ & $9.41 \%$ & 0.02 & 0.00 & 0.01 & 0.00 \\
\hline ENSGALTO00(ITGBL1 & chr1_141489|Predictability_time_spent_in_tonic_immobility & $12.58 \%$ & $8.69 \%$ & $9.41 \%$ & 0.02 & 0.00 & 0.01 & 0.00 \\
\hline ENSGALTO00(ITGBL1 & chr1_141523।Predictability_time_spent_in_tonic_immobility & $12.24 \%$ & $8.55 \%$ & $9.41 \%$ & 0.03 & 0.00 & 0.02 & 0.00 \\
\hline ENSGALT000(ITGBL1 & chr1_141264Imaximum_time_spent_in_tonic_immobility & $10.06 \%$ & $7.12 \%$ & $7.65 \%$ & 0.04 & 0.00 & 0.03 & 0.00 \\
\hline ENSGALT000(ITGBL1 & chr1_141324ımaximum_time_spent_in_tonic_immobility & $9.92 \%$ & $6.9 \%$ & $7.65 \%$ & 0.05 & 0.01 & 0.03 & 0.00 \\
\hline ENSGALT000(ITGBL1 & chr1_141523ımaximum_time_spent_in_tonic_immobility & $10.13 \%$ & $6.72 \%$ & $7.65 \%$ & 0.04 & 0.01 & 0.02 & 0.00 \\
\hline ENSGALT000(ITGBL1 & chr1_141404ımaximum_time_spent_in_tonic_immobility & $9.98 \%$ & $6.67 \%$ & $7.65 \%$ & 0.05 & 0.01 & 0.02 & 0.00 \\
\hline ENSGALT000(ITGBL1 & chr1_141524lPredictability_time_spent_in_tonic_immobility & $11.82 \%$ & $7.78 \%$ & $9.41 \%$ & 0.04 & 0.01 & 0.01 & 0.00 \\
\hline ENSGALT000(ITGBL1 & chr1_141286।Predictability_time_spent_in_tonic_immobility & $11.41 \%$ & $7.58 \%$ & $9.41 \%$ & 0.06 & 0.01 & 0.01 & 0.00 \\
\hline ENSGALT000(ITGBL1 & chr1_141472ımaximum_time_spent_in_tonic_immobility & $9.54 \%$ & $6.24 \%$ & $7.65 \%$ & 0.06 & 0.01 & 0.02 & 0.00 \\
\hline ENSGALT000(ITGBL1 & chr1_141524ımaximum_time_spent_in_tonic_immobility & $9.81 \%$ & $6.19 \%$ & $7.65 \%$ & 0.05 & 0.01 & 0.02 & 0.00 \\
\hline ENSGALTO00(ITGBL1 & chr1_141287ımaximum_time_spent_in_tonic_immobility & $9.34 \%$ & $6.05 \%$ & $7.65 \%$ & 0.08 & 0.01 & 0.02 & 0.00 \\
\hline ENSGALT000(ITGBL1 & chr1_141333ımaximum_time_spent_in_tonic_immobility & $9.64 \%$ & $5.96 \%$ & $7.65 \%$ & 0.06 & 0.01 & 0.02 & 0.00 \\
\hline ENSGALT000(ITGBL1 & chr1_141484IPredictability_time_spent_in_tonic_immobility & $10.72 \%$ & $6.81 \%$ & $9.41 \%$ & 0.10 & 0.01 & 0.01 & 0.00 \\
\hline ENSGALT000(ITGBL1 & chr1_141286ımaximum_time_spent_in_tonic_immobility & $9.13 \%$ & $5.57 \%$ & $7.65 \%$ & 0.09 & 0.01 & 0.02 & 0.00 \\
\hline ENSGALT000(ITGBL1 & chr1_141489 Imaximum_time_spent_in_tonic_immobility & $9.36 \%$ & $5.55 \%$ & $7.65 \%$ & 0.07 & 0.01 & 0.02 & 0.00 \\
\hline ENSGALT000(ITGBL1 & chr1_141634IPredictability_time_spent_in_tonic_immobility & $10.2 \%$ & $6.01 \%$ & $9.41 \%$ & 0.16 & 0.03 & 0.01 & 0.00 \\
\hline ENSGALT000(ITGBL1 & chr1_141484Imaximum_time_spent_in_tonic_immobility & $8.21 \%$ & $4.39 \%$ & $7.65 \%$ & 0.19 & 0.03 & 0.02 & 0.00 \\
\hline ENSGALT000(ITGBL1 & chr1_141634ımaximum_time_spent_in_tonic_immobility & $8.02 \%$ & $4.1 \%$ & $7.65 \%$ & 0.23 & 0.04 & 0.02 & 0.00 \\
\hline X603600179F LOC100 & 1chr10_12817।Open_field_total_movement_trial_2 & $8.65 \%$ & $4.33 \%$ & $6.85 \%$ & 0.07 & 0.01 & 0.01 & 0.00 \\
\hline
\end{tabular}




\begin{tabular}{|c|c|c|c|c|c|c|c|c|}
\hline X603600179F LOC10013 & 11chr10_12817।Predictability_open_field_velocity & $5.01 \%$ & $4.73 \%$ & $2.07 \%$ & 0.03 & 0.01 & 0.25 & 0.07 \\
\hline X603862632FADAM10 & chr10_48090।Open_field_average_velocity & $4.05 \%$ & $3.99 \%$ & $1.7 \%$ & 0.05 & 0.01 & 0.30 & 0.07 \\
\hline X603862632FADAM10 & chr10_48090।Open_field_velocity_trial_1 & $4.43 \%$ & $4.04 \%$ & $2.66 \%$ & 0.08 & 0.02 & 0.22 & 0.05 \\
\hline X603863179FCA8 & chr2_111755 maximum_time_spent_in_tonic_immobility & $10.67 \%$ & $10.31 \%$ & $4.48 \%$ & 0.00 & 0.00 & 0.23 & 0.03 \\
\hline X603863179FCA8 & chr2_111755 laverage_time_spent_in_tonic_immobility & $6.87 \%$ & $6.87 \%$ & $2.37 \%$ & 0.01 & 0.00 & 0.32 & 0.07 \\
\hline X603863179FCA8 & chr2_111734ımaximum_time_spent_in_tonic_immobility & $7.3 \%$ & $7.02 \%$ & $4.48 \%$ & 0.03 & 0.01 & 0.25 & 0.03 \\
\hline X603863179FCA8 & chr2_111734laverage_time_spent_in_tonic_immobility & $4.6 \%$ & $4.7 \%$ & $2.37 \%$ & 0.06 & 0.01 & 0.35 & 0.07 \\
\hline X603863179FCA8 & chr2_111729ı maximum_time_spent_in_tonic_immobility & $6.55 \%$ & $5.38 \%$ & $4.48 \%$ & 0.06 & 0.02 & 0.12 & 0.03 \\
\hline X603863179FCA8 & chr2_111729laverage_time_spent_in_tonic_immobility & $3.82 \%$ & $3.24 \%$ & $2.37 \%$ & 0.10 & 0.04 & 0.19 & 0.07 \\
\hline X603865613FCA8 & chr2_111755।maximum_time_spent_in_tonic_immobility & $11.13 \%$ & $10.31 \%$ & $5.44 \%$ & 0.00 & 0.00 & 0.15 & 0.02 \\
\hline X603865613FCA8 & chr2_111755 laverage_time_spent_in_tonic_immobility & $7.27 \%$ & $6.87 \%$ & $3.2 \%$ & 0.01 & 0.00 & 0.22 & 0.04 \\
\hline X603865613FCA8 & chr2_111763ımaximum_time_spent_in_tonic_immobility & $8.84 \%$ & $7.17 \%$ & $5.44 \%$ & 0.02 & 0.00 & 0.08 & 0.02 \\
\hline X603865613FCA8 & chr2_111734ımaximum_time_spent_in_tonic_immobility & $7.77 \%$ & $7.02 \%$ & $5.44 \%$ & 0.05 & 0.01 & 0.16 & 0.02 \\
\hline X603865613FCA8 & chr2_111752 Imaximum_time_spent_in_tonic_immobility & $7.98 \%$ & $6.32 \%$ & $5.44 \%$ & 0.04 & 0.01 & 0.08 & 0.02 \\
\hline X603865613FCA8 & chr2_111734laverage_time_spent_in_tonic_immobility & $4.99 \%$ & $4.7 \%$ & $3.2 \%$ & 0.08 & 0.01 & 0.25 & 0.04 \\
\hline X603865613FCA8 & chr2_111729ı maximum_time_spent_in_tonic_immobility & $7.2 \%$ & $5.38 \%$ & $5.44 \%$ & 0.07 & 0.02 & 0.07 & 0.02 \\
\hline X603865613FCA8 & chr2_111745 Imaximum_time_spent_in_tonic_immobility & $6.81 \%$ & $4.63 \%$ & $5.44 \%$ & 0.10 & 0.03 & 0.05 & 0.02 \\
\hline X603865613FCA8 & chr2_111752 laverage_time_spent_in_tonic_immobility & $4.51 \%$ & $3.44 \%$ & $3.2 \%$ & 0.11 & 0.03 & 0.13 & 0.04 \\
\hline X603865613FCA8 & chr2_111729laverage_time_spent_in_tonic_immobility & $4.39 \%$ & $3.24 \%$ & $3.2 \%$ & 0.12 & 0.04 & 0.12 & 0.04 \\
\hline X603865613FCA8 & chr2_111763laverage_time_spent_in_tonic_immobility & $4.37 \%$ & $3.06 \%$ & $3.2 \%$ & 0.12 & 0.04 & 0.11 & 0.04 \\
\hline
\end{tabular}


Supplementary Table 9. NEO directional causality analysis for the behaviours that passed the suggestive threshold for directionality with methylation.

\section{edge}

trait1 $->$ trait2

trait1 $->$ trait2

trait1 $->$ trait2

trait1 $->$ trait2

trait1 $->$ trait2

trait1 $\rightarrow$ trait2

trait1 $->$ trait2

trait1 $->$ trait2

\section{LEO.NB.OCA LEO.NB.CPA Model.P.value trait_name}

0.56

0.47

0.55

0.46

0.54

0.43

0.48

0.32
0.56

0.47

0.55

0.46

0.54

0.43

0.48

0.32
0.08 maximum_time_spent_in_tonic_immobility

0.70 Open_field_total_movement_trial_2

0.98 Predictability_open_field_velocity

methylation_phenotype

chr2_111755000

chr10_12817000

chr10_12817000

0.84 Social_reinstatement_average_latency_to_enter_stimulus_zone chr2_96553000

0.91 Social_reinstatement_latency_to_enter_stimulus_zone_trial_2 chr2_96553000

0.47 Social_reinstatement_maximum_time_in_start_zone

0.94 Social_reinstatement_maximum_time_in_start_zone

0.75 Social_reinstatement_average_time_in_start_zone chr2 91588000

chr2_96553000

chr2_96553000 
Supplementary Table 10. Methylation values and p-values for cis methQTL regions in a

\begin{tabular}{|c|c|c|c|c|c|}
\hline methylation window & RJF_highfear_ & RJF_lowfear_a & RJF_highfear_ & RJF_lowfear_s & Stest_pvalue \\
\hline chr1_1061000 & 2.2 & 4.2 & 1.4 & 1.0 & 0.015 \\
\hline chr1_3442000 & 63.9 & 49.4 & 8.7 & 12.8 & 0.047 \\
\hline chr1_3451000 & 59.4 & 44.8 & 3.9 & 13.3 & 0.042 \\
\hline chr1_10684000 & 1.5 & 2.4 & 0.7 & 0.6 & 0.038 \\
\hline chr1_131192000 & 10.4 & 6.3 & 3.0 & 2.1 & 0.022 \\
\hline chr1_137589000 & 33.5 & 43.1 & 7.4 & 4.4 & 0.025 \\
\hline chr1_137596000 & 64.9 & 96.6 & 15.1 & 27.0 & 0.037 \\
\hline chr1_137606000 & 95.0 & 139.4 & 19.2 & 36.9 & 0.033 \\
\hline chr1_137611000 & 31.7 & 51.9 & 6.4 & 13.5 & 0.012 \\
\hline chr1_137618000 & 8.8 & 13.2 & 2.6 & 3.9 & 0.046 \\
\hline chr1_137633000 & 27.2 & 45.3 & 8.5 & 11.4 & 0.012 \\
\hline chr1_137649000 & 109.6 & 153.6 & 23.1 & 37.0 & 0.038 \\
\hline chr1_137650000 & 141.0 & 205.6 & 28.1 & 38.9 & 0.009 \\
\hline chr1_137653000 & 57.3 & 84.6 & 19.8 & 21.7 & 0.046 \\
\hline chr1_137656000 & 19.2 & 28.9 & 7.2 & 7.9 & 0.050 \\
\hline chr1_137664000 & 24.5 & 35.7 & 5.3 & 9.8 & 0.042 \\
\hline chr1_137665000 & 15.7 & 23.8 & 2.8 & 3.8 & 0.002 \\
\hline chr1_137667000 & 52.9 & 83.3 & 13.5 & 21.5 & 0.018 \\
\hline chr1_137668000 & 60.0 & 92.0 & 16.1 & 22.7 & 0.020 \\
\hline chr1_137672000 & 48.7 & 67.8 & 11.6 & 15.8 & 0.040 \\
\hline chr1_137674000 & 18.3 & 25.9 & 4.4 & 5.8 & 0.030 \\
\hline chr1_137692000 & 51.8 & 75.8 & 13.4 & 20.3 & 0.040 \\
\hline chr1_137696000 & 35.1 & 52.2 & 10.1 & 14.3 & 0.041 \\
\hline chr1_137708000 & 93.1 & 129.4 & 21.5 & 24.7 & 0.022 \\
\hline chr1_137713000 & 84.6 & 119.6 & 22.1 & 30.2 & 0.047 \\
\hline chr1_137714000 & 58.7 & 87.3 & 12.8 & 23.2 & 0.030 \\
\hline chr1_137720000 & 39.5 & 54.2 & 6.2 & 10.5 & 0.018 \\
\hline chr1_137721000 & 75.7 & 102.6 & 13.9 & 17.9 & 0.017 \\
\hline
\end{tabular}




\begin{tabular}{|l|r|r|r|r|r|} 
chr1_137722000 & 79.1 & 120.3 & 18.6 & 32.3 & 0.027 \\
chr1_137726000 & 29.4 & 45.7 & 10.0 & 14.0 & 0.045 \\
chr1_137729000 & 34.4 & 52.2 & 9.6 & 9.1 & 0.008 \\
chr1_137731000 & 87.0 & 128.8 & 21.4 & 35.7 & 0.039 \\
chr1_137732000 & 36.9 & 52.5 & 8.4 & 9.1 & 0.012 \\
chr1_137745000 & 52.6 & 75.3 & 13.5 & 18.0 & 0.035 \\
chr1_137746000 & 33.7 & 48.0 & 9.3 & 9.5 & 0.025 \\
chr1_137753000 & 44.9 & 67.0 & 10.8 & 20.1 & 0.046 \\
chr1_137757000 & 37.3 & 46.9 & 4.4 & 4.4 & 0.004 \\
chr2_9640000 & 8.0 & 10.7 & 1.4 & 1.3 & 0.006 \\
chr231070000 & 5.9 & 2.7 & 1.6 & 2.0 & 0.014 \\
chr2_53174000 & 29.5 & 33.9 & 2.9 & 3.6 & 0.042 \\
chr2_138785000 & 9.3 & 15.4 & 3.0 & 4.5 & 0.022 \\
chr3_22196000 & 11.7 & 14.2 & 1.2 & 1.0 & 0.003 \\
chr3_38613000 & 15.0 & 10.4 & 4.3 & 1.3 & 0.047 \\
chr3_57251000 & 22.9 & 18.7 & 3.3 & 2.6 & 0.034 \\
chr5_14572000 & 16.9 & 9.8 & 5.9 & 1.9 & 0.030 \\
chr5_45725000 & 8.9 & 6.1 & 2.2 & 1.5 & 0.033 \\
chr61005000 & 6.9 & 10.0 & 1.9 & 2.7 & 0.047 \\
chr6_1059000 & 14.5 & 19.2 & 3.5 & 2.9 & 0.032 \\
chr6_11112000 & 26.2 & 37.4 & 4.4 & 6.9 & 0.009 \\
chr7_23633000 & 14.3 & 10.6 & 2.1 & 3.3 & 0.047 \\
chr7_24754000 & 16.7 & 18.8 & 1.2 & 1.5 & 0.027 \\
chr7_28207000 & 10.9 & 7.1 & 1.9 & 2.9 & 0.027 \\
chr811332000 & 129.3 & 160.5 & 25.0 & 16.6 & 0.032 \\
chr8_26254000 & 12.5 & 16.0 & 1.1 & 3.3 & 0.045 \\
chr13_10201000 & 3.3 & 4.9 & 1.0 & 1.4 & 0.042 \\
chr20_12649000 & 28.6 & 23.9 & 2.5 & 2.5 & 0.009 \\
chr20_12609000 & 14.7 & 18.7 & 2.7 & 1.7 & 0.016 \\
\hline
\end{tabular}




\section{allelic imbalance chi-sq}

allelic imbalance test $p$-value - only

chi-sq test $p$-value individuals with $7+$

SNP - all individuals

reads

\begin{tabular}{lll}
\hline chr1.182303631 & $1.6 \mathrm{E}-36$ & $4.1 \mathrm{E}-27$ \\
chr7.30200796 & $2.2 \mathrm{E}-07$ & $1.9 \mathrm{E}-04$ \\
chr7.30200978 & $2.5 \mathrm{E}-10$ & $1.2 \mathrm{E}-09$ \\
chr2.30963511 & $6.1 \mathrm{E}-14$ & $2.6 \mathrm{E}-14$ \\
chr7.27835914 & $3.2 \mathrm{E}-16$ & $1.2 \mathrm{E}-13$ \\
chr1.13862669 & $3.2 \mathrm{E}-33$ & $1.1 \mathrm{E}-28$ \\
chr12.2738637 & $7.1 \mathrm{E}-07$ & $6.3 \mathrm{E}-05$ \\
chr13.8901269 & $1.1 \mathrm{E}-08$ & $7.2 \mathrm{E}-07$ \\
chr7.25522361 & $7.6 \mathrm{E}-06$ & $4.9 \mathrm{E}-05$ \\
chr8.19537272 & $3.1 \mathrm{E}-02$ & $2.3 \mathrm{E}-02$ \\
\hline
\end{tabular}

Supplementary Table 11. Testing of allelic imbalance SNPs with and without low read-coverage individuals. 


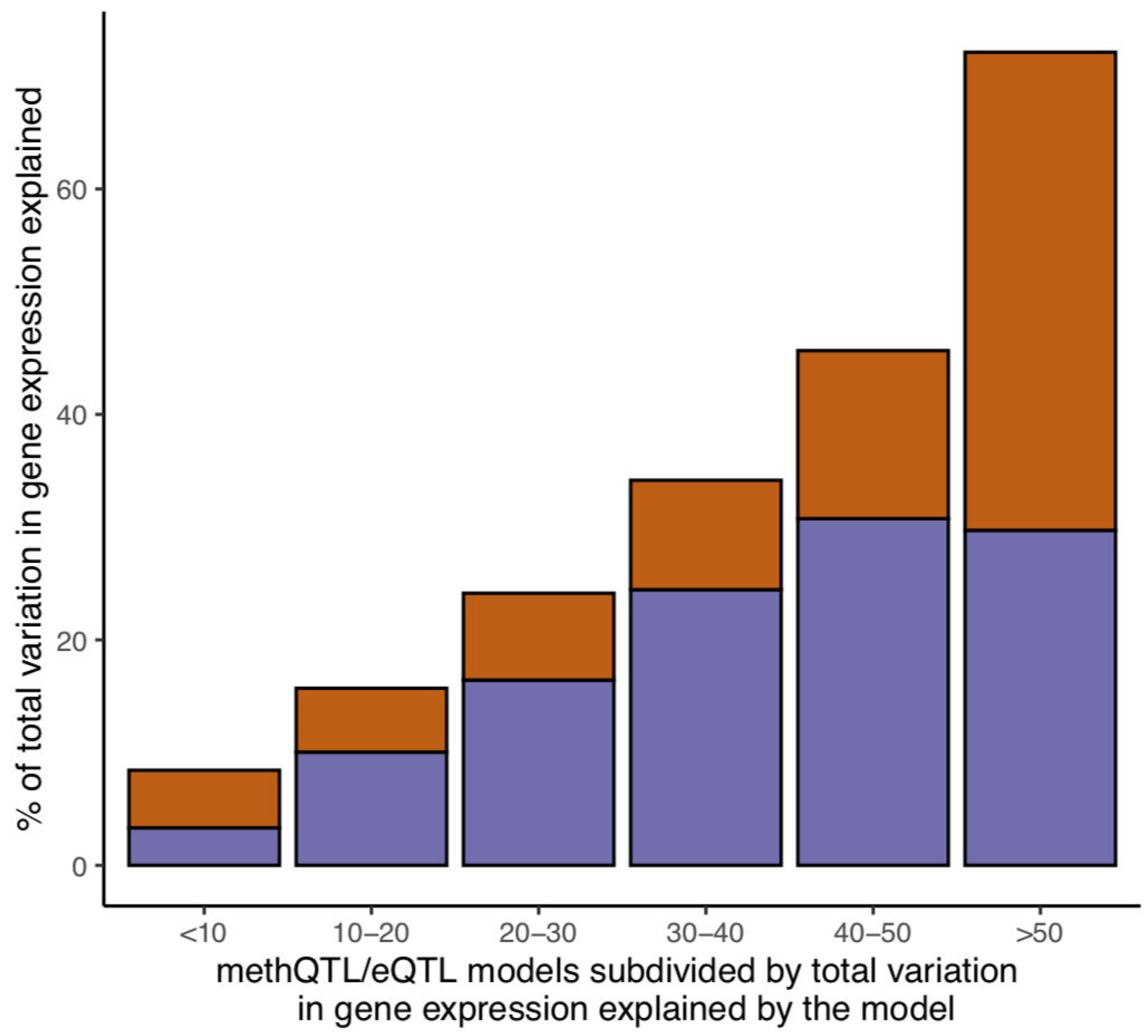

Variance explained by: $\square$ DNA methylation $\square$ eQTL genotype + sex 